\title{
PERFORMANCE OF ENERGY MANAGEMENT CONTROL SYSTEMS (EMCS) IN SELECTED TEXAS LoanSTAR BUILDINGS
}

\author{
A Report by \\ Michelle M. Schmode
}

Submitted to the Mechanical Engineering Department of Texas A\&M University in partial fulfillment of the requirement for the degree of Master of Science

August 1995

Major Subject: Mechanical Engineering 


\section{Abstract}

This report describes an investigation of energy use at various LoanSTAR sites. The effects of installing Energy Management Control Systems (EMCS) on electricity consumption was studied at four LoanSTAR sites: Stroman High School, Victoria High school, Sims Elementary School, and Zachry Engineering Center. In the course of this study, LoanSTAR monitoring data was used to analyze the changes in energy consumption based only on EMCS retrofits. The results will show that the installation of EMCS was successful in reducing energy consumption and/or changing the hourly energy consumption pattern. 


\section{Table of Contents}

Introduction

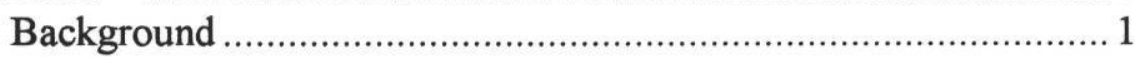

The LoanSTAR Program ........................................................ 2

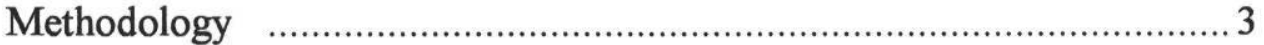

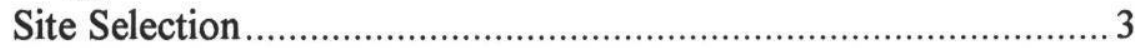

Data Reduction.................................................................... 3

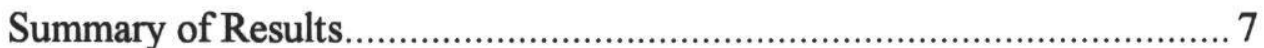

Appendix A; Stroman High School ................................................ A-1

Appendix B; Victoria High School.................................................. B-1

Appendix C; Sims Elementary School...........................................

Appendix D; Zachry Engineering Center........................................... D-1 


\section{Introduction}

\section{Background}

"Energy retrofits can cut use and costs." "Direct digital control system saves over 40 percent in energy costs." "University sheds HVAC system for state-of-the-art energy management control." "Vision and technology revolutionize Bryant College." "Control system offers remedy for hospital's energy use ailments." These are some of the headlines found in Buildings and Mechanical Engineering magazines. Many facility managers are moving toward Energy Management Control Systems (EMCS) or Direct Digital Control systems (DDC) in an effort to reduce energy costs. These efforts have claimed to be successful; however, few reports detail exacly how and/or why the savings occurred.

The following briefly describes the results reported in the above mentioned articles. In general, energy conservation retrofits can reduce the energy use of buildings by 10 to 30 percent, with paybacks typically in the 2- to 4- year range. ${ }^{1}$ The Valley Building, an 8-year-old office tower in Renton, Washington achieved a 40 percent savings in energy costs within six months of installing a DDCS. ${ }^{2}$ The C.W. Post campus of Long Island University, Brookville, New York, does not report specific monetary savings, rather they report significant maintenance staff labor savings and energy savings due to their EMCS keeping their target setpoints more effectively. ${ }^{3}$ Bryant College in Smithville, Rhode Island has achieved energy savings of about $\$ 34,000$ per year since they installed a DDCS. ${ }^{4}$ Craven Regional Medical Center in New Bern, North Carolina, saves an estimated $\$ 65,000$ in electric costs per year since the installation and proper utilization of an EMCS. ${ }^{5}$

The objective of this report is to determine how successful Energy Management Control Systems have been in reducing energy consumption.

\footnotetext{
${ }^{1}$ Claridge, D.E., et al 1994. "Energy retrofits can cut use and costs." Mechanical Engineering, August, pp. 64-67.

${ }^{2}$ Editor, 1991. Buildings, December, p.16.

${ }^{3}$ Editor, 1992. Buildings, September, p. 34.

${ }^{4}$ Mumford, S., 1994. Buildings, February, pp. 38-41.

${ }^{5}$ Editor, 1993. Buildings, May, p 38.
} 


\section{The LoanSTAR Program ${ }^{6}$}

In 1988, the Texas Governor's Energy Management Center (GEMC) ${ }^{7}$ received approval from the U.S. Department of Energy to establish a $\$ 98.6$ million statewide retrofit demonstration revolving loan

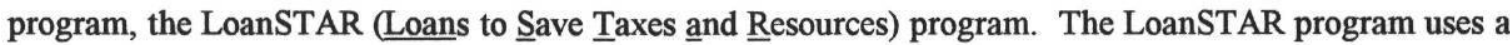
revolving loan financing mechanism to fund energy-conservation retrofits in state and local government buildings and public schools. Potential retrofit projects are identified by energy audits conducted by engineering teams under contract to the Texas SECO. Each proposed retrofit competes for funds on the basis of the estimated payback period, the ability to repay the loan through energy savings, an engineering assessment of the viability of the retrofit, and the feasibility of monitoring the project effectively.

The projects funded by the LoanSTAR program primarily include retrofits to lighting, HVAC systems, building shell, electric motors, and EMCS.

The LoanSTAR Monitoring and Analysis Program (MAP) was designed to serve the differing needs of the many participants in the LoanSTAR revolving loan program. The energy monitoring program's first objective is to determine whether retrofits save as much as estimated in audits. The second objective of the MAP is to reduce energy costs of a building by using the monitored data to evaluate its energy-using characteristics, and to diagnose opportunities for improved operations. The final major objective of energy monitoring is the establishment of an end-use database for institutional and commercial buildings in Texas.

The LoanSTAR MAP is not set up to collect monitoring data based on individual retrofits. This would be too costly; therefore, tracking the performance of groups of retrofits was chosen over tracking of specific retrofits. This report evaluates the LoanSTAR monitored data in order to isolate the changes in energy usage based only on EMCS retrofits.

\footnotetext{
${ }^{6}$ Turner, W.D., 1990. "Overview of the Texas LoanSTAR Monitoring Program," Proceedingts of the Seventh Annual Symposium on Improving Building Systems in Hot and Humid C limates, College Station, Texas: Texas A\&M University. October 9-10, pp. 28-34

${ }^{7}$ The GEMC is now called the Texas State Energy Conservation Office (SECO)
} 


\section{Methodology}

\section{Site Selection}

The LoanSTAR program currently monitors 90 sites. Sites studied in this report were chosen based on the following:

a) EMCS retrofit was completed,

b) whole building and limited sub-metered hourly data were collected (utilizing one-to fourchannel data acquisition systems or data loggers), and

c) ease of separating EMCS retrofit data from whole building data.

The final list of sites studied is:

a) Stroman High School (SHS), Victoria Independent School District,

b) Victoria High School (VHS), Victoria Independent School District,

c) Sims Elementary School (SIM), Fort Worth Independent School District, and

d) Zachry Engineering Center (ZEC), Texas A\&M University, College Station.

A data summary notebook is prepared by the Monitoring and Analysis Task of the Texas LoanSTAR program. It provides various plots, giving an historical look at all of the data that has been collected for all of the LoanSTAR sites. Zachry Engineering Center (ZEC) data summary plots are included as a comparison to the methodology used in this report. The charts and table prepared for the other sites were not prepared for ZEC.

\section{Data Reduction}

The raw data were obtained from a database maintained by the Energy Systems Lab (ESL) at Texas A\&M University under the LoanSTAR MAP. A number of data loggers were installed to collect specific consumption data for each site. The location of the data loggers is indicated on monitoring diagrams, which are included for each site in the Appendices.

The data was extracted from the database using an ESL program called "Getdatc." It is a simple program that expedites the retrieval of data from the LoanSTAR database. It allows the user to obtain columns of logger channel data and to perform calculations on the data quickly and easily. Getdatc outputs the data with timestamps and, when necessary, bad data marks $(-99){ }^{8}$ Data was retrieved for a

\footnotetext{
${ }^{8}$ Getdatc is copyrighted public domain software developed by the Energy Systems Laboratory, Texas A\&M University, Mechanical Engineering Department, College Station, TX 77843-3123
} 
period defined as the report period, which varies from site to site. In all cases, the report period commenced on the monitoring start date for the study site. The report periods for each site are as follows:

$\begin{array}{ll}\text { Stroman High School } & 6 / 5 / 91 \text { through } 6 / 4 / 94 \\ \text { Victoria High School } & 6 / 5 / 91 \text { through } 6 / 4 / 94 \\ \text { Sims Elementary School } & 10 / 1 / 91 \text { through } 5 / 31 / 95 \\ \text { Zachry Engineering Center } & 5 / 31 / 89 \text { through } 10 / 29 / 94\end{array}$

To facilitate subsequent data manipulation, the report periods were subdivided into annual blocks of data. For this report, the "whole building" data was extracted. A list showing the information contained in the whole building data set is shown in Figure 1. The output was in the form of an ASCII file, which could be edited and imported into other software for further manipulation.

In order to separate the effects of other retrofits, a list of channels and equations associated with the whole building calculations was reviewed. This list is included in each site Appendix. As an example, the listing for Stroman High School is shown in Figure 1.

Figure 1: Getdatc "listwb" output for Stroman High School

\begin{tabular}{|c|c|c|}
\hline$\underline{\text { Cp }}$ & Name & Expression \\
\hline 1 & wbele & ch0323 \\
\hline 2 & wbcool & ch0324/1000 \\
\hline 3 & wbheat & $\operatorname{ch} 0326 * 0.00103$ \\
\hline 4 & oadrybulb & $\operatorname{ch} 0827$ \\
\hline 5 & oarh & dp2rh(ch0827,ch0828) \\
\hline 6 & chiller & $\operatorname{ch} 0325$ \\
\hline 7 & windspeed & ch0829 \\
\hline \multicolumn{2}{|c|}{ channel ID } & Description \\
\hline 0323 & \multicolumn{2}{|c|}{ Whole Bldg $(\mathrm{kWh} / \mathrm{h})(126,8)$} \\
\hline 0324 & \multicolumn{2}{|c|}{ ChW (kBtu) $(126,9)$} \\
\hline 0325 & \multicolumn{2}{|c|}{ Chiller $(\mathrm{kWh} / \mathrm{h})(126,10)$} \\
\hline 0326 & \multicolumn{2}{|c|}{ Gas Meter (cuft) $(126,11)$} \\
\hline 0827 & \multicolumn{2}{|c|}{ VCT Dry Bulb (Victoria) $(850,8)$} \\
\hline 0828 & \multicolumn{2}{|c|}{ VCT Dew Point (Victoria) $(850,9)$} \\
\hline 0829 & \multicolumn{2}{|c|}{ VCT Wind Speed (Victoria) $(850,10)$} \\
\hline
\end{tabular}

The monitoring diagram in Appendix A, Tab A-3 shows the location of the channel where the consumption is measured. Electricity consumption is being examined in this report. This includes all channels which are kilowatt-hour per hour (kWh/h) (channels 0323 and 0325 in Figure 1). 
Stroman High School had three retrofits: install an EMCS, replace absorption chiller with electric chiller, and rewire hallway wiring. The effects of the new electric chiller consumption can be eliminated by subtracting chiller consumption (channel 0325 , measured at the chiller) from whole building electricity consumption (channel 0323 , measured before the electrial main panel). Similar reasoning is used for the data reductions of the other study sites.

Once the data were extracted from the LoanSTAR database, the data file was reduced to two columns of data: decimal date and consumption. This was done using a MS DOS routine called "gawk-f." A *.awk file was created using a text editor. It contains a statement which specifies the column numbers corresponding to the columns of data to be extracted from the getdatc *acs file. The output file is in ascii format. This process was repeated for all consumption categories to be studied.

Next, the output data file was transformed from columnar format to tabular format using an ESL program called "ColRow3D." 9 It is a columnar data manipulation program which processes hourly energy consumption data to produce a "new" file containing a spread sheet compatible data matrix. ColRow3D transforms each day's worth of data into one row in the matrix. For example, a leap year's worth of hourly data (8764 lines) will be compressed down to just 366 lines of data. The ColRow3D output file was opened in Microsoft Excel for further manipulation.

After each ColRow3D file was opened in MS Excel, they were combined into one .xls worksheet, covering the entire report period. At this point, the data was in consecutive date order. Columns were inserted at the beginning of the worksheet to input sort parameters, which are:
$1 / 0$ weekday/weekend
A/B pre-/post-retrofit
S/NS semester/non-semester

Ones and zeros were input into the ' $1 / 0$ ' column, where a " 1 " indicates a weekday and a " 0 " indicates a weekend. A's and B's were input into the "A/B" column, where an "A" indicates a pre- retrofit date and a "B" indicates a post- retrofit date. S's and NS's were input in the "S/NS" column, where an "S" indicates a semester day and a "NS" indicates a non-semester day.

\footnotetext{
${ }^{9}$ ColRow3D is copyrighted public domain software developed by the Energy Systems Laboratory, Texas A\&M University Mechanical Engineering Department, College Station, TX 77843-3123
} 
A school district schedule was obtained for each site which indicates holidays, breaks, and days when school was not in session. For this report, all holidays, semester breaks and summers were considered non-semester days. The summers were categorized as non-semester for the two independent school districts, even though they held summer school sessions. Consumption during the summer, even with summer school in session, was significantly less than normal school year consumption. In order to avoid falsely reducing the daily average data, the summers were categorized as non-semester.

For cells which contained a "-99" (missing data) or a " 0 ", the content of the cell was replaced with a blank cell. This allows mathematical functions to be used on the data without including erroneous data or zeroes, which would result in bad results (either too large or too small of totals or averages).

The hourly data for each date was summed to obtain total daily data. The total daily consumption was plotted against the day of year for each energy consumption category. This is the timeline plot shown in each site Appendix, Figure 3. The total monthly consumption was then calculated. The data were presented in tabular format in each site Appendix, Table 2. These calclulations were performed for each consumption category.

Next, the "other electric" data was sorted on pre-/post-retrofit (A/B) and semester/non-semester (S/NS). The daily totals were summed for post-retrofit, semester (B, S) and post-retrofit, non-semester (B, NS). The sort category totals are represented as a percentage of whole building electricity consumption in a pie chart, shown in each site Appendix, Figure 4. The sort totals were then multiplied by the appropriate cost of energy (\$/unit of energy) to obtain the total cost of energy. The data were presented in tabular form in each site Appendix, Table 1

Average hourly consumption (other electric) was calculated by sorting the data based on (1) semester/non-semester (S/NS), (2) weekday/weekend (1/0), and (3) pre-/post-retrofit (A/B). This yielded eight sort categories:
S-1-A
S-1-B
S-0-A
S-0-B
NS-1-A
NS-1-B
NS-0-A
semester/weekday/pre-retrofit semester/weekday/post-retrofit semester/weekend/pre-retrofit semester/weekend/post-retrofit non-semester/weekday/pre-retrofit non-semester/weekday/post-retrofit non-semester/weekend/pre-retrofit NS-0-B non-semester/weekend/post-retrofit 
The hourly consumption (other electric) was averaged for each sort category. This represents the average hourly consumption for each hour of the day. It was calculated for only those hours when the equipment was actually on. The average hourly consumption was plotted against hour of day to obtain daily profiles, which are shown in each site Appendix, Figure 5.

\section{Summary of Results}

All sites showed both reductions and increases in "other" electricity consumption. The term "other" is defined differently for each site. For Stroman and Victoria High Schools, it is whole building electricity minus chiller electricity consumption. For Sims Elementary Schools, it is whole building electricity minus lighting electricity consumption. For Zachry Engineering Center, whole building electric was analyzed instead of "other" electricity consumption.

Table 2 summarizes the difference in other electric consumption for all study sites except Zachry Engineering Center. Stroman and Victoria High Schools both showed reductions in other electricity consumption for each category except semester/weekend. Sims Elementary School showed reductions in other electricity consumption for each category except semester/weekday. Possible explanations for these increases are discussed in each site Appendix.

Table 3 summarizes the whole building electricity consumption for all sites, for the pre-retrofit period and the most recent year of post-retrofit period. The square footage of each site is also shown in this table. Table 4 summarizes the whole building electricity consumption for all sites, normalized on a square footage basis. From the data shown in both of these tables, one can see that Stroman and Victoria High Schools are relatively low energy use sites, while Sims Elementary School and Zachry Engineering Center are relatively high energy use sites 
Table 2: Summary of Differences in "Other Electric" Consumption

\begin{tabular}{|c|c|c|c|c|}
\hline & $\begin{array}{l}\text { \# days } \\
\text { in sort } \\
\text { category }\end{array}$ & $\begin{array}{l}\text { Average Daily } \\
\text { Consumption } \\
\text { kWh/day }\end{array}$ & $\begin{array}{l}\text { Difference in } \\
\text { Average Daily } \\
\text { Consumption } \\
\text { kWh/day }\end{array}$ & $\begin{array}{l}\text { \% Difference in } \\
\text { Average Daily } \\
\text { Consumption }\end{array}$ \\
\hline \multicolumn{5}{|c|}{ STROMAN HIGH SCHOOL. } \\
\hline \multicolumn{5}{|c|}{ Semester : } \\
\hline weekday-pre & 91 & 5,210 & \multirow[b]{2}{*}{-685} & \multirow[b]{2}{*}{$-13.15 \%$} \\
\hline weekday-post & 394 & 4,525 & & \\
\hline weekend-pre & 35 & 2,206 & \multirow[b]{2}{*}{189} & \multirow[b]{2}{*}{$8.57 \%$} \\
\hline weekend-post & 149 & 2,395 & & \\
\hline \multicolumn{5}{|l|}{ Non-semester } \\
\hline weekday-pre & 79 & 3,557 & \multirow[b]{2}{*}{-332} & \multirow[b]{2}{*}{$-9.33 \%$} \\
\hline weekday-post & 241 & 3,225 & & \\
\hline weekend-pre & 33 & 2,060 & \multirow[b]{2}{*}{-130} & \multirow[b]{2}{*}{$-6.31 \%$} \\
\hline weekend-post & 92 & 1,930 & & \\
\hline \multicolumn{5}{|c|}{ VICTORIA HIGH SCHOOL. } \\
\hline \multicolumn{5}{|c|}{ Semester: } \\
\hline weekday-pre & 91 & 7,877 & & \\
\hline weekday-post & 394 & 6,889 & -988 & $-12.54 \%$ \\
\hline weekend-pre & 35 & 3,674 & & \\
\hline weekend-post & 149 & 4,245 & 571 & $15.54 \%$ \\
\hline \multicolumn{5}{|l|}{ Non-semester } \\
\hline weekday-pre & 79 & 6,159 & & \\
\hline weekday-post & 241 & 5,182 & -977 & $-15.86 \%$ \\
\hline weekend-pre & 33 & 4,180 & & \\
\hline weekend-post & 92 & 3,017 & $-1,163$ & $-27.82 \%$ \\
\hline \multicolumn{5}{|c|}{ SIMS ELEMENTARY SCHOOL. } \\
\hline \multicolumn{5}{|l|}{ Semester: } \\
\hline weekday-pre & 514 & 1,453 & & \\
\hline weekday-post & 229 & 1,617 & 164 & $11.29 \%$ \\
\hline weekend-pre & 192 & 861 & & \\
\hline weekend-post & 88 & 444 & -417 & $-48.41 \%$ \\
\hline \multicolumn{5}{|l|}{ Non-semester } \\
\hline weekday-pre & 148 & $1-517$ & & \\
\hline weekday-post & 66 & 1,479 & -38 & $-2.48 \%$ \\
\hline weekend-pre & 72 & 1,235 & & \\
\hline weekend-post & 543 & -692 & -692 & $-56.03 \%$ \\
\hline
\end{tabular}


Table 3: Summary of Whole Building Electricity Consumption, pre- and post-retrofit periods

\begin{tabular}{|l|c|r|r|}
\hline \multicolumn{1}{|c|}{ Site } & $\begin{array}{c}\text { Pre-Retrofit Period } \\
\text { kWh }\end{array}$ & $\begin{array}{c}\text { Post-Retrofit Period } \\
\text { kWh }\end{array}$ & $\begin{array}{c}\text { Conditioned Area } \\
\text { sq. ft. }\end{array}$ \\
\hline Stroman High School & $1,207,697^{10}$ & $1,184,318^{11}$ & 210,414 \\
\hline Victoria High School & $1,499,242^{12}$ & $1,845,529^{13}$ & 257,014 \\
\hline Sims Elementary School & $1,816,566^{14}$ & $660,673^{15}$ & 62,400 \\
\hline Zachry Engineering Center & $14,727,147^{16}$ & $8,555,071^{17}$ & 324,400 \\
\hline
\end{tabular}

Table 4: Summary of Whole Building Electricity Consumption per square foot, post-retrofit

\begin{tabular}{|c|c|c|}
\hline Site & $\begin{array}{l}\text { annual consumption per square foot } \\
\mathrm{kWh} / \mathrm{SF} / \text { year }^{18}\end{array}$ & $\begin{array}{c}\text { annual cost per square foot } \\
\text { S/SF/year }\end{array}$ \\
\hline Stroman High School & 5.63 & .01570 \\
\hline Victoria High School & 7.18 & .02002 \\
\hline Sims Elementary School & 10.59 & .07138 \\
\hline Zachry Engineering Center & 26.37 & .07352 \\
\hline
\end{tabular}

Figures 2 through 5 show the average hourly profiles for each study site. The changes in other electricity consumption are easier to see in these figures. Detailed discussions of these plots are contained in the site Appendices. The nighttime consumption (5:00 p.m. to 6:00 a.m.) dropped for all sites. The increases in consumption seen in Table 2 can be seen in the average hourly profiles as well. More interesting to note is the change in the profile itself. In most cases, the nighttime consumption dropped, with a steeper increase to daytime levels in the morning and a steeper decrease to nighttime levels in the afternoon.

In looking only at Tables $2-4$, and the average hourly profiles, the conclusion can be made that EMCS retrofits at the study sites have been successful. Further study is required to pinpoint the reasons for increased semester/weekend consumption at Stroman and Victoria High Schools, and semester/weekday consumption at Sims Elementary School.

\footnotetext{
${ }^{10}$ June 1991 - January 1992 (8 month period)

11 June 1993 - May 1994 (12 month period)

12 June 1991 - January 1992 (8 month period)

13 June 1993 - May 1994 (12 month period)

${ }^{14}$ October 1991- April 14, 1994 (30-1/2 month period)

15 June 1994 - May 1995 (12 month period)

16 June 1989 - March 1991 (21 month period)

${ }^{17}$ October 1993 - September 1994 (12 month period)

${ }^{18}$ based on post-retrofit period consumption reported in Table 3
} 
Figure 2a: SHS Semester Average Hourly Profile

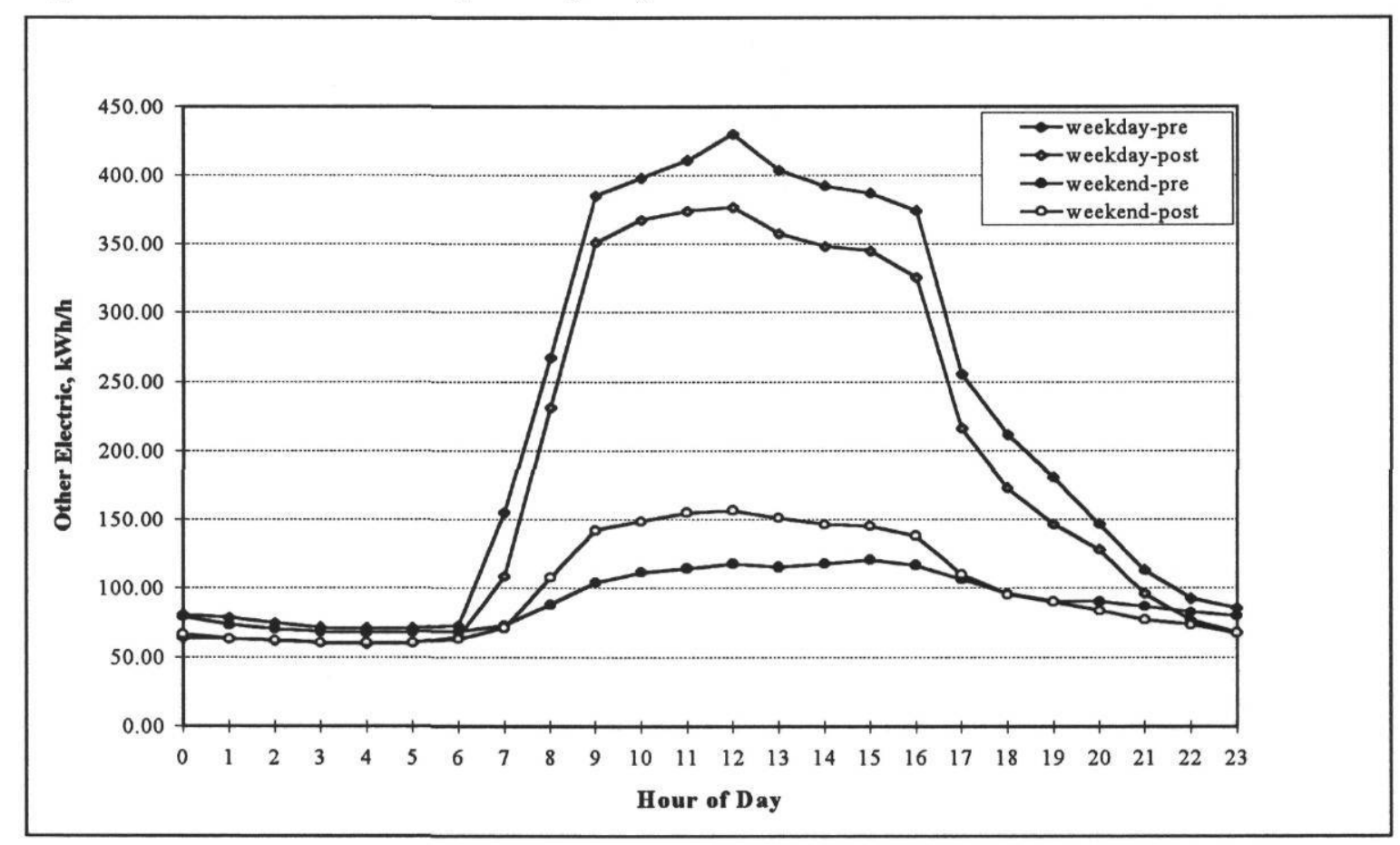

Figure 2b: SHS Non-semester Average Hourly Profile

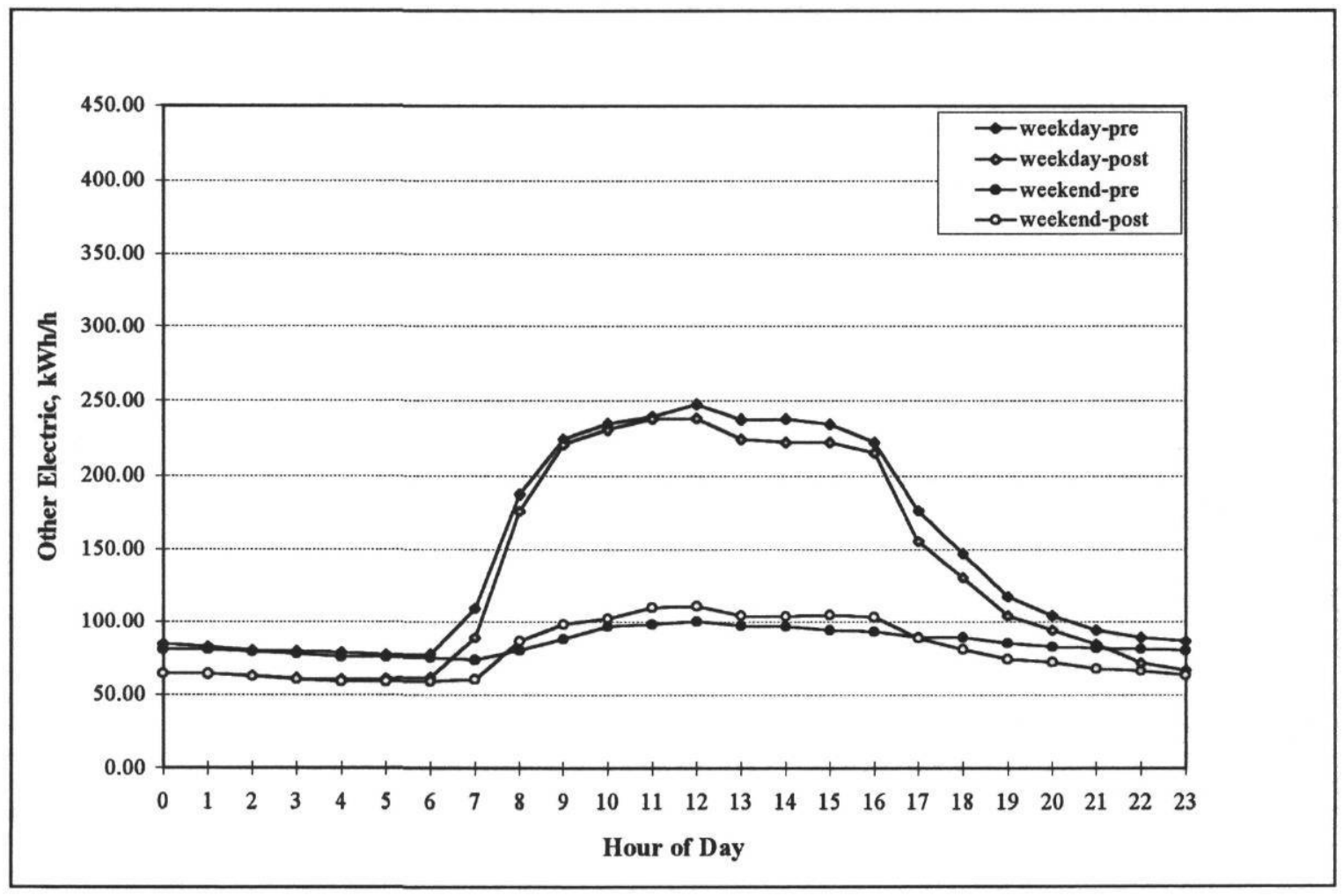


Figure 3a: VHS Semester Average Hourly Profile

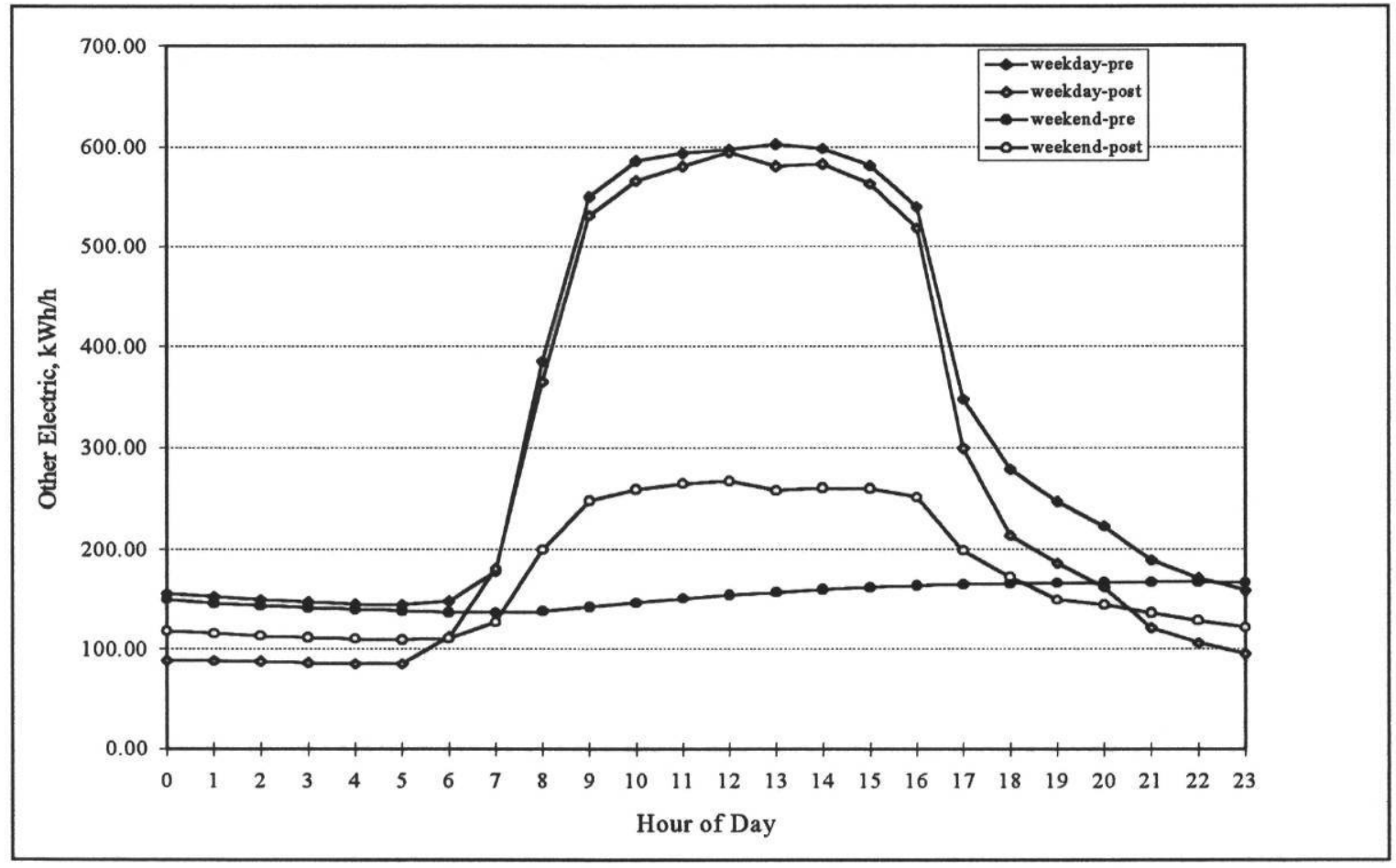

Figure 3b: VHS Non-semester Average Hourly Profile

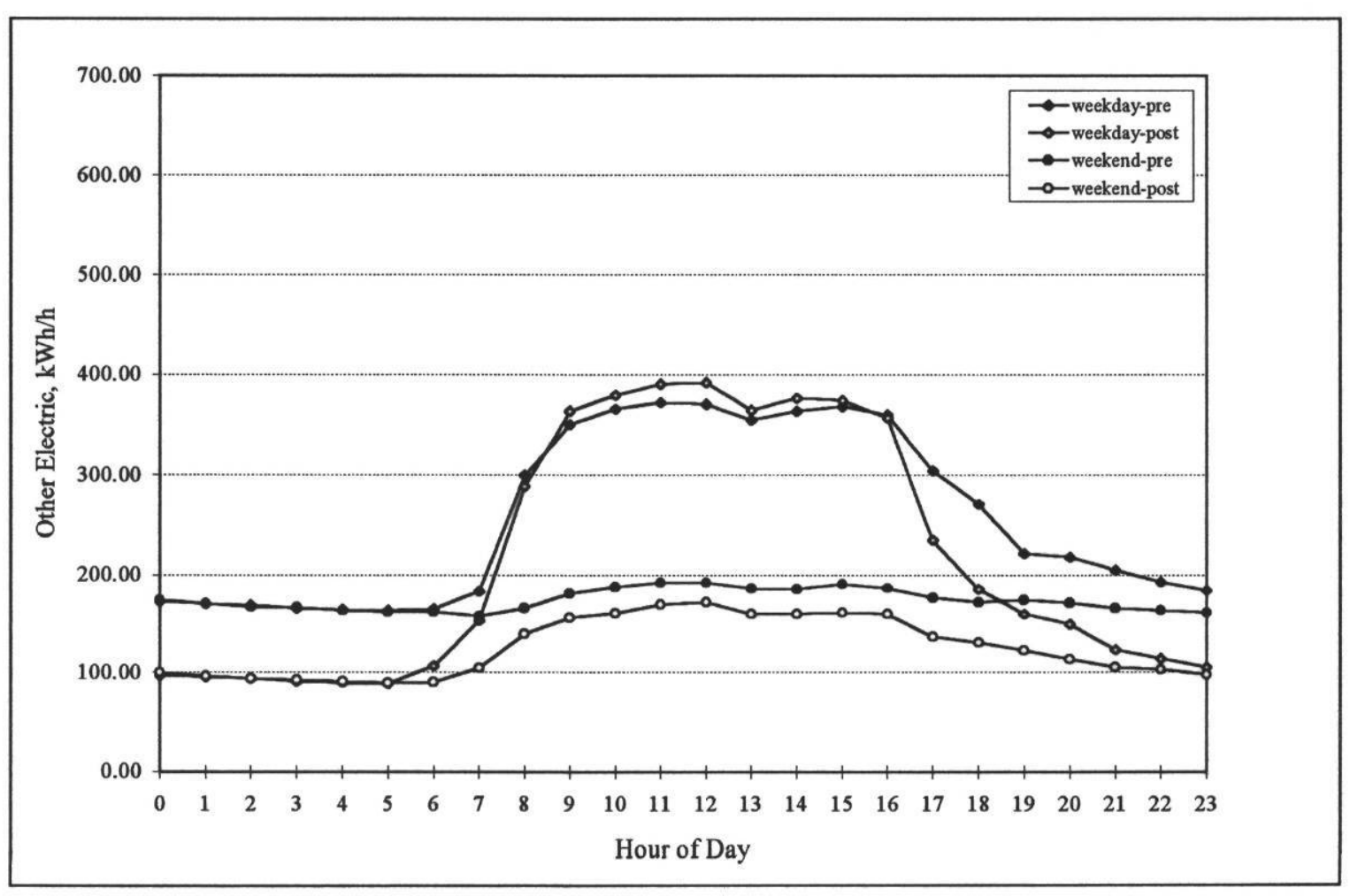


Figure 4a: SIM Semester Average Hourly Profile

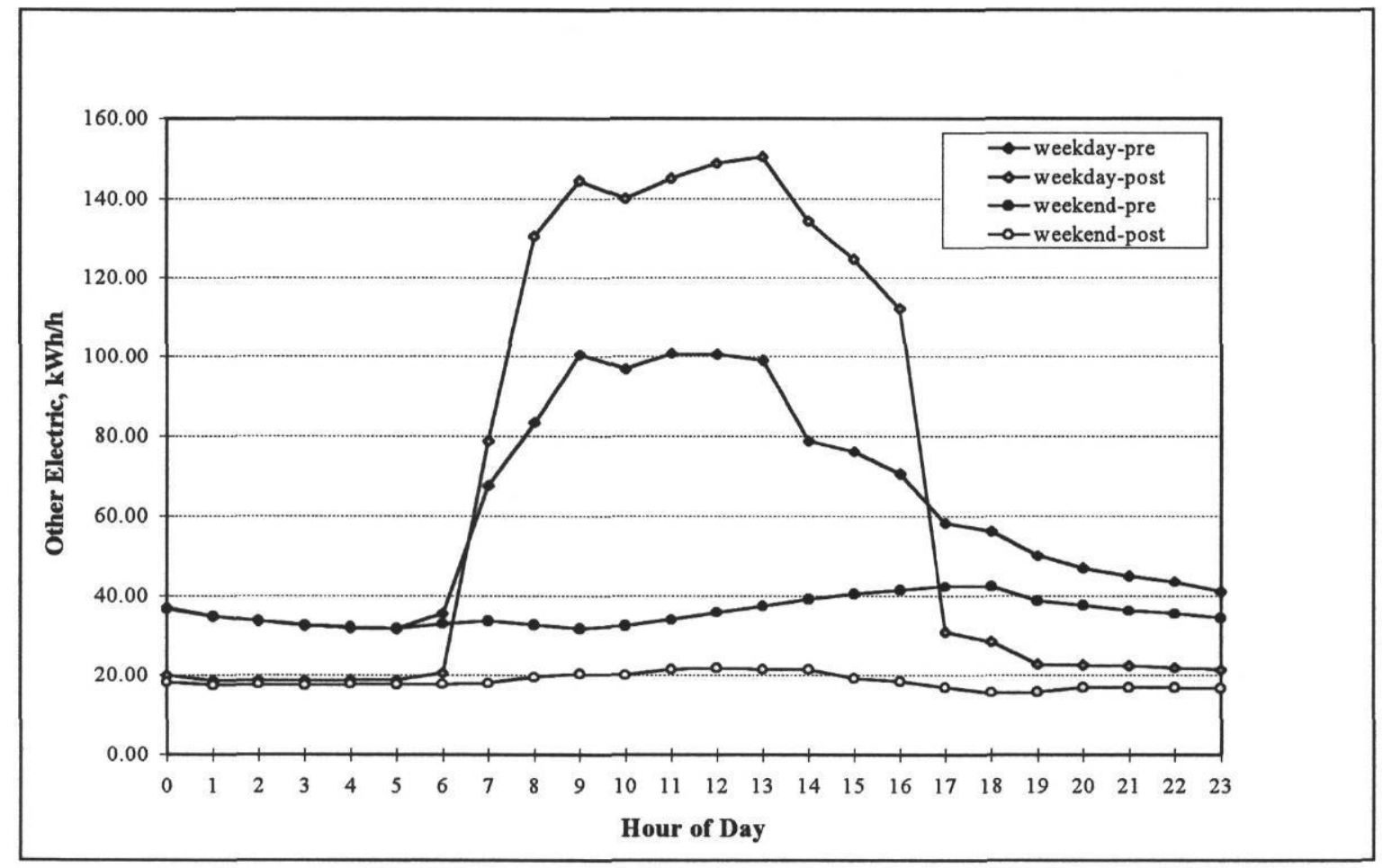

Figure 4b: SIM Non-semester Average Hourly Profile

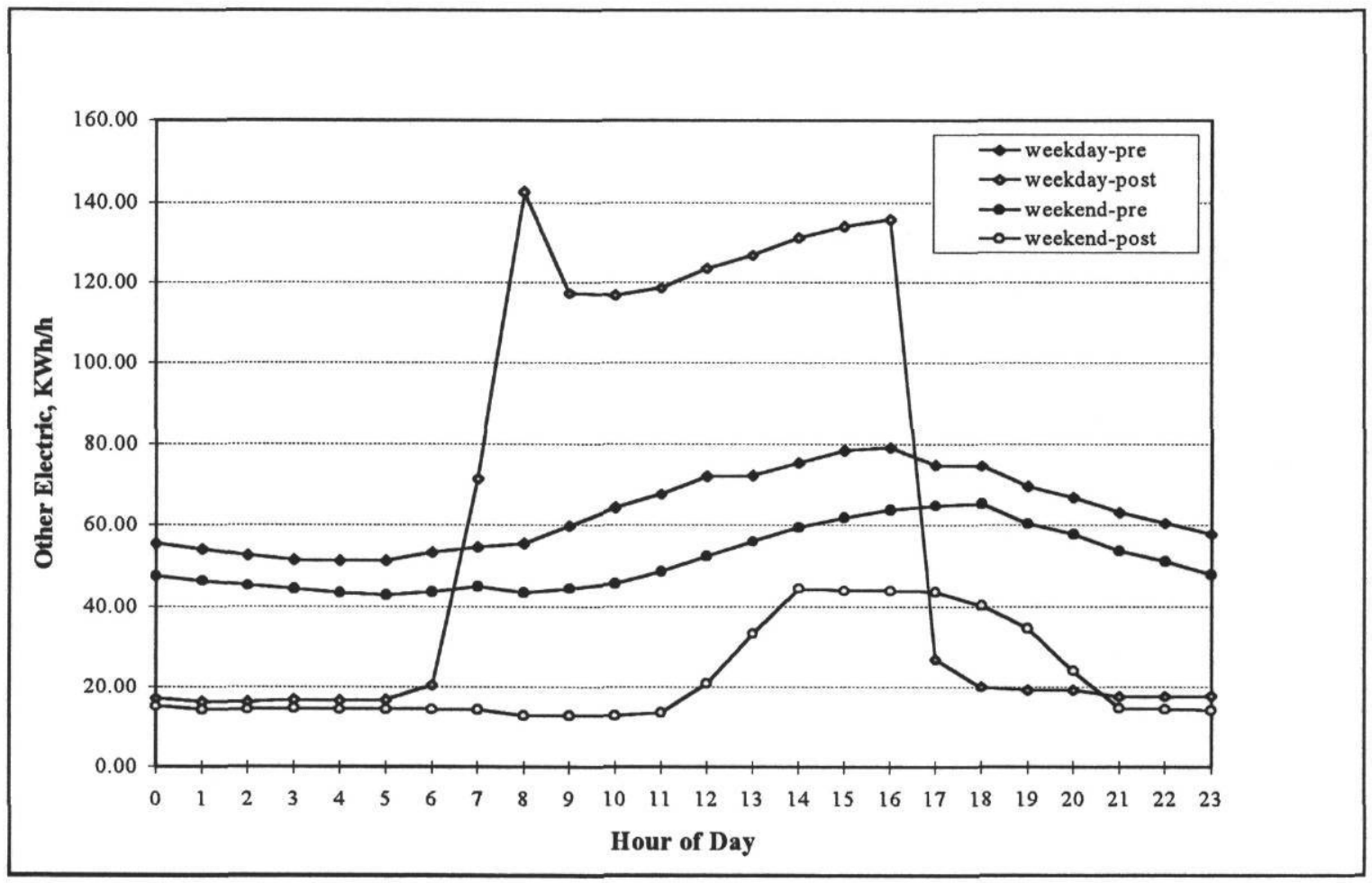


Pre-Retrofit (05/31/1989 - 11/28/1990)

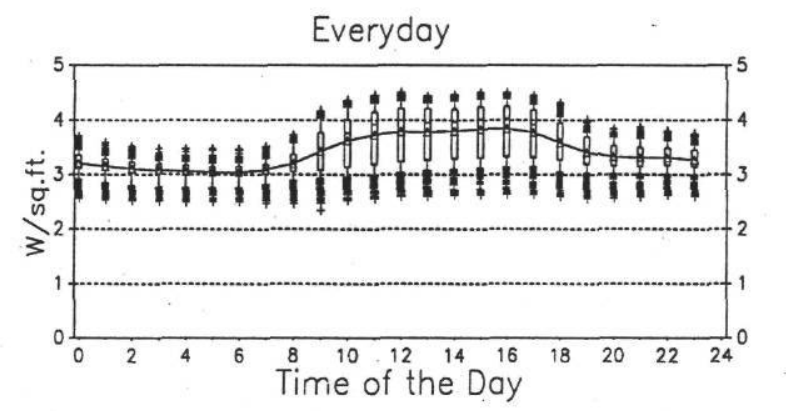

Weekdays

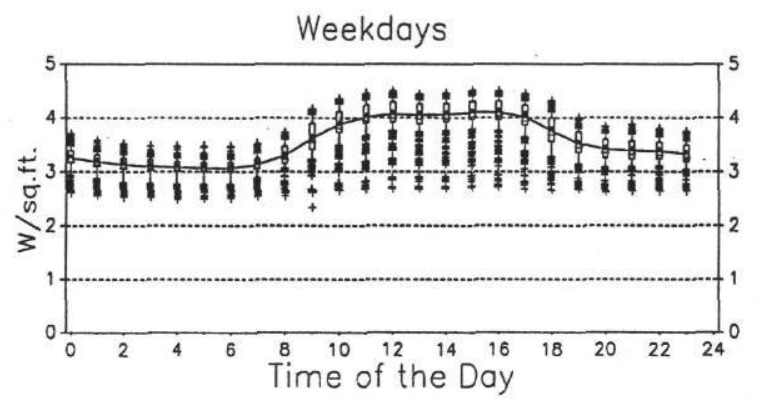

Weekends

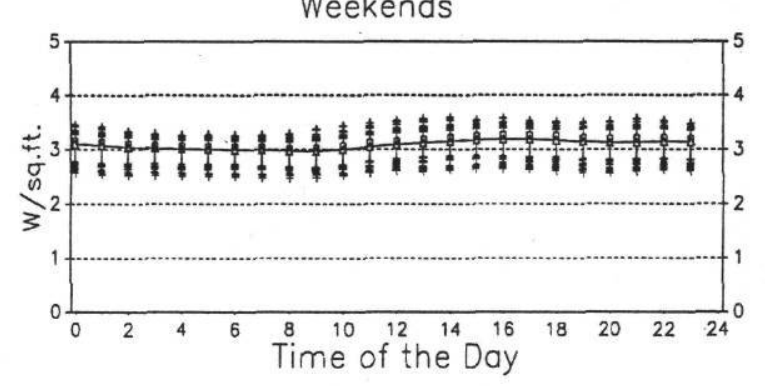

Post-Retrofit (03/06/1991 - 12/31/1994)

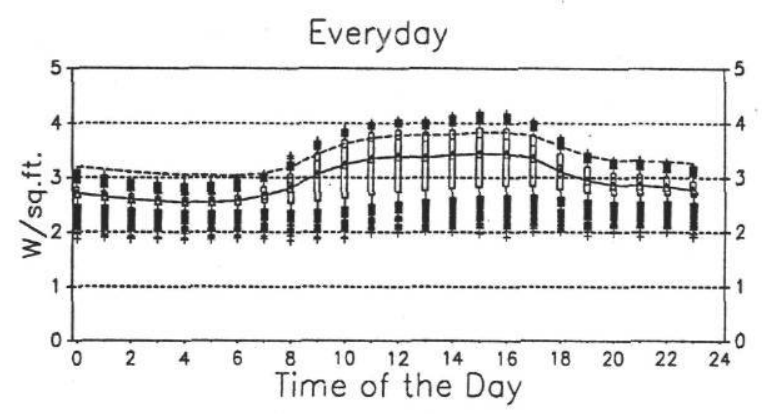

Weekdays

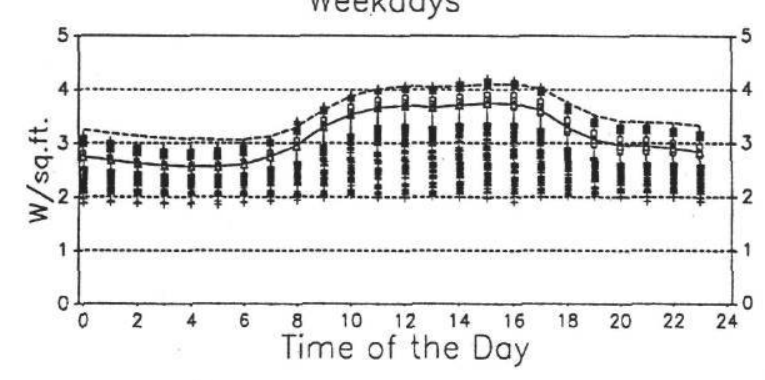

Weekends

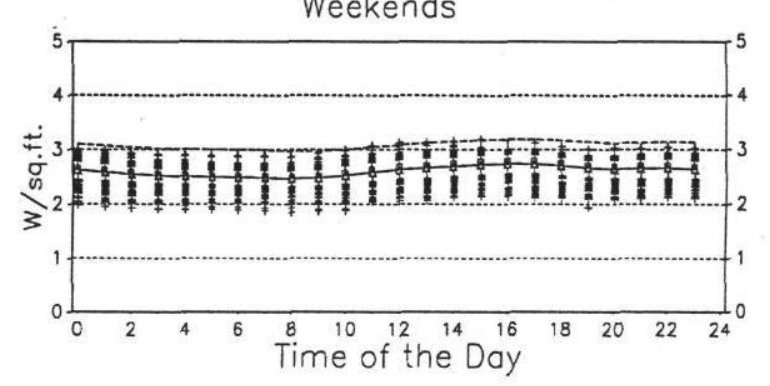




\section{A. STROMAN HIGH SCHOOL}

\section{A.1 Site Description ${ }^{1}$}

Stroman High School is located in Victoria, Texas. It consists of nine separate buildings with a total floor area of 210,414 square feet. Classrooms are heated and cooled by individual hydronic fan coil units. The first floor is heated and cooled by a hydronic air handler, and there are single air handlers on floors two through four to supply outside air to each floor. The two-story Unit B contains the auditorium, choir room, band room, and drafting classrooms. It is heated and cooled by air handlers. The band hall has direct expansion cooling as well, operating whenever the hydronic air handler does not provide cooling, in order to prevent humidity problems. Unit $\mathrm{C}$ is single story, housing the cafeteria and kitchen. It is heated and cooled by hydronic fan-coil units (six in the cafeteria, two in the kitchen). Units D and E are in one contiguous building, a two-story structure containing the library, gymnasium, locker rooms, and the main mechanical room. HVAC is provided by a hydronic air handler in the library, and by heating/ventilation units in the remaining athletic facilities. Unit $\mathrm{F}$ is a two-story building containing the science classrooms. It is heated and cooled by hydronic fan-coil units. Unit G is a single story shop building, containing several pieces of electrical equipment, from band saws to drills. It is heated and cooled by direct expansion units with gas furnaces. Chilled water and hot water for units A through $\mathrm{G}$ is provided by a 460 ton electric chiller and a 5,050 MBtu gas fired steam boiler. Auxiliary equipment includes a 50 horsepower chilled water pump, a 40 horsepower condenser water pump, a 30 horsepower cooling tower fan, and a 20 horsepower hot water pump.

There are also three athletic buildings just north of the main buildings that house the girls' gym, the field house, and the "athletic dome," in which weight training takes place. All three buildings are heated and cooled by direct expansion units with gas furnace.

\footnotetext{
${ }^{1}$ Adapted from: Landman, D.S., 1995. "Preliminary Study of Advanced Diagnostic Prescreening Methods," Energy Systems Laboratory, Mechanical Engineering Department, Texas A\&M University, College Station, TX.
} 
Air distribution is primarily through single duct multizone systems providing cooling temperatures of approximately $75^{\circ} \mathrm{F}$, and heating temperatures within the range of 70 to $72^{\circ} \mathrm{F}$. Heating and air handling systems are turned off completely during the night and are controlled from a central location through a Carrier EMCS.

The school is operated from the middle of August through the middle of May, with approximately 1,529 students and 145 faculty and staff. The maximum school occupancy is from about 8:00 a.m. until 4:00 p.m.; however, the building is occupied for much longer periods, including weekends and summers. Stroman and Victoria High School alternate as the primary location for summer school. Stroman was the site during the summer of 1993 . School district calendars for the reporting period of June 5, 1991, through June 4, 1994, are included in Tab A-1.

Large quartz lamps are used to light the tennis courts. These are shut off at 11:00 p.m. Electricity is purchased from Central Power and Light Company, and natural gas from ENTEX Gas Company.

\section{A.2 EMCS Retrofit}

The energy audit for Stroman High School determined that the HVAC operation was controlled manually, which resulted in excessive operating hours in each of the schools within the school district. Timeclock controls were installed many years ago, but were not suited for the needs of the school. See Tab A-2 for the full text technical analysis of the facility that was provided in the audit.

The proposed EMCS retrofit called for the installation of a direct digital control-based EMCS, which would control all HVAC equipment, measure exterior and interior space temperatures, and measure humidity in one or two critical locations within the school. The EMCS would have no override timers that custodial staffs could activate. Operating hours of all HVAC units would be determined by the maintenance staff, and controlled by that staff from its central headquarters via modem.

The EMCS system was installed and activated on January 31, 1992. It controls the HVAC equipment and some lights and measures the temperature and humidity at select locations. Although there are override capabilities, they are not used. 


\section{A.3 Analysis}

\section{A.3.1 Snapshot of consumption for September 1991-December 1993}

Figures A-1 and A-2 represent monthly average consumption and peak consumption versus minmax average monthly temperature and peak temperature, respectively. ${ }^{2}$ Min-max average monthly temperature is calculated by averaging the maximum and minimum temperature each day to obtain minmax average daily temperature. The daily temperatures are then averaged over all days in each month to obtain min-max average monthly temperature.

The data points reflecting high temperature and low consumption are indicative of non-semester consumption. If those data points are ignored, there is a general increase of consumption with temperature, indicating a temperature dependence of consumption. Additionally, the post-retrofit data points are generally lower than the pre-retrofit data points. When compared to similar plots for other Texas schools in the LoanSTAR program, this site is a low energy use school. The reader is referred to the referenced report for a more detailed discussion of these plots.

\footnotetext{
${ }^{2}$ Landman, D.S., 1995. "Preliminary Study of Advanced Diagnostic Prescreening Methods," Energy Systems Laboratory, Mechanical Engineering Department, Texas A\&M University, College Station, TX.
} 
Figure A-1: Monthly Average Consumption: Consumption, in W/sf, versus min-max average monthly temperature, in ${ }^{\circ} \mathrm{F}$ for September 1991 - December 1993 (Stroman High School)

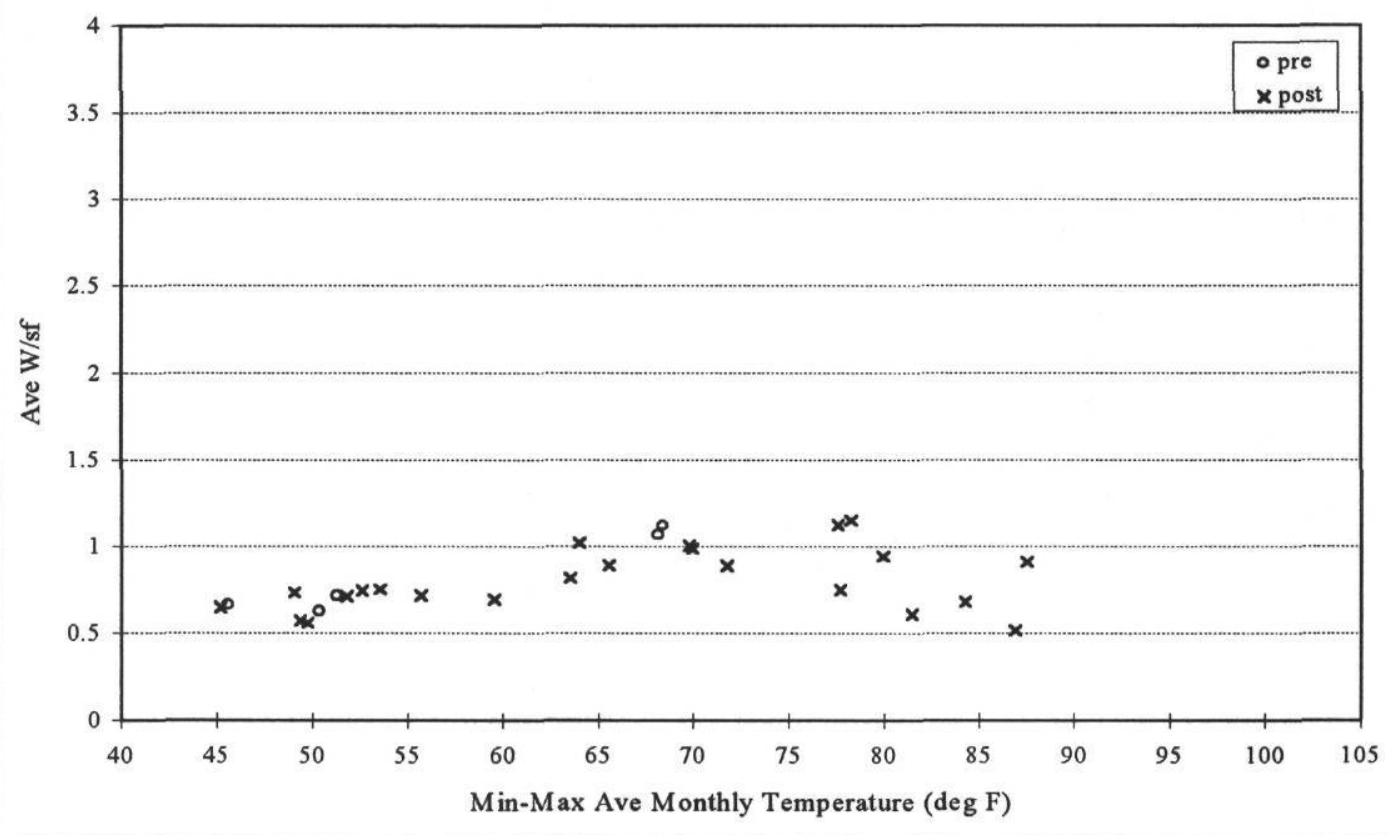

Figure A-2: Monthly Peak Consumption: Consumption, in W/sf, versus peak monthly temperatures, in ${ }^{\circ}$ F, for September 1991 through December 1993 (Stroman High School)

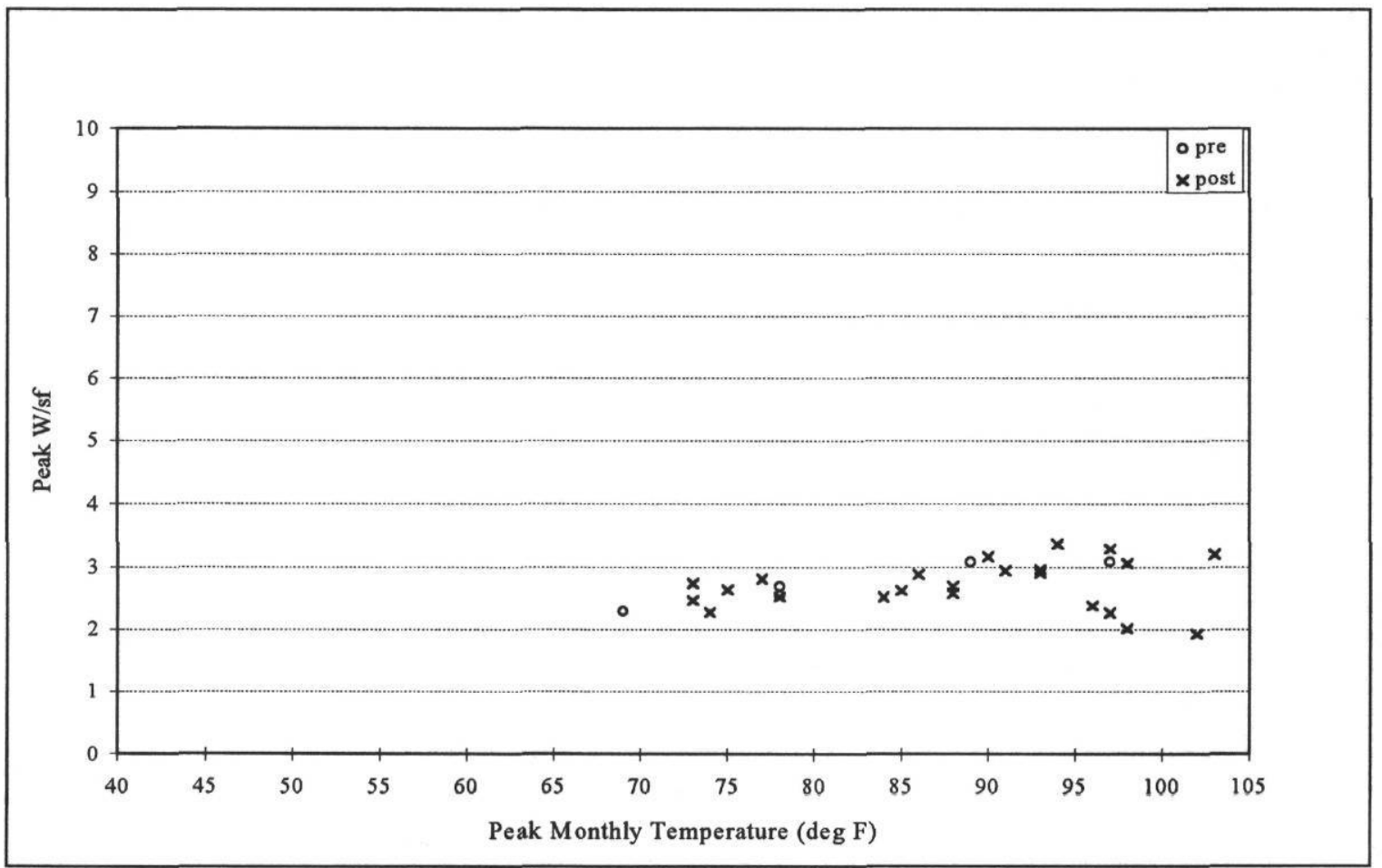




\section{A.3.2 Timeline plots}

Plots of energy consumption for the reporting period are shown in Figure A-3. The EMCS retrofit date of January 31,1992 , is shown by a vertical, bold line. Monitoring diagrams are provided in Tab A-3.

In looking at the whole building electric plot, there is no apparent decrease in consumption at any point along the timeline. There was an absorption chiller installed as a concurrent retrofit at this site. This resulted in the appearance of chiller consumption in September 1991. Any possible decrease in consumption due to the EMCS may have been offset by the increase in consumption due to the new chiller. The appropriate plot to analyze to look at effects due to EMCS only is the "other electric" plot, which is whole building electric minus the absorption chiller. Here a drop in consumption is evident between the pre-retrofit and post-retrofit time periods

The plot of whole building heat shows seasonal heating between November and April of each year. There is also a decrease in consumption evident between the pre-retrofit and post-retrofit periods. 


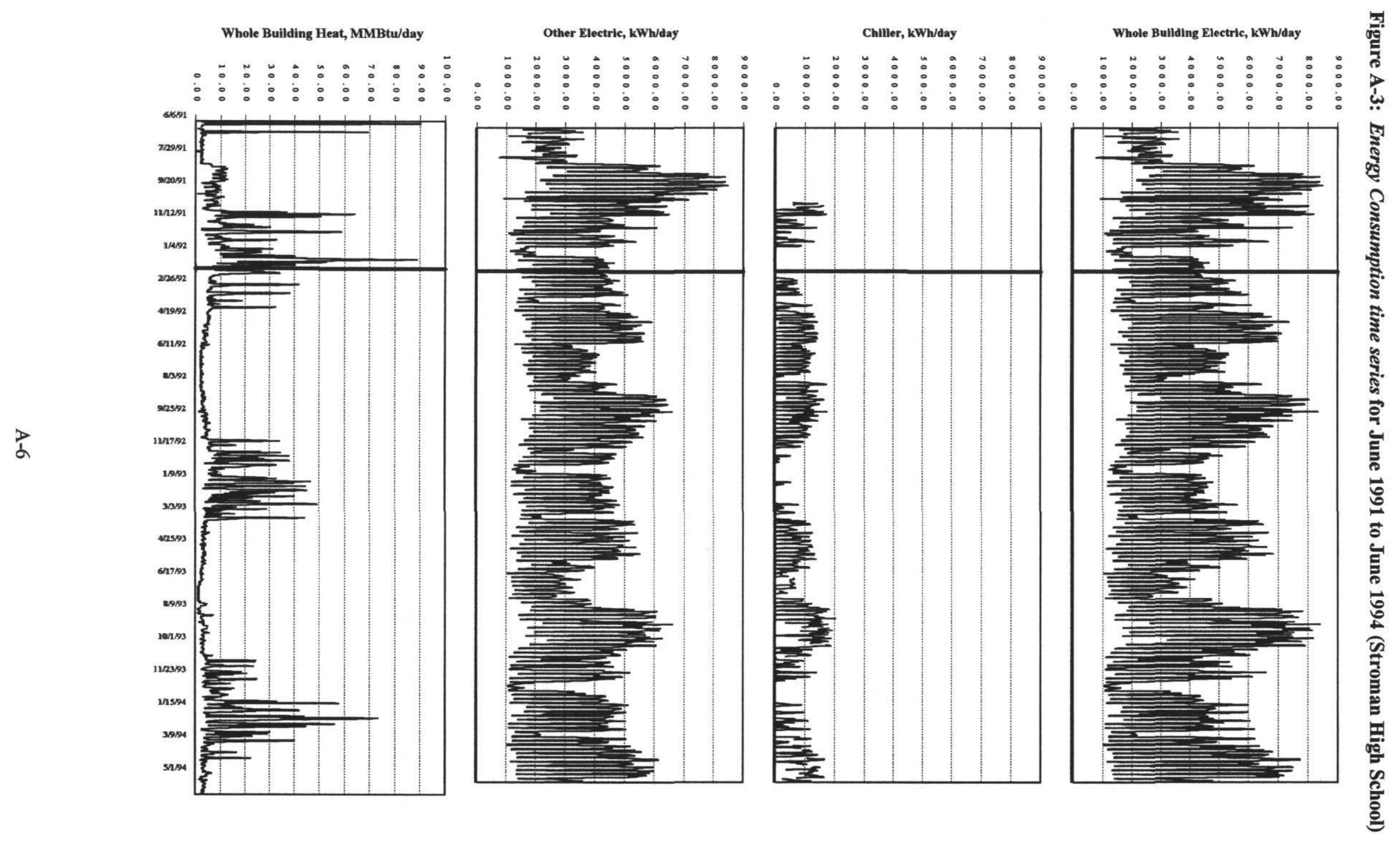




\section{A.3.3 Whole Building Electricity Consumption (Post Period)}

Table A-1 shows energy consumption for the post-retrofit period (February 1, 1992, through June $4,1994)$, broken down by semester and non-semester. Whole building electricity consumption is broken down into two components: chiller electricity consumption and other electricity consumption. The post-retrofit period is used because there is significantly more data available in the that period, and it represents current usage.

Figure A-4 graphically shows whole building electricity consumption for the post-retrofit period. For the semester period, $62 \%$ of whole building electric energy use is attributable to other electric equipment, while $7 \%$ is due to the electric chiller. For the non-semester period, other electric accounts for $28 \%$ of whole building electric energy, while the chiller accounts for $3 \%$.

For both Table A-1 and Figure A-4, it is readily apparent that chiller consumption accounts for a small portion of the whole building electricity usage. Therefore, attention for reducing energy usage should be focused on the other electricity usage. This is also the reason for focusing attention on other electric consumption in this report. In this case, other electricity consumption is mainly roof-top HVAC units and lighting.

Table A-1: Energy Consumption for post period, February 1992 - June 1994 (Stroman High School)

\begin{tabular}{|l|r|r|r|r|r|r|}
\hline & \multicolumn{2}{|c|}{ SEMESTER } & \multicolumn{2}{c|}{ NON-SEMESTER } & \multicolumn{2}{c|}{ TOTAL } \\
\cline { 2 - 7 } & \multicolumn{1}{|c|}{ ENERGY } & \multicolumn{1}{c|}{$\$$} & ENERGY & \multicolumn{1}{c|}{$\$$} & \multicolumn{1}{c|}{ ENERGY } & \multicolumn{1}{c|}{$\$$} \\
\hline wbelec, $\mathrm{kWh}$ & $2,209,234$ & $\$ 61,593$ & 952,917 & $\$ 26,567$ & $3,162,150$ & $\$ 88,161$ \\
\hline chlr, $\mathrm{kWh}$ & 236,828 & $\$ 6,603$ & 119,815 & $\$ 3,340$ & 356,643 & $\$ 9,943$ \\
\hline other, $\mathrm{kWh}$ & $1,972,405$ & $\$ 54,991$ & 833,102 & $\$ 23,227$ & $2,805,507$ & $\$ 78,218$ \\
\hline wbheat, MMBtu & 5,059 & $\$ 24,028$ & 1,352 & $\$ 6,421$ & 64,10 & $\$ 30,449$ \\
\hline & & & & & & \\
\hline
\end{tabular}


Figure A-4: Whole Building Electricity Consumption for post period, February 1992 - June 1994 Stroman High School

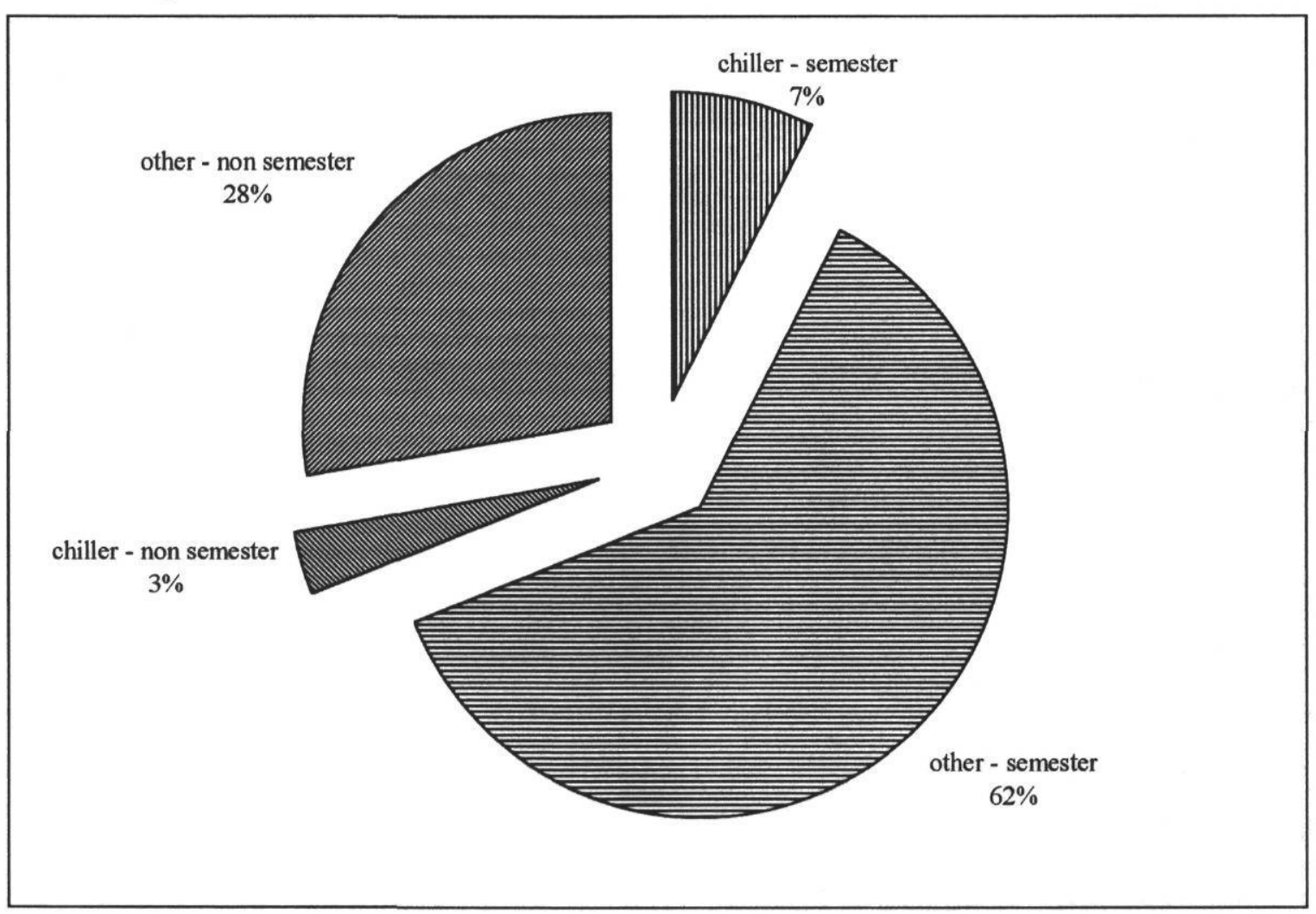

\section{A.3.4 Total Monthly Consumption}

The total monthly energy consumption is summarized in Table A-2. Again, it is readily apparent that other electric accounts for the majority of this site's electric energy use. 
Table A-2: Monthly Energy Consumption (Stroman High School)

\begin{tabular}{|c|c|c|c|c|}
\hline & $\begin{array}{c}\text { wbelec } \\
\text { kWh/month }\end{array}$ & $\begin{array}{c}\text { chiller } \\
\text { kWh/month }\end{array}$ & $\begin{array}{c}\text { other } \\
\text { kWh/month }\end{array}$ & $\begin{array}{c}\text { wbheat } \\
\text { MMBtu/month }\end{array}$ \\
\hline \multicolumn{5}{|l|}{ PRE PERIOD } \\
\hline Jun 91 & 167,040 & 0 & 167,040 & 216 \\
\hline Jul & 181,199 & 24,422 & 156,777 & 437 \\
\hline Aug & 135,033 & 6,361 & 128,672 & 783 \\
\hline Sep & 120,760 & 7,331 & 113,429 & 398 \\
\hline Oct & 140,339 & 16,726 & 123,613 & 274 \\
\hline Nov & 172,213 & 29,039 & 143,174 & 110 \\
\hline Dec & 161,017 & 22,728 & 138,289 & 171 \\
\hline Jan 92 & 130,095 & 6,118 & 123,977 & 571 \\
\hline Total Consumption & $1,207,697$ & 112,726 & $1,094,971$ & 2,960 \\
\hline Total Cost & $\$ 33,671$ & $\$ 3,143$ & $\$ 30,528$ & $\$ 14,059$ \\
\hline \multicolumn{5}{|l|}{ POST PERIOD } \\
\hline Feb 92 & 123,864 & 999 & 122,865 & 700 \\
\hline Mar & 155,836 & 16,837 & 138,999 & 222 \\
\hline Apr & 178,822 & 32,459 & 146,363 & 104 \\
\hline May & 216,629 & 45,963 & 170,666 & 111 \\
\hline Jun & 145,812 & 13,999 & 131,813 & 287 \\
\hline Jul & 139,191 & 5,025 & 134,166 & 862 \\
\hline Aug & 150,833 & 13,930 & 136,903 & 400 \\
\hline Sep & 193,871 & 34,960 & 158,912 & 107 \\
\hline Oct & 85,113 & 7,833 & 77,280 & 210 \\
\hline Nov & 107,539 & 11,146 & 96,393 & 450 \\
\hline Dec & 126,965 & 16,559 & 110,405 & 183 \\
\hline $\operatorname{Jan} 93$ & 59,302 & 770 & 58,532 & 217 \\
\hline Feb & 61,654 & 6,893 & 54,761 & 133 \\
\hline Mar & 48,803 & 2,534 & 46,269 & 164 \\
\hline Apr & 73,353 & 1,936 & 71,418 & 396 \\
\hline May & 111,118 & 0 & 111,118 & 82 \\
\hline Jun & 113,650 & 2,348 & 111,301 & 406 \\
\hline Jul & 125,016 & 22,649 & 102,366 & 101 \\
\hline Aug & 124,743 & 20,660 & 104,082 & 72 \\
\hline Sep & 125,745 & 18,271 & 107,474 & 199 \\
\hline Oct & 103,438 & 14,459 & 88,979 & 120 \\
\hline Nov & 98,266 & 11,325 & 86,941 & 52 \\
\hline Dec & 164,686 & 33,704 & 130,982 & 105 \\
\hline Jan 94 & 75,544 & 5,414 & 70,130 & 253 \\
\hline Feb & 57,468 & 43 & 57,425 & 149 \\
\hline Mar & 83,609 & 8,992 & 74,617 & 129 \\
\hline Apr & 64,819 & 4,018 & 60,802 & 154 \\
\hline May & 47,334 & 2,034 & 45,300 & 112 \\
\hline Jun 94 & 8,492 & 896 & 7,596 & 11 \\
\hline Total Consumption & $3,171,513$ & 356,656 & $2,814,857$ & 6,483 \\
\hline Total Cost & $\$ 88,422$ & $\$ 9,944$ & $\$ 78,478$ & $\$ 30,795$ \\
\hline Grand Total Consumption & $4,379,210$ & 469,382 & $3,909,828$ & 9,443 \\
\hline Grand Total Cost & $\$ 122,092$ & $\$ 13,086$ & $\$ 109,006$ & $\$ 44,854$ \\
\hline
\end{tabular}




\section{A.3.5 Average Daily Consumption}

Figures A-5a and A-5b depict the average daily consumption for the semester period and the non-semester period. From both plots, you can see that the consumption for the weekdays does not change in profile, but does decrease in magnitude.

For the semester period, Figure A-5a, the weekday consumption decreased substantially during the daytime hours, 7:00 a.m. to 5:00 p.m., and slightly decreased during the nighttime hours, 5:00 p.m. to 7:00 a.m. The weekend consumption decreased during the nighttime, but increased during the daytime hours. Why does the post-retrofit consumption exceed that of the pre-retrofit consumption for weekends? One possible explanation is that the setpoints on the new EMCS are such that the consumption is greater during the weekend than before the EMCS was installed. Another possible explanation may be due to many more data points in the post period, and periodic special events on the weekends. These two factors combined may result in higher weekend daytime consumption in the post period.

For the non-semester period, Figure A-5b, weekday consumption slightly decreased during the daytime hours and greatly decreased during the nighttime hours. Here, the weekend usage changed in a manner similar to that of the weekdays. The changes in both weekday and weekend consumption can be attributed to the EMCS retrofit.

Tab A-4 contains a summary of the hourly averages and the respective standard deviations and count of data points. The hourly averages are the data that is plotted in Figures A-5a and A-5b. For this site, the standard deviations are quite large. They do not vary much between the hours of 0 through 6 , then jump to higher levels in hours 7 through 23 . This should not be alarming, because the periods that the data were averaged over include wide ranges of temperatures. As was seen earlier, in Figures A-1 and A-2, the energy usage is temperature dependent. The count of data points represents the actual number of data points used to calculate the average, which corresponds to the amount of time that the equipment was actually operating. 
Figure A-5a: Semester Pre-/Post-retrofit Consumption (Stroman High School)

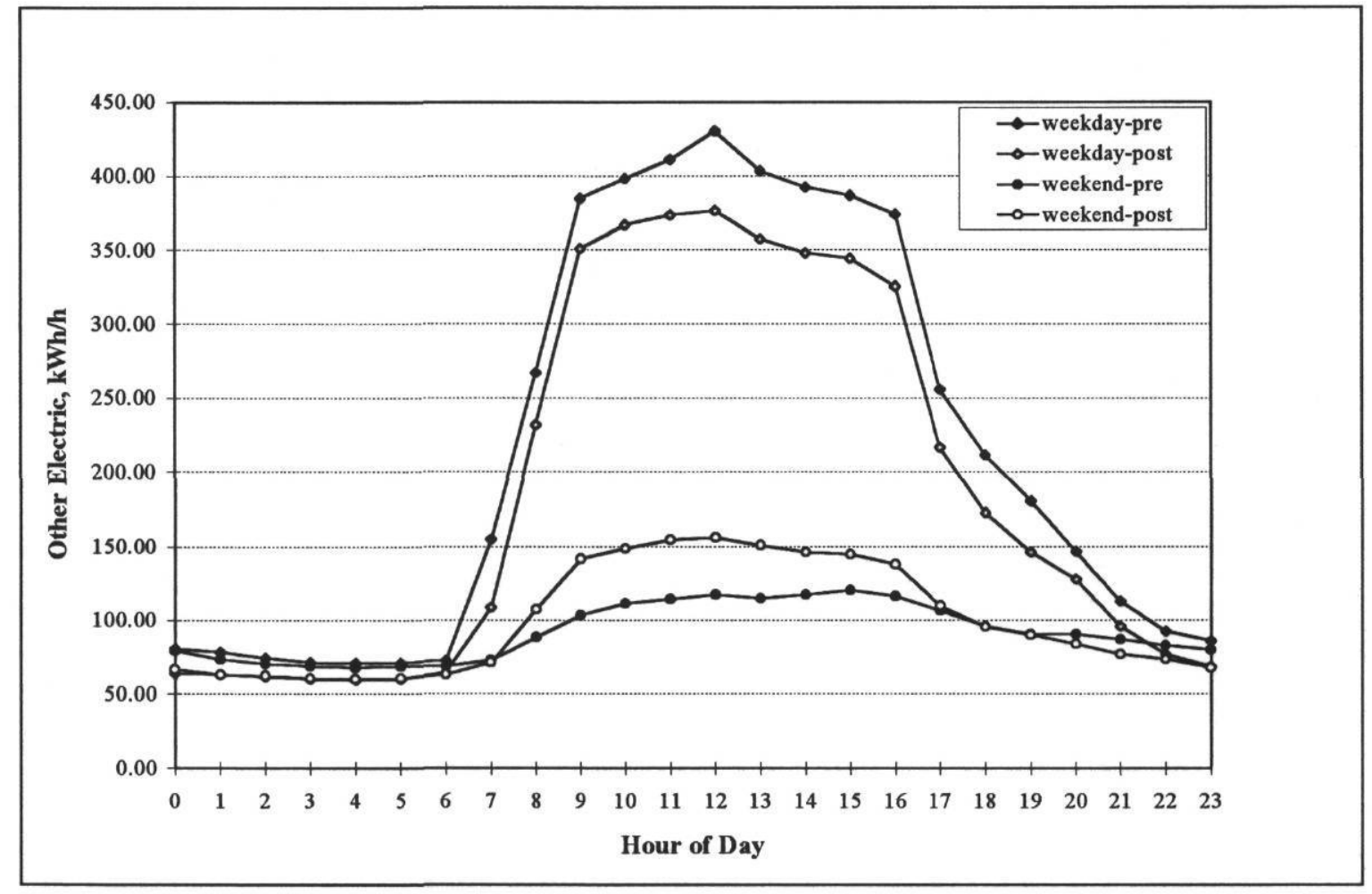

Figure A-5b: Non-semester Pre-/Post-retrofit Comparison (Stroman High School)

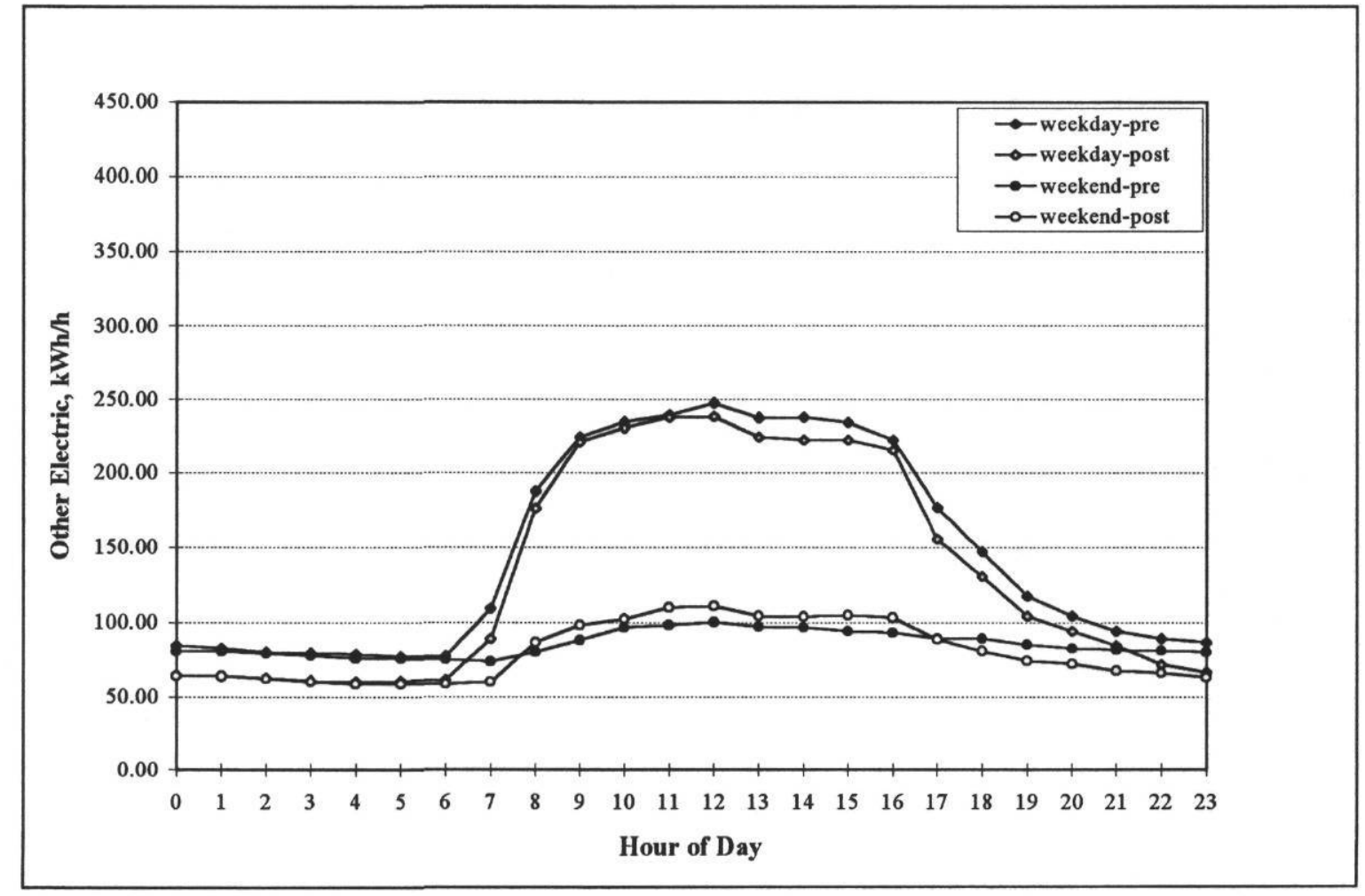


The difference in other electric energy consumption was calculated based on the average daily data. This is shown in Table A-3, both as a difference in energy and a percentage difference in energy.

Table A-3: Reduction in Other Electric Energy Consumption, based on average daily data (Stroman High School)

\begin{tabular}{|c|c|c|c|c|}
\hline & $\begin{array}{l}\text { \# days } \\
\text { in sort } \\
\text { category }\end{array}$ & $\begin{array}{l}\text { Average Daily } \\
\text { Consumption } \\
\text { kWh/day }\end{array}$ & $\begin{array}{c}\text { Difference in } \\
\text { Average Daily } \\
\text { Consumption } \\
\text { kWh/day }\end{array}$ & $\begin{array}{l}\text { \% Difference in } \\
\text { Average Daily } \\
\text { Consumption }\end{array}$ \\
\hline \multicolumn{5}{|l|}{ Semester } \\
\hline weekday-pre & 91 & 5,210 & \multirow[b]{2}{*}{-685} & \multirow[b]{2}{*}{$-13.15 \%$} \\
\hline weekday-post & 394 & 4,525 & & \\
\hline weekend-pre & 35 & 2,206 & \multirow[b]{2}{*}{189} & \multirow[b]{2}{*}{$8.57 \%$} \\
\hline weekend-post & 149 & 2,395 & & \\
\hline \multicolumn{5}{|l|}{ Non-semester } \\
\hline weekday-pre & 79 & 3,557 & \multirow[b]{2}{*}{-332} & \multirow[b]{2}{*}{$-9.33 \%$} \\
\hline weekday-post & 241 & 3,225 & & \\
\hline weekend-pre & 33 & 2,060 & \multirow[b]{2}{*}{-130} & \multirow[b]{2}{*}{$-6.31 \%$} \\
\hline weekend-post & 92 & 1,930 & & \\
\hline
\end{tabular}

There was a reduction in consumption for all categories except semester, weekend. Possible reasons for this increase were discussed in the previous section.

\section{A.3.6 Plots from MECR}

The September MECR energy use plots for four years are shown in Tab A-5. These provide a more qualitative look at the effects of the EMCS. September 1991 is a pre-retrofit plot. Note that there is generally low consumption between the hours of Midnight and 6:00 a.m., with a gradual increase to daytime levels. This is followed by a slow decrease in consumption between the hours of 4:00 p.m. and 10:00 p.m. There are many afternoons and evenings where consumption did not drop to nighttime levels. September 1992 shows a decreased nighttime consumption, with a much sharper slope up to daytime levels between 7:00 a.m. and 8:00 a.m. The consumption drops off much more quickly at 4:00 p.m., as compared to September 1991, indicating that the EMCS is controlling the consumption as expected. There are still a few days with high afternoon and evening consumption, most likely due to special events 
that required the air conditioning and lighting to remain on after hours. The profiles continue to improve for the months of September 1993 and September 1994.

It should be noted that these profiles only allow a look at weekday data. The weekend data is unreadable from these plots. Separating the data into weekdays and weekends, then plotting separately, would enable one to evaluate weekends, as well as weekdays.

\section{A.3.7 Data Summary Notebook Information}

The Data Summary Notebook information is included in Tab A-6 for information only. It is not analyzed for this site 
Tab A-1

School District Schedule 
VICTORIA PUBLIC SCHOOLS SCHOOL CALENDAR 1990 - 91

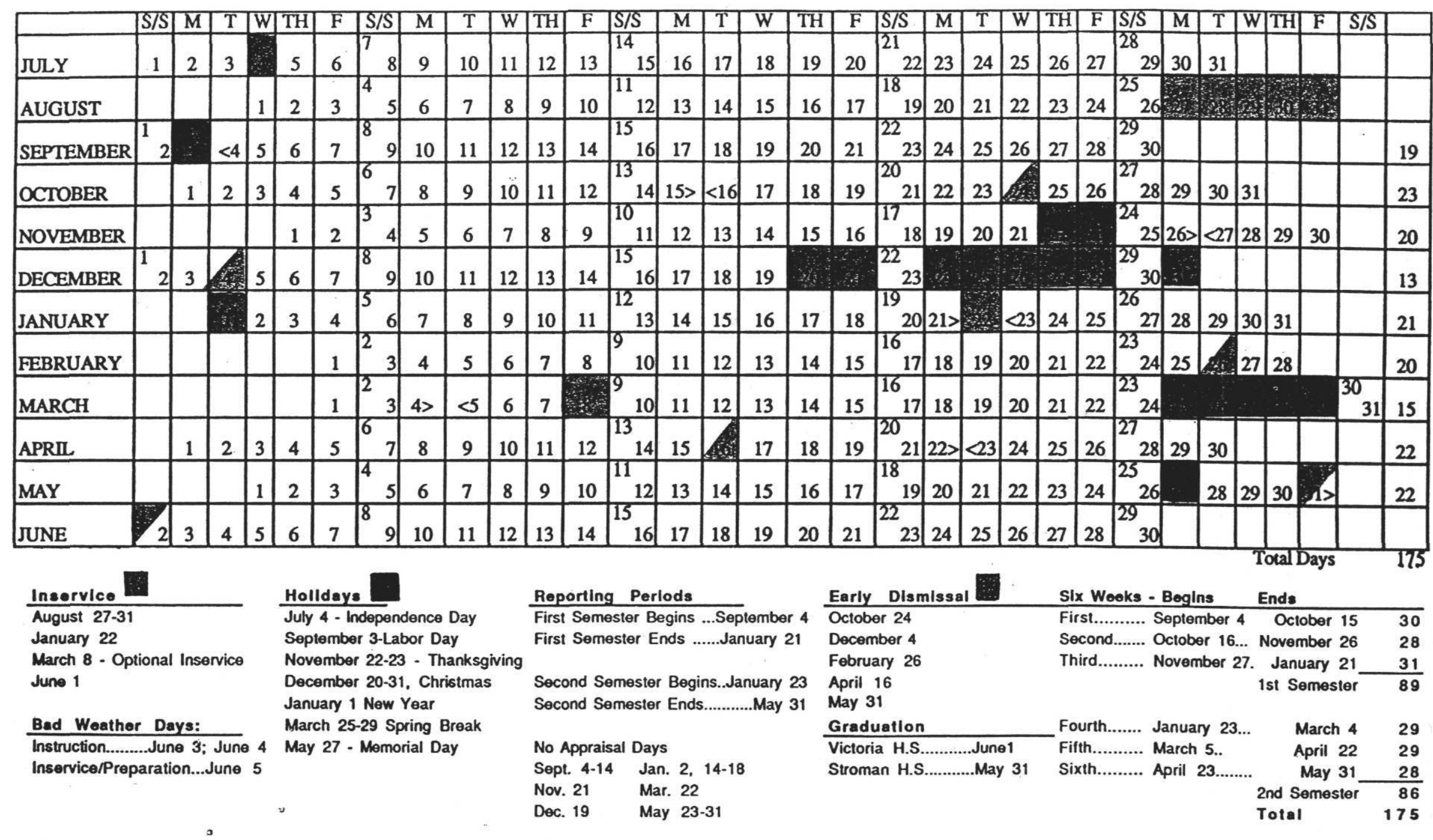


VICTORIA PUBLIC SCHOOLS SCHOOL CALENDAR 1991- 1992

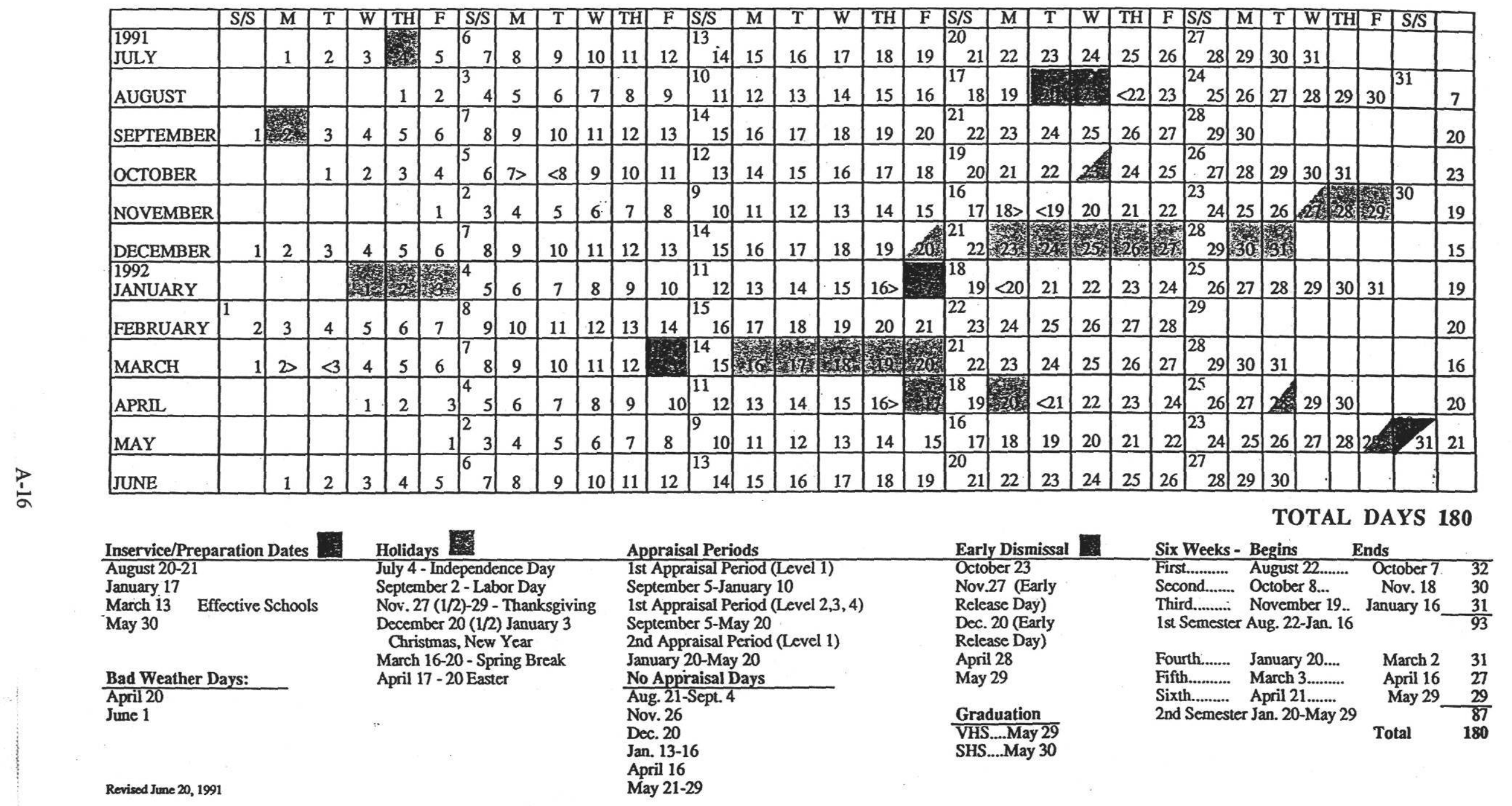


VICTORIA PUBLIC SCHOOLS

SCHOOL CALENDAR 1992 - 93

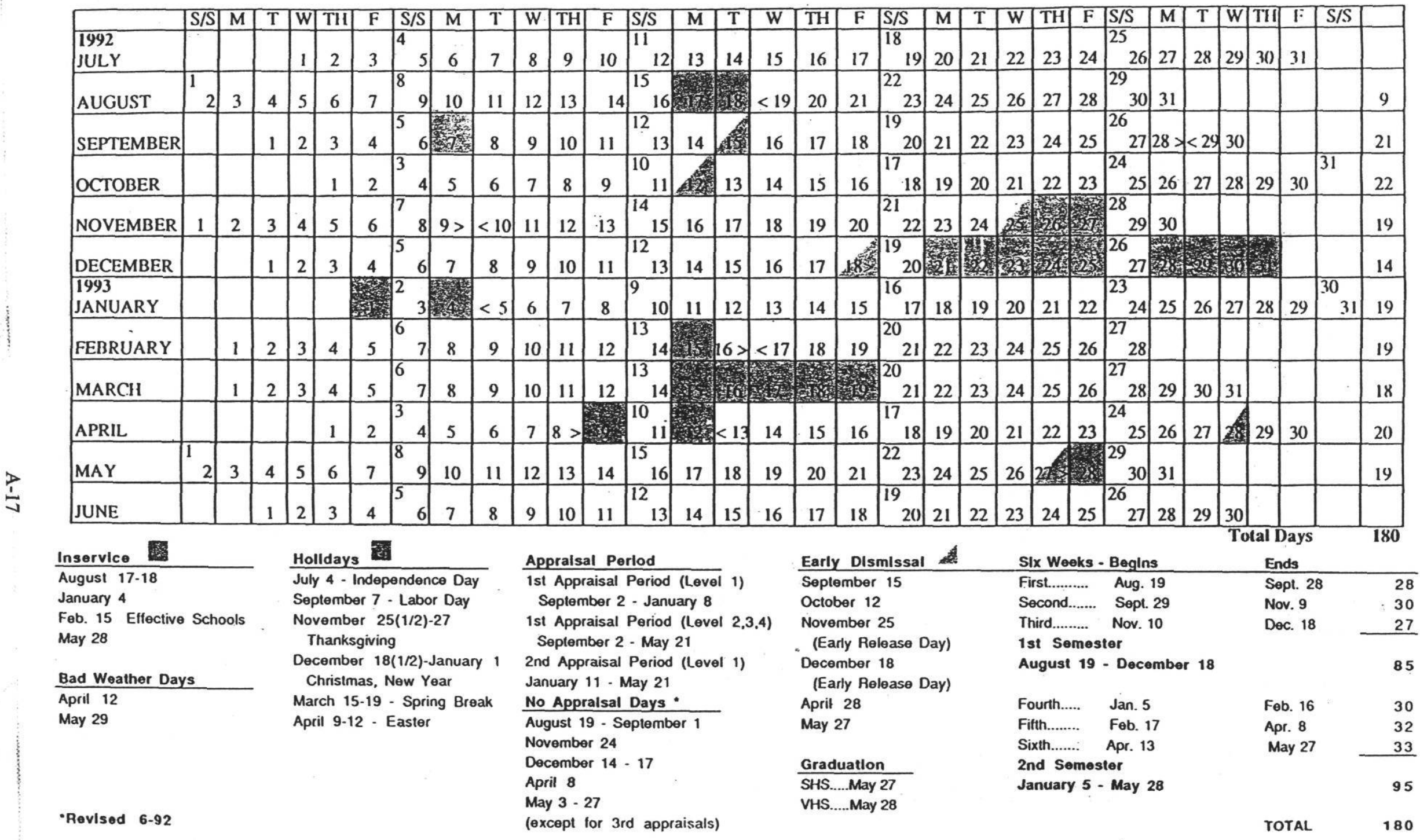




\section{VICTORIA PUBLIC SCHOOLS}

School Calendar 1993-1994

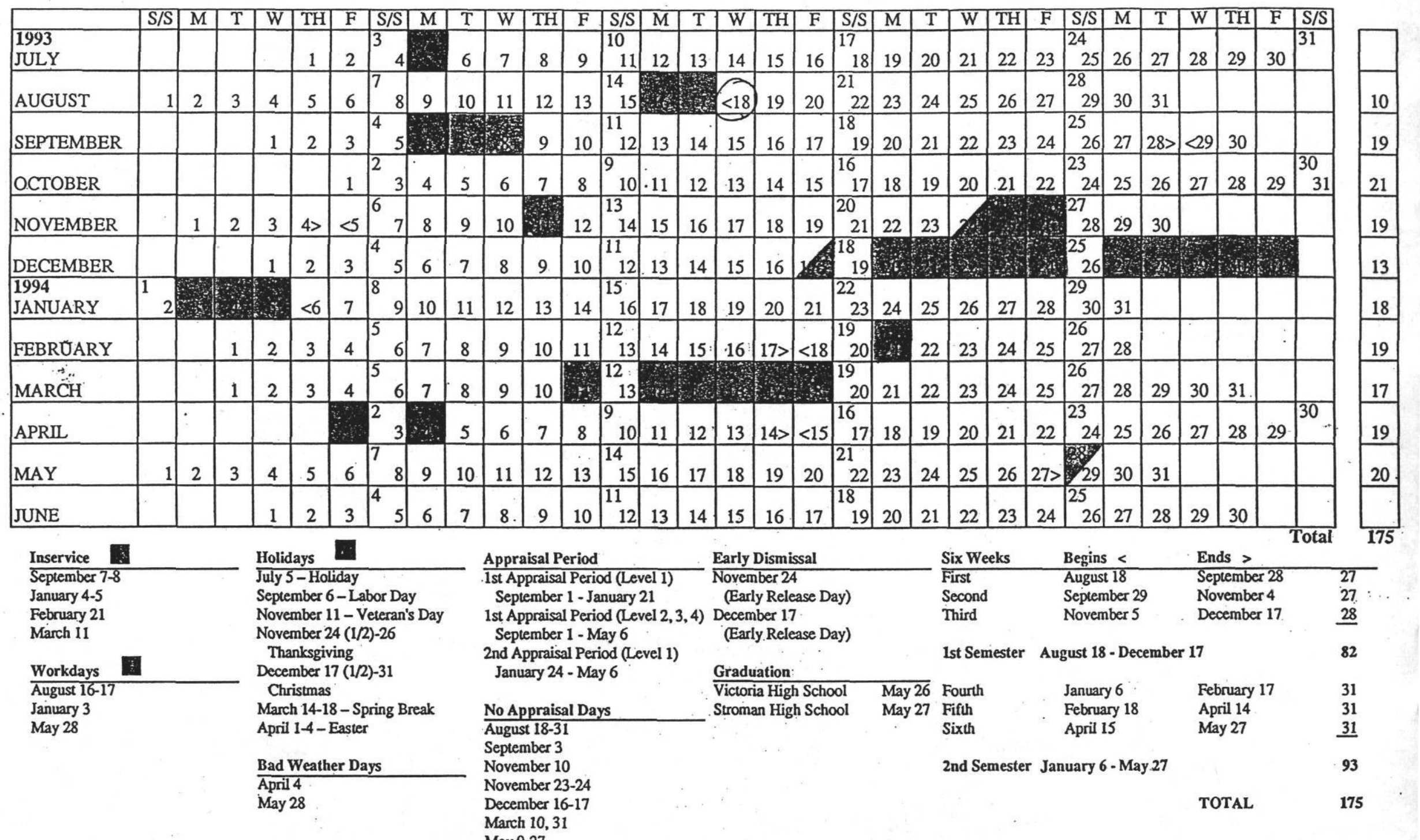


Tab A-2

Audit Technical Analysis

A-19 


\section{ECRM DESCRIPTIONS AND CALCULATIONS}

Facility Name: All Schools

ECRM No.: 1

ECRM Name: Energy Management System

a. Summary

Kwh savings:

Demand savings:

MCF savings:

Cost savings:

Implementation cost:

Simple payback:

\author{
$1,583,682 \mathrm{Kwh} / \mathrm{yr}$ \\ $898 \mathrm{KW}-\mathrm{mo} / \mathrm{yr}$ \\ $3,850 \mathrm{MCF} / \mathrm{yr}$ \\ $\$ 95,254 / \mathrm{yr}$ \\ $\$ 380,980$ \\ 4.0 years
}

\section{b. Description}

On/Off and temperature control in all of the Victoria ISD schools addressed in this report are inadequate. Typically, on/off controls consist of a) 7-day timeclocks which are controlled manually, b) manual control at thermostats or wall switches, and c) programmable thermostats in a very few locations, installed in the last two years. The great majority of on/off control is performed manually, with the result that operating hours are excessive in every school. There is not a single school addressed in this report where on/off control for the majority of HVAC equipment is performed automatically.

Timeclock controls were installed many years ago and are not suited for the needs of the schools.

- There is no way to enforce rigorous hours of HVAC operation if the custodial staff has, access to all timeclocks. Even if the timeclocks were functioning with their trippers and the timeclock cabinet were locked, override timers on the face of the timeclock cabinets would allow custodians to turn on HVAC units. The custodians work typically until 9 PM. The natural human tendency is to keep the units on to maintain most comfortable working conditions. Custodial staffs have been instructed on several occasions by the VISD maintenance staff to turn off HVAC promptly after school. Without direct and continuous supervision, one cannot reasonably expect the custodial staff to do so. And they don't.

- The timeclocks offer little flexibility. They typically control multiple HVAC units on one circuit. Often, an entire bank of HVAC units operates when in fact not all are needed. Special events may at time be held outside of normal operating hours. The existing override timers also control banks of units, so -- if the timeclocks and override timers were even used -- more units would operate than necessary.

- There is no feedback with the timeclock system, such as space temperature or humidity readings, and actual operating status of the unit. In several cases, air conditioning takes place 24 hours per day in order to prevent humidity-related problems. Also, heating units may be left on overnight when weather is cold, maintaining temperatures at comfort conditions. Feedback information on space versus outdoor conditions could save a great deal of energy by reducing operating hours. 
Summer operation of HVAC systems is also excessive. Schools are cleaned over a period of several weeks during each summer. Depending upon school size, the number of people cleaning, whether summer school is held or not, and the type of cleaning projects taking place, the cleaning process can take up to 6 weeks or more. Often the cleaning crews will turn on air conditioning for entire schools or wings of schools, regardless of how many rooms are actually being cleaned, since the method of turning units on is to flip a master timeclock switch which turns on whole banks of units. Again, virtually all control is manual through thermostats or timeclock master trippers. In addition to air conditioning schools for personal comfort, the cleaning crew operates the air conditioning to speed up drying of floors and other surfaces cleaned. Also, some teachers start coming to school by mid August. Typically, air conditioning throughout an entire school is again turned on, even though the number of teachers occupying the school is very small.

Temperature controls are virtually all open to occupant adjustment. The number of locking thermostats in all schools addressed in this report can be counted on one hand, and some of those are not locked. Typical settings are in the low 70's (deg F).

Even the programmable thermostats of the most recently installed HVAC units offer less than ideal control. The units inspected were programmed for $6 \mathrm{AM}$ to $6 \mathrm{PM}$ operation. While this schedule covers most occupancy demands, it is generally excessive. Neither teachers nor staff reprogram the thermostats as their occupancy needs differ.

Though the quantity of timeclocks and HVAC units may vary by school, the control methodology described above is typical of all the schools in this report. Controls in each school are addressed individually below. A summary of On/Off times follows (as determined by interviews with custodial staffs), starting on page 80 .

\section{Aloe Elementary}

There are four timeclocks located in a small janitorial room in the main wing. Each is a 7-day timeclock. Clock \#1 controls the library unit; \#2 the kitchen, \#3 the offices and classrooms, and \#4 the cafeteria units. There are override toggle switches in the face of the timeclock cabinet, one for each timeclock. However, as the timeclocks are not used as originally intended, the overrides are useless. On each timeclock, on/off trippers have been removed, and the custodial staff uses the master on/off tripper to control units. All units are turned on manually by custodians at about 6:30 - 7:00 AM. The custodial staff works after school until $9 \mathrm{PM}$, and turns the units off when they leave.

In the 3rd/4th grade wing and the kindergarten wing, programmable thermostats have been installed. On/off times are $6 \mathrm{AM}$ to $6 \mathrm{PM}$, Monday through Friday.

\section{De Leon Elementary}

There are two timeclock stations in the school. The first station, located behind the library, has four 7-day timeclocks. The second station, located in an electrical room in the south classroom wing, has three 7-day timeclocks. There is an override toggle switch for each timeclock. These seven timeclocks control the seven rooftop HVAC units installed with the original school. HVAC units $8-11$ were added with the new classroom addition. They are controlled directly from individual room thermostats, not by timeclock.

All units are controlled manually by the custodial staff using the timeclock master on/off tripper, and room thermostats. Operating hours are from 6 AM until 8 PM. 


\section{Dudley Elementary}

There are three 7-day timeclocks located in the electrical room across the hall from the cafeteria. The first controls classroom and office units, the second the kitchen, and the third the cafeteria. All units are controlled manually by the custodial staff using the timeclock master on/off tripper. On/off hours are typically 7 AM to 7 PM, Monday through Friday.

\section{Hopkins Elementary}

There are four rooms which contain timeclocks at Hopkins. The main mechanical room has four 7-day timeclocks, controlling direct expansion units for 1) the office area, 2) the library, 3 ) the kitchen, and 4) the cafeteria. There is a single 7-day timeclock in the north wing, one in the south wing, and one in the middle wing. Each controls HVAC fan-coil units and chillers/pump for their respective wing. Most or all trippers have been removed from all timeclocks, and all are operated manually.

All units are turned on manually by custodians at about 6:00 AM. The custodial staff works after school until 9 PM, and turns the units off when they leave.

\section{Howell Intermediate}

There is a main control panel at Howell Intermediate located in the main mechanical room. Toggle switches are located in the face of the panel for controlling virtually all HVAC units in the school. When the custodian arrives at 6:30 AM, he turns on all HVAC units via the toggle switches, and the chiller if necessary. He always turns on the boiler, no matter what the weather conditions, since the HVAC system at Howell is reheat. Another custodian turns off HVAC equipment around 7 PM.

In summer, the same procedure is followed for the approximately six weeks cleaning period.

\section{$\underline{\text { Juan Linn Elementary }}$}

All HVAC units installed with the 1986 addition are controlled by programmable thermostats. Programmed on/off times are $6 \mathrm{AM}$ on, and $6 \mathrm{PM}$ off, Monday through Friday. The one exception is the library unit. It has a programmable thermostat, but the unit remains in operation continuously out of concern for mildew on library books. The two rooftop units over the original (east) classroom wing have been replaced recently, and are controlled by programmable thermostats also.

All fan-coil units and the chiller of the stand-alone 1951 addition are controlled by 7-day timeclock located by the east entrance to the building. All trippers to the clock have been removed. The janitor operates the master timeclock tripper to control HVAC.

In the main building, the custodian turns units on manually at the thermostats when she arrives at 6:45 AM, and another custodian turns units off around 8 PM.

Summer school is held in Juan Linn for six weeks. Again, custodians turn equipment on/off manually. However, most units are turned off earlier in the day as compared to the regular school year. 


\section{$\underline{\text { O'Connor Elementary }}$}

Two rooms contain 7-day timeclocks at O'Connor, one in the north wing and one in the south. All units are turned on manually by custodians at about 6:30 AM, and off at around 8:00 PM. The east wing addition units are controlled manually by custodians via their thermostats.

There are two locking thermostats in the north wing, but neither was locked when seen.

\section{Shields Elementary}

The majority of floor area in Shields is served by hydronic fan-coil units. Control is the same as in all other elementaries: 7-day timeclocks exist, but custodial staff uses only the master trippers to turn units on and off when they arrive and depart. Units are turned on around 7 AM, and off about 6:30 PM.

\section{Stanly Elementary}

Control of HVAC units in Stanly is identical to O'Connor. The two schools originally had identical floor and HVAC plans. Timeclocks are located in exactly the same rooms as in O'Connor.

\section{Stroman High School}

Control of HVAC units at Stroman requires very intensive footwork. The custodian makes rounds to every air handling unit, most fan-coil units, many direct expansion units, and the chiller/boiler/auxiliary equipment each morning around 6:45 $\mathrm{AM}$, where he turns equipment on. Another custodian makes a similar round at about 8:30 PM to turn equipment off.

The kitchen staff turns kitchen HVAC on and off. The coaching staff turns athletic building HVAC off, and the custodial staff turns it back on in the moming, though often the coaching staff forgets to turn units off.

A small (46 ton) reciprocating chiller is located adjacent to the four story Unit A. This chiller is piped to serve only Unit A. During summer and after school hours, parts of Unit A (which contains administrative offices) are the only occupied portions of the school. At 4:30 PM during the school year, the absorption chiller is shut down and the reciprocating chiller is turned on, and continues to operate until $9 \mathrm{PM}$. In summer, the reciprocating chiller is turned on 7:00 AM, and off at 6:00 PM, unless the main chiller is operating.

Direct expansion split systems serving the Band hall are thermostatically controlled, but are left in operation continuously, summer and winter. If the main air handler serving Band has been shut off and indoor temperature starts to rise, the DX units will maintain humidity and temperature conditions. These backup DX units were installed out of concern for humidityrelated problems with Band instruments.

Summer cleaning of the high school takes about 5 to 6 weeks. During this time, the main absorption chiller operates every day, and virtually the entire school is cooled. Cleaning is finished by mid- to late-July, and only the reciprocating chiller operates after that. 


\section{TECHNICAL ANALYSIS OF FACILITY}

\section{Victoria High School}

Victoria High is another school requiring intensive footwork in turning HVAC systems on and off. The VHS campus contains numerous buildings spread out over a wide geographical area. The maintenance man starts his round at 7 AM to all mechanical rooms and thermostats/wall switches, turning on equipment. As at Stroman, the coaching staff is responsible for turning off some athletic building HVAC equipment (though they often forget) and the maintenance man turns it back on in the morning.

There are two rooftop units over the Learning Resource Center. During the regular school year, these operate from 7:15 AM until 4 PM. During summer, one of the units is shut down, but the other remains in operation 24 hours per day to prevent problems with mildew. Starting in September, HVAC for the boys dressing room is left on continuously until cold weather hits, so as to reduce odor problems which are worsened by heat and humidity.

Summer school is held in the Academic Wing of VHS, and occasionally in the main wing. The Academic Wing is served by the absorption chiller. The chiller is turned on at 6:30 AM, and off at 1:30 PM. The fan-coil units served by the chiller remain in operation continuously, both summer and winter. The on/off switches for them are located inside the units.

Fan-coil units for the main building are controlled by toggle switches mounted on the wall of each classroom. Teachers are supposed to turn these units off as they leave each day, and the maintenance staff turns them back on in the morning. However, as often as not, the fan-coil units are left on at night.

This ECRM calls for the installation of a direct digital control-based energy management system (EMS) for each school addressed in this report. The EMS will control all HVAC equipment, measure exterior and interior space temperatures, and measure humidity in one or two critical locations within each school. The EMS will have no override timers that custodial staffs can activate. Operating hours of all HVAC units will be determined by the maintenance staff, and controlled by that staff from its central headquarters via modem. (Floor plans on pages 27 through 37 show locations of the units to be controlled, and the proposed locations of new DDC controllers). 
Tab A-3

Monitoring Diagrams 


\section{THERMAL MONITORING DIAGRAM}

ЕISD - STROMAN HS

$K=K W H$ CHANNE

$A=A N A L O G$ CHANNE

$D=D I G T A L$ CHANNEL
$P C=P U M P E D$ CONDENSATE

STROMAN HIGH SCHOOL

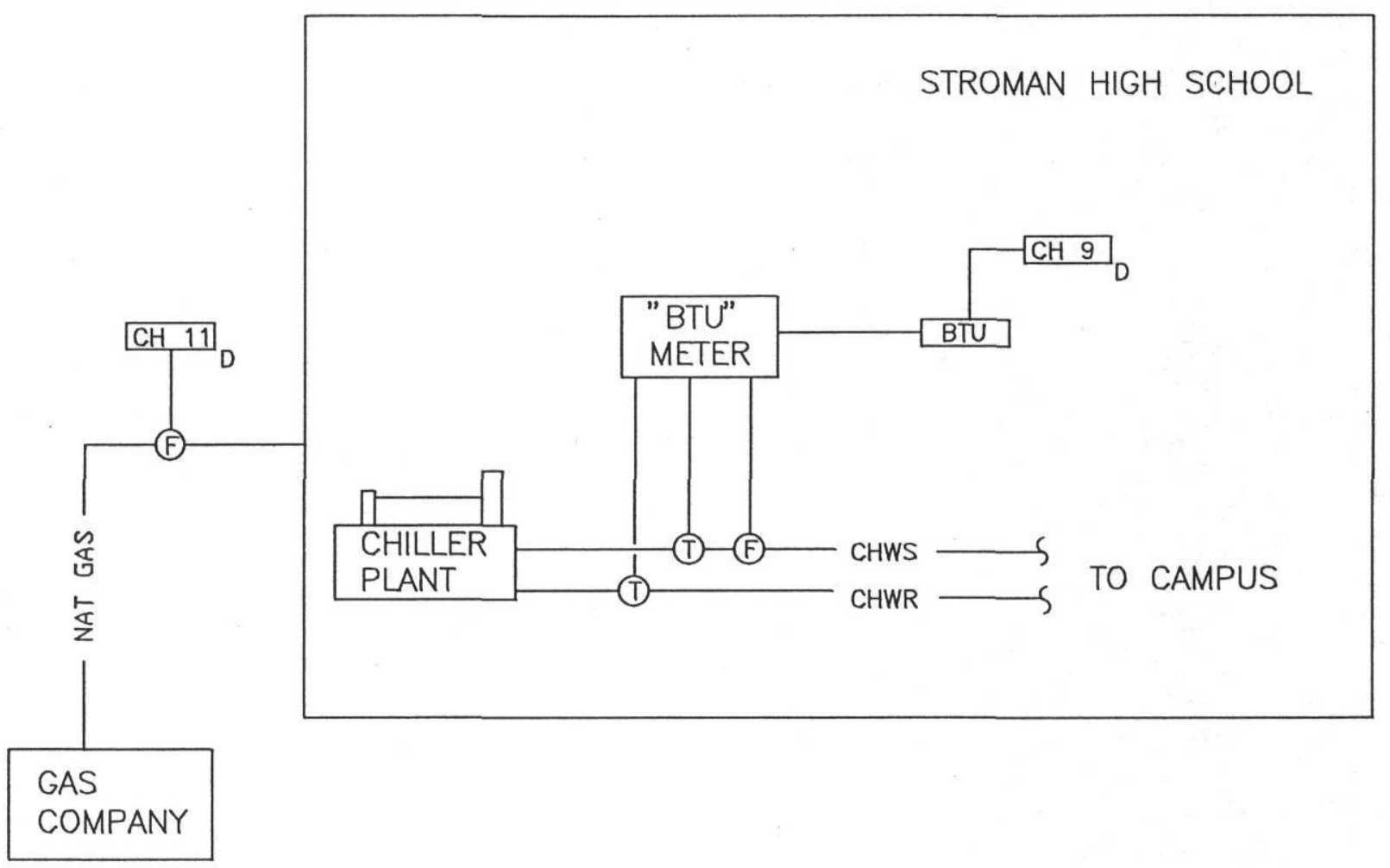

VISD/STROMAN HS - SITE 126 
Tab A-4

Average Hourly Data \& Related Statistics 

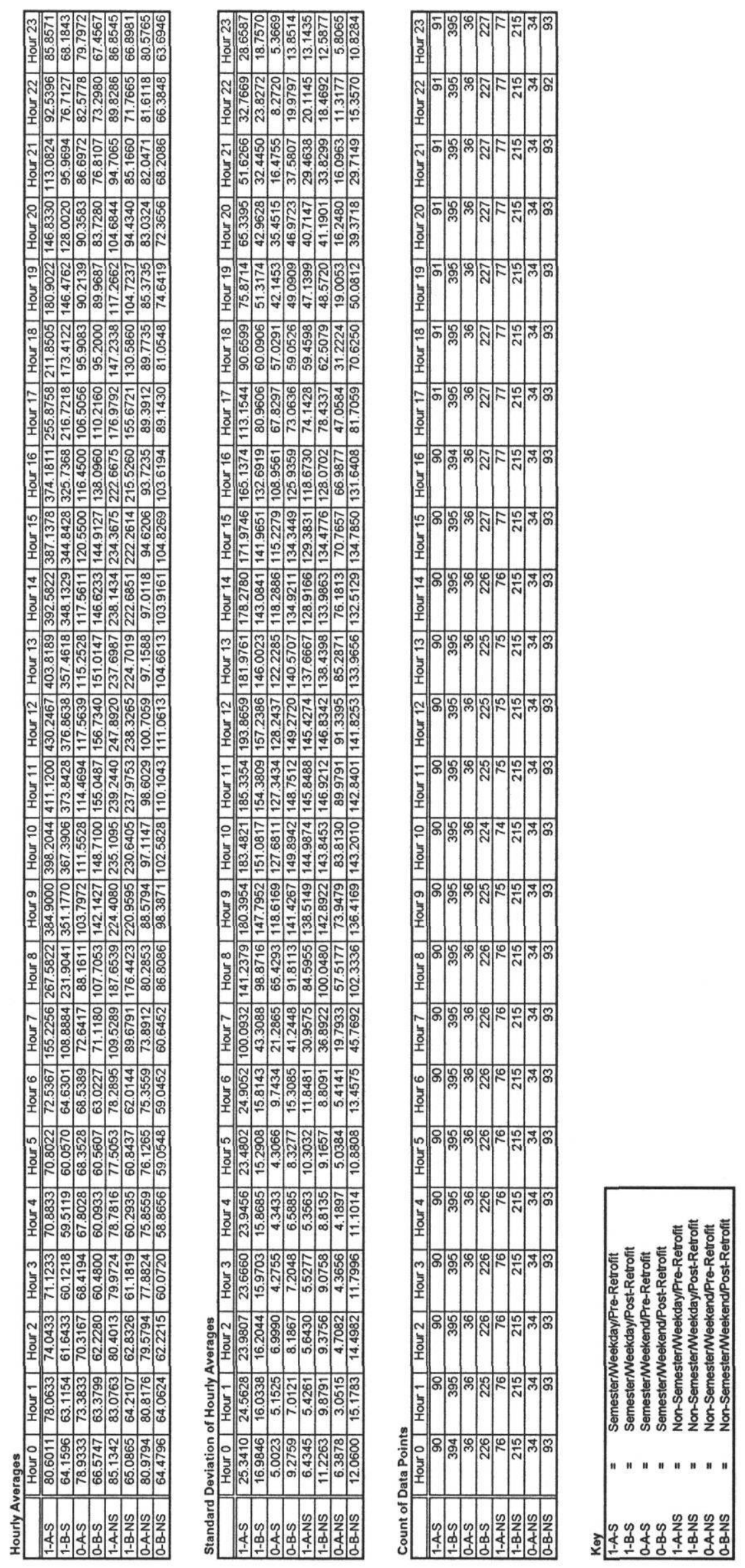
Tab A-5

MECR Plots

A-29 
Whole-Building Electric

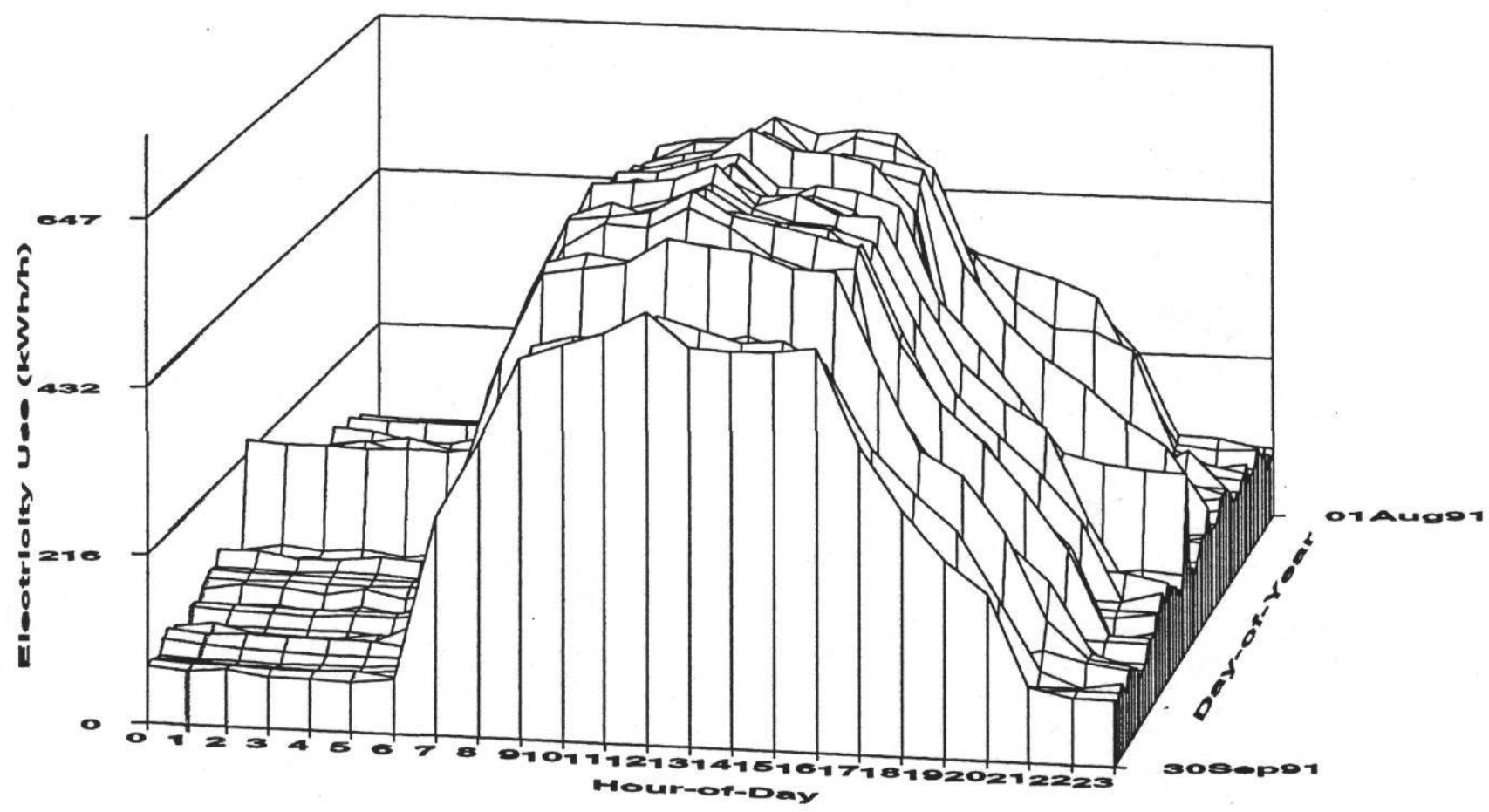

Whole-Building Electric

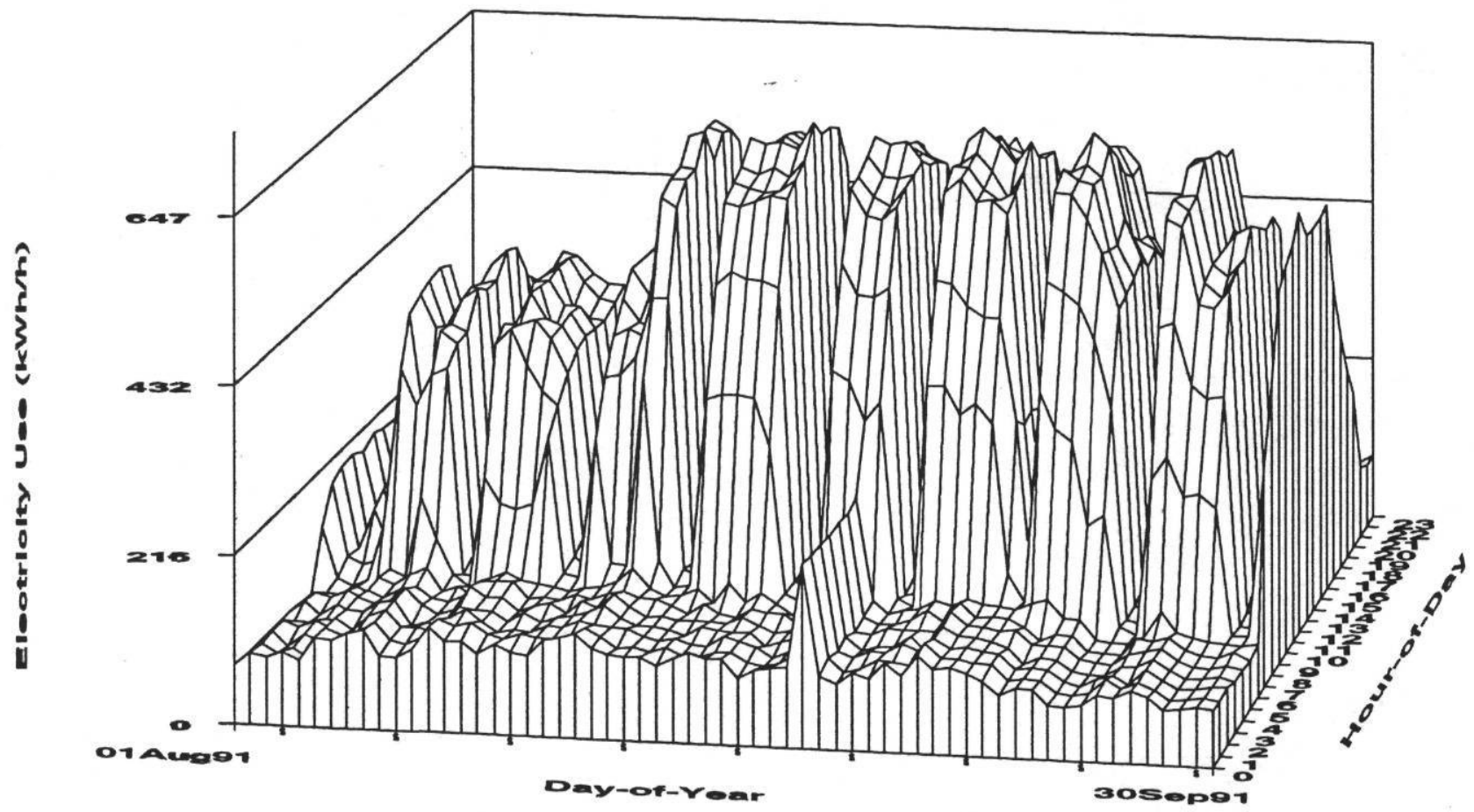

Sundays are marked with an "S"

Stroman High School - Victoria ISD - September 1991

Texas Governor's Enemsy Office LoenSTAR Monitouing \& Analysis Program
Monthly Energy Consumption Report (C)

Version 1.3
Energy Systems Lab Texas A\&M University 
Whole-Building Electric

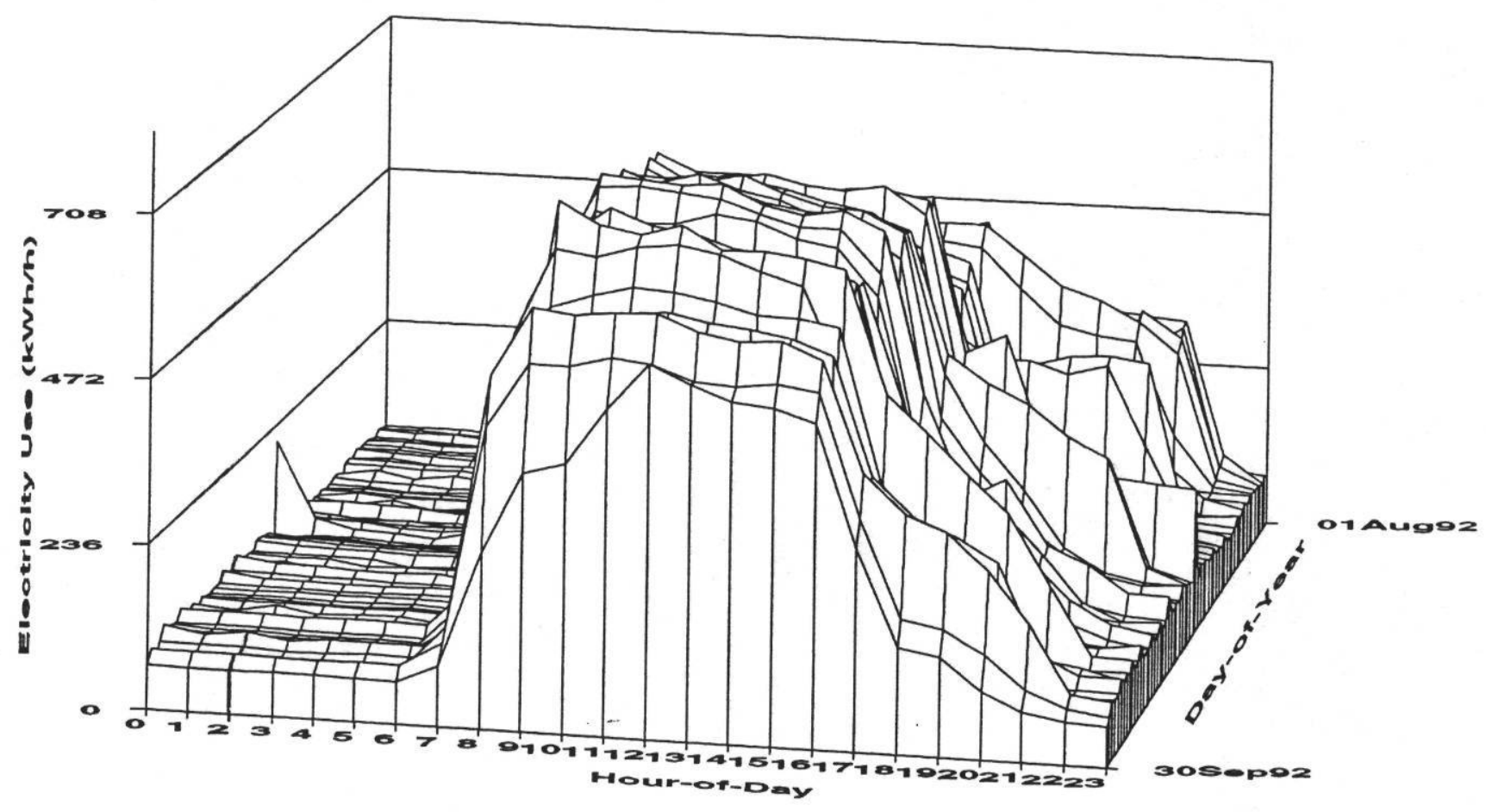

Whole-Building Electric

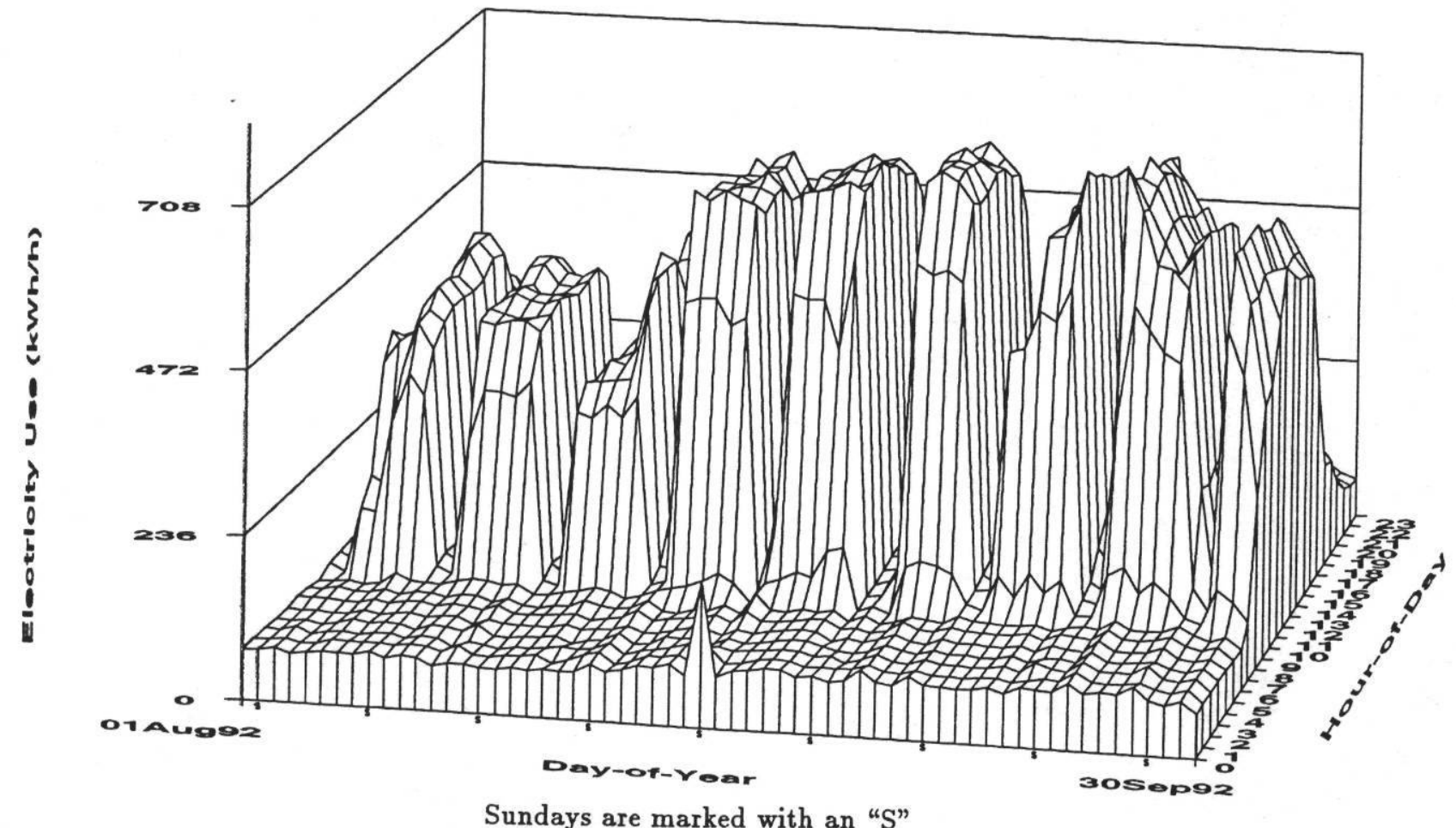

Stroman High School - Victoria ISD - September 1992

$\begin{array}{lcr}\text { Texas Governor's Energy Office } & \text { Monthly Energy Consumption Report C) } \\ \text { LosnSTAR Monitoring \& Analysis Program } & \text { Version 1.4 } & \begin{array}{r}\text { Energy Systems Lab } \\ \text { Texas A\&M University }\end{array}\end{array}$


Whole-Building Electric

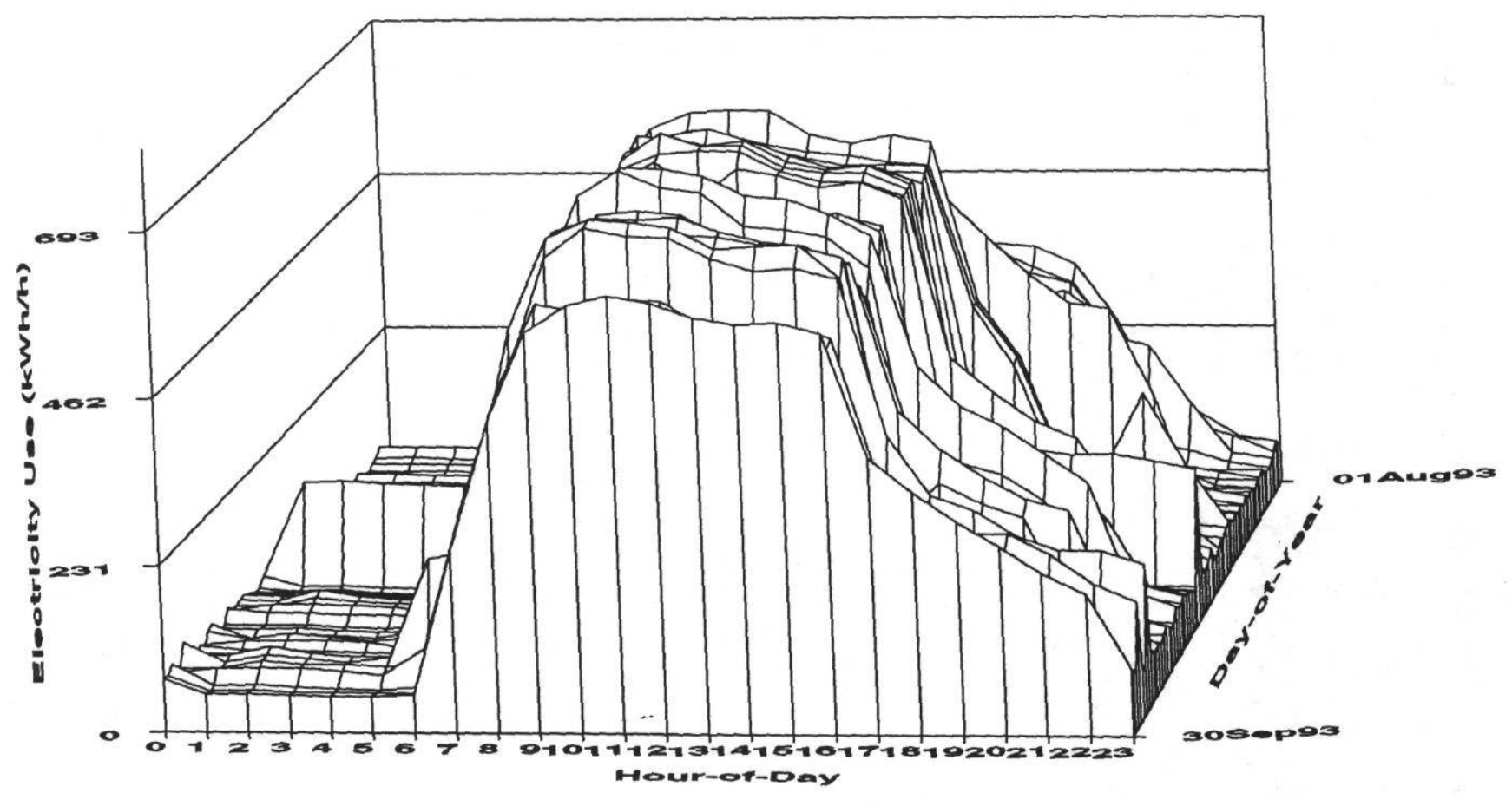

Whole-Building Electric

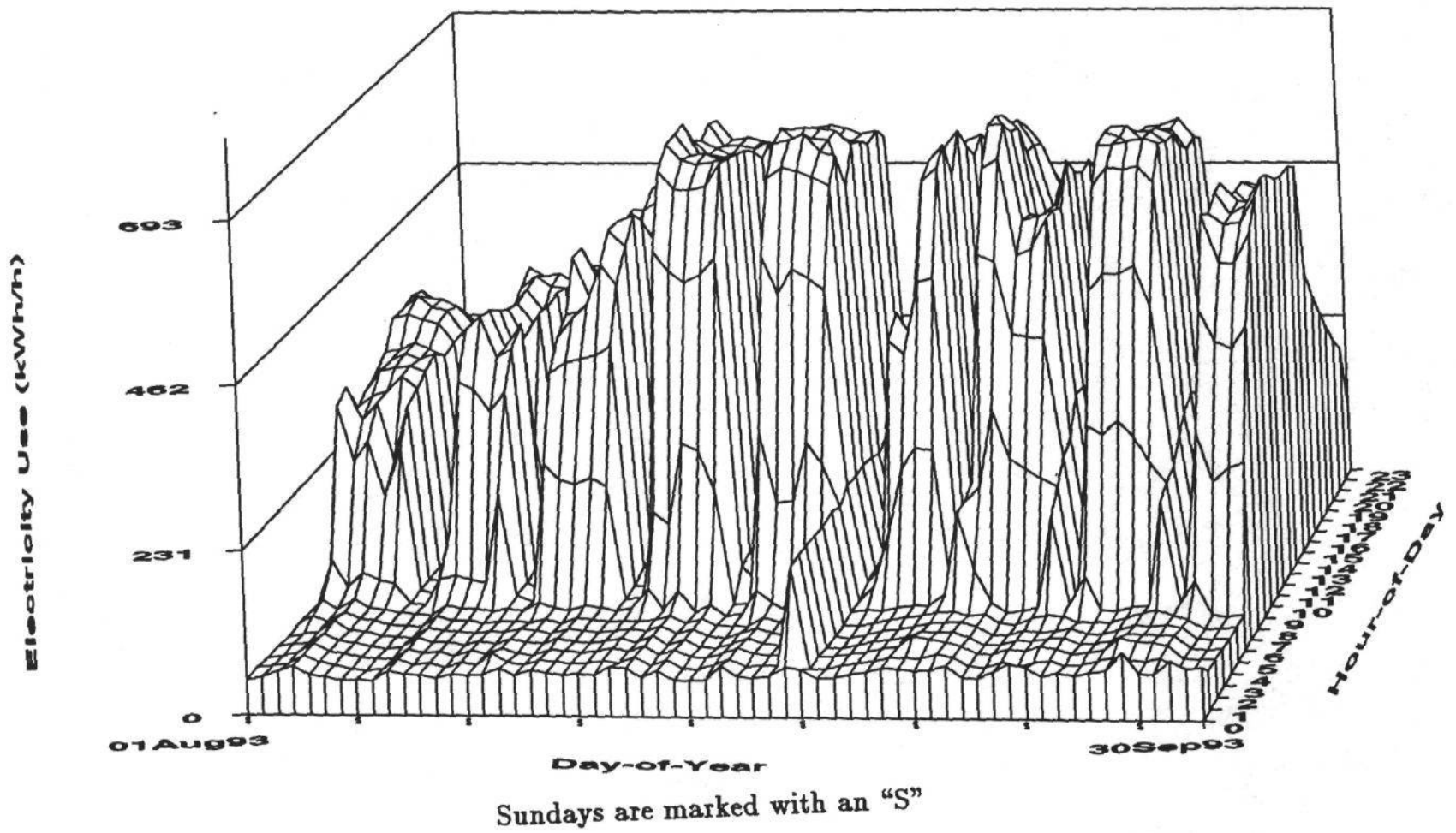

Stroman High School - Victoria ISD - September 1993
$\begin{aligned} & \text { Texas Energy Office } \\ & \text { LoanSTAR Monitoring \& Analysis Program }\end{aligned}$
Monthly Energy Consumption Report
$\begin{gathered}\text { Version } 2.2 \\ \text { A-32 }\end{gathered}$




\section{Whole-Building Electric}

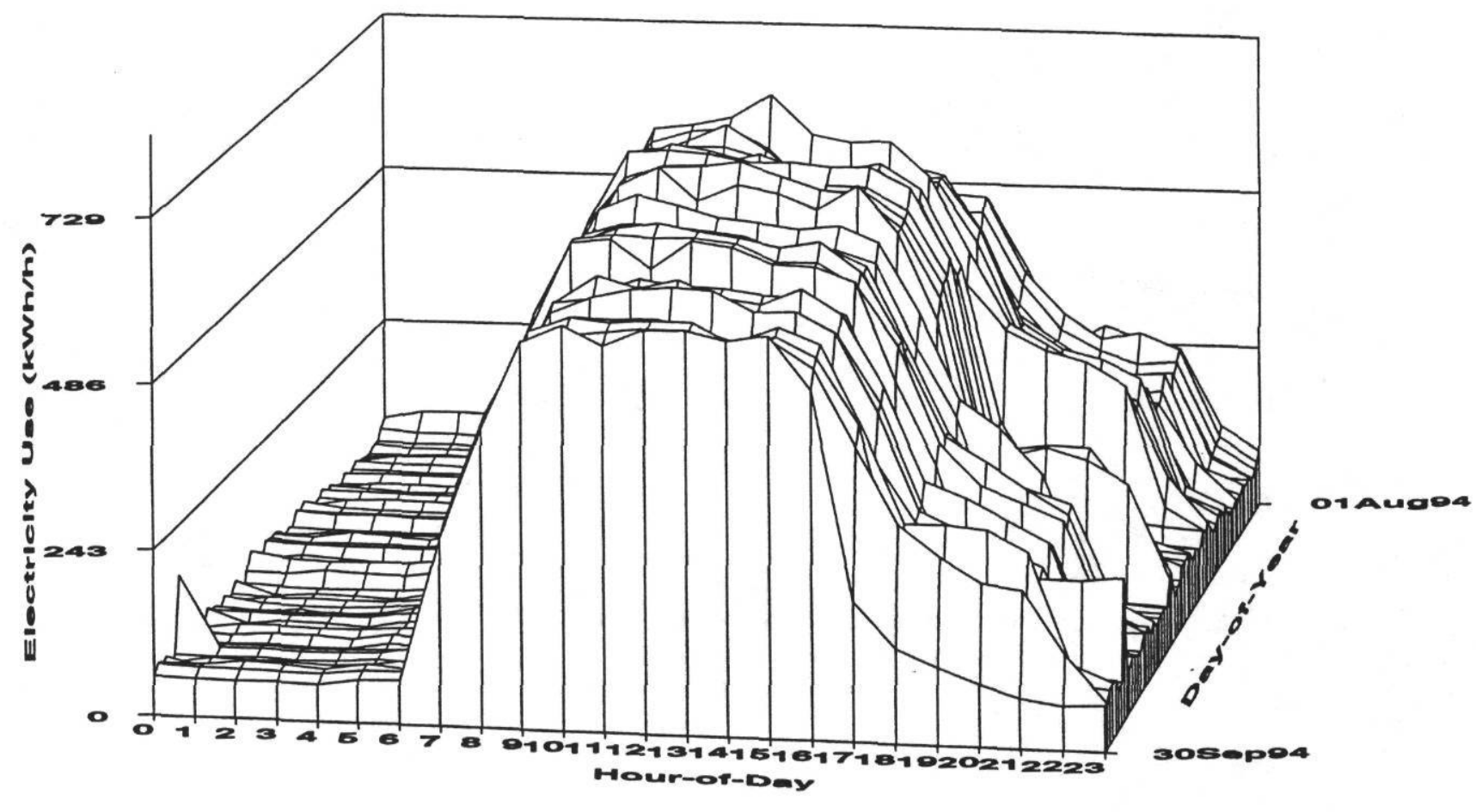

Whole-Building Electric

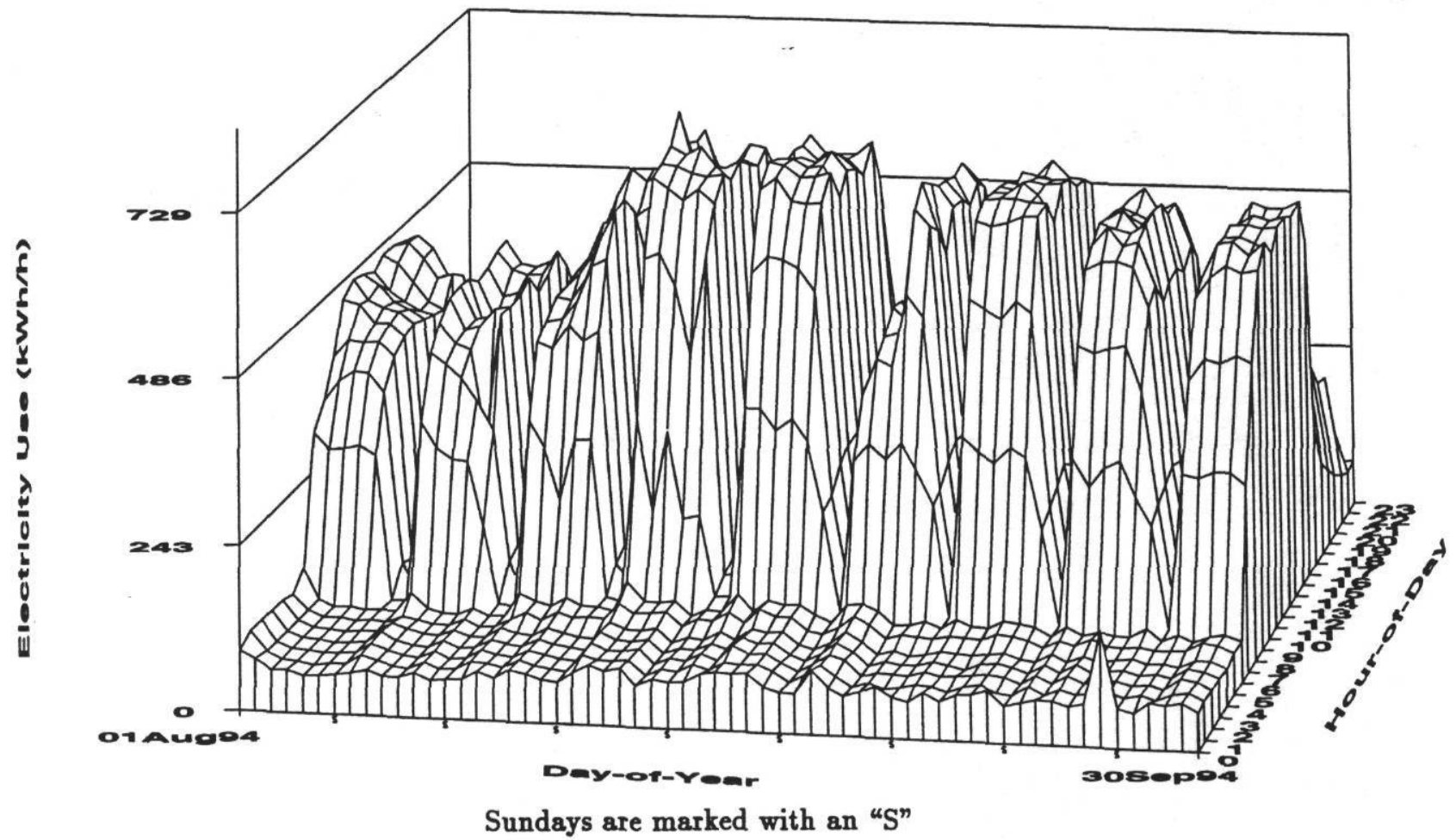

Stroman High School - Victoria ISD - September 1994

Texas State Energy Conservation Office LoanSTAR Monitoring \& Analysis Program
Monthly Energy Consumption Report (C) A-33 Version 2.2
Energy Systems Lab Texes A\&M University 
TAB A-6

Data Summary Notebook Information 


\section{Stroman High School}

Building Envelope:

- 210,500 sq.ft.

- Unit A: four storied, administrative offices on ground floor, classrooms on 2nd and 4th floors, 17,500 sq $\mathrm{ft} /$ floor.

- Unit B: 2 storied, auditorium, choir room, band room, and drafting classroom, 12,000 sq ft/floor.

- Unit C: single story, cafeteria and kitchen, 9,000 sq ft.

- Unit D and E: One contiguous building, 2 storied, library, gymnasium, locker rooms, and main mechanical room, 25,000 sq ft (Unit D), 27,000 sq ft (Unit E).

- Unit F: 2 storied, science classrooms, 23,000 sq ft.

- Unit G: single storied, shops, 7,000 sq ft.

- 3 Athletic Buildings: girls' gym, field house, and athletic dome, 25,000 sq ft.

Building Schedule:

- 7 am to $4 \mathrm{pm}(\mathrm{M}-\mathrm{F})$

Building HVAC and Other Equipment:

- Unit A: 4 AHUs (1 mutizone of $7.5 \mathrm{hp}$ and 3 single zone of $3 \mathrm{hp}$ each), 50 fan-coil units and 1-45.8 ton chiller

- Unit B: 1 single zone AHU of $3 \mathrm{hp}$ and a rooftop DX unit

- Unit C: 2 single zone units of $0.75 \mathrm{hp}$ each and 6 fan-coil units

- Unit D and E: 3 AHUs, 1 single zone of $5 \mathrm{hp} 2 \mathrm{H} \& \mathrm{~V}$ units of $2 \mathrm{hp}$ each. and 2 fan-coil units

- Unit F: 18 fan-coil units

- Unit G: 2 rooftop DX units

- 18 exhaust fans $(1 / 4 \mathrm{hp}$ each)

HVAC Schedule:

- HVAC equipment is turned on manually at 7:00a.m. and turned off at 8:00p.m., on weekdays.

Auxillary Equipment:

- 3 single zone AHUs. @ of $3 \mathrm{hp}$ each, 1 of $0.75 \mathrm{hp}$.

- 1 - $50 \mathrm{hp}$ chilled water pump.

- 1 - $20 \mathrm{hp}$ hot water pump.

- 1 - 30 hp cooling tower.

- 1 - 2 hp boiler motor.

- 1 brine pump.

- 1 refrigerant pump.

- 1 reciprocating chiller of $3 \mathrm{hp}$.

- 2 gas fired boilers.

- 1 rooftop unit serving the First Aid room.

- 1 centrifugal chiller @ 460 tons (replaced 414 ton absorption chiller in Aug 91).

- 3 hot water heaters $(500,000 \mathrm{Btu} / \mathrm{hr}$ each).

Lighting:

- mostly fluorescent (40 W). Total lighting load $260 \mathrm{~kW}$.

\section{Proposed Retrofits:}

- Energy Management System.

- replace absorption chiller.

- rewire wiring in hallways.

Stroman High School - Victoria ISD - April 1994

\begin{tabular}{lcc}
\hline Texas State Energy Conservation Office & Monthly Energy Consumption Report C) & $\begin{array}{c}\text { Energy Systems Lab } \\
\text { Texas A\&M University }\end{array}$ \\
LoanSTAR Monitoring \& Analysis Program & Version 2.2 & Texas
\end{tabular}


Date of Retrofits:

- replacement of absorption chiller was completed in August 1991. Work on the other two retrofits was completed in January 1992. 


\section{Stroman High School (SHS) W.B. Electric as W/sq.ft.}
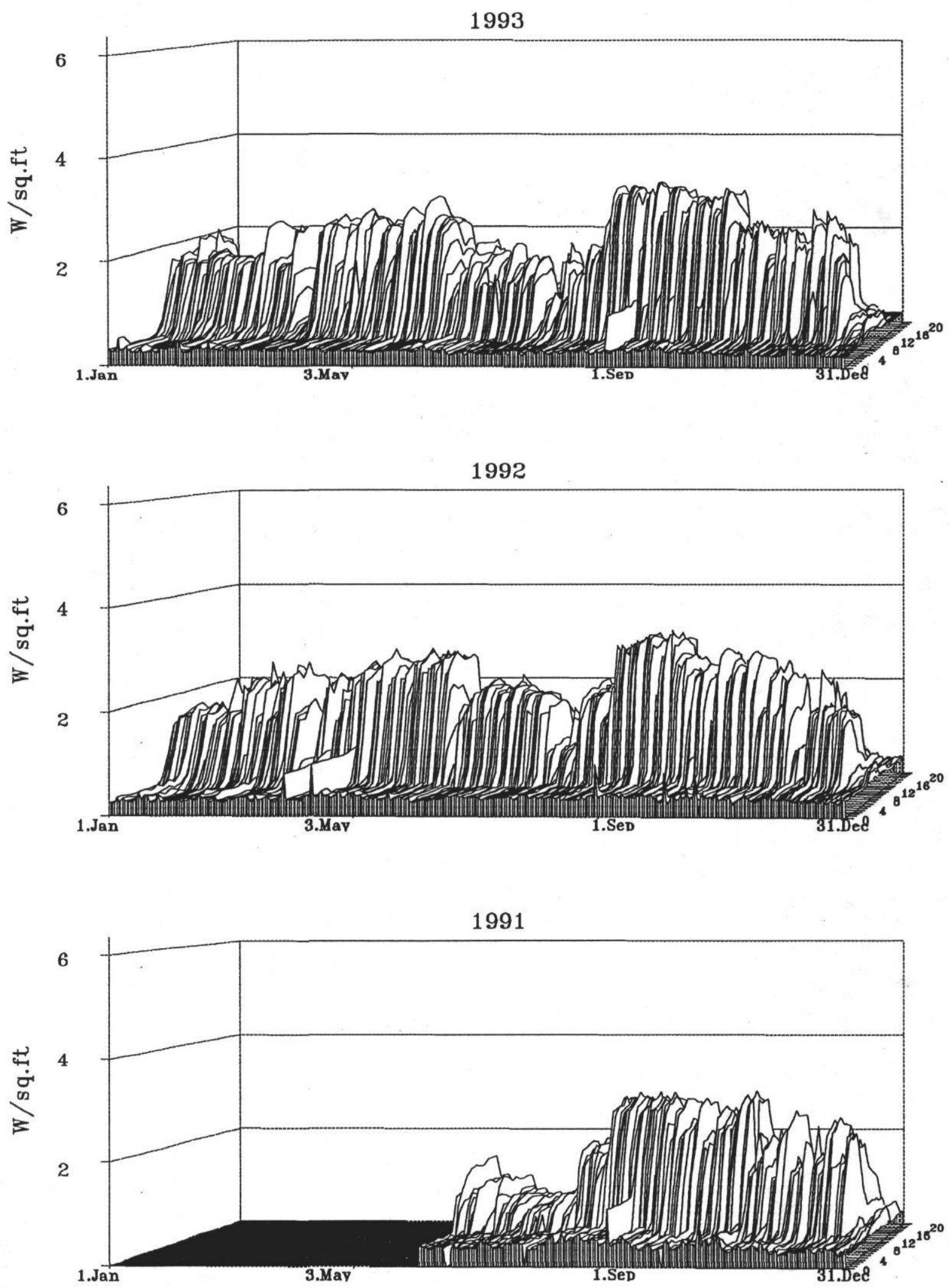


\section{Stroman High School (SHS) W.B. CHW as Btu/sq.ft.-h}
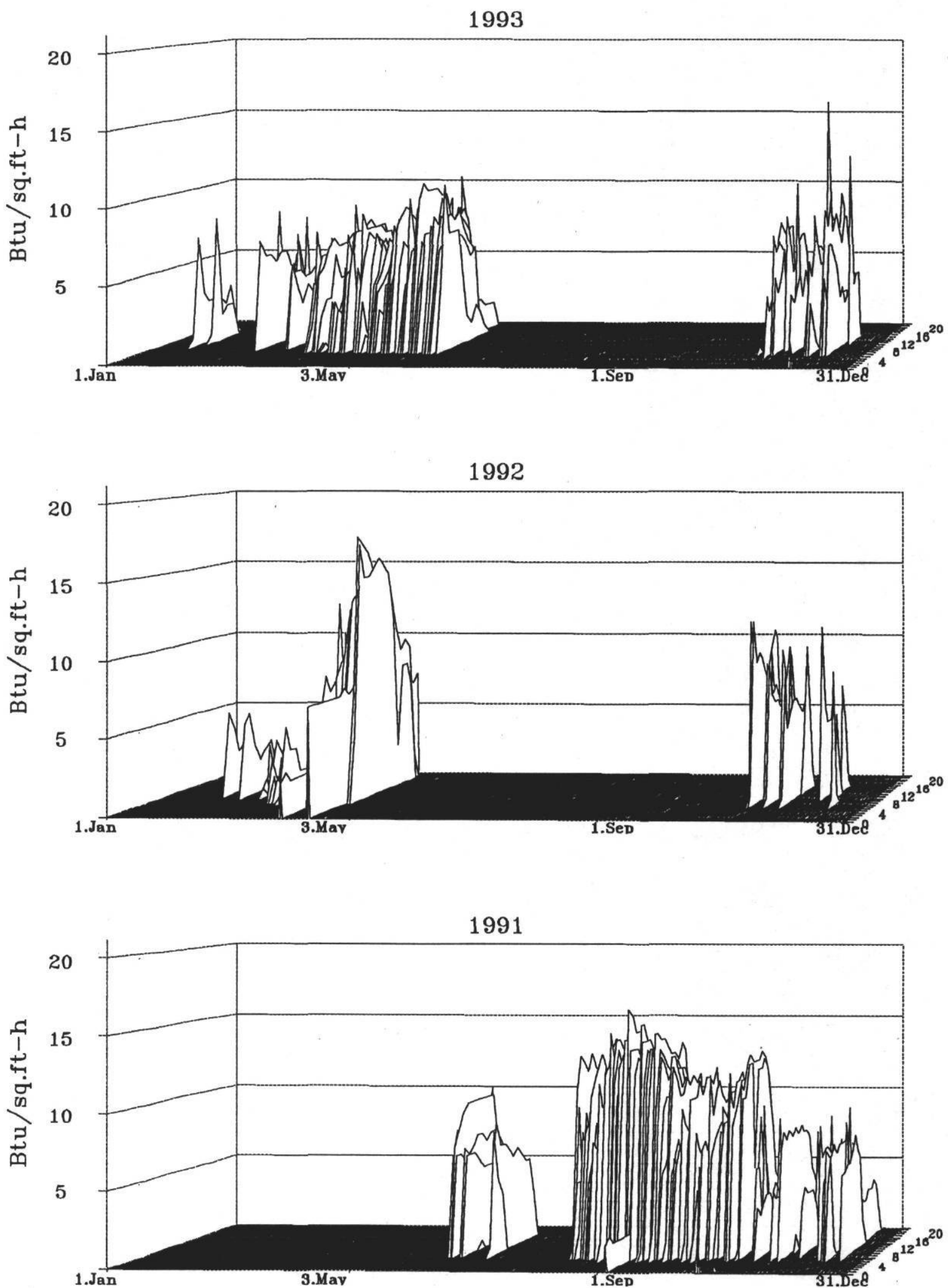


\section{Stroman High School (SHS) W.B. HW as Btu/sq.ft.-h}
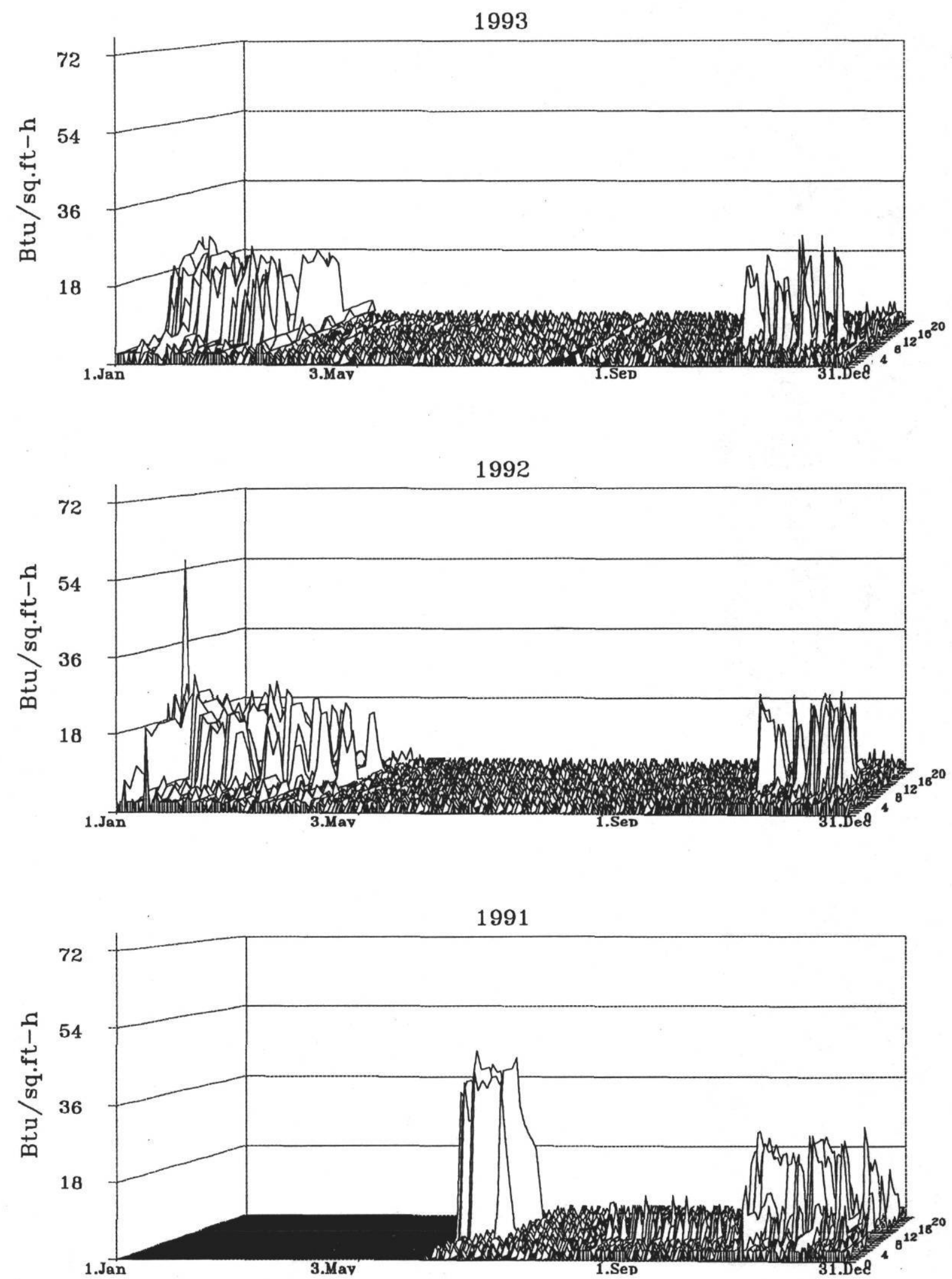


\section{Stroman High School (SHS) \\ W.B. Electric as W/sq.ft.}
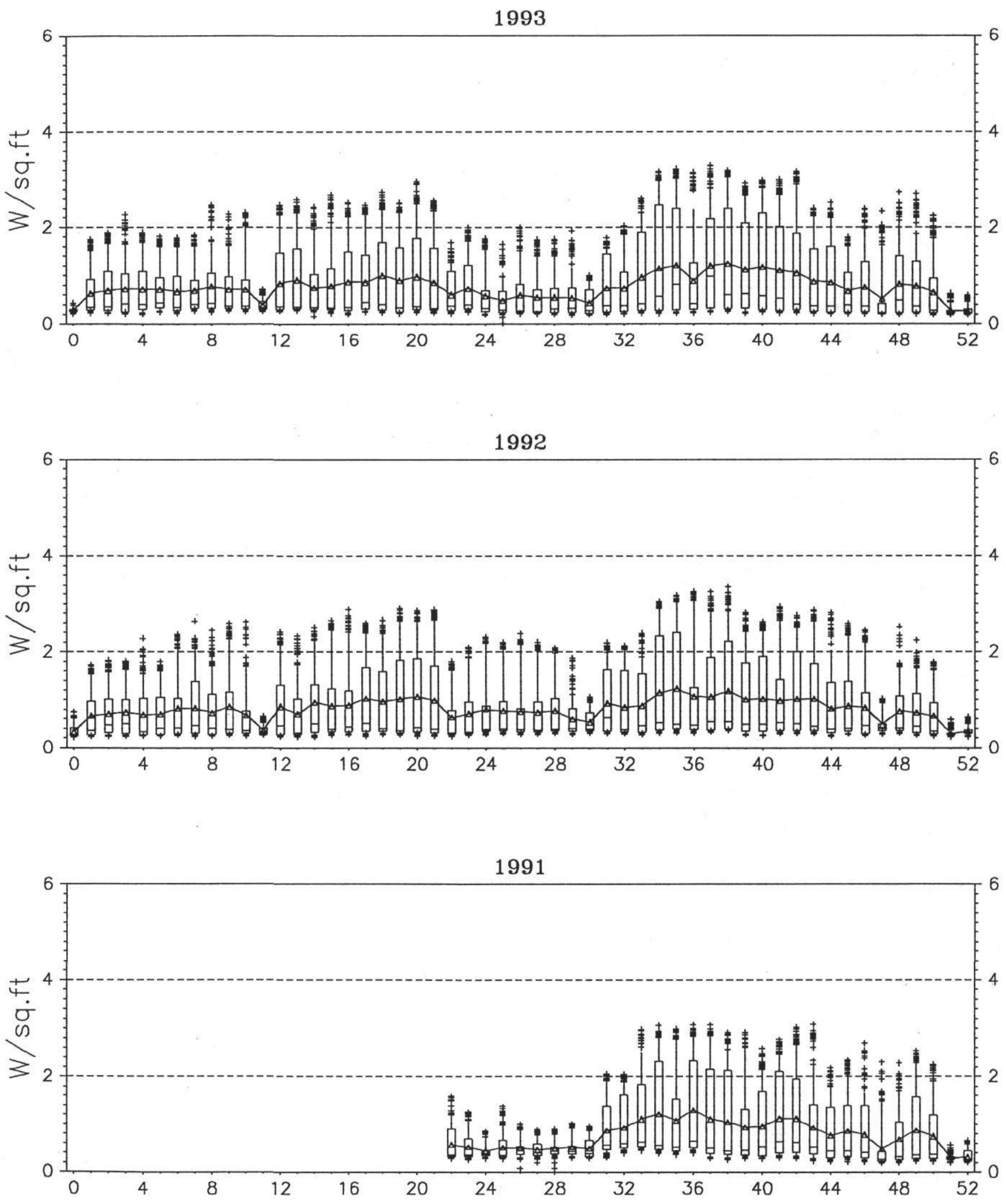


\section{Stroman High School (SHS) \\ W.B. CHW as Btu/sq.ft.-h}
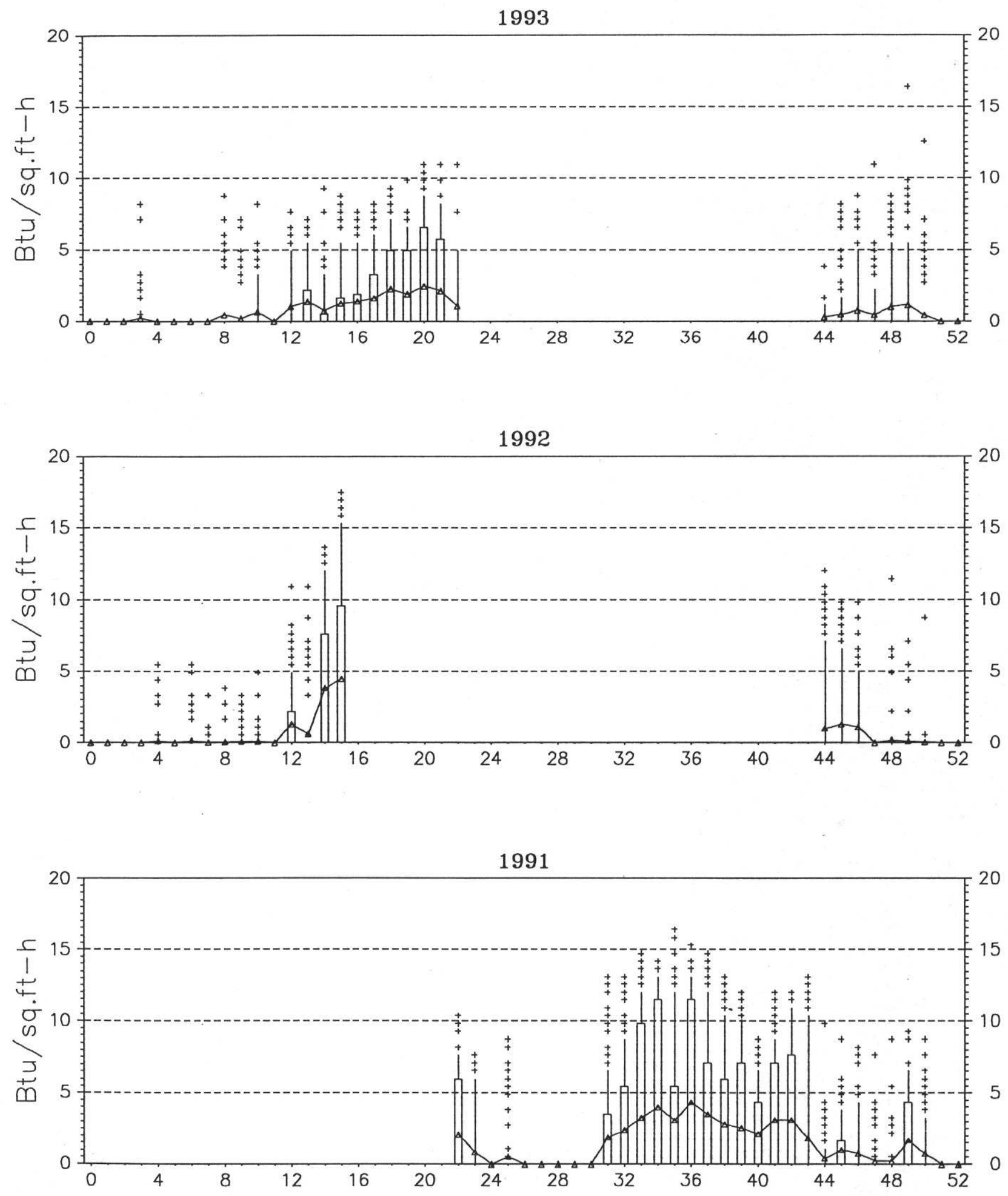


\section{Stroman High School (SHS) \\ W.B. HW as Btu/sq.ft.-h}
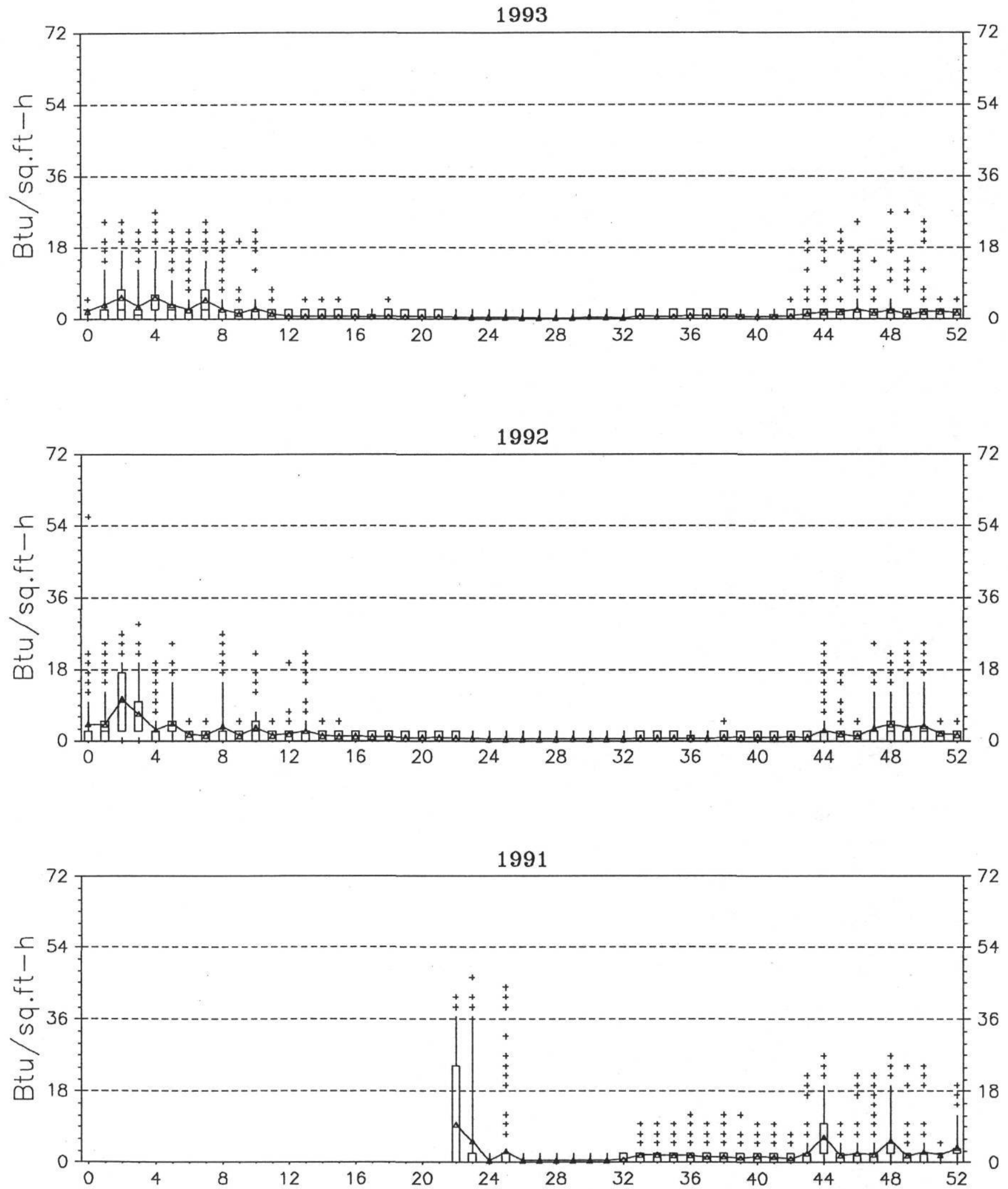


\section{Stroman High School (SHS) \\ W.B. Electric as W/sq.ft.}

Pre-Retrofit (06/04/1991 - 08/01/1991)

Everyday

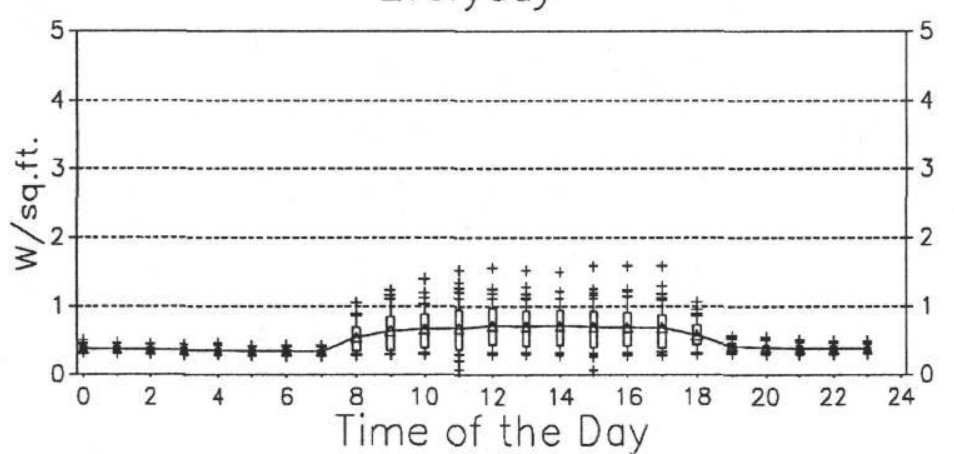

Weekdays

$\frac{p}{\omega}$

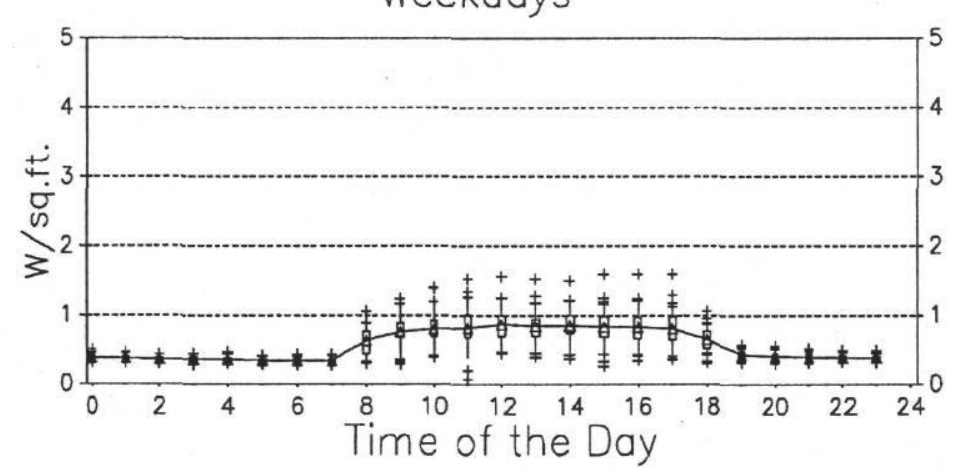

Weekends

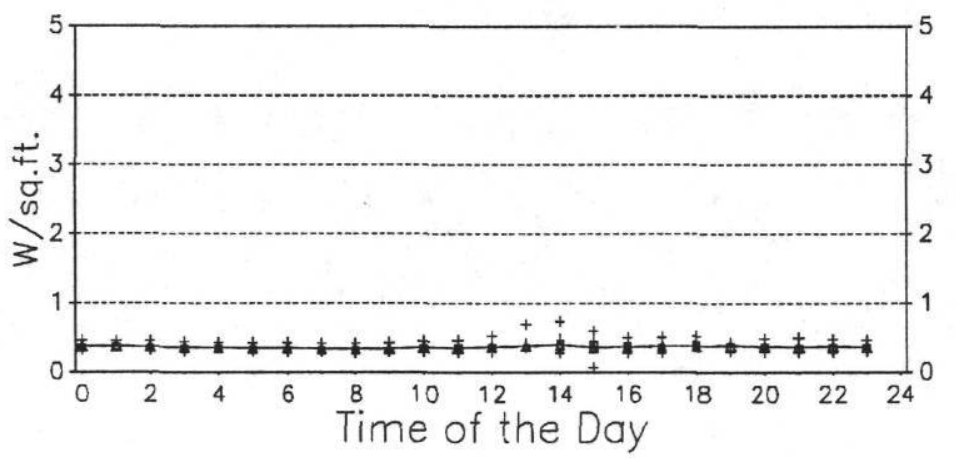

Post-Retrofit (08/15/1991 - 12/31/1993) Everyday

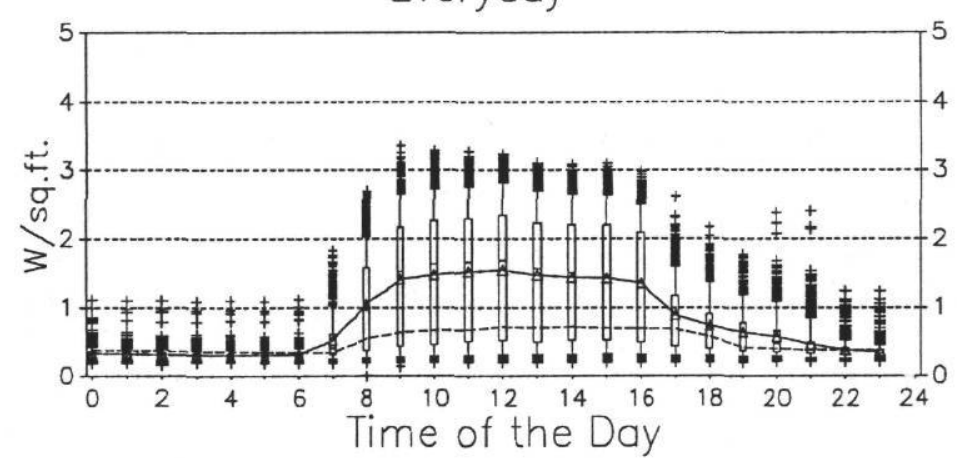

Weekdays

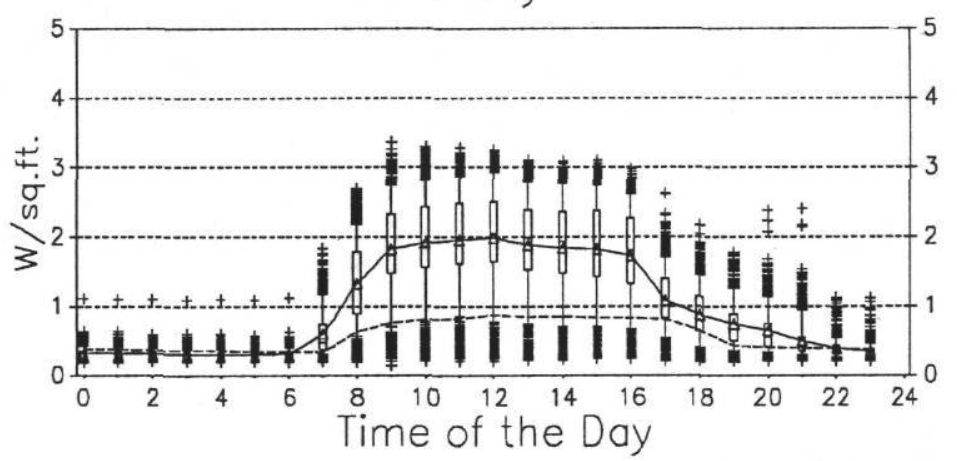

Weekends

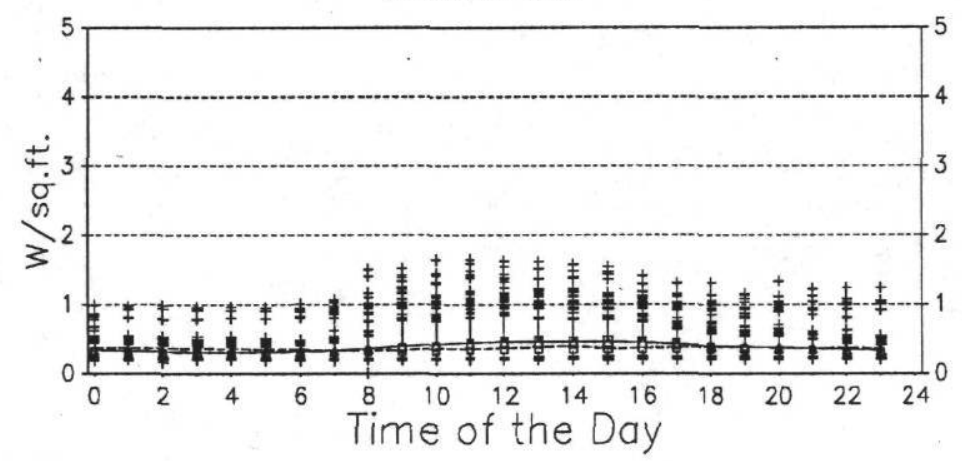




\section{Stroman High School (SHS) \\ W.B. CHW as Btu/sq.ft.-h}

Pre-Retrofit (06/04/1991 - 08/01/1991)

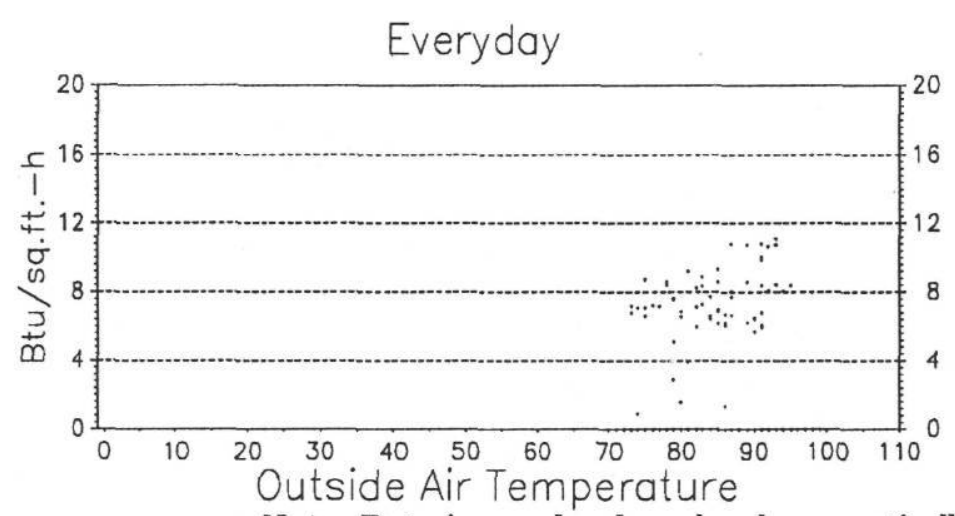

Weekdays

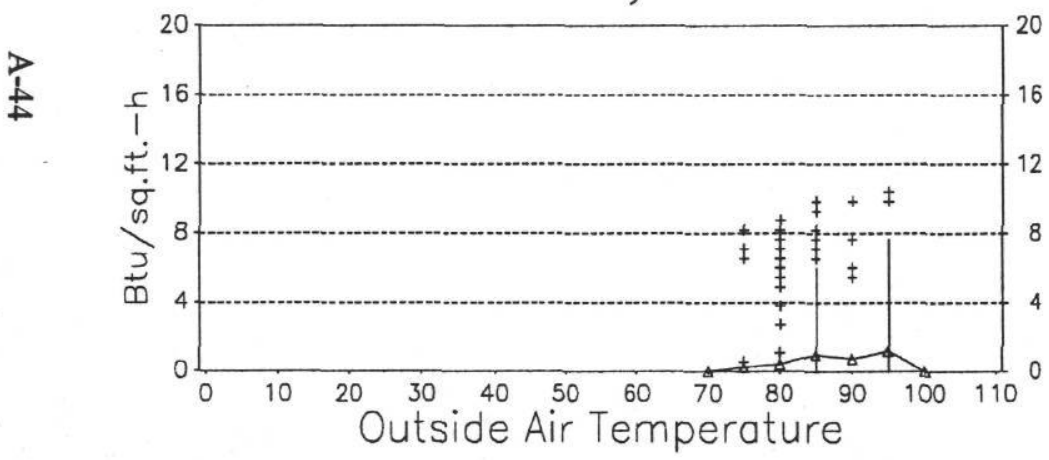

Weekends

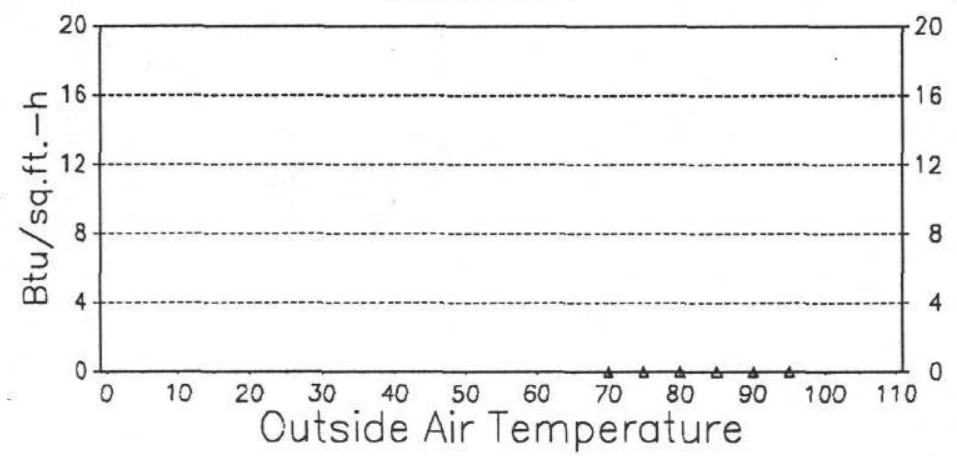

Post-Retrofit (08/15/1991 - 12/31/1993)

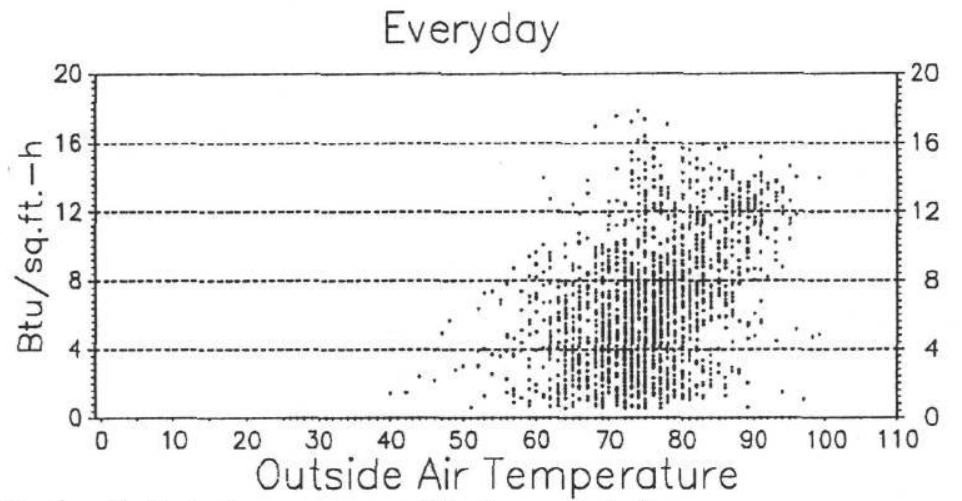

Outside Air Temperature

Weekdays

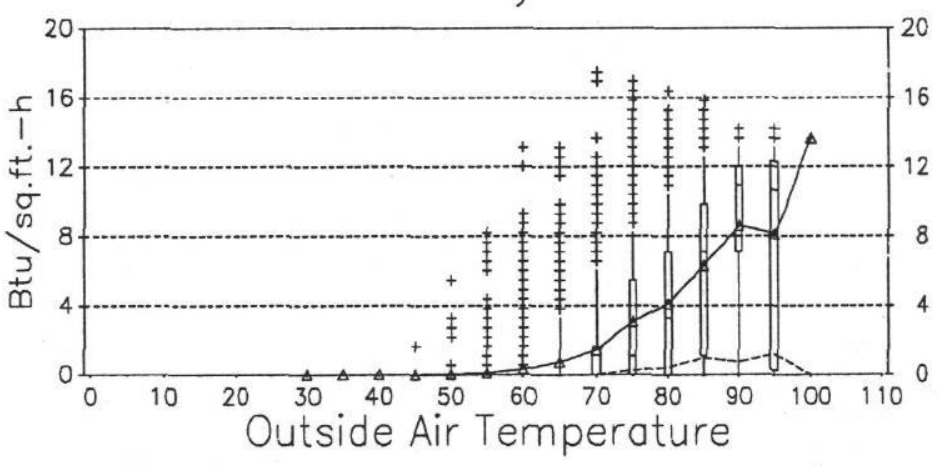

Weekends

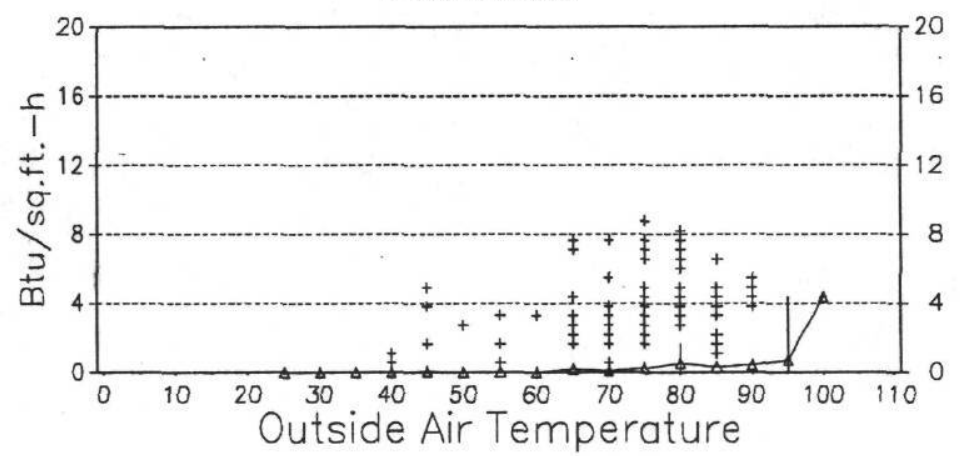




\section{Stroman High School (SHS) W.B. HW as Btu/sq.ft.-h}

Pre-Retrofit (06/04/1991 - 08/01/1991)

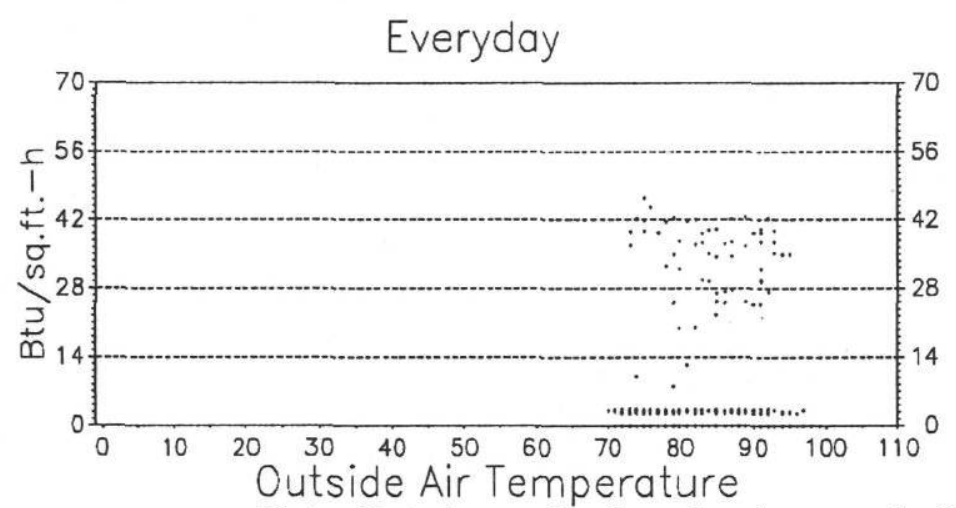

Weekdays

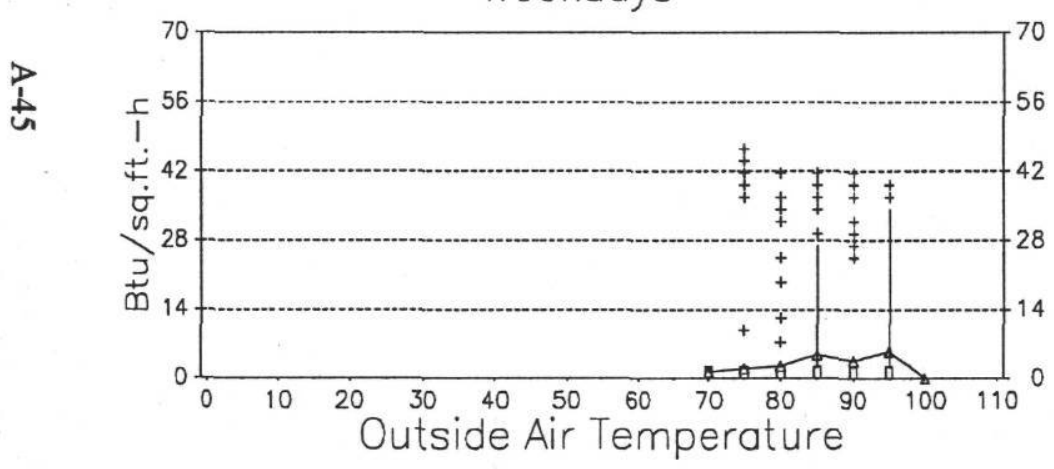

Weekends

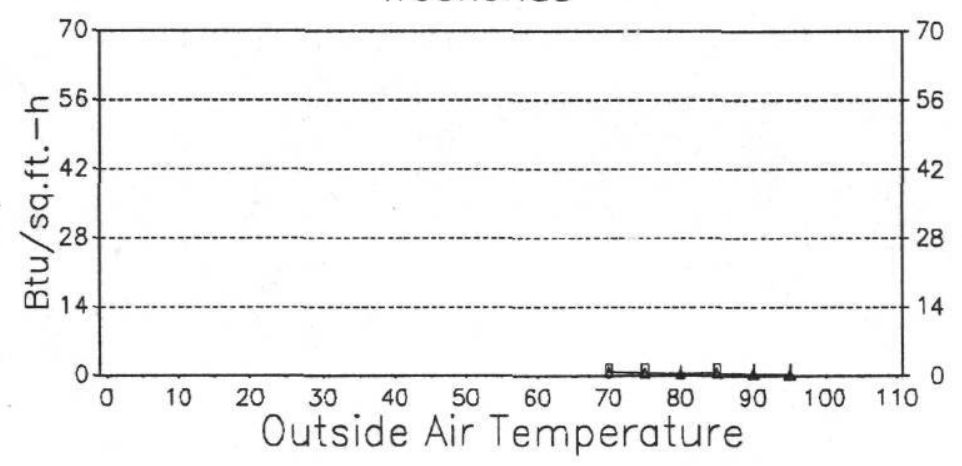

Post-Retrofit (08/15/1991 - 12/31/1993)
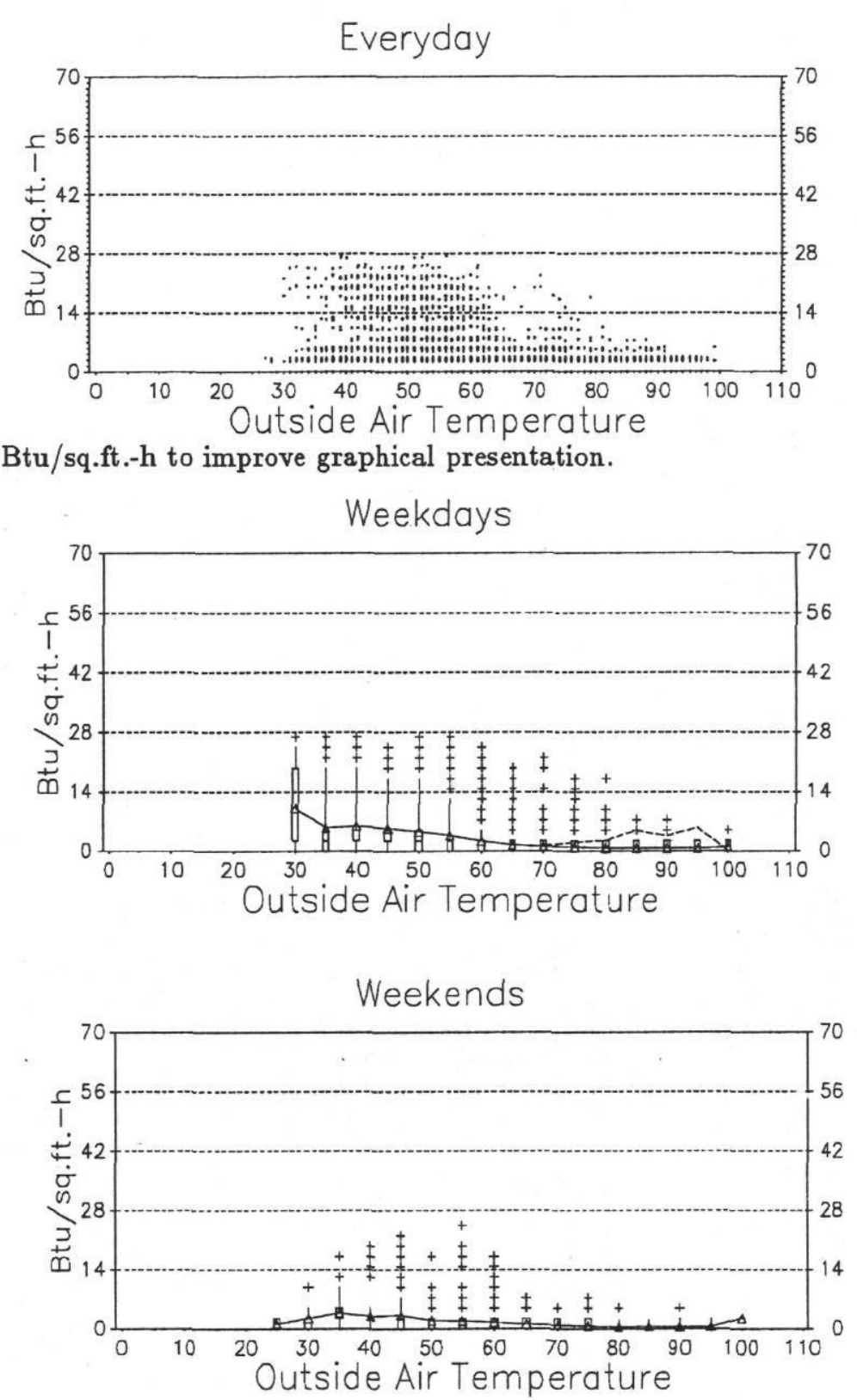


\section{Stroman High School (SHS)}

Daily Average Values

Pre-Retrofit $(\triangle)$ 06/04/1991 - 08/01/1991

Electric

$(\mathrm{kWh} / \mathrm{h})$
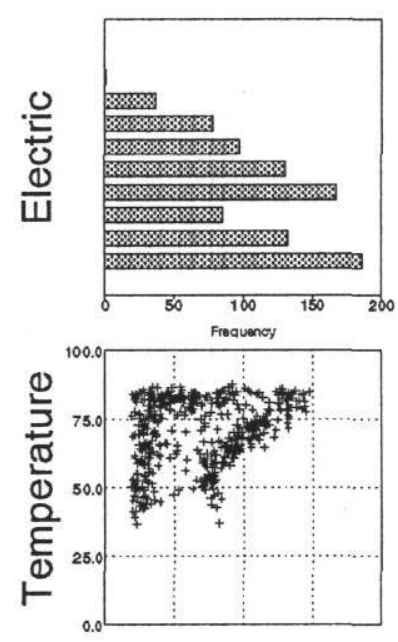

t

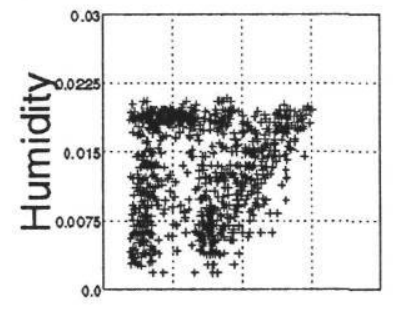

Temperature

(degrees F)
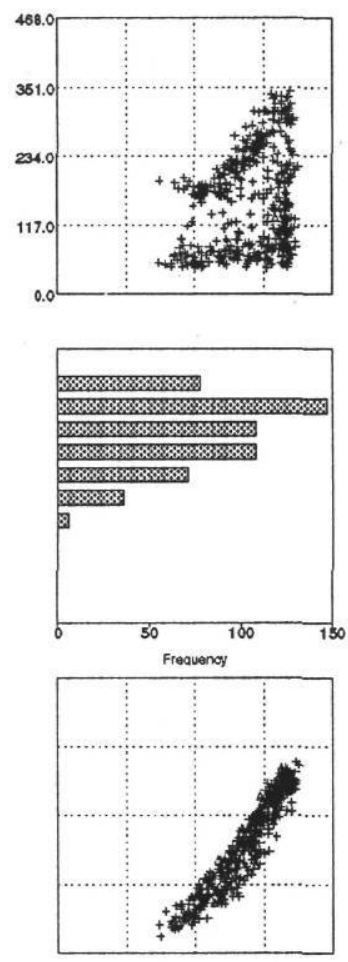

Post-Retrofit (+) 08/15/1991 - 12/31/1993

Humidity

(lbw/lba)
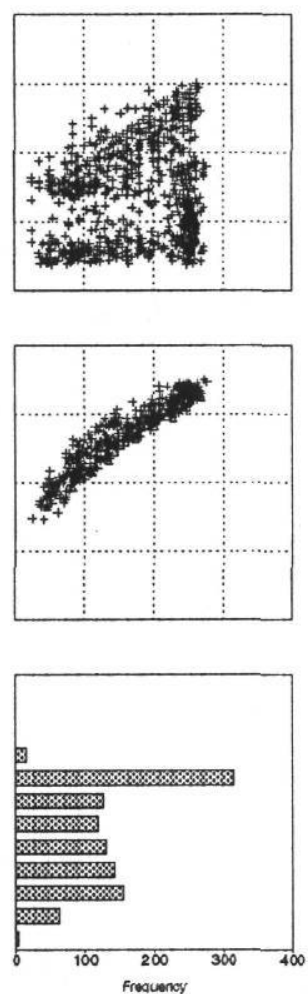

Solar Rad

(W/sq.m)

Wind Speed

$(\mathrm{mph})$
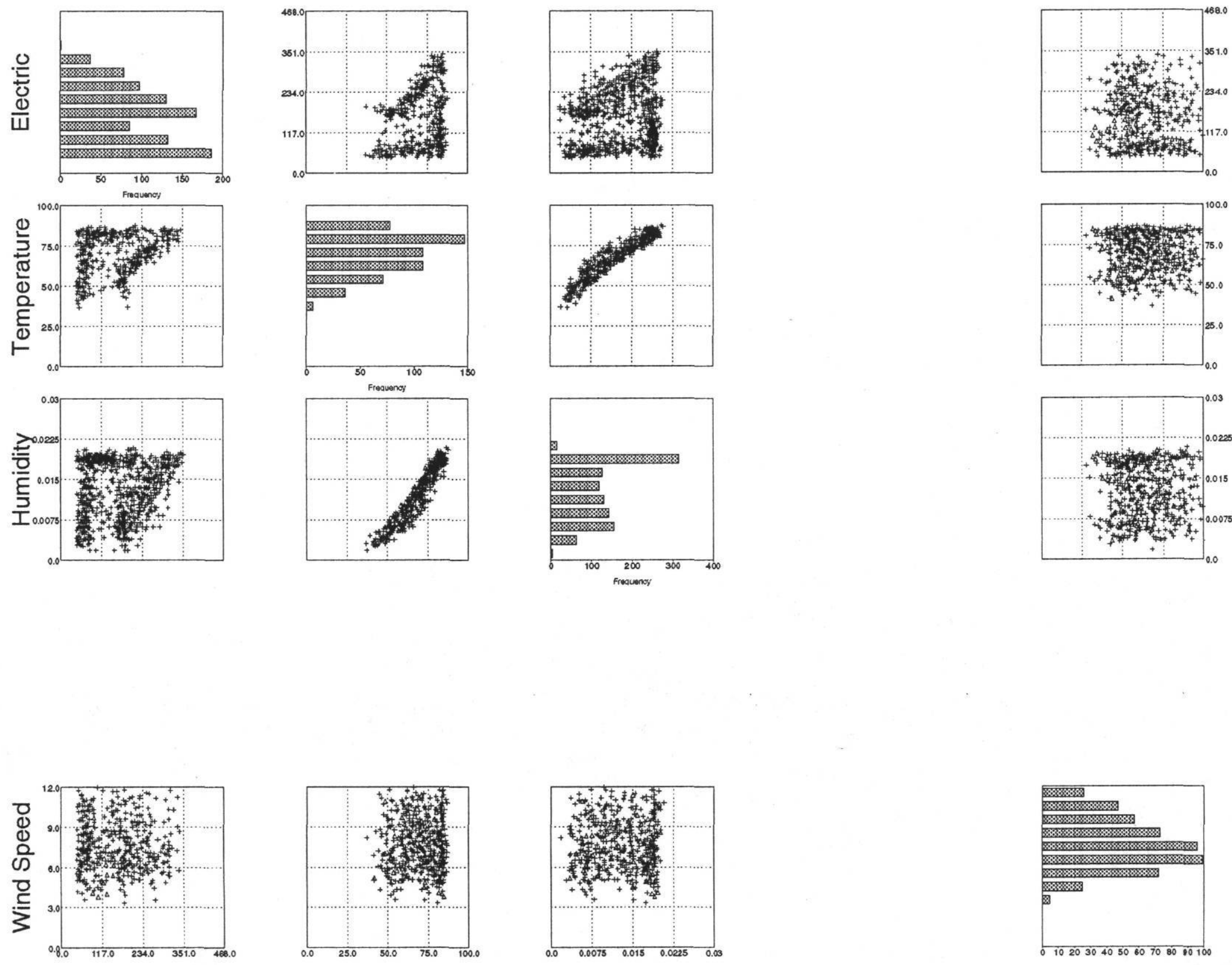


\section{Stroman High School (SHS)}

Daily Average Values

Pre-Retrofit $(\Delta) \quad$ 06/04/1991 - 08/01/1991

Chw Cons.

$(\mathrm{kBtu} / \mathrm{h})$
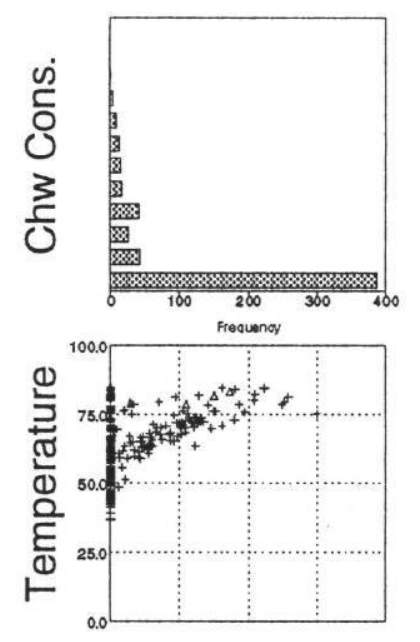

t
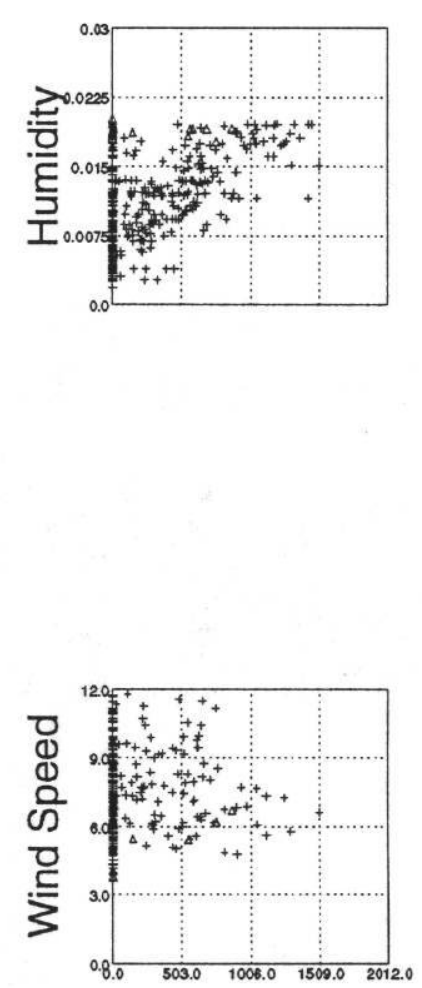

Temperature

(degrees F)
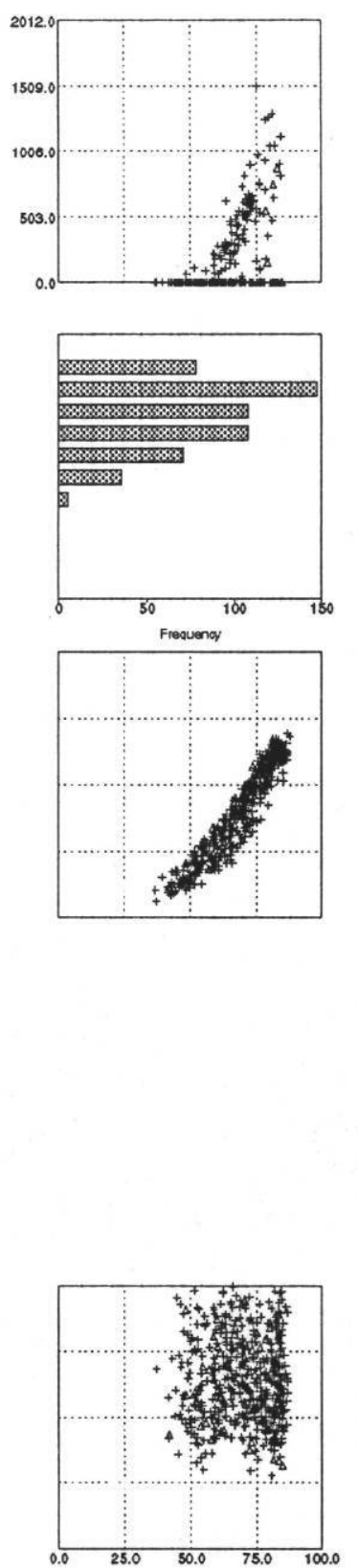

Post-Retrofit (+) 08/15/1991 - 12/31/1993

Solar Rad

Wind Speed

(mph)
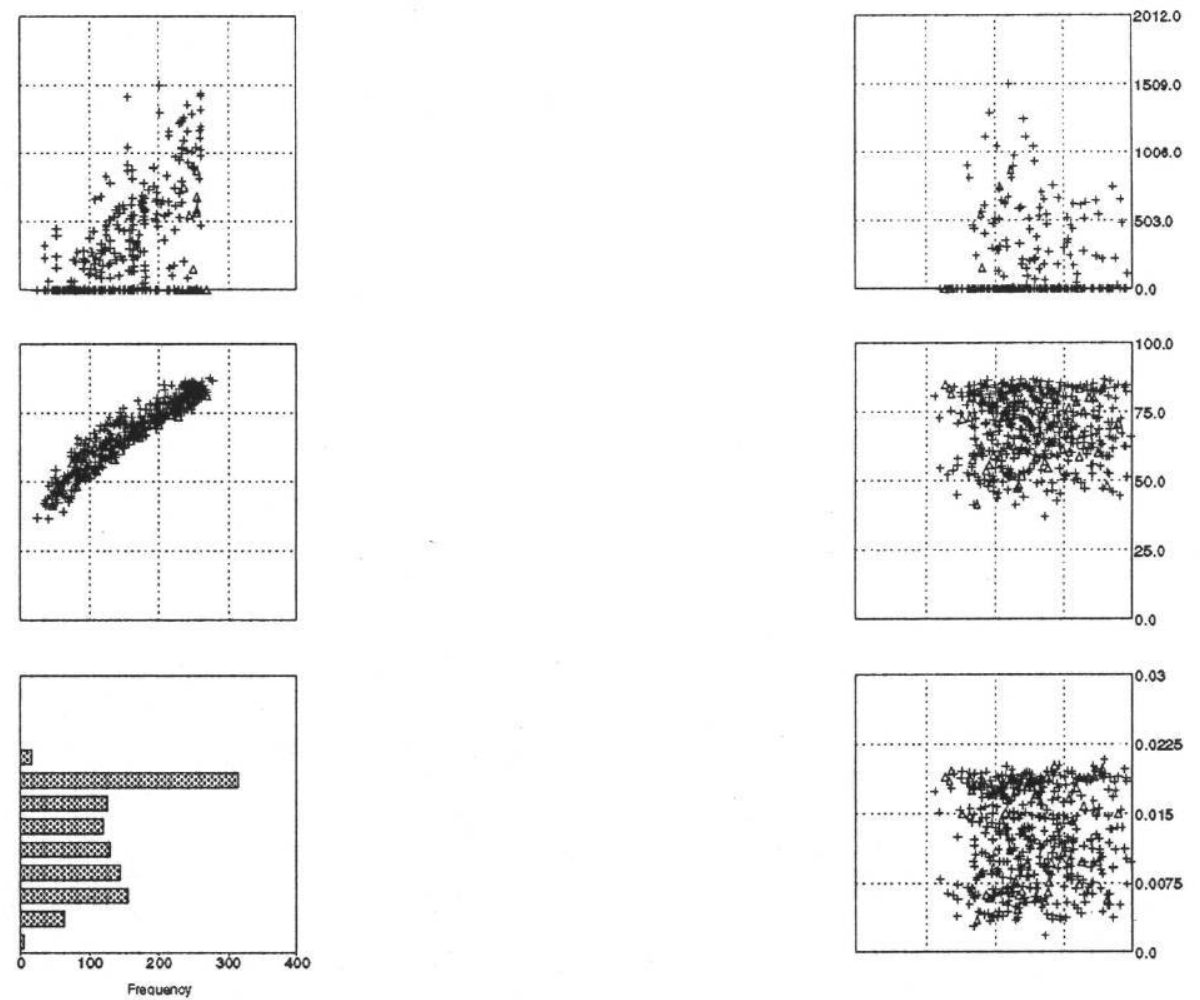
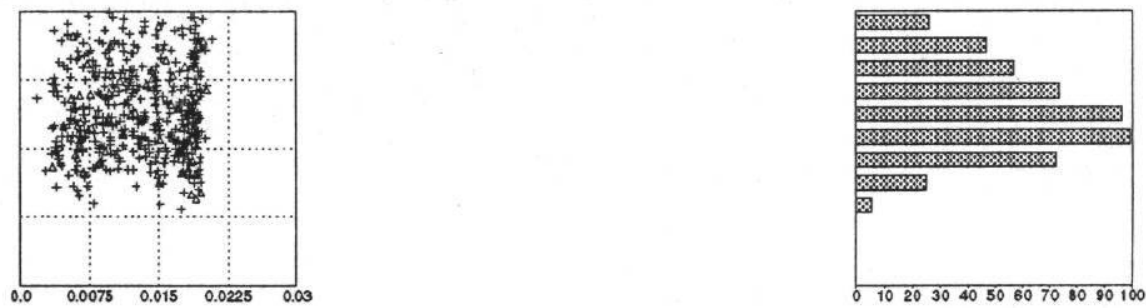


\section{Stroman High School (SHS)}

Daily Average Values

Pre-Retrofit $(\triangle)$ 06/04/1991 - 08/01/1991

HW/Steam Cons. Temperature $(\mathrm{kBtu} / \mathrm{h})$
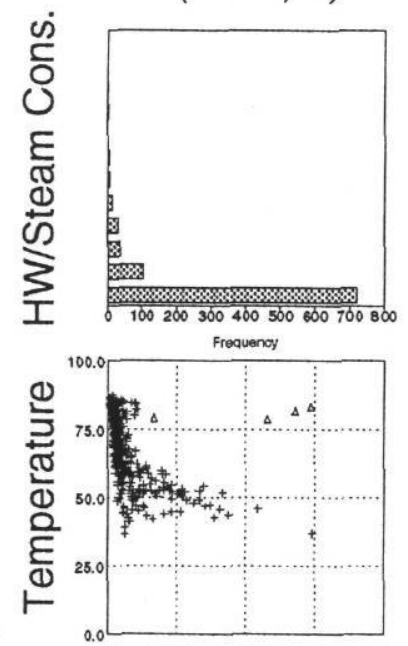

$\underset{\infty}{\stackrel{b}{+}}$
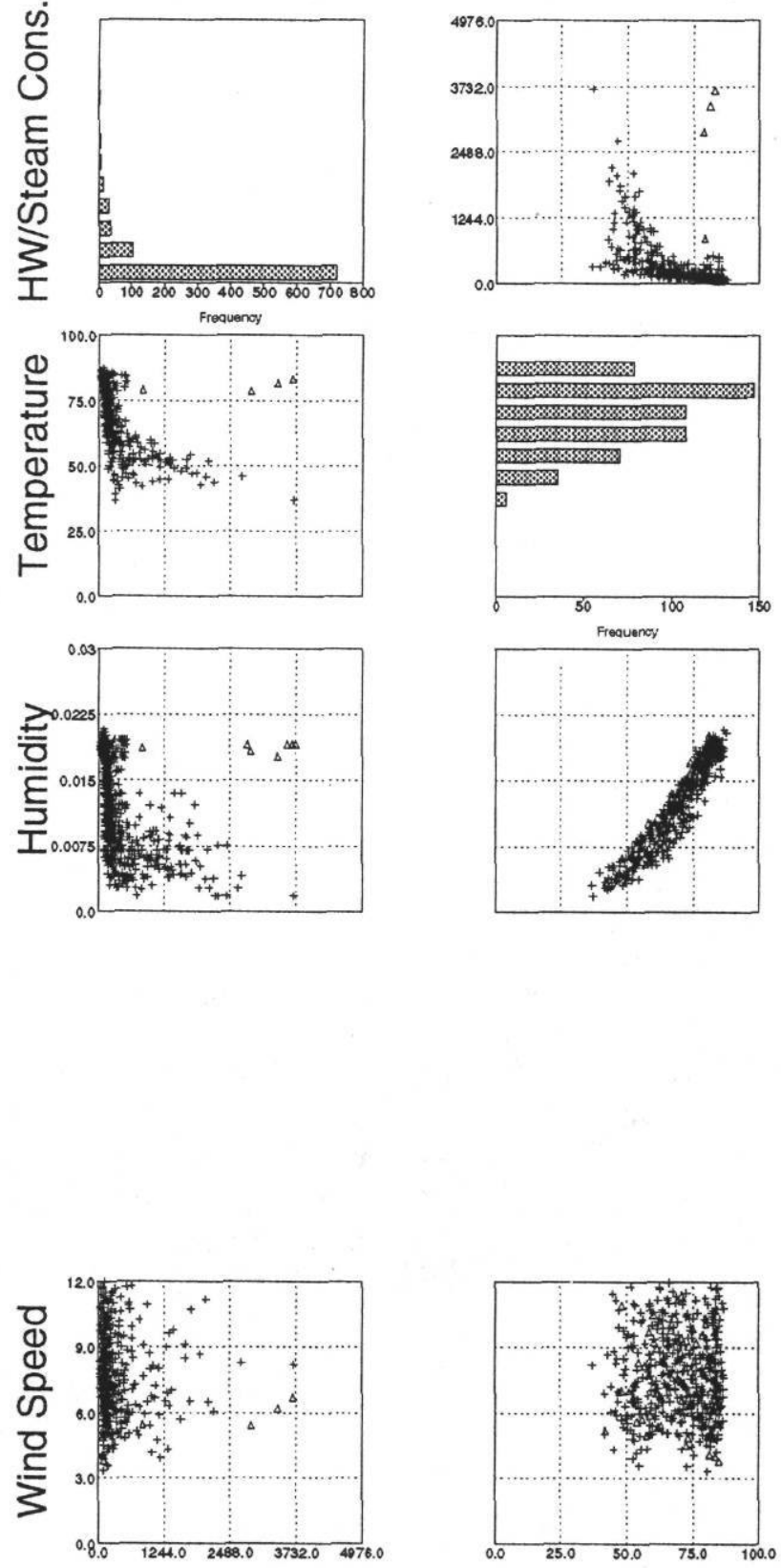

Post-Retrofit (+) 08/15/1991 - 12/31/1993

Solar Rad

(W/sq.m)

Wind Speed

(mph)
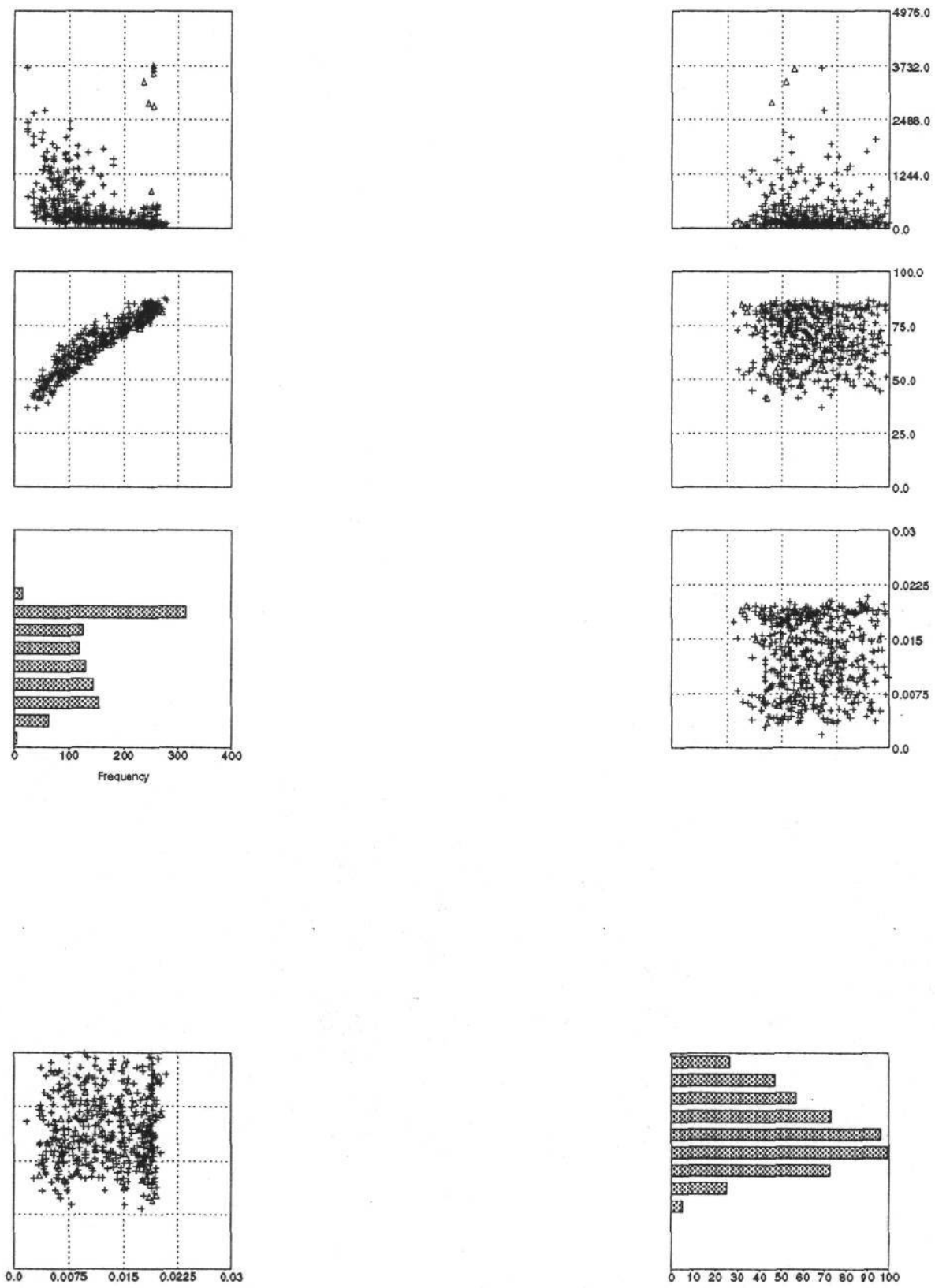


\section{B. VICTORIA HIGH SCHOOL}

\section{B.1 Site Description ${ }^{1}$}

Victoria High School is located in Victoria, Texas. It consists of ten buildings with a total floor area of 257,014 square feet. The two largest buildings are the Main Building and the Academic Wing. Both of these buildings are two-story, brick, slab on grade, with flat roofs. Both buildings are served by hydronic fan-coil units. The chiller serving the Main Building is a 192 ton centrifugal chiller, with 25 horsepower chilled water and condenser pumps, and a 15 horsepower cooling tower fan. The chiller serving the Academic Wing is a 182 ton chiller with a 20 horsepower chilled water pump, a 15 horsepower condenser water pump, and a 20 horsepower cooling tower fan. The eight remaining buildings are all single story, served by rooftop units with direct expansion cooling and gas heating. These buildings include a field house/dressing room, two shop buildings, a gymnasium, special education building, learning resource center, home economics building, and a multipurpose building with kitchen, cafeteria, band hall, and choir rooms.

Air distribution is primarily through single duct air handling systems, providing cooling temperatures of approximately $75^{\circ} \mathrm{F}$, and heating temperatures within the range of 70 to $72{ }^{\circ} \mathrm{F}$. Heating and air handling systems are turned off completely during the night and are controlled from a central location through a Carrier EMCS.

The school is operated from the middle of August through the middle of May, with approximately 2,135 students and 228 faculty and staff. The maximum school occupancy is from about 8:00 a.m. until 4:00 p.m.; however, the building is occupied for much longer periods, including weekends and summers. Stroman and Victoria High School alternate as the primary location for summer school. Victoria was the site during the summer of 1992 . School district calendars for the reporting period of June 5, 1991, through June 4, 1994, are included in Tab B-1.

\footnotetext{
${ }^{1}$ Adapted from: Landman, D.S., 1995. "Preliminary Study of Advanced Diagnostic Prescreening Methods," Energy Systems Laboratory, Mechanical Engineering Department, Texas A\&M University, College Station, TX.
} 
Electricity is purchased from Central Power and Light Company. Natural gas is purchased from ENTEX Gas Company.

\section{B.2 EMCS Retrofit}

The energy audit for Victoria High School determined that the HVAC operation was controlled manually, which resulted in excessive operating hours in each of the schools in the school district. Timeclock controls were installed many years ago, but were not suited for the needs of the school. See Tab B-2 for the full text technical analysis of the facility, which was provided in the audit.

The proposed EMCS retrofit called for the installation of a direct digital control-based EMCS, which would control all HVAC equipment, measure exterior and interior space temperatures, and measure humidity in one or two critical locations within the school. The EMCS would have no override timers that custodial staffs could activate. Operating hours of all HVAC units would be determined by the maintenance staff, and controlled by that staff from its central headquarters via modem.

The EMCS system was installed and activated on January 31, 1992. It controls the HVAC equipment and some lights, and measures the temperature and humidity at select locations. Although there are override capabilities, they are not used.

\section{B.3 Analysis}

\section{B.3.1 Snapshot of consumption for September 1991 through December 1993}

Figures B-1 and B-2 represent monthly average consumption and peak consumption versus minmax average (or peak) monthly temperature. ${ }^{2}$ Min-max average monthly temperature is calculated by averaging the maximum and minimum temperature each day to obtain min-max average daily temperature. The daily temperatures are then averaged over all days in each month to obtain min-max average monthly temperature.

\footnotetext{
${ }^{2}$ Landman, D.S., 1995. "Preliminary Study of Advanced Diagnostic Prescreening Methods," Energy Systems Laboratory, Mechanical Engineering Department, Texas A\&M University, College Station, TX.
} 
The data points reflecting high temperature and low consumption are indicative of non-semester consumption. If those data points are ignored, there is a general increase of consumption with temperature, indicating a temperature dependence of consumption. Additionally, the post-retrofit data points are generally lower than the pre-retrofit data points. When compared to similar plots for other Texas schools in the LoanSTAR program, this site is a low energy use school. However, it does have higher energy use than Stroman High School. The reader is referred to the referenced report for a more detailed discussion of these plots.

Figure B-1: Monthly Average Consumption: Consumption, in W/sf, versus min-max average monthly temperature, in ${ }^{\circ} \mathrm{F}$, for September 1991 through December 1993 (Victoria High School)

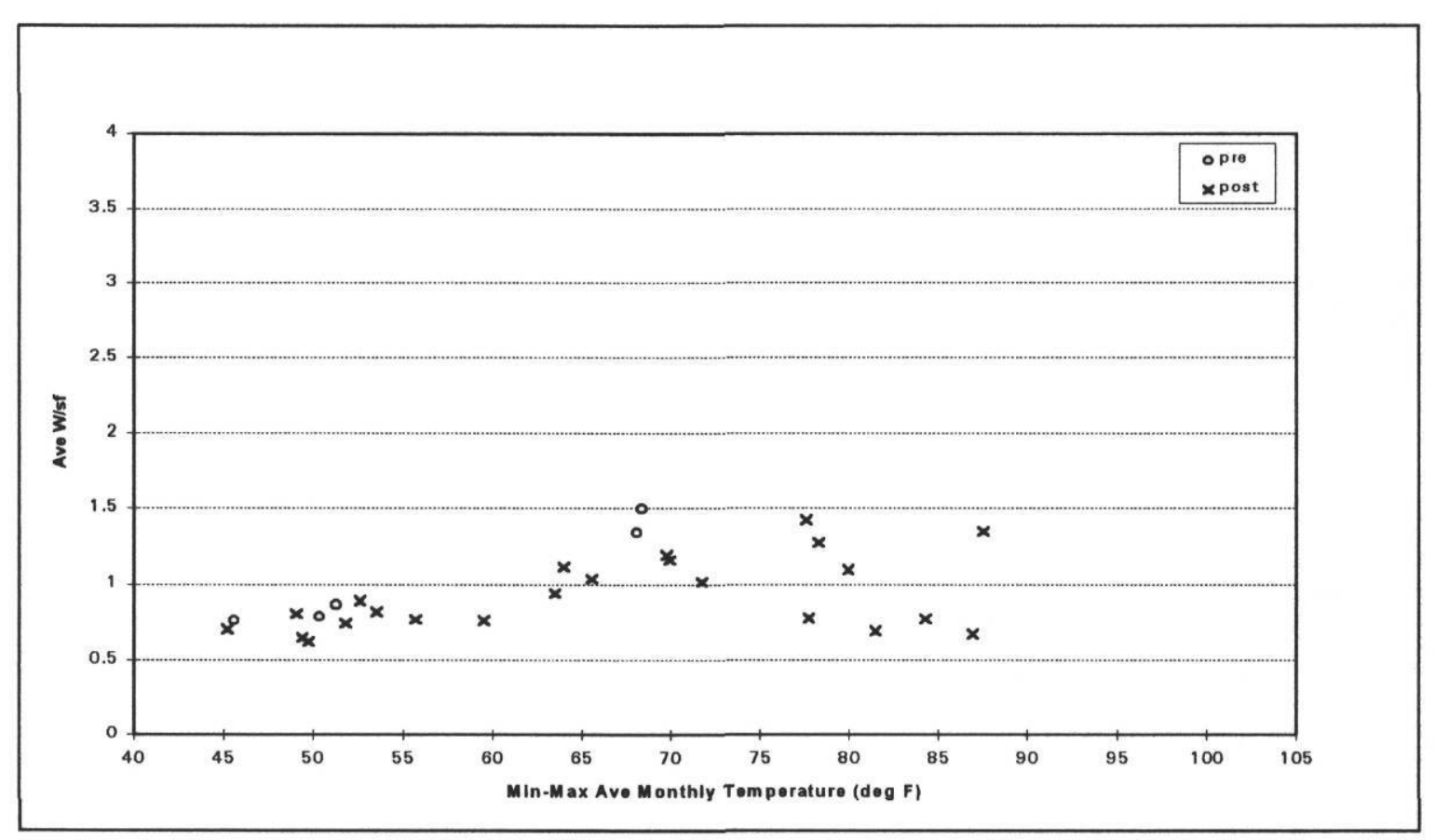


Figure B-2: Monthly Peak Consumption: Consumption, in W/sf, versus peak monthly temperature, in ${ }^{\circ}$, for September 1991 through December 1993 (Victoria High School)

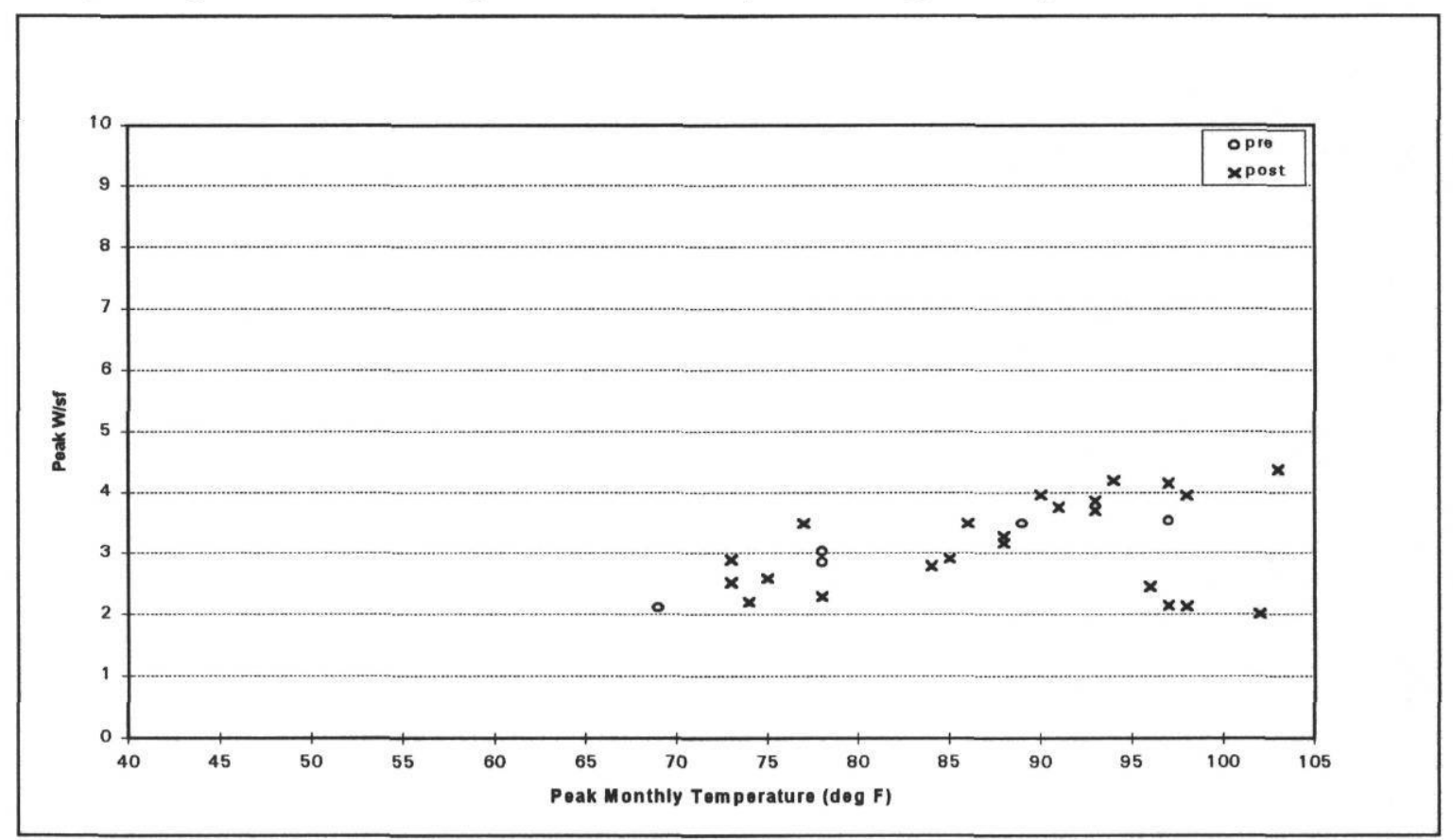

\section{B.3.2 Timeline plots}

Plots of energy consumption for the reporting period are shown in Figure B-3. The EMCS retrofit date of January 31,1992 , is shown by a vertical, bold line. Monitoring diagrams are provided in Tab B-3.

In looking at the whole building electric plot, there is no apparent decrease in consumption at any point along the timeline. There was an a chiller installed as a concurrent retrofit at this site. This resulted in the appearance of chiller consumption in September 1991. Any possible decrease in consumption due to the EMCS may have been offset by the increase in consumption due to the new chiller. The appropriate plot to analyze the effects due only to the EMCS is the "other electric" plot, which is whole building electric minus the chiller. Here, a drop in consumption is evident between the pre-retrofit and post-retrofit periods

The plot of whole building heat shows seasonal heating between November and April of each year. There is also a decrease in consumption evident between the pre-retrofit and post-retrofit periods. 
Figure B-3: Energy Consumption time series for June 1991 to June 1994 (Victoria High School)
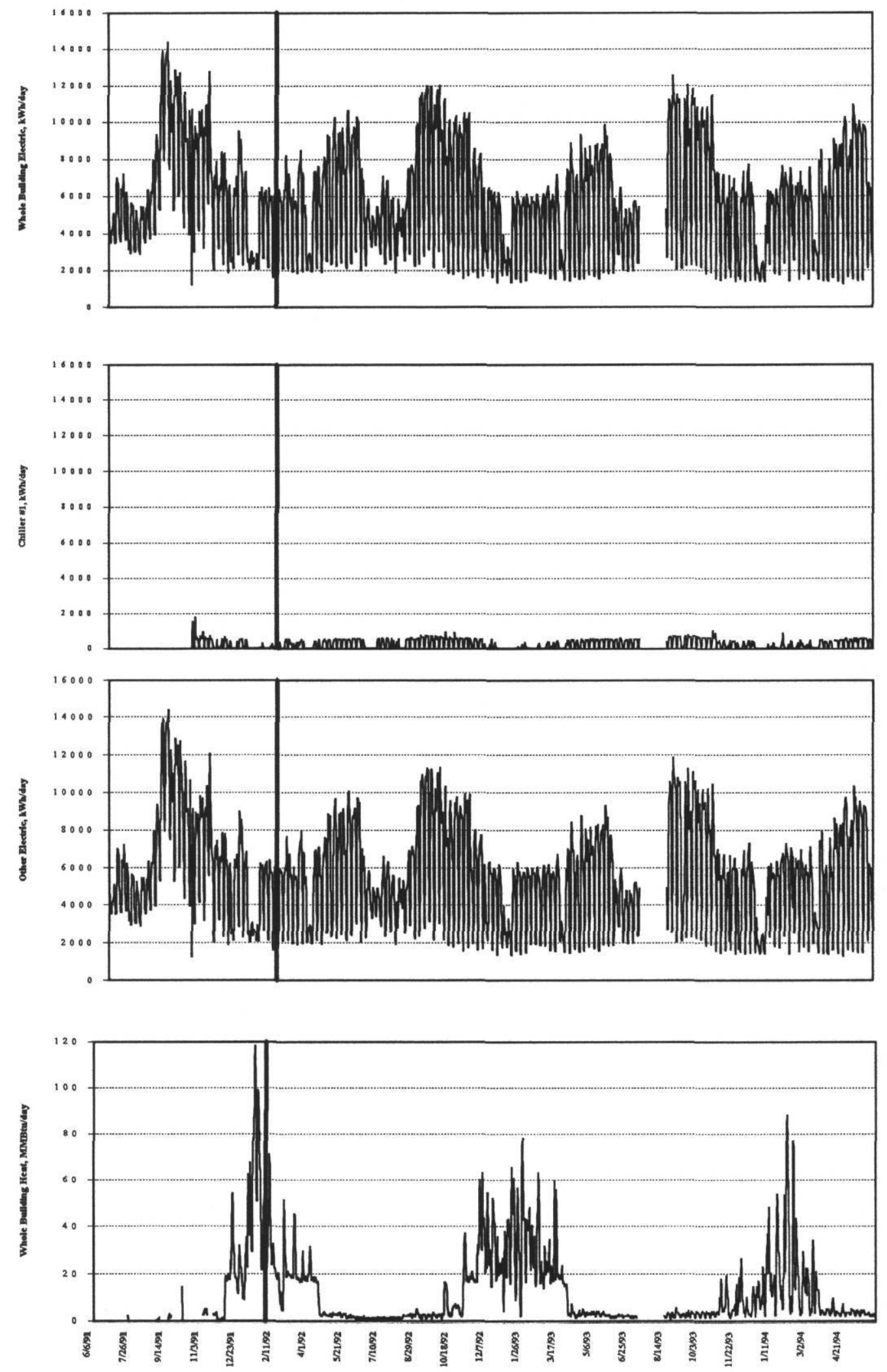


\section{B.3.3 Whole Building Electricity Consumption (Post Period)}

Table B-1 shows energy consumption for the post period (February 1, 1992, through June 4, 1994). Whole building electricity consumption is broken down into two components: chiller \#1 electricity consumption and other electricity consumption. It is further subdivided into semester period and non-semester periods. The post-retrofit period is used because there is significantly more data available in the post-retrofit period, and it represents current usage.

Figure B-4 graphically shows whole building electricity consumption for the post period. For the semester period, $66 \%$ of whole building electric energy use is attributable to other electric equipment, while $3 \%$ is due to electric chiller \#1. For the non-semester period, other electric accounts for $30 \%$ of whole building electric energy, while chiller \#1 accounts for $1 \%$.

From both Table B-1 and Figure B-4, it is readily apparent that chiller \#1 accounts for a small portion of the whole building electricity usage. Therefore, attention for reducing energy usage should be focused on the other electricity usage. In this case, other electricity consumption is primarily roof-top HVAC units and lighting.

Table B-1: Energy Consumption for post period, February 1992 through June 1994 (Victoria High School)

\begin{tabular}{|l|r|r|r|r|r|r|}
\hline \multirow{2}{*}{} & \multicolumn{2}{|c|}{ SEMESTER } & \multicolumn{2}{c|}{ NON-SEMESTER } & \multicolumn{2}{c|}{ TOTAL } \\
\cline { 2 - 7 } & ENERGY & \multicolumn{1}{c|}{$\$$} & ENERGY & \multicolumn{1}{c|}{$\$$} & \multicolumn{1}{c|}{ ENERGY } & $\$$ \\
\hline wbelec, kWh & $3,343,246$ & $\$ 93,210$ & $1,262,155$ & $\$ 35,189$ & $4,605,400$ & $\$ 128,399$ \\
\hline chlr \#1, kWh & 159,076 & $\$ 4,435$ & 60,298 & $\$ 1,681$ & 219,374 & $\$ 6,116$ \\
\hline other, kWh & $3,184,170$ & $\$ 88,775$ & $1,201,857$ & $\$ 33,508$ & $4,386,027$ & $\$ 122,282$ \\
\hline wbheat, MMBtu & 7,847 & $\$ 37,271$ & 1,888 & $\$ 8,966$ & 9,734 & $\$ 46,237$ \\
\hline
\end{tabular}


Figure B-4: Whole Building Electricity Consumption for post period, February 1992 - June 1994 (Victoria High School)

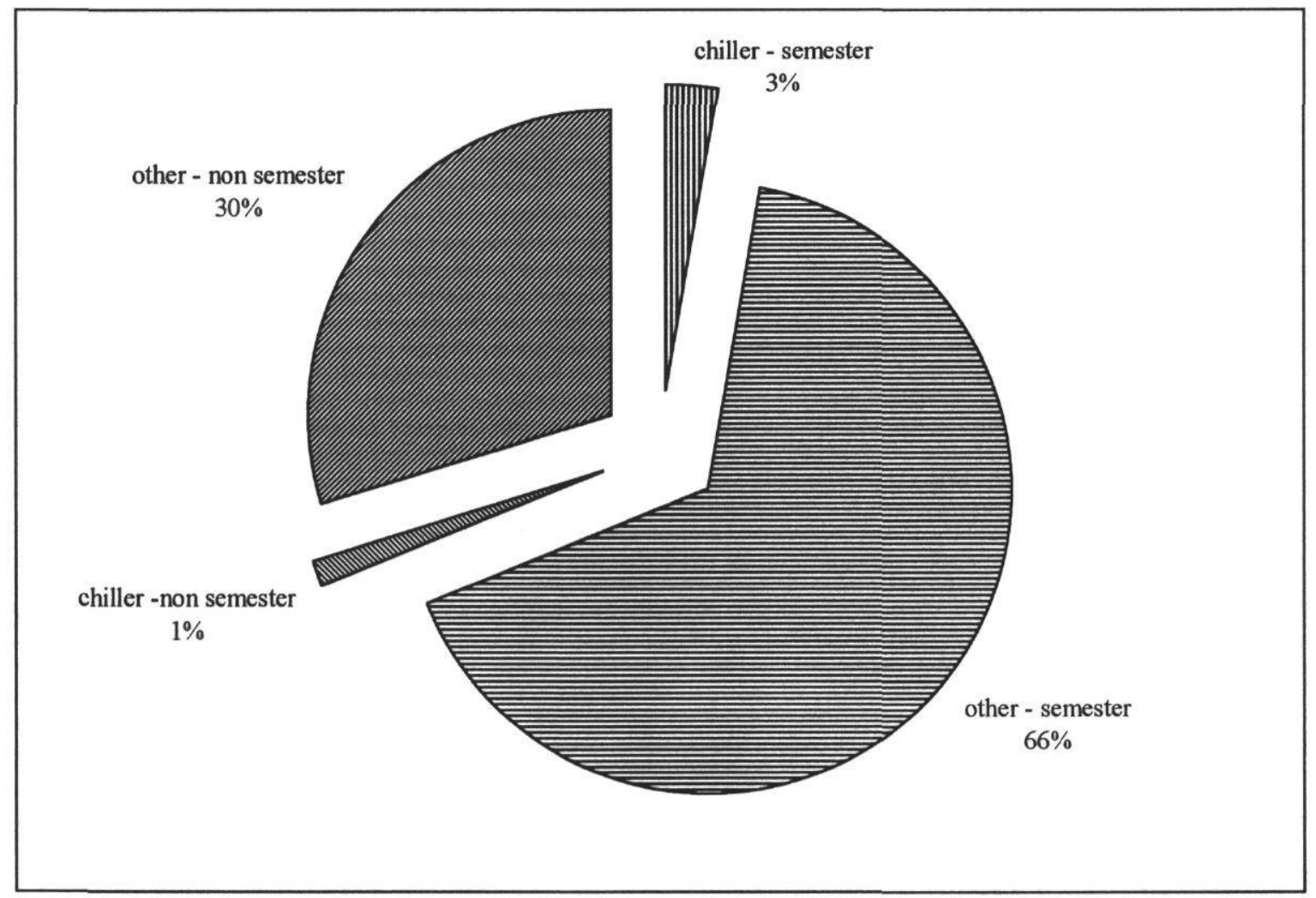

\section{B.3.4 Total Monthly Consumption}

The total monthly energy consumption is summarized in Table B-2. Again, it is readily apparent that other electric accounts for the majority of this site's electric energy use. 
Table B-2 : Monthly Energy Consumption (Victoria High School)

\begin{tabular}{|c|c|c|c|c|}
\hline & $\begin{array}{c}\text { wbelec } \\
\mathrm{kWh} / \text { month }\end{array}$ & $\begin{array}{c}\text { chlr \#1 } \\
\mathrm{kWh} / \text { month }\end{array}$ & $\begin{array}{c}\text { other } \\
\mathrm{kWh} / \mathrm{month}\end{array}$ & $\begin{array}{c}\text { wbheat } \\
\text { MMBtu/month }\end{array}$ \\
\hline \multicolumn{5}{|l|}{ PRE PERIOD } \\
\hline Jun 91 & 121,743 & 0 & 241,636 & 16 \\
\hline Jul & 137,603 & 0 & 220,847 & 21 \\
\hline Aug & 270,742 & 0 & 167,456 & 1 \\
\hline Sep & 276,575 & 0 & 167,304 & 18 \\
\hline Oct & 242,163 & 17,072 & 152,268 & 16 \\
\hline Nov & 160,325 & 5,118 & 136,391 & 45 \\
\hline Dec & 150,396 & 4,642 & 219,845 & 597 \\
\hline $\operatorname{Jan} 92$ & 139,697 & 1,225 & 165,440 & 1,640 \\
\hline Total Consumption & $1,499,242$ & 28,057 & $1,471,186$ & 2,354 \\
\hline Total Cost & $\$ 41,799$ & $\$ 782$ & $\$ 41,017$ & $\$ 10,755$ \\
\hline \multicolumn{5}{|l|}{ POST PERIOD } \\
\hline Feb 92 & 149,365 & 5,518 & 143,414 & 775 \\
\hline Mar & 141,229 & 4,906 & 161,499 & 664 \\
\hline Apr & 190,903 & 9,330 & 200,369 & 326 \\
\hline May & 227,078 & 12,205 & 244,332 & 78 \\
\hline Jun & 136,443 & 4,319 & 203,001 & 41 \\
\hline Jul & 145,357 & 6,713 & 182,240 & 40 \\
\hline Aug & 199,450 & 11,992 & 142,447 & 65 \\
\hline Sep & 263,715 & 14,623 & 139,716 & 69 \\
\hline Oct & 230,083 & 13,660 & 153,058 & 174 \\
\hline Nov & 159,136 & 7,442 & 171,351 & 685 \\
\hline Dec & 125,653 & 1,982 & 211,597 & 933 \\
\hline $\operatorname{Jan} 93$ & 134,521 & 1,253 & 225,745 & 1,113 \\
\hline Feb & 134,220 & 2,229 & 151,229 & 864 \\
\hline Mar & 147,342 & 5,880 & 155,418 & 674 \\
\hline Apr & 173,404 & 9,780 & 154,491 & 93 \\
\hline May & 194,974 & 11,349 & 159,892 & 89 \\
\hline Jun & 128,470 & 10,102 & 173,318 & 59 \\
\hline Jul & 20,765 & 1,629 & 221,511 & 9 \\
\hline Aug & 153,649 & 8,711 & 138,114 & 49 \\
\hline Sep & 233,928 & 13,954 & 131,201 & 86 \\
\hline Oct & 211,490 & 13,750 & 144,102 & 108 \\
\hline Nov & 135,042 & 5,710 & 209,575 & 258 \\
\hline Dec & 116,966 & 4,896 & 77,688 & 276 \\
\hline $\operatorname{Jan} 94$ & 145,092 & 3,929 & 126,914 & 733 \\
\hline Feb & 145,476 & 4,244 & 38,909 & 756 \\
\hline Mar & 146,811 & 6,386 & 77,542 & 290 \\
\hline Apr & 184,945 & 9,826 & 151,400 & 118 \\
\hline May & 222,895 & 12,802 & 88,572 & 104 \\
\hline Jun 94 & 20,330 & 1,033 & 19,297 & 10 \\
\hline Total Consumption & $4,618,730$ & 220,155 & $4,397,944$ & 9,538 \\
\hline Total Cost & $\$ 128,770$ & $\$ 6,138$ & $\$ 122,615$ & $\$ 45,303$ \\
\hline Grand Total Consumption & $6,117,972$ & 248,212 & $5,869,129$ & 11,891 \\
\hline Grand Total Cost & $\$ 170,569$ & $\$ 6,920$ & $\$ 163,631$ & $\$ 56,059$ \\
\hline
\end{tabular}




\section{B.3.5 Average Daily Consumption}

Figures B-5a and B-5b depict the average hourly consumption for the semester period and the non-semester period. From both figures, you can see that the consumption for the weekdays does not significantly change in profile, but decreases in magnitude, with more significant reductions in the nighttime hours.

For the semester period, Figure B-5a, the weekday consumption slightly decreased during the daytime hours, 7:00 a.m. to 5:00 p.m., and greatly decreased during the nighttime hours, 5:00 p.m. to 7:00 a.m. The weekend consumption decreased during the nighttime, but increased during the daytime hours. Why does the post consumption exceed that of the pre consumption for weekends? One possible explanation is that the setpoints on the new EMCS are such that the consumption is greater during the weekend than before the EMCS was installed. Another possible explanation is that there are many more data points in the post period, and there are periodic special events on the weekends These two factors combined may result in higher weekend daytime consumption in the post-retrofit period.

For the non-semester period, Figure B-5b, weekday consumption slightly decreased during the daytime hours and greatly decreased during the nighttime hours. Here, the weekend usage decreased in a manner similar to that of the weekdays. The changes in both weekday and weekend consumption can be attributed to the EMCS retrofit.

Tab B-4 contains a summary of the hourly averages and the respective standard deviations and count of data points. The hourly averages are the data that is plotted in Figures B-5a and B-5b. For this site, the standard deviations are quite large. They do not vary much for the hours of 0 through 7 , then jump to higher levels in hours 8 through 23 . This should not be alarming, because the periods that the data were averaged over include wide ranges of temperatures. As was seen earlier, in Figures B-1 and B2 , the energy usage is temperature dependent. The count of data points represents the actual number of data points used to calculate the average, which corresponds to the amount of time that the equipment was actually operating. 
Figure B-5a: Semester Pre-/Post-retrofit Comparison (Victoria High School)

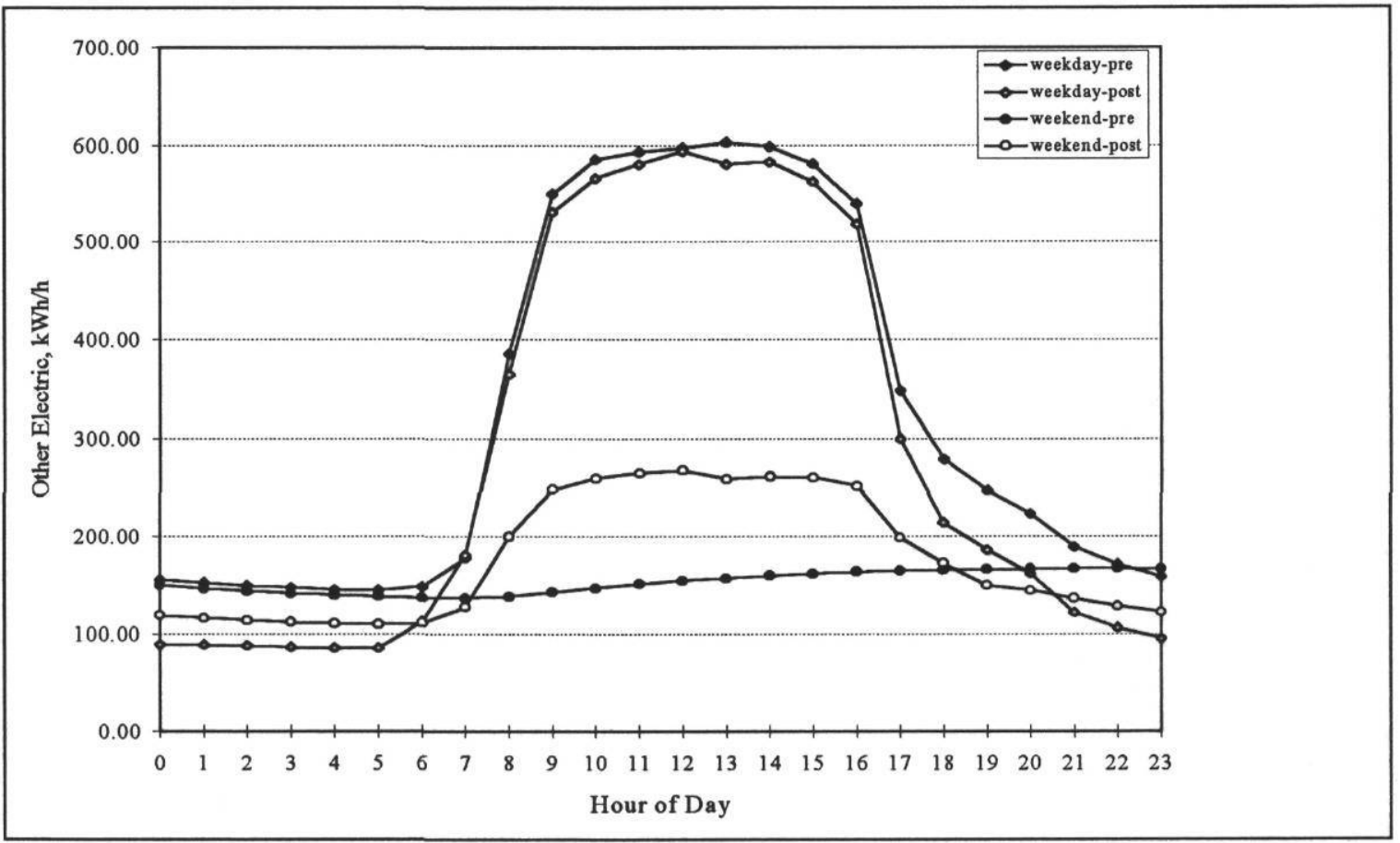

Figure B-5b: Non-semester Pre-/Post-retrofit Comparison (Victoria High School)

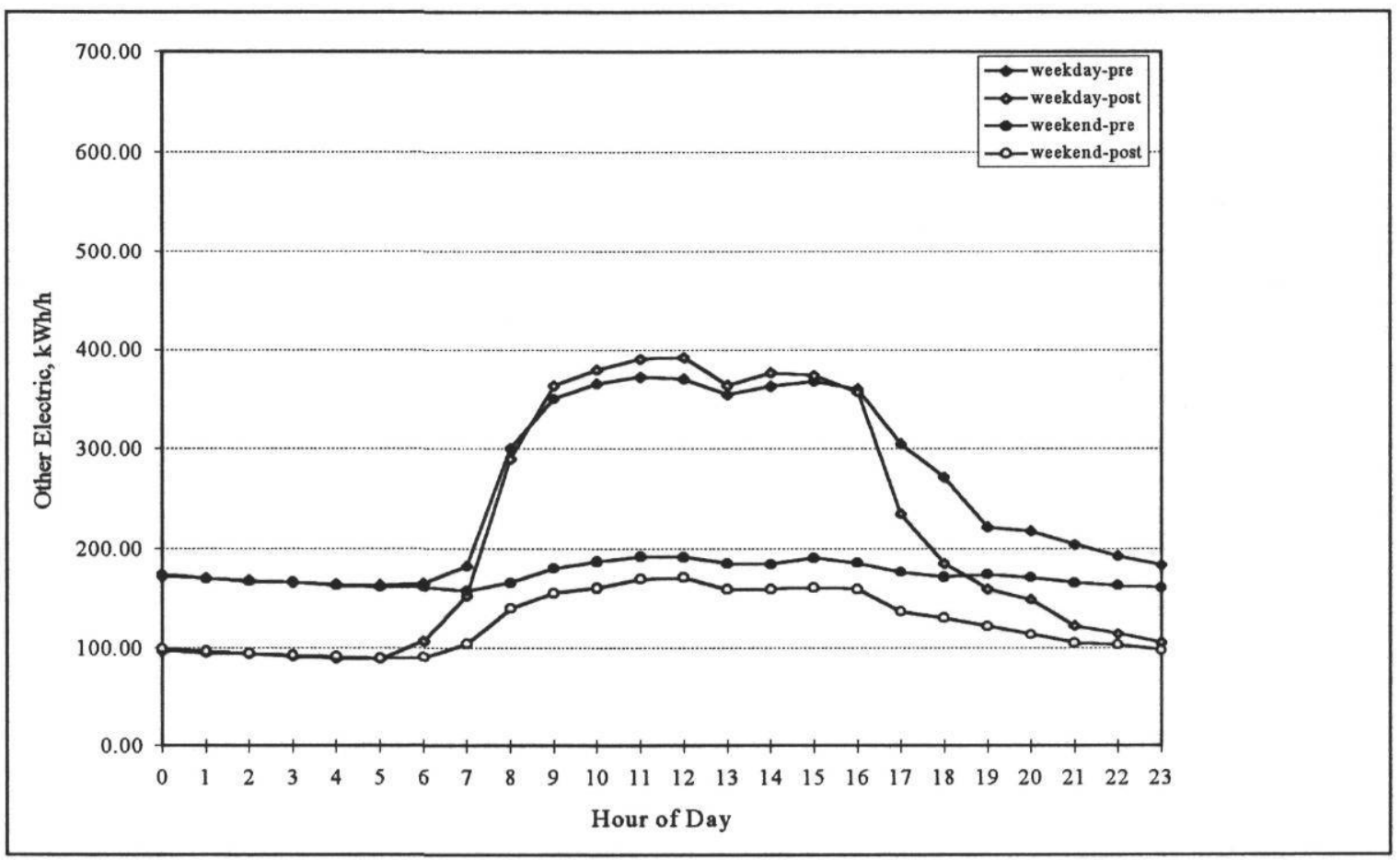


The difference in other electric energy consumption was calculated based on the average daily data. This is shown in Table B-3, both as a difference in energy and a percentage difference in energy.

Table B-3: Reduction in Other Electric Consumption based on average daily data (Victoria High School)

\begin{tabular}{|c|c|c|c|c|}
\hline & $\begin{array}{l}\text { \# days } \\
\text { in period }\end{array}$ & $\begin{array}{l}\text { Average Daily } \\
\text { Consumption } \\
\text { kWh/day }\end{array}$ & $\begin{array}{l}\text { Difference in } \\
\text { Average Daily } \\
\text { Consumption } \\
\text { kWh/period }\end{array}$ & $\begin{array}{l}\text { \% Difference in } \\
\text { Average Daily } \\
\text { Consumption }\end{array}$ \\
\hline \multicolumn{5}{|l|}{ Semester } \\
\hline weekday-pre & 91 & 7,877 & \multirow[b]{2}{*}{-988} & \multirow[b]{2}{*}{$-12.54 \%$} \\
\hline weekday-post & 394 & 6,889 & & \\
\hline weekend-pre & 35 & 3,674 & \multirow[b]{2}{*}{571} & \multirow[b]{2}{*}{$15.54 \%$} \\
\hline weekend-post & 149 & 4,245 & & \\
\hline \multicolumn{5}{|l|}{ Non-semester } \\
\hline weekday-pre & 79 & 6,159 & \multirow[b]{2}{*}{-977} & \multirow[b]{2}{*}{$-15.86 \%$} \\
\hline weekday-post & 241 & 5,182 & & \\
\hline weekend-pre & 33 & 4,180 & \multirow[b]{2}{*}{$-1,163$} & \multirow[b]{2}{*}{$-27.82 \%$} \\
\hline weekend-post & 92 & 3,017 & & \\
\hline
\end{tabular}

\section{B.3.6 Plots from MECR}

The September MECR energy use plots for four years are shown in Tab B-5. These provide a more qualitative look at the effects of the EMCS. September 1991 is a pre-retrofit plot. Note that there is relatively high consumption between the hours of midnight and 6:00 a.m., with a gradual increase to daytime levels. This is followed by a slow decrease in consumption between the hours of 4:00 p.m. and 10:00 p.m. There are many afternoons and evenings where consumption did not drop to nighttime levels. September 1992 shows dramatically reduced nighttime consumption, with a much sharper slope up to daytime levels between 7:00 a.m. and 8:00 a.m. when compared to September 1991. The consumption drops off much more quickly at 4:00 p.m. and the afternoon and evening consumption is drastically reduced as compared to September 1991.. The profiles are slightly improved between the months of September 1992 and September 1993. The profiles are slightly degraded in September 1994, although are still greatly improved when compared to September 1991. The characteristic post-retrofit shape is maintained, but there are many occurrences of increased nighttime consumption. 
Overall, the changes seen in the MECR plots can be attributed to the EMCS retrofit.

It should be noted that these profiles only allow a look at weekday data. The weekend data is unreadable from these plots. Separating the data into weekdays and weekends, then plotting separately would enable one to evaluate weekends, as well as weekdays.

\section{B.3.7 Data Summary Notebook Information}

The Data Summary Notebook information is included in Tab B-6 for information only. It is not analyzed for this site. 
Tab B-1

School District Schedules

B-13 


\section{VICTORIA PUBLIC SCHOOLS}

School Calendar 1993-1994

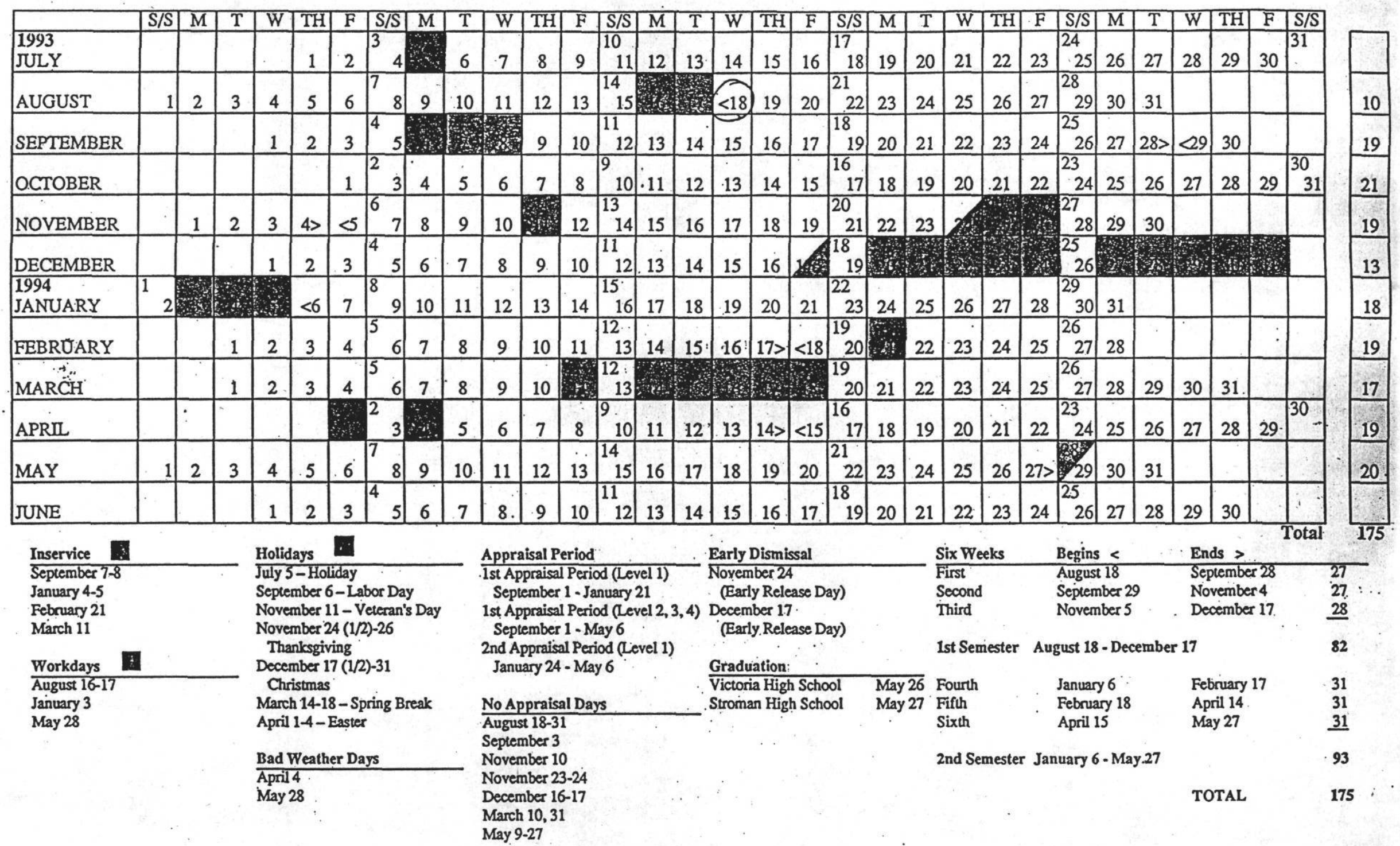


VICTORIA PUBLIC SCHOOLS

SCHOOL CALENDAR 1992 - 93

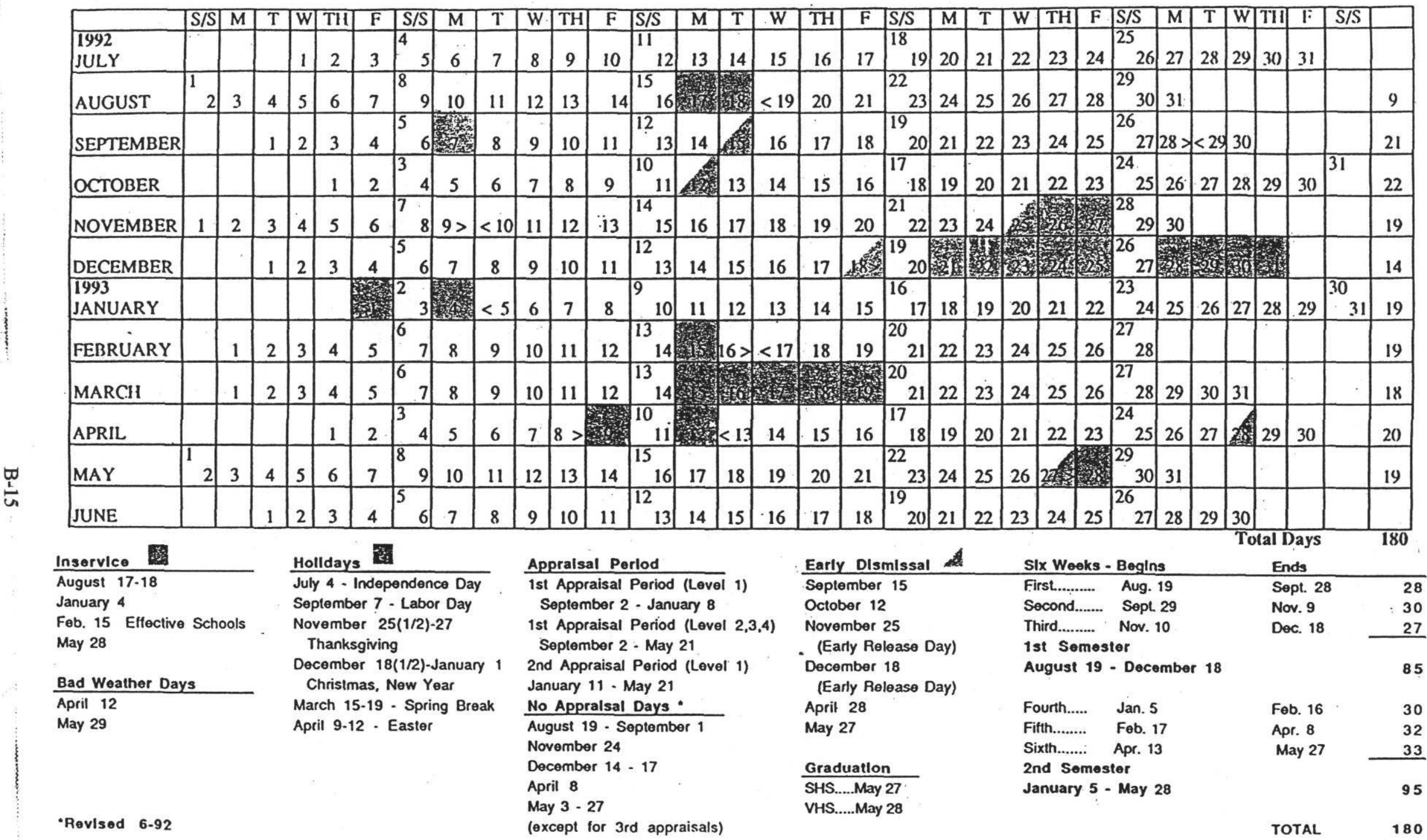


VICTORIA PUBLIC SCHOOLS

SCHOOL CALENDAR 1991- 1992

\begin{tabular}{|c|c|c|c|c|c|c|c|c|c|c|c|c|c|c|c|c|c|c|c|c|c|c|c|c|c|c|c|c|c|c|c|c|}
\hline & $\overline{S / S}$ & $\bar{M}$ & $\mathbf{T}$ & $\overline{\mathrm{W}}$ & TH & $\bar{F}$ & $S / S$ & $\bar{M}$ & $\mathrm{~T}$ & $\mathrm{~W}$ & TH] & $\mathrm{F}$ & $S / S$ & $\mathrm{M}$ & $\mathrm{T}$ & $\bar{W}$ & TH & $\mathrm{F}$ & $S / S$ & $\mathrm{M}$ & $T$ & W & TH & $\mathrm{F}$ & $S / S$ & $M$ & $\mathrm{~T}$ & $\mathrm{~W}$ & $\mathrm{TH}$ & $\mathbf{F}$ & $\mathrm{S} / \mathrm{S}$ & \\
\hline $\begin{array}{l}1991 \\
\text { UULY }\end{array}$ & & 1 & 2 & 3 & 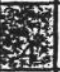 & 5 & $\begin{array}{ll}67 \\
7\end{array}$ & 8 & 9 & 10 & 11 & 12 & 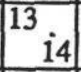 & 15 & 16 & 17 & 18 & 19 & \begin{tabular}{|c|}
20 \\
21 \\
\end{tabular} & 22 & 23 & 24 & 25 & 26 & \begin{tabular}{|r|}
27 \\
28 \\
\end{tabular} & 29 & 30 & 31 & & & & \\
\hline AUGUST & & & & & 1 & 2 & 4 & 5 & 6 & 7 & 8 & 9 & \begin{tabular}{|c|}
10 \\
11 \\
\end{tabular} & 12 & 13 & 14 & 15 & 16 & \begin{tabular}{|r|}
17 \\
18 \\
\end{tabular} & 19 & 被 & 28 & $<22$ & 23 & \begin{tabular}{r|}
24 \\
25 \\
\end{tabular} & 26 & 27 & 28 & 29 & 30 & 31 & 7 \\
\hline SEPTEMBER & & 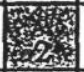 & 3 & 4 & 5 & 6 & 8 & 9 & 10 & 11 & 12 & 13 & $\begin{array}{r}14 \\
15 \\
\end{array}$ & 16 & 17. & 18 & 19 & 20 & \begin{tabular}{|r|}
21 \\
22 \\
\end{tabular} & 23 & 24 & 25 & 26 & 27 & \begin{tabular}{|r|}
28 \\
29 \\
\end{tabular} & 30 & & & & & & 20 \\
\hline OCTOBER & & & 1 & 2 & 3 & 4 & 6 & $7>$ & $<8$ & 9 & 10 & 11 & \begin{tabular}{r|}
12 \\
13 \\
\end{tabular} & 14 & 15 & 16 & 17 & 18 & \begin{tabular}{r|}
19 \\
20 \\
\end{tabular} & 21 & 22 & 23 & 24 & 25 & \begin{tabular}{|l|}
26 \\
27 \\
\end{tabular} & 28 & 29 & 30 & 31 & & & 23 \\
\hline NOVEMBER & & & & & & 1 & $\begin{array}{ll}2 \\
3\end{array}$ & 4 & 5 & 6 & 7 & 8 & 10 & 11 & 12 & 13 & 14 & 15 & \begin{tabular}{|r|}
16 \\
17 \\
\end{tabular} & $18>$ & $<19$ & 20 & 21. & 22 & \begin{tabular}{|l|}
23 \\
24 \\
\end{tabular} & 25 & 26. & & 新 & 29 & 30 & 19 \\
\hline DECEMBER & 1 & 2 & 3 & 4 & 5 & 6 & $\begin{array}{l}78 \\
\end{array}$ & 9 & 10 & 11 & 12 & 13 & \begin{tabular}{r|}
14 \\
15 \\
\end{tabular} & 16 & 17 & 18 & 19 & 20 & & & & 25 & 26 & 然得 & 29 & & 3 & & & & & 15 \\
\hline $\begin{array}{l}1992 \\
\text { JANUARY }\end{array}$ & & & & 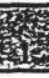 & 2 & 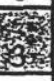 & 4 & 6 & 7 & 8 & 9 & 10 & \begin{tabular}{|l|}
11 \\
12 \\
\end{tabular} & 13 & 14 & 15 & $16>$ & & \begin{tabular}{r|}
18 \\
19 \\
\end{tabular} & $<20$ & 21 & 22 & 23 & 24 & \begin{tabular}{|c|}
25 \\
26 \\
\end{tabular} & 27 & 28 & 29 & 30 & 31 & & 19 \\
\hline FEBRUARY & 2 & 3 & 4 & 5 & 6 & 7 & 9 & 10 & 11 & 12 & 13 & 14 & \begin{tabular}{|r|}
15 \\
16 \\
\end{tabular} & 17 & 18 & 19 & 20 & 21 & 22 & 24 & 25 & 26 & 27 & 28 & 29 & & & & & & & 20 \\
\hline MARCH & 1 & $2>$ & $<3$ & 4 & 5 & 6 & 8 & 9 & 10 & 11 & 12 & 48 & \begin{tabular}{|r|}
14 \\
15 \\
\end{tabular} & 46 & 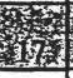 & 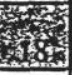 & 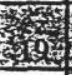 & & \begin{tabular}{|r|}
21 \\
22 \\
\end{tabular} & 23 & 24 & 25 & 26 & 27 & $\begin{array}{r}28 \\
29 \\
\end{array}$ & 30 & 31 & & & & & 16 \\
\hline APRIL & & & & 1 & 2 & 3 & 5 & 6 & 7 & 8 & 9 & 10 & \begin{tabular}{|l|}
11 \\
12 \\
\end{tabular} & 13 & 14. & 15 & $16>$ & 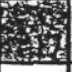 & 19 & 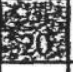 & 21 & 22 & 23 & 24 & \begin{tabular}{|l|}
25 \\
26 \\
\end{tabular} & 27 & & 29 & 30 & & & 20 \\
\hline MAY & & & & & & 1 & $\begin{array}{l}2 \\
3 \\
\end{array}$ & 4 & 5 & 6 & 7 & 8 & 10 & 11 & 12 & 13 & 14 & 15 & \begin{tabular}{|r|}
16 \\
17 \\
\end{tabular} & 18 & 19 & 20 & 21 & 22 & $\begin{array}{r}23 \\
24 \\
\end{array}$ & 25 & 26 & 27 & 28 & & & 21 \\
\hline UN & & 1 & 2 & 3 & 4 & 5 & 6 & 8 & 9 & 10 & 11 & 12 & 13 & 15 & 16 & 17 & 18 & 19 & \begin{tabular}{|c|}
20 \\
21 \\
\end{tabular} & 22 & 23 & 24 & 25 & 26 & $\begin{array}{r}27 \\
28\end{array}$ & 29 & 30 & & & & & \\
\hline
\end{tabular}

\section{Inservice/Preparation Dates} August 20-21

January 17

March i3 Effective Schools

May 30

Bad Weather Days:

April 20

Revised June 20, 1991
Holidars

July 4 - Independence Day September 2 - Labor Day Nov. $27(1 / 2)-29$ - Thanksgiving December 20 (1/2) January 3 Christmas, New Year March 16-20 - Spring Break April 17-20 Easter
Appraisal Periods

1st Appraisal Period (Level 1)

September 5-January 10

1st Appraisal Period (Level 2,3,4)

September 5-May 20

2nd Appraisal Period (Level 1)

January 20-May 20

No Appraisal Days

Aug. 21-Sept. 4

Nov. 26

Dec. 20

April 16

May 21-29
TOTAL DAYS 180

Early Dismissal October 23 Nov.27 (Early Release Dayi) Dec. 20 (Early Release Day)

April 28 May 29

Graduation VHS....May 29 SHS.....May 30

\section{Ends}

Six Weeks - Begins First........... August 22....... October 7. 32 Second....... October 8.... Nov. 18 Third.......: November 19.. January 16 $\quad 31$ 1st Semester Aug. 22-Jan. 16

Fourth....... January 20.... March 3.....

Sixth.......... April 21......... 2nd Semester Jan. 20-May 29

March 2

Total
31

16

8 


\section{VICTORIA PUBLIC SCHOOLS}

SCHOOL CALENDAR 1990 - 91

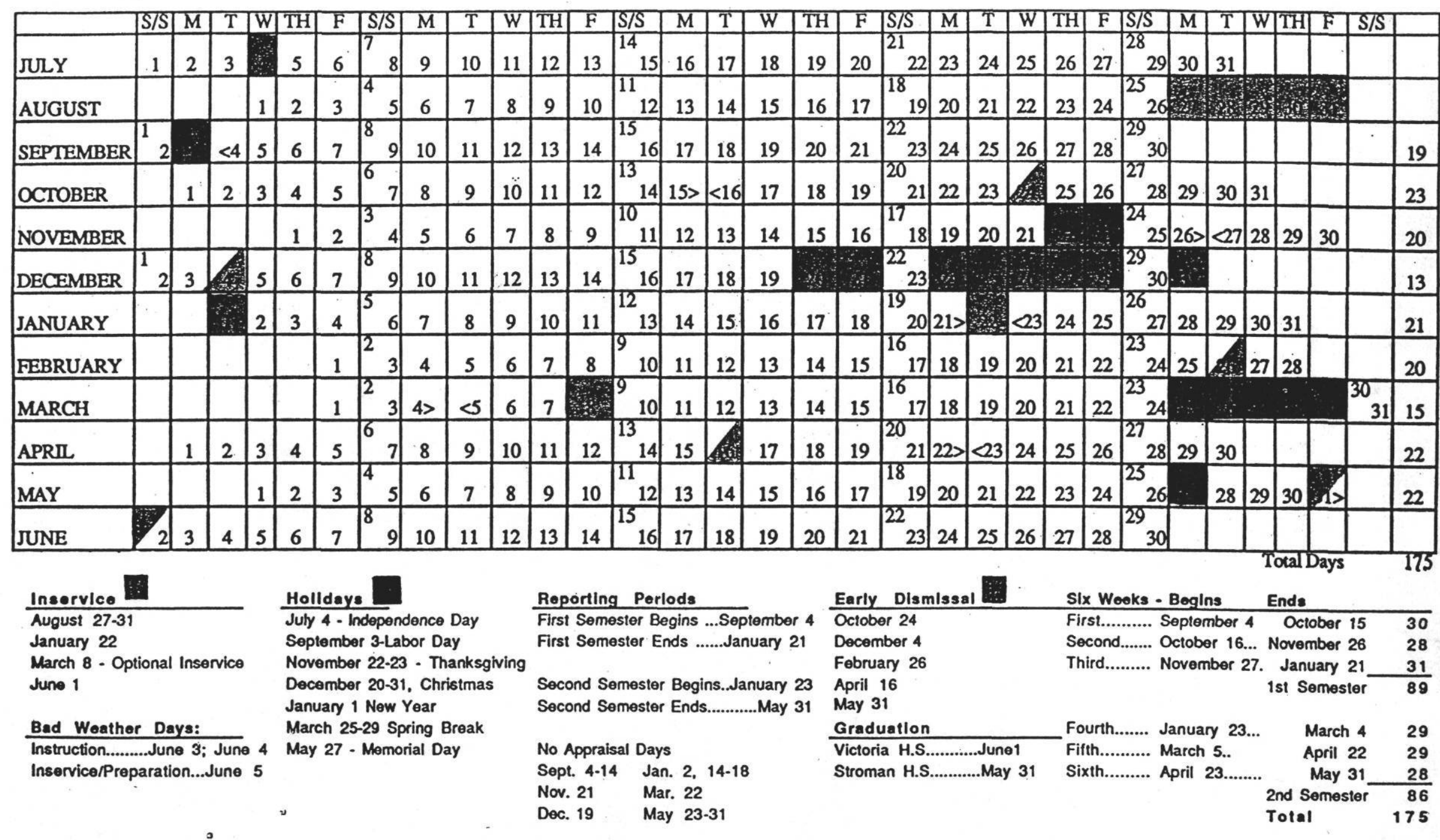


Tab B-2

Audit Technical Analysis

B-18 


\section{ECRM DESCRIPTIONS AND CALCULATIONS}

Facility Name: All Schools

ECRM No.: 1

ECRM Name: Energy Management System

a. Summary

Kwh savings:

Demand savings:

MCF savings:

Cost savings:

Implementation cost:

Simple payback:

\author{
$1,583,682 \mathrm{Kwh} / \mathrm{yr}$ \\ $898 \mathrm{KW}-\mathrm{mo} / \mathrm{yr}$ \\ $3,850 \mathrm{MCF} / \mathrm{yr}$ \\ $\$ 95,254 \mathrm{Hr}$ \\ $\$ 380,980$ \\ 4.0 years
}

\section{b. Description}

On/Off and temperature control in all of the Victoria ISD schools addressed in this report are inadequate. Typically, on/off controls consist of a) 7-day timeclocks which are controlled manually, b) manual control at thermostats or wall switches, and c) programmable thermostats in a very few locations, installed in the last two years. The great majority of on/off control is performed manually, with the result that operating hours are excessive in every school. There is not a single school addressed in this report where on/off control for the majority of HVAC equipment is performed automatically.

Timeclock controls were installed many years ago and are not suited for the needs of the schools.

- There is no way to enforce rigorous hours of HVAC operation if the custodial staff has, access to all timeclocks. Even if the timeclocks were functioning with their trippers and the timeclock cabinet were locked, override timers on the face of the timeclock cabinets would allow custodians to turn on HVAC units. The custodians work typically until 9 PM. The natural human tendency is to keep the units on to maintain most comfortable working conditions. Custodial staffs have been instructed on several occasions by the VISD maintenance staff to turn off HVAC promptly after school. Without direct and continuous supervision, one cannot reasonably expect the custodial staff to do so. And they don't.

- The timeclocks offer little flexibility. They typically control multiple HVAC units on one circuit. Often, an entire bank of HVAC units operates when in fact not all are needed. Special events may at time be held outside of normal operating hours. The existing override timers also control banks of units, so -- if the timeclocks and override timers were even used -- more units would operate than necessary.

- There is no feedback with the timeclock system, such as space temperature or humidity readings, and actual operating status of the unit. In several cases, air conditioning takes place 24 hours per day in order to prevent humidity-related problems. Also, heating units may be left on overnight when weather is cold, maintaining temperatures at comfort conditions. Feedback information on space versus outdoor conditions could save a great deal of energy by reducing operating hours. 
Summer operation of HVAC systems is also excessive. Schools are cleaned over a period of several weeks during each summer. Depending upon school size, the number of people cleaning, whether summer school is held or not, and the type of cleaning projects taking place, the cleaning process can take up to 6 weeks or more. Often the cleaning crews will turn on air conditioning for entire schools or wings of schools, regardless of how many rooms are actually being cleaned, since the method of turning units on is to flip a master timeclock switch which turns on whole banks of units. Again, virtually all control is manual through thermostats or timeclock master trippers. In addition to air conditioning schools for personal comfort, the cleaning crew operates the air conditioning to speed up drying of floors and other surfaces cleaned. Also, some teachers start coming to school by mid August. Typically, air conditioning throughout an entire school is again turned on, even though the number of teachers occupying the school is very small.

Temperature controls are virtually all open to occupant adjustment. The number of locking thermostats in all schools addressed in this report can be counted on one hand, and some of those are not locked. Typical settings are in the low 70's (deg F).

Even the programmable thermostats of the most recently installed HVAC units offer less than ideal control. The units inspected were programmed for $6 \mathrm{AM}$ to $6 \mathrm{PM}$ operation. While this schedule covers most occupancy demands, it is generally excessive. Neither teachers nor staff reprogram the thermostats as their occupancy needs differ.

Though the quantity of timeclocks and HVAC units may vary by school, the control methodology described above is typical of all the schools in this report. Controls in each school are addressed individually below. A summary of On/Off times follows (as determined by interviews with custodial staffs), starting on page 80 .

\section{Aloe Elementary}

There are four timeclocks located in a small janitorial room in the main wing. Each is a 7-day timeclock. Clock \#1 controls the library unit; \#2 the kitchen, \#3 the offices and classrooms, and \#4 the cafeteria units. There are override toggle switches in the face of the timeclock cabinet, one for each timeclock. However, as the timeclocks are not used as originally intended, the overrides are useless. On each timeclock, on/off trippers have been removed, and the custodial staff uses the master on/off tripper to control units. All units are tumed on manually by custodians at about 6:30 - 7:00 AM. The custodial staff works after school until 9 PM, and turns the units off when they leave.

In the 3rd/4th grade wing and the kindergarten wing, programmable thermostats have been installed. On/off times are $6 \mathrm{AM}$ to $6 \mathrm{PM}$, Monday through Friday.

\section{De Leon Elementary}

There are two timeclock stations in the school. The first station, located behind the library, has four 7-day timeclocks. The second station, located in an electrical room in the south classroom wing, has three 7-day timeclocks. There is an override toggle switch for each timeclock. These seven timeclocks control the seven rooftop HVAC units installed with the original school. HVAC units $8-11$ were added with the new classroom addition. They are controlled directly from individual room thermostats, not by timeclock.

All units are controlled manually by the custodial staff using the timeclock master on/off tripper, and room thermostats. Operating hours are from 6 AM until 8 PM. 


\section{TECHNICAL ANALYSIS OF FACILITY}

\section{Dudley Elementary}

There are three 7-day timeclocks located in the electrical room across the hall from the cafeteria. The first controls classroom and office units, the second the kitchen, and the third the cafeteria. All units are controlled manually by the custodial staff using the timeclock master on/off tripper. On/off hours are typically 7 AM to 7 PM, Monday through Friday.

\section{Hopkins Elementary}

There are four rooms which contain timeclocks at Hopkins. The main mechanical room has four 7-day timeclocks, controlling direct expansion units for 1) the office area, 2) the library, 3 ) the kitchen, and 4) the cafeteria. There is a single 7-day timeclock in the north wing, one in the south wing, and one in the middle wing. Each controls HVAC fan-coil units and chillers/pump for their respective wing. Most or all trippers have been removed from all timeclocks, and all are operated manually.

All units are turned on manually by custodians at about 6:00 AM. The custodial staff works after school until 9 PM, and turns the units off when they leave.

\section{Howell Intermediate}

There is a main control panel at Howell Intermediate located in the main mechanical room. Toggle switches are located in the face of the panel for controlling virtually all HVAC units in the school. When the custodian arrives at 6:30 AM, he turns on all HVAC units via the toggle switches, and the chiller if necessary. He always turns on the boiler, no matter what the weather conditions, since the HVAC system at Howell is reheat. Another custodian turns off HVAC equipment around 7 PM.

In summer, the same procedure is followed for the approximately six weeks cleaning period.

\section{$\underline{\text { Juan Linn Elementary }}$}

All HVAC units installed with the 1986 addition are controlled by programmable thermostats. Programmed on/off times are $6 \mathrm{AM}$ on, and $6 \mathrm{PM}$ off, Monday through Friday. The one exception is the library unit. It has a programmable thermostat, but the unit remains in operation continuously out of concern for mildew on library books. The two rooftop units over the original (east) classroom wing have been replaced recently, and are controlled by programmable thermostats also.

All fan-coil units and the chiller of the stand-alone 1951 addition are controlled by 7-day timeclock located by the east entrance to the building. All trippers to the clock have been removed. The janitor operates the master timeclock tripper to control HVAC.

In the main building, the custodian turns units on manually at the thermostats when she arrives at 6:45 AM, and another custodian turns units off around 8 PM.

Summer school is held in Juan Linn for six weeks. Again, custodians turn equipment on/off manually. However, most units are turned off earlier in the day as compared to the regular school year. 


\section{TECHNICAL ANALXSIS OF FACILITY}

\section{O'Connor Elementary}

Two rooms contain 7-day timeclocks at O'Connor, one in the north wing and one in the south. All units are turned on manually by custodians at about 6:30 AM, and off at around 8:00 PM. The east wing addition units are controlled manually by custodians via their thermostats.

There are two locking thermostats in the north wing, but neither was locked when seen.

\section{Shields Elementary}

The majority of floor area in Shields is served by hydronic fan-coil units. Control is the same as in all other elementaries: 7-day timeclocks exist, but custodial staff uses only the master trippers to turn units on and off when they arrive and depart. Units are turned on around 7 AM, and off about 6:30 PM.

\section{Stanly Elementary}

Control of HVAC units in Stanly is identical to O'Connor. The two schools originally had identical floor and HVAC plans. Timeclocks are located in exactly the same rooms as in O'Connor.

\section{Stroman High School}

Control of HVAC units at Stroman requires very intensive footwork. The custodian makes rounds to every air handling unit, most fan-coil units, many direct expansion units, and the chiller/boiler/auxiliary equipment each morning around 6:45 $\mathrm{AM}$, where he turns equipment on. Another custodian makes a similar round at about 8:30 PM to turn equipment off.

The kitchen staff turns kitchen HVAC on and off. The coaching staff turns athletic building HVAC off, and the custodial staff turns it back on in the morning, though often the coaching staff forgets to turn units off.

A small (46 ton) reciprocating chiller is located adjacent to the four story Unit A. This chiller is piped to serve only Unit A. During summer and after school hours, parts of Unit A (which contains administrative offices) are the only occupied portions of the school. At 4:30 PM during the school year, the absorption chiller is shut down and the reciprocating chiller is turned on, and continues to operate until 9 PM. In summer, the reciprocating chiller is tumed on 7:00 AM, and off at 6:00 PM, unless the main chiller is operating.

Direct expansion split systems serving the Band hall are thermostatically controlled, but are left in operation continuously, summer and winter. If the main air handler serving Band has been shut off and indoor temperature starts to rise, the DX units will maintain humidity and temperature conditions. These backup DX units were installed out of concern for humidityrelated problems with Band instruments.

Summer cleaning of the high school takes about 5 to 6 weeks. During this time, the main absorption chiller operates every day, and virtually the entire school is cooled. Cleaning is finished by mid- to late-July, and only the reciprocating chiller operates after that. 


\section{Victoria High School}

Victoria High is another school requiring intensive footwork in turning HVAC systems on and off. The VHS campus contains numerous buildings spread out over a wide geographical area. The maintenance man starts his round at $7 \mathrm{AM}$ to all mechanical rooms and thermostats/wall switches, turning on equipment. As at Stroman, the coaching staff is responsible for turning off some athletic building HVAC equipment (though they often forget) and the maintenance man turns it back on in the morning.

There are two rooftop units over the Learning Resource Center. During the regular school year, these operate from 7:15 AM until 4 PM. During summer, one of the units is shut down, but the other remains in operation 24 hours per day to prevent problems with mildew. Starting in September, HVAC for the boys dressing room is left on continuously until cold weather hits, so as to reduce odor problems which are worsened by heat and humidity.

Summer school is held in the Academic Wing of VHS, and occasionally in the main wing. The Academic Wing is served by the absorption chiller. The chiller is turned on at 6:30 AM, and off at 1:30 PM. The fan-coil units served by the chiller remain in operation continuously, both summer and winter. The on/off switches for them are located inside the units.

Fan-coil units for the main building are controlled by toggle switches mounted on the wall of each classroom. Teachers are supposed to turn these units off as they leave each day, and the maintenance staff turns them back on in the morning. However, as often as not, the fan-coil units are left on at night.

This ECRM calls for the installation of a direct digital control-based energy management system (EMS) for each school addressed in this report. The EMS will control all HVAC equipment, measure exterior and interior space temperatures, and measure humidity in one or two critical locations within each school. The EMS will have no override timers that custodial staffs can activate. Operating hours of all HVAC units will be determined by the maintenance staff, and controlled by that staff from its central headquarters via modem. (Floor plans on pages 27 through 37 show locations of the units to be controlled, and the proposed locations of new DDC controllers). 
Tab B-3

Monitoring Diagrams 


\section{THERMAL MONITORING DIAGRAM \\ LEGEND VISD - VICTORIA HS}

$K=K W H$ CHANNE

$A=A N A L O G$ CHANNE

$P C=P U M P E D$ CONDENSATE

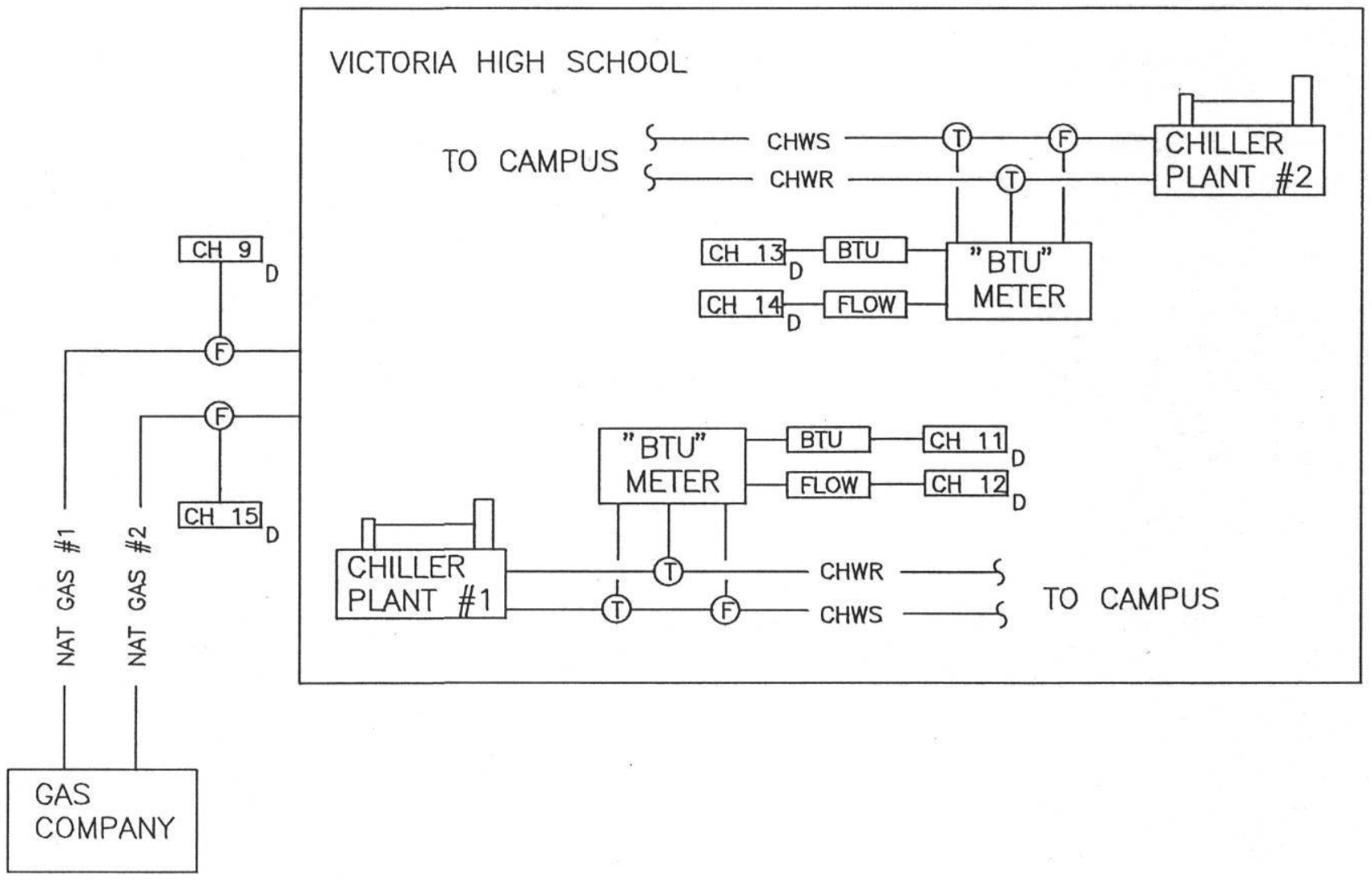

VISD/VICTORIA HS - SITE 127 


\section{ELECTRICAL MONITORING DIAGRAM}

LEGEND

$K=K W H$ CHANNE

$A=A N A L O G$ CHANNEI

VISD - VICTORIA HS

$D=$ DIGTAL CHANNEL

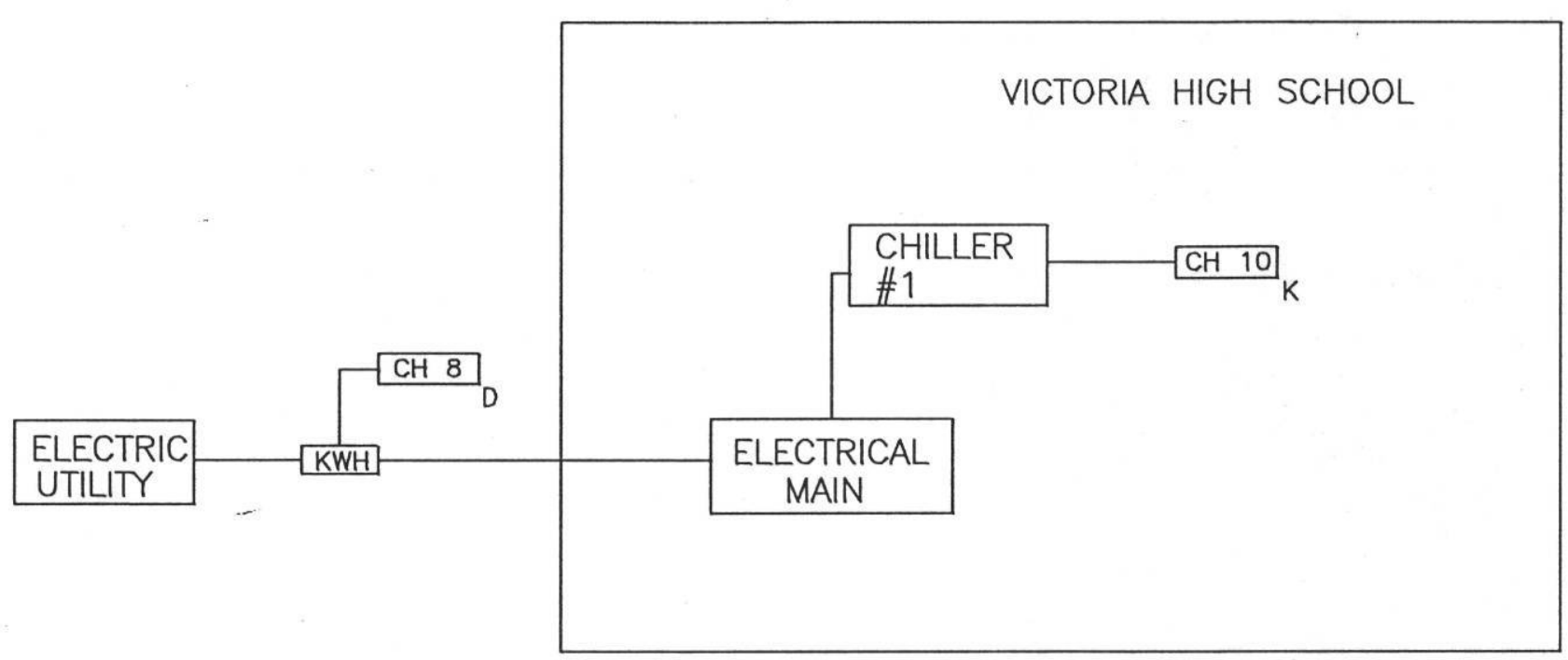

VISD/VICTORIA HS - SITE 127 
Tab B-4

Average Hourly Data \& Related Statistics 

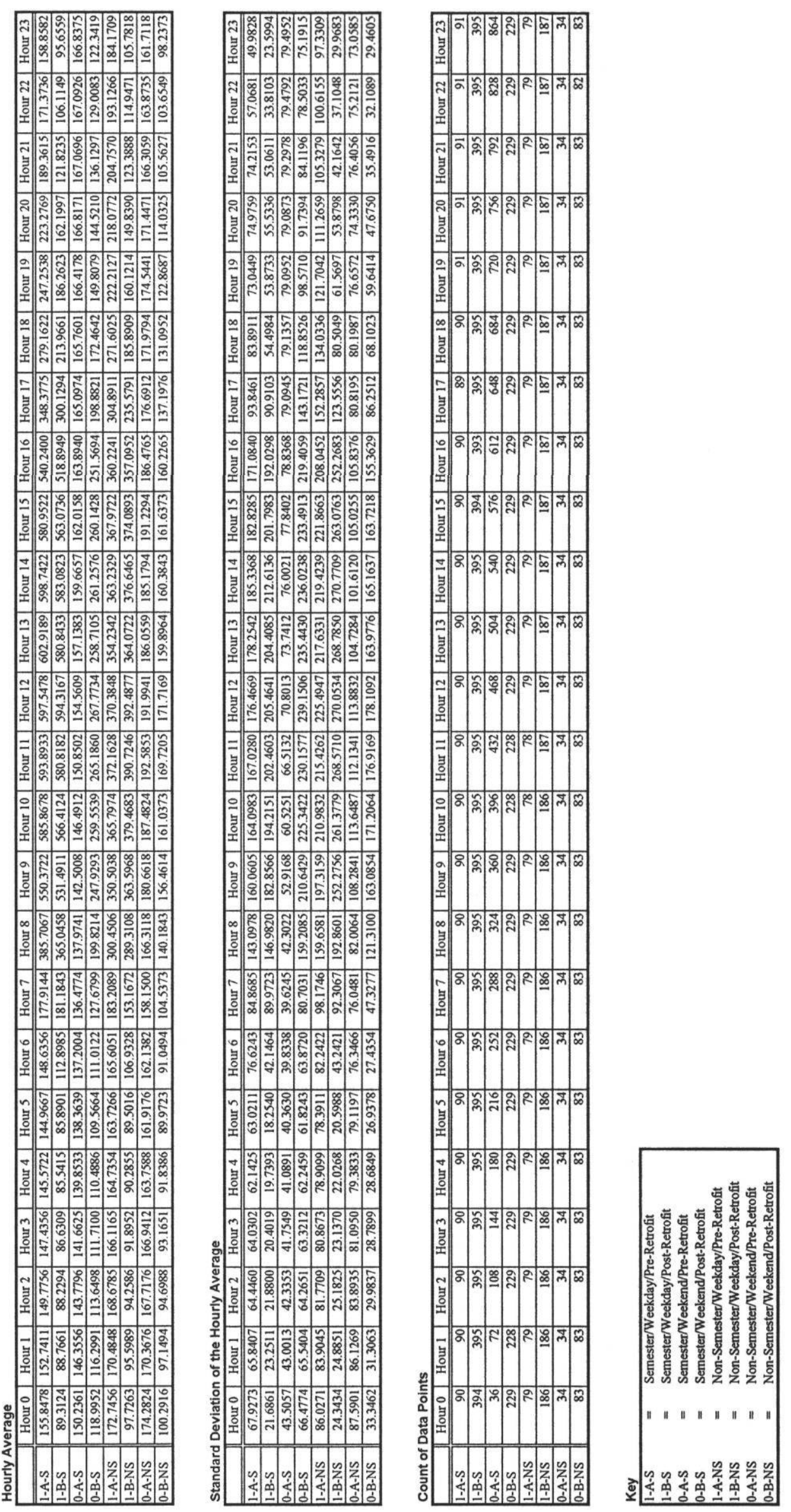
Tab B-5

MECR Plots 


\section{Whole-Building Electric}

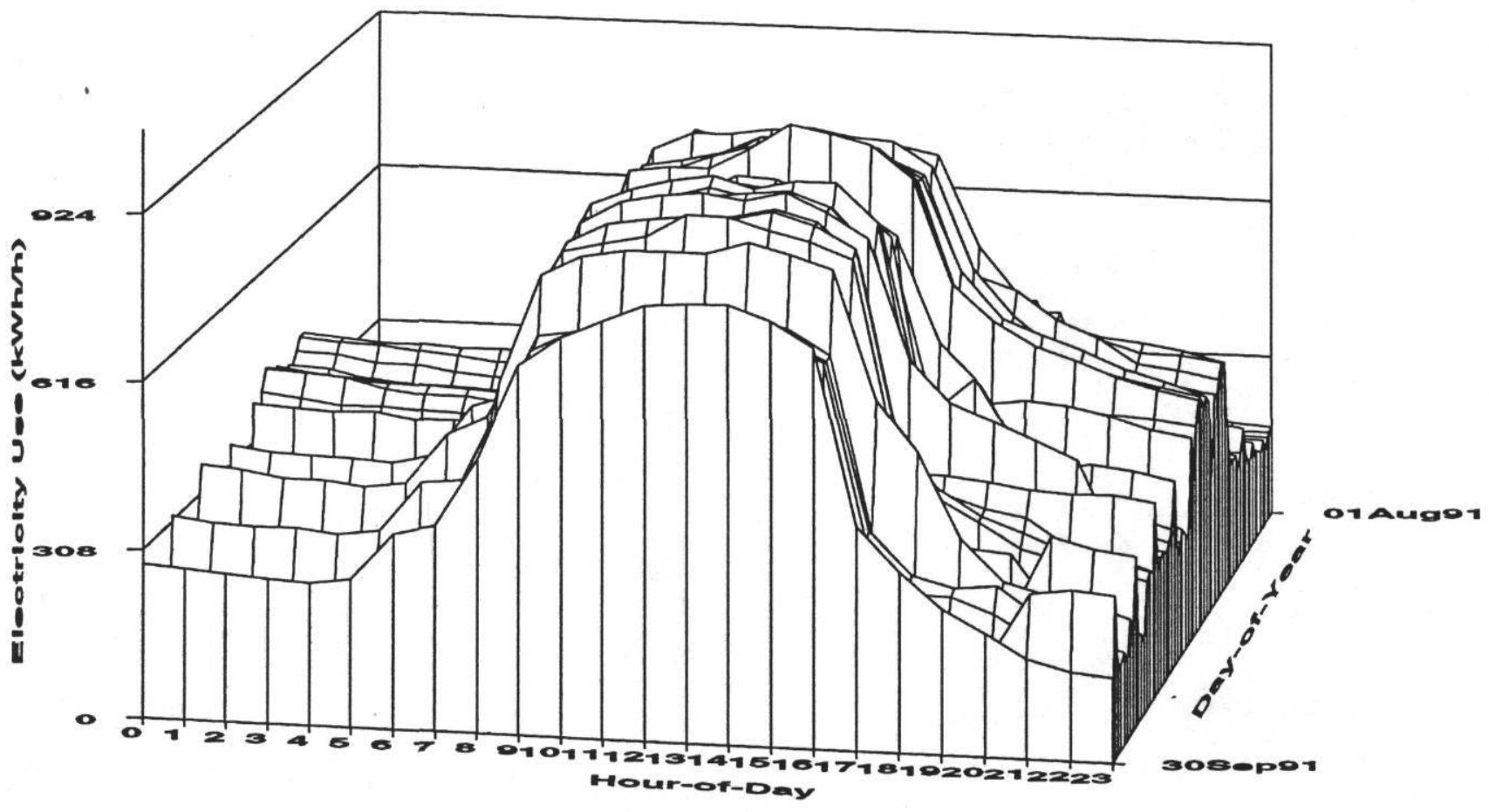

Whole-Building Electric

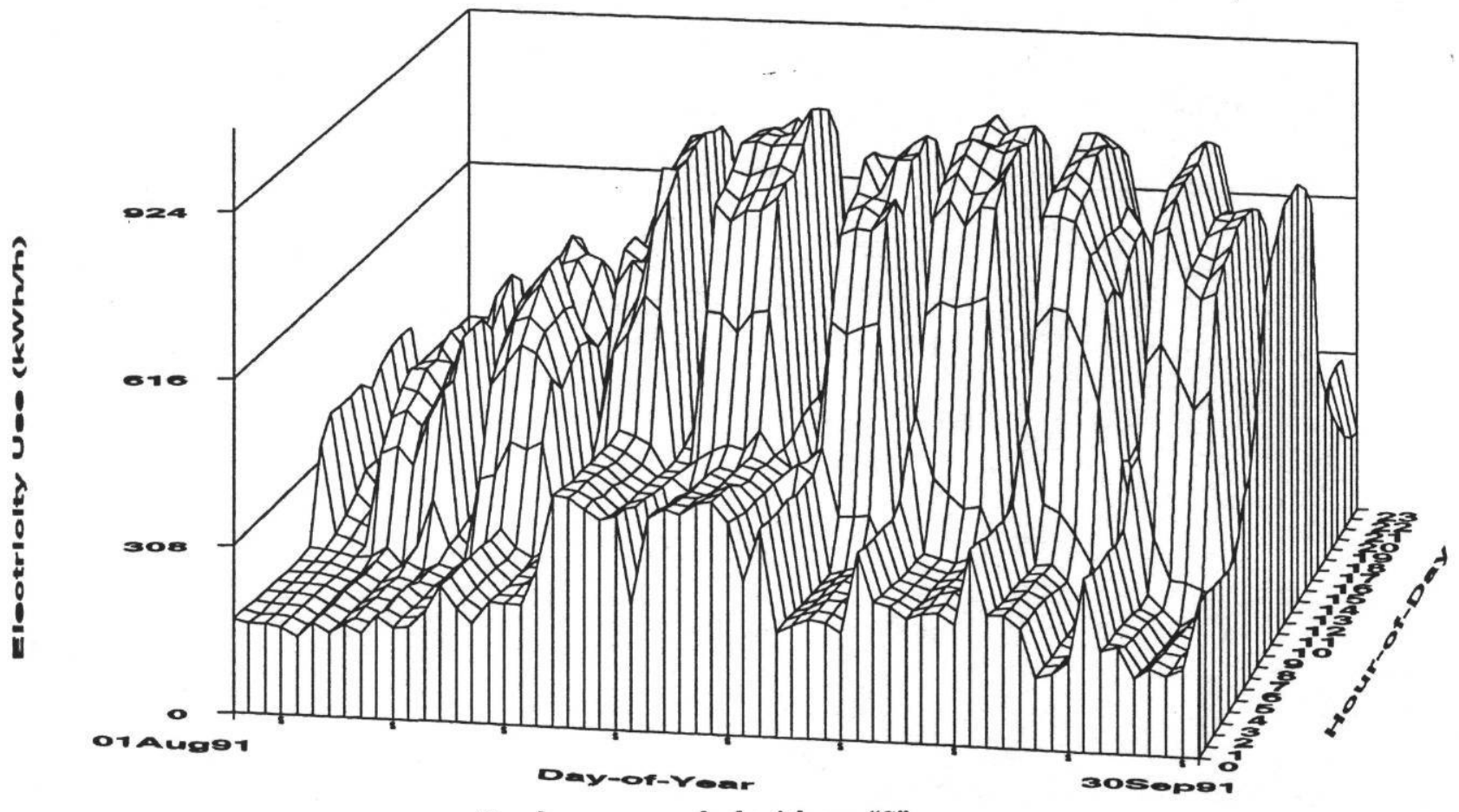

Sundays are marked with an "S"

Victoria High School - Victoria ISD - September 1991

Texas Governor's Energy Office LoanSTAR Monitoring \& Analysis Program
Monthly Energy Consumption Report (C)

B-30
Energy Systems Lab

Texes A\&M University 
Whole-Building Electric

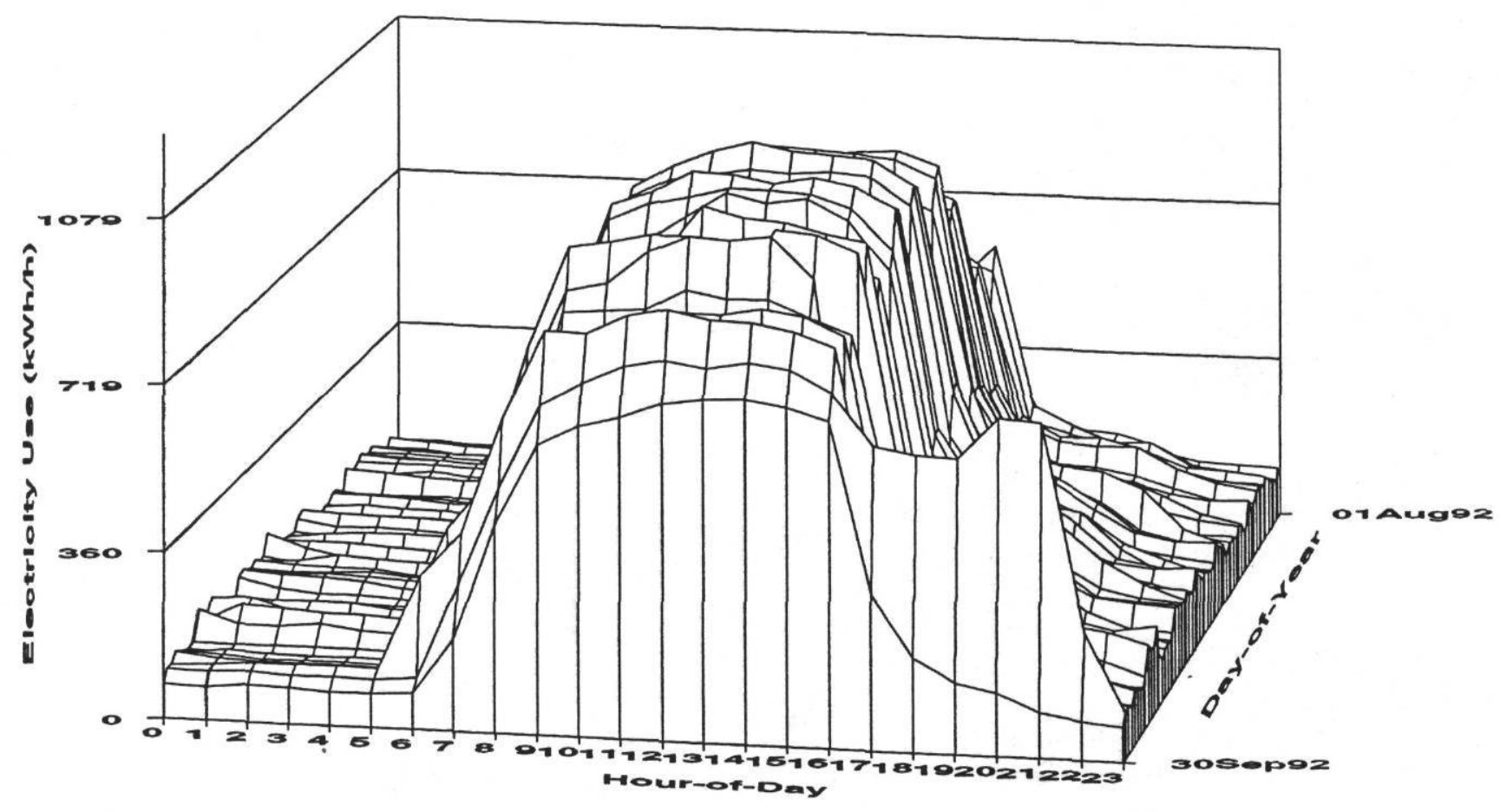

Whole-Building Electric

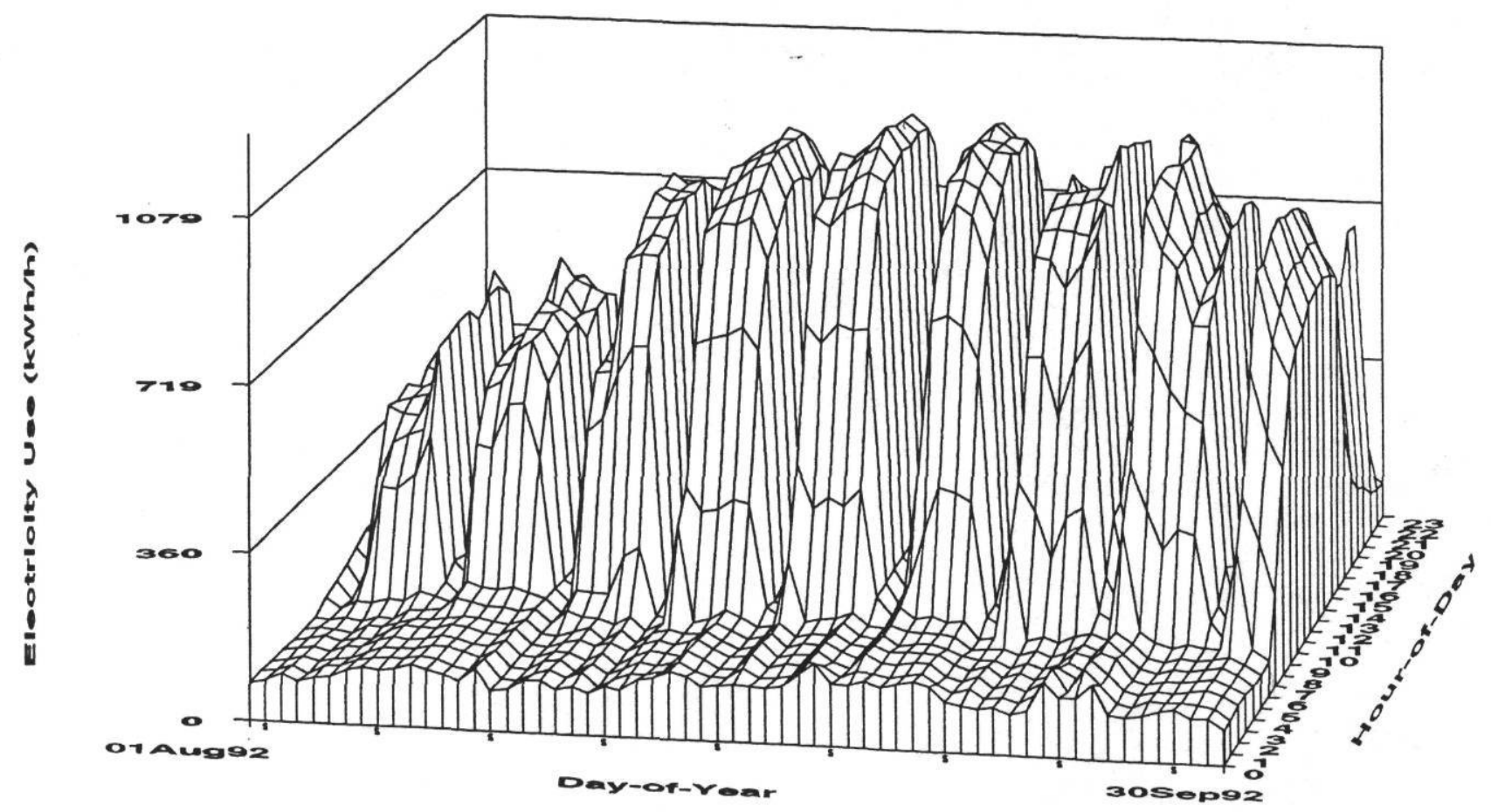

Sundays are marked with an "S"

Victoria High School - Victoria ISD - September 1992 
Whole-Building Electric

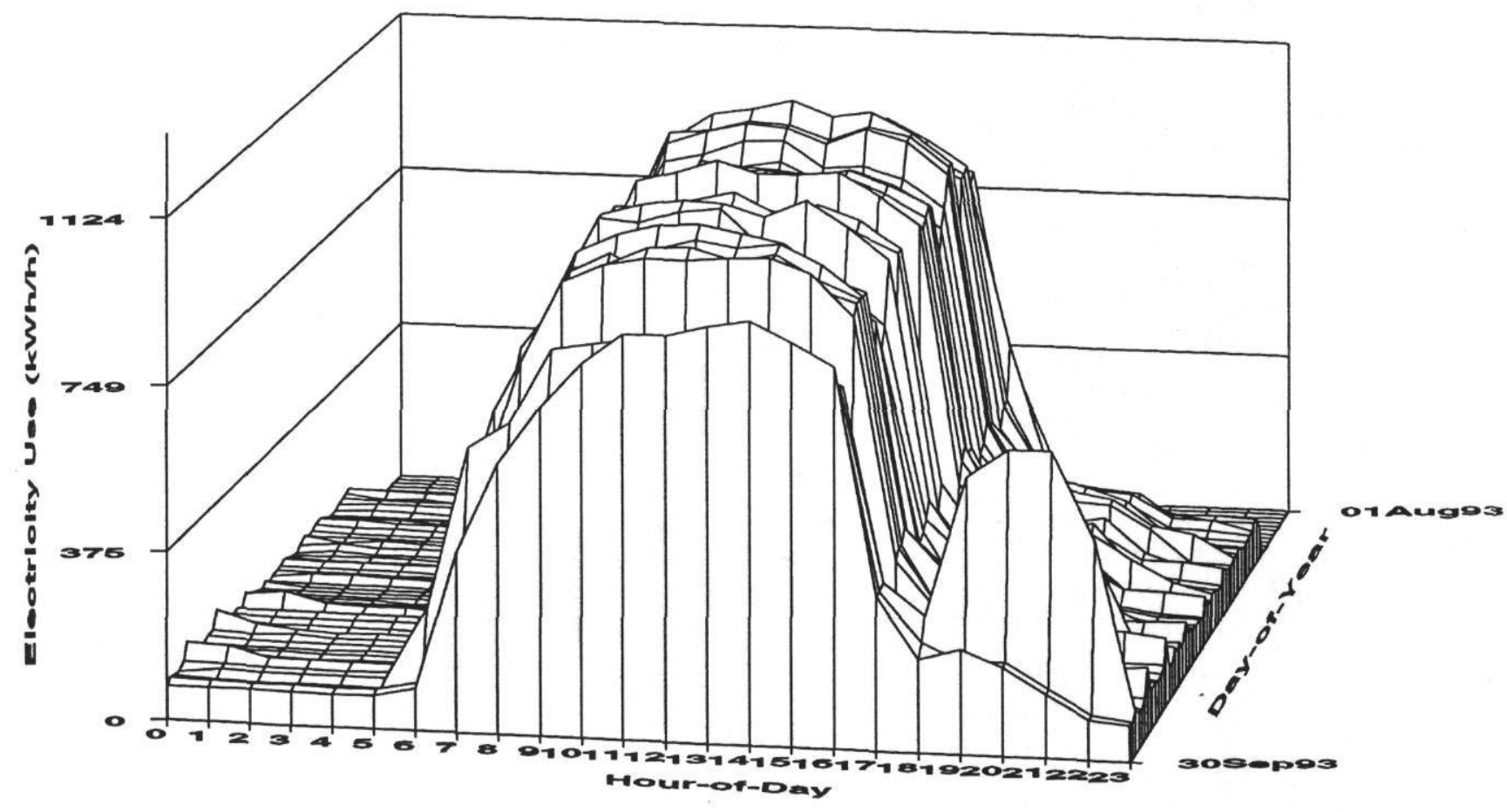

Whole-Building Electric

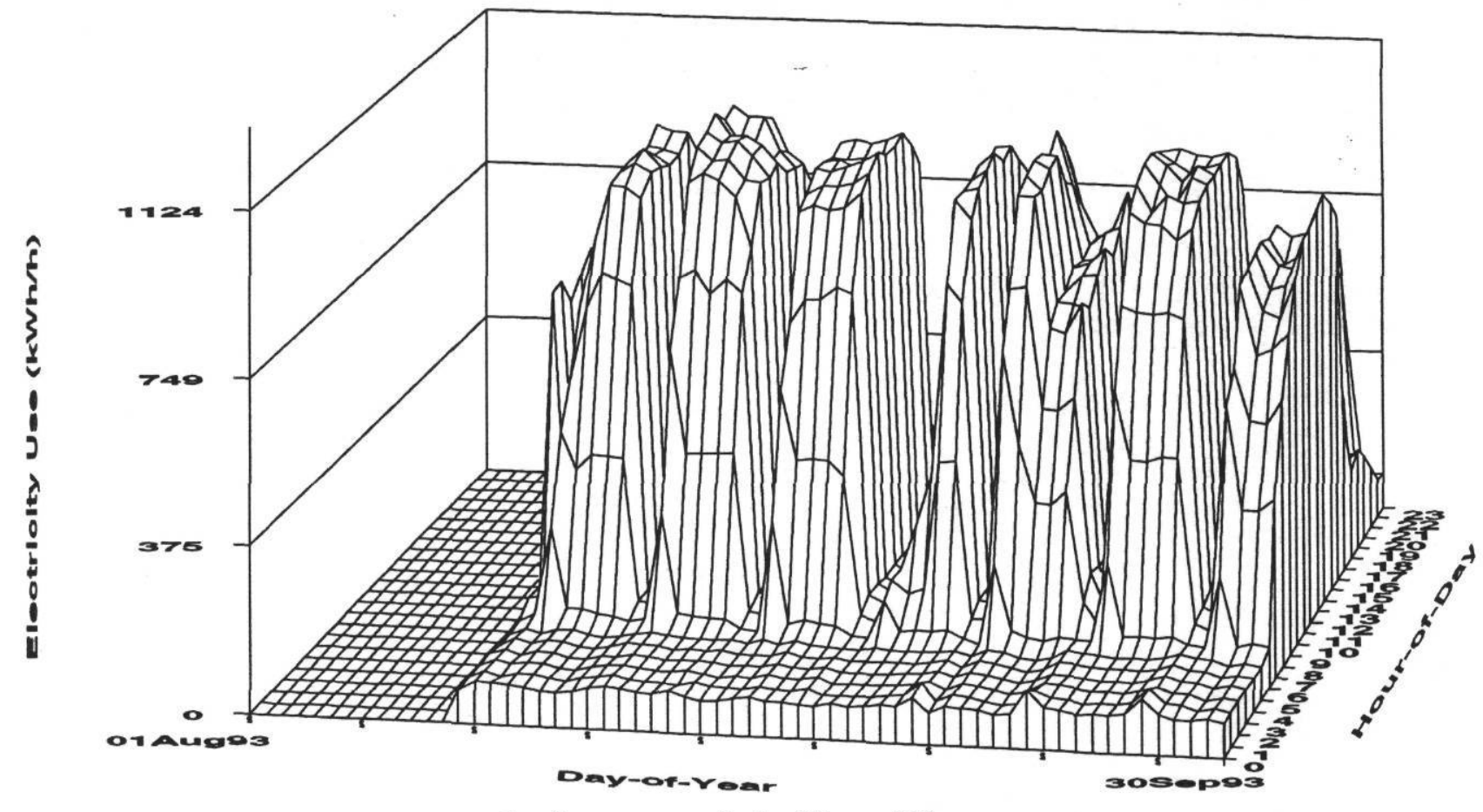

Sundays are marked with an "S"

Victoria High School - Victoria ISD - September 1993 
Whole-Building Electric

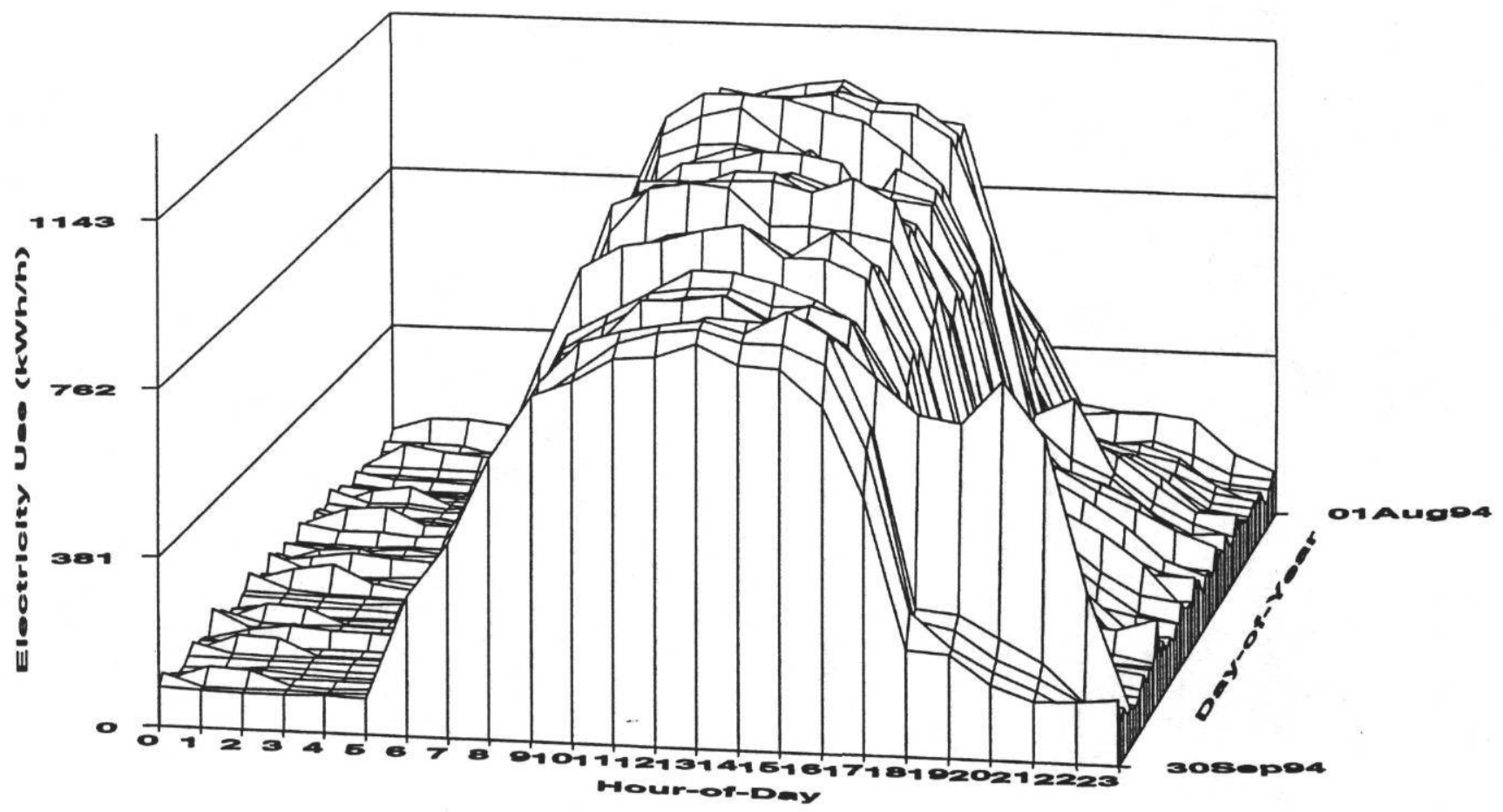

Whole-Building Electric

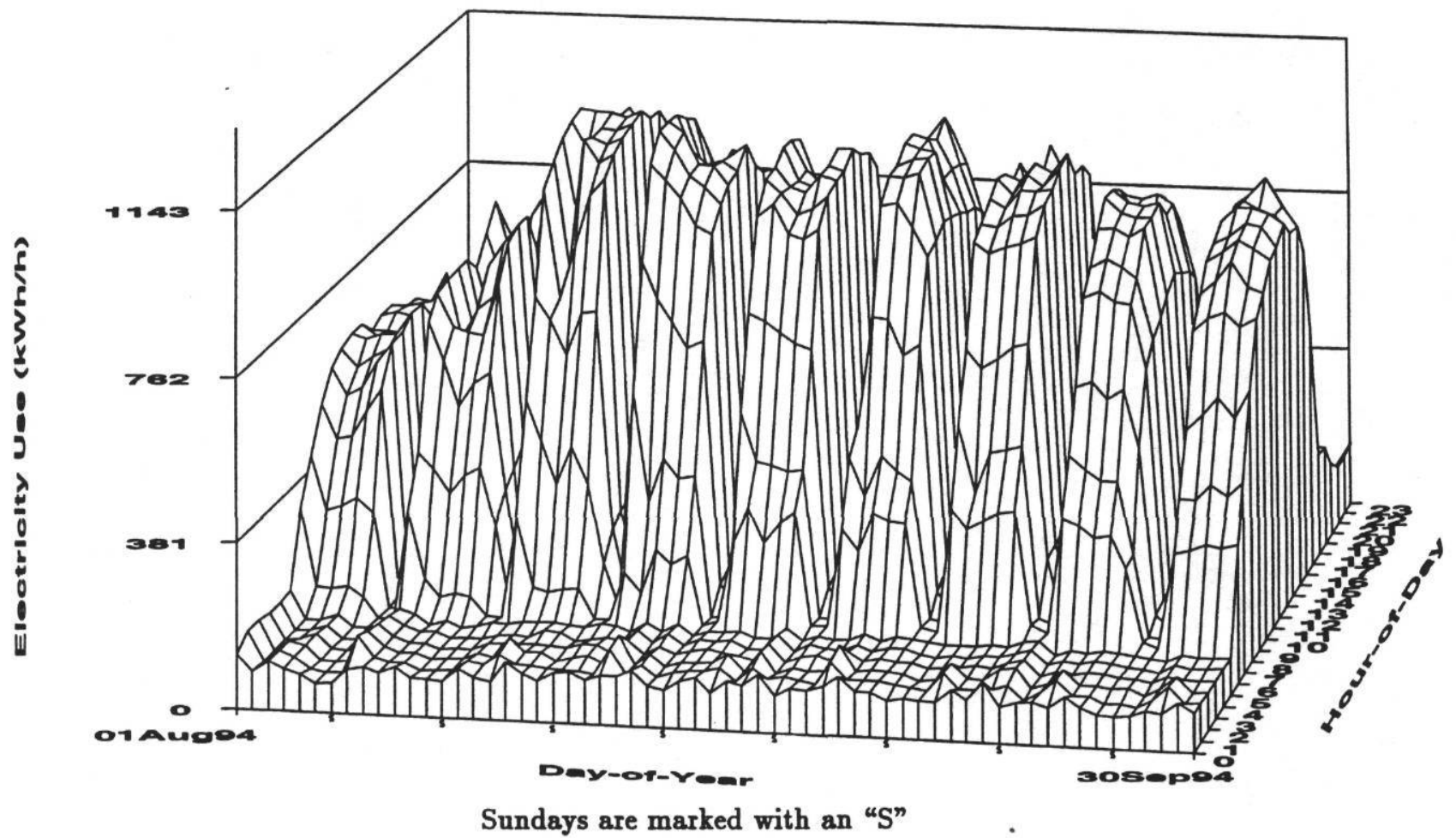

Victoria High School - Victoria ISD - September 1994 
Tab B-6

Data Summary Notebook Information 


\section{VICTORIA INDEPENDENT SCHOOL DISTRICT \\ Victoria High School}

Building Envelope:

- 257,000 sq.ft.

- Main Building: Two stories, brick slab, and grade construction with flat roof, 54,000 sq ft.

- Academic Building: same as Main Building, 60,700 sq ft.

- Field house (dressing room, 2-shop buildings, gymnasium, special education building, learning resource center, home economics building, a multi-purpose building with kitchen, cafeteria, band hall and choir room are all one story.), $142,300 \mathrm{sq} \mathrm{ft}$.

Building Schedule:

- 7 am to 4 pm (M-F)

Building's HVAC \& other equipment:

Main building:

- 17 rooftop units (4 - 7.5 tons)

- 70 fan-coil units each $0.17 \mathrm{hp}$

- 1-192 ton Trane centrifugal chiller

- 1 boiler

Academic Wing:

- 1-25\% O.A 7.5hp AHU

- $112-0.05 \mathrm{hp}$ fan-coil units and $6-0.17 \mathrm{hp}$ fan-coil units

- 1-182 ton York centrifugal chiller (replaced absorption chiller in Aug 91)

\section{Field House}

- boys dressing room: 8 rooftop units (2-5 tons and 4-7.5 tons).

- Vocational: rooftop unit

- Industrial Arts: 2 rooftop units (15 tons each).

- Gymnasium: 1 rooftop unit (3.0 tons) and 1 steam boiler.

- Home Economics: 1 rooftop unit (15 tons).

- Kitchen: 1 rooftop unit (7.5 tons).

- Cafeteria: 1 rooftop unit (7.5 tons).

- Band Hall: 3 rooftop units (1-10 tons and 1-5 tons).

- Choir: 3 rooftop units (1-5 tons).

- Special Education: rooftop unit (7.5 tons).

- Learning Resource Center: 2 rooftop units (15 tons each).

Auxillary Equipment:

- 2 CHWPs, 1 of $25 \mathrm{hp}, 1$ of $20 \mathrm{hp}$

- 2 CWPs, 1 of $25 \mathrm{hp}, 1$ of $15 \mathrm{hp}$

- 2 Cooling towers, 1 of $15 \mathrm{hp}, 1$ of $20 \mathrm{hp}$

- $1 \mathrm{HWP}$ of $5 \mathrm{hp}$

- 1 Brine pump

- 1 Refrigerant pump

- 2 Boilers

- 10 Exhaust fans (1/2 hp each)

Lighting:

- Mostly fluorescent (Total load $260 \mathrm{~kW}$ ).

Victoria High School - Victoria ISD - April 1994

\begin{tabular}{|c|c|c|}
\hline $\begin{array}{l}\text { Texas State Energy Conservation Office } \\
\text { LoanSTAR Monitoring \& Analysis Program }\end{array}$ & $\begin{array}{c}\text { Monthly Energy Consumption Report (C) } \\
\text { Version } 2.2 \\
\text { B-35 }\end{array}$ & $\begin{array}{r}\text { Energy Systems Lab } \\
\text { Texas A\&M University }\end{array}$ \\
\hline
\end{tabular}


HVAC Schedule:

- HVAC equipment is turned on manually at 6:00 a.m. and turned off at 8:00 p.m. on weekdays.

\section{Proposed Retrofits:}

- Energy Management System

- Replace Absorption chiller

Date of Retrofits:

- Replacement of absorption chiller was completed in August 1991, while work on the other retrofit was completed in January 1992. 


\section{Victoria High School (VHS) \\ W.B. Electric as W/sq.ft.}
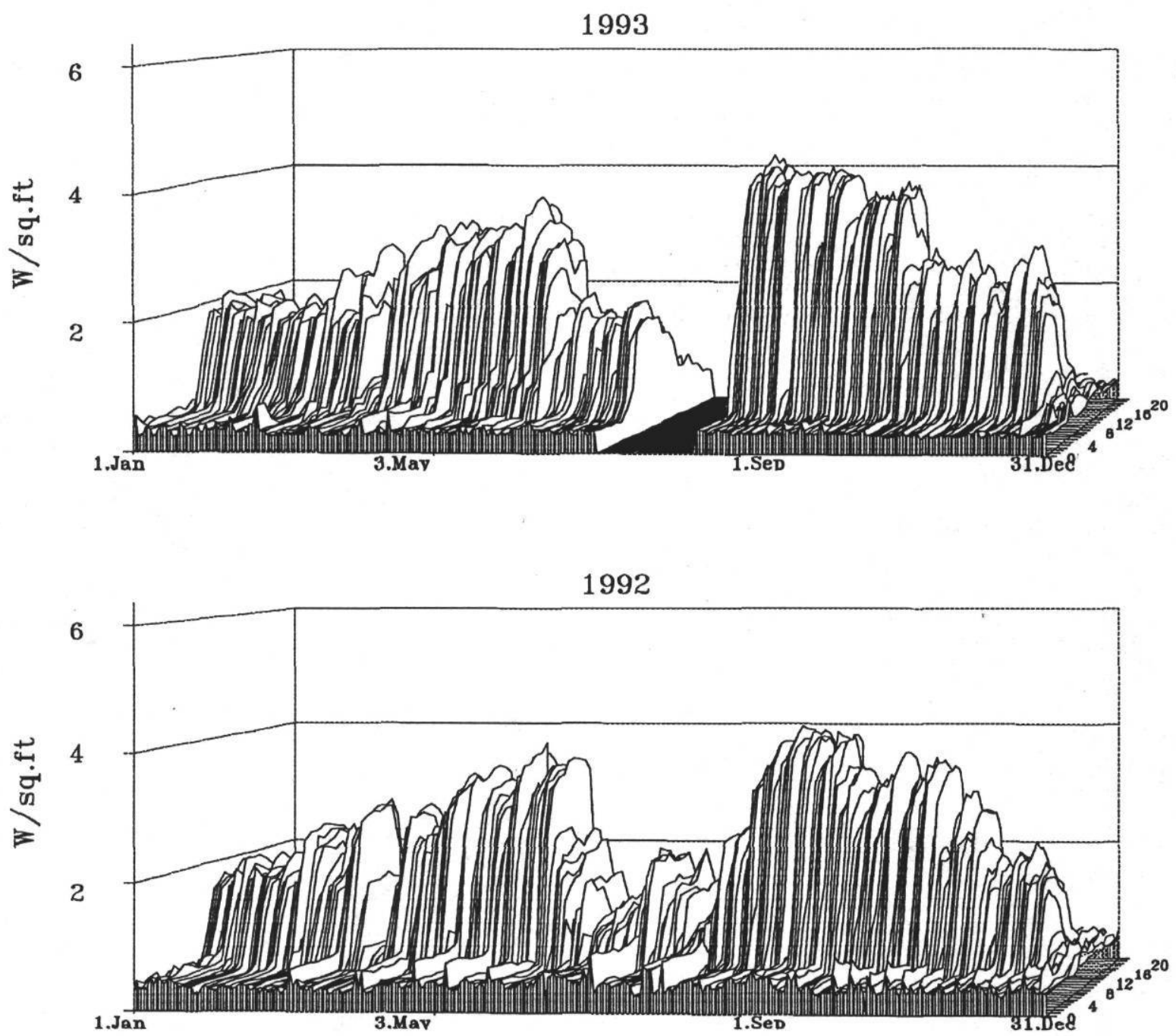

1991

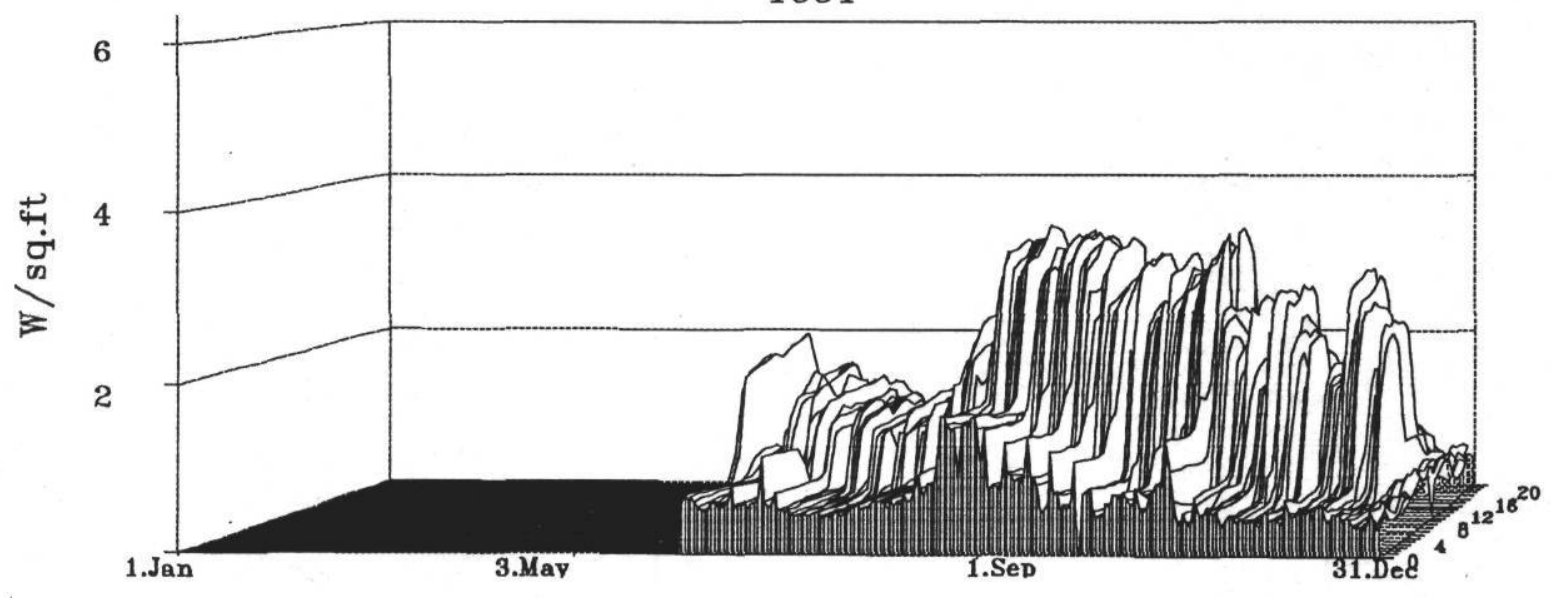




\section{Victoria High School (VHS) W.B. CHW as Btu/sq.ft.-h}
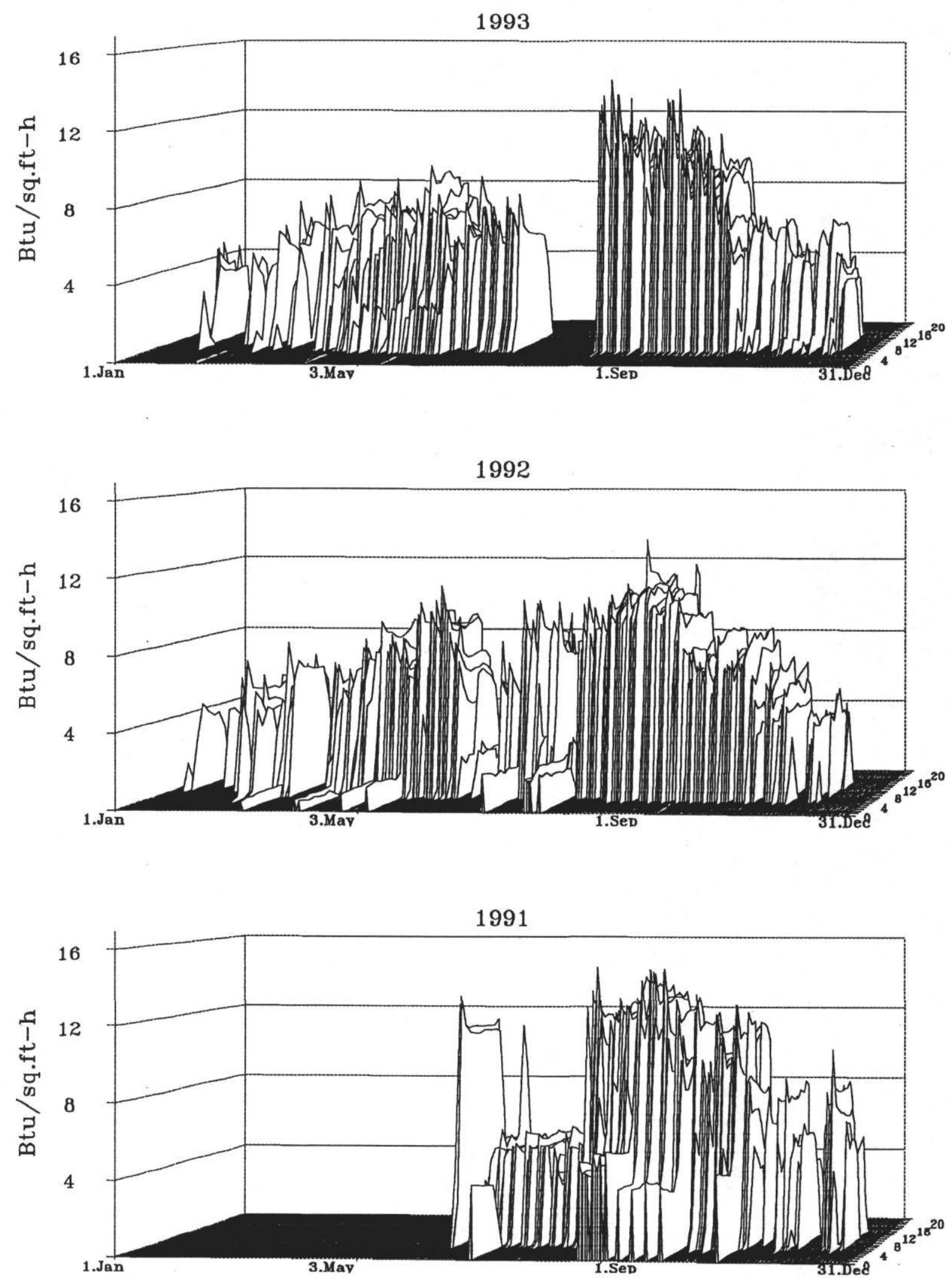

B-38 


\section{Victoria High School (VHS) \\ W.B. HW as Btu/sq.ft.-h}

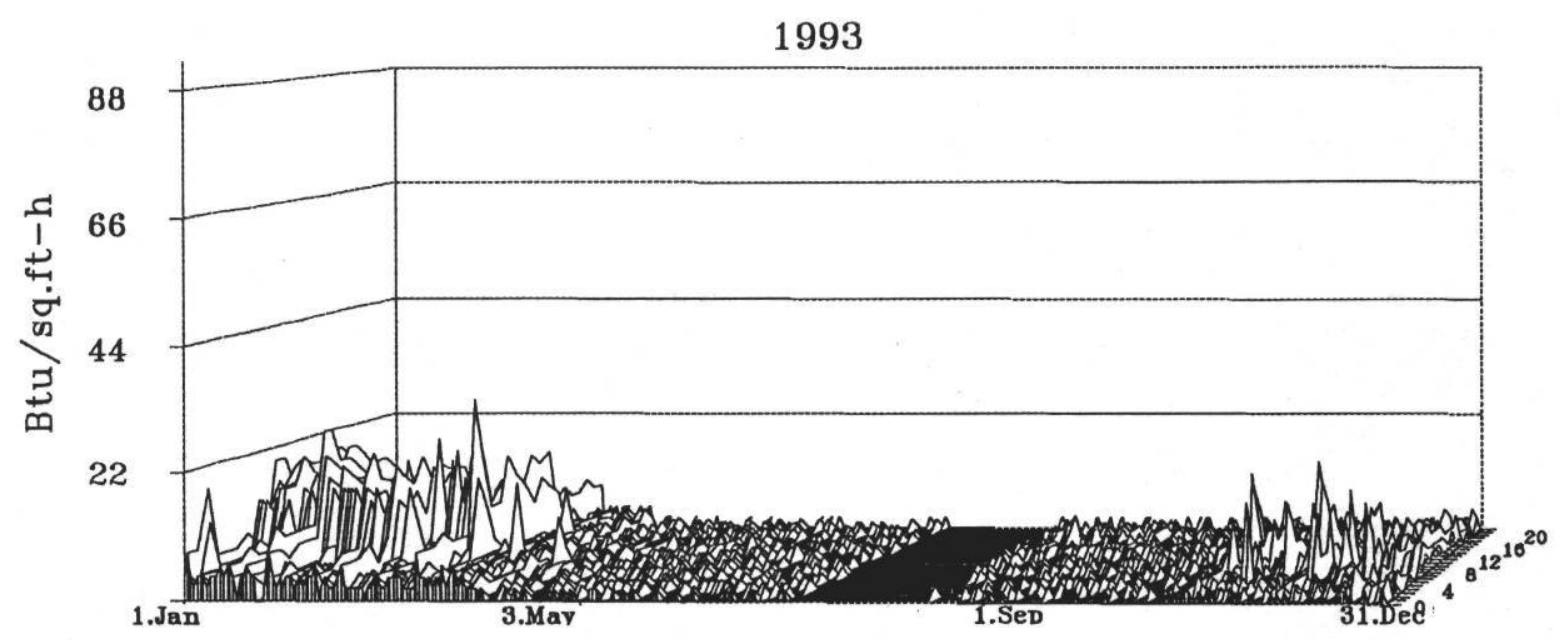

1992

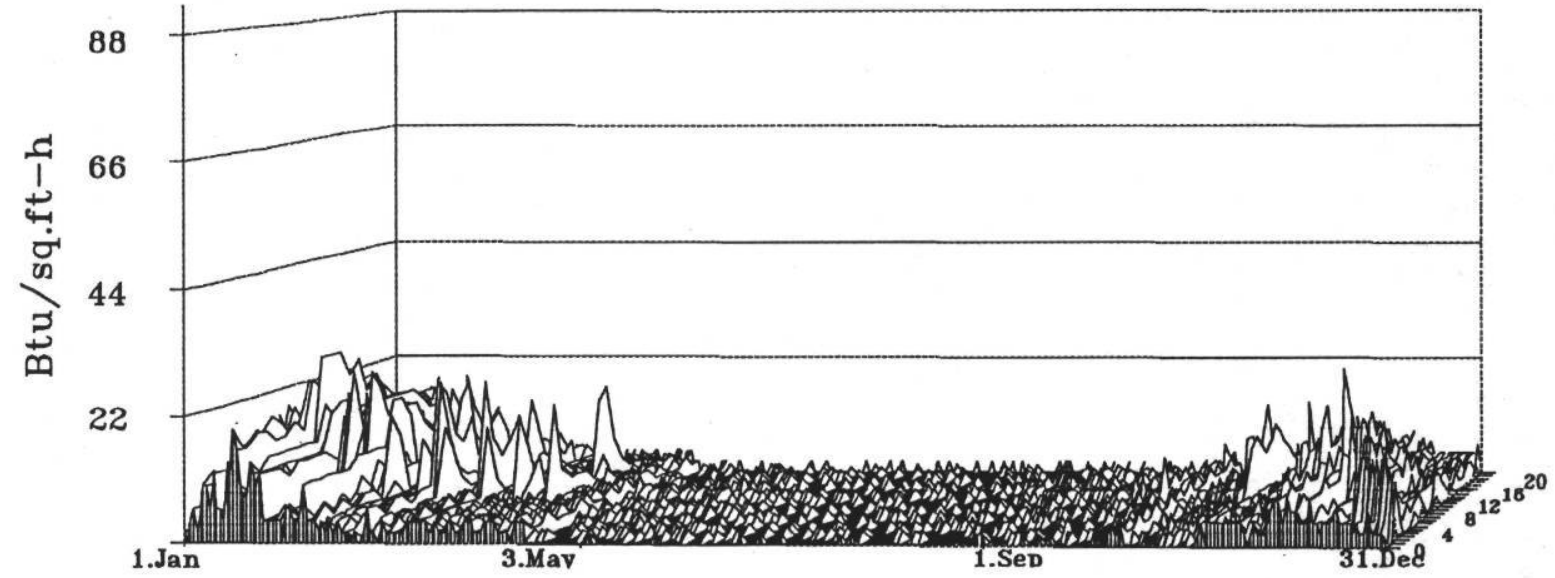

1991

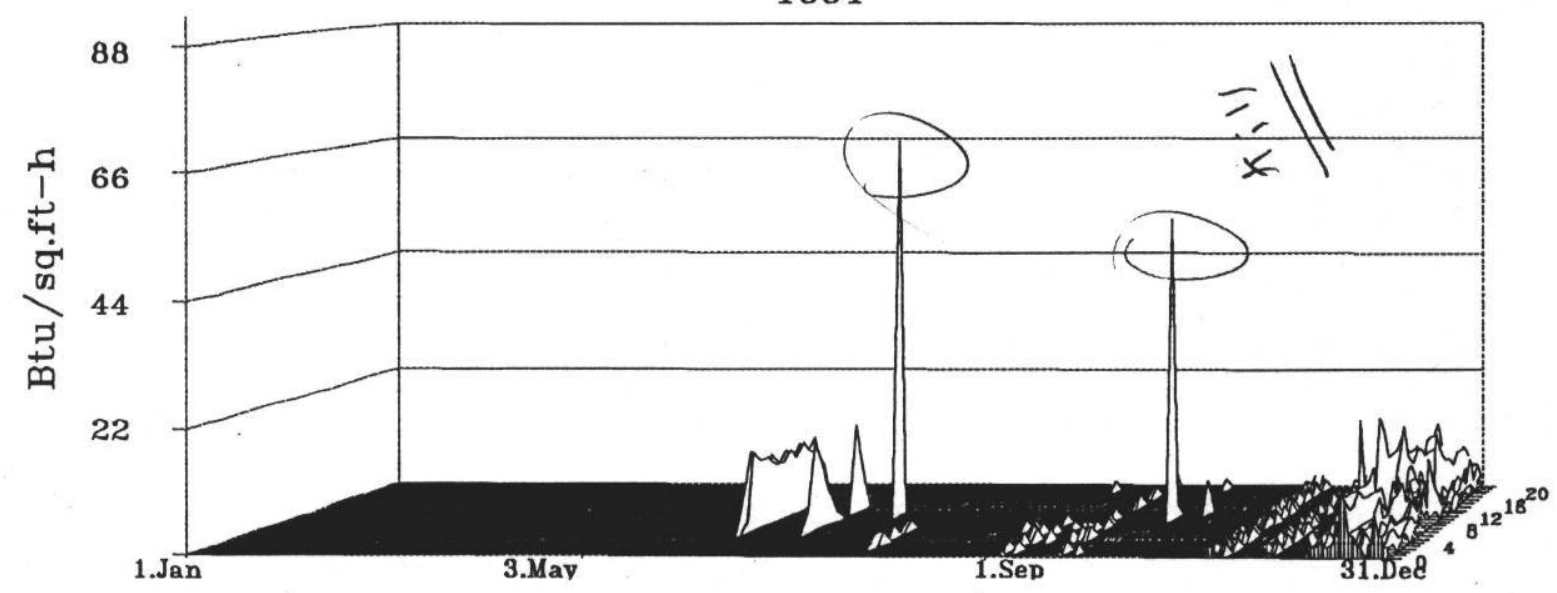

B-39 


\section{Victoria High School (VHS) \\ W.B. Electric as W/sq.ft.}
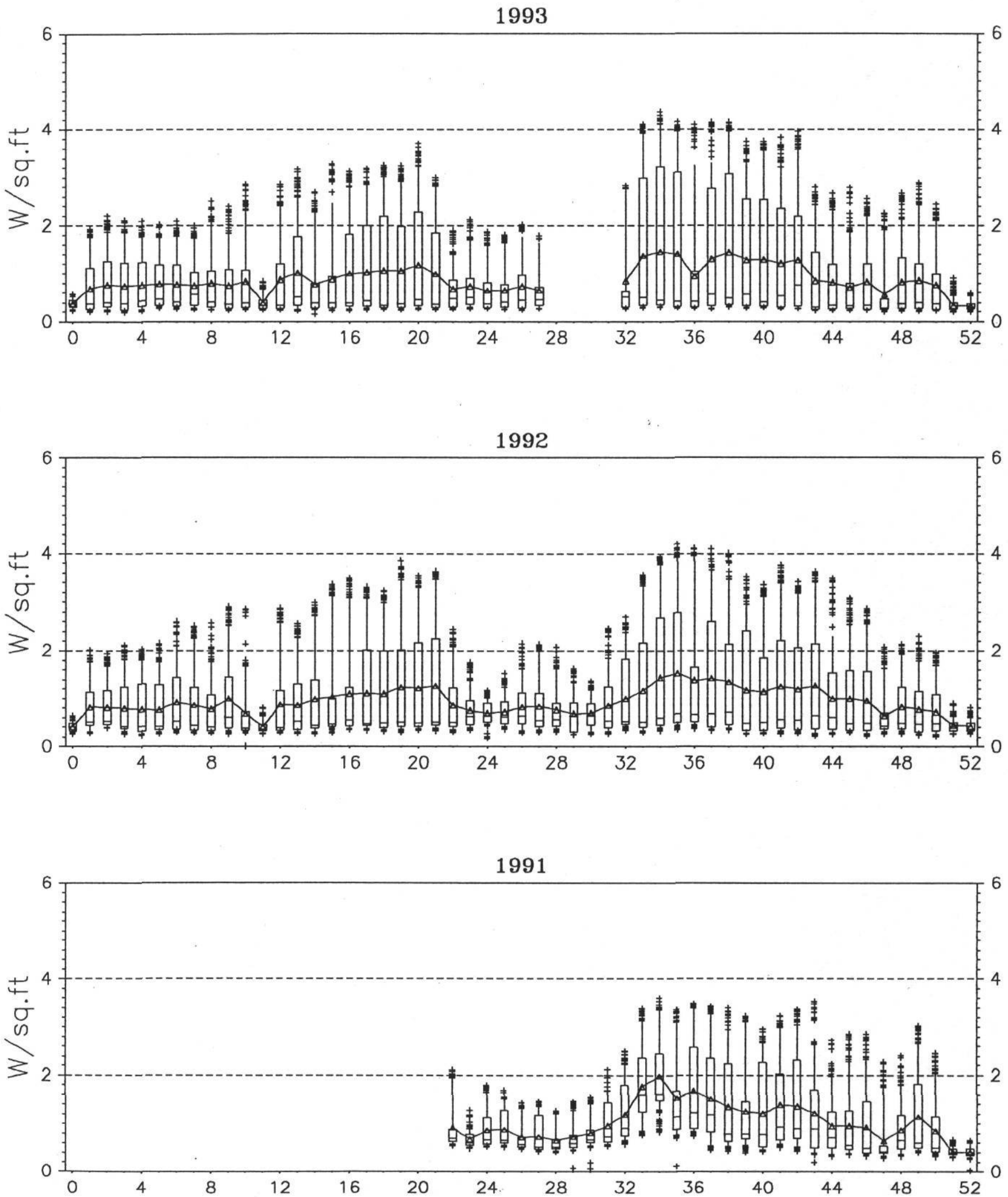


\section{Victoria High School (VHS) \\ W.B. CHW as Btu/sq.ft.-h}
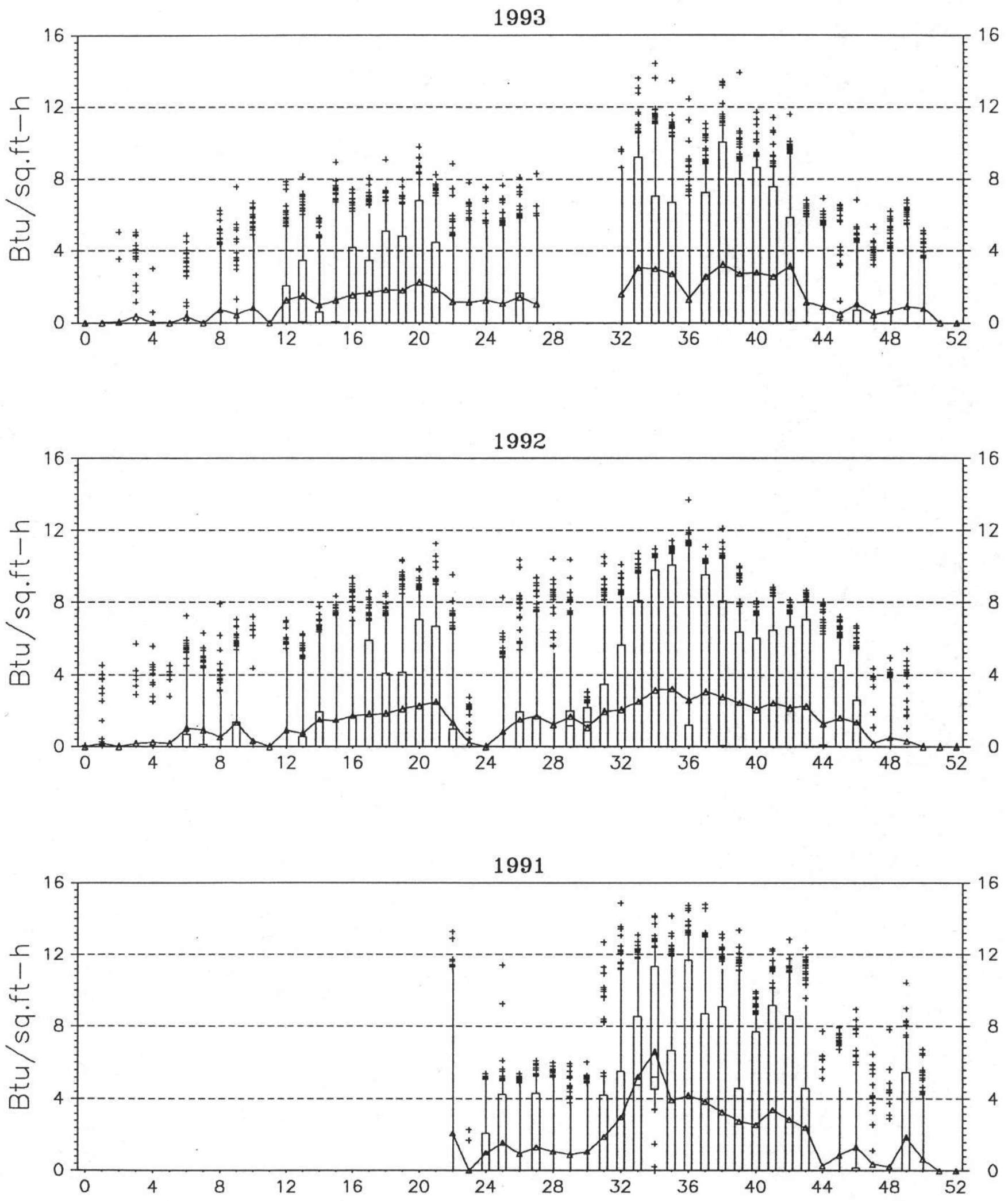


\section{Victoria High School (VHS) \\ W.B. HW as Btu/sq.ft.-h}
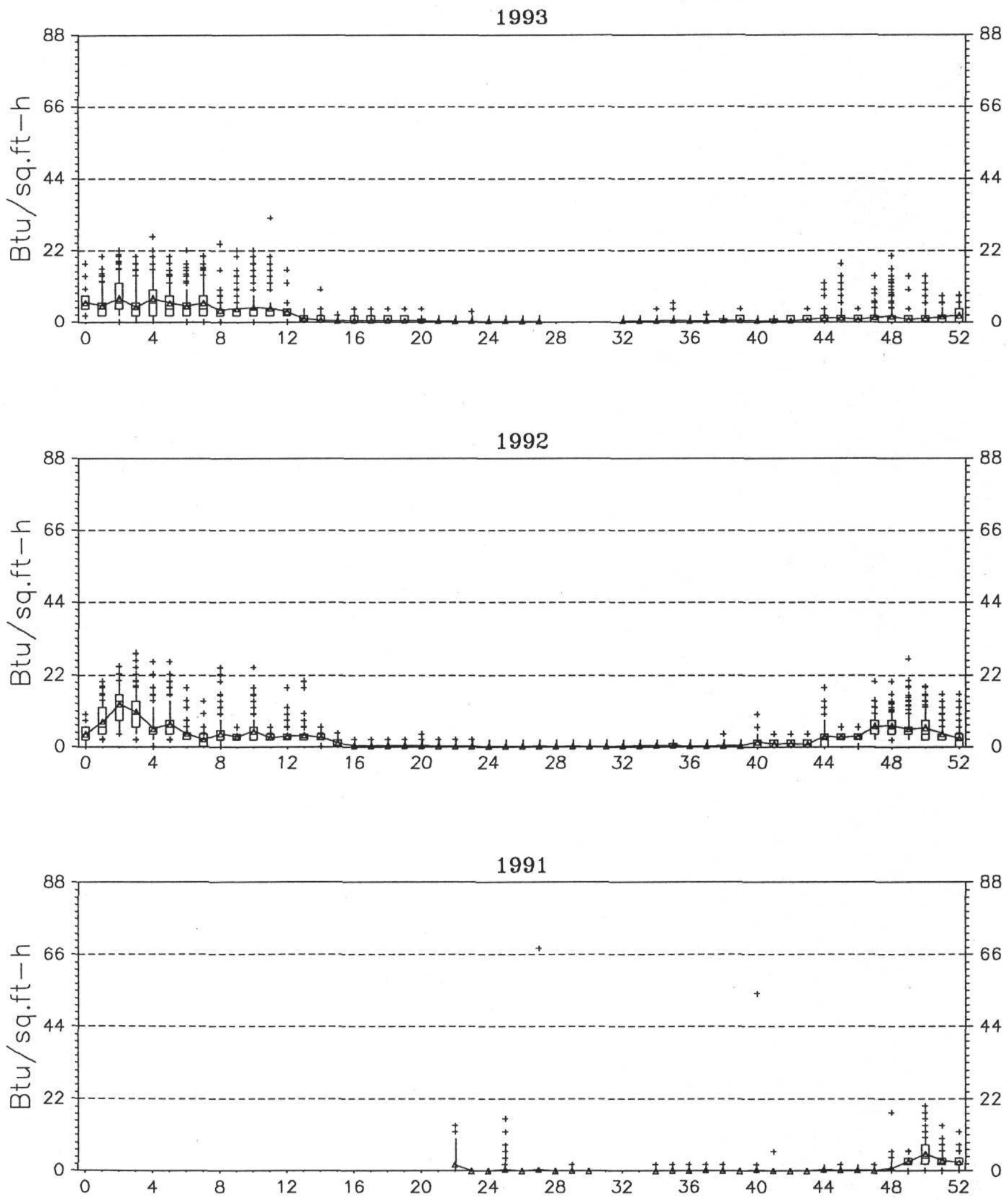


\section{Victoria High School (VHS)}

W.B. Electric as W/sq.ft.

Pre-Retrofit (06/04/1991 - 08/01/1991)

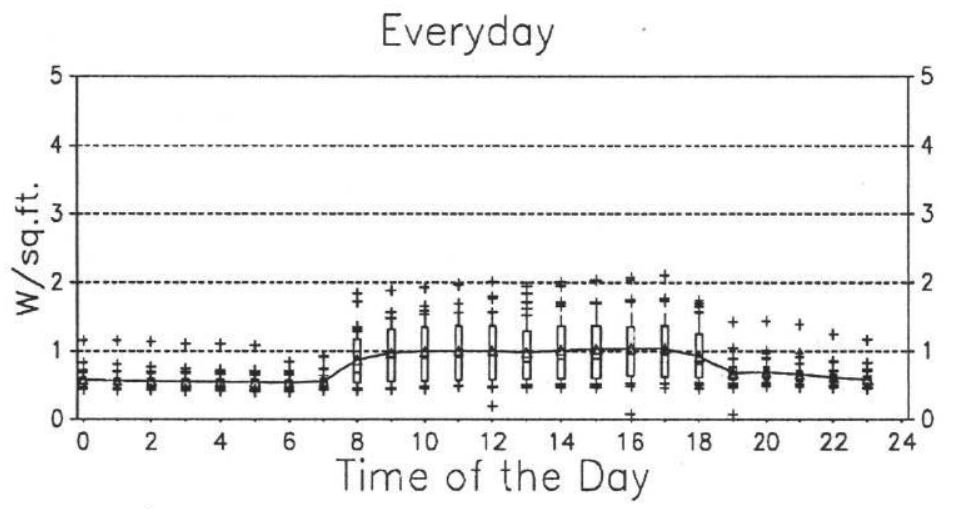

苦

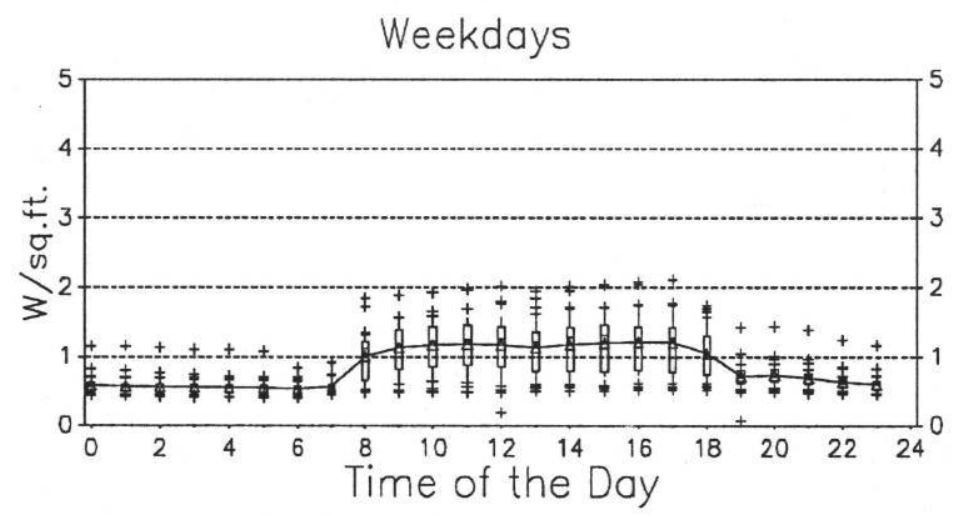

Weekends

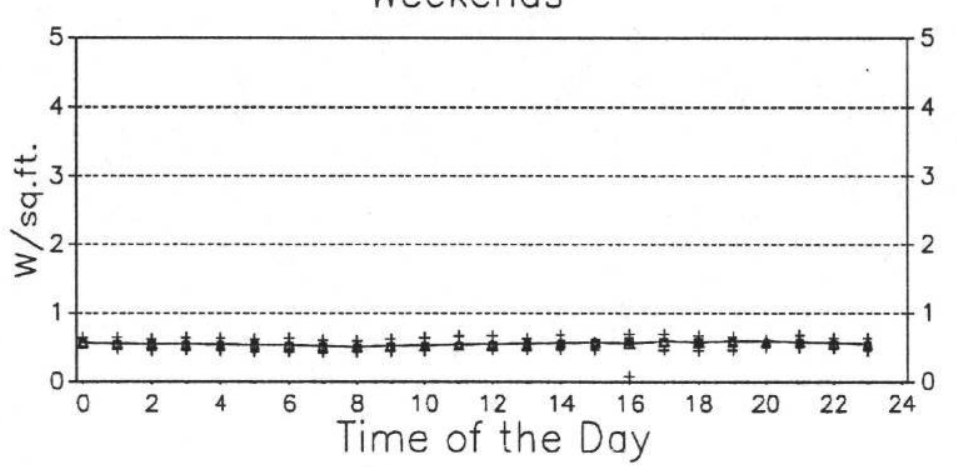

Post-Retrofit (08/15/1991 - 12/31/1993)
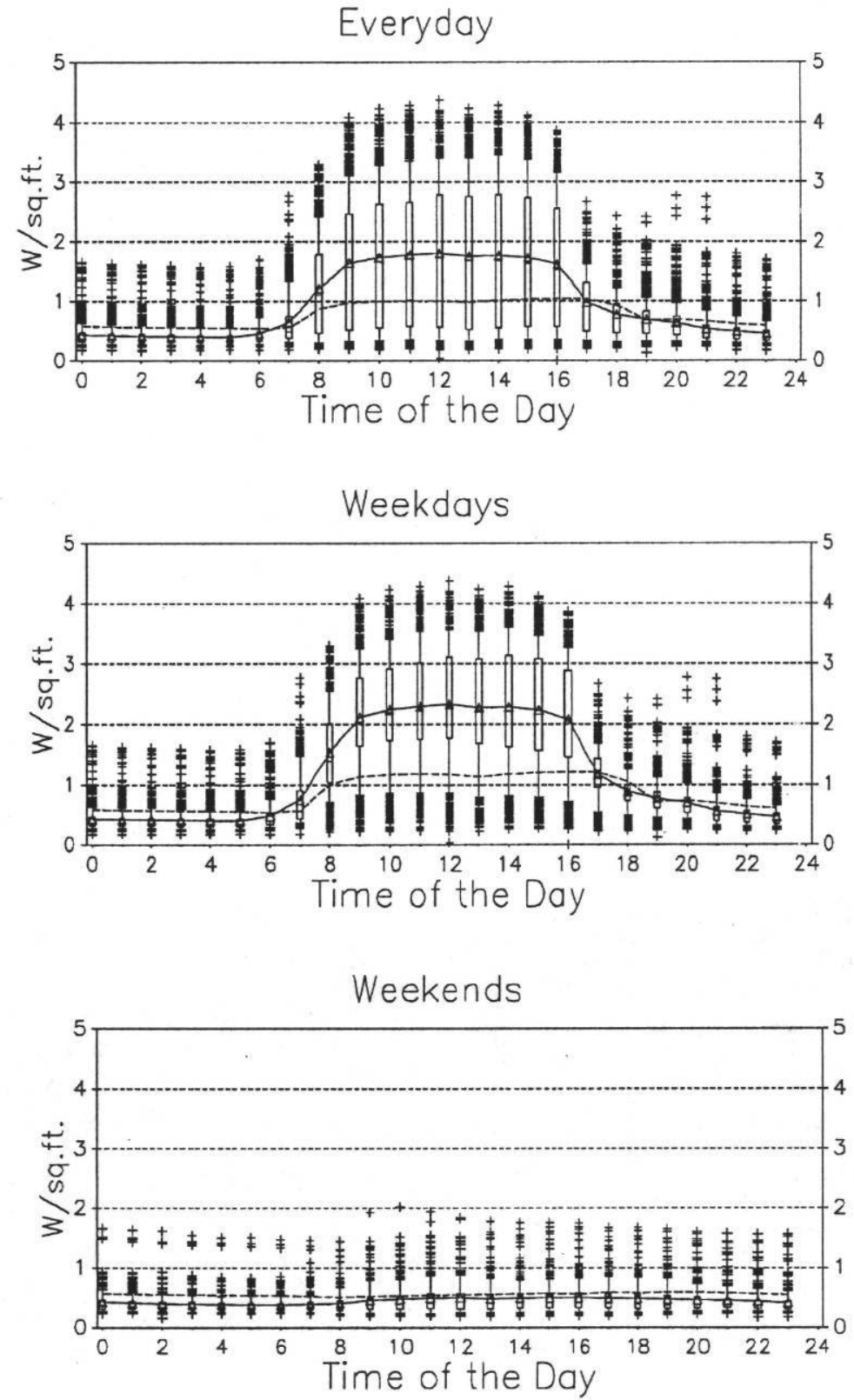


\section{Victoria High School (VHS) W.B. CHW as Btu/sq.ft.-h}

Pre-Retrofit (06/04/1991 - 08/01/1991)

Everyday

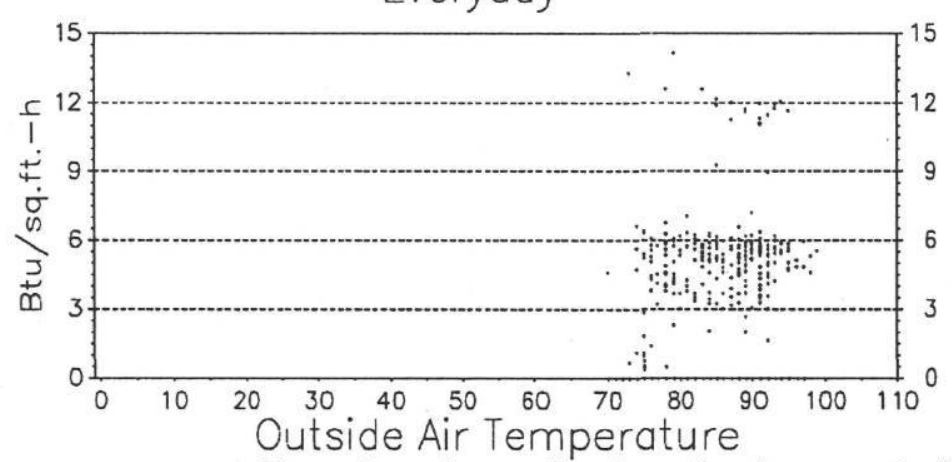

Weekdays

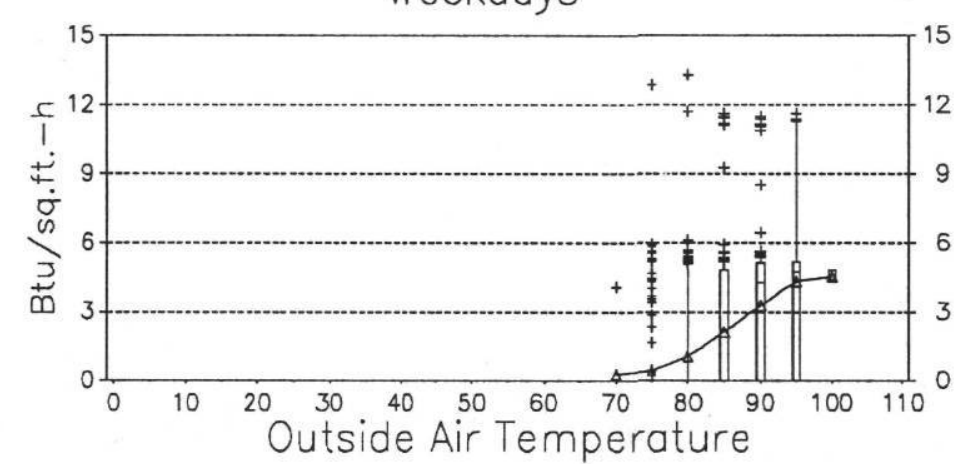

Weekends

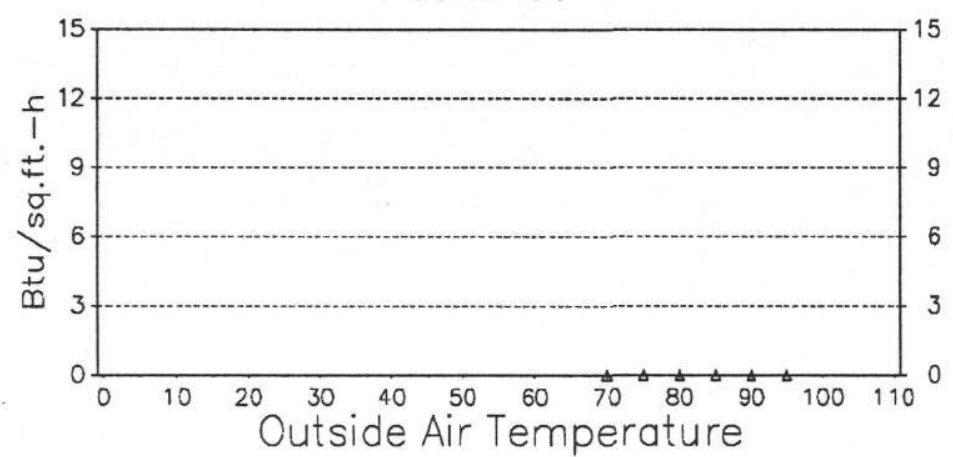

Post-Retrofit (08/15/1991 - 12/31/1993)

Everyday

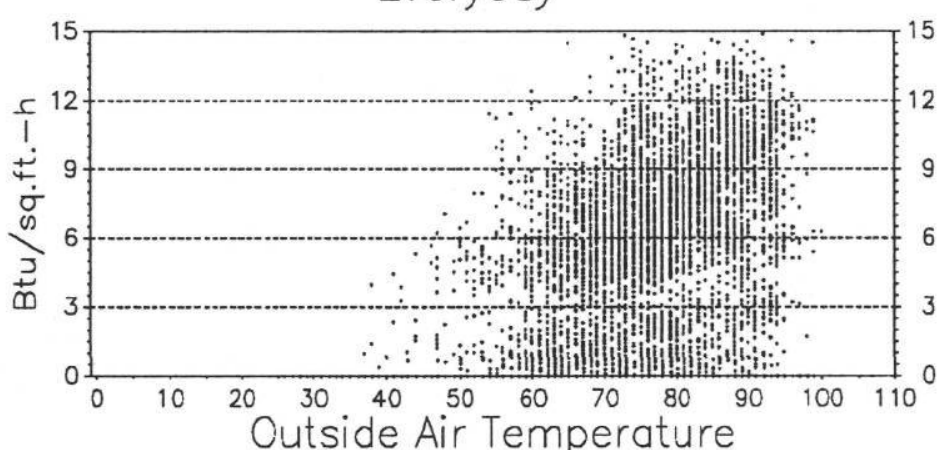

Outside Air Temperature

Weekdays

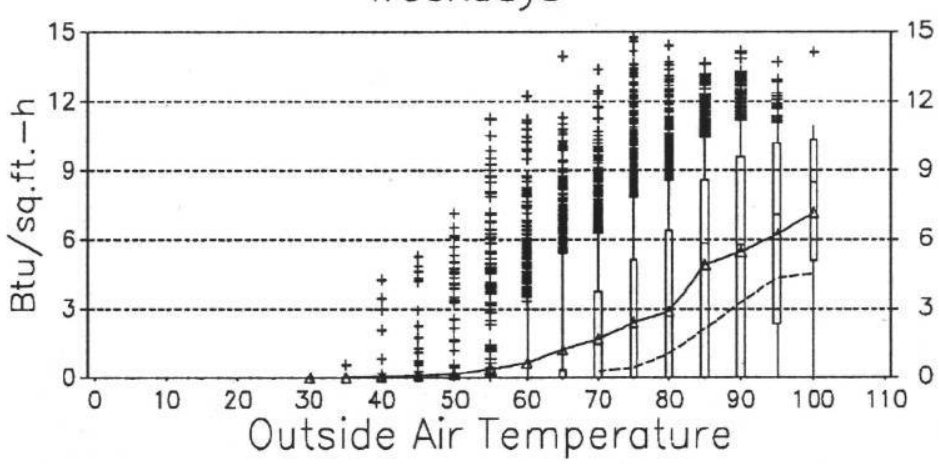

Weekends

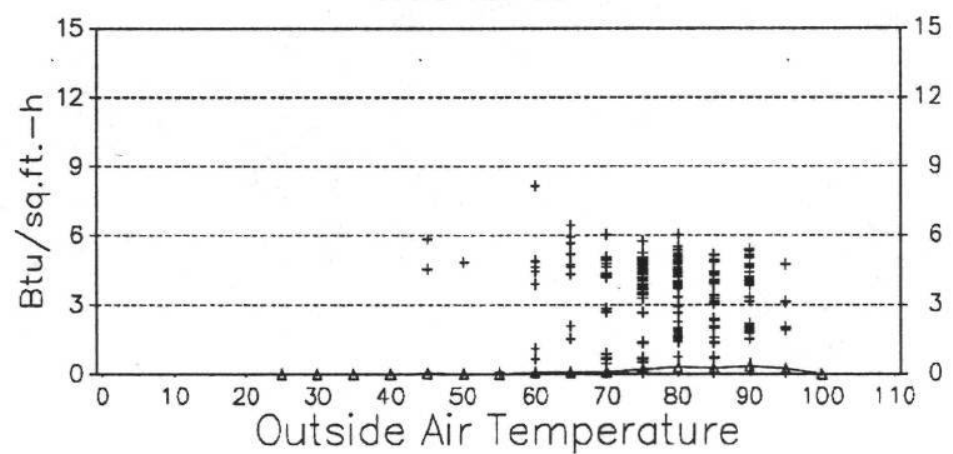




\section{Victoria High School (VHS) \\ W.B. HW as Btu/sq.ft.-h}

Pre-Retrofit (06/04/1991 - 08/01/1991)

Everyday

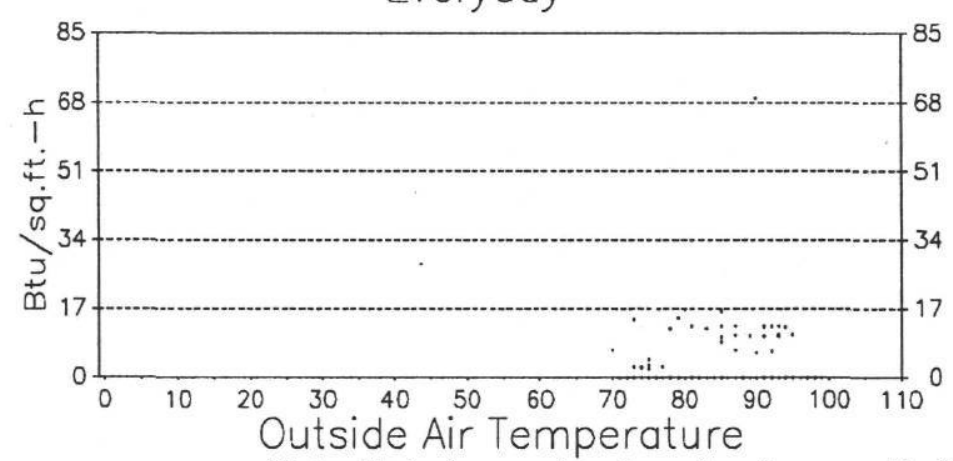

Weekdays

$\underset{1}{\infty}$

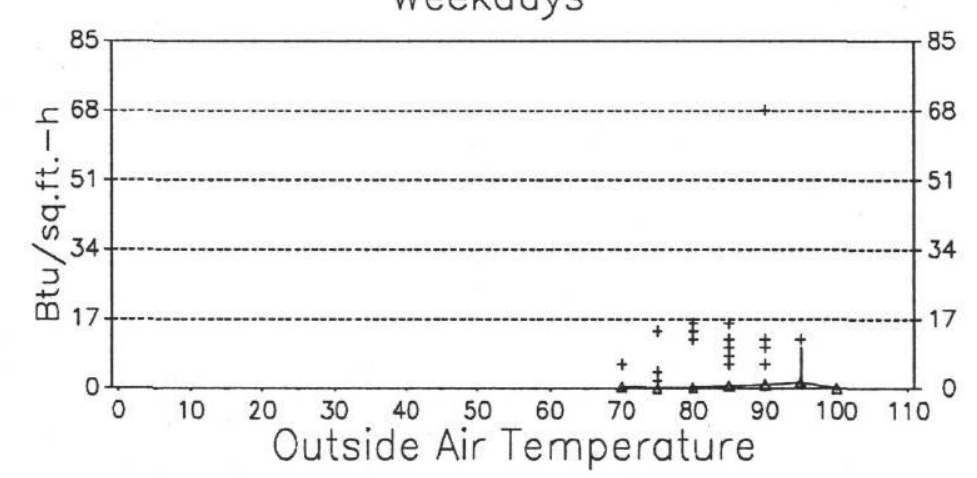

Weekends

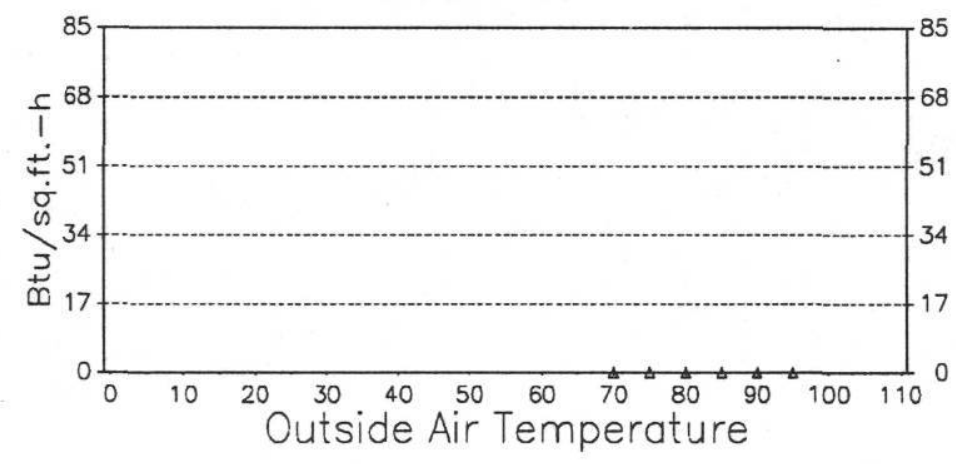

Post-Retrofit (08/15/1991 - 12/31/1993)

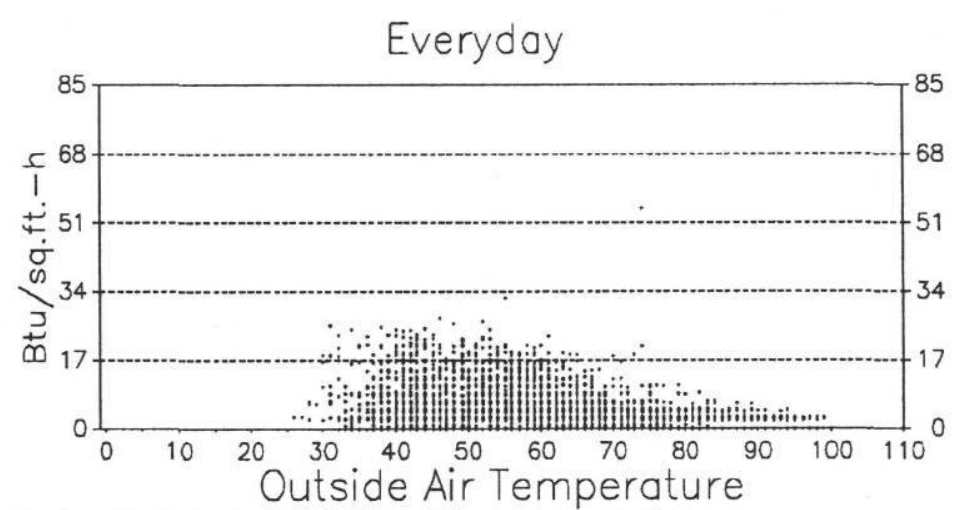

$30 \quad 40 \quad 50 \quad 60 \quad 70 \quad 80$
Outside Air Temperature

Weekdays

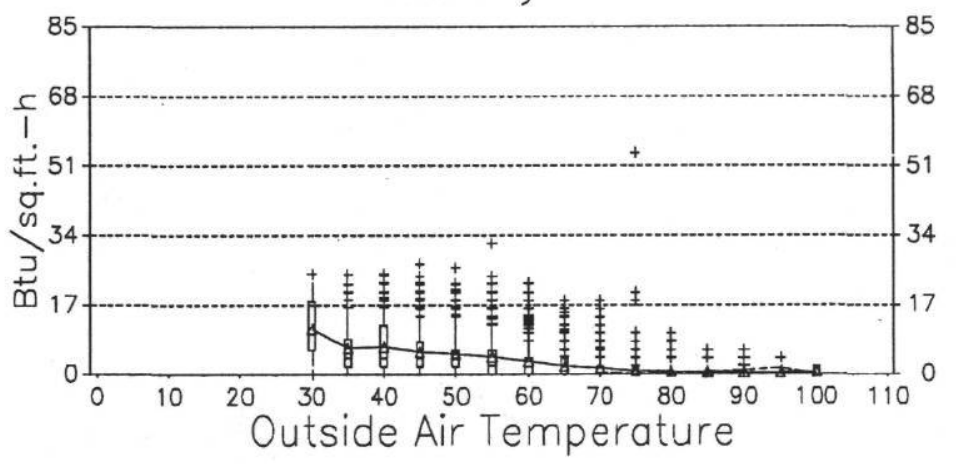

Weekends

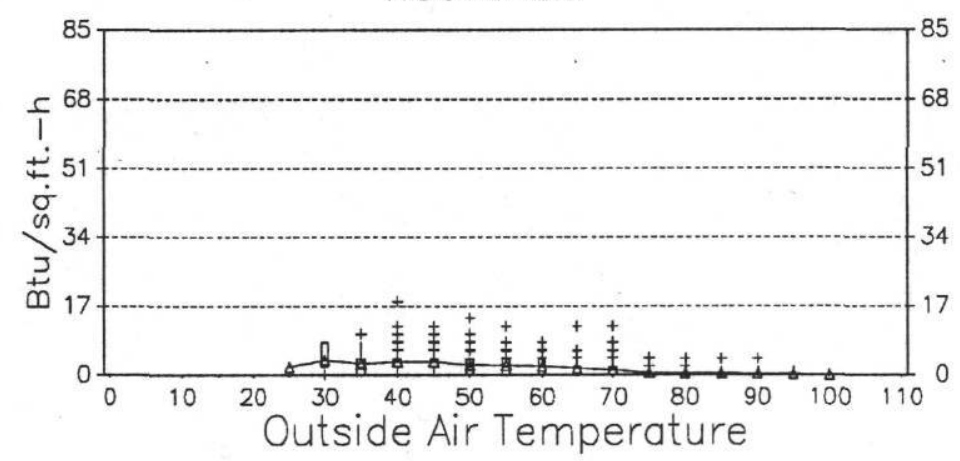




\section{Victoria High School (VHS)}

\section{Daily Average Values}

Pre-Retrofit $(\Delta)$ 06/04/1991 - 08/01/1991

Electric

$(\mathrm{kWh} / \mathrm{h})$
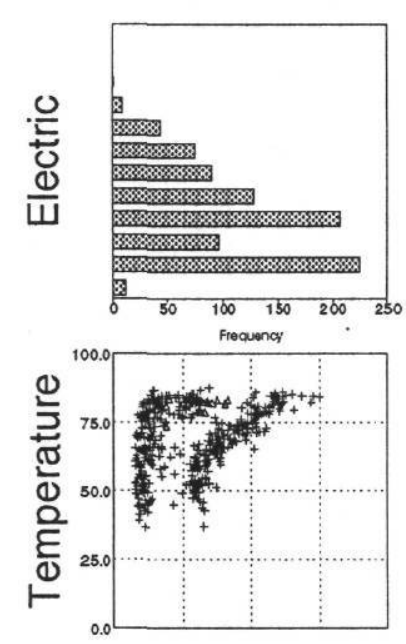

点
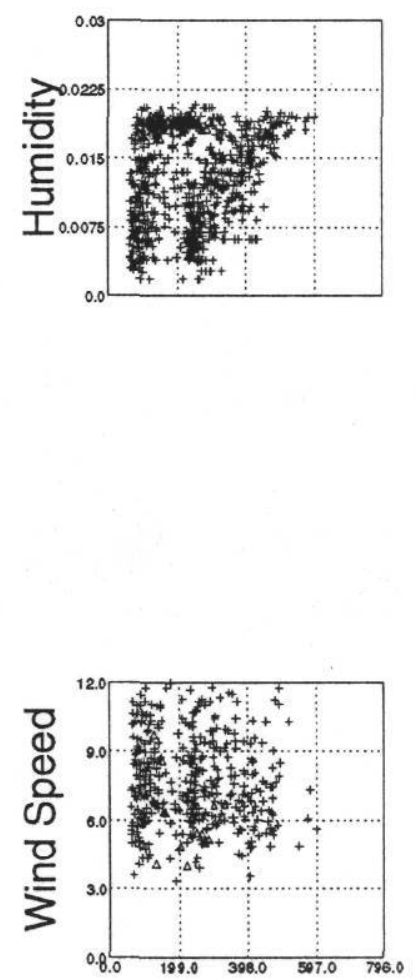

Temperature

(degrees F)
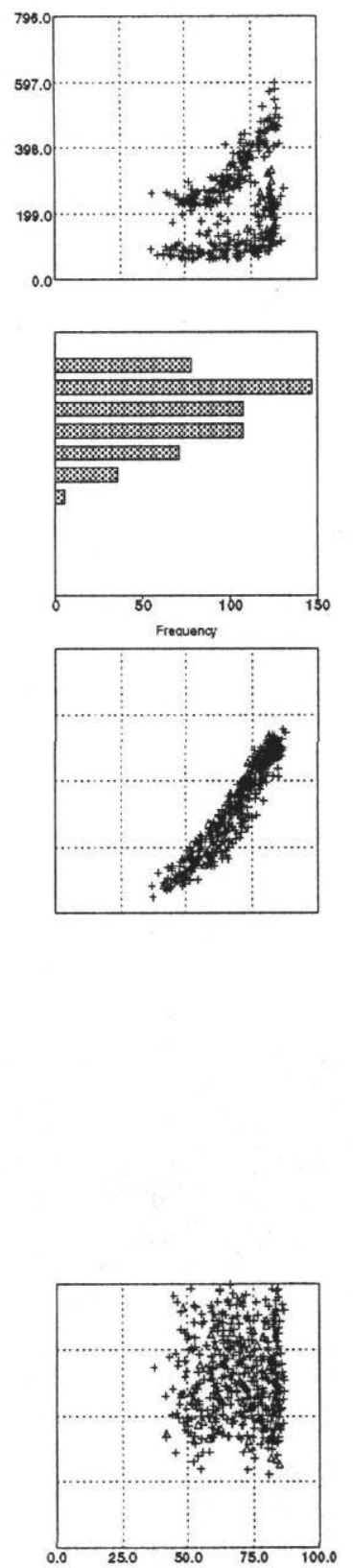

Post-Retrofit (+) 08/15/1991 - 12/31/1993

Solar Rad

(W/sq.m)

Wind Speed

$(\mathrm{mph})$
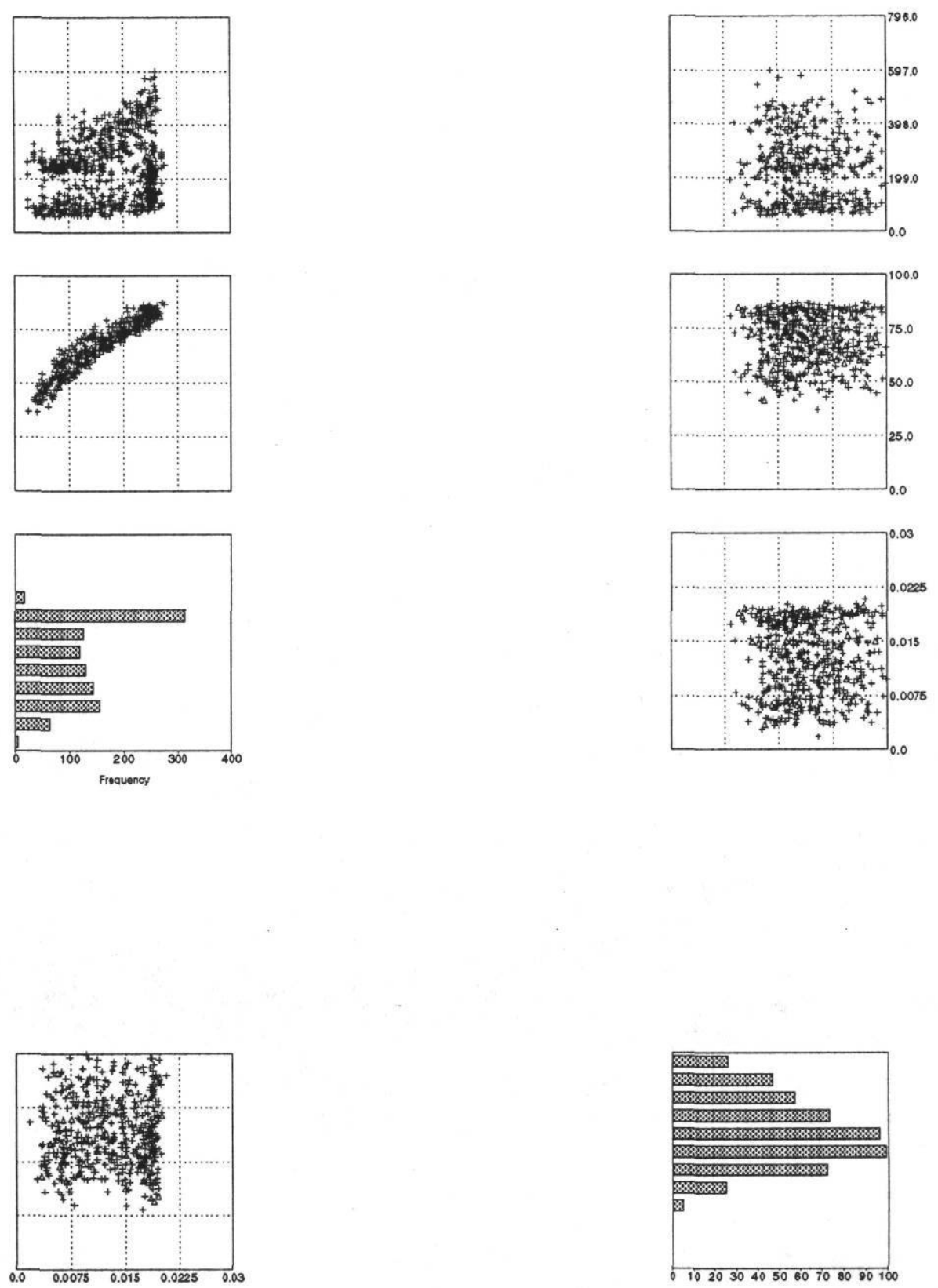


\section{Victoria High School (VHS)}

Daily Average Values

Pre-Retrofit $(\Delta)$ 06/04/1991 - 08/01/1991

Chw Cons.

$(\mathrm{kBtu} / \mathrm{h})$

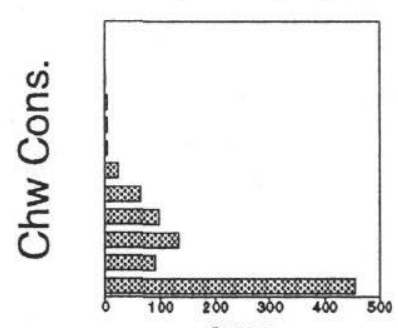

Froouenoy

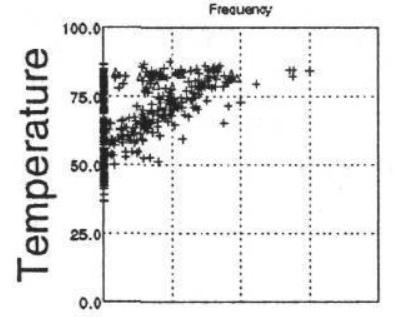

$\pm$

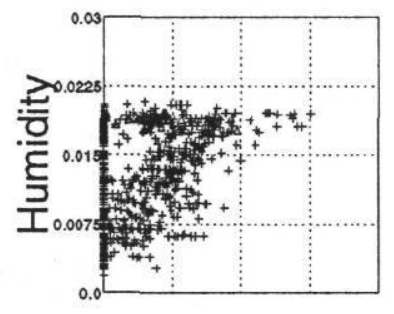

Temperature

(degrees F)
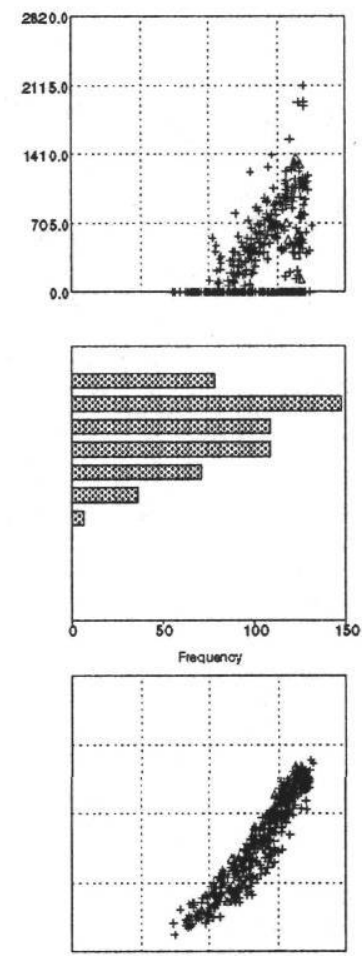

Post-Retrofit (+) 08/15/1991 - 12/31/1993

Humidity

(lbw/lba)
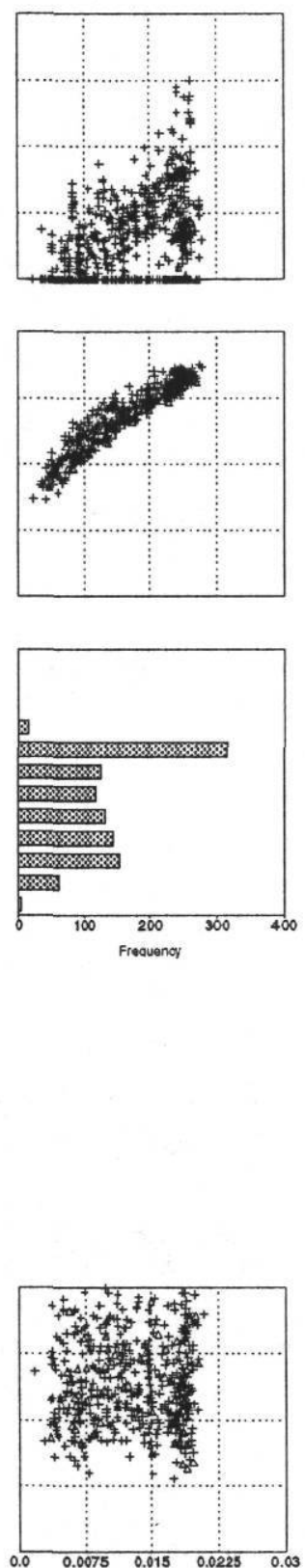

$\begin{array}{llllll}0.0 & 0.0075 & 0.015 & 0.0225 & 0.03\end{array}$
Solar Rad

(W/sq.m)

Wind Speed

$(\mathrm{mph})$
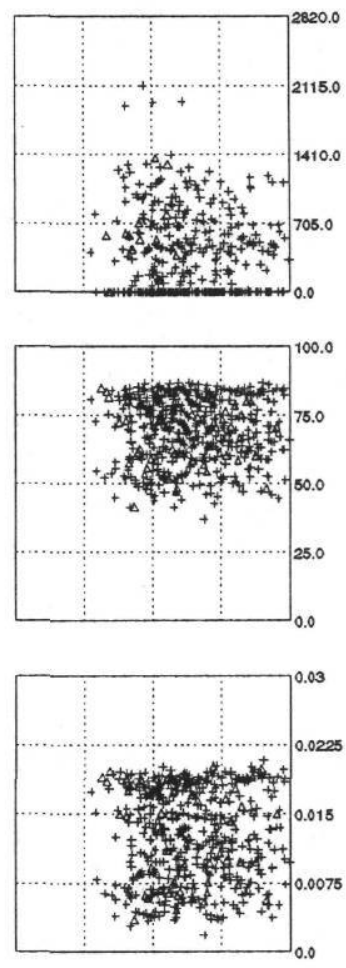
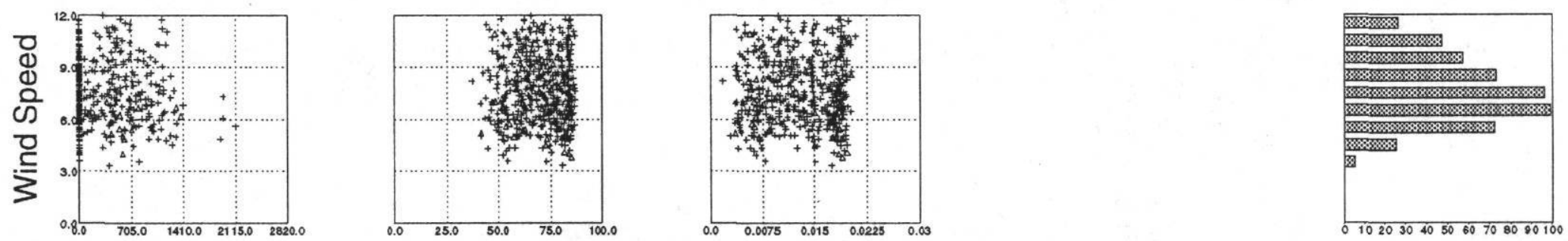


\section{Victoria High School (VHS) \\ Daily Average Values}

Pre-Retrofit $(\triangle)$ 06/04/1991 - 08/01/1991 $\mathrm{HW} /$ Steam Cons. $(\mathrm{kBtu} / \mathrm{h})$
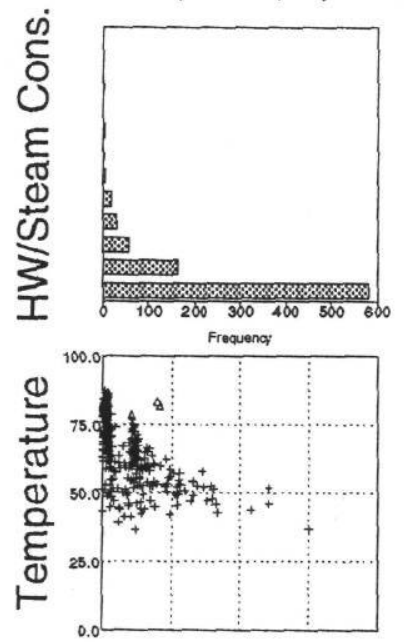

$\frac{\pi}{\infty}$
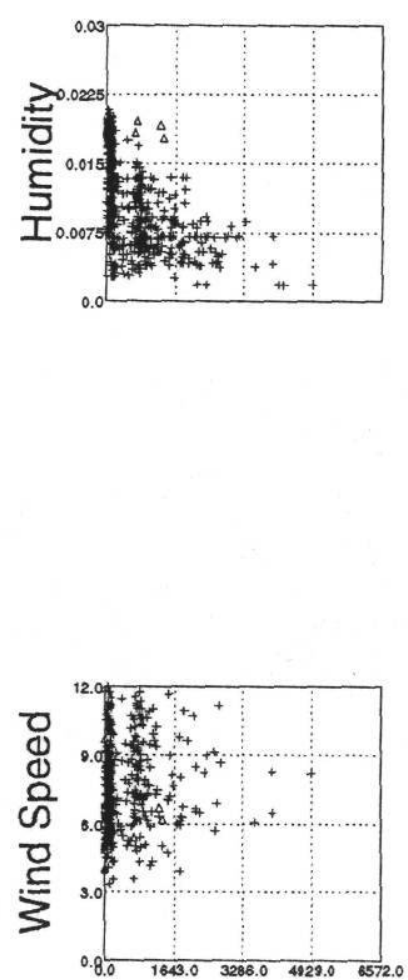
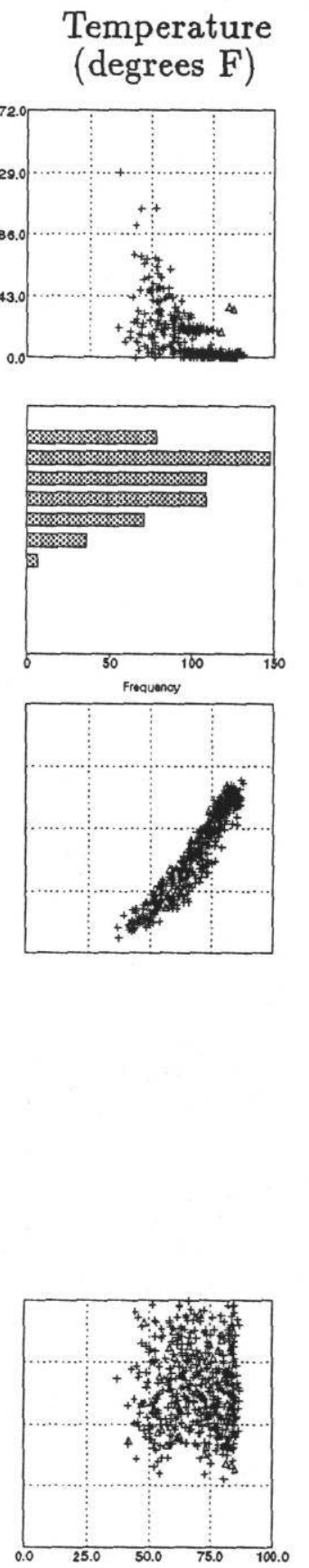

Post-Retrofit (+) 08/15/1991 - 12/31/1993

Humidity

(lbw/lba)
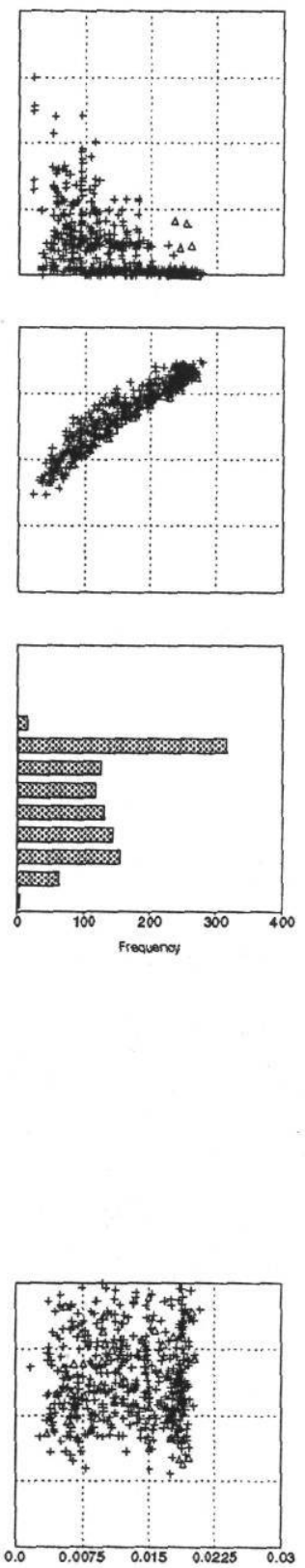

Solar Rad

$(\mathrm{W} / \mathrm{sq} \cdot \mathrm{m})$

Wind Speed (mph)
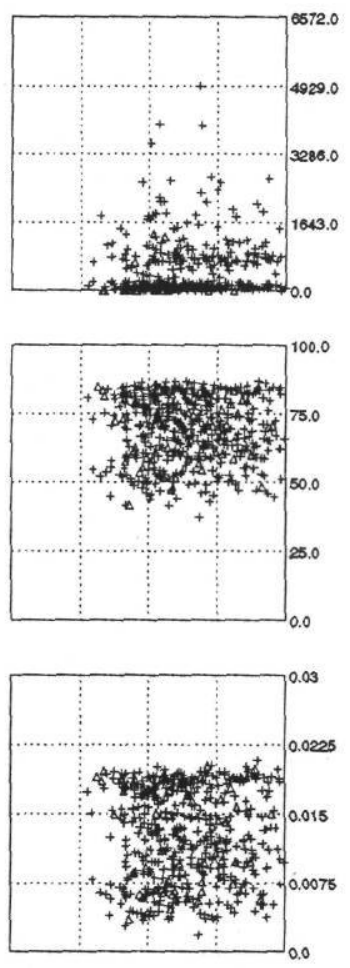

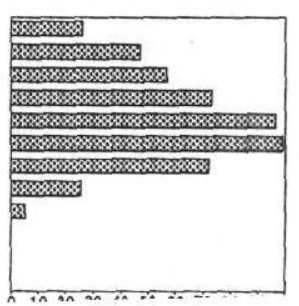




\section{SIMS ELEMENTARY SCHOOL}

\section{C.1 Site Description ${ }^{1}$}

Sims Elementary School is located in Fort Worth, Texas. It is a 62,400 square foot, single story, concrete building with single pane, tinted, operable windows.

The school is operated from August through May, with approximately 862 students and 50 faculty and staff. The maximum school occupancy is from about 7:00 a.m. through 3:00 p.m. The building has a lower occupancy during the weekend. There are also three summer sessions of three weeks duration each, during the morning in the summertime, with only about $10 \%$ of the students and staff present. The school district schedule is included under Tab C-1.

Electricity is purchased from Texas Utilities Electric Company, and natural gas from Lone Star Gas Company.

\section{C.2 EMCS Retrofit}

As part of monitoring done for other retrofits at this site and Dunbar Middle School, it was decided to fine tune the existing EMCS at Dunbar Middle School. This was done as an operation and maintenance (O\&M) project; separate from the other retrofits. This was a success at Dunbar Middle School. Meanwhile, a private company approached the school district, proposing the installation of a new EMCS. The school district purchased and installed a new EMCS at Sims Elementary School. The new system was operable on April 14, 1991, and has a few more capabilities than the existing system. The LoanSTAR staff at Texas A\&M University pointed out to the school district that their existing EMCS was able to control the majority of their equipment, and if fine tuned, would operate well for them. Based on an economic analysis, it was recommended that they fine tune their existing systems instead of buying new systems for other schools within the district.

\footnotetext{
${ }^{1}$ Adapted from: Landman, D.S., 1995. "Preliminary Study of Advanced Diagnostic Prescreening Methods," Energy Systems Laboratory, Mechanical Engineering Department, Texas A\&M University, College Station, TX.
} 


\section{C.3 Analysis}

\section{C.3.1 Snapshot of consumption for September 1991 through December 1993}

Figures C-1 and C-2 represent monthly average consumption and peak consumption versus minmax average (or peak) monthly temperature. ${ }^{2}$ Min-max average monthly temperature is calculated by averaging the maximum and minimum temperature each day to obtain min-max average daily temperature. The daily temperatures are then averaged over all days in each month to obtain min-max average monthly temperature.

The data points reflecting high temperature and low consumption are indicative of non-semester consumption. If those data points are ignored, there is a general increase of consumption with temperature, indicating a temperature dependence of consumption. Additionally, the post-retrofit data points are generally lower than the pre-retrofit data points. When compared to similar plots for other Texas schools in the LoanSTAR program, this site is a high energy use school. The reader is referred to the referenced report for a more detailed discussion of these plots.

\footnotetext{
${ }^{2}$ Landman, D.S., 1995. "Preliminary Study of Advanced Diagnostic Prescreening Methods," Energy Systems Laboratory, Mechanical Engineering Department, Texas A\&M University, College Station, TX.
} 
Figure C-1: Monthly Average Consumption: Demand, in W/sf, versus min-max average monthly temperature, in ${ }^{\circ}$ F, for September 1991 through December 1993 (Sims Elementary School)

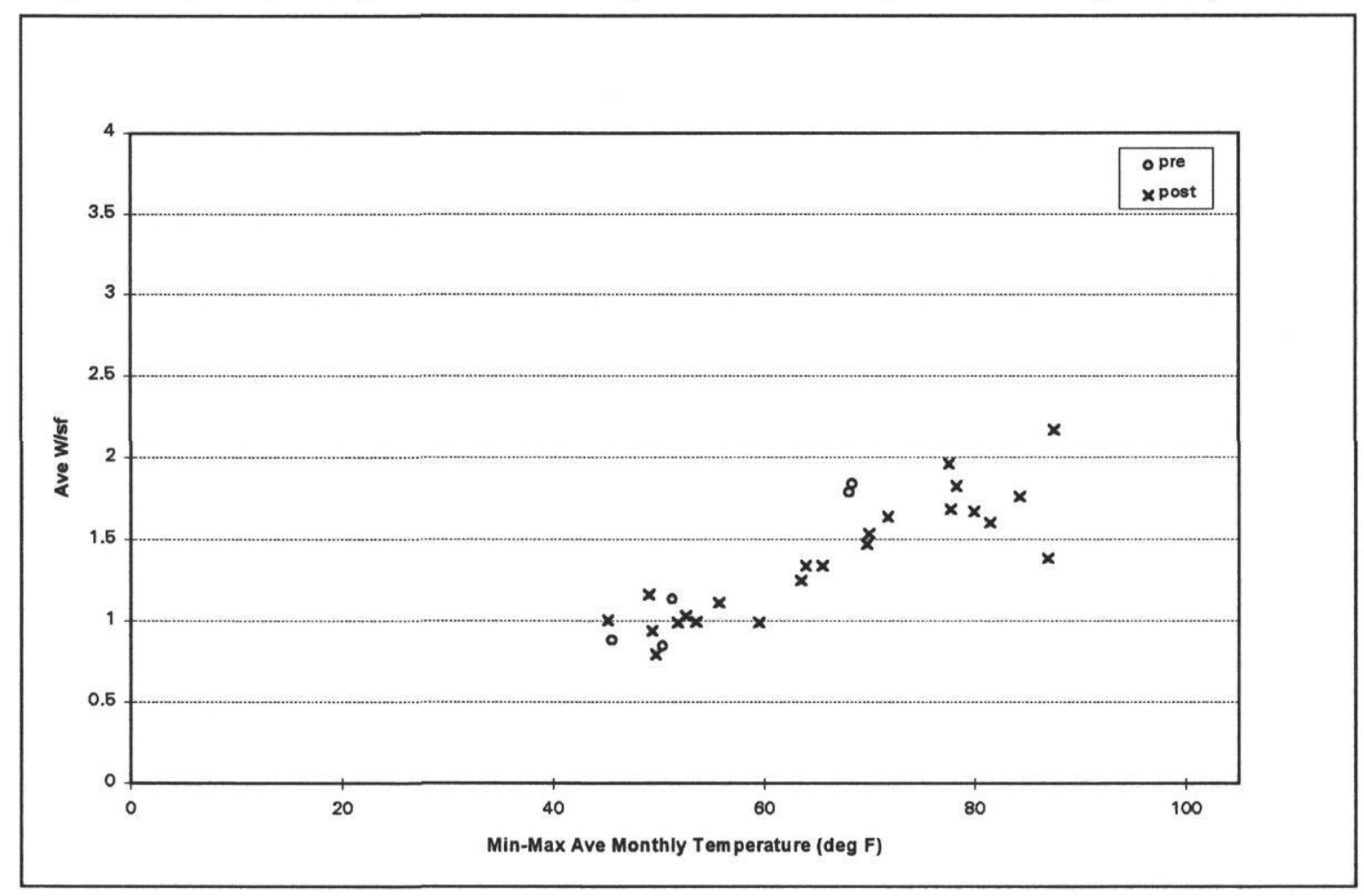

Figure C-2: Monthly Peak Consumption: Demand, in W/sf, versus peak monthly temperature, in ${ }^{\circ} \mathrm{F}$, for September 1991 through December 1993 (Sims Elementary School)

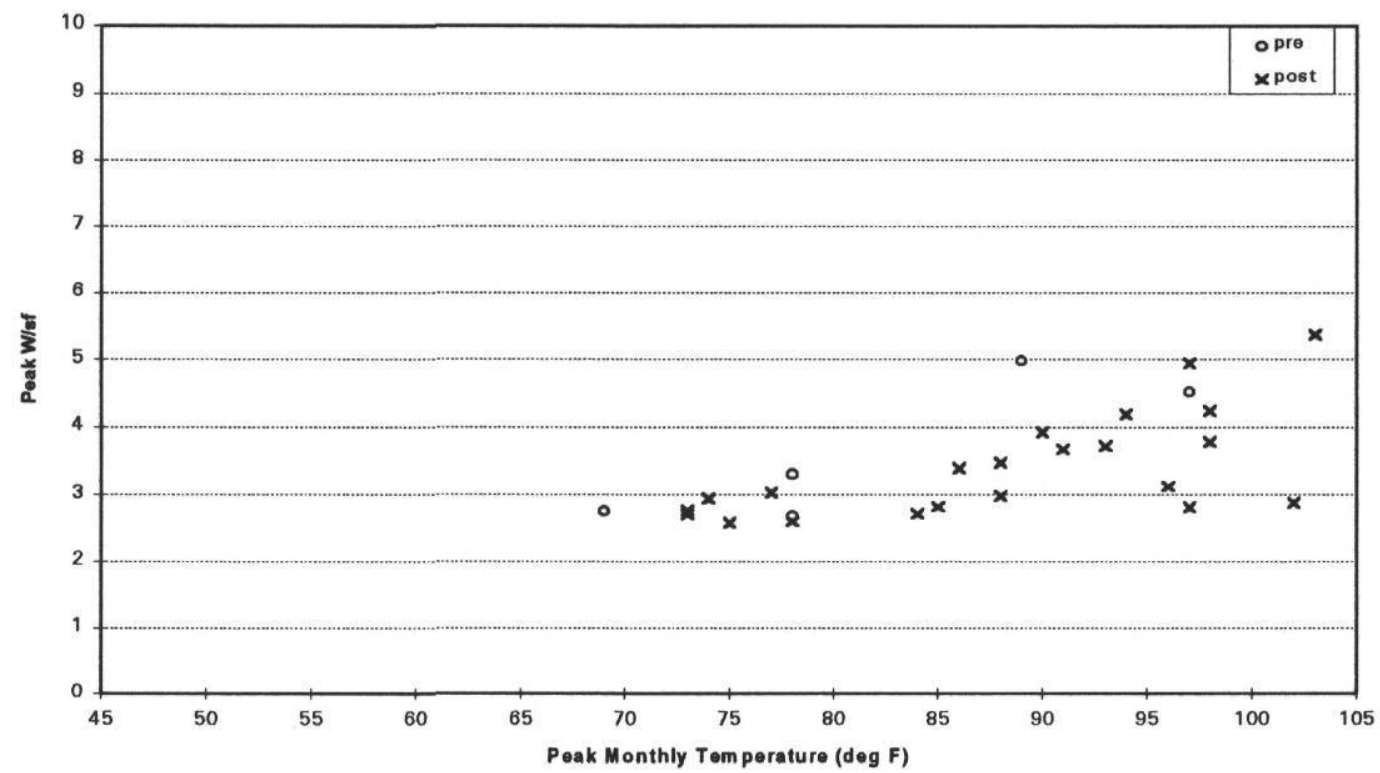




\section{C.3.2 Timeline plots}

Plots of energy consumption for the reporting period of October 1, 1991, through May 31, 1995, are shown in Figure C-3. The EMCS retrofit date of April 14, 1994, is shown by a vertical, bold line. Monitoring diagrams are provided in Tab C-2.

In looking at the whole building electric plot, there is no apparent decrease in consumption at any point along the timeline. The monitoring of electricity consumption at this site includes separate channels for whole building electric and lights. The EMCS does not control the lights, so they were subtracted from whole building electric to obtain other electric. This category consists of HVAC equipment, mostly roof top $\mathrm{A} / \mathrm{C}$ units. The timeline of other electric shows a definite pattern between October 1, 1991, and April 14, 1994, the retrofit date. After the retrofit date, the consumption actually appears to increase. If the months surrounding February are analyzed for each year on the plot, one can see an increase from approximately $1,000 \mathrm{kWh} /$ day to $1,500 \mathrm{kWh} /$ day. The plot of whole building heat shows seasonal heating between November and April of each year. 
Figure C-3: Energy Consumption: time series for October 1991 to May 1995 (Sims Elementary School)
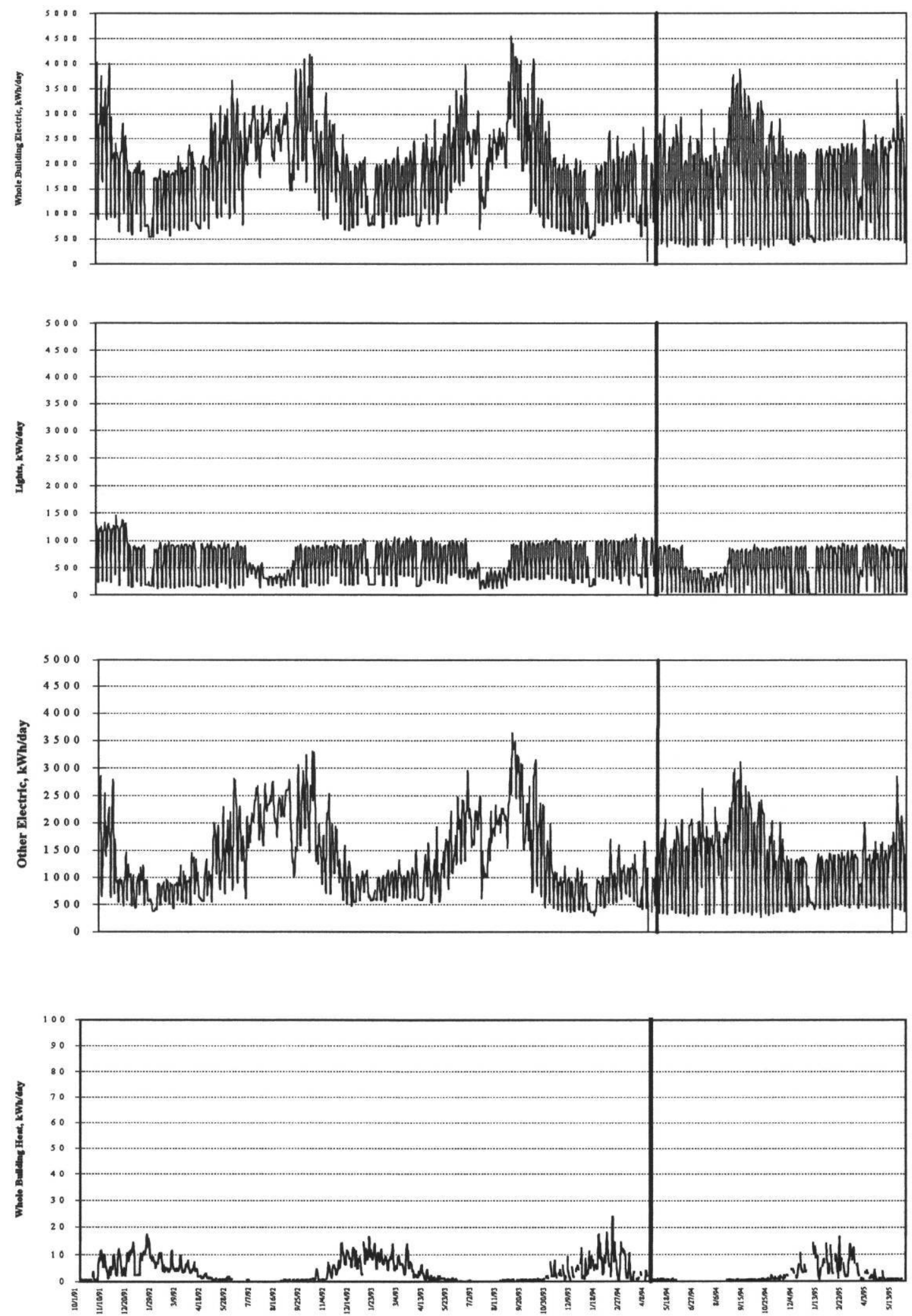


\section{C.3.3 Whole Building Electricity Consumption (Post-Retrofit Period)}

Table C-1 shows energy consumption for the post-retrofit period (April 14, 1994, through May 31, 1995). Whole building electricity consumption is broken down into two components: lighting consumption and other electricity consumption. It is further subdivided into semester period and non-semester periods. The post-retrofit period is used because there is significantly more data available in the post-retrofit period, and it represents current usage.

Figure C-4 graphically shows whole building electricity consumption for the post-retrofit period. For the semester period, $55 \%$ of whole building electric energy use is attributable to other electric equipment, while $26 \%$ is due to the lights. For the non-semester period, other electric accounts for $15 \%$ of whole building electric energy, while the lights account for $4 \%$.

From both Table $\mathrm{C}-1$ and Figure $\mathrm{C}-4$, it is readily apparent that lighting accounts for a small portion of the whole building electricity usage. Therefore, attention for reducing energy usage should be focused on the other electricity usage. In this case, other electricity consumption is mainly roof-top HVAC units.

Table C-1: Energy Consumption for post period, October 1991 through May 1995 (Sims Elementary School)

\begin{tabular}{|l|r|r|r|r|r|r|}
\hline \multirow{2}{*}{} & \multicolumn{2}{|c|}{ SEMESTER } & \multicolumn{2}{c|}{ NON-SEMESTER } & \multicolumn{2}{c|}{ TOTAL } \\
\cline { 2 - 7 } & ENERGY & \multicolumn{1}{c|}{$\$$} & ENERGY & \multicolumn{1}{c|}{$\$$} & ENERGY & $\$$ \\
\hline wbelec, kWh & 605,715 & $\$ 40,825$ & 142,510 & $\$ 9,605$ & 748,225 & $\$ 50,430$ \\
\hline lights, kWh & 196,445 & $\$ 13,240$ & 27,258 & $\$ 1,837$ & 223,703 & $\$ 15,078$ \\
\hline other, kWh & 409,270 & $\$ 27,585$ & 115,251 & $\$ 7,768$ & 524,521 & $\$ 35,353$ \\
\hline wbheat, MMBtu & 588 & $\$ 2,705$ & 49 & $\$ 225$ & 637 & $\$ 2,930$ \\
\hline
\end{tabular}


Figure C-4: Whole Building Electricity Consumption for post period, April 15, 1994, through May 1995 (Sims Elementary School)

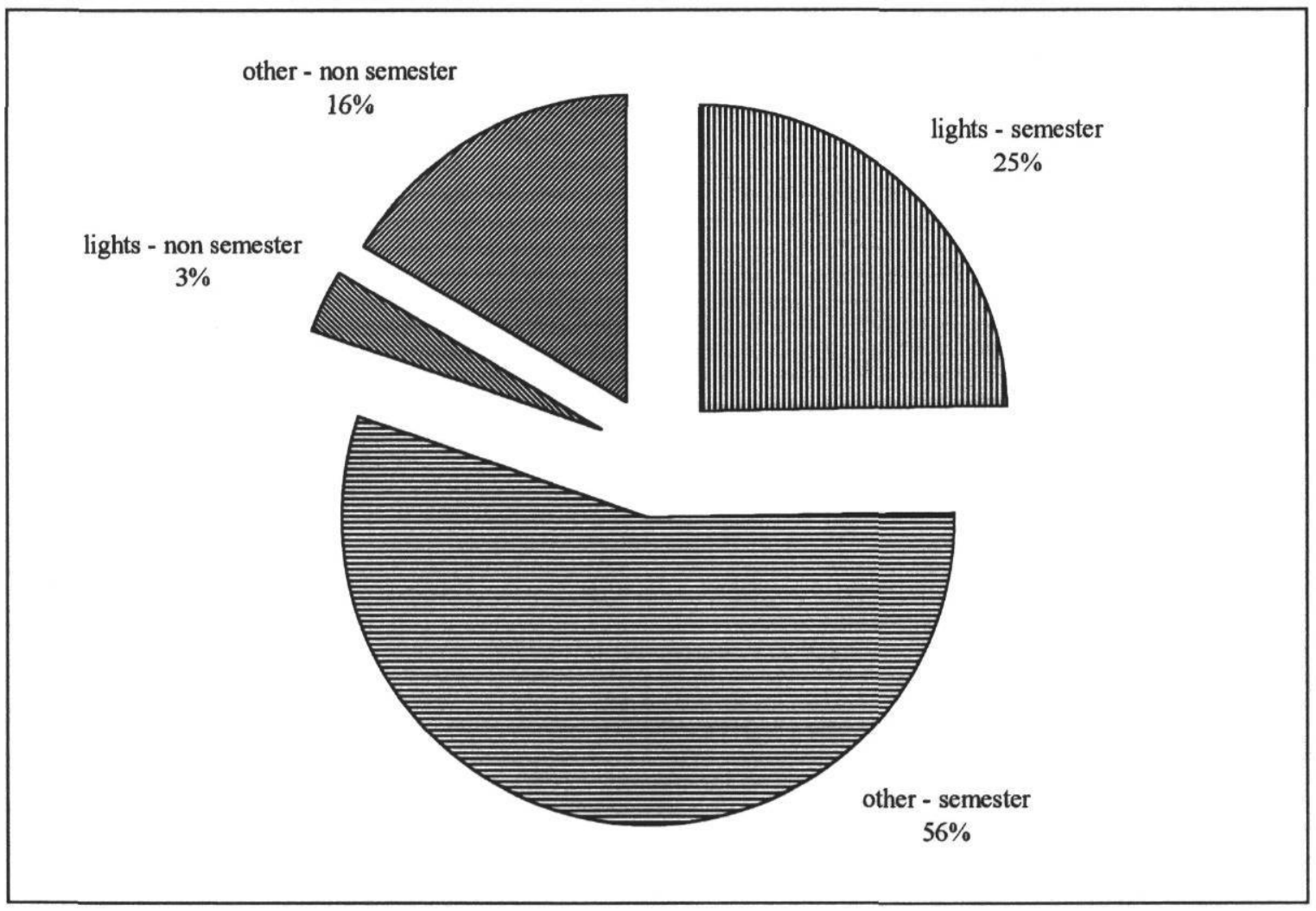

\section{C.3.4 Total Monthly Consumption}

The total monthly energy consumption is summarized in Table C-2. Again, it is readily apparent that other electric accounts for the majority of this site's electric energy use. 
Table C-2: Monthly Energy Consumption (Sims Elementary School)

\begin{tabular}{|c|c|c|c|c|}
\hline & $\begin{array}{c}\text { wbelec } \\
\text { kWh/month }\end{array}$ & $\begin{array}{c}\text { lights } \\
\text { kWh/month }\end{array}$ & $\begin{array}{c}\text { other } \\
\text { kWh/month } \\
\end{array}$ & $\begin{array}{c}\text { wbheat } \\
\text { MMBtu/month }\end{array}$ \\
\hline \multicolumn{5}{|c|}{ PRE-RETROFIT PERIOD } \\
\hline Oct 91 & 83,200 & 30,111 & 52,767 & 94 \\
\hline Nov & 50,829 & 25,299 & 30,443 & 194 \\
\hline Dec & 39,223 & 15,934 & 28,111 & 254 \\
\hline Jan 92 & 40,865 & 19,072 & 39,175 & 158 \\
\hline Feb & 43,217 & 19,463 & 53,383 & 36 \\
\hline Mar & 45,896 & 17,250 & 64,549 & 14 \\
\hline Apr & 60,103 & 20,321 & 60,523 & 25 \\
\hline May & 71,339 & 20,348 & 37,712 & 126 \\
\hline Jun & 75,790 & 14,853 & 30,594 & 301 \\
\hline Jul & 82,092 & 9,536 & 34,582 & 202 \\
\hline Aug & 77,690 & 13,810 & 42,819 & 66 \\
\hline Sep & 88,241 & 19,932 & 68,998 & 19 \\
\hline Oct & 68,149 & 21,649 & 76,339 & 18 \\
\hline Nov & 46,415 & 19,362 & 37,985 & 62 \\
\hline Dec & 42,766 & 17,975 & 28,563 & 156 \\
\hline $\operatorname{Jan} 93$ & 46,702 & 20,424 & 34,678 & 325 \\
\hline Feb & 47,163 & 21,440 & 29,892 & 62 \\
\hline Mar & 51,509 & 20,818 & 48,753 & 208 \\
\hline Apr & 56,023 & 21,790 & 71,657 & 48 \\
\hline May & 76,217 & 23,457 & 55,507 & 82 \\
\hline Jun & 72,174 & 13,640 & 39,562 & 177 \\
\hline Jul & 64,380 & 9,258 & 39,837 & 1 \\
\hline Aug & 100,835 & 18,188 & 43,885 & 123 \\
\hline Sep & 82,136 & 21,994 & 44,956 & 119 \\
\hline Oct & 62,260 & 23,622 & 35,769 & 2 \\
\hline Nov & 44,385 & 21,773 & 19,656 & 147 \\
\hline Dec & 36,881 & 17,399 & 42,318 & 2 \\
\hline $\operatorname{Jan} 94$ & 47,920 & 22,462 & 20,807 & 77 \\
\hline Feb & 47,822 & 21,114 & 34,312 & 68 \\
\hline Mar & 47,045 & 20,615 & 22,858 & 118 \\
\hline Apr (partial) & 17,300 & 8,329 & 7,468 & 12 \\
\hline Total Consumption & $1,816,566$ & 591,236 & $1,278,455$ & 3,294 \\
\hline Total Cost & $\$ 122,437$ & $\$ 39,849$ & $\$ 86,168$ & $\$ 15,151$ \\
\hline
\end{tabular}

**post-retrofit period and grand total shown on next page 
Table C-5 (continuation): Monthly Energy Consumption (Sims Elementary School)

\begin{tabular}{|c|c|c|c|c|}
\hline & $\begin{array}{c}\text { wbelec } \\
\mathrm{kWh} / \text { month }\end{array}$ & $\begin{array}{c}\text { lights } \\
\mathrm{kWh} / \text { month }\end{array}$ & $\begin{array}{c}\text { other } \\
\mathrm{kWh} / \mathrm{month}\end{array}$ & $\begin{array}{c}\text { wbheat } \\
\text { MMBtu/month }\end{array}$ \\
\hline \multicolumn{5}{|l|}{ POST PERIOD } \\
\hline Apr (partial) & 32,096 & 11,077 & 6,693 & 13 \\
\hline May & 55,455 & 18,115 & 12,136 & 12 \\
\hline Jun & 53,858 & 10,086 & 15,211 & 18 \\
\hline Jul & 49,640 & 8,130 & 14,564 & 33 \\
\hline Aug & 76,882 & 17,289 & 60,594 & 152 \\
\hline Sep & 69,274 & 18,626 & 53,163 & 215 \\
\hline Oct & 58,523 & 18,894 & 42,543 & 102 \\
\hline Nov & 47,199 & 16,682 & 55,783 & 20 \\
\hline Dec & 37,715 & 11,858 & 37,564 & 1 \\
\hline $\operatorname{Jan} 95$ & 51,821 & 18,954 & 53,586 & 13 \\
\hline Feb & 48,218 & 17,560 & 17,931 & 10 \\
\hline Mar & 54,202 & 19,076 & 46,095 & 1 \\
\hline Apr & 52,034 & 18,638 & 37,498 & 46 \\
\hline May & 61,310 & 18,720 & 18,036 & 2 \\
\hline Total Consumption & 748,224 & 223,703 & 471,396 & 637 \\
\hline Total Cost & $\$ 50,430$ & $\$ 15,078$ & $\$ 31,772$ & $\$ 2,930$ \\
\hline Grand Total Consumption & $2,564,791$ & 814,939 & $1,749,852$ & 3,931 \\
\hline Grand Total Cost & $\$ 172,867$ & $\$ 54,927$ & $\$ 117,940$ & $\$ 18,081$ \\
\hline
\end{tabular}

\section{C.3.5 Average Daily Consumption}

Figures $\mathrm{C}-5 \mathrm{a}$ and $\mathrm{C}-5 \mathrm{~b}$ depict the average daily consumption for the semester period and the non-semester period.

For the semester period (Figure C-5a) the weekday consumption greatly increased during the daytime hours (7:00 a.m. to 6:00 p.m.), and significantly decreased during the nighttime hours (6:00 p.m. to 7:00 a.m.) Although the daytime consumption increased, the profile is as expected, with the startup of an EMCS. The nighttime consumption is low, with a sharp increase to daytime levels at 7:00 a.m. At the end of the day, there is a sharp decrease in consumption, indicating that the EMCS has shut off the equipment. The reason for daytime consumption increasing has not been determined for the purposes of this report. It could be due to a number of factors, such as: installation of new equipment, repair of existing equipment that was down during the pre-retrofit period, or erroneous data. 
Figure C-5a: Semester Pre-/Post-Retrofit Comparison: based on average hourly data Elementary School)

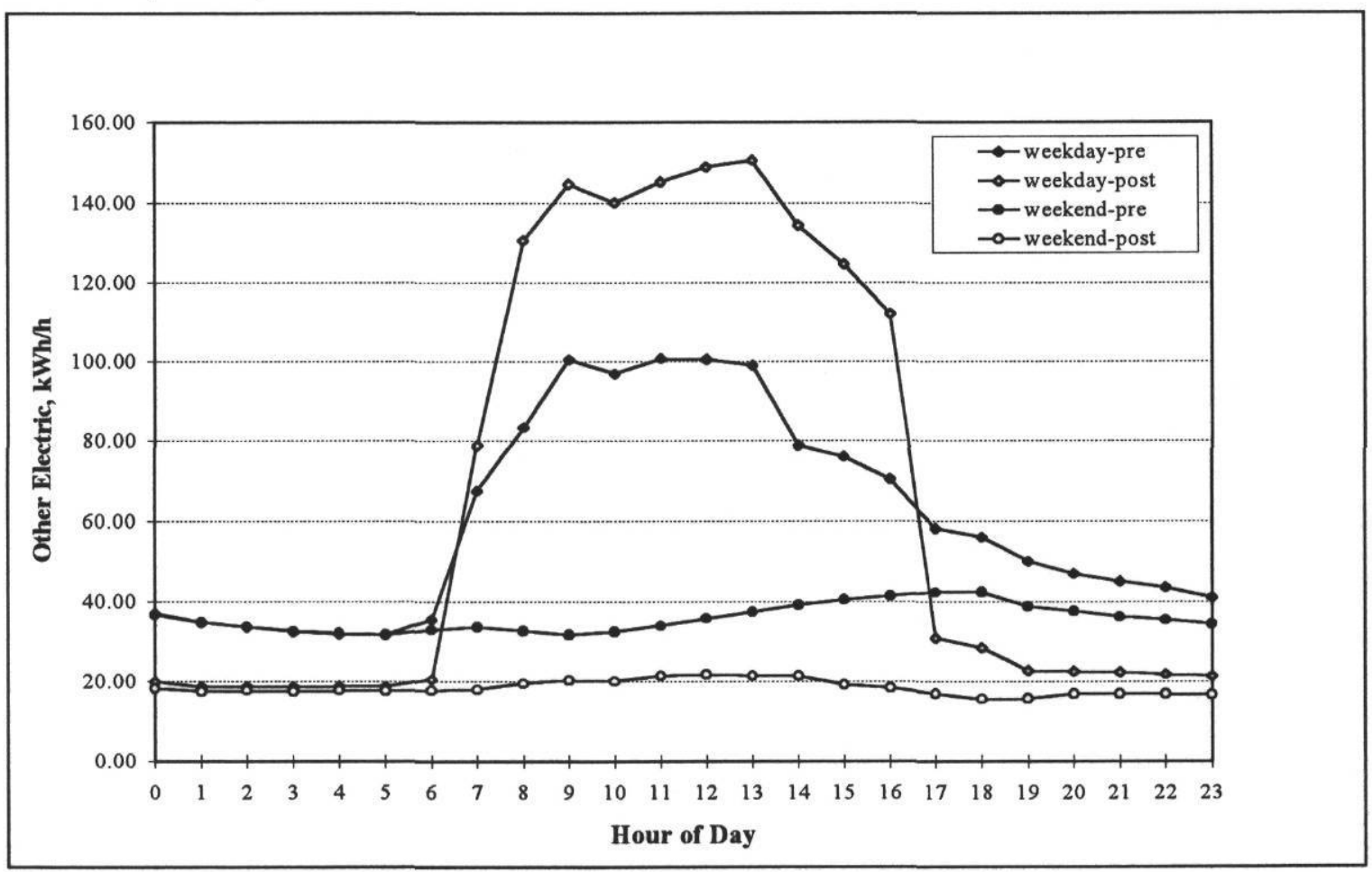

Figure C-5b: Non-semester Pre-/Post-Retrofit Comparison: based on average hourly data (Sims Elementary School)

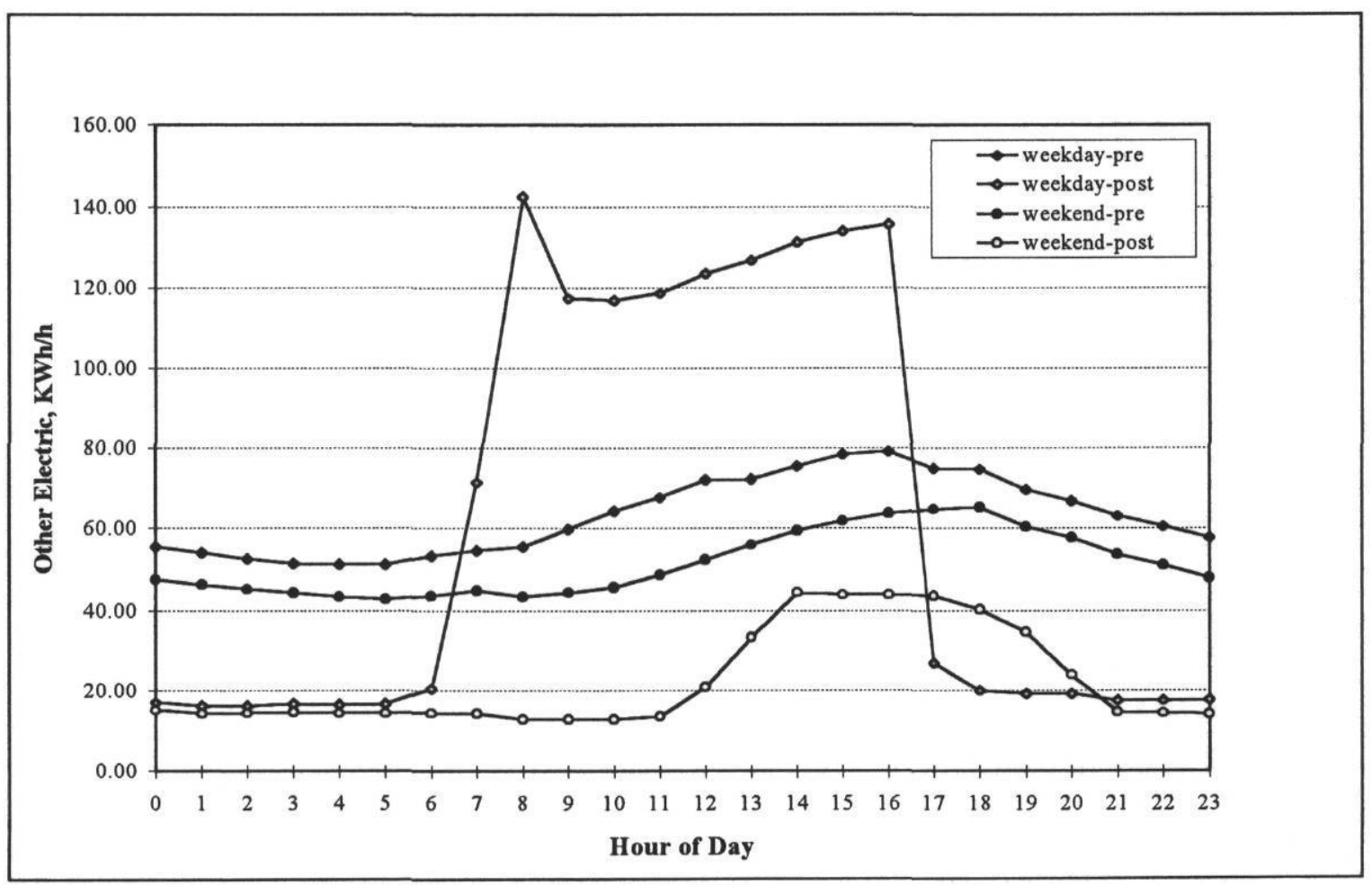


The weekend consumption decreased over all hours between the pre-retrofit and post-retrofit periods. This is indicative of equipment being left on over the weekend during the pre-retrofit period, which is now being turned off by the EMCS.

For the non-semester period, Figure C-5b, similar results as the semester period occur. The weekday consumption dramatically decreased during the nighttime hours, but dramatically increased during the daytime hours. This may be due to the same reasons stated above in the semester period analysis. For the weekends, there was an overall decrease in consumption over all hours. The consumption increases between the hours of 12:00 p.m. and 10:00 p.m. This may be due to faculty and staff working later in the day on weekends.

Tab C-3 contains a summary of the hourly averages and the respective standard deviations and count of data points. The hourly averages are the data that is plotted in Figures C-5a and C-5b. They do not vary much for the hours of 0 through 7 , then jump to higher levels in the hours of 8 through 23 . This should not be alarming, because the periods that the data was averaged over include wide ranges of temperatures. As was seen earlier in Figures $\mathrm{C}-1$ and $\mathrm{C}-2$, the energy usage is temperature dependent. The count of data points represents the actual number of data points used to calculated the average, which corresponds to the amount of time that the equipment was actually operating.

The difference in other electric energy consumption was calculated based on the average daily data. This is shown in Table C-3, both as a difference in energy and a percentage difference in energy. 
Table C-3: Difference in Other Electric Consumption based on average daily data (Sims Elementary School)

\begin{tabular}{|c|c|c|c|c|}
\hline & $\begin{array}{c}\text { \# days } \\
\text { in period }\end{array}$ & $\begin{array}{l}\text { Average Daily } \\
\text { Consumption } \\
\text { kWh/day }\end{array}$ & $\begin{array}{l}\text { Difference in } \\
\text { Average Daily } \\
\text { Consumption } \\
\text { kWh/period }\end{array}$ & $\begin{array}{l}\text { \% Difference in } \\
\text { Average Daily } \\
\text { Consumption }\end{array}$ \\
\hline \multicolumn{5}{|l|}{ Semester: } \\
\hline weekday-pre & 514 & 1,453 & \multirow[b]{2}{*}{164} & \multirow[b]{2}{*}{$11.29 \%$} \\
\hline weekday-post & 229 & 1,617 & & \\
\hline weekend-pre & 192 & 861 & \multirow[b]{2}{*}{-417} & \multirow[b]{2}{*}{$-48.41 \%$} \\
\hline weekend-post & 88 & 444 & & \\
\hline \multicolumn{5}{|l|}{ Non-Semester } \\
\hline weekday-pre & 148 & $1-517$ & \multirow[b]{2}{*}{-38} & \multirow[b]{2}{*}{$-2.48 \%$} \\
\hline weekday-post & 66 & 1,479 & & \\
\hline weekend-pre & 72 & 1,235 & \multirow[b]{2}{*}{-692} & \multirow[b]{2}{*}{$-56.03 \%$} \\
\hline weekend-post & 543 & -692 & & \\
\hline
\end{tabular}

\section{C.3.6 Plots from MECR}

The September MECR energy use plots for four years are shown in Tab C-4. These provide a more qualitative look at the effects of the EMCS. September 1992 is a pre-retrofit plot. Note that there is relatively high consumption between the hours of midnight to 6:00 a.m., with a gradual increase to daytime levels. This is followed by a slow decrease in consumption between the hours of 4:00 p.m. and 10:00 p.m. Most afternoon and evening consumption does not drop to nighttime levels. September 1993 is also a pre-retrofit plot, but shows slight improvement in afternoon, evening, and nighttime consumption. There is a measurable decrease in consumption in the afternoon. Although consumption between the hours of 7:00 p.m. and midnight is not as low as the hours of midnight to 6:00 a.m., there are greatly reduced when compared to September 1991. September 1994 is a post-retrofit plot. This plot displays all the telltale signs of an EMCS. The nighttime consumption is very low, with a sharp increase to daytime levels at 6:00 a.m. The consumption drops dramatically from daytime levels to nighttime levels at 4:00 p.m. There are two or three afternoons where the consumption id slightly higher than nighttime levels. This could be due to occasional special events in which certain areas of the school remain in use after hours. 
It should be noted that these profiles only allow a good look at weekday data only. The weekend data is virtually unreadable from these plots. The September 1994 plots shows a typical "picket fence" pattern on the Day-of-Year axis of the top plot. This shows the weekday consumption as the peaks, and the weekend consumption as the troughs. Separating the data into weekdays and weekends, then plotting separately would enable one to better evaluate weekends, as well as weekdays.

\section{C.3.7 Data Summary Notebook Information}

The Data Summary Notebook information is included in Tab C-5. It is not analyzed in this report for this site. Since it is analyzed for Zachry Engineering Center, it is provided for informational purposes only. 
Tab C-1

School District Schedules 


\section{0-91 SCHOOL CALENDAR}

AUGUST - 1990

SUN MON TUE WED THU FRI SAT

\begin{tabular}{|c|c|c|c|c|c|c|}
\hline & & & 1 & 2 & 3 & 4 \\
\hline $\begin{array}{l}3 \\
3 \\
\text { marous }\end{array}$ & 6 & 7 & 8 & 9 & $\begin{array}{l}10 \\
40 \text { and }\end{array}$ & 11 \\
\hline 12 & 13 & 14 & 15 & 16 & $\begin{array}{l}177 \\
\text { mands }\end{array}$ & 18 \\
\hline 19 & 20 मे & 210 & 220 & 230 & $24 \star$ & 25 \\
\hline 26 & $\begin{array}{ll}27 \\
\text { soma } \\
\text { simen }\end{array}$ & 28 & 29 & 30 & $\begin{array}{l}31 \\
\text { matour }\end{array}$ & \\
\hline
\end{tabular}

SEPTEMBER - 1990

\begin{tabular}{|c|c|c|c|c|c|c|}
\hline & & & & & & 1 \\
\hline $\begin{array}{l}2 \\
\text { anous } \\
\text { acosis }\end{array}$ & ${ }_{\text {uxan }}^{3}$ & ${ }^{4}$ & 5 & 6 & 7 & 8 \\
\hline 9 & 10 & 11 & 12 & 13 & 14 & 15 \\
\hline 16 & 17 & 18 & 19 & $\begin{array}{l}120 \\
\text { osimment }\end{array}$ & 21 & 22 \\
\hline 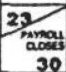 & 24 & 25 & 26 & 27 & $\begin{array}{ll}28 \\
\text { spart }\end{array}$ & 29 \\
\hline
\end{tabular}

OCTOBER - 1990

\begin{tabular}{|c|c|c|c|c|c|c|}
\hline & 1 & 2 & 3 & $\begin{array}{l}4 \\
3 \sin \times 0\end{array}$ & 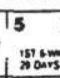 & 8 \\
\hline 7 & 81 & 9 & 10 & $\begin{array}{l}11 \\
\text { sux }\end{array}$ & 12 & 13 \\
\hline 14 & $\begin{array}{l}15 \\
2000\end{array}$ & 16 & 17 & 18 & 19 & 20 \\
\hline 21 & 22 & 23 & 24 & 25 & 26 & 27 \\
\hline 28 & 29 & 30 & 31 & & & \\
\hline
\end{tabular}

NOVEMBER - 1990

\begin{tabular}{|c|c|c|c|c|c|c|}
\hline & & & & 1 & 2 & 3 \\
\hline 4 & 5 & ${ }^{6}$ & 7 & 8 & 9 & 10 \\
\hline 11 & 12 & \begin{tabular}{|l|l}
13 \\
AMERICA
\end{tabular} & $\begin{array}{l}14 \\
\text { EDUCA }\end{array}$ & $\begin{array}{l}\text { I15 } \\
\text { TION WEE }\end{array}$ & $\begin{array}{ll}116 \\
\text { EK }\end{array}$ & 17 \\
\hline 18 & 196 & 20 & 21 & $22 \cdot$ & 23 & 24 \\
\hline $\begin{array}{l}25 \\
25 \\
\text { atosest }\end{array}$ & 26 & $\begin{array}{l}27 \\
\text { mata }\end{array}$ & \begin{tabular}{|l|}
28 \\
$x_{000}$
\end{tabular} & 29 & 30 & \\
\hline
\end{tabular}

DECEMBER - 1990

\begin{tabular}{|c|c|c|c|c|c|c|}
\hline & & & & & & 1 \\
\hline 2 & 3 & 4 & 5 & 6 & 7 & 8 \\
\hline 9 & 10 & ${ }^{11}$ & 12 & 13 & 14 & 15 \\
\hline 16 & 17 & ${ }^{18}$ & \begin{tabular}{|l|l}
19 \\
\end{tabular} & $\begin{array}{l}20^{\circ} \\
\text { WNTTER }\end{array}$ & $\begin{array}{l}210^{\circ} \\
\text { BREAA }\end{array}$ & 22 \\
\hline 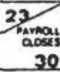 & 240 & & $\begin{array}{l}26 \\
\text { NTER }\end{array}$ & 27 & $\begin{array}{l}280^{\circ} \\
=A K\end{array}$ & 29 \\
\hline
\end{tabular}

FORT WörTH

INDERENADENT SCH(X)L DISTRICT

Quality Education For All
JANUARY - 1991

SUN MON TUE WED THU FAJ SAT

\begin{tabular}{|c|c|c|c|c|c|c|}
\hline & & 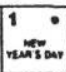 & ${ }^{2}$ max & 3 & 4 & 5 \\
\hline 6 & .7 & 8 & 9 & 10 & $\begin{array}{l}11 \\
\text { xotat }\end{array}$ & 12 \\
\hline 13 & 140 & 150 & $\begin{array}{ll}161 \\
\end{array}$ & 17 & 18 & 19 \\
\hline 20 & 210 & \begin{tabular}{|l|l|}
22 \\
250
\end{tabular} & 23 & 24 & 25 & 26 \\
\hline 27 & 28 & 29 & 30 & 31 & & \\
\hline
\end{tabular}

FEBRUARY - 1991

\begin{tabular}{|c|c|c|c|c|c|c|}
\hline & & & & & 1 & 2 \\
\hline $\begin{array}{l}3 \\
\text { acosost }\end{array}$ & 4 & $5^{5}$ & 6 & 7 & 8 & 9 \\
\hline 10 & 11 & 12 & 13 & 14 & 15 & 16 \\
\hline 17 & 18 & 19 & 20 & 21 & 22 & 23 \\
\hline 24 & 25 & 26 & 27 & 28 & & \\
\hline
\end{tabular}

MARCH - 1991

\begin{tabular}{|c|c|c|c|c|c|c|}
\hline & & & & & \begin{tabular}{|ll}
1 & 1 \\
matam \\
maxis
\end{tabular} \mid & 2 \\
\hline 3 & $\stackrel{4}{\mathrm{~A}}$ PU & & ${ }^{6} \mathrm{CH}$ & $\begin{array}{l}7 \\
0 \mathrm{~L} W\end{array}$ & $V^{8} E \in K$ & 9 \\
\hline 10 & 11 & 12 & 13 & 14 & 15 & 16 \\
\hline 17 & $18 \cdot$ & $\begin{array}{l}19{ }^{\circ} \\
P R \text { I I }\end{array}$ & & \begin{tabular}{l|}
21 \\
$R E A$
\end{tabular} & $\begin{array}{l}222 \\
\mathrm{~K}\end{array}$ & 23 \\
\hline 24 & 25 & 26 & 27 & 28 & 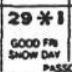 & \\
\hline
\end{tabular}

APRIL - 1991

\begin{tabular}{|c|c|c|c|c|c|c|}
\hline & 1 & 2 & 3 & 4 & S 5 & \\
\hline 7 & 8 & 9 & 10 & 11 & 12 & 13 \\
\hline 14 & 15 & 16 & 17 & 18 & $\begin{array}{l}19 \\
\operatorname{sim}^{2}\end{array}$ & 20 \\
\hline 21 & 226 & 23 & 24 & 25 & 26 & 27 \\
\hline 28 & 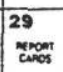 & 30 & & & & \\
\hline
\end{tabular}

O INSERVICETEACHERS' PAEPARATION Lे INSERVICE

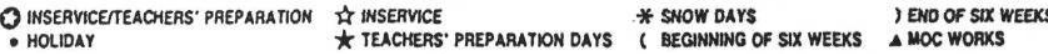

\begin{tabular}{|c|c|}
\hline \multicolumn{2}{|c|}{ 1990-91 STANOARDIZED TEST DATES } \\
\hline 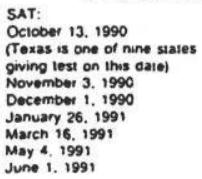 & 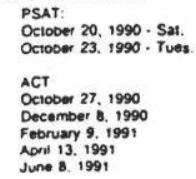 \\
\hline \multicolumn{2}{|c|}{ 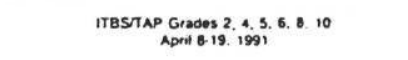 } \\
\hline 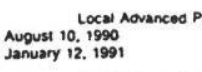 & 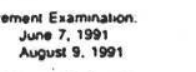 \\
\hline 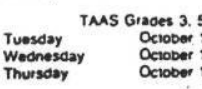 & 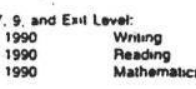 \\
\hline 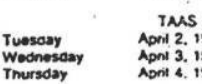 & 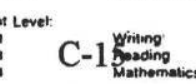 \\
\hline
\end{tabular}

MAY - 1991

SUN MON TUE WED THU FFI SAT

\begin{tabular}{|c|c|c|c|c|c|c|}
\hline . & & & 1 & 2 & 3 & 4 \\
\hline $\begin{array}{l}3 \\
\text { mamal }\end{array}$ & 6 & $7^{7}$ & 8 & 9 & 10 & 11 \\
\hline 12 & 13 & 14 & 15 & 16 & 17 & סוסس 18 \\
\hline 19 & \begin{tabular}{|l|}
20 \\
,
\end{tabular} & 21 & 22 & 23 & 24 & 25 \\
\hline 26 & $27 \Delta$ & 28 & $\begin{array}{ll}290 \\
\text { maxis }\end{array}$ & $30 \star$ mant & 31 & \\
\hline
\end{tabular}

JUNE - 1991

\begin{tabular}{|c|c|c|c|c|c|c|}
\hline & & & & & & 1 \\
\hline $\begin{array}{l}2 \\
2 \\
\text { anowit }\end{array}$ & 3 & 4 & 5 & 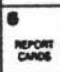 & 7 & 8 \\
\hline 9 & 10 & 11 & 12 & 13 & 14 & 15 \\
\hline 16 & 17 & 18 & 19 & 20 & 21 & 22 \\
\hline $\begin{array}{c}23 \\
\text { anos } \\
\text { and }\end{array}$ & 24 & 25 & 26 & 27 & 28 & 29 \\
\hline
\end{tabular}

$$
\text { JULY - } 1991
$$

\begin{tabular}{|c|c|c|c|c|c|c|}
\hline & 1 & ${ }_{\text {max }}^{2}$ & 3 & 4 & 5 & 6 \\
\hline 7 & 8 & 7 & 10 & 11 & 12 & 13 \\
\hline & & Vi & CAI & ON & & \\
\hline 14 & 15 & & $\begin{array}{l}17 \\
\text { CAT }\end{array}$ & $\begin{array}{l}18 \\
\text { ON }\end{array}$ & 19 & 20 \\
\hline 21 & 22 & 23 & 24 & 25 & 26 & 27 \\
\hline 28 & 29 & 30 & 31 & & & \\
\hline
\end{tabular}

\section{AUGUST - 1991}

\begin{tabular}{|c|c|c|c|c|c|c|}
\hline & & & & 1 & 2 & 3 \\
\hline 4 & 5 & & 7 & 8 & 9 & 10 \\
\hline 11 & 12 & 13 & 14 & 15 & 16 & 17 \\
\hline 18 & 19 & 20 & 21 & 22 & 23 & 24 \\
\hline 25 & 26 & 27 & 28 & 29 & 30 & 31 \\
\hline
\end{tabular}

Teaching Days

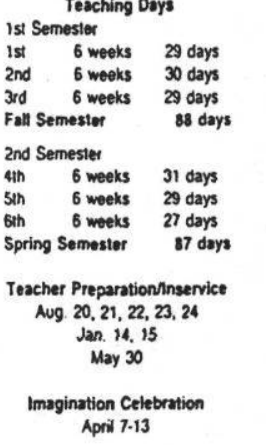

I Feb. 1. All 12 month contract personnet will work.

* Snow Day or Holiday I March 29 - All 12 month contract personnel will work. Holiday if nol a Snow Day for students, leachers. len month and oleven month coniract personnel. May 27 - A snow make-up day or holiday lor alt Porsonnel EXCEPT 260 DAY CONTRACT. 


\section{FORT WORTH INDEPENDENT SCHOOL DISTRICT}

\section{1-92 SCHOOL CALENDAR}

AUGUST - 1991

SUN MON TUE WED THU FRI SAT

\begin{tabular}{|c|c|c|c|c|c|c|}
\hline & & & & 1 & 2 & 3 \\
\hline $\begin{array}{l}4 \\
40003\end{array}$ & 5 & 8 & 7 & 8 & 9 & 10 \\
\hline 11 & 12 & 13 & 14 & $15^{*}$ & 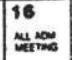 & 17 \\
\hline 18 & 19 & 20 & 21 & $\begin{array}{l}222 \\
\text { tha }\end{array}$ & $23 \star$ & 24 \\
\hline 25 & 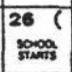 & 27 & 28 & 29 & $30 *$ & 31 \\
\hline
\end{tabular}

SEPTEMBER - 1991

\begin{tabular}{|c|c|c|c|c|c|c|}
\hline 1 & 2 & 3 & 4 & 5 & 6 & 7 \\
\hline 8 & שves & 10 & 11 & 12 & $13^{*}$ & 14 \\
\hline 15 & 16 & 17 & 18 & 19 & 20 & 21 \\
\hline 22 & 23 & 24 & 25 & 26 & 27 & 28 \\
\hline 29 & 30* & & & & & \\
\hline
\end{tabular}

OCTOBER - 1991

\begin{tabular}{|c|c|c|c|c|c|c|}
\hline & & $\begin{array}{l}1 \\
\text { sumor } \\
\text { meat }\end{array}$ & 2 & 3 & 40 & 5 \\
\hline 6 & $7^{\prime}$ & 8 & 9 & 10 & 11 & 12 \\
\hline 13 & 14 & $15^{*}$ & 16 & 17 & 18 & 19 \\
\hline 20 & 21 & 22 & 23 & 24 & 25 & 26 \\
\hline 27 & 28 & 29 & 30 & 31 sivar & & \\
\hline
\end{tabular}

NOVEMBER - 1991

\begin{tabular}{|c|c|c|c|c|c|c|}
\hline & & & & & 1 & 2 \\
\hline 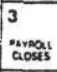 & 4 & 5 & 6 & 7 & 8 & 9 \\
\hline 10 & & $\begin{array}{l}12 \\
\text { AICAN }\end{array}$ & $\int_{\text {EDUCA }}^{13}$ & $\int_{\text {TiON W }}^{14}$ & 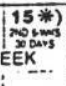 & 16 \\
\hline 17 & 18 & 19 & 20 & 21 & 22 & 23 \\
\hline 24 & \begin{tabular}{|l|}
25 \\
2500 \\
\end{tabular} & 26 & $27 *$ & 28 & $\mid 29$ & 30 \\
\hline
\end{tabular}

\section{DECEMBER - 1991}

\begin{tabular}{|c|c|c|c|c|c|c|}
\hline $\begin{array}{l}1 \\
\text { manous } \\
\text { arosest }\end{array}$ & 2 & $P_{\text {Pman }}^{3}$ & Endnuke & 5 & 6 & 7 \\
\hline 8 D. & 9 & 10 & 11 & 12 & 13 & 14 \\
\hline 15 & 16 & 17 & 18 & 19 & $20 *$ & 21 \\
\hline 22 & 23 & $\begin{array}{l}24 \\
N I N T\end{array}$ & & $\begin{array}{l}126 \\
B R E A\end{array}$ & & 28 \\
\hline 29 & 30 & 31 & & & & \\
\hline
\end{tabular}

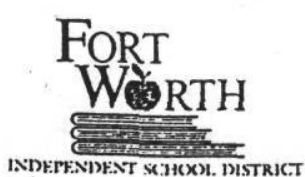

Where the Future Begins...NOW!
JANUARY - 1992

SUN MON TUE WED THU FRI SAT

\begin{tabular}{|c|c|c|c|c|c|c|}
\hline \multirow[b]{2}{*}{5} & \multirow[b]{2}{*}{6} & \multirow[b]{2}{*}{7} & \multicolumn{3}{|c|}{${ }_{1}^{1} \cdot|2 \Lambda \cdot| 3 \Lambda \cdot \mid$} & \multirow{2}{*}{\begin{tabular}{|c|}
4 \\
11
\end{tabular}} \\
\hline & & & 8 & 9 & 10 & \\
\hline 12 & 13 & 14 & $15^{*}$ & 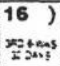 & $17^{\star}$ & 18 \\
\hline 19 & 200 & 21 & 22 & 23 & 24 & 25 \\
\hline 26 & 27 & 28 & 29 & $30^{*}$ & 316 & \\
\hline
\end{tabular}

FEBRUARY - 1992

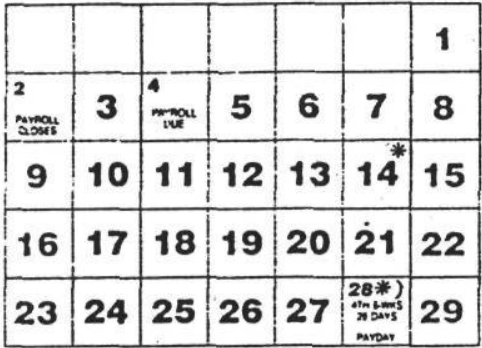

MARCH -1992

\begin{tabular}{|c|c|c|c|c|c|c|}
\hline casasis & 26 & 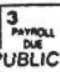 & ${ }_{\mathrm{SCHOO}}$ & 5 & 6 & 7 \\
\hline 8 & 9 & 10 & 11 & 12 & $13^{*}$ & 14 \\
\hline 15 & 16 & PR & $\begin{array}{l}18 \\
18\end{array}$ & $\begin{array}{l}19 \\
R E A\end{array}$ & $\begin{array}{l}200 \\
K\end{array}$ & 21 \\
\hline 22 & 23 & 24 & 25 & 26 & 27 & 28 \\
\hline 29 & 30 & $\begin{array}{l}31 * \\
\text { saroar }\end{array}$ & & & & \\
\hline
\end{tabular}

APRIL - 1992

\begin{tabular}{|c|c|c|c|c|c|c|}
\hline & & & 1 & 2 & 3 & 4 \\
\hline $\begin{array}{l}5 \\
\text { amasis }\end{array}$ & 6 & 7 & 8 & 9 & 10 & 11 \\
\hline 12 & 13 & 14 & $15^{*}$ & 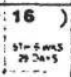 & 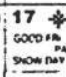 & 18 \\
\hline 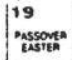 & 20 & 21 & 22 & 23 & 24 & 25 \\
\hline 26 & $\begin{array}{l}27 \\
\text { secont }\end{array}$ & 28 & 29 & $\begin{array}{l}30 * \\
70\end{array}$ & & \\
\hline
\end{tabular}

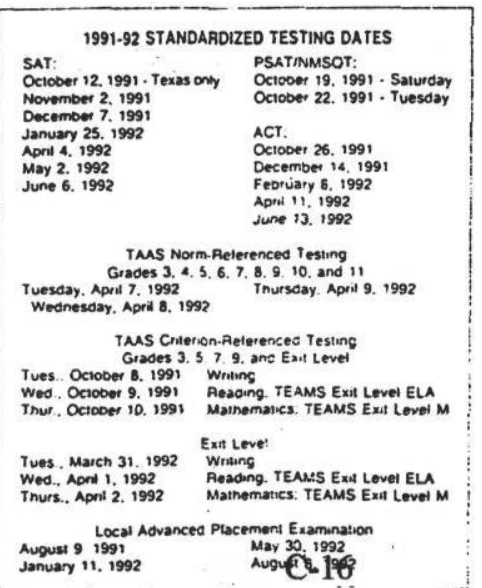

SCIE' ICE FAIR - March 31 - Aprit 5. 1992
MAY - 1992

SUN MON TUE WED THU FRI SAT

\begin{tabular}{|l|c|c|c|c|c|c|}
\hline & & & & & 1 & 2 \\
\hline 3 & 4 & $\begin{array}{l}5 \\
\text { and }\end{array}$ & 6 & 7 & 8 & 9 \\
\hline 10 & 11 & 12 & 13 & 14 & $15^{*}$ & 16 \\
\hline 17 & 18 & 19 & 20 & 21 & 22 & 23 \\
\hline 24 & \begin{tabular}{l}
25 \\
\hline
\end{tabular} & 26 & 27 & 28 & $29 *$ & 30 \\
\hline
\end{tabular}

JUNE - 1992

\begin{tabular}{|c|c|c|c|c|c|c|}
\hline & 1 & $\begin{array}{ll}20 \\
4=0\end{array}$ & $+3^{4}$ & ${ }^{4} \Delta^{\star}$ & 5 & 6 \\
\hline 7 & 8 & 9 & 10 & 11 & 12 & 13 \\
\hline 14 & $15^{*}$ & 16 & 17 & 18 & 19 & 20 \\
\hline 21 & 22 & 23 & 24 & 25 & 26 & 27 \\
\hline 28 & 29 & $30 *$ & & & & \\
\hline
\end{tabular}

JULY - 1992

\begin{tabular}{|c|c|c|c|c|c|c|}
\hline & & & 1 & 2 & $3_{\text {nam }}$ & 4 \\
\hline 5 & 6 & $\begin{array}{l}7 \\
\text { VAnas } \\
\text { VA }\end{array}$ & CAT & $\begin{array}{l}9 \\
\text { ON }\end{array}$ & 100 & 11 \\
\hline 12 & & $\sqrt{14}$ & $\begin{array}{l}115 * \\
\text { CATI }\end{array}$ & \begin{tabular}{|l|}
116 \\
$O \mathrm{~N}$
\end{tabular} & 17. & 18 \\
\hline 19 & 20 & 21 & 22 & 23 & 24 & 25 \\
\hline 26 & 27 & 28 & 29 & 30 & 310 & \\
\hline
\end{tabular}

AUGUST - 1992

\begin{tabular}{|r|r|r|r|r|r|r|}
\hline 2 & & & & & & 1 \\
\hline 9 & 10 & 11 & 12 & 13 & 14 & 15 \\
\hline 16 & 17 & 18 & 19 & 20 & 21 & 22 \\
\hline 23 & 24 & 25 & 26 & 27 & 28 & 29 \\
\hline
\end{tabular}

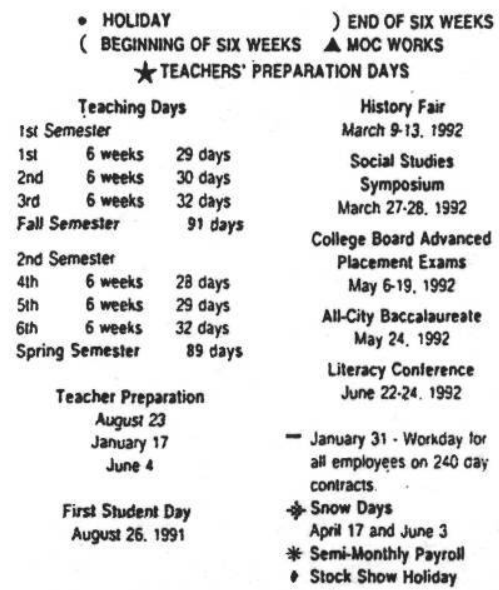

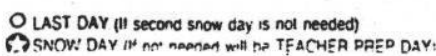




\section{Fort Worth Independent Scnool vistrici 1992-93 SCHOOL CAIENDAR}

AUGUST 1992

\begin{tabular}{|c|c|c|c|c|c|c|}
\hline SUN & MON & TUE & WED & THUR & FAI & SAT \\
\hline & & & & & & 1 \\
\hline 2 & 3 & 4 & 5 & 6 & 7 & 8 \\
\hline 9 & 10 & 11 & 12 & 13 & $\begin{array}{c}14 \\
14\end{array}$ & 15 \\
\hline 16 & 17 & 18 & 19 & $\underset{1 \in E}{20}$ & $\underset{*}{21}$ & 22 \\
\hline 20 & 24 & 25 & 26 & 27 & 28 & 29 \\
\hline
\end{tabular}

SEPTEMBER

\begin{tabular}{|c|c|c|c|c|c|c|}
\multicolumn{1}{c|}{ SUN } & \multicolumn{1}{l}{ MON } & \multicolumn{1}{l}{ TUE } & WED \\
\hline & & 1 & 2 & 3 & 4 & 5 \\
\hline 6 & 7 & 8 & 9 & 10 & 11 & 12 \\
\hline 13 & 14 & 15 & 16 & 17 & 18 & 19 \\
\hline 20 & 21 & 22 & 23 & 24 & 25 & 26 \\
\hline 27 & 28 & 29 & 30 & & & \\
\hline
\end{tabular}

\section{OCTOBER}

\begin{tabular}{|c|c|c|c|c|c|c|}
\hline SUN & MON & TUE & WED & THUR & FRII & SAT \\
\hline & & & & 1 & 2 & 3 \\
\hline 4 & 5 & 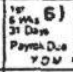 & $\{7$ & 8 & 9 & 10 \\
\hline 11 , & 12 & 13 & $\begin{array}{l}14 \\
3\end{array}$ & 15 & 16 & 17 \\
\hline 18, & $\begin{array}{r}19 \\
\times \times \times \\
\end{array}$ & 20 & 21 & 22 & 23 & 24 \\
\hline 25 & 26 & 27 & 28 & 29 & 30 & 31 \\
\hline
\end{tabular}

\section{NOVEMBER}

\begin{tabular}{|c|c|c|c|c|c|c|}
\hline SUN & MON & TUE & WED & THUR & FRI & SAT \\
\hline $\mathbf{1}$ & 2 & 3 & 4 & 5 & 6 & 7 \\
\hline 8 & 9 & 10 & 11 & 12 & 13 & 14 \\
\hline 15 & 16 & 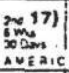 & 118 & 19 & 20 & 21 \\
\hline 22 & 23 & 24 & 25 & 26 & $\begin{array}{l}27 \\
\text { Giving }\end{array}$ & 28 \\
\hline 29 & 30 & & & & & \\
\hline
\end{tabular}

\begin{tabular}{|c|c|c|c|c|c|c|}
\hline \multicolumn{7}{|c|}{ DECEMBER } \\
\hline SUN & MON & TUE & WEO & THUR & $F R I$ & SAT \\
\hline & & 1 & 2 & 3 & 4 & 5 \\
\hline 6 & 7 & 8 & 9 & 10 & 11 & 12 \\
\hline 13 & 14 & 15 & 16 & 17 & 18 & 19 \\
\hline 20 & ${ }_{*}^{21}$ & $\begin{array}{r}22 \\
* * 1\end{array}$ & 23 & 24 & 25 & 25 \\
\hline 27 & 28 & 29 & 30 & 31 & & \\
\hline
\end{tabular}

\section{FORT}

WerTH

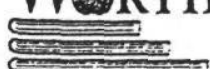

INDEPENDENT SCHOOL DISTRICT

Where the Future Begins... Now
JANUARY 1993

\begin{tabular}{|c|c|c|c|c|c|c|}
\hline SUN & MON & TUE & WED & THUR & FRI & SAT \\
\hline & & & & & $\begin{array}{c}1 \\
-\operatorname{ran}\end{array}$ & 2 \\
\hline$\underbrace{3}_{n=\infty}$ & 4 & $\underset{\substack{\text { paration. } \\
\text { and }}}{5}$ & 6 & 7 & 8 & 9 \\
\hline 10 & 11 & 12 & 13 & $\begin{array}{l}141 \mathrm{~J} \\
3 \ldots c i n s \\
\text { wents }\end{array}$ & $\begin{array}{r}15 \\
* \\
\end{array}$ & 16 \\
\hline 17 & $(18)$ & 119 & 20 & 21 & 22 & 23 \\
\hline 24 & 25 & 26 & 27 & 28 & 29 & 30 \\
\hline 21 & $\tilde{c}^{2 \infty}$ & & Dararar & & & \\
\hline
\end{tabular}

FEBRUARY

\begin{tabular}{|c|c|c|c|c|c|c|}
\hline SUN & MON & TUE & WED & THUR & $F R I$ & SAT \\
\hline & 1 & $\underset{a}{2}$ & 3 & 4 & $\begin{array}{c}\text { (5) } \\
\text { co } \\
\sin 0 \sin \end{array}$ & 6 \\
\hline 7 & 8 & 9 & 10 & 11 & 12 & 13 \\
\hline 14 & 15 & 16 & 17 & 18 & 19 & 20 \\
\hline 21 & 22 & 23 & 24 & 25 & 26 & 27 \\
\hline 28 & & & & & & \\
\hline
\end{tabular}

\section{MARCH}

SUN MON TUE WED THUR FRI SAT

\begin{tabular}{|c|c|c|c|c|c|c|c|}
\hline & 1 & 2 & 3 & & \\
\hline 7 & 8 & 9 & 10 & 11 & 125 & 13 \\
\hline 14 & 15 & 16 & 17 & 18 & 19 & 20 \\
\hline 21 & 22 & 23 & 24 & 25 & 26 & 27 \\
\hline 28 & 29 & 30 & 31 & & & \\
\hline
\end{tabular}

APRIL

\begin{tabular}{|c|c|c|c|c|c|c|}
\hline SUN & MON & TUE & WED & THUA & FAI & SAT \\
\hline & & & : & 1 & 2 & 3 \\
\hline 4 & 5 & 6 & 7 & 8 & 9 & 10 \\
\hline 11 & $\begin{array}{l}12 \\
\mathrm{~s} A \\
\end{array}$ & 13 & 14 & 15 & $\begin{array}{l}\text { 16) } \\
\text { sans }\end{array}$ & 17 \\
\hline 18 & $\begin{array}{c}19 \\
\end{array}$ & 120 & 21 & 22 & 23 & 24 \\
\hline 25 & 26 & 27 & 28 & 29 & 30 & \\
\hline
\end{tabular}

MAY

\begin{tabular}{|c|c|c|c|c|c|c|}
\hline SUN & MON & TUE & WED & THUA & FRI & SAT \\
\hline 2 & 3 & $\frac{4}{50}$ & 5 & 6 & 7 & 8 \\
\hline 9 & 10 & 11 & 12 & 13 & 14 & 15 \\
\hline 16 & 17 & 18 & 19 & 20 & 21 & 22 \\
\hline 23 & 24 & 25 & 26 & 27 & 28 & 29 \\
\hline
\end{tabular}

JUNE

\begin{tabular}{|c|c|c|c|c|c|c|}
\hline SUN & MON & TUE & WED & THUR & FRA & SAT \\
\hline & & 1 & $\sum_{0 \rightarrow 0}^{21}$ & $\begin{array}{r}3 \\
+\end{array}$ & 4 & 5 \\
\hline 6 & 7 & 8 & 9 & 10 & 11 & 12 \\
\hline 13 & 14 & 15 & 16 & 17 & 18 & 19 \\
\hline 20 & 21 & 22 & 23 & 24 & 25 & 26 \\
\hline 27 & 28 & 29 & 30 & & & \\
\hline
\end{tabular}

JULY

\begin{tabular}{|c|c|c|c|c|c|c|}
\hline SUN & MON & TUE & WED & THUR & FRI & SAT \\
\hline & & & & 1 & 2 & 5 \\
\hline$\underset{4}{4}$ & (5) & 6 & 7 & 8 & 9 & To? \\
\hline 11 & $12_{v}$ & 13 & 14 & $\begin{array}{l}15 \mathrm{~J} \\
10\end{array}$ & 16 & $3 i$ \\
\hline 18 & 19 & 20 & 21 & 22 & 23 & $2=$ \\
\hline 25 & 26 & 27 & 28 & 29 & 30 & $\approx 3$ \\
\hline
\end{tabular}

\section{AUGUST 1993}

\begin{tabular}{|c|c|c|c|c|c|c|}
\hline SUN & MON & TUE & WED & THUA & FFA & SAT \\
\hline $\begin{array}{c}1 \\
0 \\
0\end{array}$ & 2 & 3 & 4 & 5 & 6 & 7 \\
\hline 8 & 9 & 10 & 11 & 12 & 13 & 13 \\
\hline 15 & 16 & 17 & 18 & 19 & 20 & $2\}$ \\
\hline 22 & 23 & 24 & 25 & 26 & 27 & 28 \\
\hline 29 & 30 & 31 & & & & \\
\hline
\end{tabular}

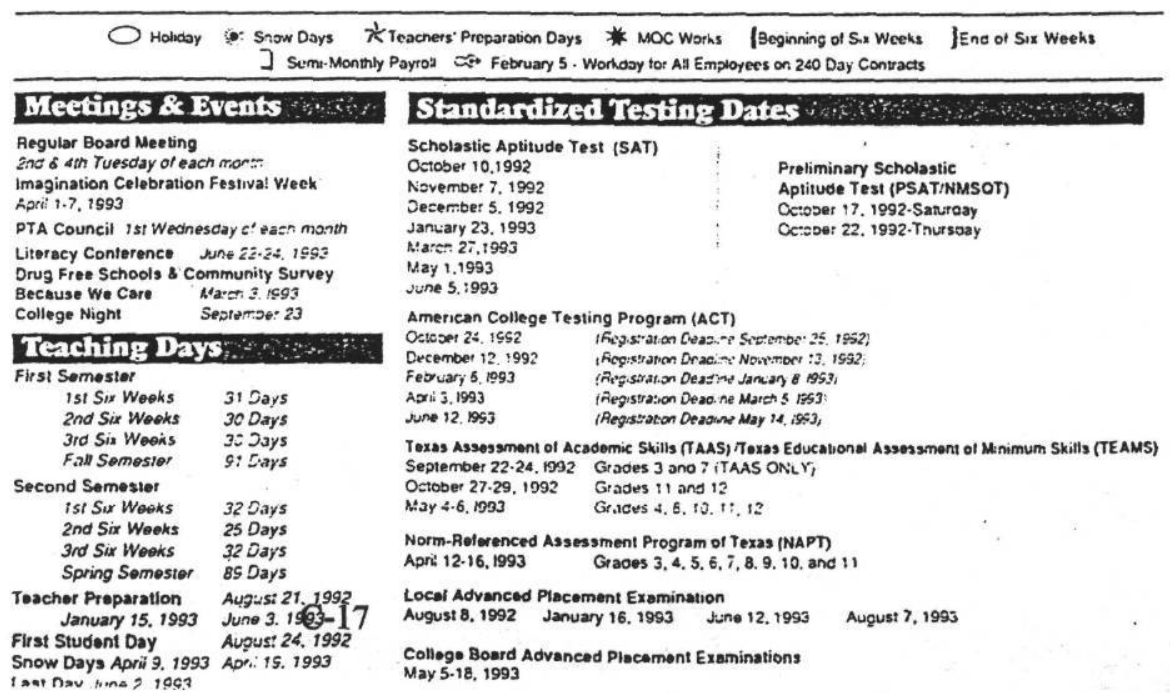




\section{Fort Worth Independent School District 1993-94 SCHOOL CALENDAR}

AUGUST 1993

\begin{tabular}{|c|c|c|c|c|c|c|}
\hline 1 & 2 & 3 & 4 & 5 & 6 & 7 \\
\hline 8 & 9 & 10 & 11 & 12 & 13 & 14 \\
\hline 15 & 16 & 17 & 18 & 19 & 20 & 21 \\
\hline 22 & 23 & 24 & 25 & 26 & 27 & 28 \\
\hline 29 & 30 & 31 & & & & \\
\hline
\end{tabular}

SEPTEMBER 1993

SUN MON TUE WED THUR FPI SAT

\begin{tabular}{|c|c|c|c|c|c|c|}
\hline & & & 1 & 2 & 3 & 4 \\
\hline 5 & 6 & 7 & 8 & 9 & 10 & 11 \\
\hline 12 & 13 & 14 & 15 & 16 & 17 & 18 \\
\hline 19 & 20 & 21 & 22 & 23 & 24 & 25 \\
\hline 26 & 27 & 28 & 29 & 30 & & \\
\hline
\end{tabular}

OCTOBER 1993

SUN MON TUE WED THUR FPA SAT

\begin{tabular}{|c|c|c|c|c|c|c|}
\hline & & & & & 1 & 2 \\
\hline 3 & 4 & 5 & 6 & 7 & 8 & 9 \\
\hline 10 & 11 & 12 & 13 & 14 & 15 & 16 \\
\hline 17 & 18 & 19 & 20 & 21 & 22 & 23 \\
\hline 24 & 25 & 26 & 27 & 28 & 29 & 30 \\
\hline
\end{tabular}

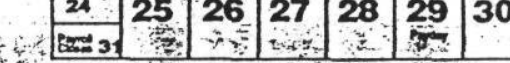

NOVEMBER 1993

OC SUW WOW TUE WED ITHUA FFA SSAT

\begin{tabular}{rl|l|l|l|l|l|l|l|l|}
\hline & 1 & 2 & 3 & 4 & 5 & 6
\end{tabular}

$\begin{array}{lllllll}7 & 7 & 9 & 10 & 11 & 12 & 13\end{array}$

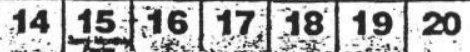

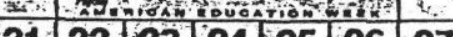

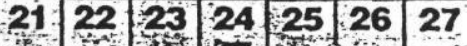

282930

$\Leftrightarrow \quad 1$,

WO O DECEMBER

SUN WON TUE WED THUR FAA SAT

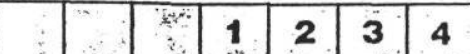

$\begin{array}{llllllll}5 & 6 & 7 & 8 & 9 & 10 & 11\end{array}$

\begin{tabular}{l|l|l|l|l|l|l|}
12 & 13 & 14 & 15 & 16 & 17 & 18
\end{tabular}

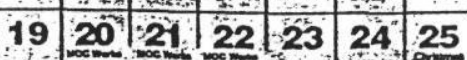

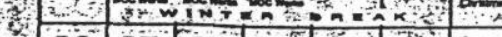

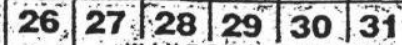

\section{FORT:}

W'WTH

7 INDEPENDENT SCHOOL DISTRIC

Where the Future Begins... Now

Student Services Department
JANUARY 1994

\begin{tabular}{|c|c|c|c|c|c|c|} 
SUN & \multicolumn{1}{l}{ MON } & \multicolumn{1}{l}{ TUE } \\
\hline & & & & & & \multicolumn{1}{l|}{1} \\
\hline 2 & $\star 3$ & 4 & 5 & 6 & 7 & 8 \\
\hline 9 & 10 & 11 & 12 & 13 & 14 & 15 \\
\hline 16 & 17 & 18 & 19 & 20 & 21 & 22 \\
\hline 20 & 24 & 25 & 26 & 27 & 28 & 29 \\
\hline
\end{tabular}

FEBRUARY 1994

SUN MON TUE WED THUR FFI SAT

\begin{tabular}{|c|c|c|c|c|c|c|}
\hline & & 1 & 2 & 3 & 4 & 5 \\
\hline 6 & 7 & 8 & 9 & 10 & 11 & 12 \\
\hline 13 & 14 & 15 & 16 & 17 & 18 & 19 \\
\hline 20 & 21 & 22 & 23 & 24 & 25 & 26 \\
\hline 27 & 28 & & & & & \\
\hline
\end{tabular}

MARCH 1994

\begin{tabular}{|r|r|r|r|r|r|r|}
\hline & & 1 & 2 & 3 & 4 & 5 \\
\hline 6 & 7 & 8 & 9 & 10 & 11 & 12 \\
\hline 13 & 14 & 15 & 16 & 17 & 18 & 19 \\
\hline 20 & 21 & 22 & 23 & 24 & 25 & 26 \\
\hline 27 & 23 & 29 & 30 & 31 & & \\
\hline & & & & & & \\
\hline
\end{tabular}

\begin{tabular}{|c|c|c|c|c|c|c|}
\hline SuN & MON & TUE & WED & THUR & FFi & SAT \\
\hline$\because$ & $\therefore$ & & & : & 1 & 2 \\
\hline-3 & & 5 & 6 & 7 & 8 & 9 \\
\hline 10 & 11 & $\begin{array}{l}12 \\
\wedge .5\end{array}$ & $\begin{array}{l}13 \\
A T\end{array}$ & $\begin{array}{r}14 \\
10\end{array}$ & N & 16 \\
\hline 17 & 18 & $\begin{array}{r}19 \\
\times \quad c\end{array}$ & 20 & 21 & 22 & 23 \\
\hline 24 & 25 & 26 & 27 & 28 & 29 & 30 \\
\hline
\end{tabular}

APRIL 1994

SUN MON TUE WED THUR IFA S SAT

\begin{tabular}{|r|r|r|r|r|r|r|}
\hline & & & & & & 2 \\
\hline 3 & 4 & 5 & 6 & 7 & 8 & 9 \\
\hline 10 & 11 & 12 & 13 & 14 & 15 & 16 \\
\hline 17 & 18 & 19 & 20 & 21 & 22 & 23 \\
\hline 24 & 25 & 26 & 27 & 28 & 29 & 30 \\
\hline
\end{tabular}

JUNE 1994

\begin{tabular}{|c|c|c|c|c|c|c|}
\hline SUN & MON & TUE & WED & THUR & $F F$ : & SAT \\
\hline & & & 1 & 2 & 3 & 4 \\
\hline 5 & 6 & $\frac{7}{26}$ & 8 & 9 & 10 & 12 \\
\hline 12 & 13 & 14 & 15 & 16 & 17 & 18 \\
\hline 19 & 20 & 21 & 22 & 23 & 24 & 25 \\
\hline & 27 & 28 & 29 & 30 & & \\
\hline
\end{tabular}

JULY 1994

$x_{3+}-$ AUGUST 1994

SUN MON JUE WED THUA FFA SAT

\begin{tabular}{|c|c|c|c|c|c|c|}
\hline 1 & 2 & 3 & 4 & 5 & 6 & 7 \\
\hline 8 & 9 & 10 & 11 & 12 & 13 & 14 \\
\hline 15 & 16 & 17 & 18 & 19 & 20 & 21 \\
\hline 22 & 23 & 24 & 25 & \multirow{2}{*}{$\begin{array}{c}26 \\
29\end{array}$} & 27 & 28 \\
\hline 29 & 30 & 31 & & & & \\
\hline
\end{tabular}

\begin{tabular}{|c|c|c|c|c|c|c|}
\hline & 1 & 2 & 3 & 4 & 5 & 6 \\
\hline 7 & 8 & 9 & 10 & 11 & 12 & 13 \\
\hline 14 & 15 & 16 & 17 & 18 & 19 & 20 \\
\hline 21 & 22 & 23 & 24 & 25 & 26 & 27 \\
\hline 28 & 29 & 30 & 31 & & & \\
\hline
\end{tabular}

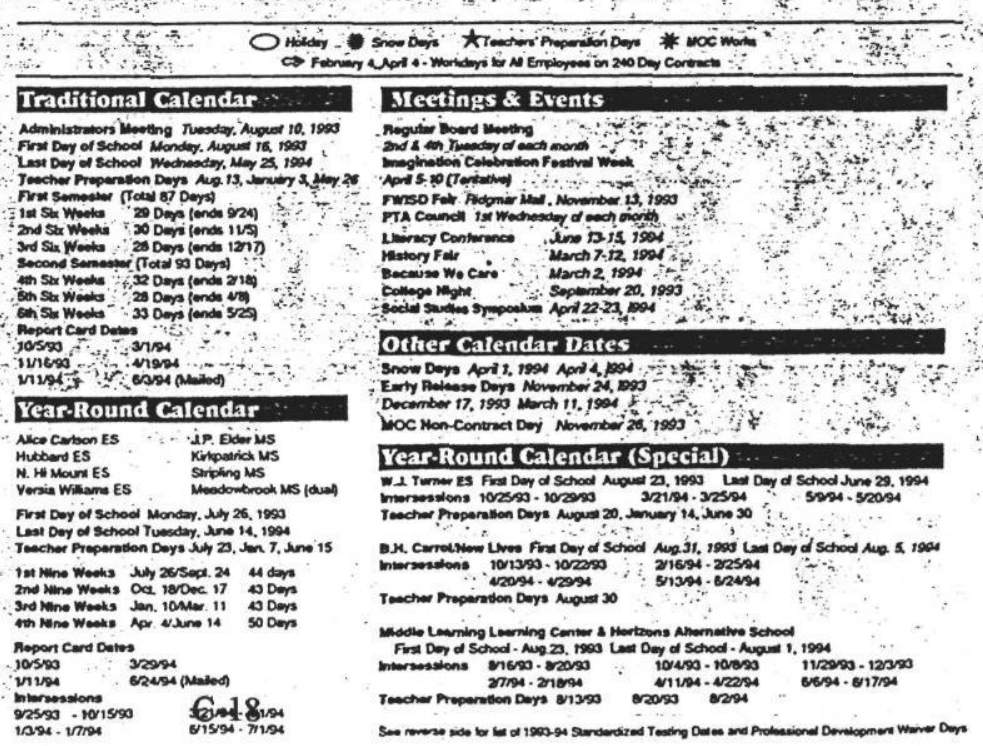


Tab C-2

Monitoring Diagrams 


\section{ELECTRICAL MONITORING DIAGRAM}

FWISD - SIMS ELEMENTARY

$\mathrm{K}=\mathrm{KWH}$ CHANNE

A=ANALOG CHANNE

$D=$ DIGITAL CHANNEL

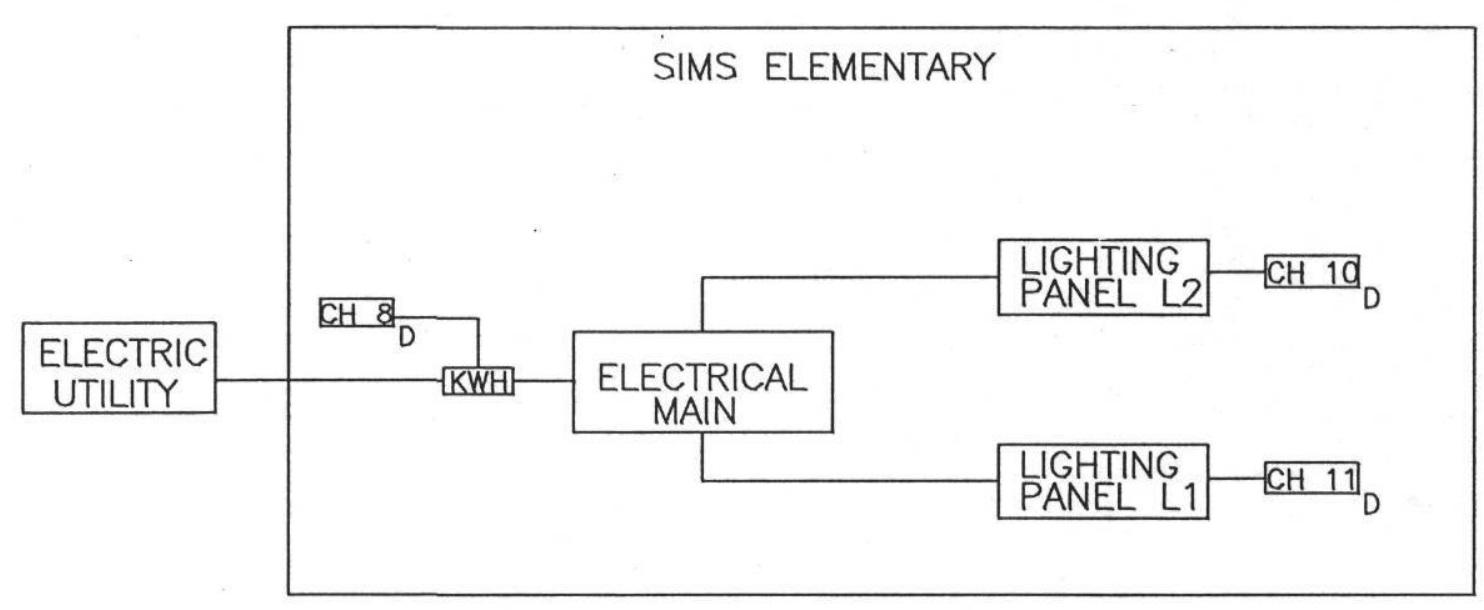

FWISD/SIMS ELEMENTARY - SITE 128 


\section{THERMAL MONITORING DIAGRAM}

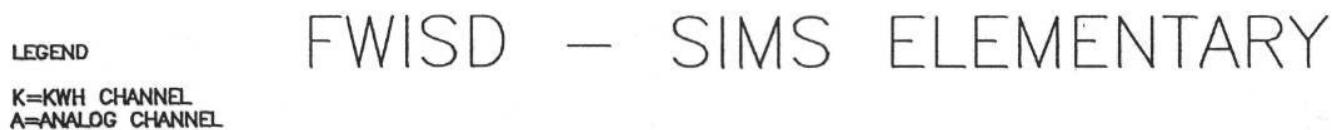

$D=D I G T A L$ CHANNE

PC=PUMPED CONDENSATE

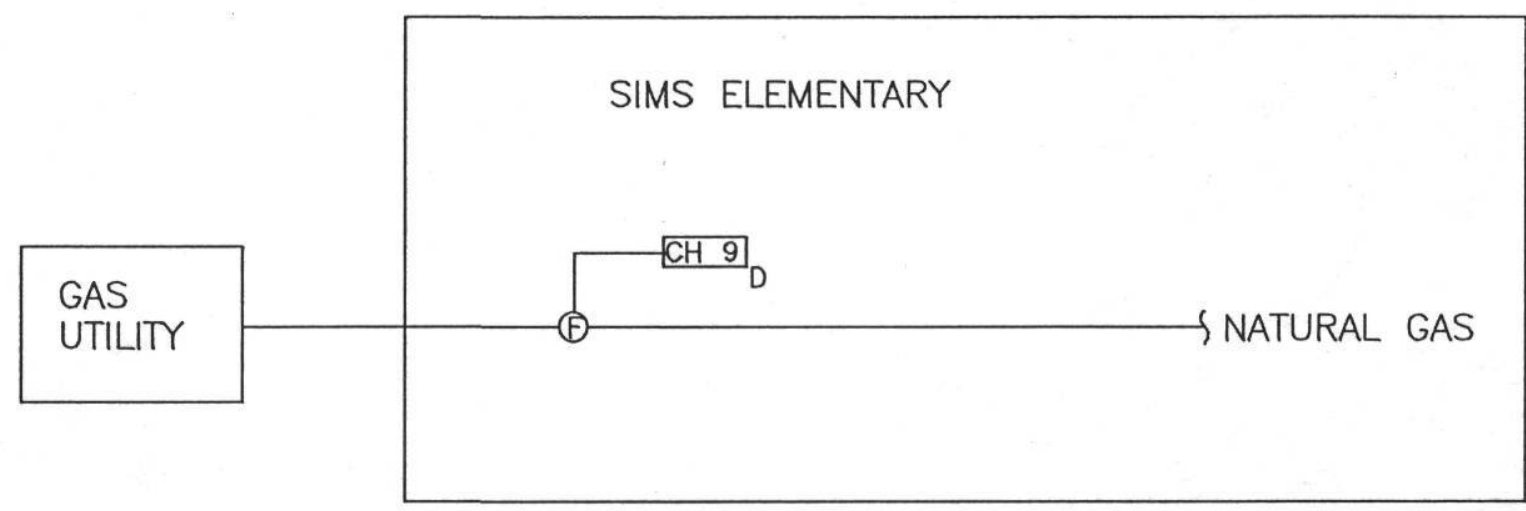

FWISD/SIMS ELEMENTARY - SITE 128 


\section{Tab C-3}

Average Hourly Data \& Related Statistics 

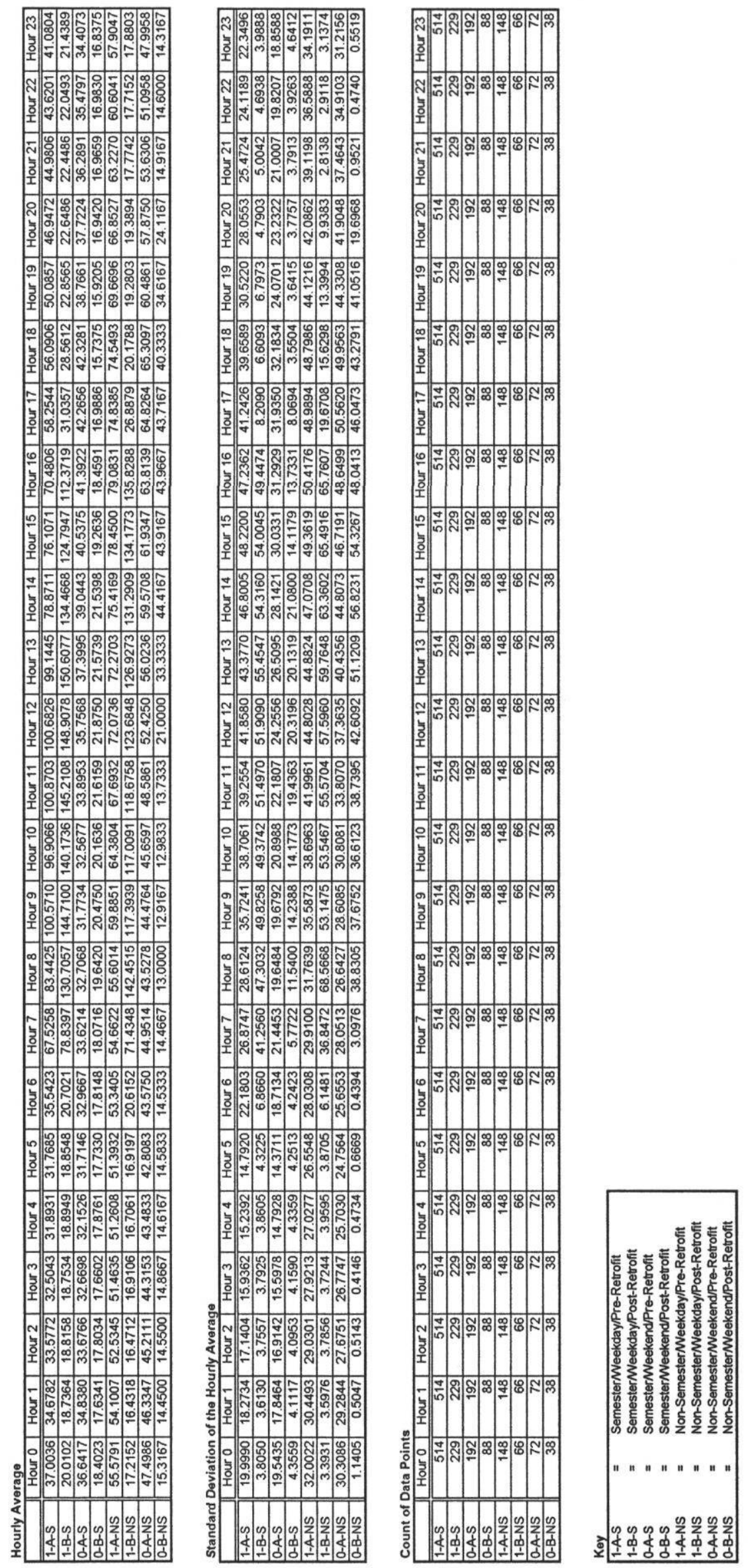
Tab C-4

MECR Plots 
Whole-Building Electric

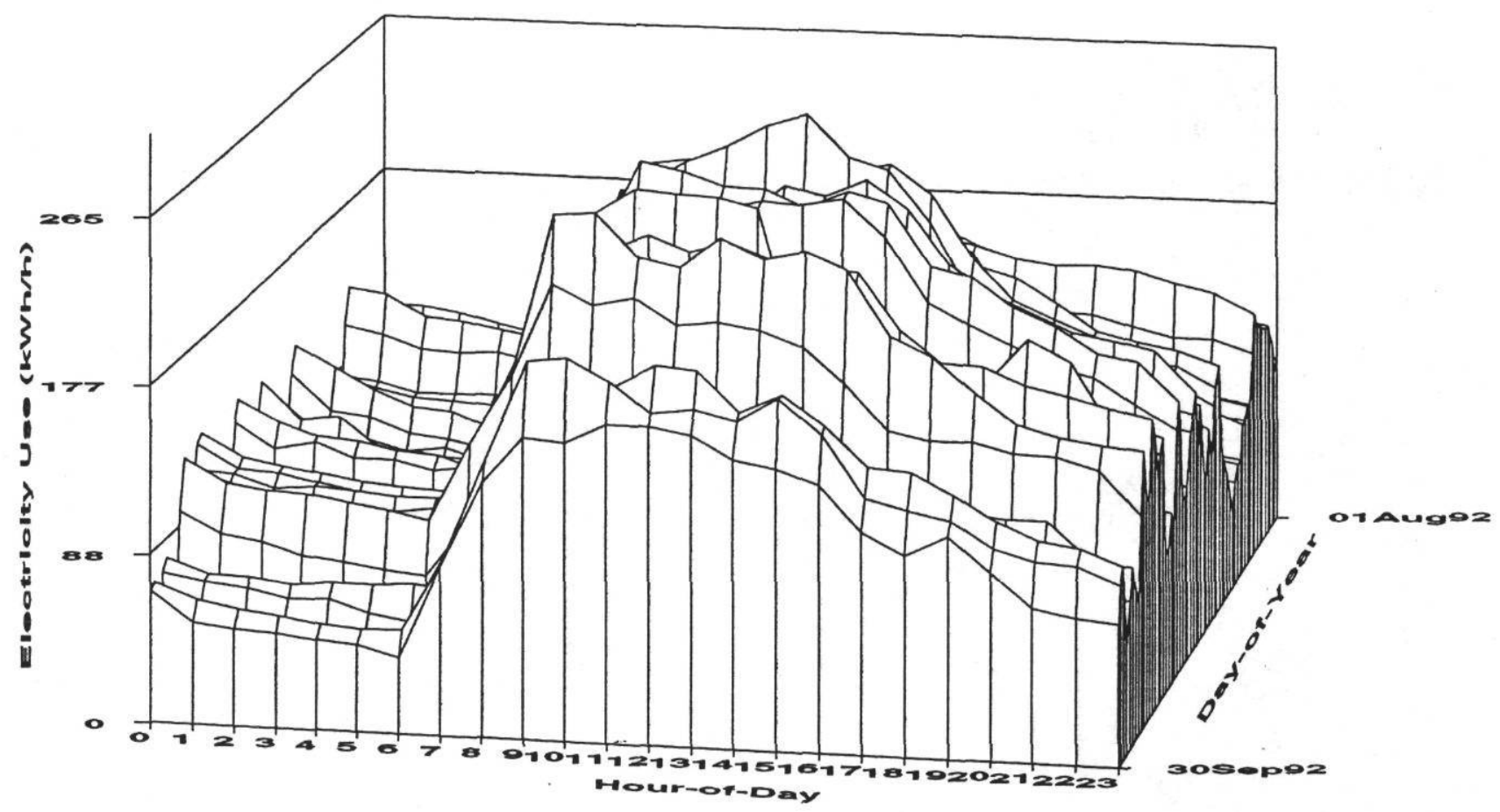

Whole-Building Electric

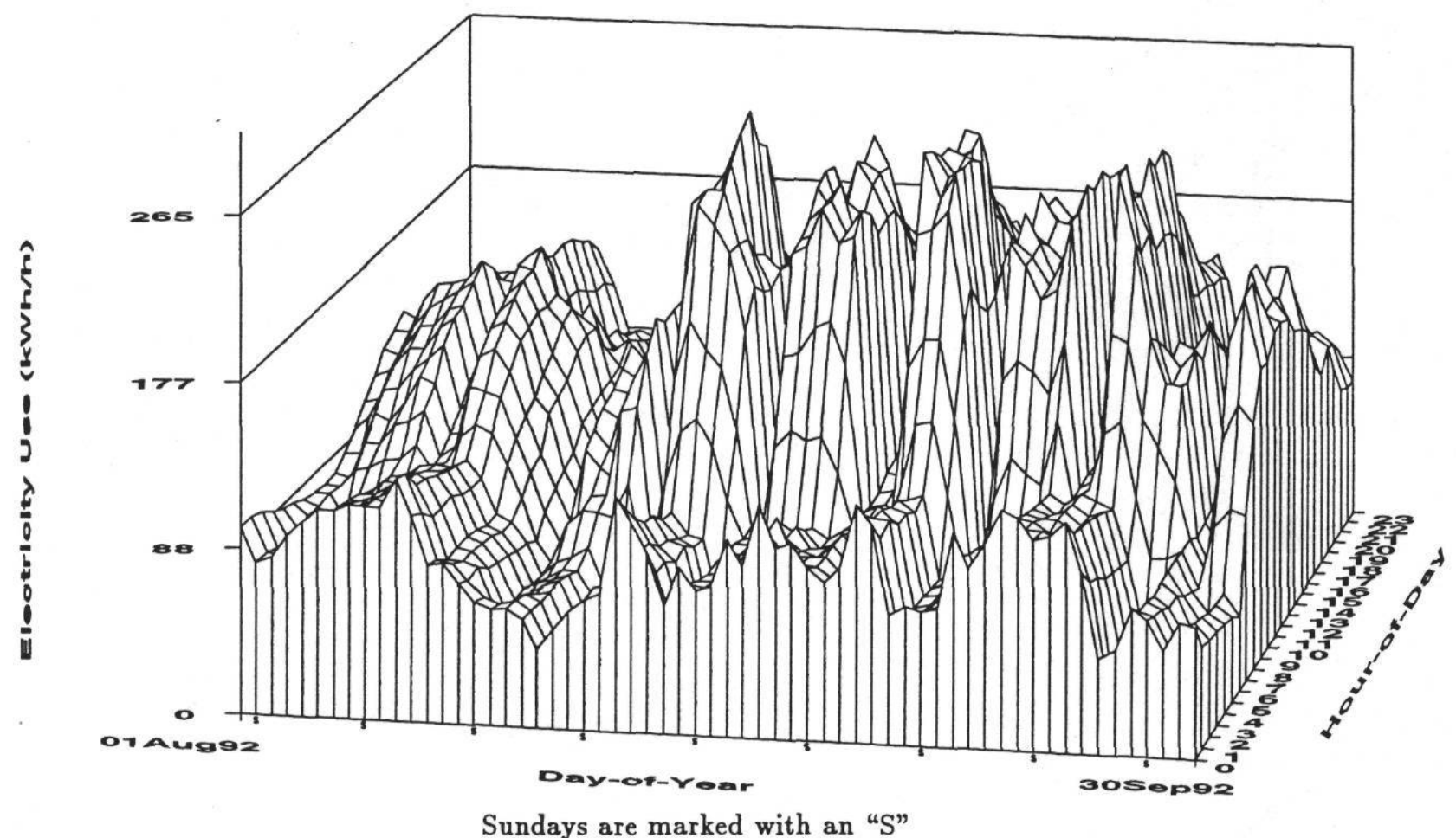

Sims Elementary School - Fort Worth ISD - September 1992

Texas Governor's Energy Office

LoanSTAR Monitoring \& Analysis Program
Monthly Energy Consumption Report (C)

Version 1.4

C-25
Energy Systems Lab Texas A\&M University 
Page 5

Whole-Building Electric

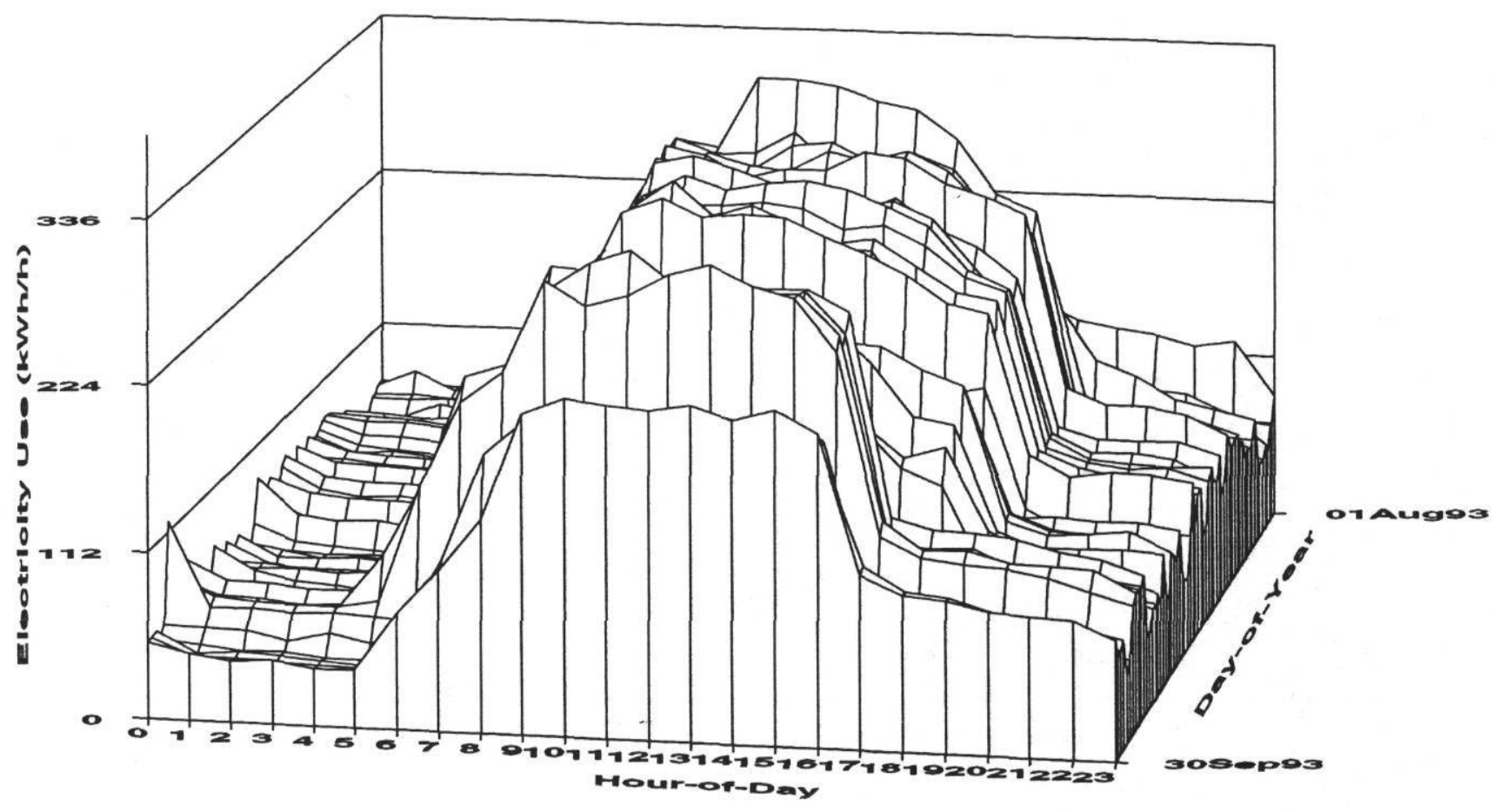

Whole-Building Electric

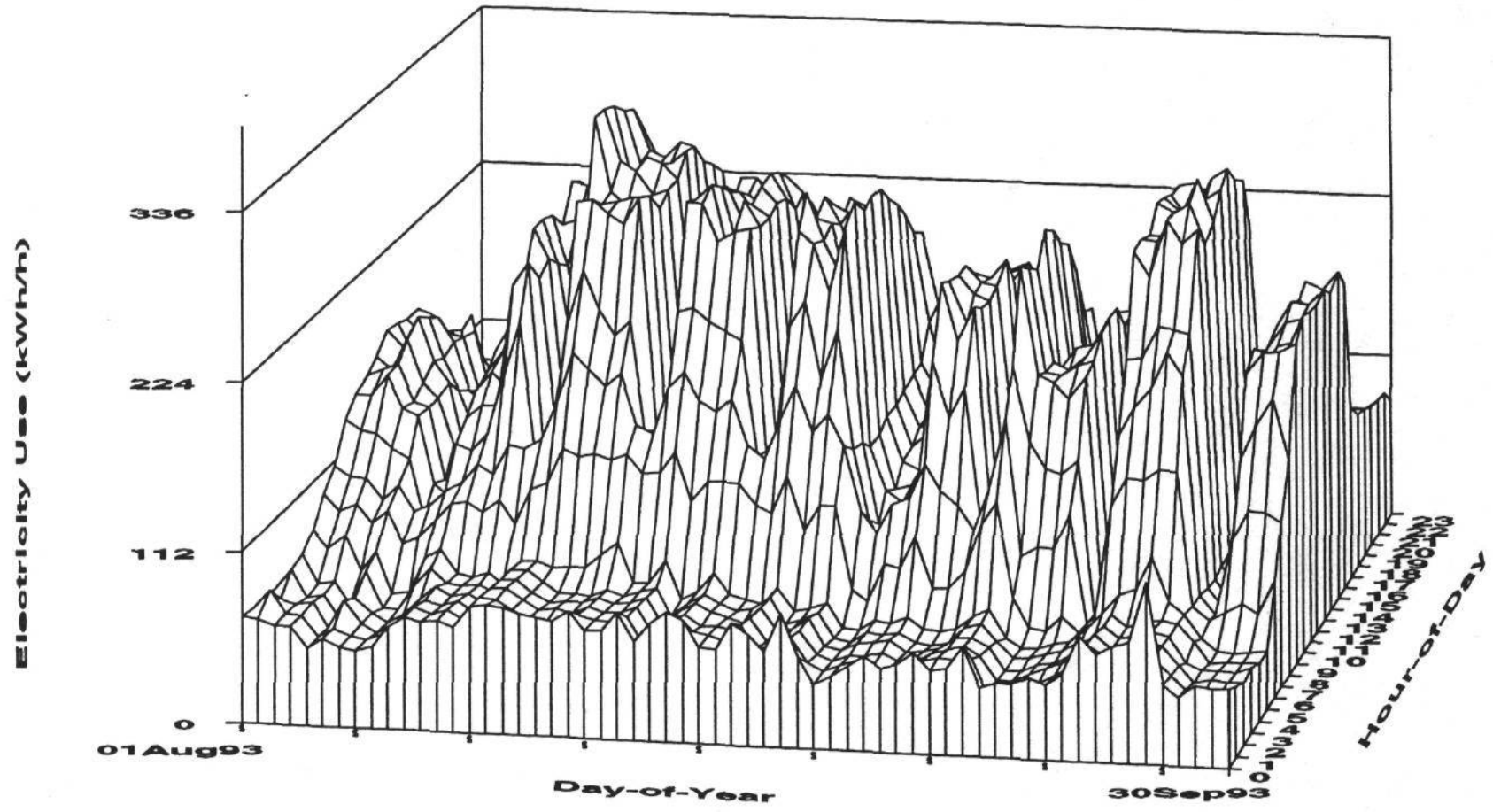

Sundays are marked with an "S"

Sims Elementary School - Fort Worth ISD - September 1993 
Whole-Building Electric

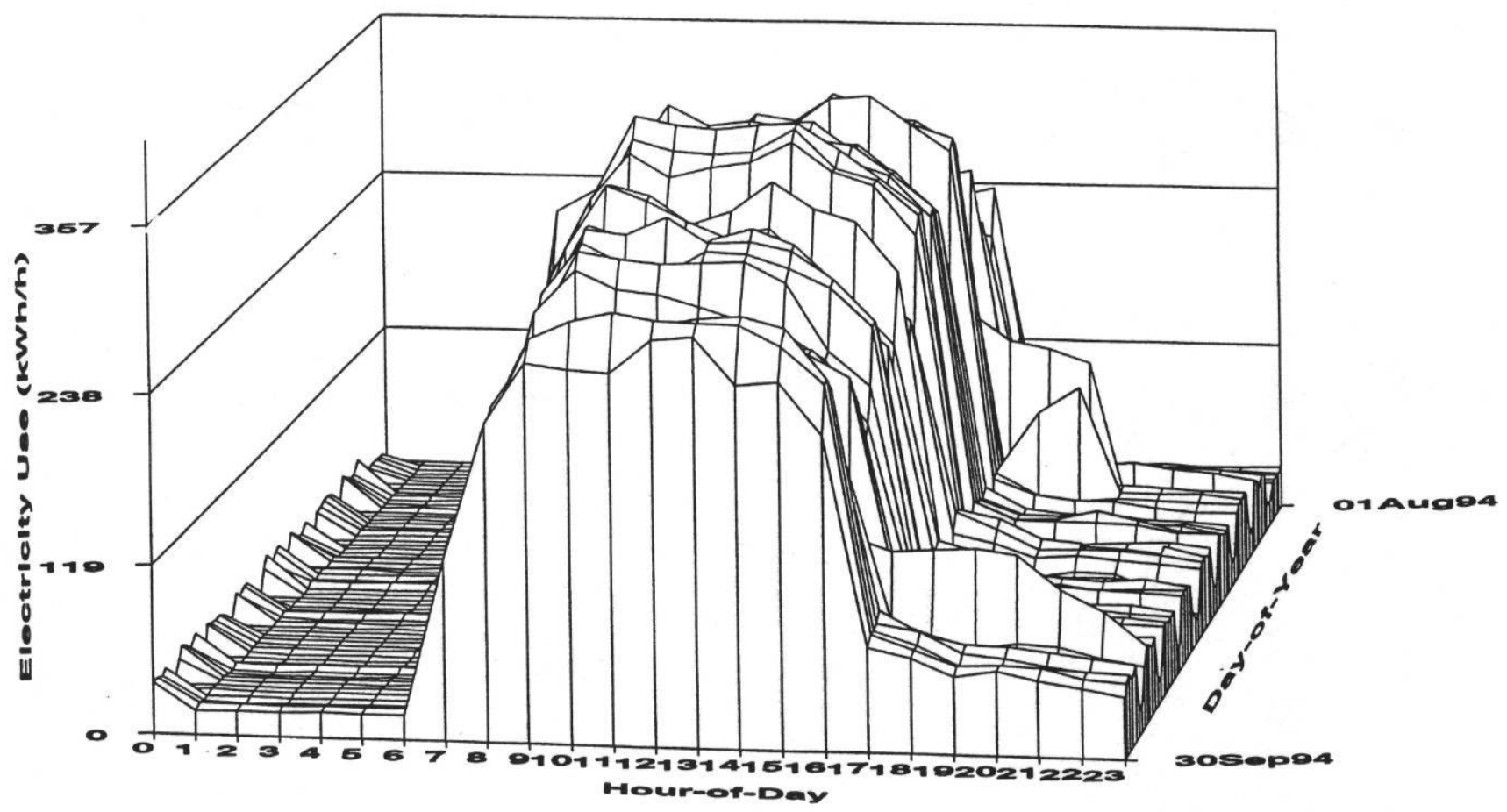

Whole-Building Electric

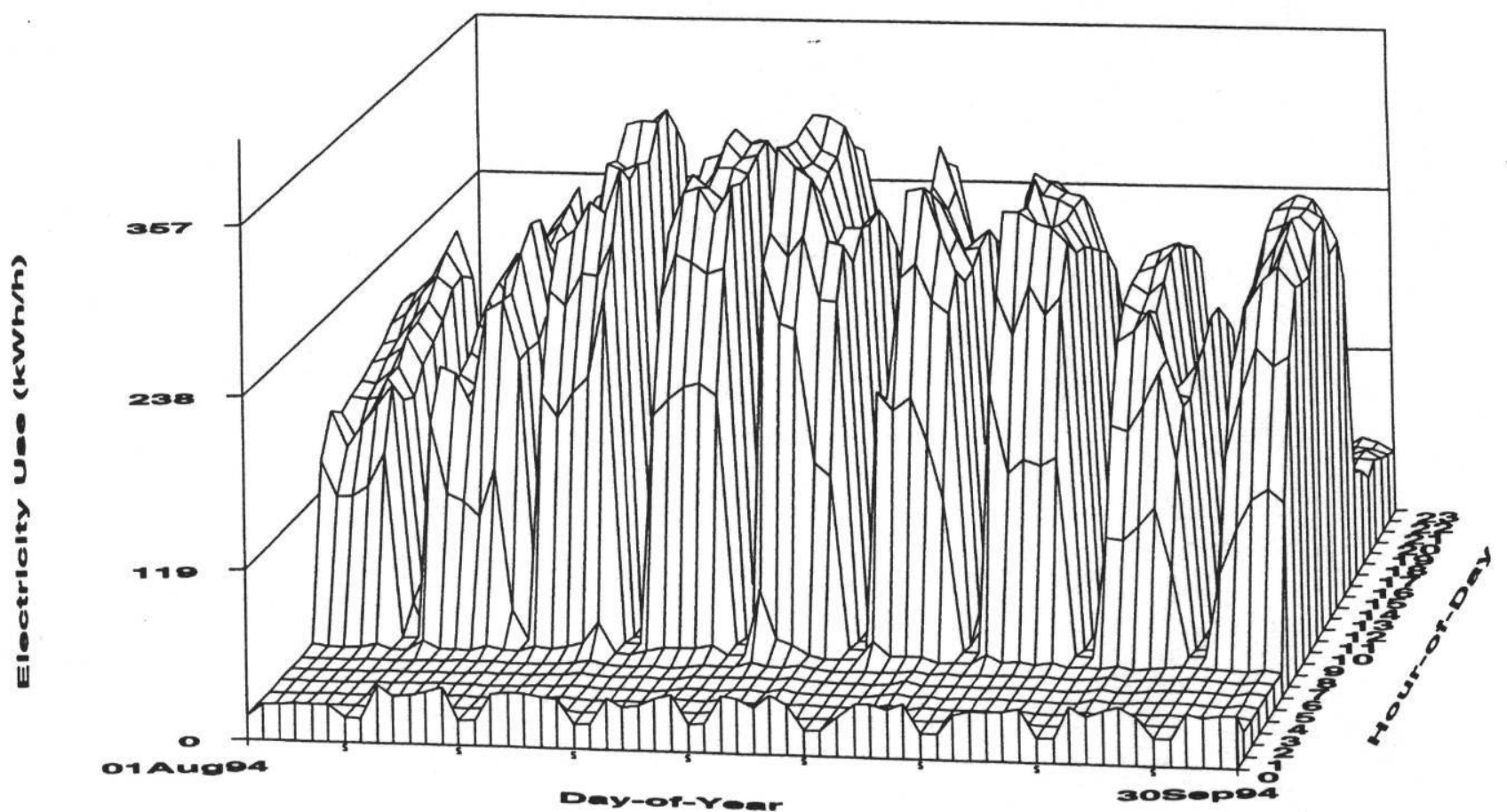

Sundays are marked with an "S"

Sims Elementary School - Fort Worth ISD - September 1994 
Tab C-5

Data Summary Notebook Information 


\section{FORT WORTH INDEPENDENT SCHOOL DISTRICT}

Building Envelope:

\section{Sims Elementary School}

- 62,400 sq. ft. built in 1988

- 1-story, walls of face brick, 1/2" scathing on 6" studs, 5/8" gypsum board, and 10" concrete.

- roof built-up with tar and gravel

- windows are single pane, operable, both tinted and clear

Building Schedule:

- 7:00 am to $5 \mathrm{pm}$ (M-F) closed (Sat./Sun.)

- 3 months summer break, $18+5$ other holidays

Building HVAC and Equipment

- About 54 rooftop units (mostly $2 / 4$ to $1 / 2$ ton)

- 2 hot water heaters each $270,000 \mathrm{Btu} / \mathrm{hr}$

- $91 / 2$ hpe a exhaust fans

\section{Lighting}

- Mostly fluorescent (40 W), few PL-13 lamps

\section{Proposed Retrofits}

- Convert 2 X 4, 4 lamp fluorescent light fixtures to 2 X 4, 2 lamp configuration to reduce energy consumption for lights by 50

\section{Completion Data of Report:}

- Lighting modification was completed in November 91. 


\section{Sims Elementary School (SIM) \\ W.B. Electric as W/sq.ft.}
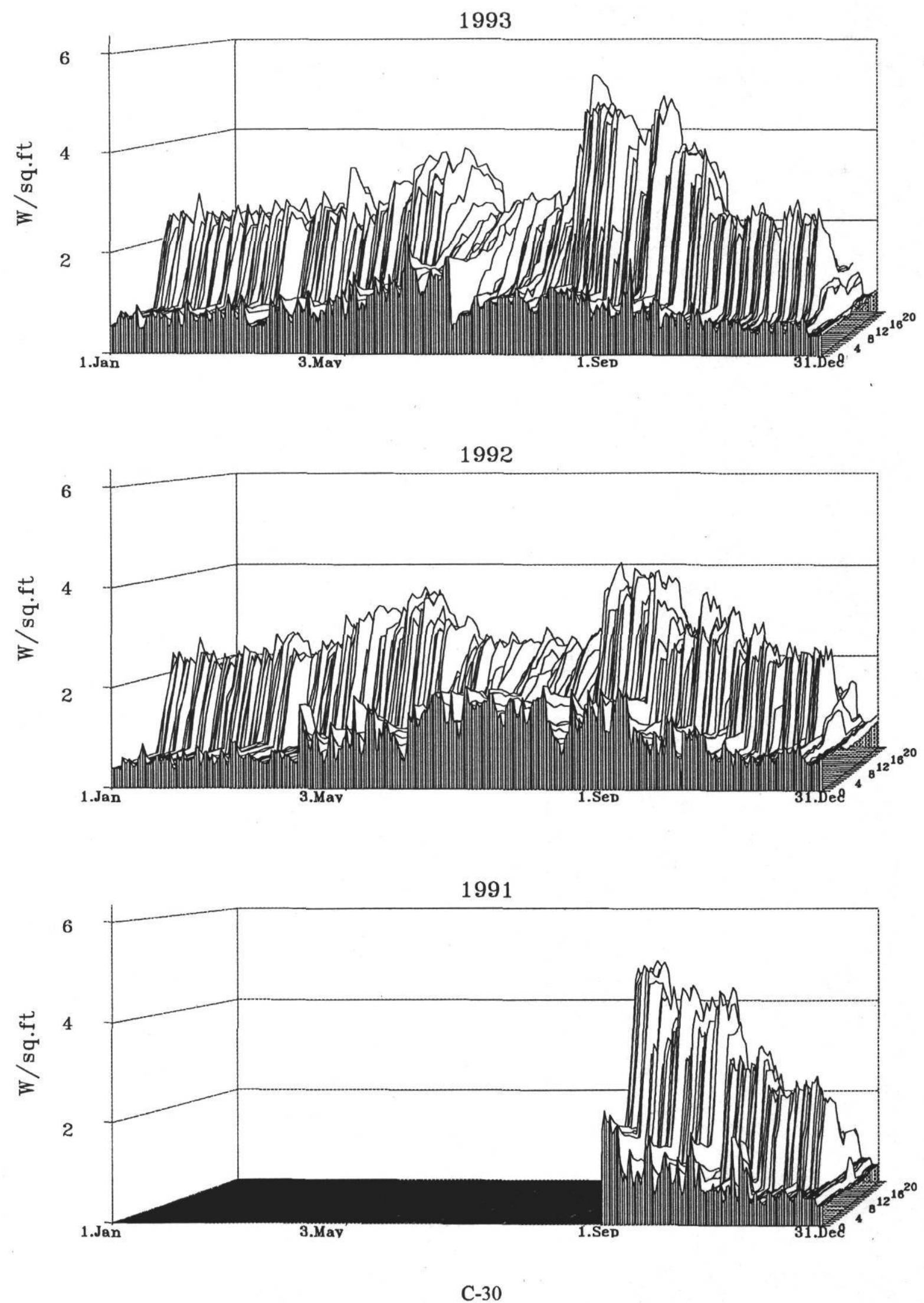


\section{Sims Elementary School (SIM) W.B. HW as Btu/sq.ft.-h}
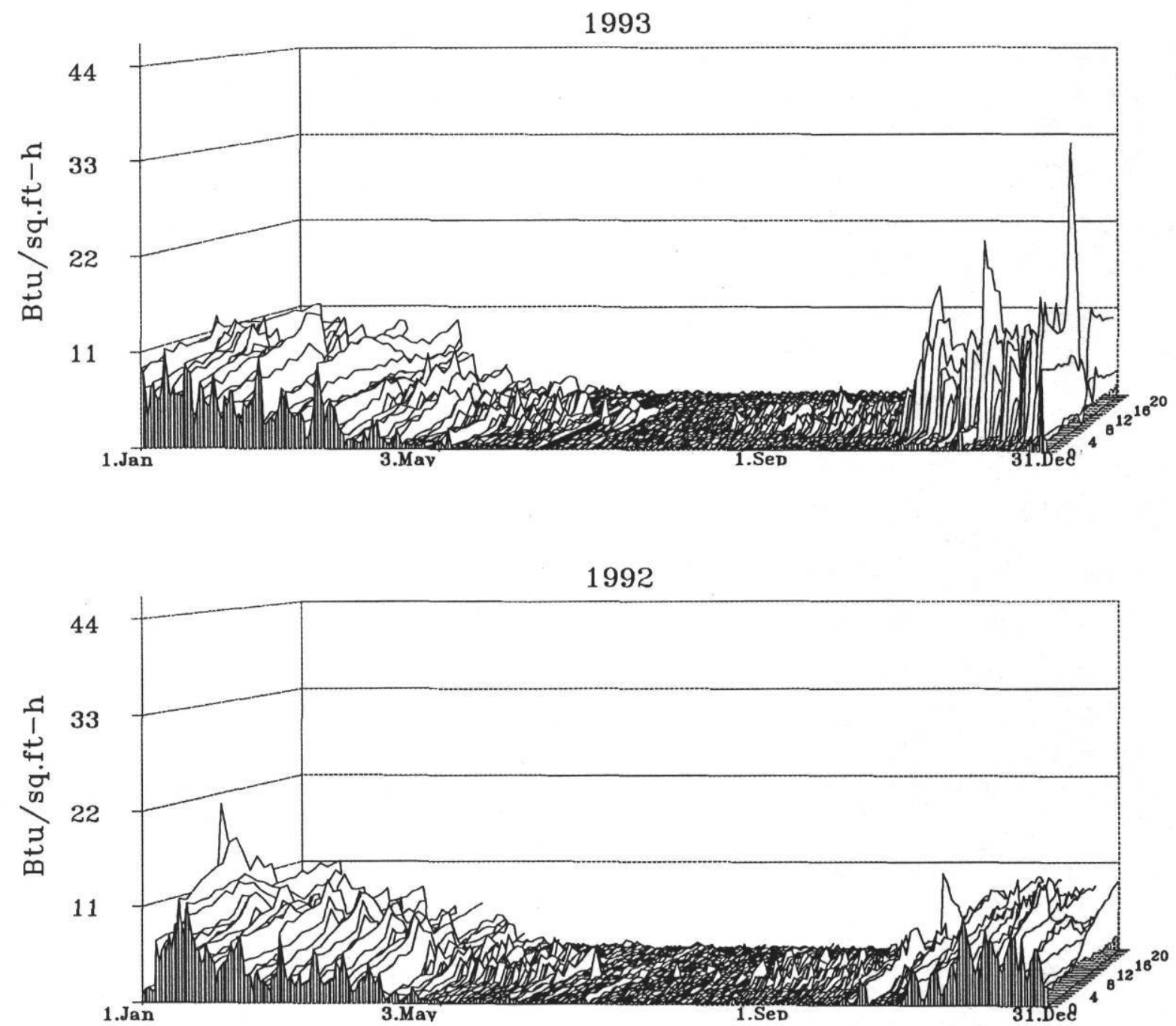

1991

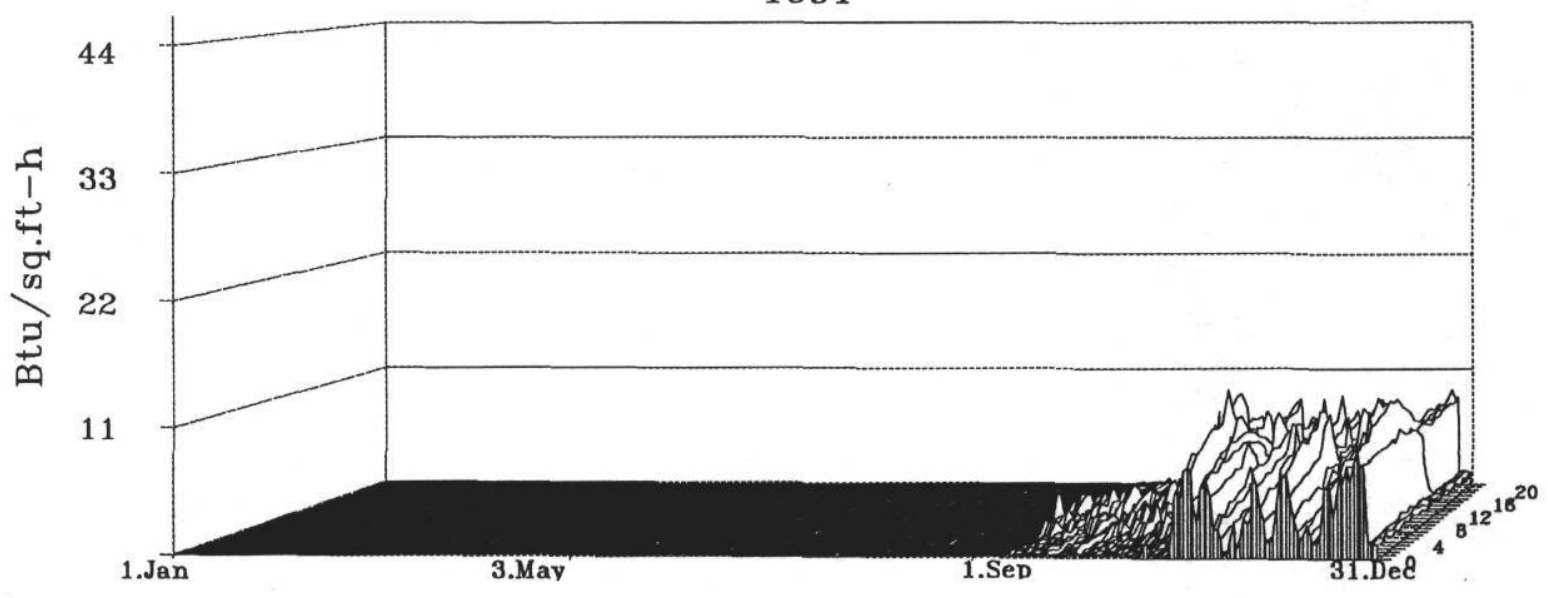

C-31 


\section{Sims Elementary School (SIM) W.B. Electric as W/sq.ft.}
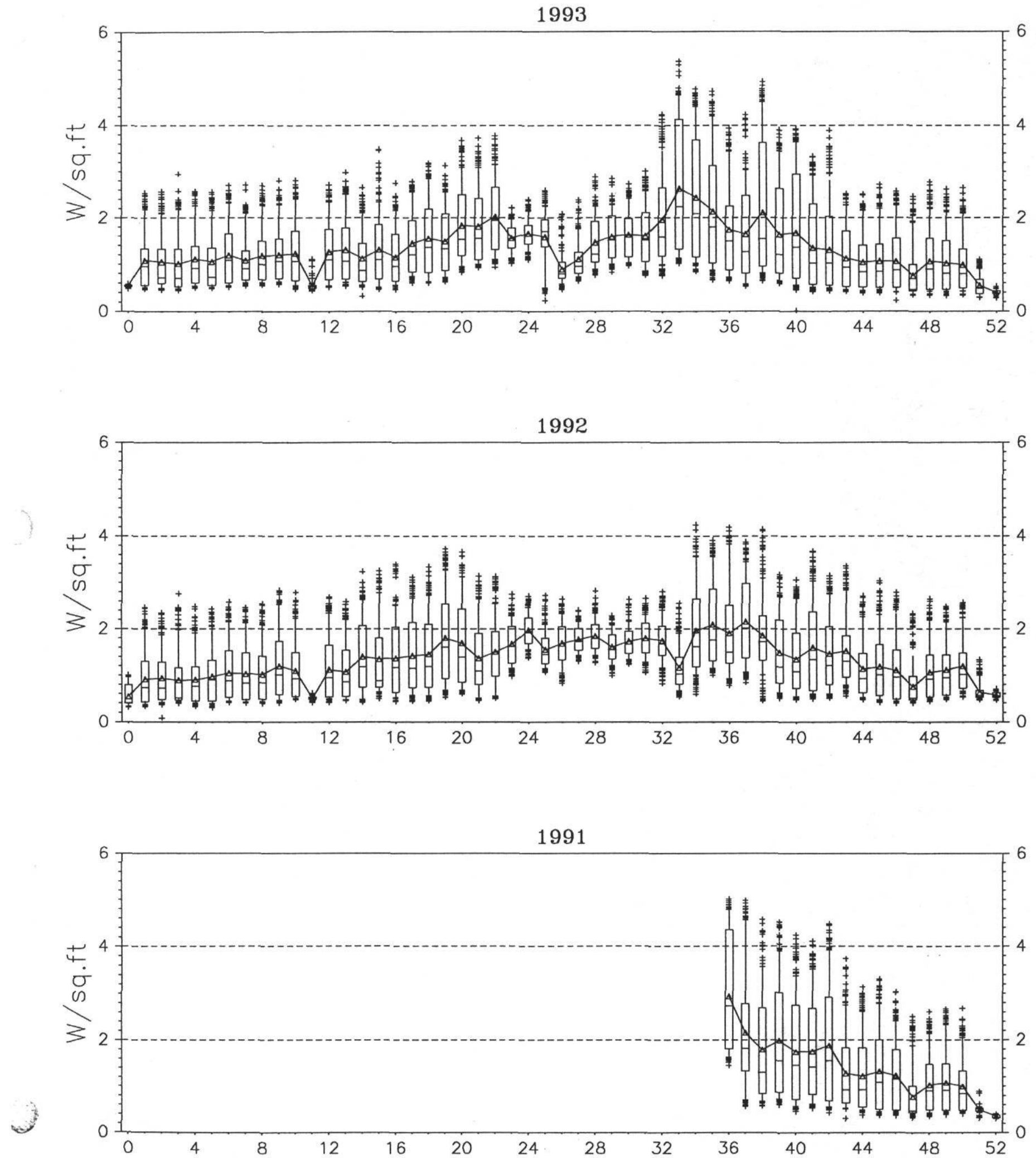


\section{Sims Elementary School (SIM) W.B. HW as Btu/sq.ft.-h}
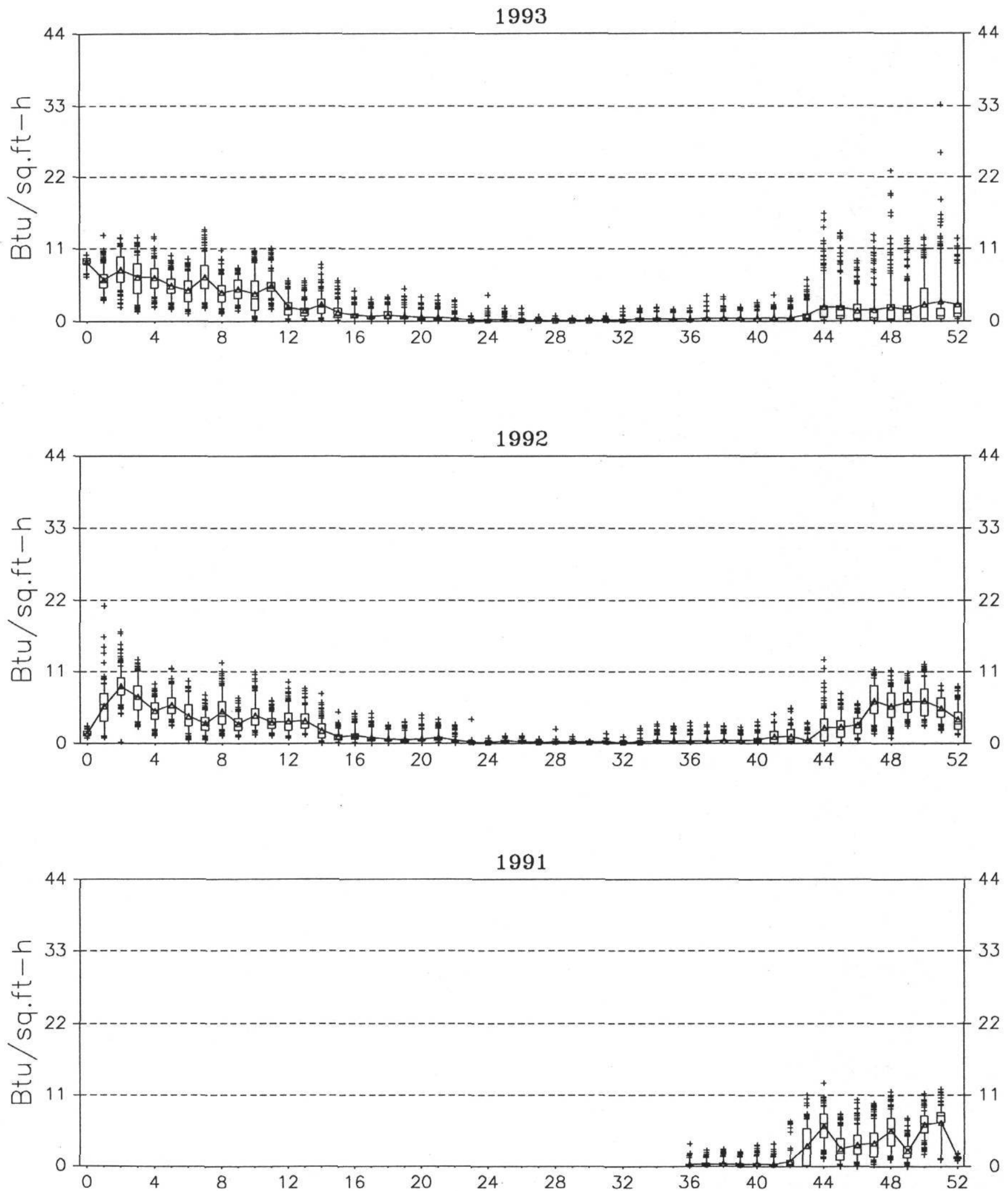
Sims Elementary School (SIM)

W.B. Electric as W/sq.ft.

Pre-Retrofit (09/10/1991 - 11/01/1991)

Everyday

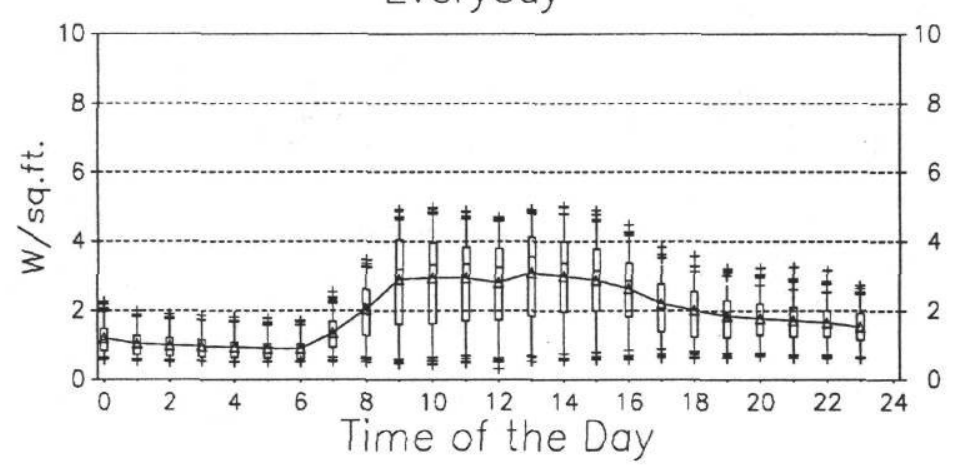

Weekdays

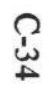

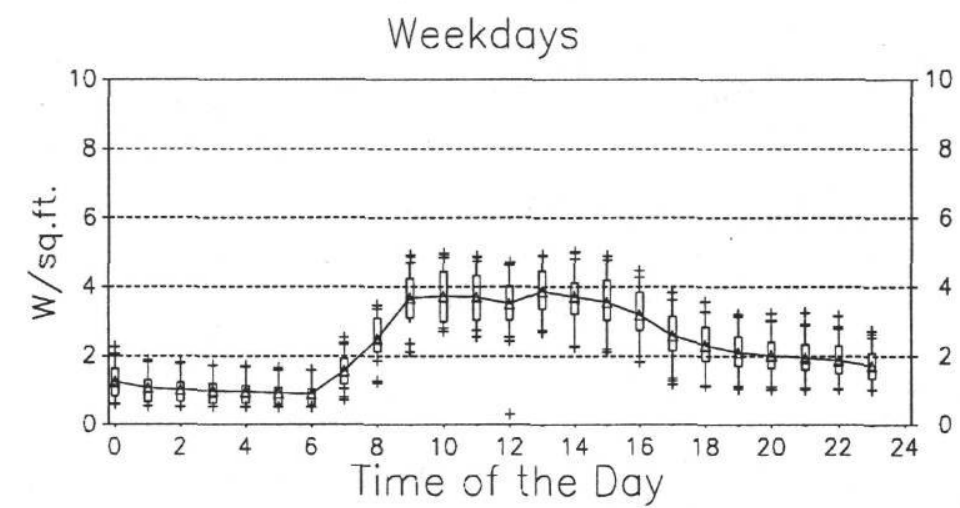

Weekends

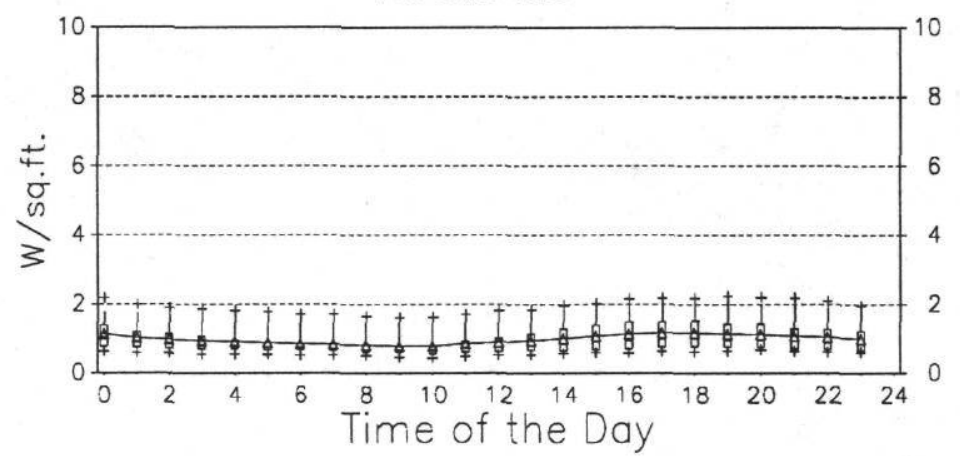

Post-Retrofit (11/23/1991 - 12/31/1993)

Everyday

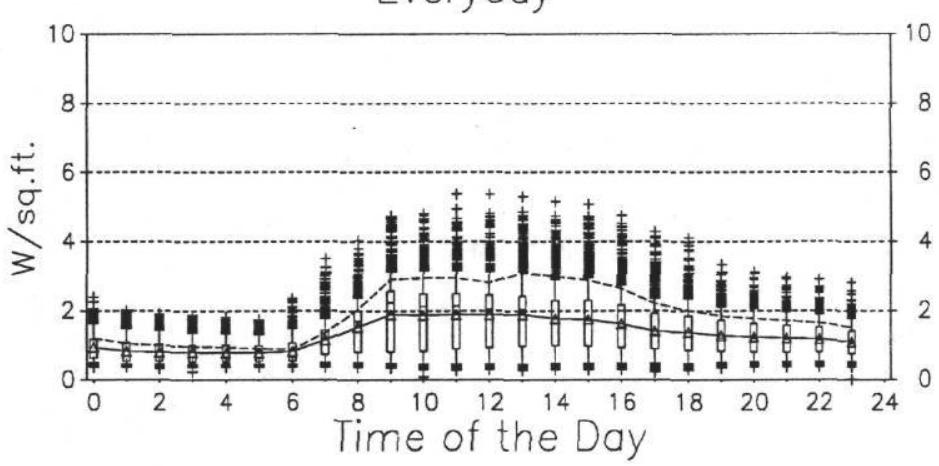

Weekdays

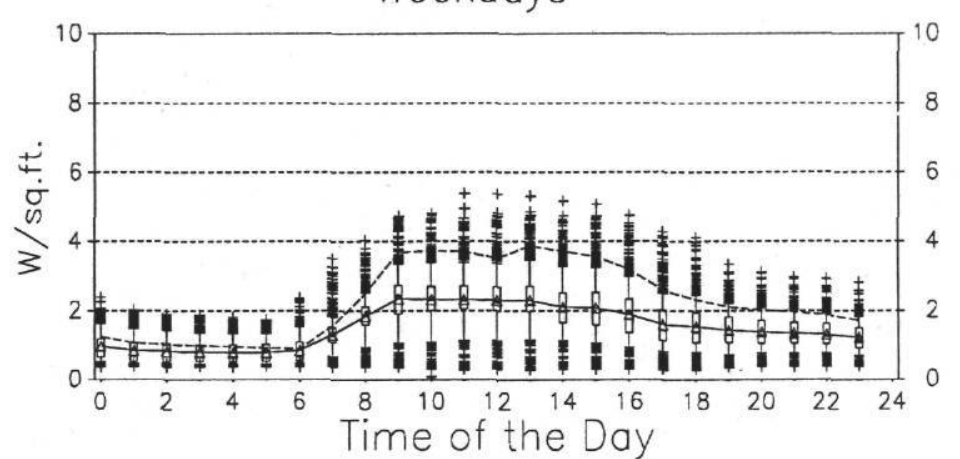

Weekends

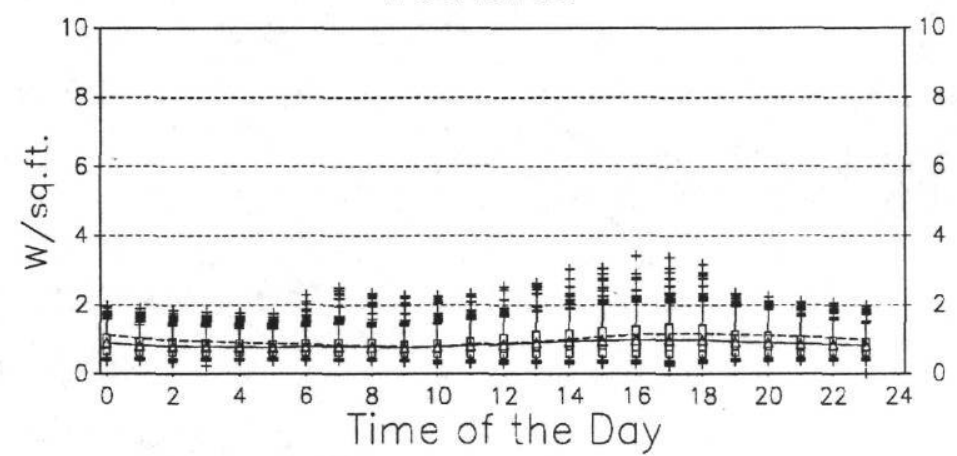




\section{Sims Elementary School (SIM) W.B. HW as Btu/sq.ft.-h}

Pre-Retrofit (09/10/1991 - 11/01/1991)

Everyday

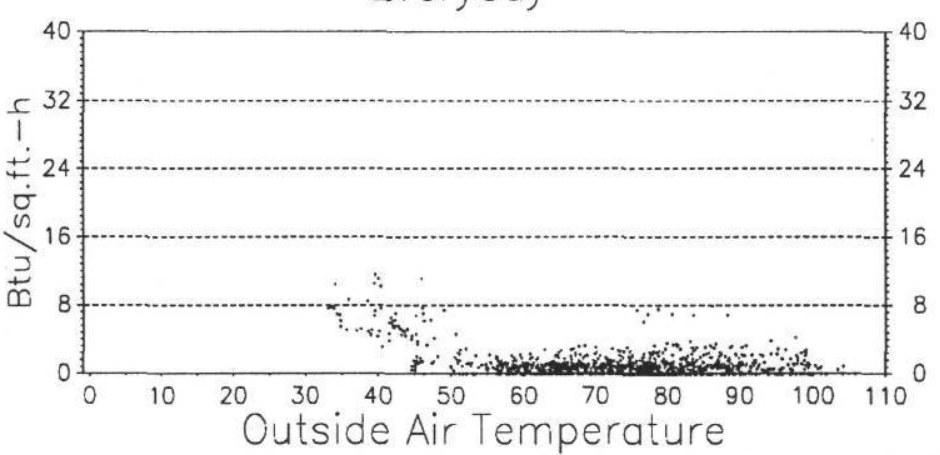

Note: Data in graphs above has been vertically offset randomly up to $1 \mathrm{Btu} / \mathrm{sq} . \mathrm{ft} . \mathrm{-h}$ to improve graphical presentation.

Weekdays

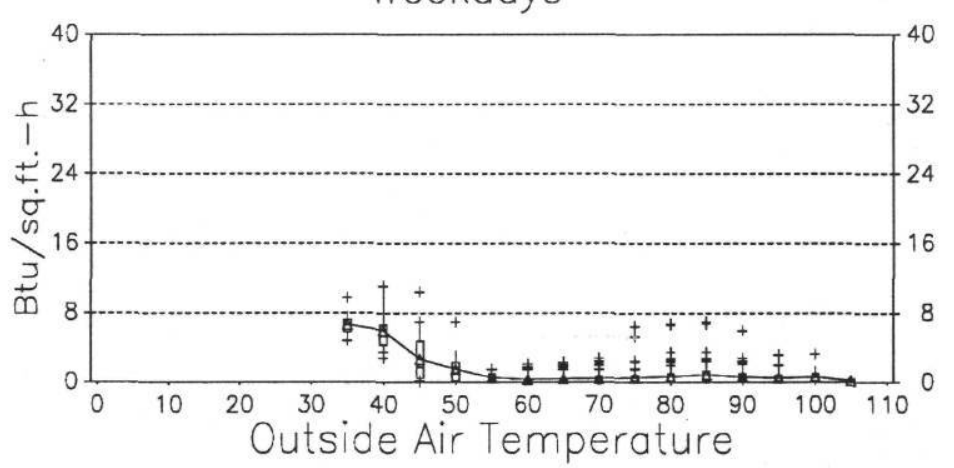

Weekends

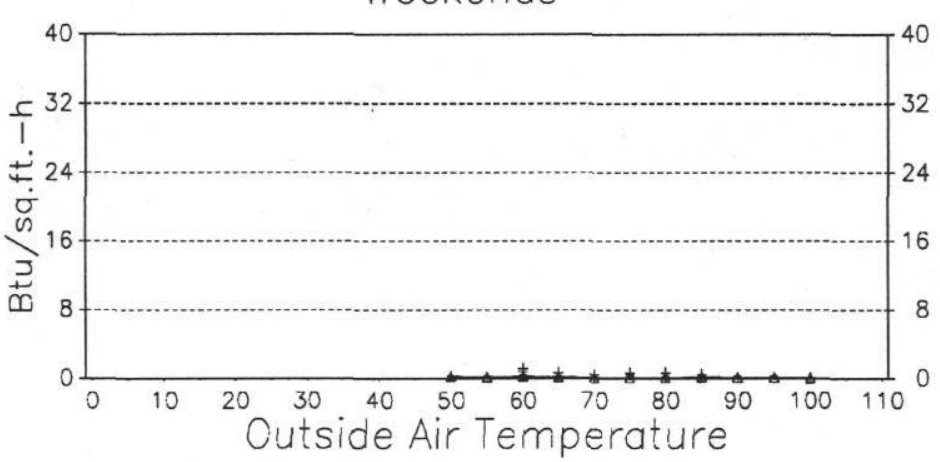

Post-Retrofit (11/23/1991 - 12/31/1993)

Everyday

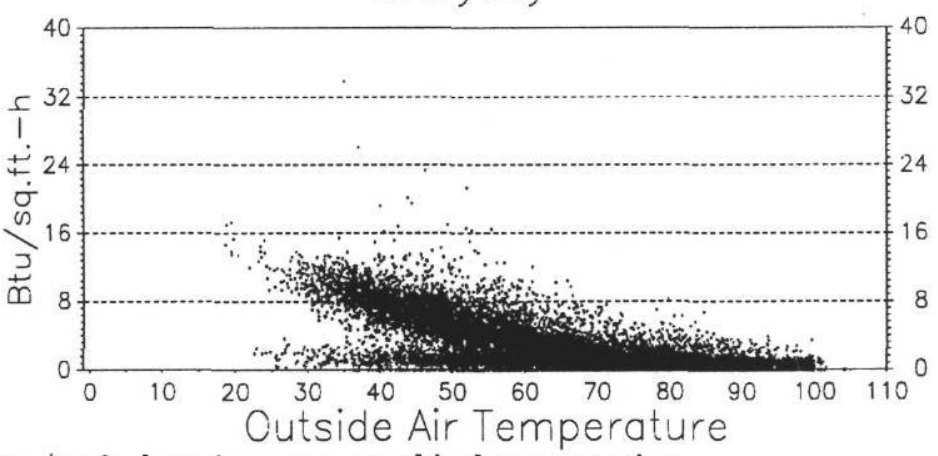

Weekdays

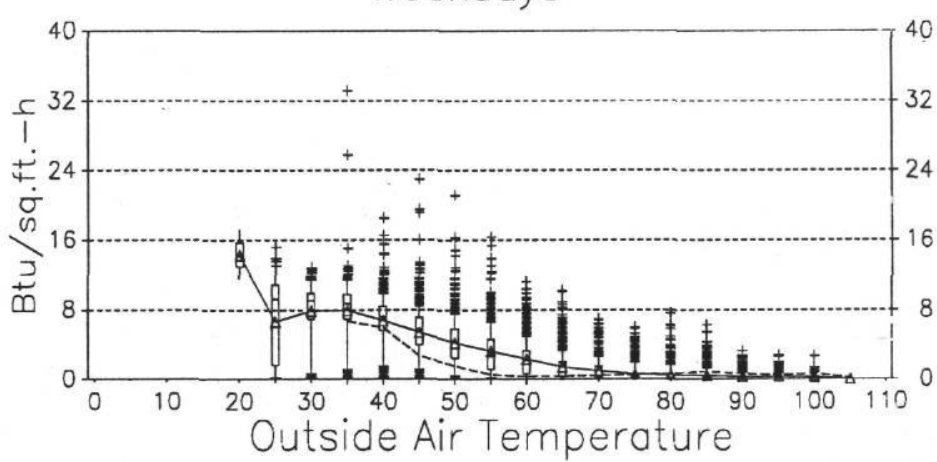

Weekends

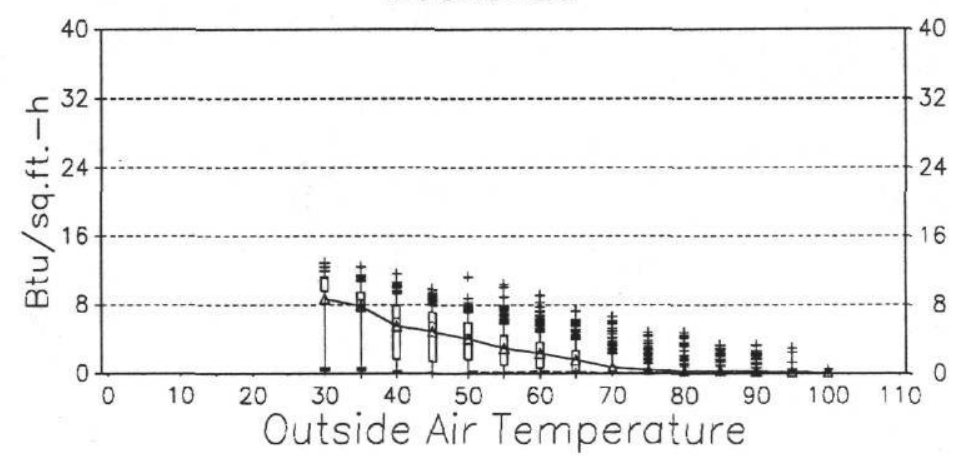




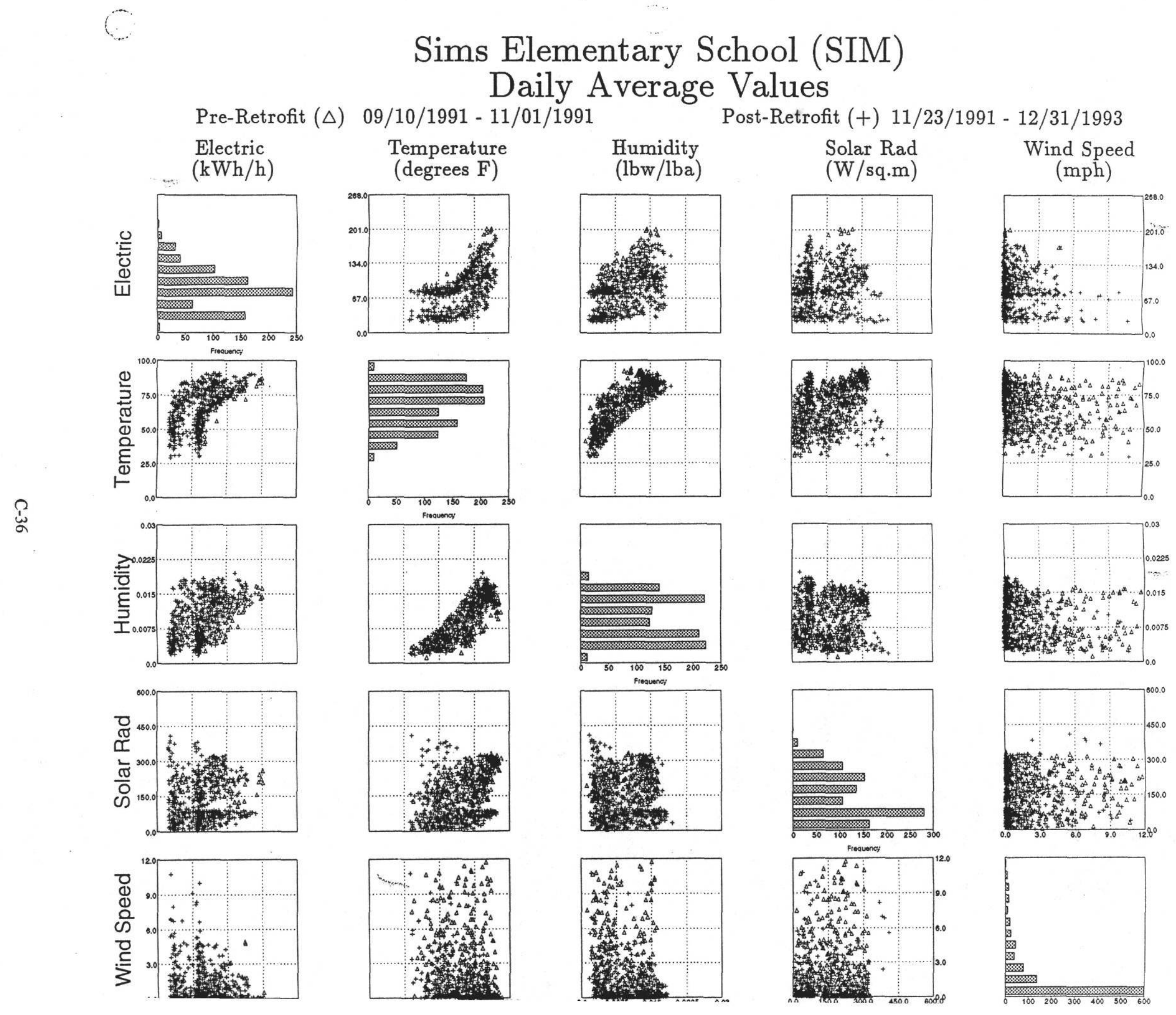




\section{Sims Elementary School (SIM) Daily Average Values}

Pre-Retrofit $(\triangle)$ 09/10/1991 - 11/01/1991 HW/Steam Cons.

$(\mathrm{kBtu} / \mathrm{h})$
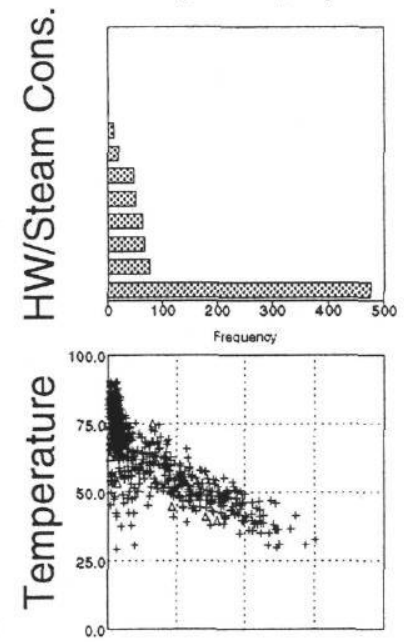

$\stackrel{\leftrightarrow}{u}$
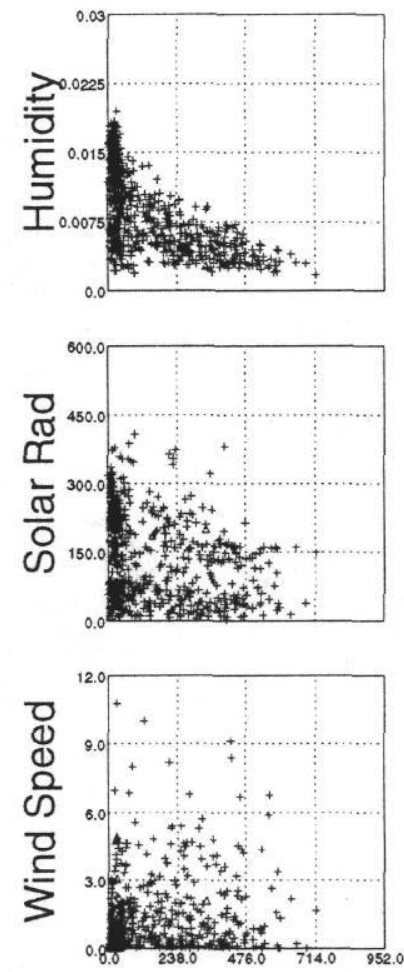

Temperature
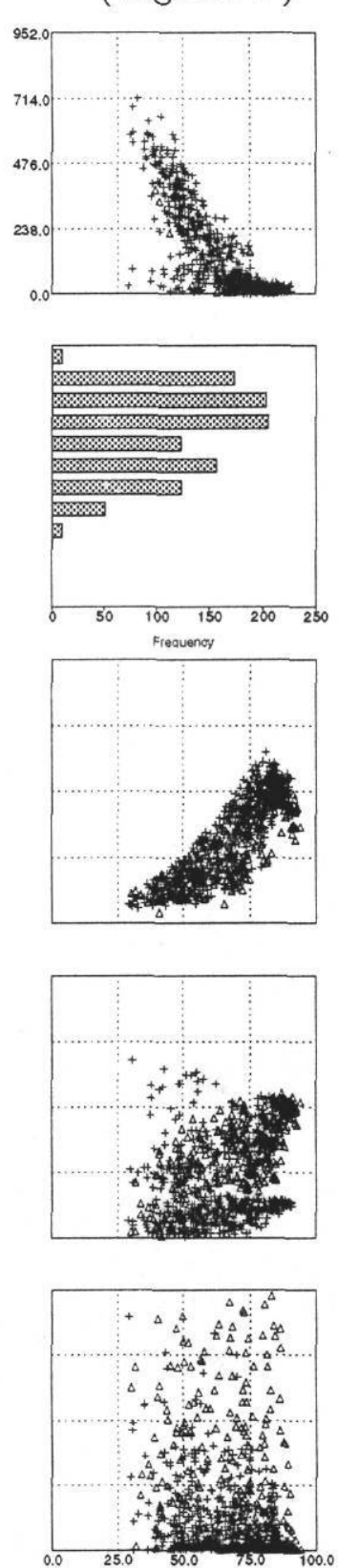

Post-Retrofit (+) 11/23/1991 - 12/31/1993

Humidity

(lbw/lba)
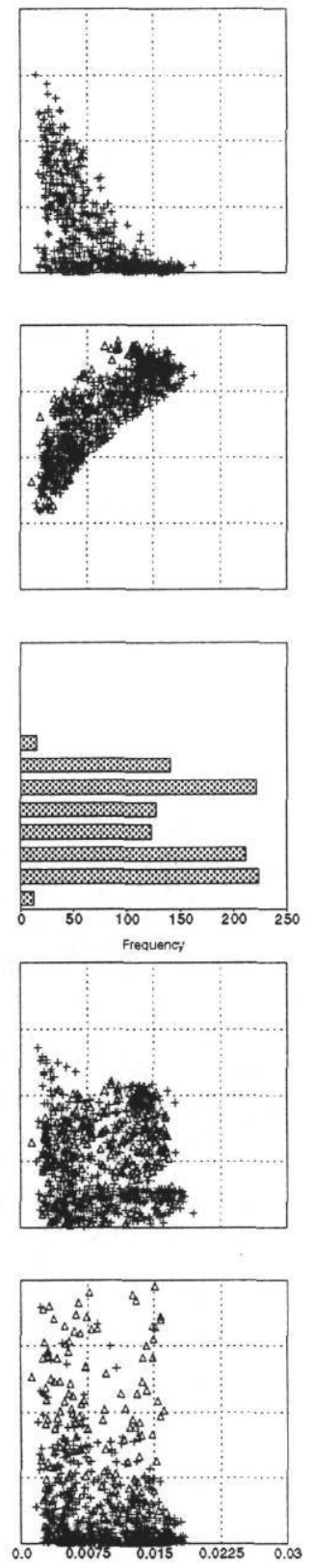
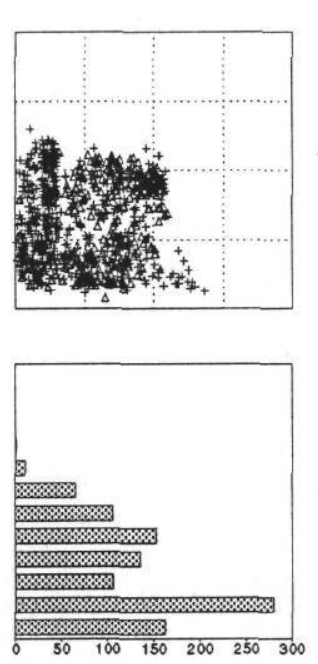

Solar Rad

(W/sq.m)
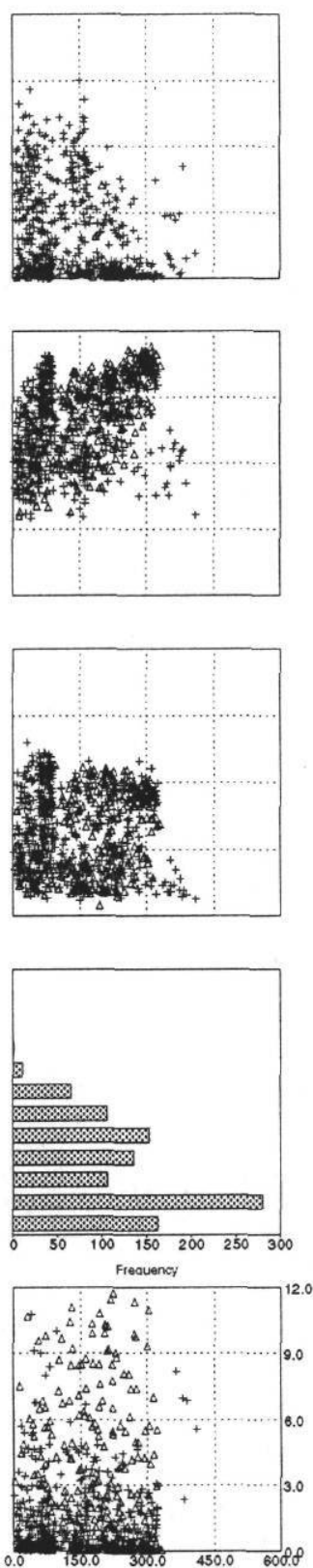

Wind Speed (mph)
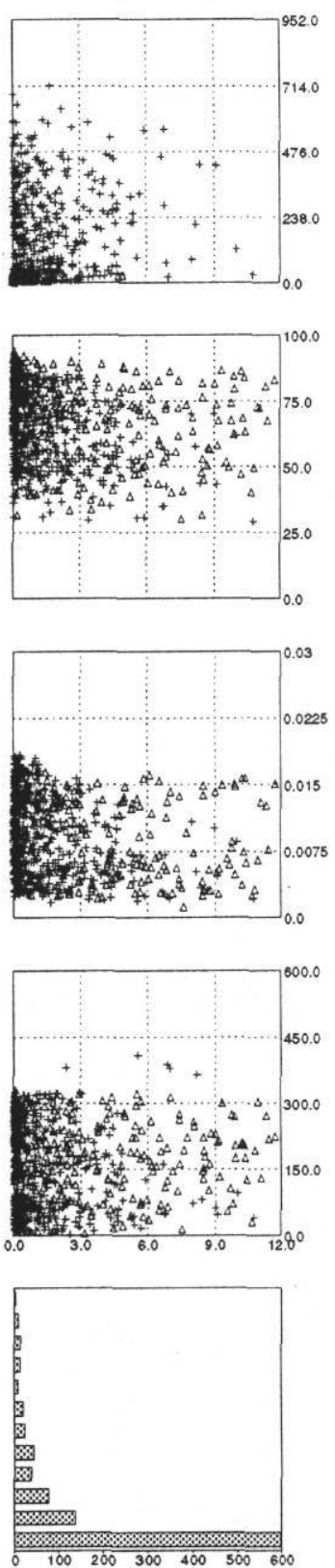


\section{ZACHRY ENGINEERING CENTER}

\section{D.1 Site Description}

The Zachry Engineering Center is located on the north side of Texas A\&M University in College Station, Texas. The 3-1/2 story structure includes 324,400 square feet of conditioned space. The main functions of the building are classrooms, laboratory research, and staff offices. All HVAC systems are operated 24 hours/day, 7 days/week, year round. The building activity is very large during regular hours (6:30 a.m. to 5:30 p.m.) and slows down to a moderate pace after hours and on weekends.

Energy using systems include: chilled water, hot water, domestic hot water, and electricity. These services are provided by the Main Campus Central Plant via an underground tunnel. There are several types of air distribution systems in the building. The main system that serves $90 \%$ of the building is a double duct variable air volume arrangement, with twelve major and some smaller air handling units located along the periphery of the basement parking garage. Each of the major AHUs is equipped with a 40 horsepower supply fan, and is serving portions of the three floors.

A site summary sheet from the September 1994 MECR is included in Tab D-1. The monitoring diagrams are included in Tab D-2.

\section{D.2 EMCS Retrofit}

The audit did not make a separate recommendation for an EMCS retrofit. An EMCS to control HVAC was installed along with the recommended retrofits for this site in March of 1991. The EMCS controls the HVAC by controlling the fan speed and nighttime setbacks. The fan speed is controlled according to the demand for cooling in a particular zone. If the occupancy of a zone drops, then there is not as great of a heat load, and the fan speed slows down, thereby providing a lesser amount of cold air to that room. The nighttime setback involves changing the thermostat setpoints to a higher temperature during nighttime hours, when occupancy is greatly reduced. 


\section{D.3 Analysis}

\section{D.3.1 Data Summary Notebook ${ }^{1}$}

The Data Summary Notebook was prepared by the Monitoring and Analysis Task of the Texas LoanSTAR program. Is was prepared to provide a historical look at all the data that have been collected for all of the LoanSTAR sites. The data are displayed in several graphical forms to show different aspects of the energy consumption behavior of a building both before and after the retrofit. These plots are analyzed for this site as a means to compare them to those prepared for the other study sites.

\section{D.3.1.1 3-D Surface Plots}

The 3-D surface plots for this site are shown in Tab D-3. The 3-D surface plots are generated using $\mathrm{SAS}^{2}$ graphics and are displayed to show hourly data over several years. The plots show the whole building (WB) energy consumption, the total electricity consumption by motor control centers (MCC), and the whole building thermal energy use in one plot per year. The 3-D surface plots show the hours in a day on the $\mathrm{X}$ axis (into the page), the days of the year from left to right on the horizontal $\mathrm{Y}$-axis in front of the plot, and the variable itself is the height of the plot above the X-Y plane. The plot, in effect, becomes a compilation of 365 daily 24 hour profiles.

Observations: A noticeable reduction in whole building electricity consumption can be seen at the retrofit date of March 1991. No comment can really be made about the hour of day axis, as it is very difficult to read, and it shows up on the pre/post, weekday/weekend, 24 hour BWM plots. A much more significant reduction is evident in the plots for the Motor Control Center (from approximately $380 \mathrm{kWh} / \mathrm{h}$ to approximately $200 \mathrm{kWh} / \mathrm{h}$ ). The daily profile changes from basically constant consumption to low nighttime consumption with higher daytime consumption. Again, this is hard to read on this plot. As chilled water and hot water usage were not studied in the other test sites, no comparisons with other sites can be made. However, the plots are included for completeness.

\footnotetext{
${ }^{1}$ Data Summary Notebook for Site 001, Zachary Engineering Center

${ }^{2}$ Base SAS Software, SAS Institute, Inc., Cary NC 27512-8000
} 
Comparison: For the other study sites, 2-D energy consumption plots were used to show daily consumption versus day of the year. The hourly consumption was averaged over certain sort parameters and plotted as hourly consumption versus hour of day.

\section{D.3.1.2 Weekly Box Whisker Mean (BWM) Plots}

Weekly Box Whisker Mean Plots for this site are shown in Tab D-4. The weekly BWM plots are arranged in the same manner as the 3-D surface plots to facilitate a comparison between pages. To generate the weekly BWM plots, the data are first grouped into 52 weeks. Each week starts on a Sunday and ends on a Saturday. The BWM symbol efficiently displays the means, the 10th, 25th, 50th, 75th, and the 90th percentiles and all the outliers above the 90th percentile and below the 10th percentile. The box extends from the 25th (first quartile) to the 75th (third quartile) percentile. The whiskers extend from the top of the box to the 90 th percentile and from the bottom of the box to the 10th percentile. The median (50th percentile) is marked inside the box with a single cross hatch. Values less than the 10th percentile and greater than the 90th percentile are marked as pluses (+), which lie below or above the whiskers. Means for each week are superimposed as triangles and joined by a line.

Observations: As would be expected, the weekly consumption also drops at the retrofit date of March 1991. More information is provided in these plots than in the 3-D surface plots. Here, statistical data is included in the form of BWM plots.

Comparison: Weekly data was not analyzed in the other sites.

\section{D.3.1.3 Pre/Post, Weekday/Weekend, 24-hour BWM Plots}

The pre/post, weekday/weekend, 24 hour BWM plots for this site are shown in Tab D-5. To generate these plots, the data are first separated into pre-retrofit and post-retrofit periods. Within each period, the data are grouped into weekdays and weekends. The whole building electricity (WB Electric) consumption, as W/sf, is plotted as 24-hour BWM plots against the time of the day for: (1) each day (regardless of weekdays or weekends), (2) weekdays only, and (3) weekends only for both the pre-retrofit 
and the post-retrofit periods. In addition, the mean lines from the pre-retrofit panels are superimposed as dashed lines on the post-retrofit panels to show the changes in the hourly profiles due to the retrofit.

Observations: In all three plots (everyday, weekdays and weekends), a reduction in whole building electricity is evident. In each post-retrofit plot, the line connecting the means of each data point is below the superimposed pre-retrofit line (dashed line). The reduction is fairly constant across all hours of the day.

Comparison: There are a number of differences between these plots and the hourly average plots used for the other sites. First, there is an additional category in the average hourly plots, which is semester/non-semester. This is an important sort category because for many sites, the average consumption during the semester periods varies greatly from the average consumption during the non-semester periods. As consumption is different between these two periods, it is useful to study them separately. Secondly, the consumption units are different (W/sf for BWM plot versus $\mathrm{kWh} / \mathrm{h}$ for the average hourly consumption plots. It is useful to convert the units to W/sf for the sake of comparing with other sites, which may be significantly different in size. Lastly, the BWM plot is more effective in showing the statistical variation in the data, but it is also difficult to read.

\section{D.3.1.4 Pre/Post, Weekday/Weekend, BWM Temperature Binned Plots}

The pre/post, weekday/weekend, BWM temperature binned plots for this site are shown in Tab D-6. For thermal loads, such as the chilled water consumption and steam/hot water consumption, the weekday and weekend energy consumption is grouped into $5{ }^{\circ} \mathrm{F}$ temperature bins and plotted as BWM plots against the ambient temperature. These same data are also plotted as scatter plots to show the general trend and density of the data points. The data for the scatter plots are slightly jittered to improve graphical presentation. Jittering is a graphical enhancement that improves a plot by adding a random noise to one of the variables. Jittering is necessary when BTU data are plotted because data are recorded in large increments, which causes severe data overlap when plotted. 
Observations: There is a noticeable reduction in whole building chilled water and whole building hot water consumption. Although these were not analyzed in the other study sites, they provide an additional way to view the data.

Comparison: Chilled water and hot water consumption were not studied in the other sites.

\section{D.3.1.5 Carpet Plots of Energy Use versus Ambient Conditions with Juxtaposed \\ Histograms}

Carpet plots of energy use versus ambient conditions are shown in Tab D-7. Carpet plots show the daily averaged pre/post data plotted against several variables. Separate symbols are used for the pre-retrofit (triangle) and post-retrofit (plus) periods. The carpet plot is arranged so that relationships between energy consumption and several weather variables (ambient temperature, humidity, global horizontal solar radiation, and wind speed) can be simultaneously viewed. In the carpet plot shown, energy consumption is shown along the top row and the left most column. Other panels show the interaction among the weather variables. The panels along the diagonal show the frequency distribution of all the data points within bins.

Observations: These plots are very small and hard to read, but show various trends in data.

Comparison: No comparisons can be made as these plots are not used for the other study sites.

\section{D.3.1.6 Carpet Plots of One Energy Channel Use Against Other Energy Use Channels}

Carpet plots of one energy channel use against other energy use channels are shown in Tab D-8. These are specialty carpet plots where one energy use is plotted against other energy use channels. In the plot shown, lights and receptacle (L\&R) electricity use is a derived channel which is obtained by subtracting the MCC electricity use from the whole building electricity use. These carpet plots are helpful in determining interactions between one energy use, such as chilled water consumption, and another, such as steam/not water consumption in both the pre-and post-retrofit periods.

Observations: These plots are very small and hard to read, but show trends in data. 
Comparison: No comparisons can be made as these plots are not used for the other study sites.

\section{D.3.1.7 Coincident Cumulative Frequency Plots}

Coincident cumulative frequency plots for this site are shown in Tab D-9. The coincident cumulative frequency plot shows the whole building electricity consumption and the coincident electricity consumption by the Motor Control Centers (MCC). To produce these plots, data are first separated into pre- and post-retrofit periods and sorted into descending order of whole building electricity consumption. The data is then plotted from the highest to the lowest consumption along with the coincident MCC electricity consumption. This plot is generally useful to show the drop in whole building and MCC electricity consumption due to a VAV retrofit.

Observations: These plots show a definite decrease in both whole building electricity and MCC electricity consumption.

Comparison: No comparisons can be made as these plots are not used for the other study sites. 
Tab D-1

Site Summary Sheet

D-7 


\section{Zachry Engineering Center}

Building Envelope:

- 324,400 sq.ft

- 3-1/2 floors and a ground floor level, erected 1973, classes, offices, labs, computer facility, and clean rooms for Solid State Electronics

- walls: cement block

- windows: $12 \%$ of total wall area

single pane with built-in-place vertical blinds

- roof: flat

\section{Building Schedule:}

- classrooms and labs: 7:30 am to 6:30 pm weekdays

- offices: 7:30 am to 5:30 pm weekdays

- computer facility: $24 \mathrm{hrs} / \mathrm{day}$

\section{Building HVAC:}

- 12 variable volume dual duct AHUs (12-40hp)

- 3 constant volume multizone AHU (1-1 hp, 1-7hp, 1-10hp)

- 4 constant volume single zone AHU (4-3hp)

- 10 fan coils (10-0.5 hp)

- 2 constant volume chilled water pump (2-30hp)

- 2 constant hot water pump (2-20hp)

- 7 misc. pumps (total of $5.8 \mathrm{hp}$ )

- 50 exhaust fans (50-0.5hp)

HVAC Schedule:

- $24 \mathrm{hrs} / \mathrm{day}$

\section{Lighting:}

- fluorescent

\section{Retrofits Implemented:}

- control modifications to the dual duct system

- variable volume dual duct system

\section{Other Information:}

- EMCS system to control HVAC was also installed along with the retrofits.

Date of Retrofits:

- date of completion for VAV and control modifications to the dual duct system: 03/05/91.

Savings Calculations:

- estimated savings are average monthly savings from the audit report (total annual savings divided by 12 ).

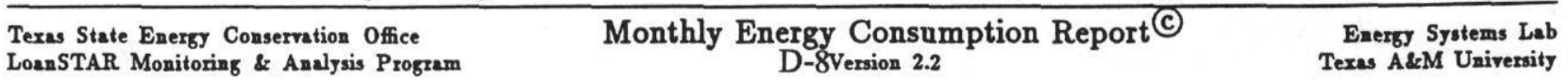


Tab D-2

Monitoring Diagrams

D-9 


\section{ELECTRICAL MONITORING DIAGRAM ZACHRY ENGINEERING CENTER}

LEGEND

$K=K W H$ CHANNE

$A=A N A L O G$ CHANNE

$D=D I G T A L$ CHANNEI

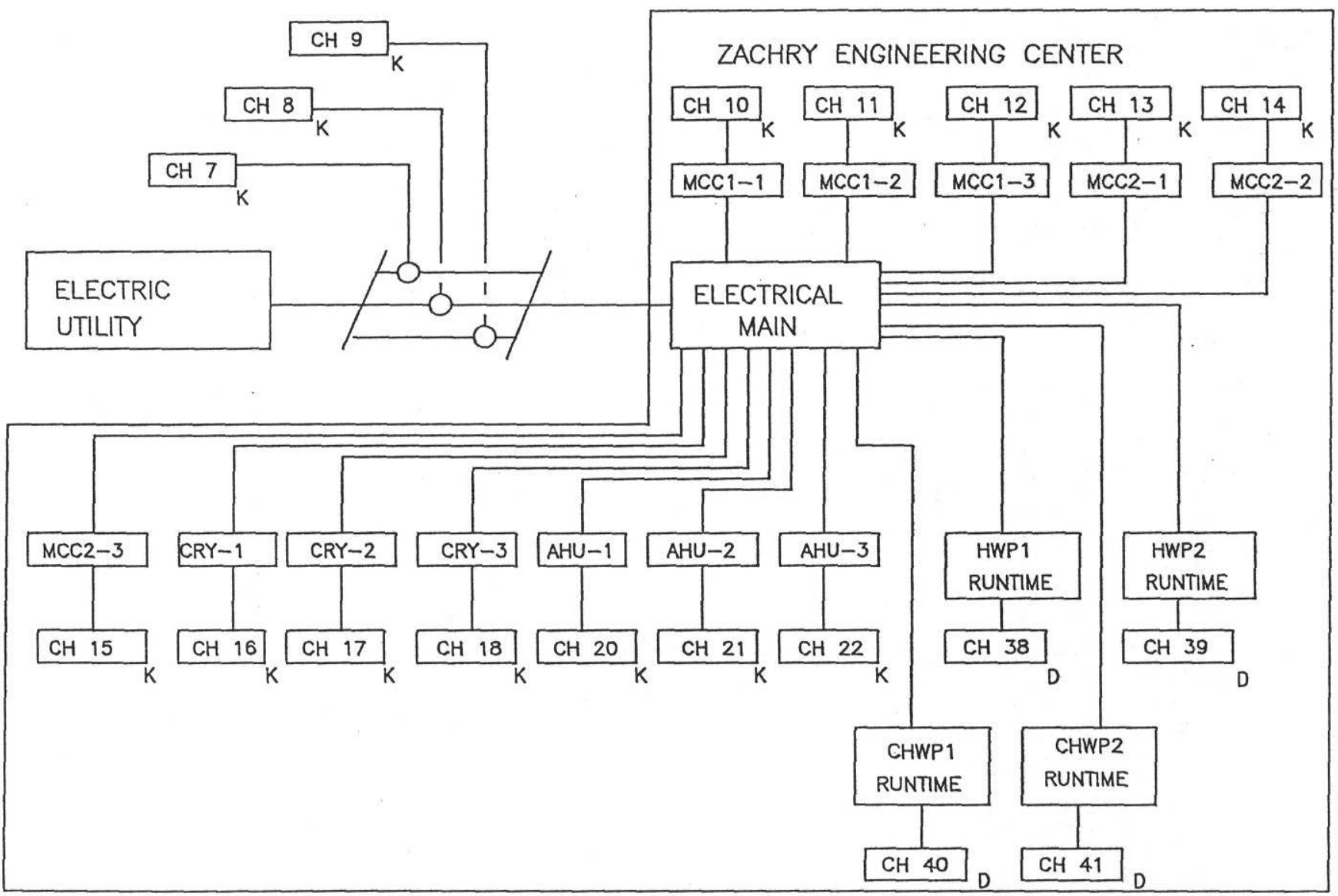

ZACHRY ENGINEERING CENTER - SITE 001 


\section{WEATHER MONITORING DIAGRAM ZACHRY ENGINEERING CENTER}

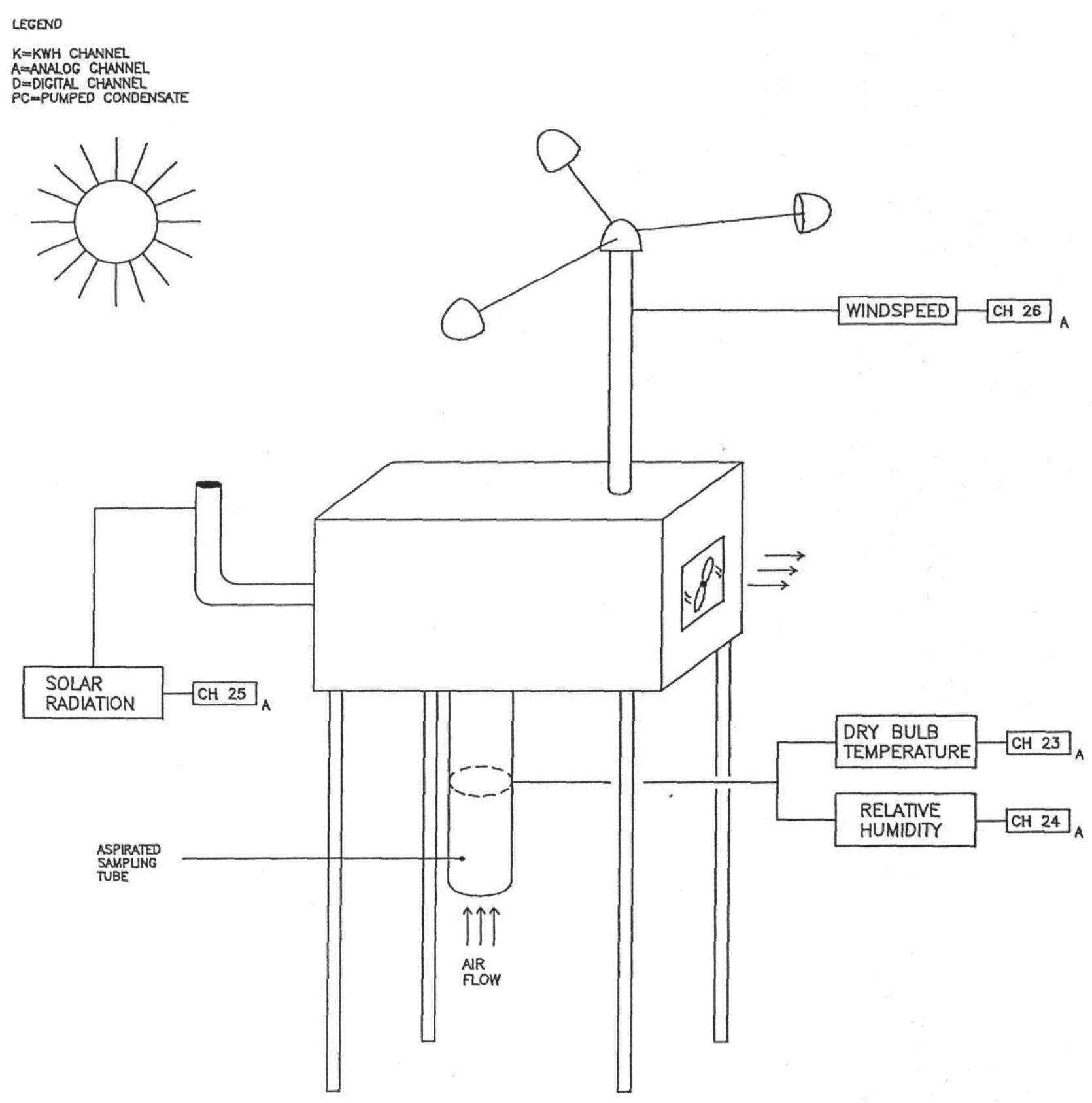




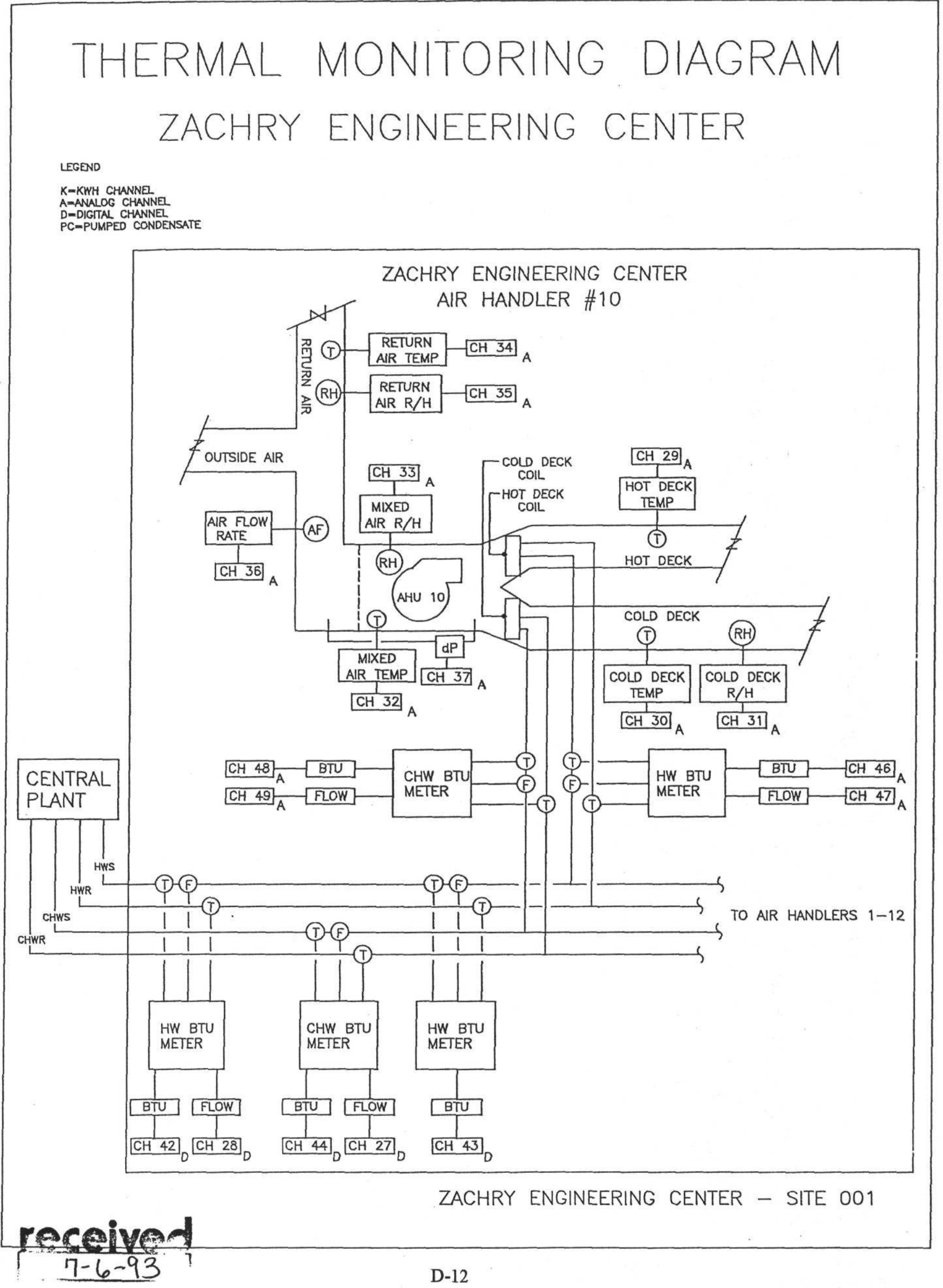




\section{THERMAL MONITORING DIAGRAM ZACHRY ENGINEERING CENTER}

LFGEND

K-KWH CHANNE

A-ANALOG CHANNE

D-DIGTAL CHANNE

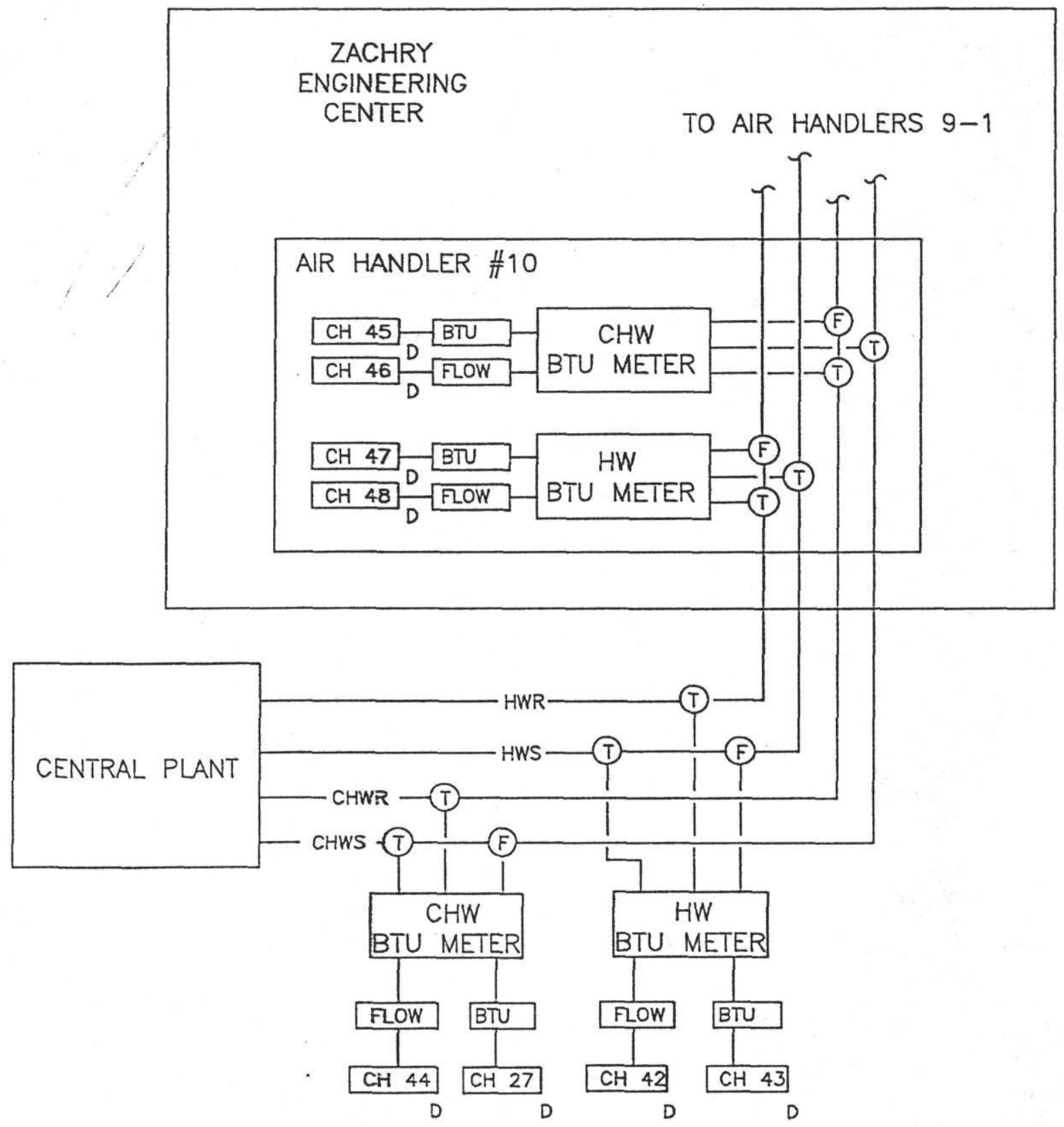

ZACHARY ENGINEERING CENTER - SITE 001 


\section{THERMAL MUNIT̈URING DIAGRAM A ZACHRY ENGINEERING CENTER}

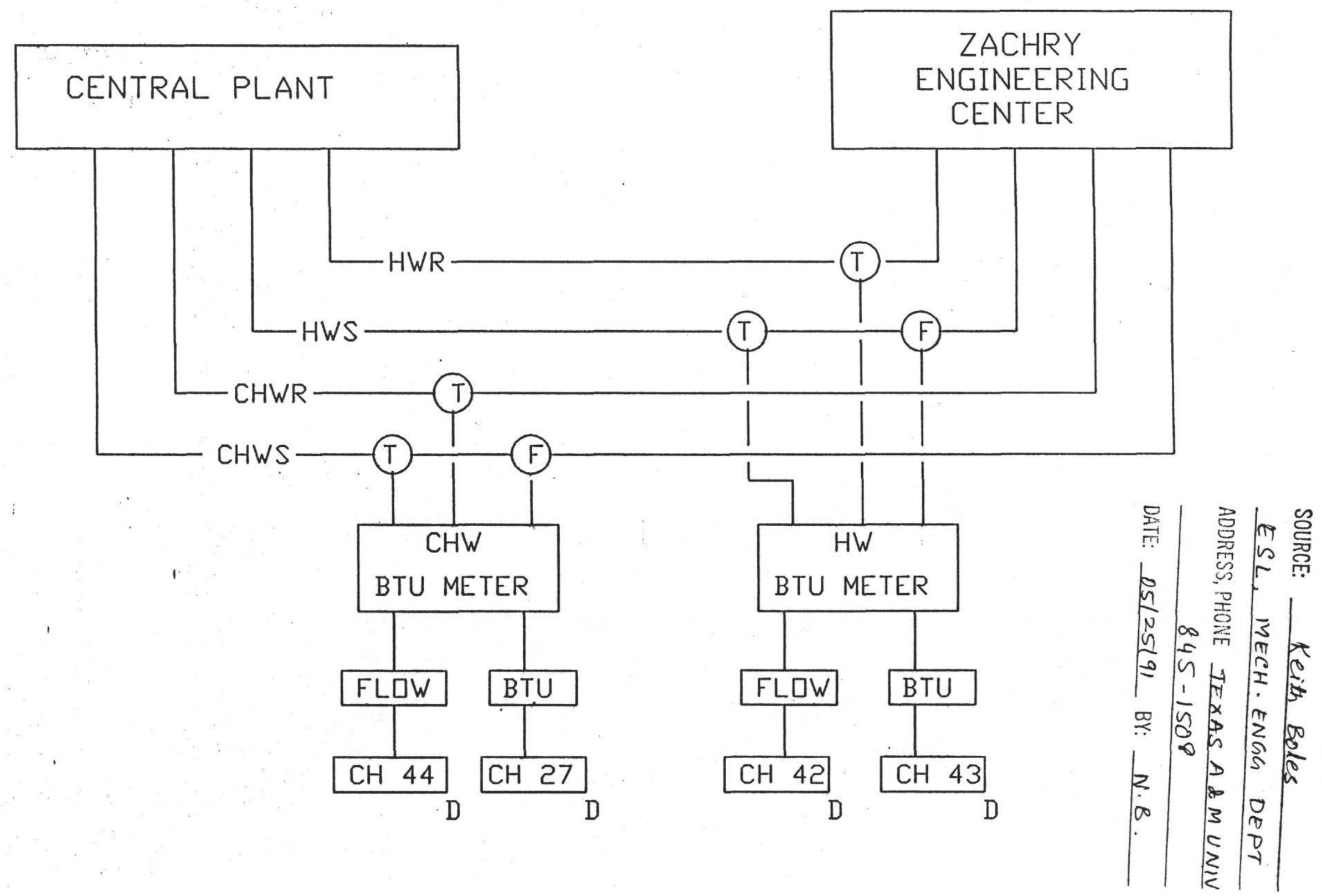




\section{KWH MUNIIGRING DIAGRAM ZACHRY ENGINEERING CENTER}

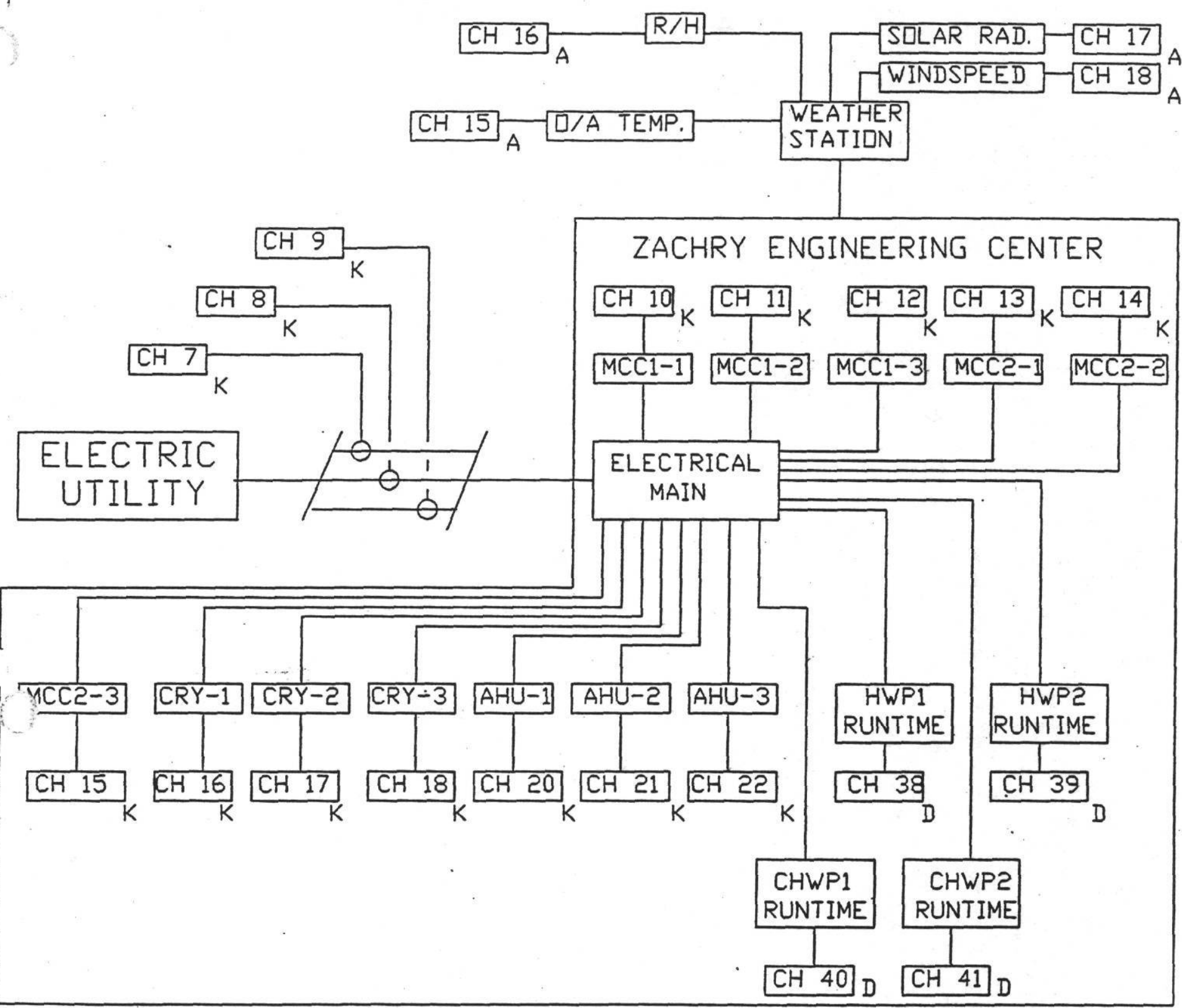




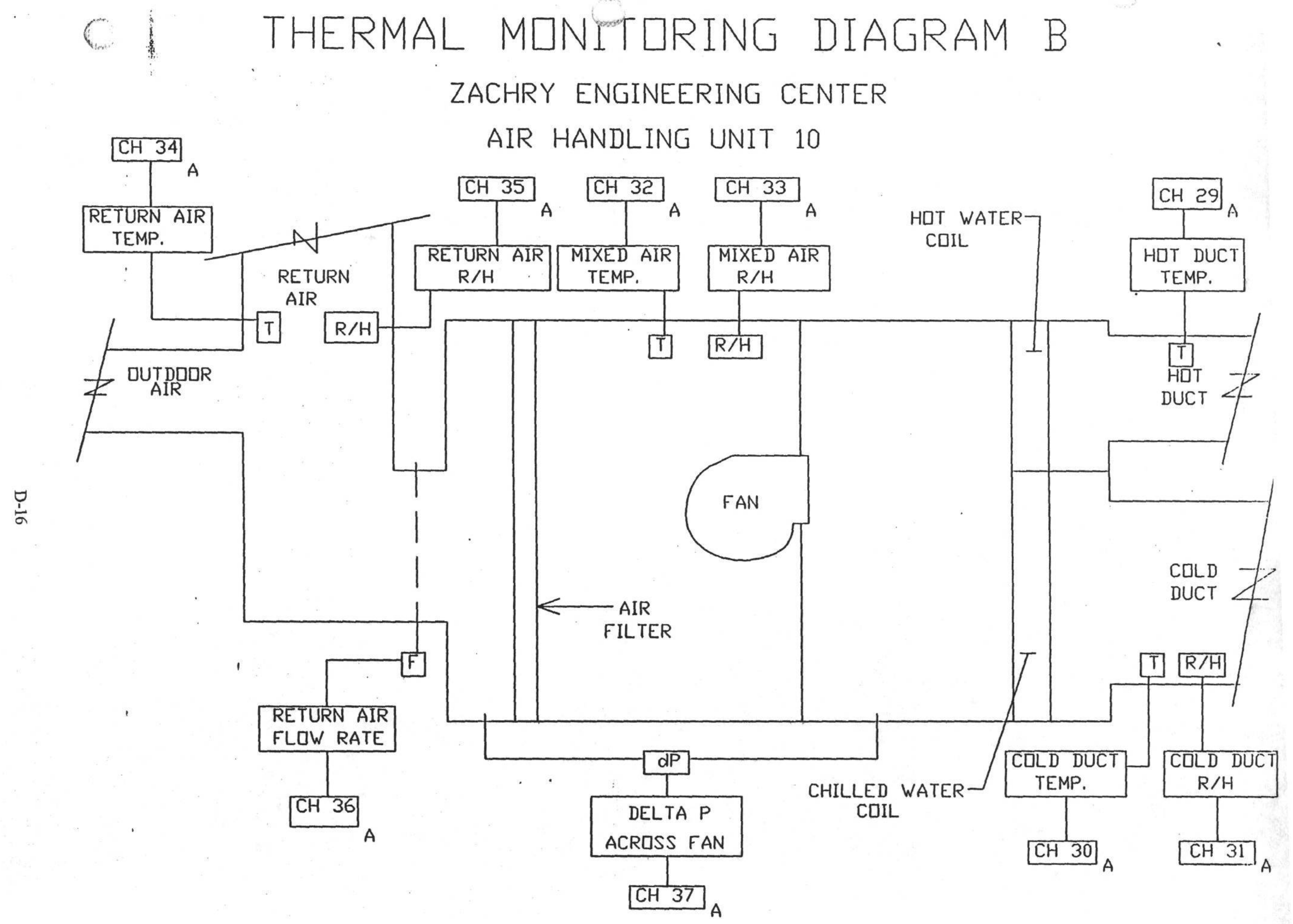


Tab D-3

3-D Surface Plots 


\section{Zachry Engineering Center (ZEC) W.B. Electric as W/sq.ft.}
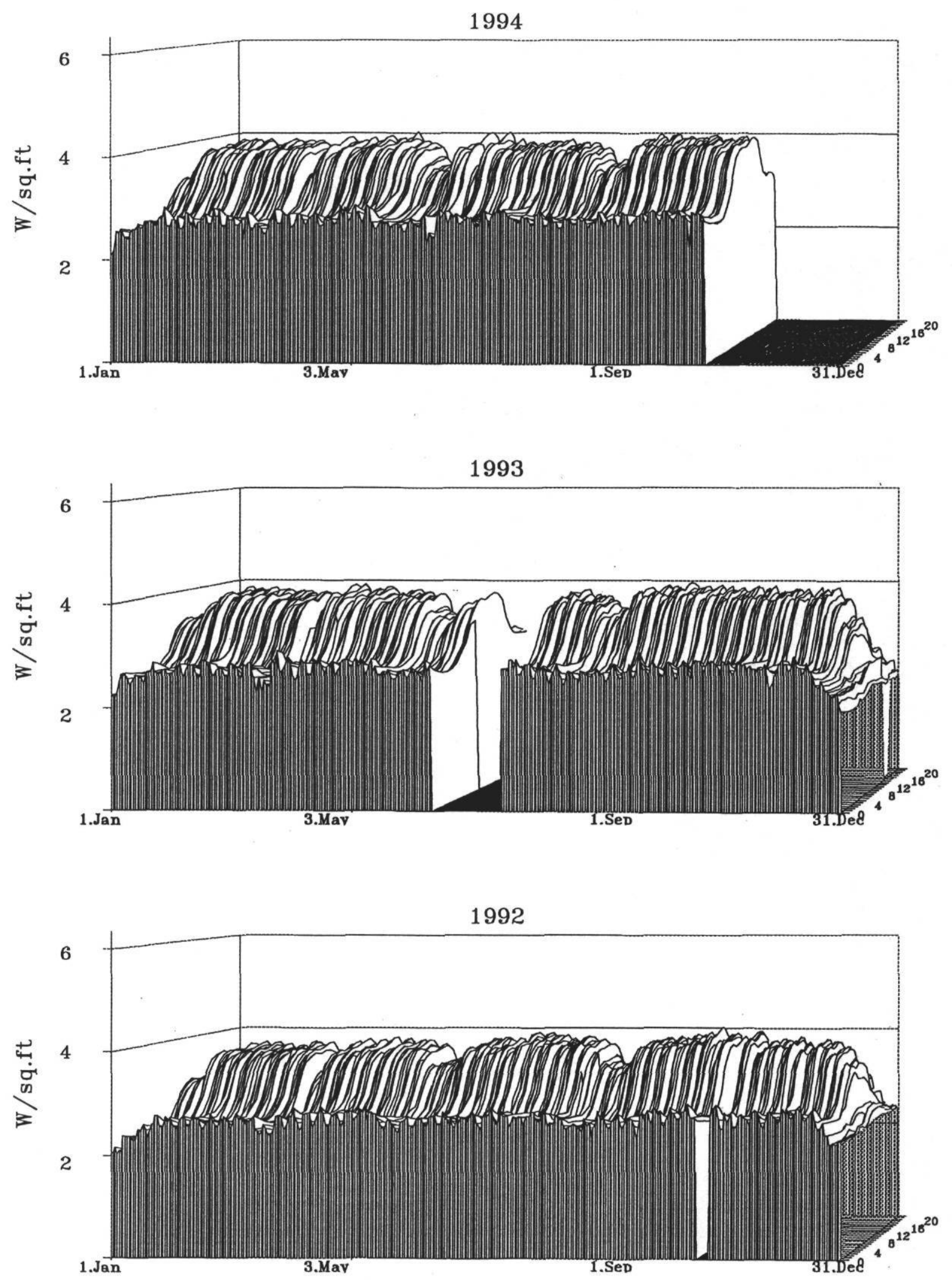

D-18 


\section{Zachry Engineering Center (ZEC) W.B. Electric as W/sq.ft.}
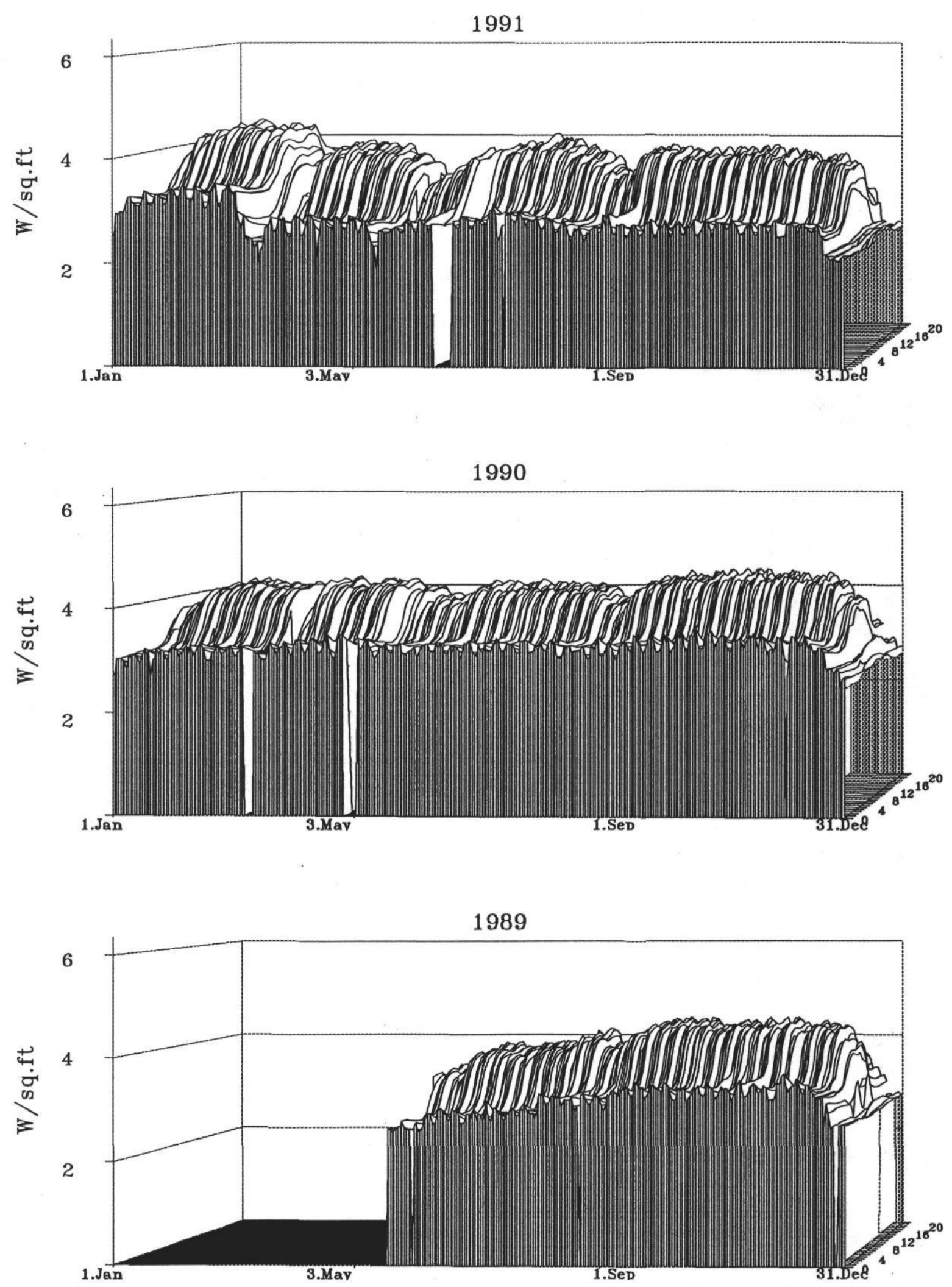

D-19 


\section{Zachry Engineering Center (ZEC) Motor Control Cen. (kWh/h)}
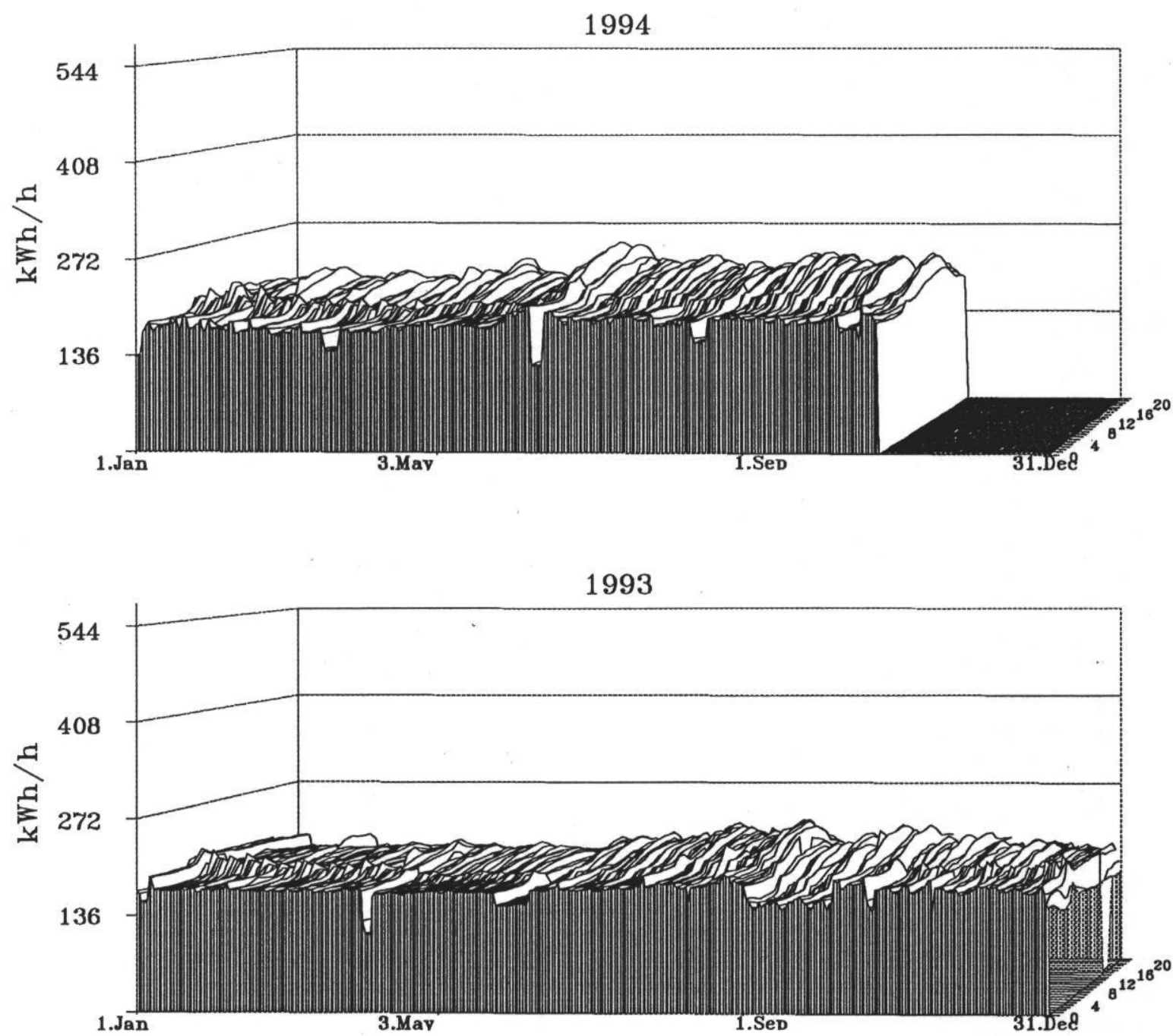

1992

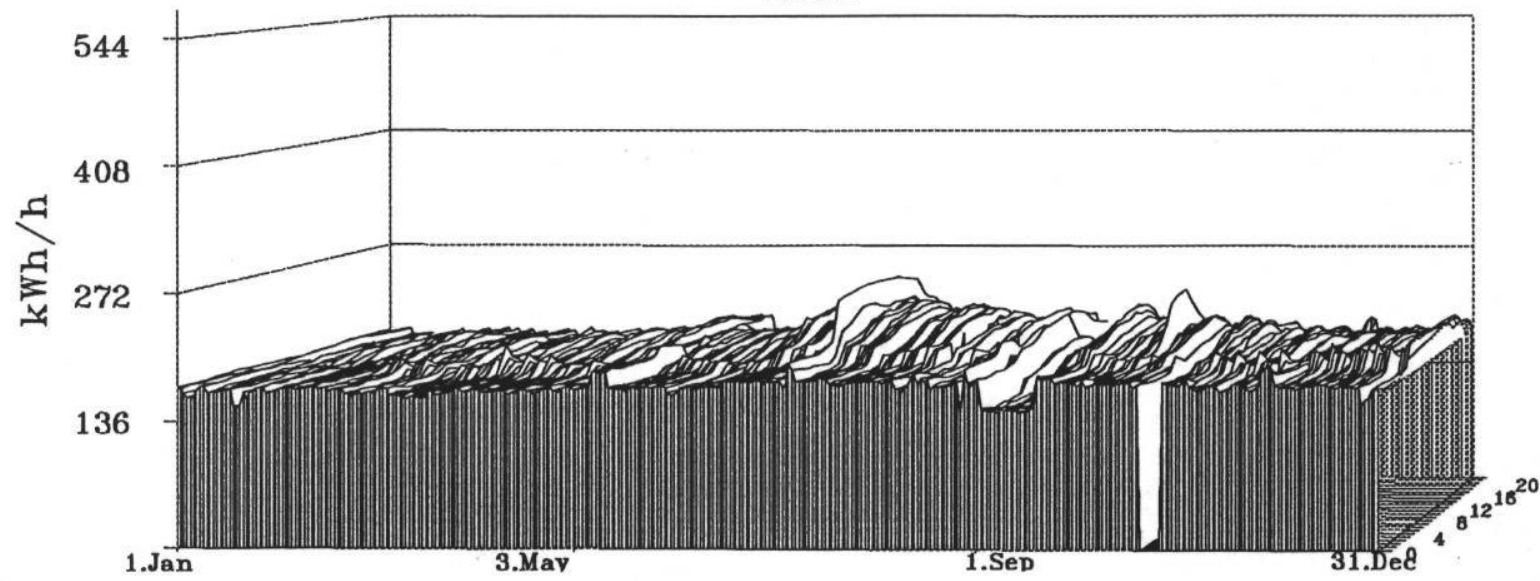

D-20 


\section{Zachry Engineering Center (ZEC) Motor Control Cen. (kWh/h)}
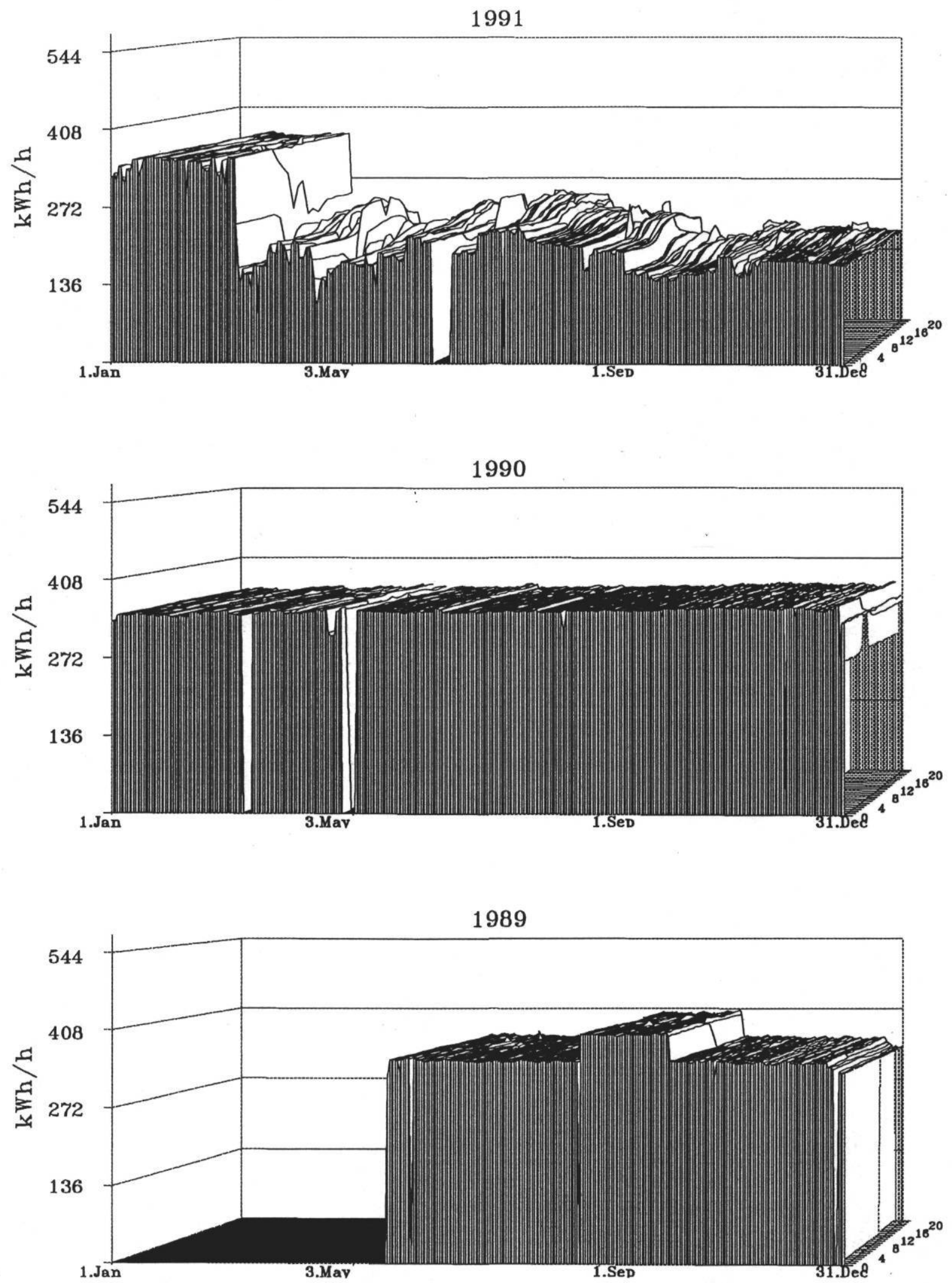

D-21 


\section{Zachry Engineering Center (ZEC) W.B. CHW as Btu/sq.ft.-h}
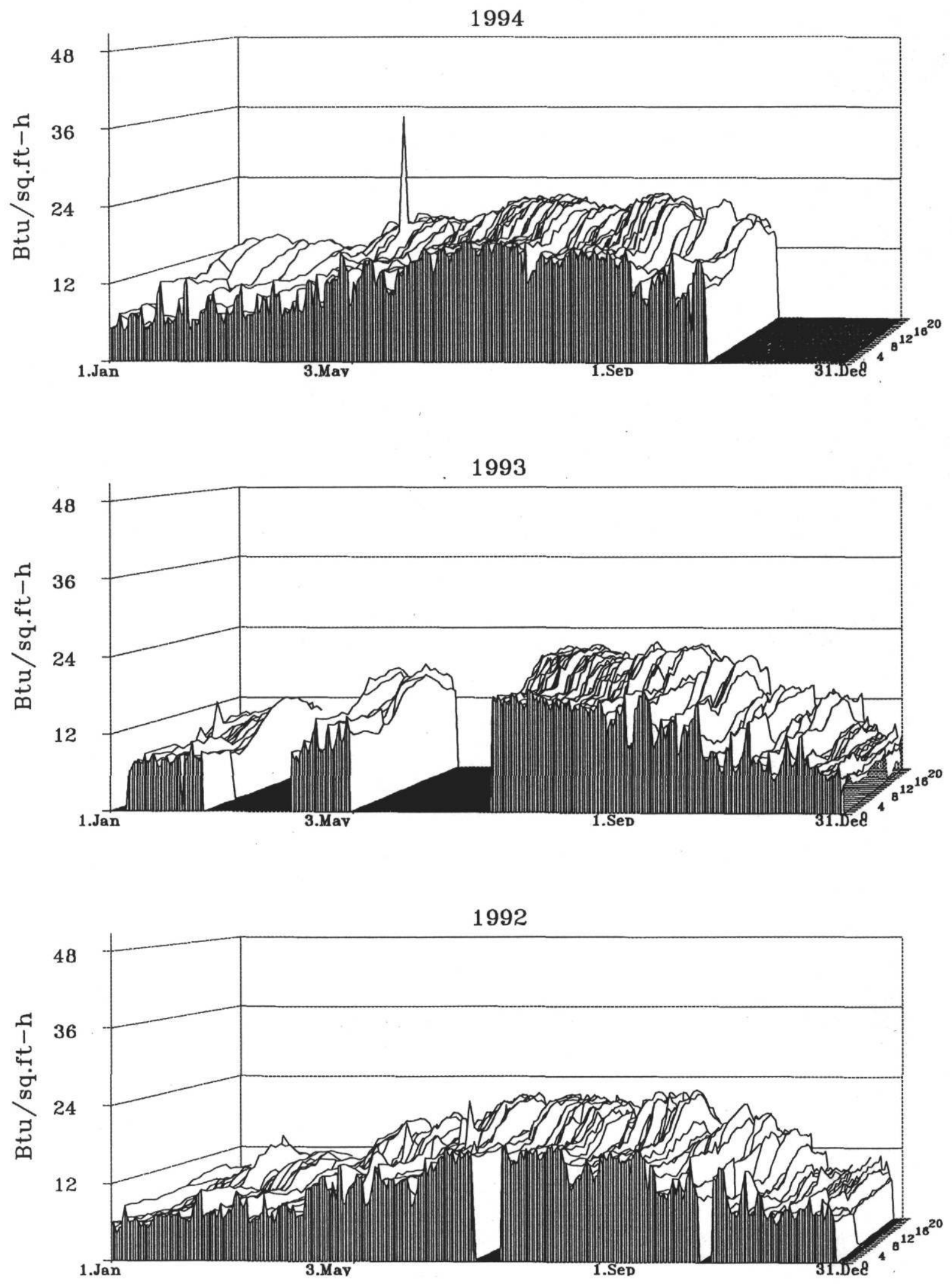

D-22 


\section{Zachry Engineering Center (ZEC) W.B. CHW as Btu/sq.ft.-h}
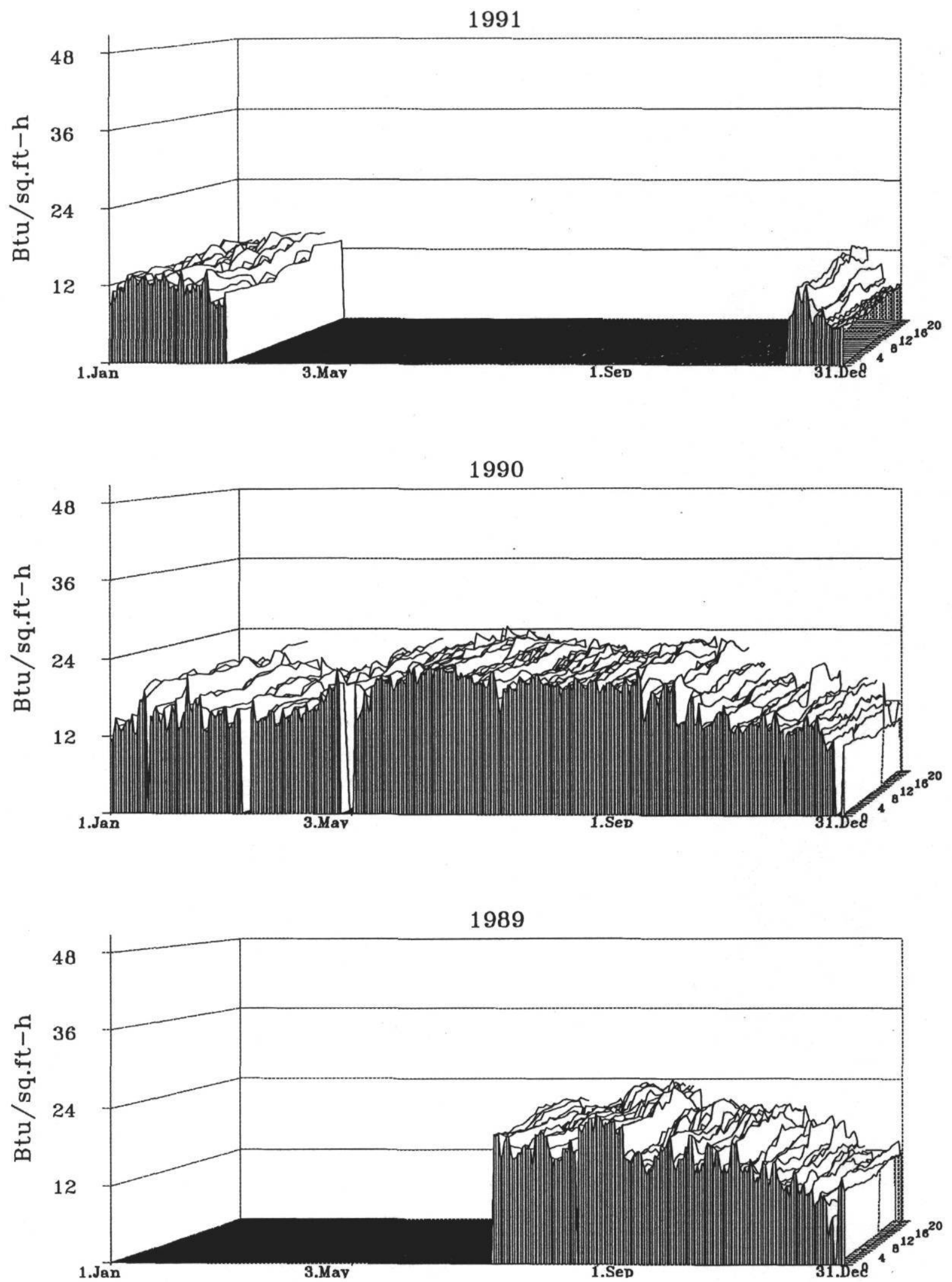

D-23 


\section{Zachry Engineering Center (ZEC) W.B. HW as Btu/sq.ft.-h}
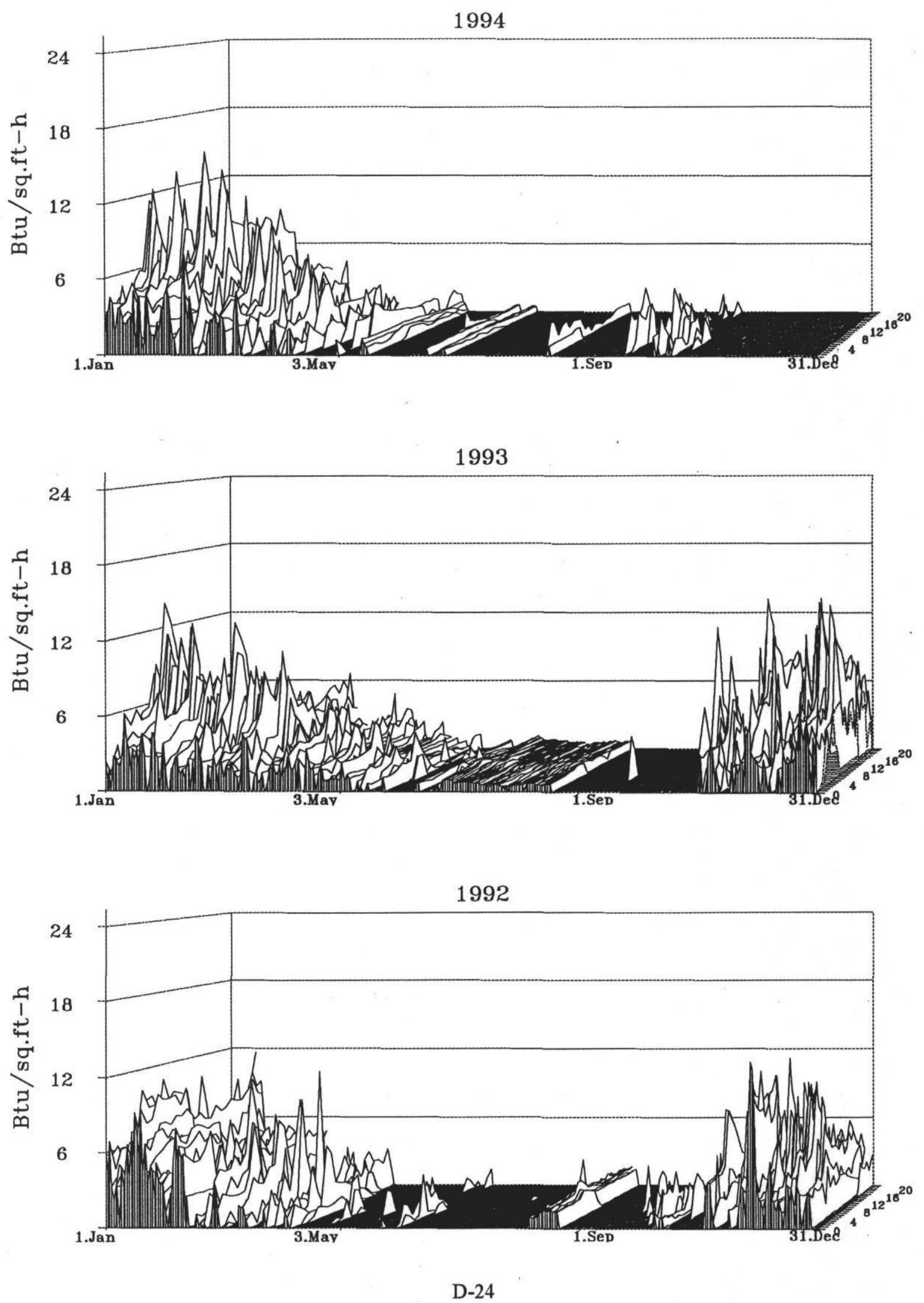


\section{Zachry Engineering Center (ZEC) \\ W.B. HW as Btu/sq.ft.-h}
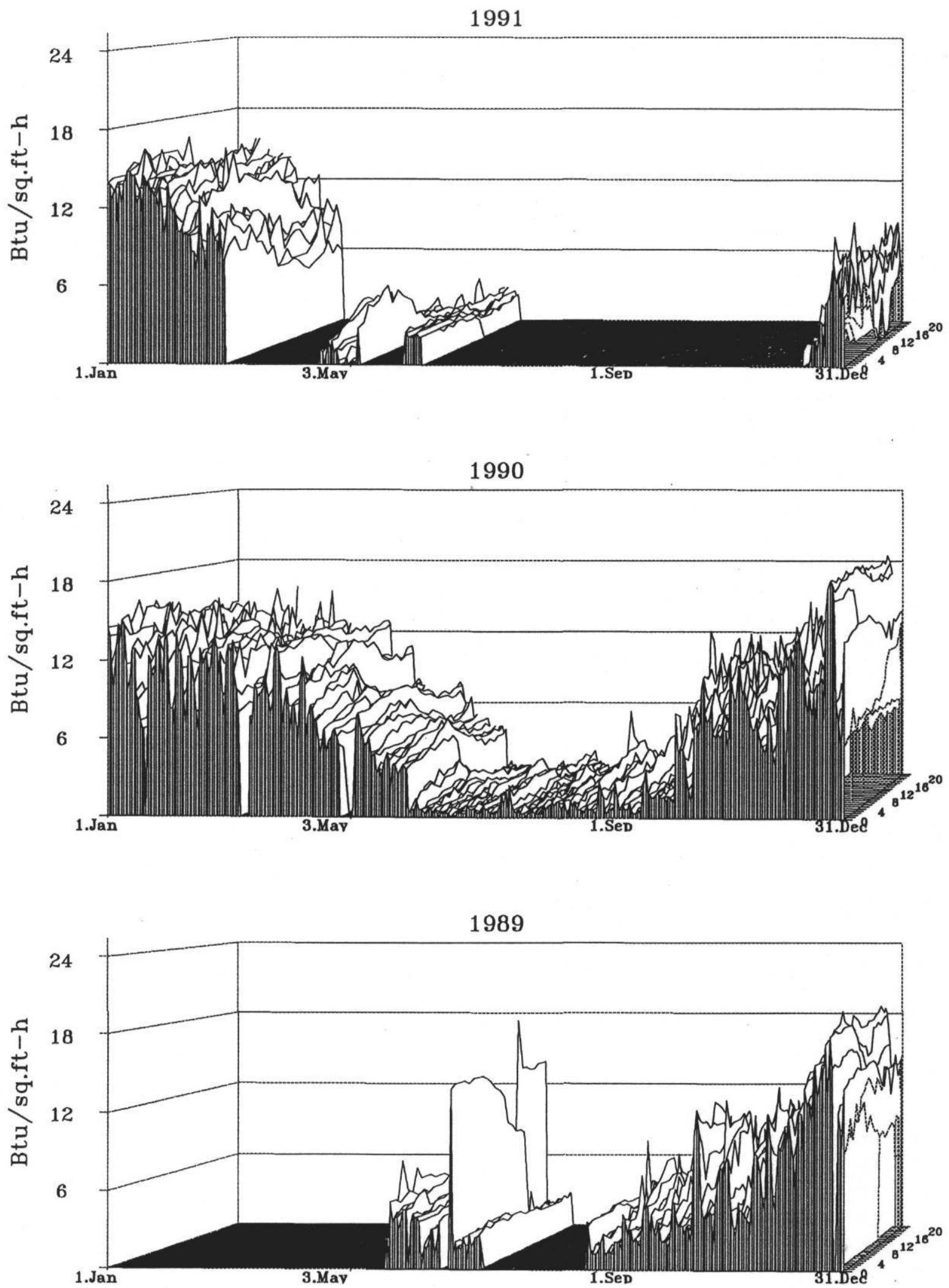

D-25 
Tab D-4

Weekly Box Whisker Mean Plots

D-26 


\section{Zachry Engineering Center (ZEC) W.B. Electric as W/sq.ft.}
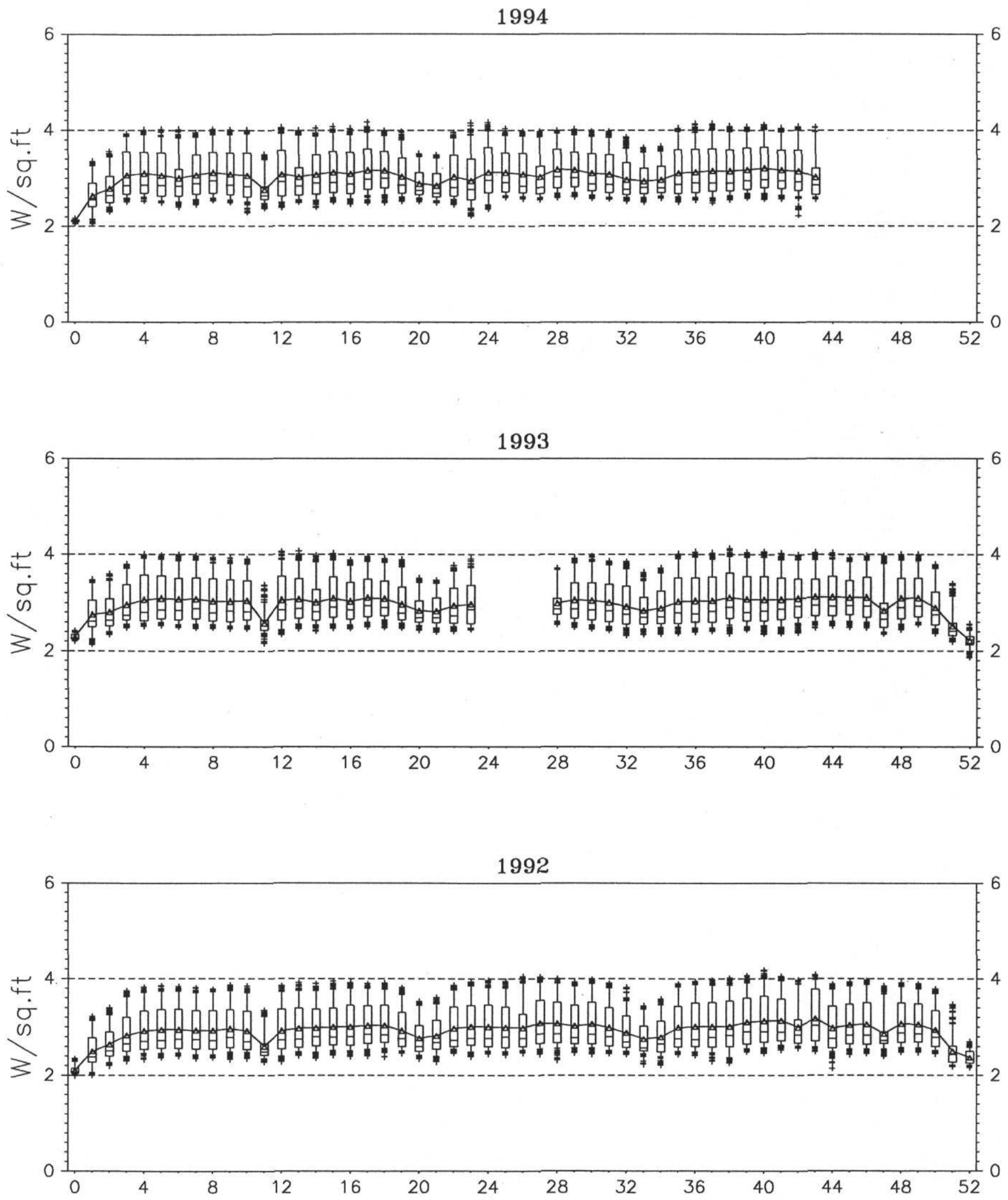


\section{Zachry Engineering Center (ZEC) W.B. Electric as W/sq.ft.}
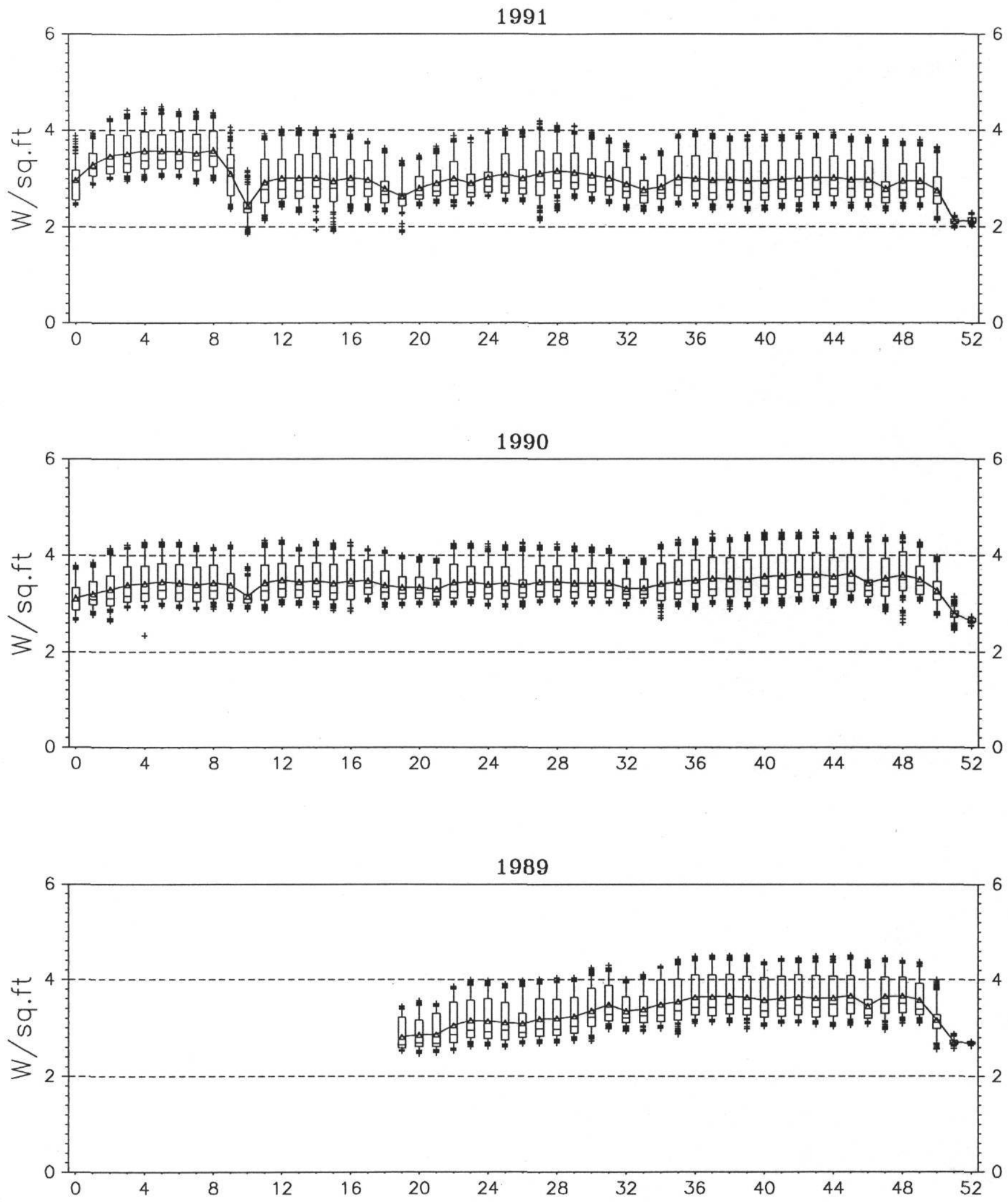


\section{Zachry Engineering Center (ZEC) Motor Control Cen. (kWh/h)}
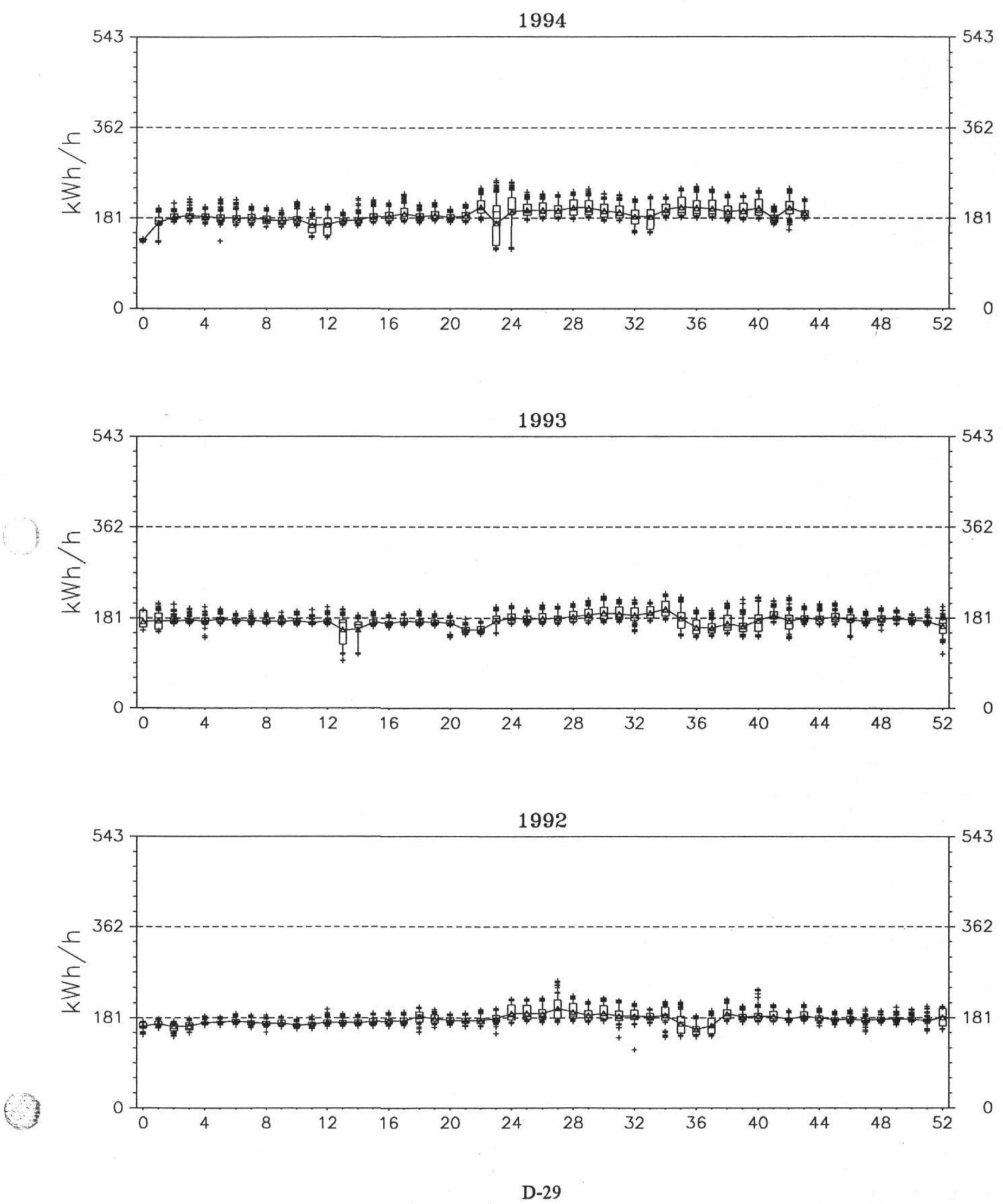


\section{Zachry Engineering Center (ZEC) Motor Control Cen. (kWh/h)}
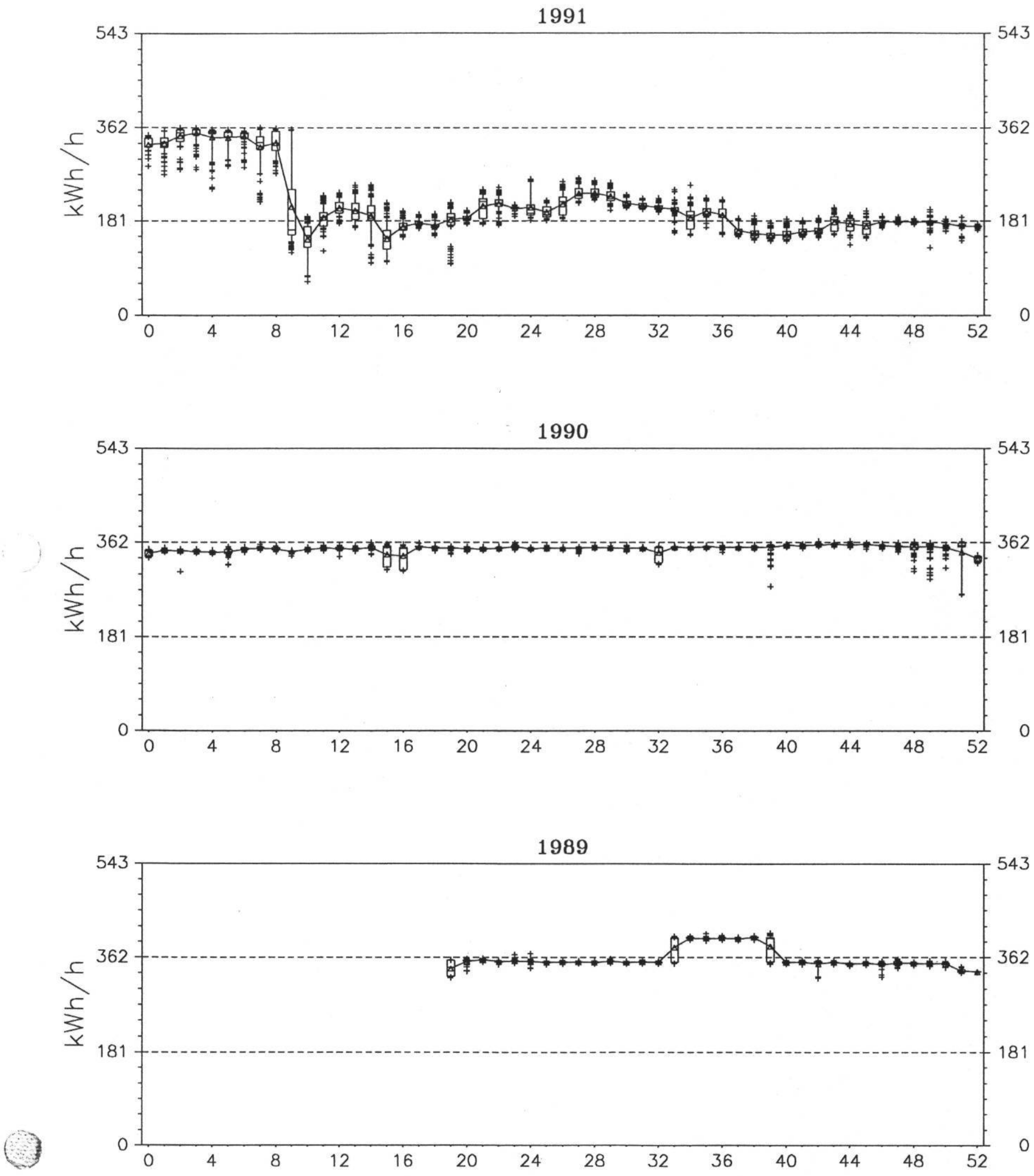

D-30 


\section{Zachry Engineering Center (ZEC) W.B. CHW as Btu/sq.ft.-h}
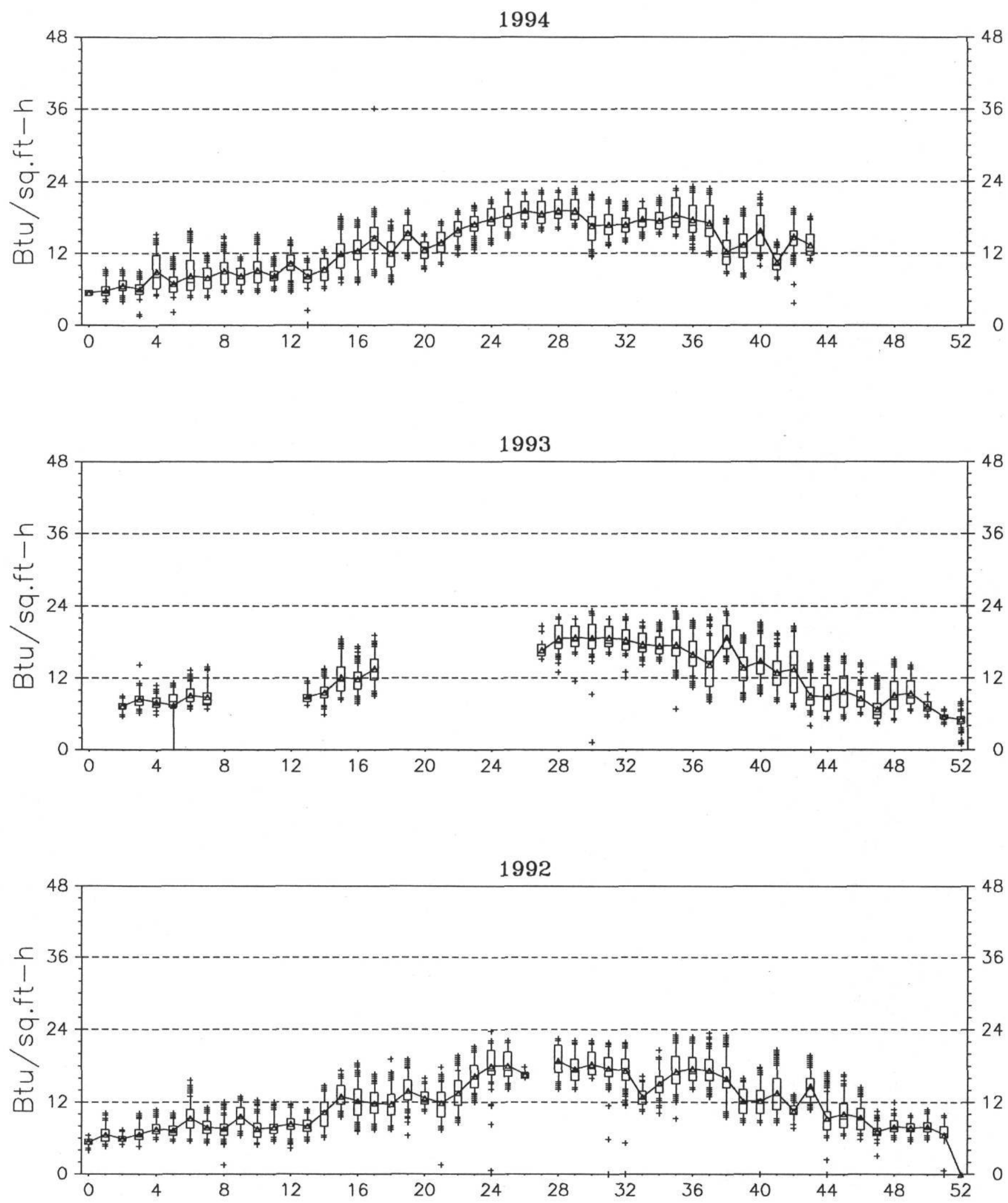


\section{Zachry Engineering Center (ZEC) W.B. CHW as Btu/sq.ft.-h}
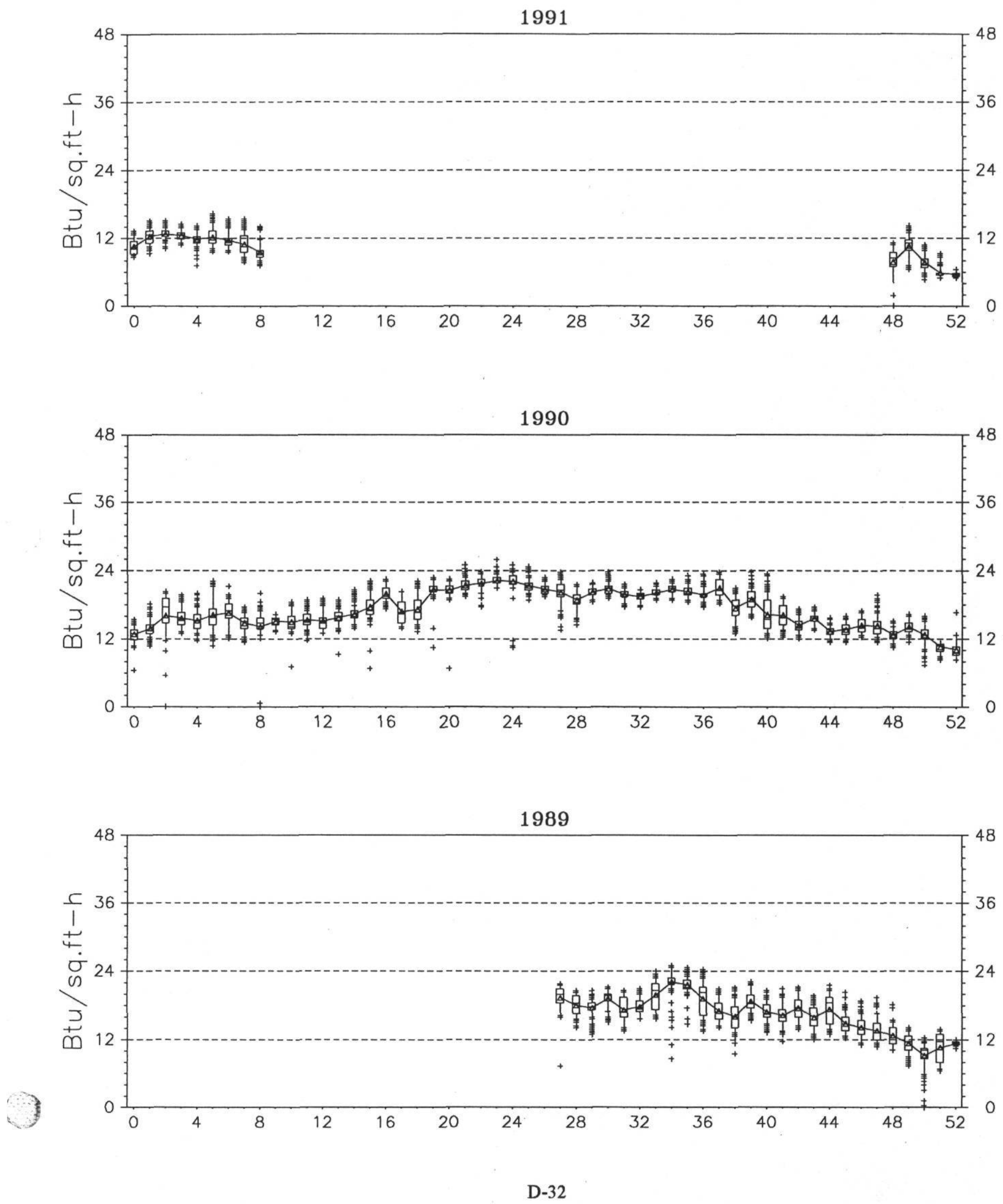


\section{Zachry Engineering Center (ZEC) W.B. HW as Btu/sq.ft.-h}
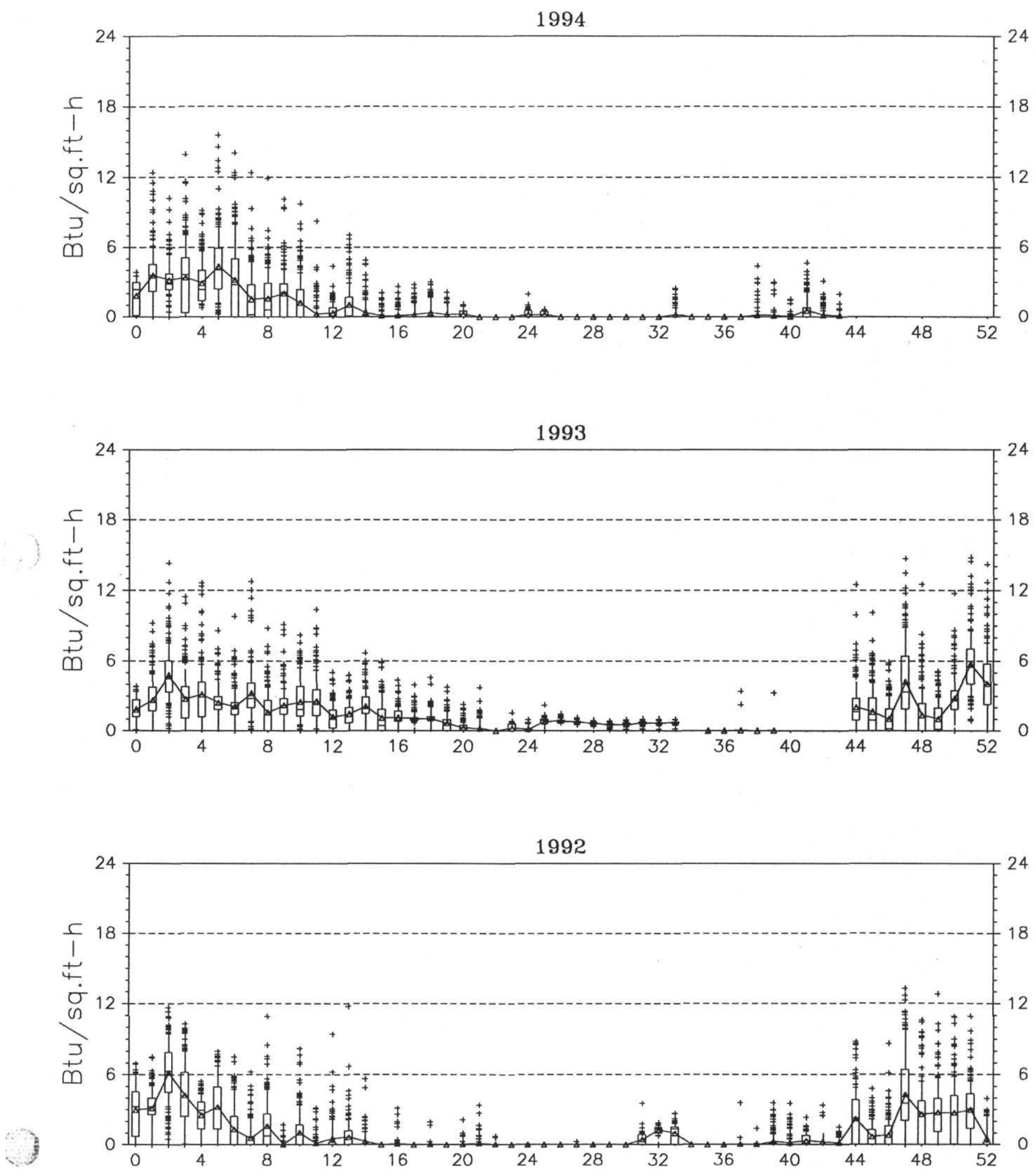


\section{Zachry Engineering Center (ZEC) W.B. HW as Btu/sq.ft.-h}
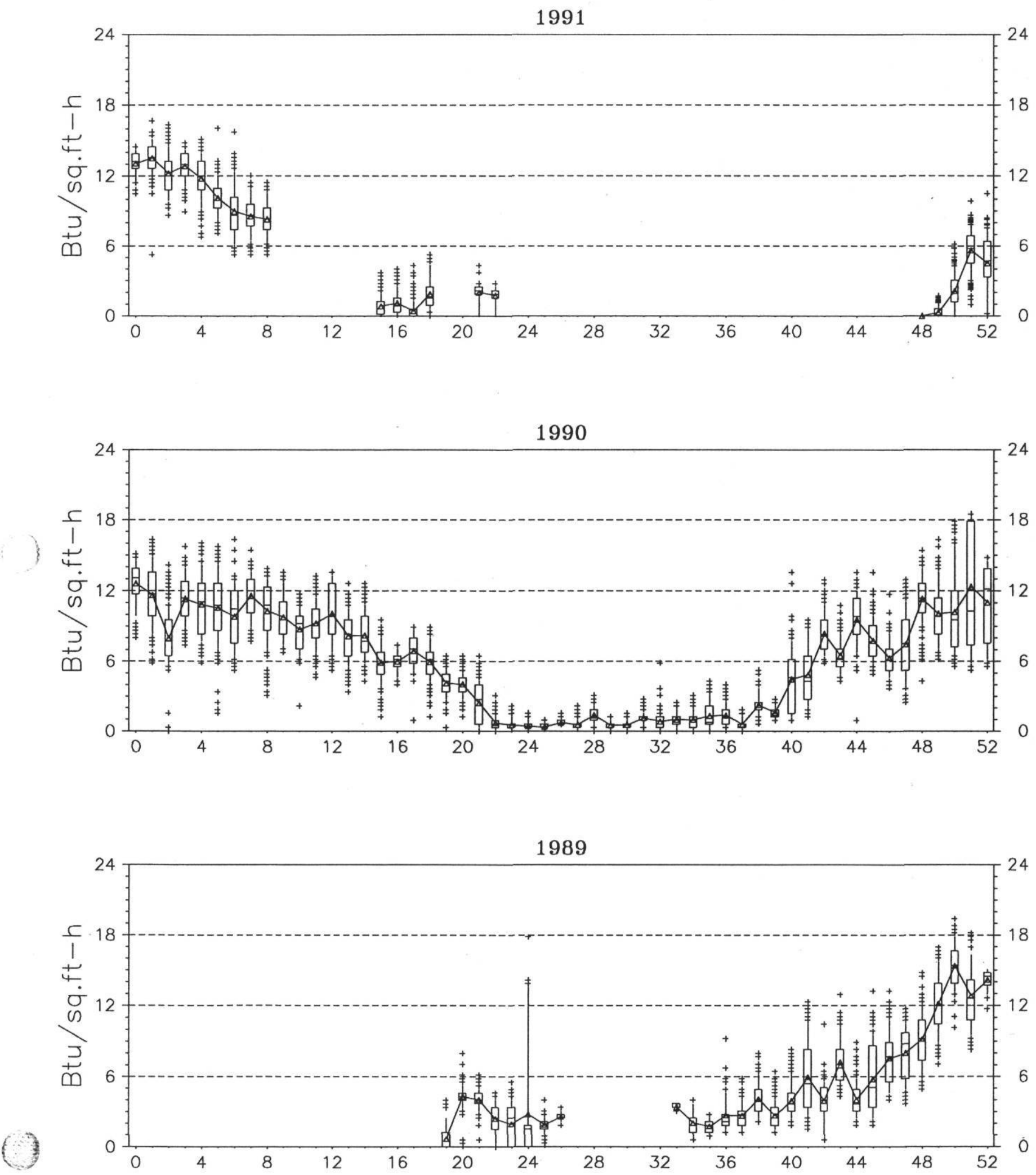
Tab D-5

Pre/Post, Weekday/Weekend, 24 Hour BWM Plots 


\section{Zachry Engineerıng Center (ZEC) \\ W.B. Electric as W/sq.ft.}

Pre-Retrofit (05/31/1989-11/28/1990)

Everyday

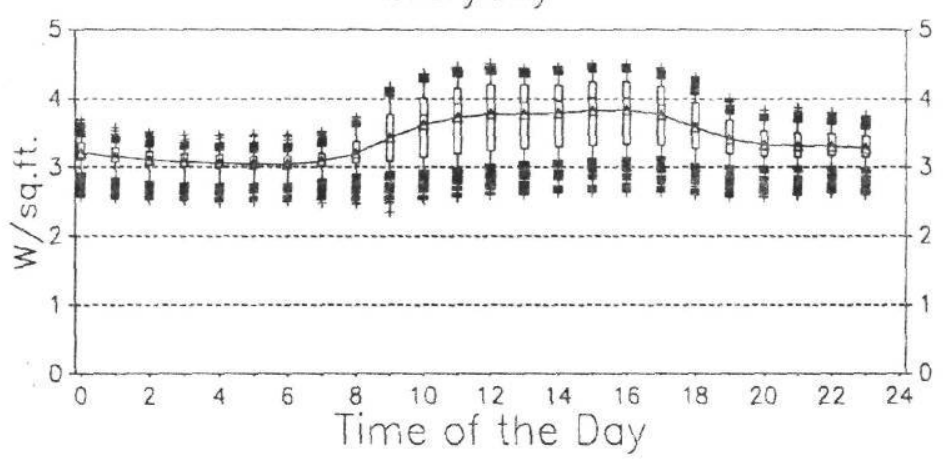

Weekdays

$\underset{\dot{u}}{\square}$

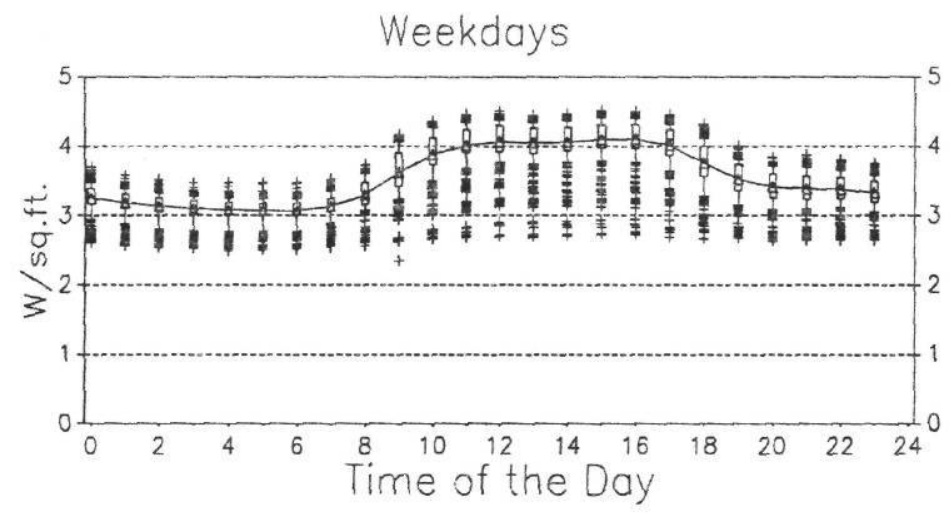

Weekends

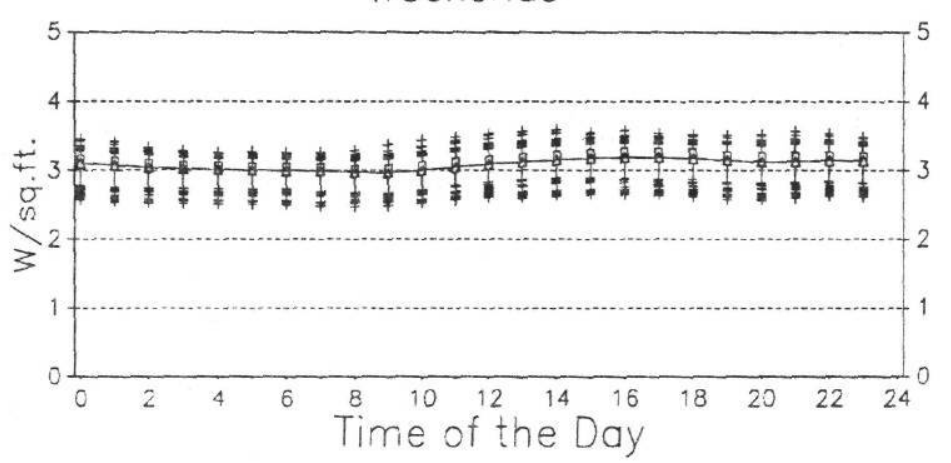

Post-Retrofit (03/06/1991-12/31/1994)

Everyday

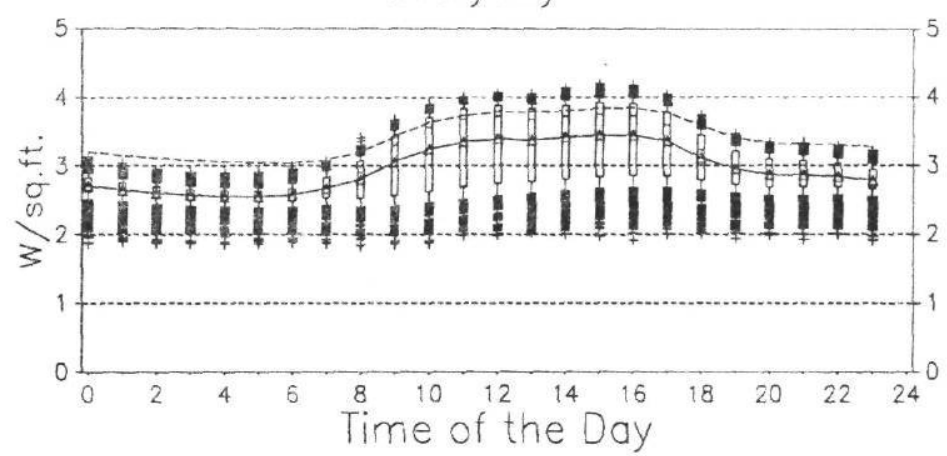

Weekdays

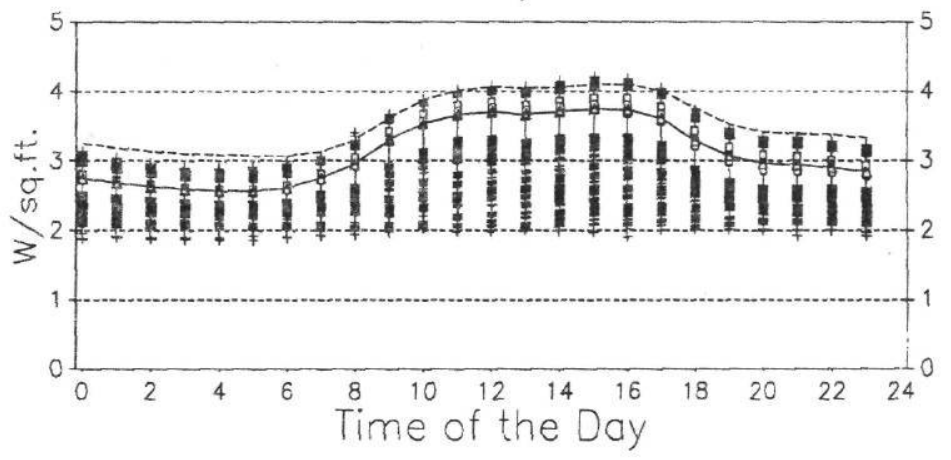

Weekends

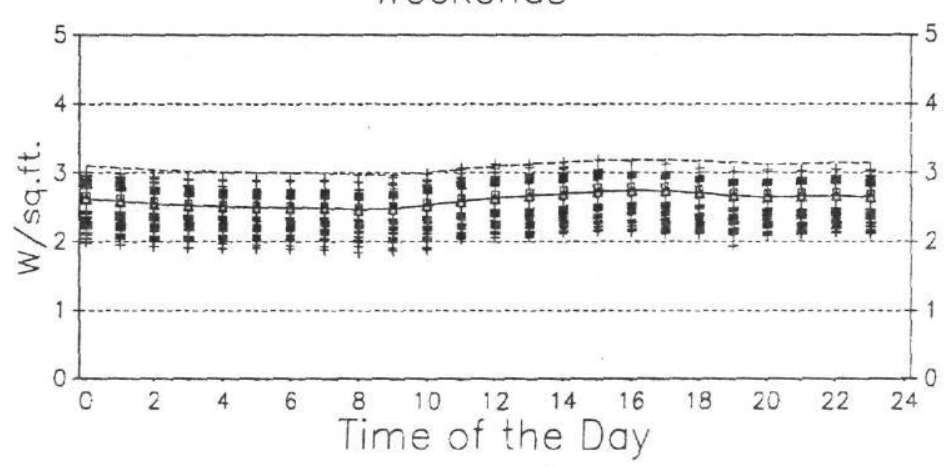


Tab D-6

Tab D-6

Pre/Post, Weekday/Weekend, BWM Temperature Binned Plots

Pre/Post, Weekday/Weekend, BWM Temperature Binned Plots 


\section{Zachry Engineering Center (ZEC) \\ W.B. CHW as Btu/sq.ft.-h}

Pre-Retrofit (05/31/1989-11/28/1990)

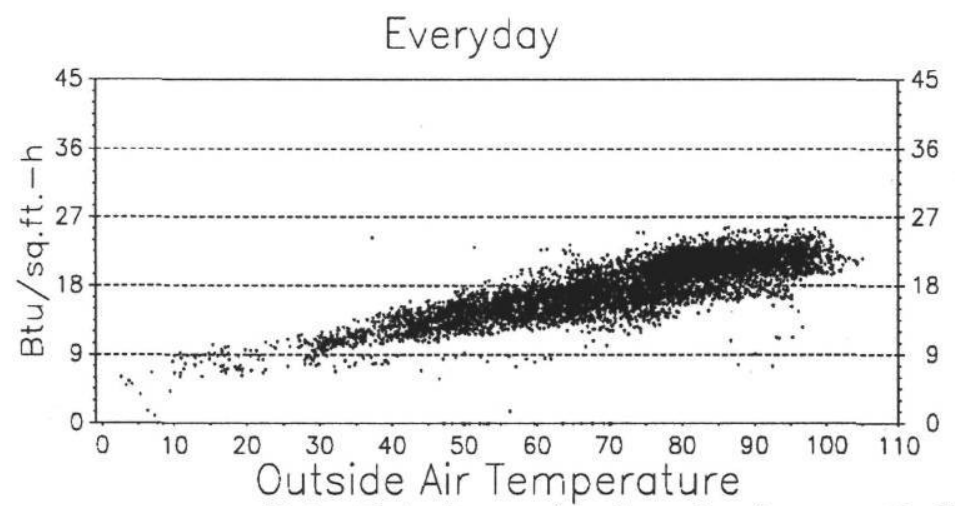

Post-Retrofit $(03 / 06 / 1991-12 / 31 / 1994)$

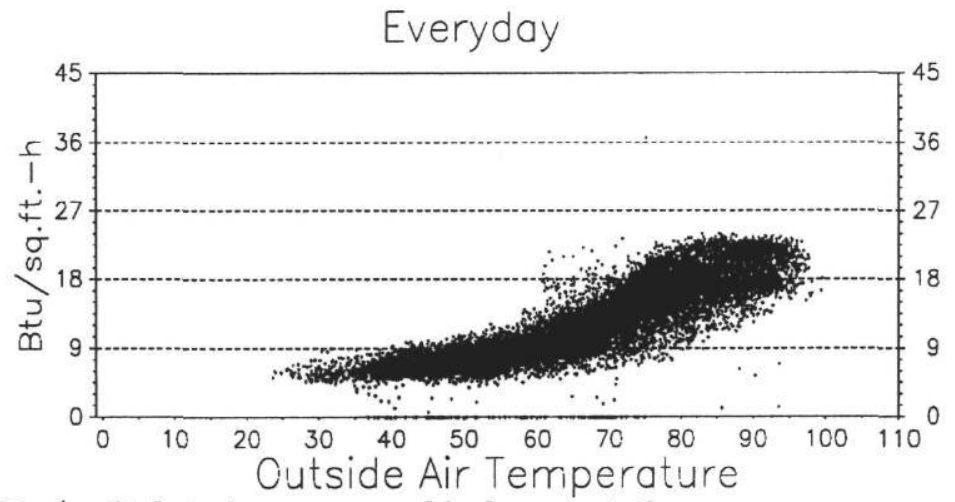

Note: Data in graphs above has been vertically offset randomly up to $1 \mathrm{Btu} / \mathrm{sq}$.ft.-h to improve graphical presentation.

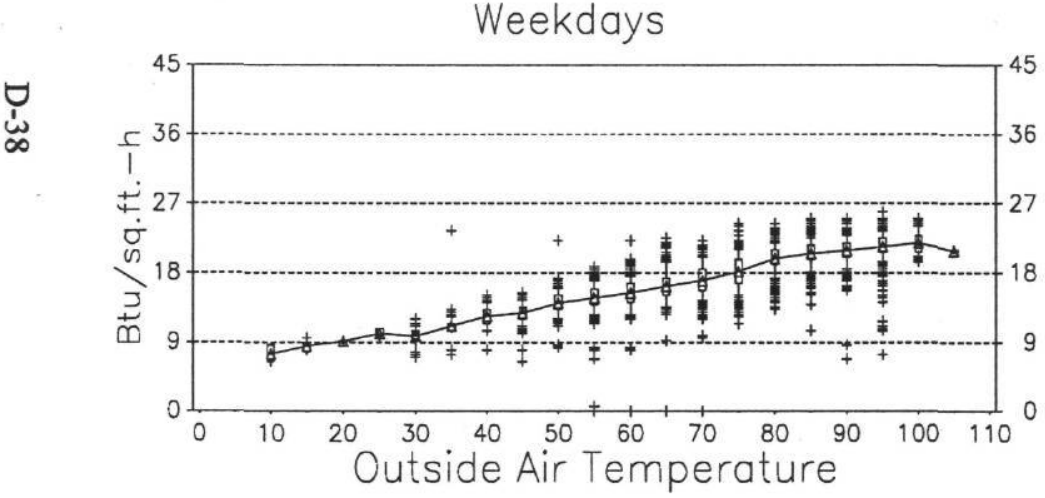

Weekends

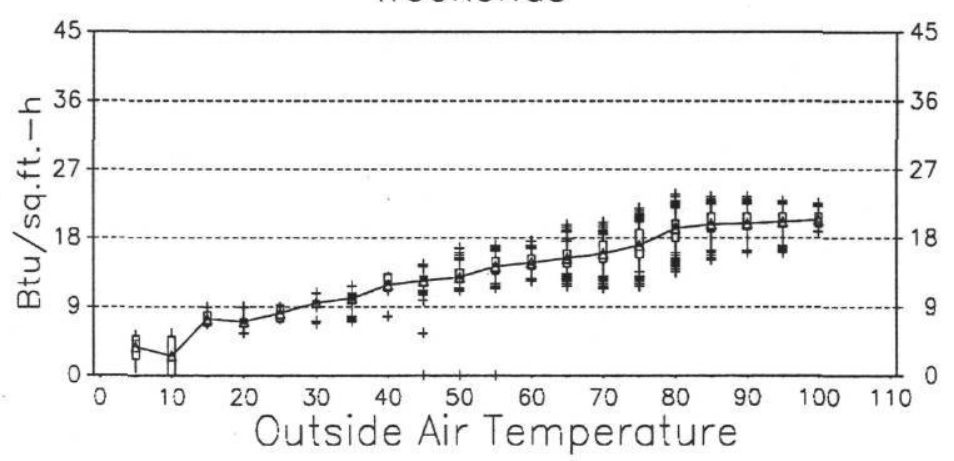

Weekdays

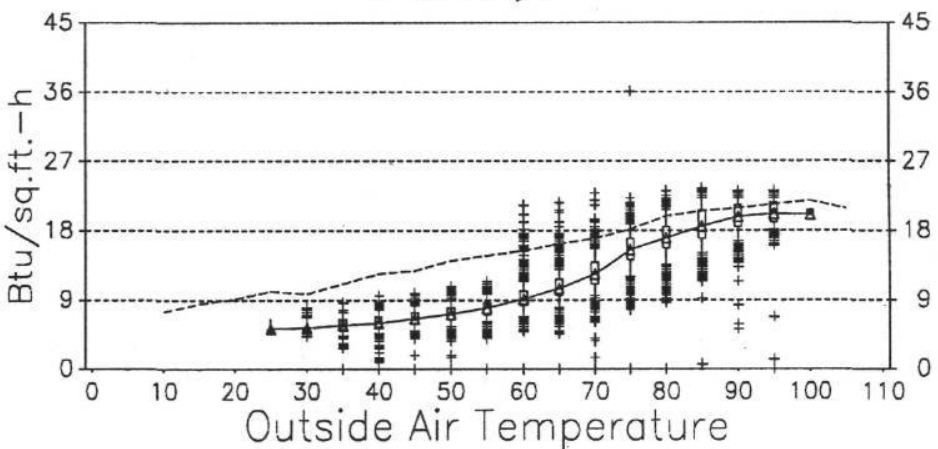

Weekends

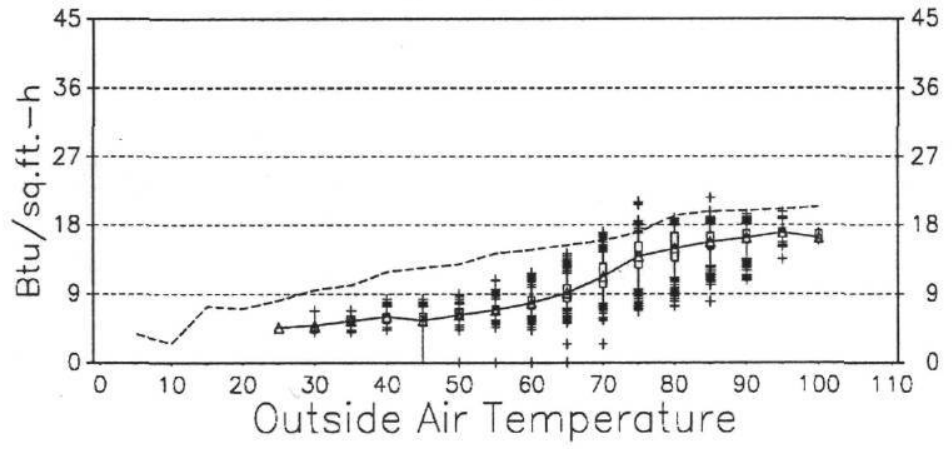




\section{Zachry Engineering Center (ZEC) \\ W.B. HW as Btu/sq.ft.-h}

Pre-Retrofit (05/31/1989-11/28/1990)

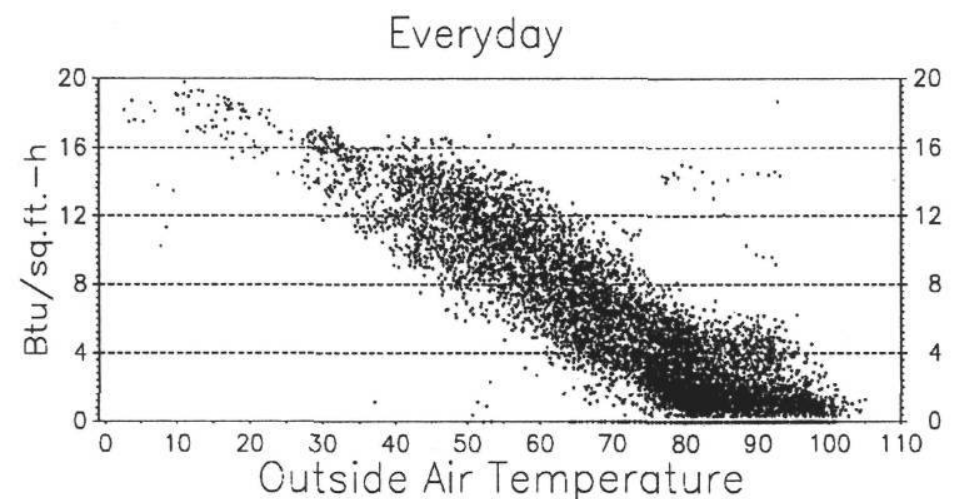

Post-Retrofit $(03 / 06 / 1991-12 / 31 / 1994)$

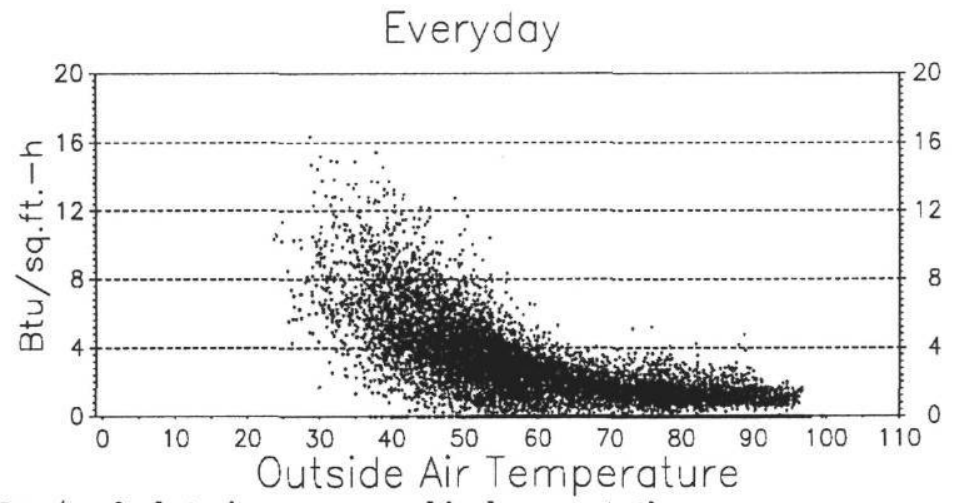

Note: Data in graphs above has been vertically offset randomly up to $1 \mathrm{Btu} / \mathrm{sq} . \mathrm{ft}$.h to improve graphical presentation.
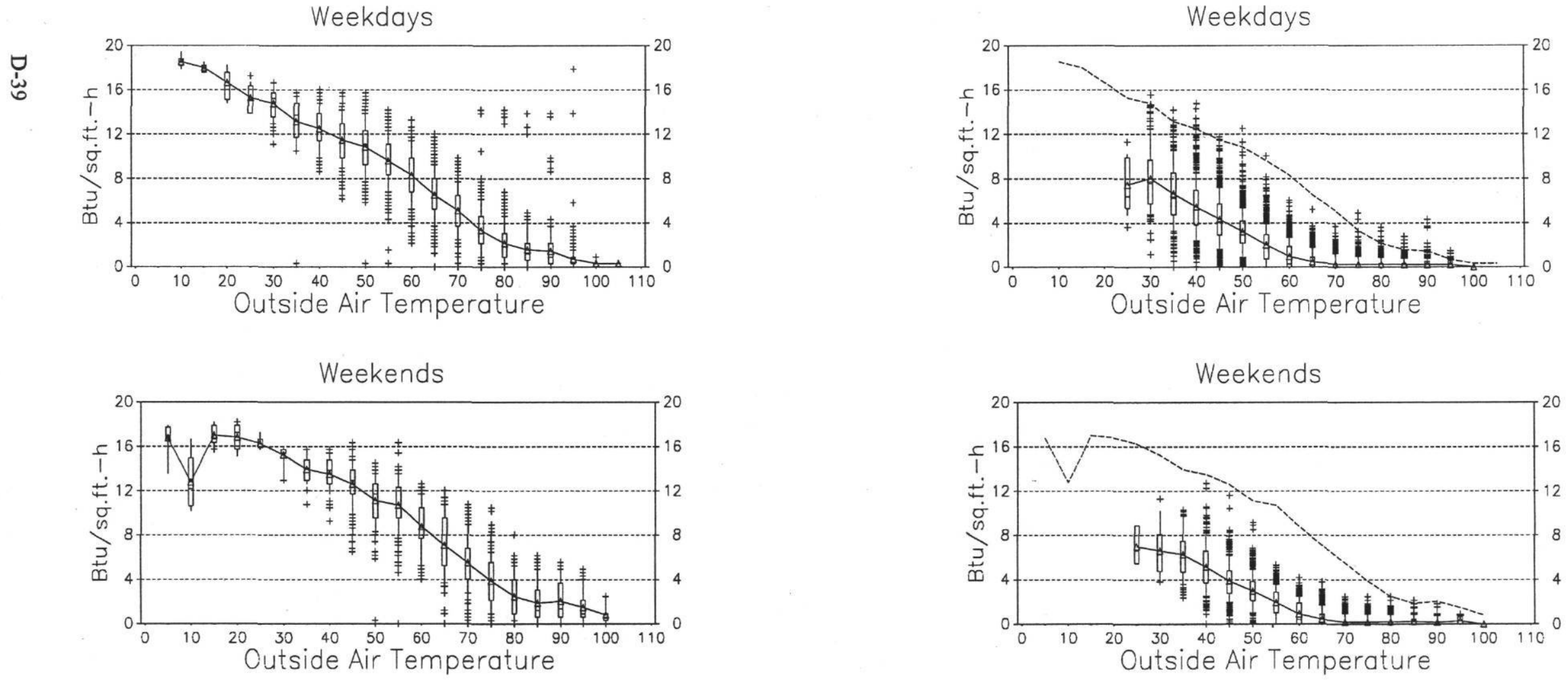
Tab D-7

Carpet Plots of Energy Use versus Ambient Conditions with Juxtaposed Histograms 


\section{Zachry Engineering Center (ZEC) Daily Average Values}

Pre-Retrofit $(\triangle)$ 05/31/1989 - 11/28/1990

Electric

$(\mathrm{kWh} / \mathrm{h})$
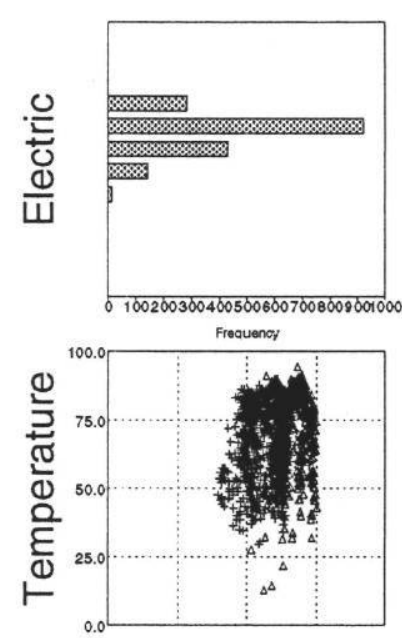

$\stackrel{Ð}{\ddagger}$
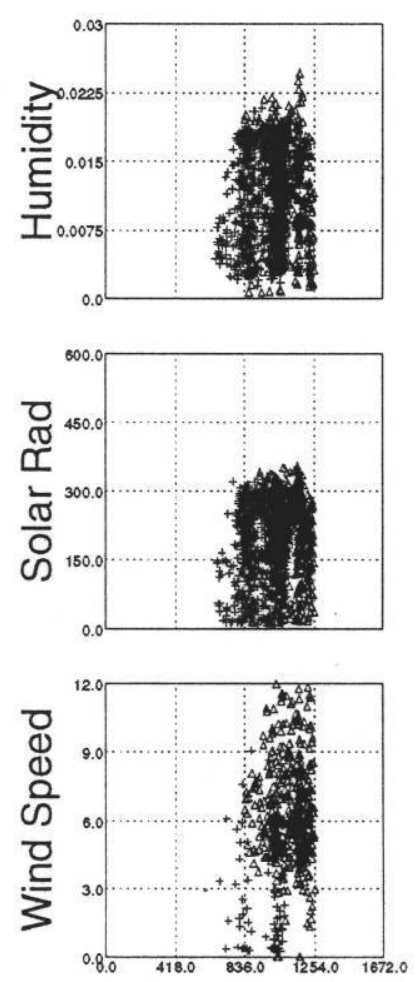

Temperature

(degrees F)
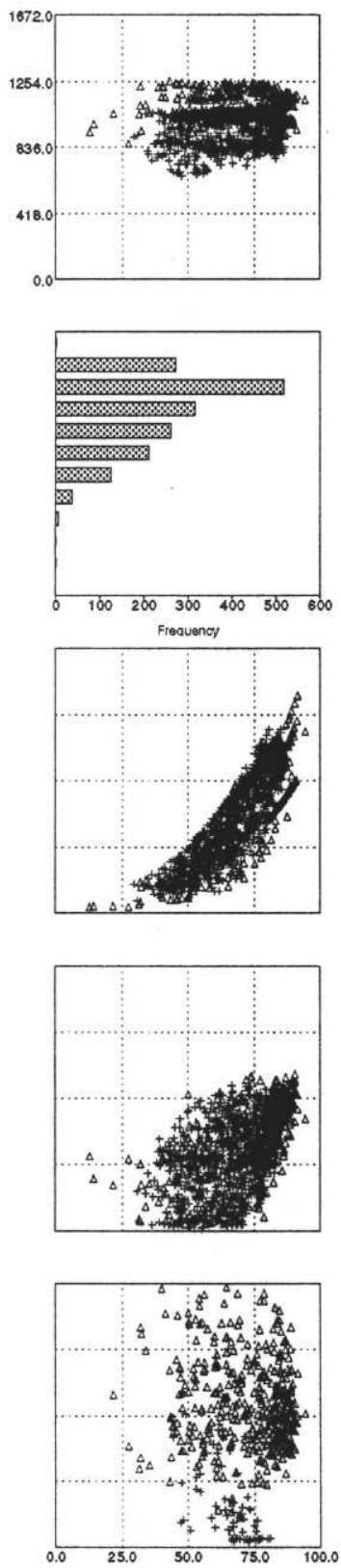

Post-Retrofit (+) 03/06/1991 - 12/31/1994

Humidity

(lbw/lba)

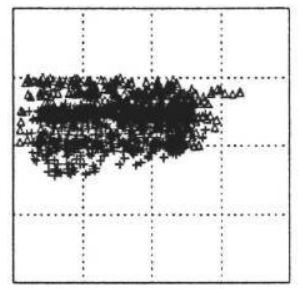

Solar Rad

(W/sq.m)
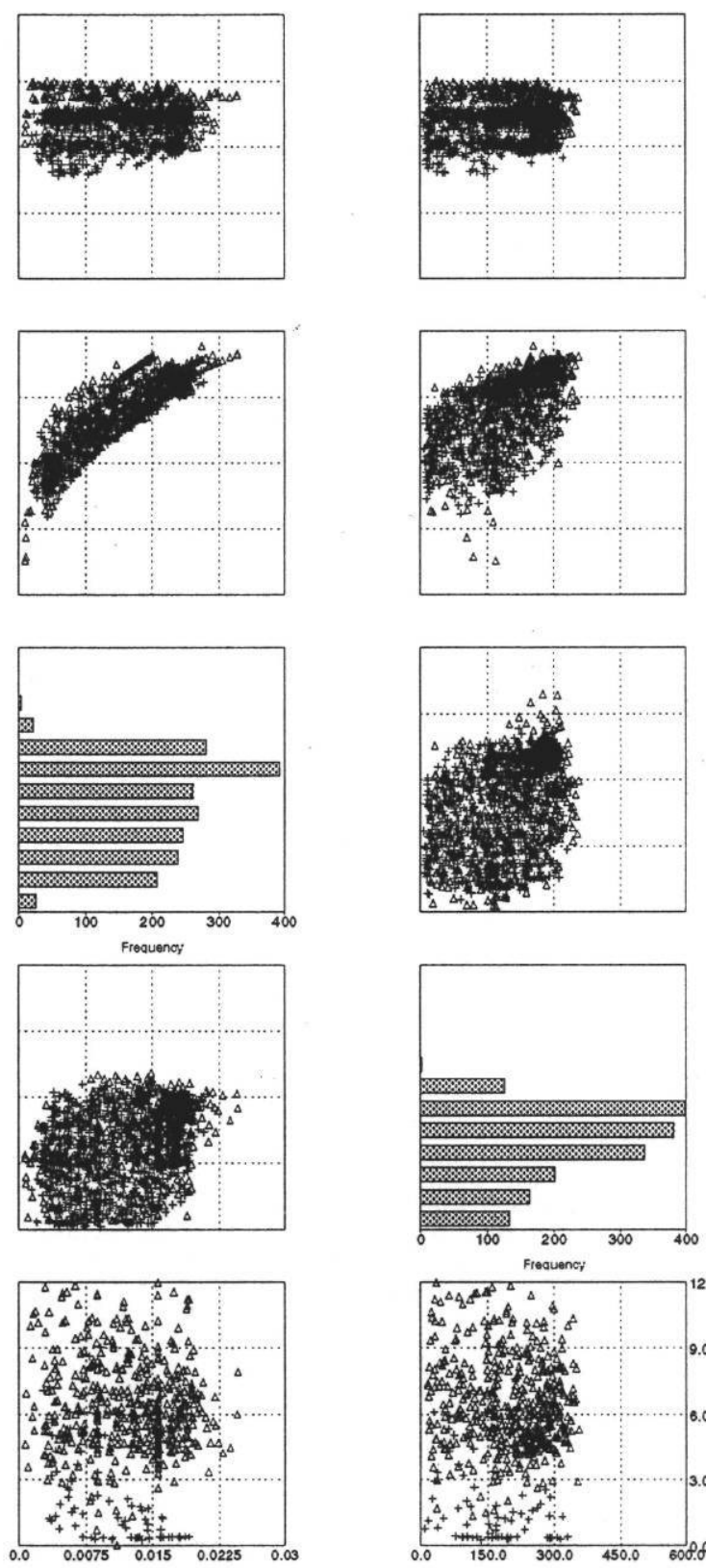

Wind Speed (mph)
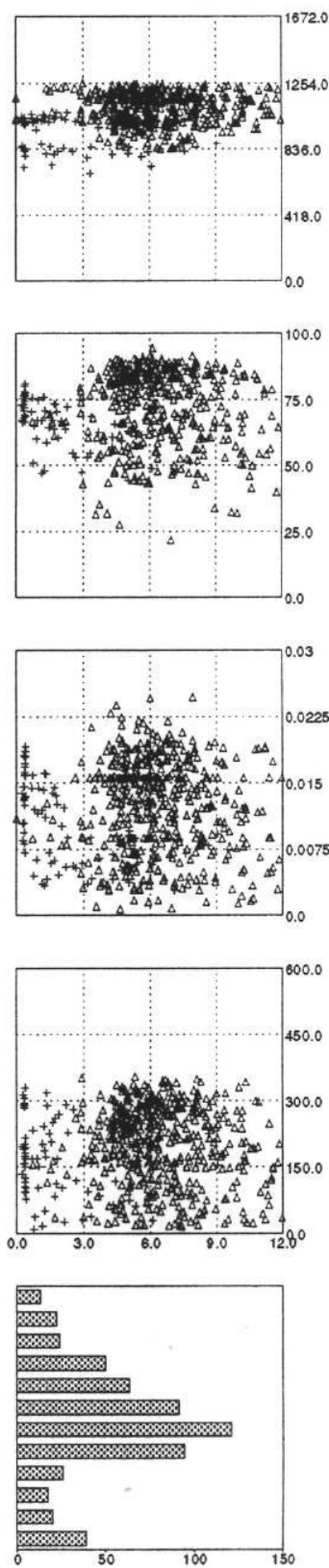


\section{Zachry Engineering Center (ZEC) Daily Average Values}

Pre-Retrofit $(\Delta) \quad 05 / 31 / 1989$ - 11/28/1990

Motor C.C.

$(\mathrm{kWh} / \mathrm{h})$
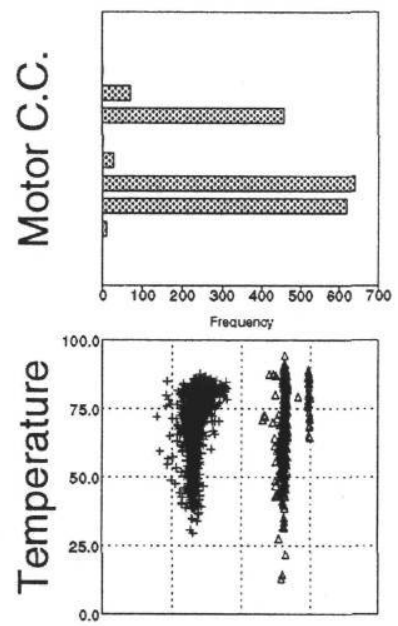

点
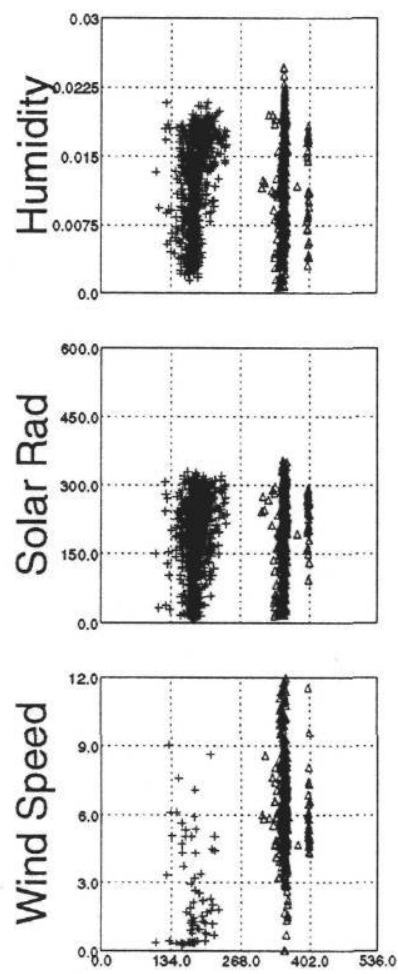

Temperature

(degrees F)
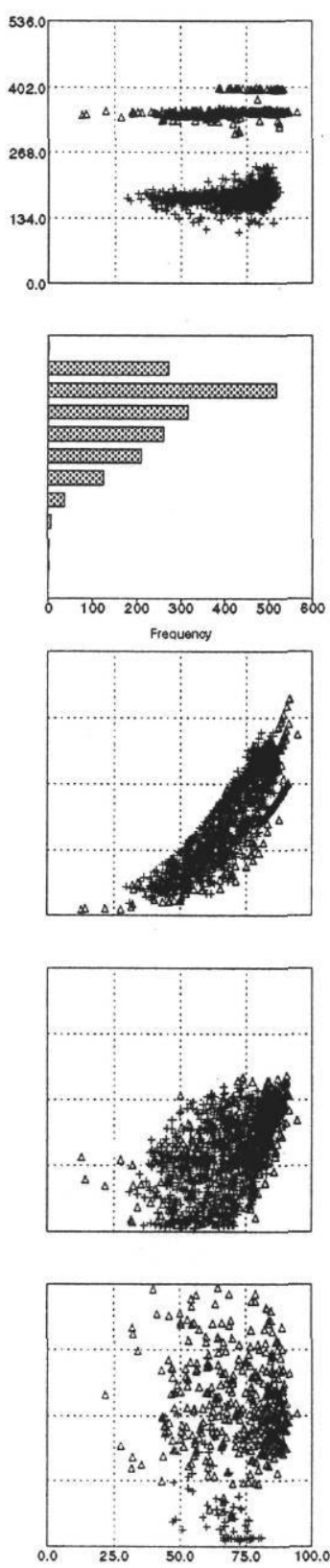

Post-Retrofit (+) 03/06/1991 - 12/31/1994

Humidity

(lbw/lba)
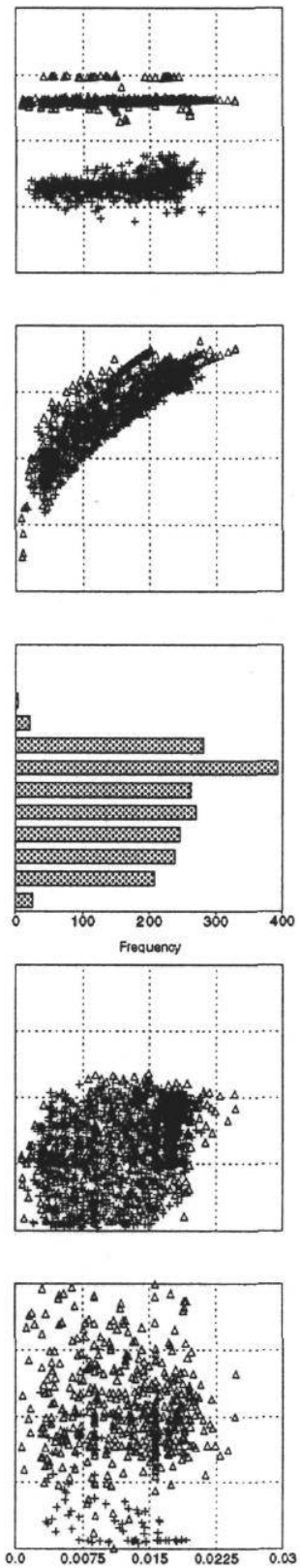

Solar Rad

$(\mathrm{W} / \mathrm{sq} \cdot \mathrm{m})$
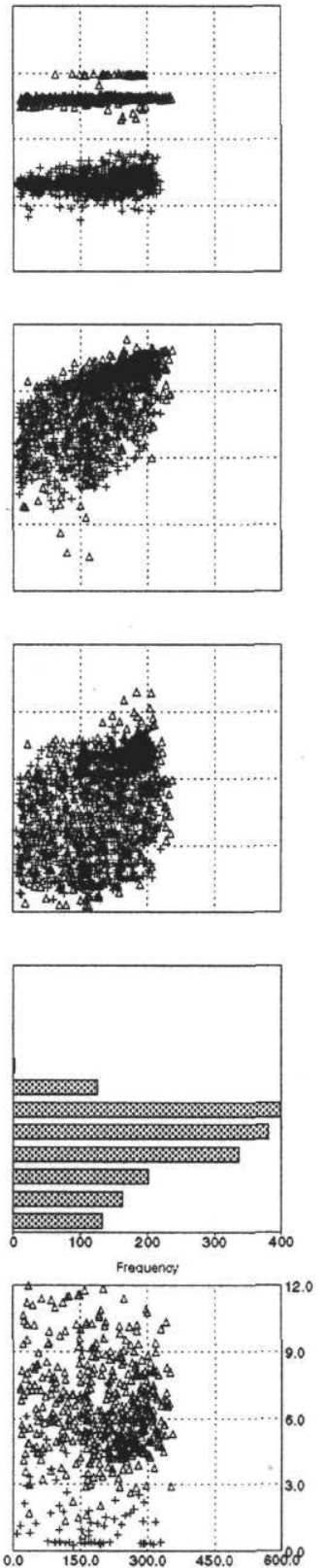

Wind Speed (mph)
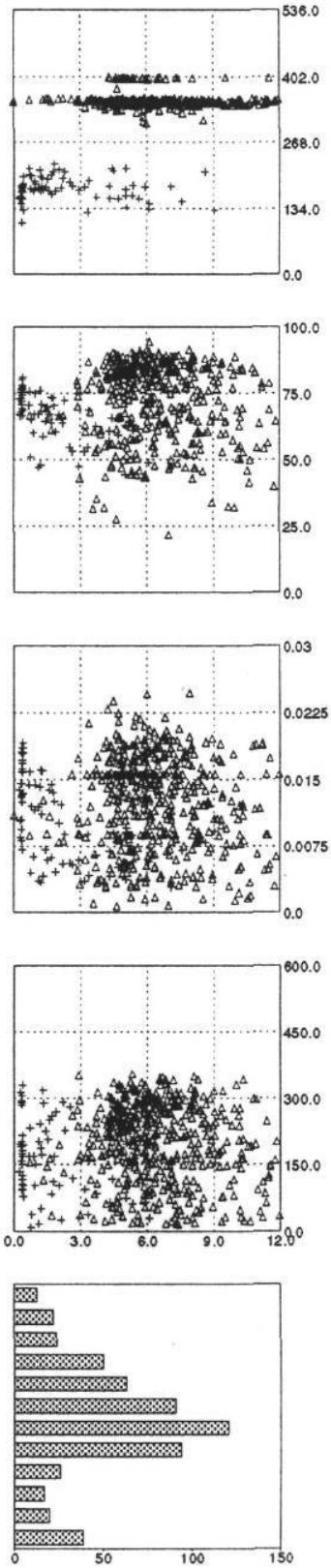
Tab D-8

Carpet Plots of One Energy Channel Use Against Other Energy Use Channels 


\section{Zachry Engineering Center (ZEC) Daily Average Values}

Pre-Retrofit $(\triangle)$ 05/31/1989 - 11/28/1990

Electric

$(\mathrm{kWh} / \mathrm{h})$
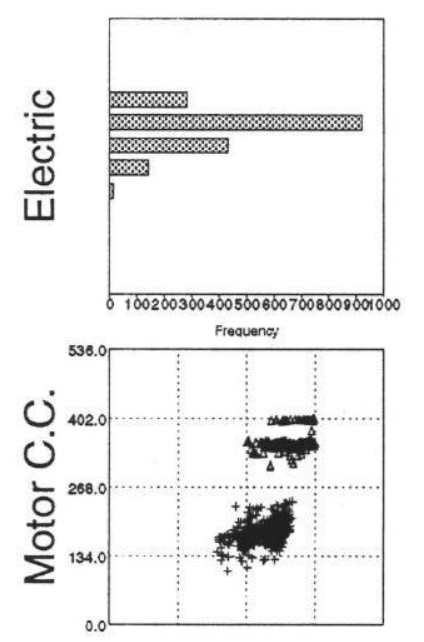

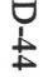
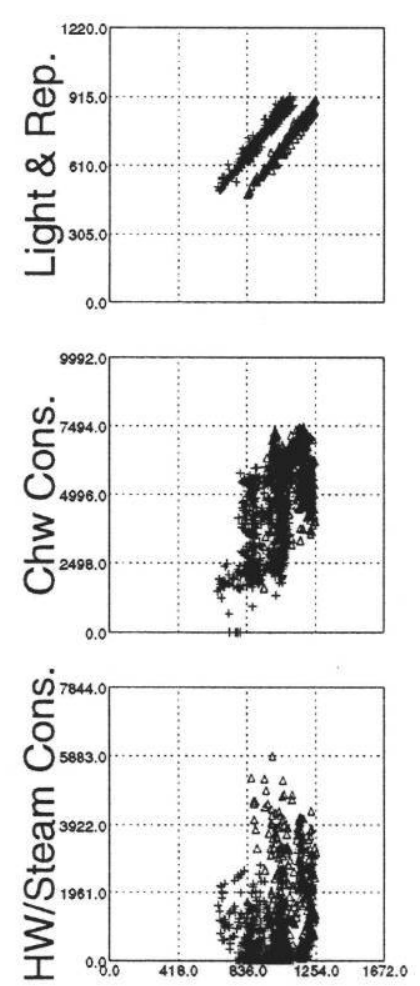

Motor C.C.

$(\mathrm{kWh} / \mathrm{h})$
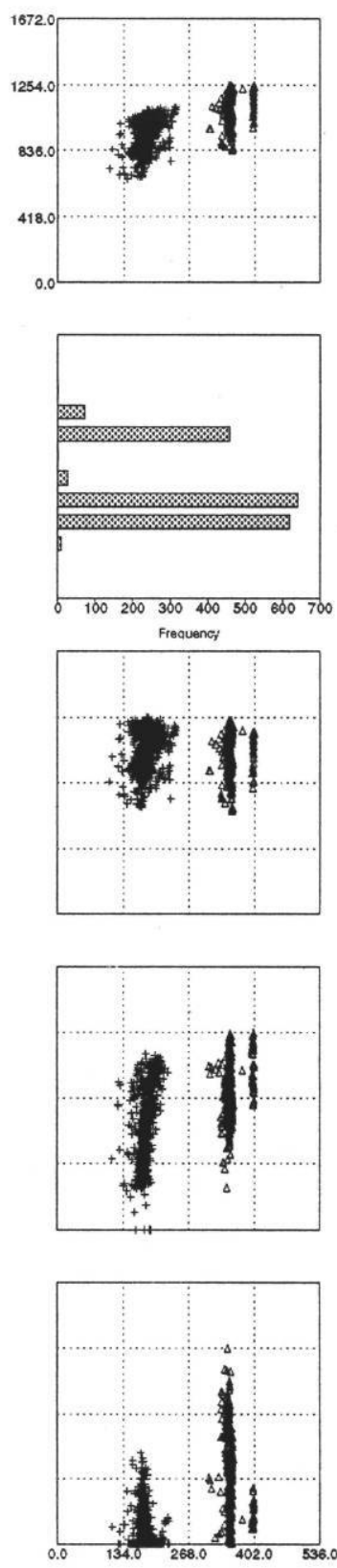

Lights \& Rep. $(\mathrm{kWh} / \mathrm{h})$
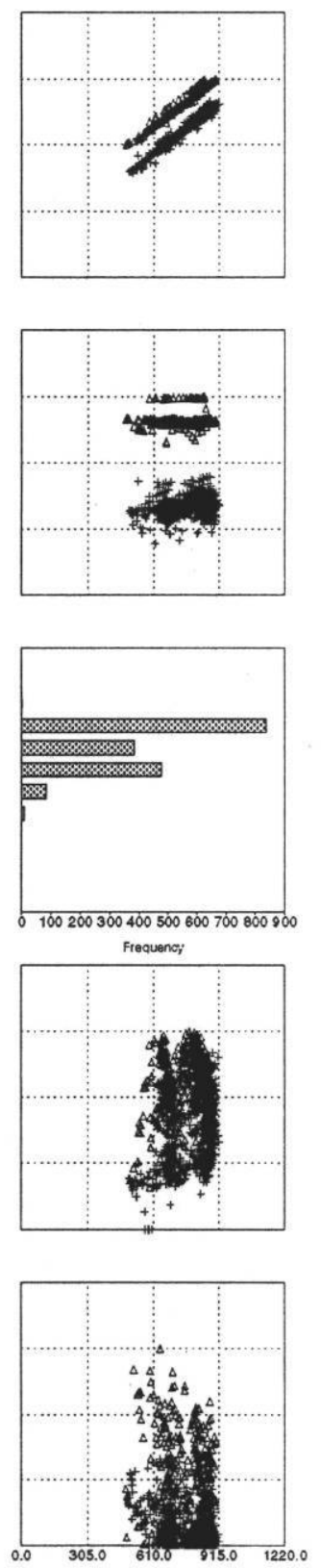

Post-Retrofit (+) 03/06/1991 - 12/31/1994

Chw Cons. $(\mathrm{kBtu} / \mathrm{h})$

$\mathrm{HW} /$ Steam Cons. $(\mathrm{kBtu} / \mathrm{h})$
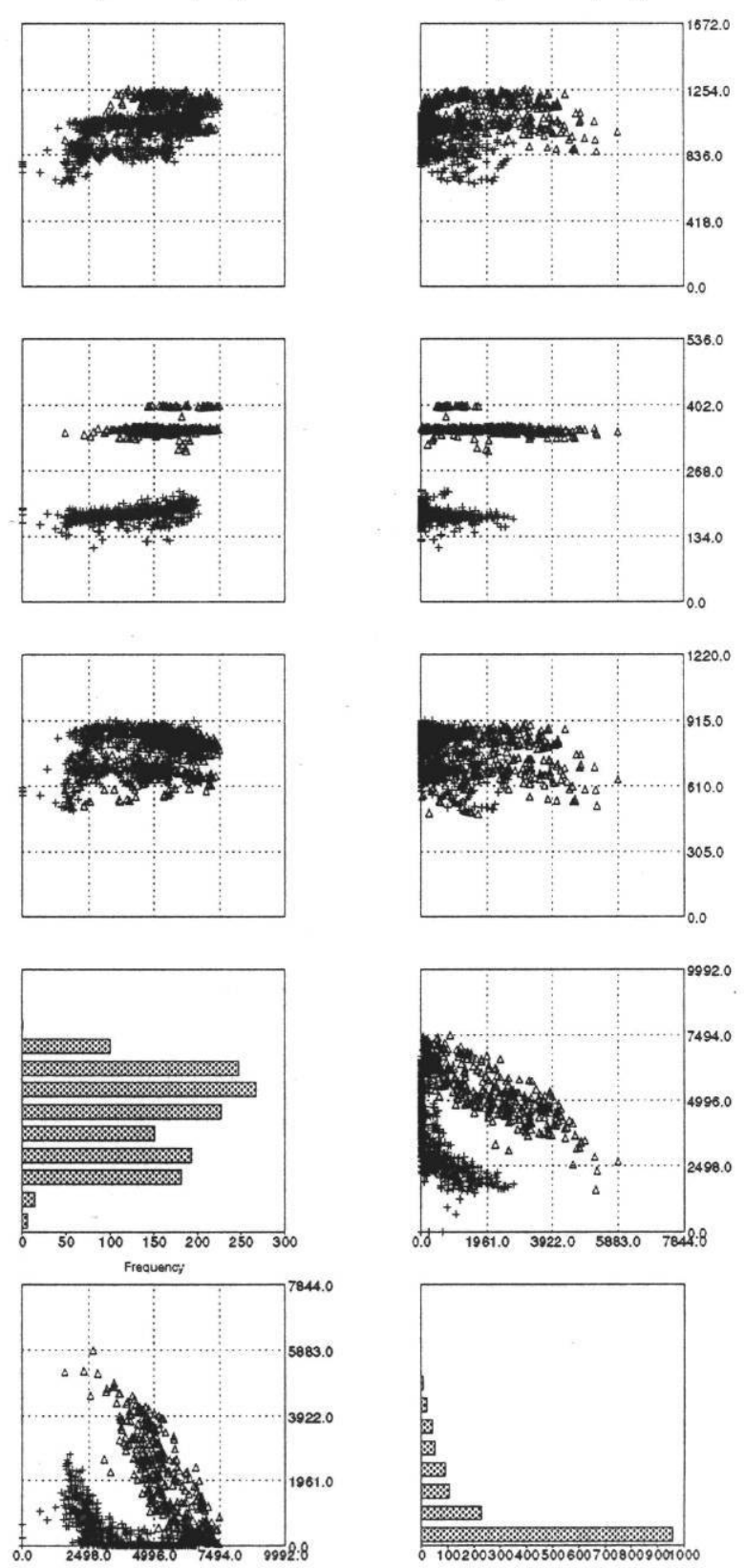


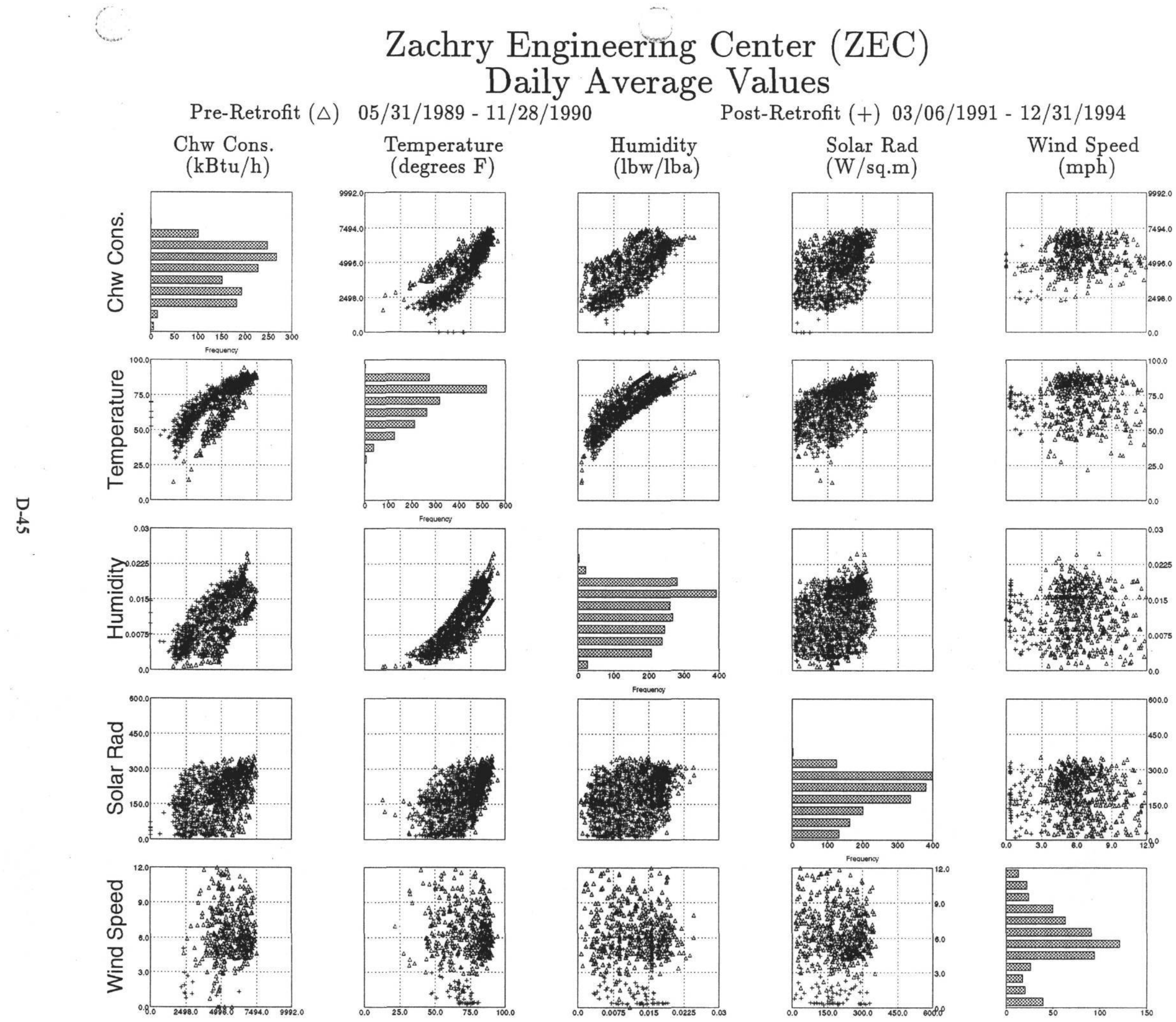




\section{Zachry Engineering Center (ZEC) \\ Daily Average Values}

Pre-Retrofit $(\Delta) \quad$ 05/31/1989 - 11/28/1990
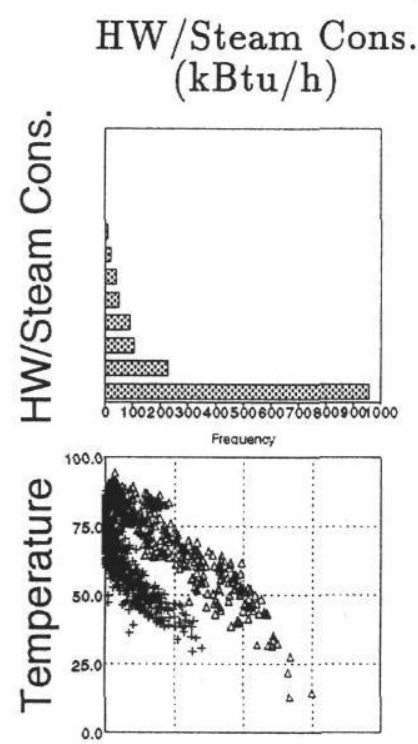

克
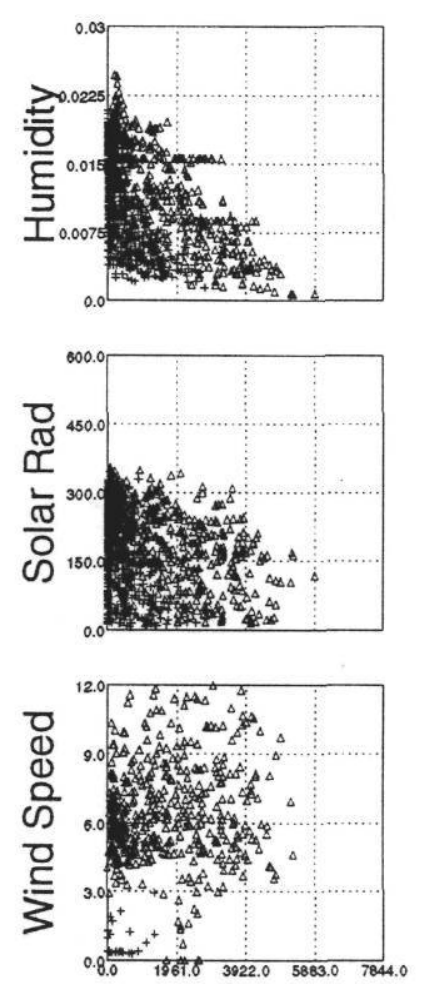

$100 \quad 200 \quad 300 \quad 400 \quad 500 \quad 600$

Post-Retrofit (+) 03/06/1991 - 12/31/1994
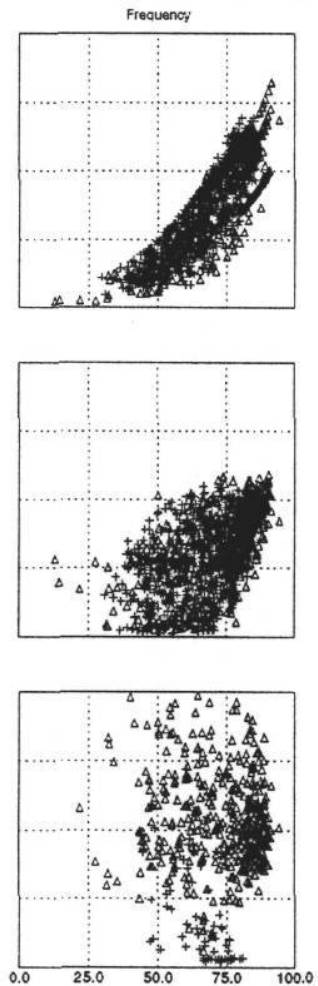

Humidity
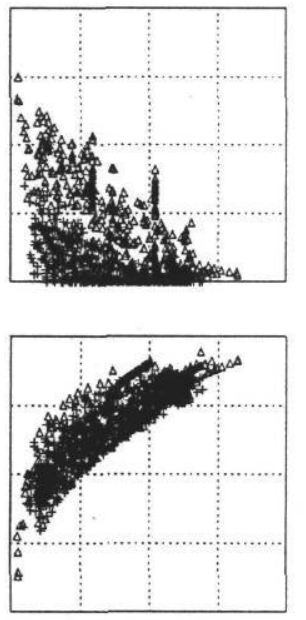

Solar Rad

(W/sq.m)
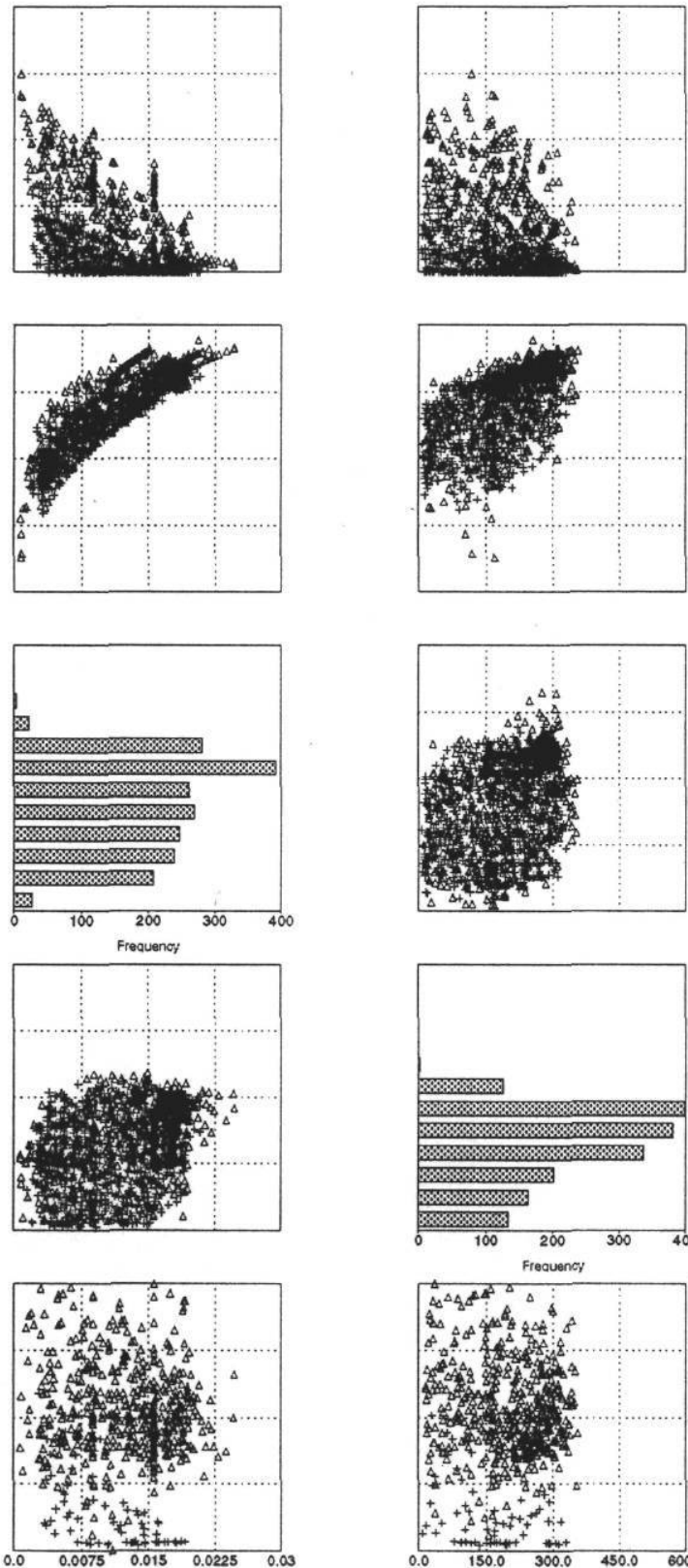

Wind Speed $(\mathrm{mph})$
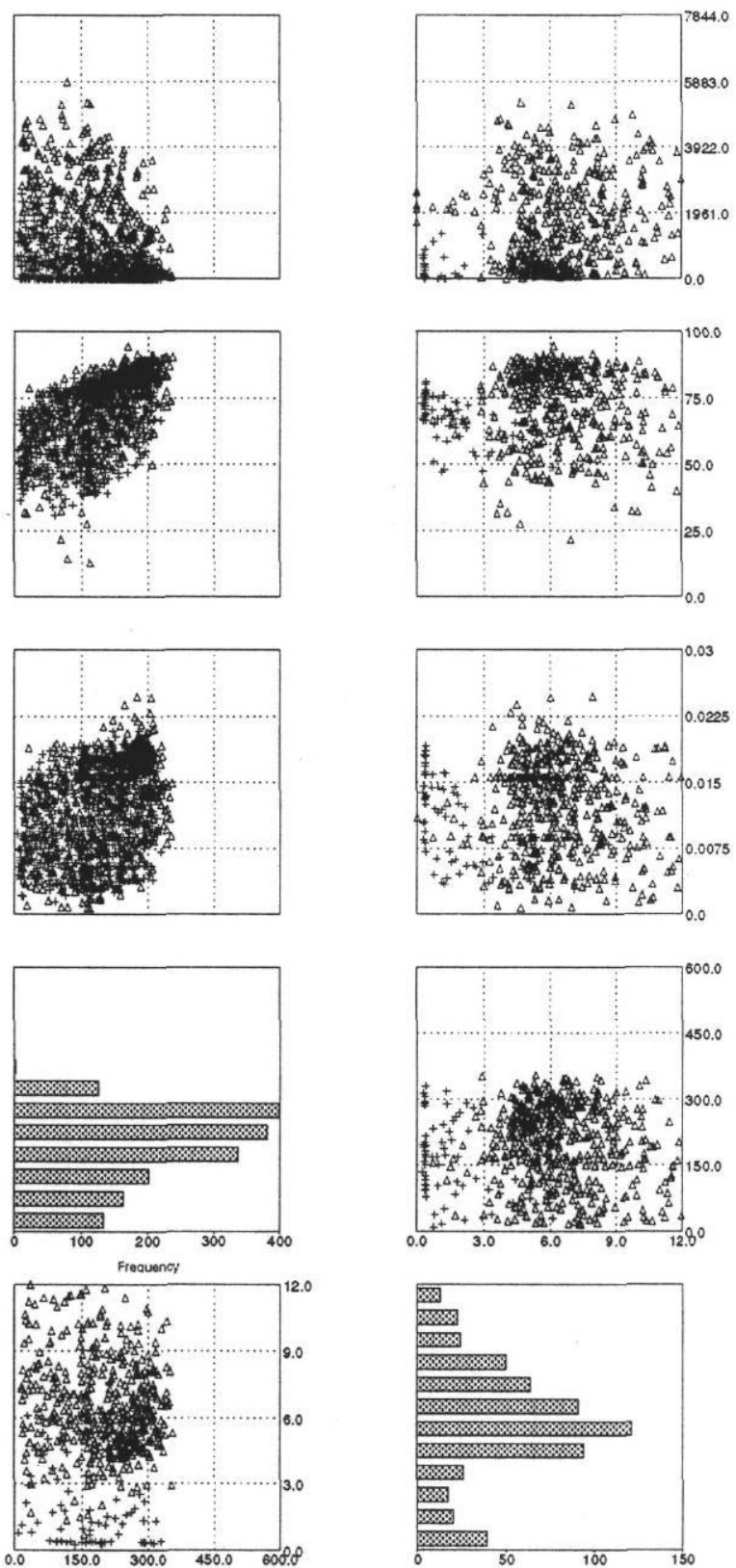
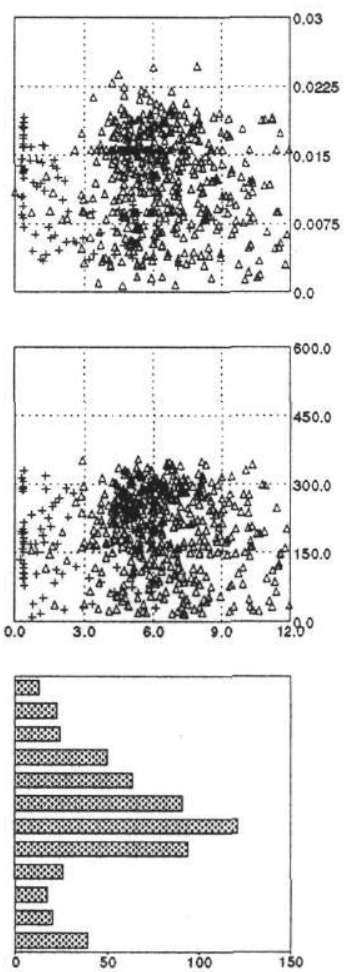
Tab D-9

Coincident Cumulative Frequency Plots 


\section{Zachry Engineering Center (ZEC) W.B. Electric \& M.C.C. as $\mathrm{kWh} / \mathrm{h}$}

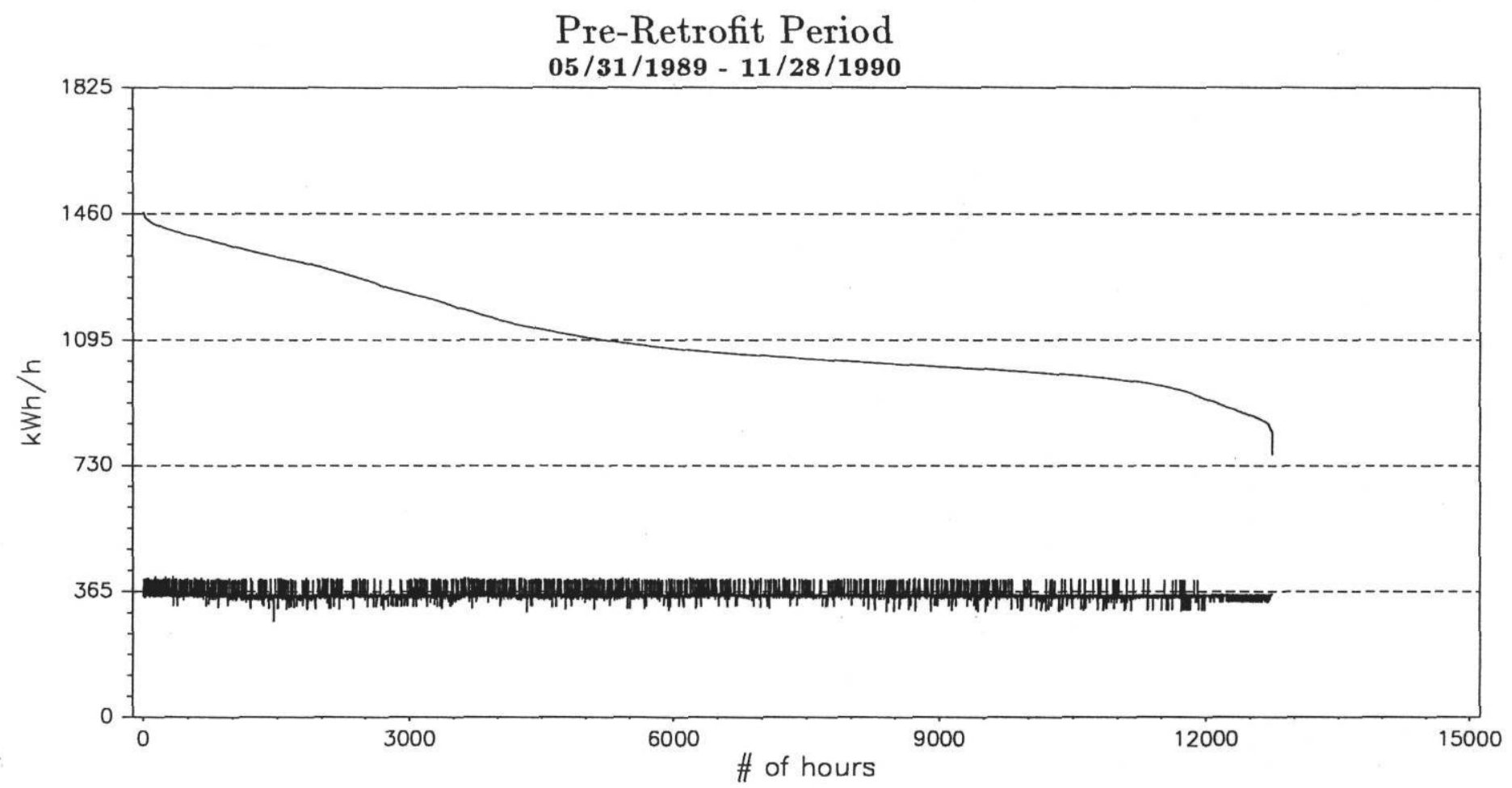

upper line $=$ W.B. Electric lower line = M.C.C.

Post-Retrofit Period

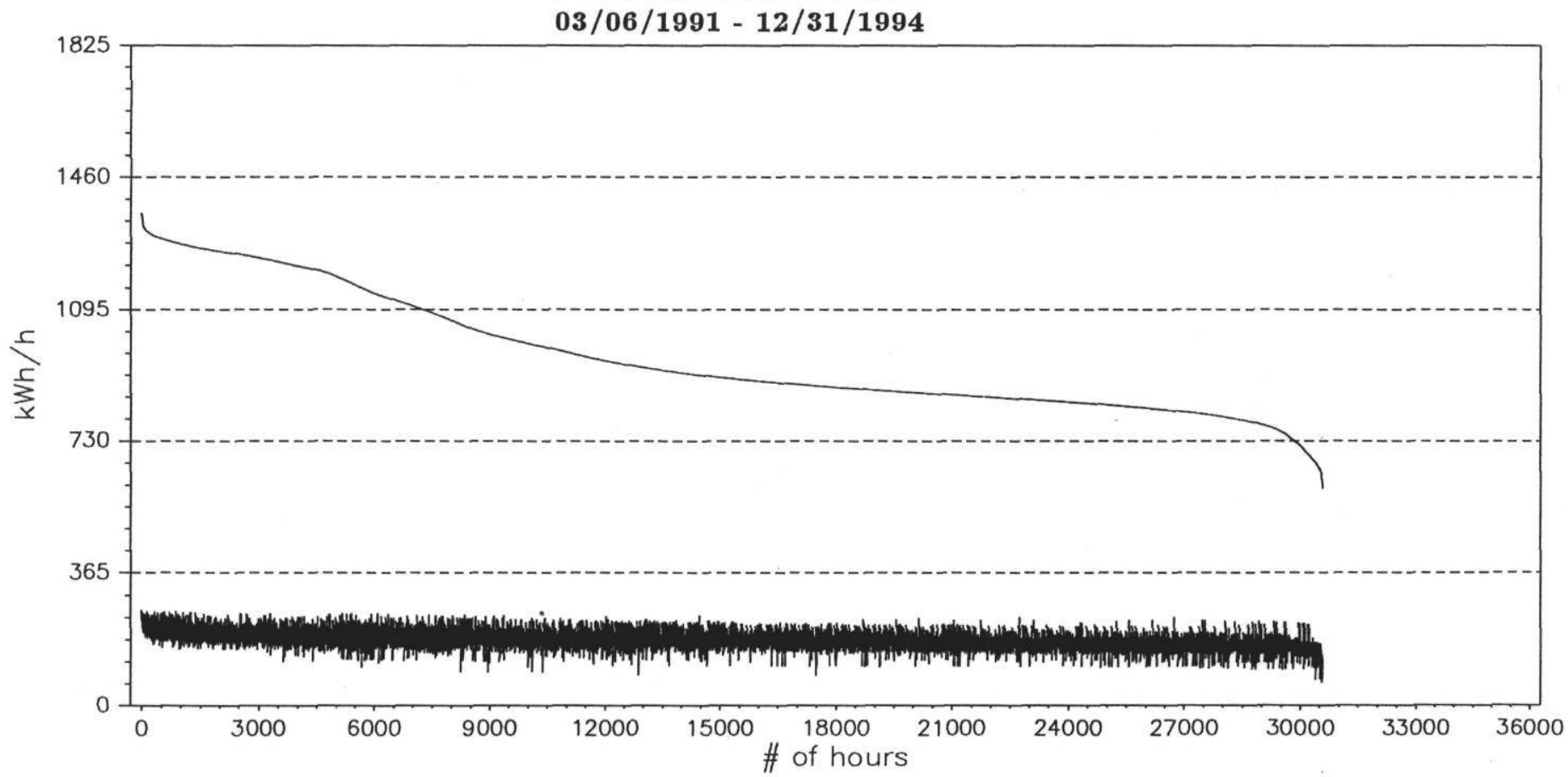

upper line $=$ W.B. Electric

D-48 lower line = M.C.C. 
I: $\backslash>$ DIR

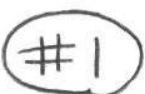

ume in drive $B$ has no label

tume Serial Number is 2643-10DB

Directory of $\mathrm{B}: \mathrm{I}$

IBSTRACT DOC $\quad 7,168$ 08-08-95 12:54p

ZONTENTS DOC $\quad 7,168$ 08-08-95 1:04p

IEPORTC DOC 139,776 08-08-95 1:06p

IHS DOC 432,128 08-08-95 10:16a

SIM DOC 318,976 08-08-95 11:14a

IITLE DOC 13,824 08-08-95 1:13p

JHS DOC 302,592 08-08-95 10:50a

ZEC DOC 23,040 08-08-95 11:31a

8 file(s) $\quad 1,244,672$ bytes 212,992 bytes free

B:\>

B: $\backslash>$ DIR

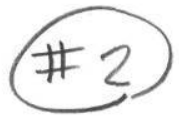

Cume in drive $B$ has no label

Volume Serial Number is 3E4F-1104

Directory of $\mathrm{B}: \mathbf{I}$

$\begin{array}{llrrr}\text { MMSPROJ } & \text { PPT } & 449,024 & 07-28-95 & 8: 39 a \\ \text { QSTNS } & \text { PPT } & 44,544 & 07-27-95 & 12: 39 p \\ \text { MO-WBE } & \text { XLS } & 191,488 & 07-06-95 & 5: 08 p \\ \text { SHS } & \text { XLS } & 492,544 & 08-10-95 & 11: 41 a \\ & 4 \text { file(s) } & 1,177,600 \text { bytes } \\ & & 280,064 \text { bytes free }\end{array}$

B:\〉 
3: \>DIR

Volume in drive $B$ has no label

ume Serial Number is 2643-10DB

rrectory of B:।

IIM XLS 524,288 08-08-95 1:52p

IHS XLS $554,496 \quad 08-10-95 \quad 11: 45 a$

2 file(s) $\quad 1,078,784$ bytes

378,880 bytes free

$3: \backslash>D I R$

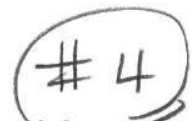

Volume in drive- $B$ has no label

Volume Serial Number is OF4C-14DA

Directory of $\mathrm{B}: \mathrm{I}$

\begin{tabular}{|c|c|c|c|}
\hline 'KUNZIP & EXE & $29,378 \quad 04-26-93$ & $1: 10 p$ \\
\hline SHS & ZIP & $387,04708-10-95$ & $12: 33 p$ \\
\hline SIM & ZIP & $510,133 \quad 08-10-95$ & $12: 34 p$ \\
\hline & ZIP & $393,30308-10-95$ & $12: 34 p$ \\
\hline & 4 file(s) & \multicolumn{2}{|c|}{$1,319,861$ bytes } \\
\hline
\end{tabular}

$3: 1>$ 


\section{Performance of Energy Management}

Control Systems (EMCS) in Selected LoanSTAR Buildings

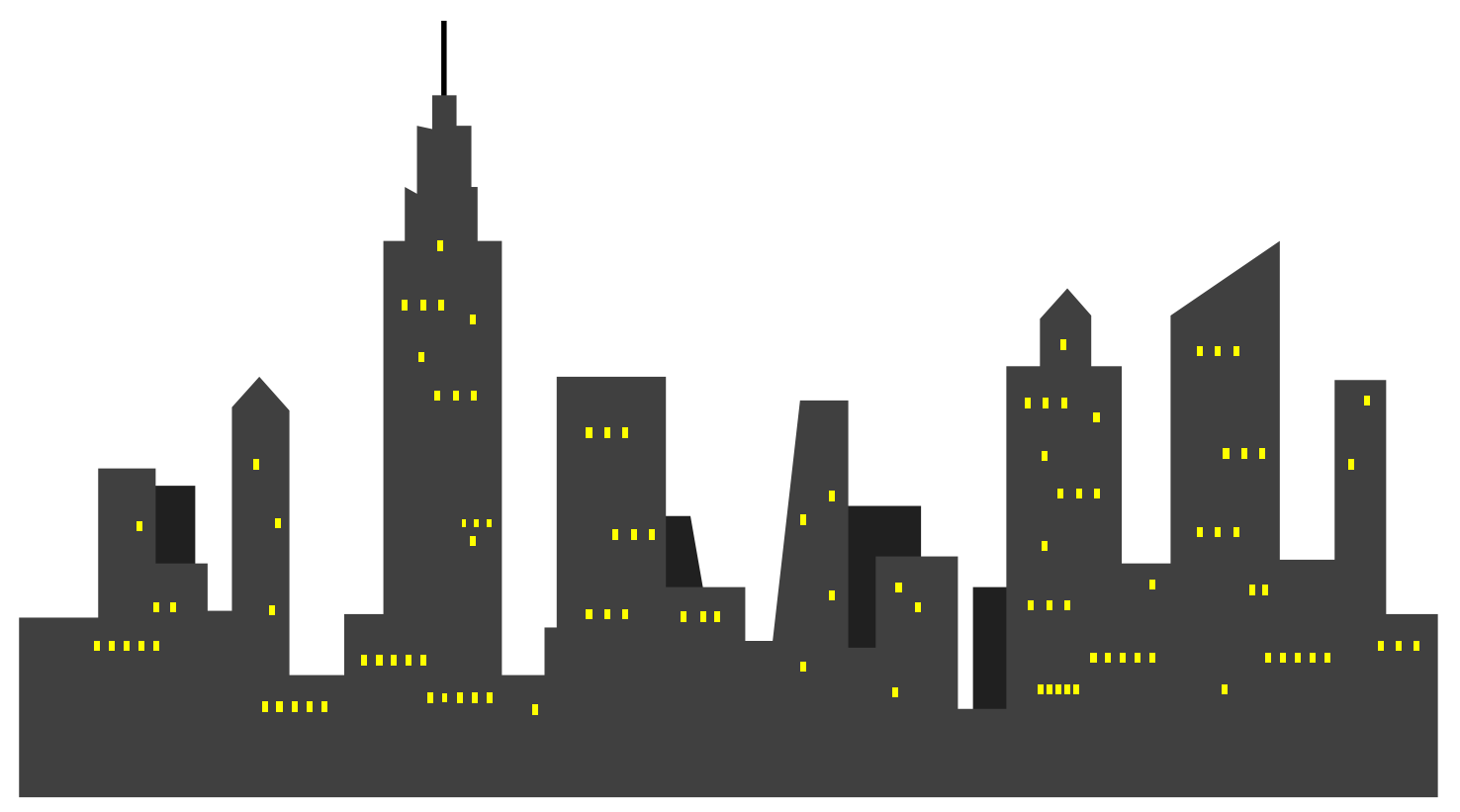




\section{Overview of Presentation}

- Objective of Report

- LoanSTAR Program

- Sites Studied

- Data Reduction

- Results 


\section{Objective of Report:}

Determine how successful Energy Management Control Systems (EMCS) have been in reducing energy consumption in selected LoanSTAR buildings 


\section{The LoanSTAR Program}

- Loans to $\underline{\text { Save }}$ Taxes and $\underline{\text { Resources (LoanSTAR) }}$

- established in 1988 by the Texas Governor's Energy Management Center

- funds energy conservation retrofits in state and local government buildings and public schools

- typical retrofit projects include lighting, HVAC systems, electric motors and EMCS

- the Energy Systems Laboratory (ESL) at Texas A\&M University provides monitoring and data analysis in order to track energy savings resulting from retrofits 


\section{Site Selection}

- the LoanSTAR program currently monitors 121 sites

- sites for this report were chosen based on the following:

- an EMCS retrofit was completed

- whole building and limited sub-metered hourly data was collected

- ease of separating EMCS retrofit data from the whole whole building data 


\section{Sites Studied}

Fort Worth ISD

Sims Elementary School

\section{Texas A\&M University}

Zachry Engineering Center

Victoria ISD

Stroman High School

Victoria High School 


\section{Data Reduction}

- data was obtained from the LoanSTAR Monitoring and Analysis Program (MAP), run by the ESL at Texas A\&M University

- ESL software was used to extract the data from the LoanSTAR database and convert it a format that is compatible with Microsoft Excel

- MS Excel was used to complete the data analysis 


\section{Data Reduction}

- building energy data categories:

- whole building electric $(\mathrm{kWh} / \mathrm{h})$

- lights $(k W h / h)$

- whole building heat (MMBtu/h)

- other $(k W h / h)$

- chiller $(k W h / h)$

- "other" is defined as:

whole building electric - (chiller + lights) 


\section{Data Reduction}

- Consumption data was sorted with three sort parameters, yielding eight sort categories:

- semester / weekday / pre-retrofit

- semester / weekday / post-retrofit

$(S-1-A)$

- semester / weekend / pre-retrofit

$(S-1-B)$

- semester / weekend / post-retrofit

- non-semester / weekday / pre-retrofit

- non-semester / weekday / post retrofit

- non-semester / weekend / pre-retrofit

- non-semester / weekend / post-retrofit 


\section{Stroman High School}

- Building Envelope

- 210,500 square feet

- 7 buildings

- HVAC \& Other Equipment

- rooftop fan-coil units and AHUs

- 45.8 ton chiller (Unit A)

- 460 ton centrifugal chiller

- Retrofits (completion date)

- replace 460 ton centrifugal chiller with 414 ton absorption chiller (Aug 1991)

- rewire wiring in hallways (Jan 1992)

- install Energy Management Control System (Jan 1992) 


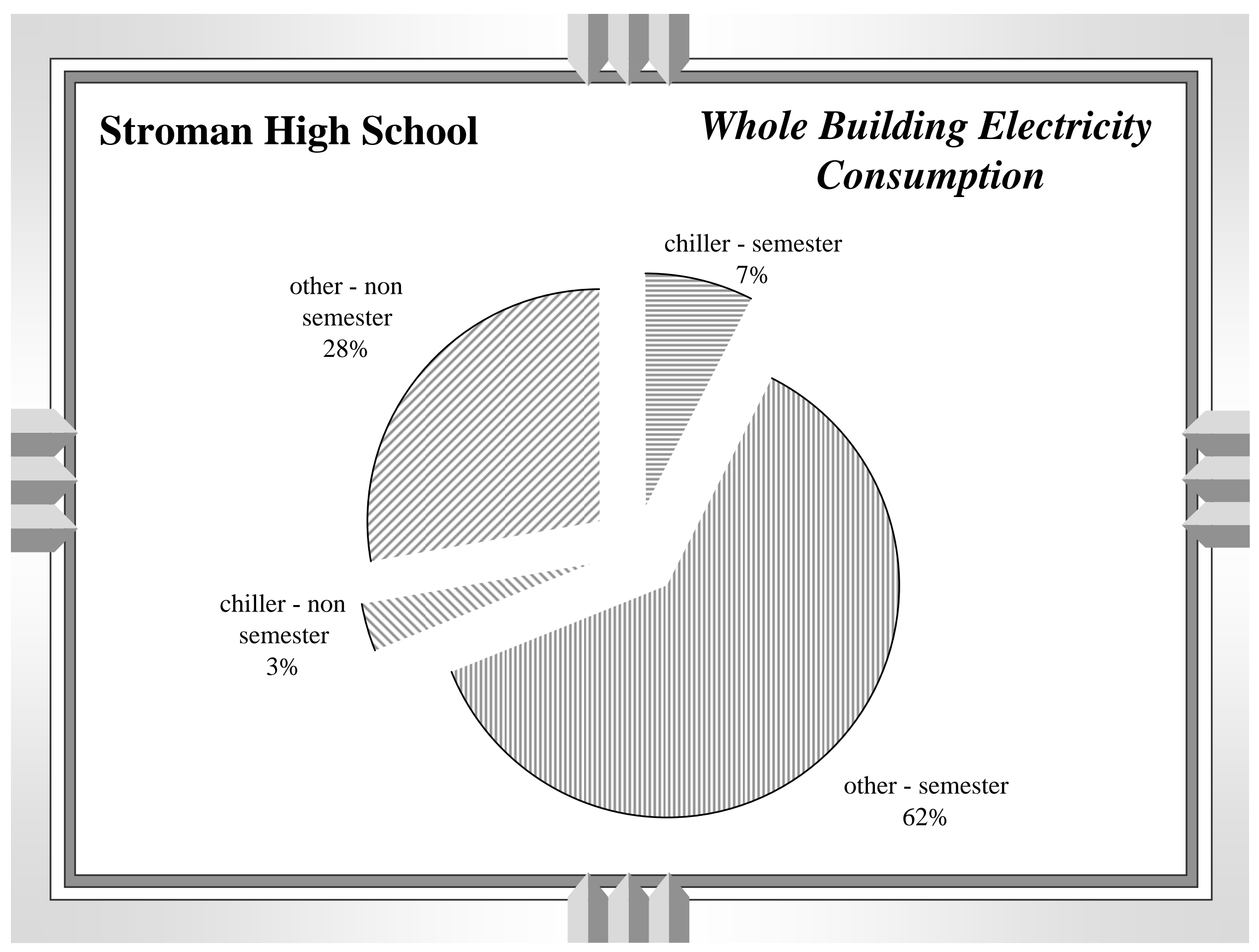




\section{Stroman High School}

\section{Total Daily Consumption Time Series}

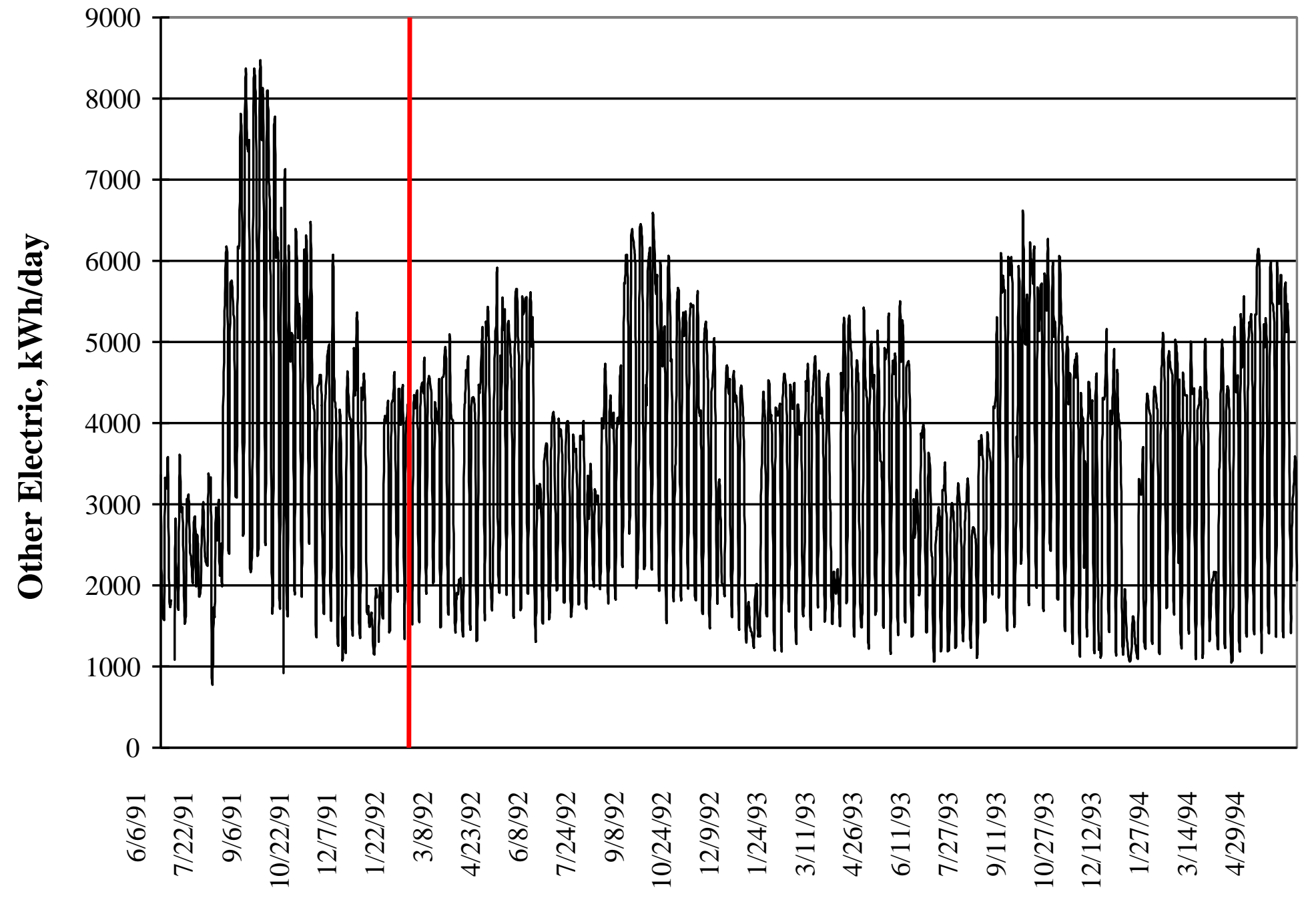




\section{Stroman High School}

Average Hourly Consumption

Semester

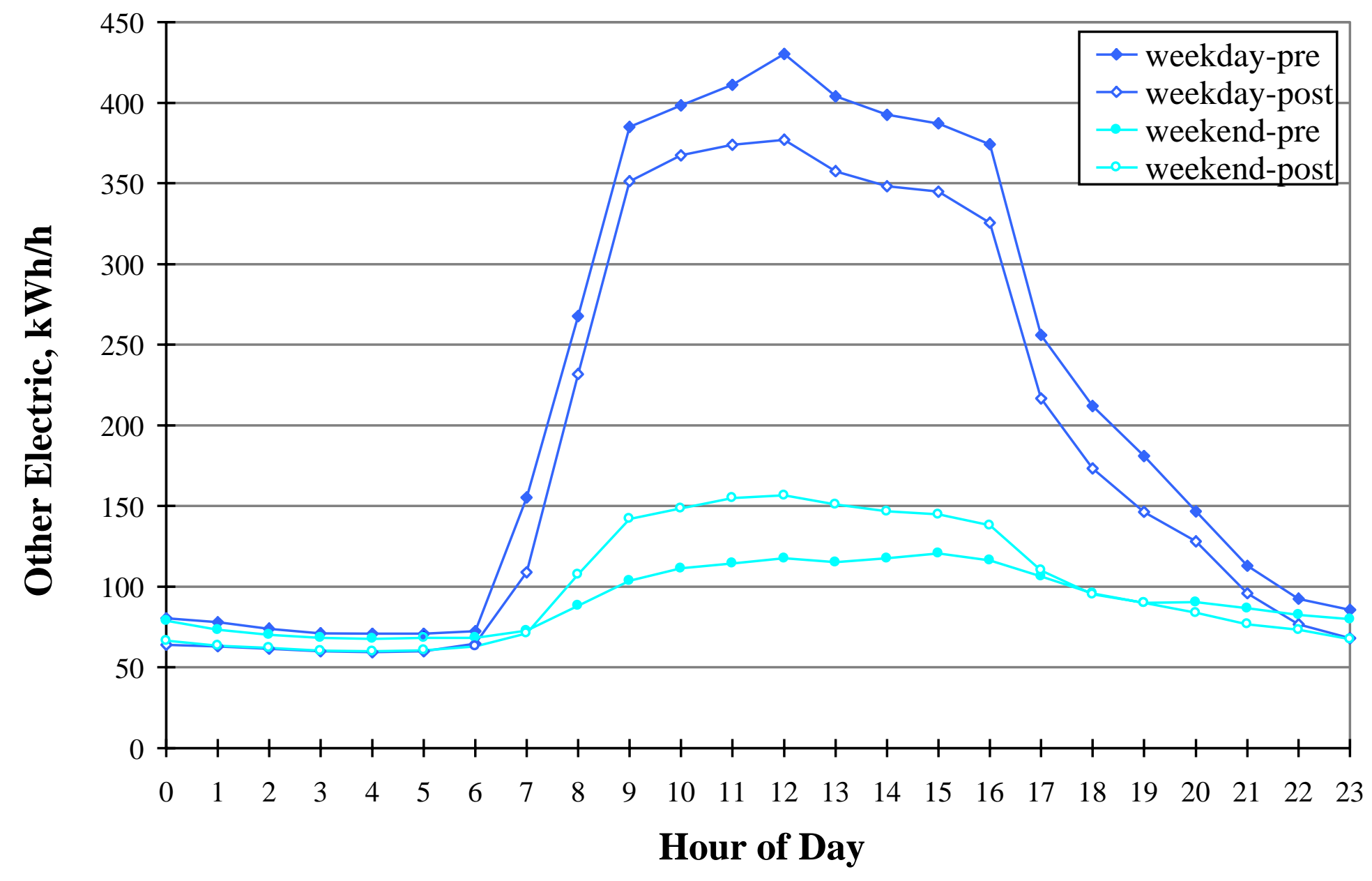




\section{Stroman High School}

\section{Average Hourly Consumption}

\section{Non-Semester}

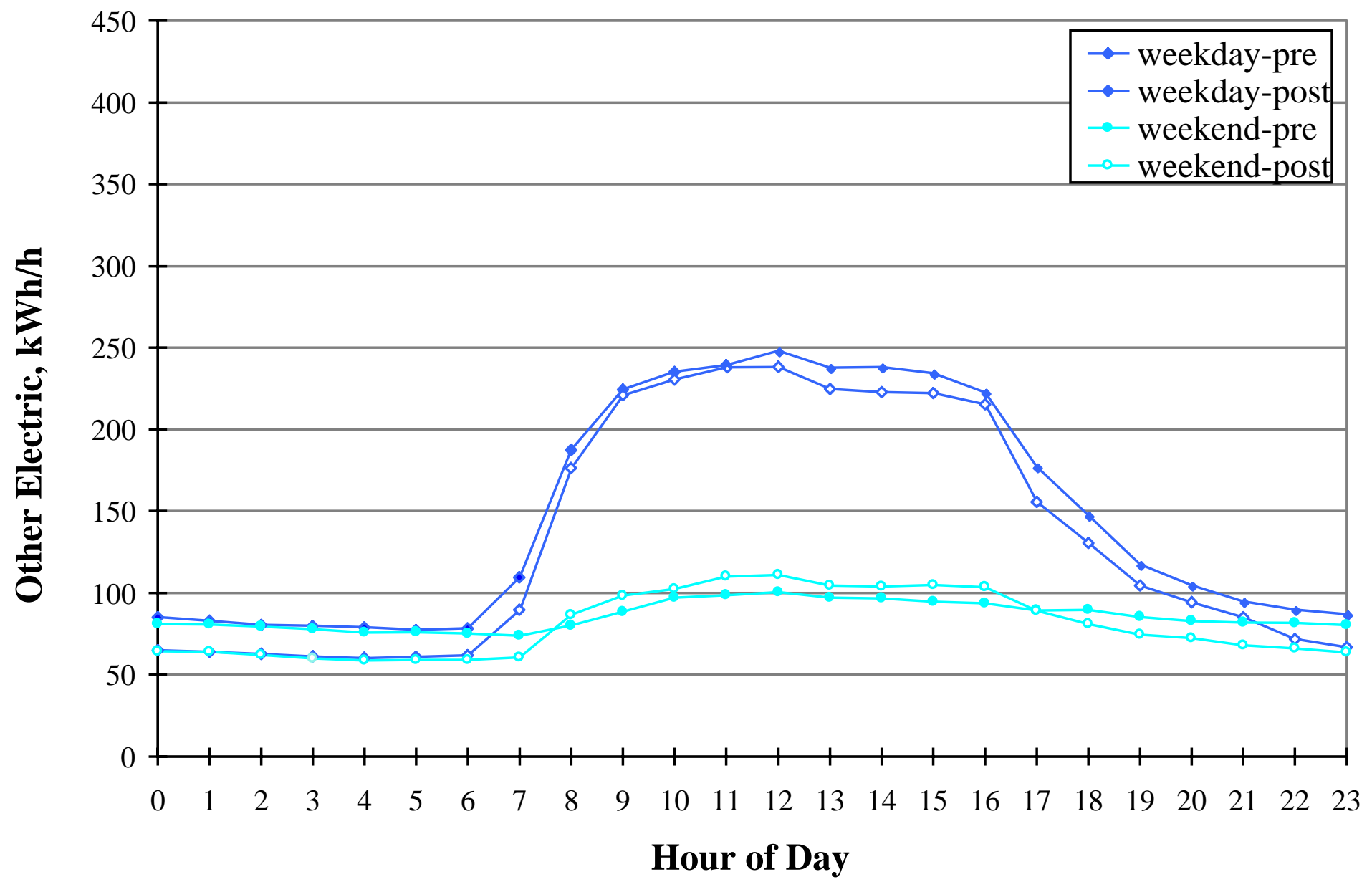




\section{Victoria High School}

- Building Envelope

- 257,000 square feet

- 3 buildings

- HVAC \& Other Equipment

- rooftop fan-coil units and AHUs

- 192 ton centrifugal chiller

- Retrofits (completion date)

- replace 192 ton centrifugal chiller with absorption chiller (Aug 1991)

- install Energy Management Control System (Jan 1992) 


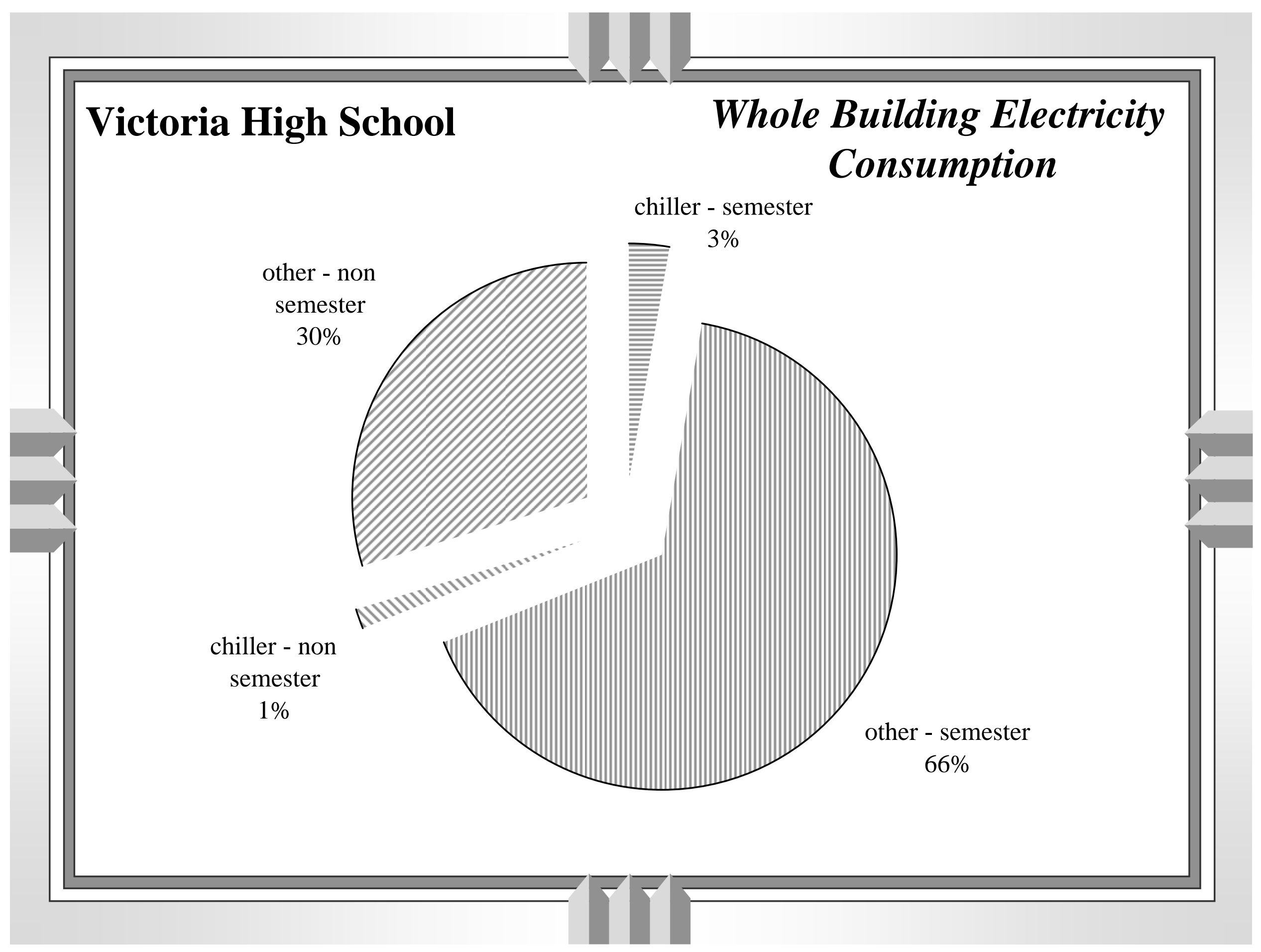




\section{Victoria High School}

\section{Total Daily Consumption Time Series}

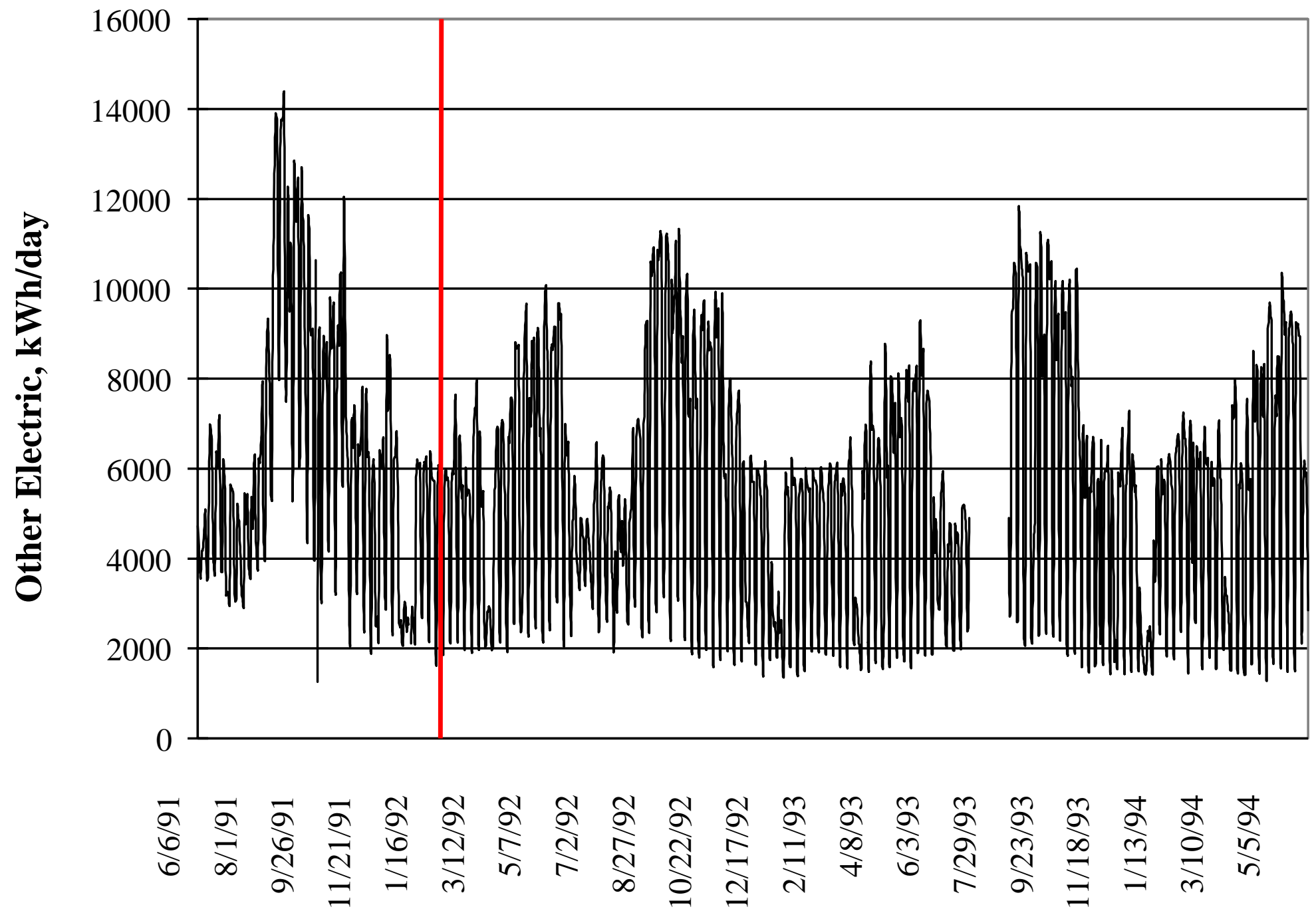




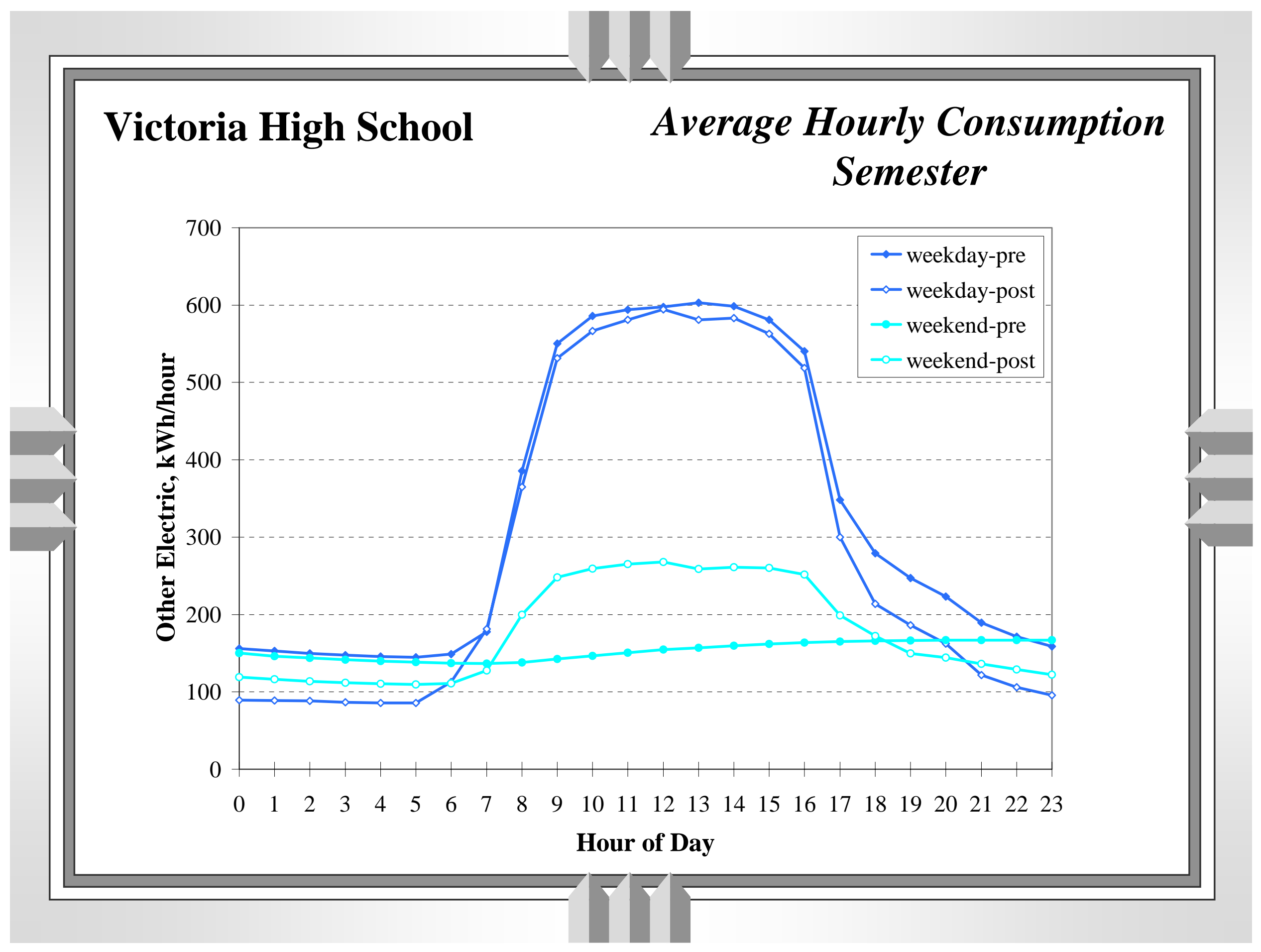




\section{Victoria High School}

\section{Average Hourly Consumption Non-Semester}

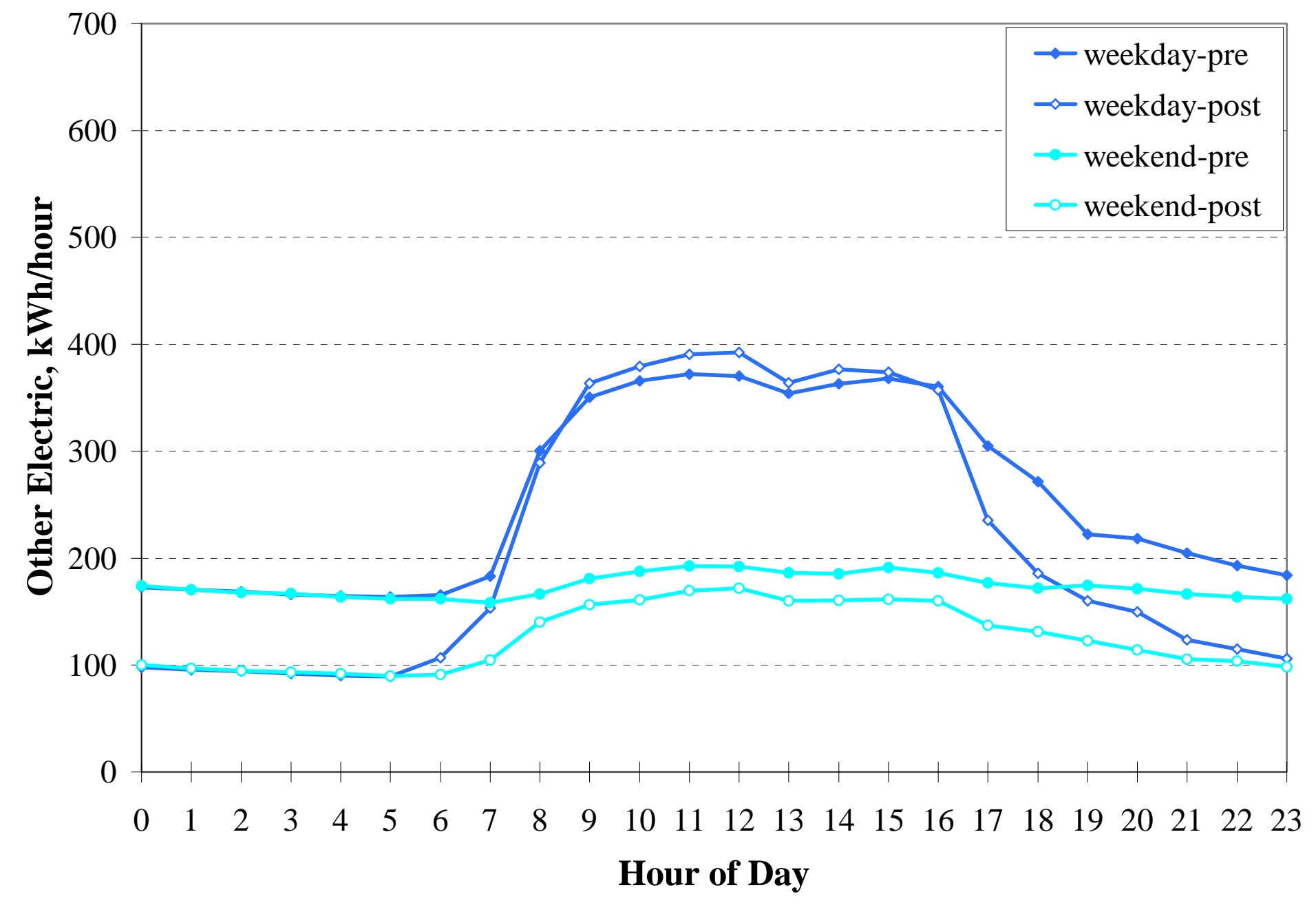




\section{Sims Elementary School}

- Building Envelope

- 62,400 square feet

- 1 building

- HVAC \& Other Equipment

- rooftop units

- Retrofits (completion date)

- convert lighting from 4 bulb to 2 bulb (Nov 1991)

- install EMCS* (Apr 1994) 


\section{Sims Elementary School}

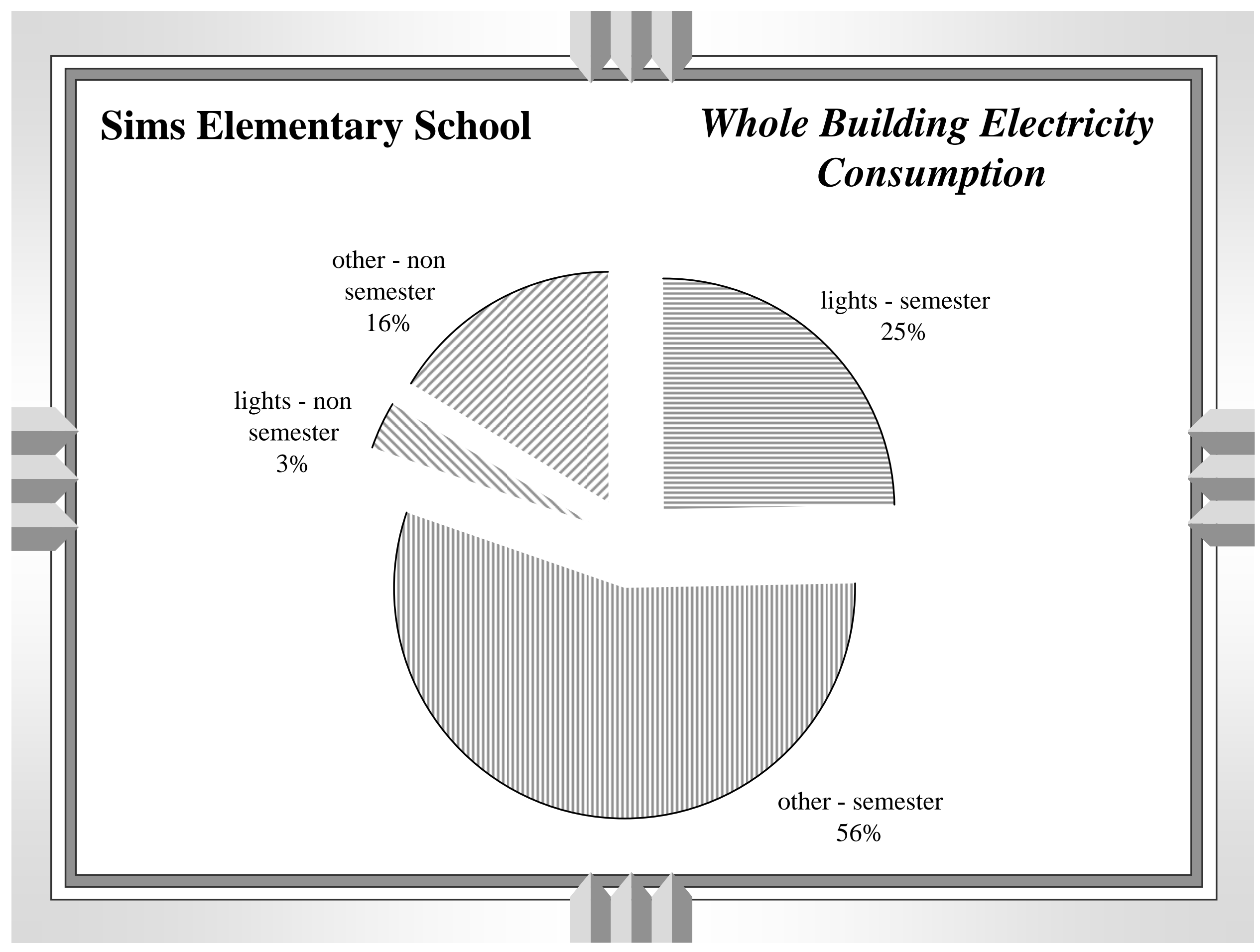

Whole Building Electricity Consumption 


\section{Sims Elementary School}

\section{Total Daily Consumption Time Series}

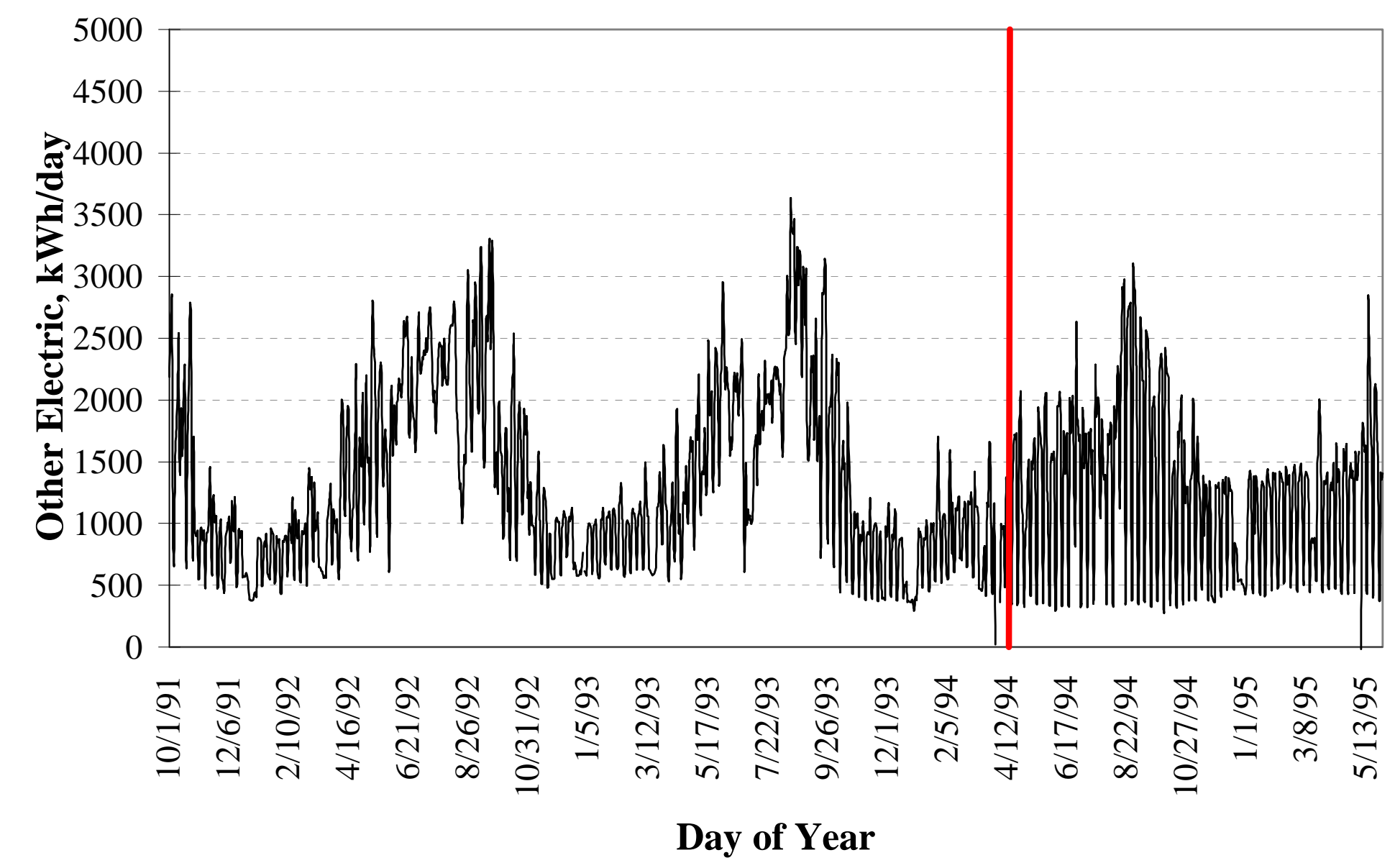




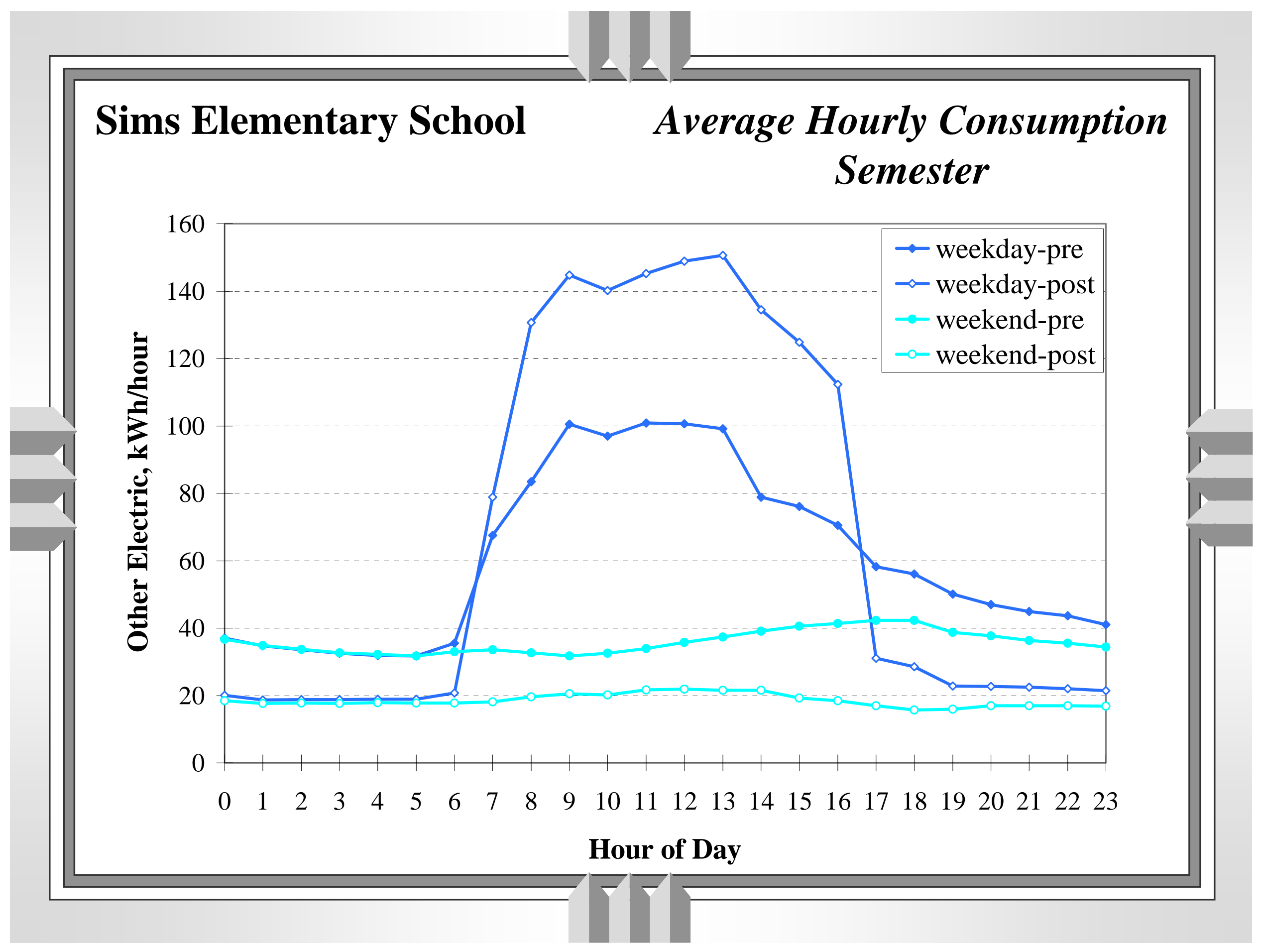




\section{Sims Elementary School}

\section{Average Hourly Consumption}

\section{Non-Semester}

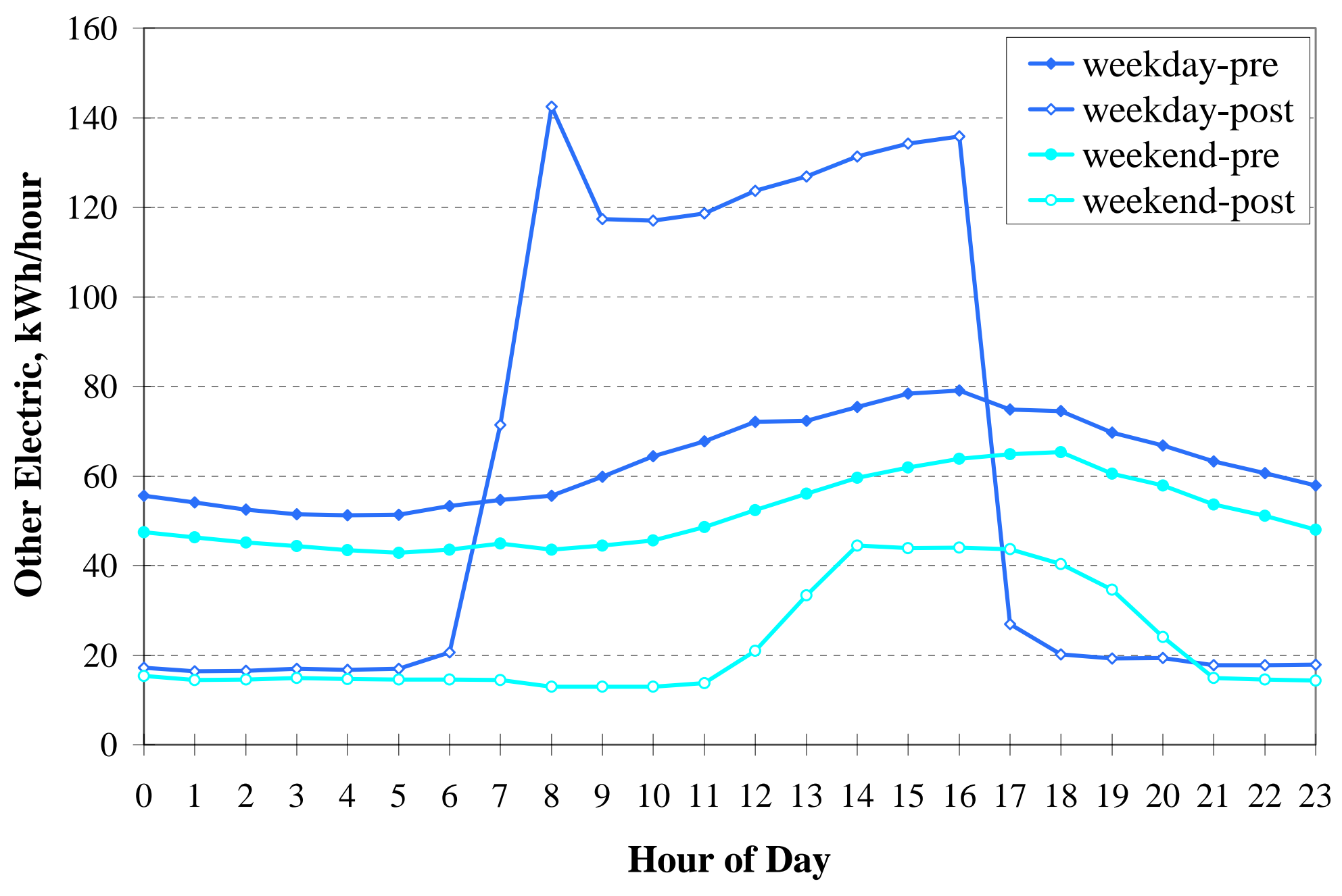




\section{Zachry Engineering Center}

- Building Envelope

- 324,400 square feet

- 1 building

- HVAC \& Other Equipment

- fan coils and AHUs

- Retrofits (completion date)

- control modifications to dual duct system (Mar 1991)

- variable volume dual duct system (Mar 1991)

- install EMCS* (Mar 1991)

* not a LoanSTAR retrofit 


\section{Zachry Engineering Center}

24-hour Box Whisker Mean (BWM) Plots 


\section{Change in "Other" consumption}

\begin{tabular}{lccc} 
& Stroman & Victoria & Sims \\
\cline { 2 - 4 } & & & \\
semester / weekday & $-13.15 \%$ & $-12.54 \%$ & $11.29 \%$ \\
semester / weekend & $8.57 \%$ & $15.54 \%$ & $-48.41 \%$ \\
non-semester / weekday & $-9.33 \%$ & $-15.86 \%$ & $-2.48 \%$ \\
non-semester / weekend & $-6.31 \%$ & $-27.82 \%$ & $-56.03 \%$
\end{tabular}




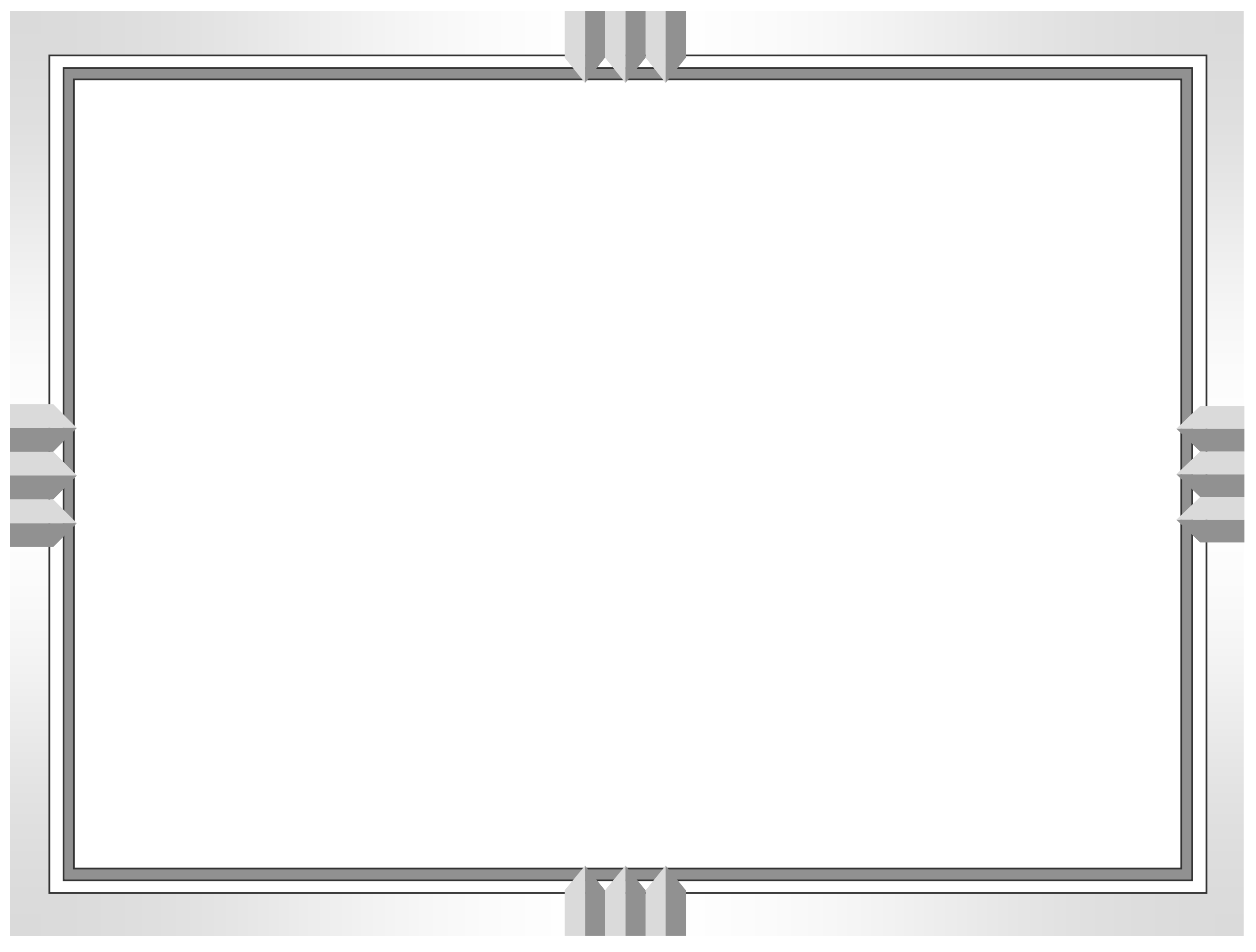




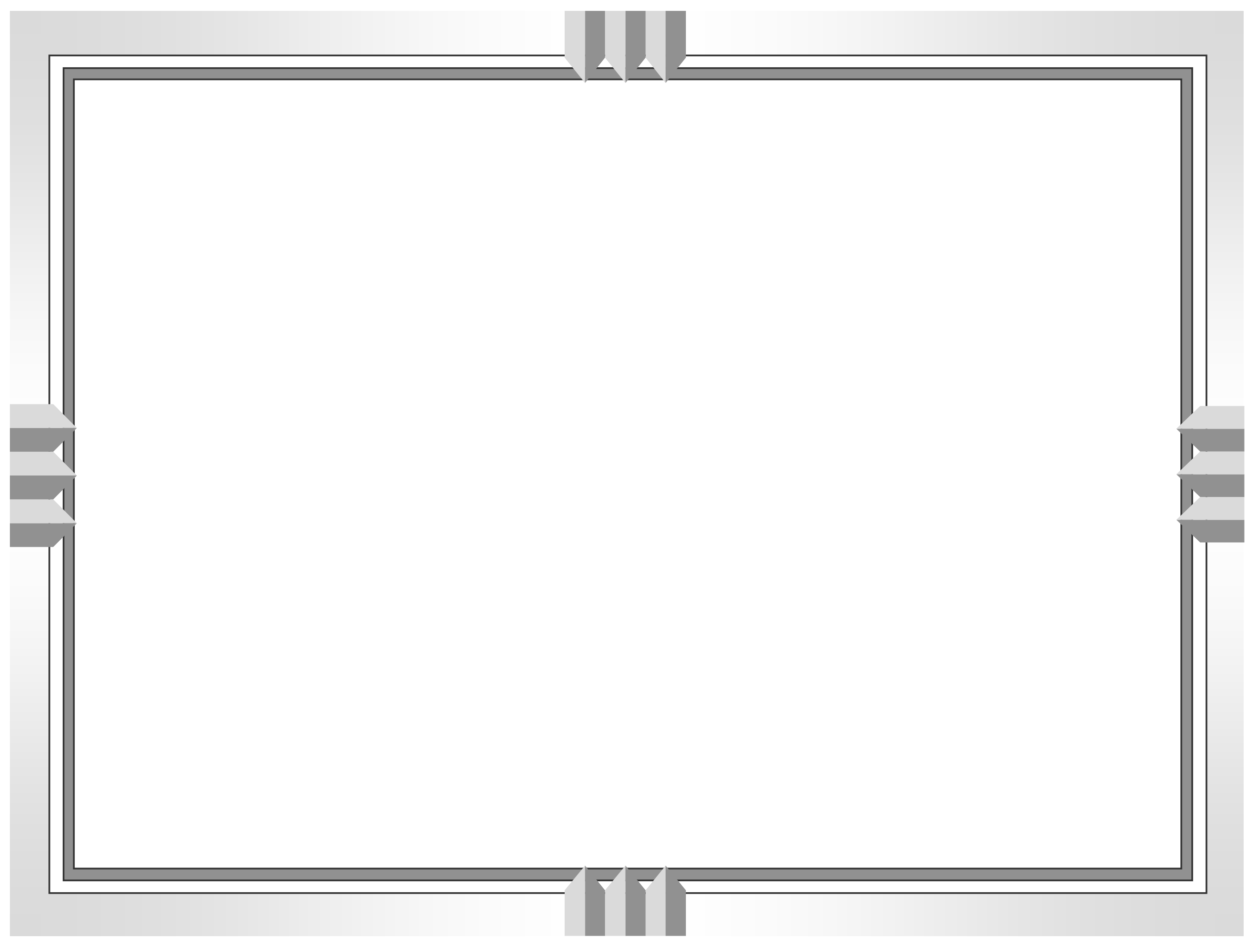




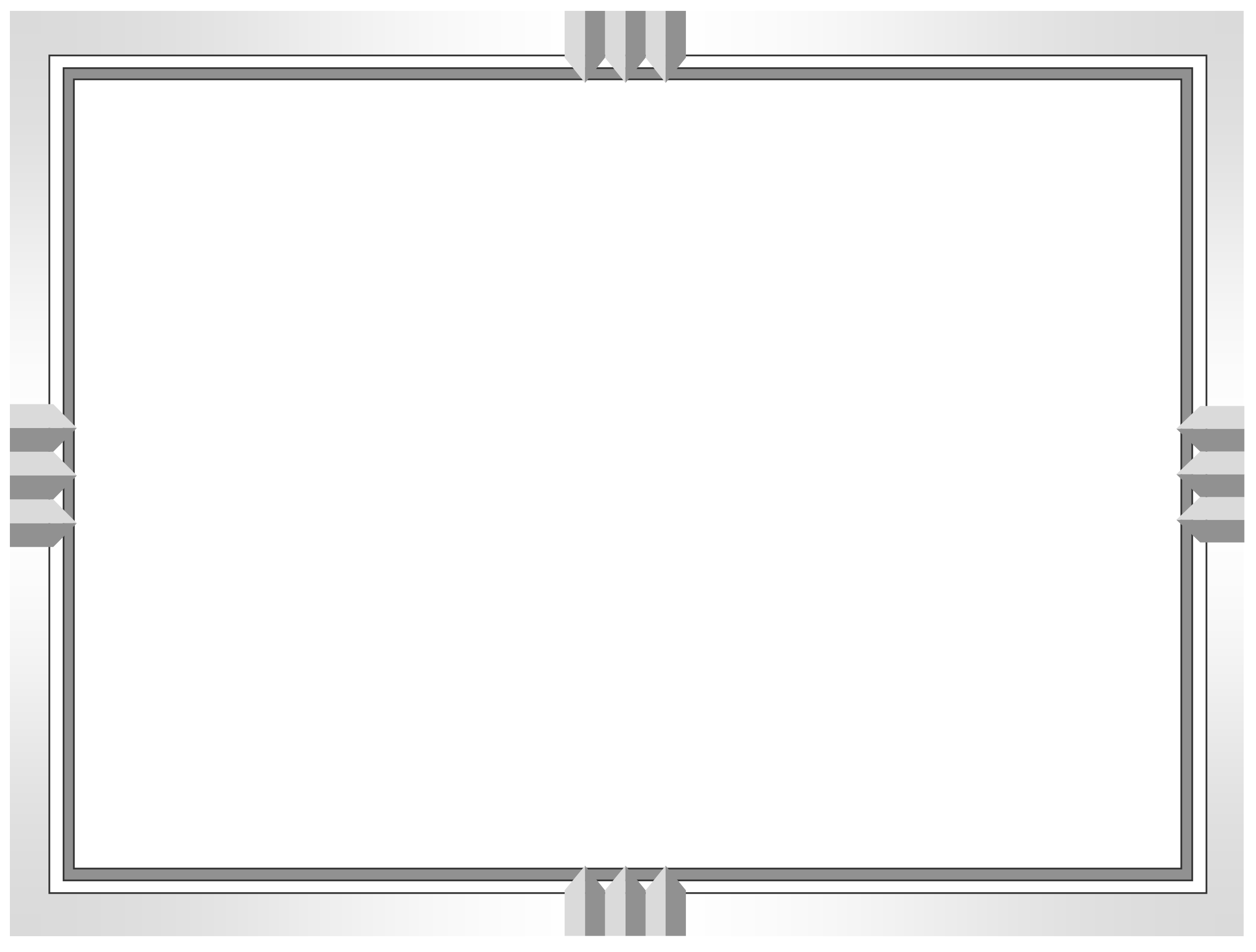




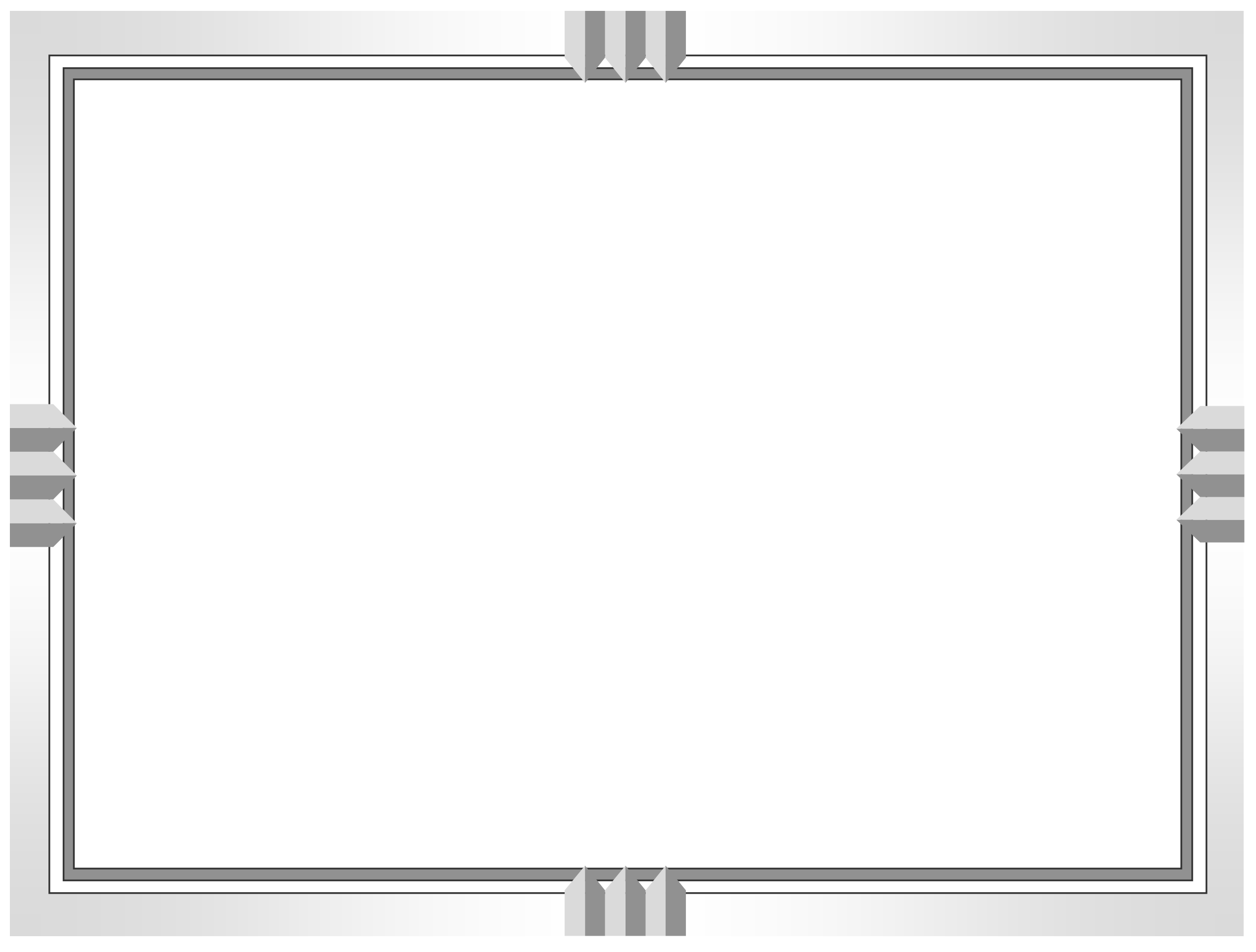




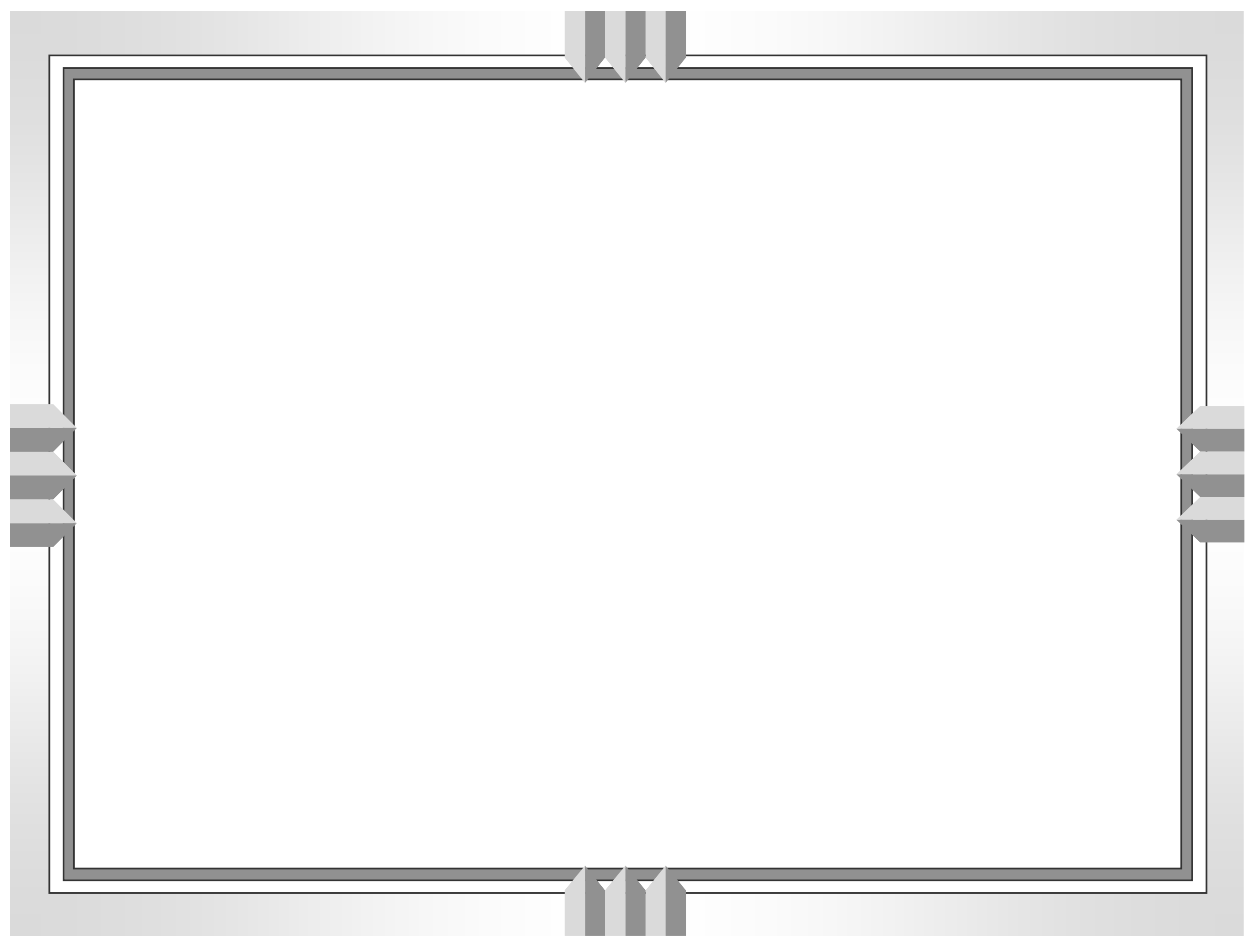


Sheet1

\begin{tabular}{|c|c|c|c|c|c|c|c|}
\hline & DFW & Sims & & DEF" & Simc & & \\
\hline & Tave & Ave W/sf & & Tpeak & Peak W/sf & & \\
\hline & & pre & post & & pre & post & \\
\hline 9/1/1991 & 68 & 1.842661 & & 89 & 4.984615 & & 9/1/1991 \\
\hline 10/1/1991 & 68 & 1.792132 & & 97 & 4.523077 & & 10/1/1991 \\
\hline 11/1/1991 & 51 & 1.132968 & & 78 & 3.311538 & & 11/1/1991 \\
\hline $12 / 1 / 1991$ & 50 & 0.844877 & & 78 & 2.684615 & & $12 / 1 / 1991$ \\
\hline $1 / 1 / 1992$ & 46 & 0.880226 & & 69 & 2.761538 & & 1/1/1992 \\
\hline $2 / 1 / 1992$ & 54 & & 0.99507 & 75 & & 2.584615 & $2 / 1 / 1992$ \\
\hline 3/1/1992 & 60 & & 0.988564 & 85 & & 2.830769 & 3/1/1992 \\
\hline 4/1/1992 & 66 & & 1.339628 & 86 & & 3.4 & 4/1/1992 \\
\hline $5 / 1 / 1992$ & 70 & & 1.536631 & 93 & & 3.726923 & $5 / 1 / 1992$ \\
\hline $6 / 1 / 1992$ & 78 & & 1.686944 & 96 & & 3.130769 & $6 / 1 / 1992$ \\
\hline $7 / 1 / 1992$ & 84 & & 1.768244 & 97 & & 2.823077 & $7 / 1 / 1992$ \\
\hline 8/1/1992 & 80 & & 1.673424 & 98 & & 4.246154 & 8/1/1992 \\
\hline 9/1/1992 & 78 & & 1.964071 & 94 & & 4.188462 & 9/1/1992 \\
\hline 10/1/1992 & 70 & & 1.469877 & 91 & & 3.676923 & 10/1/1992 \\
\hline 11/1/1992 & 53 & & 1.033093 & 77 & & 3.042308 & 11/1/1992 \\
\hline 12/1/1992 & 49 & & 0.939072 & 78 & & 2.6 & 12/1/1992 \\
\hline 1/1/1993 & 45 & & 1.005949 & 74 & & 2.946154 & 1/1/1993 \\
\hline 2/1/1993 & 49 & & 1.164878 & 73 & & 2.715385 & 2/1/1993 \\
\hline $3 / 1 / 1993$ & 56 & & 1.112512 & 88 & & 2.984615 & $3 / 1 / 1993$ \\
\hline 4/1/1993 & 64 & & 1.248674 & 88 & & 3.480769 & 4/1/1993 \\
\hline $5 / 1 / 1993$ & 72 & & 1.641693 & 93 & & 3.726923 & $5 / 1 / 1993$ \\
\hline $6 / 1 / 1993$ & 81 & & 1.606426 & 98 & & 3.784615 & $6 / 1 / 1993$ \\
\hline $7 / 1 / 1993$ & 87 & & 1.386731 & 102 & & 2.892308 & $7 / 1 / 1993$ \\
\hline 8/1/1993 & 88 & & 2.171991 & 103 & & 5.376923 & 8/1/1993 \\
\hline 9/1/1993 & 78 & & 1.828163 & 97 & & 4.946154 & 9/1/1993 \\
\hline 10/1/1993 & 64 & & 1.341073 & 90 & & 3.934615 & 10/1/1993 \\
\hline $11 / 1 / 1993$ & 52 & & 0.989297 & 84 & & 2.719231 & $11 / 1 / 1993$ \\
\hline 12/1/1993 & 50 & & 0.795471 & 73 & & 2.776923 & 12/1/1993 \\
\hline
\end{tabular}


Sheet1

\begin{tabular}{|c|c|c|c|c|c|c|c|}
\hline VCT & Stroman & & VCT & Stroman & & & VCT \\
\hline \multirow[t]{2}{*}{ Tave } & Ave W/sf & & Tpeak & Peak W/sf & & & Tave \\
\hline & pre & post & & pre & post & & \\
\hline 77.913 & 1.120738 & & 93 & 3.075482 & & 9/1/1991 & 77.913 \\
\hline 73.8065 & 1.068827 & & 99 & 3.083692 & & 10/1/1991 & 73.8065 \\
\hline 58.8167 & 0.717382 & & 83 & 2.692893 & & 11/1/1991 & 58.8167 \\
\hline 58.1 & 0.627494 & & 81 & 2.550039 & & $12 / 1 / 1991$ & 58.1 \\
\hline 52.3333 & 0.663773 & & 72 & 2.282391 & & 1/1/1992 & 52.3333 \\
\hline 60.7593 & & 0.754243 & 83 & & 2.633779 & 2/1/1992 & 60.7593 \\
\hline 66.8704 & & 0.693692 & 89 & & 2.617359 & $3 / 1 / 1992$ & 66.8704 \\
\hline 70.2679 & & 0.892356 & 88 & & 2.88829 & 4/1/1992 & 70.2679 \\
\hline 74.7 & & 0.989159 & 91 & & 2.909637 & $5 / 1 / 1992$ & 74.7 \\
\hline 82.5208 & & 0.750522 & 99 & & 2.379268 & $6 / 1 / 1992$ & 82.5208 \\
\hline 85.3 & & 0.68092 & 95 & & 2.265971 & $7 / 1 / 1992$ & 85.3 \\
\hline 82.5484 & & 0.942739 & 99 & & 3.059062 & 8/1/1992 & 82.5484 \\
\hline 81.25 & & 1.123687 & 97 & & 3.366116 & 9/1/1992 & 81.25 \\
\hline 74.0645 & & 1.00436 & 92 & & 2.942477 & 10/1/1992 & 74.0645 \\
\hline 59.5667 & & 0.744226 & 85 & & 2.811117 & 11/1/1992 & 59.5667 \\
\hline 58.8889 & & 0.571936 & 79 & & 2.525409 & 12/1/1992 & 58.8889 \\
\hline 54.4355 & & 0.647393 & 80 & & 2.270898 & 1/1/1993 & 54.4355 \\
\hline 57.9821 & & 0.732382 & 84 & & 2.467939 & 2/1/1993 & 57.9821 \\
\hline 63.1935 & & 0.716304 & 87 & & 2.581236 & 3/1/1993 & 63.1935 \\
\hline 68.0167 & & 0.820051 & 87 & & 2.68961 & $4 / 1 / 1993$ & 68.0167 \\
\hline 74.0161 & & 0.88734 & 91 & & 2.963825 & $5 / 1 / 1993$ & 74.0161 \\
\hline 80.9167 & & 0.608861 & 91 & & 2.009816 & $6 / 1 / 1993$ & 80.9167 \\
\hline 84.4839 & & 0.517645 & 98 & & 1.931003 & 7/1/1993 & 84.4839 \\
\hline 85.5484 & & 0.910049 & 99 & & 3.20356 & 8/1/1993 & 85.5484 \\
\hline 81.5 & & 1.151983 & 100 & & 3.290582 & 9/1/1993 & 81.5 \\
\hline 71.6935 & & 1.020999 & 93 & & 3.167432 & 10/1/1993 & 71.6935 \\
\hline 58.4667 & & 0.710658 & 81 & & 2.517199 & 11/1/1993 & 58.4667 \\
\hline 57.3226 & & 0.5591 & 80 & & 2.73887 & $12 / 1 / 1993$ & 57.3226 \\
\hline
\end{tabular}




\begin{tabular}{|c|c|c|c|c|}
\hline Victoria & & VCT & Victoria & \\
\hline Ave W/sf & & Tpeak & Peak W/sf & \\
\hline pre & post & & pre & post \\
\hline 1.498759 & & 93 & 3.49279 & \\
\hline 1.342185 & & 99 & 3.536492 & \\
\hline 0.866387 & & 83 & 2.85407 & \\
\hline 0.786514 & & 81 & 3.032239 & \\
\hline 0.761694 & & 72 & 2.117861 & \\
\hline & 0.818988 & 83 & & 2.591859 \\
\hline & 0.762097 & 89 & & 2.911219 \\
\hline & 1.039433 & 88 & & 3.496152 \\
\hline & 1.165073 & 91 & & 3.862576 \\
\hline & 0.778503 & 99 & & 2.45403 \\
\hline & 0.771809 & 95 & & 2.151478 \\
\hline & 1.098921 & 99 & & 3.949979 \\
\hline & 1.424144 & 97 & & 4.198744 \\
\hline & 1.197629 & 92 & & 3.761725 \\
\hline & 0.891007 & 85 & & 3.496152 \\
\hline & 0.649529 & 79 & & 2.292669 \\
\hline & 0.703494 & 80 & & 2.208627 \\
\hline & 0.804886 & 84 & & 2.521263 \\
\hline & 0.770543 & 87 & & 3.170069 \\
\hline & 0.938369 & 87 & & 3.287728 \\
\hline & 1.01964 & 91 & & 3.707938 \\
\hline & 0.69425 & 91 & & 2.131308 \\
\hline & 0.673292 & 98 & & 2.017011 \\
\hline & 1.350165 & 99 & & 4.373552 \\
\hline & 1.278022 & 100 & & 4.158404 \\
\hline & 1.118904 & 93 & & 3.956703 \\
\hline & 0.741464 & 81 & & 2.786837 \\
\hline & 0.624255 & 80 & & 2.897772 \\
\hline
\end{tabular}


Sheet1

\begin{tabular}{|c|c|c|c|c|c|c|c|}
\hline & DFW & Sims & & DEF" & Simc & & \\
\hline & Tave & Ave W/sf & & Tpeak & Peak W/sf & & \\
\hline & & pre & post & & pre & post & \\
\hline 9/1/1991 & 68 & 1.842661 & & 89 & 4.984615 & & 9/1/1991 \\
\hline 10/1/1991 & 68 & 1.792132 & & 97 & 4.523077 & & 10/1/1991 \\
\hline 11/1/1991 & 51 & 1.132968 & & 78 & 3.311538 & & 11/1/1991 \\
\hline $12 / 1 / 1991$ & 50 & 0.844877 & & 78 & 2.684615 & & $12 / 1 / 1991$ \\
\hline $1 / 1 / 1992$ & 46 & 0.880226 & & 69 & 2.761538 & & 1/1/1992 \\
\hline $2 / 1 / 1992$ & 54 & & 0.99507 & 75 & & 2.584615 & $2 / 1 / 1992$ \\
\hline 3/1/1992 & 60 & & 0.988564 & 85 & & 2.830769 & 3/1/1992 \\
\hline 4/1/1992 & 66 & & 1.339628 & 86 & & 3.4 & 4/1/1992 \\
\hline $5 / 1 / 1992$ & 70 & & 1.536631 & 93 & & 3.726923 & $5 / 1 / 1992$ \\
\hline $6 / 1 / 1992$ & 78 & & 1.686944 & 96 & & 3.130769 & $6 / 1 / 1992$ \\
\hline $7 / 1 / 1992$ & 84 & & 1.768244 & 97 & & 2.823077 & $7 / 1 / 1992$ \\
\hline 8/1/1992 & 80 & & 1.673424 & 98 & & 4.246154 & 8/1/1992 \\
\hline 9/1/1992 & 78 & & 1.964071 & 94 & & 4.188462 & 9/1/1992 \\
\hline 10/1/1992 & 70 & & 1.469877 & 91 & & 3.676923 & 10/1/1992 \\
\hline 11/1/1992 & 53 & & 1.033093 & 77 & & 3.042308 & 11/1/1992 \\
\hline 12/1/1992 & 49 & & 0.939072 & 78 & & 2.6 & 12/1/1992 \\
\hline 1/1/1993 & 45 & & 1.005949 & 74 & & 2.946154 & 1/1/1993 \\
\hline 2/1/1993 & 49 & & 1.164878 & 73 & & 2.715385 & 2/1/1993 \\
\hline $3 / 1 / 1993$ & 56 & & 1.112512 & 88 & & 2.984615 & $3 / 1 / 1993$ \\
\hline 4/1/1993 & 64 & & 1.248674 & 88 & & 3.480769 & 4/1/1993 \\
\hline $5 / 1 / 1993$ & 72 & & 1.641693 & 93 & & 3.726923 & $5 / 1 / 1993$ \\
\hline $6 / 1 / 1993$ & 81 & & 1.606426 & 98 & & 3.784615 & $6 / 1 / 1993$ \\
\hline $7 / 1 / 1993$ & 87 & & 1.386731 & 102 & & 2.892308 & $7 / 1 / 1993$ \\
\hline 8/1/1993 & 88 & & 2.171991 & 103 & & 5.376923 & 8/1/1993 \\
\hline 9/1/1993 & 78 & & 1.828163 & 97 & & 4.946154 & 9/1/1993 \\
\hline 10/1/1993 & 64 & & 1.341073 & 90 & & 3.934615 & 10/1/1993 \\
\hline $11 / 1 / 1993$ & 52 & & 0.989297 & 84 & & 2.719231 & $11 / 1 / 1993$ \\
\hline 12/1/1993 & 50 & & 0.795471 & 73 & & 2.776923 & 12/1/1993 \\
\hline
\end{tabular}


Sheet1

\begin{tabular}{|c|c|c|c|c|c|c|c|}
\hline VCT & Stroman & & VCT & Stroman & & & VCT \\
\hline \multirow[t]{2}{*}{ Tave } & Ave W/sf & & Tpeak & Peak W/sf & & & Tave \\
\hline & pre & post & & pre & post & & \\
\hline 77.913 & 1.120738 & & 93 & 3.075482 & & 9/1/1991 & 77.913 \\
\hline 73.8065 & 1.068827 & & 99 & 3.083692 & & 10/1/1991 & 73.8065 \\
\hline 58.8167 & 0.717382 & & 83 & 2.692893 & & 11/1/1991 & 58.8167 \\
\hline 58.1 & 0.627494 & & 81 & 2.550039 & & $12 / 1 / 1991$ & 58.1 \\
\hline 52.3333 & 0.663773 & & 72 & 2.282391 & & 1/1/1992 & 52.3333 \\
\hline 60.7593 & & 0.754243 & 83 & & 2.633779 & 2/1/1992 & 60.7593 \\
\hline 66.8704 & & 0.693692 & 89 & & 2.617359 & $3 / 1 / 1992$ & 66.8704 \\
\hline 70.2679 & & 0.892356 & 88 & & 2.88829 & 4/1/1992 & 70.2679 \\
\hline 74.7 & & 0.989159 & 91 & & 2.909637 & $5 / 1 / 1992$ & 74.7 \\
\hline 82.5208 & & 0.750522 & 99 & & 2.379268 & $6 / 1 / 1992$ & 82.5208 \\
\hline 85.3 & & 0.68092 & 95 & & 2.265971 & $7 / 1 / 1992$ & 85.3 \\
\hline 82.5484 & & 0.942739 & 99 & & 3.059062 & 8/1/1992 & 82.5484 \\
\hline 81.25 & & 1.123687 & 97 & & 3.366116 & 9/1/1992 & 81.25 \\
\hline 74.0645 & & 1.00436 & 92 & & 2.942477 & 10/1/1992 & 74.0645 \\
\hline 59.5667 & & 0.744226 & 85 & & 2.811117 & 11/1/1992 & 59.5667 \\
\hline 58.8889 & & 0.571936 & 79 & & 2.525409 & 12/1/1992 & 58.8889 \\
\hline 54.4355 & & 0.647393 & 80 & & 2.270898 & 1/1/1993 & 54.4355 \\
\hline 57.9821 & & 0.732382 & 84 & & 2.467939 & 2/1/1993 & 57.9821 \\
\hline 63.1935 & & 0.716304 & 87 & & 2.581236 & 3/1/1993 & 63.1935 \\
\hline 68.0167 & & 0.820051 & 87 & & 2.68961 & $4 / 1 / 1993$ & 68.0167 \\
\hline 74.0161 & & 0.88734 & 91 & & 2.963825 & $5 / 1 / 1993$ & 74.0161 \\
\hline 80.9167 & & 0.608861 & 91 & & 2.009816 & $6 / 1 / 1993$ & 80.9167 \\
\hline 84.4839 & & 0.517645 & 98 & & 1.931003 & 7/1/1993 & 84.4839 \\
\hline 85.5484 & & 0.910049 & 99 & & 3.20356 & 8/1/1993 & 85.5484 \\
\hline 81.5 & & 1.151983 & 100 & & 3.290582 & 9/1/1993 & 81.5 \\
\hline 71.6935 & & 1.020999 & 93 & & 3.167432 & 10/1/1993 & 71.6935 \\
\hline 58.4667 & & 0.710658 & 81 & & 2.517199 & 11/1/1993 & 58.4667 \\
\hline 57.3226 & & 0.5591 & 80 & & 2.73887 & $12 / 1 / 1993$ & 57.3226 \\
\hline
\end{tabular}




\begin{tabular}{|c|c|c|c|c|}
\hline Victoria & & VCT & Victoria & \\
\hline Ave W/sf & & Tpeak & Peak W/sf & \\
\hline pre & post & & pre & post \\
\hline 1.498759 & & 93 & 3.49279 & \\
\hline 1.342185 & & 99 & 3.536492 & \\
\hline 0.866387 & & 83 & 2.85407 & \\
\hline 0.786514 & & 81 & 3.032239 & \\
\hline 0.761694 & & 72 & 2.117861 & \\
\hline & 0.818988 & 83 & & 2.591859 \\
\hline & 0.762097 & 89 & & 2.911219 \\
\hline & 1.039433 & 88 & & 3.496152 \\
\hline & 1.165073 & 91 & & 3.862576 \\
\hline & 0.778503 & 99 & & 2.45403 \\
\hline & 0.771809 & 95 & & 2.151478 \\
\hline & 1.098921 & 99 & & 3.949979 \\
\hline & 1.424144 & 97 & & 4.198744 \\
\hline & 1.197629 & 92 & & 3.761725 \\
\hline & 0.891007 & 85 & & 3.496152 \\
\hline & 0.649529 & 79 & & 2.292669 \\
\hline & 0.703494 & 80 & & 2.208627 \\
\hline & 0.804886 & 84 & & 2.521263 \\
\hline & 0.770543 & 87 & & 3.170069 \\
\hline & 0.938369 & 87 & & 3.287728 \\
\hline & 1.01964 & 91 & & 3.707938 \\
\hline & 0.69425 & 91 & & 2.131308 \\
\hline & 0.673292 & 98 & & 2.017011 \\
\hline & 1.350165 & 99 & & 4.373552 \\
\hline & 1.278022 & 100 & & 4.158404 \\
\hline & 1.118904 & 93 & & 3.956703 \\
\hline & 0.741464 & 81 & & 2.786837 \\
\hline & 0.624255 & 80 & & 2.897772 \\
\hline
\end{tabular}




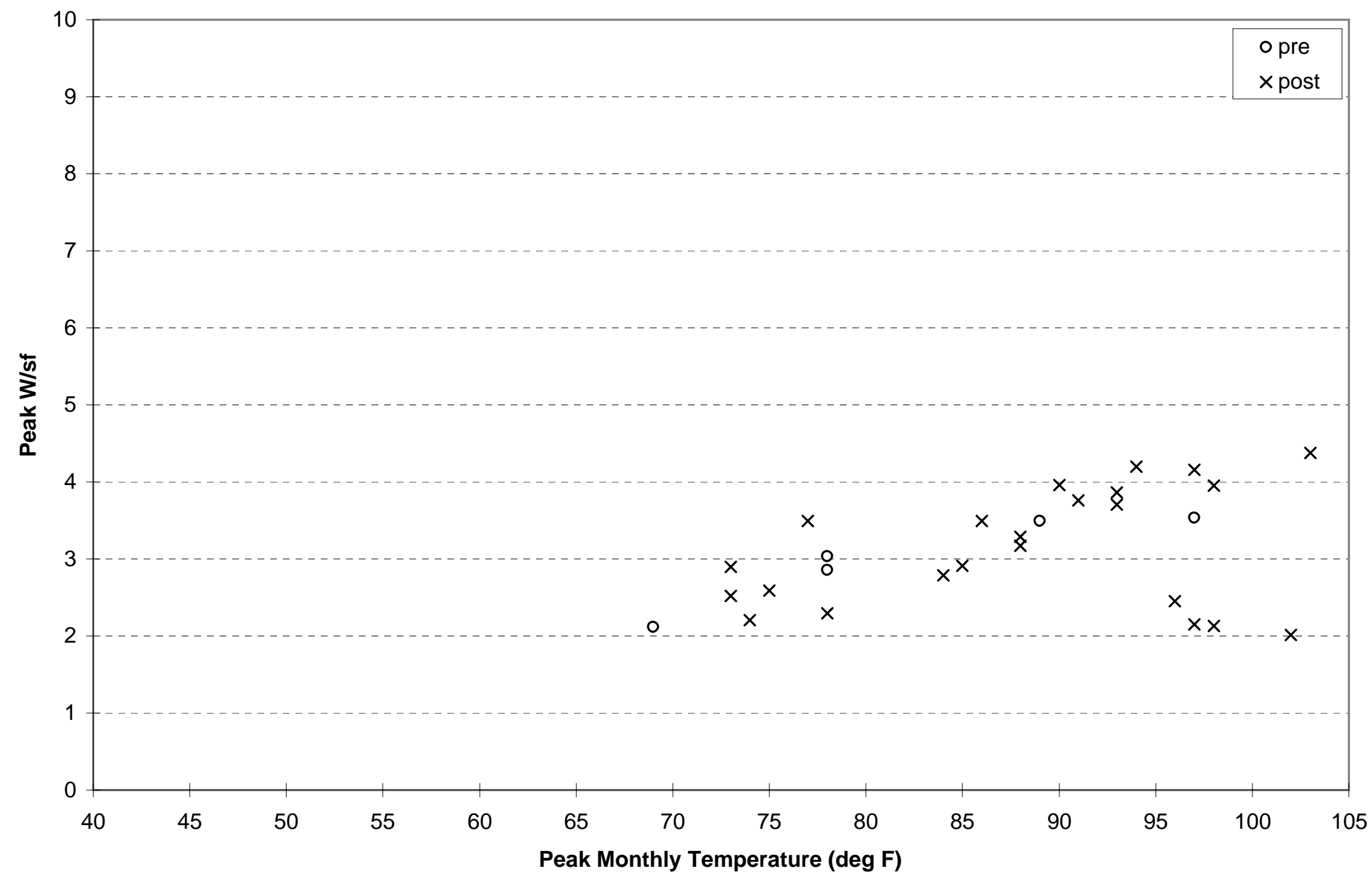

Page 1 
VHS Ave

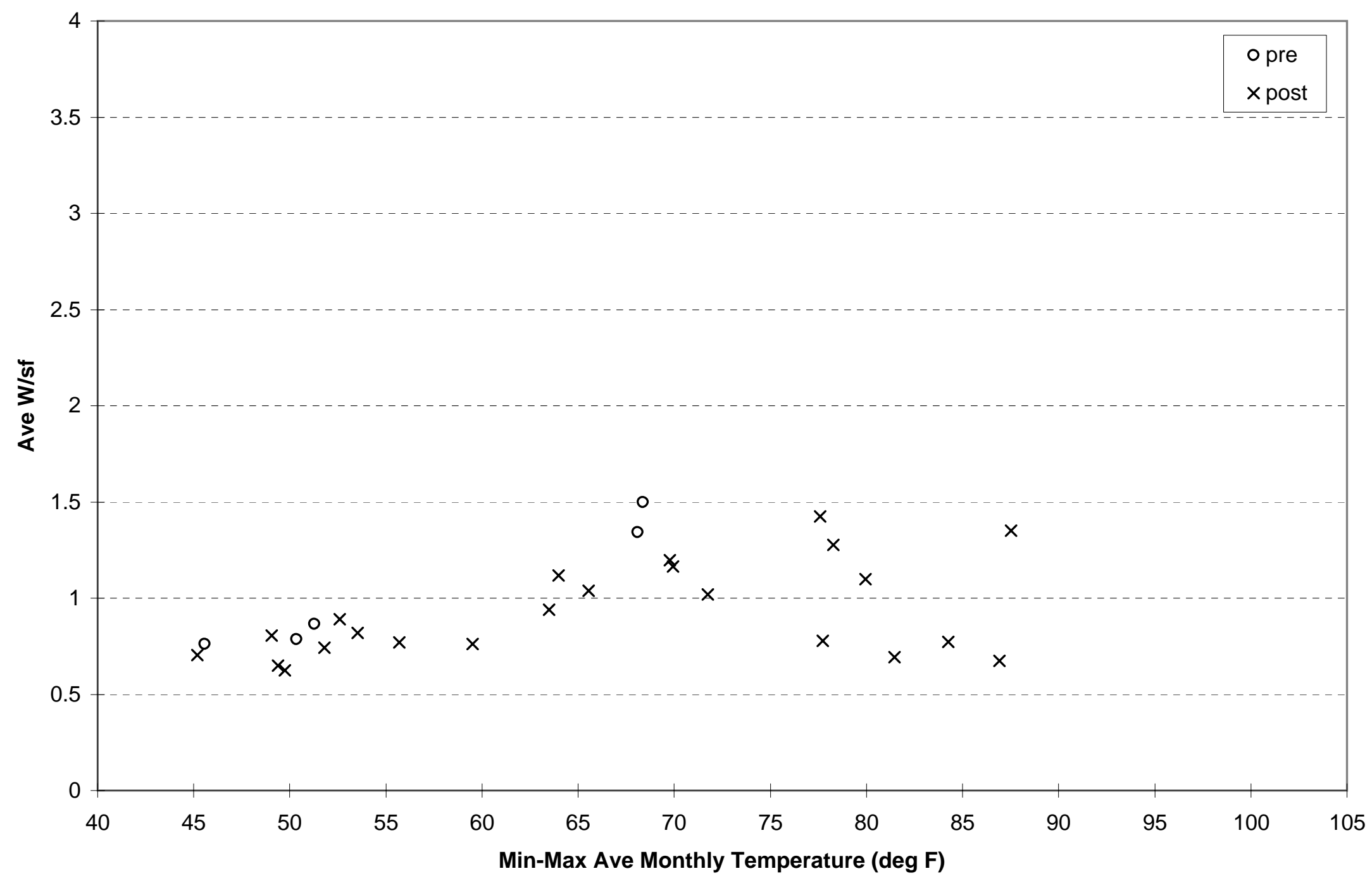

Page 1 


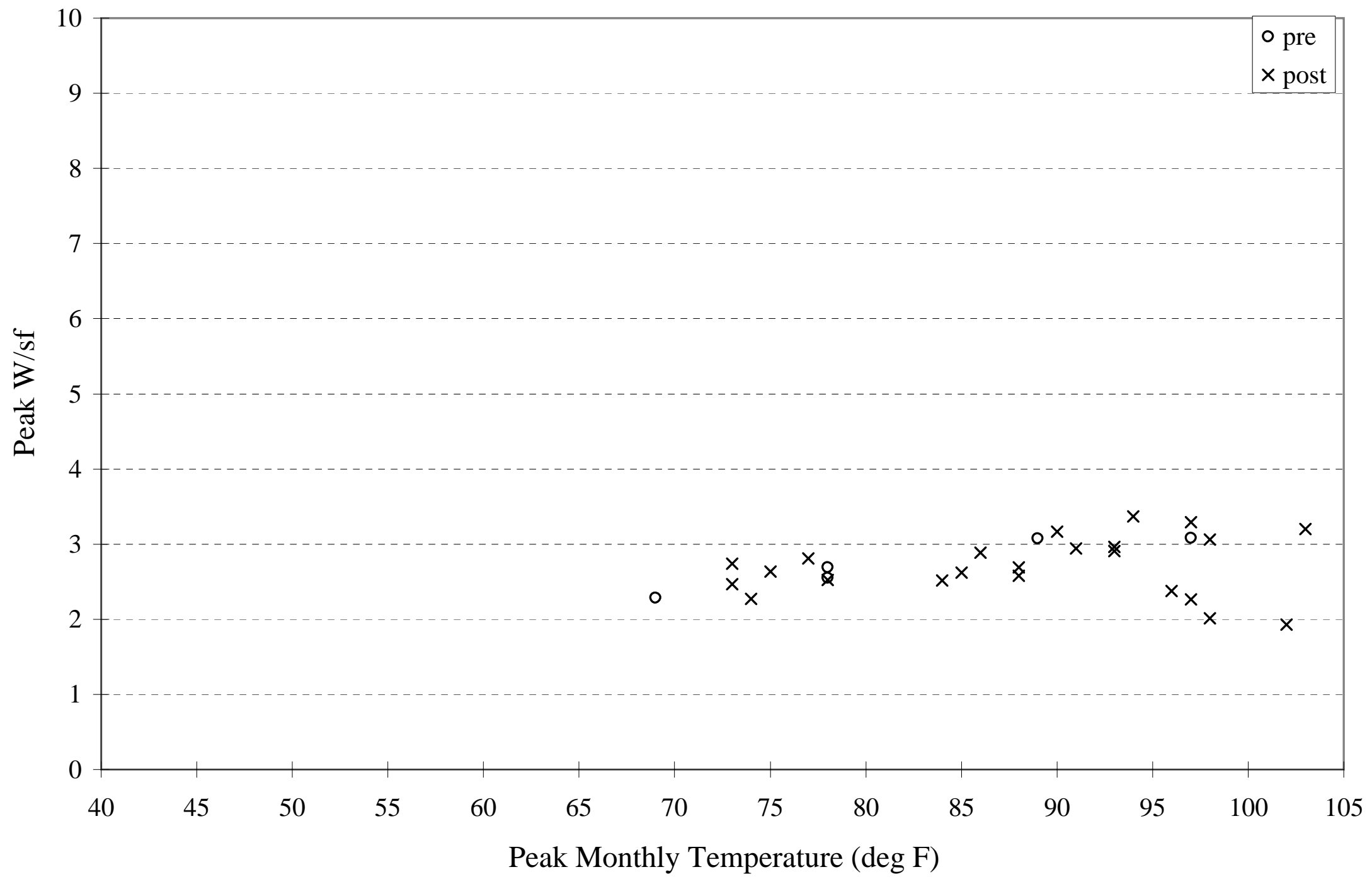

Page 1 
SHS Ave

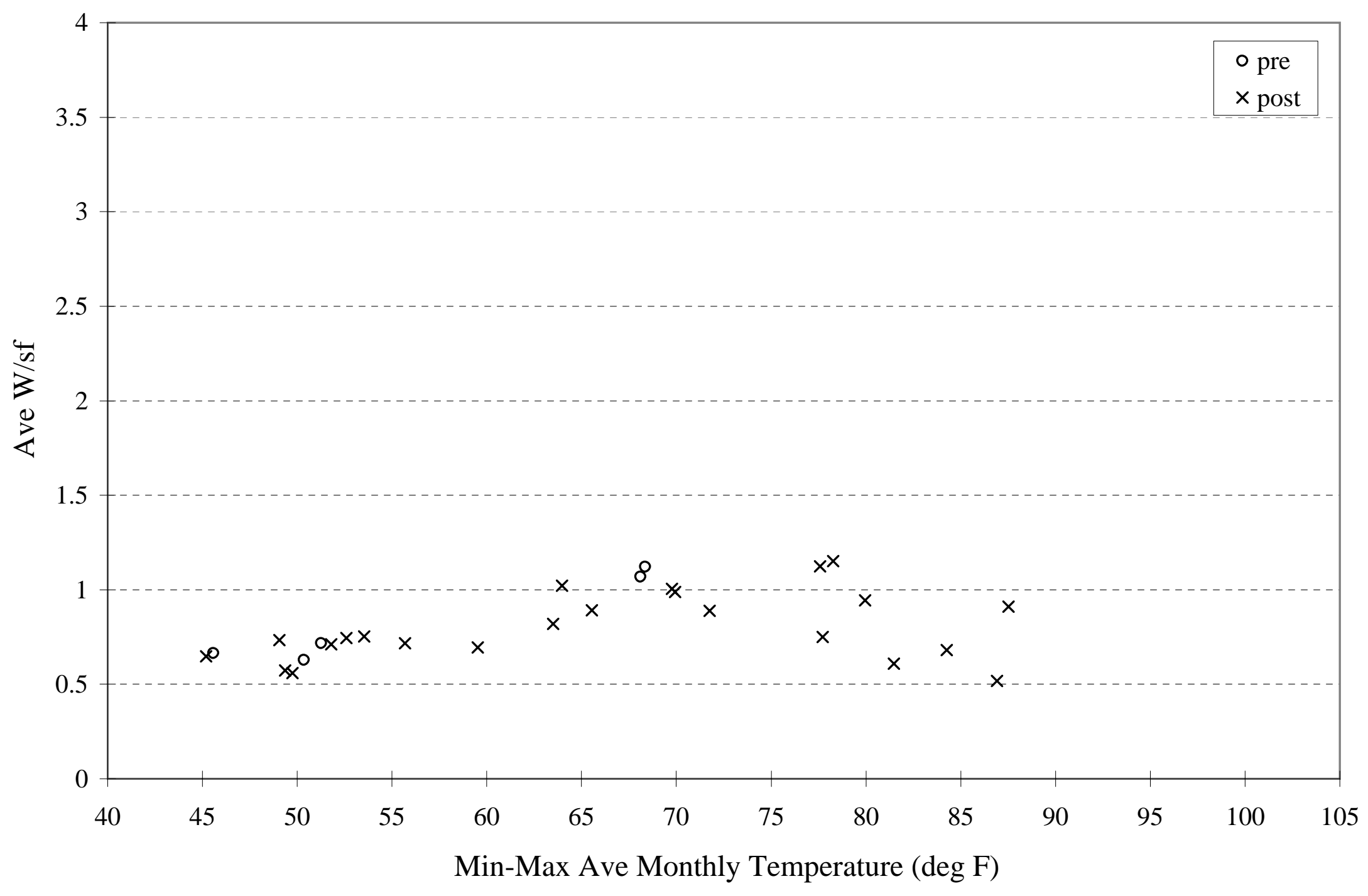

Page 1 


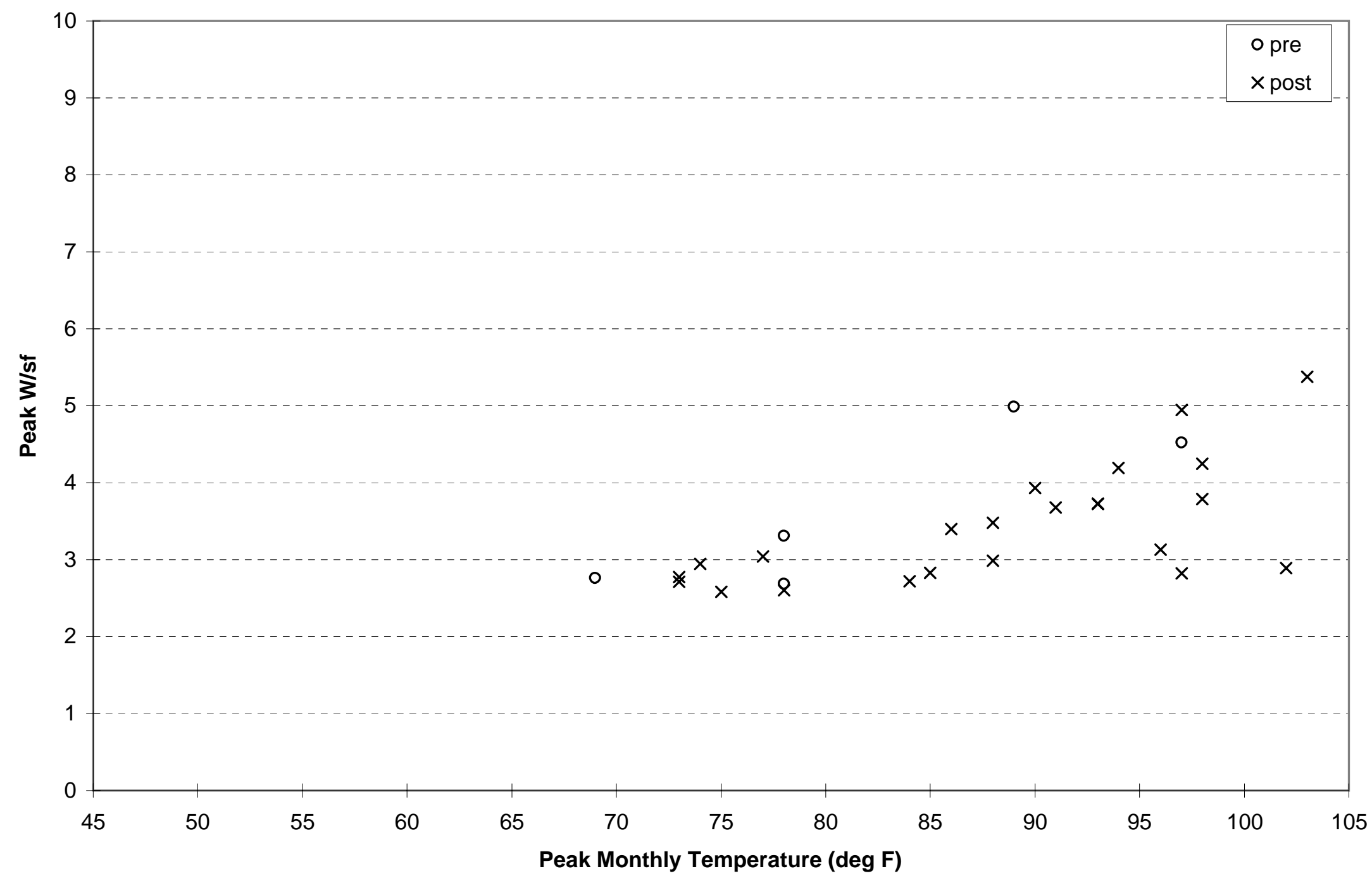

Page 1 


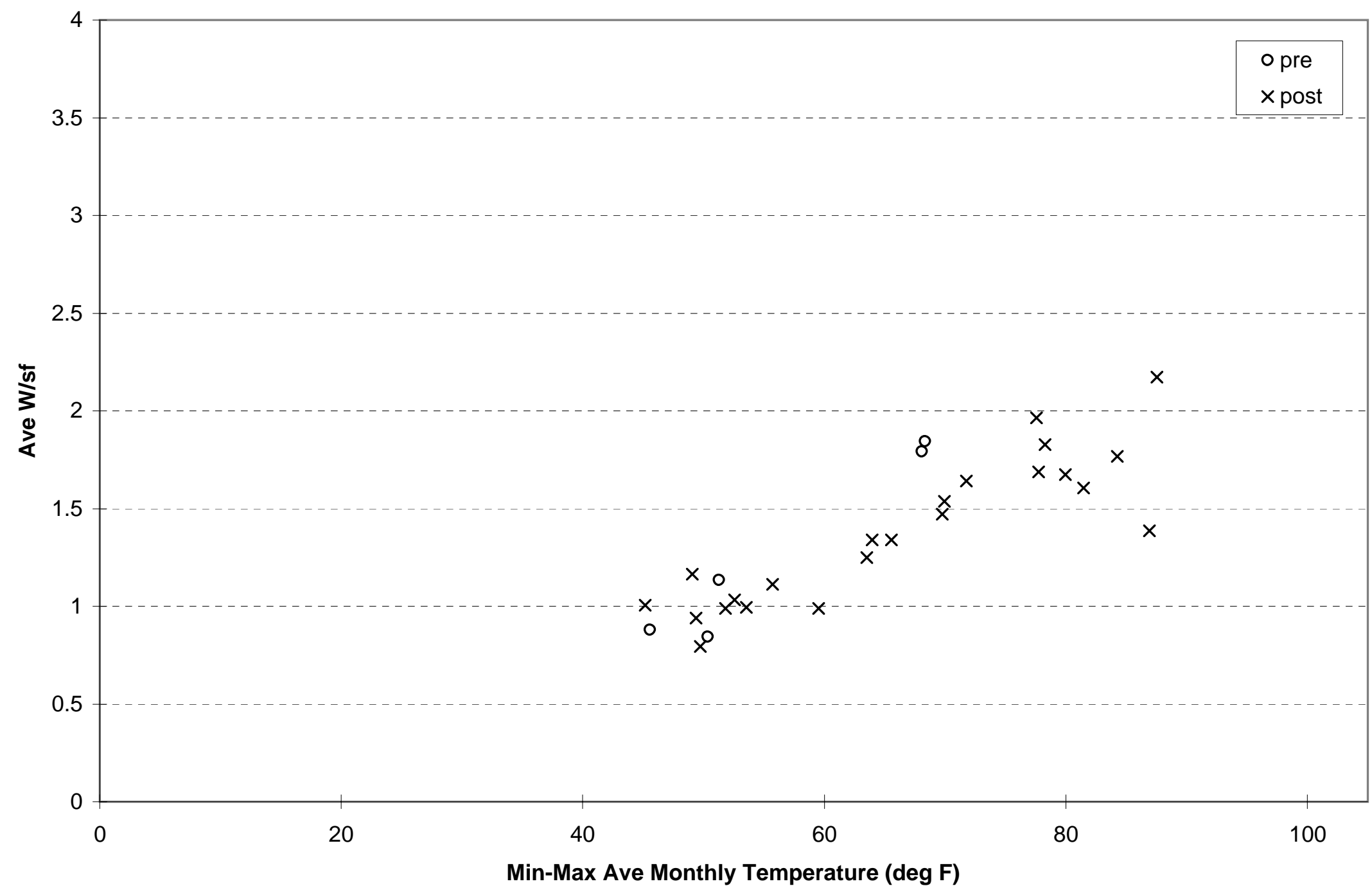

Page 1 
Calculations

\begin{tabular}{|c|c|c|c|c|c|c|c|c|c|}
\hline Model \# & $\mathrm{xcp}$ & $\mathrm{ycp}$ & Is & rs & $\mathrm{xO}$ & $\mathrm{x} 2$ & $\mathrm{y} 0$ & $y 2$ & \\
\hline CMS-sch & 72.1076 & 0.8795 & 0 & 0.1075 & 48 & 82 & 0.8795 & 1.94293 & \\
\hline CMS-smr & 81.1905 & 0.433 & 0 & 0.7274 & 75 & 82 & 0.433 & 1.02183 & \\
\hline DMS-sch & 57.852 & 1.2979 & 0 & 0.0841 & 45 & 88 & 1.2979 & 3.83335 & \\
\hline DMS-smr & & & & & & & 0 & 0 & \\
\hline NHS-sch & 60.4922 & 1.0705 & 0 & 0.0665 & 48 & 82 & 1.0705 & 2.50077 & \\
\hline NHS-smr & 80.087 & 0.7144 & 0 & 0.4117 & 75 & 82 & 0.7144 & 1.50198 & \\
\hline SES-sch & 56.9229 & 0.9985 & 0 & 0.0522 & 45 & 88 & 0.9985 & 2.62072 & \\
\hline SES-smr & & & & & & & 0 & 0 & \\
\hline SHS-sch & 61.6097 & 0.7665 & 0 & 0.0331 & 52 & 84 & 0.7665 & 1.50762 & \\
\hline SHS-smr & 85.1162 & 0.8585 & 0 & 0.1256 & 69 & 87 & 0.8585 & 1.09511 & \\
\hline VHS-sch & 57.1397 & 0.6527 & 0 & 0.0195 & 52 & 84 & 0.6527 & 1.17648 & \\
\hline VHS-smr & 86 & 0.75713 & 0 & & 69 & & 0.75713 & 0.75713 & \\
\hline OES-sch & 62.8144 & 1.1452 & 0 & 0.0294 & 57 & 84 & 1.1452 & 1.76806 & \\
\hline OES-smr & & & 0 & & 77 & 87 & 0 & 0 & \\
\hline WMS-sch & 63.5078 & 1.3406 & 0 & 0.0469 & 57 & 84 & 1.3406 & 2.30168 & \\
\hline WMS-smr & & & & & 77 & 87 & 0 & 0 & \\
\hline PES-sch & 62.2788 & 1.0485 & 0 & 0.0414 & 57 & 84 & 1.0485 & 1.94776 & \\
\hline PES-smr & & & 0 & & 77 & 87 & 0 & 0 & \\
\hline MES-sch & 63.5078 & 1.3406 & 0 & 0.0469 & 57 & 84 & 1.3406 & 2.30168 & \\
\hline MES-smr & & & & & 77 & & 0.57 & 1.38 & \\
\hline RES-sch & 59.633 & 0.9234 & 0 & 0.0442 & 57 & 84 & 0.9234 & 2.00042 & \\
\hline RES-smr & & & & & 73 & 87 & 0 & 0 & \\
\hline & & & & & & & & & \\
\hline CMS-sch & CMS-sch & CMS-smr & CMS-smr & DMS-sch & DMS-sch & DMS-smr & DMS-smr & NHS-sch & NHS-sch \\
\hline 48 & 0.8795 & 75 & 0.8 & 45 & 1.2979 & 79 & 2.38 & 48 & 1.0705 \\
\hline 72.1076 & 0.8795 & 82 & 0.8 & 57.852 & 1.2979 & 88 & 2.38 & 60.4922 & 1.0705 \\
\hline 82 & 1.94293 & & & 88 & 3.83335 & & & 82 & 2.50077 \\
\hline OES-sch & OES-sch & OES-smr & OES-smr & WMS-sch & WMS-sch & WMS-smr & WMS-smr & PES-sch & PES-sch \\
\hline 57 & 1.1452 & 77 & 0.75 & 57 & 1.3406 & 77 & 0.7 & 57 & 1.0485 \\
\hline 62.8144 & 1.1452 & 87 & 0.75 & 63.5078 & 1.3406 & 87 & 0.7 & 62.2788 & 1.0485 \\
\hline 84 & 1.76806 & & & 84 & 2.30168 & & & 84 & 1.94776 \\
\hline
\end{tabular}




\begin{tabular}{|c|c|c|c|c|c|c|c|c|}
\hline & & & & & & & & \\
\hline & & & & & & & & \\
\hline & & & & & & & & \\
\hline & & & & & & & & \\
\hline & & & & & & & & \\
\hline & & & & & & & & \\
\hline & & & & & & & & \\
\hline & & & & & & & & \\
\hline & & & & & & & & \\
\hline & & & & & & & & \\
\hline & & & & & & & & \\
\hline & & & & & & & & \\
\hline & & & & & & & & \\
\hline & & & & & & & & \\
\hline & & & & & & & & \\
\hline & & & & & & & & \\
\hline & & & & & & & & \\
\hline & & & & & & & & \\
\hline & & & & & & & & \\
\hline & & & & & & & & \\
\hline & & & & & & & & \\
\hline NHS-smr & NHS-smr & SES-sch & SES-sch & SES-smr & SES-smr & SHS-sch & SHS-sch & SHS-smr \\
\hline $\begin{array}{r}75 \\
\end{array}$ & 1.25 & 45 & 0.9985 & 79 & 1.62 & 52 & 0.7665 & $\begin{array}{l}69 \\
\end{array}$ \\
\hline 82 & 1.25 & 56.9229 & 0.9985 & 87 & 1.62 & 61.6097 & 0.7665 & 86 \\
\hline & & 88 & 2.620725 & & & 84 & 1.507619 & \\
\hline PES-smr & PES-smr & MES-sch & MES-sch & MES-smr & MES-smr & RES-sch & RES-sch & RES-smr \\
\hline $\begin{array}{l}77 \\
\end{array}$ & 0.79 & 57 & 1.3406 & 77 & 0.98 & 57 & 0.9234 & 77 \\
\hline 87 & 0.79 & 77 & 1.3406 & 87 & 0.98 & 73 & 0.9234 & 82 \\
\hline & & 84 & 2.301684 & & & 84 & 2.000421 & \\
\hline
\end{tabular}




\begin{tabular}{|c|c|c|c|c|}
\hline & & & & \\
\hline & & & & \\
\hline & & & & \\
\hline & & & & \\
\hline & & & & \\
\hline & & & & \\
\hline & & & & \\
\hline & & & & \\
\hline & & & & \\
\hline & & & & \\
\hline & & & & \\
\hline & & & & \\
\hline & & & 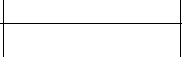 & \\
\hline & & & & \\
\hline & & & & \\
\hline & & & & \\
\hline & & & & \\
\hline & & & & \\
\hline & & & & \\
\hline & & & & \\
\hline & & & & \\
\hline & & & & \\
\hline SHS-smr & VHS-sch & VHS-sch & VHS-smr & VHS-smr \\
\hline 0.74 & 52 & 0.6527 & 69 & 0.62 \\
\hline 0.74 & 57.1397 & 0.6527 & 86 & 0.62 \\
\hline & 84 & 1.176476 & & \\
\hline RES-smr & & & & \\
\hline 0.36 & & & & \\
\hline 0.36 & & & & \\
\hline & & & & \\
\hline
\end{tabular}

Page 3 


\section{Any Questions?}

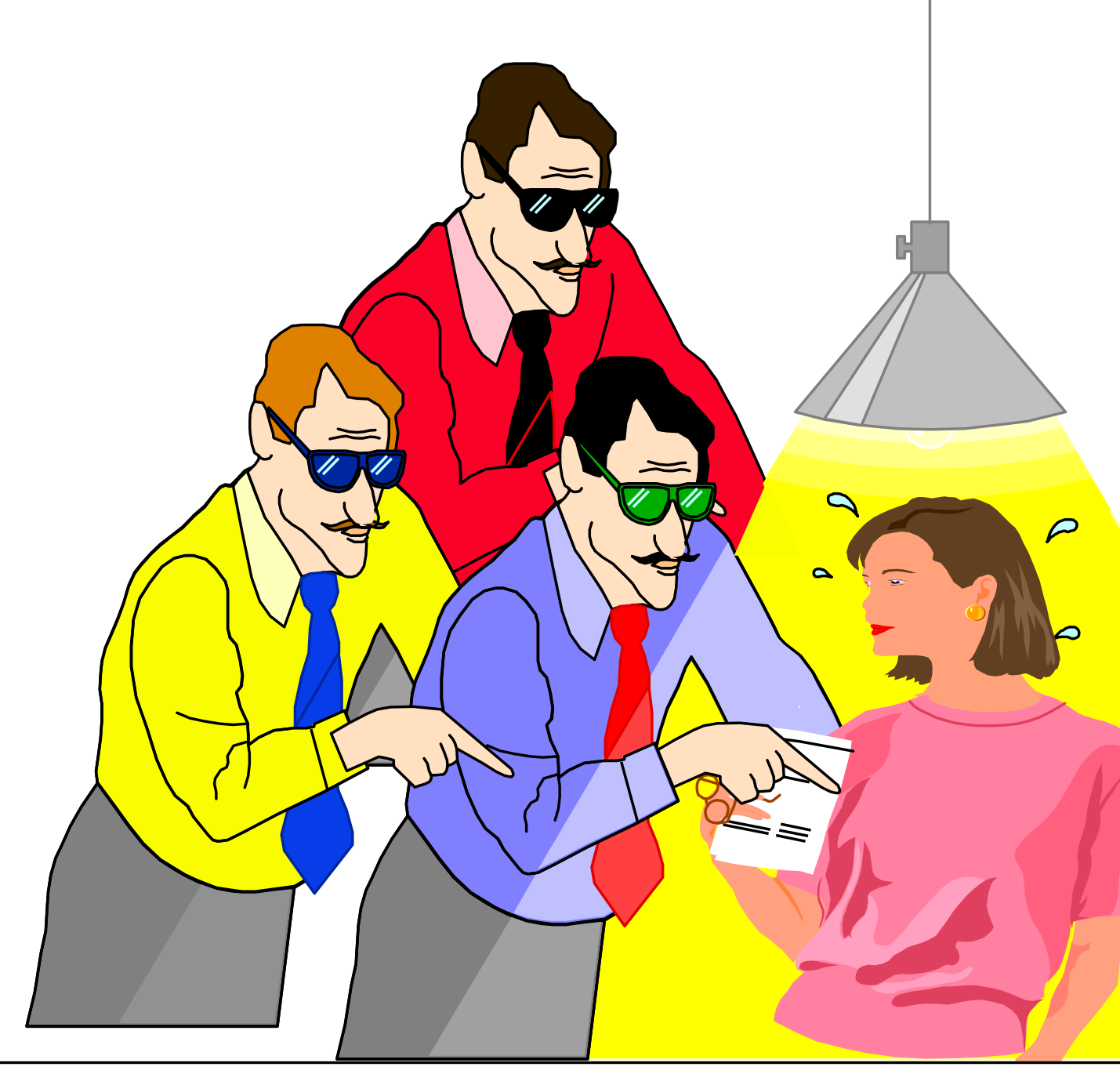



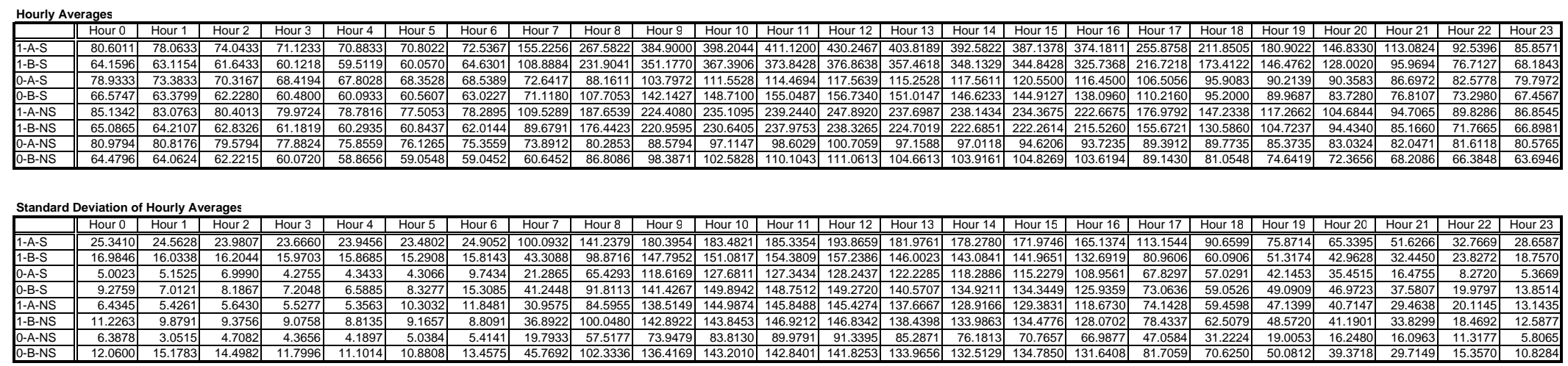

Count of Data Points
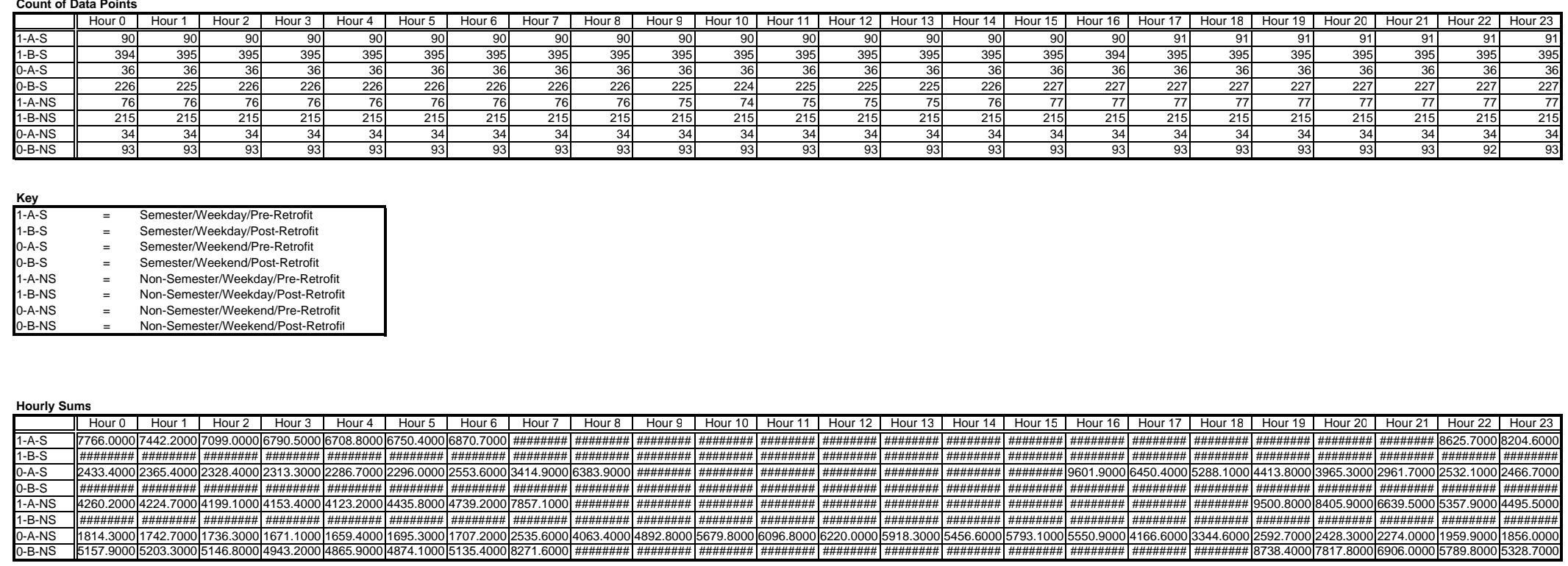
ns hourly

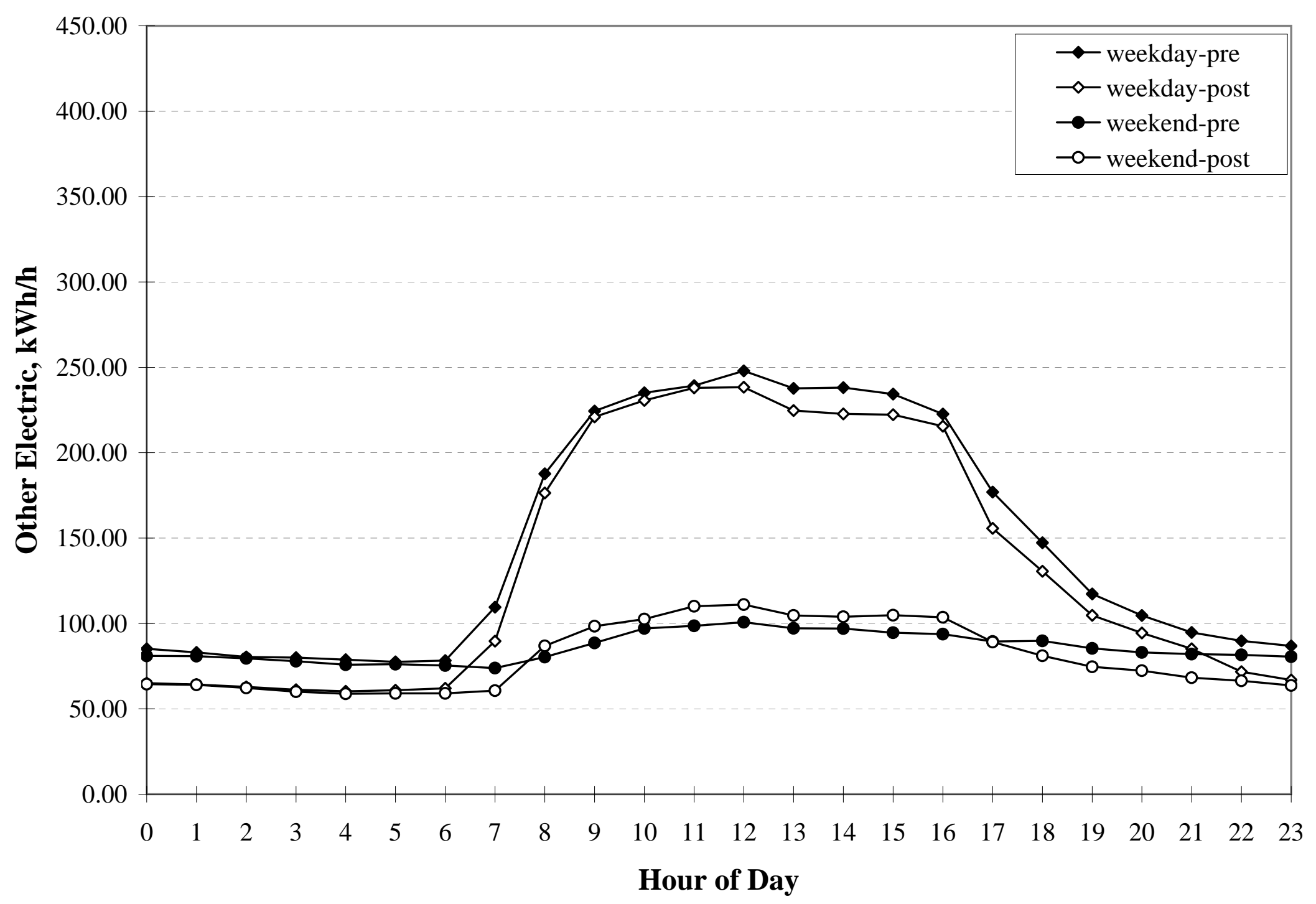

Page 1 


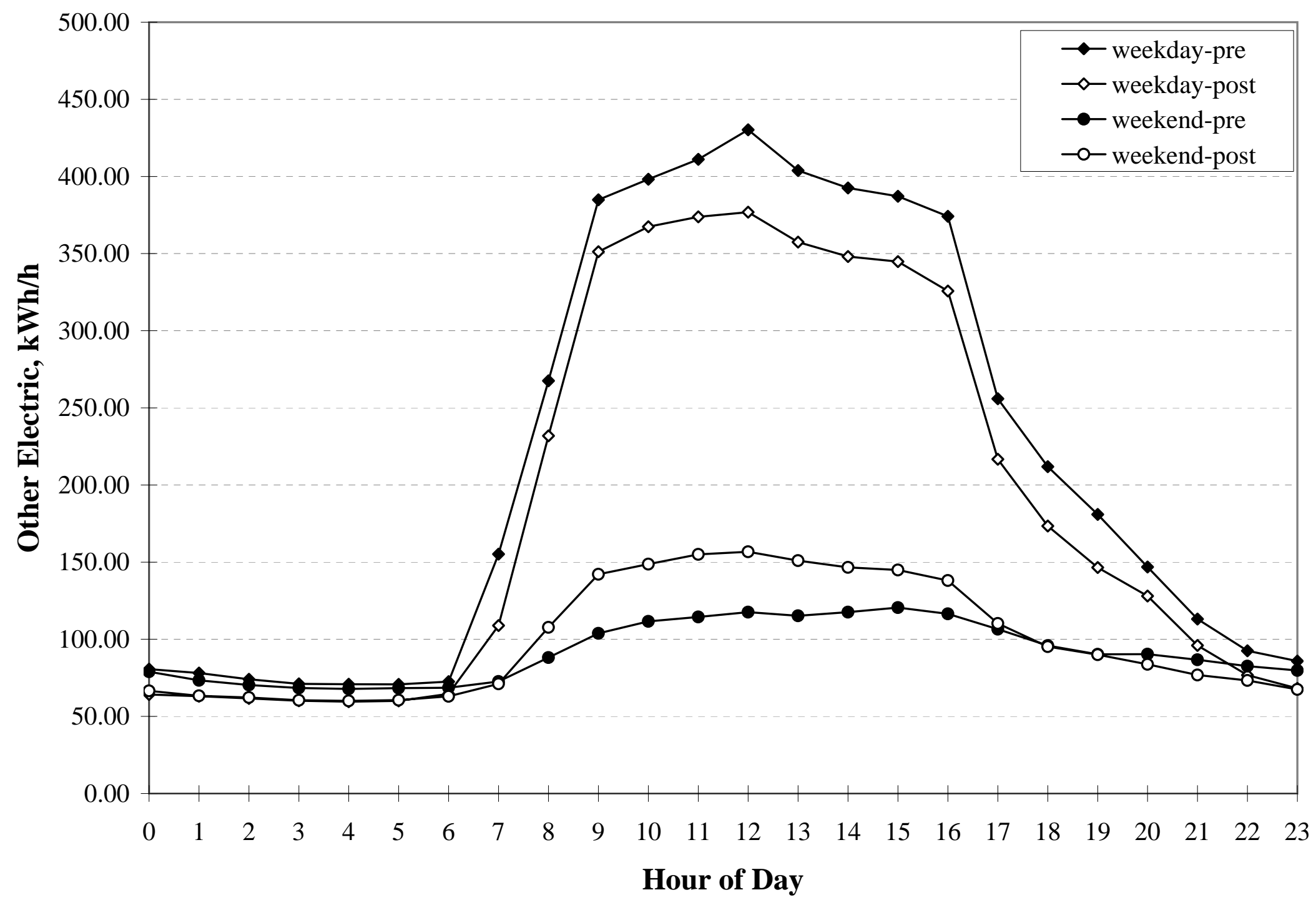

Page 1 


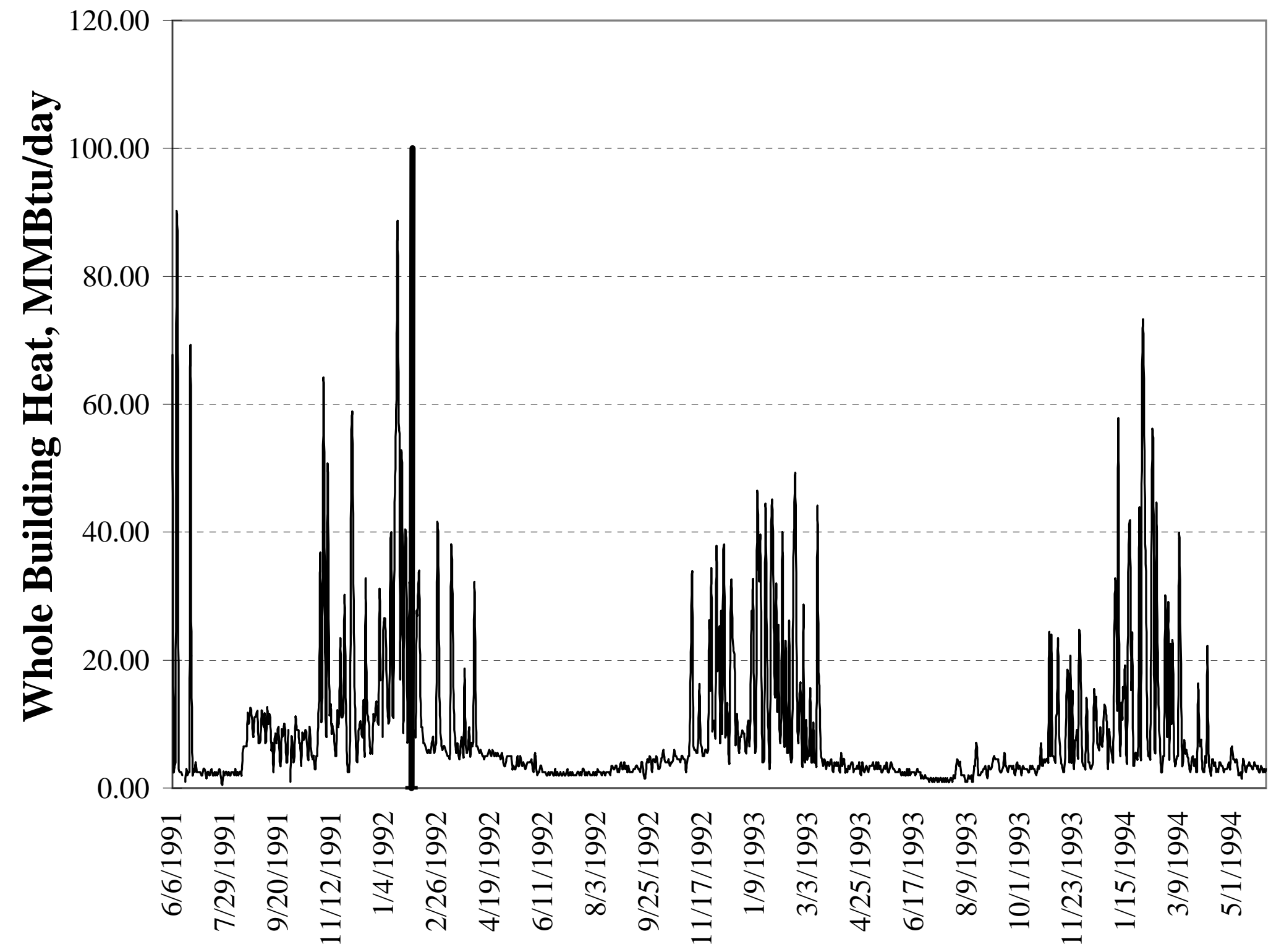




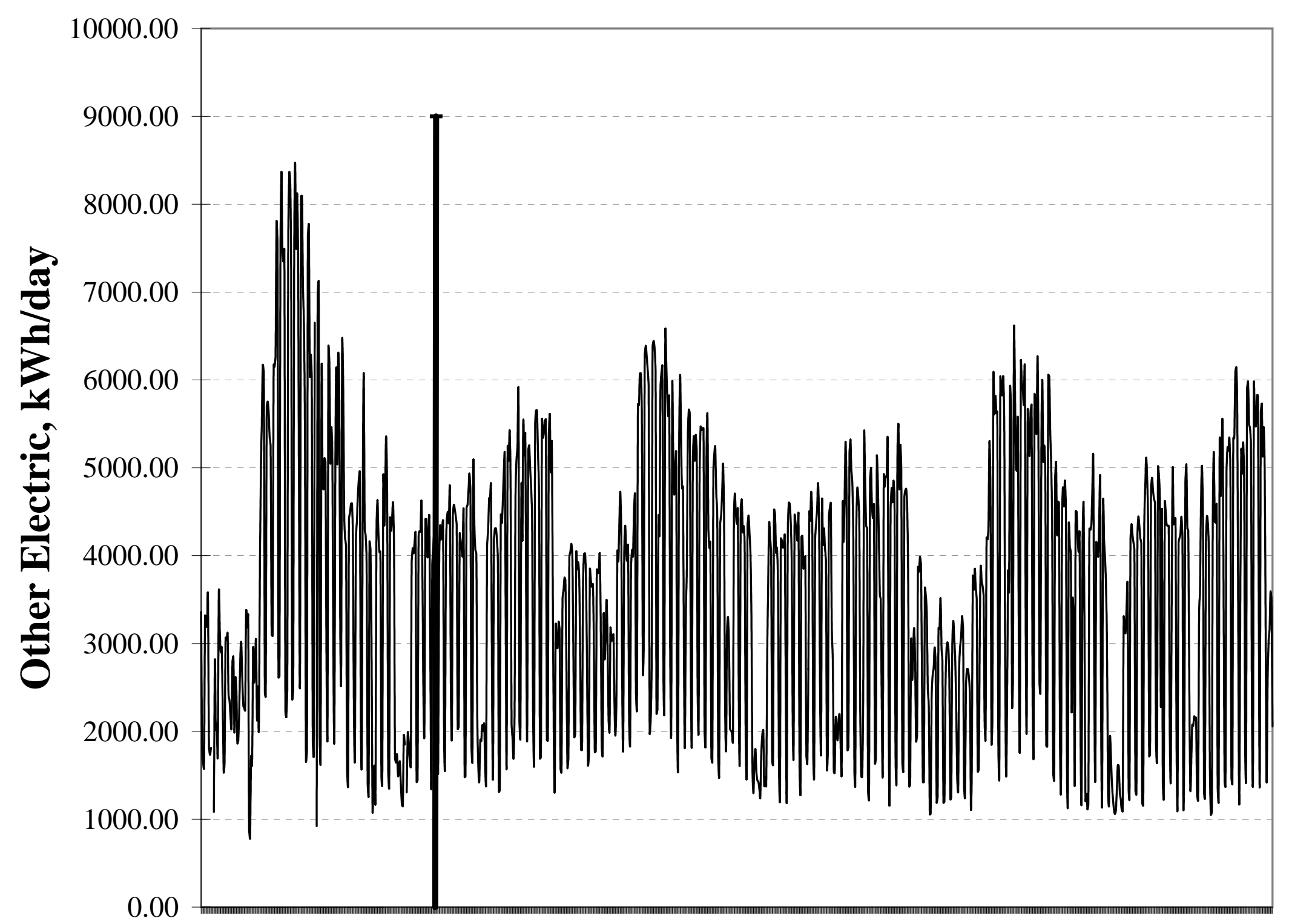




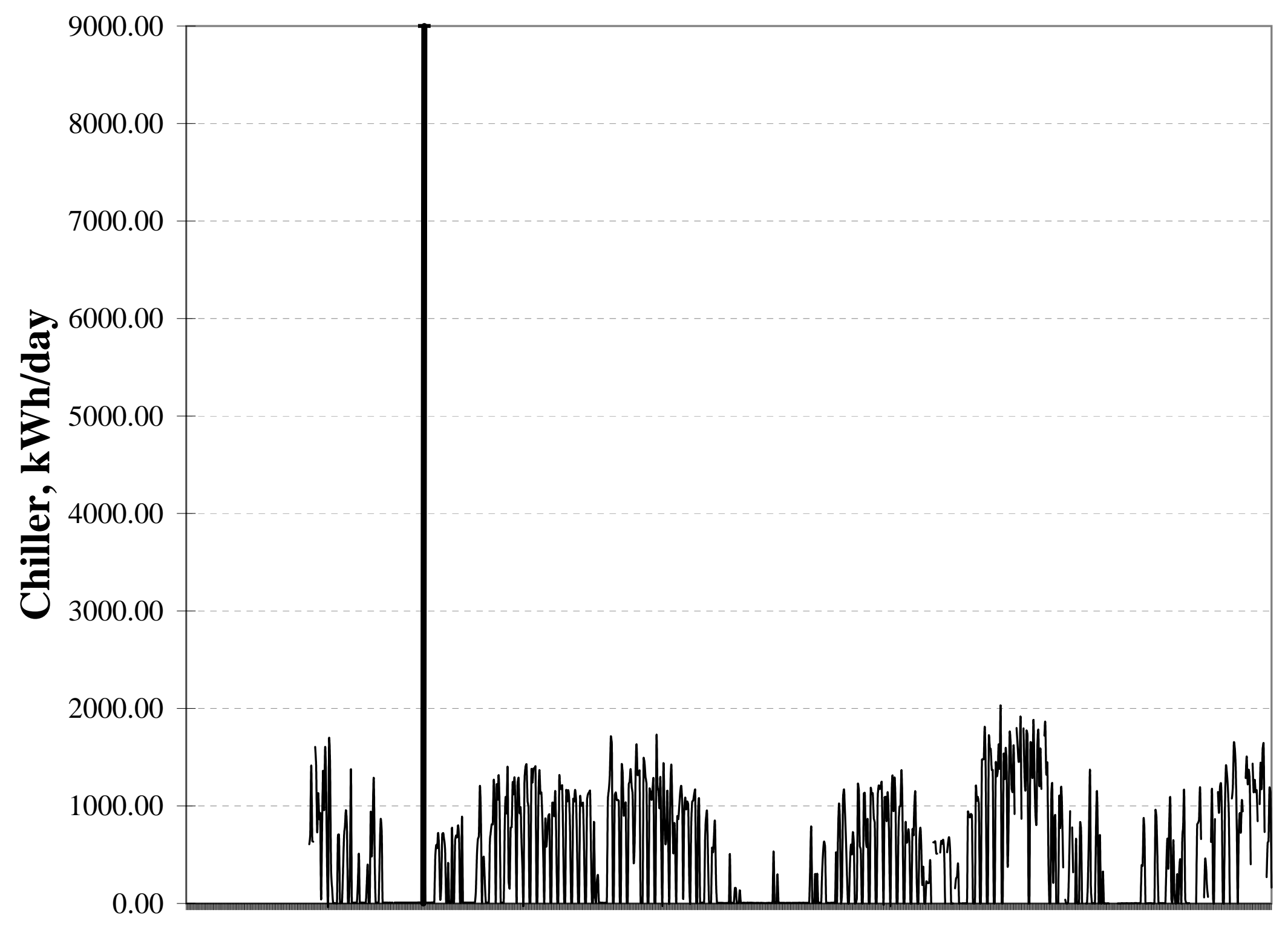

Page 1 


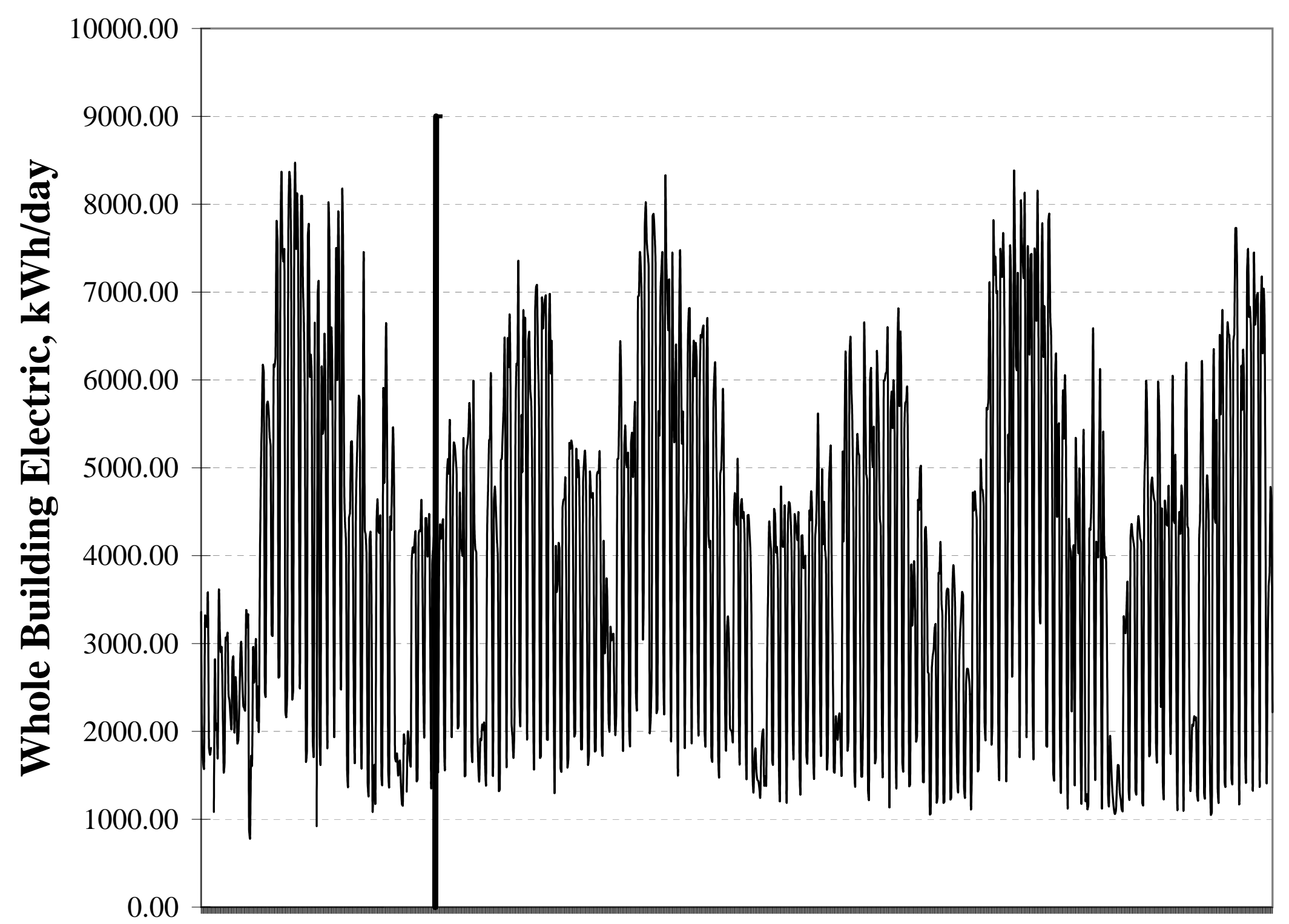




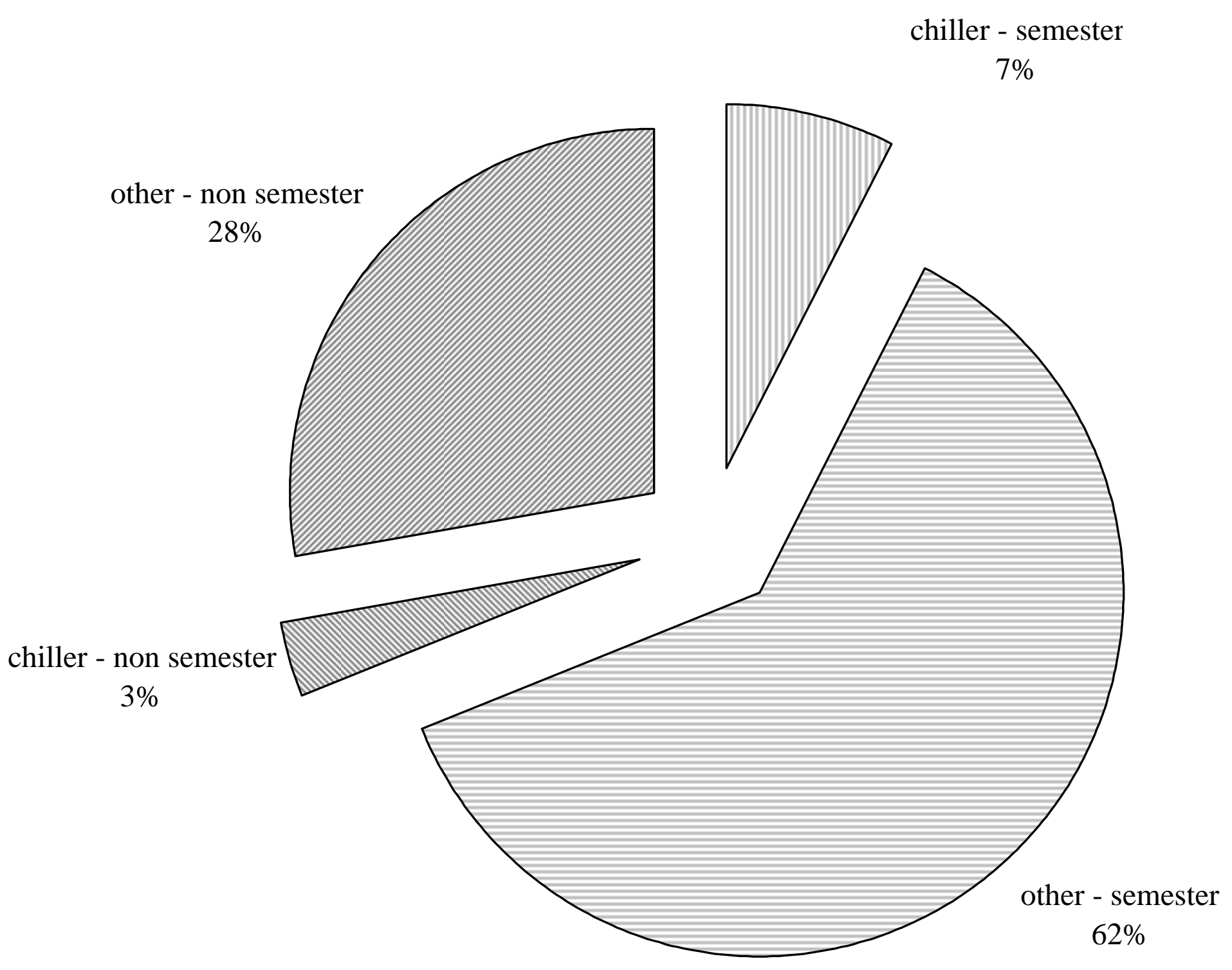




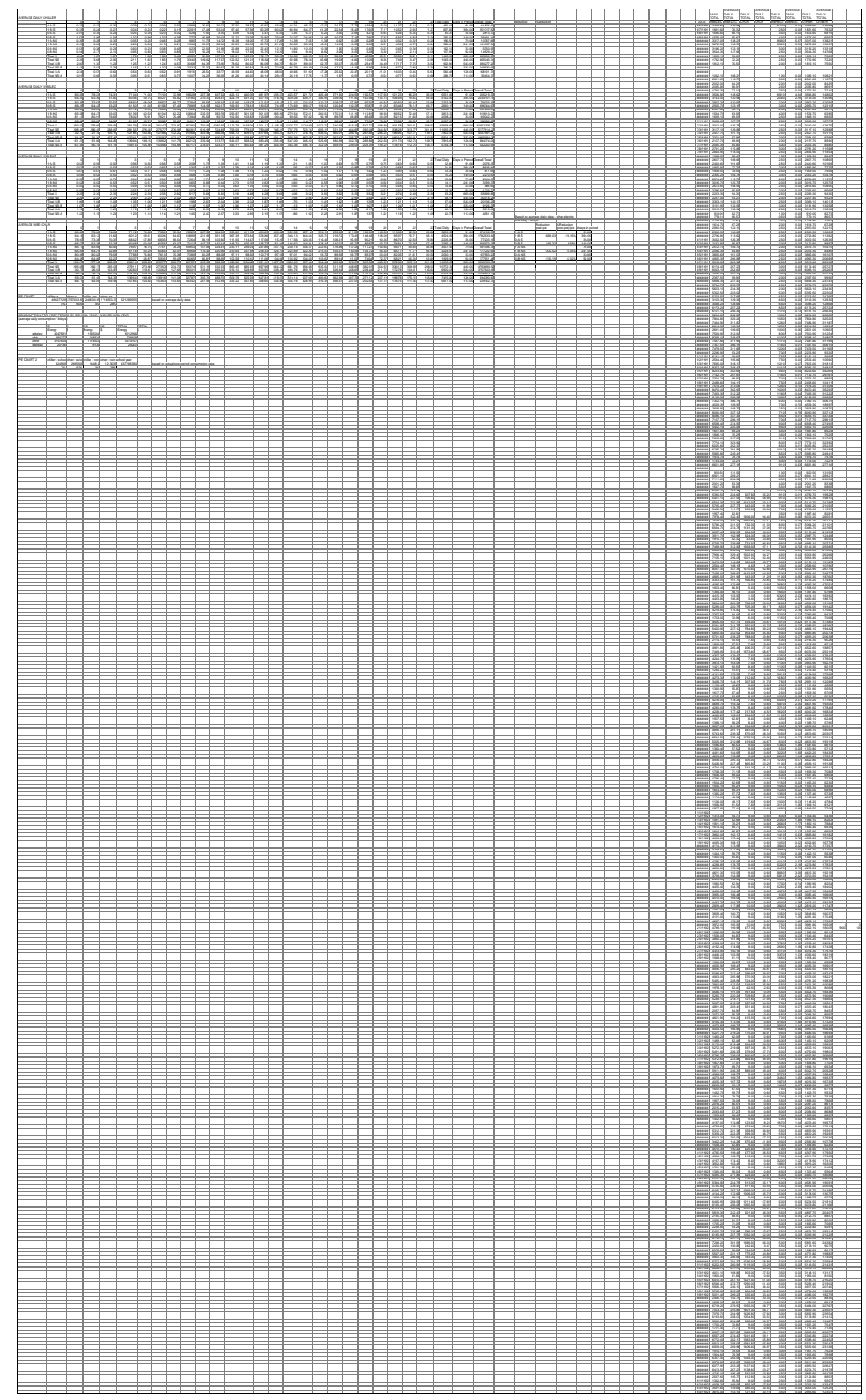




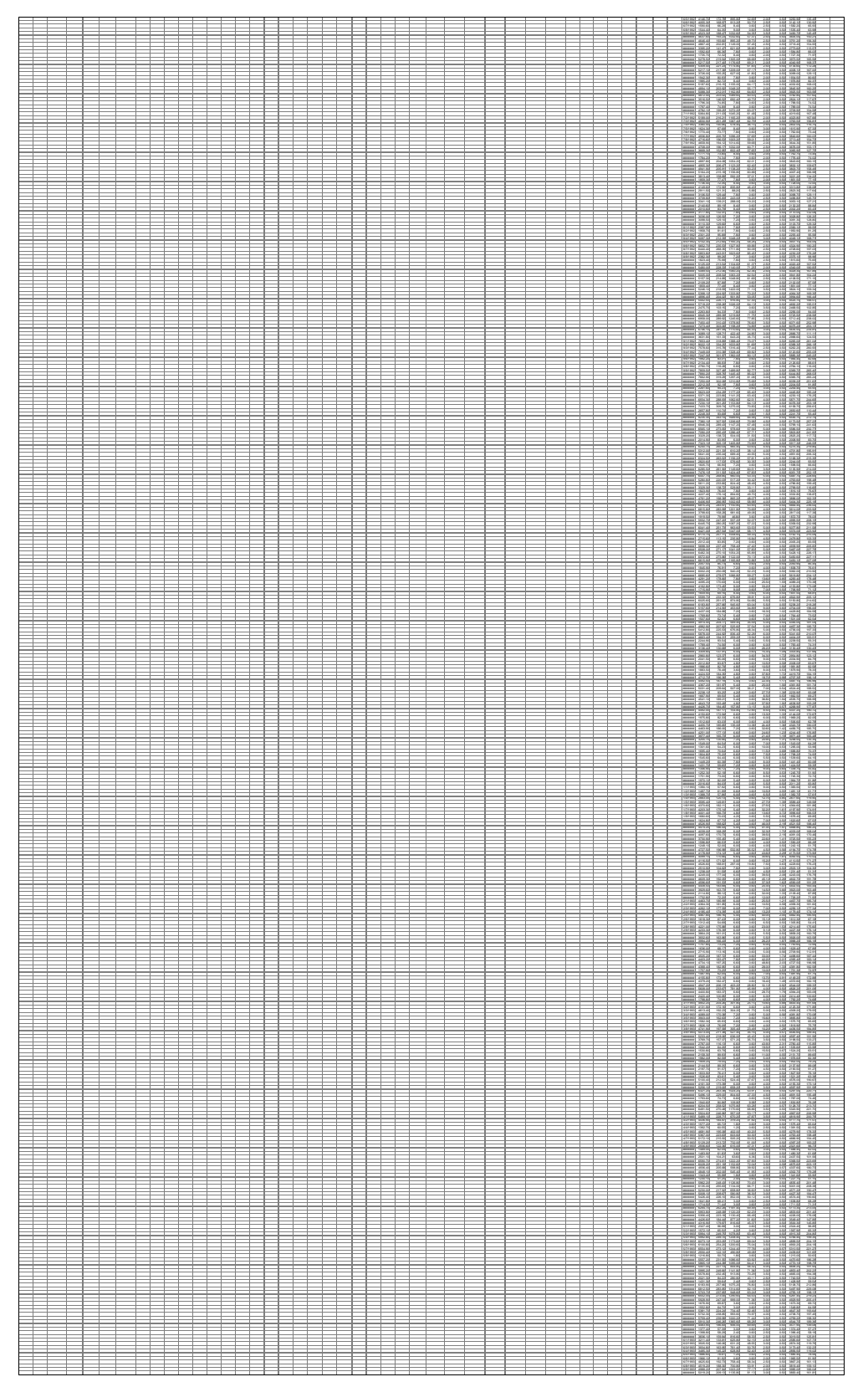




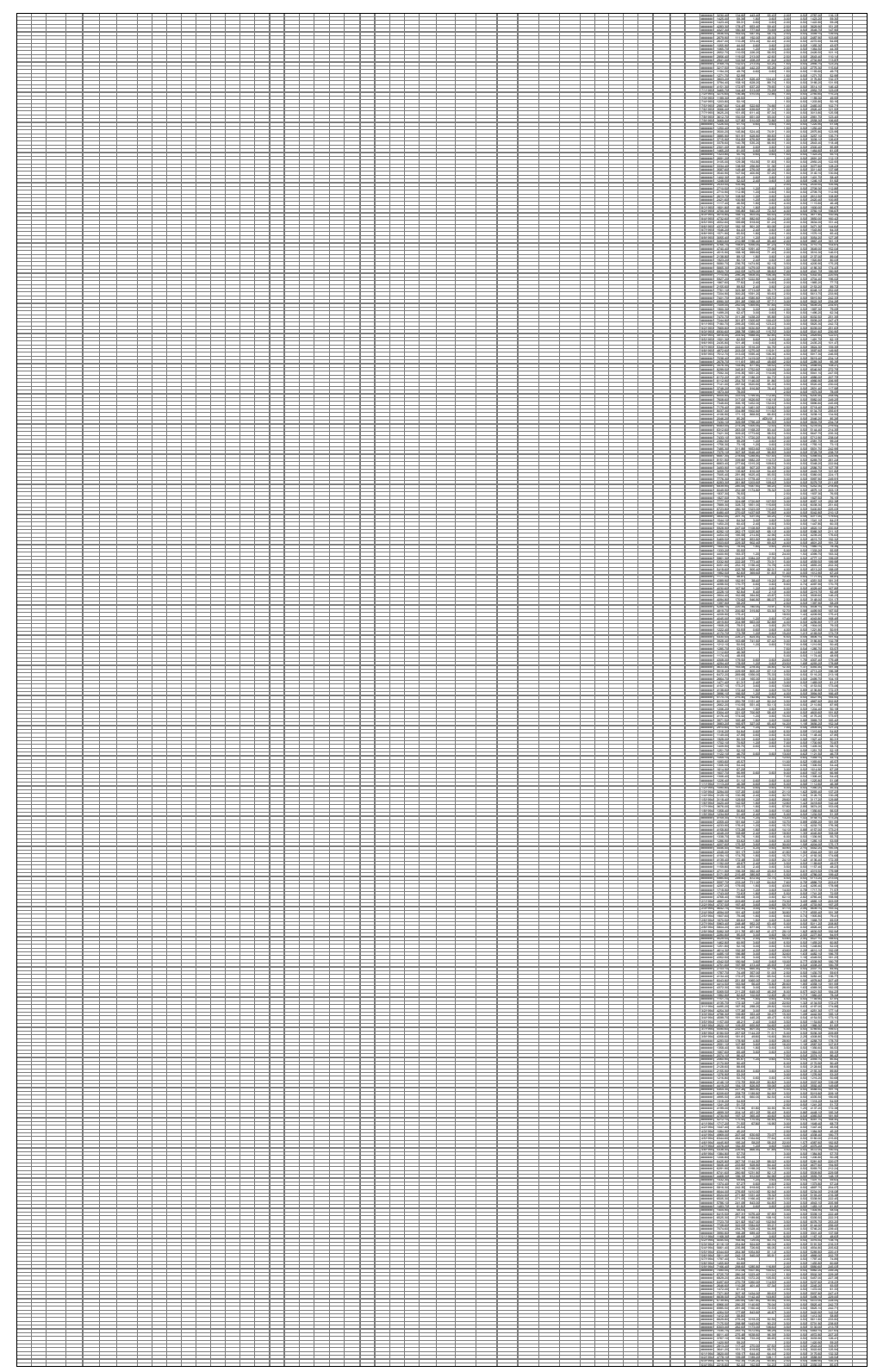


Tables

\begin{tabular}{|c|c|c|c|c|c|c|}
\hline \multicolumn{3}{|c|}{ TOTAL MONTHLY CONSUMPTION } & & & \multicolumn{2}{|c|}{ TOTAL POST PERIOD CO } \\
\hline PRE PERIOD & wbelec & chlr & other & wbheat & & $\mathrm{SCHOQ}$ \\
\hline Jun 91 & 54,919 & 0 & 54,919 & 380 & & ENERGY \\
\hline Jul & 75,348 & 0 & 75,348 & 71 & wbelec, kWh & 2209234 \\
\hline Aug & 154,573 & 0 & 154,573 & 191 & chlr, kWh & 236828 \\
\hline Sep & 169,839 & 0 & 169,839 & 244 & other, kWh & 1972405 \\
\hline Oct & 158,148 & 20,548 & 137,600 & 206 & wbheat, MMBtu & 5059 \\
\hline Nov & 108,715 & 7,583 & 101,132 & 499 & & \\
\hline Dec & 98,261 & 6,889 & 91,372 & 482 & & \\
\hline Jan 92 & 99,143 & 274 & 98,869 & 918 & \multicolumn{2}{|c|}{ TOTAL POST PERIOD CO } \\
\hline Total & 918,944 & 35,294 & 883,651 & 2,991 & & \\
\hline Rate/kWh & 0.02788 & 0.02788 & 0.02788 & & & $\mathrm{SCHOO}$ \\
\hline Rate/MMBtu & & & & 4.75000 & & ENERGY \\
\hline \multirow[t]{2}{*}{ Total \$ } & $\$ 25,620$ & $\$ 984$ & $\$ 24,636$ & $\$ 14,206$ & wbelec, kWh & 2437823 \\
\hline & & & & & chlr, kWh & 291649 \\
\hline POST PERIOD| & wbelec & chlr & other & wbheat & other, kWh & 2146174 \\
\hline \begin{tabular}{|l|l} 
Feb \\
\end{tabular} & 113,799 & 7,549 & 106,250 & 386 & wbheat, MMBtu & 5153 \\
\hline Mar & 105,333 & 8,997 & 96,336 & 280 & & \\
\hline Apr & 133,564 & 17,933 & 115,630 & 204 & & \\
\hline May & 159,814 & 27,583 & 132,232 & 125 & & \\
\hline Jun & 110,014 & 20,490 & 89,524 & 77 & & \\
\hline Jul & 109,294 & 15,226 & 94,068 & 71 & & \\
\hline Aug & 142,370 & 25,181 & 117,189 & 86 & & \\
\hline Sep & 172,628 & 27,523 & 145,105 & 97 & & \\
\hline Oct & 160,320 & 25,024 & 135,295 & 136 & & \\
\hline Nov & 110,415 & 7,948 & 102,467 & 310 & & \\
\hline Dec & 92,196 & 1,120 & 91,076 & 480 & \multicolumn{2}{|c|}{ CONSUMPTION REDUCTI } \\
\hline Jan 93 & 101,377 & 1,003 & 100,375 & 652 & & \\
\hline Feb & 100,016 & 1,351 & 98,665 & 454 & \multicolumn{2}{|c|}{ Based on average daily dat } \\
\hline Mar & 112,168 & 9,082 & 103,086 & 223 & \multicolumn{2}{|c|}{ ave daily * \#days } \\
\hline Apr & 124,100 & 16,925 & 107,175 & 100 & & Reduction \\
\hline May & 138,953 & 23,227 & 115,726 & 98 & & post-pre \\
\hline Jun & 92,268 & 12,475 & 79,794 & 68 & 1-A-S & \\
\hline Jul & 81,059 & 7,423 & 73,636 & 49 & 1-B-S & -685.03 \\
\hline Aug & 142,507 & 26,747 & 115,760 & 79 & $0-A-S$ & \\
\hline Sep & 174,574 & 36,874 & 137,700 & 105 & 0-B-S & 189.32 \\
\hline Oct & 159,880 & 29,778 & 130,102 & 127 & 1-A-NS & \\
\hline Nov & 107,693 & 9,059 & 98,634 & 302 & 1-B-NS & -332.52 \\
\hline Dec & 87,550 & 6,098 & 81,452 & 237 & $0-A-N S$ & \\
\hline Jan 94 & 102,702 & 2,415 & 100,287 & 570 & 0-B-NS & -130.19 \\
\hline Feb & 107,156 & 6,487 & 100,670 & 628 & & \\
\hline Mar & 106,874 & 9,169 & 97,706 & 263 & & \\
\hline Apr & 137,979 & 21,392 & 116,588 & 132 & & \\
\hline May & 157,004 & 26,888 & 130,115 & 105 & & \\
\hline Jun & 15,433 & 3,122 & 12,310 & 11 & & \\
\hline Total & $3,459,040$ & 434,088 & $3,024,952$ & 6,451 & & \\
\hline Rate/kWh & 0.02788 & 0.02788 & 0.02788 & & & \\
\hline Rate/MMBtu & & & & 4.75000 & & \\
\hline Total \$ & $\$ 96,438$ & $\$ 12,102$ & $\$ 84,336$ & $\$ 30,643$ & & \\
\hline & & & & & & \\
\hline
\end{tabular}


Tables

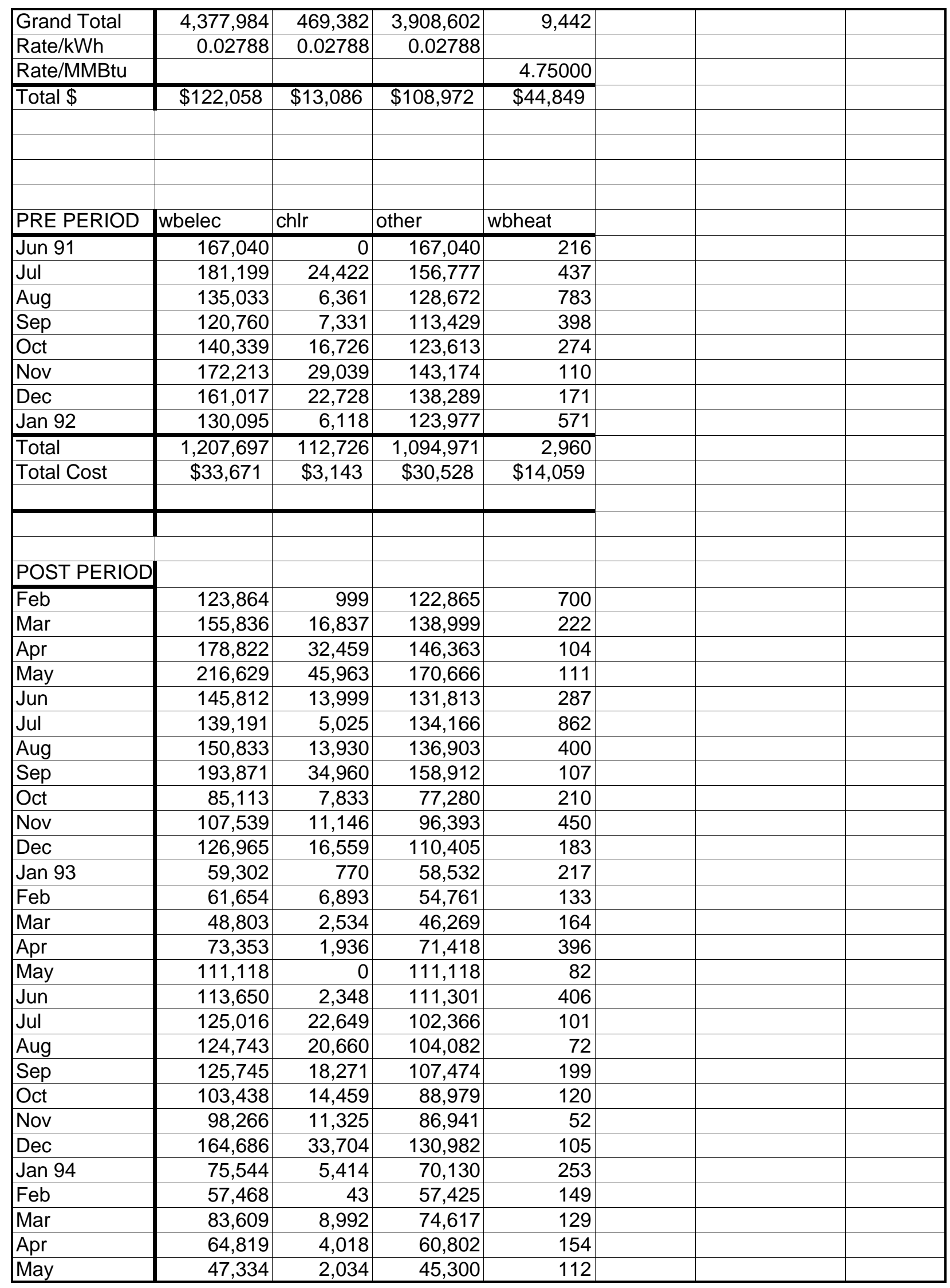


Tables

\begin{tabular}{|l|r|r|r|r|r|r|r|}
\hline Jun & 8,492 & 896 & 7,596 & 11 & & & \\
\hline Total & $3,171,513$ & 356,656 & $2,814,857$ & 6,483 & & & \\
\hline Total Cost & $\$ 88,422$ & $\$ 9,944$ & $\$ 78,478$ & $\$ 30,795$ & & & \\
\hline & & & & & & & \\
\hline & & & & & & & \\
\hline & & & & & & & \\
\hline Grand Total & $4,379,210$ & 469,382 & $3,909,828$ & 9,443 & & & \\
\hline Total Cost & $\$ 122,092$ & $\$ 13,086$ & $\$ 109,006$ & $\$ 44,854$ & & & \\
\hline & & & & & & & \\
\hline
\end{tabular}

Page 3 
Tables

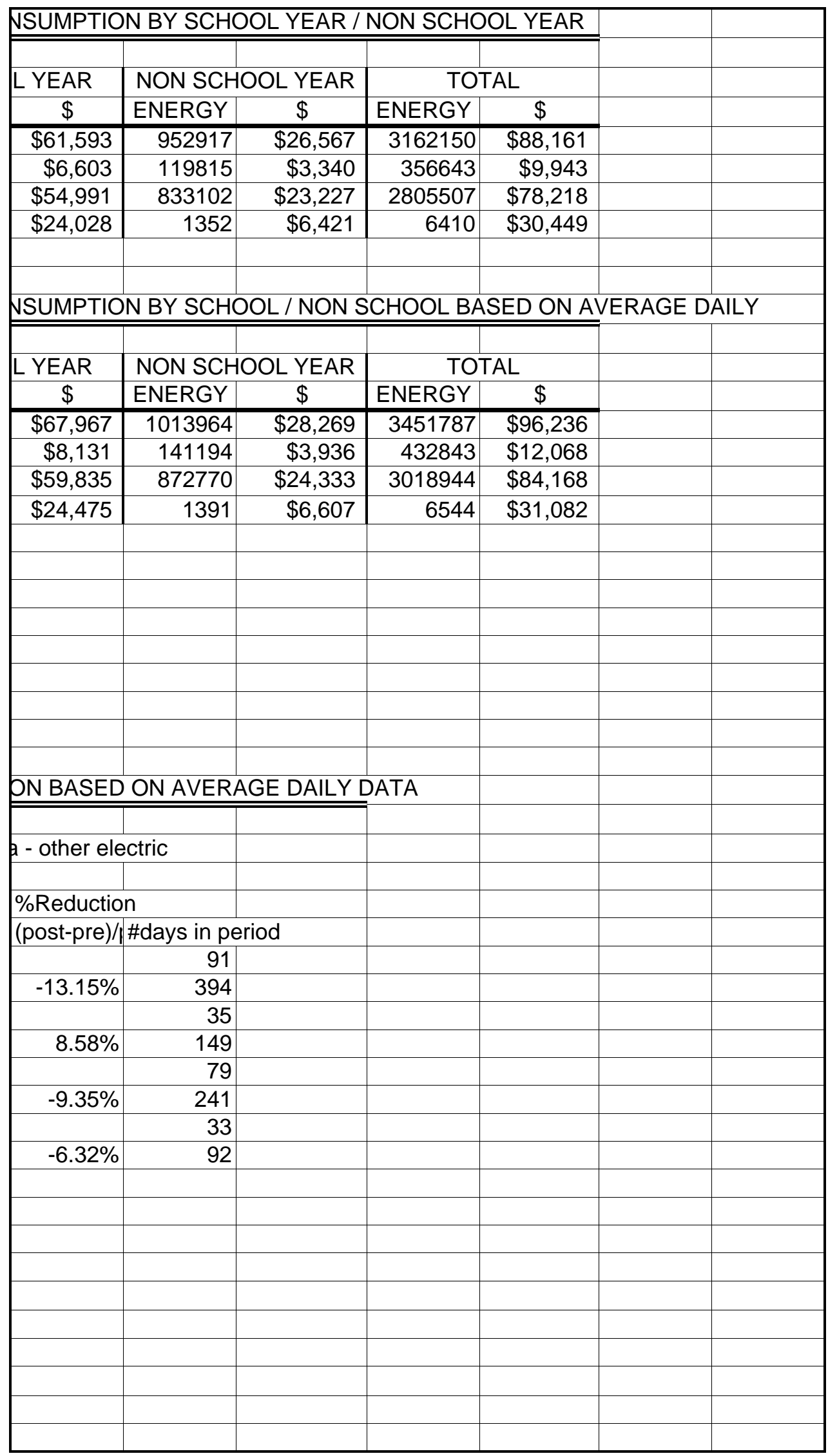



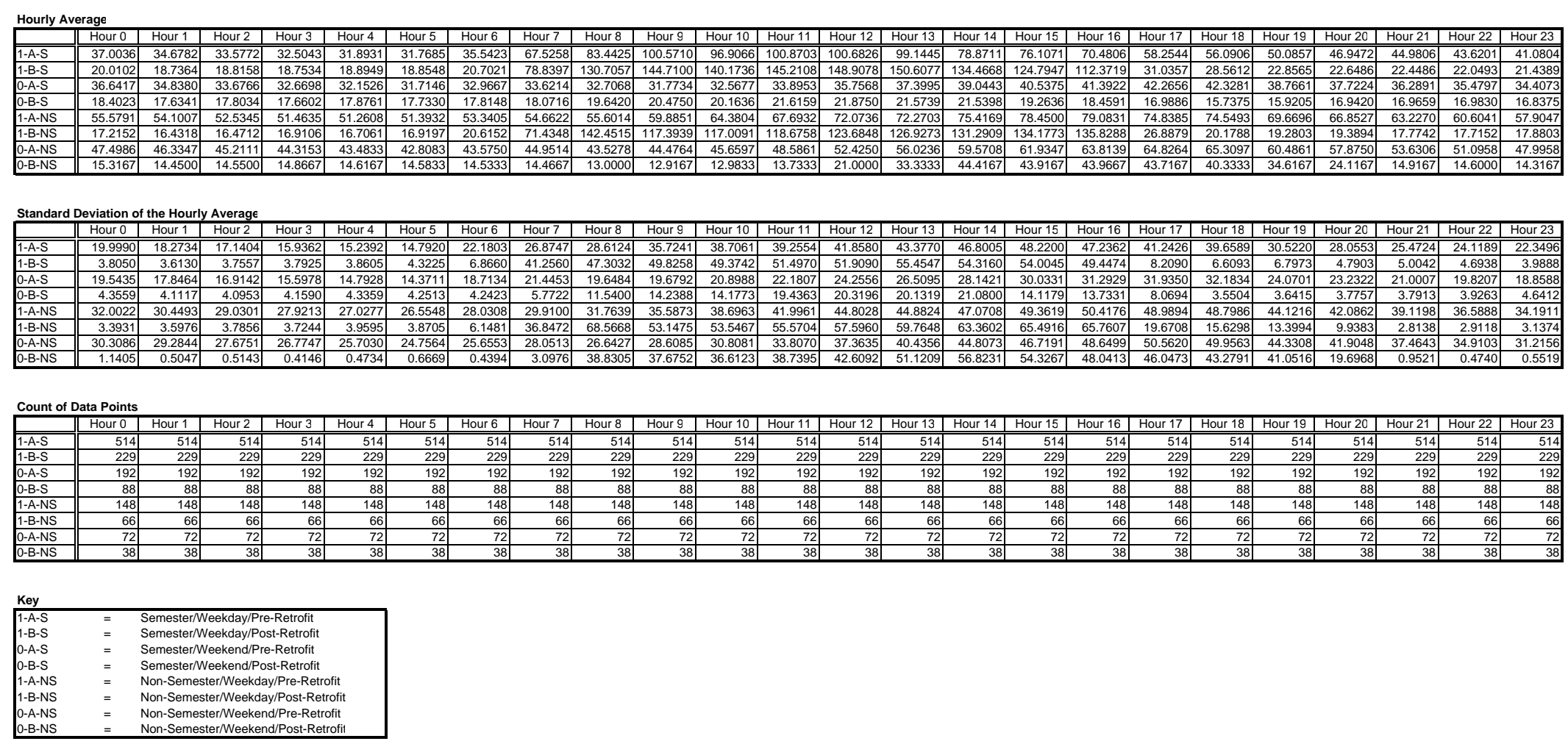

Hourly Sum (sort "Category")

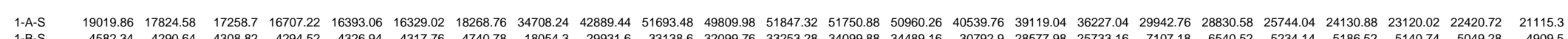

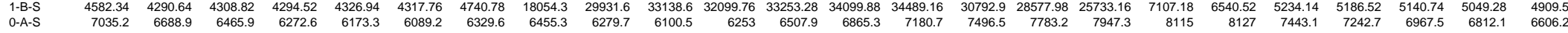

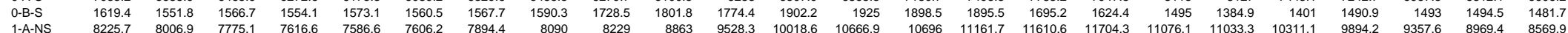

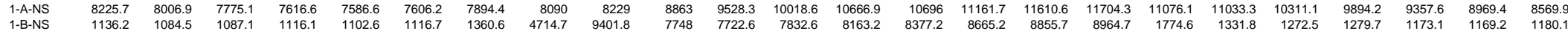

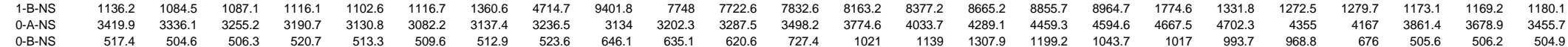




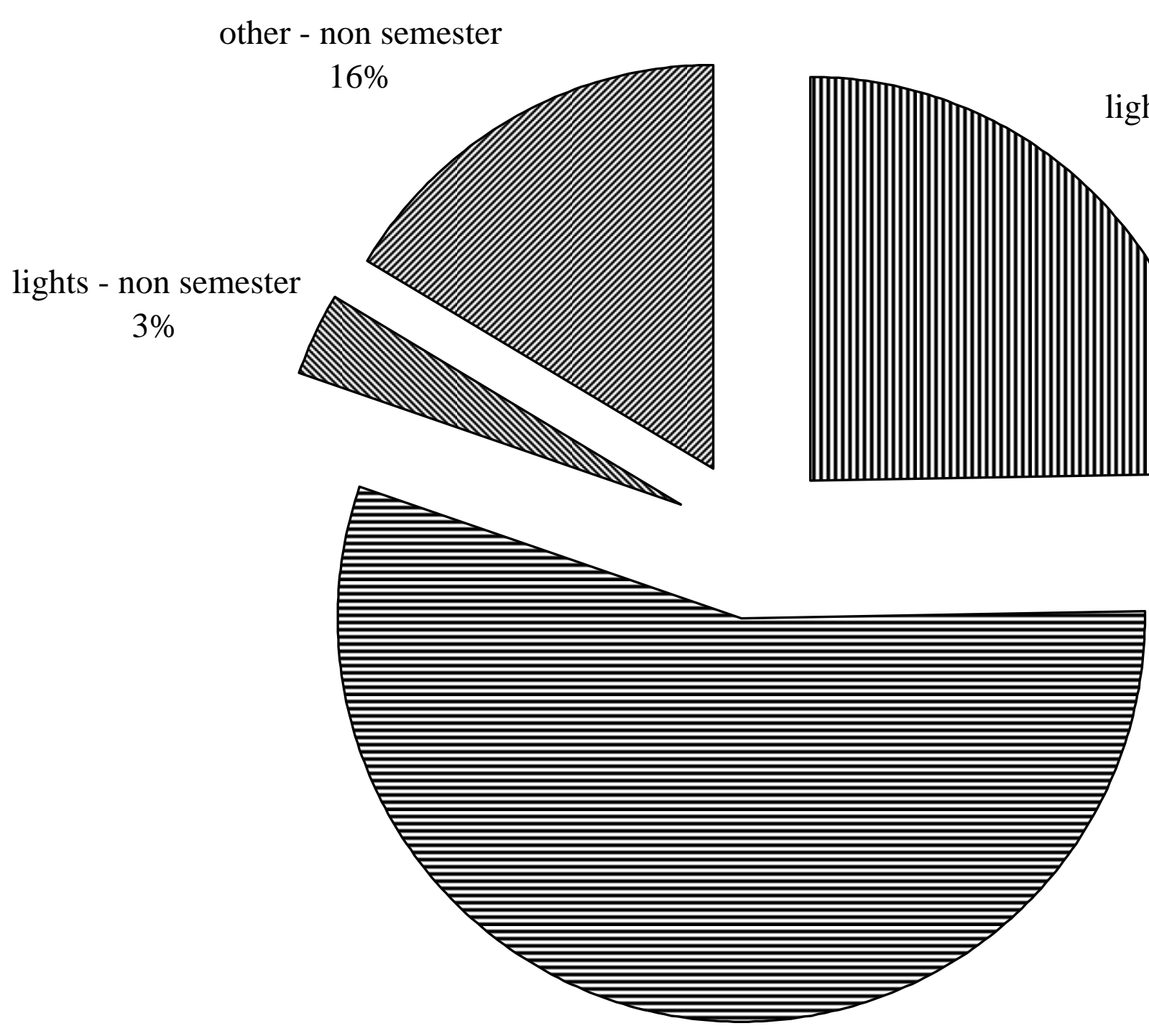

lights - semester $25 \%$

other - semester

$56 \%$ 


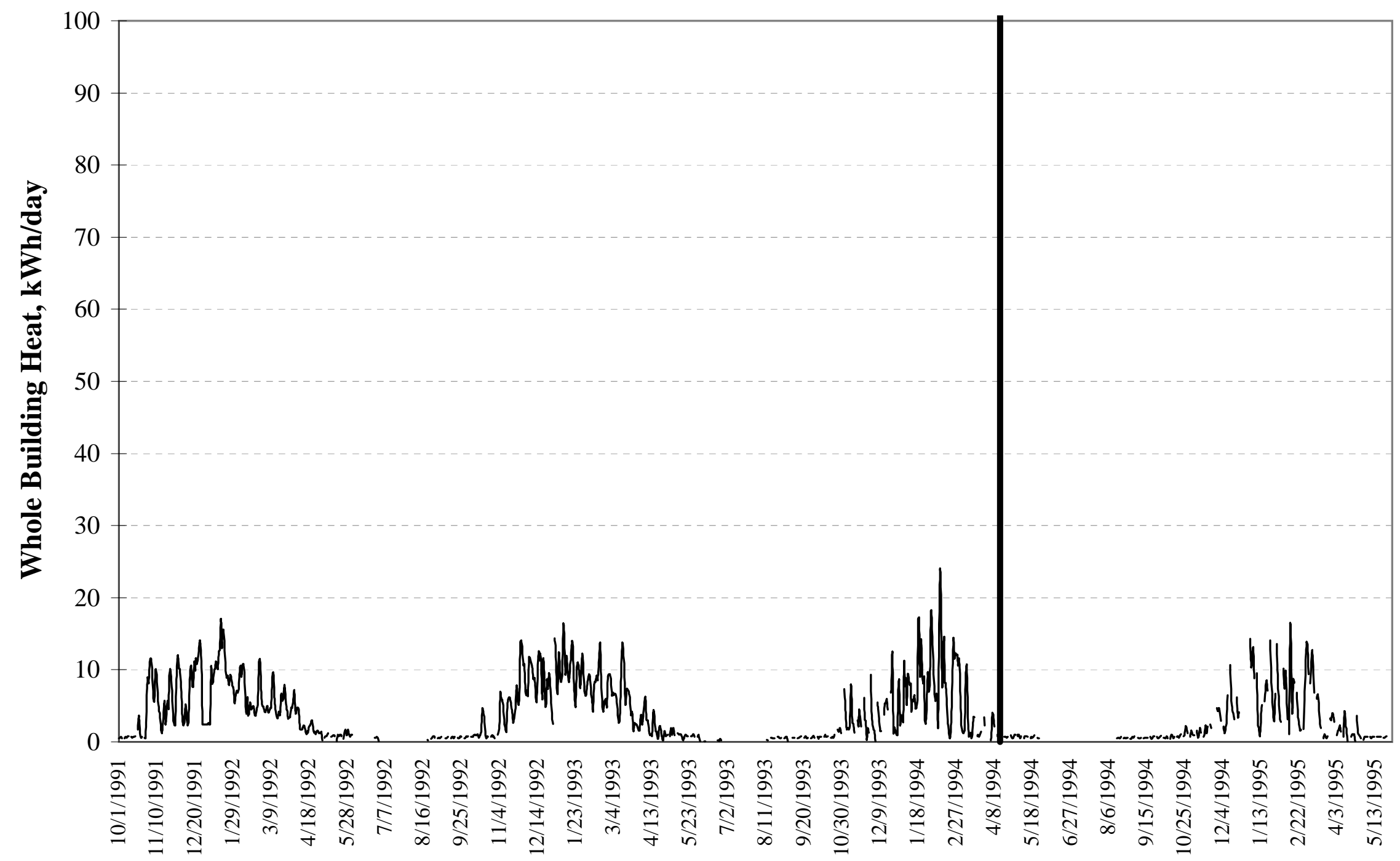




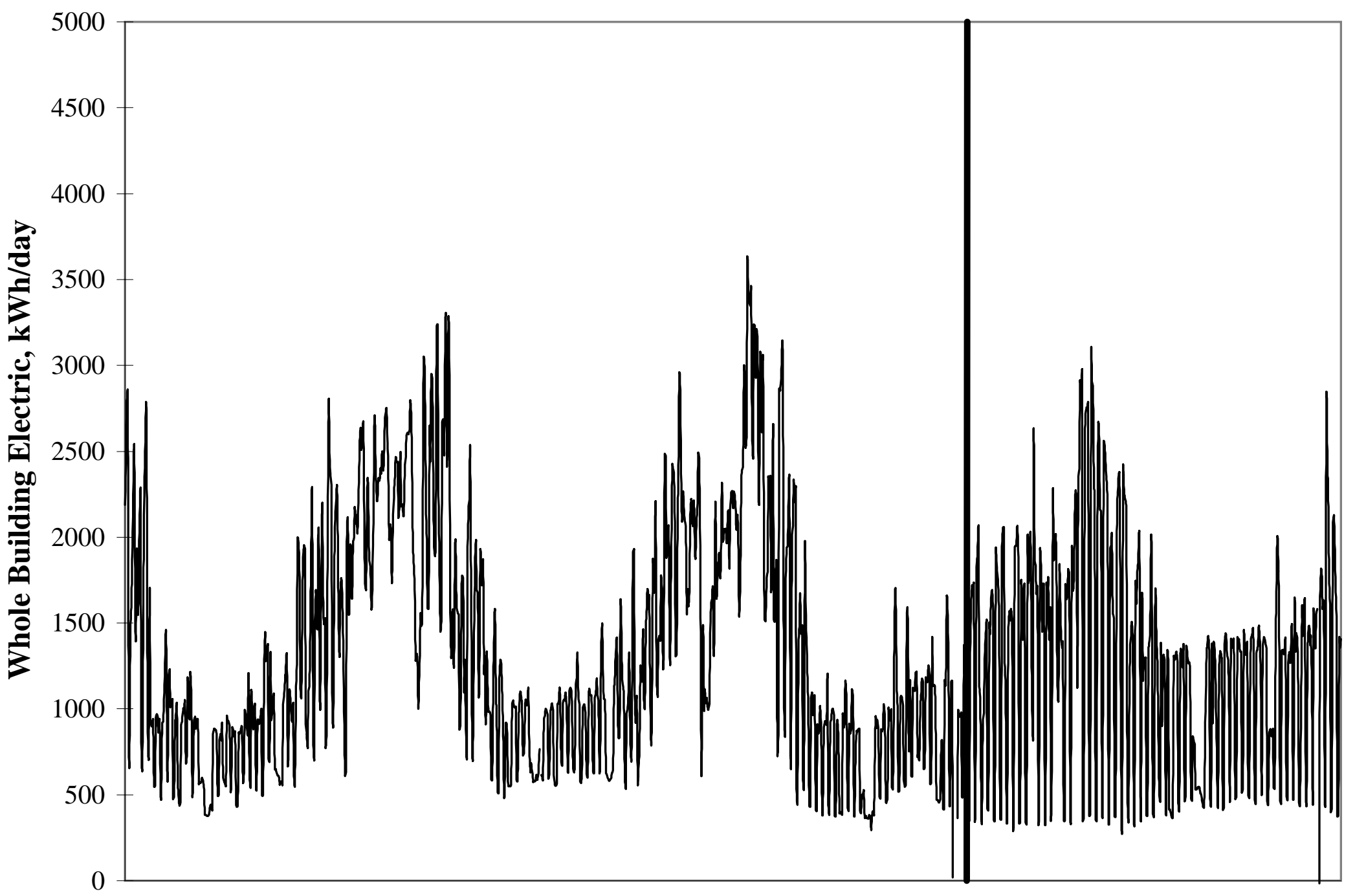




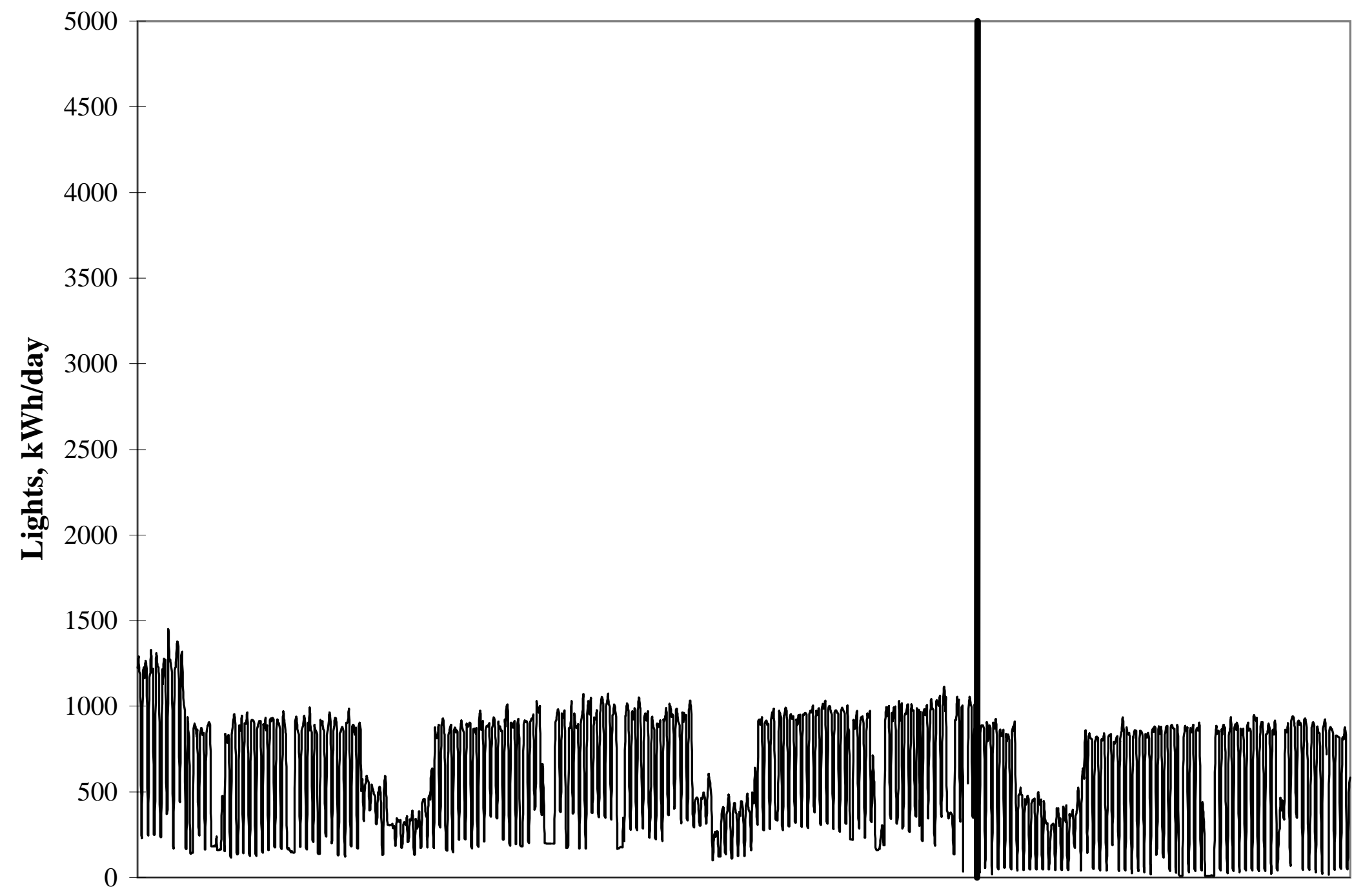

Page 1 


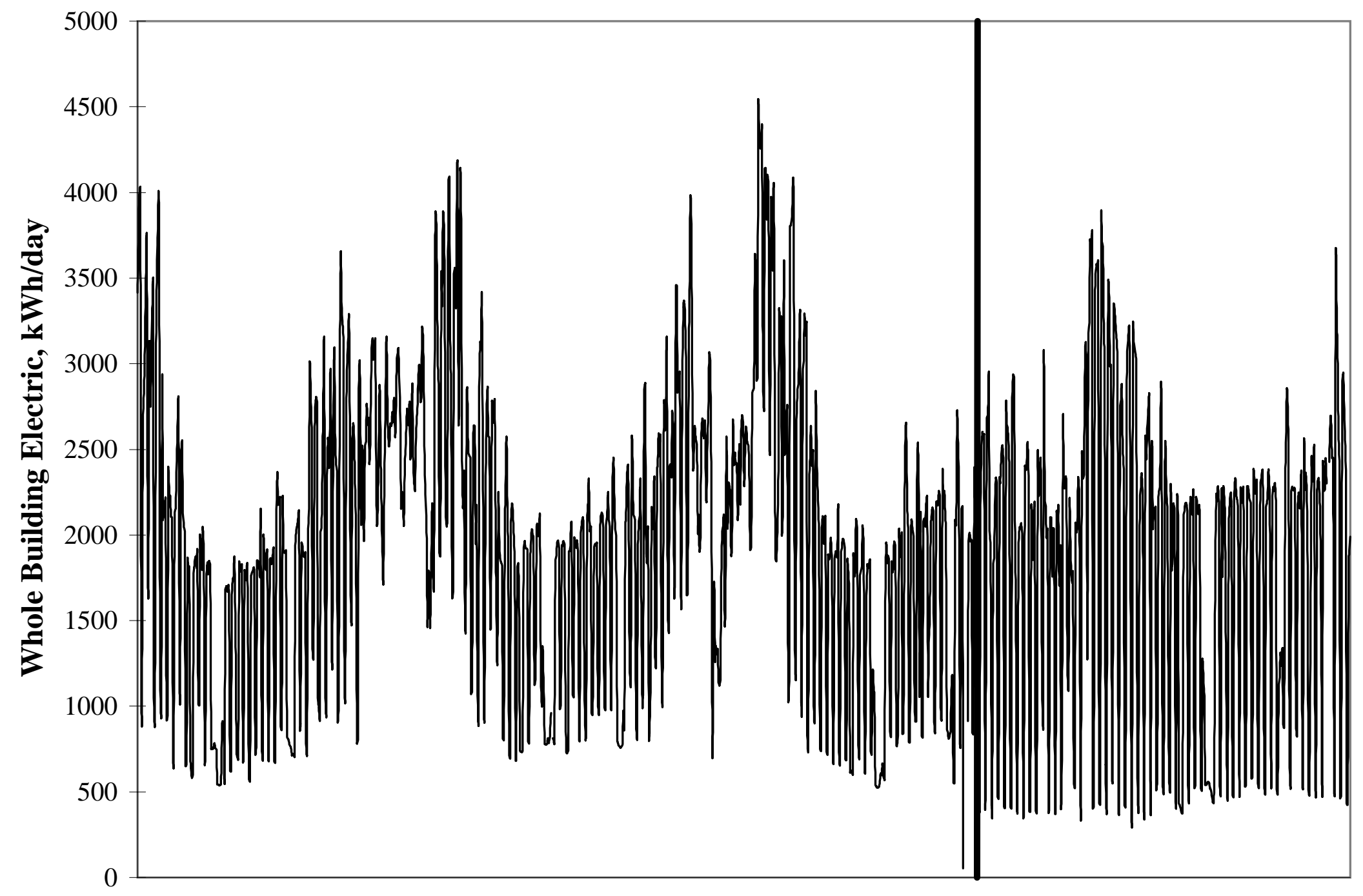




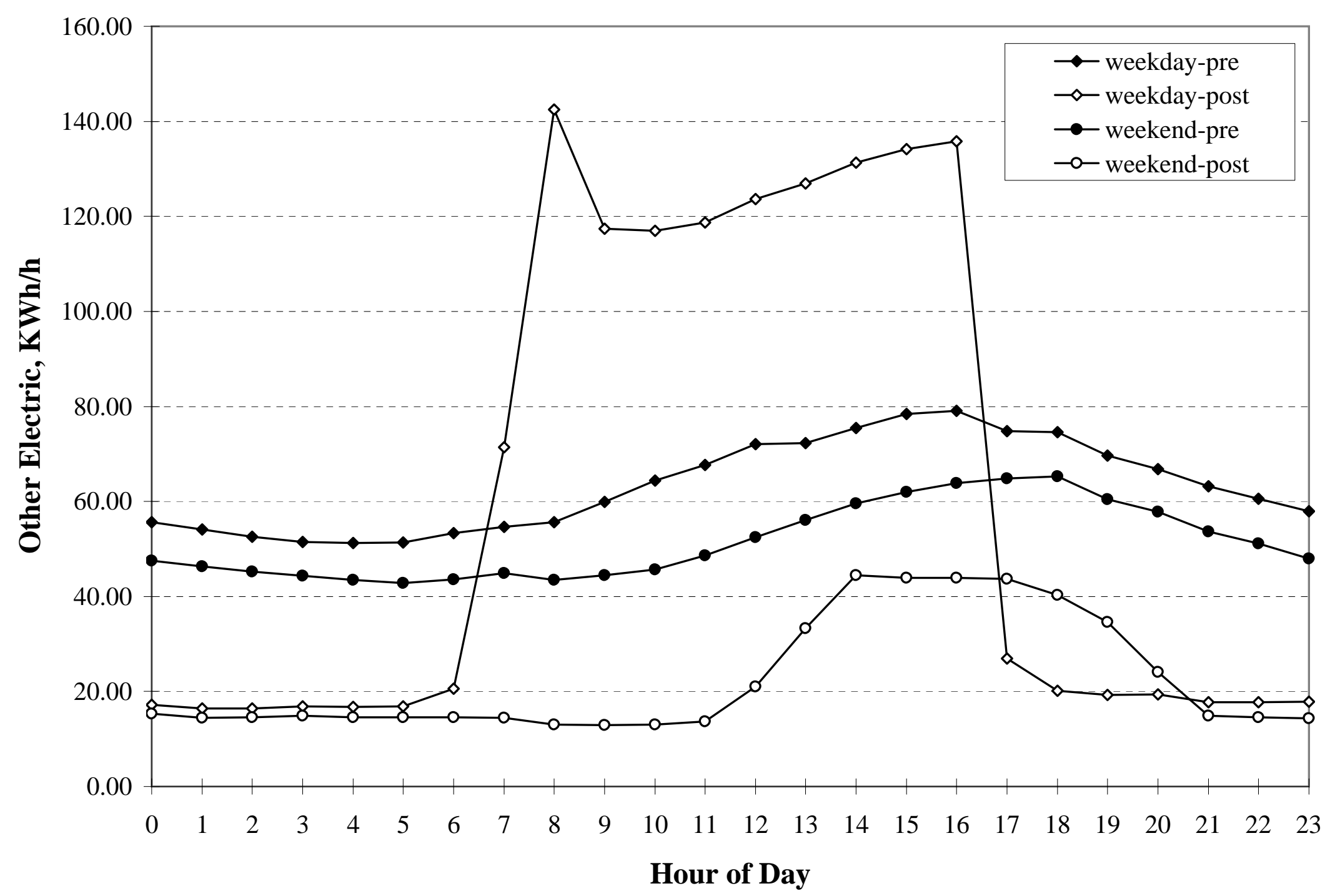

Page 1 


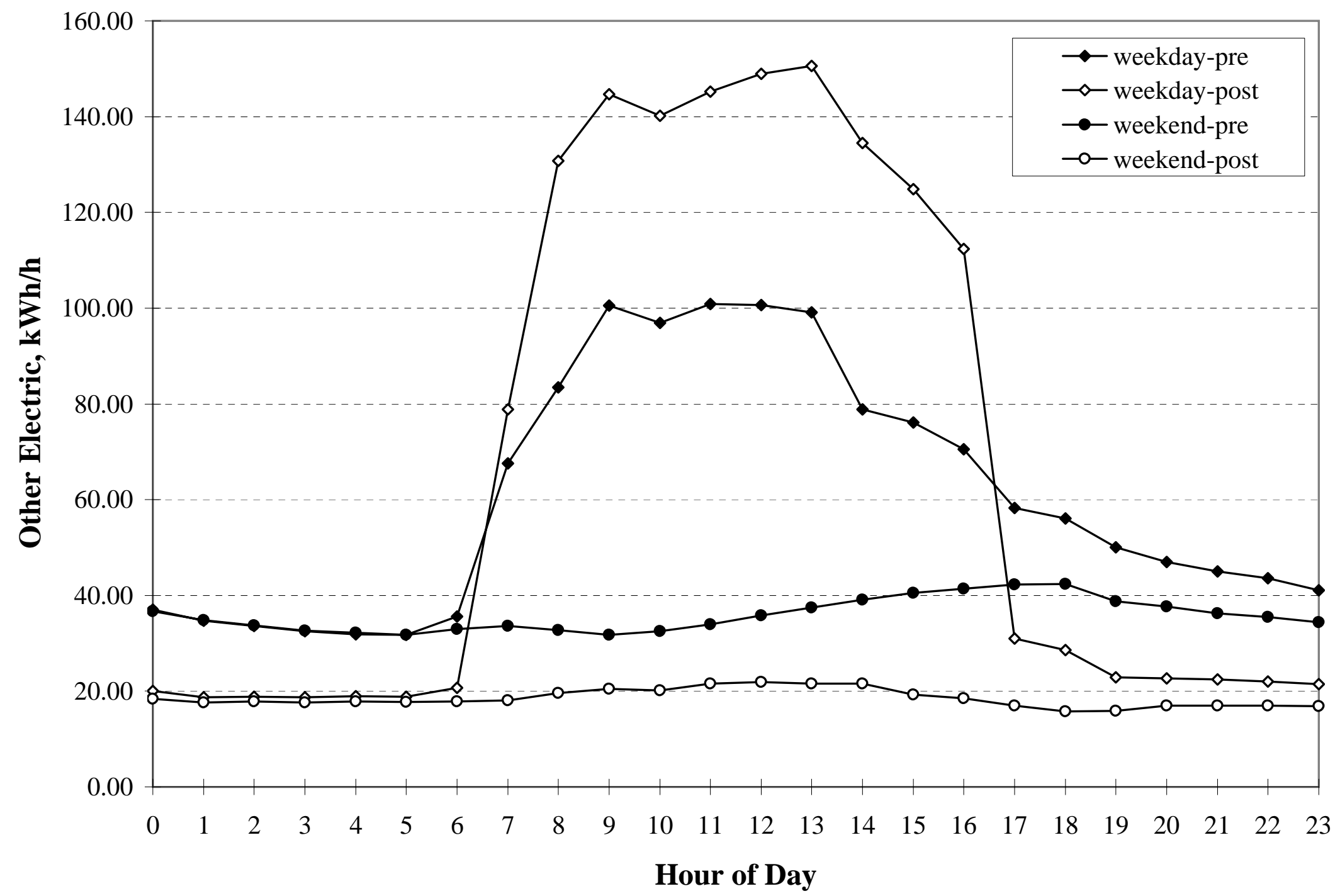

Page 1 


\begin{tabular}{|c|c|c|c|c|c|c|c|}
\hline \multicolumn{8}{|c|}{ AVERAGE DAILY WBE } \\
\hline & & 0 & 1 & 2 & 3 & 4 & 5 \\
\hline & $1-A-S$ & 56.79 & 47.65 & 46.26 & 44.73 & 43.89 & 43.69 \\
\hline & 1-B-S & 36.28 & 22.03 & 22.12 & 22.09 & 22.16 & 22.14 \\
\hline & $0-A-S$ & 52.59 & 47.25 & 45.92 & 44.61 & 44.08 & 43.67 \\
\hline & $0-B-S$ & 30.30 & 21.21 & 21.40 & 21.36 & 21.68 & 21.68 \\
\hline & 1-A-NS & 67.07 & 64.72 & 63.12 & 61.96 & 61.77 & 61.88 \\
\hline & 1-B-NS & 25.40 & 19.61 & 19.69 & 20.23 & 20.10 & 20.25 \\
\hline & O-A-NS & 57.53 & 55.96 & 54.77 & 53.72 & 52.94 & 52.17 \\
\hline & $0-B-N S$ & 21.20 & 17.85 & 17.85 & 18.27 & 17.92 & 17.88 \\
\hline & Total S & 175.95 & 138.13 & 135.70 & 132.79 & 131.81 & 131.18 \\
\hline & Total NS & 171.20 & 158.14 & 155.42 & 154.17 & 152.73 & 152.19 \\
\hline & Total S-B & 66.57 & 43.23 & 43.51 & 43.45 & 43.83 & 43.82 \\
\hline & Total NS-B & 46.60 & 37.46 & 37.54 & 38.50 & 38.02 & 38.13 \\
\hline & Total S-A & 109.38 & 94.89 & 92.18 & 89.34 & 87.97 & 87.36 \\
\hline & Total NS-A & 124.60 & 120.69 & 117.88 & 115.68 & 114.71 & 114.05 \\
\hline & & & & & & & \\
\hline & & & & & & & \\
\hline \multicolumn{8}{|c|}{ AVERAGE DAILY LIGHTS } \\
\hline & & 0 & 1 & 2 & 3 & 4 & 5 \\
\hline & $1-A-S$ & 19.50 & 12.70 & 12.42 & 11.97 & 11.75 & 11.67 \\
\hline & 1-B-S & 16.27 & 3.29 & 3.30 & 3.33 & 3.26 & 3.20 \\
\hline & $0-A-S$ & 15.56 & 11.85 & 11.71 & 11.60 & 11.59 & 11.62 \\
\hline & $0-B-S$ & 11.47 & 3.17 & 3.18 & 3.29 & 3.38 & 3.54 \\
\hline & 1-A-NS & 11.11 & 10.26 & 10.23 & 10.15 & 10.16 & 10.14 \\
\hline & 1-B-NS & 8.19 & 3.17 & 3.22 & 3.32 & 3.40 & 3.33 \\
\hline & O-A-NS & 10.04 & 9.63 & 9.56 & 9.40 & 9.45 & 9.37 \\
\hline & 0-B-NS & 5.88 & 3.40 & 3.30 & 3.40 & 3.30 & 3.30 \\
\hline & Total S & 62.79 & 31.01 & 30.61 & 30.19 & 29.99 & 30.04 \\
\hline & Total NS & 35.21 & 26.46 & 26.30 & 26.27 & 26.32 & 26.14 \\
\hline & Total S-B & 27.73 & 6.45 & 6.48 & 6.62 & 6.65 & 6.74 \\
\hline & Total NS-B & 14.07 & 6.57 & 6.52 & 6.72 & 6.70 & 6.63 \\
\hline & Total S-A & 35.06 & 24.55 & 24.13 & 23.57 & 23.34 & 23.30 \\
\hline & Total NS-A & 21.14 & 19.88 & 19.78 & 19.55 & 19.62 & 19.50 \\
\hline & & & & & & & \\
\hline & & & & & & & \\
\hline \multicolumn{8}{|c|}{ AVERAGE DAILY OTHER } \\
\hline & & 0 & 1 & 2 & 3 & 4 & 5 \\
\hline & $1-A-S$ & 37.00 & 34.68 & 33.58 & 32.50 & 31.89 & 31.77 \\
\hline & 1-B-S & 20.01 & 18.74 & 18.82 & 18.75 & 18.89 & 18.85 \\
\hline & $0-A-S$ & 36.64 & 34.84 & 33.68 & 32.67 & 32.15 & 31.71 \\
\hline & 0-B-S & 18.40 & 17.63 & 17.80 & 17.66 & 17.88 & 17.73 \\
\hline & 1-A-NS & 55.58 & 54.10 & 52.53 & 51.46 & 51.26 & 51.39 \\
\hline & 1-B-NS & 17.22 & 16.43 & 16.47 & 16.91 & 16.71 & 16.92 \\
\hline & 0-A-NS & 47.50 & 46.33 & 45.21 & 44.32 & 43.48 & 42.81 \\
\hline & 0-B-NS & 15.32 & 14.45 & 14.55 & 14.87 & 14.62 & 14.58 \\
\hline & Total S & 112.06 & 105.89 & 103.87 & 101.59 & 100.82 & 100.07 \\
\hline & Total NS & 135.61 & 131.32 & 128.77 & 127.56 & 126.07 & 125.70 \\
\hline & Total S-B & 38.41 & 36.37 & 36.62 & 36.41 & 36.77 & 36.59 \\
\hline & Total NS-B & 32.53 & 30.88 & 31.02 & 31.78 & 31.32 & 31.50 \\
\hline & Total S-A & 73.65 & 69.52 & 67.25 & 65.17 & 64.05 & 63.48 \\
\hline
\end{tabular}




\begin{tabular}{|c|c|c|c|c|c|c|c|c|}
\hline & & Total NS-A & 103.08 & 100.44 & 97.75 & 95.78 & 94.74 & 94.20 \\
\hline & & & & & & & & \\
\hline \multirow{2}{*}{\multicolumn{9}{|c|}{ AVERAGE DAILY WBHEAT }} \\
\hline & & & & & & & & \\
\hline & & & 0 & 1 & 2 & 3 & 4 & 5 \\
\hline & & $1-A-S$ & 0.26 & 0.29 & 0.28 & 0.30 & 0.31 & 0.35 \\
\hline & & 1-B-S & 0.07 & 0.06 & 0.10 & 0.10 & 0.07 & 0.17 \\
\hline & & $0-A-S$ & 0.26 & 0.27 & 0.27 & 0.29 & 0.29 & 0.30 \\
\hline & & 0-B-S & 0.00 & 0.00 & 0.00 & 0.00 & 0.10 & 0.10 \\
\hline & & 1-A-NS & 0.27 & 0.27 & 0.27 & 0.28 & 0.28 & 0.31 \\
\hline & & 1-B-NS & 0.00 & 0.00 & 0.00 & 0.00 & 0.00 & 0.00 \\
\hline & & 0-A-NS & 0.28 & 0.29 & 0.29 & 0.29 & 0.30 & 0.33 \\
\hline & & 0-B-NS & 0.00 & 0.00 & 0.00 & 0.00 & 0.00 & 0.00 \\
\hline & & Total S & 0.59 & 0.62 & 0.65 & 0.70 & 0.78 & 0.92 \\
\hline & & Total NS & 0.55 & 0.55 & 0.57 & 0.57 & 0.58 & 0.63 \\
\hline & & Total S-B & 0.07 & 0.06 & 0.10 & 0.10 & 0.17 & 0.27 \\
\hline & & Total NS-B & 0.00 & 0.00 & 0.00 & 0.00 & 0.00 & 0.00 \\
\hline & & Total S-A & 0.52 & 0.56 & 0.55 & 0.60 & 0.61 & 0.65 \\
\hline & & Total NS-A & 0.55 & 0.55 & 0.57 & 0.57 & 0.58 & 0.63 \\
\hline & & & & & & & & \\
\hline & & & & & & & & \\
\hline & & & & & & & & \\
\hline \multicolumn{9}{|c|}{ POST PERIOD CONSUMPTION } \\
\hline & & & & & & & & \\
\hline & $\begin{array}{l}\text { SCHOOL } \\
\text { YEAR }\end{array}$ & $\$$ & $\begin{array}{l}\text { NON } \\
\text { SCHOOL } \\
\text { YEAR }\end{array}$ & $\$$ & TOTAL & $\$$ & & \\
\hline WBELEC & 605714.9 & 40825.184 & 142509.5 & 9605.1403 & 748224.4 & 50430.32 & & \\
\hline LIGHTS & 196444.5 & 13240.359 & 27258.4 & 1837.2162 & 223702.9 & 15077.58 & & \\
\hline OTHER & 409270.4 & 27584.825 & 115251.1 & 7767.9241 & 524521.5 & 35352.75 & & \\
\hline WBHEAT & 588.046 & 2705.0116 & 49 & 225.4 & 637.046 & 2930.412 & & \\
\hline & & & & & & & & \\
\hline & & & & & & & & \\
\hline & & & & & & & & \\
\hline \multicolumn{9}{|c|}{ POST PERIOD PIE CHARTS } \\
\hline & & & & & & & & \\
\hline & & lights - s & other - s & lights - ns & other - ns & & & \\
\hline & & 292154 & 653202 & 40056 & 194162 & & & \\
\hline & & & & & & & & \\
\hline & & & & & & & & \\
\hline & & & & & & & & \\
\hline & & & & & & & & \\
\hline & & & & & & & & \\
\hline & & & & & & & & \\
\hline & & & & & & & & \\
\hline & & & & & & & & \\
\hline & & & & & & & & \\
\hline & & & & & & & & \\
\hline & & & & & & & & \\
\hline & & & & & & & & \\
\hline & & & & & & & & \\
\hline
\end{tabular}


CHART DATA

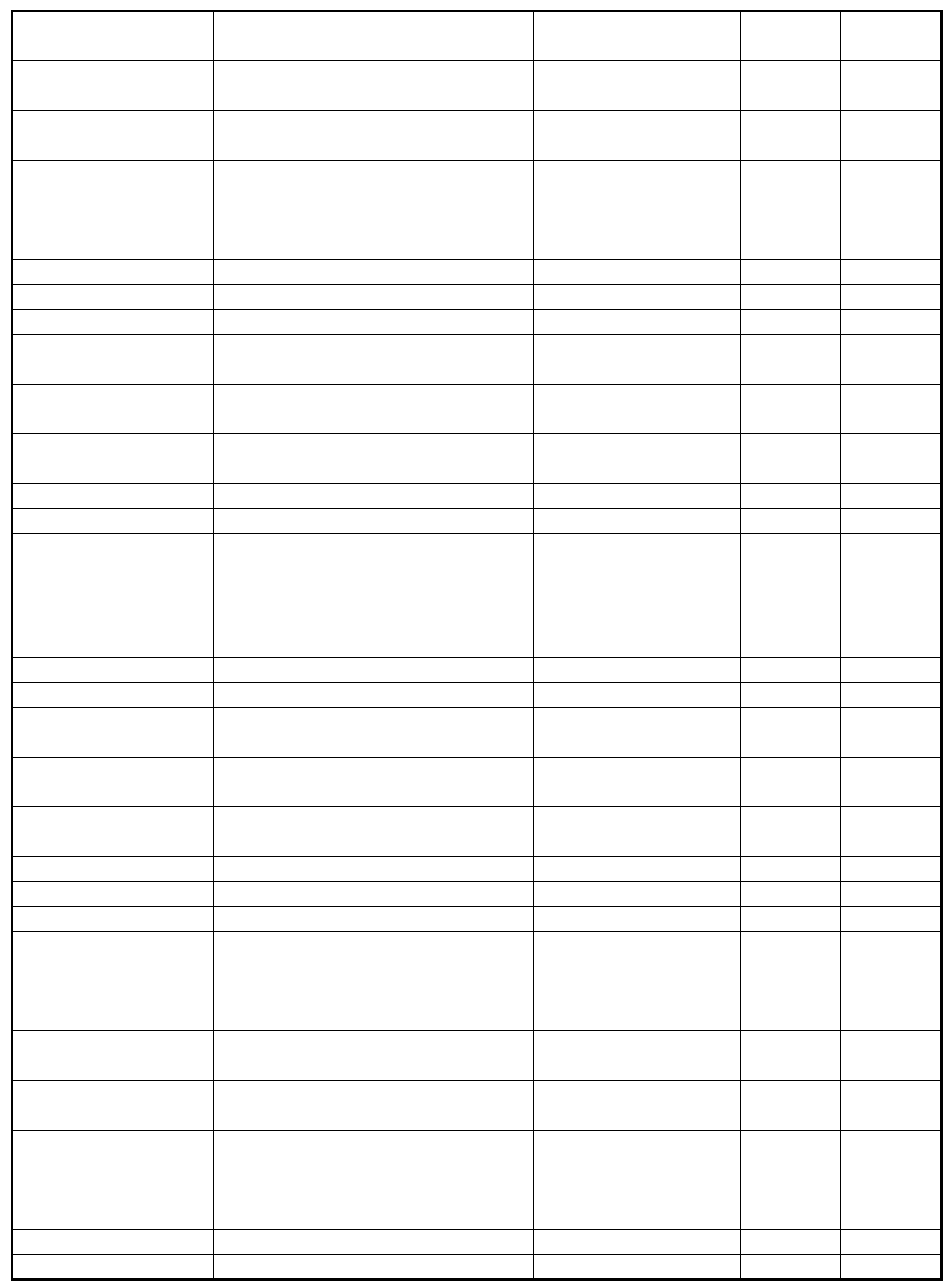

Page 3 
CHART DATA

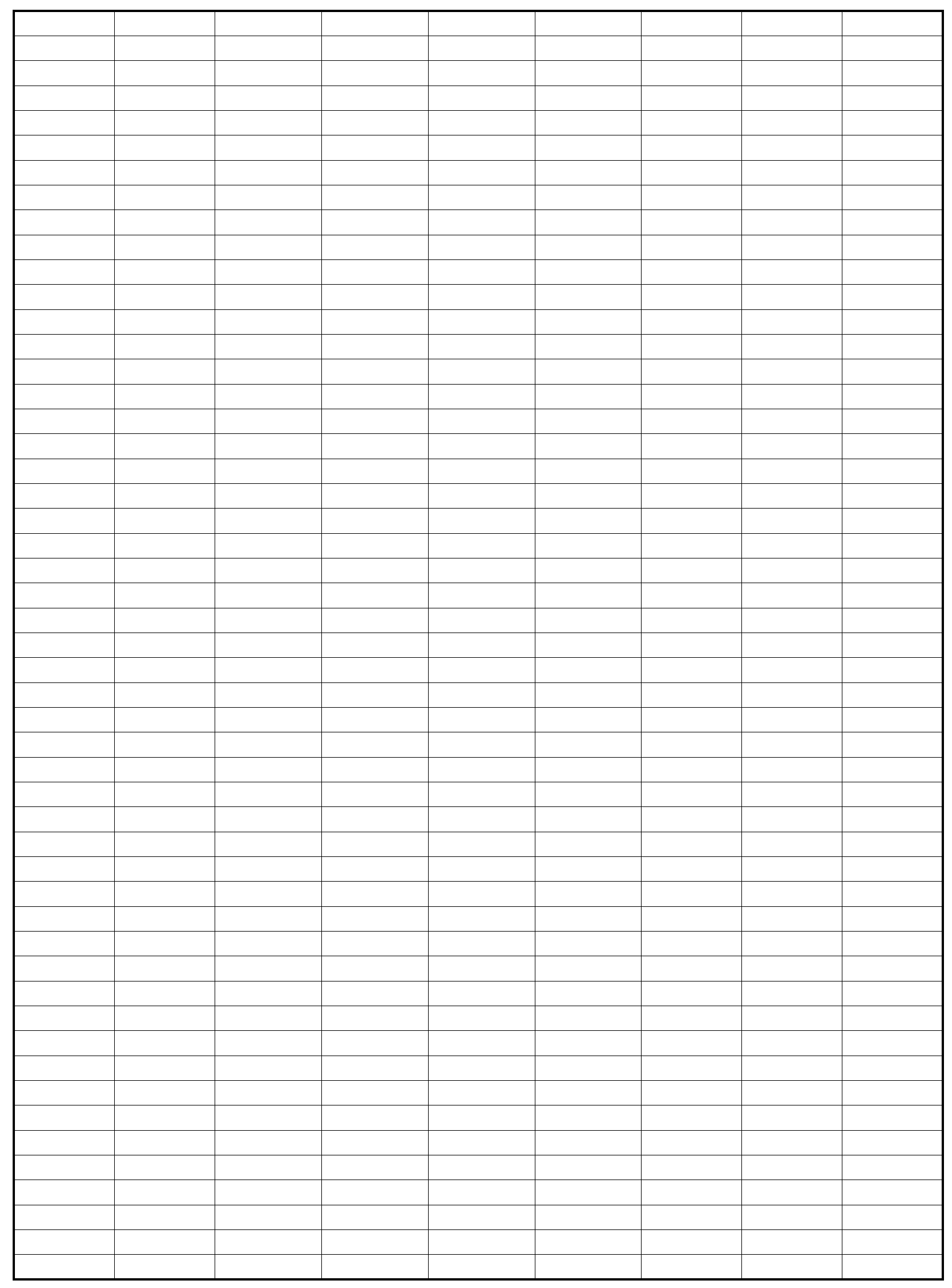

Page 4 
CHART DATA

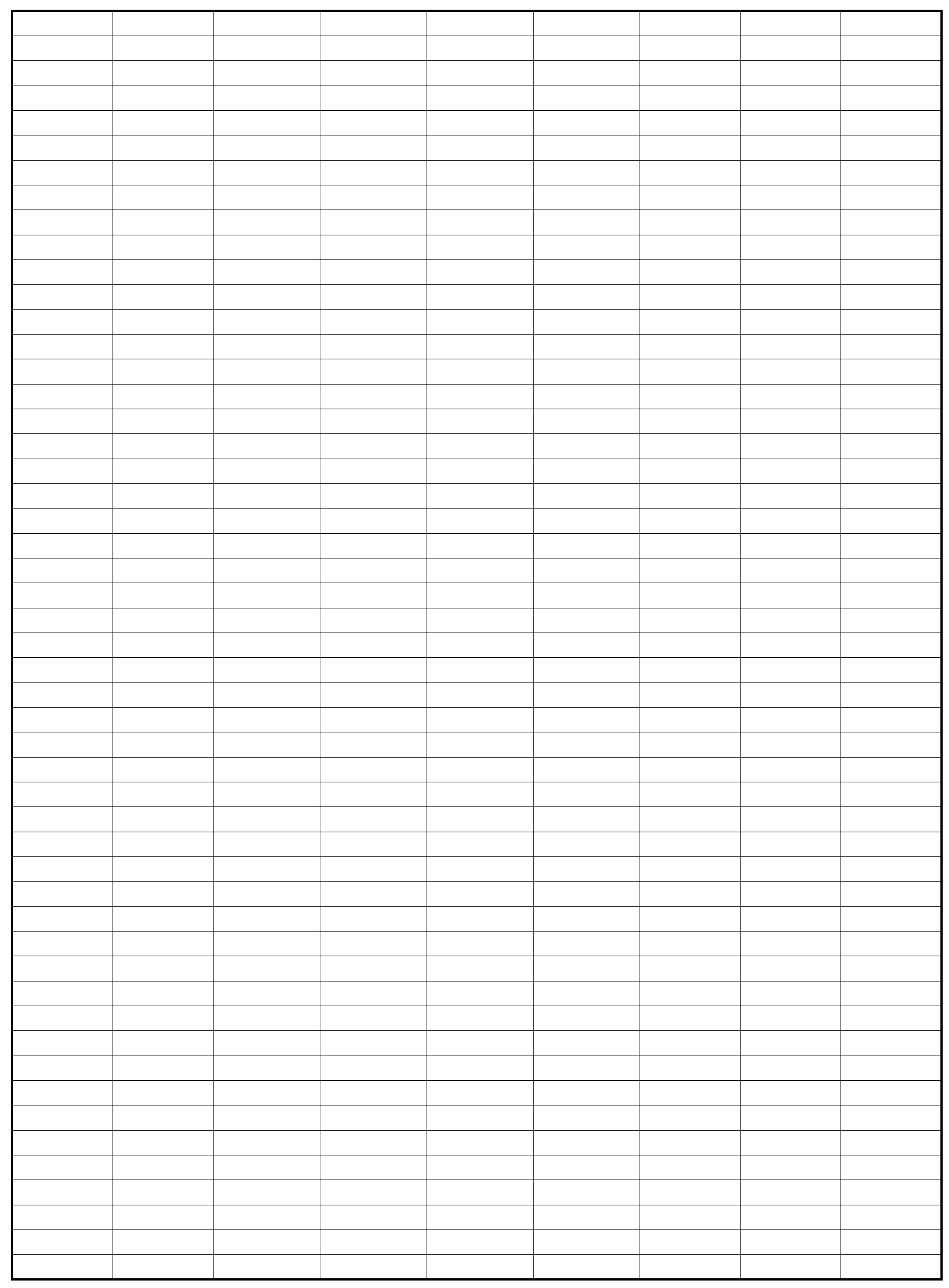

Page 5 
CHART DATA

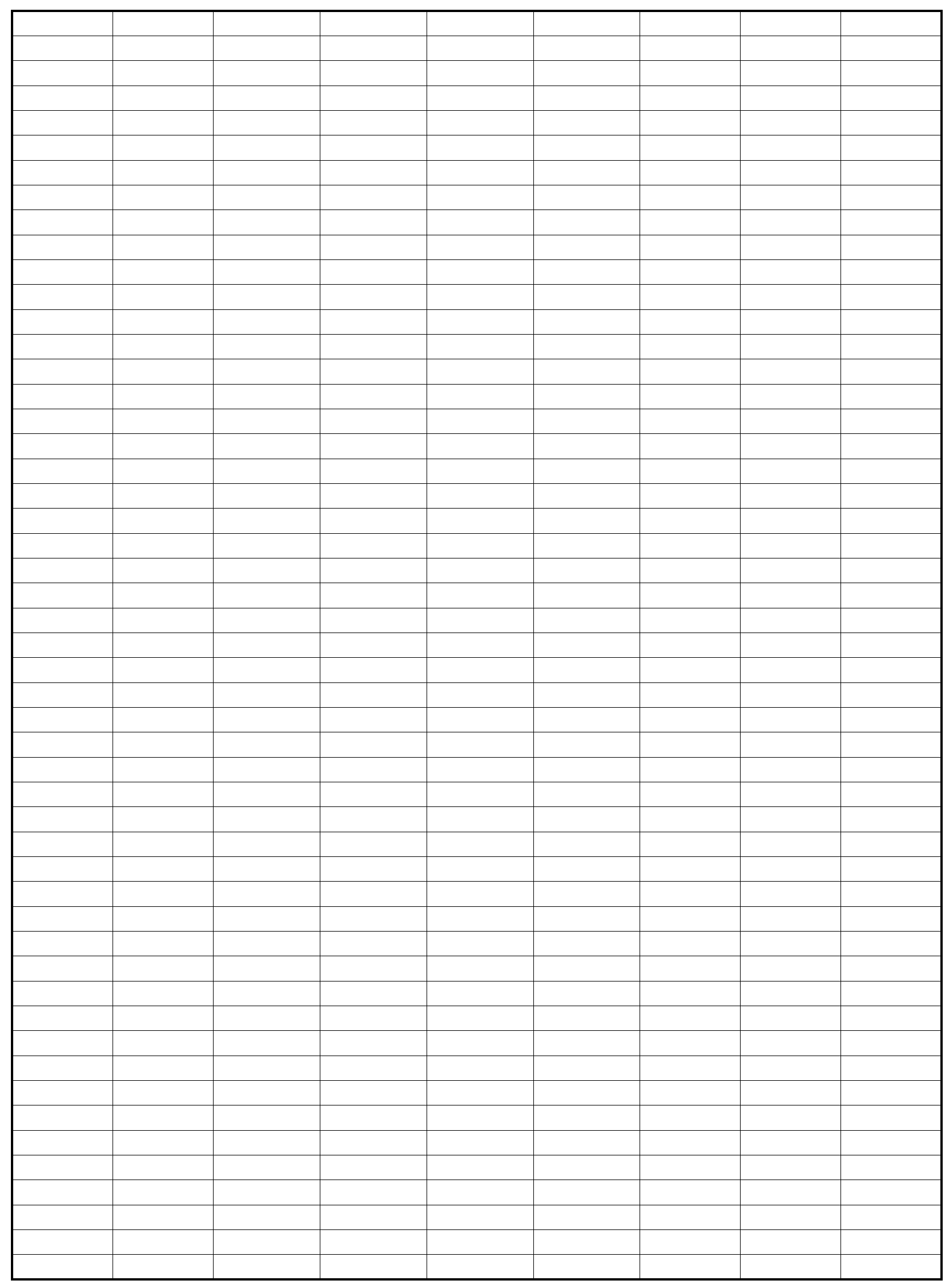

Page 6 
CHART DATA

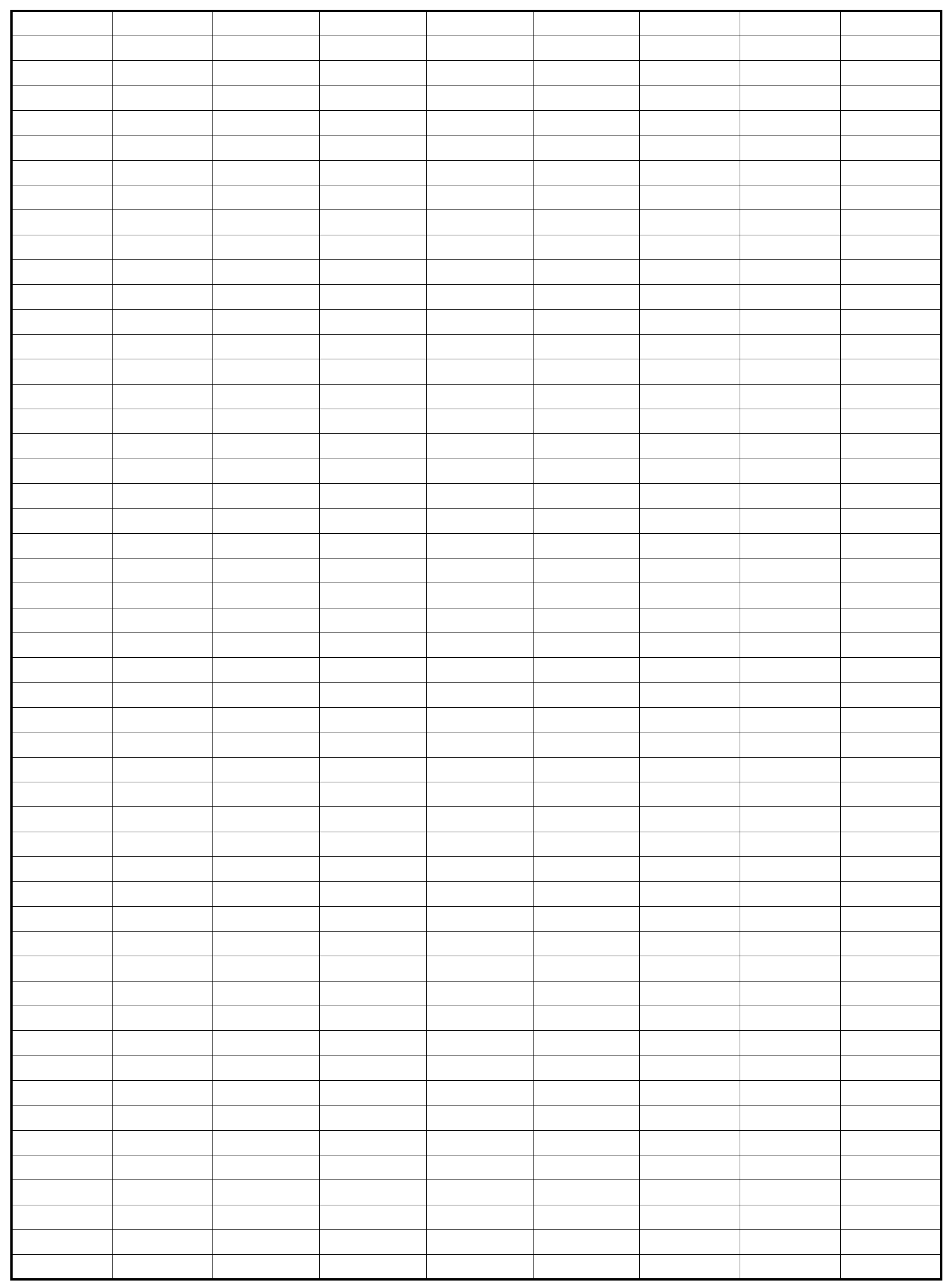

Page 7 
CHART DATA

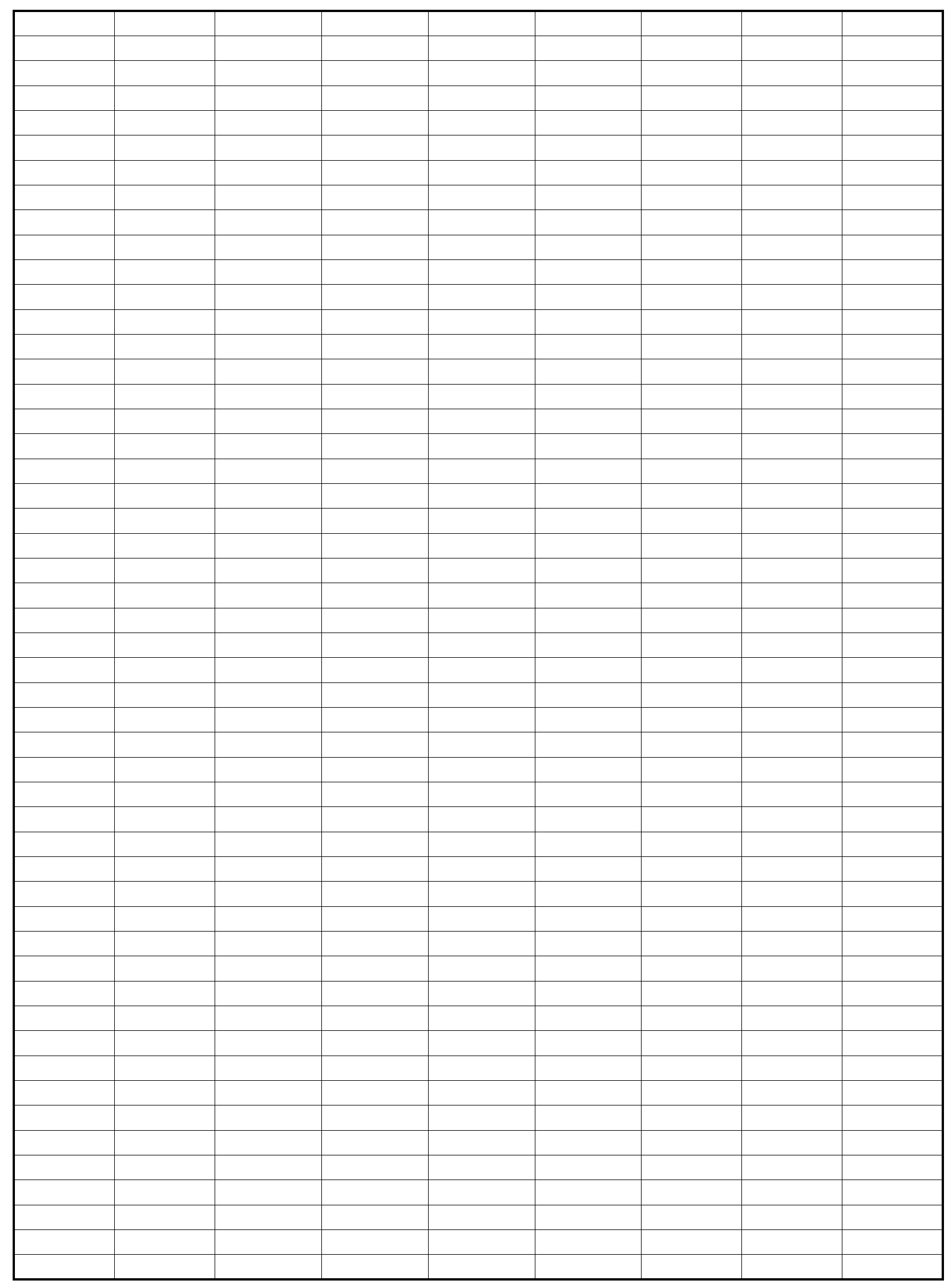

Page 8 
CHART DATA

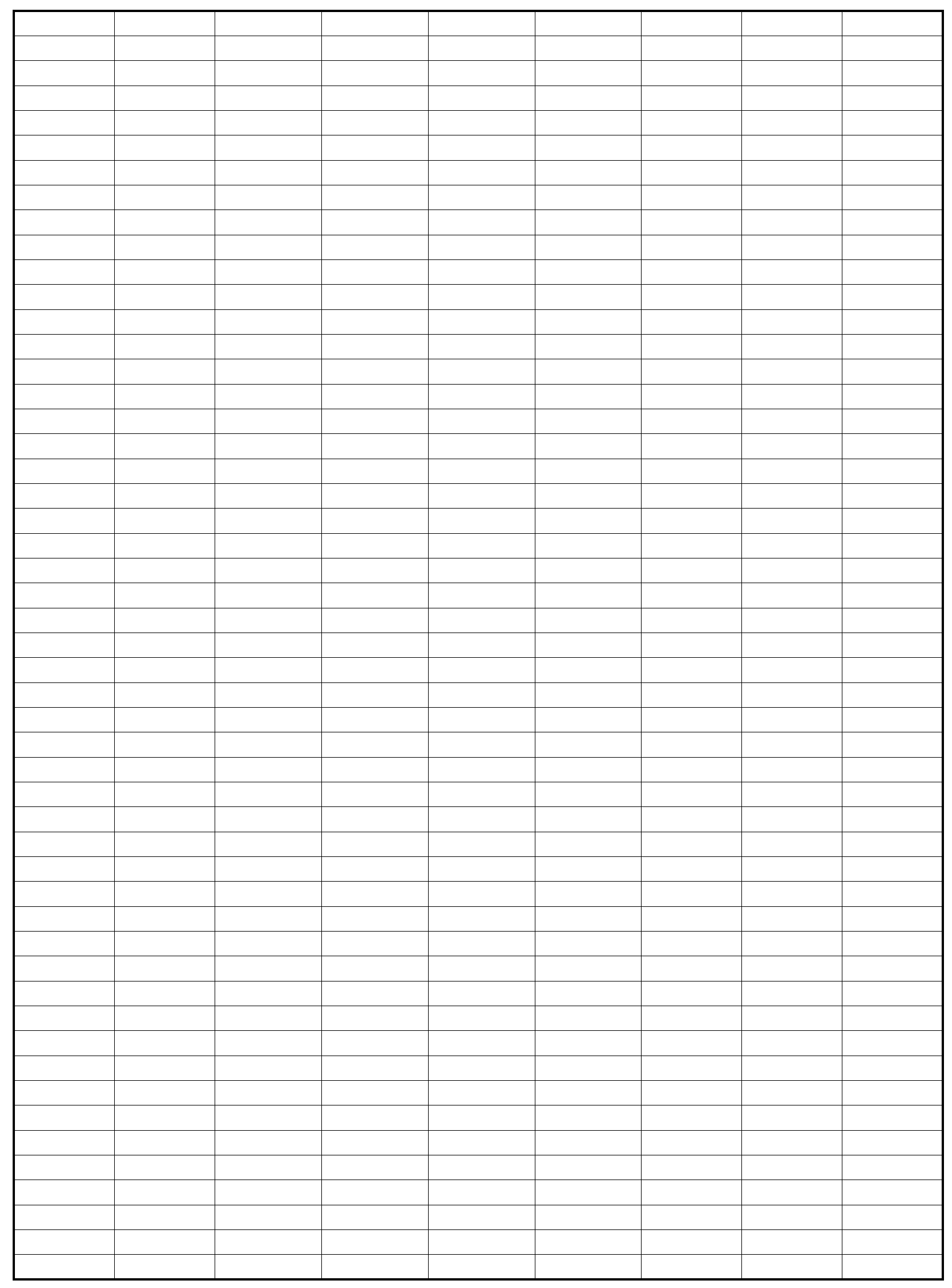

Page 9 
CHART DATA

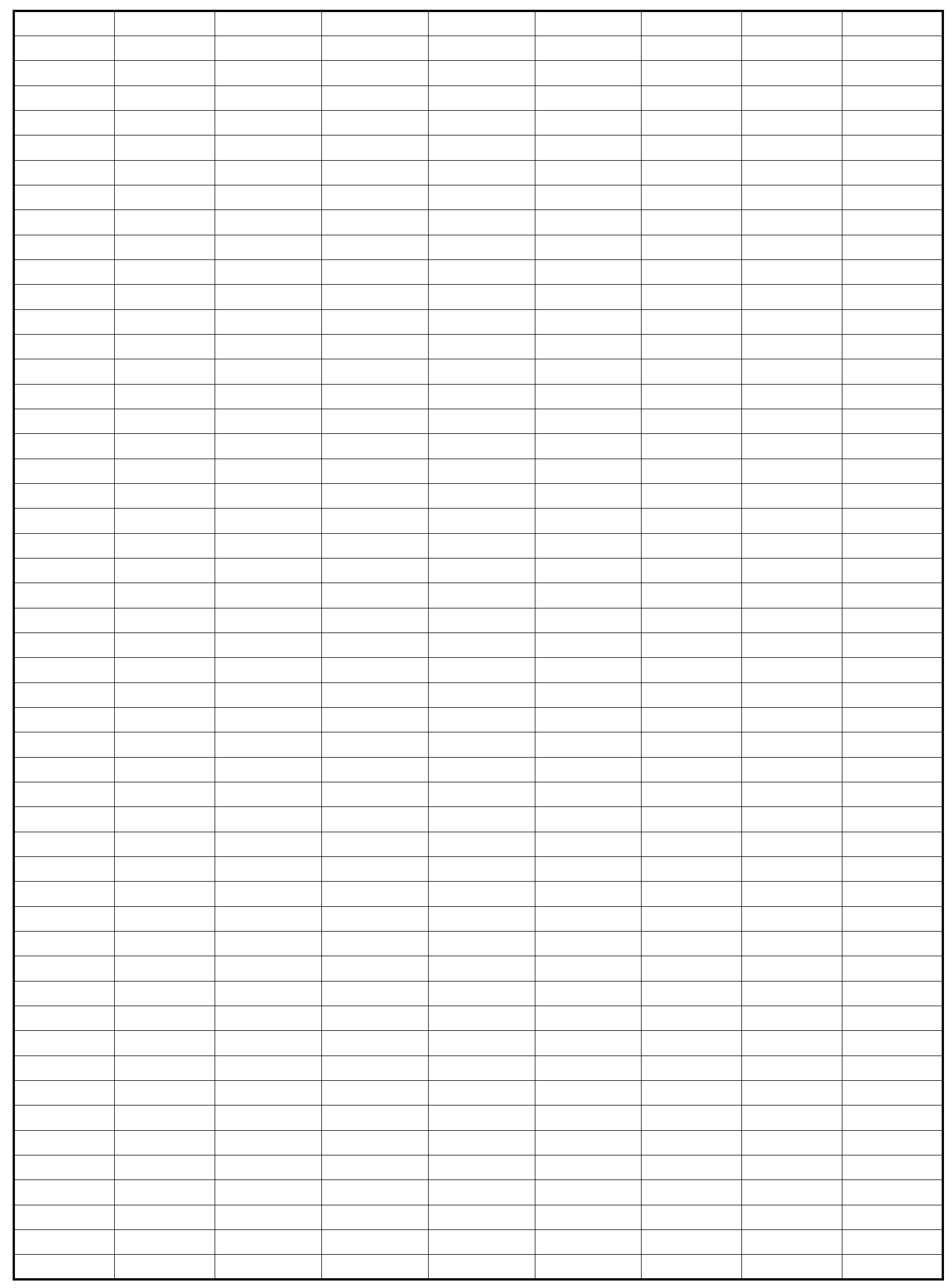

Page 10 
CHART DATA

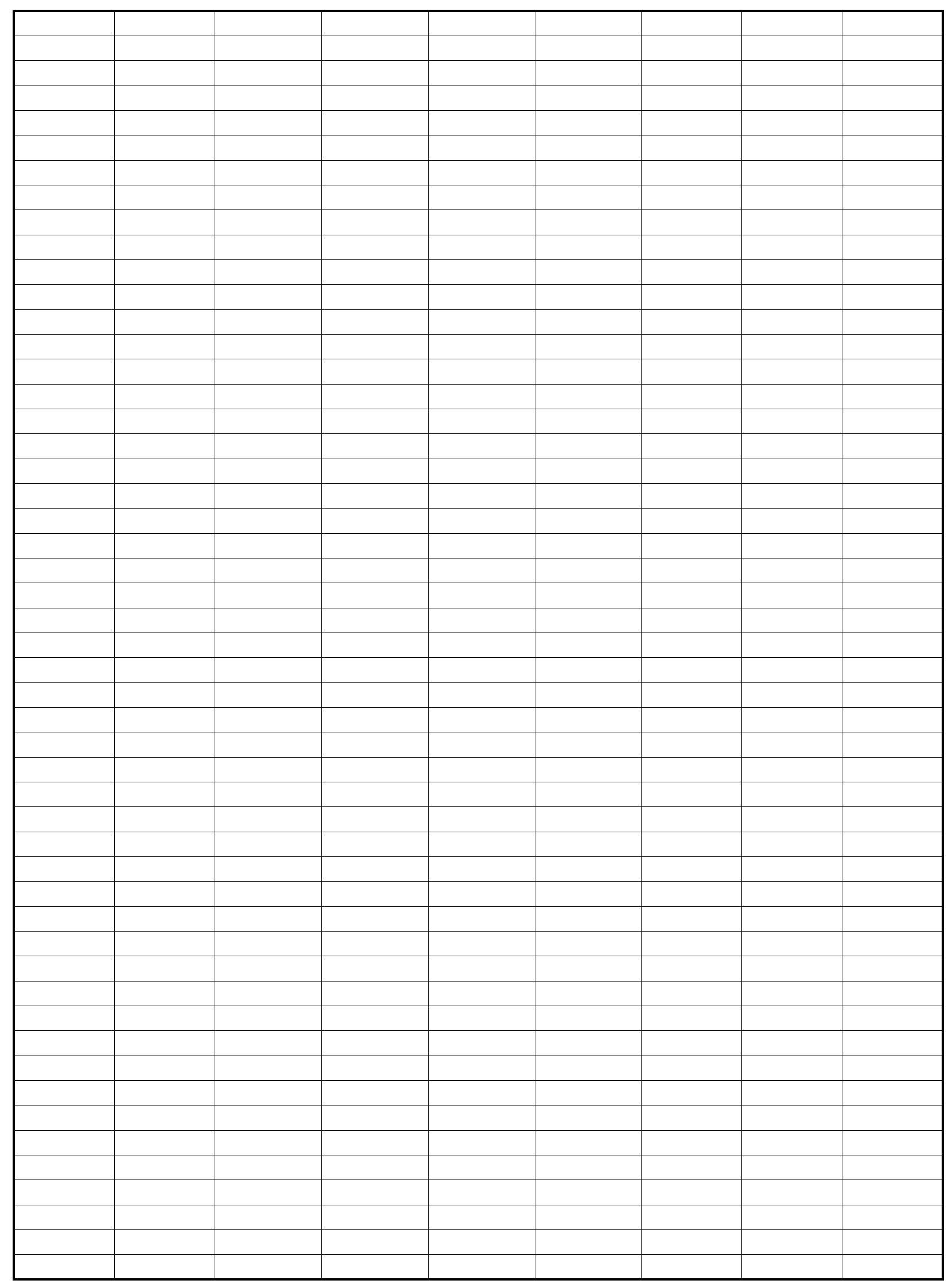

Page 11 
CHART DATA

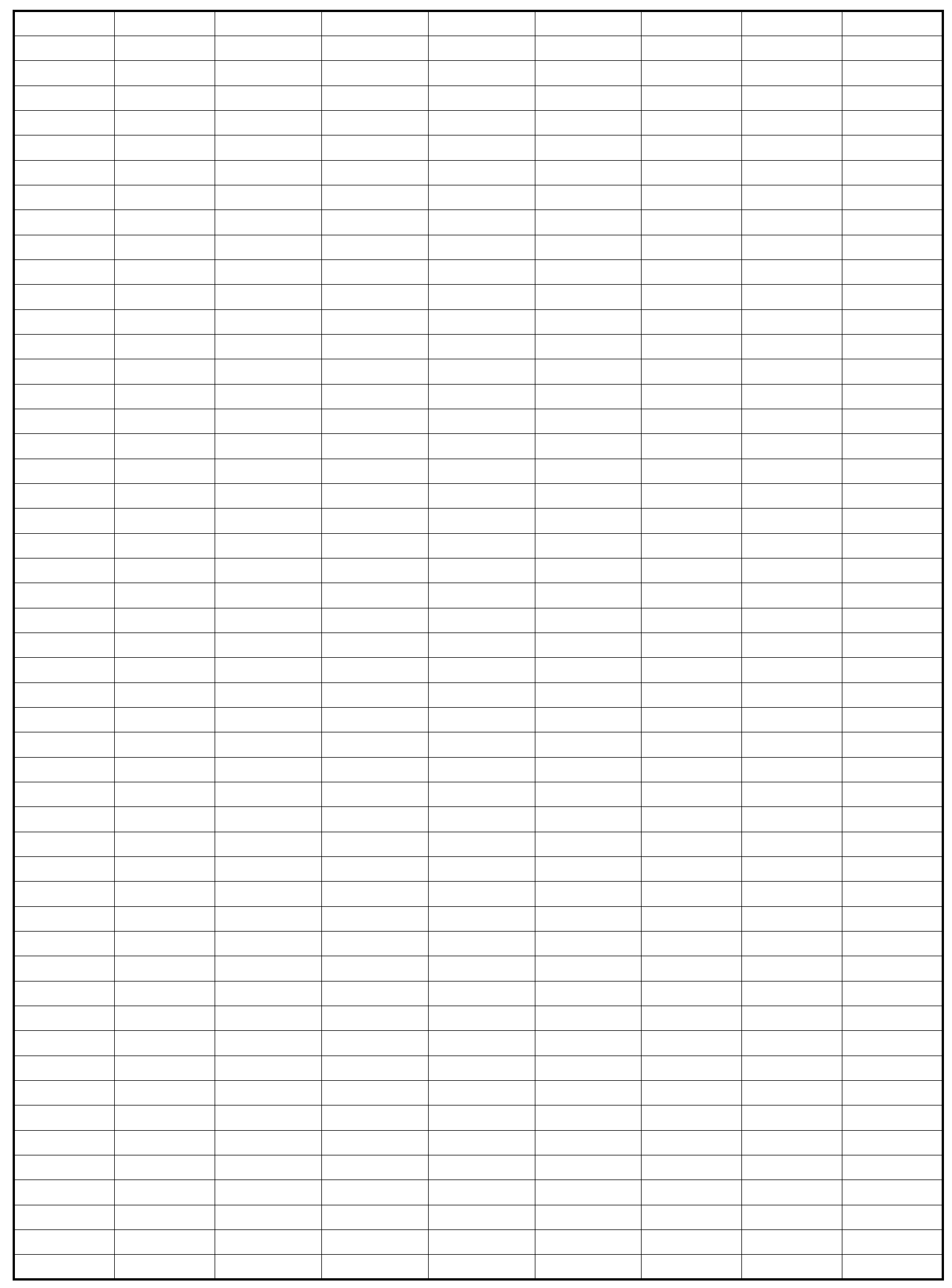

Page 12 
CHART DATA

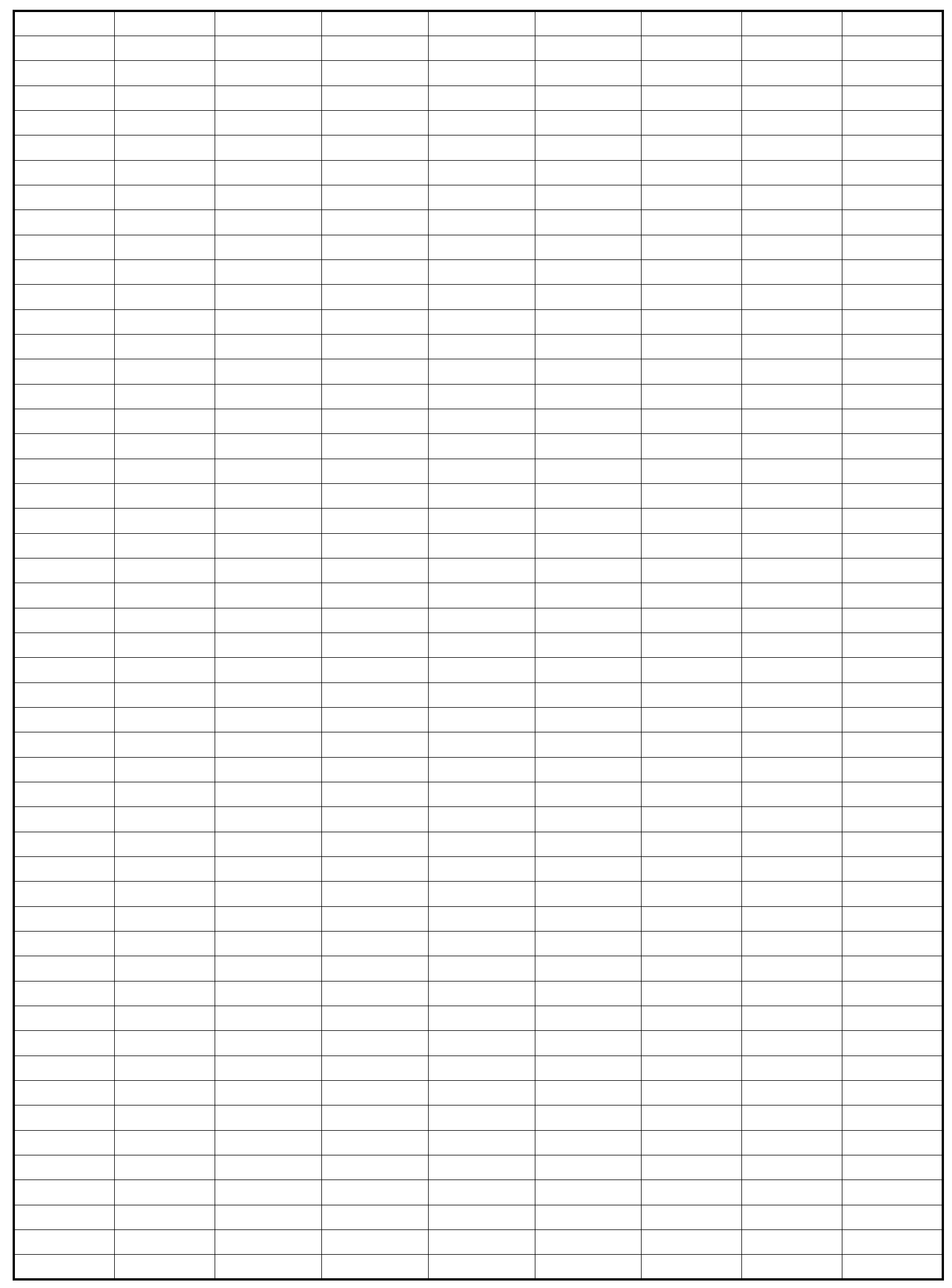

Page 13 
CHART DATA

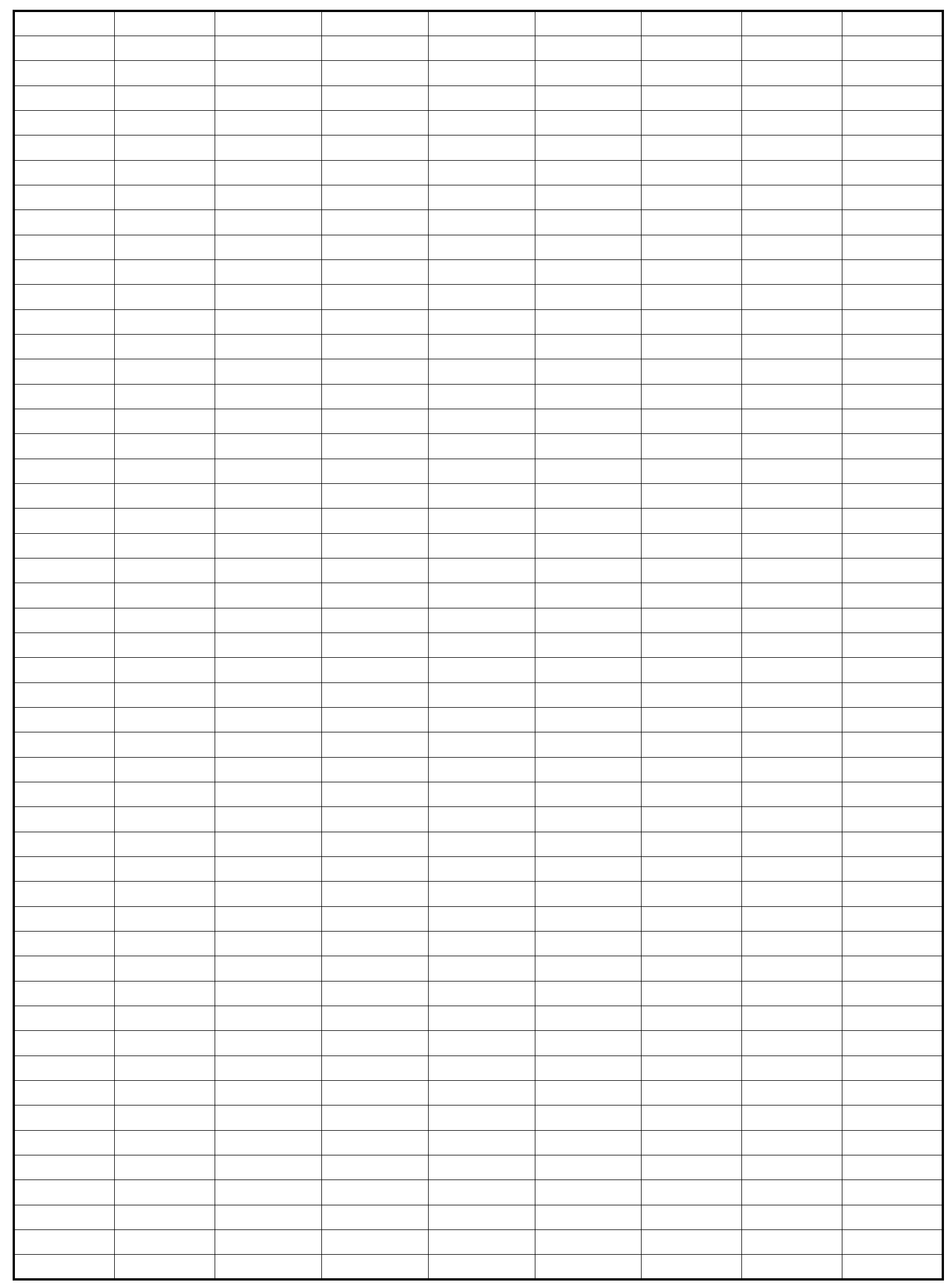

Page 14 
CHART DATA

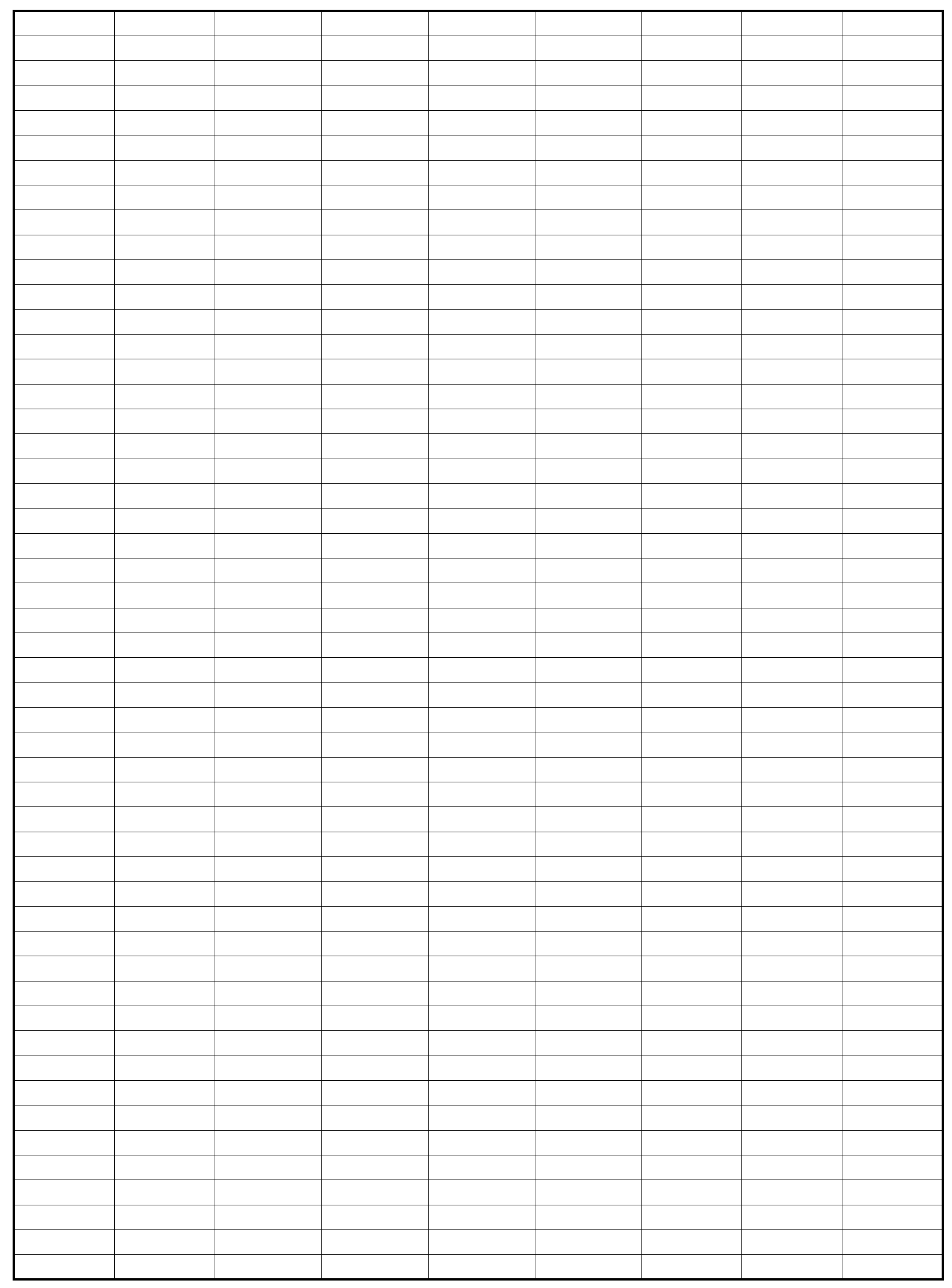

Page 15 
CHART DATA

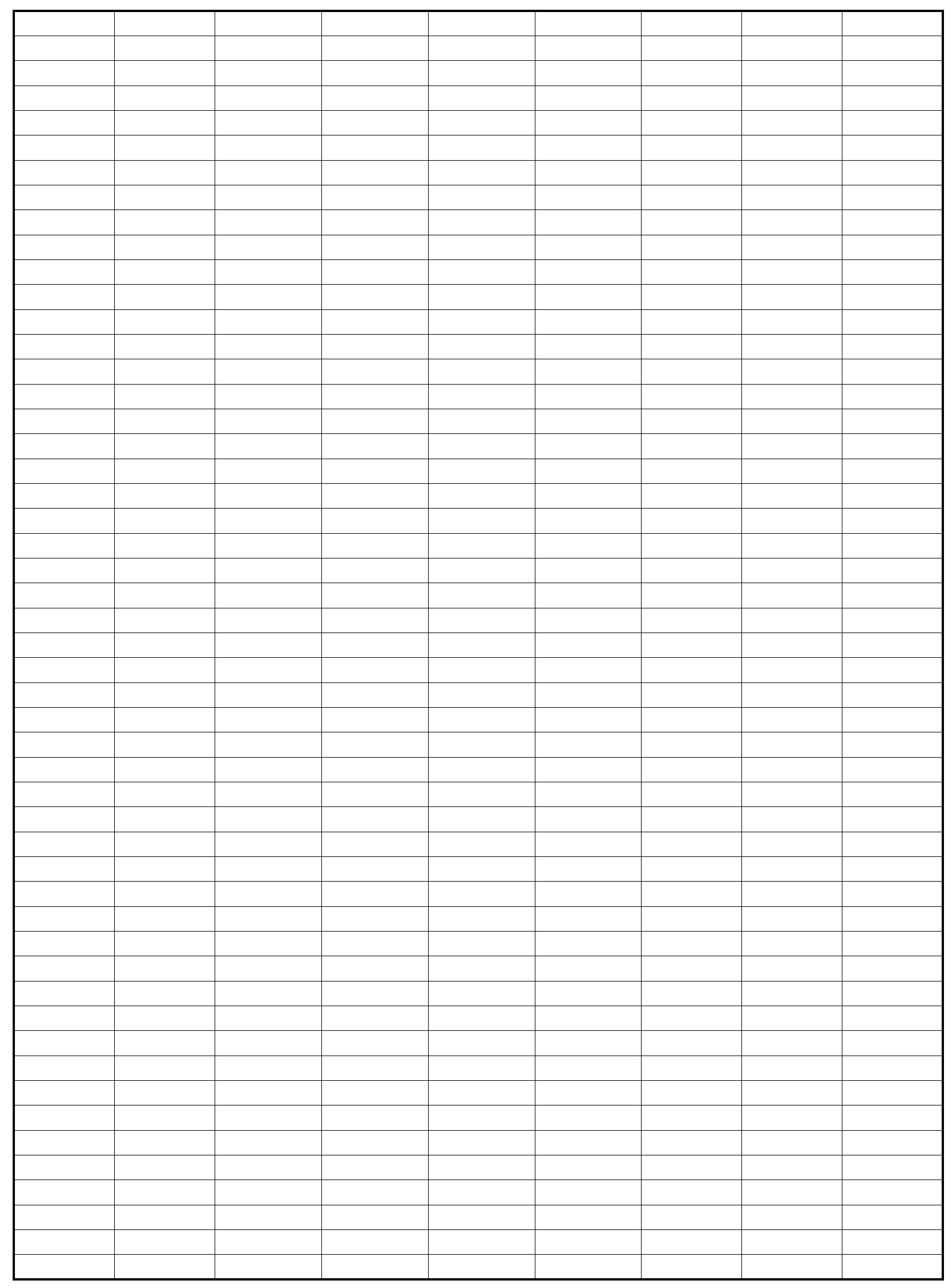

Page 16 
CHART DATA

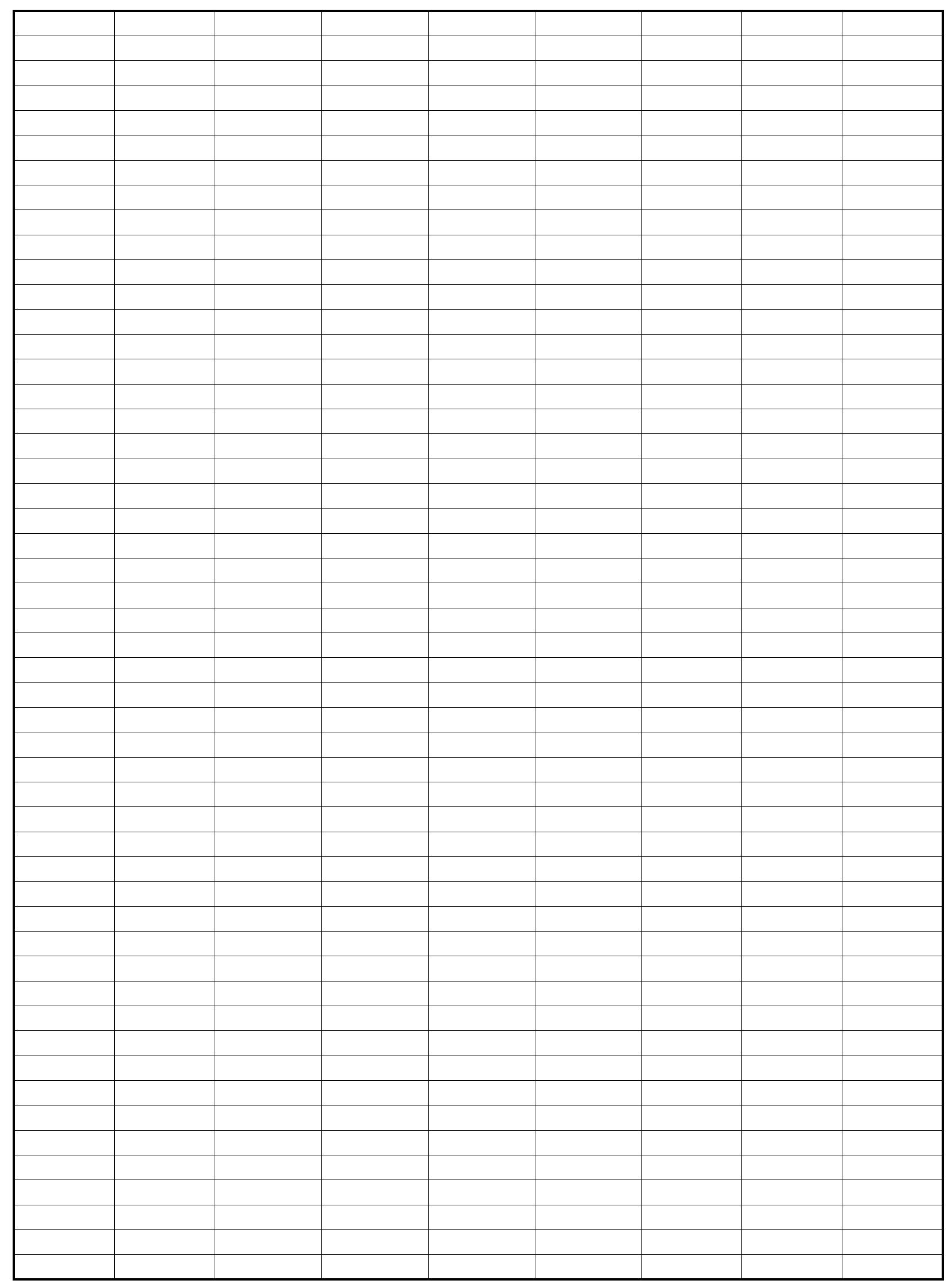

Page 17 
CHART DATA

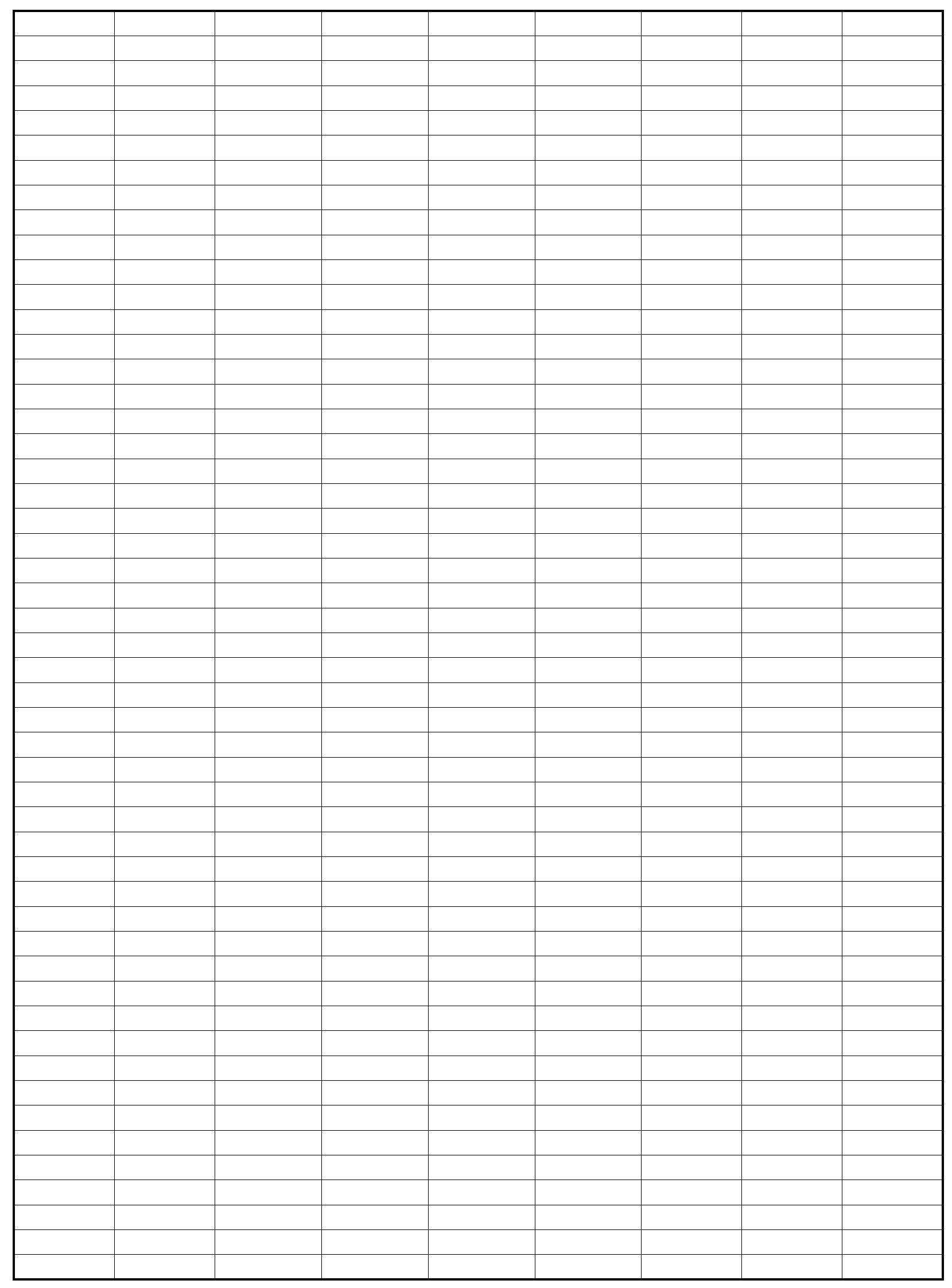

Page 18 
CHART DATA

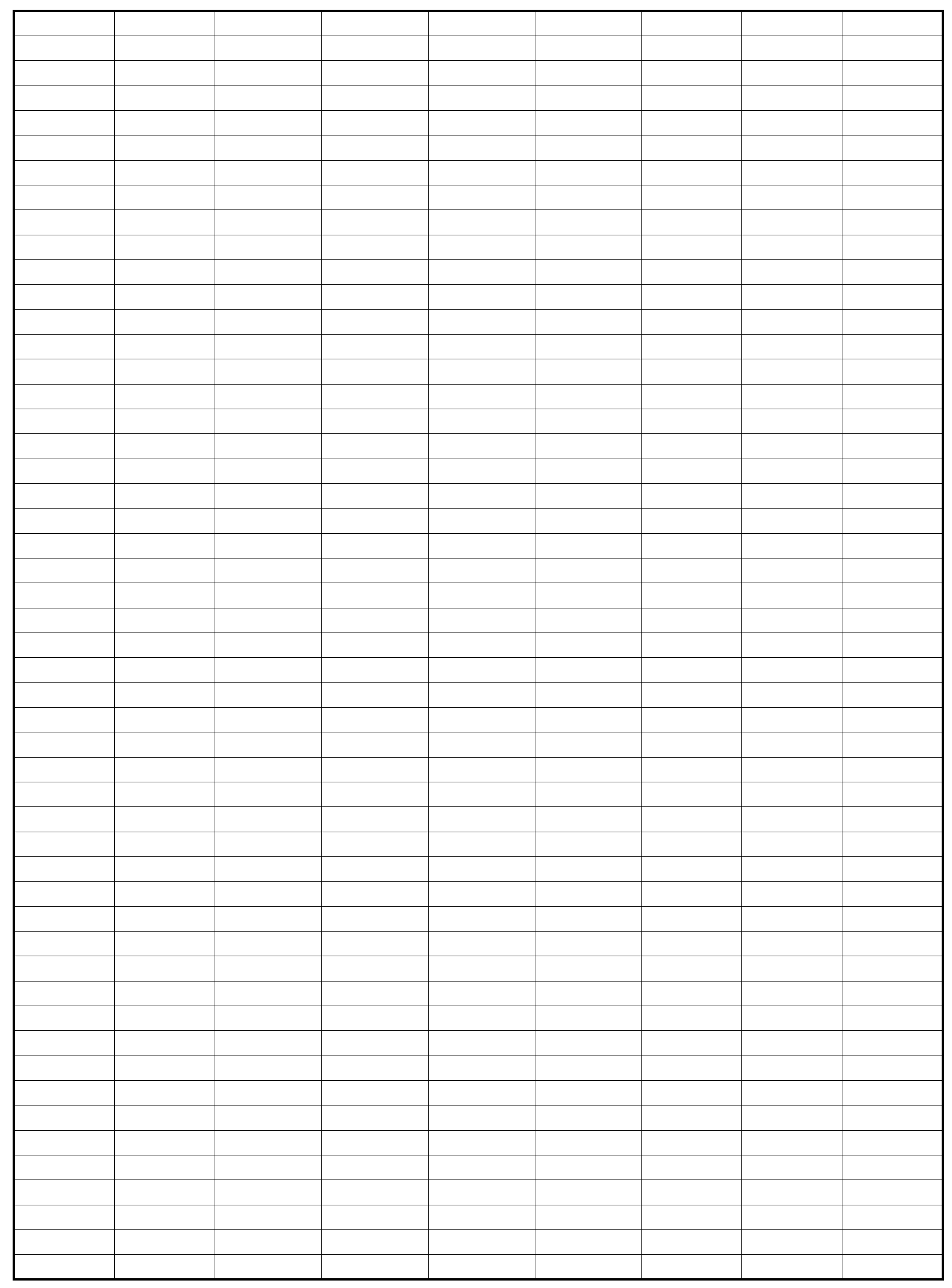

Page 19 
CHART DATA

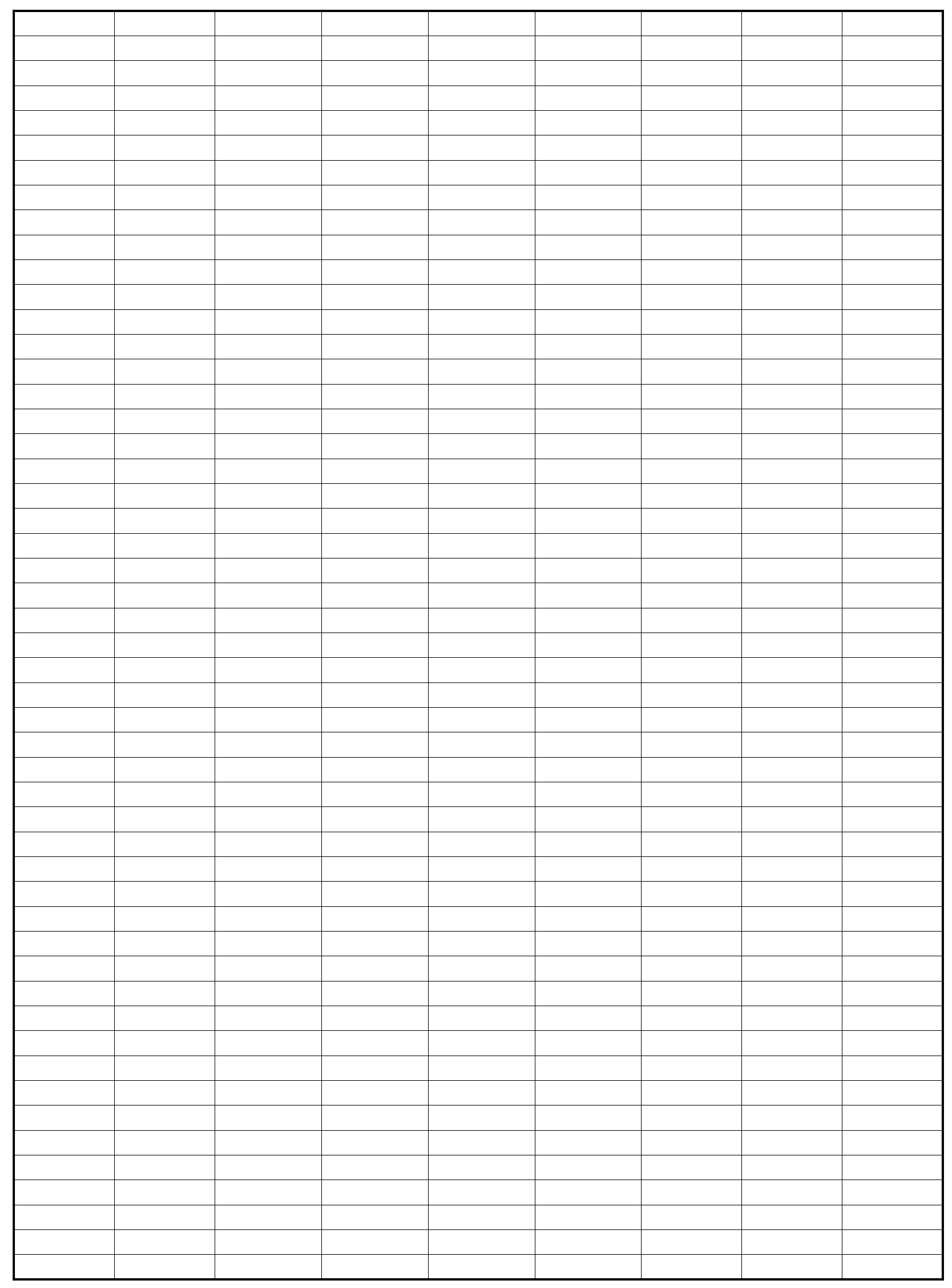

Page 20 
CHART DATA

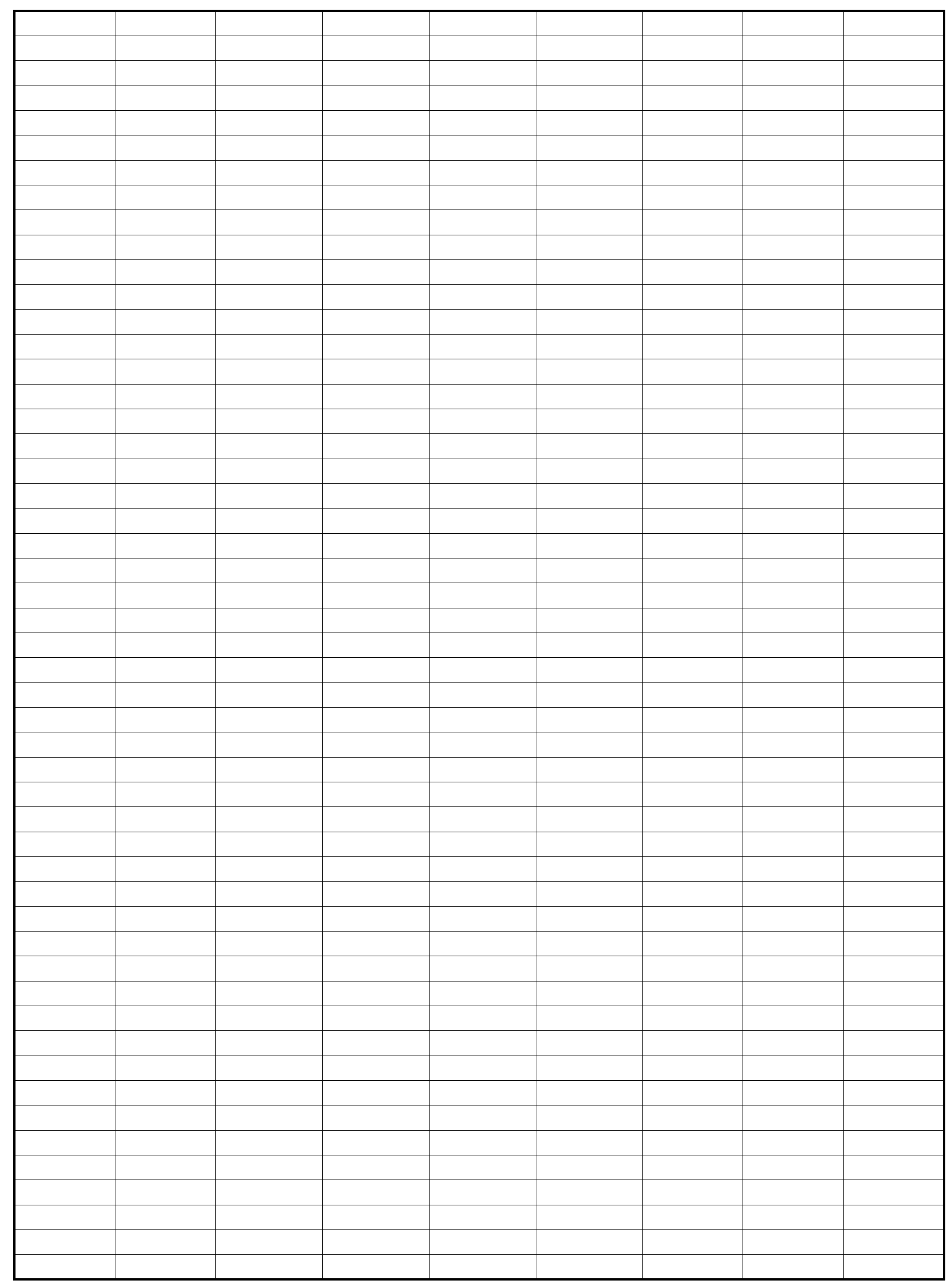

Page 21 
CHART DATA

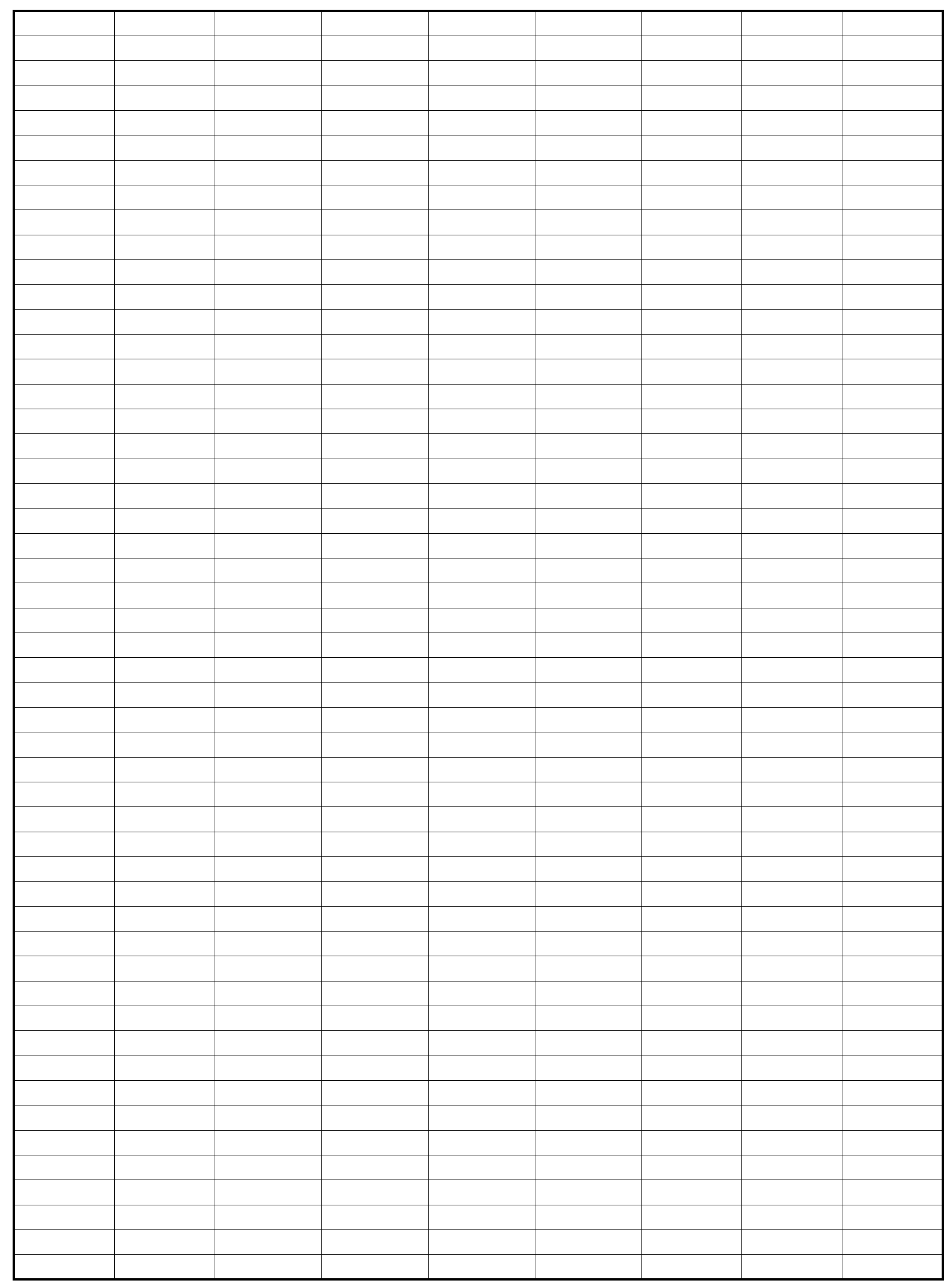

Page 22 
CHART DATA

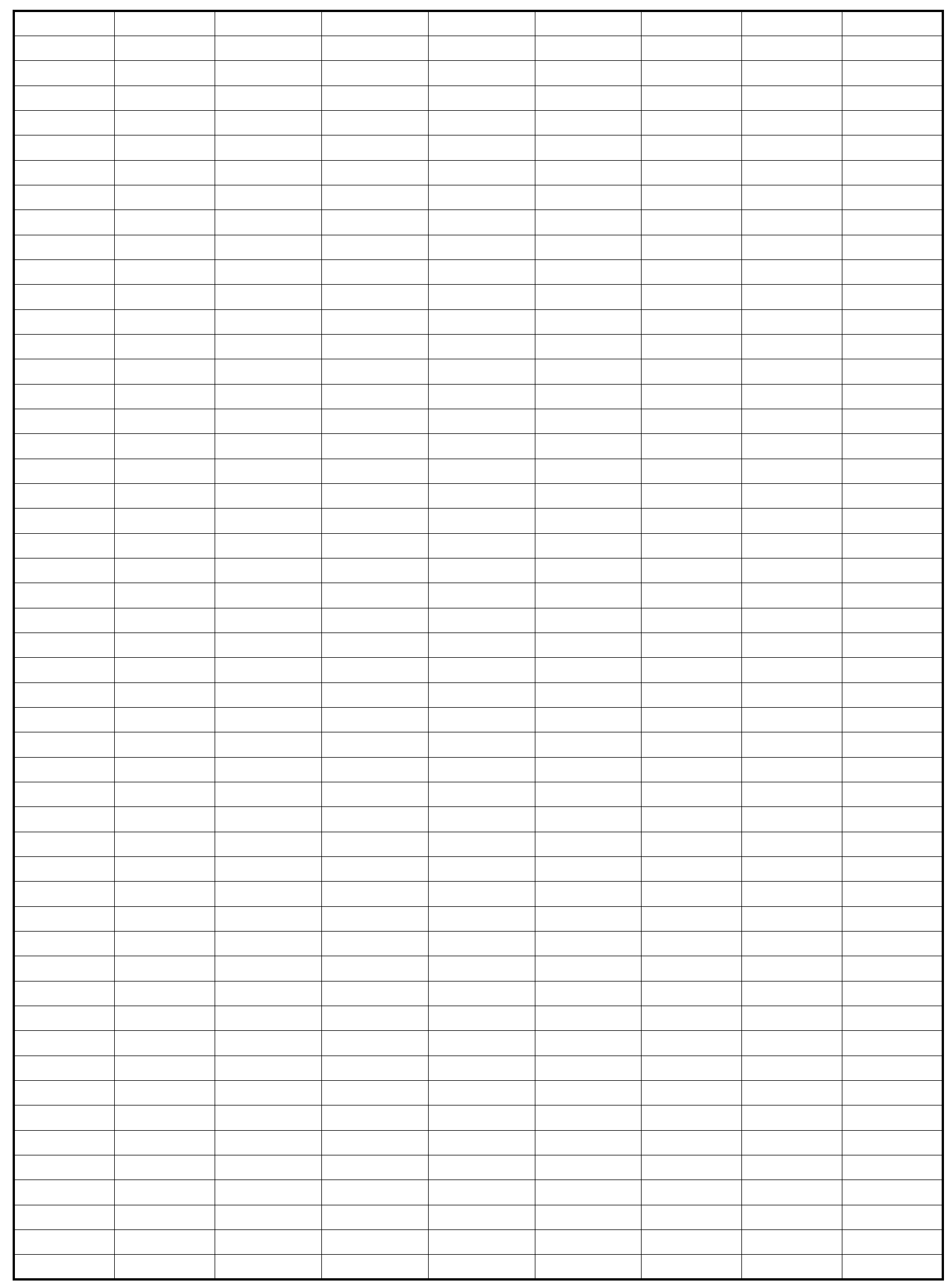

Page 23 
CHART DATA

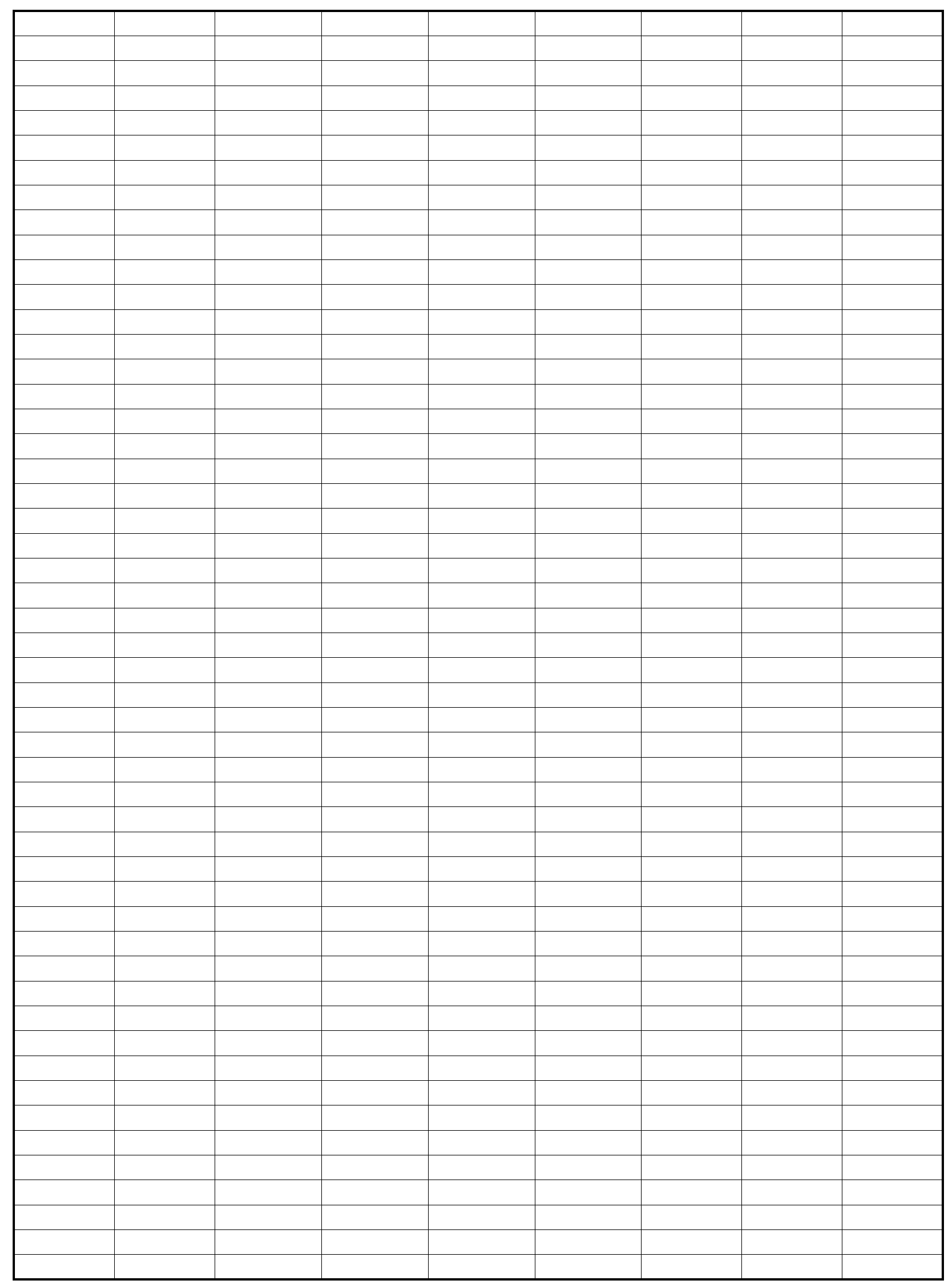

Page 24 
CHART DATA

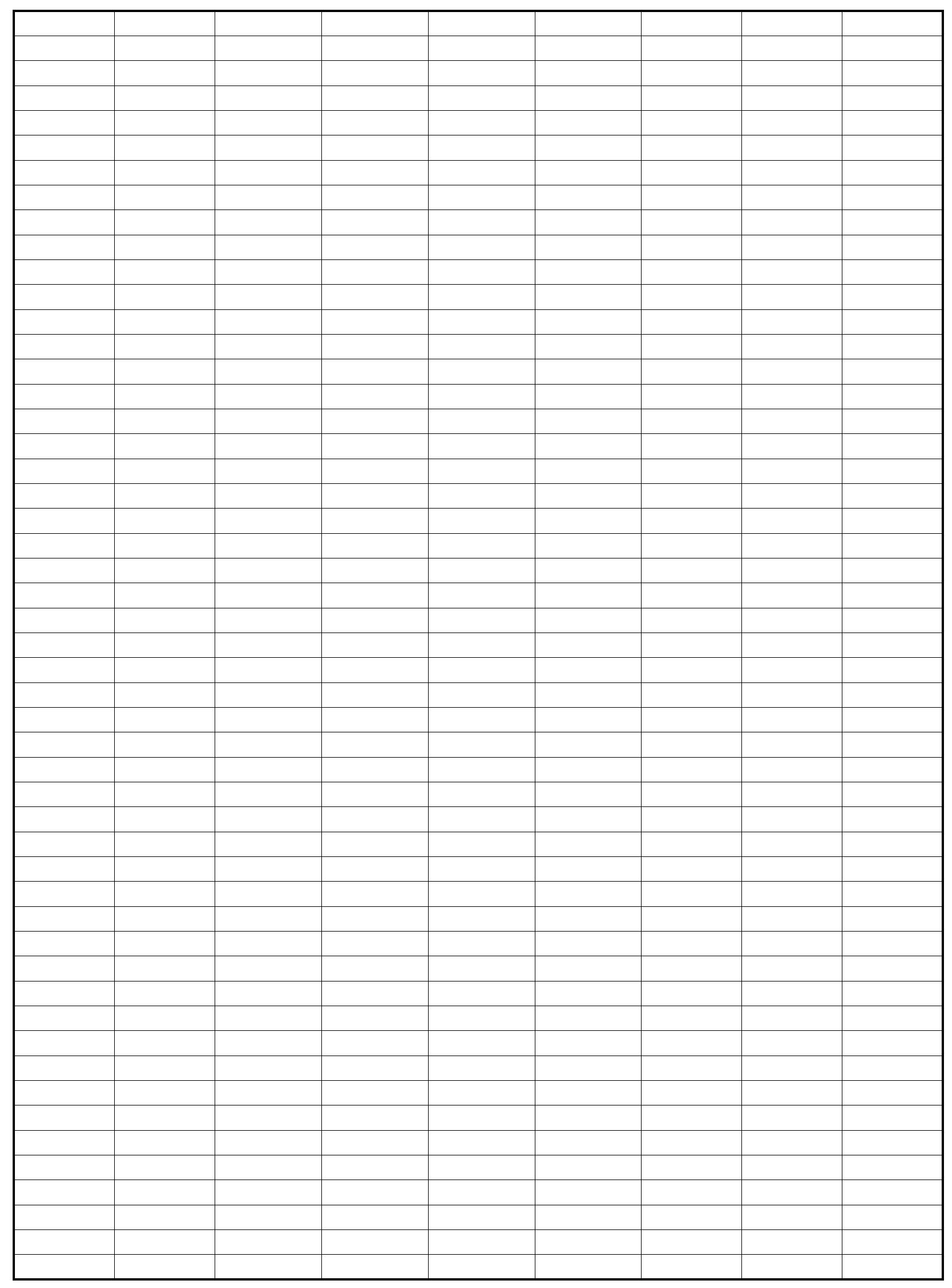

Page 25 
CHART DATA

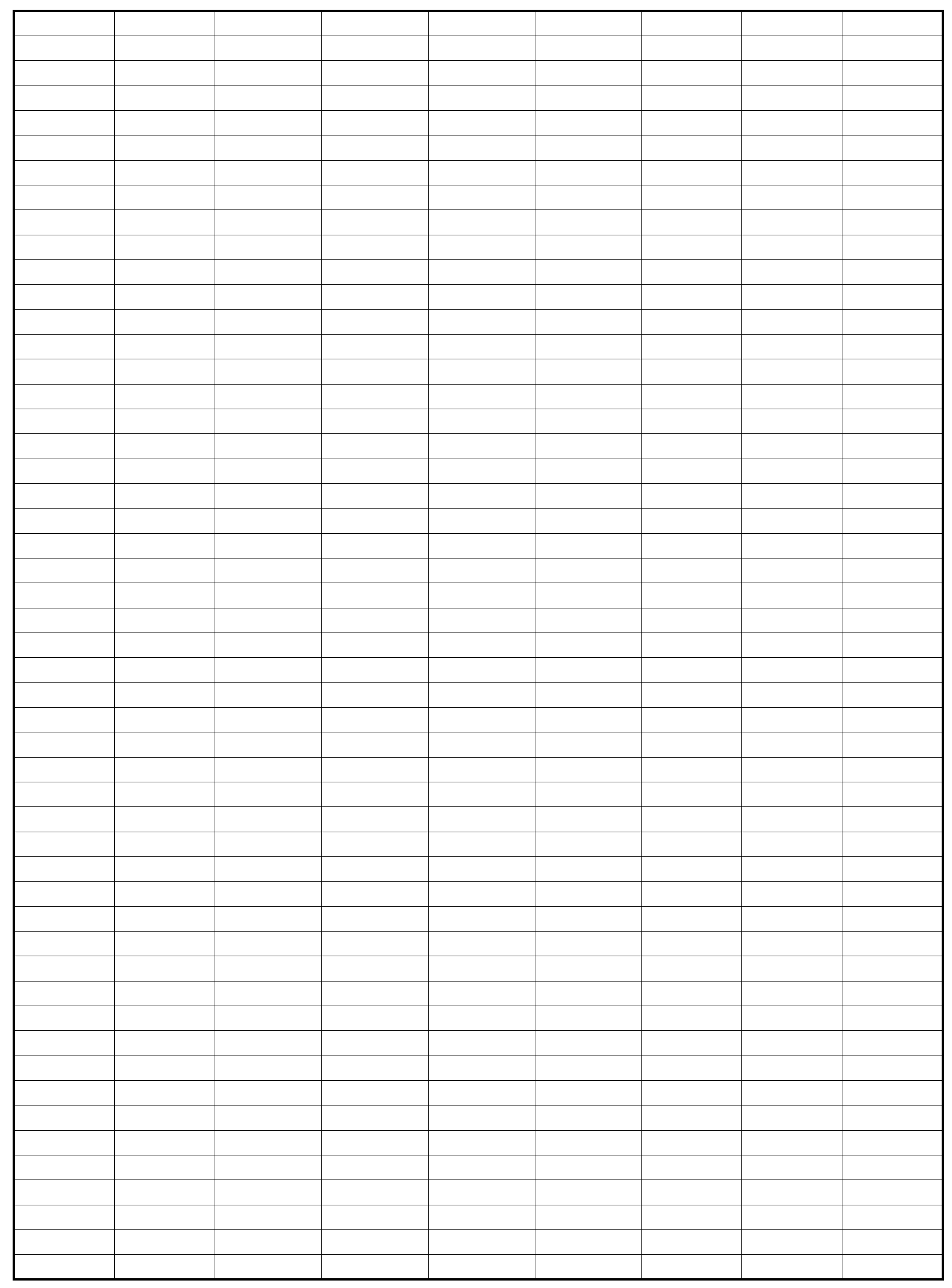

Page 26 
CHART DATA

\begin{tabular}{|l|l|l|l|l|l|l|l|l|}
\hline & & & & & & & & \\
\hline & & & & & & & & \\
\hline & & & & & & & & \\
\hline & & & & & & & & \\
\hline & & & & & & & & \\
\hline \\
\hline
\end{tabular}

Page 27 


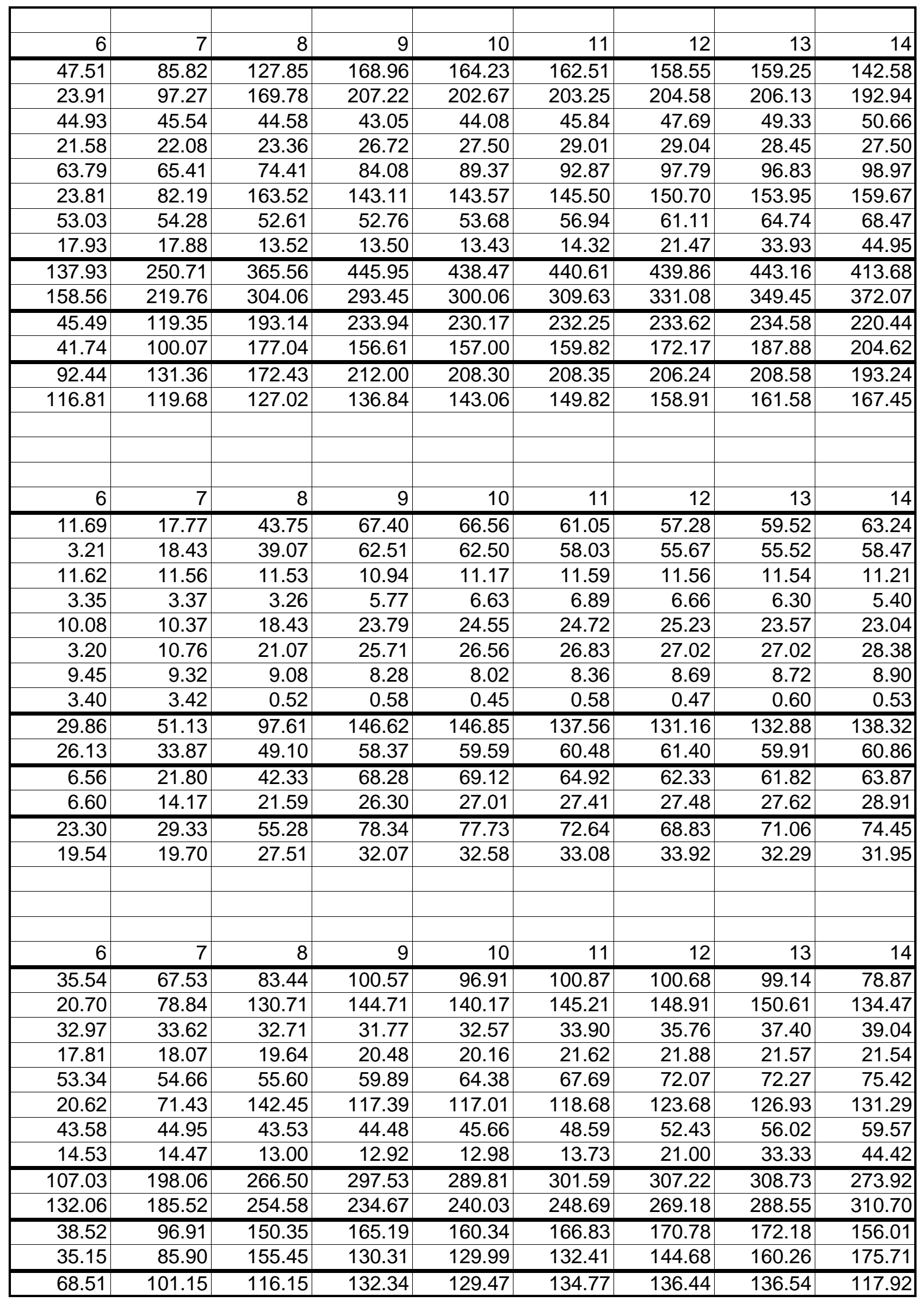


CHART DATA

\begin{tabular}{|c|c|c|c|c|c|c|c|c|}
\hline 96.92 & 99.61 & 99.13 & 104.36 & 110.04 & 116.28 & 124.50 & 128.29 & 134.99 \\
\hline & & & & & & & & \\
\hline & & & & & & & & \\
\hline 6 & 7 & 8 & 9 & 10 & 11 & 12 & 13 & 14 \\
\hline 0.39 & 0.35 & 0.37 & $\overline{0.34}$ & 0.28 & 0.26 & 0.28 & 0.28 & 0.20 \\
\hline 0.52 & 0.46 & 0.71 & 0.41 & 0.41 & 0.28 & 0.25 & 0.23 & 0.20 \\
\hline 0.30 & 0.31 & 0.31 & 0.31 & 0.31 & 0.30 & 0.28 & 0.27 & 0.27 \\
\hline 0.10 & 0.53 & 0.84 & 0.64 & 0.56 & 0.58 & 0.50 & 0.40 & 0.40 \\
\hline 0.34 & 0.32 & 0.32 & 0.29 & 0.31 & 0.30 & 0.29 & 0.30 & 0.28 \\
\hline 0.23 & 0.67 & 0.96 & 0.70 & 0.47 & 0.42 & 0.35 & 0.33 & 0.30 \\
\hline 0.31 & 0.32 & 0.32 & 0.32 & 0.32 & 0.32 & 0.29 & 0.28 & 0.27 \\
\hline 0.00 & 0.00 & 0.00 & 0.00 & 0.00 & 0.00 & 0.00 & 0.00 & 0.00 \\
\hline 1.31 & 1.65 & 2.23 & 1.70 & 1.56 & 1.41 & 1.31 & 1.18 & 1.07 \\
\hline 0.88 & 1.32 & 1.61 & 1.30 & 1.09 & 1.04 & 0.93 & 0.91 & 0.84 \\
\hline 0.62 & 0.99 & 1.55 & 1.05 & 0.97 & 0.86 & 0.75 & 0.63 & 0.60 \\
\hline 0.23 & 0.67 & 0.96 & 0.70 & 0.47 & 0.42 & 0.35 & 0.33 & 0.30 \\
\hline 0.69 & 0.66 & 0.68 & 0.65 & 0.59 & 0.56 & 0.56 & 0.55 & $\overline{0.48}$ \\
\hline 0.65 & 0.64 & 0.65 & 0.60 & 0.63 & 0.61 & 0.58 & 0.58 & 0.54 \\
\hline & & & & & & & & \\
\hline & & & & & & & & \\
\hline & & & & & & & & \\
\hline & & & & & & & & \\
\hline & & & & & & & & \\
\hline & & & & & & & & \\
\hline & & & & & & & & \\
\hline & & & & & & & & \\
\hline & & & & & & & & \\
\hline & & & & & & & & \\
\hline & & & & & & & & \\
\hline & & & & & & & & \\
\hline & & & & & & & & \\
\hline & & & & & & & & \\
\hline & & & & & & & & \\
\hline & & & & & & & & \\
\hline & & & & & & & & \\
\hline & & & & & & & & \\
\hline & & & & & & & & \\
\hline & & & & & & & & \\
\hline & & & & & & & & \\
\hline & & & & & & & & \\
\hline & & & & & & & & \\
\hline & & & & & & & & \\
\hline & & & & & & & & \\
\hline & & & & & & & & \\
\hline & & & & & & & & \\
\hline & & & & & & & & \\
\hline & & & & & & & & \\
\hline & & & & & & & & \\
\hline & & & & & & & & \\
\hline
\end{tabular}


CHART DATA

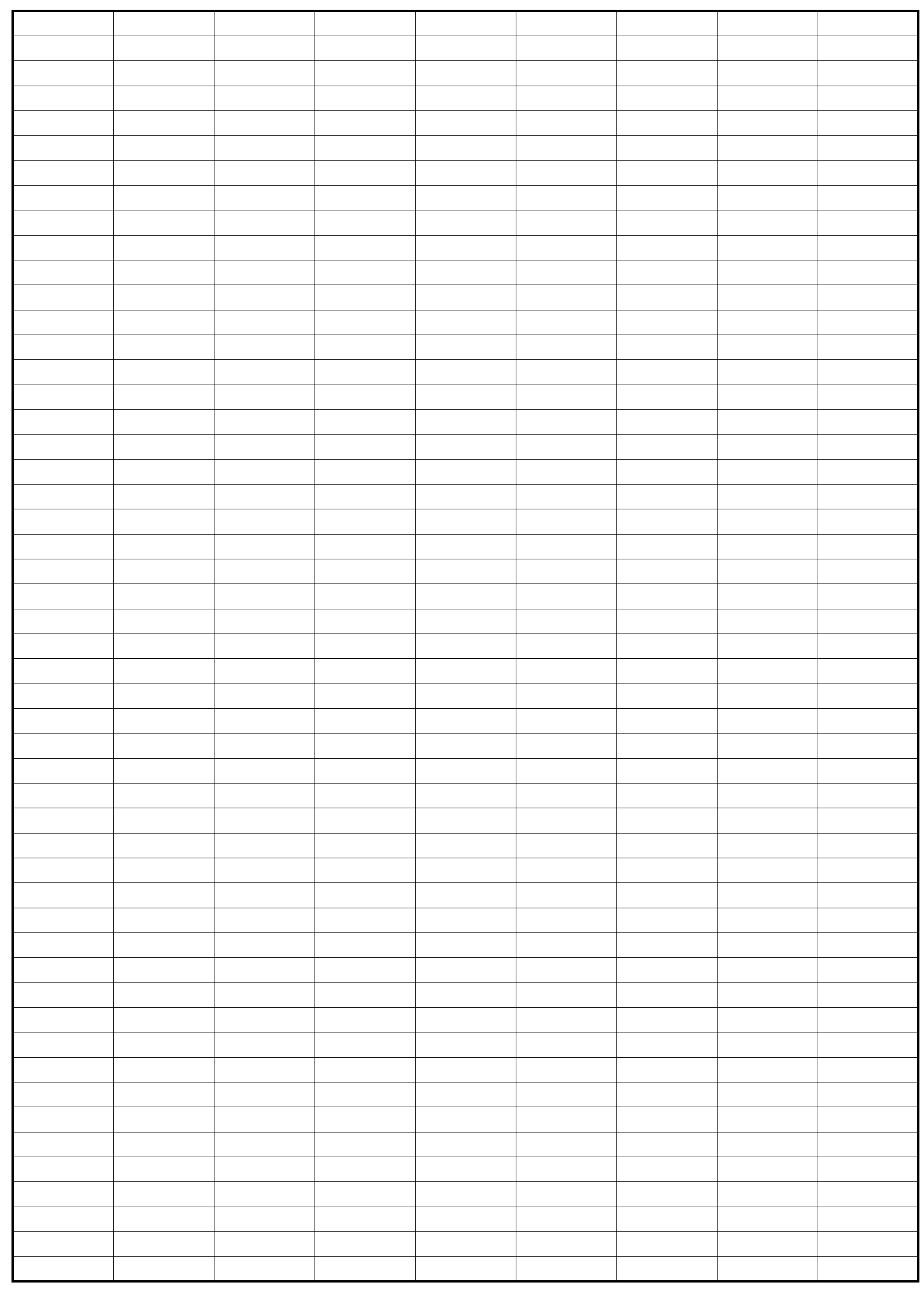

Page 30 
CHART DATA

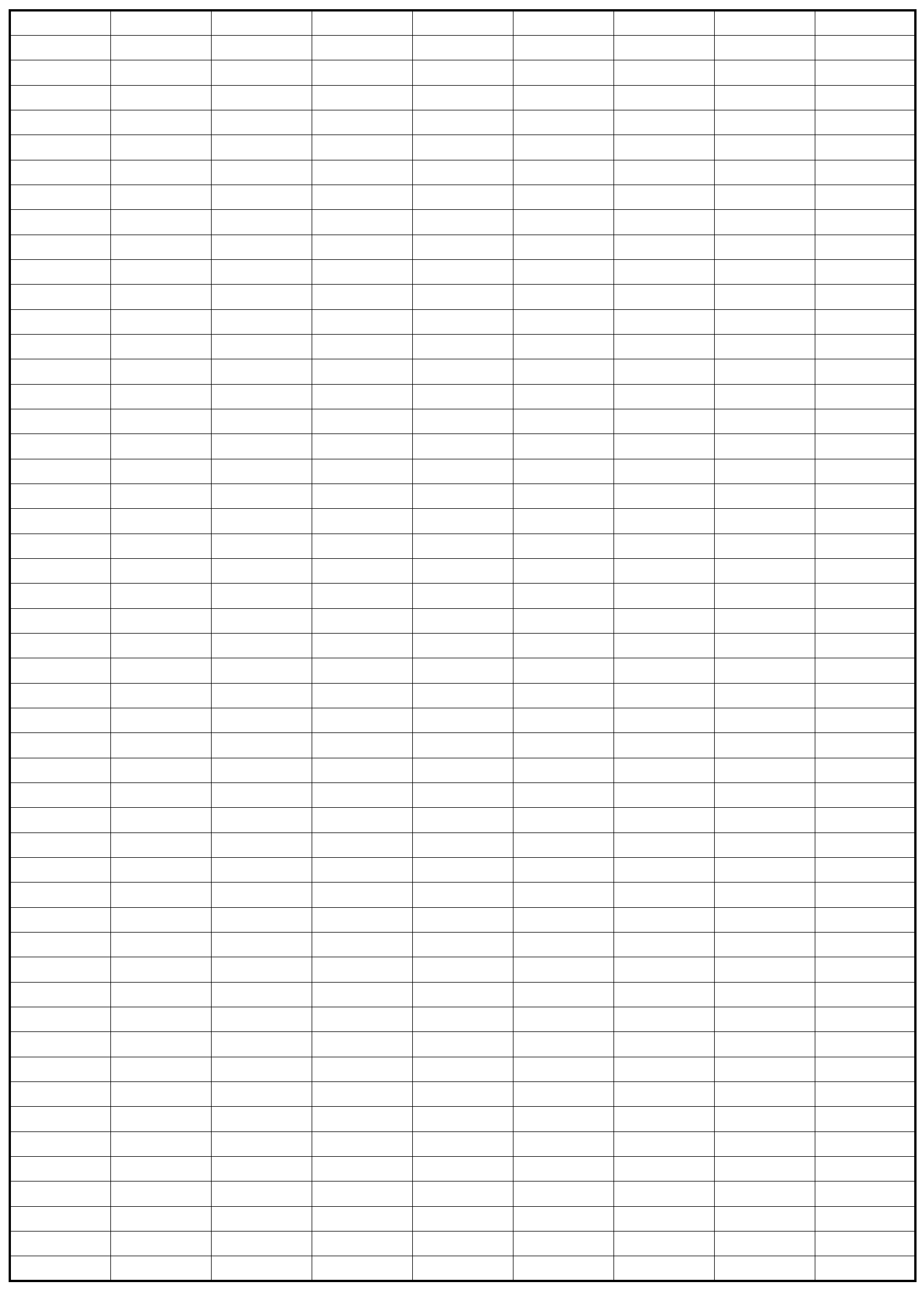

Page 31 
CHART DATA

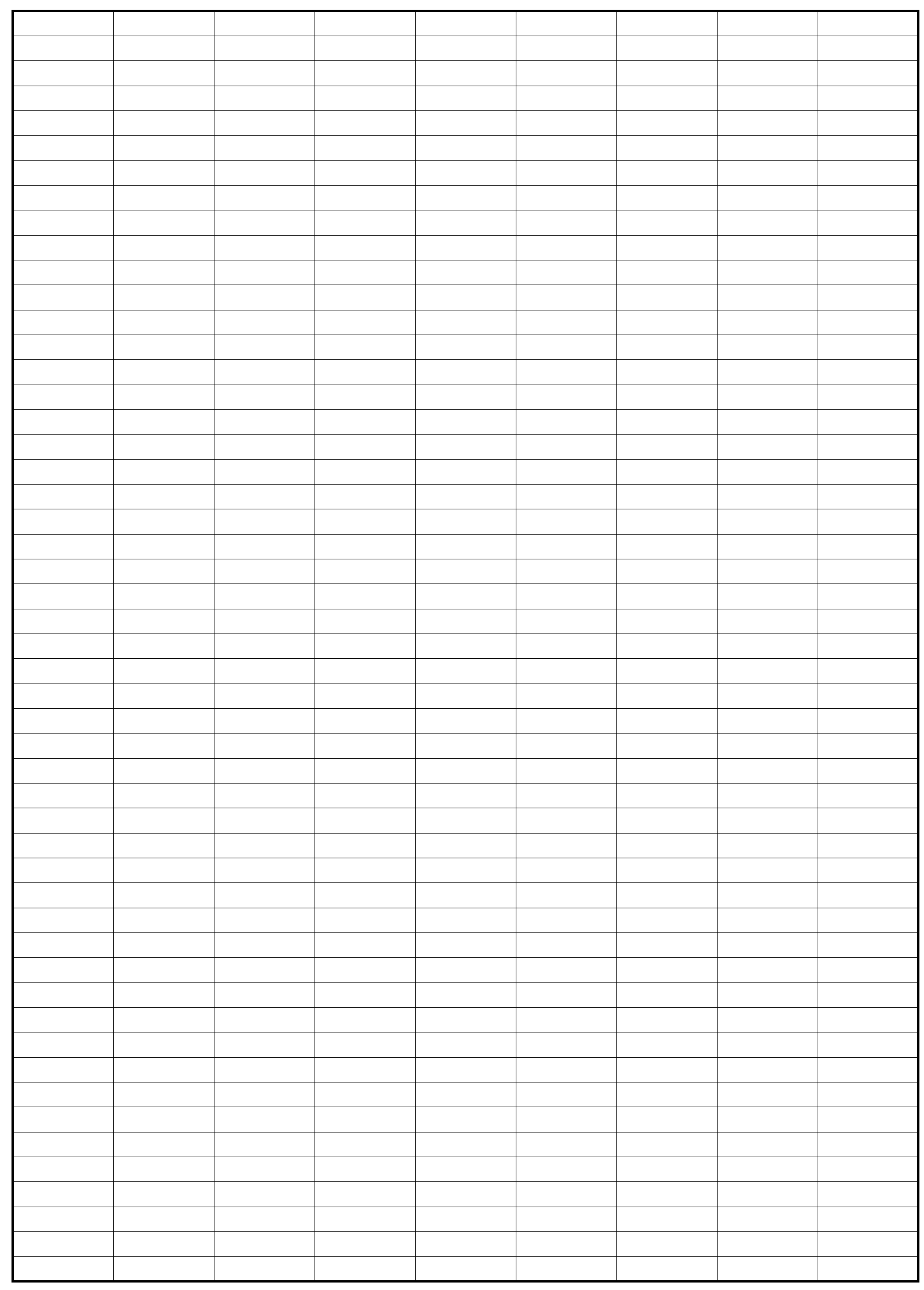

Page 32 
CHART DATA

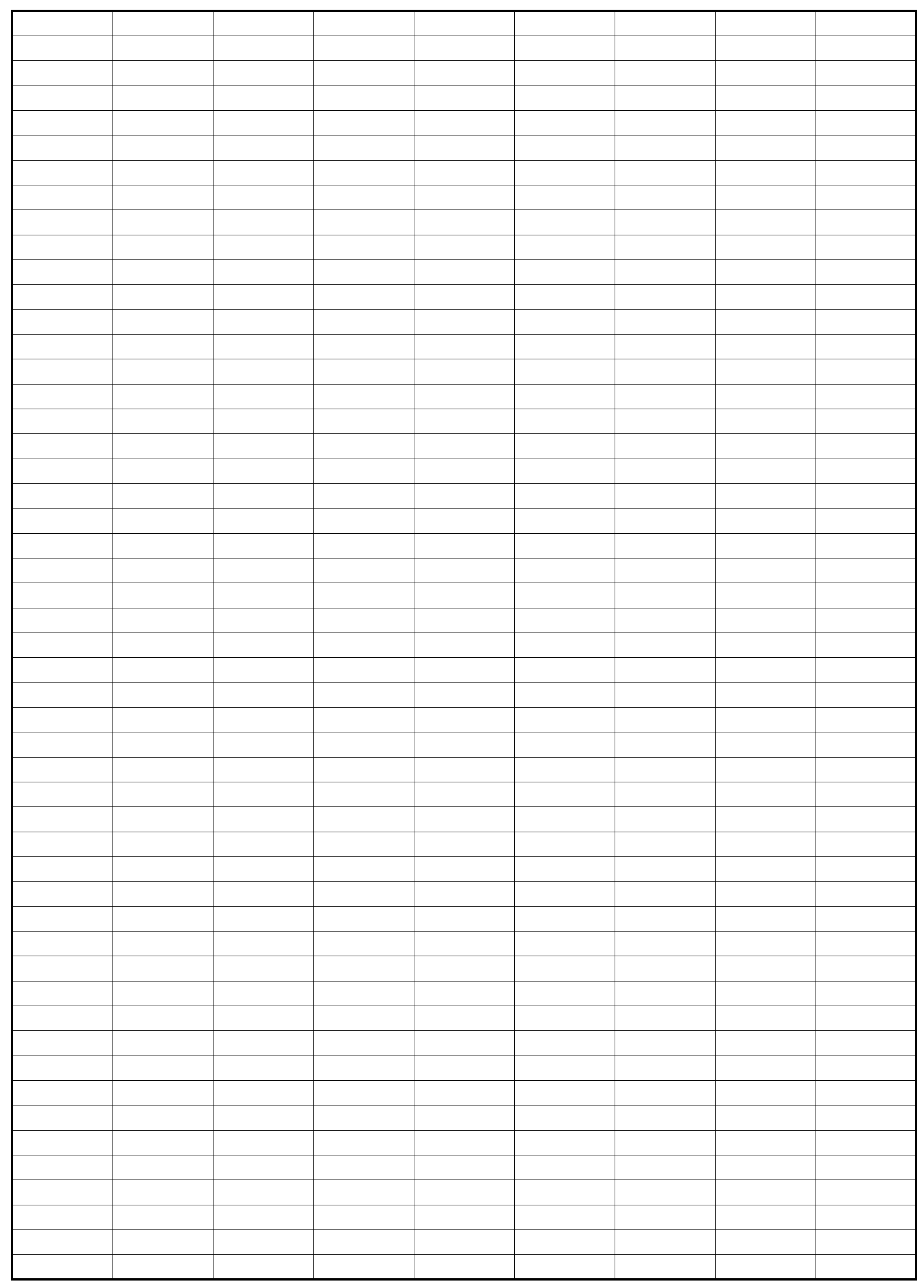

Page 33 
CHART DATA

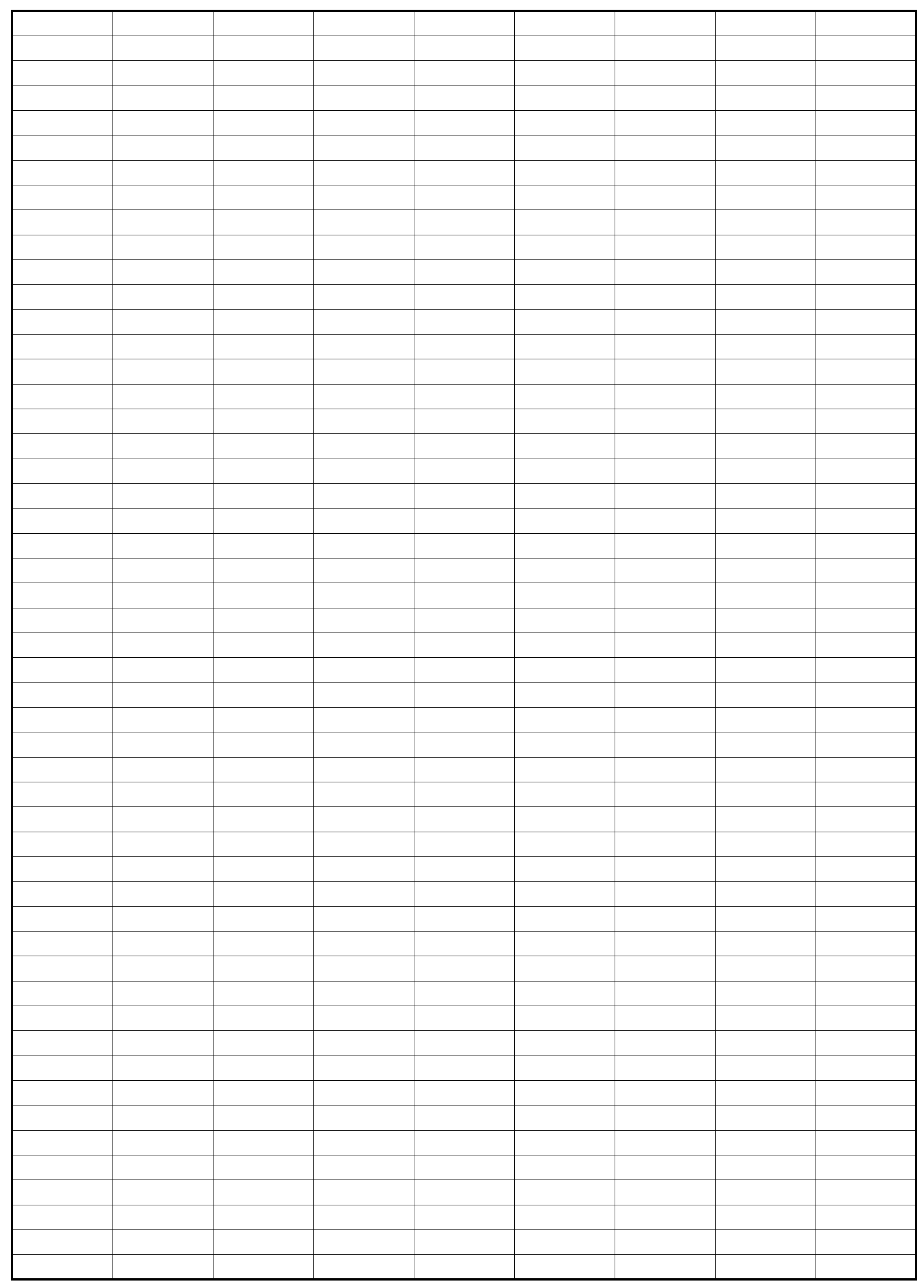

Page 34 
CHART DATA

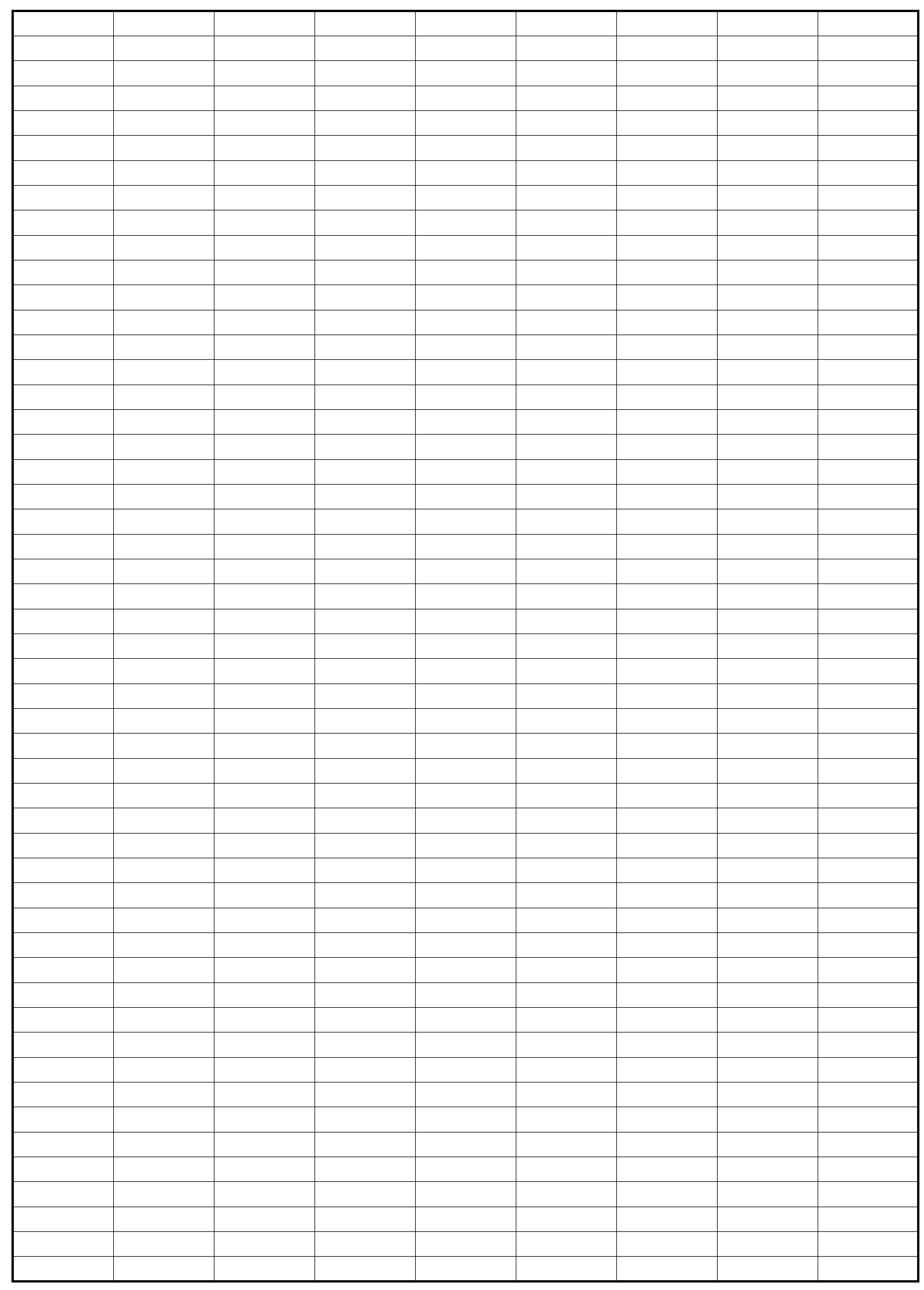

Page 35 
CHART DATA

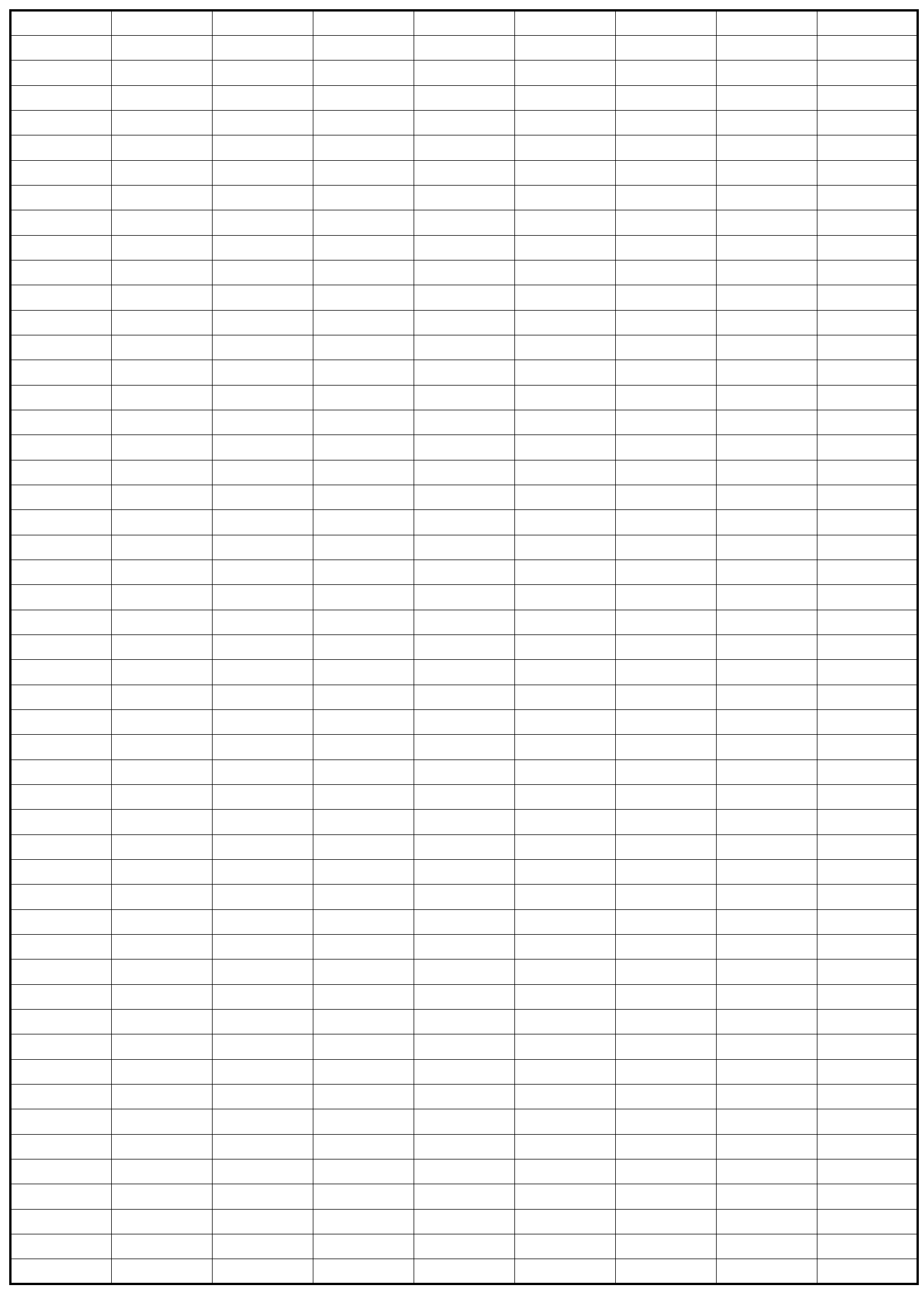

Page 36 
CHART DATA

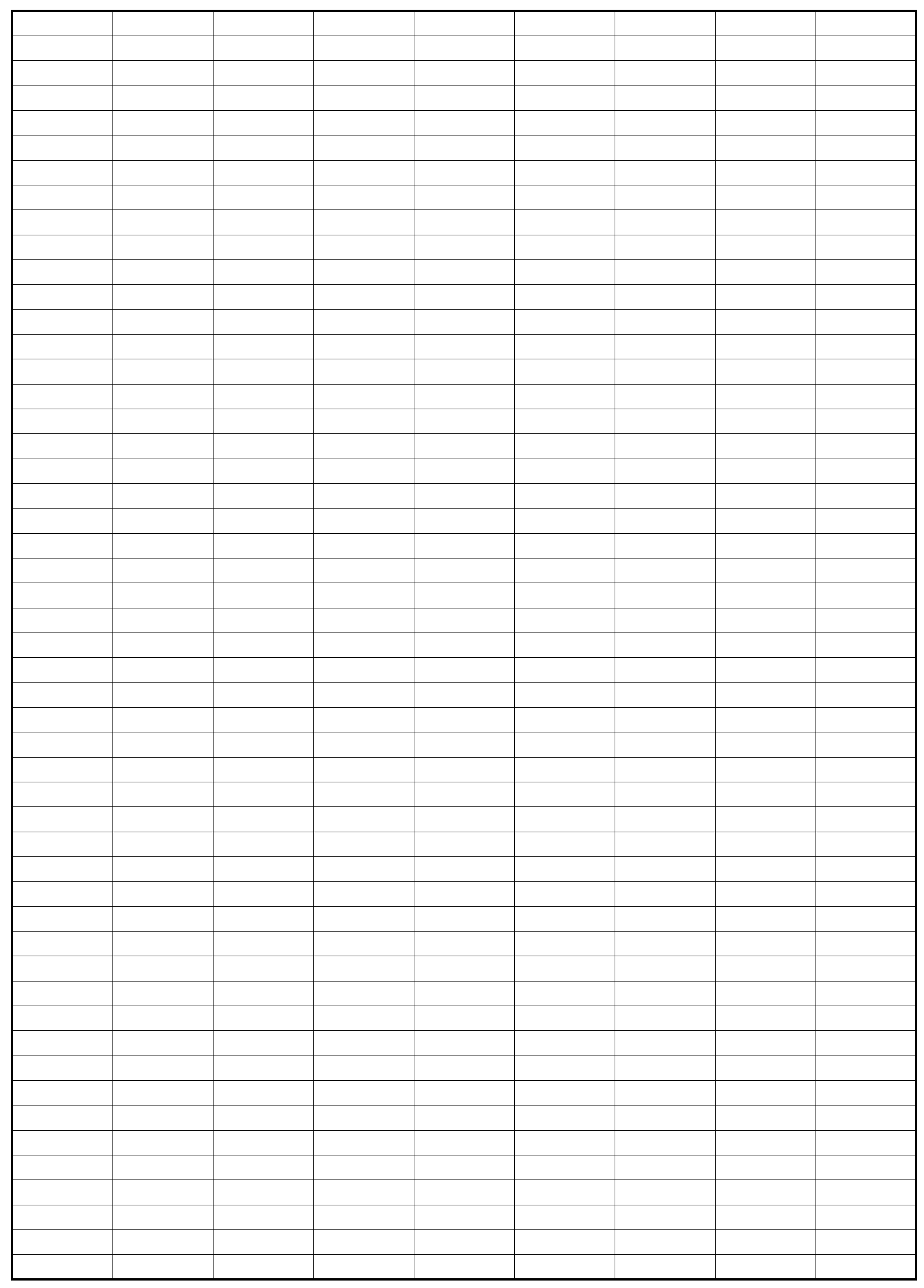

Page 37 
CHART DATA

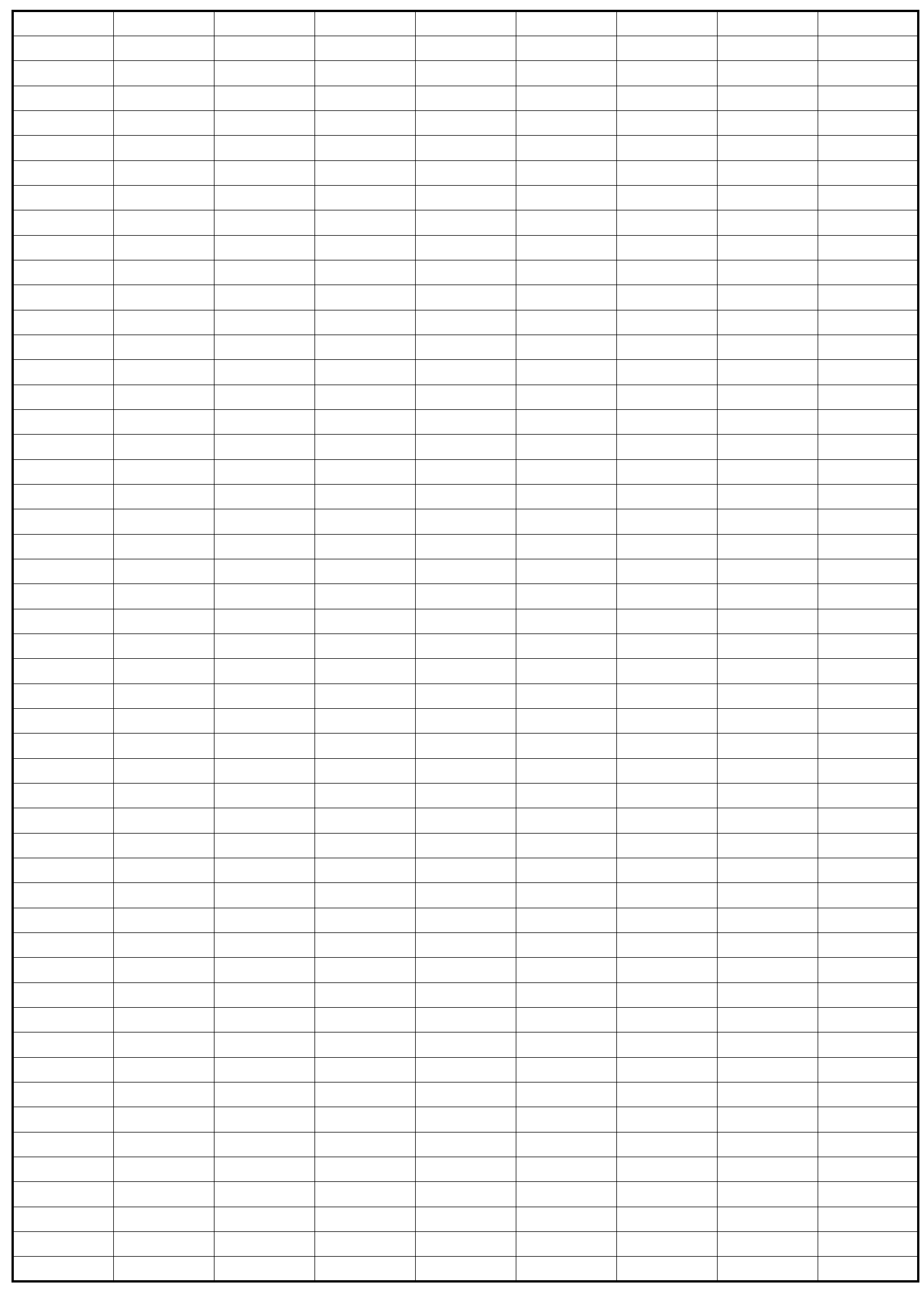

Page 38 
CHART DATA

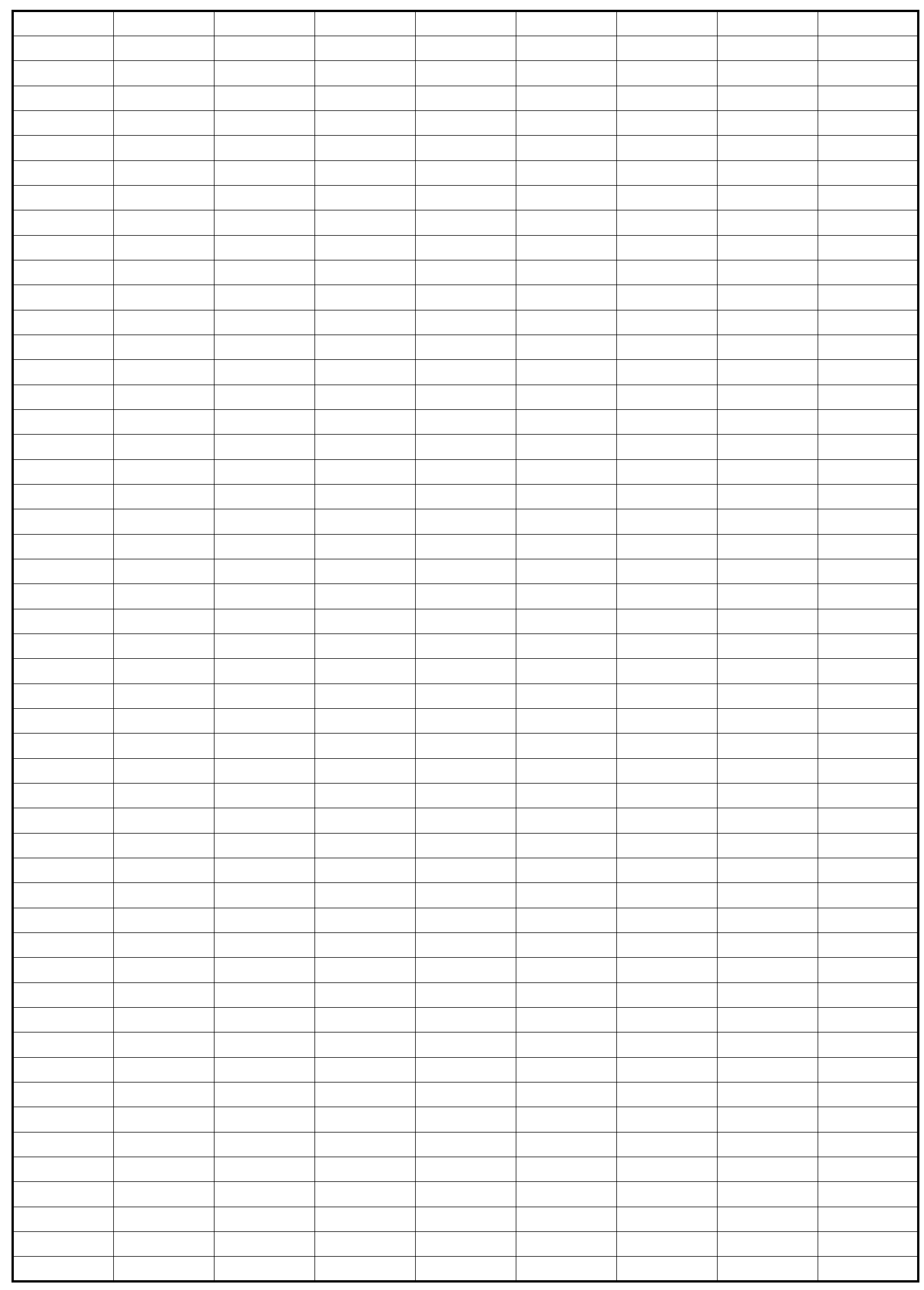

Page 39 
CHART DATA

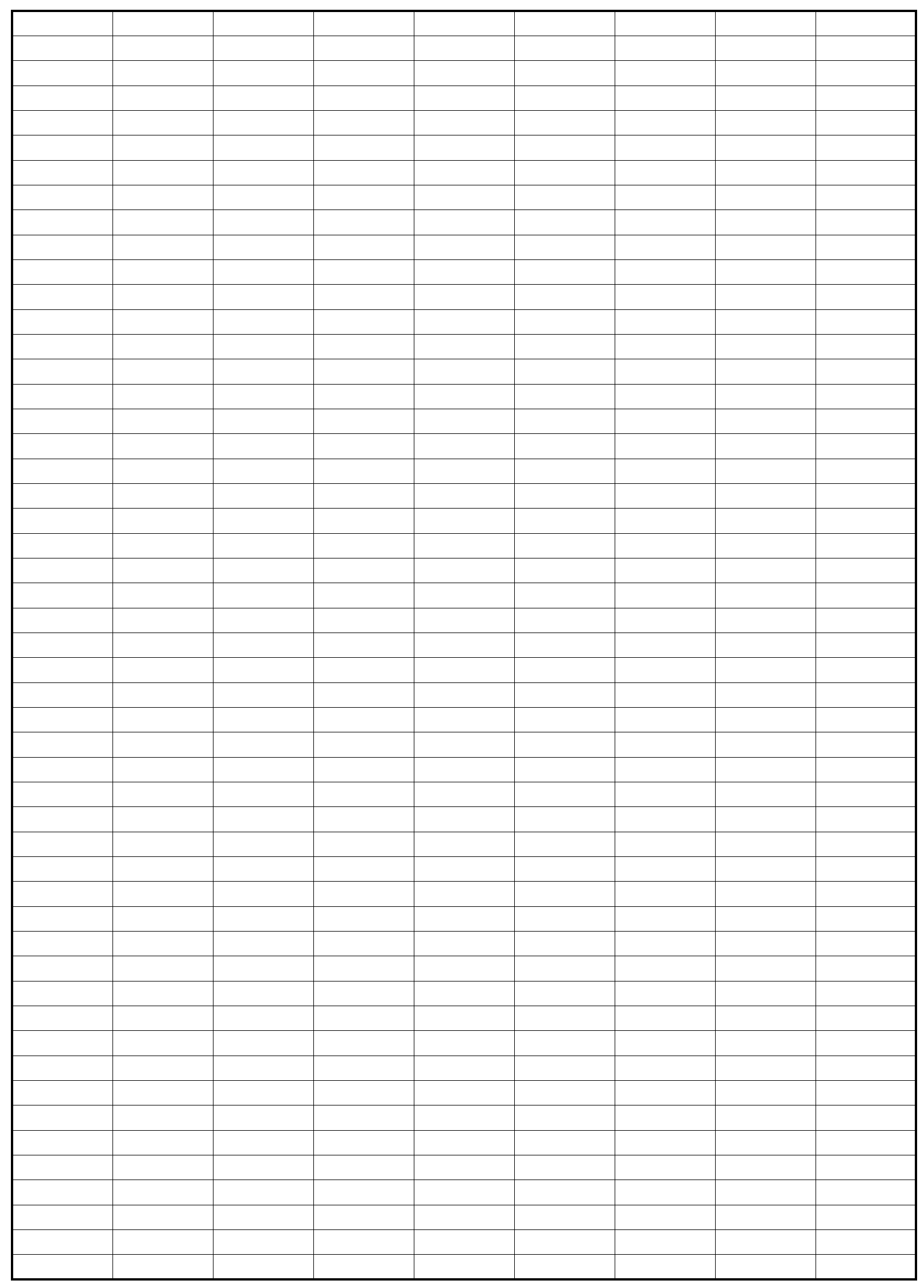

Page 40 
CHART DATA

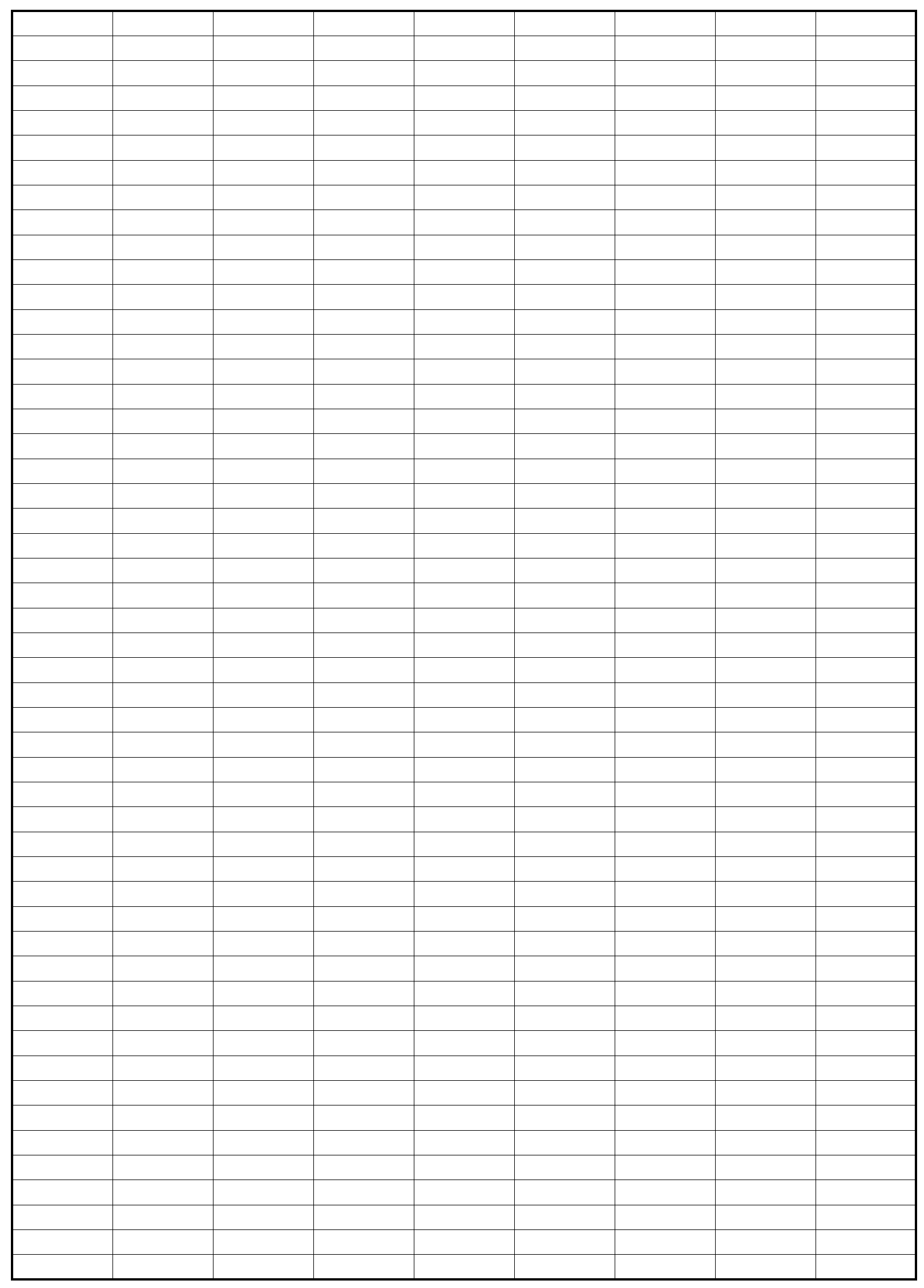

Page 41 
CHART DATA

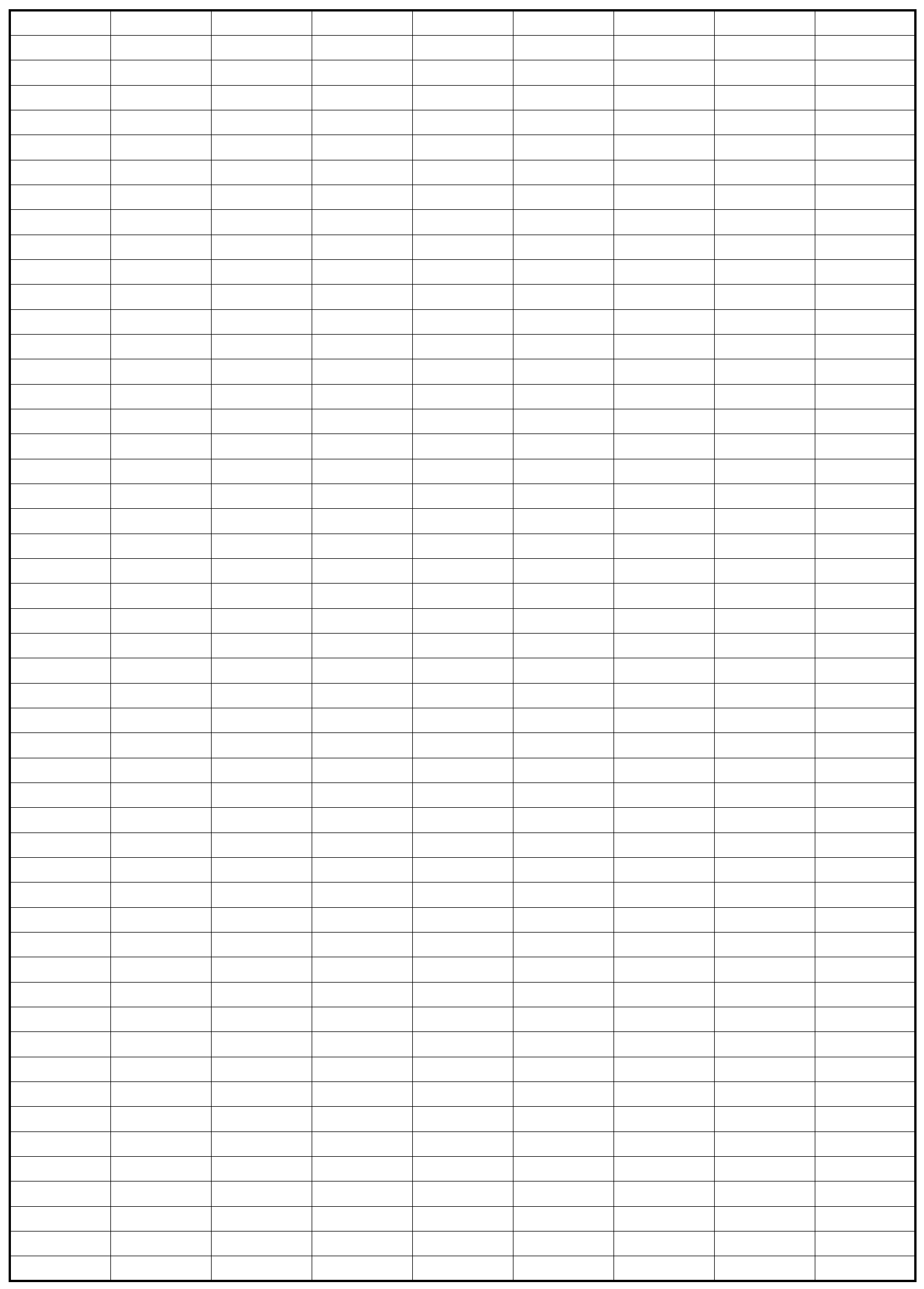

Page 42 
CHART DATA

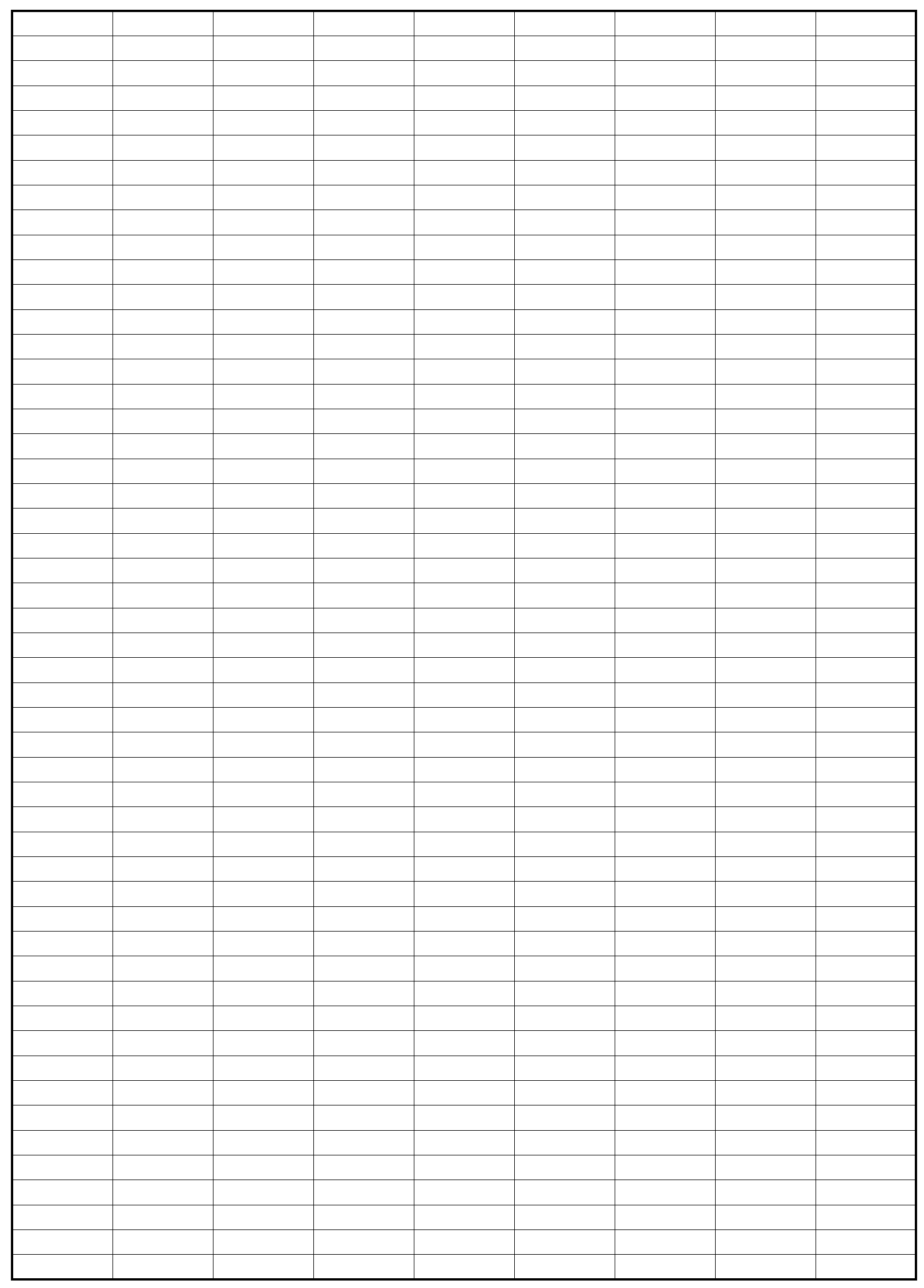

Page 43 
CHART DATA

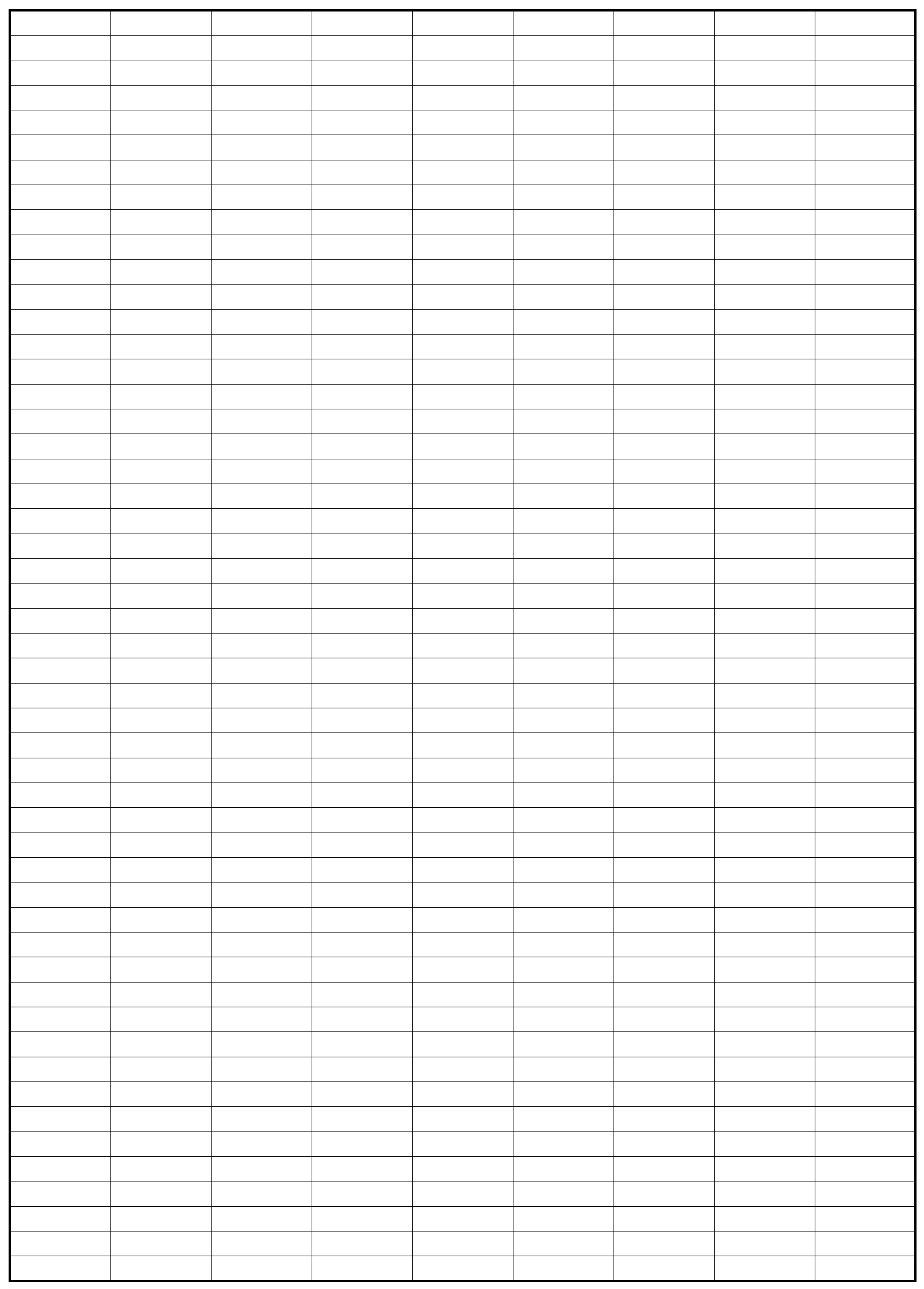

Page 44 
CHART DATA

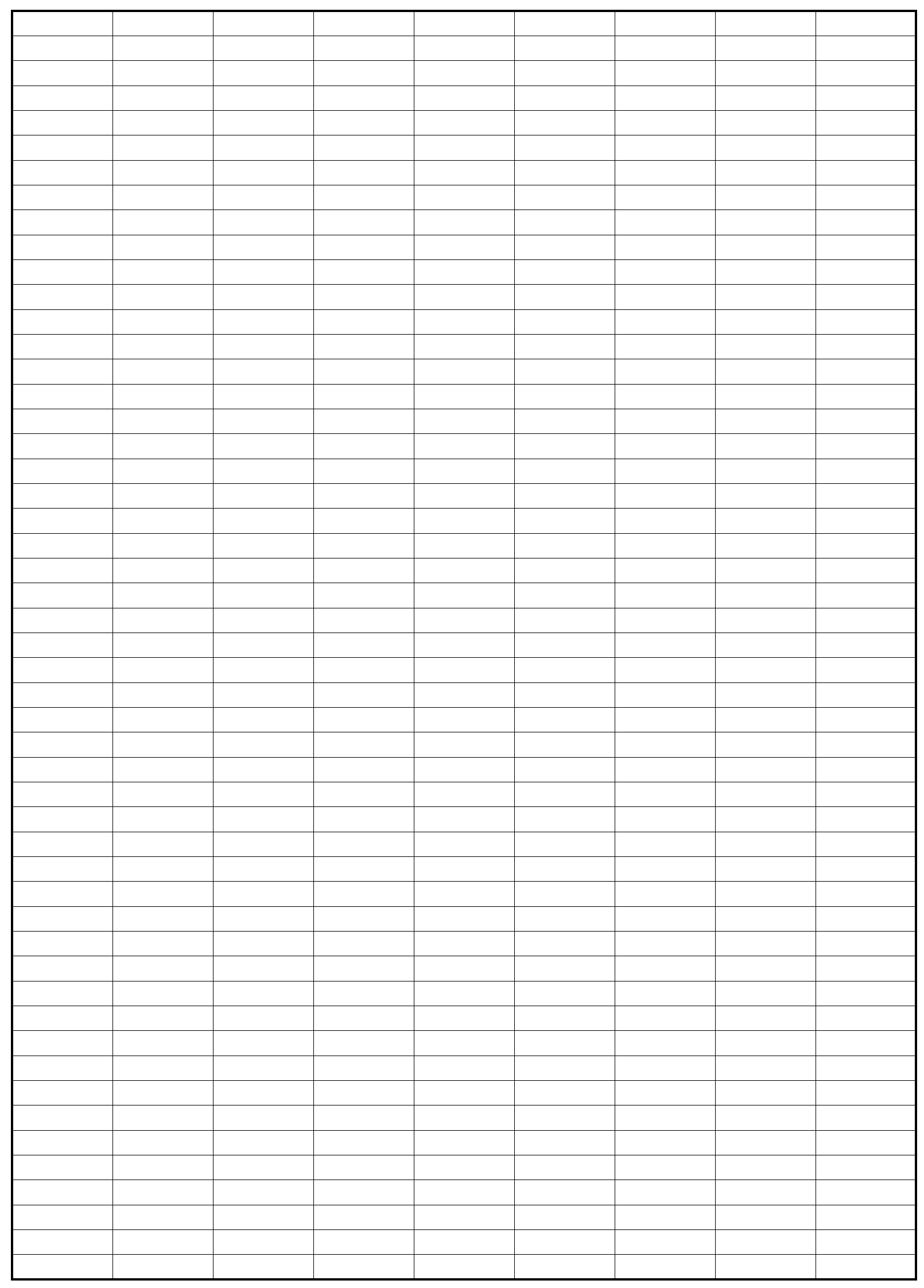

Page 45 
CHART DATA

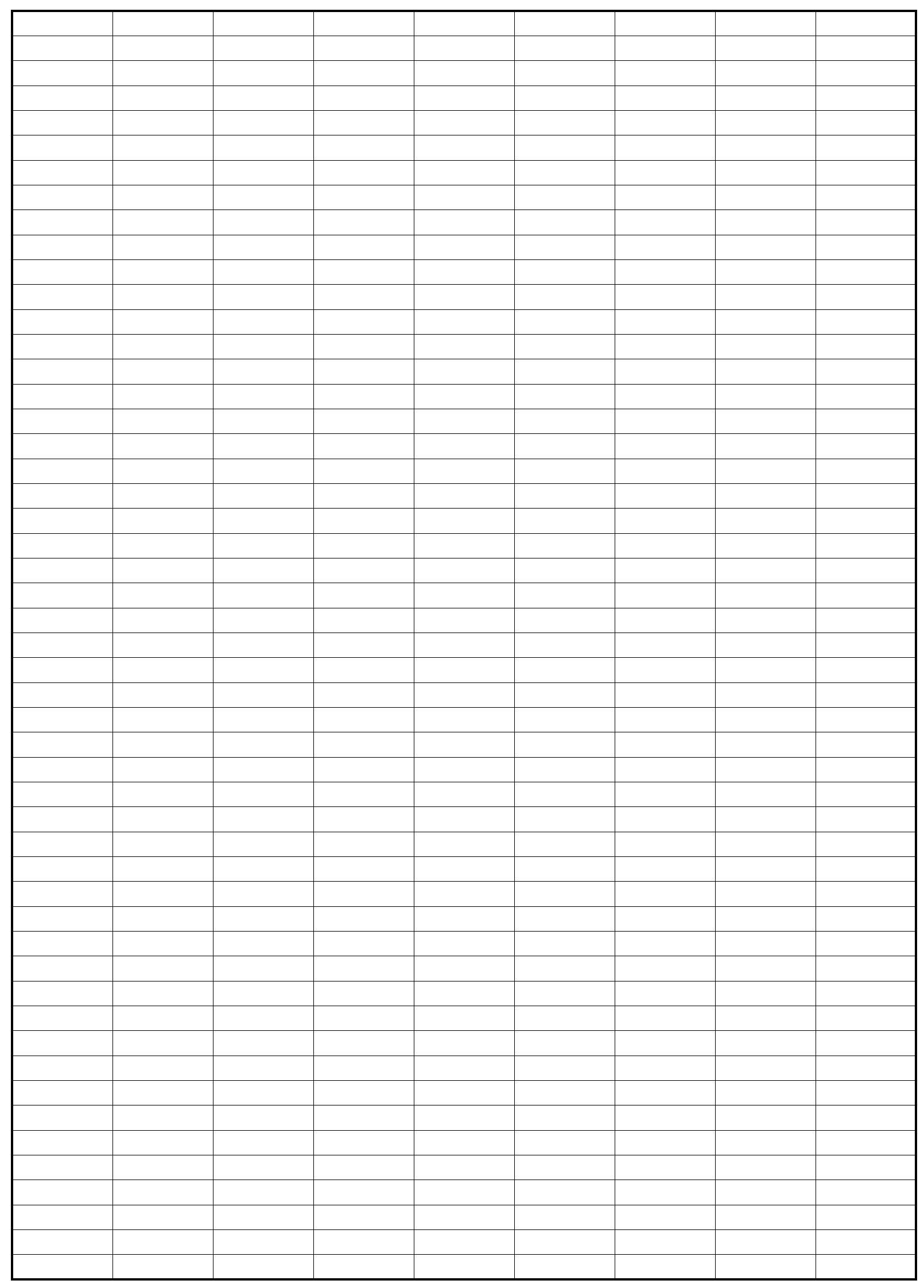

Page 46 
CHART DATA

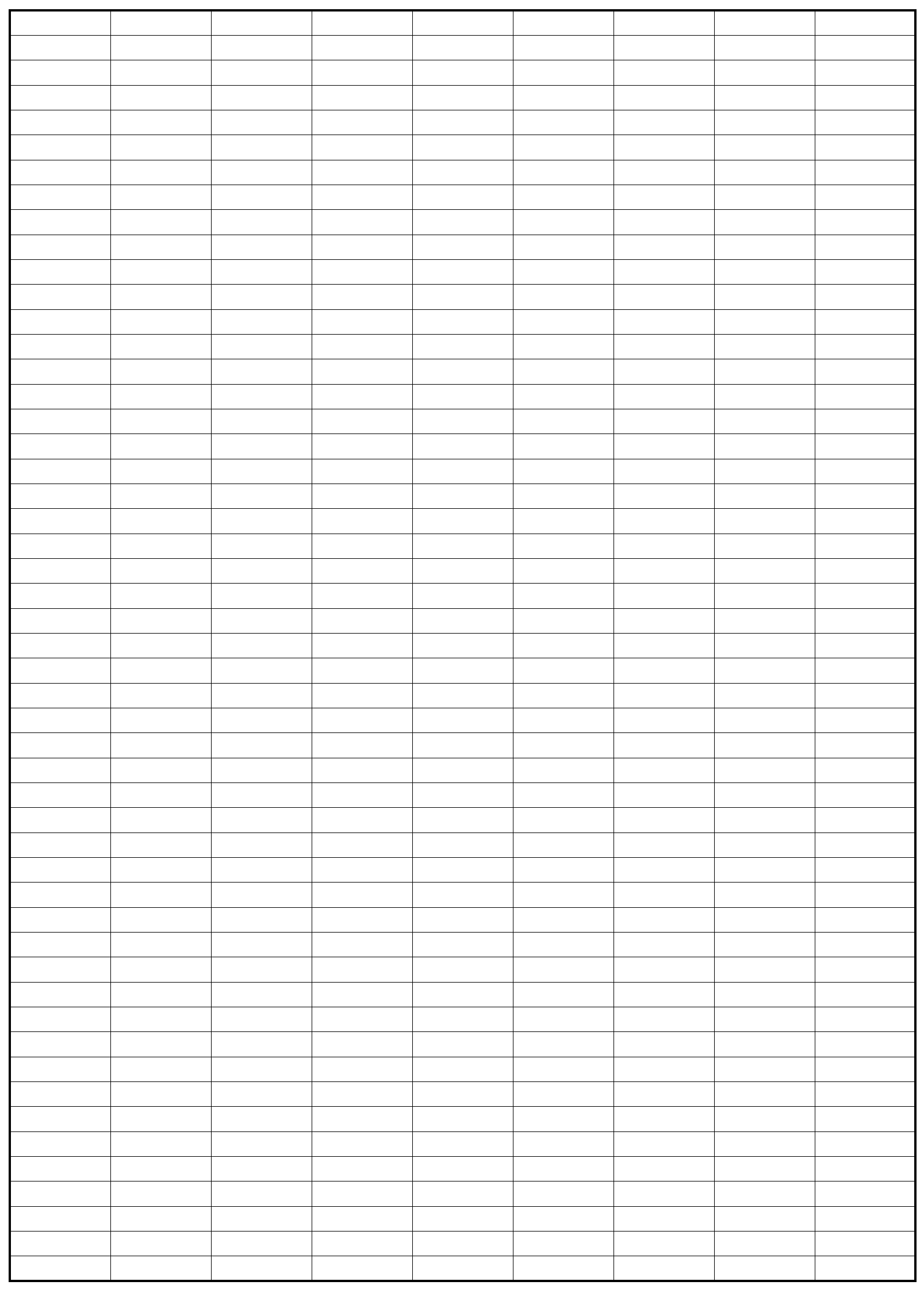

Page 47 
CHART DATA

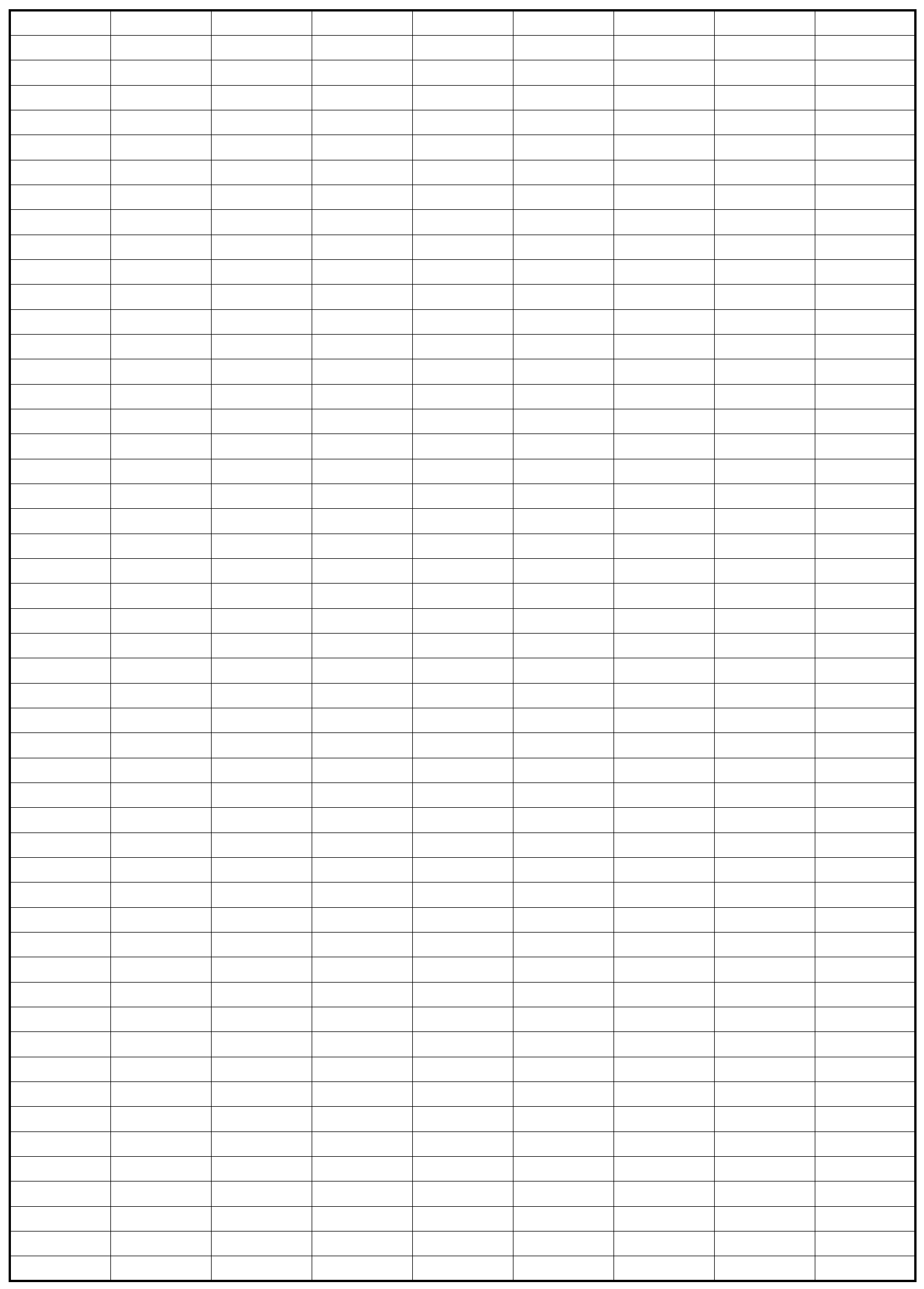

Page 48 
CHART DATA

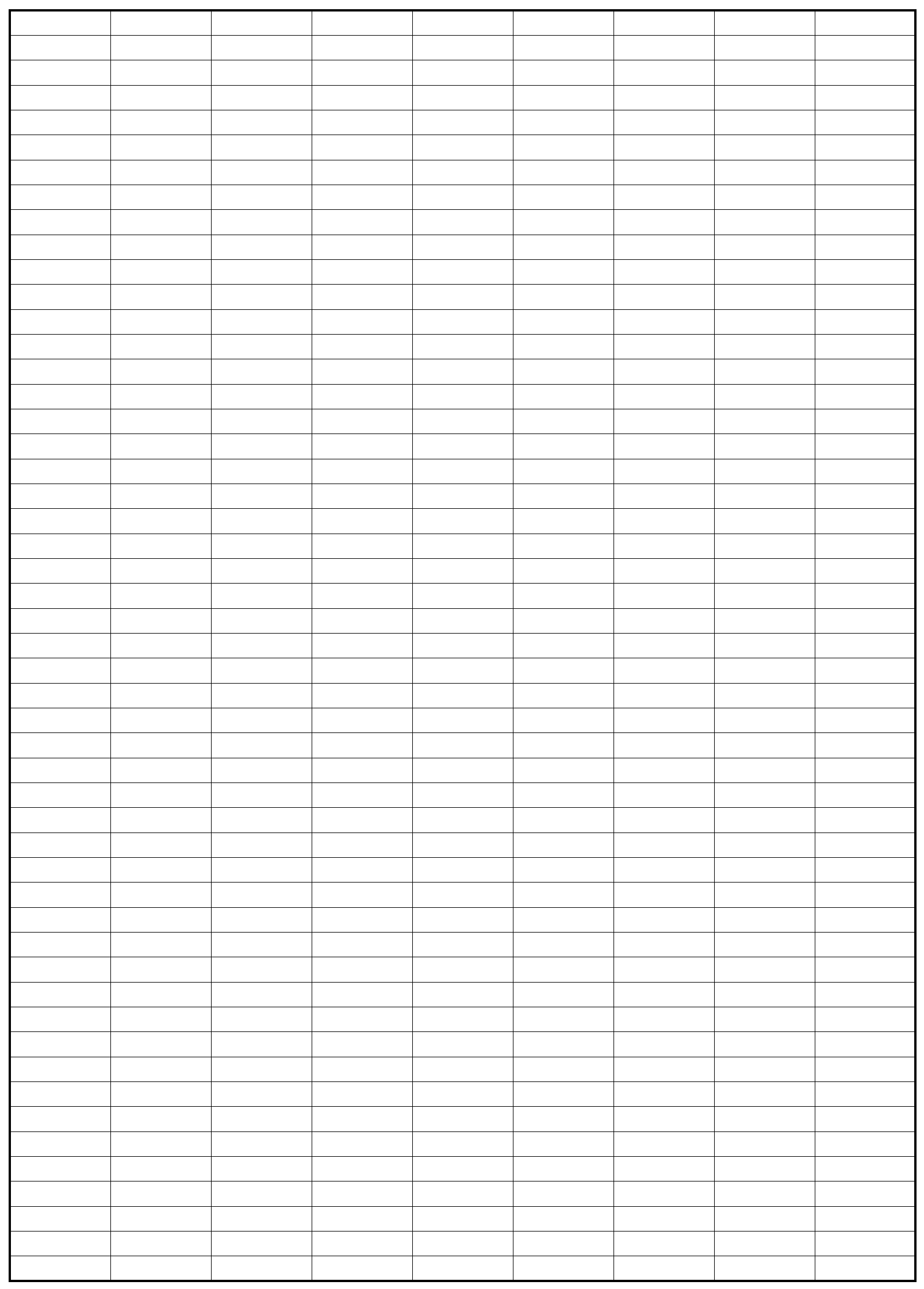

Page 49 
CHART DATA

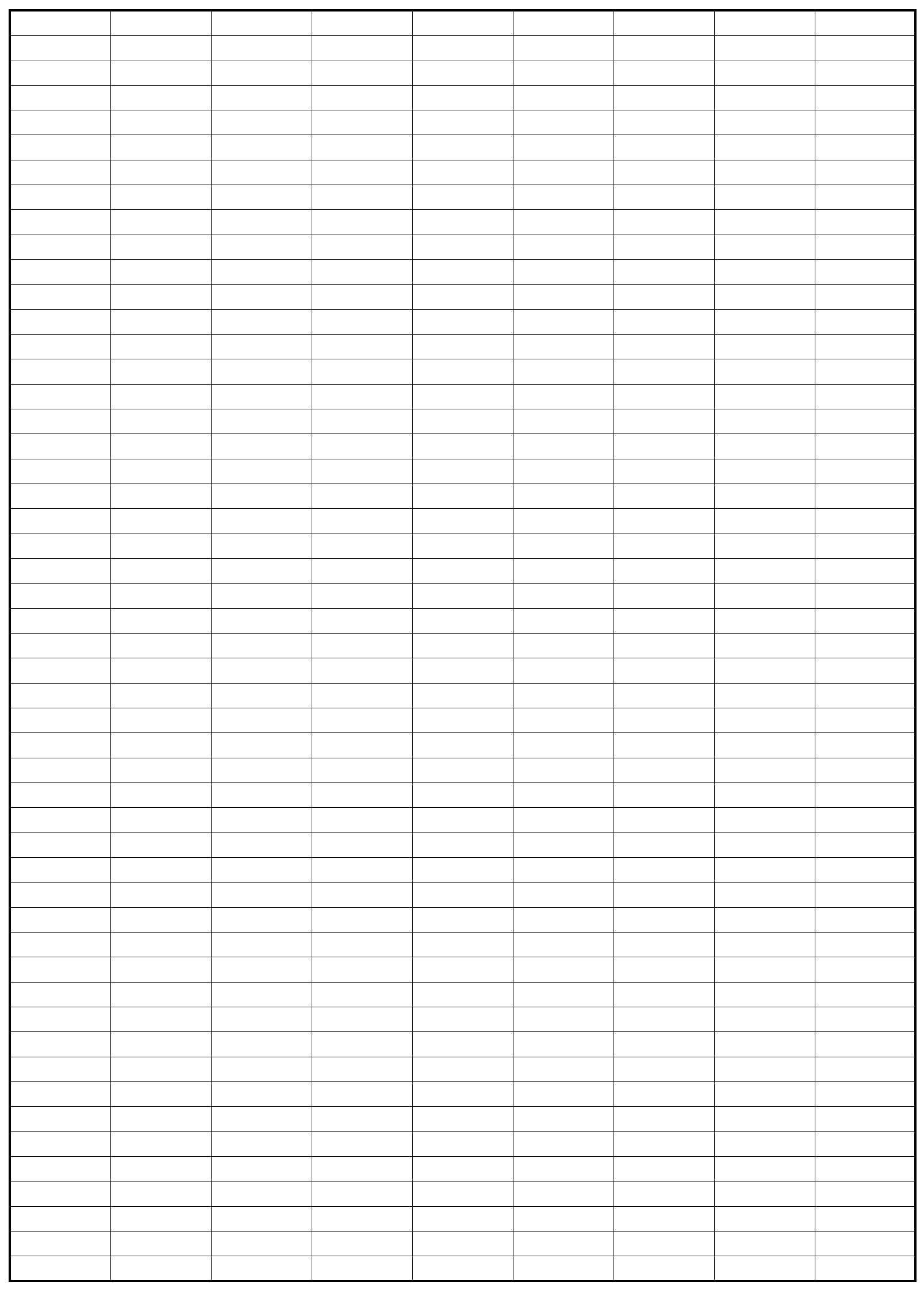

Page 50 
CHART DATA

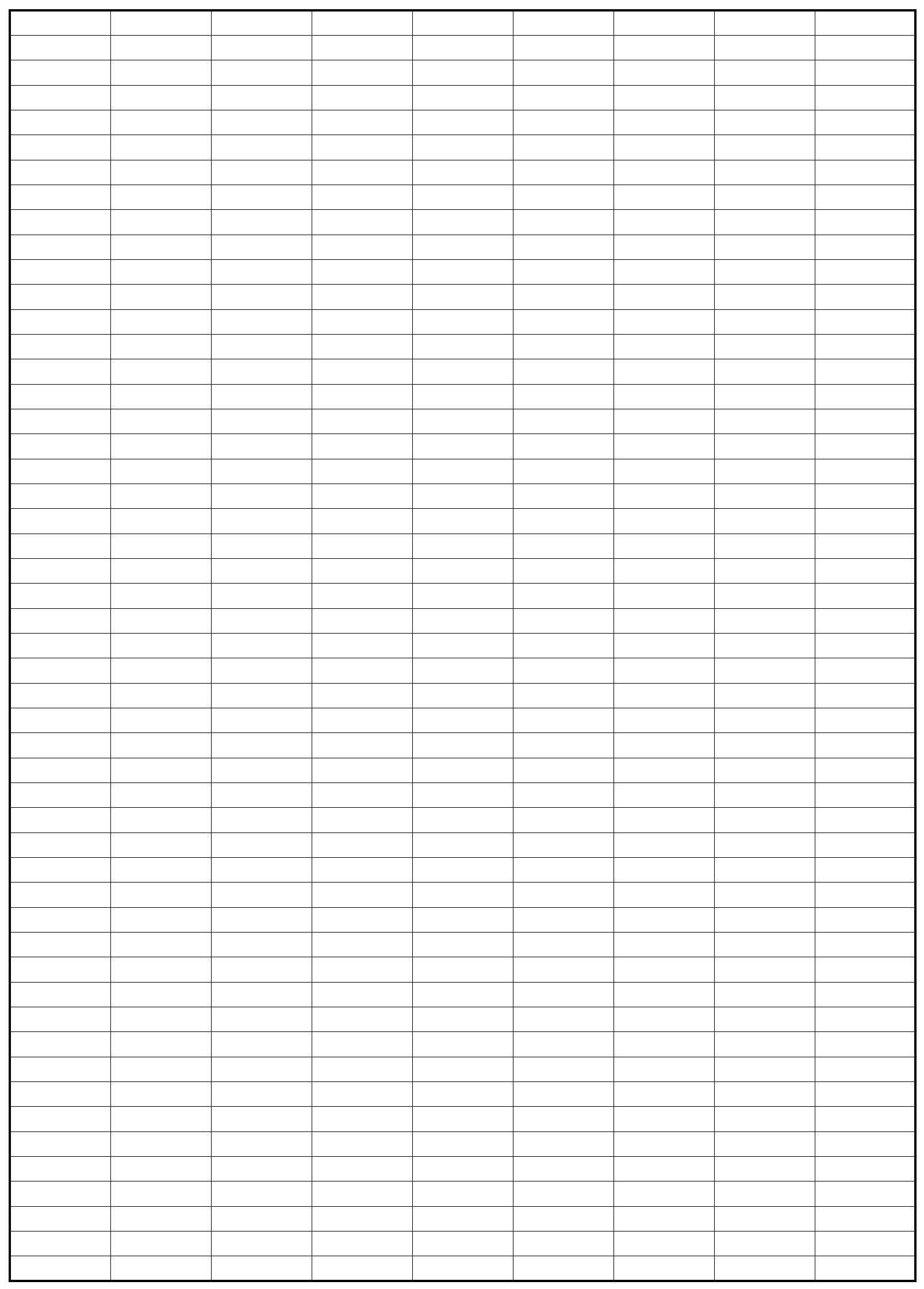

Page 51 
CHART DATA

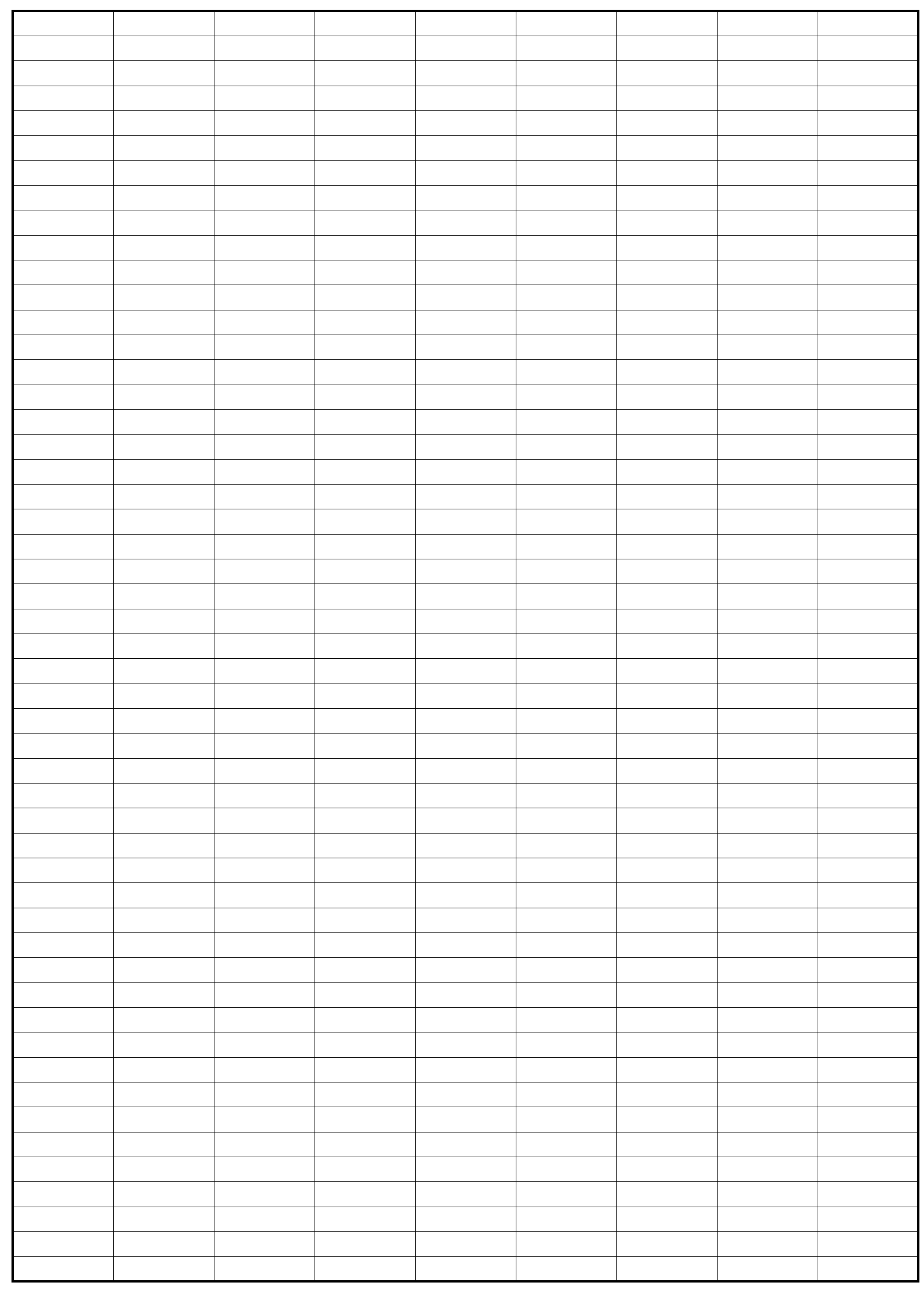

Page 52 
CHART DATA

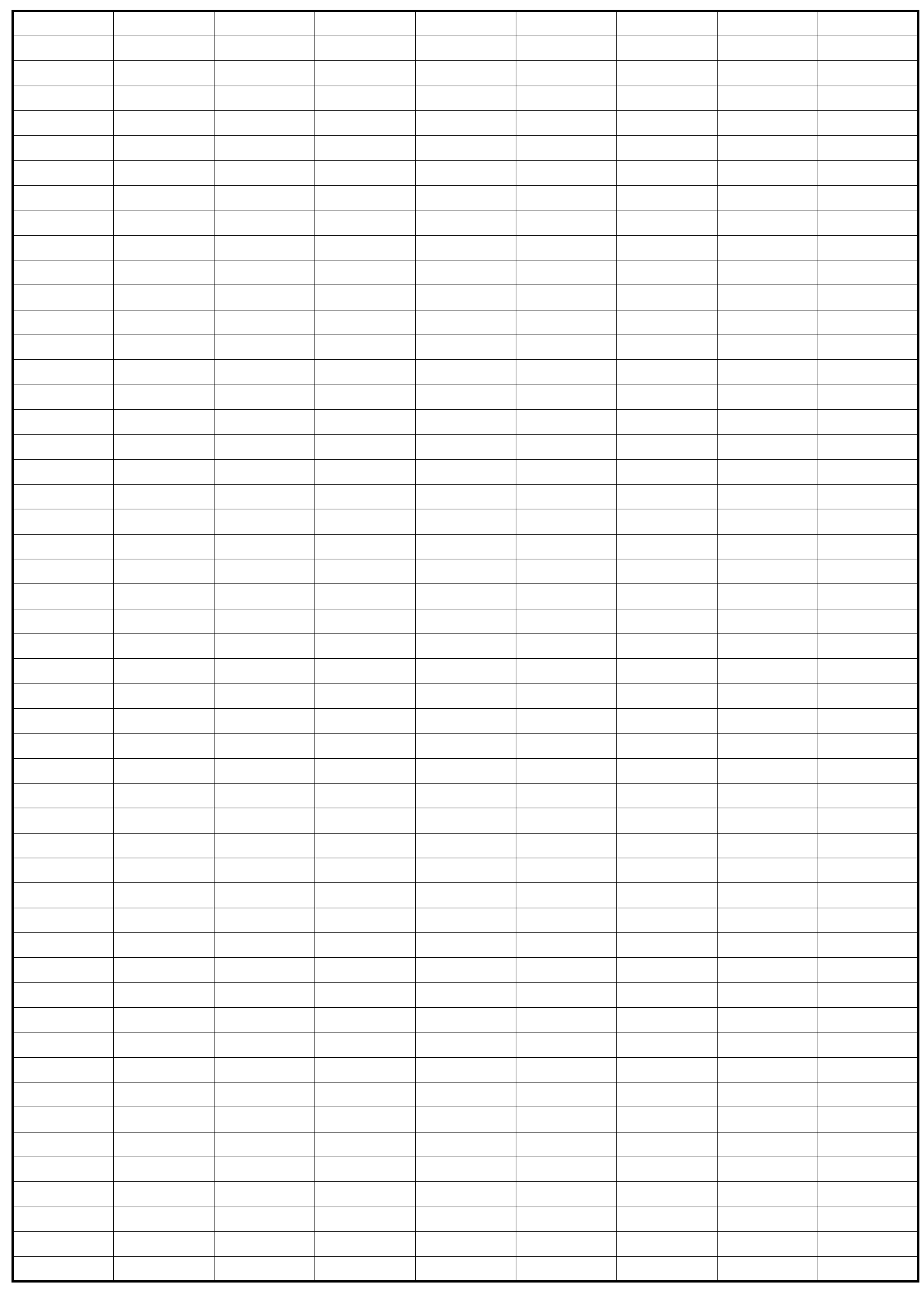

Page 53 
CHART DATA

\begin{tabular}{|l|l|l|l|l|l|l|l|l|}
\hline & & & & & & & & \\
\hline & & & & & & & & \\
\hline & & & & & & & & \\
\hline & & & & & & & & \\
\hline & & & & & & & & \\
\hline & & & & & & & & \\
\hline & & & & & & & & \\
\hline & & & & & & & & \\
\hline & & & & & & & & \\
\hline & & & & & & & & \\
\hline & & & & & & & & \\
\hline
\end{tabular}

Page 54 


\begin{tabular}{|c|c|c|c|c|c|c|c|c|}
\hline 15 & 16 & 17 & 18 & 19 & 20 & 21 & 22 & 23 \\
\hline 138.82 & 124.75 & 102.67 & 97.51 & 90.90 & 87.90 & 85.99 & 83.82 & 77.16 \\
\hline 183.30 & 164.77 & 74.27 & 67.84 & 60.01 & 61.09 & 60.05 & 57.55 & 54.35 \\
\hline 51.49 & 51.28 & 52.00 & 52.27 & 49.47 & 48.76 & 47.76 & 47.36 & 46.17 \\
\hline 24.00 & 22.19 & 19.56 & 17.42 & 17.39 & 20.84 & 20.86 & 20.68 & 20.61 \\
\hline 101.45 & 95.01 & 84.86 & 84.08 & 79.34 & 76.49 & 73.11 & 71.67 & 69.12 \\
\hline 162.21 & 161.61 & 43.95 & 31.92 & 30.87 & 32.80 & 31.17 & 30.82 & 30.79 \\
\hline 70.31 & 71.75 & 72.57 & 73.05 & 68.59 & 66.16 & 61.93 & 60.37 & 57.37 \\
\hline 44.40 & 44.55 & 44.17 & 40.80 & 35.18 & 27.38 & 18.22 & 18.00 & 17.72 \\
\hline 397.61 & 362.99 & 248.50 & 235.03 & 217.78 & 218.59 & 214.66 & 209.41 & 198.29 \\
\hline 378.37 & 372.92 & 245.54 & 229.85 & 213.98 & 202.84 & 184.43 & 180.86 & 174.99 \\
\hline 207.30 & 186.96 & 93.83 & 85.25 & 77.40 & 81.93 & 80.91 & 78.24 & 74.96 \\
\hline 206.61 & 206.16 & 88.12 & 72.72 & 66.05 & 60.18 & 49.39 & 48.82 & 48.51 \\
\hline 190.31 & 176.03 & 154.67 & 149.78 & 140.38 & 136.66 & 133.75 & 131.17 & 123.33 \\
\hline 171.76 & 166.76 & 157.42 & 157.14 & 147.93 & 142.65 & 135.04 & 132.04 & 126.48 \\
\hline & & & & & & & & \\
\hline & & & & & & & & \\
\hline 15 & 16 & 17 & 18 & 19 & 20 & 21 & 22 & 23 \\
\hline 62.27 & 53.58 & 43.84 & 41.09 & 40.53 & 40.67 & 40.74 & 39.94 & 35.84 \\
\hline 58.50 & 52.39 & 43.24 & 39.28 & 37.15 & 38.34 & 37.40 & 35.41 & 32.82 \\
\hline 10.53 & 9.46 & 9.29 & 9.50 & 10.30 & 10.64 & 11.09 & 11.50 & 11.40 \\
\hline 4.24 & 3.27 & 2.15 & 1.30 & 1.09 & 3.47 & 3.46 & 3.27 & 3.14 \\
\hline 22.47 & 15.39 & 9.51 & 9.03 & 9.20 & 9.19 & 9.45 & 10.65 & 10.82 \\
\hline 28.03 & 25.78 & 17.07 & 11.74 & 11.59 & 13.41 & 13.40 & 13.11 & 12.91 \\
\hline 8.38 & 7.93 & 7.74 & 7.74 & 8.10 & 8.28 & 8.30 & 9.28 & 9.37 \\
\hline 0.48 & 0.58 & 0.45 & 0.47 & 0.57 & 3.27 & 3.30 & 3.40 & 3.40 \\
\hline 135.53 & 118.69 & 98.52 & 91.16 & 89.07 & 93.12 & 92.70 & 90.12 & 83.20 \\
\hline 59.36 & 49.69 & 34.77 & 28.97 & 29.45 & 34.15 & 34.45 & 36.44 & 36.50 \\
\hline 62.74 & 55.66 & 45.38 & 40.57 & 38.24 & 41.80 & 40.86 & 38.68 & 35.96 \\
\hline 28.52 & 26.37 & 17.52 & 12.20 & 12.15 & 16.68 & 16.70 & 16.51 & 16.31 \\
\hline 72.79 & 63.03 & 53.14 & 50.58 & 50.82 & 51.32 & 51.83 & 51.44 & 47.24 \\
\hline 30.84 & 23.33 & 17.25 & 16.77 & 17.30 & 17.47 & 17.75 & 19.93 & 20.19 \\
\hline & & & & & & & & \\
\hline & & & & & & & & \\
\hline & & & & & & & & \\
\hline 15 & 16 & 17 & 18 & 19 & 20 & 21 & 22 & 23 \\
\hline 76.11 & 70.48 & 58.25 & 56.09 & 50.09 & 46.95 & 44.98 & 43.62 & 41.08 \\
\hline 124.79 & 112.37 & 31.04 & 28.56 & 22.86 & 22.65 & 22.45 & 22.05 & 21.44 \\
\hline 40.54 & 41.39 & 42.27 & 42.33 & 38.77 & 37.72 & 36.29 & 35.48 & 34.41 \\
\hline 19.26 & 18.46 & 16.99 & 15.74 & 15.92 & 16.94 & 16.97 & 16.98 & 16.84 \\
\hline 78.45 & 79.08 & 74.84 & 74.55 & 69.67 & 66.85 & 63.23 & 60.60 & 57.90 \\
\hline 134.18 & 135.83 & 26.89 & 20.18 & 19.28 & 19.39 & 17.77 & 17.72 & 17.88 \\
\hline 61.93 & 63.81 & 64.83 & 65.31 & 60.49 & 57.88 & 53.63 & 51.10 & 48.00 \\
\hline 43.92 & 43.97 & 43.72 & 40.33 & 34.62 & 24.12 & 14.92 & 14.60 & 14.32 \\
\hline 260.70 & 242.70 & 148.54 & 142.72 & 127.63 & 124.26 & 120.68 & 118.13 & 113.76 \\
\hline 318.48 & 322.69 & 210.27 & 200.37 & 184.05 & 168.23 & 149.55 & 144.02 & 138.10 \\
\hline 144.06 & 130.83 & 48.02 & 44.30 & 38.78 & 39.59 & 39.41 & 39.03 & 38.28 \\
\hline 178.09 & 179.80 & 70.60 & 60.51 & 53.90 & 43.51 & 32.69 & 32.32 & 32.20 \\
\hline 116.64 & 111.87 & 100.52 & 98.42 & 88.85 & 84.67 & 81.27 & 79.10 & 75.49 \\
\hline
\end{tabular}


CHART DATA

\begin{tabular}{|c|c|c|c|c|c|c|c|c|}
\hline 140.38 & 142.90 & 139.66 & 139.86 & 130.16 & 124.73 & 116.86 & 111.70 & 105.90 \\
\hline & & & & & & & & \\
\hline & & & & & & & & \\
\hline & & & & & & & & \\
\hline 15 & 16 & 17 & 18 & 19 & 20 & 21 & 22 & 23 \\
\hline 0.20 & 0.21 & 0.20 & 0.21 & 0.23 & 0.23 & 0.24 & 0.24 & 0.24 \\
\hline 0.32 & 0.35 & 0.11 & 0.00 & 0.07 & 0.00 & 0.07 & 0.10 & 0.03 \\
\hline 0.26 & 0.24 & 0.24 & 0.25 & 0.26 & 0.27 & 0.27 & 0.28 & 0.27 \\
\hline 0.33 & 0.20 & 0.20 & 0.00 & 0.00 & 0.10 & 0.00 & 0.10 & 0.10 \\
\hline 0.28 & 0.28 & 0.25 & 0.25 & 0.25 & 0.25 & 0.26 & 0.26 & 0.27 \\
\hline 0.24 & 0.23 & 0.00 & 0.00 & 0.00 & 0.00 & 0.00 & 0.00 & 0.00 \\
\hline 0.26 & 0.25 & 0.25 & 0.27 & 0.25 & 0.27 & 0.26 & 0.27 & 0.27 \\
\hline 0.00 & 0.00 & 0.00 & 0.00 & 0.00 & 0.00 & 0.00 & 0.00 & 0.00 \\
\hline 1.10 & 0.99 & 0.75 & 0.46 & 0.56 & 0.60 & 0.57 & 0.72 & 0.65 \\
\hline 0.79 & 0.76 & 0.50 & 0.52 & 0.50 & 0.52 & 0.51 & 0.53 & 0.54 \\
\hline 0.64 & 0.55 & 0.31 & 0.00 & 0.07 & $\overline{0.10}$ & 0.07 & 0.20 & 0.13 \\
\hline 0.24 & 0.23 & 0.00 & 0.00 & 0.00 & 0.00 & 0.00 & 0.00 & 0.00 \\
\hline 0.46 & 0.45 & 0.44 & 0.46 & 0.48 & 0.50 & 0.50 & 0.52 & 0.52 \\
\hline 0.55 & 0.53 & 0.50 & 0.52 & 0.50 & 0.52 & 0.51 & 0.53 & 0.54 \\
\hline & & & & & & & & \\
\hline & & & & & & & & \\
\hline & & & & & & & & \\
\hline & & & & & & & & \\
\hline & & & & & & & & \\
\hline & & & & & & & & \\
\hline & & & & & & & & \\
\hline & & & & & & & & \\
\hline & & & & & & & & \\
\hline & & & & & & & & \\
\hline & & & & & & & & \\
\hline & & & & & & & & \\
\hline & & & & & & & & \\
\hline & & & & & & & & \\
\hline & & & & & & & & \\
\hline & & & & & & & & \\
\hline & & & & & & & & \\
\hline & & & & & & & & \\
\hline & & & & & & & & \\
\hline & & & & & & & & \\
\hline & & & & & & & & \\
\hline & & & & & & & & \\
\hline & & & & & & & & \\
\hline & & & & & & & & \\
\hline & & & & & & & & \\
\hline & & & & & & & & \\
\hline & & & & & & & & \\
\hline & & & & & & & & \\
\hline & & & & & & & & \\
\hline & & & & & & & & \\
\hline & & & & & & & & \\
\hline
\end{tabular}


CHART DATA

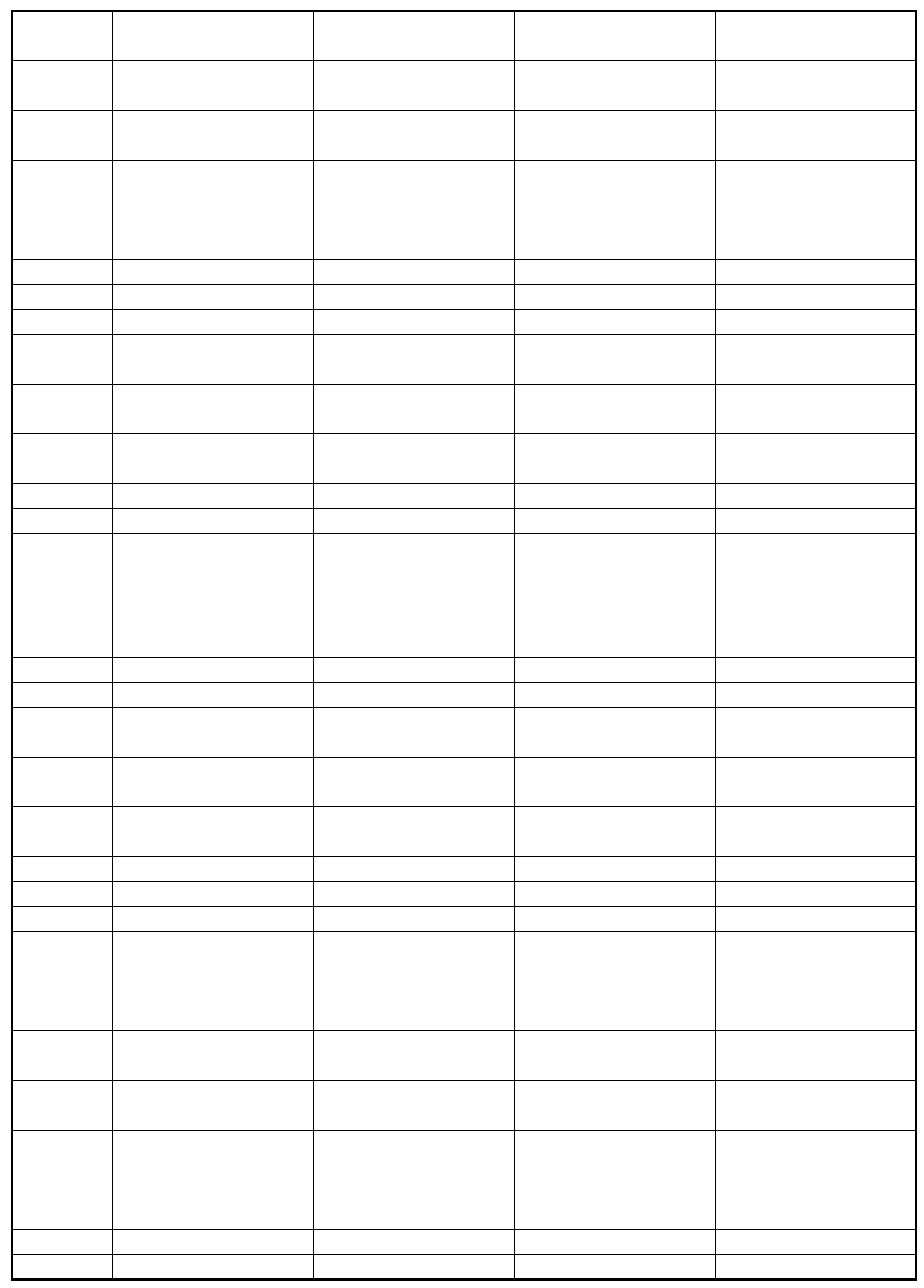

Page 57 
CHART DATA

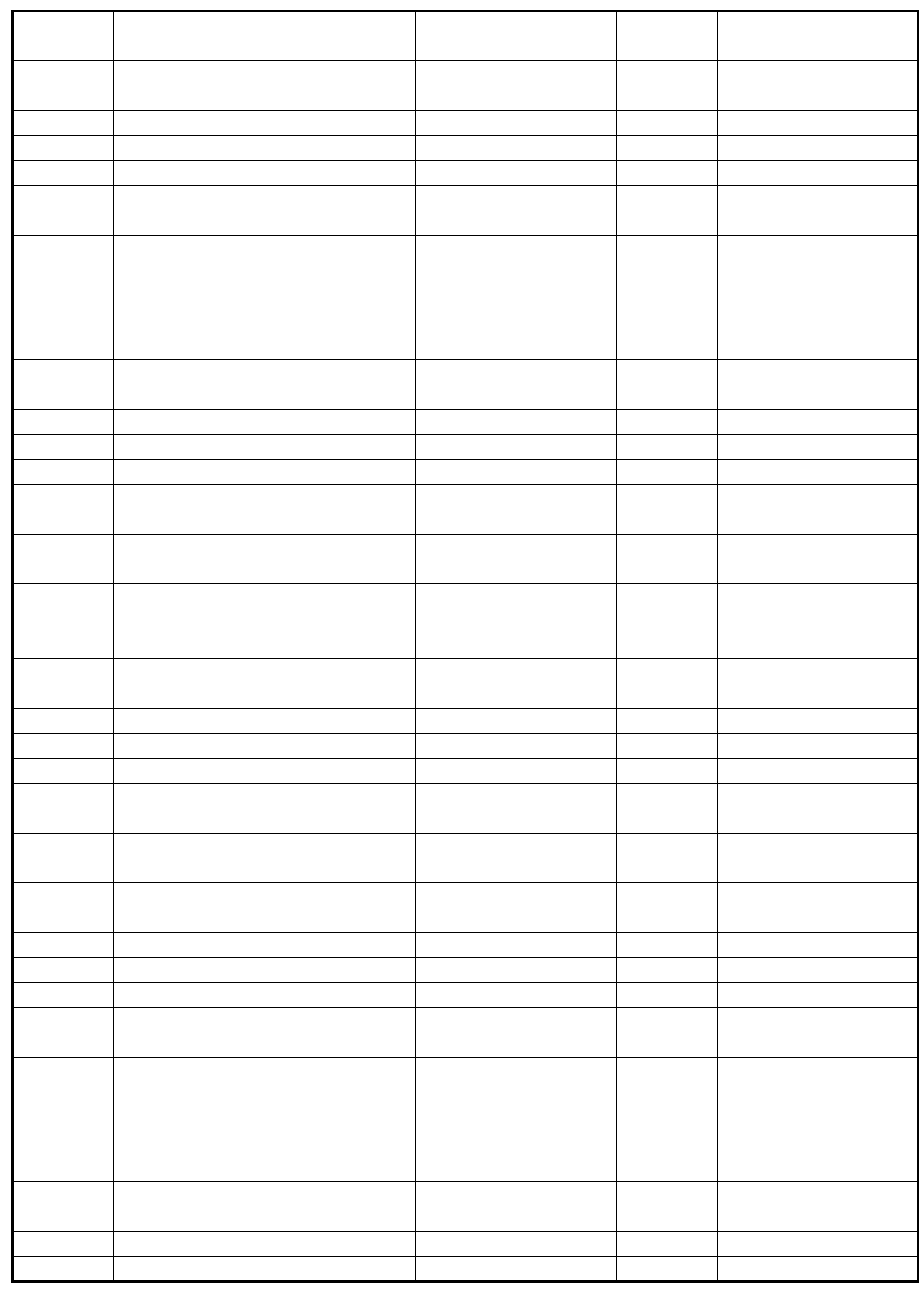

Page 58 
CHART DATA

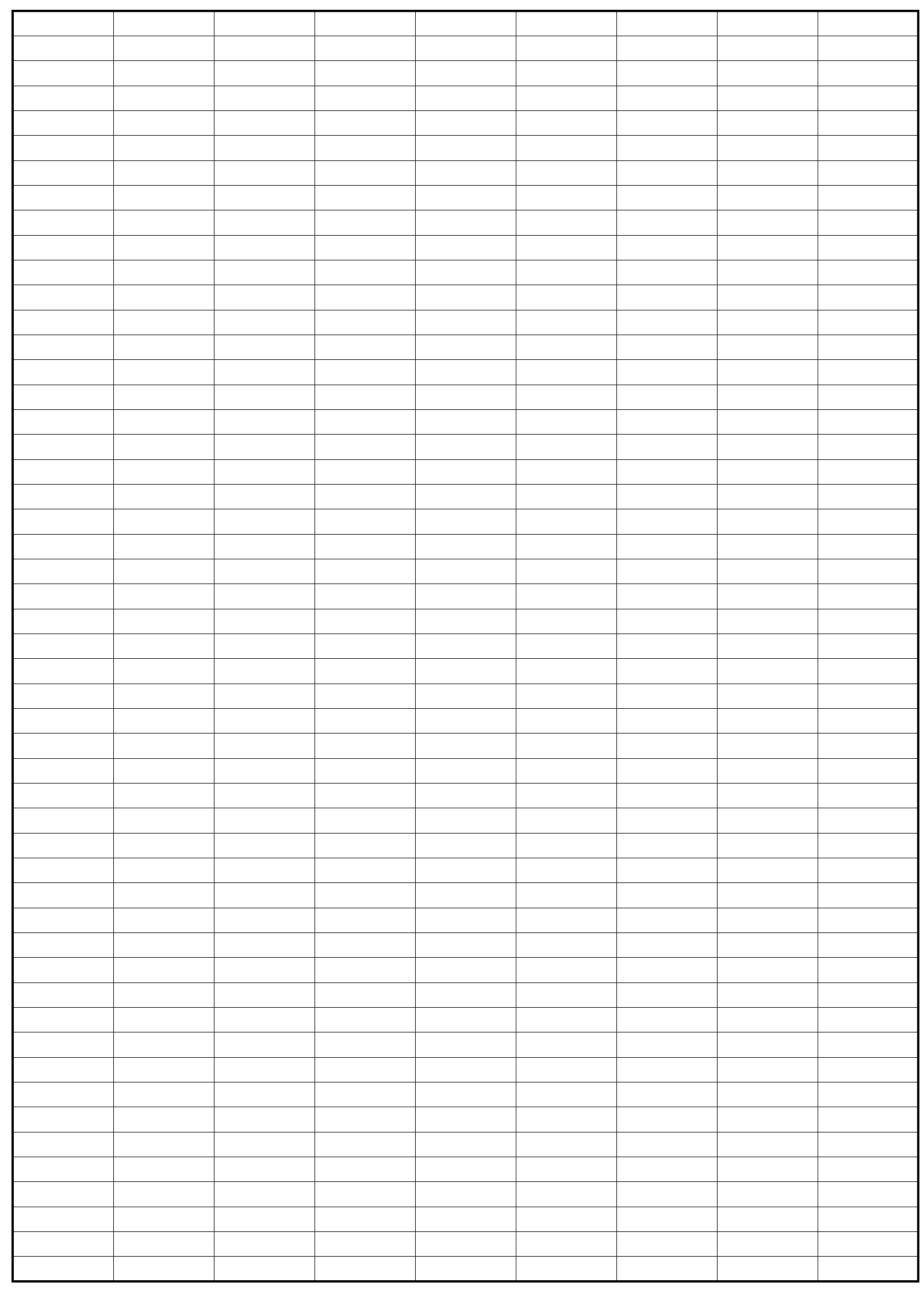

Page 59 
CHART DATA

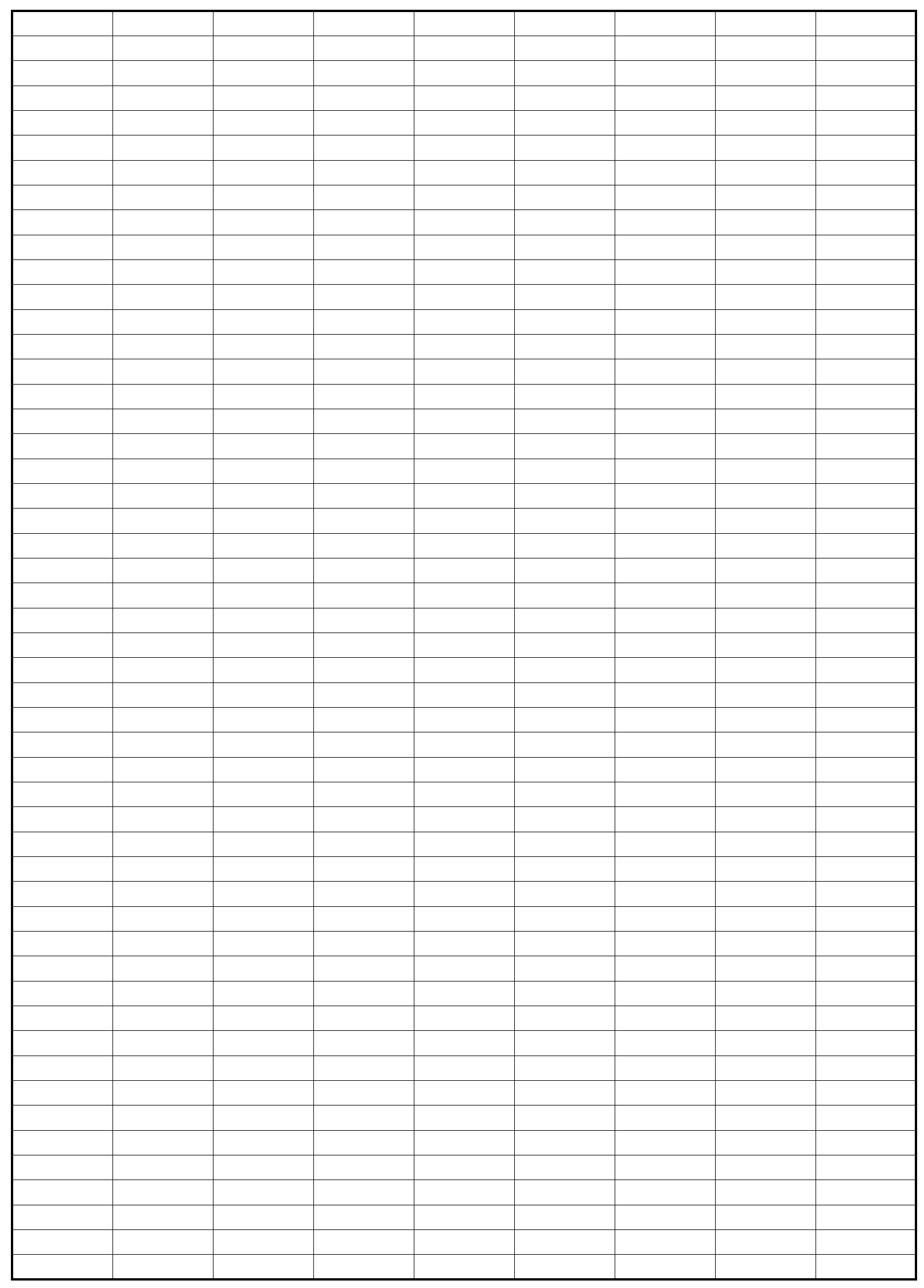

Page 60 
CHART DATA

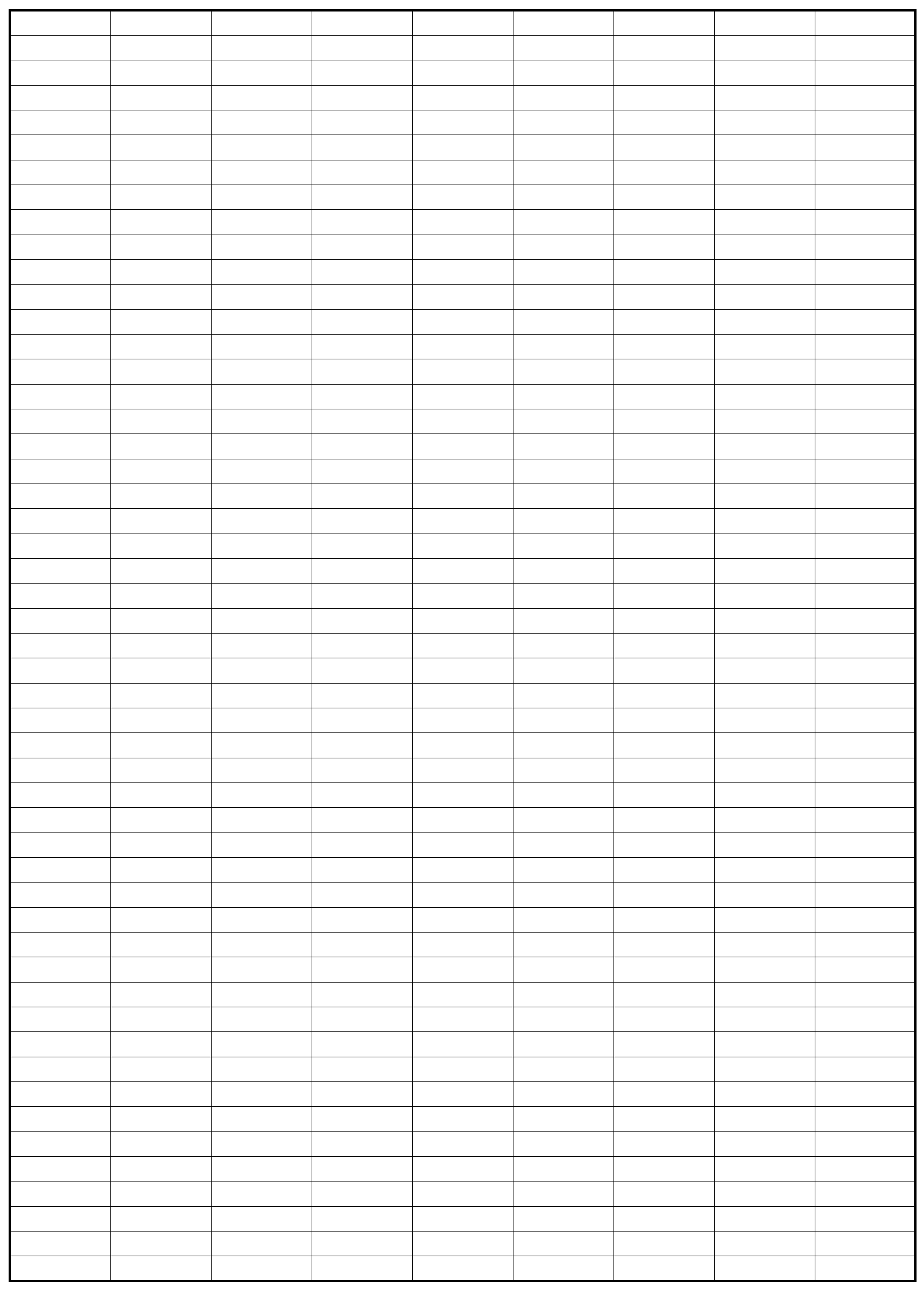

Page 61 
CHART DATA

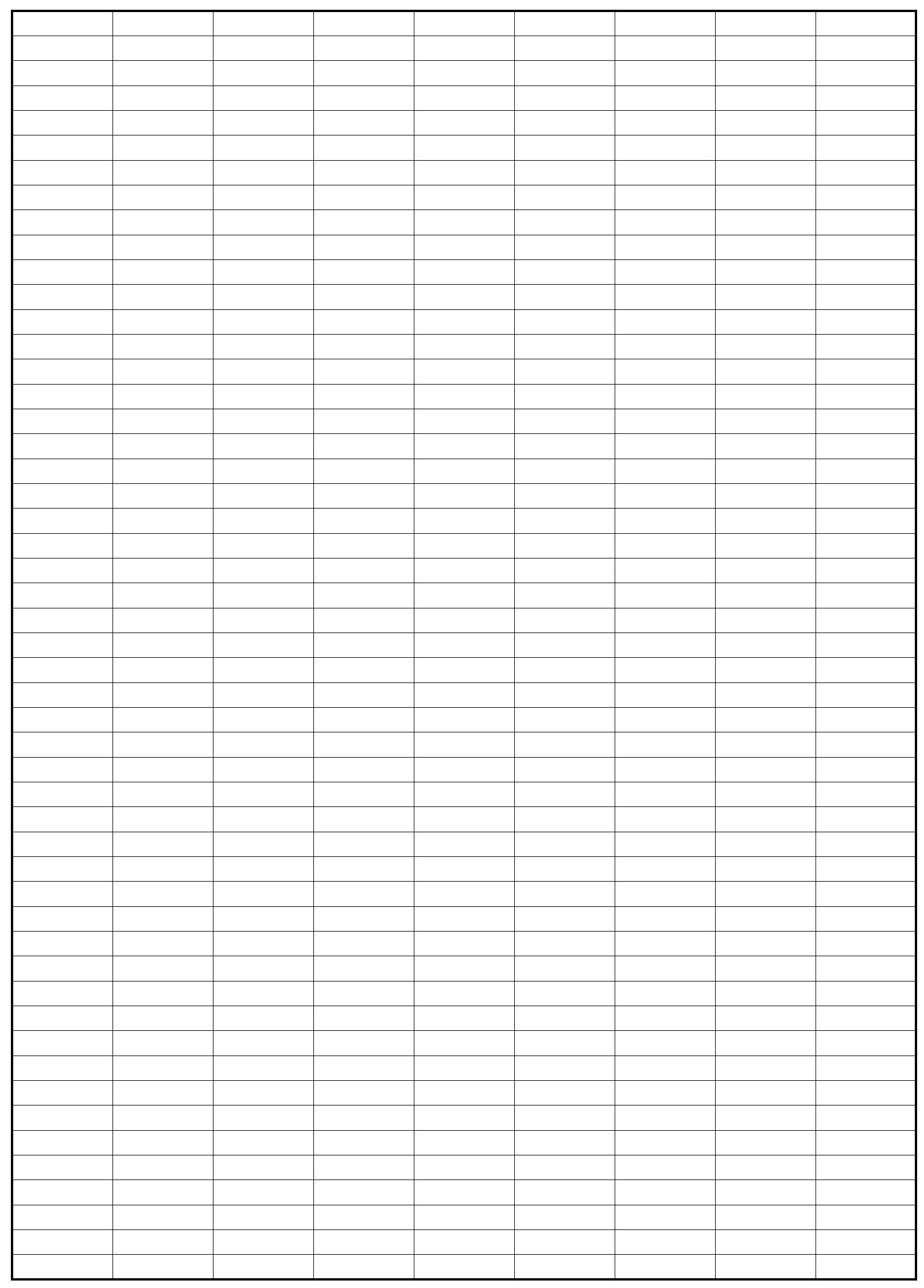

Page 62 
CHART DATA

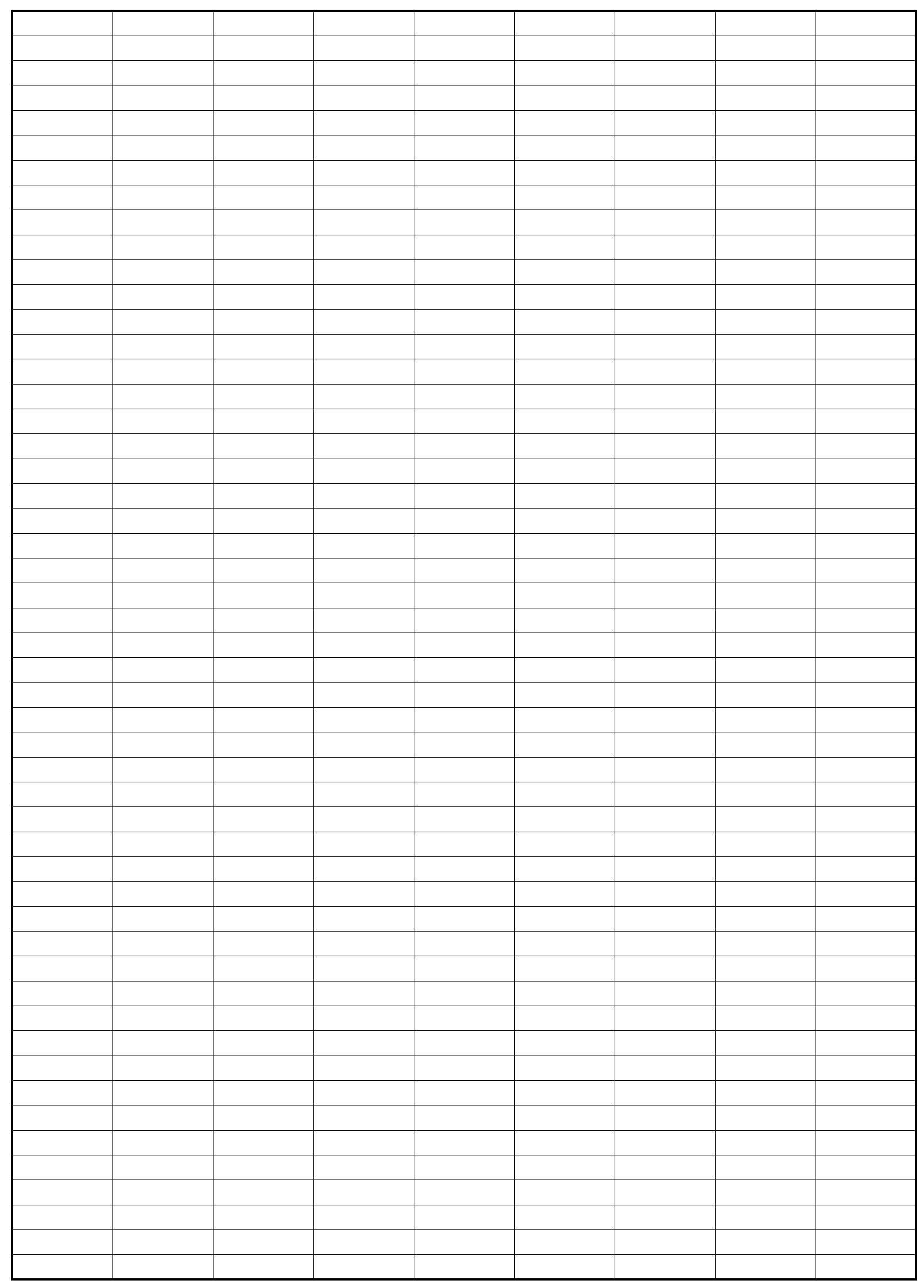

Page 63 
CHART DATA

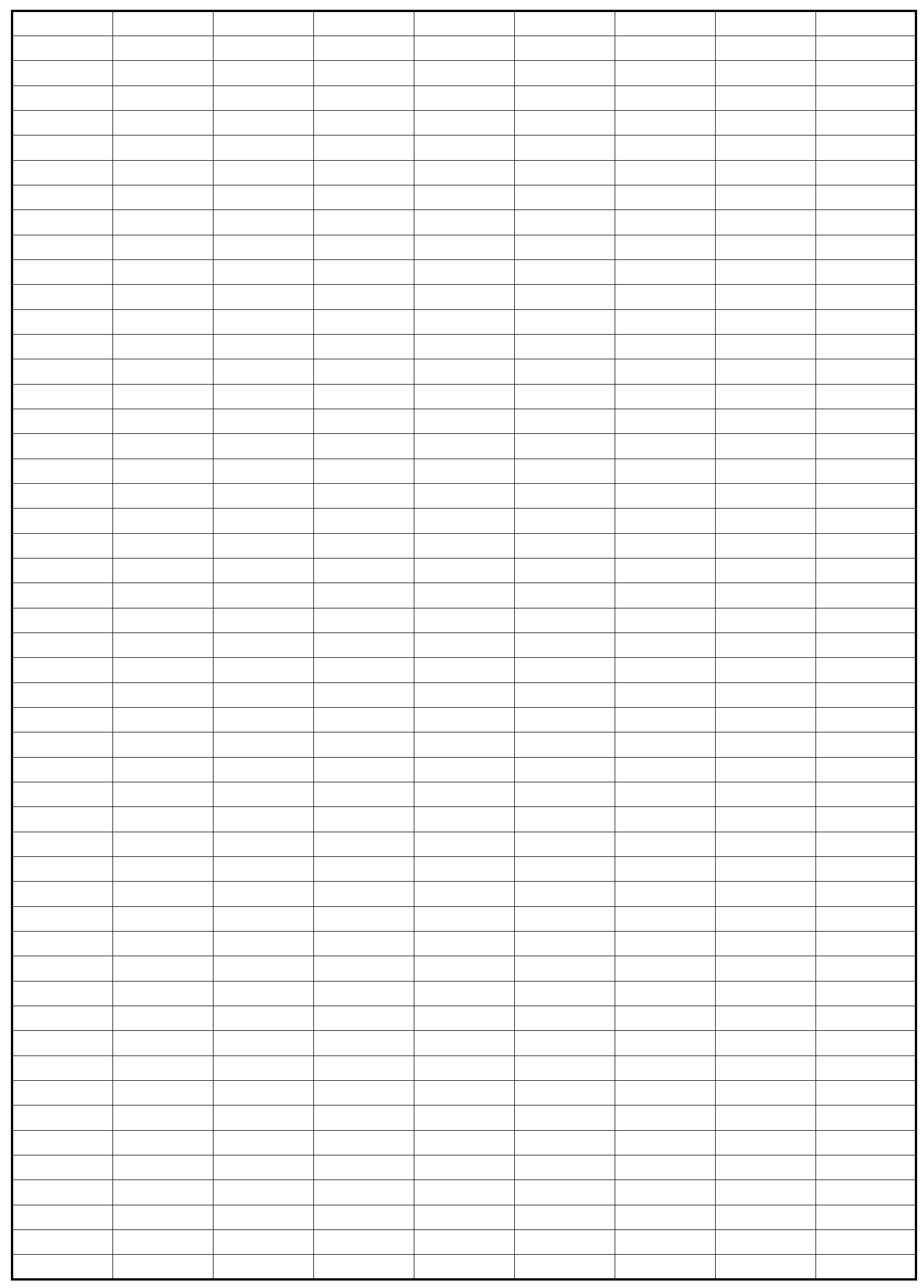

Page 64 
CHART DATA

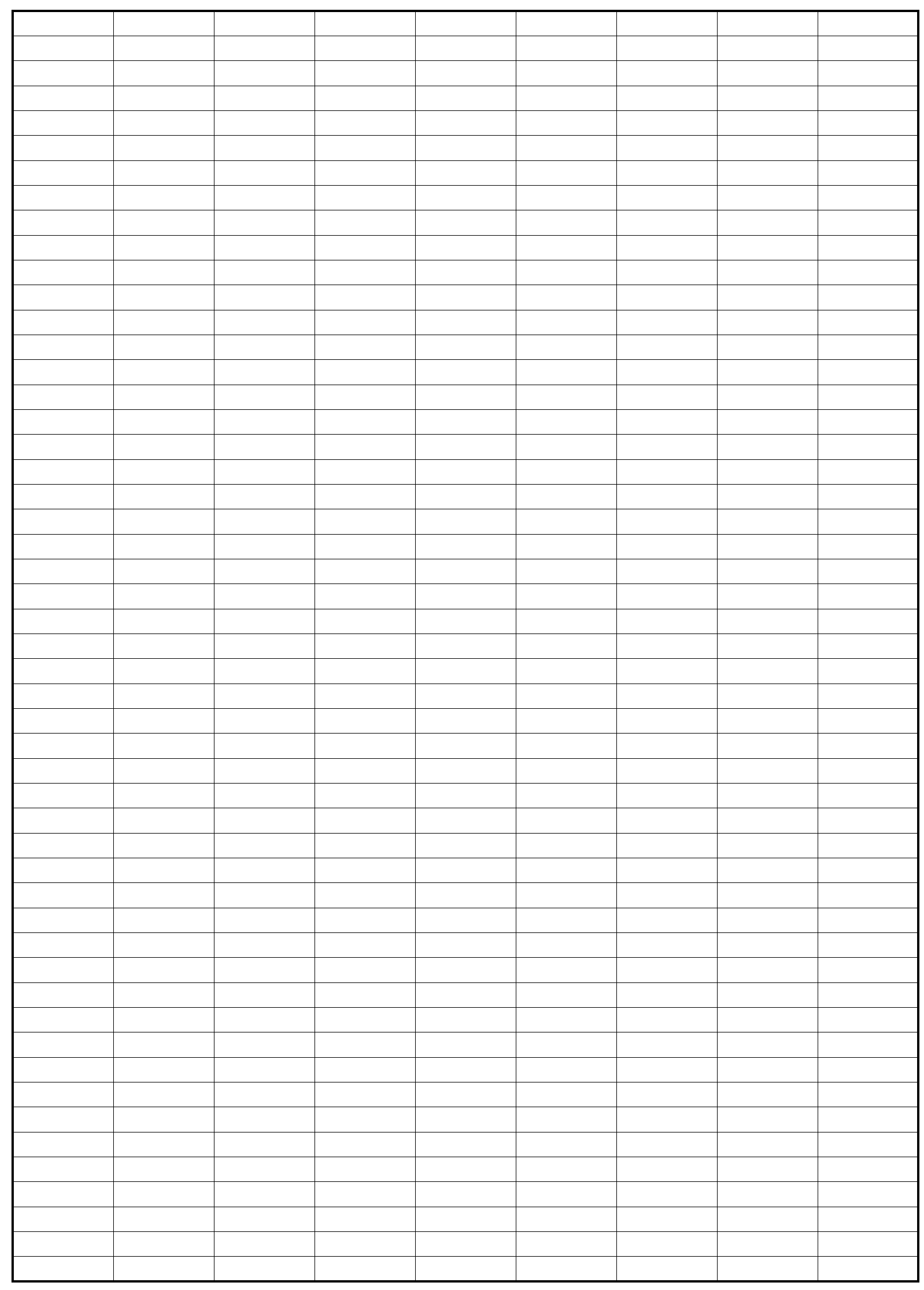

Page 65 
CHART DATA

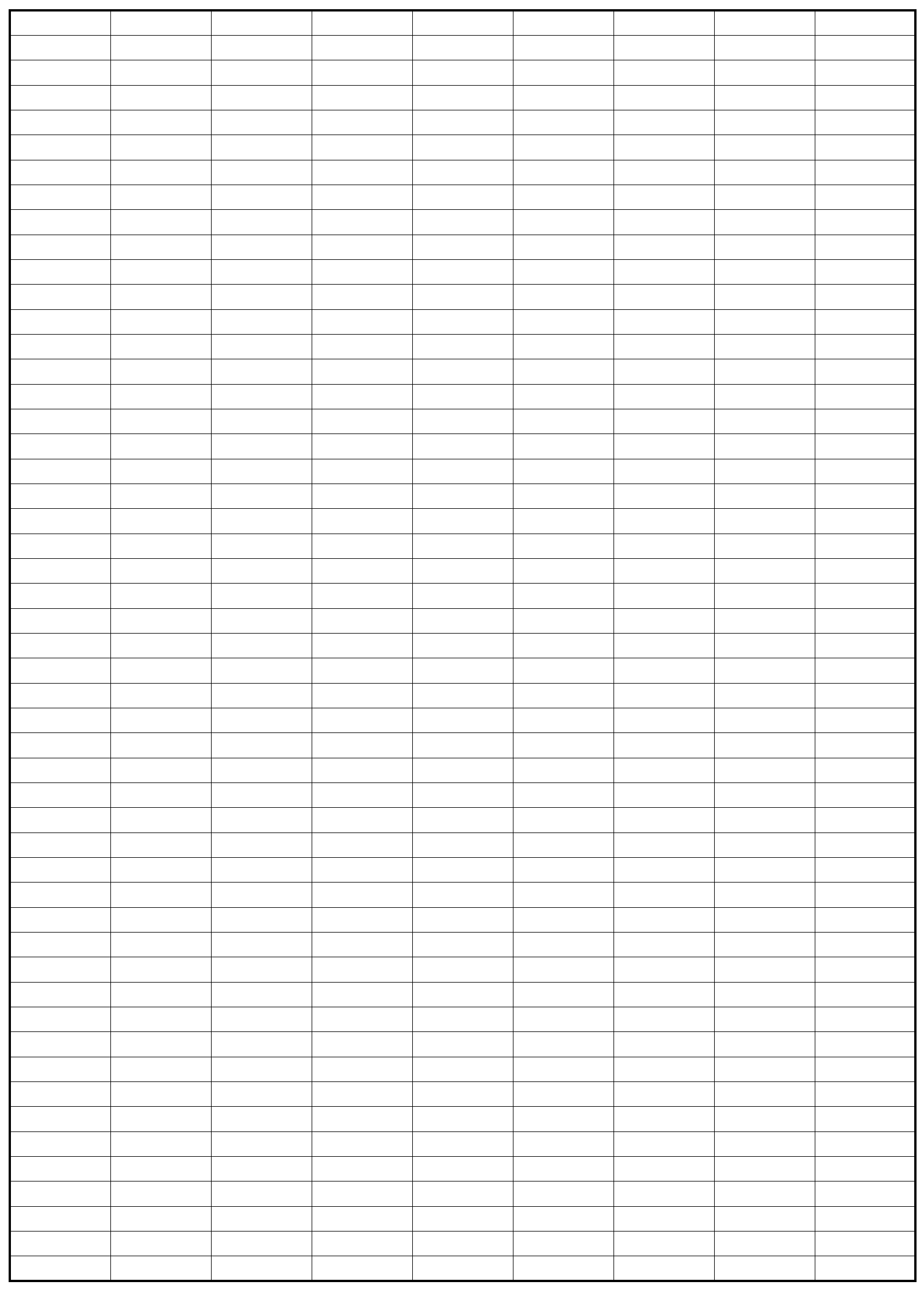

Page 66 
CHART DATA

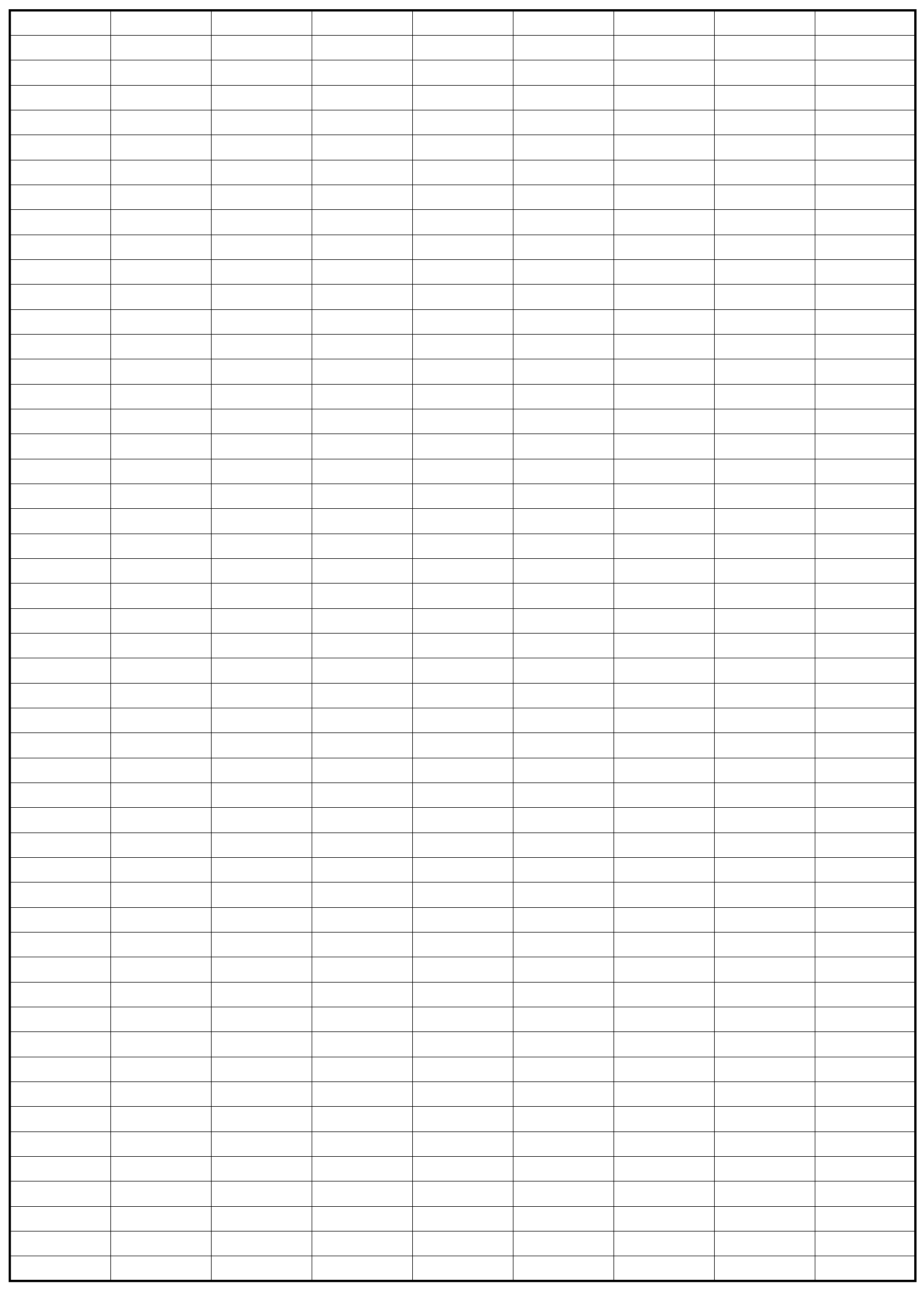

Page 67 
CHART DATA

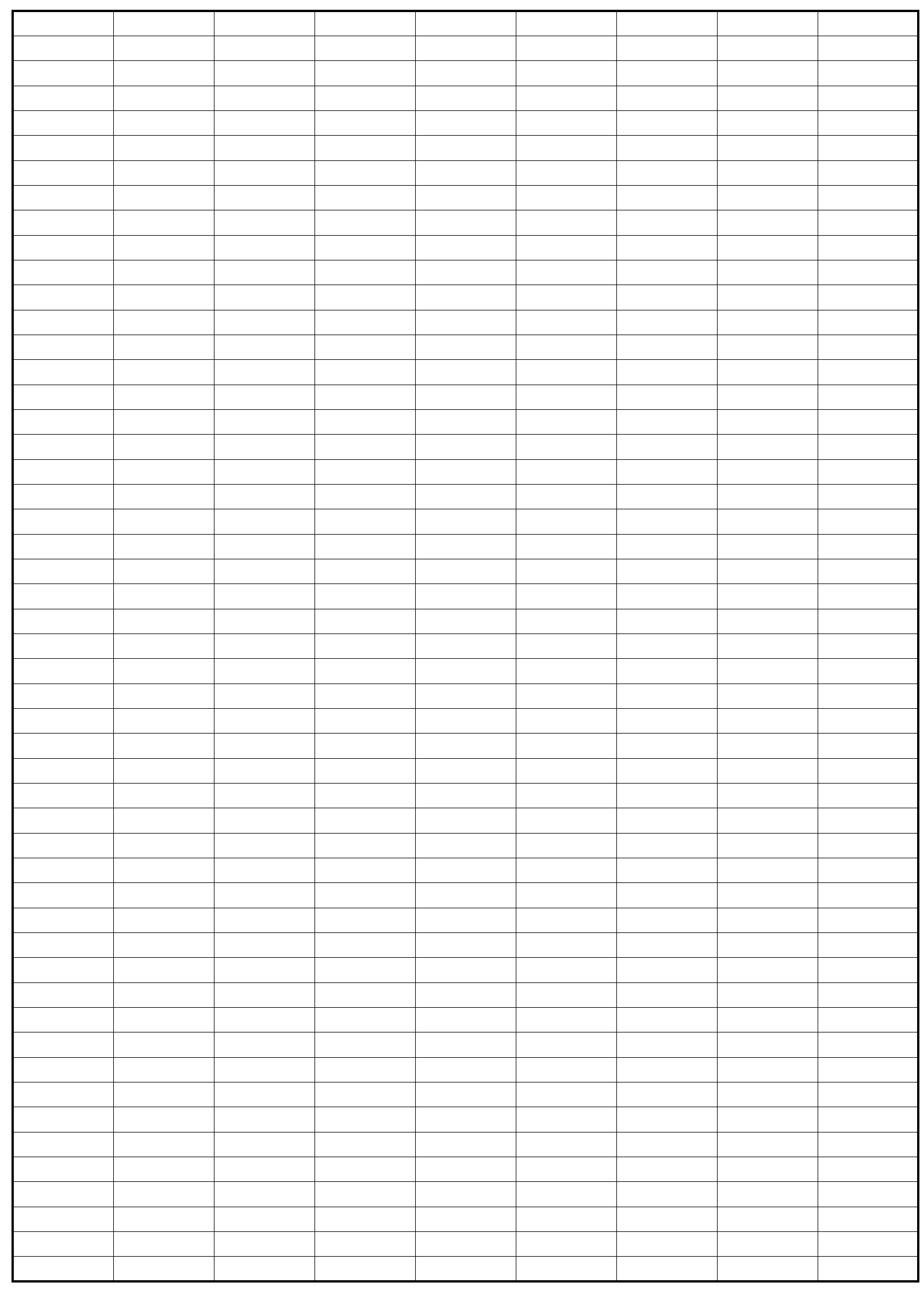

Page 68 
CHART DATA

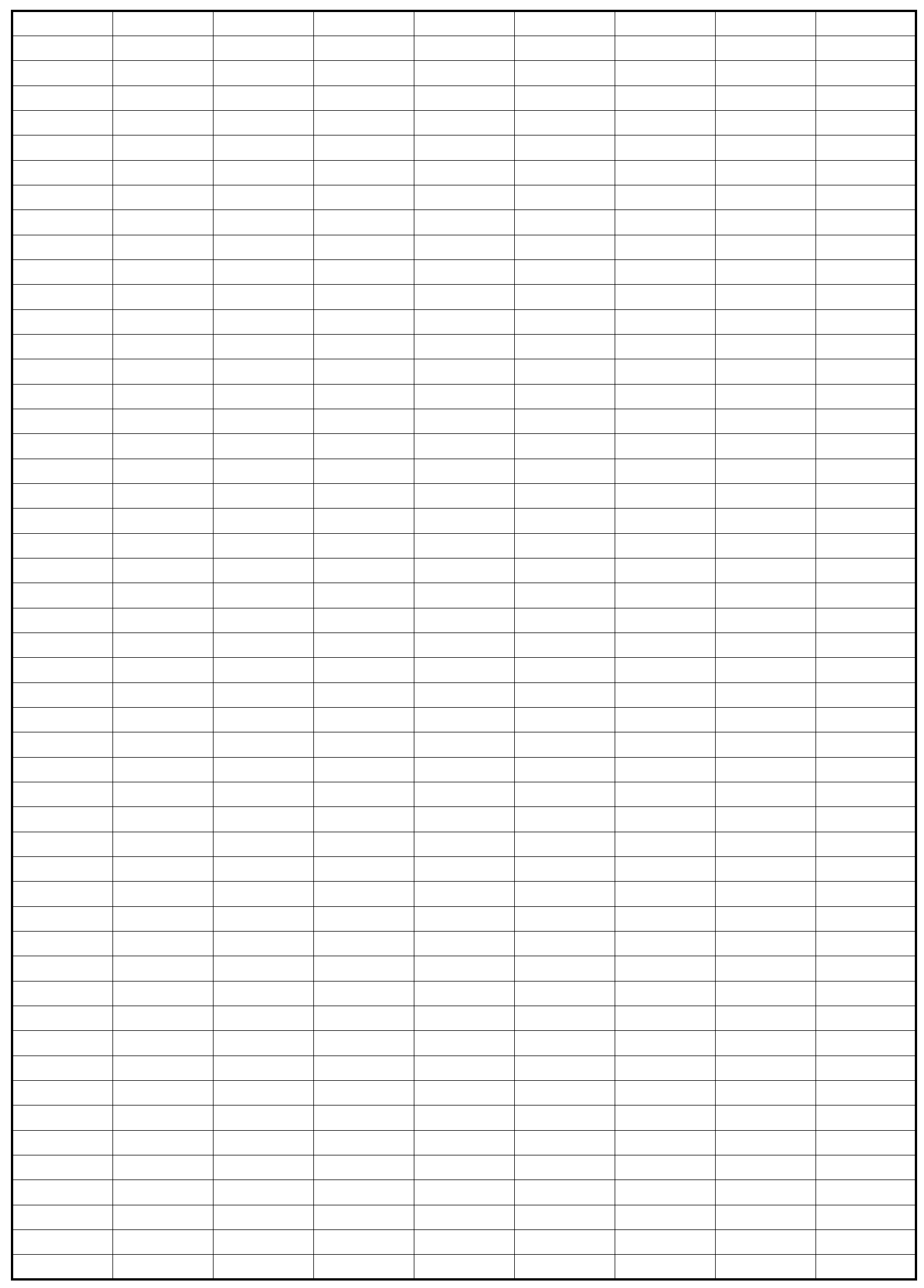

Page 69 
CHART DATA

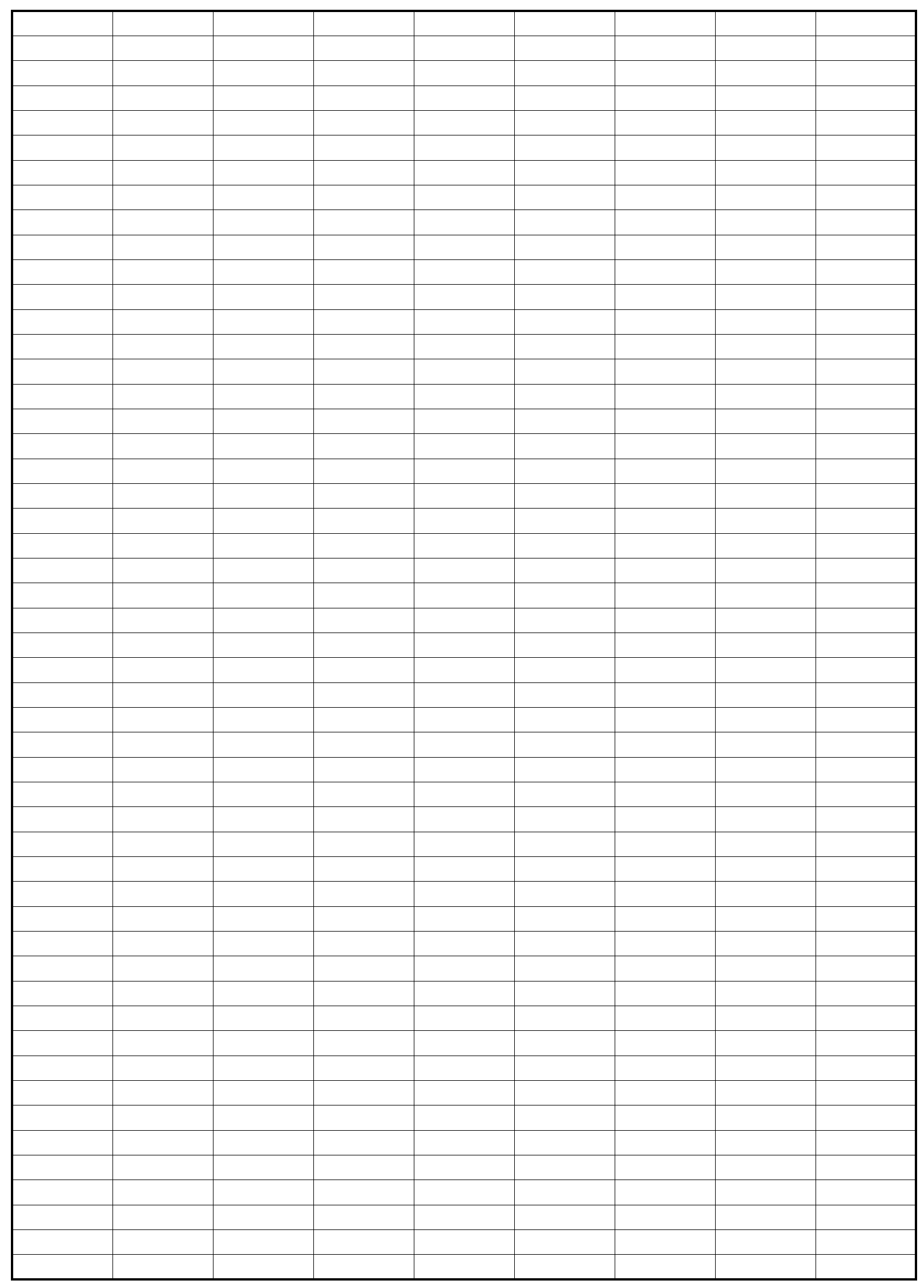

Page 70 
CHART DATA

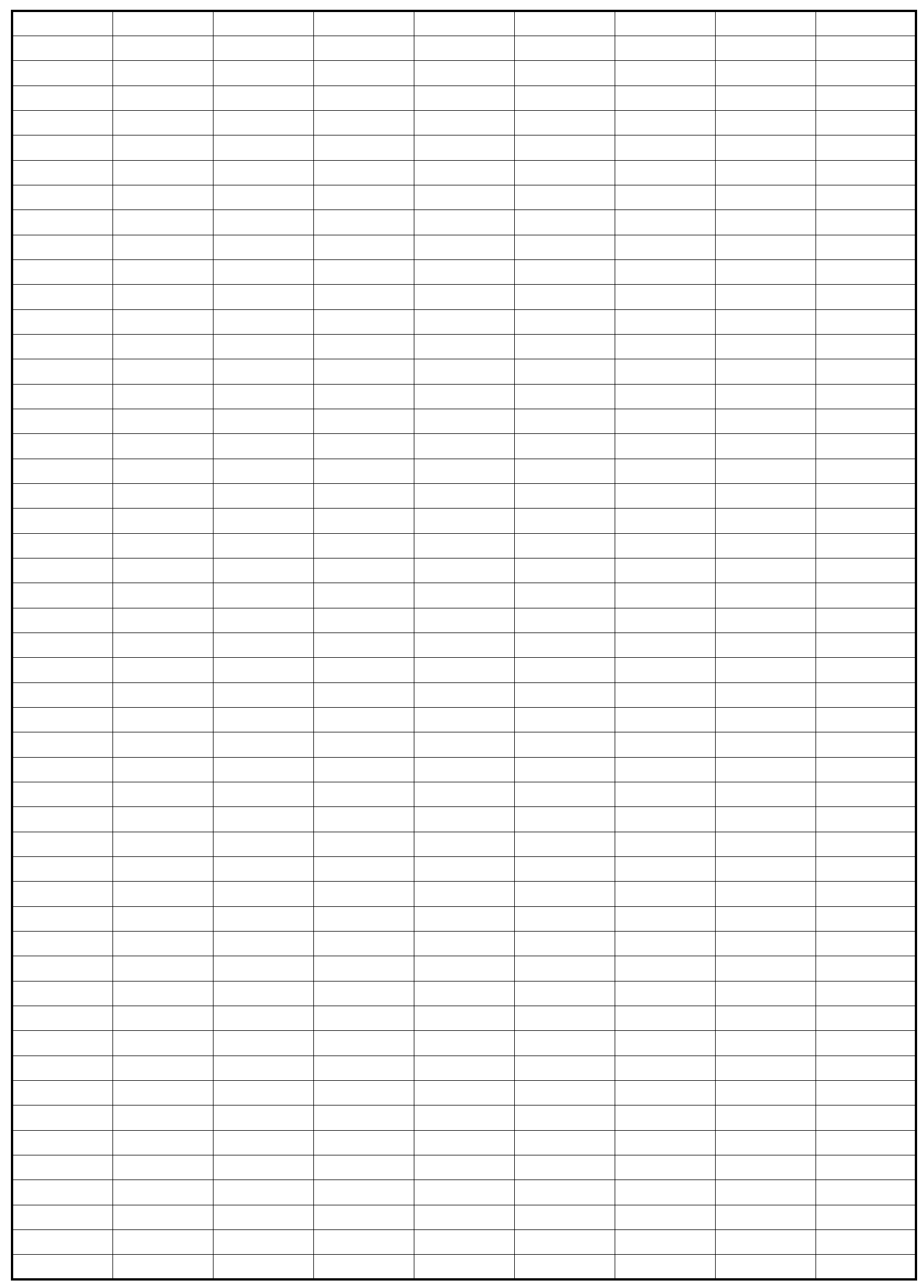

Page 71 
CHART DATA

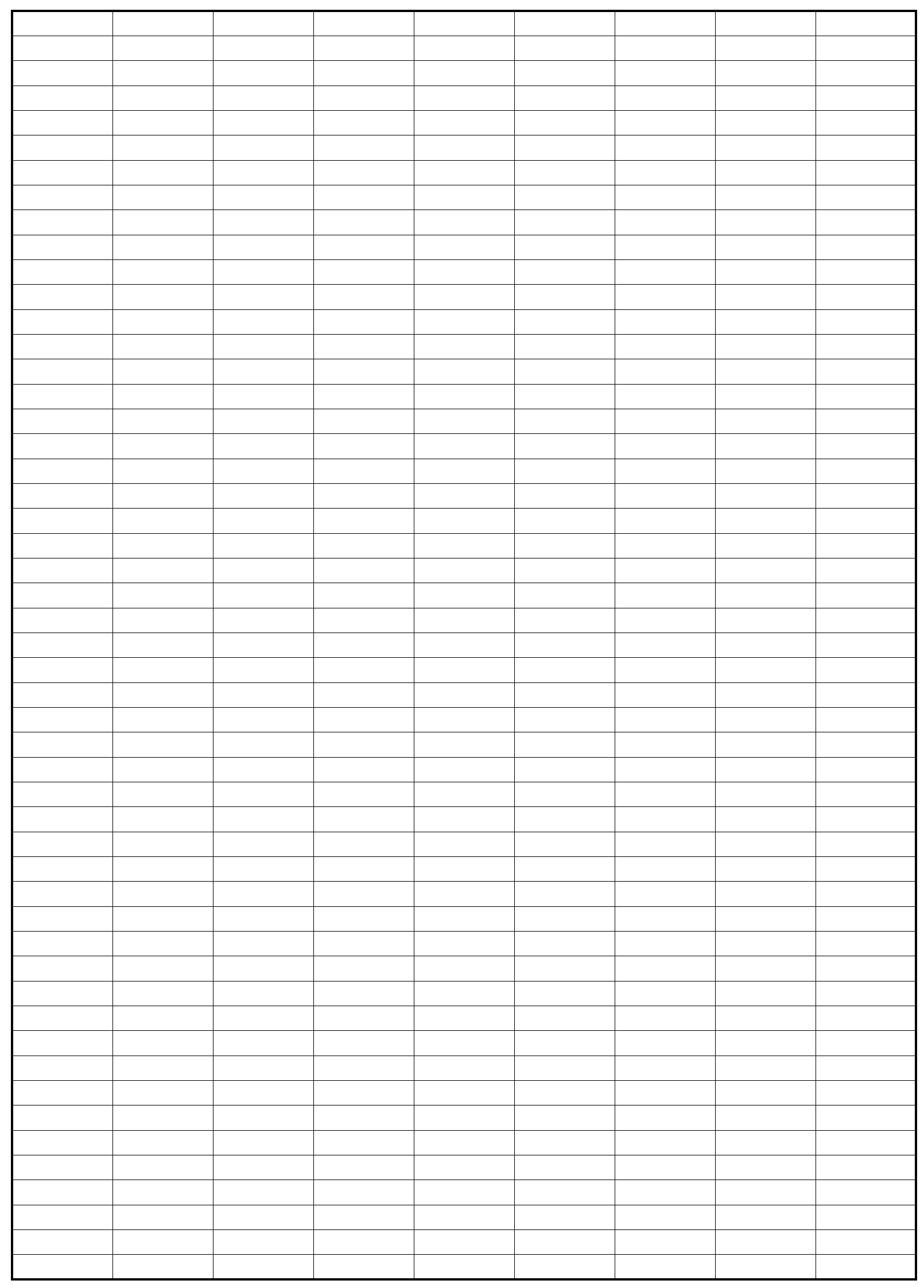

Page 72 
CHART DATA

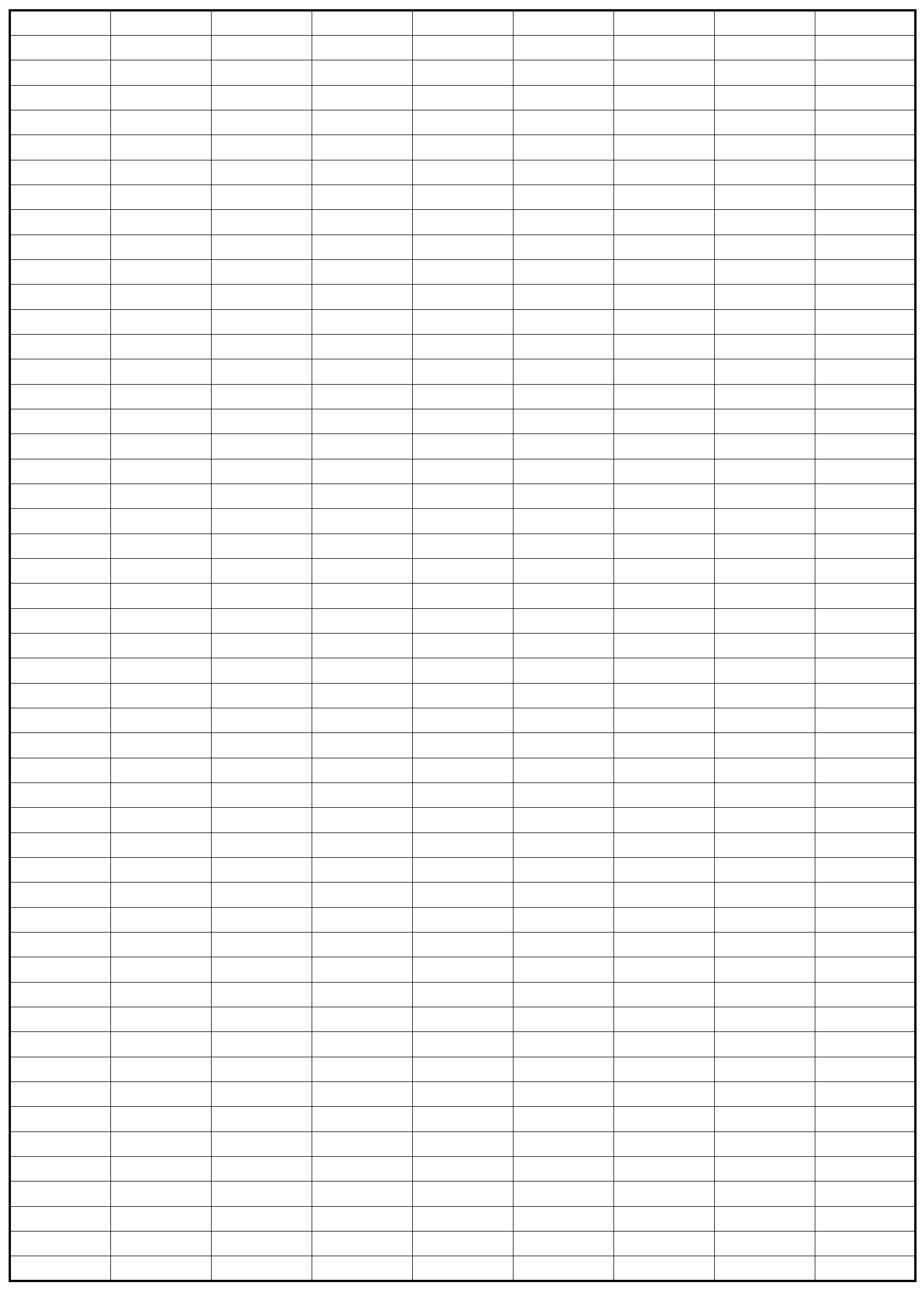

Page 73 
CHART DATA

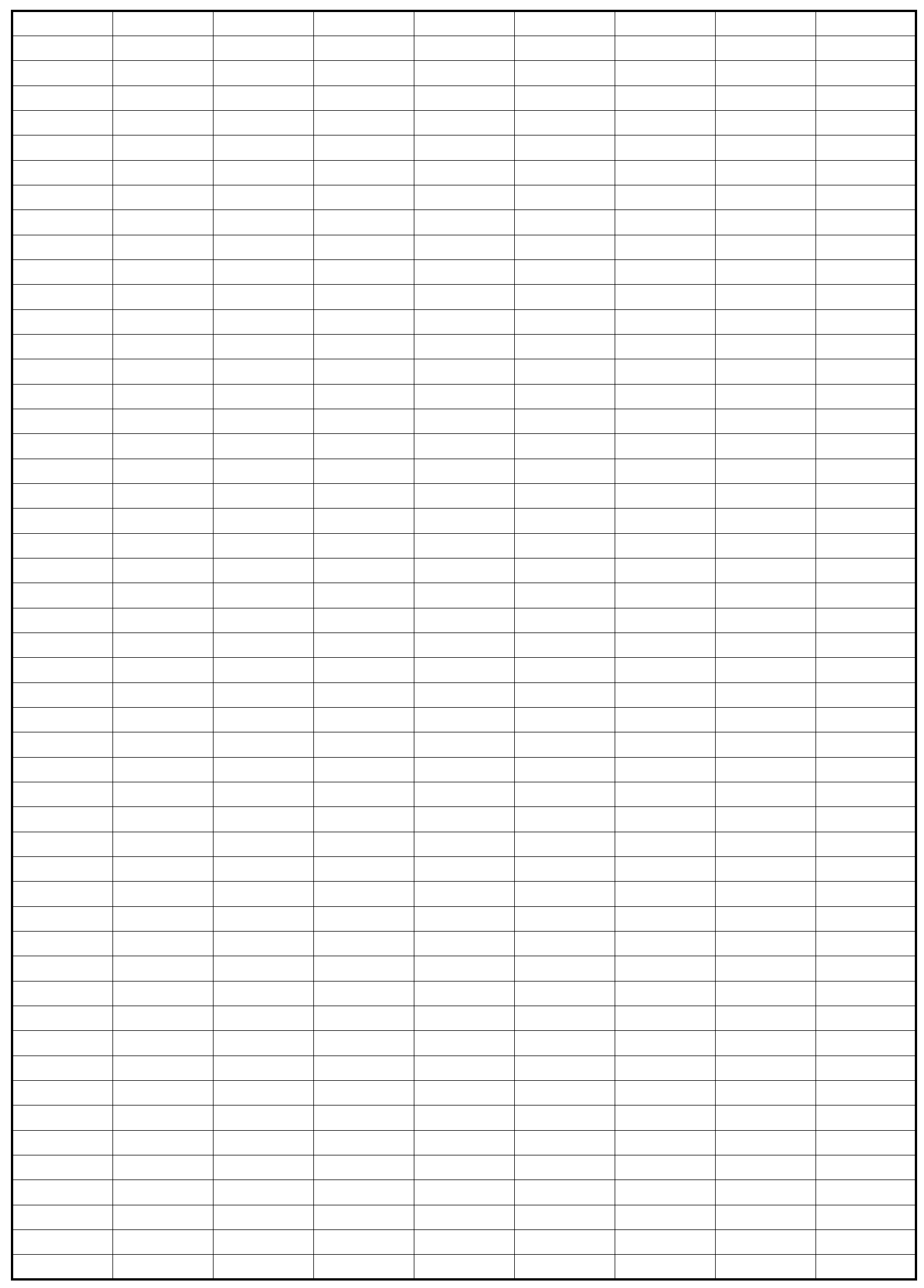

Page 74 
CHART DATA

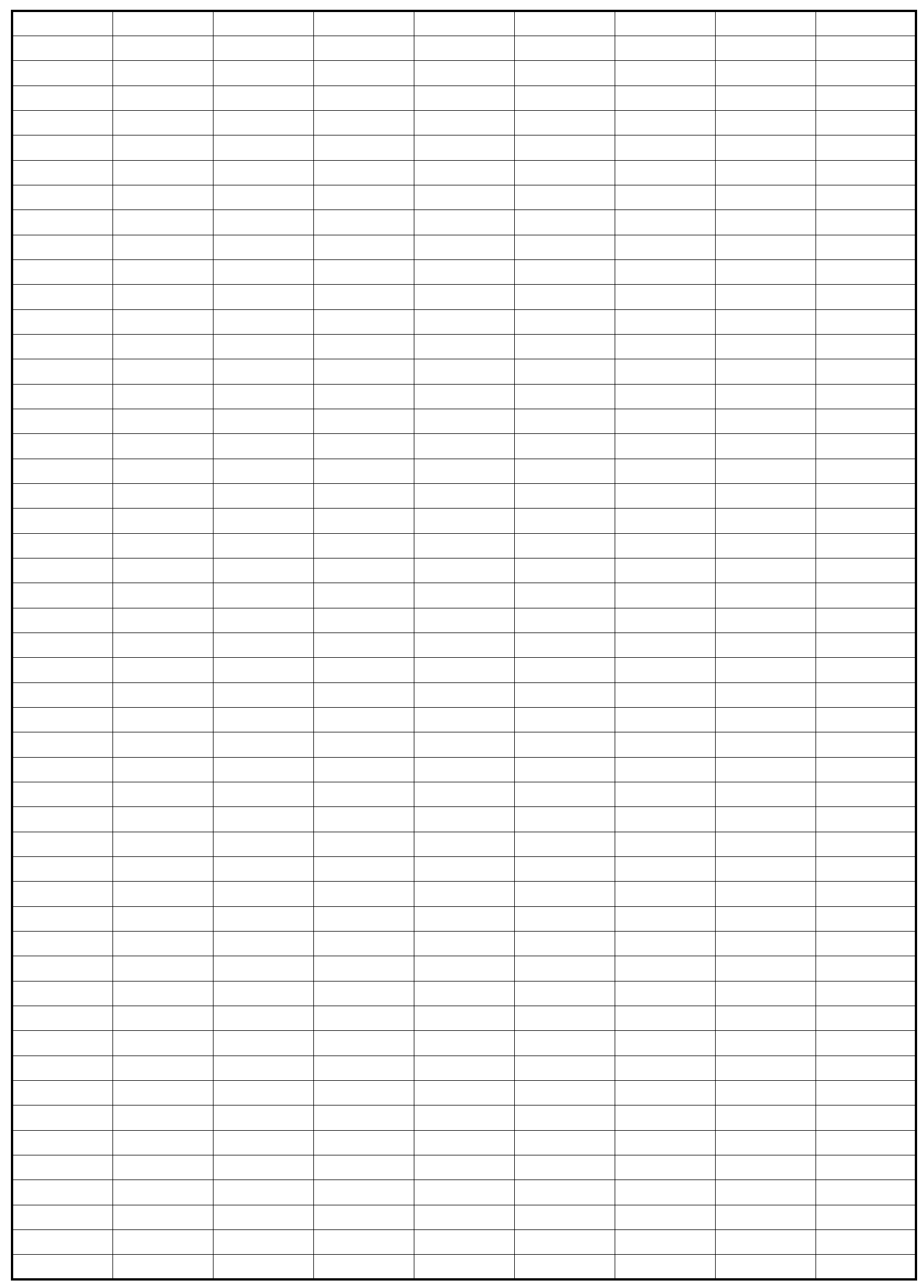

Page 75 
CHART DATA

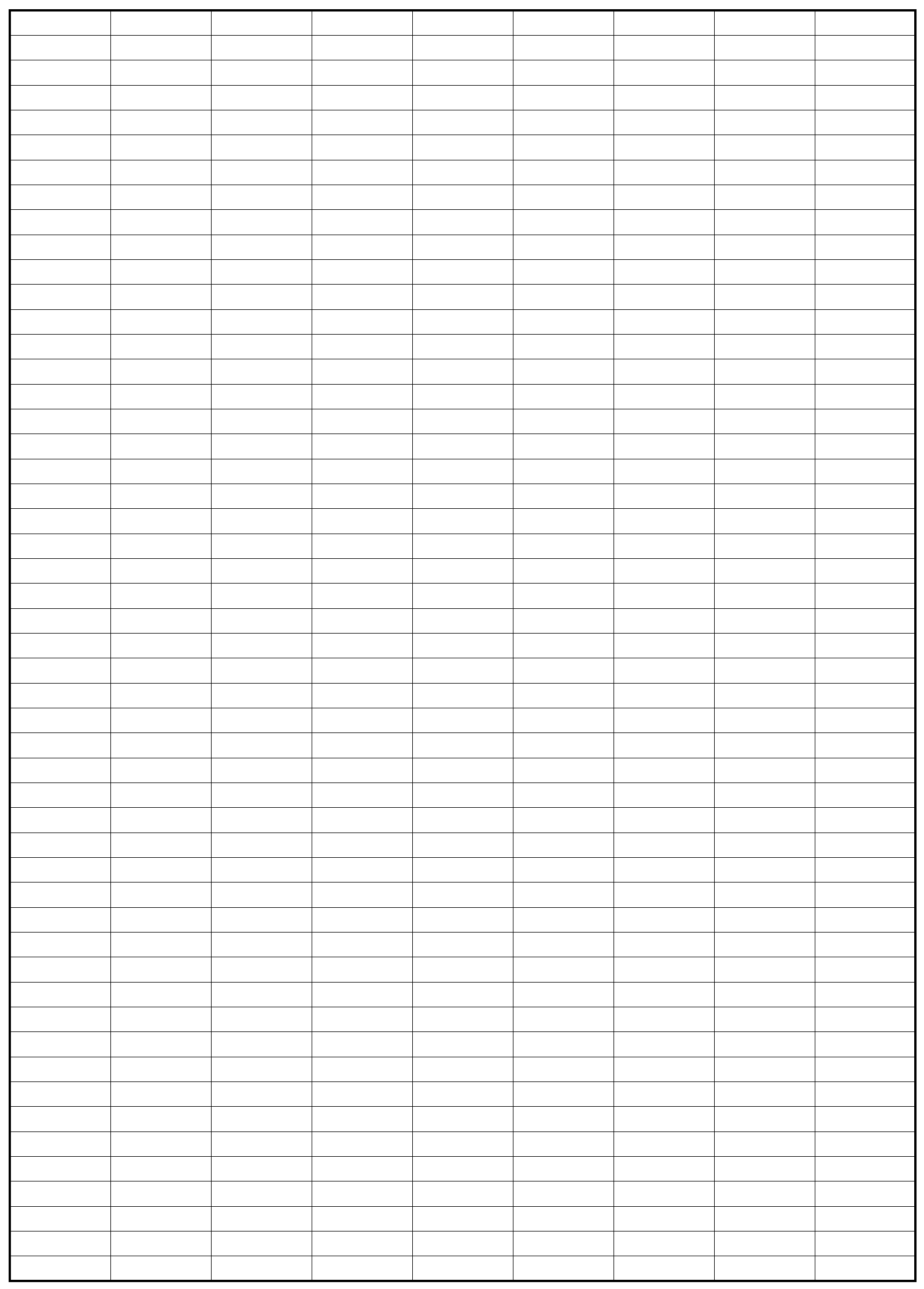

Page 76 
CHART DATA

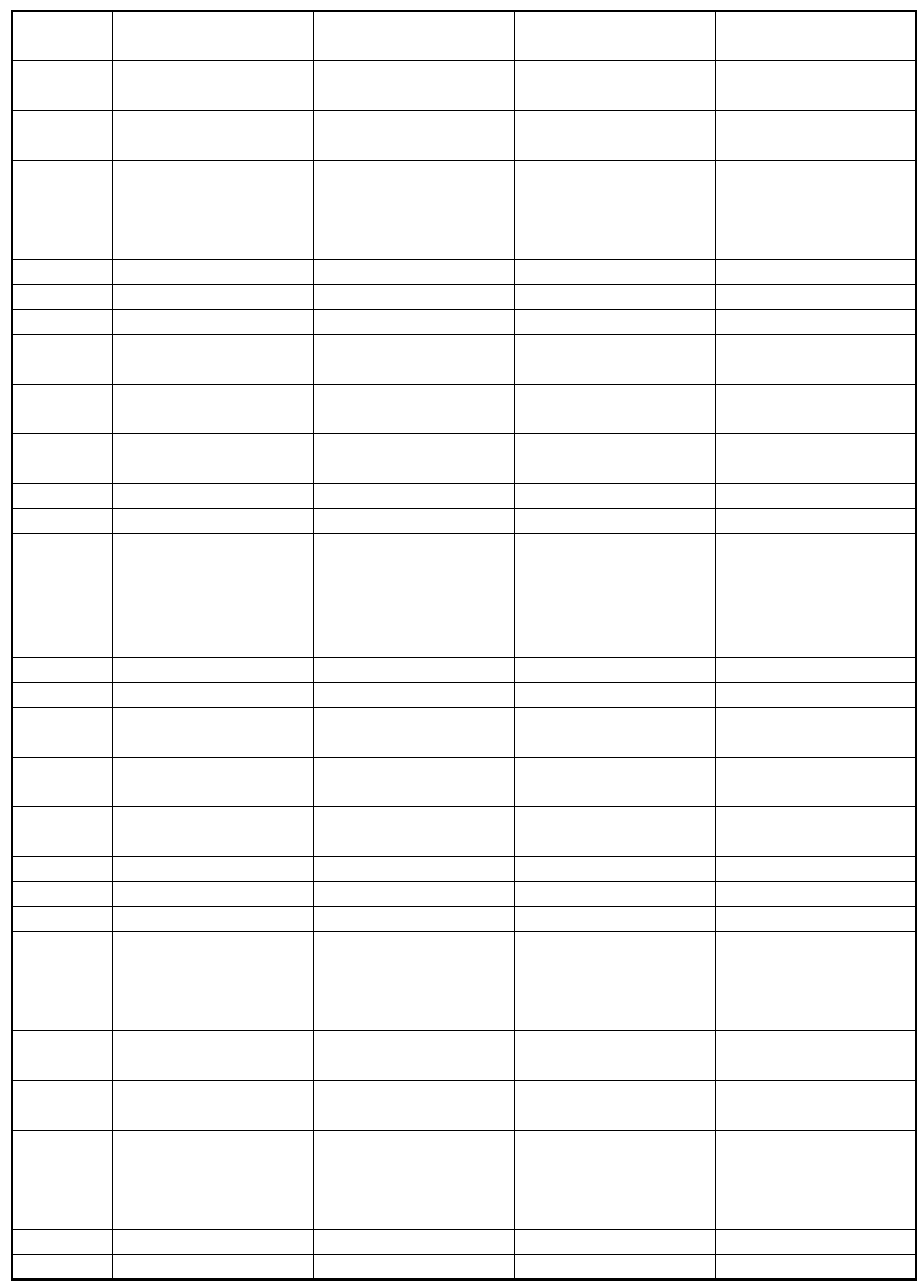

Page 77 
CHART DATA

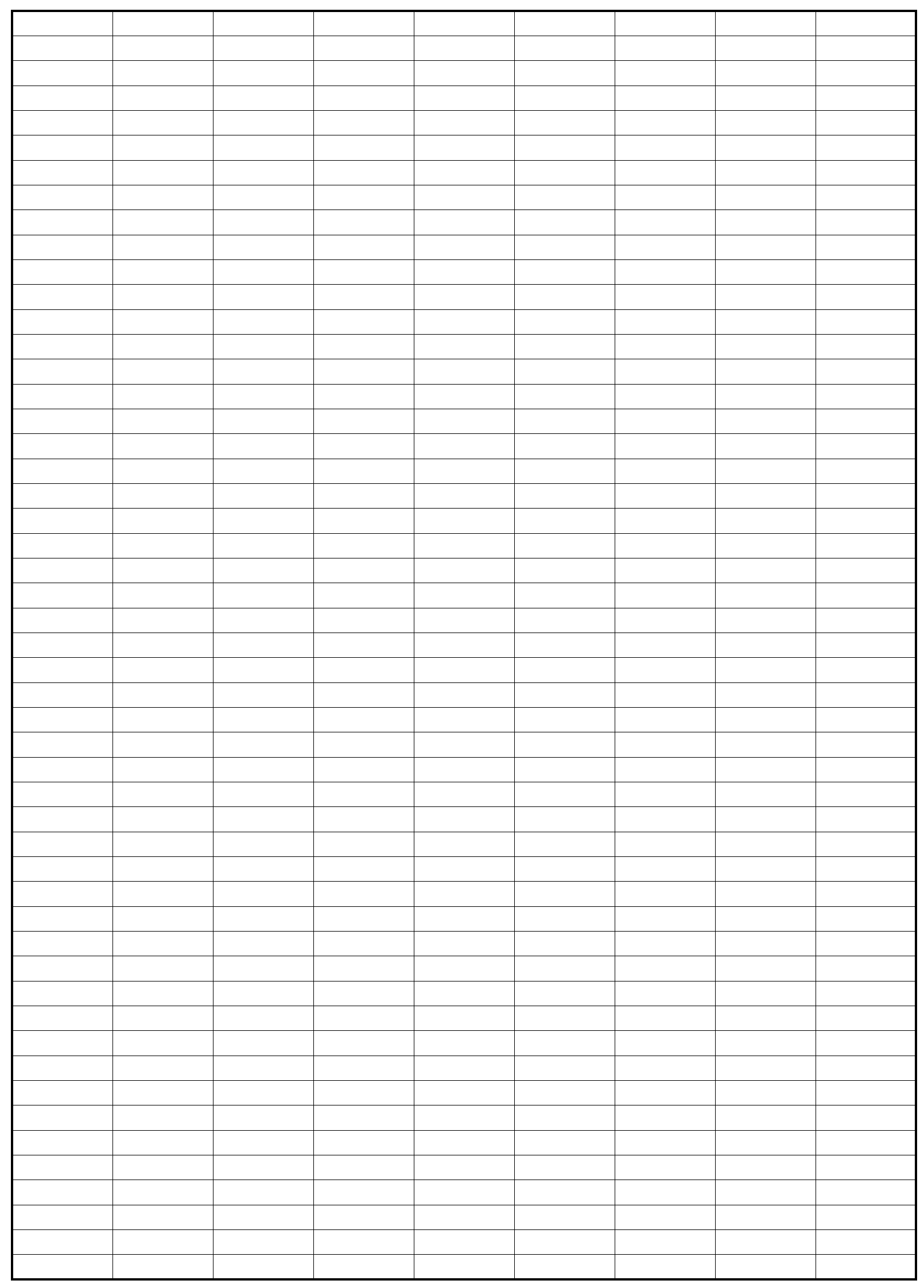

Page 78 
CHART DATA

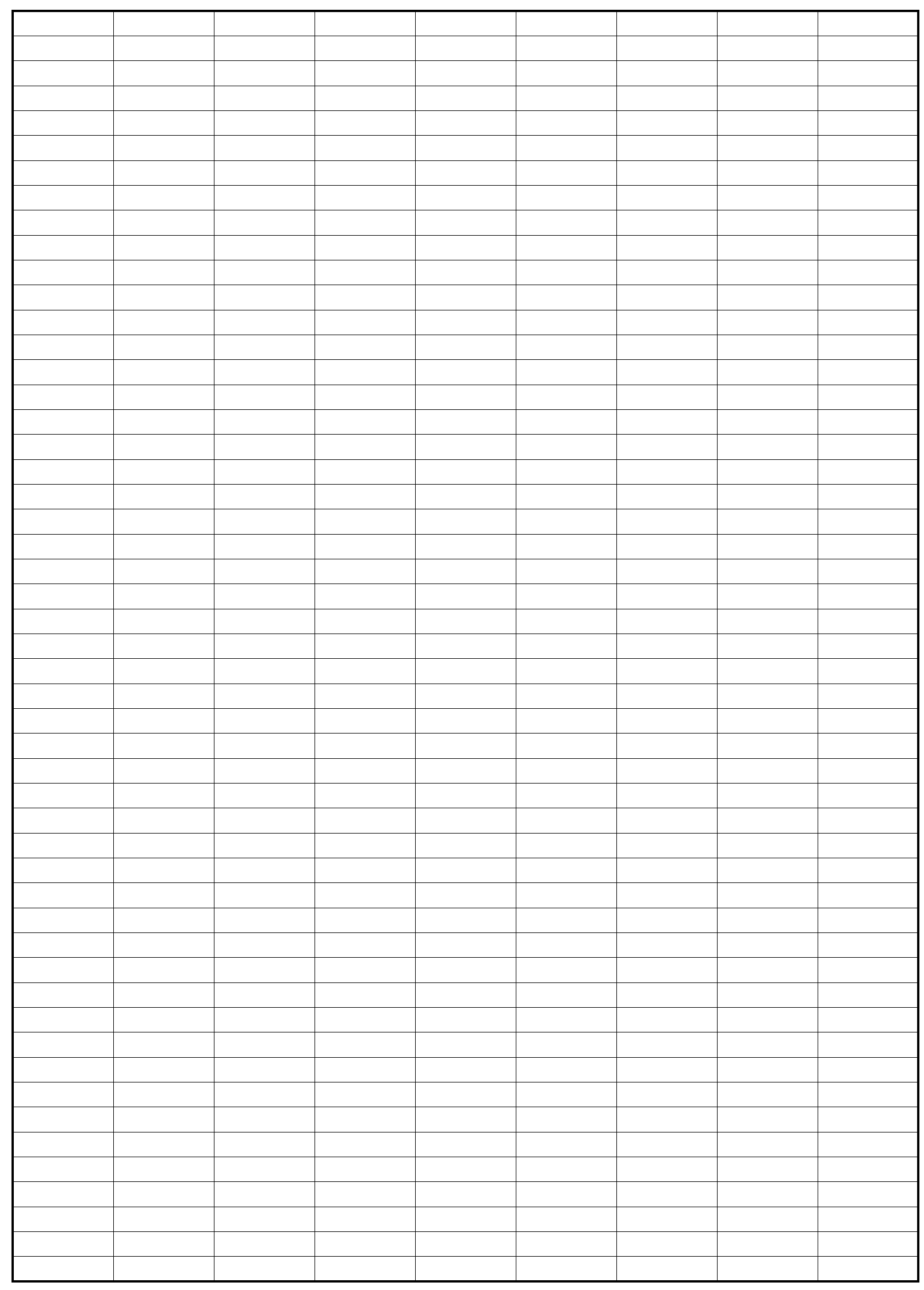

Page 79 
CHART DATA

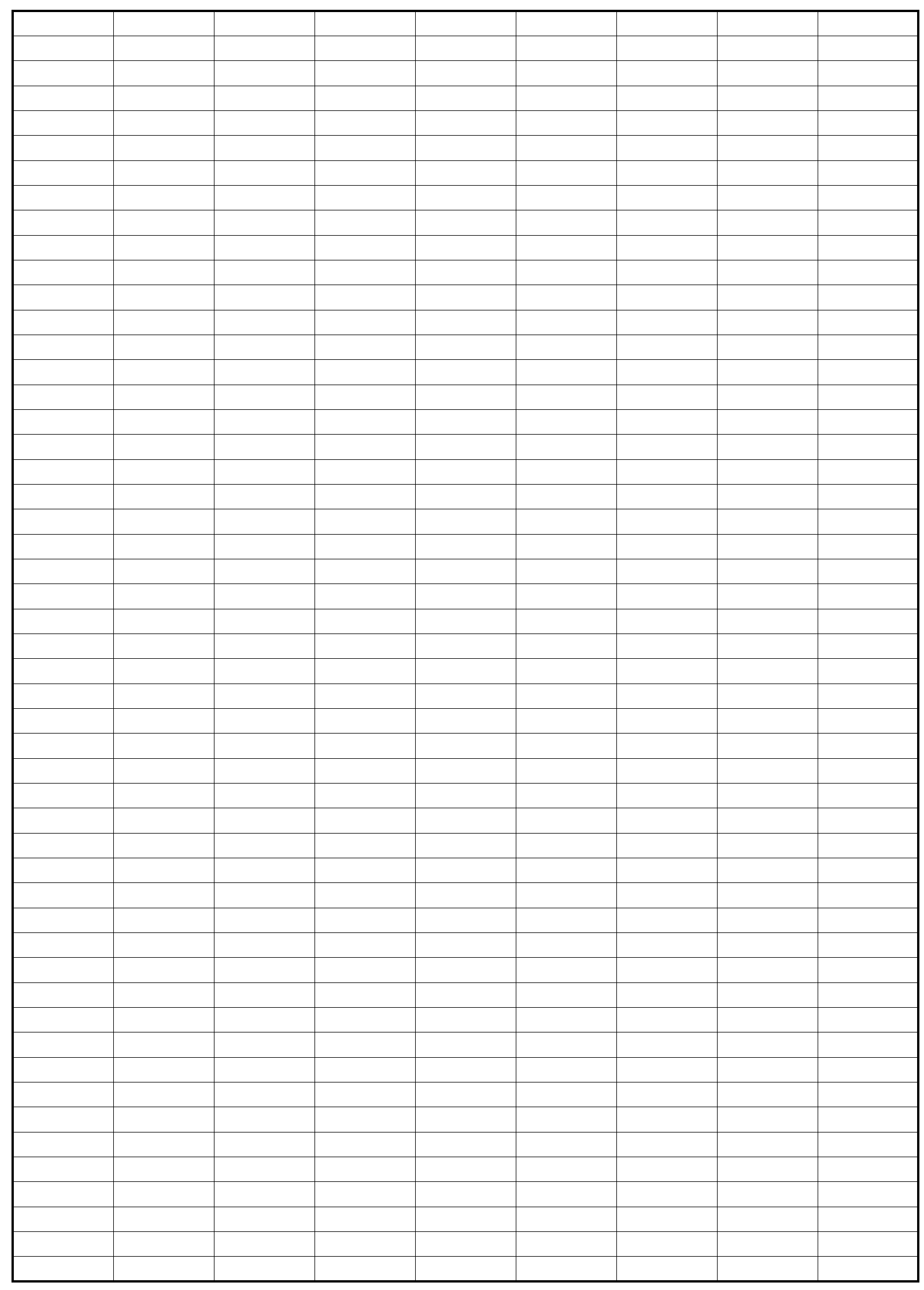

Page 80 
CHART DATA

\begin{tabular}{|l|l|l|l|l|l|l|l|l|}
\hline & & & & & & & & \\
\hline & & & & & & & & \\
\hline & & & & & & & & \\
\hline & & & & & & & & \\
\hline & & & & & & & & \\
\hline & & & & & & & & \\
\hline & & & & & & & & \\
\hline & & & & & & & & \\
\hline & & & & & & & & \\
\hline & & & & & & & & \\
\hline & & & & & & & & \\
\hline
\end{tabular}

Page 81 


\begin{tabular}{|c|c|c|c|c|c|c|}
\hline & & & \multicolumn{3}{|c|}{ Based on average daily data } & \multirow[b]{2}{*}{ \#days in period } \\
\hline Total Daily & Days in Period & Overall Total & & post-pre & (post-pre)/pre & \\
\hline 2389.77 & 514.00 & 1228342.02 & $1-A-S$ & & & 514.00 \\
\hline 2437.77 & 229.00 & 558248.22 & 1-B-S & 47.99 & $2.01 \%$ & 229.00 \\
\hline 1140.39 & 192.00 & 218955.35 & $0-A-S$ & & & 192.00 \\
\hline 556.42 & 88.00 & 48964.92 & $0-B-S$ & -583.97 & $-51.21 \%$ & 88.00 \\
\hline 1879.18 & 148.00 & 278118.51 & 1-A-NS & & & 148.00 \\
\hline 1847.45 & 66.00 & 121931.40 & 1-B-NS & -31.73 & $-1.69 \%$ & 66.00 \\
\hline 1446.82 & 72.00 & 104170.90 & $0-A-N S$ & & & 72.00 \\
\hline 592.32 & 30.00 & 17769.50 & 0-B-NS & -854.50 & $-59.06 \%$ & 30.00 \\
\hline 6524.35 & 1023.00 & 6674407.67 & & & & \\
\hline 5765.76 & 316.00 & 1821979.95 & & & & \\
\hline 2994.18 & 317.00 & 949156.57 & & & & \\
\hline 2439.76 & 96.00 & 234217.16 & & & & \\
\hline 3530.16 & 706.00 & 2492295.02 & & & & \\
\hline 3326.00 & 220.00 & 731719.39 & & & & \\
\hline & & & & & & \\
\hline & & & & & & \\
\hline & & & \multicolumn{3}{|c|}{ Based on average daily data } & \\
\hline Total Daily & Days in Period & Overall Total & & post-pre & (post-pre)/pre & \#days in period \\
\hline 926.75 & 514.00 & 476350.26 & $1-A-S$ & & & 514.00 \\
\hline 820.60 & 229.00 & 187917.26 & 1-B-S & -169.33 & $-62.63 \%$ & 229.00 \\
\hline 270.35 & 192.00 & 51907.97 & $0-A-S$ & & & 192.00 \\
\hline 101.02 & 88.00 & 8889.89 & $0-B-S$ & 16.67 & $4.74 \%$ & 88.00 \\
\hline 351.53 & 148.00 & 52026.10 & 1-A-NS & & & 148.00 \\
\hline 368.20 & 66.00 & 24300.90 & 1-B-NS & -162.35 & $-76.80 \%$ & 66.00 \\
\hline 211.40 & 72.00 & 15221.00 & 0-A-NS & & & 72.00 \\
\hline 49.05 & 30.00 & 1471.50 & 0-B-NS & -1138.55 & $-53.74 \%$ & 30.00 \\
\hline 2118.73 & 1023.00 & 2167457.13 & & & & \\
\hline 980.18 & 316.00 & 309735.60 & & & & \\
\hline 921.62 & 317.00 & 292153.83 & & & & \\
\hline 417.25 & 96.00 & 40055.56 & & & & \\
\hline 1197.11 & 706.00 & 845156.48 & & & & \\
\hline \multirow[t]{4}{*}{562.93} & 220.00 & 123844.71 & & & & \\
\hline & & & & & & \\
\hline & & & & & & \\
\hline & & & \multicolumn{3}{|c|}{ Based on average daily data } & \\
\hline Total Daily & Days in Period & Overall Total & & post-pre & (post-pre)/pre & \#days in period \\
\hline 1452.63 & 514.00 & 746650.94 & $1-\mathrm{A}-\mathrm{S}$ & & & 514.00 \\
\hline 1616.60 & 229.00 & 370200.30 & 1-B-S & -416.63 & $-48.41 \%$ & 229.00 \\
\hline 860.61 & 192.00 & 165237.70 & $0-A-S$ & & & 192.00 \\
\hline 443.98 & 88.00 & 39070.10 & 0-B-S & -37.58 & $-2.48 \%$ & 88.00 \\
\hline 1516.83 & 148.00 & 224491.50 & 1-A-NS & & & 148.00 \\
\hline 1479.25 & 66.00 & 97630.50 & 1-B-NS & -692.15 & $-56.03 \%$ & 66.00 \\
\hline 1235.42 & 72.00 & 88949.90 & $0-A-N S$ & & & 72.00 \\
\hline 543.27 & 30.00 & 16298.00 & 0-B-NS & 400.95 & $9.17 \%$ & 30.00 \\
\hline 4373.81 & 1023.00 & 4474412.66 & & & & \\
\hline 4774.77 & 316.00 & 1508826.18 & & & & \\
\hline 2060.57 & 317.00 & 653201.83 & & & & \\
\hline 2022.52 & 96.00 & 194161.60 & & & & \\
\hline 2313.24 & 706.00 & 1633148.36 & & & & \\
\hline
\end{tabular}


CHART DATA

\begin{tabular}{|c|c|c|c|c|c|c|}
\hline 2752.25 & 220.00 & 605494.94 & & & & \\
\hline & & & & & & \\
\hline & & & \multirow{2}{*}{\multicolumn{3}{|c|}{ Based on average daily data }} & \\
\hline Tatal Railv & & & & & & \\
\hline Total Daily & Days in Period & Overall Total & & post-pre & (post-pre)/pre & \#days in period \\
\hline 6.56 & 514.00 & 3369.88 & $1-A-S$ & & & 514.00 \\
\hline 5.08 & 229.00 & 1162.29 & 1-B-S & -0.92 & $-13.78 \%$ & 229.00 \\
\hline 6.69 & 192.00 & 1283.75 & $0-A-S$ & & & 192.00 \\
\hline 5.77 & 88.00 & 507.32 & $0-B-S$ & -1.86 & $-27.46 \%$ & 88.00 \\
\hline 6.77 & 148.00 & 1002.03 & $1-A-N S$ & & & 148.00 \\
\hline 4.91 & 66.00 & 324.15 & $1-B-N S$ & -6.87 & $-100.00 \%$ & 66.00 \\
\hline 6.87 & 72.00 & 494.71 & $0-A-N S$ & & & 72.00 \\
\hline 0.00 & 30.00 & 0.00 & 0-B-NS & -5.53 & $-22.96 \%$ & 30.00 \\
\hline 24.08 & 1023.00 & 24636.79 & & & & \\
\hline 18.55 & 316.00 & 5862.67 & & & & \\
\hline 10.84 & 317.00 & 3436.44 & & & & \\
\hline 4.91 & 96.00 & 471.49 & & & & \\
\hline 13.24 & 706.00 & 9349.13 & & & & \\
\hline 13.64 & 220.00 & 3001.11 & & & & \\
\hline & & & & & & \\
\hline & & & & & & \\
\hline & & & & & & \\
\hline & & & & & & \\
\hline & & & & & & \\
\hline & & & & & & \\
\hline & & & & & & \\
\hline & & & & & & \\
\hline & & & & & & \\
\hline & & & & & & \\
\hline & & & & & & \\
\hline & & & & & & \\
\hline & & & & & & \\
\hline & & & & & & \\
\hline & & & & & & \\
\hline & & & & & & \\
\hline & & & & & & \\
\hline & & & & & & \\
\hline & & & & & & \\
\hline & & & & & & \\
\hline & & & & & & \\
\hline & & & & & & \\
\hline & & & & & & \\
\hline & & & & & & \\
\hline & & & & & & \\
\hline & & & & & & \\
\hline & & & & & & \\
\hline & & & & & & \\
\hline & & & & & & \\
\hline & & & & & & \\
\hline
\end{tabular}


CHART DATA

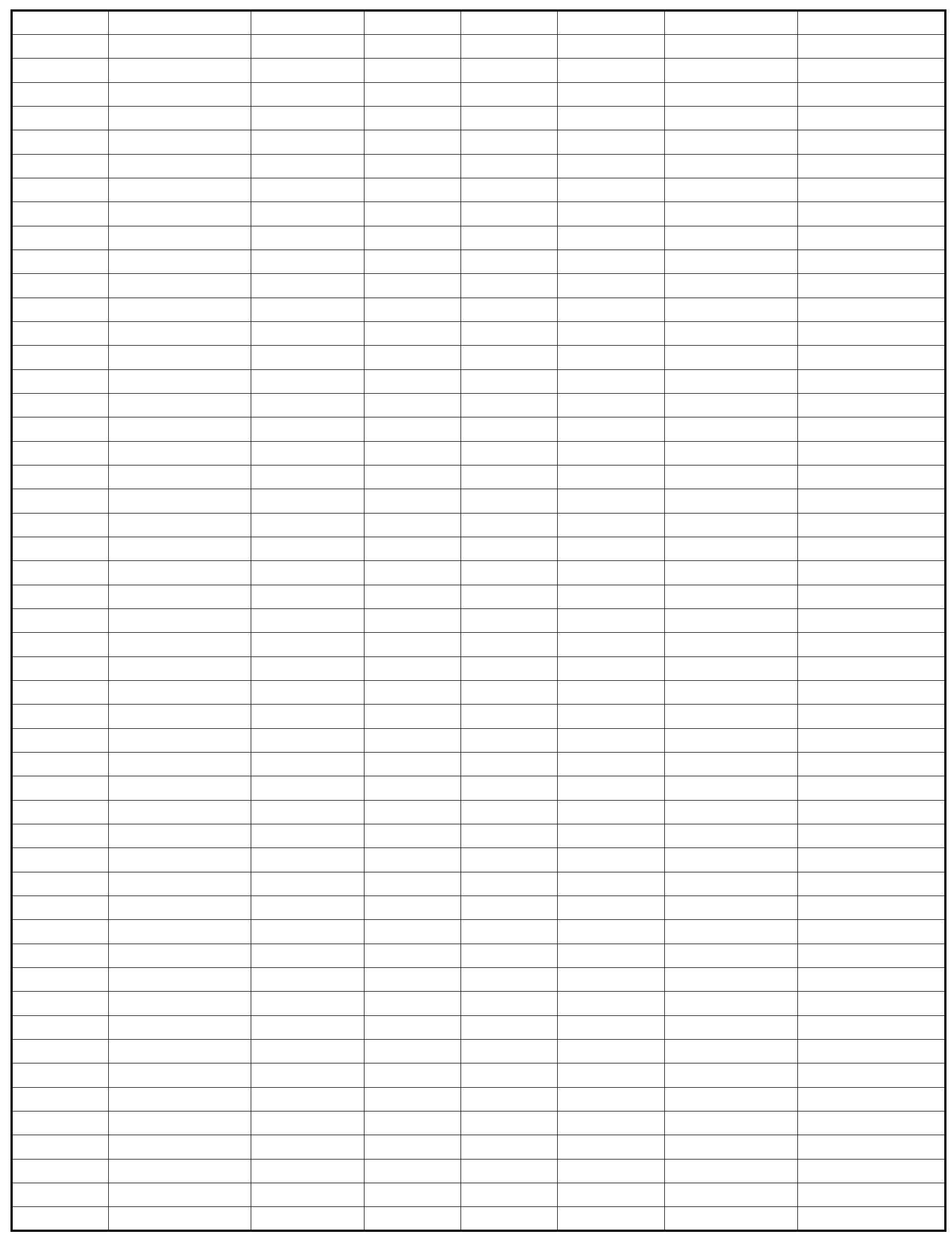

Page 84 
CHART DATA

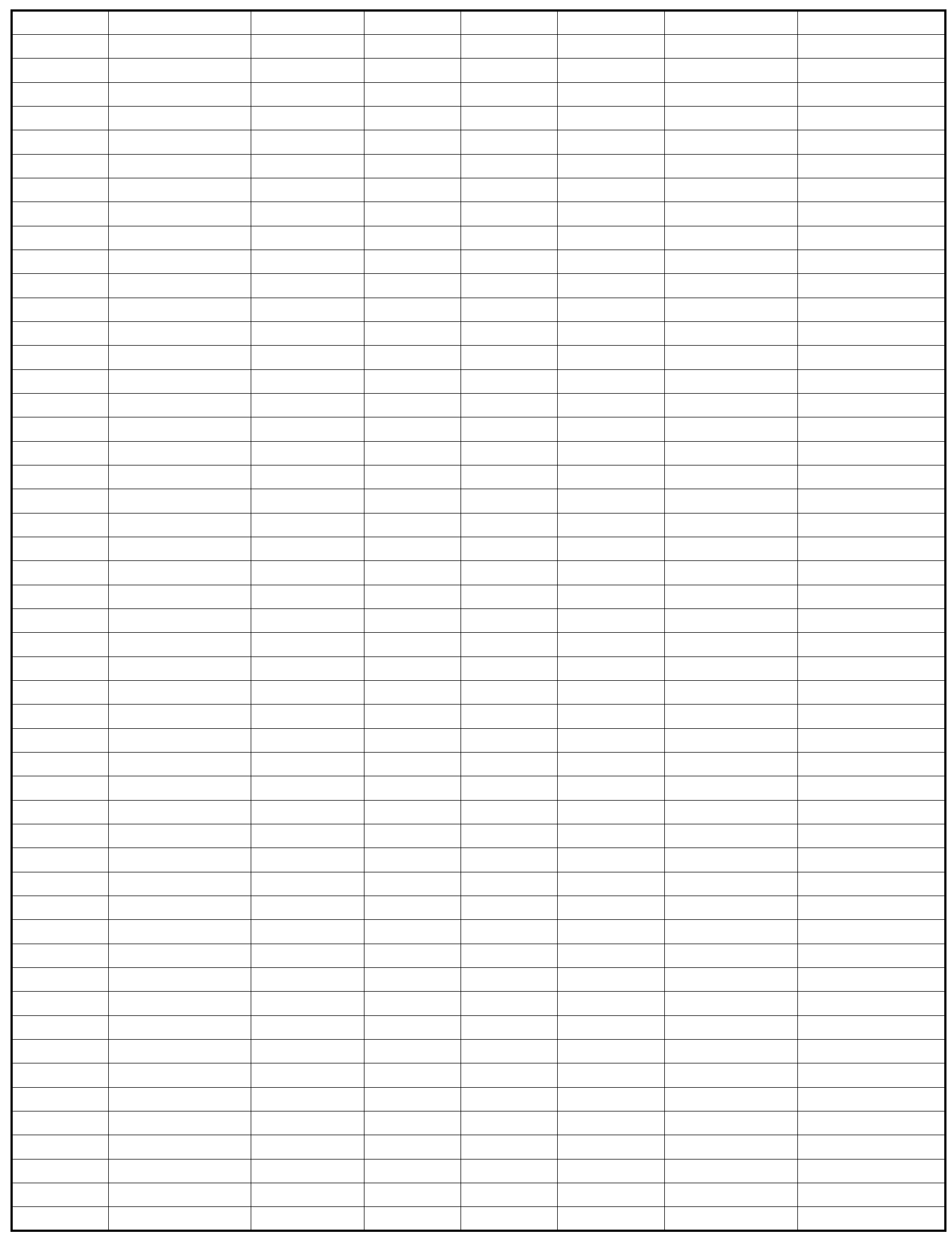

Page 85 
CHART DATA

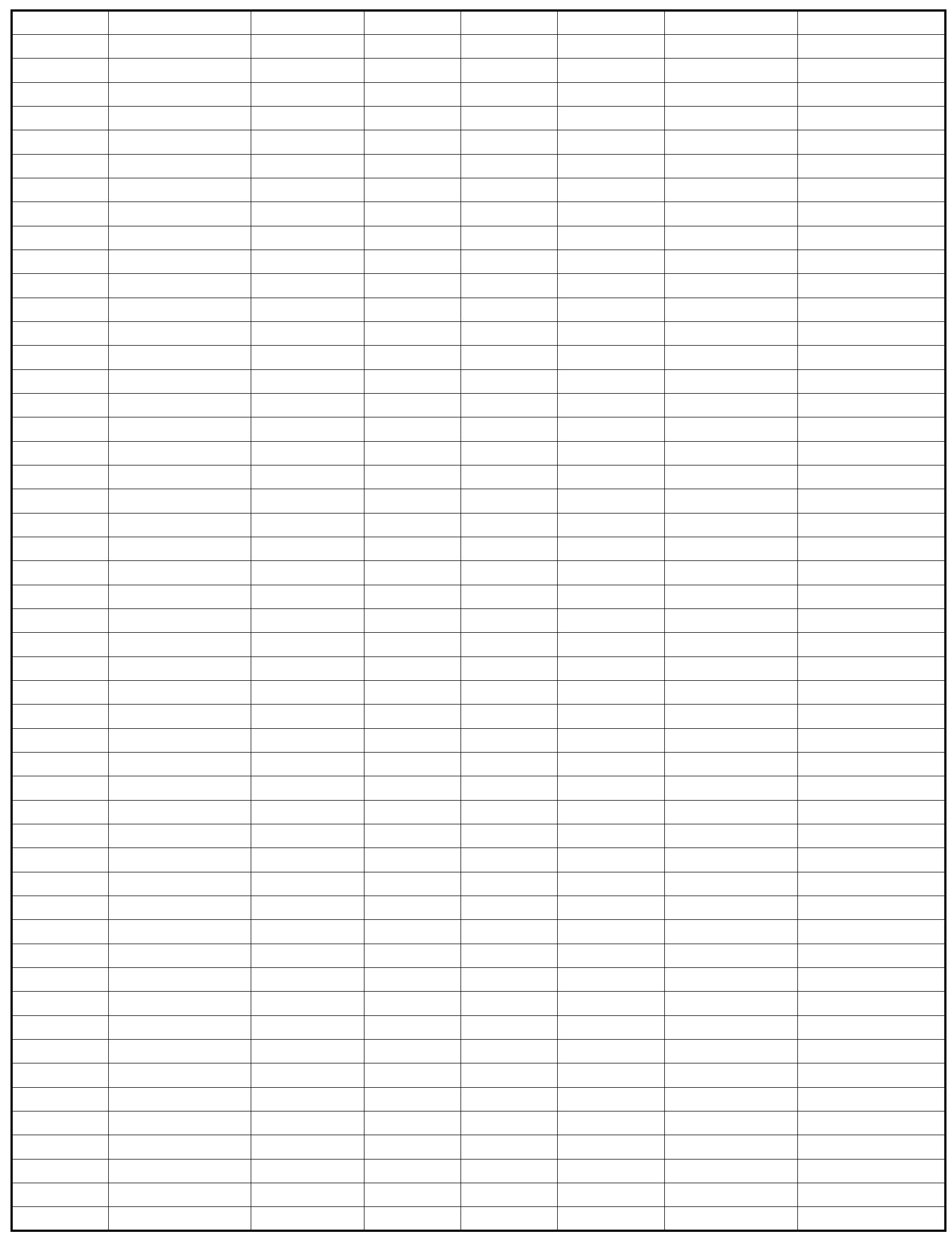

Page 86 
CHART DATA

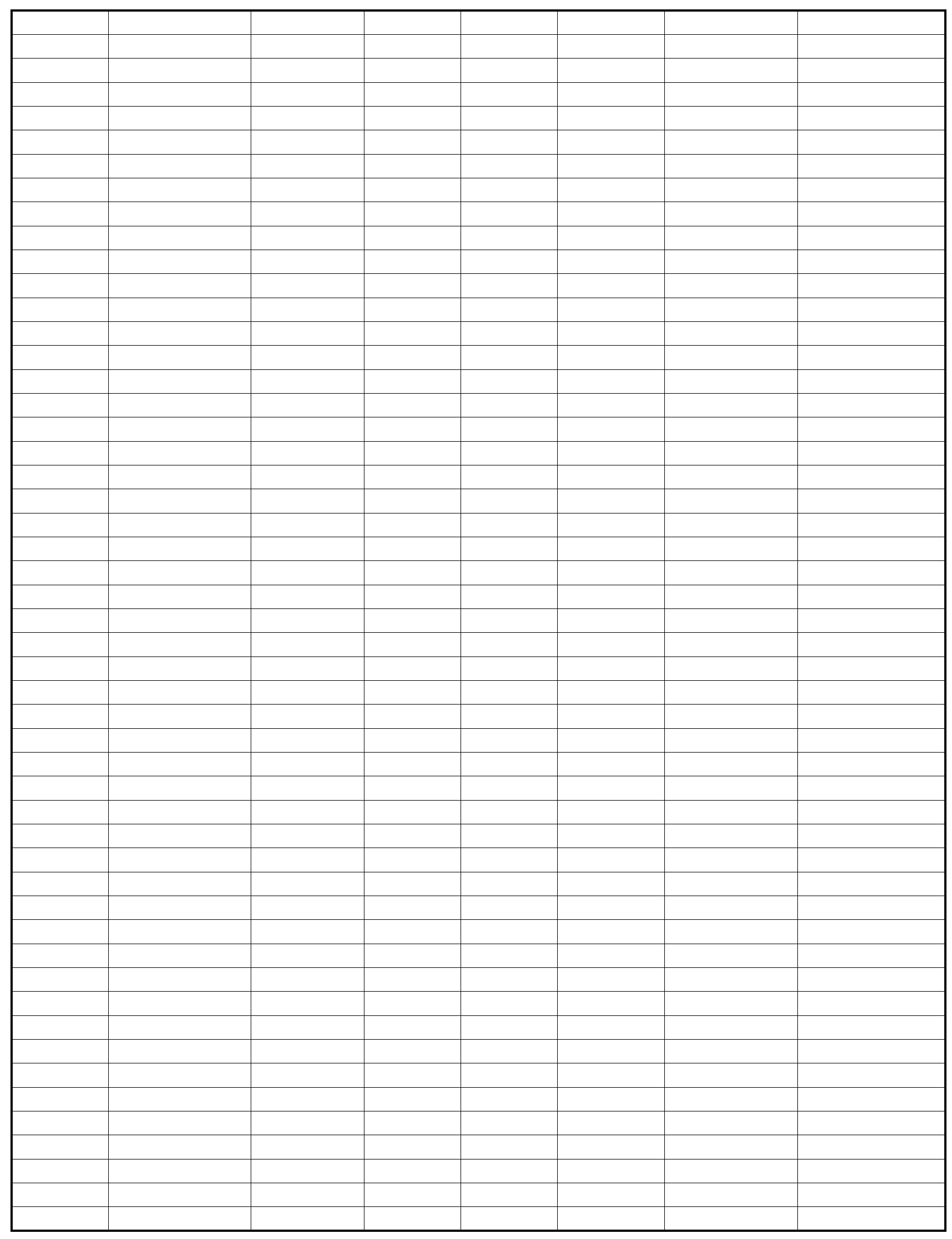

Page 87 
CHART DATA

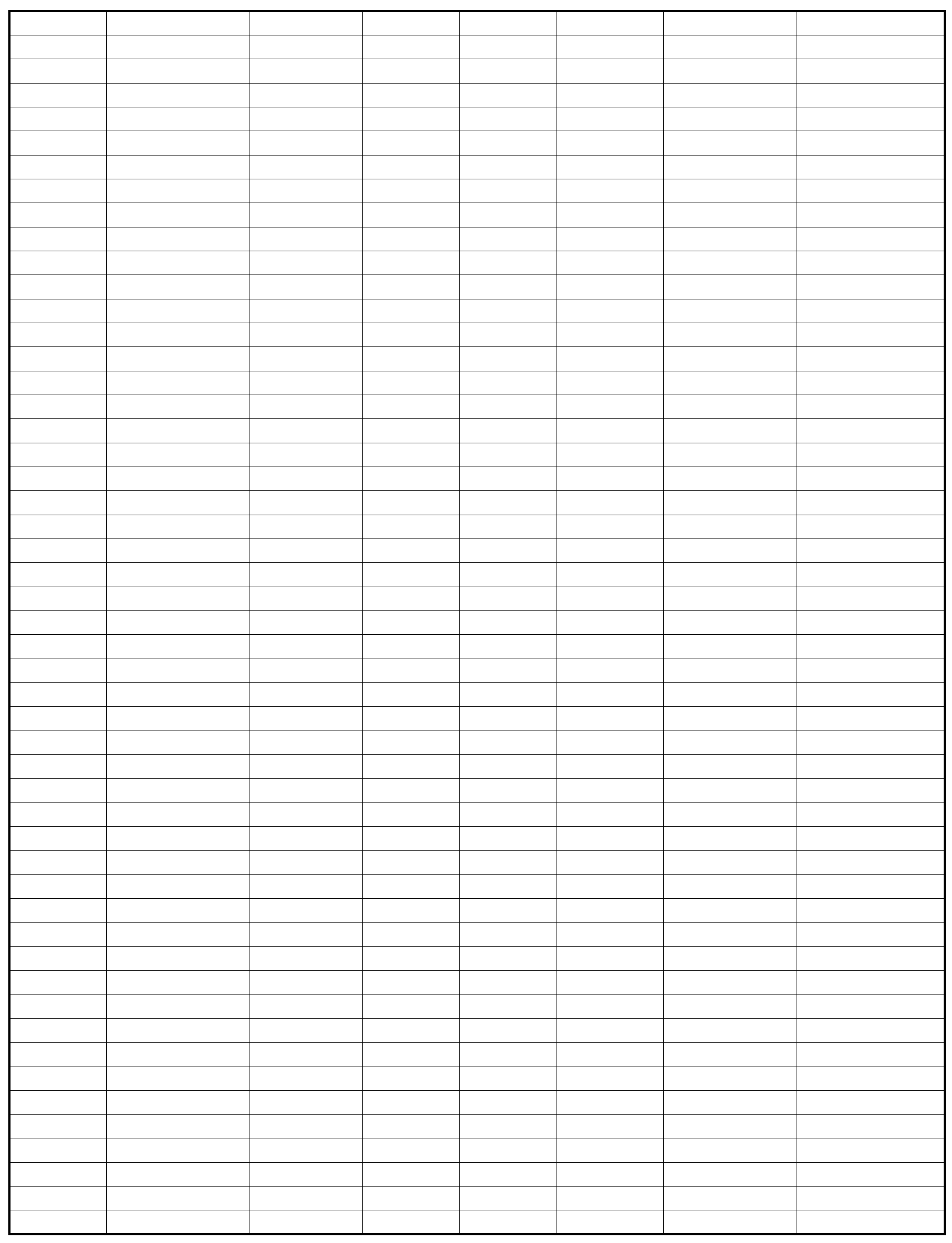

Page 88 
CHART DATA

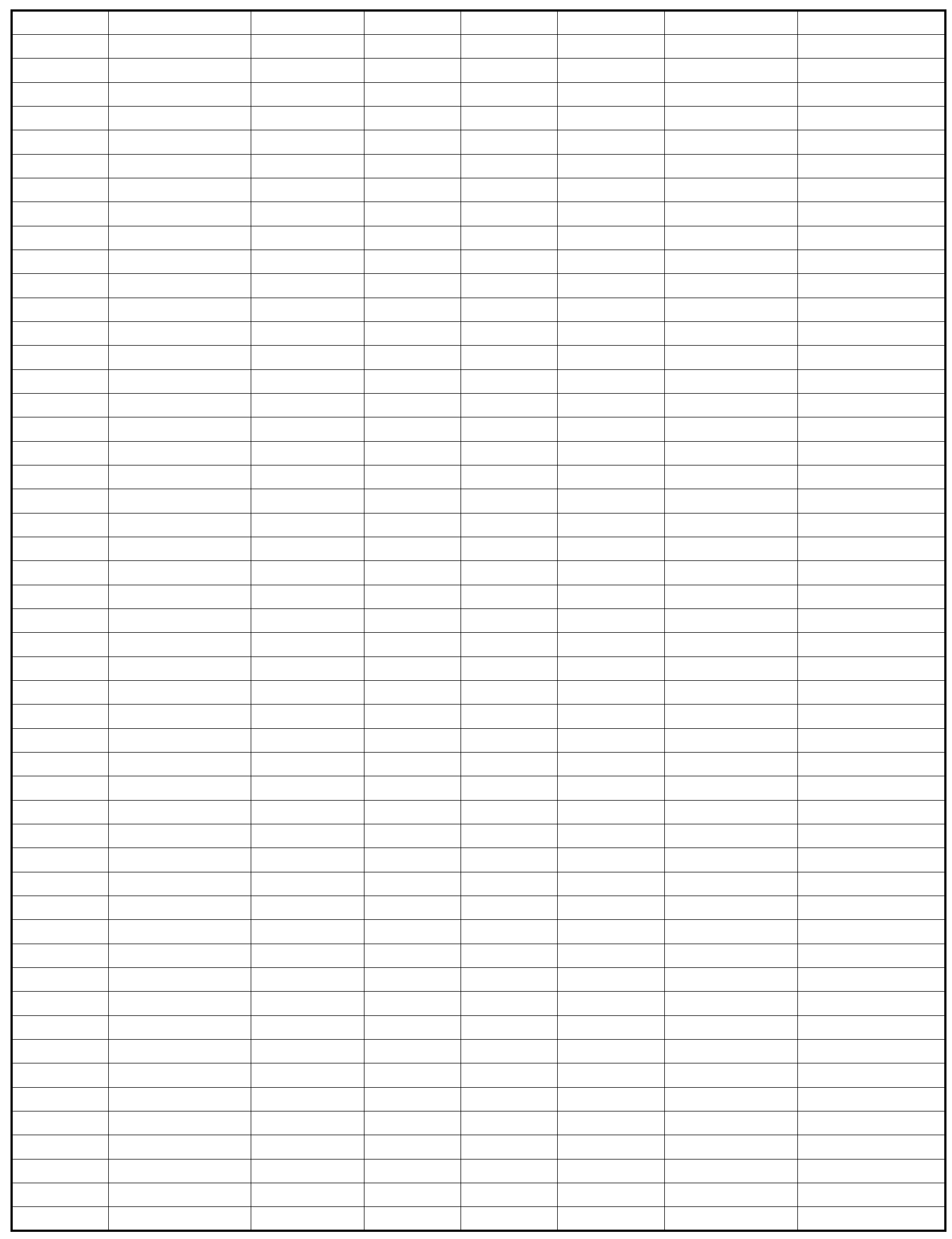

Page 89 
CHART DATA

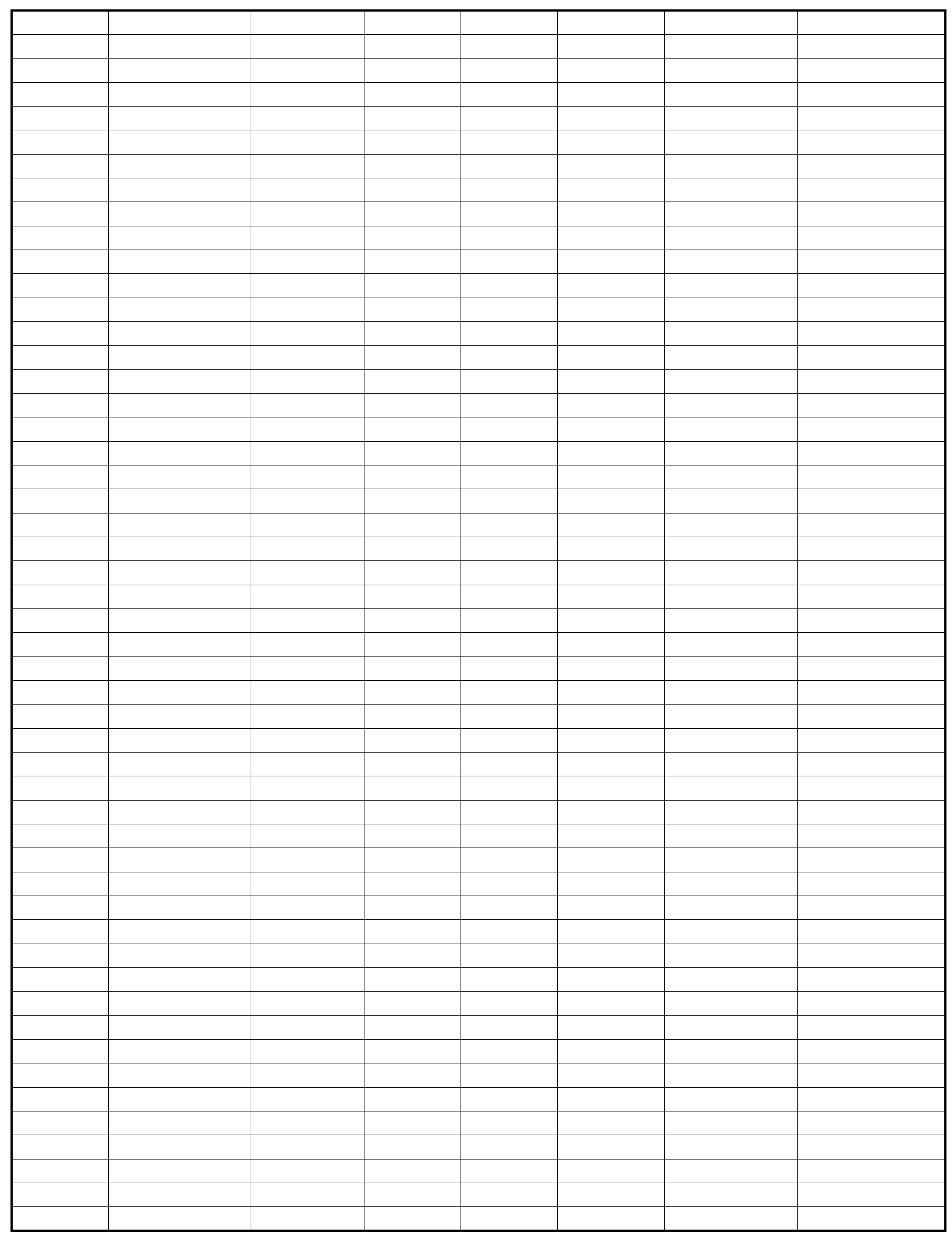

Page 90 
CHART DATA

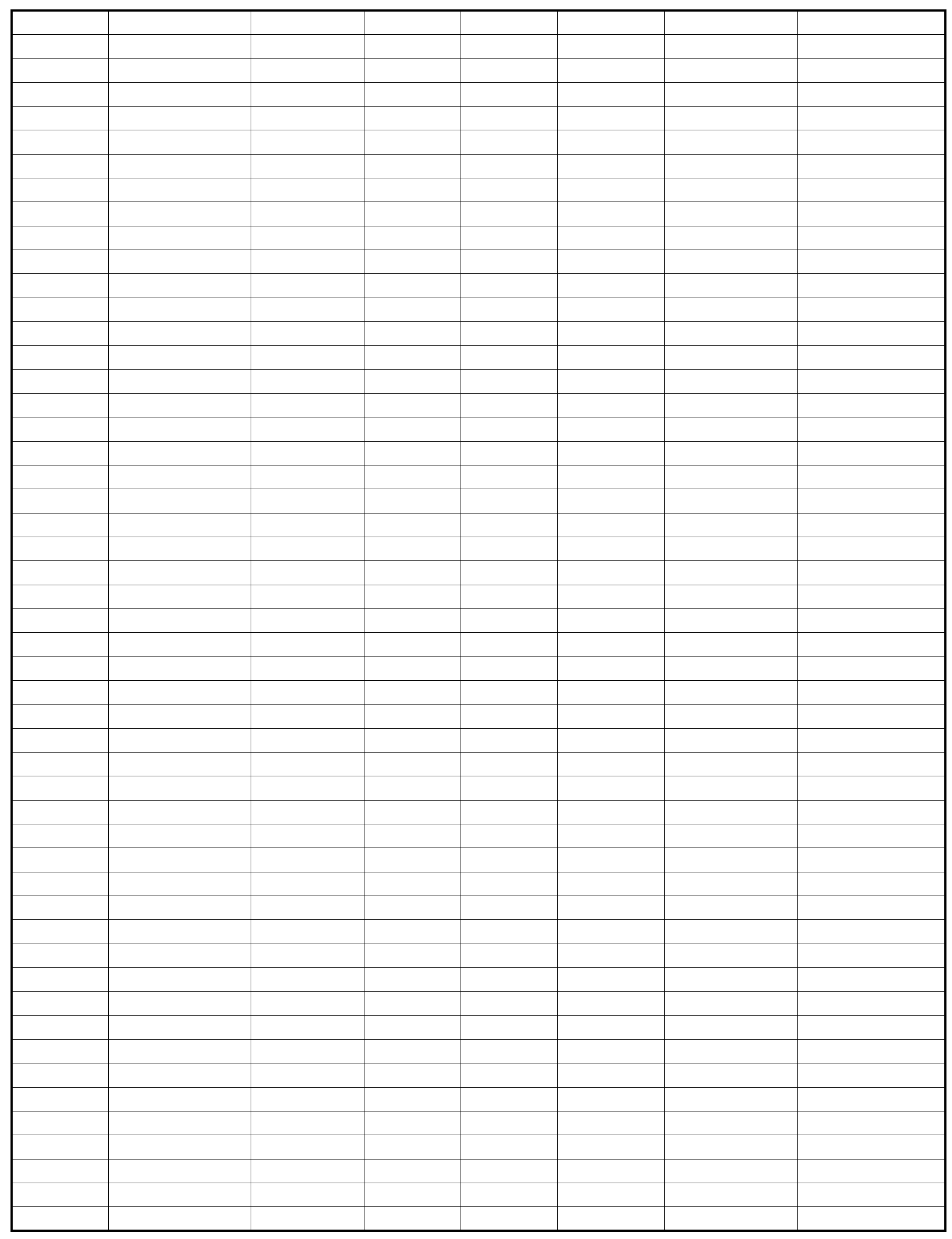

Page 91 
CHART DATA

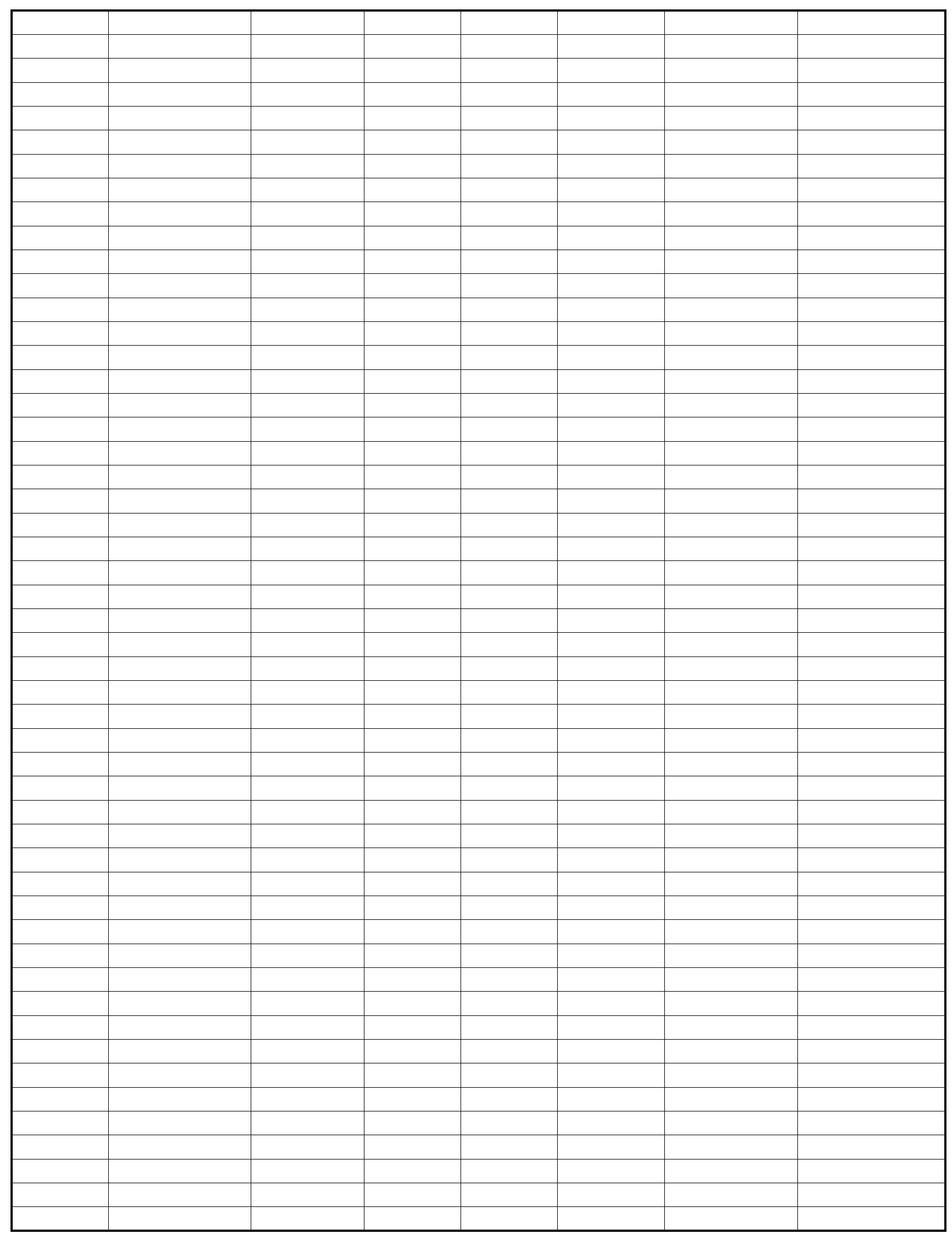

Page 92 
CHART DATA

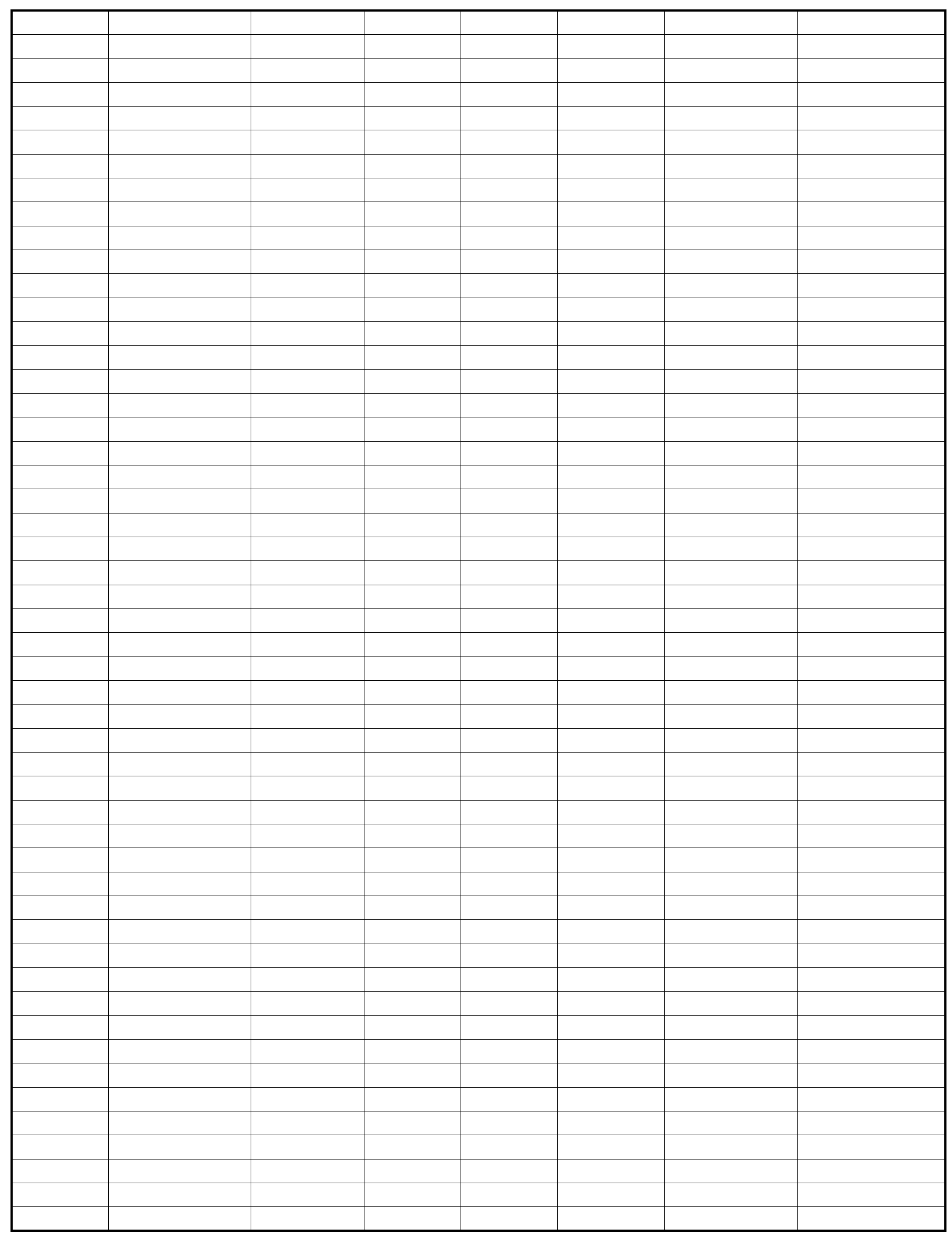

Page 93 
CHART DATA

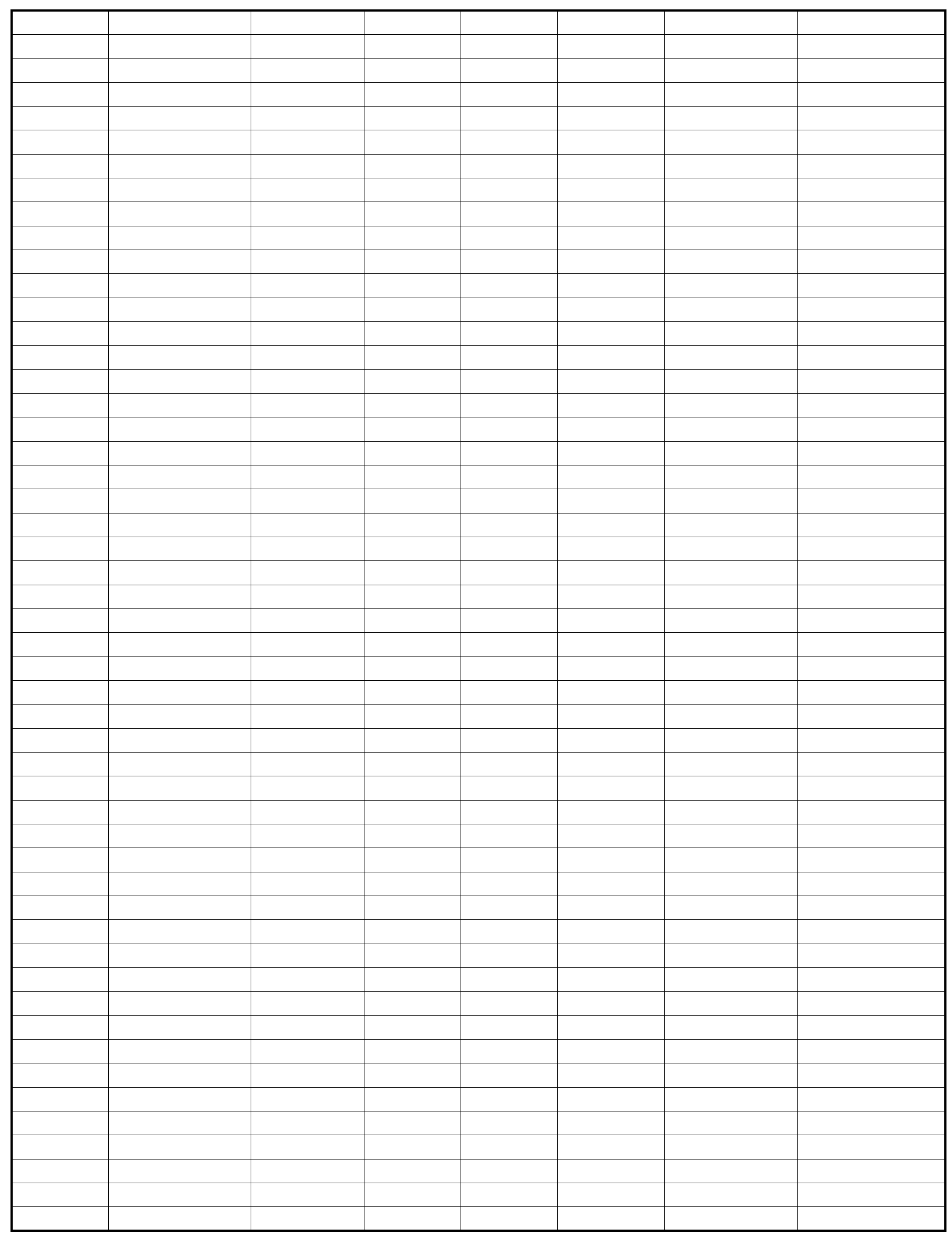

Page 94 
CHART DATA

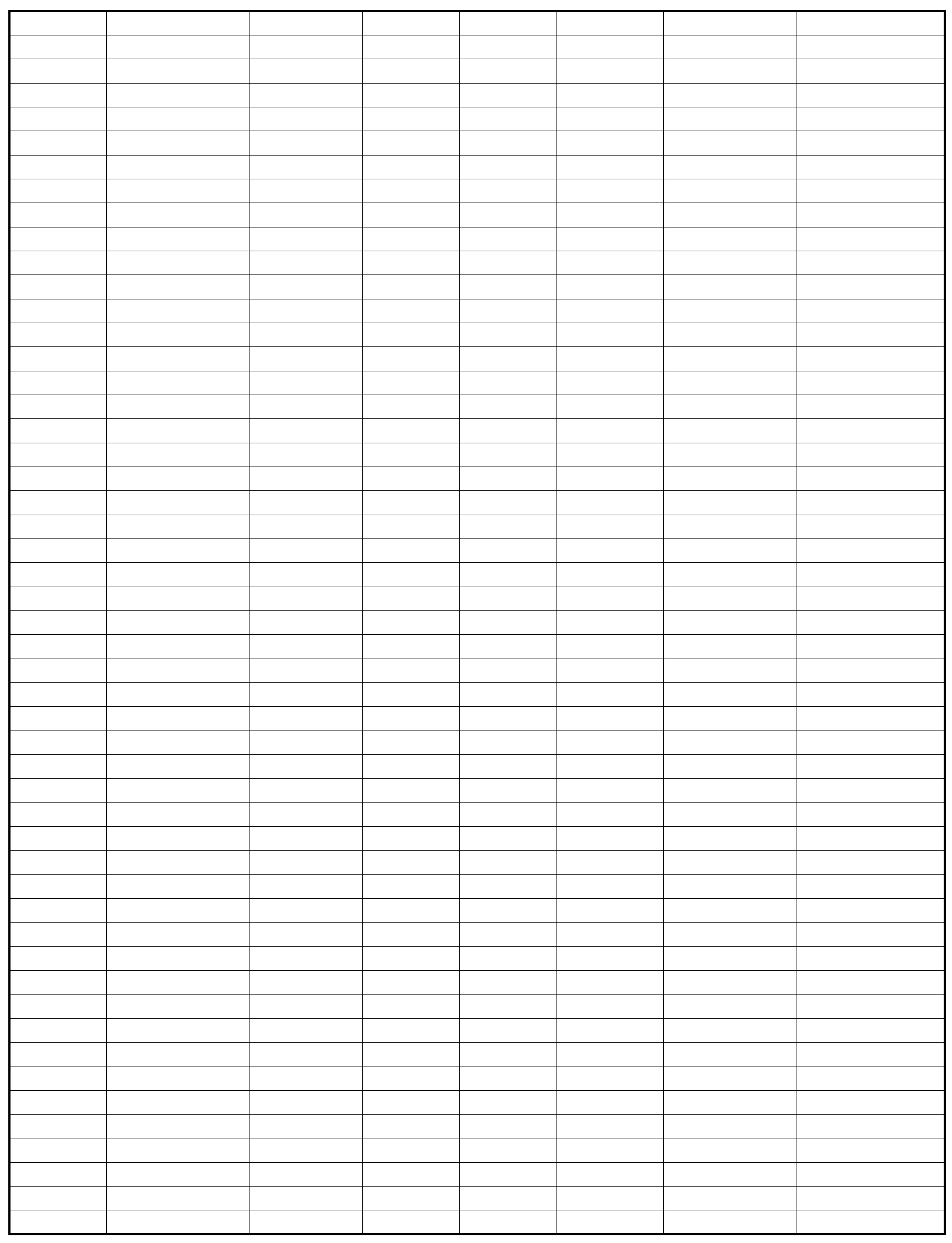

Page 95 
CHART DATA

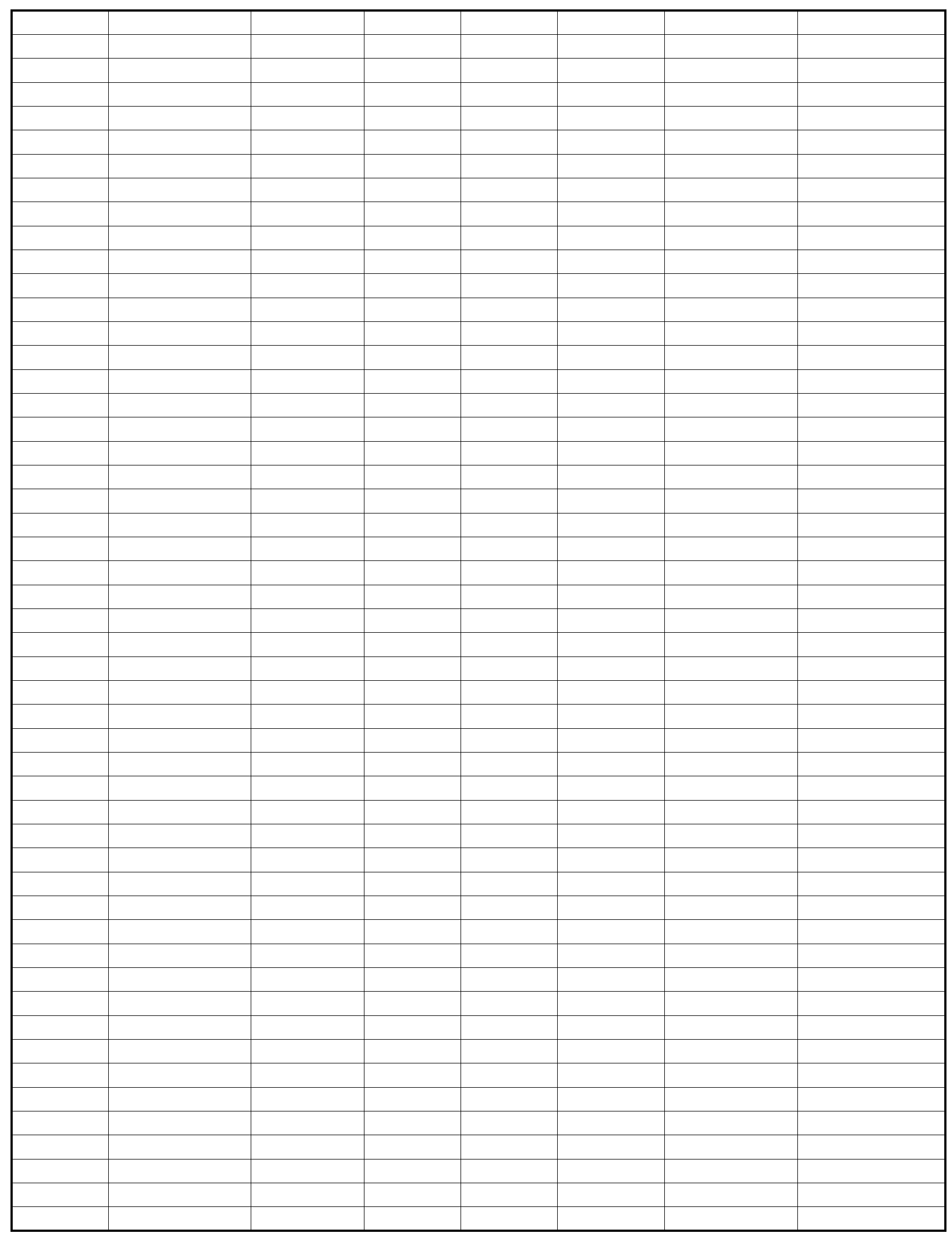

Page 96 
CHART DATA

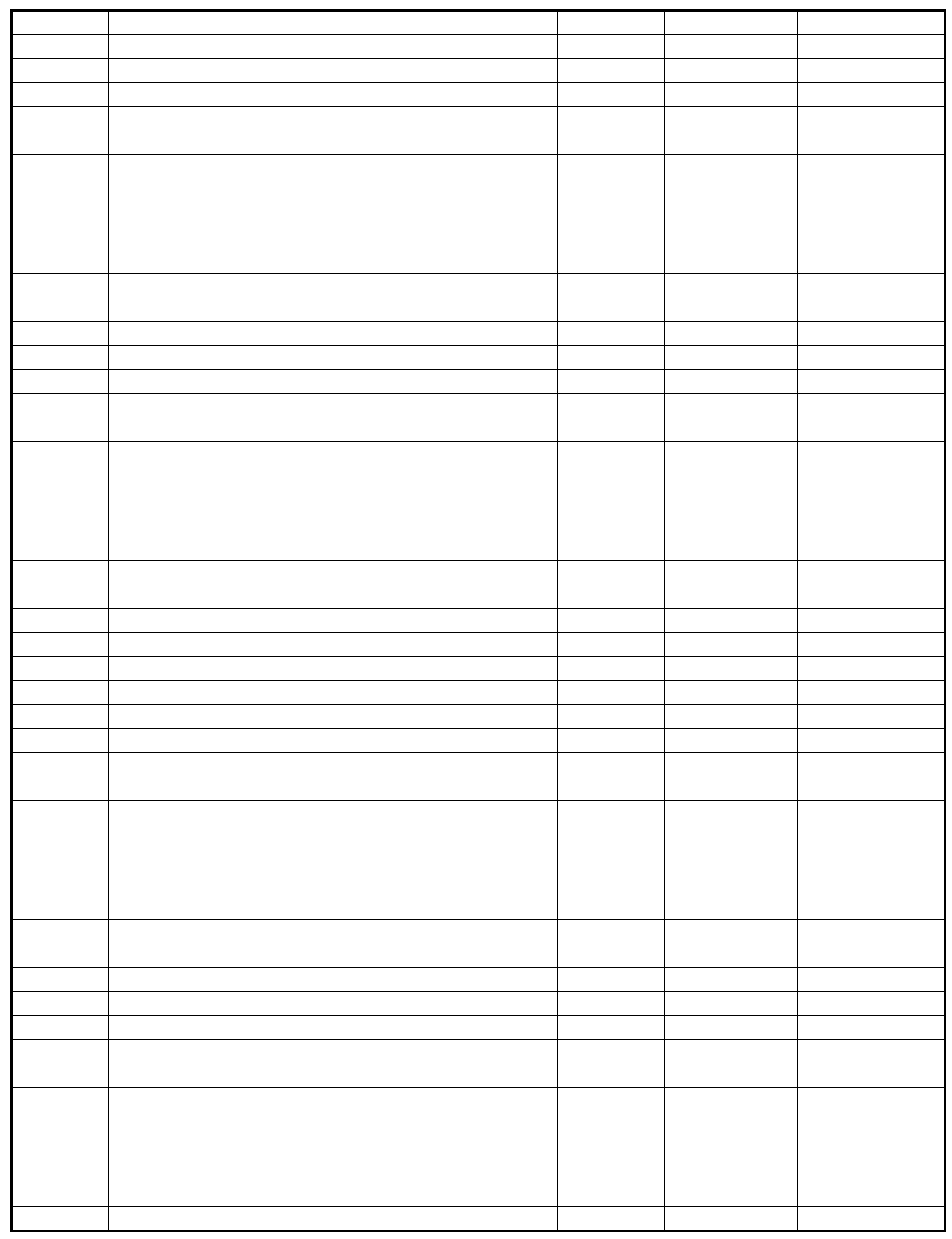

Page 97 
CHART DATA

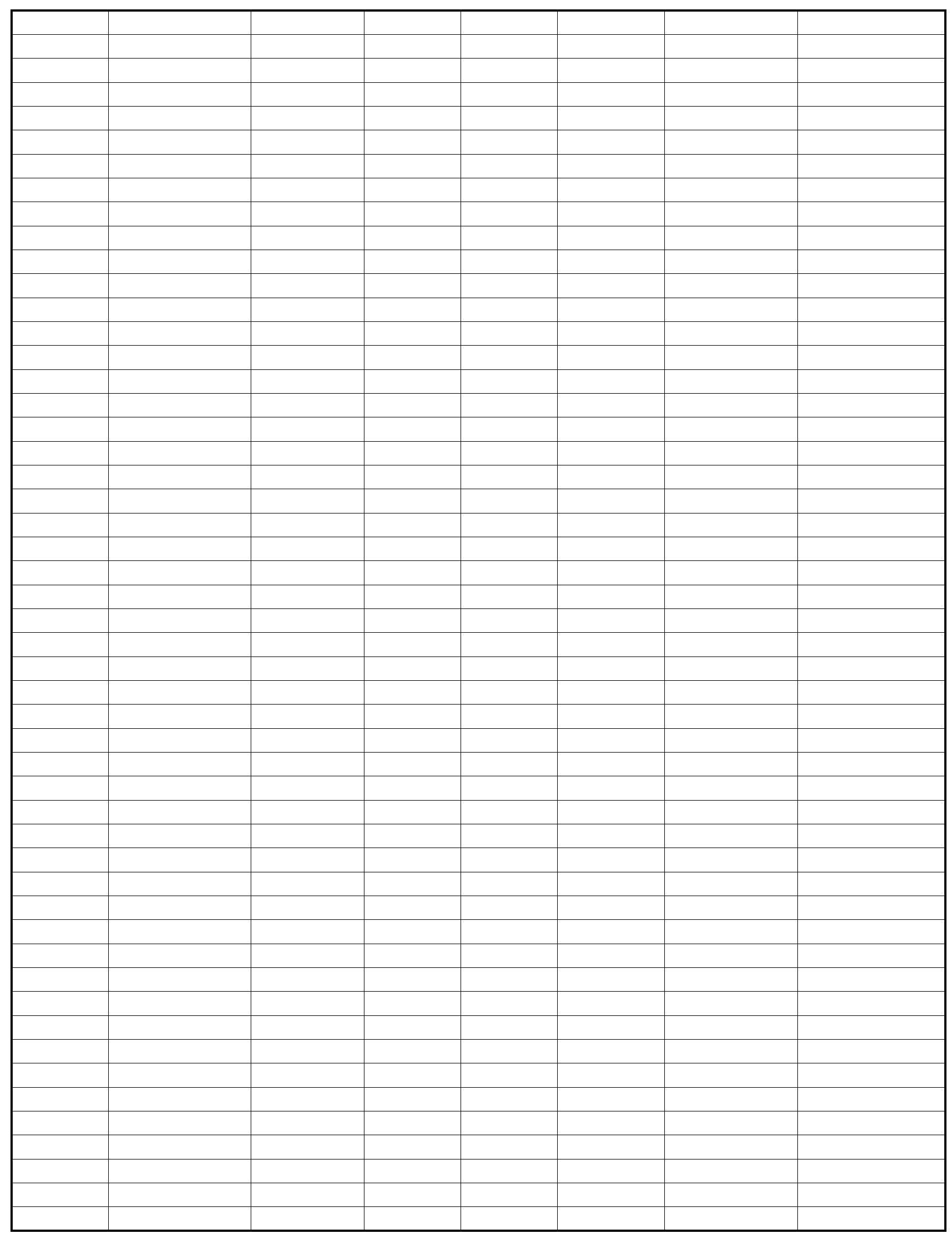

Page 98 
CHART DATA

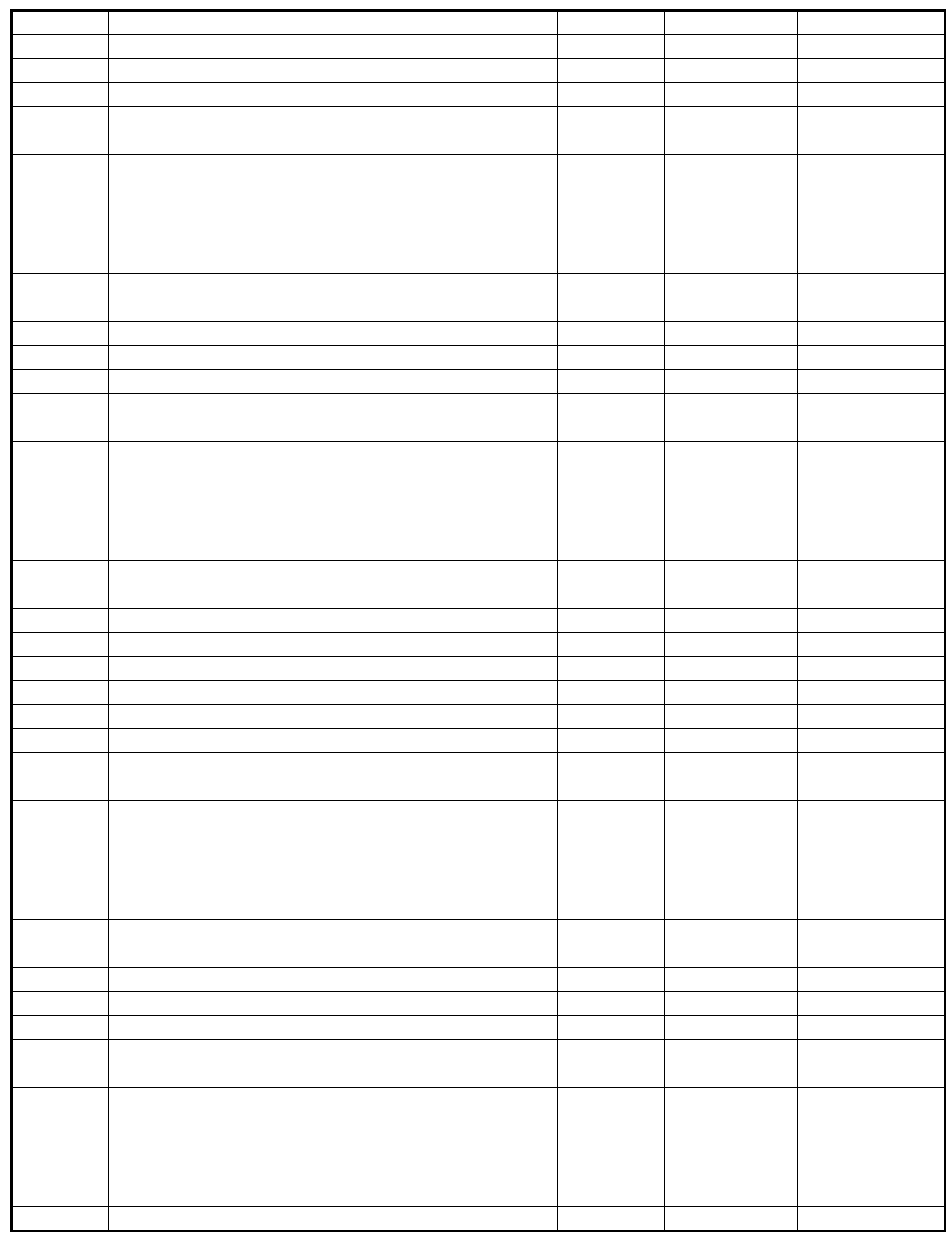

Page 99 
CHART DATA

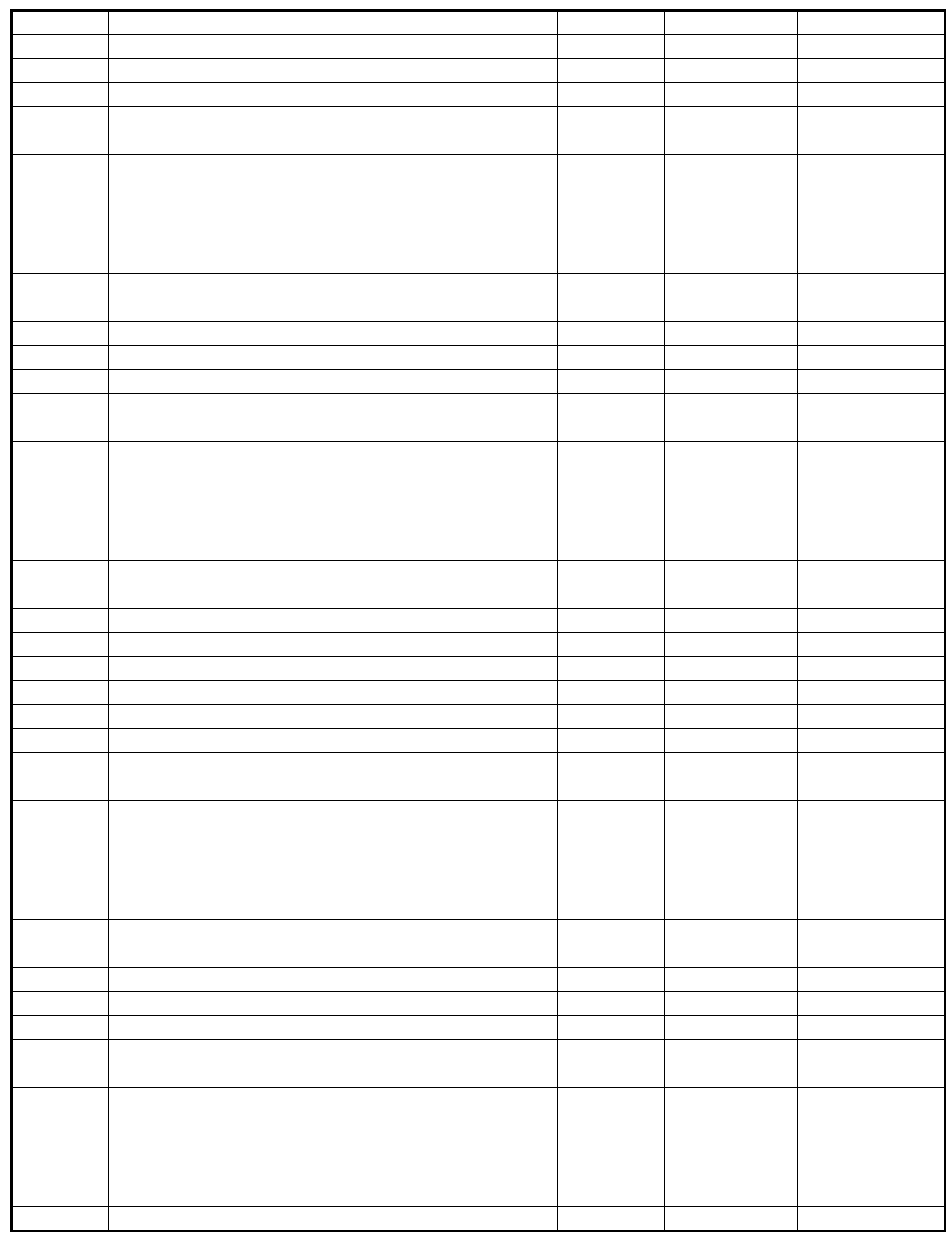

Page 100 
CHART DATA

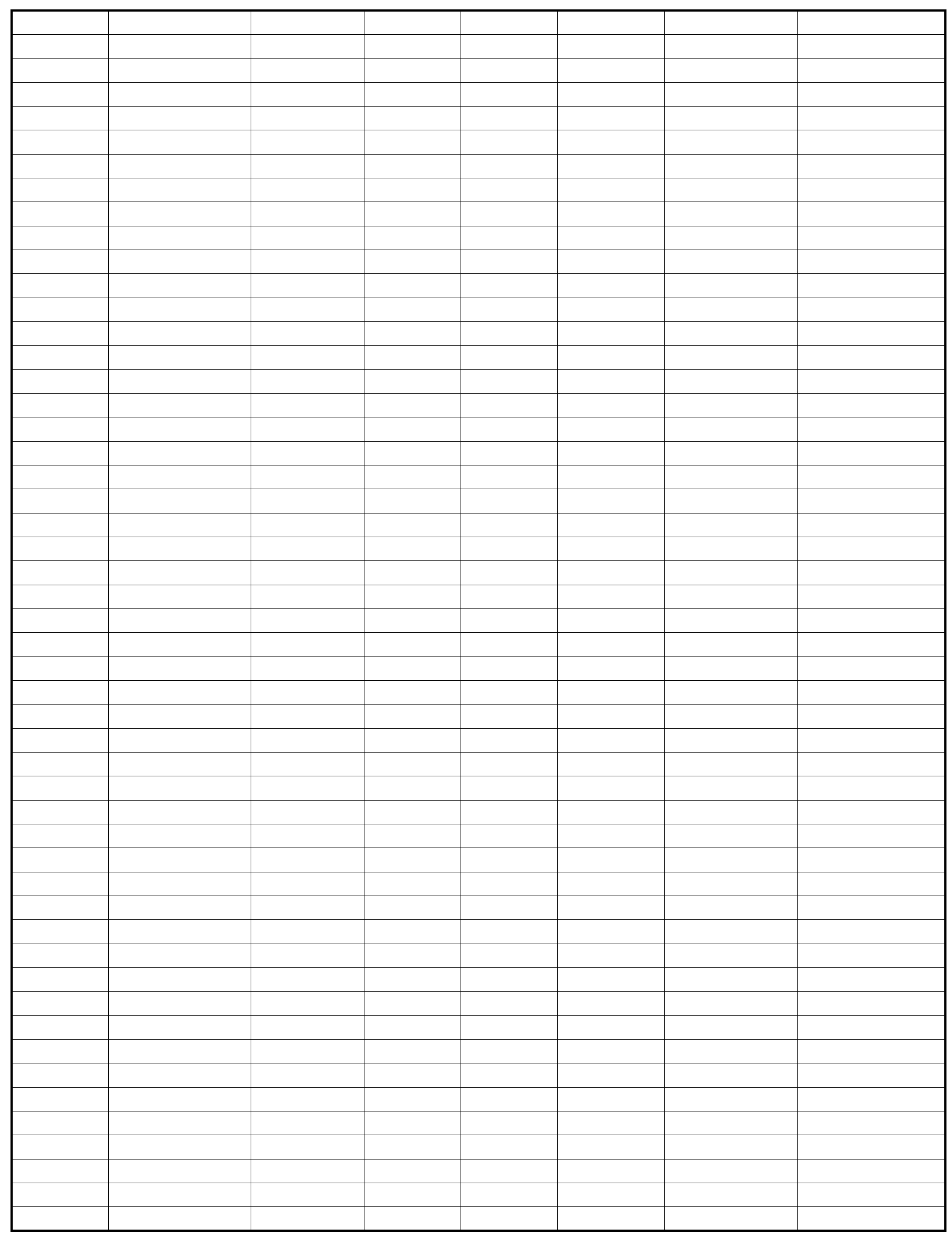

Page 101 
CHART DATA

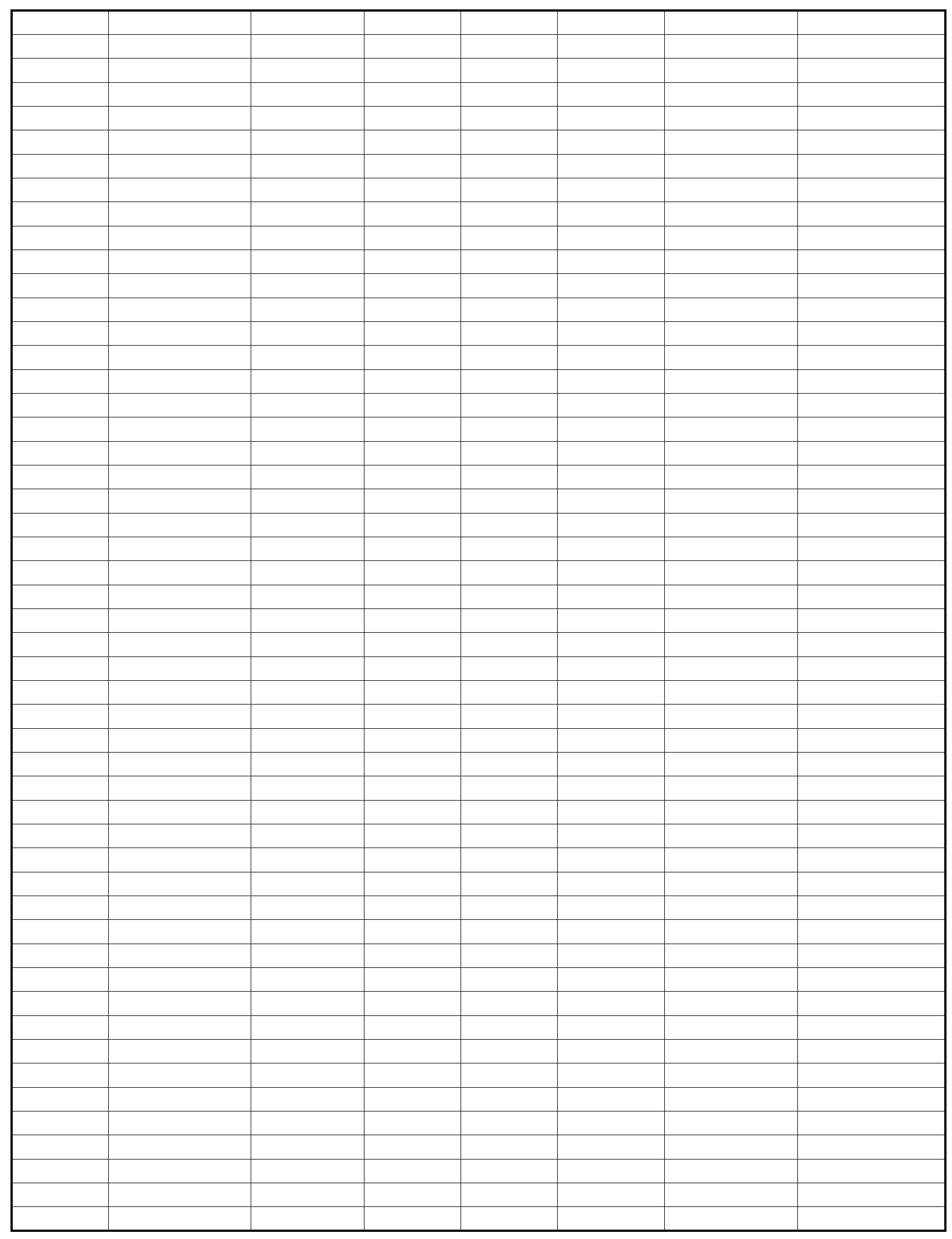

Page 102 
CHART DATA

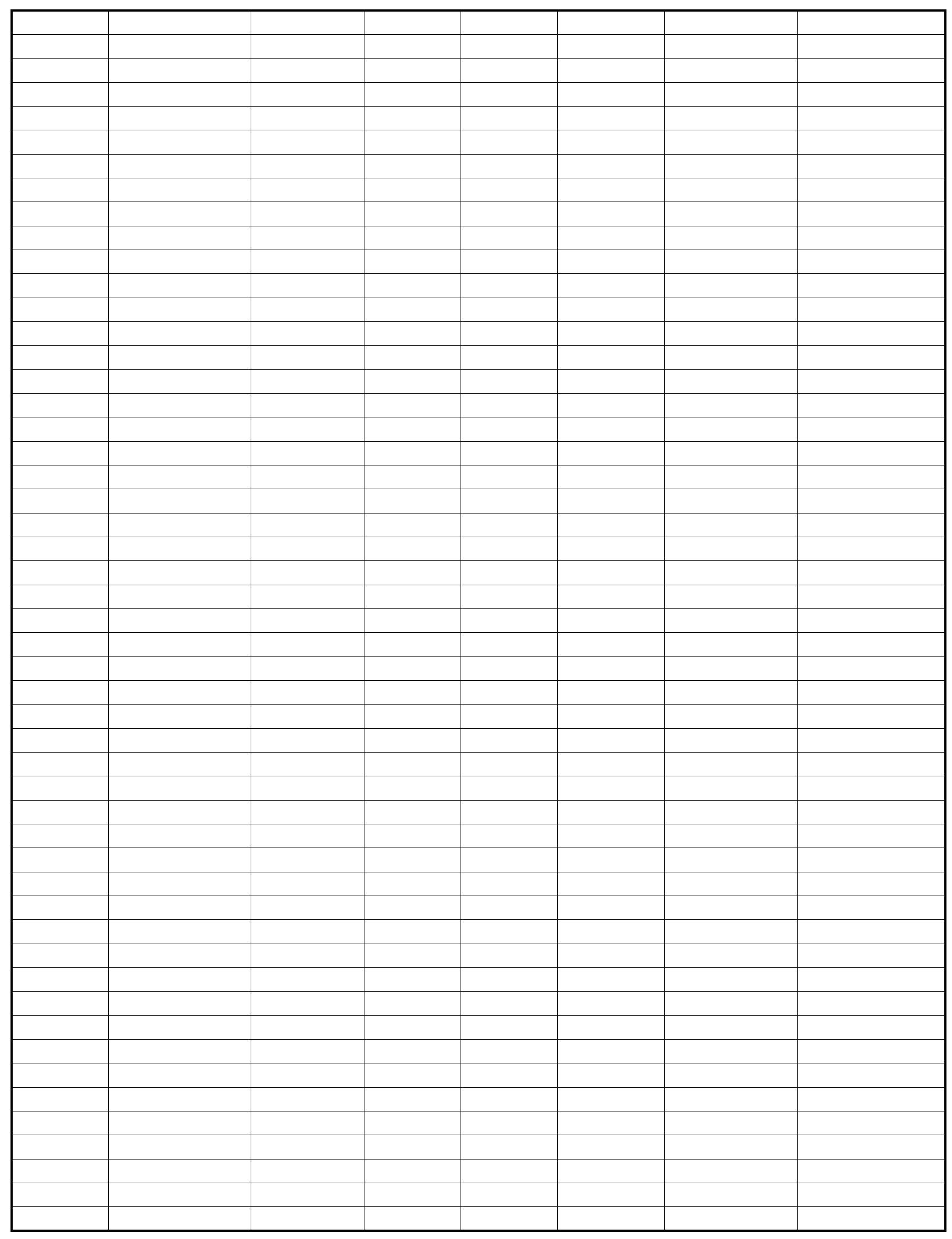

Page 103 
CHART DATA

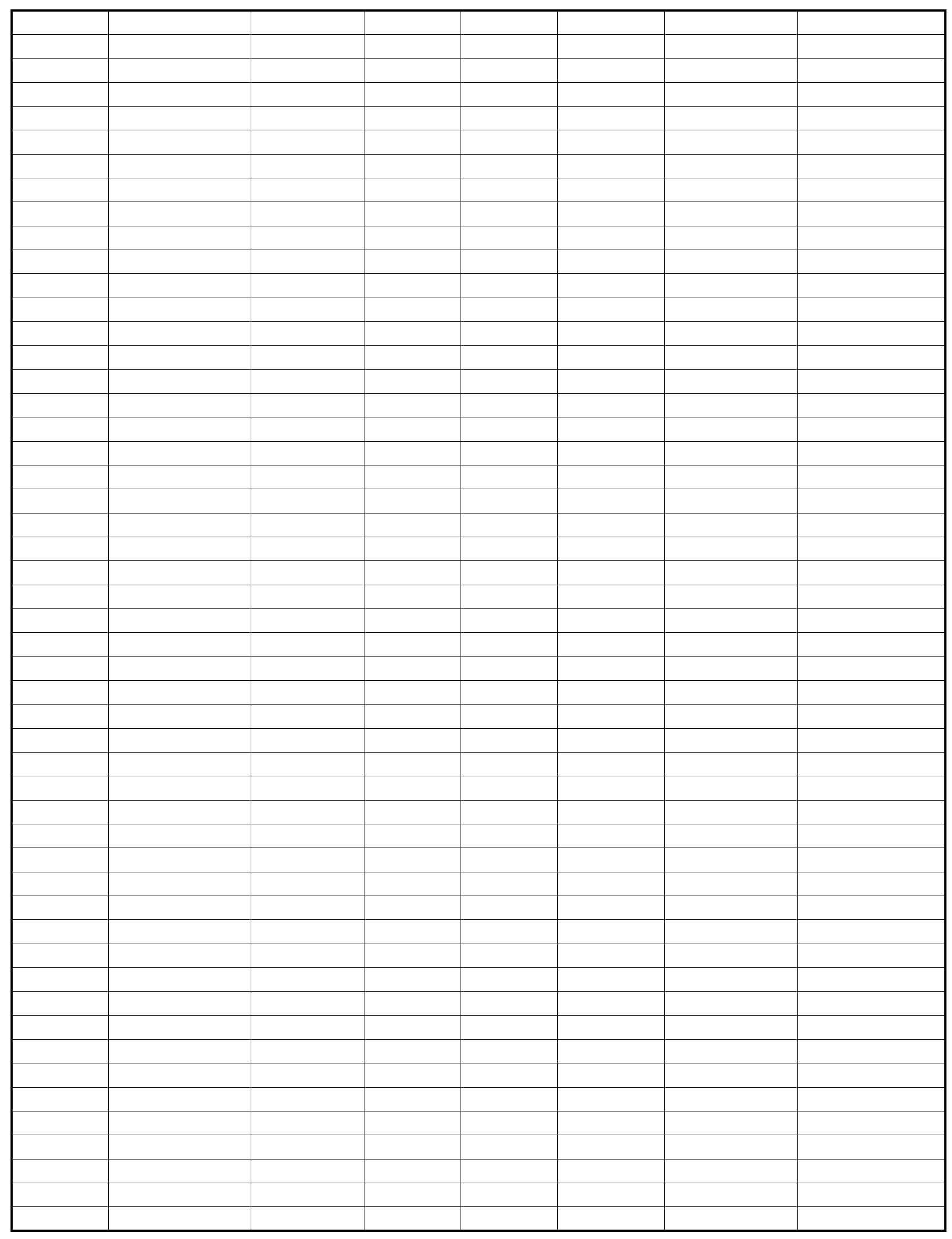

Page 104 
CHART DATA

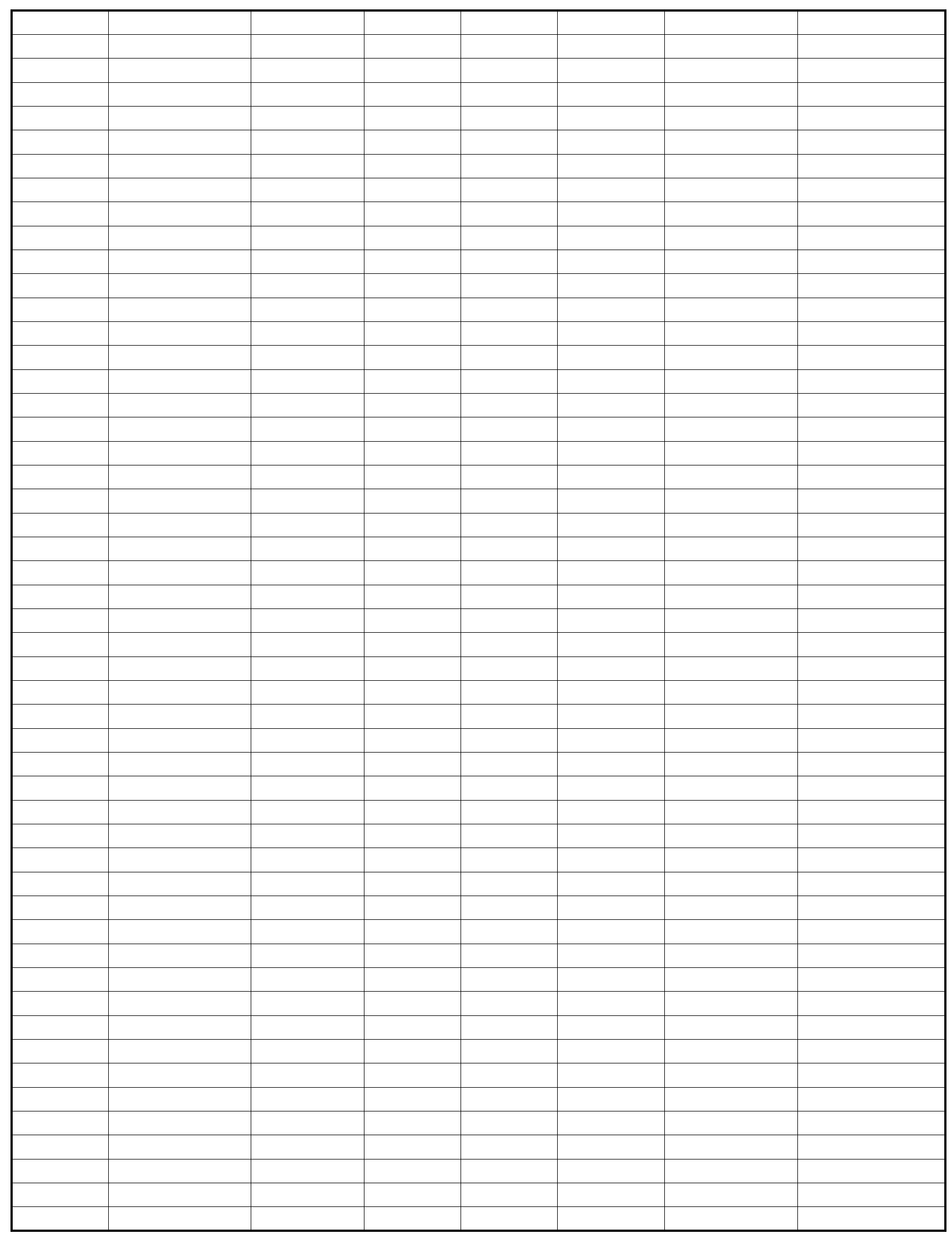

Page 105 
CHART DATA

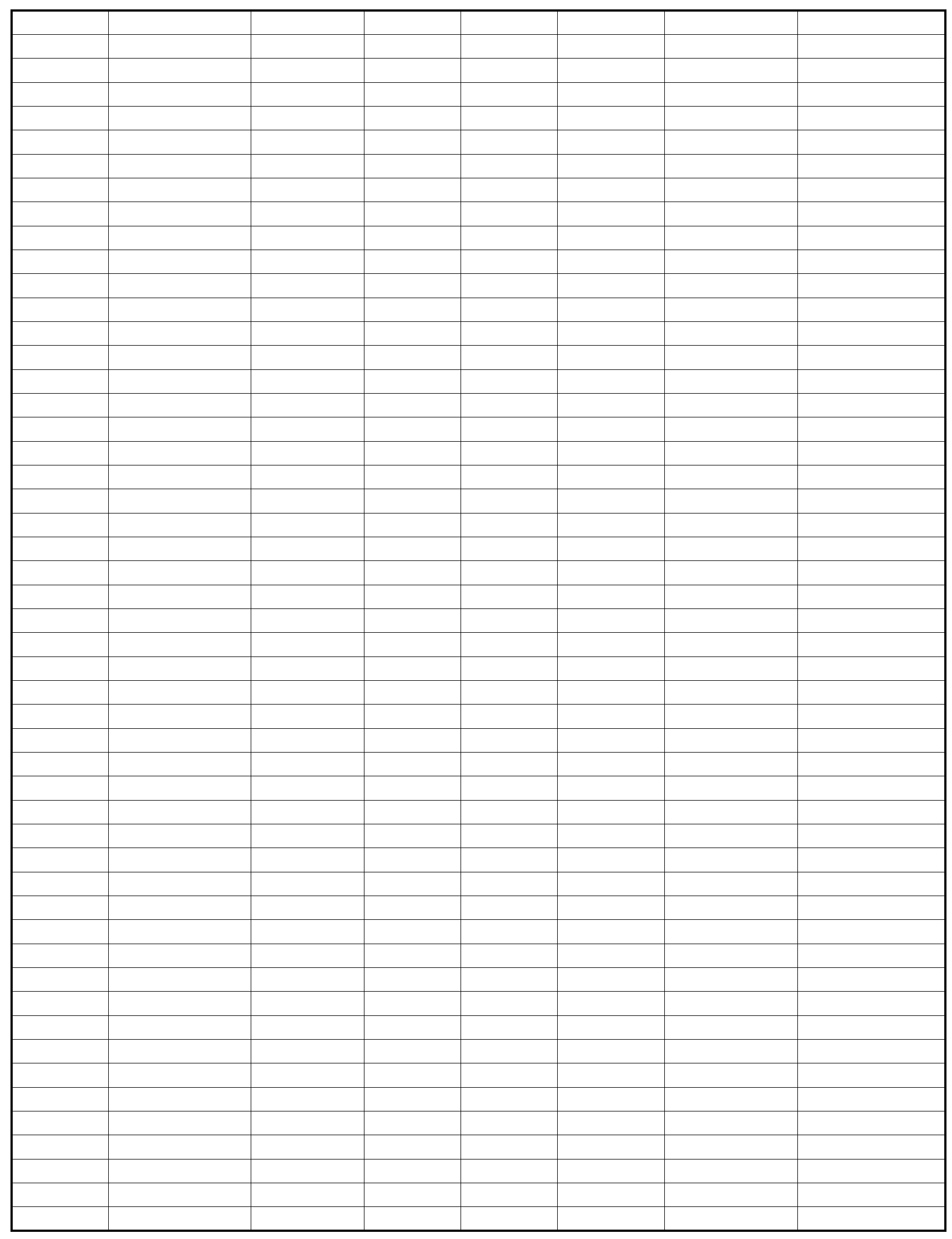

Page 106 
CHART DATA

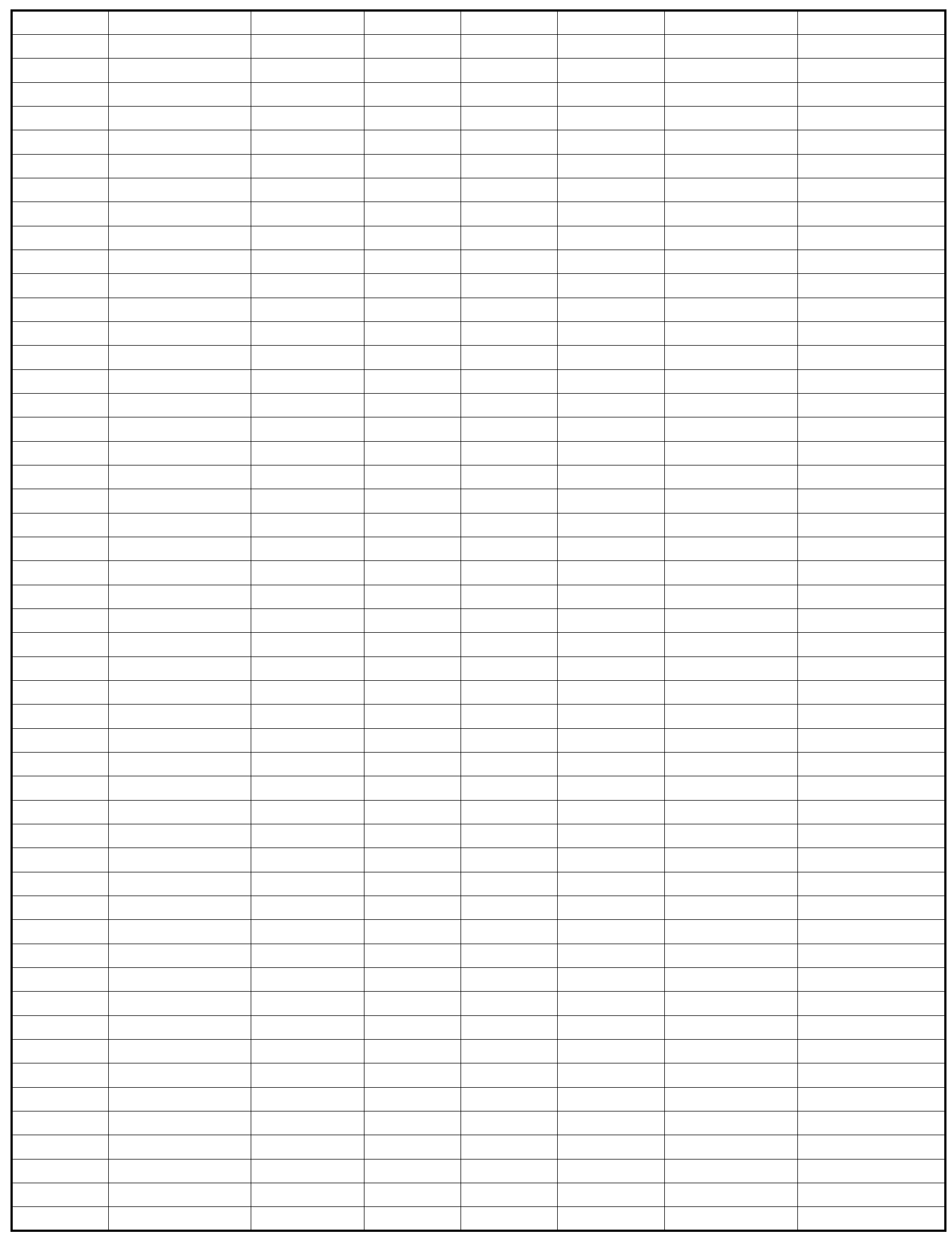

Page 107 
CHART DATA

\begin{tabular}{|l|l|l|l|l|l|l|l|}
\hline & & & & & & & \\
\hline \\
\hline
\end{tabular}




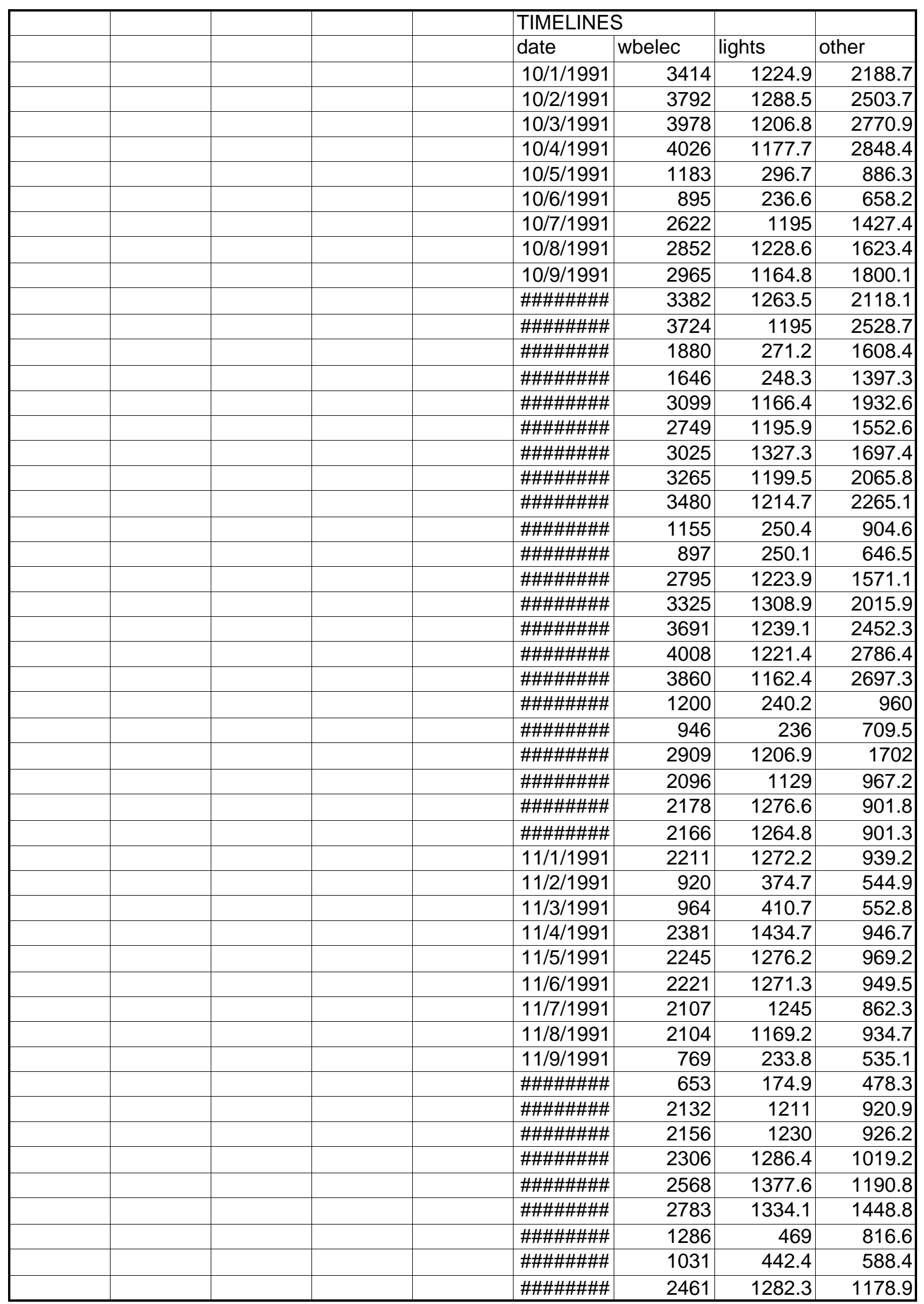


CHART DATA

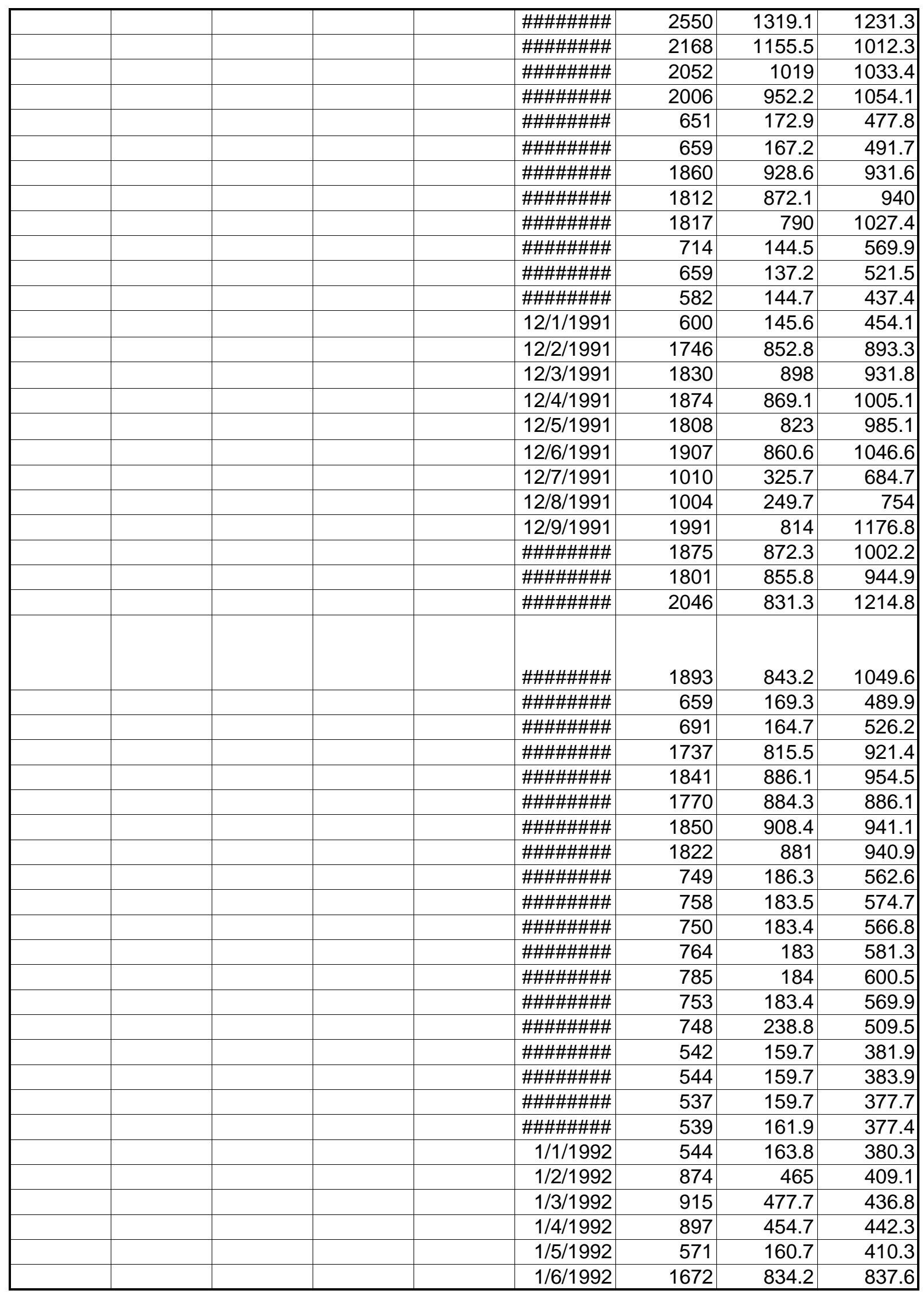




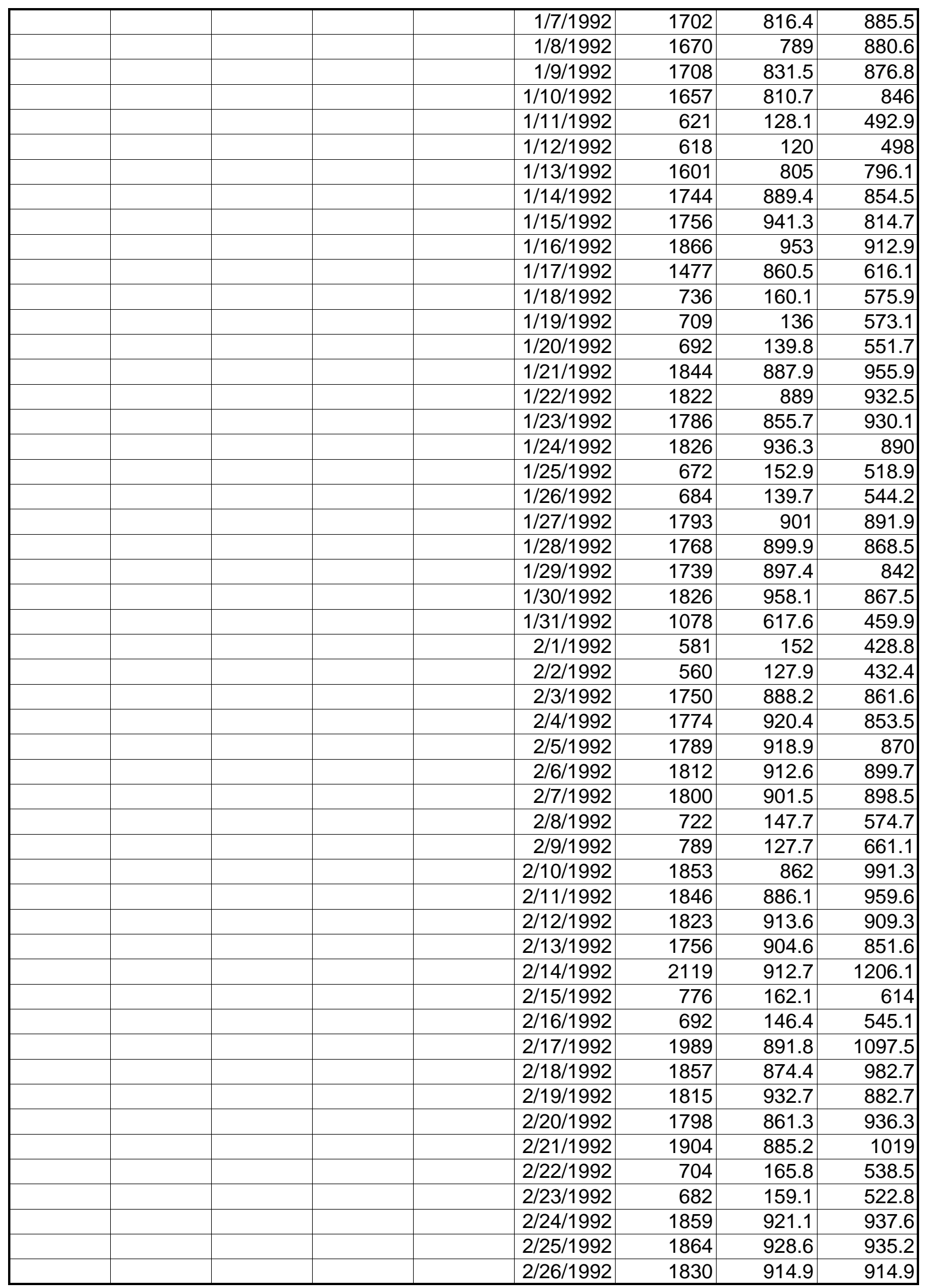


CHART DATA

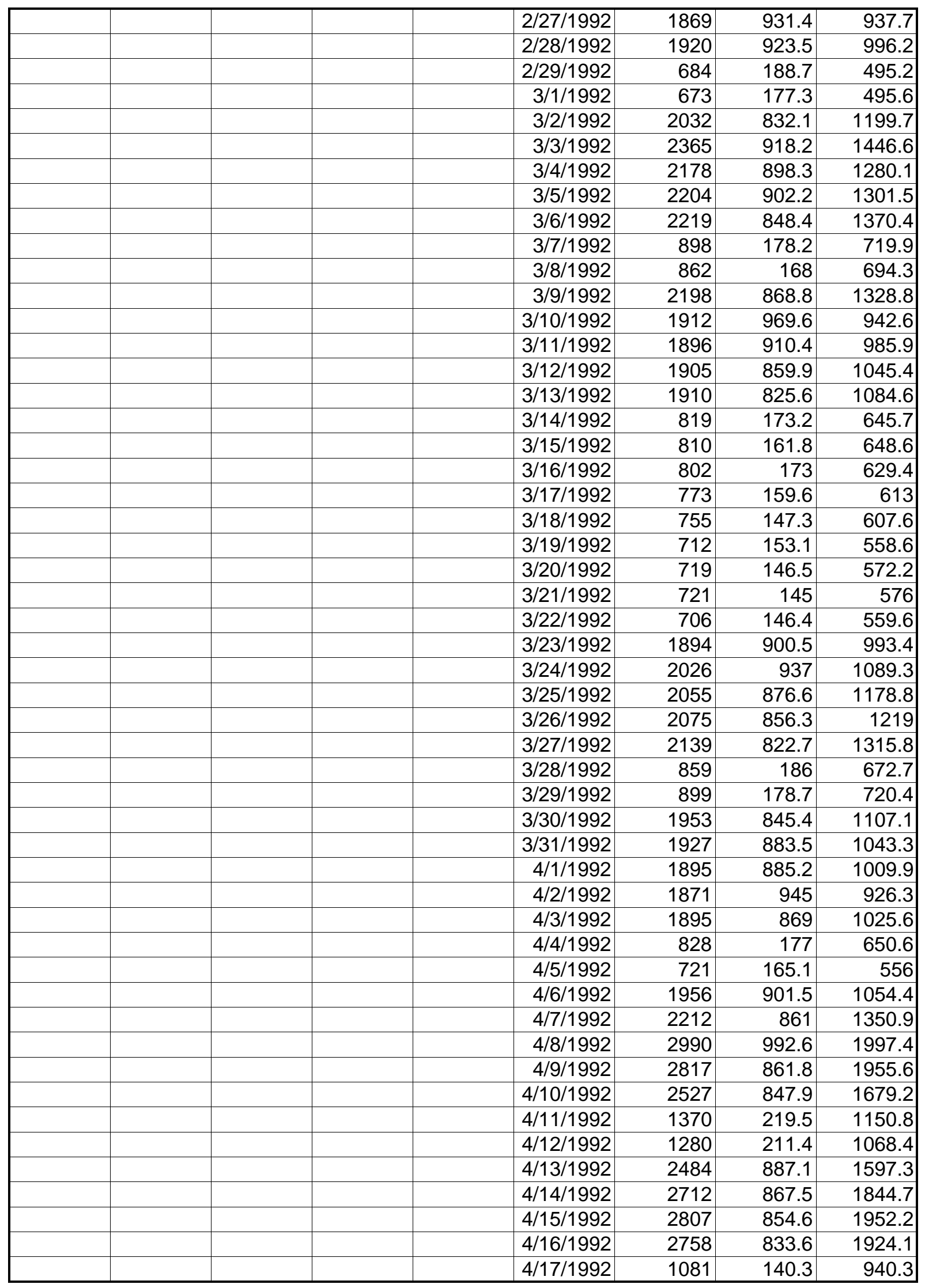


CHART DATA

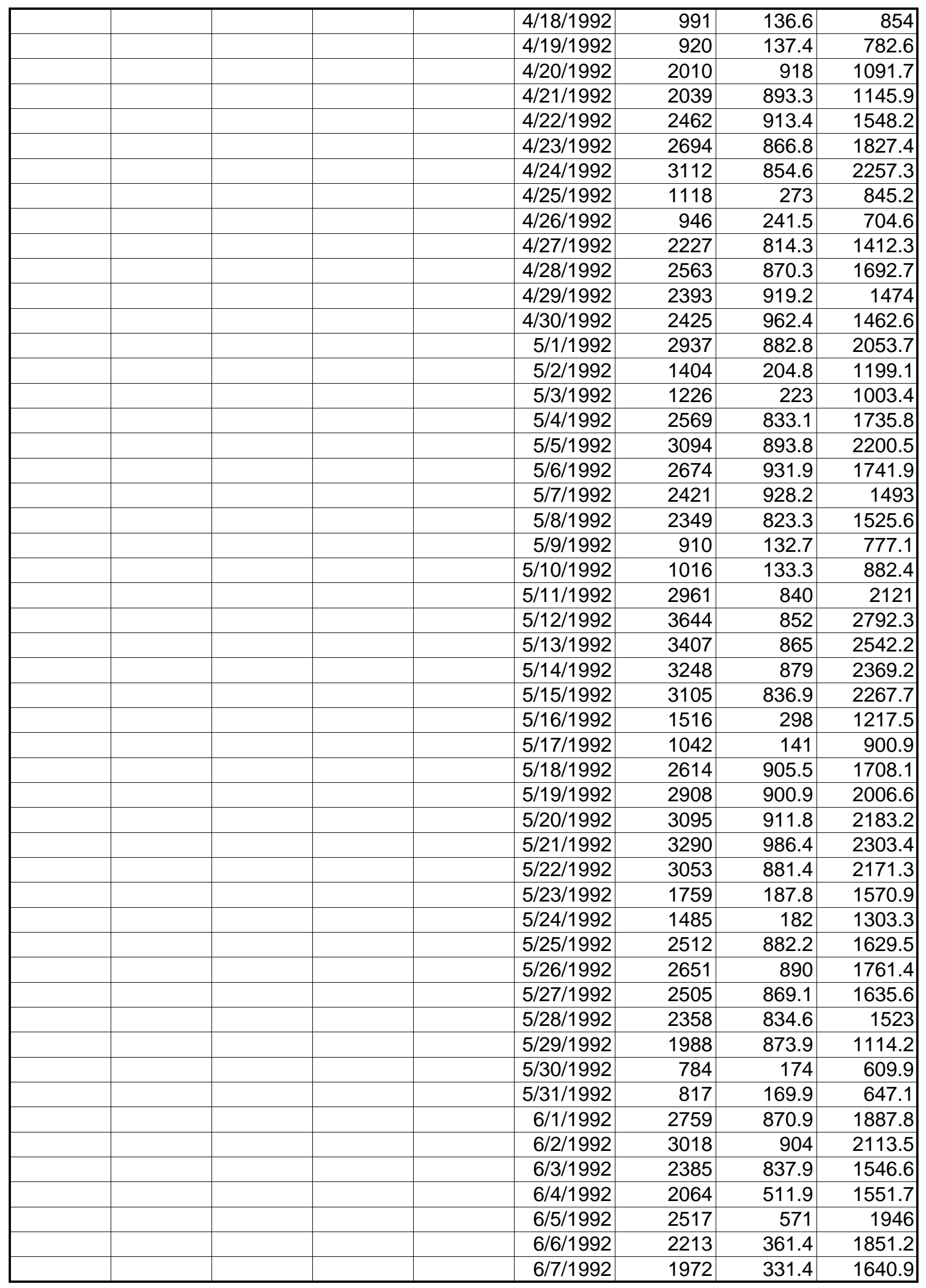


CHART DATA

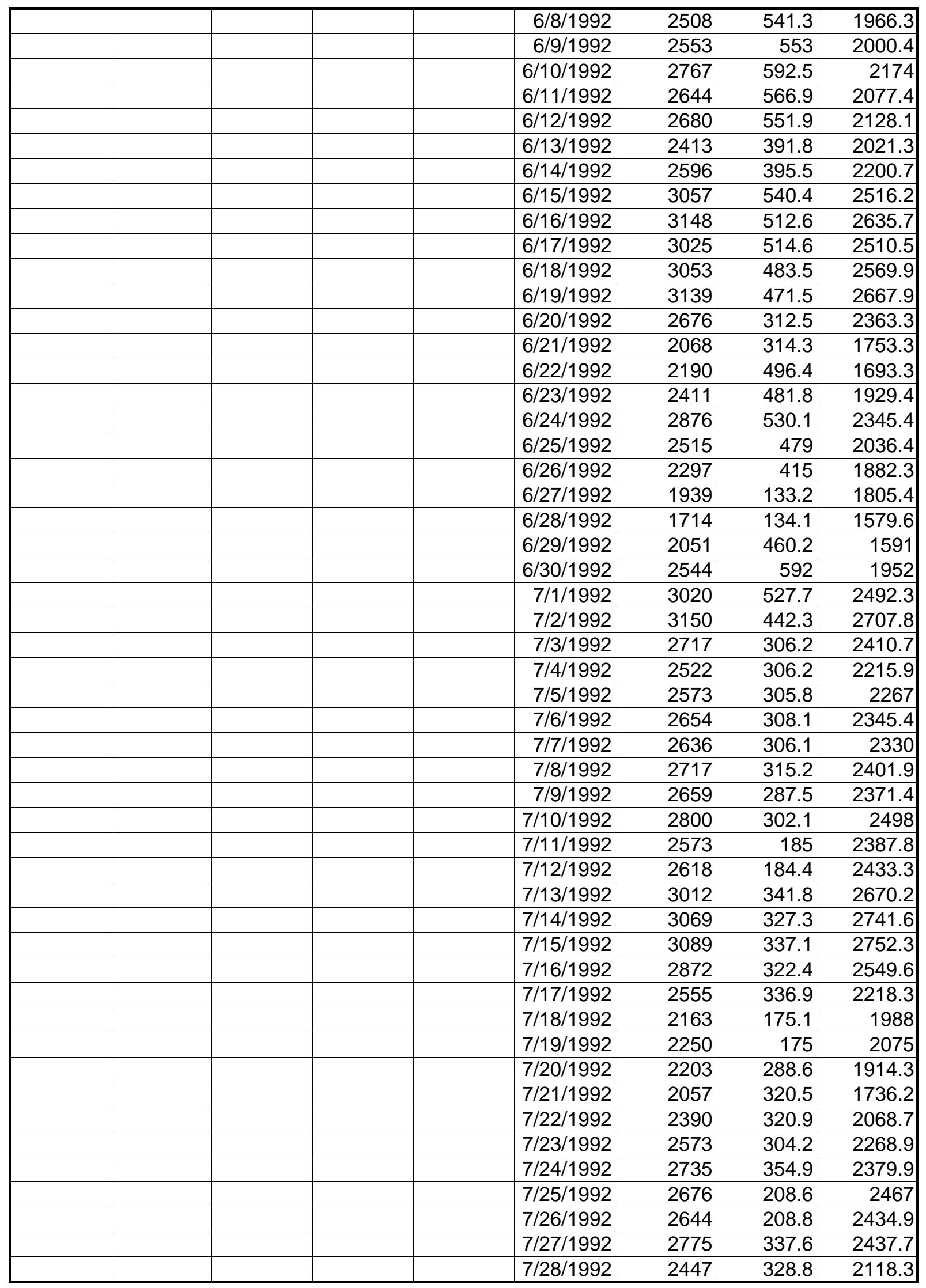


CHART DATA

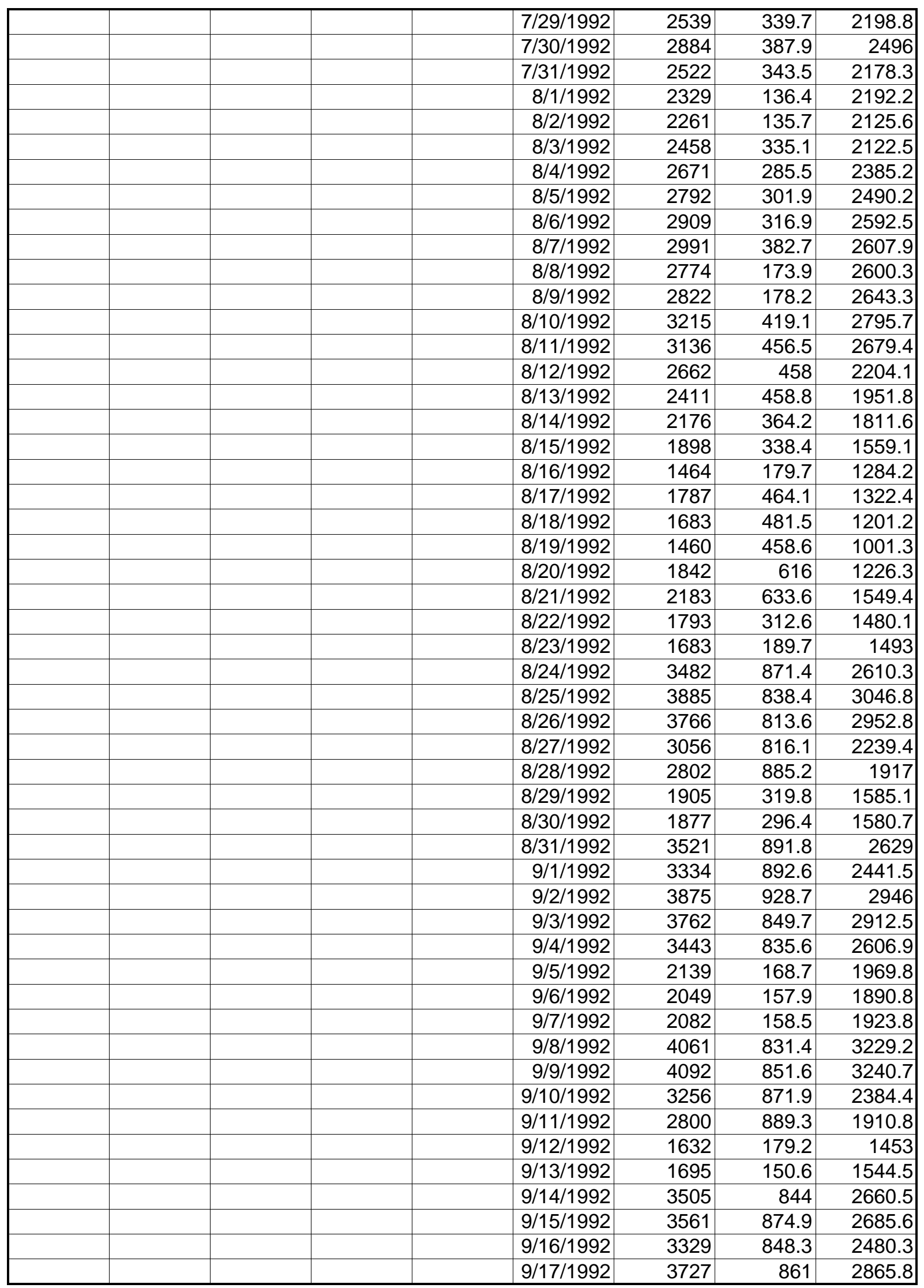




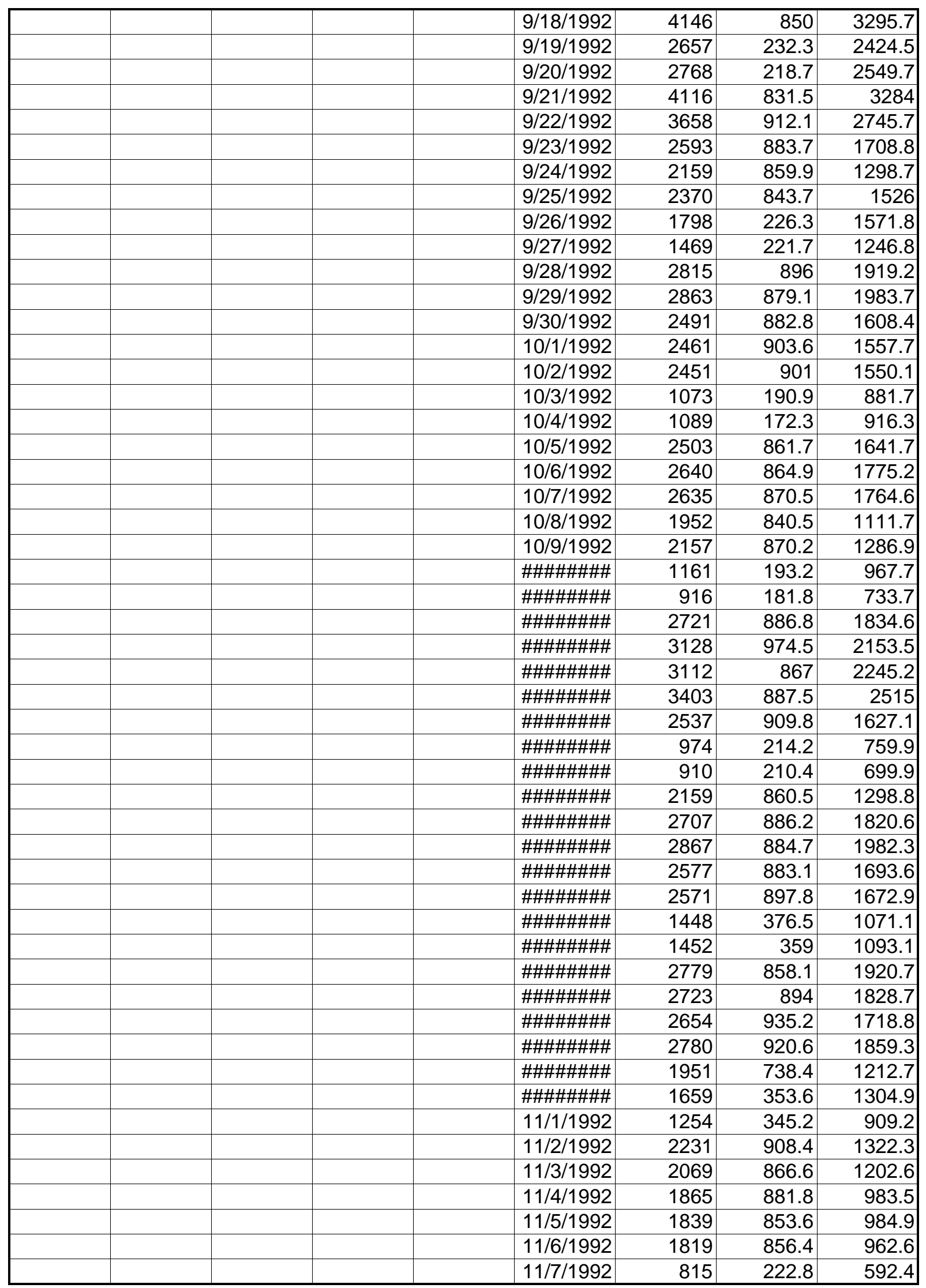


CHART DATA

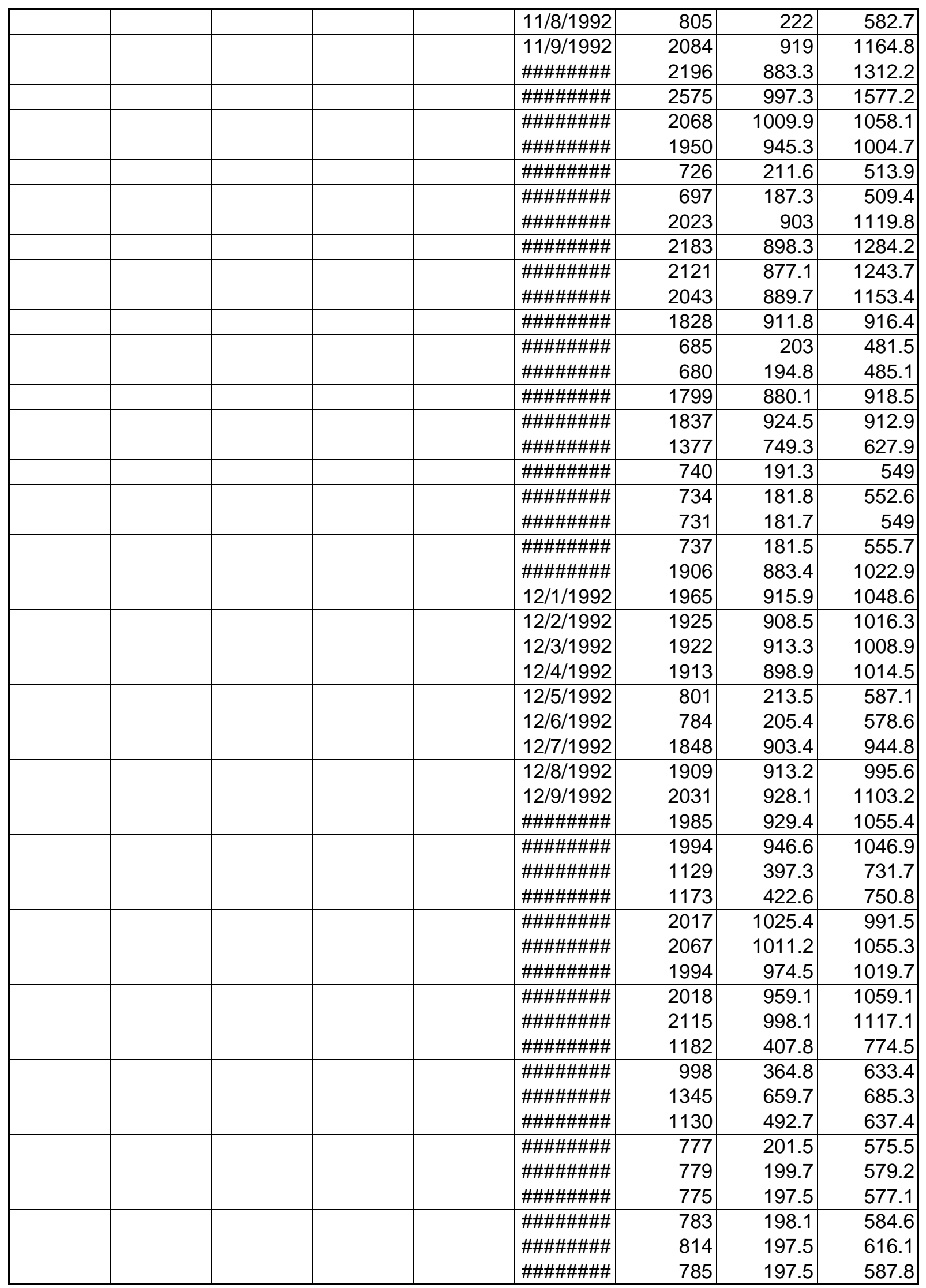


CHART DATA

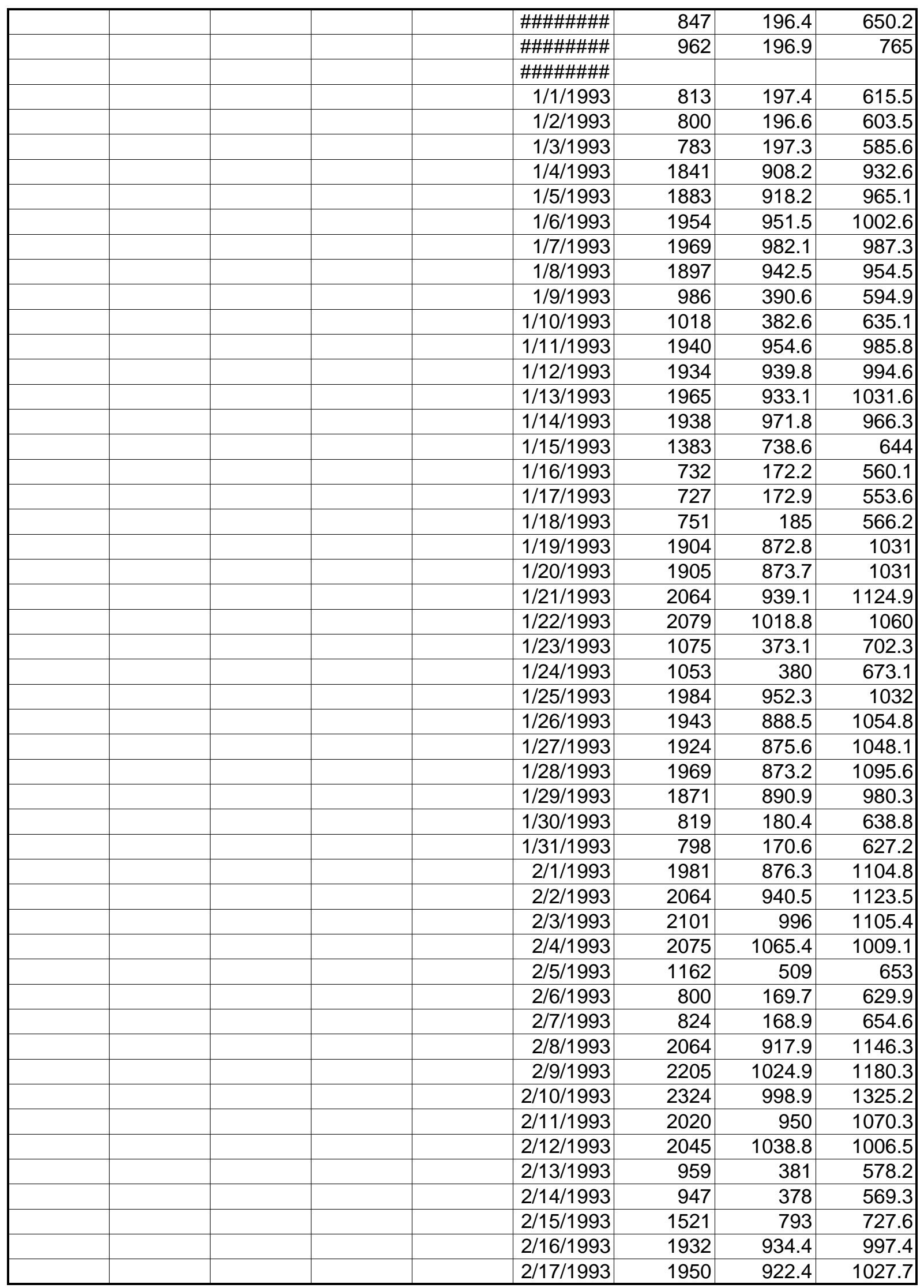


CHART DATA

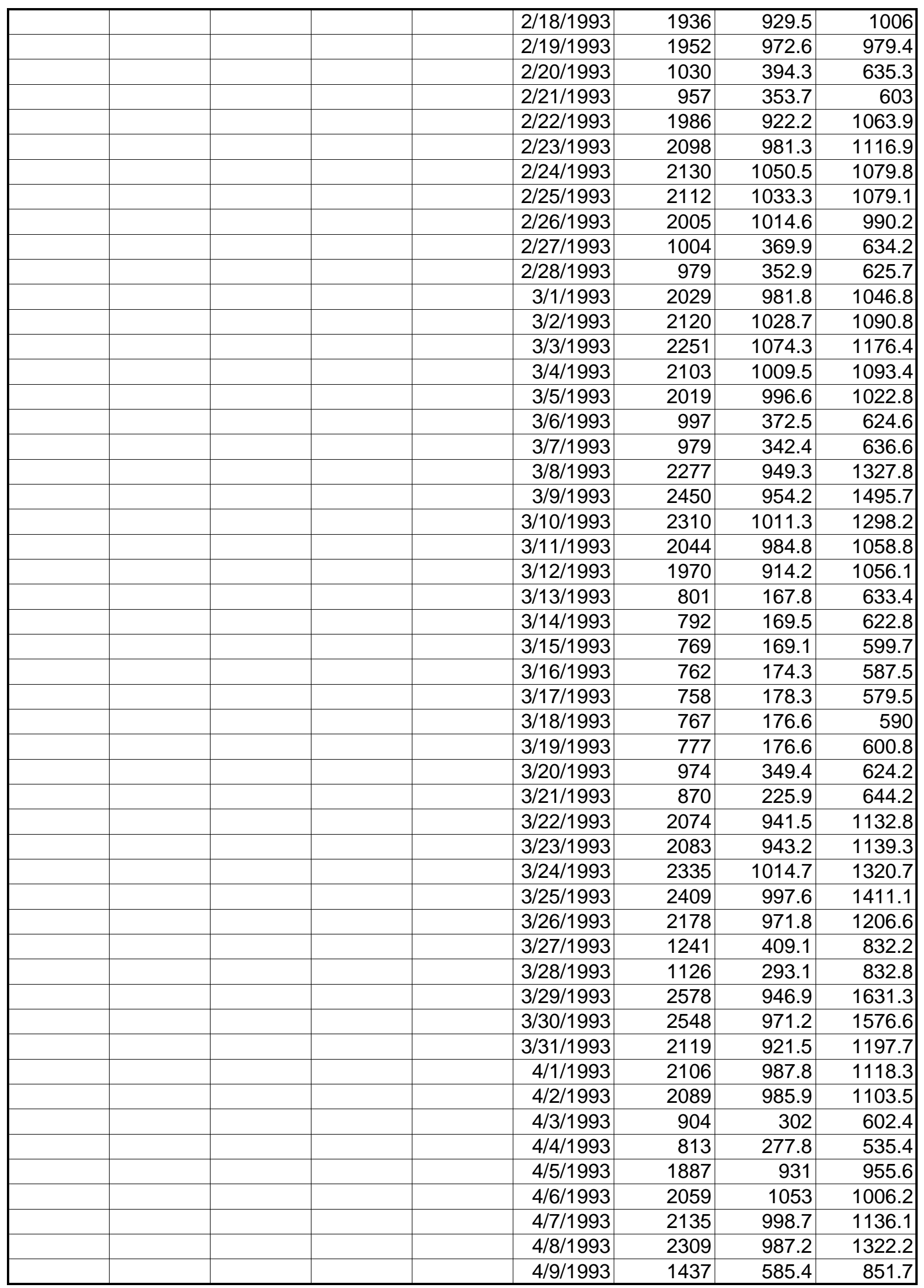


CHART DATA

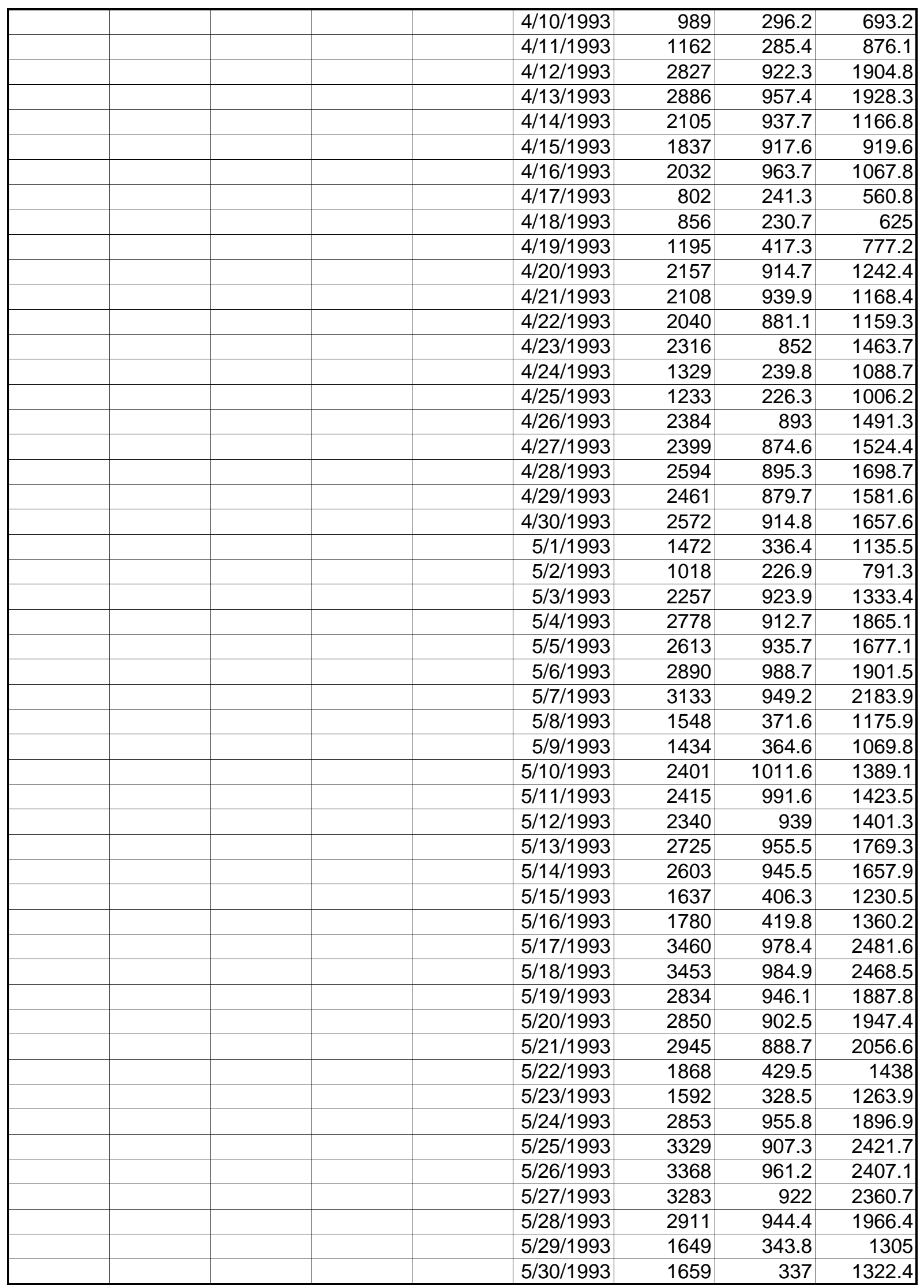


CHART DATA

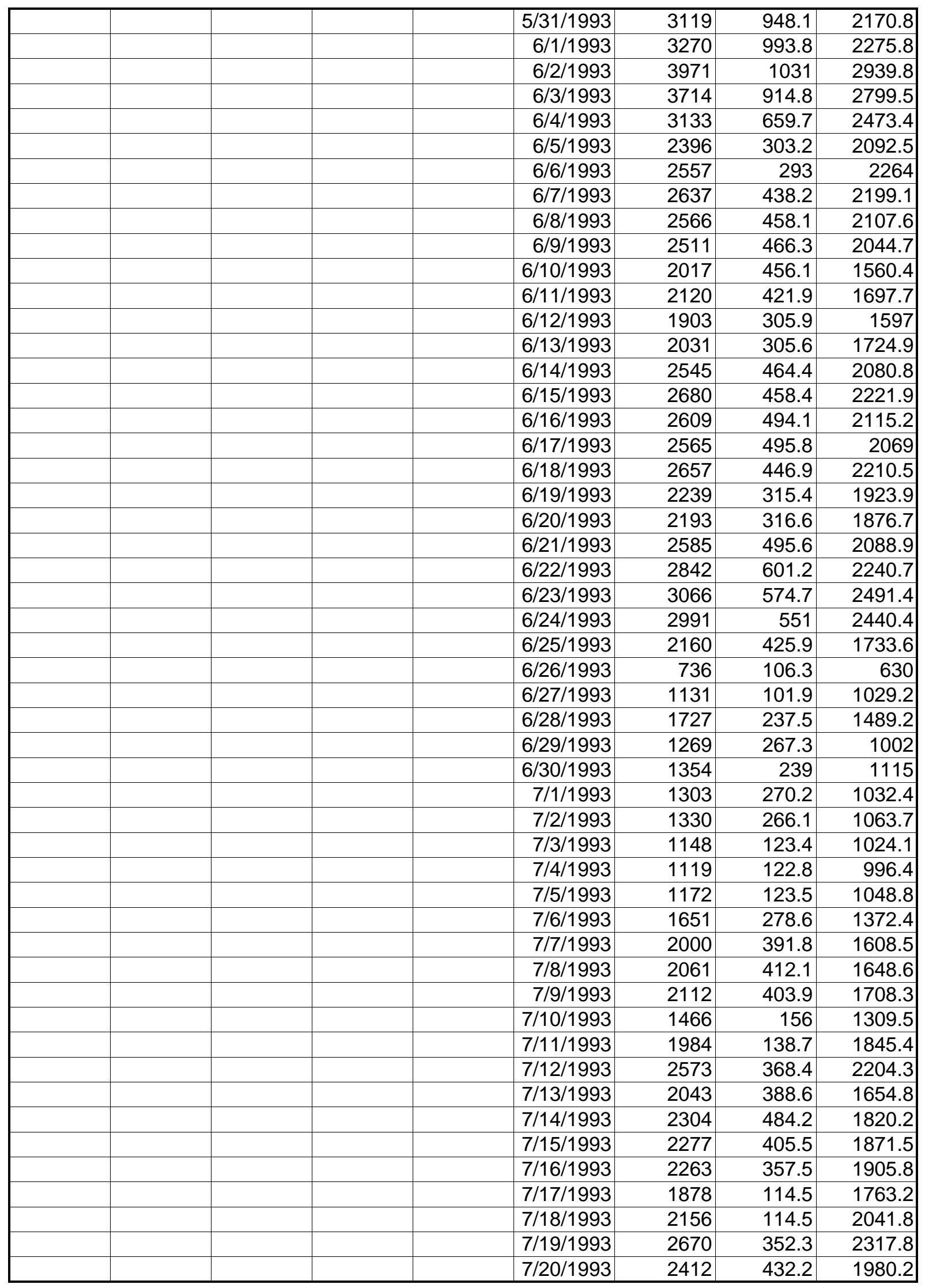


CHART DATA

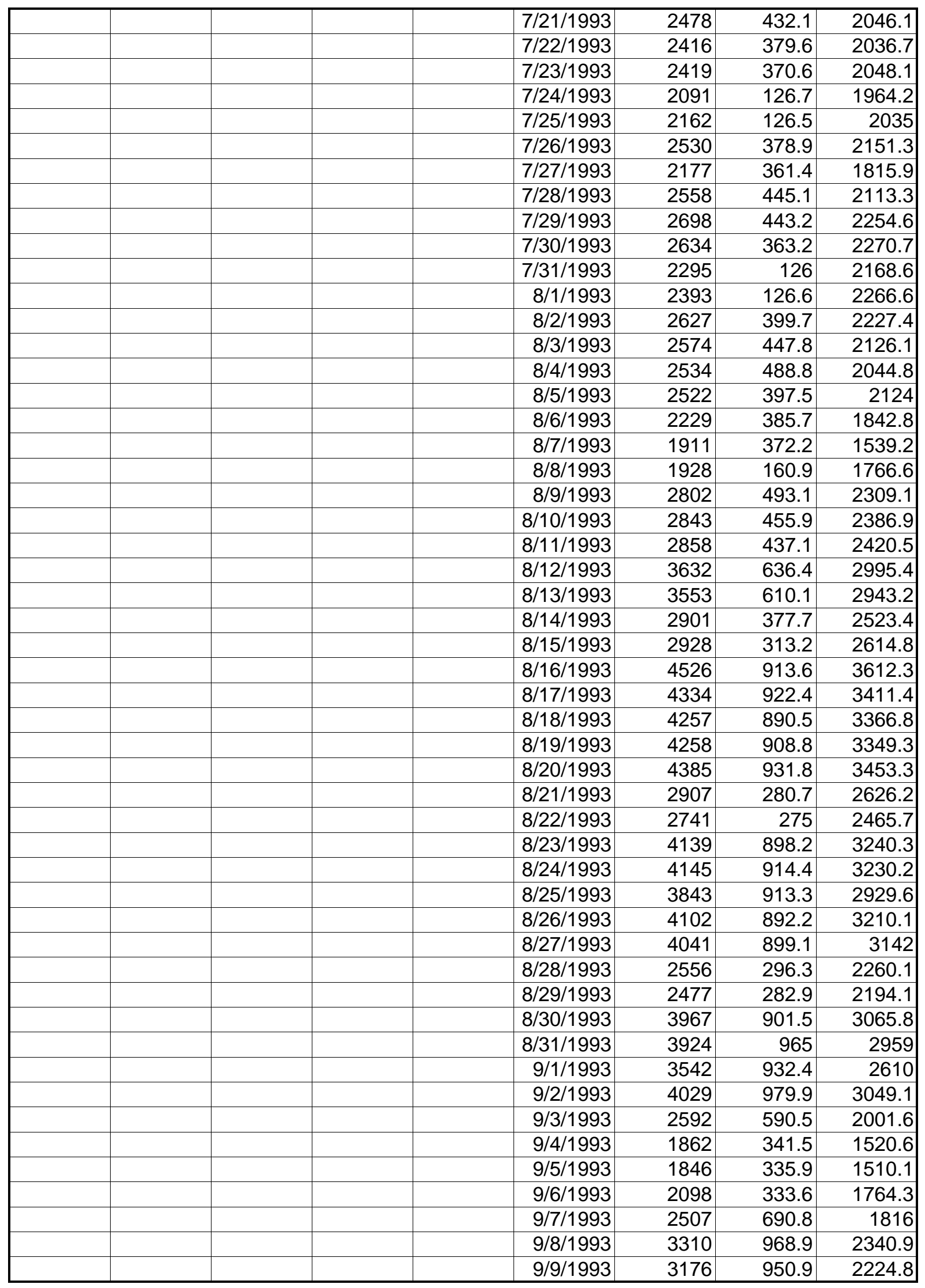


CHART DATA

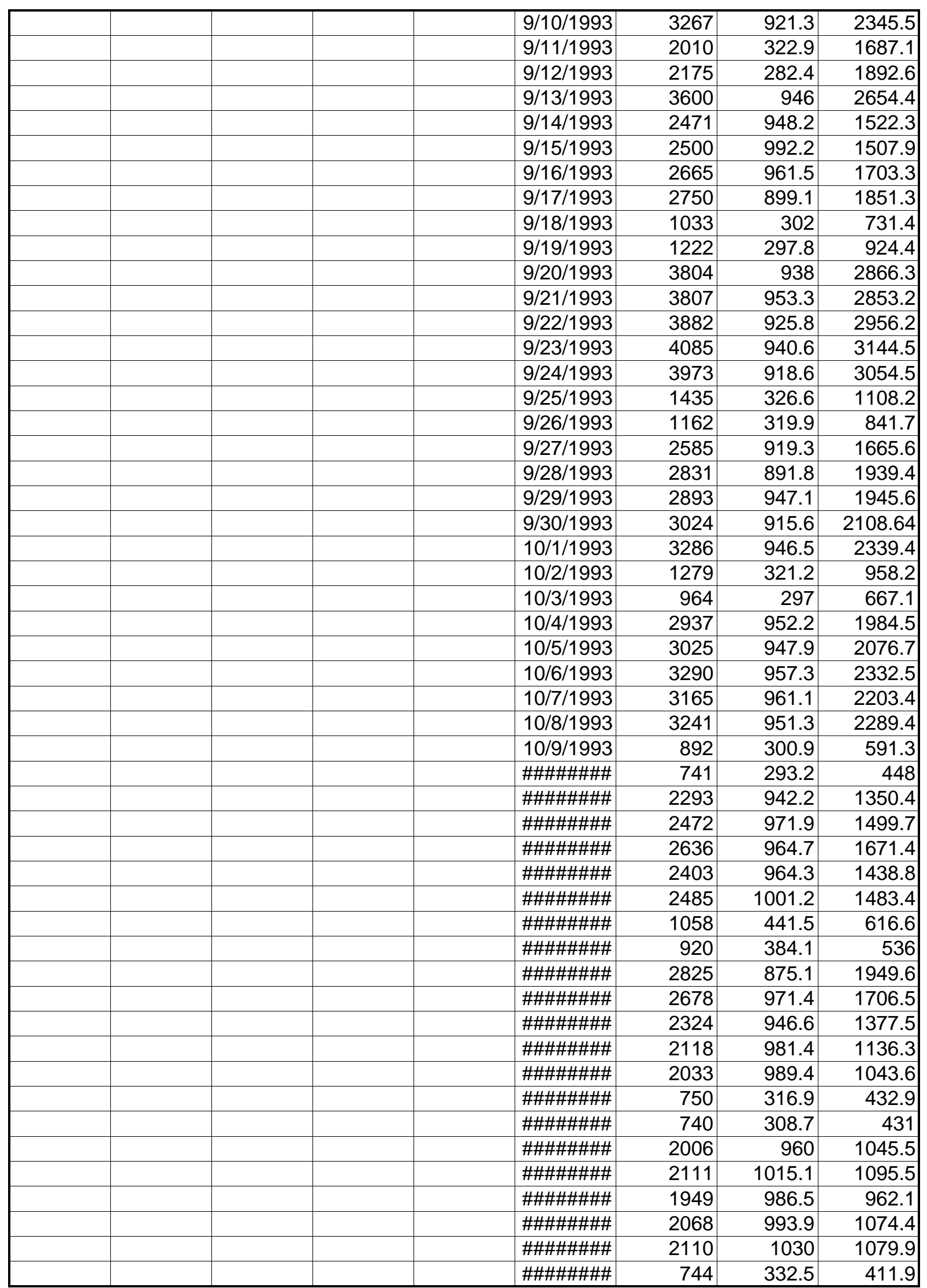


CHART DATA

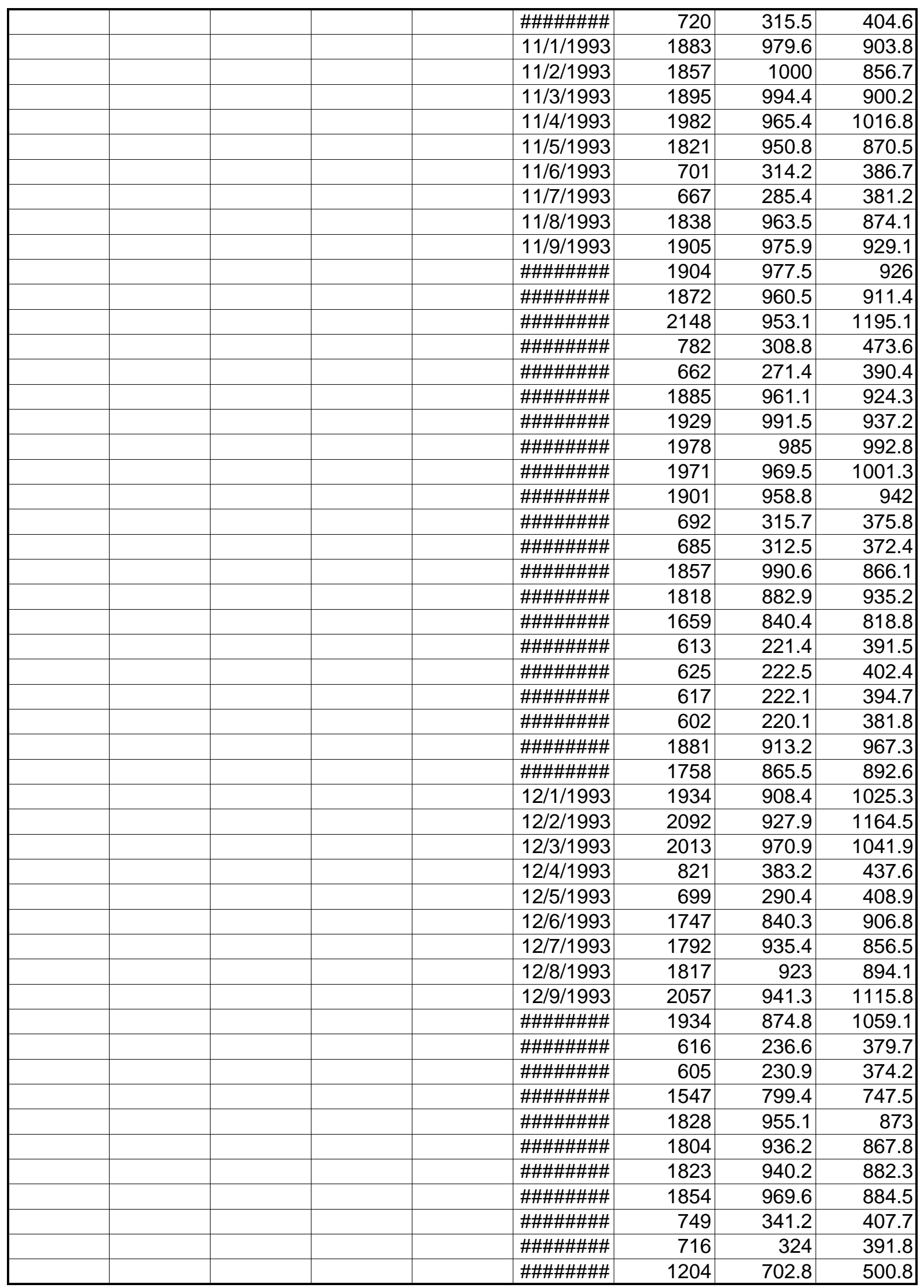


CHART DATA

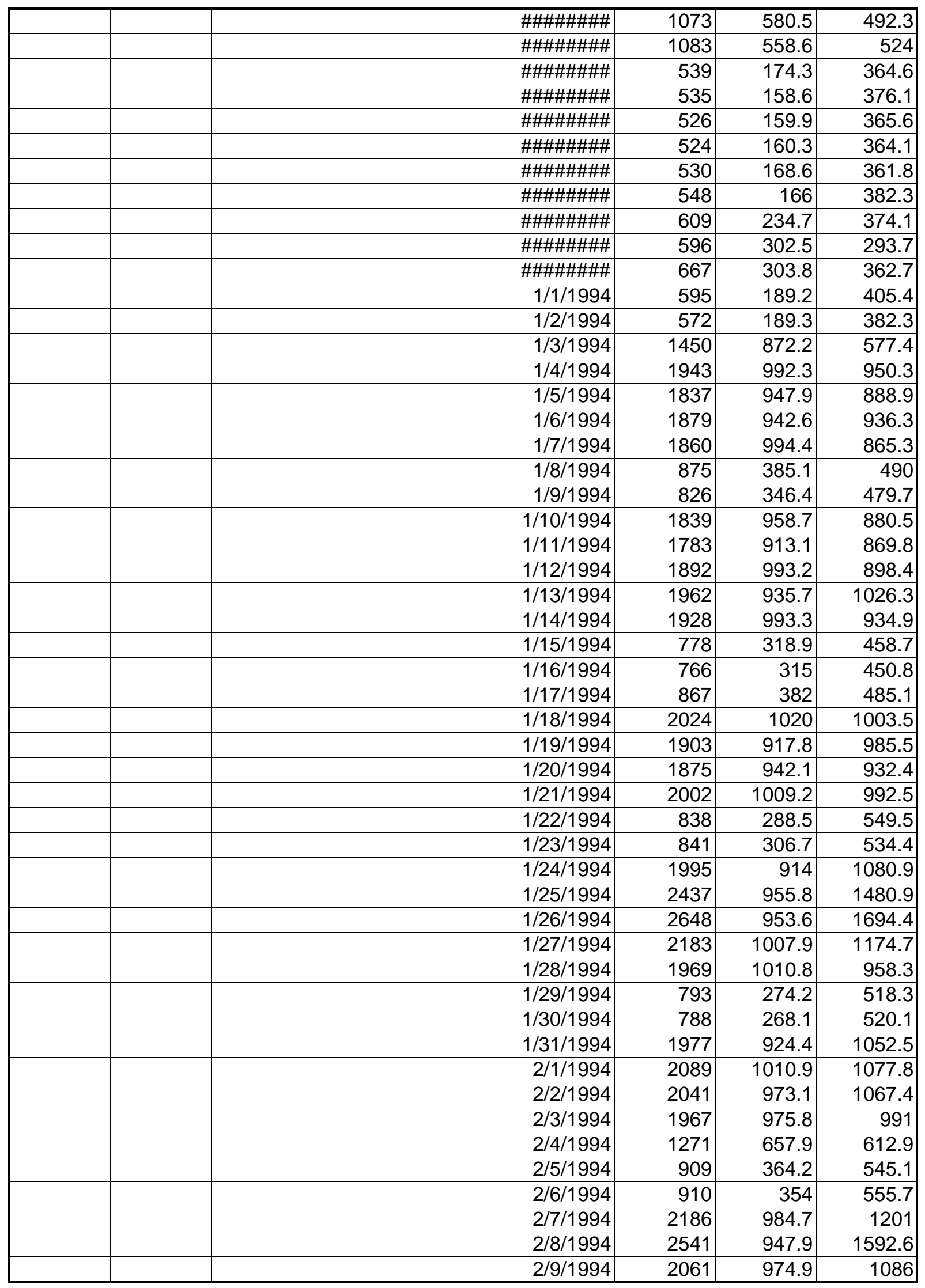


CHART DATA

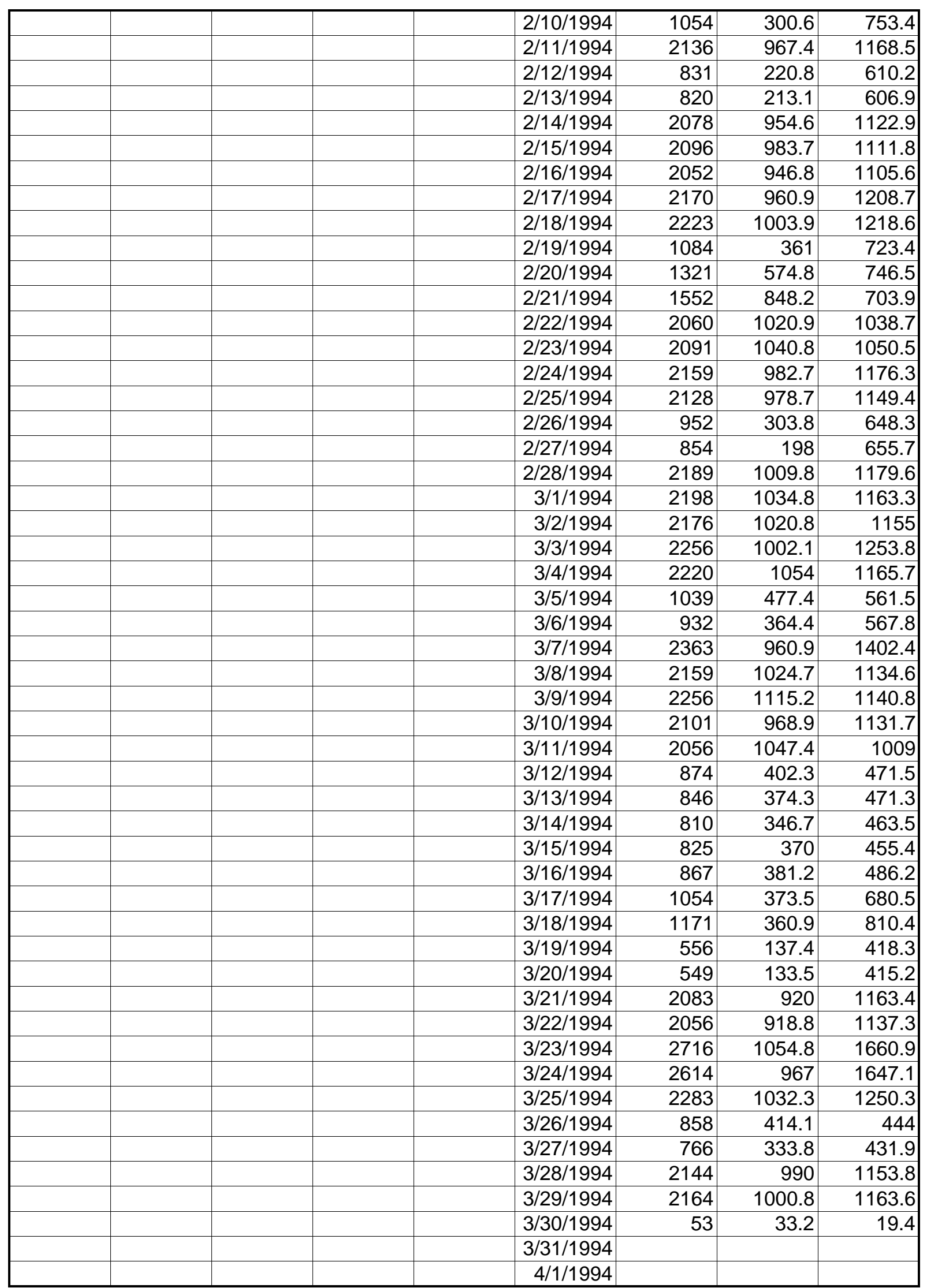


CHART DATA

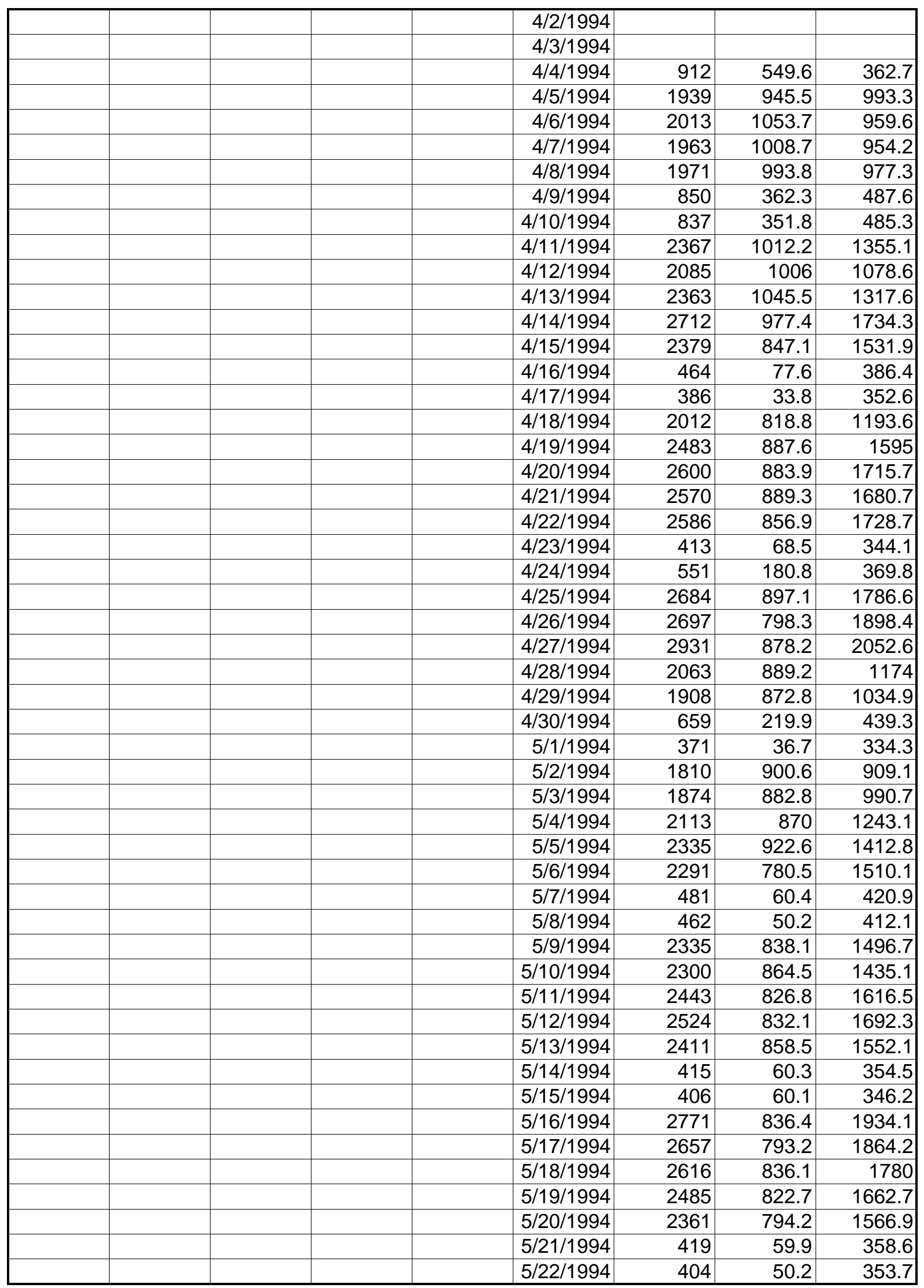


CHART DATA

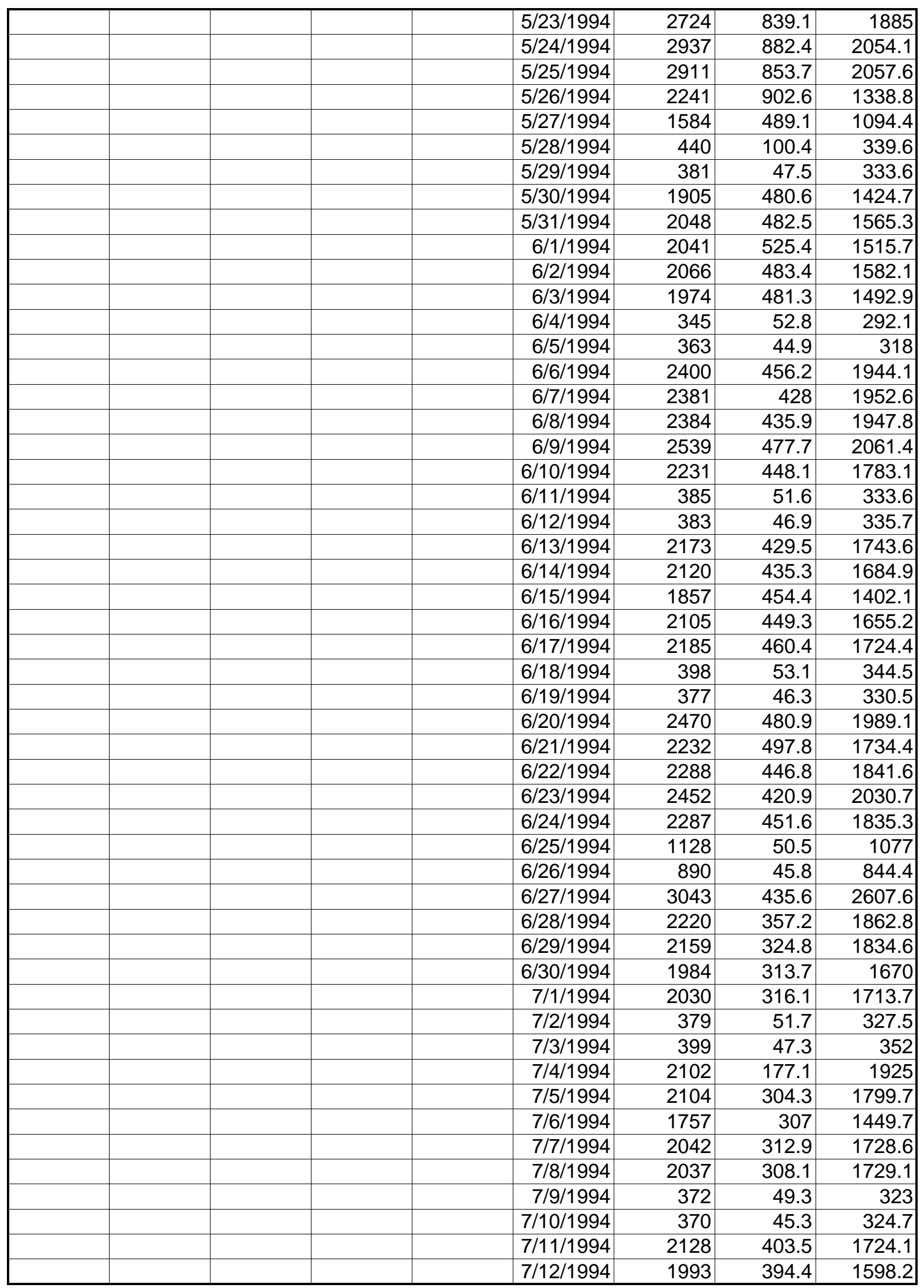


CHART DATA

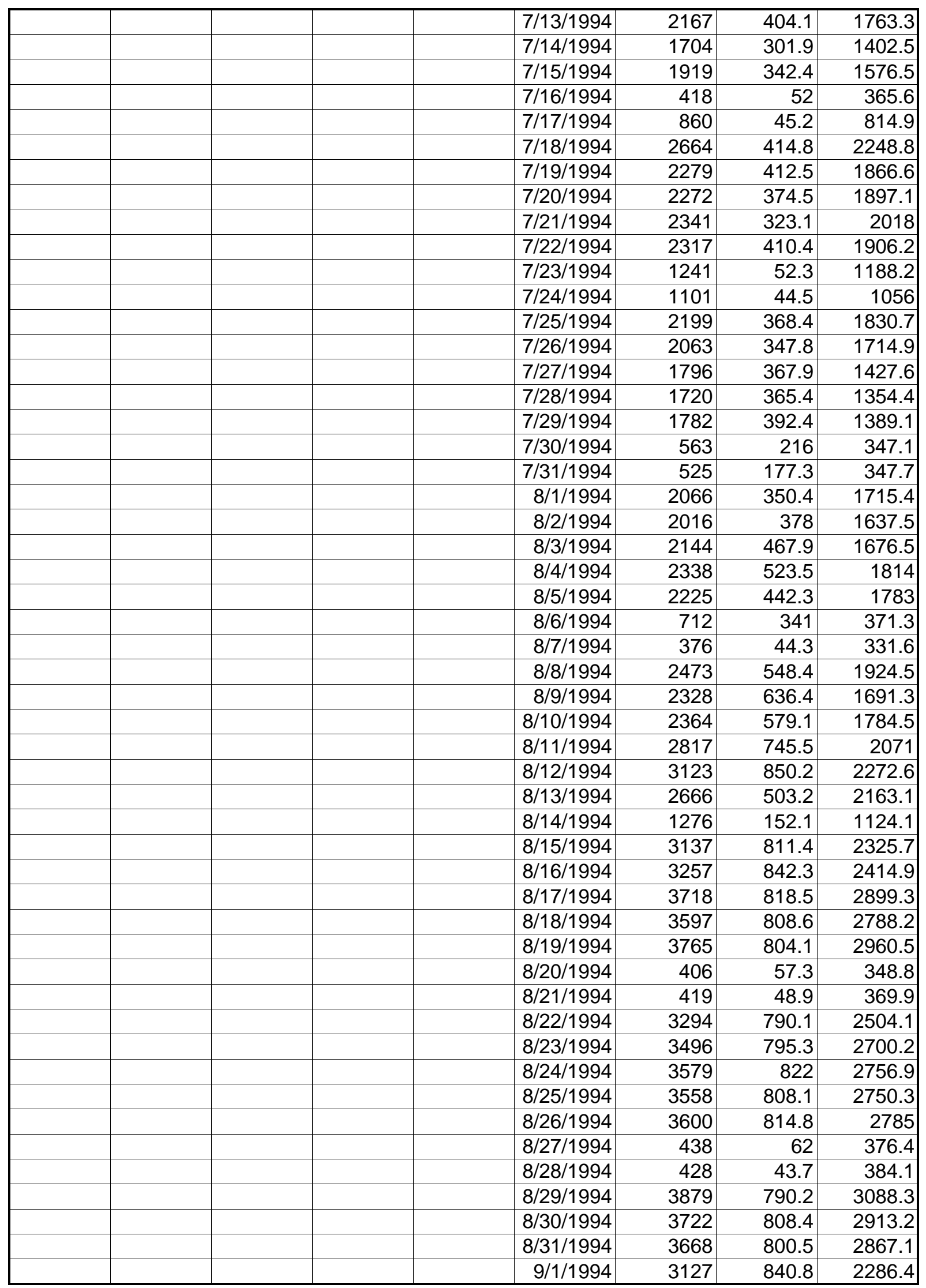


CHART DATA

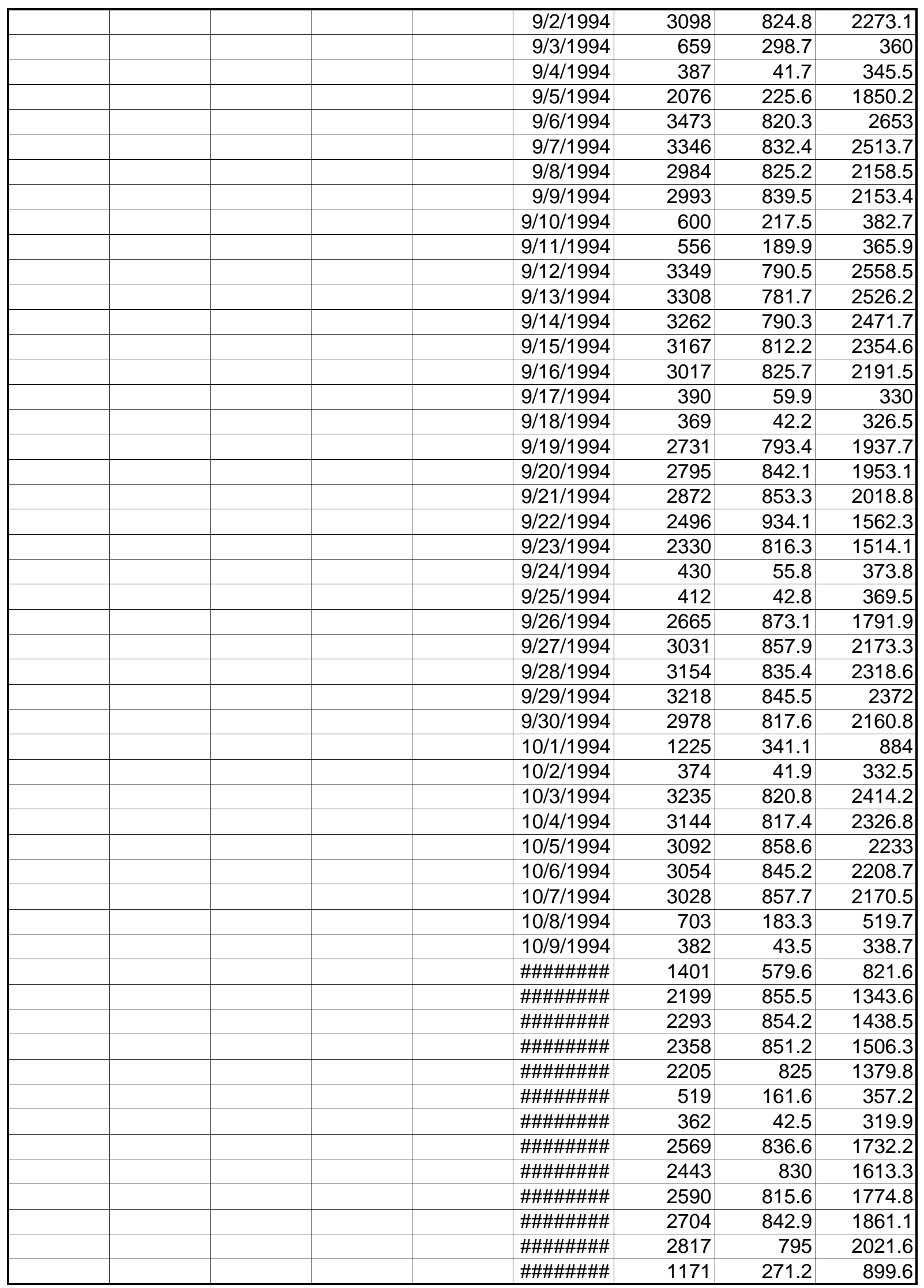


CHART DATA

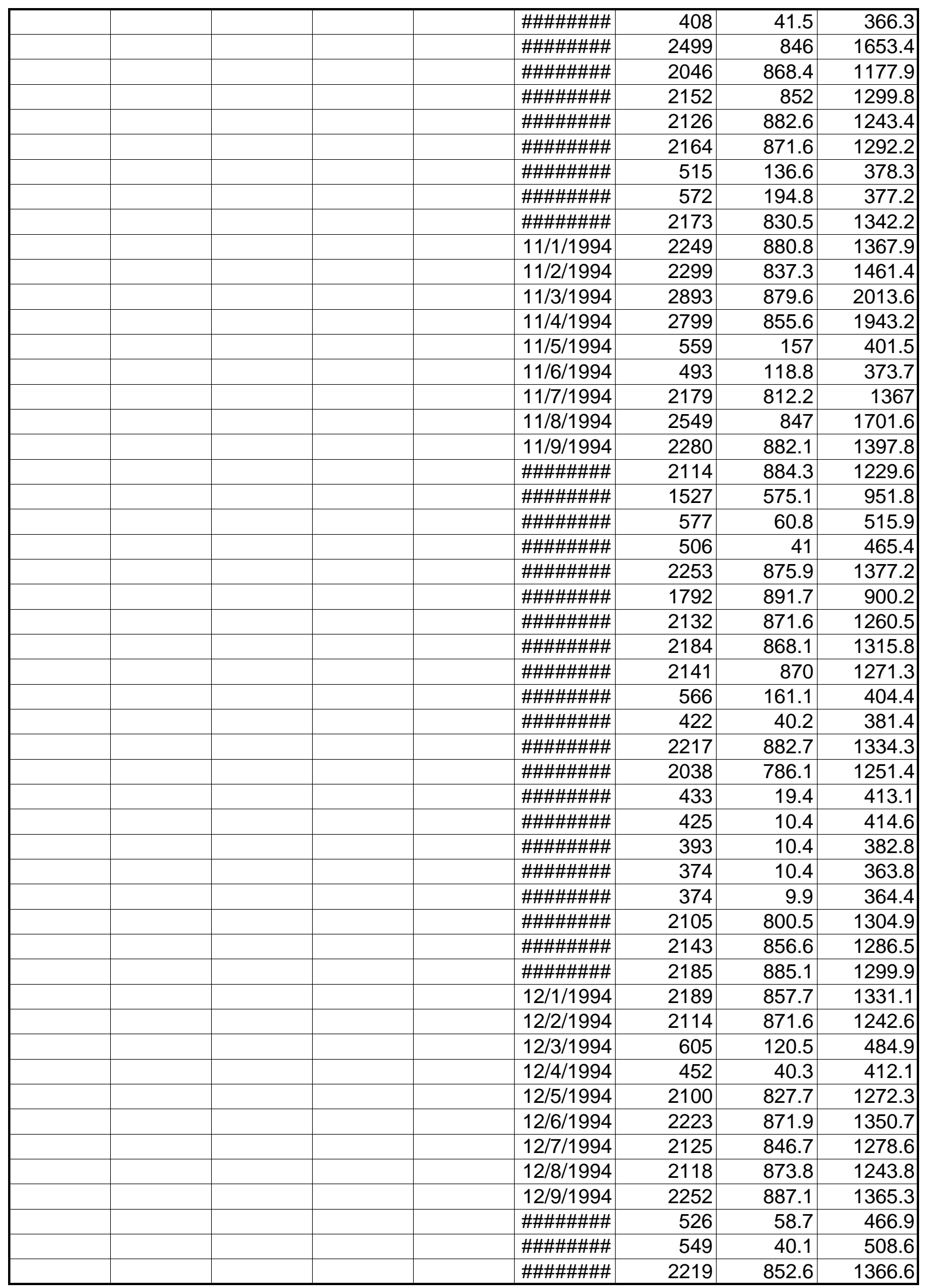


CHART DATA

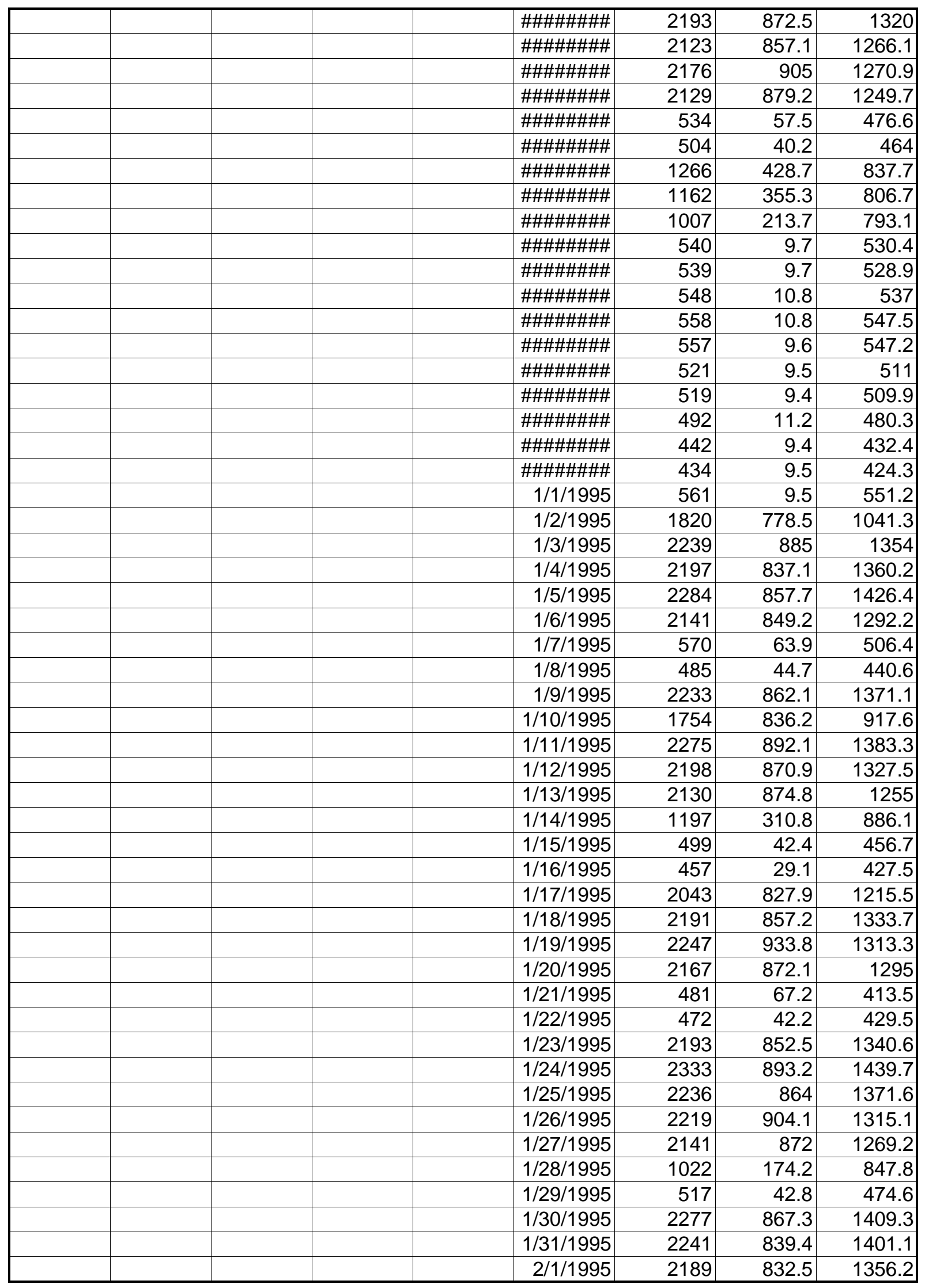


CHART DATA

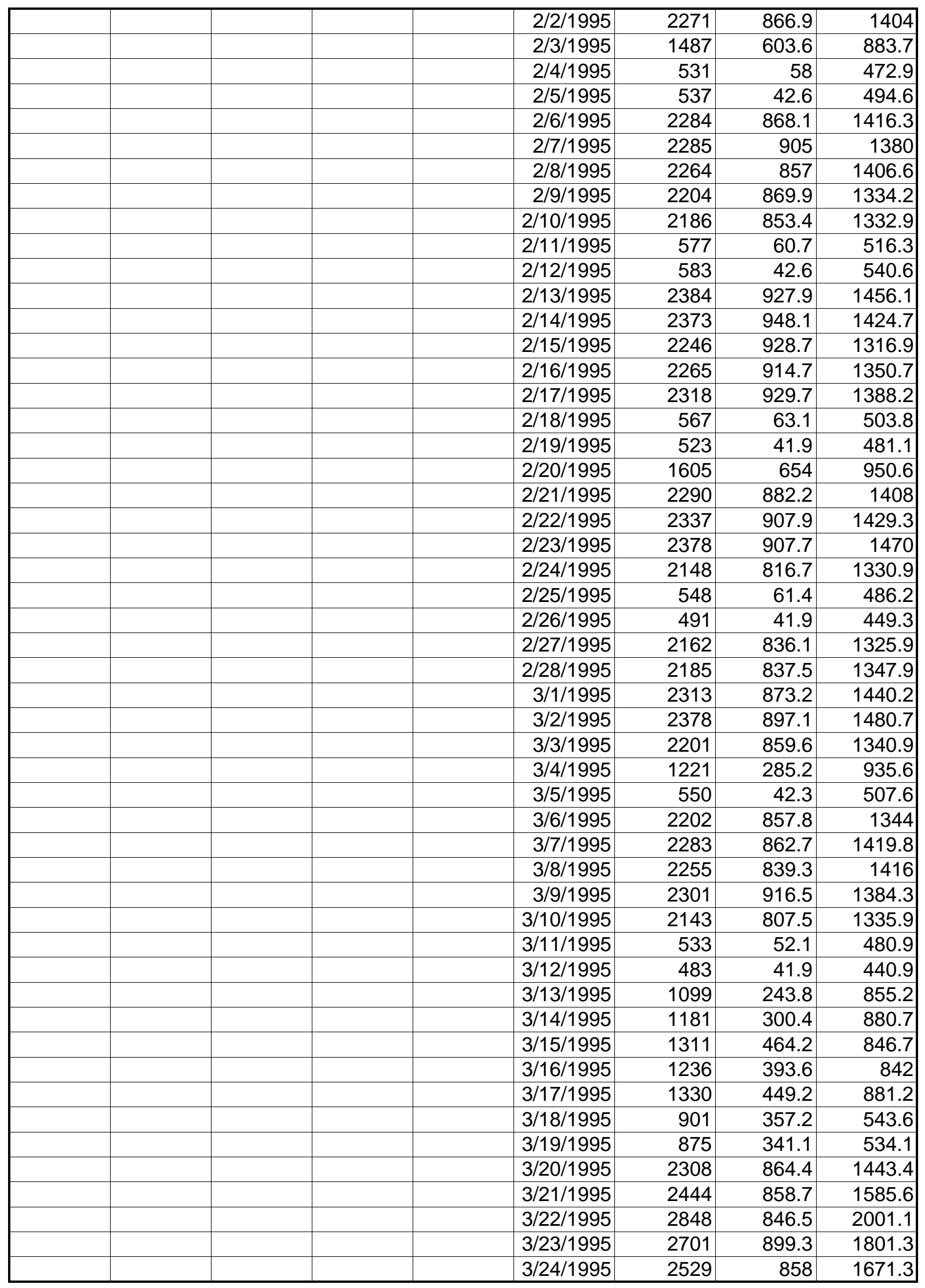


CHART DATA

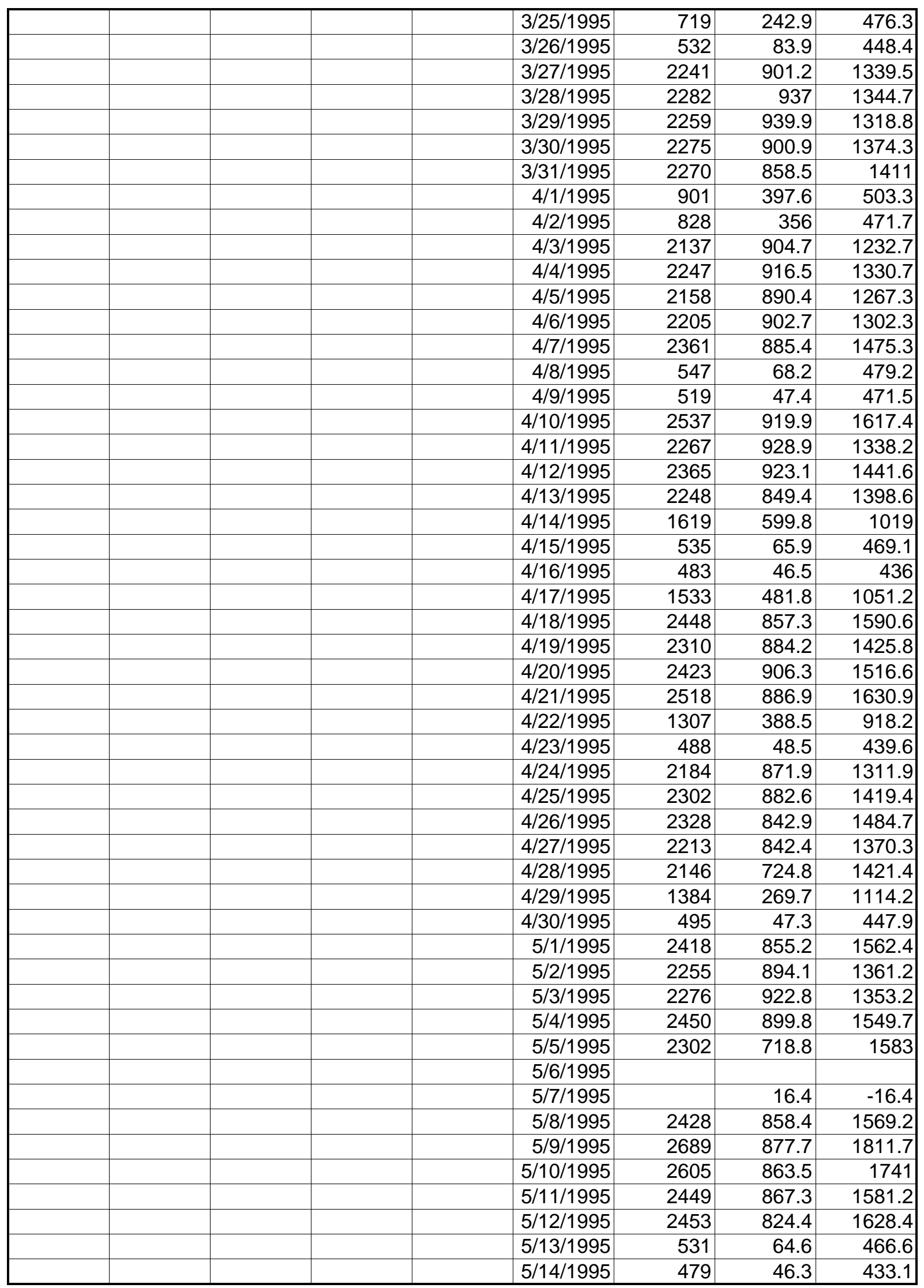


CHART DATA

\begin{tabular}{|r|r|r|r|r|r|r|r|}
\hline & & & & $5 / 15 / 1995$ & 3630 & 828.4 & 2801.8 \\
\hline & & & & $5 / 16 / 1995$ & 3218 & 829.5 & 2388.3 \\
\hline & & & & $5 / 17 / 1995$ & 3146 & 825.9 & 2320.5 \\
\hline & & & & $5 / 18 / 1995$ & 2601 & 819.9 & 1781.4 \\
\hline & & & & $5 / 19 / 1995$ & 2346 & 817.5 & 1528.5 \\
\hline & & & & $5 / 20 / 1995$ & 466 & 64.1 & 402 \\
\hline & & & & $5 / 21 / 1995$ & 483 & 54.9 & 427.6 \\
\hline & & & & $5 / 22 / 1995$ & 2874 & 811.7 & 2062.7 \\
\hline & & & & $5 / 23 / 1995$ & 2948 & 819.2 & 2129 \\
\hline & & & & $5 / 24 / 1995$ & 2833 & 807.1 & 2026 \\
\hline & & & & $5 / 25 / 1995$ & 2604 & 875.9 & 1728 \\
\hline & & & & $5 / 26 / 1995$ & 2258 & 813.2 & 1445.2 \\
\hline & & & & $5 / 27 / 1995$ & 432 & 58.1 & 373.7 \\
\hline & & & & $5 / 28 / 1995$ & 425 & 47.1 & 377.6 \\
\hline & & & & $5 / 29 / 1995$ & 1812 & 400.5 & 1411.3 \\
\hline & & & & $5 / 30 / 1995$ & 1909 & 552.6 & 1356.8 \\
\hline & & & & $5 / 31 / 1995$ & 1991 & 584.6 & 1406 \\
\hline
\end{tabular}




\begin{tabular}{|c|c|}
\hline dateline & wbheat \\
\hline & 0.5 \\
\hline & 0.6 \\
\hline & 0.7 \\
\hline & 0.5 \\
\hline & \\
\hline & \\
\hline & 0.5 \\
\hline & 0.7 \\
\hline & 0.6 \\
\hline & 0.8 \\
\hline & 0.8 \\
\hline & \\
\hline & \\
\hline & 0.7 \\
\hline & 0.6 \\
\hline & 0.7 \\
\hline & 0.7 \\
\hline & 0.8 \\
\hline & \\
\hline & \\
\hline & 1.7 \\
\hline & 3.6 \\
\hline & 1.2 \\
\hline & 0.6 \\
\hline & 0.7 \\
\hline & \\
\hline & \\
\hline & 0.6 \\
\hline & 0.5 \\
\hline & 5.4 \\
\hline & 8.9 \\
\hline & 8.1 \\
\hline & 9.4 \\
\hline & 11.5 \\
\hline & 11.5 \\
\hline & 9.5 \\
\hline & 6.4 \\
\hline & 5.6 \\
\hline & 9.9 \\
\hline & 10.1 \\
\hline & 8.9 \\
\hline & 6.2 \\
\hline & 4.5 \\
\hline & 3.8 \\
\hline & 1.9 \\
\hline & 1.2 \\
\hline & 1.9 \\
\hline & 3.5 \\
\hline & 5.7 \\
\hline
\end{tabular}

Page 136 


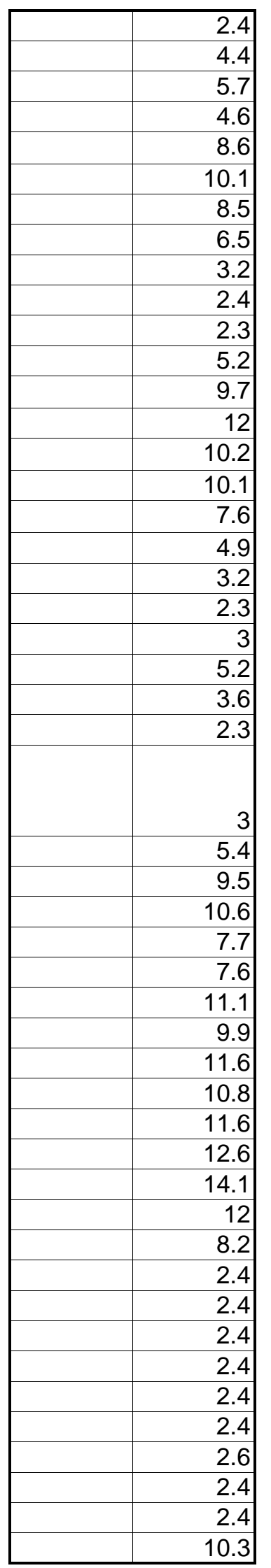

Page 137 


\begin{tabular}{|c|c|}
\hline & 8.1 \\
\hline & 8.5 \\
\hline & 10 \\
\hline & 10.1 \\
\hline & 11.2 \\
\hline & 10.9 \\
\hline & 10.2 \\
\hline & 12.6 \\
\hline & 12.7 \\
\hline & 17.1 \\
\hline & 13 \\
\hline & 15.5 \\
\hline & 15.6 \\
\hline & 13.4 \\
\hline & 10.4 \\
\hline & 8.9 \\
\hline & 9.2 \\
\hline & 8.8 \\
\hline & 7.9 \\
\hline & 9.3 \\
\hline & 9 \\
\hline & 7.8 \\
\hline & 7.5 \\
\hline & 6 \\
\hline & 5.3 \\
\hline & 6.5 \\
\hline & 7.1 \\
\hline & 6.8 \\
\hline & 7.6 \\
\hline & 10.3 \\
\hline & 10.6 \\
\hline & 9 \\
\hline & 10.6 \\
\hline & 10.8 \\
\hline & 9.1 \\
\hline & 5.5 \\
\hline & 3.9 \\
\hline & 6.2 \\
\hline & 3.7 \\
\hline & 4 \\
\hline & 5.5 \\
\hline & 4.5 \\
\hline & 4.8 \\
\hline & 5 \\
\hline & 4.5 \\
\hline & 3.7 \\
\hline & 3.6 \\
\hline & 4.7 \\
\hline & 5.1 \\
\hline & 10.8 \\
\hline & 11.5 \\
\hline
\end{tabular}

Page 138 


\begin{tabular}{|c|c|}
\hline & 8.3 \\
\hline & 6.5 \\
\hline & 5 \\
\hline & 4.9 \\
\hline & 4.2 \\
\hline & 4.1 \\
\hline & 4.8 \\
\hline & 5 \\
\hline & 4.1 \\
\hline & 4.1 \\
\hline & 4.7 \\
\hline & 4.7 \\
\hline & 8.5 \\
\hline & 9.7 \\
\hline & 6.9 \\
\hline & 5.7 \\
\hline & 4.3 \\
\hline & 3.5 \\
\hline & 3.3 \\
\hline & 4.2 \\
\hline & 3.8 \\
\hline & 5.9 \\
\hline & 6.7 \\
\hline & 5.7 \\
\hline & 6.2 \\
\hline & 7.9 \\
\hline & 6.1 \\
\hline & 4.6 \\
\hline & 4.2 \\
\hline & 3.2 \\
\hline & 3.4 \\
\hline & 3.4 \\
\hline & 4.8 \\
\hline & 4.7 \\
\hline & 5.9 \\
\hline & 7.2 \\
\hline & 6.2 \\
\hline & 4 \\
\hline & 4.7 \\
\hline & 4.8 \\
\hline & 4.5 \\
\hline & 1.8 \\
\hline & 1.7 \\
\hline & 1.7 \\
\hline & 1.8 \\
\hline & 2.3 \\
\hline & 2.2 \\
\hline & 1.4 \\
\hline & 1.1 \\
\hline & 1.5 \\
\hline & 1.3 \\
\hline
\end{tabular}

Page 139 


\begin{tabular}{|c|c|}
\hline & 2.1 \\
\hline & 2.3 \\
\hline & 2.8 \\
\hline & 3 \\
\hline & 1.8 \\
\hline & 1.3 \\
\hline & 1.4 \\
\hline & 1.1 \\
\hline & 1.5 \\
\hline & 1.6 \\
\hline & 1.2 \\
\hline & 1.2 \\
\hline & 1.2 \\
\hline & 1.4 \\
\hline & 0.2 \\
\hline & 0.6 \\
\hline & 0.8 \\
\hline & 0.8 \\
\hline & 1.1 \\
\hline & 1.2 \\
\hline & \\
\hline & 0.7 \\
\hline & 0.8 \\
\hline & 0.9 \\
\hline & 0.9 \\
\hline & 0.8 \\
\hline & 0.1 \\
\hline & 1 \\
\hline & 0.8 \\
\hline & 1 \\
\hline & 1 \\
\hline & 0.8 \\
\hline & 0.4 \\
\hline & 1.3 \\
\hline & 1.7 \\
\hline & 0.9 \\
\hline & 1.3 \\
\hline & 1.7 \\
\hline & 1.1 \\
\hline & 0.7 \\
\hline & 1 \\
\hline & 1 \\
\hline & \\
\hline & \\
\hline & \\
\hline & \\
\hline & 1 \\
\hline
\end{tabular}

Page 140 
CHART DATA

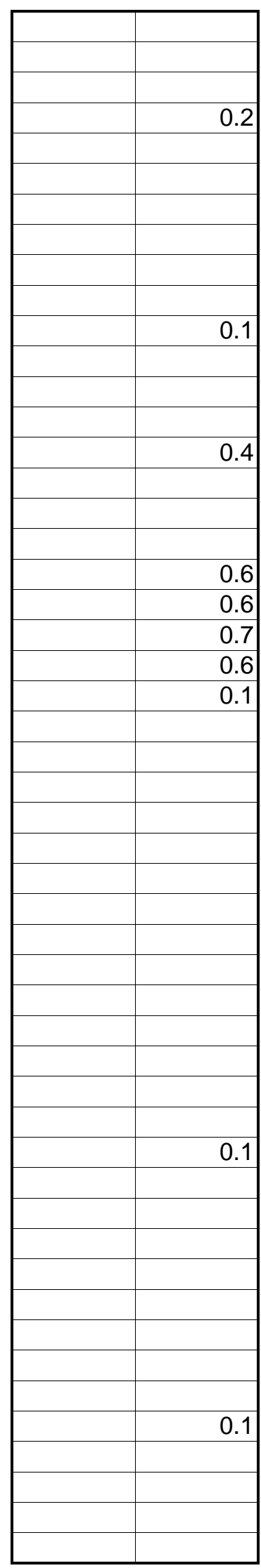

Page 141 


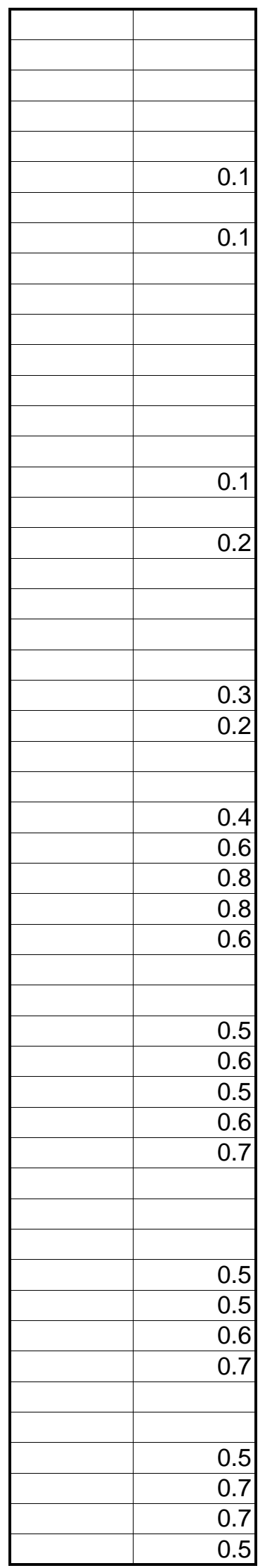

Page 142 


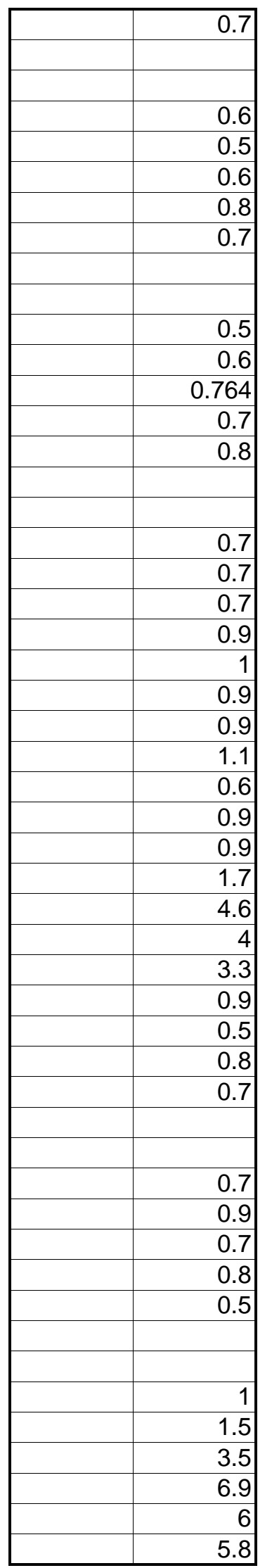

Page 143 


\begin{tabular}{|c|c|}
\hline & 4.7 \\
\hline & 2.6 \\
\hline & 1.9 \\
\hline & 1.4 \\
\hline & 3.5 \\
\hline & 5.8 \\
\hline & 6.2 \\
\hline & 6.1 \\
\hline & 5.1 \\
\hline & 4.1 \\
\hline & 2.6 \\
\hline & 2.8 \\
\hline & 3.8 \\
\hline & 5.1 \\
\hline & 7.8 \\
\hline & 5.9 \\
\hline & 5.2 \\
\hline & 8.7 \\
\hline & 13.9 \\
\hline & 14.1 \\
\hline & 12.8 \\
\hline & 10.6 \\
\hline & 10.8 \\
\hline & 7 \\
\hline & 6.5 \\
\hline & 7.1 \\
\hline & 6.4 \\
\hline & 11.8 \\
\hline & 11.6 \\
\hline & 11 \\
\hline & 10.5 \\
\hline & 8.9 \\
\hline & 8.7 \\
\hline & 8.9 \\
\hline & 6.6 \\
\hline & 5.6 \\
\hline & 9.8 \\
\hline & 12.6 \\
\hline & 11.3 \\
\hline & 12.2 \\
\hline & 10.7 \\
\hline & 5.9 \\
\hline & 11.5 \\
\hline & 8.6 \\
\hline & 4.9 \\
\hline & 5.7 \\
\hline & 8.7 \\
\hline & 7.3 \\
\hline & 9.5 \\
\hline & 8.3 \\
\hline & 6.1 \\
\hline
\end{tabular}




\begin{tabular}{|c|c|}
\hline & 3.4 \\
\hline & 2.5 \\
\hline & 14 \\
\hline & 14.4 \\
\hline & 12.9 \\
\hline & 8 \\
\hline & 6.7 \\
\hline & 12.3 \\
\hline & 10.6 \\
\hline & 9.1 \\
\hline & 8.3 \\
\hline & 9.8 \\
\hline & 16.3 \\
\hline & 14.2 \\
\hline & 9.4 \\
\hline & 11.9 \\
\hline & 11.5 \\
\hline & 9.7 \\
\hline & 8.3 \\
\hline & 10.4 \\
\hline & 11.8 \\
\hline & 14 \\
\hline & 12.7 \\
\hline & 9.7 \\
\hline & 6.4 \\
\hline & 5 \\
\hline & 9.9 \\
\hline & 11 \\
\hline & 10.3 \\
\hline & 8.4 \\
\hline & 7.5 \\
\hline & 9.5 \\
\hline & 12.2 \\
\hline & 10.2 \\
\hline & 7.4 \\
\hline & 6.4 \\
\hline & 6.6 \\
\hline & 7.4 \\
\hline & 8.6 \\
\hline & 9.4 \\
\hline & 9.1 \\
\hline & 7 \\
\hline & 5.7 \\
\hline & 4.2 \\
\hline & 6.2 \\
\hline & 8.3 \\
\hline & 8.3 \\
\hline & 9.2 \\
\hline & 8.6 \\
\hline & 11 \\
\hline & 13.3 \\
\hline
\end{tabular}




\begin{tabular}{|c|c|}
\hline & 13.7 \\
\hline & 8.5 \\
\hline & 5.2 \\
\hline & 4.2 \\
\hline & 5.6 \\
\hline & 5.8 \\
\hline & 6 \\
\hline & 4.9 \\
\hline & 9.1 \\
\hline & 9.4 \\
\hline & 9.4 \\
\hline & 8.4 \\
\hline & 6.5 \\
\hline & 6.8 \\
\hline & 6.5 \\
\hline & 6.7 \\
\hline & 6.6 \\
\hline & 5.9 \\
\hline & 4.1 \\
\hline & 2.6 \\
\hline & 2.9 \\
\hline & 4.6 \\
\hline & 9.6 \\
\hline & 13.7 \\
\hline & 12.4 \\
\hline & 10.2 \\
\hline & 5.2 \\
\hline & 7 \\
\hline & 7.4 \\
\hline & 7.2 \\
\hline & 6.9 \\
\hline & 5.8 \\
\hline & 3.8 \\
\hline & 4.2 \\
\hline & 2.2 \\
\hline & 1.5 \\
\hline & 2.6 \\
\hline & 2.4 \\
\hline & 2.4 \\
\hline & 2 \\
\hline & 1.6 \\
\hline & 1.6 \\
\hline & 2.8 \\
\hline & 3.8 \\
\hline & 2.4 \\
\hline & 4 \\
\hline & 5.1 \\
\hline & 6.2 \\
\hline & 3.2 \\
\hline & 3 \\
\hline & 3 \\
\hline
\end{tabular}




\begin{tabular}{|c|c|}
\hline & 1.6 \\
\hline & 0.9 \\
\hline & 1.1 \\
\hline & 0.8 \\
\hline & 1.9 \\
\hline & 4.4 \\
\hline & 3.1 \\
\hline & 1.5 \\
\hline & 1.4 \\
\hline & 0.4 \\
\hline & 1 \\
\hline & 2 \\
\hline & 2.2 \\
\hline & 1.3 \\
\hline & 0.4 \\
\hline & 0.2 \\
\hline & 1.5 \\
\hline & 1 \\
\hline & 0.8 \\
\hline & 0.9 \\
\hline & 1 \\
\hline & 0.8 \\
\hline & 1.9 \\
\hline & 1 \\
\hline & 1.7 \\
\hline & 1.9 \\
\hline & 0.9 \\
\hline & \\
\hline & 1.2 \\
\hline & 1.1 \\
\hline & 1 \\
\hline & 1.1 \\
\hline & 1 \\
\hline & 0.5 \\
\hline & 0.2 \\
\hline & 0.7 \\
\hline & 0.9 \\
\hline & 1 \\
\hline & 0.8 \\
\hline & 0.8 \\
\hline & \\
\hline & 0.8 \\
\hline & 0.8 \\
\hline & 0.9 \\
\hline & 1.1 \\
\hline & 0.7 \\
\hline & \\
\hline & \\
\hline
\end{tabular}


CHART DATA

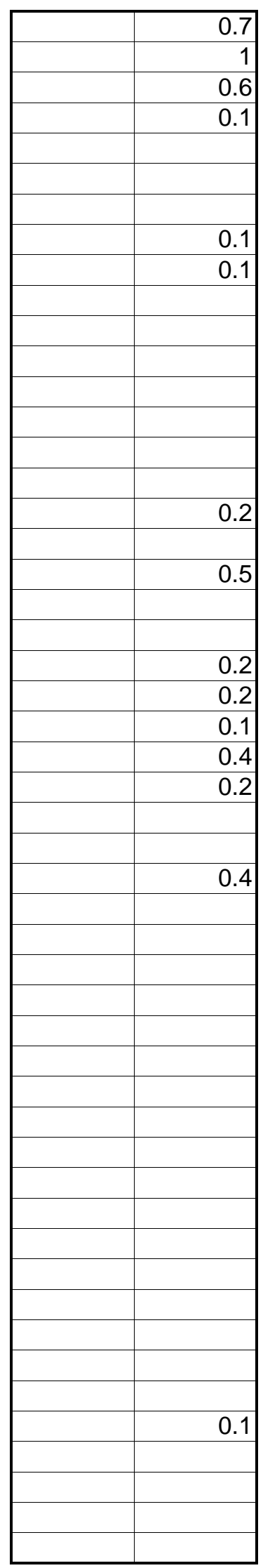

Page 148 


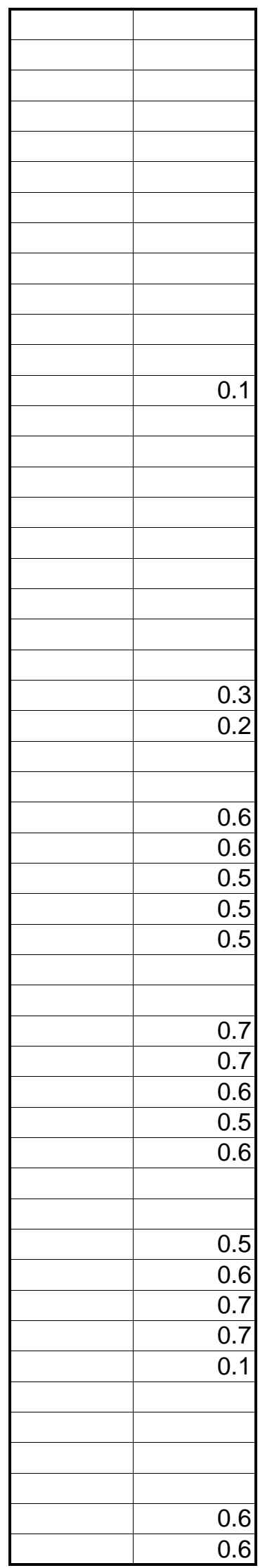

Page 149 


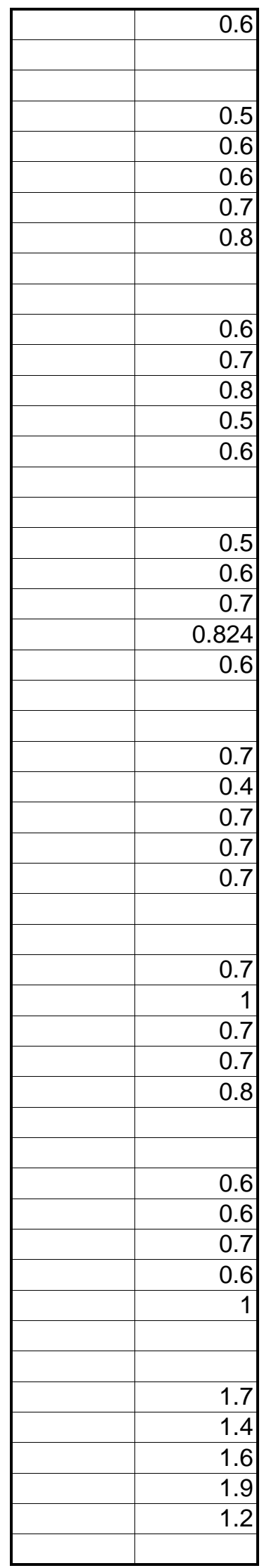

Page 150 


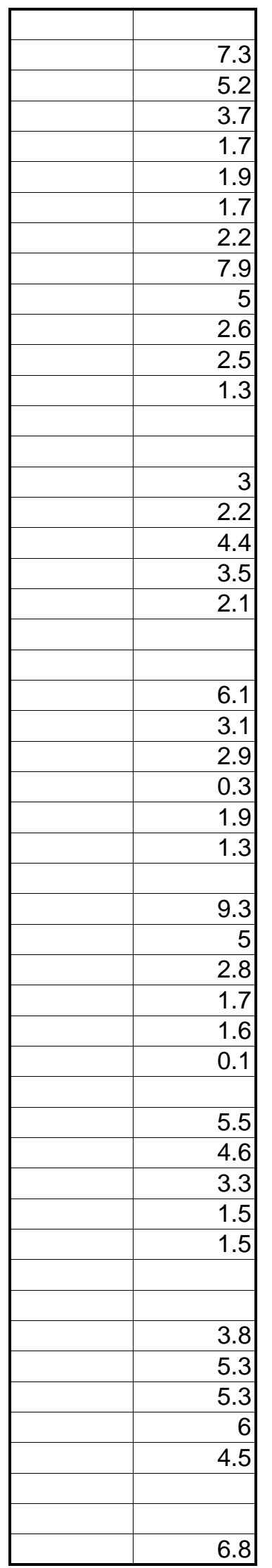

Page 151 


\begin{tabular}{|c|c|}
\hline & 7.2 \\
\hline & 12.4 \\
\hline & 1.2 \\
\hline & 2 \\
\hline & 1.4 \\
\hline & 1 \\
\hline & 0.9 \\
\hline & 6.3 \\
\hline & 8.5 \\
\hline & 2.4 \\
\hline & 3.7 \\
\hline & 3.5 \\
\hline & 2.8 \\
\hline & 11.1 \\
\hline & 7.8 \\
\hline & 8.8 \\
\hline & 5.1 \\
\hline & 9.4 \\
\hline & 8.7 \\
\hline & 7.9 \\
\hline & 8.1 \\
\hline & 4.3 \\
\hline & 5.8 \\
\hline & 5.6 \\
\hline & 6.5 \\
\hline & 4.6 \\
\hline & 4.7 \\
\hline & 6.4 \\
\hline & 16.9 \\
\hline & 17.3 \\
\hline & 9 \\
\hline & 14.2 \\
\hline & 9.5 \\
\hline & 8.1 \\
\hline & 9 \\
\hline & 3.3 \\
\hline & 2.5 \\
\hline & 5.3 \\
\hline & 9.7 \\
\hline & 7 \\
\hline & 8.4 \\
\hline & 18.1 \\
\hline & 18.3 \\
\hline & 14.6 \\
\hline & 10.3 \\
\hline & 7.1 \\
\hline & 5.7 \\
\hline & 6.7 \\
\hline & 5.7 \\
\hline & 2.1 \\
\hline & 13.3 \\
\hline
\end{tabular}

Page 152 


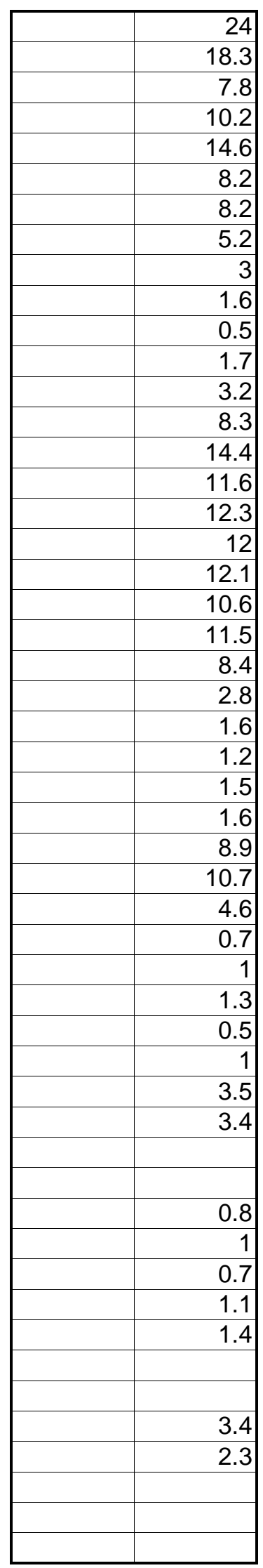

Page 153 


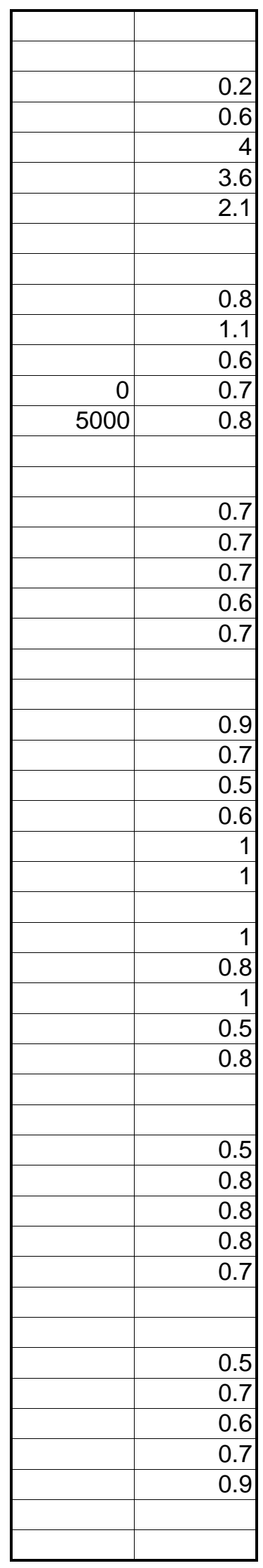

Page 154 
CHART DATA

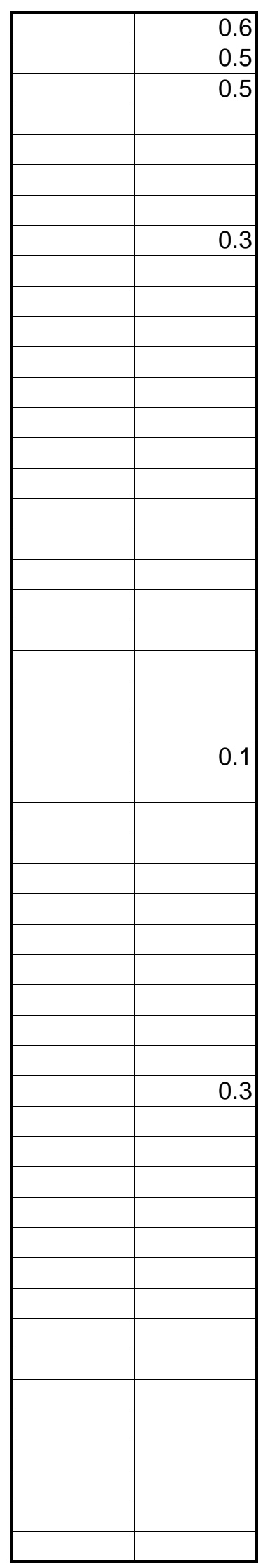

Page 155 
CHART DATA

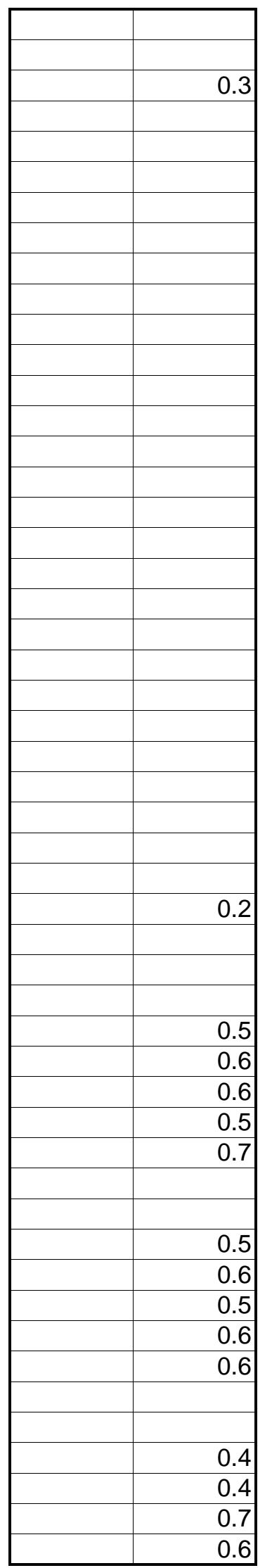

Page 156 


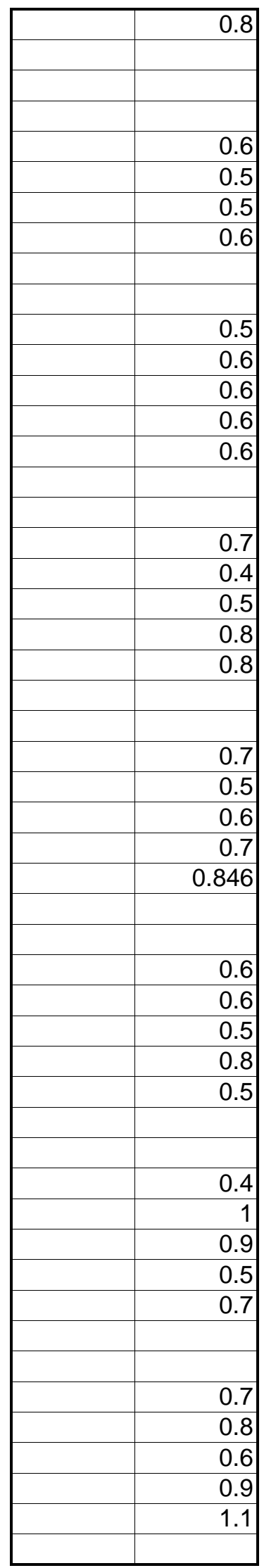

Page 157 


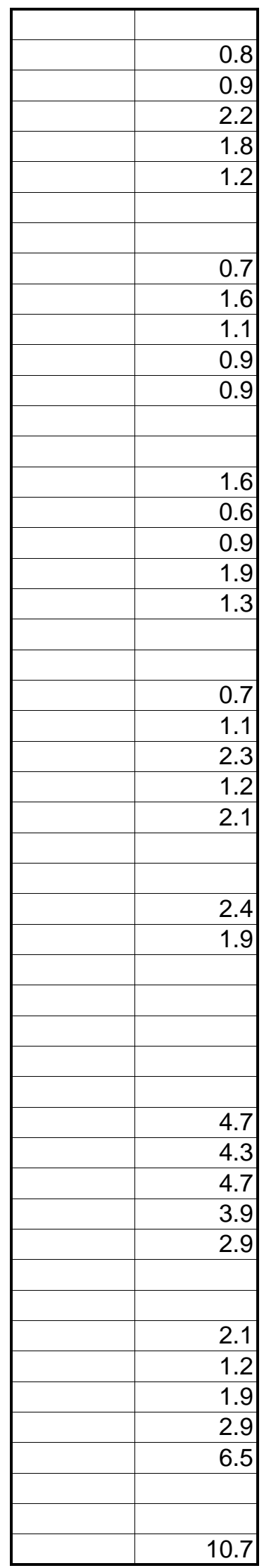

Page 158 


\begin{tabular}{|c|c|}
\hline & 6.4 \\
\hline & 4.4 \\
\hline & 4 \\
\hline & 3.1 \\
\hline & \\
\hline & \\
\hline & 6.2 \\
\hline & 3.4 \\
\hline & 4.1 \\
\hline & \\
\hline & \\
\hline & 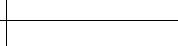 \\
\hline & \\
\hline & \\
\hline & \\
\hline & \\
\hline & \\
\hline & \\
\hline & 14.3 \\
\hline & 10.4 \\
\hline & 11.7 \\
\hline & 13.1 \\
\hline & 8.8 \\
\hline & 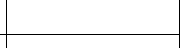 \\
\hline & 9.5 \\
\hline & 2.3 \\
\hline & 2 \\
\hline & 0.8 \\
\hline & 3.4 \\
\hline & 5.1 \\
\hline & \\
\hline & 5.6 \\
\hline & 7.5 \\
\hline & 8.5 \\
\hline & 7.1 \\
\hline & \\
\hline & 14.1 \\
\hline & 9.2 \\
\hline & 6.2 \\
\hline & 3.8 \\
\hline & 2.9 \\
\hline & 6.7 \\
\hline & 13.6 \\
\hline & 8.2 \\
\hline & 5.8 \\
\hline
\end{tabular}




\begin{tabular}{|c|c|}
\hline & 3.1 \\
\hline & 2.8 \\
\hline & \\
\hline & \\
\hline & 10.2 \\
\hline & 7.4 \\
\hline & 9.9 \\
\hline & 6.2 \\
\hline & 3.1 \\
\hline & 11 \\
\hline & 1.1 \\
\hline & 16.1 \\
\hline & 11.9 \\
\hline & 4 \\
\hline & 8.7 \\
\hline & 8.2 \\
\hline & \\
\hline & 6.8 \\
\hline & 3.8 \\
\hline & 2.8 \\
\hline & 1.6 \\
\hline & 1.7 \\
\hline & \\
\hline & 18 \\
\hline & 5.8 \\
\hline & 10.3 \\
\hline & 13.8 \\
\hline & 13.3 \\
\hline & 9.4 \\
\hline & 81 \\
\hline & 10.4 \\
\hline & 12.7 \\
\hline & 9.6 \\
\hline & 6.8 \\
\hline & \\
\hline & 6 \\
\hline & 6.6 \\
\hline & 4.9 \\
\hline & 2.5 \\
\hline & 1.9 \\
\hline & \\
\hline & 0.5 \\
\hline & 1.1 \\
\hline & 0.7 \\
\hline & 0.5 \\
\hline & 0.8 \\
\hline
\end{tabular}




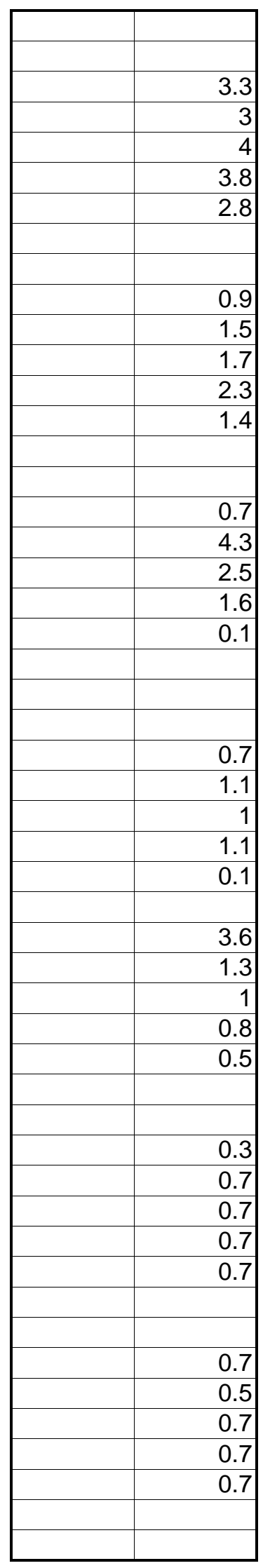

Page 161 
CHART DATA

\begin{tabular}{|l|l|}
\hline & 0.7 \\
\hline & 0.7 \\
\hline & 0.7 \\
\hline & 0.7 \\
\hline & 0.7 \\
\hline & \\
\hline & \\
\hline & 0.6 \\
\hline & 0.6 \\
\hline & 0.7 \\
\hline & 0.8 \\
\hline & \\
\hline & \\
\hline & \\
\hline & \\
\hline & \\
\hline
\end{tabular}

Page 162 
TABLES

\begin{tabular}{|c|c|c|c|c|}
\hline & $\begin{array}{c}\text { wbelec } \\
\text { kWh/month }\end{array}$ & $\begin{array}{c}\text { lights } \\
\mathrm{kWh} / \text { month }\end{array}$ & $\begin{array}{c}\text { other } \\
\mathrm{kWh} / \mathrm{month}\end{array}$ & $\begin{array}{c}\text { wbheat } \\
\text { MMBtu/month }\end{array}$ \\
\hline \multicolumn{5}{|l|}{ PRE PERIOD } \\
\hline Oct 91 & 83,200 & 30,111 & 52,767 & 94 \\
\hline Nov & 50,829 & 25,299 & 30,443 & 194 \\
\hline Dec & 39,223 & 15,934 & 28,111 & 254 \\
\hline Jan 92 & 40,865 & 19,072 & 39,175 & 158 \\
\hline Feb & 43,217 & 19,463 & 53,383 & 36 \\
\hline Mar & 45,896 & 17,250 & 64,549 & 14 \\
\hline Apr & 60,103 & 20,321 & 60,523 & 25 \\
\hline May & 71,339 & 20,348 & 37,712 & 126 \\
\hline Jun & 75,790 & 14,853 & 30,594 & 301 \\
\hline Jul & 82,092 & 9,536 & 34,582 & 202 \\
\hline Aug & 77,690 & 13,810 & 42,819 & 66 \\
\hline Sep & 88,241 & 19,932 & 68,998 & 19 \\
\hline Oct & 68,149 & 21,649 & 76,339 & 18 \\
\hline Nov & 46,415 & 19,362 & 37,985 & 62 \\
\hline Dec & 42,766 & 17,975 & 28,563 & 156 \\
\hline Jan 93 & 46,702 & 20,424 & 34,678 & 325 \\
\hline Feb & 47,163 & 21,440 & 29,892 & 62 \\
\hline Mar & 51,509 & 20,818 & 48,753 & 208 \\
\hline Apr & 56,023 & 21,790 & 71,657 & 48 \\
\hline May & 76,217 & 23,457 & 55,507 & 82 \\
\hline Jun & 72,174 & 13,640 & 39,562 & 177 \\
\hline Jul & 64,380 & 9,258 & 39,837 & 1 \\
\hline Aug & 100,835 & 18,188 & 43,885 & 123 \\
\hline Sep & 82,136 & 21,994 & 44,956 & 119 \\
\hline Oct & 62,260 & 23,622 & 35,769 & 2 \\
\hline Nov & 44,385 & 21,773 & 19,656 & 147 \\
\hline Dec & 36,881 & 17,399 & 42,318 & 2 \\
\hline Jan 94 & 47,920 & 22,462 & 20,807 & 77 \\
\hline Feb & 47,822 & 21,114 & 34,312 & 68 \\
\hline Mar & 47,045 & 20,615 & 22,858 & 118 \\
\hline Apr (partial) & 17,300 & 8,329 & 7,468 & 12 \\
\hline Total Consumption & $1,816,566$ & 591,236 & $1,278,455$ & 3,294 \\
\hline
\end{tabular}


TABLES

\begin{tabular}{|l|r|r|r|r|}
\hline Total Cost & $\$ 122,437$ & $\$ 39,849$ & $\$ 86,168$ & $\$ 15,151$ \\
\hline POST PERIOD & & & & \\
\hline Apr (partial) & 32,096 & 11,077 & 6,693 & 13 \\
\hline May & 55,455 & 18,115 & 12,136 & 12 \\
\hline Jun & 53,858 & 10,086 & 15,211 & 18 \\
\hline Jul & 49,640 & 8,130 & 14,564 & 33 \\
\hline Aug & 76,882 & 17,289 & 60,594 & 152 \\
\hline Sep & 69,274 & 18,626 & 53,163 & 215 \\
\hline Oct & 58,523 & 18,894 & 42,543 & 102 \\
\hline Nov & 47,199 & 16,682 & 55,783 & 20 \\
\hline Dec & 37,715 & 11,858 & 37,564 & 1 \\
\hline Jan 95 & 51,821 & 18,954 & 53,586 & 13 \\
\hline Feb & 48,218 & 17,560 & 17,931 & 10 \\
\hline Mar & 54,202 & 19,076 & 46,095 & 1 \\
\hline Apr & 52,034 & 18,638 & 37,498 & 46 \\
\hline May & 61,310 & 18,720 & 18,036 & 2 \\
\hline Total Consumption & 748,224 & 223,703 & 471,396 & 637 \\
\hline Total Cost & $\$ 50,430$ & $\$ 15,078$ & $\$ 31,772$ & $\$ 2,930$ \\
\hline Grand Total Consumption & $2,564,791$ & 814,939 & $1,749,852$ & 3931 \\
\hline Grand Total Cost & $\$ 172,867$ & $\$ 54,927$ & $\$ 117,940$ & $\$ 18,081$ \\
\hline
\end{tabular}




\section{Hourly Average}

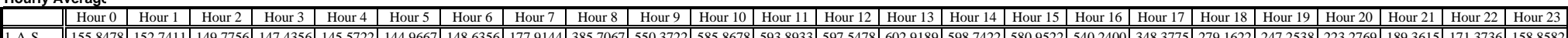

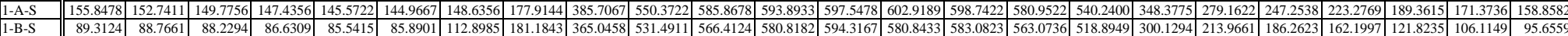

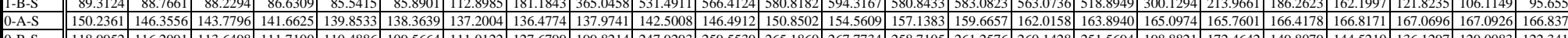

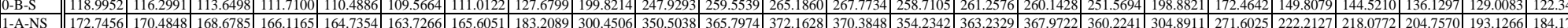

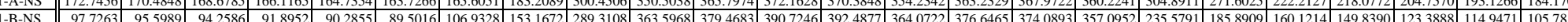

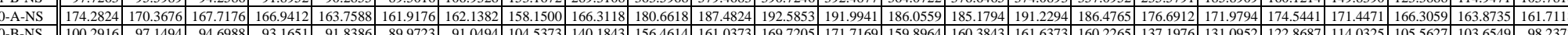
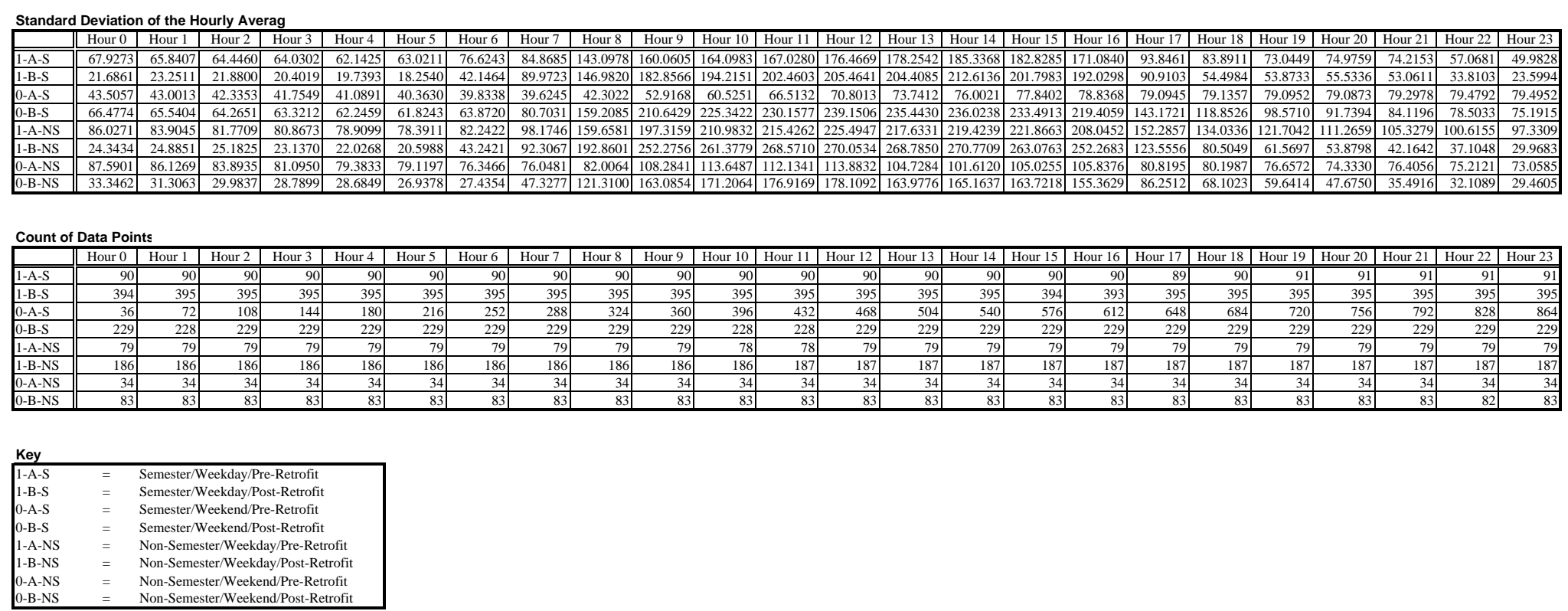

Hourly Sum (sort "Category")

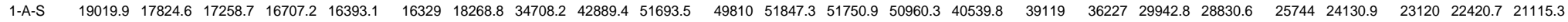

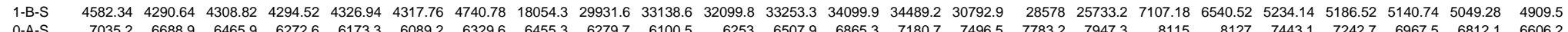

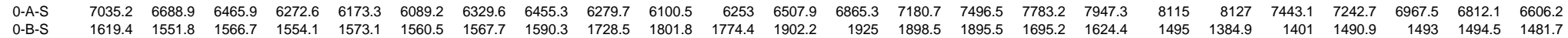

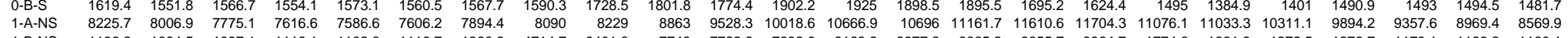

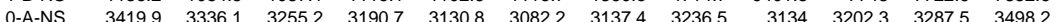

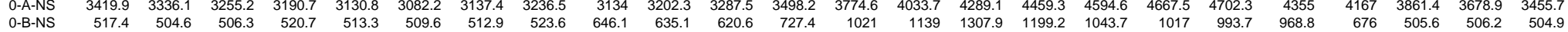




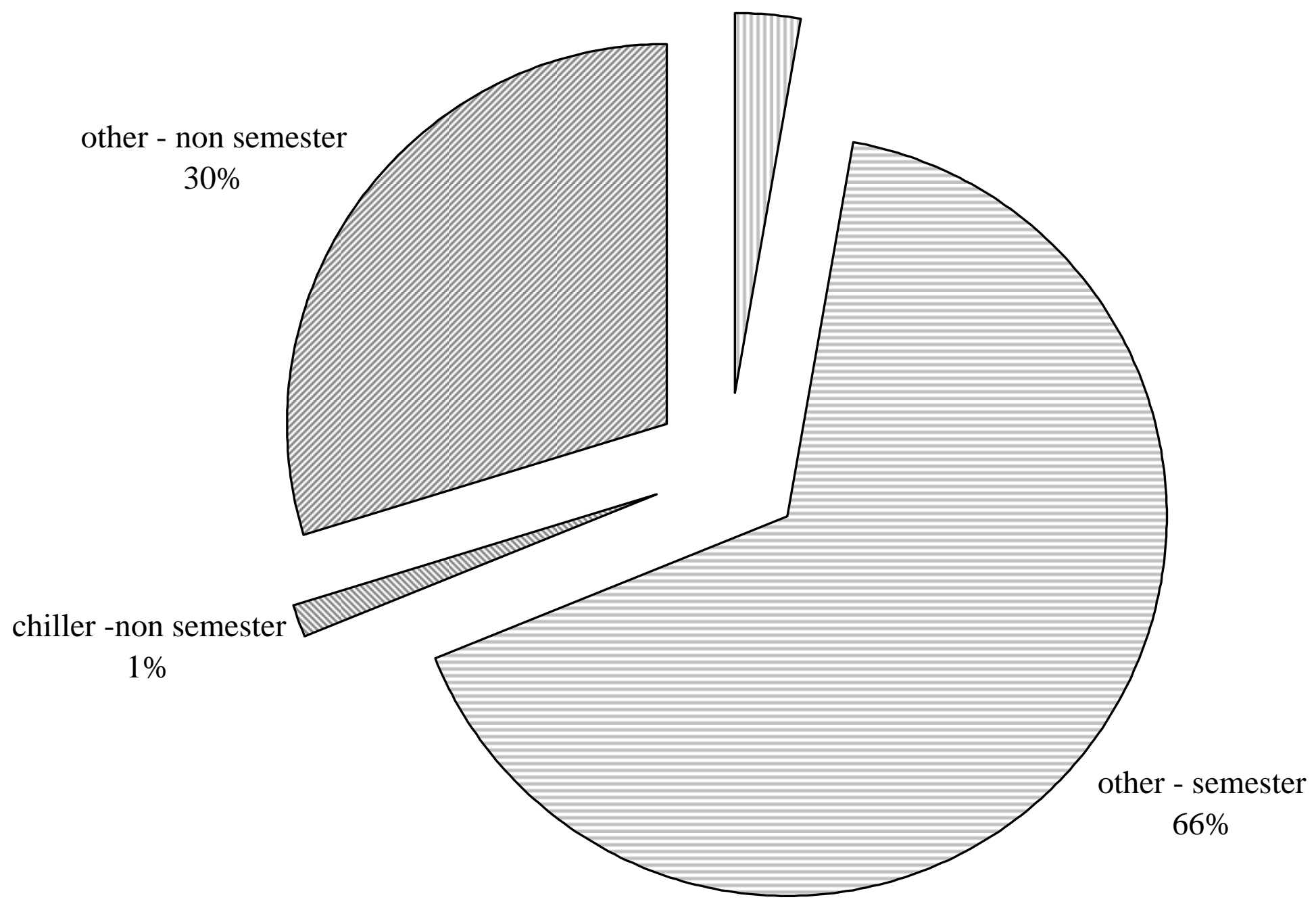




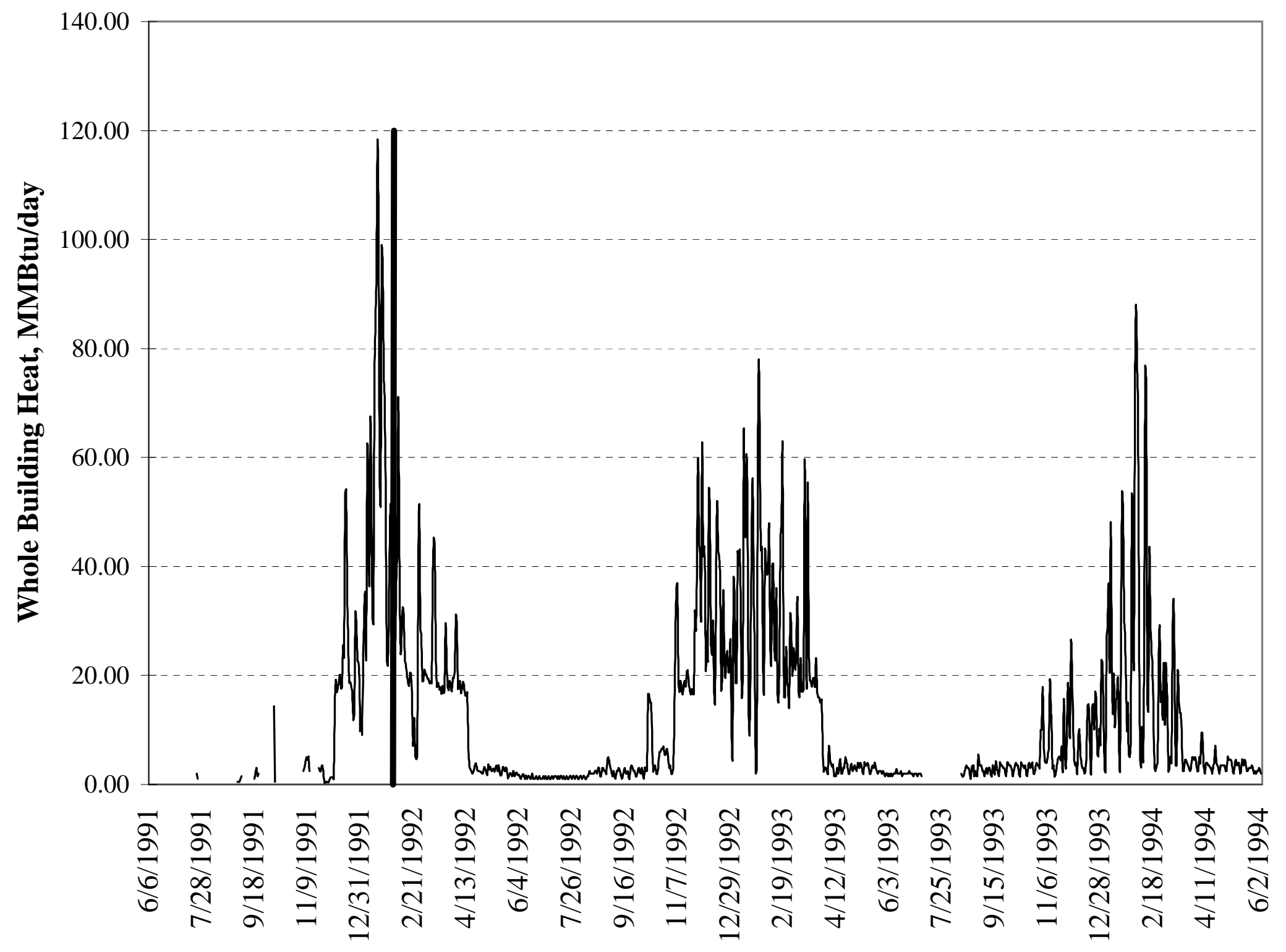


other

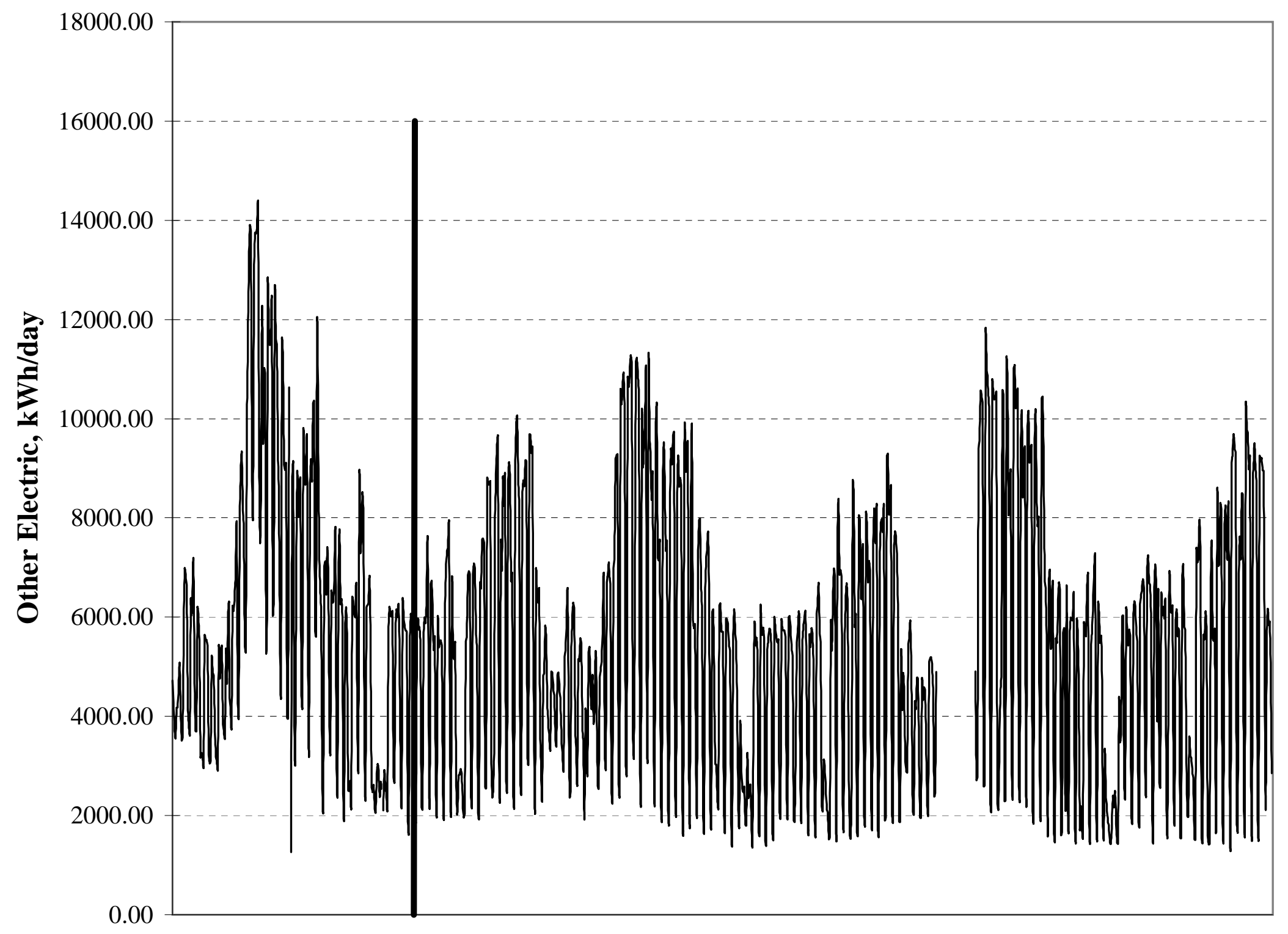

Page 1 


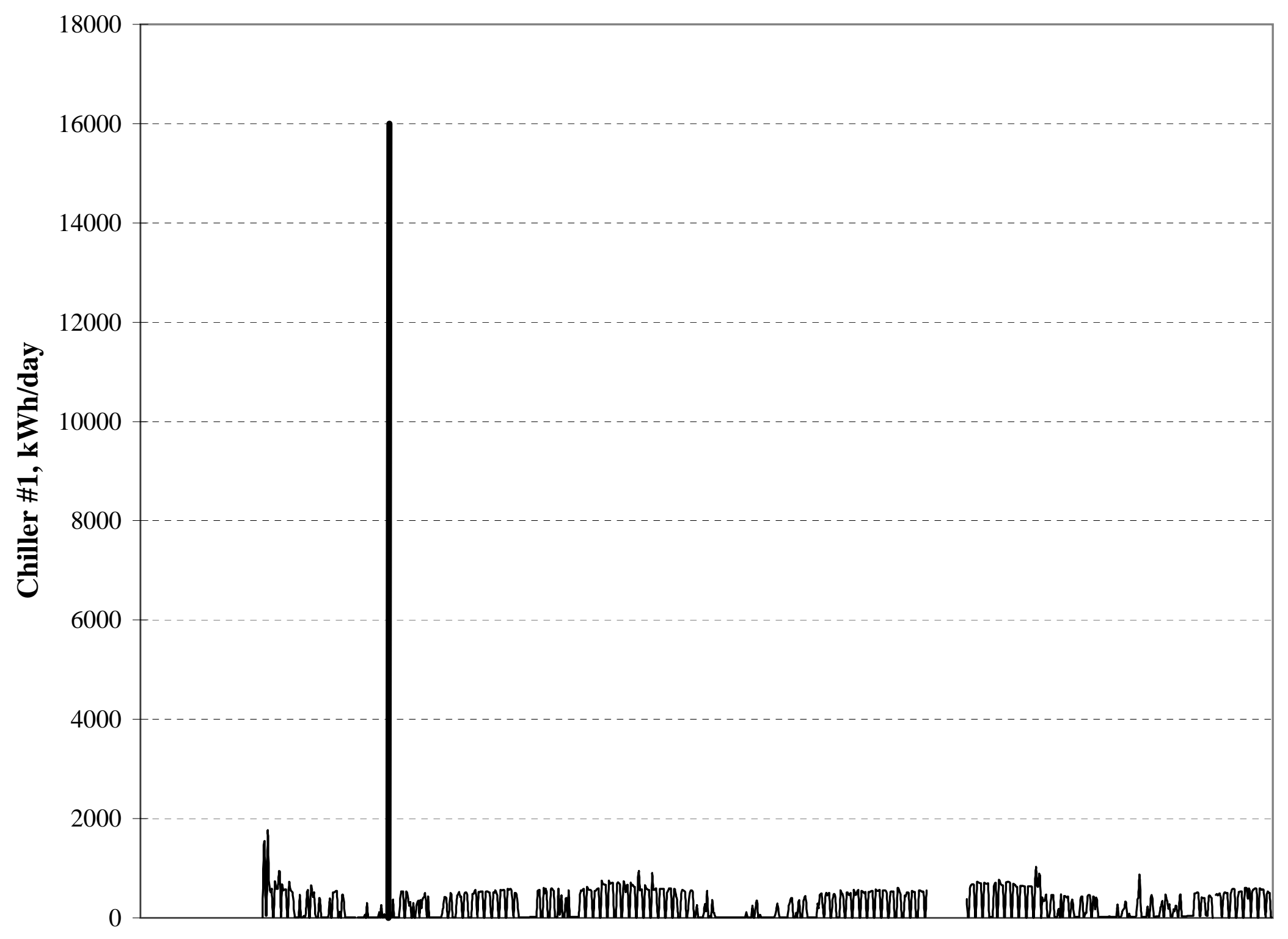

Page 1 


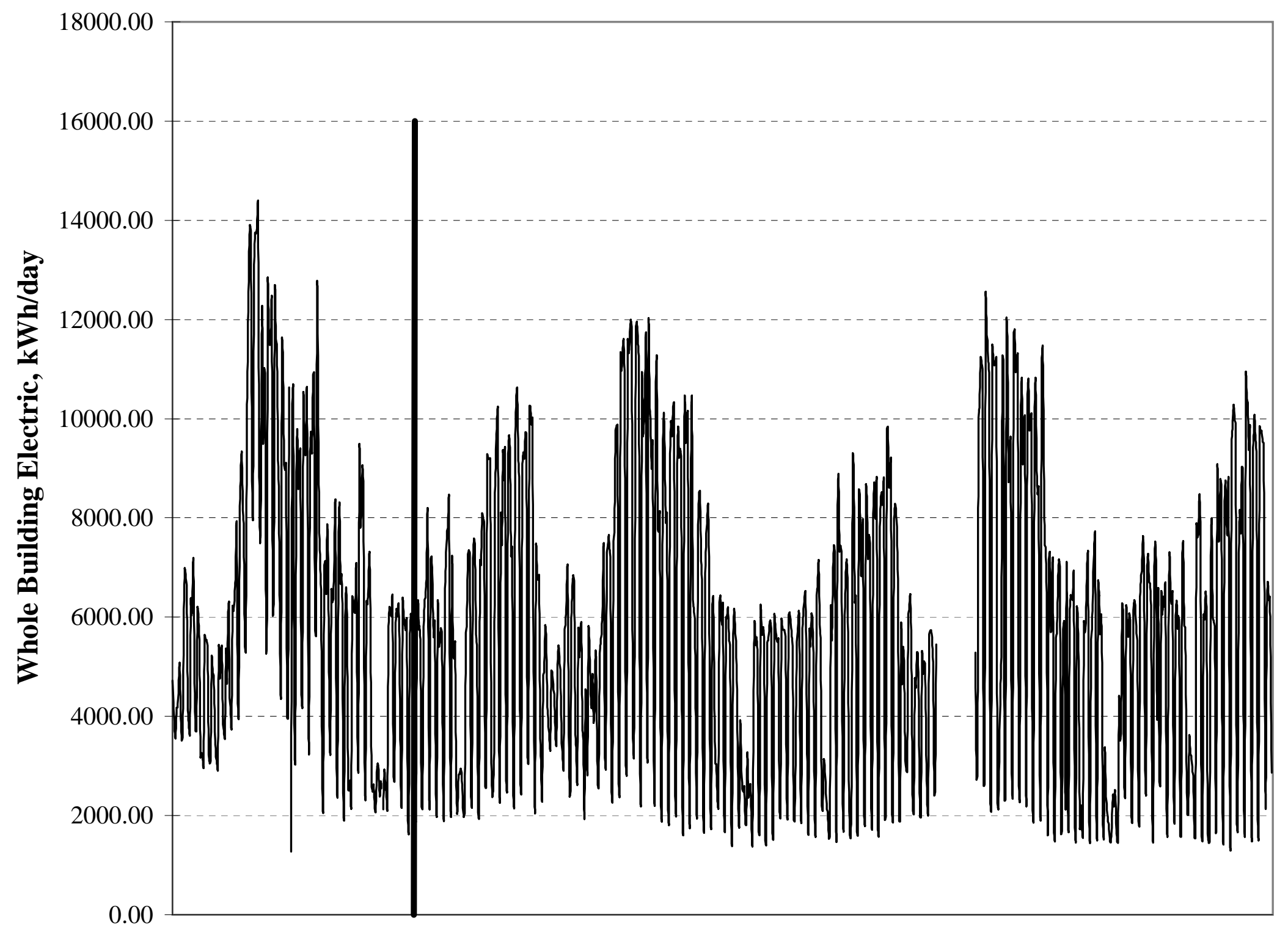

Page 1 


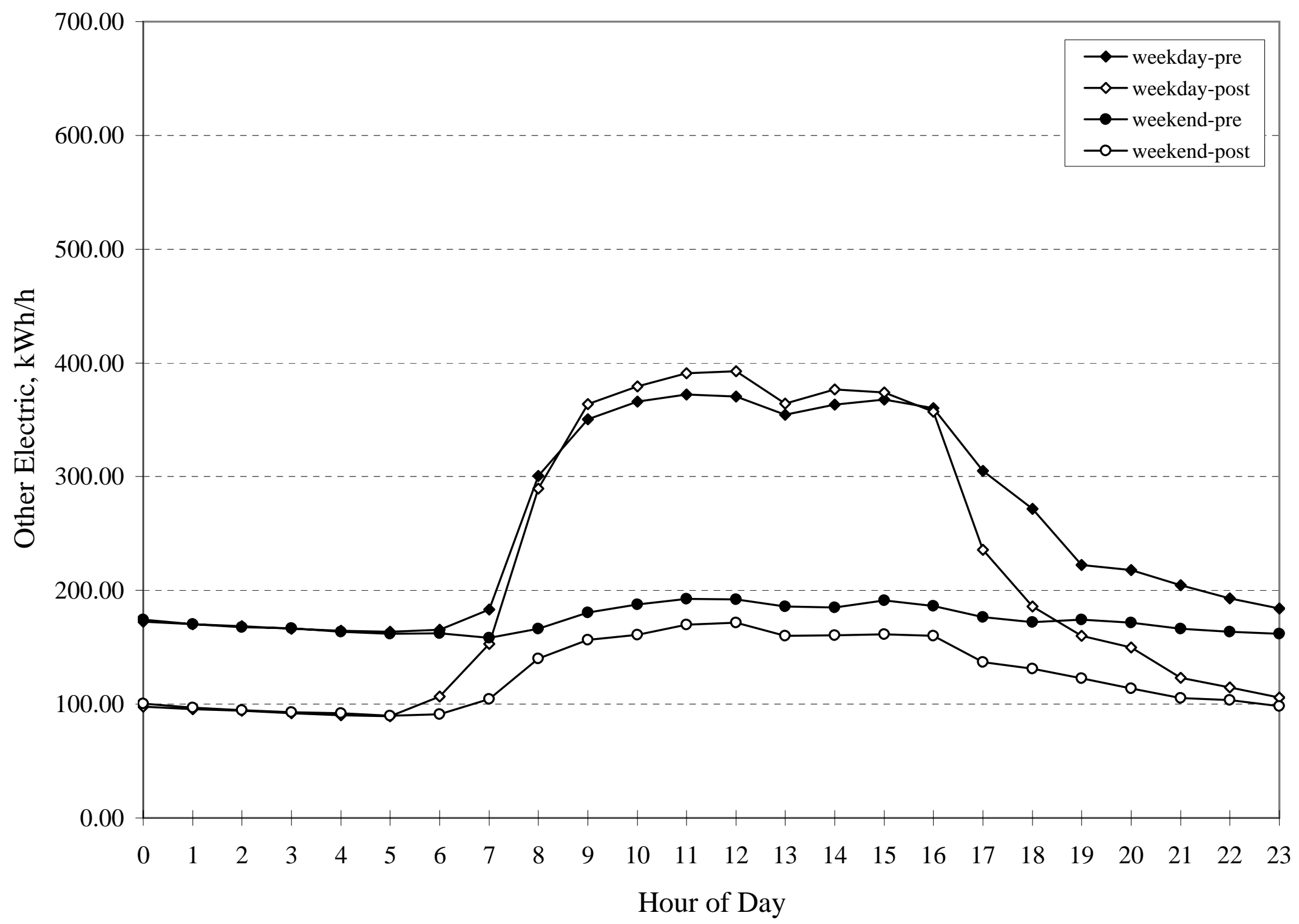

Page 1 


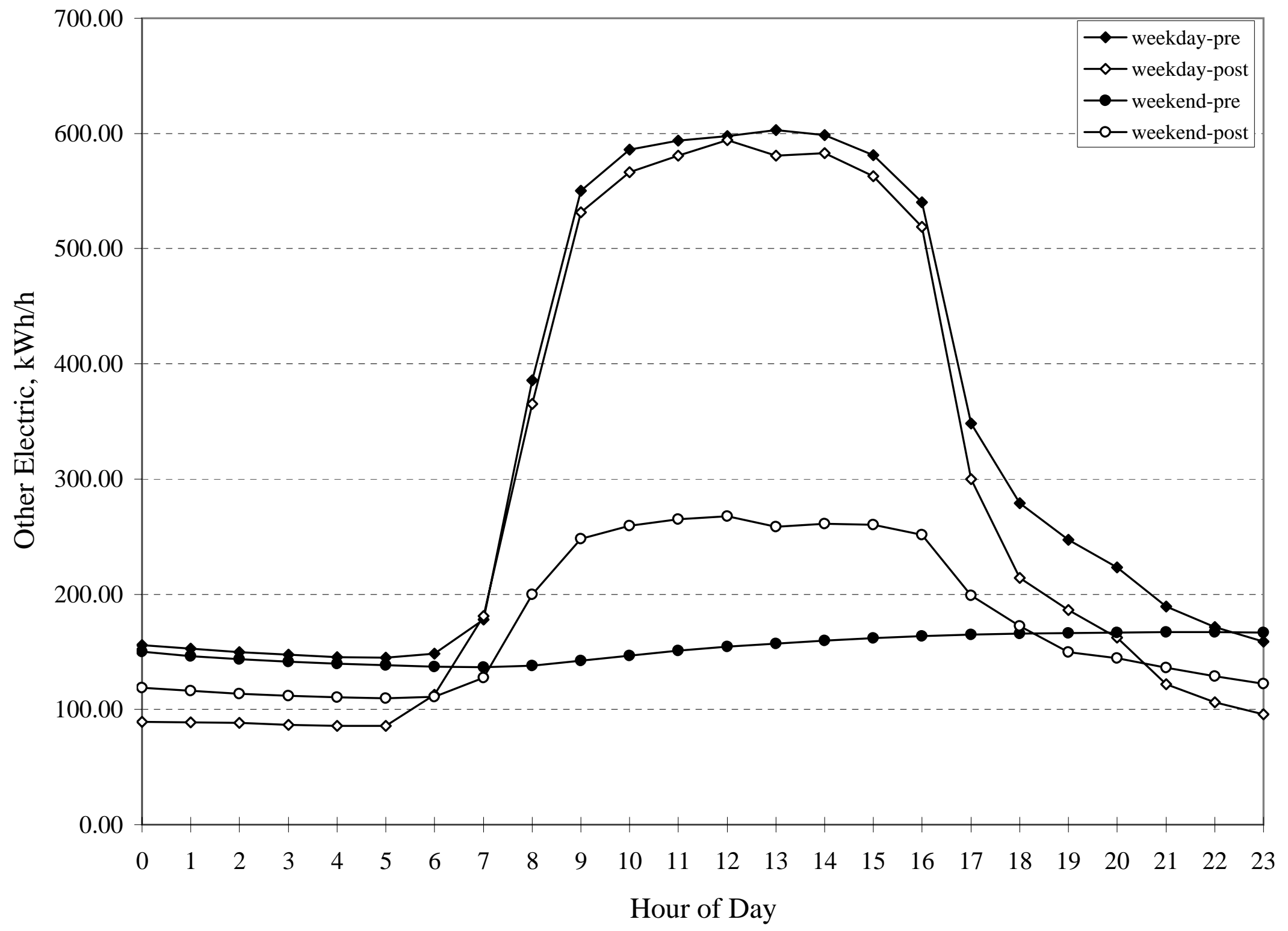

Page 1 


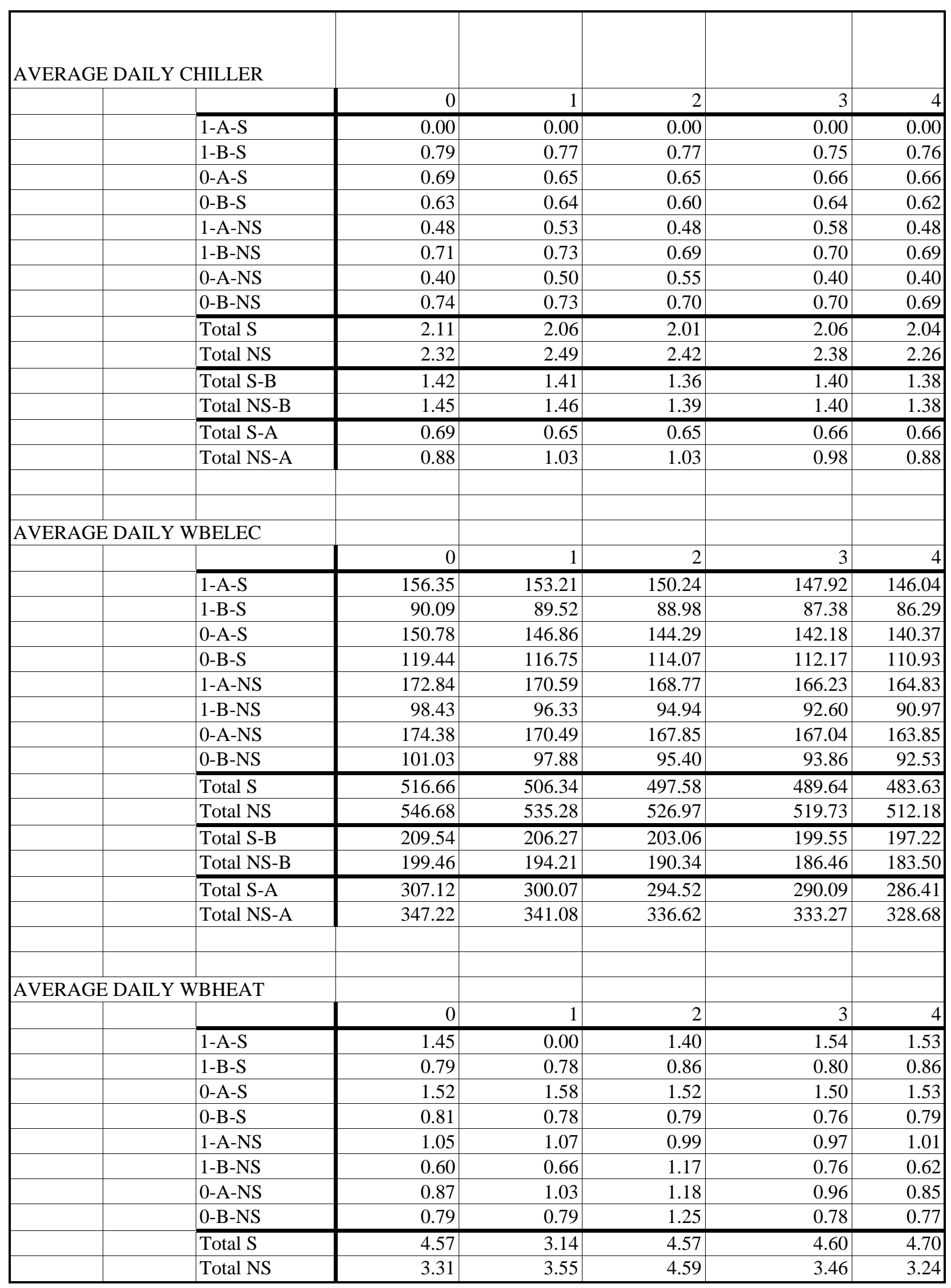


CHART DATA

\begin{tabular}{|c|c|c|c|c|c|c|c|}
\hline & & Total S-B & 1.59 & 1.57 & 1.65 & 1.56 & 1.64 \\
\hline & & Total NS-B & 1.39 & 1.45 & 2.42 & 1.53 & 1.39 \\
\hline & & Total S-A & 2.97 & 1.58 & 2.92 & 3.04 & 3.06 \\
\hline & & Total NS-A & 1.92 & 2.10 & 2.18 & 1.92 & 1.86 \\
\hline & & & & & & & \\
\hline \multicolumn{8}{|c|}{ AVERAGE WBE-CHLR } \\
\hline & & & 0 & 1 & 2 & 3 & 4 \\
\hline & & $1-\mathrm{A}-\mathrm{S}$ & 155.85 & 152.74 & 149.78 & 147.44 & 145.57 \\
\hline & & 1-B-S & 89.31 & 88.77 & 88.23 & 86.63 & 85.54 \\
\hline & & 0-A-S & 150.24 & 146.36 & 143.78 & 141.66 & 139.85 \\
\hline & & $0-B-S$ & 119.00 & 116.30 & 113.65 & 111.71 & 110.49 \\
\hline & & 1-A-NS & 172.75 & 170.48 & 168.68 & 166.12 & 164.74 \\
\hline & & 1-B-NS & 97.73 & 95.60 & 94.26 & 91.90 & 90.29 \\
\hline & & 0-A-NS & 174.28 & 170.37 & 167.72 & 166.94 & 163.76 \\
\hline & & 0-B-NS & 100.29 & 97.15 & 94.70 & 93.17 & 91.84 \\
\hline & & Total S & 514.39 & 504.16 & 495.43 & 487.44 & 481.46 \\
\hline & & Total NS & 545.05 & 533.60 & 525.35 & 518.12 & 510.62 \\
\hline & & Total S-B & 208.31 & 205.07 & 201.88 & 198.34 & 196.03 \\
\hline & & Total NS-B & 198.02 & 192.75 & 188.96 & 185.06 & 182.12 \\
\hline & & Total S-A & 306.08 & 299.10 & 293.56 & 289.10 & 285.43 \\
\hline & & Total NS-A & 347.03 & 340.85 & 336.40 & 333.06 & 328.49 \\
\hline & & & & & & & \\
\hline & & & & & & & \\
\hline \multicolumn{2}{|c|}{ PIE CHART } & chiller -s & other -s & chiller -ns & other -ns & & \\
\hline & & 257134.47 & 6045264.66 & 119059.33 & 2730274.29 & 9151732.75 & \\
\hline & & $3 \%$ & $66 \%$ & $1 \%$ & $30 \%$ & & \\
\hline & & & & & & & \\
\hline & & & & & & & \\
\hline \multicolumn{8}{|c|}{ CONSUMPTION FOR POST PERIOD BY SCHOOL YEAR / NON SCHOOL YEAR } \\
\hline \multicolumn{8}{|c|}{\begin{tabular}{|l|l|l|l|} 
(average daily consumption * \#days) & & & \\
\end{tabular}} \\
\hline & & & & & & & \\
\hline & & S & S & NS & NS & TOTAL & TOTAL \\
\hline & & Energy & $\$$ & Energy & $\$$ & Energy & $\$$ \\
\hline & wbelec & 6284114 & & 2848484 & & 9132598 & \\
\hline & chlr & 257134 & & 119059 & & 376194 & \\
\hline & other & 6045265 & & 2730274 & & 8775539 & \\
\hline & wbheat & 25762 & & 12822 & & 38583 & \\
\hline & & & & & & & \\
\hline & & & & & & & \\
\hline & & & & & & & \\
\hline & & & & & & & \\
\hline & & & & & & & \\
\hline & & & & & & & \\
\hline & & & & & & & \\
\hline & & & & & & & \\
\hline & & & & & & & \\
\hline & & & & & & & \\
\hline & & & & & & & \\
\hline
\end{tabular}


CHART DATA

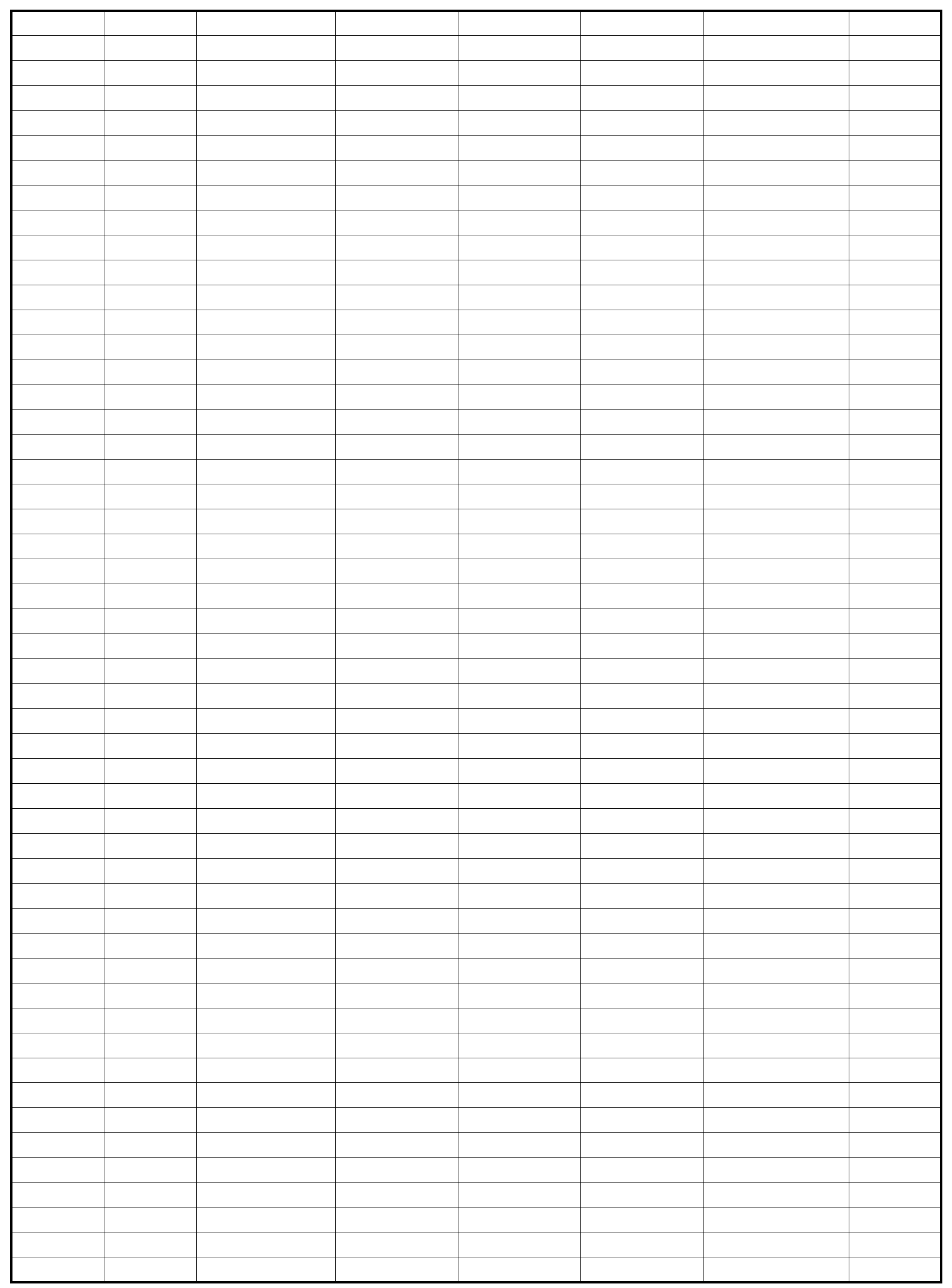

Page 3 
CHART DATA

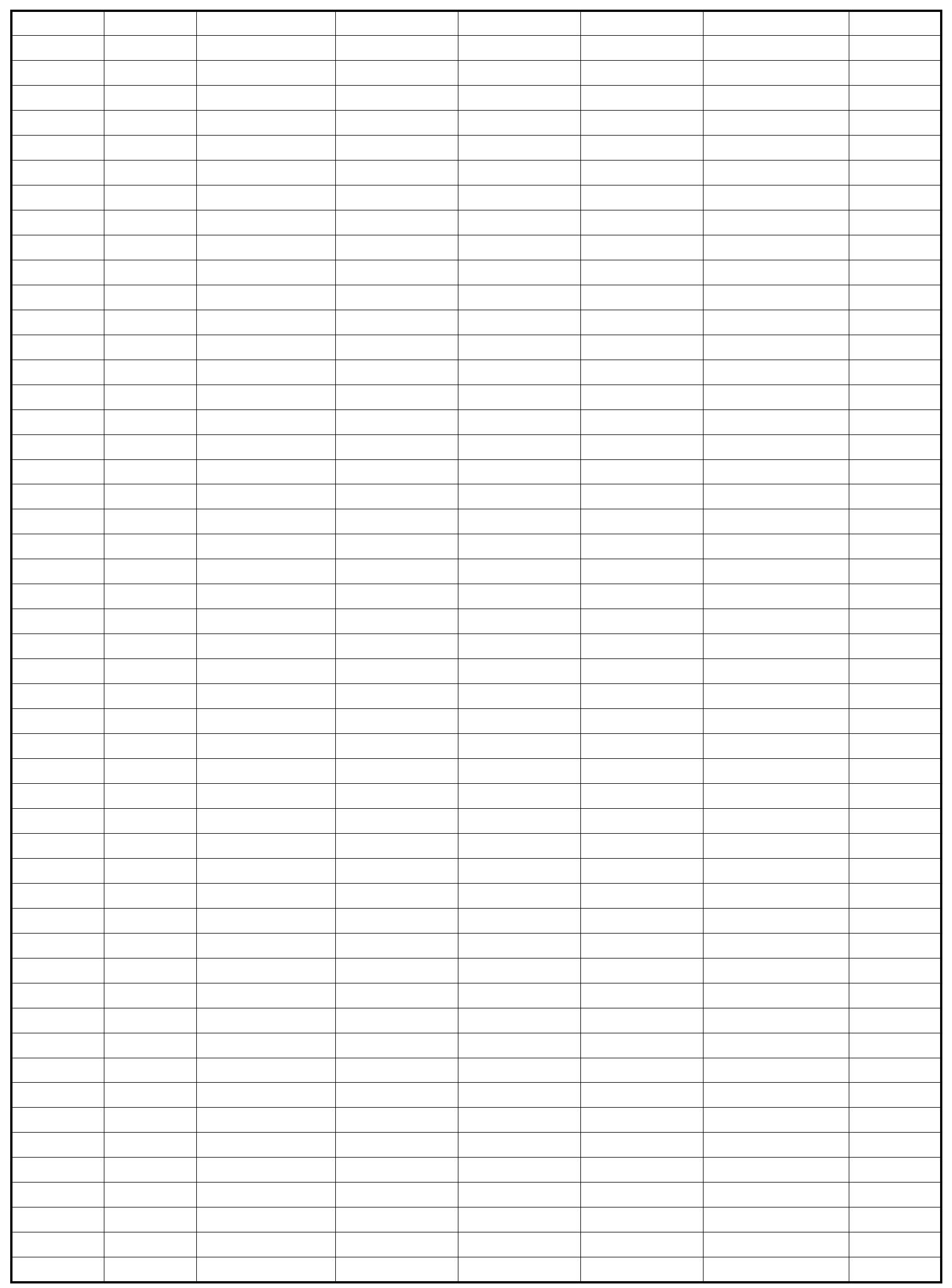

Page 4 
CHART DATA

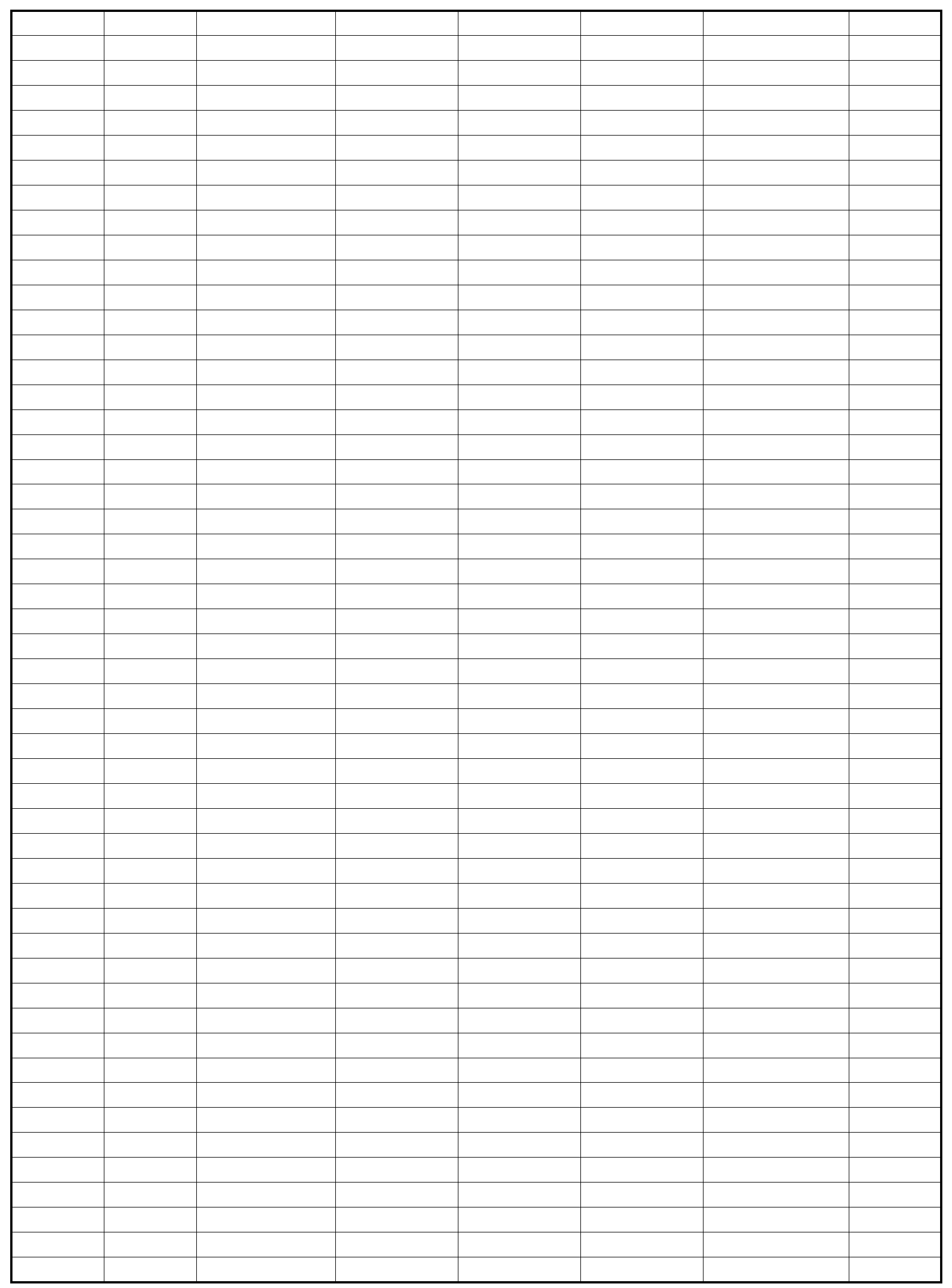

Page 5 
CHART DATA

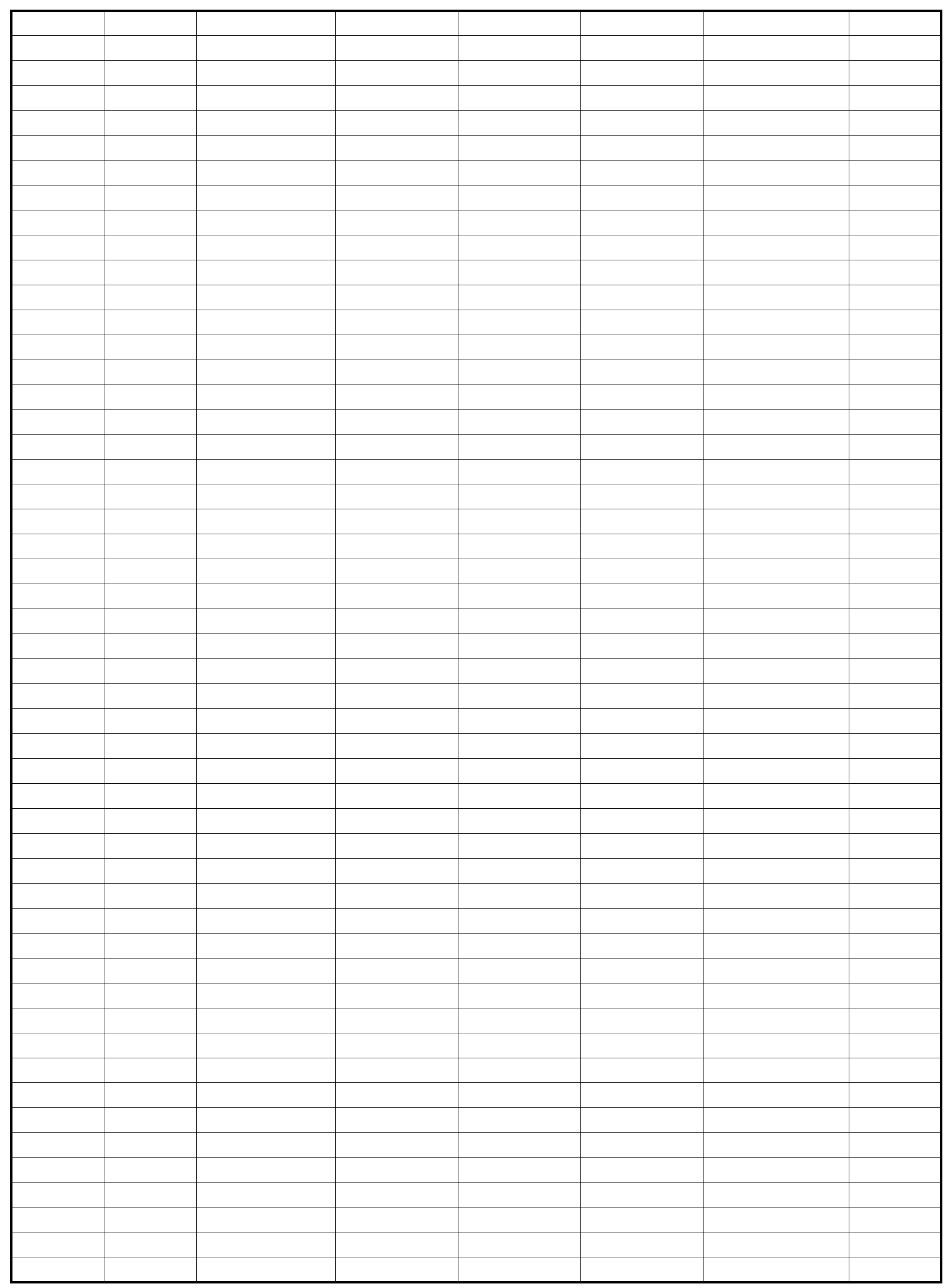

Page 6 
CHART DATA

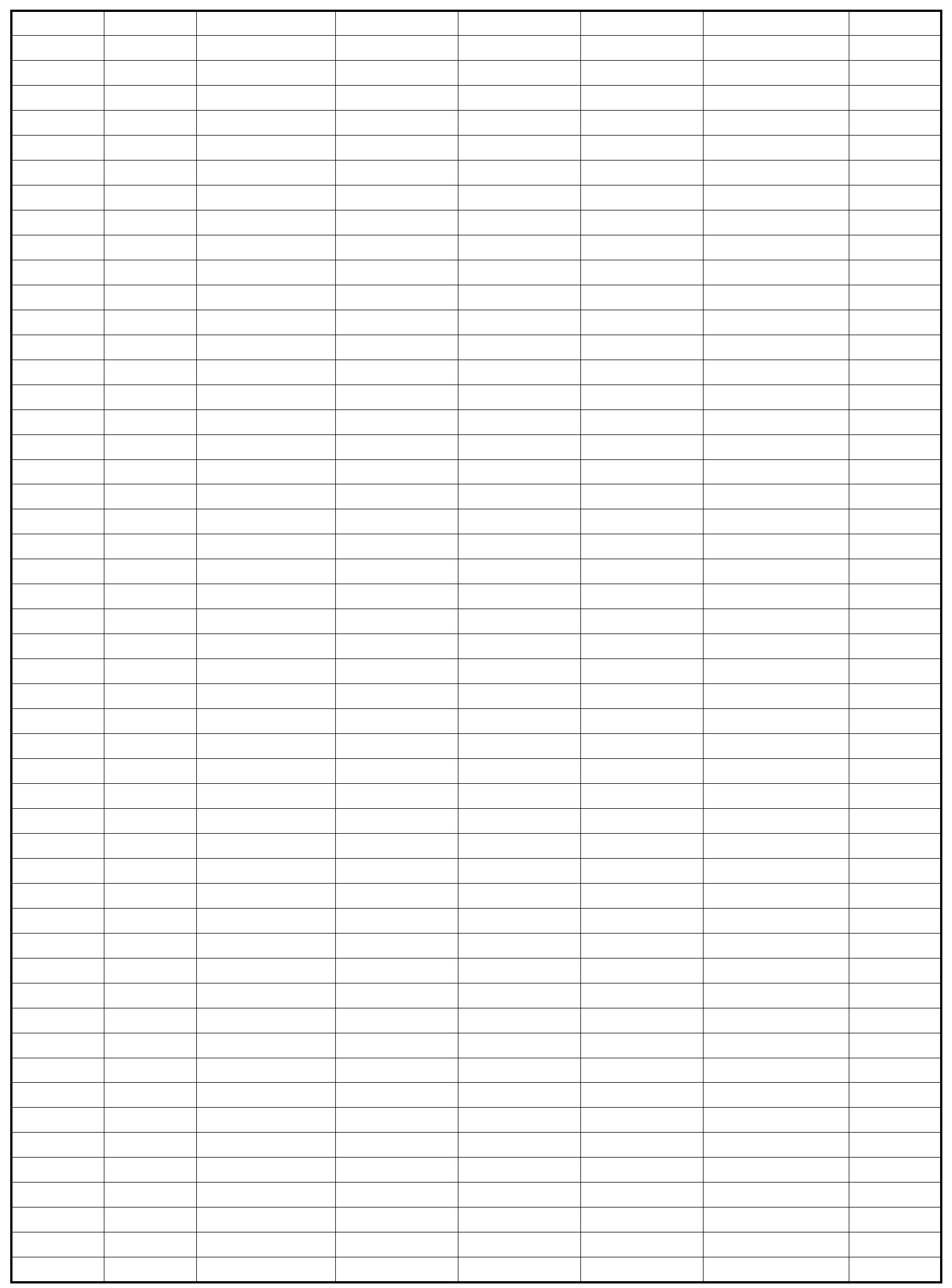

Page 7 
CHART DATA

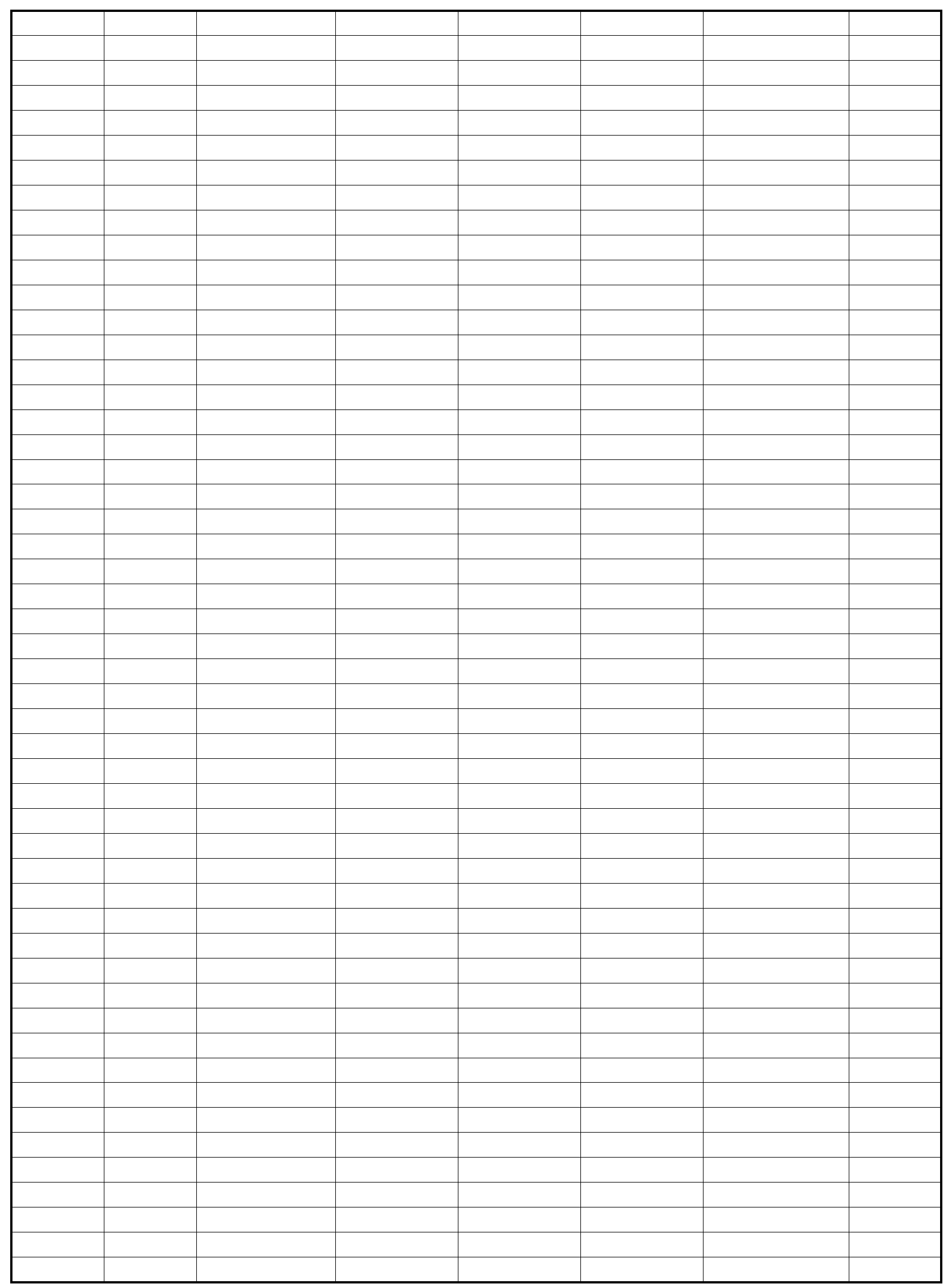

Page 8 
CHART DATA

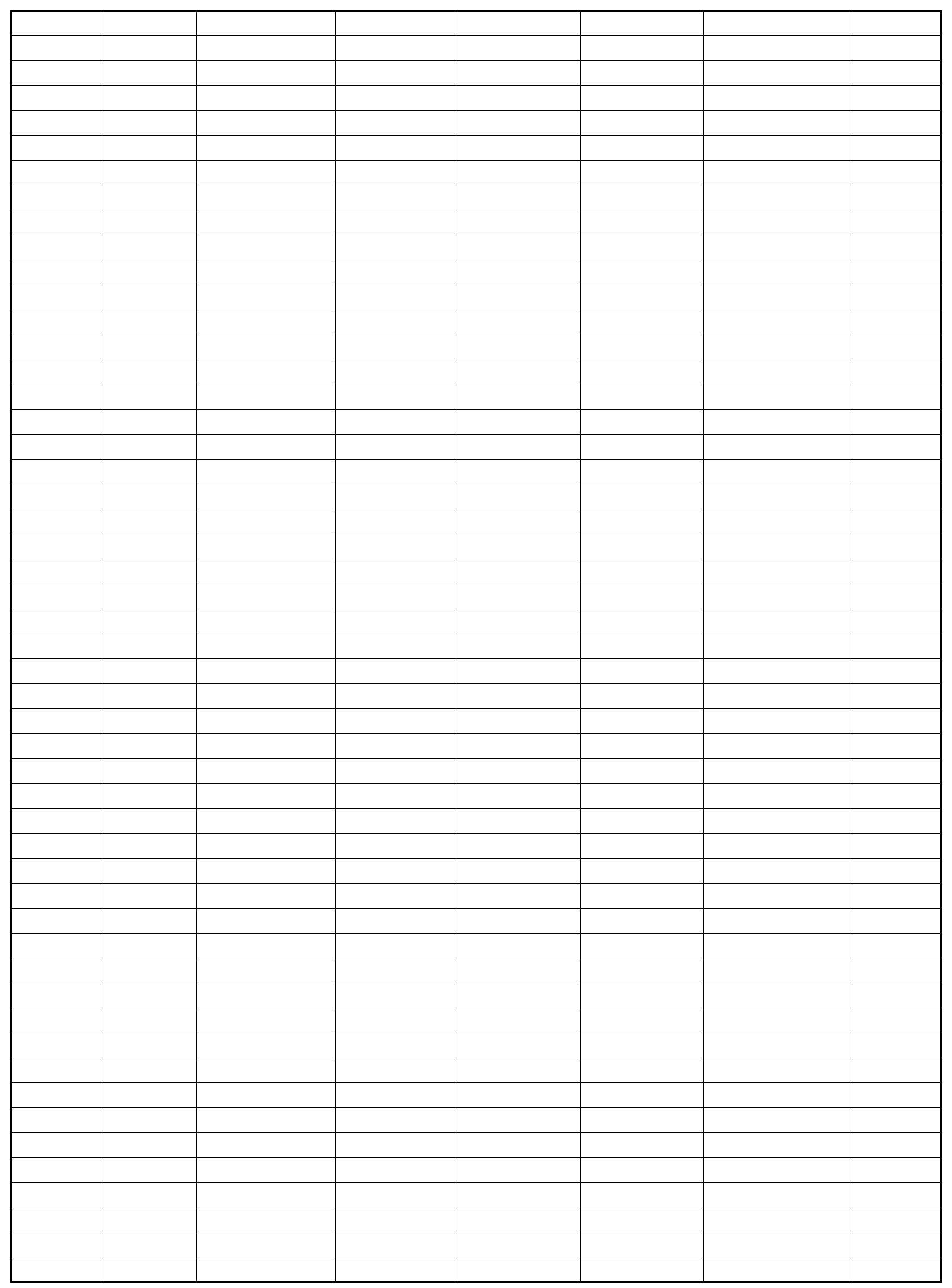

Page 9 
CHART DATA

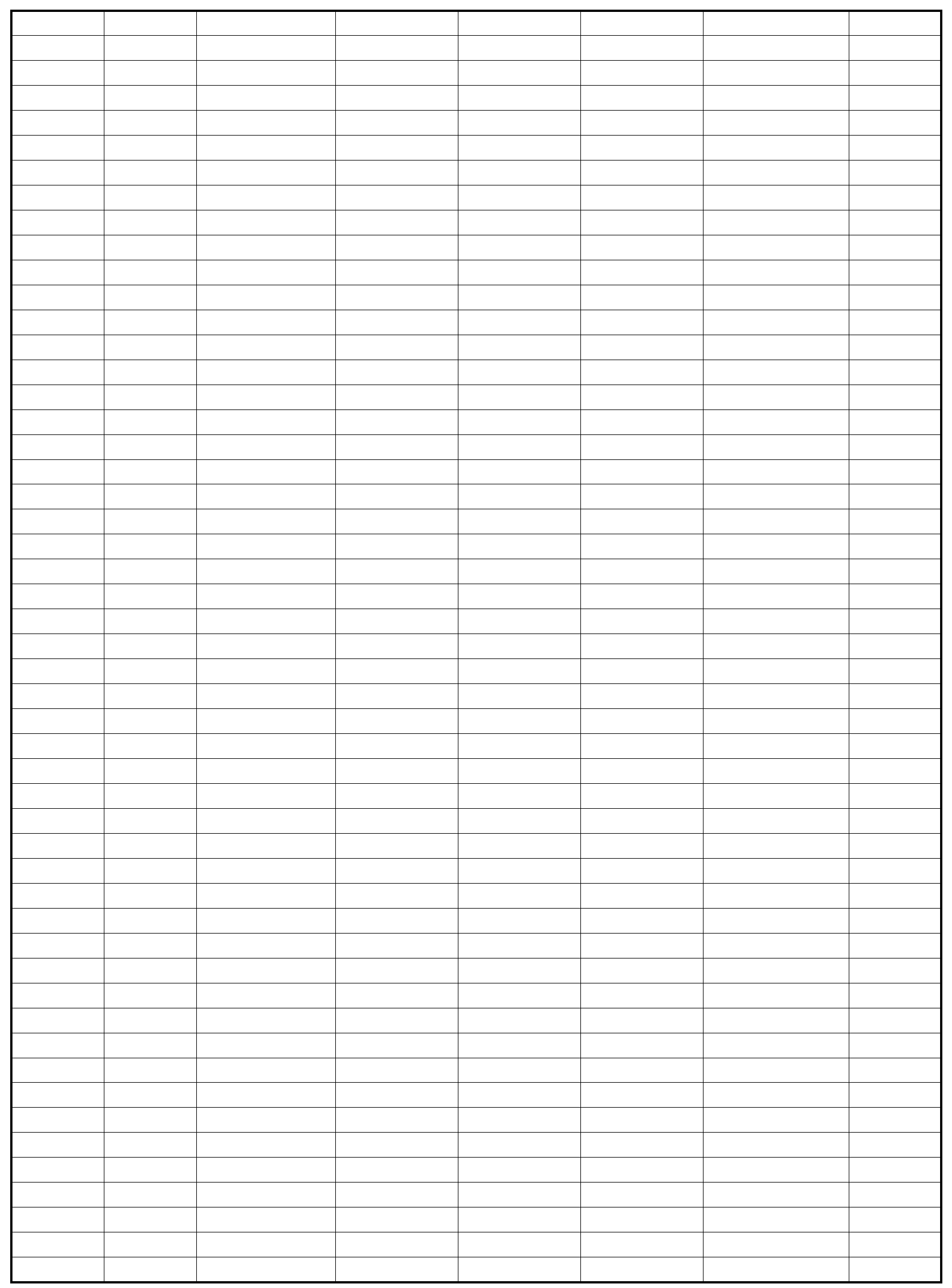

Page 10 
CHART DATA

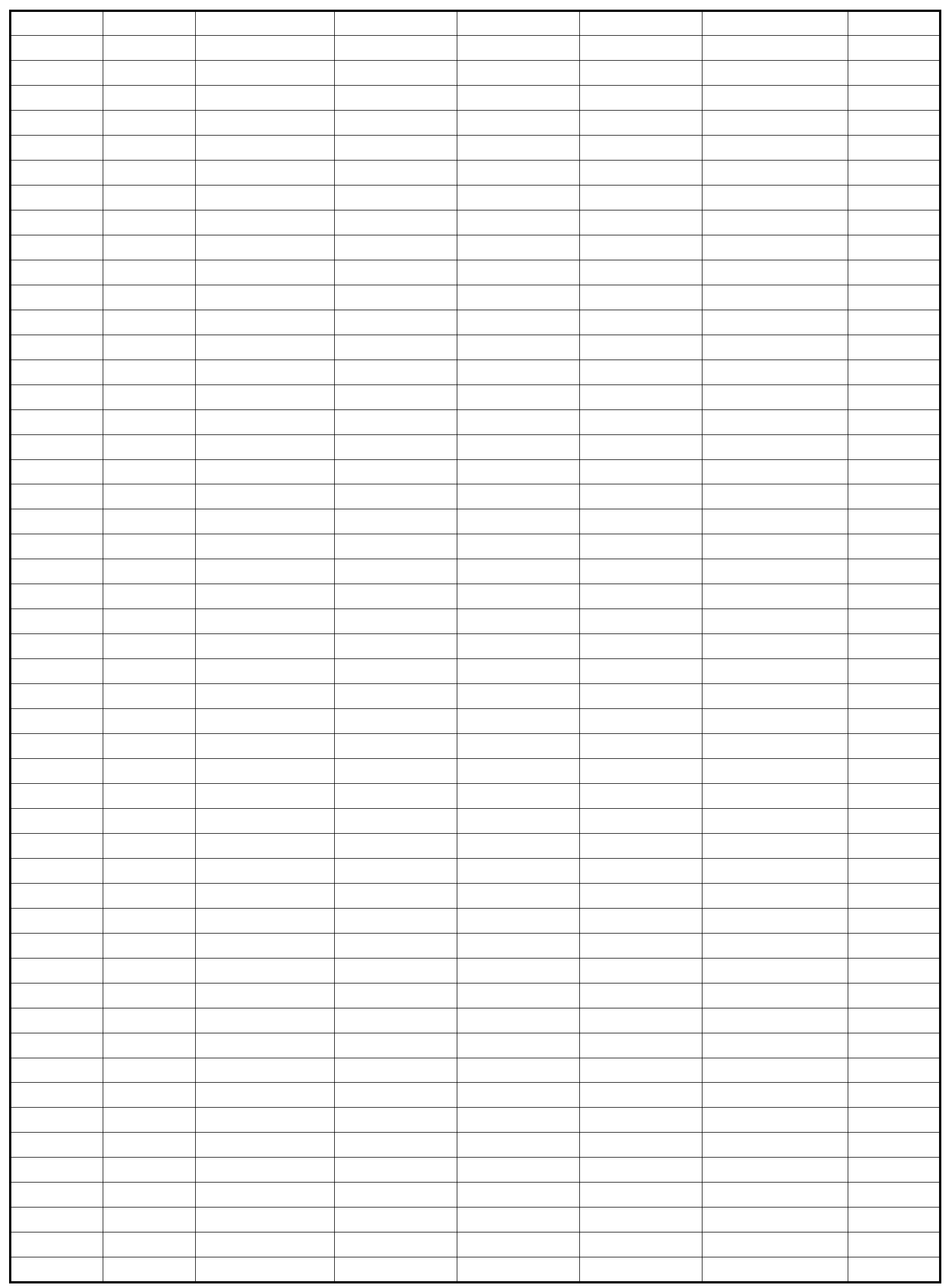

Page 11 
CHART DATA

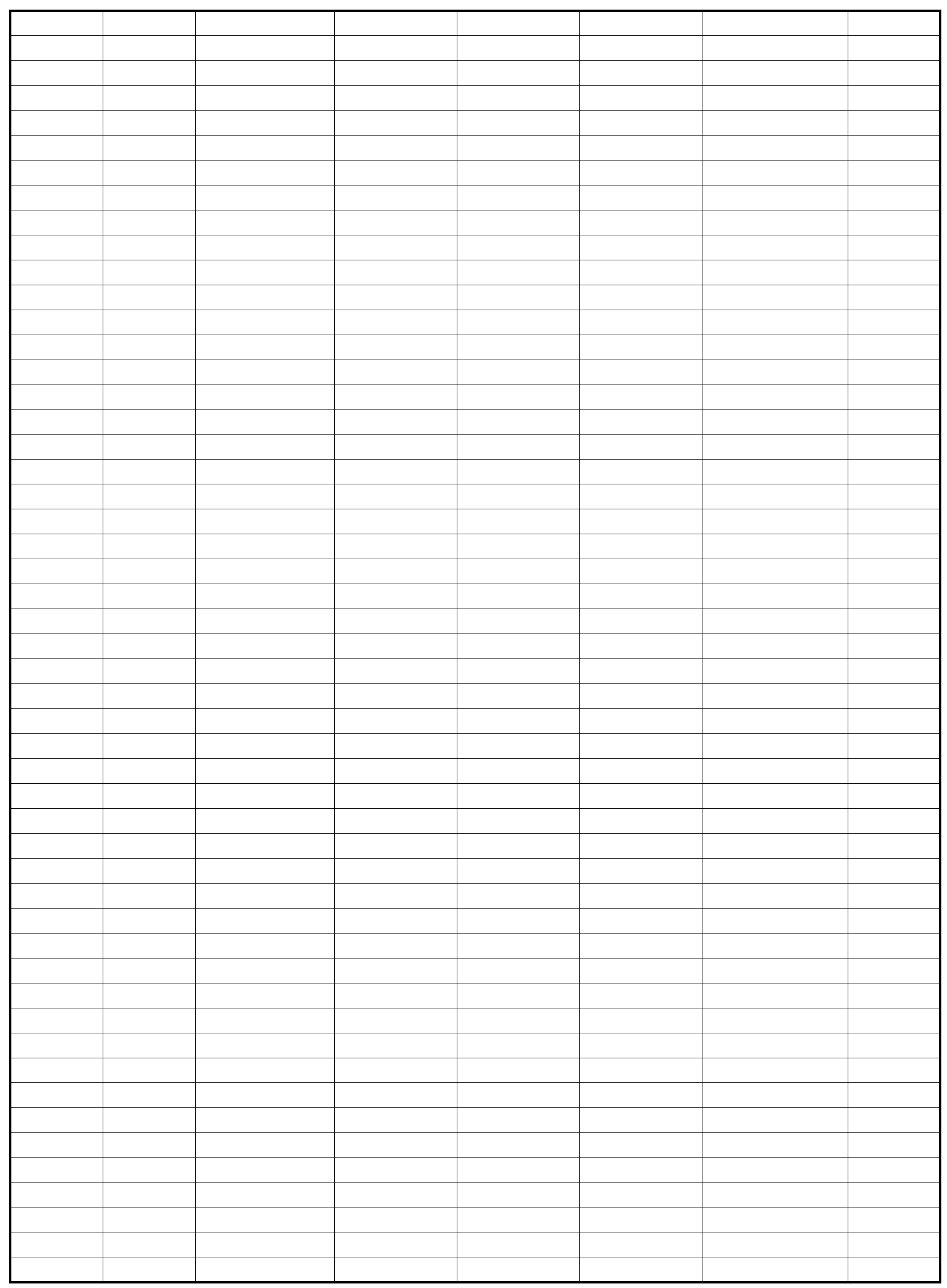

Page 12 
CHART DATA

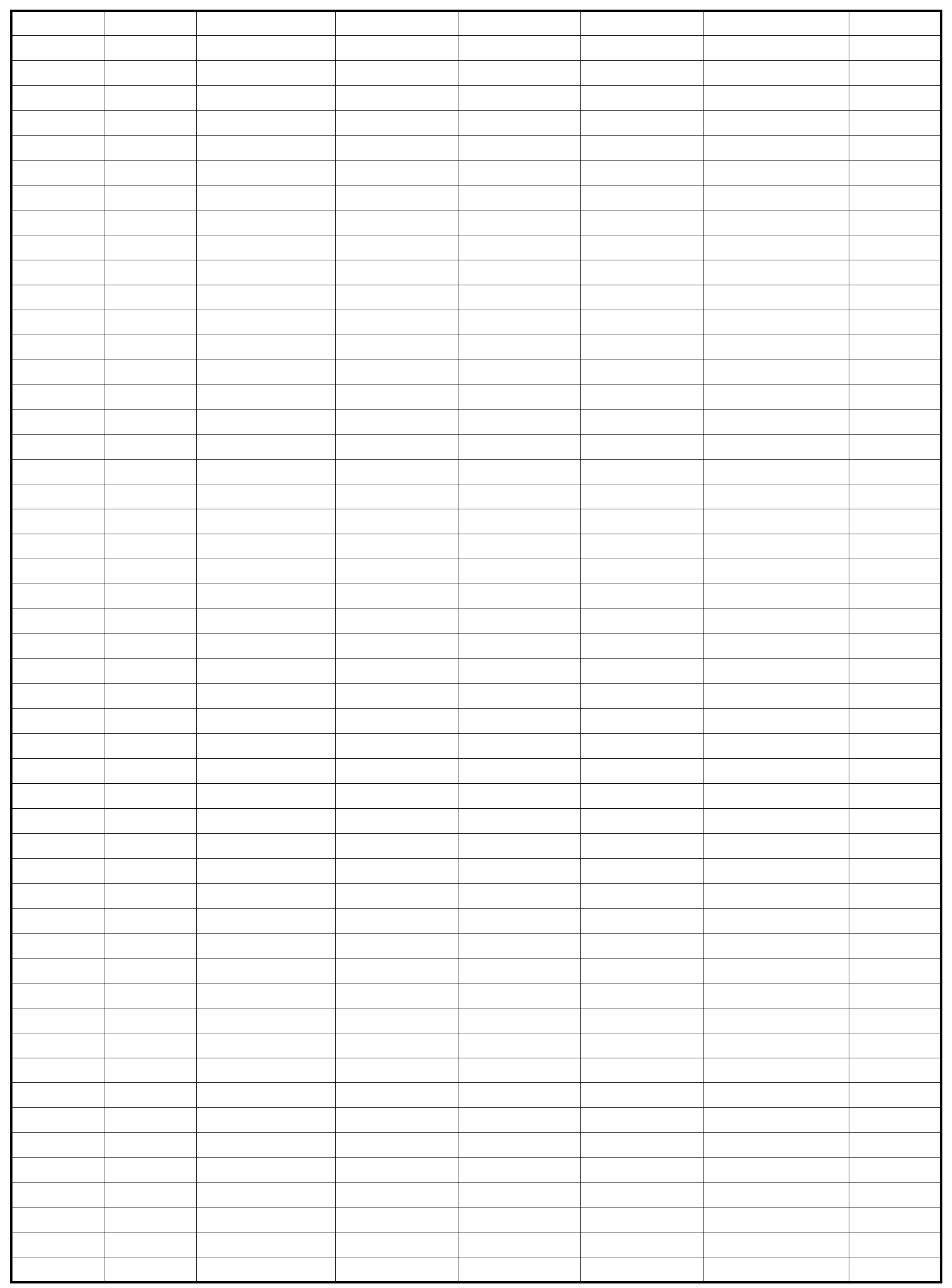

Page 13 
CHART DATA

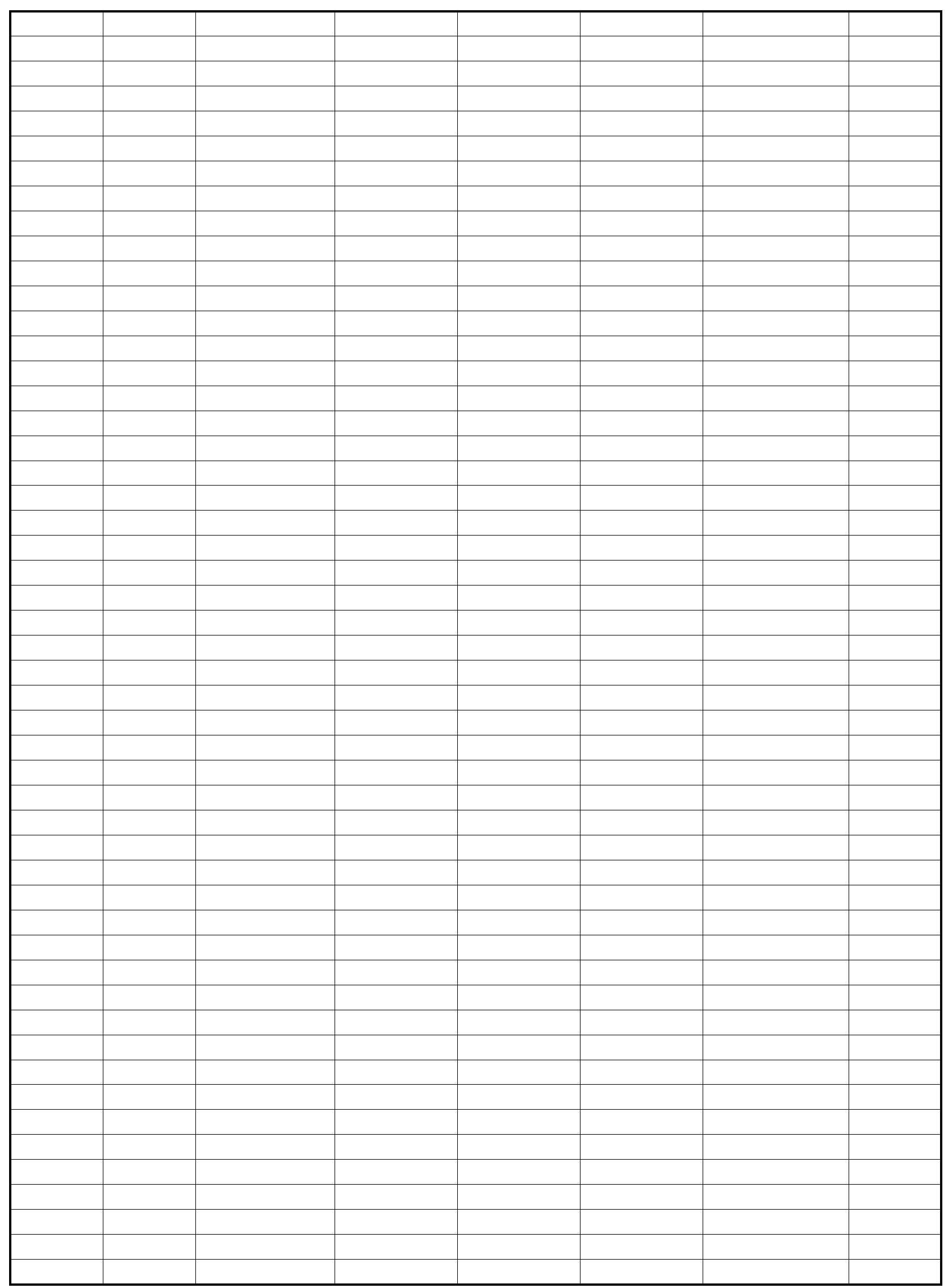

Page 14 
CHART DATA

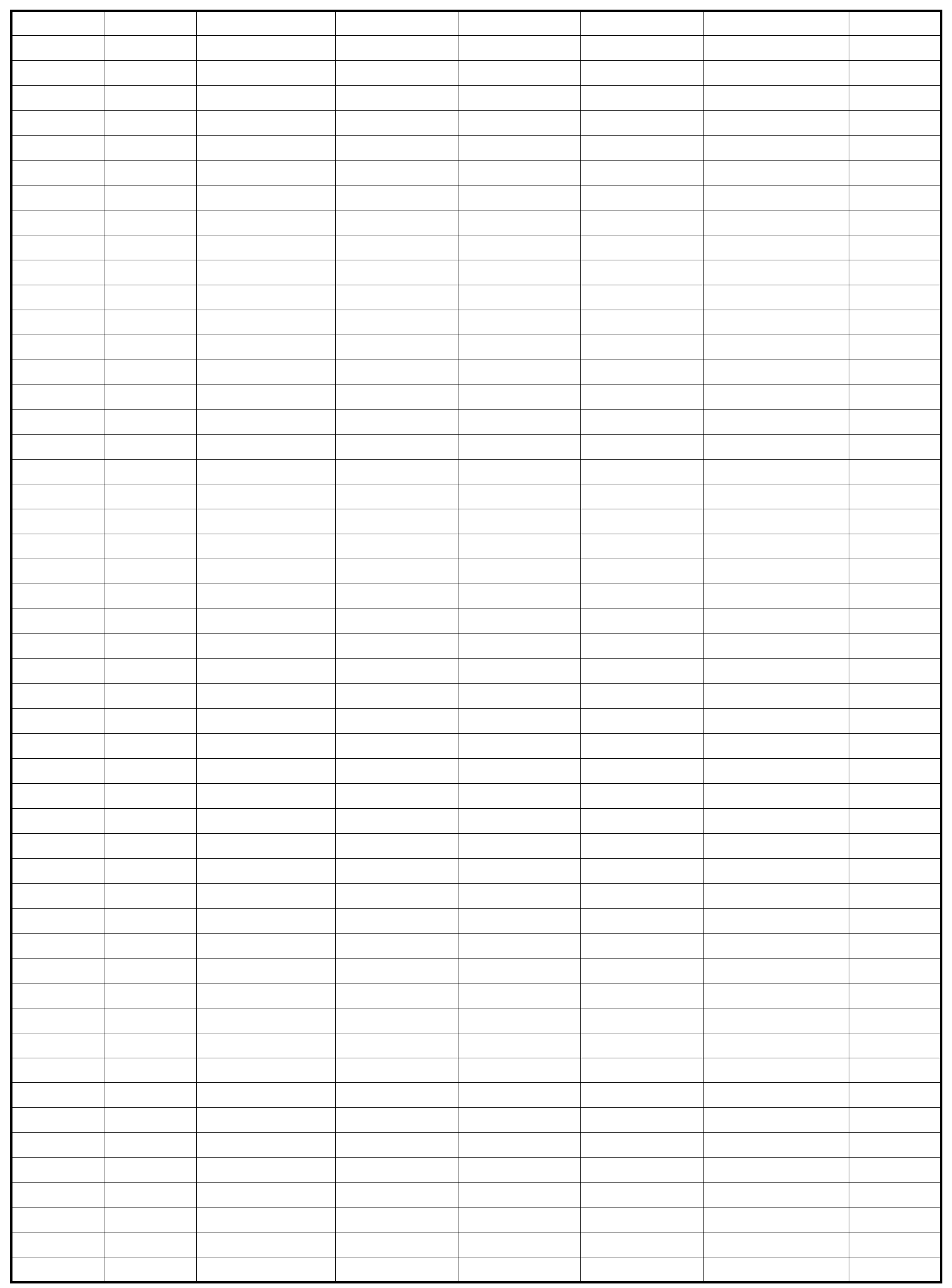

Page 15 
CHART DATA

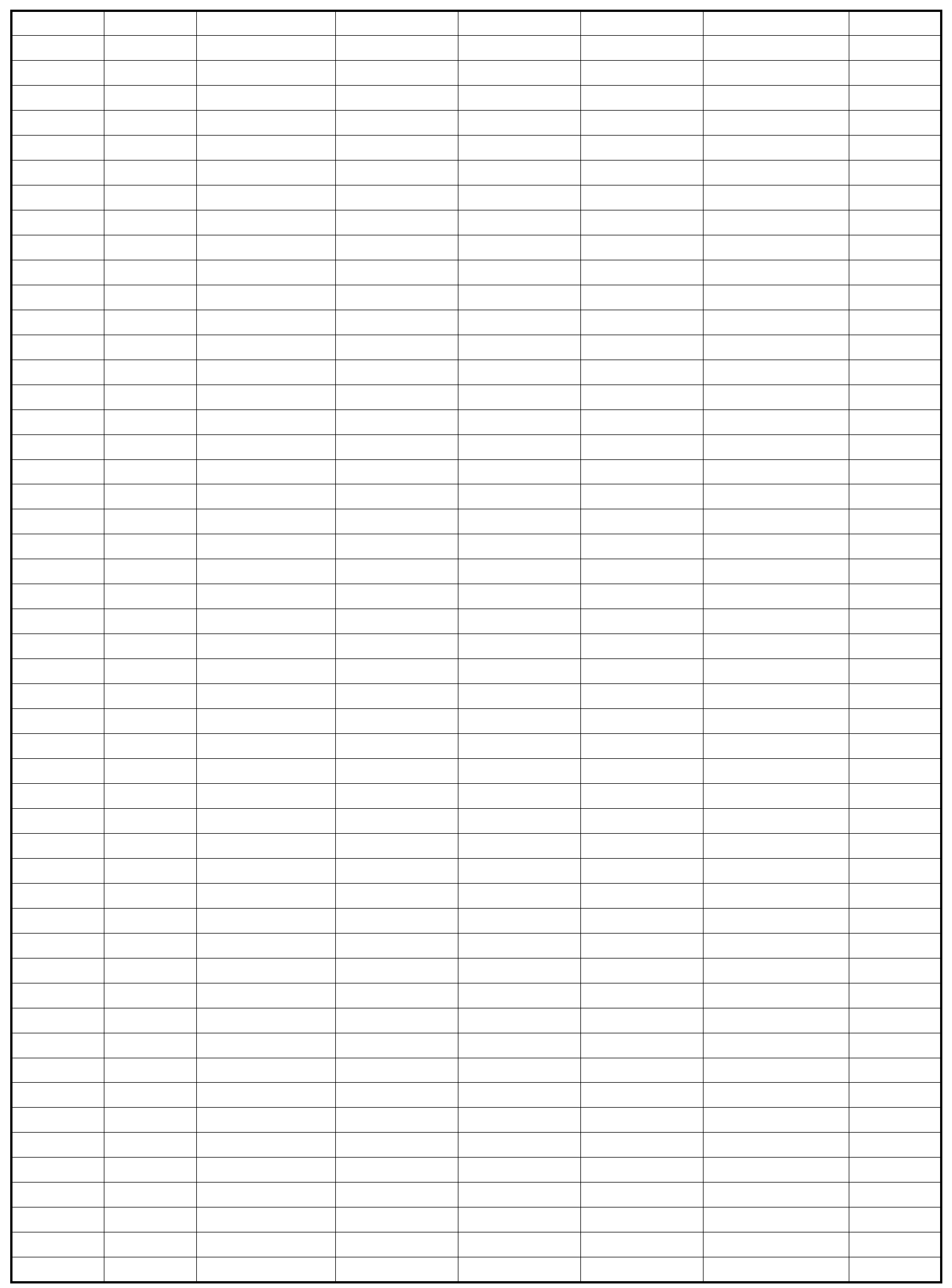

Page 16 
CHART DATA

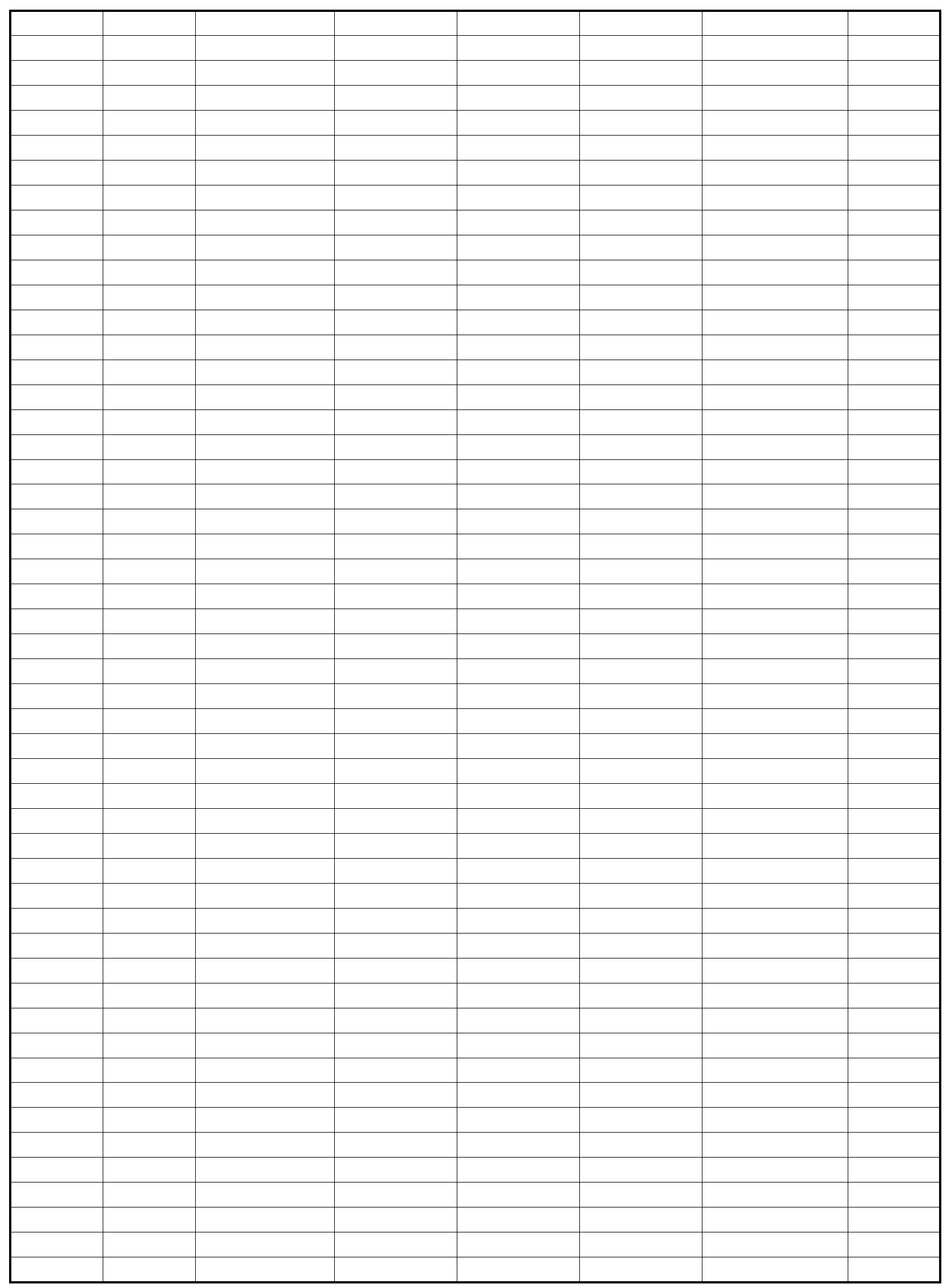

Page 17 
CHART DATA

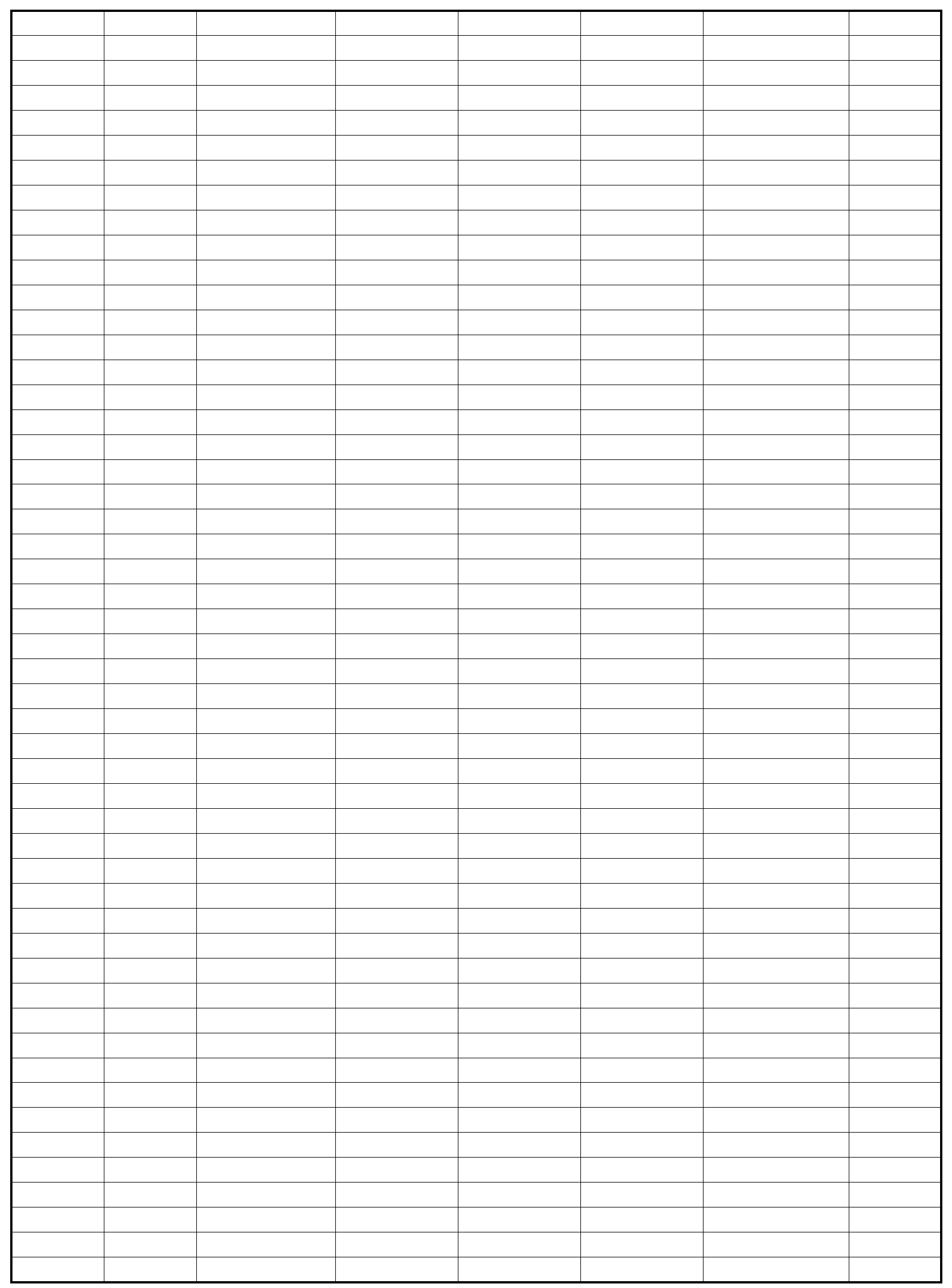

Page 18 
CHART DATA

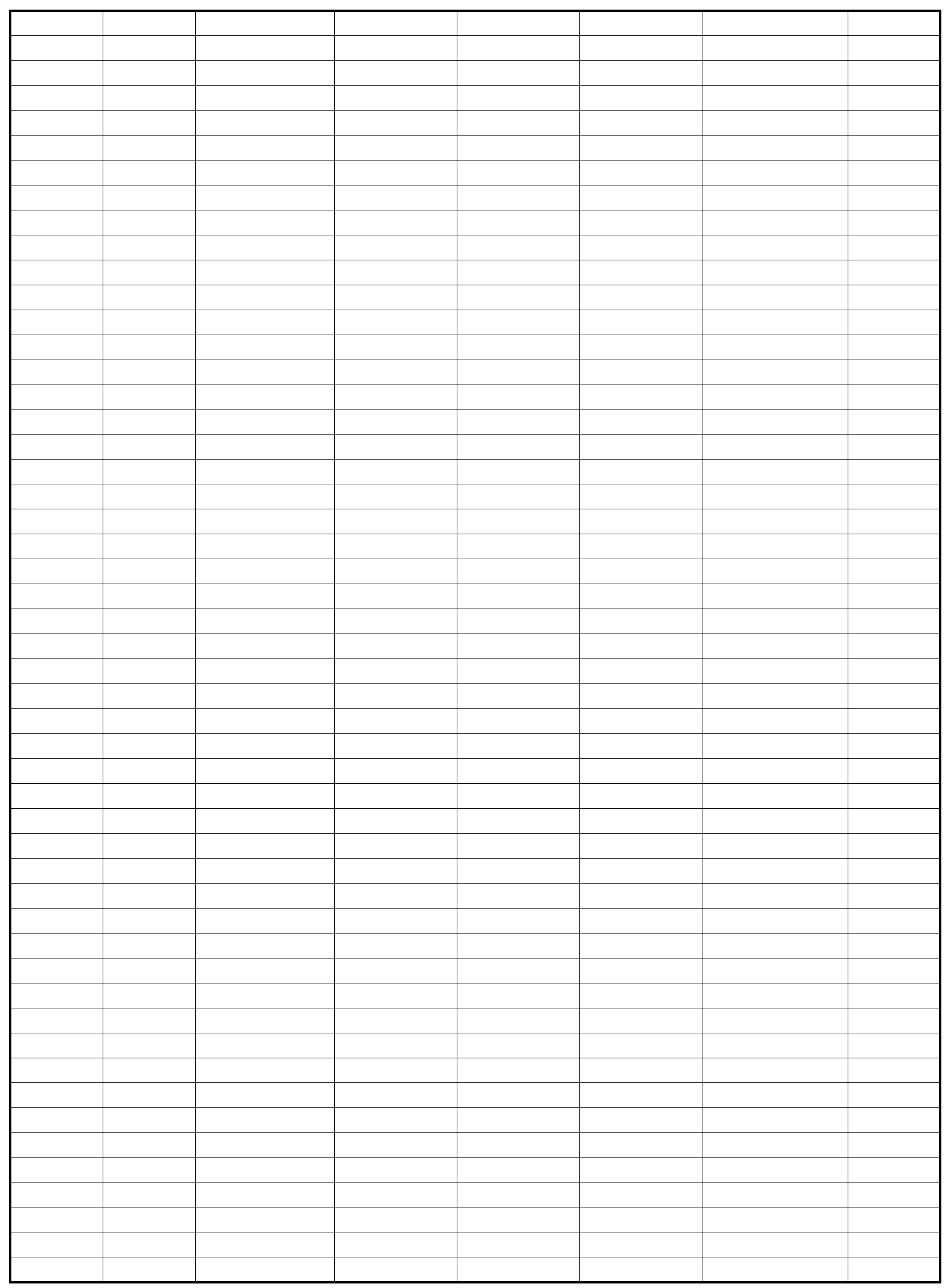

Page 19 
CHART DATA

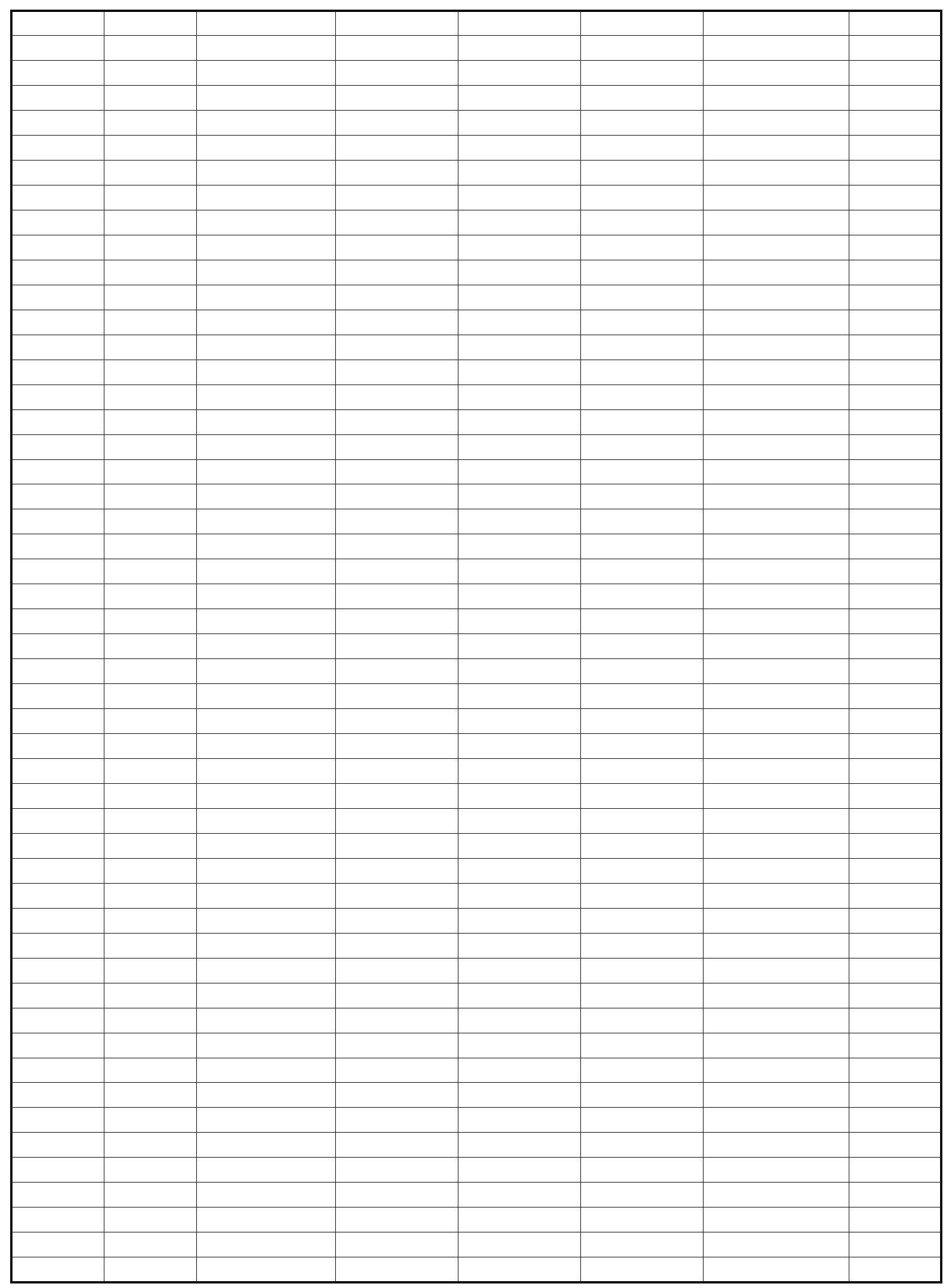

Page 20 
CHART DATA

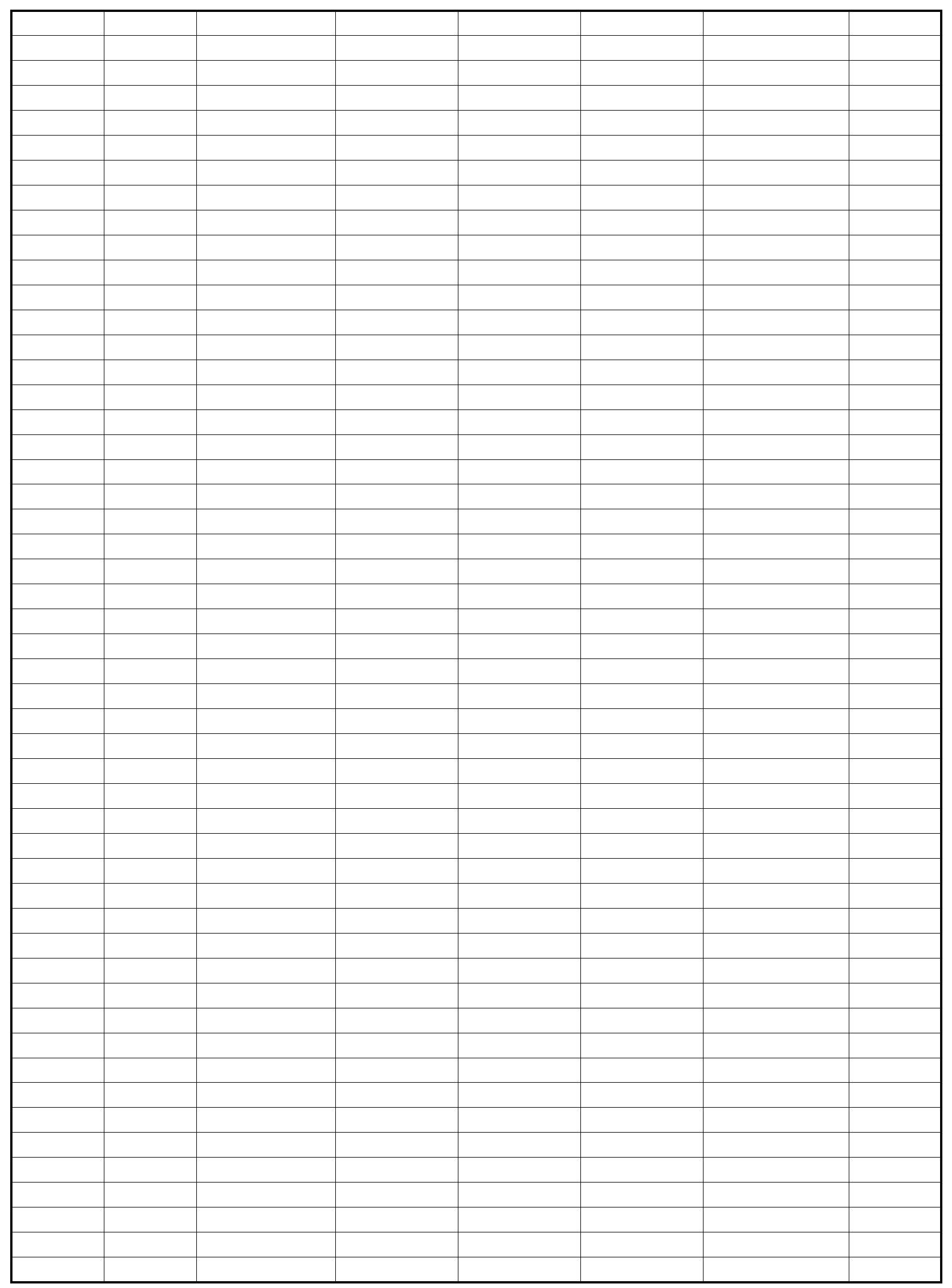

Page 21 
CHART DATA

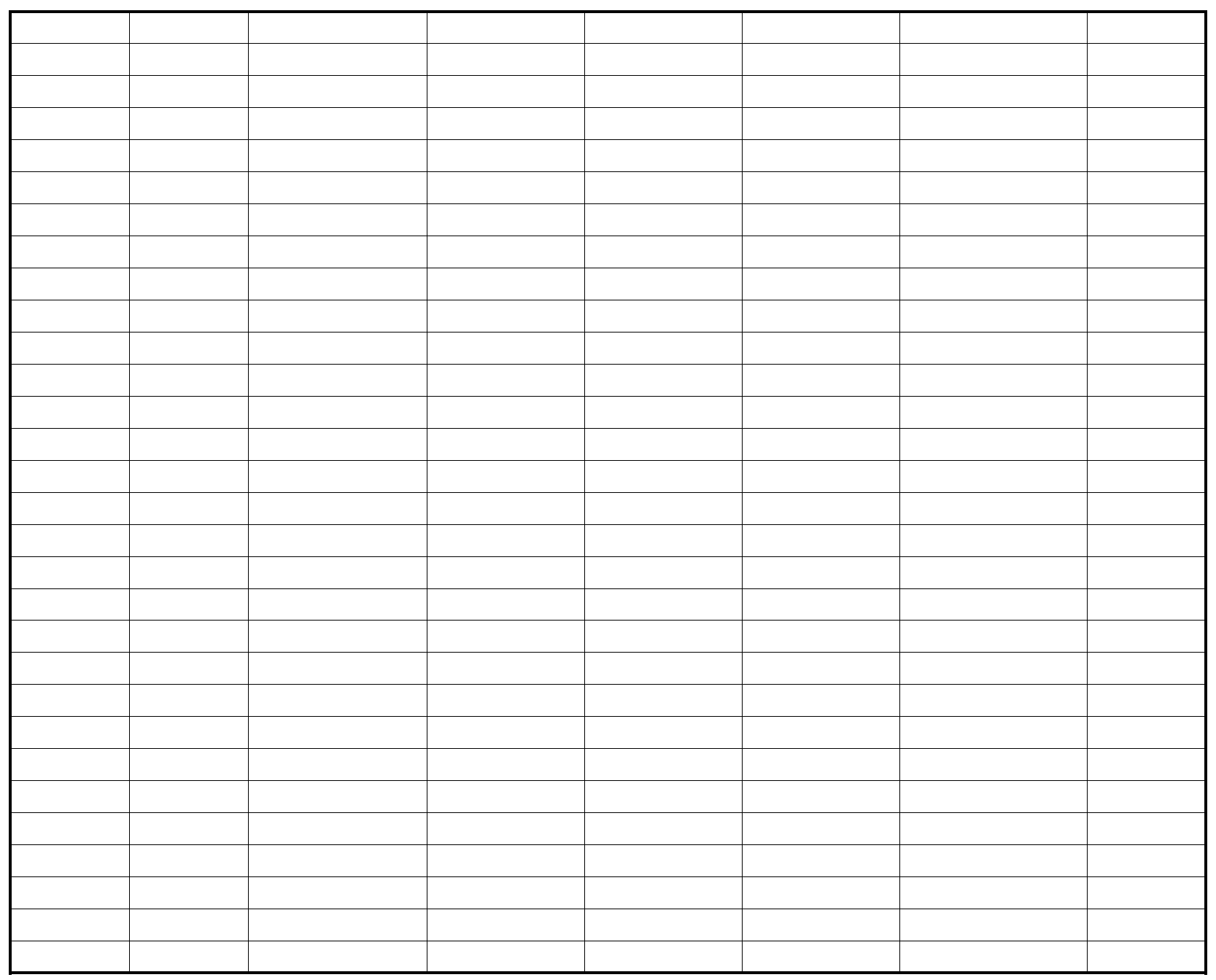

Page 22 


\begin{tabular}{|c|c|c|c|c|c|c|c|c|c|}
\hline 5 & 6 & 7 & 8 & 9 & 10 & 11 & 12 & 13 & 14 \\
\hline 0.00 & 0.00 & 0.00 & 0.00 & 0.00 & 0.00 & 0.00 & 0.00 & 0.00 & 0.00 \\
\hline 0.78 & 1.53 & 14.74 & 27.05 & 33.13 & 37.27 & 38.30 & 39.74 & 39.39 & 38.83 \\
\hline 0.66 & 0.65 & 0.90 & 1.10 & 1.25 & 1.39 & 1.57 & 1.93 & 2.27 & 2.54 \\
\hline 0.61 & 0.60 & 4.31 & 8.86 & 9.61 & 10.61 & 11.61 & 11.93 & 11.43 & 10.67 \\
\hline 0.48 & 0.53 & 1.15 & 11.25 & 11.10 & 11.20 & 11.25 & 11.12 & 7.75 & 5.40 \\
\hline 0.68 & 1.69 & 10.61 & 27.98 & 31.24 & 32.51 & 31.92 & 32.84 & 32.48 & 31.80 \\
\hline 0.45 & 0.50 & 0.50 & 9.75 & 15.95 & 15.15 & 14.95 & 14.75 & 11.15 & 7.45 \\
\hline 0.76 & 0.68 & 2.02 & 4.61 & 5.34 & 5.30 & 5.27 & 5.25 & 4.75 & 4.60 \\
\hline 2.05 & 2.78 & 19.96 & 37.01 & 43.99 & 49.26 & 51.49 & 53.60 & 53.09 & 52.04 \\
\hline 2.36 & 3.40 & 14.28 & 53.59 & 63.63 & 64.15 & 63.40 & 63.96 & 56.13 & 49.25 \\
\hline 1.39 & 2.13 & 19.05 & 35.91 & 42.73 & 47.88 & 49.92 & \begin{tabular}{|l|}
51.67 \\
\end{tabular} & 50.82 & 49.50 \\
\hline 1.43 & 2.37 & 12.63 & 32.59 & 36.58 & 37.80 & 37.20 & 38.09 & 37.23 & 36.40 \\
\hline 0.66 & 0.65 & 0.90 & 1.10 & 1.25 & 1.39 & 1.57 & 1.93 & 2.27 & 2.54 \\
\hline 0.93 & 1.03 & 1.65 & 21.00 & 27.05 & 26.35 & 26.20 & 25.87 & 18.90 & 12.85 \\
\hline & & & & & & & & & \\
\hline & & & & & & & & & \\
\hline 5 & 6 & 7 & 8 & & 10 & 11 & 12 & 12 & \\
\hline 146.16 & 154.64 & 185.45 & 410.64 & 576.64 & 614.62 & 622.74 & 624.73 & 630.77 & 627.12 \\
\hline 86.66 & 114.42 & 195.85 & 391.96 & 564.45 & 603.68 & 619.03 & 633.96 & 620.14 & 621.71 \\
\hline 138.88 & 137.71 & 137.18 & 138.83 & 143.47 & 147.57 & 152.07 & 156.06 & 158.90 & 161.64 \\
\hline 110.00 & 111.44 & 130.77 & 206.16 & 254.77 & 267.14 & 273.49 & 276.27 & 266.90 & 268.90 \\
\hline 163.82 & 165.71 & 183.44 & 302.73 & 352.75 & 368.09 & 374.47 & 372.50 & 355.80 & 364.33 \\
\hline 90.17 & 108.59 & 163.72 & 316.99 & 394.67 & 411.80 & 422.48 & 424.80 & 396.21 & 408.11 \\
\hline 162.02 & 162.26 & 158.27 & 168.61 & 184.41 & 191.05 & 196.10 & 195.46 & 188.68 & 186.93 \\
\hline 90.73 & 91.73 & 106.53 & 144.80 & 161.80 & 166.33 & 174.99 & 176.97 & 164.65 & 164.92 \\
\hline 481.70 & 518.20 & 649.25 & 1147.59 & 1539.34 & 1633.01 & 1667.32 & 1691.01 & 1676.70 & 1679.37 \\
\hline 506.75 & 528.28 & 611.96 & 933.12 & 1093.64 & 1137.28 & 1168.04 & 1169.73 & 1105.34 & 1124.29 \\
\hline 196.66 & 225.85 & 326.62 & 598.13 & 819.22 & 870.82 & 892.52 & 910.22 & 887.03 & 890.61 \\
\hline 180.90 & 200.32 & 270.25 & 461.78 & 556.47 & 578.14 & 597.47 & 601.77 & 560.86 & 573.03 \\
\hline 285.04 & 292.34 & 322.63 & 549.47 & 720.12 & 762.19 & 774.81 & 780.79 & 789.67 & 788.76 \\
\hline 325.85 & 327.97 & 341.71 & 471.33 & 537.17 & 559.14 & 570.57 & 567.96 & 544.48 & 551.26 \\
\hline & & & & & & & & & \\
\hline & & & & & & & & & \\
\hline & & & & & & & & & \\
\hline 5 & 6 & 7 & 8 & 9 & 10 & 11 & 12 & 13 & 14 \\
\hline 0.00 & 1.39 & 1.70 & 2.28 & 2.49 & 2.11 & 2.05 & 0.00 & 2.08 & 2.19 \\
\hline 0.88 & 1.57 & 1.49 & 2.01 & 1.65 & 1.59 & 1.49 & 1.45 & 1.20 & 1.22 \\
\hline 1.51 & 1.53 & 1.53 & 1.53 & 1.57 & 1.55 & 1.56 & 1.56 & 1.57 & 1.57 \\
\hline 0.78 & 0.83 & 0.78 & 1.08 & 1.09 & 0.97 & 0.99 & 0.94 & 1.07 & 0.89 \\
\hline 1.04 & 1.14 & 0.89 & 1.31 & 1.28 & 1.48 & 1.25 & 1.43 & 2.85 & 1.42 \\
\hline 0.72 & 0.99 & 1.26 & 1.05 & 1.17 & 1.12 & 1.07 & 0.96 & 0.93 & 0.85 \\
\hline 1.00 & 1.07 & 1.04 & 0.81 & 1.27 & 0.86 & 0.95 & 1.10 & 0.96 & 0.94 \\
\hline 0.74 & 0.96 & 0.73 & 0.93 & 0.84 & 0.76 & 0.76 & 0.91 & 0.68 & 0.67 \\
\hline 3.17 & 5.33 & 5.50 & 6.91 & 6.80 & 6.21 & 6.10 & 3.95 & 5.92 & 5.87 \\
\hline 3.50 & 4.16 & 3.92 & 4.10 & 4.56 & 4.23 & 4.02 & 4.41 & 5.42 & 3.88 \\
\hline
\end{tabular}


CHART DATA

\begin{tabular}{|c|c|c|c|c|c|c|c|c|c|}
\hline 1.66 & 2.41 & 2.27 & 3.09 & 2.74 & 2.56 & 2.48 & 2.39 & 2.27 & 2.11 \\
\hline 1.46 & 1.95 & 1.99 & 1.98 & 2.01 & 1.88 & 1.82 & 1.88 & 1.61 & 1.52 \\
\hline 1.51 & 2.93 & 3.23 & 3.82 & 4.06 & 3.66 & 3.61 & 1.56 & 3.65 & 3.76 \\
\hline 2.04 & 2.21 & 1.93 & 2.13 & 2.55 & 2.34 & 2.20 & 2.53 & 3.81 & 2.36 \\
\hline & & & & & & & & & \\
\hline & & & & & & & & & \\
\hline & & & & & & & & & \\
\hline 5 & 6 & 7 & 8 & 9 & 10 & 11 & 12 & 13 & 14 \\
\hline 144.97 & 148.64 & 177.91 & 385.71 & 550.37 & 585.87 & 593.89 & 597.55 & 602.92 & 598.74 \\
\hline 85.89 & 112.90 & 181.18 & 365.05 & 531.49 & 566.41 & 580.82 & 594.32 & 580.84 & 583.08 \\
\hline 138.36 & 137.20 & 136.48 & 137.97 & 142.50 & 146.49 & 150.85 & 154.56 & 157.14 & 159.67 \\
\hline 109.57 & 111.01 & 127.68 & 199.82 & 247.93 & 259.55 & 265.19 & 267.77 & 258.71 & 261.26 \\
\hline 163.73 & 165.61 & 183.21 & 300.45 & 350.50 & 365.80 & 372.16 & 370.38 & 354.23 & 363.23 \\
\hline 89.50 & 106.93 & 153.17 & 289.31 & 363.60 & 379.47 & 390.72 & 392.49 & 364.07 & 376.65 \\
\hline 161.92 & 162.14 & 158.15 & 166.31 & 180.66 & 187.48 & 192.59 & 191.99 & 186.06 & 185.18 \\
\hline 89.97 & 91.05 & 104.54 & 140.18 & 156.46 & 161.04 & 169.72 & 171.72 & 159.90 & 160.38 \\
\hline 478.79 & 509.75 & 623.26 & 1088.55 & 1472.29 & 1558.33 & 1590.75 & 1614.20 & 1599.61 & 1602.75 \\
\hline 505.12 & 525.73 & 599.06 & 896.26 & 1051.22 & 1093.79 & 1125.19 & 1126.58 & 1064.26 & 1085.44 \\
\hline 195.46 & 223.91 & 308.86 & 564.87 & 779.42 & 825.97 & 846.00 & 862.09 & 839.55 & 844.34 \\
\hline 179.47 & 197.98 & 257.70 & 429.50 & 520.06 & 540.51 & 560.45 & 564.20 & 523.97 & 537.03 \\
\hline 283.33 & 285.84 & 314.39 & 523.68 & 692.87 & 732.36 & 744.74 & 752.11 & 760.06 & 758.41 \\
\hline 325.64 & 327.74 & 341.36 & 466.76 & 531.17 & 553.28 & 564.75 & 562.38 & 540.29 & 548.41 \\
\hline & & & & & & & & & \\
\hline & & & & & & & & & \\
\hline & & & & & & & & & \\
\hline & & & & & & & & & \\
\hline & & & & & & & & & \\
\hline & & & & & & & & & \\
\hline & & & & & & & & & \\
\hline & & & & & & & & & \\
\hline & & & & & & & & & \\
\hline & & & & & & & & & \\
\hline & & & & & & & & & \\
\hline & & & & & & & & & \\
\hline & & & & & & & & & \\
\hline & & & & & & & & & \\
\hline & & & & & & & & & \\
\hline & & & & & & & & & \\
\hline & & & & & & & & & \\
\hline & & & & & & & & & \\
\hline & & & & & & & & & \\
\hline & & & & & & & & & \\
\hline & & & & & & & & & \\
\hline & & & & & & & & & \\
\hline & & & & & & & & & \\
\hline & & & & & & & & & \\
\hline & & & & & & & & & \\
\hline & & & & & & & & & \\
\hline & & & & & & & & & \\
\hline & & & & & & & & & \\
\hline
\end{tabular}


CHART DATA

\begin{tabular}{|l|l|l|l|l|l|l|l|l|}
\hline & & & & & & & & \\
\hline
\end{tabular}

Page 25 
CHART DATA

\begin{tabular}{|l|l|l|l|l|l|l|l|l|}
\hline & & & & & & & & \\
\hline
\end{tabular}

Page 26 
CHART DATA

\begin{tabular}{|l|l|l|l|l|l|l|l|l|}
\hline & & & & & & & & \\
\hline
\end{tabular}

Page 27 
CHART DATA

\begin{tabular}{|l|l|l|l|l|l|l|l|l|}
\hline & & & & & & & & \\
\hline
\end{tabular}

Page 28 
CHART DATA

\begin{tabular}{|l|l|l|l|l|l|l|l|l|}
\hline & & & & & & & & \\
\hline
\end{tabular}

Page 29 
CHART DATA

\begin{tabular}{|l|l|l|l|l|l|l|l|l|}
\hline & & & & & & & & \\
\hline & & & & & & & & \\
\hline
\end{tabular}

Page 30 
CHART DATA

\begin{tabular}{|l|l|l|l|l|l|l|l|l|}
\hline & & & & & & & & \\
\hline & & & & & & & & \\
\hline
\end{tabular}

Page 31 
CHART DATA

\begin{tabular}{|l|l|l|l|l|l|l|l|l|}
\hline & & & & & & & & \\
\hline & & & & & & & & \\
\hline
\end{tabular}

Page 32 
CHART DATA

\begin{tabular}{|l|l|l|l|l|l|l|l|l|}
\hline & & & & & & & & \\
\hline
\end{tabular}

Page 33 
CHART DATA

\begin{tabular}{|l|l|l|l|l|l|l|l|l|}
\hline & & & & & & & & \\
\hline & & & & & & & & \\
\hline
\end{tabular}

Page 34 
CHART DATA

\begin{tabular}{|l|l|l|l|l|l|l|l|l|}
\hline & & & & & & & & \\
\hline & & & & & & & & \\
\hline
\end{tabular}

Page 35 
CHART DATA

\begin{tabular}{|l|l|l|l|l|l|l|l|l|}
\hline & & & & & & & & \\
\hline & & & & & & & & \\
\hline
\end{tabular}

Page 36 
CHART DATA

\begin{tabular}{|l|l|l|l|l|l|l|l|l|}
\hline & & & & & & & & \\
\hline & & & & & & & & \\
\hline
\end{tabular}

Page 37 
CHART DATA

\begin{tabular}{|l|l|l|l|l|l|l|l|l|}
\hline & & & & & & & & \\
\hline & & & & & & & & \\
\hline
\end{tabular}

Page 38 
CHART DATA

\begin{tabular}{|l|l|l|l|l|l|l|l|l|}
\hline & & & & & & & & \\
\hline
\end{tabular}

Page 39 
CHART DATA

\begin{tabular}{|l|l|l|l|l|l|l|l|l|}
\hline & & & & & & & & \\
\hline
\end{tabular}

Page 40 
CHART DATA

\begin{tabular}{|l|l|l|l|l|l|l|l|l|}
\hline & & & & & & & & \\
\hline & & & & & & & & \\
\hline
\end{tabular}

Page 41 
CHART DATA

\begin{tabular}{|l|l|l|l|l|l|l|l|l|}
\hline & & & & & & & & \\
\hline
\end{tabular}

Page 42 
CHART DATA

\begin{tabular}{|l|l|l|l|l|l|l|l|l|}
\hline & & & & & & & & \\
\hline & & & & & & & & \\
\hline
\end{tabular}

Page 43 
CHART DATA

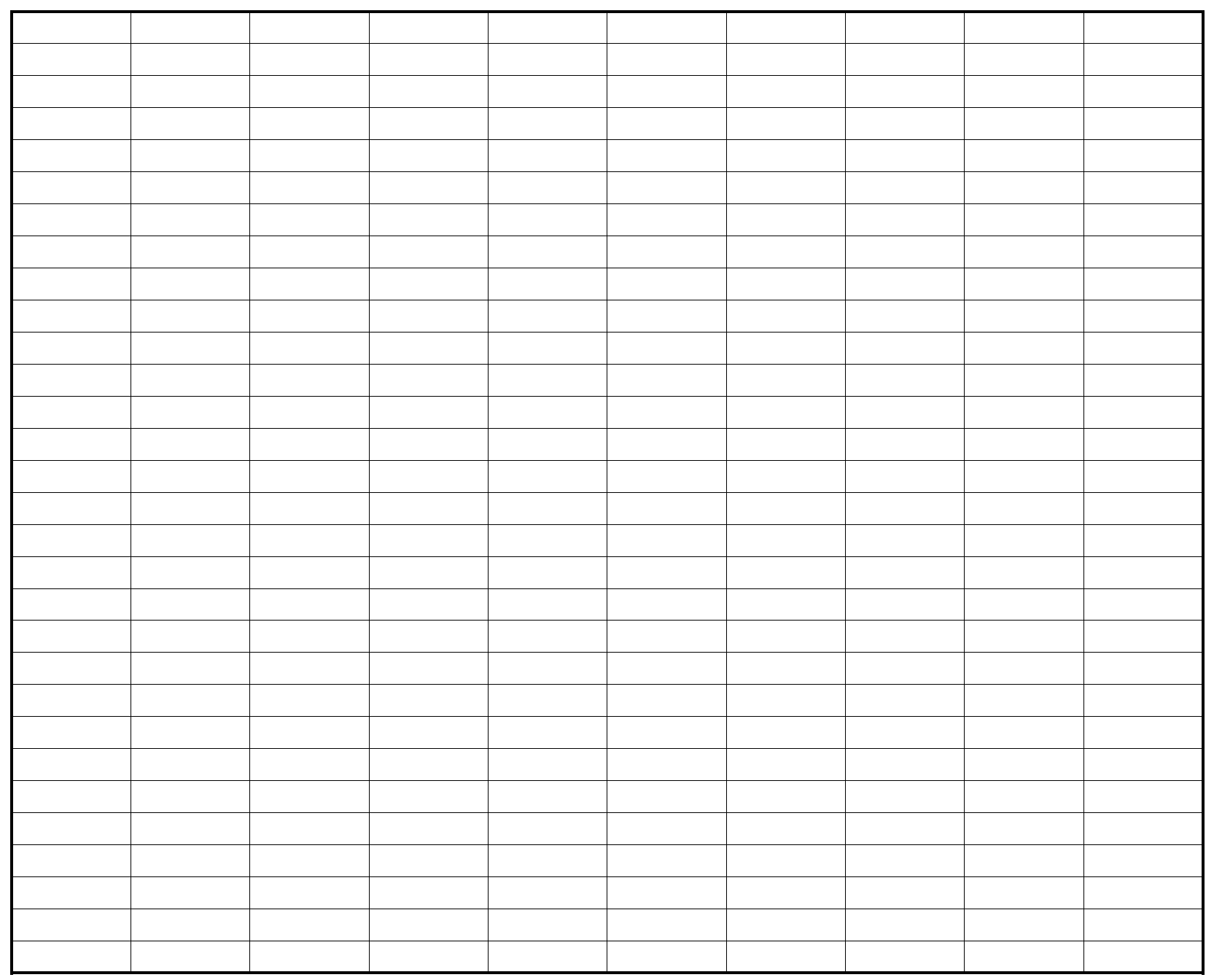




\begin{tabular}{|c|c|c|c|c|c|c|c|c|}
\hline 15 & 16 & 17 & 18 & 19 & 20 & 21 & 22 & 23 \\
\hline 0.00 & 0.00 & 0.00 & 0.00 & 0.00 & 0.00 & 0.00 & 0.00 & 0.00 \\
\hline 41.52 & 37.45 & 2.17 & 1.44 & 1.35 & 1.37 & 1.36 & 1.06 & 0.78 \\
\hline 2.80 & 2.96 & 2.94 & 2.93 & 2.92 & 2.91 & 2.90 & 2.89 & 2.81 \\
\hline 10.52 & 9.60 & 1.56 & 1.07 & 0.96 & 0.92 & 0.96 & 0.87 & 0.62 \\
\hline 4.08 & 3.71 & 0.50 & 0.55 & 0.48 & 0.50 & 0.55 & 0.45 & 0.53 \\
\hline 31.16 & 26.02 & 2.04 & 0.71 & 0.75 & 0.73 & 0.74 & 0.73 & 0.72 \\
\hline 7.40 & 7.40 & 0.70 & 0.40 & 0.45 & 0.45 & 0.60 & 0.40 & 0.45 \\
\hline 4.55 & 4.61 & 0.98 & 0.71 & 0.75 & 0.73 & 0.74 & 0.71 & 0.72 \\
\hline 54.84 & 50.02 & 6.67 & 5.44 & 5.23 & 5.20 & 5.21 & 4.82 & 4.21 \\
\hline 47.19 & 41.74 & 4.23 & 2.37 & 2.42 & 2.42 & 2.63 & 2.29 & 2.41 \\
\hline 52.04 & 47.06 & 3.72 & 2.51 & 2.31 & 2.29 & 2.31 & 1.93 & 1.40 \\
\hline 35.71 & 30.63 & 3.03 & 1.42 & 1.50 & 1.47 & 1.48 & 1.44 & 1.44 \\
\hline 2.80 & 2.96 & 2.94 & 2.93 & 2.92 & 2.91 & 2.90 & 2.89 & 2.81 \\
\hline 11.48 & 11.11 & 1.20 & 0.95 & 0.93 & 0.95 & 1.15 & 0.85 & 0.98 \\
\hline & & & & & & & & \\
\hline & & & & & & & & \\
\hline 15 & 16 & 17 & 18 & 19 & 20 & 21 & 22 & 23 \\
\hline 610.93 & 565.28 & 350.30 & 280.92 & 249.01 & 225.08 & 191.11 & 172.54 & 159.39 \\
\hline 604.07 & 555.87 & 302.29 & 215.40 & 187.60 & 163.55 & 123.17 & 107.16 & 96.42 \\
\hline 164.19 & 166.19 & 167.38 & 168.03 & 168.68 & 169.07 & 169.32 & 169.34 & 169.02 \\
\hline 267.59 & 258.40 & 200.00 & 173.22 & 150.49 & 145.18 & 136.81 & 129.63 & 122.78 \\
\hline 368.80 & 360.93 & 304.99 & 271.71 & 222.31 & 218.18 & 204.87 & 193.22 & 184.28 \\
\hline 405.09 & 382.98 & 237.61 & 186.59 & 160.87 & 150.57 & 124.13 & 115.67 & 106.49 \\
\hline 192.97 & 188.22 & 176.86 & 172.06 & 174.65 & 171.55 & 166.45 & 163.96 & 161.82 \\
\hline 166.19 & 164.84 & 138.18 & 131.81 & 123.62 & 114.77 & 106.30 & 104.37 & 98.96 \\
\hline 1646.76 & 1545.75 & 1019.97 & 837.58 & 755.79 & 702.89 & 620.41 & 578.67 & 547.61 \\
\hline 1133.04 & 1096.96 & 857.64 & 762.18 & 681.44 & 655.07 & 601.75 & 577.22 & 551.55 \\
\hline 871.65 & 814.28 & 502.29 & 388.62 & 338.10 & 308.73 & 259.98 & 236.79 & 219.21 \\
\hline 571.27 & 547.82 & 375.79 & 318.40 & 284.49 & 265.34 & 230.43 & 220.04 & 205.45 \\
\hline 775.11 & 731.48 & 517.68 & 448.96 & 417.70 & 394.16 & 360.43 & 341.88 & 328.41 \\
\hline 561.77 & 549.15 & 481.85 & 443.78 & 396.96 & 389.73 & 371.32 & 357.17 & 346.09 \\
\hline & & & & & & & & \\
\hline & & & & & & & & \\
\hline 15 & 16 & 17 & 18 & 19 & 20 & 21 & 22 & 23 \\
\hline 2.02 & 0.00 & 0.00 & 0.00 & 0.00 & 0.00 & 1.48 & 0.00 & 0.00 \\
\hline 1.17 & 1.03 & 0.96 & 0.87 & 0.85 & 0.85 & 0.84 & 0.82 & 0.82 \\
\hline 1.56 & 1.57 & 1.56 & 1.53 & 1.51 & 1.49 & 1.48 & 1.45 & 1.44 \\
\hline 0.93 & 0.89 & 0.78 & 0.80 & 0.75 & 0.79 & 0.78 & 0.75 & 0.78 \\
\hline 1.70 & 1.44 & 1.20 & 1.13 & 1.03 & 1.16 & 1.15 & 1.16 & 1.22 \\
\hline 0.77 & 0.88 & 0.69 & 0.70 & 0.65 & 0.66 & 0.66 & 0.71 & 0.66 \\
\hline 1.00 & 0.85 & 0.68 & 1.18 & 1.10 & 0.85 & 0.94 & 0.76 & 0.87 \\
\hline 0.69 & 0.65 & 0.67 & 0.65 & 0.68 & 0.58 & 0.72 & 0.57 & 0.66 \\
\hline 5.68 & 3.49 & 3.29 & 3.19 & 3.12 & 3.13 & 4.58 & 3.02 & 3.03 \\
\hline 4.16 & 3.81 & 3.24 & 3.66 & 3.45 & 3.26 & 3.48 & 3.19 & 3.40 \\
\hline
\end{tabular}


CHART DATA

\begin{tabular}{|c|c|c|c|c|c|c|c|c|}
\hline 2.10 & 1.92 & 1.74 & 1.67 & 1.61 & 1.64 & 1.63 & 1.56 & 1.59 \\
\hline 1.46 & 1.52 & 1.36 & 1.35 & 1.33 & 1.24 & 1.38 & 1.27 & 1.31 \\
\hline 3.58 & 1.57 & 1.56 & 1.53 & 1.51 & 1.49 & 2.95 & 1.45 & 1.44 \\
\hline 2.70 & 2.29 & 1.88 & 2.31 & 2.13 & 2.01 & 2.09 & 1.92 & 2.09 \\
\hline & & & & & & & & \\
\hline & & & & & & & & \\
\hline & & & & & & & & \\
\hline 15 & 16 & 17 & 18 & 19 & 20 & 21 & 22 & 23 \\
\hline 580.95 & 540.24 & 348.38 & 279.16 & 247.25 & 223.28 & 189.36 & 171.37 & 158.86 \\
\hline 563.07 & 518.89 & 300.13 & 213.97 & 186.26 & 162.20 & 121.82 & 106.11 & 95.66 \\
\hline 162.02 & 163.89 & 165.10 & 165.76 & 166.42 & 166.82 & 167.07 & 167.09 & 166.84 \\
\hline 260.14 & 251.57 & 198.88 & 172.46 & 149.81 & 144.52 & 136.13 & 129.01 & 122.34 \\
\hline 367.97 & 360.22 & 304.89 & 271.60 & 222.21 & 218.08 & 204.76 & 193.13 & 184.17 \\
\hline 374.09 & 357.10 & 235.58 & 185.89 & 160.12 & 149.84 & 123.39 & 114.95 & 105.78 \\
\hline 191.23 & 186.48 & 176.69 & 171.98 & 174.54 & 171.45 & 166.31 & 163.87 & 161.71 \\
\hline 161.64 & 160.23 & 137.20 & 131.10 & 122.87 & 114.03 & 105.56 & 103.65 & 98.24 \\
\hline 1566.18 & 1474.60 & 1012.49 & 831.35 & 749.74 & 696.81 & 614.38 & 573.59 & 543.69 \\
\hline 1094.93 & 1064.02 & 854.36 & 760.57 & 679.75 & 653.40 & 600.01 & 575.60 & 549.90 \\
\hline 823.22 & 770.46 & 499.01 & 386.43 & 336.07 & 306.72 & 257.95 & 235.12 & 218.00 \\
\hline 535.73 & 517.32 & 372.78 & 316.99 & 282.99 & 263.87 & 228.95 & 218.60 & 204.02 \\
\hline 742.97 & 704.13 & 513.47 & 444.92 & 413.67 & 390.09 & 356.43 & 338.47 & 325.70 \\
\hline 559.20 & 546.70 & 481.58 & 443.58 & 396.76 & 389.52 & 371.06 & 357.00 & 345.88 \\
\hline & & & & & & & & \\
\hline & & & & & & & & \\
\hline & & & & & & & & \\
\hline & & & & & & & & \\
\hline & & & & & & & & \\
\hline & & & & & & & & \\
\hline & & & & & & & & \\
\hline & & & & & & & & \\
\hline & & & & & & & & \\
\hline & & & & & & & & \\
\hline & & & & & & & & \\
\hline & & & & & & & & \\
\hline & & & & & & & & \\
\hline & & & & & & & & \\
\hline & & & & & & & & \\
\hline & & & & & & & & \\
\hline & & & & & & & & \\
\hline & & & & & & & & \\
\hline & & & & & & & & \\
\hline & & & & & & & & \\
\hline & & & & & & & & \\
\hline & & & & & & & & \\
\hline & & & & & & & & \\
\hline & & & & & & & & \\
\hline & & & & & & & & \\
\hline & & & & & & & & \\
\hline & & & & & & & & \\
\hline & & & & & & & & \\
\hline
\end{tabular}


CHART DATA

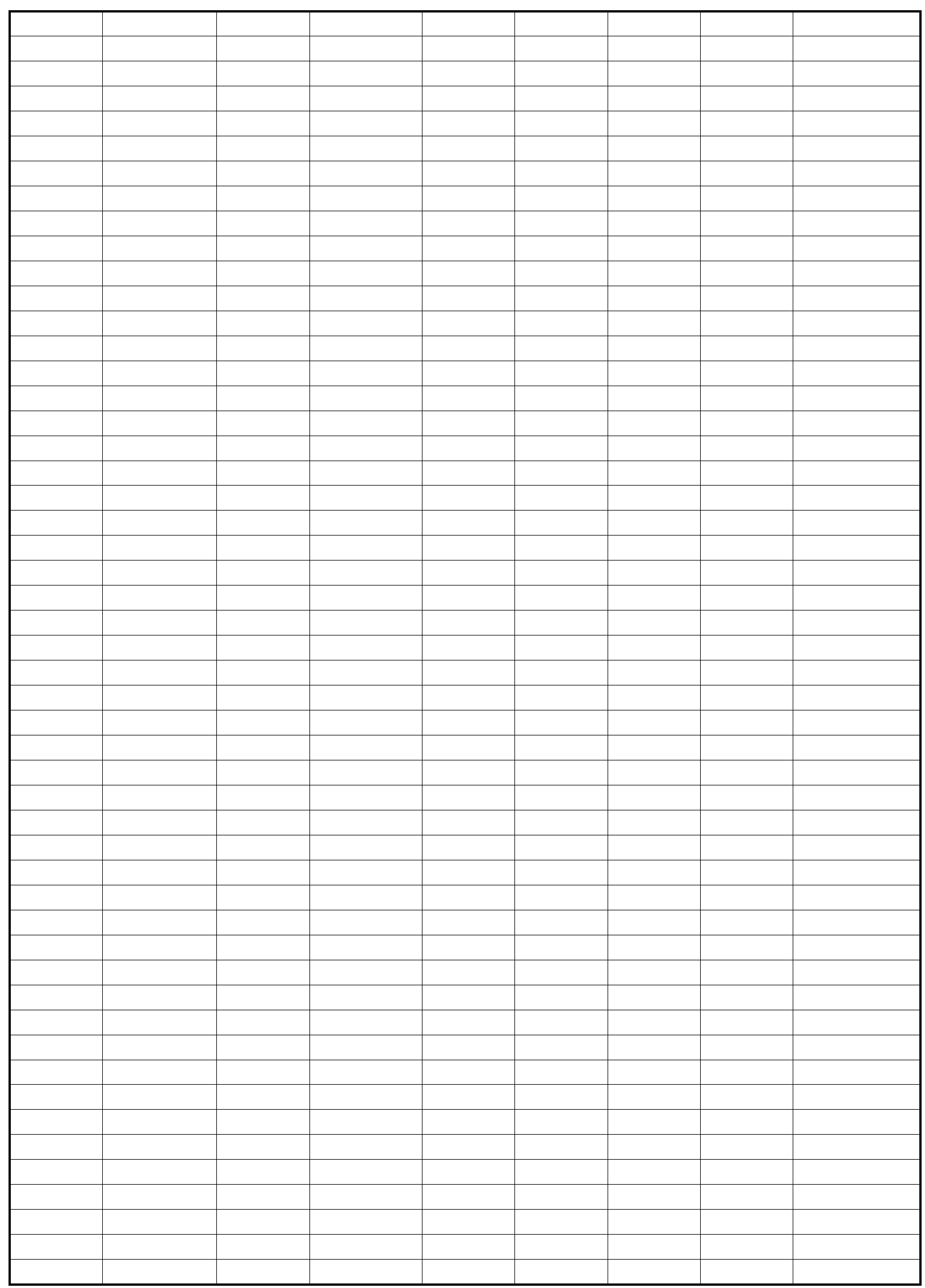

Page 47 
CHART DATA

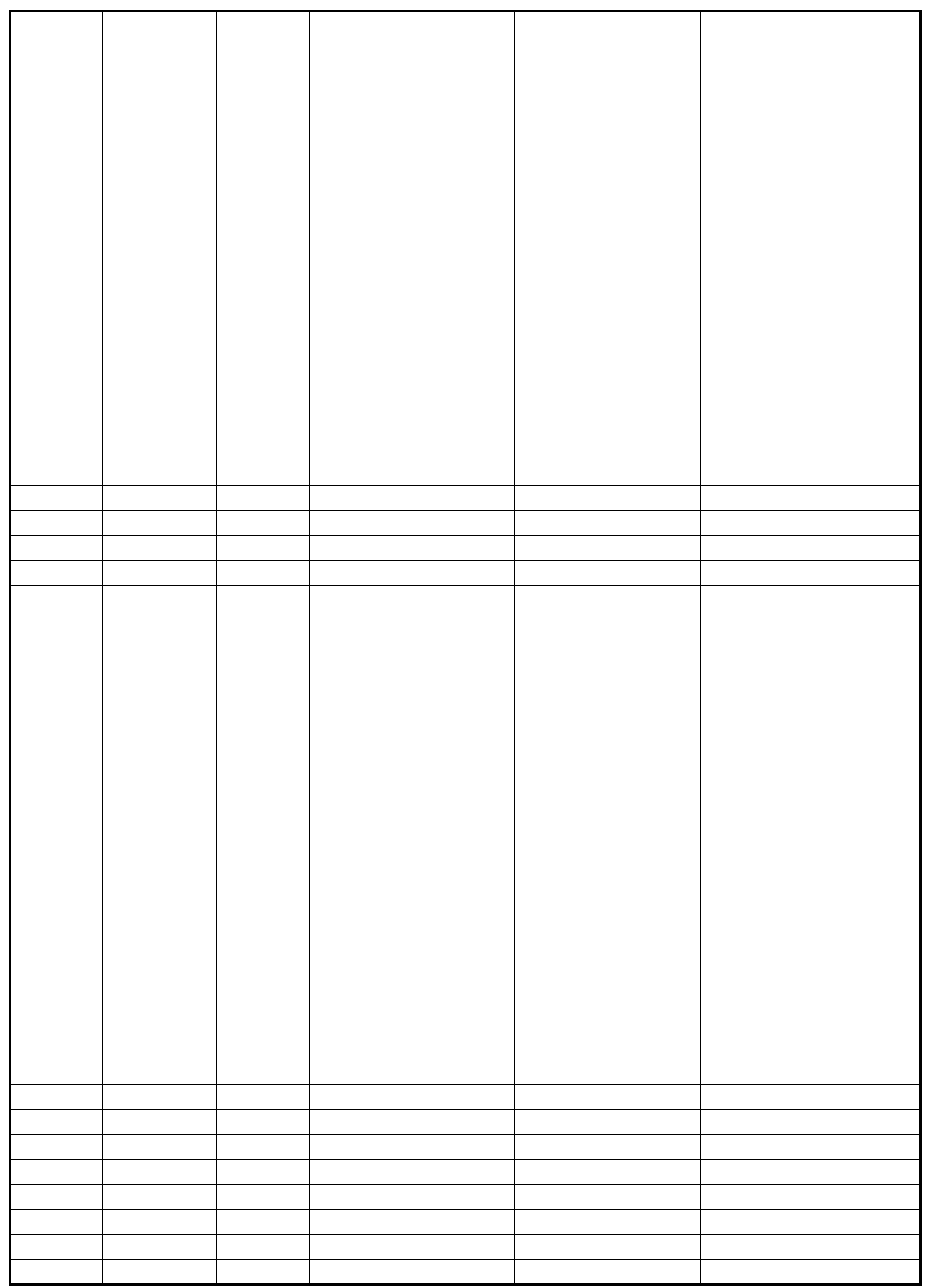

Page 48 
CHART DATA

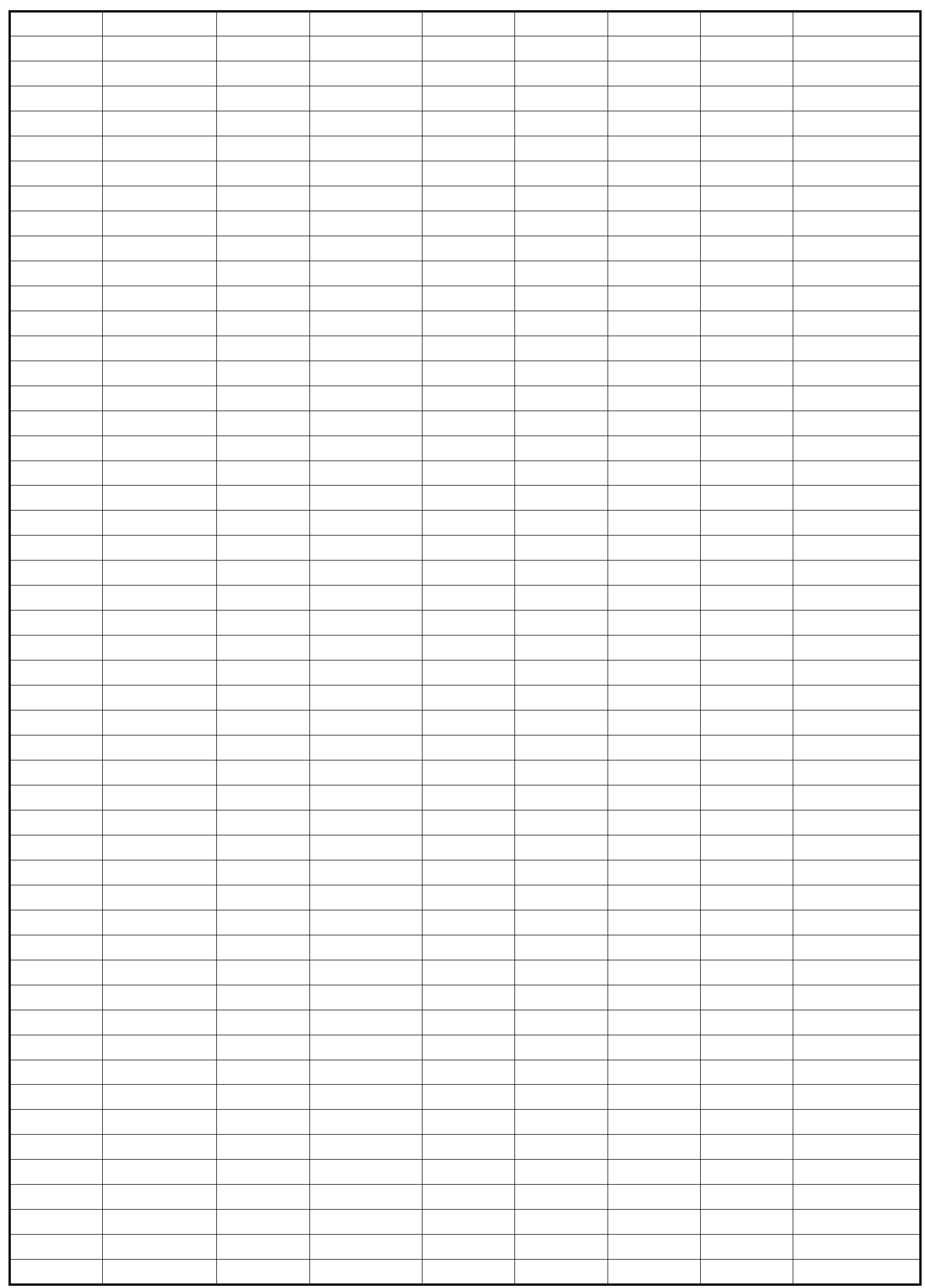

Page 49 
CHART DATA

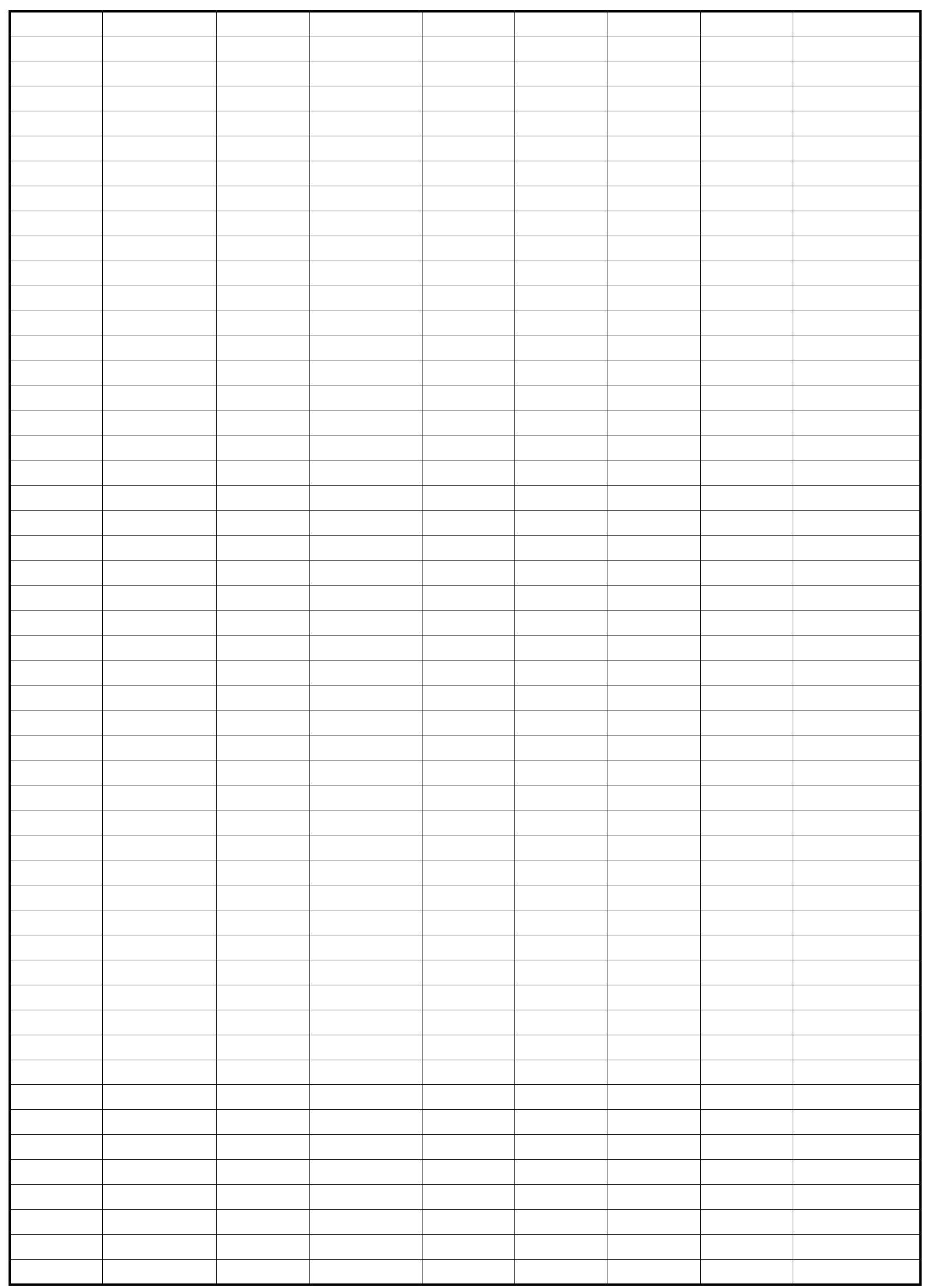

Page 50 
CHART DATA

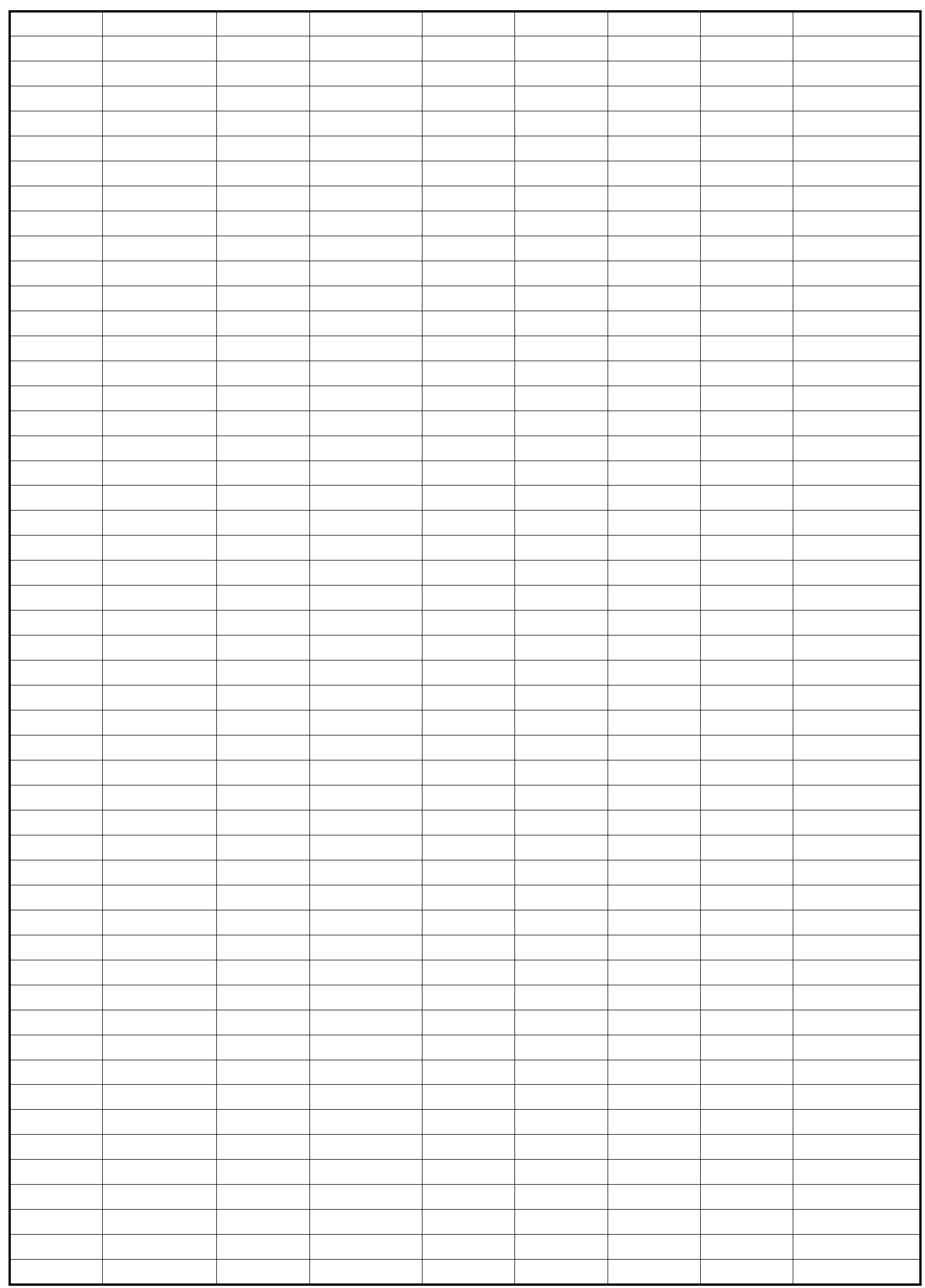

Page 51 
CHART DATA

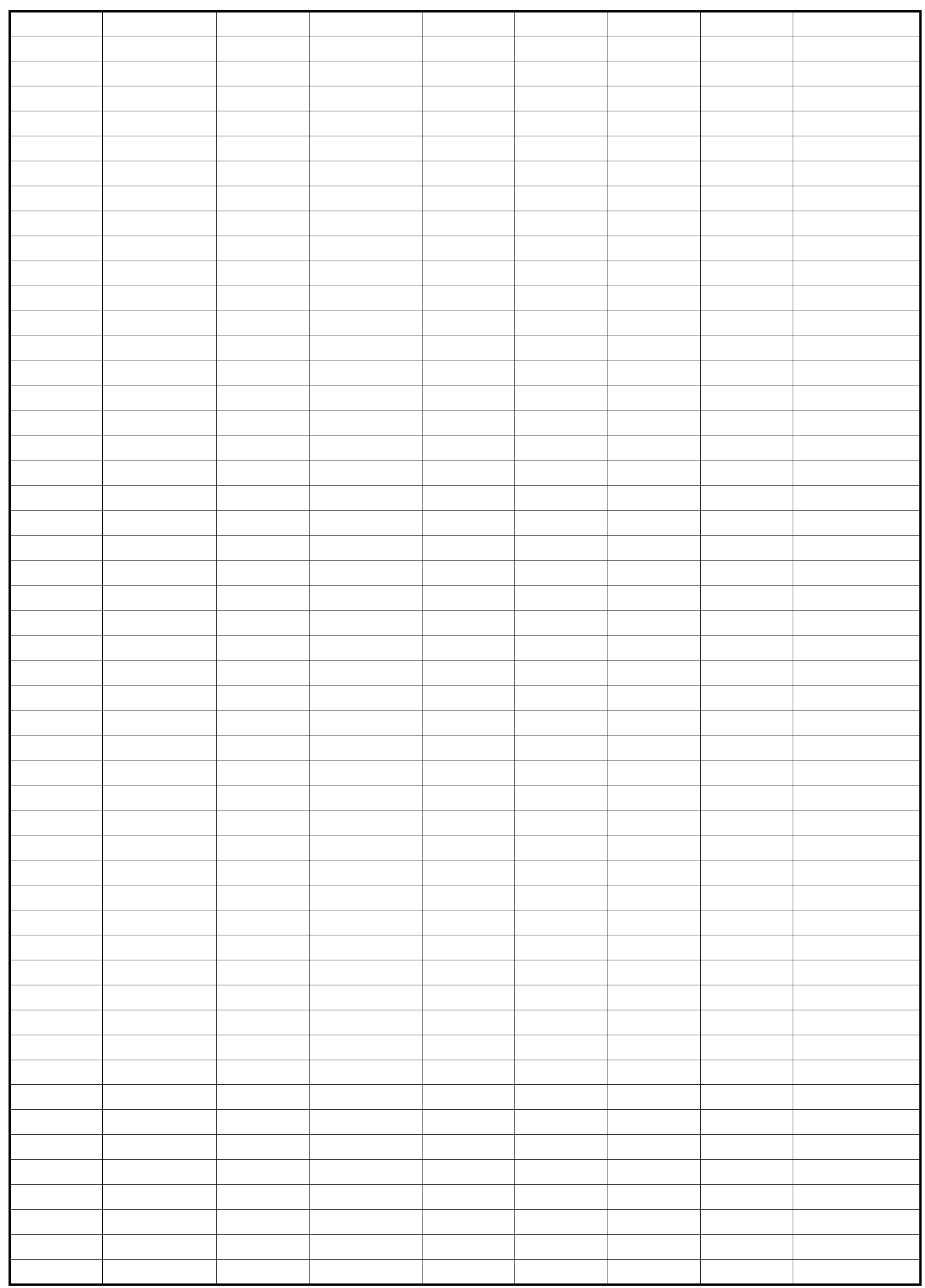

Page 52 
CHART DATA

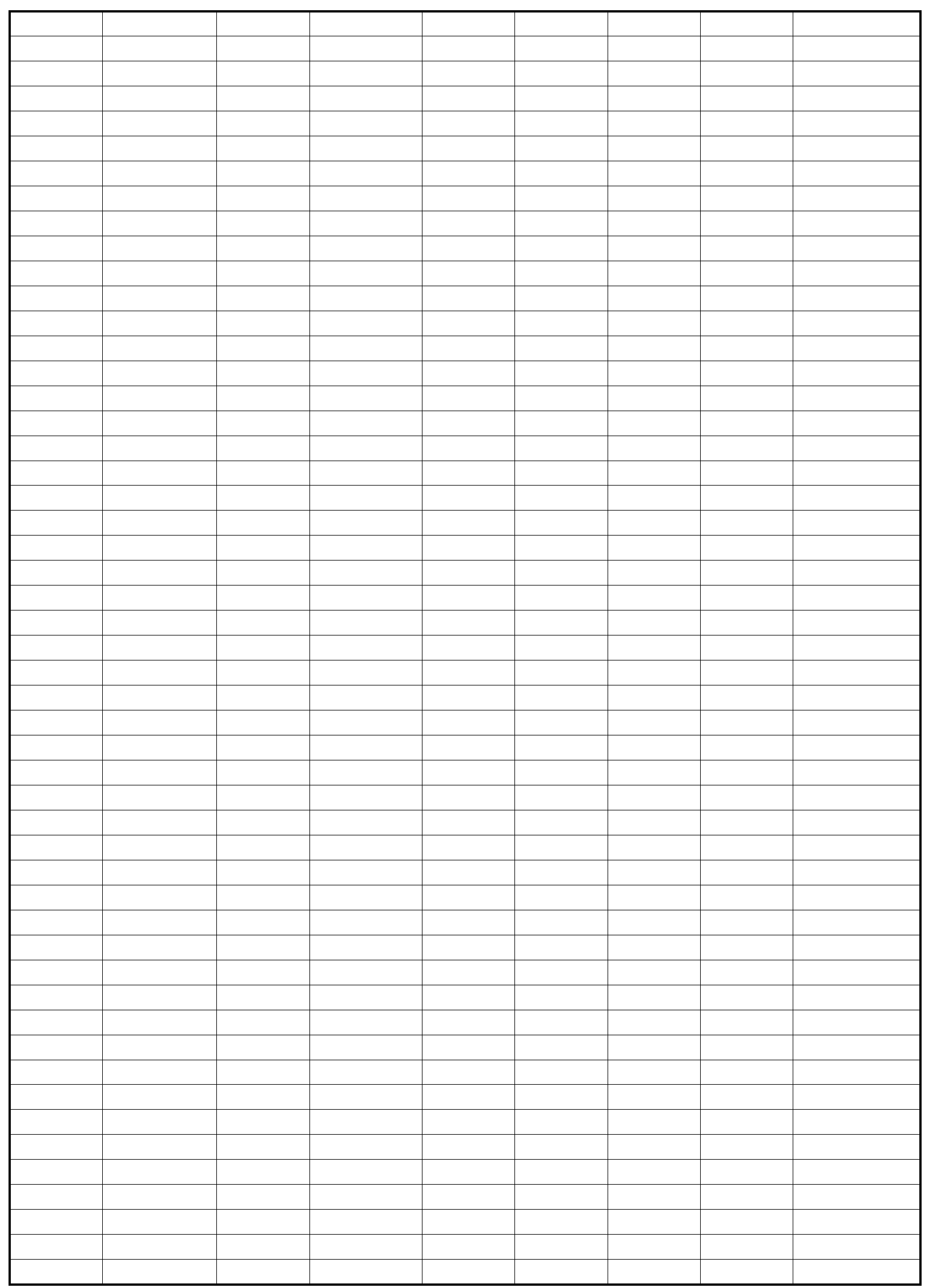

Page 53 
CHART DATA

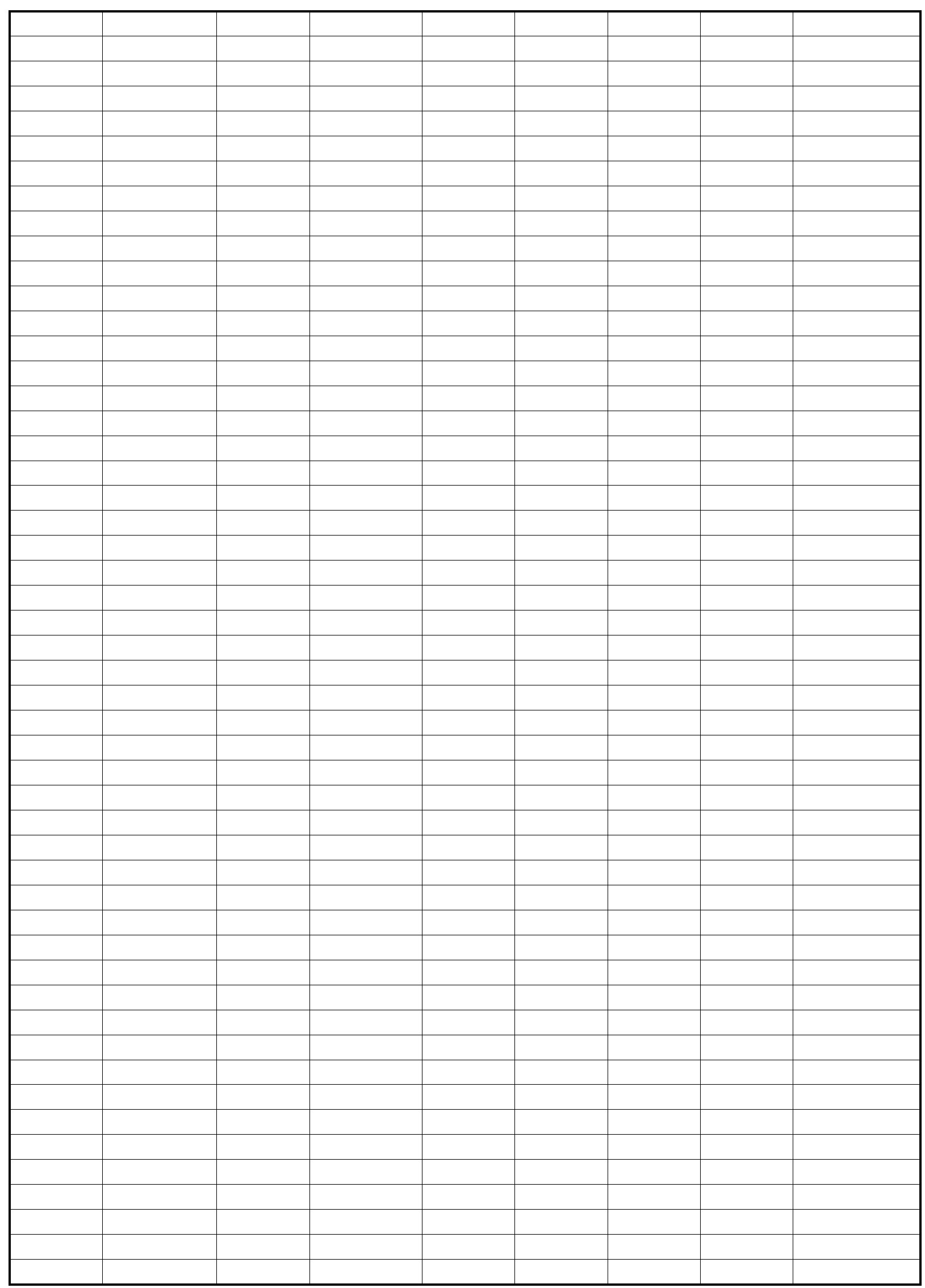

Page 54 
CHART DATA

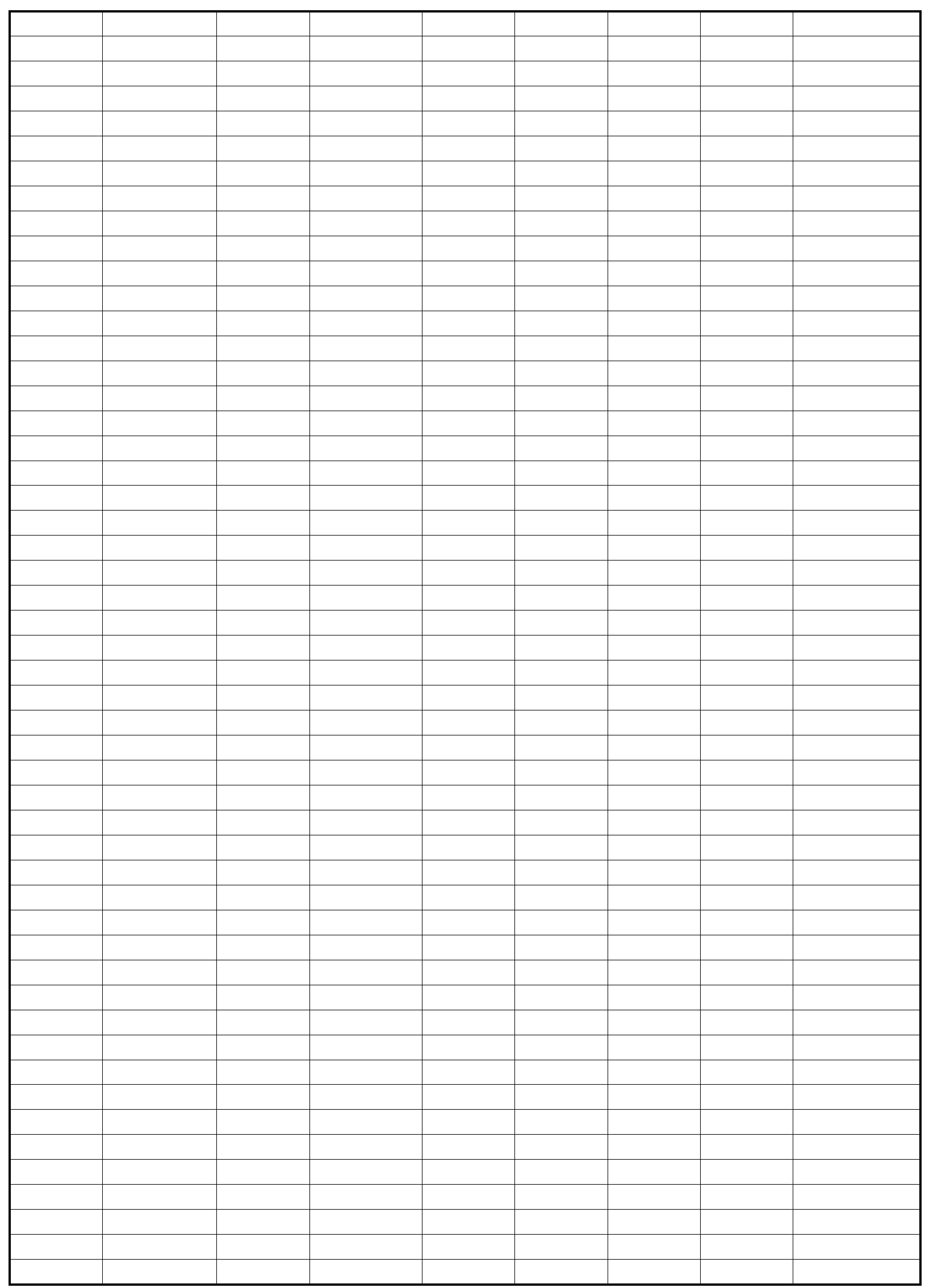

Page 55 
CHART DATA

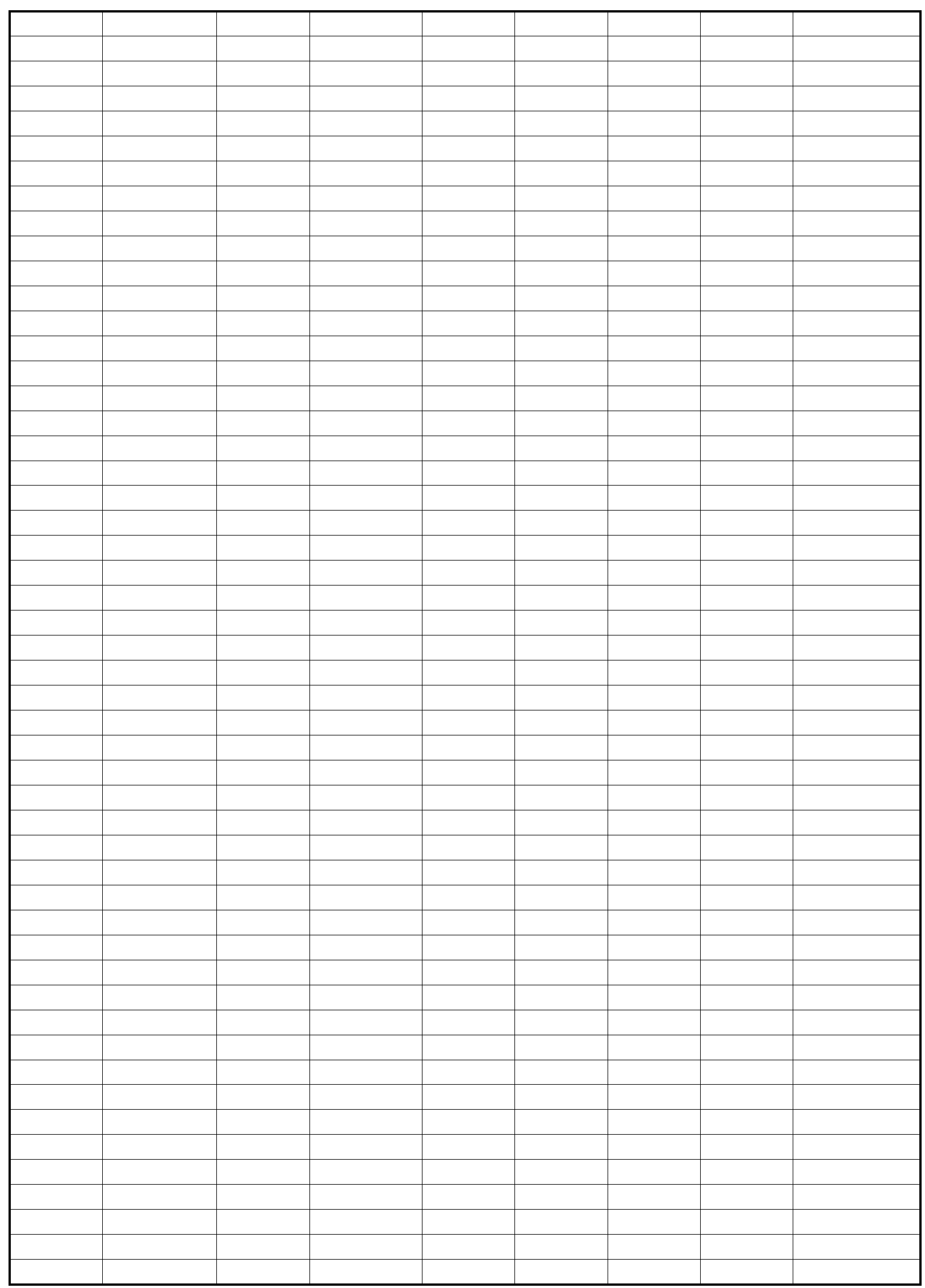

Page 56 
CHART DATA

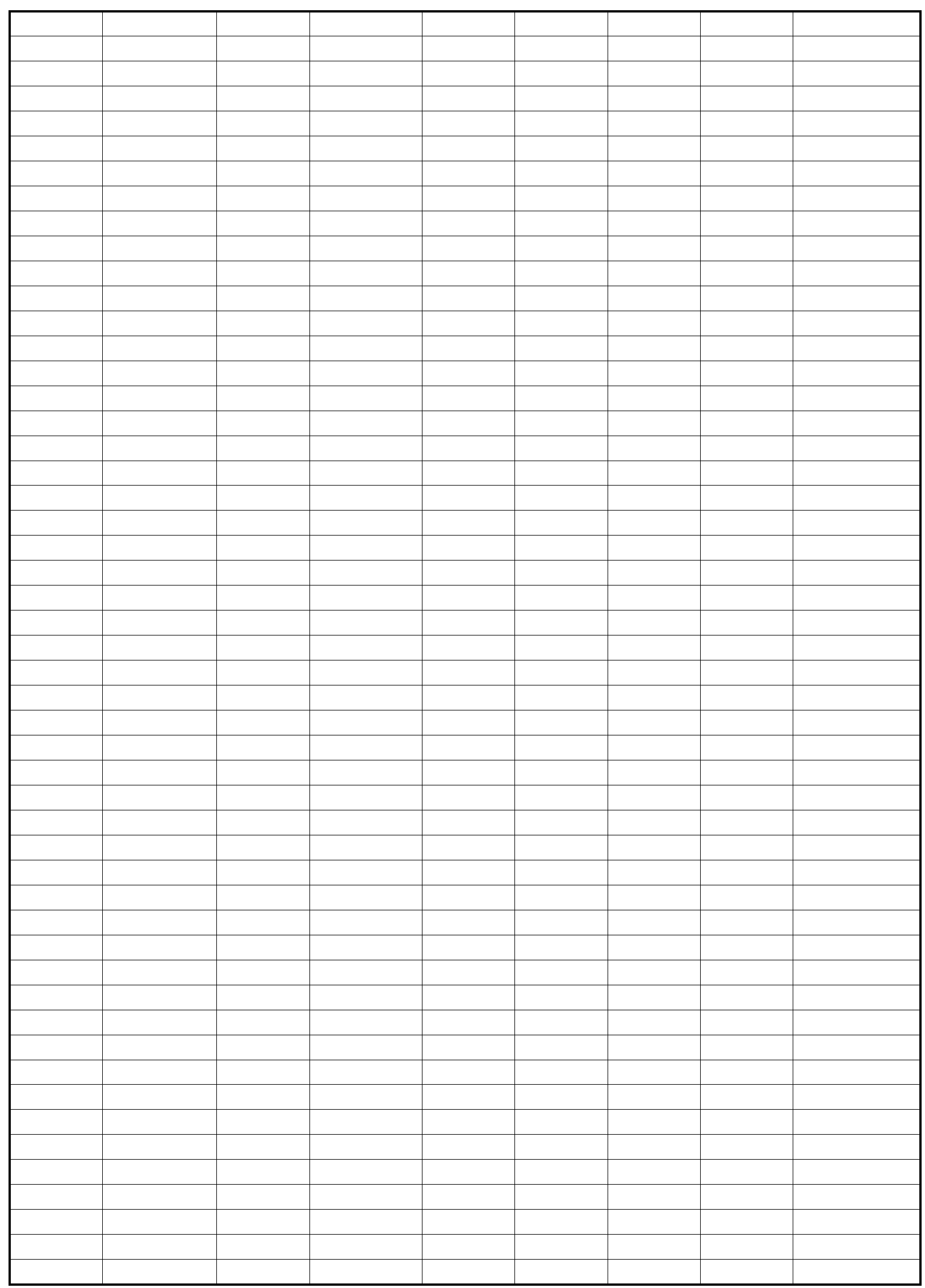

Page 57 
CHART DATA

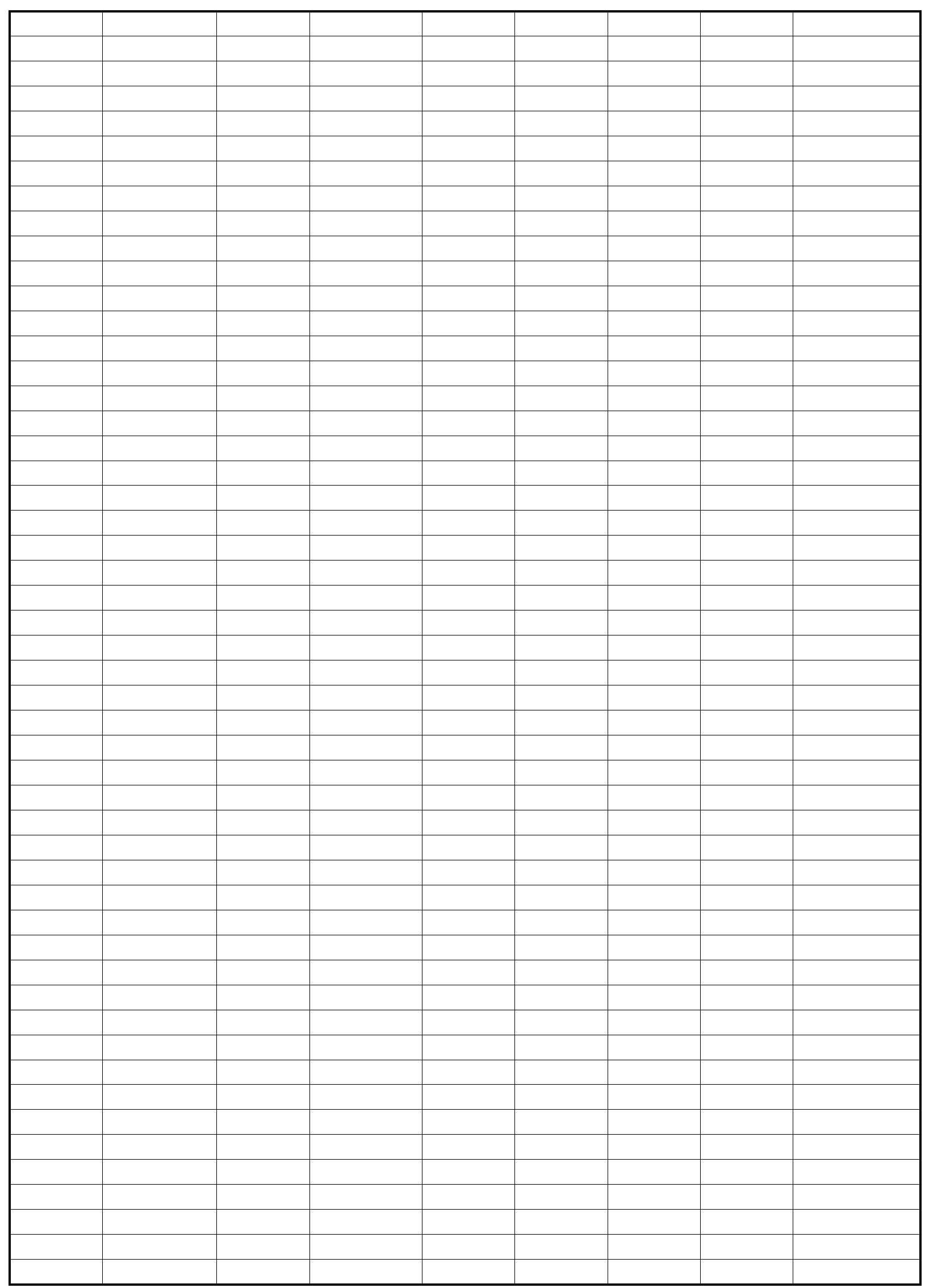

Page 58 
CHART DATA

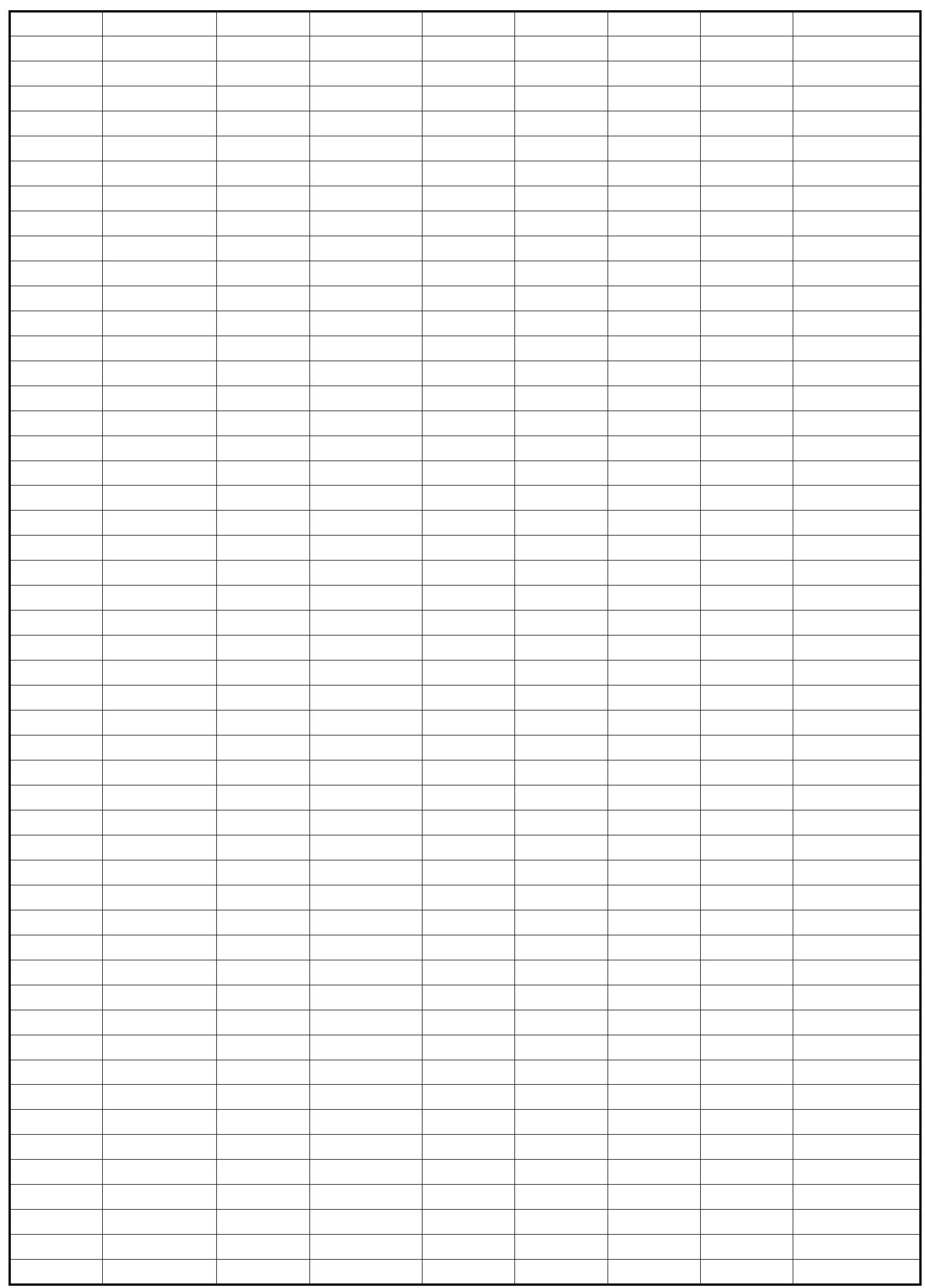

Page 59 
CHART DATA

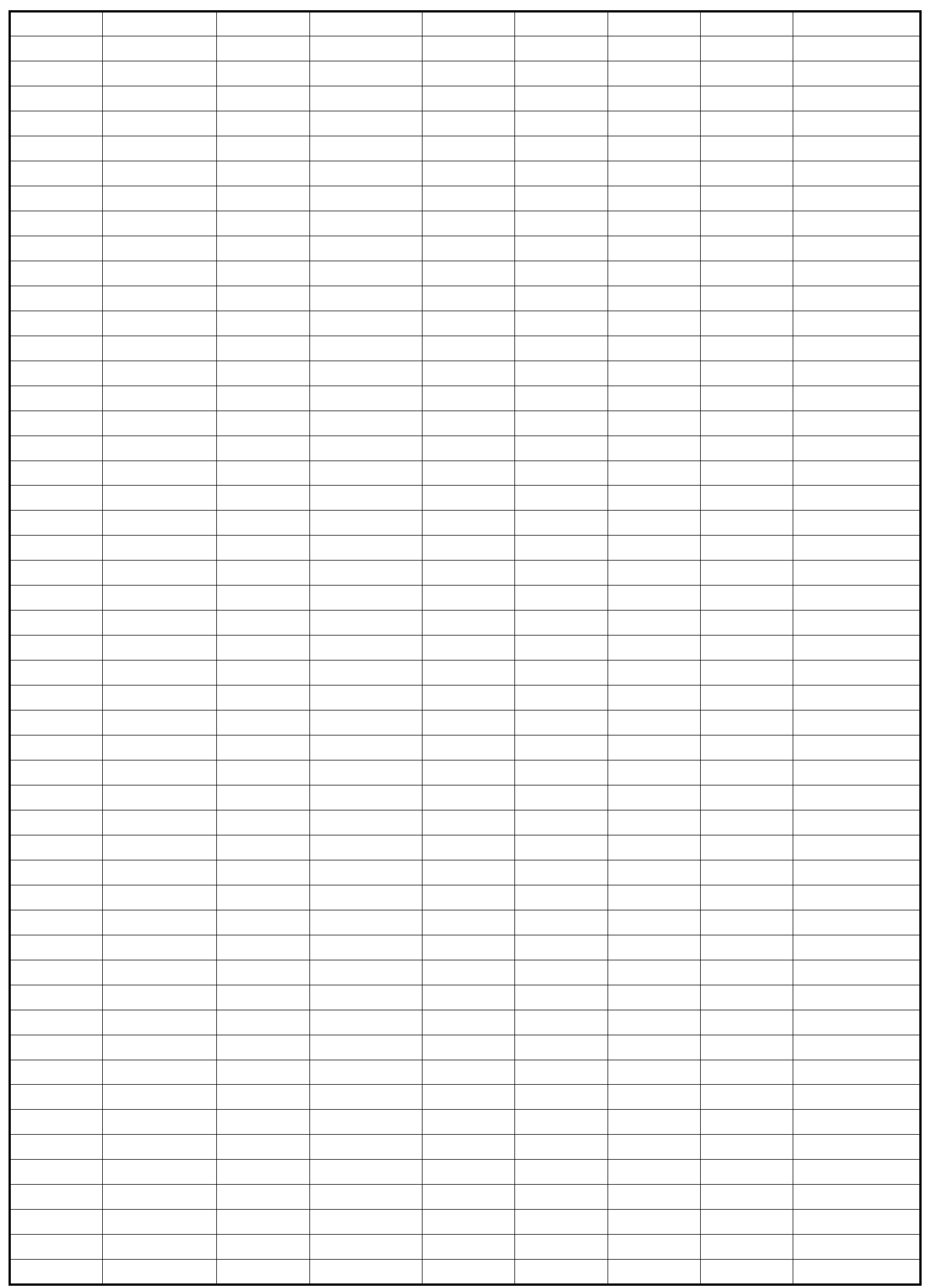

Page 60 
CHART DATA

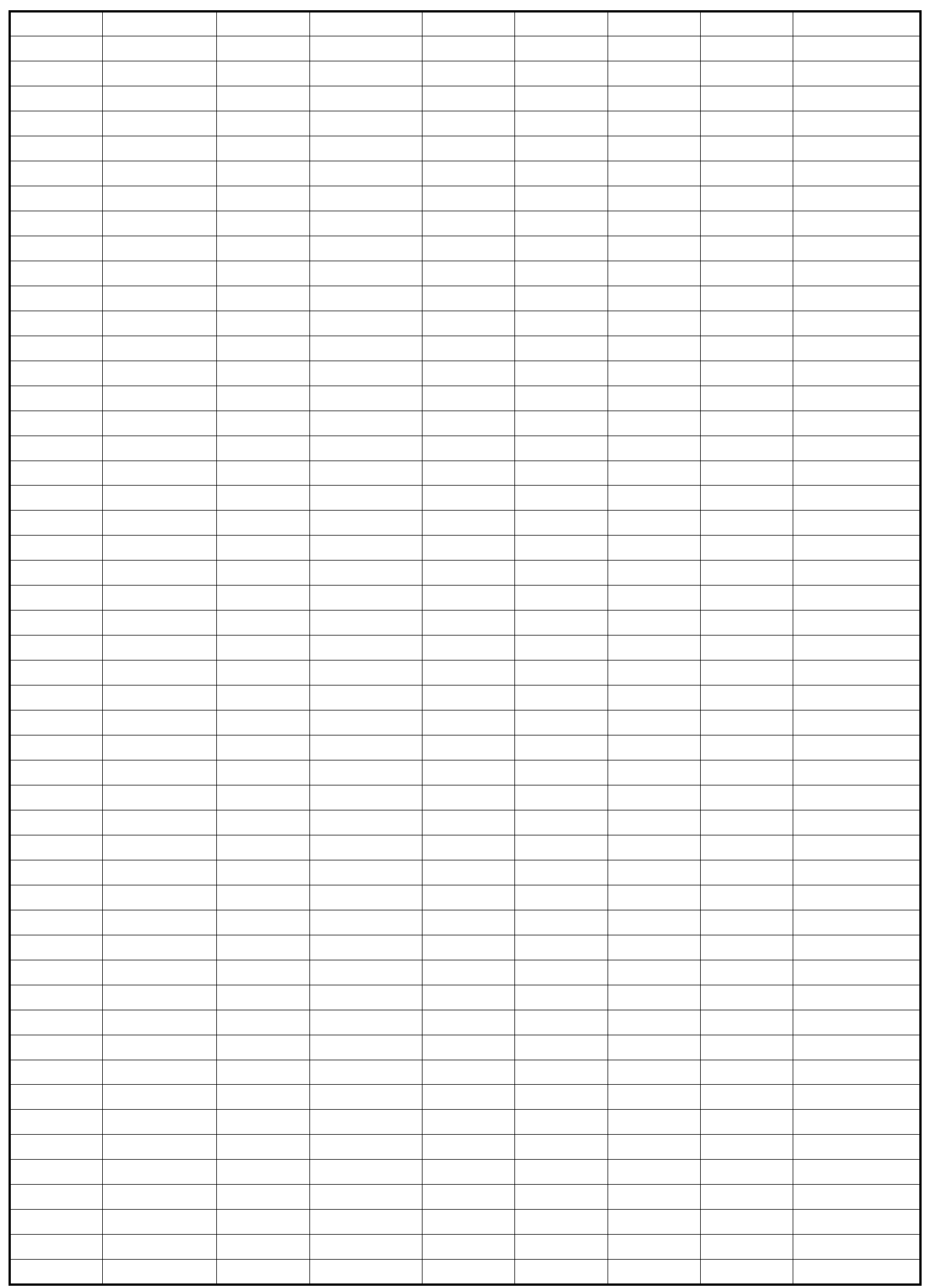

Page 61 
CHART DATA

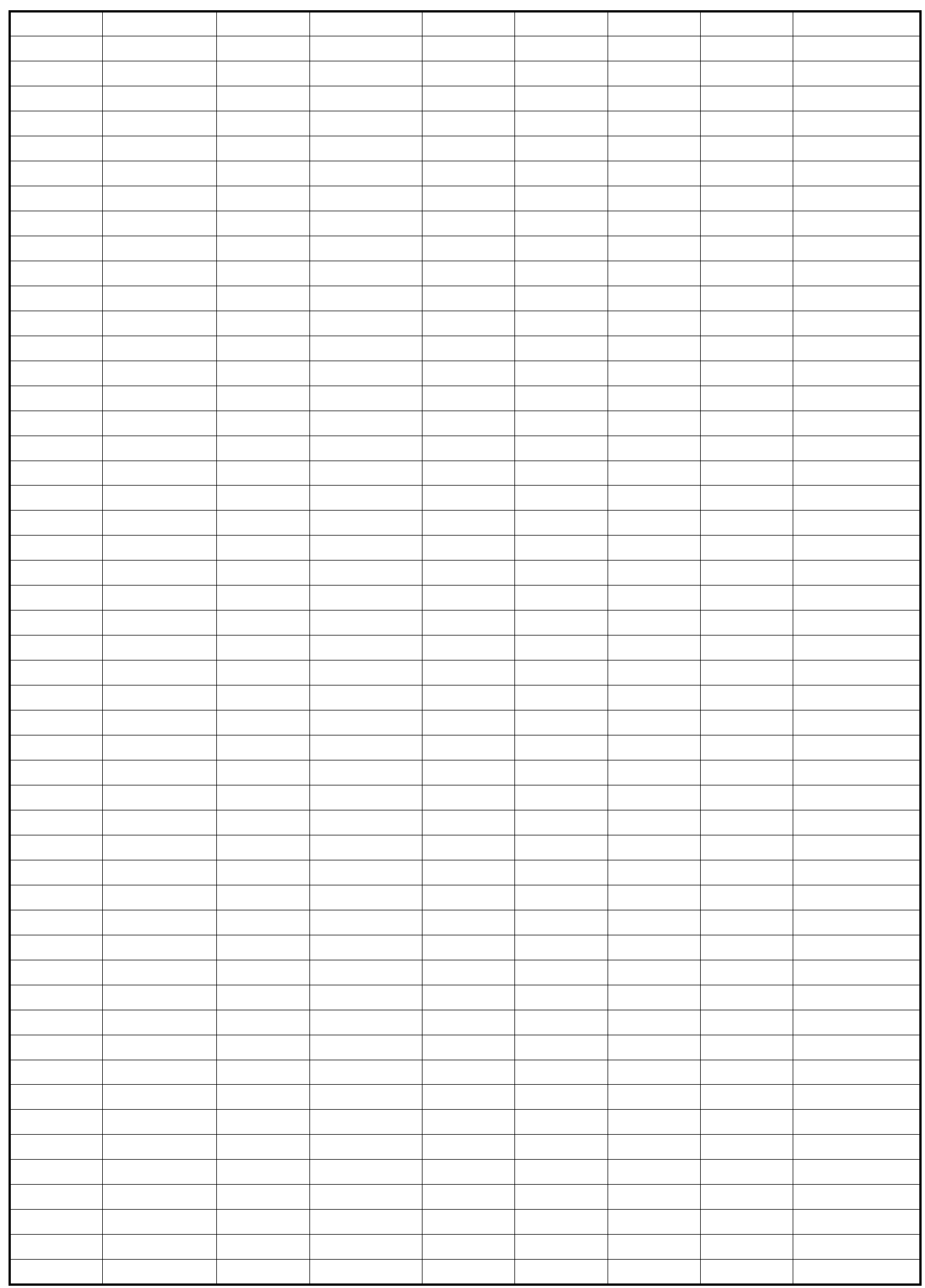

Page 62 
CHART DATA

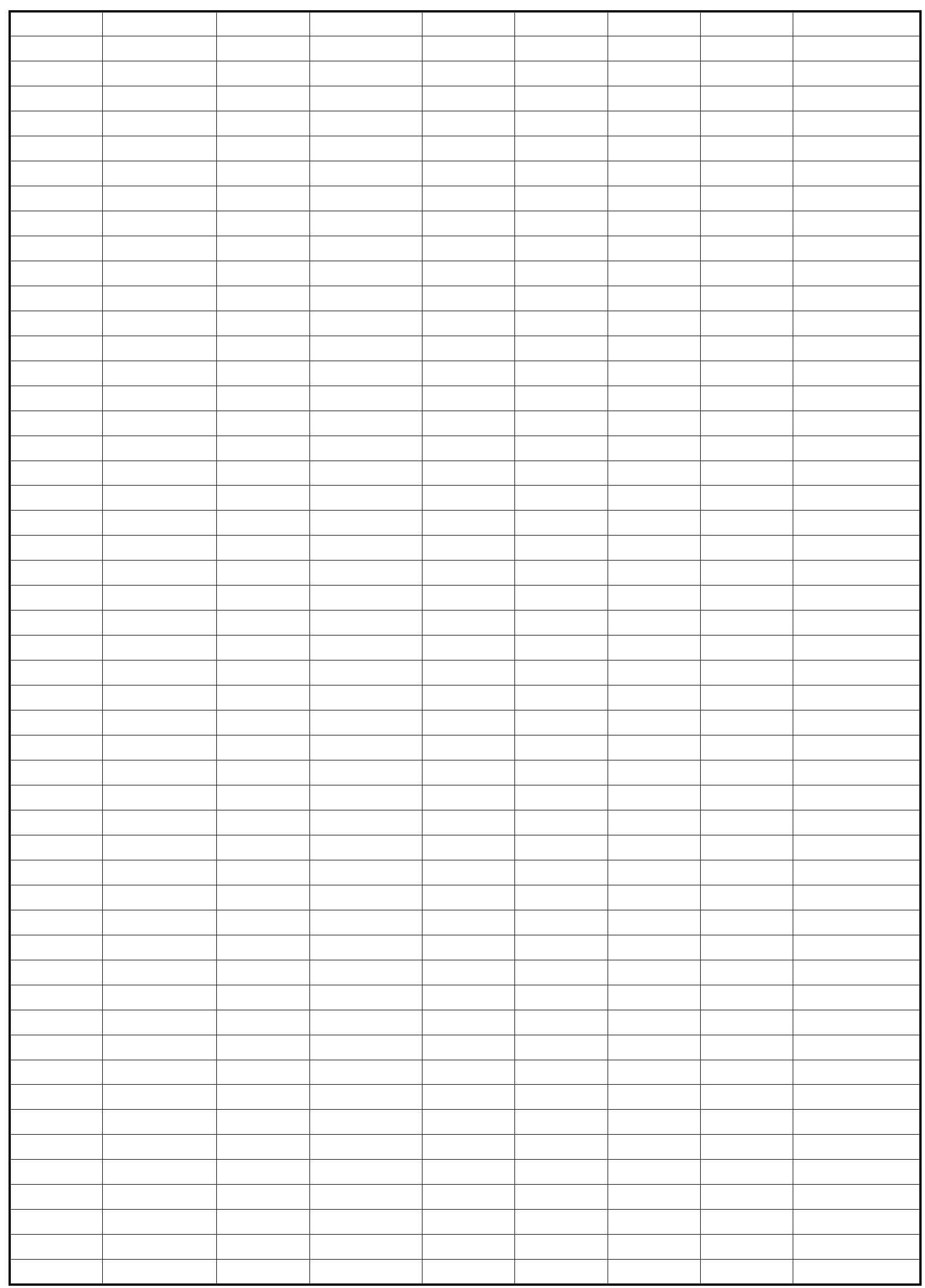

Page 63 
CHART DATA

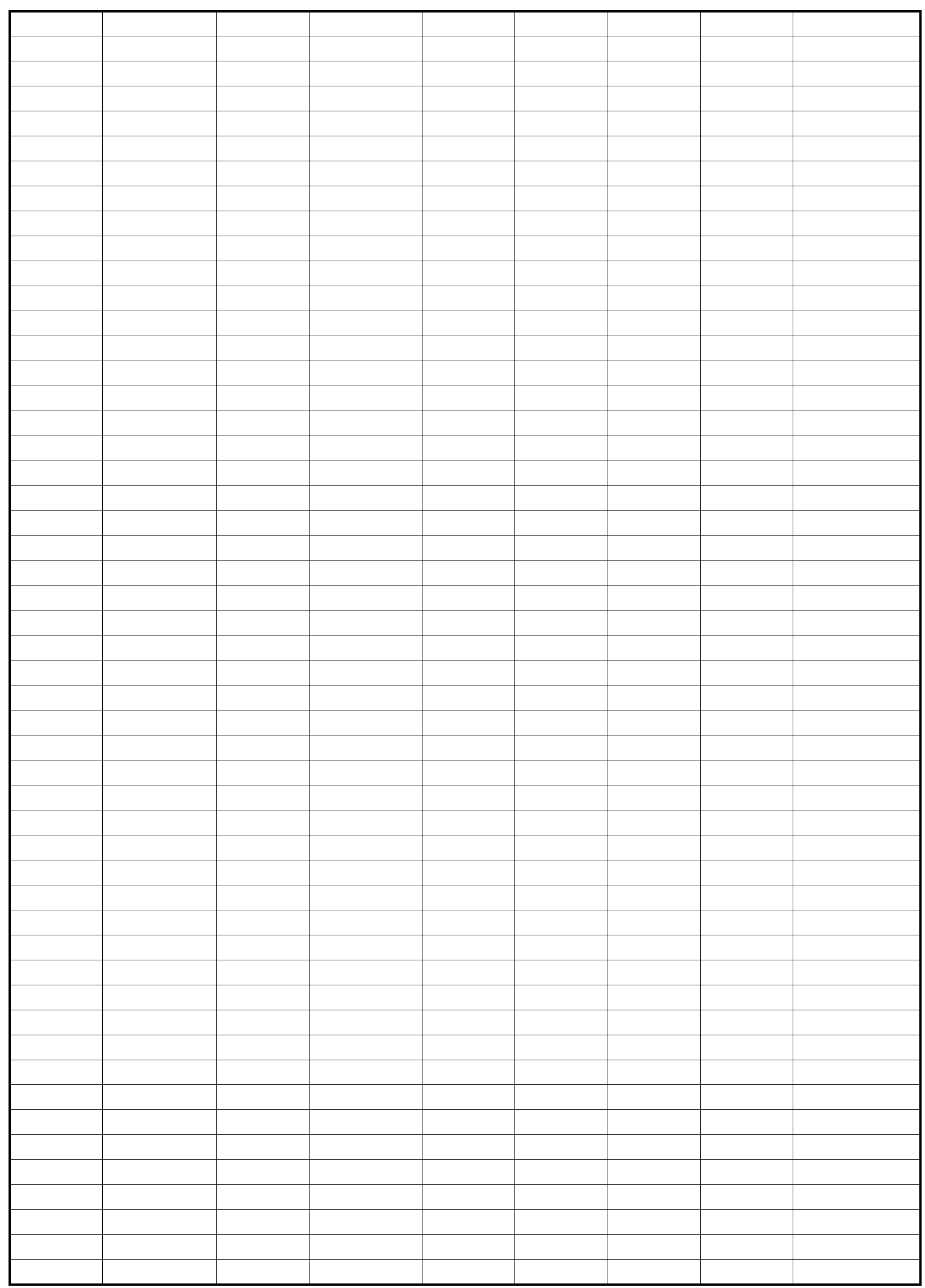

Page 64 
CHART DATA

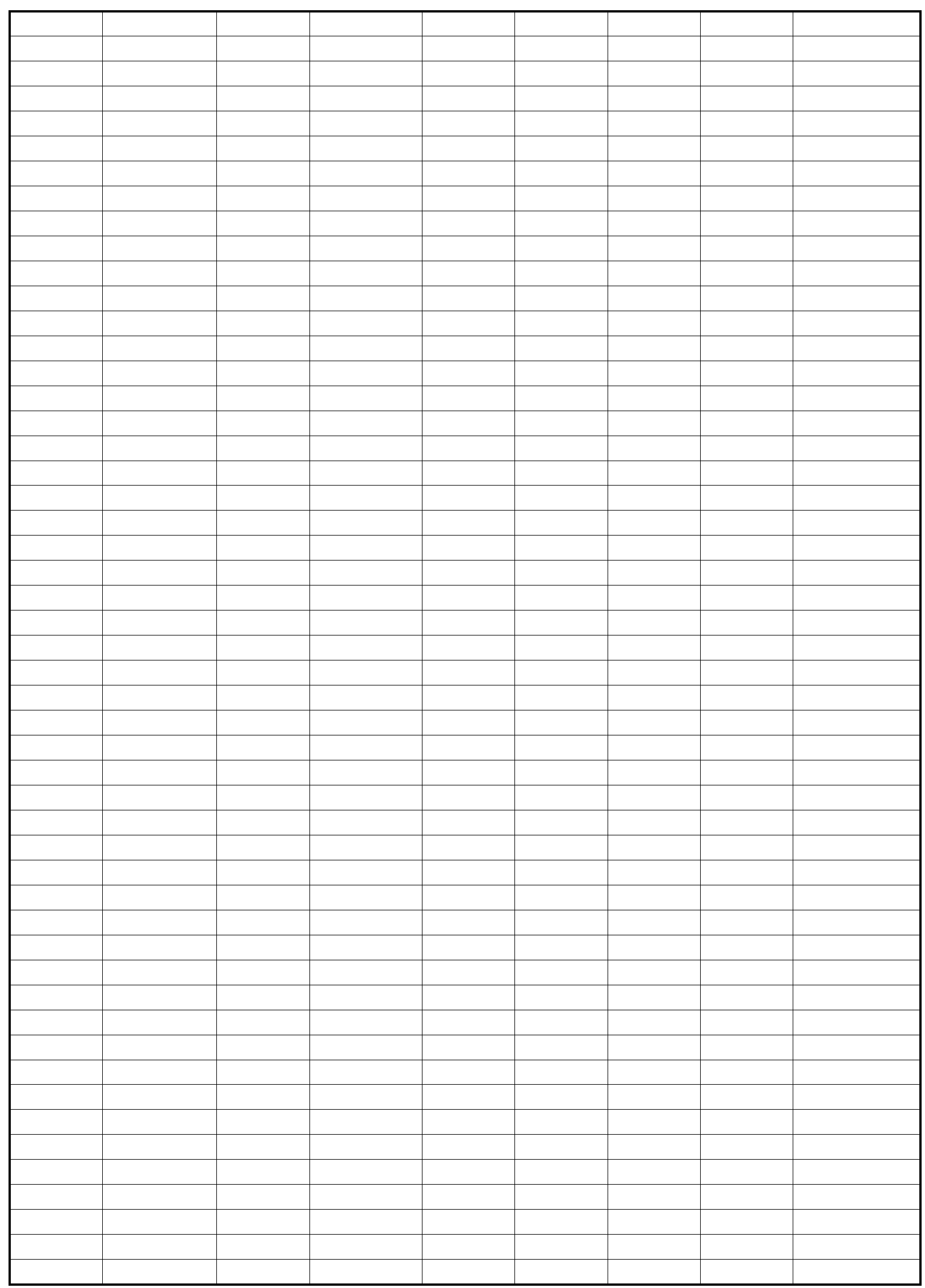

Page 65 
CHART DATA

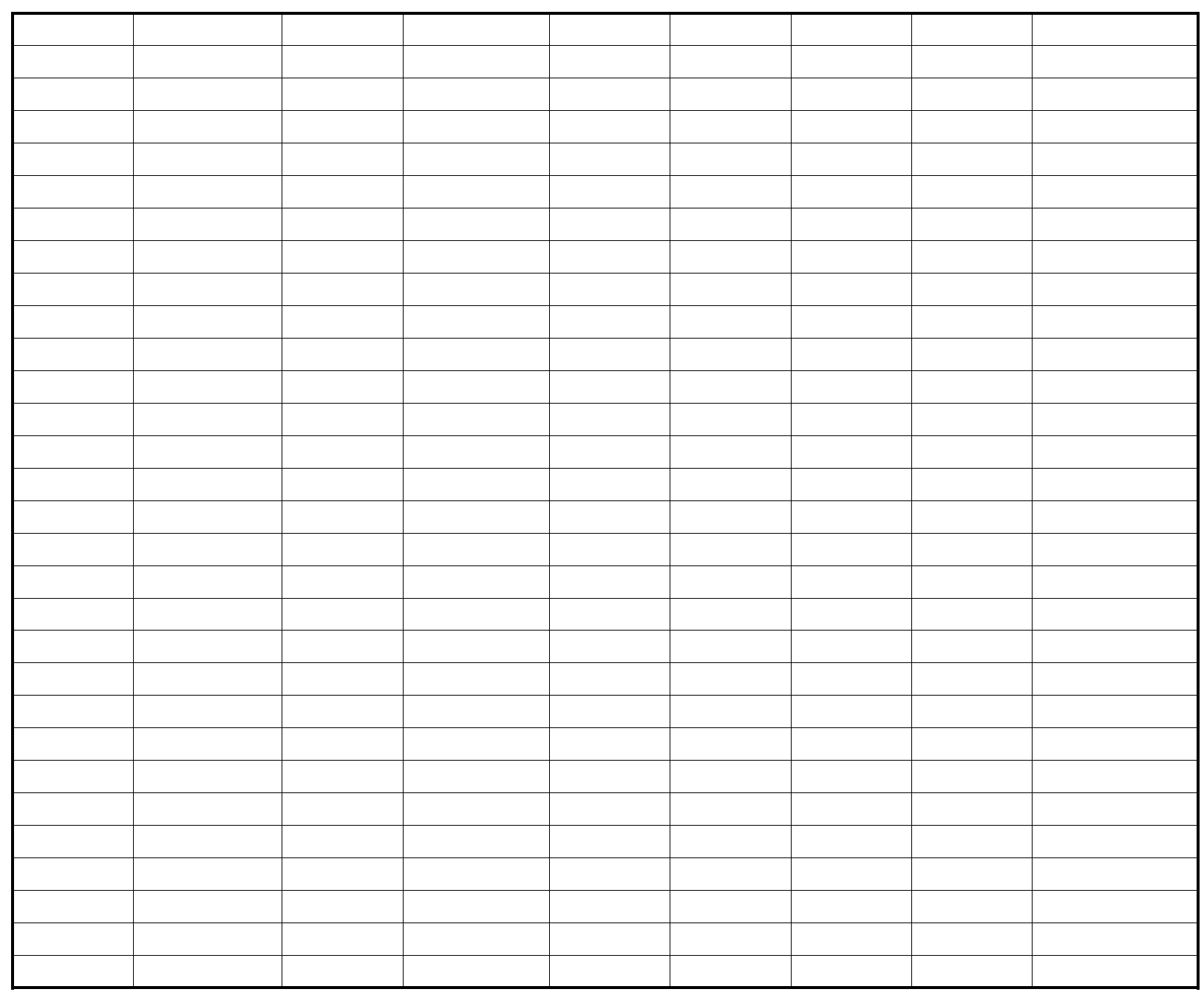




\begin{tabular}{|c|c|c|c|c|}
\hline Total Daily & Days in Period & Overall Total & reduction & \%reduction \\
\hline 0.00 & 91.00 & 0.00 & & \\
\hline 363.11 & 394.00 & 143063.46 & & \\
\hline 43.64 & 35.00 & 1527.48 & & \\
\hline 110.44 & 149.00 & 16455.40 & & \\
\hline 85.08 & 79.00 & 6721.45 & & \\
\hline 300.88 & 241.00 & 72511.74 & & \\
\hline 111.10 & 33.00 & 3666.30 & & \\
\hline 56.66 & 92.00 & 5212.44 & & \\
\hline 517.19 & 669.00 & 345997.64 & & \\
\hline 553.72 & 445.00 & 246404.15 & & \\
\hline 473.54 & 543.00 & 257134.47 & & \\
\hline 357.54 & 333.00 & 119059.33 & & \\
\hline 43.64 & 126.00 & 5498.91 & & \\
\hline 196.18 & 112.00 & 21972.35 & & \\
\hline & & & & \\
\hline & & & & \\
\hline Total Daily & Days in Period & Overall Total & & \\
\hline 8151.83 & 91.00 & 741816.19 & & \\
\hline 7249.65 & 394.00 & 2856362.15 & & \\
\hline 3708.00 & 35.00 & 129780.07 & & \\
\hline 4323.30 & 149.00 & 644172.27 & & \\
\hline 6176.20 & 79.00 & 487919.67 & & \\
\hline 5480.81 & 241.00 & 1320875.52 & & \\
\hline 4205.92 & 33.00 & 138795.38 & & \\
\hline 3073.19 & 92.00 & 282733.81 & & \\
\hline 23432.78 & 669.00 & 15676531.29 & & \\
\hline 18936.12 & 445.00 & 8426575.06 & & \\
\hline 11572.95 & 543.00 & 6284114.00 & & \\
\hline 8554.00 & 333.00 & 2848483.62 & & \\
\hline 11859.83 & 126.00 & 1494338.36 & & \\
\hline 10382.12 & 112.00 & 1162797.32 & & \\
\hline & & & & \\
\hline & & & & \\
\hline Total Daily & Days in Period & Overall Total & & \\
\hline 25.73 & 91.00 & 2341.19 & & \\
\hline 26.87 & 394.00 & 10585.15 & & \\
\hline 36.70 & 35.00 & 1284.59 & & \\
\hline 20.58 & 149.00 & 3066.02 & & \\
\hline 30.35 & 79.00 & 2397.64 & & \\
\hline 20.30 & 241.00 & 4893.44 & & \\
\hline 23.14 & 33.00 & 763.75 & & \\
\hline 18.20 & 92.00 & 1674.25 & & \\
\hline 109.87 & 669.00 & 73505.19 & & \\
\hline 92.00 & 445.00 & 40938.72 & & \\
\hline
\end{tabular}




\begin{tabular}{|c|c|c|c|c|}
\hline 47.44 & 543.00 & 25761.63 & & \\
\hline 38.50 & 333.00 & 12821.55 & & \\
\hline \multirow{5}{*}{53.49} & 126.00 & 7866.19 & & \\
\hline & 112.00 & 5991.33 & & \\
\hline & & & \multicolumn{2}{|c|}{ Based on average daily data - other $\mathrm{e}$} \\
\hline & & & ave daily * \#days & \\
\hline & & & & Reduction \\
\hline Total Daily & Days in Period & Overall Total & & post-pre \\
\hline 7876.79 & 91.00 & 716788.25 & $1-\mathrm{A}-\mathrm{S}$ & \\
\hline 6888.58 & 394.00 & 2714101.89 & 1-B-S & -988.21 \\
\hline 3674.11 & 35.00 & 128593.91 & $0-A-S$ & \\
\hline 4244.50 & 149.00 & 632430.59 & 0-B-S & 570.39 \\
\hline 6159.10 & 79.00 & 486569.06 & 1-A-NS & \\
\hline 5182.41 & 241.00 & 1248959.74 & 1-B-NS & -976.70 \\
\hline 4179.80 & 33.00 & 137933.50 & $0-\mathrm{A}-\mathrm{NS}$ & \\
\hline 3016.62 & 92.00 & 277528.70 & 0-B-NS & -1163.19 \\
\hline 22683.99 & 669.00 & 15175589.21 & & \\
\hline 18537.93 & 445.00 & 8249377.46 & & \\
\hline 11133.08 & 543.00 & 6045264.66 & & \\
\hline 8199.02 & 333.00 & 2730274.29 & & \\
\hline 11550.91 & 126.00 & 1455414.13 & & \\
\hline 10338.90 & 112.00 & 1157957.36 & & \\
\hline & & & & \\
\hline & & & & \\
\hline & & & & \\
\hline & & & & \\
\hline & & & & \\
\hline & & & & \\
\hline & & & & \\
\hline & & & & \\
\hline & & & & \\
\hline & & & & \\
\hline & & & & \\
\hline & & & & \\
\hline & & & & \\
\hline & & & & \\
\hline & & & & \\
\hline & & & & \\
\hline & & & & \\
\hline & & & & \\
\hline & & & & \\
\hline & & & & \\
\hline & & & & \\
\hline & & & & \\
\hline & & & & \\
\hline
\end{tabular}


CHART DATA

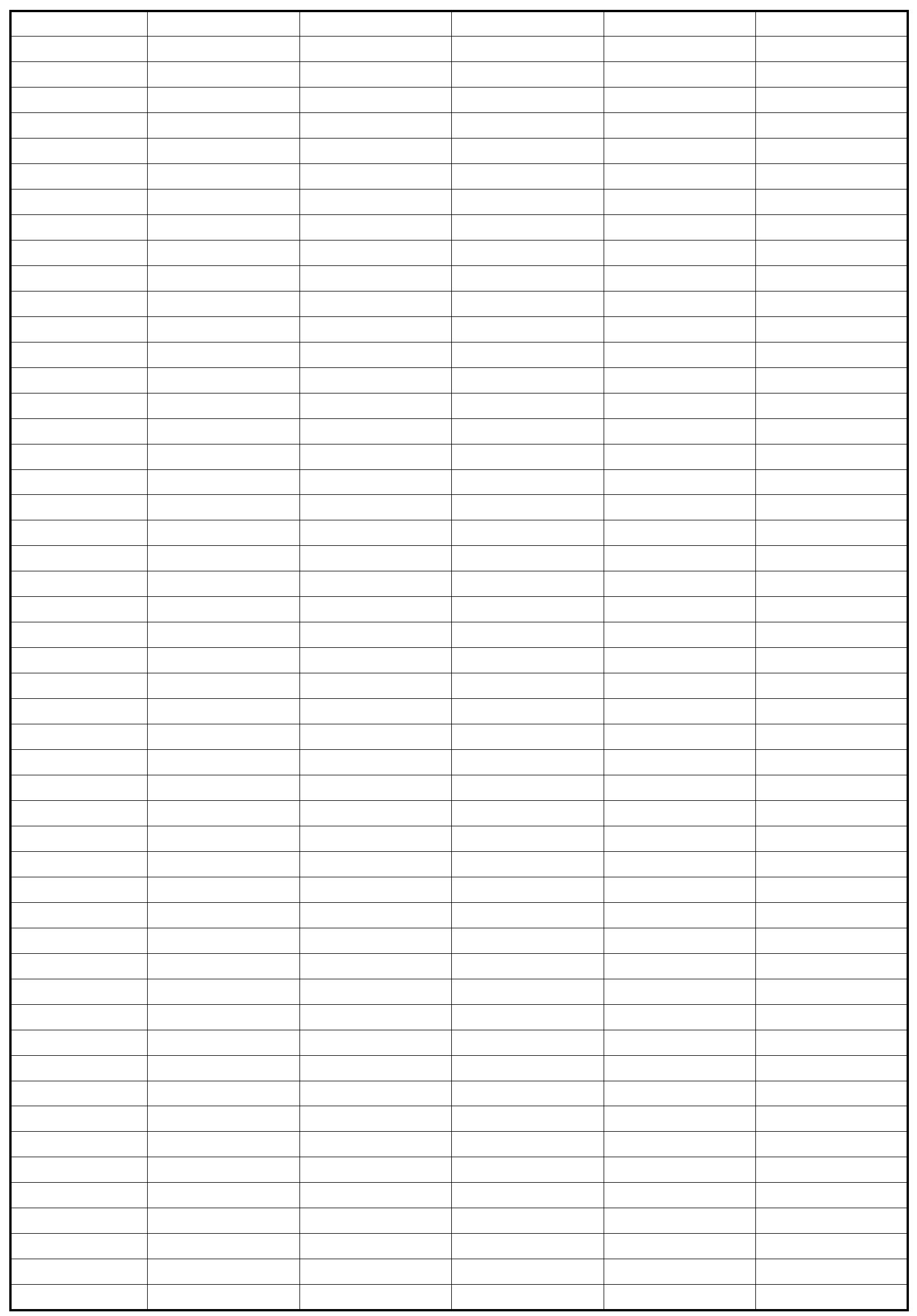

Page 69 
CHART DATA

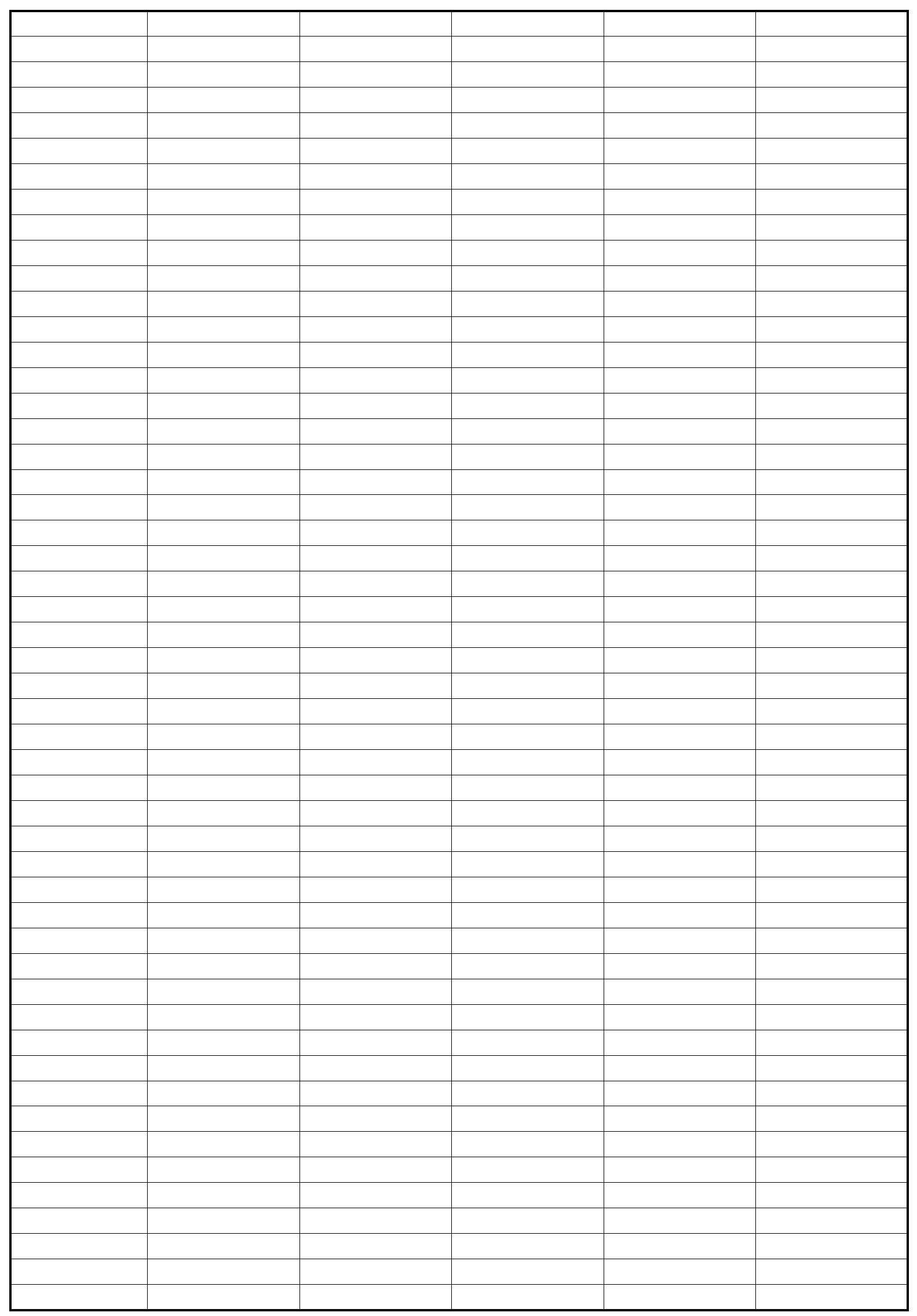

Page 70 
CHART DATA

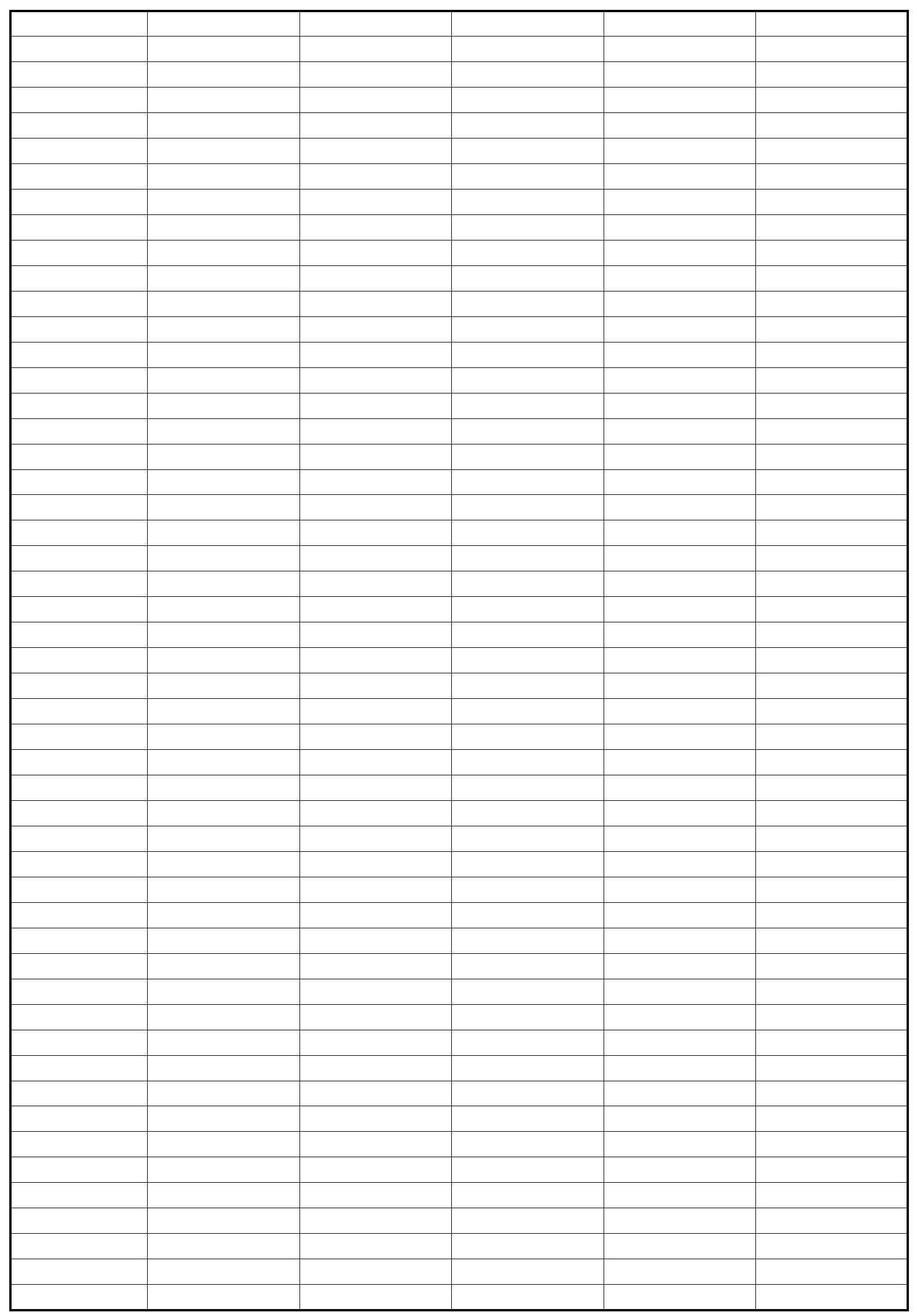

Page 71 
CHART DATA

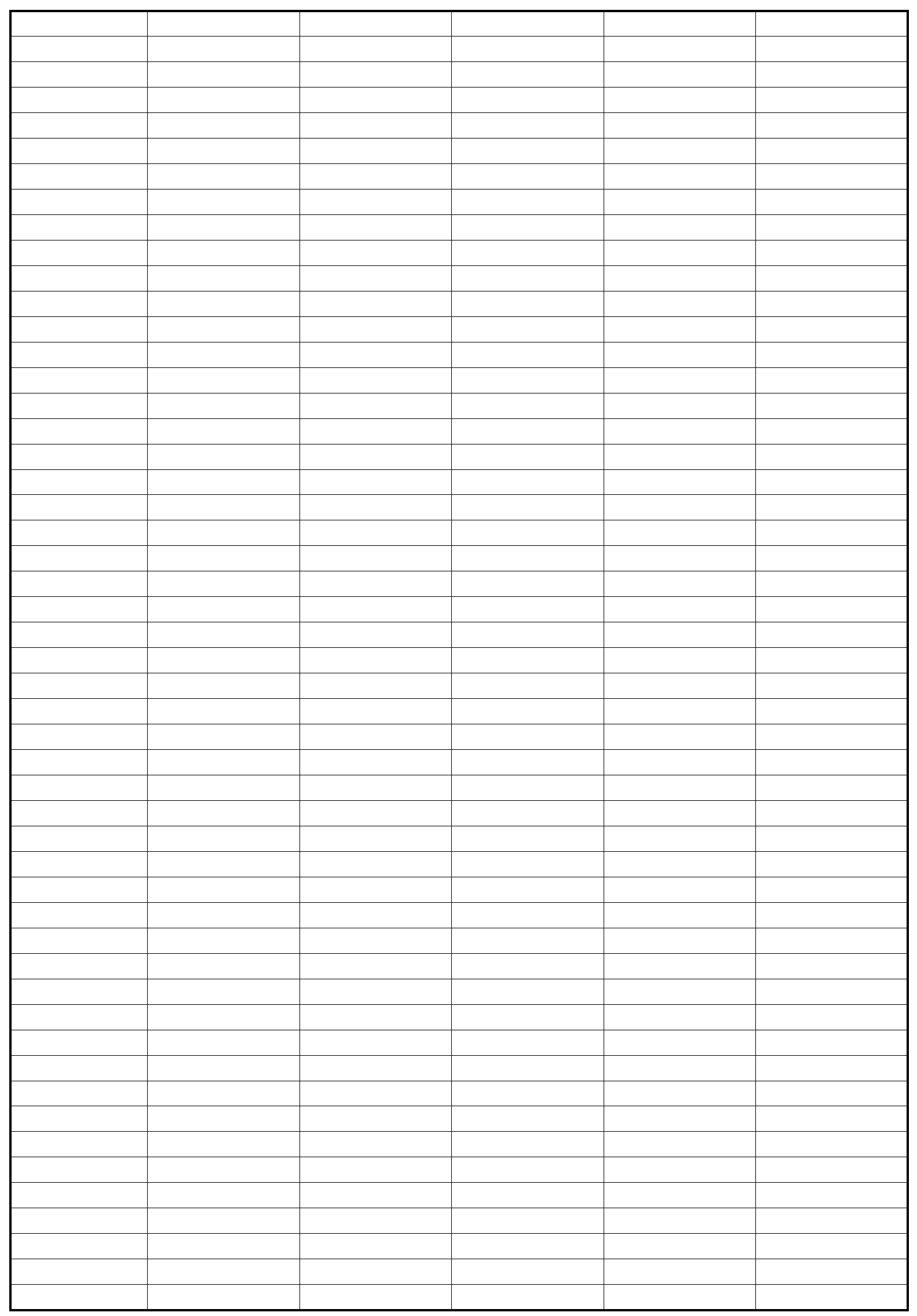

Page 72 
CHART DATA

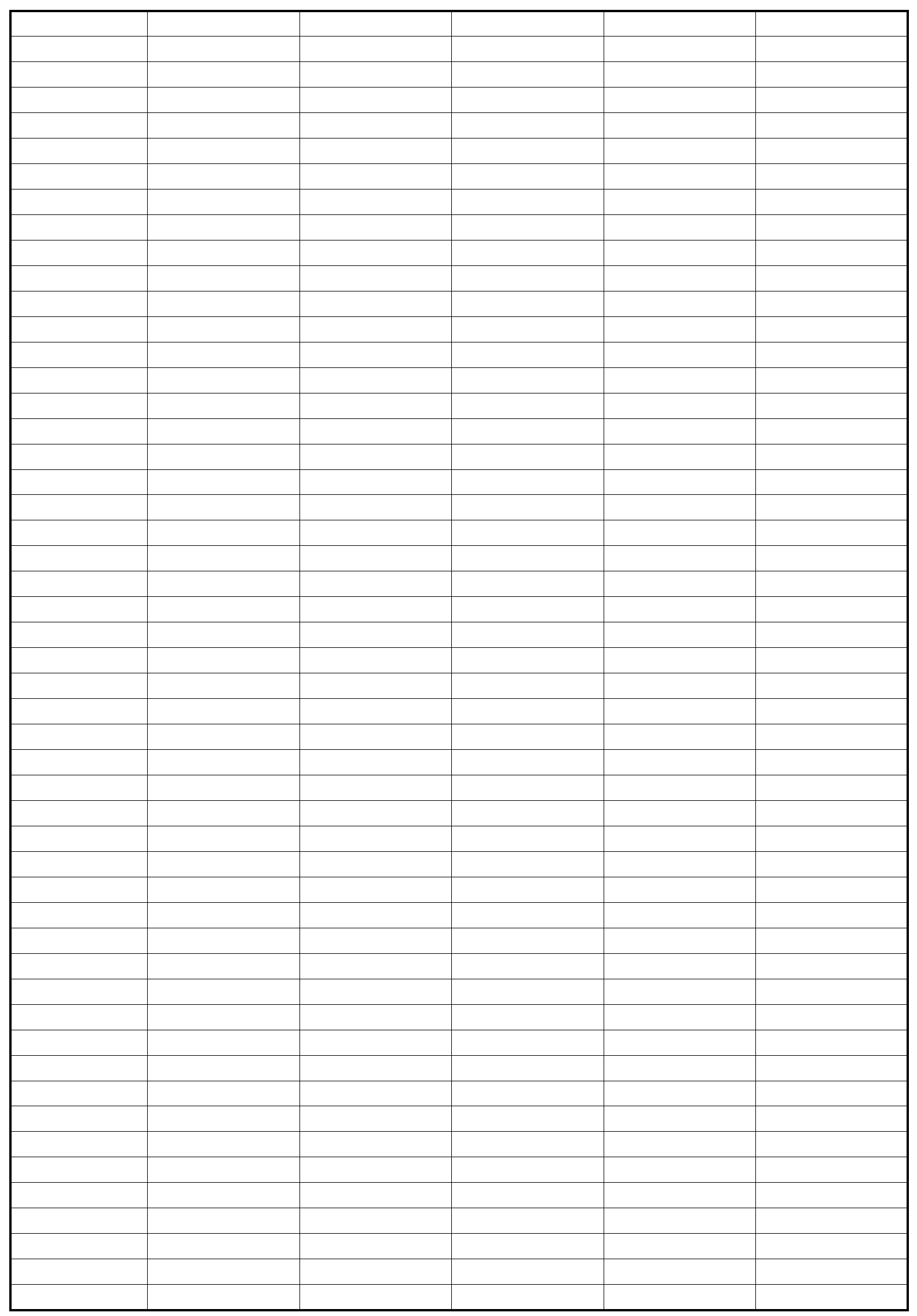

Page 73 
CHART DATA

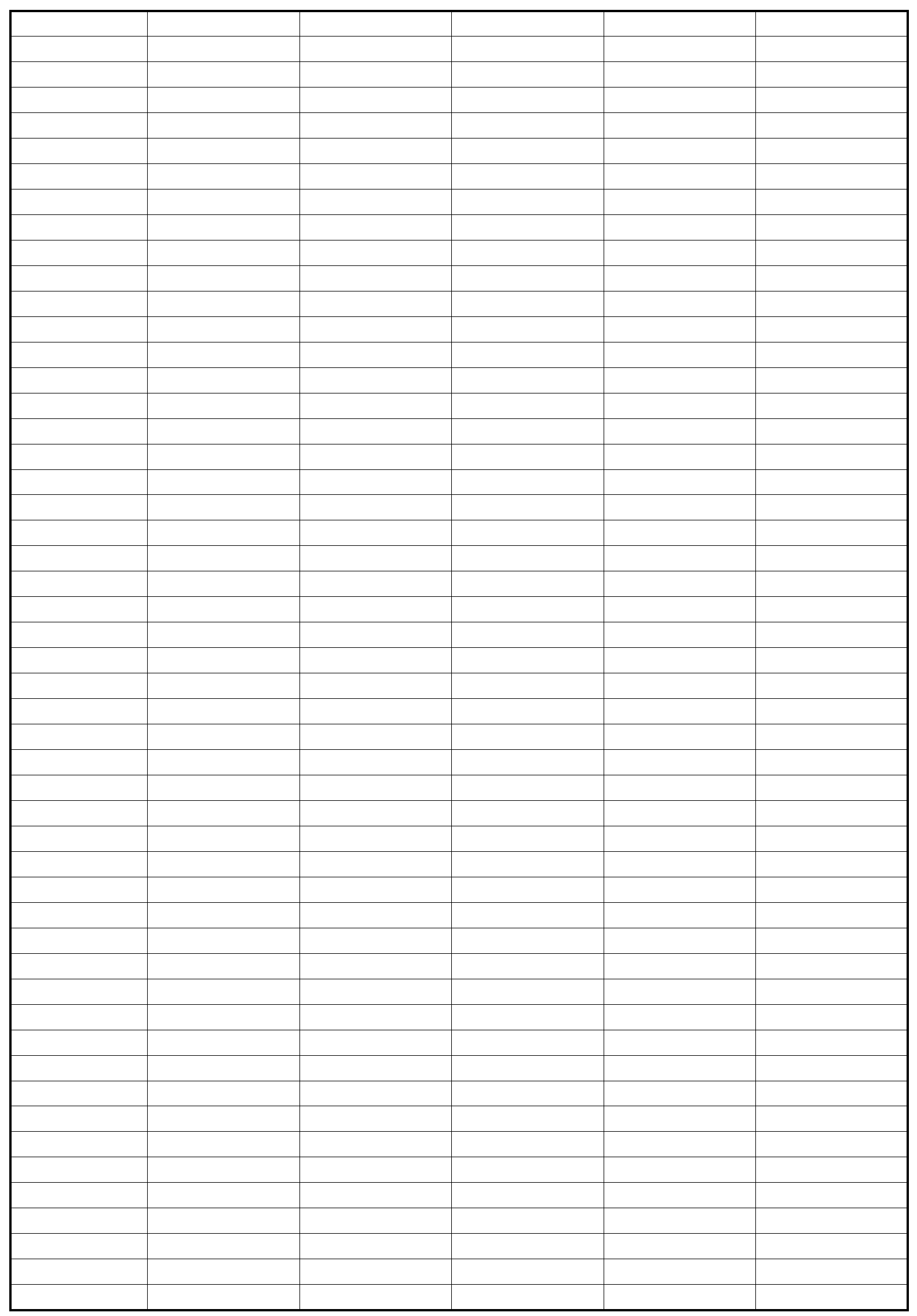

Page 74 
CHART DATA

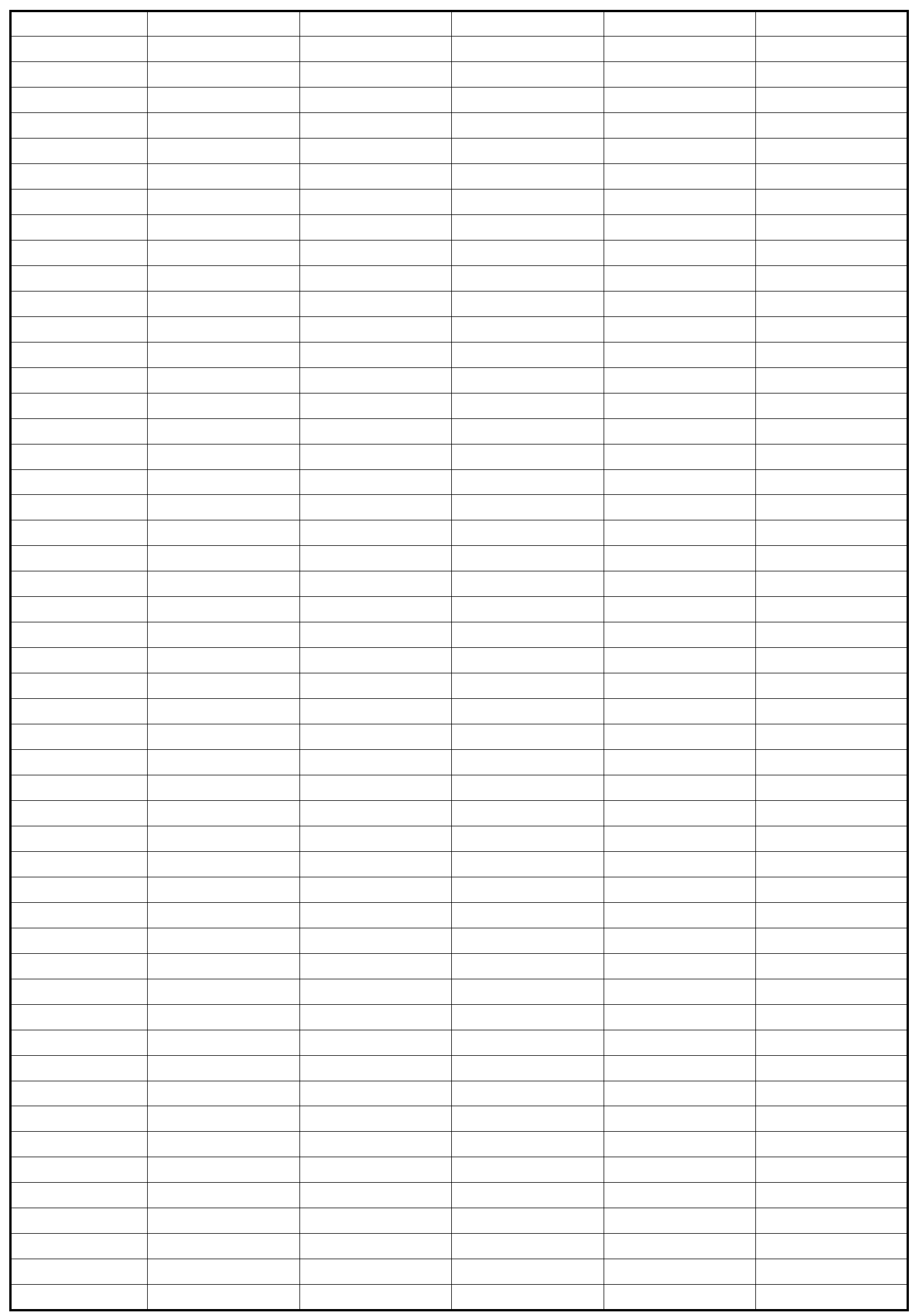

Page 75 
CHART DATA

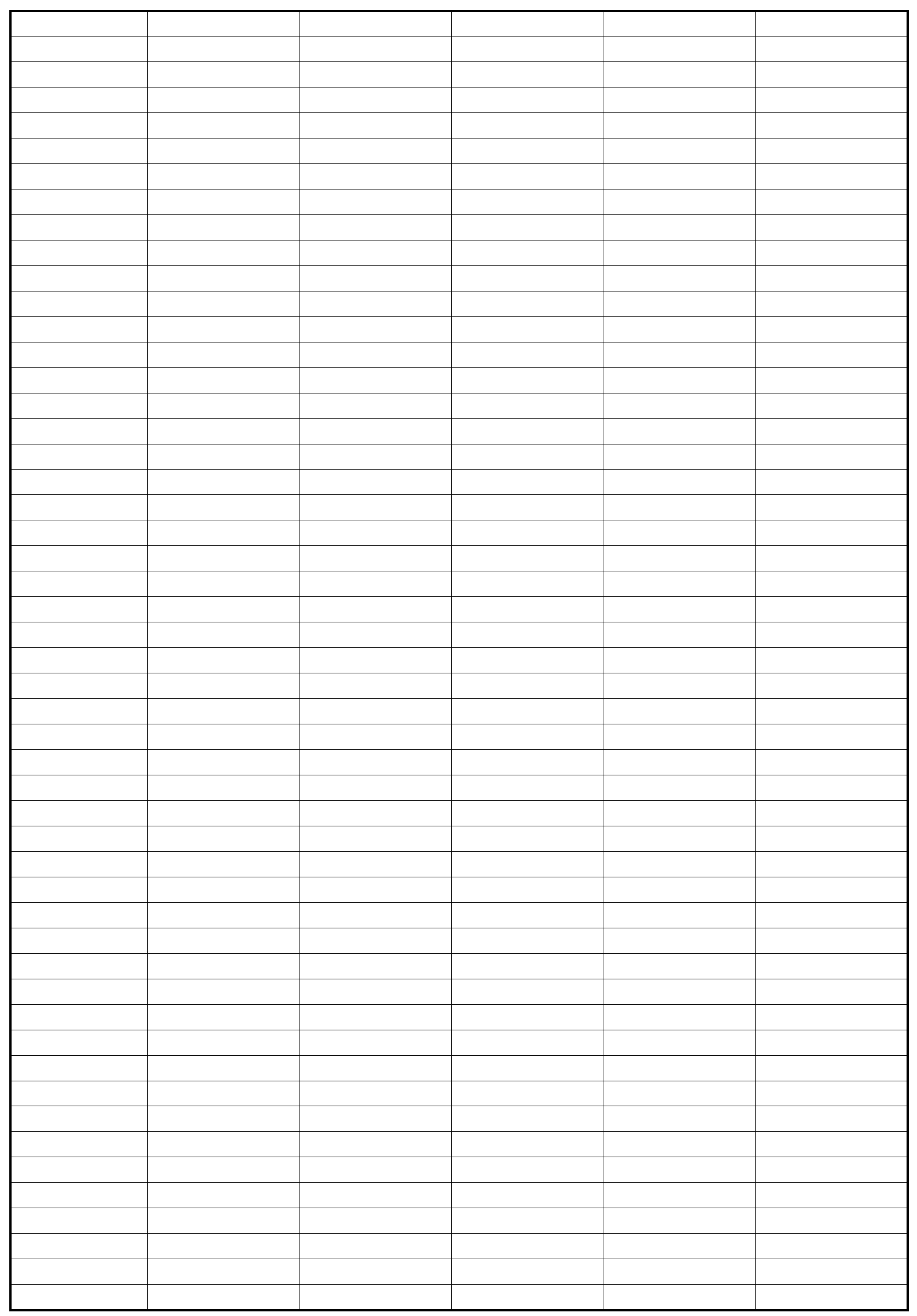

Page 76 
CHART DATA

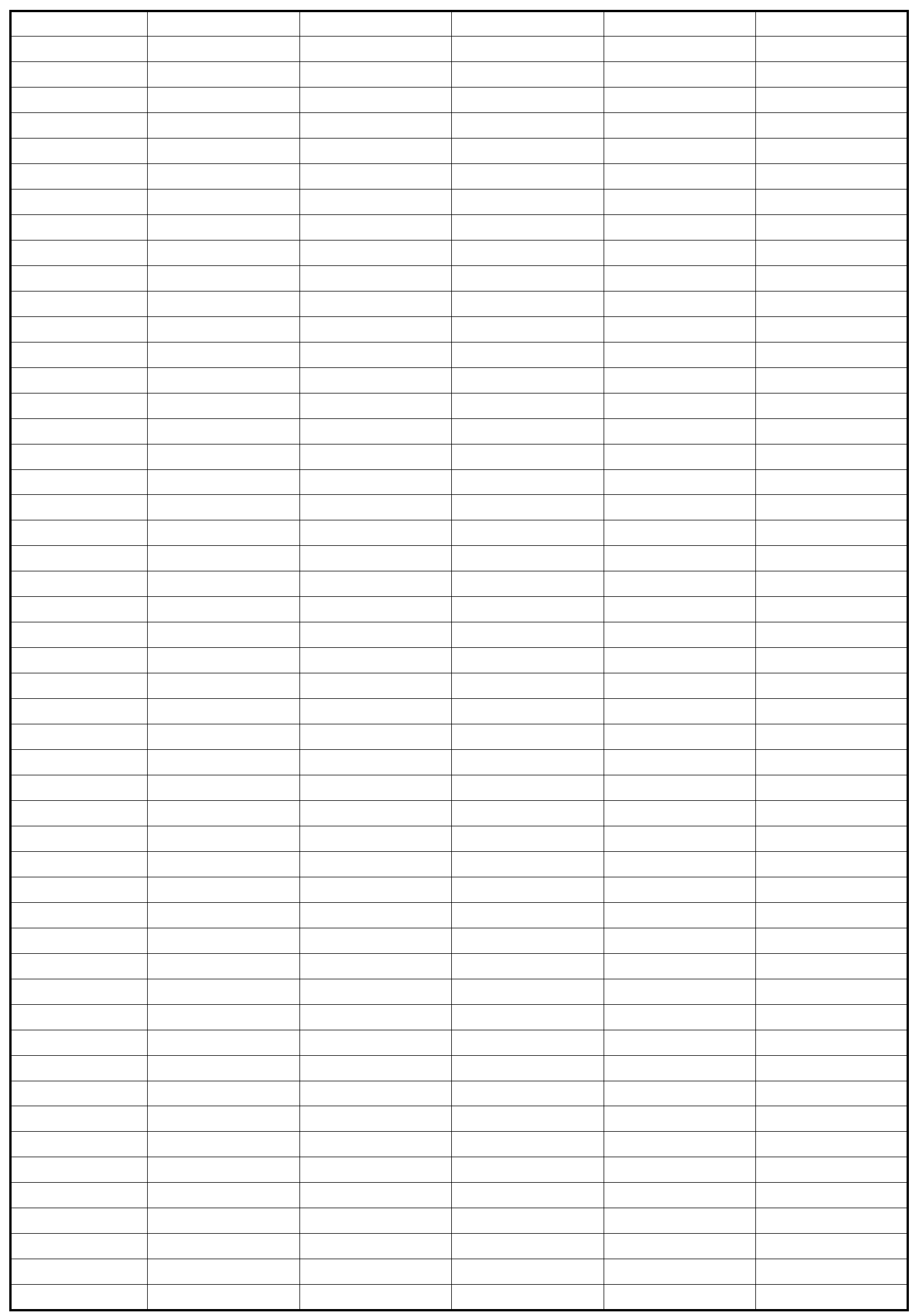

Page 77 
CHART DATA

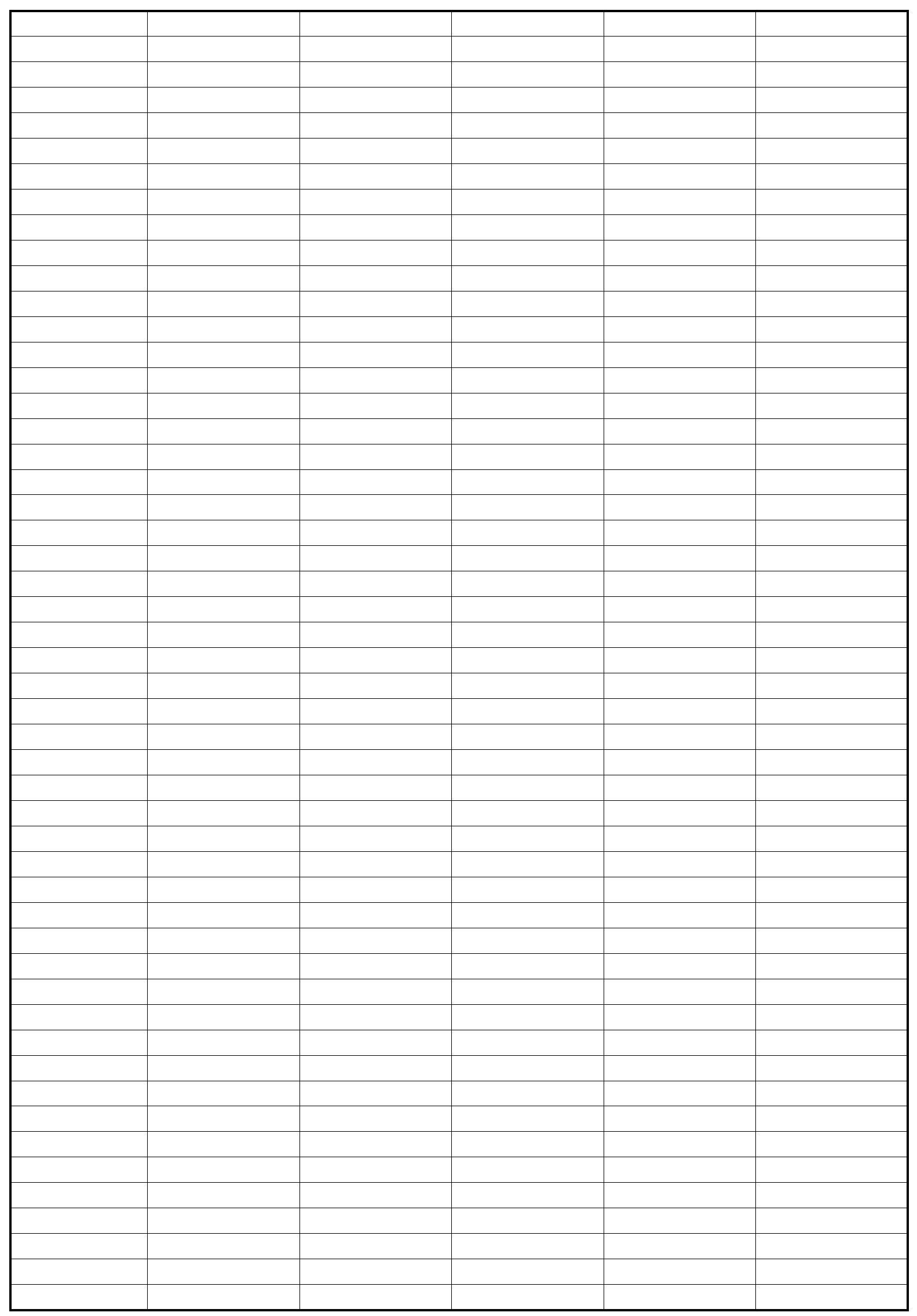

Page 78 
CHART DATA

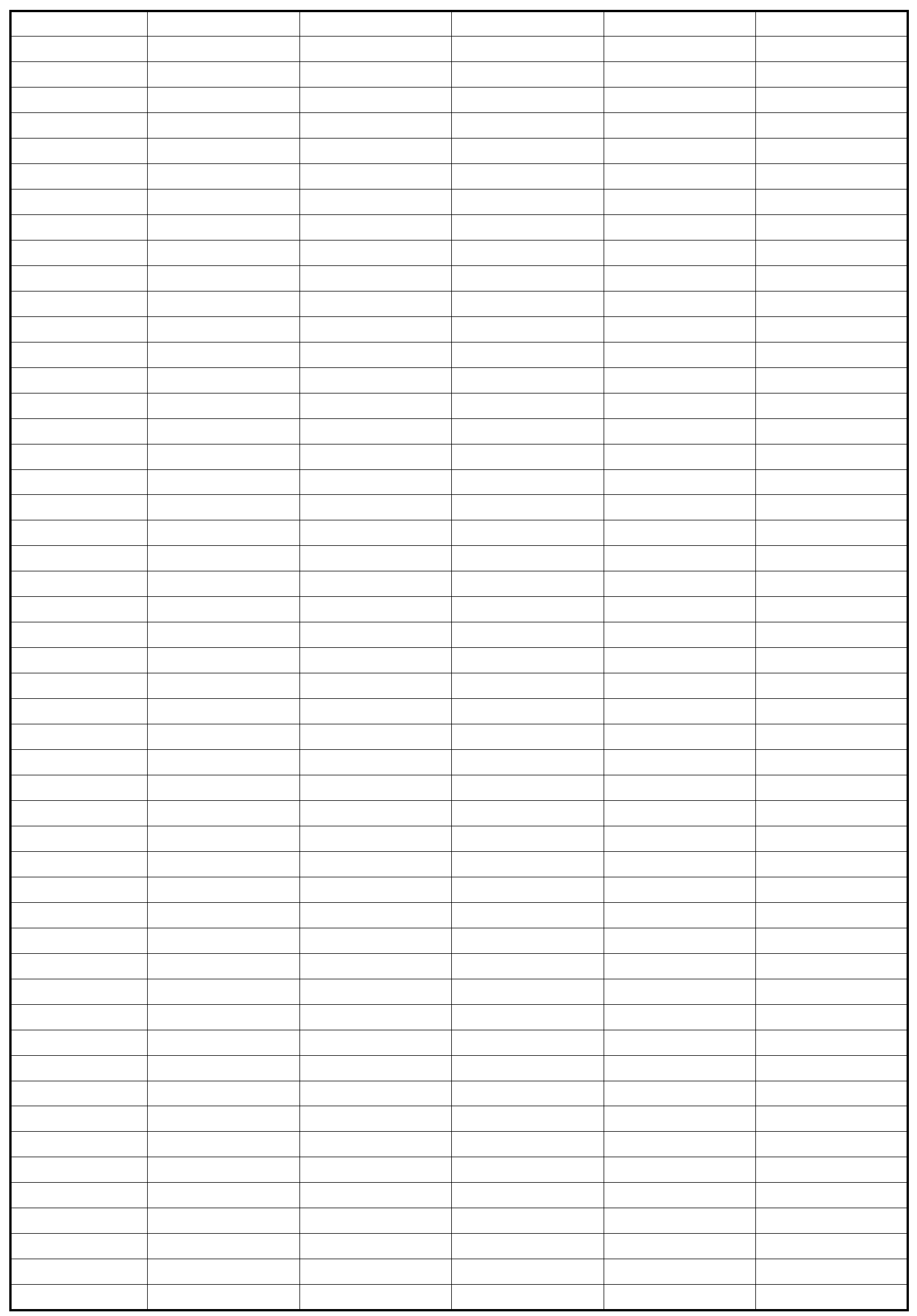

Page 79 
CHART DATA

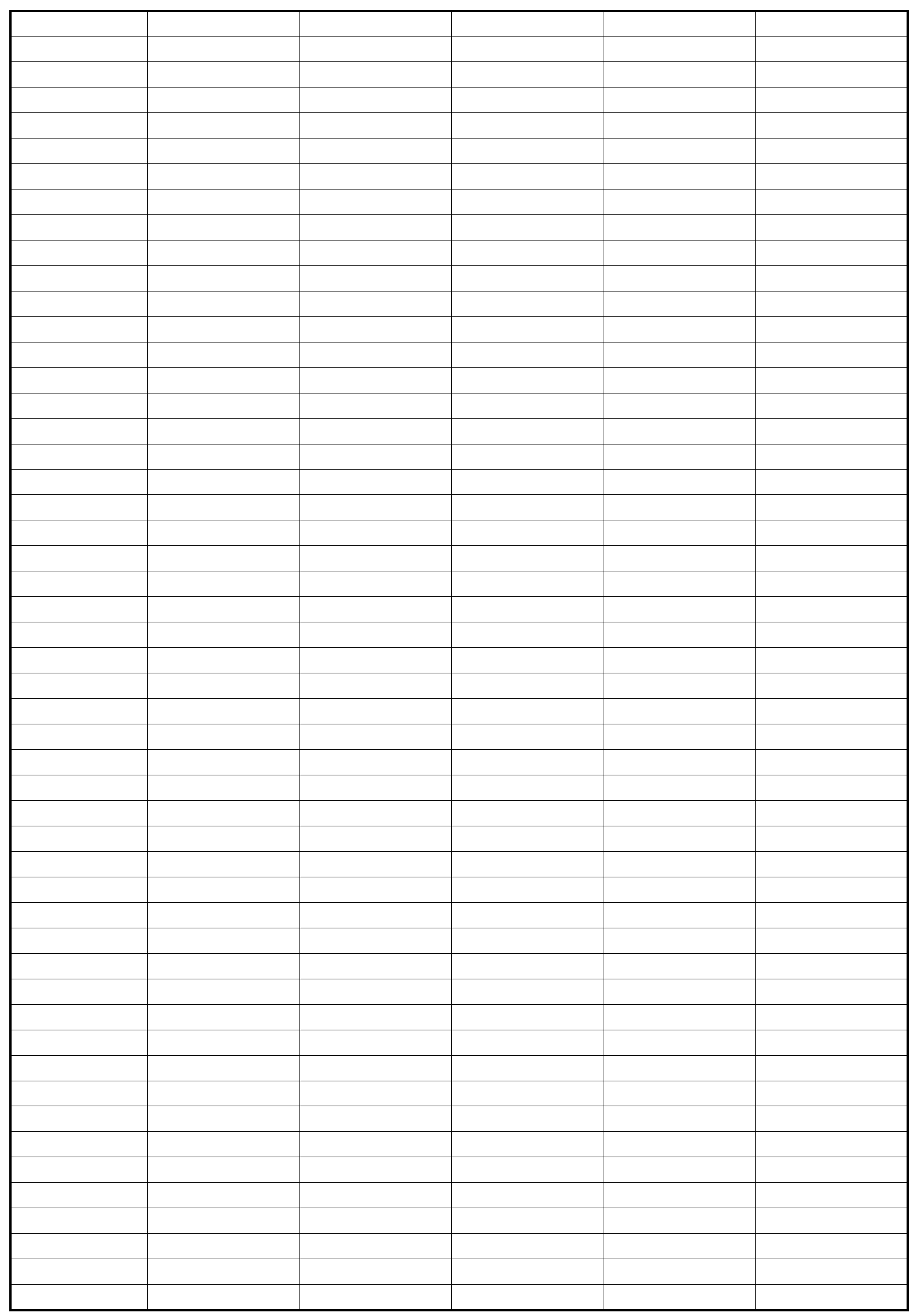

Page 80 
CHART DATA

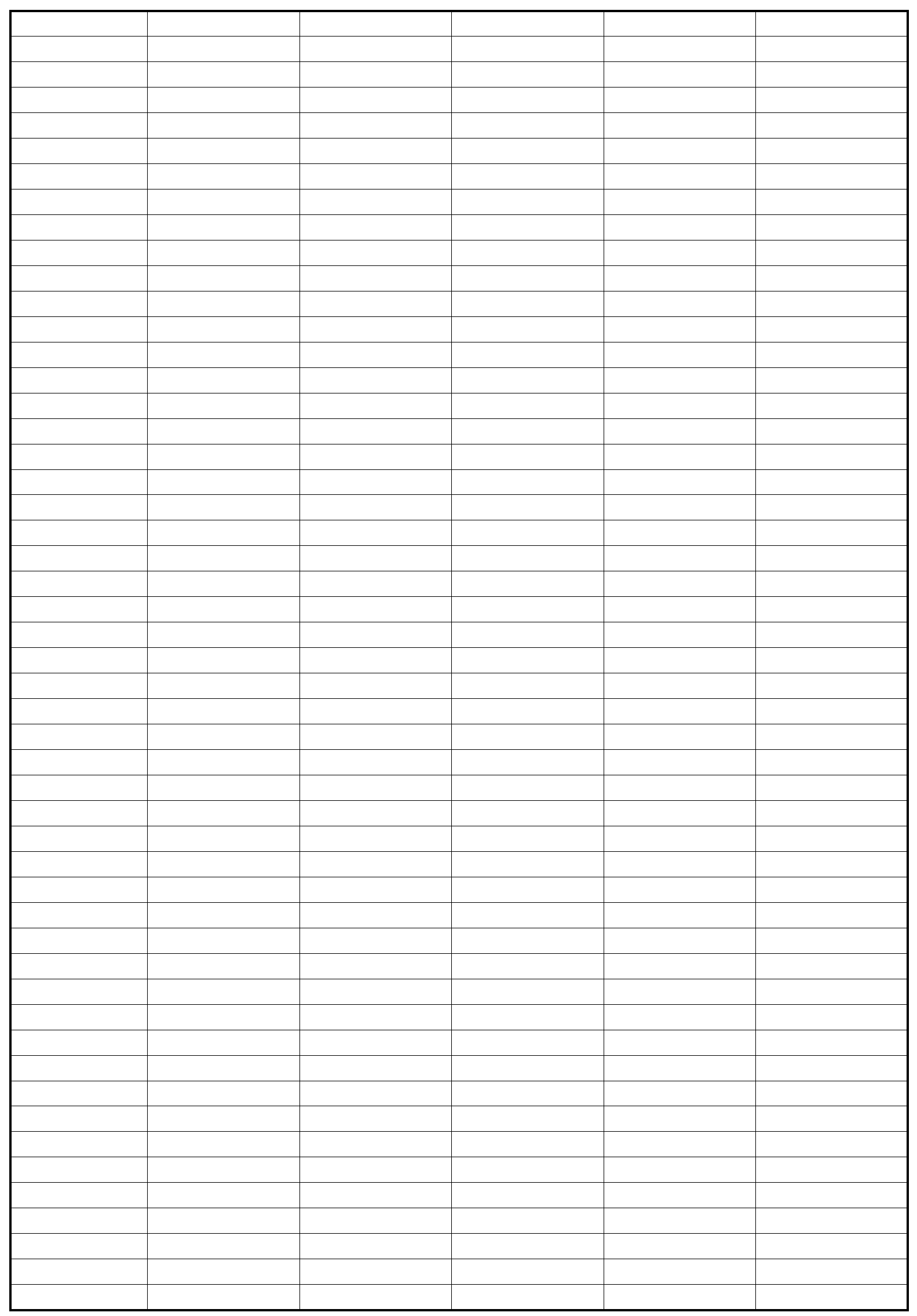

Page 81 
CHART DATA

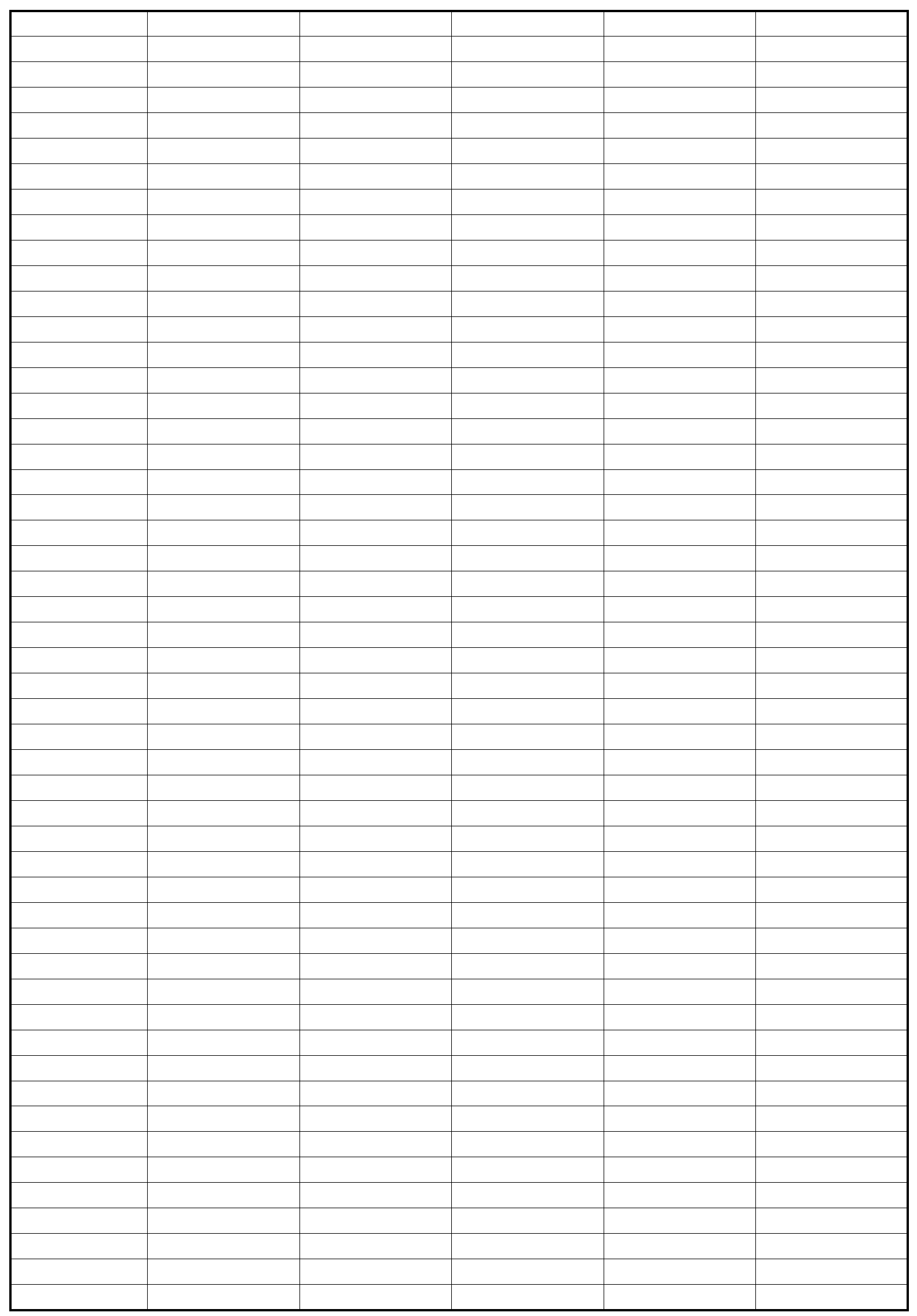

Page 82 
CHART DATA

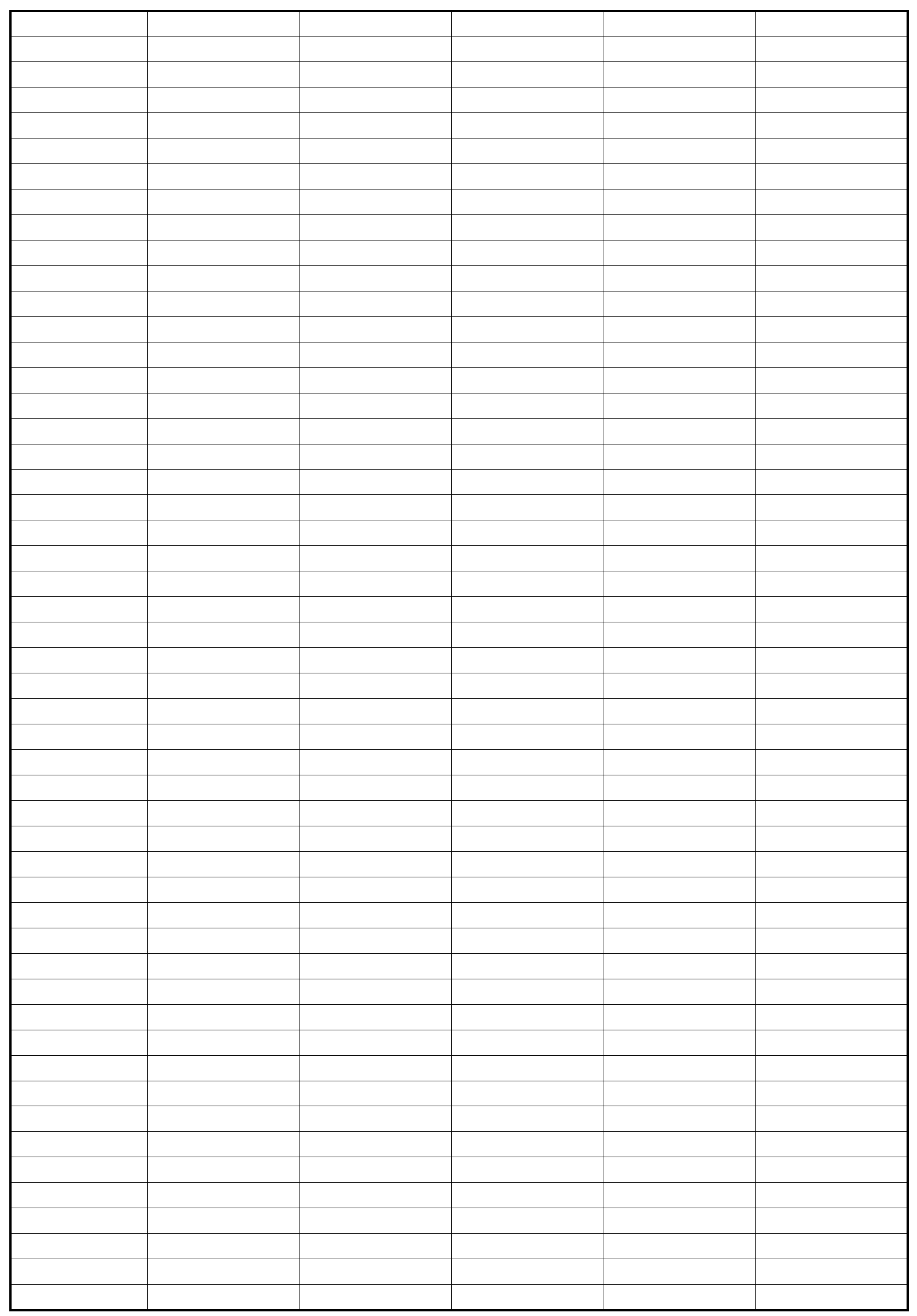

Page 83 
CHART DATA

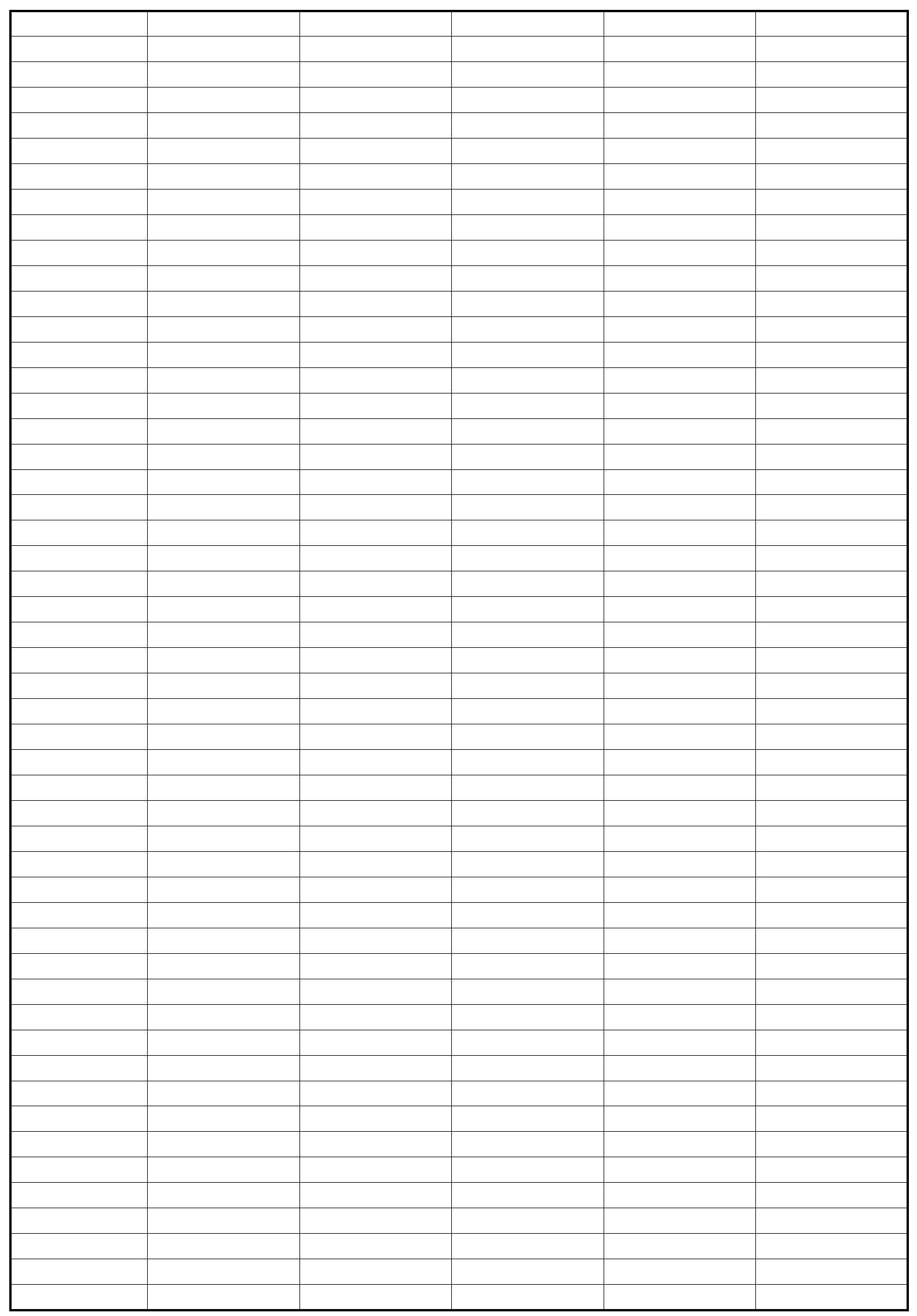

Page 84 
CHART DATA

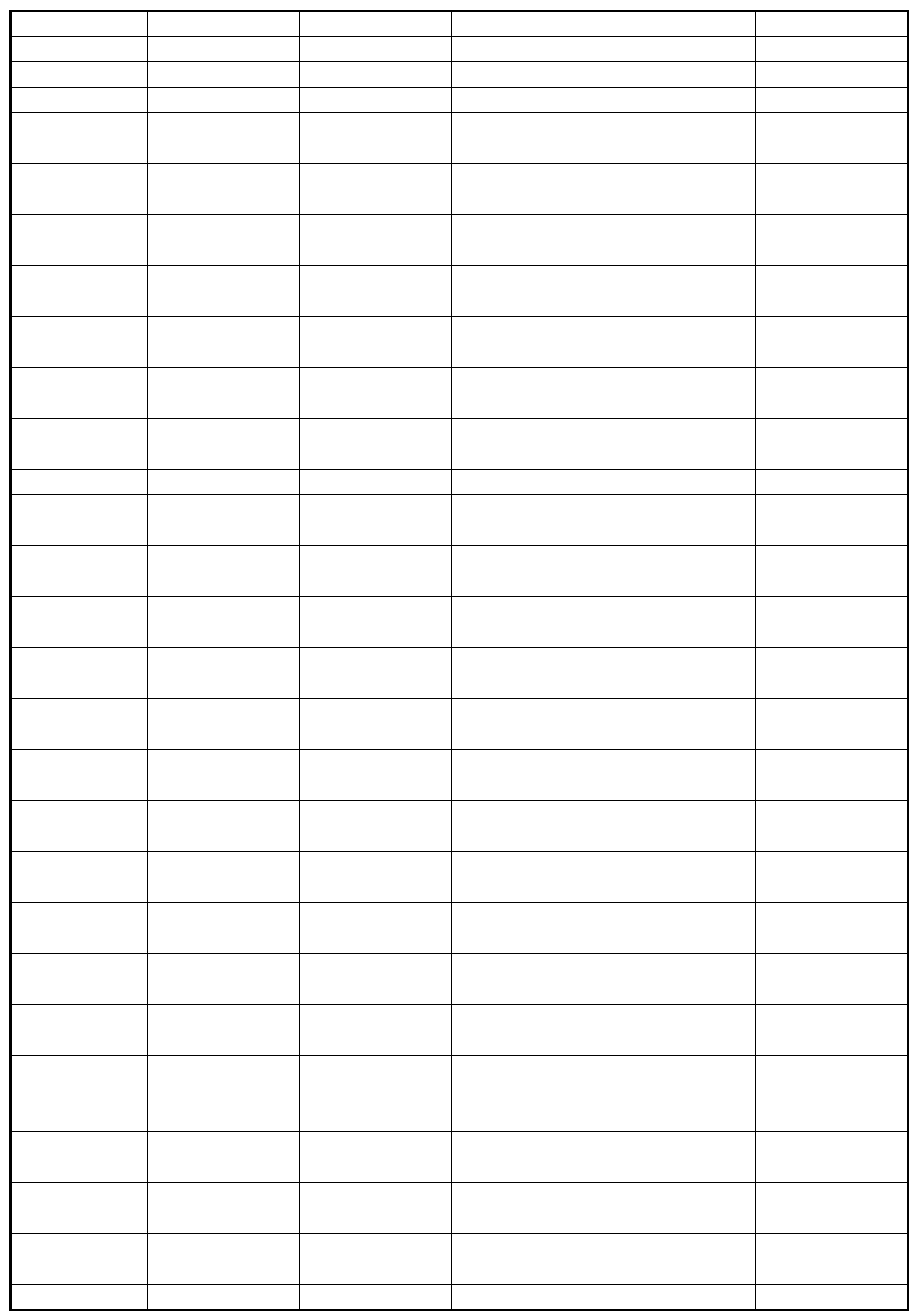

Page 85 
CHART DATA

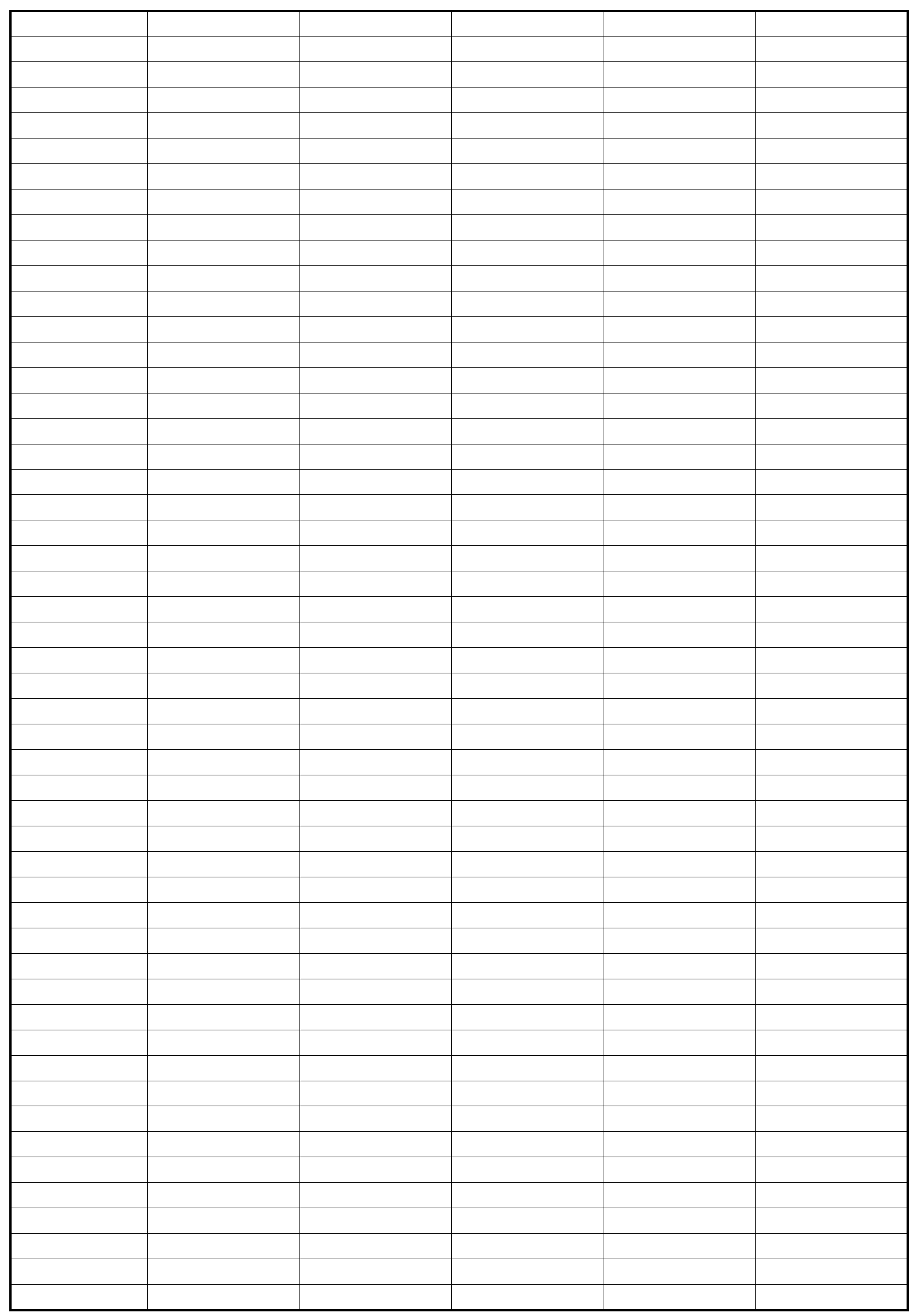

Page 86 
CHART DATA

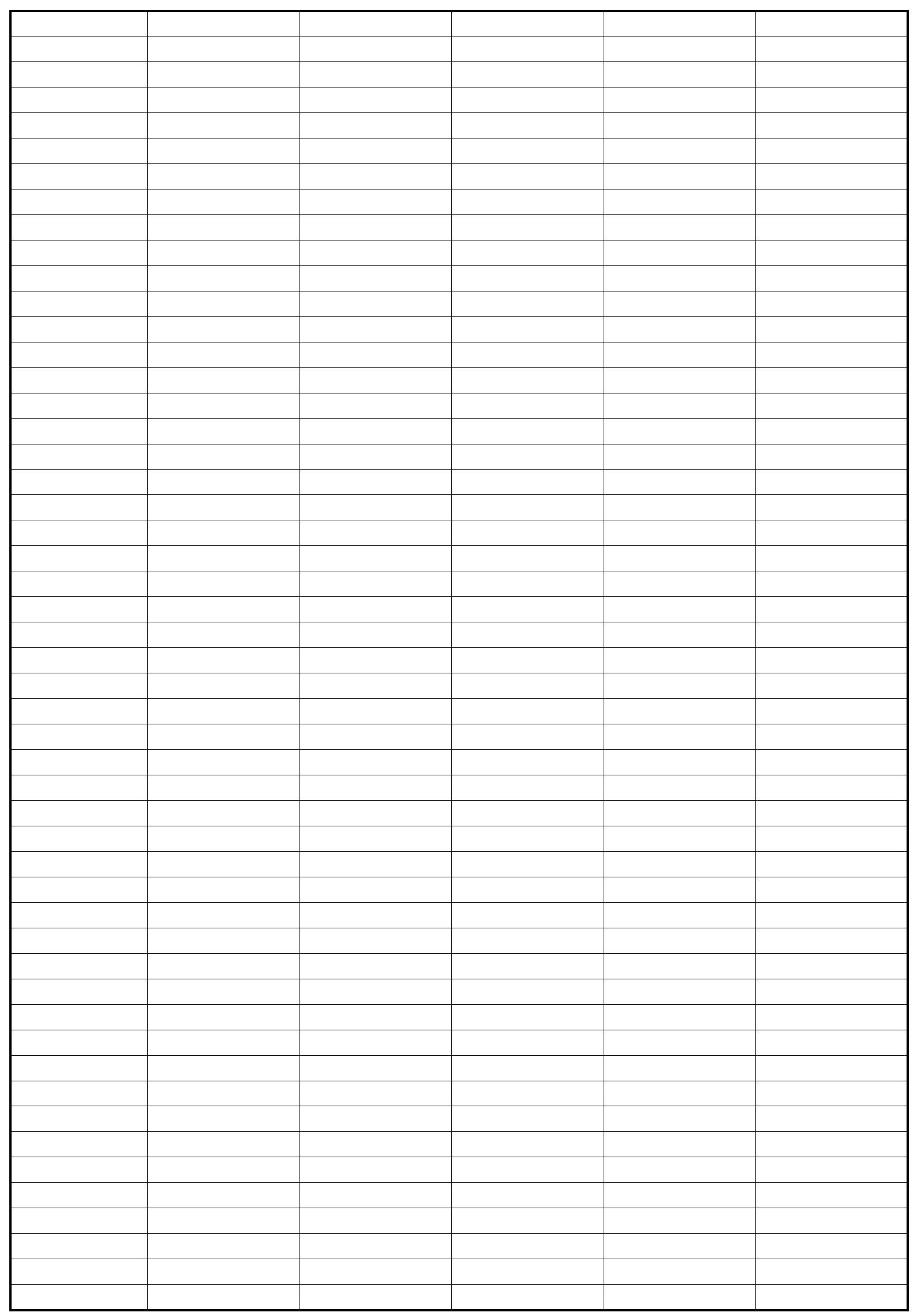

Page 87 
CHART DATA

\begin{tabular}{|l|l|l|l|l|l|}
\hline & & & & & \\
\hline & & & & & \\
\hline & & & & & \\
\hline & & & & & \\
\hline & & & & & \\
\hline & & & & & \\
\hline & & & & & \\
\hline & & & & & \\
\hline & & & & & \\
\hline & & & & & \\
\hline & & & & & \\
\hline & & & & & \\
\hline & & & & & \\
\hline & & & & & \\
\hline
\end{tabular}


CHART DATA

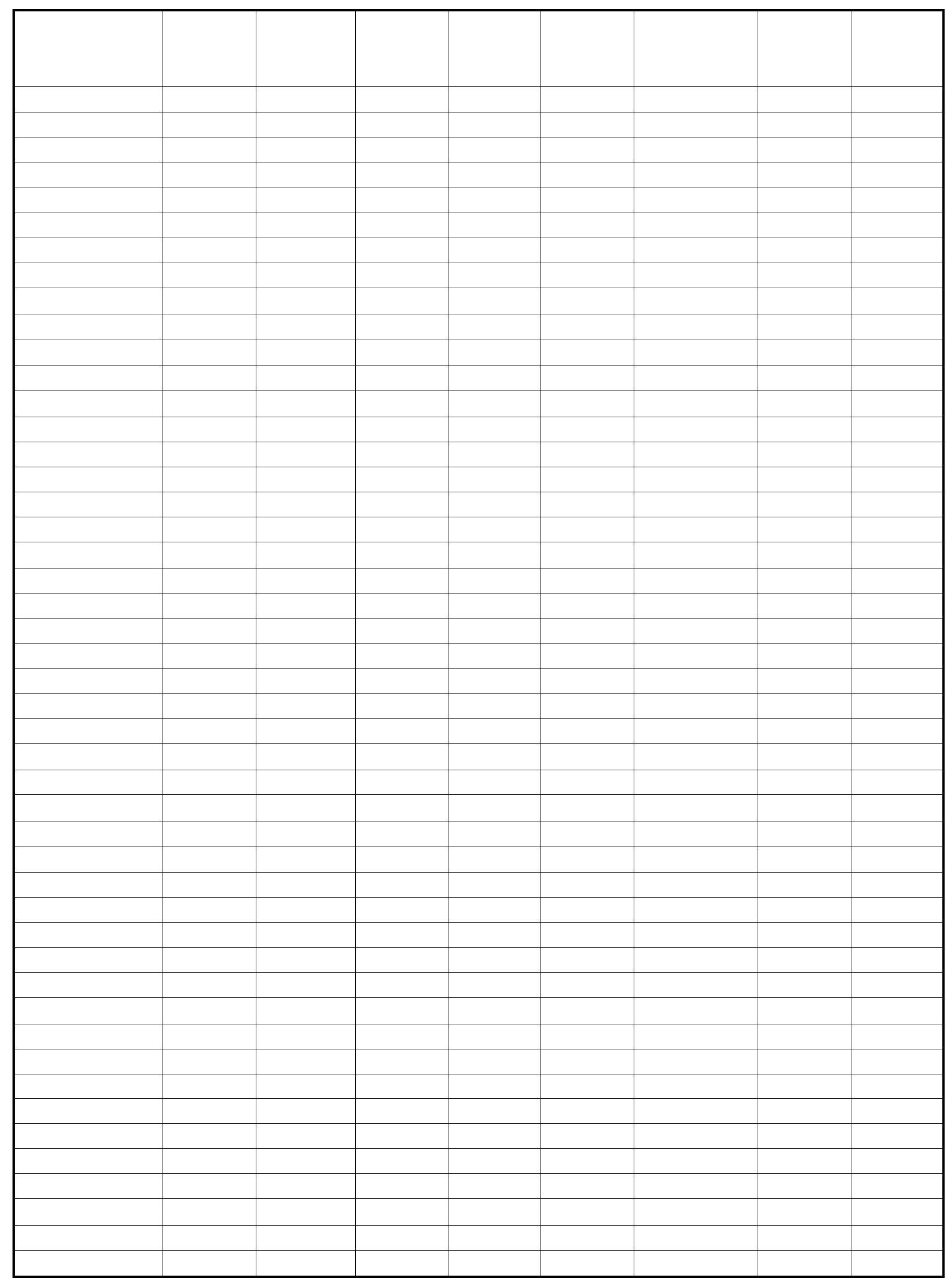

Page 89 


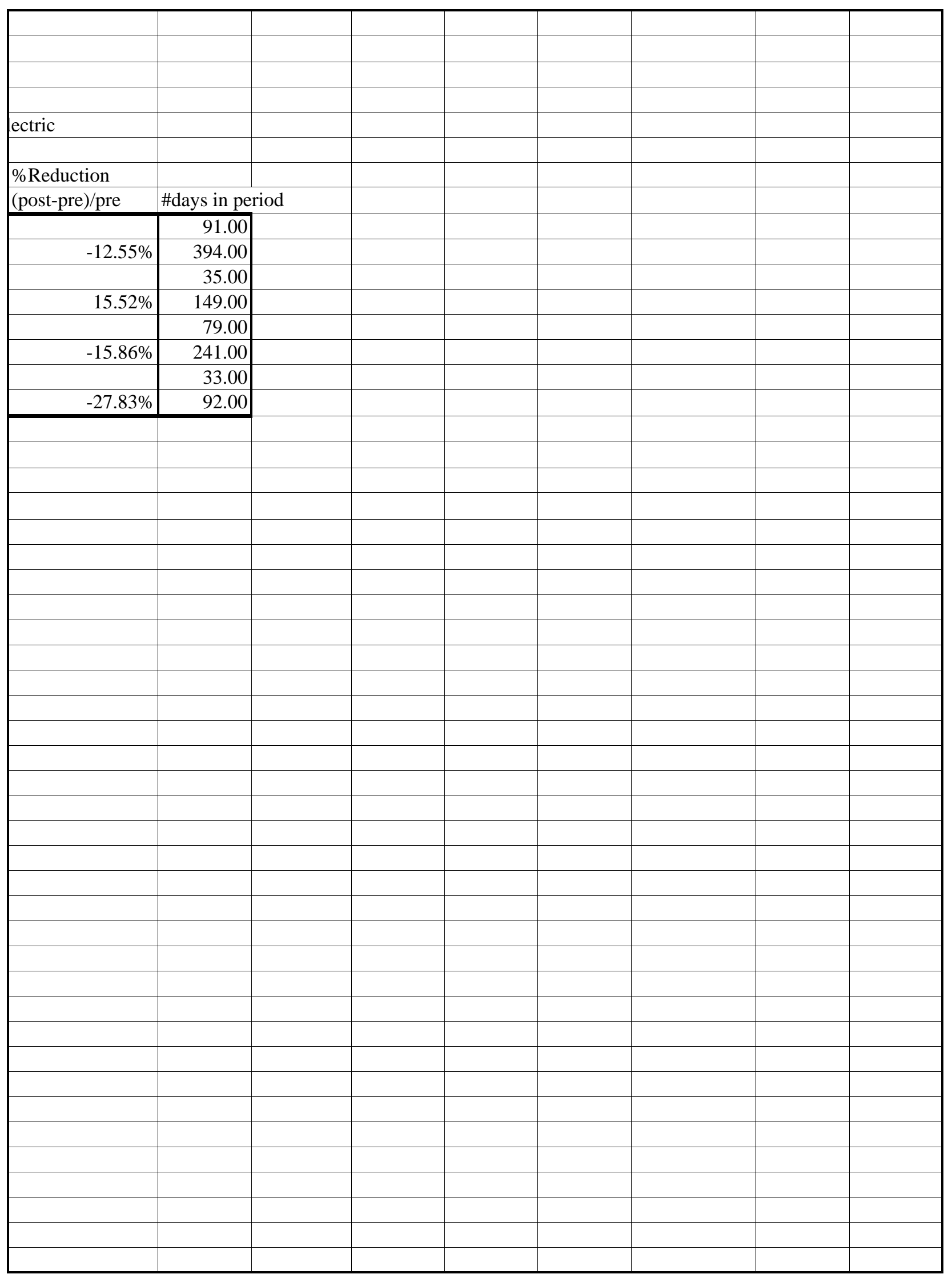


CHART DATA

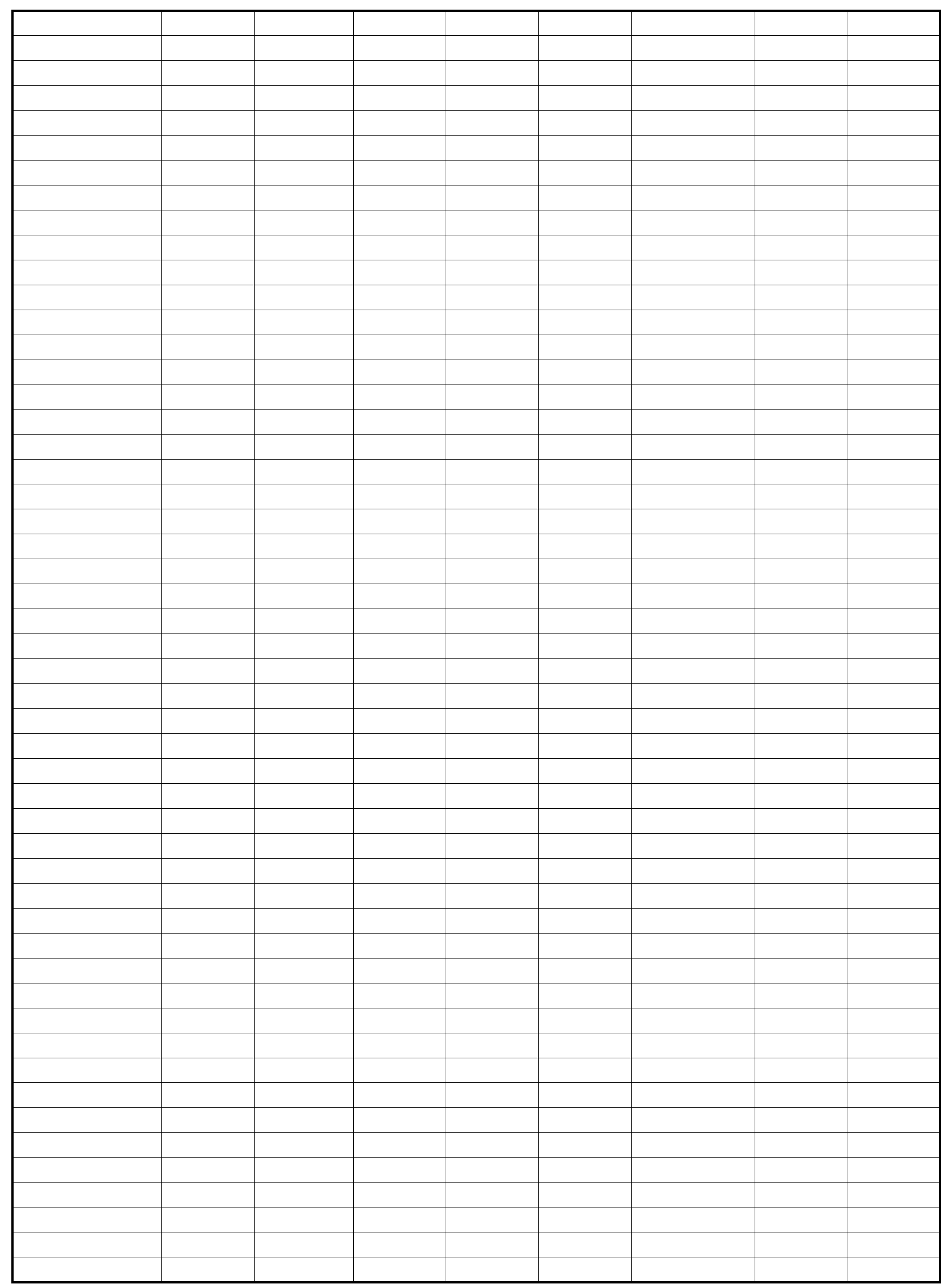

Page 91 
CHART DATA

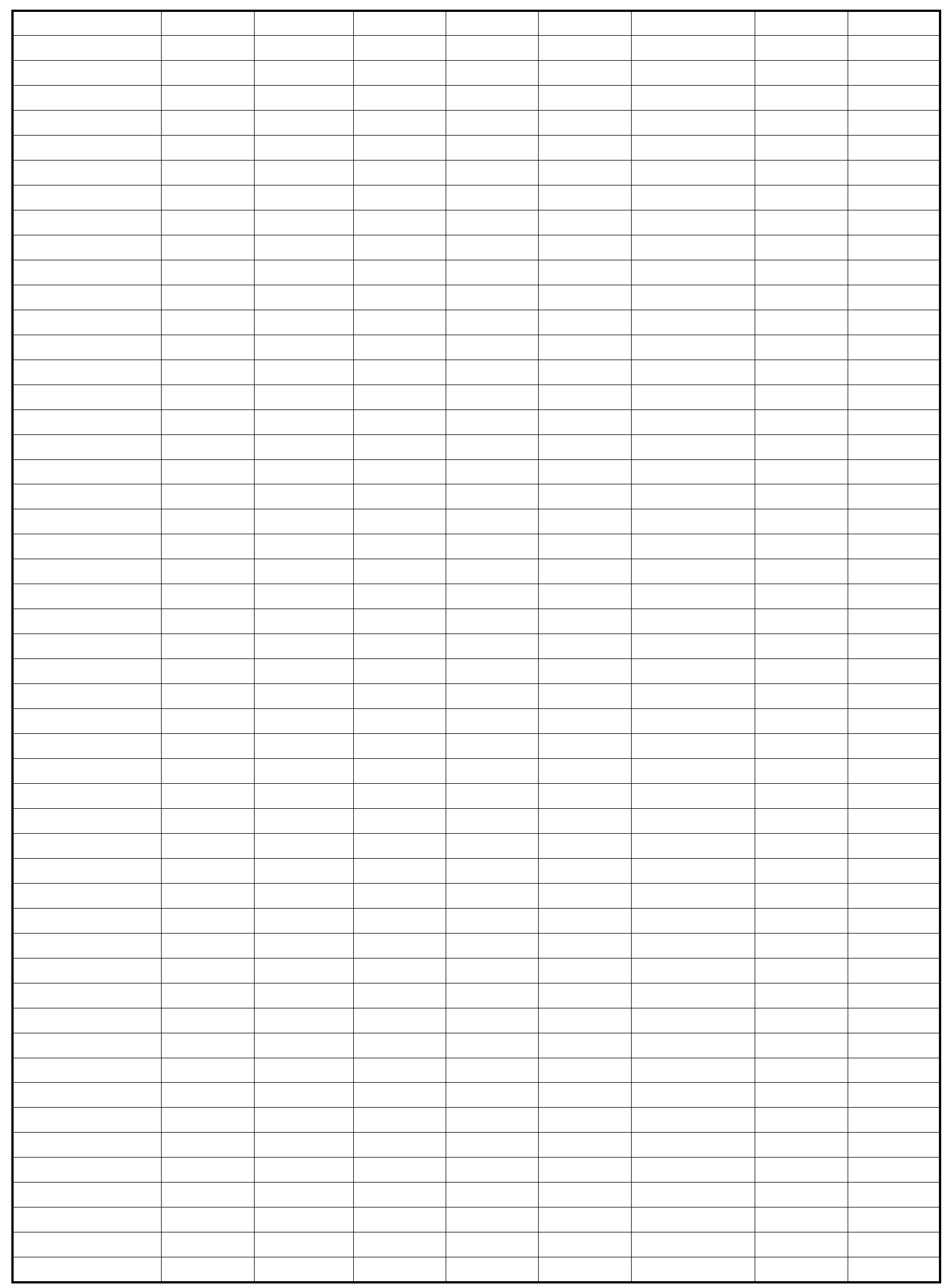

Page 92 
CHART DATA

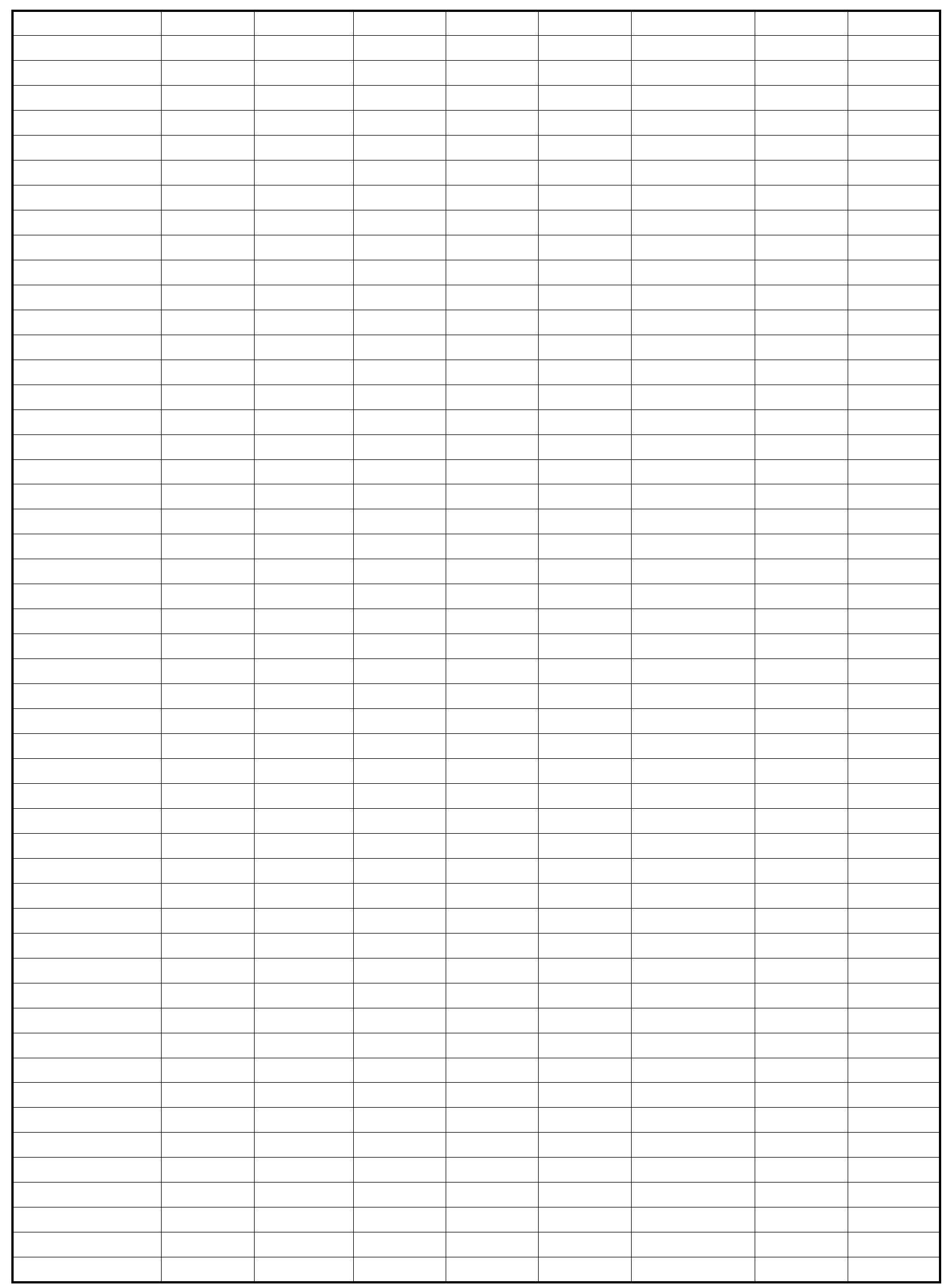

Page 93 
CHART DATA

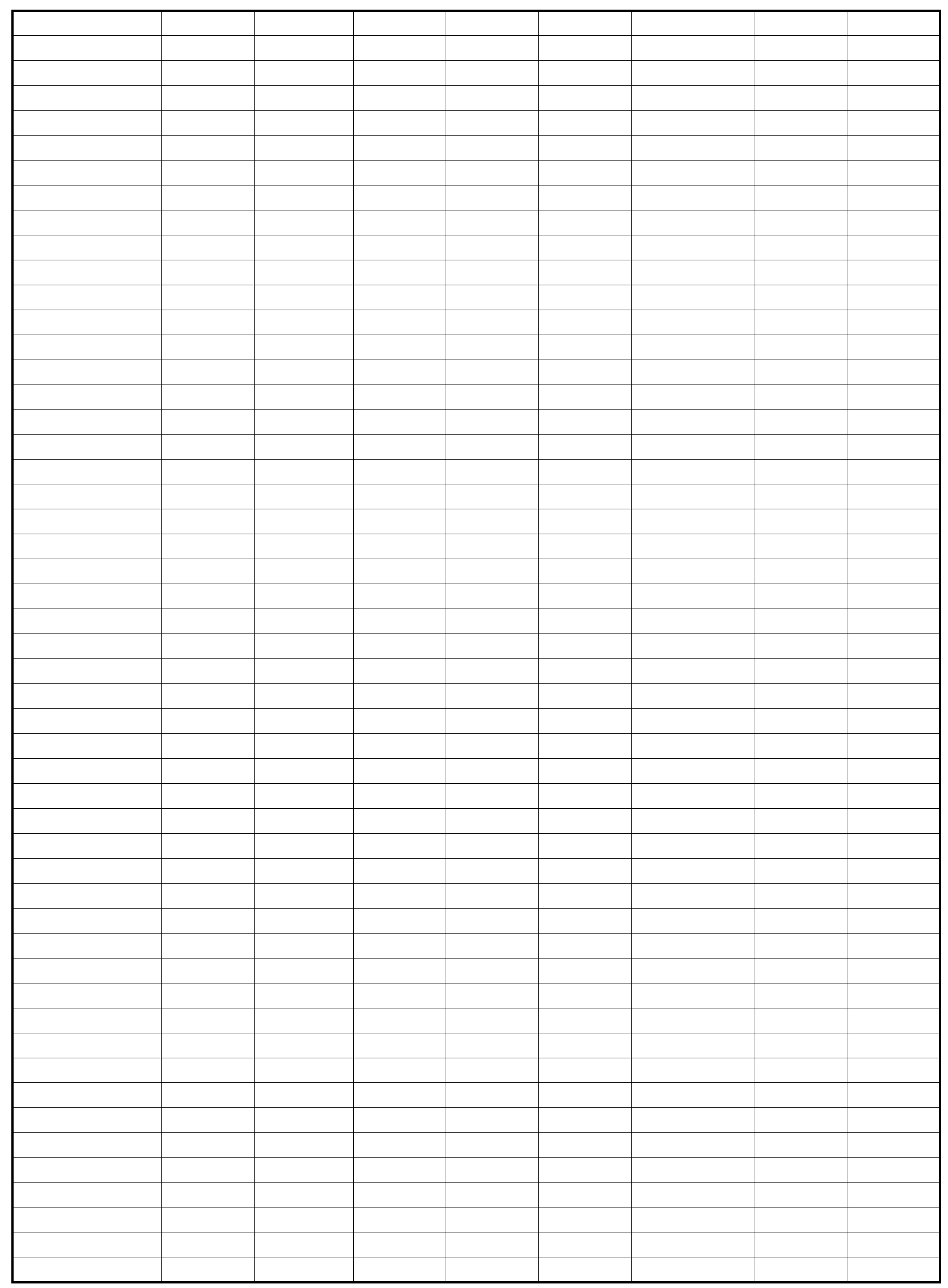


CHART DATA

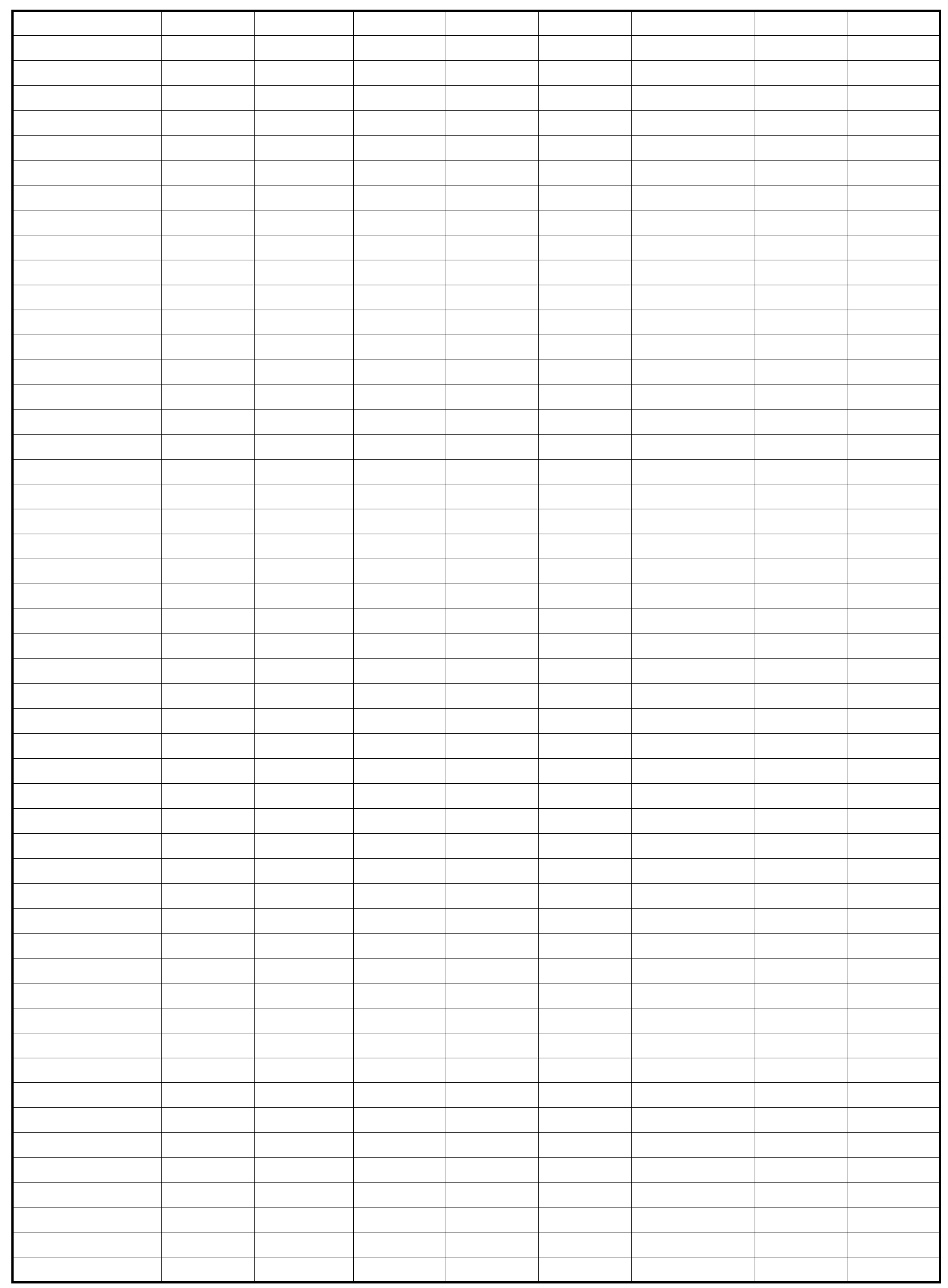

Page 95 
CHART DATA

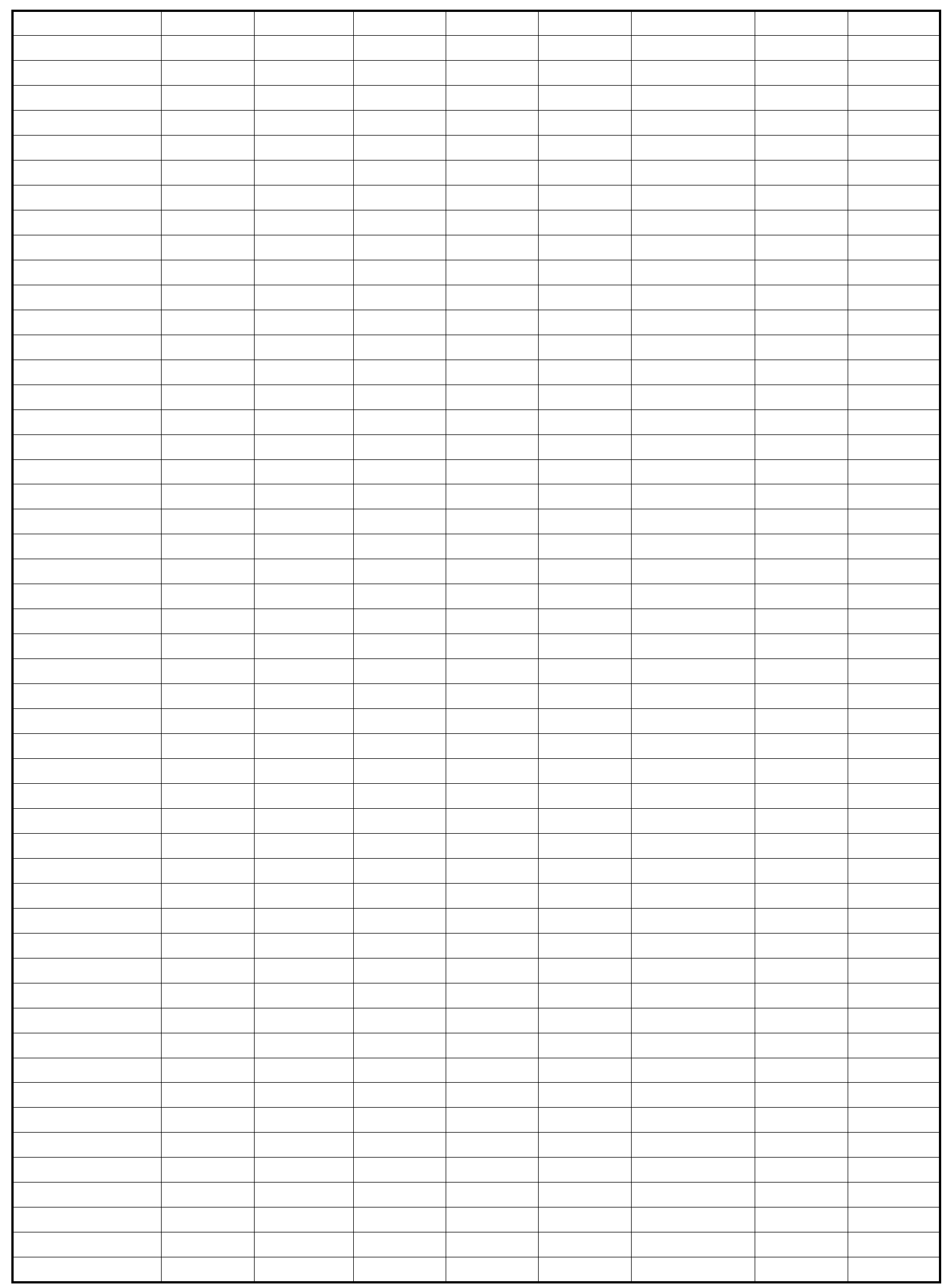

Page 96 
CHART DATA

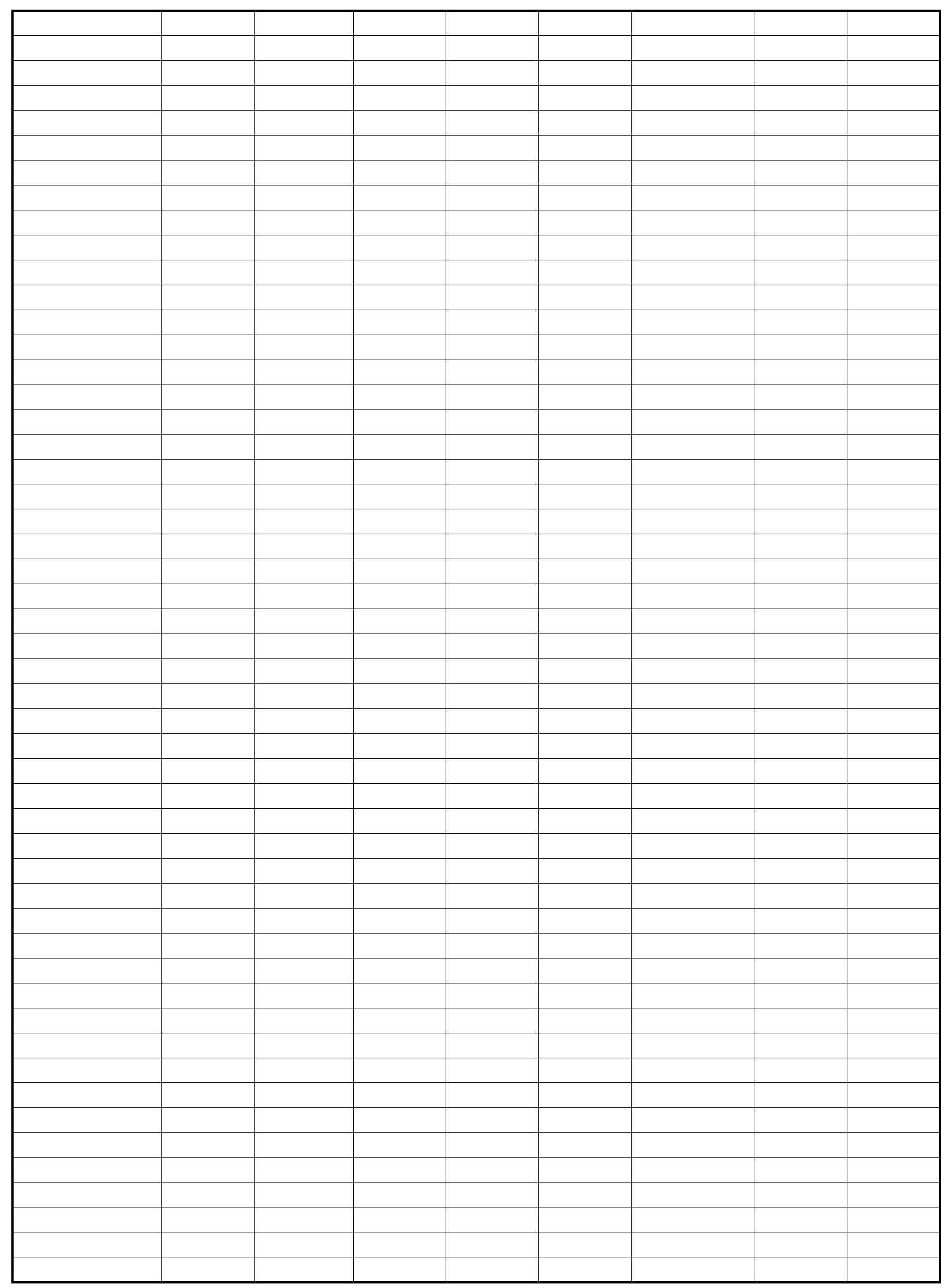

Page 97 
CHART DATA

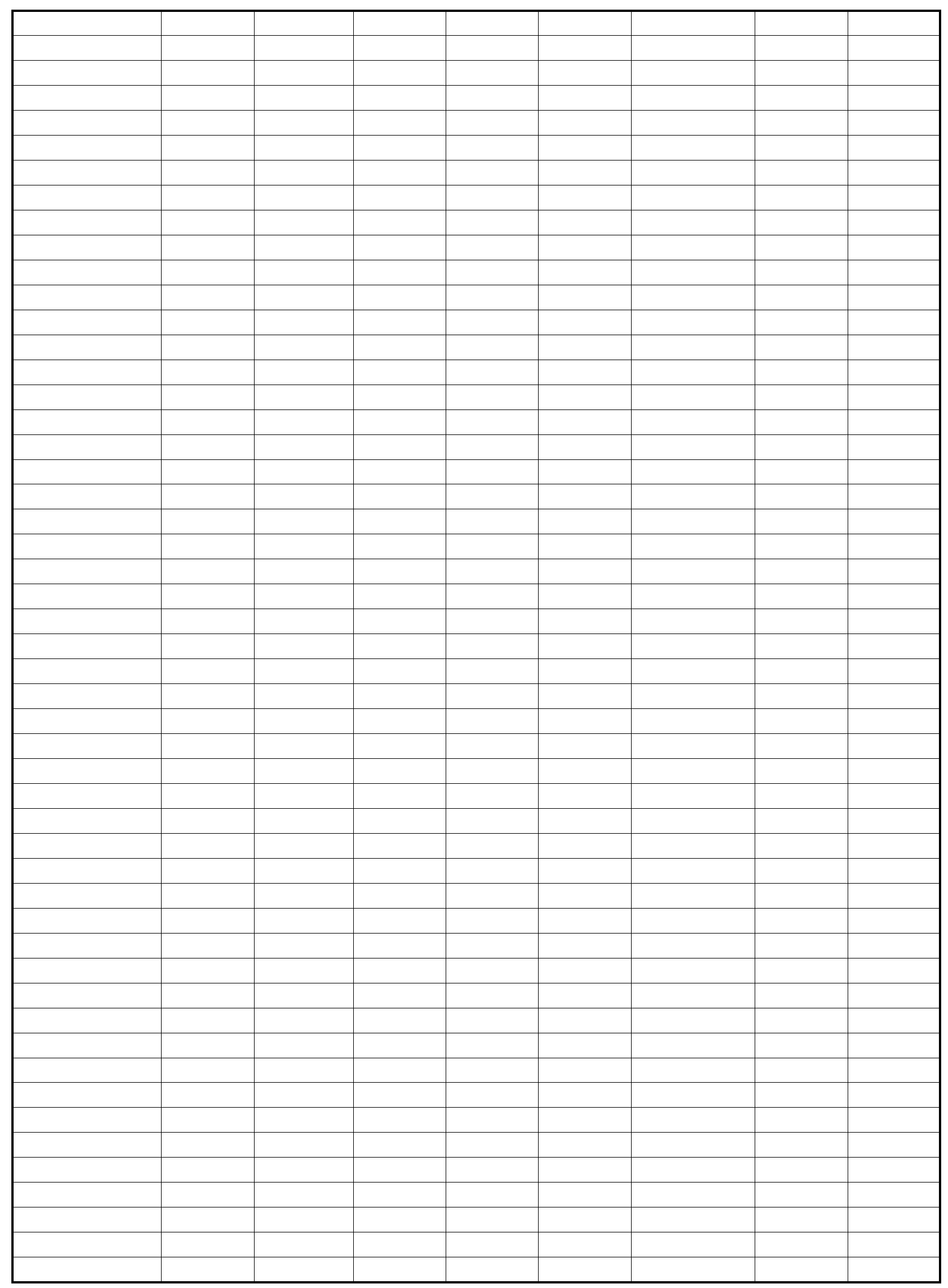


CHART DATA

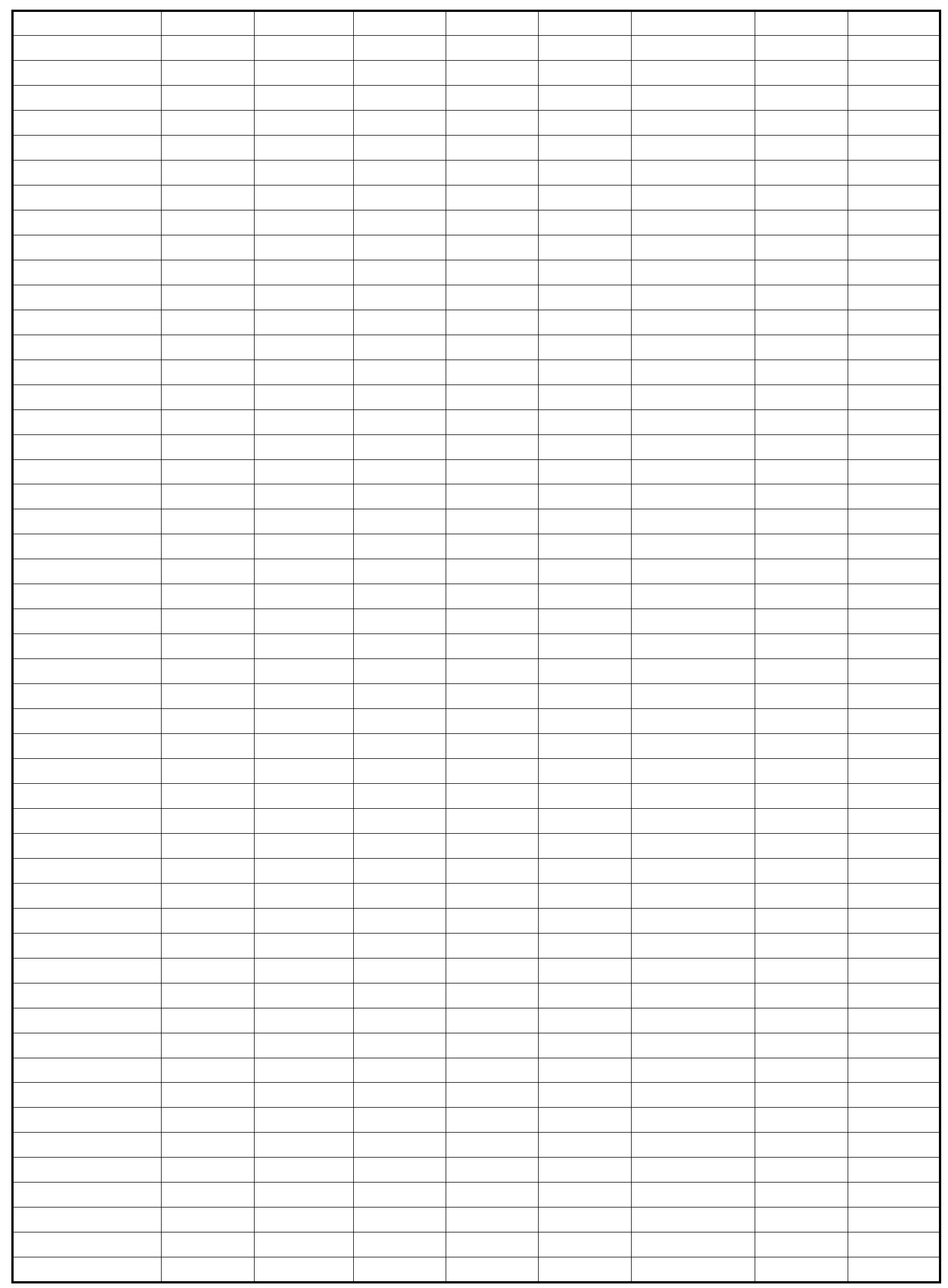

Page 99 
CHART DATA

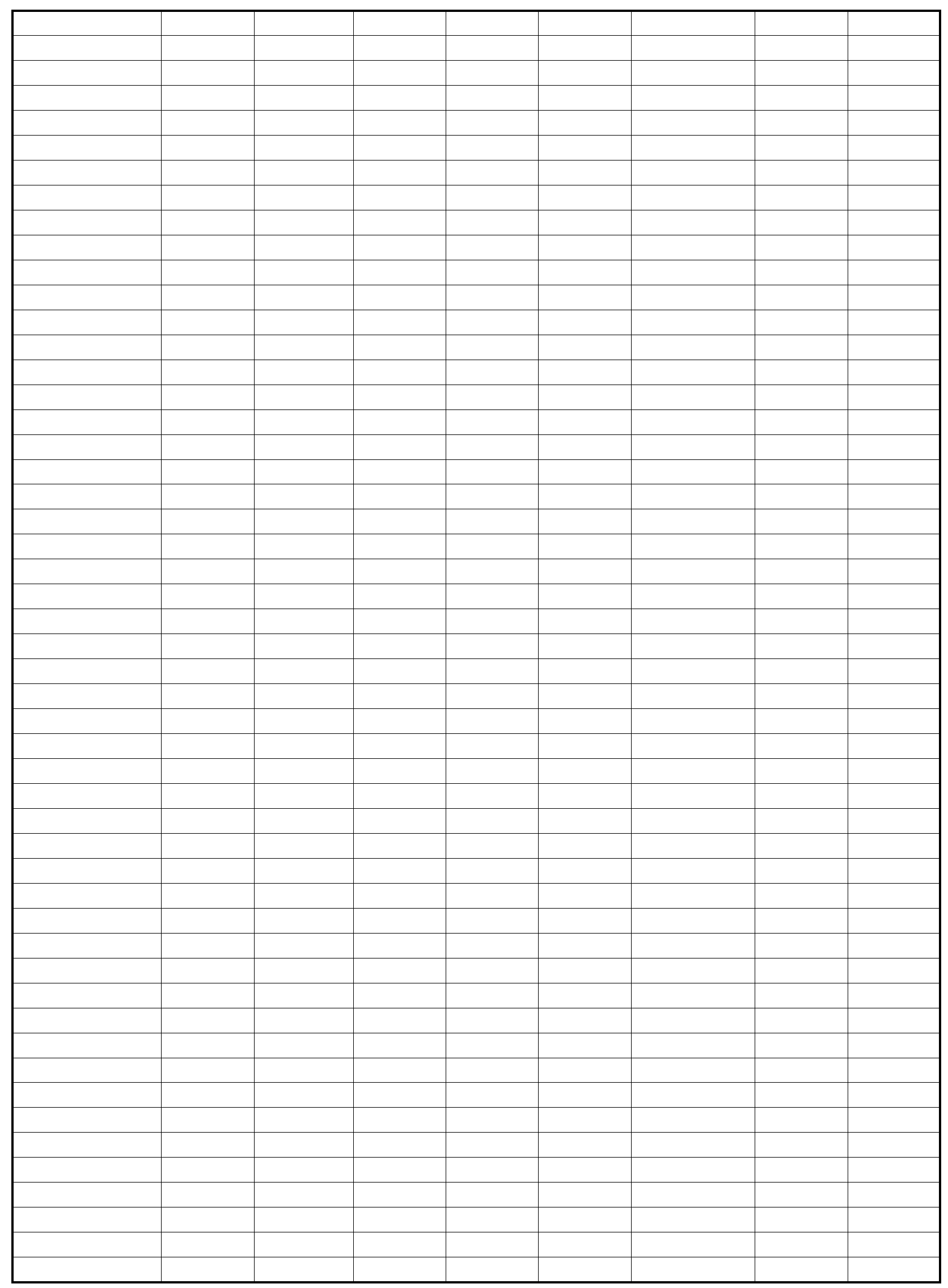

Page 100 
CHART DATA

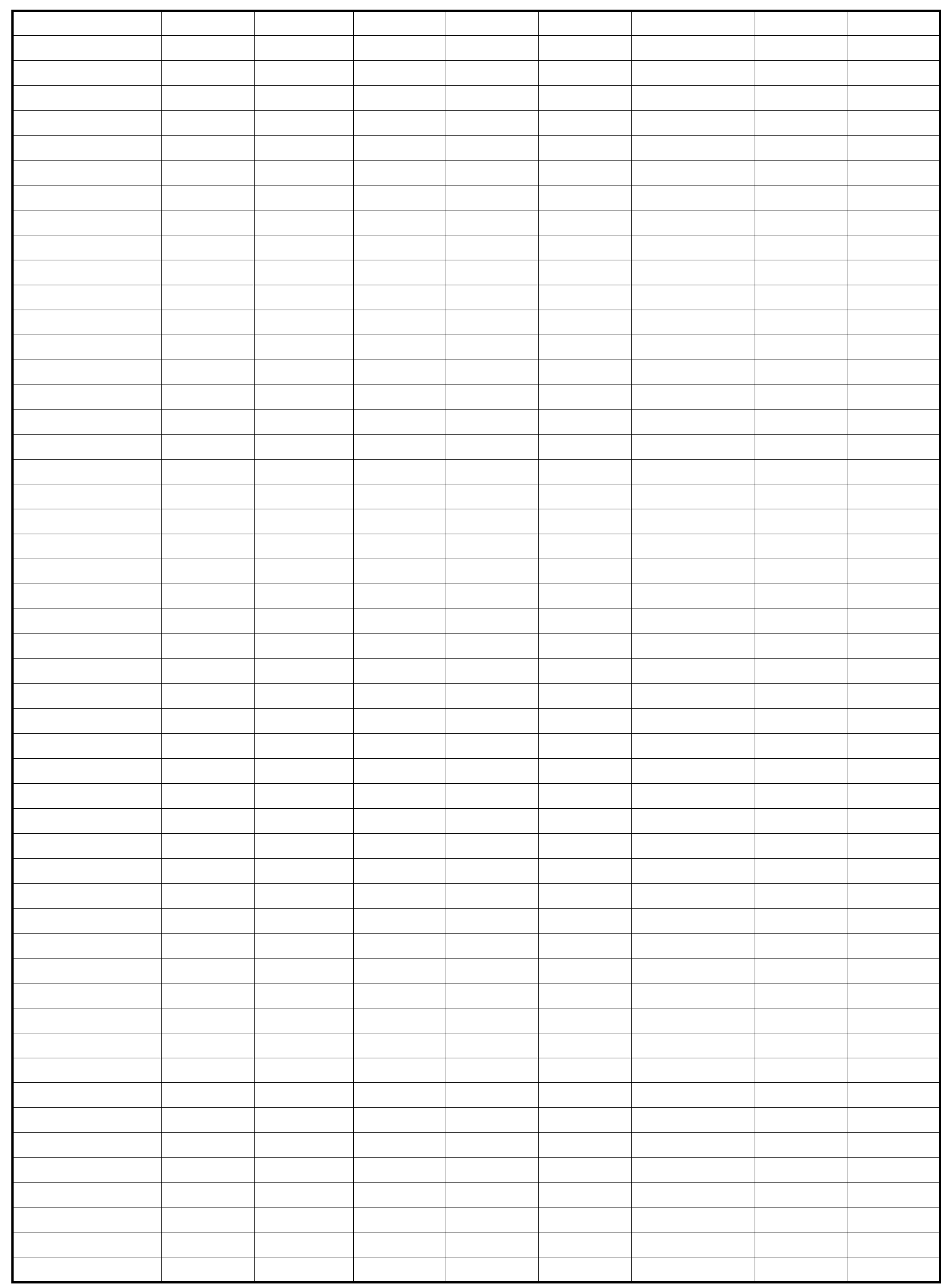

Page 101 
CHART DATA

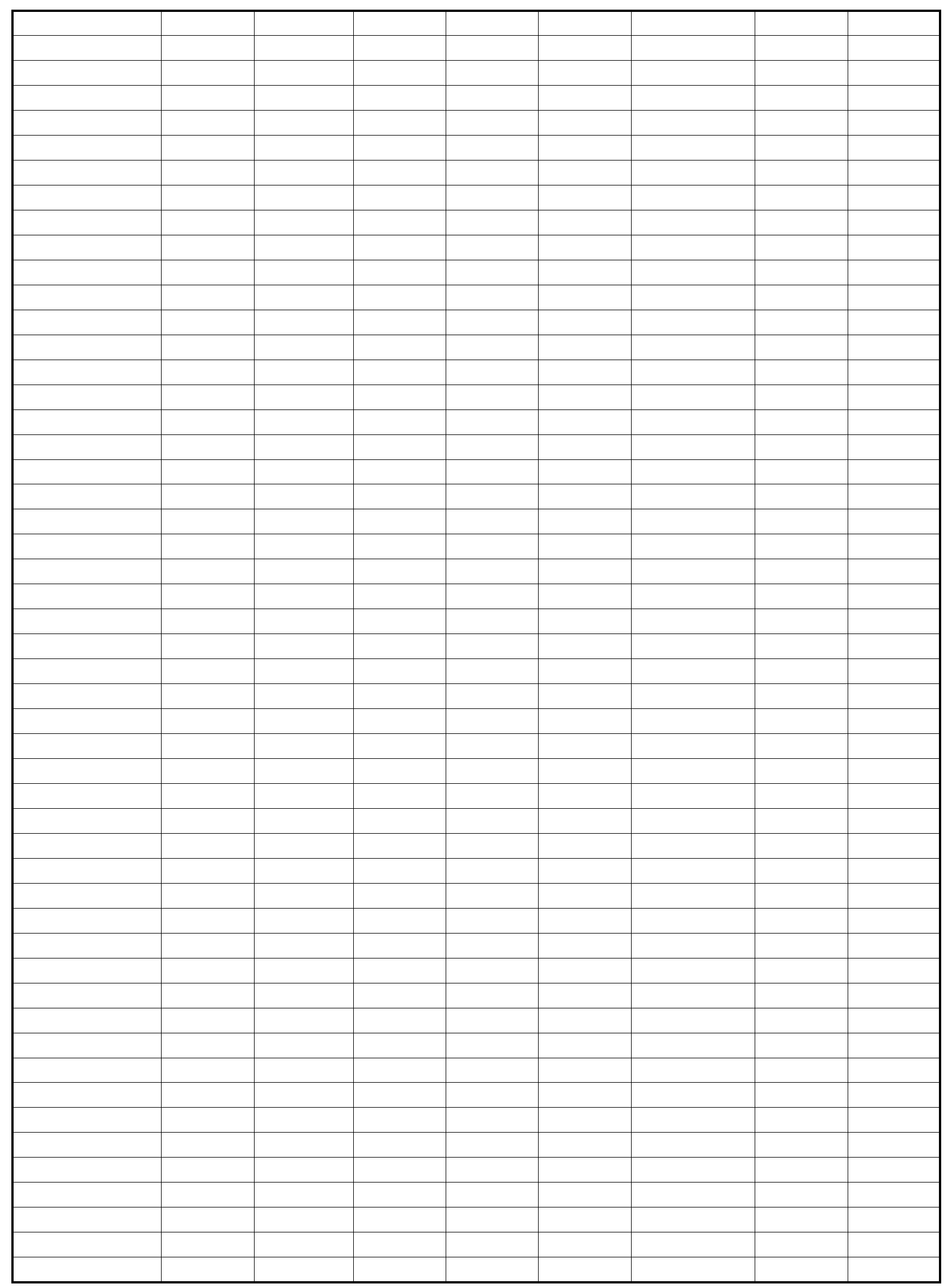

Page 102 
CHART DATA

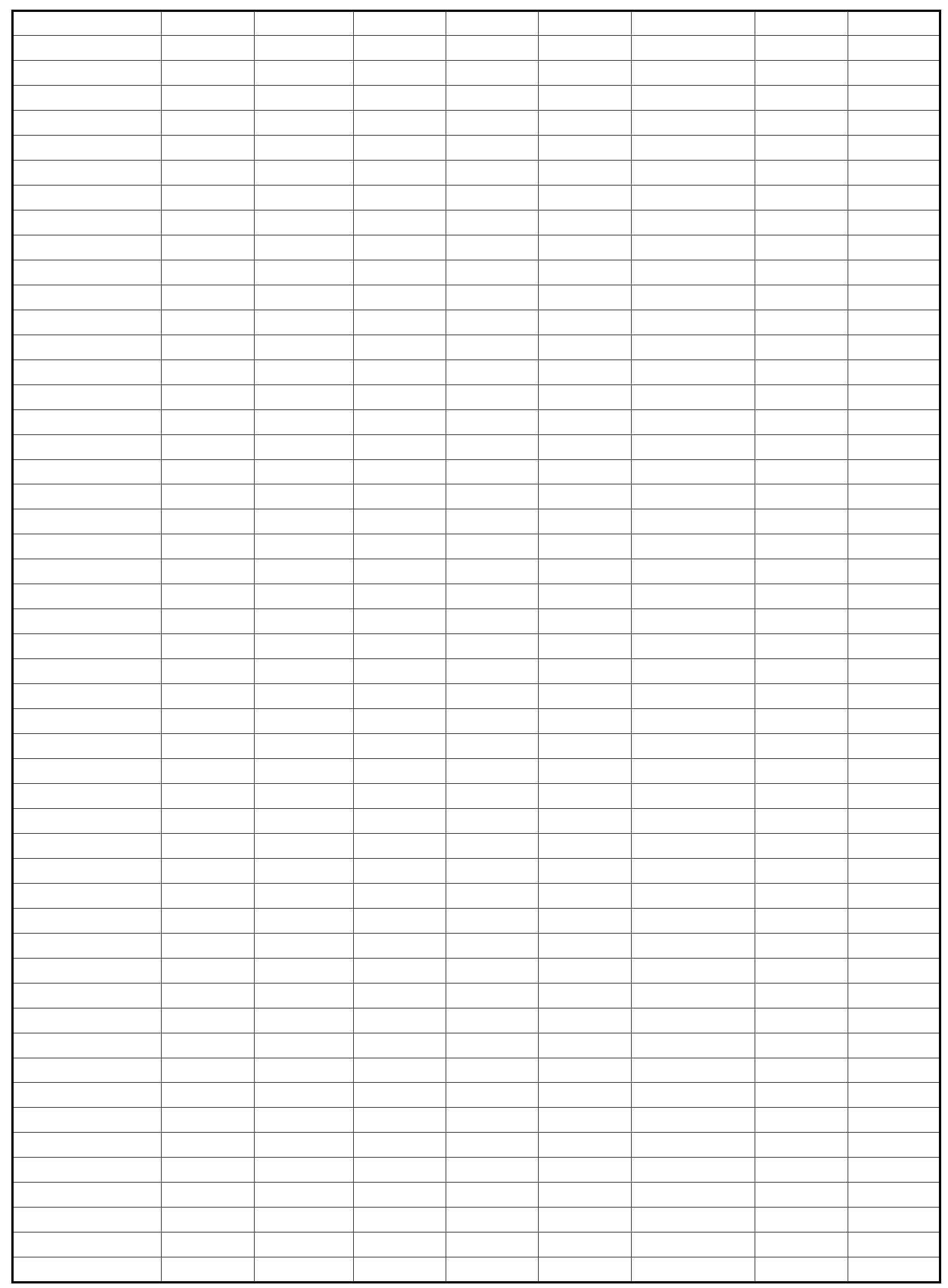

Page 103 
CHART DATA

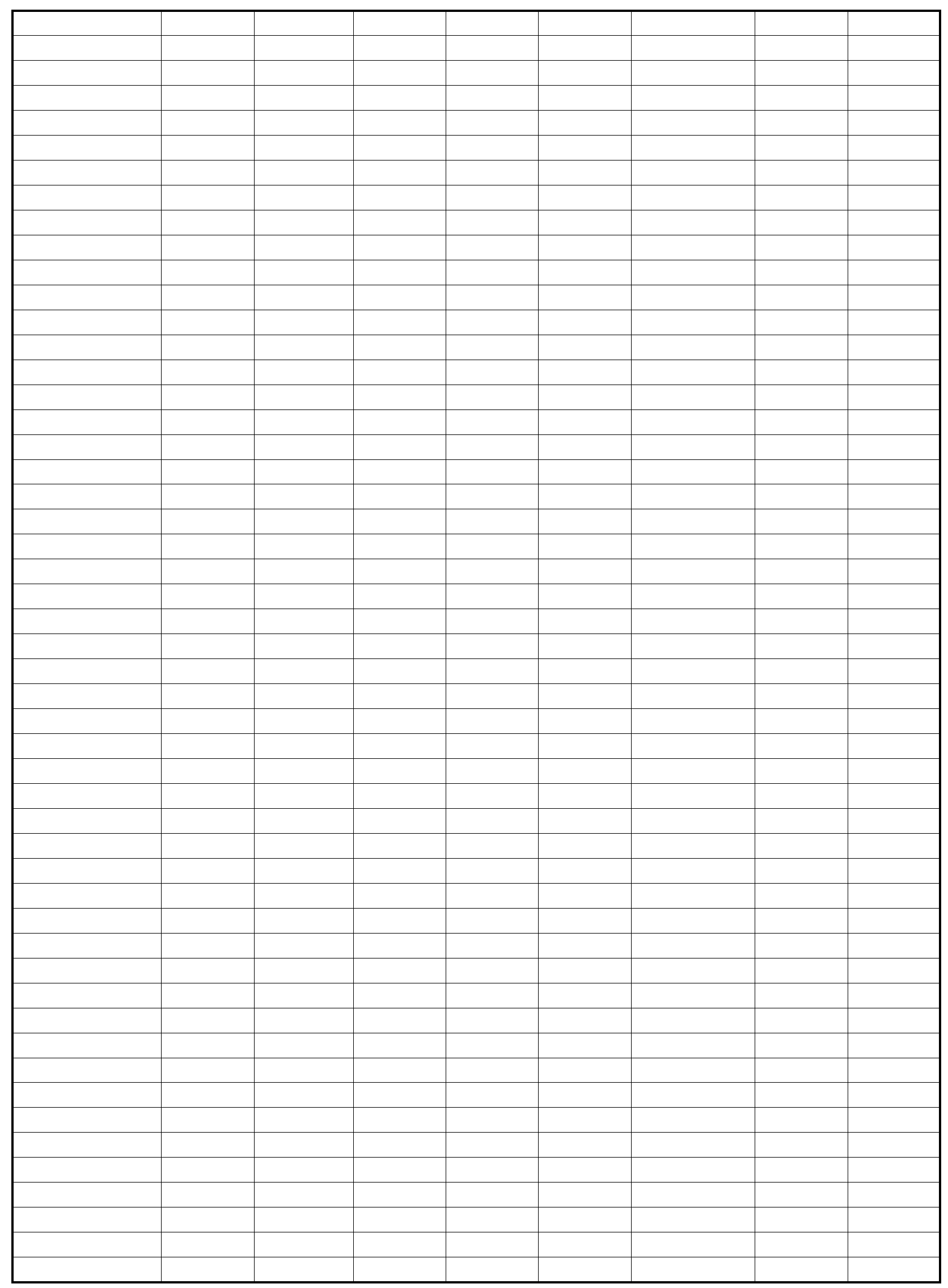

Page 104 
CHART DATA

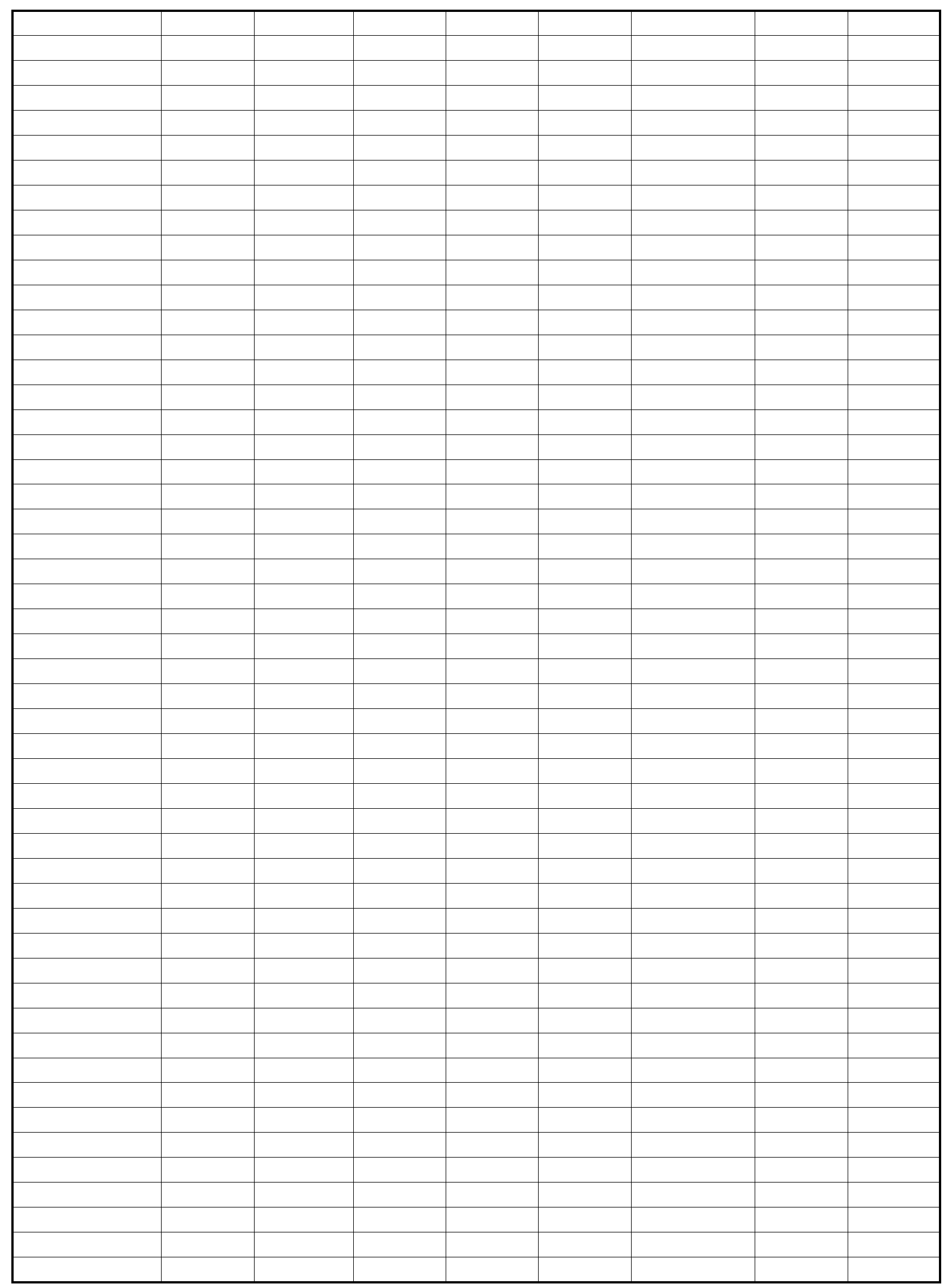

Page 105 
CHART DATA

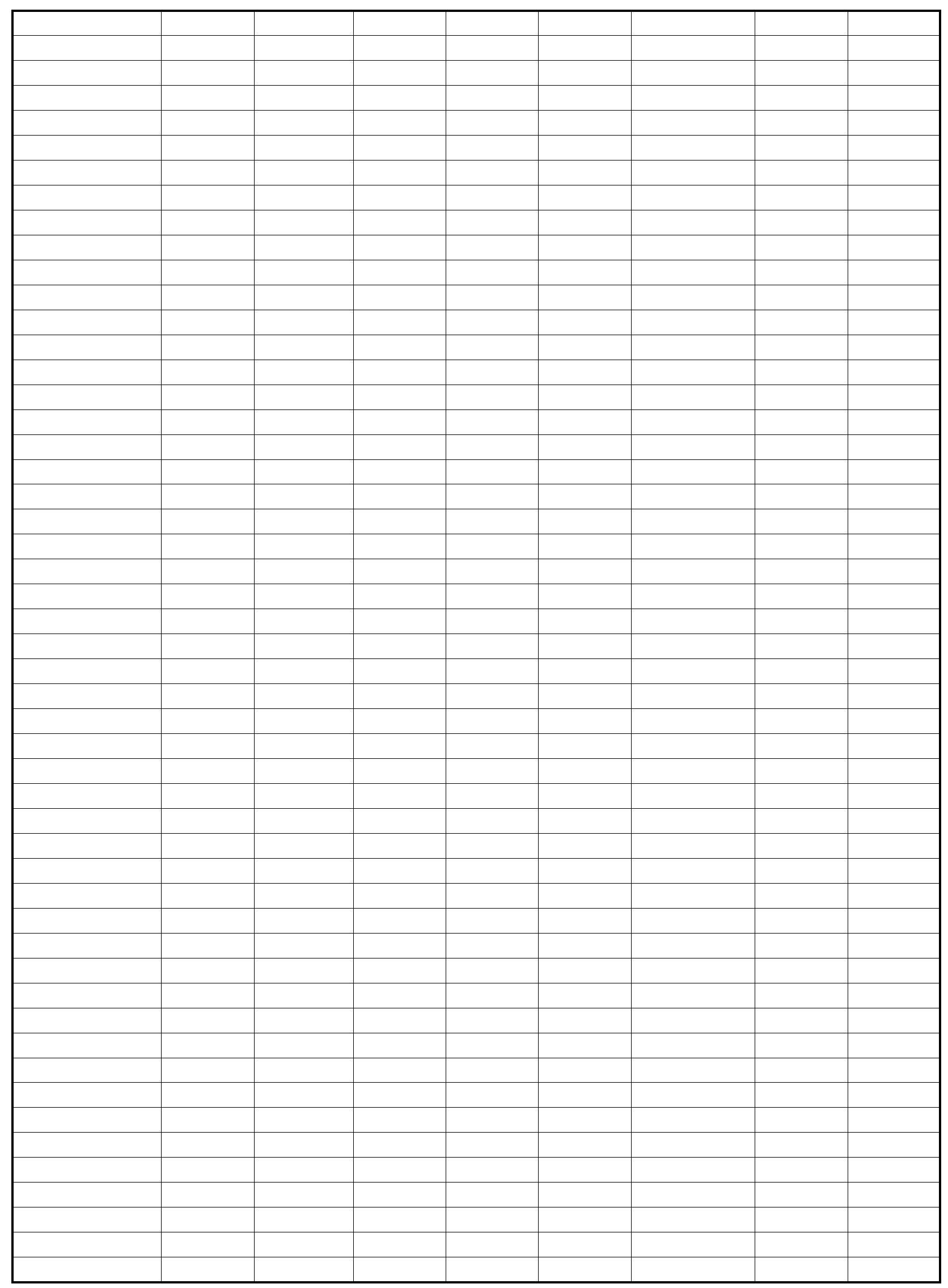

Page 106 
CHART DATA

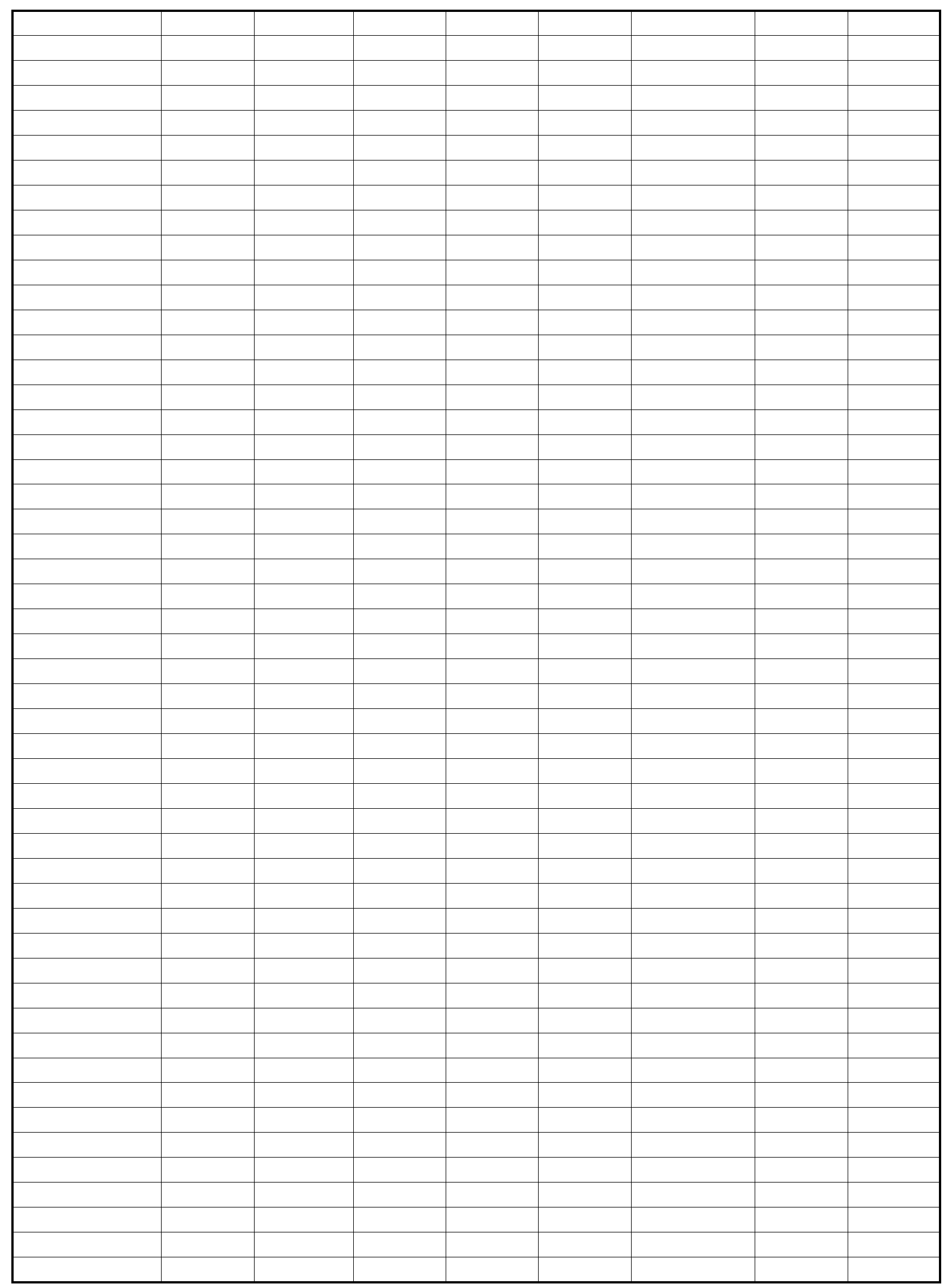

Page 107 
CHART DATA

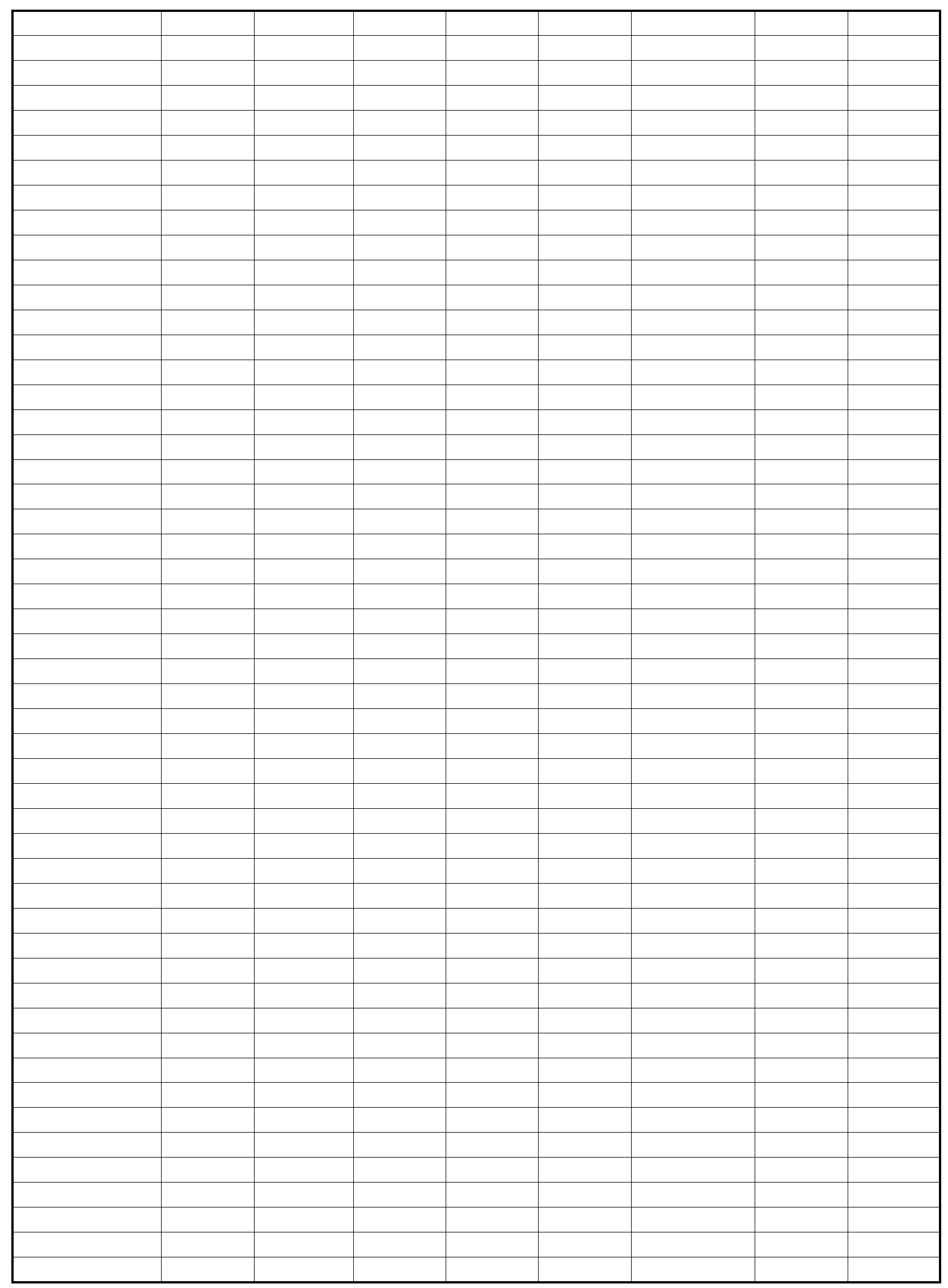

Page 108 
CHART DATA

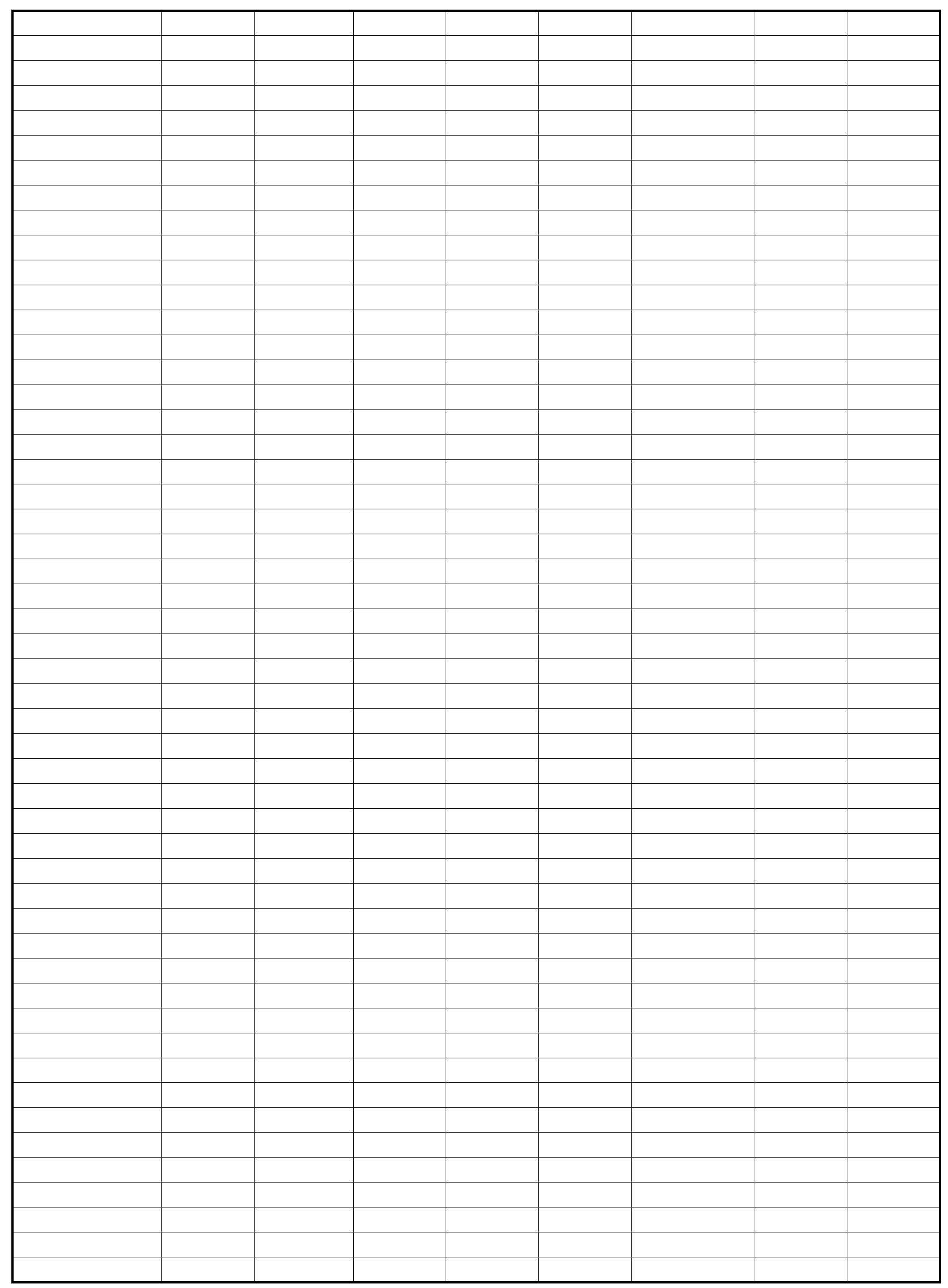

Page 109 
CHART DATA

\begin{tabular}{|l|l|l|l|l|l|l|l|l|}
\hline & & & & & & & & \\
\hline & & & & & & & & \\
\hline \\
\hline
\end{tabular}

Page 110 


\begin{tabular}{|c|c|c|c|c|c|c|c|c|}
\hline & $\begin{array}{l}\text { DAILY } \\
\text { TOTAL }\end{array}$ & $\begin{array}{l}\text { AVERAGE } \\
\text { DAILY } \\
\text { TOTAL }\end{array}$ & $\begin{array}{l}\text { DAILY } \\
\text { TOTAL }\end{array}$ & $\begin{array}{l}\text { AVERAGE } \\
\text { DAILY } \\
\text { TOTAL }\end{array}$ & $\begin{array}{l}\text { DAILY } \\
\text { TOTAL }\end{array}$ & $\begin{array}{l}\text { AVERAGE } \\
\text { DAILY } \\
\text { TOTAL }\end{array}$ & $\begin{array}{l}\text { DAILY } \\
\text { TOTAL }\end{array}$ & $\begin{array}{l}\text { AVERAGE } \\
\text { DAILY } \\
\text { TOTAL }\end{array}$ \\
\hline DATE & WBELEC & WBELEC & CHLR & CHLR & WBHEAT & WBHEAT & OTHER & OTHER \\
\hline 6/6/1991 & 4720.00 & 196.67 & & & & & 4720.00 & 196.67 \\
\hline 6/7/1991 & 4274.30 & 178.10 & & & & & 4274.30 & 178.10 \\
\hline 6/8/1991 & 3794.90 & 158.12 & & & & & 3794.90 & 158.12 \\
\hline 6/9/1991 & 3556.20 & 148.18 & & & & & 3556.20 & 148.18 \\
\hline 6/10/1991 & 4172.10 & 173.84 & & & & & 4172.10 & 173.84 \\
\hline 6/11/1991 & 4181.00 & 174.21 & & & & & 4181.00 & 174.21 \\
\hline $6 / 12 / 1991$ & 4572.40 & 190.52 & & & & & 4572.40 & 190.52 \\
\hline $6 / 13 / 1991$ & 5071.90 & 211.33 & & & & & 5071.90 & 211.33 \\
\hline $6 / 14 / 1991$ & 4167.20 & 173.63 & & & & & 4167.20 & 173.63 \\
\hline $6 / 15 / 1991$ & 3507.00 & 146.13 & & & & & 3507.00 & 146.13 \\
\hline $6 / 16 / 1991$ & 3601.90 & 150.08 & & & & & 3601.90 & 150.08 \\
\hline 6/17/1991 & 5759.30 & 239.97 & & & & & 5759.30 & 239.97 \\
\hline 6/18/1991 & 6964.90 & 290.20 & & & & & 6964.90 & 290.20 \\
\hline 6/19/1991 & 6813.50 & 283.90 & & & & & 6813.50 & 283.90 \\
\hline 6/20/1991 & 6554.40 & 273.10 & & & & & 6554.40 & 273.10 \\
\hline $6 / 21 / 1991$ & 4266.50 & 177.77 & & & & & 4266.50 & 177.77 \\
\hline $6 / 22 / 1991$ & 3813.00 & 158.88 & & & & & 3813.00 & 158.88 \\
\hline 6/23/1991 & 3633.90 & 151.41 & & & & & 3633.90 & 151.41 \\
\hline $6 / 24 / 1991$ & 6348.80 & 264.53 & & & 16.40 & 2.34 & 6348.80 & 264.53 \\
\hline 6/25/1991 & 6035.80 & 251.49 & & & & & 6035.80 & 251.49 \\
\hline $6 / 26 / 1991$ & 6275.10 & 261.46 & & & & & 6275.10 & 261.46 \\
\hline 6/27/1991 & 7169.50 & 298.73 & & & & & 7169.50 & 298.73 \\
\hline 6/28/1991 & 5096.70 & 212.36 & & & & & 5096.70 & 212.36 \\
\hline 6/29/1991 & 3701.30 & 154.22 & & & & & 3701.30 & 154.22 \\
\hline 6/30/1991 & 3691.70 & 153.82 & & & & & 3691.70 & 153.82 \\
\hline 7/1/1991 & 6183.80 & 257.66 & & & & & 6183.80 & 257.66 \\
\hline 7/2/1991 & 6004.00 & 250.17 & & & & & 6004.00 & 250.17 \\
\hline 7/3/1991 & 5319.60 & 221.65 & & & & & 5319.60 & 221.65 \\
\hline 7/4/1991 & 3182.90 & 132.62 & & & & & 3182.90 & 132.62 \\
\hline 7/5/1991 & 3260.70 & 135.86 & & & & & 3260.70 & 135.86 \\
\hline 7/6/1991 & 3198.20 & 133.26 & & & & & 3198.20 & 133.26 \\
\hline 7/7/1991 & 2971.20 & 123.80 & & & & & 2971.20 & 123.80 \\
\hline 7/8/1991 & 5629.70 & 234.57 & & & & & 5629.70 & 234.57 \\
\hline 7/9/1991 & 5542.20 & 230.93 & & & & & 5542.20 & 230.93 \\
\hline 7/10/1991 & 5541.00 & 230.88 & & & 17.50 & 17.50 & 5541.00 & 230.88 \\
\hline 7/11/1991 & 5364.60 & 223.53 & & & & & 5364.60 & 223.53 \\
\hline 7/12/1991 & 3317.10 & 138.21 & & & & & 3317.10 & 138.21 \\
\hline 7/13/1991 & 3047.30 & 126.97 & & & & & 3047.30 & 126.97 \\
\hline 7/14/1991 & 3096.60 & 129.03 & & & & & 3096.60 & 129.03 \\
\hline $7 / 15 / 1991$ & 5188.20 & 216.18 & & & & & 5188.20 & 216.18 \\
\hline 7/16/1991 & 4870.20 & 202.93 & & & & & 4870.20 & 202.93 \\
\hline 7/17/1991 & 4825.50 & 201.06 & & & & & 4825.50 & 201.06 \\
\hline 7/18/1991 & 3894.20 & 162.26 & & & & & 3894.20 & 162.26 \\
\hline 7/19/1991 & 3227.90 & 134.50 & & & & & 3227.90 & 134.50 \\
\hline 7/20/1991 & 3087.10 & 128.63 & & & & & 3087.10 & 128.63 \\
\hline 7/21/1991 & 2928.10 & 122.00 & & & & & 2928.10 & 122.00 \\
\hline
\end{tabular}


CHART DATA

\begin{tabular}{|c|c|c|c|c|c|c|}
\hline 7/22/1991 & 5375.00 & 223.96 & & & 5375.00 & 223.96 \\
\hline 7/23/1991 & 4759.70 & 198.32 & 2.00 & 0.50 & 4759.70 & 198.32 \\
\hline 7/24/1991 & 5229.80 & 217.91 & 1.00 & 0.50 & 5229.80 & 217.91 \\
\hline 7/25/1991 & 5412.10 & 225.50 & & & 5412.10 & 225.50 \\
\hline 7/26/1991 & 3919.30 & 163.30 & & & 3919.30 & 163.30 \\
\hline 7/27/1991 & 3686.60 & 153.61 & & & 3686.60 & 153.61 \\
\hline 7/28/1991 & 3549.30 & 147.89 & & & 3549.30 & 147.89 \\
\hline 7/29/1991 & 5344.00 & 222.67 & & & 5344.00 & 222.67 \\
\hline 7/30/1991 & 4671.40 & 212.34 & & & 4671.40 & 212.34 \\
\hline 7/31/1991 & 5975.40 & 248.98 & & & 5975.40 & 248.98 \\
\hline 8/1/1991 & 6274.10 & 261.42 & & & 6274.10 & 261.42 \\
\hline 8/2/1991 & 4491.00 & 187.13 & & & 4491.00 & 187.13 \\
\hline 8/3/1991 & 4135.20 & 172.30 & & & 4135.20 & 172.30 \\
\hline 8/4/1991 & 3780.90 & 157.54 & & & 3780.90 & 157.54 \\
\hline 8/5/1991 & 6214.10 & 258.92 & & & 6214.10 & 258.92 \\
\hline 8/6/1991 & 6126.90 & 255.29 & & & 6126.90 & 255.29 \\
\hline 8/7/1991 & 6430.70 & 267.95 & & & 6430.70 & 267.95 \\
\hline 8/8/1991 & 6904.30 & 287.68 & & & 6904.30 & 287.68 \\
\hline 8/9/1991 & 7849.40 & 327.06 & & & 7849.40 & 327.06 \\
\hline 8/10/1991 & 4454.70 & 185.61 & & & 4454.70 & 185.61 \\
\hline 8/11/1991 & 3995.30 & 166.47 & & & 3995.30 & 166.47 \\
\hline $8 / 12 / 1991$ & 7891.00 & 328.79 & & & 7891.00 & 328.79 \\
\hline $8 / 13 / 1991$ & 8888.70 & 370.36 & & & 8888.70 & 370.36 \\
\hline 8/14/1991 & 9313.30 & 388.05 & & & 9313.30 & 388.05 \\
\hline $8 / 15 / 1991$ & 8276.10 & 344.84 & & & 8276.10 & 344.84 \\
\hline 8/16/1991 & 7712.00 & 321.33 & & & 7712.00 & 321.33 \\
\hline 8/17/1991 & 5464.60 & 227.69 & & & 5464.60 & 227.69 \\
\hline 8/18/1991 & 5305.80 & 221.08 & & & 5305.80 & 221.08 \\
\hline 8/19/1991 & 9698.50 & 404.10 & & & 9698.50 & 404.10 \\
\hline 8/20/1991 & 11502.50 & 479.27 & & & 11502.50 & 479.27 \\
\hline 8/21/1991 & 13090.50 & 545.44 & & & 13090.50 & 545.44 \\
\hline 8/22/1991 & 13902.50 & 579.27 & & & 13902.50 & 579.27 \\
\hline 8/23/1991 & 13719.30 & 571.64 & & & 13719.30 & 571.64 \\
\hline 8/24/1991 & 9237.70 & 384.90 & & & 9237.70 & 384.90 \\
\hline 8/25/1991 & 8039.40 & 334.98 & & & 8039.40 & 334.98 \\
\hline 8/26/1991 & 12757.00 & 531.54 & & & 12757.00 & 531.54 \\
\hline 8/27/1991 & 13755.90 & 573.16 & & & 13755.90 & 573.16 \\
\hline 8/28/1991 & 13733.30 & 572.22 & & & 13733.30 & 572.22 \\
\hline 8/29/1991 & 13887.10 & 578.63 & 0.50 & 0.50 & 13887.10 & 578.63 \\
\hline 8/30/1991 & 14345.30 & 597.72 & & & 14345.30 & 597.72 \\
\hline 8/31/1991 & 9564.50 & 398.52 & & & 9564.50 & 398.52 \\
\hline 9/1/1991 & 7496.90 & 312.37 & 0.50 & 0.50 & 7496.90 & 312.37 \\
\hline 9/2/1991 & 8885.40 & 370.23 & 0.50 & 0.50 & 8885.40 & 370.23 \\
\hline 9/3/1991 & 12268.80 & 511.20 & 0.50 & 0.50 & 12268.80 & 511.20 \\
\hline 9/4/1991 & 9516.30 & 432.56 & 1.00 & 0.50 & 9516.30 & 432.56 \\
\hline 9/5/1991 & 11021.00 & 459.21 & 1.50 & 0.50 & 11021.00 & 459.21 \\
\hline 9/6/1991 & 10852.60 & 452.19 & & & 10852.60 & 452.19 \\
\hline 9/7/1991 & 5333.40 & 222.23 & 1.50 & 0.50 & 5333.40 & 222.23 \\
\hline 9/8/1991 & 6423.10 & 267.63 & & & 6423.10 & 267.63 \\
\hline 9/9/1991 & 12764.70 & 531.86 & 0.50 & 0.50 & 12764.70 & 531.86 \\
\hline
\end{tabular}




\begin{tabular}{|c|c|c|c|c|c|c|c|c|}
\hline $9 / 10 / 1991$ & 11985.30 & 499.39 & & & & & 11985.30 & 499.39 \\
\hline 9/11/1991 & 11483.40 & 478.48 & & & 0.50 & 0.50 & 11483.40 & 478.48 \\
\hline 9/12/1991 & 11522.30 & 480.10 & & & & & 11522.30 & 480.10 \\
\hline 9/13/1991 & 12388.80 & 516.20 & & & 0.50 & 0.50 & 12388.80 & 516.20 \\
\hline 9/14/1991 & 6062.00 & 252.58 & & & & & 6062.00 & 252.58 \\
\hline 9/15/1991 & 6401.70 & 266.74 & & & & & 6401.70 & 266.74 \\
\hline 9/16/1991 & 12590.30 & 524.60 & & & 0.50 & 0.50 & 12590.30 & 524.60 \\
\hline 9/17/1991 & 11606.90 & 483.62 & & & & & 11606.90 & 483.62 \\
\hline 9/18/1991 & 11455.60 & 477.32 & & & 1.00 & 0.50 & 11455.60 & 477.32 \\
\hline 9/19/1991 & 9480.60 & 395.03 & & & 2.00 & 0.50 & 9480.60 & 395.03 \\
\hline 9/20/1991 & 8258.20 & 344.09 & & & 3.00 & 0.50 & 8258.20 & 344.09 \\
\hline 9/21/1991 & 5708.20 & 237.84 & & & 1.50 & 0.50 & 5708.20 & 237.84 \\
\hline 9/22/1991 & 4494.30 & 187.26 & & & 2.00 & 0.50 & 4494.30 & 187.26 \\
\hline 9/23/1991 & 11591.80 & 482.99 & & & & & 11591.80 & 482.99 \\
\hline 9/24/1991 & 11229.40 & 467.89 & & & & & 11229.40 & 467.89 \\
\hline $9 / 25 / 1991$ & 9132.40 & 380.52 & & & & & 9132.40 & 380.52 \\
\hline 9/26/1991 & 8964.10 & 373.50 & & & & & 8964.10 & 373.50 \\
\hline 9/27/1991 & 9100.40 & 379.18 & & & 0.50 & 0.50 & 9100.40 & 379.18 \\
\hline 9/28/1991 & 3973.40 & 165.56 & & & & & 3973.40 & 165.56 \\
\hline 9/29/1991 & 3951.20 & 164.63 & & & & & 3951.20 & 164.63 \\
\hline 9/30/1991 & 10632.20 & 443.01 & & & & & 10632.20 & 443.01 \\
\hline 10/1/1991 & & & & & & & & \\
\hline $10 / 2 / 1991$ & 1270.00 & 211.67 & 11.00 & 1.83 & & & 1259.00 & 209.83 \\
\hline 10/3/1991 & 10068.40 & 419.52 & 1418.00 & 59.08 & & & 8650.40 & 360.43 \\
\hline 10/4/1991 & 10652.20 & 443.84 & 1535.00 & 63.96 & & & 9117.20 & 379.88 \\
\hline 10/5/1991 & 4367.60 & 181.98 & 53.00 & 2.21 & & & 4314.60 & 179.78 \\
\hline 10/6/1991 & 3099.40 & 129.14 & 48.00 & 2.00 & & & 3051.40 & 127.14 \\
\hline 10/7/1991 & 8434.50 & 351.44 & 1758.00 & 73.25 & 14.40 & 7.20 & 6676.50 & 278.19 \\
\hline 10/8/1991 & 9786.40 & 407.77 & 864.80 & 36.03 & 0.50 & 0.50 & 8921.60 & 371.73 \\
\hline 10/9/1991 & 8605.50 & 358.56 & 562.80 & 23.45 & & & 8042.70 & 335.11 \\
\hline 10/10/1991 & 8868.10 & 369.50 & 513.60 & 21.40 & & & 8354.50 & 348.10 \\
\hline 10/11/1991 & 9345.30 & 389.39 & 572.40 & 23.85 & & & 8772.90 & 365.54 \\
\hline 10/12/1991 & 4576.00 & 190.67 & 19.20 & 0.80 & & & 4556.80 & 189.87 \\
\hline $10 / 13 / 1991$ & 4217.20 & 175.72 & 19.60 & 0.82 & & & 4197.60 & 174.90 \\
\hline 10/14/1991 & 10462.00 & 435.92 & 728.00 & 30.33 & & & 9734.00 & 405.58 \\
\hline 10/15/1991 & 9655.30 & 402.30 & 599.20 & 24.97 & 1.50 & 1.50 & 9056.10 & 377.34 \\
\hline 10/16/1991 & 9263.00 & 385.96 & 587.60 & 24.48 & & & 8675.40 & 361.48 \\
\hline 10/17/1991 & 9400.20 & 391.68 & 589.20 & 24.55 & & & 8811.00 & 367.13 \\
\hline 10/18/1991 & 10550.30 & 439.60 & 948.80 & 39.53 & & & 9601.50 & 400.06 \\
\hline 10/19/1991 & 6676.10 & 278.17 & 938.40 & 39.10 & & & 5737.70 & 239.07 \\
\hline 10/20/1991 & 3273.10 & 136.38 & 16.40 & 0.68 & & & 3256.70 & 135.70 \\
\hline 10/21/1991 & 9115.10 & 379.80 & 665.60 & 27.73 & & & 8449.50 & 352.06 \\
\hline 10/22/1991 & 9739.80 & 405.83 & 559.20 & 23.30 & & & 9180.60 & 382.53 \\
\hline $10 / 23 / 1991$ & 9329.50 & 388.73 & 564.40 & 23.52 & & & 8765.10 & 365.21 \\
\hline 10/24/1991 & 10869.30 & 452.89 & 574.00 & 23.92 & & & 10295.30 & 428.97 \\
\hline $10 / 25 / 1991$ & 10930.40 & 455.43 & 579.20 & 24.13 & & & 10351.20 & 431.30 \\
\hline $10 / 26 / 1991$ & 5786.30 & 241.10 & 16.40 & 0.68 & & & 5769.90 & 240.41 \\
\hline 10/27/1991 & 5635.90 & 234.83 & 16.00 & 0.67 & & & 5619.90 & 234.16 \\
\hline 10/28/1991 & 12589.20 & 524.55 & 708.40 & 29.52 & & & 11880.80 & 495.03 \\
\hline $10 / 29 / 1991$ & 10510.60 & 437.94 & 555.60 & 23.15 & & & 9955.00 & 414.79 \\
\hline 10/30/1991 & 7993.50 & 333.06 & 534.40 & 22.27 & & & 7459.10 & 310.80 \\
\hline
\end{tabular}




\begin{tabular}{|c|c|c|c|c|c|c|c|c|}
\hline 10/31/1991 & 7092.40 & 295.52 & 516.00 & 21.50 & & & 6576.40 & 274.02 \\
\hline 11/1/1991 & 6276.20 & 261.51 & 255.60 & 10.65 & & & 6020.60 & 250.86 \\
\hline 11/2/1991 & 2830.60 & 117.94 & 12.80 & 0.53 & & & 2817.80 & 117.41 \\
\hline 11/3/1991 & 2142.60 & 89.28 & 12.80 & 0.53 & & & 2129.80 & 88.74 \\
\hline 11/4/1991 & 6994.00 & 291.42 & 12.80 & 0.53 & & & 6981.20 & 290.88 \\
\hline 11/5/1991 & 7136.70 & 297.36 & 13.60 & 0.57 & 2.50 & 0.50 & 7123.10 & 296.80 \\
\hline 11/6/1991 & 6470.70 & 269.61 & 12.80 & 0.53 & 3.00 & 0.50 & 6457.90 & 269.08 \\
\hline $11 / 7 / 1991$ & 7873.60 & 328.07 & 464.40 & 19.35 & 4.00 & 0.50 & 7409.20 & 308.72 \\
\hline 11/8/1991 & 6741.20 & 280.88 & 12.80 & 0.53 & 5.00 & 0.50 & 6728.40 & 280.35 \\
\hline 11/9/1991 & 4168.00 & 173.67 & 13.60 & 0.57 & 4.50 & 0.50 & 4154.40 & 173.10 \\
\hline 11/10/1991 & 3296.10 & 137.34 & 14.00 & 0.58 & 5.00 & 0.50 & 3282.10 & 136.75 \\
\hline 11/11/1991 & 6551.60 & 272.98 & 38.40 & 1.60 & 2.50 & 0.50 & 6513.20 & 271.38 \\
\hline 11/12/1991 & 6300.90 & 262.54 & 14.40 & 0.60 & & & 6286.50 & 261.94 \\
\hline 11/13/1991 & 6403.90 & 266.83 & 96.00 & 4.00 & & & 6307.90 & 262.83 \\
\hline 11/14/1991 & 7417.20 & 309.05 & 498.00 & 21.65 & & & 6919.20 & 288.30 \\
\hline 11/15/1991 & 8281.40 & 345.06 & 550.00 & 22.92 & & & 7731.40 & 322.14 \\
\hline 11/16/1991 & 3323.80 & 138.49 & 10.00 & 0.42 & & & 3313.80 & 138.08 \\
\hline 11/17/1991 & 2432.20 & 101.34 & 10.80 & 0.45 & & & 2421.40 & 100.89 \\
\hline 11/18/1991 & 7355.10 & 306.46 & 646.00 & 26.92 & & & 6709.10 & 279.55 \\
\hline 11/19/1991 & 8315.20 & 346.47 & 545.60 & 22.73 & & & 7769.60 & 323.73 \\
\hline 11/20/1991 & 6693.30 & 278.89 & 423.20 & 17.63 & 3.00 & 0.50 & 6270.10 & 261.25 \\
\hline 11/21/1991 & 6869.50 & 286.23 & 503.60 & 20.98 & 2.50 & 0.50 & 6365.90 & 265.25 \\
\hline 11/22/1991 & 5938.20 & 247.43 & 120.00 & 5.00 & 2.50 & 0.50 & 5818.20 & 242.43 \\
\hline 11/23/1991 & 2786.30 & 116.10 & 12.00 & 0.50 & 3.00 & 0.50 & 2774.30 & 115.60 \\
\hline 11/24/1991 & 1973.60 & 82.23 & 11.20 & 0.47 & 3.50 & 0.50 & 1962.40 & 81.77 \\
\hline 11/25/1991 & 5698.80 & 237.45 & 11.60 & 0.48 & 2.00 & 0.50 & 5687.20 & 236.97 \\
\hline 11/26/1991 & 6599.30 & 274.97 & 401.20 & 16.72 & 0.30 & 0.10 & 6198.10 & 258.25 \\
\hline 11/27/1991 & 5739.70 & 239.15 & 367.20 & 15.30 & 0.50 & 0.10 & 5372.50 & 223.85 \\
\hline 11/28/1991 & 2511.70 & 104.65 & 11.20 & 0.49 & 0.50 & 0.10 & 2500.50 & 104.19 \\
\hline 11/29/1991 & 2502.10 & 104.25 & 11.20 & 0.49 & 0.40 & 0.10 & 2490.90 & 103.79 \\
\hline 11/30/1991 & 2701.20 & 112.55 & 10.80 & 0.45 & 0.40 & 0.10 & 2690.40 & 112.10 \\
\hline $12 / 1 / 1991$ & 2189.30 & 91.22 & 12.40 & 0.52 & 0.90 & 0.10 & 2176.90 & 90.70 \\
\hline $12 / 2 / 1991$ & 6411.60 & 267.15 & 11.20 & 0.47 & 1.30 & 0.11 & 6400.40 & 266.68 \\
\hline $12 / 3 / 1991$ & 6306.30 & 262.76 & 11.60 & 0.48 & 1.30 & 0.12 & 6294.70 & 262.28 \\
\hline 12/4/1991 & 6091.30 & 253.80 & 11.60 & 0.50 & 1.30 & 0.12 & 6079.70 & 253.32 \\
\hline $12 / 5 / 1991$ & 6081.60 & 253.40 & 77.20 & 3.22 & 0.90 & 0.10 & 6004.40 & 250.18 \\
\hline $12 / 6 / 1991$ & 7018.30 & 292.43 & 390.80 & 16.28 & 14.30 & 0.89 & 6627.50 & 276.15 \\
\hline 12/7/1991 & 3959.00 & 164.96 & 10.40 & 0.43 & 19.20 & 0.80 & 3948.60 & 164.53 \\
\hline $12 / 8 / 1991$ & 2990.30 & 124.60 & 10.40 & 0.43 & 16.90 & 0.70 & 2979.90 & 124.16 \\
\hline 12/9/1991 & 9325.90 & 388.58 & 514.40 & 21.43 & 18.00 & 0.75 & 8811.50 & 367.15 \\
\hline 12/10/1991 & 7837.50 & 326.56 & 514.00 & 21.42 & 18.60 & 0.78 & 7323.50 & 305.15 \\
\hline 12/11/1991 & 8176.10 & 340.67 & 519.20 & 21.63 & 20.10 & 0.87 & 7656.90 & 319.04 \\
\hline $12 / 12 / 1991$ & 9061.50 & 377.56 & 541.60 & 23.55 & 17.60 & 0.73 & 8519.90 & 355.00 \\
\hline 12/13/1991 & 8554.60 & 356.44 & 545.20 & 23.70 & 17.80 & 0.74 & 8009.40 & 333.73 \\
\hline 12/14/1991 & 3540.60 & 147.53 & 11.20 & 0.47 & 25.50 & 1.06 & 3529.40 & 147.06 \\
\hline 12/15/1991 & 2360.20 & 98.34 & 11.20 & 0.47 & 23.40 & 0.98 & 2349.00 & 97.88 \\
\hline 12/16/1991 & 6314.30 & 263.10 & 120.80 & 5.03 & 52.90 & 2.20 & 6193.50 & 258.06 \\
\hline 12/17/1991 & 6256.00 & 260.67 & 12.00 & 0.50 & 54.10 & 2.25 & 6244.00 & 260.17 \\
\hline 12/18/1991 & 6756.30 & 281.51 & 466.80 & 20.30 & 36.10 & 1.50 & 6289.50 & 262.06 \\
\hline 12/19/1991 & 7272.50 & 303.02 & 464.40 & 19.35 & 26.20 & 1.09 & 6808.10 & 283.67 \\
\hline $12 / 20 / 1991$ & 5602.80 & 233.45 & 261.60 & 10.90 & 18.60 & 0.78 & 5341.20 & 222.55 \\
\hline
\end{tabular}


CHART DATA

\begin{tabular}{|c|c|c|c|c|c|c|c|c|}
\hline $12 / 21 / 1991$ & 2639.50 & 109.98 & 10.80 & 0.47 & 18.80 & 0.78 & 2628.70 & 109.53 \\
\hline 12/22/1991 & 2482.00 & 103.42 & 10.00 & 0.42 & 18.00 & 0.75 & 2472.00 & 103.00 \\
\hline 12/23/1991 & 2616.80 & 109.03 & 11.20 & 0.47 & 16.60 & 0.75 & 2605.60 & 108.57 \\
\hline 12/24/1991 & 2193.10 & 91.38 & 11.60 & 0.48 & 11.80 & 0.59 & 2181.50 & 90.90 \\
\hline $12 / 25 / 1991$ & 2073.90 & 86.41 & 11.20 & 0.47 & 13.40 & 0.61 & 2062.70 & 85.95 \\
\hline 12/26/1991 & 2766.80 & 115.28 & 11.60 & 0.48 & 31.50 & 1.31 & 2755.20 & 114.80 \\
\hline 12/27/1991 & 3045.60 & 126.90 & 11.60 & 0.48 & 29.20 & 1.27 & 3034.00 & 126.42 \\
\hline 12/28/1991 & 2848.60 & 118.69 & 11.20 & 0.47 & 22.90 & 0.95 & 2837.40 & 118.23 \\
\hline 12/29/1991 & 2385.60 & 99.40 & 11.20 & 0.47 & 22.30 & 0.97 & 2374.40 & 98.93 \\
\hline 12/30/1991 & 2692.10 & 112.17 & 11.20 & 0.47 & 17.80 & 0.85 & 2680.90 & 111.70 \\
\hline 12/31/1991 & 2546.00 & 106.08 & 12.40 & 0.54 & 9.70 & 0.61 & 2533.60 & 105.57 \\
\hline \multicolumn{9}{|l|}{ 1/1/1992 } \\
\hline 1/2/1992 & 2121.90 & 88.41 & 14.40 & 0.60 & 9.10 & 0.51 & 2107.50 & 87.81 \\
\hline 1/3/1992 & 2919.60 & 121.65 & 12.40 & 0.52 & 21.40 & 1.07 & 2907.20 & 121.13 \\
\hline $1 / 4 / 1992$ & 2571.30 & 107.14 & 11.20 & 0.47 & 30.90 & 1.29 & 2560.10 & 106.67 \\
\hline 1/5/1992 & 2452.90 & 102.20 & 11.20 & 0.47 & 35.20 & 1.47 & 2441.70 & 101.74 \\
\hline 1/6/1992 & 2127.90 & 88.66 & 11.20 & 0.47 & 23.60 & 0.98 & 2116.70 & 88.20 \\
\hline 1/7/1992 & 5604.00 & 233.50 & 11.20 & 0.47 & 61.80 & 2.58 & 5592.80 & 233.03 \\
\hline 1/8/1992 & 6203.40 & 258.48 & 10.80 & 0.47 & 56.10 & 2.34 & 6192.60 & 258.03 \\
\hline 1/9/1992 & 6091.20 & 253.80 & 59.20 & 2.57 & 36.50 & 1.52 & 6032.00 & 251.33 \\
\hline $1 / 10 / 1992$ & 6025.60 & 251.07 & 11.60 & 0.48 & 67.00 & 2.79 & 6014.00 & 250.58 \\
\hline 1/11/1992 & 6409.10 & 267.05 & 296.80 & 12.37 & 53.50 & 2.23 & 6112.30 & 254.68 \\
\hline $1 / 12 / 1992$ & 2853.70 & 118.90 & 11.20 & 0.47 & 31.00 & 1.29 & 2842.50 & 118.44 \\
\hline $1 / 13 / 1992$ & 2693.80 & 112.24 & 10.80 & 0.45 & 29.60 & 1.23 & 2683.00 & 111.79 \\
\hline $1 / 14 / 1992$ & 5748.30 & 239.51 & 11.20 & 0.47 & 73.60 & 3.07 & 5737.10 & 239.05 \\
\hline $1 / 15 / 1992$ & 6156.70 & 256.53 & 12.00 & 0.50 & 84.80 & 3.53 & 6144.70 & 256.03 \\
\hline $1 / 16 / 1992$ & 6014.30 & 250.60 & 11.60 & 0.48 & 95.30 & 3.97 & 6002.70 & 250.11 \\
\hline 1/17/1992 & 6257.00 & 260.71 & 11.60 & 0.48 & 118.30 & 4.93 & 6245.40 & 260.23 \\
\hline 1/18/1992 & 5178.60 & 215.78 & 10.80 & 0.45 & 101.70 & 4.24 & 5167.80 & 215.33 \\
\hline $1 / 19 / 1992$ & 2895.10 & 120.63 & 11.20 & 0.47 & 57.40 & 2.39 & 2883.90 & 120.16 \\
\hline 1/20/1992 & 2240.40 & 93.35 & 11.20 & 0.47 & 51.50 & 2.15 & 2229.20 & 92.88 \\
\hline 1/21/1992 & 6362.60 & 265.11 & 10.00 & 0.42 & 98.60 & 4.11 & 6352.60 & 264.69 \\
\hline $1 / 22 / 1992$ & 6035.10 & 251.46 & 10.80 & 0.45 & 95.60 & 3.98 & 6024.30 & 251.01 \\
\hline $1 / 23 / 1992$ & 5904.60 & 246.03 & 108.40 & 4.52 & 76.20 & 3.18 & 5796.20 & 241.51 \\
\hline 1/24/1992 & 5734.30 & 238.93 & 11.20 & 0.47 & 69.30 & 2.89 & 5723.10 & 238.46 \\
\hline $1 / 25 / 1992$ & 5966.00 & 248.58 & 255.60 & 11.11 & 50.10 & 2.09 & 5710.40 & 237.93 \\
\hline $1 / 26 / 1992$ & 2059.40 & 85.81 & 10.80 & 0.45 & 23.10 & 0.96 & 2048.60 & 85.36 \\
\hline 1/27/1992 & 1664.80 & 69.37 & 10.40 & 0.43 & 21.70 & 0.90 & 1654.40 & 68.93 \\
\hline $1 / 28 / 1992$ & 5437.20 & 226.55 & 69.20 & 2.88 & 31.50 & 1.31 & 5368.00 & 223.67 \\
\hline $1 / 29 / 1992$ & 6059.30 & 252.47 & 10.80 & 0.45 & 47.60 & 1.98 & 6048.50 & 252.02 \\
\hline 1/30/1992 & 5875.00 & 244.79 & 10.40 & 0.43 & 51.40 & 2.14 & 5864.60 & 244.36 \\
\hline 1/31/1992 & 6033.40 & 251.39 & 165.60 & 6.90 & 36.70 & 1.60 & 5867.80 & 244.49 \\
\hline 2/1/1992 & 5952.10 & 248.00 & 264.00 & 11.00 & 25.90 & 1.18 & 5688.10 & 237.00 \\
\hline 2/2/1992 & 2341.80 & 97.58 & 10.80 & 0.45 & 22.10 & 0.92 & 2331.00 & 97.13 \\
\hline 2/3/1992 & 1910.30 & 79.60 & 10.80 & 0.45 & 23.10 & 0.96 & 1899.50 & 79.15 \\
\hline 2/4/1992 & 5587.30 & 232.80 & 11.20 & 0.47 & 35.50 & 1.48 & 5576.10 & 232.34 \\
\hline 2/5/1992 & 6345.10 & 264.38 & 372.00 & 15.50 & 43.80 & 1.83 & 5973.10 & 248.88 \\
\hline 2/6/1992 & 5761.90 & 240.08 & 11.20 & 0.47 & 70.70 & 2.95 & 5750.70 & 239.61 \\
\hline 2/7/1992 & 5813.90 & 242.25 & 11.60 & 0.48 & 63.80 & 2.66 & 5802.30 & 241.76 \\
\hline 2/8/1992 & 5411.00 & 225.46 & 11.20 & 0.47 & 35.60 & 1.48 & 5399.80 & 224.99 \\
\hline 2/9/1992 & 2205.80 & 91.91 & 11.60 & 0.48 & 24.00 & 1.00 & 2194.20 & 91.43 \\
\hline
\end{tabular}


CHART DATA

\begin{tabular}{|c|c|c|c|c|c|c|c|c|}
\hline 2/10/1992 & 2136.70 & 89.03 & 11.20 & 0.47 & 29.20 & 1.22 & 2125.50 & 88.56 \\
\hline $2 / 11 / 1992$ & 5539.10 & 230.80 & 11.20 & 0.47 & 32.50 & 1.35 & 5527.90 & 230.33 \\
\hline 2/12/1992 & 6307.20 & 262.80 & 324.40 & 13.52 & 30.40 & 1.27 & 5982.80 & 249.28 \\
\hline 2/13/1992 & 6424.50 & 267.69 & 534.40 & 23.23 & 22.70 & 0.95 & 5890.10 & 245.42 \\
\hline $2 / 14 / 1992$ & 6936.40 & 289.02 & 528.00 & 22.96 & 22.10 & 0.92 & 6408.40 & 267.02 \\
\hline 2/15/1992 & 8128.30 & 338.68 & 530.40 & 23.06 & 20.00 & 0.83 & 7597.90 & 316.58 \\
\hline 2/16/1992 & 4638.10 & 193.25 & 10.00 & 0.42 & 19.00 & 0.79 & 4628.10 & 192.84 \\
\hline $2 / 17 / 1992$ & 2182.30 & 90.93 & 10.40 & 0.43 & 18.10 & 0.75 & 2171.90 & 90.50 \\
\hline 2/18/1992 & 7169.50 & 298.73 & 530.40 & 22.10 & 20.50 & 0.85 & 6639.10 & 276.63 \\
\hline 2/19/1992 & 7227.50 & 301.15 & 492.80 & 20.53 & 20.30 & 0.85 & 6734.70 & 280.61 \\
\hline $2 / 20 / 1992$ & 6441.20 & 268.38 & 376.80 & 15.70 & 15.20 & 0.72 & 6064.40 & 252.68 \\
\hline 2/21/1992 & 5588.30 & 232.85 & 246.80 & 10.28 & 7.10 & 0.47 & 5341.50 & 222.56 \\
\hline 2/22/1992 & 5902.70 & 245.95 & 310.00 & 12.92 & 12.20 & 0.81 & 5592.70 & 233.03 \\
\hline 2/23/1992 & 2719.90 & 113.33 & 10.40 & 0.43 & 5.60 & 0.40 & 2709.50 & 112.90 \\
\hline 2/24/1992 & 2050.10 & 85.42 & 10.00 & 0.43 & 4.60 & 0.38 & 2040.10 & 85.00 \\
\hline 2/25/1992 & 6254.50 & 260.60 & 293.20 & 12.75 & 5.10 & 0.43 & 5961.30 & 248.39 \\
\hline 2/26/1992 & 5413.00 & 225.54 & 11.20 & 0.47 & 38.70 & 1.94 & 5401.80 & 225.08 \\
\hline 2/27/1992 & 5532.90 & 230.54 & 11.20 & 0.47 & 51.20 & 2.23 & 5521.70 & 230.07 \\
\hline 2/28/1992 & 5775.20 & 240.63 & 245.20 & 10.22 & 28.90 & 1.26 & 5530.00 & 230.42 \\
\hline $2 / 29 / 1992$ & 5668.70 & 236.20 & 305.60 & 12.73 & 27.30 & 1.14 & 5363.10 & 223.46 \\
\hline 3/1/1992 & 3088.70 & 128.70 & 346.80 & 14.45 & 18.90 & 0.86 & 2741.90 & 114.25 \\
\hline 3/2/1992 & 2007.80 & 83.66 & 10.40 & 0.43 & 19.00 & 0.79 & 1997.40 & 83.23 \\
\hline 3/3/1992 & 6283.00 & 261.79 & 364.00 & 15.83 & 21.10 & 0.88 & 5919.00 & 246.63 \\
\hline 3/4/1992 & 7142.70 & 297.61 & 191.20 & 7.97 & 20.20 & 0.84 & 6951.50 & 289.65 \\
\hline 3/5/1992 & 7682.60 & 320.11 & 375.20 & 15.63 & 20.10 & 0.84 & 7307.40 & 304.48 \\
\hline 3/6/1992 & 7852.30 & 327.18 & 420.80 & 17.53 & 19.40 & 0.81 & 7431.50 & 309.65 \\
\hline 3/7/1992 & 8400.60 & 350.03 & 498.00 & 20.75 & 19.40 & 0.81 & 7902.60 & 329.28 \\
\hline 3/8/1992 & 3902.00 & 162.58 & 263.60 & 10.98 & 18.50 & 0.77 & 3638.40 & 151.60 \\
\hline 3/9/1992 & 2063.30 & 85.97 & 10.40 & 0.45 & 18.90 & 0.79 & 2052.90 & 85.54 \\
\hline 3/10/1992 & 7156.50 & 311.15 & 430.00 & 18.70 & 18.50 & 0.77 & 6726.50 & 292.46 \\
\hline 3/11/1992 & 5238.60 & 218.28 & 11.20 & 0.47 & 36.10 & 1.50 & 5227.40 & 217.81 \\
\hline 3/12/1992 & 5164.00 & 215.17 & 10.80 & 0.45 & 45.20 & 1.88 & 5153.20 & 214.72 \\
\hline 3/13/1992 & 5479.50 & 228.31 & 13.60 & 0.57 & 43.60 & 1.82 & 5465.90 & 227.75 \\
\hline 3/14/1992 & 2909.90 & 121.25 & 11.20 & 0.47 & 25.10 & 1.05 & 2898.70 & 120.78 \\
\hline 3/15/1992 & 2041.70 & 85.07 & 10.80 & 0.45 & 17.90 & 0.75 & 2030.90 & 84.62 \\
\hline 3/16/1992 & 2109.70 & 87.90 & 10.80 & 0.45 & 18.00 & 0.75 & 2098.90 & 87.45 \\
\hline 3/17/1992 & 2827.10 & 117.80 & 11.20 & 0.47 & 18.60 & 0.78 & 2815.90 & 117.33 \\
\hline 3/18/1992 & 2829.20 & 117.88 & 10.40 & 0.43 & 17.40 & 0.73 & 2818.80 & 117.45 \\
\hline 3/19/1992 & 2943.10 & 122.63 & 10.80 & 0.45 & 17.90 & 0.75 & 2932.30 & 122.18 \\
\hline 3/20/1992 & 2824.30 & 117.68 & 10.80 & 0.47 & 16.60 & 0.69 & 2813.50 & 117.23 \\
\hline 3/21/1992 & 2255.00 & 93.96 & 11.60 & 0.48 & 18.10 & 0.75 & 2243.40 & 93.48 \\
\hline 3/22/1992 & 1968.40 & 82.02 & 10.40 & 0.43 & 16.70 & 0.70 & 1958.00 & 81.58 \\
\hline 3/23/1992 & 2085.70 & 86.90 & 10.80 & 0.47 & 17.10 & 0.71 & 2074.90 & 86.45 \\
\hline 3/24/1992 & 5595.10 & 233.13 & 130.80 & 5.45 & 29.50 & 1.23 & 5464.30 & 227.68 \\
\hline 3/25/1992 & 5863.00 & 244.29 & 240.00 & 10.00 & 22.80 & 0.95 & 5623.00 & 234.29 \\
\hline 3/26/1992 & 7219.70 & 300.82 & 420.80 & 17.53 & 18.20 & 0.76 & 6798.90 & 283.29 \\
\hline 3/27/1992 & 7345.70 & 306.07 & 416.40 & 18.10 & 17.30 & 0.72 & 6929.30 & 288.72 \\
\hline 3/28/1992 & 7263.70 & 302.65 & 398.80 & 16.62 & 19.00 & 0.79 & 6864.90 & 286.04 \\
\hline 3/29/1992 & 2514.20 & 104.76 & 10.00 & 0.42 & 18.30 & 0.76 & 2504.20 & 104.34 \\
\hline 3/30/1992 & 2191.20 & 91.30 & 10.40 & 0.43 & 17.10 & 0.71 & 2180.80 & 90.87 \\
\hline 3/31/1992 & 6980.20 & 290.84 & 223.60 & 9.32 & 19.60 & 0.82 & 6756.60 & 281.53 \\
\hline
\end{tabular}


CHART DATA

\begin{tabular}{|c|c|c|c|c|c|c|c|c|}
\hline 4/1/1992 & 7586.70 & 316.11 & 504.00 & 21.00 & 19.50 & 0.81 & 7082.70 & 295.11 \\
\hline 4/2/1992 & 7413.20 & 308.88 & 475.60 & 19.82 & 21.50 & 0.90 & 6937.60 & 289.07 \\
\hline $4 / 3 / 1992$ & 5751.70 & 239.65 & 12.00 & 0.50 & 31.10 & 1.30 & 5739.70 & 239.15 \\
\hline 4/4/1992 & 5285.30 & 220.22 & 11.20 & 0.47 & 29.00 & 1.21 & 5274.10 & 219.75 \\
\hline 4/5/1992 & 2131.50 & 88.81 & 11.20 & 0.47 & 17.60 & 0.73 & 2120.30 & 88.35 \\
\hline 4/6/1992 & 1953.30 & 81.39 & 10.80 & 0.45 & 18.50 & 0.77 & 1942.50 & 80.94 \\
\hline 4/7/1992 & 7139.10 & 297.46 & 452.40 & 18.85 & 18.90 & 0.79 & 6686.70 & 278.61 \\
\hline 4/8/1992 & 7047.70 & 306.42 & 471.60 & 20.50 & 16.70 & 0.73 & 6576.10 & 285.92 \\
\hline 4/9/1992 & 8085.20 & 336.88 & 525.60 & 21.90 & 18.00 & 0.75 & 7559.60 & 314.98 \\
\hline 4/10/1992 & 7998.90 & 333.29 & 416.80 & 17.37 & 18.80 & 0.78 & 7582.10 & 315.92 \\
\hline 4/11/1992 & 7885.60 & 328.57 & 434.00 & 18.87 & 18.00 & 0.75 & 7451.60 & 310.48 \\
\hline 4/12/1992 & 2590.40 & 107.93 & 9.60 & 0.40 & 16.40 & 0.68 & 2580.80 & 107.53 \\
\hline 4/13/1992 & 2558.00 & 106.58 & 10.40 & 0.45 & 16.30 & 0.71 & 2547.60 & 106.15 \\
\hline 4/14/1992 & 9277.60 & 386.57 & 476.40 & 19.85 & 16.80 & 0.70 & 8801.20 & 366.72 \\
\hline 4/15/1992 & 9180.20 & 382.51 & 510.40 & 21.27 & 8.30 & 0.64 & 8669.80 & 361.24 \\
\hline $4 / 16 / 1992$ & 9193.80 & 383.08 & 512.00 & 21.33 & 3.40 & 0.38 & 8681.80 & 361.74 \\
\hline 4/17/1992 & 9202.50 & 383.44 & 466.80 & 19.45 & 2.80 & 0.35 & 8735.70 & 363.99 \\
\hline 4/18/1992 & 3437.20 & 143.22 & 10.40 & 0.43 & 2.50 & 0.28 & 3426.80 & 142.78 \\
\hline $4 / 19 / 1992$ & 2383.00 & 99.29 & 10.40 & 0.43 & 2.00 & 0.25 & 2372.60 & 98.86 \\
\hline 4/20/1992 & 2572.80 & 107.20 & 10.40 & 0.43 & 2.40 & 0.34 & 2562.40 & 106.77 \\
\hline 4/21/1992 & 3628.80 & 151.20 & 10.80 & 0.47 & 3.10 & 0.28 & 3618.00 & 150.75 \\
\hline 4/22/1992 & 8173.30 & 340.55 & 486.00 & 20.25 & 3.40 & 0.34 & 7687.30 & 320.30 \\
\hline 4/23/1992 & 9152.40 & 381.35 & 479.20 & 19.97 & 3.90 & 0.35 & 8673.20 & 361.38 \\
\hline 4/24/1992 & 9948.90 & 414.54 & 520.00 & 21.67 & 2.80 & 0.35 & 9428.90 & 392.87 \\
\hline 4/25/1992 & 10209.20 & 425.38 & 565.20 & 23.55 & 2.50 & 0.50 & 9644.00 & 401.83 \\
\hline 4/26/1992 & 3955.70 & 164.82 & 344.40 & 14.35 & 2.50 & 0.31 & 3611.30 & 150.47 \\
\hline 4/27/1992 & 2337.30 & 97.39 & 10.40 & 0.45 & 2.40 & 0.30 & 2326.90 & 96.95 \\
\hline 4/28/1992 & 8042.00 & 335.08 & 525.20 & 21.88 & 2.30 & 0.33 & 7516.80 & 313.20 \\
\hline $4 / 29 / 1992$ & 7452.80 & 310.53 & 516.00 & 22.43 & 2.00 & 0.50 & 6936.80 & 289.03 \\
\hline 4/30/1992 & 9328.60 & 388.69 & 531.20 & 22.13 & 2.20 & 0.44 & 8797.40 & 366.56 \\
\hline 5/1/1992 & 8764.30 & 365.18 & 525.60 & 22.85 & 3.20 & 0.40 & 8238.70 & 343.28 \\
\hline $5 / 2 / 1992$ & 9374.30 & 390.60 & 529.20 & 22.05 & 2.80 & 0.35 & 8845.10 & 368.55 \\
\hline 5/3/1992 & 2856.40 & 119.02 & 10.00 & 0.43 & 2.30 & 0.38 & 2846.40 & 118.60 \\
\hline $5 / 4 / 1992$ & 2500.60 & 104.19 & 10.00 & 0.43 & 1.80 & 0.30 & 2490.60 & 103.78 \\
\hline $5 / 5 / 1992$ & 9080.90 & 378.37 & 522.80 & 21.78 & 3.70 & 0.41 & 8558.10 & 356.59 \\
\hline 5/6/1992 & 9663.70 & 402.65 & 536.80 & 22.37 & 2.70 & 0.45 & 9126.90 & 380.29 \\
\hline 5/7/1992 & 9271.00 & 386.29 & 536.80 & 22.37 & 3.20 & 0.46 & 8734.20 & 363.93 \\
\hline $5 / 8 / 1992$ & 7226.40 & 301.10 & 508.80 & 21.20 & 2.50 & 0.50 & 6717.60 & 279.90 \\
\hline 5/9/1992 & 7394.90 & 308.12 & 513.20 & 21.38 & 2.60 & 0.43 & 6881.70 & 286.74 \\
\hline 5/10/1992 & 2529.70 & 105.40 & 10.40 & 0.43 & 2.90 & 0.32 & 2519.30 & 104.97 \\
\hline $5 / 11 / 1992$ & 2186.70 & 91.11 & 10.00 & 0.42 & 2.40 & 0.34 & 2176.70 & 90.70 \\
\hline $5 / 12 / 1992$ & 9297.70 & 387.40 & 517.20 & 21.55 & 3.00 & 0.50 & 8780.50 & 365.85 \\
\hline $5 / 13 / 1992$ & 10362.20 & 431.76 & 507.60 & 21.15 & 3.50 & 0.50 & 9854.60 & 410.61 \\
\hline $5 / 14 / 1992$ & 10609.20 & 442.05 & 557.60 & 23.23 & 2.50 & 0.50 & 10051.60 & 418.82 \\
\hline 5/15/1992 & 9257.50 & 402.50 & 494.80 & 21.51 & 3.50 & 0.50 & 8762.70 & 380.99 \\
\hline 5/16/1992 & 8604.70 & 358.53 & 447.60 & 20.35 & 2.30 & 0.38 & 8157.10 & 339.88 \\
\hline 5/17/1992 & 2759.60 & 114.98 & 10.00 & 0.42 & 1.60 & 0.40 & 2749.60 & 114.57 \\
\hline $5 / 18 / 1992$ & 2458.20 & 102.43 & 10.00 & 0.42 & 1.90 & 0.27 & 2448.20 & 102.01 \\
\hline $5 / 19 / 1992$ & 8970.90 & 373.79 & 566.40 & 24.63 & 3.20 & 0.46 & 8404.50 & 350.19 \\
\hline $5 / 20 / 1992$ & 9316.70 & 388.20 & 559.20 & 23.30 & 3.00 & 0.60 & 8757.50 & 364.90 \\
\hline $5 / 21 / 1992$ & 9204.40 & 383.52 & 557.20 & 23.22 & 2.50 & 0.50 & 8647.20 & 360.30 \\
\hline
\end{tabular}


CHART DATA

\begin{tabular}{|c|c|c|c|c|c|c|c|c|}
\hline $5 / 22 / 1992$ & 9725.20 & 405.22 & 563.20 & 23.47 & 3.10 & 0.44 & 9162.00 & 381.75 \\
\hline $5 / 23 / 1992$ & 9704.60 & 404.36 & 556.80 & 23.20 & 2.70 & 0.39 & 9147.80 & 381.16 \\
\hline $5 / 24 / 1992$ & 3252.20 & 135.51 & 11.20 & 0.47 & 1.10 & 0.37 & 3241.00 & 135.04 \\
\hline $5 / 25 / 1992$ & 3054.20 & 127.26 & 12.00 & 0.50 & 1.70 & 0.34 & 3042.20 & 126.76 \\
\hline 5/26/1992 & 10265.20 & 427.72 & 582.00 & 24.25 & 2.10 & 0.53 & 9683.20 & 403.47 \\
\hline 5/27/1992 & 10240.10 & 426.67 & 563.60 & 23.48 & 2.00 & 0.50 & 9676.50 & 403.19 \\
\hline 5/28/1992 & 9885.00 & 411.88 & 579.20 & 24.13 & 1.50 & 0.50 & 9305.80 & 387.74 \\
\hline 5/29/1992 & 10012.90 & 417.20 & 582.40 & 24.27 & 2.30 & 0.33 & 9430.50 & 392.94 \\
\hline $5 / 30 / 1992$ & 7021.00 & 292.54 & 541.20 & 23.53 & 2.50 & 0.31 & 6479.80 & 269.99 \\
\hline 5/31/1992 & 4261.10 & 177.55 & 281.20 & 11.72 & 1.50 & 0.21 & 3979.90 & 165.83 \\
\hline 6/1/1992 & 2138.50 & 89.10 & 10.40 & 0.43 & 2.00 & 0.29 & 2128.10 & 88.67 \\
\hline 6/2/1992 & 7434.60 & 309.78 & 498.80 & 20.78 & 2.20 & 0.37 & 6935.80 & 288.99 \\
\hline 6/3/1992 & 6907.40 & 287.81 & 505.60 & 21.07 & 1.70 & 0.43 & 6401.80 & 266.74 \\
\hline 6/4/1992 & 6753.80 & 281.41 & 460.80 & 19.20 & 1.80 & 0.30 & 6293.00 & 262.21 \\
\hline 6/5/1992 & 6841.00 & 285.04 & 278.40 & 11.60 & 1.50 & 0.50 & 6562.60 & 273.44 \\
\hline 6/6/1992 & 3863.00 & 167.96 & 14.80 & 0.64 & 1.00 & 0.50 & 3848.20 & 167.31 \\
\hline 6/7/1992 & 2963.40 & 128.84 & 14.00 & 0.61 & 1.50 & 0.50 & 2949.40 & 128.23 \\
\hline 6/8/1992 & 2363.10 & 102.74 & 10.80 & 0.47 & 1.90 & 0.32 & 2352.30 & 102.27 \\
\hline 6/9/1992 & 4831.50 & 210.07 & 11.20 & 0.53 & 1.70 & 0.34 & 4820.30 & 209.58 \\
\hline 6/10/1992 & 4880.00 & 212.17 & 13.60 & 0.59 & 1.00 & 0.50 & 4866.40 & 211.58 \\
\hline $6 / 11 / 1992$ & 5833.60 & 253.63 & 14.00 & 0.61 & 1.60 & 0.40 & 5819.60 & 253.03 \\
\hline 6/12/1992 & 5539.90 & 240.87 & 14.40 & 0.63 & 1.00 & 0.50 & 5525.50 & 240.24 \\
\hline 6/13/1992 & 4345.80 & 188.95 & 14.40 & 0.63 & 1.50 & 0.50 & 4331.40 & 188.32 \\
\hline $6 / 14 / 1992$ & 3868.20 & 168.18 & 15.20 & 0.66 & 1.50 & 0.50 & 3853.00 & 167.52 \\
\hline 6/15/1992 & 3599.30 & 156.49 & 13.60 & 0.65 & 1.00 & 0.50 & 3585.70 & 155.90 \\
\hline 6/16/1992 & 3338.60 & 145.16 & 5.60 & 0.93 & 1.00 & 0.50 & 3333.00 & 144.91 \\
\hline $6 / 17 / 1992$ & 4923.00 & 214.04 & 16.00 & 0.70 & 2.00 & 0.50 & 4907.00 & 213.35 \\
\hline 6/18/1992 & 4888.30 & 212.53 & 16.00 & 0.70 & 1.00 & 0.50 & 4872.30 & 211.84 \\
\hline 6/19/1992 & 4577.50 & 199.02 & 16.00 & 0.70 & 1.00 & 0.50 & 4561.50 & 198.33 \\
\hline 6/20/1992 & 4370.80 & 190.03 & 16.00 & 0.70 & 1.00 & 0.50 & 4354.80 & 189.34 \\
\hline $6 / 21 / 1992$ & 3609.60 & 156.94 & 16.40 & 0.71 & 1.50 & 0.50 & 3593.20 & 156.23 \\
\hline $6 / 22 / 1992$ & 3421.50 & 148.76 & 16.80 & 0.73 & 1.00 & 0.50 & 3404.70 & 148.03 \\
\hline $6 / 23 / 1992$ & 4848.60 & 210.81 & 17.60 & 0.77 & 1.00 & 0.50 & 4831.00 & 210.04 \\
\hline 6/24/1992 & 5426.90 & 235.95 & 542.80 & 23.60 & 1.50 & 0.50 & 4884.10 & 212.35 \\
\hline 6/25/1992 & 5114.00 & 222.35 & 555.60 & 24.16 & 1.00 & 0.50 & 4558.40 & 198.19 \\
\hline 6/26/1992 & 4848.10 & 210.79 & 567.60 & 24.68 & 1.00 & 0.50 & 4280.50 & 186.11 \\
\hline $6 / 27 / 1992$ & 3579.50 & 155.63 & 16.80 & 0.73 & 1.00 & 0.50 & 3562.70 & 154.90 \\
\hline 6/28/1992 & 3218.30 & 139.93 & 16.80 & 0.73 & 1.50 & 0.50 & 3201.50 & 139.20 \\
\hline 6/29/1992 & 2933.30 & 127.53 & 16.40 & 0.71 & 1.00 & 0.50 & 2916.90 & 126.82 \\
\hline $6 / 30 / 1992$ & 5702.70 & 247.94 & 594.80 & 25.86 & 1.50 & 0.50 & 5107.90 & 222.08 \\
\hline 7/1/1992 & 5862.40 & 254.89 & 496.80 & 21.60 & 1.00 & 0.50 & 5365.60 & 233.29 \\
\hline 7/2/1992 & 6500.20 & 282.62 & 584.00 & 25.39 & 1.00 & 0.50 & 5916.20 & 257.23 \\
\hline $7 / 3 / 1992$ & 7001.70 & 304.42 & 480.40 & 20.89 & 1.50 & 0.50 & 6521.30 & 283.53 \\
\hline 7/4/1992 & 4197.20 & 182.49 & 17.20 & 0.75 & 1.00 & 0.50 & 4180.00 & 181.74 \\
\hline 7/5/1992 & 2387.90 & 103.82 & 15.20 & 0.66 & 1.00 & 0.50 & 2372.70 & 103.16 \\
\hline 7/6/1992 & 2566.90 & 111.60 & 16.00 & 0.70 & 1.50 & 0.50 & 2550.90 & 110.91 \\
\hline 7/7/1992 & 5710.10 & 248.27 & 571.20 & 24.83 & 1.50 & 0.50 & 5138.90 & 223.43 \\
\hline 7/8/1992 & 6494.80 & 282.38 & 594.00 & 25.83 & 1.00 & 0.50 & 5900.80 & 256.56 \\
\hline 7/9/1992 & 6845.60 & 297.63 & 559.60 & 24.33 & 1.50 & 0.50 & 6286.00 & 273.30 \\
\hline 7/10/1992 & 6662.20 & 289.66 & 540.40 & 23.50 & 1.50 & 0.50 & 6121.80 & 266.17 \\
\hline 7/11/1992 & 3806.50 & 165.50 & 16.80 & 0.73 & 1.50 & 0.50 & 3789.70 & 164.77 \\
\hline
\end{tabular}


CHART DATA

\begin{tabular}{|c|c|c|c|c|c|c|c|c|}
\hline 7/12/1992 & 3298.00 & 143.39 & 16.00 & 0.70 & 1.00 & 0.50 & 3282.00 & 142.70 \\
\hline $7 / 13 / 1992$ & 2688.00 & 116.87 & 16.40 & 0.71 & 1.50 & 0.50 & 2671.60 & 116.16 \\
\hline 7/14/1992 & 5733.40 & 249.28 & 585.20 & 25.44 & 1.00 & 0.50 & 5148.20 & 223.83 \\
\hline $7 / 15 / 1992$ & 5190.10 & 225.66 & 74.80 & 3.25 & 1.50 & 0.50 & 5115.30 & 222.40 \\
\hline 7/16/1992 & 5721.40 & 248.76 & 151.60 & 6.59 & 1.50 & 0.50 & 5569.80 & 242.17 \\
\hline 7/17/1992 & 5882.00 & 255.74 & 452.80 & 19.69 & 1.00 & 0.50 & 5429.20 & 236.05 \\
\hline 7/18/1992 & 3850.00 & 167.39 & 16.80 & 0.73 & 1.50 & 0.50 & 3833.20 & 166.66 \\
\hline 7/19/1992 & 3417.90 & 148.60 & 16.40 & 0.71 & 1.00 & 0.50 & 3401.50 & 147.89 \\
\hline $7 / 20 / 1992$ & 1946.80 & 84.64 & 16.00 & 0.70 & 1.00 & 0.50 & 1930.80 & 83.95 \\
\hline 7/21/1992 & 4482.10 & 194.87 & 386.40 & 16.80 & 1.50 & 0.50 & 4095.70 & 178.07 \\
\hline 7/22/1992 & 3971.10 & 172.66 & 399.60 & 17.37 & 1.00 & 0.50 & 3571.50 & 155.28 \\
\hline $7 / 23 / 1992$ & 2867.80 & 124.69 & 15.60 & 0.68 & 1.00 & 0.50 & 2852.20 & 124.01 \\
\hline 7/24/1992 & 5775.00 & 251.09 & 555.20 & 24.14 & 1.50 & 0.50 & 5219.80 & 226.95 \\
\hline 7/25/1992 & 5415.60 & 235.46 & 17.20 & 0.75 & 1.50 & 0.50 & 5398.40 & 234.71 \\
\hline $7 / 26 / 1992$ & 4612.00 & 200.52 & 18.00 & 0.78 & 1.00 & 0.50 & 4594.00 & 199.74 \\
\hline 7/27/1992 & 4162.80 & 180.99 & 17.20 & 0.75 & 1.50 & 0.50 & 4145.60 & 180.24 \\
\hline 7/28/1992 & 4850.60 & 210.90 & 17.60 & 0.77 & 1.50 & 0.50 & 4833.00 & 210.13 \\
\hline $7 / 29 / 1992$ & 3875.10 & 168.48 & 16.40 & 0.71 & 1.00 & 0.50 & 3858.70 & 167.77 \\
\hline 7/30/1992 & 4254.30 & 184.97 & 16.00 & 0.70 & 1.50 & 0.50 & 4238.30 & 184.27 \\
\hline 7/31/1992 & 5327.40 & 231.63 & 16.00 & 0.70 & 1.50 & 0.50 & 5311.40 & 230.93 \\
\hline $8 / 1 / 1992$ & 4364.00 & 189.74 & 17.20 & 0.75 & 1.00 & 0.50 & 4346.80 & 188.99 \\
\hline 8/2/1992 & 2654.20 & 115.40 & 16.00 & 0.70 & 1.00 & 0.50 & 2638.20 & 114.70 \\
\hline 8/3/1992 & 2560.10 & 111.31 & 15.60 & 0.68 & 1.50 & 0.50 & 2544.50 & 110.63 \\
\hline $8 / 4 / 1992$ & 5136.50 & 223.33 & 422.80 & 18.38 & 1.50 & 0.50 & 4713.70 & 204.94 \\
\hline 8/5/1992 & 5509.60 & 239.55 & 558.40 & 24.28 & 1.00 & 0.50 & 4951.20 & 215.27 \\
\hline 8/6/1992 & 5729.90 & 249.13 & 545.60 & 23.72 & 1.50 & 0.50 & 5184.30 & 225.40 \\
\hline $8 / 7 / 1992$ & 6428.00 & 279.48 & 570.00 & 24.78 & 1.50 & 0.50 & 5858.00 & 254.70 \\
\hline 8/8/1992 & 7380.30 & 320.88 & 580.00 & 25.22 & 1.00 & 0.50 & 6800.30 & 295.67 \\
\hline 8/9/1992 & 3304.30 & 143.67 & 16.80 & 0.73 & 1.00 & 0.50 & 3287.50 & 142.93 \\
\hline 8/10/1992 & 2968.00 & 129.04 & 16.00 & 0.70 & 1.50 & 0.50 & 2952.00 & 128.35 \\
\hline 8/11/1992 & 7225.60 & 314.16 & 623.20 & 27.10 & 1.50 & 0.50 & 6602.40 & 287.06 \\
\hline 8/12/1992 & 7465.70 & 324.60 & 586.40 & 25.50 & 2.50 & 0.50 & 6879.30 & 299.10 \\
\hline 8/13/1992 & 7660.30 & 333.06 & 560.00 & 24.35 & 2.00 & 0.50 & 7100.30 & 308.71 \\
\hline 8/14/1992 & 7405.30 & 321.97 & 560.40 & 24.37 & 2.00 & 0.50 & 6844.90 & 297.60 \\
\hline 8/15/1992 & 7101.30 & 308.75 & 536.40 & 23.32 & 2.00 & 0.50 & 6564.90 & 285.43 \\
\hline 8/16/1992 & 2611.80 & 113.56 & 15.60 & 0.68 & 2.00 & 0.50 & 2596.20 & 112.88 \\
\hline $8 / 17 / 1992$ & 2294.90 & 99.78 & 14.80 & 0.64 & 2.00 & 0.50 & 2280.10 & 99.13 \\
\hline 8/18/1992 & 7412.20 & 322.27 & 539.60 & 23.46 & 2.50 & 0.50 & 6872.60 & 298.81 \\
\hline 8/19/1992 & 7771.00 & 337.87 & 548.40 & 23.84 & 2.50 & 0.50 & 7222.60 & 314.03 \\
\hline $8 / 20 / 1992$ & 9730.20 & 423.05 & 572.80 & 24.90 & 2.00 & 0.50 & 9157.40 & 398.15 \\
\hline 8/21/1992 & 9847.00 & 428.13 & 589.60 & 25.63 & 3.00 & 0.50 & 9257.40 & 402.50 \\
\hline 8/22/1992 & 9884.30 & 429.75 & 593.20 & 25.79 & 3.00 & 0.50 & 9291.10 & 403.96 \\
\hline 8/23/1992 & 2792.30 & 121.40 & 15.20 & 0.66 & 1.50 & 0.50 & 2777.10 & 120.74 \\
\hline 8/24/1992 & 2421.80 & 105.30 & 15.20 & 0.66 & 1.50 & 0.50 & 2406.60 & 104.63 \\
\hline 8/25/1992 & 11305.70 & 491.55 & 735.60 & 31.98 & 3.00 & 0.50 & 10570.10 & 459.57 \\
\hline $8 / 26 / 1992$ & 10967.80 & 476.86 & 679.60 & 29.55 & 3.00 & 0.50 & 10288.20 & 447.31 \\
\hline 8/27/1992 & 11330.50 & 492.63 & 674.40 & 29.32 & 2.50 & 0.50 & 10656.10 & 463.31 \\
\hline 8/28/1992 & 11575.10 & 503.27 & 671.60 & 29.20 & 2.50 & 0.50 & 10903.50 & 474.07 \\
\hline $8 / 29 / 1992$ & 10634.00 & 462.35 & 670.80 & 29.17 & 2.00 & 0.50 & 9963.20 & 433.18 \\
\hline 8/30/1992 & 3130.50 & 136.11 & 15.60 & 0.68 & 4.50 & 0.50 & 3114.90 & 135.43 \\
\hline 8/31/1992 & 2847.60 & 123.81 & 15.60 & 0.68 & 5.00 & 0.50 & 2832.00 & 123.13 \\
\hline
\end{tabular}


CHART DATA

\begin{tabular}{|c|c|c|c|c|c|c|c|c|}
\hline 9/1/1992 & 11573.00 & 503.17 & 741.20 & 32.23 & 4.00 & 0.50 & 10831.80 & 470.95 \\
\hline 9/2/1992 & 11323.50 & 492.33 & 683.60 & 29.72 & 3.00 & 0.50 & 10639.90 & 462.60 \\
\hline 9/3/1992 & 11640.60 & 506.11 & 692.80 & 30.12 & 2.50 & 0.50 & 10947.80 & 475.99 \\
\hline 9/4/1992 & 11992.40 & 521.41 & 712.80 & 30.99 & 1.50 & 0.50 & 11279.60 & 490.42 \\
\hline 9/5/1992 & 11770.30 & 511.75 & 708.40 & 30.80 & 2.50 & 0.50 & 11061.90 & 480.95 \\
\hline 9/6/1992 & 3817.10 & 165.96 & 16.00 & 0.70 & 1.50 & 0.50 & 3801.10 & 165.27 \\
\hline 9/7/1992 & 3164.00 & 137.57 & 16.00 & 0.70 & 1.00 & 0.50 & 3148.00 & 136.87 \\
\hline 9/8/1992 & 6236.20 & 271.14 & 15.60 & 0.68 & 2.50 & 0.50 & 6220.60 & 270.46 \\
\hline 9/9/1992 & 11803.30 & 513.19 & 689.60 & 29.98 & 2.50 & 0.50 & 11113.70 & 483.20 \\
\hline 9/10/1992 & 11952.70 & 519.68 & 722.40 & 31.41 & 3.00 & 0.50 & 11230.30 & 488.27 \\
\hline 9/11/1992 & 11559.50 & 502.59 & 698.40 & 30.37 & 2.50 & 0.50 & 10861.10 & 472.22 \\
\hline 9/12/1992 & 11108.70 & 482.99 & 666.40 & 28.97 & 2.00 & 0.50 & 10442.30 & 454.01 \\
\hline 9/13/1992 & 3268.30 & 142.10 & 15.60 & 0.68 & 1.00 & 0.50 & 3252.70 & 141.42 \\
\hline 9/14/1992 & 2288.60 & 99.50 & 14.80 & 0.64 & 1.50 & 0.50 & 2273.80 & 98.86 \\
\hline 9/15/1992 & 10816.50 & 470.28 & 731.60 & 31.81 & 2.50 & 0.50 & 10084.90 & 438.47 \\
\hline 9/16/1992 & 9670.70 & 420.47 & 644.00 & 28.00 & 3.00 & 0.50 & 9026.70 & 392.47 \\
\hline 9/17/1992 & 10098.60 & 439.07 & 524.80 & 22.82 & 2.00 & 0.50 & 9573.80 & 416.25 \\
\hline 9/18/1992 & 10600.20 & 460.88 & 652.00 & 28.35 & 2.00 & 0.50 & 9948.20 & 432.53 \\
\hline 9/19/1992 & 11627.60 & 505.55 & 670.00 & 29.13 & 2.50 & 0.50 & 10957.60 & 476.42 \\
\hline 9/20/1992 & 5802.90 & 252.30 & 16.40 & 0.71 & 1.50 & 0.50 & 5786.50 & 251.59 \\
\hline 9/21/1992 & 3280.60 & 142.63 & 16.00 & 0.70 & 1.00 & 0.50 & 3264.60 & 141.94 \\
\hline 9/22/1992 & 11859.00 & 515.61 & 692.80 & 30.12 & 3.00 & 0.50 & 11166.20 & 485.49 \\
\hline 9/23/1992 & 10361.00 & 450.48 & 649.60 & 28.24 & 3.50 & 0.70 & 9711.40 & 422.23 \\
\hline 9/24/1992 & 9878.20 & 429.49 & 666.40 & 28.97 & 3.00 & 0.50 & 9211.80 & 400.51 \\
\hline 9/25/1992 & 8986.50 & 390.72 & 627.20 & 27.27 & 2.50 & 0.63 & 8359.30 & 363.45 \\
\hline 9/26/1992 & 9517.80 & 413.82 & 629.60 & 27.37 & 2.50 & 0.50 & 8888.20 & 386.44 \\
\hline 9/27/1992 & 3673.90 & 159.73 & 14.40 & 0.63 & 1.50 & 0.50 & 3659.50 & 159.11 \\
\hline 9/28/1992 & 2362.30 & 102.71 & 15.20 & 0.66 & 1.50 & 0.50 & 2347.10 & 102.05 \\
\hline 9/29/1992 & 10425.80 & 453.30 & 739.60 & 32.16 & 2.50 & 0.50 & 9686.20 & 421.14 \\
\hline 9/30/1992 & 11255.20 & 489.36 & 939.60 & 40.85 & 3.00 & 0.50 & 10315.60 & 448.50 \\
\hline 10/1/1992 & 7771.60 & 337.90 & 549.20 & 23.88 & 2.50 & 0.50 & 7222.40 & 314.02 \\
\hline 10/2/1992 & 7718.10 & 335.57 & 571.20 & 25.96 & 2.00 & 0.50 & 7146.90 & 310.73 \\
\hline 10/3/1992 & 8092.10 & 351.83 & 574.00 & 24.96 & 3.00 & 0.50 & 7518.10 & 326.87 \\
\hline 10/4/1992 & 2381.50 & 103.54 & 11.60 & 0.50 & 2.00 & 0.50 & 2369.90 & 103.04 \\
\hline 10/5/1992 & 1940.50 & 84.37 & 12.40 & 0.54 & 1.00 & 0.50 & 1928.10 & 83.83 \\
\hline 10/6/1992 & 9282.10 & 403.57 & 646.80 & 28.12 & 3.00 & 0.60 & 8635.30 & 375.45 \\
\hline 10/7/1992 & 10115.60 & 439.81 & 591.20 & 25.70 & 2.50 & 0.50 & 9524.40 & 414.10 \\
\hline 10/8/1992 & 9104.60 & 395.85 & 583.20 & 25.36 & 2.50 & 0.50 & 8521.40 & 370.50 \\
\hline 10/9/1992 & 7899.50 & 343.46 & 562.40 & 24.45 & 16.60 & 1.04 & 7337.10 & 319.00 \\
\hline 10/10/1992 & 8087.80 & 351.64 & 566.40 & 24.63 & 16.50 & 0.72 & 7521.40 & 327.02 \\
\hline 10/11/1992 & 2300.00 & 100.00 & 11.20 & 0.49 & 15.00 & 0.65 & 2288.80 & 99.51 \\
\hline 10/12/1992 & 1866.10 & 81.13 & 12.80 & 0.56 & 15.00 & 0.68 & 1853.30 & 80.58 \\
\hline 10/13/1992 & 8940.70 & 388.73 & 897.20 & 39.01 & 8.00 & 0.62 & 8043.50 & 349.72 \\
\hline 10/14/1992 & 9934.90 & 431.95 & 561.20 & 24.40 & 2.50 & 0.50 & 9373.70 & 407.55 \\
\hline 10/15/1992 & 9643.00 & 419.26 & 564.40 & 24.54 & 3.00 & 0.50 & 9078.60 & 394.72 \\
\hline 10/16/1992 & 10217.60 & 444.24 & 571.60 & 24.85 & 3.50 & 0.50 & 9646.00 & 419.39 \\
\hline 10/17/1992 & 10324.80 & 448.90 & 596.40 & 25.93 & 2.00 & 0.50 & 9728.40 & 422.97 \\
\hline 10/18/1992 & 2663.00 & 115.78 & 10.40 & 0.45 & 2.00 & 0.50 & 2652.60 & 115.33 \\
\hline 10/19/1992 & 2035.60 & 88.50 & 10.40 & 0.45 & 3.50 & 0.58 & 2025.20 & 88.05 \\
\hline 10/20/1992 & 8671.00 & 377.00 & 588.80 & 25.60 & 6.00 & 0.55 & 8082.20 & 351.40 \\
\hline 10/21/1992 & 9834.90 & 427.60 & 584.00 & 25.39 & 6.00 & 0.50 & 9250.90 & 402.21 \\
\hline
\end{tabular}


CHART DATA

\begin{tabular}{|c|c|c|c|c|c|c|c|c|}
\hline $10 / 22 / 1992$ & 9223.40 & 401.02 & 583.20 & 25.36 & 6.50 & 0.50 & 8640.20 & 375.66 \\
\hline 10/23/1992 & 9386.60 & 408.11 & 582.80 & 25.34 & 6.50 & 0.54 & 8803.80 & 382.77 \\
\hline 10/24/1992 & 9166.20 & 398.53 & 585.60 & 25.46 & 7.00 & 0.50 & 8580.60 & 373.07 \\
\hline 10/25/1992 & 2514.30 & 109.32 & 11.60 & 0.50 & 5.50 & 0.50 & 2502.70 & 108.81 \\
\hline 10/26/1992 & 1694.10 & 73.66 & 11.60 & 0.50 & 5.50 & 0.50 & 1682.50 & 73.15 \\
\hline 10/27/1992 & 9159.20 & 398.23 & 503.20 & 21.88 & 6.50 & 0.54 & 8656.00 & 376.35 \\
\hline 10/28/1992 & 10466.30 & 455.06 & 538.00 & 23.39 & 6.50 & 0.50 & 9928.30 & 431.67 \\
\hline 10/29/1992 & 9526.40 & 414.19 & 590.40 & 25.67 & 5.00 & 0.50 & 8936.00 & 388.52 \\
\hline 10/30/1992 & 9980.00 & 433.91 & 582.00 & 25.30 & 3.00 & 0.50 & 9398.00 & 408.61 \\
\hline 10/31/1992 & 10141.90 & 440.95 & 594.40 & 25.84 & 3.50 & 0.50 & 9547.50 & 415.11 \\
\hline 11/1/1992 & 3375.50 & 146.76 & 13.20 & 0.57 & 2.00 & 0.50 & 3362.30 & 146.19 \\
\hline $11 / 2 / 1992$ & 1851.60 & 80.50 & 11.60 & 0.50 & 2.00 & 0.50 & 1840.00 & 80.00 \\
\hline 11/3/1992 & 8424.10 & 366.27 & 582.00 & 25.30 & 3.50 & 0.50 & 7842.10 & 340.96 \\
\hline 11/4/1992 & 10439.80 & 453.90 & 570.80 & 24.82 & 11.60 & 0.97 & 9869.00 & 429.09 \\
\hline 11/5/1992 & 6465.20 & 281.10 & 432.40 & 18.80 & 26.80 & 1.17 & 6032.80 & 262.30 \\
\hline 11/6/1992 & 6149.90 & 267.39 & 368.80 & 16.03 & 35.70 & 1.70 & 5781.10 & 251.35 \\
\hline $11 / 7 / 1992$ & 5865.20 & 255.01 & 11.60 & 0.50 & 36.90 & 1.94 & 5853.60 & 254.50 \\
\hline 11/8/1992 & 3405.00 & 148.04 & 11.20 & 0.49 & 19.00 & 0.83 & 3393.80 & 147.56 \\
\hline 11/9/1992 & 2115.20 & 91.97 & 10.80 & 0.49 & 17.00 & 0.74 & 2104.40 & 91.50 \\
\hline 11/10/1992 & 7500.10 & 326.09 & 485.20 & 21.10 & 19.00 & 0.83 & 7014.90 & 305.00 \\
\hline 11/11/1992 & 8500.70 & 369.60 & 568.00 & 25.82 & 17.50 & 0.76 & 7932.70 & 344.90 \\
\hline 11/12/1992 & 8543.20 & 371.44 & 556.80 & 24.21 & 16.50 & 0.72 & 7986.40 & 347.23 \\
\hline 11/13/1992 & 7252.50 & 315.33 & 547.60 & 23.81 & 18.00 & 0.78 & 6704.90 & 291.52 \\
\hline 11/14/1992 & 6507.80 & 282.95 & 524.80 & 22.82 & 19.00 & 0.83 & 5983.00 & 260.13 \\
\hline 11/15/1992 & 2173.70 & 94.51 & 10.80 & 0.47 & 18.00 & 0.78 & 2162.90 & 94.04 \\
\hline 11/16/1992 & 1705.50 & 74.15 & 10.80 & 0.47 & 20.50 & 0.89 & 1694.70 & 73.68 \\
\hline 11/17/1992 & 6531.70 & 283.99 & 234.80 & 10.21 & 21.00 & 0.91 & 6296.90 & 273.78 \\
\hline 11/18/1992 & 7552.00 & 328.35 & 430.00 & 18.70 & 18.50 & 0.80 & 7122.00 & 309.65 \\
\hline 11/19/1992 & 7956.40 & 345.93 & 554.40 & 24.10 & 17.50 & 0.76 & 7402.00 & 321.83 \\
\hline 11/20/1992 & 8257.30 & 359.01 & 558.00 & 24.26 & 16.50 & 0.75 & 7699.30 & 334.75 \\
\hline 11/21/1992 & 6642.40 & 288.80 & 528.40 & 22.97 & 17.50 & 0.76 & 6114.00 & 265.83 \\
\hline $11 / 22 / 1992$ & 2393.30 & 104.06 & 10.40 & 0.45 & 16.50 & 0.72 & 2382.90 & 103.60 \\
\hline 11/23/1992 & 1783.10 & 77.53 & 10.80 & 0.47 & 16.50 & 0.72 & 1772.30 & 77.06 \\
\hline $11 / 24 / 1992$ & 6156.70 & 267.68 & 81.60 & 3.55 & 31.70 & 1.38 & 6075.10 & 264.13 \\
\hline 11/25/1992 & 6416.80 & 278.99 & 260.80 & 11.34 & 28.40 & 1.23 & 6156.00 & 267.65 \\
\hline 11/26/1992 & 4593.10 & 199.70 & 11.20 & 0.49 & 41.60 & 1.81 & 4581.90 & 199.21 \\
\hline 11/27/1992 & 3049.80 & 132.60 & 11.60 & 0.50 & 59.80 & 2.60 & 3038.20 & 132.10 \\
\hline 11/28/1992 & 3036.80 & 132.03 & 11.60 & 0.50 & 45.00 & 1.96 & 3025.20 & 131.53 \\
\hline 11/29/1992 & 2337.10 & 101.61 & 11.20 & 0.49 & 41.40 & 1.80 & 2325.90 & 101.13 \\
\hline 11/30/1992 & 2154.80 & 93.69 & 11.20 & 0.49 & 30.50 & 1.33 & 2143.60 & 93.20 \\
\hline 12/1/1992 & 6220.90 & 270.47 & 10.40 & 0.45 & 62.70 & 2.73 & 6210.50 & 270.02 \\
\hline 12/2/1992 & 6435.20 & 279.79 & 150.80 & 6.85 & 42.10 & 1.83 & 6284.40 & 273.23 \\
\hline 12/3/1992 & 5980.40 & 260.02 & 276.00 & 12.00 & 43.70 & 1.90 & 5704.40 & 248.02 \\
\hline 12/4/1992 & 5852.90 & 254.47 & 142.80 & 6.21 & 36.20 & 1.57 & 5710.10 & 248.27 \\
\hline 12/5/1992 & 6252.80 & 271.86 & 547.60 & 23.81 & 21.00 & 0.91 & 5705.20 & 248.05 \\
\hline $12 / 6 / 1992$ & 2251.60 & 97.90 & 11.20 & 0.49 & 28.10 & 1.22 & 2240.40 & 97.41 \\
\hline 12/7/1992 & 1712.40 & 74.45 & 11.20 & 0.49 & 23.00 & 1.00 & 1701.20 & 73.97 \\
\hline $12 / 8 / 1992$ & 6007.30 & 261.19 & 36.40 & 1.58 & 54.00 & 2.35 & 5970.90 & 259.60 \\
\hline 12/9/1992 & 5967.60 & 259.46 & 10.80 & 0.47 & 50.40 & 2.19 & 5956.80 & 258.99 \\
\hline 12/10/1992 & 6185.40 & 268.93 & 356.00 & 15.48 & 26.70 & 1.16 & 5829.40 & 253.45 \\
\hline 12/11/1992 & 5534.60 & 240.63 & 112.00 & 4.87 & 23.80 & 1.03 & 5422.60 & 235.77 \\
\hline
\end{tabular}


CHART DATA

\begin{tabular}{|c|c|c|c|c|c|c|c|c|}
\hline $12 / 12 / 1992$ & 5434.70 & 236.29 & 105.20 & 4.57 & 30.00 & 1.30 & 5329.50 & 231.72 \\
\hline 12/13/1992 & 2139.30 & 93.01 & 10.40 & 0.45 & 18.00 & 0.78 & 2128.90 & 92.56 \\
\hline 12/14/1992 & 1459.10 & 63.44 & 10.40 & 0.45 & 15.00 & 0.65 & 1448.70 & 62.99 \\
\hline 12/15/1992 & 5375.80 & 233.73 & 10.80 & 0.47 & 36.30 & 1.58 & 5365.00 & 233.26 \\
\hline 12/16/1992 & 6159.30 & 267.80 & 10.40 & 0.45 & 51.90 & 2.26 & 6148.90 & 267.34 \\
\hline 12/17/1992 & 5735.10 & 249.35 & 10.40 & 0.45 & 43.20 & 1.88 & 5724.70 & 248.90 \\
\hline $12 / 18 / 1992$ & 5407.60 & 235.11 & 10.80 & 0.47 & 42.00 & 1.83 & 5396.80 & 234.64 \\
\hline 12/19/1992 & 4172.50 & 181.41 & 11.20 & 0.49 & 37.60 & 1.63 & 4161.30 & 180.93 \\
\hline 12/20/1992 & 2365.90 & 102.87 & 10.80 & 0.47 & 17.60 & 0.77 & 2355.10 & 102.40 \\
\hline 12/21/1992 & 1795.30 & 78.06 & 11.60 & 0.50 & 20.10 & 0.87 & 1783.70 & 77.55 \\
\hline 12/22/1992 & 3894.10 & 169.31 & 11.20 & 0.49 & 35.60 & 1.55 & 3882.90 & 168.82 \\
\hline 12/23/1992 & 3071.50 & 133.54 & 10.80 & 0.47 & 23.30 & 1.01 & 3060.70 & 133.07 \\
\hline 12/24/1992 & 2560.90 & 111.34 & 11.20 & 0.49 & 19.60 & 0.85 & 2549.70 & 110.86 \\
\hline 12/25/1992 & 2485.70 & 108.07 & 11.60 & 0.50 & 23.30 & 1.01 & 2474.10 & 107.57 \\
\hline 12/26/1992 & 2586.70 & 112.47 & 11.20 & 0.49 & 24.40 & 1.06 & 2575.50 & 111.98 \\
\hline 12/27/1992 & 1830.50 & 79.59 & 11.60 & 0.50 & 20.60 & 0.90 & 1818.90 & 79.08 \\
\hline 12/28/1992 & 1804.70 & 78.47 & 11.20 & 0.49 & 20.60 & 0.90 & 1793.50 & 77.98 \\
\hline 12/29/1992 & 3259.70 & 141.73 & 10.80 & 0.47 & 26.40 & 1.15 & 3248.90 & 141.26 \\
\hline 12/30/1992 & 2384.70 & 103.68 & 10.40 & 0.50 & 11.10 & 0.93 & 2374.30 & 103.23 \\
\hline 12/31/1992 & 2535.90 & 110.26 & 10.40 & 0.45 & 5.10 & 0.85 & 2525.50 & 109.80 \\
\hline $1 / 1 / 1993$ & 2626.60 & 114.20 & 12.00 & 0.52 & 37.60 & 1.63 & 2614.60 & 113.68 \\
\hline 1/2/1993 & 1892.30 & 82.27 & 10.80 & 0.47 & 34.00 & 1.48 & 1881.50 & 81.80 \\
\hline $1 / 3 / 1993$ & 1421.90 & 61.82 & 11.20 & 0.49 & 18.60 & 0.81 & 1410.70 & 61.33 \\
\hline $1 / 4 / 1993$ & 3939.50 & 171.28 & 10.80 & 0.47 & 18.60 & 0.81 & 3928.70 & 170.81 \\
\hline 1/5/1993 & 5876.00 & 255.48 & 10.40 & 0.45 & 42.50 & 1.85 & 5865.60 & 255.03 \\
\hline 1/6/1993 & 5452.60 & 237.07 & 10.40 & 0.45 & 40.20 & 1.75 & 5442.20 & 236.62 \\
\hline $1 / 7 / 1993$ & 5592.90 & 243.17 & 10.40 & 0.47 & 42.80 & 1.86 & 5582.50 & 242.72 \\
\hline 1/8/1993 & 5206.40 & 226.37 & 10.40 & 0.47 & 31.80 & 1.38 & 5196.00 & 225.91 \\
\hline 1/9/1993 & 1709.10 & 74.31 & 10.00 & 0.48 & 16.00 & 0.70 & 1699.10 & 73.87 \\
\hline $1 / 10 / 1993$ & 1606.10 & 69.83 & 11.60 & 0.50 & 20.50 & 0.89 & 1594.50 & 69.33 \\
\hline $1 / 11 / 1993$ & 6227.70 & 270.77 & 10.40 & 0.47 & 64.50 & 2.80 & 6217.30 & 270.32 \\
\hline 1/12/1993 & 6072.10 & 264.00 & 116.00 & 5.52 & 49.50 & 2.15 & 5956.10 & 258.96 \\
\hline $1 / 13 / 1993$ & 5655.80 & 245.90 & 10.40 & 0.45 & 45.70 & 1.99 & 5645.40 & 245.45 \\
\hline $1 / 14 / 1993$ & 5800.10 & 252.18 & 10.40 & 0.45 & 60.50 & 2.63 & 5789.70 & 251.73 \\
\hline 1/15/1993 & 5471.60 & 237.90 & 10.00 & 0.45 & 52.10 & 2.27 & 5461.60 & 237.46 \\
\hline $1 / 16 / 1993$ & 1570.80 & 68.30 & 9.60 & 0.44 & 15.00 & 0.88 & 1561.20 & 67.88 \\
\hline $1 / 17 / 1993$ & 1421.00 & 61.78 & 10.00 & 0.43 & 9.10 & 0.83 & 1411.00 & 61.35 \\
\hline 1/18/1993 & 5491.40 & 238.76 & 243.20 & 10.57 & 24.20 & 1.05 & 5248.20 & 228.18 \\
\hline $1 / 19 / 1993$ & 5529.00 & 240.39 & 10.80 & 0.47 & 44.30 & 1.93 & 5518.20 & 239.92 \\
\hline $1 / 20 / 1993$ & 5781.70 & 251.38 & 10.40 & 0.45 & 56.10 & 2.44 & 5771.30 & 250.93 \\
\hline 1/21/1993 & 5935.70 & 258.07 & 190.00 & 8.26 & 36.60 & 1.59 & 5745.70 & 249.81 \\
\hline $1 / 22 / 1993$ & 5665.30 & 246.32 & 346.40 & 15.06 & 23.60 & 1.39 & 5318.90 & 231.26 \\
\hline $1 / 23 / 1993$ & 1726.40 & \begin{tabular}{|l|}
75.06 \\
\end{tabular} & 10.80 & 0.47 & 2.00 & 0.50 & 1715.60 & 74.59 \\
\hline $1 / 24 / 1993$ & 1530.20 & 66.53 & 11.60 & 0.50 & 3.00 & 0.50 & 1518.60 & 66.03 \\
\hline $1 / 25 / 1993$ & 6045.30 & 262.84 & 60.00 & 2.61 & 57.90 & 3.05 & 5985.30 & 260.23 \\
\hline $1 / 26 / 1993$ & 5795.80 & 251.99 & 11.60 & 0.50 & 78.00 & 3.39 & 5784.20 & 251.49 \\
\hline 1/27/1993 & 5590.30 & 243.06 & 11.60 & 0.50 & 60.80 & 2.64 & 5578.70 & 242.55 \\
\hline $1 / 28 / 1993$ & 5498.40 & 239.06 & 11.60 & 0.50 & 42.90 & 1.87 & 5486.80 & 238.56 \\
\hline $1 / 29 / 1993$ & 5558.00 & 241.65 & 11.60 & 0.50 & 43.50 & 1.89 & 5546.40 & 241.15 \\
\hline $1 / 30 / 1993$ & 2164.50 & 94.11 & 11.60 & 0.50 & 24.00 & 1.04 & 2152.90 & 93.60 \\
\hline 1/31/1993 & 1962.70 & 85.33 & 12.00 & 0.52 & 17.00 & 0.74 & 1950.70 & 84.81 \\
\hline
\end{tabular}


CHART DATA

\begin{tabular}{|c|c|c|c|c|c|c|c|c|}
\hline $2 / 1 / 1993$ & 5956.50 & 258.98 & 11.20 & 0.51 & 42.90 & 1.87 & 5945.30 & 258.49 \\
\hline 2/2/1993 & 5751.60 & 250.07 & 10.40 & 0.45 & 39.60 & 1.72 & 5741.20 & 249.62 \\
\hline 2/3/1993 & 5745.80 & 249.82 & 10.40 & 0.45 & 38.50 & 1.67 & 5735.40 & 249.37 \\
\hline 2/4/1993 & 5736.90 & 249.43 & 11.20 & 0.49 & 39.00 & 1.70 & 5725.70 & 248.94 \\
\hline $2 / 5 / 1993$ & 5541.90 & 240.95 & 11.60 & 0.50 & 47.60 & 2.07 & 5530.30 & 240.45 \\
\hline 2/6/1993 & 2593.00 & 112.74 & 11.20 & 0.49 & 26.60 & 1.16 & 2581.80 & 112.25 \\
\hline 2/7/1993 & 2006.10 & 87.22 & 11.60 & 0.50 & 22.00 & 0.96 & 1994.50 & 86.72 \\
\hline 2/8/1993 & 6014.10 & 261.48 & 21.20 & 0.92 & 40.30 & 1.75 & 5992.90 & 260.56 \\
\hline 2/9/1993 & 6099.80 & 265.21 & 70.80 & 3.08 & 40.60 & 1.77 & 6029.00 & 262.13 \\
\hline 2/10/1993 & 5892.50 & 256.20 & 161.60 & 7.03 & 25.70 & 1.51 & 5730.90 & 249.17 \\
\hline 2/11/1993 & 5651.50 & 245.72 & 287.20 & 12.49 & 23.00 & 1.21 & 5364.30 & 233.23 \\
\hline $2 / 12 / 1993$ & 5291.00 & 230.04 & 141.20 & 6.14 & 35.90 & 1.63 & 5149.80 & 223.90 \\
\hline 2/13/1993 & 1908.40 & 82.97 & 10.80 & 0.47 & 17.50 & 0.76 & 1897.60 & 82.50 \\
\hline 2/14/1993 & 1875.60 & 81.55 & 10.40 & 0.45 & 15.00 & 0.68 & 1865.20 & 81.10 \\
\hline 2/15/1993 & 3475.20 & 151.10 & 10.00 & 0.43 & 23.20 & 1.01 & 3465.20 & 150.66 \\
\hline 2/16/1993 & 5362.80 & 233.17 & 11.60 & 0.50 & 44.60 & 2.03 & 5351.20 & 232.66 \\
\hline 2/17/1993 & 5771.70 & 250.94 & 12.00 & 0.52 & 48.50 & 2.11 & 5759.70 & 250.42 \\
\hline 2/18/1993 & 6129.20 & 266.49 & 11.60 & 0.50 & 62.90 & 2.73 & 6117.60 & 265.98 \\
\hline 2/19/1993 & 6047.10 & 262.92 & 10.80 & 0.47 & 42.60 & 2.03 & 6036.30 & 262.45 \\
\hline 2/20/1993 & 2988.40 & 129.93 & 10.00 & 0.43 & 16.00 & 0.80 & 2978.40 & 129.50 \\
\hline $2 / 21 / 1993$ & 1914.70 & 83.25 & 10.80 & 0.47 & 16.00 & 0.70 & 1903.90 & 82.78 \\
\hline 2/22/1993 & 5615.90 & 244.17 & 220.80 & 9.60 & 25.20 & 1.10 & 5395.10 & 234.57 \\
\hline $2 / 23 / 1993$ & 6089.40 & 264.76 & 300.40 & 13.06 & 19.50 & 0.85 & 5789.00 & 251.70 \\
\hline $2 / 24 / 1993$ & 6360.70 & 276.55 & 403.20 & 17.53 & 17.30 & 0.75 & 5957.50 & 259.02 \\
\hline $2 / 25 / 1993$ & 6511.00 & 283.09 & 400.40 & 18.20 & 14.30 & 0.79 & 6110.60 & 265.68 \\
\hline 2/26/1993 & 5039.60 & 219.11 & 11.20 & 0.49 & 31.20 & 1.36 & 5028.40 & 218.63 \\
\hline $2 / 27 / 1993$ & 2551.20 & 110.92 & 11.60 & 0.50 & 28.70 & 1.25 & 2539.60 & 110.42 \\
\hline 2/28/1993 & 1710.70 & 74.38 & 10.80 & 0.47 & 20.00 & 0.87 & 1699.90 & 73.91 \\
\hline 3/1/1993 & 5738.80 & 249.51 & 101.60 & 4.42 & 25.00 & 1.09 & 5637.20 & 245.10 \\
\hline 3/2/1993 & 5412.20 & 235.31 & 11.60 & 0.50 & 23.40 & 1.02 & 5400.60 & 234.81 \\
\hline 3/3/1993 & 6068.90 & 263.87 & 289.20 & 12.57 & 21.10 & 0.92 & 5779.70 & 251.29 \\
\hline 3/4/1993 & 5950.20 & 258.70 & 348.40 & 15.15 & 26.00 & 1.13 & 5601.80 & 243.56 \\
\hline 3/5/1993 & 4928.10 & 214.27 & 10.80 & 0.47 & 34.20 & 1.49 & 4917.30 & 213.80 \\
\hline 3/6/1993 & 2119.20 & 92.14 & 10.40 & 0.45 & 17.50 & 0.76 & 2108.80 & 91.69 \\
\hline 3/7/1993 & 1632.20 & 70.97 & 10.80 & 0.47 & 16.00 & 0.70 & 1621.40 & 70.50 \\
\hline 3/8/1993 & 6330.40 & 275.23 & 343.20 & 14.92 & 23.20 & 1.01 & 5987.20 & 260.31 \\
\hline 3/9/1993 & 6642.50 & 288.80 & 390.00 & 17.73 & 17.00 & 0.77 & 6252.50 & 271.85 \\
\hline 3/10/1993 & 7130.50 & 310.02 & 446.00 & 19.39 & 17.00 & 0.74 & 6684.50 & 290.63 \\
\hline 3/11/1993 & 5955.60 & 258.94 & 243.60 & 11.07 & 17.50 & 0.76 & 5712.00 & 248.35 \\
\hline $3 / 12 / 1993$ & 5399.90 & 234.78 & 12.00 & 0.52 & 59.30 & 2.58 & 5387.90 & 234.26 \\
\hline 3/13/1993 & 2272.10 & 98.79 & 11.60 & 0.50 & 36.70 & 1.60 & 2260.50 & 98.28 \\
\hline 3/14/1993 & 2090.10 & 90.87 & 11.20 & 0.49 & 18.00 & 0.78 & 2078.90 & 90.39 \\
\hline $3 / 15 / 1993$ & 3122.60 & 135.77 & 10.80 & 0.47 & 55.40 & 2.41 & 3111.80 & 135.30 \\
\hline 3/16/1993 & 2969.70 & 129.12 & 11.20 & 0.49 & 25.70 & 1.12 & 2958.50 & 128.63 \\
\hline 3/17/1993 & 2567.90 & 111.65 & 11.20 & 0.49 & 19.10 & 0.83 & 2556.70 & 111.16 \\
\hline $3 / 18 / 1993$ & 2156.80 & 93.77 & 10.80 & 0.47 & 19.00 & 0.83 & 2146.00 & 93.30 \\
\hline 3/19/1993 & 2046.20 & 88.97 & 10.40 & 0.47 & 18.00 & 0.78 & 2035.80 & 88.51 \\
\hline 3/20/1993 & 1531.70 & 66.60 & 10.80 & 0.47 & 19.50 & 0.85 & 1520.90 & 66.13 \\
\hline 3/21/1993 & 1574.20 & 68.44 & 10.80 & 0.47 & 19.50 & 0.85 & 1563.40 & 67.97 \\
\hline 3/22/1993 & 6182.00 & 268.78 & 290.00 & 13.18 & 18.00 & 0.82 & 5892.00 & 256.17 \\
\hline 3/23/1993 & 5527.90 & 240.34 & 200.00 & 8.70 & 23.20 & 1.01 & 5327.90 & 231.65 \\
\hline
\end{tabular}


CHART DATA

\begin{tabular}{|c|c|c|c|c|c|c|c|c|}
\hline $3 / 24 / 1993$ & 6768.60 & 294.29 & 462.40 & 20.10 & 17.50 & 0.76 & 6306.20 & 274.18 \\
\hline $3 / 25 / 1993$ & 7447.60 & 323.81 & 471.60 & 20.50 & 16.00 & 0.70 & 6976.00 & 303.30 \\
\hline $3 / 26 / 1993$ & 7241.20 & 314.83 & 492.00 & 21.39 & 16.00 & 0.70 & 6749.20 & 293.44 \\
\hline $3 / 27 / 1993$ & 2372.70 & 103.16 & 242.40 & 10.54 & 15.00 & 0.65 & 2130.30 & 92.62 \\
\hline 3/28/1993 & 1570.50 & 68.28 & 10.40 & 0.45 & 15.00 & 0.65 & 1560.10 & 67.83 \\
\hline 3/29/1993 & 7887.30 & 342.93 & 418.00 & 18.17 & 15.50 & 0.67 & 7469.30 & 324.75 \\
\hline $3 / 30 / 1993$ & 8889.00 & 386.48 & 507.60 & 24.17 & 7.00 & 0.58 & 8381.40 & 364.41 \\
\hline 3/31/1993 & 7323.10 & 318.40 & 454.00 & 19.74 & 2.50 & 0.50 & 6869.10 & 298.66 \\
\hline 4/1/1993 & 7443.60 & 323.63 & 497.60 & 21.63 & 3.00 & 0.60 & 6946.00 & 302.00 \\
\hline 4/2/1993 & 7259.30 & 315.62 & 506.00 & 22.00 & 3.00 & 0.50 & 6753.30 & 293.62 \\
\hline 4/3/1993 & 2585.90 & 112.43 & 10.40 & 0.45 & 2.50 & 0.50 & 2575.50 & 111.98 \\
\hline 4/4/1993 & 1728.80 & 78.58 & 10.40 & 0.47 & 2.00 & 0.50 & 1718.40 & 78.11 \\
\hline 4/5/1993 & 5653.40 & 245.80 & 312.00 & 13.57 & 7.10 & 0.89 & 5341.40 & 232.23 \\
\hline 4/6/1993 & 6747.80 & 293.38 & 461.20 & 20.05 & 4.60 & 0.51 & 6286.60 & 273.33 \\
\hline 4/7/1993 & 7165.30 & 311.53 & 485.60 & 21.11 & 3.90 & 0.35 & 6679.70 & 290.42 \\
\hline 4/8/1993 & 6590.60 & 286.55 & 414.00 & 18.00 & 3.30 & 0.47 & 6176.60 & 268.55 \\
\hline 4/9/1993 & 3258.30 & 141.67 & 10.80 & 0.47 & 3.70 & 0.37 & 3247.50 & 141.20 \\
\hline 4/10/1993 & 1658.90 & 72.13 & 10.40 & 0.45 & 1.50 & 0.50 & 1648.50 & 71.67 \\
\hline $4 / 11 / 1993$ & 1553.60 & 67.55 & 10.40 & 0.45 & 1.50 & 0.50 & 1543.20 & 67.10 \\
\hline $4 / 12 / 1993$ & 3129.50 & 136.07 & 18.00 & 0.78 & 1.50 & 0.50 & 3111.50 & 135.28 \\
\hline 4/13/1993 & 9276.60 & 403.33 & 542.80 & 23.60 & 3.00 & 0.50 & 8733.80 & 379.73 \\
\hline $4 / 14 / 1993$ & 8964.10 & 389.74 & 488.00 & 22.18 & 2.00 & 0.50 & 8476.10 & 368.53 \\
\hline $4 / 15 / 1993$ & 6286.40 & 273.32 & 478.00 & 20.78 & 3.10 & 0.52 & 5808.40 & 252.54 \\
\hline 4/16/1993 & 6423.00 & 279.26 & 379.60 & 16.50 & 4.60 & 0.46 & 6043.40 & 262.76 \\
\hline $4 / 17 / 1993$ & 1710.00 & 74.35 & 10.00 & 0.48 & 2.00 & 0.50 & 1700.00 & 73.91 \\
\hline $4 / 18 / 1993$ & 1601.70 & 69.64 & 10.40 & 0.47 & 2.00 & 0.50 & 1591.30 & 69.19 \\
\hline 4/19/1993 & 8564.70 & 372.38 & 516.40 & 22.45 & 3.10 & 0.52 & 8048.30 & 349.93 \\
\hline 4/20/1993 & 8450.70 & 367.42 & 517.60 & 22.50 & 3.00 & 0.50 & 7933.10 & 344.92 \\
\hline $4 / 21 / 1993$ & 6834.10 & 297.13 & 465.60 & 20.24 & 5.00 & 0.56 & 6368.50 & 276.89 \\
\hline 4/22/1993 & 6826.40 & 296.80 & 463.20 & 20.14 & 4.10 & 0.51 & 6363.20 & 276.66 \\
\hline 4/23/1993 & 7861.60 & 341.81 & 506.80 & 22.03 & 3.60 & 0.45 & 7354.80 & 319.77 \\
\hline $4 / 24 / 1993$ & 2109.20 & 91.70 & 10.00 & 0.43 & 2.00 & 0.50 & 2099.20 & 91.27 \\
\hline $4 / 25 / 1993$ & 1824.70 & 79.33 & 10.00 & 0.43 & 2.00 & 0.50 & 1814.70 & 78.90 \\
\hline 4/26/1993 & 8652.90 & 376.21 & 554.40 & 24.10 & 3.30 & 0.41 & 8098.50 & 352.11 \\
\hline 4/27/1993 & 8350.50 & 363.07 & 506.40 & 22.02 & 3.80 & 0.48 & 7844.10 & 341.05 \\
\hline $4 / 28 / 1993$ & 7187.70 & 312.51 & 466.00 & 21.18 & 2.50 & 0.50 & 6721.70 & 292.25 \\
\hline 4/29/1993 & 7657.50 & 332.93 & 529.60 & 26.48 & 3.00 & 0.50 & 7127.90 & 309.91 \\
\hline 4/30/1993 & 7398.30 & 321.67 & 566.00 & 24.61 & 3.50 & 0.50 & 6832.30 & 297.06 \\
\hline $5 / 1 / 1993$ & 2409.00 & 104.74 & 10.40 & 0.45 & 3.00 & 0.50 & 2398.60 & 104.29 \\
\hline $5 / 2 / 1993$ & 1796.30 & 78.10 & 10.40 & 0.45 & 2.50 & 0.50 & 1785.90 & 77.65 \\
\hline 5/3/1993 & 7747.30 & 336.84 & 546.40 & 24.84 & 4.00 & 0.57 & 7200.90 & 313.08 \\
\hline $5 / 4 / 1993$ & 8683.10 & 377.53 & 526.00 & 22.87 & 3.50 & 0.50 & 8157.10 & 354.66 \\
\hline $5 / 5 / 1993$ & 8443.10 & 367.09 & 523.20 & 23.78 & 3.00 & 0.50 & 7919.90 & 344.34 \\
\hline 5/6/1993 & 7980.70 & 346.99 & 487.60 & 21.20 & 4.00 & 0.50 & 7493.10 & 325.79 \\
\hline $5 / 7 / 1993$ & 8738.50 & 379.93 & 526.00 & 22.87 & 3.50 & 0.50 & 8212.50 & 357.07 \\
\hline 5/8/1993 & 1803.30 & 78.40 & 9.60 & 0.44 & 2.50 & 0.50 & 1793.70 & 77.99 \\
\hline 5/9/1993 & 1594.90 & 69.34 & 10.00 & 0.45 & 2.00 & 0.50 & 1584.90 & 68.91 \\
\hline $5 / 10 / 1993$ & 7526.10 & 327.22 & 521.20 & 22.66 & 4.00 & 0.57 & 7004.90 & 304.56 \\
\hline $5 / 11 / 1993$ & 8367.20 & 363.79 & 510.00 & 23.18 & 3.50 & 0.50 & 7857.20 & 341.62 \\
\hline $5 / 12 / 1993$ & 8513.90 & 370.17 & 535.60 & 23.29 & 3.50 & 0.58 & 7978.30 & 346.88 \\
\hline $5 / 13 / 1993$ & 8263.30 & 359.27 & 542.80 & 23.60 & 4.00 & 0.50 & 7720.50 & 335.67 \\
\hline
\end{tabular}


CHART DATA

\begin{tabular}{|c|c|c|c|c|c|c|c|c|}
\hline $5 / 14 / 1993$ & 8764.60 & 381.07 & 539.20 & 23.44 & 3.50 & 0.50 & 8225.40 & 357.63 \\
\hline $5 / 15 / 1993$ & 1920.60 & 83.50 & 12.40 & 0.54 & 2.00 & 0.50 & 1908.20 & 82.97 \\
\hline $5 / 16 / 1993$ & 2016.50 & 87.67 & 12.80 & 0.56 & 2.00 & 0.50 & 2003.70 & 87.12 \\
\hline $5 / 17 / 1993$ & 9796.90 & 425.95 & 574.00 & 24.96 & 3.00 & 0.50 & 9222.90 & 401.00 \\
\hline 5/18/1993 & 9843.70 & 427.99 & 541.60 & 23.55 & 3.50 & 0.50 & 9302.10 & 404.44 \\
\hline $5 / 19 / 1993$ & 8608.20 & 374.27 & 543.60 & 23.63 & 3.00 & 0.50 & 8064.60 & 350.63 \\
\hline $5 / 20 / 1993$ & 8618.10 & 374.70 & 538.80 & 23.43 & 4.00 & 0.50 & 8079.30 & 351.27 \\
\hline $5 / 21 / 1993$ & 9168.10 & 398.61 & 562.40 & 24.45 & 3.00 & 0.50 & 8605.70 & 374.16 \\
\hline $5 / 22 / 1993$ & 2099.70 & 91.29 & 11.60 & 0.50 & 2.50 & 0.63 & 2088.10 & 90.79 \\
\hline 5/23/1993 & 1876.80 & 81.60 & 10.40 & 0.45 & 2.00 & 0.50 & 1866.40 & 81.15 \\
\hline $5 / 24 / 1993$ & 7842.50 & 340.98 & 549.20 & 24.96 & 2.50 & 0.50 & 7293.30 & 317.10 \\
\hline $5 / 25 / 1993$ & 8276.20 & 359.83 & 554.00 & 24.09 & 2.50 & 0.50 & 7722.20 & 335.75 \\
\hline $5 / 26 / 1993$ & 8124.20 & 353.23 & 549.20 & 23.88 & 2.50 & 0.50 & 7575.00 & 329.35 \\
\hline $5 / 27 / 1993$ & 7660.30 & 333.06 & 540.80 & 23.51 & 2.00 & 0.50 & 7119.50 & 309.54 \\
\hline $5 / 28 / 1993$ & 6300.30 & 273.93 & 495.20 & 21.53 & 2.50 & 0.50 & 5805.10 & 252.40 \\
\hline 5/29/1993 & 1884.40 & 81.93 & 10.40 & 0.47 & 2.00 & 0.50 & 1874.00 & 81.48 \\
\hline $5 / 30 / 1993$ & 1879.30 & 81.71 & 11.60 & 0.50 & 1.50 & 0.50 & 1867.70 & 81.20 \\
\hline $5 / 31 / 1993$ & 5812.00 & 252.70 & 518.80 & 22.56 & 2.00 & 0.50 & 5293.20 & 230.14 \\
\hline 6/1/1993 & 4652.90 & 202.30 & 532.00 & 23.13 & 2.00 & 0.50 & 4120.90 & 179.17 \\
\hline $6 / 2 / 1993$ & 5392.90 & 234.47 & 521.20 & 22.66 & 1.50 & 0.50 & 4871.70 & 211.81 \\
\hline 6/3/1993 & 4959.10 & 215.61 & 536.80 & 23.34 & 2.00 & 0.50 & 4422.30 & 192.27 \\
\hline 6/4/1993 & 3211.30 & 139.62 & 13.60 & 0.62 & 1.50 & 0.50 & 3197.70 & 139.03 \\
\hline 6/5/1993 & 2909.90 & 121.25 & 14.80 & 0.62 & 2.00 & 0.50 & 2895.10 & 120.63 \\
\hline 6/6/1993 & 2876.10 & 119.84 & 15.60 & 0.65 & 1.50 & 0.50 & 2860.50 & 119.19 \\
\hline 6/7/1993 & 6001.40 & 250.06 & 606.80 & 25.28 & 2.00 & 0.50 & 5394.60 & 224.78 \\
\hline 6/8/1993 & 6170.70 & 257.11 & 591.20 & 24.63 & 2.00 & 0.50 & 5579.50 & 232.48 \\
\hline 6/9/1993 & 6431.00 & 267.96 & 523.20 & 21.80 & 2.00 & 0.50 & 5907.80 & 246.16 \\
\hline 6/10/1993 & 5353.20 & 223.05 & 474.00 & 19.75 & 2.80 & 0.56 & 4879.20 & 203.30 \\
\hline 6/11/1993 & 2969.30 & 123.72 & 11.60 & 0.50 & 2.00 & 0.50 & 2957.70 & 123.24 \\
\hline $6 / 12 / 1993$ & 2117.80 & 88.24 & 10.00 & 0.43 & 2.00 & 0.50 & 2107.80 & 87.83 \\
\hline 6/13/1993 & 2031.30 & 84.64 & 10.40 & 0.43 & 2.00 & 0.50 & 2020.90 & 84.20 \\
\hline $6 / 14 / 1993$ & 4750.20 & 197.93 & 452.40 & 18.85 & 2.00 & 0.50 & 4297.80 & 179.08 \\
\hline $6 / 15 / 1993$ & 4574.70 & 190.61 & 433.60 & 18.07 & 2.50 & 0.50 & 4141.10 & 172.55 \\
\hline 6/16/1993 & 5292.70 & 220.53 & 509.60 & 21.23 & 1.50 & 0.50 & 4783.10 & 199.30 \\
\hline 6/17/1993 & 5272.40 & 219.68 & 515.20 & 21.47 & 2.00 & 0.50 & 4757.20 & 198.22 \\
\hline 6/18/1993 & 3908.70 & 162.86 & 498.40 & 20.77 & 2.00 & 0.50 & 3410.30 & 142.10 \\
\hline $6 / 19 / 1993$ & 1971.70 & 82.15 & 12.00 & 0.50 & 2.00 & 0.50 & 1959.70 & 81.65 \\
\hline 6/20/1993 & 1958.60 & 81.61 & 10.80 & 0.45 & 2.00 & 0.50 & 1947.80 & 81.16 \\
\hline 6/21/1993 & 5275.80 & 219.83 & 541.20 & 22.55 & 2.00 & 0.50 & 4734.60 & 197.28 \\
\hline $6 / 22 / 1993$ & 4854.90 & 202.29 & 519.20 & 21.63 & 2.00 & 0.50 & 4335.70 & 180.65 \\
\hline 6/23/1993 & 5107.70 & 212.82 & 523.60 & 21.82 & 2.50 & 0.50 & 4584.10 & 191.00 \\
\hline 6/24/1993 & 5036.30 & 209.85 & 528.40 & 22.02 & 2.00 & 0.50 & 4507.90 & 187.83 \\
\hline $6 / 25 / 1993$ & 3573.30 & 148.89 & 15.60 & 0.65 & 2.00 & 0.50 & 3557.70 & 148.24 \\
\hline 6/26/1993 & 2307.00 & 96.13 & 14.00 & 0.58 & 2.00 & 0.50 & 2293.00 & 95.54 \\
\hline 6/27/1993 & 2030.90 & 84.62 & 13.60 & 0.57 & 1.50 & 0.50 & 2017.30 & 84.05 \\
\hline $6 / 28 / 1993$ & 5655.80 & 235.66 & 556.80 & 23.20 & 2.00 & 0.50 & 5099.00 & 212.46 \\
\hline 6/29/1993 & 5718.70 & 238.28 & 552.80 & 23.03 & 2.00 & 0.50 & 5165.90 & 215.25 \\
\hline 6/30/1993 & 5738.80 & 239.12 & 541.60 & 22.57 & 2.00 & 0.50 & 5197.20 & 216.55 \\
\hline 7/1/1993 & 5517.60 & 229.90 & 527.20 & 21.97 & 1.50 & 0.50 & 4990.40 & 207.93 \\
\hline 7/2/1993 & 4867.00 & 202.79 & 519.20 & 21.63 & 2.00 & 0.50 & 4347.80 & 181.16 \\
\hline 7/3/1993 & 2409.50 & 100.40 & 16.40 & 0.68 & 2.00 & 0.50 & 2393.10 & 99.71 \\
\hline
\end{tabular}


CHART DATA

\begin{tabular}{|c|c|c|c|c|c|c|c|c|}
\hline \begin{tabular}{|l|}
$7 / 4 / 1993$ \\
\end{tabular} & 2522.00 & 105.08 & 16.80 & 0.70 & 2.00 & 0.50 & 2505.20 & 104.38 \\
\hline 7/5/1993 & 5449.20 & 227.05 & 549.20 & 22.88 & 1.50 & 0.50 & 4900.00 & 204.17 \\
\hline 7/6/1993 & & & & & & & & \\
\hline 7/7/1993 & & & & & & & & \\
\hline 7/8/1993 & & & & & & & & \\
\hline 7/9/1993 & & & & & & & & \\
\hline 7/10/1993 & & & & & & & & \\
\hline 7/11/1993 & & & & & & & & \\
\hline $7 / 12 / 1993$ & & & & & & & & \\
\hline 7/13/1993 & & & & & & & & \\
\hline 7/14/1993 & & & & & & & & \\
\hline 7/15/1993 & & & & & & & & \\
\hline 7/16/1993 & & & & & & & & \\
\hline 7/17/1993 & & & & & & & & \\
\hline 7/18/1993 & & & & & & & & \\
\hline 7/19/1993 & & & & & & & & \\
\hline 7/20/1993 & & & & & & & & \\
\hline 7/21/1993 & & & & & & & & \\
\hline 7/22/1993 & & & & & & & & \\
\hline 7/23/1993 & & & & & & & & \\
\hline 7/24/1993 & & & & & & & & \\
\hline 7/25/1993 & & & & & & & & \\
\hline $7 / 26 / 1993$ & & & & & & & & \\
\hline 7/27/1993 & & & & & & & & \\
\hline 7/28/1993 & & & & & & & & \\
\hline 7/29/1993 & & & & & & & & \\
\hline 7/30/1993 & & & & & & & & \\
\hline 7/31/1993 & & & & & & & & \\
\hline 8/1/1993 & & & & & & & & \\
\hline 8/2/1993 & & & & & & & & \\
\hline 8/3/1993 & & & & & & & & \\
\hline 8/4/1993 & & & & & & & & \\
\hline 8/5/1993 & & & & & & & & \\
\hline 8/6/1993 & & & & & & & & \\
\hline 8/7/1993 & & & & & & & & \\
\hline 8/8/1993 & & & & & & & & \\
\hline 8/9/1993 & & & & & & & & \\
\hline 8/10/1993 & & & & & & & & \\
\hline 8/11/1993 & & & & & & & & \\
\hline $8 / 12 / 1993$ & & & & & & & & \\
\hline $8 / 13 / 1993$ & 5284.20 & 406.48 & 379.60 & 29.20 & 2.00 & 0.50 & 4904.60 & 377.28 \\
\hline 8/14/1993 & 2723.20 & 113.47 & 17.60 & 0.73 & 1.50 & 0.50 & 2705.60 & 112.73 \\
\hline $8 / 15 / 1993$ & 2827.90 & 117.83 & 18.00 & 0.75 & 1.50 & 0.50 & 2809.90 & 117.08 \\
\hline 8/16/1993 & 9895.30 & 412.30 & 594.80 & 24.78 & 2.10 & 0.42 & 9300.50 & 387.52 \\
\hline $8 / 17 / 1993$ & 10257.40 & 427.39 & 660.00 & 27.50 & 3.00 & 0.50 & 9597.40 & 399.89 \\
\hline 8/18/1993 & 11236.40 & 468.18 & 674.00 & 28.08 & 3.50 & 0.50 & 10562.40 & 440.10 \\
\hline 8/19/1993 & 11134.60 & 463.94 & 681.20 & 28.38 & 3.00 & 0.50 & 10453.40 & 435.56 \\
\hline 8/20/1993 & 10931.10 & 455.46 & 668.00 & 27.83 & 3.00 & 0.50 & 10263.10 & 427.63 \\
\hline $8 / 21 / 1993$ & 2599.80 & 108.33 & 17.20 & 0.72 & 2.50 & 0.50 & 2582.60 & 107.61 \\
\hline 8/22/1993 & 2624.00 & 109.33 & 17.20 & 0.72 & 1.00 & 0.50 & 2606.80 & 108.62 \\
\hline $8 / 23 / 1993$ & 12486.50 & 520.27 & 730.40 & 30.43 & 3.00 & 0.50 & 11756.10 & 489.84 \\
\hline
\end{tabular}


CHART DATA

\begin{tabular}{|c|c|c|c|c|c|c|c|c|}
\hline 8/24/1993 & 11766.10 & 490.25 & 722.40 & 30.10 & 3.50 & 0.58 & 11043.70 & 460.15 \\
\hline 8/25/1993 & 11526.80 & 480.28 & 712.00 & 29.67 & 3.50 & 0.50 & 10814.80 & 450.62 \\
\hline $8 / 26 / 1993$ & 11197.50 & 466.56 & 693.60 & 28.90 & 1.50 & 0.50 & 10503.90 & 437.66 \\
\hline $8 / 27 / 1993$ & 10789.60 & 449.57 & 700.00 & 29.17 & 2.50 & 0.50 & 10089.60 & 420.40 \\
\hline 8/28/1993 & 2330.40 & 97.10 & 15.60 & 0.65 & 1.50 & 0.50 & 2314.80 & 96.45 \\
\hline 8/29/1993 & 2105.50 & 87.73 & 15.60 & 0.65 & 1.50 & 0.50 & 2089.90 & 87.08 \\
\hline 8/30/1993 & 11476.60 & 478.19 & 704.40 & 29.35 & 5.50 & 0.69 & 10772.20 & 448.84 \\
\hline $8 / 31 / 1993$ & 11227.70 & 467.82 & 696.40 & 29.02 & 3.50 & 0.50 & 10531.30 & 438.80 \\
\hline 9/1/1993 & 11068.90 & 461.20 & 687.20 & 28.63 & 3.50 & 0.50 & 10381.70 & 432.57 \\
\hline 9/2/1993 & 11125.00 & 463.54 & 689.20 & 28.72 & 3.50 & 0.58 & 10435.80 & 434.83 \\
\hline 9/3/1993 & 11236.30 & 468.18 & 701.60 & 29.23 & 2.50 & 0.50 & 10534.70 & 438.95 \\
\hline 9/4/1993 & 2327.80 & 96.99 & 16.80 & 0.70 & 2.50 & 0.50 & 2311.00 & 96.29 \\
\hline 9/5/1993 & 2126.40 & 88.60 & 16.40 & 0.68 & 1.50 & 0.50 & 2110.00 & 87.92 \\
\hline 9/6/1993 & 3430.70 & 142.95 & 16.40 & 0.68 & 2.50 & 0.50 & 3414.30 & 142.26 \\
\hline 9/7/1993 & 4491.80 & 187.16 & 16.40 & 0.68 & 3.00 & 0.50 & 4475.40 & 186.48 \\
\hline 9/8/1993 & 5560.00 & 231.67 & 597.20 & 24.88 & 2.00 & 0.50 & 4962.80 & 206.78 \\
\hline 9/9/1993 & 11266.70 & 469.45 & 699.20 & 29.13 & 3.00 & 0.50 & 10567.50 & 440.31 \\
\hline 9/10/1993 & 11132.50 & 463.85 & 710.40 & 29.60 & 3.00 & 0.50 & 10422.10 & 434.25 \\
\hline 9/11/1993 & 2301.80 & 95.91 & 16.40 & 0.68 & 2.00 & 0.50 & 2285.40 & 95.23 \\
\hline 9/12/1993 & 2316.90 & 96.54 & 16.00 & 0.67 & 1.50 & 0.50 & 2300.90 & 95.87 \\
\hline 9/13/1993 & 11986.40 & 499.43 & 766.80 & 31.95 & 3.50 & 0.50 & 11219.60 & 467.48 \\
\hline 9/14/1993 & 11516.50 & 479.85 & 724.00 & 30.17 & 2.50 & 0.50 & 10792.50 & 449.69 \\
\hline 9/15/1993 & 8746.50 & 364.44 & 672.40 & 28.02 & 1.90 & 0.27 & 8074.10 & 336.42 \\
\hline 9/16/1993 & 8917.40 & 371.56 & 661.60 & 27.57 & 4.30 & 0.43 & 8255.80 & 343.99 \\
\hline 9/17/1993 & 9560.00 & 398.33 & 653.60 & 27.23 & 2.80 & 0.40 & 8906.40 & 371.10 \\
\hline 9/18/1993 & 3142.30 & 130.93 & 16.00 & 0.67 & 2.50 & 0.50 & 3126.30 & 130.26 \\
\hline 9/19/1993 & 2428.10 & 101.17 & 16.00 & 0.67 & 1.50 & 0.50 & 2412.10 & 100.50 \\
\hline 9/20/1993 & 11705.60 & 487.73 & 719.60 & 29.98 & 4.00 & 0.50 & 10986.00 & 457.75 \\
\hline 9/21/1993 & 11803.20 & 491.80 & 718.80 & 29.95 & 3.50 & 0.50 & 11084.40 & 461.85 \\
\hline 9/22/1993 & 10960.70 & 456.70 & 730.40 & 30.43 & 3.50 & 0.50 & 10230.30 & 426.26 \\
\hline 9/23/1993 & 11234.70 & 468.11 & 722.00 & 30.08 & 3.00 & 0.50 & 10512.70 & 438.03 \\
\hline 9/24/1993 & 11305.60 & 471.07 & 708.80 & 29.53 & 3.00 & 0.50 & 10596.80 & 441.53 \\
\hline 9/25/1993 & 2806.20 & 116.93 & 16.40 & 0.68 & 2.50 & 0.50 & 2789.80 & 116.24 \\
\hline 9/26/1993 & 2321.70 & 96.74 & 16.40 & 0.68 & 1.50 & 0.50 & 2305.30 & 96.05 \\
\hline 9/27/1993 & 9923.20 & 413.47 & 691.60 & 28.82 & 4.10 & 0.46 & 9231.60 & 384.65 \\
\hline 9/28/1993 & 10828.40 & 451.18 & 662.40 & 27.60 & 4.00 & 0.57 & 10166.00 & 423.58 \\
\hline 9/29/1993 & 9102.20 & 379.26 & 668.00 & 27.83 & 3.50 & 0.50 & 8434.20 & 351.43 \\
\hline 9/30/1993 & 9826.10 & 409.42 & 628.80 & 26.20 & 3.50 & 0.50 & 9197.30 & 383.22 \\
\hline 10/1/1993 & 10047.50 & 418.65 & 630.40 & 26.27 & 3.00 & 0.50 & 9417.10 & 392.38 \\
\hline $10 / 2 / 1993$ & 3094.70 & 128.95 & 16.00 & 0.67 & 2.50 & 0.50 & 3078.70 & 128.28 \\
\hline 10/3/1993 & 2291.40 & 95.48 & 15.60 & 0.65 & 1.50 & 0.50 & 2275.80 & 94.83 \\
\hline 10/4/1993 & 10325.60 & 430.23 & 648.00 & 27.00 & 2.50 & 0.50 & 9677.60 & 403.23 \\
\hline 10/5/1993 & 10802.50 & 450.10 & 639.20 & 26.63 & 3.50 & 0.50 & 10163.30 & 423.47 \\
\hline $10 / 6 / 1993$ & 9768.40 & 407.02 & 642.00 & 26.75 & 4.00 & 0.50 & 9126.40 & 380.27 \\
\hline 10/7/1993 & 9855.70 & 410.65 & 638.00 & 26.58 & 3.50 & 0.50 & 9217.70 & 384.07 \\
\hline $10 / 8 / 1993$ & 10089.90 & 420.41 & 636.00 & 26.50 & 3.50 & 0.50 & 9453.90 & 393.91 \\
\hline 10/9/1993 & 2267.30 & 94.47 & 14.80 & 0.62 & 2.00 & 0.50 & 2252.50 & 93.85 \\
\hline 10/10/1993 & 1893.90 & 78.91 & 13.60 & 0.57 & 1.50 & 0.50 & 1880.30 & 78.35 \\
\hline 10/11/1993 & 9884.50 & 411.85 & 639.60 & 26.65 & 4.00 & 0.50 & 9244.90 & 385.20 \\
\hline 10/12/1993 & 10830.30 & 451.26 & 636.40 & 26.52 & 3.50 & 0.50 & 10193.90 & 424.75 \\
\hline 10/13/1993 & 9209.60 & 383.73 & 636.40 & 26.52 & 3.50 & 0.50 & 8573.20 & 357.22 \\
\hline
\end{tabular}


CHART DATA

\begin{tabular}{|c|c|c|c|c|c|c|c|c|}
\hline $10 / 14 / 1993$ & 8472.30 & 353.01 & 638.40 & 27.76 & 3.00 & 0.50 & 7833.90 & 326.41 \\
\hline 10/15/1993 & 8633.10 & 359.71 & 624.40 & 26.02 & 3.50 & 0.50 & 8008.70 & 333.70 \\
\hline 10/16/1993 & 3329.80 & 138.74 & 13.20 & 0.57 & 2.00 & 0.50 & 3316.60 & 138.19 \\
\hline 10/17/1993 & 2065.30 & 86.05 & 14.00 & 0.58 & 1.50 & 0.50 & 2051.30 & 85.47 \\
\hline 10/18/1993 & 11086.90 & 461.95 & 668.40 & 27.85 & 3.50 & 0.50 & 10418.50 & 434.10 \\
\hline 10/19/1993 & 11471.20 & 477.97 & 1023.20 & 42.63 & 4.00 & 0.50 & 10448.00 & 435.33 \\
\hline 10/20/1993 & 9631.30 & 401.30 & 646.40 & 26.93 & 3.00 & 0.50 & 8984.90 & 374.37 \\
\hline 10/21/1993 & 7450.30 & 310.43 & 624.40 & 27.15 & 3.50 & 0.50 & 6825.90 & 284.41 \\
\hline 10/22/1993 & 7411.40 & 308.81 & 897.20 & 37.38 & 4.00 & 0.50 & 6514.20 & 271.43 \\
\hline 10/23/1993 & 6003.00 & 250.13 & 822.80 & 34.28 & 2.00 & 0.50 & 5180.20 & 215.84 \\
\hline $10 / 24 / 1993$ & 1604.20 & 66.84 & 11.20 & 0.47 & 2.00 & 0.50 & 1593.00 & 66.38 \\
\hline 10/25/1993 & 7062.20 & 294.26 & 400.40 & 16.68 & 3.00 & 0.50 & 6661.80 & 277.58 \\
\hline 10/26/1993 & 7314.60 & 304.78 & 354.00 & 14.75 & 4.00 & 0.50 & 6960.60 & 290.03 \\
\hline 10/27/1993 & 5724.90 & 238.54 & 339.60 & 14.15 & 3.50 & 0.50 & 5385.30 & 224.39 \\
\hline 10/28/1993 & 5990.00 & 249.58 & 370.80 & 15.45 & 3.50 & 0.50 & 5619.20 & 234.13 \\
\hline 10/29/1993 & 7083.90 & 295.16 & 464.40 & 19.35 & 3.00 & 0.50 & 6619.50 & 275.81 \\
\hline 10/30/1993 & 1758.30 & 73.26 & 28.40 & 1.18 & 10.00 & 0.59 & 1729.90 & 72.08 \\
\hline 10/31/1993 & 1501.70 & 62.57 & 23.20 & 0.97 & 10.00 & 0.50 & 1478.50 & 61.60 \\
\hline 11/1/1993 & 5585.10 & 232.71 & 23.60 & 0.98 & 17.90 & 0.94 & 5561.50 & 231.73 \\
\hline $11 / 2 / 1993$ & 5680.00 & 236.67 & 201.20 & 8.38 & 8.10 & 0.68 & 5478.80 & 228.28 \\
\hline 11/3/1993 & 6657.90 & 277.41 & 460.00 & 19.17 & 4.30 & 0.43 & 6197.90 & 258.25 \\
\hline 11/4/1993 & 7168.00 & 298.67 & 462.40 & 19.27 & 4.00 & 0.50 & 6705.60 & 279.40 \\
\hline $11 / 5 / 1993$ & 6931.10 & 288.80 & 460.80 & 19.20 & 4.00 & 0.50 & 6470.30 & 269.60 \\
\hline $11 / 6 / 1993$ & 1636.30 & 68.18 & 26.40 & 1.10 & 5.50 & 0.50 & 1609.90 & 67.08 \\
\hline 11/7/1993 & 1722.90 & 71.79 & 23.20 & 0.97 & 7.00 & 0.50 & 1699.70 & 70.82 \\
\hline 11/8/1993 & 5655.00 & 235.63 & 23.60 & 0.98 & 19.20 & 1.13 & 5631.40 & 234.64 \\
\hline 11/9/1993 & 5919.20 & 246.63 & 144.80 & 6.03 & 13.20 & 0.94 & 5774.40 & 240.60 \\
\hline 11/10/1993 & 5559.00 & 231.63 & 188.80 & 7.87 & 12.70 & 1.15 & 5370.20 & 223.76 \\
\hline 11/11/1993 & 2131.60 & 88.82 & 24.40 & 1.02 & 3.00 & 0.50 & 2107.20 & 87.80 \\
\hline 11/12/1993 & 7110.10 & 296.25 & 471.60 & 19.65 & 3.50 & 0.50 & 6638.50 & 276.60 \\
\hline 11/13/1993 & 2454.60 & 102.28 & 26.00 & 1.08 & 1.50 & 0.50 & 2428.60 & 101.19 \\
\hline 11/14/1993 & 1717.80 & 71.58 & 25.60 & 1.07 & 2.00 & 0.50 & 1692.20 & 70.51 \\
\hline 11/15/1993 & 5997.20 & 249.88 & 446.40 & 18.60 & 3.00 & 0.50 & 5550.80 & 231.28 \\
\hline 11/16/1993 & 6430.80 & 267.95 & 439.20 & 18.30 & 4.50 & 0.50 & 5991.60 & 249.65 \\
\hline 11/17/1993 & 6384.00 & 266.00 & 422.40 & 17.60 & 5.00 & 0.56 & 5961.60 & 248.40 \\
\hline 11/18/1993 & 6344.50 & 264.35 & 415.20 & 17.30 & 5.00 & 0.50 & 5929.30 & 247.05 \\
\hline 11/19/1993 & 6886.10 & 286.92 & 434.00 & 18.08 & 4.50 & 0.50 & 6452.10 & 268.84 \\
\hline 11/20/1993 & 1949.20 & 81.22 & 23.60 & 0.98 & 7.00 & 0.70 & 1925.60 & 80.23 \\
\hline 11/21/1993 & 1496.30 & 62.35 & 22.00 & 0.92 & 2.50 & 0.50 & 1474.30 & 61.43 \\
\hline 11/22/1993 & 6199.40 & 258.31 & 259.60 & 10.82 & 15.70 & 1.31 & 5939.80 & 247.49 \\
\hline 11/23/1993 & 6021.20 & 250.88 & 368.00 & 15.33 & 6.00 & 0.55 & 5653.20 & 235.55 \\
\hline 11/24/1993 & 4658.70 & 194.11 & 221.60 & 9.23 & 3.00 & 0.50 & 4437.10 & 184.88 \\
\hline 11/25/1993 & 1765.20 & 73.55 & 24.80 & 1.03 & 9.70 & 0.61 & 1740.40 & 72.52 \\
\hline 11/26/1993 & 2209.10 & 92.05 & 21.20 & 0.88 & 18.60 & 0.85 & 2187.90 & 91.16 \\
\hline 11/27/1993 & 1762.50 & 73.44 & 18.00 & 0.75 & 12.50 & 0.63 & 1744.50 & 72.69 \\
\hline 11/28/1993 & 1572.40 & 65.52 & 18.40 & 0.77 & 9.00 & 0.53 & 1554.00 & 64.75 \\
\hline 11/29/1993 & 5905.20 & 246.05 & 21.20 & 0.88 & 26.20 & 1.54 & 5884.00 & 245.17 \\
\hline 11/30/1993 & 5698.20 & 237.43 & 22.40 & 0.93 & 19.80 & 1.32 & 5675.80 & 236.49 \\
\hline 12/1/1993 & 5980.10 & 249.17 & 361.20 & 15.05 & 11.70 & 1.06 & 5618.90 & 234.12 \\
\hline $12 / 2 / 1993$ & 6983.50 & 290.98 & 429.20 & 17.88 & 5.00 & 0.50 & 6554.30 & 273.10 \\
\hline 12/3/1993 & 7303.50 & 304.31 & 433.20 & 18.05 & 3.50 & 0.50 & 6870.30 & 286.26 \\
\hline
\end{tabular}


CHART DATA

\begin{tabular}{|c|c|c|c|c|c|c|c|c|}
\hline $12 / 4 / 1993$ & 2120.30 & 88.35 & 22.40 & 0.93 & 4.00 & 0.57 & 2097.90 & 87.41 \\
\hline 12/5/1993 & 1490.40 & 62.10 & 20.80 & 0.87 & 2.00 & 0.50 & 1469.60 & 61.23 \\
\hline $12 / 6 / 1993$ & 5407.70 & 225.32 & 100.40 & 4.18 & 8.10 & 0.90 & 5307.30 & 221.14 \\
\hline $12 / 7 / 1993$ & 5823.40 & 242.64 & 106.00 & 4.42 & 10.10 & 0.84 & 5717.40 & 238.23 \\
\hline 12/8/1993 & 6709.80 & 279.58 & 426.40 & 17.77 & 6.00 & 0.55 & 6283.40 & 261.81 \\
\hline 12/9/1993 & 7412.90 & 308.87 & 464.40 & 19.35 & 4.00 & 0.50 & 6948.50 & 289.52 \\
\hline $12 / 10 / 1993$ & 7697.50 & 320.73 & 447.20 & 18.63 & 3.00 & 0.50 & 7250.30 & 302.10 \\
\hline 12/11/1993 & 2279.30 & 94.97 & 22.80 & 0.95 & 3.00 & 0.50 & 2256.50 & 94.02 \\
\hline 12/12/1993 & 1558.10 & 64.92 & 22.00 & 0.92 & 2.00 & 0.50 & 1536.10 & 64.00 \\
\hline 12/13/1993 & 6714.00 & 279.75 & 434.80 & 18.12 & 2.50 & 0.50 & 6279.20 & 261.63 \\
\hline $12 / 14 / 1993$ & 6439.30 & 268.30 & 416.40 & 17.35 & 6.00 & 0.67 & 6022.90 & 250.95 \\
\hline 12/15/1993 & 5655.90 & 235.66 & 176.80 & 7.37 & 14.30 & 1.10 & 5479.10 & 228.30 \\
\hline 12/16/1993 & 6021.80 & 250.91 & 413.20 & 17.22 & 14.70 & 1.13 & 5608.60 & 233.69 \\
\hline $12 / 17 / 1993$ & 4601.60 & 191.73 & 314.00 & 13.08 & 9.70 & 0.88 & 4287.60 & 178.65 \\
\hline 12/18/1993 & 1996.70 & 83.20 & 25.60 & 1.07 & 6.50 & 0.65 & 1971.10 & 82.13 \\
\hline 12/19/1993 & 1539.70 & 64.15 & 22.80 & 0.95 & 2.00 & 0.50 & 1516.90 & 63.20 \\
\hline $12 / 20 / 1993$ & 3351.20 & 139.63 & 22.40 & 0.93 & 14.40 & 0.63 & 3328.80 & 138.70 \\
\hline $12 / 21 / 1993$ & 2482.10 & 103.42 & 21.20 & 0.88 & 14.70 & 0.77 & 2460.90 & 102.54 \\
\hline $12 / 22 / 1993$ & 2187.70 & 91.15 & 24.40 & 1.02 & 10.10 & 0.53 & 2163.30 & 90.14 \\
\hline $12 / 23 / 1993$ & 1907.00 & 79.46 & 21.20 & 0.88 & 16.90 & 0.73 & 1885.80 & 78.58 \\
\hline $12 / 24 / 1993$ & 1794.00 & 74.75 & 22.80 & 0.95 & 15.10 & 0.66 & 1771.20 & 73.80 \\
\hline $12 / 25 / 1993$ & 1476.70 & 61.53 & 19.60 & 0.82 & 5.80 & 0.41 & 1457.10 & 60.71 \\
\hline $12 / 26 / 1993$ & 1452.40 & 60.52 & 22.00 & 0.92 & 5.10 & 0.51 & 1430.40 & 59.60 \\
\hline $12 / 27 / 1993$ & 1880.10 & 78.34 & 22.00 & 0.92 & 10.20 & 0.93 & 1858.10 & 77.42 \\
\hline 12/28/1993 & 2412.40 & 100.52 & 22.40 & 0.93 & 7.50 & 0.63 & 2390.00 & 99.58 \\
\hline 12/29/1993 & 2190.90 & 91.29 & 26.00 & 1.08 & 22.80 & 1.14 & 2164.90 & 90.20 \\
\hline 12/30/1993 & 2511.40 & 104.64 & 21.20 & 0.88 & 22.20 & 0.97 & 2490.20 & 103.76 \\
\hline 12/31/1993 & 1987.90 & 82.83 & 20.00 & 0.83 & 13.50 & 0.64 & 1967.90 & 82.00 \\
\hline 1/1/1994 & 1475.50 & 61.48 & 20.40 & 0.85 & 2.80 & 0.40 & 1455.10 & 60.63 \\
\hline $1 / 2 / 1994$ & 1443.70 & 60.15 & 21.60 & 0.90 & 2.30 & 0.33 & 1422.10 & 59.25 \\
\hline $1 / 3 / 1994$ & 4349.70 & 181.24 & 22.80 & 0.95 & 26.20 & 1.54 & 4326.90 & 180.29 \\
\hline $1 / 4 / 1994$ & 3504.90 & 146.04 & 22.80 & 0.95 & 29.20 & 1.83 & 3482.10 & 145.09 \\
\hline $1 / 5 / 1994$ & 3747.10 & 156.13 & 23.60 & 0.98 & 36.70 & 1.75 & 3723.50 & 155.15 \\
\hline 1/6/1994 & 6269.90 & 261.25 & 265.20 & 11.05 & 20.70 & 1.09 & 6004.70 & 250.20 \\
\hline $1 / 7 / 1994$ & 6065.20 & 252.72 & 26.80 & 1.12 & 48.10 & 2.09 & 6038.40 & 251.60 \\
\hline $1 / 8 / 1994$ & 2701.00 & 112.54 & 22.00 & 0.92 & 25.90 & 1.08 & 2679.00 & 111.63 \\
\hline 1/9/1994 & 2386.40 & 99.43 & 22.80 & 0.95 & 13.00 & 0.54 & 2363.60 & 98.48 \\
\hline $1 / 10 / 1994$ & 6200.10 & 258.34 & 55.20 & 2.30 & 20.30 & 1.27 & 6144.90 & 256.04 \\
\hline $1 / 11 / 1994$ & 5788.80 & 241.20 & 139.20 & 5.80 & 10.60 & 0.88 & 5649.60 & 235.40 \\
\hline $1 / 12 / 1994$ & 5615.10 & 233.96 & 183.20 & 7.63 & 15.20 & 0.89 & 5431.90 & 226.33 \\
\hline 1/13/1994 & 6079.00 & 253.29 & 330.80 & 13.78 & 16.10 & 1.15 & 5748.20 & 239.51 \\
\hline $1 / 14 / 1994$ & 5870.70 & 244.61 & 310.40 & 12.93 & 19.40 & 1.39 & 5560.30 & 231.68 \\
\hline $1 / 15 / 1994$ & 2045.30 & 85.22 & 22.40 & 0.93 & 8.50 & 0.61 & 2022.90 & 84.29 \\
\hline $1 / 16 / 1994$ & 1867.10 & 77.80 & 23.20 & 0.97 & 3.00 & 0.50 & 1843.90 & 76.83 \\
\hline $1 / 17 / 1994$ & 6018.10 & 250.75 & 85.60 & 3.57 & 27.70 & 1.46 & 5932.50 & 247.19 \\
\hline $1 / 18 / 1994$ & 6344.70 & 264.36 & 23.60 & 0.98 & 53.30 & 2.22 & 6321.10 & 263.38 \\
\hline 1/19/1994 & 6168.70 & 257.03 & 22.00 & 0.92 & 50.00 & 2.08 & 6146.70 & 256.11 \\
\hline $1 / 20 / 1994$ & 5704.90 & 237.70 & 21.60 & 0.90 & 31.30 & 1.65 & 5683.30 & 236.80 \\
\hline $1 / 21 / 1994$ & 5438.00 & 226.58 & 24.00 & 1.00 & 26.70 & 1.34 & 5414.00 & 225.58 \\
\hline $1 / 22 / 1994$ & 1963.90 & 81.83 & 22.40 & 0.93 & 16.70 & 0.76 & 1941.50 & 80.90 \\
\hline $1 / 23 / 1994$ & 1797.00 & 74.88 & 21.60 & 0.90 & 9.70 & 0.49 & 1775.40 & 73.98 \\
\hline
\end{tabular}


CHART DATA

\begin{tabular}{|c|c|c|c|c|c|c|c|c|}
\hline $1 / 24 / 1994$ & 6204.30 & 258.51 & 36.00 & 1.50 & 14.90 & 0.83 & 6168.30 & 257.01 \\
\hline 1/25/1994 & 6786.80 & 282.78 & 373.60 & 15.57 & 5.50 & 0.55 & 6413.20 & 267.22 \\
\hline $1 / 26 / 1994$ & 6992.80 & 291.37 & 424.80 & 17.70 & 5.00 & 0.56 & 6568.00 & 273.67 \\
\hline $1 / 27 / 1994$ & 7634.20 & 318.09 & 873.60 & 36.40 & 8.50 & 0.61 & 6760.60 & 281.69 \\
\hline 1/28/1994 & 6917.10 & 288.21 & 435.20 & 18.13 & 52.00 & 2.17 & 6481.90 & 270.08 \\
\hline $1 / 29 / 1994$ & 3231.20 & 134.63 & 20.80 & 0.87 & 40.90 & 1.70 & 3210.40 & 133.77 \\
\hline $1 / 30 / 1994$ & 2481.50 & 103.40 & 18.80 & 0.78 & 21.80 & 0.91 & 2462.70 & 102.61 \\
\hline $1 / 31 / 1994$ & 6903.40 & 287.64 & 23.20 & 0.97 & 70.60 & 2.94 & 6880.20 & 286.68 \\
\hline 2/1/1994 & 7272.20 & 303.01 & 25.60 & 1.07 & 87.90 & 3.66 & 7246.60 & 301.94 \\
\hline 2/2/1994 & 6699.50 & 279.15 & 22.40 & 0.93 & 77.70 & 3.24 & 6677.10 & 278.21 \\
\hline 2/3/1994 & 6680.30 & 278.35 & 26.00 & 1.08 & 68.50 & 2.85 & 6654.30 & 277.26 \\
\hline 2/4/1994 & 5995.30 & 249.80 & 229.60 & 9.57 & 30.30 & 1.26 & 5765.70 & 240.24 \\
\hline 2/5/1994 & 2242.70 & 93.45 & 28.00 & 1.17 & 5.50 & 0.55 & 2214.70 & 92.28 \\
\hline 2/6/1994 & 1551.80 & 64.66 & 27.60 & 1.15 & 3.10 & 0.44 & 1524.20 & 63.51 \\
\hline 2/7/1994 & 6740.80 & 280.87 & 386.00 & 16.08 & 10.60 & 0.82 & 6354.80 & 264.78 \\
\hline 2/8/1994 & 7523.70 & 313.49 & 460.40 & 19.18 & 4.50 & 0.56 & 7063.30 & 294.30 \\
\hline 2/9/1994 & 6912.00 & 288.00 & 338.00 & 14.08 & 26.90 & 1.79 & 6574.00 & 273.92 \\
\hline $2 / 10 / 1994$ & 3932.20 & 163.84 & 27.60 & 1.15 & 76.60 & 3.19 & 3904.60 & 162.69 \\
\hline 2/11/1994 & 6580.30 & 274.18 & 25.20 & 1.05 & 73.00 & 3.04 & 6555.10 & 273.13 \\
\hline $2 / 12 / 1994$ & 2743.20 & 114.30 & 26.40 & 1.10 & 15.50 & 0.67 & 2716.80 & 113.20 \\
\hline $2 / 13 / 1994$ & 2603.20 & 108.47 & 29.60 & 1.23 & 13.40 & 0.56 & 2573.60 & 107.23 \\
\hline 2/14/1994 & 6483.60 & 270.15 & 27.60 & 1.15 & 43.20 & 1.96 & 6456.00 & 269.00 \\
\hline $2 / 15 / 1994$ & 6058.20 & 252.43 & 27.20 & 1.13 & 30.20 & 1.89 & 6031.00 & 251.29 \\
\hline $2 / 16 / 1994$ & 6411.50 & 267.15 & 201.60 & 8.40 & 25.00 & 1.67 & 6209.90 & 258.75 \\
\hline 2/17/1994 & 6207.00 & 258.63 & 235.20 & 9.80 & 21.50 & 1.43 & 5971.80 & 248.83 \\
\hline 2/18/1994 & 6661.50 & 277.56 & 328.80 & 13.70 & 12.70 & 0.91 & 6332.70 & 263.86 \\
\hline $2 / 19 / 1994$ & 2498.50 & 104.10 & 28.80 & 1.20 & 3.00 & 0.60 & 2469.70 & 102.90 \\
\hline 2/20/1994 & 1609.70 & 67.07 & 28.80 & 1.20 & 2.50 & 0.50 & 1580.90 & 65.87 \\
\hline 2/21/1994 & 5089.90 & 212.08 & 462.00 & 19.25 & 3.50 & 0.50 & 4627.90 & 192.83 \\
\hline $2 / 22 / 1994$ & 7265.30 & 302.72 & 378.40 & 15.77 & 4.00 & 0.57 & 6886.90 & 286.95 \\
\hline $2 / 23 / 1994$ & 6465.30 & 269.39 & 324.80 & 13.53 & 20.90 & 1.31 & 6140.50 & 255.85 \\
\hline 2/24/1994 & 6270.00 & 261.25 & 183.20 & 7.63 & 29.10 & 1.46 & 6086.80 & 253.62 \\
\hline $2 / 25 / 1994$ & 6505.30 & 271.05 & 279.60 & 11.65 & 15.30 & 1.02 & 6225.70 & 259.40 \\
\hline $2 / 26 / 1994$ & 2723.30 & 113.47 & 29.60 & 1.23 & 17.00 & 0.89 & 2693.70 & 112.24 \\
\hline 2/27/1994 & 1894.60 & 78.94 & 27.60 & 1.15 & 12.00 & 0.67 & 1867.00 & 77.79 \\
\hline $2 / 28 / 1994$ & 5854.70 & 243.95 & 28.00 & 1.17 & 22.30 & 1.31 & 5826.70 & 242.78 \\
\hline 3/1/1994 & 6328.00 & 263.67 & 175.60 & 7.32 & 11.00 & 0.79 & 6152.40 & 256.35 \\
\hline $3 / 2 / 1994$ & 5763.60 & 240.15 & 134.80 & 5.62 & 22.00 & 1.57 & 5628.80 & 234.53 \\
\hline $3 / 3 / 1994$ & 6025.50 & 251.06 & 247.20 & 10.30 & 19.90 & 1.24 & 5778.30 & 240.76 \\
\hline $3 / 4 / 1994$ & 5952.90 & 248.04 & 231.60 & 9.65 & 14.20 & 1.18 & 5721.30 & 238.39 \\
\hline 3/5/1994 & 1575.90 & 65.66 & 28.00 & 1.17 & 2.50 & 0.50 & 1547.90 & 64.50 \\
\hline $3 / 6 / 1994$ & 1570.70 & 65.45 & 27.60 & 1.15 & 3.00 & 0.50 & 1543.10 & 64.30 \\
\hline $3 / 7 / 1994$ & 7151.30 & 297.97 & 443.20 & 18.47 & 5.10 & 0.51 & 6708.10 & 279.50 \\
\hline $3 / 8 / 1994$ & 7534.00 & 313.92 & 467.20 & 19.47 & 4.00 & 0.50 & 7066.80 & 294.45 \\
\hline 3/9/1994 & 5827.10 & 242.80 & 32.80 & 1.37 & 26.70 & 1.48 & 5794.30 & 241.43 \\
\hline $3 / 10 / 1994$ & 5777.80 & 240.74 & 31.60 & 1.32 & 33.90 & 1.70 & 5746.20 & 239.43 \\
\hline 3/11/1994 & 3575.40 & 148.98 & 31.60 & 1.32 & 20.00 & 1.11 & 3543.80 & 147.66 \\
\hline 3/12/1994 & 2022.30 & 84.26 & 31.60 & 1.32 & 3.50 & 0.50 & 1990.70 & 82.95 \\
\hline 3/13/1994 & 2007.40 & 83.64 & 35.20 & 1.47 & 3.50 & 0.50 & 1972.20 & 82.18 \\
\hline $3 / 14 / 1994$ & 3584.80 & 149.37 & 35.20 & 1.47 & 20.50 & 1.03 & 3549.60 & 147.90 \\
\hline 3/15/1994 & 3242.60 & 135.11 & 38.40 & 1.60 & 16.30 & 0.86 & 3204.20 & 133.51 \\
\hline
\end{tabular}


CHART DATA

\begin{tabular}{|c|c|c|c|c|c|c|c|c|}
\hline 3/16/1994 & 3202.10 & 133.42 & 40.00 & 1.67 & 13.10 & 0.82 & 3162.10 & 131.75 \\
\hline 3/17/1994 & 2914.10 & 121.42 & 43.20 & 1.80 & 13.10 & 0.77 & 2870.90 & 119.62 \\
\hline 3/18/1994 & 2738.20 & 114.09 & 42.00 & 1.75 & 8.60 & 0.72 & 2696.20 & 112.34 \\
\hline 3/19/1994 & 1555.30 & 64.80 & 38.80 & 1.62 & 2.50 & 0.50 & 1516.50 & 63.19 \\
\hline $3 / 20 / 1994$ & 1541.50 & 64.23 & 36.80 & 1.53 & 2.50 & 0.50 & 1504.70 & 62.70 \\
\hline $3 / 21 / 1994$ & 7859.80 & 327.49 & 490.40 & 20.43 & 4.50 & 0.56 & 7369.40 & 307.06 \\
\hline $3 / 22 / 1994$ & 7600.50 & 316.69 & 495.60 & 20.65 & 4.50 & 0.50 & 7104.90 & 296.04 \\
\hline $3 / 23 / 1994$ & 7707.00 & 321.13 & 505.60 & 21.07 & 4.00 & 0.57 & 7201.40 & 300.06 \\
\hline 3/24/1994 & 8476.60 & 353.19 & 508.80 & 21.20 & 3.50 & 0.50 & 7967.80 & 331.99 \\
\hline $3 / 25 / 1994$ & 7609.20 & 317.05 & 495.20 & 20.63 & 3.00 & 0.50 & 7114.00 & 296.42 \\
\hline $3 / 26 / 1994$ & 1637.20 & 68.22 & 38.80 & 1.62 & 2.50 & 0.50 & 1598.40 & 66.60 \\
\hline $3 / 27 / 1994$ & 1487.70 & 61.99 & 40.00 & 1.67 & 3.00 & 0.50 & 1447.70 & 60.32 \\
\hline $3 / 28 / 1994$ & 6040.90 & 251.70 & 411.20 & 17.13 & 5.00 & 0.56 & 5629.70 & 234.57 \\
\hline 3/29/1994 & 5955.70 & 248.15 & 402.40 & 16.77 & 5.00 & 0.50 & 5553.30 & 231.39 \\
\hline 3/30/1994 & 6517.30 & 271.55 & 404.80 & 16.87 & 4.50 & 0.50 & 6112.50 & 254.69 \\
\hline 3/31/1994 & 6028.80 & 251.20 & 401.20 & 16.72 & 5.00 & 0.50 & 5627.60 & 234.48 \\
\hline 4/1/1994 & 1749.60 & 72.90 & 59.60 & 2.48 & 4.00 & 0.50 & 1690.00 & 70.42 \\
\hline 4/2/1994 & 1449.80 & 63.03 & 38.40 & 1.67 & 2.50 & 0.50 & 1411.40 & 61.37 \\
\hline $4 / 3 / 1994$ & 1464.40 & 61.02 & 41.20 & 1.72 & 2.50 & 0.50 & 1423.20 & 59.30 \\
\hline $4 / 4 / 1994$ & 6790.20 & 282.93 & 429.20 & 17.88 & 5.00 & 0.50 & 6361.00 & 265.04 \\
\hline $4 / 5 / 1994$ & 7986.80 & 332.78 & 454.00 & 18.92 & 4.00 & 0.50 & 7532.80 & 313.87 \\
\hline 4/6/1994 & 5989.30 & 249.55 & 416.40 & 17.35 & 9.50 & 0.68 & 5572.90 & 232.20 \\
\hline 4/7/1994 & 5920.10 & 246.67 & 399.20 & 19.01 & 9.50 & 0.68 & 5520.90 & 230.04 \\
\hline 4/8/1994 & 5754.10 & 239.75 & 3.20 & 1.07 & 4.00 & 0.50 & 5750.90 & 239.62 \\
\hline 4/9/1994 & 1645.20 & 68.55 & & & 2.50 & 0.50 & 1645.20 & 68.55 \\
\hline $4 / 10 / 1994$ & 1666.50 & 69.44 & & & 2.00 & 0.50 & 1666.50 & 69.44 \\
\hline $4 / 11 / 1994$ & 8924.00 & 371.83 & 463.60 & 28.98 & 4.00 & 0.50 & 8460.40 & 352.52 \\
\hline $4 / 12 / 1994$ & 7522.20 & 313.43 & 477.60 & 20.77 & 4.00 & 0.50 & 7044.60 & 293.53 \\
\hline $4 / 13 / 1994$ & 7539.20 & 314.13 & 456.40 & 19.84 & 3.50 & 0.50 & 7082.80 & 295.12 \\
\hline $4 / 14 / 1994$ & 8781.60 & 365.90 & 483.20 & 21.01 & 3.50 & 0.50 & 8298.40 & 345.77 \\
\hline $4 / 15 / 1994$ & 8605.30 & 358.55 & 494.40 & 20.60 & 3.00 & 0.50 & 8110.90 & 337.95 \\
\hline $4 / 16 / 1994$ & 2465.90 & 102.75 & 357.20 & 14.88 & 3.00 & 0.50 & 2108.70 & 87.86 \\
\hline $4 / 17 / 1994$ & 1512.90 & 63.04 & 10.80 & 0.45 & 2.00 & 0.50 & 1502.10 & 62.59 \\
\hline 4/18/1994 & 8108.10 & 337.84 & 440.40 & 18.35 & 3.50 & 0.50 & 7667.70 & 319.49 \\
\hline $4 / 19 / 1994$ & 8757.30 & 364.89 & 508.40 & 23.11 & 4.00 & 0.57 & 8248.90 & 343.70 \\
\hline $4 / 20 / 1994$ & 7989.50 & 332.90 & 513.60 & 22.33 & 7.10 & 0.89 & 7475.90 & 311.50 \\
\hline $4 / 21 / 1994$ & 7667.80 & 319.49 & 496.00 & 20.67 & 4.00 & 0.50 & 7171.80 & 298.83 \\
\hline $4 / 22 / 1994$ & 8715.00 & 363.13 & 499.60 & 21.72 & 3.50 & 0.50 & 8215.40 & 342.31 \\
\hline $4 / 23 / 1994$ & 3418.80 & 142.45 & 12.80 & 0.53 & 3.00 & 0.50 & 3406.00 & 141.92 \\
\hline 4/24/1994 & 1495.60 & 62.32 & 10.80 & 0.45 & 2.00 & 0.50 & 1484.80 & 61.87 \\
\hline $4 / 25 / 1994$ & 9455.60 & 393.98 & 434.00 & 18.87 & 3.50 & 0.50 & 9021.60 & 375.90 \\
\hline $4 / 26 / 1994$ & 9828.60 & 409.53 & 551.20 & 22.97 & 3.50 & 0.50 & 9277.40 & 386.56 \\
\hline $4 / 27 / 1994$ & 10282.40 & 428.43 & 590.00 & 24.58 & 3.50 & 0.50 & 9692.40 & 403.85 \\
\hline 4/28/1994 & 9974.30 & 415.60 & 587.60 & 24.48 & 3.50 & 0.58 & 9386.70 & 391.11 \\
\hline 4/29/1994 & 9872.70 & 411.36 & 573.60 & 23.90 & 3.50 & 0.50 & 9299.10 & 387.46 \\
\hline 4/30/1994 & 1930.90 & 80.45 & 12.00 & 0.50 & 2.50 & 0.50 & 1918.90 & 79.95 \\
\hline 5/1/1994 & 1680.80 & 70.03 & 11.60 & 0.48 & 2.50 & 0.50 & 1669.20 & 69.55 \\
\hline $5 / 2 / 1994$ & 6824.60 & 284.36 & 334.40 & 13.93 & 5.00 & 0.63 & 6490.20 & 270.43 \\
\hline $5 / 3 / 1994$ & 8136.30 & 339.01 & 526.40 & 21.93 & 4.50 & 0.50 & 7609.90 & 317.08 \\
\hline $5 / 4 / 1994$ & 7705.20 & 321.05 & 521.60 & 22.68 & 4.50 & 0.50 & 7183.60 & 299.32 \\
\hline 5/5/1994 & 9034.00 & 376.42 & 537.20 & 22.38 & 4.50 & 0.50 & 8496.80 & 354.03 \\
\hline
\end{tabular}


CHART DATA

\begin{tabular}{|r|r|r|r|r|r|r|r|r|}
\hline $5 / 6 / 1994$ & 9011.50 & 375.48 & 532.80 & 22.20 & 4.00 & 0.50 & 8478.70 & 353.28 \\
\hline $5 / 7 / 1994$ & 2424.30 & 101.01 & 12.40 & 0.52 & 2.50 & 0.50 & 2411.90 & 100.50 \\
\hline $5 / 8 / 1994$ & 1671.90 & 69.66 & 13.60 & 0.57 & 2.00 & 0.50 & 1658.30 & 69.10 \\
\hline $5 / 9 / 1994$ & 10898.60 & 454.11 & 604.80 & 25.20 & 4.00 & 0.50 & 10293.80 & 428.91 \\
\hline $5 / 10 / 1994$ & 10407.70 & 433.65 & 605.20 & 25.22 & 4.50 & 0.50 & 9802.50 & 408.44 \\
\hline $5 / 11 / 1994$ & 10287.50 & 428.65 & 597.60 & 24.90 & 3.50 & 0.50 & 9689.90 & 403.75 \\
\hline $5 / 12 / 1994$ & 9372.90 & 390.54 & 392.80 & 16.37 & 4.00 & 0.50 & 8980.10 & 374.17 \\
\hline $5 / 13 / 1994$ & 9831.60 & 409.65 & 593.60 & 24.73 & 4.00 & 0.50 & 9238.00 & 384.92 \\
\hline $5 / 14 / 1994$ & 3788.00 & 157.83 & 508.80 & 21.20 & 2.00 & 0.50 & 3279.20 & 136.63 \\
\hline $5 / 15 / 1994$ & 1638.00 & 68.25 & 10.40 & 0.43 & 2.50 & 0.50 & 1627.60 & 67.82 \\
\hline $5 / 16 / 1994$ & 9230.80 & 384.62 & 495.60 & 20.65 & 4.50 & 0.56 & 8735.20 & 363.97 \\
\hline $5 / 17 / 1994$ & 9770.90 & 407.12 & 561.60 & 23.40 & 4.00 & 0.57 & 9209.30 & 383.72 \\
\hline $5 / 18 / 1994$ & 10073.30 & 419.72 & 575.20 & 23.97 & 3.50 & 0.50 & 9498.10 & 395.75 \\
\hline $5 / 19 / 1994$ & 9610.10 & 400.42 & 591.60 & 24.65 & 4.50 & 0.50 & 9018.50 & 375.77 \\
\hline $5 / 20 / 1994$ & 9190.40 & 382.93 & 581.60 & 24.23 & 4.00 & 0.50 & 8608.80 & 358.70 \\
\hline $5 / 21 / 1994$ & 2181.60 & 90.90 & 13.60 & 0.57 & 2.50 & 0.50 & 2168.00 & 90.33 \\
\hline $5 / 22 / 1994$ & 1581.00 & 65.88 & 13.20 & 0.55 & 2.50 & 0.50 & 1567.80 & 65.33 \\
\hline $5 / 23 / 1994$ & 9846.00 & 410.25 & 596.80 & 24.87 & 3.00 & 0.50 & 9249.20 & 385.38 \\
\hline $5 / 24 / 1994$ & 9721.60 & 405.07 & 575.60 & 23.98 & 3.00 & 0.50 & 9146.00 & 381.08 \\
\hline $5 / 25 / 1994$ & 9762.30 & 406.76 & 563.20 & 23.47 & 3.00 & 0.50 & 9199.10 & 383.30 \\
\hline $5 / 26 / 1994$ & 9535.80 & 397.33 & 578.40 & 24.10 & 3.50 & 0.50 & 8957.40 & 373.23 \\
\hline $5 / 27 / 1994$ & 9515.10 & 396.46 & 568.00 & 23.67 & 3.00 & 0.50 & 8947.10 & 372.80 \\
\hline $5 / 28 / 1994$ & 5408.30 & 225.35 & 298.80 & 12.45 & 2.00 & 0.50 & 5109.50 & 212.90 \\
\hline $5 / 29 / 1994$ & 2132.20 & 88.84 & 16.00 & 0.67 & 2.50 & 0.50 & 2116.20 & 88.18 \\
\hline $5 / 30 / 1994$ & 5924.60 & 246.86 & 433.60 & 18.07 & 2.00 & 0.50 & 5491.00 & 228.79 \\
\hline $5 / 31 / 1994$ & 6697.90 & 279.08 & 535.60 & 22.32 & 2.50 & 0.50 & 6162.30 & 256.76 \\
\hline $6 / 1 / 1994$ & 6337.40 & 264.06 & 500.40 & 20.85 & 2.50 & 0.50 & 5837.00 & 243.21 \\
\hline $6 / 2 / 1994$ & 6406.70 & 266.95 & 502.00 & 20.92 & 3.00 & 0.60 & 5904.70 & 246.03 \\
\hline $6 / 3 / 1994$ & 4719.00 & 196.63 & 16.00 & 0.67 & 2.50 & 0.50 & 4703.00 & 195.96 \\
\hline $6 / 4 / 1994$ & 2867.00 & 119.46 & 14.80 & 0.62 & 2.00 & 0.50 & 2852.20 & 118.84 \\
\hline
\end{tabular}


CHART DATA

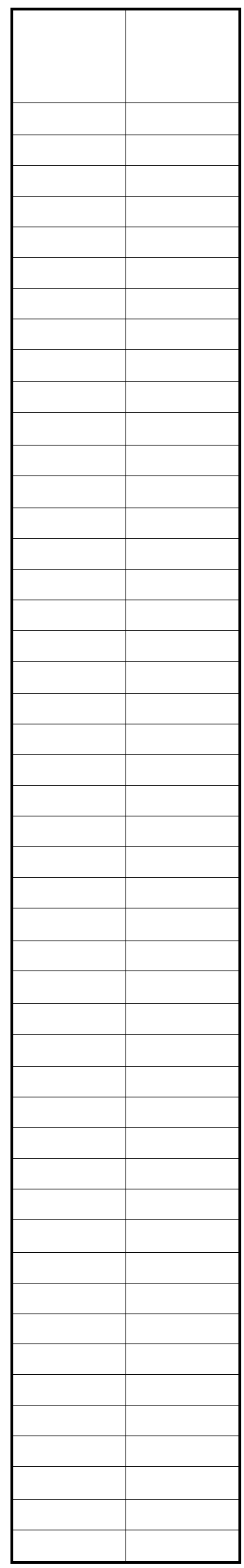

Page 133 
CHART DATA

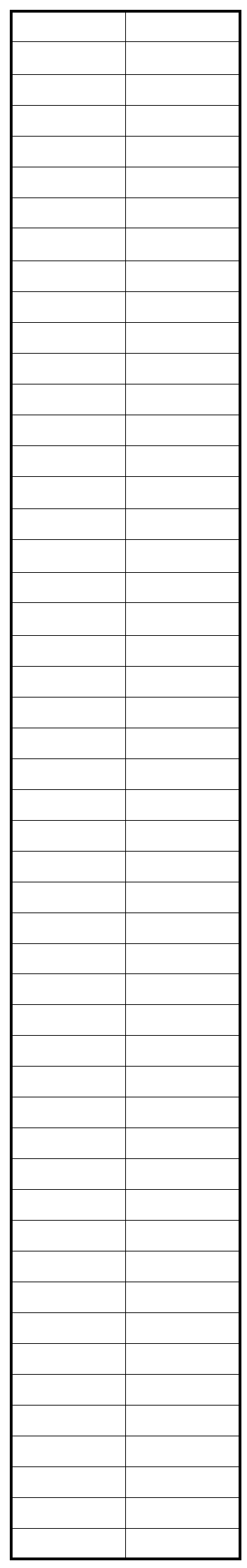

Page 134 
CHART DATA

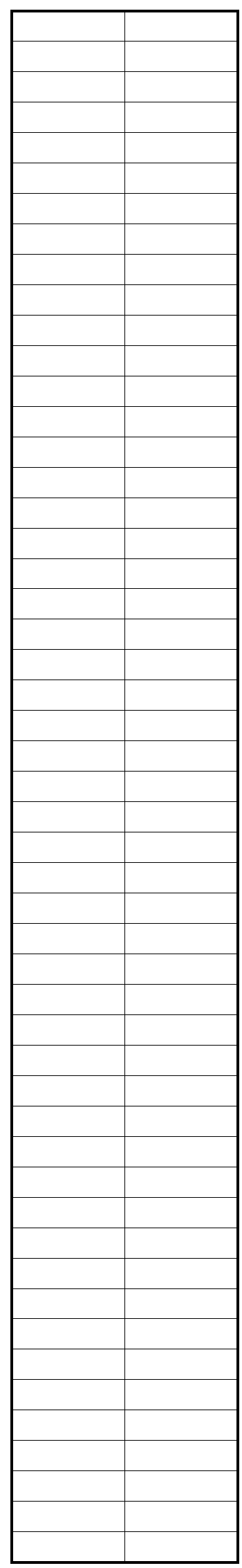

Page 135 
CHART DATA

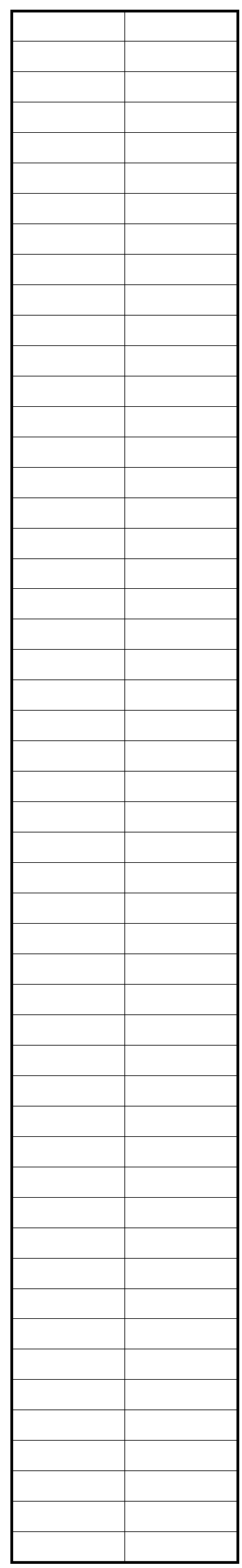

Page 136 
CHART DATA

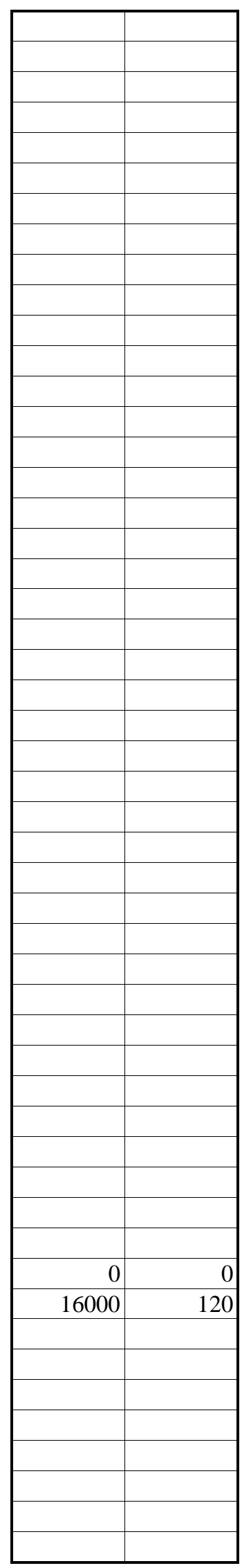

Page 137 
Tables

\begin{tabular}{|c|c|c|c|c|c|}
\hline \multicolumn{2}{|c|}{ TOTAL MONTHLY CONSUMPTION } & \multirow[b]{2}{*}{ chlr } & \multirow[b]{2}{*}{ other } & \multirow[b]{2}{*}{ wbheat } & \multirow[t]{2}{*}{ TOTAL POST PEH } \\
\hline & wbelec & & & & \\
\hline \multicolumn{6}{|l|}{ PRE PERIOD } \\
\hline Jun 91 & 121,743 & 0 & 241,636 & 16 & \\
\hline Jul & 137,603 & 0 & 220,847 & 21 & wbelec, kWh \\
\hline Aug & 270,742 & 0 & 167,456 & 1 & chlr, kWh \\
\hline Sep & 276,575 & 0 & 167,304 & 18 & other, kWh \\
\hline Oct & 242,163 & 17,072 & 152,268 & 16 & wbheat, MMBtu \\
\hline Nov & 160,325 & 5,118 & 136,391 & 45 & \\
\hline Dec & 150,396 & 4,642 & 219,845 & 597 & \\
\hline Jan 92 & 139,697 & 1,225 & 165,440 & 1,640 & TOTAL POST PEH \\
\hline Total Consumption & $1,499,242$ & 28,057 & $1,471,186$ & 2,354 & \\
\hline Total Cost & $\$ 41,799$ & $\$ 782$ & $\$ 41,017$ & $\$ 10,755$ & \\
\hline \multicolumn{6}{|l|}{ POST PERIOD } \\
\hline Feb 92 & 149,365 & 5,518 & 143,414 & 775 & wbelec, kWh \\
\hline Mar & 141,229 & 4,906 & 161,499 & 664 & chlr, kWh \\
\hline Apr & 190,903 & 9,330 & 200,369 & 326 & other, kWh \\
\hline May & 227,078 & 12,205 & 244,332 & 78 & wbheat, MMBtu \\
\hline Jun & 136,443 & 4,319 & 203,001 & 41 & \\
\hline Jul & 145,357 & 6,713 & 182,240 & 40 & \\
\hline Aug & 199,450 & 11,992 & 142,447 & 65 & \\
\hline Sep & 263,715 & 14,623 & 139,716 & 69 & \\
\hline Oct & 230,083 & 13,660 & 153,058 & 174 & \\
\hline Nov & 159,136 & 7,442 & 171,351 & 685 & \\
\hline Dec & 125,653 & 1,982 & 211,597 & 933 & \\
\hline Jan 93 & 134,521 & 1,253 & 225,745 & 1,113 & \\
\hline Feb & 134,220 & 2,229 & 151,229 & 864 & \\
\hline Mar & 147,342 & 5,880 & 155,418 & 674 & \\
\hline Apr & 173,404 & 9,780 & 154,491 & 93 & \\
\hline May & 194,974 & 11,349 & 159,892 & 89 & \\
\hline Jun & 128,470 & 10,102 & 173,318 & 59 & \\
\hline Jul & 20,765 & 1,629 & 221,511 & 9 & \\
\hline Aug & 153,649 & 8,711 & 138,114 & 49 & \\
\hline Sep & 233,928 & 13,954 & 131,201 & 86 & \\
\hline Oct & 211,490 & 13,750 & 144,102 & 108 & \\
\hline Nov & 135,042 & 5,710 & 209,575 & 258 & \\
\hline Dec & 116,966 & 4,896 & 77,688 & 276 & \\
\hline Jan 94 & 145,092 & 3,929 & 126,914 & 733 & \\
\hline Feb & 145,476 & 4,244 & 38,909 & 756 & \\
\hline Mar & 146,811 & 6,386 & 77,542 & 290 & \\
\hline Apr & 184,945 & 9,826 & 151,400 & 118 & \\
\hline May & 222,895 & 12,802 & 88,572 & 104 & \\
\hline Jun 94 & 20,330 & 1,033 & 19,297 & 10 & \\
\hline Total Consumption & $4,618,730$ & 220,155 & $4,397,944$ & 9,538 & \\
\hline Total Cost & $\$ 128,770$ & $\$ 6,138$ & $\$ 122,615$ & $\$ 45,303$ & \\
\hline Grand Total Consumption & $6,117,972$ & 248,212 & $5,869,129$ & 11,891 & \\
\hline Grand Total Cost & $\$ 170,569$ & $\$ 6,920$ & $\$ 163,631$ & $\$ 56,059$ & \\
\hline & & & & & \\
\hline
\end{tabular}


Tables

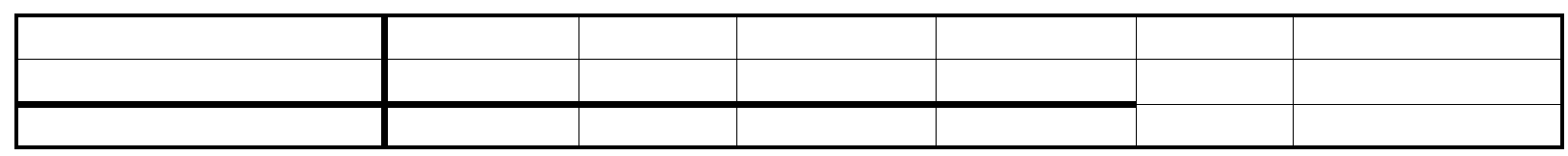

Page 2 


\begin{tabular}{|c|c|c|c|c|c|c|}
\hline IOD CONS & MPTION E & Y SCHOO & $\mathrm{AR} / \mathrm{NOI}$ & SCHOOL & $\mathrm{AF}$ & \\
\hline & & & & & & \\
\hline SCHOC & YEAR & NON SC & L YEAR & $\mathrm{TO}$ & & \\
\hline ENERGY & $\$$ & ENERGY & $\$$ & ENERGY & $\$$ & \\
\hline $3,343,246$ & $\$ 93,210$ & $1,262,155$ & $\$ 35,189$ & $4,605,400$ & $\$ 128,399$ & \\
\hline 159,076 & $\$ 4,435$ & 60,298 & $\$ 1,681$ & 219,374 & $\$ 6,116$ & \\
\hline $3,184,170$ & $\$ 88,775$ & $1,201,857$ & $\$ 33,508$ & $4,386,027$ & $\$ 122,282$ & \\
\hline 7,847 & $\$ 37,271$ & 1,888 & $\$ 8,966$ & 9,734 & $\$ 46,237$ & \\
\hline & & & & & & \\
\hline IOD CONS & MPTION E & Y SCHOOI & ON SCHC & OL BASED & N AVERA & AGE DAIL I \\
\hline & & & & & & \\
\hline $\mathrm{SCHOC}$ & YEAR & NON SC & L YEAR & TO & & \\
\hline ENERGY & $\$$ & ENERGY & $\$$ & ENERGY & $\$$ & \\
\hline 6284114 & $\$ 175,201$ & 2848484 & $\$ 79,416$ & 9132598 & $\$ 254,617$ & \\
\hline 257134 & $\$ 7,169$ & 119059 & $\$ 3,319$ & 376194 & $\$ 10,488$ & \\
\hline 6045265 & $\$ 168,542$ & 2730274 & $\$ 76,120$ & 8775539 & $\$ 244,662$ & \\
\hline 25762 & $\$ 122,368$ & 12822 & $\$ 60,902$ & 38583 & $\$ 183,270$ & \\
\hline & & & & & & \\
\hline & & & & & & \\
\hline & & & & & & \\
\hline & & & & & & \\
\hline & & & & & & \\
\hline & & & & & & \\
\hline & & & & & & \\
\hline & & & & & & \\
\hline & & & & & & \\
\hline & & & & & & \\
\hline & & & & & & \\
\hline & & & & & & \\
\hline & & & & & & \\
\hline & & & & & & \\
\hline & & & & & & \\
\hline & & & & & & \\
\hline & & & & & & \\
\hline & & & & & & \\
\hline & & & & & & \\
\hline & & & & & & \\
\hline & & & & & & \\
\hline & & & & & & \\
\hline & & & & & & \\
\hline & & & & & & \\
\hline & & & & & & \\
\hline & & & & & & \\
\hline & & & & & & \\
\hline & & & & & & \\
\hline & & & & & & \\
\hline & & & & & & \\
\hline & & & & & & \\
\hline
\end{tabular}




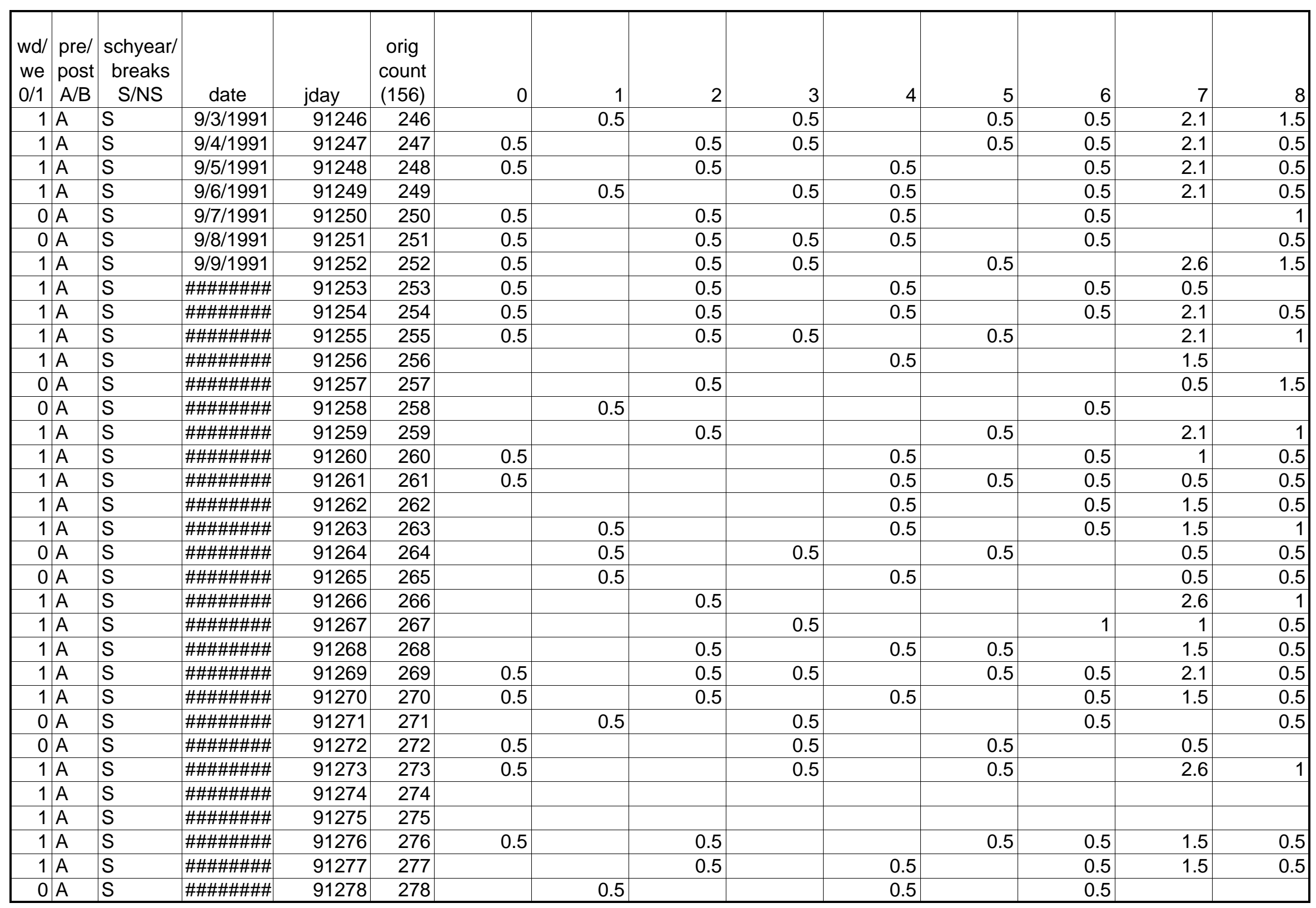




\begin{tabular}{|c|c|c|c|c|c|c|c|c|c|c|c|c|c|}
\hline $0 \mathrm{~A}$ & S & \#\#\#\#\#\#\# & 91279 & 279 & & 0.5 & 0.5 & & & 0.5 & & 0.5 & 0.5 \\
\hline $1 \mathrm{~A}$ & S & \#\#\#\#\#\#\#\# & 91282 & 282 & & 0.5 & & 0.5 & & 0.5 & 0.5 & 2.1 & 0.5 \\
\hline $0 \mathrm{~A}$ & S & \#\#\#\#\#\#\#\# & 91285 & 285 & & & 0.5 & & 0.5 & & & 0.5 & 1.5 \\
\hline $0 \mathrm{~A}$ & S & \#\#\#\#\#\#\#\# & 91286 & 286 & & 0.5 & & 0.5 & & & 0.5 & & 0.5 \\
\hline $1 \mathrm{~A}$ & S & \#\#\#\#\#\#\#\# & 91287 & 287 & 0.5 & & & 0.5 & & 0.5 & & 2.1 & 0.5 \\
\hline $1 \mathrm{~A}$ & S & \#\#\#\#\#\#\#\# & 91290 & 290 & & & 0.5 & & 0.5 & & & 2.1 & 0.5 \\
\hline $1 \mathrm{~A}$ & S & \#\#\#\#\#\#\#\# & 91291 & 291 & 0.5 & & 0.5 & 0.5 & 0.5 & & 0.5 & 0.5 & 0.5 \\
\hline $0 \mathrm{~A}$ & S & \#\#\#\#\#\#\#\# & 91292 & 292 & 0.5 & & 1 & & 0.5 & 0.5 & & 0.5 & 0.5 \\
\hline $0 \mathrm{~A}$ & S & \#\#\#\#\#\#\#\# & 91293 & 293 & 0.5 & & 0.5 & & & 0.5 & & 0.5 & \\
\hline $1 \mathrm{~A}$ & $\mathrm{~S}$ & \#\#\#\#\#\#\#\# & 91294 & 294 & & & 0.5 & & 0.5 & & & 1.5 & 1 \\
\hline $1 \mathrm{~A}$ & $\mathrm{~S}$ & \#\#\#\#\#\#\#\# & 91295 & 295 & & & 0.5 & & & 0.5 & & 0.5 & 1.5 \\
\hline $1 \mathrm{~A}$ & S & \#\#\#\#\#\#\#\# & 91302 & 302 & & & & 0.5 & & & & 0.5 & 1.5 \\
\hline $1 \mathrm{~A}$ & S & \#\#\#\#\#\#\#\# & 91303 & 303 & 0.5 & & 0.5 & & 0.5 & & 1.5 & 0.5 & 1.5 \\
\hline $1 \mathrm{~A}$ & $S$ & \#\#\#\#\#\#\#\# & 91304 & 304 & 0.5 & & 0.5 & 0.5 & & 0.5 & 0.5 & 1.5 & 1 \\
\hline $1 \mathrm{~A}$ & S & \#\#\#\#\#\#\#\# & 91305 & 305 & 0.5 & 0.5 & 0.5 & 0.5 & 0.5 & 0.5 & 0.5 & 2.1 & 4.1 \\
\hline $0 \mathrm{~A}$ & S & \#\#\#\#\#\#\#\# & 91306 & 306 & 0.5 & & 0.5 & 0.5 & & 0.5 & 0.5 & 0.5 & 0.5 \\
\hline $0 \mathrm{~A}$ & S & \#\#\#\#\#\#\#\# & 91307 & 307 & 0.5 & 0.5 & 0.5 & 0.5 & 1 & 0.5 & 1 & 0.5 & 1 \\
\hline $1 \mathrm{~A}$ & S & \#\#\#\#\#\#\#\# & 91308 & 308 & 0.5 & 1 & 0.5 & 1 & 0.5 & 1 & 0.5 & 2.6 & 5.7 \\
\hline $1 \mathrm{~A}$ & S & \#\#\#\#\#\#\#\# & 91309 & 309 & 0.5 & 0.5 & 1 & 0.5 & 1 & 0.5 & 1 & 4.6 & 3.1 \\
\hline $1 \mathrm{~A}$ & S & \#\#\#\#\#\#\#\# & 91310 & 310 & & 0.5 & 0.5 & 0.5 & 0.5 & 0.5 & 0.5 & 0.5 & 0.5 \\
\hline $1 \mathrm{~A}$ & S & \#\#\#\#\#\#\#\# & 91311 & 311 & & 0.5 & & 0.5 & & & 0.5 & 1 & 0.5 \\
\hline $1 \mathrm{~A}$ & S & \#\#\#\#\#\#\#\# & 91312 & 312 & 0.5 & & 0.5 & 0.5 & 0.5 & 0.5 & 0.5 & 4.6 & 5.2 \\
\hline $0 \mathrm{~A}$ & S & \#\#\#\#\#\#\#\# & 91313 & 313 & 2.6 & 3.6 & 2.1 & 2.1 & 2.1 & 1.5 & 2.1 & 2.1 & 2.6 \\
\hline
\end{tabular}




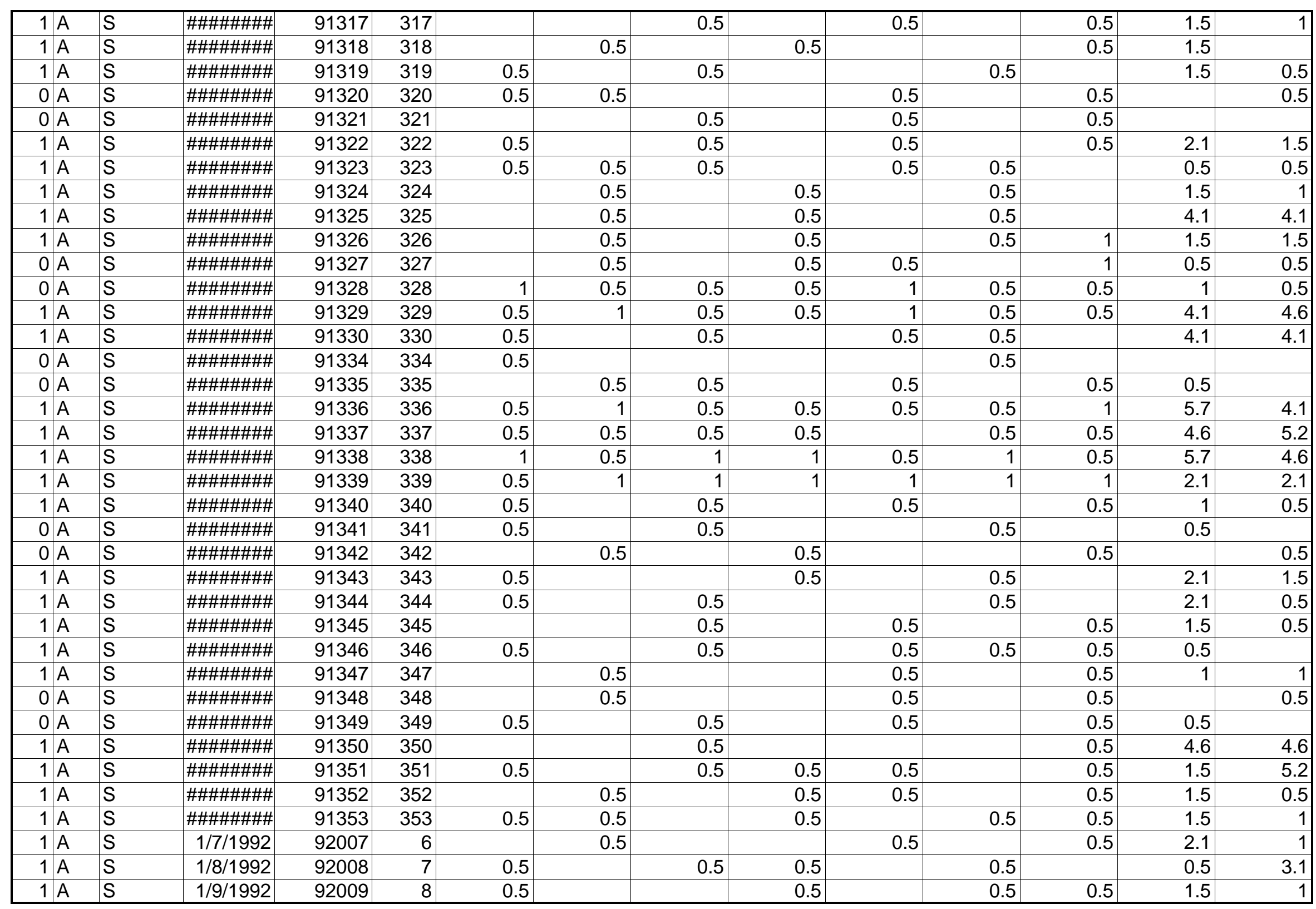




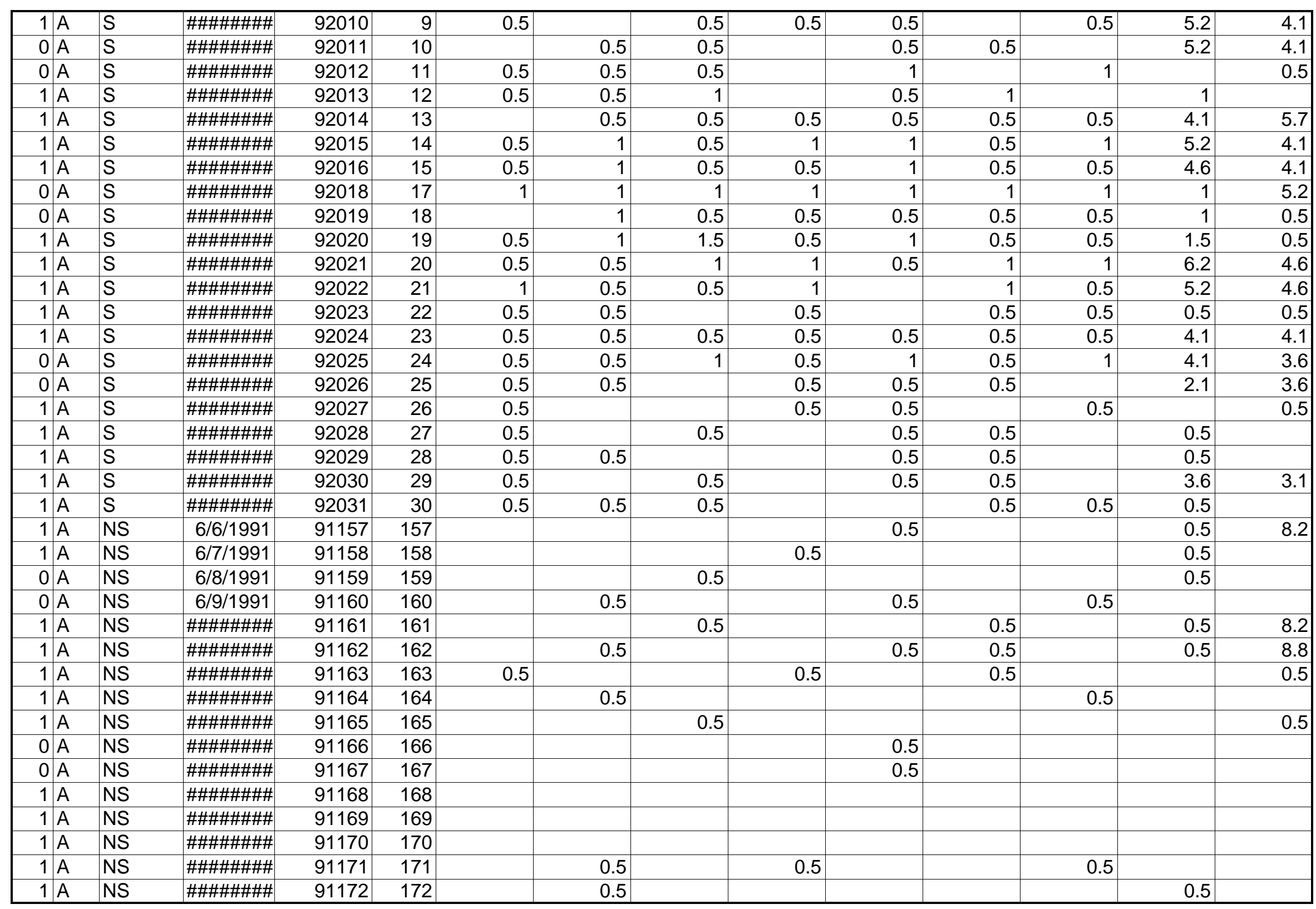




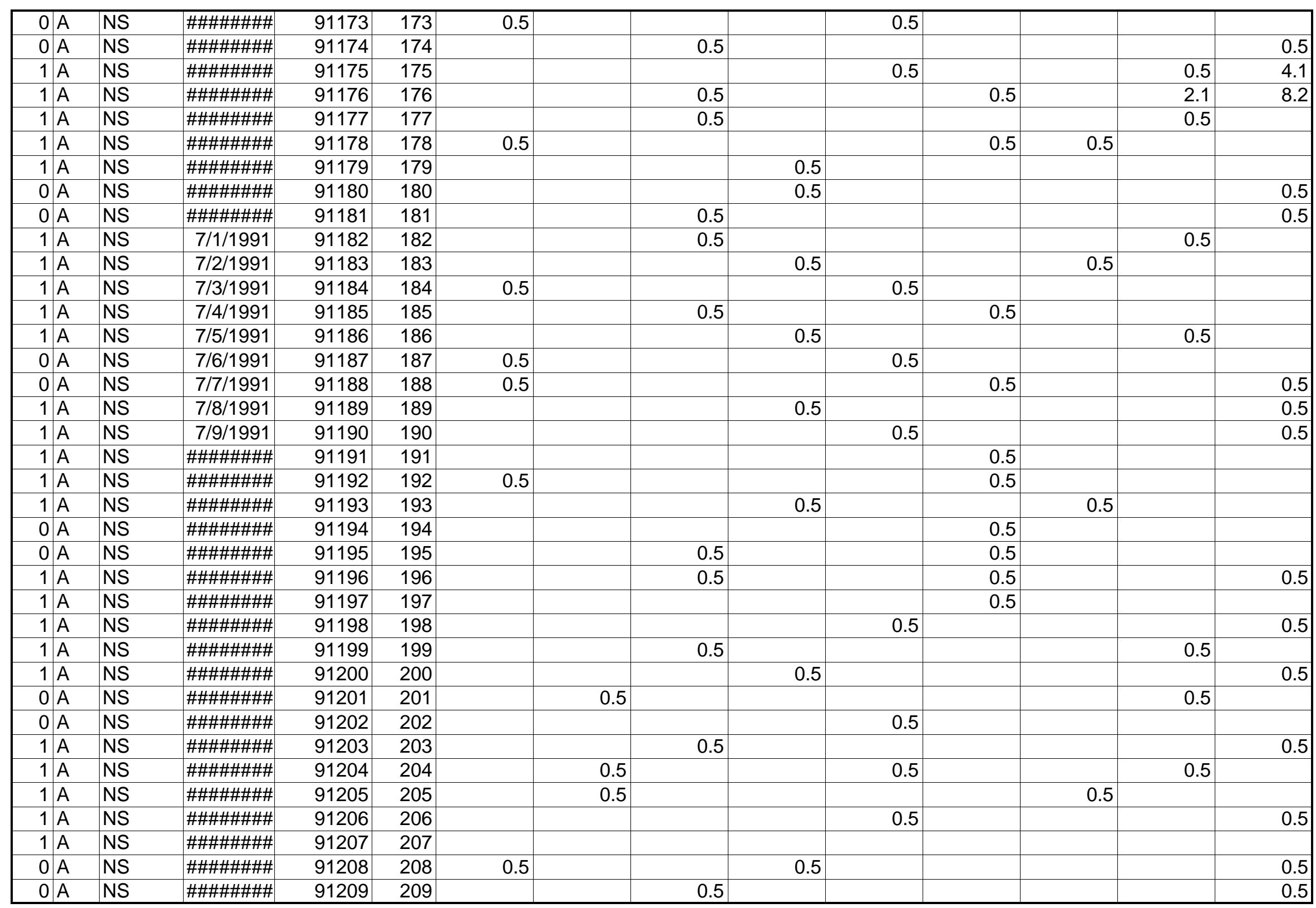




\begin{tabular}{|c|c|c|c|c|c|c|c|c|c|c|c|c|c|}
\hline $1 \mathrm{~A}$ & NS & \#\#\#\#\#\#\#\# & 91210 & 210 & & 0.5 & & & & & & 0.5 & \\
\hline $1 \mathrm{~A}$ & NS & 8/1/1991 & 91213 & 213 & & 0.5 & & & & & 0.5 & & \\
\hline $0 \mathrm{~A}$ & NS & $8 / 4 / 1991$ & 91216 & 216 & & 0.5 & & & & 0.5 & & & \\
\hline $1 \mathrm{~A}$ & NS & $8 / 5 / 1991$ & 91217 & 217 & & 0.5 & & & & 0.5 & & & \\
\hline $1 \mathrm{~A}$ & NS & 8/6/1991 & 91218 & 218 & & 0.5 & & & & & 0.5 & & \\
\hline $1 \mathrm{~A}$ & NS & 8/9/1991 & 91221 & 221 & & & & & & 0.5 & & & \\
\hline $0 \mathrm{~A}$ & NS & \#\#\#\#\#\#\#\# & 91222 & 222 & & & 0.5 & & & & & & 0.5 \\
\hline $0 \mathrm{~A}$ & NS & \#\#\#\#\#\#\#\# & 91223 & 223 & & & & & 0.5 & & & & \\
\hline $1 \mathrm{~A}$ & NS & \#\#\#\#\#\#\#\# & 91224 & 224 & 0.5 & & & & & 0.5 & & & \\
\hline $1 \mathrm{~A}$ & NS & \#\#\#\#\#\#\#\# & 91225 & 225 & & & & 0.5 & & & & & 0.5 \\
\hline $1 \mathrm{~A}$ & NS & \#\#\#\#\#\#\#\# & 91226 & 226 & & & & & 0.5 & & & & \\
\hline $1 \mathrm{~A}$ & NS & \#\#\#\#\#\#\#\# & 91232 & 232 & & 0.5 & & 0.5 & 0.5 & & 0.5 & & 2.1 \\
\hline $1 \mathrm{~A}$ & NS & \#\#\#\#\#\#\#\# & 91233 & 233 & 0.5 & & 0.5 & & 0.5 & & 0.5 & 0.5 & 1.5 \\
\hline $1 \mathrm{~A}$ & NS & \#\#\#\#\#\#\#\# & 91234 & 234 & 0.5 & & 0.5 & & 0.5 & & 0.5 & 1.5 & 0.5 \\
\hline $1 \mathrm{~A}$ & NS & \#\#\#\#\#\#\#\# & 91235 & 235 & 0.5 & & 0.5 & & 0.5 & 0.5 & & 0.5 & 2.1 \\
\hline $0 \mathrm{~A}$ & NS & \#\#\#\#\#\#\#\# & 91236 & 236 & 0.5 & 0.5 & 0.5 & 0.5 & 0.5 & 0.5 & 0.5 & 0.5 & 0.5 \\
\hline $0 \mathrm{~A}$ & NS & \#\#\#\#\#\#\#\# & 91237 & 237 & 0.5 & 0.5 & 0.5 & 1 & & 0.5 & 0.5 & 1 & 0.5 \\
\hline $1 \mathrm{~A}$ & NS & \#\#\#\#\#\#\#\# & 91238 & 238 & 0.5 & & 0.5 & 0.5 & 0.5 & & 0.5 & 0.5 & 0.5 \\
\hline $1 \mathrm{~A}$ & NS & \#\#\#\#\#\#\#\# & 91239 & 239 & & 0.5 & & 0.5 & & 0.5 & 0.5 & 0.5 & \\
\hline $1 \mathrm{~A}$ & NS & \#\#\#\#\#\#\#\# & 91240 & 240 & & 0.5 & 0.5 & & 0.5 & 0.5 & & 2.1 & 0.5 \\
\hline $1 \mathrm{~A}$ & NS & \#\#\#\#\#\#\#\# & 91241 & 241 & 0.5 & 0.5 & & 0.5 & & 0.5 & & 2.1 & 0.5 \\
\hline $1 \mathrm{~A}$ & NS & \#\#\#\#\#\#\#\# & 91242 & 242 & & 0.5 & & 0.5 & & 0.5 & 0.5 & & 1 \\
\hline $0 \mathrm{~A}$ & NS & \#\#\#\#\#\#\#\# & 91243 & 243 & 0.5 & & 0.5 & & 0.5 & 0.5 & & 0.5 & 0.5 \\
\hline
\end{tabular}




\begin{tabular}{|c|c|c|c|c|c|c|c|c|c|c|c|c|c|}
\hline $1 \mathrm{~A}$ & NS & \#\#\#\#\#\#\#\# & 91331 & 331 & & & & 0.5 & & 0.5 & & 2.1 & 1.5 \\
\hline $1 \mathrm{~A}$ & NS & \#\#\#\#\#\#\#\# & 91354 & 354 & & 0.5 & & 0.5 & & 0.5 & 0.5 & 2.1 & 0.5 \\
\hline $1 \mathrm{~A}$ & NS & \#\#\#\#\#\#\#\# & 91357 & 357 & 0.5 & & & 0.5 & & 0.5 & & 0.5 & \\
\hline $1 \mathrm{~A}$ & NS & \#\#\#\#\#\#\#\# & 91358 & 358 & 0.5 & 0.5 & 0.5 & 0.5 & 0.5 & 0.5 & 0.5 & 0.5 & 0.5 \\
\hline $1 \mathrm{~A}$ & NS & \#\#\#\#\#\#\#\# & 91359 & 359 & 0.5 & 0.5 & & 0.5 & 0.5 & 0.5 & 0.5 & 0.5 & 1 \\
\hline $0 \mathrm{~A}$ & NS & \#\#\#\#\#\#\#\# & 91362 & 362 & 0.5 & 0.5 & 0.5 & 1 & 0.5 & 0.5 & 0.5 & 0.5 & 0.5 \\
\hline $0 \mathrm{~A}$ & NS & \#\#\#\#\#\#\#\# & 91363 & 363 & 0.5 & 0.5 & 0.5 & 0.5 & 0.5 & 0.5 & 1 & 0.5 & 0.5 \\
\hline $1 \mathrm{~A}$ & NS & \#\#\#\#\#\#\#\# & 91364 & 364 & & 0.5 & & 0.5 & 0.5 & & 0.5 & 0.5 & 1 \\
\hline $1 \mathrm{~A}$ & NS & \#\#\#\#\#\#\#\# & 91365 & 365 & 0.5 & & 0.5 & 0.5 & 0.5 & 0.5 & & 0.5 & 2.6 \\
\hline $1 \mathrm{~A}$ & NS & $1 / 1 / 1992$ & 92001 & 366 & & & & & & & & & \\
\hline $1 \mathrm{~A}$ & NS & $1 / 2 / 1992$ & 92002 & 1 & 0.5 & & 0.5 & 0.5 & 0.5 & 0.5 & 0.5 & 0.5 & 0.5 \\
\hline $0 \mathrm{~A}$ & NS & \#\#\#\#\#\#\#\# & 92151 & 150 & 0.5 & & 0.5 & & & 0.5 & 0.5 & & \\
\hline $0 \mathrm{~A}$ & NS & \#\#\#\#\#\#\#\# & 92152 & 151 & & 0.5 & & & & 0.5 & & & \\
\hline 0 B & $\mathrm{S}$ & 2/1/1992 & 92032 & 31 & 0.5 & 0.5 & & 0.5 & 0.5 & & & 0.5 & 0.5 \\
\hline 0 B & $S$ & 2/2/1992 & 92033 & 32 & 0.5 & & 0.5 & 0.5 & 0.5 & & 0.5 & 0.5 & 0.5 \\
\hline $1 \mathrm{~B}$ & S & $2 / 3 / 1992$ & 92034 & 33 & & 0.5 & 0.5 & 0.5 & & 0.5 & 0.5 & 0.5 & \\
\hline $1 \mathrm{~B}$ & S & $2 / 4 / 1992$ & 92035 & 34 & 0.5 & & 0.5 & 0.5 & 0.5 & & 0.5 & 0.5 & 0.5 \\
\hline $1 \mathrm{~B}$ & S & $2 / 5 / 1992$ & 92036 & 35 & 0.5 & & 0.5 & 0.5 & & 0.5 & & 0.5 & 0.5 \\
\hline $1 \mathrm{~B}$ & S & 2/6/1992 & 92037 & 36 & 0.5 & 1 & 1 & 0.5 & 0.5 & 1 & 0.5 & 2.6 & 4.1 \\
\hline $1 \mathrm{~B}$ & S & $2 / 7 / 1992$ & 92038 & 37 & 1 & 0.5 & 0.5 & 1 & 0.5 & 0.5 & 1 & 4.1 & 4.1 \\
\hline \begin{tabular}{l|l}
0 & $B$ \\
\end{tabular} & S & 2/8/1992 & 92039 & 38 & 0.5 & 1 & & 1 & 0.5 & 1 & 0.5 & 4.1 & 4.1 \\
\hline $\begin{array}{ll}0 & B \\
\end{array}$ & $S$ & 2/9/1992 & 92040 & 39 & 0.5 & & 1 & 0.5 & & 1 & 0.5 & 1 & 3.1 \\
\hline $1 B$ & $S$ & \#\#\#\#\#\#\#\# & 92041 & 40 & 0.5 & 0.5 & 0.5 & 0.5 & 0.5 & 0.5 & 0.5 & 0.5 & 0.5 \\
\hline
\end{tabular}




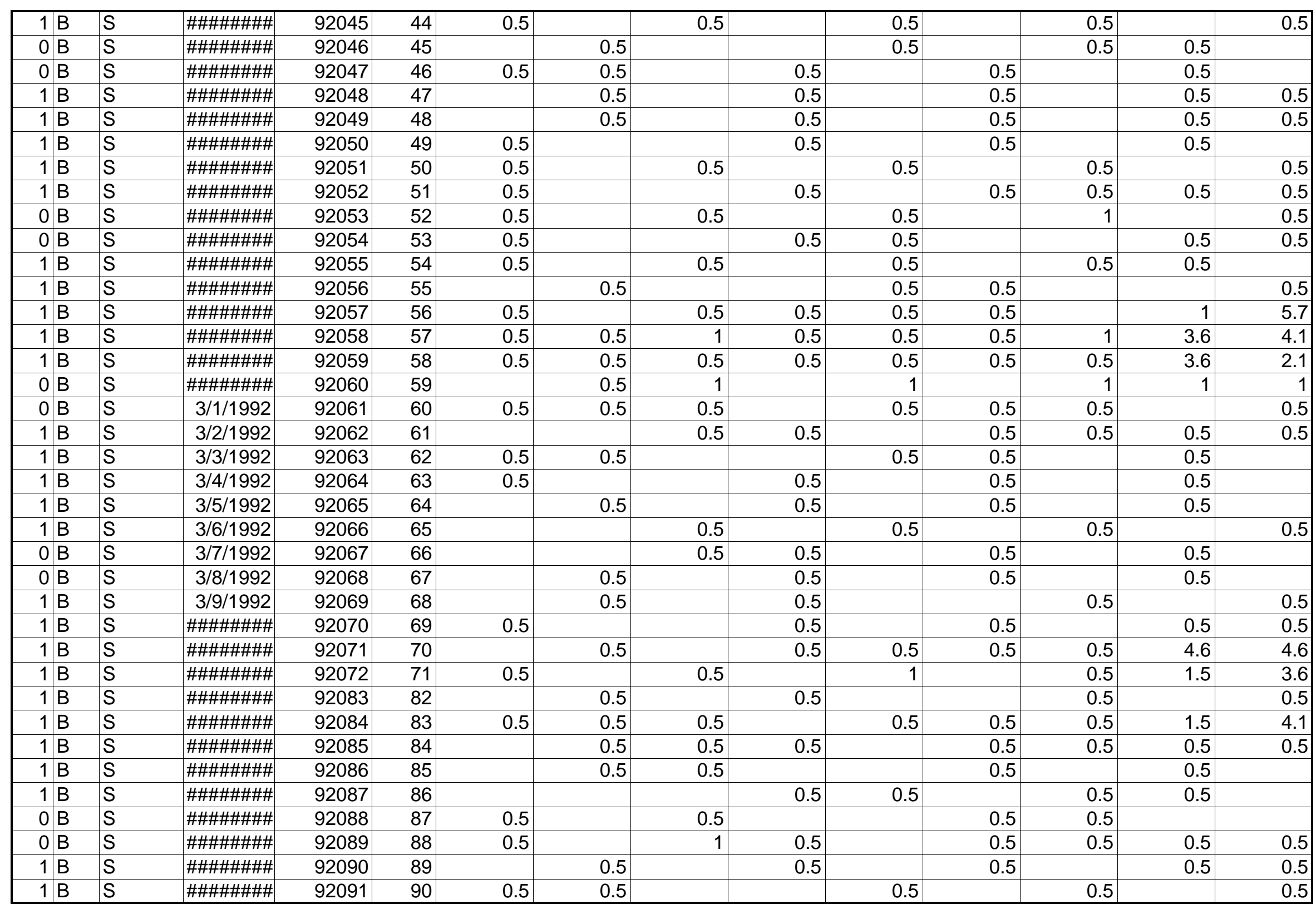




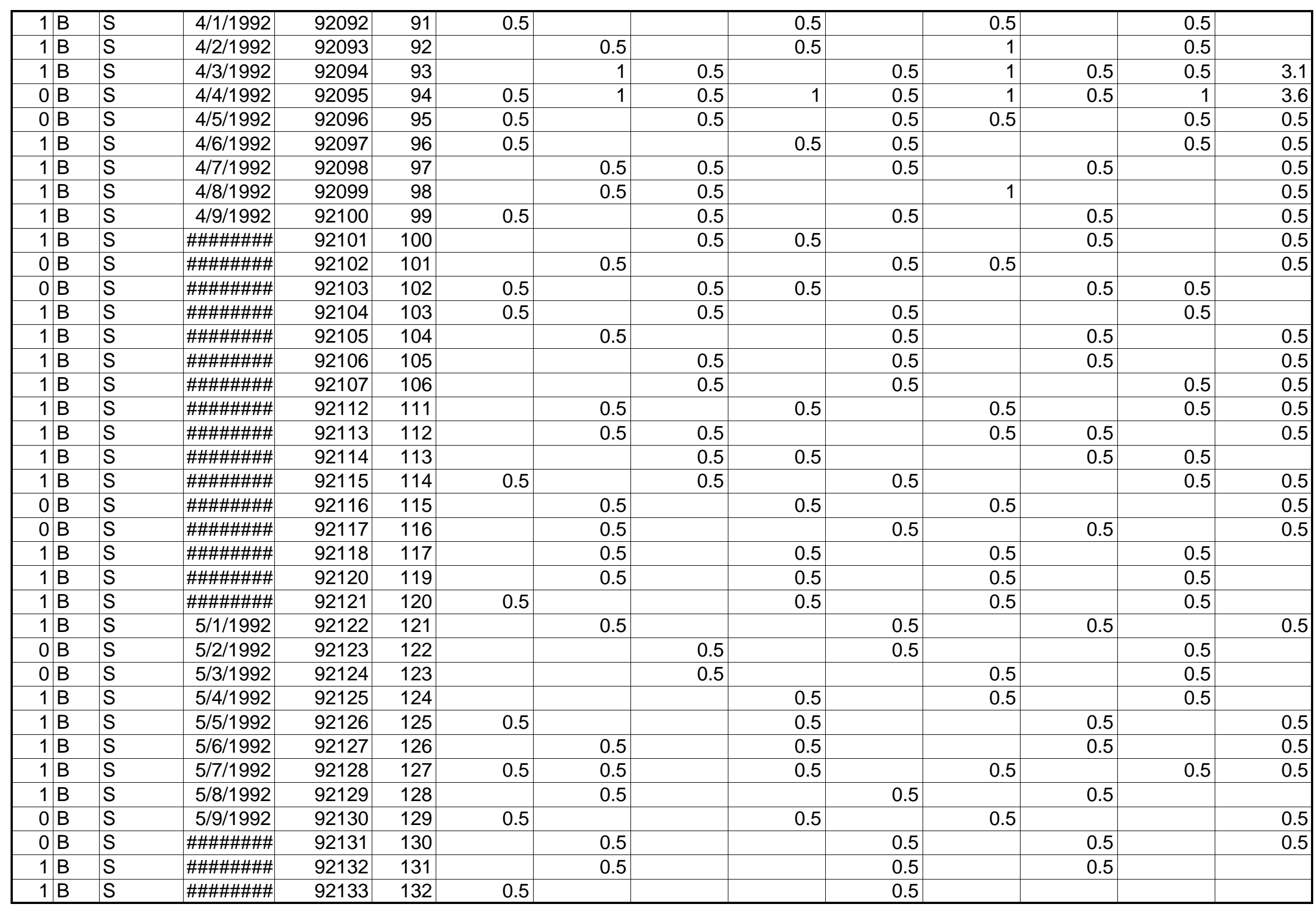




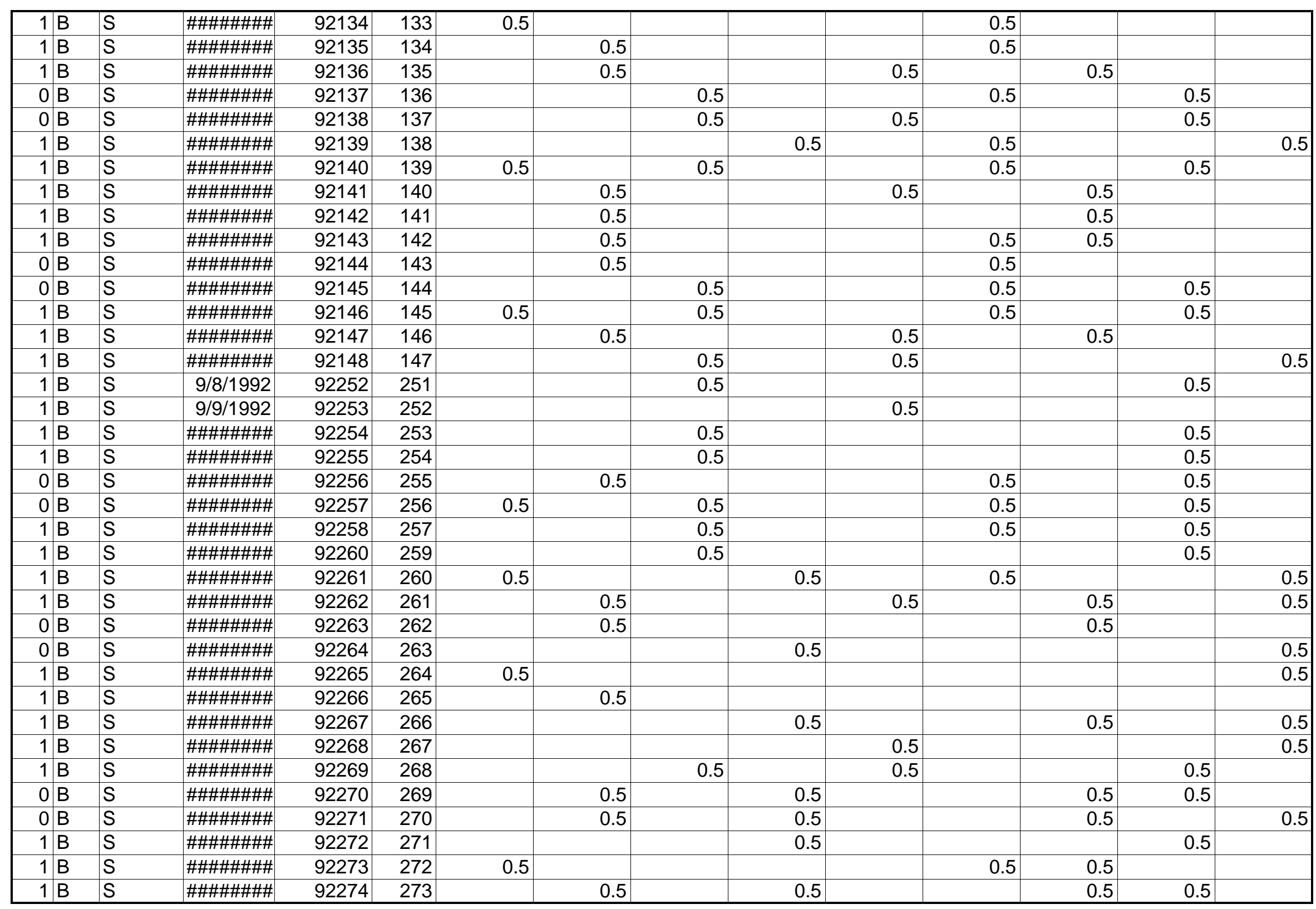

Page 10 


\begin{tabular}{|c|c|c|c|c|c|c|c|c|c|c|c|c|c|}
\hline $1 \mathrm{~B}$ & S & \#\#\#\#\#\#\# & 92275 & 274 & & & 0.5 & & & 0.5 & & 0.5 & 0.5 \\
\hline $0 \mathrm{~B}$ & S & \#\#\#\#\#\#\#\# & 92278 & 277 & & & 0.5 & & & & 0.5 & & 0.5 \\
\hline $1 \mathrm{~B}$ & S & \#\#\#\#\#\#\#\# & 92281 & 280 & & 0.5 & & 0.5 & & & 0.5 & & 0.5 \\
\hline $1 \mathrm{~B}$ & S & \#\#\#\#\#\#\#\# & 92282 & 281 & 0.5 & & & 0.5 & & 0.5 & & 0.5 & \\
\hline $1 \mathrm{~B}$ & S & \#\#\#\#\#\#\#\# & 92283 & 282 & 0.5 & & 0.5 & & 0.5 & & & 0.5 & 0.5 \\
\hline $1 \mathrm{~B}$ & S & \#\#\#\#\#\#\#\# & 92287 & 286 & 0.5 & & & 0.5 & & 0.5 & & 0.5 & \\
\hline $1 \mathrm{~B}$ & S & \#\#\#\#\#\#\#\# & 92288 & 287 & 0.5 & & & 0.5 & & 0.5 & & 0.5 & \\
\hline $1 B$ & S & \#\#\#\#\#\#\#\# & 92289 & 288 & & 0.5 & & & 0.5 & & 0.5 & & \\
\hline $1 \mathrm{~B}$ & S & \#\#\#\#\#\#\#\# & 92290 & 289 & & & 0.5 & & & & 0.5 & & \\
\hline $0 \mathrm{~B}$ & $\mathrm{~S}$ & \#\#\#\#\#\#\#\# & 92291 & 290 & 0.5 & & & & & 0.5 & & & \\
\hline $0 \mathrm{~B}$ & $\mathrm{~S}$ & \#\#\#\#\#\#\#\# & 92292 & 291 & & 0.5 & & & 0.5 & & 0.5 & & 0.5 \\
\hline $0 \mathrm{~B}$ & S & \#\#\#\#\#\#\#\# & 92298 & 297 & 0.5 & & & 0.5 & & 0.5 & & & 0.5 \\
\hline 0 B & S & \#\#\#\#\#\#\#\# & 92299 & 298 & 0.5 & & & 0.5 & & 0.5 & & & 0.5 \\
\hline $1 B$ & $S$ & \#\#\#\#\#\#\#\# & 92300 & 299 & & 0.5 & & & 0.5 & & 0.5 & & 0.5 \\
\hline $1 \mathrm{~B}$ & S & \#\#\#\#\#\#\#\# & 92301 & 300 & & 0.5 & & & 0.5 & & 0.5 & 0.5 & \\
\hline $1 \mathrm{~B}$ & S & \#\#\#\#\#\#\#\# & 92302 & 301 & & 0.5 & & 0.5 & & 0.5 & & & 0.5 \\
\hline $1 \mathrm{~B}$ & S & \#\#\#\#\#\#\#\# & 92303 & 302 & & 0.5 & & 0.5 & & 0.5 & & & 0.5 \\
\hline $1 \mathrm{~B}$ & S & \#\#\#\#\#\#\#\# & 92304 & 303 & 0.5 & & 0.5 & & & 0.5 & & 0.5 & \\
\hline $0 \mathrm{~B}$ & S & \#\#\#\#\#\#\#\# & 92305 & 304 & & & 0.5 & & & & 0.5 & & \\
\hline $0 \mathrm{~B}$ & S & \#\#\#\#\#\#\#\# & 92306 & 305 & & 0.5 & & & & & & 0.5 & \\
\hline $1 B$ & S & \#\#\#\#\#\#\#\# & 92307 & 306 & & 0.5 & & & & & 0.5 & & 0.5 \\
\hline \begin{tabular}{l|l}
11 & $B$ \\
\end{tabular} & S & \#\#\#\#\#\#\#\# & 92308 & 307 & & 0.5 & & 0.5 & & & 0.5 & 0.5 & \\
\hline \begin{tabular}{l|l}
11 & $B$ \\
\end{tabular} & S & \#\#\#\#\#\#\#\# & 92309 & 308 & & 0.5 & & 0.5 & & & 0.5 & 0.5 & \\
\hline
\end{tabular}

Page 11 


\begin{tabular}{|c|c|c|c|c|c|c|c|c|c|c|c|c|c|}
\hline 0 B & S & \#\#\#\#\#\#\# & 92313 & 312 & 0.5 & 0.5 & & & 0.5 & 0.5 & 0.5 & & 0.5 \\
\hline $1 \mathrm{~B}$ & S & \#\#\#\#\#\#\#\# & 92316 & 315 & 0.5 & & & & 0.5 & 0.5 & & & 0.5 \\
\hline $0 \mathrm{~B}$ & S & \#\#\#\#\#\#\#\# & 92319 & 318 & & 0.5 & 0.5 & & & 0.5 & 0.5 & & 2.1 \\
\hline 0 B & S & \#\#\#\#\#\#\# & 92320 & 319 & 0.5 & & & 0.5 & 0.5 & & 0.5 & 0.5 & 1 \\
\hline $1 \mathrm{~B}$ & S & \#\#\#\#\#\#\#\# & 92321 & 320 & 0.5 & & 0.5 & & 0.5 & 0.5 & & & 0.5 \\
\hline $1 \mathrm{~B}$ & S & \#\#\#\#\#\#\#\# & 92324 & 323 & 0.5 & & 0.5 & & 0.5 & & 0.5 & & 0.5 \\
\hline $1 \mathrm{~B}$ & S & \#\#\#\#\#\#\#\# & 92325 & 324 & 0.5 & & & 0.5 & 0.5 & & 0.5 & & \\
\hline $0 B$ & S & \#\#\#\#\#\#\#\# & 92326 & 325 & & & 0.5 & & 0.5 & 0.5 & & & 0.5 \\
\hline $0 \mathrm{~B}$ & S & \#\#\#\#\#\#\#\# & 92327 & 326 & & & 0.5 & & 0.5 & & 0.5 & & 0.5 \\
\hline $1 \mathrm{~B}$ & $\mathrm{~S}$ & \#\#\#\#\#\#\#\# & 92328 & 327 & 0.5 & & 0.5 & & 0.5 & & 0.5 & & 0.5 \\
\hline $1 B$ & $\mathrm{~S}$ & \#\#\#\#\#\#\#\# & 92329 & 328 & 0.5 & & & 0.5 & 0.5 & & & 0.5 & 5.2 \\
\hline $1 \mathrm{~B}$ & S & \#\#\#\#\#\#\# & 92338 & 337 & & 0.5 & & 0.5 & & 0.5 & & 1.5 & 4.1 \\
\hline $1 \mathrm{~B}$ & S & \#\#\#\#\#\#\#\# & 92339 & 338 & & 0.5 & 0.5 & 0.5 & & 1 & & 2.6 & 4.6 \\
\hline $0 \mathrm{~B}$ & S & \#\#\#\#\#\#\#\# & 92340 & 339 & & 0.5 & & & 0.5 & & 0.5 & & 0.5 \\
\hline $0 \mathrm{~B}$ & S & \#\#\#\#\#\#\#\# & 92341 & 340 & 0.5 & 0.5 & 0.5 & & 0.5 & 0.5 & & 4.6 & 3.6 \\
\hline $1 \mathrm{~B}$ & S & \#\#\#\#\#\#\#\# & 92342 & 341 & 0.5 & 0.5 & 0.5 & & 0.5 & 0.5 & & 0.5 & \\
\hline $1 \mathrm{~B}$ & S & \#\#\#\#\#\#\#\# & 92343 & 342 & & 1 & & 0.5 & & 0.5 & 0.5 & 3.1 & 4.6 \\
\hline $1 \mathrm{~B}$ & S & \#\#\#\#\#\#\#\# & 92344 & 343 & 0.5 & 0.5 & & 0.5 & 0.5 & 0.5 & 0.5 & 2.6 & 4.1 \\
\hline $1 \mathrm{~B}$ & S & \#\#\#\#\#\#\#\# & 92345 & 344 & 0.5 & & 0.5 & & 0.5 & 0.5 & & 1 & 0.5 \\
\hline $1 \mathrm{~B}$ & S & \#\#\#\#\#\#\#\# & 92346 & 345 & & 0.5 & & 0.5 & & 0.5 & 0.5 & & 1.5 \\
\hline $0 \mathrm{~B}$ & S & \#\#\#\#\#\#\#\# & 92347 & 346 & 0.5 & & & 0.5 & & 0.5 & & & 5.2 \\
\hline \begin{tabular}{l|l}
0 & $B$ \\
\end{tabular} & S & \#\#\#\#\#\#\#\# & 92348 & 347 & 0.5 & & 0.5 & & & & 0.5 & & \\
\hline $1 B$ & S & \#\#\#\#\#\#\#\# & 92349 & 348 & & & 0.5 & & 0.5 & & & 0.5 & \\
\hline
\end{tabular}




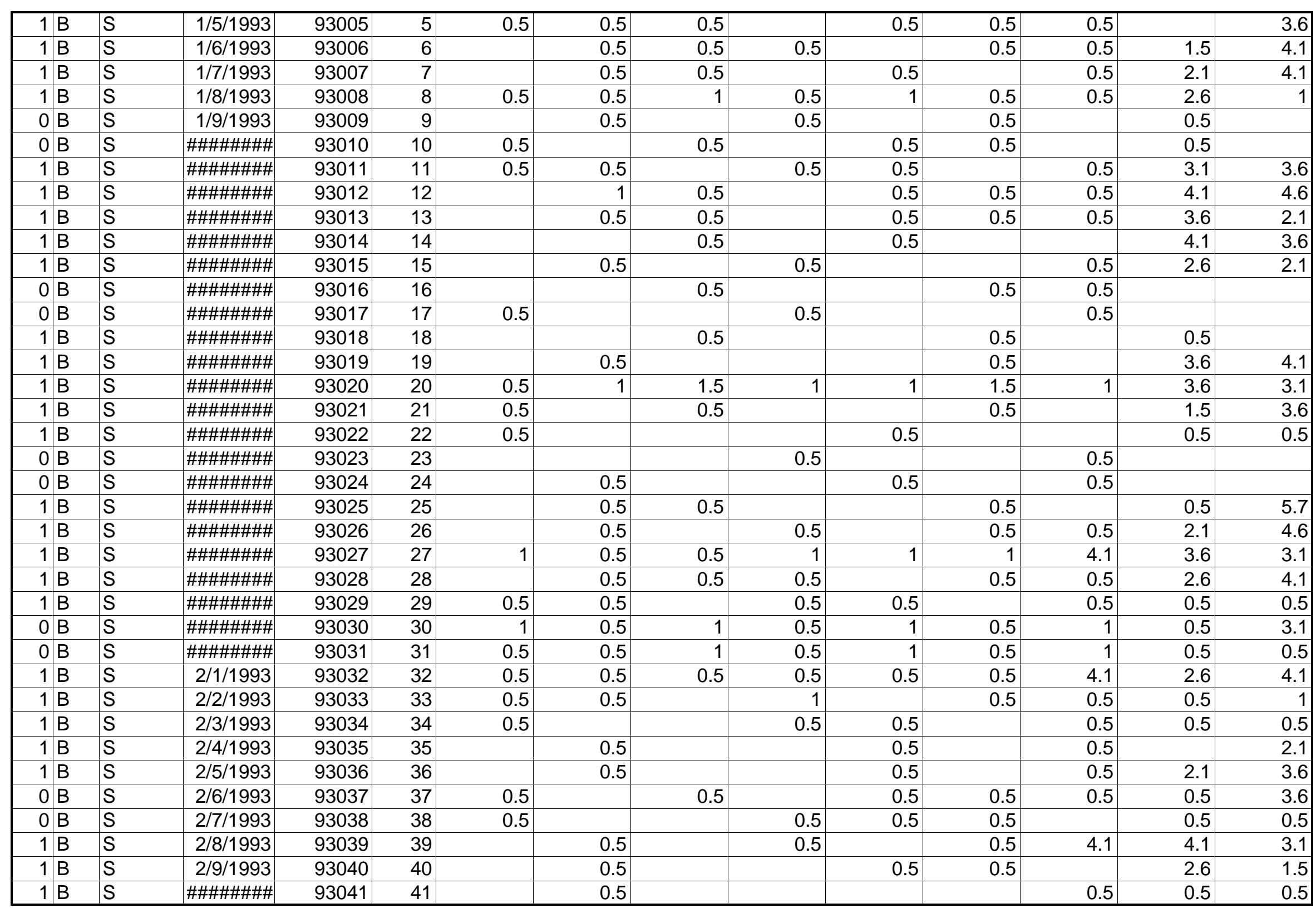

Page 13 


\begin{tabular}{|c|c|c|c|c|c|c|c|c|c|c|c|c|c|}
\hline $1 \mathrm{~B}$ & $S$ & \#\#\#\#\#\#\# & 93042 & 42 & & 0.5 & & & & 0.5 & & & 0.5 \\
\hline $0 \mathrm{~B}$ & S & \#\#\#\#\#\#\#\# & 93045 & 45 & & 0.5 & & 0.5 & & & & 0.5 & 0.5 \\
\hline $1 \mathrm{~B}$ & S & \#\#\#\#\#\#\#\# & 93049 & 49 & 0.5 & 0.5 & 0.5 & 0.5 & 0.5 & 0.5 & 3.1 & 2.1 & 3.1 \\
\hline $1 \mathrm{~B}$ & S & \#\#\#\#\#\#\#\# & 93050 & 50 & 0.5 & & 1 & 0.5 & 0.5 & 0.5 & 2.6 & 1.5 & 2.6 \\
\hline $0 \mathrm{~B}$ & S & \#\#\#\#\#\#\#\# & 93051 & 51 & 0.5 & 0.5 & 0.5 & 0.5 & 0.5 & 0.5 & 0.5 & 0.5 & \\
\hline $1 \mathrm{~B}$ & S & \#\#\#\#\#\#\#\# & 93054 & 54 & 0.5 & & 0.5 & & & & & 3.1 & 3.6 \\
\hline $1 \mathrm{~B}$ & $S$ & \#\#\#\#\#\#\#\# & 93055 & 55 & & & 0.5 & & 0.5 & & 4.1 & 1.5 & 0.5 \\
\hline $1 \mathrm{~B}$ & $\mathrm{~S}$ & \#\#\#\#\#\#\#\# & 93056 & 56 & & & 0.5 & & & & & 0.5 & \\
\hline $1 \mathrm{~B}$ & $S$ & \#\#\#\#\#\#\#\# & 93057 & 57 & 0.5 & & & & 0.5 & & & 2.6 & 4.6 \\
\hline $0 \mathrm{~B}$ & $S$ & \#\#\#\#\#\#\#\# & 93058 & 58 & & 0.5 & & & 0.5 & & 0.5 & & 0.5 \\
\hline $0 \mathrm{~B}$ & $S$ & \#\#\#\#\#\#\#\# & 93059 & 59 & & 0.5 & & & & 0.5 & 0.5 & & \\
\hline 0 B & $\mathrm{S}$ & $3 / 6 / 1993$ & 93065 & 65 & & 0.5 & & & & 0.5 & 0.5 & & \\
\hline 0 B & $\mathrm{S}$ & $3 / 7 / 1993$ & 93066 & 66 & & & & 0.5 & & 0.5 & & & 0.5 \\
\hline $1 B$ & $S$ & $3 / 8 / 1993$ & 93067 & 67 & & & 0.5 & & & 0.5 & 4.6 & 2.6 & \\
\hline $1 \mathrm{~B}$ & S & $3 / 9 / 1993$ & 93068 & 68 & & & 0.5 & & & & & & 0.5 \\
\hline $1 \mathrm{~B}$ & $S$ & \#\#\#\#\#\#\#\# & 93069 & 69 & 0.5 & & & & 0.5 & & & & 0.5 \\
\hline $1 \mathrm{~B}$ & S & \#\#\#\#\#\#\#\# & 93070 & 70 & & & & & & 0.5 & & & 0.5 \\
\hline $1 \mathrm{~B}$ & $S$ & \#\#\#\#\#\#\#\# & 93071 & 71 & 0.5 & & & & 0.5 & & & 0.5 & 2.6 \\
\hline \begin{tabular}{l|l}
11 & $B$ \\
\end{tabular} & $S$ & \#\#\#\#\#\#\#\# & 93081 & 81 & 0.5 & & & & & 0.5 & & & 0.5 \\
\hline $1 \mathrm{~B}$ & $S$ & \#\#\#\#\#\#\#\# & 93082 & 82 & & & & 0.5 & & & & 0.5 & \\
\hline \begin{tabular}{l|l}
11 & $B$ \\
\end{tabular} & $S$ & \#\#\#\#\#\#\#\# & 93083 & 83 & & & 0.5 & & & & & 0.5 & \\
\hline \begin{tabular}{l|l}
11 & $B$ \\
\end{tabular} & $S$ & \#\#\#\#\#\#\#\# & 93084 & 84 & 0.5 & & & & & 0.5 & & & \\
\hline \begin{tabular}{l|l}
11 & $B$ \\
\end{tabular} & $S$ & \#\#\#\#\#\#\#\# & 93085 & 85 & & 0.5 & & & 0.5 & & & & \\
\hline
\end{tabular}




\begin{tabular}{|c|c|c|c|c|c|c|c|c|c|c|c|c|c|}
\hline $1 \mathrm{~B}$ & $\mathrm{~S}$ & \#\#\#\#\#\#\#\# & 93089 & 89 & & 0.5 & & & & & & 0.5 & \\
\hline $1 \mathrm{~B}$ & S & 4/2/1993 & 93092 & 92 & & 0.5 & & & 0.5 & & & & \\
\hline $1 \mathrm{~B}$ & S & 4/5/1993 & 93095 & 95 & & 0.5 & & & 0.5 & & & & 0.5 \\
\hline $1 \mathrm{~B}$ & S & 4/6/1993 & 93096 & 96 & & 0.5 & & & & 0.5 & & & 0.5 \\
\hline $1 \mathrm{~B}$ & S & $4 / 7 / 1993$ & 93097 & 97 & & & 0.5 & & & 0.5 & & & \\
\hline $1 \mathrm{~B}$ & S & \#\#\#\#\#\#\#\# & 93104 & 104 & 0.5 & & & & & & 0.5 & & \\
\hline $1 \mathrm{~B}$ & S & \#\#\#\#\#\#\#\# & 93105 & 105 & & & 0.5 & & & 0.5 & & & 1 \\
\hline $1 \mathrm{~B}$ & S & \#\#\#\#\#\#\#\# & 93106 & 106 & 0.5 & & & & 0.5 & & & & 0.5 \\
\hline $0 \mathrm{~B}$ & S & \#\#\#\#\#\#\#\# & 93107 & 107 & & & 0.5 & & & & 0.5 & & \\
\hline $0 \mathrm{~B}$ & $\mathrm{~S}$ & \#\#\#\#\#\#\#\# & 93108 & 108 & 0.5 & & & & 0.5 & & & & \\
\hline $1 B$ & $\mathrm{~S}$ & \#\#\#\#\#\#\#\# & 93109 & 109 & & & & & 0.5 & & & & 0.5 \\
\hline $0 \mathrm{~B}$ & S & \#\#\#\#\#\#\#\# & 93115 & 115 & & & & & 0.5 & & & & 0.5 \\
\hline $1 \mathrm{~B}$ & S & \#\#\#\#\#\#\#\# & 93116 & 116 & & & 0.5 & & & 0.5 & & & \\
\hline $1 B$ & $S$ & \#\#\#\#\#\#\#\# & 93117 & 117 & & & & 0.5 & & & & & \\
\hline $1 \mathrm{~B}$ & S & \#\#\#\#\#\#\#\# & 93119 & 119 & & 0.5 & & & 0.5 & & & & \\
\hline $1 \mathrm{~B}$ & S & \#\#\#\#\#\#\#\# & 93120 & 120 & 0.5 & & & & & 0.5 & & & \\
\hline $0 \mathrm{~B}$ & S & $5 / 1 / 1993$ & 93121 & 121 & 0.5 & & & & 0.5 & & & & \\
\hline $0 \mathrm{~B}$ & S & $5 / 2 / 1993$ & 93122 & 122 & & & & & 0.5 & & & & 0.5 \\
\hline $1 \mathrm{~B}$ & S & 5/3/1993 & 93123 & 123 & & & & & 0.5 & & & 0.5 & \\
\hline $1 \mathrm{~B}$ & S & $5 / 4 / 1993$ & 93124 & 124 & & 0.5 & & & & 0.5 & & & 0.5 \\
\hline \begin{tabular}{l|l}
11 & $B$ \\
\end{tabular} & S & $5 / 5 / 1993$ & 93125 & 125 & & & & & 0.5 & & & & 0.5 \\
\hline \begin{tabular}{l|l}
11 & $B$ \\
\end{tabular} & S & $5 / 6 / 1993$ & 93126 & 126 & & 0.5 & & & 0.5 & & & & \\
\hline \begin{tabular}{l|l}
11 & $B$ \\
\end{tabular} & S & $5 / 7 / 1993$ & 93127 & 127 & & & 0.5 & & & & 0.5 & & \\
\hline
\end{tabular}




\begin{tabular}{|c|c|c|c|c|c|c|c|c|c|c|c|c|c|c|}
\hline 1 & B & $\mathrm{S}$ & \#\#\#\#\#\#\#\# & 93131 & 131 & & & & & & 0.5 & & & 0.5 \\
\hline 1 & B & S & \#\#\#\#\#\#\#\# & 93132 & 132 & & 0.5 & & & & & & & 0.5 \\
\hline 1 & B & S & \#\#\#\#\#\#\#\# & 93133 & 133 & & & & & 0.5 & & & 0.5 & \\
\hline 1 & B & S & \#\#\#\#\#\#\#\# & 93134 & 134 & & & & & 0.5 & & & & 0.5 \\
\hline 0 & B & S & \#\#\#\#\#\#\#\# & 93135 & 135 & & 0.5 & & & & & 0.5 & & \\
\hline 0 & $B$ & S & \#\#\#\#\#\#\#\# & 93136 & 136 & & & 0.5 & & & & & 0.5 & \\
\hline 1 & B & S & \#\#\#\#\#\#\#\# & 93137 & 137 & & & & 0.5 & & & & & 0.5 \\
\hline 1 & B & S & \#\#\#\#\#\#\#\# & 93138 & 138 & & 0.5 & & & & & & 0.5 & \\
\hline 1 & B & S & \#\#\#\#\#\#\#\# & 93139 & 139 & 0.5 & & & & & 0.5 & & & \\
\hline 1 & B & S & \#\#\#\#\#\#\#\# & 93140 & 140 & 0.5 & & & & 0.5 & & & & 0.5 \\
\hline 1 & B & S & \#\#\#\#\#\#\#\# & 93141 & 141 & & & 0.5 & & & & 0.5 & & \\
\hline 0 & $B$ & S & \#\#\#\#\#\#\#\# & 93142 & 142 & & 0.5 & & & & & 0.5 & & \\
\hline 0 & B & S & \#\#\#\#\#\#\#\# & 93143 & 143 & & & & 0.5 & & & & & 0.5 \\
\hline 1 & B & S & \#\#\#\#\#\#\#\# & 93144 & 144 & 0.5 & & & & 0.5 & & & & 0.5 \\
\hline 1 & $B$ & S & \#\#\#\#\#\#\#\# & 93145 & 145 & & 0.5 & & & & 0.5 & & & \\
\hline 1 & B & S & \#\#\#\#\#\#\#\# & 93146 & 146 & & 0.5 & & & & 0.5 & & & \\
\hline 1 & B & S & 9/9/1993 & 93252 & 252 & 0.5 & & 0.5 & & & 0.5 & & 0.5 & \\
\hline 1 & B & S & \#\#\#\#\#\#\#\# & 93253 & 253 & & & & 0.5 & & & & & 0.5 \\
\hline 0 & $B$ & S & \#\#\#\#\#\#\#\# & 93254 & 254 & & 0.5 & & & & & 0.5 & & \\
\hline 0 & B & S & \#\#\#\#\#\#\#\# & 93255 & 255 & & & & 0.5 & & & & 0.5 & \\
\hline 1 & B & S & \#\#\#\#\#\#\#\# & 93256 & 256 & & & & 0.5 & & & & & \\
\hline 1 & B & S & \#\#\#\#\#\#\#\# & 93257 & 257 & & 0.5 & & & & & 0.5 & & \\
\hline 1 & B & S & \#\#\#\#\#\#\#\# & 93258 & 258 & & & 0.5 & & & 0.5 & 0.5 & 0.5 & 0.5 \\
\hline 1 & B & S & \#\#\#\#\#\#\#\# & 93259 & 259 & & 0.5 & & & & 0.5 & & & \\
\hline 1 & B & S & \#\#\#\#\#\#\#\# & 93260 & 260 & & 0.5 & & & & 0.5 & & & \\
\hline 0 & B & S & \#\#\#\#\#\#\#\# & 93261 & 261 & & & & 0.5 & & & & 0.5 & \\
\hline 0 & B & S & \#\#\#\#\#\#\#\# & 93262 & 262 & 0.5 & & & & & & 0.5 & & \\
\hline 1 & B & S & \#\#\#\#\#\#\#\# & 93263 & 263 & & & & & 0.5 & & & & 0.5 \\
\hline 1 & $B$ & S & \#\#\#\#\#\#\#\# & 93264 & 264 & & & & 0.5 & & & & 0.5 & \\
\hline 1 & B & S & \#\#\#\#\#\#\#\# & 93265 & 265 & & & 0.5 & & & & & 0.5 & \\
\hline 1 & B & S & \#\#\#\#\#\#\#\# & 93266 & 266 & & & & & 0.5 & & & & 0.5 \\
\hline 1 & $B$ & S & \#\#\#\#\#\#\#\# & 93267 & 267 & & 0.5 & & & & & 0.5 & & \\
\hline 0 & B & S & \#\#\#\#\#\#\#\# & 93268 & 268 & & & & & 0.5 & & & 0.5 & \\
\hline 0 & B & S & \#\#\#\#\#\#\#\# & 93269 & 269 & & 0.5 & & & & & & 0.5 & \\
\hline 1 & $B$ & S & \#\#\#\#\#\#\#\# & 93270 & 270 & 0.5 & & & & & 0.5 & & & \\
\hline 1 & $B$ & S & \#\#\#\#\#\#\#\# & 93271 & 271 & 0.5 & & & & & 0.5 & & & 0.5 \\
\hline 1 & $B$ & $S$ & \#\#\#\#\#\#\#\# & 93272 & 272 & & & & 0.5 & & & & 0.5 & \\
\hline
\end{tabular}




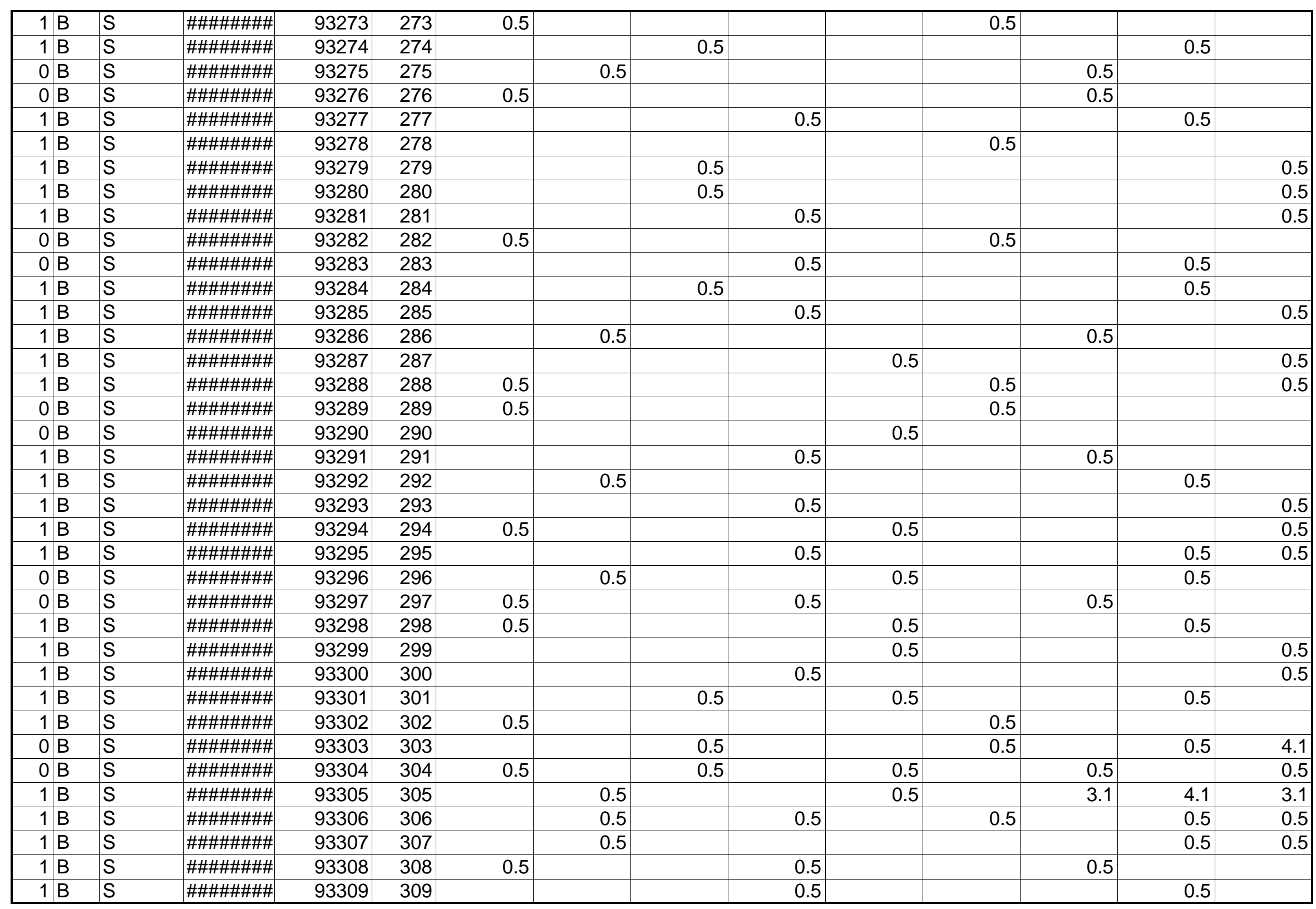




\begin{tabular}{|c|c|c|c|c|c|c|c|c|c|c|c|c|c|}
\hline 0 B & $S$ & \#\#\#\#\#\#\# & 93310 & 310 & 0.5 & & & 0.5 & & & 0.5 & 0.5 & 1 \\
\hline $1 \mathrm{~B}$ & S & \#\#\#\#\#\#\#\# & 93313 & 313 & 0.5 & & & & 0.5 & & 0.5 & & 3.6 \\
\hline $0 \mathrm{~B}$ & S & \#\#\#\#\#\#\#\# & 93317 & 317 & & & & & 0.5 & & & & 0.5 \\
\hline 0 B & S & \#\#\#\#\#\#\#\# & 93318 & 318 & & & 0.5 & & & & & & 0.5 \\
\hline $1 \mathrm{~B}$ & S & \#\#\#\#\#\#\# & 93319 & 319 & & 0.5 & & & 0.5 & & & 0.5 & \\
\hline $1 \mathrm{~B}$ & $S$ & \#\#\#\#\#\#\#\# & 93322 & 322 & 0.5 & & 0.5 & & & 0.5 & 3.6 & 3.1 & 2.1 \\
\hline $1 \mathrm{~B}$ & $S$ & \#\#\#\#\#\#\#\# & 93323 & 323 & & & 0.5 & & & & 0.5 & & \\
\hline $0 \mathrm{~B}$ & $S$ & \#\#\#\#\#\#\#\# & 93324 & 324 & & & & 0.5 & & & & 3.6 & 3.6 \\
\hline $0 \mathrm{~B}$ & $S$ & \#\#\#\#\#\#\#\# & 93325 & 325 & 0.5 & & 0.5 & & & 0.5 & & 0.5 & \\
\hline $1 \mathrm{~B}$ & $\mathrm{~S}$ & \#\#\#\#\#\#\#\# & 93326 & 326 & 0.5 & & & 0.5 & & 0.5 & 3.1 & 3.1 & 1.5 \\
\hline $1 B$ & $S$ & \#\#\#\#\#\#\#\# & 93327 & 327 & & & 0.5 & & 0.5 & & & 0.5 & 1.5 \\
\hline $0 \mathrm{~B}$ & $S$ & \#\#\#\#\#\#\#\# & 93338 & 338 & 0.5 & & & & & 0.5 & & & \\
\hline $0 \mathrm{~B}$ & S & \#\#\#\#\#\#\#\# & 93339 & 339 & & & & & 0.5 & & 0.5 & & \\
\hline $1 \mathrm{~B}$ & S & \#\#\#\#\#\#\#\# & 93340 & 340 & 0.5 & & & & 0.5 & & & 0.5 & 1.5 \\
\hline $1 \mathrm{~B}$ & S & \#\#\#\#\#\#\#\# & 93341 & 341 & & & & 0.5 & 0.5 & & & 2.6 & 3.1 \\
\hline $1 \mathrm{~B}$ & S & \#\#\#\#\#\#\#\# & 93342 & 342 & & & 0.5 & & & & 0.5 & & 0.5 \\
\hline $1 \mathrm{~B}$ & S & \#\#\#\#\#\#\#\# & 93343 & 343 & 0.5 & & & & & 0.5 & & & 0.5 \\
\hline $1 \mathrm{~B}$ & $S$ & \#\#\#\#\#\#\#\# & 93344 & 344 & & 0.5 & & & & 0.5 & & & \\
\hline $0 \mathrm{~B}$ & $S$ & \#\#\#\#\#\#\#\# & 93345 & 345 & & & 0.5 & & & 0.5 & & & 0.5 \\
\hline $0 \mathrm{~B}$ & $S$ & \#\#\#\#\#\#\#\# & 93346 & 346 & & 0.5 & & 0.5 & & & & 0.5 & \\
\hline \begin{tabular}{l|l}
11 & $B$ \\
\end{tabular} & $S$ & \#\#\#\#\#\#\#\# & 93347 & 347 & & & 0.5 & & & & & 0.5 & 0.5 \\
\hline \begin{tabular}{l|l}
11 & $B$ \\
\end{tabular} & $S$ & \#\#\#\#\#\#\#\# & 93348 & 348 & & 0.5 & & & & & 0.5 & & 5.2 \\
\hline $1 B$ & $S$ & \#\#\#\#\#\#\#\# & 93349 & 349 & & 0.5 & 0.5 & & & 0.5 & & & 2.6 \\
\hline
\end{tabular}




\begin{tabular}{|c|c|c|c|c|c|c|c|c|c|c|c|c|c|}
\hline 0 B & $S$ & $1 / 8 / 1994$ & 94008 & 8 & 2.1 & 0.5 & 0.5 & 0.5 & 0.5 & 0.5 & 0.5 & 0.5 & 0.5 \\
\hline $1 \mathrm{~B}$ & $S$ & \#\#\#\#\#\#\# & 94011 & 11 & & 0.5 & & & 0.5 & & 3.6 & 2.1 & 0.5 \\
\hline $1 \mathrm{~B}$ & S & \#\#\#\#\#\#\#\# & 94014 & 14 & 0.5 & & 0.5 & & 0.5 & & 3.6 & 3.1 & 3.6 \\
\hline $0 \mathrm{~B}$ & $\mathrm{~S}$ & \#\#\#\#\#\#\#\# & 94015 & 15 & 0.5 & & & 0.5 & & 0.5 & & 0.5 & 0.5 \\
\hline 0 B & S & \#\#\#\#\#\#\# & 94016 & 16 & & & 0.5 & & 0.5 & & & 0.5 & \\
\hline $1 \mathrm{~B}$ & $S$ & \#\#\#\#\#\#\#\# & 94019 & 19 & 0.5 & 0.5 & 0.5 & 0.5 & 0.5 & & 0.5 & 3.6 & 4.1 \\
\hline $1 \mathrm{~B}$ & S & \#\#\#\#\#\#\#\# & 94020 & 20 & 0.5 & & & 0.5 & 0.5 & & & 1 & 1 \\
\hline $1 \mathrm{~B}$ & S & \#\#\#\#\#\#\#\# & 94021 & 21 & 0.5 & & & 0.5 & & & 1 & 1.5 & 2.6 \\
\hline $0 \mathrm{~B}$ & $S$ & \#\#\#\#\#\#\#\# & 94022 & 22 & 0.5 & & & & 0.5 & & & & 0.5 \\
\hline 0 B & S & \#\#\#\#\#\#\#\# & 94023 & 23 & 0.5 & & & & 0.5 & & & & 0.5 \\
\hline $1 B$ & $S$ & \#\#\#\#\#\#\#\# & 94024 & 24 & & & 0.5 & & & 0.5 & & & \\
\hline $0 \mathrm{~B}$ & $S$ & \#\#\#\#\#\#\#\# & 94030 & 30 & & 0.5 & 0.5 & & & 0.5 & & 0.5 & \\
\hline $1 \mathrm{~B}$ & $\mathrm{~S}$ & \#\#\#\#\#\#\#\# & 94031 & 31 & 0.5 & 0.5 & & & 0.5 & 0.5 & 0.5 & 0.5 & 4.6 \\
\hline $1 B$ & $S$ & $2 / 1 / 1994$ & 94032 & 32 & 0.5 & 0.5 & 0.5 & 0.5 & 0.5 & 0.5 & 4.1 & 3.1 & 4.1 \\
\hline $1 \mathrm{~B}$ & $S$ & $2 / 2 / 1994$ & 94033 & 33 & 1 & 1 & 1 & 1 & 1 & 2.1 & 2.1 & 2.1 & 5.2 \\
\hline $1 \mathrm{~B}$ & $\mathrm{~S}$ & 2/3/1994 & 94034 & 34 & & & 0.5 & 0.5 & & 3.6 & 2.6 & 2.6 & 1.5 \\
\hline $1 \mathrm{~B}$ & S & 2/4/1994 & 94035 & 35 & & 0.5 & & & 0.5 & 3.6 & 2.6 & 2.1 & 1 \\
\hline $0 \mathrm{~B}$ & $S$ & $2 / 5 / 1994$ & 94036 & 36 & & & 0.5 & & 0.5 & 2.6 & 1 & 1 & 0.5 \\
\hline $0 \mathrm{~B}$ & $S$ & 2/6/1994 & 94037 & 37 & 0.5 & & 0.5 & & & 0.5 & & 0.5 & \\
\hline $1 \mathrm{~B}$ & $S$ & $2 / 7 / 1994$ & 94038 & 38 & 0.5 & & & & 0.5 & 0.5 & & & 0.5 \\
\hline $1 B$ & $S$ & 2/8/1994 & 94039 & 39 & & 0.5 & & & & 0.5 & & & 0.5 \\
\hline $1 \mathrm{~B}$ & $S$ & $2 / 9 / 1994$ & 94040 & 40 & 0.5 & & & & & & 0.5 & & \\
\hline $1 \mathrm{~B}$ & $S$ & \#\#\#\#\#\#\#\# & 94041 & 41 & 1 & 1 & 1 & 1 & 1 & 1 & 4.6 & 3.6 & 4.1 \\
\hline
\end{tabular}




\begin{tabular}{|c|c|c|c|c|c|c|c|c|c|c|c|c|c|}
\hline $1 \mathrm{~B}$ & $S$ & \#\#\#\#\#\#\# & 94045 & 45 & & 0.5 & 0.5 & & & 1.5 & 2.1 & 1.5 & 5.7 \\
\hline $1 \mathrm{~B}$ & $\mathrm{~S}$ & \#\#\#\#\#\#\#\# & 94048 & 48 & & & 0.5 & & & & 1.5 & 1.5 & 1 \\
\hline $0 \mathrm{~B}$ & S & \#\#\#\#\#\#\#\# & 94051 & 51 & & & & & 0.5 & & & 0.5 & \\
\hline $1 \mathrm{~B}$ & S & \#\#\#\#\#\#\#\# & 94053 & 53 & 0.5 & 0.5 & & & & & & 0.5 & \\
\hline $1 \mathrm{~B}$ & S & \#\#\#\#\#\#\#\# & 94054 & 54 & 0.5 & & & & & 0.5 & & & 5.2 \\
\hline $0 \mathrm{~B}$ & S & \#\#\#\#\#\#\#\# & 94057 & 57 & & & & 0.5 & 0.5 & & & 0.5 & \\
\hline $0 \mathrm{~B}$ & $S$ & \#\#\#\#\#\#\#\# & 94058 & 58 & & 0.5 & 0.5 & & & & 0.5 & 0.5 & \\
\hline $1 B$ & $\mathrm{~S}$ & \#\#\#\#\#\#\#\# & 94059 & 59 & & 0.5 & & 0.5 & & & 1.5 & 1.5 & 0.5 \\
\hline $1 \mathrm{~B}$ & $S$ & 3/1/1994 & 94060 & 60 & & 0.5 & & & 0.5 & 1.5 & 1 & 1 & 0.5 \\
\hline $1 \mathrm{~B}$ & $\mathrm{~S}$ & $3 / 2 / 1994$ & 94061 & 61 & & & 0.5 & & 0.5 & & & 0.5 & 5.2 \\
\hline $1 B$ & $S$ & $3 / 3 / 1994$ & 94062 & 62 & 0.5 & & 1 & & 0.5 & & & 0.5 & 5.2 \\
\hline $1 B$ & $\mathrm{~S}$ & $3 / 9 / 1994$ & 94068 & 68 & & 0.5 & & & & & 0.5 & 0.5 & 5.2 \\
\hline $1 B$ & $\mathrm{~S}$ & \#\#\#\#\#\#\#\# & 94069 & 69 & & 0.5 & & 0.5 & & 1.5 & 1.5 & 1.5 & 4.1 \\
\hline $1 B$ & $S$ & \#\#\#\#\#\#\#\# & 94080 & 80 & & 0.5 & & & & & 0.5 & & \\
\hline $1 \mathrm{~B}$ & S & \#\#\#\#\#\#\#\# & 94081 & 81 & & & 0.5 & & & & 0.5 & & \\
\hline $1 \mathrm{~B}$ & $S$ & \#\#\#\#\#\#\#\# & 94082 & 82 & 0.5 & & & & 0.5 & & & & 0.5 \\
\hline $1 \mathrm{~B}$ & S & \#\#\#\#\#\#\#\# & 94083 & 83 & & 0.5 & & & & & & 0.5 & \\
\hline $1 \mathrm{~B}$ & $S$ & \#\#\#\#\#\#\#\# & 94084 & 84 & & 0.5 & 0.5 & & & & & & 0.5 \\
\hline 0 B & $S$ & \#\#\#\#\#\#\#\# & 94085 & 85 & & 0.5 & & & & & & 0.5 & \\
\hline $0 \mathrm{~B}$ & $S$ & \#\#\#\#\#\#\#\# & 94086 & 86 & & & 0.5 & & & & & & 0.5 \\
\hline \begin{tabular}{l|l}
11 & $B$ \\
\end{tabular} & $S$ & \#\#\#\#\#\#\#\# & 94087 & 87 & & 0.5 & & 0.5 & & & & 0.5 & 0.5 \\
\hline \begin{tabular}{l|l}
11 & $B$ \\
\end{tabular} & $S$ & \#\#\#\#\#\#\#\# & 94088 & 88 & & 0.5 & & 0.5 & & & 0.5 & & 3.6 \\
\hline $1 B$ & $S$ & \#\#\#\#\#\#\#\# & 94089 & 89 & 0.5 & & 0.5 & & & & 0.5 & & 0.5 \\
\hline
\end{tabular}




\begin{tabular}{|c|c|c|c|c|c|c|c|c|c|c|c|c|c|}
\hline $1 \mathrm{~B}$ & $S$ & 4/7/1994 & 94097 & 97 & & & 0.5 & 0.5 & & & & 0.5 & 5.2 \\
\hline $0 \mathrm{~B}$ & S & \#\#\#\#\#\#\#\# & 94100 & 100 & & & & & & 0.5 & & & \\
\hline $1 \mathrm{~B}$ & S & \#\#\#\#\#\#\#\# & 94103 & 103 & & & & & 0.5 & & & & 0.5 \\
\hline $1 \mathrm{~B}$ & $\mathrm{~S}$ & \#\#\#\#\#\#\#\# & 94104 & 104 & & 0.5 & & & & & 0.5 & & \\
\hline $1 \mathrm{~B}$ & S & \#\#\#\#\#\#\#\# & 94105 & 105 & & & & & 0.5 & & & & \\
\hline $1 \mathrm{~B}$ & S & \#\#\#\#\#\#\#\# & 94108 & 108 & & & 0.5 & & & & & 0.5 & 0.5 \\
\hline $1 \mathrm{~B}$ & $S$ & \#\#\#\#\#\#\#\# & 94109 & 109 & & 0.5 & & & & 0.5 & & & \\
\hline $1 B$ & $\mathrm{~S}$ & \#\#\#\#\#\#\#\# & 94110 & 110 & & & & & & 0.5 & & 0.5 & \\
\hline $1 \mathrm{~B}$ & $S$ & \#\#\#\#\#\#\#\# & 94111 & 111 & & & 0.5 & & & & & & 0.5 \\
\hline $1 B$ & $S$ & \#\#\#\#\#\#\#\# & 94112 & 112 & & & & 0.5 & & & & & 0.5 \\
\hline $0 \mathrm{~B}$ & $\mathrm{~S}$ & \#\#\#\#\#\#\#\# & 94113 & 113 & & 0.5 & & & & 0.5 & & & \\
\hline $1 \mathrm{~B}$ & S & \#\#\#\#\#\#\# & 94119 & 119 & & & & & & 0.5 & & & \\
\hline $0 \mathrm{~B}$ & S & \#\#\#\#\#\#\#\# & 94120 & 120 & & & 0.5 & & & & 0.5 & & \\
\hline $0 \mathrm{~B}$ & S & 5/1/1994 & 94121 & 121 & 0.5 & 0.5 & & 0.5 & 0.5 & 0.5 & & 0.5 & \\
\hline $1 \mathrm{~B}$ & S & $5 / 2 / 1994$ & 94122 & 122 & & 0.5 & 0.5 & & 0.5 & & & 0.5 & \\
\hline $1 \mathrm{~B}$ & S & $5 / 3 / 1994$ & 94123 & 123 & & & & 0.5 & & & 0.5 & & 0.5 \\
\hline $1 \mathrm{~B}$ & S & $5 / 4 / 1994$ & 94124 & 124 & & & & 0.5 & & & & 0.5 & \\
\hline $1 \mathrm{~B}$ & $S$ & $5 / 5 / 1994$ & 94125 & 125 & & 0.5 & & & 0.5 & & & & 0.5 \\
\hline $1 \mathrm{~B}$ & $S$ & $5 / 6 / 1994$ & 94126 & 126 & 0.5 & & & & & 0.5 & & & \\
\hline $0 \mathrm{~B}$ & $S$ & $5 / 7 / 1994$ & 94127 & 127 & & 0.5 & & & & & & 0.5 & \\
\hline $0 \mathrm{~B}$ & $S$ & $5 / 8 / 1994$ & 94128 & 128 & 0.5 & & & & & & 0.5 & & \\
\hline \begin{tabular}{l|l}
11 & $B$ \\
\end{tabular} & $S$ & $5 / 9 / 1994$ & 94129 & 129 & & & & & 0.5 & & & & \\
\hline \begin{tabular}{l|l}
11 & $B$ \\
\end{tabular} & $S$ & \#\#\#\#\#\#\#\# & 94130 & 130 & & & & 0.5 & & & & & \\
\hline
\end{tabular}




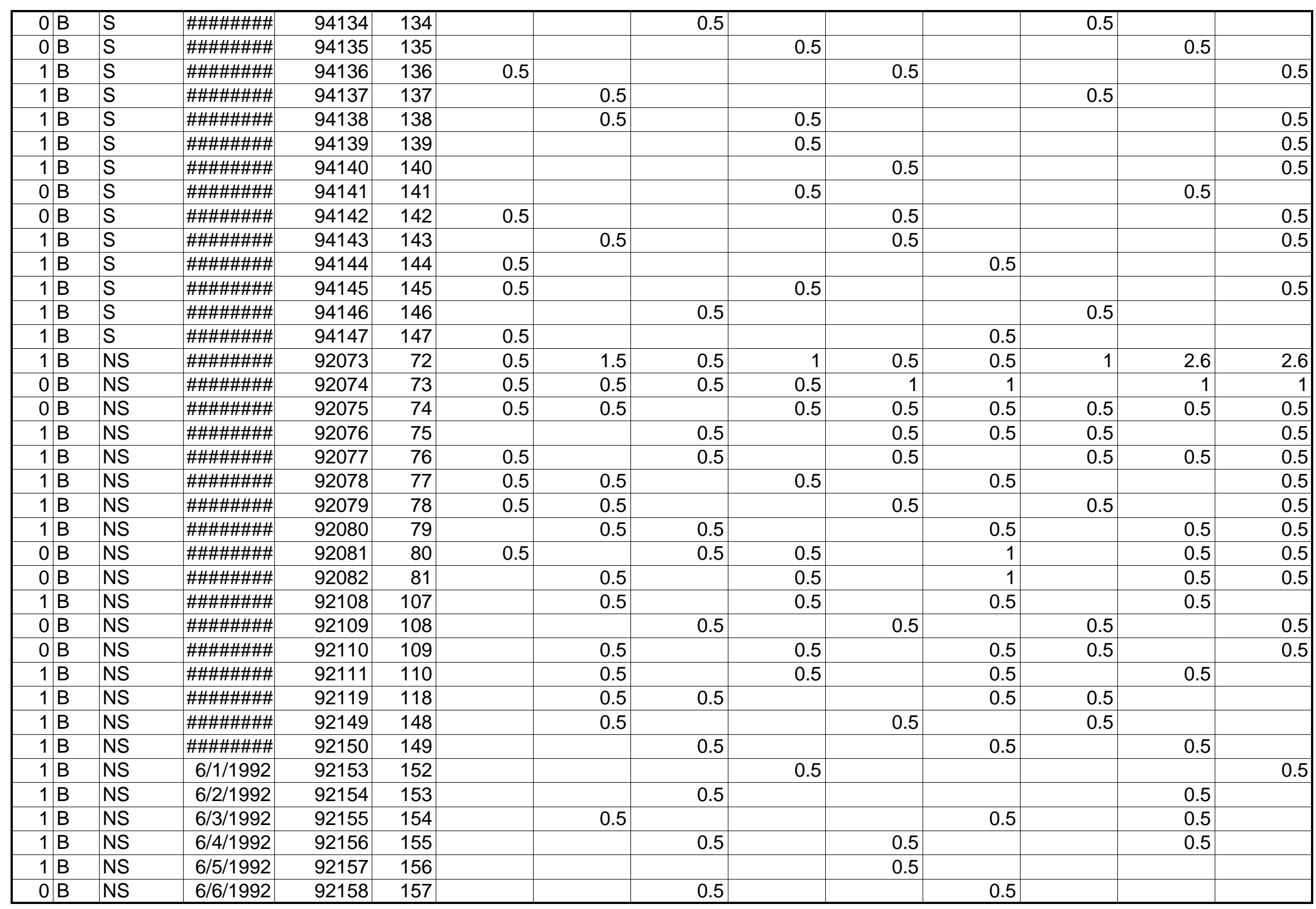




\begin{tabular}{|c|c|c|c|c|c|c|c|c|c|c|c|c|c|c|}
\hline 0 & & NS & 6/7/1992 & 92159 & 158 & & 0.5 & & & & & 0.5 & & \\
\hline 1 & B & NS & 6/8/1992 & 92160 & 159 & & & & 0.5 & & 0.5 & & & 0.5 \\
\hline 1 & B & NS & $6 / 9 / 1992$ & 92161 & 160 & 0.5 & & & 0.5 & & & 0.5 & & 0.5 \\
\hline 1 & B & NS & \#\#\#\#\#\#\#\# & 92162 & 161 & & & & 0.5 & & & 0.5 & & \\
\hline 1 & B & NS & \#\#\#\#\#\#\#\# & 92163 & 162 & 0.5 & & & & & & 0.5 & & \\
\hline 1 & $B$ & NS & \#\#\#\#\#\#\#\# & 92164 & 163 & & & & 0.5 & & 0.5 & & & \\
\hline 0 & B & NS & \#\#\#\#\#\#\#\# & 92165 & 164 & & 0.5 & & & & & 0.5 & & \\
\hline 0 & B & NS & \#\#\#\#\#\#\#\# & 92166 & 165 & & & & 0.5 & & & & & \\
\hline 1 & B & NS & \#\#\#\#\#\#\#\# & 92167 & 166 & & & 0.5 & & & & & 0.5 & \\
\hline 1 & B & NS & \#\#\#\#\#\#\#\# & 92168 & 167 & & 0.5 & & & & & & 0.5 & \\
\hline 1 & B & NS & \#\#\#\#\#\#\#\# & 92169 & 168 & & & & & 0.5 & & & & \\
\hline 1 & $B$ & NS & \#\#\#\#\#\#\#\# & 92170 & 169 & & & & 0.5 & & & & & 0.5 \\
\hline 1 & B & NS & \#\#\#\#\#\#\#\# & 92171 & 170 & 0.5 & & & & & & 0.5 & & \\
\hline 0 & B & NS & \#\#\#\#\#\#\#\# & 92172 & 171 & & & 0.5 & & & & & 0.5 & \\
\hline 0 & B & NS & \#\#\#\#\#\#\#\# & 92173 & 172 & & 0.5 & & & & & 0.5 & & \\
\hline 1 & B & NS & \#\#\#\#\#\#\#\# & 92174 & 173 & & & 0.5 & & & & & & 0.5 \\
\hline 1 & B & NS & \#\#\#\#\#\#\#\# & 92175 & 174 & 0.5 & & & & & 0.5 & & & 0.5 \\
\hline 1 & B & NS & \#\#\#\#\#\#\#\# & 92176 & 175 & & & & 0.5 & & & & 0.5 & \\
\hline 1 & $B$ & NS & \#\#\#\#\#\#\#\# & 92177 & 176 & 0.5 & & & & & & 0.5 & & \\
\hline 1 & B & NS & \#\#\#\#\#\#\#\# & 92178 & 177 & & & & & 0.5 & & & & \\
\hline 0 & B & NS & \#\#\#\#\#\#\#\# & 92179 & 178 & 0.5 & & & & & & 0.5 & & \\
\hline 0 & B & NS & \#\#\#\#\#\#\#\# & 92180 & 179 & 0.5 & & & & & & 0.5 & & \\
\hline 1 & B & NS & \#\#\#\#\#\#\#\# & 92181 & 180 & & & & & 0.5 & & & & \\
\hline 1 & B & NS & \#\#\#\#\#\#\#\# & 92182 & 181 & & & & & 0.5 & & & & \\
\hline 1 & B & NS & 7/1/1992 & 92183 & 182 & & 0.5 & & & & & & 0.5 & \\
\hline 1 & B & NS & 7/2/1992 & 92184 & 183 & & & & & & 0.5 & & & \\
\hline 1 & B & NS & $7 / 3 / 1992$ & 92185 & 184 & & & 0.5 & & & & & & 0.5 \\
\hline 0 & B & NS & 7/4/1992 & 92186 & 185 & & & 0.5 & & & & & 0.5 & \\
\hline 0 & $B$ & NS & $7 / 5 / 1992$ & 92187 & 186 & 0.5 & & & 0.5 & & & 0.5 & & 0.5 \\
\hline 1 & B & NS & $7 / 6 / 1992$ & 92188 & 187 & & 0.5 & & & & & 0.5 & & \\
\hline 1 & B & NS & $7 / 7 / 1992$ & 92189 & 188 & & 0.5 & & & & & & 0.5 & \\
\hline 1 & B & NS & $7 / 8 / 1992$ & 92190 & 189 & 0.5 & & & & & 0.5 & & & \\
\hline 1 & B & NS & $7 / 9 / 1992$ & 92191 & 190 & & & & 0.5 & & & & & 0.5 \\
\hline 1 & B & NS & \#\#\#\#\#\#\#\# & 92192 & 191 & & 0.5 & & & & & & 0.5 & \\
\hline 0 & $B$ & NS & \#\#\#\#\#\#\#\# & 92193 & 192 & & & & & & 0.5 & & & \\
\hline 0 & $B$ & NS & \#\#\#\#\#\#\#\# & 92194 & 193 & & & & 0.5 & & & & & \\
\hline 1 & $B$ & NS & \#\#\#\#\#\#\#\# & 92195 & 194 & & & 0.5 & & & & & 0.5 & \\
\hline
\end{tabular}




\begin{tabular}{|c|c|c|c|c|c|c|c|c|c|c|c|c|c|c|}
\hline 1 & B & NS & \#\#\#\#\#\#\#\# & 92196 & 195 & & 0.5 & & & & & & 0.5 & \\
\hline 1 & B & NS & \#\#\#\#\#\#\#\# & 92197 & 196 & & 0.5 & & & & & 0.5 & & \\
\hline 1 & B & NS & \#\#\#\#\#\#\#\# & 92198 & 197 & & & & 0.5 & & & & 0.5 & \\
\hline 1 & B & NS & \#\#\#\#\#\#\#\# & 92199 & 198 & & & & & 0.5 & & & & \\
\hline 0 & B & NS & \#\#\#\#\#\#\#\# & 92200 & 199 & & 0.5 & & & & & 0.5 & & \\
\hline 0 & B & NS & \#\#\#\#\#\#\#\# & 92201 & 200 & & & & 0.5 & & & & & 0.5 \\
\hline 1 & B & NS & \#\#\#\#\#\#\#\# & 92202 & 201 & & 0.5 & & & & & 0.5 & & \\
\hline 1 & B & NS & \#\#\#\#\#\#\#\# & 92203 & 202 & & & 0.5 & & 0.5 & & & & 0.5 \\
\hline 1 & B & NS & \#\#\#\#\#\#\#\# & 92204 & 203 & & & 0.5 & & & & & 0.5 & \\
\hline 1 & B & NS & \#\#\#\#\#\#\#\# & 92205 & 204 & & & & & 0.5 & & & & \\
\hline 1 & B & NS & \#\#\#\#\#\#\#\# & 92206 & 205 & 0.5 & & & & & 0.5 & & & \\
\hline 0 & $B$ & NS & \#\#\#\#\#\#\#\# & 92207 & 206 & & & 0.5 & & & & & 0.5 & \\
\hline 0 & B & NS & \#\#\#\#\#\#\#\# & 92208 & 207 & 0.5 & & & & & 0.5 & & & \\
\hline 1 & B & NS & \#\#\#\#\#\#\#\# & 92209 & 208 & & 0.5 & & & & & 0.5 & & \\
\hline 1 & $B$ & NS & \#\#\#\#\#\#\#\# & 92210 & 209 & & & & & & 0.5 & & & \\
\hline 1 & B & NS & \#\#\#\#\#\#\#\# & 92211 & 210 & & & 0.5 & & & & & & 0.5 \\
\hline 1 & B & NS & \#\#\#\#\#\#\#\# & 92212 & 211 & & 0.5 & & & & & & 0.5 & \\
\hline 1 & $B$ & NS & \#\#\#\#\#\#\#\# & 92213 & 212 & & 0.5 & & & & & & 0.5 & \\
\hline 0 & $B$ & NS & $8 / 1 / 1992$ & 92214 & 213 & & & & & & 0.5 & & & \\
\hline 0 & B & NS & $8 / 2 / 1992$ & 92215 & 214 & & & 0.5 & & & & 0.5 & & \\
\hline 1 & B & NS & 8/3/1992 & 92216 & 215 & & 0.5 & & & & & & 0.5 & \\
\hline 1 & B & NS & 8/4/1992 & 92217 & 216 & 0.5 & & & & & 0.5 & & 0.5 & \\
\hline 1 & B & NS & 8/5/1992 & 92218 & 217 & & & & 0.5 & & & & 0.5 & \\
\hline 1 & B & NS & 8/6/1992 & 92219 & 218 & & & & 0.5 & & & & & 0.5 \\
\hline 1 & B & NS & 8/7/1992 & 92220 & 219 & & & 0.5 & & & & & 0.5 & \\
\hline 0 & B & NS & 8/8/1992 & 92221 & 220 & & & & & 0.5 & & & & \\
\hline 0 & B & NS & 8/9/1992 & 92222 & 221 & & & 0.5 & & & & & 0.5 & \\
\hline 1 & B & NS & \#\#\#\#\#\#\#\# & 92223 & 222 & 0.5 & & & & & 0.5 & & & \\
\hline 1 & $B$ & NS & \#\#\#\#\#\#\#\# & 92224 & 223 & & 0.5 & & & & & & 0.5 & \\
\hline 1 & B & NS & \#\#\#\#\#\#\#\# & 92225 & 224 & & & & & 0.5 & & & & \\
\hline 1 & B & NS & \#\#\#\#\#\#\#\# & 92226 & 225 & & & 0.5 & & & & 0.5 & & 0.5 \\
\hline 1 & $B$ & NS & \#\#\#\#\#\#\#\# & 92227 & 226 & & 0.5 & & & & & 0.5 & & \\
\hline 0 & B & NS & \#\#\#\#\#\#\#\# & 92228 & 227 & & & 0.5 & & & & & 0.5 & \\
\hline 0 & B & NS & \#\#\#\#\#\#\#\# & 92229 & 228 & & & & & 0.5 & & & & \\
\hline 1 & $B$ & NS & \#\#\#\#\#\#\#\# & 92230 & 229 & & & & & 0.5 & & & & \\
\hline 1 & $B$ & NS & \#\#\#\#\#\#\#\# & 92231 & 230 & & 0.5 & & 0.5 & & & 0.5 & & \\
\hline 1 & $B$ & NS & \#\#\#\#\#\#\#\# & 92232 & 231 & 0.5 & & & 0.5 & & 0.5 & & & \\
\hline
\end{tabular}




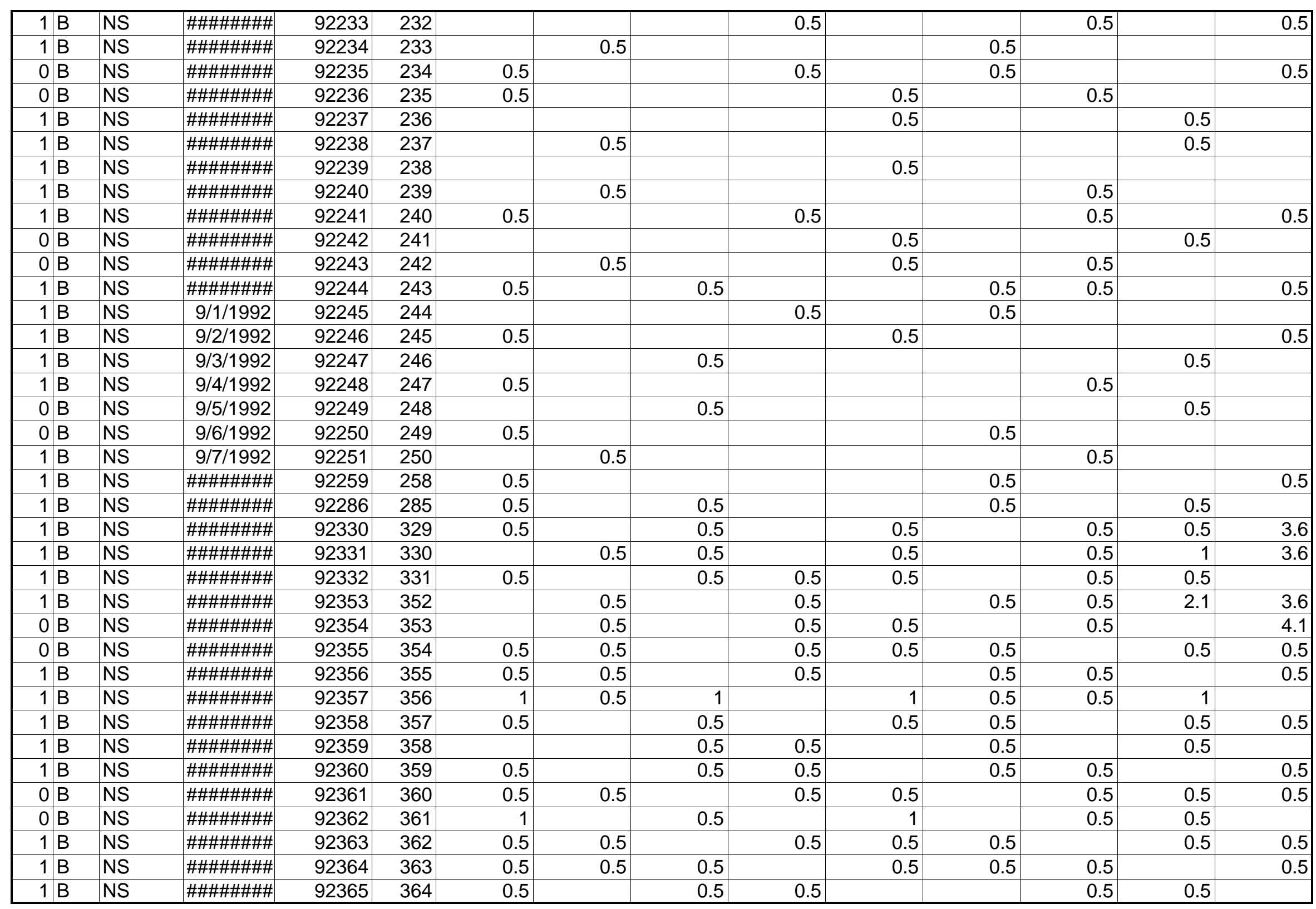




\begin{tabular}{|c|c|c|c|c|c|c|c|c|c|c|c|c|c|}
\hline $1 \mathrm{~B}$ & NS & \#\#\#\#\#\#\#\# & 92366 & 365 & & 0.5 & 0.5 & & 0.5 & & 0.5 & 0.5 & \\
\hline $0 \mathrm{~B}$ & NS & $1 / 3 / 1993$ & 93003 & 3 & 0.5 & 0.5 & & 0.5 & & 0.5 & & 0.5 & 0.5 \\
\hline $0 \mathrm{~B}$ & NS & \#\#\#\#\#\#\#\# & 93072 & 72 & 1 & 1 & 1 & 1 & 1 & 1 & 1 & 1 & 1 \\
\hline 0 B & NS & \#\#\#\#\#\#\#\# & 93073 & 73 & 1 & 1 & 0.5 & 1 & 1 & 1 & 0.5 & 0.5 & 1.5 \\
\hline $1 \mathrm{~B}$ & NS & \#\#\#\#\#\#\#\# & 93074 & 74 & 1 & 0.5 & 0.5 & 0.5 & 0.5 & 0.5 & 0.5 & 0.5 & 1 \\
\hline $1 \mathrm{~B}$ & NS & \#\#\#\#\#\#\#\# & 93077 & 77 & 0.5 & & 0.5 & & & & & 0.5 & \\
\hline $1 \mathrm{~B}$ & NS & \#\#\#\#\#\#\#\# & 93078 & 78 & 0.5 & & & 0.5 & & & & 0.5 & \\
\hline $0 \mathrm{~B}$ & NS & \#\#\#\#\#\#\#\# & 93079 & 79 & & 0.5 & & & 0.5 & & & 0.5 & \\
\hline $0 \mathrm{~B}$ & NS & \#\#\#\#\#\#\#\# & 93080 & 80 & & & & 0.5 & & 0.5 & & & \\
\hline $1 \mathrm{~B}$ & NS & $4 / 9 / 1993$ & 93099 & 99 & & & 0.5 & & & & 0.5 & & \\
\hline $0 \mathrm{~B}$ & NS & \#\#\#\#\#\#\#\# & 93100 & 100 & 0.5 & & & & 0.5 & & & & \\
\hline $0 \mathrm{~B}$ & NS & \#\#\#\#\#\#\#\# & 93149 & 149 & & 0.5 & & & & 0.5 & & & \\
\hline $0 \mathrm{~B}$ & NS & \#\#\#\#\#\#\#\# & 93150 & 150 & & & 0.5 & & & & & 0.5 & \\
\hline $1 \mathrm{~B}$ & NS & \#\#\#\#\#\#\#\# & 93151 & 151 & & & & 0.5 & & & & 0.5 & \\
\hline $1 \mathrm{~B}$ & NS & 6/1/1993 & 93152 & 152 & & & & 0.5 & & & & & 0.5 \\
\hline $1 \mathrm{~B}$ & NS & $6 / 2 / 1993$ & 93153 & 153 & & & 0.5 & & & & & 0.5 & \\
\hline $1 \mathrm{~B}$ & NS & $6 / 3 / 1993$ & 93154 & 154 & & & & & 0.5 & & & & \\
\hline $1 \mathrm{~B}$ & NS & 6/4/1993 & 93155 & 155 & & & & & 0.5 & & & & \\
\hline $0 \mathrm{~B}$ & NS & 6/5/1993 & 93156 & 156 & 0.5 & & & & & 0.5 & & & \\
\hline $0 \mathrm{~B}$ & NS & 6/6/1993 & 93157 & 157 & & & & 0.5 & & & & & 0.5 \\
\hline $1 B$ & NS & 6/7/1993 & 93158 & 158 & & 0.5 & & & & & 0.5 & & \\
\hline \begin{tabular}{l|l}
11 & $B$ \\
\end{tabular} & NS & $6 / 8 / 1993$ & 93159 & 159 & & & & & 0.5 & & & & \\
\hline \begin{tabular}{l|l}
11 & $B$ \\
\end{tabular} & NS & $6 / 9 / 1993$ & 93160 & 160 & 0.5 & & & & & 0.5 & & & \\
\hline
\end{tabular}




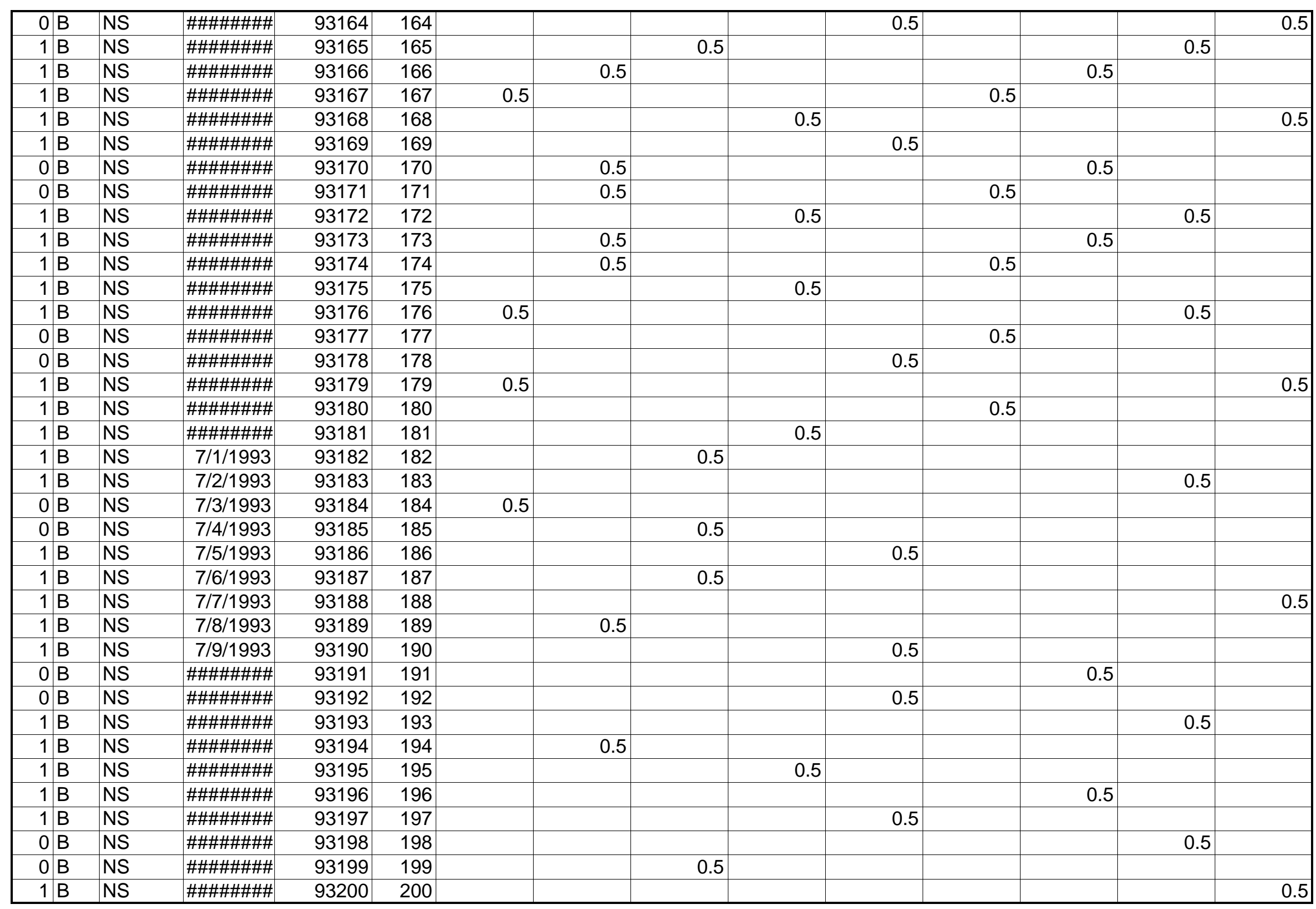




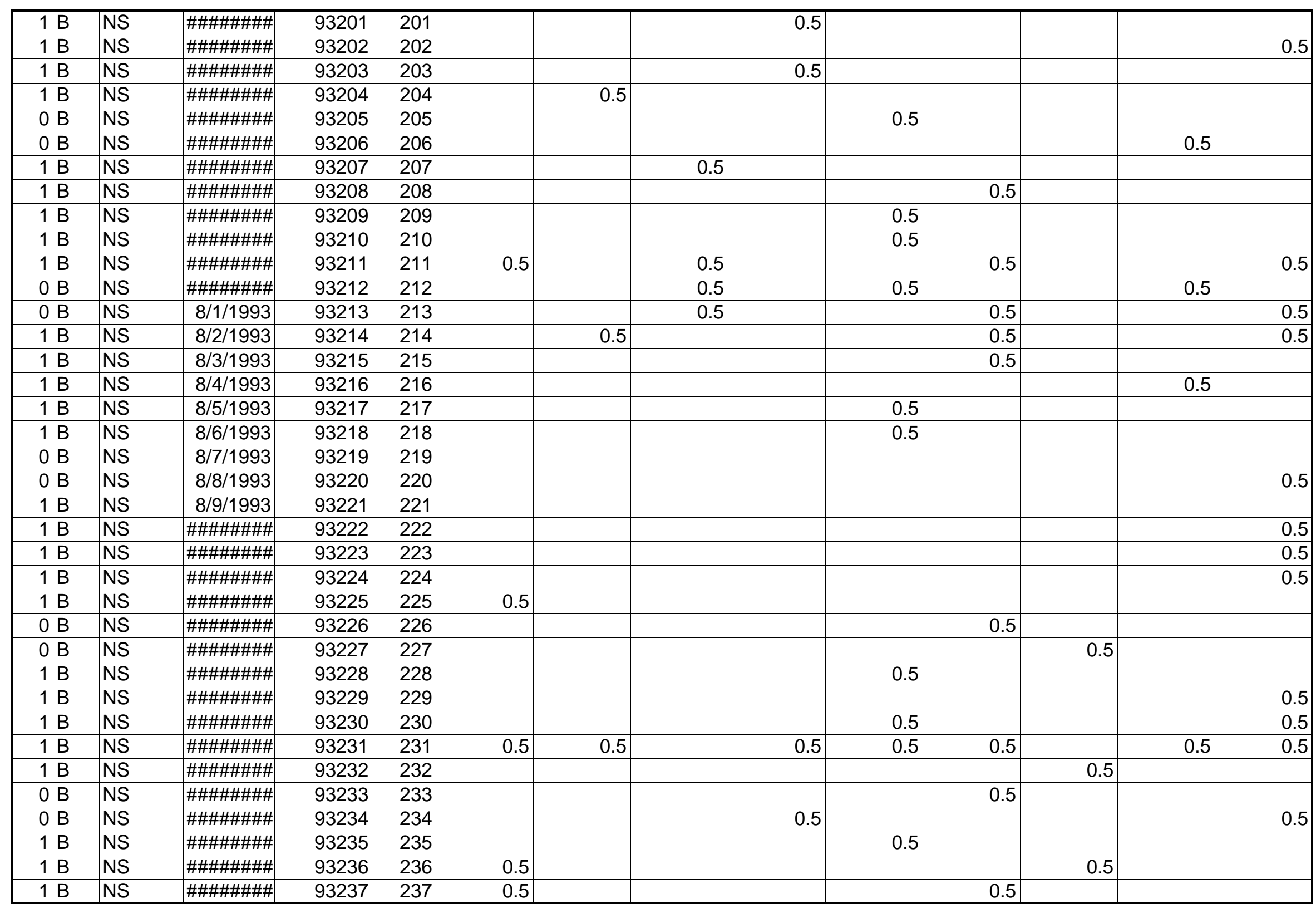




\begin{tabular}{|c|c|c|c|c|c|c|c|c|c|c|c|c|c|}
\hline $1 \mathrm{~B}$ & NS & \#\#\#\#\#\#\#\# & 93238 & 238 & & & & & 0.5 & & & & \\
\hline $0 \mathrm{~B}$ & NS & \#\#\#\#\#\#\#\# & 93241 & 241 & & & & & & 0.5 & & & \\
\hline $1 \mathrm{~B}$ & NS & 9/1/1993 & 93244 & 244 & & & & 0.5 & & & & & 0.5 \\
\hline $1 \mathrm{~B}$ & NS & 9/2/1993 & 93245 & 245 & & & & 0.5 & & & & 0.5 & \\
\hline $1 \mathrm{~B}$ & NS & 9/3/1993 & 93246 & 246 & & & & & 0.5 & & & & \\
\hline $1 \mathrm{~B}$ & NS & 9/6/1993 & 93249 & 249 & & & 0.5 & & 0.5 & & & & 0.5 \\
\hline $1 \mathrm{~B}$ & NS & 9/7/1993 & 93250 & 250 & & 0.5 & & & 0.5 & & & 0.5 & \\
\hline $1 \mathrm{~B}$ & NS & 9/8/1993 & 93251 & 251 & & 0.5 & & 0.5 & & & 0.5 & & 0.5 \\
\hline $1 B$ & NS & \#\#\#\#\#\#\#\# & 93315 & 315 & 0.5 & & & 0.5 & & 0.5 & & & 0.5 \\
\hline $1 \mathrm{~B}$ & NS & \#\#\#\#\#\#\#\# & 93328 & 328 & & & & & 0.5 & & & & 0.5 \\
\hline $1 B$ & NS & \#\#\#\#\#\#\#\# & 93329 & 329 & & 0.5 & & & & 0.5 & & 0.5 & \\
\hline $0 \mathrm{~B}$ & NS & \#\#\#\#\#\#\#\# & 93353 & 353 & & 0.5 & & 0.5 & & & 0.5 & & 0.5 \\
\hline $1 \mathrm{~B}$ & NS & \#\#\#\#\#\#\#\# & 93354 & 354 & 0.5 & 0.5 & & 0.5 & 0.5 & & 0.5 & 0.5 & \\
\hline $1 \mathrm{~B}$ & NS & \#\#\#\#\#\#\#\# & 93355 & 355 & & 0.5 & & & 0.5 & & 0.5 & 0.5 & 0.5 \\
\hline $1 \mathrm{~B}$ & NS & \#\#\#\#\#\#\#\# & 93356 & 356 & & & 0.5 & & 0.5 & & & 1 & \\
\hline $1 \mathrm{~B}$ & NS & \#\#\#\#\#\#\#\# & 93357 & 357 & 0.5 & 0.5 & 1 & & 0.5 & & 0.5 & 0.5 & \\
\hline $1 \mathrm{~B}$ & NS & \#\#\#\#\#\#\#\# & 93358 & 358 & & 1 & & 1 & 0.5 & 0.5 & 0.5 & 1 & 0.5 \\
\hline $0 \mathrm{~B}$ & NS & \#\#\#\#\#\#\#\# & 93359 & 359 & 0.5 & 0.5 & 1 & 0.5 & 0.5 & 0.5 & 0.5 & 0.5 & 0.5 \\
\hline $0 \mathrm{~B}$ & NS & \#\#\#\#\#\#\#\# & 93360 & 360 & 0.5 & 0.5 & & 1 & 0.5 & 0.5 & 0.5 & 0.5 & 0.5 \\
\hline $1 \mathrm{~B}$ & NS & \#\#\#\#\#\#\#\# & 93361 & 361 & 0.5 & 0.5 & 0.5 & 0.5 & 0.5 & 0.5 & 0.5 & 1 & 0.5 \\
\hline \begin{tabular}{l|l}
11 & $B$ \\
\end{tabular} & NS & \#\#\#\#\#\#\#\# & 93362 & 362 & & 0.5 & & & & & & 0.5 & \\
\hline \begin{tabular}{l|l}
11 & $B$ \\
\end{tabular} & NS & \#\#\#\#\#\#\#\# & 93363 & 363 & & & 0.5 & & & 0.5 & & 0.5 & 0.5 \\
\hline $1 \mathrm{~B}$ & NS & \#\#\#\#\#\#\#\# & 93364 & 364 & & & & 0.5 & 0.5 & & 0.5 & 0.5 & \\
\hline
\end{tabular}




\begin{tabular}{|c|c|c|c|c|c|c|c|c|c|c|c|c|c|c|}
\hline $1 \mathrm{~B}$ & & NS & $1 / 3 / 1994$ & 94003 & 3 & & 0.5 & & 0.5 & & 0.5 & & 2.1 & 5.2 \\
\hline $1 \mathrm{~B}$ & $B$ & NS & \#\#\#\#\#\#\#\# & 94052 & 52 & & 0.5 & & & & & 0.5 & & 0.5 \\
\hline $1 \mathrm{~B}$ & B & NS & \#\#\#\#\#\#\#\# & 94070 & 70 & 0.5 & & 0.5 & & 0.5 & 0.5 & & 0.5 & 4.6 \\
\hline $0 \mathrm{~B}$ & $B$ & NS & \#\#\#\#\#\#\#\# & 94072 & 72 & & 0.5 & & & 0.5 & & & 0.5 & \\
\hline $1 \mathrm{~B}$ & & NS & \#\#\#\#\#\#\#\# & 94073 & 73 & & 0.5 & 0.5 & 0.5 & 0.5 & 0.5 & & 0.5 & 0.5 \\
\hline $1 \mathrm{~B}$ & & NS & \#\#\#\#\#\#\#\# & 94074 & 74 & 0.5 & & 0.5 & 0.5 & & & 0.5 & & 0.5 \\
\hline $1 \mathrm{~B}$ & B & NS & \#\#\#\#\#\#\#\# & 94077 & 77 & & 0.5 & 0.5 & & & 0.5 & & 0.5 & \\
\hline $0 \mathrm{~B}$ & $B$ & NS & \#\#\#\#\#\#\#\# & 94078 & 78 & 0.5 & & & & & 0.5 & & & \\
\hline $0 \mathrm{~B}$ & $B$ & NS & \#\#\#\#\#\#\#\# & 94079 & 79 & & & & & 0.5 & & & 0.5 & \\
\hline $1 \mathrm{~B}$ & $B$ & NS & $4 / 1 / 1994$ & 94091 & 91 & & & & 0.5 & & & & 0.5 & \\
\hline $0 \mathrm{~B}$ & $B$ & NS & $4 / 2 / 1994$ & 94092 & 92 & & & & 0.5 & & & & & 0.5 \\
\hline $0 \mathrm{~B}$ & $B$ & NS & $4 / 3 / 1994$ & 94093 & 93 & & & & 0.5 & & & & 0.5 & \\
\hline $1 \mathrm{~B}$ & & NS & \#\#\#\#\#\#\#\# & 94151 & 151 & & & & & 0.5 & & & & \\
\hline $1 \mathrm{~B}$ & & NS & $6 / 1 / 1994$ & 94152 & 152 & & & 0.5 & & & & & 0.5 & \\
\hline $1 \mathrm{~B}$ & & NS & $6 / 2 / 1994$ & 94153 & 153 & 0.5 & & & & & 0.5 & & & \\
\hline $1 \mathrm{~B}$ & & NS & $6 / 3 / 1994$ & 94154 & 154 & & & & 0.5 & & & & 0.5 & \\
\hline \multirow[t]{9}{*}{$0 \mathrm{~B}$} & B & NS & $6 / 4 / 1994$ & 94155 & 155 & 0.5 & & & & 0.5 & & & 0.5 & \\
\hline & & & & & & & & & & & & & & \\
\hline & & & & & \multicolumn{3}{|c|}{ sort S/NS - 0/1 - A/B } & & & & & & & \\
\hline & & & & & \multicolumn{2}{|c|}{ Daily Averages } & & & & & & & & \\
\hline & & & & & $1-A-S$ & 0.518182 & 0.589744 & 0.561404 & 0.561224 & 0.557692 & 0.564815 & 0.580645 & 2.081818 & 1.74625 \\
\hline & & & & & 1-B-S & 0.52069 & 0.521875 & 0.536765 & 0.528571 & 0.525316 & 0.645963 & 1.098235 & 1.295477 & 1.618072 \\
\hline & & & & & $0-A-S$ & 0.672222 & 0.670833 & 0.671429 & 0.63125 & 0.709091 & 0.578947 & 0.686364 & 1.113636 & 1.244444 \\
\hline & & & & & $0-B-S$ & 0.535593 & 0.517544 & 0.546296 & 0.517544 & 0.534483 & 0.562121 & 0.556452 & 0.682192 & 1.047059 \\
\hline & & & & & $1-A-N S$ & 0.719048 & 0.646429 & 0.736364 & 0.635294 & 0.664286 & 0.639394 & 0.658621 & 0.905556 & 1.7375 \\
\hline
\end{tabular}




\begin{tabular}{|c|c|c|c|c|c|c|c|c|c|c|c|c|c|}
\hline sort & & & & & & & & & & & & & \\
\hline Tabl & f Monthly & nptio & & sort S/I & $\mathrm{VS}-0 / 1-\mathrm{A}$ & & & & & & & & \\
\hline & & & & Daily S & \begin{tabular}{l|l} 
ums \\
\end{tabular} & & & & & & & & \\
\hline & Jun 91 & 216 & 216 & & & & & & & & & & \\
\hline & Jul & 437 & 437 & $1-A-S$ & 26.6 & 25.1 & 31.1 & 25.1 & 28.6 & 27.5 & 35.1 & 130.7 & 99 \\
\hline & Aug & 783 & 783 & 1-B-S & 94.1 & 106.6 & 100.7 & 103.6 & 105.1 & 116.1 & 128.7 & 283.3 & 404.1 \\
\hline & Sep & 398 & 398 & $0-A-S$ & 5.5 & 5.5 & 5.5 & 2.5 & 5.5 & 7 & 20.4 & 16.4 & 20.3 \\
\hline & Oct & 274 & 274 & $0-B-S$ & 38.6 & 34.5 & 34 & 33 & 41 & 57.6 & 108.2 & 121 & 201.6 \\
\hline & Nov & 110 & 110 & $1-A-N S$ & 15.5 & 15.5 & 16 & 13 & 16 & 36.6 & 41.2 & 48.9 & 107.2 \\
\hline & Dec & 171 & 171 & 1-B-NS & 37.5 & 37 & 30.5 & 34.5 & 34 & 37 & 35.5 & 45.7 & 50.5 \\
\hline & Jan 92 & 571 & 571 & $0-A-N S$ & 1.5 & 2 & 2.5 & 2.5 & 3 & 1.5 & 1 & 2.5 & 2 \\
\hline & Feb & 700 & 700 & $0-B-N S$ & 11.5 & 15.5 & 13 & 15.5 & 18 & 14.5 & 17.6 & 25.8 & 37 \\
\hline & Mar & 222 & 222 & & & & & & & & & & \\
\hline & Apr & 104 & 104 & & & & & & & & & & \\
\hline & May & 111 & 111 & sort S/R & VS - $0 / 1-A /$ & & & & & & & & \\
\hline & Jun & 287 & 287 & Standa & rd Deviation & of Daily $A$ & verages & & & & & & \\
\hline & Jul & 862 & 862 & & & & & & & & & & \\
\hline & Aug & 400 & 400 & $1-A-S$ & 0.319835 & 0.50535 & 0.25693 & 0.268137 & 0.277495 & 0.194525 & 0.292642 & 1.236899 & 1.339552 \\
\hline & Sep & 107 & 107 & 1-B-S & 0.296551 & 0.255985 & 0.339754 & 0.299855 & 0.302448 & 0.29081 & 0.572708 & 1.275519 & 1.814697 \\
\hline & Oct & 210 & 210 & $0-A-S$ & 0 & 0 & 0 & 0 & 0 & 0 & 1.785293 & 1.005741 & 1.151231 \\
\hline & Nov & 450 & 450 & $0-B-S$ & 0.21296 & 0.08573 & 0.105726 & 0.107335 & 0.096178 & 0.769332 & 1.398108 & 1.043243 & 1.615214 \\
\hline & Dec & 183 & 183 & $1-A-N S$ & 0.12894 & 0.12894 & 0.154967 & 0.141165 & 0.126854 & 1.058757 & 1.034193 & 0.928138 & 1.874318 \\
\hline & Jan 93 & 217 & 217 & 1-B-NS & 0.129699 & 0.144606 & 0.09126 & 0.104948 & 0.136239 & 0.130577 & 0.084497 & 0.309427 & 0.816275 \\
\hline & Feb & 133 & 133 & $0-A-N S$ & 0 & 0 & 0 & 0 & 0 & 0 & 0 & 0 & 0 \\
\hline & Mar & 164 & 164 & $0-B-N S$ & 0 & 0.091287 & 0.141165 & 0.12894 & 0 & 0 & 0.80613 & 0.604507 & 1.110273 \\
\hline & Apr & 396 & 396 & & & & & & & & & & \\
\hline & May & 82 & 82 & & & & & & & & & & \\
\hline & Jun & 406 & 406 & & & & & & & & & & \\
\hline & Jul & 101 & 101 & & & & & & & & & & \\
\hline & Aug & 72 & 72 & & & & & & & & & & \\
\hline & Sep & 199 & 199 & & & & & & & & & & \\
\hline & Oct & 120 & 120 & & & & & & & & & & \\
\hline & Nov & 52 & 52 & & & & & & & & & & \\
\hline & Dec & 105 & 105 & & & & & & & & & & \\
\hline & Jan 94 & 253 & 253 & & & & & & & & & & \\
\hline & Feb & 149 & 149 & & & & & & & & & & \\
\hline & Mar & 129 & 129 & & & & & & & & & & \\
\hline
\end{tabular}


wbheat

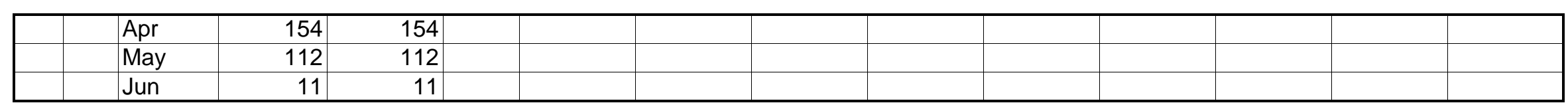




\begin{tabular}{|c|c|c|c|c|c|c|c|c|c|c|c|c|}
\hline 9 & 10 & 11 & 12 & 13 & 14 & 15 & 16 & 17 & 18 & 19 & 20 & 21 \\
\hline 0.5 & 0.5 & 0.5 & 0.5 & 0.5 & 1 & 0.5 & 0.5 & 0.5 & & 0.5 & & 0.5 \\
\hline 1 & & 1 & 0.5 & 1 & 0.5 & 0.5 & 0.5 & 0.5 & & & & \\
\hline 0.5 & 0.5 & 0.5 & 0.5 & 0.5 & 1 & 0.5 & & 0.5 & & 0.5 & & \\
\hline \multirow[t]{3}{*}{0.5} & 0.5 & 0.5 & 0.5 & 1 & 0.5 & 0.5 & 0.5 & 0.5 & 0.5 & & 0.5 & \\
\hline & 0.5 & & 0.5 & 0.5 & & 0.5 & & 0.5 & & 0.5 & 0.5 & \\
\hline & 0.5 & 0.5 & & 0.5 & & 0.5 & & 0.5 & 0.5 & 0.5 & & 0.5 \\
\hline 0.5 & 0.5 & 0.5 & 0.5 & 0.5 & 1 & 0.5 & 0.5 & & 0.5 & & 0.5 & 0.5 \\
\hline 0.5 & 1.5 & 1.5 & 0.5 & 0.5 & & 0.5 & 0.5 & & 0.5 & & 0.5 & 0.5 \\
\hline 1 & 0.5 & 0.5 & 0.5 & 1 & 0.5 & 0.5 & 0.5 & & 0.5 & 0.5 & & 0.5 \\
\hline 0.5 & 0.5 & 0.5 & & 0.5 & 0.5 & 0.5 & & 0.5 & 0.5 & 0.5 & & 0.5 \\
\hline 0.5 & 0.5 & & 0.5 & 0.5 & 0.5 & 0.5 & & & 0.5 & & & 0.5 \\
\hline \multirow{4}{*}{1} & & 0.5 & 0.5 & & 0.5 & & 1 & 0.5 & & & & 0.5 \\
\hline & & 0.5 & & & & & 0.5 & & & & & 0.5 \\
\hline & 0.5 & & 0.5 & & 1 & 0.5 & & & & & 0.5 & \\
\hline & 0.5 & 1 & & 0.5 & & 0.5 & 1 & & 0.5 & & 0.5 & 0.5 \\
\hline 0.5 & & 0.5 & 0.5 & 0.5 & 0.5 & & 0.5 & & 0.5 & & & \\
\hline 0.5 & 1 & & 0.5 & 1 & 0.5 & 0.5 & & 0.5 & 0.5 & & 0.5 & \\
\hline \multirow[t]{3}{*}{0.5} & 0.5 & 0.5 & 0.5 & 0.5 & 1 & 0.5 & & & 0.5 & & 0.5 & \\
\hline & 0.5 & & & & 0.5 & & & 0.5 & & & & \\
\hline & & & 0.5 & & & & 0.5 & & & & & 0.5 \\
\hline 0.5 & 0.5 & & 0.5 & & 1 & 0.5 & 0.5 & & 0.5 & & 0.5 & \\
\hline 0.5 & & 0.5 & 0.5 & 0.5 & 0.5 & & 0.5 & 0.5 & 0.5 & & 0.5 & \\
\hline 0.5 & 1 & 0.5 & 0.5 & 0.5 & & 0.5 & & 0.5 & & & & 0.5 \\
\hline 0.5 & 0.5 & 0.5 & 0.5 & 0.5 & & 0.5 & & 0.5 & 0.5 & 0.5 & & 0.5 \\
\hline 0.5 & 0.5 & & 0.5 & 0.5 & 0.5 & 0.5 & 0.5 & & & & & 0.5 \\
\hline 0.5 & & & & 0.5 & & & & & 0.5 & & & \\
\hline 0.5 & & & 0.5 & & & & & 0.5 & & & & \\
\hline \multirow[t]{3}{*}{1} & 0.5 & & 0.5 & & 1 & & 0.5 & & & 0.5 & & \\
\hline & & & & & & & & & & & & \\
\hline & & & & & & & & 0.5 & & & & \\
\hline \multirow[t]{2}{*}{0.5} & & 0.5 & & 0.5 & 0.5 & 0.5 & 0.5 & & & 0.5 & & \\
\hline & 0.5 & & 0.5 & 0.5 & 0.5 & & 0.5 & & & & & 0.5 \\
\hline 0.5 & & 0.5 & & & & 0.5 & & & & & 0.5 & \\
\hline
\end{tabular}


wbheat

\begin{tabular}{|c|c|c|c|c|c|c|c|c|c|c|c|c|}
\hline & 0.5 & & & 0.5 & & 0.5 & & & & 0.5 & & \\
\hline 0.5 & 0.5 & & 0.5 & 1.5 & & 0.5 & 0.5 & & & 0.5 & & 0.5 \\
\hline 0.5 & 0.5 & & 0.5 & 0.5 & 0.5 & 0.5 & & 0.5 & & & 0.5 & \\
\hline 0.5 & 0.5 & & 0.5 & 0.5 & & 0.5 & 0.5 & 0.5 & & & 0.5 & \\
\hline \multirow{2}{*}{0.5} & & 0.5 & 0.5 & 0.5 & 0.5 & & 0.5 & 0.5 & & 0.5 & & 0.5 \\
\hline & 0.5 & & 0.5 & 0.5 & 0.5 & & 0.5 & & & 0.5 & & \\
\hline \multirow[t]{2}{*}{1} & 0.5 & & 0.5 & & & 0.5 & 0.5 & 0.5 & & & & \\
\hline & & 0.5 & & & & & 0.5 & & & & & 0.5 \\
\hline \multirow[t]{2}{*}{0.5} & & 0.5 & & 1 & & 0.5 & & 0.5 & 0.5 & 0.5 & & 0.5 \\
\hline & 0.5 & 0.5 & & 0.5 & 0.5 & & 0.5 & & 0.5 & 0.5 & & 0.5 \\
\hline 0.5 & 0.5 & & 1 & & 0.5 & & 0.5 & 0.5 & & & & 0.5 \\
\hline 0.5 & 0.5 & & 0.5 & 0.5 & & 0.5 & 0.5 & 0.5 & 0.5 & & 0.5 & \\
\hline 0.5 & 0.5 & & & 1 & 0.5 & & 0.5 & & 0.5 & & 0.5 & 0.5 \\
\hline \multirow{2}{*}{0.5} & & & & 0.5 & & & & & 0.5 & & & 0.5 \\
\hline & 0.5 & & & 0.5 & & & & 0.5 & & & & 0.5 \\
\hline 0.5 & 0.5 & 0.5 & 0.5 & & 1 & & 1 & 0.5 & & 0.5 & 0.5 & \\
\hline 1 & 0.5 & & & 0.5 & 1 & & & 0.5 & & & 0.5 & \\
\hline 0.5 & & 0.5 & & 0.5 & & & & & 0.5 & & & \\
\hline \multirow[t]{5}{*}{1} & 0.5 & & 0.5 & & & & 0.5 & & & & 0.5 & \\
\hline & & & 0.5 & & & & 0.5 & & & & 0.5 & \\
\hline & & & 0.5 & & & & & 0.5 & & & & \\
\hline & 0.5 & 1.5 & 0.5 & 0.5 & & & & & 0.5 & & & \\
\hline & 0.5 & & & 0.5 & & & & 0.5 & & & 0.5 & \\
\hline 0.5 & 0.5 & 0.5 & 0.5 & 0.5 & & 1 & 0.5 & & 0.5 & 0.5 & & 0.5 \\
\hline 0.5 & 0.5 & 1 & & 1 & 1.5 & 0.5 & 1.5 & 0.5 & 0.5 & 0.5 & 0.5 & 0.5 \\
\hline \multirow[t]{2}{*}{4.6} & 4.6 & 3.6 & 3.6 & 3.6 & 2.1 & 0.5 & 1 & 0.5 & 0.5 & 0.5 & 0.5 & 0.5 \\
\hline & 0.5 & 1 & 1 & 0.5 & & 0.5 & 0.5 & & 0.5 & 0.5 & 0.5 & 0.5 \\
\hline 1 & 0.5 & 1 & 1 & 0.5 & 1 & 0.5 & 0.5 & 0.5 & 0.5 & 1 & 0.5 & 1 \\
\hline 5.7 & 4.6 & 4.6 & 5.2 & 4.1 & 4.6 & 4.1 & 3.6 & 3.6 & 2.6 & 2.6 & 2.1 & 1 \\
\hline 4.6 & 4.1 & 3.6 & 4.1 & 4.1 & 3.6 & 3.1 & 2.6 & 0.5 & 0.5 & & 1 & \\
\hline 2.1 & 1 & 1 & 0.5 & & 0.5 & 1 & 0.5 & 0.5 & 0.5 & & 0.5 & \\
\hline 1 & 0.5 & 0.5 & & 0.5 & & 0.5 & & 0.5 & 0.5 & & 0.5 & \\
\hline 4.6 & 4.6 & 4.1 & 4.1 & 3.6 & 4.1 & 4.1 & 2.6 & 0.5 & 1 & 0.5 & 1 & 0.5 \\
\hline 2.6 & 2.1 & 1.5 & 0.5 & 0.5 & & 0.5 & 0.5 & & 0.5 & 0.5 & & 0.5 \\
\hline 0.5 & 0.5 & 0.5 & & 0.5 & & 0.5 & & 0.5 & & 0.5 & 0.5 & 0.5 \\
\hline 1 & 1 & 0.5 & 1.5 & 0.5 & & 1 & 0.5 & & 0.5 & & 0.5 & \\
\hline 0.5 & 0.5 & 0.5 & 1 & & 0.5 & 0.5 & & 0.5 & & 0.5 & & 0.5 \\
\hline
\end{tabular}




\begin{tabular}{|c|c|c|c|c|c|c|c|c|c|c|c|c|}
\hline & 0.5 & 0.5 & & 0.5 & 0.5 & & 1 & 0.5 & 0.5 & 0.5 & & 0.5 \\
\hline 0.5 & 0.5 & 0.5 & 0.5 & 0.5 & 0.5 & & 0.5 & 1 & & 0.5 & 0.5 & \\
\hline 0.5 & 0.5 & 0.5 & & 0.5 & 0.5 & 0.5 & & 0.5 & & 0.5 & & 0.5 \\
\hline & & 0.5 & & & 0.5 & & & & 0.5 & & & 0.5 \\
\hline 0.5 & & 0.5 & & 0.5 & & & 0.5 & & 0.5 & 0.5 & & 0.5 \\
\hline 1 & & 0.5 & 0.5 & 0.5 & 0.5 & 0.5 & 0.5 & & 0.5 & 0.5 & 0.5 & 0.5 \\
\hline 0.5 & 0.5 & & 0.5 & 0.5 & 1 & & 0.5 & 0.5 & 0.5 & & 0.5 & 0.5 \\
\hline 1 & 0.5 & 1 & 0.5 & 0.5 & 1.5 & 1 & 0.5 & & 0.5 & 0.5 & & 0.5 \\
\hline 4.6 & 3.6 & 1 & 0.5 & 1 & 0.5 & 0.5 & & & 0.5 & 0.5 & & 0.5 \\
\hline 1 & 0.5 & 0.5 & 0.5 & 0.5 & 0.5 & & 0.5 & & & 0.5 & & 0.5 \\
\hline 1 & 0.5 & 0.5 & 1 & 0.5 & 0.5 & 0.5 & 0.5 & & 0.5 & & 0.5 & 0.5 \\
\hline 1 & 0.5 & 0.5 & & 0.5 & 0.5 & 0.5 & & 0.5 & & 0.5 & & 0.5 \\
\hline 3.1 & 2.1 & 1 & 0.5 & 1.5 & 0.5 & 2.6 & 3.6 & 0.5 & 0.5 & & & 0.5 \\
\hline 3.6 & 0.5 & & 0.5 & 0.5 & 0.5 & & & & 0.5 & & & \\
\hline & 0.5 & & & & & 0.5 & & & & & 0.5 & \\
\hline 0.5 & 0.5 & 0.5 & 0.5 & 0.5 & & 1 & & 0.5 & 0.5 & 1 & & 0.5 \\
\hline 4.6 & 4.6 & 5.2 & 4.6 & 5.2 & 4.6 & 4.1 & 4.1 & 1 & 0.5 & 0.5 & & 0.5 \\
\hline 5.2 & 5.2 & 5.2 & 3.6 & 4.1 & 3.6 & 2.1 & 1 & 1 & 4.1 & 4.1 & 2.6 & 2.1 \\
\hline 4.1 & 4.1 & 2.6 & 1 & 1 & 1 & 1 & 1 & 1 & 1 & 1 & 0.5 & 0.5 \\
\hline 1.5 & 1 & 0.5 & 0.5 & 1 & 1 & 0.5 & & & 0.5 & & 0.5 & \\
\hline 1.5 & & 0.5 & 1.5 & & 1 & 0.5 & & 0.5 & 0.5 & 0.5 & 0.5 & 0.5 \\
\hline 0.5 & & & & 0.5 & & & 0.5 & & & & 0.5 & \\
\hline & & 0.5 & & 0.5 & & & & & 0.5 & & & \\
\hline 0.5 & & 0.5 & & 0.5 & 0.5 & 0.5 & & & 0.5 & & & 0.5 \\
\hline 0.5 & 0.5 & 0.5 & & 1 & 0.5 & 0.5 & & 0.5 & & 0.5 & & 0.5 \\
\hline 0.5 & 0.5 & & 0.5 & 1 & & 0.5 & 1 & 0.5 & 0.5 & & 0.5 & 0.5 \\
\hline 0.5 & 1 & 0.5 & 0.5 & 0.5 & & 1 & & & 0.5 & & & 0.5 \\
\hline 0.5 & 0.5 & & 0.5 & 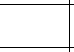 & 1 & 0.5 & & & 0.5 & & 0.5 & \\
\hline & & 0.5 & & 0.5 & & & 0.5 & & & 5.2 & 4.1 & 1 \\
\hline 0.5 & & 0.5 & & & & 0.5 & & & & 0.5 & 0.5 & \\
\hline 4.1 & 5.2 & 4.1 & 1.5 & 1 & 2.1 & 1 & 0.5 & & 0.5 & 0.5 & & 0.5 \\
\hline 4.1 & 2.1 & 1 & 1 & 1 & 1 & 1 & & 0.5 & 0.5 & & & 0.5 \\
\hline 1 & 0.5 & 0.5 & 0.5 & 0.5 & 1 & 1.5 & 0.5 & 0.5 & & 0.5 & & 0.5 \\
\hline 0.5 & 0.5 & 0.5 & 0.5 & 0.5 & 0.5 & 0.5 & 0.5 & & 0.5 & 0.5 & & 0.5 \\
\hline 1 & 1.5 & 0.5 & 1 & 1 & 1 & 0.5 & 1 & & 0.5 & 0.5 & & 0.5 \\
\hline 1 & 0.5 & 0.5 & & 0.5 & & 0.5 & & & 0.5 & & 0.5 & \\
\hline 0.5 & & 0.5 & 0.5 & 0.5 & 0.5 & 0.5 & 1 & & 0.5 & & 0.5 & 0.5 \\
\hline
\end{tabular}


wbheat

\begin{tabular}{|c|c|c|c|c|c|c|c|c|c|c|c|c|}
\hline 4.6 & 4.6 & 3.6 & 4.1 & 3.6 & 2.6 & 1 & 0.5 & & 0.5 & 0.5 & & 0.5 \\
\hline 4.6 & 3.6 & 3.6 & 2.6 & 2.6 & 1 & 1 & 1.5 & 3.1 & 1.5 & 1.5 & 1 & \\
\hline 0.5 & 0.5 & 0.5 & 0.5 & 0.5 & 0.5 & 0.5 & & 0.5 & 0.5 & 0.5 & 0.5 & 0.5 \\
\hline 0.5 & 1 & & & 0.5 & 1 & & 0.5 & 0.5 & & 0.5 & 0.5 & \\
\hline 4.1 & 3.6 & 2.1 & 2.1 & 3.1 & 2.6 & 2.6 & 3.1 & 1.5 & & 0.5 & 0.5 & 1 \\
\hline 3.6 & 4.1 & 4.1 & 3.6 & 4.6 & 3.6 & 2.6 & 3.1 & 2.1 & 1.5 & 1.5 & 1.5 & 0.5 \\
\hline 4.6 & 4.1 & 3.6 & 3.6 & 3.1 & 2.1 & 4.1 & 3.1 & 3.1 & 3.1 & 2.6 & 3.1 & 3.6 \\
\hline 4.1 & 4.6 & 3.1 & 4.1 & 2.1 & 2.1 & 3.6 & 3.6 & 2.6 & 2.6 & 3.1 & 3.1 & 3.1 \\
\hline 4.6 & 3.6 & 4.1 & 4.1 & 2.6 & 3.6 & 3.6 & 3.1 & 3.6 & 4.1 & 3.1 & 3.6 & 3.6 \\
\hline 1 & 1 & 1 & 0.5 & 0.5 & 1 & 0.5 & 0.5 & 0.5 & & 0.5 & 0.5 & 0.5 \\
\hline 4.6 & 4.6 & 4.6 & 4.1 & 4.6 & 3.6 & 4.1 & 3.1 & 0.5 & 0.5 & 0.5 & & 0.5 \\
\hline \multirow{2}{*}{4.1} & 3.6 & 3.6 & 4.1 & 3.1 & 2.1 & 0.5 & 1 & 4.6 & 3.6 & 2.1 & 1.5 & 0.5 \\
\hline & 1 & 0.5 & & 1 & 0.5 & & 0.5 & & 0.5 & 0.5 & & 0.5 \\
\hline 4.1 & 3.6 & 1.5 & 1.5 & 1 & 1 & 0.5 & 1 & 0.5 & 0.5 & 0.5 & & 0.5 \\
\hline 3.6 & 3.6 & 1 & 0.5 & 0.5 & 0.5 & 0.5 & 0.5 & 3.1 & 3.1 & 3.6 & 2.1 & 2.1 \\
\hline 3.6 & 3.1 & 3.1 & 2.6 & 2.1 & 2.6 & 2.1 & 2.6 & 2.1 & 1.5 & 2.1 & 1.5 & \\
\hline 0.5 & 0.5 & & 0.5 & & 0.5 & 0.5 & & 0.5 & 0.5 & 0.5 & & 0.5 \\
\hline 0.5 & 0.5 & 1 & 1 & 0.5 & 1 & 0.5 & 1 & 0.5 & & 0.5 & 0.5 & \\
\hline 0.5 & 0.5 & 0.5 & 0.5 & 1 & 3.6 & 4.1 & 3.6 & 3.6 & 3.1 & 2.1 & 2.6 & 2.1 \\
\hline \multirow[t]{2}{*}{3.6} & 1.5 & 1 & 2.6 & 3.6 & 1 & 0.5 & 1 & 0.5 & & 0.5 & 0.5 & \\
\hline & 0.5 & 0.5 & 0.5 & & 0.5 & 0.5 & & 0.5 & & 0.5 & 0.5 & \\
\hline \multirow[t]{3}{*}{6.2} & 6.2 & 5.7 & 5.7 & 5.2 & 6.2 & 5.7 & 6.2 & 5.2 & 5.2 & 0.5 & & 0.5 \\
\hline & & 0.5 & & & & & & 0.5 & & & & \\
\hline & & & 0.5 & & & 0.5 & & & 0.5 & & & 0.5 \\
\hline 0.5 & & & 0.5 & & & & 0.5 & & & 0.5 & & \\
\hline 9.8 & 8.8 & 8.8 & 8.8 & 7.7 & 7.7 & 7.2 & 8.2 & 7.2 & 5.2 & & 0.5 & \\
\hline \multirow[t]{4}{*}{7.7} & 8.2 & 7.7 & 8.2 & 8.2 & 7.7 & 8.2 & 7.7 & 7.2 & 3.1 & 0.5 & & \\
\hline & & & & & 0.5 & & & & & & 0.5 & \\
\hline & 0.5 & & & & & & 0.5 & & & & & 0.5 \\
\hline & & & & 0.5 & & & & 0.5 & & & & \\
\hline \multirow[t]{7}{*}{0.5} & & & 0.5 & & & & & 0.5 & & & 0.5 & \\
\hline & 0.5 & & & & & & 0.5 & & & 0.5 & & \\
\hline & & & & & & & & & & & & \\
\hline & & & & & & & & & & & & \\
\hline & & & & & & & 0.5 & & & & & 0.5 \\
\hline & & 0.5 & & & & 0.5 & & & & & 0.5 & \\
\hline & & & & 0.5 & & & & & & 0.5 & & \\
\hline
\end{tabular}


wbheat

\begin{tabular}{|c|c|c|c|c|c|c|c|c|c|c|c|c|}
\hline & & & 0.5 & & & 0.5 & & & & & & 0.5 \\
\hline & & & & & 0.5 & & & & & 0.5 & & \\
\hline 8.8 & 8.8 & 8.8 & 9.3 & 6.7 & 5.7 & 5.2 & 4.6 & 4.1 & 1.5 & & 0.5 & \\
\hline \multirow[t]{3}{*}{5.2} & 2.6 & 0.5 & & & & 0.5 & & & & & & 0.5 \\
\hline & & & & 0.5 & & & 0.5 & & & & & \\
\hline & & 0.5 & & & & & 0.5 & & & & & \\
\hline \multirow[t]{8}{*}{0.5} & & & & & 0.5 & & & 0.5 & & & & \\
\hline & & 0.5 & & 0.5 & 0.5 & & 0.5 & 0.5 & & & & 0.5 \\
\hline & & & & 0.5 & & & & & 0.5 & & & \\
\hline & & & & 0.5 & & & & & 0.5 & & & \\
\hline & 0.5 & & & & 0.5 & & & & & 0.5 & & \\
\hline & 0.5 & & & & & 0.5 & & & & & & 0.5 \\
\hline & & 0.5 & & & & & 0.5 & & & & & \\
\hline & & & & 0.5 & & & & & & 0.5 & & \\
\hline \multirow[t]{2}{*}{0.5} & & & & & & & & & & & 0.5 & \\
\hline & & 0.5 & & & & & 0.5 & & & & & \\
\hline \multirow[t]{5}{*}{0.5} & & & & & 0.5 & & & & 0.5 & & & \\
\hline & & & & 0.5 & & & & & 0.5 & & & \\
\hline & & & & & 0.5 & & & & & 0.5 & & \\
\hline & & 0.5 & & & & & 0.5 & & & & & \\
\hline & & & 0.5 & & & & & & 0.5 & & & \\
\hline \multirow[t]{16}{*}{0.5} & & & & & & 0.5 & & & & & 0.5 & \\
\hline & 0.5 & & & & & 0.5 & & & & & & 0.5 \\
\hline & & & 0.5 & & & & & & 0.5 & & & \\
\hline & & 0.5 & & & & & & 0.5 & & & & \\
\hline & & & & & 0.5 & & & & & & 0.5 & \\
\hline & & & 0.5 & & & 0.5 & & & & & & 0.5 \\
\hline & & & & & 0.5 & & & & & & 0.5 & \\
\hline & & 0.5 & & & & & & 0.5 & & & & \\
\hline & 0.5 & & & & & & 0.5 & & & & 0.5 & \\
\hline & & & & 0.5 & & & & 0.5 & & & & \\
\hline & & 0.5 & & & 0.5 & & & & & & 0.5 & \\
\hline & & & 0.5 & & & & & & 0.5 & & & \\
\hline & & & & & & & & & & & & \\
\hline & & & & & & & & & 0.5 & & & \\
\hline & & & & & 0.5 & & & & & & 0.5 & \\
\hline & & & & & 0.5 & & & & & & 0.5 & \\
\hline
\end{tabular}

Page 37 
wbheat

\begin{tabular}{|c|c|c|c|c|c|c|c|c|c|c|c|c|}
\hline & & & & 0.5 & & & & & & 0.5 & & \\
\hline & & 0.5 & & & & & 0.5 & & & & & \\
\hline \multirow[t]{8}{*}{0.5} & & & & 0.5 & & & & & & 0.5 & & \\
\hline & & & 0.5 & & & & & & 0.5 & & & \\
\hline & & 0.5 & & & & 0.5 & & & & & 0.5 & \\
\hline & & & & & 0.5 & & & & & & 0.5 & \\
\hline & & 0.5 & & & & & & 0.5 & & & 0.5 & \\
\hline & & 0.5 & & & 0.5 & & & & & 0.5 & & \\
\hline & & & 0.5 & & & & & 0.5 & & & & \\
\hline & & & & 0.5 & & & & & 0.5 & & & \\
\hline \multirow[t]{3}{*}{0.5} & & & & & & 0.5 & & & & & 0.5 & \\
\hline & 0.5 & & & & & & 0.5 & & & & & 0.5 \\
\hline & & 0.5 & & & & & & 0.5 & & & & \\
\hline \multirow[t]{3}{*}{0.5} & & & & & & 0.5 & & & & & & 0.5 \\
\hline & & 0.5 & & & & & 0.5 & & & & & \\
\hline & & & & & 0.5 & & & 0.5 & & & & \\
\hline \multirow[t]{6}{*}{0.5} & & & & & & 0.5 & & & & & 0.5 & \\
\hline & & 0.5 & & 0.5 & & 0.5 & & 0.5 & & 0.5 & & 0.5 \\
\hline & 0.5 & & 0.5 & & 0.5 & 0.5 & & 0.5 & & 0.5 & & 0.5 \\
\hline & 0.5 & & 0.5 & & 0.5 & & 0.5 & & 0.5 & & 0.5 & 0.5 \\
\hline & 0.5 & & & 0.5 & 0.5 & & 0.5 & & 0.5 & & 0.5 & 0.5 \\
\hline & 0.5 & & 0.5 & & 0.5 & & 0.5 & 0.5 & & 0.5 & & 0.5 \\
\hline 2.1 & 1 & 0.5 & 0.5 & & 0.5 & 0.5 & 0.5 & 0.5 & & 0.5 & & 0.5 \\
\hline 0.5 & 0.5 & 0.5 & 0.5 & 0.5 & 0.5 & 0.5 & 0.5 & & 0.5 & 0.5 & & 0.5 \\
\hline 1 & 0.5 & 0.5 & 0.5 & 0.5 & 0.5 & 0.5 & 0.5 & 0.5 & & 0.5 & 0.5 & \\
\hline 0.5 & 0.5 & 0.5 & 0.5 & 0.5 & 0.5 & 0.5 & 1 & 0.5 & 0.5 & 0.5 & 0.5 & 0.5 \\
\hline 0.5 & 0.5 & 0.5 & 0.5 & 0.5 & 0.5 & 0.5 & 0.5 & 0.5 & 0.5 & 0.5 & 0.5 & 0.5 \\
\hline \multirow[t]{2}{*}{0.5} & 0.5 & 0.5 & 0.5 & & 0.5 & 0.5 & & 0.5 & & 0.5 & & 0.5 \\
\hline & 0.5 & & 0.5 & 0.5 & & 0.5 & 0.5 & & 0.5 & & 0.5 & 0.5 \\
\hline 0.5 & 1 & 1.5 & 1 & 1 & 0.5 & 0.5 & 0.5 & & 0.5 & 0.5 & & 0.5 \\
\hline 0.5 & 0.5 & 0.5 & 1 & 0.5 & 0.5 & 0.5 & 0.5 & 0.5 & & 0.5 & & 0.5 \\
\hline 1 & 0.5 & 0.5 & 0.5 & 0.5 & 0.5 & 0.5 & 0.5 & 0.5 & & 0.5 & 0.5 & \\
\hline \multirow[t]{4}{*}{1.5} & 0.5 & 0.5 & 0.5 & 0.5 & 0.5 & 1 & 0.5 & 0.5 & 0.5 & 0.5 & 0.5 & 0.5 \\
\hline & 0.5 & & 0.5 & & 0.5 & & 0.5 & 0.5 & & 0.5 & 0.5 & \\
\hline & 0.5 & 0.5 & & 0.5 & & 0.5 & 0.5 & & 0.5 & & 0.5 & \\
\hline & 0.5 & & 0.5 & 0.5 & & 0.5 & & 0.5 & & 0.5 & 0.5 & \\
\hline 0.5 & 0.5 & & & 0.5 & & & & & 0.5 & & & \\
\hline
\end{tabular}


wbheat

\begin{tabular}{|c|c|c|c|c|c|c|c|c|c|c|c|c|}
\hline & 0.5 & & 0.5 & \begin{tabular}{|c|}
0.5 \\
\end{tabular} & 0.5 & & & & 0.5 & & & \\
\hline & & & 0.5 & & & & & 0.5 & & & & \\
\hline & 0.5 & & & & & 0.5 & & & & & 0.5 & \\
\hline & 0.5 & 0.5 & 0.5 & & 1 & 0.5 & & 0.5 & & 0.5 & & 0.5 \\
\hline 0.5 & & 0.5 & & & 0.5 & & 0.5 & & 0.5 & & 0.5 & \\
\hline 0.5 & & 0.5 & & & 0.5 & & 0.5 & & 0.5 & & 0.5 & \\
\hline 0.5 & & 0.5 & & 0.5 & & 0.5 & & & 0.5 & & 0.5 & \\
\hline & 0.5 & 0.5 & 0.5 & 0.5 & 0.5 & 0.5 & 0.5 & 0.5 & 0.5 & 0.5 & 0.5 & 0.5 \\
\hline 0.5 & 0.5 & 0.5 & & 0.5 & 0.5 & 0.5 & 0.5 & 0.5 & 0.5 & 0.5 & & 0.5 \\
\hline 0.5 & 0.5 & 0.5 & 0.5 & 1 & 0.5 & 0.5 & 0.5 & 0.5 & 0.5 & 0.5 & 0.5 & 0.5 \\
\hline 0.5 & 0.5 & 1 & 0.5 & 0.5 & 0.5 & 1 & 0.5 & 0.5 & & 0.5 & 0.5 & 0.5 \\
\hline 1 & & 0.5 & 0.5 & 0.5 & 0.5 & & 0.5 & & 0.5 & & 0.5 & \\
\hline 0.5 & 0.5 & 0.5 & 0.5 & 0.5 & & 0.5 & & 0.5 & & 0.5 & & 0.5 \\
\hline 4.1 & 3.6 & 4.1 & 3.1 & 0.5 & 1 & 0.5 & 1.5 & 3.1 & 2.1 & 1.5 & 1.5 & \\
\hline 2.6 & 0.5 & 1 & 1 & 3.1 & 1 & & 0.5 & & 0.5 & & 0.5 & \\
\hline & & & & & & & & & & & & \\
\hline 0.5 & 0.5 & & 0.5 & & 0.5 & & 0.5 & & & 0.5 & & 0.5 \\
\hline 0.5 & 0.5 & 0.5 & 0.5 & & 0.5 & 2.6 & 4.6 & 3.6 & 2.1 & 2.1 & 1.5 & 1.5 \\
\hline 2.1 & 2.1 & 11.8 & 1 & & & 0.5 & & & 0.5 & & & 0.5 \\
\hline 0.5 & 0.5 & 0.5 & & 0.5 & & 0.5 & 3.6 & 4.1 & 3.1 & 2.1 & 1.5 & 1.5 \\
\hline 1.5 & 1.5 & 0.5 & 0.5 & & 0.5 & & & 0.5 & & 0.5 & & 0.5 \\
\hline 5.2 & 5.2 & 5.2 & 4.1 & 4.1 & 4.6 & 3.6 & 3.6 & 2.6 & 2.6 & 3.1 & 2.6 & 2.1 \\
\hline 0.5 & & 0.5 & & 0.5 & & & 0.5 & & & & 0.5 & \\
\hline 0.5 & & & & & 0.5 & & & & 0.5 & & & \\
\hline & 0.5 & 0.5 & & 0.5 & & 0.5 & & 0.5 & 0.5 & 0.5 & & 0.5 \\
\hline 0.5 & 0.5 & & 0.5 & & 0.5 & & 0.5 & 0.5 & & 0.5 & 0.5 & \\
\hline 0.5 & & 0.5 & 0.5 & 0.5 & & 0.5 & 1 & & 0.5 & 0.5 & 0.5 & \\
\hline & & 0.5 & 0.5 & 0.5 & & 0.5 & 0.5 & 0.5 & & 0.5 & & 0.5 \\
\hline & 0.5 & 1 & & 1.5 & 0.5 & 1 & 2.1 & 5.2 & 3.6 & 3.6 & 2.1 & 1.5 \\
\hline 4.1 & 3.6 & 1.5 & 0.5 & 1 & & 1 & 0.5 & & 0.5 & 0.5 & 0.5 & 0.5 \\
\hline 4.1 & 3.1 & 3.1 & 2.1 & 0.5 & 0.5 & 0.5 & 0.5 & 0.5 & 0.5 & 0.5 & 0.5 & 0.5 \\
\hline 3.6 & 3.6 & 1.5 & 0.5 & 0.5 & 0.5 & 0.5 & 0.5 & 3.6 & 2.6 & 2.1 & 0.5 & \\
\hline 3.1 & 2.1 & 0.5 & 0.5 & 0.5 & & 0.5 & & 0.5 & & 0.5 & 0.5 & \\
\hline 0.5 & 0.5 & & 0.5 & 0.5 & 0.5 & & & 0.5 & 0.5 & & 0.5 & 0.5 \\
\hline 1 & 0.5 & & 0.5 & 0.5 & 0.5 & & 0.5 & & 0.5 & 0.5 & & 0.5 \\
\hline 0.5 & 0.5 & 0.5 & & & 0.5 & 0.5 & 0.5 & & 0.5 & & 0.5 & 0.5 \\
\hline 0.5 & & 0.5 & 0.5 & & 0.5 & & 0.5 & 0.5 & & 0.5 & 0.5 & \\
\hline
\end{tabular}




\begin{tabular}{|c|c|c|c|c|c|c|c|c|c|c|c|c|}
\hline & 0.5 & & 0.5 & & 0.5 & & 0.5 & & 0.5 & 0.5 & & 0.5 \\
\hline 0.5 & & 0.5 & & 0.5 & 0.5 & & & 0.5 & & 0.5 & & 0.5 \\
\hline \multirow{3}{*}{0.5} & & & 0.5 & & & 0.5 & & & 0.5 & & 0.5 & \\
\hline & 0.5 & & 0.5 & & & 0.5 & & 0.5 & & 0.5 & & 0.5 \\
\hline & & 0.5 & & & 0.5 & & & 0.5 & & 0.5 & 0.5 & \\
\hline \multirow{5}{*}{0.5} & 0.5 & & & 0.5 & & & 0.5 & & 0.5 & & 0.5 & \\
\hline & 1 & 0.5 & & 0.5 & & 0.5 & & 0.5 & 0.5 & & 0.5 & \\
\hline & 0.5 & 0.5 & 0.5 & & 0.5 & 0.5 & & 0.5 & & 0.5 & 0.5 & \\
\hline & 1 & & 0.5 & 0.5 & & 0.5 & & 0.5 & 0.5 & & 0.5 & 0.5 \\
\hline & 0.5 & & 0.5 & & & 0.5 & & & 0.5 & & 0.5 & \\
\hline 0.5 & & 0.5 & 0.5 & & & 0.5 & & 0.5 & & & 0.5 & 0.5 \\
\hline 0.5 & & 0.5 & & 0.5 & 0.5 & & 1 & 0.5 & & 0.5 & 0.5 & \\
\hline 4.6 & 3.6 & 4.1 & 3.1 & 1 & 3.6 & 3.6 & 3.1 & 1 & 1 & 0.5 & 1 & 0.5 \\
\hline 3.1 & 1 & 5.2 & 4.6 & 1 & 1 & 1 & 0.5 & 1 & 0.5 & 0.5 & 3.6 & 3.1 \\
\hline 3.1 & 0.5 & 0.5 & 0.5 & 0.5 & & 0.5 & & & 0.5 & 0.5 & & \\
\hline 0.5 & 0.5 & & 0.5 & 0.5 & & 0.5 & & 0.5 & & 0.5 & & 0.5 \\
\hline 0.5 & & 0.5 & & 0.5 & & & 0.5 & & & 0.5 & & \\
\hline 0.5 & & 0.5 & & 0.5 & & & & 0.5 & & 0.5 & & 0.5 \\
\hline 0.5 & & 0.5 & & 0.5 & & 0.5 & & 0.5 & 0.5 & & & 0.5 \\
\hline 0.5 & 0.5 & & 0.5 & & 0.5 & 0.5 & & 0.5 & & 0.5 & & 0.5 \\
\hline \multirow[t]{2}{*}{0.5} & & 0.5 & & 0.5 & & 1 & & & & 0.5 & & 0.5 \\
\hline & 0.5 & 0.5 & & 0.5 & & 0.5 & & & & 0.5 & & 0.5 \\
\hline \multirow[t]{4}{*}{0.5} & & 0.5 & & & 0.5 & & & 0.5 & & & 0.5 & \\
\hline & 0.5 & & & 0.5 & & & & 0.5 & & & 0.5 & \\
\hline & & 0.5 & & & 0.5 & & & 0.5 & & 0.5 & & \\
\hline & & 0.5 & 0.5 & & 0.5 & & 0.5 & & & 0.5 & & 0.5 \\
\hline 4.6 & 3.6 & 3.1 & 1 & 1 & 0.5 & 1 & 1.5 & 3.6 & 2.6 & 1 & 1 & 1 \\
\hline 2.6 & 1 & 4.6 & 4.6 & 3.1 & 3.1 & 1 & 1 & 1 & 1.5 & 0.5 & 1 & 1 \\
\hline 0.5 & & & & 0.5 & & & 0.5 & & & 0.5 & & 0.5 \\
\hline 4.1 & 1.5 & 0.5 & 1 & & 0.5 & 0.5 & & 0.5 & & 0.5 & & \\
\hline \multirow[t]{2}{*}{0.5} & 0.5 & 0.5 & & 0.5 & & & 0.5 & & 0.5 & & & 0.5 \\
\hline & 0.5 & 0.5 & & & & 0.5 & & 0.5 & & 0.5 & & \\
\hline 0.5 & 0.5 & & & 0.5 & & 0.5 & & & 0.5 & & 0.5 & \\
\hline 0.5 & 0.5 & & & 0.5 & 0.5 & & 0.5 & 0.5 & & 0.5 & & 0.5 \\
\hline 0.5 & 0.5 & & & 1 & & 0.5 & & 0.5 & 0.5 & 0.5 & & 1 \\
\hline 0.5 & & & 0.5 & & 0.5 & & & & & 0.5 & & 0.5 \\
\hline 0.5 & 0.5 & 0.5 & & 0.5 & 0.5 & & & 0.5 & & 0.5 & & 0.5 \\
\hline
\end{tabular}


wbheat

\begin{tabular}{|c|c|c|c|c|c|c|c|c|c|c|c|c|}
\hline 0.5 & 0.5 & & 0.5 & & 0.5 & & 0.5 & 0.5 & & 0.5 & & 0.5 \\
\hline 0.5 & 0.5 & & 0.5 & 0.5 & & 0.5 & & 0.5 & & 0.5 & 0.5 & 0.5 \\
\hline 4.6 & 4.1 & 1 & 1 & 1 & 3.6 & 3.6 & 1.5 & 0.5 & & 1 & 0.5 & 1 \\
\hline 2.1 & 3.1 & 0.5 & 0.5 & & 0.5 & 0.5 & 0.5 & & & 0.5 & 0.5 & \\
\hline \multirow{2}{*}{0.5} & 0.5 & & & 0.5 & & & & 0.5 & 0.5 & & 0.5 & \\
\hline & 0.5 & & 0.5 & & 0.5 & & 0.5 & 0.5 & & 0.5 & 0.5 & \\
\hline \multirow[t]{4}{*}{0.5} & & 0.5 & & & 0.5 & & 0.5 & & 0.5 & & & 0.5 \\
\hline & 0.5 & & 0.5 & & 0.5 & 0.5 & & & & & 0.5 & \\
\hline & & 0.5 & & 0.5 & & 0.5 & 0.5 & & & 0.5 & & \\
\hline & 0.5 & 0.5 & & & 0.5 & 0.5 & & & 0.5 & & & 0.5 \\
\hline 0.5 & & & 0.5 & & & 0.5 & & 0.5 & & 0.5 & & 0.5 \\
\hline 0.5 & & 0.5 & & & & & 0.5 & & & 0.5 & & \\
\hline \multirow[t]{6}{*}{0.5} & & 0.5 & & & & 0.5 & & & & & 0.5 & \\
\hline & 0.5 & & 0.5 & & & 0.5 & & & 0.5 & & & 0.5 \\
\hline & 0.5 & & & 0.5 & & 0.5 & & & 0.5 & & & 0.5 \\
\hline & 0.5 & & 0.5 & & 0.5 & & & 0.5 & & & 0.5 & \\
\hline & & 0.5 & & 0.5 & & & 0.5 & 0.5 & 0.5 & & & 0.5 \\
\hline & & 0.5 & & 0.5 & & 0.5 & & & & 0.5 & & 0.5 \\
\hline \multirow[t]{2}{*}{0.5} & & & 0.5 & & 0.5 & & 0.5 & & & 0.5 & & \\
\hline & & 0.5 & & 0.5 & & 0.5 & 0.5 & & 0.5 & & & \\
\hline \multirow[t]{2}{*}{0.5} & & & 0.5 & & & 0.5 & & 0.5 & & & 0.5 & \\
\hline & 0.5 & & 0.5 & 0.5 & & & 0.5 & & 0.5 & & & 0.5 \\
\hline \multirow[t]{2}{*}{0.5} & & 0.5 & & 0.5 & & & & 0.5 & & & 0.5 & \\
\hline & 0.5 & & 0.5 & & & 0.5 & & & & 0.5 & & \\
\hline 0.5 & & 0.5 & & & 0.5 & & 0.5 & & & & 0.5 & \\
\hline \multirow[t]{7}{*}{0.5} & & 0.5 & & 0.5 & & & 0.5 & 0.5 & & & 0.5 & \\
\hline & & 0.5 & & & & 0.5 & & & 0.5 & & & 0.5 \\
\hline & 0.5 & & & & & 0.5 & & & & 0.5 & & \\
\hline & & 0.5 & & & & & 0.5 & & & & 0.5 & \\
\hline & & 0.5 & 0.5 & & & 0.5 & & & 0.5 & & & \\
\hline & 0.5 & & 0.5 & & 0.5 & & 0.5 & & & 0.5 & & 0.5 \\
\hline & & 0.5 & & & 0.5 & & & & & & & 0.5 \\
\hline \multirow[t]{3}{*}{0.5} & & 0.5 & 0.5 & & & 0.5 & & 0.5 & & & 0.5 & \\
\hline & 0.5 & & & 0.5 & & 0.5 & & & 0.5 & & & 0.5 \\
\hline & & 0.5 & & & 0.5 & & 0.5 & & & 0.5 & & 0.5 \\
\hline 0.5 & & & & & 0.5 & & & & & 0.5 & & \\
\hline 0.5 & & & 0.5 & & & 0.5 & & 0.5 & & 0.5 & & \\
\hline
\end{tabular}


wbheat

\begin{tabular}{|c|c|c|c|c|c|c|c|c|c|c|c|c|}
\hline 0.5 & & & 0.5 & & & 0.5 & & & & & 0.5 & \\
\hline & 0.5 & & & 0.5 & & 0.5 & & & & & 0.5 & \\
\hline & 0.5 & & & 0.5 & & & 0.5 & & 0.5 & & & \\
\hline 0.5 & & 0.5 & & & 0.5 & & 0.5 & & 0.5 & & & 0.5 \\
\hline \multirow[t]{2}{*}{0.5} & & & & 0.5 & & & & 0.5 & & & & \\
\hline & 0.5 & & & 0.5 & & 0.5 & & & & & 0.5 & \\
\hline 0.5 & 0.5 & & & 0.5 & & & 0.5 & & & & 0.5 & \\
\hline \multirow[t]{2}{*}{0.5} & 0.5 & & & 0.5 & & & 0.5 & & & & & 0.5 \\
\hline & 0.5 & & & 0.5 & & & 0.5 & & & & 0.5 & \\
\hline 0.5 & & 0.5 & & 0.5 & & & & 0.5 & & & & \\
\hline \multirow[t]{3}{*}{0.5} & & & 0.5 & & & 0.5 & & & & 0.5 & & \\
\hline & 0.5 & & & & & 0.5 & & & & & 0.5 & \\
\hline & & 0.5 & & & & & & & 0.5 & & & \\
\hline \multirow[t]{3}{*}{0.5} & & & 0.5 & & & 0.5 & & 0.5 & & & & \\
\hline & 0.5 & & 0.5 & & & 0.5 & & & 0.5 & & & \\
\hline & & & 0.5 & & & & & & 0.5 & & & \\
\hline \multirow[t]{3}{*}{0.5} & & & 0.5 & & & & 0.5 & 0.5 & & & & 0.5 \\
\hline & 0.5 & & & 0.5 & & & & 0.5 & & & & 0.5 \\
\hline & & 0.5 & & & 0.5 & & 0.5 & & & & 0.5 & \\
\hline \multirow[t]{4}{*}{0.5} & & & 0.5 & & & 0.5 & & & & & 0.5 & \\
\hline & & 0.5 & & & & & 0.5 & & & & & 0.5 \\
\hline & 0.5 & & & & 0.5 & & & & & 0.5 & & \\
\hline & & 0.5 & & & & 0.5 & & & & 0.5 & & \\
\hline \multirow[t]{5}{*}{0.5} & & & & & 0.5 & & & & 0.5 & & & \\
\hline & 0.5 & & & 0.5 & & & & 0.5 & & & 0.5 & \\
\hline & 0.5 & & & & & 0.5 & & & & 0.5 & & \\
\hline & & & & & & & 0.5 & & & & & \\
\hline & & & & & & & & 0.5 & & & & \\
\hline \multirow[t]{2}{*}{0.5} & & & 0.5 & 0.5 & 0.5 & & & & 0.5 & & & \\
\hline & 0.5 & & & & & 0.5 & 0.5 & & & 0.5 & & 0.5 \\
\hline 0.5 & & 0.5 & & & 0.5 & & & 0.5 & & & 0.5 & \\
\hline 0.5 & 1 & & 0.5 & & & 0.5 & & & & 0.5 & & \\
\hline \multirow[t]{3}{*}{0.5} & & & 0.5 & & & 0.5 & & & 0.5 & & & \\
\hline & & & & 0.5 & & & & 0.5 & & & & \\
\hline & 0.5 & & & & & 0.5 & & & & 0.5 & & \\
\hline 0.5 & & 0.5 & & 0.5 & & & & 0.5 & & 0.5 & & \\
\hline 0.5 & 0.5 & & & & 0.5 & & & 0.5 & & & & 0.5 \\
\hline
\end{tabular}


wbheat

\begin{tabular}{|c|c|c|c|c|c|c|c|c|c|c|c|c|}
\hline & 0.5 & 0.5 & & & & 0.5 & & & & 0.5 & & \\
\hline & 0.5 & & & 0.5 & & & 0.5 & & & & 0.5 & \\
\hline 0.5 & & & 0.5 & & & 0.5 & & & 0.5 & & & \\
\hline & & 0.5 & & & & & 0.5 & & & & 0.5 & \\
\hline 0.5 & & & & & 0.5 & & & & & 0.5 & & \\
\hline 0.5 & & & 0.5 & & & & 0.5 & & & 0.5 & & \\
\hline 0.5 & & & 0.5 & & & & 0.5 & & & 0.5 & & \\
\hline 0.5 & & 0.5 & & & 0.5 & & 0.5 & 0.5 & & & 0.5 & \\
\hline 0.5 & 0.5 & 0.5 & & 0.5 & & & 0.5 & & & & 0.5 & \\
\hline 0.5 & & 0.5 & & & 0.5 & & & & 0.5 & & & \\
\hline 0.5 & & 0.5 & & & & 0.5 & & & & 0.5 & & \\
\hline & 0.5 & & & & 0.5 & & & & & 0.5 & & \\
\hline 0.5 & & & & 0.5 & & & & & 0.5 & & & \\
\hline 0.5 & & 0.5 & & 0.5 & & & & 0.5 & & & & 0.5 \\
\hline 0.5 & & & 0.5 & & & & 0.5 & 0.5 & & 0.5 & & \\
\hline 0.5 & & & 0.5 & & & & 0.5 & & 0.5 & & 0.5 & \\
\hline & & 0.5 & & 0.5 & & & & & 0.5 & & & 0.5 \\
\hline 0.5 & & 0.5 & & 0.5 & & & & 0.5 & & & & 0.5 \\
\hline 0.5 & 0.5 & & 0.5 & & & 0.5 & 0.5 & 0.5 & & & 0.5 & \\
\hline 0.5 & & & & 0.5 & & 0.5 & & 0.5 & & & & 0.5 \\
\hline & 0.5 & & 0.5 & & & 0.5 & 0.5 & 0.5 & & & & 0.5 \\
\hline 0.5 & & 0.5 & & & 0.5 & & & & 0.5 & & & \\
\hline 0.5 & & & 0.5 & & & 0.5 & & & & & 0.5 & \\
\hline 0.5 & & & & 0.5 & & & & 0.5 & & & & 0.5 \\
\hline & & & 0.5 & & & & 0.5 & & & & & 0.5 \\
\hline & & 0.5 & & & 0.5 & & & 0.5 & 0.5 & & 0.5 & \\
\hline 0.5 & & & 0.5 & & & 0.5 & & & 0.5 & & 0.5 & \\
\hline & 0.5 & & 0.5 & & & 0.5 & & & 0.5 & & & \\
\hline & 0.5 & & 0.5 & & & 0.5 & & & 0.5 & & & \\
\hline & 0.5 & & & & 0.5 & & 0.5 & & & 0.5 & & 0.5 \\
\hline & & 0.5 & & & & 0.5 & & & & & 0.5 & \\
\hline & 0.5 & & & & 0.5 & & & & 0.5 & & & 0.5 \\
\hline & 0.5 & & 0.5 & & & & 0.5 & & 0.5 & & 0.5 & \\
\hline & 0.5 & & & 0.5 & & & 0.5 & & 0.5 & & & 0.5 \\
\hline 1 & 0.5 & 1 & 1.5 & 1 & 2.1 & 1 & 1 & 1 & & & 0.5 & \\
\hline 4.6 & 4.1 & 4.1 & 1 & 1 & 0.5 & 1 & & 0.5 & 0.5 & & 0.5 & 0.5 \\
\hline 4.1 & 3.6 & 3.6 & 3.6 & 1.5 & 1 & 3.1 & 2.6 & 1 & 0.5 & & & 0.5 \\
\hline
\end{tabular}


wbheat

\begin{tabular}{|c|c|c|c|c|c|c|c|c|c|c|c|c|}
\hline 0.5 & & 0.5 & & 0.5 & & 0.5 & 0.5 & & & 0.5 & 0.5 & \\
\hline & 0.5 & & 0.5 & & 0.5 & & 0.5 & & & 0.5 & 0.5 & \\
\hline 0.5 & & 0.5 & & & & 1 & & 1 & & & 0.5 & \\
\hline 0.5 & & 0.5 & & 0.5 & & 0.5 & & 0.5 & & 0.5 & & 0.5 \\
\hline 0.5 & 0.5 & & & 0.5 & & 0.5 & & 1 & & & & \\
\hline 1 & 1 & 0.5 & 1 & 0.5 & 0.5 & 0.5 & 0.5 & & 0.5 & 0.5 & & \\
\hline 3.6 & 3.1 & 1.5 & & 0.5 & 0.5 & 0.5 & & 0.5 & 0.5 & 0.5 & & 0.5 \\
\hline 0.5 & & 0.5 & & 0.5 & & & 0.5 & & & 0.5 & 0.5 & \\
\hline \multirow[t]{4}{*}{0.5} & & & 1 & & & & 0.5 & 0.5 & & & 0.5 & 0.5 \\
\hline & 0.5 & & 0.5 & & & 0.5 & & 0.5 & & & 0.5 & \\
\hline & & & 0.5 & 0.5 & & 0.5 & 0.5 & 0.5 & & & 0.5 & 0.5 \\
\hline & 0.5 & & 0.5 & & & 0.5 & & 0.5 & & & 0.5 & \\
\hline 0.5 & & 0.5 & & 0.5 & & 0.5 & & 0.5 & 0.5 & & & 0.5 \\
\hline 0.5 & & & 0.5 & 0.5 & & & 0.5 & & 0.5 & & 0.5 & 0.5 \\
\hline \multirow{2}{*}{0.5} & 0.5 & & & 0.5 & & & 0.5 & & 0.5 & & 0.5 & \\
\hline & 0.5 & & 0.5 & & 0.5 & & & 0.5 & & 0.5 & 0.5 & \\
\hline 3.1 & 3.1 & 1.5 & 0.5 & 0.5 & 1.5 & 2.6 & 2.6 & 2.6 & & & & 0.5 \\
\hline 0.5 & 0.5 & & 0.5 & 1 & 1 & 1 & 0.5 & 0.5 & & 0.5 & 0.5 & 0.5 \\
\hline 1 & 0.5 & 1 & 1 & & 0.5 & & 0.5 & 0.5 & & 0.5 & 0.5 & 0.5 \\
\hline 0.5 & & 0.5 & 0.5 & & 0.5 & 0.5 & 0.5 & & & 1 & & 0.5 \\
\hline 4.6 & 4.1 & 3.6 & 2.6 & 2.1 & 4.1 & 4.1 & 4.1 & 1 & 0.5 & 0.5 & 1 & 0.5 \\
\hline 2.6 & 1 & & 1 & 0.5 & 0.5 & 1 & & 0.5 & 0.5 & & & 0.5 \\
\hline 4.1 & 1.5 & 2.1 & 1.5 & 1 & 1 & 0.5 & 0.5 & 0.5 & 0.5 & & 0.5 & 0.5 \\
\hline 3.6 & 3.6 & 3.1 & 1.5 & & 1 & 0.5 & & & 0.5 & 0.5 & & \\
\hline 0.5 & & 0.5 & 0.5 & & 0.5 & & 0.5 & 0.5 & 0.5 & 0.5 & & 1 \\
\hline 2.6 & 2.1 & 2.1 & 1.5 & 2.1 & 1.5 & 2.1 & 0.5 & 0.5 & 0.5 & 0.5 & & 0.5 \\
\hline 1 & & 0.5 & & 0.5 & 0.5 & 0.5 & & 0.5 & 0.5 & & 0.5 & 0.5 \\
\hline 5.2 & 4.6 & 4.6 & 4.1 & 3.6 & 0.5 & 1 & 0.5 & 0.5 & 0.5 & & 0.5 & 0.5 \\
\hline 4.6 & 3.6 & 3.1 & 4.1 & 4.1 & 3.1 & 0.5 & 0.5 & 3.1 & & 0.5 & 0.5 & \\
\hline 1 & & 0.5 & & & 0.5 & 0.5 & & & 0.5 & 0.5 & & \\
\hline 1 & 1 & 0.5 & 0.5 & 0.5 & & & 0.5 & & & 0.5 & & \\
\hline 3.1 & 1.5 & & 0.5 & & 0.5 & & & 0.5 & & & 0.5 & \\
\hline \multirow[t]{2}{*}{0.5} & 0.5 & & 0.5 & 1 & 0.5 & & & & 0.5 & & 0.5 & \\
\hline & 0.5 & & & 0.5 & & & 0.5 & & 0.5 & & & \\
\hline 1 & 5.2 & 3.1 & 1 & 1 & 1 & 4.1 & 1 & 1 & 1 & 0.5 & 0.5 & 1 \\
\hline 3.6 & 4.1 & 1 & 1 & 4.1 & 3.6 & 1 & 0.5 & 0.5 & 0.5 & 0.5 & 0.5 & 0.5 \\
\hline 3.6 & 3.1 & 3.1 & 2.1 & 0.5 & 0.5 & 0.5 & & 0.5 & 0.5 & & 0.5 & \\
\hline
\end{tabular}


wbheat

\begin{tabular}{|c|c|c|c|c|c|c|c|c|c|c|c|c|}
\hline 3.6 & 3.1 & 2.6 & 2.6 & 2.6 & 2.6 & 1 & 0.5 & 0.5 & 0.5 & & 0.5 & 0.5 \\
\hline 3.1 & 2.6 & 2.6 & 2.6 & 1 & 1 & 1.5 & 1 & 1 & 0.5 & 0.5 & 1 & \\
\hline 3.1 & 4.1 & 3.1 & 3.1 & 3.6 & 1.5 & 1 & 0.5 & 0.5 & & 0.5 & 0.5 & 1 \\
\hline 0.5 & & 0.5 & & 0.5 & 0.5 & 1 & 0.5 & & 0.5 & 0.5 & & 0.5 \\
\hline 0.5 & & 0.5 & & 0.5 & & 0.5 & & & 0.5 & & 0.5 & \\
\hline 0.5 & 0.5 & & 0.5 & & 0.5 & & 0.5 & 0.5 & & & 0.5 & 0.5 \\
\hline 4.6 & 4.6 & 5.2 & 4.6 & 4.6 & 4.6 & 2.6 & 1.5 & 1 & 0.5 & 1 & 1 & \\
\hline 4.6 & 3.6 & 4.1 & 3.1 & 1 & & 0.5 & 0.5 & 4.1 & 2.6 & 1.5 & 1.5 & 1.5 \\
\hline 3.1 & 3.6 & 3.1 & 3.1 & 3.6 & 3.1 & 1 & 1 & 1 & 0.5 & & & 0.5 \\
\hline 3.6 & 4.1 & 3.6 & 2.6 & 3.1 & 2.6 & 2.1 & 3.1 & 1.5 & 1.5 & 1 & 1 & 0.5 \\
\hline 3.1 & 3.6 & 3.6 & 2.1 & 0.5 & 1 & 0.5 & 0.5 & 0.5 & & & & 0.5 \\
\hline 0.5 & & 0.5 & & & 0.5 & & & & 0.5 & & & \\
\hline 0.5 & & 0.5 & & & 0.5 & & & & 0.5 & & & 0.5 \\
\hline & 0.5 & 0.5 & & & & 1 & & & 0.5 & & & 0.5 \\
\hline 4.6 & 4.6 & 3.6 & 3.1 & 3.6 & 2.6 & 2.1 & 0.5 & 3.1 & 1 & 2.6 & 1 & 1 \\
\hline 3.6 & 2.6 & 2.6 & 3.6 & 0.5 & 1.5 & 0.5 & 0.5 & 0.5 & & & 0.5 & \\
\hline 4.6 & 1.5 & 0.5 & & 0.5 & 0.5 & & 0.5 & & & & & 0.5 \\
\hline 1 & 1.5 & 0.5 & 0.5 & & 0.5 & & & & 0.5 & & 0.5 & \\
\hline 0.5 & & & 0.5 & & & & & 0.5 & & & & 0.5 \\
\hline 0.5 & 0.5 & & & 0.5 & & & 0.5 & & & & 0.5 & 0.5 \\
\hline 5.2 & 4.1 & 4.6 & 3.1 & 1 & 2.6 & 4.1 & 3.1 & 1 & 0.5 & & 0.5 & 1 \\
\hline 4.6 & 4.6 & 4.6 & 4.1 & 4.1 & 3.1 & 2.6 & 2.6 & 2.1 & 1 & & 1 & 0.5 \\
\hline 3.6 & 4.1 & 3.6 & 2.6 & 2.1 & 1 & 0.5 & 0.5 & 1 & 0.5 & & 0.5 & 0.5 \\
\hline 3.6 & 1.5 & 1 & 1 & 0.5 & & 0.5 & 0.5 & 0.5 & 0.5 & & 0.5 & 0.5 \\
\hline 0.5 & 0.5 & 0.5 & 0.5 & 1 & 1.5 & 1 & 1 & 1 & 0.5 & 0.5 & 0.5 & 0.5 \\
\hline 4.1 & 3.6 & 2.1 & 2.1 & 1.5 & 1.5 & 1.5 & 1 & 1.5 & 1.5 & 0.5 & 0.5 & 0.5 \\
\hline 0.5 & 0.5 & 1 & & 0.5 & & 0.5 & 0.5 & & 0.5 & & 1 & 0.5 \\
\hline 3.6 & 2.6 & 1 & 0.5 & 0.5 & & 0.5 & 0.5 & 0.5 & 0.5 & & 0.5 & 0.5 \\
\hline 1 & 0.5 & 0.5 & 0.5 & 0.5 & & 0.5 & 0.5 & 0.5 & & 0.5 & 0.5 & \\
\hline & & 0.5 & 0.5 & 0.5 & 0.5 & & 0.5 & 0.5 & 0.5 & & & \\
\hline 4.6 & 1 & & 0.5 & 0.5 & & 1 & 1 & & & & 0.5 & 0.5 \\
\hline 3.1 & 4.1 & 3.1 & 3.1 & 3.6 & 3.6 & 2.6 & 2.1 & 1.5 & 1 & 1.5 & 1 & 1 \\
\hline 1 & 1 & 1.5 & 1 & 1 & & 1 & & 0.5 & & 0.5 & & 0.5 \\
\hline & 0.5 & & 0.5 & 0.5 & & & 0.5 & & 0.5 & & & 0.5 \\
\hline 4.1 & 2.1 & 1 & 0.5 & & & 0.5 & 0.5 & 0.5 & & & 0.5 & \\
\hline 0.5 & & 0.5 & & 0.5 & & & 0.5 & 0.5 & & & & 0.5 \\
\hline & 0.5 & & & 0.5 & 0.5 & & 0.5 & & 0.5 & & 0.5 & \\
\hline
\end{tabular}




\begin{tabular}{|c|c|c|c|c|c|c|c|c|c|c|c|c|}
\hline & 0.5 & 0.5 & 0.5 & 0.5 & 0.5 & & 0.5 & 0.5 & & & & 0.5 \\
\hline 4.6 & 3.6 & 2.1 & 2.6 & 2.1 & 1.5 & & 0.5 & 0.5 & & 0.5 & & 0.5 \\
\hline \multirow[t]{2}{*}{0.5} & 1 & & 0.5 & & 0.5 & & & 0.5 & & 0.5 & & \\
\hline & & & 0.5 & & & 0.5 & & & & & 0.5 & 0.5 \\
\hline 3.6 & 3.1 & 1.5 & 2.1 & 3.1 & 2.1 & 2.1 & 2.1 & 0.5 & 1 & 1 & 1 & 0.5 \\
\hline 4.1 & 3.6 & 3.1 & 3.6 & 2.6 & 3.1 & 3.1 & 2.1 & 2.1 & 1.5 & 1 & 1 & 1.5 \\
\hline 4.6 & 4.1 & 4.1 & 3.6 & 3.1 & 3.1 & 3.1 & 2.1 & 2.1 & 0.5 & 2.1 & 1.5 & 1.5 \\
\hline 3.6 & 3.6 & 3.6 & 1 & 1 & 0.5 & 1 & 0.5 & 1 & 0.5 & & 1 & \\
\hline 1 & & 0.5 & 0.5 & 0.5 & 0.5 & & 0.5 & 0.5 & & 0.5 & 0.5 & 0.5 \\
\hline 0.5 & & & 0.5 & & & & & & 0.5 & 0.5 & & 0.5 \\
\hline 1 & 1 & & 0.5 & 0.5 & & & 0.5 & 0.5 & & 0.5 & & \\
\hline \multirow[t]{2}{*}{3.6} & 2.1 & 1 & 0.5 & & & 0.5 & & & 0.5 & & & \\
\hline & & 0.5 & & 0.5 & & & 0.5 & 0.5 & 0.5 & & & \\
\hline 0.5 & & & 0.5 & & 0.5 & & 0.5 & 0.5 & & & & 0.5 \\
\hline 4.1 & 2.1 & 2.1 & 0.5 & 1 & & 0.5 & 1 & & 1.5 & 3.6 & 2.1 & 1 \\
\hline \multirow[t]{4}{*}{0.5} & 0.5 & 0.5 & 0.5 & & & 0.5 & & 0.5 & & 0.5 & & \\
\hline & 0.5 & & 0.5 & & & 0.5 & & & 0.5 & & & \\
\hline & 0.5 & 0.5 & & 0.5 & 0.5 & & 0.5 & 0.5 & & & 0.5 & \\
\hline & 0.5 & & 0.5 & & & 0.5 & 0.5 & & & & 0.5 & \\
\hline 0.5 & 0.5 & & 0.5 & & & 0.5 & 0.5 & & 0.5 & & & 0.5 \\
\hline 1 & 0.5 & & & 0.5 & & & 0.5 & & & 0.5 & & \\
\hline 1.5 & 1.5 & 1.5 & 1.5 & 1 & 1 & 0.5 & 0.5 & 4.1 & & & 0.5 & \\
\hline 0.5 & & & 0.5 & & 0.5 & & & & & 0.5 & & \\
\hline 0.5 & & & 0.5 & & & 0.5 & & & & 0.5 & & \\
\hline \multirow[t]{4}{*}{0.5} & 0.5 & & & & & & 0.5 & & & & & 0.5 \\
\hline & & 0.5 & 1 & 0.5 & & & 0.5 & & & 0.5 & & \\
\hline & & 0.5 & 0.5 & & 0.5 & & 0.5 & 0.5 & & & & 0.5 \\
\hline & & & 0.5 & & & 0.5 & 0.5 & 0.5 & & & & 0.5 \\
\hline \multirow[t]{2}{*}{4.6} & 4.1 & 4.1 & 4.6 & 4.1 & 3.6 & 4.1 & 3.6 & 1 & 1 & 1 & 1 & 1 \\
\hline & 0.5 & & 0.5 & & & & 0.5 & & & 0.5 & & \\
\hline 0.5 & & & 0.5 & & & 0.5 & 0.5 & & & & 0.5 & \\
\hline 0.5 & & 0.5 & & 0.5 & & & 0.5 & & & & 0.5 & \\
\hline 0.5 & & 0.5 & & 0.5 & & & 0.5 & 0.5 & & & & 0.5 \\
\hline \multirow[t]{4}{*}{0.5} & & 0.5 & & & 0.5 & & 0.5 & 0.5 & 0.5 & & & \\
\hline & 0.5 & & 0.5 & & & & & 0.5 & & & 0.5 & \\
\hline & & & & & & 0.5 & & & 0.5 & & & \\
\hline & & 0.5 & & & & 0.5 & & 1 & & & & 0.5 \\
\hline
\end{tabular}


wbheat

\begin{tabular}{|c|c|c|c|c|c|c|c|c|c|c|c|c|}
\hline & 0.5 & & & 0.5 & 0.5 & & & 0.5 & & & 0.5 & \\
\hline & & & 0.5 & & & 0.5 & & 0.5 & 0.5 & & & 0.5 \\
\hline 0.5 & 0.5 & & & & 0.5 & & 0.5 & & & 0.5 & & \\
\hline \multirow[t]{3}{*}{0.5} & 0.5 & & & & & 0.5 & 0.5 & 0.5 & & & 0.5 & \\
\hline & & & 0.5 & & & & & 0.5 & & & & 0.5 \\
\hline & & & & 0.5 & & & & 0.5 & & & & \\
\hline \multirow[t]{3}{*}{0.5} & 1 & & & 0.5 & 0.5 & & 0.5 & 0.5 & & & & 0.5 \\
\hline & 0.5 & & & & 0.5 & & & 0.5 & & & 0.5 & \\
\hline & 0.5 & 0.5 & & & & 0.5 & 0.5 & 0.5 & & & 0.5 & \\
\hline \multirow[t]{6}{*}{0.5} & & & & 0.5 & & & 0.5 & 0.5 & 0.5 & & & 0.5 \\
\hline & 0.5 & & & & 0.5 & & & 0.5 & & & 0.5 & \\
\hline & & & & & 0.5 & 0.5 & & 1 & & & & \\
\hline & & 0.5 & & 0.5 & & & 0.5 & & & & 0.5 & \\
\hline & & 0.5 & & & & 0.5 & & 0.5 & 0.5 & & & 0.5 \\
\hline & 0.5 & & & & & 0.5 & & & & & 0.5 & \\
\hline \multirow[t]{3}{*}{0.5} & & & & 0.5 & & & & & & 0.5 & & \\
\hline & & 0.5 & & & & 0.5 & & & 0.5 & & & \\
\hline & 0.5 & & & 0.5 & & & 0.5 & & & & 0.5 & \\
\hline \multirow[t]{3}{*}{0.5} & & 0.5 & & & & 0.5 & & & 0.5 & & & \\
\hline & 0.5 & & & 0.5 & & 0.5 & & & & & & \\
\hline & 0.5 & & & 0.5 & & 0.5 & 0.5 & & & 0.5 & & \\
\hline \multirow[t]{3}{*}{0.5} & & & & & 0.5 & & & & 0.5 & & & \\
\hline & & & & & & 0.5 & & & 0.5 & & & \\
\hline & 0.5 & & 0.5 & & & 0.5 & & 0.5 & 0.5 & & & \\
\hline \multirow[t]{2}{*}{0.5} & & 0.5 & & 0.5 & & & & & & 0.5 & & \\
\hline & 0.5 & & 0.5 & & & & 0.5 & & & & 0.5 & \\
\hline 0.5 & & & 0.5 & & & & 0.5 & 0.5 & & 0.5 & & \\
\hline \multirow[t]{5}{*}{0.5} & & & & & 0.5 & & & & & 0.5 & & \\
\hline & & & 0.5 & & & & & & 0.5 & & & \\
\hline & 0.5 & & & 0.5 & & & 0.5 & 0.5 & & 0.5 & & \\
\hline & & & 0.5 & & & 0.5 & & & & 0.5 & & \\
\hline & & 0.5 & & & & 0.5 & 0.5 & 0.5 & & & 0.5 & \\
\hline \multirow[t]{3}{*}{0.5} & & & 0.5 & & 0.5 & & & & 0.5 & & 0.5 & \\
\hline & 0.5 & & 0.5 & & 0.5 & & & 1 & & & & 0.5 \\
\hline & & & & 0.5 & & & 0.5 & & 0.5 & & & \\
\hline \multirow[t]{2}{*}{0.5} & & & 0.5 & & & & & & & 0.5 & & \\
\hline & 0.5 & & & 0.5 & & & 0.5 & 0.5 & & & 0.5 & \\
\hline
\end{tabular}


wbheat

\begin{tabular}{|c|c|c|c|c|c|c|c|c|c|c|c|c|}
\hline & & 0.5 & & & 0.5 & & & & & 0.5 & & \\
\hline & 0.5 & & & 0.5 & & & 0.5 & 0.5 & 0.5 & & & \\
\hline & & 0.5 & & 0.5 & & & 0.5 & & & & & \\
\hline & & 0.5 & & & 0.5 & & 0.5 & 0.5 & & & 0.5 & \\
\hline & & 0.5 & & & & & & & 0.5 & & & 0.5 \\
\hline & & & 0.5 & & & & & & 0.5 & & & \\
\hline & & & 0.5 & & & 0.5 & 0.5 & & & & 0.5 & \\
\hline & 0.5 & & 0.5 & & & 0.5 & & 0.5 & & 0.5 & & \\
\hline \multirow[t]{6}{*}{0.5} & & & 0.5 & & & 0.5 & & & & 0.5 & & \\
\hline & & 0.5 & & & & 0.5 & & 0.5 & 0.5 & & & 0.5 \\
\hline & 0.5 & & & 0.5 & & & 0.5 & & & & 0.5 & \\
\hline & & 0.5 & & & & 0.5 & & & & & & \\
\hline & & & & 0.5 & & & & 0.5 & & & & 0.5 \\
\hline & & & & & & 0.5 & 0.5 & 0.5 & & & & 0.5 \\
\hline 0.5 & 0.5 & & & 0.5 & & & 0.5 & 0.5 & & & & 0.5 \\
\hline 0.5 & 0.5 & & & 0.5 & & & 0.5 & & & & & \\
\hline \multirow[t]{4}{*}{0.5} & & 0.5 & & & & 0.5 & & & & 0.5 & & \\
\hline & & 0.5 & & & & 0.5 & & & 0.5 & & & 0.5 \\
\hline & & & 0.5 & & & & & 0.5 & & & & \\
\hline & & & 0.5 & & & & 0.5 & & & & & \\
\hline 0.5 & & 0.5 & & & & 0.5 & & & 0.5 & & & 0.5 \\
\hline 0.5 & & & 0.5 & & & & & 0.5 & & 0.5 & & \\
\hline \multirow[t]{2}{*}{0.5} & 0.5 & & & 0.5 & & & 0.5 & & 0.5 & & & 0.5 \\
\hline & 0.5 & & 0.5 & & & & 0.5 & & 0.5 & & & 0.5 \\
\hline \multirow[t]{5}{*}{0.5} & & 0.5 & & & & & 0.5 & & & 0.5 & & \\
\hline & 0.5 & & 0.5 & & & & 0.5 & & & 0.5 & & \\
\hline & & & & 0.5 & & & & & 0.5 & & & \\
\hline & 0.5 & & & 0.5 & & & 0.5 & & & & 0.5 & \\
\hline & 0.5 & & & 0.5 & & 0.5 & & & 0.5 & & 0.5 & \\
\hline \multirow[t]{5}{*}{0.5} & & & 0.5 & & & 0.5 & & & & 0.5 & & \\
\hline & & & & 0.5 & & & 0.5 & & 0.5 & & & 0.5 \\
\hline & 0.5 & & & 0.5 & & & & 0.5 & & & 0.5 & \\
\hline & & 0.5 & & & & 0.5 & & & & & 0.5 & \\
\hline & & & & & 0.5 & & & & & 0.5 & & \\
\hline \multirow[t]{3}{*}{0.5} & & 0.5 & & & 0.5 & & & 0.5 & & & & 0.5 \\
\hline & 0.5 & & & & 0.5 & & & 0.5 & & 0.5 & & \\
\hline & & 0.5 & & & & 0.5 & & & 0.5 & & 0.5 & \\
\hline
\end{tabular}


wbheat

\begin{tabular}{|c|c|c|c|c|c|c|c|c|c|c|c|c|}
\hline 0.5 & & 0.5 & & & & 0.5 & & & 0.5 & & 0.5 & \\
\hline & & 0.5 & & & & 0.5 & & & 0.5 & & & 0.5 \\
\hline & & 0.5 & & & & & & & & 0.5 & & \\
\hline & & 0.5 & & & & & 0.5 & & & & & 0.5 \\
\hline & 0.5 & & & & 0.5 & & & 0.5 & & & 0.5 & \\
\hline \multirow[t]{6}{*}{0.5} & 0.5 & & 0.5 & & 0.5 & & & & & & 0.5 & \\
\hline & & 0.5 & & & & 0.5 & & & 0.5 & & & 0.5 \\
\hline & & 0.5 & & & 0.5 & & & & 0.5 & & & 0.5 \\
\hline & & & 0.5 & & & 0.5 & & & 0.5 & & 0.5 & \\
\hline & & 0.5 & & & & & & 0.5 & & & & \\
\hline & & 0.5 & & & & & 0.5 & & & & & 0.5 \\
\hline \multirow[t]{6}{*}{0.5} & & & 0.5 & & & & 0.5 & & & 0.5 & & \\
\hline & & 0.5 & & & & 0.5 & & & 0.5 & & & 0.5 \\
\hline & 0.5 & & & & 0.5 & & & 0.5 & & 0.5 & & \\
\hline & 0.5 & & & & 0.5 & & & 0.5 & & & 0.5 & \\
\hline & & & 0.5 & & & & 0.5 & & & 0.5 & & \\
\hline & & 0.5 & & & & & 0.5 & & & & & \\
\hline \multirow[t]{5}{*}{0.5} & & & & & & 0.5 & & & & & & 0.5 \\
\hline & 0.5 & & & 0.5 & & & & 0.5 & & & 0.5 & \\
\hline & 0.5 & & & 0.5 & & & & 0.5 & & 0.5 & & \\
\hline & & 0.5 & & 0.5 & & & 0.5 & & & 0.5 & & \\
\hline & & 0.5 & & 0.5 & & & 0.5 & & & 0.5 & & \\
\hline 0.5 & 0.5 & 1 & 1 & & 0.5 & 0.5 & & & 0.5 & & 0.5 & \\
\hline 0.5 & & & 0.5 & & 0.5 & & & & & 0.5 & & \\
\hline 0.5 & & & & 0.5 & & & & 0.5 & & & & 0.5 \\
\hline \multirow[t]{3}{*}{0.5} & & 0.5 & & 0.5 & & & & 0.5 & & & 0.5 & \\
\hline & 0.5 & & & & 0.5 & & & 0.5 & & 0.5 & & 0.5 \\
\hline & 0.5 & & 0.5 & & 0.5 & & 0.5 & & 0.5 & & 0.5 & \\
\hline 0.5 & & 0.5 & & 0.5 & & & 0.5 & & & 0.5 & & 0.5 \\
\hline 0.5 & & & 0.5 & & & & 0.5 & & 0.5 & & 0.5 & \\
\hline \multirow[t]{2}{*}{4.1} & 3.6 & 2.6 & 1 & 1 & 1.5 & 1 & 1 & 1 & 1 & 0.5 & & 0.5 \\
\hline & & 0.5 & & & 0.5 & & 0.5 & & & & 0.5 & \\
\hline \multirow[t]{3}{*}{3.1} & 3.6 & 1.5 & 1 & 0.5 & 0.5 & 0.5 & 0.5 & & 0.5 & & 0.5 & \\
\hline & 0.5 & & 0.5 & & & & 0.5 & & 0.5 & & & 0.5 \\
\hline & 0.5 & 0.5 & & 0.5 & & 0.5 & & 0.5 & & & 0.5 & 0.5 \\
\hline \multirow[t]{2}{*}{0.5} & 0.5 & & & 0.5 & & & 0.5 & & & 0.5 & & 0.5 \\
\hline & 0.5 & & & 0.5 & & 0.5 & & & 0.5 & & 0.5 & \\
\hline
\end{tabular}


wbheat

\begin{tabular}{|c|c|c|c|c|c|c|c|c|c|c|c|c|}
\hline 0.5 & 0.5 & 0.5 & 0.5 & 0.5 & 1 & 0.5 & 0.5 & 0.5 & 0.5 & 0.5 & 0.5 & 0.5 \\
\hline 0.5 & 0.5 & 1 & & 0.5 & 0.5 & 0.5 & 0.5 & 0.5 & 0.5 & & 1 & \\
\hline 4.6 & 4.1 & 3.6 & 2.1 & 0.5 & 0.5 & 0.5 & 0.5 & 0.5 & & 0.5 & & 0.5 \\
\hline 0.5 & 0.5 & 0.5 & & 0.5 & & 0.5 & 0.5 & & 0.5 & 0.5 & & \\
\hline \multirow[t]{4}{*}{0.5} & & 0.5 & 0.5 & & 0.5 & 0.5 & 0.5 & & 0.5 & 0.5 & & \\
\hline & 0.5 & 0.5 & & & & 0.5 & & & & 0.5 & & 0.5 \\
\hline & & & & & 0.5 & & & & 0.5 & & & \\
\hline & & & & 0.5 & & & & & 0.5 & & 0.5 & \\
\hline 0.5 & 0.5 & 0.5 & 0.5 & & 0.5 & 0.5 & 0.5 & & & & 0.5 & \\
\hline 0.5 & 0.5 & & 1 & 0.5 & 0.5 & 1 & 5.2 & 1 & & 0.5 & & \\
\hline 3.1 & 3.1 & 0.5 & 0.5 & & 0.5 & 0.5 & & 0.5 & & & 0.5 & \\
\hline 3.1 & 1.5 & & 0.5 & 0.5 & & & & 1 & & & & \\
\hline 0.5 & & 0.5 & & & & 0.5 & 0.5 & & & & 0.5 & \\
\hline \multirow[t]{2}{*}{1.5} & 1 & 1 & 1 & 0.5 & 1 & 0.5 & 1 & 1 & 1.5 & 1 & 1 & 1 \\
\hline & & 0.5 & 0.5 & & & & & 0.5 & & & & 0.5 \\
\hline 1 & 1 & 1 & 0.5 & & 0.5 & & 0.5 & 0.5 & & & 0.5 & \\
\hline 0.5 & 0.5 & & & 0.5 & & 0.5 & 0.5 & & & & & 0.5 \\
\hline 4.1 & 2.1 & 1 & 1 & 0.5 & & 1 & & & 0.5 & 0.5 & & \\
\hline 4.6 & 2.1 & 0.5 & 0.5 & & & 0.5 & 1 & 0.5 & & & & \\
\hline \multirow[t]{2}{*}{0.5} & 1 & 0.5 & & 0.5 & & & & 0.5 & & 0.5 & & \\
\hline & 0.5 & & 0.5 & & & 0.5 & 0.5 & & 0.5 & & & \\
\hline 0.5 & 0.5 & & & & & 0.5 & 0.5 & 0.5 & & & & \\
\hline 0.5 & & & 0.5 & & & & 0.5 & & & & 0.5 & \\
\hline 0.5 & & & & & 0.5 & & & 0.5 & & & & 0.5 \\
\hline 5.7 & 2.1 & 0.5 & 0.5 & & & 0.5 & 0.5 & & & & 0.5 & \\
\hline \multirow[t]{3}{*}{0.5} & 0.5 & 0.5 & & 0.5 & 0.5 & 0.5 & & & 0.5 & & & \\
\hline & 0.5 & 0.5 & & & 0.5 & & 0.5 & & & 0.5 & & \\
\hline & 0.5 & 0.5 & & & & 0.5 & 0.5 & & & & & 0.5 \\
\hline \multirow[t]{4}{*}{0.5} & 0.5 & & & & 0.5 & 0.5 & & & & & 0.5 & \\
\hline & & & & & 0.5 & 0.5 & & & & & 0.5 & \\
\hline & 0.5 & & & & 0.5 & & & & & & 0.5 & 0.5 \\
\hline & & 0.5 & & & 0.5 & 0.5 & & 0.5 & & & & 0.5 \\
\hline 4.1 & 1 & 0.5 & 1 & & 0.5 & 1 & & 0.5 & & & & 0.5 \\
\hline 1.5 & 1 & 1 & 1 & & & 0.5 & 0.5 & & & 0.5 & & \\
\hline \multirow[t]{2}{*}{4.1} & 1 & 0.5 & & & 0.5 & 0.5 & & 0.5 & & & & 0.5 \\
\hline & 0.5 & & 0.5 & & 0.5 & & & & & & 0.5 & \\
\hline 5.2 & 4.6 & 4.1 & 4.1 & 4.1 & 3.6 & 4.6 & 3.1 & 3.1 & 2.1 & 2.6 & 2.1 & 2.6 \\
\hline
\end{tabular}


wbheat

\begin{tabular}{|c|c|c|c|c|c|c|c|c|c|c|c|c|}
\hline 0.5 & 0.5 & 0.5 & 1 & 1 & & & 0.5 & & 0.5 & & & 0.5 \\
\hline & 0.5 & 0.5 & & & & 0.5 & 0.5 & & & 0.5 & & \\
\hline & 1 & 0.5 & & 0.5 & & 0.5 & & 0.5 & 0.5 & & & \\
\hline 0.5 & & 0.5 & 0.5 & & & 0.5 & 0.5 & & 0.5 & & & \\
\hline 1.5 & & 0.5 & 0.5 & 0.5 & & 0.5 & 0.5 & 4.6 & 3.1 & & & 1 \\
\hline 1.5 & 1 & 1 & & 0.5 & 0.5 & 0.5 & 0.5 & 0.5 & 0.5 & & & 0.5 \\
\hline 3.1 & 1 & 0.5 & & 0.5 & & 0.5 & 0.5 & & & 0.5 & & \\
\hline \multirow[t]{2}{*}{0.5} & & 0.5 & & 0.5 & & & 0.5 & & & 0.5 & & \\
\hline & 0.5 & & & 0.5 & & & 0.5 & & 0.5 & & & \\
\hline 1.5 & 1.5 & 1.5 & 1.5 & 1.5 & 1 & 1 & 0.5 & 3.6 & 3.1 & 1.5 & 1 & 0.5 \\
\hline 4.6 & 4.6 & 3.6 & 3.1 & 1.5 & 1 & 1.5 & 3.6 & 3.1 & 1.5 & 1 & 1 & 0.5 \\
\hline \multirow[t]{2}{*}{4.6} & 4.6 & 4.6 & 3.1 & 1 & 1 & 0.5 & 1.5 & 4.1 & 3.6 & 1 & 0.5 & 0.5 \\
\hline & 1 & & 0.5 & & 0.5 & 0.5 & & 4.6 & 2.6 & 1.5 & 1 & \\
\hline \multirow[t]{3}{*}{2.6} & 1 & 2.1 & 2.1 & 1 & 0.5 & 0.5 & 1 & 3.6 & 2.1 & 1 & & 0.5 \\
\hline & & 0.5 & & 0.5 & & & & 0.5 & & & & \\
\hline & & 0.5 & & & & 0.5 & & & 0.5 & & & 0.5 \\
\hline \multirow[t]{3}{*}{1} & & 0.5 & 0.5 & 0.5 & & & 1 & & 0.5 & & & \\
\hline & 1 & & 0.5 & & 0.5 & & 0.5 & 0.5 & 0.5 & & & 0.5 \\
\hline & 0.5 & 0.5 & & & 0.5 & 0.5 & & 0.5 & & & 0.5 & \\
\hline 0.5 & & 0.5 & & 0.5 & & 1 & 0.5 & 2.1 & & & & 0.5 \\
\hline 4.6 & 4.6 & 4.1 & 4.6 & 3.6 & 3.6 & 4.1 & 3.1 & 1.5 & 1.5 & 1 & 1.5 & 1 \\
\hline 1.5 & 1 & 0.5 & 1.5 & 0.5 & 1 & 0.5 & 0.5 & & 0.5 & & & 0.5 \\
\hline 0.5 & & 0.5 & & 0.5 & & 0.5 & & & 0.5 & 0.5 & & \\
\hline 4.6 & 4.6 & 5.2 & 4.1 & 4.1 & 4.1 & 4.1 & 3.6 & 3.6 & 3.1 & 4.1 & 3.6 & 2.6 \\
\hline 4.6 & 4.6 & 5.2 & 4.6 & 4.1 & 3.6 & 4.6 & 3.6 & 4.1 & 4.1 & 4.1 & 3.6 & 3.6 \\
\hline 5.2 & 4.6 & 4.1 & 4.6 & 3.6 & 1.5 & 1 & 1 & 4.1 & 3.1 & 3.1 & 2.6 & 2.1 \\
\hline 3.1 & 1 & 1.5 & 1 & 1.5 & 0.5 & 0.5 & 2.1 & 4.1 & 3.1 & 3.6 & 2.6 & 2.6 \\
\hline 1 & 0.5 & 0.5 & 0.5 & & 1 & & 2.1 & 3.6 & 3.6 & 2.6 & 1.5 & 1.5 \\
\hline \multirow[t]{2}{*}{0.5} & 0.5 & & 0.5 & & 0.5 & & 0.5 & & & 0.5 & & \\
\hline & 0.5 & 0.5 & & & & & 0.5 & 0.5 & & & & 0.5 \\
\hline \multirow[t]{2}{*}{0.5} & 0.5 & 0.5 & & & & 0.5 & 0.5 & & & & 0.5 & \\
\hline & 0.5 & 0.5 & & 0.5 & & & 0.5 & & 0.5 & & 0.5 & \\
\hline 0.5 & & 0.5 & 2.6 & 4.6 & 3.6 & 4.1 & 5.2 & 1 & 1 & 1 & 1 & 1 \\
\hline 4.6 & 4.6 & 4.1 & 4.1 & 4.1 & 4.6 & 3.6 & 3.6 & 1 & 1 & 1 & 1 & \\
\hline \multirow[t]{3}{*}{4.6} & 4.6 & 5.2 & 4.1 & 4.6 & 4.6 & 2.1 & 1 & 1.5 & 1.5 & 1.5 & 1.5 & 2.1 \\
\hline & 0.5 & & 0.5 & 0.5 & & & 0.5 & 0.5 & & & 0.5 & \\
\hline & 0.5 & & 0.5 & & 0.5 & & & 0.5 & & 0.5 & & 0.5 \\
\hline
\end{tabular}


wbheat

\begin{tabular}{|c|c|c|c|c|c|c|c|c|c|c|c|c|}
\hline 4.1 & 4.6 & 4.6 & 1.5 & 0.5 & 0.5 & 0.5 & 1 & 3.6 & 3.6 & 3.1 & 2.1 & 2.1 \\
\hline 3.6 & 1 & 1 & 1.5 & 1 & & 0.5 & 1.5 & 4.1 & 3.6 & 1.5 & 1 & 0.5 \\
\hline \multirow{2}{*}{1} & 0.5 & 1 & 0.5 & & & 0.5 & 0.5 & 3.6 & 3.1 & 1.5 & 1 & 0.5 \\
\hline & 1 & 0.5 & 0.5 & 0.5 & & 0.5 & 0.5 & & 1 & & 0.5 & \\
\hline \multirow[t]{4}{*}{1} & 0.5 & 0.5 & 0.5 & & & 0.5 & 0.5 & & 0.5 & & & \\
\hline & & & & 0.5 & & & & 0.5 & & & & \\
\hline & & 0.5 & & & & & & 0.5 & & & & \\
\hline & 1 & & 0.5 & 0.5 & & 0.5 & & & & 0.5 & 0.5 & \\
\hline 4.1 & 3.6 & 1 & 1.5 & 1 & 1.5 & 1 & 1 & 4.1 & 3.1 & 0.5 & & \\
\hline 4.1 & 4.1 & 2.6 & 1.5 & 1 & 0.5 & 0.5 & 0.5 & & & & 0.5 & 0.5 \\
\hline 0.5 & 0.5 & 0.5 & 0.5 & & 0.5 & 0.5 & 0.5 & & & 0.5 & & \\
\hline \multirow{2}{*}{1.5} & 4.1 & 3.6 & 3.6 & 2.1 & 2.1 & 2.6 & 1.5 & 1.5 & 1.5 & 1.5 & 0.5 & 1 \\
\hline & 0.5 & & 0.5 & & & & & 0.5 & 0.5 & & & 0.5 \\
\hline 1 & 0.5 & 1 & 0.5 & 2.1 & 2.1 & 2.1 & 0.5 & 3.6 & 3.6 & & & 0.5 \\
\hline 0.5 & 0.5 & 0.5 & 0.5 & & & 0.5 & 0.5 & 0.5 & 0.5 & 0.5 & & \\
\hline 4.6 & 2.6 & 2.6 & 1.5 & 0.5 & 0.5 & 0.5 & 1 & & 0.5 & 1 & 0.5 & \\
\hline 3.1 & 1 & 0.5 & 0.5 & & & 0.5 & 0.5 & 0.5 & & & & 0.5 \\
\hline \multirow[t]{2}{*}{1} & 0.5 & & & 0.5 & & & 0.5 & 0.5 & 0.5 & 0.5 & 0.5 & \\
\hline & & 0.5 & & & & & & 0.5 & & 0.5 & & \\
\hline \multirow[t]{2}{*}{0.5} & 0.5 & & & & 0.5 & 0.5 & & & 0.5 & & & \\
\hline & 0.5 & 0.5 & 0.5 & & & 0.5 & & & 0.5 & 0.5 & & \\
\hline 0.5 & & 0.5 & & 0.5 & 0.5 & & & 0.5 & & 0.5 & 0.5 & \\
\hline 4.1 & 4.6 & 3.6 & 3.1 & 2.6 & 3.6 & 3.1 & 3.1 & 1.5 & 1.5 & & 0.5 & \\
\hline 4.6 & 4.1 & 3.1 & 1 & 1.5 & 0.5 & 1 & 0.5 & 0.5 & 0.5 & & 0.5 & 0.5 \\
\hline 0.5 & & & & 0.5 & & & & 0.5 & & & & \\
\hline \multirow[t]{2}{*}{0.5} & 0.5 & & & & 0.5 & 0.5 & 0.5 & 0.5 & 0.5 & & & \\
\hline & 0.5 & 0.5 & & 0.5 & 0.5 & 0.5 & & & 0.5 & & & \\
\hline \multirow[t]{4}{*}{0.5} & & 0.5 & 0.5 & & & 0.5 & & & & 0.5 & & \\
\hline & & 0.5 & 0.5 & & & & 0.5 & 0.5 & 0.5 & & & \\
\hline & & & & 0.5 & & & & 0.5 & & & & 0.5 \\
\hline & & & & 0.5 & 0.5 & & & & & & 0.5 & \\
\hline 6.2 & 3.6 & 1 & 0.5 & 1 & & & 0.5 & 0.5 & & & 0.5 & \\
\hline 1 & 0.5 & 0.5 & & 0.5 & & & 0.5 & & & 0.5 & & \\
\hline 0.5 & 1 & 0.5 & & & 0.5 & & 0.5 & & 0.5 & & 0.5 & \\
\hline 1 & 1 & 1 & 0.5 & 0.5 & & & 0.5 & & 0.5 & & & \\
\hline 0.5 & & 0.5 & & 0.5 & & & 0.5 & 0.5 & & & & \\
\hline 4.6 & 3.1 & 2.6 & 1.5 & 0.5 & 1 & 0.5 & 0.5 & & & 0.5 & & 0.5 \\
\hline
\end{tabular}

Page 52 
wbheat

\begin{tabular}{|c|c|c|c|c|c|c|c|c|c|c|c|c|}
\hline 3.1 & 1 & 1 & & 0.5 & & & 0.5 & & & & 0.5 & \\
\hline & 0.5 & 0.5 & & & & 0.5 & & & & & 0.5 & \\
\hline & & 0.5 & & & 0.5 & & & & & & 0.5 & \\
\hline \multirow[t]{4}{*}{0.5} & & & & 0.5 & & & & & 0.5 & & & \\
\hline & & 0.5 & & 0.5 & & & 0.5 & 0.5 & 0.5 & 0.5 & & \\
\hline & 0.5 & & 0.5 & & & 0.5 & & 0.5 & & & 0.5 & \\
\hline & 0.5 & 0.5 & & & & 0.5 & & & 0.5 & & & 0.5 \\
\hline 0.5 & 0.5 & & 0.5 & & & 0.5 & & & 0.5 & & & \\
\hline \multirow[t]{4}{*}{0.5} & & 0.5 & & & & 0.5 & & & & & 0.5 & \\
\hline & & & & 0.5 & & & & & & 0.5 & & \\
\hline & & 0.5 & & & & 0.5 & & & & & 0.5 & \\
\hline & 0.5 & & 0.5 & & & 0.5 & 0.5 & & & & & 0.5 \\
\hline \multirow[t]{4}{*}{0.5} & & 0.5 & & & 0.5 & & & 0.5 & 0.5 & & & \\
\hline & 0.5 & 0.5 & & & & 0.5 & & & 0.5 & & & 0.5 \\
\hline & 0.5 & & 0.5 & & & & 0.5 & & & & 0.5 & \\
\hline & 0.5 & & & & 0.5 & 0.5 & & & & & 0.5 & \\
\hline 0.5 & & & & & & 0.5 & & & & & & 0.5 \\
\hline \multirow[t]{2}{*}{0.5} & & & & & & 0.5 & & & & 0.5 & & \\
\hline & 0.5 & & 0.5 & & 0.5 & & & & 0.5 & & & 0.5 \\
\hline 0.5 & 0.5 & & & 0.5 & & & & 0.5 & & & 0.5 & \\
\hline 0.5 & & 0.5 & & & 0.5 & & 0.5 & & & & 0.5 & \\
\hline 0.5 & 0.5 & & & & & 0.5 & & 0.5 & 0.5 & & & \\
\hline 0.5 & & 0.5 & & & 0.5 & 0.5 & & & & & 0.5 & \\
\hline 0.5 & 0.5 & & 0.5 & 0.5 & 0.5 & & 0.5 & & 1 & & 0.5 & \\
\hline 0.5 & 0.5 & & 0.5 & & & & 0.5 & & 0.5 & & 0.5 & \\
\hline 0.5 & & & 0.5 & & & 0.5 & & & 0.5 & & & \\
\hline 0.5 & & 0.5 & 0.5 & & & 0.5 & & 0.5 & & & & \\
\hline \multirow[t]{8}{*}{0.5} & & 0.5 & 0.5 & & & 0.5 & 0.5 & & & & & 0.5 \\
\hline & 0.5 & 0.5 & & & & 0.5 & 0.5 & 0.5 & & & 0.5 & \\
\hline & 0.5 & & & 0.5 & & 0.5 & 0.5 & 0.5 & & & & 0.5 \\
\hline & & & & 0.5 & & & & & & & 0.5 & \\
\hline & & & & & 0.5 & & & & & & & 0.5 \\
\hline & 0.5 & & & & & & 0.5 & & & & 0.5 & \\
\hline & 0.5 & & & & & & 0.5 & & & 0.5 & & \\
\hline & 0.5 & & & & & 0.5 & & & & & & \\
\hline \multirow[t]{2}{*}{0.5} & 0.5 & & 0.5 & & 0.5 & & & 0.5 & & 0.5 & & \\
\hline & 0.5 & & & 0.5 & & & 0.5 & & 0.5 & 0.5 & & \\
\hline
\end{tabular}


wbheat

\begin{tabular}{|c|c|c|c|c|c|c|c|c|c|c|c|c|}
\hline & & 0.5 & & & & 0.5 & & & 0.5 & & & \\
\hline & & & 0.5 & & & & 0.5 & & & 0.5 & & \\
\hline & & 0.5 & & & & 0.5 & & 0.5 & & & & 0.5 \\
\hline \multirow[t]{4}{*}{0.5} & & 0.5 & & 0.5 & & & & 0.5 & & & 0.5 & \\
\hline & & 0.5 & & & 0.5 & & & 0.5 & & 0.5 & & \\
\hline & 0.5 & & & 0.5 & & & 0.5 & & & 0.5 & & \\
\hline & & 0.5 & & & 0.5 & & & 0.5 & & 0.5 & & \\
\hline \multirow[t]{3}{*}{0.5} & & & & 0.5 & & & 0.5 & & & & 0.5 & \\
\hline & & & 0.5 & & & & & 0.5 & & & & 0.5 \\
\hline & & 0.5 & & & 0.5 & & 0.5 & & 0.5 & & & 0.5 \\
\hline \multirow[t]{3}{*}{0.5} & & 0.5 & & & & 0.5 & & & 0.5 & & 0.5 & \\
\hline & & 0.5 & & & 0.5 & & & 0.5 & & & & 0.5 \\
\hline & 0.5 & & & 0.5 & & & 0.5 & & & & 0.5 & \\
\hline 0.5 & & & 0.5 & & & & 0.5 & & & 0.5 & & \\
\hline \multirow[t]{3}{*}{1} & 0.5 & 0.5 & 0.5 & 1 & 0.5 & 1 & & 0.5 & 0.5 & 0.5 & & 0.5 \\
\hline & 1 & 0.5 & & 0.5 & & & & 0.5 & & 0.5 & & 0.5 \\
\hline & 0.5 & 0.5 & & & & 0.5 & & 0.5 & & 0.5 & & \\
\hline \multirow[t]{4}{*}{0.5} & 0.5 & & & 0.5 & & & & & 0.5 & 0.5 & & \\
\hline & 0.5 & 0.5 & & 0.5 & & 0.5 & 0.5 & 0.5 & & 0.5 & & 0.5 \\
\hline & 0.5 & & 0.5 & & & 0.5 & 0.5 & & & 0.5 & 0.5 & \\
\hline & & & 0.5 & & 0.5 & & & & & & 0.5 & \\
\hline 0.5 & & & & 0.5 & & 0.5 & 0.5 & 0.5 & & & & 0.5 \\
\hline 0.5 & 0.5 & & 0.5 & 0.5 & & 0.5 & 0.5 & & & 0.5 & & 0.5 \\
\hline 0.5 & 0.5 & & 0.5 & & 0.5 & & & 0.5 & 0.5 & & & 0.5 \\
\hline \multirow[t]{3}{*}{0.5} & & 0.5 & & & 0.5 & & 0.5 & & 0.5 & & & 0.5 \\
\hline & & 0.5 & & 0.5 & & 0.5 & 0.5 & & & 1 & & 0.5 \\
\hline & 0.5 & & 0.5 & & & 0.5 & & & & 0.5 & & 0.5 \\
\hline 0.5 & & 0.5 & & & & 0.5 & & & 0.5 & & & 0.5 \\
\hline 0.5 & & 0.5 & & & 0.5 & & 0.5 & & & & 0.5 & \\
\hline \multirow[t]{4}{*}{0.5} & & 0.5 & & & & 0.5 & & & & 0.5 & & \\
\hline & 0.5 & & & 0.5 & & & 0.5 & & 0.5 & & & 0.5 \\
\hline & & & 0.5 & & & & & 0.5 & & & & 0.5 \\
\hline & 0.5 & & 0.5 & & & 0.5 & & 0.5 & & & & 0.5 \\
\hline 0.5 & & 0.5 & & 0.5 & 0.5 & & & 0.5 & 0.5 & & & 0.5 \\
\hline 0.5 & & & & 0.5 & & & & & 0.5 & & & \\
\hline \multirow[t]{2}{*}{0.5} & & & & & & & 0.5 & & & & 0.5 & \\
\hline & 0.5 & & & & & 0.5 & & & & & 0.5 & \\
\hline
\end{tabular}


wbheat

\begin{tabular}{|c|c|c|c|c|c|c|c|c|c|c|c|c|}
\hline & & 0.5 & & & & & 0.5 & & & & & \\
\hline & 0.5 & & & 0.5 & & & 0.5 & & & & 0.5 & \\
\hline & & & 0.5 & & & & 0.5 & & & & & \\
\hline \multirow[t]{2}{*}{0.5} & & & & & 0.5 & & & & & 0.5 & & \\
\hline & & 0.5 & & & & & 0.5 & & & & & \\
\hline \multirow{2}{*}{0.5} & & & & & 0.5 & & & & & & 0.5 & \\
\hline & & 0.5 & & & & & & 0.5 & & & & \\
\hline \multirow[t]{3}{*}{0.5} & & & & & 0.5 & & & & & & 0.5 & \\
\hline & & & & & 0.5 & & & & & & 0.5 & \\
\hline & & & 0.5 & & & & & 0.5 & & & & \\
\hline \multirow[t]{9}{*}{0.5} & & & & & 0.5 & & & & & & & 0.5 \\
\hline & & & 0.5 & & & 0.5 & & & & 0.5 & & \\
\hline & 0.5 & & & & & 0.5 & & & & & 0.5 & \\
\hline & & 0.5 & & & & & & 0.5 & & & & \\
\hline & & & 0.5 & & & & & & & & 0.5 & \\
\hline & & & & & 0.5 & & & & & 0.5 & & \\
\hline & & & & 0.5 & & & & & & 0.5 & & \\
\hline & & 0.5 & & & & & & 0.5 & & & & \\
\hline & & 0.5 & & & & & & 0.5 & & & & \\
\hline \multirow[t]{4}{*}{0.5} & & & & & 0.5 & & & & & 0.5 & & \\
\hline & & & 0.5 & & & & & & 0.5 & & & \\
\hline & & & 0.5 & & & & & & 0.5 & & & \\
\hline & 0.5 & & & & & & 0.5 & & & & & \\
\hline \multirow[t]{12}{*}{0.5} & & & & & 0.5 & & & & & & 0.5 & \\
\hline & & & 0.5 & & & & & 0.5 & & & & \\
\hline & 0.5 & & & & & 0.5 & & & & & & 0.5 \\
\hline & & & & 0.5 & & & & & & & 0.5 & \\
\hline & & & & 0.5 & & & & & 0.5 & & & 0.5 \\
\hline & & & & & 0.5 & & & & & & 0.5 & \\
\hline & & & 0.5 & & & & & & & 0.5 & & \\
\hline & & & 0.5 & & & & & & 0.5 & & & \\
\hline & & 0.5 & & & & & 0.5 & & & & & \\
\hline & & & & 0.5 & & & & & & & 0.5 & \\
\hline & & & 0.5 & & & & & & 0.5 & & & \\
\hline & & 0.5 & & & & & & 0.5 & & & & \\
\hline \multirow[t]{2}{*}{0.5} & & & & & & 0.5 & & & & & 0.5 & \\
\hline & & & & 0.5 & & & & & & 0.5 & & \\
\hline
\end{tabular}

Page 55 
wbheat

\begin{tabular}{|c|c|c|c|c|c|c|c|c|c|c|c|c|}
\hline & & & 0.5 & & & & & & & 0.5 & & \\
\hline & & 0.5 & & & & & & 0.5 & & & & \\
\hline & & & 0.5 & & & & & 0.5 & & & & \\
\hline & 0.5 & & & & & 0.5 & & & & & 0.5 & \\
\hline & & & 0.5 & & & & & 0.5 & & & & \\
\hline & & & & & 0.5 & & & & & & 0.5 & \\
\hline & 0.5 & & & & & 0.5 & & & & & 0.5 & \\
\hline & 0.5 & & & & & 0.5 & & & & & 0.5 & \\
\hline & & 0.5 & & & & & 0.5 & & & & & \\
\hline \multirow[t]{24}{*}{0.5} & & & 0.5 & & & & & & 0.5 & & & \\
\hline & 0.5 & & & & & 0.5 & & & & & & 0.5 \\
\hline & & & & 0.5 & & & & & & 0.5 & & \\
\hline & 0.5 & & & & & 0.5 & & & & & 0.5 & \\
\hline & & & & 0.5 & & & & & 0.5 & & & \\
\hline & 0.5 & & & & & & 0.5 & & & & & 0.5 \\
\hline & & & & & 0.5 & & & & & & 0.5 & \\
\hline & & & 0.5 & & & & & & & 0.5 & & \\
\hline & & & & 0.5 & & & & & 0.5 & & & \\
\hline & 0.5 & & & & & & 0.5 & & & & & 0.5 \\
\hline & 0.5 & & & & & 0.5 & & & & & 0.5 & \\
\hline & & & 0.5 & & & & & & 0.5 & & & \\
\hline & & 0.5 & & & & & & 0.5 & & & & \\
\hline & & 0.5 & & & & & 0.5 & & & & & 0.5 \\
\hline & & & & 0.5 & & & & 0.5 & & & & 0.5 \\
\hline & & & 0.5 & & & & & & 0.5 & & & \\
\hline & 0.5 & & & & & & 0.5 & & & & & 0.5 \\
\hline & & & & 0.5 & & & & & & 0.5 & & \\
\hline & 0.5 & & & & & 0.5 & & & & & 0.5 & \\
\hline & & 0.5 & & & & & & 0.5 & & & & \\
\hline & 0.5 & & & & & 0.5 & & & & & & 0.5 \\
\hline & & & & & 0.5 & & & & & 0.5 & & \\
\hline & & & 0.5 & & & & & 0.5 & & & & 0.5 \\
\hline & & & 0.5 & & & & & 0.5 & & & & \\
\hline 0.5 & & & & & 0.5 & & & & 0.5 & & & \\
\hline 0.5 & & & & 0.5 & & & & & & 0.5 & & \\
\hline 0.5 & & & & & 0.5 & & & & 0.5 & & & 0.5 \\
\hline 0.5 & & & 0.5 & & & & & 0.5 & & & & \\
\hline
\end{tabular}


wbheat

\begin{tabular}{|c|c|c|c|c|c|c|c|c|c|c|c|c|}
\hline & & 0.5 & & & & & 0.5 & & & & 0.5 & \\
\hline \multirow[t]{2}{*}{0.5} & & & 0.5 & & & & 0.5 & & & & & 0.5 \\
\hline & & 0.5 & & & & 0.5 & & & & & 0.5 & \\
\hline \multirow[t]{3}{*}{0.5} & & & & 0.5 & & & & & 0.5 & & & \\
\hline & 0.5 & & & & & & 0.5 & & & & 0.5 & \\
\hline & 0.5 & & & & 0.5 & & & & 0.5 & & & \\
\hline \multirow[t]{3}{*}{0.5} & & & 0.5 & & & & 0.5 & & & & 0.5 & \\
\hline & 0.5 & & & 0.5 & & & 0.5 & & 0.5 & & & \\
\hline & & 0.5 & & & & 0.5 & & & 0.5 & & & \\
\hline 0.5 & & & & 0.5 & & & & & 0.5 & & & \\
\hline \multirow[t]{5}{*}{0.5} & & & & & 0.5 & & & & & & 0.5 & \\
\hline & & & 0.5 & & & & & 0.5 & & & & \\
\hline & 0.5 & & & 0.5 & & & & 0.5 & & 0.5 & & \\
\hline & & 0.5 & & & & 0.5 & & 0.5 & & & & 0.5 \\
\hline & 0.5 & & & & 0.5 & & & & & 0.5 & & \\
\hline \multirow[t]{6}{*}{0.5} & & 0.5 & & & & 0.5 & & 0.5 & & & & 0.5 \\
\hline & & 0.5 & & & 0.5 & & & & 0.5 & & & \\
\hline & 0.5 & & & & & 0.5 & & & & & 0.5 & \\
\hline & & 0.5 & & & & & 0.5 & & & & & 0.5 \\
\hline & & & 0.5 & & & & & 0.5 & & & & 0.5 \\
\hline & 0.5 & & & & 0.5 & & & & & 0.5 & & \\
\hline 3.1 & 3.1 & 0.5 & 0.5 & & & & & 0.5 & 0.5 & & & 0.5 \\
\hline 3.6 & 1.5 & 0.5 & 1 & 1 & 0.5 & 0.5 & 2.6 & 3.6 & 3.1 & 3.6 & 3.1 & 2.6 \\
\hline 0.5 & 0.5 & 0.5 & & 0.5 & 0.5 & 0.5 & & 0.5 & 0.5 & 0.5 & & 0.5 \\
\hline 4.6 & 1.5 & 2.1 & 1 & 0.5 & 1 & 0.5 & 0.5 & & 0.5 & 0.5 & & 0.5 \\
\hline \multirow[t]{2}{*}{4.1} & 3.6 & 2.1 & 1 & 0.5 & & 0.5 & 0.5 & 0.5 & 0.5 & & 1 & \\
\hline & & 0.5 & 0.5 & & 0.5 & & 0.5 & & 0.5 & & 0.5 & 0.5 \\
\hline 0.5 & & 0.5 & 0.5 & 0.5 & 0.5 & 0.5 & 0.5 & 0.5 & 0.5 & 0.5 & 0.5 & 0.5 \\
\hline 1 & 0.5 & 0.5 & 1 & & 0.5 & 0.5 & & 0.5 & & 0.5 & 0.5 & \\
\hline 0.5 & 0.5 & & 1 & & & 0.5 & & 0.5 & & 0.5 & & 0.5 \\
\hline \multirow[t]{3}{*}{0.5} & 0.5 & & 0.5 & 0.5 & & & & 0.5 & & & 0.5 & \\
\hline & 0.5 & 0.5 & 0.5 & & 0.5 & 0.5 & & 0.5 & 0.5 & 0.5 & & 0.5 \\
\hline & 0.5 & 0.5 & & 0.5 & 0.5 & & 0.5 & 0.5 & & 0.5 & 0.5 & \\
\hline \multirow[t]{2}{*}{0.5} & 0.5 & & 1 & & 0.5 & 0.5 & 0.5 & & 0.5 & 0.5 & & 0.5 \\
\hline & 0.5 & 0.5 & 0.5 & & 0.5 & 0.5 & 0.5 & & 0.5 & & 1 & \\
\hline 0.5 & & 0.5 & 0.5 & 0.5 & 0.5 & & 0.5 & & 0.5 & 0.5 & & \\
\hline 0.5 & & 0.5 & & 0.5 & & & 0.5 & & 0.5 & & 0.5 & \\
\hline
\end{tabular}


wbheat

\begin{tabular}{|c|c|c|c|c|c|c|c|c|c|c|c|c|}
\hline 0.5 & & 0.5 & & & & 0.5 & & & 0.5 & & & 0.5 \\
\hline & 0.5 & 0.5 & 0.5 & 0.5 & & 0.5 & 0.5 & 0.5 & & 0.5 & 0.5 & 0.5 \\
\hline \multirow[t]{2}{*}{0.5} & 0.5 & & 1 & 1 & 0.5 & 0.5 & 0.5 & 0.5 & 0.5 & 0.5 & 0.5 & \\
\hline & 0.5 & & 0.5 & 0.5 & & & 0.5 & 0.5 & & & 0.5 & 0.5 \\
\hline \multirow[t]{2}{*}{0.5} & 0.5 & & & 1 & 5.2 & 0.5 & 0.5 & 0.5 & & 0.5 & & 0.5 \\
\hline & 0.5 & & 0.5 & 1 & & & 0.5 & & & 0.5 & & 0.5 \\
\hline 1 & 1 & 0.5 & 0.5 & 1 & 1 & 0.5 & 0.5 & 0.5 & 1 & 0.5 & 0.5 & 0.5 \\
\hline 0.5 & 0.5 & 0.5 & 0.5 & 1 & 0.5 & 0.5 & 0.5 & & 0.5 & 0.5 & 0.5 & 0.5 \\
\hline \multirow{2}{*}{0.5} & 0.5 & 0.5 & 0.5 & 0.5 & 0.5 & 0.5 & & 0.5 & & 0.5 & 0.5 & \\
\hline & 0.5 & & & 0.5 & & & 0.5 & 0.5 & & & & 0.5 \\
\hline 0.5 & & & & & 0.5 & & 0.5 & & & & & 0.5 \\
\hline \multirow{2}{*}{0.5} & & & & 0.5 & & & 0.5 & & & & 0.5 & \\
\hline & 0.5 & & 0.5 & 0.5 & 0.5 & & & & 0.5 & & & 0.5 \\
\hline \multirow[t]{8}{*}{0.5} & & & 0.5 & & & 0.5 & & & & & 0.5 & \\
\hline & 0.5 & & 0.5 & & & & 0.5 & & & & & 0.5 \\
\hline & & 0.5 & & & 0.5 & & & & & & & 0.5 \\
\hline & 0.5 & & & 0.5 & & & & & & 0.5 & & \\
\hline & & & 0.5 & & & & & & 0.5 & & & 0.5 \\
\hline & & & & & 0.5 & & 0.5 & 0.5 & 0.5 & & & \\
\hline & & 0.5 & & & & 0.5 & & & & & & 0.5 \\
\hline & & 0.5 & & & 0.5 & 0.5 & & & & & 0.5 & \\
\hline \multirow[t]{6}{*}{0.5} & & & 0.5 & & & 0.5 & & & & & 0.5 & \\
\hline & 0.5 & & & & & & & 0.5 & & & & 0.5 \\
\hline & & & 0.5 & & & & & 0.5 & & & & \\
\hline & & & 0.5 & & & & & & 0.5 & & & \\
\hline & & & 0.5 & & & & 0.5 & & & & & 0.5 \\
\hline & & 0.5 & & & 0.5 & & & & 0.5 & & & \\
\hline 0.5 & & & & 0.5 & & & & & 0.5 & & & \\
\hline \multirow[t]{4}{*}{0.5} & & & & 0.5 & & & & & & 0.5 & & \\
\hline & 0.5 & & & & & & 0.5 & & & & & 0.5 \\
\hline & & & & 0.5 & & & & & & 0.5 & & \\
\hline & & & & 0.5 & & & & & 0.5 & & & \\
\hline \multirow[t]{2}{*}{0.5} & & & & 0.5 & & & & & 0.5 & & & \\
\hline & & 0.5 & & & & & & & 0.5 & & & \\
\hline \multirow[t]{2}{*}{0.5} & & & & 0.5 & & & & 0.5 & & & & 0.5 \\
\hline & & 0.5 & & & & & & & & 0.5 & & \\
\hline 0.5 & & & & & 0.5 & & & & & 0.5 & & \\
\hline
\end{tabular}


wbheat

\begin{tabular}{|c|c|c|c|c|c|c|c|c|c|c|c|c|}
\hline & & & & & & & 0.5 & & & & 0.5 & \\
\hline & & 0.5 & & & & 0.5 & & & & & 0.5 & \\
\hline & 0.5 & & & & 0.5 & & & & & 0.5 & & \\
\hline \multirow[t]{2}{*}{0.5} & & & & & & & & 0.5 & & & & \\
\hline & & & & 0.5 & & & & & 0.5 & & & \\
\hline \multirow[t]{2}{*}{0.5} & & & & & 0.5 & & & & & & 0.5 & \\
\hline & 0.5 & & & & & 0.5 & & & & & 0.5 & \\
\hline \multirow[t]{3}{*}{0.5} & & & & & & 0.5 & & & & 0.5 & & \\
\hline & & & 0.5 & & & & & 0.5 & & & & 0.5 \\
\hline & & & & 0.5 & 0.5 & & & & 0.5 & & & \\
\hline \multirow[t]{10}{*}{0.5} & & & & & & 0.5 & & & & & 0.5 & \\
\hline & 0.5 & & & & & & & 0.5 & & & & \\
\hline & & & & & 0.5 & & & & & & & 0.5 \\
\hline & & 0.5 & & & & & & & 0.5 & & & \\
\hline & 0.5 & & & & & & & & 0.5 & & & \\
\hline & & & & & & 0.5 & & & & & & \\
\hline & & & 0.5 & & & & & & & & 0.5 & \\
\hline & 0.5 & & & & & & & & 0.5 & & & \\
\hline & & 0.5 & & & & & & & & & & \\
\hline & & & & & & & 0.5 & & & & & \\
\hline \multirow[t]{10}{*}{0.5} & & & & & & & & & 0.5 & & & \\
\hline & & 0.5 & & & & & & & & & 0.5 & \\
\hline & & & & 0.5 & & & & & & & & \\
\hline & & 0.5 & & & & & & & & & & \\
\hline & & & & & & & & 0.5 & & & & \\
\hline & 0.5 & & & & & & & & & 0.5 & & \\
\hline & & & & 0.5 & & & & & & & & \\
\hline & & & & & & & 0.5 & & & & & \\
\hline & & & & 0.5 & & & & & & & & \\
\hline & & & & & & & 0.5 & & & & & \\
\hline \multirow[t]{7}{*}{0.5} & & & & & & & & & 0.5 & & & \\
\hline & & & & 0.5 & & & & & & & & \\
\hline & & & & & & & 0.5 & & & & & \\
\hline & & & & 0.5 & & & & & & & & \\
\hline & & & & & & & & 0.5 & & & & \\
\hline & & & 0.5 & & & & & & & & & \\
\hline & & & & & & & & & 0.5 & & & \\
\hline
\end{tabular}


wbheat

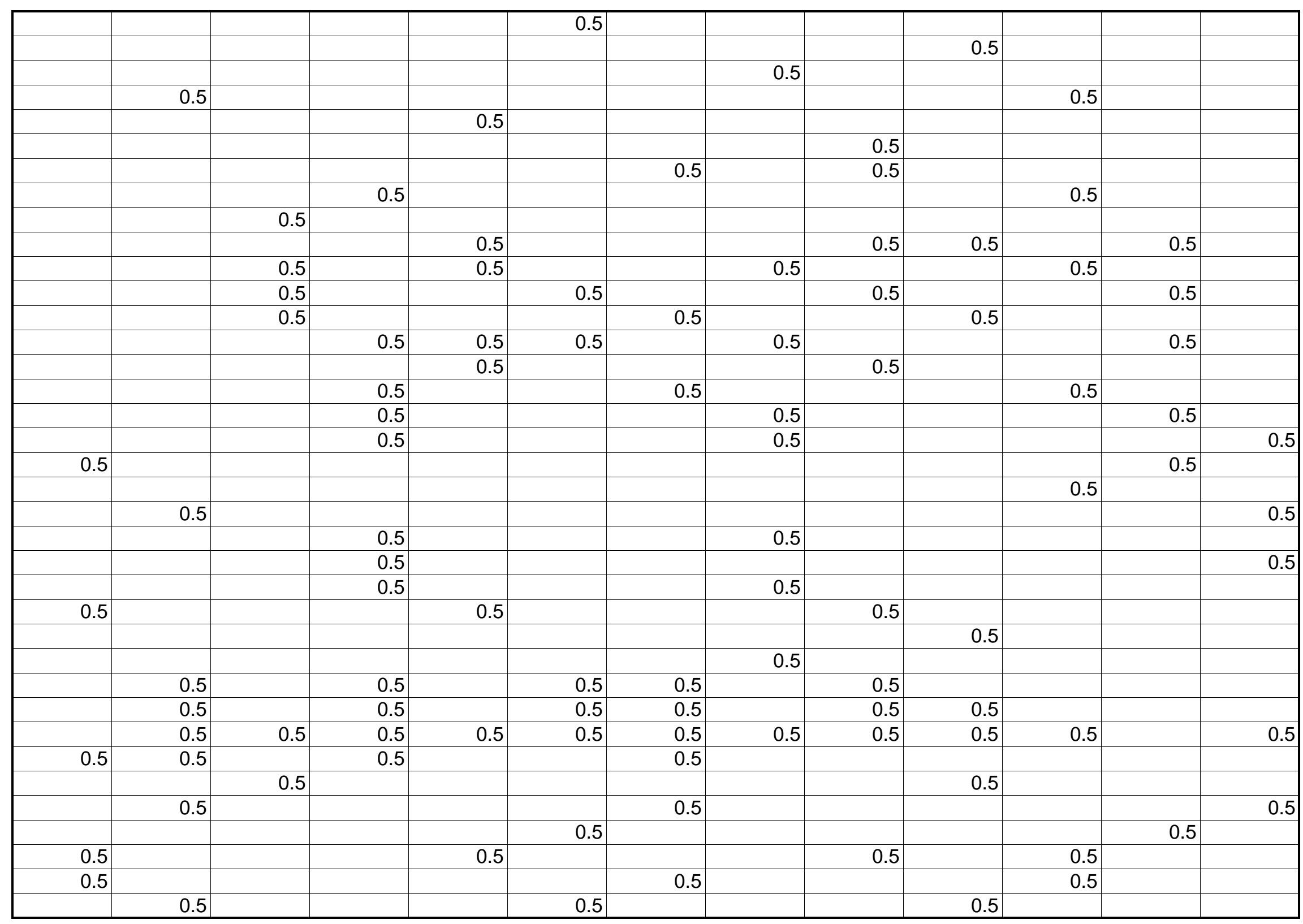




\begin{tabular}{|c|c|c|c|c|c|c|c|c|c|c|c|c|}
\hline & 0.5 & & & 0.5 & & & & 0.5 & & 0.5 & & 0.5 \\
\hline \multirow[t]{7}{*}{0.5} & & & 0.5 & & & & 0.5 & & & & 0.5 & \\
\hline & & & 0.5 & & & & & & 0.5 & & & \\
\hline & & & & 0.5 & & & & & 0.5 & & & \\
\hline & 0.5 & & & 0.5 & & & 0.5 & & 0.5 & & & \\
\hline & 0.5 & & & 0.5 & & & 0.5 & & & 0.5 & & \\
\hline & & 0.5 & & & & 0.5 & & & 0.5 & & & \\
\hline & 0.5 & & & 0.5 & & & & 0.5 & & & & \\
\hline \multirow[t]{4}{*}{0.5} & & & 0.5 & & & & 0.5 & & 0.5 & & 0.5 & \\
\hline & 0.5 & & 0.5 & & 0.5 & & 0.5 & & & 0.5 & & 0.5 \\
\hline & & 0.5 & & 0.5 & & & 0.5 & & 0.5 & & & 0.5 \\
\hline & 0.5 & & & 0.5 & & 0.5 & & 0.5 & & & 0.5 & \\
\hline \multirow[t]{3}{*}{0.5} & & & 0.5 & & 0.5 & & & 0.5 & & 0.5 & & \\
\hline & & 0.5 & & 0.5 & & 0.5 & & & 0.5 & & & 0.5 \\
\hline & 0.5 & & 0.5 & & & & & & 0.5 & & & 0.5 \\
\hline 0.5 & & & & & 0.5 & & & & 0.5 & & & \\
\hline 1 & & 0.5 & 0.5 & 0.5 & 0.5 & & 1 & & 0.5 & & 0.5 & \\
\hline \multirow[t]{3}{*}{1} & 0.5 & & 0.5 & 0.5 & 0.5 & 0.5 & & 0.5 & & 0.5 & 0.5 & \\
\hline & 0.5 & 0.5 & 1 & 1 & 0.5 & 1 & 0.5 & 0.5 & & & & 0.5 \\
\hline & & 0.5 & & 0.5 & & & & 0.5 & & & 0.5 & \\
\hline \multirow[t]{3}{*}{0.5} & & 0.5 & & & 0.5 & & 1 & 1 & 0.5 & & 0.5 & 0.5 \\
\hline & & 0.5 & & & 0.5 & & & 1.5 & 0.5 & 0.5 & 0.5 & \\
\hline & & 0.5 & & 0.5 & & 0.5 & 0.5 & & 0.5 & 0.5 & 0.5 & \\
\hline 0.5 & 0.5 & 0.5 & & 0.5 & 0.5 & 0.5 & & 0.5 & 1 & 0.5 & 1 & \\
\hline 0.5 & 0.5 & & 0.5 & 0.5 & 0.5 & & & 0.5 & & 0.5 & & \\
\hline 0.5 & & 1 & 0.5 & & 0.5 & 0.5 & & & 0.5 & & 0.5 & 0.5 \\
\hline 0.5 & & & 0.5 & 0.5 & & & 0.5 & & 0.5 & 0.5 & 1 & 0.5 \\
\hline 0.5 & 0.5 & 0.5 & 0.5 & 1 & & 1 & 0.5 & 0.5 & 0.5 & 0.5 & 0.5 & 0.5 \\
\hline 1 & & 0.5 & 1 & 0.5 & & 0.5 & 0.5 & 0.5 & 0.5 & 0.5 & & 0.5 \\
\hline 0.5 & 0.5 & 0.5 & 0.5 & 0.5 & 0.5 & 0.5 & & 0.5 & 0.5 & & 0.5 & 0.5 \\
\hline 0.5 & 0.5 & 1 & 0.5 & & 0.5 & & 0.5 & & 0.5 & 0.5 & & \\
\hline 0.5 & & & & & & 0.5 & & & & 0.5 & & \\
\hline 1 & & 0.5 & 1 & 1 & 0.5 & 0.5 & 0.5 & & 0.5 & & 0.5 & \\
\hline 0.5 & 0.5 & & 1 & 0.5 & 0.5 & 0.5 & & 0.5 & & 0.5 & & 0.5 \\
\hline \multirow[t]{3}{*}{0.5} & 0.5 & & 0.5 & & 0.5 & & 0.5 & & & 0.5 & & \\
\hline & & 1 & 0.5 & & & & 0.5 & & & 0.5 & & 0.5 \\
\hline & & 0.5 & & 0.5 & & & & & 0.5 & & & \\
\hline
\end{tabular}




\begin{tabular}{|c|c|c|c|c|c|c|c|c|c|c|c|c|}
\hline 4.1 & 3.6 & 2.1 & & 0.5 & & 0.5 & 0.5 & & & 0.5 & & \\
\hline 3.6 & 4.1 & 4.6 & 3.1 & 1 & 1 & 0.5 & 0.5 & 0.5 & & 0.5 & & 0.5 \\
\hline 4.6 & 4.6 & 4.1 & 4.1 & 0.5 & 1 & 0.5 & 0.5 & & & 0.5 & & 0.5 \\
\hline 0.5 & 0.5 & 1 & 0.5 & & 0.5 & & & & & 0.5 & & \\
\hline \multirow[t]{2}{*}{3.1} & 1.5 & 1 & 1 & 1 & & 0.5 & 0.5 & & & & 0.5 & \\
\hline & & & 0.5 & & 0.5 & & & & & 0.5 & & \\
\hline 0.5 & & 0.5 & & 0.5 & & 0.5 & & 0.5 & & & & 0.5 \\
\hline \multirow[t]{2}{*}{1} & & 0.5 & & 0.5 & & 0.5 & & 0.5 & & & 0.5 & 0.5 \\
\hline & 0.5 & & & 0.5 & & 0.5 & & 0.5 & & 0.5 & & \\
\hline 0.5 & & 0.5 & & & 0.5 & 0.5 & & & 0.5 & & 0.5 & \\
\hline 0.5 & & 0.5 & & & & & 0.5 & & & 0.5 & & 0.5 \\
\hline \multirow[t]{6}{*}{0.5} & & & & 0.5 & & & 0.5 & & & & 0.5 & \\
\hline & 0.5 & & & & & 0.5 & & & & & 0.5 & \\
\hline & & 0.5 & & & & & 0.5 & & & & & \\
\hline & 0.5 & & & & & & 0.5 & & 0.5 & & & \\
\hline & & & & 0.5 & & 0.5 & & & & & & 0.5 \\
\hline & 0.5 & & & & & 0.5 & & & & & & 0.5 \\
\hline \multirow[t]{4}{*}{0.5} & 0.5 & & 0.5 & & & 0.5 & 0.5 & & 0.5 & & & 0.5 \\
\hline & & & 0.5 & & & & 0.5 & & & & & 0.5 \\
\hline & 0.5 & & & & & 0.5 & & & & & 0.5 & \\
\hline & & & 0.5 & & & 0.5 & & & & 0.5 & & \\
\hline \multirow[t]{7}{*}{0.5} & & & 0.5 & & & 0.5 & & & 0.5 & & & \\
\hline & & 0.5 & & & & & 0.5 & & & & 0.5 & \\
\hline & 0.5 & & & & 0.5 & & & & 0.5 & & & \\
\hline & & 0.5 & & & & 0.5 & & & & 0.5 & & \\
\hline & & & & 0.5 & & & & & 0.5 & & & \\
\hline & & & & & & & & & & & & \\
\hline & & & & & & & & & & & & \\
\hline 1.585 & 1.405063 & 1.337313 & 1.193056 & 1.203846 & 1.206944 & 1.052941 & 1.07377 & 0.830189 & 0.771186 & 0.75 & 0.747826 & 0.630357 \\
\hline 1.750644 & 1.378313 & 1.242489 & 1.179293 & 1.025258 & 1.052381 & 0.871749 & 0.826667 & 0.99951 & 0.876647 & 0.788235 & 0.703448 & 0.679114 \\
\hline 1.57619 & 1.38 & 1.126087 & 1.216667 & 0.821739 & 1.15 & 0.94 & 1.016667 & 1.166667 & 1.105882 & 1.422222 & 1.229412 & 0.895238 \\
\hline 0.88481 & 0.998214 & 0.775 & 0.695238 & 0.636667 & 0.651064 & 0.654386 & 0.585185 & 0.62 & 0.607018 & 0.558491 & 0.528846 & 0.534483 \\
\hline 2.231034 & 1.697143 & 1.591667 & 1.608824 & 1.470588 & 1.358333 & 1.424242 & 1.54375 & 1.451613 & 1.153571 & 0.667742 & 0.664 & 0.6 \\
\hline 0.835897 & 0.730986 & 0.695455 & 0.627632 & 0.556338 & 0.601639 & 0.515385 & 0.54697 & 0.559016 & 0.54697 & 0.55 & 0.577358 & 0.535 \\
\hline 0.64 & 0.633333 & 1.253333 & 0.545455 & 0.5 & 0.5 & 0.5 & 0.706667 & 0.86 & 0.716667 & 0.7 & 0.555556 & 0.583333 \\
\hline 0.719048 & 0.632258 & 0.572414 & 0.589286 & 0.568966 & 0.52381 & 0.517241 & 0.5 & 0.541667 & 0.517241 & 0.521739 & 0.516129 & 0.5 \\
\hline
\end{tabular}

Page 62 


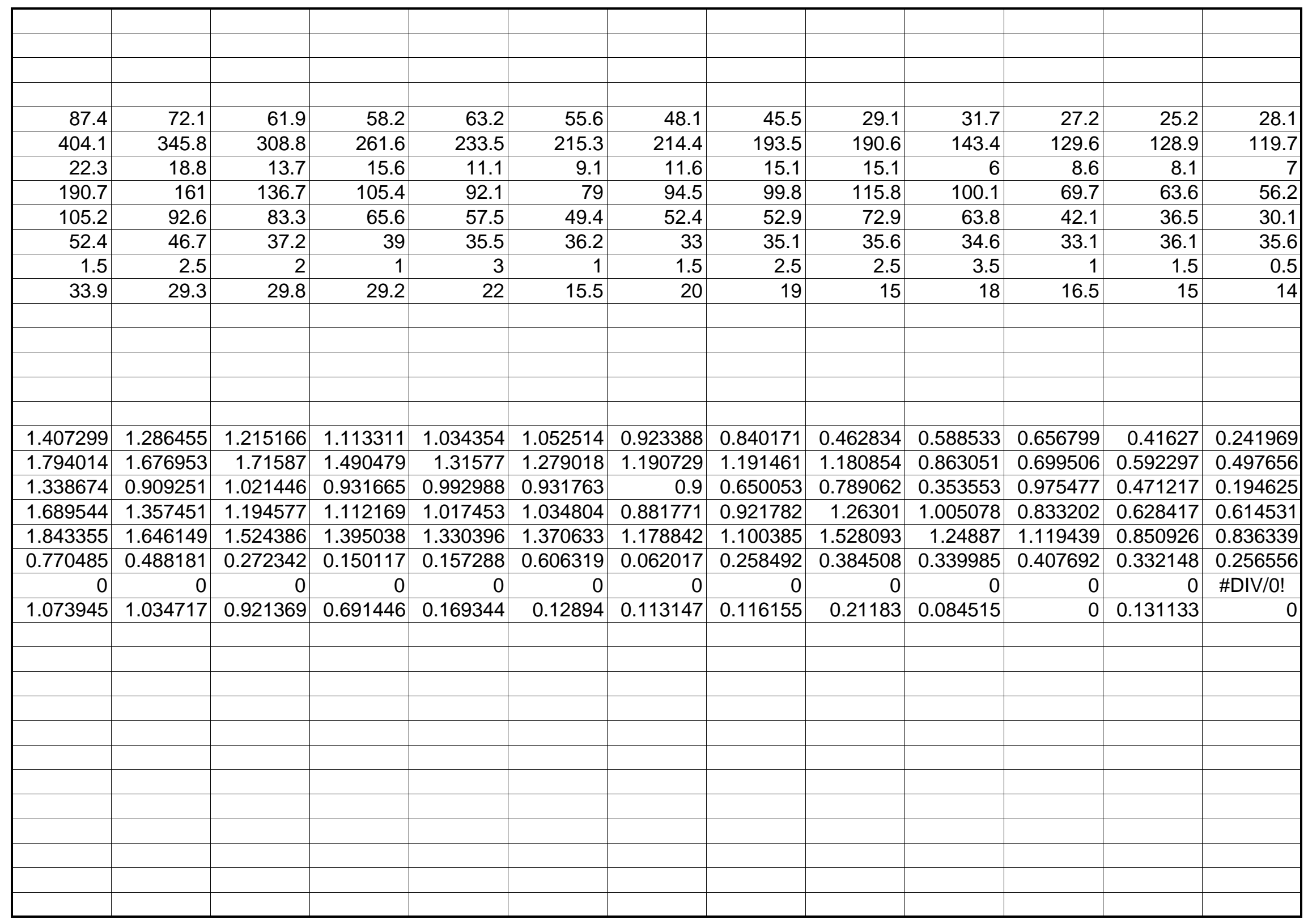


wbheat

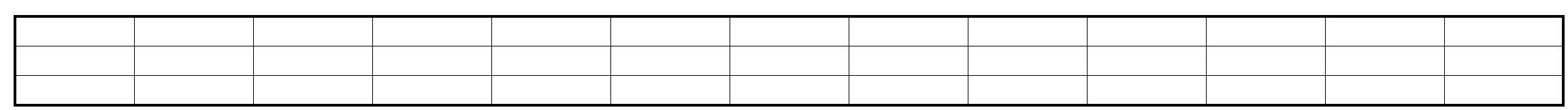

Page 64 


\begin{tabular}{|c|c|c|c|c|c|c|c|c|}
\hline 22 & 23 & $\begin{array}{l}\text { DAILY } \\
\text { TOTAL }\end{array}$ & $\begin{array}{l}\text { AVERAGE } \\
\text { DAILY } \\
\text { TOTAL }\end{array}$ & $\begin{array}{l}\text { MONTHLY } \\
\text { TOTAL formula }\end{array}$ & $\begin{array}{l}\text { MONTHLY } \\
\text { TOTAL } \\
\text { value }\end{array}$ & & & \\
\hline 0.5 & & 12.1 & 0.67 & & & & & \\
\hline \multirow[t]{2}{*}{0.5} & & 11.1 & 0.69 & & & & & \\
\hline & & 9.6 & 0.64 & & & & & \\
\hline 0.5 & 0.5 & 11.6 & 0.61 & & & & & \\
\hline \multirow[t]{4}{*}{0.5} & & 12.6 & 0.70 & & & & & \\
\hline & 0.5 & 10 & 0.63 & & & & & \\
\hline & 0.5 & 11.6 & 0.64 & & & & & \\
\hline & 0.5 & 10.6 & 0.62 & & & \multicolumn{2}{|c|}{ sort $A / B, S / N S$} & \\
\hline \multirow[t]{10}{*}{0.5} & & 6 & 0.60 & & & B-S & 5058.50 & \\
\hline & 0.5 & 7.1 & 0.79 & & & B-NS & 1351.80 & \\
\hline & & 8.5 & 0.61 & & & & & \\
\hline & & 7 & 0.50 & & & A-S & 1971.60 & \\
\hline & & 9 & 0.64 & & & A-NS & 1060.00 & \\
\hline & & 9.5 & 0.63 & & & & & \\
\hline & 0.5 & 9.1 & 0.76 & & & & 9441.90 & 0.00 \\
\hline & 0.5 & 8 & 0.57 & & & & & \\
\hline & 0.5 & 8.5 & 0.61 & & & & & \\
\hline & 0.5 & 10.1 & 0.59 & & & & & \\
\hline \multirow[t]{7}{*}{0.5} & & 8.5 & 0.57 & & & & & \\
\hline & & 9.1 & 0.83 & & 0 & & & \\
\hline & 0.5 & 0 & \#DIV/0! & & & & & \\
\hline & 0.5 & 1 & 0.50 & & & & & \\
\hline & 0.5 & 8 & 0.57 & & & & & \\
\hline & & 6.5 & 0.59 & & & & & \\
\hline & 0.5 & 11.1 & 0.74 & 216.20 & & & & \\
\hline 0.5 & & 9.1 & 0.61 & & & & & \\
\hline \multirow[t]{3}{*}{0.5} & & 9.1 & 0.61 & & & & & \\
\hline & & 9 & 0.60 & & & & & \\
\hline & & 7 & 0.54 & & & & & \\
\hline 0.5 & & 8.6 & 0.66 & & & & & \\
\hline \multirow[t]{2}{*}{0.5} & & 7.5 & 0.58 & & & & & \\
\hline & & 8 & 0.57 & & & & & \\
\hline 0.5 & & 9.1 & 0.61 & & & & & \\
\hline
\end{tabular}


wbheat

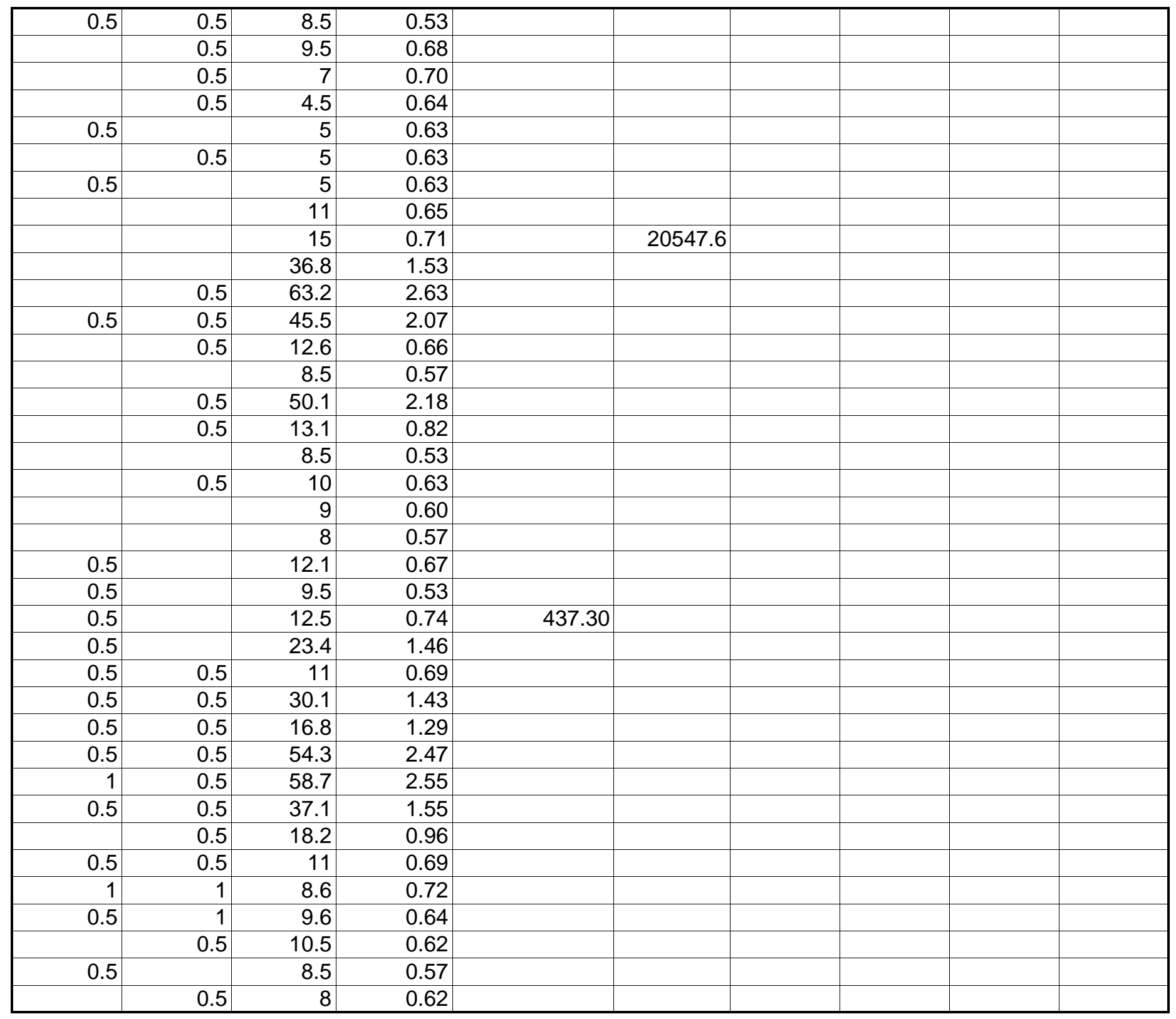

Page 66 
wbheat

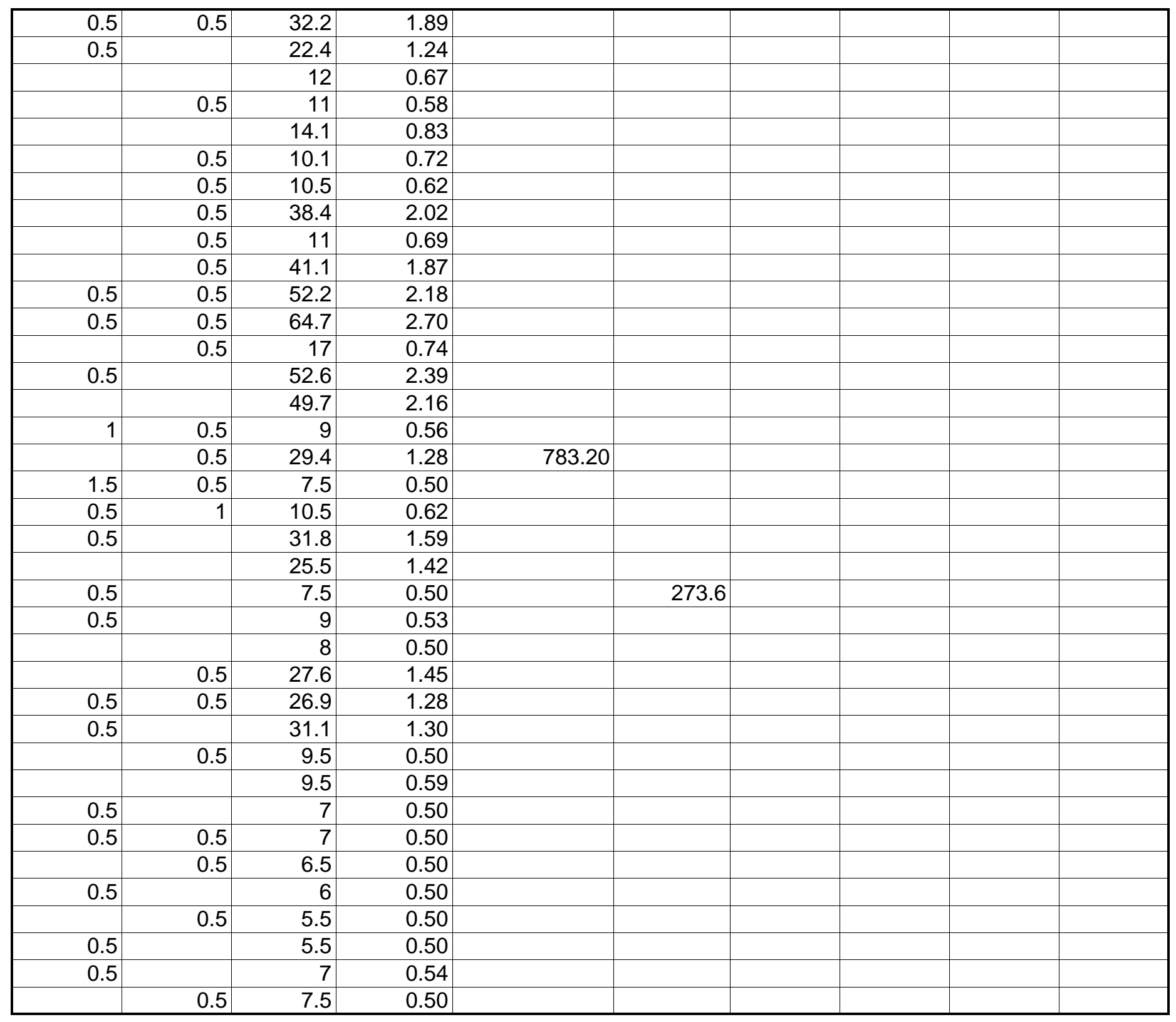

Page 67 
wbheat

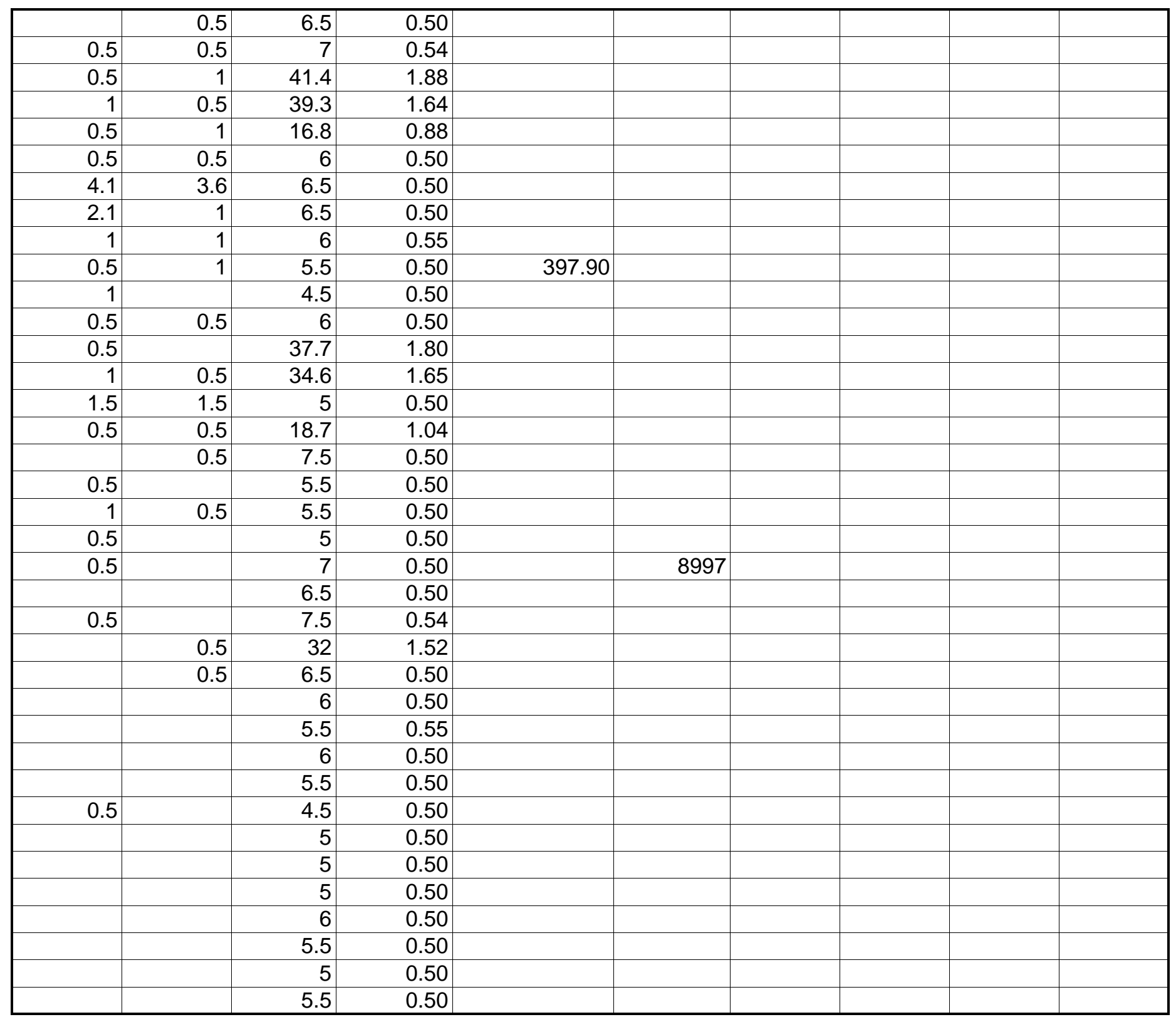

Page 68 
wbheat

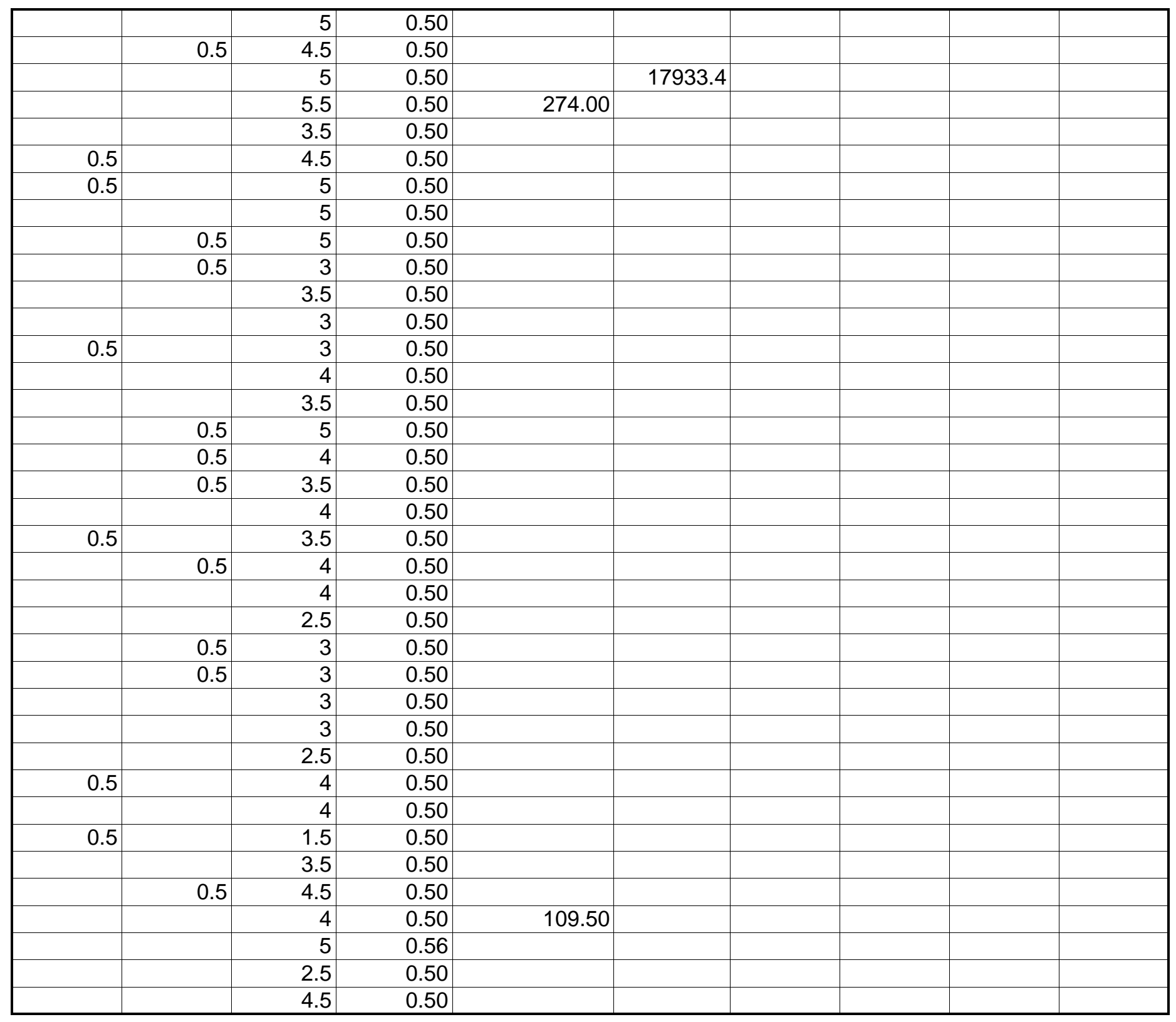

Page 69 
wbheat

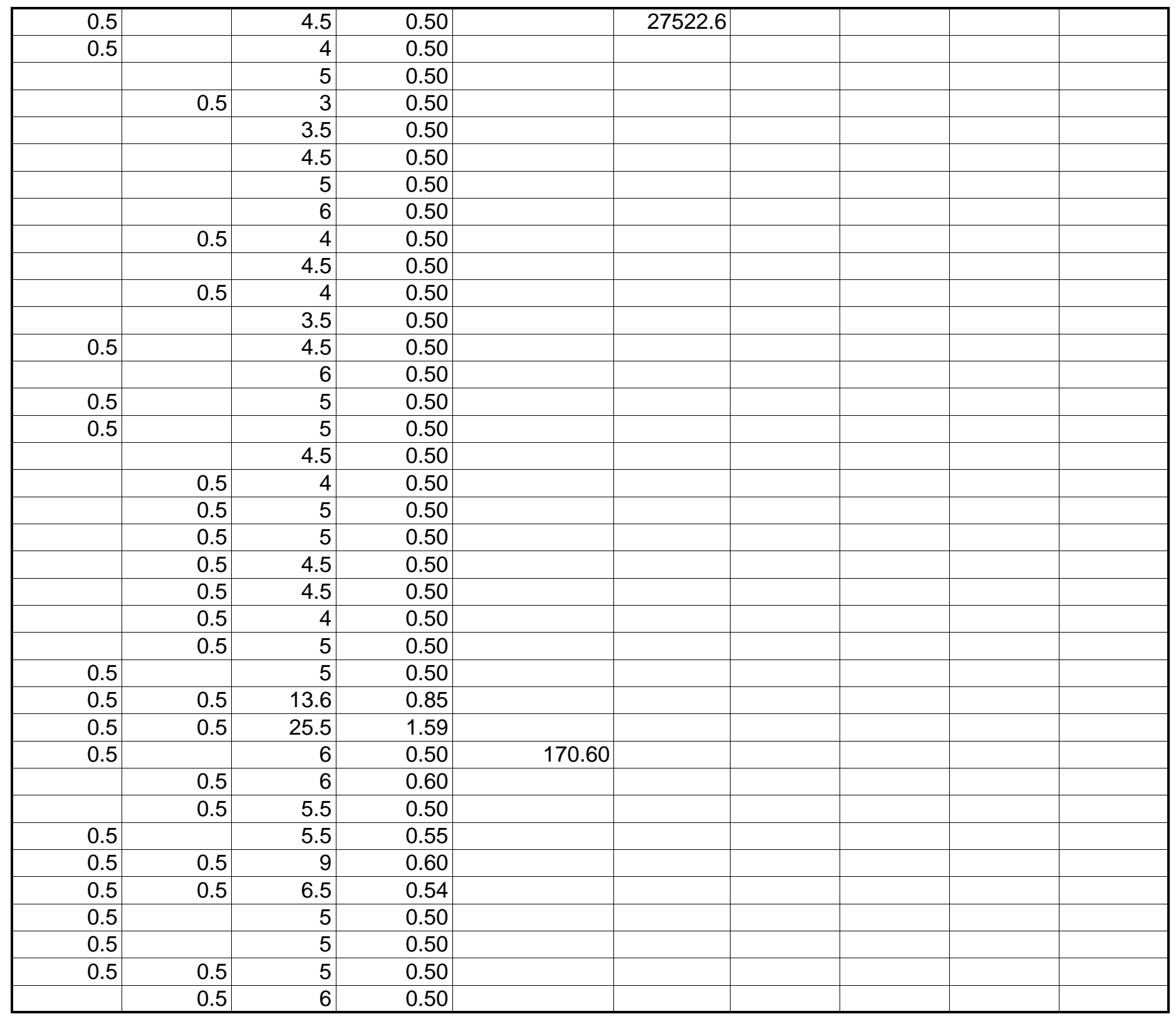

Page 70 
wbheat

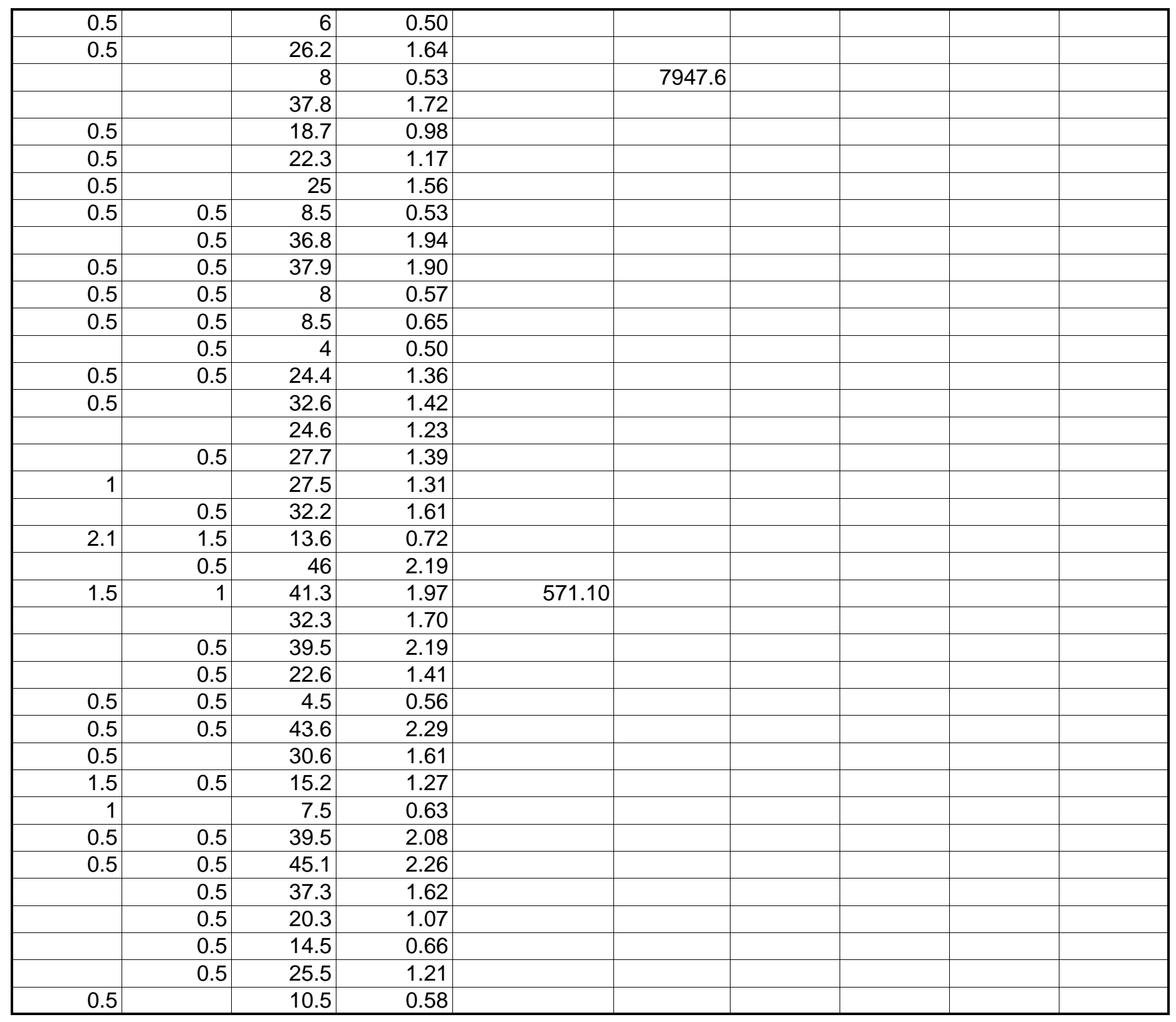

Page 71 
wbheat

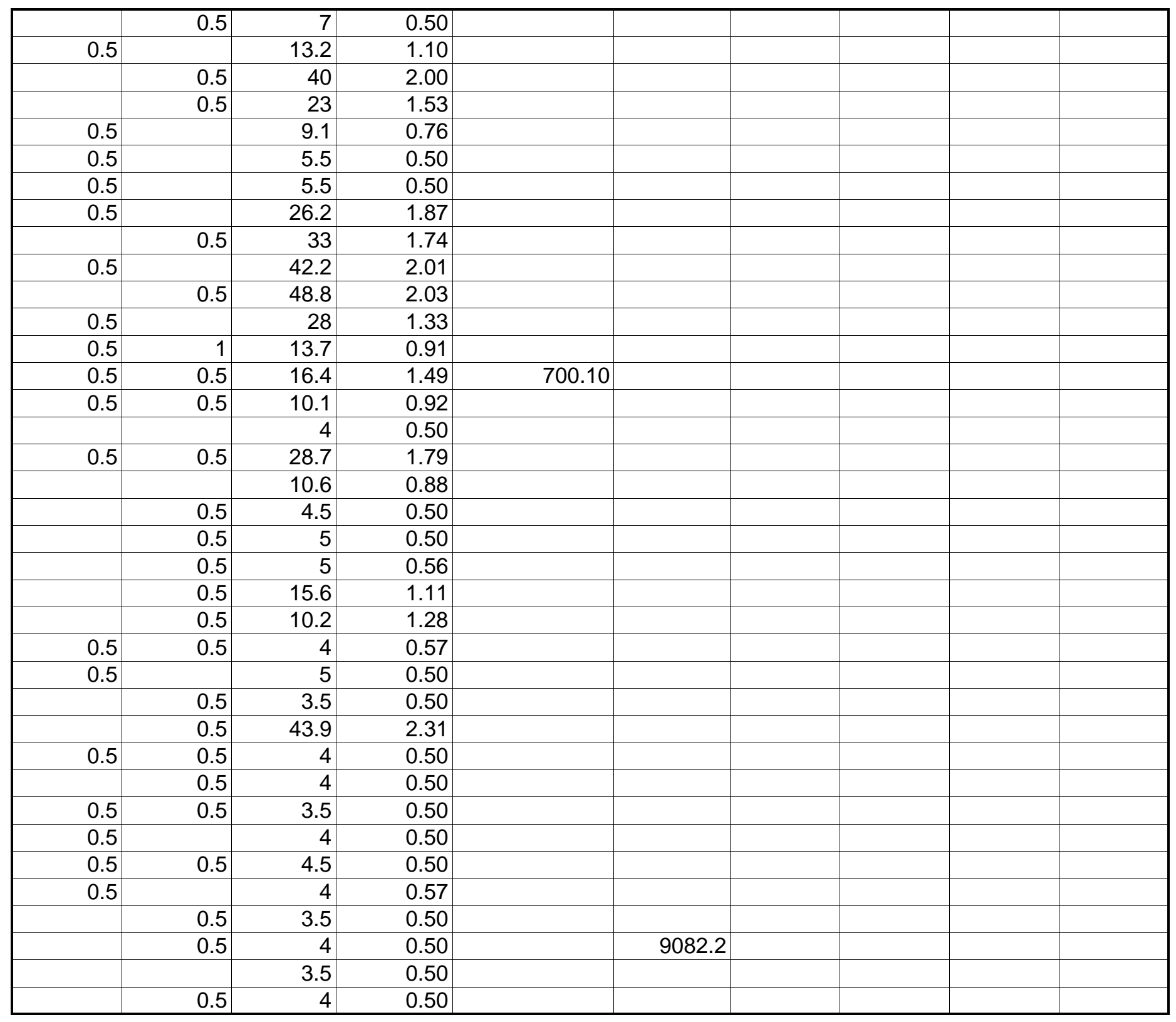

Page 72 
wbheat

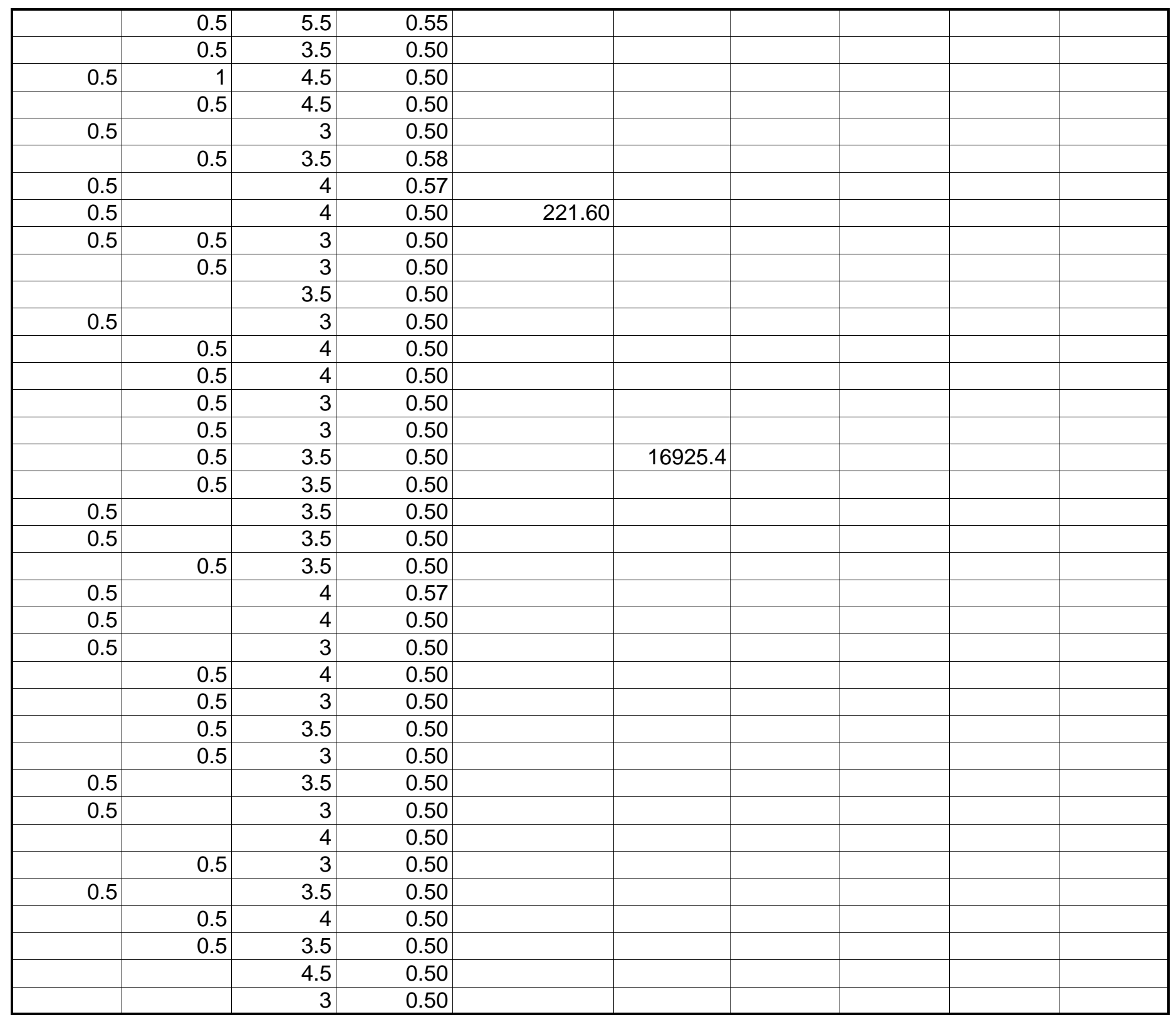

Page 73 
wbheat

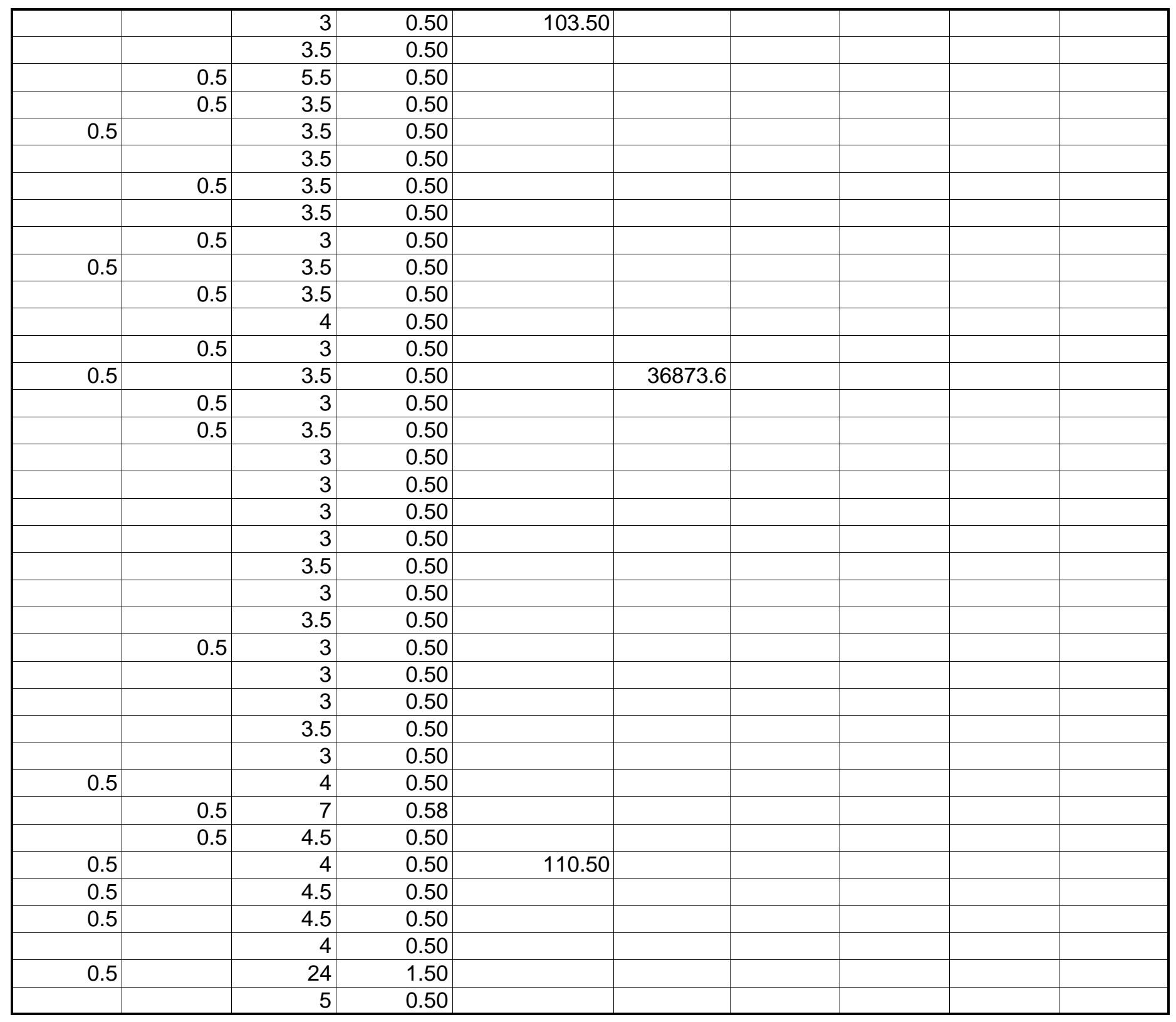

Page 74 
wbheat

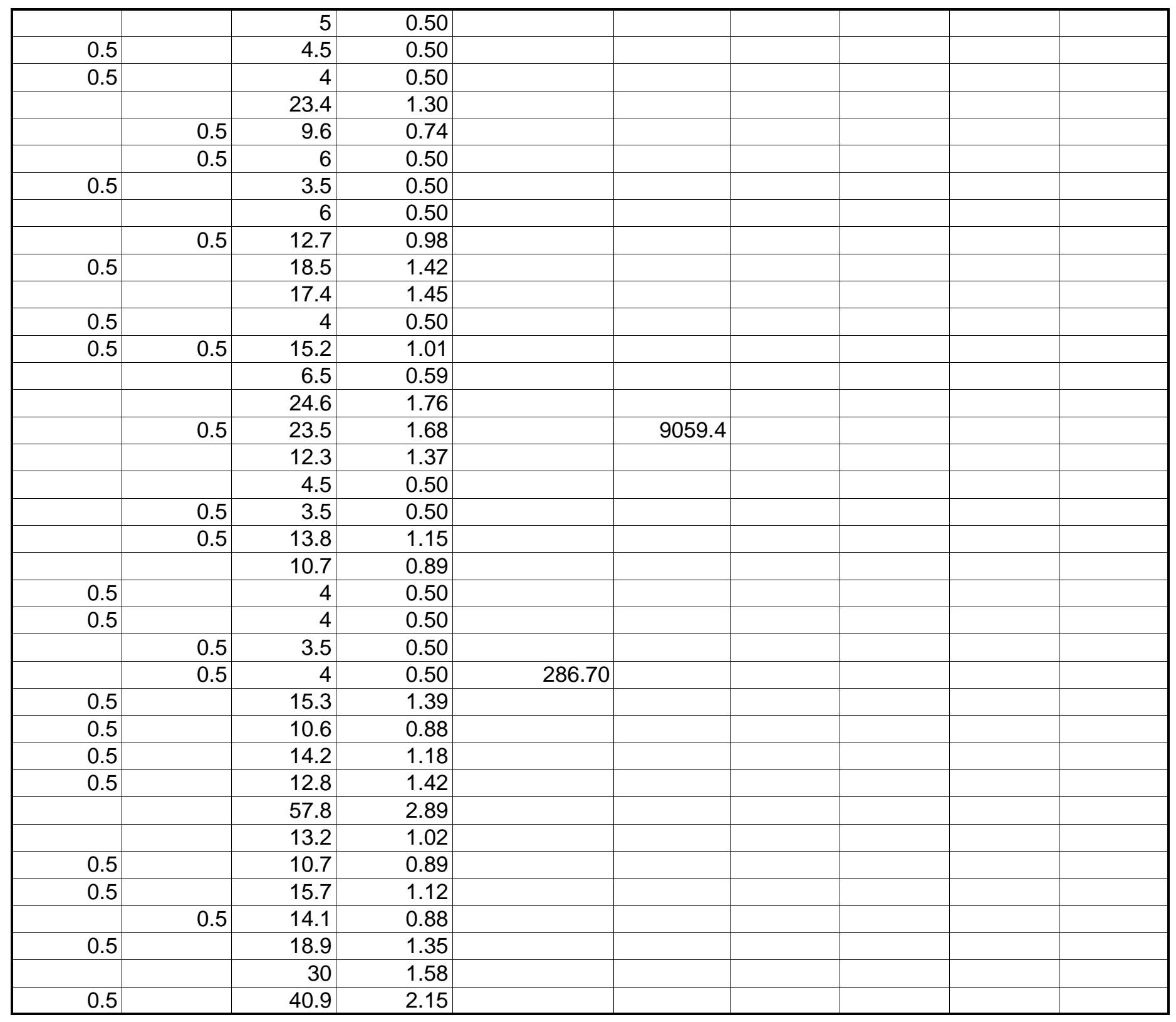

Page 75 
wbheat

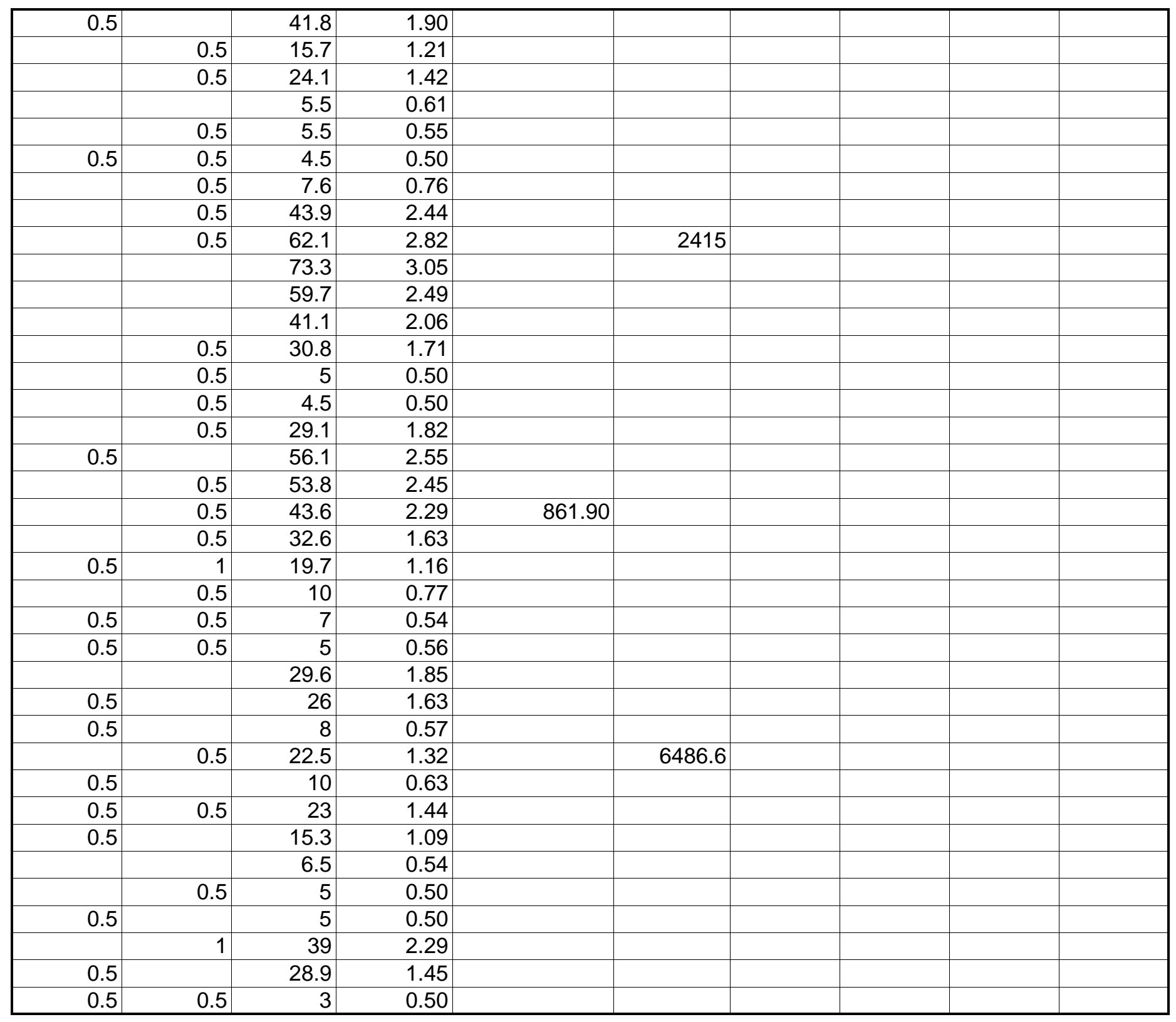

Page 76 
wbheat

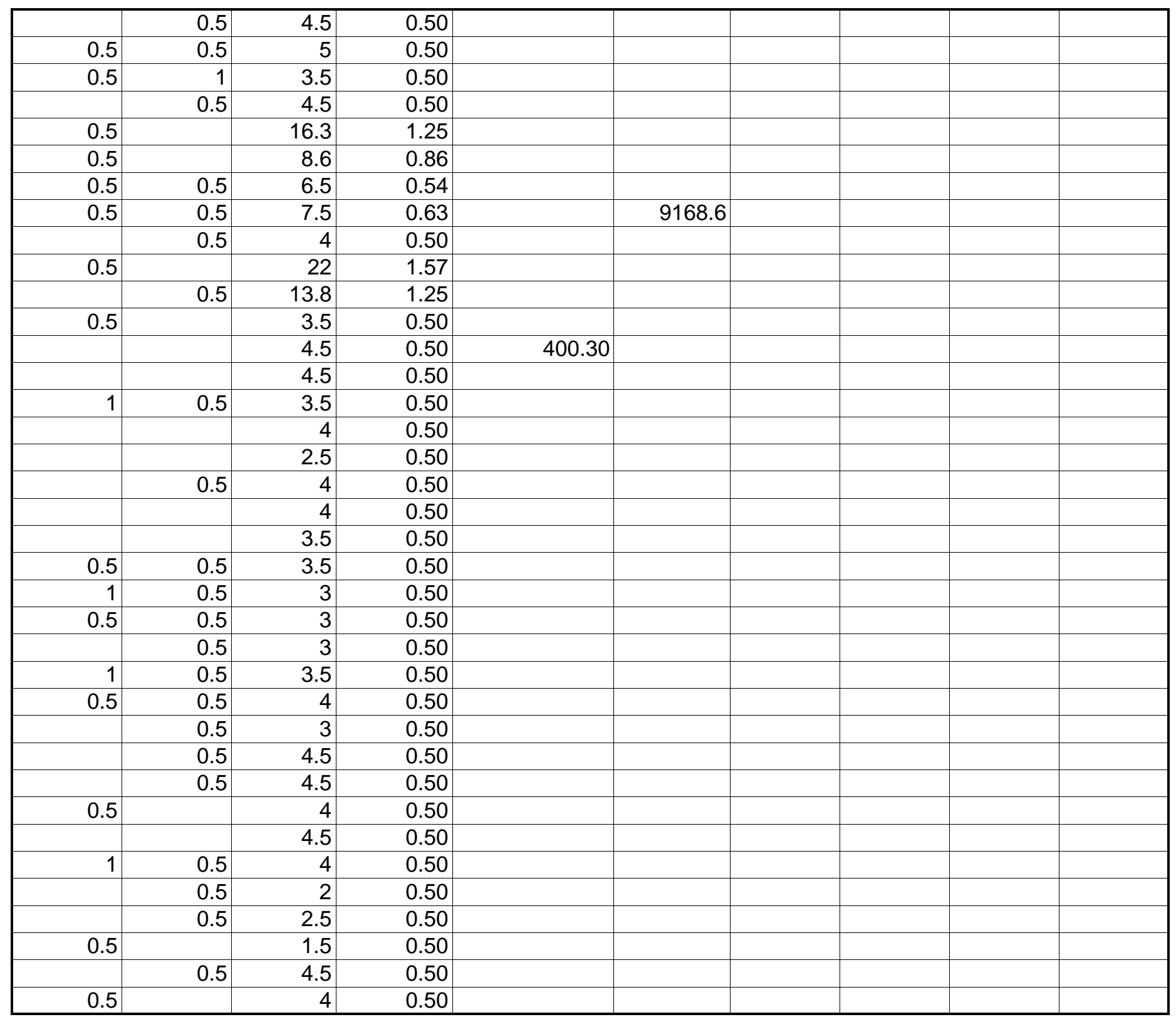

Page 77 
wbheat

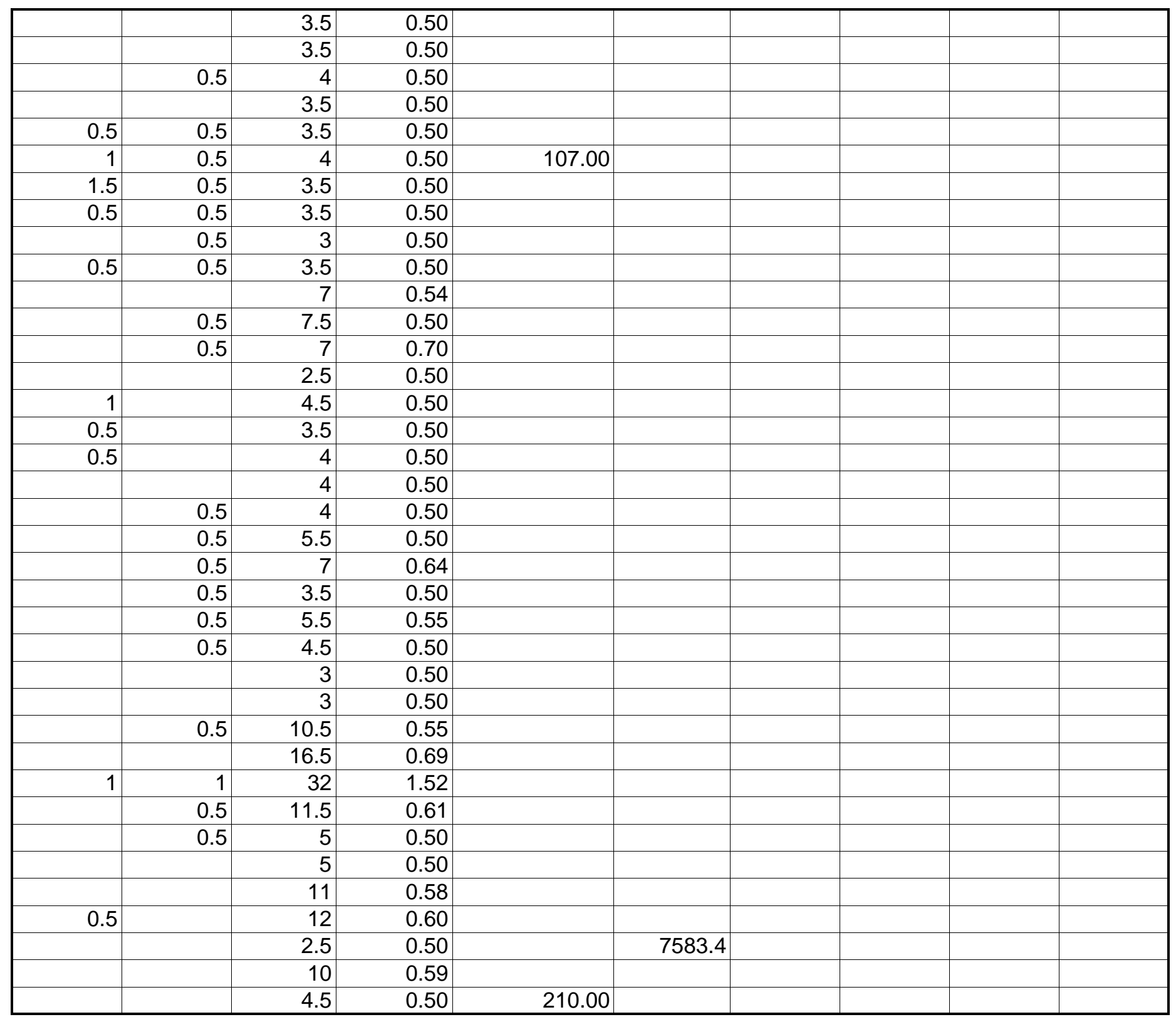

Page 78 
wbheat

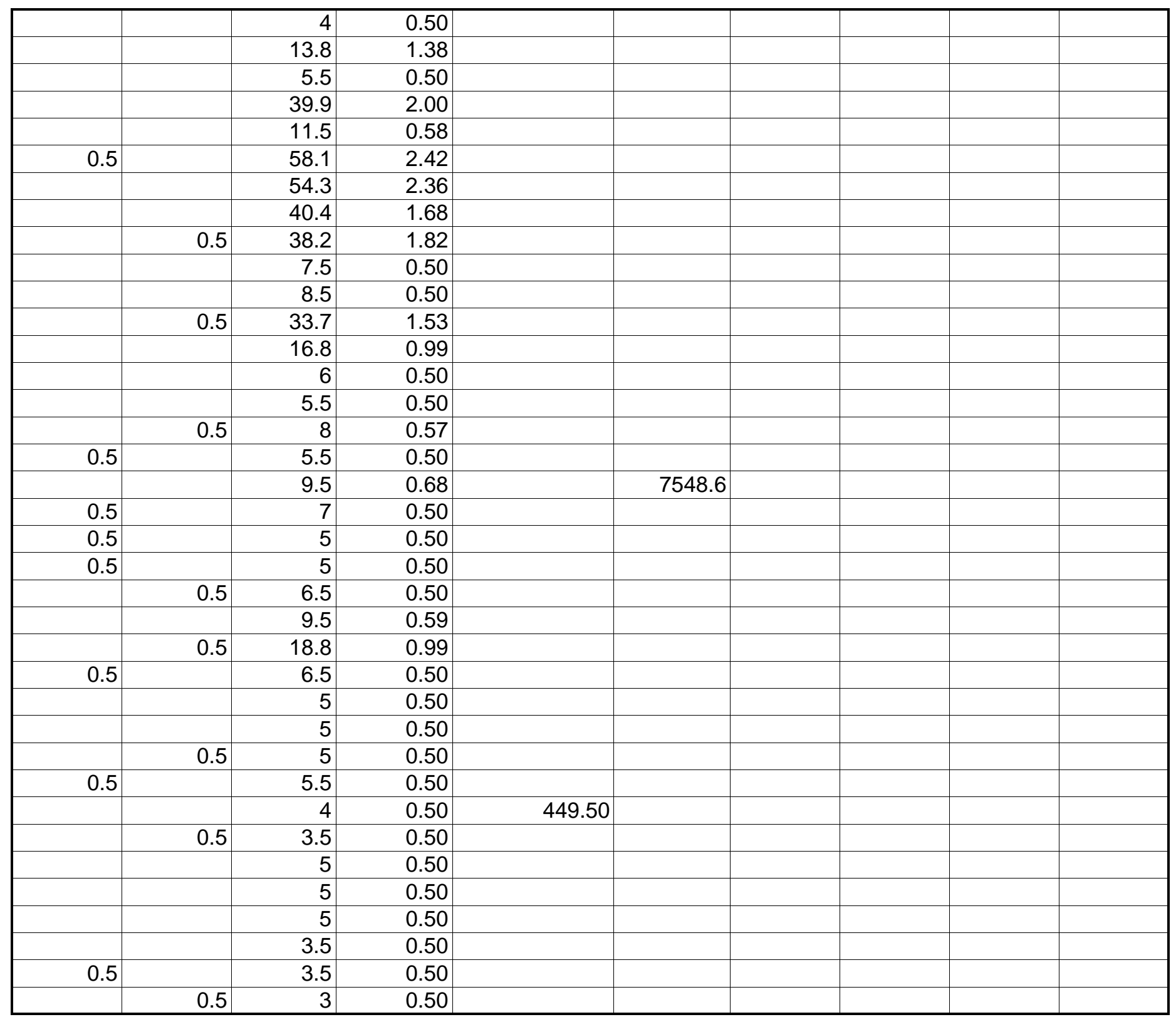

Page 79 
wbheat

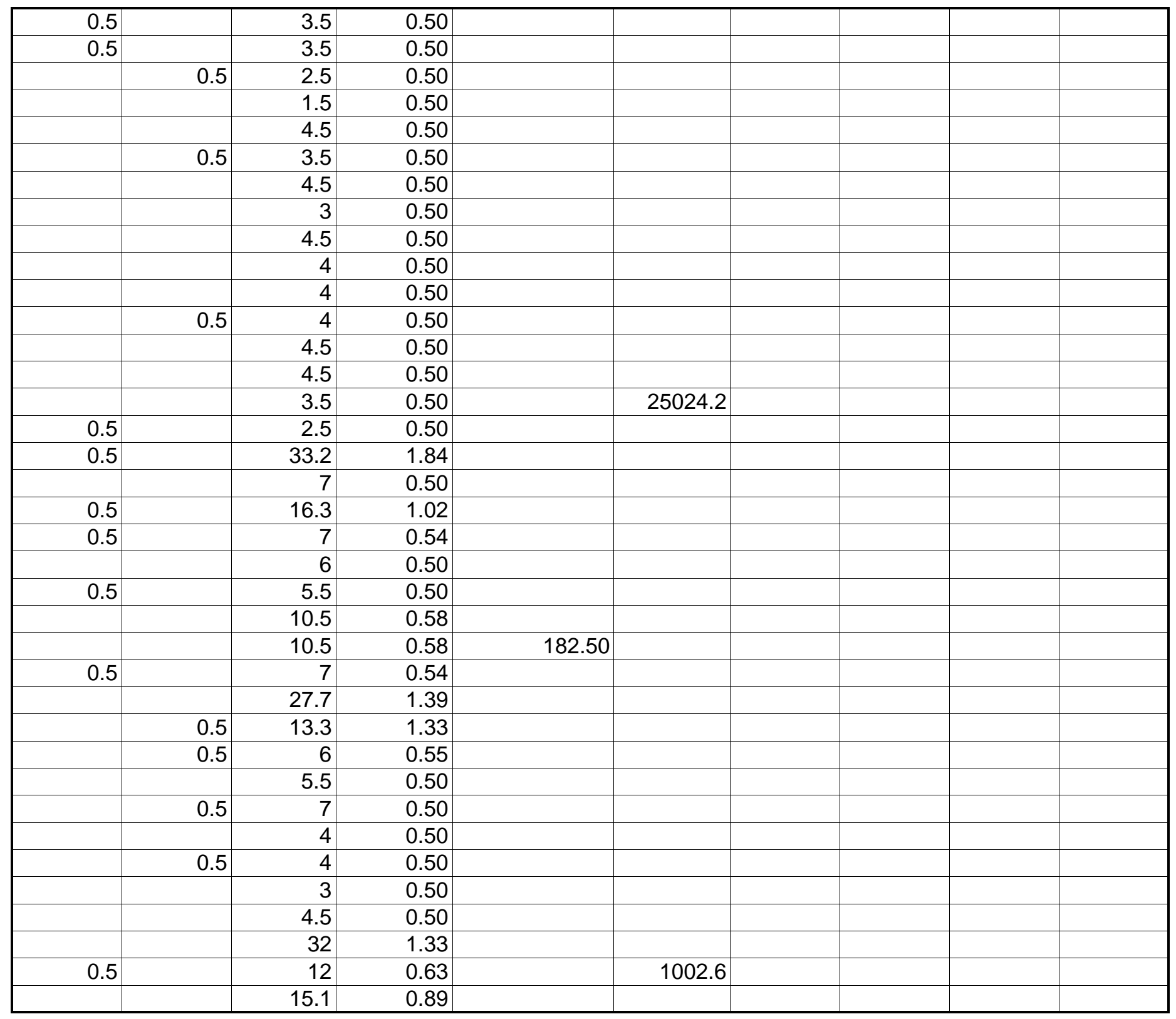

Page 80 
wbheat

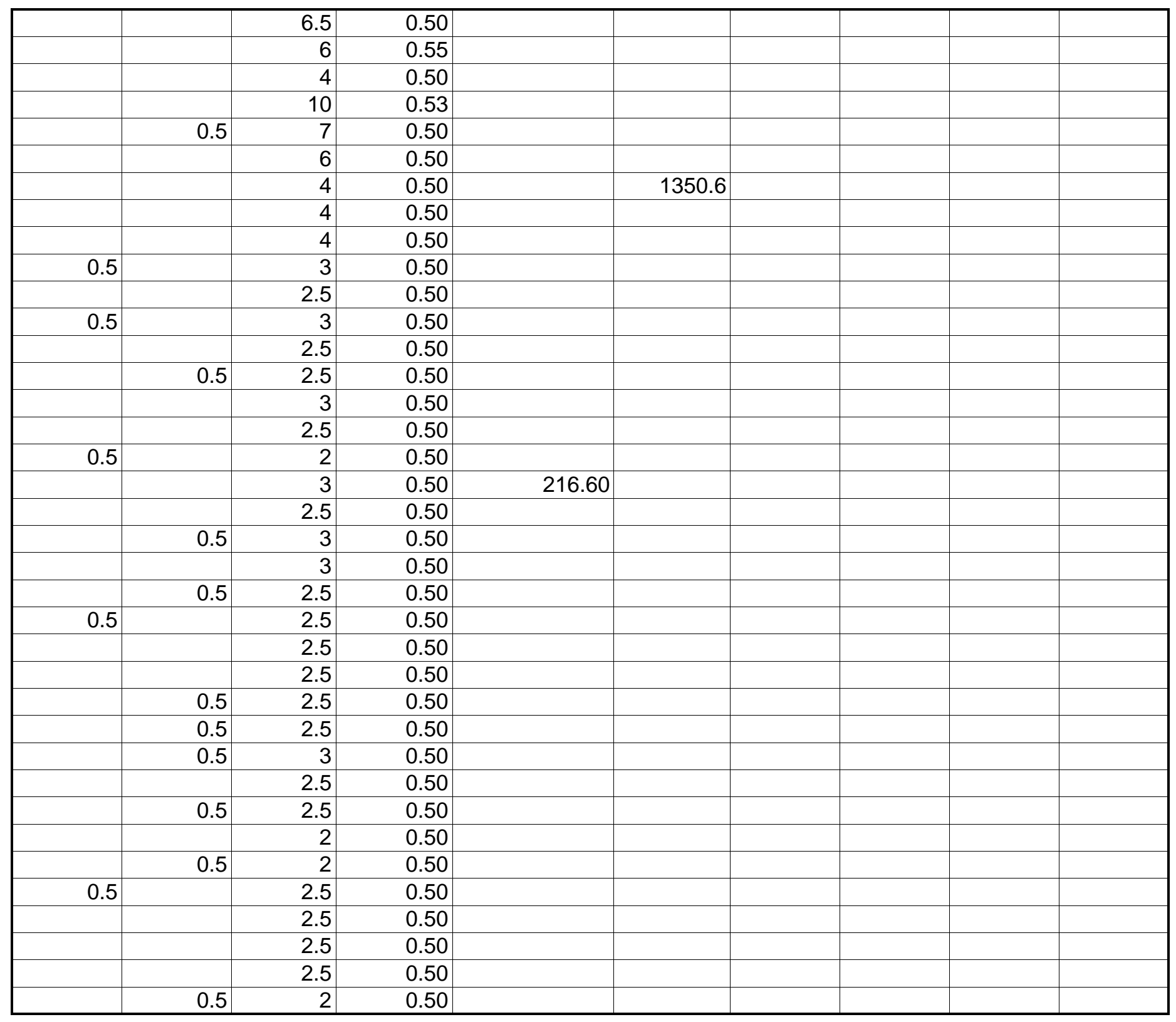

Page 81 
wbheat

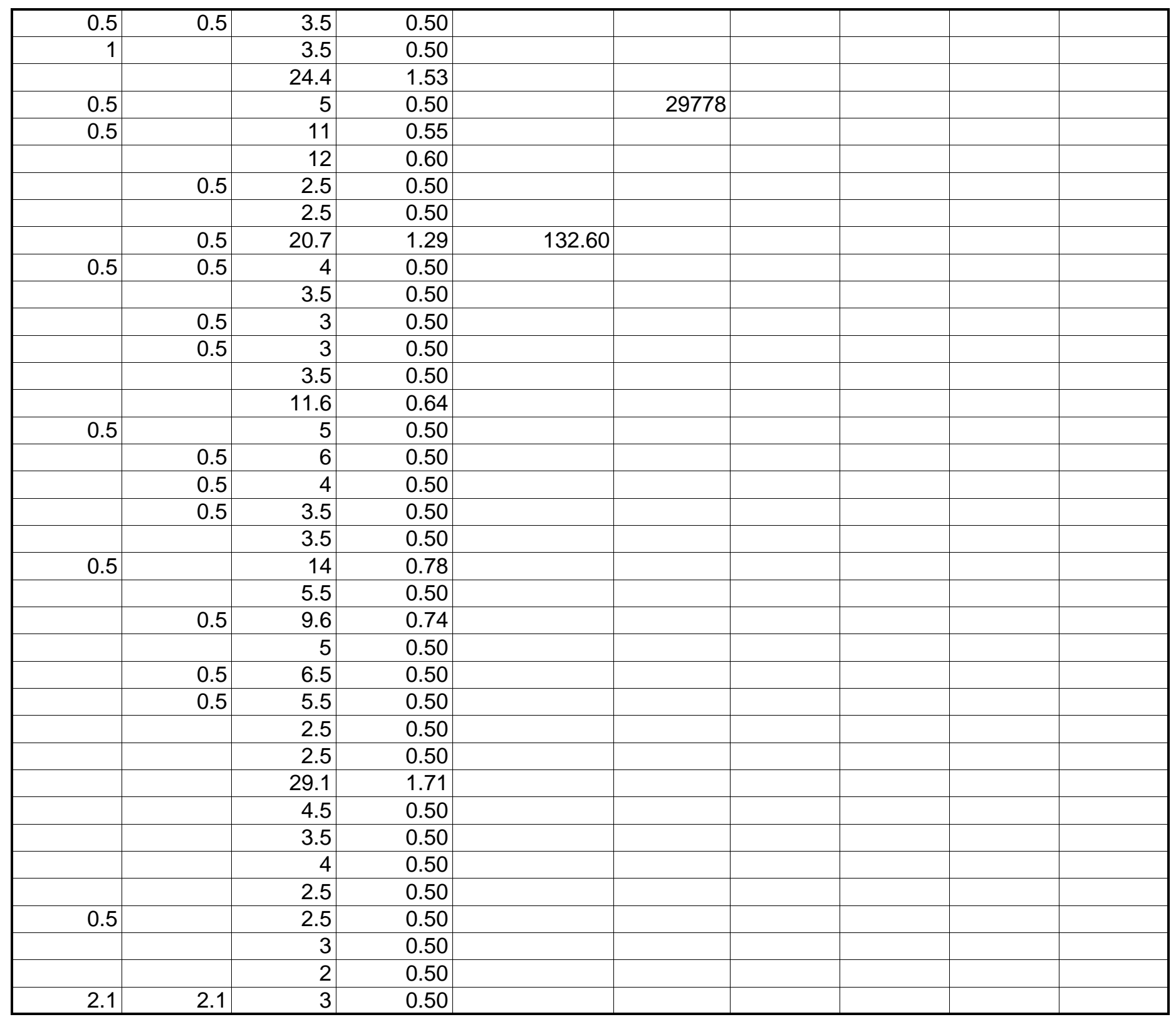

Page 82 
wbheat

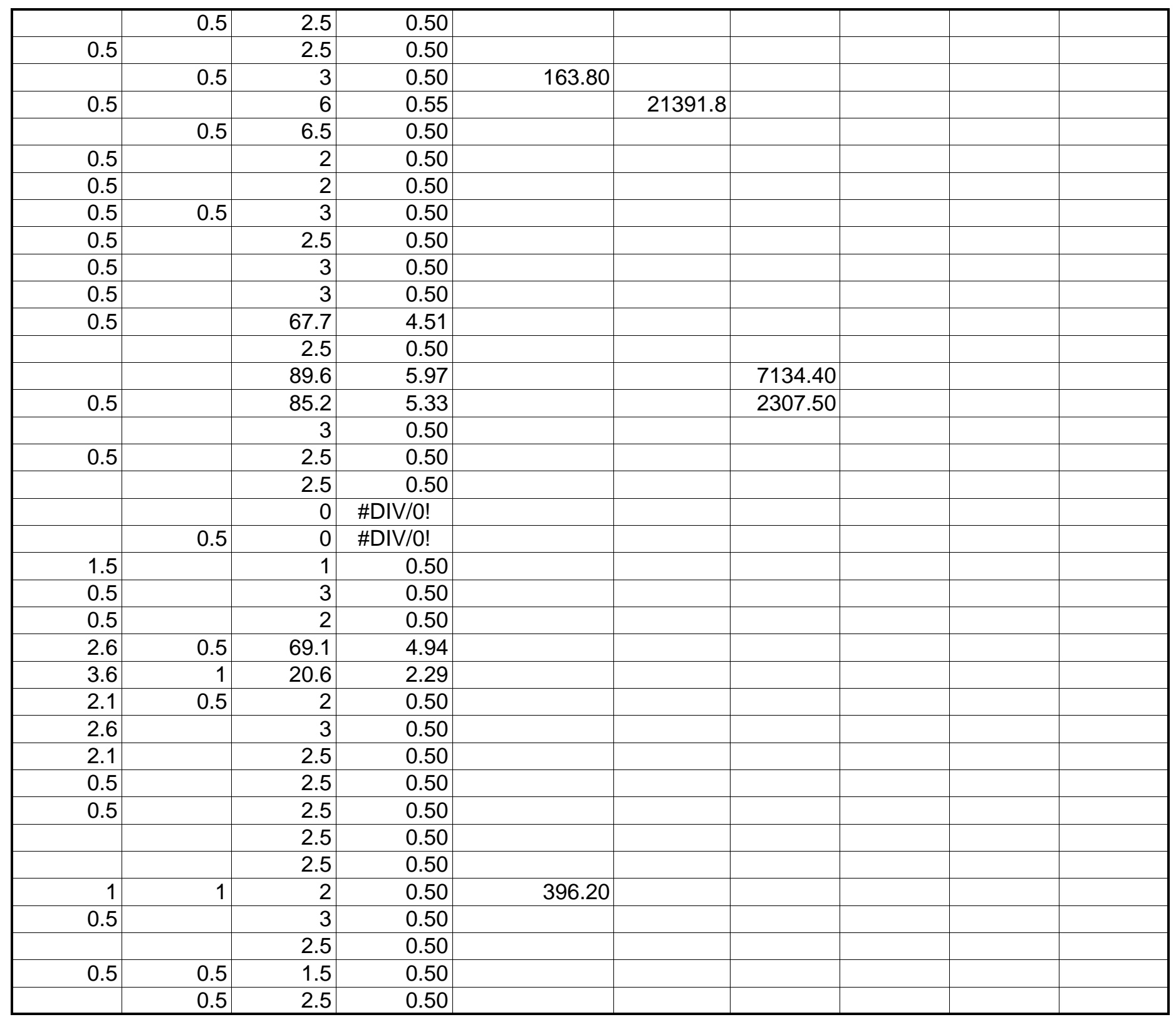

Page 83 
wbheat

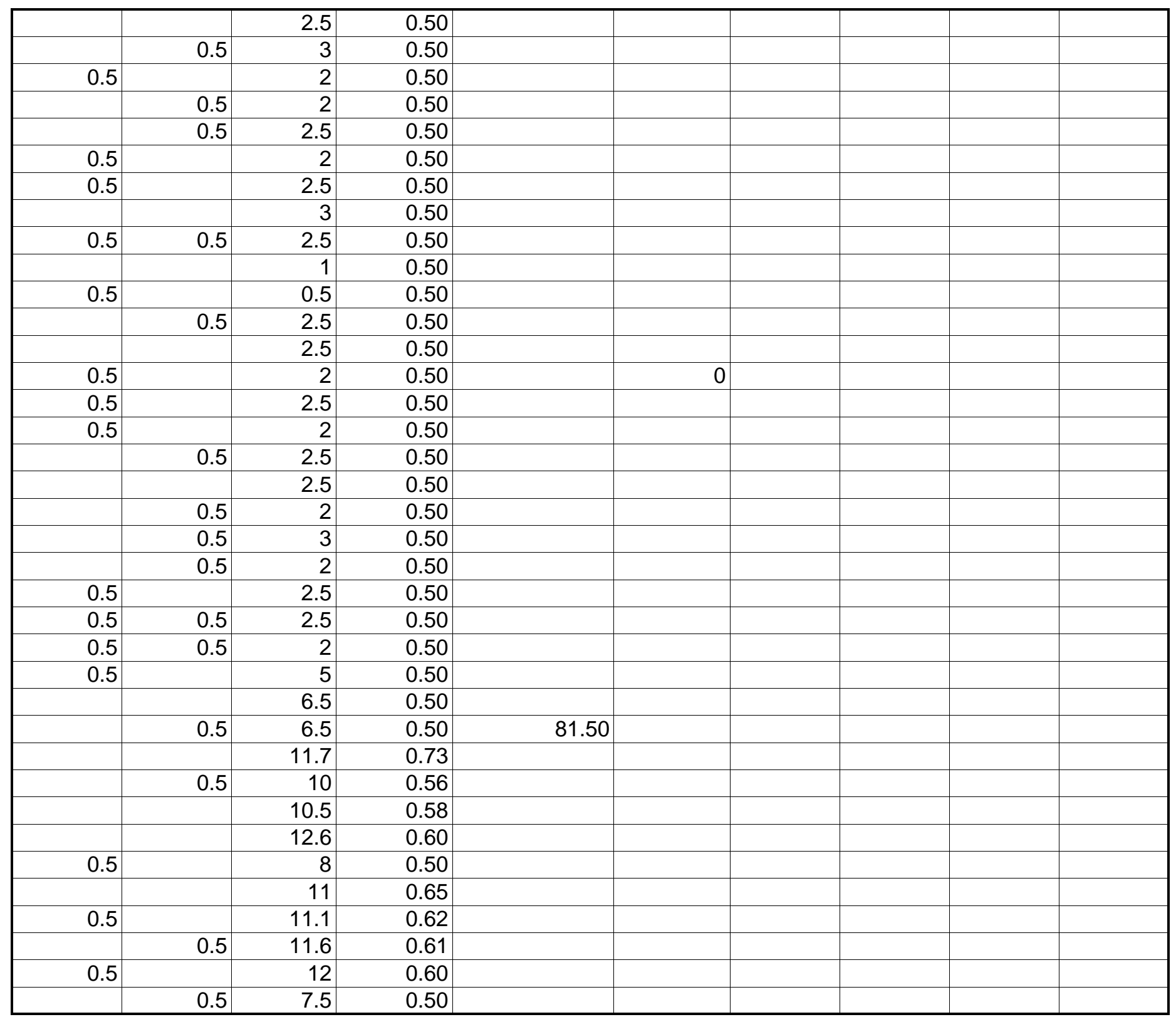

Page 84 
wbheat

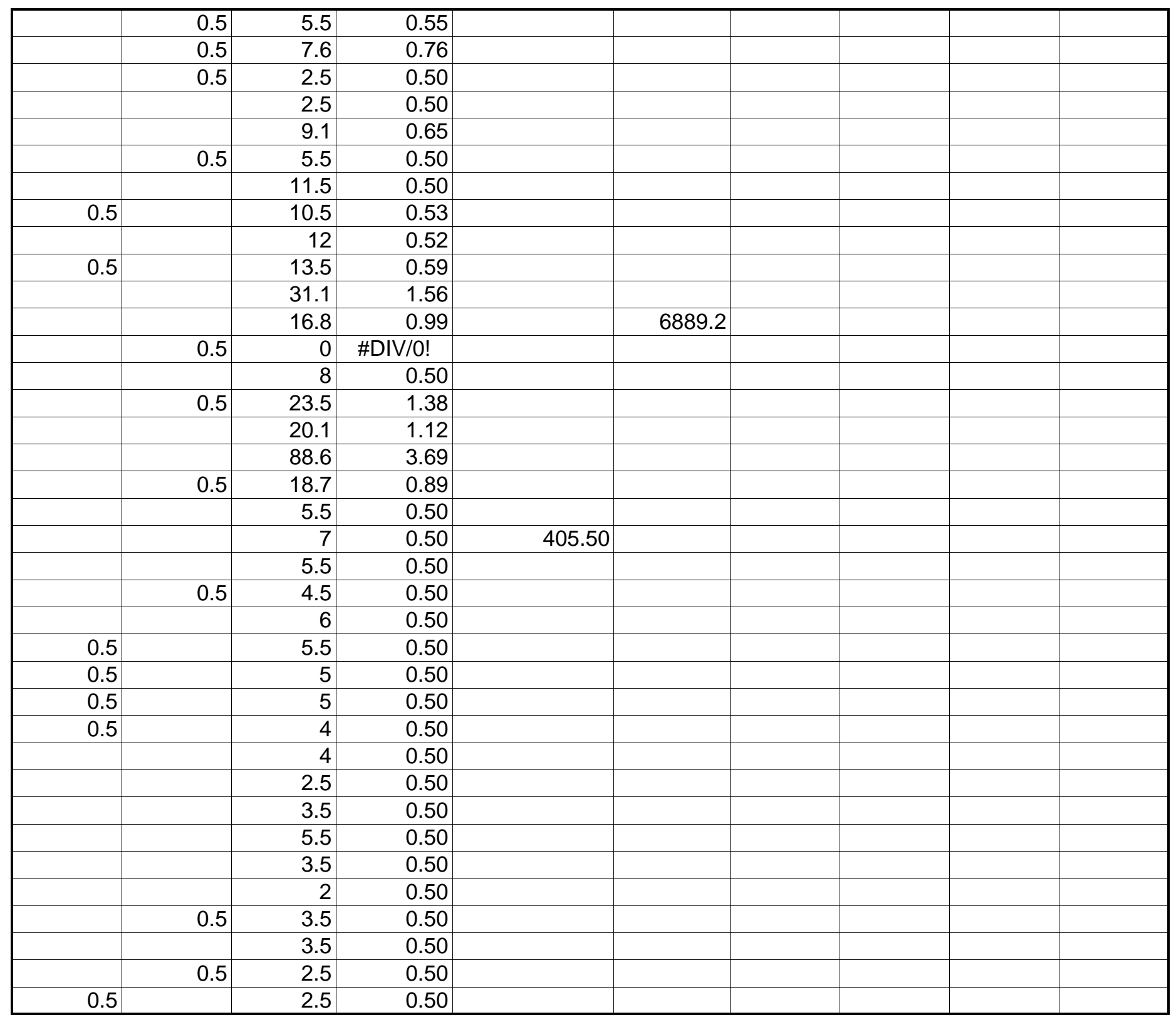

Page 85 
wbheat

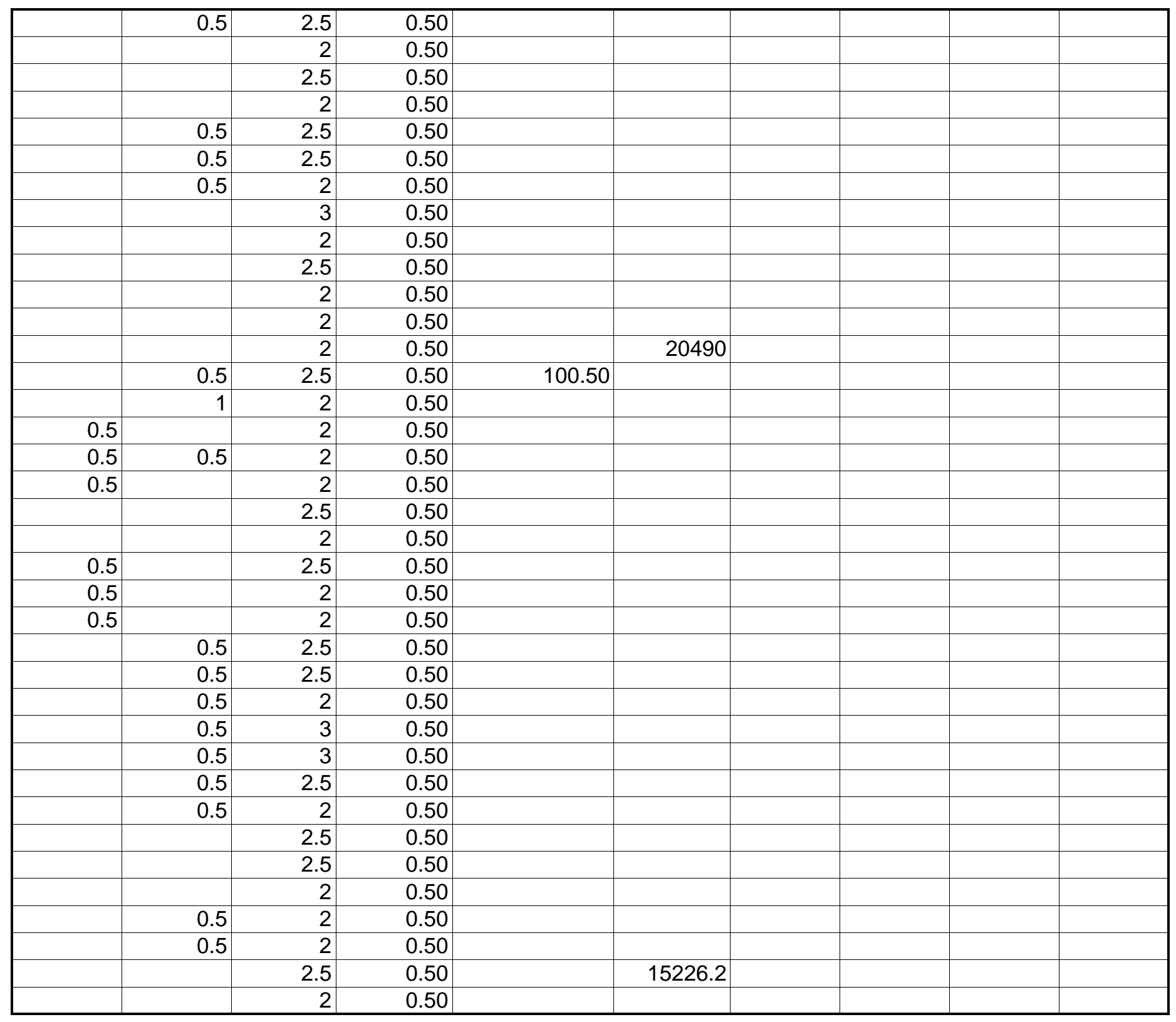

Page 86 
wbheat

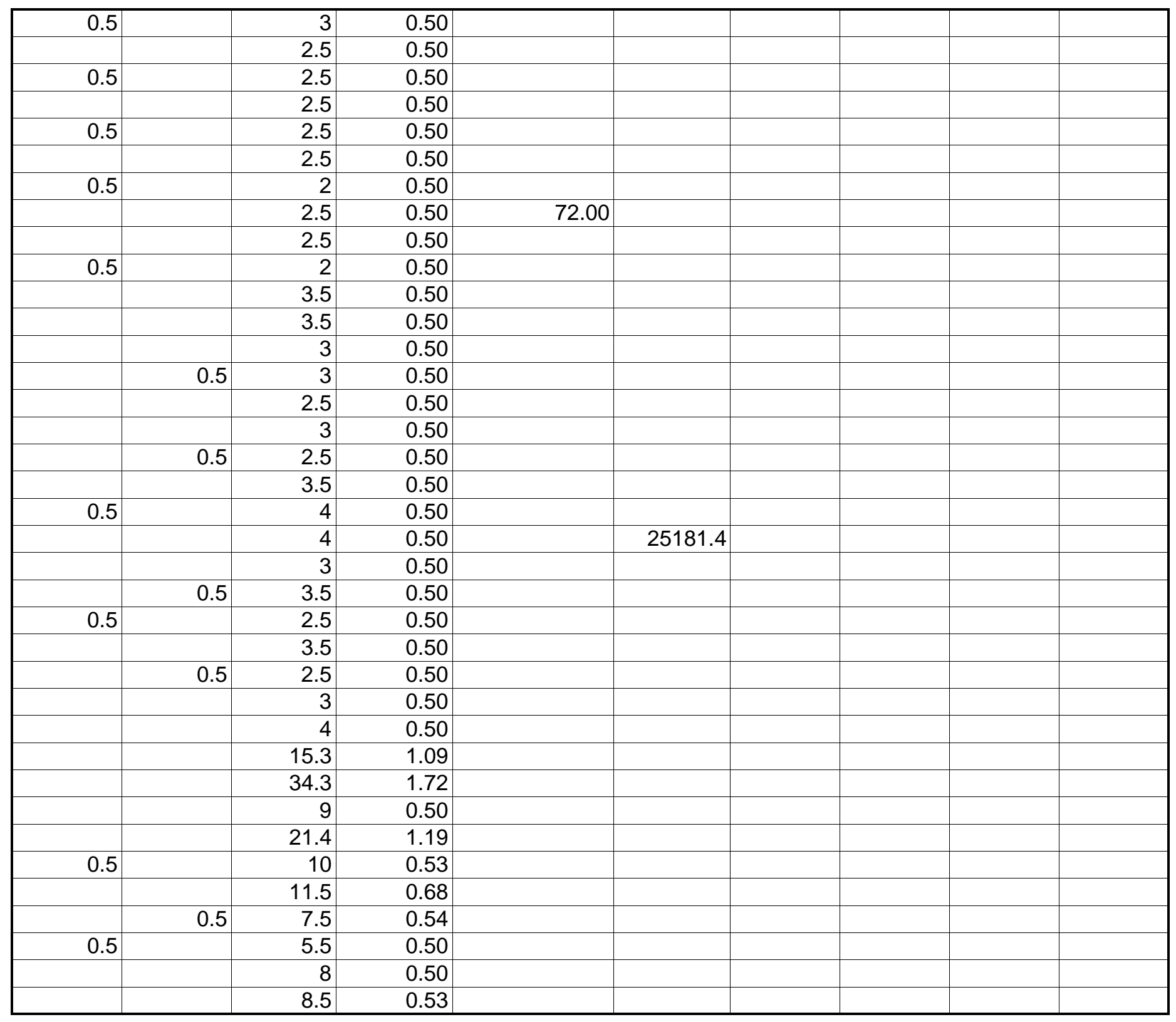

Page 87 
wbheat

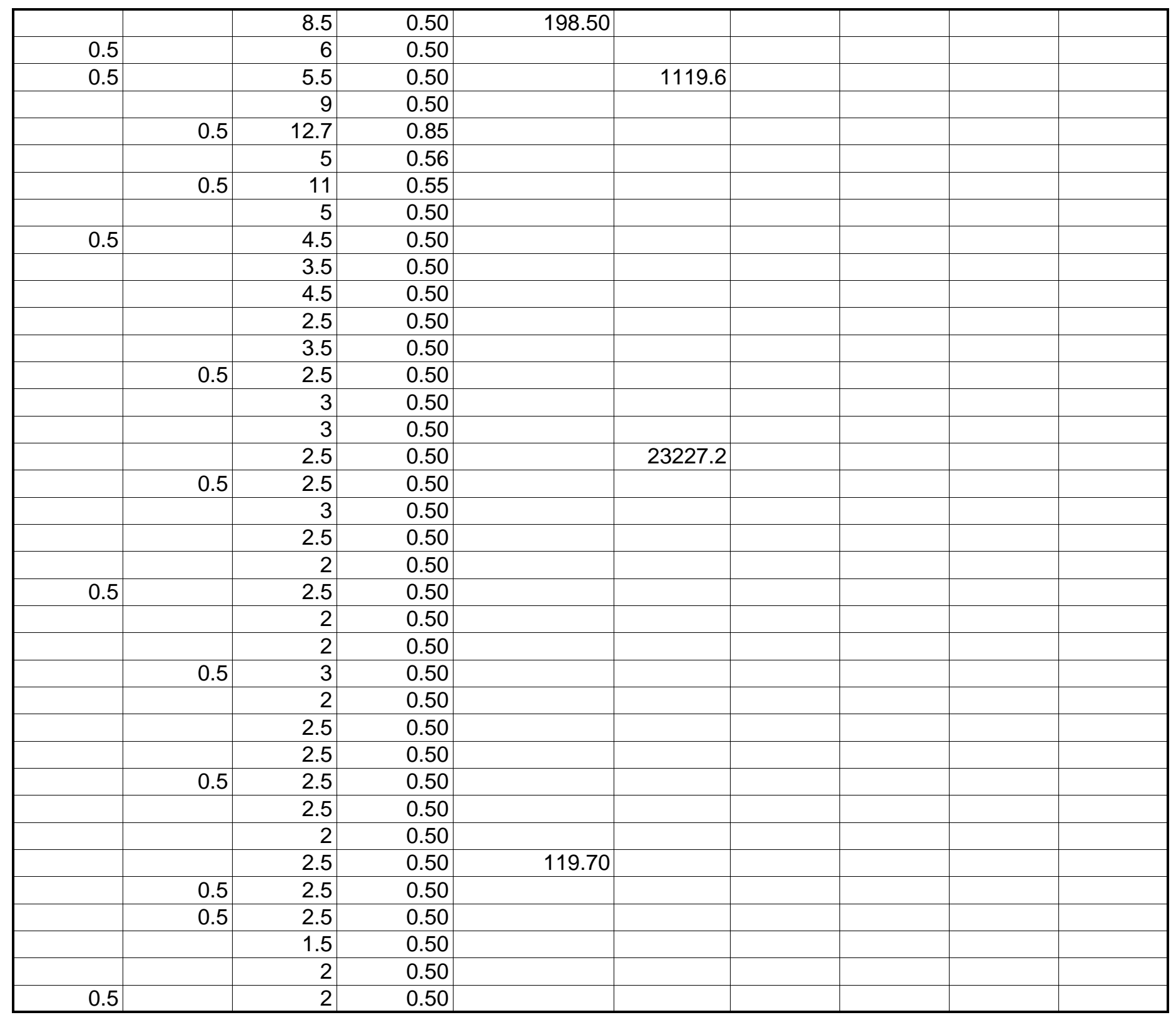

Page 88 
wbheat

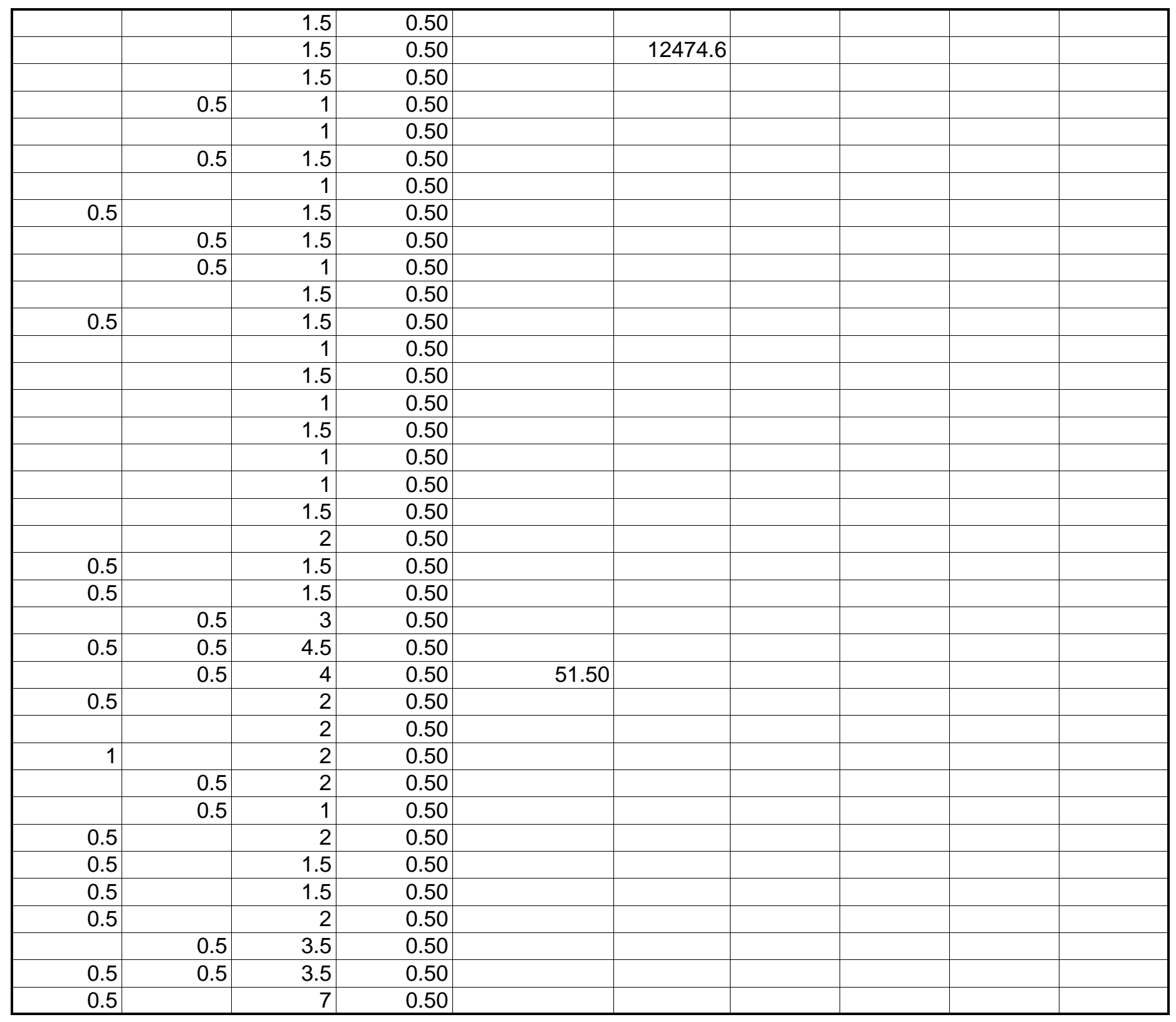

Page 89 
wbheat

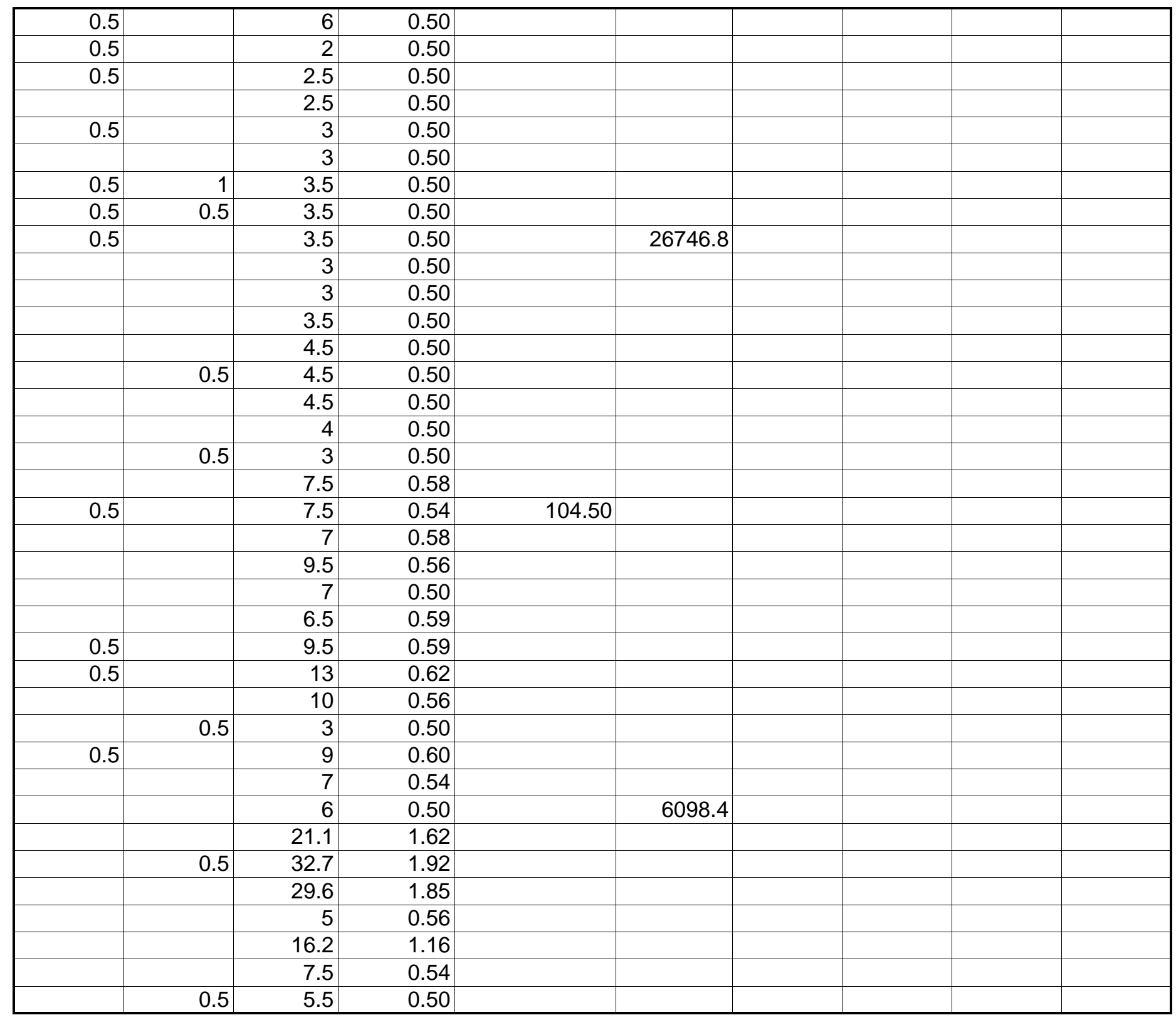

Page 90 
wbheat

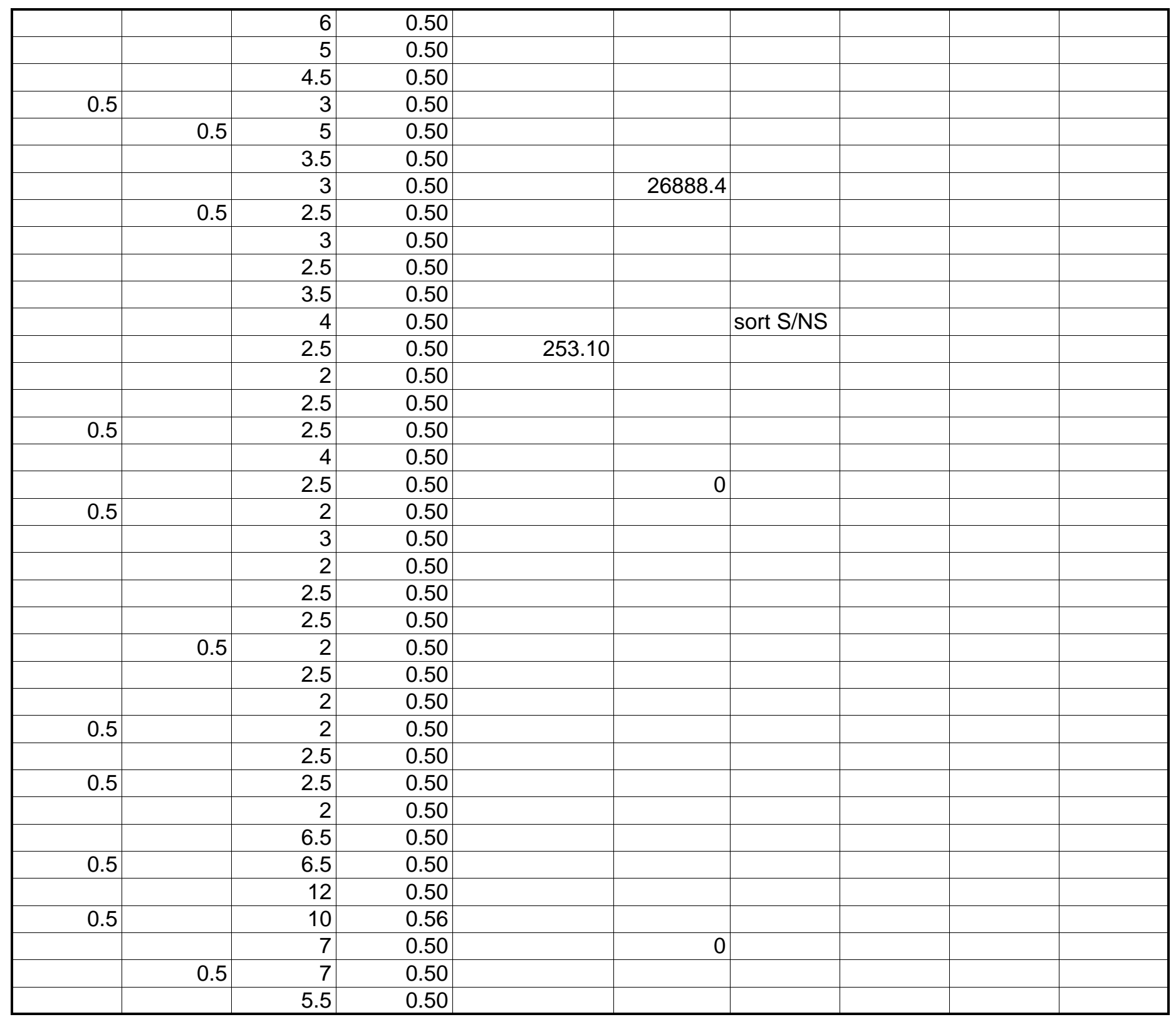

Page 91 
wbheat

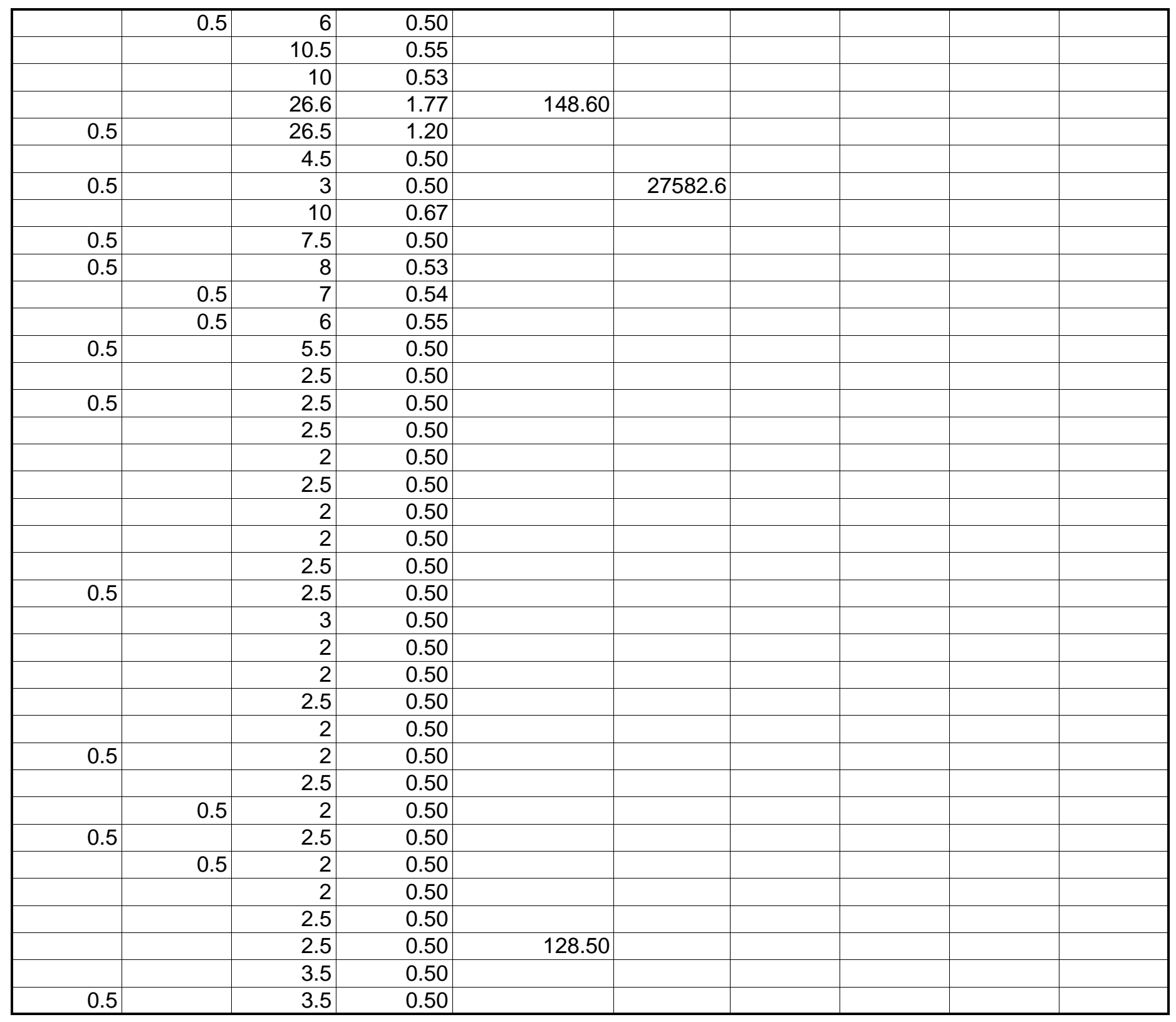

Page 92 
wbheat

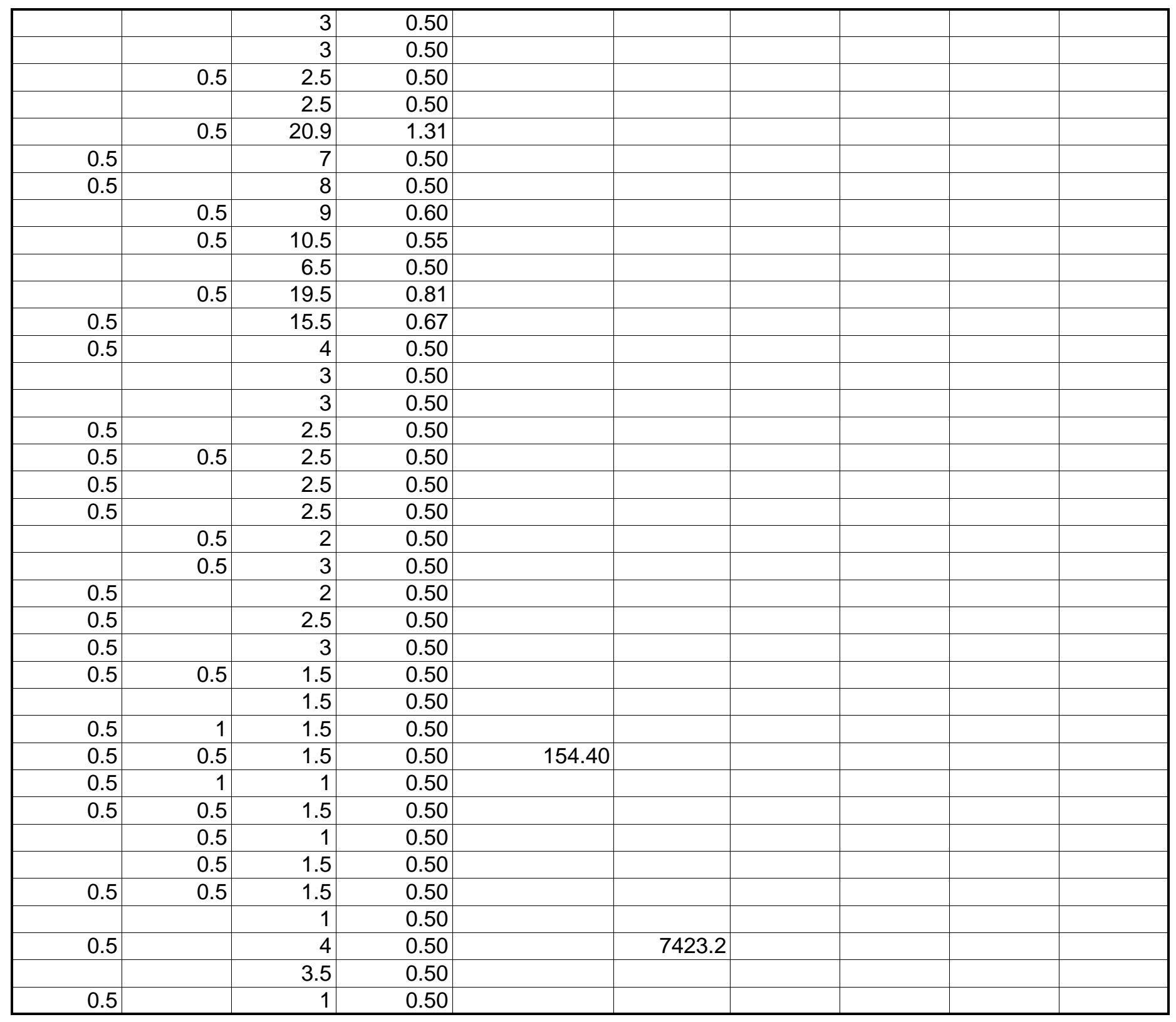

Page 93 


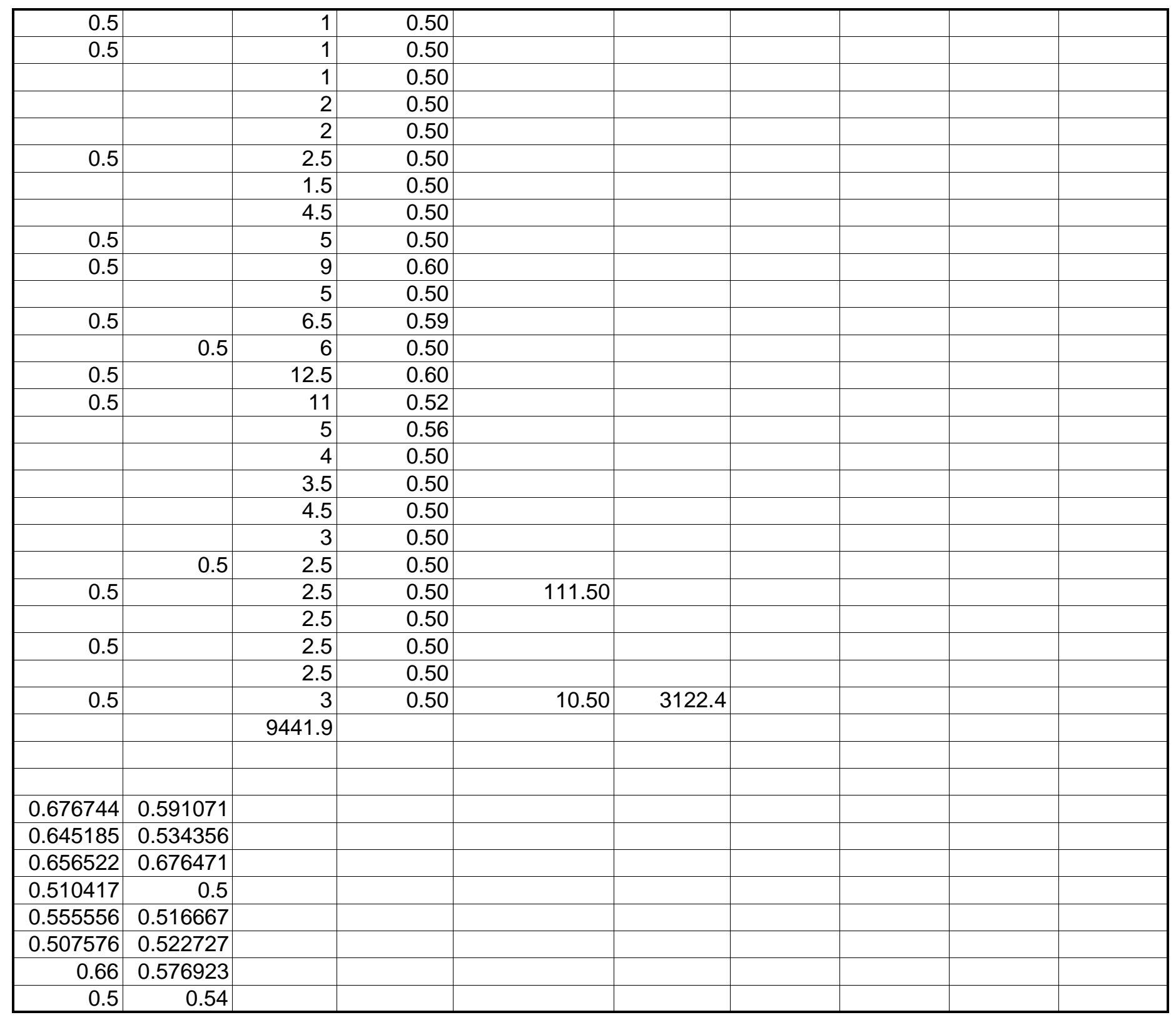




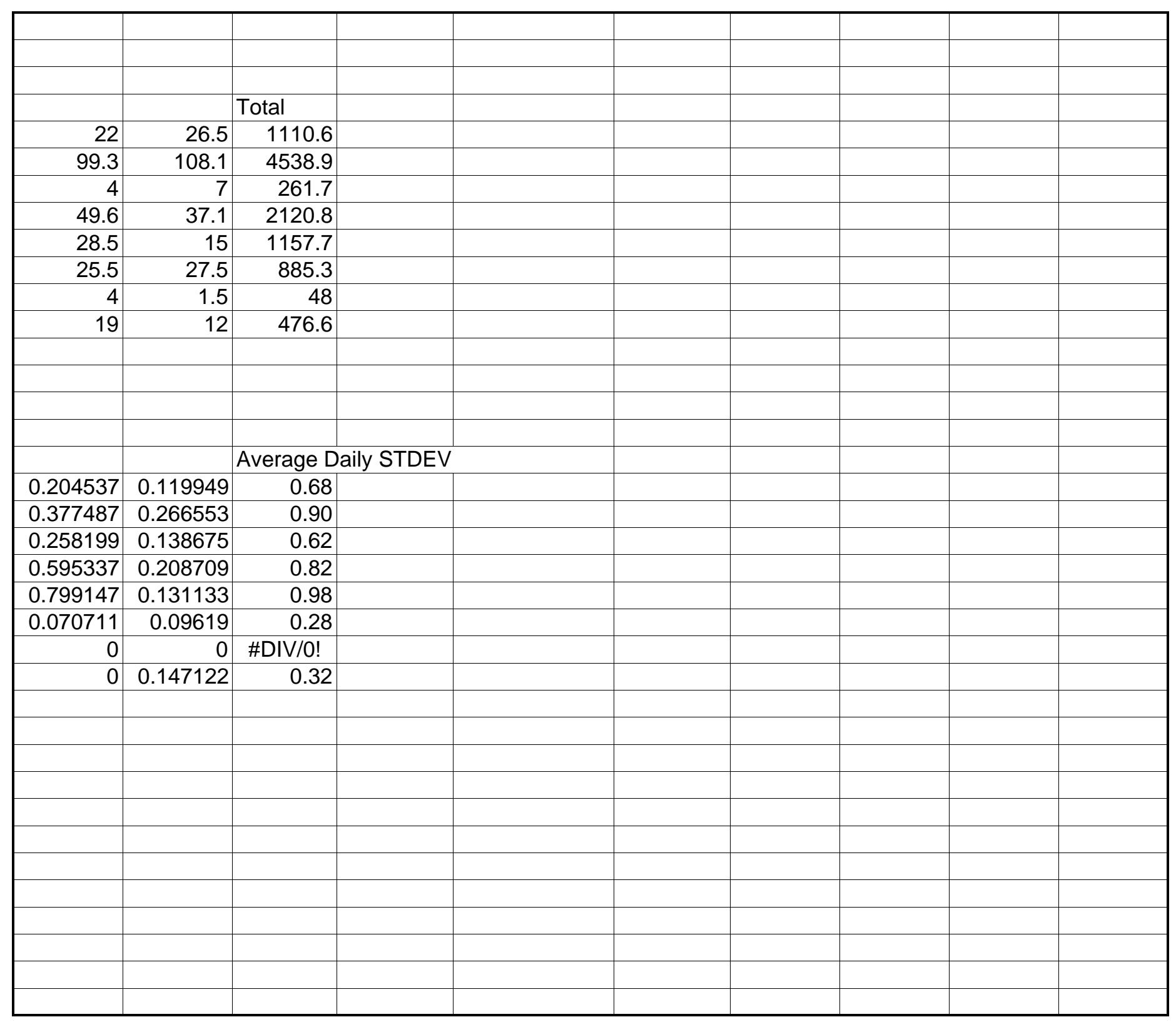

Page 95 


\begin{tabular}{|c|c|c|c|c|c|c|c|c|c|c|c|c|c|c|}
\hline $\begin{array}{l}\text { wd/ } \\
\text { we } \\
0 / 1\end{array}$ & $\begin{array}{l}\text { pre/ } \\
\text { post } \\
\text { A/B }\end{array}$ & $\begin{array}{c}\text { schyear/ } \\
\text { breaks } \\
\text { S/NS }\end{array}$ & date & jday & $\begin{array}{c}\text { orig } \\
\text { count } \\
(156)\end{array}$ & 0 & 1 & 2 & 3 & 4 & 5 & 6 & 7 & 8 \\
\hline 1 & A & S & 9/3/1991 & 91246 & 246 & 99.9 & 92.3 & 91.2 & 95.4 & 99.2 & 91.2 & 98.8 & 385.7 & 512.2 \\
\hline 1 & A & $S$ & 9/4/1991 & 91247 & 247 & 102 & 103.7 & 97.5 & 96.4 & 95 & 103.7 & 101.6 & 371.5 & 472.4 \\
\hline 1 & A & $S$ & 9/5/1991 & 91248 & 248 & 234.7 & 231.6 & 231.6 & 229.1 & 231.9 & 229.1 & 235.4 & 321.8 & 470 \\
\hline 1 & $A$ & $S$ & 9/6/1991 & 91249 & 249 & 92.6 & 102 & 95.4 & 99.9 & 90.9 & 97.1 & 103 & 347.3 & 483.8 \\
\hline 1 & A & S & 9/9/1991 & 91252 & 252 & 91.6 & 101.6 & 96.4 & 89.2 & 96.8 & 95.4 & 96.1 & 370.5 & 495.2 \\
\hline 1 & $A$ & S & 9/10/1991 & 91253 & 253 & 113.4 & 100.2 & 109.2 & 104 & 97.1 & 109.2 & 107.1 & 371.2 & 491.8 \\
\hline 1 & A & $S$ & 9/11/1991 & 91254 & 254 & 97.8 & 96.8 & 101.3 & 86.7 & 97.1 & 95.4 & 101.3 & 335.6 & 463.5 \\
\hline 1 & $A$ & $S$ & 9/12/1991 & 91255 & 255 & 125.1 & 125.8 & 117.2 & 118.2 & 123.7 & 120.3 & 111.3 & 350.1 & 449.3 \\
\hline 1 & A & $S$ & 9/13/1991 & 91256 & 256 & 107.8 & 107.5 & 105.1 & 107.1 & 106.4 & 102.6 & 105.1 & 325.2 & 449.3 \\
\hline 1 & A & S & 9/16/1991 & 91259 & 259 & 95 & 103 & 101.3 & 94.3 & 103.3 & 91.9 & 108.5 & 369.4 & 500.1 \\
\hline 1 & A & S & 9/17/1991 & 91260 & 260 & 78.5 & 82.3 & 83.3 & 74.7 & 85.7 & 76.4 & 115.1 & 322.1 & 487 \\
\hline 1 & A & S & 9/18/1991 & 91261 & 261 & 88.8 & 86.7 & 85.7 & 84.7 & 85.4 & 84.7 & 91.9 & 306.2 & 471.1 \\
\hline 1 & $A$ & $S$ & 9/19/1991 & 91262 & 262 & 88.1 & 85 & 83.6 & 81.9 & 84.7 & 84.3 & 90.9 & 198.4 & 425.4 \\
\hline 1 & $A$ & $S$ & 9/20/1991 & 91263 & 263 & 68.1 & 69.8 & 70.2 & 68.4 & 69.8 & 65 & 65.3 & 212.5 & 338.7 \\
\hline 1 & A & S & 9/23/1991 & 91266 & 266 & 84.7 & 90.5 & 80.2 & 77.4 & 78.1 & 84.7 & 70.8 & 262 & 444.8 \\
\hline 1 & A & S & 9/24/1991 & 91267 & 267 & 81.6 & 79.1 & 69.5 & 77.8 & 82.9 & 81.9 & 106.4 & 291.7 & 452.4 \\
\hline 1 & A & $S$ & 9/25/1991 & 91268 & 268 & 92.3 & 87.8 & 89.9 & 88.1 & 88.1 & 87.8 & 80.2 & 241.2 & 371.9 \\
\hline 1 & A & S & 9/26/1991 & 91269 & 269 & 84.7 & 79.8 & 76.4 & 75 & 72.9 & 72.9 & 73.6 & 246.4 & 355.6 \\
\hline 1 & A & $S$ & 9/27/1991 & 91270 & 270 & 69.1 & 66.7 & 66.7 & 64.6 & 65.7 & 66 & 69.5 & 228.1 & 359.1 \\
\hline 1 & A & $S$ & 9/30/1991 & 91273 & 273 & 71.9 & 68.4 & 72.6 & 64.6 & 65.3 & 62.6 & 69.1 & 278.6 & 377.4 \\
\hline 1 & A & S & 10/1/1991 & 91274 & 274 & & & & & & & & & \\
\hline 1 & A & S & 10/2/1991 & 91275 & 275 & & & & & & & & & \\
\hline 1 & A & S & 10/3/1991 & 91276 & 276 & 102 & 90.5 & 82.9 & 81.9 & 78.8 & 78.1 & 94 & 275.1 & 401.6 \\
\hline 1 & A & S & 10/4/1991 & 91277 & 277 & 115.1 & 114 & 107.5 & 94.7 & 98.8 & 99.2 & 104 & 320.7 & 416.1 \\
\hline 1 & A & $\mathrm{S}$ & 10/7/1991 & 91280 & 280 & 70.8 & 69.1 & 67.4 & 66.7 & 65.7 & 65.7 & 80.2 & 264.7 & 359.4 \\
\hline 1 & A & S & 10/8/1991 & 91281 & 281 & 69.5 & 73.3 & 71.5 & 60.8 & 61.2 & 60.8 & 72.3 & 166 & 282.1 \\
\hline 1 & A & S & 10/9/1991 & 91282 & 282 & 82.9 & 67.7 & 66.4 & 64.3 & 62.6 & 62.9 & 61.5 & 169.9 & 261.8 \\
\hline 1 & A & S & \#\#\#\#\#\#\#\# & 91283 & 283 & 77.1 & 74.7 & 70.8 & 68.8 & 65 & 66.4 & 67 & 161.6 & 266.2 \\
\hline 1 & A & $\mathrm{S}$ & \#\#\#\#\#\#\#\# & 91284 & 284 & 84.7 & 80.2 & 81.2 & 70.5 & 71.5 & 71.2 & 69.5 & 106.4 & 217.7 \\
\hline 1 & A & $S$ & \#\#\#\#\#\#\#\# & 91287 & 287 & 90.5 & 81.9 & 81.9 & 89.2 & 76.4 & 80.9 & 86.1 & 195.1 & 299.7 \\
\hline 1 & A & S & \#\#\#\#\#\#\#\# & 91288 & 288 & 117.2 & 96.4 & 89.2 & 94.3 & 85.4 & 86.4 & 83.3 & 174.2 & 288.8 \\
\hline 1 & $A$ & S & \#\#\#\#\#\#\#\# & 91289 & 289 & 90.9 & 82.3 & 72.2 & 68.8 & 67.7 & 65.7 & 65.3 & 108.2 & 210.8 \\
\hline 1 & A & $\mathrm{S}$ & \#\#\#\#\#\#\#\# & 91290 & 290 & 95 & 82.6 & 75.7 & 74.3 & 74.3 & 75.3 & 68.4 & 93.7 & 208.1 \\
\hline
\end{tabular}




\begin{tabular}{|c|c|c|c|c|c|c|c|c|c|c|c|c|c|c|}
\hline 1 & $A$ & $\mathrm{~S}$ & \#\#\#\#\#\#\#\# & 91291 & 291 & 84 & 85 & 78.8 & 78.1 & 75 & 74.7 & 70.5 & 111.3 & 247.2 \\
\hline 1 & $A$ & S & \#\#\#\#\#\#\#\# & 91294 & 294 & 79.8 & 71.2 & 74.7 & 67 & 65.3 & 71.5 & 62.9 & 103 & 217.4 \\
\hline 1 & A & S & \#\#\#\#\#\#\#\# & 91295 & 295 & 109.2 & 108.2 & 98.8 & 85 & 81.2 & 80.2 & 78.5 & 117.8 & 281.6 \\
\hline 1 & $A$ & S & \#\#\#\#\#\#\#\# & 91297 & 297 & 116.5 & 110 & 107.8 & 103.7 & 93.3 & 95.4 & 93 & 194.4 & 327.7 \\
\hline 1 & $A$ & S & \#\#\#\#\#\#\#\# & 91298 & 298 & 84.3 & 80.9 & 79.1 & 70.9 & 65.3 & 69.9 & 71.5 & 169.4 & 303.3 \\
\hline 1 & $A$ & S & \#\#\#\#\#\#\#\# & 91301 & 301 & 100.6 & 98.5 & 90.3 & 92.6 & 91.2 & 92.4 & 95 & 93.4 & 213.7 \\
\hline 1 & $A$ & S & \#\#\#\#\#\#\#\# & 91302 & 302 & 129.7 & 134.8 & 123.1 & 118.5 & 99.5 & 103.7 & 97.5 & 102.4 & 206.4 \\
\hline 1 & A & S & \#\#\#\#\#\#\#\# & 91303 & 303 & 118.9 & 104.8 & 74 & 70.2 & 72.9 & 65.8 & 84.3 & 113.8 & 194.6 \\
\hline 1 & A & S & \#\#\#\#\#\#\#\# & 91304 & 304 & 71.5 & 66.8 & 63.6 & 53.3 & 52.9 & 54 & 52.5 & 88.1 & 189.4 \\
\hline 1 & A & S & 11/1/1991 & 91305 & 305 & 77.2 & 77.8 & 69.9 & 57 & 56.1 & 55.6 & 56 & 75 & 195.6 \\
\hline 1 & $A$ & S & 11/4/1991 & 91308 & 308 & 59.4 & 55 & 56.4 & 55.3 & 51.8 & 59.4 & 56.7 & 77.4 & 227.8 \\
\hline 1 & $A$ & S & 11/5/1991 & 91309 & 309 & 68.5 & 64.6 & 62.6 & 63.7 & 64.3 & 63 & 64.3 & 115.8 & 241.9 \\
\hline 1 & A & S & 11/6/1991 & 91310 & 310 & 73.6 & 69.9 & 68.8 & 67.8 & 68.8 & 67.1 & 69.1 & 100.2 & 199.5 \\
\hline 1 & $A$ & S & 11/7/1991 & 91311 & 311 & 73.4 & 69.5 & 58.2 & 50.8 & 49.2 & 51.9 & 52.2 & 121.4 & 233.7 \\
\hline 1 & A & S & 11/8/1991 & 91312 & 312 & 90.3 & 91.2 & 90.3 & 68.4 & 58.8 & 59.4 & 58.2 & 98.8 & 223.9 \\
\hline 1 & $A$ & $\mathrm{~S}$ & \#\#\#\#\#\#\#\# & 91315 & 315 & 72.9 & 70.2 & 70.5 & 67.8 & 67.8 & 68.8 & 67.5 & 93.7 & 188 \\
\hline 1 & A & S & \#\#\#\#\#\#\#\# & 91316 & 316 & 63.9 & 64.4 & 46 & 46.1 & 46.3 & 45 & 45.7 & 71.5 & 170.1 \\
\hline 1 & $A$ & S & \#\#\#\#\#\#\#\# & 91317 & 317 & 76.8 & 75.7 & 74.7 & 68.8 & 55 & 53.7 & 55.6 & 83.7 & 189.4 \\
\hline 1 & A & S & \#\#\#\#\#\#\#\# & 91318 & 318 & 78.9 & 75 & 65.8 & 67.4 & 65.1 & 63.7 & 64.6 & 88.6 & 198.6 \\
\hline 1 & A & S & \#\#\#\#\#\#\#\# & 91319 & 319 & 85.8 & 103.7 & 84.4 & 69.5 & 65.4 & 69.2 & 66 & 149.6 & 242.7 \\
\hline 1 & $A$ & S & \#\#\#\#\#\#\#\# & 91322 & 322 & 64.7 & 62.2 & 58.8 & 58.2 & 59.1 & 59.5 & 57.4 & 81.7 & 187 \\
\hline 1 & A & S & \#\#\#\#\#\#\#\# & 91323 & 323 & 101 & 101.3 & 83.7 & 83.3 & 80.6 & 81.2 & 85.1 & 163.8 & 281.6 \\
\hline 1 & $A$ & S & \#\#\#\#\#\#\#\# & 91324 & 324 & 93.7 & 85.8 & 82.6 & 77.5 & 76.5 & 76.4 & 74.1 & 101.3 & 185.7 \\
\hline 1 & $A$ & S & \#\#\#\#\#\#\#\# & 91325 & 325 & 67.8 & 66.1 & 59.4 & 55 & 53.2 & 51.9 & 51.2 & 98.8 & 198.5 \\
\hline 1 & A & $\mathrm{S}$ & \#\#\#\#\#\#\#\# & 91326 & 326 & 83 & 87.8 & 86.1 & 73.3 & 66.1 & 64 & 67 & 94.1 & 173.5 \\
\hline 1 & $A$ & S & \#\#\#\#\#\#\#\# & 91329 & 329 & 56.8 & 51.1 & 54.7 & 48.1 & 54.6 & 50.9 & 50.5 & 92 & 208.4 \\
\hline 1 & $A$ & S & \#\#\#\#\#\#\#\# & 91330 & 330 & 78.5 & 79.8 & 73.4 & 58.4 & 60.2 & 59.1 & 58.8 & 88.9 & 192.8 \\
\hline 1 & $A$ & S & 12/2/1991 & 91336 & 336 & 48.8 & 48.4 & 43.3 & 53.7 & 43.2 & 51.2 & 49.4 & 76.5 & 219.1 \\
\hline 1 & $A$ & S & $12 / 3 / 1991$ & 91337 & 337 & 59.4 & 57.5 & 54 & 52.9 & 56.8 & 65.3 & 53.7 & 92 & 221.5 \\
\hline 1 & $A$ & S & 12/4/1991 & 91338 & 338 & 68.5 & 57 & 54.4 & 51.6 & 55.3 & 54.4 & 53.3 & 96.8 & 225.4 \\
\hline 1 & $A$ & S & $12 / 5 / 1991$ & 91339 & 339 & 56.7 & 54.4 & 51.9 & 50.1 & 54 & 68.2 & 66.4 & 72.7 & 179.7 \\
\hline 1 & A & S & 12/6/1991 & 91340 & 340 & 56.1 & 56.7 & 54.4 & 52.5 & 51.6 & 53.7 & 50.1 & 76.5 & 163.8 \\
\hline 1 & $A$ & S & $12 / 9 / 1991$ & 91343 & 343 & 62.6 & 60.9 & 58.1 & 57.1 & 58.8 & 60.5 & 57.5 & 142.4 & 243.5 \\
\hline 1 & $A$ & S & \#\#\#\#\#\#\#\# & 91344 & 344 & 59.2 & 57.8 & 56.3 & 54.4 & 55 & 56 & 53.3 & 74.3 & 183.6 \\
\hline 1 & A & S & \#\#\#\#\#\#\#\# & 91345 & 345 & 62.3 & 62.2 & 59.9 & 55.6 & 57.1 & 58.5 & 57.4 & 116.2 & 236 \\
\hline 1 & $A$ & S & \#\#\#\#\#\#\#\# & 91346 & 346 & 79.2 & 78.1 & 74.4 & 73.3 & 73.4 & 75.8 & 74 & 145.6 & 265 \\
\hline 1 & $A$ & $\mathrm{~S}$ & \#\#\#\#\#\#\#\# & 91347 & 347 & 74.7 & 76.4 & 65.8 & 67.8 & 65 & 65.4 & 63.3 & 125.9 & 257.1 \\
\hline
\end{tabular}




\begin{tabular}{|c|c|c|c|c|c|c|c|c|c|c|c|c|c|c|}
\hline 1 & $A$ & $\mathrm{~S}$ & \#\#\#\#\#\#\#\# & 91350 & 350 & 56.8 & 56 & 50.5 & 61.6 & 63.6 & 51.2 & 50.2 & 90.5 & 227.2 \\
\hline 1 & $A$ & S & \#\#\#\#\#\#\#\# & 91351 & 351 & 65.1 & 63.3 & 61.2 & 60.2 & 60.9 & 61.3 & 60.1 & 77.2 & 203.6 \\
\hline 1 & $A$ & S & \#\#\#\#\#\#\#\# & 91352 & 352 & 79.8 & 67.1 & 65.1 & 65 & 65.8 & 65.4 & 65 & 81.7 & 182.5 \\
\hline 1 & A & S & \#\#\#\#\#\#\#\# & 91353 & 353 & 59.2 & 57.7 & 53.3 & 51.6 & 52.9 & 54 & 53.6 & 77.2 & 171.5 \\
\hline 1 & $A$ & S & 1/7/1992 & 92007 & 6 & 57 & 53 & 50.2 & 47.7 & 46.7 & 49.5 & 61.9 & 89.9 & 184.9 \\
\hline 1 & $A$ & S & 1/8/1992 & 92008 & 7 & 59.8 & 55 & 53 & 53.6 & 53.7 & 52.6 & 53.2 & 66.4 & 178.1 \\
\hline 1 & A & S & 1/9/1992 & 92009 & 8 & 70.6 & 69.1 & 60.6 & 60.6 & 62.9 & 60.9 & 63.2 & 96.2 & 179.1 \\
\hline 1 & A & S & $1 / 10 / 1992$ & 92010 & 9 & 59.2 & 57.4 & 54.7 & 51.2 & 52.9 & 53 & 54 & 98.8 & 227.5 \\
\hline 1 & $A$ & S & $1 / 13 / 1992$ & 92013 & 12 & 55.4 & 55.6 & 52.3 & 53 & 56.3 & 50.2 & 51.2 & 52.9 & 56.1 \\
\hline 1 & $A$ & S & $1 / 14 / 1992$ & 92014 & 13 & 58.2 & 54.6 & 51.6 & 51.6 & 51.5 & 48.1 & 51.6 & 94.7 & 217.1 \\
\hline 1 & $A$ & S & $1 / 15 / 1992$ & 92015 & 14 & 69.6 & 74.4 & 65 & 62.6 & 66.4 & 61.6 & 70.6 & 94.7 & 182.9 \\
\hline 1 & A & S & 1/16/1992 & 92016 & 15 & 69.5 & 64 & 59.5 & 59.4 & 56.8 & 58.8 & 60.5 & 95.8 & 181.8 \\
\hline 1 & $A$ & S & 1/20/1992 & 92020 & 19 & 85.5 & 78.1 & 73.7 & 78.5 & 89.2 & 74.4 & 83.7 & 111.6 & 95.8 \\
\hline 1 & $A$ & S & 1/21/1992 & 92021 & 20 & 82.9 & 69.9 & 76.5 & 65.7 & 77.2 & 65.1 & 81.2 & 112.4 & 236.8 \\
\hline 1 & $A$ & S & $1 / 22 / 1992$ & 92022 & 21 & 55.7 & 57 & 54.7 & 50.2 & 52.2 & 62 & 53.3 & 92 & 209.1 \\
\hline 1 & $A$ & S & $1 / 23 / 1992$ & 92023 & 22 & 58.8 & 57.1 & 53.2 & 51.9 & 51.9 & 50.9 & 52.5 & 91 & 180.5 \\
\hline 1 & $A$ & S & 1/24/1992 & 92024 & 23 & 59.2 & 56.4 & 55 & 52.3 & 51.9 & 51.6 & 52.5 & 81 & 186.7 \\
\hline 1 & $A$ & S & 1/27/1992 & 92027 & 26 & 58.8 & 58.1 & 54.7 & 53 & 54.3 & 53.3 & 55.4 & 53.3 & 52.5 \\
\hline 1 & $A$ & S & $1 / 28 / 1992$ & 92028 & 27 & 61.2 & 56.8 & 55 & 53 & 54.3 & 53 & 55 & 87.9 & 186.6 \\
\hline 1 & $A$ & S & 1/29/1992 & 92029 & 28 & 56 & 53 & 50.2 & 49.5 & 50.5 & 50.9 & 51.2 & 67.1 & 157.9 \\
\hline 1 & $A$ & S & $1 / 30 / 1992$ & 92030 & 29 & 61.2 & 57.5 & 55.4 & 54.7 & 56.3 & 54.7 & 54.7 & 94 & 201.2 \\
\hline 1 & $A$ & S & 1/31/1992 & 92031 & 30 & 63 & 60.6 & 58.5 & 57.7 & 58.2 & 58.5 & 57 & 84.1 & 185 \\
\hline 1 & B & S & 2/3/1992 & 92034 & 33 & 61.2 & 57.8 & 54.7 & 54.3 & 53.7 & 54.7 & 56.4 & 56.7 & 53.3 \\
\hline 1 & B & S & 2/4/1992 & 92035 & 34 & 62.3 & 57.1 & 54 & 53.6 & 55.7 & 55.7 & 57 & 79.9 & 175.3 \\
\hline 1 & $B$ & S & 2/5/1992 & 92036 & 35 & 67.5 & 65.1 & 59.2 & 56.7 & 58.8 & 59.5 & 59.4 & 69.2 & 160.8 \\
\hline 1 & B & S & 2/6/1992 & 92037 & 36 & 63.3 & 62.3 & 57.7 & 56.1 & 53.3 & 57 & 55.7 & 84.8 & 204.6 \\
\hline 1 & B & $\mathrm{S}$ & 2/7/1992 & 92038 & 37 & 55.4 & 53.2 & 49.5 & 49.5 & 52.3 & 56.7 & 53.7 & 86.5 & 218.4 \\
\hline 1 & $B$ & S & 2/10/1992 & 92041 & 40 & 62.3 & 60.6 & 60.8 & 58.2 & 55.7 & 57.5 & 61.9 & 59.2 & 56.1 \\
\hline 1 & B & S & 2/11/1992 & 92042 & 41 & 67.1 & 63 & 59.4 & 57.8 & 59.5 & 59.9 & 61.2 & 78.9 & 176 \\
\hline 1 & B & S & 2/12/1992 & 92043 & 42 & 66.1 & 67.5 & 61.5 & 58.2 & 57.5 & 59.1 & 61.3 & 75.4 & 153.4 \\
\hline 1 & B & S & 2/13/1992 & 92044 & 43 & 61.6 & 62.3 & 58.1 & 57.5 & 56.4 & 54 & 58.4 & 86.1 & 178.4 \\
\hline 1 & B & S & 2/14/1992 & 92045 & 44 & 67 & 62.6 & 63.3 & 59.9 & 61.2 & 61.6 & 62 & 87.1 & 176 \\
\hline 1 & B & S & 2/17/1992 & 92048 & 47 & 72 & 70.2 & 64.4 & 64.7 & 64.7 & 64.3 & 65.4 & 62.6 & 69.8 \\
\hline 1 & $B$ & S & 2/18/1992 & 92049 & 48 & 70.2 & 66.8 & 64.6 & 63 & 62.6 & 63.2 & 64.7 & 84.1 & 171.1 \\
\hline 1 & $B$ & $S$ & 2/19/1992 & 92050 & 49 & 69.2 & 66.4 & 60.5 & 56.4 & 56.8 & 57.7 & 68.2 & 96.2 & 209.8 \\
\hline 1 & B & S & 2/20/1992 & 92051 & 50 & 63.6 & 61.6 & 58.5 & 58.4 & 56.1 & 57.1 & 66.7 & 99.6 & 183.3 \\
\hline 1 & $B$ & S & 2/21/1992 & 92052 & 51 & 54.7 & 52.5 & 48.1 & 47.4 & 48.7 & 47.4 & 49.2 & 72.2 & 177.7 \\
\hline
\end{tabular}




\begin{tabular}{|c|c|c|c|c|c|c|c|c|c|c|c|c|c|c|}
\hline 1 & B & $\mathrm{S}$ & $2 / 24 / 1992$ & 92055 & 54 & 77.9 & 93 & 92.7 & 87.2 & 87.4 & 91.3 & 79.6 & 95 & 101.4 \\
\hline 1 & $B$ & S & $2 / 25 / 1992$ & 92056 & 55 & 61.6 & 59.9 & 59.4 & 54.7 & 55 & 60.1 & 56.8 & 78.8 & 179.5 \\
\hline 1 & B & S & $2 / 26 / 1992$ & 92057 & 56 & 56.8 & 57.7 & 54.4 & 50.2 & 49.8 & 52.6 & 53.7 & 73.3 & 178.8 \\
\hline 1 & B & S & 2/27/1992 & 92058 & 57 & 59.9 & 55.6 & 55 & 50.9 & 50.8 & 53.3 & 53 & 91.2 & 189.1 \\
\hline 1 & B & $S$ & 2/28/1992 & 92059 & 58 & 60.6 & 57.8 & 56.7 & 50.9 & 53.7 & 54.6 & 54.4 & 85.8 & 193.2 \\
\hline 1 & B & S & 3/2/1992 & 92062 & 61 & 72.2 & 63 & 61.3 & 56.7 & 55.7 & 59.5 & 60.8 & 53.3 & 53.7 \\
\hline 1 & $B$ & S & $3 / 3 / 1992$ & 92063 & 62 & 65.1 & 64.7 & 62.9 & 58.8 & 61.6 & 62.2 & 59.5 & 79.2 & 193.9 \\
\hline 1 & $B$ & S & 3/4/1992 & 92064 & 63 & 67 & 59.9 & 57.8 & 57 & 53 & 58.5 & 61.5 & 84.4 & 192.7 \\
\hline 1 & B & S & $3 / 5 / 1992$ & 92065 & 64 & 73 & 72 & 71.9 & 66.4 & 64.4 & 65.7 & 64 & 88.6 & 188.4 \\
\hline 1 & B & S & $3 / 6 / 1992$ & 92066 & 65 & 73.7 & 72.6 & 68.5 & 69.1 & 67.1 & 63.7 & 66.7 & 74.7 & 226.3 \\
\hline 1 & B & S & $3 / 9 / 1992$ & 92069 & 68 & 77.2 & 67.1 & 63.6 & 67.8 & 61.3 & 65 & 68.9 & 62 & 62.9 \\
\hline 1 & $B$ & S & 3/10/1992 & 92070 & 69 & 75.3 & 72.3 & 69.6 & 66 & 69.6 & 65.4 & 71.5 & 97.8 & 246.3 \\
\hline 1 & B & S & $3 / 11 / 1992$ & 92071 & 70 & 81 & 79.2 & 71.9 & 67.8 & 60.9 & 56 & 59.2 & 92.4 & 205.6 \\
\hline 1 & B & S & $3 / 12 / 1992$ & 92072 & 71 & 64.7 & 61.9 & 55.7 & 57.1 & 58.4 & 54.7 & 62 & 92.6 & 198.5 \\
\hline 1 & B & S & 3/23/1992 & 92083 & 82 & 64.3 & 62 & 58.5 & 58.8 & 58.2 & 57 & 58.8 & 53.7 & 53.9 \\
\hline 1 & B & S & $3 / 24 / 1992$ & 92084 & 83 & 58.1 & 55.7 & 53 & 51.8 & 51.9 & 51.6 & 52.5 & 72.7 & 188.1 \\
\hline 1 & B & S & $3 / 25 / 1992$ & 92085 & 84 & 62 & 54.7 & 48 & 47.4 & 48.5 & 47 & 51.9 & 72.3 & 169 \\
\hline 1 & $B$ & S & $3 / 26 / 1992$ & 92086 & 85 & 68.8 & 64 & 58.5 & 58.4 & 55.4 & 56 & 57.5 & 75.1 & 216.4 \\
\hline 1 & B & $\mathrm{S}$ & $3 / 27 / 1992$ & 92087 & 86 & 73.4 & 69.6 & 61.3 & 59.1 & 59.2 & 58.2 & 60.5 & 72.3 & 162.6 \\
\hline 1 & B & S & 3/30/1992 & 92090 & 89 & 65.8 & 62.6 & 65 & 63 & 64.4 & 63.9 & 64.7 & 59.2 & 59.8 \\
\hline 1 & B & S & 3/31/1992 & 92091 & 90 & 68.4 & 59.9 & 56.4 & 58.1 & 53.7 & 51.9 & 53.9 & 65.4 & 159.8 \\
\hline 1 & B & S & 4/1/1992 & 92092 & 91 & 66.1 & 63.6 & 55.7 & 55.7 & 56 & 52.6 & 47.1 & 61.5 & 155.6 \\
\hline 1 & B & S & 4/2/1992 & 92093 & 92 & 67.4 & 64.4 & 58.5 & 56 & 56.8 & 55.7 & 55.6 & 72.3 & 173.2 \\
\hline 1 & B & $\mathrm{S}$ & 4/3/1992 & 92094 & 93 & 67 & 61.3 & 51.9 & 51.6 & 53.2 & 50.9 & 54.4 & 67.4 & 187.8 \\
\hline 1 & B & S & 4/6/1992 & 92097 & 96 & 59.9 & 55.7 & 49.8 & 50.2 & 50.2 & 51.1 & 51.6 & 45.7 & 44.9 \\
\hline 1 & B & S & 4/7/1992 & 92098 & 97 & 68.2 & 68.8 & 66.8 & 65.4 & 62.2 & 54.7 & 71.3 & 154.6 & 358.3 \\
\hline 1 & B & S & 4/8/1992 & 92099 & 98 & 75.3 & 64.7 & 60.6 & 62.2 & 60.6 & 59.5 & 74.7 & 190.8 & 338.5 \\
\hline 1 & B & S & 4/9/1992 & 92100 & 99 & 85 & 85.1 & 65.1 & 60.8 & 59.5 & 61.6 & 58.8 & 76.8 & 205.8 \\
\hline 1 & B & $\mathrm{S}$ & 4/10/1992 & 92101 & 100 & 82.3 & 80.3 & 78.8 & 75.8 & 74.7 & 74.7 & 71.6 & 83.4 & 219.4 \\
\hline 1 & B & S & $4 / 13 / 1992$ & 92104 & 103 & 79.9 & 80.5 & 68.5 & 62.3 & 62.2 & 60.9 & 60.2 & 56.4 & 57.7 \\
\hline 1 & $B$ & S & 4/14/1992 & 92105 & 104 & 77.2 & 75.7 & 75.8 & 72 & 72.6 & 68.9 & 65.1 & 81.2 & 190.3 \\
\hline 1 & B & S & 4/15/1992 & 92106 & 105 & 80.5 & 79.2 & 71.3 & 68.9 & 65.3 & 64 & 66.4 & 73.6 & 187.6 \\
\hline 1 & B & S & 4/16/1992 & 92107 & 106 & 91.7 & 85.1 & 71.6 & 68.4 & 69.6 & 69.2 & 68.4 & 78.5 & 219.3 \\
\hline 1 & B & S & 4/21/1992 & 92112 & 111 & 73.6 & 71.6 & 74.7 & 72.2 & 70.9 & 71.2 & 68.2 & 67.8 & 89.2 \\
\hline 1 & $B$ & S & 4/22/1992 & 92113 & 112 & 73.6 & 71.6 & 64 & 62.2 & 60.6 & 60.9 & 61.5 & 72.7 & 182.8 \\
\hline 1 & $B$ & S & 4/23/1992 & 92114 & 113 & 84.8 & 77.4 & 70.6 & 67.8 & 66 & 65.4 & 64.7 & 78.5 & 194.2 \\
\hline 1 & B & S & $4 / 24 / 1992$ & 92115 & 114 & 85.4 & 82.7 & 64.7 & 60.8 & 60.9 & 57.1 & 61.9 & 66.4 & 189.4 \\
\hline
\end{tabular}




\begin{tabular}{|c|c|c|c|c|c|c|c|c|c|c|c|c|c|c|}
\hline 1 & $B$ & $\mathrm{~S}$ & $4 / 27 / 1992$ & 92118 & 117 & 72.9 & 67.8 & 68.2 & 67.1 & 66 & 66.4 & 65.8 & 62.2 & 60.2 \\
\hline 1 & B & S & $4 / 29 / 1992$ & 92120 & 119 & 80.5 & 80.6 & 71.3 & 66.7 & 65.4 & 63.7 & 65.3 & 77.9 & 185.3 \\
\hline 1 & B & S & $4 / 30 / 1992$ & 92121 & 120 & 93.1 & 88.9 & 78.8 & 74.1 & 73 & 74 & 72.3 & 82.7 & 188.4 \\
\hline 1 & B & S & 5/1/1992 & 92122 & 121 & 88.9 & 85.8 & 67.7 & 64 & 61.6 & 60.2 & 61.9 & 70.2 & 186 \\
\hline 1 & B & S & 5/4/1992 & 92125 & 124 & 79.6 & 79.5 & 77.9 & 76 & 75.4 & 74.7 & 76 & 72.3 & 73 \\
\hline 1 & B & S & $5 / 5 / 1992$ & 92126 & 125 & 82.6 & 81 & 76.8 & 75.7 & 73.4 & 74.7 & 78.8 & 84.8 & 196.8 \\
\hline 1 & B & S & $5 / 6 / 1992$ & 92127 & 126 & 84.4 & 82 & 64.6 & 63.7 & 57.8 & 63.2 & 60.9 & 73.4 & 202.5 \\
\hline 1 & B & S & $5 / 7 / 1992$ & 92128 & 127 & 72 & 70.6 & 69.1 & 65.4 & 65.4 & 65.3 & 63 & 70.9 & 246.5 \\
\hline 1 & B & S & $5 / 8 / 1992$ & 92129 & 128 & 71.2 & 70.6 & 67.5 & 67 & 66.4 & 65.8 & 67 & 85.8 & 175.7 \\
\hline 1 & B & S & $5 / 11 / 1992$ & 92132 & 131 & 73 & 71.6 & 71.2 & 69.2 & 67.5 & 69.5 & 70.2 & 67.1 & 67 \\
\hline 1 & B & S & $5 / 12 / 1992$ & 92133 & 132 & 67.8 & 69.8 & 66.1 & 64.4 & 66 & 65.8 & 67.7 & 77.9 & 235.5 \\
\hline 1 & $B$ & S & $5 / 13 / 1992$ & 92134 & 133 & 70.2 & 70.2 & 68.1 & 69.2 & 62.3 & 69.1 & 64.4 & 76.1 & 258.1 \\
\hline 1 & B & S & $5 / 14 / 1992$ & 92135 & 134 & 77.2 & 74.7 & 72.9 & 69.9 & 65.8 & 69.8 & 71.6 & 66.1 & 253 \\
\hline 1 & B & S & $5 / 15 / 1992$ & 92136 & 135 & 67.1 & 64 & 63.9 & 60.6 & 64.7 & 67.4 & 70.2 & 75.8 & 245 \\
\hline 1 & B & S & $5 / 18 / 1992$ & 92139 & 138 & 72 & 71.2 & 69.6 & 67.5 & 65.7 & 65.8 & 67.1 & 66 & 65.8 \\
\hline 1 & $B$ & $\mathrm{~S}$ & $5 / 19 / 1992$ & 92140 & 139 & 70.9 & 74.7 & 72.2 & 72 & 70.2 & 71.9 & 72.7 & 76.5 & 238.1 \\
\hline 1 & B & S & $5 / 20 / 1992$ & 92141 & 140 & 101.7 & 79.9 & 75.7 & 73.4 & 72 & 72.2 & 74.1 & 79.2 & 232.5 \\
\hline 1 & $B$ & S & $5 / 21 / 1992$ & 92142 & 141 & 62.2 & 52.6 & 51.9 & 51.5 & 49.2 & 49.5 & 50.8 & 53.3 & 263.1 \\
\hline 1 & $B$ & S & $5 / 22 / 1992$ & 92143 & 142 & 70.2 & 68.2 & 68.8 & 66.1 & 66.8 & 70.8 & 71.3 & 80.6 & 236.5 \\
\hline 1 & B & S & $5 / 25 / 1992$ & 92146 & 145 & 81.7 & 79.2 & 74 & 73.4 & 71.3 & 71.5 & 73.4 & 69.2 & 68.8 \\
\hline 1 & B & S & $5 / 26 / 1992$ & 92147 & 146 & 75.1 & 75.4 & 77.1 & 75.1 & 75.1 & 72.9 & 74.1 & 79.8 & 246.4 \\
\hline 1 & B & S & $5 / 27 / 1992$ & 92148 & 147 & 72.6 & 74.7 & 74.7 & 66 & 64 & 65.4 & 66.7 & 80.8 & 296.2 \\
\hline 1 & B & S & 9/8/1992 & 92252 & 251 & 76 & 78.9 & 71.9 & 69.6 & 70.5 & 67.8 & 65 & 64.4 & 110.6 \\
\hline 1 & B & S & 9/9/1992 & 92253 & 252 & 78.9 & 80.2 & 78.2 & 74.3 & 75.8 & 72.2 & 70.2 & 125.3 & 391.4 \\
\hline 1 & B & $\mathrm{S}$ & 9/10/1992 & 92254 & 253 & 97.1 & 87.2 & 87.4 & 83.7 & 84.3 & 86.5 & 79.1 & 126.6 & 399.6 \\
\hline 1 & B & S & 9/11/1992 & 92255 & 254 & 80.9 & 78.9 & 78.5 & 78.2 & 76.4 & 76.5 & 75.3 & 120.2 & 411.4 \\
\hline 1 & B & S & 9/14/1992 & 92258 & 257 & 79.5 & 77.9 & 78.1 & 75.8 & 77.1 & 78.5 & 77.8 & 78.2 & 92.6 \\
\hline 1 & B & S & 9/16/1992 & 92260 & 259 & 75.8 & 76.7 & 75.8 & 75 & 74.4 & 76.7 & 73.7 & 86.7 & 303.5 \\
\hline 1 & $B$ & S & 9/17/1992 & 92261 & 260 & 77.8 & 77.9 & 77.4 & 75.1 & 75.3 & 77.5 & 75.7 & 119.7 & 389.3 \\
\hline 1 & B & $\mathrm{S}$ & 9/18/1992 & 92262 & 261 & 81.7 & 79.6 & 81.2 & 79.9 & 79.1 & 80.3 & 77.8 & 108.3 & 380.2 \\
\hline 1 & B & S & 9/21/1992 & 92265 & 264 & 83.3 & 83 & 82.3 & 79.9 & 79.1 & 80.3 & 80.5 & 79.6 & 80.2 \\
\hline 1 & $B$ & S & 9/22/1992 & 92266 & 265 & 80.6 & 80.9 & 78.2 & 77.4 & 76.1 & 78.5 & 76.5 & 127.8 & 254.9 \\
\hline 1 & B & S & 9/23/1992 & 92267 & 266 & 96.1 & 95.5 & 95.4 & 92.7 & 94 & 93.7 & 91.6 & 140.2 & 390.1 \\
\hline 1 & B & S & 9/24/1992 & 92268 & 267 & 81.2 & 81.7 & 81.6 & 79.6 & 79.5 & 80.3 & 78.2 & 103.5 & 339.2 \\
\hline 1 & $B$ & S & 9/25/1992 & 92269 & 268 & 84.8 & 81.6 & 81.3 & 79.6 & 79.5 & 79.9 & 78.5 & 122.5 & 334.3 \\
\hline 1 & B & S & 9/28/1992 & 92272 & 271 & 78.5 & 77.2 & 77.4 & 74.4 & 74.7 & 76.8 & 76.7 & 76.7 & 76.8 \\
\hline 1 & $B$ & $\mathrm{~S}$ & 9/29/1992 & 92273 & 272 & 72.6 & 72.6 & 73 & 71.2 & 71.3 & 71.5 & 68.2 & 116.6 & 355.7 \\
\hline
\end{tabular}




\begin{tabular}{|c|c|c|c|c|c|c|c|c|c|c|c|c|c|c|}
\hline 1 & $B$ & $\mathrm{~S}$ & 9/30/1992 & 92274 & 273 & 79.8 & 79.2 & 79.2 & 78.1 & 76.1 & 78.8 & 76.5 & 99.6 & 317.5 \\
\hline 1 & $B$ & S & 10/1/1992 & 92275 & 274 & 63.6 & 62 & 62.2 & 62.3 & 62 & 61.5 & 59.5 & 85.4 & 250 \\
\hline 1 & B & S & 10/2/1992 & 92276 & 275 & 57.4 & 57.8 & 59.1 & 56.4 & 57.7 & 58.2 & 56.8 & 81.2 & 249.3 \\
\hline 1 & B & S & 10/5/1992 & 92279 & 278 & 57.4 & 56.8 & 57.7 & 54.7 & 54.3 & 56.4 & 56.7 & 56.1 & 55.7 \\
\hline 1 & B & S & 10/6/1992 & 92280 & 279 & 56 & 56.1 & 57 & 53.7 & 53 & 55.3 & 52.3 & 79.1 & 279.4 \\
\hline 1 & $B$ & $S$ & 10/7/1992 & 92281 & 280 & 78.5 & 79.1 & 78.5 & 76.4 & 74.7 & 75.8 & 75.3 & 97 & 293.8 \\
\hline 1 & B & $S$ & 10/8/1992 & 92282 & 281 & 84 & 81.7 & 81 & 79.5 & 79.6 & 82.3 & 77.5 & 109.5 & 312.4 \\
\hline 1 & B & S & 10/9/1992 & 92283 & 282 & 81.6 & 81.7 & 78.8 & 75.4 & 75.7 & 73.7 & 71.2 & 90.8 & 243.5 \\
\hline 1 & B & S & \#\#\#\#\#\#\#\# & 92287 & 286 & 77.4 & 75.8 & 77.1 & 75.1 & 70.6 & 75.7 & 70.9 & 94.5 & 212.5 \\
\hline 1 & B & S & \#\#\#\#\#\#\#\# & 92288 & 287 & 75.8 & 106.1 & 96.2 & 89.5 & 89.9 & 84.1 & 82.3 & 103.2 & 196.8 \\
\hline 1 & B & S & \#\#\#\#\#\#\#\# & 92289 & 288 & 105.1 & 84.1 & 78.5 & 78.9 & 75.1 & 78.1 & 78.2 & 98.7 & 296.6 \\
\hline 1 & $B$ & S & \#\#\#\#\#\#\#\# & 92290 & 289 & 69.2 & 68.8 & 65.4 & 65.7 & 65.4 & 66.4 & 61.9 & 86 & 316 \\
\hline 1 & B & S & \#\#\#\#\#\#\#\# & 92293 & 292 & 124.9 & 86.8 & 81.6 & 70.6 & 70.5 & 70.6 & 69.8 & 69.2 & 74 \\
\hline 1 & B & S & \#\#\#\#\#\#\#\# & 92294 & 293 & 69.5 & 66.8 & 71.9 & 66.8 & 66.1 & 66.7 & 63.7 & 87.2 & 277.4 \\
\hline 1 & B & S & \#\#\#\#\#\#\#\# & 92295 & 294 & & 97.9 & 91.2 & 87.2 & 82.6 & 79.9 & 78.5 & 96.6 & 287.3 \\
\hline 1 & $B$ & $\mathrm{~S}$ & \#\#\#\#\#\#\#\# & 92296 & 295 & 89.2 & 76.8 & 72.2 & 70.6 & 69.9 & 71.9 & 68.5 & 92.7 & 284 \\
\hline 1 & B & S & \#\#\#\#\#\#\#\# & 92297 & 296 & 76.5 & 75 & 75.1 & 72.9 & 70.9 & 72.9 & 69.6 & 94.9 & 299.4 \\
\hline 1 & B & $\mathrm{S}$ & \#\#\#\#\#\#\#\# & 92300 & 299 & 82.6 & 73.7 & 75 & 72.3 & 68.9 & 70.5 & 69.6 & 69.1 & 66.4 \\
\hline 1 & B & S & \#\#\#\#\#\#\#\# & 92301 & 300 & 75.7 & 74.4 & 75.3 & 73.4 & 72.9 & 71.6 & 71.6 & 69.5 & 99.6 \\
\hline 1 & B & S & \#\#\#\#\#\#\#\# & 92302 & 301 & 74 & 94.8 & 94.3 & 89.3 & 88.9 & 84.7 & 79.9 & 123.8 & 298.4 \\
\hline 1 & B & S & \#\#\#\#\#\#\#\# & 92303 & 302 & 93.3 & 80.6 & 78.5 & 77.2 & 77.1 & 76.8 & 75 & 127.9 & 310.2 \\
\hline 1 & B & S & \#\#\#\#\#\#\#\# & 92304 & 303 & 82.3 & 81.3 & 79.8 & 77.5 & 79.5 & 77.2 & 75 & 122.5 & 314.6 \\
\hline 1 & B & S & 11/2/1992 & 92307 & 306 & 73.6 & 74.4 & 69.5 & 68.9 & 72.6 & 67.8 & 62.9 & 62.3 & 76 \\
\hline 1 & B & S & $11 / 3 / 1992$ & 92308 & 307 & 70.5 & 67.8 & 67.1 & 66.4 & 64 & 63.2 & 62.3 & 98.5 & 269.3 \\
\hline 1 & B & $\mathrm{S}$ & $11 / 4 / 1992$ & 92309 & 308 & 73.7 & 86.1 & 82.7 & 77.8 & 75.8 & 75.3 & 74.7 & 106.8 & 294.8 \\
\hline 1 & B & S & 11/5/1992 & 92310 & 309 & 83.3 & 76.8 & 73 & 71.5 & 69.9 & 69.8 & 66.4 & 90.9 & 238.6 \\
\hline 1 & B & S & 11/6/1992 & 92311 & 310 & 57.1 & 57.7 & 56.4 & 55.3 & 55.4 & 55.4 & 53.9 & 83 & 227.1 \\
\hline 1 & B & S & 11/9/1992 & 92314 & 313 & 69.5 & 71.3 & 71.3 & 67.7 & 65.4 & 65.1 & 65 & 59.9 & 60.9 \\
\hline 1 & $B$ & S & \#\#\#\#\#\#\#\# & 92315 & 314 & 73 & 72.6 & 70.9 & 70.8 & 74.4 & 74 & 69.6 & 91 & 230.2 \\
\hline 1 & B & S & \#\#\#\#\#\#\#\# & 92316 & 315 & 77.1 & 93.7 & 89.9 & 83.7 & 86.4 & 84.1 & 79.5 & 113.4 & 284.5 \\
\hline 1 & B & S & \#\#\#\#\#\#\#\# & 92317 & 316 & 88.6 & 76 & 75.4 & 75 & 74.1 & 72.2 & 74.7 & 105.3 & 274.6 \\
\hline 1 & $B$ & S & \#\#\#\#\#\#\#\# & 92318 & 317 & 76.5 & 72.6 & 73.3 & 72 & 76 & 73.7 & 72.6 & 105.4 & 236.5 \\
\hline 1 & B & S & \#\#\#\#\#\#\#\# & 92321 & 320 & 64 & 63.2 & 59.2 & 60.8 & 61.3 & 58.1 & 57.8 & 55.3 & 53 \\
\hline 1 & B & S & \#\#\#\#\#\#\#\# & 92322 & 321 & 64.4 & 64.6 & 63.7 & 63.6 & 62 & 61.9 & 59.2 & 74.7 & 192.6 \\
\hline 1 & $B$ & S & \#\#\#\#\#\#\#\# & 92323 & 322 & 57.5 & 69.8 & 63.3 & 61.2 & 60.2 & 60.5 & 56.4 & 94.3 & 226.8 \\
\hline 1 & B & S & \#\#\#\#\#\#\#\# & 92324 & 323 & 76.7 & 66.1 & 62.6 & 60.9 & 59.8 & 59.2 & 59.1 & 96.2 & 226.4 \\
\hline 1 & $B$ & $\mathrm{~S}$ & \#\#\#\#\#\#\#\# & 92325 & 324 & 64 & 65 & 62.6 & 62.9 & 62.3 & 63.6 & 62 & 98.6 & 259.9 \\
\hline
\end{tabular}




\begin{tabular}{|c|c|c|c|c|c|c|c|c|c|c|c|c|c|c|}
\hline 1 & $B$ & $\mathrm{~S}$ & \#\#\#\#\#\#\#\# & 92328 & 327 & 73.3 & 70.9 & 69.1 & 68.5 & 67.7 & 67.5 & 64.6 & 60.2 & 58.1 \\
\hline 1 & B & S & \#\#\#\#\#\#\#\# & 92329 & 328 & 72.7 & 70.2 & 67.8 & 68.4 & 67.8 & 67.4 & 66.4 & 78.8 & 151.1 \\
\hline 1 & B & S & \#\#\#\#\#\#\#\# & 92335 & 334 & 67.7 & 73 & 77.4 & 76.5 & 76.4 & 76 & 74.3 & 69.8 & 69.9 \\
\hline 1 & B & S & 12/1/1992 & 92336 & 335 & 70.8 & 77.9 & 79.1 & 79.1 & 79.6 & 79.8 & 76.7 & 87.5 & 215.3 \\
\hline 1 & B & S & 12/2/1992 & 92337 & 336 & 72.6 & 79.2 & 81.2 & 77.9 & 78.8 & 76.8 & 75 & 100.6 & 227.2 \\
\hline 1 & B & S & 12/3/1992 & 92338 & 337 & 69.9 & 72.9 & 76.1 & 75 & 75.1 & 74 & 72.9 & 101.7 & 237.8 \\
\hline 1 & B & S & 12/4/1992 & 92339 & 338 & 68.5 & 75.3 & 92.4 & 87.8 & 87.5 & 85.7 & 83.7 & 105.8 & 226.8 \\
\hline 1 & B & S & 12/7/1992 & 92342 & 341 & 62.3 & 68.8 & 73.4 & 70.2 & 72.7 & 72.6 & 68.5 & 68.4 & 64.6 \\
\hline 1 & B & S & 12/8/1992 & 92343 & 342 & 60.2 & 69.1 & 70.6 & 69.8 & 71.5 & 67.1 & 68.4 & 96.8 & 243.4 \\
\hline 1 & B & S & 12/9/1992 & 92344 & 343 & 69.9 & 76.4 & 79.8 & 77.2 & 80.9 & 77.5 & 76 & 95.7 & 256.5 \\
\hline 1 & B & S & \#\#\#\#\#\#\#\# & 92345 & 344 & 64.4 & 63.2 & 60.6 & 61.2 & 62.2 & 59.5 & 56 & 70.2 & 208.7 \\
\hline 1 & B & S & \#\#\#\#\#\#\#\# & 92346 & 345 & 56.8 & 57.7 & 71.3 & 65 & 64.7 & 65.7 & 59.2 & 71.5 & 195.4 \\
\hline 1 & B & S & \#\#\#\#\#\#\#\# & 92349 & 348 & 59.9 & 62.2 & 59.9 & 60.1 & 61.3 & 59.1 & 56.4 & 54.6 & 52.6 \\
\hline 1 & B & S & \#\#\#\#\#\#\#\# & 92350 & 349 & 65.7 & 67.1 & 66 & 61.6 & 66.4 & 63.3 & 61.5 & 105.2 & 241.4 \\
\hline 1 & $B$ & S & \#\#\#\#\#\#\#\# & 92351 & 350 & 63 & 65 & 61.3 & 61.9 & 64.4 & 62.2 & 79.6 & 118.9 & 246.8 \\
\hline 1 & B & S & \#\#\#\#\#\#\#\# & 92352 & 351 & 65.3 & 60.9 & 60.5 & 59.2 & 61.9 & 61.6 & 75.7 & 101.4 & 196.3 \\
\hline 1 & B & S & $1 / 5 / 1993$ & 93005 & 5 & 57.1 & 57.7 & 55.7 & 54.6 & 55 & 55 & 58.4 & 60.2 & 145.8 \\
\hline 1 & B & $\mathrm{S}$ & 1/6/1993 & 93006 & 6 & 112 & 109 & 65 & 60.2 & 61.2 & 60.6 & 63.6 & 99.6 & 232.2 \\
\hline 1 & $B$ & S & 1/7/1993 & 93007 & 7 & 64.6 & 62.6 & 63.6 & 60.6 & 65.3 & 69.9 & 72.2 & 104.8 & 210.5 \\
\hline 1 & B & S & 1/8/1993 & 93008 & 8 & 64.6 & 65.1 & 63.9 & 63.3 & 63.9 & 61.9 & 64.4 & 82.3 & 179.7 \\
\hline 1 & B & S & 1/11/1993 & 93011 & 11 & 61.3 & 61.9 & 58.8 & 57.7 & 58.4 & 57.8 & 62.9 & 100.7 & 224.6 \\
\hline 1 & B & S & $1 / 12 / 1993$ & 93012 & 12 & 67.4 & 67.8 & 66.4 & 64.7 & 65 & 64.3 & 64 & 96.1 & 234 \\
\hline 1 & B & S & 1/13/1993 & 93013 & 13 & 53.2 & 62.2 & 50.9 & 50.5 & 51.2 & 49.4 & 49.5 & 95.4 & 227.2 \\
\hline 1 & B & S & $1 / 14 / 1993$ & 93014 & 14 & 53.7 & 53.2 & 51.2 & 51.5 & 49.5 & 49.1 & 48.5 & 101.6 & 217.8 \\
\hline 1 & B & $\mathrm{S}$ & $1 / 15 / 1993$ & 93015 & 15 & 52.2 & 50.9 & 50.5 & 48.5 & 48.4 & 48.7 & 46.1 & 71.9 & 182.2 \\
\hline 1 & B & S & 1/18/1993 & 93018 & 18 & 53 & 52.5 & 50.5 & 50.1 & 49.5 & 52.2 & 55.6 & 89.9 & 188.4 \\
\hline 1 & B & S & 1/19/1993 & 93019 & 19 & 54.6 & 54.4 & 53.2 & 51.6 & 51.8 & 50.5 & 50.5 & 69.9 & 188 \\
\hline 1 & B & S & 1/20/1993 & 93020 & 20 & 57.1 & 59.4 & 54.4 & 53.9 & 54.4 & 54.6 & 52.6 & 99.5 & 232.3 \\
\hline 1 & $B$ & S & 1/21/1993 & 93021 & 21 & 55.3 & 53.7 & 52.2 & 52.3 & 50.8 & 50.5 & 50.5 & 84.4 & 225.7 \\
\hline 1 & B & S & $1 / 22 / 1993$ & 93022 & 22 & 58.5 & 56.8 & 56.3 & 53.7 & 53.6 & 52.9 & 51.6 & 73.6 & 160.5 \\
\hline 1 & B & S & $1 / 25 / 1993$ & 93025 & 25 & 51.8 & 50.9 & 50.1 & 47.4 & 48 & 48.1 & 47 & 84 & 213.7 \\
\hline 1 & $B$ & S & 1/26/1993 & 93026 & 26 & 55.4 & 58.1 & 54 & 54.3 & 53.3 & 55.6 & 84.4 & 105.8 & 227.8 \\
\hline 1 & B & S & 1/27/1993 & 93027 & 27 & 71.9 & 67.1 & 66 & 65.4 & 66 & 68.2 & 113.4 & 140 & 249.3 \\
\hline 1 & B & S & 1/28/1993 & 93028 & 28 & 68.1 & 68.2 & 66 & 64.7 & 65.7 & 68.5 & 97.1 & 122.1 & 227.4 \\
\hline 1 & $B$ & S & 1/29/1993 & 93029 & 29 & 67.4 & 67.1 & 65.7 & 63.7 & 64.3 & 65.8 & 72.2 & 85.5 & 182.5 \\
\hline 1 & B & S & 2/1/1993 & 93032 & 32 & 71.6 & 72.9 & 69.9 & 68.1 & 69.6 & 72.9 & 119.2 & 143.9 & 247.1 \\
\hline 1 & $B$ & $S$ & 2/2/1993 & 93033 & 33 & 69.5 & 70.2 & 68.4 & 68.2 & 68.8 & 68.5 & 85 & 103.7 & 196.7 \\
\hline
\end{tabular}




\begin{tabular}{|c|c|c|c|c|c|c|c|c|c|c|c|c|c|c|}
\hline 1 & $B$ & $\mathrm{~S}$ & $2 / 3 / 1993$ & 93034 & 34 & 96.1 & 79.2 & 68.4 & 66.4 & 65.4 & 67 & 84.1 & 107.1 & 196.7 \\
\hline 1 & B & $S$ & 2/4/1993 & 93035 & 35 & 93.7 & 56.1 & 55.6 & 53.7 & 53.9 & 54 & 65 & 96.8 & 193.6 \\
\hline 1 & B & S & 2/5/1993 & 93036 & 36 & 99.2 & 69.9 & 58.1 & 55 & 56.3 & 57 & 72.7 & 76.7 & 201.9 \\
\hline 1 & B & S & $2 / 8 / 1993$ & 93039 & 39 & 52.3 & 53.2 & 51.6 & 51.8 & 50.2 & 55.3 & 99.9 & 109.6 & 221.2 \\
\hline 1 & B & S & $2 / 9 / 1993$ & 93040 & 40 & 53.2 & 53.7 & 50.8 & 52.3 & 51.5 & 51.9 & 78.1 & 99.6 & 214.3 \\
\hline 1 & B & S & 2/10/1993 & 93041 & 41 & 53.9 & 52.3 & 50.8 & 48.8 & 49.4 & 49.5 & 61.2 & 81.2 & 171.9 \\
\hline 1 & B & S & $2 / 11 / 1993$ & 93042 & 42 & 62.3 & 63.6 & 60.6 & 60.5 & 58.8 & 61.5 & 61.3 & 73.3 & 178.8 \\
\hline 1 & B & S & $2 / 12 / 1993$ & 93043 & 43 & 64.3 & 63.7 & 61.2 & 60.6 & 60.1 & 59.9 & 61.9 & 74.7 & 198.7 \\
\hline 1 & B & S & $2 / 16 / 1993$ & 93047 & 47 & 82.7 & 84 & 81.7 & 80.5 & 79.6 & 78.8 & 84.4 & 103.7 & 199.8 \\
\hline 1 & B & S & 2/17/1993 & 93048 & 48 & 66.4 & 65.3 & 63.3 & 63.7 & 63.6 & 63 & 85 & 91.6 & 219.5 \\
\hline 1 & $B$ & S & 2/18/1993 & 93049 & 49 & 69.1 & 67.1 & 66 & 63.7 & 64.3 & 65.1 & 82.9 & 98.6 & 237.4 \\
\hline 1 & B & S & $2 / 19 / 1993$ & 93050 & 50 & 70.5 & 71.3 & 69.5 & 66.8 & 67.4 & 67.5 & 86.1 & 97.9 & 224.6 \\
\hline 1 & B & S & $2 / 22 / 1993$ & 93053 & 53 & 73.7 & 74.7 & 66.8 & 62.2 & 62 & 64.3 & 91 & 102 & 201.6 \\
\hline 1 & B & S & 2/23/1993 & 93054 & 54 & 65.8 & 66 & 62.6 & 63.6 & 60.6 & 61.9 & 69.6 & 94.4 & 218.4 \\
\hline 1 & B & S & $2 / 24 / 1993$ & 93055 & 55 & 67.1 & 66.4 & 63.3 & 65 & 63 & 64.6 & 88.2 & 100.9 & 194 \\
\hline 1 & B & S & $2 / 25 / 1993$ & 93056 & 56 & 70.6 & 70.2 & 67.8 & 65.3 & 66.8 & 66 & 72.2 & 103.1 & 263.6 \\
\hline 1 & B & S & $2 / 26 / 1993$ & 93057 & 57 & 62.9 & 61.6 & 60.8 & 61.3 & 59.8 & 58.2 & 64.3 & 85.8 & 219.5 \\
\hline 1 & $B$ & $\mathrm{~S}$ & $3 / 1 / 1993$ & 93060 & 60 & 72.3 & 72.9 & 71.6 & 69.8 & 69.6 & 71.5 & 101 & 116.5 & 210.9 \\
\hline 1 & B & S & $3 / 2 / 1993$ & 93061 & 61 & 65.8 & 68.1 & 65.8 & 64.3 & 64.7 & 63.9 & 70.6 & 89.5 & 181.9 \\
\hline 1 & B & S & $3 / 3 / 1993$ & 93062 & 62 & 63.3 & 63.9 & 61.3 & 59.8 & 57.8 & 58.4 & 68.9 & 88.5 & 190.9 \\
\hline 1 & $B$ & S & 3/4/1993 & 93063 & 63 & 72.9 & 72.3 & 70.2 & 69.1 & 67.8 & 67.4 & 66.8 & 88.5 & 197.4 \\
\hline 1 & B & S & $3 / 5 / 1993$ & 93064 & 64 & 66.7 & 66.1 & 65.7 & 64.4 & 63.9 & 63.3 & 63.2 & 77.5 & 193.2 \\
\hline 1 & B & S & $3 / 8 / 1993$ & 93067 & 67 & 63.6 & 65.4 & 61.2 & 59.5 & 60.8 & 61.3 & 95 & 106.2 & 205.3 \\
\hline 1 & B & S & $3 / 9 / 1993$ & 93068 & 68 & 67.5 & 68.1 & 66.1 & 64.6 & 63.7 & 65 & 73 & 111.6 & 177.7 \\
\hline 1 & $B$ & $\mathrm{~S}$ & $3 / 10 / 1993$ & 93069 & 69 & 68.1 & 66.8 & 66.7 & 65.1 & 65 & 65.4 & 73.6 & 96.2 & 188.7 \\
\hline 1 & B & S & $3 / 11 / 1993$ & 93070 & 70 & 64 & 63.2 & 61.6 & 61.2 & 60.9 & 62.2 & 66.8 & 81.9 & 135.2 \\
\hline 1 & B & $\mathrm{S}$ & $3 / 12 / 1993$ & 93071 & 71 & 68.5 & 68.8 & 65.4 & 65 & 63.7 & 63.9 & 62.3 & 76 & 130.7 \\
\hline 1 & B & S & $3 / 22 / 1993$ & 93081 & 81 & 63.6 & 63.9 & 62 & 59.8 & 58.2 & 59.4 & 78.8 & 91.3 & 180.7 \\
\hline 1 & B & S & $3 / 23 / 1993$ & 93082 & 82 & 61.2 & 60.6 & 59.4 & 59.2 & 58.1 & 59.5 & 68.4 & 90.2 & 184.6 \\
\hline 1 & B & S & $3 / 24 / 1993$ & 93083 & 83 & 60.5 & 60.9 & 60.8 & 59.1 & 57.5 & 59.1 & 68.9 & 91.9 & 194.7 \\
\hline 1 & B & S & $3 / 25 / 1993$ & 93084 & 84 & 62.6 & 63.2 & 59.9 & 59.1 & 57.5 & 58.4 & 62.3 & 105.7 & 249 \\
\hline 1 & B & S & $3 / 26 / 1993$ & 93085 & 85 & 73.4 & 76 & 72 & 70.8 & 62.3 & 62.9 & 67.5 & 113.9 & 277.8 \\
\hline 1 & B & S & $3 / 29 / 1993$ & 93088 & 88 & 65.7 & 69.2 & 65 & 63 & 61.9 & 63.7 & 80.2 & 128.7 & 269.6 \\
\hline 1 & $B$ & S & $3 / 30 / 1993$ & 93089 & 89 & 72.7 & 71.9 & 68.4 & 66.8 & 68.1 & 67.4 & 72.2 & 138 & 268.7 \\
\hline 1 & B & $S$ & $3 / 31 / 1993$ & 93090 & 90 & 71.5 & 70.2 & 67.4 & 66.1 & 66 & 63.2 & 66.4 & 124.3 & 248.8 \\
\hline 1 & B & S & 4/1/1993 & 93091 & 91 & 62.2 & 60.5 & 58.8 & 62.2 & 62.6 & 62.3 & 61.9 & 110.5 & 200.9 \\
\hline 1 & $B$ & S & 4/2/1993 & 93092 & 92 & 69.8 & 68.9 & 69.5 & 68.9 & 68.4 & 67.4 & 66.1 & 95.3 & 188.7 \\
\hline
\end{tabular}




\begin{tabular}{|c|c|c|c|c|c|c|c|c|c|c|c|c|c|c|}
\hline 1 & $B$ & $\mathrm{~S}$ & $4 / 5 / 1993$ & 93095 & 95 & 64.7 & 65.7 & 66.4 & 65.7 & 62.9 & 62.6 & 75.7 & 111.6 & 186.3 \\
\hline 1 & B & $S$ & $4 / 6 / 1993$ & 93096 & 96 & 68.8 & 66.7 & 66.4 & 64.4 & 66 & 65 & 67 & 120.6 & 191.7 \\
\hline 1 & B & S & 4/7/1993 & 93097 & 97 & 73.3 & 68.1 & 68.2 & 68.4 & 67 & 67.7 & 71.2 & 130.1 & 240.6 \\
\hline 1 & B & S & $4 / 8 / 1993$ & 93098 & 98 & 69.5 & 61.9 & 62.6 & 61.3 & 61.2 & 60.5 & 58.8 & 83.7 & 185.2 \\
\hline 1 & B & S & 4/13/1993 & 93103 & 103 & 65.4 & 66.7 & 66.7 & 64.4 & 62.6 & 63 & 65.7 & 125 & 263.7 \\
\hline 1 & B & S & 4/14/1993 & 93104 & 104 & 70.5 & 69.8 & 68.5 & 67 & 65.7 & 65.4 & 77.4 & 143.7 & 272.5 \\
\hline 1 & B & S & 4/15/1993 & 93105 & 105 & 70.2 & 62.9 & 64.7 & 61.9 & 60.6 & 60.8 & 60.8 & 103.2 & 185.3 \\
\hline 1 & B & S & $4 / 16 / 1993$ & 93106 & 106 & 67.4 & 65.3 & 65.7 & 63.3 & 61.5 & 61.9 & 67.4 & 81.6 & 169.4 \\
\hline 1 & B & S & $4 / 19 / 1993$ & 93109 & 109 & 51.5 & 51.5 & 51.1 & 50.1 & 47.4 & 50.1 & 65 & 107.7 & 237.3 \\
\hline 1 & B & S & 4/20/1993 & 93110 & 110 & 62.9 & 63.3 & 64.3 & 61.2 & 60.6 & 60.8 & 66.1 & 132.9 & 269.1 \\
\hline 1 & $B$ & S & 4/21/1993 & 93111 & 111 & 62.3 & 60.1 & 60.2 & 59.4 & 57 & 56.4 & 56.7 & 106.3 & 202.4 \\
\hline 1 & B & S & $4 / 22 / 1993$ & 93112 & 112 & 61.2 & 58.5 & 60.1 & 57.1 & 56 & 56.8 & 57.4 & 95.7 & 167 \\
\hline 1 & B & S & 4/23/1993 & 93113 & 113 & 58.5 & 59.8 & 58.5 & 58.4 & 56.1 & 56 & 58.2 & 97.2 & 170.7 \\
\hline 1 & B & S & 4/26/1993 & 93116 & 116 & 69.1 & 67.4 & 67.1 & 64.6 & 64 & 64.3 & 93.7 & 150.5 & 246.6 \\
\hline 1 & B & S & $4 / 27 / 1993$ & 93117 & 117 & 58.8 & 63.6 & 57.7 & 57.5 & 52.9 & 53.7 & 57.7 & 118.8 & 233.6 \\
\hline 1 & B & S & $4 / 29 / 1993$ & 93119 & 119 & 53 & 56.3 & 56.4 & 53.2 & 55.6 & 54.4 & 59.8 & 108 & 205.4 \\
\hline 1 & B & S & $4 / 30 / 1993$ & 93120 & 120 & 67.8 & 66.7 & 68.9 & 66 & 65.1 & 64.6 & 65.1 & 98.7 & 179.1 \\
\hline 1 & B & S & $5 / 3 / 1993$ & 93123 & 123 & 58.8 & 58.4 & 59.1 & 56.8 & 56.3 & 57.1 & 73.3 & 117.5 & 232.6 \\
\hline 1 & B & S & $5 / 4 / 1993$ & 93124 & 124 & 60.2 & 53.9 & 53.9 & 51.6 & 50.8 & 50.2 & 55 & 134.7 & 236.8 \\
\hline 1 & B & S & $5 / 5 / 1993$ & 93125 & 125 & 58.4 & 53.9 & 57.5 & 53.6 & 55 & 51.6 & 55.3 & 142 & 267.2 \\
\hline 1 & $B$ & S & $5 / 6 / 1993$ & 93126 & 126 & 53.2 & 50.9 & 54.3 & 51.8 & 49.9 & 49.8 & 50.8 & 97.5 & 218.9 \\
\hline 1 & B & S & $5 / 7 / 1993$ & 93127 & 127 & 57.4 & 54.7 & 66.7 & 64.6 & 64 & 62.9 & 64.3 & 124.6 & 290.2 \\
\hline 1 & B & S & $5 / 10 / 1993$ & 93130 & 130 & 52.3 & 52.2 & 53.6 & 50.5 & 51.5 & 50.2 & 83.6 & 116.7 & 233.3 \\
\hline 1 & B & S & $5 / 11 / 1993$ & 93131 & 131 & 51.8 & 51.2 & 53.2 & 50.2 & 48.4 & 48.7 & 48.1 & 85 & 208.3 \\
\hline 1 & B & S & $5 / 12 / 1993$ & 93132 & 132 & 54 & 55 & 55 & 51.1 & 52.2 & 49.9 & 51.8 & 106 & 250.5 \\
\hline 1 & B & S & $5 / 13 / 1993$ & 93133 & 133 & 50.5 & 53.2 & 50.2 & 50.5 & 50.1 & 47.8 & 49.1 & 104.9 & 239.9 \\
\hline 1 & B & $\mathrm{S}$ & 5/14/1993 & 93134 & 134 & 62.9 & 61.6 & 64.3 & 61.2 & 58.5 & 57.7 & 59.1 & 113.1 & 246.6 \\
\hline 1 & B & S & $5 / 17 / 1993$ & 93137 & 137 & 55.4 & 66.4 & 67 & 63 & 62.2 & 63.7 & 91.2 & 134.6 & 261.2 \\
\hline 1 & B & S & $5 / 18 / 1993$ & 93138 & 138 & 68.4 & 63.3 & 65.3 & 62.9 & 60.5 & 57.8 & 60.8 & 137 & 307.7 \\
\hline 1 & B & S & $5 / 19 / 1993$ & 93139 & 139 & 62.3 & 65.3 & 63 & 61.9 & 61.2 & 58.5 & 60.5 & 122.7 & 250.2 \\
\hline 1 & B & S & $5 / 20 / 1993$ & 93140 & 140 & 60.9 & 55.3 & 56 & 55.4 & 55 & 53.3 & 52.9 & 103.7 & 264.6 \\
\hline 1 & B & S & $5 / 21 / 1993$ & 93141 & 141 & 72.2 & 64.7 & 64.3 & 62.3 & 61.2 & 62.3 & 60.1 & 110.2 & 266.3 \\
\hline 1 & B & S & $5 / 24 / 1993$ & 93144 & 144 & 61.5 & 67.5 & 68.1 & 66.1 & 63.6 & 63.6 & 88.9 & 112.6 & 254.6 \\
\hline 1 & $B$ & S & $5 / 25 / 1993$ & 93145 & 145 & 69.2 & 68.1 & 65.7 & 61.6 & 60.8 & 59.9 & 49.8 & 106 & 254.4 \\
\hline 1 & B & $S$ & $5 / 26 / 1993$ & 93146 & 146 & 57.1 & 58.4 & 58.4 & 56.4 & 55.3 & 54.7 & 53.9 & 104.7 & 246.2 \\
\hline 1 & B & S & 9/9/1993 & 93252 & 252 & 64.3 & 63.2 & 65.3 & 61.9 & 59.5 & 60.5 & 62.9 & 181.4 & 356.6 \\
\hline 1 & $B$ & S & 9/10/1993 & 93253 & 253 & 69.5 & 68.1 & 67.5 & 66.4 & 64.3 & 63.9 & 62.9 & 178.2 & 357.1 \\
\hline
\end{tabular}




\begin{tabular}{|c|c|c|c|c|c|c|c|c|c|c|c|c|c|c|}
\hline 1 & B & S & 9/13/1993 & 93256 & 256 & 66.7 & 74.3 & 75.3 & 73 & 71.5 & 64.3 & 113.7 & 217 & 391.6 \\
\hline 1 & B & S & 9/14/1993 & 93257 & 257 & 74.3 & 68.8 & 69.1 & 66.4 & 65.3 & 65.7 & 67.7 & 237.1 & 377.3 \\
\hline 1 & B & S & 9/15/1993 & 93258 & 258 & 57.4 & 57.7 & 57.8 & 55 & 53 & 54.3 & 52.3 & 174.7 & 295.3 \\
\hline 1 & B & $\mathrm{S}$ & 9/16/1993 & 93259 & 259 & 56 & 55.4 & 57 & 55.7 & 53.6 & 52.5 & 54.7 & 162.8 & 299.5 \\
\hline 1 & B & $\mathrm{S}$ & 9/17/1993 & 93260 & 260 & 65.3 & 63.9 & 65.3 & 61.3 & 60.1 & 60.8 & 60.5 & 148.4 & 296.2 \\
\hline 1 & $B$ & S & 9/20/1993 & 93263 & 263 & 60.1 & 65 & 66 & 66.4 & 63.9 & 56.3 & 104.4 & 204.9 & 344.7 \\
\hline 1 & B & S & 9/21/1993 & 93264 & 264 & 65 & 58.8 & 59.1 & 57.7 & 55.6 & 56 & 57.4 & 206.7 & 365.1 \\
\hline 1 & B & S & 9/22/1993 & 93265 & 265 & 60.5 & 59.4 & 60.8 & 58.1 & 56 & 56.7 & 55.6 & 220.9 & 361.6 \\
\hline 1 & B & S & 9/23/1993 & 93266 & 266 & 64.6 & 65.3 & 65.3 & 62.2 & 62.2 & 62.6 & 62.2 & 196.3 & 372.8 \\
\hline 1 & B & S & 9/24/1993 & 93267 & 267 & 67.7 & 70.8 & 82.9 & 72.9 & 69.1 & 69.5 & 63.6 & 162.2 & 316.2 \\
\hline 1 & B & $\mathrm{S}$ & 9/27/1993 & 93270 & 270 & 62.6 & 70.2 & 71.2 & 70.2 & 66.4 & 60.2 & 98.5 & 200.5 & 312.9 \\
\hline 1 & B & $\mathrm{S}$ & 9/28/1993 & 93271 & 271 & 76.8 & 52.2 & 52.6 & 51.1 & 48.7 & 46.4 & 51.5 & 151.4 & 294.8 \\
\hline 1 & B & S & 9/29/1993 & 93272 & 272 & 71.2 & 50.9 & 52.9 & 49.4 & 48.7 & 48.1 & 48.7 & 162.7 & 291.4 \\
\hline 1 & B & S & 9/30/1993 & 93273 & 273 & 73.3 & 53.6 & 53.9 & 51.5 & 49.5 & 50.5 & 52.2 & 159.9 & 309.5 \\
\hline 1 & B & S & 10/1/1993 & 93274 & 274 & 80.2 & 61.5 & 68.8 & 64 & 60.8 & 64.3 & 58.5 & 144.7 & 322.4 \\
\hline 1 & B & S & $10 / 4 / 1993$ & 93277 & 277 & 57 & 62.9 & 65.3 & 61.5 & 59.4 & 56.3 & 89.9 & 189.6 & 335.6 \\
\hline 1 & $B$ & S & $10 / 5 / 1993$ & 93278 & 278 & 59.1 & 56 & 56.7 & 53.6 & 51.9 & 52.5 & 57.4 & 187 & 333.3 \\
\hline 1 & $B$ & $\mathrm{~S}$ & $10 / 6 / 1993$ & 93279 & 279 & 60.8 & 61.2 & 60.5 & 58.8 & 57.4 & 56.8 & 56.7 & 188.4 & 327.7 \\
\hline 1 & B & S & $10 / 7 / 1993$ & 93280 & 280 & 55 & 53.9 & 55 & 52.9 & 51.8 & 51.6 & 54.3 & 174.9 & 327.3 \\
\hline 1 & B & S & 10/8/1993 & 93281 & 281 & 73.3 & 55.6 & 56.3 & 53.9 & 52.5 & 51.6 & 51.8 & 151.8 & 317.4 \\
\hline 1 & B & S & \#\#\#\#\#\#\#\# & 93284 & 284 & 52.5 & 56.4 & 57.4 & 53.7 & 53.6 & 50.5 & 71.3 & 165.6 & 297 \\
\hline 1 & B & S & \#\#\#\#\#\#\#\# & 93285 & 285 & 56.3 & 53.2 & 54.6 & 52.2 & 50.8 & 50.1 & 55 & 180.9 & 317.6 \\
\hline 1 & B & $\mathrm{S}$ & \#\#\#\#\#\#\#\# & 93286 & 286 & 52.2 & 50.8 & 51.8 & 49.4 & 48.4 & 49.8 & 47.7 & 153.9 & 310 \\
\hline 1 & $B$ & S & \#\#\#\#\#\#\#\# & 93287 & 287 & 52.5 & 62.9 & 61.9 & 56.4 & 55.6 & 57.4 & 60.2 & 169.1 & 300.1 \\
\hline 1 & B & S & \#\#\#\#\#\#\#\# & 93288 & 288 & 61.6 & 51.8 & 51.9 & 51.5 & 50.1 & 48.1 & 49.4 & 132.6 & 298.9 \\
\hline 1 & B & S & \#\#\#\#\#\#\#\# & 93291 & 291 & 55.6 & 61.2 & 62.9 & 62.2 & 59.1 & 53.6 & 81.2 & 185.5 & 352.4 \\
\hline 1 & $B$ & S & \#\#\#\#\#\#\#\# & 93292 & 292 & 67.7 & 61.9 & 62.6 & 62.9 & 61.2 & 60.1 & 60.8 & 221.2 & 360.4 \\
\hline 1 & B & $\mathrm{S}$ & \#\#\#\#\#\#\#\# & 93293 & 293 & 58.8 & 59.4 & 59.8 & 57.7 & 56.7 & 56 & 55.6 & 221 & 371.1 \\
\hline 1 & $B$ & S & \#\#\#\#\#\#\#\# & 93294 & 294 & 58.1 & 58.1 & 58.2 & 58.4 & 56.7 & 55.4 & 58.4 & 164 & 292.7 \\
\hline 1 & B & $\mathrm{S}$ & \#\#\#\#\#\#\#\# & 93295 & 295 & 65.3 & 65.3 & 63.3 & 63.6 & 62.6 & 62 & 61.9 & 126.7 & 240.7 \\
\hline 1 & B & S & \#\#\#\#\#\#\#\# & 93298 & 298 & 55.3 & 55.6 & 55.6 & 54.7 & 53.2 & 54.3 & 53.2 & 89.9 & 219.1 \\
\hline 1 & B & S & \#\#\#\#\#\#\#\# & 93299 & 299 & 62.9 & 59.9 & 60.1 & 59.1 & 58.8 & 59.1 & 59.2 & 137 & 258.2 \\
\hline 1 & B & $\mathrm{S}$ & \#\#\#\#\#\#\#\# & 93300 & 300 & 52.2 & 50.8 & 52.2 & 49.2 & 48.7 & 49.4 & 50.5 & 138.7 & 262.5 \\
\hline 1 & $B$ & S & \#\#\#\#\#\#\#\# & 93301 & 301 & 50.1 & 49.5 & 51.5 & 50.1 & 49.1 & 48.7 & 53.2 & 119.7 & 241.6 \\
\hline 1 & B & $\mathrm{S}$ & \#\#\#\#\#\#\#\# & 93302 & 302 & 46.3 & 47 & 48.1 & 47 & 46.3 & 45.4 & 47.3 & 119.5 & 251.8 \\
\hline 1 & B & $S$ & $11 / 1 / 1993$ & 93305 & 305 & 50.8 & 51.8 & 51.5 & 50.8 & 50.1 & 50.5 & 67 & 120.3 & 261.6 \\
\hline 1 & $B$ & $\mathrm{~S}$ & $11 / 2 / 1993$ & 93306 & 306 & 48.4 & 48.4 & 48.4 & 46.3 & 46.1 & 47 & 48.7 & 97.5 & 268.9 \\
\hline
\end{tabular}




\begin{tabular}{|c|c|c|c|c|c|c|c|c|c|c|c|c|c|c|}
\hline 1 & B & S & $11 / 3 / 1993$ & 93307 & 307 & 47.3 & 47.3 & 45.3 & 46.3 & 44.6 & 46 & 44.2 & 130.2 & 277.1 \\
\hline 1 & B & S & 11/4/1993 & 93308 & 308 & 45.3 & 46.3 & 45.6 & 44.6 & 44.6 & 46.3 & 46 & 120.6 & 268.9 \\
\hline 1 & $B$ & S & 11/5/1993 & 93309 & 309 & 48.7 & 48.7 & 47.3 & 46.7 & 46.3 & 45.6 & 50.8 & 131.4 & 267.6 \\
\hline 1 & B & S & 11/8/1993 & 93312 & 312 & 44.2 & 46.3 & 45.6 & 45.6 & 45.6 & 46 & 46.3 & 111.5 & 235 \\
\hline 1 & B & S & 11/9/1993 & 93313 & 313 & 48.4 & 48.7 & 48 & 46.7 & 47 & 46.7 & 60.1 & 82.3 & 227.1 \\
\hline 1 & $B$ & S & \#\#\#\#\#\#\#\# & 93314 & 314 & 48 & 47.8 & 47.3 & 48.4 & 46.3 & 47.3 & 43.9 & 90.5 & 218.9 \\
\hline 1 & B & S & \#\#\#\#\#\#\#\# & 93316 & 316 & 76 & 67.7 & 64.6 & 62.9 & 63.6 & 62.9 & 61.9 & 102 & 189.4 \\
\hline 1 & $B$ & S & \#\#\#\#\#\#\#\# & 93319 & 319 & 53.6 & 55.6 & 55.6 & 55.3 & 55.6 & 53.2 & 56 & 147.9 & 290.8 \\
\hline 1 & B & S & \#\#\#\#\#\#\#\# & 93320 & 320 & 48.4 & 48 & 48.7 & 47 & 46.7 & 46.7 & 47.3 & 119.5 & 266.3 \\
\hline 1 & B & S & \#\#\#\#\#\#\#\# & 93321 & 321 & 48.7 & 47.3 & 47.7 & 47 & 47.3 & 46.3 & 60.1 & 102.6 & 251.6 \\
\hline 1 & B & S & \#\#\#\#\#\#\#\# & 93322 & 322 & 50.5 & 50.5 & 50.8 & 50.1 & 49.8 & 49.8 & 63.9 & 94.3 & 232 \\
\hline 1 & $B$ & S & \#\#\#\#\#\#\#\# & 93323 & 323 & 51.8 & 51.1 & 50.1 & 49.4 & 49.8 & 49.4 & 47.3 & 81.2 & 227.8 \\
\hline 1 & B & S & \#\#\#\#\#\#\#\# & 93326 & 326 & 44.9 & 44.9 & 45.7 & 44.9 & 44.2 & 42.6 & 67.7 & 107.8 & 254 \\
\hline 1 & B & S & \#\#\#\#\#\#\#\# & 93327 & 327 & 50.1 & 48.4 & 47.3 & 46.7 & 47.3 & 47 & 46 & 102.6 & 231.6 \\
\hline 1 & B & S & \#\#\#\#\#\#\#\# & 93333 & 333 & 42.9 & 44.2 & 43.9 & 43.5 & 42.9 & 42.9 & 77.4 & 107.1 & 259.2 \\
\hline 1 & B & S & \#\#\#\#\#\#\#\# & 93334 & 334 & 48 & 47.3 & 47.7 & 46.3 & 47.1 & 47.7 & 64.6 & 105.1 & 255.1 \\
\hline 1 & B & S & 12/1/1993 & 93335 & 335 & 49.4 & 49.1 & 48.1 & 48.7 & 48 & 48.5 & 66.4 & 103.3 & 225.7 \\
\hline 1 & $B$ & S & $12 / 2 / 1993$ & 93336 & 336 & 50.1 & 52.9 & 48.5 & 50.1 & 48.4 & 48.7 & 47.7 & 112.7 & 244.8 \\
\hline 1 & $B$ & S & $12 / 3 / 1993$ & 93337 & 337 & 50.1 & 48.8 & 48.4 & 49.1 & 49.1 & 49.1 & 49.8 & 129.4 & 274.7 \\
\hline 1 & B & S & 12/6/1993 & 93340 & 340 & 50.1 & 50.2 & 51.1 & 50.1 & 49.2 & 49.8 & 51.8 & 82.3 & 230.2 \\
\hline 1 & B & S & 12/7/1993 & 93341 & 341 & 51.8 & 50.5 & 49.4 & 46.3 & 46.7 & 46.7 & 62.2 & 94.4 & 241.2 \\
\hline 1 & B & S & $12 / 8 / 1993$ & 93342 & 342 & 49.1 & 53.9 & 50.1 & 47.8 & 48 & 49.8 & 46.3 & 73 & 219.8 \\
\hline 1 & B & S & 12/9/1993 & 93343 & 343 & 46.7 & 46.7 & 45.6 & 44.9 & 44.2 & 44.6 & 43.5 & 78.5 & 229.6 \\
\hline 1 & $B$ & S & \#\#\#\#\#\#\#\# & 93344 & 344 & 66.7 & 53.9 & 52.9 & 56.7 & 47.4 & 47.3 & 45.3 & 118.6 & 287.5 \\
\hline 1 & B & S & \#\#\#\#\#\#\#\# & 93347 & 347 & 49.8 & 50.5 & 49.5 & 49.1 & 49.4 & 48.7 & 52.9 & 140.9 & 290 \\
\hline 1 & B & S & \#\#\#\#\#\#\#\# & 93348 & 348 & 48.4 & 47.3 & 46.4 & 46.7 & 46.3 & 47 & 44 & 105.1 & 238.5 \\
\hline 1 & B & S & \#\#\#\#\#\#\#\# & 93349 & 349 & 46 & 47.1 & 47 & 46 & 46.3 & 46.4 & 63.9 & 85 & 222.2 \\
\hline 1 & B & S & \#\#\#\#\#\#\#\# & 93350 & 350 & 46.3 & 46.3 & 45.3 & 44.7 & 45.3 & 44.6 & 60.8 & 82.3 & 207.4 \\
\hline 1 & B & S & $1 / 6 / 1994$ & 94006 & 6 & 50.1 & 48.8 & 49.4 & 48.7 & 49.1 & 49.2 & 67.4 & 93 & 187.7 \\
\hline 1 & $B$ & S & 1/7/1994 & 94007 & 7 & 69.5 & 70.8 & 69.6 & 69.1 & 69.8 & 70.8 & 86.5 & 102.6 & 174.5 \\
\hline 1 & $B$ & S & 1/10/1994 & 94010 & 10 & 47.3 & 50.5 & 52.2 & 49.5 & 46.7 & 47.3 & 81.6 & 110.6 & 231.9 \\
\hline 1 & B & S & $1 / 11 / 1994$ & 94011 & 11 & 47.3 & 46.4 & 46 & 46 & 47 & 45.4 & 62.6 & 97.1 & 238.1 \\
\hline 1 & B & S & $1 / 12 / 1994$ & 94012 & 12 & 47 & 48 & 46.7 & 45.7 & 47.7 & 47 & 60.8 & 91.9 & 231.9 \\
\hline 1 & B & $S$ & $1 / 13 / 1994$ & 94013 & 13 & 49.5 & 48.7 & 49.4 & 47.3 & 48.1 & 49.4 & 65.7 & 98.5 & 240.2 \\
\hline 1 & $B$ & $S$ & $1 / 14 / 1994$ & 94014 & 14 & 52.9 & 46.7 & 46.7 & 45.3 & 46.3 & 46.7 & 62.9 & 84.4 & 219.1 \\
\hline 1 & B & S & $1 / 17 / 1994$ & 94017 & 17 & 50.5 & 48 & 44.7 & 44.9 & 45.6 & 44.7 & 62.9 & 92.3 & 223.3 \\
\hline 1 & $B$ & $S$ & $1 / 18 / 1994$ & 94018 & 18 & 57.4 & 47.4 & 49.1 & 48 & 48.5 & 49.1 & 50.1 & 115.8 & 264.8 \\
\hline
\end{tabular}

Page 11 


\begin{tabular}{|c|c|c|c|c|c|c|c|c|c|c|c|c|c|c|}
\hline 1 & $B$ & $\mathrm{~S}$ & $1 / 19 / 1994$ & 94019 & 19 & 44.9 & 45.3 & 43.3 & 43.9 & 44.2 & 43.9 & 44.7 & 109.2 & 265.4 \\
\hline 1 & $B$ & S & 1/20/1994 & 94020 & 20 & 50.5 & 49.8 & 48.7 & 47.8 & 49.8 & 49.4 & 47.7 & 100.2 & 227.5 \\
\hline 1 & B & S & 1/21/1994 & 94021 & 21 & 46.7 & 47 & 45.6 & 45.3 & 46.1 & 46 & 62.2 & 96.4 & 242.7 \\
\hline 1 & B & S & 1/24/1994 & 94024 & 24 & 44.6 & 47.3 & 45.6 & 46.3 & 46.7 & 46.3 & 47 & 99.9 & 109.8 \\
\hline 1 & B & S & 1/25/1994 & 94025 & 25 & 73.3 & 72.9 & 71.6 & 71.9 & 71.9 & 71.6 & 72.2 & 109.2 & 228.4 \\
\hline 1 & B & $S$ & $1 / 26 / 1994$ & 94026 & 26 & 56.3 & 58.4 & 55.4 & 56.3 & 56.7 & 54.6 & 59.8 & 94.3 & 281 \\
\hline 1 & B & $S$ & 1/27/1994 & 94027 & 27 & 59.2 & 59.1 & 59.1 & 58.1 & 58.8 & 57.7 & 62.6 & 151.8 & 304.6 \\
\hline 1 & B & S & $1 / 28 / 1994$ & 94028 & 28 & 47.7 & 48.8 & 49.4 & 47.3 & 46.7 & 49.8 & 51.5 & 81.2 & 257.6 \\
\hline 1 & B & S & 1/31/1994 & 94031 & 31 & 53.6 & 55 & 52.3 & 54.6 & 54 & 76 & 87.1 & 116.5 & 263.3 \\
\hline 1 & B & S & 2/1/1994 & 94032 & 32 & 57.7 & 52.6 & 56.7 & 56.1 & 56.3 & 65.3 & 81.6 & 110.6 & 259.6 \\
\hline 1 & B & S & 2/2/1994 & 94033 & 33 & 57.4 & 53.9 & 56.1 & 57.4 & 57 & 81.9 & 81.6 & 109.6 & 264.7 \\
\hline 1 & B & S & $2 / 3 / 1994$ & 94034 & 34 & 63.6 & 60.1 & 64.6 & 62.3 & 64.3 & 88.1 & 88.1 & 116.8 & 259.5 \\
\hline 1 & B & S & 2/4/1994 & 94035 & 35 & 53.9 & 50.8 & 55.6 & 56.1 & 54.6 & 80.5 & 82.3 & 108.9 & 235.4 \\
\hline 1 & B & S & 2/7/1994 & 94038 & 38 & 54.3 & 53.9 & 58.8 & 57 & 58.1 & 75.1 & 78.1 & 105.8 & 229.5 \\
\hline 1 & B & S & 2/8/1994 & 94039 & 39 & 50.1 & 48.1 & 48.7 & 47.3 & 47.4 & 49.1 & 52.5 & 136.9 & 269.7 \\
\hline 1 & $B$ & $\mathrm{~S}$ & 2/9/1994 & 94040 & 40 & 53 & 53.6 & 52.5 & 53.2 & 53.2 & 50.5 & 57 & 144.2 & 296.1 \\
\hline 1 & B & S & $2 / 10 / 1994$ & 94041 & 41 & 53.6 & 53.3 & 53.2 & 53.2 & 52.6 & 53.6 & 70.8 & 91.2 & 111.3 \\
\hline 1 & B & $\mathrm{S}$ & $2 / 11 / 1994$ & 94042 & 42 & 47.8 & 49.1 & 48 & 47.1 & 47.7 & 65.3 & 70.8 & 104.4 & 257.5 \\
\hline 1 & B & S & 2/14/1994 & 94045 & 45 & 49.1 & 51.6 & 50.5 & 50.1 & 49.5 & 66.4 & 68.8 & 110 & 260.2 \\
\hline 1 & B & S & 2/15/1994 & 94046 & 46 & 53 & 53.2 & 51.8 & 52.5 & 51.5 & 69.6 & 68.8 & 121.7 & 243.3 \\
\hline 1 & B & S & 2/16/1994 & 94047 & 47 & 46.7 & 48.7 & 47.7 & 47.1 & 47 & 65.3 & 65 & 98.2 & 224.4 \\
\hline 1 & B & S & 2/17/1994 & 94048 & 48 & 52.6 & 52.2 & 51.5 & 50.2 & 51.5 & 51.5 & 67.5 & 102.6 & 215.3 \\
\hline 1 & B & S & 2/18/1994 & 94049 & 49 & 51.1 & 52.5 & 51.8 & 49.2 & 51.1 & 50.5 & 68.1 & 98.5 & 227.8 \\
\hline 1 & B & S & $2 / 22 / 1994$ & 94053 & 53 & 56.7 & 55.3 & 54.3 & 53.3 & 52.5 & 53.6 & 53.6 & 105.4 & 256.4 \\
\hline 1 & B & $\mathrm{S}$ & 2/23/1994 & 94054 & 54 & 55.6 & 55.6 & 56 & 53.3 & 53.9 & 52.9 & 55.6 & 121.4 & 223.3 \\
\hline 1 & B & S & 2/24/1994 & 94055 & 55 & 50.9 & 50.5 & 50.1 & 50.1 & 48.8 & 66.7 & 67 & 101.6 & 250.6 \\
\hline 1 & B & S & $2 / 25 / 1994$ & 94056 & 56 & 47.4 & 48 & 47.3 & 47 & 46.1 & 62.6 & 62.9 & 92.6 & 222.9 \\
\hline 1 & B & S & $2 / 28 / 1994$ & 94059 & 59 & 47.7 & 48.8 & 49.1 & 49.1 & 49.4 & 48.4 & 70.2 & 103.7 & 219.1 \\
\hline 1 & $B$ & S & 3/1/1994 & 94060 & 60 & 48 & 46.7 & 46.7 & 47 & 47.1 & 63.6 & 65.3 & 107.8 & 212.2 \\
\hline 1 & B & $\mathrm{S}$ & $3 / 2 / 1994$ & 94061 & 61 & 45.6 & 46.3 & 44.7 & 44.9 & 43.9 & 44.6 & 46.7 & 93.7 & 234.1 \\
\hline 1 & B & S & $3 / 3 / 1994$ & 94062 & 62 & 71.5 & 71.5 & 69.1 & 63.2 & 53.3 & 67.4 & 68.4 & 114.4 & 240.6 \\
\hline 1 & $B$ & S & $3 / 4 / 1994$ & 94063 & 63 & 52.3 & 52.9 & 51.1 & 51.5 & 50.2 & 50.8 & 74.7 & 100.3 & 217.7 \\
\hline 1 & B & S & 3/7/1994 & 94066 & 66 & 47.3 & 51.5 & 48.7 & 49.9 & 49.8 & 48 & 53.6 & 98.8 & 251.7 \\
\hline 1 & B & S & $3 / 8 / 1994$ & 94067 & 67 & 57.7 & 53.2 & 51.9 & 52.2 & 52.9 & 52.2 & 56 & 115.3 & 281.1 \\
\hline 1 & $B$ & S & $3 / 9 / 1994$ & 94068 & 68 & 49.1 & 47.1 & 46.3 & 45.6 & 46 & 46.3 & 47.3 & 110.4 & 234.3 \\
\hline 1 & $B$ & S & $3 / 10 / 1994$ & 94069 & 69 & 57.1 & 57.4 & 57 & 55.4 & 57 & 71.9 & 71.9 & 110 & 237.1 \\
\hline 1 & $B$ & $\mathrm{~S}$ & $3 / 21 / 1994$ & 94080 & 80 & 47.7 & 49.4 & 47 & 47 & 47 & 45.6 & 46.3 & 64.3 & 174.5 \\
\hline
\end{tabular}

Page 12 


\begin{tabular}{|c|c|c|c|c|c|c|c|c|c|c|c|c|c|c|}
\hline 1 & B & $\mathrm{S}$ & $3 / 22 / 1994$ & 94081 & 81 & 54.4 & 53.9 & 53.6 & 53.9 & 54.3 & 52.5 & 53.2 & 69.5 & 145.8 \\
\hline 1 & B & S & 3/23/1994 & 94082 & 82 & 59.4 & 60.8 & 58.1 & 59.4 & 59.8 & 58.1 & 59.4 & 144.9 & 275.2 \\
\hline 1 & B & S & $3 / 24 / 1994$ & 94083 & 83 & 49.4 & 51.5 & 49.8 & 50.1 & 49.8 & 50.5 & 53.6 & 78.1 & 251.7 \\
\hline 1 & B & S & $3 / 25 / 1994$ & 94084 & 84 & 53.6 & 57 & 54.6 & 54.6 & 53.2 & 53.6 & 50.5 & 121 & 245.2 \\
\hline 1 & B & S & $3 / 28 / 1994$ & 94087 & 87 & 48.4 & 48.8 & 50.8 & 50.5 & 48.7 & 48 & 52.9 & 123.4 & 217 \\
\hline 1 & $B$ & $\mathrm{~S}$ & 3/29/1994 & 94088 & 88 & 65.7 & 65.3 & 65.7 & 65 & 63.6 & 63.6 & 65.3 & 99.6 & 218.4 \\
\hline 1 & B & S & 3/30/1994 & 94089 & 89 & 45.3 & 45.6 & 46.3 & 45.3 & 44.6 & 44.9 & 44.9 & 110.5 & 222.3 \\
\hline 1 & B & S & $3 / 31 / 1994$ & 94090 & 90 & 55.6 & 55 & 55 & 49.8 & 47.1 & 46.7 & 47.3 & 95.7 & 231.2 \\
\hline 1 & B & S & $4 / 5 / 1994$ & 94095 & 95 & 56 & 52.2 & 52.2 & 51.5 & 52.2 & 51.5 & 54.3 & 126.1 & 262.3 \\
\hline 1 & B & S & 4/6/1994 & 94096 & 96 & 56.3 & 56 & 53.2 & 48.4 & 48 & 48 & 50.8 & 115.3 & 227.8 \\
\hline 1 & B & S & 4/7/1994 & 94097 & 97 & 55.6 & 55 & 59.8 & 59.4 & 59.4 & 60.1 & 74.7 & 95 & 215.7 \\
\hline 1 & B & S & $4 / 8 / 1994$ & 94098 & 98 & 58.1 & 56.3 & 57.7 & 57 & 56.3 & 49.8 & 64.3 & 90.5 & 202.2 \\
\hline 1 & B & S & 4/11/1994 & 94101 & 101 & 47.7 & 52.2 & 51.1 & 49.4 & 50.8 & 47.7 & 57.7 & 148 & 280.6 \\
\hline 1 & B & S & 4/12/1994 & 94102 & 102 & 66 & 62.2 & 62.2 & 62.6 & 62.2 & 61.5 & 57.7 & 137 & 274.5 \\
\hline 1 & B & S & 4/13/1994 & 94103 & 103 & 48.4 & 46 & 46.7 & 46 & 47.3 & 45.6 & 48 & 104.9 & 250 \\
\hline 1 & B & S & 4/14/1994 & 94104 & 104 & 57.7 & 56.3 & 57.4 & 58.1 & 51.8 & 49.4 & 52.9 & 150.2 & 281.8 \\
\hline 1 & B & S & $4 / 15 / 1994$ & 94105 & 105 & 52.5 & 51.5 & 51.5 & 51.8 & 50.1 & 49.4 & 53.2 & 154.4 & 246.5 \\
\hline 1 & B & $\mathrm{S}$ & 4/18/1994 & 94108 & 108 & 56.3 & 61.2 & 61.9 & 62.2 & 61.9 & 60.8 & 67 & 147.4 & 252.9 \\
\hline 1 & B & S & $4 / 19 / 1994$ & 94109 & 109 & 51.5 & 53.9 & 53.6 & 50.1 & 47.7 & 47.7 & 52.2 & 135.3 & 275.9 \\
\hline 1 & B & S & $4 / 20 / 1994$ & 94110 & 110 & 73.5 & 61.5 & 62.6 & 62.9 & 62.2 & 62.2 & 61.5 & 148.5 & 290.4 \\
\hline 1 & B & S & $4 / 21 / 1994$ & 94111 & 111 & 56.3 & 55.3 & 56 & 49.8 & 47.7 & 47.7 & 47.7 & 115.9 & 252.4 \\
\hline 1 & B & S & $4 / 22 / 1994$ & 94112 & 112 & 66.8 & 53.6 & 52.3 & 51.8 & 52.2 & 52.9 & 53.2 & 136.5 & 260.5 \\
\hline 1 & B & S & $4 / 25 / 1994$ & 94115 & 115 & 56 & 59.4 & 52.9 & 52.9 & 52.5 & 47.7 & 65.7 & 164.1 & 296.7 \\
\hline 1 & B & S & $4 / 26 / 1994$ & 94116 & 116 & 53.6 & 49.8 & 50.1 & 51.1 & 51.1 & 50.1 & 52.9 & 168.9 & 316.9 \\
\hline 1 & B & $\mathrm{S}$ & 4/27/1994 & 94117 & 117 & 52.2 & 55.6 & 56 & 56.3 & 53.6 & 54.6 & 58.1 & 185.2 & 333.4 \\
\hline 1 & B & S & $4 / 28 / 1994$ & 94118 & 118 & 71.2 & 70.8 & 69.8 & 62.6 & 63.2 & 60.8 & 66.4 & 193.2 & 323.4 \\
\hline 1 & B & S & 4/29/1994 & 94119 & 119 & 71.2 & 70.2 & 69.5 & 67.4 & 68.1 & 63.6 & 60.5 & 186.5 & 308.2 \\
\hline 1 & B & S & $5 / 2 / 1994$ & 94122 & 122 & 49.4 & 50.8 & 50.1 & 49.8 & 48.8 & 48.4 & 50.1 & 141.6 & 214.2 \\
\hline 1 & $B$ & S & $5 / 3 / 1994$ & 94123 & 123 & 53.2 & 50.1 & 49.8 & 49.8 & 50.1 & 49.4 & 51.8 & 136.8 & 270.8 \\
\hline 1 & B & S & $5 / 4 / 1994$ & 94124 & 124 & 57 & 57.4 & 56.3 & 55.6 & 56.3 & 55 & 57.4 & 150.4 & 282.7 \\
\hline 1 & B & S & $5 / 5 / 1994$ & 94125 & 125 & 55.3 & 55 & 54.3 & 53.9 & 53.9 & 53.6 & 54.6 & 140.9 & 272.4 \\
\hline 1 & $B$ & S & $5 / 6 / 1994$ & 94126 & 126 & 60.1 & 61.5 & 60.5 & 60.8 & 59.8 & 59.1 & 61.9 & 150.5 & 273.8 \\
\hline 1 & B & S & $5 / 9 / 1994$ & 94129 & 129 & 55 & 63.2 & 59.8 & 58.4 & 55.6 & 51.5 & 78.1 & 194.5 & 329.3 \\
\hline 1 & B & S & 5/10/1994 & 94130 & 130 & 78.8 & 72.2 & 74 & 69.5 & 72.9 & 69.5 & 75.7 & 205 & 340.4 \\
\hline 1 & $B$ & S & $5 / 11 / 1994$ & 94131 & 131 & 57.4 & 57 & 55.6 & 54.3 & 53.9 & 53.6 & 59.1 & 187.1 & 324.7 \\
\hline 1 & $B$ & S & $5 / 12 / 1994$ & 94132 & 132 & 52.9 & 53.2 & 51.1 & 51.1 & 49.1 & 50.5 & 55.3 & 183.2 & 326.7 \\
\hline 1 & $B$ & $\mathrm{~S}$ & 5/13/1994 & 94133 & 133 & 59.4 & 61.5 & 59.1 & 59.4 & 58.8 & 58.1 & 61.9 & 175.3 & 300.9 \\
\hline
\end{tabular}




\begin{tabular}{|c|c|c|c|c|c|c|c|c|c|c|c|c|c|c|}
\hline 1 & B & $S$ & $5 / 16 / 1994$ & 94136 & 136 & 59.1 & 69.5 & 63.6 & 66.7 & 61.2 & 61.2 & 75.3 & 178.1 & 305.9 \\
\hline 1 & $B$ & S & $5 / 17 / 1994$ & 94137 & 137 & 65 & 68.1 & 62.2 & 64.6 & 60.5 & 61.5 & 63.6 & 170.1 & 308.4 \\
\hline 1 & B & S & $5 / 18 / 1994$ & 94138 & 138 & 58.8 & 53.6 & 50.5 & 50.8 & 49.8 & 50.1 & 51.8 & 173.3 & 319.4 \\
\hline 1 & B & S & $5 / 19 / 1994$ & 94139 & 139 & 59.1 & 58.8 & 58.4 & 56.7 & 56.7 & 54.6 & 59.1 & 177.9 & 311.7 \\
\hline 1 & B & S & $5 / 20 / 1994$ & 94140 & 140 & 63.2 & 63.9 & 61.2 & 61.9 & 61.5 & 60.8 & 65.7 & 165.8 & 298.6 \\
\hline 1 & $B$ & S & $5 / 23 / 1994$ & 94143 & 143 & 55 & 77.8 & 66.4 & 68.1 & 63.9 & 62.6 & 75 & 187.6 & 313.5 \\
\hline 1 & B & S & $5 / 24 / 1994$ & 94144 & 144 & 73.6 & 72.6 & 71.5 & 68.4 & 62.9 & 63.6 & 69.8 & 167.7 & 276.1 \\
\hline 1 & $B$ & S & $5 / 25 / 1994$ & 94145 & 145 & 62.9 & 60.1 & 60.5 & 59.1 & 57.7 & 58.1 & 61.2 & 169.8 & 285.5 \\
\hline 1 & B & S & $5 / 26 / 1994$ & 94146 & 146 & 66.7 & 67 & 63.6 & 63.6 & 61.9 & 62.6 & 66.4 & 184 & 294.2 \\
\hline 1 & $B$ & S & $5 / 27 / 1994$ & 94147 & 147 & 55.3 & 55 & 55.6 & 54.3 & 53.9 & 53.9 & 59.1 & 164.1 & 271.7 \\
\hline 0 & $A$ & S & 9/7/1991 & 91250 & 250 & 83.6 & 84.3 & 87.8 & 82.6 & 89.2 & 88.5 & 86.7 & 92.6 & 82.6 \\
\hline 0 & $A$ & S & 9/8/1991 & 91251 & 251 & 98.5 & 89.2 & 97.5 & 88.1 & 98.2 & 88.8 & 92.6 & 83.6 & 89.2 \\
\hline 0 & $A$ & S & 9/14/1991 & 91257 & 257 & 110.6 & 107.5 & 107.5 & 106.1 & 105.1 & 104.7 & 103.7 & 130.6 & 315.5 \\
\hline 0 & $A$ & S & 9/15/1991 & 91258 & 258 & 104 & 103 & 103.7 & 102 & 100.9 & 103 & 100.6 & 96.1 & 96.8 \\
\hline 0 & $A$ & S & 9/21/1991 & 91264 & 264 & 66.4 & 68.1 & 64.6 & 65.3 & 66 & 63.9 & 62.9 & 68.8 & 64.3 \\
\hline 0 & $A$ & S & 9/22/1991 & 91265 & 265 & 70.8 & 71.5 & 74.3 & 64.6 & 63.9 & 62.6 & 62.9 & 59.8 & 59.4 \\
\hline 0 & $A$ & S & 9/28/1991 & 91271 & 271 & 70.8 & 69.1 & 67.4 & 65.7 & 64.6 & 64.3 & 68.1 & 63.6 & 61.2 \\
\hline 0 & $A$ & S & 9/29/1991 & 91272 & 272 & 75 & 72.2 & 71.9 & 70.2 & 69.1 & 72.2 & 65.7 & 64.6 & 61.9 \\
\hline 0 & $A$ & S & 10/5/1991 & 91278 & 278 & 98.2 & 101.3 & 84.3 & 84 & 78.5 & 72.9 & 72.9 & 81.2 & 70.8 \\
\hline 0 & $A$ & S & 10/6/1991 & 91279 & 279 & 75.3 & 80.9 & 71.9 & 68.4 & 66.4 & 66.7 & 64.3 & 62.9 & 62.6 \\
\hline 0 & $A$ & S & \#\#\#\#\#\#\#\# & 91285 & 285 & 113.7 & 103.3 & 92.3 & 81.2 & 79.1 & 79.8 & 80.9 & 86.7 & 113.3 \\
\hline 0 & A & S & \#\#\#\#\#\#\#\# & 91286 & 286 & 97.5 & 81.9 & 85.7 & 79.8 & 74.3 & 75.7 & 78.8 & 72.9 & 69.5 \\
\hline 0 & $A$ & S & \#\#\#\#\#\#\#\# & 91292 & 292 & 98.5 & 91.2 & 91.6 & 83.6 & 82.6 & 84.3 & 80.2 & 84 & 79.8 \\
\hline 0 & $A$ & S & \#\#\#\#\#\#\#\# & 91293 & 293 & 136.3 & 93.7 & 77.8 & 75 & 70.5 & 72.6 & 74.7 & 65.3 & 65.7 \\
\hline 0 & A & S & \#\#\#\#\#\#\#\# & 91299 & 299 & 109.2 & 95.4 & 96.8 & 94.8 & 93.7 & 99.9 & 96.2 & 107.5 & 112.3 \\
\hline 0 & A & S & \#\#\#\#\#\#\#\# & 91300 & 300 & 128 & 112 & 93.7 & 91.6 & 91.7 & 91.9 & 91.7 & 91.6 & 99.9 \\
\hline 0 & A & S & 11/2/1991 & 91306 & 306 & 68.1 & 63.6 & 59.5 & 65.7 & 60.6 & 61.5 & 68.9 & 68.8 & 70.9 \\
\hline 0 & $A$ & S & 11/3/1991 & 91307 & 307 & 60.5 & 48.8 & 56.3 & 46.4 & 55.3 & 48.5 & 57 & 49.4 & 55.7 \\
\hline 0 & $A$ & S & 11/9/1991 & 91313 & 313 & 82 & 79.5 & 76.8 & 75.3 & 77.2 & 81.2 & 88.9 & 102 & 102.3 \\
\hline 0 & $A$ & S & \#\#\#\#\#\#\#\# & 91314 & 314 & 70.8 & 66.4 & 65.3 & 64.4 & 65.7 & 65.8 & 65.3 & 66.1 & 64.3 \\
\hline 0 & A & S & \#\#\#\#\#\#\#\# & 91320 & 320 & 86.7 & 69.9 & 67.5 & 71.9 & 66.8 & 67 & 67.8 & 75.7 & 88.6 \\
\hline 0 & A & S & \#\#\#\#\#\#\#\# & 91321 & 321 & 75 & 67.8 & 71.9 & 66.1 & 63.7 & 69.5 & 65.4 & 61.9 & 59.2 \\
\hline 0 & $A$ & S & \#\#\#\#\#\#\#\# & 91327 & 327 & 58.2 & 56.7 & 54.7 & 51.8 & 53.3 & 51.6 & 50.5 & 51.6 & 48 \\
\hline 0 & A & $\mathrm{S}$ & \#\#\#\#\#\#\#\# & 91328 & 328 & 53.6 & 50.9 & 45.3 & 52.3 & 45 & 50.1 & 47.8 & 47.3 & 48.1 \\
\hline 0 & A & S & \#\#\#\#\#\#\#\# & 91334 & 334 & 52.9 & 50.5 & 50.1 & 47.8 & 48.1 & 49.4 & 47.8 & 48.8 & 49.4 \\
\hline 0 & A & S & 12/1/1991 & 91335 & 335 & 50.1 & 47.4 & 44 & 44.6 & 43.6 & 44.7 & 45.3 & 44 & 41.6 \\
\hline 0 & $A$ & $\mathrm{~S}$ & 12/7/1991 & 91341 & 341 & 58.1 & 57.5 & 53.3 & 52.9 & 51.9 & 54.3 & 51.9 & 51.6 & 61.9 \\
\hline
\end{tabular}




\begin{tabular}{|c|c|c|c|c|c|c|c|c|c|c|c|c|c|c|}
\hline 0 & A & S & $12 / 8 / 1991$ & 91342 & 342 & 60.9 & 59.8 & 56.8 & 54.7 & 57.4 & 58.2 & 54.4 & 57 & 53.3 \\
\hline 0 & A & S & \#\#\#\#\#\#\#\# & 91348 & 348 & 68.5 & 72.6 & 66.1 & 64.4 & 63.2 & 64.4 & 63 & 71.5 & 62 \\
\hline 0 & $A$ & S & \#\#\#\#\#\#\#\# & 91349 & 349 & 54.7 & 53.3 & 50.5 & 49.9 & 50.2 & 51.8 & 49.9 & 49.2 & 47.4 \\
\hline 0 & $A$ & S & 1/11/1992 & 92011 & 10 & 61.9 & 58.5 & 56.4 & 57 & 56.4 & 56 & 65.4 & 99.6 & 205.6 \\
\hline 0 & $A$ & S & 1/12/1992 & 92012 & 11 & 59.1 & 54.4 & 51.2 & 49.1 & 51.9 & 51.2 & 53.6 & 50.5 & 52.3 \\
\hline 0 & $A$ & S & 1/18/1992 & 92018 & 17 & 63 & 74.1 & 57 & 56.4 & 57.5 & 70.2 & 56.4 & 58.2 & 124.1 \\
\hline 0 & A & S & 1/19/1992 & 92019 & 18 & 76.8 & 75.1 & 72.7 & 71.5 & 72.3 & 71.3 & 72.9 & 72 & 72 \\
\hline 0 & A & S & 1/25/1992 & 92025 & 24 & 55 & 51.6 & 51.6 & 63.2 & 52.3 & 48.5 & 56.3 & 103.4 & 204.3 \\
\hline 0 & A & S & 1/26/1992 & 92026 & 25 & 89.3 & 58.8 & 55.6 & 54.7 & 54.7 & 53.7 & 56 & 74.7 & 158 \\
\hline 0 & $B$ & S & 2/1/1992 & 92032 & 31 & 57.7 & 56.1 & 53.3 & 52.3 & 52.5 & 50.2 & 53.3 & 69.8 & 170.8 \\
\hline 0 & B & S & 2/2/1992 & 92033 & 32 & 61.3 & 59.8 & 57.8 & 54.7 & 54.4 & 53.2 & 57.1 & 55.7 & 78.8 \\
\hline 0 & $B$ & S & 2/8/1992 & 92039 & 38 & 67.1 & 63.9 & 62.6 & 62 & 76.8 & 61.2 & 62.3 & 90.6 & 198.7 \\
\hline 0 & $B$ & S & 2/9/1992 & 92040 & 39 & 61.6 & 57.1 & 57.5 & 56 & 53.3 & 55.4 & 54.7 & 56 & 67.8 \\
\hline 0 & B & S & $2 / 15 / 1992$ & 92046 & 45 & 68.4 & 60.6 & 59.5 & 61.5 & 57.5 & 60.2 & 59.4 & 90.6 & 207 \\
\hline 0 & B & $\mathrm{S}$ & 2/16/1992 & 92047 & 46 & 90.3 & 77.2 & 75.3 & 73 & 72 & 73.6 & 71.6 & 81.3 & 119.5 \\
\hline 0 & B & S & 2/22/1992 & 92053 & 52 & 59.9 & 57.7 & 54.4 & 53.3 & 54.3 & 51.6 & 54 & 80.9 & 167.2 \\
\hline 0 & B & S & 2/23/1992 & 92054 & 53 & 64.3 & 62.3 & 60.6 & 59.4 & 57.8 & 57.1 & 56.7 & 69.9 & 89.6 \\
\hline 0 & $B$ & S & 2/29/1992 & 92060 & 59 & 55.7 & 53 & 50.8 & 49.9 & 52.6 & 51.1 & 53 & 67.8 & 156.9 \\
\hline 0 & $B$ & S & 3/1/1992 & 92061 & 60 & 69.2 & 60.1 & 54.7 & 51.2 & 52.5 & 53.3 & 58.2 & 50.8 & 49.5 \\
\hline 0 & $B$ & S & 3/7/1992 & 92067 & 66 & 77.2 & 72.2 & 70.2 & 64 & 66.4 & 66.4 & 64 & 88.5 & 170.8 \\
\hline 0 & B & S & 3/8/1992 & 92068 & 67 & 75.8 & 69.5 & 66.1 & 59.9 & 63.6 & 64.4 & 63.3 & 66.4 & 61.6 \\
\hline 0 & $B$ & S & $3 / 28 / 1992$ & 92088 & 87 & 79.6 & 75.4 & 65.7 & 65.8 & 63 & 62.9 & 56.8 & 71.2 & 190.8 \\
\hline 0 & B & S & $3 / 29 / 1992$ & 92089 & 88 & 113.6 & 112.8 & 107.9 & 108.2 & 110.5 & 109.1 & 112.2 & 125.1 & 138.9 \\
\hline 0 & B & S & 4/4/1992 & 92095 & 94 & 65.1 & 57.5 & 50.2 & 49.4 & 48.5 & 46.4 & 51.1 & 62.6 & 202.2 \\
\hline 0 & $B$ & S & 4/5/1992 & 92096 & 95 & 66.7 & 63.3 & 54.4 & 50.5 & 51.2 & 51.6 & 52.9 & 51.6 & 46.4 \\
\hline 0 & $B$ & S & 4/11/1992 & 92102 & 101 & 78.9 & 82.6 & 71.6 & 65.4 & 66.8 & 67.7 & 65.4 & 83.3 & 174 \\
\hline 0 & B & S & $4 / 12 / 1992$ & 92103 & 102 & 149.1 & 146 & 147 & 140.8 & 141.8 & 139.4 & 139.5 & 140.6 & 144.9 \\
\hline 0 & B & S & $4 / 25 / 1992$ & 92116 & 115 & 100.9 & 95.5 & 75.1 & 71.9 & 68.5 & 67.8 & 68.8 & 74.4 & 208.5 \\
\hline 0 & $B$ & S & $4 / 26 / 1992$ & 92117 & 116 & 93.4 & 93 & 70.9 & 66.4 & 65.7 & 65.1 & 63.3 & 62.2 & 77.2 \\
\hline 0 & $B$ & S & $5 / 2 / 1992$ & 92123 & 122 & 68.8 & 75.1 & 54 & 50.2 & 50.1 & 48.8 & 50.2 & 65 & 200.2 \\
\hline 0 & $B$ & S & $5 / 3 / 1992$ & 92124 & 123 & 98.2 & 96.8 & 76.8 & 73.7 & 72.6 & 74.1 & 75.1 & 71.5 & 90.2 \\
\hline 0 & B & S & $5 / 9 / 1992$ & 92130 & 129 & 65.8 & 64.4 & 62.9 & 60.9 & 62.3 & 62.6 & 61.6 & 73.6 & 159.1 \\
\hline 0 & B & S & $5 / 10 / 1992$ & 92131 & 130 & 72.9 & 73 & 70.9 & 69.5 & 67.1 & 69.2 & 71.2 & 65.8 & 65.3 \\
\hline 0 & B & S & $5 / 16 / 1992$ & 92137 & 136 & 64.4 & 56.4 & 62.2 & 61.3 & 60.2 & 65.3 & 66.1 & 63.3 & 222.1 \\
\hline 0 & $B$ & S & $5 / 17 / 1992$ & 92138 & 137 & 67.1 & 66 & 65.4 & 63 & 61.9 & 62 & 64.4 & 62.9 & 59.2 \\
\hline 0 & B & S & $5 / 23 / 1992$ & 92144 & 143 & 74.7 & 74.7 & 71.6 & 69.6 & 68.4 & 72 & 73.6 & 70.6 & 233.1 \\
\hline 0 & $B$ & $\mathrm{~S}$ & 5/24/1992 & 92145 & 144 & 70.5 & 67.8 & 66.1 & 66 & 64 & 63.3 & 65 & 62 & 61.6 \\
\hline
\end{tabular}




\begin{tabular}{|c|c|c|c|c|c|c|c|c|c|c|c|c|c|c|}
\hline 0 & $B$ & $\mathrm{~S}$ & 9/12/1992 & 92256 & 255 & 76.7 & 76.8 & 76 & 74.1 & 72.9 & 75.1 & 72.9 & 118.3 & 398.4 \\
\hline 0 & $B$ & S & 9/13/1992 & 92257 & 256 & 91.7 & 84.3 & 83.4 & 82.3 & 81.3 & 83.3 & 83.7 & 86.4 & 90.3 \\
\hline 0 & B & $S$ & 9/19/1992 & 92263 & 262 & 75 & 74.4 & 74 & 72 & 71.6 & 73.3 & 69.9 & 115.4 & 397.9 \\
\hline 0 & B & S & 9/20/1992 & 92264 & 263 & 84.8 & 79.5 & 88.9 & 80.5 & 82 & 83.6 & 76.5 & 78.5 & 86.5 \\
\hline 0 & B & S & 9/26/1992 & 92270 & 269 & 82.7 & 83.3 & 81.3 & 81.3 & 80.9 & 81.3 & 78.8 & 101.4 & 319.3 \\
\hline 0 & $B$ & $S$ & 9/27/1992 & 92271 & 270 & 95.5 & 86.1 & 93.1 & 84.3 & 88.2 & 88.8 & 87.5 & 84.7 & 113.4 \\
\hline 0 & B & $S$ & 10/3/1992 & 92277 & 276 & 68.8 & 67.5 & 68.1 & 65.4 & 65.4 & 66.7 & 67.1 & 89.5 & 286.4 \\
\hline 0 & B & S & 10/4/1992 & 92278 & 277 & 117.5 & 64.4 & 61.6 & 57 & 57.1 & 55.3 & 61.3 & 112.3 & 120.2 \\
\hline 0 & B & S & \#\#\#\#\#\#\#\# & 92284 & 283 & 69.8 & 69.2 & 70.2 & 67.5 & 66 & 68.2 & 66.8 & 86.7 & 267.6 \\
\hline 0 & B & S & \#\#\#\#\#\#\#\# & 92285 & 284 & 73.7 & 75.1 & 89.9 & 73.7 & 72.6 & 71.3 & 90.2 & 92 & 102.3 \\
\hline 0 & B & S & \#\#\#\#\#\#\#\# & 92291 & 290 & 79.1 & 79.2 & 73.3 & 75.4 & 70.2 & 72 & 71.9 & 118.1 & 376.1 \\
\hline 0 & $B$ & S & \#\#\#\#\#\#\#\# & 92292 & 291 & 73.4 & 71.5 & 77.9 & 70.2 & 70.2 & 73.3 & 71.3 & 69.5 & 82.3 \\
\hline 0 & B & S & \#\#\#\#\#\#\#\# & 92298 & 297 & 86.4 & 87.5 & 86.1 & 81.3 & 80.9 & 80.3 & 78.8 & 102.5 & 304.9 \\
\hline 0 & B & S & \#\#\#\#\#\#\#\# & 92299 & 298 & 72.9 & 72 & 77.1 & 69.9 & 70.2 & 70.2 & 72.2 & 82.3 & 99.9 \\
\hline 0 & B & S & \#\#\#\#\#\#\#\# & 92305 & 304 & 67.1 & 66.7 & 66.8 & 65.3 & 63.2 & 61.3 & 61.5 & 102.8 & 328.4 \\
\hline 0 & $B$ & $\mathrm{~S}$ & 11/1/1992 & 92306 & 305 & 75.8 & 78.8 & 75.8 & 68.4 & 73.4 & 74.3 & 64.4 & 69.8 & 92 \\
\hline 0 & B & S & 11/7/1992 & 92312 & 311 & 54 & 54.3 & 52.3 & 50.9 & 51.8 & 51.2 & 49.4 & 69.2 & 223.3 \\
\hline 0 & B & S & 11/8/1992 & 92313 & 312 & 66.4 & 59.9 & 59.5 & 62.2 & 59.9 & 57.8 & 61.9 & 64.4 & 73.7 \\
\hline 0 & $B$ & S & \#\#\#\#\#\#\#\# & 92319 & 318 & 77.2 & 74.3 & 72.7 & 73 & 74 & 74.1 & 72.6 & 104.1 & 237.4 \\
\hline 0 & B & S & \#\#\#\#\#\#\#\# & 92320 & 319 & 66.4 & 66.1 & 65.7 & 62.6 & 63.9 & 62.6 & 60.5 & 59.2 & 76.7 \\
\hline 0 & B & S & \#\#\#\#\#\#\#\# & 92326 & 325 & 72.3 & 67.4 & 61.3 & 61.9 & 59.5 & 60.5 & 57.8 & 76 & 190.5 \\
\hline 0 & B & S & \#\#\#\#\#\#\#\# & 92327 & 326 & 89.5 & 66.8 & 65.7 & 66.1 & 65 & 64.4 & 63.9 & 60.6 & 72.6 \\
\hline 0 & B & S & \#\#\#\#\#\#\#\# & 92333 & 332 & 68.8 & 74.7 & 76.8 & 78.1 & 77.5 & 76.7 & 74.3 & 71.3 & 82.9 \\
\hline 0 & B & S & \#\#\#\#\#\#\#\# & 92334 & 333 & 68.4 & 73.4 & 77.1 & 76.8 & 78.8 & 76.8 & 74.7 & 72.2 & 71.3 \\
\hline 0 & B & S & 12/5/1992 & 92340 & 339 & 67.4 & 69.6 & 69.5 & 69.2 & 71.5 & 69.6 & 68.1 & 80.2 & 202.6 \\
\hline 0 & B & S & 12/6/1992 & 92341 & 340 & 63 & 69.1 & 70.6 & 70.2 & 70.2 & 70.5 & 67.4 & 84.1 & 125.5 \\
\hline 0 & B & S & \#\#\#\#\#\#\#\# & 92347 & 346 & 78.8 & 61.6 & 58.1 & 56.8 & 59.1 & 56.8 & 55.3 & 62.6 & 198.4 \\
\hline 0 & B & S & \#\#\#\#\#\#\#\# & 92348 & 347 & 61.9 & 60.6 & 59.1 & 57.8 & 61.2 & 59.5 & 57.4 & 53 & 59.1 \\
\hline 0 & $B$ & S & 1/9/1993 & 93009 & 9 & 67.1 & 74.3 & 62.6 & 61.5 & 62 & 62.2 & 58.5 & 58.8 & 58.4 \\
\hline 0 & B & S & 1/10/1993 & 93010 & 10 & 78.5 & 76.4 & 73.7 & 74.3 & 73 & 74 & 70.2 & 69.5 & 65.8 \\
\hline 0 & B & S & 1/16/1993 & 93016 & 16 & 56.1 & 55.6 & 51.6 & 50.5 & 50.1 & 49.9 & 49.4 & 47.4 & 63.6 \\
\hline 0 & B & S & 1/17/1993 & 93017 & 17 & 73.6 & 75.8 & 54.3 & 49.9 & 50.5 & 50.2 & 50.8 & 47.1 & 44.9 \\
\hline 0 & B & S & 1/23/1993 & 93023 & 23 & 68.2 & 59.1 & 55.7 & 52.9 & 53.3 & 53.6 & 52.6 & 67.4 & 157.7 \\
\hline 0 & B & S & 1/24/1993 & 93024 & 24 & 56 & 55.4 & 53.6 & 50.2 & 52.5 & 50.2 & 50.1 & 48.8 & 43.9 \\
\hline 0 & $B$ & S & 1/30/1993 & 93030 & 30 & 66.8 & 67 & 64 & 63.9 & 62.6 & 62.6 & 62 & 61.9 & 77.2 \\
\hline 0 & B & S & 1/31/1993 & 93031 & 31 & 76.1 & 77.1 & 75.1 & 74.3 & 72.3 & 71.9 & 71.3 & 70.2 & 66.1 \\
\hline 0 & $B$ & $S$ & 2/6/1993 & 93037 & 37 & 65.3 & 55.4 & 53.9 & 53 & 53.6 & 51.9 & 55 & 59.8 & 84.4 \\
\hline
\end{tabular}




\begin{tabular}{|c|c|c|c|c|c|c|c|c|c|c|c|c|c|c|}
\hline 0 & B & $\mathrm{S}$ & 2/7/1993 & 93038 & 38 & 55.4 & 55.6 & 53.3 & 53.9 & 54 & 52.3 & 51.5 & 50.2 & 49.8 \\
\hline 0 & B & S & 2/13/1993 & 93044 & 44 & 69.2 & 70.5 & 66.1 & 65.8 & 66 & 65.4 & 65 & 63.7 & 65.7 \\
\hline 0 & B & S & 2/14/1993 & 93045 & 45 & 71.2 & 69.9 & 70.2 & 67.5 & 67.4 & 68.9 & 67.4 & 67.1 & 61.9 \\
\hline 0 & B & S & 2/20/1993 & 93051 & 51 & 93.1 & 81.2 & 59.2 & 59.4 & 59.5 & 59.4 & 58.5 & 57 & 70.9 \\
\hline 0 & B & S & 2/21/1993 & 93052 & 52 & 62.2 & 61.6 & 58.8 & 59.2 & 57.7 & 58.2 & 57 & 55 & 54 \\
\hline 0 & $B$ & $\mathrm{~S}$ & 2/27/1993 & 93058 & 58 & 68.4 & 68.2 & 67 & 65.8 & 66 & 65.8 & 64.3 & 81.3 & 117.5 \\
\hline 0 & B & S & $2 / 28 / 1993$ & 93059 & 59 & 82 & 82.6 & 79.9 & 72.2 & 72 & 72.2 & 70.6 & 69.8 & 67.1 \\
\hline 0 & B & S & 3/6/1993 & 93065 & 65 & 63.6 & 62.6 & 60.9 & 60.1 & 60.2 & 60.1 & 60.2 & 60.5 & 57.1 \\
\hline 0 & B & S & 3/7/1993 & 93066 & 66 & 79.2 & 79.1 & 77.2 & 77.8 & 77.2 & 78.8 & 76.1 & 94.3 & 92 \\
\hline 0 & B & S & $3 / 27 / 1993$ & 93086 & 86 & 67 & 67.1 & 63.9 & 61.3 & 63.6 & 62.3 & 62.6 & 62 & 84.7 \\
\hline 0 & B & S & $3 / 28 / 1993$ & 93087 & 87 & 64.6 & 63.3 & 63.6 & 62 & 61.5 & 61.6 & 61.2 & 57.8 & 58.1 \\
\hline 0 & $B$ & S & 4/3/1993 & 93093 & 93 & 61.9 & 62.2 & 60.2 & 58.4 & 58.1 & 58.5 & 65.3 & 59.4 & 58.2 \\
\hline 0 & B & S & 4/4/1993 & 93094 & 94 & 63.9 & & 34.6 & 62 & 61.5 & 60.8 & 60.2 & 59.1 & 58.1 \\
\hline 0 & B & S & 4/17/1993 & 93107 & 107 & 58.1 & 58.4 & 58.8 & 57.4 & 56 & 54.7 & 55 & 53 & 56.3 \\
\hline 0 & B & S & 4/18/1993 & 93108 & 108 & 54.4 & 49.1 & 49.8 & 46.1 & 45.3 & 44.2 & 44.3 & 44.2 & 42.9 \\
\hline 0 & B & S & 4/24/1993 & 93114 & 114 & 61.6 & 61.2 & 61.6 & 59.8 & 59.1 & 57.1 & 57 & 55 & 62.6 \\
\hline 0 & B & S & $4 / 25 / 1993$ & 93115 & 115 & 66.7 & 66 & 66.8 & 66.7 & 63.6 & 63.7 & 64.3 & 61.9 & 61.6 \\
\hline 0 & $B$ & S & $5 / 1 / 1993$ & 93121 & 121 & 63.6 & 61.3 & 63.6 & 62.3 & 60.1 & 66.4 & 70.2 & 70.2 & 108.2 \\
\hline 0 & $B$ & S & $5 / 2 / 1993$ & 93122 & 122 & 59.8 & 63.7 & 64.6 & 61.9 & 57.8 & 57.7 & 56.8 & 55.3 & 54.6 \\
\hline 0 & B & S & $5 / 8 / 1993$ & 93128 & 128 & 69.8 & 68.1 & 67 & 65.1 & 65 & 64.6 & 68.9 & 69.8 & 71.9 \\
\hline 0 & B & S & $5 / 9 / 1993$ & 93129 & 129 & 62.2 & 53.6 & 51.2 & 49.8 & 48 & 48.4 & 46.7 & 47.3 & 46 \\
\hline 0 & B & S & $5 / 15 / 1993$ & 93135 & 135 & 57.4 & 57.7 & 56.7 & 53.7 & 51.8 & 51.8 & 53 & 48 & 51.5 \\
\hline 0 & B & S & 5/16/1993 & 93136 & 136 & 59.1 & 58.1 & 57.8 & 56.7 & 56.7 & 54 & 51.1 & 53.6 & 51.6 \\
\hline 0 & B & S & $5 / 22 / 1993$ & 93142 & 142 & 65.1 & 63.2 & 61.3 & 62.6 & 60.1 & 57.8 & 62.2 & 54.7 & 65 \\
\hline 0 & B & S & $5 / 23 / 1993$ & 93143 & 143 & 66.7 & 67.4 & 66.1 & 64.3 & 61.9 & 60.8 & 59.5 & 61.2 & 61.5 \\
\hline 0 & B & S & 9/11/1993 & 93254 & 254 & 68.2 & 67.7 & 83.6 & 74.3 & 69.8 & 68.8 & 78.8 & 74 & 84.4 \\
\hline 0 & B & S & 9/12/1993 & 93255 & 255 & 66.4 & 66.8 & 79.1 & 72.9 & 69.6 & 69.8 & 120.5 & 123.4 & 135.1 \\
\hline 0 & B & S & 9/18/1993 & 93261 & 261 & 77.1 & 56 & 62.6 & 60.8 & 57.7 & 65 & 117.8 & 144.8 & 168 \\
\hline 0 & $B$ & S & 9/19/1993 & 93262 & 262 & 71.5 & 62.9 & 69.8 & 65.3 & 59.8 & 64.6 & 66 & 61.5 & 80.5 \\
\hline 0 & B & S & 9/25/1993 & 93268 & 268 & 88.5 & 66.4 & 77.1 & 71.5 & 66.7 & 74 & 137.8 & 150.7 & 159.2 \\
\hline 0 & B & S & 9/26/1993 & 93269 & 269 & 63.9 & 64.6 & 75.7 & 70.2 & 69.1 & 67 & 68.8 & 69.5 & 81.6 \\
\hline 0 & B & S & 10/2/1993 & 93275 & 275 & 76.1 & 55.3 & 62.2 & 59.1 & 53.6 & 58.1 & 77.4 & 84 & 129.3 \\
\hline 0 & B & S & $10 / 3 / 1993$ & 93276 & 276 & 66 & 68.4 & 82.9 & 63.9 & 60.9 & 60.5 & 62.2 & 61.9 & 82 \\
\hline 0 & B & S & 10/9/1993 & 93282 & 282 & 68.8 & 54.6 & 60.8 & 54.6 & 56 & 58.1 & 109.9 & 113.2 & 136.8 \\
\hline 0 & $B$ & S & \#\#\#\#\#\#\#\# & 93283 & 283 & 73.3 & 60.6 & 62.2 & 59.8 & 55.7 & 55.6 & 108.8 & 120.1 & 152.6 \\
\hline 0 & B & S & \#\#\#\#\#\#\#\# & 93289 & 289 & 53.9 & 52.5 & 59.4 & 54.3 & 54.6 & 57.4 & 51.8 & 56 & 71.5 \\
\hline 0 & $B$ & $\mathrm{~S}$ & \#\#\#\#\#\#\#\# & 93290 & 290 & 55.6 & 53.2 & 61.5 & 58.8 & 52.2 & 56.7 & 56.7 & 52.5 & 82.9 \\
\hline
\end{tabular}




\begin{tabular}{|c|c|c|c|c|c|c|c|c|c|c|c|c|c|c|}
\hline 0 & B & $\mathrm{S}$ & \#\#\#\#\#\#\#\# & 93296 & 296 & 55 & 51.2 & 51.8 & 50.5 & 49.4 & 50.8 & 49.2 & 52.2 & 58.8 \\
\hline 0 & $B$ & S & \#\#\#\#\#\#\#\# & 93297 & 297 & 62.9 & 53 & 55 & 54.3 & 52.2 & 51.6 & 52.5 & 51.6 & 58.8 \\
\hline 0 & $B$ & S & \#\#\#\#\#\#\#\# & 93303 & 303 & 55.3 & 51.6 & 54.6 & 53.6 & 56.8 & 58.4 & 65 & 73.3 & 102 \\
\hline 0 & B & S & \#\#\#\#\#\#\#\# & 93304 & 304 & 53.2 & 49.4 & 49.8 & 49.8 & 49.1 & 49.4 & 49.1 & 48.4 & 51.8 \\
\hline 0 & B & S & 11/6/1993 & 93310 & 310 & 56.3 & 48.7 & 48.7 & 47.7 & 47.7 & 47.3 & 46.7 & 54.3 & 71.5 \\
\hline 0 & $B$ & $\mathrm{~S}$ & 11/7/1993 & 93311 & 311 & 45.3 & 46.7 & 47 & 46.7 & 47 & 47.3 & 46 & 46.3 & 50.8 \\
\hline 0 & B & S & \#\#\#\#\#\#\#\# & 93317 & 317 & 67.7 & 68.1 & 68.4 & 68.4 & 66.7 & 68.1 & 106.7 & 132.6 & 203.3 \\
\hline 0 & B & S & \#\#\#\#\#\#\#\# & 93318 & 318 & 58.4 & 60.1 & 59.1 & 58.1 & 58.1 & 59.1 & 57.4 & 59.4 & 56.7 \\
\hline 0 & B & S & \#\#\#\#\#\#\#\# & 93324 & 324 & 47 & 46.4 & 46.7 & 46 & 47 & 45.7 & 44.2 & 72.2 & 82.7 \\
\hline 0 & B & S & \#\#\#\#\#\#\#\# & 93325 & 325 & 45.6 & 45.6 & 46.3 & 45.3 & 48.4 & 45.6 & 46 & 48.4 & 46.3 \\
\hline 0 & B & S & $12 / 4 / 1993$ & 93338 & 338 & 107 & 56 & 56.3 & 56.3 & 54.7 & 54.3 & 54.3 & 135.6 & 188.5 \\
\hline 0 & B & S & $12 / 5 / 1993$ & 93339 & 339 & 53.9 & 51.5 & 52.6 & 52.5 & 52.5 & 50.9 & 50.8 & 50.5 & 50.5 \\
\hline 0 & B & S & \#\#\#\#\#\#\#\# & 93345 & 345 & 53.7 & 48.7 & 48.7 & 47.3 & 48.1 & 48.4 & 46.7 & 109.4 & 125 \\
\hline 0 & B & S & \#\#\#\#\#\#\#\# & 93346 & 346 & 50.1 & 48.1 & 49.8 & 49.4 & 48.5 & 49.1 & 48.1 & 49.4 & 49.1 \\
\hline 0 & B & S & $1 / 8 / 1994$ & 94008 & 8 & 78.5 & 53 & 52.2 & 52.9 & 52.9 & 52.2 & 51.5 & 53.2 & 53.9 \\
\hline 0 & B & S & $1 / 9 / 1994$ & 94009 & 9 & 47.7 & 47.3 & 48.7 & 47.4 & 48.7 & 49.4 & 48.5 & 49.8 & 50.5 \\
\hline 0 & B & S & $1 / 15 / 1994$ & 94015 & 15 & 46 & 47.3 & 44.3 & 44.9 & 44.9 & 45.3 & 43.9 & 44.7 & 66 \\
\hline 0 & $B$ & S & $1 / 16 / 1994$ & 94016 & 16 & 49.8 & 49.9 & 48.7 & 48.7 & 47.8 & 49.8 & 48 & 49.8 & 50.9 \\
\hline 0 & $B$ & S & $1 / 22 / 1994$ & 94022 & 22 & 46.7 & 46.7 & 45.7 & 45.6 & 45.3 & 44.7 & 45.3 & 42.9 & 45.6 \\
\hline 0 & B & S & 1/23/1994 & 94023 & 23 & 47.3 & 47.1 & 48.4 & 48.4 & 45.7 & 47 & 46.7 & 43.6 & 45.6 \\
\hline 0 & B & S & $1 / 29 / 1994$ & 94029 & 29 & 51.8 & 53.9 & 52.2 & 53 & 54.3 & 70.5 & 70.5 & 70.2 & 73.3 \\
\hline 0 & B & S & 1/30/1994 & 94030 & 30 & 54.3 & 57 & 56 & 56.1 & 56 & 72.6 & 73.6 & 73.6 & 77.4 \\
\hline 0 & B & S & 2/5/1994 & 94036 & 36 & 51.2 & 51.5 & 48.4 & 47.7 & 47.7 & 66.4 & 63.9 & 67 & 88.1 \\
\hline 0 & B & S & 2/6/1994 & 94037 & 37 & 56.3 & 55.3 & 53.3 & 53.6 & 52.5 & 68.5 & 69.1 & 70.8 & 74.7 \\
\hline 0 & B & S & $2 / 12 / 1994$ & 94043 & 43 & 48.4 & 49.1 & 48.4 & 48.1 & 48.7 & 48.8 & 48.4 & 47 & 59.9 \\
\hline 0 & B & S & 2/13/1994 & 94044 & 44 & 49.4 & 48.8 & 51.8 & 50.1 & 49.9 & 51.1 & 50.5 & 49.2 & 49.8 \\
\hline 0 & B & S & 2/19/1994 & 94050 & 50 & 47.7 & 48 & 49.1 & 46.1 & 48 & 47.3 & 46 & 90.7 & 119.7 \\
\hline 0 & B & S & 2/20/1994 & 94051 & 51 & 48.7 & 49.1 & 47.4 & 49.1 & 49.1 & 48 & 48.5 & 47.3 & 46.3 \\
\hline 0 & $B$ & S & 2/26/1994 & 94057 & 57 & 45.6 & 46 & 44.6 & 42.9 & 44.6 & 44.2 & 91.6 & 102.9 & 113.8 \\
\hline 0 & $B$ & S & 2/27/1994 & 94058 & 58 & 49.1 & 48.7 & 46.7 & 47 & 46.3 & 46.4 & 46.7 & 44 & 45.6 \\
\hline 0 & B & S & 3/5/1994 & 94064 & 64 & 45 & 45.6 & 44.9 & 46 & 44.3 & 44.9 & 44.9 & 43.2 & 45.4 \\
\hline 0 & B & S & $3 / 6 / 1994$ & 94065 & 65 & 49.8 & 50.8 & 51.5 & 50.5 & 49.8 & 50.5 & 48.4 & 116 & 116.4 \\
\hline 0 & B & $S$ & $3 / 26 / 1994$ & 94085 & 85 & 49.4 & 48.7 & 49.1 & 48.4 & 48.7 & 47.7 & 50.1 & 46 & 50.1 \\
\hline 0 & B & $S$ & $3 / 27 / 1994$ & 94086 & 86 & 49.1 & 50.8 & 52.2 & 52.5 & 52.5 & 52.2 & 50.1 & 49.1 & 50.1 \\
\hline 0 & $B$ & S & 4/9/1994 & 94099 & 99 & 53.2 & 53.6 & 50.8 & 51.5 & 50.1 & 49.4 & 50.5 & 49.4 & 48.4 \\
\hline 0 & B & S & 4/10/1994 & 94100 & 100 & 48.7 & 45.6 & 47.3 & 47 & 48 & 46 & 46.3 & 46.3 & 43.2 \\
\hline 0 & $B$ & $S$ & 4/16/1994 & 94106 & 106 & 61.5 & 56 & 56.7 & 56.4 & 51.8 & 50.1 & 48.4 & 47 & 49.1 \\
\hline
\end{tabular}




\begin{tabular}{|c|c|c|c|c|c|c|c|c|c|c|c|c|c|c|}
\hline 0 & B & S & 4/17/1994 & 94107 & 107 & 57.4 & 52.5 & 53.2 & 53.2 & 52.9 & 53.6 & 51.9 & 52.2 & 48.4 \\
\hline 0 & B & S & 4/23/1994 & 94113 & 113 & 59.8 & 57.4 & 51.5 & 50.8 & 51.5 & 51.1 & 50.2 & 53.2 & 90.2 \\
\hline 0 & B & S & 4/24/1994 & 94114 & 114 & 48.7 & 49.4 & 51.5 & 50.5 & 49.1 & 51.1 & 50.8 & 49.8 & 49.1 \\
\hline 0 & B & S & 4/30/1994 & 94120 & 120 & 61.2 & 62.9 & 62.2 & 60.8 & 61.2 & 60.1 & 61.2 & 141.2 & 216 \\
\hline 0 & B & S & 5/1/1994 & 94121 & 121 & 52.2 & 46.7 & 48 & 48 & 47.7 & 47.3 & 48.1 & 48 & 43.5 \\
\hline 0 & $B$ & S & $5 / 7 / 1994$ & 94127 & 127 & 58.8 & 56.7 & 54.3 & 53.9 & 53.9 & 54.3 & 52.5 & 51.5 & 83.6 \\
\hline 0 & $B$ & $\mathrm{~S}$ & $5 / 8 / 1994$ & 94128 & 128 & 55.3 & 56 & 55.6 & 53.9 & 53.9 & 54.6 & 53.2 & 54.6 & 50.8 \\
\hline 0 & $B$ & $\mathrm{~S}$ & $5 / 14 / 1994$ & 94134 & 134 & 65.7 & 65.7 & 57.7 & 57.4 & 59.4 & 54.6 & 55.6 & 59.4 & 85 \\
\hline 0 & B & S & $5 / 15 / 1994$ & 94135 & 135 & 60.8 & 59.8 & 59.4 & 58.8 & 57 & 55 & 56 & 56 & 51.8 \\
\hline 0 & B & $S$ & $5 / 21 / 1994$ & 94141 & 141 & 71.8 & 59.4 & 58.8 & 58.1 & 58.1 & 57.7 & 57.7 & 126.3 & 180.2 \\
\hline 0 & B & S & $5 / 22 / 1994$ & 94142 & 142 & 61.5 & 61.5 & 59.8 & 55 & 55 & 54.3 & 53.9 & 53.2 & 51.1 \\
\hline 1 & $A$ & NS & 6/6/1991 & 91157 & 157 & 75.7 & 85 & 71.5 & 75.7 & 72.2 & 68.1 & 76.4 & 62.9 & 165.9 \\
\hline 1 & A & NS & 6/7/1991 & 91158 & 158 & 75.7 & 77.8 & 65 & 77.8 & 62.6 & 68.4 & 65.3 & 65.7 & 77.8 \\
\hline 1 & A & NS & 6/10/1991 & 91161 & 161 & 68.1 & 64.6 & 63.6 & 72.6 & 62.2 & 59.4 & 64.6 & 66.4 & 186.6 \\
\hline 1 & $A$ & NS & 6/11/1991 & 91162 & 162 & 79.5 & 77.1 & 68.4 & 80.2 & 68.4 & 69.5 & 74.7 & 65.3 & 181.1 \\
\hline 1 & $A$ & NS & 6/12/1991 & 91163 & 163 & 77.1 & 72.2 & 75.3 & 76.4 & 66.7 & 66.4 & 77.4 & 72.6 & 143.4 \\
\hline 1 & A & NS & 6/13/1991 & 91164 & 164 & 82.6 & 68.8 & 71.5 & 79.1 & 69.8 & 63.9 & 72.2 & 72.9 & 156.6 \\
\hline 1 & $A$ & NS & 6/14/1991 & 91165 & 165 & 77.8 & 77.8 & 77.1 & 72.2 & 76.4 & 68.4 & 75.7 & 64.6 & 75 \\
\hline 1 & A & NS & 6/17/1991 & 91168 & 168 & & & & & & & & & \\
\hline 1 & A & NS & $6 / 18 / 1991$ & 91169 & 169 & & & & & & & & & \\
\hline 1 & $A$ & NS & 6/19/1991 & 91170 & 170 & & & & & & & & & \\
\hline 1 & A & NS & 6/20/1991 & 91171 & 171 & 67.4 & 78.8 & 77.8 & 65.7 & 67.7 & 74.3 & 64.3 & 60.1 & 121.7 \\
\hline 1 & $A$ & NS & $6 / 21 / 1991$ & 91172 & 172 & 93.7 & 88.8 & 79.5 & 87.8 & 83.3 & 74.7 & 84 & 72.9 & 63.2 \\
\hline 1 & $A$ & NS & 6/24/1991 & 91175 & 175 & 71.5 & 75 & 66 & 76.7 & 63.9 & 70.2 & 68.1 & 61.5 & 104.7 \\
\hline 1 & A & NS & 6/25/1991 & 91176 & 176 & 66 & 72.6 & 69.1 & 65 & 71.9 & 60.5 & 68.4 & 70.5 & 222.2 \\
\hline 1 & A & NS & $6 / 26 / 1991$ & 91177 & 177 & 74 & 73.3 & 74.3 & 66 & 74 & 64.3 & 67.4 & 66.7 & 133.4 \\
\hline 1 & A & NS & $6 / 27 / 1991$ & 91178 & 178 & 71.9 & 79.5 & 75 & 69.8 & 76.7 & 65 & 73.3 & 65.3 & 136.5 \\
\hline 1 & $A$ & NS & 6/28/1991 & 91179 & 179 & 69.8 & 69.8 & 76.4 & 71.2 & 65.3 & 63.6 & 63.2 & 68.1 & 88.5 \\
\hline 1 & A & NS & 7/1/1991 & 91182 & 182 & 74 & 84 & 71.2 & 68.4 & 74.7 & 71.2 & 63.2 & 65.7 & 161.1 \\
\hline 1 & $A$ & NS & $7 / 2 / 1991$ & 91183 & 183 & 70.5 & 68.4 & 73.6 & 62.2 & 68.8 & 67 & 57.4 & 69.5 & 147.2 \\
\hline 1 & A & NS & $7 / 3 / 1991$ & 91184 & 184 & 82.3 & 85 & 91.2 & 88.1 & 76.4 & 82.3 & 85 & 74.3 & 146.2 \\
\hline 1 & A & NS & 7/4/1991 & 91185 & 185 & 106.4 & 91.2 & 85.7 & 92.6 & 87.8 & 77.1 & 83.3 & 87.8 & 86.4 \\
\hline 1 & $A$ & NS & 7/5/1991 & 91186 & 186 & 98.8 & 86.1 & 91.6 & 92.3 & 90.5 & 81.2 & 78.8 & 88.5 & 92.3 \\
\hline 1 & $A$ & NS & $7 / 8 / 1991$ & 91189 & 189 & 88.5 & 84 & 75.3 & 82.9 & 80.5 & 70.8 & 79.8 & 79.5 & 135.8 \\
\hline 1 & A & NS & $7 / 9 / 1991$ & 91190 & 190 & 84.3 & 88.1 & 77.1 & 76 & 76 & 74.3 & 71.9 & 71.5 & 128.9 \\
\hline 1 & A & NS & 7/10/1991 & 91191 & 191 & 77.1 & 74.7 & 76.4 & 61.9 & 66 & 72.6 & 70.2 & 59.1 & 93 \\
\hline 1 & $A$ & NS & $7 / 11 / 1991$ & 91192 & 192 & 71.9 & 73.3 & 67.4 & 59.1 & 68.4 & 69.5 & 60.1 & 57 & 144.5 \\
\hline
\end{tabular}




\begin{tabular}{|c|c|c|c|c|c|c|c|c|c|c|c|c|c|c|}
\hline 1 & $A$ & NS & $7 / 12 / 1991$ & 91193 & 193 & 76.4 & 77.8 & 71.5 & 63.2 & 74 & 72.6 & 59.1 & 63.6 & 71.2 \\
\hline 1 & A & NS & $7 / 15 / 1991$ & 91196 & 196 & 74.3 & 77.8 & 86.7 & 73.6 & 72.2 & 75 & 77.4 & 90.5 & 140.3 \\
\hline 1 & $A$ & NS & $7 / 16 / 1991$ & 91197 & 197 & 85.7 & 77.1 & 73.3 & 77.4 & 78.1 & 67.4 & 66 & 77.4 & 132.7 \\
\hline 1 & $A$ & NS & $7 / 17 / 1991$ & 91198 & 198 & 91.9 & 99.2 & 88.1 & 85 & 93.7 & 86.1 & 81.6 & 79.8 & 162.8 \\
\hline 1 & $A$ & NS & $7 / 18 / 1991$ & 91199 & 199 & 95 & 93.7 & 80.9 & 81.9 & 89.9 & 80.9 & 74 & 79.5 & 145.2 \\
\hline 1 & $A$ & NS & $7 / 19 / 1991$ & 91200 & 200 & 85.4 & 78.8 & 91.2 & 90.2 & 75.7 & 76.7 & 85.4 & 73.3 & 70.5 \\
\hline 1 & $A$ & NS & $7 / 22 / 1991$ & 91203 & 203 & 93.7 & 98.8 & 89.9 & 86.1 & 97.5 & 84 & 85 & 85.7 & 165.5 \\
\hline 1 & A & NS & $7 / 23 / 1991$ & 91204 & 204 & 95.7 & 86.7 & 89.9 & 93.3 & 82.3 & 82.6 & 91.2 & 78.8 & 150.3 \\
\hline 1 & A & NS & $7 / 24 / 1991$ & 91205 & 205 & 91.6 & 83.6 & 81.6 & 84.3 & 91.2 & 79.8 & 77.8 & 75.3 & 162.4 \\
\hline 1 & $A$ & NS & $7 / 25 / 1991$ & 91206 & 206 & 93 & 87.8 & 87.4 & 90.2 & 80.2 & 88.1 & 77.1 & 74 & 66 \\
\hline 1 & $A$ & NS & $7 / 26 / 1991$ & 91207 & 207 & & & & & & & & & \\
\hline 1 & $A$ & NS & $7 / 29 / 1991$ & 91210 & 210 & 66.7 & 69.5 & 68.1 & 67 & 68.4 & 70.8 & 67.7 & 66 & 143.8 \\
\hline 1 & $A$ & NS & $7 / 30 / 1991$ & 91211 & 211 & 79.8 & 78.1 & 79.8 & 79.5 & 78.5 & 76.4 & 76.4 & 75.3 & 140 \\
\hline 1 & $A$ & NS & $7 / 31 / 1991$ & 91212 & 212 & 68.8 & 69.1 & 68.4 & 67.4 & 67 & 68.1 & 65.7 & 63.2 & 122.7 \\
\hline 1 & $A$ & NS & 8/1/1991 & 91213 & 213 & 76.4 & 71.5 & 72.6 & 72.2 & 72.9 & 71.2 & 68.8 & 66 & 140.3 \\
\hline 1 & $A$ & NS & $8 / 2 / 1991$ & 91214 & 214 & 91.9 & 89.2 & 90.5 & 90.2 & 74.7 & 86.7 & 84 & 68.8 & 74.3 \\
\hline 1 & $A$ & NS & 8/5/1991 & 91217 & 217 & 85 & 72.2 & 77.8 & 82.9 & 72.6 & 74 & 76.4 & 66.4 & 144.8 \\
\hline 1 & A & NS & 8/6/1991 & 91218 & 218 & 114.7 & 112.7 & 110.9 & 107.8 & 109.2 & 104.4 & 106.1 & 103.3 & 351.5 \\
\hline 1 & $A$ & NS & 8/7/1991 & 91219 & 219 & 111.6 & 103.7 & 96.4 & 106.1 & 102 & 95 & 98.5 & 93.7 & 304.5 \\
\hline 1 & A & NS & 8/8/1991 & 91220 & 220 & 121 & 109.6 & 115.4 & 118.2 & 106.4 & 107.5 & 112 & 199.1 & 344.2 \\
\hline 1 & $A$ & NS & 8/9/1991 & 91221 & 221 & 126.8 & 123.4 & 123.4 & 121.7 & 123.4 & 110.6 & 120.3 & 144.8 & 353.9 \\
\hline 1 & $A$ & NS & 8/12/1991 & 91224 & 224 & 104.7 & 105.8 & 96.4 & 91.9 & 97.1 & 101.3 & 86.4 & 227.4 & 387.8 \\
\hline 1 & $A$ & NS & $8 / 13 / 1991$ & 91225 & 225 & 133.4 & 129.9 & 116.5 & 116.5 & 128.9 & 121.3 & 110.6 & 194.9 & 409.5 \\
\hline 1 & $A$ & NS & 8/14/1991 & 91226 & 226 & 116.1 & 121.3 & 128.9 & 113.4 & 115.1 & 121.7 & 119.9 & 188 & 363.2 \\
\hline 1 & A & NS & 8/15/1991 & 91227 & 227 & 117.5 & 126.8 & 113.4 & 112.3 & 116.8 & 123 & 109.9 & 195.6 & 329.7 \\
\hline 1 & $A$ & NS & 8/16/1991 & 91228 & 228 & 102.3 & 112.7 & 100.2 & 99.9 & 99.2 & 100.9 & 105.8 & 282 & 310.7 \\
\hline 1 & $A$ & NS & 8/19/1991 & 91231 & 231 & 102.6 & 110.9 & 111.6 & 107.5 & 99.5 & 110.2 & 104.4 & 281.7 & 350.4 \\
\hline 1 & $A$ & NS & 8/20/1991 & 91232 & 232 & 122.7 & 109.9 & 110.9 & 111.3 & 116.5 & 104 & 106.4 & 124.1 & 354.9 \\
\hline 1 & $A$ & NS & $8 / 21 / 1991$ & 91233 & 233 & 127.2 & 111.3 & 119.9 & 117.2 & 108.2 & 112.3 & 113.7 & 121 & 378.8 \\
\hline 1 & A & NS & $8 / 22 / 1991$ & 91234 & 234 & 133.1 & 126.1 & 116.5 & 126.8 & 126.8 & 116.5 & 132 & 364.6 & 457.9 \\
\hline 1 & $A$ & NS & $8 / 23 / 1991$ & 91235 & 235 & 115.1 & 115.4 & 113.4 & 112 & 111.3 & 108.9 & 107.1 & 129.6 & 458.3 \\
\hline 1 & A & NS & $8 / 26 / 1991$ & 91238 & 238 & 107.1 & 98.8 & 98.8 & 98.8 & 96.4 & 90.5 & 108.9 & 341.8 & 477.3 \\
\hline 1 & $A$ & NS & $8 / 27 / 1991$ & 91239 & 239 & 96.4 & 106.1 & 103 & 91.6 & 97.8 & 104 & 91.2 & 348.7 & 495.9 \\
\hline 1 & A & NS & 8/28/1991 & 91240 & 240 & 107.1 & 96.8 & 96.1 & 104.4 & 96.4 & 92.6 & 118.5 & 349.7 & 487.3 \\
\hline 1 & A & NS & $8 / 29 / 1991$ & 91241 & 241 & 116.1 & 94.3 & 94 & 100.6 & 95.7 & 88.8 & 93.7 & 345.3 & 486.3 \\
\hline 1 & A & NS & 8/30/1991 & 91242 & 242 & 107.8 & 102 & 108.2 & 99.2 & 104 & 99.9 & 107.1 & 283 & 477.3 \\
\hline 1 & $A$ & NS & 9/2/1991 & 91245 & 245 & 96.4 & 87.1 & 83.3 & 89.5 & 88.8 & 81.6 & 78.5 & 89.5 & 77.1 \\
\hline
\end{tabular}




\begin{tabular}{|c|c|c|c|c|c|c|c|c|c|c|c|c|c|c|}
\hline 1 & $A$ & NS & \#\#\#\#\#\#\#\# & 91296 & 296 & 107.8 & 87.4 & 83.6 & 80.2 & 82.3 & 82 & 84 & 180.7 & 289.4 \\
\hline 1 & $A$ & NS & \#\#\#\#\#\#\#\# & 91331 & 331 & 56.4 & 67.5 & 56.3 & 47.1 & 48.4 & 47.1 & 46.7 & 65.4 & 151 \\
\hline 1 & $A$ & NS & \#\#\#\#\#\#\#\# & 91332 & 332 & 48.5 & 47.1 & 45.6 & 43.6 & 43.5 & 41.2 & 42.3 & 42.5 & 40.2 \\
\hline 1 & A & NS & \#\#\#\#\#\#\#\# & 91333 & 333 & 49.2 & 47.1 & 44.9 & 42.9 & 46.1 & 43.2 & 43.6 & 43.9 & 46.1 \\
\hline 1 & $A$ & NS & \#\#\#\#\#\#\#\# & 91354 & 354 & 72 & 61.5 & 57.8 & 58.1 & 57.1 & 57.5 & 83.6 & 143.5 & 220.1 \\
\hline 1 & $A$ & NS & \#\#\#\#\#\#\#\# & 91357 & 357 & 72.2 & 65.1 & 63.2 & 63 & 63 & 62.3 & 63.9 & 62 & 74 \\
\hline 1 & A & NS & \#\#\#\#\#\#\#\# & 91358 & 358 & 67.7 & 67.1 & 60.6 & 59.1 & 59.5 & 59.2 & 59.4 & 58.8 & 55.7 \\
\hline 1 & A & NS & \#\#\#\#\#\#\#\# & 91359 & 359 & 64.7 & 65 & 65.1 & 65.4 & 60.8 & 62.3 & 69.2 & 60.8 & 59.9 \\
\hline 1 & $A$ & NS & \#\#\#\#\#\#\#\# & 91360 & 360 & 66.1 & 65.3 & 67.8 & 63.3 & 61.5 & 64.7 & 68.5 & 58.1 & 77.9 \\
\hline 1 & $A$ & NS & \#\#\#\#\#\#\#\# & 91361 & 361 & 46.4 & 59.1 & 42.9 & 51.9 & 44.9 & 53.7 & 45.3 & 51.2 & 55.6 \\
\hline 1 & A & NS & \#\#\#\#\#\#\#\# & 91364 & 364 & 54.6 & 47.1 & 50.2 & 45.3 & 44.3 & 52.5 & 44 & 44.9 & 56.1 \\
\hline 1 & A & NS & \#\#\#\#\#\#\#\# & 91365 & 365 & 53.9 & 49.9 & 46.1 & 47 & 48.5 & 54.4 & 55.6 & 51.2 & 64.6 \\
\hline 1 & $A$ & NS & 1/1/1992 & 92001 & 366 & 0 & 0 & 0 & 0 & 0 & 0 & 0 & 0 & 0 \\
\hline 1 & $A$ & NS & 1/2/1992 & 92002 & 1 & 52.3 & 50.5 & 49.5 & 49.2 & 49.4 & 55.7 & 47.1 & 47 & 49.2 \\
\hline 1 & $A$ & NS & $1 / 3 / 1992$ & 92003 & 2 & 51.9 & 49.4 & 46.1 & 45.3 & 43.6 & 44.7 & 48 & 46.4 & 73 \\
\hline 1 & $A$ & NS & 1/6/1992 & 92006 & 5 & 74.7 & 74.7 & 67.1 & 66.4 & 68.4 & 69.2 & 69.9 & 68.1 & 64 \\
\hline 1 & $A$ & NS & 1/17/1992 & 92017 & 16 & 96.2 & 98.6 & 88.8 & 99.3 & 87.5 & 102.6 & 89.6 & 119.9 & 203.6 \\
\hline 1 & $B$ & NS & $3 / 13 / 1992$ & 92073 & 72 & 56.8 & 54.3 & 50.9 & 50.2 & 53.6 & 50.9 & 58.2 & 98.2 & 201.9 \\
\hline 1 & B & NS & $3 / 16 / 1992$ & 92076 & 75 & 66.4 & 61.6 & 59.5 & 58.8 & 57.5 & 58.2 & 59.8 & 54 & 55.7 \\
\hline 1 & B & NS & $3 / 17 / 1992$ & 92077 & 76 & 67.1 & 64 & 58.1 & 58.5 & 59.2 & 57.7 & 57.1 & 55.4 & 72.9 \\
\hline 1 & $B$ & NS & $3 / 18 / 1992$ & 92078 & 77 & 67.8 & 69.9 & 60.8 & 59.9 & 58.8 & 59.8 & 62 & 53.3 & 77.4 \\
\hline 1 & B & NS & $3 / 19 / 1992$ & 92079 & 78 & 71.3 & 72.9 & 61.3 & 59.2 & 59.1 & 60.2 & 58.5 & 59.1 & 84.1 \\
\hline 1 & B & NS & $3 / 20 / 1992$ & 92080 & 79 & 77.2 & 79.1 & 61.6 & 57.8 & 57.4 & 56.1 & 58.5 & 54.6 & 74.7 \\
\hline 1 & B & NS & 4/17/1992 & 92108 & 107 & 100.7 & 103.3 & 79.6 & 72.7 & 72 & 65.3 & 63.7 & 74.4 & 192.9 \\
\hline 1 & B & NS & $4 / 20 / 1992$ & 92111 & 110 & 86.5 & 86.8 & 77.4 & 73 & 67.5 & 66.7 & 66.8 & 67.5 & 62.9 \\
\hline 1 & B & NS & $4 / 28 / 1992$ & 92119 & 118 & 75 & 68.5 & 68.9 & 67.4 & 66.8 & 66.1 & 66.7 & 73.4 & 195.3 \\
\hline 1 & B & NS & $5 / 28 / 1992$ & 92149 & 148 & 67.8 & 67.1 & 67 & 67.1 & 64.6 & 60.2 & 63 & 87.5 & 249.8 \\
\hline 1 & B & NS & $5 / 29 / 1992$ & 92150 & 149 & 67 & 65.8 & 65.3 & 61.6 & 61.6 & 62.2 & 64.4 & 95.2 & 248.8 \\
\hline 1 & B & NS & 6/1/1992 & 92153 & 152 & 60.2 & 59.9 & 60.1 & 55.7 & 56.4 & 57.7 & 59.2 & 53.7 & 53.6 \\
\hline 1 & B & NS & $6 / 2 / 1992$ & 92154 & 153 & 54 & 55 & 53.7 & 52.3 & 51.1 & 50.9 & 53.9 & 51.2 & 120.4 \\
\hline 1 & B & NS & $6 / 3 / 1992$ & 92155 & 154 & 75.7 & 74.1 & 73.6 & 72.3 & 70.6 & 71.2 & 72.3 & 70.2 & 138 \\
\hline 1 & B & NS & 6/4/1992 & 92156 & 155 & 66.7 & 65.8 & 63 & 63.9 & 61.3 & 62.6 & 64.4 & 58.8 & 120.6 \\
\hline 1 & B & NS & $6 / 5 / 1992$ & 92157 & 156 & 62.9 & 63.3 & 62 & 60.8 & 59.2 & 60.8 & 61.3 & 59.1 & 199.3 \\
\hline 1 & $B$ & NS & 6/8/1992 & 92160 & 159 & 62.6 & 62 & 62.6 & 59.5 & 59.8 & 59.5 & 62 & 57.4 & 54.4 \\
\hline 1 & $B$ & NS & $6 / 9 / 1992$ & 92161 & 160 & 62.6 & 62.9 & 60.9 & 61.2 & 59.2 & 57.8 & 60.8 & 62.6 & 246.4 \\
\hline 1 & B & NS & $6 / 10 / 1992$ & 92162 & 161 & 66.7 & 65.4 & 65 & 60.9 & 60.2 & 62.6 & 63.3 & 109.7 & 255.4 \\
\hline 1 & $B$ & NS & $6 / 11 / 1992$ & 92163 & 162 & 68.8 & 69.6 & 69.9 & 66.4 & 65.8 & 66.1 & 69.8 & 105.7 & 258.3 \\
\hline
\end{tabular}




\begin{tabular}{|c|c|c|c|c|c|c|c|c|c|c|c|c|c|c|}
\hline 1 & B & NS & 6/12/1992 & 92164 & 163 & 70.2 & 68.2 & 69.1 & 68.2 & 66 & 64.7 & 66.4 & 109.4 & 272.3 \\
\hline 1 & B & NS & $6 / 15 / 1992$ & 92167 & 166 & 71.3 & 70.9 & 69.8 & 66.1 & 66.7 & 66.8 & 66.8 & 62.2 & 57.1 \\
\hline 1 & $B$ & NS & 6/16/1992 & 92168 & 167 & 75.3 & 74.7 & 75.1 & 70.5 & 66.4 & 68.8 & 68.2 & 111.5 & 273.8 \\
\hline 1 & B & NS & 6/17/1992 & 92169 & 168 & 77.1 & 74.7 & 71.9 & 70.6 & 68.9 & 70.8 & 70.2 & 115.6 & 282.1 \\
\hline 1 & B & NS & 6/18/1992 & 92170 & 169 & 81.3 & 82.7 & 81.6 & 77.9 & 76.7 & 76.8 & 77.5 & 114.1 & 284.8 \\
\hline 1 & $B$ & NS & 6/19/1992 & 92171 & 170 & 78.1 & 76.1 & 74.7 & 72.7 & 73 & 73.6 & 70.2 & 112.4 & 278.3 \\
\hline 1 & $B$ & NS & $6 / 22 / 1992$ & 92174 & 173 & 86.8 & 88.9 & 80.9 & 79.9 & 78.8 & 77.9 & 74.4 & 71.2 & 68.9 \\
\hline 1 & $B$ & NS & 6/23/1992 & 92175 & 174 & 84.7 & 82.7 & 80.9 & 78.2 & 73.4 & 78.5 & 73 & 108.4 & 285.6 \\
\hline 1 & B & NS & 6/24/1992 & 92176 & 175 & 75.8 & 74.7 & 73 & 72 & 71.2 & 68.2 & 66.8 & 102.8 & 270 \\
\hline 1 & B & NS & $6 / 25 / 1992$ & 92177 & 176 & 79.5 & 77.9 & 74.7 & 76 & 72.7 & 71.9 & 70.9 & 109.6 & 268 \\
\hline 1 & B & NS & 6/26/1992 & 92178 & 177 & 81 & 83.3 & 77.9 & 79.5 & 75.4 & 73.4 & 73.6 & 109.9 & 256.9 \\
\hline 1 & $B$ & NS & 6/29/1992 & 92181 & 180 & 79.6 & 77.1 & 74.7 & 72.2 & 70.6 & 69.2 & 68.8 & 68.9 & 65 \\
\hline 1 & B & NS & $6 / 30 / 1992$ & 92182 & 181 & 76 & 73.7 & 72.7 & 66.7 & 64 & 65 & 63 & 85.8 & 254.4 \\
\hline 1 & B & NS & 7/1/1992 & 92183 & 182 & 74.4 & 75.3 & 71.3 & 68.2 & 70.2 & 68.2 & 67.7 & 104 & 274.7 \\
\hline 1 & B & NS & 7/2/1992 & 92184 & 183 & 84.3 & 82.3 & 81.9 & 79.9 & 81 & 78.5 & 77.5 & 114.3 & 255.8 \\
\hline 1 & B & NS & $7 / 3 / 1992$ & 92185 & 184 & 76.5 & 76.7 & 73.7 & 75.1 & 69.8 & 72 & 70.2 & 106.5 & 262.6 \\
\hline 1 & B & NS & 7/6/1992 & 92188 & 187 & 70.2 & 70.8 & 68.5 & 65.3 & 64.4 & 66 & 65.1 & 62 & 59.8 \\
\hline 1 & $B$ & NS & $7 / 7 / 1992$ & 92189 & 188 & 73 & 72.7 & 70.2 & 67.8 & 68.4 & 67.8 & 65.4 & 125.5 & 262.6 \\
\hline 1 & $B$ & NS & $7 / 8 / 1992$ & 92190 & 189 & 71.5 & 69.2 & 68.1 & 65.1 & 65.4 & 64.6 & 64.7 & 102.3 & 255.9 \\
\hline 1 & $B$ & NS & 7/9/1992 & 92191 & 190 & 72.9 & 72 & 69.1 & 67.8 & 66.8 & 67 & 66.1 & 105.7 & 251.6 \\
\hline 1 & B & NS & $7 / 10 / 1992$ & 92192 & 191 & 74.4 & 73.3 & 70.9 & 69.8 & 67.1 & 66.1 & 65 & 105.1 & 252.5 \\
\hline 1 & B & NS & $7 / 13 / 1992$ & 92195 & 194 & 76 & 74.7 & 74.3 & 73.7 & 69.8 & 70.2 & 68.5 & 65 & 64.4 \\
\hline 1 & B & NS & $7 / 14 / 1992$ & 92196 & 195 & 76.8 & 74.7 & 75.1 & 70.8 & 67.8 & 67.1 & 66 & 112.1 & 254.8 \\
\hline 1 & $B$ & NS & $7 / 15 / 1992$ & 92197 & 196 & 75.8 & 75.7 & 73 & 71.9 & 70.6 & 70.6 & 71.9 & 100.9 & 254.2 \\
\hline 1 & $B$ & NS & $7 / 16 / 1992$ & 92198 & 197 & 75.7 & 75.1 & 73.4 & 73.3 & 69.6 & 70.5 & 69.6 & 107.2 & 250.5 \\
\hline 1 & $B$ & NS & $7 / 17 / 1992$ & 92199 & 198 & 76.4 & 77.5 & 74.3 & 73.4 & 71.6 & 69.8 & 69.9 & 108.9 & 257.5 \\
\hline 1 & B & NS & $7 / 20 / 1992$ & 92202 & 201 & 77.9 & 79.2 & 75.7 & 72.7 & 69.5 & 70.2 & 70.6 & 66.4 & 64.4 \\
\hline 1 & B & NS & $7 / 21 / 1992$ & 92203 & 202 & 68.5 & 69.1 & 66.8 & 65.8 & 65.7 & 66.4 & 63.3 & 63.9 & 173.7 \\
\hline 1 & B & NS & $7 / 22 / 1992$ & 92204 & 203 & 66.7 & 65.8 & 65.4 & 59.1 & 62.6 & 64.7 & 61.9 & 60.2 & 139 \\
\hline 1 & $B$ & NS & $7 / 23 / 1992$ & 92205 & 204 & 72 & 72.6 & 71.6 & 71.2 & 70.9 & 71.6 & 68.8 & 66.1 & 173.8 \\
\hline 1 & $B$ & NS & $7 / 24 / 1992$ & 92206 & 205 & 82.7 & 80.9 & 82 & 84 & 78.9 & 79.6 & 79.5 & 75.8 & 188.4 \\
\hline 1 & B & NS & $7 / 27 / 1992$ & 92209 & 208 & 83 & 84 & 82.3 & 79.1 & 80.3 & 82 & 79.1 & 76.8 & 72.3 \\
\hline 1 & B & NS & $7 / 28 / 1992$ & 92210 & 209 & 90.9 & 84.8 & 81.7 & 84.7 & 82.3 & 84.7 & 80.3 & 78.9 & 165.2 \\
\hline 1 & B & NS & $7 / 29 / 1992$ & 92211 & 210 & 73.6 & 75.8 & 76.4 & 70.6 & 69.9 & 73.3 & 70.2 & 69.8 & 154.9 \\
\hline 1 & $B$ & NS & $7 / 30 / 1992$ & 92212 & 211 & 75.8 & 74.7 & 73.4 & 74 & 70.9 & 73.6 & 70.2 & 68.1 & 160.5 \\
\hline 1 & B & NS & $7 / 31 / 1992$ & 92213 & 212 & 77.1 & 76.8 & 72.9 & 71.3 & 72.9 & 71.6 & 70.5 & 67.5 & 156.2 \\
\hline 1 & $B$ & NS & 8/3/1992 & 92216 & 215 & 74.3 & 73.4 & 70.6 & 68.4 & 68.5 & 71.5 & 67.8 & 64.6 & 62.6 \\
\hline
\end{tabular}




\begin{tabular}{|c|c|c|c|c|c|c|c|c|c|c|c|c|c|c|}
\hline 1 & B & NS & 8/4/1992 & 92217 & 216 & 78.8 & 75.1 & 77.1 & 70.9 & 69.8 & 70.9 & 72.9 & 66.8 & 157.9 \\
\hline 1 & B & NS & 8/5/1992 & 92218 & 217 & 70.9 & 70.5 & 67.5 & 65.7 & 66.4 & 67.8 & 68.4 & 62.6 & 154.5 \\
\hline 1 & B & NS & 8/6/1992 & 92219 & 218 & 74 & 71.3 & 72.2 & 69.9 & 68.1 & 68.2 & 68.4 & 65.4 & 217.1 \\
\hline 1 & B & NS & 8/7/1992 & 92220 & 219 & 76.7 & 77.5 & 73.7 & 76 & 73.4 & 74 & 72.7 & 81.9 & 262.2 \\
\hline 1 & B & NS & 8/10/1992 & 92223 & 222 & 79.9 & 76.8 & 75.7 & 72.7 & 73.6 & 74.4 & 72.2 & 68.5 & 68.2 \\
\hline 1 & $B$ & NS & $8 / 11 / 1992$ & 92224 & 223 & 71.3 & 75.3 & 69.2 & 65.7 & 68.2 & 69.6 & 66.7 & 75.4 & 221.7 \\
\hline 1 & $B$ & NS & $8 / 12 / 1992$ & 92225 & 224 & 77.4 & 77.2 & 74.3 & 74.1 & 72.2 & 73.4 & 71.9 & 78.5 & 263.4 \\
\hline 1 & $B$ & NS & $8 / 13 / 1992$ & 92226 & 225 & 76 & 75.1 & 71.6 & 73.3 & 72.3 & 71.9 & 72 & 77.4 & 265.6 \\
\hline 1 & $B$ & NS & $8 / 14 / 1992$ & 92227 & 226 & 65.3 & 69.6 & 60.9 & 68.4 & 61.3 & 67.7 & 62.6 & 73.3 & 252.8 \\
\hline 1 & B & NS & 8/17/1992 & 92230 & 229 & 66.4 & 65.8 & 61.5 & 62 & 64.6 & 63.7 & 59.2 & 62.6 & 59.9 \\
\hline 1 & B & NS & 8/18/1992 & 92231 & 230 & 66 & 68.5 & 65.4 & 65.3 & 64 & 66.4 & 63 & 67.5 & 204.4 \\
\hline 1 & $B$ & NS & 8/19/1992 & 92232 & 231 & 69.8 & 69.2 & 66 & 65.4 & 64.4 & 67.7 & 64.7 & 71.2 & 211.4 \\
\hline 1 & B & NS & $8 / 20 / 1992$ & 92233 & 232 & 66.4 & 73.3 & 67.5 & 69.5 & 69.6 & 70.5 & 73.4 & 77.8 & 229.5 \\
\hline 1 & B & NS & $8 / 21 / 1992$ & 92234 & 233 & 69.5 & 61.3 & 70.6 & 59.1 & 63.3 & 61.9 & 67.8 & 73.6 & 219.3 \\
\hline 1 & B & NS & $8 / 24 / 1992$ & 92237 & 236 & 80.3 & 82.9 & 79.9 & 79.1 & 73.4 & 81 & 79.8 & 77.5 & 78.1 \\
\hline 1 & B & NS & $8 / 25 / 1992$ & 92238 & 237 & 71.5 & 65.4 & 64.6 & 64 & 65.7 & 66.4 & 65.1 & 84.3 & 364.6 \\
\hline 1 & B & NS & $8 / 26 / 1992$ & 92239 & 238 & 75.4 & 71.5 & 72 & 67 & 65.8 & 71.2 & 63.7 & 87.8 & 368 \\
\hline 1 & $B$ & NS & $8 / 27 / 1992$ & 92240 & 239 & 83.6 & 81.3 & 74.7 & 78.1 & 71.3 & 73.6 & 70.9 & 97.5 & 367.9 \\
\hline 1 & $B$ & NS & $8 / 28 / 1992$ & 92241 & 240 & 82.6 & 81 & 79.5 & 76.1 & 75.8 & 76 & 70.2 & 109.2 & 364.5 \\
\hline 1 & B & NS & $8 / 31 / 1992$ & 92244 & 243 & 144.3 & 90.2 & 84.3 & 81 & 77.4 & 88.2 & 98.2 & 97.9 & 136.3 \\
\hline 1 & B & NS & 9/1/1992 & 92245 & 244 & 74 & 76.5 & 79.1 & 74.7 & 73 & 75.7 & 76.1 & 106.4 & 383.8 \\
\hline 1 & B & NS & 9/2/1992 & 92246 & 245 & 84 & 78.9 & 81.6 & 76.5 & 77.8 & 76.1 & 75.1 & 116.8 & 377.7 \\
\hline 1 & B & NS & 9/3/1992 & 92247 & 246 & 85.1 & 85 & 83 & 81.6 & 80.6 & 78.5 & 79.9 & 111.3 & 376.2 \\
\hline 1 & $B$ & NS & 9/4/1992 & 92248 & 247 & 90.2 & 88.2 & 85.1 & 84 & 82.3 & 82.9 & 77.5 & 132.7 & 368 \\
\hline 1 & B & NS & 9/7/1992 & 92251 & 250 & 83.3 & 79.2 & 81.2 & 71.3 & 74.1 & 73.6 & 76.5 & 74.3 & 74.4 \\
\hline 1 & B & NS & 9/15/1992 & 92259 & 258 & 78.2 & 77.4 & 77.9 & 77.4 & 77.2 & 78.1 & 76.5 & 112.5 & 290.5 \\
\hline 1 & B & NS & \#\#\#\#\#\#\#\# & 92286 & 285 & 91.3 & 88.1 & 81.3 & 81.2 & 78.5 & 79.5 & 76.8 & 74.1 & 76.7 \\
\hline 1 & B & NS & \#\#\#\#\#\#\#\# & 92330 & 329 & 96.2 & 82.9 & 93.4 & 93.3 & 94.1 & 93.7 & 90.3 & 95 & 160 \\
\hline 1 & B & NS & \#\#\#\#\#\#\#\# & 92331 & 330 & 71.5 & 68.2 & 67.7 & 64.7 & 66.4 & 64 & 61.9 & 70.2 & 139.3 \\
\hline 1 & $B$ & NS & \#\#\#\#\#\#\#\# & 92332 & 331 & 74.1 & 79.8 & 84.4 & 82.9 & 84 & 83.4 & 81.6 & 78.2 & 82.6 \\
\hline 1 & $B$ & NS & \#\#\#\#\#\#\#\# & 92353 & 352 & 59.8 & 59.5 & 58.1 & 58.2 & 59.1 & 58.2 & 78.8 & 103.4 & 178 \\
\hline 1 & B & NS & \#\#\#\#\#\#\#\# & 92356 & 355 & 80.9 & 62.2 & 60.6 & 61.9 & 61.3 & 58.8 & 57 & 51.8 & 48.5 \\
\hline 1 & B & NS & \#\#\#\#\#\#\#\# & 92357 & 356 & 56.8 & 65.7 & 58.8 & 64.3 & 58.8 & 64.3 & 56.4 & 59.4 & 62.6 \\
\hline 1 & B & NS & \#\#\#\#\#\#\#\# & 92358 & 357 & 58.2 & 58.1 & 55.7 & 55.6 & 57.1 & 56.7 & 54.4 & 65 & 86.1 \\
\hline 1 & $B$ & NS & \#\#\#\#\#\#\#\# & 92359 & 358 & 63.7 & 66 & 63.9 & 62.6 & 65.1 & 61.9 & 60.6 & 57.4 & 66.4 \\
\hline 1 & B & NS & \#\#\#\#\#\#\#\# & 92360 & 359 & 67.8 & 67.4 & 63.7 & 65.3 & 64.7 & 64.3 & 60.6 & 59.1 & 55.7 \\
\hline 1 & $B$ & NS & \#\#\#\#\#\#\#\# & 92363 & 362 & 55.7 & 56.3 & 55 & 54.6 & 54.7 & 53.9 & 51.9 & 50.1 & 47.4 \\
\hline
\end{tabular}




\begin{tabular}{|c|c|c|c|c|c|c|c|c|c|c|c|c|c|c|}
\hline 1 & B & NS & \#\#\#\#\#\#\#\# & 92364 & 363 & 56.4 & 73.3 & 65.4 & 65.3 & 63.7 & 65.3 & 58.5 & 62.9 & 63.7 \\
\hline 1 & B & NS & \#\#\#\#\#\#\#\# & 92365 & 364 & 69.6 & 68.8 & 68.9 & 66.7 & 69.2 & 69.5 & 65.3 & 62.6 & 83.6 \\
\hline 1 & B & NS & \#\#\#\#\#\#\#\# & 92366 & 365 & 72.6 & 72.3 & 71.5 & 68.9 & 72.6 & 70.6 & 67.7 & 65.7 & 77.9 \\
\hline 1 & B & NS & 1/1/1993 & 93001 & 1 & 61.6 & 61.5 & 58.5 & 56.8 & 58.1 & 56.8 & 56.7 & 53 & 56 \\
\hline 1 & B & NS & 1/4/1993 & 93004 & 4 & 62.2 & 61.6 & 59.1 & 58.5 & 60.5 & 59.1 & 65.4 & 72.9 & 121.7 \\
\hline 1 & B & NS & 2/15/1993 & 93046 & 46 & 68.1 & 68.9 & 68.4 & 65.4 & 65.3 & 65.8 & 75.7 & 79.6 & 122 \\
\hline 1 & B & NS & $3 / 15 / 1993$ & 93074 & 74 & 67.5 & 68.4 & 66.4 & 65.3 & 64.4 & 65.7 & 84.7 & 87.2 & 108.9 \\
\hline 1 & B & NS & $3 / 16 / 1993$ & 93075 & 75 & 65.7 & 64.4 & 61.2 & 58.8 & 60.1 & 59.4 & 68.9 & 76.7 & 98.2 \\
\hline 1 & B & NS & $3 / 17 / 1993$ & 93076 & 76 & 65.8 & 67 & 59.5 & 58.8 & 58.2 & 59.1 & 61.3 & 67 & 87.5 \\
\hline 1 & B & NS & $3 / 18 / 1993$ & 93077 & 77 & 61.9 & 62.6 & 57.4 & 56.4 & 55.6 & 56.4 & 56.7 & 62.3 & 94.1 \\
\hline 1 & B & NS & $3 / 19 / 1993$ & 93078 & 78 & 74.4 & 74 & 66.1 & 65 & 63.3 & 65 & 62.3 & 71.9 & 95.8 \\
\hline 1 & $B$ & NS & 4/9/1993 & 93099 & 99 & 73 & 81.9 & 75.3 & 72.2 & 71.6 & 68.8 & 64.3 & 94.6 & 129.7 \\
\hline 1 & B & NS & 4/12/1993 & 93102 & 102 & 67.1 & 72.9 & 67.1 & 69.1 & 65.3 & 67.1 & 75.3 & 117 & 119.3 \\
\hline 1 & B & NS & $4 / 28 / 1993$ & 93118 & 118 & 66.4 & 57.1 & 57 & 54 & 52.2 & 54.4 & 58.4 & 121.1 & 240.1 \\
\hline 1 & B & NS & $5 / 27 / 1993$ & 93147 & 147 & 67.8 & 63.6 & 59.8 & 56.8 & 56.7 & 57.5 & 57.4 & 96 & 237.6 \\
\hline 1 & $B$ & NS & $5 / 28 / 1993$ & 93148 & 148 & 62.2 & 59.9 & 68.4 & 70.2 & 58.8 & 59.8 & 56.7 & 90.3 & 205.9 \\
\hline 1 & B & NS & $5 / 31 / 1993$ & 93151 & 151 & 56.4 & 63.9 & 56.7 & 50.9 & 52.2 & 50.1 & 89.6 & 119.8 & 191.1 \\
\hline 1 & B & NS & 6/1/1993 & 93152 & 152 & 57.7 & 49.4 & 55.3 & 53.6 & 46 & 46.7 & 46.1 & 67.4 & 137.6 \\
\hline 1 & $B$ & NS & 6/2/1993 & 93153 & 153 & 58.1 & 54 & 65 & 58.8 & 50.9 & 52.5 & 61.9 & 75.4 & 139.7 \\
\hline 1 & B & NS & $6 / 3 / 1993$ & 93154 & 154 & 64 & 55 & 65 & 61.9 & 50.9 & 62.2 & 58.4 & 76.5 & 159.3 \\
\hline 1 & B & NS & 6/4/1993 & 93155 & 155 & 65 & 62.9 & 58.8 & 64.6 & 55 & 59.4 & 58.8 & 75.4 & 145.8 \\
\hline 1 & B & NS & 6/7/1993 & 93158 & 158 & 85.8 & 88.1 & 88.1 & 83.3 & 82.3 & 82 & 83.3 & 125.7 & 203.5 \\
\hline 1 & B & NS & 6/8/1993 & 93159 & 159 & 68.8 & 67.5 & 66.7 & 67.4 & 63.9 & 62.6 & 62.6 & 85.9 & 181.9 \\
\hline 1 & B & NS & $6 / 9 / 1993$ & 93160 & 160 & 72 & 72.6 & 70.5 & 69.1 & 66.8 & 67.4 & 63.6 & 92.3 & 187.2 \\
\hline 1 & B & NS & 6/10/1993 & 93161 & 161 & 68.4 & 68.4 & 69.1 & 61.3 & 65 & 61.9 & 62.6 & 85.6 & 194.8 \\
\hline 1 & B & NS & 6/11/1993 & 93162 & 162 & 62.9 & 54.3 & 55 & 60.1 & 54.3 & 51.2 & 58.8 & 75.7 & 182.8 \\
\hline 1 & B & NS & 6/14/1993 & 93165 & 165 & 53.9 & 60.8 & 60.1 & 51.9 & 57 & 60.5 & 55.6 & 85 & 173.8 \\
\hline 1 & B & NS & 6/15/1993 & 93166 & 166 & 56 & 57.4 & 61.9 & 54.6 & 52.9 & 58.4 & 49.4 & 79.6 & 174.8 \\
\hline 1 & $B$ & NS & 6/16/1993 & 93167 & 167 & 74.7 & 75.7 & 75 & 66.4 & 73.3 & 70.2 & 64.6 & 98.4 & 190.6 \\
\hline 1 & B & NS & $6 / 17 / 1993$ & 93168 & 168 & 67.7 & 67.7 & 66.7 & 65.7 & 65.3 & 63.2 & 56.3 & 105.3 & 182.4 \\
\hline 1 & B & NS & 6/18/1993 & 93169 & 169 & 55 & 55.3 & 55.3 & 53.9 & 52.2 & 52.2 & 52.2 & 92.2 & 163.5 \\
\hline 1 & $B$ & NS & $6 / 21 / 1993$ & 93172 & 172 & 45.3 & 45.6 & 47 & 45.3 & 43.5 & 43.2 & 46.7 & 88.7 & 179.1 \\
\hline 1 & B & NS & 6/22/1993 & 93173 & 173 & 51.5 & 51.2 & 53.2 & 51.5 & 50.5 & 50.5 & 50.5 & 87.2 & 166.7 \\
\hline 1 & B & NS & 6/23/1993 & 93174 & 174 & 53.2 & 54.7 & 55 & 52.5 & 51.8 & 51.8 & 50.1 & 87.6 & 169.2 \\
\hline 1 & $B$ & NS & 6/24/1993 & 93175 & 175 & 58.4 & 58.1 & 65 & 63.6 & 61.2 & 60.5 & 60.5 & 95.5 & 188.6 \\
\hline 1 & B & NS & $6 / 25 / 1993$ & 93176 & 176 & 55 & 54.4 & 60.8 & 59.8 & 55.6 & 56.7 & 55.6 & 94.1 & 182.4 \\
\hline 1 & $B$ & NS & 6/28/1993 & 93179 & 179 & 50.8 & 50.5 & 49.4 & 50.1 & 50.1 & 48.4 & 51.5 & 75.7 & 168.2 \\
\hline
\end{tabular}




\begin{tabular}{|c|c|c|c|c|c|c|c|c|c|c|c|c|c|c|}
\hline 1 & B & NS & $6 / 29 / 1993$ & 93180 & 180 & 53.6 & 55.3 & 54.3 & 51.8 & 50.8 & 49.1 & 53.6 & 87.7 & 171.2 \\
\hline 1 & B & NS & 6/30/1993 & 93181 & 181 & 55.3 & 57.4 & 56.3 & 52.9 & 52.9 & 50.9 & 50.8 & 96.4 & 174 \\
\hline 1 & $B$ & NS & 7/1/1993 & 93182 & 182 & 53.6 & 52.5 & 51.8 & 52.2 & 50.5 & 50.5 & 49.8 & 92.5 & 168.6 \\
\hline 1 & B & NS & $7 / 2 / 1993$ & 93183 & 183 & 51.8 & 49.8 & 50.8 & 49.4 & 48 & 48 & 46.3 & 90.1 & 163.7 \\
\hline 1 & B & NS & 7/5/1993 & 93186 & 186 & 52.9 & 51.1 & 55 & 50.1 & 49.4 & 47 & 51.1 & 92 & 153 \\
\hline 1 & $B$ & NS & 7/6/1993 & 93187 & 187 & 47.7 & 52.2 & 48 & 46.3 & 49.4 & 47.3 & 44.9 & 90.7 & 165.9 \\
\hline 1 & $B$ & NS & $7 / 7 / 1993$ & 93188 & 188 & 51.5 & 50.5 & 50.8 & 49.1 & 50.5 & 49.8 & 48.4 & 91.8 & 167.8 \\
\hline 1 & $B$ & NS & 7/8/1993 & 93189 & 189 & 50.8 & 46 & 50.1 & 46 & 43.9 & 43.9 & 44.2 & 83.7 & 159.6 \\
\hline 1 & B & NS & $7 / 9 / 1993$ & 93190 & 190 & 49.4 & 50.8 & 49.4 & 47.3 & 47.7 & 47 & 48 & 86.8 & 145.3 \\
\hline 1 & $B$ & NS & $7 / 12 / 1993$ & 93193 & 193 & 53.9 & 48.7 & 50.8 & 46 & 50.8 & 48 & 48 & 90.7 & 164.8 \\
\hline 1 & B & NS & $7 / 13 / 1993$ & 93194 & 194 & 53.2 & 51.8 & 50.1 & 48.7 & 49.8 & 47.7 & 47.7 & 93.9 & 182 \\
\hline 1 & $B$ & NS & $7 / 14 / 1993$ & 93195 & 195 & 49.4 & 51.8 & 46.7 & 48.4 & 46.7 & 46.3 & 44.6 & 90.1 & 168.8 \\
\hline 1 & $B$ & NS & $7 / 15 / 1993$ & 93196 & 196 & 46.1 & 50.1 & 47.7 & 48 & 45.6 & 44.6 & 45.6 & 93.7 & 163.5 \\
\hline 1 & B & NS & $7 / 16 / 1993$ & 93197 & 197 & 62.9 & 51.1 & 48.7 & 45.4 & 48.4 & 58.4 & 57.7 & 69.8 & 75.7 \\
\hline 1 & $B$ & NS & $7 / 19 / 1993$ & 93200 & 200 & 47.7 & 48.4 & 42.9 & 50.5 & 41.8 & 54.3 & 55 & 67.7 & 89.5 \\
\hline 1 & B & NS & $7 / 20 / 1993$ & 93201 & 201 & 63.6 & 53.9 & 52.2 & 47.7 & 50.5 & 58.4 & 59.8 & 84.7 & 137.2 \\
\hline 1 & B & NS & $7 / 21 / 1993$ & 93202 & 202 & 67.4 & 54 & 52.2 & 49.8 & 50.5 & 58.8 & 62.2 & 84.3 & 126.1 \\
\hline 1 & $B$ & NS & $7 / 22 / 1993$ & 93203 & 203 & 65.3 & 51.8 & 52.2 & 51.5 & 49.9 & 50.5 & 49.8 & 75.3 & 140.3 \\
\hline 1 & $B$ & NS & $7 / 23 / 1993$ & 93204 & 204 & 63.6 & 50.5 & 52.5 & 47 & 49.1 & 57.7 & 60.5 & 76.4 & 86.4 \\
\hline 1 & B & NS & $7 / 26 / 1993$ & 93207 & 207 & 43.2 & 45.3 & 42.2 & 42.2 & 41.8 & 52.5 & 51.8 & 79.5 & 134.4 \\
\hline 1 & B & NS & $7 / 27 / 1993$ & 93208 & 208 & 58.8 & 48 & 47 & 46 & 44.6 & 55.6 & 57.4 & 85.7 & 143.1 \\
\hline 1 & B & NS & $7 / 28 / 1993$ & 93209 & 209 & 58.4 & 46.3 & 46.7 & 43.6 & 44.6 & 53.9 & 55.3 & 82.9 & 160.7 \\
\hline 1 & B & NS & $7 / 29 / 1993$ & 93210 & 210 & 63.2 & 52.2 & 51.6 & 50.8 & 48.4 & 59.1 & 60.5 & 89.2 & 146.5 \\
\hline 1 & $B$ & NS & $7 / 30 / 1993$ & 93211 & 211 & 59.4 & 47.8 & 46.3 & 44.9 & 44.7 & 54.3 & 57 & 71.9 & 86.1 \\
\hline 1 & B & NS & $8 / 2 / 1993$ & 93214 & 214 & 53.6 & 53.6 & 50.2 & 51.5 & 50.1 & 59.1 & 66 & 106.8 & 221.4 \\
\hline 1 & B & NS & $8 / 3 / 1993$ & 93215 & 215 & 62.2 & 56 & 55.3 & 51.2 & 52.5 & 51.1 & 52.6 & 68.4 & 155.7 \\
\hline 1 & B & NS & 8/4/1993 & 93216 & 216 & 69.1 & 68.4 & 67.5 & 66.7 & 68.1 & 64.3 & 67 & 89.9 & 182.1 \\
\hline 1 & B & NS & 8/5/1993 & 93217 & 217 & 56.7 & 59.1 & 57 & 56 & 55 & 54.4 & 55.6 & 78.8 & 145.6 \\
\hline 1 & B & NS & 8/6/1993 & 93218 & 218 & 54.6 & 54.7 & 55 & 52.9 & 53.3 & 52.9 & 51.9 & 74 & 169.2 \\
\hline 1 & $B$ & NS & 8/9/1993 & 93221 & 221 & 49.1 & 49.1 & 47.1 & 47 & 43.5 & 44.9 & 43.5 & 81.6 & 127.5 \\
\hline 1 & $B$ & NS & 8/10/1993 & 93222 & 222 & 62.9 & 63.2 & 59.9 & 60.1 & 59.1 & 59.4 & 59.4 & 86.4 & 246.5 \\
\hline 1 & B & NS & $8 / 11 / 1993$ & 93223 & 223 & 57.7 & 55 & 56 & 54.3 & 55 & 52.3 & 54.3 & 80.2 & 236.1 \\
\hline 1 & B & NS & 8/12/1993 & 93224 & 224 & 57.4 & 58.8 & 57.7 & 55 & 54.6 & 54.3 & 54.3 & 76 & 204.8 \\
\hline 1 & B & NS & $8 / 13 / 1993$ & 93225 & 225 & 52.2 & 51.5 & 51.8 & 50.1 & 50.5 & 50.5 & 49.2 & 131 & 226.9 \\
\hline 1 & $B$ & NS & 8/16/1993 & 93228 & 228 & 57.4 & 72.6 & 62.3 & 59.4 & 57.5 & 50.8 & 63.2 & 148.3 & 238 \\
\hline 1 & B & NS & $8 / 17 / 1993$ & 93229 & 229 & 67 & 60.1 & 56.1 & 56 & 55 & 55.4 & 55.3 & 141.1 & 247.8 \\
\hline 1 & $B$ & NS & 8/18/1993 & 93230 & 230 & 56.7 & 54.7 & 53.6 & 53.6 & 51.1 & 49.9 & 51.8 & 134.8 & 247.8 \\
\hline
\end{tabular}




\begin{tabular}{|c|c|c|c|c|c|c|c|c|c|c|c|c|c|c|}
\hline 1 & B & NS & 8/19/1993 & 93231 & 231 & 63.3 & 62.9 & 61.2 & 60.5 & 58.2 & 57.7 & 59.1 & 144.2 & 255.4 \\
\hline 1 & B & NS & $8 / 20 / 1993$ & 93232 & 232 & 60.1 & 60.1 & 59.5 & 57 & 55.6 & 56.1 & 56 & 136 & 278.7 \\
\hline 1 & B & NS & 8/23/1993 & 93235 & 235 & 58.1 & 62.2 & 64 & 61.5 & 59.1 & 54 & 85 & 166.9 & 379.6 \\
\hline 1 & B & NS & 8/24/1993 & 93236 & 236 & 68.5 & 59.1 & 59.1 & 55.7 & 55.3 & 55.4 & 54.6 & 126.3 & 354.6 \\
\hline 1 & B & NS & $8 / 25 / 1993$ & 93237 & 237 & 67.7 & 67.7 & 67 & 65.4 & 63.6 & 62.2 & 81.6 & 192.5 & 354.6 \\
\hline 1 & $B$ & NS & $8 / 26 / 1993$ & 93238 & 238 & 54.6 & 55.3 & 54.4 & 52.9 & 52.2 & 51.2 & 51.5 & 182.5 & 366.2 \\
\hline 1 & B & NS & $8 / 27 / 1993$ & 93239 & 239 & 64.3 & 63.3 & 62.9 & 60.8 & 58.2 & 58.4 & 61.5 & 165.5 & 371 \\
\hline 1 & B & NS & 8/30/1993 & 93242 & 242 & 51.6 & 57.4 & 57.7 & 54.4 & 54.3 & 48 & 82.6 & 234.7 & 374 \\
\hline 1 & B & NS & 8/31/1993 & 93243 & 243 & 72.2 & 65 & 65.4 & 62.9 & 61.2 & 60.2 & 64.3 & 192.2 & 380 \\
\hline 1 & B & NS & 9/1/1993 & 93244 & 244 & 57 & 54.6 & 57.4 & 56.3 & 55 & 53.2 & 54.3 & 165 & 355 \\
\hline 1 & $B$ & NS & 9/2/1993 & 93245 & 245 & 54.4 & 56.3 & 54.6 & 52.6 & 50.8 & 51.1 & 51.5 & 192.5 & 362.2 \\
\hline 1 & $B$ & NS & 9/3/1993 & 93246 & 246 & 57.7 & 56 & 56.7 & 54.6 & 52.3 & 51.1 & 51.5 & 170.6 & 346.4 \\
\hline 1 & B & NS & 9/6/1993 & 93249 & 249 & 54.6 & 51.8 & 53.9 & 49.8 & 47.7 & 48.7 & 56.3 & 72.9 & 89.9 \\
\hline 1 & B & NS & 9/7/1993 & 93250 & 250 & 55.3 & 56.3 & 56.8 & 54.3 & 53.2 & 52.5 & 53.9 & 134.5 & 252.3 \\
\hline 1 & B & NS & 9/8/1993 & 93251 & 251 & 60.8 & 60.5 & 60.5 & 59.4 & 58.1 & 58.1 & 59.1 & 157 & 265 \\
\hline 1 & B & NS & \#\#\#\#\#\#\#\# & 93315 & 315 & 49.8 & 51.5 & 52.5 & 50.2 & 50.5 & 49.8 & 48 & 62.9 & 95.1 \\
\hline 1 & $B$ & NS & \#\#\#\#\#\#\#\# & 93328 & 328 & 49.1 & 47.8 & 47.7 & 47 & 47 & 47.3 & 48.7 & 117.6 & 232.3 \\
\hline 1 & B & NS & \#\#\#\#\#\#\#\# & 93329 & 329 & 44.2 & 44.6 & 44 & 42.9 & 43.9 & 43.6 & 42.9 & 56.3 & 56.7 \\
\hline 1 & B & NS & \#\#\#\#\#\#\#\# & 93330 & 330 & 42.5 & 43.9 & 43.2 & 42.2 & 42.2 & 41.8 & 41.5 & 63.2 & 67 \\
\hline 1 & B & NS & \#\#\#\#\#\#\#\# & 93351 & 351 & 47.3 & 47.7 & 44.2 & 42.3 & 41.8 & 42.9 & 41.1 & 63.9 & 163.8 \\
\hline 1 & B & NS & \#\#\#\#\#\#\#\# & 93354 & 354 & 43.9 & 55.6 & 53.3 & 55 & 61.2 & 65 & 61.5 & 64.6 & 92.6 \\
\hline 1 & B & NS & \#\#\#\#\#\#\#\# & 93355 & 355 & 52.2 & 48 & 47.3 & 47.1 & 47 & 47 & 45.6 & 71.2 & 94.7 \\
\hline 1 & B & NS & \#\#\#\#\#\#\#\# & 93356 & 356 & 40.8 & 42.5 & 44.2 & 44.2 & 43.3 & 43.9 & 42.9 & 56.3 & 71.5 \\
\hline 1 & $B$ & NS & \#\#\#\#\#\#\#\# & 93357 & 357 & 40.8 & 51.8 & 54.6 & 43.5 & 43.5 & 43.9 & 42.2 & 53.6 & 65.7 \\
\hline 1 & B & NS & \#\#\#\#\#\#\#\# & 93358 & 358 & 41.5 & 42.2 & 43.9 & 43.9 & 43.5 & 43.9 & 43.2 & 53.6 & 53.2 \\
\hline 1 & B & NS & \#\#\#\#\#\#\#\# & 93361 & 361 & 40.1 & 41.5 & 44.9 & 44.2 & 44.2 & 44.9 & 43.9 & 54.6 & 52.2 \\
\hline 1 & $B$ & NS & \#\#\#\#\#\#\#\# & 93362 & 362 & 41.5 & 42.2 & 44.2 & 43.5 & 43.5 & 45.6 & 43.9 & 44.9 & 67.7 \\
\hline 1 & B & NS & \#\#\#\#\#\#\#\# & 93363 & 363 & 40.1 & 42.2 & 44 & 43.5 & 44.2 & 43.9 & 43.5 & 53.9 & 70.2 \\
\hline 1 & $B$ & NS & \#\#\#\#\#\#\#\# & 93364 & 364 & 40.4 & 39.1 & 42.2 & 41.5 & 42.5 & 41.5 & 41.5 & 53.9 & 70.8 \\
\hline 1 & B & NS & \#\#\#\#\#\#\#\# & 93365 & 365 & 39.1 & 39.7 & 43.5 & 42.5 & 42.5 & 42.3 & 42.2 & 55.3 & 59.8 \\
\hline 1 & B & NS & $1 / 3 / 1994$ & 94003 & 3 & 47 & 46.3 & 43.9 & 42.2 & 44.2 & 41.1 & 75.3 & 102.7 & 157.6 \\
\hline 1 & B & NS & $1 / 4 / 1994$ & 94004 & 4 & 55.6 & 57.1 & 56.3 & 55.6 & 56.3 & 57.4 & 73.6 & 108.5 & 161.8 \\
\hline 1 & B & NS & $1 / 5 / 1994$ & 94005 & 5 & 53.2 & 54.3 & 54.3 & 53.9 & 53.2 & 53.6 & 69.5 & 107.1 & 167.3 \\
\hline 1 & B & NS & 2/21/1994 & 94052 & 52 & 50.5 & 53.2 & 51.8 & 50.5 & 51.1 & 50.5 & 53.9 & 123.1 & 191.6 \\
\hline 1 & B & NS & 3/11/1994 & 94070 & 70 & 55 & 55.3 & 45 & 45.6 & 46.3 & 44.7 & 61.5 & 85.7 & 132.1 \\
\hline 1 & B & NS & $3 / 14 / 1994$ & 94073 & 73 & 52.9 & 68.4 & 69.5 & 69.5 & 67.7 & 68.1 & 74.3 & 78.5 & 102.6 \\
\hline 1 & $B$ & NS & $3 / 15 / 1994$ & 94074 & 74 & 65 & 62.6 & 63.6 & 63.9 & 61.9 & 62.9 & 61.9 & 69.8 & 86.7 \\
\hline
\end{tabular}




\begin{tabular}{|c|c|c|c|c|c|c|c|c|c|c|c|c|c|c|}
\hline 1 & B & NS & $3 / 16 / 1994$ & 94075 & 75 & 67.7 & 65 & 66 & 63.9 & 66 & 67.4 & 63.2 & 72.9 & 91.6 \\
\hline 1 & B & NS & $3 / 17 / 1994$ & 94076 & 76 & 81.9 & 80.9 & 80.5 & 73.6 & 71.9 & 73.6 & 70.2 & 78.1 & 94 \\
\hline 1 & B & NS & $3 / 18 / 1994$ & 94077 & 77 & 66.7 & 67.7 & 66.4 & 64.7 & 65.3 & 65.3 & 64.6 & 71.9 & 87.8 \\
\hline 1 & B & NS & 4/1/1994 & 94091 & 91 & 44.9 & 47 & 44.9 & 44.9 & 43.5 & 43.2 & 46.7 & 57.4 & 88.5 \\
\hline 1 & B & NS & 4/4/1994 & 94094 & 94 & 45.6 & 45.6 & 46.7 & 46.3 & 46.7 & 44.6 & 51.5 & 84.3 & 182.1 \\
\hline 1 & $B$ & NS & $5 / 30 / 1994$ & 94150 & 150 & 59.8 & 82.3 & 75.7 & 73.3 & 69.8 & 65 & 72.9 & 116.8 & 154.1 \\
\hline 1 & B & NS & $5 / 31 / 1994$ & 94151 & 151 & 59.8 & 58.4 & 57.4 & 56 & 52.9 & 55.6 & 56 & 87.4 & 136.9 \\
\hline 1 & B & NS & 6/1/1994 & 94152 & 152 & 56.7 & 51.5 & 50.5 & 51.8 & 49.8 & 49.4 & 49.4 & 103.3 & 150.5 \\
\hline 1 & B & NS & $6 / 2 / 1994$ & 94153 & 153 & 56.3 & 57 & 56 & 56.7 & 57 & 55 & 59.8 & 174.2 & 226 \\
\hline 1 & B & NS & $6 / 3 / 1994$ & 94154 & 154 & 61.5 & 61.9 & 60.8 & 60.1 & 62.2 & 60.1 & 64.3 & 170.8 & 208 \\
\hline 0 & $A$ & NS & $6 / 8 / 1991$ & 91159 & 159 & 74.7 & 69.5 & 70.2 & 78.5 & 65.7 & 65 & 74.3 & 65 & 61.2 \\
\hline 0 & $A$ & NS & 6/9/1991 & 91160 & 160 & 66.4 & 66 & 72.2 & 68.1 & 63.6 & 63.2 & 72.6 & 60.5 & 60.1 \\
\hline 0 & $A$ & NS & 6/15/1991 & 91166 & 166 & 80.2 & 76 & 79.5 & 72.6 & 81.6 & 69.1 & 77.8 & 67 & 71.9 \\
\hline 0 & $A$ & NS & 6/16/1991 & 91167 & 167 & 71.9 & 76.4 & 72.2 & 73.3 & 70.2 & 69.8 & 69.5 & 66.7 & 68.8 \\
\hline 0 & A & NS & $6 / 22 / 1991$ & 91173 & 173 & 73.3 & 85.4 & 82.6 & 81.2 & 72.9 & 69.1 & 78.5 & 75.7 & 67.4 \\
\hline 0 & $A$ & NS & $6 / 23 / 1991$ & 91174 & 174 & 74.3 & 86.4 & 75.7 & 72.9 & 75.7 & 66.4 & 80.2 & 70.8 & 66.4 \\
\hline 0 & A & NS & $6 / 29 / 1991$ & 91180 & 180 & 79.1 & 68.4 & 68.1 & 65 & 65.7 & 73.3 & 63.2 & 62.9 & 60.5 \\
\hline 0 & A & NS & 6/30/1991 & 91181 & 181 & 71.9 & 67 & 66.4 & 64.6 & 63.6 & 69.5 & 62.9 & 61.5 & 61.5 \\
\hline 0 & A & NS & $7 / 6 / 1991$ & 91187 & 187 & 90.9 & 87.4 & 78.1 & 81.6 & 85.7 & 80.9 & 71.9 & 77.8 & 87.4 \\
\hline 0 & A & NS & $7 / 7 / 1991$ & 91188 & 188 & 94 & 90.9 & 86.1 & 77.1 & 87.8 & 84.7 & 73.6 & 81.2 & 85 \\
\hline 0 & $A$ & NS & 7/13/1991 & 91194 & 194 & 76 & 86.7 & 81.2 & 75 & 75.7 & 78.8 & 69.8 & 67 & 77.8 \\
\hline 0 & A & NS & $7 / 14 / 1991$ & 91195 & 195 & 77.8 & 74.3 & 82.9 & 82.9 & 71.2 & 70.2 & 75 & 74.7 & 67.4 \\
\hline 0 & $A$ & NS & $7 / 20 / 1991$ & 91201 & 201 & 94 & 93.7 & 93.3 & 90.2 & 90.2 & 89.2 & 76.7 & 79.5 & 85.7 \\
\hline 0 & $A$ & NS & $7 / 21 / 1991$ & 91202 & 202 & 96.4 & 95.7 & 95 & 93.3 & 80.5 & 84.7 & 89.2 & 87.4 & 76.4 \\
\hline 0 & A & NS & $7 / 27 / 1991$ & 91208 & 208 & 77.4 & 78.1 & 78.8 & 78.5 & 78.5 & 78.8 & 77.8 & 75.3 & 72.9 \\
\hline 0 & $A$ & NS & $7 / 28 / 1991$ & 91209 & 209 & 66 & 66 & 65.7 & 67 & 66.4 & 68.4 & 66 & 63.9 & 62.9 \\
\hline 0 & $A$ & NS & $8 / 3 / 1991$ & 91215 & 215 & 87.1 & 77.4 & 71.9 & 83.3 & 74.7 & 69.5 & 77.8 & 68.1 & 64.3 \\
\hline 0 & $A$ & NS & 8/4/1991 & 91216 & 216 & 94.7 & 85.4 & 89.2 & 91.2 & 79.8 & 83.6 & 87.8 & 78.8 & 72.9 \\
\hline 0 & $A$ & NS & $8 / 10 / 1991$ & 91222 & 222 & 94 & 100.6 & 103.3 & 90.5 & 102.3 & 96.1 & 90.9 & 99.2 & 85 \\
\hline 0 & A & NS & 8/11/1991 & 91223 & 223 & 93.3 & 95.7 & 103.7 & 94.3 & 90.5 & 93 & 98.5 & 84.7 & 84 \\
\hline 0 & $A$ & NS & 8/17/1991 & 91229 & 229 & 115.1 & 107.1 & 105.1 & 113 & 106.8 & 103.7 & 102.6 & 106.1 & 115.8 \\
\hline 0 & A & NS & 8/18/1991 & 91230 & 230 & 106.1 & 114.4 & 102.6 & 100.9 & 111.6 & 101.6 & 98.2 & 102.6 & 99.2 \\
\hline 0 & $A$ & NS & 8/24/1991 & 91236 & 236 & 109.2 & 102.3 & 112.7 & 102 & 99.2 & 108.2 & 97.8 & 94.7 & 105.1 \\
\hline 0 & $A$ & NS & $8 / 25 / 1991$ & 91237 & 237 & 106.4 & 104.7 & 103.3 & 102.3 & 101.3 & 100.6 & 99.5 & 98.5 & 95 \\
\hline 0 & A & NS & $8 / 31 / 1991$ & 91243 & 243 & 109.6 & 103.7 & 102.6 & 99.9 & 94 & 96.8 & 93.3 & 92.6 & 93.7 \\
\hline 0 & $A$ & NS & 9/1/1991 & 91244 & 244 & 85.4 & 91.2 & 96.1 & 82.9 & 85.4 & 96.4 & 80.9 & 81.9 & 91.2 \\
\hline 0 & $A$ & NS & \#\#\#\#\#\#\#\# & 91355 & 355 & 86.5 & 84 & 77.5 & 76.1 & 71.5 & 73.4 & 76.1 & 71.5 & 71.3 \\
\hline
\end{tabular}




\begin{tabular}{|c|c|c|c|c|c|c|c|c|c|c|c|c|c|c|}
\hline 0 & $A$ & NS & \#\#\#\#\#\#\#\# & 91356 & 356 & 70.2 & 70.8 & 66.1 & 67.1 & 68.1 & 68.2 & 68.8 & 68.9 & 64.4 \\
\hline 0 & $A$ & NS & \#\#\#\#\#\#\#\# & 91362 & 362 & 46.7 & 53 & 44.9 & 49.9 & 44.9 & 51.6 & 41.6 & 50.8 & 42.9 \\
\hline 0 & $A$ & NS & \#\#\#\#\#\#\#\# & 91363 & 363 & 48 & 56.4 & 43.2 & 52.3 & 42.3 & 54.3 & 42.3 & 49.1 & 41.9 \\
\hline 0 & $A$ & NS & 1/4/1992 & 92004 & 3 & 55.7 & 53.7 & 46.3 & 44.3 & 44.7 & 44.2 & 55 & 44.9 & 70.2 \\
\hline 0 & $A$ & NS & 1/5/1992 & 92005 & 4 & 52.3 & 50.5 & 63.6 & 54.7 & 46.7 & 47.3 & 49.5 & 55.4 & 57.4 \\
\hline 0 & $A$ & NS & $5 / 30 / 1992$ & 92151 & 150 & 72.3 & 72.3 & 71.2 & 69.9 & 69.5 & 68.2 & 69.6 & 71.9 & 217.5 \\
\hline 0 & $A$ & NS & $5 / 31 / 1992$ & 92152 & 151 & 56.4 & 56.7 & 54.4 & 51.6 & 50.8 & 50.5 & 52.9 & 55.7 & 124.6 \\
\hline 0 & B & NS & $3 / 14 / 1992$ & 92074 & 73 & 68.8 & 66.1 & 54.7 & 54.3 & 53 & 51.6 & 54.6 & 51.6 & 89.3 \\
\hline 0 & B & NS & $3 / 15 / 1992$ & 92075 & 74 & 86.8 & 77.4 & 69.2 & 68.2 & 68.8 & 67.5 & 68.5 & 63.9 & 62 \\
\hline 0 & B & NS & 3/21/1992 & 92081 & 80 & 73.3 & 71.6 & 61.3 & 60.1 & 59.2 & 58.8 & 59.5 & 57.4 & 86.1 \\
\hline 0 & B & NS & $3 / 22 / 1992$ & 92082 & 81 & 64.7 & 62.3 & 56.7 & 54.4 & 53.7 & 56.7 & 56.8 & 54 & 57.4 \\
\hline 0 & $B$ & NS & 4/18/1992 & 92109 & 108 & 80.3 & 81.2 & 72.7 & 69.9 & 69.6 & 69.5 & 68.2 & 69.2 & 82.3 \\
\hline 0 & B & NS & 4/19/1992 & 92110 & 109 & 78.9 & 76 & 70.9 & 72 & 69.1 & 70.2 & 67.5 & 66.7 & 62.6 \\
\hline 0 & B & NS & 6/6/1992 & 92158 & 157 & 67.1 & 65.8 & 65.7 & 64.7 & 62.6 & 63.2 & 64.7 & 59.8 & 190.7 \\
\hline 0 & B & NS & 6/7/1992 & 92159 & 158 & 67.7 & 65.1 & 65.8 & 63.2 & 63.7 & 63 & 66.4 & 58.2 & 60.5 \\
\hline 0 & $B$ & NS & $6 / 13 / 1992$ & 92165 & 164 & 68.1 & 68.2 & 65.7 & 64 & 62.3 & 64.6 & 63.7 & 85.9 & 205.2 \\
\hline 0 & B & NS & $6 / 14 / 1992$ & 92166 & 165 & 68.2 & 68.1 & 64.4 & 62.6 & 61.2 & 62.3 & 62.6 & 59.9 & 57.1 \\
\hline 0 & $B$ & NS & 6/20/1992 & 92172 & 171 & 78.5 & 78.9 & 76.7 & 73.7 & 72 & 71.9 & 69.6 & 93.4 & 222.7 \\
\hline 0 & $B$ & NS & $6 / 21 / 1992$ & 92173 & 172 & 79.6 & 77.4 & 75.1 & 75.8 & 73.3 & 72.3 & 72.6 & 68.2 & 67.8 \\
\hline 0 & B & NS & 6/27/1992 & 92179 & 178 & 72.7 & 71.5 & 69.6 & 68.8 & 67.5 & 67.5 & 67.7 & 79.5 & 195.5 \\
\hline 0 & B & NS & 6/28/1992 & 92180 & 179 & 77.8 & 75.1 & 72.2 & 69.9 & 69.9 & 68.1 & 67.1 & 62.6 & 61.3 \\
\hline 0 & B & NS & 7/4/1992 & 92186 & 185 & 75.7 & 75.8 & 74.4 & 72.9 & 70.6 & 71.2 & 69.2 & 65.1 & 194.2 \\
\hline 0 & B & NS & $7 / 5 / 1992$ & 92187 & 186 & 67.4 & 65.8 & 63.3 & 64.6 & 61.6 & 61.6 & 62.2 & 58.5 & 57.8 \\
\hline 0 & B & NS & $7 / 11 / 1992$ & 92193 & 192 & 74.3 & 74.1 & 72.3 & 69.5 & 66.4 & 68.8 & 67.5 & 71.1 & 231.6 \\
\hline 0 & $B$ & NS & $7 / 12 / 1992$ & 92194 & 193 & 74.7 & 72 & 70.2 & 71.5 & 69.9 & 70.8 & 68.2 & 64.4 & 61.9 \\
\hline 0 & B & NS & $7 / 18 / 1992$ & 92200 & 199 & 78.8 & 79.2 & 77.5 & 76 & 74.7 & 73.6 & 75.4 & 85.6 & 234 \\
\hline 0 & B & NS & 7/19/1992 & 92201 & 200 & 77.1 & 72.7 & 73.7 & 71.9 & 68.5 & 70.5 & 67.5 & 67 & 63.3 \\
\hline 0 & B & NS & $7 / 25 / 1992$ & 92207 & 206 & 84 & 83 & 83.6 & 76.1 & 78.5 & 79.5 & 79.2 & 74.7 & 104 \\
\hline 0 & $B$ & NS & $7 / 26 / 1992$ & 92208 & 207 & 88.9 & 83.3 & 85.1 & 84 & 81.3 & 82.3 & 80.2 & 78.2 & 85 \\
\hline 0 & B & NS & 8/1/1992 & 92214 & 213 & 83.3 & 77.5 & 77.1 & 73.7 & 76.8 & 74.3 & 70.9 & 69.1 & 98.6 \\
\hline 0 & B & NS & $8 / 2 / 1992$ & 92215 & 214 & 73.3 & 72 & 72.9 & 69.2 & 69.6 & 72.2 & 68.5 & 64.3 & 74.1 \\
\hline 0 & B & NS & 8/8/1992 & 92221 & 220 & 76.4 & 75.8 & 71.9 & 72 & 69.5 & 69.6 & 67.4 & 70.2 & 235.7 \\
\hline 0 & B & NS & 8/9/1992 & 92222 & 221 & 76 & 76.1 & 74 & 73.7 & 70.9 & 74 & 71.3 & 70.2 & 78.9 \\
\hline 0 & B & NS & $8 / 15 / 1992$ & 92228 & 227 & 70.5 & 79.2 & 69.5 & 77.5 & 69.5 & 76.1 & 70.2 & 75.1 & 262.3 \\
\hline 0 & $B$ & NS & 8/16/1992 & 92229 & 228 & 70.8 & 67.1 & 67 & 63 & 65.1 & 65 & 60.9 & 61.9 & 66.8 \\
\hline 0 & $B$ & NS & $8 / 22 / 1992$ & 92235 & 234 & 67.8 & 68.1 & 66.8 & 65.7 & 65.4 & 66.4 & 64 & 63.9 & 235 \\
\hline 0 & $B$ & NS & 8/23/1992 & 92236 & 235 & 68.5 & 78.1 & 70.6 & 68.1 & 66.8 & 69.1 & 67.8 & 69.8 & 70.2 \\
\hline
\end{tabular}




\begin{tabular}{|c|c|c|c|c|c|c|c|c|c|c|c|c|c|c|}
\hline 0 & B & NS & 8/29/1992 & 92242 & 241 & 88.2 & 86.1 & 84.8 & 78.1 & 75.8 & 81.2 & 78.5 & 96.8 & 359.4 \\
\hline 0 & B & NS & 8/30/1992 & 92243 & 242 & 73 & 75.7 & 71.6 & 70.6 & 68.8 & 70.2 & 68.8 & 67.5 & 71.3 \\
\hline 0 & B & NS & 9/5/1992 & 92249 & 248 & 90.2 & 86.1 & 84.7 & 83.7 & 82.3 & 83.4 & 82.3 & 138.6 & 417.5 \\
\hline 0 & B & NS & 9/6/1992 & 92250 & 249 & 79.2 & 79.5 & 78.5 & 77.4 & 67.8 & 71.9 & 65.4 & 65.8 & 71.9 \\
\hline 0 & $B$ & NS & \#\#\#\#\#\#\#\# & 92354 & 353 & 59.5 & 59.4 & 55.7 & 55.7 & 55 & 56.1 & 64.3 & 78.5 & 155.5 \\
\hline 0 & $B$ & NS & \#\#\#\#\#\#\#\# & 92355 & 354 & 64 & 61.5 & 56.8 & 57 & 58.2 & 57.4 & 54.7 & 54.3 & 50.8 \\
\hline 0 & B & NS & \#\#\#\#\#\#\#\# & 92361 & 360 & 62.6 & 63.7 & 60.1 & 63 & 63.2 & 63 & 57.5 & 58.1 & 53 \\
\hline 0 & B & NS & \#\#\#\#\#\#\#\# & 92362 & 361 & 64.7 & 59.5 & 59.8 & 56.4 & 57.4 & 56.4 & 55 & 50.9 & 49.8 \\
\hline 0 & B & NS & $1 / 2 / 1993$ & 93002 & 2 & 67.1 & 59.1 & 50.5 & 51.8 & 51.6 & 54.6 & 48.5 & 49.1 & 44.7 \\
\hline 0 & B & NS & 1/3/1993 & 93003 & 3 & 58.8 & 57.1 & 56.7 & 55.7 & 58.4 & 54.4 & 54.3 & 52.2 & 49.5 \\
\hline 0 & $B$ & NS & $3 / 13 / 1993$ & 93072 & 72 & 68.2 & 69.5 & 66.8 & 66.7 & 66.1 & 65.3 & 65.1 & 63.2 & 60.2 \\
\hline 0 & $B$ & NS & $3 / 14 / 1993$ & 93073 & 73 & 66.4 & 67 & 65.4 & 65 & 64 & 65 & 63 & 61.5 & 59.9 \\
\hline 0 & B & NS & 3/20/1993 & 93079 & 79 & 68.4 & 66.7 & 60.2 & 60.5 & 59.2 & 59.4 & 74.1 & 78.8 & 71.3 \\
\hline 0 & B & NS & $3 / 21 / 1993$ & 93080 & 80 & 63.2 & 63.7 & 61.5 & 59.9 & 60.1 & 59.9 & 58.8 & 57.1 & 55.6 \\
\hline 0 & B & NS & 4/10/1993 & 93100 & 100 & 69.1 & 62.2 & 61.9 & 60.5 & 59.4 & 59.8 & 58.8 & 57.5 & 56.7 \\
\hline 0 & B & NS & 4/11/1993 & 93101 & 101 & 65.3 & 63.3 & 63.9 & 62.6 & 63.2 & 62.3 & 62.9 & 61.2 & 58.2 \\
\hline 0 & $B$ & NS & 5/29/1993 & 93149 & 149 & 65.3 & 60.2 & 55.3 & 54.4 & 52.5 & 53.2 & 54.4 & 59.8 & 58.1 \\
\hline 0 & $B$ & NS & $5 / 30 / 1993$ & 93150 & 150 & 65 & 62.2 & 54.4 & 53.6 & 53.2 & 57.1 & 62.2 & 52.2 & 49.9 \\
\hline 0 & B & NS & $6 / 5 / 1993$ & 93156 & 156 & 78.5 & 79.1 & 76.4 & 76 & 76.5 & 75 & 69.1 & 74 & 75 \\
\hline 0 & B & NS & 6/6/1993 & 93157 & 157 & 83.3 & 84 & 84.3 & 82 & 75.3 & 78.8 & 80.2 & 70.2 & 78.8 \\
\hline 0 & B & NS & 6/12/1993 & 93163 & 163 & 57.4 & 65 & 59.4 & 54.4 & 62.2 & 52.9 & 56 & 57.7 & 51.2 \\
\hline 0 & B & NS & 6/13/1993 & 93164 & 164 & 63.6 & 58.4 & 58.1 & 63.6 & 54.6 & 56.7 & 62.2 & 50.2 & 55.3 \\
\hline 0 & B & NS & 6/19/1993 & 93170 & 170 & 47 & 47 & 48.5 & 46 & 43.5 & 43.9 & 43.5 & 42.9 & 44.2 \\
\hline 0 & $B$ & NS & $6 / 20 / 1993$ & 93171 & 171 & 46.7 & 46.4 & 47.7 & 46.3 & 45.6 & 45.6 & 44.9 & 44.6 & 42.3 \\
\hline 0 & B & NS & $6 / 26 / 1993$ & 93177 & 177 & 55.3 & 53.9 & 60.5 & 56 & 57.4 & 53.7 & 56.3 & 54.6 & 1.4 \\
\hline 0 & B & NS & $6 / 27 / 1993$ & 93178 & 178 & 46 & 54.3 & 51.5 & 50.8 & 50.1 & 51.5 & 49.8 & 47.3 & 48.4 \\
\hline 0 & $B$ & NS & 7/3/1993 & 93184 & 184 & 48.7 & 51.8 & 50.5 & 46.3 & 47.3 & 46 & 47.3 & 45.3 & 43.9 \\
\hline 0 & B & NS & $7 / 4 / 1993$ & 93185 & 185 & 50.8 & 50.5 & 50.1 & 46.7 & 50.1 & 49.1 & 45.6 & 46 & 46.7 \\
\hline 0 & $B$ & NS & $7 / 10 / 1993$ & 93191 & 191 & 52.2 & 51.8 & 51.1 & 45.3 & 46.3 & 46 & 44.6 & 45.3 & 43.5 \\
\hline 0 & B & NS & 7/11/1993 & 93192 & 192 & 47.7 & 50.1 & 47 & 49.4 & 43.9 & 46 & 46.3 & 43.9 & 42.9 \\
\hline 0 & B & NS & $7 / 17 / 1993$ & 93198 & 198 & 63.9 & 48.7 & 48 & 46.1 & 43.5 & 47.3 & 48.4 & 55 & 56 \\
\hline 0 & B & NS & 7/18/1993 & 93199 & 199 & 46.7 & 45.6 & 50.8 & 43.2 & 45.3 & 39.4 & 42.5 & 39.4 & 41.5 \\
\hline 0 & B & NS & $7 / 24 / 1993$ & 93205 & 205 & 59.1 & 47.3 & 46.3 & 46.7 & 45.3 & 44 & 44.6 & 43.5 & 48.7 \\
\hline 0 & $B$ & NS & $7 / 25 / 1993$ & 93206 & 206 & 43.6 & 48 & 45.6 & 44.3 & 43.5 & 44.2 & 44.3 & 44.6 & 44.2 \\
\hline 0 & $B$ & NS & $7 / 31 / 1993$ & 93212 & 212 & 56.8 & 44.6 & 45.3 & 42.9 & 41.9 & 41.1 & 41.8 & 40.8 & 40.8 \\
\hline 0 & B & NS & 8/1/1993 & 93213 & 213 & 49.4 & 50.8 & 48 & 47.4 & 44.9 & 47 & 46 & 44.7 & 43.2 \\
\hline 0 & $B$ & NS & 8/7/1993 & 93219 & 219 & 49.1 & 48.1 & 50.1 & 47.7 & 47 & 46.1 & 48 & 45.7 & 48.7 \\
\hline
\end{tabular}




\begin{tabular}{|c|c|c|c|c|c|c|c|c|c|c|c|c|c|c|}
\hline 0 & $B$ & NS & $8 / 8 / 1993$ & 93220 & 220 & 49.5 & 47 & 47.3 & 45.3 & 46 & 45.6 & 45.6 & 43.5 & 43.9 \\
\hline 0 & B & NS & 8/14/1993 & 93226 & 226 & 58.1 & 59.4 & 58.8 & 56.7 & 54.3 & 51.2 & 53.9 & 57 & 60.5 \\
\hline 0 & B & NS & 8/15/1993 & 93227 & 227 & 55 & 55.3 & 54.6 & 52.3 & 53.2 & 53.2 & 49.9 & 49.4 & 48.7 \\
\hline 0 & B & NS & $8 / 21 / 1993$ & 93233 & 233 & 57 & 56.8 & 57.4 & 54.6 & 52.6 & 53.2 & 52.5 & 53.6 & 55.4 \\
\hline 0 & B & NS & $8 / 22 / 1993$ & 93234 & 234 & 59.1 & 59.1 & 58.8 & 58.4 & 55.6 & 53.3 & 56.3 & 55 & 57 \\
\hline 0 & B & NS & $8 / 28 / 1993$ & 93240 & 240 & 51.1 & 50.5 & 52.2 & 49.4 & 48.8 & 48.7 & 46.1 & 46.3 & 86.4 \\
\hline 0 & B & NS & $8 / 29 / 1993$ & 93241 & 241 & 50.8 & 49.5 & 50.5 & 48.7 & 46.1 & 47.3 & 50.1 & 48.5 & 65.3 \\
\hline 0 & B & NS & 9/4/1993 & 93247 & 247 & 70.2 & 120.6 & 136.5 & 133.9 & 132.2 & 130.8 & 137.2 & 129.1 & 144.3 \\
\hline 0 & B & NS & 9/5/1993 & 93248 & 248 & 59 & 51.8 & 53.2 & 50.8 & 49.8 & 49.5 & 52.5 & 51.8 & 63.9 \\
\hline 0 & B & NS & \#\#\#\#\#\#\#\# & 93331 & 331 & 42.9 & 44.6 & 43.5 & 42.9 & 42.5 & 42.2 & 42.2 & 42.5 & 41.1 \\
\hline 0 & $B$ & NS & \#\#\#\#\#\#\#\# & 93332 & 332 & 43.9 & 43.2 & 42.9 & 42.9 & 43.2 & 43.2 & 43.2 & 42.9 & 41.8 \\
\hline 0 & $B$ & NS & \#\#\#\#\#\#\#\# & 93352 & 352 & 65 & 45.6 & 43.2 & 42.2 & 41.8 & 41.9 & 39.1 & 40.8 & 43.9 \\
\hline 0 & $B$ & NS & \#\#\#\#\#\#\#\# & 93353 & 353 & 49.1 & 50.8 & 45.3 & 43.9 & 44.2 & 44.2 & 42.9 & 43.9 & 47.7 \\
\hline 0 & $B$ & NS & \#\#\#\#\#\#\#\# & 93359 & 359 & 40.1 & 41.1 & 44.6 & 44.2 & 43.5 & 43.5 & 42.5 & 42.9 & 44.2 \\
\hline 0 & $B$ & NS & \#\#\#\#\#\#\#\# & 93360 & 360 & 41.8 & 42.5 & 47 & 46.7 & 47 & 46.7 & 45.3 & 44.9 & 46 \\
\hline 0 & B & NS & $1 / 1 / 1994$ & 94001 & 1 & 41.8 & 40.1 & 43.2 & 42.2 & 42.5 & 42.5 & 42.5 & 44.9 & 44.7 \\
\hline 0 & $B$ & NS & $1 / 2 / 1994$ & 94002 & 2 & 41.8 & 40.4 & 46.3 & 45.3 & 45.3 & 45.6 & 45.3 & 44.9 & 46 \\
\hline 0 & $B$ & NS & $3 / 12 / 1994$ & 94071 & 71 & 55.3 & 54.6 & 55 & 54 & 54.6 & 53.9 & 70.2 & 66 & 63 \\
\hline 0 & $B$ & NS & $3 / 13 / 1994$ & 94072 & 72 & 50.5 & 51.8 & 50.5 & 50.2 & 51.5 & 51.8 & 66 & 79.6 & 88.1 \\
\hline 0 & $B$ & NS & $3 / 19 / 1994$ & 94078 & 78 & 74 & 67.7 & 50.1 & 49.1 & 50.1 & 49.4 & 48.7 & 55 & 48.7 \\
\hline 0 & $B$ & NS & $3 / 20 / 1994$ & 94079 & 79 & 55 & 55 & 53 & 53.2 & 52.5 & 53.6 & 46.3 & 44.6 & 44.6 \\
\hline 0 & $B$ & NS & $4 / 2 / 1994$ & 94092 & 92 & 45.3 & 45.3 & 45.6 & 44.6 & 45.3 & 44.9 & 43.9 & 41.5 & 42.5 \\
\hline 0 & $B$ & NS & $4 / 3 / 1994$ & 94093 & 93 & 45.6 & 44.2 & 44.9 & 44.9 & 45.6 & 45.3 & 44.6 & 44.6 & 41.8 \\
\hline 0 & $B$ & NS & $5 / 28 / 1994$ & 94148 & 148 & 126.5 & 123 & 116.8 & 80.2 & 59.1 & 59.1 & 59.1 & 121.1 & 230.4 \\
\hline 0 & B & NS & $5 / 29 / 1994$ & 94149 & 149 & 58.8 & 58.8 & 58.1 & 57 & 57.4 & 57 & 57.7 & 57 & 54.3 \\
\hline 0 & B & NS & $6 / 4 / 1994$ & 94155 & 155 & 85.4 & 126.7 & 108.9 & 68.4 & 58.4 & 57.4 & 57.7 & 70.5 & 135.1 \\
\hline & & & & & \multicolumn{3}{|c|}{ sort S/NS - 0/1 - A/B } & & & & & & & \\
\hline & & & & & \multicolumn{2}{|c|}{ Hourly Averages } & & & & & & & & \\
\hline & & & & & $1-A-S$ & 80.60111 & 78.06333 & 74.04333 & 71.12333 & 70.88333 & 70.80222 & 72.53667 & 155.2256 & 267.5822 \\
\hline & & & & & 1-B-S & 64.15964 & 63.11544 & 61.64329 & 60.12177 & 59.5119 & 60.05696 & 64.63013 & 108.8884 & 231.9041 \\
\hline & & & & & $0-A-S$ & 78.93333 & 73.38333 & 70.31667 & 68.41944 & 67.80278 & 68.35278 & 68.53889 & 72.64167 & 88.16111 \\
\hline & & & & & 0-B-S & 66.57467 & 63.37987 & 62.228 & 60.48 & 60.09333 & 60.56067 & 63.02267 & 71.118 & 107.7053 \\
\hline & & & & & $1-\mathrm{A}-\mathrm{NS}$ & 85.13421 & 83.07632 & 80.40132 & 79.97237 & 78.78158 & 77.50526 & 78.28947 & 109.5289 & 187.6539 \\
\hline & & & & & 1-B-NS & 65.08651 & 64.2107 & 62.83256 & 61.18186 & 60.29349 & 60.84372 & 62.01442 & 89.67907 & 176.4423 \\
\hline & & & & & $0-A-N S$ & 80.97941 & 80.81765 & 79.57941 & 77.88235 & 75.85588 & 76.12647 & 75.35588 & 73.89118 & 80.28529 \\
\hline & & & & & $0-\mathrm{B}-\mathrm{NS}$ & 64.47957 & 64.06237 & 62.22151 & 60.07204 & 58.86559 & 59.05484 & 59.04516 & 60.64516 & 86.8086 \\
\hline
\end{tabular}




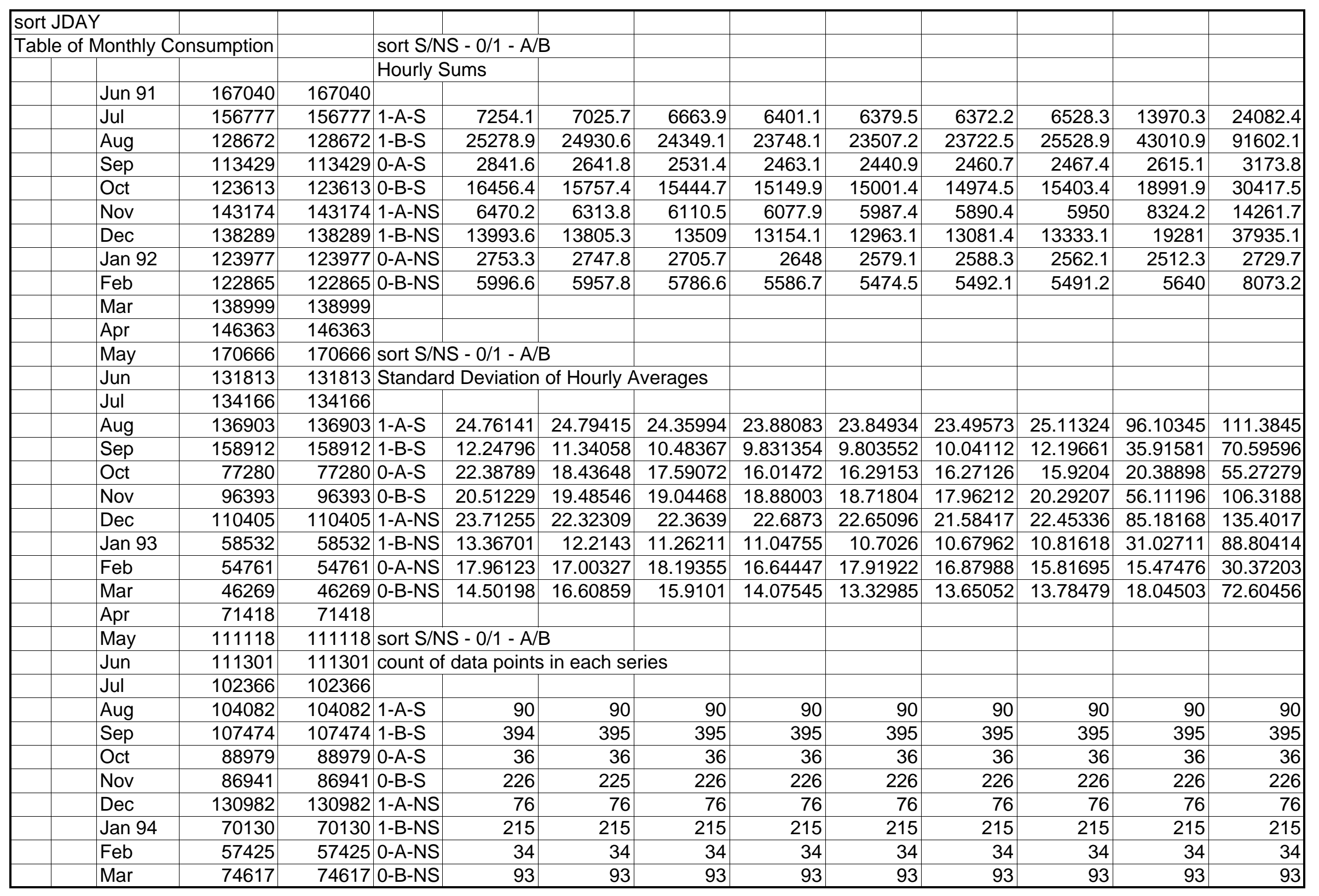

Page 31 


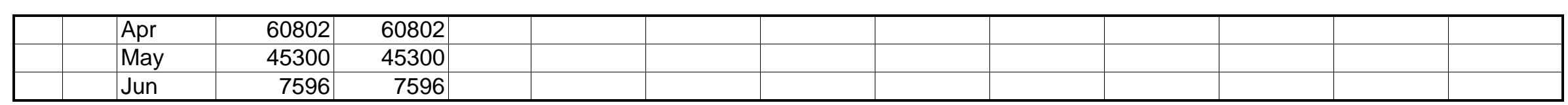




\begin{tabular}{|c|c|c|c|c|c|c|c|c|c|c|c|c|}
\hline 9 & 10 & 11 & 12 & 13 & 14 & 15 & 16 & 17 & 18 & 19 & 20 & 21 \\
\hline 598.9 & 601.3 & 616.9 & 630 & 586.8 & 585.8 & 565.1 & 522.9 & 313.8 & 253.7 & 210.5 & 183.9 & 175.2 \\
\hline 563.3 & 576.1 & 591.3 & 620.4 & 569.9 & 595.8 & 589.2 & 572.3 & 450 & 391.9 & 324.9 & 256.8 & 244 \\
\hline 558.8 & 564.4 & 588.9 & 625.9 & 578.9 & 585.8 & 568.9 & 499 & 317.6 & 256.4 & 204.9 & 137.2 & 105.4 \\
\hline 577.8 & 590.3 & 594.8 & 600 & 582.7 & 575.8 & 560.6 & 501.5 & 279.2 & 232.9 & 162.1 & 96.4 & 98.2 \\
\hline 597.5 & 620 & 610.3 & 629.3 & 602.7 & 605.8 & 584.8 & 539.5 & 277.5 & 220.8 & 188 & 151.7 & 134.1 \\
\hline 617.2 & 619.7 & 625.9 & 647.3 & 594.8 & 612.1 & 586.5 & 586.5 & 487 & 399.2 & 351.5 & 319 & 203.2 \\
\hline 587.5 & 591.3 & 603.4 & 626.9 & 595.8 & 580.6 & 576.1 & 538.4 & 297.2 & 249.9 & 212.9 & 149.3 & 141.7 \\
\hline 590.6 & 577.5 & 566.8 & 600.7 & 582.7 & 583 & 577.2 & 562.3 & 461 & 369.1 & 318 & 270.6 & 210.1 \\
\hline 573 & 589.2 & 604.8 & 630.7 & 598.2 & 586.8 & 582 & 534.6 & 278.2 & 226 & 176.6 & 116.1 & 112 \\
\hline 603.1 & 621.4 & 605.5 & 611 & 615.9 & 605.1 & 507.7 & 578.5 & 465.5 & 367.7 & 326.9 & 250.2 & 198 \\
\hline 602 & 608.3 & 616.2 & 645.6 & 614.5 & 604.8 & 592.7 & 579.9 & 458.3 & 383.3 & 333.9 & 272.7 & 199.8 \\
\hline 570.2 & 603.1 & 600 & 628.6 & 603.8 & 568.9 & 552.6 & 556.1 & 300.7 & 228.4 & 153.4 & 91.9 & 88.5 \\
\hline 526 & 516.7 & 511.8 & 535 & 499.4 & 480.4 & 475.9 & 456.9 & 366.3 & 311.7 & 258.2 & 180.7 & 120.3 \\
\hline 454.1 & 460.3 & 469.7 & 482.8 & 459.6 & 468.6 & 443.8 & 419.6 & 183.9 & 142 & 106.8 & 79.5 & 70.5 \\
\hline 574.4 & 588.2 & 579.6 & 605.1 & 604.1 & 588.6 & 568.9 & 541.6 & 434.8 & 356.3 & 282.4 & 237.4 & 189.7 \\
\hline 564.7 & 573.3 & 581.6 & 616.2 & 607.6 & 603.1 & 583.7 & 581.3 & 461 & 359.4 & 327.6 & 255.4 & 145.5 \\
\hline 463.8 & 480.4 & 491.4 & 491.8 & 487.3 & 500.1 & 478.7 & 453.8 & 242.6 & 191.8 & 140 & 101.3 & 112.7 \\
\hline 460 & 460.3 & 475.9 & 519.4 & 480.4 & 465.2 & 460.3 & 462.8 & 368.4 & 313.1 & 267.1 & 180.7 & 92.6 \\
\hline 485.9 & 500.4 & 501.5 & 531.2 & 516.3 & 501.5 & 505.6 & 477.3 & 247.8 & 208.4 & 132.7 & 78.8 & 78.1 \\
\hline \multirow[t]{2}{*}{485.6} & 504.9 & 521.2 & 550.5 & 508.4 & 505.3 & 506.6 & 512.9 & 392.6 & 312.1 & 250.6 & 209.4 & 94.7 \\
\hline & & & & & & & & 161.7 & 201.1 & 165.5 & 104.7 & 106.8 \\
\hline 525.7 & 542.6 & 563.3 & 592.4 & 569.2 & 554 & 554.7 & 499.4 & 299.3 & 240.2 & 189.7 & 142.7 & 144.1 \\
\hline 538.4 & 589.9 & 604.8 & 617.6 & 584.4 & 586.8 & 584.4 & 510.8 & 272.3 & 213.9 & 144.5 & 105.8 & 92.3 \\
\hline 452 & 453.8 & 468.6 & 484.2 & 448.2 & 447.9 & 434.8 & 426.1 & 343.5 & 285.1 & 247.4 & 194.2 & 140.7 \\
\hline 369 & 333 & 337.3 & 385.3 & 388.8 & 383.6 & 372.8 & 371.5 & 216.3 & 168.7 & 148.3 & 114.4 & 100.6 \\
\hline 341.3 & 307.2 & 326.1 & 383.7 & 398.4 & 355 & 364.2 & 367.6 & 281.2 & 209.1 & 153.4 & 105.1 & 91.6 \\
\hline 352.8 & 325.4 & 334.6 & 392.1 & 388.3 & 386.7 & 377.1 & 381.5 & 292.9 & 243.3 & 196.1 & 163.9 & 150.1 \\
\hline 334.5 & 377.7 & 417.5 & 447.5 & 417.4 & 420.3 & 412.1 & 379.5 & 208.7 & 180.7 & 138.2 & 123.7 & 108.2 \\
\hline 438.4 & 441.9 & 462.3 & 474.1 & 454.3 & 442.7 & 452.4 & 425.1 & 359 & 305.1 & 267.5 & 239.7 & 222.2 \\
\hline 404.6 & 405.9 & 424.1 & 430.8 & 396.2 & 399.1 & 403.3 & 399.7 & 328 & 263 & 272.5 & 345.8 & 356.6 \\
\hline 384.6 & 396.7 & 417.9 & 437 & 404.2 & 409.3 & 405.3 & 392.6 & 257.5 & 196 & 147.6 & 85 & 102.3 \\
\hline 386.6 & 416.3 & 438.8 & 467.8 & 425 & 413.3 & 402.7 & 396.1 & 310.5 & 245.7 & 214.5 & 178.3 & 137.2 \\
\hline
\end{tabular}


other

\begin{tabular}{|c|c|c|c|c|c|c|c|c|c|c|c|c|}
\hline 395.1 & 409.7 & 406.7 & 449.7 & 427.3 & 413.9 & 414.2 & 386.2 & 237.8 & 190.4 & 139.6 & 97.8 & 101.3 \\
\hline 380.7 & 395 & 397.6 & 430.9 & 403.6 & 393.9 & 370.1 & 373 & 220.5 & 187.7 & 163.8 & 118.5 & 121.7 \\
\hline 396.9 & 417 & 445.1 & 472.2 & 444.3 & 437.9 & 443.6 & 452 & 351.1 & 289.2 & 272.5 & 234.7 & 191.7 \\
\hline 448.1 & 452.2 & 478.3 & 502.7 & 469 & 444.5 & 455.6 & 439.4 & 346.5 & 299.1 & 241 & 196 & 122.2 \\
\hline 440.5 & 478.1 & 495.3 & 511.8 & 461.8 & 451.1 & 449 & 432.5 & 287.5 & 229.1 & 170.4 & 133.1 & 121 \\
\hline 333.9 & 464.8 & 478.1 & 486.8 & 514.6 & 476.3 & 465.5 & 441.8 & 420.9 & 344.9 & 285.5 & 243 & 209.2 \\
\hline 324.8 & 476.1 & 480.1 & 204.3 & 470.9 & 452.6 & 455.9 & 460.8 & 373.4 & 333.8 & 241.4 & 193.1 & 142.7 \\
\hline 324.9 & 323.1 & 346.6 & 363.4 & 358.9 & 359.6 & 345.6 & 330.1 & 222.9 & 180.1 & 136.2 & 84.4 & 77.1 \\
\hline 298.6 & 310.7 & 361.5 & 401 & 336.6 & 300.1 & 287.5 & 286.8 & 203.9 & 165.9 & 136.2 & 108.9 & 105.2 \\
\hline 318.6 & 322.1 & 347.7 & 367.7 & 322.1 & 293.1 & 276.8 & 274.8 & 200.2 & 162.8 & 147.6 & 98.2 & 80.6 \\
\hline 365 & 361.2 & 362.9 & 389.5 & 357.7 & 334.9 & 321.8 & 315.9 & 231.6 & 191.8 & 170.7 & 108.9 & 72.9 \\
\hline 360.5 & 360.8 & 379.5 & 378.1 & 339.4 & 324.2 & 298.6 & 296.2 & 219.8 & 184.6 & 171.4 & 111.7 & 99.2 \\
\hline 292.4 & 333.2 & 371.9 & 372.8 & 344.2 & 292 & 297.7 & 320.6 & 252.7 & 229 & 205.4 & 151.4 & 131 \\
\hline 348.9 & 352.7 & 370.1 & 391.9 & 373.5 & 348.5 & 346.2 & 343.8 & 227.2 & 173.6 & 152.4 & 116.6 & 102.3 \\
\hline 338.7 & 345.3 & 331.5 & 360.5 & 328 & 309.7 & 301.8 & 292.4 & 184.9 & 147.7 & 122.3 & 72 & 74.3 \\
\hline 295.6 & 293.8 & 305.2 & 387.9 & 359.5 & 332.9 & 342.2 & 316.2 & 197.4 & 160.4 & 139 & 78.5 & 65.4 \\
\hline 285.5 & 295.8 & 333.9 & 393.5 & 362.7 & 343.1 & 339.9 & 328.5 & 240.7 & 201.4 & 175.2 & 168.8 & 141.3 \\
\hline 302.1 & 358.8 & 366.8 & 396.5 & 360.8 & 340.5 & 333.1 & 337.8 & 264.6 & 216.1 & 180.6 & 156.8 & 139.1 \\
\hline 347.5 & 353.7 & 385 & 404.3 & 374.2 & 347.5 & 346.3 & 344.3 & 265.6 & 225.3 & 187.4 & 167.2 & 138.6 \\
\hline 364.3 & 388.1 & 404.6 & 414.9 & 397.5 & 390.2 & 389.4 & 363.2 & 223.3 & 146.5 & 135.9 & 120.3 & 92.7 \\
\hline 297 & 300.7 & 323.8 & 375.7 & 363.2 & 349.9 & 353.1 & 359.4 & 227.8 & 166.7 & 170.7 & 168.3 & 131.8 \\
\hline 404.6 & 414.4 & 436.6 & 468.4 & 439.4 & 436 & 430.5 & 426.1 & 321 & 261.5 & 271.1 & 254.1 & 209.3 \\
\hline 297.9 & 322.2 & 323.5 & 369.2 & 335.6 & 313.9 & 307.2 & 304.9 & 199.8 & 177.7 & 149.6 & 122.1 & 72.2 \\
\hline 324.2 & 320.5 & 333.2 & 351.5 & 316 & 309.7 & 311.5 & 316.9 & 196.7 & 168.3 & 175 & 153.1 & 91.7 \\
\hline 305.6 & 312.1 & 324.9 & 340.5 & 301 & 291.4 & 285.8 & 255.1 & 166.9 & 142.8 & 132.7 & 56.4 & 74.7 \\
\hline 340.1 & 340.5 & 320.4 & 357.1 & 319.7 & 299.3 & 298.7 & 304.5 & 207.5 & 175.6 & 148 & 127.9 & 82 \\
\hline 305.5 & 312.9 & 341.8 & 339.8 & 309 & 320.2 & 338.3 & 291.5 & 172.5 & 144.2 & 133.1 & 121.7 & 62.9 \\
\hline 345 & 352.2 & 346.4 & 375 & 340.1 & 327.4 & 314.1 & 309.1 & 196.3 & 172.5 & 144.5 & 144.9 & 99.2 \\
\hline 355 & 370.1 & 375.4 & 389.1 & 341.9 & 331.1 & 321.5 & 307.6 & 223 & 196.3 & 189.5 & 180.1 & 141.4 \\
\hline 353.5 & 358.8 & 359.8 & 371.3 & 331.4 & 322.2 & 320.7 & 308 & 210.5 & 176 & 143.8 & 115.9 & 78.1 \\
\hline 308 & 320.4 & 337.4 & 360.8 & 326 & 336.4 & 317 & 327 & 186.6 & 148 & 127.2 & 104.5 & 72 \\
\hline 299.4 & 310.8 & 322.2 & 386.4 & 348.4 & 342.9 & 342.8 & 334.7 & 177.2 & 139 & 118.5 & 97.2 & 85.4 \\
\hline 381.9 & 393.7 & 412.1 & 429.5 & 400.6 & 377.8 & 384.1 & 367.7 & 228.9 & 186.7 & 159.3 & 132.8 & 77.4 \\
\hline 319.8 & 321.4 & 375.6 & 418.8 & 380.7 & 362.2 & 364.1 & 319.9 & 183.6 & 156.9 & 141.8 & 120.6 & 81.7 \\
\hline 373.6 & 382.1 & 418.1 & 416.6 & 379.9 & 371.4 & 378 & 382 & 219.9 & 183.2 & 164.6 & 145.5 & 115.5 \\
\hline 397.8 & 395.8 & 425.7 & 442.5 & 405.5 & 400.5 & 390.1 & 381.1 & 259.7 & 223.2 & 228.4 & 199.1 & 105.3 \\
\hline 340.4 & 346 & 353.9 & 383.7 & 342.5 & 362.1 & 375.7 & 366.5 & 203.6 & 170.1 & 157.2 & 137.3 & 78.5 \\
\hline
\end{tabular}

Page 34 
other

\begin{tabular}{|c|c|c|c|c|c|c|c|c|c|c|c|c|}
\hline 360.1 & 350.5 & 364.3 & 383.4 & 335 & 319 & 320.5 & 318.3 & 243.7 & 186.7 & 162.1 & 129.3 & 96.4 \\
\hline 326.7 & 339.1 & 362.5 & 372.3 & 329.7 & 326 & 325.6 & 316.3 & 202.2 & 176.7 & 149 & 131.1 & 76.7 \\
\hline 305.6 & 372.1 & 392.6 & 400.1 & 363.4 & 342.9 & 349.1 & 326.6 & 207.5 & 163.1 & 143.2 & 132.7 & 68.2 \\
\hline 297.9 & 347.1 & 375.4 & 393.5 & 369.5 & 332.2 & 329.4 & 337.5 & 257.4 & 216.9 & 187.3 & 172 & 145.6 \\
\hline 302.1 & 315.6 & 326.9 & 346.4 & 303.4 & 291.8 & 290.6 & 286.2 & 189.4 & 157.7 & 133.5 & 116.5 & 61.3 \\
\hline 310 & 318.7 & 325.2 & 336 & 296.2 & 298.3 & 303.4 & 295.2 & 185.7 & 163.1 & 166.3 & 157.9 & 116.2 \\
\hline 311.7 & 319.8 & 337.3 & 345.7 & 306.2 & 290 & 289.3 & 282.4 & 197.1 & 159 & 136.3 & 118.2 & 79.6 \\
\hline 342.5 & 339.8 & 355.6 & 361.9 & 327 & 313.1 & 308 & 289.3 & 189.8 & 159.1 & 153.4 & 130 & 65.7 \\
\hline 55.3 & 65.8 & 58.5 & 62.6 & 64.7 & 66.8 & 70.2 & 73.4 & 75.3 & 73 & 75.1 & 60.5 & 55.4 \\
\hline 341.1 & 337.1 & 340.1 & 368.5 & 332.8 & 321.2 & 328.7 & 303.5 & 204 & 171.1 & 141.8 & 130.3 & 75.1 \\
\hline 335.6 & 351.9 & 360.9 & 353.5 & 319.4 & 314.8 & 307 & 290.6 & 214.7 & 180.5 & 166.9 & 138.3 & 68.1 \\
\hline 332.9 & 349.8 & 359.1 & 340.9 & 301.7 & 295.2 & 310 & 293.9 & 214 & 179.4 & 169.8 & 147.6 & 90.6 \\
\hline 86.1 & 98.8 & 97.2 & 90.5 & 88.2 & 86.5 & 91.9 & 73.7 & 63 & 70.2 & 73.7 & 69.9 & 77.8 \\
\hline 369.8 & 360.2 & 369.8 & 377.5 & 351.9 & 323.1 & 311.5 & 306.5 & 196 & 165.3 & 139.3 & 112.4 & 54.4 \\
\hline 343.3 & 345.3 & 373.7 & 374 & 332.1 & 316.3 & 306.2 & 297.7 & 209.2 & 188.4 & 172.5 & 165.2 & 121.4 \\
\hline 317.6 & 327.7 & 339.8 & 367 & 328.8 & 296.2 & 295.2 & 270.4 & 193.9 & 171.5 & 143.5 & 112.3 & 57.8 \\
\hline 335.9 & 346.4 & 344.9 & 379.6 & 333.9 & 316.9 & 305.9 & 280.7 & 193.5 & 162.2 & 137.6 & 103.7 & 54.7 \\
\hline 50.9 & 50.9 & 52.2 & 53 & 60.2 & 64.7 & 61.5 & 62.6 & 55.7 & 53.9 & 58.2 & 57.8 & 58.1 \\
\hline 301.8 & 311 & 323.2 & 343.3 & 300.7 & 286.2 & 279.9 & 265.2 & 198.1 & 164.2 & 138 & 112.4 & 54.3 \\
\hline 288.7 & 318.4 & 304.1 & 351.6 & 313.8 & 297 & 303.9 & 295.1 & 221.3 & 198.1 & 168.3 & 158.4 & 116.1 \\
\hline 325.3 & 340.4 & 356.4 & 375.7 & 335.7 & 317.7 & 326.2 & 322.2 & 216.1 & 181.4 & 150.1 & 111.6 & 64.7 \\
\hline 305.9 & 327.7 & 349.1 & 348 & 297.7 & 273.8 & 283.7 & 263.1 & 188.8 & 162.8 & 152.5 & 130 & 76.7 \\
\hline 53 & 50.5 & 53.6 & 74.7 & 77.9 & 79.5 & 80.6 & 84.1 & 82.9 & 82 & 81.3 & 67 & 58.5 \\
\hline 312.8 & 51.8 & 330.1 & 358.7 & 322.2 & 310.8 & 311 & 297 & 232.2 & 204.7 & 168.1 & 125.5 & 68.9 \\
\hline 316.6 & 329.8 & 339.5 & 352.5 & 307.7 & 317.3 & 303.2 & 290 & 233.6 & 196 & 189.8 & 173.8 & 139.4 \\
\hline 360.2 & 359.8 & 353.6 & 361.9 & 343.2 & 327.7 & 315.3 & 309.3 & 185.3 & 160.5 & 140.3 & 106.2 & 55.7 \\
\hline 336.7 & 345.6 & 355.4 & 377.5 & 330.4 & 325.3 & 319.8 & 302.7 & 220.9 & 186.7 & 160.4 & 139.4 & 92.7 \\
\hline 53 & 63.6 & 60.9 & 64.7 & 67 & 76.8 & 74.4 & 80.3 & 84 & 82.3 & 67.1 & 68.8 & 65.8 \\
\hline 310 & 322.5 & 323.6 & 349.7 & 309.7 & 307.9 & 303.9 & 290 & 208.4 & 173.6 & 148.4 & 125.5 & 72 \\
\hline 308.4 & 358.1 & 404.7 & 403.9 & 356.6 & 344.8 & 352.3 & 316.4 & 184.2 & 164.3 & 151.1 & 143.8 & 105.8 \\
\hline 376.3 & 366.5 & 404.5 & 411 & 360.9 & 355.1 & 345.1 & 314.8 & 216.4 & 183.9 & 157.3 & 124.2 & 73.3 \\
\hline 342.7 & 360.6 & 384.1 & 400 & 347.6 & 337.5 & 343.2 & 312.5 & 199.2 & 158.3 & 153.5 & 124.9 & 76.7 \\
\hline 72.7 & 77.5 & 104.4 & 122.9 & 109.6 & 106.4 & 108.3 & 96.5 & 87.8 & 88.2 & 75.8 & 73.3 & 70.9 \\
\hline 317.4 & 344.6 & 345.3 & 393.4 & 338 & 343.7 & 369.2 & 351.8 & 209.5 & 175.9 & 158.7 & 152.8 & 82 \\
\hline 355 & 370.8 & 395.1 & 402.7 & 358.3 & 355.6 & 351.9 & 343.6 & 187.9 & 141.4 & 147.7 & 150.8 & 111.3 \\
\hline 341.5 & 347.1 & 362.8 & 426.5 & 361 & 362 & 354.6 & 331.5 & 244.6 & 198.9 & 171.9 & 153.9 & 60.5 \\
\hline 309.7 & 326.9 & 372.9 & 402.2 & 360.8 & 348.1 & 331.2 & 323.9 & 248.4 & 215.4 & 193.1 & 181.9 & 105.8 \\
\hline
\end{tabular}




\begin{tabular}{|c|c|c|c|c|c|c|c|c|c|c|c|c|}
\hline 104.1 & 106.4 & 101.7 & 109 & 112.7 & 104.5 & 90.2 & 78.2 & 65.4 & 62.9 & 70.6 & 67.4 & 60.6 \\
\hline 318.4 & 333.2 & 367 & 416.4 & 375.8 & 350.8 & 334.7 & 291.4 & 194.2 & 167.4 & 141.4 & 117.8 & 59.2 \\
\hline 319.7 & 339.8 & 344.9 & 360.2 & 330.7 & 319.8 & 309.7 & 306.9 & 211.6 & 183.2 & 153.5 & 136.9 & 87.5 \\
\hline 317.6 & 351.9 & 366.3 & 371.6 & 329 & 311.5 & 287.3 & 270.6 & 199.2 & 150.3 & 118.3 & 113.1 & 83.3 \\
\hline 332.9 & 336.6 & 356.8 & 358.4 & 312.9 & 312.2 & 289.3 & 278 & 183.5 & 142.5 & 131.1 & 119.9 & 63.7 \\
\hline 58.1 & 55.7 & 58.8 & 64.6 & 59.5 & 63.7 & 69.1 & 66.1 & 64.4 & 65.7 & 61.6 & 67.4 & 63.7 \\
\hline 376.3 & 372.8 & 385.2 & 402.3 & 365 & 359.2 & 341.2 & 321.8 & 206.7 & 163.9 & 145.8 & 140.1 & 93.3 \\
\hline 368.2 & 370.1 & 403.5 & 413.7 & 366.4 & 363.2 & 347.6 & 325.2 & 208 & 164.5 & 147 & 146.5 & 110.7 \\
\hline 377.4 & 399.3 & 413.3 & 438 & 393.3 & 381.7 & 377.8 & 354.1 & 209.4 & 146.6 & 146.5 & 132.5 & 80.3 \\
\hline 387.6 & 409.5 & 420 & 437.3 & 408.2 & 389.6 & 389.1 & 368.9 & 232.1 & 178 & 147.3 & 148.3 & 86.1 \\
\hline 64.7 & 69.2 & 70.5 & 70.6 & 74.4 & 75.3 & 67.8 & 80.5 & 63.3 & 64 & 74.7 & 73 & 72.9 \\
\hline 408 & 422.3 & 393.7 & 450.7 & 398.2 & 397.3 & 392.8 & 372.4 & 223 & 170.7 & 149.4 & 135.5 & 102.4 \\
\hline 354.7 & 356.3 & 371.3 & 368.1 & 331.2 & 328.8 & 319.7 & 300.4 & 201.8 & 167.4 & 148.7 & 137.9 & 89.9 \\
\hline 345 & 345.6 & 355.4 & 361.2 & 319.4 & 310.3 & 295.2 & 283.1 & 185.2 & 152.5 & 126.5 & 113.4 & 56.1 \\
\hline 54 & 57.1 & 64.6 & 64 & 60.1 & 55 & 57.8 & 58.8 & 53.7 & 58.1 & 54.4 & 58.5 & 58.8 \\
\hline 326.6 & 320.5 & 338 & 352.9 & 325.2 & 321.2 & 317.3 & 334.7 & 199.3 & 147.6 & 133.1 & 115.8 & 75.4 \\
\hline 321.5 & 346.1 & 382.2 & 384.1 & 346.5 & 357.9 & 347.1 & 332.2 & 199.6 & 164.2 & 139.4 & 117.6 & 90.9 \\
\hline 341.3 & 371.2 & 383.7 & 391.6 & 359 & 374.9 & 366.6 & 362.5 & 255.5 & 200.8 & 159.1 & 138 & 89.2 \\
\hline 361 & 372.6 & 399.3 & 422.5 & 374.7 & 390 & 372 & 339.9 & 209.8 & 164.3 & 156 & 134.1 & 97.6 \\
\hline 65.1 & 61.6 & 66.4 & 53.7 & 51.9 & 58.8 & 54 & 63.6 & 65.8 & 63 & 67.4 & 61.6 & 72.2 \\
\hline 304.5 & 300.4 & 322.1 & 371.4 & 354.9 & 356.3 & 344.5 & 343.7 & 203.7 & 163.2 & 136.2 & 113.4 & 68.9 \\
\hline 319.8 & 329 & 369.7 & 390.7 & 343.7 & 377.5 & 363.6 & 340.4 & 216 & 164.2 & 138 & 121.1 & 78.8 \\
\hline 331.8 & 349.1 & 358.4 & 354.3 & 315.9 & 347.7 & 366.9 & 344.5 & 217.1 & 183.3 & 159 & 112.4 & 74 \\
\hline 354.2 & 356.1 & 364.4 & 372.2 & 333.6 & 323.5 & 315.6 & 295.8 & 210.9 & 161.7 & 136.3 & 112.4 & 71.9 \\
\hline 47.4 & 46.7 & 47.3 & 47.8 & 47.8 & 51.8 & 58.5 & 56.8 & 63.6 & 60.6 & 66.4 & 73.7 & 72 \\
\hline 369.5 & 377.6 & 396.7 & 352 & 353.8 & 356.4 & 335.2 & 199.1 & 163.9 & 134.9 & 124.4 & 85.5 & 81.2 \\
\hline 328.4 & 352.4 & 338.8 & 355.7 & 381.3 & 379.9 & 361.2 & & 140.3 & 186.6 & 144.2 & 136.9 & 99.9 \\
\hline 362.8 & 345.4 & 365.4 & 361.2 & 366 & 380.8 & 376.4 & 365.7 & 229.5 & 190.9 & 126.2 & 105.8 & 81.7 \\
\hline 401.3 & 409.7 & 419.1 & 434.6 & 399.3 & 381.7 & 378.7 & 369.8 & 229.4 & 180.8 & 130 & 106.1 & 83.7 \\
\hline 61.6 & 53.3 & 56 & 62.6 & 60.9 & 75 & 69.2 & 73.3 & 79.9 & 76.5 & 71.2 & 69.6 & 77.9 \\
\hline 409.5 & 412.6 & 431.9 & 449.1 & 399.8 & 410.9 & 403.2 & 381.2 & 288.5 & 235.2 & 194.5 & 174.1 & 100.9 \\
\hline 385.7 & 409.3 & 433.9 & 437.1 & 401.8 & 407.7 & 389.3 & 372 & 237.9 & 229.8 & 198.9 & 164.5 & 81.2 \\
\hline 417.2 & 423.3 & 417.3 & 426.8 & 398.3 & 404.2 & 396.8 & 379.9 & 298.1 & 245.1 & 212.5 & 202.3 & 164.9 \\
\hline 117.6 & 115.5 & 115.4 & 113.1 & 97.8 & 129 & 134.9 & 137.2 & 112.8 & 85.1 & 84 & 86.5 & 85.7 \\
\hline 379.3 & 363.4 & 332.9 & 407.8 & 382.3 & 394.5 & 375.9 & 334.4 & 231 & 183.9 & 141.1 & 125.8 & 101 \\
\hline 383.6 & 399.4 & 410 & 422.3 & 380.1 & 395.2 & 378.1 & 371 & 265.7 & 223.6 & 201.4 & 184.5 & 122.1 \\
\hline 388.2 & 409.6 & 418.8 & 443.8 & 420 & 443.9 & 439.2 & 411 & 251.3 & 207.8 & 176.6 & 160.5 & 127.6 \\
\hline
\end{tabular}


other

\begin{tabular}{|c|c|c|c|c|c|c|c|c|c|c|c|c|}
\hline 60.6 & 61.9 & 70.9 & 70.6 & 77.1 & 86.1 & 91 & 149.2 & 151.3 & 99.1 & 80.3 & 78.2 & 83.3 \\
\hline 344.7 & 349.3 & 347.7 & 350.1 & 298.6 & 272.3 & 284 & 271.4 & 161.2 & 130.6 & 123.5 & 127.3 & 132 \\
\hline 392.3 & 401.2 & 417.9 & 429.2 & 410.1 & 423.6 & 408.5 & 413.5 & 334.1 & 278 & 221.1 & 202.2 & 164.2 \\
\hline 391.5 & 397.2 & 399.5 & 429.6 & 404.8 & 393.2 & 389 & 376.5 & 269.2 & 225.7 & 217.6 & 194.7 & 131.7 \\
\hline 78.8 & 79.2 & 83.7 & 85 & 77.5 & 84 & 86.1 & 89.3 & 89.9 & 86.1 & 100.6 & 77.9 & 87.1 \\
\hline 383.9 & 384.2 & 393.6 & 417.9 & 387 & 410.3 & 407.2 & 386.2 & 283.1 & 225.6 & 200.7 & 182.4 & 131.2 \\
\hline 376.4 & 373.9 & 414 & 432.9 & 422.3 & 398.6 & 401.1 & 388.1 & 304.7 & 237.3 & 210.2 & 199.3 & 143.3 \\
\hline 403.5 & 431.2 & 454.6 & 454.2 & 418.9 & 385.9 & 383.9 & 379.3 & 249.5 & 163.2 & 125.8 & 120 & 72.9 \\
\hline 360 & 381.9 & 404.9 & 409.8 & 383.4 & 372.6 & 352.3 & 356.6 & 248.5 & 181.1 & 155.7 & 154 & 115.8 \\
\hline 65.8 & 61.9 & 60.6 & 58.5 & 65.7 & 67.1 & 71.2 & 78.5 & 72.3 & 76 & 72.7 & 69.8 & 70.2 \\
\hline 438.5 & 430.2 & 431.9 & 454.4 & 431.9 & 437.4 & 420 & 422.1 & 320 & 246.3 & 216 & 190.2 & 100.8 \\
\hline 450.7 & 447.9 & 459 & 469.5 & 439.9 & 440.4 & 435.7 & 419.7 & 299.2 & 231.9 & 200.2 & 176 & 149.7 \\
\hline 422.7 & 450.9 & 467.3 & 494.8 & 447.3 & 440.1 & 436.5 & 415.8 & 341 & 249.7 & 211.9 & 175.7 & 140.9 \\
\hline 435.9 & 446.7 & 472.1 & 481.6 & 442.2 & 430.8 & 417.5 & 407.1 & 260.9 & 152.4 & 107.6 & 92.7 & 76.7 \\
\hline 67.8 & 69.5 & 67.8 & 69.2 & 64.3 & 67.1 & 69.5 & 76.1 & 89.6 & 91.2 & 70.2 & 73 & 84.3 \\
\hline 421.9 & 420.5 & 424.7 & 456.8 & 431.3 & 424.9 & 413.9 & 401.4 & 316.2 & 240.4 & 212 & 193.8 & 156.1 \\
\hline 418.3 & 432.5 & 450.3 & 473.4 & 426.5 & 422.7 & 416.6 & 406.4 & 278 & 199.1 & 166.6 & 174 & 91.3 \\
\hline 425.9 & 435.5 & 434.9 & 468.5 & 443.5 & 435.2 & 406 & 390.9 & 310.8 & 257.9 & 213.9 & 198.4 & 140.7 \\
\hline 437.8 & 438.4 & 454.5 & 481.5 & 440.3 & 434.6 & 430.5 & 423.4 & 314.1 & 208.8 & 179.3 & 167.7 & 145.5 \\
\hline 67.1 & 77.2 & 88.8 & 83 & 81.9 & 84.1 & 84.7 & 88.2 & 87.5 & 84.3 & 82.7 & 82.3 & 85.7 \\
\hline 444.6 & 458.2 & 469.7 & 481.9 & 441.2 & 452 & 435 & 413.3 & 238.1 & 155.3 & 124.1 & 105.5 & 80.9 \\
\hline 469.7 & 462 & 471.7 & 477.4 & 445.7 & 445 & 437.2 & 421.4 & 311.7 & 228.6 & 181.4 & 165.1 & 98.2 \\
\hline 120 & 128.2 & 134.9 & 196.6 & 199.2 & 203.9 & 215.3 & 186 & 131.7 & 122.8 & 114.7 & 94.4 & 94.7 \\
\hline 515.8 & 501.8 & 512.9 & 512 & 495.8 & 486.1 & 484.7 & 459.7 & 306.3 & 235.7 & 267.6 & 174.9 & 149 \\
\hline 546.1 & 528.4 & 520.4 & 527.7 & 499.9 & 488.8 & 498.8 & 479.4 & 327.6 & 258.2 & 222.7 & 152.4 & 96.9 \\
\hline 534.9 & 535.5 & 524.8 & 518.4 & 501.1 & 485.1 & 484.1 & 441.5 & 310.7 & 280.6 & 233.7 & 173.8 & 106.5 \\
\hline 86.5 & 86.5 & 138.6 & 137.5 & 136.3 & 127.5 & 117.2 & 136.6 & 100.6 & 78.2 & 79.1 & 76.5 & 80.5 \\
\hline 316.6 & 291.2 & 283 & 281.1 & 271.7 & 284.8 & 285.3 & 299.9 & 262.9 & 204.9 & 142.1 & 120.6 & 105.2 \\
\hline 481.5 & 470 & 478.5 & 484.3 & 470.1 & 443.8 & 413.8 & 442.5 & 332.9 & 235.4 & 185.7 & 135.8 & 88.2 \\
\hline 483.1 & 477.5 & 497.2 & 493.7 & 484.6 & 466 & 476.4 & 455.8 & 322.7 & 251 & 216 & 163.1 & 85.8 \\
\hline 81.7 & 94 & 96.5 & 89.5 & 111.7 & 126.5 & 133.1 & 132.1 & 113.7 & 93.1 & 96.8 & 88.6 & 89.9 \\
\hline 553.1 & 520.4 & 505.4 & 526.1 & 496 & 495.9 & 488.3 & 478.9 & 367.3 & 333.7 & 276 & 226.7 & 152.7 \\
\hline 511.2 & 502.5 & 512.7 & 517.1 & 507 & 484.5 & 467.9 & 406.8 & 276.5 & 223.6 & 186.7 & 128.6 & 94.1 \\
\hline 438.8 & 460.3 & 477.3 & 475.6 & 464.5 & 448.6 & 445.9 & 444.7 & 331.5 & 242.6 & 175.3 & 112.7 & 94.7 \\
\hline 455.4 & 433.1 & 441.6 & 453 & 431.5 & 415 & 407.1 & 406.4 & 292.1 & 229.5 & 172.9 & 133.4 & 108.2 \\
\hline 79.1 & 85.5 & 86.7 & 107.6 & 133.1 & 102.6 & 79.8 & 85.1 & 88.5 & 86.1 & 84 & 75 & 76.5 \\
\hline 473 & 464.3 & 465.6 & 473.5 & 451.3 & 439.3 & 443.7 & 425 & 306.6 & 262.7 & 241.3 & 189 & 143 \\
\hline
\end{tabular}


other

\begin{tabular}{|c|c|c|c|c|c|c|c|c|c|c|c|c|}
\hline 415.6 & 420.2 & 434.7 & 428.4 & 406.4 & 398.8 & 407.7 & 396.4 & 271.4 & 184.9 & 172.2 & 141.7 & 95.1 \\
\hline 366.4 & 383.6 & 408.1 & 414.8 & 407 & 393.7 & 385.1 & 362.8 & 236.8 & 151 & 140.4 & 95.4 & 71.2 \\
\hline 356.4 & 404.8 & 423.6 & 420.2 & 391.6 & 398 & 394.9 & 376.4 & 283.9 & 207.4 & 191.2 & 136.9 & 87.5 \\
\hline 52.5 & 62.6 & 62.9 & 63 & 65.3 & 101 & 110.6 & 109.9 & 104.8 & 73.3 & 59.5 & 57.4 & 57.1 \\
\hline 389.4 & 415.2 & 405.7 & 440.1 & 401.7 & 380.3 & 398.5 & 386.8 & 242.3 & 162.8 & 181.7 & 207.8 & 177.8 \\
\hline 423.1 & 422.5 & 434 & 432.9 & 404.1 & 407.2 & 408.9 & 396.9 & 305 & 272.2 & 283 & 369 & 359.2 \\
\hline 430.8 & 432.3 & 451.2 & 446.8 & 442.9 & 426.8 & 437.9 & 404.7 & 233.8 & 167.6 & 184.6 & 157.9 & 114.1 \\
\hline 360.6 & 356.3 & 390 & 396.9 & 371.5 & 354.9 & 369.5 & 361.7 & 218.9 & 158.4 & 145.8 & 144.2 & 116.5 \\
\hline 219.9 & 214.3 & 199.8 & 200.9 & 194.9 & 203.2 & 211.7 & 226.4 & 172.8 & 138.2 & 131.1 & 123.7 & 109.6 \\
\hline 224.8 & 235.8 & 260.7 & 257.8 & 243.3 & 252.5 & 265.9 & 269.9 & 200.5 & 157.3 & 151.4 & 128.9 & 116.2 \\
\hline 421.3 & 434.3 & 444.6 & 460.9 & 452.8 & 443.8 & 441.9 & 418.8 & 262.7 & 163.1 & 145.6 & 118.5 & 83.6 \\
\hline 434.9 & 463.3 & 477.4 & 488.5 & 466.2 & 452.2 & 457.6 & 438.5 & 303.4 & 215.7 & 184.3 & 153.4 & 104.8 \\
\hline 71.6 & 77.4 & 72 & 71.5 & 69.9 & 78.1 & 115.2 & 112.3 & 78.9 & 62.6 & 62.3 & 71.5 & 71.6 \\
\hline 385.7 & 406.1 & 411.9 & 436.5 & 401.9 & 394.9 & 388.3 & 368.1 & 219.2 & 174.2 & 166.7 & 138.9 & 114.5 \\
\hline 404.4 & 424.7 & 446.1 & 418.3 & 392.6 & 408.8 & 405.9 & 381.1 & 231.7 & 185.2 & 174.6 & 218.2 & 168.4 \\
\hline 389.5 & 428 & 442.4 & 429.9 & 402.3 & 403.5 & 408.1 & 388.3 & 232.4 & 167.7 & 141 & 113.8 & 80.2 \\
\hline 430.2 & 454.6 & 465.8 & 464.8 & 435.9 & 421.8 & 424.9 & 397 & 246.5 & 178.1 & 147.2 & 127.9 & 99.6 \\
\hline 72.6 & 73.7 & 82.3 & 82.7 & 103 & 135.6 & 130.3 & 121.7 & 80.5 & 76.8 & 70.5 & 78.5 & 83.7 \\
\hline 274.1 & 237.2 & 448.3 & 451.7 & 443.4 & 437.1 & 443.3 & 412 & 265.2 & 200.4 & 216.8 & 162.4 & 112.1 \\
\hline 410.5 & 417.8 & 417.6 & 437.9 & 420.4 & 421.9 & 414.8 & 387.4 & 239.5 & 194.7 & 187.3 & 162.4 & 133.1 \\
\hline 413.7 & 431.6 & 444.6 & 454.4 & 430.6 & 420.2 & 426 & 396.9 & 247.2 & 203.2 & 184.2 & 127.5 & 85.5 \\
\hline 420.4 & 443.1 & 454.9 & 458.1 & 447.5 & 445.8 & 435.1 & 405.8 & 249.9 & 188 & 170.1 & 122 & 79.5 \\
\hline 74.1 & 82.6 & 76.5 & 71.5 & 60.9 & 118.5 & 117.9 & 110.9 & 73.4 & 66 & 71.3 & 74.7 & 71.3 \\
\hline 390.3 & 403.8 & 414.9 & 426.1 & 407.7 & 395.7 & 394.1 & 361.9 & 243.9 & 187 & 184.6 & 154.8 & 112.8 \\
\hline 412.9 & 449 & 481.3 & 478 & 462.3 & 446.3 & 432.9 & 420.1 & 274.8 & 215.1 & 153.1 & 148.6 & 115.9 \\
\hline 340.1 & 351.6 & 370.2 & 351.8 & 334.3 & 331.4 & 325.3 & 303.4 & 214.7 & 149 & 117.2 & 84.1 & 56.3 \\
\hline 343.9 & 343.3 & 359.8 & 342.5 & 328.7 & 300 & 301.8 & 284.1 & 201.2 & 155.5 & 136.9 & 112.8 & 66 \\
\hline 61.2 & 61.6 & 63.9 & 64 & 69.1 & 66.4 & 66.8 & 68.1 & 77.2 & 81.2 & 79.2 & 77.8 & 75.4 \\
\hline 369.2 & 391.8 & 414.8 & 418.5 & 400.4 & 402.8 & 389.8 & 365.6 & 226 & 145.5 & 159.4 & 141.4 & 112.1 \\
\hline 404 & 412.9 & 433 & 358.1 & 405.2 & 398.8 & 388.2 & 369.3 & 244.3 & 178.7 & 143.8 & 137.3 & 101.3 \\
\hline 391.2 & 407 & 423.4 & 443.7 & 415.2 & 417.6 & 429.6 & 410.8 & 263.1 & 193.5 & 158.3 & 139.3 & 76 \\
\hline 349.1 & 353.3 & 373.6 & 392.2 & 382.3 & 359 & 354.8 & 341.2 & 237.5 & 172.1 & 156.9 & 132.8 & 85 \\
\hline 54.6 & 57.8 & 59.1 & 55.4 & 58.8 & 60.9 & 63.2 & 65 & 79.6 & 77.1 & 75.7 & 66.4 & 65.7 \\
\hline 332.1 & 384.1 & 361.9 & 375.1 & 368.1 & 358.4 & 347.1 & 312.5 & 187 & 123.1 & 146.9 & 134.9 & 88.1 \\
\hline 329.4 & 362.3 & 349.5 & 314.3 & 378.1 & 354 & 364.2 & 336 & 225.4 & 173.5 & 140 & 117.8 & 93 \\
\hline 374.5 & 413.7 & 414.1 & 401.4 & 404.6 & 387 & 382.1 & 341.9 & 228 & 161.4 & 144.8 & 115.4 & 67.1 \\
\hline 397.1 & 415.8 & 423.5 & 417.3 & 395.8 & 390.7 & 394.5 & 354.7 & 222.5 & 179.7 & 163.1 & 148.6 & 124.5 \\
\hline
\end{tabular}




\begin{tabular}{|c|c|c|c|c|c|c|c|c|c|c|c|c|}
\hline 59.2 & 61.2 & 63.7 & 77.4 & 78.2 & 88.5 & 91.3 & 90.5 & 90.5 & 88.9 & 90.9 & 83.6 & 77.2 \\
\hline 201.1 & 194.9 & 200.9 & 204.9 & 180.8 & 198.7 & 210.8 & 214 & 179.4 & 128.2 & 121.4 & 98.8 & 94.1 \\
\hline 69.1 & 71.5 & 72.2 & 74.7 & 75.3 & 82.7 & 93 & 90.9 & 84.1 & 80.5 & 89.2 & 89.9 & 84.7 \\
\hline 344.6 & 360.6 & 361.2 & 363.2 & 343.9 & 323.2 & 325.9 & 286.5 & 202.3 & 169.3 & 138.2 & 114.1 & 80.5 \\
\hline 357.4 & 362.6 & 362.2 & 360.9 & 330.7 & 323.8 & 311.5 & 292.4 & 204 & 180.7 & 184.2 & 179 & 153.1 \\
\hline 351.6 & 359.1 & 348.8 & 363.9 & 336.3 & 323.2 & 317.3 & 304.2 & 208.4 & 175.2 & 154.8 & 133.1 & 93.4 \\
\hline 329.4 & 346.3 & 344.3 & 338.3 & 315.9 & 311 & 305.6 & 298.6 & 205.4 & 166.2 & 138.2 & 107.8 & 83.6 \\
\hline 64.4 & 65.3 & 69.2 & 67 & 67 & 65.7 & 61.3 & 64.3 & 67 & 70.6 & 72.9 & 80.2 & 76.8 \\
\hline 370.5 & 384.1 & 371.5 & 380.2 & 354.7 & 345.9 & 309.3 & 291.8 & 199.1 & 169.3 & 159.3 & 134.9 & 87.1 \\
\hline 369.4 & 379.1 & 372 & 373.2 & 351.5 & 347.7 & 300.4 & 282 & 210.8 & 163.1 & 151.5 & 144.1 & 123 \\
\hline 323.9 & 342.1 & 342.1 & 343.6 & 330.4 & 317 & 322.3 & 330.4 & 216.9 & 166.9 & 152.2 & 133.7 & 97.6 \\
\hline 314.8 & 313.2 & 329.4 & 385.3 & 352.7 & 317.3 & 289.4 & 283.7 & 195.4 & 169.3 & 153.8 & 148.3 & 138 \\
\hline 52.2 & 54.3 & 55.7 & 57.7 & 56.7 & 60.9 & 59.1 & 70.5 & 70.9 & 71.5 & 79.8 & 82 & 77.1 \\
\hline 342.5 & 360.6 & 357.4 & 373.7 & 342.8 & 335 & 335.6 & 291.8 & 196.3 & 151.5 & 135.5 & 103.1 & 69.8 \\
\hline 376.4 & 372.3 & 370.5 & 366.4 & 341.8 & 318 & 322.1 & 312.9 & 209.4 & 161.5 & 140.7 & 128.9 & 83 \\
\hline 360.6 & 366 & 354.7 & 346.3 & 333.5 & 317.7 & 319.3 & 305.6 & 208.4 & 141.1 & 128.9 & 119.9 & 74.4 \\
\hline 255.8 & 280.6 & 291.4 & 267.5 & 202.6 & 232.6 & 232 & 191.5 & 181.1 & 152.5 & 150 & 145.5 & 143.4 \\
\hline 367.5 & 354.6 & 354.9 & 361.6 & 337.3 & 321.2 & 310.3 & 291.1 & 194.6 & 167.7 & 133.4 & 110.9 & 65.3 \\
\hline 298.9 & 345.3 & 360.5 & 366.4 & 338.7 & 321.8 & 302.8 & 286.5 & 193.6 & 170 & 133.4 & 109.9 & 64.6 \\
\hline 328.8 & 337 & 335 & 335.2 & 320.7 & 317.7 & 310.3 & 282.4 & 167.7 & 136.2 & 111.6 & 98.2 & 66.1 \\
\hline 380.5 & 385.4 & 379.1 & 397.1 & 353.3 & 355.6 & 334.6 & 324.5 & 204.9 & 175.6 & 153.1 & 120.7 & 74.7 \\
\hline 387.2 & 393.3 & 372.9 & 368.8 & 346.6 & 317.7 & 312.8 & 289 & 202.5 & 171.1 & 159.7 & 143.8 & 103.4 \\
\hline 357.7 & 363 & 353.2 & 351.9 & 322.4 & 306.3 & 295.8 & 277.9 & 189.1 & 150 & 128.2 & 88.9 & 52.9 \\
\hline 343.2 & 348 & 339.1 & 352.5 & 325.9 & 314.9 & 301 & 275.5 & 196.6 & 176.3 & 150 & 112 & 70.9 \\
\hline 277.2 & 355.7 & 346.3 & 339.7 & 323.9 & 303.4 & 284.1 & 258.9 & 162.1 & 122.8 & 108.9 & 79.5 & 53.3 \\
\hline 308.6 & 365.4 & 369.7 & 383.5 & 357.6 & 364.9 & 352.4 & 332 & 198.9 & 147.2 & 124.1 & 84.7 & 54.7 \\
\hline 320.1 & 350.1 & 340.2 & 346.6 & 323.5 & 329.8 & 315.5 & 290 & 201.2 & 155.9 & 157.9 & 143.4 & 118.2 \\
\hline 351.5 & 358.1 & 351.5 & 347.1 & 341.8 & 314.2 & 308.3 & 287.6 & 195.3 & 157.6 & 110.9 & 87.5 & 52.9 \\
\hline 349.1 & 346.6 & 348.8 & 352.2 & 323.2 & 319.3 & 316.7 & 297.2 & 205 & 163.1 & 125.8 & 103.7 & 67.5 \\
\hline 298.6 & 306.6 & 331.4 & 375.4 & 351.4 & 350.3 & 336.2 & 278.3 & 193.2 & 146.6 & 143.1 & 138.7 & 134.8 \\
\hline 378.8 & 398.1 & 380.9 & 379.8 & 352.3 & 338 & 310.4 & 296.5 & 185 & 156.9 & 129 & 114.4 & 65.3 \\
\hline 367.4 & 384.4 & 389.8 & 391 & 355.6 & 340.9 & 329 & 302.1 & 214.6 & 166.7 & 144.5 & 138.6 & 121.3 \\
\hline 365.6 & 381.6 & 384.7 & 379.6 & 351.8 & 332.6 & 323.1 & 302.8 & 216.7 & 176 & 145.5 & 115.4 & 67.5 \\
\hline 352.3 & 365 & 362.3 & 373.6 & 345.7 & 326.6 & 320.7 & 295.9 & 189 & 159.1 & 135.8 & 116.1 & 76.7 \\
\hline 307.3 & 324.2 & 335.7 & 342.8 & 327.7 & 297.9 & 284.2 & 265.8 & 151.8 & 133.7 & 114.7 & 96.9 & 70.5 \\
\hline 361.9 & 380.2 & 362.5 & 365.7 & 337 & 328.8 & 304.8 & 284.9 & 194.6 & 151.7 & 129.3 & 108.5 & 76 \\
\hline 337.7 & 349.8 & 345.9 & 328.1 & 319 & 299 & 293.8 & 270.3 & 187.1 & 157.9 & 155.2 & 156.6 & 131.4 \\
\hline
\end{tabular}


other

\begin{tabular}{|c|c|c|c|c|c|c|c|c|c|c|c|c|}
\hline 323.1 & 354.2 & 340.9 & 349.4 & 323.9 & 309.3 & 299.4 & 279.6 & 189.1 & 147.2 & 123.4 & 108.5 & 93.1 \\
\hline 328 & 348.8 & 344.9 & 337 & 322.5 & 290 & 285.2 & 263 & 185.7 & 159.7 & 152.1 & 137.5 & 111.7 \\
\hline 360.1 & 370.8 & 367.1 & 357.4 & 332.8 & 314.6 & 296.5 & 271.3 & 190.2 & 156.6 & 179.7 & 153.8 & 149 \\
\hline 361.6 & 375 & 368.2 & 363.6 & 339 & 323.6 & 311.8 & 278.9 & 190.9 & 148.6 & 129.6 & 104.4 & 62.3 \\
\hline 343.3 & 351.8 & 362.3 & 356 & 337.7 & 300 & 289.3 & 271 & 191.1 & 158.6 & 155.3 & 153.8 & 106.8 \\
\hline 307.6 & 336.4 & 336.3 & 325.3 & 314.1 & 311.1 & 302.7 & 282.1 & 174.9 & 135.5 & 121.3 & 100.3 & 65 \\
\hline 315.2 & 326.2 & 332.6 & 334.5 & 313.2 & 297.9 & 289.7 & 271 & 176.7 & 139.6 & 128.2 & 110.2 & 79.9 \\
\hline 340.2 & 352.9 & 345.9 & 348.1 & 330.7 & 315.3 & 303.8 & 271.4 & 153.4 & 116.5 & 113.4 & 87.9 & 65.7 \\
\hline 340.4 & 344 & 338.3 & 337.4 & 325.6 & 317.7 & 302.4 & 271.4 & 196.6 & 173.9 & 182.8 & 170.4 & 134.5 \\
\hline 354 & 362.2 & 363.7 & 366.3 & 337.4 & 314.8 & 306.6 & 285.5 & 215.7 & 184.9 & 151.5 & 127.9 & 88.9 \\
\hline 368.2 & 387.1 & 382.7 & 379.8 & 350.9 & 334.9 & 323.6 & 301 & 213.6 & 186.7 & 188.7 & 171.4 & 145.9 \\
\hline 352.3 & 385.3 & 363.7 & 350.1 & 331.4 & 317.4 & 313.1 & 296.6 & 193.5 & 130 & 122.3 & 109.3 & 96.8 \\
\hline 333.9 & 350.9 & 342.8 & 336.4 & 317.3 & 309.7 & 294.8 & 280.4 & 183.5 & 131.3 & 121.1 & 114.7 & 87.1 \\
\hline 353.6 & 362.2 & 361.2 & 352.3 & 335.6 & 328.1 & 327.6 & 302.8 & 217.4 & 164.9 & 140 & 123.7 & 90.9 \\
\hline 341.1 & 366.1 & 358.7 & 397.6 & 383.1 & 375.8 & 371.4 & 353.9 & 198.8 & 154.1 & 132 & 106.9 & 82.9 \\
\hline 403.9 & 418.8 & 428.8 & 422.4 & 398.6 & 385.4 & 374.8 & 348.9 & 200.5 & 147.9 & 123.1 & 110.6 & 89.6 \\
\hline 344 & 358.4 & 357.8 & 350.1 & 343.6 & 318.3 & 306.9 & 291.4 & 202.5 & 168.1 & 168.7 & 147.6 & 123.8 \\
\hline 352.5 & 369.9 & 359.4 & 371.5 & 368.9 & 382.9 & 376 & 343.9 & 199.8 & 157.9 & 124.5 & 114.4 & 86.1 \\
\hline 323.8 & 343.3 & 335.2 & 334.2 & 319.4 & 307.2 & 299.7 & 280.6 & 190.2 & 162.8 & 147.2 & 130.4 & 82.6 \\
\hline 325.9 & 331.5 & 334.5 & 341.2 & 368.1 & 350.2 & 345.2 & 331.1 & 205 & 172.1 & 141.7 & 118.3 & 79.8 \\
\hline 318.3 & 325.3 & 335.6 & 341.9 & 314.8 & 297.7 & 296.9 & 274.2 & 201.1 & 152.5 & 122.7 & 112.3 & 81.3 \\
\hline 317.7 & 347.4 & 336.6 & 333.6 & 309.7 & 299.4 & 287.5 & 254.5 & 181.8 & 122.8 & 105.8 & 85.8 & 62.9 \\
\hline 340.2 & 364.3 & 347.8 & 355.3 & 371.3 & 368.9 & 361.4 & 333.4 & 203 & 174.2 & 123.7 & 100.3 & 76.4 \\
\hline 320 & 399.4 & 398.4 & 402.3 & 386.9 & 383.9 & 371.1 & 337.4 & 192.9 & 134.1 & 117.5 & 113.1 & 87.8 \\
\hline 364.4 & 396.6 & 386.8 & 408.7 & 400.7 & 394.5 & 384.6 & 352.4 & 200.4 & 154.5 & 111 & 93.7 & 67 \\
\hline 229.5 & 239.7 & 242.1 & 234.7 & 227.3 & 230.6 & 235.7 & 229.5 & 127.5 & 110.7 & 95.4 & 108.6 & 94.3 \\
\hline 209.4 & 224.4 & 238.5 & 217.1 & 180.4 & 196.7 & 196.6 & 163.9 & 87.1 & 63.9 & 65.8 & 68.4 & 67.7 \\
\hline 332.8 & 373.2 & 400.4 & 415.1 & 390.8 & 381.3 & 380.1 & 351.9 & 210.5 & 179.4 & 132.4 & 102.6 & 77.8 \\
\hline 331.1 & 351.9 & 347 & 346.7 & 335.2 & 319 & 308.4 & 286.8 & 199.8 & 169 & 127.2 & 118.9 & 88.9 \\
\hline 384.5 & 398.9 & 406.9 & 401.7 & 371.8 & 363.6 & 365.1 & 348.3 & 225 & 183.9 & 126.5 & 107.1 & 72.6 \\
\hline 403.1 & 428.3 & 426.9 & 437.4 & 401.2 & 401.4 & 404 & 373.3 & 276.4 & 265 & 215.3 & 196.1 & 136.7 \\
\hline 411.5 & 426.1 & 415.8 & 367.4 & 397.4 & 380.3 & 365.5 & 337.3 & 195.3 & 130.4 & 100.2 & 85.4 & 67.1 \\
\hline 388.5 & 415.3 & 420.5 & 424.3 & 405 & 406.7 & 390.1 & 367.5 & 264.4 & 220.6 & 170.4 & 151 & 93.7 \\
\hline 392.5 & 419.7 & 434.7 & 446.7 & 409 & 414.6 & 396.2 & 380 & 282.4 & 247.3 & 201 & 168.9 & 89.3 \\
\hline 380.6 & 419.4 & 411.4 & 404.5 & 381.1 & 382.3 & 367.5 & 351.8 & 256.4 & 227 & 179 & 152.8 & 81.6 \\
\hline 336.3 & 347 & 356.1 & 413.9 & 402.6 & 390.3 & 378.3 & 363.7 & 261.4 & 220 & 193.7 & 176.3 & 94.3 \\
\hline 316.6 & 332.1 & 332.9 & 343.9 & 378.1 & 355.9 & 343.8 & 322.4 & 163.8 & 112.3 & 97.1 & 72.9 & 61.5 \\
\hline
\end{tabular}




\begin{tabular}{|c|c|c|c|c|c|c|c|c|c|c|c|c|}
\hline 314.2 & 325.6 & 334.9 & 338.7 & 313.1 & 297.2 & 321.8 & 319.4 & 238.7 & 200.2 & 155.2 & 144 & 81.2 \\
\hline 314.8 & 326.6 & 387.5 & 397.1 & 363.3 & 365.5 & 364.5 & 357.8 & 259.3 & 225.3 & 184.6 & 170.9 & 111.3 \\
\hline 369.9 & 402.8 & 412.1 & 428.2 & 388.7 & 330.6 & 319.7 & 298.3 & 217.7 & 165.9 & 132.1 & 113 & 88.5 \\
\hline 317.6 & 329.7 & 396.8 & 390.9 & 368.4 & 353.6 & 341 & 312.3 & 210.4 & 181.8 & 142.7 & 122.3 & 81.9 \\
\hline 404.6 & 436.9 & 451.7 & 461.7 & 424.4 & 419.1 & 401.8 & 386.5 & 273.2 & 238.5 & 189.2 & 186 & 102.4 \\
\hline 81.9 & 371.9 & 381.2 & 442.6 & 412.2 & 407 & 403.9 & 370.8 & 258.4 & 230 & 195.7 & 175.6 & 94.3 \\
\hline 292 & 281.1 & 307.2 & 369.5 & 363.7 & 348.4 & 328 & 316.8 & 232.6 & 194.1 & 171.2 & 162.4 & 94 \\
\hline 306.5 & 342.5 & 365.5 & 355.3 & 344 & 339.1 & 333.7 & 301.9 & 194.3 & 179.8 & 167.1 & 146.9 & 87.4 \\
\hline 369.4 & 397.7 & 414.1 & 403.8 & 388 & 390.3 & 386.6 & 357.1 & 251 & 216.6 & 176.1 & 155.6 & 86.7 \\
\hline 402.7 & 426.3 & 431.4 & 430.8 & 399.8 & 378.9 & 375.3 & 349.7 & 226.5 & 202.8 & 166.5 & 155.5 & 86.1 \\
\hline 318.6 & 353.8 & 389.4 & 389.5 & 370.9 & 366.2 & 363 & 341.9 & 236.1 & 194.1 & 167.5 & 154 & 80.6 \\
\hline 319.7 & 322.9 & 379 & 375.8 & 364 & 354.3 & 346.4 & 322.6 & 231.1 & 193.9 & 170 & 160.3 & 91.6 \\
\hline 327 & 398.3 & 400.3 & 381.7 & 394.7 & 377.6 & 378 & 344.3 & 230.8 & 182 & 156.9 & 132.3 & 71.9 \\
\hline 382.4 & 407.3 & 402.5 & 428 & 415 & 415.7 & 405.7 & 384.6 & 248.5 & 201.6 & 161 & 153.1 & 94.4 \\
\hline 385.4 & 397.9 & 402.4 & 411.8 & 399.4 & 388 & 373.5 & 357.1 & 226.1 & 195.8 & 152.1 & 145.7 & 99.9 \\
\hline 264.1 & 288.8 & 286.6 & 278.4 & 237.4 & 245.8 & 252 & 234.9 & 157.7 & 118.7 & 122.4 & 133.7 & 90.6 \\
\hline 227.8 & 251.5 & 255.8 & 257.7 & 219.1 & 262.5 & 281.9 & 264.3 & 166.9 & 141.4 & 121.2 & 110.1 & 73.3 \\
\hline 374.8 & 395.6 & 407 & 430.2 & 393 & 382.9 & 384.1 & 357.7 & 251.2 & 212.4 & 186.6 & 161.5 & 87.1 \\
\hline 335.2 & 351.8 & 378.2 & 408.7 & 410.5 & 386.9 & 360.9 & 360.8 & 269.6 & 224.5 & 184.3 & 161.2 & 84 \\
\hline 361.9 & 379.5 & 394.4 & 438.4 & 418.7 & 402.3 & 398.1 & 371.8 & 259.7 & 203 & 163.5 & 138.4 & 68.8 \\
\hline 365.4 & 413.3 & 432.5 & 426.6 & 402.4 & 391.2 & 392.1 & 384.3 & 268.1 & 221 & 173.8 & 160 & 72.2 \\
\hline 433 & 454.2 & 468.3 & 452.4 & 435.3 & 415.9 & 412.7 & 378 & 236.5 & 188.1 & 180.4 & 164.1 & 94.3 \\
\hline 367.9 & 392.1 & 171.7 & 396.4 & 379.7 & 370.6 & 360.3 & 346.7 & 235.9 & 196.7 & 171.9 & 148.5 & 77.4 \\
\hline 362.4 & 381.9 & 404.5 & 395.6 & 383.8 & 377.9 & 373.9 & 347.6 & 241.5 & 218 & 180.3 & 173.8 & 136.1 \\
\hline 367.1 & 419.8 & 425 & 404.2 & 411.3 & 377.4 & 381.5 & 366.4 & 224 & 157.6 & 103.7 & 79.8 & 57 \\
\hline 368.8 & 379.1 & 407.1 & 402.6 & 395.9 & 397.2 & 379.1 & 369.7 & 267 & 213.3 & 174.5 & 155.8 & 98.5 \\
\hline 387.7 & 431.3 & 434.2 & 416.3 & 408 & 398.1 & 384.4 & 362.9 & 179.7 & 127.9 & 97.8 & 79.5 & 57.8 \\
\hline 402.6 & 420.5 & 441 & 449.2 & 423.5 & 408.1 & 413.1 & 397.4 & 212.2 & 156.2 & 140.4 & 123.4 & 100.6 \\
\hline 461.4 & 487.1 & 490.9 & 499.6 & 486.4 & 437.6 & 391.6 & 371.6 & 216 & 216.7 & 176.2 & 166.4 & 83.4 \\
\hline 376.9 & 400.9 & 416.9 & 415.3 & 397.3 & 404.2 & 390.3 & 380.6 & 235.6 & 149.3 & 107.9 & 88.8 & 63.2 \\
\hline 389.9 & 415.9 & 440.1 & 432.4 & 421 & 400.7 & 406.7 & 391.2 & 284 & 228.9 & 188.7 & 155.3 & 127.9 \\
\hline 382.6 & 427.8 & 443.4 & 435 & 419.4 & 410.9 & 406.4 & 389.3 & 224.6 & 154.8 & 113.4 & 93.7 & 73.6 \\
\hline 386.1 & 421.8 & 438.6 & 420 & 218.2 & 367.3 & 389.9 & 376.6 & 202 & 139.3 & 112.7 & 90.5 & 81.2 \\
\hline 382.7 & 405.2 & 427.8 & 404.4 & 388.8 & 387.5 & 384.5 & 360.9 & 209.7 & 174.5 & 111.3 & 97.1 & 74 \\
\hline 379.1 & 400 & 423.1 & 410.9 & 390.1 & 398.4 & 388 & 372.6 & 232.9 & 171.8 & 132.7 & 120.6 & 72.9 \\
\hline 476.1 & 493.3 & 490 & 486.3 & 467.7 & 461.4 & 454.9 & 443.1 & 264.5 & 246.3 & 215.6 & 170.5 & 125.1 \\
\hline 492.1 & 512.4 & 459.7 & 510.9 & 472.2 & 451.4 & 463.7 & 439.5 & 202.4 & 141.7 & 131.3 & 101.6 & 84 \\
\hline
\end{tabular}


other

\begin{tabular}{|c|c|c|c|c|c|c|c|c|c|c|c|c|}
\hline 480.6 & 531.3 & 533.3 & 523 & 495.4 & 490.8 & 475.4 & 447.3 & 319.8 & 283.3 & 255.1 & 219.3 & 180 \\
\hline 499.1 & 534.8 & 519.5 & 523.3 & 469 & 462.1 & 451.1 & 416.2 & 218 & 199.6 & 183.1 & 155 & 100.6 \\
\hline 394.9 & 432.2 & 429.4 & 410.8 & 401.7 & 400.5 & 413.1 & 394 & 225.4 & 167.3 & 151.7 & 116.1 & 75.3 \\
\hline 403.7 & 440.8 & 433.9 & 414.4 & 421.3 & 406.4 & 391.4 & 376.5 & 219.3 & 158.3 & 129.6 & 102.6 & 86.7 \\
\hline 435.9 & 437 & 430.9 & 479.9 & 451.7 & 439.8 & 442.9 & 414.3 & 261 & 232 & 182.3 & 149.2 & 129 \\
\hline 477.9 & 507.6 & 513.2 & 509.3 & 481.2 & 464.7 & 461.3 & 447.2 & 300.3 & 255.9 & 223.5 & 194.6 & 179.1 \\
\hline 494.7 & 509.2 & 507.6 & 515.4 & 493.9 & 480 & 475.3 & 451.6 & 229.9 & 225 & 191.5 & 181.5 & 115.4 \\
\hline 485.3 & 512.9 & 498.8 & 496.3 & 490.7 & 480.3 & 472.7 & 458.8 & 269.8 & 230.2 & 163.5 & 116.8 & 91.6 \\
\hline 488.6 & 514.6 & 516.4 & 512.4 & 480.7 & 480 & 481.8 & 435.6 & 189 & 148.3 & 125.1 & 99.5 & 90.9 \\
\hline 467.5 & 509.8 & 507.3 & 506.4 & 477.6 & 462.1 & 463.4 & 442.9 & 285.9 & 225.5 & 202.6 & 169.1 & 152.2 \\
\hline 428.9 & 424.4 & 443 & 451.7 & 420.5 & 416.3 & 414 & 395.2 & 256.7 & 221.2 & 182 & 184.5 & 157.2 \\
\hline 389 & 430.7 & 437.1 & 440.8 & 414.1 & 407 & 413 & 402.4 & 236.6 & 204.2 & 192.9 & 183.6 & 131.3 \\
\hline 388.5 & 436.4 & 443.3 & 437.1 & 420.5 & 410.7 & 400.2 & 382.6 & 240 & 189.7 & 163.5 & 138.6 & 106.1 \\
\hline 418.9 & 453.8 & 471.8 & 460.3 & 440.6 & 426.6 & 423.3 & 410.6 & 289.2 & 252.7 & 211.8 & 179.3 & 147 \\
\hline 444.6 & 475.3 & 470.5 & 466.9 & 456.2 & 438.4 & 446.8 & 428.9 & 256.2 & 217.3 & 190.6 & 156.7 & 127.5 \\
\hline 451.9 & 485.2 & 491.7 & 481.9 & 459.9 & 451.2 & 451.1 & 432.3 & 283.1 & 231.6 & 180.7 & 159.5 & 145.6 \\
\hline 451.2 & 467.5 & 476.6 & 483.4 & 459.1 & 450.4 & 436.7 & 413.7 & 245.3 & 230.6 & 207.1 & 200.7 & 154.8 \\
\hline 447.8 & 461.7 & 477.7 & 466.1 & 440.5 & 429.1 & 437.6 & 429.2 & 251.2 & 180.7 & 150.3 & 105.8 & 76.7 \\
\hline 431.2 & 470.7 & 478.3 & 462.4 & 451.1 & 434.3 & 440.9 & 417.3 & 312.6 & 260.1 & 256.5 & 383.2 & 381.5 \\
\hline 441.8 & 483.5 & 440.6 & 477.9 & 452.1 & 440.6 & 440.6 & 416.2 & 227.1 & 165.2 & 149.6 & 112 & 80.2 \\
\hline 416.5 & 455.2 & 464.4 & 457.4 & 440.6 & 419.9 & 415 & 386.3 & 266.6 & 220.8 & 182.7 & 150.8 & 120.9 \\
\hline 451.7 & 491.4 & 478.4 & 486.7 & 468.3 & 453.6 & 449.2 & 435.8 & 306.5 & 243.2 & 231.2 & 244.6 & 213.1 \\
\hline 428.1 & 451.2 & 448.7 & 444.9 & 441.1 & 431.8 & 429.7 & 396.7 & 241.3 & 170.4 & 116.1 & 91.6 & 68.4 \\
\hline 413.1 & 437.3 & 439.3 & 434.7 & 410.9 & 387.5 & 398.6 & 373.8 & 243.6 & 204.7 & 179.7 & 174.8 & 137.2 \\
\hline 428.2 & 447.7 & 448.9 & 432.8 & 413.1 & 408.6 & 414.8 & 376.9 & 205.3 & 131.8 & 99.5 & 83.3 & 64.3 \\
\hline 481.6 & 518.5 & 507.5 & 496.6 & 473.9 & 471 & 466.6 & 443.8 & 310.3 & 242.6 & 205.3 & 173.2 & 142.7 \\
\hline 468.8 & 512.8 & 489.6 & 487.4 & 483.1 & 474.1 & 481.8 & 449.2 & 278 & 220 & 194 & 188 & 158.1 \\
\hline 484.9 & 503 & 502.8 & 473.9 & 445.4 & 423.2 & 406.7 & 381.2 & 230.9 & 159.3 & 116.5 & 88.8 & 70.5 \\
\hline 382.7 & 400.9 & 413.6 & 398.8 & 386.2 & 366.8 & 362.3 & 346 & 237.9 & 195.5 & 187.8 & 172.3 & 155 \\
\hline 347.3 & 369.4 & 331.4 & 393 & 368.7 & 363 & 361.9 & 333.3 & 170.5 & 121.3 & 85 & 73.6 & 57.7 \\
\hline 355.2 & 391.3 & 391.1 & 395.9 & 379 & 376.5 & 372.2 & 354.6 & 258 & 227.2 & 196.7 & 159.6 & 132.6 \\
\hline 361.5 & 406.6 & 385.5 & 411.2 & 394.1 & 393.2 & 396.4 & 367.1 & 252 & 221.5 & 209.4 & 192.1 & 140.4 \\
\hline 357.4 & 380.9 & 367.4 & 351.5 & 345.3 & 326.9 & 325.9 & 312.1 & 215.1 & 154.5 & 116.5 & 76 & 56.3 \\
\hline 355.3 & 354.6 & 387.9 & 383.5 & 370 & 352.4 & 347.2 & 328.9 & 232 & 198.9 & 168 & 153.7 & 140.3 \\
\hline 371.6 & 403.4 & 364.8 & 416.8 & 390.3 & 375.3 & 377.7 & 358 & 223.2 & 179 & 125.8 & 100.9 & 80.9 \\
\hline 392.6 & 396.7 & 391.2 & 379.5 & 359.4 & 331.8 & 321.4 & 297.6 & 200.9 & 164.9 & 133.1 & 90.2 & 76.4 \\
\hline 376.2 & 406.2 & 392.3 & 395 & 381.9 & 366.5 & 376.5 & 346.5 & 230.9 & 193.8 & 201 & 164.6 & 125.6 \\
\hline
\end{tabular}


other

\begin{tabular}{|c|c|c|c|c|c|c|c|c|c|c|c|c|}
\hline 383.9 & 400.9 & 404.4 & 400.1 & 375.8 & 350.8 & 338.4 & 332.9 & 227.8 & 185.2 & 147.6 & 108.5 & 73.6 \\
\hline 383.9 & 398.8 & 407.9 & 395.9 & 395.9 & 358 & 363.8 & 360.7 & 262.7 & 217.1 & 176.3 & 160.8 & 147.4 \\
\hline 396.2 & 417 & 382.1 & 400.5 & 370.3 & 349.1 & 348.8 & 326.5 & 202.5 & 160.4 & 112.3 & 99.5 & 79.8 \\
\hline 344.2 & 377 & 377 & 372.9 & 341.1 & 325.6 & 330 & 310.7 & 215.7 & 179 & 146.2 & 134.1 & 101.3 \\
\hline 345.6 & 366 & 365 & 360.8 & 344.9 & 337.7 & 332.8 & 311.7 & 179.4 & 113.4 & 95 & 91.2 & 82.6 \\
\hline 328 & 337.7 & 351.5 & 342.8 & 328.3 & 314.1 & 317.3 & 310.7 & 216.7 & 166.9 & 124.4 & 84 & 65.3 \\
\hline 243 & 263.9 & 239.4 & 216.7 & 221.2 & 240.9 & 251 & 87.4 & 201.3 & 189.8 & 158.9 & 153.9 & 151.5 \\
\hline 374.7 & 395.5 & 390.1 & 397.4 & 386.3 & 362.6 & 352.7 & 336.1 & 199.8 & 154.5 & 110.9 & 74.7 & 52.5 \\
\hline 384.3 & 412.7 & 378.9 & 365.3 & 347 & 335.7 & 330.7 & 305.9 & 192.2 & 156.6 & 151.4 & 138.2 & 121 \\
\hline 360.5 & 382.6 & 363.6 & 349.7 & 349.4 & 319 & 322.4 & 304.8 & 204.2 & 160 & 128.2 & 99.2 & 57.4 \\
\hline 345.6 & 361.2 & 358.4 & 343.2 & 329.4 & 315.9 & 316.2 & 291 & 197.3 & 135.5 & 105.4 & 83.6 & 61.5 \\
\hline 372.1 & 412.9 & 396.2 & 391.7 & 382.8 & 359.4 & 358.8 & 328.2 & 194.9 & 119.6 & 77.4 & 59.8 & 49.4 \\
\hline 354.9 & 359.1 & 373.6 & 360.5 & 330.7 & 315.5 & 303.8 & 288.6 & 184.6 & 141.4 & 106.8 & 116.5 & 96.8 \\
\hline 338 & 386.6 & 368.7 & 362.6 & 356.6 & 338.5 & 336.1 & 318.9 & 228.7 & 210.2 & 188.8 & 180.8 & 168.4 \\
\hline 382.9 & 406.1 & 401.9 & 387.8 & 372.6 & 336.3 & 325.6 & 298.3 & 193.2 & 150.7 & 108.2 & 91.2 & 51.1 \\
\hline 353.5 & 367.7 & 358.4 & 370.5 & 343.3 & 320 & 316.2 & 301 & 195.3 & 150 & 142.4 & 133.7 & 107.5 \\
\hline 337 & 369.8 & 373.6 & 379.2 & 368.2 & 352.2 & 362.8 & 365.2 & 247.3 & 164.9 & 113.7 & 70.8 & 60.5 \\
\hline 389.2 & 446.9 & 421.1 & 431.6 & 430.6 & 411.3 & 400.5 & 382.9 & 246.2 & 121.3 & 86.1 & 74 & 67 \\
\hline 387.1 & 415.2 & 426.2 & 420.2 & 401.2 & 370.9 & 381.8 & 346 & 233.6 & 194 & 170.4 & 165.2 & 152.5 \\
\hline 351.1 & 377.1 & 378.1 & 377.7 & 355.3 & 321.2 & 316.9 & 291.3 & 175.7 & 129.6 & 107.1 & 103 & 95.7 \\
\hline 345.3 & 382.9 & 365 & 364.3 & 350.8 & 329.4 & 315.2 & 287.9 & 186.6 & 125.5 & 95.4 & 90.5 & 81.6 \\
\hline 342.8 & 355.6 & 366 & 361.5 & 335.2 & 314.8 & 311 & 298.6 & 202.2 & 145.2 & 88.5 & 75 & 59.4 \\
\hline 349.1 & 425.7 & 416.3 & 417.8 & 387.5 & 373.8 & 382.9 & 364.3 & 232.2 & 138.9 & 91.2 & 69.8 & 49.8 \\
\hline 409.2 & 436.3 & 430.4 & 438.2 & 404.8 & 376.9 & 379.4 & 346.2 & 231.4 & 181 & 163.8 & 88.8 & 71.2 \\
\hline 384.3 & 391.5 & 384 & 393.3 & 373.1 & 353.4 & 353 & 336.7 & 225.1 & 189.4 & 150 & 106.1 & 78.5 \\
\hline 355.6 & 375.7 & 373.2 & 372.9 & 353.2 & 316.2 & 314.8 & 298.6 & 185.2 & 153.4 & 124.1 & 98.8 & 76 \\
\hline 358.4 & 370.1 & 385.4 & 354.9 & 326.9 & 307.6 & 310.7 & 290.6 & 171.4 & 126.5 & 103 & 68.8 & 52.2 \\
\hline 332.8 & 348.7 & 354.9 & 54.3 & 338.2 & 322.8 & 328.3 & 315.9 & 161.4 & 124.1 & 104.7 & 95 & 62.6 \\
\hline 246.1 & 267.5 & 271 & 263.3 & 244.7 & 267.1 & 292.1 & 265.8 & 149 & 108.9 & 102.6 & 82.9 & 69.8 \\
\hline 260.6 & 274.8 & 278.9 & 261.6 & 255.1 & 232.9 & 211.5 & 213.9 & 173.8 & 142 & 128.6 & 128.9 & 126.5 \\
\hline 325.9 & 356 & 356.3 & 353.5 & 338.7 & 334.9 & 330.7 & 301.8 & 197.3 & 163.1 & 124.1 & 97.5 & 57.7 \\
\hline 342.1 & 355.3 & 367.7 & 362.5 & 346.6 & 325.6 & 328 & 302.1 & 221.5 & 181.1 & 151 & 145.5 & 107.1 \\
\hline 350.8 & 360.8 & 357.7 & 367.4 & 352.2 & 321.8 & 320 & 308.6 & 229.5 & 175.9 & 134.4 & 103.7 & 75 \\
\hline 352.9 & 358 & 357.4 & 350.1 & 340.2 & 318.6 & 322.4 & 307.2 & 205.3 & 157.9 & 119.6 & 98.2 & 60.1 \\
\hline 346.6 & 351.8 & 352.3 & 349.1 & 320.7 & 308.6 & 298.3 & 290.6 & 180.7 & 140.3 & 129.9 & 111.7 & 96.4 \\
\hline 350.1 & 349.1 & 363.2 & 366.1 & 336.3 & 328 & 321.2 & 292.4 & 211.9 & 177.7 & 146.9 & 117.2 & 78.1 \\
\hline 366.3 & 390.5 & 373.3 & 386.7 & 362.2 & 327.3 & 317.7 & 285.1 & 200.2 & 171.4 & 144.1 & 139.4 & 104.4 \\
\hline
\end{tabular}


other

\begin{tabular}{|c|c|c|c|c|c|c|c|c|c|c|c|c|}
\hline 394.4 & 405.7 & 397.4 & 380.2 & 351.5 & 317.7 & 326.2 & 306.2 & 216.8 & 165.2 & 112.1 & 96.8 & 68.1 \\
\hline 340.1 & 349.4 & 371.5 & 358.4 & 337 & 312.8 & 312.8 & 294.1 & 219.1 & 184 & 150 & 126.8 & 66.4 \\
\hline 337.7 & 347 & 349.1 & 353.2 & 337.7 & 315.5 & 318.7 & 288.2 & 177.3 & 155.2 & 141 & 128.9 & 87.8 \\
\hline 336.4 & 363.2 & 368.1 & 368.1 & 345.3 & 289.5 & 384.9 & 358.3 & 219.3 & 180.8 & 164.9 & 142.8 & 107.1 \\
\hline 331.4 & 370.5 & 365.3 & 373.9 & 401.1 & 395.8 & 396.2 & 361.4 & 204.2 & 160.7 & 149 & 147.7 & 125.5 \\
\hline 406.4 & 424.7 & 442.2 & 437.4 & 408.8 & 397.7 & 400 & 374.8 & 287.1 & 226 & 178.3 & 144.8 & 87.1 \\
\hline 417.8 & 435.5 & 430.6 & 444.4 & 417 & 379.6 & 359.6 & 330.4 & 201.8 & 161.4 & 140.4 & 118.9 & 78.8 \\
\hline 350.1 & 343.2 & 369.4 & 348 & 341.1 & 321.8 & 302.7 & 284.1 & 199.4 & 177.3 & 149 & 138.2 & 124.8 \\
\hline 391.3 & 399.2 & 402.3 & 395.7 & 372.2 & 343.2 & 325.3 & 309.7 & 219.1 & 184.2 & 169.4 & 161.1 & 123.4 \\
\hline 378.4 & 375.3 & 384.3 & 398.8 & 396.1 & 371.5 & 348.4 & 320.7 & 228.8 & 192.8 & 180.1 & 174.2 & 148.4 \\
\hline 415.5 & 400.9 & 386.7 & 373.6 & 357.8 & 336.3 & 322.8 & 310.7 & 220.9 & 192.5 & 163.8 & 150 & 114.4 \\
\hline 381.3 & 375.7 & 367.4 & 379.5 & 356.8 & 337 & 328.3 & 297.2 & 218.5 & 178.3 & 156.3 & 135.1 & 107.8 \\
\hline 365.6 & 367.4 & 375 & 373.2 & 350.8 & 330 & 324.2 & 293.4 & 214.6 & 192.2 & 181.1 & 162.4 & 119.2 \\
\hline 357.6 & 427.3 & 428.7 & 432.2 & 395.6 & 375.8 & 376.4 & 357.6 & 265.6 & 226.4 & 193.7 & 172.8 & 122 \\
\hline 395.9 & 426.2 & 424.2 & 433.1 & 417.8 & 394.9 & 397.4 & 365.4 & 214.3 & 188 & 151.7 & 137.9 & 99.9 \\
\hline 421.3 & 439.6 & 439.9 & 392.3 & 371.9 & 342.2 & 333.2 & 307.9 & 196.7 & 162.1 & 127.2 & 96.9 & 74 \\
\hline 125.9 & 127.5 & 149.3 & 151 & 130.6 & 159.7 & 169.8 & 167.6 & 110.2 & 75.7 & 85.5 & 79.8 & 52.2 \\
\hline 389.5 & 380.6 & 407.8 & 394 & 375 & 348.4 & 327.3 & 284.8 & 194.2 & 177 & 148.6 & 133.1 & 106.4 \\
\hline 387.8 & 387.9 & 388.5 & 390.9 & 361.9 & 335.6 & 325.2 & 299.6 & 219.5 & 190.4 & 188.4 & 156 & 107.8 \\
\hline 354.2 & 366.3 & 367 & 373.2 & 350.2 & 335.9 & 327.3 & 307.9 & 215.7 & 181.4 & 152.2 & 141.4 & 121.3 \\
\hline 338 & 356.7 & 362.5 & 355.3 & 331.1 & 311.7 & 319.7 & 307.2 & 224.7 & 191.5 & 178.7 & 162.5 & 104.4 \\
\hline 353.9 & 351.2 & 369.1 & 367 & 339.7 & 324.9 & 339.4 & 320.4 & 228.9 & 203.2 & 154.8 & 115.8 & 65.7 \\
\hline 340.1 & 403.4 & 395.2 & 412.2 & 391.3 & 361.4 & 364.8 & 329.6 & 173.1 & 135.1 & 98.5 & 77.8 & 51.5 \\
\hline 386 & 401.8 & 418.7 & 421.3 & 393.1 & 371.7 & 370.5 & 354.6 & 263.6 & 210 & 184.9 & 167.6 & 154.3 \\
\hline 362.9 & 367.7 & 359.8 & 356.3 & 339.4 & 316.6 & 316.9 & 303.8 & 228.1 & 199.4 & 135.8 & 106.4 & 97.1 \\
\hline 373.3 & 368.1 & 370.1 & 366 & 343.5 & 321.4 & 315.5 & 295.1 & 229.5 & 192.2 & 149.3 & 119.3 & 88.1 \\
\hline 331.5 & 354.9 & 371.5 & 370.4 & 364.4 & 348.6 & 352.3 & 325.6 & 210 & 181.5 & 161.1 & 144.8 & 123.7 \\
\hline 324.2 & 352.2 & 366.7 & 364.6 & 334.2 & 316.6 & 315.9 & 293.8 & 223.6 & 174.2 & 114.8 & 98.5 & 69.5 \\
\hline 348.7 & 363.7 & 367 & 373.1 & 376.7 & 364.5 & 354.7 & 327.7 & 173.1 & 124.4 & 92.4 & 85.4 & 65.4 \\
\hline 353.9 & 365.3 & 360.5 & 361.2 & 343.3 & 319.7 & 315.2 & 310.8 & 221.9 & 182.5 & 146.2 & 96.1 & 84 \\
\hline 336.6 & 353.2 & 355.6 & 370.6 & 370.4 & 341.3 & 331.3 & 320.7 & 212 & 168 & 136.3 & 117.8 & 95 \\
\hline 346.3 & 375 & 392.4 & 381.8 & 364.7 & 338.8 & 346.7 & 321.9 & 182.1 & 117.8 & 82.3 & 58.8 & 48 \\
\hline 383 & 421.4 & 427.8 & 426.3 & 411.9 & 384.7 & 390.4 & 372.3 & 260.3 & 184.6 & 132 & 99.9 & 77.8 \\
\hline 401.4 & 430.7 & 423 & 432.8 & 399.1 & 395.4 & 389.8 & 362 & 259.6 & 206.1 & 171.8 & 156.1 & 124.1 \\
\hline 354.2 & 391.9 & 379.1 & 370.8 & 356.3 & 343.9 & 337.3 & 322.5 & 222.2 & 172.5 & 110.2 & 87.8 & 72.2 \\
\hline 375.7 & 365.6 & 376.4 & 355.3 & 337 & 327.7 & 318 & 295.1 & 200.2 & 143.1 & 123.5 & 100.9 & 81.6 \\
\hline 295.6 & 300.9 & 309 & 254.4 & 224.2 & 281.2 & 295.1 & 253 & 142.7 & 106.8 & 67.4 & 69.1 & 57.4 \\
\hline
\end{tabular}


other

\begin{tabular}{|c|c|c|c|c|c|c|c|c|c|c|c|c|}
\hline 222.6 & 278 & 295.2 & 271.1 & 237.1 & 287.4 & 267.9 & 252.2 & 173.3 & 151 & 145.7 & 146.3 & 144.2 \\
\hline 398.1 & 419.6 & 427 & 406.9 & 393.8 & 378.2 & 369 & 310.3 & 191.2 & 136.5 & 97.1 & 71.2 & 53.6 \\
\hline 395.1 & 418.8 & 434 & 448.9 & 422.6 & 391.8 & 394.5 & 387.4 & 268.2 & 216.6 & 177 & 141 & 123.6 \\
\hline 359.6 & 377.4 & 372.2 & 406.7 & 372.3 & 376.2 & 398.2 & 359.8 & 178.3 & 105.1 & 68.4 & 63.2 & 53.9 \\
\hline 341.1 & 359.1 & 366 & 352.5 & 330.4 & 316.9 & 315.5 & 299.3 & 185.6 & 141 & 119.2 & 108.2 & 81.2 \\
\hline 364.3 & 389.4 & 413 & 399.1 & 371.8 & 372.9 & 354.2 & 329.5 & 184.2 & 129.3 & 100.6 & 100.6 & 69.8 \\
\hline 348.4 & 373.2 & 378.5 & 387.5 & 363.5 & 326.5 & 364.3 & 342.6 & 228.6 & 178.3 & 117.5 & 100.2 & 83.6 \\
\hline 350.8 & 352.2 & 366 & 356 & 340.8 & 355.5 & 361.9 & 290.3 & 173.5 & 123.4 & 80.2 & 67.4 & 47 \\
\hline 413 & 428 & 442.9 & 462.5 & 425.4 & 404.4 & 402.8 & 384 & 283.8 & 225.2 & 185.8 & 161.7 & 81.9 \\
\hline 391.2 & 398.5 & 389.1 & 372.9 & 340.1 & 318.6 & 327.3 & 305.5 & 225.7 & 169 & 140.7 & 109.2 & 78.5 \\
\hline 367.4 & 365.6 & 364.3 & 359.4 & 336 & 318 & 318.3 & 304.5 & 218.8 & 172.5 & 141.4 & 128.9 & 95.7 \\
\hline 321.8 & 365 & 409.4 & 401.8 & 382 & 374.6 & 358.8 & 342.3 & 205.5 & 177.4 & 130.2 & 117.4 & 113.8 \\
\hline 464.9 & 470.9 & 461 & 463.3 & 440.3 & 427.5 & 420 & 393.2 & 269.6 & 241.1 & 128.3 & 105.1 & 84 \\
\hline 406.5 & 416.8 & 414.2 & 406.8 & 395 & 367.3 & 358.5 & 345.4 & 204.4 & 153.8 & 101.3 & 98.2 & 64.3 \\
\hline 391.1 & 421.3 & 413.4 & 407 & 406.1 & 379.7 & 408.9 & 390.6 & 290.1 & 244.4 & 203.5 & 173.8 & 127.7 \\
\hline 462.1 & 473.3 & 473.5 & 478.3 & 452.1 & 424.9 & 432.3 & 412.3 & 299.5 & 254.1 & 186.5 & 163.3 & 77.6 \\
\hline 286.6 & 290.9 & 290.7 & 285 & 266.6 & 297.2 & 291.7 & 272.3 & 114.7 & 83.3 & 67 & 66 & 62.9 \\
\hline 405.7 & 425.2 & 430.9 & 428 & 417 & 391.2 & 397.7 & 386.5 & 220.2 & 159 & 116.5 & 106.8 & 71.5 \\
\hline 411.5 & 432.9 & 426.7 & 448 & 421.3 & 398.7 & 417.7 & 397.2 & 271.1 & 204.1 & 154.3 & 140.5 & 118.7 \\
\hline 423.5 & 423.7 & 426.1 & 431 & 403.5 & 386.2 & 387 & 362.1 & 263.5 & 212.3 & 169.5 & 152 & 114.7 \\
\hline 397.3 & 399.8 & 437.2 & 413.2 & 416.7 & 402.3 & 409.9 & 397.3 & 283.4 & 237.8 & 199.6 & 186.7 & 145.8 \\
\hline 431.8 & 436.1 & 452.8 & 435.9 & 435.8 & 415.6 & 421.4 & 400.2 & 207.4 & 146.2 & 104.7 & 82.9 & 67.4 \\
\hline 454.8 & 466.4 & 481.1 & 460.6 & 455.6 & 433.1 & 445.2 & 409.5 & 229.9 & 178 & 137.2 & 112.3 & 108.9 \\
\hline 455.9 & 462.6 & 489.8 & 492.3 & 454.6 & 454.1 & 454.5 & 426.3 & 217.2 & 178 & 109.2 & 108.2 & 68.4 \\
\hline 485.8 & 487.2 & 502.4 & 498.7 & 470.1 & 449.3 & 459.2 & 436 & 323.5 & 273.5 & 224.1 & 198.4 & 167.7 \\
\hline 466.7 & 467.2 & 485.5 & 507.5 & 469 & 451.3 & 465.4 & 443 & 299.7 & 272.1 & 220.2 & 199.7 & 178.9 \\
\hline 457 & 469.7 & 486.5 & 477.2 & 466.6 & 447.4 & 450.7 & 427.6 & 312.6 & 268.9 & 223.6 & 99.7 & 65 \\
\hline 242.3 & 252.6 & 260.7 & 258.8 & 240.9 & 282 & 286.7 & 259.2 & 125.4 & 88.8 & 67 & 73.3 & 61.9 \\
\hline 395.2 & 405.4 & 438.7 & 437.3 & 426.7 & 415.1 & 413 & 398.6 & 289 & 218.4 & 182.5 & 176.5 & 100.2 \\
\hline 418.9 & 424.6 & 432 & 434.5 & 423 & 401 & 400.3 & 376 & 222.9 & 168.7 & 115.8 & 88.8 & 77.8 \\
\hline 426.5 & 426.2 & 451 & 473.1 & 457.9 & 429.5 & 428.3 & 429.8 & 297.6 & 253.8 & 155.1 & 81.2 & 65.3 \\
\hline 412.1 & 436.4 & 451.4 & 443.1 & 427.2 & 398.2 & 413.8 & 399.1 & 178.1 & 120.3 & 82.9 & 74 & 59.1 \\
\hline 478.7 & 491.1 & 506.9 & 507 & 486.4 & 458 & 464.5 & 435.9 & 247.5 & 203.6 & 169 & 152.4 & 133.4 \\
\hline 473.4 & 485.4 & 486.3 & 502 & 464 & 456.4 & 450.1 & 424.7 & 311.3 & 270.7 & 214.3 & 176 & 77.6 \\
\hline 455 & 481.7 & 507 & 507.1 & 476.6 & 448.6 & 457.4 & 447.6 & 261.1 & 187.7 & 126.1 & 82.9 & 54.3 \\
\hline 458.8 & 471.7 & 477.7 & 486.2 & 456.7 & 427.9 & 431.8 & 413.6 & 282.9 & 244.3 & 166.9 & 82.3 & 61.5 \\
\hline 455.1 & 472.6 & 482 & 484.1 & 467.8 & 445.6 & 448.5 & 420.4 & 185.3 & 132 & 80.5 & 72.6 & 65 \\
\hline
\end{tabular}


other

\begin{tabular}{|c|c|c|c|c|c|c|c|c|c|c|c|c|}
\hline 436.2 & 462.7 & 479.3 & 483.3 & 453.3 & 441.3 & 442.1 & 432.5 & 328.7 & 258.2 & 208.6 & 202.5 & 169 \\
\hline 440.8 & 456.8 & 492.5 & 498 & 469.2 & 444.8 & 453.1 & 431.1 & 252.3 & 180.7 & 136.5 & 125.8 & 71.9 \\
\hline 448.4 & 452.7 & 475.2 & 504.3 & 472.1 & 438.6 & 444.9 & 431.1 & 254.5 & 165.9 & 120.6 & 131.2 & 143.5 \\
\hline 446.5 & 474.1 & 492.5 & 494.1 & 471.1 & 452.7 & 445.7 & 417.7 & 303.3 & 254 & 205.3 & 180.2 & 148.3 \\
\hline 449.3 & 454.2 & 478.7 & 453.8 & 455.5 & 435.6 & 438.4 & 432.4 & 261.7 & 231.5 & 205.8 & 176.5 & 159.4 \\
\hline 435.5 & 481.9 & 489.6 & 488.9 & 462.6 & 440.2 & 448.1 & 439 & 242.2 & 189.4 & 143.8 & 129.6 & 93.3 \\
\hline 446.1 & 488.4 & 474 & 485.9 & 466.4 & 445.5 & 440.8 & 430 & 284.4 & 227.9 & 182 & 167.9 & 127.7 \\
\hline 421.4 & 444.3 & 461.7 & 469.5 & 423.1 & 432.5 & 441.5 & 402.2 & 213.2 & 154.5 & 95.7 & 84 & 75 \\
\hline 414.7 & 432.9 & 454.1 & 445.3 & 403.2 & 421.4 & 459.8 & 432.7 & 262.2 & 202.9 & 178.6 & 159.2 & 125 \\
\hline 389.5 & 423.5 & 420 & 400.8 & 403.9 & 389 & 360.8 & 324.2 & 223.7 & 189.4 & 134.4 & 122.7 & 118.3 \\
\hline 93.3 & 105.4 & 121.3 & 131 & 110.2 & 115.8 & 111.6 & 120.6 & 115.4 & 108.2 & 91.2 & 95.4 & 89.9 \\
\hline 92.6 & 96.4 & 112.3 & 110.6 & 123.7 & 116.1 & 132.4 & 120.3 & 131 & 117.8 & 104 & 93.3 & 104.7 \\
\hline 298.6 & 303.8 & 293.1 & 289.6 & 287.9 & 290 & 302.1 & 298.9 & 275.8 & 192.5 & 109.9 & 107.5 & 106.8 \\
\hline 96.1 & 102.6 & 108.5 & 117.2 & 119.9 & 130.6 & 125.5 & 132.7 & 121 & 105.8 & 114.7 & 110.2 & 103 \\
\hline 68.4 & 75.3 & 81.2 & 78.1 & 70.2 & 80.5 & 80.9 & 69.1 & 68.4 & 67.7 & 71.5 & 61.5 & 71.5 \\
\hline 57.7 & 55.6 & 58.1 & 58.8 & 74.3 & 106.1 & 103.7 & 99.2 & 106.1 & 84.7 & 77.4 & 87.1 & 87.8 \\
\hline 66.4 & 80.5 & 83.6 & 93 & 101.3 & 99.5 & 109.6 & 91.9 & 92.6 & 91.9 & 89.5 & 79.1 & 78.5 \\
\hline 61.5 & 63.2 & 67 & 72.2 & 72.9 & 77.1 & 78.1 & 79.1 & 90.2 & 77.4 & 70.8 & 72.9 & 81.6 \\
\hline 81.9 & 93.7 & 88.5 & 87.4 & 94.7 & 102.6 & 98.2 & 86.7 & 72.6 & 75 & 69.8 & 74 & 77.8 \\
\hline 60.5 & 65 & 70.8 & 73.3 & 72.9 & 71.9 & 73.6 & 65.7 & 67.7 & 62.9 & 63.6 & 70.2 & 70.5 \\
\hline 169.8 & 165 & 155.4 & 160 & 177.5 & 163.9 & 165.8 & 151.5 & 108.4 & 95.7 & 86.1 & 82.3 & 90.9 \\
\hline 69.1 & 70.5 & 77.8 & 74 & 77.4 & 90.2 & 92.3 & 85.4 & 108.9 & 100.6 & 82.6 & 88.5 & 88.1 \\
\hline 82.9 & 134.3 & 150.9 & 164.9 & 158.5 & 152.6 & 153.1 & 156.5 & 149.6 & 146.7 & 153.4 & 152.1 & 152.1 \\
\hline 61.5 & 63.9 & 70.2 & 71.9 & 75 & 95.7 & 98.5 & 85.4 & 96.1 & 86.1 & 71.5 & 82.3 & 82.9 \\
\hline 118.2 & 116.1 & 123.7 & 133.1 & 138.6 & 137.5 & 144.5 & 157.2 & 131.7 & 131.7 & 134.1 & 194 & 193.5 \\
\hline 94.1 & 96.8 & 103.7 & 109.2 & 117.5 & 121 & 124.1 & 138.6 & 133.4 & 130.3 & 112.3 & 112 & 98.2 \\
\hline 75.7 & 80.5 & 87.2 & 87.8 & 95.7 & 82 & 66.4 & 58.8 & 52.3 & 50.1 & 54.7 & 60.5 & 52.9 \\
\hline 50.8 & 58.1 & 62.6 & 59.8 & 66.4 & 56 & 72.2 & 60.1 & 72.9 & 56.3 & 56.7 & 63.2 & 57.4 \\
\hline 123.1 & 132.4 & 113.8 & 110.6 & 106.8 & 116.9 & 129.6 & 120.4 & 112.7 & 75.3 & 69.9 & 71.9 & 70.6 \\
\hline 65.1 & 67 & 69.1 & 67.8 & 63.2 & 63.7 & 75.3 & 85.5 & 83.6 & 81.9 & 78.5 & 80.5 & 75.1 \\
\hline 102.4 & 126.8 & 138 & 144.1 & 130 & 111.3 & 121.7 & 96.4 & 95.5 & 77.1 & 69.9 & 71.9 & 74.7 \\
\hline 61.6 & 63.6 & 64 & 60.5 & 59.5 & 67.7 & 75.8 & 73.3 & 72 & 74.7 & 74.4 & 69.1 & 65.4 \\
\hline 62.6 & 68.1 & 67.5 & 70.2 & 88.1 & 87.2 & 85.7 & 86.5 & 60.5 & 45.4 & 52.2 & 48.5 & 48.7 \\
\hline 48 & 49.9 & 56.4 & 54.6 & 48.5 & 57 & 53.3 & 58.1 & 62.6 & 65.4 & 59.1 & 55.7 & 60.8 \\
\hline 57.5 & 77.2 & 80.5 & 76.8 & 98.5 & 106.5 & 110.2 & 106.9 & 105.1 & 70.6 & 57.5 & 57.7 & 56.4 \\
\hline 43.2 & 41.2 & 47.7 & 57.1 & 53.3 & 58.1 & 60.6 & 59.8 & 58.8 & 53.9 & 55.7 & 61.2 & 53.3 \\
\hline 53.7 & 63.6 & 57.8 & 58.8 & 56.3 & 56.4 & 67.7 & 81 & 84.8 & 86.7 & 86.5 & 67 & 61.6 \\
\hline
\end{tabular}




\begin{tabular}{|c|c|c|c|c|c|c|c|c|c|c|c|c|}
\hline 54.6 & 55 & 66.7 & 67.5 & 56.8 & 49.1 & 52.6 & 56.3 & 57.5 & 64.3 & 55.4 & 53.3 & 60.5 \\
\hline 62.3 & 66 & 64.7 & 65.4 & 70.8 & 60.6 & 71.2 & 70.9 & 60.2 & 59.1 & 78.2 & 78.5 & 68.2 \\
\hline 48.7 & 50.5 & 54.7 & 67.4 & 67.1 & 58.1 & 58.2 & 59.9 & 70.2 & 60.9 & 64.4 & 73.3 & 63.3 \\
\hline 331.9 & 339 & 344.7 & 355.3 & 327 & 311 & 301.1 & 286.9 & 185.6 & 185.3 & 170.4 & 148.4 & 120.6 \\
\hline 56 & 66.1 & 79.6 & 90.2 & 76.5 & 65.7 & 56.8 & 57.4 & 62 & 54.7 & 54.6 & 54.4 & 55.7 \\
\hline 235.8 & 288.7 & 281 & 264.5 & 209.1 & 270.7 & 286.2 & 261.6 & 196.7 & 156.2 & 142.5 & 135.6 & 134.4 \\
\hline 107.8 & 119.3 & 106.8 & 106.9 & 112.4 & 129.6 & 131.4 & 127.9 & 128 & 135.2 & 146.5 & 137.3 & 127.6 \\
\hline 355.3 & 342.9 & 349.1 & 369.2 & 324.3 & 304.8 & 303.9 & 282.1 & 195.3 & 181.9 & 178.1 & 174.2 & 133.1 \\
\hline 168 & 171.9 & 162.9 & 173.5 & 166.3 & 168.7 & 165.9 & 163.6 & 149 & 142.7 & 140.1 & 128.3 & 103 \\
\hline 301.1 & 306.9 & 341.5 & 388.6 & 339 & 351.4 & 354.8 & 330.2 & 204.2 & 188.8 & 168 & 151.5 & 109.9 \\
\hline 113.1 & 122.8 & 113.7 & 115.9 & 109.3 & 115.8 & 115.2 & 111.4 & 100.9 & 67.5 & 61.3 & 60.5 & 60.9 \\
\hline 342.6 & 347.1 & 361.5 & 362.6 & 333.3 & 316.2 & 302.8 & 278 & 201.8 & 189.8 & 178.8 & 149.6 & 113.4 \\
\hline 111.7 & 113 & 109 & 108.6 & 91.6 & 97.6 & 95.1 & 97.5 & 95.1 & 95.1 & 92.7 & 94.7 & 89.3 \\
\hline 357.3 & 373.6 & 397.9 & 413 & 388 & 370.3 & 387.1 & 352.6 & 213.1 & 160.8 & 148.4 & 140.7 & 117.6 \\
\hline 127.2 & 127.9 & 141.9 & 145.8 & 146.2 & 141.1 & 140.3 & 141 & 101.7 & 93.7 & 88.1 & 75.8 & 72.3 \\
\hline 304.8 & 361.7 & 374.6 & 386.3 & 344 & 342 & 332.7 & 320.2 & 190.5 & 160.7 & 148.4 & 149.4 & 112.3 \\
\hline 109.6 & 112.1 & 125.5 & 131.7 & 126.2 & 120.6 & 104.1 & 81.3 & 69.1 & 72.7 & 81.7 & 81.9 & 76.8 \\
\hline 302.1 & 338.5 & 394.5 & 401.3 & 345.7 & 339.8 & 345.7 & 332.6 & 234.5 & 189.7 & 166.6 & 154.5 & 117.4 \\
\hline 52.3 & 59.8 & 65.4 & 70.2 & 68.1 & 67.5 & 69.6 & 64.3 & 68.2 & 67.5 & 63.9 & 70.2 & 65.7 \\
\hline 332.5 & 391 & 434.3 & 434.3 & 402.5 & 391.8 & 376.3 & 368.2 & 203.4 & 145.9 & 138.2 & 122.8 & 77.2 \\
\hline 90.6 & 80.9 & 77.5 & 82.3 & 91.3 & 90.3 & 97.5 & 89.6 & 90.6 & 80.2 & 81.7 & 77.1 & 79.9 \\
\hline 386.1 & 341.3 & 402.1 & 404.8 & 377 & 373.3 & 354.5 & 329.5 & 227.2 & 194.7 & 171.7 & 158.6 & 124.5 \\
\hline 138.5 & 152.7 & 162.8 & 152.1 & 140.3 & 141.7 & 93.6 & 92.7 & 74.3 & 63.7 & 66.4 & 71.9 & 70.2 \\
\hline 331.2 & 334.3 & 354.9 & 350.9 & 310.3 & 313.5 & 298.9 & 272.4 & 160.1 & 131.7 & 126.6 & 98.9 & 66 \\
\hline 57.7 & 64 & 58.8 & 51.1 & 45.7 & 49.2 & 48.7 & 47.1 & 50.9 & 53.9 & 55 & 59.4 & 60.2 \\
\hline 368.3 & 388.5 & 405.5 & 414.5 & 384 & 381.6 & 407.3 & 385.6 & 266 & 200.4 & 183.8 & 166.5 & 149.7 \\
\hline 154.1 & 160.3 & 164.6 & 168.5 & 172.2 & 161.7 & 160.1 & 120.3 & 95.2 & 85.5 & 84 & 77.5 & 77.9 \\
\hline 436.9 & 449.9 & 463.9 & 495.7 & 449.8 & 436.2 & 421.9 & 412.8 & 293.7 & 246.6 & 241.4 & 208.2 & 169.6 \\
\hline 93.4 & 99.2 & 105.8 & 103.8 & 108.5 & 93.7 & 83.4 & 87.1 & 75.8 & 72.3 & 88 & 139.6 & 138.7 \\
\hline 399.2 & 436.7 & 436 & 407.3 & 426.8 & 393.7 & 414.4 & 402.4 & 285.1 & 253.2 & 230.1 & 207.3 & 180.9 \\
\hline 199.9 & 244.5 & 229.4 & 223.5 & 219.8 & 210 & 212.5 & 200.4 & 186.6 & 86.1 & 78.9 & 80.5 & 82 \\
\hline 331.3 & 372.3 & 390.7 & 398.2 & 378.2 & 369.1 & 361.3 & 341.2 & 197.5 & 137.6 & 114 & 94.8 & 76.4 \\
\hline 72.3 & 82 & 81.9 & 82 & 82.9 & 76.8 & 76.8 & 78.1 & 81.3 & 133.5 & 135.8 & 138.7 & 138.7 \\
\hline 405.7 & 402.8 & 429.4 & 434 & 397.2 & 386.5 & 368.2 & 376.1 & 201.8 & 127.6 & 102.3 & 91.3 & 77.5 \\
\hline 65.3 & 69.2 & 70.6 & 75.7 & 81.7 & 77.5 & 78.8 & 83 & 82 & 78.1 & 68.2 & 68.8 & 75.1 \\
\hline 412.9 & 439.5 & 452 & 473.1 & 433.7 & 435.5 & 437.9 & 408.6 & 288 & 211.8 & 182.6 & 164.3 & 155.9 \\
\hline 66.7 & 72.3 & 83.3 & 100.3 & 97.6 & 92.6 & 94.1 & 97.8 & 97.6 & 92.3 & 89.9 & 79.9 & 82.6 \\
\hline
\end{tabular}




\begin{tabular}{|c|c|c|c|c|c|c|c|c|c|c|c|c|}
\hline 517.8 & 492.9 & 490.9 & 507.5 & 501.3 & 477.7 & 474.9 & 432 & 313.1 & 253.4 & 149.3 & 116.2 & 94 \\
\hline 97.8 & 103.4 & 108.6 & 106.4 & 99.3 & 100.2 & 104.1 & 99.9 & 96.5 & 100.9 & 94.4 & 85.7 & 82.3 \\
\hline 519.6 & 551.3 & 521.1 & 513.7 & 498.2 & 487.7 & 485.1 & 459.4 & 312.6 & 219.5 & 157.6 & 121.7 & 95.4 \\
\hline 98.2 & 131.4 & 168.7 & 165.3 & 171.1 & 128.9 & 141.1 & 171.8 & 169.1 & 126.1 & 100.7 & 84.3 & 86.1 \\
\hline 426.5 & 458.2 & 462.4 & 466.2 & 449.9 & 443.1 & 435.2 & 420.5 & 289.2 & 246.5 & 222.5 & 187.7 & 115.9 \\
\hline 171.3 & 176.2 & 187.6 & 185.2 & 176.7 & 172.1 & 154.2 & 153.1 & 121.1 & 96.4 & 93 & 76.5 & 80.5 \\
\hline 387.4 & 413.3 & 425.1 & 432.7 & 408.8 & 381.1 & 400.6 & 380.8 & 220.4 & 177.6 & 185 & 153.3 & 116.6 \\
\hline 128.7 & 129.7 & 139.6 & 140.3 & 140.8 & 135.5 & 139.2 & 121.9 & 94.2 & 75 & 63 & 57 & 57.4 \\
\hline 374.6 & 410.1 & 416.5 & 418.4 & 427.6 & 410.9 & 404.2 & 365.2 & 186.6 & 122 & 114.5 & 96.4 & 69.9 \\
\hline 185.1 & 172.3 & 167.3 & 175.7 & 179.4 & 176.1 & 169.7 & 167.6 & 107.8 & 101.3 & 95 & 89.3 & 101.6 \\
\hline 471 & 488.6 & 488.5 & 479.8 & 467.9 & 451.1 & 448.6 & 363.6 & 229.9 & 173.9 & 158.3 & 117.2 & 85 \\
\hline 139.7 & 149.6 & 154 & 155.7 & 148.6 & 188.6 & 159 & 149.5 & 132.7 & 129.8 & 121.4 & 127.2 & 135.2 \\
\hline 416.1 & 439.4 & 445 & 439.1 & 425.3 & 412.4 & 416.7 & 388.8 & 205.6 & 137.6 & 124.4 & 100.2 & 78.2 \\
\hline 102.4 & 103.3 & 106.5 & 106.1 & 122.1 & 147.2 & 136.3 & 134.1 & 118.6 & 106.4 & 140.8 & 140.4 & 135.9 \\
\hline 451.5 & 471.3 & 479.1 & 478.3 & 458.3 & 440.9 & 432.8 & 415.7 & 249.8 & 170 & 155.3 & 119.2 & 86.4 \\
\hline 100.9 & 119 & 135.5 & 115.8 & 111 & 88.5 & 88.6 & 78.8 & 82 & 74.7 & 87.4 & 77.2 & 71.5 \\
\hline 349.1 & 364.4 & 380.2 & 368.8 & 351.8 & 338.4 & 315.6 & 286.2 & 176.3 & 139.4 & 120.6 & 105.2 & 69.8 \\
\hline 69.8 & 77.2 & 81.9 & 89.3 & 86.8 & 88.1 & 90.6 & 76.1 & 64.3 & 64.7 & 72.2 & 70.2 & 70.9 \\
\hline 345 & 356 & 382.2 & 372 & 345.6 & 335.7 & 323.1 & 301 & 184.6 & 134.8 & 118.3 & 122.3 & 105.2 \\
\hline 101 & 100.6 & 98.8 & 100.3 & 99.9 & 90.9 & 82 & 63.2 & 61.9 & 65.1 & 67.7 & 63.6 & 61.6 \\
\hline 314.1 & 334.9 & 365 & 368.4 & 350.1 & 330.3 & 347.1 & 321.2 & 207.7 & 155.6 & 137.5 & 138 & 113.4 \\
\hline 105.8 & 116.5 & 121 & 125.1 & 122.4 & 123.7 & 127.5 & 124.8 & 110.7 & 111.3 & 113.4 & 100.7 & 76 \\
\hline 84.8 & 84.3 & 83.7 & 88.8 & 100.6 & 99.6 & 109.2 & 103.4 & 96.4 & 74.4 & 77.8 & 80.9 & 81.2 \\
\hline 91.2 & 88.5 & 89.9 & 91.9 & 86.1 & 100 & 115.8 & 100.9 & 78.2 & 75 & 80.9 & 78.2 & 78.5 \\
\hline 329.4 & 369.1 & 405.5 & 404.6 & 396.6 & 364.8 & 373 & 321.5 & 202.3 & 135.5 & 115.1 & 103.1 & 79.5 \\
\hline 160.8 & 154.5 & 146.5 & 134.1 & 122 & 110.9 & 104.7 & 87.8 & 68.5 & 74 & 77.4 & 76.7 & 76.1 \\
\hline 315.3 & 349.1 & 357.8 & 344.2 & 321.8 & 309.1 & 301.4 & 279 & 200.4 & 140.1 & 139.3 & 125.1 & 98.6 \\
\hline 94.4 & 105.8 & 101.7 & 95.4 & 96.5 & 95.4 & 84.8 & 86.1 & 87.9 & 100.6 & 102 & 107.2 & 103.3 \\
\hline 65.1 & 72.2 & 74.3 & 75.1 & 71.9 & 71.5 & 71.9 & 72.9 & 74 & 71.9 & 79.1 & 79.1 & 77.8 \\
\hline 65 & 67.5 & 67.4 & 65.3 & 67.4 & 72.2 & 62.6 & 57.1 & 61.9 & 63.9 & 63.2 & 63.6 & 61.3 \\
\hline 72.6 & 70.9 & 86.4 & 86.8 & 84.7 & 73.6 & 65.4 & 63.6 & 65.4 & 64.6 & 72.2 & 85.8 & 74.3 \\
\hline 43.3 & 44.6 & 46.4 & 49.1 & 44.7 & 53.6 & 57.1 & 48.4 & 48.7 & 43.9 & 51.1 & 51.2 & 55.3 \\
\hline 168.7 & 154.5 & 150.1 & 155.9 & 144.6 & 145.8 & 146.6 & 146.5 & 124.5 & 114.4 & 110.7 & 100.2 & 88.6 \\
\hline 42.9 & 43.2 & 43.6 & 50.1 & 50.1 & 51.2 & 54.3 & 55 & 53.9 & 53.3 & 57 & 58.8 & 53.2 \\
\hline 122.7 & 111.6 & 125.5 & 127.9 & 131.8 & 120.3 & 117.2 & 108.5 & 90.2 & 86.1 & 74.7 & 76.4 & 76 \\
\hline 67.7 & 68.4 & 68.2 & 70.2 & 74.1 & 75.3 & 74.1 & 78.1 & 70.2 & 68.4 & 70.6 & 72.2 & 71.5 \\
\hline 91.2 & 105.2 & 105.4 & 100.6 & 87.9 & 65.7 & 64 & 63.2 & 57.5 & 58.1 & 58.2 & 56.3 & 55.3 \\
\hline
\end{tabular}


other

\begin{tabular}{|c|c|c|c|c|c|c|c|c|c|c|c|c|}
\hline 46.7 & 48 & 50.9 & 55.3 & 51.9 & 57.7 & 59.5 & 59.8 & 60.1 & 60.9 & 60.5 & 56.7 & 54 \\
\hline 78.9 & 91.6 & 98.9 & 96.8 & 78.9 & 74 & 73.3 & 73 & 69.8 & 66.4 & 69.8 & 68.9 & 71.2 \\
\hline 61.6 & 64.3 & 62.6 & 62.2 & 65.4 & 71.5 & 71.5 & 70.9 & 71.2 & 70.9 & 75.3 & 66.8 & 69.5 \\
\hline 82.9 & 84.8 & 87.8 & 89.9 & 93.7 & 93.7 & 87.5 & 79.8 & 78.8 & 68.5 & 60.8 & 61.3 & 60.8 \\
\hline 53.6 & 51.9 & 55.3 & 52.6 & 54.3 & 56.8 & 62.6 & 70.2 & 68.8 & 68.4 & 70.2 & 73.3 & 73.3 \\
\hline 136.9 & 159.1 & 157.2 & 152.4 & 132.1 & 131 & 128.3 & 127.5 & 124.8 & 96.2 & 79.8 & 77.9 & 81.9 \\
\hline 67 & 65.4 & 65.7 & 65.1 & 66 & 78.9 & 85.7 & 85.7 & 84.8 & 84.7 & 82.7 & 77.8 & 71.9 \\
\hline 62.6 & 65.1 & 69.8 & 73.3 & 67.1 & 68.1 & 70.2 & 71.2 & 67.1 & 62.9 & 68.2 & 70.5 & 72.2 \\
\hline 88.5 & 85.1 & 91.2 & 88.9 & 92.3 & 86.8 & 80.2 & 56.4 & 55 & 56 & 57.8 & 62.2 & 62.3 \\
\hline 93.7 & 92.6 & 101.4 & 95.7 & 104 & 93.4 & 85.7 & 73 & 60.5 & 63.9 & 68.9 & 64.6 & 65.1 \\
\hline 58.8 & 60.6 & 66.7 & 118.1 & 126.2 & 131.7 & 124.9 & 118.3 & 79.1 & 67 & 66.4 & 66.7 & 64.3 \\
\hline 61.5 & 63.6 & 65.3 & 69.1 & 72.6 & 69.8 & 69.1 & 68.4 & 70.8 & 75 & 71.9 & 74.3 & 74 \\
\hline 57 & 56.7 & 57 & 57 & 60.1 & 60.8 & 63.9 & 64.6 & 65.7 & 64.3 & 63.2 & 62.6 & 65.3 \\
\hline 58.8 & 56 & 60.1 & 61.9 & 66.4 & 65.7 & 61.9 & 47.7 & 49.4 & 49.1 & 48.4 & 50.5 & 52.2 \\
\hline 44.2 & 44.6 & 50.8 & 60.2 & 62.6 & 55.6 & 53.6 & 56 & 59.1 & 62.2 & 50.8 & 49.4 & 53.9 \\
\hline 75 & 76 & 79.5 & 86.4 & 80.9 & 71.5 & 68.4 & 68.4 & 69.1 & 74.3 & 74.3 & 71.6 & 70.2 \\
\hline 61.9 & 67.4 & 74 & 73.7 & 70.5 & 71.5 & 78.8 & 75 & 74.7 & 77.8 & 84 & 81 & 86.1 \\
\hline 134.8 & 137.9 & 136.5 & 141 & 145.9 & 140 & 136.9 & 127.5 & 109.2 & 99.2 & 93.4 & 82.9 & 77.1 \\
\hline 53.7 & 60.1 & 78.8 & 74.3 & 77.4 & 83 & 74.3 & 71.5 & 69.9 & 74 & 69.1 & 64 & 64.6 \\
\hline 102.3 & 106.8 & 117.5 & 152.5 & 156.3 & 148.8 & 147 & 144.7 & 141.5 & 139.4 & 139.8 & 84.3 & 86.4 \\
\hline 45.6 & 45.6 & 55 & 46.3 & 47.7 & 45 & 46.7 & 46.3 & 51.5 & 53.2 & 54.3 & 56.3 & 59.1 \\
\hline 59.4 & 68.9 & 87.4 & 85 & 75.3 & 67.7 & 61.5 & 62.2 & 68.8 & 126.9 & 121.3 & 133.6 & 120.6 \\
\hline 51.8 & 57.4 & 68.8 & 58.2 & 59.1 & 59.8 & 58.4 & 62.6 & 64.3 & 70.5 & 70.5 & 63.9 & 66 \\
\hline 72 & 77.8 & 94 & 83.6 & 80.5 & 74.7 & 76 & 77.4 & 78.5 & 70.8 & 67.1 & 67.4 & 68.8 \\
\hline 60.8 & 59.4 & 79.8 & 70.5 & 72.6 & 67.7 & 63.6 & 61.9 & 60.6 & 62.6 & 63.6 & 62.6 & 66.7 \\
\hline 84 & 93.3 & 133.4 & 137.9 & 132.4 & 93 & 91.2 & 84.7 & 133.8 & 127.1 & 127.6 & 121.2 & 122.6 \\
\hline 138.1 & 141.3 & 141.9 & 143 & 147 & 146.3 & 140.6 & 141.2 & 141.8 & 97.5 & 115.8 & 93.3 & 69.9 \\
\hline 179.4 & 222.6 & 222 & 205.4 & 173.1 & 169.1 & 159.5 & 156.7 & 138 & 75 & 69.1 & 61.2 & 62.2 \\
\hline 79.1 & 79.1 & 84.7 & 77.8 & 79.8 & 78.5 & 79.8 & 117.2 & 128.6 & 118.5 & 100.9 & 63.6 & 62.9 \\
\hline 188 & 206.1 & 214.7 & 203.9 & 213 & 218.4 & 212.7 & 201.9 & 180.9 & 94.3 & 114.4 & 105.1 & 65 \\
\hline 81.6 & 86.7 & 88.8 & 129.3 & 129.6 & 127.5 & 127.9 & 128.9 & 110.9 & 82.6 & 67.7 & 61.5 & 62.2 \\
\hline 158.3 & 160 & 165.2 & 175.6 & 152.1 & 125.5 & 127.2 & 138.9 & 112.3 & 75.3 & 70.8 & 67 & 66.7 \\
\hline 81.2 & 85.4 & 89.9 & 82.9 & 86.7 & 84.3 & 91.6 & 85 & 82.6 & 75 & 65 & 59.8 & 61.2 \\
\hline 144.3 & 157.1 & 163.5 & 184.7 & 184.4 & 184 & 149.2 & 150.8 & 143 & 89.5 & 72.6 & 63.2 & 62.2 \\
\hline 147.5 & 139.2 & 142.9 & 145.8 & 151.9 & 153.7 & 174.7 & 161.9 & 137.1 & 69 & 55.6 & 52.2 & 54.6 \\
\hline 75.7 & 103.7 & 109.2 & 100.9 & 94 & 95 & 97.8 & 100.6 & 117.8 & 104 & 86.7 & 61.9 & 62.2 \\
\hline 77.4 & 79.8 & 94.7 & 124.1 & 129.9 & 109.9 & 97.5 & 90.9 & 93.7 & 77.8 & 67 & 63.9 & 68.1 \\
\hline
\end{tabular}




\begin{tabular}{|c|c|c|c|c|c|c|c|c|c|c|c|c|}
\hline 76.5 & 86.1 & 90.2 & 91.2 & 85.7 & 81.6 & 73.6 & 62.6 & 59.4 & 55.3 & 61.5 & 60.1 & 62.6 \\
\hline 61.5 & 65.7 & 69.1 & 68.4 & 64.6 & 66.7 & 69.8 & 67.8 & 59.4 & 58.4 & 60.5 & 66 & 64.3 \\
\hline 123.7 & 119.6 & 129.3 & 123.7 & 111.3 & 95 & 97.5 & 94 & 75.3 & 72.9 & 58.4 & 51.8 & 52.2 \\
\hline 55.6 & 51.1 & 50.5 & 52.9 & 62.2 & 60.5 & 70.2 & 74.3 & 72.2 & 63.2 & 55.3 & 55.6 & 56.7 \\
\hline 82.6 & 82.6 & 82.6 & 80.5 & 67 & 50.1 & 52.5 & 54.3 & 51.1 & 71.3 & 97.9 & 104.1 & 104 \\
\hline 50.5 & 50.1 & 49.8 & 49.8 & 53.6 & 50.8 & 57.4 & 57 & 50.1 & 50.1 & 44.9 & 47 & 46.7 \\
\hline 223.3 & 243.5 & 243.9 & 241.8 & 240.1 & 232 & 227.2 & 166.8 & 105.1 & 73.3 & 64.3 & 59.4 & 60.1 \\
\hline 57.7 & 57.4 & 57.7 & 56.3 & 59.1 & 61.9 & 63.2 & 60.1 & 60.1 & 61.2 & 59.4 & 56.3 & 54.3 \\
\hline 103 & 109.3 & 108.2 & 102.3 & 99.9 & 98.6 & 103.7 & 106.8 & 106.2 & 99.5 & 96.1 & 96.9 & 90.2 \\
\hline 44.9 & 55 & 59.9 & 60.5 & 59.4 & 55.6 & 60.5 & 59.1 & 59.4 & 56 & 48 & 48.4 & 49.1 \\
\hline 151.3 & 141.7 & 134.1 & 131.8 & 127.5 & 123.4 & 120.4 & 125.5 & 128.6 & 118.6 & 109.2 & 113.7 & 88.8 \\
\hline 53.2 & 51.8 & 71.9 & 75 & 75.7 & 72.3 & 76 & 82.3 & 75.3 & 74 & 72.9 & 67 & 54.6 \\
\hline 143.8 & 152.3 & 150.3 & 150.9 & 148.1 & 143 & 134.8 & 79.5 & 65.7 & 66.4 & 67.7 & 60.8 & 64.3 \\
\hline 47.7 & 47.7 & 47.3 & 48.4 & 53.6 & 50.5 & 52.9 & 57.4 & 49.8 & 48.7 & 50.8 & 53.9 & 51.8 \\
\hline 51.9 & 54.3 & 57.7 & 66.7 & 74.7 & 75.7 & 57 & 58.8 & 58.8 & 57.1 & 52.2 & 47 & 47.3 \\
\hline 51.1 & 47.7 & 47.3 & 50.8 & 55.3 & 58.2 & 55.6 & 60.1 & 58.4 & 59.1 & 52.3 & 48.7 & 51.8 \\
\hline 74.7 & 73.6 & 76.4 & 71.9 & 64.6 & 62.6 & 61.2 & 65 & 57.4 & 49.1 & 48.5 & 51.5 & 50.5 \\
\hline 48.7 & 48.7 & 47.3 & 53.9 & 50.5 & 52.2 & 59.1 & 66.4 & 63.2 & 61.5 & 66.4 & 63.2 & 60.1 \\
\hline 50.5 & 53.2 & 56 & 59.1 & 61.2 & 56.7 & 55 & 55 & 51.8 & 47.7 & 46 & 46 & 47.7 \\
\hline 47.7 & 43.9 & 41.8 & 44.9 & 44.2 & 49.8 & 52.5 & 54.6 & 55 & 56.1 & 52.5 & 52.5 & 49.8 \\
\hline 80.2 & 80.9 & 80.5 & 84.3 & 84.7 & 83.3 & 76 & 84.7 & 82.3 & 77.1 & 72 & 74 & 76 \\
\hline 76.4 & 75.7 & 78.8 & 85 & 88.8 & 84.8 & 80.9 & 85.7 & 83.6 & 72.6 & 72.7 & 75.7 & 76 \\
\hline 108.5 & 113 & 116.1 & 107.5 & 101.7 & 97.8 & 100.2 & 97.8 & 93.1 & 79.5 & 73.6 & 75 & 75.7 \\
\hline 70.8 & 68.8 & 70.5 & 73.3 & 73 & 79.1 & 76.4 & 79.8 & 78.1 & 78.1 & 77.4 & 83.3 & 76.7 \\
\hline 83.6 & 90.2 & 95 & 93.3 & 83 & 67.7 & 63.6 & 63.9 & 54.4 & 56.3 & 56.7 & 51.5 & 52.2 \\
\hline 51.1 & 48.7 & 48 & 49.1 & 49.8 & 55 & 62 & 57.4 & 58.1 & 59.1 & 55.6 & 53.2 & 50.8 \\
\hline 128.6 & 129 & 130 & 129.9 & 134.6 & 135 & 128.4 & 115.2 & 102.3 & 101.1 & 53.6 & 52.2 & 54.3 \\
\hline 44.2 & 44.6 & 46.7 & 48.4 & 93.1 & 102 & 104.9 & 108.6 & 104.1 & 48.7 & 52.5 & 49.4 & 49.4 \\
\hline 135.5 & 109.6 & 121.7 & 116.6 & 114.7 & 105.5 & 96.1 & 90.5 & 70.6 & 66.4 & 68.4 & 57.7 & 50.5 \\
\hline 43.2 & 43.5 & 43.5 & 43.5 & 46 & 46.7 & 48.7 & 49.1 & 51.1 & 51.8 & 51.1 & 51.1 & 52.2 \\
\hline 56 & 59.4 & 59.4 & 58.4 & 54.6 & 45.3 & 47.3 & 46 & 44.6 & 43.2 & 44.6 & 46 & 47.7 \\
\hline 112.9 & 122.6 & 125.3 & 125.2 & 122.2 & 118.5 & 113.2 & 112.6 & 82.7 & 77.8 & 61.2 & 57.4 & 53.2 \\
\hline 61.9 & 65.7 & 68.4 & 69.1 & 66 & 55.6 & 53.2 & 54.3 & 56 & 58.1 & 56.7 & 53.6 & 52.9 \\
\hline 47 & 47 & 46.7 & 49.8 & 52.2 & 54.6 & 55 & 59.1 & 53.6 & 56 & 57.4 & 56.3 & 49.1 \\
\hline 59.8 & 60.1 & 65.7 & 66.7 & 60.5 & 66 & 71.9 & 73.6 & 67.7 & 59.4 & 56.7 & 52.5 & 56 \\
\hline 47.3 & 43.5 & 47.7 & 48.7 & 46.7 & 43.9 & 49.4 & 79.8 & 84.7 & 52.9 & 52.9 & 45.3 & 46.7 \\
\hline 69.1 & 68.4 & 74.3 & 73.6 & 69.5 & 62.9 & 63.6 & 63.6 & 65.1 & 64.6 & 58.8 & 56.3 & 54.3 \\
\hline
\end{tabular}


other

\begin{tabular}{|c|c|c|c|c|c|c|c|c|c|c|c|c|}
\hline 50.8 & 49.1 & 54.6 & 51.8 & 51.1 & 66.4 & 69.8 & 71.2 & 67.4 & 68.4 & 66 & 62.2 & 56 \\
\hline 93.7 & 95 & 95.4 & 84.3 & 58.1 & 50.1 & 55 & 57.7 & 60.5 & 57.7 & 56.7 & 53.9 & 48.7 \\
\hline 56.7 & 53.6 & 53.6 & 58.1 & 58.4 & 66.7 & 70.5 & 70.8 & 75.3 & 75.7 & 72.2 & 69.5 & 60.1 \\
\hline 252 & 251.4 & 252.9 & 248.3 & 228.5 & 217.4 & 212.1 & 179.4 & 168.6 & 157.2 & 137.3 & 61.2 & 47.7 \\
\hline 44.2 & 42.5 & 43.5 & 50.8 & 57 & 57.4 & 53.9 & 51.1 & 48 & 47 & 46.3 & 45.3 & 49.4 \\
\hline 99.2 & 104.7 & 101.3 & 112.7 & 101.6 & 99.9 & 93 & 95.7 & 84.7 & 68.1 & 69.1 & 68.8 & 61.2 \\
\hline 50.5 & 51.8 & 53.2 & 53.2 & 56.3 & 58.8 & 59.1 & 62.6 & 72.6 & 110.2 & 111.6 & 60.8 & 55.3 \\
\hline 102.3 & 109.6 & 109.6 & 106.1 & 79.1 & 72.6 & 76.4 & 63.6 & 126.2 & 147.7 & 155.3 & 156.2 & 152.9 \\
\hline 74.7 & 80.5 & 58.1 & 58.4 & 56.3 & 57.4 & 61.5 & 62.2 & 68.8 & 73.6 & 64.3 & 64.6 & 60.1 \\
\hline 212 & 214.3 & 219 & 212.1 & 189.3 & 178.5 & 180 & 180.2 & 175.7 & 163.7 & 163.2 & 192.3 & 177.7 \\
\hline 51.8 & 53.2 & 56.7 & 63.6 & 68.8 & 61.9 & 63.6 & 62.9 & 65.7 & 70.2 & 63.2 & 59.8 & 56.7 \\
\hline 197.7 & 198.4 & 234 & 225.7 & 229.1 & 234.3 & 228.8 & 240.9 & 236.7 & 187.7 & 99.2 & 76 & 66.7 \\
\hline 77.8 & 92.6 & 96.4 & 102.3 & 95.4 & 88.5 & 71.2 & 72.9 & 75 & 65 & 67.7 & 78.8 & 69.5 \\
\hline 233.6 & 217.7 & 231.6 & 234 & 231.9 & 229.8 & 225.7 & 223.9 & 218.4 & 179.7 & 83.6 & 70.5 & 77.8 \\
\hline 210.5 & 202.2 & 220.8 & 220.1 & 197.7 & 220.1 & 234.7 & 229.1 & 210.1 & 163.5 & 90.5 & 82.3 & 71.5 \\
\hline 168.7 & 184.9 & 212.2 & 234.7 & 228.8 & 230.2 & 213.2 & 218.1 & 221.2 & 162.4 & 85.7 & 85.4 & 75.3 \\
\hline 245 & 251.3 & 266.1 & 264.7 & 247.8 & 256.1 & 263.7 & 261.6 & 230.5 & 134.1 & 81.2 & 69.8 & 81.9 \\
\hline \multirow[t]{3}{*}{67} & 82.3 & 80.2 & 93.7 & 82.6 & 90.2 & 55.3 & 86.4 & 79.5 & 70.5 & 81.9 & 72.9 & 78.8 \\
\hline & & & & & & & & & & & & \\
\hline & & & & & 158.3 & 147.9 & 146.2 & 147.6 & 123.7 & 77.4 & 63.6 & 67 \\
\hline 145.2 & 166.6 & 180.7 & 177.6 & 169.3 & 180.1 & 171.8 & 171.8 & 170.4 & 141.4 & 96.8 & 92.6 & 93.3 \\
\hline 75 & 94.7 & 85.4 & 93.7 & 96.4 & 77.1 & 91.6 & 91.6 & 86.4 & 90.2 & 85 & 82.3 & 82.9 \\
\hline 260.9 & 292.4 & 282 & 262.3 & 269.9 & 254.7 & 251.9 & 255.1 & 248.5 & 180.4 & 65.7 & 71.5 & 70.5 \\
\hline 225 & 215.7 & 196.6 & 198.4 & 187.7 & 211.5 & 213.9 & 205.3 & 209.1 & 141.7 & 80.5 & 70.2 & 78.8 \\
\hline 186.3 & 182.5 & 182.5 & 200.8 & 184.2 & 188.4 & 193.5 & 181.8 & 173.8 & 145.5 & 72.6 & 81.2 & 78.1 \\
\hline 171.4 & 189.7 & 208.4 & 203.9 & 199.1 & 202.9 & 196.3 & 201.5 & 183.9 & 127.5 & 73.3 & 67.7 & 69.5 \\
\hline 145.8 & 144.8 & 143.8 & 146.2 & 134.8 & 124.4 & 123.7 & 133.7 & 127.2 & 97.1 & 69.5 & 78.5 & 64.6 \\
\hline 187 & 194.6 & 213.2 & 201.5 & 198 & 193.2 & 202.9 & 200.1 & 193.2 & 156.9 & 84 & 81.2 & 77.1 \\
\hline 176.3 & 193.9 & 199.4 & 211.2 & 198.7 & 191.8 & 188.4 & 174.9 & 178.7 & 162.8 & 95.7 & 104 & 95 \\
\hline 178.3 & 178.3 & 173.5 & 186.6 & 187 & 178.7 & 176.6 & 164.9 & 171.8 & 160.4 & 115.8 & 116.8 & 108.5 \\
\hline 107.8 & 108.9 & 108.9 & 122 & 114.4 & 96.8 & 107.8 & 109.9 & 110.9 & 110.9 & 112 & 108.5 & 107.1 \\
\hline 95.4 & 98.5 & 114.7 & 117.5 & 117.2 & 110.6 & 107.5 & 100.6 & 98.2 & 101.3 & 102.3 & 99.2 & 99.2 \\
\hline 148.6 & 154.8 & 161.1 & 170.4 & 176.6 & 169.3 & 156.9 & 149.3 & 151 & 123.7 & 92.3 & 89.5 & 86.4 \\
\hline 163.5 & 155.5 & 169.7 & 181.4 & 189 & 173.5 & 175.2 & 183.9 & 179.7 & 128.9 & 86.1 & 82.3 & 76 \\
\hline 109.9 & & 42.5 & 98.8 & 112.3 & 132.7 & 127.9 & 120.6 & 114.4 & 97.1 & 83.6 & 84.3 & 70.8 \\
\hline 168 & 163.1 & 169 & 164.2 & 158.6 & 156.2 & 141 & 149.3 & 150.7 & 128.2 & 89.9 & 88.8 & 75.7 \\
\hline
\end{tabular}




\begin{tabular}{|c|c|c|c|c|c|c|c|c|c|c|c|c|}
\hline 143.8 & 153.1 & 163.1 & 159.3 & 163.5 & 165.2 & 166.2 & 90.5 & 77.4 & 95 & 98.5 & 86.7 & 77.8 \\
\hline & & 15.6 & 174.2 & 184.9 & 177.6 & 169 & 149 & 160.4 & 116.1 & 74 & 86.1 & 83.3 \\
\hline 160 & 158.3 & 174.5 & 162.4 & 161.1 & 168 & 164.5 & 171.8 & 174.5 & 165.9 & 106.4 & 91.2 & 98.8 \\
\hline 161.4 & 163.8 & 159.7 & 172.1 & 175.2 & 166.6 & 161.7 & 178.3 & 182.8 & 144.1 & 106.8 & 106.1 & 96.8 \\
\hline 60.5 & 83.6 & 41.5 & 145.8 & 143.4 & 143.1 & 191.8 & 179 & 170.7 & 147.6 & 109.2 & 88.8 & 98.5 \\
\hline 92.3 & 99.5 & 89.5 & 99.5 & 108.2 & 112 & 116.5 & 109.9 & 109.2 & 110.9 & 108.5 & 109.2 & 105.4 \\
\hline 194.9 & 194.9 & 212.2 & 208.7 & 195.6 & 203.6 & 196 & 195.3 & 214.6 & 165.5 & 110.2 & 112.3 & 97.5 \\
\hline 174.2 & 182.1 & 189 & 211.9 & 190.8 & 206 & 203.6 & 185.2 & 174.9 & 142 & 104.7 & 104.4 & 87.4 \\
\hline 181.8 & 185.6 & 202.2 & 208.4 & 196.3 & 216 & 210.5 & 211.9 & 204.9 & 182.8 & 120.6 & 106.4 & 86.4 \\
\hline \multirow[t]{2}{*}{78.8} & 87.4 & & & & & & & & & & & \\
\hline & & & & & & 68.8 & 102 & 93.7 & 101.6 & 94 & 81.2 & 79.5 \\
\hline 168.7 & 178.3 & 202.2 & 203.6 & 190.1 & 198 & 192.5 & 194.9 & 190.1 & 128.2 & 82.3 & 78.8 & 77.8 \\
\hline 142.7 & 143.1 & 146.9 & 143.4 & 147.6 & 156.6 & 156.6 & 155.5 & 146.5 & 110.6 & 73.6 & 67.7 & 67.7 \\
\hline 150 & 160 & 168 & 175.2 & 166.6 & 173.1 & 183.2 & 185.6 & 180.7 & 143.1 & 80.9 & 75.7 & 72.6 \\
\hline 184.6 & 192.2 & 194.9 & 184.9 & 196.6 & 189.7 & 188.4 & 179.4 & 178.7 & 159 & 98.2 & 93.7 & 94 \\
\hline 89.2 & 85.4 & 89.2 & 99.2 & 98.8 & 95.4 & 99.9 & 100.6 & 104.4 & 100.9 & 90.9 & 86.4 & 87.1 \\
\hline 174.9 & 203.6 & 275.8 & 247.1 & 230.9 & 409.2 & 336.6 & 307.2 & 158.3 & 154.8 & 140.3 & 133.4 & 107.1 \\
\hline 411.3 & 423.4 & 428.2 & 433.4 & 424.1 & 194.6 & 239.8 & 228.4 & 178.3 & 169.3 & 144.5 & 132.7 & 125.1 \\
\hline 246.8 & 379.1 & 362.9 & 369.8 & 351.1 & 376.4 & 383.3 & 362.5 & 320 & 305.9 & 302.1 & 248.1 & 137.9 \\
\hline 389.1 & 385 & 406.4 & 409.5 & 401.2 & 413.7 & 410.2 & 379.5 & 362.5 & 348 & 326.2 & 227.1 & 130.3 \\
\hline 372.6 & 375.3 & 421.6 & 406.8 & 406.8 & 434.8 & 425.4 & 380.9 & 321.1 & 285.1 & 283.4 & 259.5 & 128.6 \\
\hline 402.3 & 380.2 & 398.1 & 418.9 & 414.7 & 413.3 & 423 & 393.3 & 256.4 & 203.2 & 154.5 & 151.7 & 141.4 \\
\hline 401.6 & 431.7 & 427.5 & 423 & 427.9 & 428.2 & 418.2 & 340.1 & 175.9 & 147.2 & 140.7 & 144.8 & 132.4 \\
\hline 409.2 & 397.8 & 367.7 & 390.5 & 375.7 & 396.7 & 394.3 & 349.7 & 252.6 & 202.5 & 183.5 & 134.1 & 142.7 \\
\hline 384.7 & 389.5 & 392.9 & 390.5 & 361.2 & 375.7 & 381.2 & 351.1 & 235.4 & 179.4 & 125.5 & 115.1 & 122 \\
\hline 340.8 & 357.7 & 363.6 & 360.8 & 358.4 & 370.8 & 381.9 & 340.8 & 219.8 & 190.4 & 144.1 & 134.4 & 124.8 \\
\hline 392.3 & 428.5 & 456.5 & 456.5 & 447.9 & 473.1 & 476.2 & 418.9 & 234.7 & 204.6 & 183.5 & 158.3 & 153.4 \\
\hline 402.6 & 399.2 & 421.6 & 475.5 & 442.4 & 490.4 & 526.3 & 522.9 & 261.3 & 200.1 & 180.7 & 149.6 & 144.5 \\
\hline 392.3 & 382.9 & 395 & 407.5 & 414.4 & 503.5 & 528.4 & 482.8 & 279.6 & 239.5 & 245.4 & 226 & 203.9 \\
\hline 577.8 & 589.6 & 592.7 & 622.4 & 602 & 609.6 & 606.5 & 551.9 & 365 & 258.5 & 199.4 & 135.8 & 132 \\
\hline 565.1 & 590.3 & 592.4 & 628.3 & 578.2 & 604.5 & 594.4 & 567.1 & 431.3 & 258.2 & 207.4 & 136.2 & 122.7 \\
\hline 574 & 588.2 & 596.2 & 622.1 & 596.5 & 582.3 & 582.3 & 559.2 & 340.1 & 264.7 & 208.7 & 128.2 & 123.7 \\
\hline 569.5 & 581 & 587.5 & 615.2 & 577.2 & 601.7 & 593.7 & 583.7 & 459.3 & 385.3 & 338.3 & 305.5 & 259.5 \\
\hline 576.1 & 576.5 & 597.2 & 619 & 585.1 & 590.3 & 582.7 & 567.1 & 314.5 & 241.9 & 196 & 135.1 & 125.5 \\
\hline 584.8 & 578.9 & 585.4 & 610 & 593.4 & 592 & 583 & 534 & 304.1 & 235.4 & 176.9 & 108.2 & 119.9 \\
\hline 581 & 593.7 & 607.9 & 644.5 & 618.6 & 615.5 & 602.7 & 541.6 & 282.7 & 235.4 & 185.9 & 130.3 & 117.2 \\
\hline 86.4 & 138.2 & 144.5 & 139.6 & 150 & 131.7 & 134.4 & 110.9 & 100.6 & 109.2 & 109.2 & 109.6 & 104.4 \\
\hline
\end{tabular}




\begin{tabular}{|c|c|c|c|c|c|c|c|c|c|c|c|c|}
\hline 407.3 & 423.2 & 421.7 & 420.6 & 357.3 & 313.6 & 313.3 & 271.9 & 213.6 & 157.6 & 149.6 & 142.7 & 132 \\
\hline 295.7 & 340.7 & 343.9 & 321.6 & 263.1 & 186.6 & 123.5 & 114 & 85.8 & 48.4 & 49.5 & 49.2 & 48.4 \\
\hline 43.5 & 43.3 & 43.5 & 42.9 & 45.4 & 47.7 & 53.7 & 50.5 & 45.4 & 46.7 & 49.4 & 49.9 & 48.4 \\
\hline 65.8 & 68.8 & 68.9 & 71.2 & 72 & 65 & 59.9 & 61.3 & 58.4 & 62 & 57 & 53.7 & 51.6 \\
\hline 358.2 & 372.2 & 351.4 & 373.6 & 302.7 & 218.9 & 178.3 & 138.3 & 134.5 & 140.7 & 130.4 & 132.7 & 115.5 \\
\hline 91.7 & 88.6 & 119.9 & 105.5 & 67 & 63.7 & 63.7 & 71.2 & 65.1 & 64.4 & 72.2 & 68.5 & 69.5 \\
\hline 56.3 & 57.8 & 63.9 & 60.9 & 61.3 & 59.8 & 59.5 & 63 & 65.3 & 61.3 & 67 & 65.4 & 65.8 \\
\hline 65.4 & 59.4 & 62 & 64.3 & 60.6 & 61.6 & 68.4 & 60.6 & 58.8 & 68.8 & 71.6 & 69.1 & 68.5 \\
\hline 90.9 & 85.1 & 90.5 & 81.7 & 75 & 88.9 & 90.9 & 95.5 & 42.6 & 50.1 & 51.2 & 52.9 & 56.8 \\
\hline 76.5 & 70.5 & 87.5 & 70.2 & 52.6 & 70.2 & 73.6 & 70.9 & 43.5 & 48.8 & 51.8 & 50.2 & 56 \\
\hline 76.5 & 80.5 & 94.3 & 106.2 & 103.3 & 117.9 & 118.3 & 130.3 & 122.4 & 120.6 & 124.5 & 119.9 & 89.6 \\
\hline 93.4 & 111.4 & 138.2 & 141.4 & 139.6 & 134.5 & 134.8 & 113.8 & 51.8 & 48.8 & 50.5 & 56 & 54.4 \\
\hline 0 & 0 & 0 & 0 & 0 & 0 & 0 & 0 & 0 & 0 & 0 & 0 & 0 \\
\hline 63 & 68.8 & 66.1 & 67.7 & 63.3 & 56.1 & 51.5 & 56.8 & 56.3 & 47.8 & 52.5 & 52.3 & 50.2 \\
\hline 68.8 & 80.6 & 88.1 & 75.4 & 88.5 & 99.6 & 117.6 & 131.7 & 127.6 & 125.1 & 129.3 & 127.6 & 122.7 \\
\hline 64.3 & 66.4 & 77.2 & 81.9 & 74.1 & 77.4 & 79.9 & 75.1 & 60.5 & 49.9 & 53.9 & 52.6 & 51.8 \\
\hline 350.5 & 379.8 & 383.4 & 355.6 & 318.7 & 309.1 & 318.3 & 304.6 & 211.9 & 183.5 & 161.8 & 141.7 & 79.6 \\
\hline 326.6 & 322.2 & 372.2 & 395.5 & 345 & 320 & 302.5 & 280.6 & 164.9 & 116.2 & 97.8 & 83 & 82.3 \\
\hline 57.4 & 58.8 & 55.4 & 54.6 & 54.4 & 56.1 & 57.7 & 54.4 & 57.5 & 60.1 & 63.3 & 66.7 & 66.1 \\
\hline 98.9 & 108.9 & 106.5 & 102.4 & 102 & 112.4 & 109.6 & 100.7 & 80.3 & 72.6 & 70.2 & 75 & 73 \\
\hline 94.8 & 98.6 & 101.6 & 98.9 & 93.3 & 111.4 & 110.2 & 101 & 76.1 & 71.5 & 74.7 & 72.6 & 71.3 \\
\hline 109.6 & 97.9 & 107.2 & 108.9 & 96.9 & 133.7 & 126.9 & 121.4 & 97.5 & 85.1 & 82.6 & 75.8 & 78.9 \\
\hline 94.1 & 108.9 & 107.9 & 106.9 & 103.7 & 127.3 & 110 & 110.9 & 88.2 & 81.3 & 80.5 & 76.1 & 79.5 \\
\hline 381.3 & 389.8 & 418 & 426 & 393.3 & 419 & 431 & 381.8 & 201.9 & 145.6 & 134.4 & 110.3 & 80.6 \\
\hline 61.3 & 62.3 & 62.9 & 63.3 & 62.6 & 71.3 & 68.9 & 72.2 & 72.3 & 71.6 & 72.2 & 74.1 & 76 \\
\hline 379.8 & 381.2 & 389.7 & 393.7 & 362.9 & 392.4 & 390.2 & 362.5 & 241.3 & 178.8 & 146.2 & 131.1 & 99.2 \\
\hline 402.1 & 418.3 & 432.5 & 432.7 & 406 & 412.4 & 420.4 & 398.9 & 248.7 & 150.4 & 119.2 & 100.7 & 75.1 \\
\hline 411.9 & 456.6 & 485.3 & 462.5 & 439.1 & 439 & 446.2 & 427.3 & 276.1 & 173.8 & 136.9 & 97.2 & 77.1 \\
\hline 52.3 & 55 & 58.4 & 57.8 & 55.6 & 47.8 & 49.8 & 54.7 & 54 & 53.9 & 50.9 & 54.6 & 57.5 \\
\hline 167.3 & 237.6 & 264.9 & 258.8 & 238.8 & 253.7 & 260.1 & 252.7 & 220 & 126.6 & 78.1 & 74.1 & 74.7 \\
\hline 156.9 & 162.9 & 205.1 & 223.1 & 219.8 & 230.7 & 236 & 231.9 & 134.2 & 104.5 & 83.4 & 82.3 & 76.8 \\
\hline 154.9 & 169.2 & 222.6 & 223.4 & 210.4 & 223.9 & 225.2 & 231.9 & 178 & 145.1 & 84.2 & 62.9 & 63 \\
\hline 241.7 & 265.2 & 265.4 & 262.2 & 239.5 & 250.9 & 250.6 & 239.9 & 130.1 & 87.1 & 62.6 & 63.7 & 66 \\
\hline 54.4 & 68.4 & 71.6 & 68.2 & 69.5 & 57.8 & 63.2 & 63.3 & 75.4 & 77.4 & 72.3 & 63.2 & 62.6 \\
\hline 274.2 & 278.2 & 282.3 & 285.4 & 282.2 & 264.3 & 248 & 247.5 & 158.8 & 95.5 & 69.8 & 67.1 & 67.7 \\
\hline 297.6 & 302.5 & 304.1 & 317.1 & 298.9 & 264.4 & 246 & 211.5 & 121.7 & 88.9 & 66.1 & 66.7 & 68.2 \\
\hline 294.6 & 300.1 & 319.9 & 317.8 & 302.1 & 286.6 & 262.4 & 253.9 & 123.5 & 97.1 & 74.1 & 66.8 & 68.1 \\
\hline
\end{tabular}


other

\begin{tabular}{|c|c|c|c|c|c|c|c|c|c|c|c|c|}
\hline 303.3 & 307.6 & 304.7 & 300.6 & 290 & 276.9 & 253.7 & 252.8 & 127 & 97.2 & 74.7 & 66.8 & 69.2 \\
\hline 61.5 & 62 & 75.1 & 71.9 & 75.4 & 75 & 72.6 & 82.7 & 78.8 & 80.3 & 74.1 & 80.2 & 79.2 \\
\hline 312.5 & 304.6 & 329.6 & 340.5 & 322.6 & 319.8 & 272.4 & 262.4 & 138.4 & 101.4 & 79.8 & 77.2 & 74.7 \\
\hline 327.8 & 325.5 & 326 & 331.9 & 322.4 & 304.8 & 278.9 & 247.1 & 138.9 & 107.9 & 89.9 & 84.8 & 84.7 \\
\hline 332.4 & 333.5 & 343.9 & 352.7 & 328.2 & 307.7 & 281.4 & 247.2 & 142.7 & 106.9 & 86.1 & 80.6 & 79.1 \\
\hline 326.9 & 329.8 & 327.7 & 324.2 & 318.8 & 301.1 & 273.1 & 263.2 & 137.5 & 102.4 & 79.1 & 80.6 & 80.2 \\
\hline 74.3 & 76.5 & 76.7 & 81.7 & 80.9 & 77.2 & 88.8 & 92.7 & 90.5 & 86.8 & 90.5 & 85.1 & 93 \\
\hline 333.4 & 326.8 & 335.2 & 332 & 312.7 & 298.1 & 271.1 & 256.5 & 117.9 & 101.3 & 84.4 & 78.9 & 81.2 \\
\hline 322.9 & 322 & 318.4 & 304.9 & 296.5 & 287.6 & 265.7 & 220.3 & 118.5 & 101.7 & 84.7 & 86.5 & 82 \\
\hline 308.3 & 304.2 & 327.9 & 314.6 & 286.7 & 274.5 & 249.5 & 235.2 & 171.6 & 122.3 & 88.9 & 88.5 & 83 \\
\hline 307.4 & 309.1 & 303.8 & 303.7 & 274.6 & 266.8 & 241.9 & 225.4 & 143 & 114 & 82.3 & 77.4 & 74.4 \\
\hline 62.6 & 67.7 & 68.2 & 73.7 & 70.5 & 73.4 & 82.6 & 79.2 & 79.8 & 78.2 & 85.4 & 79.6 & 83 \\
\hline 309.9 & 312.6 & 322.7 & 321.3 & 314.7 & 271.7 & 205.6 & 169 & 135.9 & 123.4 & 89.6 & 83.6 & 75.8 \\
\hline 312.9 & 313.8 & 312.9 & 359.2 & 329.4 & 313.9 & 227.1 & 209.9 & 175.6 & 157.3 & 91.2 & 88.9 & 86.7 \\
\hline 308.5 & 313.9 & 329.3 & 316.3 & 289.9 & 289 & 260.4 & 222.6 & 182.6 & 168 & 97.9 & 79.1 & 75.8 \\
\hline 307.9 & 314.9 & 307.5 & 305.1 & 296.5 & 272.5 & 208.4 & 184 & 149.3 & 140.1 & 81.2 & 81.3 & 77.5 \\
\hline 62 & 67.4 & 86.1 & 75.3 & 85.5 & 82 & 75.3 & 75.4 & 87.4 & 83 & 86.7 & 77.2 & 77.8 \\
\hline 311.6 & 312.2 & 322.1 & 314.7 & 291.8 & 289.3 & 238 & 201.9 & 166.9 & 151.1 & 77.1 & 73 & 73.6 \\
\hline 303.4 & 305.4 & 302.6 & 293.1 & 286.4 & 261.3 & 217.7 & 214.7 & 183.5 & 145.9 & 76.4 & 74.4 & 74.7 \\
\hline 295.4 & 296.1 & 295.8 & 288.2 & 279.9 & 253.4 & 214 & 188.7 & 159.1 & 147.6 & 81 & 77.4 & 76.5 \\
\hline 290.9 & 297 & 293.4 & 288.9 & 273.6 & 260.3 & 222.6 & 197.3 & 161.7 & 156 & 79.8 & 78.9 & 76.1 \\
\hline 64.7 & 67.4 & 72 & 72.6 & 70.2 & 74 & 78.5 & 77.4 & 81.7 & 76 & 80.3 & 81.2 & 82 \\
\hline 290.7 & 287.6 & 298.6 & 297.3 & 297.2 & 267.6 & 221.6 & 202.5 & 175.3 & 174.2 & 112.4 & 96.1 & 98.2 \\
\hline 296.2 & 289.7 & 291.9 & 284.1 & 276.7 & 265.7 & 229.3 & 244.9 & 184.6 & 156.9 & 95.8 & 98.5 & 95.5 \\
\hline 290.1 & 296.5 & 289.2 & 290.5 & 281.1 & 268.2 & 234.9 & 242.1 & 160.4 & 117.9 & 108.5 & 100 & 98.6 \\
\hline 299.3 & 293.7 & 294.3 & 282.7 & 270.1 & 256.8 & 287 & 278.4 & 250.9 & 173.6 & 89.3 & 88.8 & 89.3 \\
\hline 67.4 & 66.1 & 73.4 & 69.1 & 84.1 & 80.2 & 79.2 & 83.3 & 77.5 & 68.1 & 61.3 & 67.1 & 68.4 \\
\hline 249.8 & 259.1 & 266.2 & 278.4 & 269.5 & 250.7 & 191.1 & 162.9 & 157.9 & 133.5 & 86.5 & 83.3 & 84.1 \\
\hline 182.2 & 194 & 202.9 & 203 & 170.1 & 182.8 & 147.7 & 166.6 & 169.8 & 155.2 & 94.8 & 86.7 & 77.2 \\
\hline 204.3 & 182.1 & 178.8 & 176.9 & 175 & 187.7 & 185.3 & 201.5 & 173.2 & 148.6 & 98.2 & 92.3 & 128 \\
\hline 221.3 & 219.5 & 232.3 & 234.3 & 216.8 & 232.3 & 227.8 & 212.6 & 207.4 & 173.6 & 133.7 & 91.3 & 88.9 \\
\hline 76.4 & 75.8 & 77.1 & 77.5 & 82.3 & 85.1 & 90.2 & 87.2 & 91 & 91.9 & 93.7 & 86.4 & 94.8 \\
\hline 193.3 & 206.3 & 203.6 & 209.4 & 146.3 & 189.4 & 197 & 192.6 & 169.3 & 154.2 & 143.1 & 92.4 & 81.9 \\
\hline 189 & 195.7 & 193.2 & 195.4 & 182.1 & 188.4 & 193.2 & 205.6 & 164.9 & 151 & 116.2 & 81.2 & 81 \\
\hline 195.7 & 198.7 & 204.2 & 207.8 & 171.8 & 151.8 & 217.7 & 222.6 & 190.9 & 175.2 & 90.6 & 81.6 & 81.7 \\
\hline 179.5 & 205.6 & 215.7 & 217.7 & 205.6 & 159.8 & 210.5 & 208.7 & 176.7 & 164.9 & 91.3 & 85 & 82.3 \\
\hline 64 & 64.6 & 66.8 & 96.1 & 142.8 & 136.9 & 143.5 & 142.4 & 133.1 & 133.8 & 133.4 & 148 & 103.8 \\
\hline
\end{tabular}




\begin{tabular}{|c|c|c|c|c|c|c|c|c|c|c|c|c|}
\hline 190.2 & 260.1 & 251 & 249.6 & 241.5 & 257.6 & 269.7 & 260.7 & 216.3 & 236.4 & 237.8 & 233.3 & 228.1 \\
\hline 177.7 & 262.4 & 241.6 & 239.3 & 242 & 250.6 & 267.9 & 280.2 & 291.4 & 208.6 & 199 & 191.1 & 223.8 \\
\hline 260.8 & 258.7 & 282.1 & 284.2 & 270 & 301.5 & 310.9 & 323.1 & 274.4 & 199.8 & 182.2 & 224.3 & 203.2 \\
\hline 292.1 & 285.8 & 307.9 & 307.4 & 296.9 & 319.4 & 323 & 322.9 & 280.5 & 263.6 & 253.7 & 231.1 & 207.5 \\
\hline 69.1 & 70.2 & 78.5 & 78.5 & 76.4 & 78.2 & 76.4 & 74.4 & 87.1 & 76.5 & 76.7 & 80.3 & 78.5 \\
\hline 285.7 & 292 & 292.4 & 295.7 & 289.7 & 310.9 & 310.2 & 304.6 & 176.7 & 147.9 & 141.8 & 120.3 & 102.7 \\
\hline 284.9 & 290 & 309.2 & 307.9 & 297.3 & 314.9 & 313.2 & 312.4 & 222.9 & 200.8 & 179.1 & 136.9 & 141.4 \\
\hline 297.2 & 297 & 310.2 & 306.1 & 281.2 & 293.6 & 296.6 & 300.3 & 170.5 & 132.4 & 137.3 & 105.4 & 104.5 \\
\hline 300.6 & 296.9 & 302.8 & 303.4 & 290.8 & 288.8 & 292 & 294.2 & 174.3 & 143.4 & 130 & 100.9 & 89.9 \\
\hline 56.3 & 77.5 & 119.7 & 116.5 & 112.1 & 123 & 88.2 & 91.2 & 83.7 & 85.4 & 67.8 & 66.4 & 69.9 \\
\hline 229.5 & 222.3 & 226.5 & 219.7 & 218.4 & 217.1 & 226.8 & 239.8 & 209.5 & 222.1 & 241.9 & 245 & 237 \\
\hline 239.4 & 258 & 250.7 & 251.8 & 253.1 & 268.7 & 273.4 & 262.5 & 230 & 235.6 & 223.4 & 238 & 166 \\
\hline 250.7 & 261.1 & 268.3 & 287.4 & 269.8 & 272.9 & 302.3 & 327.7 & 240.9 & 203 & 147.9 & 119.3 & 100.9 \\
\hline 254.4 & 300.2 & 374.6 & 395 & 308.5 & 356.2 & 384.9 & 387.4 & 248.5 & 203.6 & 163.8 & 139 & 107.5 \\
\hline 79.6 & 88.1 & 99.6 & 98.8 & 99.3 & 111.6 & 114.8 & 122.3 & 110.7 & 109.9 & 114.8 & 124.4 & 100.7 \\
\hline 503.8 & 477.3 & 477.3 & 476.3 & 453.9 & 462.4 & 449.9 & 462.6 & 303 & 198.1 & 177.6 & 126.2 & 93 \\
\hline 489.7 & 456.5 & 469.9 & 467.6 & 437.5 & 447.3 & 445.5 & 449.4 & 287.5 & 237.8 & 175.7 & 136.2 & 100.7 \\
\hline 505 & 471.1 & 483.8 & 475.1 & 456.8 & 472.3 & 472.5 & 463.4 & 325.9 & 323.4 & 219.9 & 136.2 & 99.6 \\
\hline 492.7 & 464.8 & 481.5 & 493.2 & 479.3 & 469.7 & 478.4 & 460.4 & 308.6 & 262.7 & 203.6 & 159.8 & 125.1 \\
\hline 210.7 & 204.9 & 179.4 & 177.6 & 189.2 & 183.6 & 171.9 & 182.8 & 93.3 & 85.4 & 85.5 & 80.5 & 84.1 \\
\hline 515.5 & 483.4 & 505.5 & 500.7 & 474.9 & 469 & 466.3 & 438.4 & 320.8 & 271 & 225.9 & 214.1 & 183.3 \\
\hline 516.3 & 497.5 & 493 & 499.1 & 487.7 & 469.5 & 470 & 448.2 & 322 & 286.4 & 260.4 & 215.4 & 193.1 \\
\hline 514.4 & 504.9 & 510.7 & 508.8 & 498 & 482.6 & 476.6 & 452.3 & 330.4 & 284.8 & 217.8 & 136.5 & 102 \\
\hline 507 & 483.5 & 495.8 & 511.7 & 491.2 & 473.6 & 472 & 447.7 & 297 & 245.1 & 176.6 & 134.2 & 104.1 \\
\hline 81.2 & 82 & 87.1 & 86.1 & 83.3 & 82.3 & 80.9 & 83.7 & 109.2 & 157.3 & 120.3 & 122.4 & 102.4 \\
\hline 307 & 279.3 & 276 & 273.5 & 269.8 & 265.2 & 281.4 & 321.8 & 258.8 & 230.5 & 223.7 & 187.8 & 134.1 \\
\hline 73 & 82.3 & 65.4 & 76 & 73.7 & 71.9 & 74.4 & 68.1 & 70.9 & 72.6 & 65.4 & 69.1 & 70.2 \\
\hline 207.8 & 197 & 212.3 & 202.9 & 182.1 & 196.4 & 193.5 & 177.4 & 126.5 & 138 & 142.4 & 134.4 & 121.3 \\
\hline 214.4 & 208.7 & 209.5 & 209.8 & 176.7 & 164.5 & 159.1 & 163.5 & 141.1 & 137.5 & 119.6 & 105.1 & 100.7 \\
\hline 84.4 & 84.7 & 83 & 84.7 & 84.1 & 91.2 & 100.7 & 100.9 & 88.9 & 80.9 & 83 & 84.7 & 84 \\
\hline 328.1 & 340.8 & 366.1 & 338 & 310.3 & 305.9 & 312.1 & 279.7 & 170 & 120.3 & 97.6 & 97.1 & 69.6 \\
\hline 48.7 & 47.1 & 48.7 & 48.1 & 45.6 & 46.1 & 50.1 & 46.4 & 46 & 48.5 & 55 & 54.4 & 56 \\
\hline 95.4 & 94.3 & 100.3 & 96.4 & 85.8 & 89.5 & 89.9 & 67.8 & 59.8 & 58.8 & 62.6 & 55.7 & 58.1 \\
\hline 105.8 & 108.3 & 123.7 & 122.7 & 93.4 & 91.6 & 75.4 & 66.4 & 54.7 & 68.4 & 67.7 & 68.5 & 68.1 \\
\hline 66.8 & 66.7 & 73 & 72.2 & 63.9 & 68.5 & 56 & 53 & 54.6 & 56 & 66.1 & 69.8 & 69.5 \\
\hline 55 & 51.6 & 61.9 & 59.5 & 53.6 & 55.4 & 58.4 & 51.9 & 53.6 & 55.4 & 61.5 & 63 & 64.3 \\
\hline 47 & 46.7 & 47.7 & 45.4 & 46.3 & 53.9 & 48.8 & 47 & 46.7 & 48.8 & 55.6 & 57.5 & 57.4 \\
\hline
\end{tabular}




\begin{tabular}{|c|c|c|c|c|c|c|c|c|c|c|c|c|}
\hline 73.6 & 70.9 & 80.2 & 92.4 & 91.6 & 91.3 & 90.2 & 90.3 & 74.3 & 65.1 & 69.5 & 70.2 & 70.5 \\
\hline 113.4 & 118.6 & 128.6 & 122.7 & 80.6 & 87.1 & 89.5 & 76.8 & 64.6 & 61.9 & 77.1 & 88.6 & 85.4 \\
\hline 116.1 & 111.3 & 128.7 & 135.1 & 99.9 & 102.4 & 97.1 & 91.6 & 70.6 & 67.4 & 70.8 & 70.8 & 70.2 \\
\hline 53 & 51.8 & 54.3 & 51.6 & 54.3 & 55 & 55.6 & 55 & 57.7 & 56 & 61.6 & 63.6 & 61.5 \\
\hline 217.4 & 233 & 240.9 & 235.7 & 160.1 & 212.9 & 212.3 & 202.9 & 129.3 & 89.3 & 78.1 & 67.4 & 60.8 \\
\hline 148.4 & 145.5 & 154.9 & 157.2 & 131.4 & 163.5 & 217.5 & 189.4 & 149.7 & 126.5 & 104.4 & 87.1 & 84.7 \\
\hline 117.9 & 121.7 & 122.1 & 124.4 & 120.7 & 132.4 & 137.6 & 125.5 & 86.8 & 63.6 & 62.6 & 63.6 & 63.9 \\
\hline 105.1 & 107.8 & 110.7 & 111.3 & 102.4 & 112.3 & 106.2 & 103.7 & 76.5 & 76 & 75.4 & 69.1 & 69.5 \\
\hline 102.6 & 105.2 & 109.6 & 109.6 & 102 & 110.3 & 120.3 & 108.6 & 72.6 & 61.3 & 62.9 & 61.6 & 62.2 \\
\hline 116.5 & 128.7 & 132 & 129 & 134.4 & 145.6 & 137.5 & 122.1 & 76.4 & 75.8 & 80.5 & 77.1 & 73.6 \\
\hline 122 & 136.9 & 150 & 146.5 & 130.7 & 138 & 123.4 & 113.8 & 66.7 & 66 & 73.7 & 76 & 72.7 \\
\hline 130.9 & 135.1 & 140.7 & 144 & 143.7 & 139.1 & 138 & 131.2 & 77.8 & 66.4 & 69.2 & 67.4 & 68.1 \\
\hline 126.8 & 130 & 144.5 & 147.2 & 132.8 & 143.8 & 135.6 & 140 & 129 & 126.1 & 86.1 & 74.3 & 68.4 \\
\hline 380.7 & 404.7 & 402.2 & 376.7 & 323.5 & 271.8 & 251.7 & 240.7 & 180.1 & 166.7 & 149.5 & 129.7 & 70.8 \\
\hline 368.2 & 377.5 & 400.1 & 403.9 & 340.5 & 284.5 & 324.2 & 320.4 & 218.5 & 161.2 & 136.1 & 142.6 & 128.6 \\
\hline 282 & 323.3 & 336.4 & 337.7 & 261.1 & 283.7 & 285.7 & 246.8 & 121.3 & 79.8 & 64.6 & 55.6 & 52.9 \\
\hline 204.1 & 224.8 & 254.5 & 231.6 & 189.9 & 193.2 & 187.7 & 198.5 & 143.4 & 120.3 & 88.5 & 81.2 & 59.8 \\
\hline 151.5 & 152.1 & 182 & 180.4 & 193.3 & 187.1 & 191.6 & 190.6 & 143.4 & 99.2 & 87.1 & 83.6 & 65 \\
\hline 185.2 & 197.4 & 200.6 & 201.7 & 195.1 & 207.5 & 206.2 & 216.2 & 149.3 & 114.7 & 101.6 & 96.1 & 65.7 \\
\hline 199.7 & 204.8 & 205.8 & 213.2 & 204.9 & 207.9 & 225.2 & 231.2 & 225.6 & 197.8 & 121.1 & 95.4 & 66 \\
\hline 189.4 & 197.6 & 196.1 & 197.9 & 190 & 191.7 & 202.8 & 205.2 & 146.6 & 96.8 & 83.3 & 82.9 & 79.5 \\
\hline 246.3 & 251.1 & 264.1 & 271.5 & 265.3 & 272.1 & 276.7 & 277.4 & 188.1 & 144.8 & 128.6 & 118.9 & 91.2 \\
\hline 234.3 & 270.6 & 280.6 & 287.6 & 286.6 & 281.4 & 284.6 & 284.2 & 219.1 & 154.5 & 131.3 & 125.1 & 94.3 \\
\hline 250.7 & 280.1 & 299.8 & 303.3 & 293.7 & 293.8 & 286.3 & 292.9 & 240.8 & 176.6 & 128.2 & 131.3 & 100.9 \\
\hline 258.5 & 288.1 & 273.6 & 269.2 & 275 & 270.7 & 289.7 & 268 & 271.3 & 245.6 & 123.1 & 109.2 & 84 \\
\hline 236.2 & 251.9 & 245.9 & 202.8 & 173.5 & 160 & 155.5 & 144.8 & 118.9 & 82.6 & 86.1 & 83.3 & 64.6 \\
\hline 229.6 & 274.9 & 278.1 & 299.4 & 294.1 & 285.6 & 286.9 & 277.5 & 190.5 & 128.6 & 110.9 & 105.8 & 80.5 \\
\hline 211.6 & 255.8 & 259.4 & 266.2 & 269.5 & 271.6 & 273.7 & 267.4 & 176.5 & 142 & 130.3 & 126.1 & 99.5 \\
\hline 235 & 257.6 & 217.7 & 159.7 & 211.4 & 244.7 & 261.6 & 257.1 & 167.4 & 138.2 & 98.5 & 97.1 & 77.4 \\
\hline 184.4 & 149.4 & 148.3 & 133.4 & 126.8 & 124.4 & 144.5 & 156.9 & 129.9 & 108.5 & 81.9 & 76.4 & 72.2 \\
\hline 181.7 & 165.6 & 202.7 & 140.9 & 126.1 & 124.1 & 118.5 & 106.4 & 90.9 & 91.9 & 72.6 & 71.9 & 52.5 \\
\hline 197.1 & 158.7 & 143.1 & 141.7 & 140.3 & 141.4 & 141 & 143.1 & 134.8 & 125.8 & 92.3 & 84.7 & 80.9 \\
\hline 185 & 173.9 & 188.7 & 182.1 & 178.3 & 170.7 & 168.7 & 166.9 & 127.5 & 108.9 & 89.2 & 86.1 & 82.6 \\
\hline 193.1 & 180.3 & 190.8 & 187.3 & 181.1 & 186.3 & 185.6 & 176.6 & 135.8 & 119.9 & 89.5 & 84.3 & 79.5 \\
\hline 213.9 & 195.1 & 203.6 & 206.3 & 202.2 & 203.9 & 203.2 & 203.6 & 145.5 & 105.4 & 85 & 83.3 & 77.1 \\
\hline 21.3 & 241.9 & 271.8 & 271.5 & 234.5 & 190.1 & 178.7 & 163.1 & 117.8 & 91.9 & 73.6 & 73.3 & 62.6 \\
\hline 211.5 & 265.8 & 289.2 & 277.2 & 273.6 & 205.6 & 201.1 & 193.2 & 151.7 & 125.5 & 90.5 & 89.9 & 78.8 \\
\hline
\end{tabular}


other

\begin{tabular}{|c|c|c|c|c|c|c|c|c|c|c|c|c|}
\hline 194.1 & 240.1 & 282.5 & 283.7 & 274.9 & 198.7 & 212.5 & 217.4 & 146.2 & 122 & 94.7 & 85.7 & 74 \\
\hline 232.7 & 297.9 & 328.2 & 321.6 & 311 & 245 & 246.4 & 237.1 & 159 & 136.9 & 91.9 & 88.1 & 66.4 \\
\hline 190 & 238.7 & 268.3 & 258 & 250.5 & 180.4 & 174.2 & 171.1 & 138.9 & 111.6 & 91.6 & 79.5 & 70.5 \\
\hline 194.9 & 221.1 & 248 & 247.5 & 238.6 & 165.2 & 175.6 & 164.9 & 114.7 & 85.4 & 72.9 & 74.7 & 64.3 \\
\hline 147 & 187.4 & 211.4 & 225.5 & 218.8 & 141.7 & 143.8 & 139.6 & 105.4 & 85 & 57 & 53.2 & 50.1 \\
\hline 208.5 & 239.4 & 265.1 & 223.7 & 191.3 & 164.5 & 201.8 & 193.5 & 157.9 & 126.8 & 102.3 & 79.1 & 69.8 \\
\hline 215.1 & 252.1 & 249.5 & 248.5 & 219.1 & 207.4 & 223.6 & 210.8 & 136.5 & 115.1 & 80.5 & 73.6 & 61.9 \\
\hline 213.1 & 241.9 & 258 & 263.5 & 234.6 & 206.3 & 203.2 & 210.5 & 134.8 & 116.8 & 81.6 & 75.7 & 53.6 \\
\hline 195.1 & 210.2 & 212.6 & 205.5 & 183.3 & 146.2 & 149.6 & 157.2 & 102.6 & 92.3 & 87.4 & 72.9 & 66 \\
\hline 203.4 & 226.4 & 243.2 & 234.4 & 219.4 & 198.4 & 210.8 & 214.3 & 153.8 & 118.5 & 86.1 & 83.6 & 75 \\
\hline 227.2 & 254.8 & 264.6 & 250.3 & 237.4 & 219.1 & 227.1 & 230.5 & 173.5 & 140.7 & 93.3 & 91.9 & 94.3 \\
\hline 221.6 & 238.8 & 255 & 257 & 228.3 & 207.7 & 208.4 & 213.6 & 165.5 & 124.8 & 79.5 & 78.5 & 67 \\
\hline 208.3 & 219.9 & 214.3 & 230.6 & 208.9 & 177.6 & 190.8 & 187.3 & 138.9 & 108.5 & 78.5 & 87.1 & 83.3 \\
\hline 105.4 & 112.7 & 155.5 & 173.5 & 150.3 & 132.7 & 180.4 & 176.9 & 144.1 & 88.8 & 74 & 74 & 77.1 \\
\hline 187 & 197.3 & 196.6 & 200.4 & 190.1 & 194.9 & 197.3 & 186.6 & 146.5 & 111.6 & 84.3 & 78.8 & 81.6 \\
\hline 170.5 & 181.4 & 187.7 & 191.1 & 200.8 & 259.3 & 277.3 & 211.9 & 162.1 & 118.5 & 87.1 & 84 & 77.1 \\
\hline 163.1 & 191.8 & 193.5 & 254.3 & 265 & 275.2 & 275.4 & 207.7 & 152.8 & 118.2 & 81.2 & 79.5 & 82.9 \\
\hline 198.4 & 223.6 & 190.1 & 245 & 236.7 & 314 & 301.5 & 293.1 & 236.3 & 130.7 & 78.1 & 75.7 & 76.7 \\
\hline 125.1 & 135.8 & 201.8 & 263.7 & 258 & 266 & 248.7 & 241.2 & 200.4 & 154 & 114.7 & 108.2 & 118.5 \\
\hline 172.8 & 180.1 & 183.5 & 184.2 & 180.7 & 185.9 & 199.4 & 207.7 & 124.4 & 92.3 & 58.8 & 51.8 & 60.1 \\
\hline 188.4 & 202.2 & 209.1 & 205.3 & 195.6 & 209.8 & 206.3 & 204.2 & 126.8 & 95.7 & 57 & 48.7 & 55.4 \\
\hline 188 & 204.9 & 208.4 & 209.1 & 195.7 & 189.4 & 192.5 & 201.5 & 126.5 & 95.4 & 59.4 & 55 & 62.9 \\
\hline 186.4 & 190.4 & 187 & 185.2 & 173.1 & 173.1 & 167.6 & 167.6 & 117.8 & 102 & 77.1 & 67 & 72.6 \\
\hline 153.1 & 166.9 & 164.5 & 162.1 & 153.8 & 110.9 & 154.5 & 176.6 & 164.9 & 149.6 & 94.7 & 63.2 & 72.2 \\
\hline 250.4 & 212.8 & 217 & 222.9 & 234 & 268.8 & 267.7 & 270.3 & 276.7 & 258.4 & 196.6 & 124.4 & 110.2 \\
\hline 208.2 & 221.3 & 239.7 & 239.1 & 241.2 & 282.6 & 295.1 & 279.3 & 254.5 & 236.4 & 176.2 & 93.7 & 90.5 \\
\hline 227.9 & 231.5 & 247.9 & 249.9 & 255.6 & 278.1 & 283.6 & 280.8 & 255.6 & 244 & 189.7 & 110 & 105.4 \\
\hline 200.2 & 227.5 & 249.3 & 242.1 & 244.6 & 272.1 & 275.7 & 266.8 & 246.8 & 213.9 & 173.7 & 159.4 & 120.6 \\
\hline 218.2 & 232.2 & 243.3 & 253.1 & 236.4 & 241.9 & 243 & 240.6 & 222.1 & 242.2 & 215.8 & 82.9 & 75 \\
\hline 197.7 & 218.8 & 227.4 & 230.9 & 210.1 & 174.2 & 206.3 & 241.2 & 212.5 & 175.2 & 143.4 & 91 & 65.7 \\
\hline 273.1 & 282.2 & 280.3 & 272.7 & 266.6 & 261.7 & 267.1 & 281.1 & 225.7 & 208.7 & 151.6 & 139.3 & 76.7 \\
\hline 293.9 & 293.3 & 287.7 & 281.7 & 277.9 & 287.6 & 294.5 & 278.5 & 151.9 & 121.3 & 141 & 108.9 & 70.2 \\
\hline 248 & 257.7 & 253.4 & 263.9 & 261.6 & 264.8 & 281.6 & 281.7 & 239.3 & 221.8 & 112.2 & 92.3 & 85.7 \\
\hline 265.6 & 288.8 & 159.6 & 285.3 & 281.7 & 287.3 & 284.2 & 274.9 & 149.5 & 118.9 & 85.7 & 131.3 & 72.2 \\
\hline 267.7 & 285 & 353.1 & 348.3 & 308.1 & 295.1 & 300.1 & 313.9 & 267.9 & 224.6 & 109.8 & 89.9 & 89.5 \\
\hline 283.9 & 275.5 & 284.6 & 297.1 & 293.2 & 321.5 & 334.4 & 353.8 & 259.4 & 222.3 & 133.3 & 118.9 & 87.1 \\
\hline 304.8 & 310.6 & 315.1 & 313.5 & 306.4 & 318.4 & 330.3 & 353.2 & 263.3 & 274.9 & 176.2 & 122.3 & 69.5 \\
\hline
\end{tabular}


other

\begin{tabular}{|c|c|c|c|c|c|c|c|c|c|c|c|c|}
\hline 362.7 & 399.7 & 400.4 & 382.5 & 351.8 & 389.3 & 414.5 & 396.6 & 332.6 & 280.1 & 244.8 & 235.3 & 162.8 \\
\hline 341.4 & 382.9 & 421.4 & 404.4 & 367.8 & 408 & 422.7 & 408.3 & 211.4 & 137.2 & 89.9 & 80.2 & 76.7 \\
\hline 479 & 503.7 & 509.8 & 510 & 476.2 & 462 & 473.8 & 464.1 & 298.5 & 237 & 149 & 126.8 & 178.2 \\
\hline 452.9 & 481.6 & 480.1 & 468.9 & 457.6 & 451.8 & 441.8 & 438.8 & 302.5 & 243.9 & 157.5 & 110.6 & 95.7 \\
\hline 459.7 & 479.6 & 485 & 475.1 & 462.8 & 450.2 & 449.2 & 445.4 & 296.8 & 231.6 & 149.3 & 159.3 & 131 \\
\hline 465.8 & 487.9 & 485.2 & 473.9 & 461.8 & 440.4 & 453 & 452.4 & 243 & 234.7 & 184.6 & 99.2 & 84.7 \\
\hline 503.4 & 491.1 & 489.9 & 485.8 & 471.1 & 451.7 & 455.2 & 435.6 & 202.3 & 157.6 & 129.3 & 119 & 121.7 \\
\hline 472 & 507.1 & 519.5 & 510.8 & 488.2 & 470.1 & 470.7 & 448.8 & 249.8 & 210.1 & 190.1 & 171.2 & 151 \\
\hline 484.7 & 512.1 & 503.8 & 520.6 & 488.6 & 477 & 478.3 & 448.3 & 226.3 & 172.5 & 151.4 & 145.2 & 149.4 \\
\hline 483.1 & 523.4 & 512.1 & 520.4 & 493 & 481.8 & 478.8 & 455.2 & 248 & 169.3 & 128.2 & 159.7 & 127.9 \\
\hline 481.1 & 504.5 & 512.5 & 516.7 & 489.7 & 481.2 & 478.9 & 459.8 & 293.5 & 250.9 & 211.3 & 186.6 & 129.5 \\
\hline 467 & 496.2 & 447.9 & 493.9 & 484.6 & 466.9 & 473 & 449.1 & 197.3 & 171.8 & 143.8 & 118.5 & 81.2 \\
\hline 97.8 & 130.6 & 140.3 & 143.8 & 149.3 & 152.1 & 162.8 & 159.3 & 141.4 & 150.3 & 137.6 & 138.6 & 94 \\
\hline 286.7 & 251.1 & 261.7 & 261.4 & 238.4 & 244.8 & 258.9 & 252.9 & 200.9 & 209.6 & 186.8 & 152.4 & 122.4 \\
\hline 261.6 & 241.8 & 244.9 & 238.5 & 243.1 & 240.3 & 264.7 & 269.1 & 180 & 134.4 & 113.4 & 95 & 95.4 \\
\hline 115.1 & 112.7 & 124.1 & 143.4 & 147.6 & 146.5 & 149 & 141.4 & 109.2 & 92.3 & 88.1 & 97.4 & 88.8 \\
\hline 328.6 & 336.2 & 327.7 & 333.9 & 253.4 & 192.2 & 180.9 & 177 & 91.2 & 52.2 & 44.9 & 47.3 & 44.9 \\
\hline 58.1 & 58.1 & 58.1 & 59.1 & 60.1 & 59.1 & 60.1 & 63.2 & 55.3 & 43.2 & 43.5 & 42.9 & 44.2 \\
\hline 68.8 & 65.7 & 65 & 66.4 & 70.2 & 69.5 & 67.7 & 63.6 & 59.1 & 43.5 & 44.2 & 43.2 & 43.9 \\
\hline 269.3 & 293.4 & 291.7 & 275.1 & 226.7 & 149 & 136.9 & 115.8 & 95.7 & 91.2 & 92.3 & 94.3 & 93.3 \\
\hline 99.5 & 125.1 & 130.6 & 127.9 & 120.6 & 119.2 & 121.7 & 99.2 & 65.3 & 57.7 & 68.8 & 67.7 & 57 \\
\hline 119.9 & 115.1 & 119.2 & 119.6 & 100.6 & 112.3 & 94.7 & 84.8 & 58.1 & 50.5 & 44.6 & 45.3 & 44.9 \\
\hline 79.8 & 83.6 & 89.2 & 82.9 & 69.8 & 78.1 & 87.1 & 89.2 & 51.5 & 43.9 & 43.5 & 46 & 44.9 \\
\hline 76 & 70.2 & 62.9 & 60.5 & 51.8 & 49.4 & 51.1 & 55 & 45.6 & 43.2 & 53.2 & 56.7 & 45.3 \\
\hline 50.8 & 50.1 & 51.1 & 53.2 & 49.1 & 48.4 & 51.1 & 51.5 & 42.9 & 42.2 & 43.2 & 44.6 & 44.9 \\
\hline 51.1 & 51.8 & 67 & 79.8 & 81.2 & 79.8 & 80.9 & 57 & 49.1 & 45.3 & 57.7 & 54.3 & 47.7 \\
\hline 97.8 & 110.9 & 108.2 & 123.7 & 109.9 & 113.4 & 120.6 & 102.3 & 44.9 & 44.2 & 45.3 & 42.9 & 44.6 \\
\hline 106.1 & 123 & 116.8 & 123 & 104.7 & 96.1 & 105.8 & 95.4 & 43.9 & 42.9 & 43.5 & 43.9 & 46 \\
\hline 77.4 & 76.7 & 74.7 & 71.2 & 66.7 & 70.2 & 75 & 72.9 & 47.7 & 41.8 & 42.5 & 43.5 & 43.9 \\
\hline 74.3 & 72.6 & 66.4 & 68.8 & 57.7 & 52.5 & 56.3 & 56 & 44.9 & 43.5 & 44.2 & 44.2 & 44.9 \\
\hline 271.3 & 295.8 & 304.8 & 264.7 & 222.9 & 264.7 & 283.7 & 249.9 & 128.9 & 77.8 & 86.4 & 71.5 & 56.7 \\
\hline 207.7 & 211.5 & 219.1 & 204.2 & 191.9 & 202.5 & 216.7 & 239.2 & 140.3 & 122.4 & 110.2 & 111.3 & 105.4 \\
\hline 225.8 & 230.5 & 264.4 & 247.1 & 225.7 & 248.9 & 257.1 & 241.6 & 137.9 & 90.5 & 70.2 & 60.1 & 50.1 \\
\hline 213.2 & 221.2 & 225.5 & 244.1 & 223.5 & 254.6 & 267.5 & 261.4 & 191.6 & 165.6 & 90.2 & 70.2 & 60.1 \\
\hline 191.1 & 201.6 & 203.6 & 197.7 & 176.7 & 204.2 & 206.8 & 172.1 & 94 & 69.5 & 60.8 & 57.4 & 62.9 \\
\hline 115.8 & 128.6 & 118.9 & 122 & 122.3 & 115.1 & 117.8 & 97.5 & 82.6 & 74.7 & 61.5 & 65 & 63.2 \\
\hline \begin{tabular}{|l|}
93.3 \\
\end{tabular} & 96.8 & 105.1 & 114 & 113.4 & 105.8 & 119.6 & 118.2 & 102.4 & 90.5 & 88.1 & 88.5 & 88.8 \\
\hline
\end{tabular}




\begin{tabular}{|c|c|c|c|c|c|c|c|c|c|c|c|c|}
\hline 105.1 & 126.1 & 129.3 & 132.7 & 112.3 & 106.8 & 104 & 107.5 & 94.7 & 92.6 & 93.3 & 88.1 & 87.4 \\
\hline 97.8 & 117.5 & 119.9 & 109.2 & 90.5 & 94.3 & 93.3 & 95.7 & 99.2 & 88.8 & 96.4 & 91.2 & 91.6 \\
\hline 105.8 & 122 & 132 & 114.4 & 100.9 & 104.4 & 106.4 & 102.6 & 96.8 & 90.9 & 90.9 & 98.5 & 99.9 \\
\hline 90.2 & 100.9 & 95.1 & 87.8 & 91.9 & 119.2 & 129 & 106.8 & 69.6 & 57.4 & 51.1 & 48.4 & 47 \\
\hline 299.3 & 331.4 & 393.9 & 400.5 & 380.6 & 354.9 & 361.5 & 341.4 & 259.8 & 213.4 & 115.5 & 89.9 & 75 \\
\hline 209.7 & 222.6 & 201.8 & 177.6 & 99.2 & 103 & 100.6 & 106.4 & 92.6 & 78.8 & 85.4 & 83.6 & 78.8 \\
\hline 221.2 & 232 & 246.2 & 248.5 & 231.7 & 234.1 & 228.1 & 224.1 & 136.1 & 103.3 & 64.6 & 61.2 & 61.2 \\
\hline 255.9 & 252 & 258.9 & 254.2 & 232.8 & 241.9 & 253.8 & 244.3 & 144.5 & 103.3 & 69.5 & 62.9 & 62.9 \\
\hline 256 & 276.2 & 279 & 274.6 & 274.5 & 277.6 & 287.2 & 278.4 & 168.8 & 98.2 & 67.4 & 65 & 64.3 \\
\hline 262.8 & 248.7 & 260.9 & 264.4 & 249 & 244.8 & 229.1 & 229.5 & 140.4 & 116.1 & 93.3 & 86.1 & 84.7 \\
\hline 64.3 & 76.7 & 66 & 66 & 78.1 & 65.7 & 63.9 & 69.1 & 61.2 & 60.8 & 68.8 & 69.1 & 63.2 \\
\hline 61.9 & 68.1 & 63.2 & 69.8 & 69.1 & 65.7 & 64.6 & 62.9 & 68.8 & 60.8 & 63.6 & 71.9 & 61.2 \\
\hline 64.6 & 68.1 & 64.3 & 68.4 & 69.1 & 68.4 & 69.5 & 69.1 & 75.3 & 70.2 & 78.8 & 67.4 & 77.1 \\
\hline 64.6 & 80.9 & 65.3 & 81.9 & 70.8 & 81.6 & 75 & 81.6 & 83.3 & 75 & 89.2 & 86.7 & 76 \\
\hline 68.4 & 76.4 & 77.4 & 110.2 & 145.8 & 155.2 & 125.5 & 78.5 & 72.2 & 84 & 83.6 & 80.2 & 75 \\
\hline 71.5 & 63.2 & 74.7 & 67 & 67.4 & 76 & 71.5 & 68.8 & 77.1 & 72.9 & 64.6 & 76 & 64.6 \\
\hline 61.2 & 61.2 & 62.2 & 62.9 & 61.5 & 58.4 & 58.4 & 58.8 & 58.8 & 66.4 & 63.9 & 62.6 & 64.3 \\
\hline 61.9 & 78.5 & 61.2 & 62.2 & 61.5 & 64.6 & 74 & 63.9 & 82.3 & 84.7 & 83.3 & 72.6 & 75.3 \\
\hline 89.2 & 82.3 & 97.1 & 103.7 & 107.5 & 111.6 & 13.8 & 96.1 & 106.8 & 110.6 & 104 & 101.3 & 100.2 \\
\hline 73.3 & 84.3 & 86.7 & 80.5 & 77.1 & 95 & 97.8 & 90.5 & 84.3 & 95.4 & 92.6 & 77.8 & 84.7 \\
\hline 72.9 & 70.2 & 70.2 & 80.2 & 79.8 & 72.9 & 77.1 & 88.1 & 78.1 & 74 & 87.1 & 88.8 & 74 \\
\hline 70.5 & 75.7 & 81.9 & 71.9 & 72.6 & 82.6 & 91.2 & 79.5 & 83.3 & 94 & 95 & 84.7 & 80.9 \\
\hline 87.4 & 91.9 & 90.5 & 86.4 & 88.1 & 101.3 & 105.8 & 105.4 & 106.4 & 105.4 & 104 & 103 & 99.2 \\
\hline 83.3 & 94 & 93.7 & 92.6 & 97.5 & 116.8 & 104.4 & 97.8 & 100.9 & 95.4 & 83.3 & 90.9 & 104 \\
\hline 75 & 78.5 & 68.1 & 64.6 & 67.4 & 68.1 & 65.7 & 66.7 & 69.1 & 68.8 & 66.7 & 67 & 65.3 \\
\hline 66.4 & 66.4 & 64.3 & 67.7 & 69.1 & 69.5 & 68.8 & 70.2 & 70.2 & 69.1 & 69.1 & 69.1 & 67.7 \\
\hline 79.1 & 137.5 & 150 & 162.1 & 164.5 & 159 & 170 & 135.5 & 106.8 & 99.5 & 96.8 & 97.5 & 87.8 \\
\hline 86.1 & 90.5 & 84.7 & 101.6 & 105.1 & 85.4 & 87.1 & 87.4 & 84 & 80.5 & 70.8 & 84.3 & 77.8 \\
\hline 97.1 & 96.1 & 97.5 & 108.9 & 104.7 & 121.3 & 128.6 & 118.5 & 109.6 & 107.8 & 102.3 & 91.6 & 100.6 \\
\hline 92.3 & 97.1 & 89.2 & 93 & 105.8 & 112 & 113.4 & 108.2 & 116.8 & 109.2 & 107.8 & 107.8 & 115.4 \\
\hline 125.1 & 168 & 170 & 181.4 & 168.3 & 165.9 & 175.2 & 173.5 & 124.8 & 122.3 & 119.9 & 104.7 & 106.4 \\
\hline 96.4 & 100.6 & 182.5 & 155.9 & 121 & 184.2 & 173.8 & 160.4 & 158.6 & 179.7 & 170 & 117.8 & 117.5 \\
\hline 99.2 & 105.8 & 114.7 & 103.7 & 110.6 & 124.4 & 115.4 & 119.6 & 129.9 & 119.6 & 110.6 & 106.4 & 107.1 \\
\hline 98.8 & 100.6 & 102.3 & 99.5 & 103 & 100.2 & 96.1 & 98.5 & 143.1 & 208.7 & 109.6 & 118.2 & 120.3 \\
\hline 95.4 & 92.3 & 86.1 & 84.7 & 86.7 & 82.9 & 84.3 & 98.5 & 87.8 & 84.7 & 94.7 & 96.8 & 87.1 \\
\hline 80.9 & 82.9 & 98.2 & 89.9 & 85 & 92.6 & 94.7 & 86.4 & 94.7 & 103.7 & 87.1 & 93 & 103 \\
\hline 69.8 & 79.2 & 62.2 & 61.3 & 63 & 61.2 & 58.8 & 61.6 & 72.6 & 68.9 & 70.2 & 70.9 & 70.2 \\
\hline
\end{tabular}




\begin{tabular}{|c|c|c|c|c|c|c|c|c|c|c|c|c|}
\hline 65.3 & 68.2 & 64.7 & 75 & 69.2 & 65.3 & 69.9 & 74.3 & 72 & 69.1 & 69.2 & 68.5 & 68.1 \\
\hline 46.7 & 45.7 & 45.6 & 43.6 & 53.6 & 42.9 & 46 & 50.5 & 46.4 & 49.8 & 54 & 58.8 & 48.5 \\
\hline 44.2 & 48.8 & 47 & 43.6 & 50.5 & 42.3 & 49.5 & 44.2 & 43.3 & 52.5 & 50.2 & 48.7 & 53.7 \\
\hline 113.8 & 137.5 & 139.7 & 156.9 & 156.6 & 138 & 126.8 & 86.1 & 48.8 & 49.8 & 56.4 & 57.7 & 56.4 \\
\hline 48.1 & 47.8 & 47 & 46.4 & 47.3 & 51.9 & 58.5 & 74.7 & 100.3 & 122.3 & 125.2 & 120.3 & 121.1 \\
\hline 352.9 & 372 & 355 & 361 & 310.4 & 247 & 279.4 & 298.1 & 158 & 80.3 & 55.7 & 56 & 57.1 \\
\hline 184.1 & 214.9 & 229.3 & 219.5 & 115.7 & 68.5 & 62.6 & 63.6 & 63.7 & 56.4 & 54.6 & 55 & 58.8 \\
\hline 150.7 & 152.5 & 146.9 & 141.8 & 120.7 & 144.1 & 141.4 & 110 & 93.3 & 83.7 & 82.3 & 78.5 & 78.2 \\
\hline 65.4 & 63.6 & 58.8 & 64.7 & 64.3 & 74.4 & 67.5 & 66.4 & 63 & 61.9 & 64 & 65.4 & 73.6 \\
\hline 124.1 & 131.4 & 138 & 128.2 & 118.3 & 127.6 & 125.5 & 106.9 & 78.1 & 69.2 & 69.2 & 71.5 & 68.9 \\
\hline 56.1 & 60.6 & 80.5 & 80.6 & 82.9 & 82.3 & 76.1 & 78.1 & 76.5 & 67.5 & 66.4 & 65.4 & 66.4 \\
\hline 104.1 & 106.8 & 116.9 & 112.4 & 102 & 126.2 & 117.6 & 110.6 & 83.7 & 82.7 & 77.4 & 81 & 82.7 \\
\hline 65.4 & 65.3 & 73.4 & 77.5 & 80.9 & 89.9 & 92.7 & 95.7 & 95.5 & 96.4 & 95.1 & 94.1 & 87.4 \\
\hline 229.7 & 238.5 & 249.2 & 246.5 & 234.7 & 238.3 & 240.8 & 209.2 & 105.1 & 92.7 & 78.2 & 66 & 68.2 \\
\hline 75.8 & 81.3 & 83.6 & 77.9 & 75.3 & 59.2 & 55 & 59.8 & 64 & 62.9 & 62 & 59.9 & 61.9 \\
\hline 221.2 & 216.7 & 232.9 & 236.4 & 176.4 & 150.8 & 151.4 & 155.9 & 89.9 & 62.6 & 60.2 & 63.6 & 67.5 \\
\hline 58.8 & 59.5 & 64.6 & 59.2 & 63.9 & 66.1 & 66.7 & 67.8 & 70.5 & 70.6 & 72.6 & 73 & 71.9 \\
\hline 251.2 & 246.1 & 248.8 & 232.2 & 181.4 & 182.6 & 180.7 & 170 & 95.8 & 79.5 & 77.2 & 77.5 & 79.5 \\
\hline 70.2 & 71.3 & 70.8 & 72.3 & 76.7 & 81 & 87.4 & 92 & 97.5 & 91.7 & 95.4 & 95.8 & 90.2 \\
\hline 230.4 & 229.3 & 232.9 & 233.7 & 173.8 & 150.1 & 132 & 129.3 & 86.1 & 77.2 & 79.1 & 78.2 & 76.7 \\
\hline 64.6 & 67.8 & 69.6 & 72.9 & 76.1 & 75.3 & 77.2 & 76 & 79.2 & 88.5 & 83.4 & 86.7 & 82.3 \\
\hline 235 & 214.4 & 233.6 & 228.9 & 164.5 & 160 & 139.4 & 134.8 & 125.2 & 70.9 & 63.2 & 65.1 & 64.3 \\
\hline 58.4 & 60.2 & 71.9 & 79.9 & 78.5 & 63 & 68.5 & 68.4 & 70.6 & 72.6 & 68.5 & 73.3 & 72.3 \\
\hline 221.7 & 216.8 & 231.8 & 211.1 & 212.3 & 206.7 & 185.3 & 177.3 & 144.8 & 89.9 & 74.1 & 76 & 74.1 \\
\hline 65.8 & 66.7 & 69.6 & 74.1 & 72.9 & 72.7 & 77.4 & 75.1 & 81.9 & 79.6 & 78.8 & 85.1 & 78.5 \\
\hline 232 & 230.7 & 238.3 & 229 & 214 & 217 & 193.5 & 195.4 & 147.9 & 88.9 & 75.3 & 76.5 & 75.4 \\
\hline 78.2 & 86.1 & 78.5 & 89.5 & 89.6 & 77.2 & 77.1 & 76.8 & 80.5 & 78.2 & 80.5 & 82 & 84.3 \\
\hline 131.1 & 185.6 & 201.2 & 198.4 & 219.2 & 222.4 & 223.3 & 236.1 & 144.9 & 95.7 & 88.9 & 91.9 & 92 \\
\hline 89.6 & 95.1 & 99.9 & 109 & 104.7 & 103.1 & 92.3 & 88.6 & 82.6 & 83.7 & 85.1 & 88.8 & 87.5 \\
\hline 118.9 & 128 & 123.4 & 123.8 & 125.5 & 126.6 & 150.7 & 190.2 & 140.8 & 78.5 & 75.4 & 76 & 78.5 \\
\hline 75.8 & 77.1 & 80.6 & 81.2 & 84.1 & 80.9 & 126.6 & 116.8 & 106.9 & 107.1 & 76.1 & 75 & 76.8 \\
\hline 250.6 & 273.8 & 281.9 & 282.5 & 278.9 & 283.3 & 299.4 & 290.7 & 241.2 & 218.4 & 210.7 & 203.3 & 143.8 \\
\hline 82.7 & 82.6 & 143.5 & 148.3 & 141 & 141.4 & 152.8 & 136.3 & 135.1 & 119 & 95.7 & 83.4 & 84.3 \\
\hline 295 & 309.3 & 317.7 & 307.8 & 297.3 & 305 & 310.7 & 303 & 188.8 & 157.6 & 151.1 & 96.4 & 80.6 \\
\hline 77.1 & 75.8 & 146.5 & 154.6 & 155.9 & 142.8 & 96.4 & 122.8 & 120.6 & 93.1 & 66.7 & 65.1 & 67.7 \\
\hline 344.4 & 377 & 377.8 & 376.9 & 321.9 & 359.3 & 380.3 & 384.6 & 276.4 & 205.6 & 160.4 & 124.9 & 97.1 \\
\hline 82.3 & 137.9 & 141.1 & 155.5 & 157.7 & 143.8 & 113.4 & 143.4 & 156.6 & 151.1 & 103.7 & 87.5 & 94.1 \\
\hline
\end{tabular}


other

\begin{tabular}{|c|c|c|c|c|c|c|c|c|c|c|c|c|}
\hline 500.1 & 469.9 & 482.8 & 485.3 & 456.8 & 447 & 453.7 & 421.4 & 231.3 & 168.1 & 157.6 & 110.3 & 77.8 \\
\hline 81.2 & 83.7 & 131.1 & 141.4 & 140.1 & 105.1 & 99.3 & 146.2 & 139.4 & 185.2 & 170.6 & 154.1 & 156.5 \\
\hline 508.1 & 498.5 & 516 & 497.4 & 485 & 464 & 456.3 & 419.4 & 230.3 & 161.5 & 128.6 & 115.9 & 92.6 \\
\hline 72.7 & 78.8 & 84.8 & 89.9 & 91.7 & 88.5 & 94.4 & 97.8 & 97.2 & 93.3 & 94.8 & 86.1 & 87.5 \\
\hline 298 & 313.1 & 316 & 306.5 & 236.1 & 163.1 & 152.8 & 139.6 & 99.6 & 102 & 109.6 & 111 & 98.5 \\
\hline 53.3 & 55.6 & 73.4 & 73.6 & 76.7 & 62.9 & 59.5 & 63.2 & 64.4 & 66.7 & 72.6 & 78.9 & 79.5 \\
\hline 54.3 & 51.2 & 60.5 & 53.7 & 56.3 & 57.8 & 57 & 56.1 & 55.3 & 57.5 & 62.6 & 64.7 & 65 \\
\hline 48.1 & 48.7 & 50.2 & 78.8 & 62.6 & 48.7 & 50.5 & 58.4 & 51.6 & 50.1 & 55.6 & 56.4 & 56.7 \\
\hline 44.6 & 46.1 & 55.6 & 81.6 & 82 & 81.6 & 74.7 & 90.5 & 76 & 70.9 & 67 & 57.4 & 58.5 \\
\hline 48 & 47.1 & 51.1 & 55.3 & 53.3 & 57 & 65 & 66.1 & 68.1 & 71.3 & 61.2 & 61.9 & 60.2 \\
\hline 60.5 & 64 & 62.6 & 61.9 & 61.6 & 60.5 & 59.5 & 60.8 & 59.9 & 61.9 & 63 & 68.1 & 65.8 \\
\hline 59.8 & 59.2 & 60.5 & 59.5 & 62.2 & 61.6 & 62.6 & 61.3 & 62.6 & 62.6 & 65.1 & 68.4 & 66.1 \\
\hline 81.6 & 90.5 & 97.9 & 98.5 & 98.5 & 87.9 & 88.1 & 78.9 & 74.3 & 76.8 & 76.4 & 69.6 & 68.1 \\
\hline 55.4 & 58.1 & 60.5 & 63.7 & 69.1 & 80.3 & 70.8 & 70.2 & 70.6 & 66 & 60.1 & 65 & 65 \\
\hline 54.6 & 55.3 & 59.4 & 62.6 & 63.2 & 62.6 & 61.9 & 63.9 & 68.8 & 70.2 & 63.2 & 63.2 & 63.6 \\
\hline 59.1 & 60.1 & 61.9 & 57.5 & 57.7 & 59.8 & 57.8 & 60.5 & 62.6 & 61.9 & 61.5 & 63.2 & 63.9 \\
\hline 48.8 & 50.5 & 68.8 & 59.8 & 60.1 & 49.4 & 54.6 & 60.5 & 61.5 & 61.5 & 56.7 & 56.1 & 64.3 \\
\hline 49.1 & 58.8 & 71.3 & 59.1 & 56 & 51.8 & 58.8 & 63.9 & 66 & 60.8 & 60.8 & 58.8 & 56.3 \\
\hline 77.8 & 75.7 & 76.4 & 76.7 & 76 & 78.8 & 79.1 & 80.2 & 81.9 & 82.3 & 85.7 & 82.6 & 84 \\
\hline 77.8 & 78.1 & 78.1 & 82.3 & 79.8 & 80.5 & 84.3 & 82.3 & 84 & 84.3 & 85 & 89.5 & 88.1 \\
\hline 56 & 61.2 & 62.9 & 65.3 & 66.4 & 60.8 & 59.4 & 53.9 & 56 & 62.6 & 60.9 & 62.2 & 64.3 \\
\hline 61.9 & 62.6 & 61.5 & 61.5 & 57.7 & 52.2 & 68.1 & 69.5 & 65 & 51.5 & 57 & 59.4 & 52.9 \\
\hline 42.5 & 41.5 & 43.9 & 42.9 & 40.4 & 41.5 & 41.1 & 41.5 & 41.5 & 43.5 & 42.5 & 46.3 & 46.3 \\
\hline 42.9 & 47.7 & 41.5 & 41.5 & 42.5 & 42.9 & 42.2 & 41.5 & 41.8 & 44.6 & 44.6 & 43.2 & 45.6 \\
\hline 54.3 & 52.9 & 53.2 & 51.5 & 50.1 & 54.3 & 51.5 & 51.5 & 50.8 & 47 & 44.6 & 44.2 & 44.9 \\
\hline 46 & 48.7 & 51.1 & 51.8 & 53.2 & 51.8 & 54.3 & 51.8 & 51.1 & 64.3 & 62.6 & 66.4 & 64.6 \\
\hline 47.3 & 44.9 & 47.3 & 47 & 46.7 & 58.8 & 58.1 & 50.1 & 50.5 & 52.9 & 51.5 & 50.8 & 52.2 \\
\hline 43.9 & 45.6 & 49.4 & 46.7 & 48 & 49.8 & 52.2 & 55.3 & 52.2 & 54.6 & 56.3 & 51.8 & 53.9 \\
\hline 45.3 & 46.3 & 51.8 & 50.8 & 49.8 & 49.8 & 72.2 & 53.9 & 62.6 & 60.1 & 53.6 & 52.2 & 51.9 \\
\hline 42.9 & 50.5 & 64.6 & 66.4 & 67.4 & 67 & 57.7 & 51.1 & 50.8 & 53.2 & 52.9 & 52.5 & 51.8 \\
\hline 64.3 & 73.3 & 71.5 & 66 & 65.3 & 61.5 & 121.7 & 130.6 & 58.8 & 51.1 & 47.7 & 48 & 50.1 \\
\hline 42.6 & 45.3 & 48 & 44.2 & 42.2 & 58.4 & 121.7 & 134.1 & 88.1 & 58.1 & 50.1 & 50.5 & 47.3 \\
\hline 52.9 & 53.2 & 50.5 & 49.1 & 65 & 65.7 & 61.2 & 53.9 & 94 & 118.9 & 113 & 62.6 & 44.9 \\
\hline 40.2 & 44.6 & 44.6 & 42.2 & 41.5 & 67.4 & 126.1 & 109.2 & 62.6 & 43.2 & 46.3 & 42.9 & 44.2 \\
\hline 41.1 & 41.5 & 42.2 & 42.5 & 46.7 & 49.4 & 50.5 & 48.7 & 52.5 & 52.9 & 54.6 & 51.6 & 47.3 \\
\hline 44.6 & 45.3 & 51.1 & 50.8 & 50.1 & 50.5 & 60.1 & 145.2 & 148.3 & 108.5 & 100.6 & 128.2 & 86.4 \\
\hline 49.1 & 49.5 & 51.1 & 53.6 & 90.5 & 121 & 124.1 & 90.2 & 116.5 & 112.7 & 59.4 & 49.8 & 48.4 \\
\hline
\end{tabular}




\begin{tabular}{|c|c|c|c|c|c|c|c|c|c|c|c|c|}
\hline 46.3 & 45 & 47.3 & 46.7 & 61.5 & 134.1 & 124.4 & 142 & 138.9 & 103.3 & 62.6 & 52.2 & 51.1 \\
\hline 62.6 & 71.2 & 82.6 & 142.7 & 144.8 & 145.5 & 141.4 & 139.6 & 140 & 135.8 & 129.9 & 116.8 & 56 \\
\hline 50.1 & 75.3 & 96.1 & 124.8 & 130.3 & 136.9 & 142.7 & 87.4 & 124.1 & 142.4 & 83.7 & 78.5 & 62.2 \\
\hline 68.1 & 77.1 & 135.5 & 141 & 132.7 & 114.4 & 88.8 & 90.9 & 86.4 & 85 & 77.8 & 71.5 & 72.2 \\
\hline 56 & 57.8 & 83.3 & 132.4 & 146.9 & 153.1 & 146.5 & 105.8 & 112.7 & 155.2 & 140.7 & 133.1 & 77.4 \\
\hline 90.3 & 92.6 & 144.1 & 128.2 & 122 & 124.8 & 112.3 & 119.2 & 127.9 & 98.8 & 55.7 & 50.5 & 50.5 \\
\hline 61.5 & 63.9 & 65.8 & 70.5 & 67.7 & 69.1 & 78.1 & 62.2 & 63.6 & 61.9 & 59.9 & 117.2 & 93.7 \\
\hline 142.8 & 142.2 & 140.7 & 121.6 & 119.6 & 117.5 & 121.7 & 120 & 119.6 & 110.8 & 113 & 106.3 & 108.9 \\
\hline 67 & 70.6 & 74 & 76.7 & 72.2 & 72.2 & 76 & 74.3 & 75.3 & 68.4 & 62.9 & 54.6 & 54.6 \\
\hline 46.7 & 46.7 & 48 & 48 & 47.7 & 65 & 67 & 52.2 & 44.6 & 42.5 & 42.2 & 45.3 & 44.9 \\
\hline 43.2 & 42.5 & 43.2 & 46.3 & 67 & 70.2 & 58.8 & 70.8 & 62.2 & 54.3 & 43.2 & 45.6 & 50.1 \\
\hline 42.2 & 42.5 & 61.5 & 54.3 & 44.6 & 43.2 & 44.2 & 47 & 85.7 & 92.6 & 90.9 & 81.9 & 74.7 \\
\hline 44.6 & 43.9 & 43.5 & 50.1 & 51.5 & 53.2 & 52.2 & 53.6 & 51.5 & 46.7 & 46.3 & 50.1 & 51.1 \\
\hline 42.9 & 42.9 & 44.2 & 43.9 & 45.6 & 44.2 & 44.2 & 45.6 & 43.5 & 43.2 & 44.9 & 46.3 & 46.7 \\
\hline 44.6 & 43.9 & 47 & 48.7 & 45.3 & 44.6 & 45.3 & 48 & 45.3 & 44.2 & 44.2 & 46.7 & 47 \\
\hline 42.9 & 42.5 & 55 & 61.2 & 63.2 & 56.3 & 45.6 & 47.3 & 44.6 & 44.9 & 44.2 & 44.6 & 46 \\
\hline 45.3 & 41.8 & 41.1 & 42.2 & 44.9 & 44.2 & 42.3 & 46.7 & 46.7 & 49.4 & 50.8 & 51.1 & 49.8 \\
\hline 69.5 & 73.6 & 68.5 & 62.2 & 47 & 48 & 48 & 51.1 & 49.4 & 48 & 50.8 & 54.3 & 52.5 \\
\hline 88.9 & 96.1 & 95.7 & 93 & 91.7 & 96.1 & 79.2 & 69.5 & 67 & 67.5 & 56 & 58.4 & 57.7 \\
\hline 53.2 & 62.9 & 60.5 & 46 & 44.9 & 46.3 & 49.4 & 53.2 & 52.5 & 51.5 & 50.8 & 52.5 & 52.2 \\
\hline 42.5 & 42.5 & 43.2 & 44.9 & 47.3 & 48 & 60.1 & 63.2 & 56 & 50.8 & 50.1 & 51.8 & 53.9 \\
\hline 45.6 & 48.4 & 49.8 & 46 & 46.7 & 50.5 & 51.5 & 48 & 43.2 & 42.5 & 41.5 & 44.6 & 46 \\
\hline 41.8 & 41.8 & 41.1 & 40.4 & 41.1 & 41.1 & 43.5 & 48.7 & 50.8 & 52.2 & 51.5 & 49.8 & 44.6 \\
\hline 264.6 & 334.6 & 331.7 & 313.6 & 159.5 & 95.4 & 79.5 & 71.2 & 60.5 & 58.4 & 57 & 57 & 57.7 \\
\hline 53.6 & 55.3 & 55 & 59.4 & 72.9 & 67 & 66.4 & 59.4 & 59.8 & 57.4 & 59.1 & 59.8 & 60.1 \\
\hline 136.2 & 137.2 & 132 & 138.2 & 81.2 & 78.5 & 77.8 & 78.5 & 67 & 58.8 & 59.4 & 58.8 & 60.1 \\
\hline & & & & & & & & & & & & \\
\hline & & & & & & & & & & & & \\
\hline 384.9 & 398.2044 & 411.12 & 430.2467 & 403.8189 & 392.5822 & 387.1378 & 374.1811 & 255.8758 & 211.8505 & 180.9022 & 146.833 & 113.0824 \\
\hline 351.177 & 367.3906 & 373.8428 & 376.8638 & 357.4618 & 348.1329 & 344.8428 & 325.7368 & 216.7218 & 173.4122 & 146.4762 & 128.002 & 95.96937 \\
\hline 103.7972 & 111.5528 & 114.4694 & 117.5639 & 115.2528 & 117.5611 & 120.55 & 116.45 & 106.5056 & 95.90833 & 90.21389 & 90.35833 & 86.69722 \\
\hline 142.1427 & 148.71 & 155.0487 & 156.734 & 151.0147 & 146.6233 & 144.9127 & 138.096 & 110.216 & 95.2 & 89.96867 & 83.728 & 76.81067 \\
\hline 224.408 & 235.1095 & 239.244 & 247.892 & 237.6987 & 238.1434 & 234.3675 & 222.6675 & 176.9792 & 147.2338 & 117.2662 & 104.6844 & 94.70649 \\
\hline 220.9595 & 230.6405 & 237.9753 & 238.3265 & 224.7019 & 222.6851 & 222.2614 & 215.526 & 155.6721 & 130.586 & 104.7237 & 94.43395 & 85.16605 \\
\hline 88.57941 & 97.11471 & 98.60294 & 100.7059 & 97.15882 & 97.01176 & 94.62059 & 93.72353 & 89.39118 & 89.77353 & 85.37353 & 83.03235 & 82.04706 \\
\hline 98.3871 & 102.5828 & 110.1043 & 111.0613 & 104.6613 & 103.9161 & 104.8269 & 103.6194 & 89.14301 & 81.05484 & 74.64194 & 72.36559 & 68.2086 \\
\hline
\end{tabular}


other

\begin{tabular}{|c|c|c|c|c|c|c|c|c|c|c|c|c|}
\hline & & & & & & & & & & & & \\
\hline & & & & & & & & & & & & \\
\hline & & & & & & & & & & & & \\
\hline & & & & & & & & & & & & \\
\hline 34641 & 35838.4 & 37000.8 & 38722.2 & 36343.7 & 35332.4 & 34842.4 & 33676.3 & 23284.7 & 19278.4 & 16462.1 & 13361.8 & 10290.5 \\
\hline 138714.9 & 145119.3 & 147667.9 & 148861.2 & 141197.4 & 137512.5 & 136212.9 & 128340.3 & 85605.1 & 68497.8 & 57858.1 & 50560.8 & 37907.9 \\
\hline 3736.7 & 4015.9 & 4120.9 & 4232.3 & 4149.1 & 4232.2 & 4339.8 & 4192.2 & 3834.2 & 3452.7 & 3247.7 & 3252.9 & 3121.1 \\
\hline 38152 & 39704.6 & 41200.6 & 42102 & 40479.6 & 40092.4 & 39783.2 & 37859.8 & 30159.8 & 25617 & 22524.8 & 20619.9 & 18814 \\
\hline 16830.6 & 17398.1 & 17943.3 & 18591.9 & 17827.4 & 18098.9 & 18046.3 & 17145.4 & 13627.4 & 11337 & 9029.5 & 8060.7 & 7292.4 \\
\hline 47506.3 & 49587.7 & 51164.7 & 51240.2 & 48310.9 & 47877.3 & 47786.2 & 46338.1 & 33469.5 & 28076 & 22515.6 & 20303.3 & 18310.7 \\
\hline 3011.7 & 3301.9 & 3352.5 & 3424 & 3303.4 & 3298.4 & 3217.1 & 3186.6 & 3039.3 & 3052.3 & 2902.7 & 2823.1 & 2789.6 \\
\hline \multirow[t]{5}{*}{9150} & 9540.2 & 10239.7 & 10328.7 & 9733.5 & 9664.2 & 9748.9 & 9636.6 & 8290.3 & 7538.1 & 6941.7 & 6730 & 6343.4 \\
\hline & & & & & & & & & & & & \\
\hline & & & & & & & & & & & & \\
\hline & & & & & & & & & & & & \\
\hline & & & & & & & & & & & & \\
\hline 115.5651 & 116.7788 & 115.7481 & 119.808 & 117.1722 & 119.3651 & 114.4832 & 109.3913 & 87.97309 & 70.96112 & 60.86419 & 58.11876 & 51.01319 \\
\hline 99.27754 & 103.3231 & 102.8409 & 104.5463 & 98.12985 & 92.33413 & 91.89161 & 87.81563 & 56.3976 & 47.15482 & 41.3248 & 40.94434 & 35.95676 \\
\hline 79.63247 & 80.95905 & 79.15862 & 80.63207 & 71.31832 & 70.91502 & 71.14038 & 67.48176 & 48.57183 & 40.39862 & 35.51544 & 35.97112 & 31.77818 \\
\hline 139.9818 & 145.3313 & 148.6741 & 150.9317 & 142.3959 & 142.4667 & 141.88 & 131.0278 & 81.90155 & 61.75965 & 51.69071 & 42.2816 & 32.21847 \\
\hline 159.1126 & 162.034 & 165.0933 & 167.6213 & 159.1395 & 163.2263 & 163.1088 & 149.8432 & 93.71881 & 72.05408 & 64.76728 & 50.35044 & 36.76939 \\
\hline 119.7567 & 119.6067 & 121.0561 & 121.072 & 115.1473 & 113.2039 & 112.8733 & 109.8672 & 72.31233 & 61.41636 & 47.18331 & 39.31023 & 32.63853 \\
\hline 53.34454 & 59.06621 & 60.8982 & 60.94502 & 49.32412 & 44.63547 & 48.93487 & 46.04778 & 29.31806 & 33.94997 & 25.18545 & 19.68522 & 21.14398 \\
\hline 92.36865 & 93.71299 & 95.49931 & 92.97061 & 81.80382 & 80.03592 & 79.81325 & 76.0649 & 46.35299 & 36.80709 & 31.10717 & 26.66895 & 19.84325 \\
\hline & & & & & & & & & & & & \\
\hline & & & & & & & & & & & & \\
\hline & & & & & & & & & & & & \\
\hline 90 & 90 & 90 & 90 & 90 & 90 & 90 & 90 & 91 & 91 & 91 & 91 & 91 \\
\hline 395 & 395 & 395 & 395 & 395 & 395 & 395 & 394 & 395 & 395 & 395 & 395 & 395 \\
\hline 36 & 36 & 36 & 36 & 36 & 36 & 36 & 36 & 36 & 36 & 36 & 36 & 36 \\
\hline 225 & 224 & 225 & 225 & 225 & 226 & 227 & 227 & 227 & 227 & 227 & 227 & 227 \\
\hline 75 & 74 & 75 & 75 & 75 & 76 & 77 & 77 & 77 & 77 & 77 & 77 & 77 \\
\hline 215 & 215 & 215 & 215 & 215 & 215 & 215 & 215 & 215 & 215 & 215 & 215 & 215 \\
\hline 34 & 34 & 34 & 34 & 34 & 34 & 34 & 34 & 34 & 34 & 34 & 34 & 34 \\
\hline 93 & 93 & 93 & 93 & 93 & 93 & 93 & 93 & 93 & 93 & 93 & 93 & 93 \\
\hline
\end{tabular}




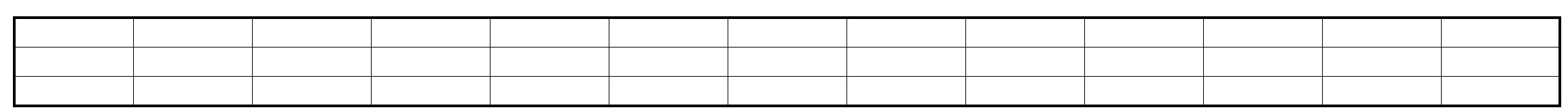




\begin{tabular}{|c|c|c|c|c|c|c|c|c|}
\hline 22 & 23 & $\begin{array}{l}\text { DAILY } \\
\text { TOTAL }\end{array}$ & $\begin{array}{l}\text { AVERAGE } \\
\text { DAILY } \\
\text { TOTAL }\end{array}$ & $\begin{array}{l}\text { MONTHLY } \\
\text { TOTAL formula }\end{array}$ & $\begin{array}{l}\text { MONTHLY } \\
\text { TOTAL } \\
\text { value }\end{array}$ & & & \\
\hline 126.1 & 98.2 & 7635.00 & 318.13 & & & & & \\
\hline 237.1 & 235.4 & 8362.20 & 348.43 & & & & & \\
\hline 110.2 & 106.1 & 8223.60 & 342.65 & & & & & \\
\hline 86.1 & 92.3 & 7142.70 & 297.61 & & & & & \\
\hline 109.2 & 110.2 & 7514.20 & 313.09 & & & & & \\
\hline 110.9 & 106.4 & 8470.40 & 352.93 & & & & & \\
\hline 138.9 & 127.9 & 7493.30 & 312.22 & & & & & \\
\hline 107.1 & 107.8 & 8125.50 & 338.56 & & & \multicolumn{2}{|c|}{ sort $A / B, S / N S$} & \\
\hline 112 & 125.8 & 7362.10 & 306.75 & & & B-S & 1972405.20 & \\
\hline 78.1 & 89.5 & 8090.90 & 337.12 & & & B-NS & 833102.00 & \\
\hline 90.2 & 88.8 & 8096.10 & 337.34 & & & & & \\
\hline 88.5 & 87.8 & 7107.70 & 296.15 & & & A-S & 605718.00 & \\
\hline 69.1 & 67.7 & 6598.40 & 274.93 & & & A-NS & 497376.80 & \\
\hline 68.1 & 65 & 5402.10 & 225.09 & & & & & \\
\hline 101.3 & 84 & 7609.60 & 317.07 & & & & 3908602.00 & 0.00 \\
\hline 98.5 & 91.9 & 7774.10 & 323.92 & & & & & \\
\hline 99.5 & 93.3 & 6055.80 & 252.33 & & & & & \\
\hline 72.2 & 69.5 & 6285.20 & 261.88 & & & & & \\
\hline 78.1 & 86.7 & 5985.80 & 249.41 & & & & & \\
\hline 83.6 & 82.9 & 6651.80 & 277.16 & & 0 & & & \\
\hline & & & & & & & & \\
\hline 86.4 & 94.3 & 920.50 & 131.50 & & & & & \\
\hline 125.5 & 113.4 & 6941.10 & 289.21 & & & & & \\
\hline 102.6 & 93 & 7111.60 & 296.32 & & & & & \\
\hline 71.9 & 72.6 & 6080.70 & 253.36 & 167040.40 & & & & \\
\hline 86.4 & 89.2 & 4782.70 & 199.28 & & & & & \\
\hline 89.5 & 80.9 & 4754.30 & 198.10 & & & & & \\
\hline 124.3 & 84 & 5110.70 & 212.95 & & & & & \\
\hline 119.9 & 123.4 & 5062.20 & 210.93 & & & & & \\
\hline 175.9 & 129.9 & 6372.20 & 265.51 & & & & & \\
\hline 147.8 & 92.6 & 6185.20 & 257.72 & & & & & \\
\hline 99.2 & 97.1 & 5064.20 & 211.01 & & & & & \\
\hline 96.8 & 86.7 & 5463.70 & 227.65 & & & & & \\
\hline
\end{tabular}


other

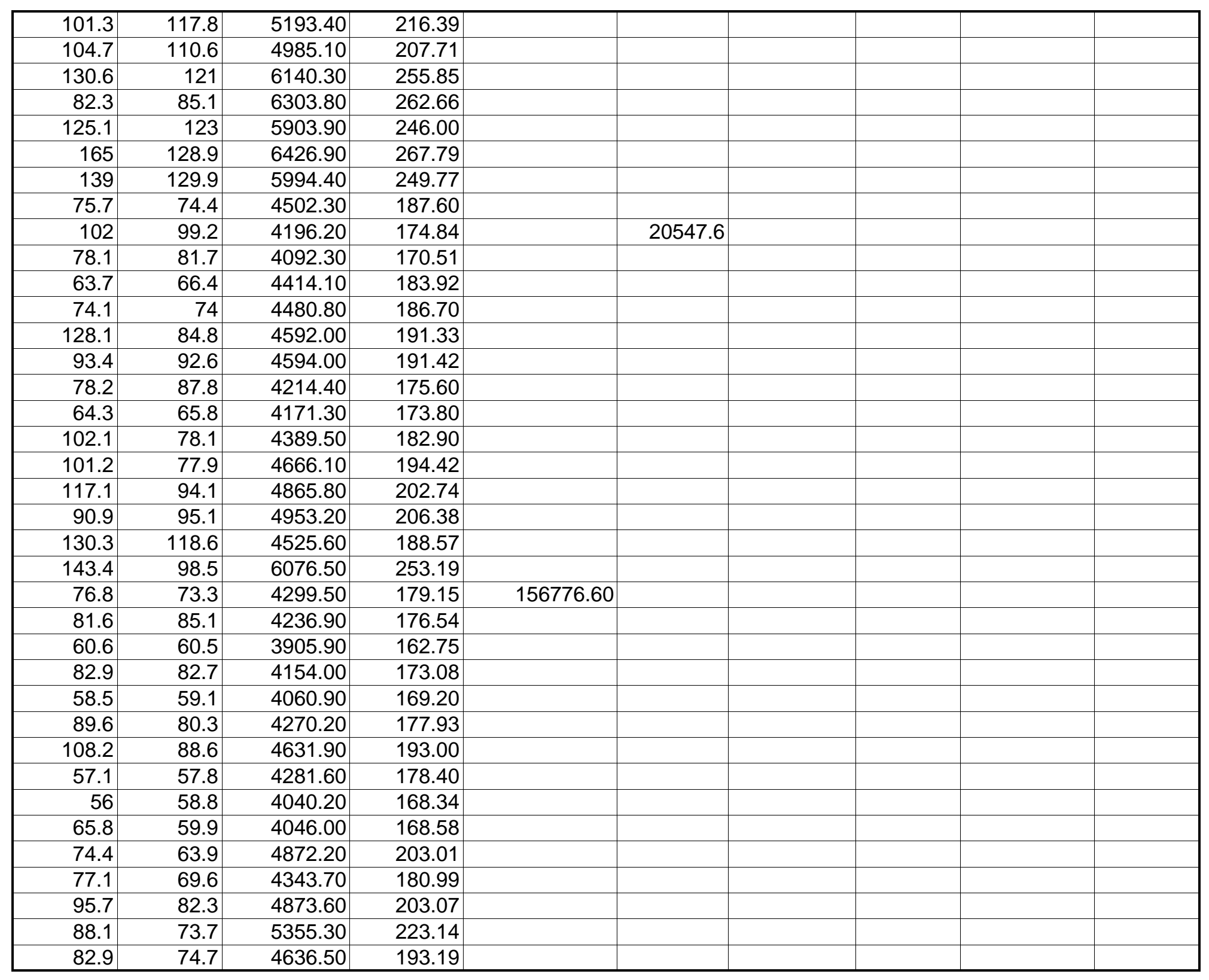


other

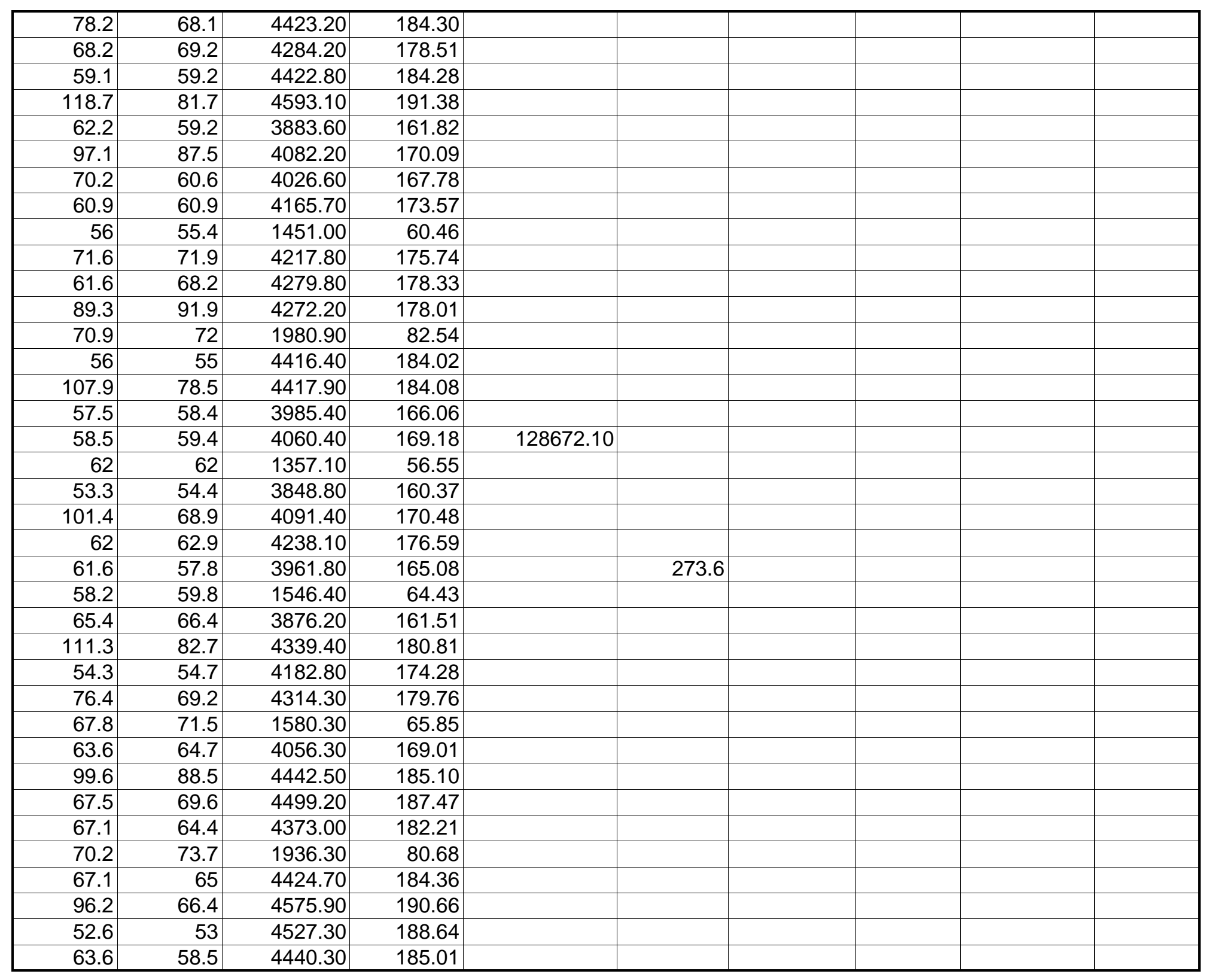


other

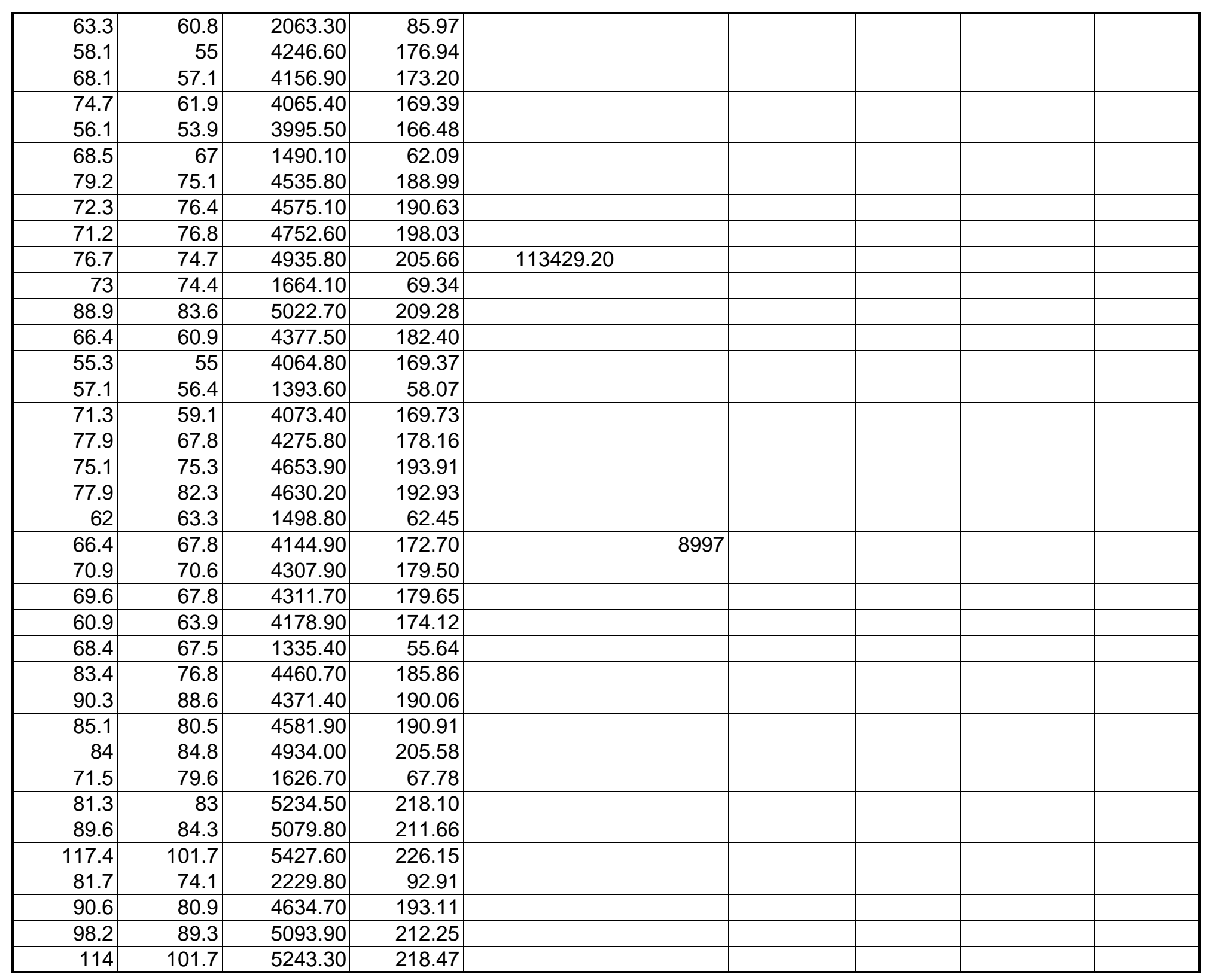


other

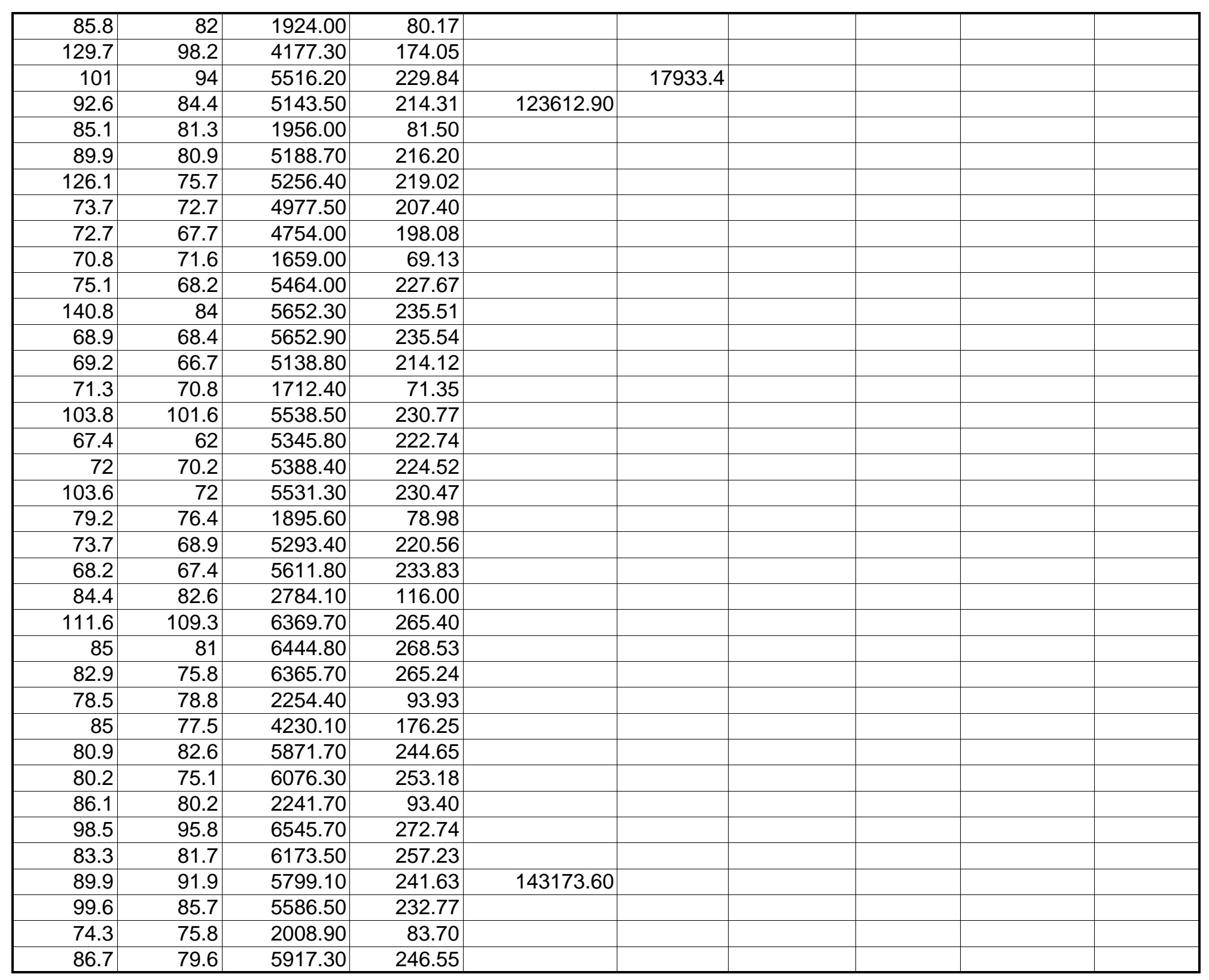


other

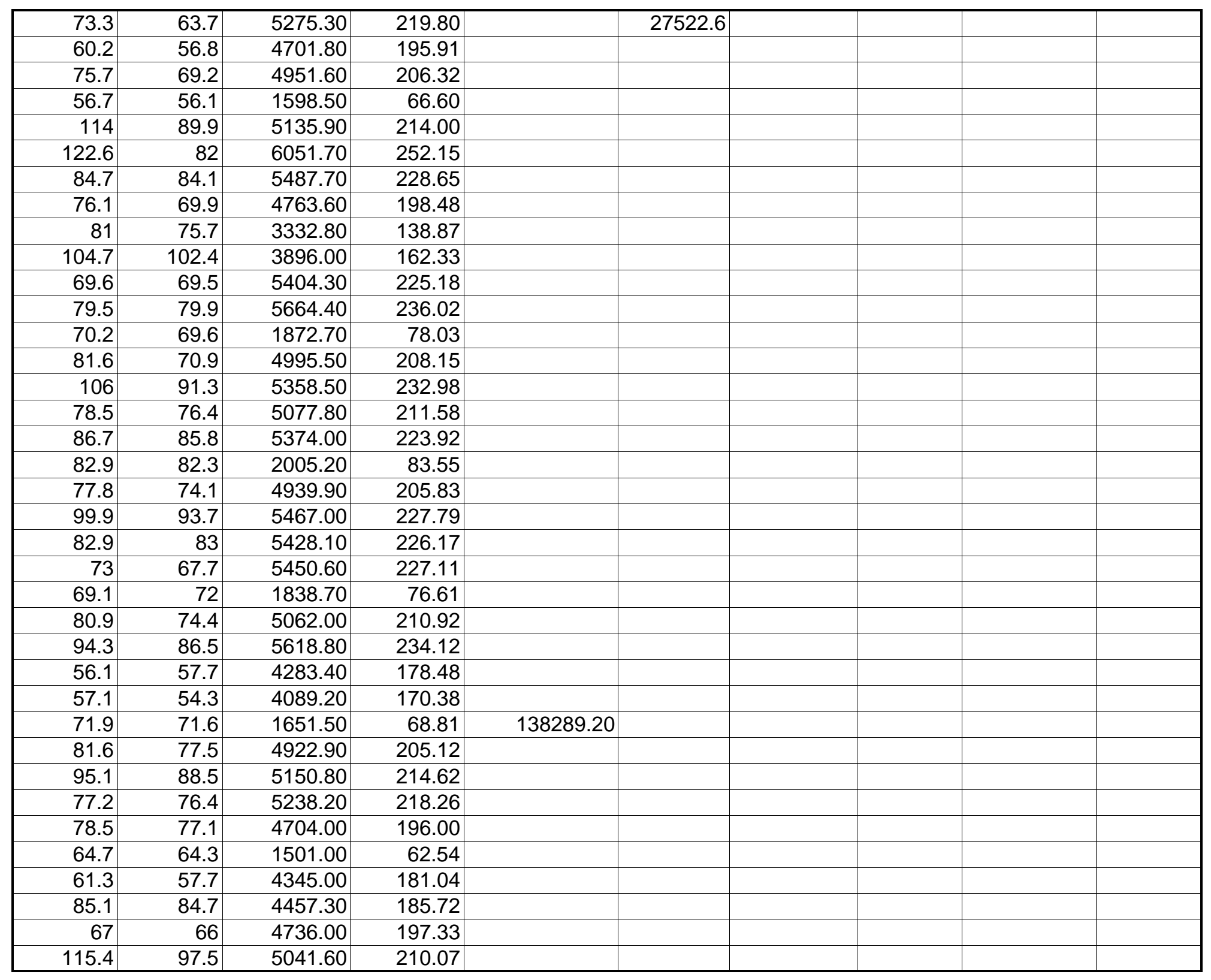

Page 70 
other

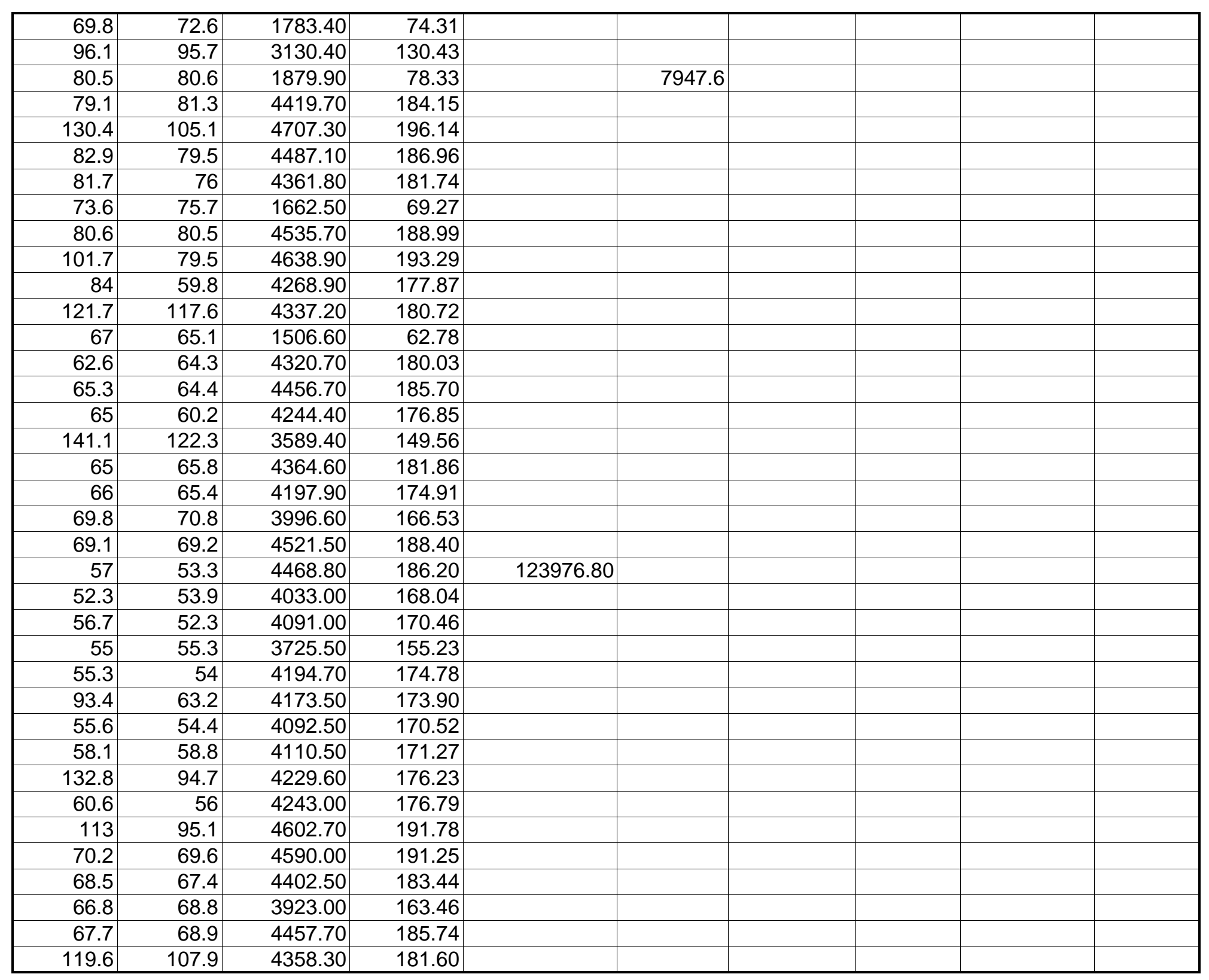

Page 71 
other

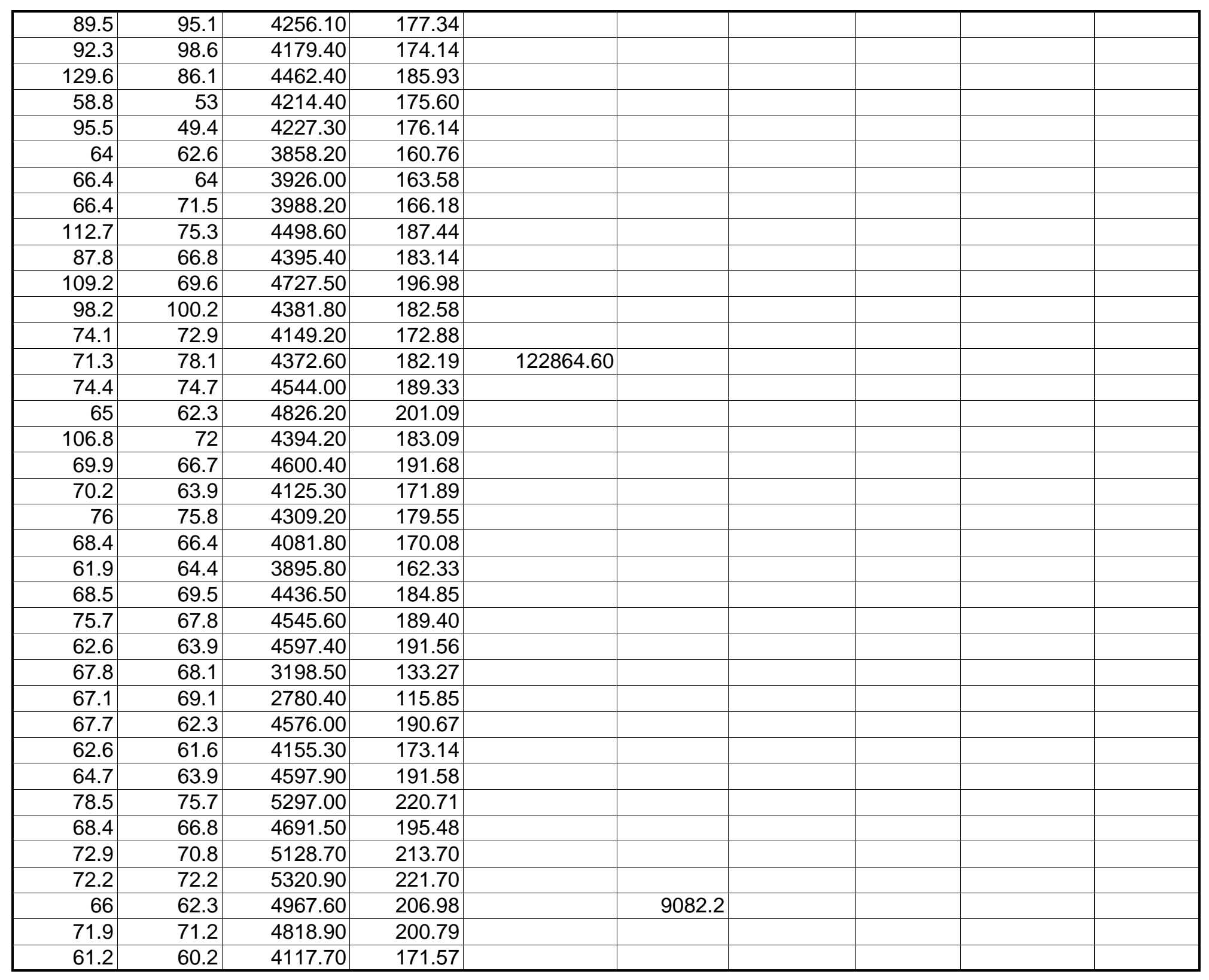


other

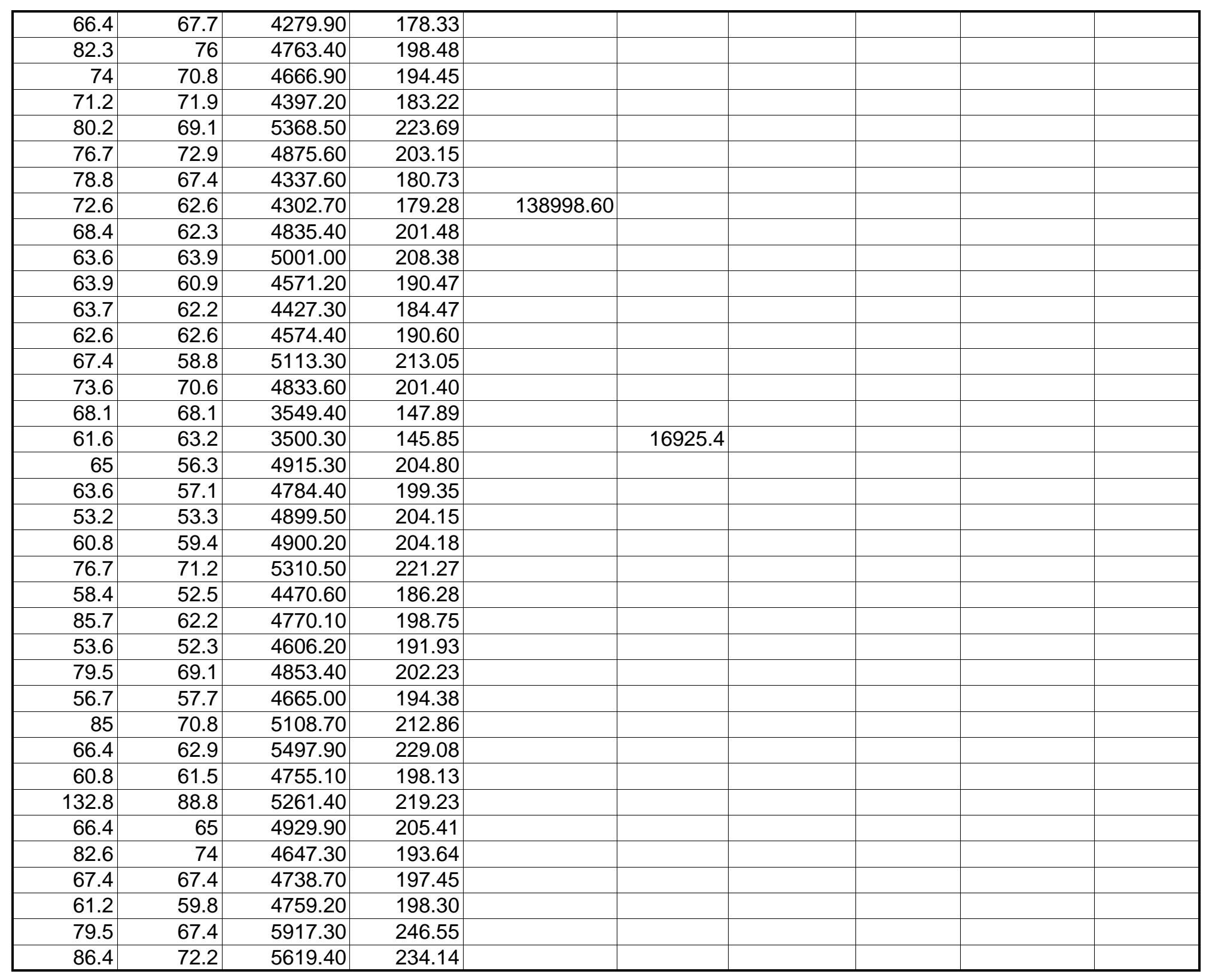


other

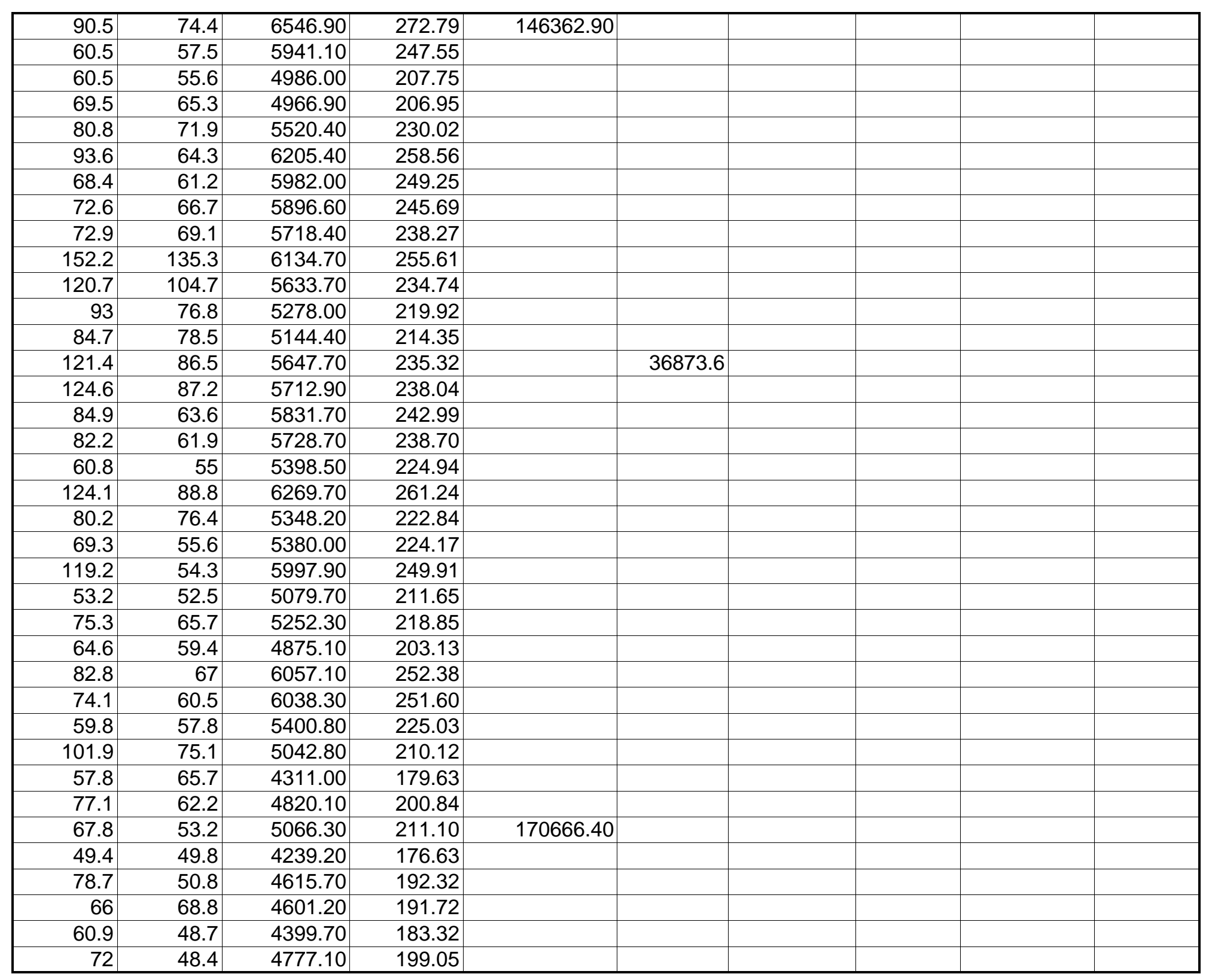


other

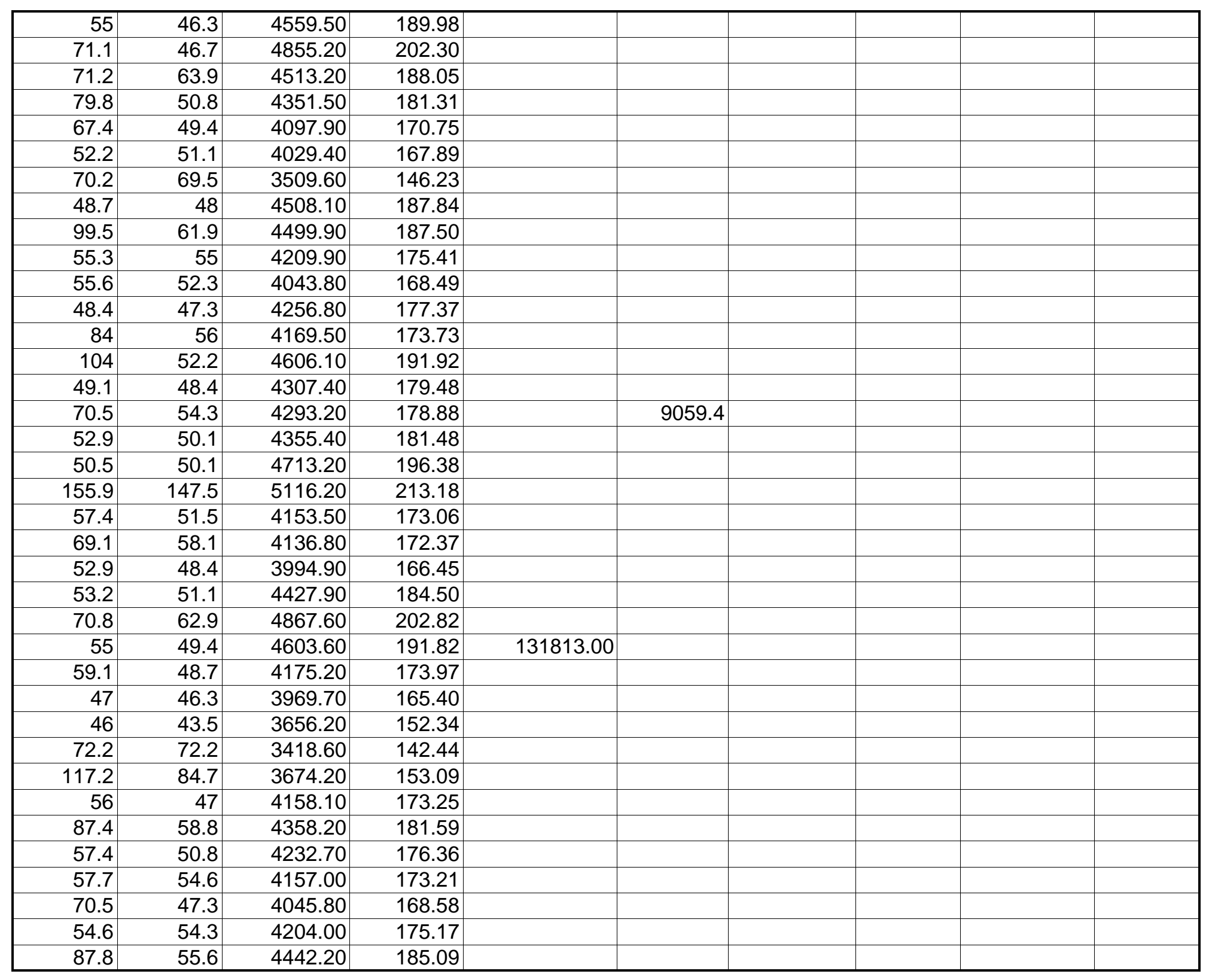


other

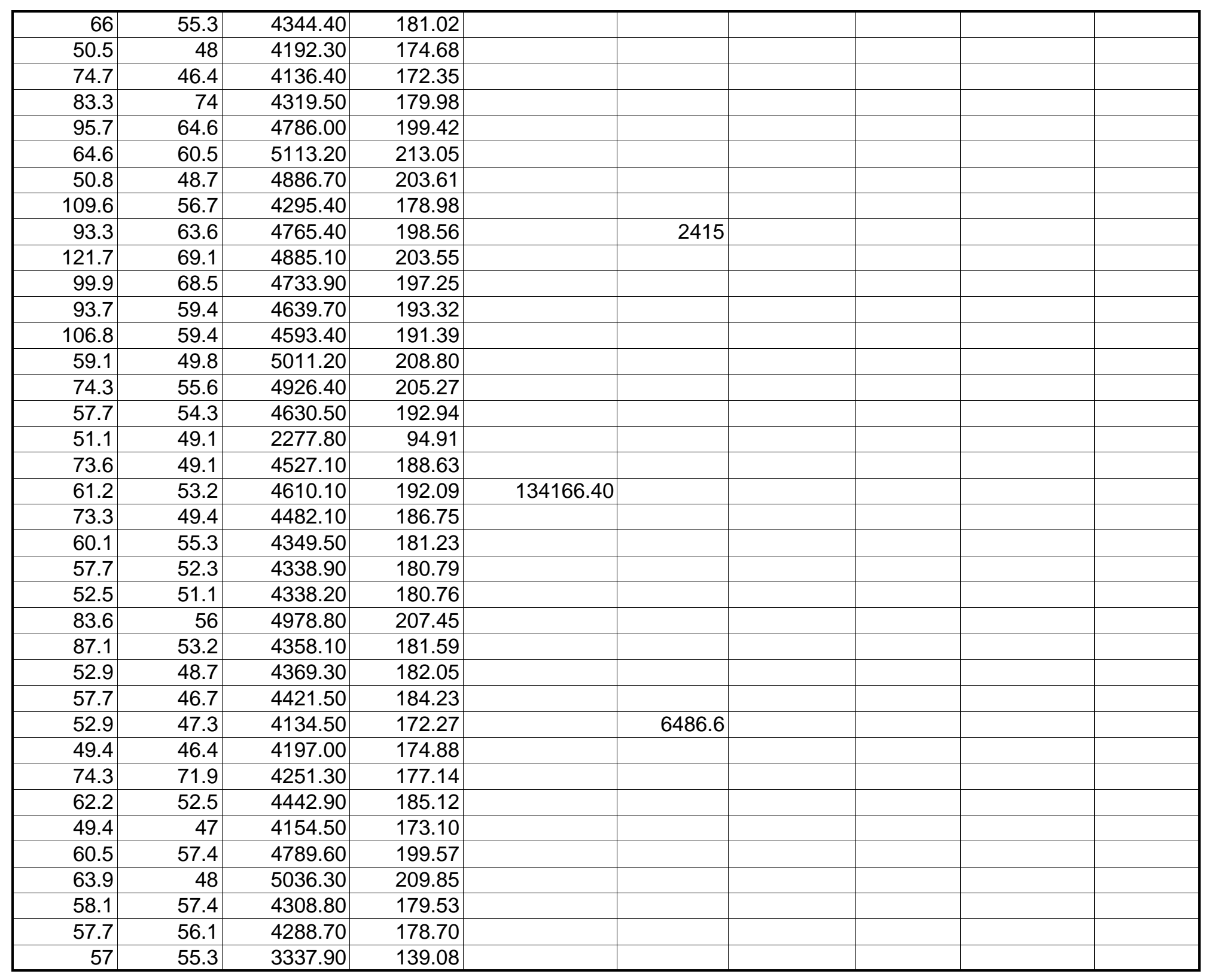

Page 76 
other

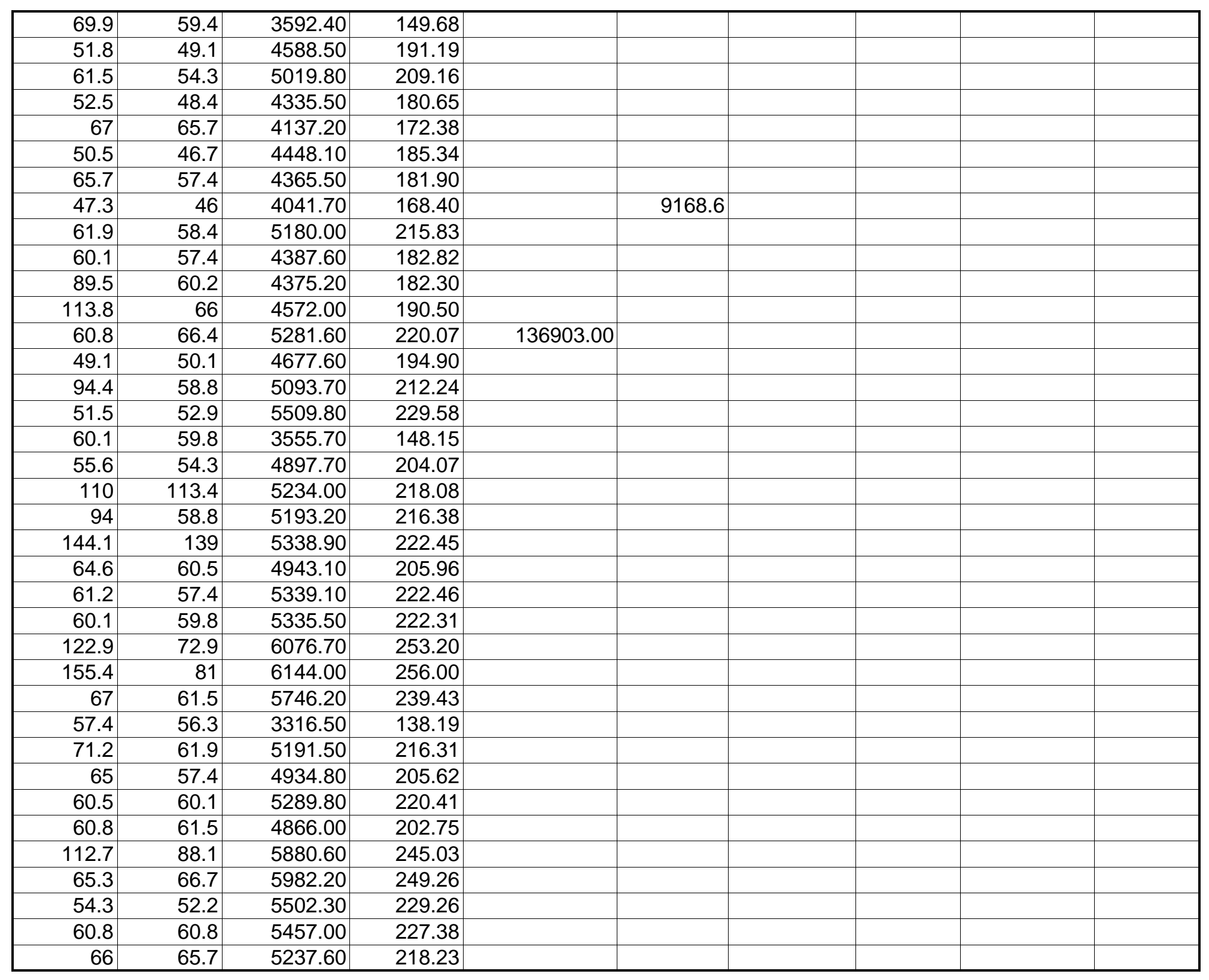


other

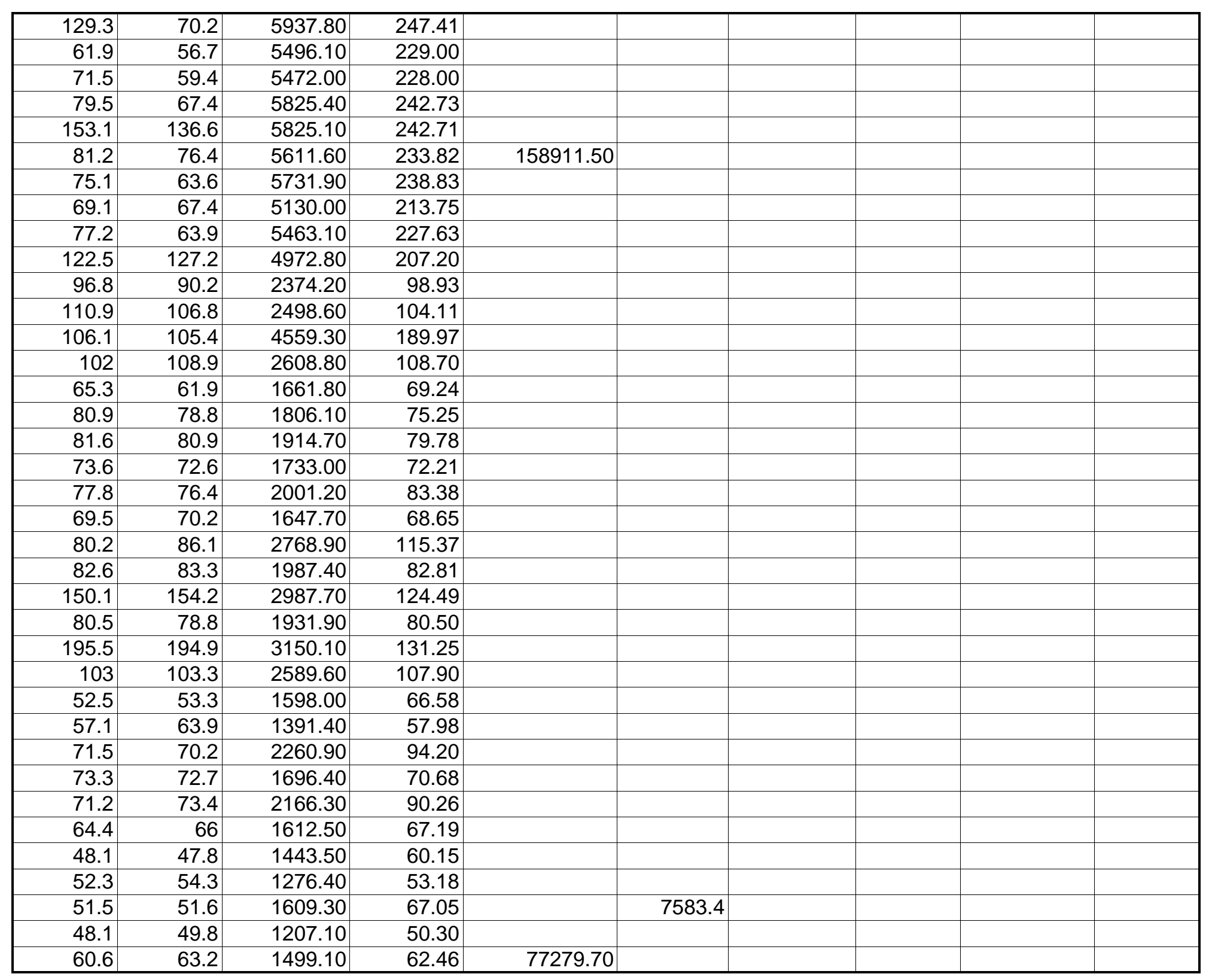

Page 78 


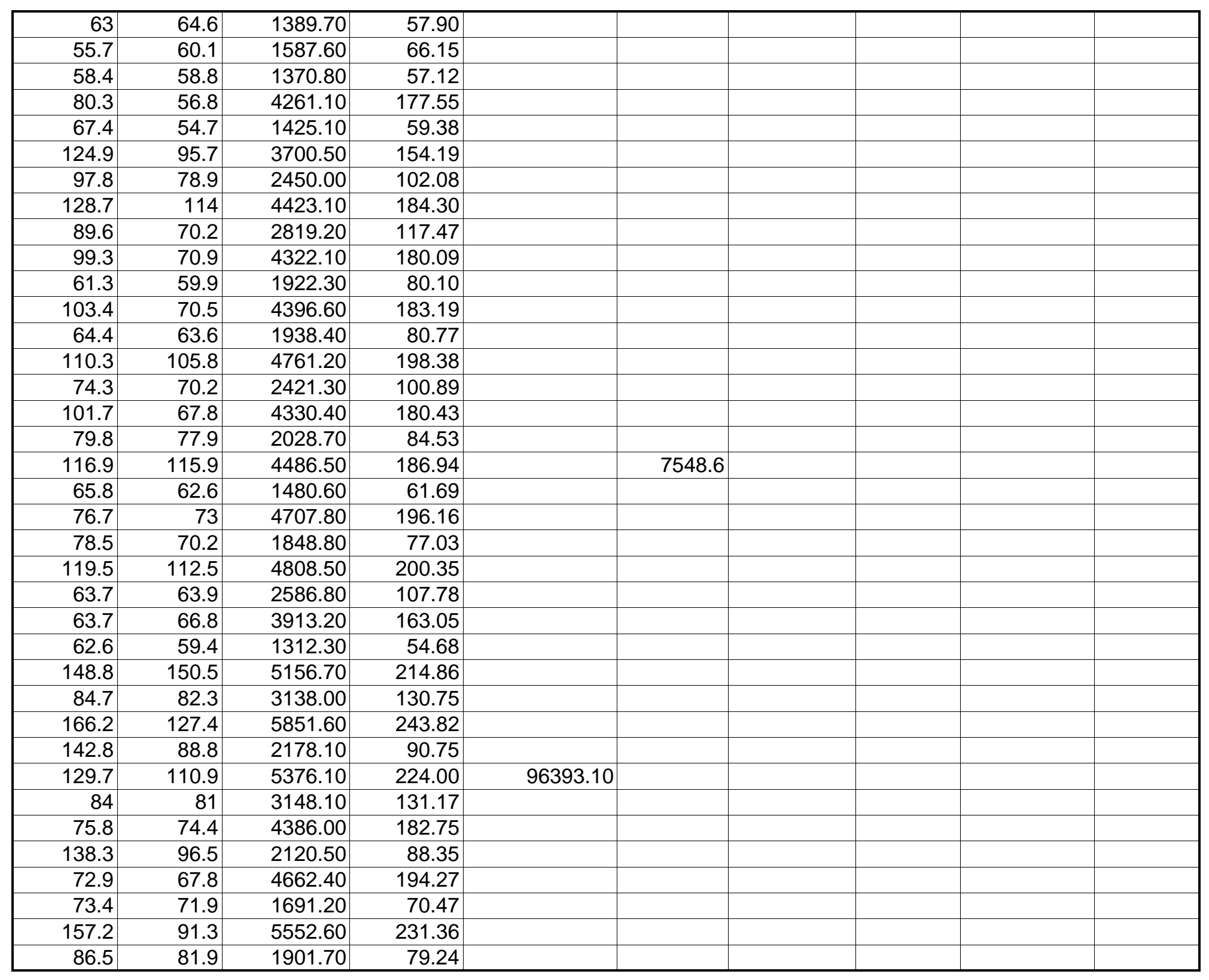


other

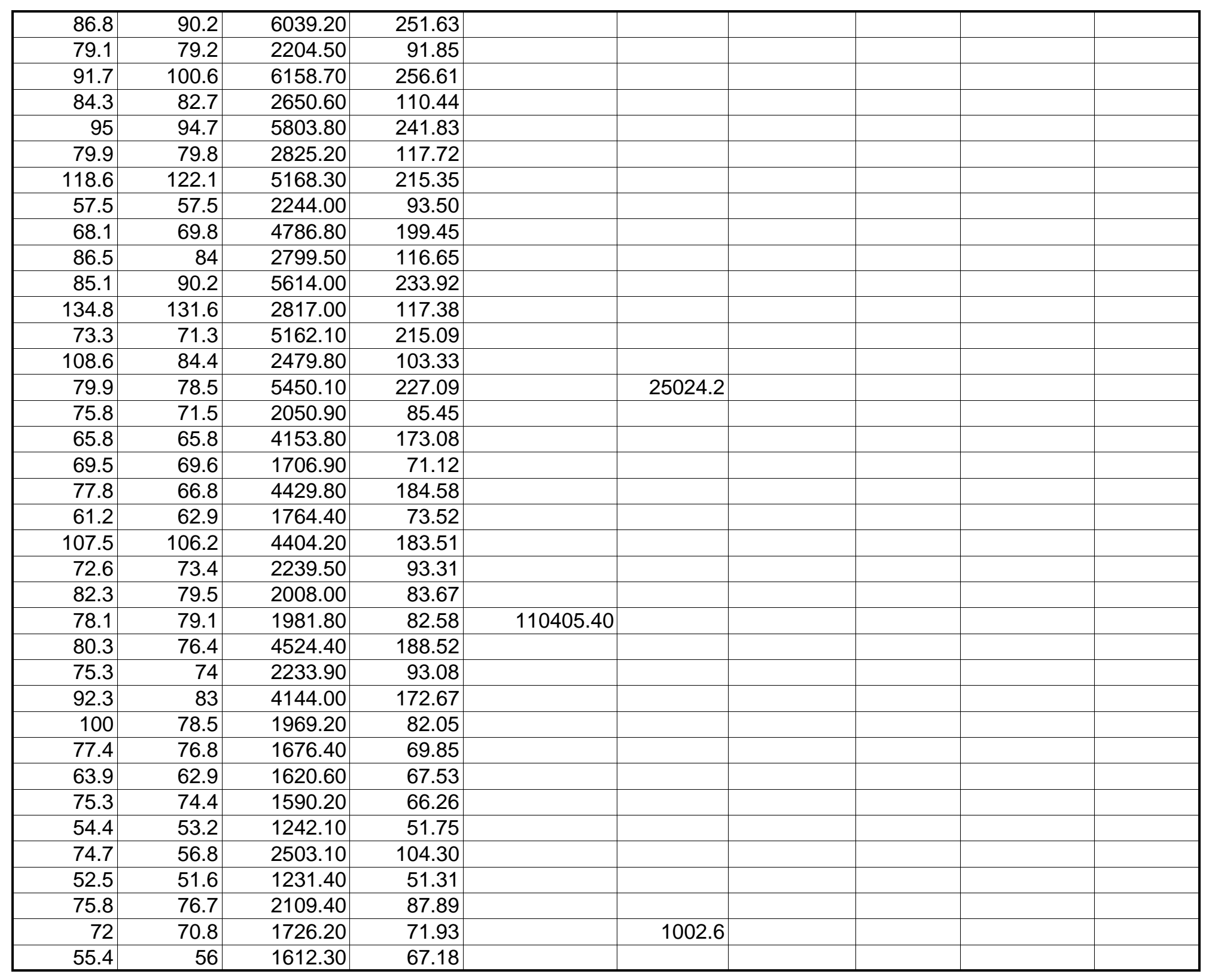


other

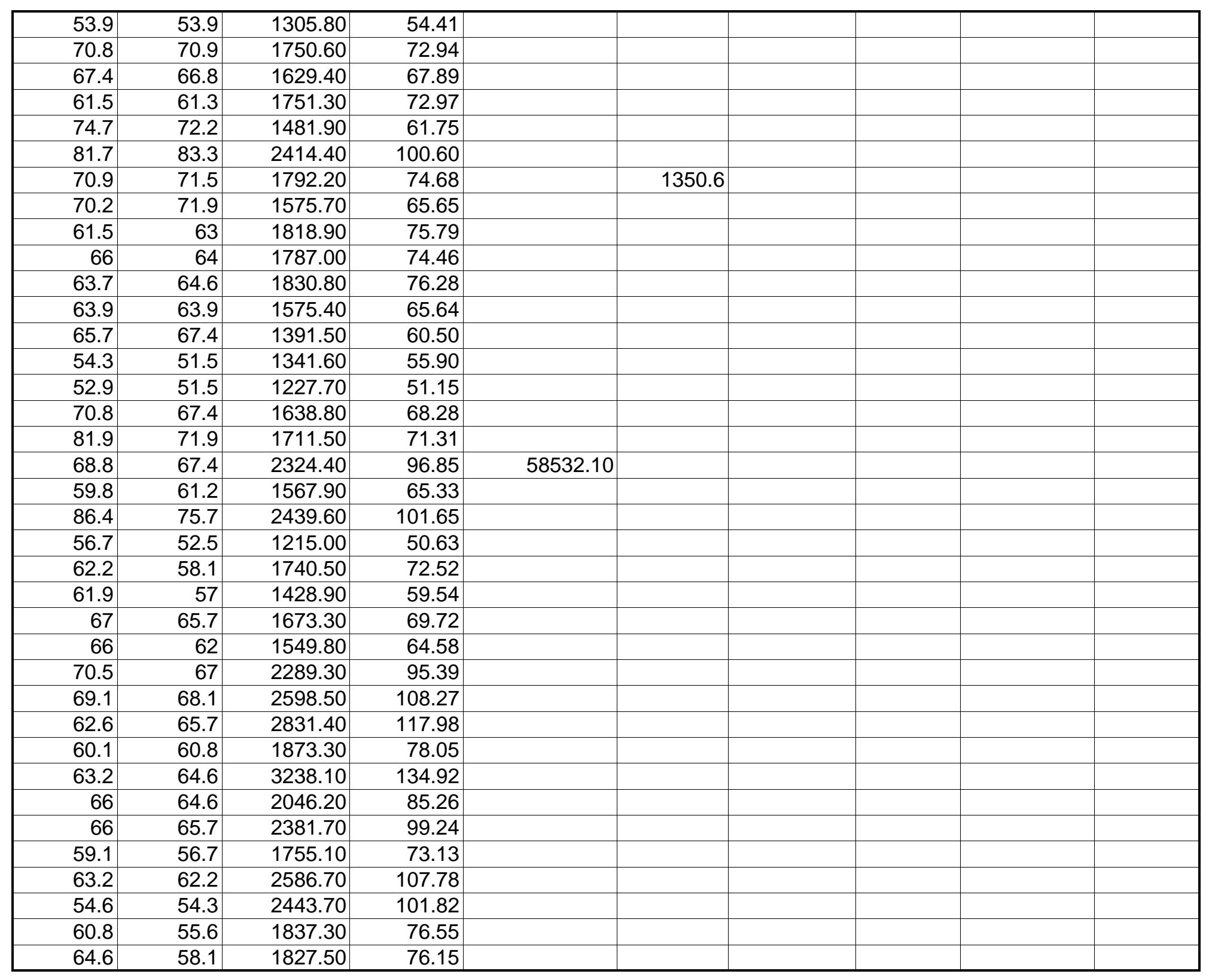

Page 81 
other

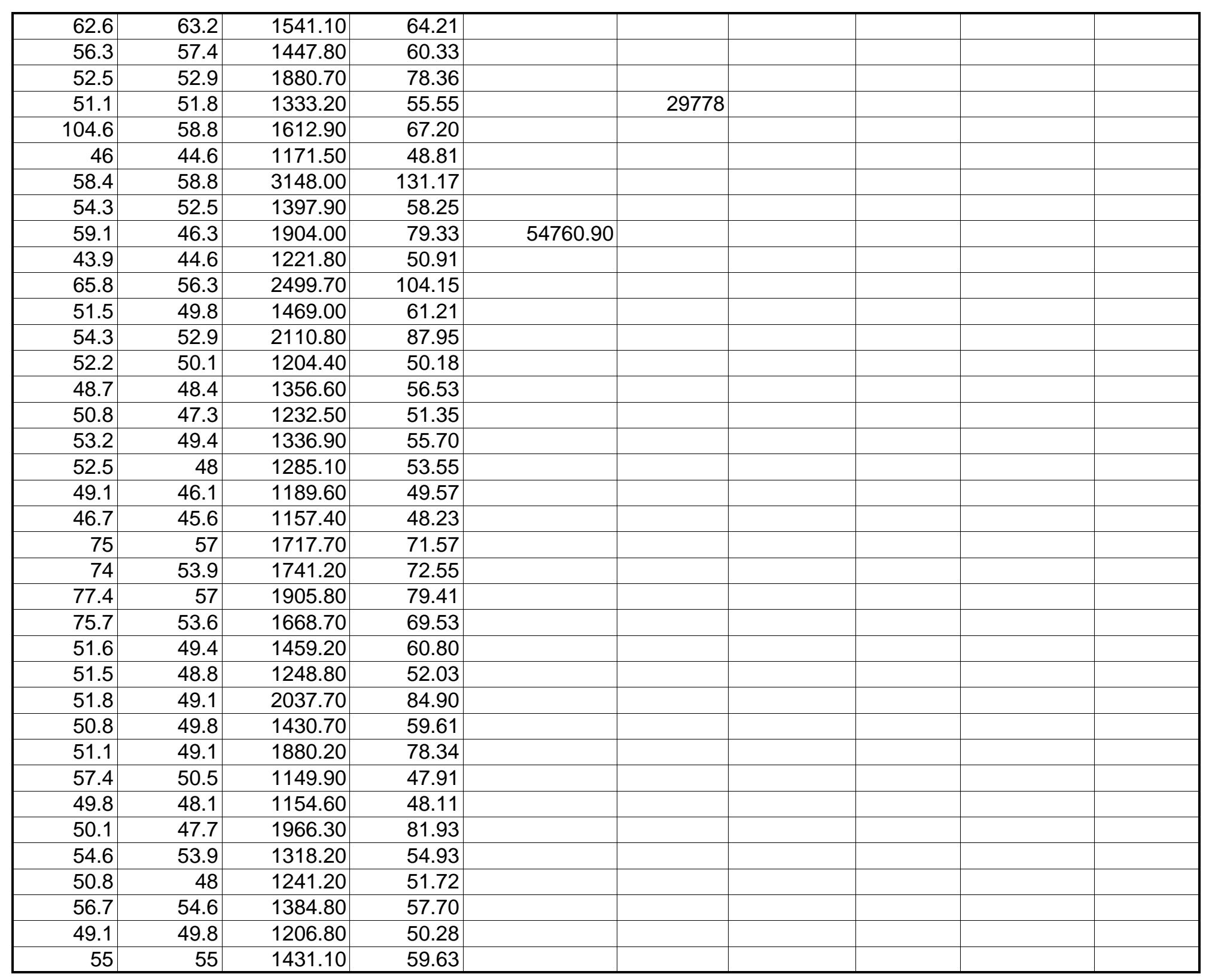


other

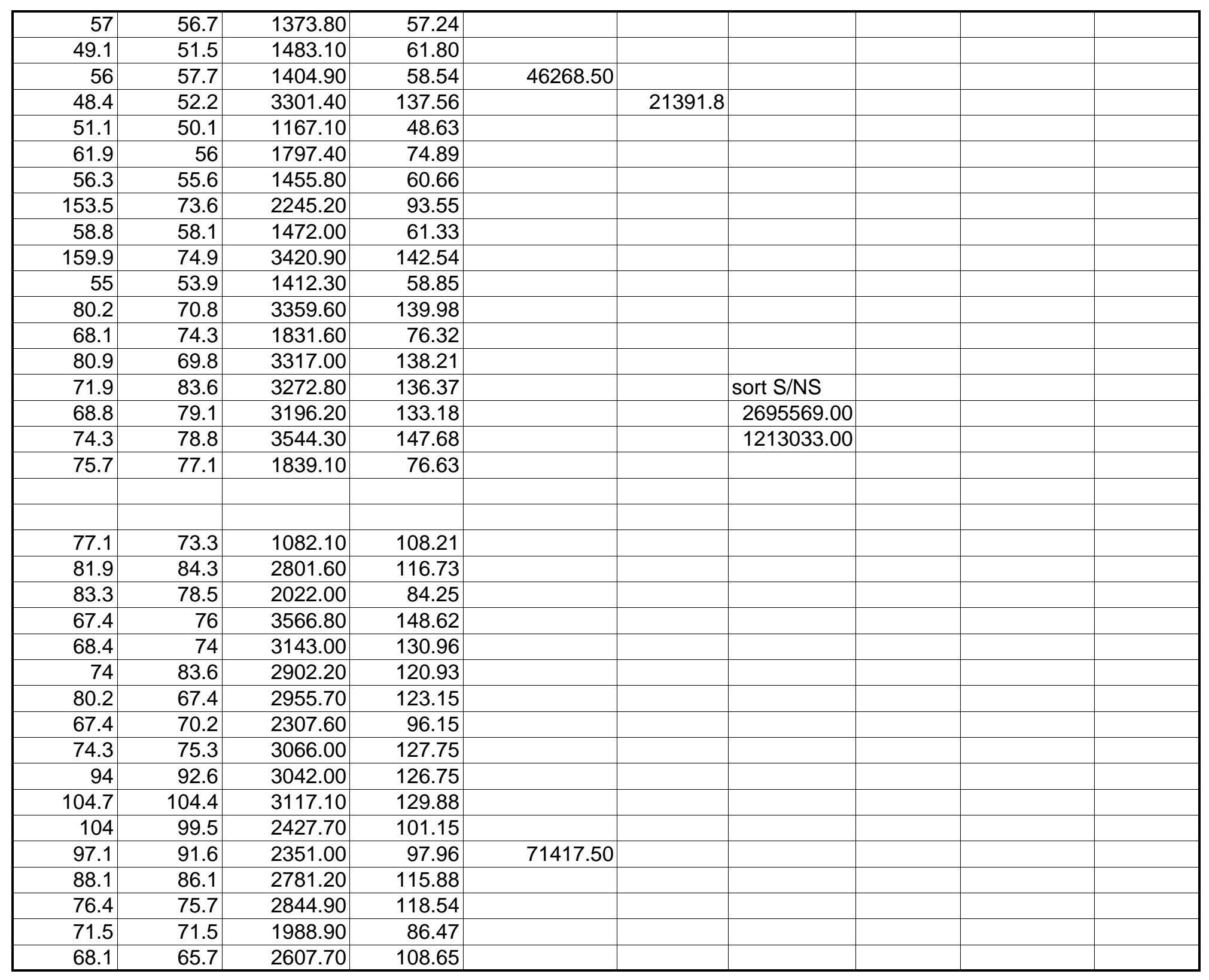


other

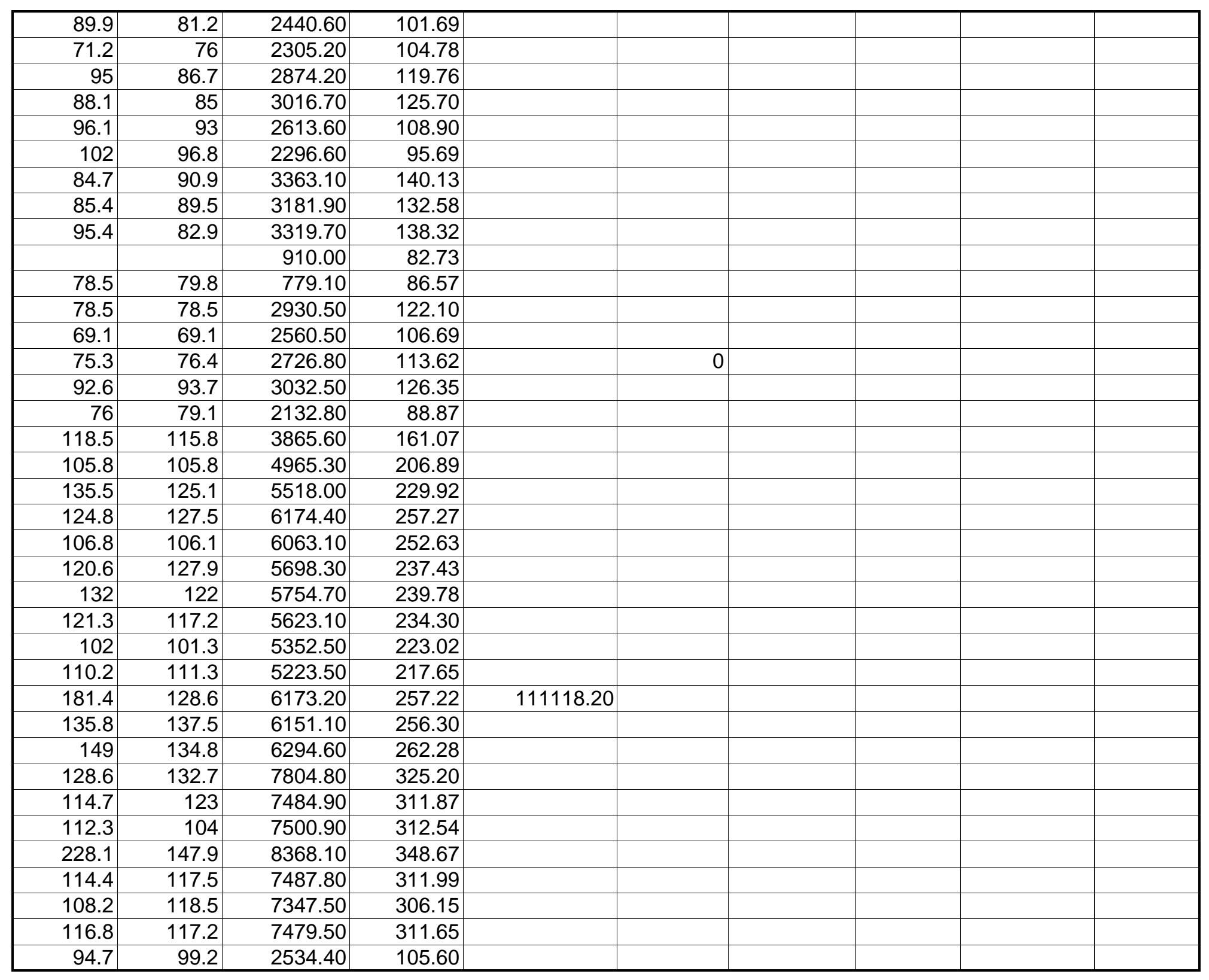


other

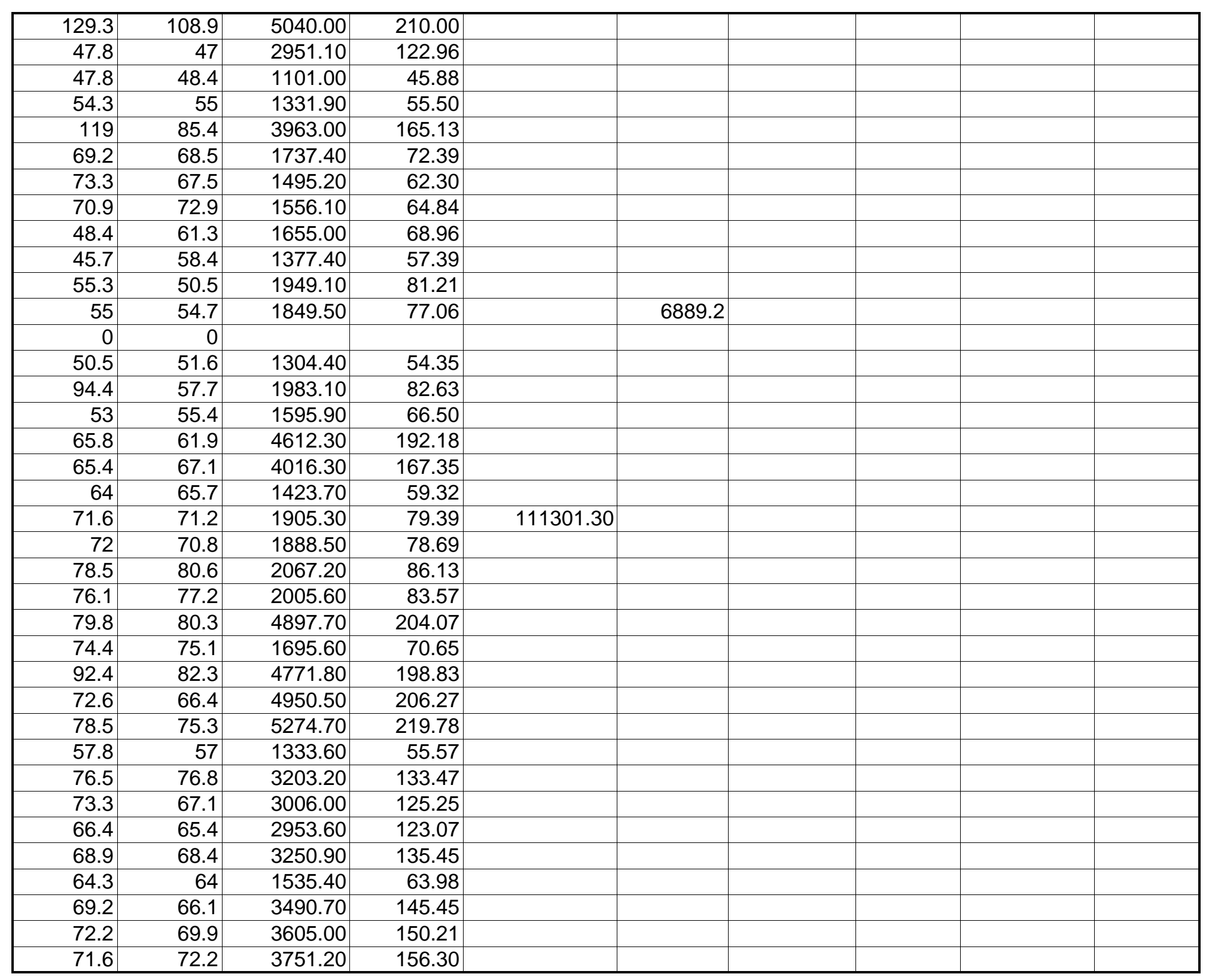

Page 85 
other

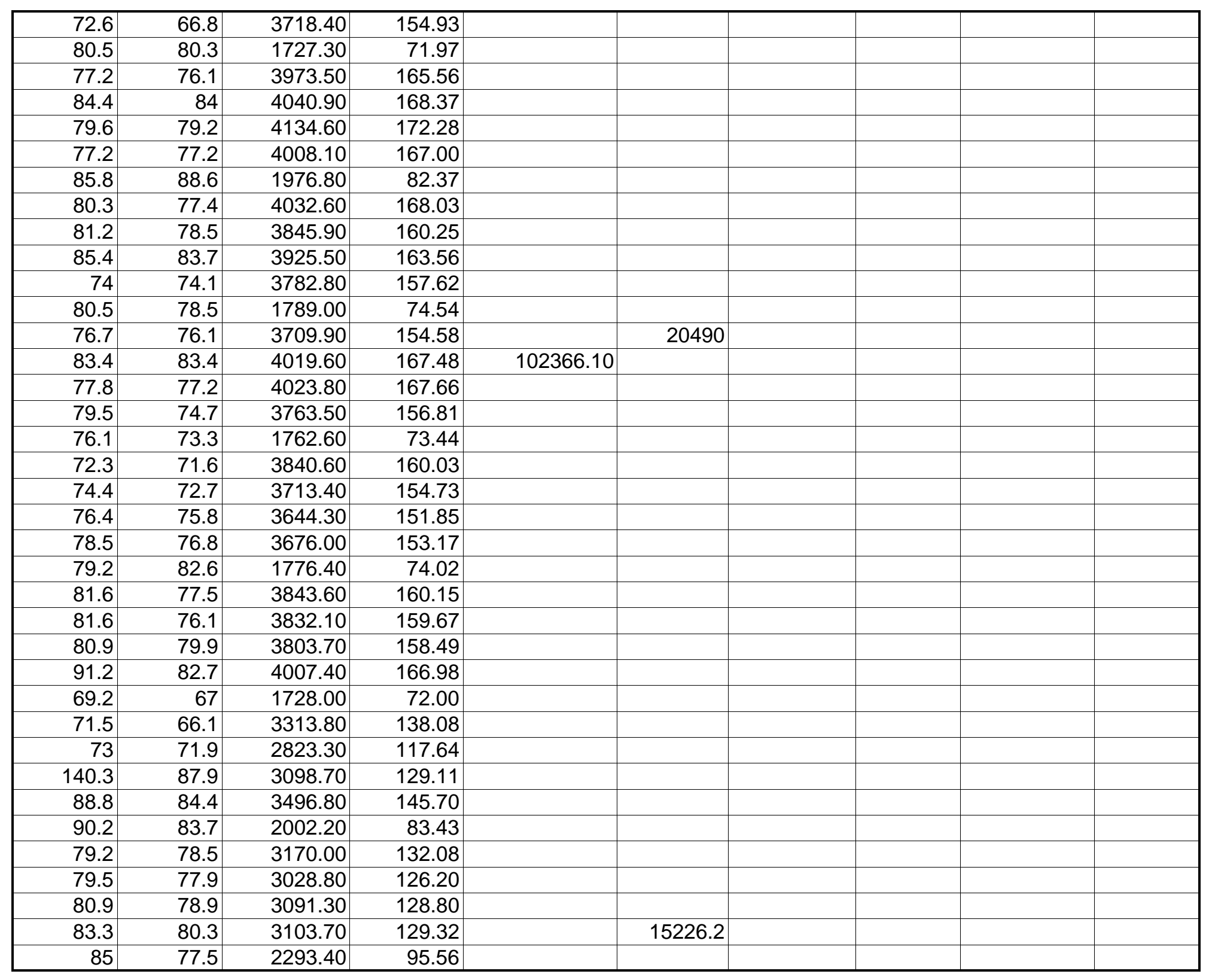


other

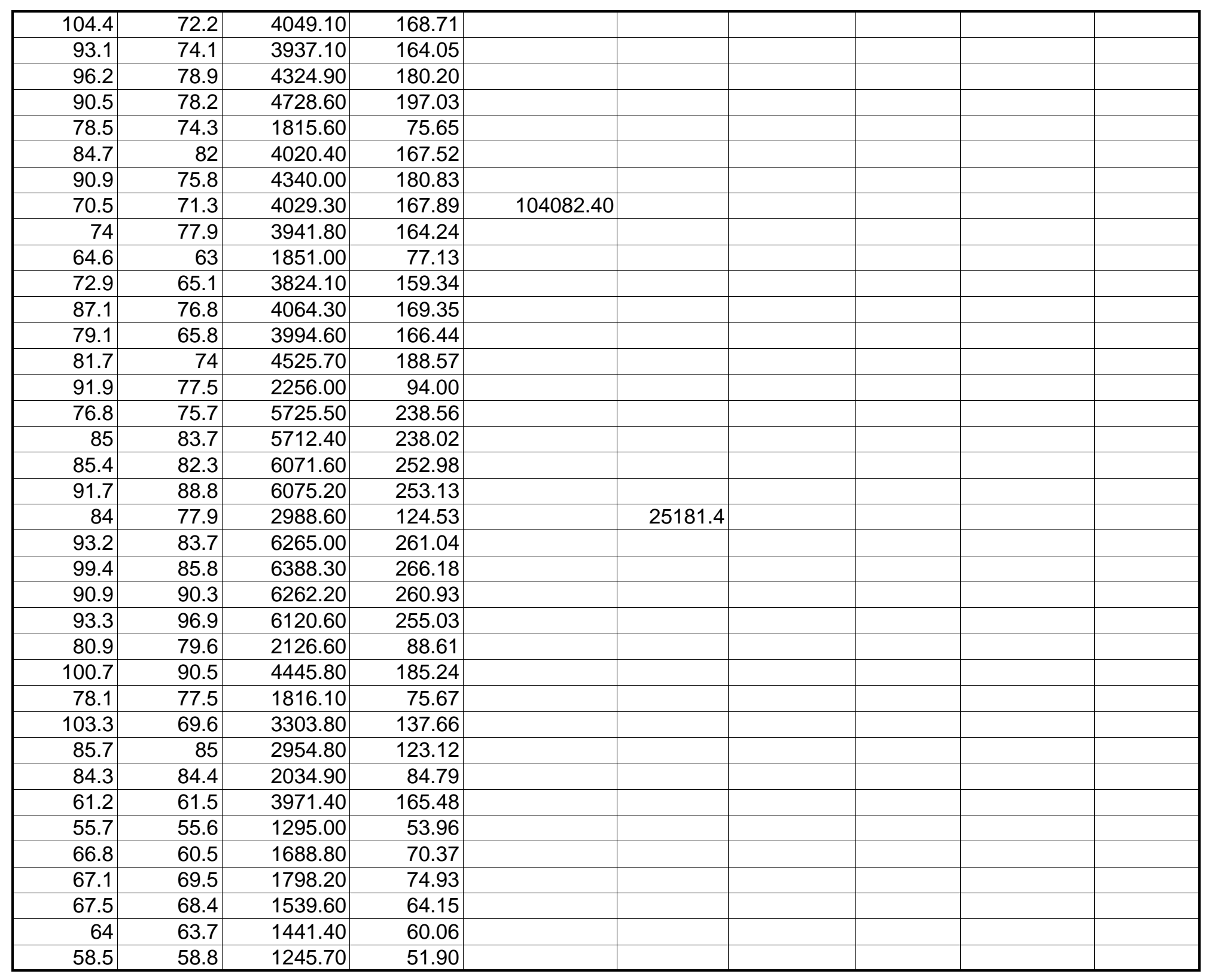


other

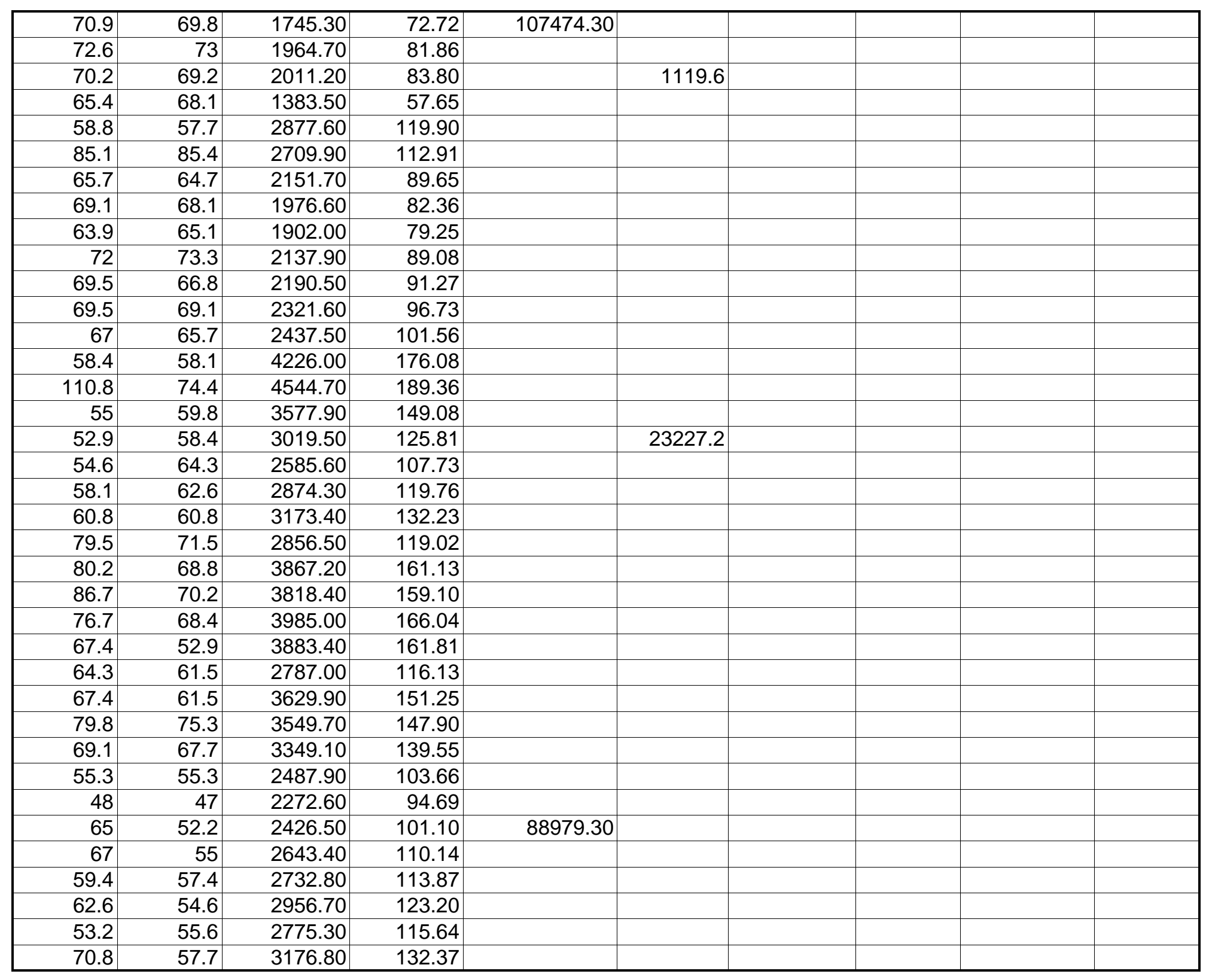


other

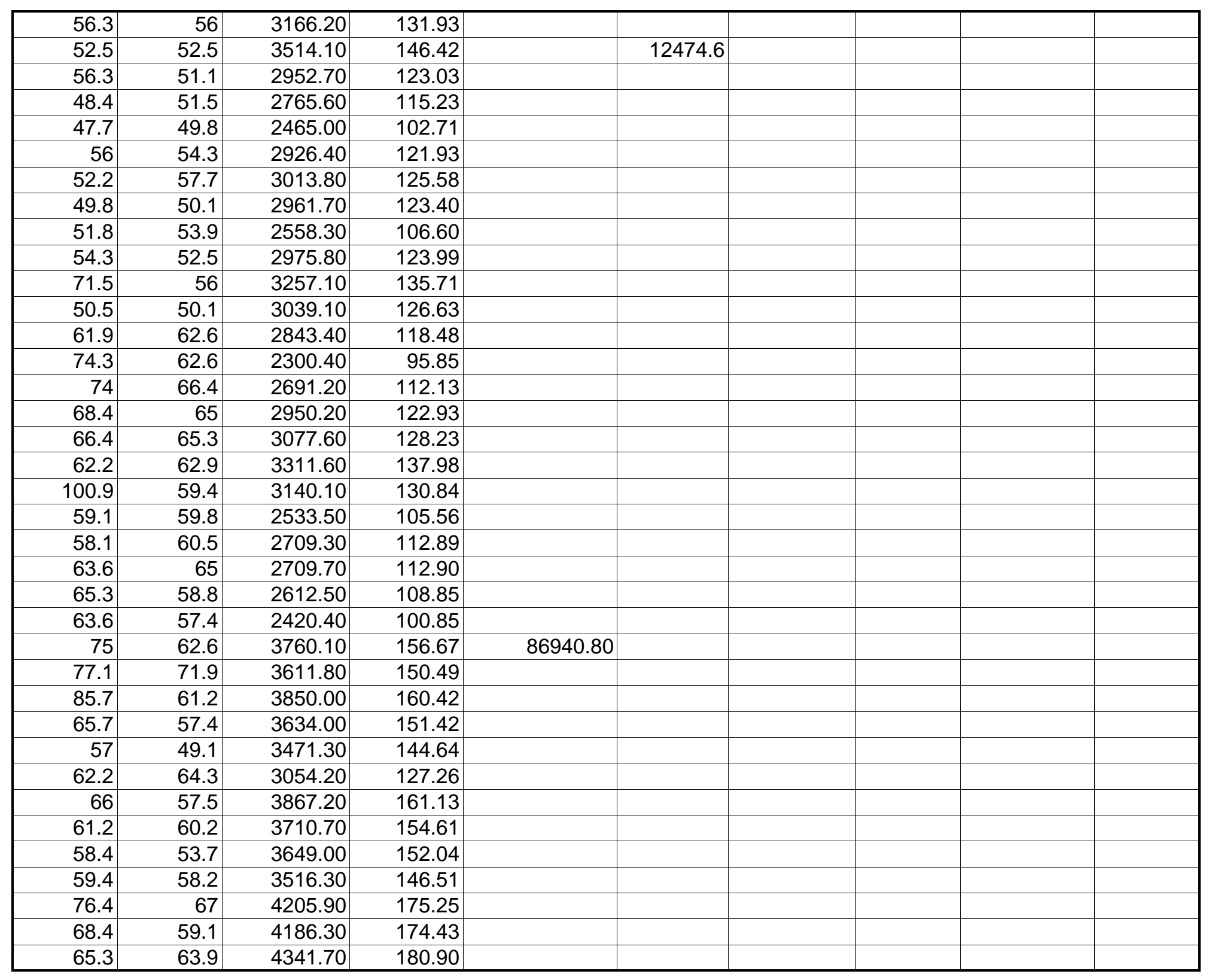

Page 89 
other

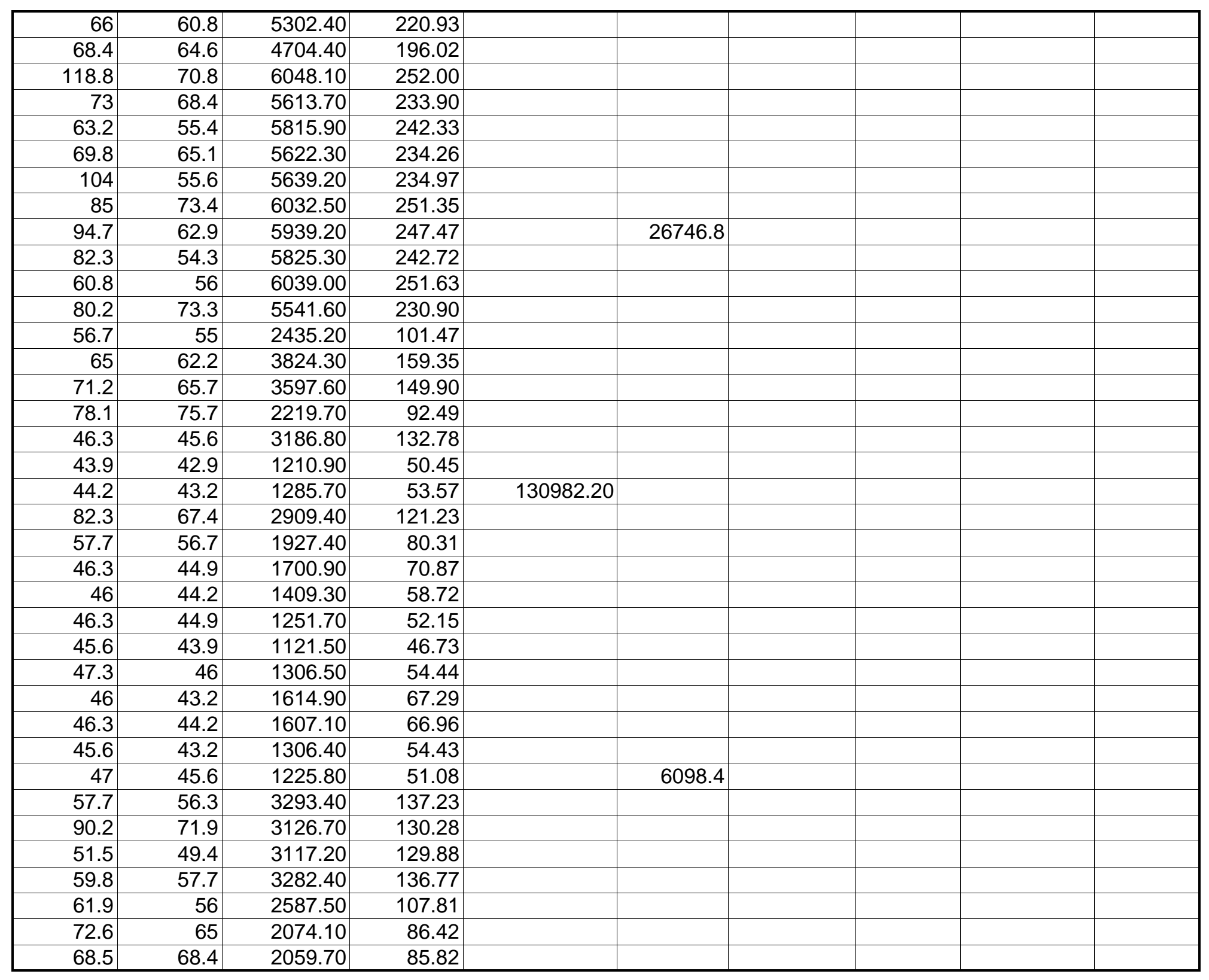

Page 90 
other

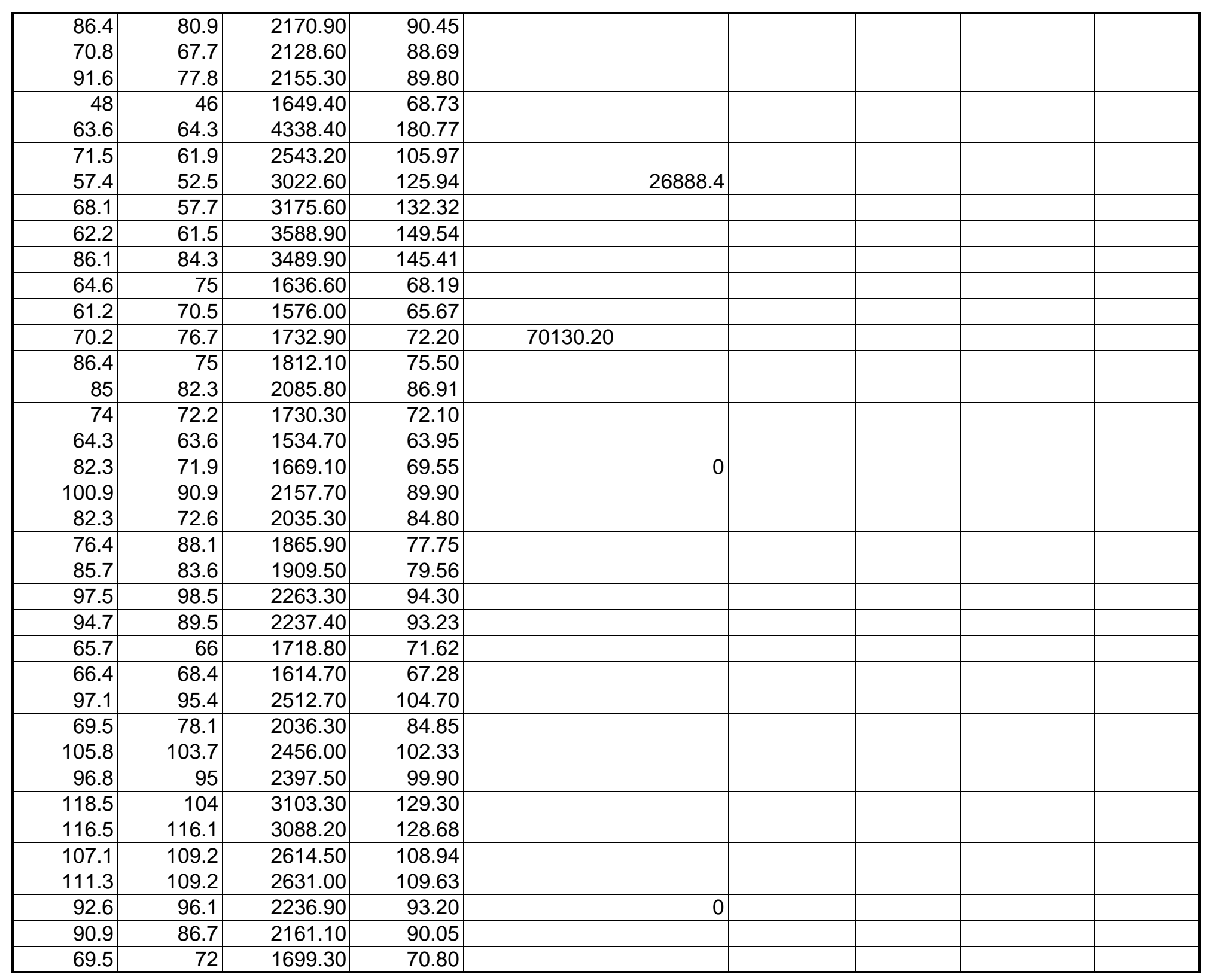

Page 91 
other

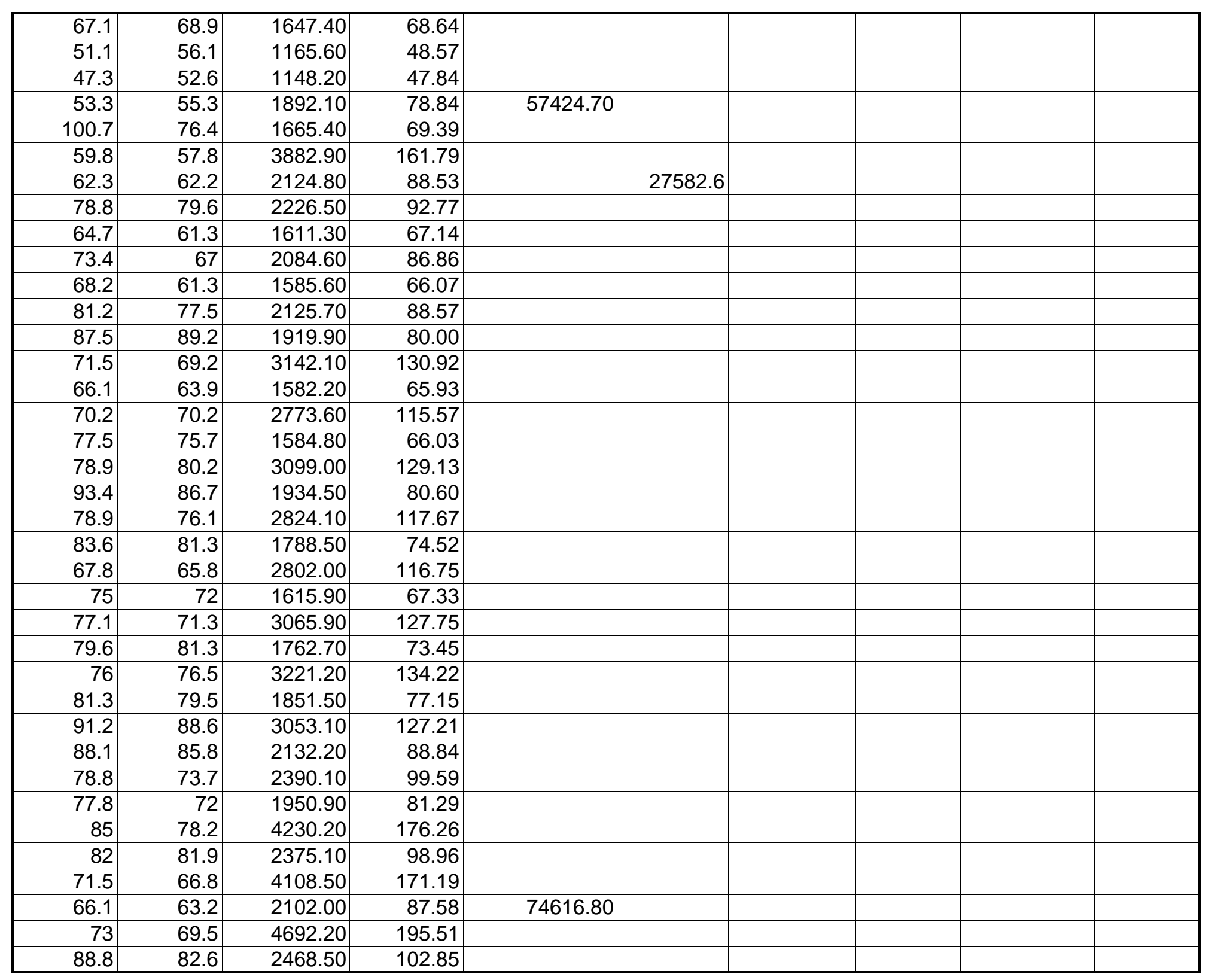

Page 92 
other

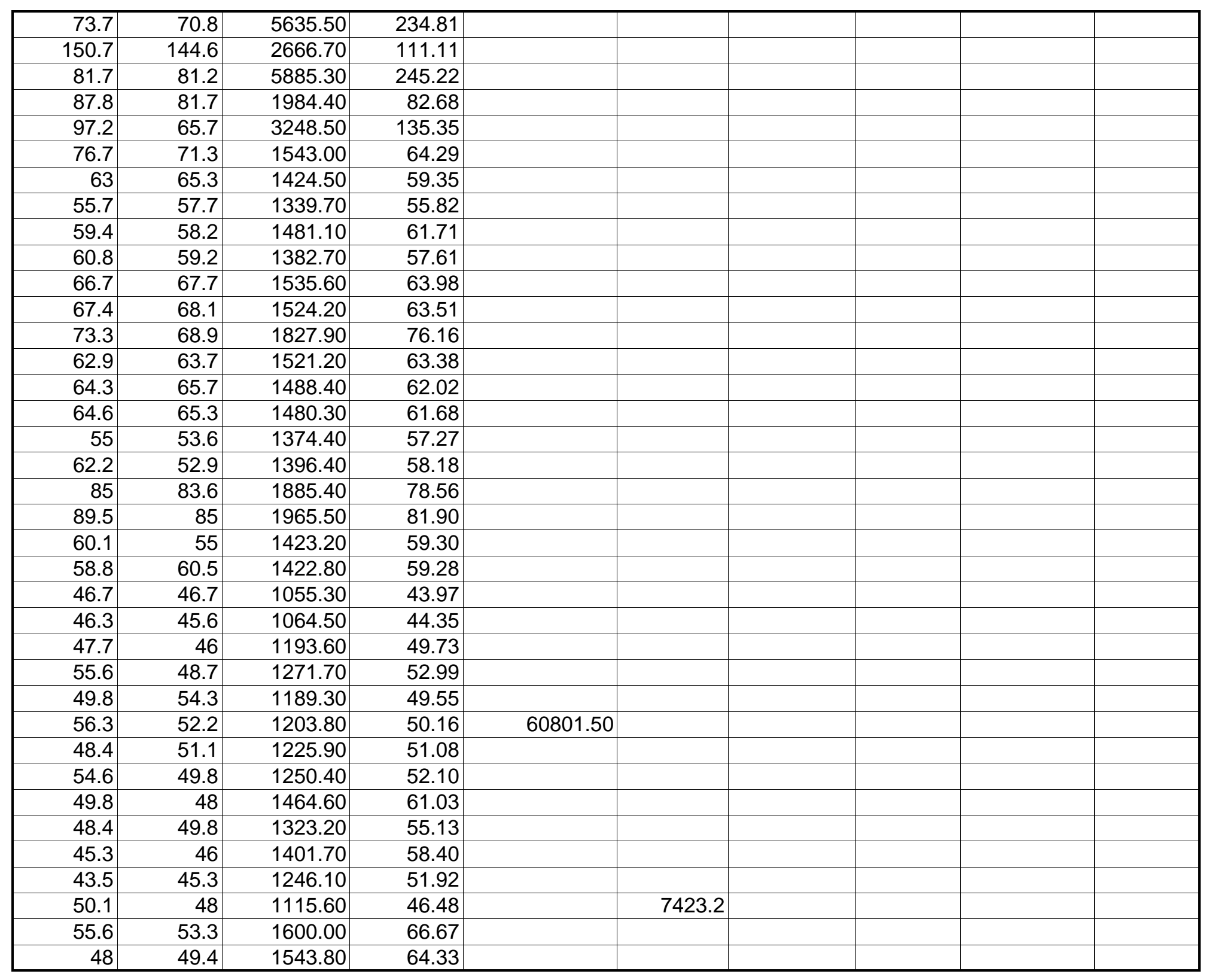

Page 93 
other

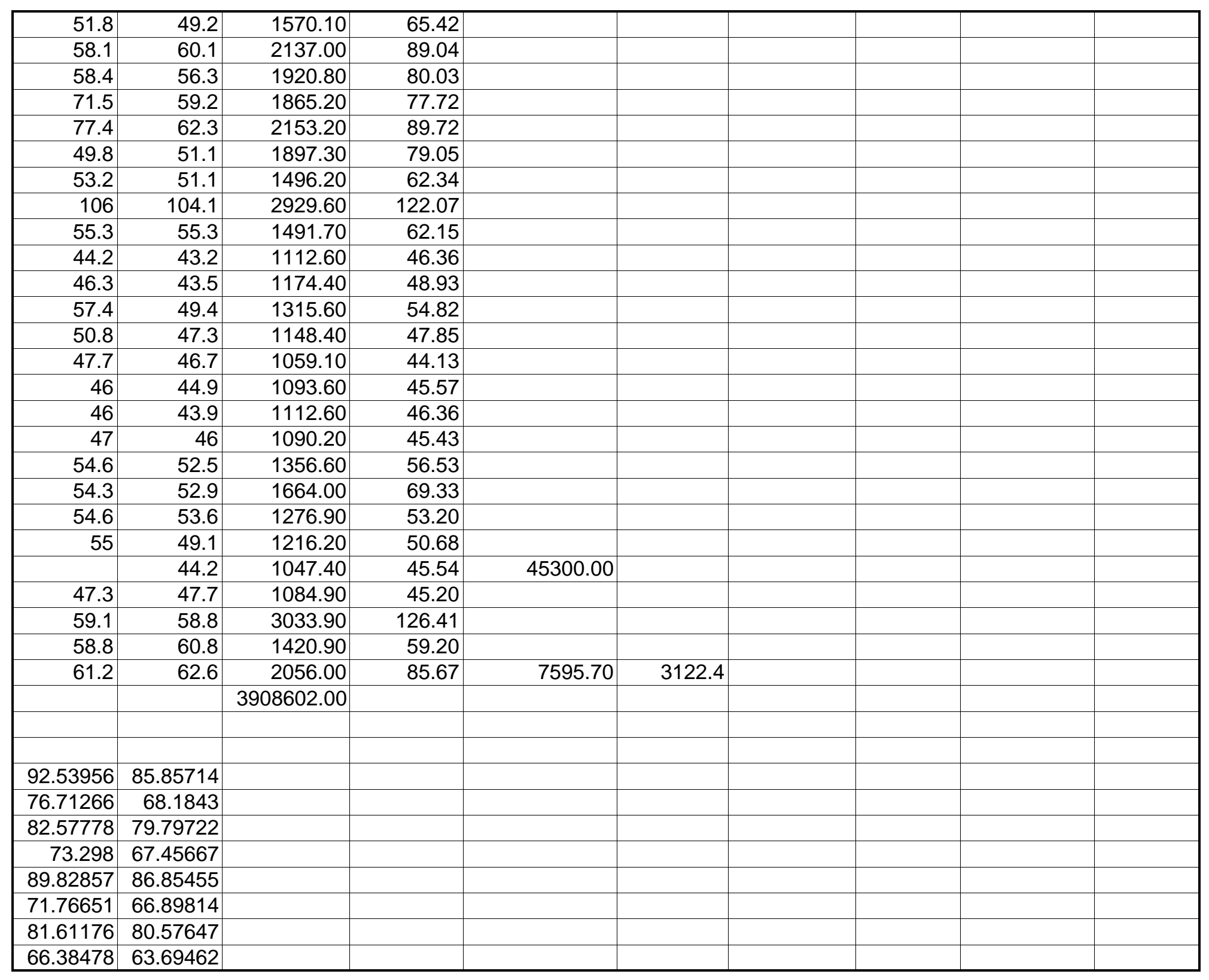


other

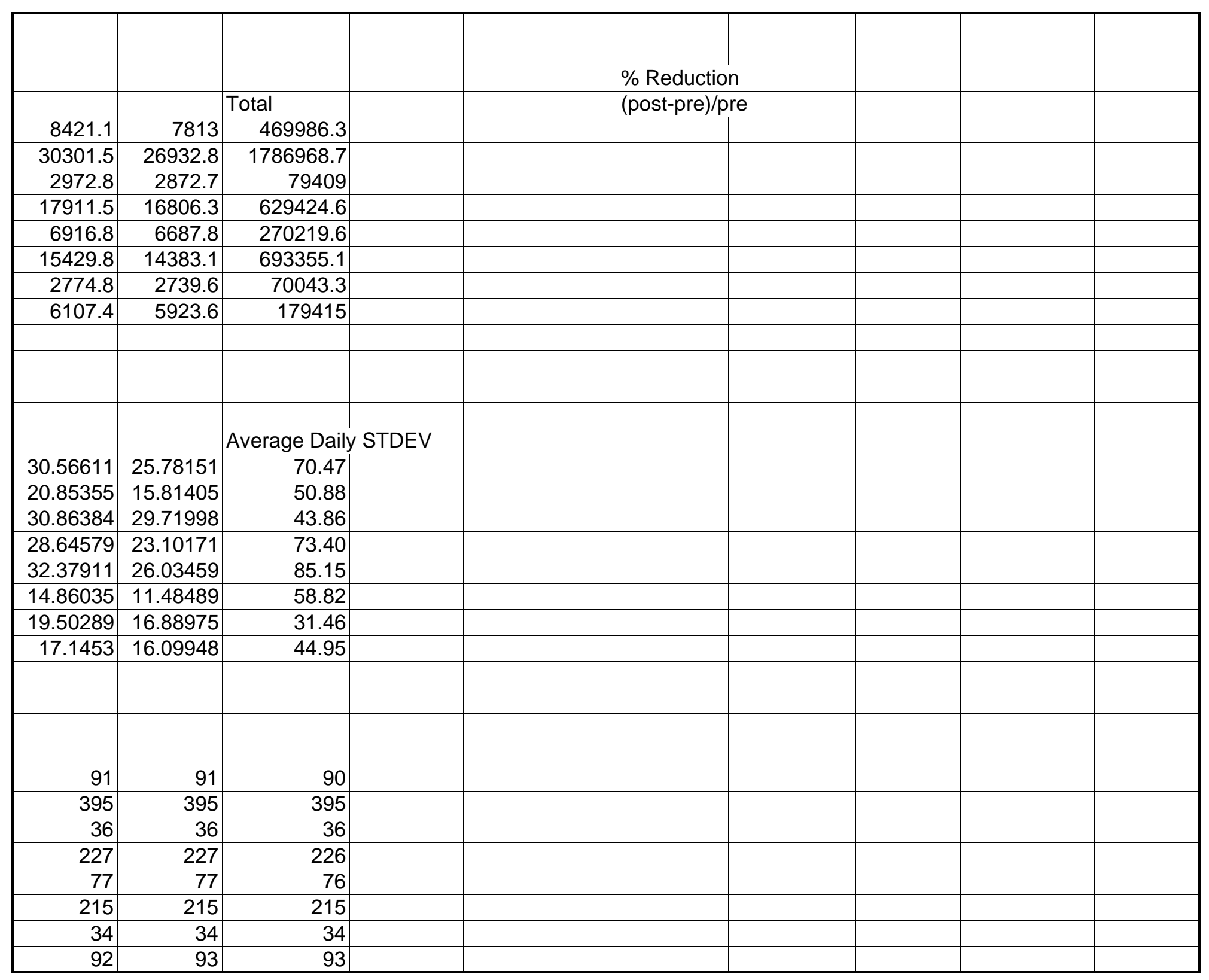

Page 95 


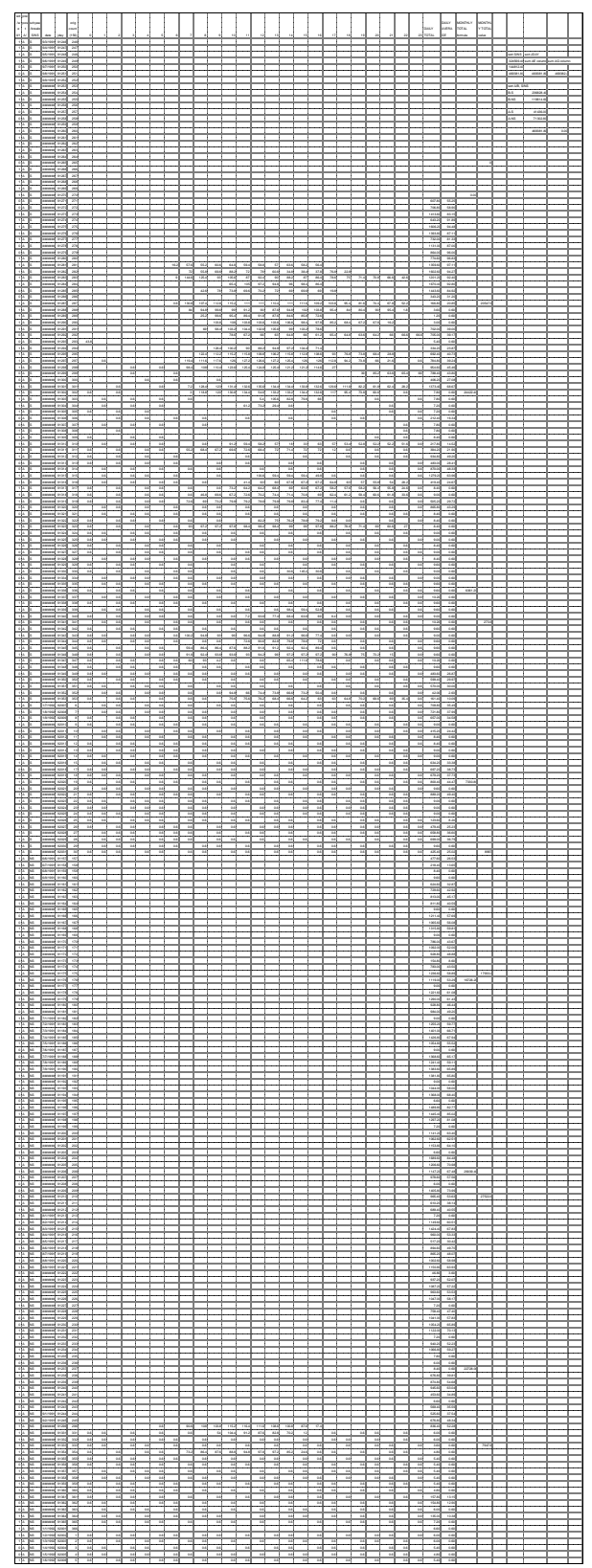




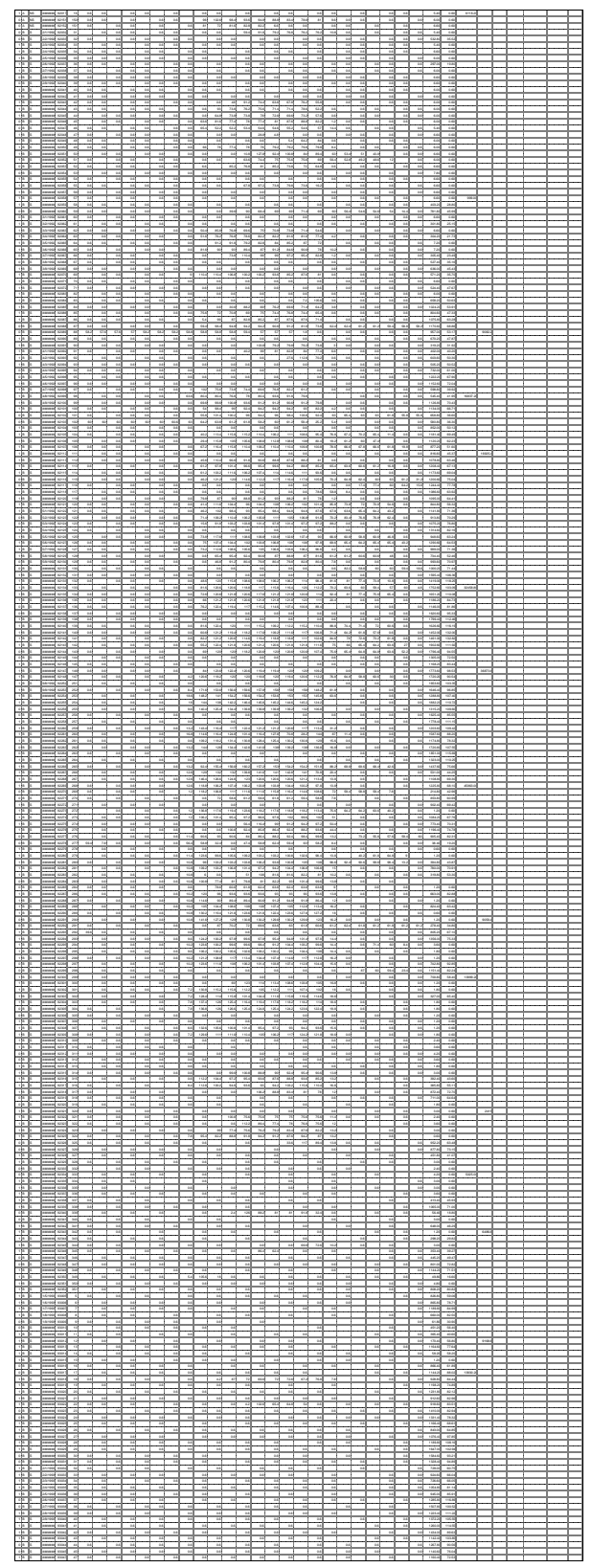




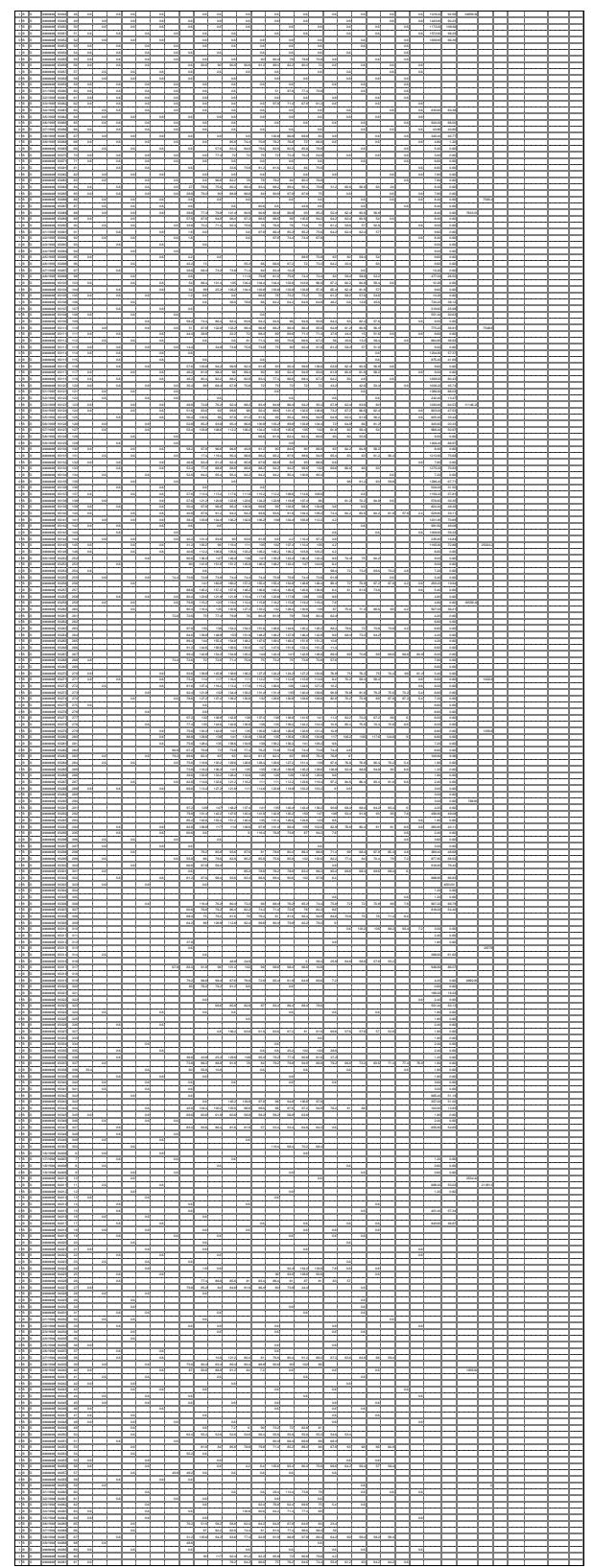




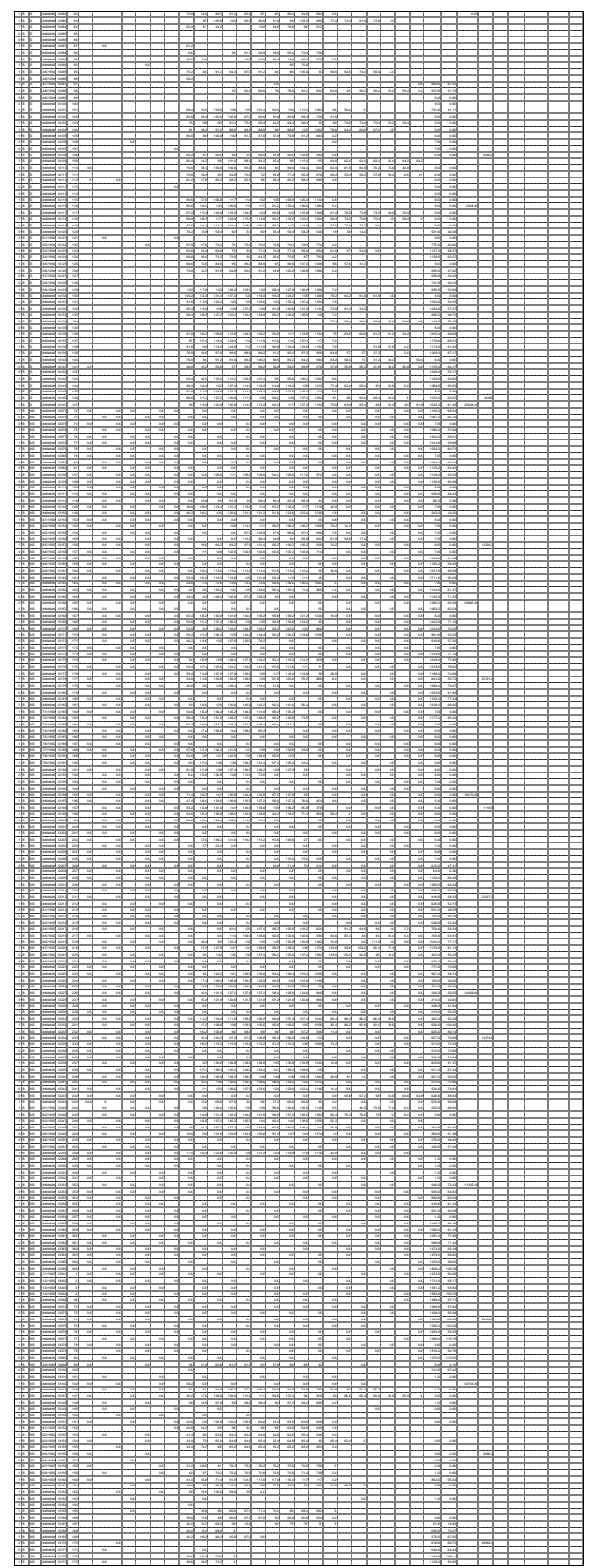




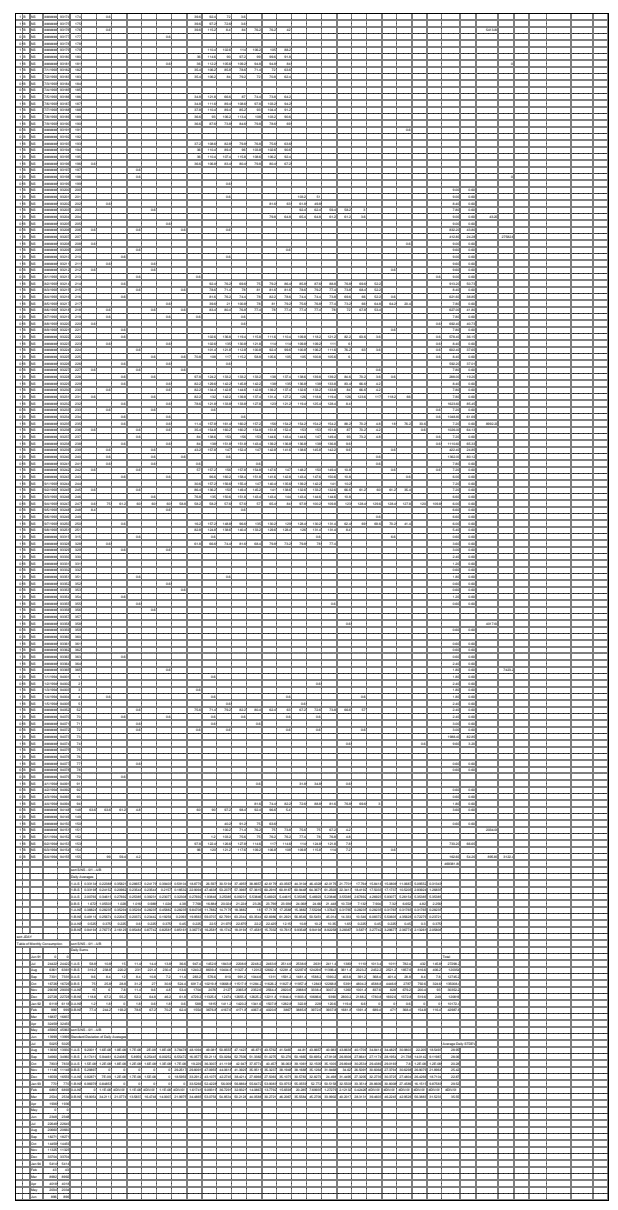




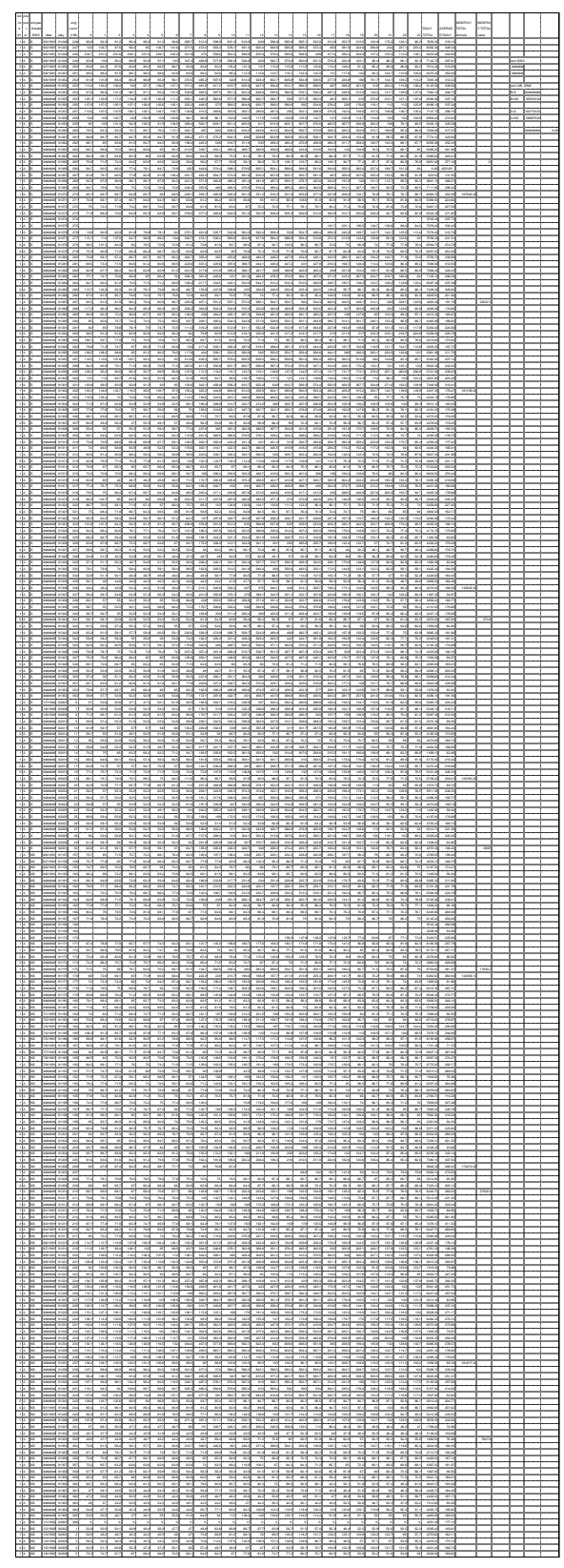




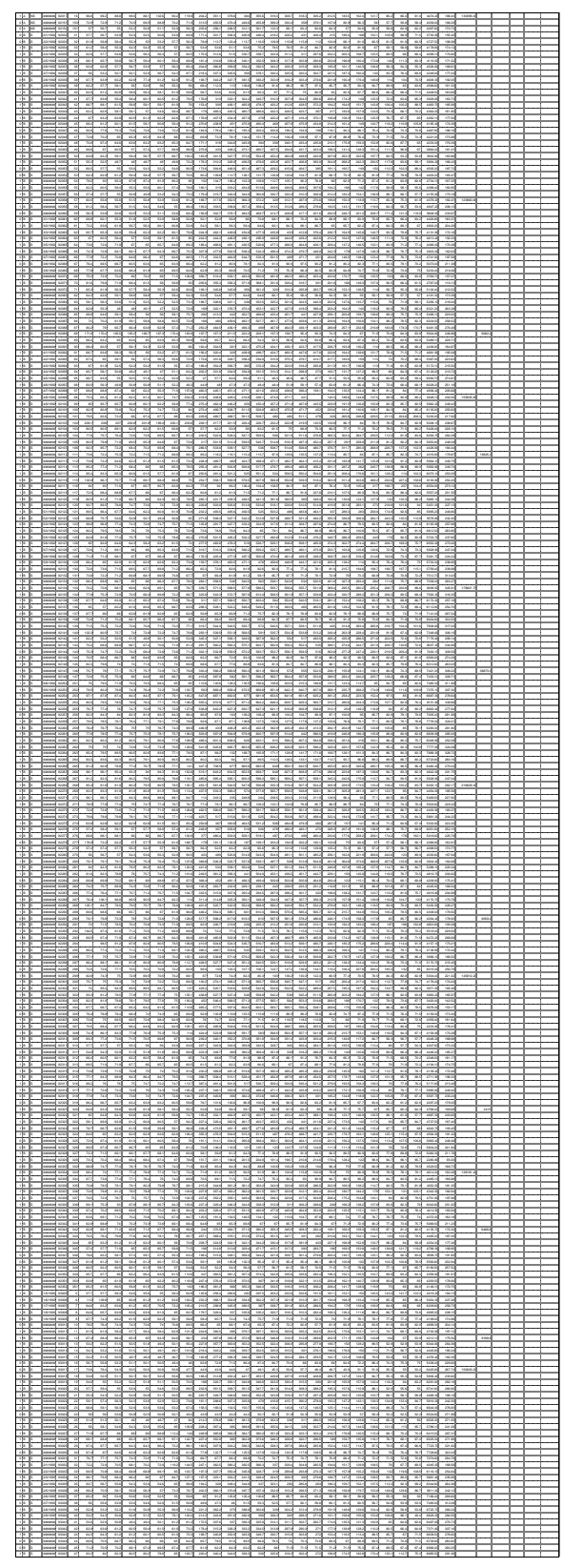




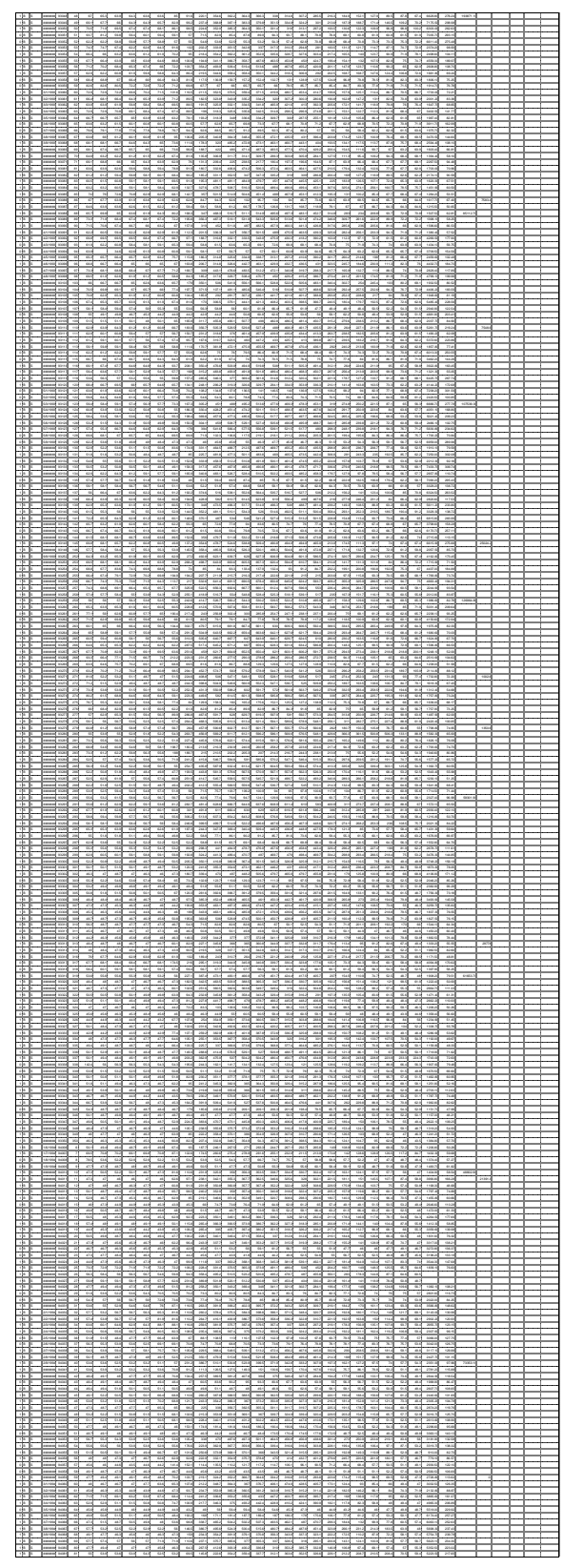




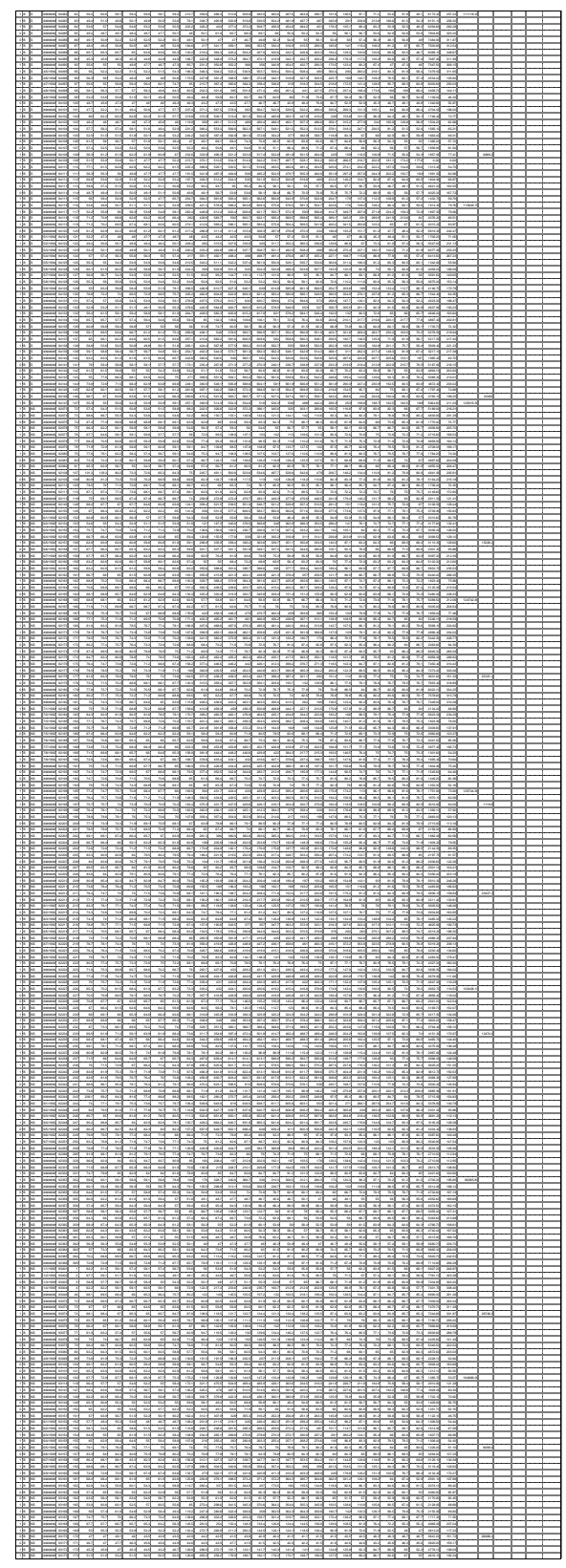




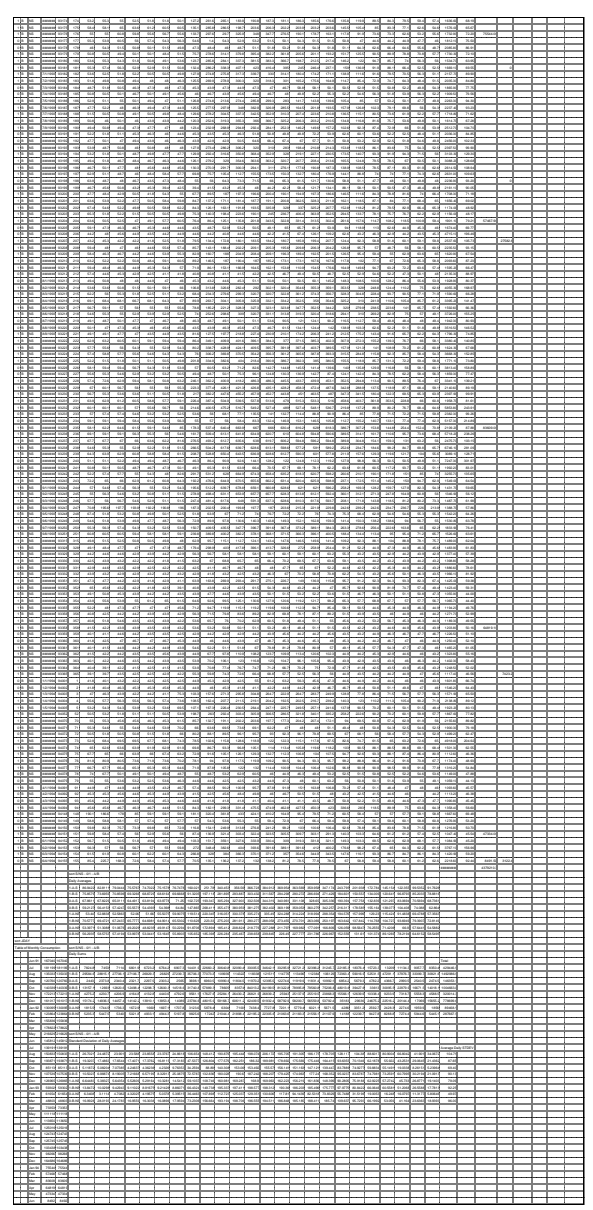


wbheat

\begin{tabular}{|c|c|c|c|c|c|c|c|c|c|c|}
\hline $\begin{array}{l}\text { wd/ } \\
\text { we } \\
0 / 1\end{array}$ & $\begin{array}{l}\text { pre/ } \\
\text { post } \\
\text { A/B }\end{array}$ & $\begin{array}{c}\text { schyearl } \\
\text { breaks } \\
\text { S/NS }\end{array}$ & date & jday & $\begin{array}{c}\text { orig } \\
\text { count }\end{array}$ & 0 & 1 & 2 & 3 & 4 \\
\hline 1 & A & $\mathrm{S}$ & $10 / 1 / 1991$ & 91274 & 274 & & & & & \\
\hline 1 & A & $\mathrm{S}$ & $10 / 2 / 1991$ & 91275 & 275 & & & & & \\
\hline 1 & A & $\mathrm{S}$ & 10/3/1991 & 91276 & 276 & & & & & \\
\hline 1 & A & $\mathrm{S}$ & 10/4/1991 & 91277 & 277 & & & & & \\
\hline 1 & $A$ & $\mathrm{~S}$ & 10/7/1991 & 91280 & 280 & & & & & \\
\hline 1 & A & $\mathrm{S}$ & $10 / 8 / 1991$ & 91281 & 281 & & & & & \\
\hline 1 & A & $\mathrm{S}$ & 10/9/1991 & 91282 & 282 & & & & & \\
\hline 1 & A & $\mathrm{S}$ & \#\#\#\#\#\#\#\# & 91283 & 283 & & & & & \\
\hline 1 & A & $\mathrm{S}$ & \#\#\#\#\#\#\#\# & 91284 & 284 & & & & & \\
\hline 1 & A & $\mathrm{S}$ & \#\#\#\#\#\#\#\# & 91287 & 287 & & & & & \\
\hline 1 & $A$ & $S$ & \#\#\#\#\#\#\#\# & 91288 & 288 & & & & & \\
\hline 1 & A & $\mathrm{S}$ & \#\#\#\#\#\#\#\# & 91289 & 289 & & & & & \\
\hline 1 & A & $\mathrm{S}$ & \#\#\#\#\#\#\#\# & 91290 & 290 & & & & & \\
\hline 1 & A & $\mathrm{S}$ & \#\#\#\#\#\#\#\# & 91291 & 291 & & & & & \\
\hline 1 & A & $\mathrm{S}$ & \#\#\#\#\#\#\#\# & 91294 & 294 & & & & & \\
\hline 1 & A & $\mathrm{S}$ & \#\#\#\#\#\#\# & 91295 & 295 & 0.1 & & & & \\
\hline 1 & A & $\mathrm{S}$ & \#\#\#\#\#\#\#\# & 91296 & 296 & 0.1 & & & & \\
\hline 1 & A & $S$ & \#\#\#\#\#\#\#\# & 91297 & 297 & 0.1 & & & & \\
\hline 1 & A & $\mathrm{S}$ & \#\#\#\#\#\#\#\# & 91298 & 298 & & & & & \\
\hline 1 & A & $\mathrm{S}$ & \#\#\#\#\#\#\#\# & 91301 & 301 & & & & & \\
\hline 1 & A & $\mathrm{S}$ & \#\#\#\#\#\#\#\# & 91302 & 302 & & & & & \\
\hline 1 & A & $\mathrm{S}$ & \#\#\#\#\#\#\#\# & 91303 & 303 & 0.1 & & & 0.1 & \\
\hline 1 & A & $\mathrm{S}$ & \#\#\#\#\#\#\#\# & 91304 & 304 & 0.3 & 0.3 & 0.3 & 0.4 & 0.3 \\
\hline 1 & A & $\mathrm{S}$ & $11 / 1 / 1991$ & 91305 & 305 & 0.4 & 0.4 & 0.4 & 0.5 & 0.4 \\
\hline 1 & A & $\mathrm{S}$ & $11 / 4 / 1991$ & 91308 & 308 & 0.5 & 0.5 & 0.5 & 0.5 & 0.6 \\
\hline 1 & A & $\mathrm{S}$ & 11/5/1991 & 91309 & 309 & 0.5 & 0.5 & 0.5 & 0.6 & 0.6 \\
\hline 1 & A & $\mathrm{S}$ & $11 / 6 / 1991$ & 91310 & 310 & 0.3 & 0.3 & 0.3 & 0.4 & 0.4 \\
\hline 1 & A & $\mathrm{S}$ & $11 / 7 / 1991$ & 91311 & 311 & 0.2 & 0.2 & 0.2 & 0.3 & 0.2 \\
\hline 1 & A & $S$ & $11 / 8 / 1991$ & 91312 & 312 & 0.4 & 0.4 & 0.4 & 0.4 & 0.5 \\
\hline 1 & A & $\mathrm{S}$ & \#\#\#\#\#\#\#\# & 91315 & 315 & 0.3 & 0.3 & 0.3 & 0.4 & 0.4 \\
\hline 1 & $A$ & $S$ & \#\#\#\#\#\#\#\# & 91316 & 316 & 0.2 & 0.2 & 0.2 & 0.2 & 0.2 \\
\hline 1 & A & $\mathrm{S}$ & \#\#\#\#\#\#\#\# & 91317 & 317 & 0.2 & 0.2 & 0.2 & 0.2 & 0.2 \\
\hline 1 & $A$ & $S$ & \#\#\#\#\#\#\#\# & 91318 & 318 & 0.1 & 0.1 & 0.1 & 0.1 & 0.1 \\
\hline 1 & A & $\mathrm{S}$ & \#\#\#\#\#\#\#\# & 91319 & 319 & & & & 0.1 & 0.1 \\
\hline 1 & A & $\mathrm{S}$ & \#\#\#\#\#\#\#\# & 91322 & 322 & 0.2 & 0.2 & 0.2 & 0.2 & 0.2 \\
\hline 1 & A & $S$ & \#\#\#\#\#\#\#\# & 91323 & 323 & & & & 0.1 & 0.1 \\
\hline 1 & A & $\mathrm{S}$ & \#\#\#\#\#\#\#\# & 91324 & 324 & 0.1 & 0.1 & 0.1 & 0.2 & 0.2 \\
\hline 1 & A & $\mathrm{S}$ & \#\#\#\#\#\#\#\# & 91325 & 325 & 0.2 & 0.2 & 0.2 & 0.3 & 0.3 \\
\hline 1 & A & $\mathrm{S}$ & \#\#\#\#\#\#\#\# & 91326 & 326 & 0.2 & 0.2 & 0.2 & 0.2 & 0.2 \\
\hline 1 & A & $S$ & \#\#\#\#\#\#\#\# & 91329 & 329 & 0.4 & 0.4 & 0.4 & 0.4 & 0.5 \\
\hline 1 & A & $\mathrm{S}$ & \#\#\#\#\#\#\#\# & 91330 & 330 & 0.3 & 0.3 & 0.3 & 0.3 & 0.4 \\
\hline 1 & A & $S$ & \#\#\#\#\#\#\#\# & 91331 & 331 & 0.2 & 0.2 & 0.2 & 0.2 & 0.2 \\
\hline 1 & A & $\mathrm{S}$ & $12 / 2 / 1991$ & 91336 & 336 & 0.5 & 0.5 & 0.5 & 0.5 & 0.5 \\
\hline 1 & A & $\mathrm{S}$ & $12 / 3 / 1991$ & 91337 & 337 & 0.4 & 0.4 & 0.5 & 0.5 & 0.5 \\
\hline 1 & A & $S$ & $12 / 4 / 1991$ & 91338 & 338 & 0.5 & 0.5 & 0.5 & 0.6 & 0.5 \\
\hline 1 & A & $\mathrm{S}$ & $12 / 5 / 1991$ & 91339 & 339 & 0.3 & 0.4 & 0.4 & 0.4 & 0.4 \\
\hline 1. & A & S & $12 / 6 / 1991$ & 91340 & 340 & 0.2 & 0.2 & 0.3 & 0.3 & 0.3 \\
\hline
\end{tabular}


wbheat

\begin{tabular}{|c|c|c|c|c|c|c|c|c|c|}
\hline $1 \mathrm{~A}$ & $S$ & $12 / 9 / 1991$ & 91343 & 343 & 0.1 & 0.1 & 0.1 & 0.1 & 0.1 \\
\hline $1 \mathrm{~A}$ & S & \#\#\#\#\#\#\#\# & 91344 & 344 & 0.2 & 0.2 & 0.2 & 0.2 & 0.2 \\
\hline $1 \mathrm{~A}$ & S & \#\#\#\#\#\#\#\# & 91345 & 345 & 0.1 & 0.1 & 0.1 & 0.2 & 0.1 \\
\hline $1 \mathrm{~A}$ & $\mathrm{~S}$ & \#\#\#\#\#\#\#\# & 91346 & 346 & 0.1 & 0.1 & 0.1 & 0.1 & 0.1 \\
\hline $1 \mathrm{~A}$ & $S$ & \#\#\#\#\#\#\#\# & 91347 & 347 & 0.1 & 0.1 & 0.1 & 0.1 & 0.1 \\
\hline $1 \mathrm{~A}$ & $S$ & \#\#\#\#\#\#\#\# & 91350 & 350 & 0.4 & 0.4 & 0.4 & 0.4 & 0.5 \\
\hline $1 \mathrm{~A}$ & $\mathrm{~S}$ & \#\#\#\#\#\#\#\# & 91351 & 351 & 0.4 & 0.4 & 0.4 & 0.4 & 0.4 \\
\hline $1 \mathrm{~A}$ & S & \#\#\#\#\#\#\#\# & 91352 & 352 & 0.2 & 0.2 & 0.3 & 0.3 & 0.3 \\
\hline $1 \mathrm{~A}$ & S & \#\#\#\#\#\#\#\# & 91353 & 353 & 0.4 & 0.4 & 0.4 & 0.5 & 0.5 \\
\hline $1 \mathrm{~A}$ & $\mathrm{~S}$ & \#\#\#\#\#\#\#\# & 91354 & 354 & 0.5 & 0.5 & 0.5 & 0.5 & 0.5 \\
\hline $1 \mathrm{~A}$ & S & 1/6/1992 & 92006 & 6 & 0.1 & 0.1 & 0.1 & 0.1 & 0.1 \\
\hline $1 \mathrm{~A}$ & S & 1/7/1992 & 92007 & 7 & 0.4 & 0.5 & 0.5 & 0.5 & 0.5 \\
\hline $1 \mathrm{~A}$ & S & $1 / 8 / 1992$ & 92008 & 8 & 0.3 & 0.3 & 0.3 & 0.3 & 0.4 \\
\hline $1 \mathrm{~A}$ & $\mathrm{~S}$ & $1 / 9 / 1992$ & 92009 & 9 & 0.4 & 0.4 & 0.4 & 0.5 & 0.5 \\
\hline $1 \mathrm{~A}$ & $\mathrm{~S}$ & $1 / 10 / 1992$ & 92010 & 10 & 0.4 & 0.5 & 0.5 & 0.5 & 0.5 \\
\hline $1 \mathrm{~A}$ & $\mathrm{~S}$ & $1 / 13 / 1992$ & 92013 & 13 & 0.4 & 0.4 & 0.4 & 0.4 & 0.4 \\
\hline $1 \mathrm{~A}$ & S & $1 / 14 / 1992$ & 92014 & 14 & 0.6 & 0.6 & 0.6 & 0.6 & 0.7 \\
\hline $1 \mathrm{~A}$ & S & $1 / 15 / 1992$ & 92015 & 15 & 0.5 & 0.5 & 0.5 & 0.5 & 0.6 \\
\hline $1 \mathrm{~A}$ & S & $1 / 16 / 1992$ & 92016 & 16 & 0.7 & 0.7 & 0.8 & 0.8 & 0.9 \\
\hline $1 \mathrm{~A}$ & S & 1/17/1992 & 92017 & 17 & 0.6 & 0.6 & 0.6 & 0.6 & 0.7 \\
\hline $1 \mathrm{~A}$ & $S$ & 1/21/1992 & 92021 & 21 & 0.5 & 0.5 & 0.5 & 0.5 & 0.5 \\
\hline $1 \mathrm{~A}$ & S & $1 / 22 / 1992$ & 92022 & 22 & 0.4 & 0.5 & 0.4 & 0.5 & 0.5 \\
\hline $1 \mathrm{~A}$ & $S$ & 1/23/1992 & 92023 & 23 & 0.4 & 0.4 & 0.4 & 0.5 & 0.4 \\
\hline $1 \mathrm{~A}$ & $S$ & $1 / 24 / 1992$ & 92024 & 24 & 0.4 & 0.4 & 0.4 & 0.4 & 0.5 \\
\hline $1 \mathrm{~A}$ & $\mathrm{~S}$ & $1 / 27 / 1992$ & 92027 & 27 & 0.4 & 0.4 & 0.4 & 0.4 & 0.4 \\
\hline $1 \mathrm{~A}$ & S & $1 / 28 / 1992$ & 92028 & 28 & 0.3 & 0.3 & 0.4 & 0.3 & 0.4 \\
\hline $1 \mathrm{~A}$ & S & 1/29/1992 & 92029 & 29 & 0.3 & 0.3 & 0.3 & 0.4 & 0.4 \\
\hline $1 \mathrm{~A}$ & S & $1 / 30 / 1992$ & 92030 & 30 & 0.3 & 0.3 & 0.3 & 0.3 & 0.3 \\
\hline $1 \mathrm{~A}$ & S & 2/3/1992 & 92034 & 34 & 0.3 & 0.3 & 0.3 & 0.3 & 0.3 \\
\hline $1 \mathrm{~A}$ & $S$ & 2/4/1992 & 92035 & 35 & 0.3 & 0.3 & 0.3 & 0.4 & 0.3 \\
\hline $1 \mathrm{~A}$ & S & $2 / 5 / 1992$ & 92036 & 36 & 0.3 & 0.4 & 0.4 & 0.4 & 0.5 \\
\hline $1 \mathrm{~A}$ & S & 2/6/1992 & 92037 & 37 & 0.4 & 0.4 & 0.5 & 0.5 & 0.6 \\
\hline $1 \mathrm{~A}$ & S & 2/7/1992 & 92038 & 38 & 0.3 & 0.4 & 0.4 & 0.4 & 0.4 \\
\hline $1 \mathrm{~A}$ & S & $2 / 10 / 1992$ & 92041 & 41 & 0.5 & 0.5 & 0.5 & 0.5 & 0.5 \\
\hline $1 \mathrm{~A}$ & S & 2/11/1992 & 92042 & 42 & 0.3 & 0.3 & 0.3 & 0.3 & 0.3 \\
\hline $1 \mathrm{~A}$ & S & 2/12/1992 & 92043 & 43 & 0.2 & 0.2 & 0.2 & 0.2 & 0.2 \\
\hline $1 \mathrm{~A}$ & S & 2/13/1992 & 92044 & 44 & 0.2 & 0.2 & 0.2 & 0.2 & 0.3 \\
\hline $1 \mathrm{~A}$ & $S$ & 2/14/1992 & 92045 & 45 & 0.2 & 0.3 & 0.2 & 0.3 & 0.2 \\
\hline $1 \mathrm{~A}$ & $S$ & 2/17/1992 & 92048 & 48 & 0.2 & 0.2 & 0.2 & 0.2 & 0.2 \\
\hline $1 A$ & S & $2 / 18 / 1992$ & 92049 & 49 & 0.1 & 0.2 & 0.2 & 0.2 & 0.2 \\
\hline $1 \mathrm{~A}$ & S & 2/19/1992 & 92050 & 50 & 0.2 & 0.2 & 0.2 & 0.2 & 0.2 \\
\hline $1 A$ & S & 2/20/1992 & 92051 & 51 & 0.1 & 0.2 & 0.2 & 0.2 & 0.2 \\
\hline $1 \mathrm{~A}$ & S & 2/21/1992 & 92052 & 52 & 0.1 & 0.1 & 0.2 & 0.1 & 0.2 \\
\hline $1 A$ & S & 2/24/1992 & 92055 & 55 & 0.2 & 0.2 & 0.2 & 0.2 & 0.2 \\
\hline $1 \mathrm{~A}$ & S & 2/25/1992 & 92056 & 56 & 0.2 & 0.3 & 0.3 & 0.3 & 0.3 \\
\hline $1 A$ & S & $2 / 26 / 1992$ & 92057 & 57 & 0.5 & 0.6 & 0.5 & 0.6 & 0.6 \\
\hline $1 \mathrm{~A}$ & S & 2/27/1992 & 92058 & 58 & 0.4 & 0.4 & 0.4 & 0.5 & 0.5 \\
\hline $1 A$ & $S$ & 2/28/1992 & 92059 & 59 & 0.3 & 0.3 & 0.3 & 0.3 & 0.3 \\
\hline $1 A$ & S & 3/2/1992 & 92062 & 62 & 0.2 & 0.2 & 0.2 & 0.2 & 0.2 \\
\hline $1 \mathrm{~A}$ & $\mathrm{~S}$ & $3 / 3 / 1992$ & 92063 & 63 & 0.1 & 0.2 & 0.2 & 0.2 & 0.2 \\
\hline $1 A$ & $\mathrm{~S}$ & $3 / 4 / 1992$ & 92064 & 64 & 0.1 & 0.2 & 0.2 & 0.2 & 0.2 \\
\hline
\end{tabular}


wbheat

\begin{tabular}{|c|c|c|c|c|c|c|c|c|c|}
\hline $1 \mathrm{~A}$ & $S$ & $3 / 5 / 1992$ & 92065 & 65 & 0.2 & 0.2 & 0.3 & 0.3 & 0.3 \\
\hline $1 \mathrm{~A}$ & $\mathrm{~S}$ & $3 / 6 / 1992$ & 92066 & 66 & 0.1 & 0.1 & 0.2 & 0.2 & 0.2 \\
\hline $1 \mathrm{~A}$ & $S$ & 3/9/1992 & 92069 & 69 & 0.2 & 0.2 & 0.2 & 0.2 & 0.2 \\
\hline $1 \mathrm{~A}$ & S & 3/10/1992 & 92070 & 70 & 0.2 & 0.3 & 0.3 & 0.3 & 0.3 \\
\hline $1 \mathrm{~A}$ & S & 3/11/1992 & 92071 & 71 & 0.4 & 0.4 & 0.4 & 0.5 & 0.5 \\
\hline $1 \mathrm{~A}$ & $S$ & 3/12/1992 & 92072 & 72 & 0.3 & 0.3 & 0.3 & 0.4 & 0.4 \\
\hline $1 \mathrm{~A}$ & $S$ & 3/13/1992 & 92073 & 73 & 0.2 & 0.2 & 0.2 & 0.3 & 0.3 \\
\hline $1 \mathrm{~A}$ & S & 3/23/1992 & 92083 & 83 & 0.4 & 0.4 & 0.4 & 0.4 & 0.4 \\
\hline $1 \mathrm{~A}$ & $S$ & 3/24/1992 & 92084 & 84 & 0.2 & 0.2 & 0.3 & 0.3 & 0.3 \\
\hline $1 \mathrm{~A}$ & $S$ & $3 / 25 / 1992$ & 92085 & 85 & 0.2 & 0.2 & 0.2 & 0.2 & 0.3 \\
\hline $1 \mathrm{~A}$ & $S$ & 3/26/1992 & 92086 & 86 & 0.1 & 0.2 & 0.2 & 0.2 & 0.2 \\
\hline $1 \mathrm{~A}$ & $S$ & $3 / 27 / 1992$ & 92087 & 87 & 0.1 & 0.1 & 0.1 & 0.1 & 0.1 \\
\hline $1 \mathrm{~A}$ & S & 3/30/1992 & 92090 & 90 & 0.2 & 0.2 & 0.2 & 0.2 & 0.2 \\
\hline $1 \mathrm{~A}$ & S & 3/31/1992 & 92091 & 91 & 0.2 & 0.2 & 0.2 & 0.2 & 0.2 \\
\hline $1 \mathrm{~A}$ & $S$ & 4/1/1992 & 92092 & 92 & 0.2 & 0.1 & 0.2 & 0.2 & 0.2 \\
\hline $1 \mathrm{~A}$ & $S$ & $4 / 2 / 1992$ & 92093 & 93 & 0.3 & 0.3 & 0.3 & 0.3 & 0.3 \\
\hline $1 \mathrm{~A}$ & S & 4/3/1992 & 92094 & 94 & 0.2 & 0.2 & 0.3 & 0.3 & 0.3 \\
\hline $1 \mathrm{~A}$ & $S$ & 4/6/1992 & 92097 & 97 & 0.2 & 0.2 & 0.2 & 0.2 & 0.2 \\
\hline $1 \mathrm{~A}$ & $S$ & 4/7/1992 & 92098 & 98 & 0.2 & 0.2 & 0.2 & 0.3 & 0.3 \\
\hline $1 \mathrm{~A}$ & $S$ & $4 / 8 / 1992$ & 92099 & 99 & 0.1 & & 0.1 & 0.1 & \\
\hline $1 \mathrm{~A}$ & $S$ & 4/9/1992 & 92100 & 100 & & & 0.1 & & 0.1 \\
\hline $1 \mathrm{~A}$ & S & 4/10/1992 & 92101 & 101 & & & 0.1 & & 0.1 \\
\hline $1 \mathrm{~A}$ & S & 4/13/1992 & 92104 & 104 & 0.1 & 0.1 & 0.1 & 0.1 & 0.1 \\
\hline $1 \mathrm{~A}$ & $S$ & $4 / 14 / 1992$ & 92105 & 105 & 0.1 & & & 0.1 & \\
\hline $1 \mathrm{~A}$ & $S$ & 4/15/1992 & 92106 & 106 & & & & & \\
\hline $1 \mathrm{~A}$ & $S$ & 4/16/1992 & 92107 & 107 & & & 0.1 & & \\
\hline $1 \mathrm{~A}$ & $S$ & 4/17/1992 & 92108 & 108 & & & & & 0.1 \\
\hline $1 \mathrm{~A}$ & $S$ & 4/20/1992 & 92111 & 111 & 0.1 & 0.1 & 0.1 & 0.1 & 0.1 \\
\hline $1 \mathrm{~A}$ & $S$ & 4/21/1992 & 92112 & 112 & 0.1 & 0.1 & 0.1 & 0.1 & 0.1 \\
\hline $1 \mathrm{~A}$ & $S$ & $4 / 22 / 1992$ & 92113 & 113 & 0.1 & 0.1 & 0.1 & 0.1 & 0.1 \\
\hline $1 \mathrm{~A}$ & $S$ & 4/23/1992 & 92114 & 114 & & & & & \\
\hline $1 \mathrm{~A}$ & $S$ & 4/24/1992 & 92115 & 115 & & & & & \\
\hline $1 \mathrm{~A}$ & $S$ & 4/27/1992 & 92118 & 118 & & 0.1 & & 0.1 & \\
\hline $1 \mathrm{~A}$ & S & 4/28/1992 & 92119 & 119 & & & & & \\
\hline $1 \mathrm{~A}$ & $S$ & $4 / 29 / 1992$ & 92120 & 120 & & & & & 0.1 \\
\hline $1 \mathrm{~A}$ & $S$ & 4/30/1992 & 92121 & 121 & & & & & \\
\hline $1 \mathrm{~A}$ & $S$ & 5/1/1992 & 92122 & 122 & & & & & \\
\hline $1 \mathrm{~A}$ & $S$ & $5 / 4 / 1992$ & 92125 & 125 & & & & & \\
\hline $1 \mathrm{~A}$ & $\mathrm{~S}$ & $5 / 5 / 1992$ & 92126 & 126 & & & & & \\
\hline $1 \mathrm{~A}$ & $S$ & $5 / 6 / 1992$ & 92127 & 127 & & & & & \\
\hline $1 \mathrm{~A}$ & $S$ & $5 / 7 / 1992$ & 92128 & 128 & & & 0.1 & & 0.1 \\
\hline $1 \mathrm{~A}$ & $S$ & $5 / 8 / 1992$ & 92129 & 129 & & & 0.1 & & \\
\hline $1 \mathrm{~A}$ & $S$ & 5/11/1992 & 92132 & 132 & & & & & \\
\hline $1 \mathrm{~A}$ & $S$ & $5 / 12 / 1992$ & 92133 & 133 & & & & & \\
\hline $1 \mathrm{~A}$ & $S$ & 5/13/1992 & 92134 & 134 & & & & & \\
\hline $1 \mathrm{~A}$ & $S$ & 5/14/1992 & 92135 & 135 & & & & & \\
\hline $1 \mathrm{~A}$ & $S$ & $5 / 15 / 1992$ & 92136 & 136 & & & & & \\
\hline $1 \mathrm{~A}$ & $\mathrm{~S}$ & $5 / 18 / 1992$ & 92139 & 139 & & & & & \\
\hline $1 \mathrm{~A}$ & $S$ & 5/19/1992 & 92140 & 140 & & & & & \\
\hline $1 \mathrm{~A}$ & S & $5 / 20 / 1992$ & 92141 & 141 & & & & & \\
\hline $1 \mathrm{~A}$ & $S$ & $5 / 21 / 1992$ & 92142 & 142 & & & & & \\
\hline
\end{tabular}


wbheat

\begin{tabular}{|c|c|c|c|c|c|c|c|c|c|}
\hline $1 \mathrm{~A}$ & $\mathrm{~S}$ & $5 / 22 / 1992$ & 92143 & 143 & & & & & \\
\hline $1 A$ & $\mathrm{~S}$ & $5 / 25 / 1992$ & 92146 & 146 & 0.1 & & & 0.1 & \\
\hline $1 A$ & S & 5/26/1992 & 92147 & 147 & & & 0.1 & 0.1 & \\
\hline $1 A$ & S & 5/27/1992 & 92148 & 148 & & & & & \\
\hline $1 A$ & S & $5 / 28 / 1992$ & 92149 & 149 & & & & & \\
\hline $1 A$ & S & $5 / 29 / 1992$ & 92150 & 150 & 0.1 & 0.1 & 0.1 & 0.1 & 0.1 \\
\hline $1 A$ & $\mathrm{~S}$ & 6/1/1992 & 92153 & 153 & & & 0.1 & & \\
\hline $1 A$ & S & 6/2/1992 & 92154 & 154 & & & & & \\
\hline $1 A$ & S & 6/3/1992 & 92155 & 155 & & & & & \\
\hline $1 A$ & S & 6/4/1992 & 92156 & 156 & & & & & \\
\hline $1 A$ & S & 6/5/1992 & 92157 & 157 & & & & & \\
\hline $1 A$ & $\mathrm{~S}$ & 6/8/1992 & 92160 & 160 & & & & & \\
\hline $1 A$ & S & $6 / 9 / 1992$ & 92161 & 161 & & & & & \\
\hline $1 A$ & S & 6/10/1992 & 92162 & 162 & & & & & \\
\hline $1 A$ & S & $6 / 11 / 1992$ & 92163 & 163 & & & & & \\
\hline $1 A$ & S & 6/12/1992 & 92164 & 164 & & & & & \\
\hline $1 A$ & S & 8/14/1992 & 92227 & 227 & & & & & \\
\hline $1 A$ & S & 8/17/1992 & 92230 & 230 & & & & & \\
\hline $1 A$ & S & 8/18/1992 & 92231 & 231 & & & & & \\
\hline $1 A$ & S & 8/19/1992 & 92232 & 232 & & & & & \\
\hline $1 A$ & S & 8/20/1992 & 92233 & 233 & & & & & \\
\hline $1 A$ & S & 8/21/1992 & 92234 & 234 & & & & & \\
\hline $1 A$ & S & 8/24/1992 & 92237 & 237 & & & & & \\
\hline $1 A$ & S & $8 / 25 / 1992$ & 92238 & 238 & & & & & \\
\hline $1 A$ & $\mathrm{~S}$ & $8 / 26 / 1992$ & 92239 & 239 & & & & & \\
\hline $1 A$ & S & 8/27/1992 & 92240 & 240 & 0.1 & & & & \\
\hline $1 A$ & S & 8/28/1992 & 92241 & 241 & & & & & \\
\hline $1 A$ & S & 8/31/1992 & 92244 & 244 & & & & & \\
\hline $1 A$ & S & 9/1/1992 & 92245 & 245 & & & & & \\
\hline $1 A$ & S & 9/2/1992 & 92246 & 246 & & & & & \\
\hline $1 A$ & S & 9/3/1992 & 92247 & 247 & & & & & \\
\hline $1 A$ & S & 9/4/1992 & 92248 & 248 & & & & & \\
\hline $1 A$ & S & 9/8/1992 & 92252 & 252 & & & & & \\
\hline $1 A$ & S & 9/9/1992 & 92253 & 253 & & & & & \\
\hline $1 A$ & S & 9/10/1992 & 92254 & 254 & & & & & \\
\hline $1 A$ & S & 9/11/1992 & 92255 & 255 & & & & & \\
\hline $1 A$ & S & 9/14/1992 & 92258 & 258 & & & & & \\
\hline $1 A$ & S & 9/15/1992 & 92259 & 259 & & & & & \\
\hline $1 A$ & $S$ & 9/16/1992 & 92260 & 260 & & & & & \\
\hline $1 A$ & S & 9/17/1992 & 92261 & 261 & & & & & \\
\hline $1 A$ & S & 9/18/1992 & 92262 & 262 & & & & & \\
\hline $1 A$ & S & 9/21/1992 & 92265 & 265 & & & & & \\
\hline $1 A$ & S & 9/22/1992 & 92266 & 266 & & & & & \\
\hline $1 A$ & S & 9/23/1992 & 92267 & 267 & & & & & \\
\hline $1 A$ & S & 9/24/1992 & 92268 & 268 & & & & & \\
\hline $1 A$ & S & 9/25/1992 & 92269 & 269 & & & & & \\
\hline $1 A$ & S & 9/28/1992 & 92272 & 272 & & & & & \\
\hline $1 A$ & $S$ & 9/29/1992 & 92273 & 273 & & & & & \\
\hline $1 A$ & S & 9/30/1992 & 92274 & 274 & 0.01 & & 0.021 & & 0.01 \\
\hline $1 A$ & S & 10/1/1992 & 92275 & 275 & & & & & \\
\hline $1 A$ & $\mathrm{~S}$ & $10 / 2 / 1992$ & 92276 & 276 & & & & & \\
\hline
\end{tabular}


wbheat

\begin{tabular}{|c|c|c|c|c|c|c|c|c|c|}
\hline $1 \mathrm{~A}$ & $S$ & $10 / 5 / 1992$ & 92279 & 279 & & & & & \\
\hline $1 \mathrm{~A}$ & $\mathrm{~S}$ & $10 / 6 / 1992$ & 92280 & 280 & & & & & \\
\hline $1 \mathrm{~A}$ & S & $10 / 7 / 1992$ & 92281 & 281 & & & & & \\
\hline $1 \mathrm{~A}$ & S & 10/8/1992 & 92282 & 282 & & & & & \\
\hline \begin{tabular}{l|l}
$1 \mathrm{~A}$ \\
\end{tabular} & S & 10/9/1992 & 92283 & 283 & & & & & \\
\hline \begin{tabular}{l|l}
$A$ \\
$A$
\end{tabular} & S & \#\#\#\#\#\#\#\# & 92286 & 286 & 0.1 & & & & 0.1 \\
\hline $1 \mathrm{~A}$ & S & \#\#\#\#\#\#\#\# & 92287 & 287 & & & & & \\
\hline $1 \mathrm{~A}$ & S & \#\#\#\#\#\#\#\# & 92288 & 288 & & & & & \\
\hline $1 \mathrm{~A}$ & S & \#\#\#\#\#\#\#\# & 92289 & 289 & & & & & \\
\hline $1 \mathrm{~A}$ & S & \#\#\#\#\#\#\#\# & 92290 & 290 & & & & & \\
\hline $1 \mathrm{~A}$ & S & \#\#\#\#\#\#\#\# & 92293 & 293 & 0.1 & 0.2 & 0.1 & 0.1 & 0.1 \\
\hline $1 \mathrm{~A}$ & S & \#\#\#\#\#\#\#\# & 92294 & 294 & & & & & \\
\hline $1 \mathrm{~A}$ & S & \#\#\#\#\#\#\#\# & 92295 & 295 & & & & & \\
\hline $1 \mathrm{~A}$ & S & \#\#\#\#\#\#\#\# & 92296 & 296 & & & & & \\
\hline $1 \mathrm{~A}$ & S & \#\#\#\#\#\#\#\# & 92297 & 297 & & & & & \\
\hline $1 \mathrm{~A}$ & S & \#\#\#\#\#\#\#\# & 92300 & 300 & & & & & \\
\hline $1 \mathrm{~A}$ & S & \#\#\#\#\#\#\#\# & 92301 & 301 & & & & & \\
\hline $1 \mathrm{~A}$ & S & \#\#\#\#\#\#\#\# & 92302 & 302 & & & & & \\
\hline $1 \mathrm{~A}$ & S & \#\#\#\#\#\#\#\# & 92303 & 303 & & & & & \\
\hline $1 \mathrm{~A}$ & S & \#\#\#\#\#\#\#\# & 92304 & 304 & & & & & \\
\hline $1 \mathrm{~A}$ & S & $11 / 2 / 1992$ & 92307 & 307 & & & & & \\
\hline $1 \mathrm{~A}$ & S & 11/3/1992 & 92308 & 308 & & & & & \\
\hline $1 \mathrm{~A}$ & S & $11 / 4 / 1992$ & 92309 & 309 & 0.1 & & 0.1 & 0.1 & 0.1 \\
\hline $1 \mathrm{~A}$ & S & $11 / 5 / 1992$ & 92310 & 310 & 0.1 & 0.1 & 0.1 & 0.2 & 0.2 \\
\hline $1 \mathrm{~A}$ & S & $11 / 6 / 1992$ & 92311 & 311 & 0.3 & 0.3 & 0.3 & 0.3 & 0.3 \\
\hline $1 \mathrm{~A}$ & S & $11 / 9 / 1992$ & 92314 & 314 & 0.2 & 0.2 & 0.1 & 0.2 & 0.2 \\
\hline $1 \mathrm{~A}$ & S & \#\#\#\#\#\#\#\# & 92315 & 315 & 0.1 & & 0.1 & 0.1 & 0.1 \\
\hline $1 \mathrm{~A}$ & S & \#\#\#\#\#\#\#\# & 92316 & 316 & & & 0.1 & & \\
\hline $1 \mathrm{~A}$ & S & \#\#\#\#\#\#\#\# & 92317 & 317 & & 0.1 & 0.1 & 0.1 & 0.1 \\
\hline $1 \mathrm{~A}$ & S & \#\#\#\#\#\#\#\# & 92318 & 318 & 0.1 & 0.1 & 0.1 & 0.2 & 0.2 \\
\hline $1 \mathrm{~A}$ & S & \#\#\#\#\#\#\#\# & 92321 & 321 & 0.2 & 0.2 & 0.3 & 0.2 & 0.3 \\
\hline $1 \mathrm{~A}$ & S & \#\#\#\#\#\#\#\# & 92322 & 322 & 0.2 & 0.2 & 0.2 & 0.3 & 0.3 \\
\hline $1 \mathrm{~A}$ & S & \#\#\#\#\#\#\#\# & 92323 & 323 & 0.1 & 0.1 & 0.1 & 0.1 & 0.1 \\
\hline $1 \mathrm{~A}$ & S & \#\#\#\#\#\#\#\# & 92324 & 324 & 0.1 & 0.1 & 0.1 & 0.1 & 0.1 \\
\hline $1 \mathrm{~A}$ & S & \#\#\#\#\#\#\#\# & 92325 & 325 & 0.1 & 0.1 & 0.1 & 0.2 & 0.1 \\
\hline $1 \mathrm{~A}$ & S & \#\#\#\#\#\#\#\# & 92328 & 328 & 0.3 & 0.3 & 0.3 & 0.3 & 0.3 \\
\hline $1 \mathrm{~A}$ & S & \#\#\#\#\#\#\#\# & 92329 & 329 & 0.2 & 0.2 & 0.2 & 0.2 & 0.2 \\
\hline $1 \mathrm{~A}$ & S & \#\#\#\#\#\#\#\# & 92330 & 330 & 0.3 & 0.3 & 0.3 & 0.4 & 0.4 \\
\hline $1 \mathrm{~A}$ & $S$ & \#\#\#\#\#\#\#\# & 92335 & 335 & 0.4 & 0.4 & 0.4 & 0.5 & 0.5 \\
\hline $1 A$ & S & 12/1/1992 & 92336 & 336 & 0.3 & 0.3 & 0.4 & 0.4 & 0.4 \\
\hline $1 A$ & S & $12 / 2 / 1992$ & 92337 & 337 & 0.2 & 0.2 & 0.2 & 0.3 & 0.3 \\
\hline $1 A$ & S & $12 / 3 / 1992$ & 92338 & 338 & 0.3 & 0.4 & 0.3 & 0.4 & 0.4 \\
\hline $1 \mathrm{~A}$ & S & $12 / 4 / 1992$ & 92339 & 339 & 0.2 & 0.2 & 0.2 & 0.2 & 0.2 \\
\hline $1 A$ & S & $12 / 7 / 1992$ & 92342 & 342 & 0.4 & 0.4 & 0.4 & 0.5 & 0.5 \\
\hline $1 A$ & S & $12 / 8 / 1992$ & 92343 & 343 & 0.5 & 0.5 & 0.5 & 0.5 & 0.5 \\
\hline $1 \mathrm{~A}$ & S & $12 / 9 / 1992$ & 92344 & 344 & 0.4 & 0.4 & 0.4 & 0.5 & 0.4 \\
\hline $1 \mathrm{~A}$ & S & \#\#\#\#\#\#\#\# & 92345 & 345 & 0.3 & 0.4 & 0.4 & 0.4 & 0.4 \\
\hline $1 A$ & $S$ & \#\#\#\#\#\#\#\# & 92346 & 346 & 0.3 & 0.4 & 0.4 & 0.4 & 0.5 \\
\hline $1 A$ & S & \#\#\#\#\#\#\#\# & 92349 & 349 & 0.3 & 0.3 & 0.4 & 0.4 & 0.4 \\
\hline $1 A$ & S & \#\#\#\#\#\#\#\# & 92350 & 350 & 0.4 & 0.4 & 0.4 & 0.5 & 0.5 \\
\hline $1 A$ & $\mathrm{~S}$ & \#\#\#\#\#\#\# & 92351 & 351 & 0.5 & 0.5 & 0.5 & 0.6 & 0.6 \\
\hline
\end{tabular}


wbheat

\begin{tabular}{|c|c|c|c|c|c|c|c|c|c|}
\hline $1 \mathrm{~A}$ & $S$ & \#\#\#\#\#\#\#\# & 92352 & 352 & 0.5 & 0.5 & 0.5 & 0.5 & 0.6 \\
\hline $1 \mathrm{~A}$ & S & \#\#\#\#\#\#\#\# & 92353 & 353 & 0.5 & 0.5 & 0.5 & 0.6 & 0.6 \\
\hline $1 \mathrm{~A}$ & S & $1 / 4 / 1993$ & 93004 & 4 & 0.2 & 0.2 & 0.2 & 0.2 & 0.2 \\
\hline $1 \mathrm{~A}$ & S & $1 / 5 / 1993$ & 93005 & 5 & 0.4 & 0.5 & 0.5 & 0.5 & 0.5 \\
\hline $1 \mathrm{~A}$ & $\mathrm{~S}$ & $1 / 6 / 1993$ & 93006 & 6 & 0.5 & 0.5 & 0.5 & 0.5 & 0.5 \\
\hline $1 \mathrm{~A}$ & $S$ & $1 / 7 / 1993$ & 93007 & 7 & 0.4 & 0.4 & 0.4 & 0.5 & 0.5 \\
\hline $1 \mathrm{~A}$ & $\mathrm{~S}$ & 1/8/1993 & 93008 & 8 & 0.3 & 0.4 & 0.4 & 0.4 & 0.4 \\
\hline $1 \mathrm{~A}$ & S & $1 / 11 / 1993$ & 93011 & 11 & 0.7 & 0.7 & 0.7 & 0.7 & 0.7 \\
\hline $1 \mathrm{~A}$ & S & $1 / 12 / 1993$ & 93012 & 12 & 0.5 & 0.5 & 0.5 & 0.5 & 0.5 \\
\hline $1 \mathrm{~A}$ & $\mathrm{~S}$ & $1 / 13 / 1993$ & 93013 & 13 & 0.4 & 0.4 & 0.4 & 0.5 & 0.5 \\
\hline $1 \mathrm{~A}$ & $\mathrm{~S}$ & $1 / 14 / 1993$ & 93014 & 14 & 0.4 & 0.5 & 0.5 & 0.5 & 0.6 \\
\hline $1 \mathrm{~A}$ & S & $1 / 15 / 1993$ & 93015 & 15 & 0.4 & 0.4 & 0.4 & 0.4 & 0.4 \\
\hline $1 \mathrm{~A}$ & S & 1/19/1993 & 93019 & 19 & 0.6 & 0.6 & 0.6 & 0.6 & 0.6 \\
\hline $1 \mathrm{~A}$ & $S$ & $1 / 20 / 1993$ & 93020 & 20 & 0.6 & 0.6 & 0.6 & 0.6 & 0.6 \\
\hline $1 \mathrm{~A}$ & $\mathrm{~S}$ & $1 / 21 / 1993$ & 93021 & 21 & 0.5 & 0.6 & 0.5 & 0.6 & 0.6 \\
\hline $1 \mathrm{~A}$ & $\mathrm{~S}$ & $1 / 22 / 1993$ & 93022 & 22 & 0.2 & 0.3 & 0.3 & 0.3 & 0.3 \\
\hline $1 \mathrm{~A}$ & S & $1 / 25 / 1993$ & 93025 & 25 & 0.5 & 0.5 & 0.5 & 0.5 & 0.6 \\
\hline $1 \mathrm{~A}$ & S & $1 / 26 / 1993$ & 93026 & 26 & 0.4 & 0.4 & 0.5 & 0.5 & 0.6 \\
\hline $1 \mathrm{~A}$ & S & $1 / 27 / 1993$ & 93027 & 27 & 0.3 & 0.4 & 0.4 & 0.4 & 0.5 \\
\hline $1 \mathrm{~A}$ & S & $1 / 28 / 1993$ & 93028 & 28 & 0.3 & 0.3 & 0.3 & 0.3 & 0.3 \\
\hline $1 \mathrm{~A}$ & $S$ & 1/29/1993 & 93029 & 29 & 0.3 & 0.3 & 0.3 & 0.3 & 0.3 \\
\hline $1 \mathrm{~A}$ & S & 2/1/1993 & 93032 & 32 & 0.3 & 0.4 & 0.4 & 0.4 & 0.4 \\
\hline $1 \mathrm{~A}$ & S & $2 / 2 / 1993$ & 93033 & 33 & 0.2 & 0.3 & 0.2 & 0.3 & 0.3 \\
\hline $1 \mathrm{~A}$ & $S$ & $2 / 3 / 1993$ & 93034 & 34 & 0.2 & 0.2 & 0.2 & 0.2 & 0.2 \\
\hline $1 \mathrm{~A}$ & $\mathrm{~S}$ & 2/4/1993 & 93035 & 35 & 0.4 & 0.3 & 0.3 & 0.3 & 0.3 \\
\hline $1 \mathrm{~A}$ & S & 2/8/1993 & 93039 & 39 & 0.3 & 0.3 & 0.3 & 0.4 & 0.4 \\
\hline $1 \mathrm{~A}$ & S & 2/9/1993 & 93040 & 40 & 0.2 & 0.2 & 0.2 & 0.3 & 0.3 \\
\hline $1 \mathrm{~A}$ & S & 2/10/1993 & 93041 & 41 & 0.2 & 0.2 & 0.2 & 0.2 & 0.2 \\
\hline $1 \mathrm{~A}$ & S & 2/11/1993 & 93042 & 42 & 0.1 & 0.1 & 0.2 & 0.2 & 0.2 \\
\hline $1 \mathrm{~A}$ & $S$ & 2/12/1993 & 93043 & 43 & 0.3 & 0.3 & 0.4 & 0.4 & 0.4 \\
\hline $1 \mathrm{~A}$ & S & $2 / 15 / 1993$ & 93046 & 46 & 0.3 & 0.4 & 0.4 & 0.4 & 0.3 \\
\hline $1 \mathrm{~A}$ & S & 2/16/1993 & 93047 & 47 & 0.3 & 0.4 & 0.4 & 0.5 & 0.5 \\
\hline $1 \mathrm{~A}$ & S & 2/17/1993 & 93048 & 48 & 0.5 & 0.5 & 0.5 & 0.5 & 0.6 \\
\hline $1 \mathrm{~A}$ & S & 2/18/1993 & 93049 & 49 & 0.6 & 0.6 & 0.7 & 0.7 & 0.7 \\
\hline $1 \mathrm{~A}$ & S & 2/19/1993 & 93050 & 50 & 0.5 & 0.5 & 0.5 & 0.5 & 0.6 \\
\hline $1 \mathrm{~A}$ & S & 2/22/1993 & 93053 & 53 & 0.2 & 0.2 & 0.2 & 0.3 & 0.3 \\
\hline $1 \mathrm{~A}$ & S & 2/23/1993 & 93054 & 54 & 0.2 & 0.3 & 0.3 & 0.3 & 0.3 \\
\hline $1 \mathrm{~A}$ & $S$ & 2/24/1993 & 93055 & 55 & 0.2 & 0.2 & 0.2 & 0.2 & 0.3 \\
\hline $1 \mathrm{~A}$ & $S$ & $2 / 25 / 1993$ & 93056 & 56 & 0.2 & 0.3 & 0.2 & 0.2 & 0.2 \\
\hline $1 A$ & S & 2/26/1993 & 93057 & 57 & 0.3 & 0.3 & 0.3 & 0.4 & 0.4 \\
\hline $1 A$ & S & 3/1/1993 & 93060 & 60 & 0.4 & 0.4 & 0.4 & 0.4 & 0.4 \\
\hline $1 A$ & S & $3 / 2 / 1993$ & 93061 & 61 & 0.3 & 0.3 & 0.3 & 0.3 & 0.3 \\
\hline $1 A$ & $\mathrm{~S}$ & $3 / 3 / 1993$ & 93062 & 62 & 0.2 & 0.2 & 0.3 & 0.3 & 0.3 \\
\hline $1 A$ & S & $3 / 4 / 1993$ & 93063 & 63 & 0.2 & 0.2 & 0.3 & 0.3 & 0.3 \\
\hline $1 \mathrm{~A}$ & S & $3 / 5 / 1993$ & 93064 & 64 & 0.2 & 0.2 & 0.3 & 0.3 & 0.3 \\
\hline $1 A$ & S & $3 / 8 / 1993$ & 93067 & 67 & 0.2 & 0.2 & 0.2 & 0.2 & 0.2 \\
\hline $1 \mathrm{~A}$ & S & 3/9/1993 & 93068 & 68 & 0.1 & 0.1 & 0.1 & 0.1 & 0.1 \\
\hline $1 A$ & $S$ & 3/10/1993 & 93069 & 69 & 0.1 & & 0.1 & 0.1 & 0.1 \\
\hline $1 A$ & S & 3/11/1993 & 93070 & 70 & 0.2 & 0.1 & 0.1 & 0.1 & 0.2 \\
\hline $1 \mathrm{~A}$ & S & $3 / 12 / 1993$ & 93071 & 71 & 0.3 & 0.3 & 0.4 & 0.3 & 0.4 \\
\hline $1 A$ & S & $3 / 22 / 1993$ & 93081 & 81 & 0.2 & 0.2 & 0.2 & 0.2 & 0.2 \\
\hline
\end{tabular}


wbheat

\begin{tabular}{|c|c|c|c|c|c|c|c|c|c|}
\hline $1 \mathrm{~A}$ & $S$ & $3 / 23 / 1993$ & 93082 & 82 & 0.1 & 0.2 & 0.2 & 0.2 & 0.2 \\
\hline $1 \mathrm{~A}$ & $\mathrm{~S}$ & $3 / 24 / 1993$ & 93083 & 83 & 0.1 & 0.1 & 0.1 & 0.1 & 0.1 \\
\hline $1 \mathrm{~A}$ & $S$ & $3 / 25 / 1993$ & 93084 & 84 & & & & & 0.1 \\
\hline $1 \mathrm{~A}$ & S & 3/26/1993 & 93085 & 85 & 0.1 & & 0.1 & 0.1 & 0.1 \\
\hline $1 \mathrm{~A}$ & S & 3/29/1993 & 93088 & 88 & 0.1 & 0.1 & 0.1 & 0.1 & 0.1 \\
\hline $1 \mathrm{~A}$ & $S$ & 3/30/1993 & 93089 & 89 & 0.1 & & 0.1 & & 0.1 \\
\hline $1 \mathrm{~A}$ & $S$ & 3/31/1993 & 93090 & 90 & & & & & 0.1 \\
\hline $1 \mathrm{~A}$ & $S$ & 4/1/1993 & 93091 & 91 & 0.1 & 0.1 & 0.1 & 0.1 & 0.1 \\
\hline $1 \mathrm{~A}$ & $S$ & $4 / 2 / 1993$ & 93092 & 92 & 0.1 & 0.1 & 0.1 & 0.2 & 0.2 \\
\hline $1 \mathrm{~A}$ & $S$ & $4 / 5 / 1993$ & 93095 & 95 & 0.2 & 0.2 & 0.2 & 0.2 & 0.2 \\
\hline $1 \mathrm{~A}$ & $S$ & 4/6/1993 & 93096 & 96 & 0.1 & 0.2 & 0.2 & 0.2 & 0.2 \\
\hline $1 \mathrm{~A}$ & $S$ & 4/7/1993 & 93097 & 97 & 0.2 & 0.2 & 0.2 & 0.2 & 0.2 \\
\hline $1 \mathrm{~A}$ & $S$ & 4/8/1993 & 93098 & 98 & 0.1 & 0.1 & 0.1 & 0.1 & 0.1 \\
\hline $1 \mathrm{~A}$ & $S$ & $4 / 9 / 1993$ & 93099 & 99 & 0.1 & 0.1 & 0.1 & 0.1 & 0.1 \\
\hline $1 \mathrm{~A}$ & $S$ & $4 / 12 / 1993$ & 93102 & 102 & & & & & \\
\hline $1 \mathrm{~A}$ & $S$ & 4/13/1993 & 93103 & 103 & 0.1 & & & & \\
\hline $1 \mathrm{~A}$ & $S$ & 4/14/1993 & 93104 & 104 & & & 0.1 & & \\
\hline $1 \mathrm{~A}$ & $S$ & 4/15/1993 & 93105 & 105 & 0.1 & 0.1 & 0.2 & 0.2 & 0.2 \\
\hline $1 \mathrm{~A}$ & $S$ & 4/16/1993 & 93106 & 106 & 0.1 & 0.1 & 0.2 & 0.1 & 0.2 \\
\hline $1 \mathrm{~A}$ & $S$ & 4/19/1993 & 93109 & 109 & & & & & \\
\hline $1 \mathrm{~A}$ & $S$ & $4 / 20 / 1993$ & 93110 & 110 & & & & & \\
\hline $1 \mathrm{~A}$ & S & 4/21/1993 & 93111 & 111 & & 0.1 & & 0.1 & 0.1 \\
\hline $1 \mathrm{~A}$ & S & $4 / 22 / 1993$ & 93112 & 112 & 0.1 & & 0.1 & 0.1 & 0.1 \\
\hline $1 \mathrm{~A}$ & $S$ & $4 / 23 / 1993$ & 93113 & 113 & & & 0.1 & 0.1 & \\
\hline $1 \mathrm{~A}$ & $S$ & 4/26/1993 & 93116 & 116 & & & & 0.1 & 0.1 \\
\hline $1 \mathrm{~A}$ & $S$ & 4/27/1993 & 93117 & 117 & & & & & 0.1 \\
\hline $1 \mathrm{~A}$ & $S$ & 4/28/1993 & 93118 & 118 & & & & & \\
\hline $1 \mathrm{~A}$ & $S$ & 4/29/1993 & 93119 & 119 & & & & & \\
\hline $1 \mathrm{~A}$ & $S$ & 4/30/1993 & 93120 & 120 & & & & & \\
\hline $1 \mathrm{~A}$ & $S$ & $5 / 3 / 1993$ & 93123 & 123 & 0.1 & 0.1 & 0.1 & 0.1 & 0.1 \\
\hline $1 \mathrm{~A}$ & $S$ & $5 / 4 / 1993$ & 93124 & 124 & & & & & \\
\hline $1 \mathrm{~A}$ & $S$ & $5 / 5 / 1993$ & 93125 & 125 & & & & & \\
\hline $1 \mathrm{~A}$ & $S$ & $5 / 6 / 1993$ & 93126 & 126 & 0.2 & 0.1 & 0.1 & 0.1 & 0.1 \\
\hline $1 \mathrm{~A}$ & $S$ & 5/7/1993 & 93127 & 127 & & & & & \\
\hline $1 \mathrm{~A}$ & $S$ & 5/10/1993 & 93130 & 130 & & & & & \\
\hline $1 \mathrm{~A}$ & $S$ & $5 / 11 / 1993$ & 93131 & 131 & & & & & \\
\hline $1 \mathrm{~A}$ & $S$ & $5 / 12 / 1993$ & 93132 & 132 & & & & & \\
\hline $1 \mathrm{~A}$ & $S$ & $5 / 13 / 1993$ & 93133 & 133 & & & & & \\
\hline $1 \mathrm{~A}$ & $\mathrm{~S}$ & $5 / 14 / 1993$ & 93134 & 134 & & & & & \\
\hline $1 \mathrm{~A}$ & $S$ & $5 / 17 / 1993$ & 93137 & 137 & & & & & \\
\hline $1 \mathrm{~A}$ & $S$ & 5/18/1993 & 93138 & 138 & & & & & \\
\hline $1 \mathrm{~A}$ & $S$ & 5/19/1993 & 93139 & 139 & & & & & \\
\hline $1 \mathrm{~A}$ & $S$ & $5 / 20 / 1993$ & 93140 & 140 & & & & & \\
\hline $1 \mathrm{~A}$ & $S$ & $5 / 21 / 1993$ & 93141 & 141 & & & & & \\
\hline $1 \mathrm{~A}$ & $S$ & $5 / 24 / 1993$ & 93144 & 144 & & & & & \\
\hline $1 \mathrm{~A}$ & $S$ & $5 / 25 / 1993$ & 93145 & 145 & & & & & \\
\hline $1 \mathrm{~A}$ & $S$ & $5 / 26 / 1993$ & 93146 & 146 & & & & & \\
\hline $1 \mathrm{~A}$ & $\mathrm{~S}$ & $5 / 27 / 1993$ & 93147 & 147 & & & & & \\
\hline $1 \mathrm{~A}$ & $S$ & $5 / 28 / 1993$ & 93148 & 148 & & & & & \\
\hline $1 \mathrm{~A}$ & S & $5 / 31 / 1993$ & 93151 & 151 & & & & & \\
\hline $1 \mathrm{~A}$ & $S$ & $6 / 1 / 1993$ & 93152 & 152 & & & & & \\
\hline
\end{tabular}


wbheat

\begin{tabular}{|c|c|c|c|c|c|c|c|}
\hline $1 A$ & $S$ & $6 / 2 / 1993$ & 93153 & 153 & & & \\
\hline $1 A$ & $\mathrm{~S}$ & $6 / 3 / 1993$ & 93154 & 154 & & & \\
\hline $1 A$ & $S$ & 6/4/1993 & 93155 & 155 & & & \\
\hline $1 A$ & S & 6/7/1993 & 93158 & 158 & & & \\
\hline $1 A$ & S & 6/8/1993 & 93159 & 159 & & & \\
\hline $1 A$ & S & 6/9/1993 & 93160 & 160 & & & \\
\hline $1 A$ & S & 6/10/1993 & 93161 & 161 & & & \\
\hline $1 A$ & S & $6 / 11 / 1993$ & 93162 & 162 & & & \\
\hline $1 A$ & S & 8/16/1993 & 93228 & 228 & & & \\
\hline $1 A$ & S & 8/17/1993 & 93229 & 229 & & & \\
\hline $1 A$ & S & 8/18/1993 & 93230 & 230 & & & \\
\hline $1 A$ & $S$ & 8/19/1993 & 93231 & 231 & & & \\
\hline $1 A$ & S & 8/20/1993 & 93232 & 232 & & & \\
\hline $1 A$ & S & 8/23/1993 & 93235 & 235 & & & \\
\hline $1 A$ & S & $8 / 24 / 1993$ & 93236 & 236 & & & \\
\hline $1 A$ & S & 8/25/1993 & 93237 & 237 & & & \\
\hline $1 A$ & S & 8/26/1993 & 93238 & 238 & & & \\
\hline $1 A$ & S & 8/27/1993 & 93239 & 239 & & & \\
\hline $1 A$ & S & 8/30/1993 & 93242 & 242 & & & \\
\hline $1 A$ & S & 8/31/1993 & 93243 & 243 & & & \\
\hline $1 A$ & S & 9/1/1993 & 93244 & 244 & & & \\
\hline $1 A$ & S & 9/2/1993 & 93245 & 245 & & & \\
\hline $1 A$ & S & 9/3/1993 & 93246 & 246 & & & \\
\hline $1 A$ & S & 9/7/1993 & 93250 & 250 & & & \\
\hline $1 A$ & S & 9/8/1993 & 93251 & 251 & & & \\
\hline $1 A$ & S & 9/9/1993 & 93252 & 252 & & & \\
\hline $1 A$ & S & 9/10/1993 & 93253 & 253 & & & \\
\hline $1 A$ & S & 9/13/1993 & 93256 & 256 & & & \\
\hline $1 A$ & S & 9/14/1993 & 93257 & 257 & & & \\
\hline $1 A$ & S & 9/15/1993 & 93258 & 258 & & & \\
\hline $1 A$ & S & 9/16/1993 & 93259 & 259 & & & \\
\hline $1 A$ & S & 9/17/1993 & 93260 & 260 & & & \\
\hline $1 A$ & S & 9/20/1993 & 93263 & 263 & & & \\
\hline $1 A$ & S & 9/21/1993 & 93264 & 264 & & & \\
\hline $1 A$ & S & 9/22/1993 & 93265 & 265 & & & \\
\hline $1 A$ & S & 9/23/1993 & 93266 & 266 & & & \\
\hline $1 \mathrm{~A}$ & S & 9/24/1993 & 93267 & 267 & & & \\
\hline $1 A$ & S & 9/27/1993 & 93270 & 270 & & & \\
\hline $1 A$ & $S$ & 9/28/1993 & 93271 & 271 & & & \\
\hline $1 A$ & S & 9/29/1993 & 93272 & 272 & & & \\
\hline $1 A$ & S & 9/30/1993 & 93273 & 273 & 0.031 & 0.01 & 0.021 \\
\hline $1 A$ & S & $10 / 1 / 1993$ & 93274 & 274 & & & \\
\hline $1 A$ & S & $10 / 4 / 1993$ & 93277 & 277 & & & \\
\hline $1 A$ & S & $10 / 5 / 1993$ & 93278 & 278 & & & \\
\hline $1 A$ & S & $10 / 6 / 1993$ & 93279 & 279 & & & \\
\hline $1 A$ & S & $10 / 7 / 1993$ & 93280 & 280 & & & \\
\hline $1 A$ & S & $10 / 8 / 1993$ & 93281 & 281 & & & \\
\hline $1 A$ & $S$ & \#\#\#\#\#\#\#\# & 93284 & 284 & & & \\
\hline $1 A$ & S & \#\#\#\#\#\#\#\# & 93285 & 285 & & & \\
\hline $1 A$ & S & \#\#\#\#\#\#\#\# & 93286 & 286 & & & \\
\hline $1 A$ & $\mathrm{~S}$ & \#\#\#\#\#\#\#\# & 93287 & 287 & & & \\
\hline
\end{tabular}


wbheat

\begin{tabular}{|c|c|c|c|c|c|c|c|c|c|c|}
\hline $1 A$ & $A$ & $S$ & \#\#\#\#\#\#\#\# & 93288 & 288 & & & & & \\
\hline $1 A$ & $A$ & $\mathrm{~S}$ & \#\#\#\#\#\#\#\# & 93291 & 291 & & & & & \\
\hline $1 A$ & A & $\mathrm{S}$ & 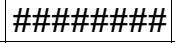 & 93292 & 292 & & & & & \\
\hline $1 A$ & $A$ & $\mathrm{~S}$ & \#\#\#\#\#\#\#\# & 93293 & 293 & & & & & \\
\hline $1 A$ & $A$ & $S$ & \#\#\#\#\#\#\#\# & 93294 & 294 & & & & & \\
\hline $1 A$ & $A$ & $S$ & \#\#\#\#\#\#\#\# & 93295 & 295 & & & & & \\
\hline $1 A$ & $A$ & $S$ & \#\#\#\#\#\#\#\# & 93298 & 298 & & & & & \\
\hline $1 A$ & $A$ & $S$ & \#\#\#\#\#\#\#\# & 93299 & 299 & & & & & \\
\hline $1 A$ & $A$ & $S$ & \#\#\#\#\#\#\#\# & 93300 & 300 & & & & & \\
\hline $1 A$ & A & $\mathrm{S}$ & \#\#\#\#\#\#\#\# & 93301 & 301 & & & & & \\
\hline $1 A$ & $A$ & $S$ & \#\#\#\#\#\#\#\# & 93302 & 302 & & & & & \\
\hline $1 A$ & A & $\mathrm{S}$ & $11 / 1 / 1993$ & 93305 & 305 & & & & & 0.1 \\
\hline $1 A$ & $A$ & $S$ & 11/2/1993 & 93306 & 306 & & & & & \\
\hline $1 A$ & $A$ & $S$ & 11/3/1993 & 93307 & 307 & 0.1 & & 0.1 & 0.1 & 0.1 \\
\hline $1 A$ & $A$ & $S$ & 11/4/1993 & 93308 & 308 & & & & & \\
\hline $1 A$ & $A$ & $S$ & 11/5/1993 & 93309 & 309 & & & & & \\
\hline $1 A$ & $A$ & $\mathrm{~S}$ & 11/8/1993 & 93312 & 312 & 0.1 & 0.1 & 0.1 & 0.1 & 0.2 \\
\hline $1 A$ & $A$ & $S$ & 11/9/1993 & 93313 & 313 & 0.1 & & & & 0.1 \\
\hline $1 A$ & $A$ & $S$ & \#\#\#\#\#\#\#\# & 93314 & 314 & & & 0.1 & & 0.1 \\
\hline $1 A$ & A & $\mathrm{S}$ & \#\#\#\#\#\#\#\# & 93315 & 315 & 0.1 & & & & \\
\hline $1 A$ & A & $\mathrm{S}$ & 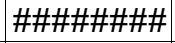 & 93316 & 316 & & & & & \\
\hline $1 A$ & $A$ & $S$ & \#\#\#\#\#\#\#\# & 93319 & 319 & & & & & \\
\hline $1 A$ & $A$ & $S$ & \#\#\#\#\#\#\#\# & 93320 & 320 & & & & & \\
\hline $1 A$ & $A$ & $S$ & \#\#\#\#\#\#\#\# & 93321 & 321 & 0.1 & & & & 0.1 \\
\hline $1 A$ & $A$ & $S$ & \#\#\#\#\#\#\#\# & 93322 & 322 & & & & & \\
\hline $1 A$ & A & $S$ & \#\#\#\#\#\#\#\# & 93323 & 323 & & & & & \\
\hline $1 A$ & $A$ & $S$ & \#\#\#\#\#\#\#\# & 93326 & 326 & & & & & 0.2 \\
\hline $1 A$ & $A$ & $S$ & \#\#\#\#\#\#\#\# & 93327 & 327 & & & & & \\
\hline $1 A$ & $A$ & $S$ & \#\#\#\#\#\#\#\# & 93328 & 328 & & & & & \\
\hline $1 A$ & $A$ & $S$ & \#\#\#\#\#\#\#\# & 93333 & 333 & & & & & 0.5 \\
\hline $1 A$ & $A$ & $\mathrm{~S}$ & \#\#\#\#\#\#\#\# & 93334 & 334 & & & & & \\
\hline $1 \mathrm{~A}$ & $A$ & $S$ & 12/1/1993 & 93335 & 335 & & & & & \\
\hline $1 A$ & $A$ & $S$ & 12/2/1993 & 93336 & 336 & & & & & \\
\hline $1 A$ & $A$ & $S$ & $12 / 3 / 1993$ & 93337 & 337 & & & & & \\
\hline $1 A$ & A & $S$ & $12 / 6 / 1993$ & 93340 & 340 & & & & & \\
\hline $1 A$ & A & $\mathrm{S}$ & $12 / 7 / 1993$ & 93341 & 341 & & & & & \\
\hline $1 \mathrm{~A}$ & A & $S$ & $12 / 8 / 1993$ & 93342 & 342 & & & & & \\
\hline $1 A$ & $A$ & $S$ & $12 / 9 / 1993$ & 93343 & 343 & & & & & \\
\hline $1 A$ & $A$ & $S$ & \#\#\#\#\#\#\#\# & 93344 & 344 & 0.1 & & & & \\
\hline $1 A$ & $A$ & $\mathrm{~S}$ & \#\#\#\#\#\#\#\# & 93347 & 347 & & & & & \\
\hline $1 A$ & $A$ & $S$ & \#\#\#\#\#\#\#\# & 93348 & 348 & & & & & \\
\hline $1 A$ & $A$ & $\mathrm{~S}$ & \#\#\#\#\#\#\#\# & 93349 & 349 & & & & & \\
\hline $1 A$ & $A$ & $S$ & \#\#\#\#\#\#\#\# & 93350 & 350 & & & & & \\
\hline $1 \mathrm{~A}$ & $A$ & $S$ & \#\#\#\#\#\#\#\# & 93351 & 351 & & & & & \\
\hline $1 A$ & $A$ & $S$ & $1 / 3 / 1994$ & 94003 & 3 & 0.1 & 0.1 & 0.1 & 0.1 & 0.3 \\
\hline $1 A$ & $A$ & $S$ & $1 / 4 / 1994$ & 94004 & 4 & 0.2 & 0.2 & 0.1 & 0.2 & 0.2 \\
\hline $1 A$ & A & $\mathrm{S}$ & 1/5/1994 & 94005 & 5 & 0.2 & 0.1 & 0.2 & 0.1 & 0.2 \\
\hline $1 A$ & $A$ & $S$ & $1 / 6 / 1994$ & 94006 & 6 & 0.2 & 0.2 & 0.2 & 0.2 & 0.2 \\
\hline $1 \mathrm{~A}$ & $A$ & $S$ & 1/7/1994 & 94007 & 7 & 0.1 & 0.1 & 0.1 & 0.1 & 0.1 \\
\hline $1 \mathrm{~A}$ & A & $S$ & $1 / 10 / 1994$ & 94010 & 10 & 0.3 & 0.3 & 0.3 & 0.3 & 0.3 \\
\hline $1 A$ & $A$ & $S$ & $1 / 11 / 1994$ & 94011 & 11 & 0.1 & & 0.1 & 0.1 & 0.1 \\
\hline
\end{tabular}


wbheat

\begin{tabular}{|c|c|c|c|c|c|c|c|c|c|}
\hline $1 \mathrm{~A}$ & $S$ & $1 / 12 / 1994$ & 94012 & 12 & 0.1 & 0.1 & 0.2 & 0.2 & 0.2 \\
\hline $1 \mathrm{~A}$ & $\mathrm{~S}$ & $1 / 13 / 1994$ & 94013 & 13 & 0.1 & 0.1 & 0.1 & 0.1 & 0.1 \\
\hline $1 \mathrm{~A}$ & $S$ & $1 / 14 / 1994$ & 94014 & 14 & 0.2 & 0.2 & 0.2 & 0.2 & 0.2 \\
\hline $1 \mathrm{~A}$ & S & 1/18/1994 & 94018 & 18 & 0.3 & 0.3 & 0.5 & 0.7 & 0.6 \\
\hline $1 \mathrm{~A}$ & S & $1 / 19 / 1994$ & 94019 & 19 & 0.5 & 0.6 & 0.5 & 0.9 & 1 \\
\hline $1 \mathrm{~A}$ & $S$ & $1 / 20 / 1994$ & 94020 & 20 & 0.2 & 0.2 & 0.2 & 0.2 & 0.2 \\
\hline $1 \mathrm{~A}$ & $S$ & $1 / 21 / 1994$ & 94021 & 21 & 0.4 & 0.4 & 0.4 & 0.4 & 0.5 \\
\hline $1 \mathrm{~A}$ & $S$ & $1 / 24 / 1994$ & 94024 & 24 & 0.3 & 0.3 & 0.3 & 0.3 & 0.3 \\
\hline $1 \mathrm{~A}$ & $S$ & $1 / 25 / 1994$ & 94025 & 25 & 0.2 & 0.2 & 0.2 & 0.2 & 0.2 \\
\hline $1 \mathrm{~A}$ & $S$ & $1 / 26 / 1994$ & 94026 & 26 & & 0.1 & & 0.1 & 0.1 \\
\hline $1 \mathrm{~A}$ & $S$ & $1 / 27 / 1994$ & 94027 & 27 & 0.1 & 0.1 & 0.1 & 0.1 & 0.1 \\
\hline $1 \mathrm{~A}$ & $S$ & $1 / 28 / 1994$ & 94028 & 28 & 0.2 & 0.2 & 0.2 & 0.2 & 0.3 \\
\hline $1 \mathrm{~A}$ & $S$ & $1 / 31 / 1994$ & 94031 & 31 & 0.4 & 0.4 & 0.4 & 0.4 & 0.8 \\
\hline $1 \mathrm{~A}$ & $S$ & 2/1/1994 & 94032 & 32 & 0.5 & 0.6 & 0.6 & 0.6 & 1.1 \\
\hline $1 \mathrm{~A}$ & S & $2 / 2 / 1994$ & 94033 & 33 & 0.5 & 0.4 & 0.5 & 0.5 & 0.5 \\
\hline $1 \mathrm{~A}$ & $S$ & 2/3/1994 & 94034 & 34 & 0.3 & 0.3 & 0.4 & 0.4 & 0.4 \\
\hline $1 \mathrm{~A}$ & S & 2/7/1994 & 94038 & 38 & 0.3 & 0.2 & 0.2 & 0.2 & 0.2 \\
\hline $1 \mathrm{~A}$ & $S$ & 2/8/1994 & 94039 & 39 & 0.1 & 0.1 & 0.1 & 0.1 & 0.1 \\
\hline $1 \mathrm{~A}$ & $S$ & 2/9/1994 & 94040 & 40 & 0.1 & & 0.1 & 0.1 & 0.1 \\
\hline $1 \mathrm{~A}$ & $S$ & 2/10/1994 & 94041 & 41 & 0.6 & 0.6 & 0.7 & 1 & 1.2 \\
\hline $1 \mathrm{~A}$ & $S$ & 2/11/1994 & 94042 & 42 & 0.6 & 0.6 & 0.6 & 0.6 & 1 \\
\hline $1 \mathrm{~A}$ & $S$ & 2/14/1994 & 94045 & 45 & 0.4 & 0.5 & 0.5 & 0.5 & 0.9 \\
\hline $1 \mathrm{~A}$ & S & $2 / 15 / 1994$ & 94046 & 46 & 0.3 & 0.3 & 0.3 & 0.3 & 0.3 \\
\hline $1 \mathrm{~A}$ & $S$ & 2/16/1994 & 94047 & 47 & 0.2 & 0.2 & 0.3 & 0.3 & 0.3 \\
\hline $1 \mathrm{~A}$ & $S$ & 2/17/1994 & 94048 & 48 & 0.2 & 0.2 & 0.2 & 0.2 & 0.3 \\
\hline $1 \mathrm{~A}$ & $S$ & 2/18/1994 & 94049 & 49 & 0.1 & 0.1 & 0.1 & 0.1 & 0.1 \\
\hline $1 \mathrm{~A}$ & $S$ & $2 / 21 / 1994$ & 94052 & 52 & & & & & \\
\hline $1 \mathrm{~A}$ & $S$ & $2 / 22 / 1994$ & 94053 & 53 & 0.1 & 0.1 & 0.1 & 0.1 & 0.1 \\
\hline $1 \mathrm{~A}$ & $S$ & 2/23/1994 & 94054 & 54 & 0.1 & 0.1 & 0.1 & 0.1 & 0.1 \\
\hline $1 \mathrm{~A}$ & $\mathrm{~S}$ & $2 / 24 / 1994$ & 94055 & 55 & 0.5 & 0.5 & 0.5 & 0.5 & 0.5 \\
\hline $1 \mathrm{~A}$ & $S$ & $2 / 25 / 1994$ & 94056 & 56 & 0.4 & 0.4 & 0.4 & 0.4 & 0.4 \\
\hline $1 \mathrm{~A}$ & $S$ & $2 / 28 / 1994$ & 94059 & 59 & 0.5 & 0.5 & 0.5 & 0.5 & 0.5 \\
\hline $1 \mathrm{~A}$ & $\mathrm{~S}$ & $3 / 1 / 1994$ & 94060 & 60 & 0.3 & 0.3 & 0.3 & 0.4 & 0.3 \\
\hline $1 \mathrm{~A}$ & $S$ & $3 / 2 / 1994$ & 94061 & 61 & 0.4 & 0.4 & 0.4 & 0.4 & 0.4 \\
\hline $1 \mathrm{~A}$ & $S$ & $3 / 3 / 1994$ & 94062 & 62 & 0.3 & 0.3 & 0.3 & 0.4 & 0.3 \\
\hline $1 \mathrm{~A}$ & $S$ & $3 / 4 / 1994$ & 94063 & 63 & 0.1 & 0.1 & 0.1 & 0.1 & 0.1 \\
\hline $1 \mathrm{~A}$ & $S$ & $3 / 7 / 1994$ & 94066 & 66 & 0.1 & & 0.1 & & \\
\hline $1 \mathrm{~A}$ & $S$ & $3 / 8 / 1994$ & 94067 & 67 & & & & & \\
\hline $1 \mathrm{~A}$ & $\mathrm{~S}$ & 3/9/1994 & 94068 & 68 & 0.1 & & & & \\
\hline $1 \mathrm{~A}$ & $S$ & 3/10/1994 & 94069 & 69 & 0.4 & 0.4 & 0.4 & 0.4 & 0.5 \\
\hline $1 \mathrm{~A}$ & $S$ & 3/11/1994 & 94070 & 70 & 0.1 & & 0.1 & & 0.1 \\
\hline $1 \mathrm{~A}$ & S & 3/21/1994 & 94080 & 80 & & & & & \\
\hline $1 \mathrm{~A}$ & $S$ & 3/22/1994 & 94081 & 81 & & & & & \\
\hline $1 \mathrm{~A}$ & $S$ & $3 / 23 / 1994$ & 94082 & 82 & & & & & \\
\hline $1 \mathrm{~A}$ & $S$ & 3/24/1994 & 94083 & 83 & & & & & \\
\hline $1 \mathrm{~A}$ & $S$ & $3 / 25 / 1994$ & 94084 & 84 & & & & & \\
\hline $1 \mathrm{~A}$ & $S$ & $3 / 28 / 1994$ & 94087 & 87 & & & & & \\
\hline $1 \mathrm{~A}$ & $\mathrm{~S}$ & 3/29/1994 & 94088 & 88 & & & & & \\
\hline $1 \mathrm{~A}$ & $S$ & 3/30/1994 & 94089 & 89 & & & & & \\
\hline $1 \mathrm{~A}$ & S & 3/31/1994 & 94090 & 90 & & & & & \\
\hline $1 \mathrm{~A}$ & $S$ & $4 / 1 / 1994$ & 94091 & 91 & & & & & \\
\hline
\end{tabular}


wbheat

\begin{tabular}{|c|c|c|c|c|c|c|c|c|c|}
\hline $1 A$ & $S$ & $4 / 4 / 1994$ & 94094 & 94 & & & & & \\
\hline $1 A$ & $\mathrm{~S}$ & $4 / 5 / 1994$ & 94095 & 95 & & & & & \\
\hline $1 A$ & S & $4 / 6 / 1994$ & 94096 & 96 & & & & & \\
\hline $1 A$ & S & $4 / 7 / 1994$ & 94097 & 97 & & & & & \\
\hline $1 A$ & S & $4 / 8 / 1994$ & 94098 & 98 & & & & & \\
\hline $1 A$ & $\mathrm{~S}$ & 4/11/1994 & 94101 & 101 & & & & & \\
\hline $1 A$ & S & $4 / 12 / 1994$ & 94102 & 102 & & & & & \\
\hline $1 A$ & S & 4/13/1994 & 94103 & 103 & & & & & \\
\hline $0 A$ & S & 10/5/1991 & 91278 & 278 & & & & & \\
\hline $0 A$ & S & $10 / 6 / 1991$ & 91279 & 279 & & & & & \\
\hline $0 A$ & S & \#\#\#\#\#\#\#\# & 91285 & 285 & & & & & \\
\hline $0 A$ & $S$ & \#\#\#\#\#\#\#\# & 91286 & 286 & & & & & \\
\hline $0 A$ & S & \#\#\#\#\#\#\#\# & 91292 & 292 & & & & & \\
\hline $0 A$ & S & \#\#\#\#\#\#\#\# & 91293 & 293 & & & & & \\
\hline $0 A$ & S & \#\#\#\#\#\#\#\# & 91299 & 299 & & & & & \\
\hline $0 A$ & S & \#\#\#\#\#\#\#\# & 91300 & 300 & & & & & \\
\hline $0 A$ & S & $11 / 2 / 1991$ & 91306 & 306 & 0.3 & 0.3 & 0.3 & 0.4 & 0.4 \\
\hline $0 A$ & S & $11 / 3 / 1991$ & 91307 & 307 & 0.5 & 0.5 & 0.5 & 0.5 & 0.5 \\
\hline $0 A$ & S & 11/9/1991 & 91313 & 313 & 0.4 & 0.4 & 0.4 & 0.5 & 0.5 \\
\hline $0 A$ & S & \#\#\#\#\#\#\#\# & 91314 & 314 & 0.4 & 0.4 & 0.4 & 0.5 & 0.5 \\
\hline $0 A$ & S & \#\#\#\#\#\#\#\# & 91320 & 320 & & 0.1 & & 0.1 & \\
\hline $0 A$ & S & \#\#\#\#\#\#\#\# & 91321 & 321 & 0.1 & 0.1 & 0.1 & 0.1 & 0.2 \\
\hline $0 A$ & S & \#\#\#\#\#\#\#\# & 91327 & 327 & 0.2 & 0.2 & 0.3 & 0.3 & 0.3 \\
\hline $0 A$ & S & \#\#\#\#\#\#\#\# & 91328 & 328 & 0.5 & 0.5 & 0.5 & 0.5 & 0.5 \\
\hline $0 A$ & S & $12 / 7 / 1991$ & 91341 & 341 & 0.1 & 0.1 & 0.2 & 0.2 & 0.2 \\
\hline $0 A$ & S & $12 / 8 / 1991$ & 91342 & 342 & 0.1 & 0.1 & 0.1 & 0.1 & 0.1 \\
\hline $0 A$ & S & \#\#\#\#\#\#\#\# & 91348 & 348 & 0.1 & 0.1 & 0.1 & 0.2 & 0.2 \\
\hline $0 A$ & S & \#\#\#\#\#\#\#\# & 91349 & 349 & 0.4 & 0.4 & 0.4 & 0.4 & 0.5 \\
\hline $0 A$ & S & $1 / 11 / 1992$ & 92011 & 11 & 0.3 & 0.4 & 0.4 & 0.4 & 0.5 \\
\hline $0 A$ & S & 1/12/1992 & 92012 & 12 & 0.5 & 0.5 & 0.5 & 0.5 & 0.5 \\
\hline $0 A$ & S & $1 / 18 / 1992$ & 92018 & 18 & 0.5 & 0.6 & 0.6 & 0.6 & 0.6 \\
\hline $0 A$ & S & $1 / 19 / 1992$ & 92019 & 19 & 0.7 & 0.7 & 0.7 & 0.7 & 0.7 \\
\hline $0 A$ & S & $1 / 25 / 1992$ & 92025 & 25 & 0.3 & 0.3 & 0.3 & 0.3 & 0.4 \\
\hline $0 A$ & S & $1 / 26 / 1992$ & 92026 & 26 & 0.3 & 0.3 & 0.3 & 0.4 & 0.3 \\
\hline $0 A$ & S & 2/1/1992 & 92032 & 32 & 0.2 & 0.3 & 0.3 & 0.3 & 0.3 \\
\hline $0 A$ & S & 2/2/1992 & 92033 & 33 & 0.3 & 0.3 & 0.3 & 0.3 & 0.3 \\
\hline $0 A$ & S & 2/8/1992 & 92039 & 39 & 0.4 & 0.4 & 0.4 & 0.5 & 0.5 \\
\hline $0 A$ & S & 2/9/1992 & 92040 & 40 & 0.4 & 0.4 & 0.5 & 0.5 & 0.5 \\
\hline $0 A$ & $S$ & 2/15/1992 & 92046 & 46 & 0.1 & 0.1 & 0.1 & 0.2 & 0.2 \\
\hline $0 A$ & S & $2 / 16 / 1992$ & 92047 & 47 & 0.2 & 0.2 & 0.2 & 0.2 & 0.2 \\
\hline $0 A$ & S & 2/22/1992 & 92053 & 53 & 0.1 & 0.1 & 0.1 & 0.1 & 0.1 \\
\hline $0 A$ & S & 2/23/1992 & 92054 & 54 & 0.2 & 0.2 & 0.2 & 0.2 & 0.2 \\
\hline $0 A$ & S & 2/29/1992 & 92060 & 60 & 0.2 & 0.2 & 0.2 & 0.2 & 0.3 \\
\hline $0 A$ & S & 3/1/1992 & 92061 & 61 & 0.2 & 0.2 & 0.2 & 0.3 & 0.3 \\
\hline $0 A$ & S & 3/7/1992 & 92067 & 67 & 0.1 & 0.2 & 0.2 & 0.2 & 0.2 \\
\hline $0 A$ & S & 3/8/1992 & 92068 & 68 & 0.1 & 0.2 & 0.2 & 0.2 & 0.2 \\
\hline $0 A$ & S & 3/28/1992 & 92088 & 88 & 0.1 & 0.1 & 0.1 & 0.1 & 0.1 \\
\hline $0 A$ & $S$ & 3/29/1992 & 92089 & 89 & 0.2 & 0.2 & 0.2 & 0.1 & 0.2 \\
\hline $0 A$ & S & $4 / 4 / 1992$ & 92095 & 95 & 0.2 & 0.2 & 0.2 & 0.2 & 0.2 \\
\hline $0 A$ & S & 4/5/1992 & 92096 & 96 & 0.1 & 0.2 & 0.2 & 0.2 & 0.2 \\
\hline $0 A$ & S & 4/11/1992 & 92102 & 102 & 0.1 & 0.1 & 0.1 & 0.1 & 0.1 \\
\hline
\end{tabular}


wbheat

\begin{tabular}{|c|c|c|c|c|c|c|c|c|c|}
\hline$\overline{O A A}$ & $S$ & $4 / 12 / 1992$ & 92103 & 103 & 0.1 & 0.1 & 0.1 & 0.1 & 0.1 \\
\hline $0 A$ & S & 4/18/1992 & 92109 & 109 & 0.1 & 0.1 & 0.1 & 0.1 & 0.1 \\
\hline $0 A$ & $\mathrm{~S}$ & $4 / 19 / 1992$ & 92110 & 110 & 0.1 & & 0.1 & 0.1 & 0.1 \\
\hline $0 A$ & $\mathrm{~S}$ & 4/25/1992 & 92116 & 116 & & & 0.1 & & 0.1 \\
\hline $0 A$ & S & 4/26/1992 & 92117 & 117 & & 0.1 & 0.1 & 0.1 & 0.1 \\
\hline $0 A$ & S & $5 / 2 / 1992$ & 92123 & 123 & & & & & \\
\hline$O A$ & $\mathrm{~S}$ & $5 / 3 / 1992$ & 92124 & 124 & & & & & \\
\hline$O A$ & S & $5 / 9 / 1992$ & 92130 & 130 & & & & & \\
\hline $0 A$ & S & 5/10/1992 & 92131 & 131 & & & & & \\
\hline $0 A$ & S & $5 / 16 / 1992$ & 92137 & 137 & & & & & \\
\hline $0 A$ & S & $5 / 17 / 1992$ & 92138 & 138 & & & & & \\
\hline $0 \mathrm{~A}$ & $\mathrm{~S}$ & $5 / 23 / 1992$ & 92144 & 144 & & & & & \\
\hline $0 A$ & S & $5 / 24 / 1992$ & 92145 & 145 & & & & & \\
\hline $0 A$ & S & $5 / 30 / 1992$ & 92151 & 151 & & & 0.1 & 0.1 & 0.1 \\
\hline $0 \mathrm{~A}$ & $\mathrm{~S}$ & $5 / 31 / 1992$ & 92152 & 152 & & & 0.1 & & 0.1 \\
\hline $0 A$ & $\mathrm{~S}$ & 6/6/1992 & 92158 & 158 & & & & & \\
\hline $0 A$ & S & 6/7/1992 & 92159 & 159 & & & & & \\
\hline $0 A$ & S & 6/13/1992 & 92165 & 165 & & & & & \\
\hline $0 A$ & S & 8/15/1992 & 92228 & 228 & & & & & \\
\hline $0 \mathrm{~A}$ & S & 8/16/1992 & 92229 & 229 & & & & & \\
\hline $0 A$ & S & 8/22/1992 & 92235 & 235 & & & & & \\
\hline $0 A$ & S & 8/23/1992 & 92236 & 236 & & & & & \\
\hline $0 A$ & S & 8/29/1992 & 92242 & 242 & & & & & \\
\hline $0 A$ & S & 8/30/1992 & 92243 & 243 & & & & & \\
\hline $0 A$ & S & 9/5/1992 & 92249 & 249 & & & & & \\
\hline $0 A$ & S & 9/6/1992 & 92250 & 250 & & & & & \\
\hline $0 A$ & S & 9/12/1992 & 92256 & 256 & & & & & \\
\hline $0 A$ & S & 9/13/1992 & 92257 & 257 & & & & & \\
\hline $0 A$ & S & 9/19/1992 & 92263 & 263 & & & & & \\
\hline $0 A$ & S & 9/20/1992 & 92264 & 264 & & & & & \\
\hline $0 A$ & S & 9/26/1992 & 92270 & 270 & & & & & \\
\hline $0 \mathrm{~A}$ & S & 9/27/1992 & 92271 & 271 & & & & & \\
\hline $0 A$ & S & $10 / 3 / 1992$ & 92277 & 277 & & & & & \\
\hline $0 A$ & S & 10/4/1992 & 92278 & 278 & & & & & \\
\hline $0 A$ & S & 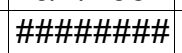 & 92284 & 284 & 0.1 & & 0.1 & 0.1 & \\
\hline $0 A$ & S & \#\#\#\#\#\#\#\# & 92285 & 285 & & & 0.1 & & 0.1 \\
\hline $0 A$ & S & \#\#\#\#\#\#\#\# & 92291 & 291 & 0.2 & 0.2 & 0.2 & 0.2 & 0.2 \\
\hline $0 A$ & S & \#\#\#\#\#\#\#\# & 92292 & 292 & 0.1 & 0.2 & 0.2 & 0.2 & 0.2 \\
\hline $0 A$ & $S$ & \#\#\#\#\#\#\#\# & 92298 & 298 & & & & & \\
\hline $0 A$ & S & \#\#\#\#\#\#\#\# & 92299 & 299 & & & & & \\
\hline $0 A$ & S & \#\#\#\#\#\#\#\# & 92305 & 305 & & & & & \\
\hline$O A$ & S & $11 / 1 / 1992$ & 92306 & 306 & & & & & \\
\hline $0 \mathrm{~A}$ & S & $11 / 7 / 1992$ & 92312 & 312 & 0.3 & 0.3 & 0.3 & 0.3 & 0.3 \\
\hline $0 \mathrm{~A}$ & S & $11 / 8 / 1992$ & 92313 & 313 & 0.2 & 0.2 & 0.2 & 0.2 & 0.2 \\
\hline $0 A$ & S & \#\#\#\#\#\#\#\# & 92319 & 319 & 0.2 & 0.2 & 0.3 & 0.3 & 0.3 \\
\hline $0 \mathrm{~A}$ & S & \#\#\#\#\#\#\#\# & 92320 & 320 & 0.2 & 0.2 & 0.3 & 0.3 & 0.3 \\
\hline $0 A$ & S & 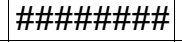 & 92326 & 326 & 0.2 & 0.2 & 0.2 & 0.2 & 0.2 \\
\hline $0 A$ & $S$ & \#\#\#\#\#\#\#\# & 92327 & 327 & 0.3 & 0.3 & 0.3 & 0.3 & 0.3 \\
\hline $0 \mathrm{~A}$ & S & $12 / 5 / 1992$ & 92340 & 340 & 0.4 & 0.4 & 0.4 & 0.4 & 0.5 \\
\hline $0 \mathrm{~A}$ & S & $12 / 6 / 1992$ & 92341 & 341 & 0.5 & 0.6 & 0.6 & 0.6 & 0.6 \\
\hline $0 \mathrm{~A}$ & $\mathrm{~S}$ & \#\#\#\#\#\#\#\# & 92347 & 347 & 0.3 & 0.3 & 0.3 & 0.3 & 0.3 \\
\hline
\end{tabular}


wbheat

\begin{tabular}{|c|c|c|c|c|c|c|c|c|c|}
\hline $0 \mathrm{~A}$ & $\mathrm{~S}$ & \#\#\#\#\#\#\#\# & 92348 & 348 & 0.2 & 0.2 & 0.2 & 0.2 & 0.3 \\
\hline $0 A$ & $\mathrm{~S}$ & $1 / 9 / 1993$ & 93009 & 9 & 0.4 & 0.4 & 0.4 & 0.4 & 0.4 \\
\hline $0 A$ & S & $1 / 10 / 1993$ & 93010 & 10 & 0.6 & 0.6 & 0.6 & 0.6 & 0.6 \\
\hline $0 A$ & S & 1/16/1993 & 93016 & 16 & 0.4 & 0.4 & 0.4 & 0.4 & 0.4 \\
\hline $0 A$ & $S$ & $1 / 17 / 1993$ & 93017 & 17 & 0.4 & 0.4 & 0.4 & 0.4 & 0.4 \\
\hline $0 A$ & S & $1 / 23 / 1993$ & 93023 & 23 & 0.2 & 0.2 & 0.2 & 0.2 & 0.2 \\
\hline$O A$ & $\mathrm{~S}$ & $1 / 24 / 1993$ & 93024 & 24 & 0.3 & 0.3 & 0.4 & 0.4 & 0.4 \\
\hline $0 \mathrm{~A}$ & S & $1 / 30 / 1993$ & 93030 & 30 & 0.5 & 0.6 & 0.6 & 0.6 & 0.6 \\
\hline $0 A$ & S & $1 / 31 / 1993$ & 93031 & 31 & 0.5 & 0.4 & 0.5 & 0.5 & 0.5 \\
\hline $0 A$ & S & 2/6/1993 & 93037 & 37 & 0.4 & 0.4 & 0.4 & 0.4 & 0.4 \\
\hline $0 A$ & S & 2/7/1993 & 93038 & 38 & 0.4 & 0.4 & 0.5 & 0.4 & 0.5 \\
\hline $0 A$ & $\mathrm{~S}$ & 2/13/1993 & 93044 & 44 & 0.3 & 0.3 & 0.3 & 0.4 & 0.4 \\
\hline $0 A$ & S & 2/14/1993 & 93045 & 45 & 0.3 & 0.3 & 0.3 & 0.4 & 0.4 \\
\hline $0 A$ & S & $2 / 20 / 1993$ & 93051 & 51 & 0.2 & 0.2 & 0.2 & 0.3 & 0.2 \\
\hline $0 A$ & S & 2/21/1993 & 93052 & 52 & 0.2 & 0.2 & 0.2 & 0.2 & 0.2 \\
\hline $0 A$ & S & $2 / 27 / 1993$ & 93058 & 58 & 0.4 & 0.4 & 0.4 & 0.4 & 0.4 \\
\hline $0 A$ & S & 2/28/1993 & 93059 & 59 & 0.3 & 0.4 & 0.4 & 0.4 & 0.4 \\
\hline $0 A$ & S & 3/6/1993 & 93065 & 65 & 0.2 & 0.3 & 0.3 & 0.3 & 0.3 \\
\hline $0 A$ & S & $3 / 7 / 1993$ & 93066 & 66 & 0.3 & 0.3 & 0.3 & 0.3 & 0.3 \\
\hline $0 \mathrm{~A}$ & S & 3/27/1993 & 93086 & 86 & 0.1 & 0.1 & 0.1 & 0.1 & 0.1 \\
\hline $0 A$ & S & 3/28/1993 & 93087 & 87 & 0.1 & 0.1 & 0.1 & 0.1 & 0.1 \\
\hline $0 A$ & S & 4/3/1993 & 93093 & 93 & 0.1 & 0.1 & 0.1 & 0.1 & 0.1 \\
\hline $0 A$ & S & 4/4/1993 & 93094 & 94 & 0.1 & & 0.1 & 0.1 & 0.2 \\
\hline $0 A$ & S & 4/10/1993 & 93100 & 100 & 0.1 & 0.1 & 0.1 & 0.1 & 0.1 \\
\hline $0 A$ & S & $4 / 11 / 1993$ & 93101 & 101 & & & 0.1 & & 0.1 \\
\hline $0 A$ & S & 4/17/1993 & 93107 & 107 & & 0.1 & & 0.1 & 0.1 \\
\hline $0 A$ & S & 4/18/1993 & 93108 & 108 & 0.1 & & 0.1 & 0.1 & 0.1 \\
\hline $0 A$ & S & 4/24/1993 & 93114 & 114 & & & & & \\
\hline $0 A$ & S & 4/25/1993 & 93115 & 115 & & & & & \\
\hline $0 A$ & S & $5 / 1 / 1993$ & 93121 & 121 & & & & & \\
\hline $0 A$ & S & $5 / 2 / 1993$ & 93122 & 122 & & & & & \\
\hline $0 \mathrm{~A}$ & S & $5 / 8 / 1993$ & 93128 & 128 & & & & & \\
\hline $0 A$ & S & $5 / 9 / 1993$ & 93129 & 129 & & & & & \\
\hline $0 A$ & S & $5 / 15 / 1993$ & 93135 & 135 & & 0.1 & 0.1 & & \\
\hline $0 A$ & S & $5 / 16 / 1993$ & 93136 & 136 & & 0.1 & & & \\
\hline $0 A$ & S & $5 / 22 / 1993$ & 93142 & 142 & & & & & \\
\hline $0 A$ & S & $5 / 23 / 1993$ & 93143 & 143 & & & & & \\
\hline $0 A$ & S & $5 / 29 / 1993$ & 93149 & 149 & & & & & \\
\hline $0 A$ & $S$ & $5 / 30 / 1993$ & 93150 & 150 & & & & & \\
\hline $0 A$ & S & $6 / 5 / 1993$ & 93156 & 156 & & & & & \\
\hline $0 A$ & S & 6/6/1993 & 93157 & 157 & & & & & \\
\hline $0 \mathrm{~A}$ & S & $6 / 12 / 1993$ & 93163 & 163 & & & & & \\
\hline $0 \mathrm{~A}$ & S & 8/21/1993 & 93233 & 233 & & & & & \\
\hline $0 A$ & S & $8 / 22 / 1993$ & 93234 & 234 & & & & & \\
\hline $0 A$ & S & 8/28/1993 & 93240 & 240 & & & & & \\
\hline $0 \mathrm{~A}$ & S & $8 / 29 / 1993$ & 93241 & 241 & & & & & \\
\hline $0 A$ & S & 9/4/1993 & 93247 & 247 & & & & & \\
\hline $0 A$ & $S$ & 9/5/1993 & 93248 & 248 & & & & & \\
\hline $0 A$ & S & 9/11/1993 & 93254 & 254 & & & & & \\
\hline $0 \mathrm{~A}$ & S & 9/12/1993 & 93255 & 255 & & & & & \\
\hline $0 A$ & $S$ & 9/18/1993 & 93261 & 261 & & & & & \\
\hline
\end{tabular}


wbheat

\begin{tabular}{|c|c|c|c|c|c|c|c|c|c|}
\hline $0 A$ & $S$ & 9/19/1993 & 93262 & 262 & & & & & \\
\hline $0 A$ & $\mathrm{~S}$ & 9/25/1993 & 93268 & 268 & & & & & \\
\hline $0 A$ & S & 9/26/1993 & 93269 & 269 & & & & & \\
\hline $0 \mathrm{~A}$ & $\mathrm{~S}$ & $10 / 2 / 1993$ & 93275 & 275 & & & & & \\
\hline $0 A$ & S & 10/3/1993 & 93276 & 276 & & & & & \\
\hline $0 A$ & $\mathrm{~S}$ & 10/9/1993 & 93282 & 282 & & & & & \\
\hline $0 A$ & $\mathrm{~S}$ & \#\#\#\#\#\#\#\# & 93283 & 283 & & & & & \\
\hline $0 A$ & S & \#\#\#\#\#\#\#\# & 93289 & 289 & & & & & \\
\hline $0 A$ & S & \#\#\#\#\#\#\#\# & 93290 & 290 & & & & & \\
\hline $0 A$ & S & \#\#\#\#\#\#\#\# & 93296 & 296 & & & & & \\
\hline $0 A$ & S & \#\#\#\#\#\#\#\# & 93297 & 297 & & & & & \\
\hline $0 A$ & $S$ & \#\#\#\#\#\#\#\# & 93303 & 303 & & & & & \\
\hline $0 A$ & S & \#\#\#\#\#\#\#\# & 93304 & 304 & & & & & \\
\hline $0 A$ & S & $11 / 6 / 1993$ & 93310 & 310 & & & 0.1 & & 0.1 \\
\hline $0 A$ & S & $11 / 7 / 1993$ & 93311 & 311 & 0.1 & 0.1 & 0.1 & 0.1 & 0.1 \\
\hline $0 A$ & $\mathrm{~S}$ & \#\#\#\#\#\#\#\# & 93317 & 317 & & & & & \\
\hline $0 A$ & S & \#\#\#\#\#\#\#\# & 93318 & 318 & & & & & \\
\hline $0 A$ & S & \#\#\#\#\#\#\#\# & 93324 & 324 & & & & & \\
\hline $0 A$ & S & \#\#\#\#\#\#\#\# & 93325 & 325 & & & & & \\
\hline $0 A$ & S & $12 / 4 / 1993$ & 93338 & 338 & & & & & \\
\hline $0 A$ & $S$ & 12/5/1993 & 93339 & 339 & & & & & \\
\hline $0 A$ & S & \#\#\#\#\#\#\#\# & 93345 & 345 & & & & & \\
\hline $0 A$ & $\mathrm{~S}$ & \#\#\#\#\#\#\#\# & 93346 & 346 & & & & & \\
\hline $0 A$ & S & $1 / 8 / 1994$ & 94008 & 8 & 0.3 & 0.3 & 0.4 & 0.4 & 0.4 \\
\hline $0 A$ & S & $1 / 9 / 1994$ & 94009 & 9 & 0.3 & 0.4 & 0.3 & 0.4 & 0.3 \\
\hline $0 A$ & S & $1 / 15 / 1994$ & 94015 & 15 & 0.1 & 0.1 & 0.2 & 0.2 & 0.2 \\
\hline $0 A$ & S & $1 / 16 / 1994$ & 94016 & 16 & 0.2 & 0.2 & 0.2 & 0.2 & 0.2 \\
\hline $0 A$ & S & $1 / 22 / 1994$ & 94022 & 22 & 0.4 & 0.4 & 0.4 & 0.4 & 0.4 \\
\hline $0 A$ & S & $1 / 23 / 1994$ & 94023 & 23 & 0.3 & 0.4 & 0.4 & 0.4 & 0.3 \\
\hline $0 A$ & $S$ & 1/29/1994 & 94029 & 29 & 0.2 & 0.3 & 0.2 & 0.3 & 0.3 \\
\hline $0 A$ & S & $1 / 30 / 1994$ & 94030 & 30 & 0.3 & 0.3 & 0.4 & 0.4 & 0.4 \\
\hline $0 A$ & S & 2/5/1994 & 94036 & 36 & 0.2 & 0.2 & 0.2 & 0.2 & 0.3 \\
\hline $0 A$ & S & $2 / 6 / 1994$ & 94037 & 37 & 0.3 & 0.3 & 0.3 & 0.3 & 0.3 \\
\hline $0 A$ & S & $2 / 12 / 1994$ & 94043 & 43 & 0.3 & 0.2 & 0.3 & 0.3 & 0.3 \\
\hline $0 A$ & S & 2/13/1994 & 94044 & 44 & 0.4 & 0.4 & 0.4 & 0.4 & 0.4 \\
\hline $0 A$ & S & 2/19/1994 & 94050 & 50 & 0.1 & 0.1 & 0.1 & 0.1 & 0.1 \\
\hline $0 A$ & S & 2/20/1994 & 94051 & 51 & & 0.1 & & 0.1 & \\
\hline $0 A$ & S & 2/26/1994 & 94057 & 57 & 0.5 & 0.5 & 0.5 & 0.5 & 0.5 \\
\hline $0 A$ & $S$ & 2/27/1994 & 94058 & 58 & 0.5 & 0.5 & 0.5 & 0.5 & 0.5 \\
\hline $0 A$ & S & 3/5/1994 & 94064 & 64 & 0.1 & & 0.1 & & \\
\hline $0 A$ & S & 3/6/1994 & 94065 & 65 & & & 0.1 & & 0.1 \\
\hline $0 A$ & S & $3 / 26 / 1994$ & 94085 & 85 & & & & & \\
\hline $0 A$ & $\mathrm{~S}$ & $3 / 27 / 1994$ & 94086 & 86 & & & & & \\
\hline $0 A$ & S & $4 / 2 / 1994$ & 94092 & 92 & & & & & \\
\hline $0 A$ & S & $4 / 3 / 1994$ & 94093 & 93 & & & & & \\
\hline $0 A$ & S & 4/9/1994 & 94099 & 99 & & & & & \\
\hline $0 A$ & S & 4/10/1994 & 94100 & 100 & & & & & \\
\hline $1 A$ & NS & \#\#\#\#\#\#\#\# & 91332 & 332 & 0.1 & 0.1 & 0.1 & 0.1 & 0.1 \\
\hline $1 A$ & NS & \#\#\#\#\#\#\#\# & 91333 & 333 & 0.1 & 0.1 & 0.1 & 0.1 & 0.1 \\
\hline $1 A$ & NS & \#\#\#\#\#\#\#\# & 91357 & 357 & 0.4 & 0.4 & 0.4 & 0.4 & 0.5 \\
\hline $1 A$ & NS & \#\#\#\#\#\#\#\# & 91358 & 358 & 0.5 & 0.5 & 0.5 & 0.6 & 0.6 \\
\hline
\end{tabular}


wbheat

\begin{tabular}{|c|c|c|c|c|c|c|c|c|c|}
\hline $1 \mathrm{~A}$ & NS & \#\#\#\#\#\#\#\# & 91359 & 359 & 0.6 & 0.6 & 0.6 & 0.7 & 0.7 \\
\hline $1 \mathrm{~A}$ & NS & \#\#\#\#\#\#\#\# & 91360 & 360 & 0.5 & 0.5 & 0.5 & 0.5 & 0.5 \\
\hline $1 \mathrm{~A}$ & NS & \#\#\#\#\#\#\# & 91361 & 361 & 0.5 & 0.5 & 0.5 & 0.5 & 0.5 \\
\hline $1 \mathrm{~A}$ & NS & \#\#\#\#\#\#\#\# & 91364 & 364 & 0.1 & 0.1 & 0.1 & 0.1 & 0.1 \\
\hline $1 \mathrm{~A}$ & NS & \#\#\#\#\#\#\#\# & 91365 & 365 & 0.1 & 0.1 & 0.1 & 0.1 & 0.1 \\
\hline $1 \mathrm{~A}$ & NS & $1 / 1 / 1992$ & 92001 & 1 & 0.1 & 0.1 & 0.1 & 0.1 & 0.1 \\
\hline $1 \mathrm{~A}$ & NS & $1 / 2 / 1992$ & 92002 & 2 & 0.1 & 0.1 & 0.1 & 0.1 & 0.1 \\
\hline $1 \mathrm{~A}$ & NS & $1 / 3 / 1992$ & 92003 & 3 & 0.1 & 0.1 & 0.1 & 0.1 & 0.1 \\
\hline $1 \mathrm{~A}$ & NS & 1/20/1992 & 92020 & 20 & 0.6 & 0.6 & 0.6 & 0.6 & 0.7 \\
\hline $1 \mathrm{~A}$ & NS & 1/31/1992 & 92031 & 31 & 0.1 & 0.2 & 0.2 & 0.2 & 0.3 \\
\hline $1 \mathrm{~A}$ & NS & 3/16/1992 & 92076 & 76 & 0.1 & 0.1 & 0.1 & 0.1 & 0.2 \\
\hline $1 \mathrm{~A}$ & NS & $3 / 17 / 1992$ & 92077 & 77 & 0.2 & 0.1 & 0.2 & 0.2 & 0.2 \\
\hline $1 \mathrm{~A}$ & NS & $3 / 18 / 1992$ & 92078 & 78 & 0.2 & 0.2 & 0.2 & 0.2 & 0.2 \\
\hline $1 \mathrm{~A}$ & NS & 3/19/1992 & 92079 & 79 & 0.2 & 0.2 & 0.2 & 0.2 & 0.2 \\
\hline $1 \mathrm{~A}$ & NS & 3/20/1992 & 92080 & 80 & 0.3 & 0.3 & 0.3 & 0.3 & 0.3 \\
\hline $1 \mathrm{~A}$ & NS & 6/15/1992 & 92167 & 167 & & & & & \\
\hline $1 \mathrm{~A}$ & NS & 6/16/1992 & 92168 & 168 & & & & & \\
\hline $1 \mathrm{~A}$ & NS & 6/17/1992 & 92169 & 169 & & & & & \\
\hline $1 \mathrm{~A}$ & NS & 6/18/1992 & 92170 & 170 & & & & & \\
\hline $1 \mathrm{~A}$ & NS & 6/19/1992 & 92171 & 171 & & & & & \\
\hline $1 \mathrm{~A}$ & NS & $6 / 22 / 1992$ & 92174 & 174 & & & & & \\
\hline $1 \mathrm{~A}$ & NS & 6/23/1992 & 92175 & 175 & & & & & \\
\hline $1 \mathrm{~A}$ & NS & 6/24/1992 & 92176 & 176 & & & & & \\
\hline $1 \mathrm{~A}$ & NS & $6 / 25 / 1992$ & 92177 & 177 & & & & & \\
\hline $1 \mathrm{~A}$ & NS & $6 / 26 / 1992$ & 92178 & 178 & & & & & \\
\hline $1 \mathrm{~A}$ & NS & $6 / 29 / 1992$ & 92181 & 181 & 0.1 & 0.1 & & 0.1 & 0.1 \\
\hline $1 \mathrm{~A}$ & NS & 6/30/1992 & 92182 & 182 & & & & & \\
\hline $1 \mathrm{~A}$ & NS & 7/1/1992 & 92183 & 183 & & & & & \\
\hline $1 \mathrm{~A}$ & NS & $7 / 2 / 1992$ & 92184 & 184 & & & & & \\
\hline $1 \mathrm{~A}$ & NS & 7/3/1992 & 92185 & 185 & & & & & \\
\hline $1 \mathrm{~A}$ & NS & $7 / 6 / 1992$ & 92188 & 188 & & & & & \\
\hline $1 \mathrm{~A}$ & NS & $7 / 7 / 1992$ & 92189 & 189 & & & & & \\
\hline $1 \mathrm{~A}$ & NS & $7 / 8 / 1992$ & 92190 & 190 & & & & & \\
\hline $1 \mathrm{~A}$ & NS & $7 / 9 / 1992$ & 92191 & 191 & & & & & \\
\hline $1 \mathrm{~A}$ & NS & $7 / 10 / 1992$ & 92192 & 192 & & & & & \\
\hline $1 \mathrm{~A}$ & NS & $7 / 13 / 1992$ & 92195 & 195 & & & & & \\
\hline $1 \mathrm{~A}$ & NS & 7/14/1992 & 92196 & 196 & & & & & \\
\hline $1 \mathrm{~A}$ & NS & 7/15/1992 & 92197 & 197 & & & & & \\
\hline $1 \mathrm{~A}$ & NS & 7/16/1992 & 92198 & 198 & & & & & \\
\hline $1 A$ & NS & 7/17/1992 & 92199 & 199 & & & & & \\
\hline $1 A$ & NS & $7 / 20 / 1992$ & 92202 & 202 & & & & & \\
\hline $1 A$ & NS & $7 / 21 / 1992$ & 92203 & 203 & & & & & \\
\hline $1 A$ & NS & $7 / 22 / 1992$ & 92204 & 204 & & & & & \\
\hline $1 A$ & NS & $7 / 23 / 1992$ & 92205 & 205 & & & & & \\
\hline $1 A$ & NS & $7 / 24 / 1992$ & 92206 & 206 & & & & & \\
\hline $1 A$ & NS & $7 / 27 / 1992$ & 92209 & 209 & & & & & \\
\hline $1 A$ & NS & 7/28/1992 & 92210 & 210 & & & & & \\
\hline $1 A$ & NS & 7/29/1992 & 92211 & 211 & & & & & \\
\hline $1 A$ & NS & 7/30/1992 & 92212 & 212 & & & & & \\
\hline $1 A$ & NS & 7/31/1992 & 92213 & 213 & & & & & \\
\hline $1 A$ & NS & $8 / 3 / 1992$ & 92216 & 216 & & & & & \\
\hline
\end{tabular}


wbheat

\begin{tabular}{|c|c|c|c|c|c|c|c|c|c|}
\hline $1 \mathrm{~A}$ & NS & $8 / 4 / 1992$ & 92217 & 217 & & & & & \\
\hline $1 \mathrm{~A}$ & NS & 8/5/1992 & 92218 & 218 & & & & & \\
\hline $1 \mathrm{~A}$ & NS & 8/6/1992 & 92219 & 219 & & & & & \\
\hline $1 \mathrm{~A}$ & NS & 8/7/1992 & 92220 & 220 & & & & & \\
\hline $1 \mathrm{~A}$ & NS & 8/10/1992 & 92223 & 223 & & & & & \\
\hline $1 \mathrm{~A}$ & NS & $8 / 11 / 1992$ & 92224 & 224 & & & & & \\
\hline $1 \mathrm{~A}$ & NS & 8/12/1992 & 92225 & 225 & & & & & \\
\hline $1 \mathrm{~A}$ & NS & 8/13/1992 & 92226 & 226 & & & & & \\
\hline $1 \mathrm{~A}$ & NS & 9/7/1992 & 92251 & 251 & & & & & \\
\hline $1 \mathrm{~A}$ & NS & \#\#\#\#\#\#\#\# & 92331 & 331 & 0.5 & 0.5 & 0.5 & 0.6 & 0.5 \\
\hline $1 \mathrm{~A}$ & NS & \#\#\#\#\#\#\#\# & 92332 & 332 & 0.6 & 0.6 & 0.7 & 0.6 & 0.6 \\
\hline $1 \mathrm{~A}$ & NS & \#\#\#\#\#\#\#\# & 92356 & 356 & 0.5 & 0.5 & 0.5 & 0.5 & 0.5 \\
\hline $1 \mathrm{~A}$ & NS & \#\#\#\#\#\#\#\# & 92357 & 357 & 0.2 & 0.2 & 0.2 & 0.2 & 0.2 \\
\hline $1 \mathrm{~A}$ & NS & \#\#\#\#\#\#\#\# & 92358 & 358 & 0.2 & 0.2 & 0.2 & 0.2 & 0.2 \\
\hline $1 \mathrm{~A}$ & NS & \#\#\#\#\#\#\#\# & 92359 & 359 & 0.3 & 0.3 & 0.3 & 0.3 & 0.4 \\
\hline $1 A$ & NS & \#\#\#\#\#\#\#\# & 92360 & 360 & 0.3 & 0.3 & 0.3 & 0.3 & 0.3 \\
\hline $1 \mathrm{~A}$ & NS & \#\#\#\#\#\#\#\# & 92363 & 363 & 0.3 & 0.3 & 0.3 & 0.3 & 0.3 \\
\hline $1 \mathrm{~A}$ & NS & \#\#\#\#\#\#\#\# & 92364 & 364 & 0.2 & 0.2 & 0.2 & 0.2 & 0.2 \\
\hline $1 \mathrm{~A}$ & NS & \#\#\#\#\#\#\#\# & 92365 & 365 & 0.1 & 0.1 & 0.1 & 0.1 & 0.1 \\
\hline $1 \mathrm{~A}$ & NS & \#\#\#\#\#\#\#\# & 92366 & 366 & & & & & \\
\hline $1 \mathrm{~A}$ & NS & 1/1/1993 & 93001 & 1 & 0.6 & 0.6 & 0.6 & 0.6 & 0.6 \\
\hline $1 \mathrm{~A}$ & NS & 1/18/1993 & 93018 & 18 & 0.4 & 0.3 & 0.4 & 0.4 & 0.4 \\
\hline $1 \mathrm{~A}$ & NS & 2/5/1993 & 93036 & 36 & 0.3 & 0.3 & 0.3 & 0.3 & 0.3 \\
\hline $1 \mathrm{~A}$ & NS & $3 / 15 / 1993$ & 93074 & 74 & 0.4 & 0.5 & 0.4 & 0.5 & 0.4 \\
\hline $1 A$ & NS & $3 / 16 / 1993$ & 93075 & 75 & 0.3 & 0.3 & 0.3 & 0.3 & 0.3 \\
\hline $1 \mathrm{~A}$ & NS & 3/17/1993 & 93076 & 76 & 0.2 & 0.2 & 0.2 & 0.2 & 0.2 \\
\hline $1 \mathrm{~A}$ & NS & 3/18/1993 & 93077 & 77 & 0.3 & 0.3 & 0.3 & 0.3 & 0.3 \\
\hline $1 \mathrm{~A}$ & NS & 3/19/1993 & 93078 & 78 & 0.3 & 0.3 & 0.3 & 0.3 & 0.3 \\
\hline $1 \mathrm{~A}$ & NS & $6 / 14 / 1993$ & 93165 & 165 & & & & & \\
\hline $1 \mathrm{~A}$ & NS & 6/15/1993 & 93166 & 166 & & & & & \\
\hline $1 \mathrm{~A}$ & NS & 6/16/1993 & 93167 & 167 & & & & & \\
\hline $1 \mathrm{~A}$ & NS & 6/17/1993 & 93168 & 168 & & & & & \\
\hline $1 \mathrm{~A}$ & NS & 6/18/1993 & 93169 & 169 & & & & & \\
\hline $1 \mathrm{~A}$ & NS & $6 / 21 / 1993$ & 93172 & 172 & & & & & \\
\hline $1 \mathrm{~A}$ & NS & $6 / 22 / 1993$ & 93173 & 173 & & & & & \\
\hline $1 \mathrm{~A}$ & NS & 6/23/1993 & 93174 & 174 & & & & & \\
\hline $1 \mathrm{~A}$ & NS & $6 / 24 / 1993$ & 93175 & 175 & & & & & \\
\hline $1 \mathrm{~A}$ & NS & $6 / 25 / 1993$ & 93176 & 176 & & & & & \\
\hline $1 \mathrm{~A}$ & NS & $6 / 28 / 1993$ & 93179 & 179 & & & & & \\
\hline $1 A$ & NS & 6/29/1993 & 93180 & 180 & & & & & \\
\hline $1 \mathrm{~A}$ & NS & 6/30/1993 & 93181 & 181 & & & & & \\
\hline $1 A$ & NS & 7/1/1993 & 93182 & 182 & & & & & \\
\hline $1 \mathrm{~A}$ & NS & $7 / 2 / 1993$ & 93183 & 183 & & & & & \\
\hline $1 A$ & NS & $7 / 5 / 1993$ & 93186 & 186 & & & & & \\
\hline $1 \mathrm{~A}$ & NS & $7 / 6 / 1993$ & 93187 & 187 & & & & & \\
\hline $1 A$ & NS & 7/7/1993 & 93188 & 188 & & & & & \\
\hline $1 \mathrm{~A}$ & NS & $7 / 8 / 1993$ & 93189 & 189 & & & & & \\
\hline $1 A$ & NS & 7/9/1993 & 93190 & 190 & & & & & \\
\hline $1 A$ & NS & $7 / 12 / 1993$ & 93193 & 193 & & & & & \\
\hline $1 A$ & NS & 7/13/1993 & 93194 & 194 & & & & & \\
\hline $1 A$ & NS & $7 / 14 / 1993$ & 93195 & 195 & & & & & \\
\hline
\end{tabular}


wbheat

\begin{tabular}{|c|c|c|c|c|c|c|c|c|c|}
\hline $1 \mathrm{~A}$ & NS & $7 / 15 / 1993$ & 93196 & 196 & & & & & \\
\hline $1 \mathrm{~A}$ & NS & $7 / 16 / 1993$ & 93197 & 197 & & & & & \\
\hline $1 \mathrm{~A}$ & NS & $7 / 19 / 1993$ & 93200 & 200 & & & & & \\
\hline $1 \mathrm{~A}$ & NS & 7/20/1993 & 93201 & 201 & & & & & \\
\hline $1 \mathrm{~A}$ & NS & 7/21/1993 & 93202 & 202 & & & & & \\
\hline $1 \mathrm{~A}$ & NS & $7 / 22 / 1993$ & 93203 & 203 & & & & & \\
\hline $1 \mathrm{~A}$ & NS & $7 / 23 / 1993$ & 93204 & 204 & & & & & \\
\hline $1 \mathrm{~A}$ & NS & 7/26/1993 & 93207 & 207 & & & & & \\
\hline $1 \mathrm{~A}$ & NS & 7/27/1993 & 93208 & 208 & & & & & \\
\hline $1 \mathrm{~A}$ & NS & 7/28/1993 & 93209 & 209 & & & & & \\
\hline $1 \mathrm{~A}$ & NS & $7 / 29 / 1993$ & 93210 & 210 & & & & & \\
\hline $1 \mathrm{~A}$ & NS & 7/30/1993 & 93211 & 211 & & & & & \\
\hline $1 \mathrm{~A}$ & NS & $8 / 2 / 1993$ & 93214 & 214 & & & & & \\
\hline $1 \mathrm{~A}$ & NS & 8/3/1993 & 93215 & 215 & & & & & \\
\hline $1 \mathrm{~A}$ & NS & 8/4/1993 & 93216 & 216 & & & & & \\
\hline $1 \mathrm{~A}$ & NS & 8/5/1993 & 93217 & 217 & & & & & \\
\hline $1 \mathrm{~A}$ & NS & $8 / 6 / 1993$ & 93218 & 218 & & & & & \\
\hline $1 \mathrm{~A}$ & NS & 8/9/1993 & 93221 & 221 & & & & & \\
\hline $1 \mathrm{~A}$ & NS & 8/10/1993 & 93222 & 222 & & & & & \\
\hline $1 \mathrm{~A}$ & NS & 8/11/1993 & 93223 & 223 & & & & & \\
\hline $1 \mathrm{~A}$ & NS & 8/12/1993 & 93224 & 224 & & & & & \\
\hline $1 \mathrm{~A}$ & NS & 8/13/1993 & 93225 & 225 & & & & & \\
\hline $1 \mathrm{~A}$ & NS & 9/6/1993 & 93249 & 249 & & & & & \\
\hline $1 \mathrm{~A}$ & NS & \#\#\#\#\#\#\#\# & 93329 & 329 & & & & & \\
\hline $1 \mathrm{~A}$ & NS & \#\#\#\#\#\#\#\# & 93330 & 330 & 0.1 & 0.1 & 0.1 & 0.1 & 0.1 \\
\hline $1 \mathrm{~A}$ & NS & \#\#\#\#\#\#\#\# & 93354 & 354 & & & & & \\
\hline $1 \mathrm{~A}$ & NS & \#\#\#\#\#\#\#\# & 93355 & 355 & & & & & 0.3 \\
\hline $1 \mathrm{~A}$ & NS & \#\#\#\#\#\#\#\# & 93356 & 356 & & & & & 0.1 \\
\hline $1 \mathrm{~A}$ & NS & \#\#\#\#\#\#\#\# & 93357 & 357 & & 0.1 & & 0.1 & \\
\hline $1 \mathrm{~A}$ & NS & \#\#\#\#\#\#\#\# & 93358 & 358 & 0.1 & & 0.1 & & 0.1 \\
\hline $1 \mathrm{~A}$ & NS & \#\#\#\#\#\#\#\# & 93361 & 361 & 0.1 & & 0.1 & & 0.1 \\
\hline $1 \mathrm{~A}$ & NS & \#\#\#\#\#\#\#\# & 93362 & 362 & & & & & \\
\hline $1 \mathrm{~A}$ & NS & \#\#\#\#\#\#\#\# & 93363 & 363 & 0.6 & 0.6 & 0.5 & 0.5 & 0.5 \\
\hline $1 \mathrm{~A}$ & NS & \#\#\#\#\#\#\#\# & 93364 & 364 & 0.1 & 0.1 & 0.1 & 0.1 & 0.1 \\
\hline $1 \mathrm{~A}$ & NS & \#\#\#\#\#\#\#\# & 93365 & 365 & 0.1 & 0.1 & 0.1 & 0.1 & 0.1 \\
\hline $1 \mathrm{~A}$ & NS & $1 / 17 / 1994$ & 94017 & 17 & 0.2 & 0.2 & 0.2 & 0.2 & 0.2 \\
\hline $1 \mathrm{~A}$ & NS & 2/4/1994 & 94035 & 35 & 0.3 & 0.2 & 0.3 & 0.3 & 0.3 \\
\hline $1 \mathrm{~A}$ & NS & $3 / 14 / 1994$ & 94073 & 73 & & 0.1 & 0.1 & 0.1 & 0.1 \\
\hline $1 \mathrm{~A}$ & NS & 3/15/1994 & 94074 & 74 & & 0.1 & & 0.1 & \\
\hline $1 \mathrm{~A}$ & NS & 3/16/1994 & 94075 & 75 & & & & & \\
\hline $1 \mathrm{~A}$ & NS & 3/17/1994 & 94076 & 76 & & & & & \\
\hline $1 \mathrm{~A}$ & NS & $3 / 18 / 1994$ & 94077 & 77 & 0.2 & 0.2 & 0.2 & 0.3 & 0.2 \\
\hline $0 \mathrm{~A}$ & NS & \#\#\#\#\#\#\#\# & 91334 & 334 & 0.1 & 0.1 & 0.2 & 0.1 & 0.2 \\
\hline $0 \mathrm{~A}$ & NS & $12 / 1 / 1991$ & 91335 & 335 & 0.3 & 0.3 & 0.3 & 0.3 & 0.3 \\
\hline $0 \mathrm{~A}$ & NS & \#\#\#\#\#\#\#\# & 91355 & 355 & 0.4 & 0.4 & 0.4 & 0.4 & 0.5 \\
\hline $0 \mathrm{~A}$ & NS & \#\#\#\#\#\#\#\# & 91356 & 356 & 0.5 & 0.5 & 0.5 & 0.5 & 0.5 \\
\hline $0 \mathrm{~A}$ & NS & \#\#\#\#\#\#\#\# & 91362 & 362 & 0.1 & 0.1 & 0.1 & 0.1 & 0.1 \\
\hline $0 \mathrm{~A}$ & NS & \#\#\#\#\#\#\#\# & 91363 & 363 & 0.1 & 0.1 & 0.1 & 0.1 & 0.1 \\
\hline $0 \mathrm{~A}$ & NS & $1 / 4 / 1992$ & 92004 & 4 & 0.1 & 0.1 & 0.1 & 0.1 & 0.1 \\
\hline $0 \mathrm{~A}$ & NS & 1/5/1992 & 92005 & 5 & 0.1 & 0.1 & 0.1 & 0.1 & 0.1 \\
\hline $0 \mathrm{~A}$ & NS & $3 / 14 / 1992$ & 92074 & 74 & 0.2 & 0.2 & 0.2 & 0.2 & 0.3 \\
\hline
\end{tabular}


wbheat

\begin{tabular}{|c|c|c|c|c|c|c|c|c|c|}
\hline $0 A$ & NS & $3 / 15 / 1992$ & 92075 & 75 & 0.1 & 0.2 & 0.1 & 0.2 & 0.2 \\
\hline $0 A$ & NS & $3 / 21 / 1992$ & 92081 & 81 & 0.2 & 0.2 & 0.2 & 0.3 & 0.3 \\
\hline $0 A$ & NS & 3/22/1992 & 92082 & 82 & 0.2 & 0.2 & 0.2 & 0.2 & 0.2 \\
\hline $0 A$ & NS & 6/14/1992 & 92166 & 166 & & & & & \\
\hline $0 A$ & NS & $6 / 20 / 1992$ & 92172 & 172 & & & & & \\
\hline $0 A$ & NS & $6 / 21 / 1992$ & 92173 & 173 & & & & & \\
\hline $0 A$ & NS & 6/27/1992 & 92179 & 179 & & 0.1 & & & \\
\hline $0 A$ & NS & $6 / 28 / 1992$ & 92180 & 180 & & & & 0.1 & \\
\hline $0 A$ & NS & 7/4/1992 & 92186 & 186 & & & & & \\
\hline $0 A$ & NS & $7 / 5 / 1992$ & 92187 & 187 & & & & & \\
\hline $0 A$ & NS & 7/11/1992 & 92193 & 193 & & & & & \\
\hline $0 A$ & NS & 7/12/1992 & 92194 & 194 & & & & & \\
\hline $0 A$ & NS & 7/18/1992 & 92200 & 200 & & & & & \\
\hline $0 A$ & NS & 7/19/1992 & 92201 & 201 & & & & & \\
\hline $0 A$ & NS & $7 / 25 / 1992$ & 92207 & 207 & & & & & \\
\hline $0 A$ & NS & $7 / 26 / 1992$ & 92208 & 208 & & & & & \\
\hline $0 A$ & NS & 8/1/1992 & 92214 & 214 & & & & & \\
\hline $0 A$ & NS & $8 / 2 / 1992$ & 92215 & 215 & & & & & \\
\hline $0 A$ & NS & 8/8/1992 & 92221 & 221 & & & & & \\
\hline $0 A$ & NS & 8/9/1992 & 92222 & 222 & & & & & \\
\hline $0 A$ & NS & \#\#\#\#\#\#\#\# & 92333 & 333 & 0.6 & 0.6 & 0.6 & 0.6 & 0.6 \\
\hline $0 A$ & NS & \#\#\#\#\#\#\#\# & 92334 & 334 & 0.5 & 0.5 & 0.5 & 0.5 & 0.5 \\
\hline $0 A$ & NS & \#\#\#\#\#\#\#\# & 92354 & 354 & 0.2 & 0.2 & 0.3 & 0.2 & 0.3 \\
\hline $0 A$ & NS & \#\#\#\#\#\#\#\# & 92355 & 355 & 0.3 & 0.4 & 0.4 & 0.4 & 0.4 \\
\hline $0 A$ & NS & \#\#\#\#\#\#\#\# & 92361 & 361 & 0.4 & 0.4 & 0.4 & 0.4 & 0.5 \\
\hline $0 A$ & NS & \#\#\#\#\#\#\#\# & 92362 & 362 & 0.4 & 0.4 & 0.4 & 0.4 & 0.4 \\
\hline $0 A$ & NS & $1 / 2 / 1993$ & 93002 & 2 & 0.5 & 0.6 & 0.6 & 0.6 & 0.5 \\
\hline $0 A$ & NS & $1 / 3 / 1993$ & 93003 & 3 & 0.4 & 0.4 & 0.4 & 0.4 & 0.4 \\
\hline $0 A$ & NS & $3 / 13 / 1993$ & 93072 & 72 & 0.5 & 0.5 & 0.6 & 0.6 & 0.6 \\
\hline $0 A$ & NS & 3/14/1993 & 93073 & 73 & 0.6 & 0.6 & 0.6 & 0.6 & 0.6 \\
\hline $0 A$ & NS & 3/20/1993 & 93079 & 79 & 0.3 & 0.3 & 0.3 & 0.3 & 0.3 \\
\hline $0 A$ & NS & $3 / 21 / 1993$ & 93080 & 80 & 0.3 & 0.3 & 0.3 & 0.3 & 0.3 \\
\hline $0 A$ & NS & $6 / 13 / 1993$ & 93164 & 164 & & & & & \\
\hline $0 A$ & NS & 6/19/1993 & 93170 & 170 & & & & & \\
\hline $0 A$ & NS & $6 / 20 / 1993$ & 93171 & 171 & & & & & \\
\hline $0 A$ & NS & 6/26/1993 & 93177 & 177 & & & & & \\
\hline $0 A$ & NS & 6/27/1993 & 93178 & 178 & & & & & \\
\hline $0 A$ & NS & 7/3/1993 & 93184 & 184 & & & & & \\
\hline $0 A$ & NS & 7/4/1993 & 93185 & 185 & & & & & \\
\hline $0 A$ & NS & 7/10/1993 & 93191 & 191 & & & & & \\
\hline $0 A$ & NS & 7/11/1993 & 93192 & 192 & & & & & \\
\hline $0 A$ & NS & $7 / 17 / 1993$ & 93198 & 198 & & & & & \\
\hline $0 A$ & NS & 7/18/1993 & 93199 & 199 & & & & & \\
\hline $0 A$ & NS & 7/24/1993 & 93205 & 205 & & & & & \\
\hline $0 A$ & NS & 7/25/1993 & 93206 & 206 & & & & & \\
\hline $0 A$ & NS & 7/31/1993 & 93212 & 212 & & & & & \\
\hline $0 A$ & NS & 8/1/1993 & 93213 & 213 & & & & & \\
\hline $0 A$ & NS & 8/7/1993 & 93219 & 219 & & & & & \\
\hline $0 A$ & NS & 8/8/1993 & 93220 & 220 & & & & & \\
\hline $0 A$ & NS & 8/14/1993 & 93226 & 226 & & & & & \\
\hline $0 A$ & NS & $8 / 15 / 1993$ & 93227 & 227 & & & & & \\
\hline
\end{tabular}


wbheat

\begin{tabular}{|c|c|c|c|c|c|c|c|c|c|}
\hline $0 \mathrm{~A}$ & NS & 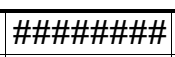 & 93331 & 331 & 0.1 & 0.1 & 0.1 & 0.1 & 0.1 \\
\hline $0 A$ & NS & \#\#\#\#\#\#\#\# & 93332 & 332 & & & & & \\
\hline $0 A$ & NS & \#\#\#\#\#\#\#\# & 93352 & 352 & & & & & \\
\hline $0 A$ & NS & \#\#\#\#\#\#\#\# & 93353 & 353 & & & & & \\
\hline $0 A$ & NS & \#\#\#\#\#\#\#\# & 93359 & 359 & 0.1 & & 0.1 & & 0.1 \\
\hline $0 A$ & NS & \#\#\#\#\#\#\#\# & 93360 & 360 & & 0.1 & & 0.1 & 0.1 \\
\hline $0 A$ & NS & 1/1/1994 & 94001 & 1 & 0.2 & 0.2 & 0.2 & 0.2 & 0.2 \\
\hline $0 A$ & NS & $1 / 2 / 1994$ & 94002 & 2 & 0.1 & 0.1 & 0.1 & 0.1 & 0.2 \\
\hline $0 A$ & NS & 3/12/1994 & 94071 & 71 & & & & & \\
\hline $0 A$ & NS & 3/13/1994 & 94072 & 72 & 0.1 & & 0.1 & & 0.1 \\
\hline $0 A$ & NS & 3/19/1994 & 94078 & 78 & & & & & \\
\hline $0 A$ & NS & 3/20/1994 & 94079 & 79 & & & & & \\
\hline $1 \mathrm{~B}$ & $S$ & 4/14/1994 & 94104 & 104 & & & & & \\
\hline $1 \mathrm{~B}$ & $S$ & $4 / 15 / 1994$ & 94105 & 105 & & & & & \\
\hline $1 \mathrm{~B}$ & $S$ & 4/18/1994 & 94108 & 108 & & & & & \\
\hline $1 \mathrm{~B}$ & $S$ & $4 / 19 / 1994$ & 94109 & 109 & & & & & \\
\hline $1 \mathrm{~B}$ & $S$ & $4 / 20 / 1994$ & 94110 & 110 & & & & & \\
\hline $1 \mathrm{~B}$ & $S$ & $4 / 21 / 1994$ & 94111 & 111 & & & & & \\
\hline $1 \mathrm{~B}$ & $S$ & $4 / 22 / 1994$ & 94112 & 112 & & & & & \\
\hline $1 \mathrm{~B}$ & $\mathrm{~S}$ & $4 / 25 / 1994$ & 94115 & 115 & & & & & \\
\hline $1 \mathrm{~B}$ & $S$ & $4 / 26 / 1994$ & 94116 & 116 & & & & & \\
\hline $1 \mathrm{~B}$ & $S$ & 4/27/1994 & 94117 & 117 & & & & & \\
\hline $1 \mathrm{~B}$ & $S$ & 4/28/1994 & 94118 & 118 & & & & & \\
\hline $1 \mathrm{~B}$ & $S$ & 4/29/1994 & 94119 & 119 & & & & & \\
\hline $1 \mathrm{~B}$ & $S$ & 5/2/1994 & 94122 & 122 & & & & & \\
\hline $1 \mathrm{~B}$ & $S$ & $5 / 3 / 1994$ & 94123 & 123 & & & & & \\
\hline $1 \mathrm{~B}$ & $S$ & 5/4/1994 & 94124 & 124 & & & & & \\
\hline $1 \mathrm{~B}$ & $S$ & $5 / 5 / 1994$ & 94125 & 125 & & & & & \\
\hline $1 \mathrm{~B}$ & $S$ & $5 / 6 / 1994$ & 94126 & 126 & & & & & \\
\hline $1 \mathrm{~B}$ & $S$ & $5 / 9 / 1994$ & 94129 & 129 & & & & & \\
\hline $1 \mathrm{~B}$ & $S$ & $5 / 10 / 1994$ & 94130 & 130 & & & & & \\
\hline $1 \mathrm{~B}$ & $S$ & $5 / 11 / 1994$ & 94131 & 131 & & & & & \\
\hline $1 \mathrm{~B}$ & $S$ & $5 / 12 / 1994$ & 94132 & 132 & & & & & \\
\hline $1 \mathrm{~B}$ & $S$ & $5 / 13 / 1994$ & 94133 & 133 & & & & & \\
\hline $1 \mathrm{~B}$ & $S$ & $5 / 16 / 1994$ & 94136 & 136 & & & & & \\
\hline $1 \mathrm{~B}$ & $S$ & $5 / 17 / 1994$ & 94137 & 137 & & & & & \\
\hline $1 \mathrm{~B}$ & $S$ & $5 / 18 / 1994$ & 94138 & 138 & & & & & \\
\hline $1 \mathrm{~B}$ & $S$ & 5/19/1994 & 94139 & 139 & & & & & \\
\hline $1 \mathrm{~B}$ & $S$ & $5 / 20 / 1994$ & 94140 & 140 & & & & & \\
\hline $1 \mathrm{~B}$ & $S$ & $5 / 23 / 1994$ & 94143 & 143 & & & & & \\
\hline $1 \mathrm{~B}$ & $S$ & $5 / 24 / 1994$ & 94144 & 144 & & & & & \\
\hline $1 \mathrm{~B}$ & $S$ & $5 / 25 / 1994$ & 94145 & 145 & & & & & \\
\hline $1 \mathrm{~B}$ & $S$ & $5 / 26 / 1994$ & 94146 & 146 & & & & & \\
\hline $1 \mathrm{~B}$ & $S$ & $5 / 27 / 1994$ & 94147 & 147 & & & & & \\
\hline $1 \mathrm{~B}$ & $S$ & 5/30/1994 & 94150 & 150 & & & & & \\
\hline $1 \mathrm{~B}$ & $\mathrm{~S}$ & $5 / 31 / 1994$ & 94151 & 151 & & & & & \\
\hline $1 \mathrm{~B}$ & $S$ & 6/1/1994 & 94152 & 152 & & & & & \\
\hline $1 \mathrm{~B}$ & $S$ & $6 / 2 / 1994$ & 94153 & 153 & & & & & \\
\hline $1 \mathrm{~B}$ & $S$ & $6 / 3 / 1994$ & 94154 & 154 & & & & & \\
\hline $1 \mathrm{~B}$ & $S$ & 6/6/1994 & 94157 & 157 & & & & & \\
\hline $1 \mathrm{~B}$ & $S$ & 6/7/1994 & 94158 & 158 & & & & & \\
\hline
\end{tabular}


wbheat

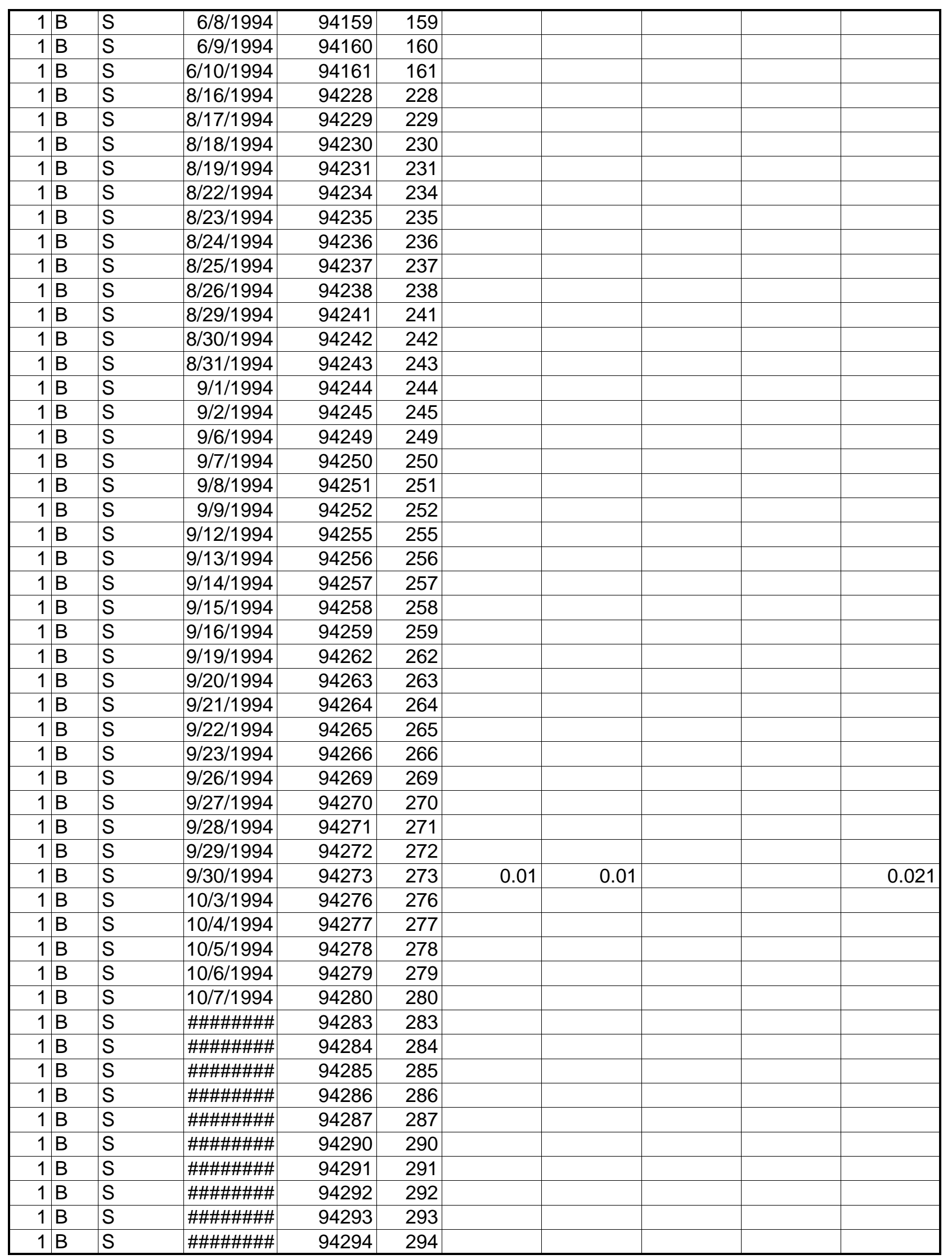


wbheat

\begin{tabular}{|c|c|c|c|c|c|c|c|c|c|}
\hline $1 \mathrm{~B}$ & $\mathrm{~S}$ & \#\#\#\#\#\#\#\# & 94297 & 297 & & & & & \\
\hline $1 \mathrm{~B}$ & $S$ & \#\#\#\#\#\#\#\# & 94298 & 298 & & & & & \\
\hline $1 \mathrm{~B}$ & $S$ & \#\#\#\#\#\#\#\# & 94299 & 299 & & & & & \\
\hline $1 \mathrm{~B}$ & S & \#\#\#\#\#\#\#\# & 94300 & 300 & & & & & \\
\hline $1 \mathrm{~B}$ & $\mathrm{~S}$ & \#\#\#\#\#\#\#\# & 94301 & 301 & & & & & \\
\hline $1 \mathrm{~B}$ & S & \#\#\#\#\#\#\#\# & 94304 & 304 & & & & & \\
\hline $1 \mathrm{~B}$ & $\mathrm{~S}$ & $11 / 1 / 1994$ & 94305 & 305 & & & & & \\
\hline $1 \mathrm{~B}$ & $\mathrm{~S}$ & $11 / 2 / 1994$ & 94306 & 306 & & & & & \\
\hline $1 \mathrm{~B}$ & $S$ & $11 / 3 / 1994$ & 94307 & 307 & & & & & \\
\hline $1 \mathrm{~B}$ & $S$ & $11 / 4 / 1994$ & 94308 & 308 & & & & & \\
\hline $1 \mathrm{~B}$ & $S$ & $11 / 7 / 1994$ & 94311 & 311 & & & & & \\
\hline $1 \mathrm{~B}$ & $S$ & $11 / 8 / 1994$ & 94312 & 312 & & & & & \\
\hline $1 \mathrm{~B}$ & $S$ & 11/9/1994 & 94313 & 313 & & & & & \\
\hline $1 \mathrm{~B}$ & $\mathrm{~S}$ & \#\#\#\#\#\#\#\# & 94314 & 314 & 0.1 & & & & \\
\hline $1 \mathrm{~B}$ & $S$ & \#\#\#\#\#\#\#\# & 94315 & 315 & & & & & \\
\hline $1 \mathrm{~B}$ & S & \#\#\#\#\#\#\#\# & 94318 & 318 & & & & & \\
\hline $1 \mathrm{~B}$ & S & \#\#\#\#\#\#\#\# & 94319 & 319 & & & & & \\
\hline $1 \mathrm{~B}$ & S & \#\#\#\#\#\#\#\# & 94320 & 320 & & & & & \\
\hline $1 \mathrm{~B}$ & $S$ & \#\#\#\#\#\#\#\# & 94321 & 321 & & & & & \\
\hline $1 \mathrm{~B}$ & $S$ & \#\#\#\#\#\#\#\# & 94322 & 322 & & & & & \\
\hline $1 \mathrm{~B}$ & $S$ & \#\#\#\#\#\#\#\# & 94325 & 325 & & & & & \\
\hline $1 \mathrm{~B}$ & $S$ & \#\#\#\#\#\#\#\# & 94326 & 326 & & & & & \\
\hline $1 \mathrm{~B}$ & S & \#\#\#\#\#\#\#\# & 94327 & 327 & & & & & \\
\hline $1 \mathrm{~B}$ & S & \#\#\#\#\#\#\#\# & 94332 & 332 & & & & & \\
\hline $1 \mathrm{~B}$ & $S$ & \#\#\#\#\#\#\#\# & 94333 & 333 & & & & & \\
\hline $1 \mathrm{~B}$ & $S$ & \#\#\#\#\#\#\#\# & 94334 & 334 & & & & & \\
\hline $1 \mathrm{~B}$ & $S$ & $12 / 1 / 1994$ & 94335 & 335 & & & & & \\
\hline $1 \mathrm{~B}$ & $S$ & $12 / 2 / 1994$ & 94336 & 336 & & & & & \\
\hline $1 \mathrm{~B}$ & S & $12 / 5 / 1994$ & 94339 & 339 & & & & & \\
\hline $1 \mathrm{~B}$ & $S$ & $12 / 6 / 1994$ & 94340 & 340 & & & & & \\
\hline $1 \mathrm{~B}$ & $\mathrm{~S}$ & $12 / 7 / 1994$ & 94341 & 341 & & & & & \\
\hline $1 \mathrm{~B}$ & $S$ & $12 / 8 / 1994$ & 94342 & 342 & & & & & \\
\hline $1 \mathrm{~B}$ & $S$ & $12 / 9 / 1994$ & 94343 & 343 & & & & & \\
\hline $1 \mathrm{~B}$ & $S$ & \#\#\#\#\#\#\#\# & 94346 & 346 & & & & & \\
\hline $1 \mathrm{~B}$ & $S$ & \#\#\#\#\#\#\#\# & 94347 & 347 & & & & & \\
\hline $1 \mathrm{~B}$ & $S$ & \#\#\#\#\#\#\#\# & 94348 & 348 & & & & & \\
\hline $1 \mathrm{~B}$ & $S$ & \#\#\#\#\#\#\#\# & 94349 & 349 & & & & & \\
\hline $1 \mathrm{~B}$ & $S$ & \#\#\#\#\#\#\#\# & 94350 & 350 & & & & & \\
\hline $1 \mathrm{~B}$ & $S$ & $1 / 2 / 1995$ & 94367 & 2 & & & 0.1 & 0.1 & 0.1 \\
\hline $1 \mathrm{~B}$ & $S$ & $1 / 3 / 1995$ & 94368 & 3 & & & & & \\
\hline $1 \mathrm{~B}$ & $S$ & 1/4/1995 & 94369 & 4 & & & & & \\
\hline $1 \mathrm{~B}$ & $S$ & $1 / 5 / 1995$ & 94370 & 5 & & & & & \\
\hline $1 \mathrm{~B}$ & $\mathrm{~S}$ & $1 / 6 / 1995$ & 94371 & 6 & & & & & \\
\hline $1 \mathrm{~B}$ & $S$ & $1 / 9 / 1995$ & 94374 & 9 & & & & & \\
\hline $1 \mathrm{~B}$ & $S$ & $1 / 10 / 1995$ & 94375 & 10 & & & & & \\
\hline $1 \mathrm{~B}$ & $S$ & $1 / 11 / 1995$ & 94376 & 11 & & & & & \\
\hline $1 \mathrm{~B}$ & $S$ & $1 / 12 / 1995$ & 94377 & 12 & & & & & \\
\hline $1 \mathrm{~B}$ & $S$ & $1 / 13 / 1995$ & 94378 & 13 & & & & & \\
\hline $1 \mathrm{~B}$ & $S$ & $1 / 16 / 1995$ & 94381 & 16 & & & & & \\
\hline $1 \mathrm{~B}$ & $S$ & $1 / 17 / 1995$ & 94382 & 17 & & & & & \\
\hline $1 \mathrm{~B}$ & $S$ & $1 / 18 / 1995$ & 94383 & 18 & & & & & \\
\hline
\end{tabular}


wbheat

\begin{tabular}{|c|c|c|c|c|c|c|c|c|c|}
\hline $1 B$ & $\mathrm{~S}$ & $1 / 19 / 1995$ & 94384 & 19 & & & & & \\
\hline $1 \mathrm{~B}$ & $\mathrm{~S}$ & $1 / 20 / 1995$ & 94385 & 20 & & & & & \\
\hline $1 \mathrm{~B}$ & S & $1 / 23 / 1995$ & 94388 & 23 & & & & & \\
\hline $1 \mathrm{~B}$ & S & 1/25/1995 & 94390 & 25 & & & & & \\
\hline $1 \mathrm{~B}$ & S & 1/26/1995 & 94391 & 26 & & & & & \\
\hline $1 \mathrm{~B}$ & S & $1 / 27 / 1995$ & 94392 & 27 & & & & & \\
\hline $1 \mathrm{~B}$ & $\mathrm{~S}$ & $1 / 30 / 1995$ & 94395 & 30 & & & & & \\
\hline $1 \mathrm{~B}$ & S & 1/31/1995 & 94396 & 31 & & & & & \\
\hline $1 \mathrm{~B}$ & S & 2/1/1995 & 94397 & 32 & & & & & \\
\hline $1 \mathrm{~B}$ & S & $2 / 2 / 1995$ & 94398 & 33 & & & & & \\
\hline $1 \mathrm{~B}$ & S & 2/6/1995 & 94402 & 37 & & & & & \\
\hline $1 \mathrm{~B}$ & $\mathrm{~S}$ & 2/7/1995 & 94403 & 38 & & & & & \\
\hline $1 \mathrm{~B}$ & S & 2/8/1995 & 94404 & 39 & & & & & \\
\hline $1 \mathrm{~B}$ & S & 2/9/1995 & 94405 & 40 & & & & & \\
\hline $1 \mathrm{~B}$ & S & 2/10/1995 & 94406 & 41 & & & & & \\
\hline $1 \mathrm{~B}$ & S & 2/13/1995 & 94409 & 44 & 0.1 & 0.1 & 0.1 & 0.1 & 0.1 \\
\hline $1 \mathrm{~B}$ & S & 2/14/1995 & 94410 & 45 & & & & & \\
\hline $1 \mathrm{~B}$ & S & 2/15/1995 & 94411 & 46 & & & & & \\
\hline $1 \mathrm{~B}$ & S & 2/16/1995 & 94412 & 47 & & & & & \\
\hline $1 \mathrm{~B}$ & S & 2/17/1995 & 94413 & 48 & & & & & \\
\hline $1 \mathrm{~B}$ & S & 2/20/1995 & 94416 & 51 & & & & & \\
\hline $1 \mathrm{~B}$ & S & 2/21/1995 & 94417 & 52 & & & & & \\
\hline $1 \mathrm{~B}$ & S & 2/22/1995 & 94418 & 53 & & & & & \\
\hline $1 \mathrm{~B}$ & S & 2/23/1995 & 94419 & 54 & & & & & \\
\hline $1 \mathrm{~B}$ & S & 2/24/1995 & 94420 & 55 & & & & & \\
\hline $1 \mathrm{~B}$ & S & 2/27/1995 & 94423 & 58 & & & & & \\
\hline $1 \mathrm{~B}$ & S & 2/28/1995 & 94424 & 59 & & & & & \\
\hline $1 \mathrm{~B}$ & S & 3/1/1995 & 94425 & 60 & & & & & \\
\hline $1 \mathrm{~B}$ & S & 3/2/1995 & 94426 & 61 & & & & & \\
\hline $1 \mathrm{~B}$ & S & 3/3/1995 & 94427 & 62 & & & & & \\
\hline $1 \mathrm{~B}$ & S & $3 / 6 / 1995$ & 94430 & 65 & & & & & \\
\hline $1 \mathrm{~B}$ & S & $3 / 7 / 1995$ & 94431 & 66 & & & & & \\
\hline $1 \mathrm{~B}$ & S & $3 / 8 / 1995$ & 94432 & 67 & & & & & \\
\hline $1 \mathrm{~B}$ & S & 3/9/1995 & 94433 & 68 & & & & & \\
\hline $1 \mathrm{~B}$ & S & 3/10/1995 & 94434 & 69 & & & & & \\
\hline $1 \mathrm{~B}$ & S & 3/20/1995 & 94444 & 79 & & & & & \\
\hline $1 \mathrm{~B}$ & S & 3/21/1995 & 94445 & 80 & & & & & \\
\hline $1 \mathrm{~B}$ & S & 3/22/1995 & 94446 & 81 & & & & & \\
\hline $1 \mathrm{~B}$ & S & 3/23/1995 & 94447 & 82 & & & & & \\
\hline $1 \mathrm{~B}$ & S & 3/24/1995 & 94448 & 83 & & & & & \\
\hline $1 \mathrm{~B}$ & S & 3/27/1995 & 94451 & 86 & & & & & \\
\hline $1 \mathrm{~B}$ & S & $3 / 28 / 1995$ & 94452 & 87 & & & & & \\
\hline $1 \mathrm{~B}$ & S & 3/29/1995 & 94453 & 88 & & & & & \\
\hline $1 \mathrm{~B}$ & S & $3 / 30 / 1995$ & 94454 & 89 & & & & & \\
\hline $1 \mathrm{~B}$ & S & 3/31/1995 & 94455 & 90 & & & & & \\
\hline $1 \mathrm{~B}$ & S & 4/3/1995 & 94458 & 93 & & & & & \\
\hline $1 \mathrm{~B}$ & S & 4/4/1995 & 94459 & 94 & & & & & \\
\hline $1 \mathrm{~B}$ & $S$ & 4/5/1995 & 94460 & 95 & & & & & \\
\hline $1 \mathrm{~B}$ & S & 4/6/1995 & 94461 & 96 & & & & & \\
\hline $1 \mathrm{~B}$ & S & 4/7/1995 & 94462 & 97 & & & & & \\
\hline $1 \mathrm{~B}$ & $\mathrm{~S}$ & 4/10/1995 & 94465 & 100 & & & & & \\
\hline
\end{tabular}


wbheat

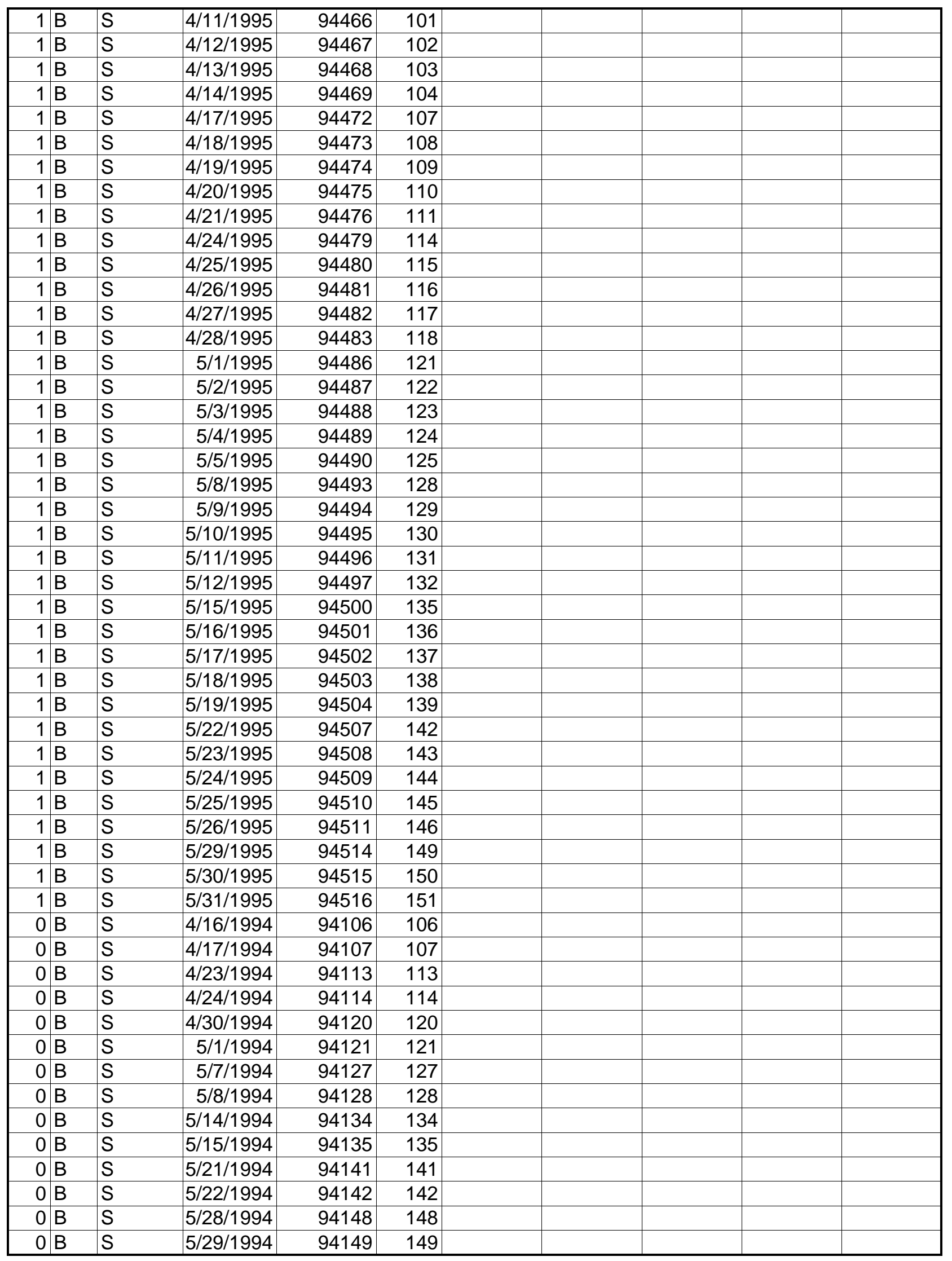


wbheat

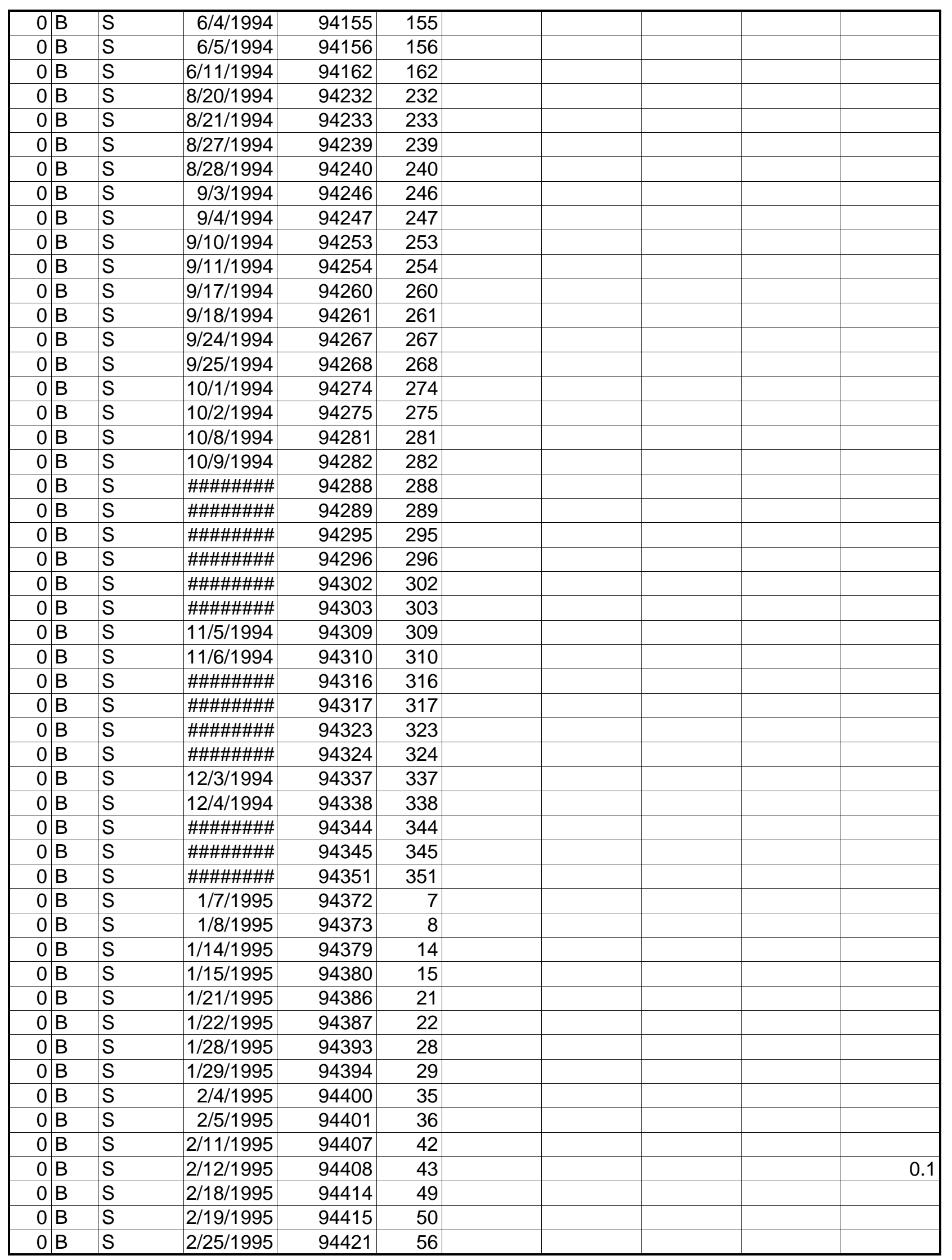


wbheat

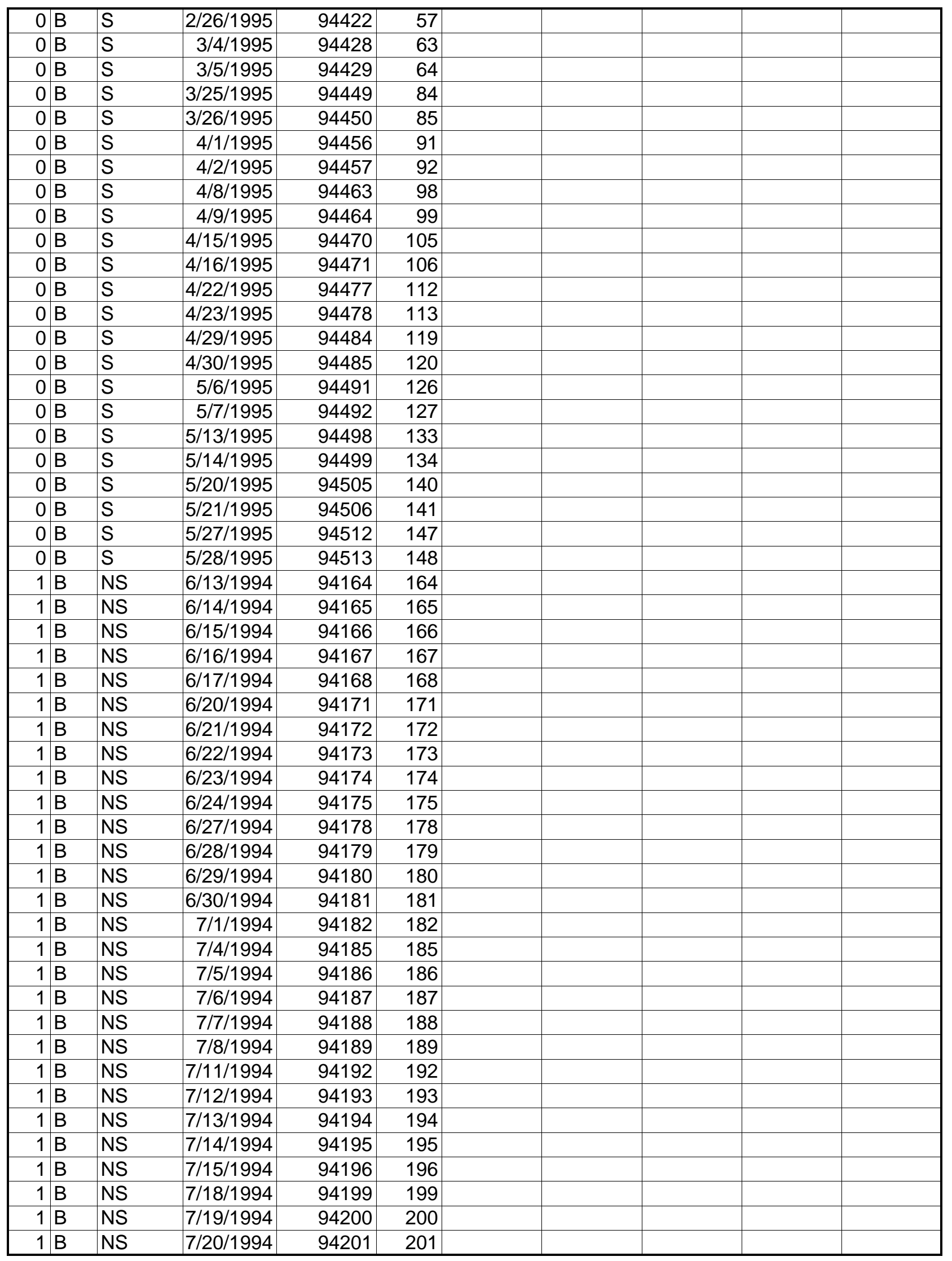


wbheat

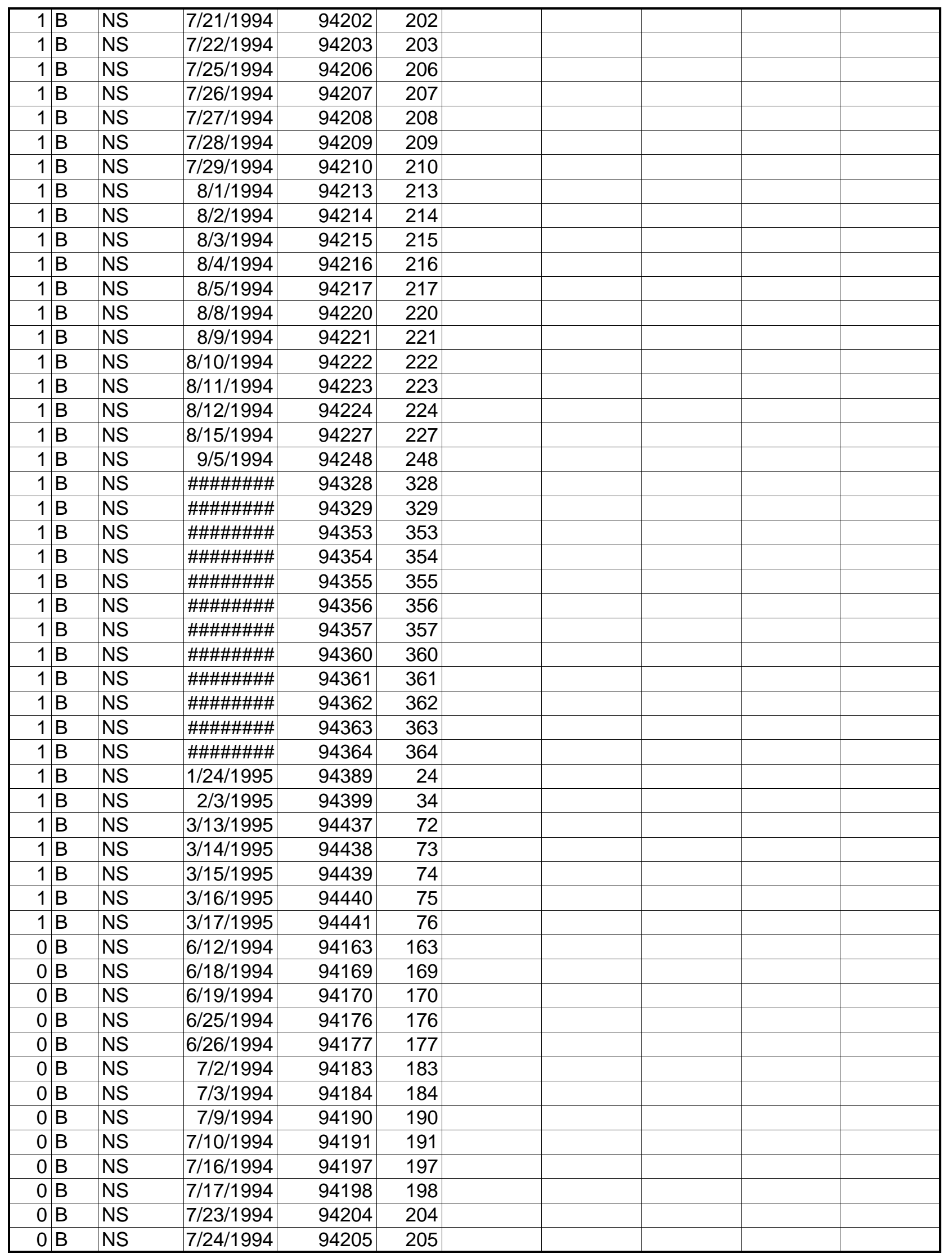


wbheat

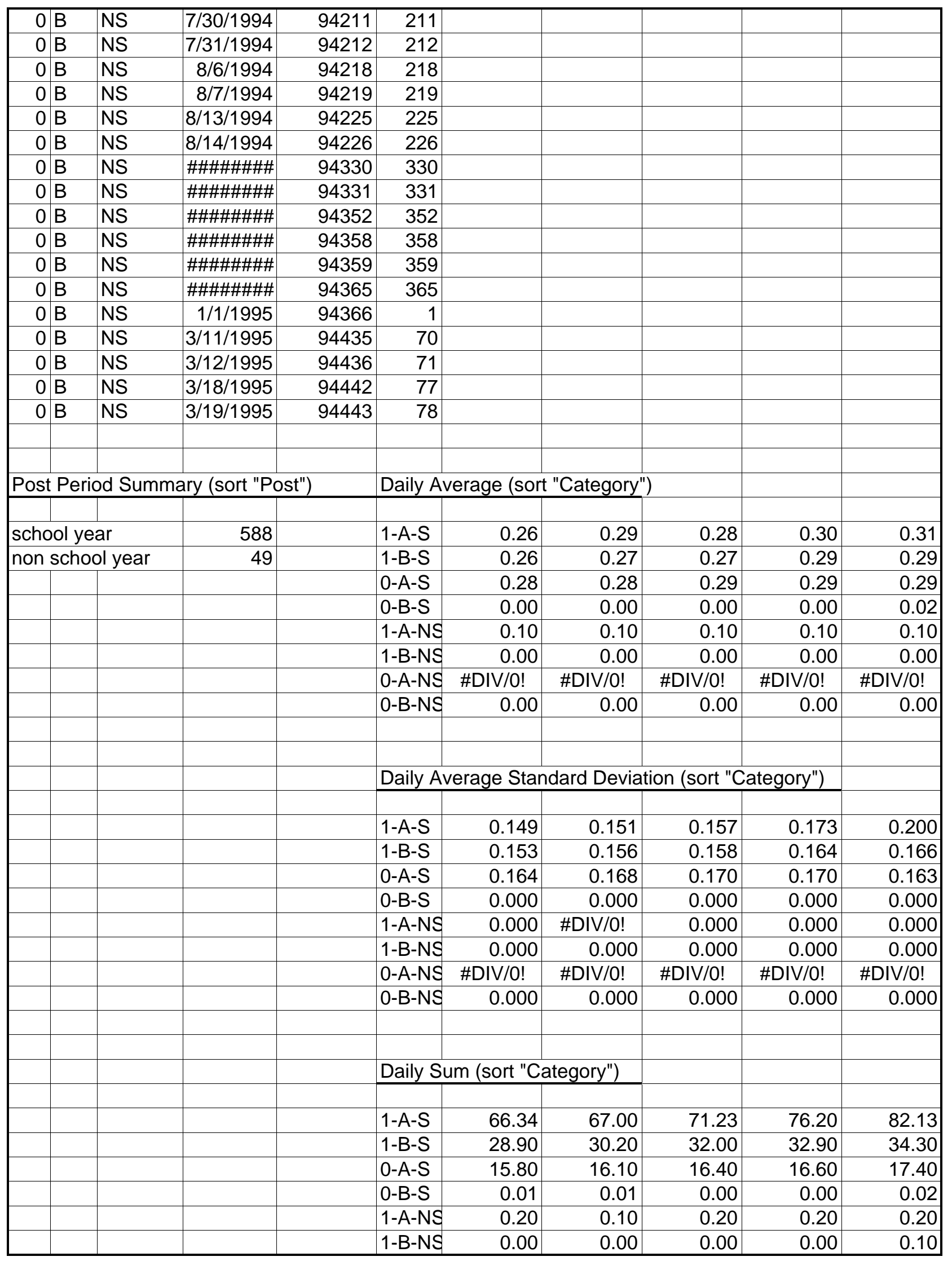


wbheat

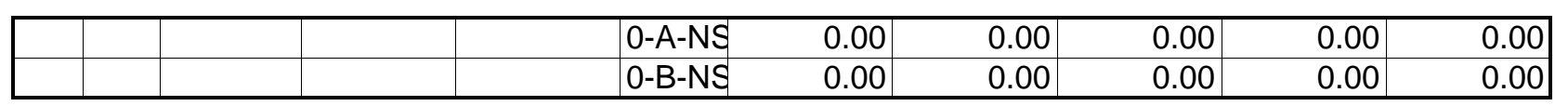

Page 28 


\begin{tabular}{|c|c|c|c|c|c|c|c|c|}
\hline 5 & 6 & 7 & 8 & 9 & 10 & 11 & 12 & 13 \\
\hline & & 0.1 & & 0.1 & & 0.1 & 0.1 & 0.1 \\
\hline & & 0.1 & & 0.1 & 0.1 & 0.1 & 0.1 & 0.1 \\
\hline & & 0.1 & & 0.1 & 0.1 & 0.1 & 0.1 & 0.1 \\
\hline & & 0.1 & & 0.1 & & 0.1 & & 0.1 \\
\hline & & 0.1 & & 0.1 & & 0.1 & 0.1 & 0.1 \\
\hline & & 0.1 & 0.1 & 0.1 & & 0.1 & 0.1 & 0.1 \\
\hline & & 0.1 & & 0.1 & & 0.1 & 0.2 & 0.1 \\
\hline & & 0.1 & & 0.1 & & 0.1 & 0.2 & 0.2 \\
\hline & & 0.1 & 0.1 & 0.1 & & 0.1 & 0.1 & 0.2 \\
\hline & & 0.1 & & 0.1 & 0.1 & 0.1 & 0.1 & 0.1 \\
\hline & & 0.1 & & 0.1 & & 0.1 & 0.1 & 0.1 \\
\hline & & 0.1 & & 0.1 & 0.1 & 0.1 & 0.1 & 0.2 \\
\hline & & 0.1 & 0.1 & 0.1 & 0.1 & 0.1 & 0.1 & 0.1 \\
\hline & & 0.1 & & 0.1 & 0.1 & 0.1 & 0.1 & 0.2 \\
\hline & & 0.1 & & 0.1 & & 0.1 & 0.1 & 0.2 \\
\hline & & 0.1 & 0.1 & 0.1 & 0.1 & & 0.1 & 0.1 \\
\hline & & 0.1 & & 0.1 & 0.1 & 0.1 & 0.1 & 0.1 \\
\hline & & 0.1 & & 0.1 & & & 0.1 & 0.2 \\
\hline & & 0.1 & & 0.1 & & 0.1 & 0.1 & 0.2 \\
\hline & & & 0.1 & & 0.1 & & 0.1 & 0.1 \\
\hline & & & 0.1 & 0.1 & 0.1 & & & 0.2 \\
\hline 0.1 & 0.1 & 0.1 & 0.1 & 0.6 & 0.4 & 0.4 & 0.4 & 0.4 \\
\hline 0.4 & 0.3 & 0.4 & 0.4 & 0.4 & 0.3 & 0.6 & 0.7 & 0.7 \\
\hline 0.5 & 0.5 & 0.6 & 0.5 & 0.5 & 0.3 & 0.3 & 0.4 & 0.4 \\
\hline 0.6 & 0.6 & 0.7 & 0.6 & 0.6 & 0.5 & 0.4 & 0.5 & 0.4 \\
\hline 0.6 & 0.6 & 0.8 & 0.7 & 0.6 & 0.4 & 0.4 & 0.4 & 0.4 \\
\hline 0.4 & 0.4 & 0.5 & 0.5 & 0.4 & 0.3 & 0.3 & 0.4 & 0.3 \\
\hline 0.3 & 0.2 & 0.3 & 0.3 & 0.2 & 0.2 & 0.2 & 0.3 & 0.3 \\
\hline 0.5 & 0.5 & 0.6 & 0.6 & 0.6 & 0.4 & 0.5 & 0.5 & 0.6 \\
\hline 0.4 & 0.4 & 0.5 & 0.4 & 0.4 & 0.3 & 0.3 & 0.2 & 0.3 \\
\hline 0.3 & 0.3 & 0.4 & 0.3 & 0.3 & 0.2 & 0.2 & 0.1 & 0.3 \\
\hline 0.2 & 0.3 & 0.3 & 0.3 & 0.2 & 0.1 & 0.2 & 0.1 & 0.2 \\
\hline 0.1 & 0.1 & 0.2 & 0.2 & 0.2 & & 0.1 & 0.1 & 0.2 \\
\hline 0.1 & 0.1 & 0.1 & 0.1 & 0.1 & & 0.1 & 0.1 & 0.2 \\
\hline 0.3 & 0.3 & 0.4 & 0.6 & 0.6 & 0.6 & 0.5 & 0.5 & 0.5 \\
\hline 0.1 & 0.1 & 0.2 & 0.1 & 0.2 & 0.1 & 0.1 & 0.2 & 0.2 \\
\hline 0.2 & 0.2 & 0.3 & 0.3 & 0.3 & 0.2 & 0.2 & 0.2 & 0.3 \\
\hline 0.4 & 0.4 & 0.5 & 0.5 & 0.4 & 0.2 & 0.3 & 0.3 & 0.3 \\
\hline 0.3 & 0.3 & 0.4 & 0.4 & 0.3 & 0.2 & 0.2 & 0.2 & 0.2 \\
\hline 0.5 & 0.5 & 0.6 & 0.6 & 0.6 & 0.4 & 0.4 & 0.4 & 0.4 \\
\hline 0.4 & 0.4 & 0.6 & 0.4 & 0.4 & 0.3 & 0.3 & 0.2 & 0.3 \\
\hline 0.2 & 0.2 & 0.3 & 0.2 & 0.2 & 0.1 & 0.1 & 0.1 & 0.2 \\
\hline 0.5 & 0.5 & 0.6 & 0.5 & 0.7 & 0.5 & 0.5 & 0.6 & 0.7 \\
\hline 0.5 & 0.5 & 0.6 & 0.6 & 0.5 & 0.4 & 0.5 & 0.5 & 0.5 \\
\hline 0.6 & 0.6 & 0.7 & 0.7 & 0.6 & 0.5 & 0.4 & 0.4 & 0.6 \\
\hline 0.4 & 0.4 & 0.6 & 0.6 & 0.5 & 0.4 & 0.3 & 0.3 & 0.3 \\
\hline 0.3 & 0.3 & 0.4 & 0.3 & 0.4 & 0.2 & 0.2 & 0.2 & 0.3 \\
\hline
\end{tabular}


wbheat

\begin{tabular}{|c|c|c|c|c|c|c|c|c|}
\hline 0.1 & 0.2 & 0.2 & 0.2 & 0.2 & 0.1 & 0.1 & 0.2 & 0.3 \\
\hline 0.3 & 0.3 & 0.5 & 0.4 & 0.5 & 0.4 & 0.3 & 0.2 & 0.3 \\
\hline 0.2 & 0.2 & 0.3 & 0.2 & 0.3 & 0.1 & 0.2 & 0.3 & 0.3 \\
\hline 0.1 & 0.1 & 0.2 & 0.2 & 0.2 & & 0.1 & 0.2 & 0.2 \\
\hline 0.2 & 0.2 & 0.2 & 0.3 & 0.2 & 0.1 & 0.2 & 0.1 & 0.3 \\
\hline 0.5 & 0.5 & 0.6 & 0.7 & 0.6 & 0.6 & 0.7 & 0.6 & 0.6 \\
\hline 0.4 & 0.5 & 0.6 & 0.5 & 0.5 & 0.4 & 0.3 & 0.4 & 0.4 \\
\hline 0.3 & 0.3 & 0.5 & 0.4 & 0.4 & 0.3 & 0.3 & 0.4 & 0.5 \\
\hline 0.5 & 0.5 & 0.7 & 0.5 & 0.5 & 0.5 & 0.5 & 0.5 & 0.6 \\
\hline 0.5 & 0.5 & 0.6 & 0.5 & 0.4 & 0.3 & 0.4 & 0.4 & 0.6 \\
\hline 0.1 & 0.1 & 0.2 & 0.2 & 1.3 & 1 & 0.9 & 0.9 & 0.8 \\
\hline 0.4 & 0.4 & 0.5 & 0.5 & 0.4 & 0.3 & 0.3 & 0.3 & 0.4 \\
\hline 0.4 & 0.4 & 0.5 & 0.5 & 0.4 & 0.3 & 0.4 & 0.6 & 0.6 \\
\hline 0.5 & 0.5 & 0.6 & 0.6 & 0.6 & 0.4 & 0.5 & 0.5 & 0.6 \\
\hline 0.5 & 0.6 & 0.6 & 0.6 & 0.6 & 0.5 & 0.6 & 0.6 & 0.5 \\
\hline 0.4 & 0.4 & 0.5 & 0.5 & 0.6 & & 0.4 & 0.4 & 0.5 \\
\hline 0.6 & 0.7 & 0.8 & 0.7 & 0.7 & 0.6 & 0.6 & 0.6 & 0.6 \\
\hline 0.5 & 0.6 & 0.6 & 0.5 & 0.6 & 0.4 & 0.5 & 0.5 & 0.6 \\
\hline 0.9 & 0.9 & 1.1 & 1.1 & 0.9 & 0.8 & 0.9 & 0.8 & 0.8 \\
\hline 0.6 & 0.7 & 0.6 & 0.6 & 0.5 & 0.5 & 0.5 & 0.5 & 0.5 \\
\hline 0.5 & 0.5 & 0.7 & 0.6 & 0.5 & 0.4 & 0.4 & 0.4 & 0.4 \\
\hline 0.5 & 0.5 & 0.6 & 0.6 & 0.5 & 0.4 & 0.3 & 0.4 & 0.4 \\
\hline 0.5 & 0.5 & 0.6 & 0.6 & 0.5 & 0.5 & 0.5 & 0.4 & 0.5 \\
\hline 0.5 & 0.5 & 0.6 & 0.7 & 0.6 & 0.5 & 0.4 & 0.4 & 0.4 \\
\hline 0.4 & 0.4 & 0.6 & 0.5 & 0.4 & 0.4 & 0.4 & 0.4 & 0.5 \\
\hline 0.4 & 0.4 & 0.5 & 0.4 & 0.5 & 0.3 & 0.3 & 0.4 & 0.4 \\
\hline 0.3 & 0.4 & 0.5 & 0.5 & 0.4 & 0.3 & 0.3 & 0.4 & 0.4 \\
\hline 0.4 & 0.4 & 0.5 & 0.5 & 0.4 & 0.3 & 0.3 & 0.3 & 0.4 \\
\hline 0.3 & 0.3 & 0.4 & 0.3 & 0.4 & 0.3 & 0.3 & 0.3 & 0.4 \\
\hline 0.4 & 0.4 & 0.5 & 0.5 & 0.4 & 0.2 & 0.3 & 0.4 & 0.4 \\
\hline 0.5 & 0.5 & 0.6 & 0.6 & 0.6 & 0.4 & 0.4 & 0.6 & 0.5 \\
\hline 0.6 & 0.6 & 0.7 & 0.7 & 0.6 & 0.6 & 0.4 & 0.6 & 0.6 \\
\hline 0.5 & 0.5 & 0.6 & 0.6 & 0.5 & 0.4 & 0.4 & 0.5 & 0.5 \\
\hline 0.5 & 0.5 & 0.6 & 0.5 & 0.5 & 0.4 & 0.4 & 0.4 & 0.4 \\
\hline 0.3 & 0.3 & 0.4 & 0.4 & 0.3 & 0.2 & 0.2 & 0.3 & 0.2 \\
\hline 0.2 & 0.2 & 0.3 & 0.2 & 0.3 & 0.1 & 0.1 & 0.2 & 0.2 \\
\hline 0.3 & 0.3 & 0.4 & 0.4 & 0.4 & 0.2 & 0.3 & 0.3 & 0.5 \\
\hline 0.2 & 0.2 & 0.3 & 0.3 & 0.2 & 0.1 & 0.2 & 0.2 & 0.2 \\
\hline 0.2 & 0.3 & 0.4 & 0.3 & 0.3 & 0.2 & 0.2 & 0.2 & 0.3 \\
\hline 0.2 & 0.2 & 0.4 & 0.4 & 0.4 & 0.2 & 0.3 & 0.4 & 0.3 \\
\hline 0.3 & 0.3 & 0.5 & 0.4 & 0.4 & 0.2 & 0.2 & 0.3 & 0.3 \\
\hline 0.2 & 0.3 & 0.4 & 0.3 & 0.4 & 0.3 & 0.2 & 0.3 & 0.3 \\
\hline 0.2 & 0.2 & 0.4 & 0.2 & 0.3 & 0.1 & 0.2 & 0.3 & 0.3 \\
\hline 0.2 & 0.2 & 0.4 & 0.3 & 0.3 & 0.2 & 0.2 & 0.2 & 0.3 \\
\hline 0.3 & 0.4 & 0.5 & 0.5 & 0.7 & 0.8 & 0.6 & 0.6 & 0.6 \\
\hline 0.6 & 0.6 & 0.8 & 0.7 & 0.7 & 0.5 & 0.5 & 0.6 & 0.5 \\
\hline 0.5 & 0.5 & 0.6 & 0.5 & 0.5 & 0.4 & 0.4 & 0.4 & 0.4 \\
\hline 0.4 & 0.4 & 0.6 & 0.4 & 0.5 & 0.3 & 0.3 & 0.4 & 0.2 \\
\hline 0.2 & 0.2 & 0.4 & 0.2 & 0.3 & 0.1 & 0.2 & 0.2 & 0.3 \\
\hline 0.2 & 0.2 & 0.4 & 0.2 & 0.3 & 0.2 & 0.2 & 0.2 & 0.3 \\
\hline 0.2 & 0.2 & 0.3 & 0.3 & 0.3 & 0.2 & 0.2 & 0.3 & 0.3 \\
\hline
\end{tabular}


wbheat

\begin{tabular}{|c|c|c|c|c|c|c|c|c|}
\hline 0.3 & 0.3 & 0.4 & 0.3 & 0.3 & 0.2 & 0.2 & 0.3 & 0.3 \\
\hline 0.2 & 0.2 & 0.3 & 0.3 & 0.2 & 0.2 & 0.2 & 0.2 & 0.4 \\
\hline 0.2 & 0.2 & 0.4 & 0.2 & 0.3 & 0.2 & 0.2 & 0.3 & 0.3 \\
\hline 0.4 & 0.4 & 0.5 & 0.5 & 0.5 & 0.4 & 0.4 & 0.5 & 0.5 \\
\hline 0.5 & 0.6 & 0.7 & 0.7 & 0.6 & 0.5 & 0.4 & 0.5 & 0.5 \\
\hline 0.4 & 0.4 & 0.6 & 0.5 & 0.4 & 0.3 & 0.3 & 0.4 & 0.3 \\
\hline 0.3 & 0.4 & 0.5 & 0.4 & 0.4 & 0.3 & 0.2 & 0.4 & 0.3 \\
\hline 0.5 & 0.5 & 0.6 & 0.5 & 0.5 & 0.4 & 0.4 & 0.4 & 0.4 \\
\hline 0.3 & 0.4 & 0.5 & 0.5 & 0.4 & 0.3 & 0.3 & 0.4 & 0.3 \\
\hline 0.3 & 0.3 & 0.4 & 0.3 & 0.4 & 0.2 & 0.2 & 0.2 & 0.2 \\
\hline 0.2 & 0.2 & 0.4 & 0.4 & 0.4 & 0.1 & 0.2 & 0.2 & 0.2 \\
\hline 0.1 & 0.1 & 0.2 & 0.2 & 0.3 & 0.2 & 0.2 & 0.2 & 0.2 \\
\hline 0.2 & 0.2 & 0.4 & 0.2 & 0.3 & 0.2 & 0.2 & 0.3 & 0.3 \\
\hline 0.2 & 0.2 & 0.4 & 0.3 & 0.4 & 0.2 & 0.2 & 0.3 & 0.4 \\
\hline 0.2 & 0.2 & 0.4 & 0.3 & 0.3 & 0.2 & 0.3 & 0.3 & 0.4 \\
\hline 0.4 & 0.4 & 0.5 & 0.5 & 0.5 & 0.3 & 0.3 & 0.4 & 0.4 \\
\hline 0.3 & 0.4 & 0.5 & 0.4 & 0.5 & 0.3 & 0.3 & 0.4 & 0.4 \\
\hline 0.2 & 0.4 & 0.2 & 0.3 & 0.2 & 0.3 & 0.3 & 0.3 & 0.2 \\
\hline 0.3 & 0.5 & 0.4 & 0.4 & 0.2 & 0.2 & 0.3 & 0.2 & 0.1 \\
\hline 0.1 & 0.1 & 0.2 & 0.1 & 0.2 & 0.1 & 0.1 & 0.1 & 0.2 \\
\hline \multirow{2}{*}{0.1} & 0.1 & 0.3 & 0.2 & 0.2 & 0.1 & 0.1 & 0.1 & 0.2 \\
\hline & 0.1 & 0.2 & 0.1 & 0.2 & & 0.1 & 0.1 & 0.2 \\
\hline 0.1 & 0.1 & 0.3 & 0.2 & 0.2 & 0.1 & 0.1 & 0.2 & 0.1 \\
\hline \multirow[t]{4}{*}{0.1} & 0.1 & 0.2 & 0.1 & 0.2 & & 0.1 & 0.2 & 0.2 \\
\hline & & 0.2 & 0.1 & 0.1 & 0.1 & 0.1 & 0.2 & 0.2 \\
\hline & 0.1 & 0.1 & 0.1 & 0.2 & 0.1 & 0.1 & 0.2 & 0.2 \\
\hline & 0.1 & 0.1 & 0.1 & 0.1 & 0.1 & 0.1 & & 0.1 \\
\hline 0.1 & 0.1 & 0.3 & 0.3 & 0.2 & 0.1 & 0.1 & 0.2 & 0.2 \\
\hline 0.1 & 0.2 & 0.3 & 0.2 & 0.3 & 0.1 & 0.2 & 0.3 & 0.2 \\
\hline 0.1 & 0.1 & 0.2 & 0.1 & 0.2 & 0.1 & 0.1 & 0.2 & 0.2 \\
\hline \multirow[t]{2}{*}{0.1} & 0.1 & 0.2 & 0.1 & 0.1 & 0.1 & 0.2 & 0.2 & 0.2 \\
\hline & & 0.2 & 0.1 & 0.2 & 0.1 & 0.1 & 0.2 & 0.2 \\
\hline \multirow[t]{9}{*}{0.1} & 0.1 & 0.2 & 0.1 & 0.2 & & 0.1 & 0.2 & 0.1 \\
\hline & 0.1 & 0.2 & 0.1 & 0.2 & 0.1 & 0.1 & 0.1 & 0.2 \\
\hline & & 0.2 & 0.1 & 0.1 & 0.1 & 0.1 & 0.2 & 0.2 \\
\hline & 0.1 & 0.2 & 0.1 & 0.1 & & 0.1 & 0.3 & 0.2 \\
\hline & 0.1 & 0.1 & 0.1 & 0.2 & 0.1 & 0.1 & 0.2 & 0.2 \\
\hline & & 0.1 & 0.1 & 0.1 & & 0.1 & 0.1 & 0.1 \\
\hline & & 0.1 & 0.1 & 0.2 & 0.1 & 0.1 & 0.1 & 0.1 \\
\hline & 0.1 & 0.1 & 0.1 & 0.1 & & 0.1 & 0.1 & 0.2 \\
\hline & 0.1 & 0.1 & 0.1 & 0.2 & & 0.1 & 0.1 & 0.2 \\
\hline \multirow[t]{6}{*}{0.1} & & 0.2 & 0.1 & 0.1 & 0.1 & 0.1 & 0.1 & 0.1 \\
\hline & & 0.1 & & 0.1 & 0.1 & 0.1 & 0.1 & 0.2 \\
\hline & & 0.1 & & 0.1 & 0.1 & 0.1 & 0.2 & 0.2 \\
\hline & & 0.1 & 0.1 & 0.1 & 0.1 & 0.1 & 0.2 & 0.2 \\
\hline & & 0.1 & 0.1 & 0.2 & & 0.1 & 0.2 & 0.2 \\
\hline & & 0.2 & & 0.2 & 0.1 & & 0.1 & 0.1 \\
\hline \multirow[t]{4}{*}{0.1} & & 0.2 & 0.1 & 0.1 & & 0.1 & 0.2 & 0.2 \\
\hline & & 0.1 & & 0.2 & 0.1 & & 0.2 & 0.2 \\
\hline & & 0.1 & 0.1 & 0.1 & 0.1 & 0.1 & 0.3 & 0.1 \\
\hline & & 0.1 & 0.1 & 0.2 & 0.1 & 0.1 & 0.2 & 0.2 \\
\hline
\end{tabular}


wbheat

\begin{tabular}{|c|c|c|c|c|c|c|c|c|}
\hline & & 0.1 & 0.1 & 0.1 & & 0.1 & 0.1 & 0.2 \\
\hline 0.1 & 0.1 & 0.1 & & 0.2 & 0.1 & 0.1 & 0.2 & 0.2 \\
\hline \multirow[t]{3}{*}{0.1} & 0.1 & 0.2 & 0.2 & 0.2 & 0.1 & 0.1 & 0.2 & 0.2 \\
\hline & & 0.2 & 0.1 & 0.1 & & 0.1 & 0.2 & 0.1 \\
\hline & & 0.2 & 0.1 & 0.2 & 0.1 & 0.1 & 0.1 & 0.1 \\
\hline 0.1 & 0.1 & 0.2 & 0.1 & 0.1 & 0.1 & 0.1 & 0.1 & 0.1 \\
\hline \multirow[t]{2}{*}{0.1} & & 0.1 & 0.1 & 0.2 & & 0.1 & 0.2 & 0.1 \\
\hline & & 0.1 & 0.1 & 0.1 & 0.1 & 0.1 & 0.1 & 0.2 \\
\hline & & & & & & & & \\
\hline & & & & & & & & \\
\hline & & & & & & & & \\
\hline & & & & & & & & \\
\hline & & & & & & & & \\
\hline & & & & & & & & \\
\hline & & & & & 0.2 & & & \\
\hline & & & & & & & & \\
\hline & & & & & & & & \\
\hline & & & & & & & & \\
\hline & & & & & & & & \\
\hline & & & & & & & & \\
\hline & & & 0.1 & 0.1 & 0.1 & & & \\
\hline & & & 0.1 & & & 0.1 & & \\
\hline & & 0.1 & & & & 0.1 & & 0.1 \\
\hline & & 0.1 & & 0.1 & 0.1 & & 0.1 & 0.1 \\
\hline & & 0.1 & & 0.1 & & 0.1 & 0.2 & 0.2 \\
\hline & & 0.1 & & 0.2 & & 0.1 & 0.1 & 0.1 \\
\hline & & 0.1 & & 0.1 & & 0.1 & 0.1 & 0.1 \\
\hline & & & & 0.1 & & 0.1 & 0.1 & 0.1 \\
\hline & & 0.1 & & 0.1 & & 0.1 & 0.1 & 0.2 \\
\hline & & 0.1 & & 0.1 & & 0.1 & 0.1 & 0.1 \\
\hline & & 0.1 & & 0.1 & & 0.1 & 0.1 & 0.1 \\
\hline & & 0.1 & & 0.2 & & 0.1 & 0.1 & 0.1 \\
\hline & & 0.1 & & 0.1 & & 0.1 & 0.1 & 0.1 \\
\hline & & 0.1 & & 0.1 & & 0.1 & 0.1 & 0.1 \\
\hline & & & & 0.1 & & 0.1 & 0.1 & 0.2 \\
\hline & & 0.1 & & 0.2 & & 0.1 & 0.1 & 0.1 \\
\hline & & & & 0.1 & & 0.1 & 0.1 & 0.1 \\
\hline & & 0.1 & 0.1 & 0.1 & & 0.1 & 0.1 & 0.1 \\
\hline & & 0.1 & & 0.1 & 0.1 & 0.1 & 0.1 & 0.2 \\
\hline & & & & 0.1 & & 0.1 & 0.1 & 0.1 \\
\hline & & & & 0.1 & 0.1 & 0.1 & 0.1 & 0.2 \\
\hline & & 0.1 & & 0.1 & & 0.1 & 0.1 & 0.1 \\
\hline & & 0.1 & & 0.1 & & & 0.1 & 0.1 \\
\hline & & 0.1 & & 0.1 & & 0.1 & & 0.1 \\
\hline & 0.1 & 0.1 & 0.1 & 0.1 & & 0.1 & 0.1 & 0.1 \\
\hline & & & & 0.1 & & 0.1 & 0.1 & 0.2 \\
\hline & & 0.1 & 0.1 & 0.1 & & & 0.1 & 0.1 \\
\hline & & 0.1 & & 0.1 & & 0.1 & 0.1 & 0.1 \\
\hline 0.021 & & 0.082 & 0.01 & 0.124 & 0.041 & 0.062 & 0.093 & 0.113 \\
\hline & & 0.1 & & 0.1 & 0.1 & 0.1 & 0.1 & 0.1 \\
\hline & & 0.1 & 0.1 & 0.2 & & 0.1 & 0.1 & 0.1 \\
\hline
\end{tabular}


wbheat

\begin{tabular}{|c|c|c|c|c|c|c|c|c|}
\hline & & 0.1 & & 0.1 & 0.1 & 0.1 & 0.1 & 0.1 \\
\hline & & 0.1 & & 0.1 & & 0.1 & 0.1 & 0.1 \\
\hline & & 0.1 & & 0.1 & 0.1 & 0.1 & 0.1 & 0.1 \\
\hline & & 0.1 & & 0.2 & 0.1 & 0.2 & 0.1 & 0.1 \\
\hline & & 0.1 & & 0.2 & & 0.1 & 0.1 & 0.1 \\
\hline & & 0.1 & 0.1 & 0.1 & 0.1 & & 0.2 & 0.2 \\
\hline & & & & 0.1 & & 0.1 & 0.2 & 0.1 \\
\hline & & 0.1 & & 0.1 & & 0.1 & 0.2 & 0.3 \\
\hline & & 0.1 & & 0.1 & & 0.2 & 0.2 & 0.2 \\
\hline & & & 0.1 & 0.2 & 0.1 & 0.2 & 0.2 & 0.2 \\
\hline 0.2 & 0.1 & 0.2 & 0.2 & 0.3 & 0.2 & 0.3 & 0.3 & 0.3 \\
\hline & & 0.1 & & 0.1 & 0.1 & 0.1 & 0.1 & 0.2 \\
\hline & & 0.1 & & 0.1 & & 0.1 & 0.1 & 0.1 \\
\hline & & 0.1 & 0.1 & 0.1 & & 0.1 & 0.2 & 0.1 \\
\hline & & 0.1 & & 0.1 & 0.1 & 0.1 & 0.1 & 0.1 \\
\hline & & & 0.1 & & & 0.1 & 0.2 & 0.2 \\
\hline & & 0.1 & 0.1 & 0.1 & & 0.1 & 0.2 & 0.2 \\
\hline & & 0.1 & & 0.1 & & 0.1 & 0.1 & 0.2 \\
\hline & & 0.1 & & 0.2 & 0.1 & 0.1 & 0.1 & 0.1 \\
\hline & & & 0.1 & 0.1 & 0.1 & 0.1 & & 0.1 \\
\hline & & 0.1 & 0.1 & 0.2 & 0.1 & 0.1 & 0.2 & 0.2 \\
\hline & & 0.1 & 0.1 & 0.1 & & 0.1 & 0.2 & 0.2 \\
\hline 0.1 & 0.1 & 0.1 & 0.1 & 0.3 & 0.1 & 0.2 & 0.4 & 0.4 \\
\hline 0.2 & 0.2 & 0.2 & 0.3 & 0.8 & 0.7 & 0.6 & 0.5 & 0.5 \\
\hline 0.3 & 0.3 & 0.4 & 0.4 & 0.4 & 0.2 & 0.2 & 0.3 & 0.3 \\
\hline 0.2 & 0.2 & 0.2 & 0.2 & 0.2 & 0.1 & 0.1 & 0.2 & 0.2 \\
\hline 0.1 & 0.1 & 0.2 & 0.1 & 0.2 & 0.1 & 0.1 & 0.2 & 0.2 \\
\hline 0.1 & & 0.1 & 0.1 & 0.2 & 0.1 & 0.1 & 0.1 & 0.1 \\
\hline 0.1 & 0.1 & 0.2 & 0.2 & 0.3 & 0.2 & 0.2 & 0.4 & 0.3 \\
\hline 0.2 & 0.2 & 0.3 & 0.3 & 0.5 & 0.5 & 0.4 & 0.4 & 0.3 \\
\hline 0.3 & 0.3 & 0.3 & 0.4 & 0.3 & 0.2 & 0.2 & 0.2 & 0.2 \\
\hline 0.3 & 0.3 & 0.4 & 0.3 & 0.2 & 0.1 & 0.1 & 0.2 & 0.2 \\
\hline 0.1 & 0.1 & 0.2 & 0.1 & 0.2 & 0.1 & 0.1 & 0.1 & 0.2 \\
\hline 0.1 & 0.1 & 0.2 & 0.1 & 0.2 & 0.1 & 0.1 & 0.2 & 0.2 \\
\hline 0.2 & 0.2 & 0.3 & 0.2 & 0.2 & 0.1 & 0.2 & 0.2 & 0.2 \\
\hline 0.3 & 0.3 & 0.4 & 0.4 & 0.4 & 0.2 & 0.3 & 0.4 & 0.3 \\
\hline 0.2 & 0.2 & 0.3 & 0.3 & 0.3 & 0.2 & 0.2 & 0.3 & 0.3 \\
\hline 0.4 & 0.4 & 0.5 & 0.5 & 0.5 & 0.4 & 0.4 & 0.4 & 0.3 \\
\hline 0.5 & 0.5 & 0.6 & 0.6 & 0.6 & 0.6 & 0.6 & 0.6 & 0.7 \\
\hline 0.4 & 0.5 & 0.5 & 0.5 & 0.5 & 0.3 & 0.3 & 0.3 & 0.3 \\
\hline 0.3 & 0.3 & 0.4 & 0.4 & 0.5 & 0.3 & 0.3 & 0.3 & 0.3 \\
\hline 0.4 & 0.4 & 0.5 & 0.5 & 0.5 & 0.3 & 0.3 & 0.3 & 0.4 \\
\hline 0.2 & 0.2 & 0.4 & 0.4 & 0.4 & 0.2 & 0.3 & 0.4 & 0.3 \\
\hline 0.5 & 0.5 & 0.6 & 0.6 & 0.5 & 0.3 & 0.4 & 0.5 & 0.6 \\
\hline 0.5 & 0.5 & 0.6 & 0.5 & 0.5 & 0.4 & 0.4 & 0.4 & 0.5 \\
\hline 0.5 & 0.4 & 0.6 & 0.5 & 0.5 & 0.3 & 0.4 & 0.6 & 0.4 \\
\hline 0.5 & 0.5 & 0.6 & 0.5 & 0.6 & 0.4 & 0.4 & 0.4 & 0.4 \\
\hline 0.5 & 0.5 & 0.7 & 0.6 & 0.6 & 0.4 & 0.4 & 0.3 & 0.4 \\
\hline 0.4 & 0.4 & 0.5 & 0.5 & 0.6 & 0.3 & 0.5 & 0.4 & 0.4 \\
\hline 0.5 & 0.5 & 0.6 & 0.6 & 0.7 & 0.6 & 0.7 & 0.7 & 0.7 \\
\hline 0.6 & 0.6 & 0.7 & 0.6 & 0.6 & 0.4 & 0.5 & 0.5 & 0.5 \\
\hline
\end{tabular}




\begin{tabular}{|c|c|c|c|c|c|c|c|c|}
\hline 0.6 & 0.6 & 0.8 & 0.7 & 0.7 & 0.5 & 0.6 & 0.6 & 0.6 \\
\hline 0.6 & 0.6 & 0.7 & 0.7 & 0.7 & 0.5 & 0.5 & 0.5 & 0.5 \\
\hline 0.2 & 0.2 & 0.3 & 0.3 & 0.3 & 0.3 & 0.4 & 0.3 & 0.4 \\
\hline 0.5 & 0.6 & 0.7 & 0.6 & 0.8 & 0.6 & 0.6 & 0.6 & 0.7 \\
\hline 0.5 & 0.5 & 0.6 & 0.6 & 0.6 & 0.4 & 0.4 & 0.5 & 0.5 \\
\hline 0.4 & 0.5 & 0.6 & 0.5 & 0.6 & 0.3 & 0.4 & 0.4 & 0.4 \\
\hline 0.4 & 0.4 & 0.5 & 0.5 & 0.4 & 0.3 & 0.4 & 0.4 & 0.4 \\
\hline 0.7 & 0.7 & 0.8 & 0.7 & 0.7 & 0.6 & 0.6 & 0.6 & 0.7 \\
\hline 0.5 & 0.5 & 0.7 & 0.6 & 0.6 & 0.4 & 0.5 & 0.4 & 0.3 \\
\hline 0.6 & 0.6 & 0.7 & 0.7 & 0.8 & 0.6 & 0.5 & 0.6 & 0.6 \\
\hline 0.6 & 0.6 & 0.7 & 0.7 & 0.7 & 0.5 & 0.5 & 0.5 & 0.6 \\
\hline 0.5 & 0.5 & 0.5 & 0.5 & 0.4 & 0.4 & 0.5 & 0.4 & 0.3 \\
\hline 0.6 & 0.7 & 0.8 & 0.7 & 0.7 & 0.6 & 0.7 & 0.6 & 0.7 \\
\hline 0.6 & 0.6 & 0.7 & 0.7 & 0.6 & 0.4 & 0.5 & 0.5 & 0.6 \\
\hline 0.6 & 0.6 & 0.7 & 0.7 & 0.8 & 0.4 & 0.5 & 0.4 & 0.4 \\
\hline 0.4 & 0.4 & 0.5 & 0.5 & 0.5 & 0.3 & 0.3 & 0.3 & 0.4 \\
\hline 0.6 & 0.6 & 0.7 & 0.7 & 0.8 & 0.5 & 0.5 & 0.5 & 0.5 \\
\hline 0.6 & 0.6 & 0.8 & 0.7 & 0.7 & 0.5 & 0.5 & 0.5 & 0.5 \\
\hline 0.5 & 0.5 & 0.6 & 0.6 & 0.6 & 0.4 & 0.4 & 0.3 & 0.4 \\
\hline 0.3 & 0.4 & 0.5 & 0.5 & 0.4 & 0.3 & 0.3 & 0.3 & 0.4 \\
\hline 0.3 & 0.3 & 0.5 & 0.4 & 0.6 & 0.3 & 0.4 & 0.4 & 0.5 \\
\hline 0.5 & 0.5 & 0.6 & 0.5 & 0.5 & 0.3 & 0.3 & 0.3 & 0.3 \\
\hline 0.3 & 0.4 & 0.5 & 0.4 & 0.5 & 0.3 & 0.4 & 0.3 & 0.3 \\
\hline 0.2 & 0.3 & 0.3 & 0.3 & 0.3 & 0.3 & 0.4 & 0.4 & 0.5 \\
\hline 0.3 & 0.3 & 0.4 & 0.4 & 0.6 & 0.3 & 0.3 & 0.5 & 0.4 \\
\hline 0.4 & 0.5 & 0.5 & 0.5 & 0.5 & 0.3 & 0.4 & 0.3 & 0.4 \\
\hline 0.3 & 0.3 & 0.5 & 0.4 & 0.5 & 0.2 & 0.3 & 0.2 & 0.4 \\
\hline 0.2 & 0.2 & 0.3 & 0.2 & 0.3 & 0.2 & 0.3 & 0.2 & 0.3 \\
\hline 0.2 & 0.2 & 0.4 & 0.4 & 0.5 & 0.2 & 0.4 & 0.4 & 0.4 \\
\hline 0.4 & 0.4 & 0.5 & 0.5 & 0.6 & 0.3 & 0.5 & 0.5 & 0.5 \\
\hline 0.4 & 0.3 & 0.4 & 0.5 & 0.5 & 0.6 & 0.5 & 0.5 & 0.4 \\
\hline 0.5 & 0.5 & 0.6 & 0.7 & 0.6 & 0.4 & 0.5 & 0.6 & 0.7 \\
\hline 0.6 & 0.6 & 0.8 & 0.7 & 0.8 & 0.5 & 0.6 & 0.7 & 0.7 \\
\hline 0.7 & 0.8 & 0.9 & 0.8 & 0.8 & 0.5 & 0.6 & 0.6 & 0.6 \\
\hline 0.5 & 0.6 & 0.6 & 0.6 & 0.5 & 0.3 & 0.4 & 0.3 & 0.4 \\
\hline 0.3 & 0.3 & 0.4 & 0.4 & 0.4 & 0.2 & 0.4 & 0.4 & 0.3 \\
\hline 0.3 & 0.3 & 0.4 & 0.4 & 0.5 & 0.3 & 0.3 & 0.3 & 0.3 \\
\hline 0.3 & 0.3 & 0.4 & 0.3 & 0.4 & 0.2 & 0.3 & 0.4 & 0.3 \\
\hline 0.2 & 0.2 & 0.3 & 0.3 & 0.3 & 0.2 & 0.2 & 0.2 & 0.3 \\
\hline 0.4 & 0.4 & 0.5 & 0.6 & 0.7 & 0.4 & 0.5 & 0.5 & 0.6 \\
\hline 0.4 & 0.4 & 0.5 & 0.5 & 0.5 & 0.3 & 0.4 & 0.4 & 0.5 \\
\hline 0.3 & 0.3 & 0.4 & 0.4 & 0.5 & 0.3 & 0.3 & 0.4 & 0.3 \\
\hline 0.3 & 0.3 & 0.5 & 0.5 & 0.4 & 0.3 & 0.4 & 0.4 & 0.4 \\
\hline 0.3 & 0.3 & 0.5 & 0.4 & 0.5 & 0.2 & 0.3 & 0.3 & 0.4 \\
\hline 0.3 & 0.4 & 0.5 & 0.4 & 0.5 & 0.3 & 0.4 & 0.3 & 0.4 \\
\hline 0.2 & 0.2 & 0.3 & 0.3 & 0.4 & 0.1 & 0.2 & 0.1 & 0.3 \\
\hline 0.1 & 0.1 & 0.2 & 0.2 & 0.3 & 0.2 & 0.2 & 0.2 & 0.2 \\
\hline 0.1 & 0.1 & 0.2 & 0.2 & 0.3 & 0.1 & 0.2 & 0.2 & 0.2 \\
\hline 0.2 & 0.2 & 0.3 & 0.2 & 0.4 & 0.2 & 0.3 & 0.2 & 0.3 \\
\hline 0.4 & 0.4 & 0.5 & 0.5 & 0.6 & 0.4 & 0.5 & 0.6 & 0.4 \\
\hline 0.2 & 0.2 & 0.3 & 0.2 & 0.2 & 0.1 & 0.2 & 0.3 & 0.2 \\
\hline
\end{tabular}


wbheat

\begin{tabular}{|c|c|c|c|c|c|c|c|c|}
\hline 0.3 & 0.2 & 0.4 & 0.3 & 0.3 & 0.2 & 0.2 & 0.3 & 0.2 \\
\hline 0.1 & 0.1 & 0.3 & 0.2 & 0.2 & 0.1 & 0.2 & 0.2 & 0.1 \\
\hline 0.1 & 0.1 & 0.2 & 0.1 & 0.2 & & 0.1 & 0.2 & 0.2 \\
\hline 0.1 & 0.1 & 0.2 & 0.1 & 0.3 & 0.1 & 0.2 & 0.2 & 0.3 \\
\hline \multirow{2}{*}{0.1} & 0.1 & 0.2 & 0.1 & 0.2 & 0.1 & 0.1 & 0.1 & 0.2 \\
\hline & 0.1 & 0.2 & 0.1 & 0.2 & 0.1 & 0.2 & 0.2 & 0.1 \\
\hline 0.1 & 0.1 & 0.1 & 0.1 & 0.2 & 0.1 & 0.1 & 0.3 & 0.2 \\
\hline 0.1 & 0.1 & 0.2 & 0.2 & 0.3 & 0.1 & 0.1 & 0.2 & 0.2 \\
\hline 0.2 & 0.2 & 0.3 & 0.3 & 0.4 & 0.2 & 0.3 & 0.4 & 0.3 \\
\hline 0.2 & 0.3 & 0.3 & 0.4 & 0.5 & 0.3 & 0.4 & 0.3 & 0.3 \\
\hline 0.2 & 0.3 & 0.4 & 0.3 & 0.4 & 0.4 & 0.4 & 0.4 & 0.5 \\
\hline 0.2 & 0.2 & 0.3 & 0.2 & 0.3 & 0.1 & 0.2 & 0.2 & 0.3 \\
\hline 0.1 & 0.1 & 0.2 & 0.2 & 0.2 & 0.1 & 0.2 & 0.3 & 0.2 \\
\hline \multirow[t]{4}{*}{0.2} & 0.2 & 0.2 & 0.2 & 0.2 & 0.2 & 0.2 & 0.1 & 0.2 \\
\hline & 0.1 & 0.1 & 0.1 & 0.2 & 0.1 & 0.1 & 0.2 & 0.1 \\
\hline & & 0.1 & 0.1 & 0.1 & & 0.1 & 0.1 & 0.1 \\
\hline & 0.1 & 0.1 & 0.1 & 0.2 & 0.1 & 0.1 & 0.2 & 0.1 \\
\hline 0.2 & 0.2 & 0.3 & 0.2 & 0.4 & 0.4 & 0.3 & 0.4 & 0.3 \\
\hline 0.2 & 0.2 & 0.3 & 0.3 & 0.3 & 0.2 & 0.2 & 0.3 & 0.2 \\
\hline \multirow[t]{2}{*}{0.1} & & & & 0.1 & 0.1 & & 0.1 & \\
\hline & & 0.1 & 0.1 & 0.1 & 0.1 & 0.2 & 0.1 & 0.1 \\
\hline 0.1 & 0.1 & 0.2 & 0.1 & 0.2 & 0.1 & 0.2 & 0.2 & 0.2 \\
\hline \multirow[t]{2}{*}{0.1} & 0.1 & 0.2 & 0.2 & 0.3 & 0.1 & 0.2 & 0.2 & 0.1 \\
\hline & 0.1 & 0.2 & 0.1 & 0.2 & 0.1 & 0.1 & 0.1 & 0.2 \\
\hline \multirow[t]{5}{*}{0.1} & 0.1 & 0.1 & 0.1 & 0.2 & 0.1 & 0.1 & 0.1 & 0.2 \\
\hline & & 0.1 & 0.1 & 0.2 & 0.1 & 0.1 & 0.1 & 0.1 \\
\hline & & 0.1 & & 0.2 & 0.1 & 0.1 & 0.1 & 0.1 \\
\hline & & 0.1 & 0.1 & 0.2 & & 0.1 & 0.1 & 0.1 \\
\hline & 0.1 & 0.1 & 0.1 & 0.1 & & 0.2 & 0.2 & 0.1 \\
\hline \multirow[t]{3}{*}{0.1} & 0.1 & 0.2 & 0.1 & 0.2 & 0.1 & 0.1 & 0.2 & 0.2 \\
\hline & & 0.1 & 0.1 & 0.2 & 0.1 & 0.1 & 0.2 & 0.1 \\
\hline & & 0.1 & & 0.1 & 0.1 & 0.1 & 0.1 & 0.2 \\
\hline \multirow[t]{2}{*}{0.1} & 0.1 & 0.2 & 0.1 & 0.1 & 0.1 & 0.1 & 0.2 & 0.1 \\
\hline & & 0.1 & 0.1 & 0.1 & 0.1 & 0.1 & 0.2 & 0.1 \\
\hline \multirow[t]{17}{*}{0.1} & 0.1 & 0.1 & 0.1 & 0.2 & 0.1 & 0.1 & 0.1 & 0.2 \\
\hline & & 0.1 & 0.1 & 0.2 & & 0.1 & 0.2 & 0.2 \\
\hline & & 0.1 & 0.1 & 0.3 & 0.1 & 0.1 & 0.1 & 0.1 \\
\hline & & 0.1 & & 0.1 & 0.1 & 0.1 & 0.2 & 0.1 \\
\hline & & 0.1 & 0.1 & 0.2 & & 0.1 & 0.1 & 0.2 \\
\hline & & 0.1 & 0.1 & 0.1 & & 0.1 & 0.1 & 0.1 \\
\hline & & 0.1 & 0.1 & 0.1 & 0.1 & 0.1 & 0.2 & 0.1 \\
\hline & & 0.1 & 0.1 & 0.2 & 0.1 & 0.1 & 0.1 & 0.2 \\
\hline & & 0.1 & 0.1 & 0.1 & 0.1 & 0.1 & 0.2 & 0.1 \\
\hline & & 0.1 & & 0.1 & & 0.2 & 0.1 & 0.2 \\
\hline & & 0.1 & & 0.1 & 0.1 & 0.1 & 0.1 & 0.2 \\
\hline & & 0.1 & & 0.1 & 0.1 & 0.1 & 0.2 & 0.2 \\
\hline & & 0.1 & & 0.1 & 0.2 & 0.1 & 0.1 & 0.1 \\
\hline & & 0.1 & 0.1 & 0.1 & 0.2 & 0.1 & 0.2 & 0.2 \\
\hline & & 0.1 & & 0.1 & 0.1 & 0.1 & 0.1 & 0.2 \\
\hline & & 0.1 & & 0.1 & & 0.1 & 0.2 & 0.1 \\
\hline & & 0.1 & 0.1 & 0.1 & 0.1 & 0.1 & 0.2 & 0.2 \\
\hline
\end{tabular}


wbheat

\begin{tabular}{|c|c|c|c|c|c|c|c|}
\hline & 0.1 & & 0.1 & 0.1 & 0.1 & & 0.2 \\
\hline & & & 0.1 & & & & \\
\hline & & & & & & & \\
\hline & & & & & 0.1 & & \\
\hline & & 0.1 & & & & & \\
\hline & & & & & & & \\
\hline & & & & & & & \\
\hline & & & & & & & \\
\hline & 0.1 & & & 0.1 & 0.1 & 0.1 & 0.1 \\
\hline & 0.1 & & 0.1 & & 0.1 & 0.1 & 0.1 \\
\hline & & & 0.1 & & 0.1 & 0.1 & 0.1 \\
\hline & 0.1 & & 0.1 & & & 0.1 & 0.1 \\
\hline & & & 0.1 & & 0.1 & 0.1 & 0.1 \\
\hline & 0.1 & 0.1 & 0.1 & & 0.1 & 0.1 & 0.1 \\
\hline & 0.1 & & 0.1 & 0.1 & 0.1 & 0.1 & 0.1 \\
\hline & 0.1 & & 0.1 & & 0.1 & 0.1 & 0.1 \\
\hline & 0.1 & & 0.1 & & 0.1 & 0.1 & 0.1 \\
\hline & 0.1 & & 0.1 & & 0.1 & 0.1 & 0.1 \\
\hline & 0.1 & & 0.1 & & & 0.1 & 0.1 \\
\hline & 0.1 & & 0.1 & & 0.1 & 0.1 & 0.1 \\
\hline & 0.1 & & 0.1 & 0.1 & 0.1 & 0.1 & 0.1 \\
\hline & 0.1 & & 0.1 & 0.1 & 0.1 & 0.1 & 0.1 \\
\hline & & & & & & & \\
\hline & & & & & & & \\
\hline & 0.1 & & 0.1 & & 0.1 & 0.1 & 0.1 \\
\hline & & 0.1 & 0.1 & & 0.1 & 0.1 & 0.1 \\
\hline & 0.1 & & 0.1 & & 0.1 & 0.1 & 0.1 \\
\hline & 0.1 & & 0.1 & & 0.1 & 0.1 & 0.1 \\
\hline & & 0.1 & 0.1 & 0.1 & 0.1 & 0.1 & 0.1 \\
\hline & & & 0.1 & 0.1 & 0.1 & 0.1 & 0.1 \\
\hline & 0.1 & & 0.1 & & 0.1 & 0.2 & 0.1 \\
\hline & 0.1 & 0.1 & 0.1 & & 0.1 & 0.1 & 0.2 \\
\hline & 0.1 & & 0.1 & & 0.1 & 0.1 & 0.1 \\
\hline & 0.1 & & 0.1 & & 0.1 & 0.2 & 0.1 \\
\hline & 0.1 & & 0.1 & 0.1 & 0.2 & 0.1 & 0.1 \\
\hline & 0.1 & & 0.1 & & 0.1 & 0.1 & 0.1 \\
\hline & 0.1 & & 0.1 & & 0.1 & 0.1 & 0.1 \\
\hline & & & 0.1 & 0.1 & & 0.1 & 0.1 \\
\hline & 0.1 & 0.1 & 0.1 & & & 0.1 & 0.1 \\
\hline & 0.1 & & 0.1 & 0.1 & 0.1 & 0.1 & 0.1 \\
\hline 0.021 & 0.031 & 0.072 & 0.113 & 0.031 & 0.103 & 0.134 & 0.072 \\
\hline & 0.1 & & 0.1 & & 0.1 & 0.1 & 0.1 \\
\hline & 0.1 & & 0.2 & & 0.1 & 0.1 & 0.1 \\
\hline & & & 0.1 & & & 0.1 & 0.1 \\
\hline & 0.1 & & 0.1 & & 0.1 & 0.2 & 0.2 \\
\hline & 0.1 & & 0.1 & 0.1 & 0.1 & 0.1 & 0.1 \\
\hline & 0.1 & & 0.1 & 0.1 & 0.1 & 0.1 & 0.1 \\
\hline & 0.1 & & 0.2 & & 0.1 & 0.1 & 0.1 \\
\hline & 0.1 & & 0.2 & 0.1 & 0.1 & 0.1 & 0.1 \\
\hline & 0.2 & 0.1 & 0.1 & & 0.1 & 0.1 & 0.1 \\
\hline & 0.1 & 0.1 & 0.1 & & 0.1 & 0.1 & 0.1 \\
\hline
\end{tabular}




\begin{tabular}{|c|c|c|c|c|c|c|c|c|}
\hline & & 0.1 & & 0.1 & 0.1 & 0.1 & 0.1 & 0.2 \\
\hline & & 0.1 & & 0.1 & & 0.1 & 0.1 & 0.1 \\
\hline & & 0.1 & & 0.1 & & 0.1 & 0.1 & 0.1 \\
\hline & & 0.1 & & 0.2 & & 0.1 & 0.1 & 0.1 \\
\hline & & 0.1 & & 0.1 & & 0.1 & 0.2 & 0.1 \\
\hline & & 0.1 & 0.1 & 0.2 & 0.1 & 0.1 & 0.1 & 0.2 \\
\hline & 0.2 & 0.3 & 0.3 & 0.2 & 0.3 & 0.1 & 0.1 & 0.1 \\
\hline & 0.1 & 0.3 & 0.2 & 0.2 & 0.1 & 0.1 & 0.1 & 0.1 \\
\hline & 0.1 & 0.3 & 0.2 & 0.2 & 0.1 & 0.2 & 0.2 & 0.2 \\
\hline & 0.2 & 0.4 & 0.3 & 0.3 & 0.2 & 0.1 & 0.2 & 0.1 \\
\hline & & 0.2 & 0.1 & 0.1 & 0.1 & 0.2 & 0.2 & 0.1 \\
\hline \multirow[t]{2}{*}{0.4} & 0.5 & 0.5 & 0.6 & 0.9 & 1 & 1 & 0.8 & 0.6 \\
\hline & 0.7 & 0.7 & 0.5 & 0.6 & 0.3 & 0.4 & 0.5 & 0.4 \\
\hline 0.1 & 0.5 & 0.7 & 0.5 & 0.4 & 0.3 & 0.2 & 0.2 & 0.2 \\
\hline \multirow{2}{*}{0.1} & 0.3 & 0.3 & 0.3 & 0.2 & 0.1 & 0.1 & 0.1 & 0.1 \\
\hline & 0.1 & 0.2 & 0.1 & 0.2 & 0.2 & 0.3 & 0.2 & 0.2 \\
\hline 0.6 & 0.8 & 0.8 & 0.8 & 0.8 & 0.6 & 0.6 & 0.6 & 0.6 \\
\hline \multirow[t]{2}{*}{0.1} & 0.6 & 0.7 & 0.5 & 0.5 & 0.4 & 0.4 & 0.5 & 0.4 \\
\hline & 0.5 & 0.5 & 0.3 & 0.3 & 0.1 & 0.2 & 0.2 & 0.2 \\
\hline \multirow[t]{3}{*}{0.1} & 0.4 & 0.4 & 0.4 & 0.3 & 0.1 & 0.1 & 0.3 & 0.1 \\
\hline & 0.2 & 0.3 & 0.1 & 0.2 & & 0.1 & 0.2 & 0.1 \\
\hline & 0.3 & 0.3 & 0.3 & 0.4 & 0.4 & 0.3 & 0.3 & 0.3 \\
\hline 0.1 & 0.2 & 0.3 & 0.2 & 0.3 & 0.1 & 0.2 & 0.3 & 0.2 \\
\hline 0.2 & 0.4 & 0.3 & 0.3 & 0.5 & 0.6 & 0.5 & 0.5 & 0.4 \\
\hline \multirow[t]{2}{*}{0.1} & 0.5 & 0.5 & 0.4 & 0.4 & 0.2 & 0.3 & 0.4 & 0.3 \\
\hline & 0.3 & 0.3 & 0.3 & 0.2 & 0.2 & 0.2 & 0.3 & 0.2 \\
\hline \multirow[t]{3}{*}{0.6} & 0.8 & 0.7 & 0.6 & 0.6 & 0.8 & 0.5 & 0.5 & 0.3 \\
\hline & 0.6 & 0.6 & 0.5 & 0.3 & 0.2 & 0.2 & 0.3 & 0.2 \\
\hline & 0.2 & 0.3 & 0.3 & 0.4 & 0.2 & 0.3 & 0.3 & 0.3 \\
\hline 1.4 & 1.2 & 1.2 & 1 & 1 & 0.7 & 0.6 & 0.5 & 0.5 \\
\hline \multirow[t]{4}{*}{0.4} & 0.7 & 0.8 & 0.6 & 0.6 & 0.3 & 0.3 & 0.4 & 0.3 \\
\hline & 0.5 & 0.5 & 0.3 & 0.3 & 0.2 & 0.2 & 0.3 & 0.2 \\
\hline & 0.2 & 0.3 & 0.3 & 0.2 & 0.1 & 0.1 & 0.2 & 0.2 \\
\hline & 0.1 & 0.3 & 0.2 & 0.2 & 0.1 & 0.1 & 0.2 & 0.2 \\
\hline 0.4 & 0.8 & 0.7 & 0.7 & 0.8 & 0.5 & 0.3 & 0.4 & 0.4 \\
\hline 0.4 & 0.7 & 0.6 & 0.6 & 0.6 & 0.3 & 0.3 & 0.4 & 0.3 \\
\hline \multirow[t]{3}{*}{0.1} & 0.6 & 0.5 & 0.4 & 0.4 & 0.2 & 0.2 & 0.2 & 0.3 \\
\hline & 0.2 & 0.3 & 0.2 & 0.1 & 0.1 & 0.1 & 0.2 & 0.2 \\
\hline & 0.1 & 0.2 & 0.1 & 0.2 & 0.1 & 0.1 & 0.2 & 0.2 \\
\hline 0.1 & 0.7 & 0.6 & 0.5 & & 0.3 & 0.4 & 0.3 & 0.3 \\
\hline 0.4 & 0.8 & 0.8 & 0.6 & 0.5 & 0.3 & 0.4 & 0.6 & 0.3 \\
\hline 0.5 & 0.7 & 0.6 & 0.6 & 0.5 & 0.4 & 0.5 & 0.5 & 0.3 \\
\hline 0.6 & 0.7 & 0.7 & 0.6 & 0.6 & 0.4 & 0.6 & 0.5 & 0.5 \\
\hline 0.3 & 0.8 & 0.7 & 0.5 & 0.6 & 0.3 & 0.3 & 0.3 & 0.4 \\
\hline 1 & 1.5 & 1.2 & 0.9 & 0.6 & 0.9 & 0.8 & 0.7 & 0.5 \\
\hline 0.5 & 0.8 & 0.9 & 0.7 & 0.7 & 0.4 & 0.5 & 0.5 & 0.4 \\
\hline 0.5 & 0.6 & 0.6 & 0.5 & 0.5 & 0.4 & 0.8 & 0.8 & 0.6 \\
\hline 0.2 & 0.5 & 0.6 & 0.4 & 0.4 & 0.2 & 0.2 & 0.3 & 0.3 \\
\hline 0.5 & 0.5 & 0.6 & 0.5 & 0.8 & 0.6 & 0.6 & 0.8 & 0.6 \\
\hline 0.6 & 0.9 & 0.7 & 0.6 & 0.7 & 0.4 & 0.4 & 0.5 & 0.4 \\
\hline 0.1 & 0.4 & 0.4 & 0.3 & 0.4 & 0.2 & 0.3 & 0.3 & 0.3 \\
\hline
\end{tabular}


wbheat

\begin{tabular}{|c|c|c|c|c|c|c|c|c|}
\hline 0.4 & 0.5 & 0.5 & 0.5 & 0.5 & 0.3 & 0.3 & 0.3 & 0.3 \\
\hline 0.2 & 0.5 & 0.5 & 0.4 & 0.4 & 0.3 & 0.4 & 0.4 & 0.4 \\
\hline 0.5 & 0.5 & 0.6 & 0.6 & 0.6 & 0.4 & 0.4 & 0.4 & 0.3 \\
\hline 0.6 & 0.6 & 0.7 & 0.9 & 1.1 & 1 & 1 & 1.1 & 1.2 \\
\hline 1.1 & 1.3 & 1.5 & 1.3 & 1.2 & 1.1 & 0.9 & 1 & 0.9 \\
\hline 0.6 & 0.8 & 0.8 & 0.7 & 0.5 & 0.5 & 0.5 & 0.5 & 0.5 \\
\hline 0.9 & 1 & 0.9 & 0.9 & 1 & 0.7 & 0.8 & 0.8 & 0.7 \\
\hline 0.5 & 1 & 0.9 & 0.8 & 0.8 & 0.4 & 0.4 & 0.5 & 0.3 \\
\hline 0.2 & 0.3 & 0.3 & 0.3 & 0.2 & 0.1 & 0.2 & 0.2 & 0.2 \\
\hline 0.1 & 0.2 & 0.2 & 0.1 & 0.1 & 0.1 & 0.2 & 0.3 & 0.2 \\
\hline 0.1 & 0.2 & 0.3 & 0.3 & 0.5 & 0.3 & 0.3 & 0.5 & 0.4 \\
\hline 0.5 & 0.6 & 0.6 & 0.6 & 0.7 & 0.6 & 0.7 & 0.8 & 0.7 \\
\hline 0.9 & 1.1 & 1.1 & 1 & 1.2 & 1.1 & 1.2 & 1.2 & 1 \\
\hline 1.4 & 1.3 & 1.4 & 1.3 & 1.2 & 0.9 & 1 & 1 & 1 \\
\hline 1.1 & 1.2 & 1.2 & 1.2 & 1.1 & 0.9 & 0.8 & 0.8 & 0.7 \\
\hline 0.6 & 0.9 & 0.8 & 0.8 & 0.8 & 0.6 & 0.6 & 0.6 & 0.5 \\
\hline 0.3 & 0.7 & 0.5 & 0.5 & 0.6 & 0.3 & 0.3 & 0.2 & 0.2 \\
\hline 0.1 & 0.2 & 0.2 & 0.2 & 0.2 & 0.1 & 0.1 & 0.2 & 0.2 \\
\hline 0.2 & 0.4 & 0.6 & 0.7 & 0.9 & 0.7 & 0.8 & 0.8 & 1 \\
\hline 1.4 & 1.6 & 1.5 & 1.4 & 1.4 & 1.3 & 1.3 & 1.3 & 1.2 \\
\hline 1.3 & 1.6 & 1.6 & 1.4 & 1.3 & 0.9 & 0.8 & 0.7 & 0.8 \\
\hline 1.3 & 1.2 & 1.2 & 1.2 & 1.1 & 0.9 & 0.7 & 0.7 & 0.6 \\
\hline 0.3 & 0.8 & 0.7 & 0.6 & 0.7 & 0.4 & 0.4 & 0.4 & 0.4 \\
\hline 0.3 & 0.6 & 0.6 & 0.8 & 0.8 & 0.4 & 0.7 & 0.5 & 0.3 \\
\hline 0.2 & 0.6 & 0.6 & 0.5 & 0.4 & 0.3 & 0.3 & 0.3 & 0.3 \\
\hline \multirow[t]{2}{*}{0.1} & 0.2 & 0.3 & 0.2 & 0.2 & 0.1 & 0.2 & 0.3 & 0.2 \\
\hline & & 0.1 & & 0.1 & 0.1 & 0.2 & 0.1 & 0.1 \\
\hline 0.1 & 0.1 & 0.2 & 0.2 & 0.4 & 0.2 & 0.3 & 0.3 & 0.2 \\
\hline 0.1 & 0.2 & 0.3 & 0.3 & 0.6 & 0.5 & 0.6 & 0.6 & 0.5 \\
\hline 0.7 & 0.7 & 0.8 & 1.2 & 1.3 & 0.9 & 0.8 & 0.8 & 0.7 \\
\hline 0.4 & 0.9 & 0.8 & 0.7 & 0.6 & 0.4 & 0.5 & 0.6 & 0.6 \\
\hline 0.7 & 0.8 & 0.8 & 0.7 & 0.8 & 0.5 & 0.6 & 0.6 & 0.6 \\
\hline 0.4 & 0.6 & 0.6 & 0.5 & 0.7 & 0.5 & 0.5 & 0.6 & 0.6 \\
\hline 0.8 & 0.8 & 0.8 & 0.7 & 0.9 & 0.6 & 0.7 & 0.6 & 0.5 \\
\hline 0.5 & 0.7 & 0.7 & 0.7 & 0.7 & 0.5 & 0.5 & 0.6 & 0.5 \\
\hline 0.1 & 0.2 & 0.3 & 0.2 & 0.3 & 0.1 & 0.2 & 0.3 & 0.2 \\
\hline \multirow[t]{2}{*}{0.1} & 0.1 & 0.2 & 0.1 & 0.2 & & 0.1 & 0.1 & 0.2 \\
\hline & 0.1 & 0.2 & 0.2 & 0.3 & 0.1 & 0.2 & 0.2 & 0.2 \\
\hline 0.3 & 0.3 & 0.3 & 0.8 & 0.9 & 0.8 & 0.8 & 0.7 & 0.7 \\
\hline 0.9 & 1.1 & 1.1 & 1.2 & 1 & 0.8 & 0.6 & 0.6 & 0.5 \\
\hline \multirow[t]{11}{*}{0.1} & 0.4 & 0.6 & 0.7 & 0.8 & 0.5 & 0.3 & 0.3 & 0.2 \\
\hline & & 0.1 & 0.1 & 0.1 & & 0.1 & 0.2 & 0.1 \\
\hline & & 0.1 & & 0.2 & 0.1 & & 0.2 & 0.3 \\
\hline & & & 0.1 & 0.1 & & 0.1 & 0.1 & 0.2 \\
\hline & & 0.1 & & 0.2 & 0.1 & 0.2 & 0.2 & 0.2 \\
\hline & & 0.1 & & 0.1 & 0.1 & 0.1 & 0.3 & 0.5 \\
\hline & & 0.1 & 0.4 & 0.9 & 0.6 & 0.5 & 0.4 & 0.2 \\
\hline & 0.1 & 0.6 & 0.5 & 0.5 & 0.1 & 0.1 & 0.2 & 0.1 \\
\hline & & & & & & & & \\
\hline & & & & & & & & \\
\hline & & & & & & & & \\
\hline
\end{tabular}




\begin{tabular}{|c|c|c|c|c|c|c|c|c|}
\hline & & & & & & & 0.1 & 0.1 \\
\hline & & 0.1 & & 0.1 & & 0.1 & 0.2 & 0.1 \\
\hline & 0.1 & 0.3 & 1.1 & 0.7 & 0.4 & 0.4 & 0.4 & 0.3 \\
\hline & 0.1 & 0.5 & 1.2 & 0.8 & 0.3 & 0.3 & 0.2 & 0.1 \\
\hline & & 0.3 & 0.7 & 0.3 & 0.2 & 0.1 & 0.2 & 0.2 \\
\hline & & 0.1 & & 0.1 & 0.2 & 0.1 & 0.1 & 0.1 \\
\hline & & 0.1 & 0.3 & 0.2 & 0.1 & 0.1 & 0.1 & 0.1 \\
\hline & & 0.1 & 0.1 & 0.1 & & 0.1 & 0.1 & 0.1 \\
\hline & & & & & & & & \\
\hline & & & & & & & & \\
\hline & & & & & & & & \\
\hline & & & & & & & & \\
\hline & & & & & & & & \\
\hline & & & & & & & & \\
\hline & & & & & & & & \\
\hline & & & & & & & & \\
\hline 0.4 & 0.5 & 0.5 & 0.5 & 0.5 & 0.5 & 0.4 & 0.4 & 0.4 \\
\hline 0.5 & 0.5 & 0.5 & 0.5 & 0.5 & 0.6 & 0.6 & 0.5 & 0.5 \\
\hline 0.5 & 0.5 & 0.5 & 0.5 & 0.5 & 0.5 & 0.5 & 0.5 & 0.4 \\
\hline 0.5 & 0.5 & 0.5 & 0.5 & 0.5 & 0.5 & 0.4 & 0.3 & 0.3 \\
\hline 0.1 & 0.1 & 0.1 & 0.1 & 0.1 & 0.1 & 0.1 & 0.1 & 0.1 \\
\hline 0.2 & 0.2 & 0.2 & 0.2 & 0.2 & 0.2 & 0.2 & 0.1 & 0.1 \\
\hline 0.3 & 0.4 & 0.4 & 0.4 & 0.4 & 0.4 & 0.4 & 0.4 & 0.4 \\
\hline 0.5 & 0.6 & 0.6 & 0.6 & 0.5 & 0.5 & 0.5 & 0.4 & 0.4 \\
\hline 0.2 & 0.2 & 0.2 & 0.2 & 0.2 & 0.1 & 0.1 & 0.1 & 0.1 \\
\hline 0.1 & 0.1 & 0.1 & 0.1 & 0.1 & 0.1 & 0.1 & 0.1 & 0.1 \\
\hline 0.2 & 0.2 & 0.2 & 0.3 & 0.3 & 0.3 & 0.3 & 0.3 & 0.2 \\
\hline 0.5 & 0.5 & 0.5 & 0.5 & 0.5 & 0.5 & 0.5 & 0.4 & 0.4 \\
\hline 0.5 & 0.5 & 0.5 & 0.5 & 0.5 & 0.5 & 0.5 & 0.4 & 0.5 \\
\hline 0.5 & 0.5 & 0.5 & 0.5 & 0.5 & 0.5 & 0.5 & 0.5 & 0.5 \\
\hline 0.6 & 0.6 & 0.6 & 0.6 & 0.6 & 0.6 & 0.7 & 0.6 & 0.7 \\
\hline 0.7 & 0.8 & 0.8 & 0.8 & 0.8 & 0.7 & 0.7 & 0.6 & 0.6 \\
\hline 0.4 & 0.4 & 0.5 & 0.5 & 0.4 & 0.4 & 0.4 & 0.4 & 0.3 \\
\hline 0.4 & 0.4 & 0.4 & 0.4 & 0.4 & 0.4 & 0.4 & 0.4 & 0.4 \\
\hline 0.3 & 0.4 & 0.4 & 0.4 & 0.4 & 0.3 & 0.3 & 0.3 & 0.3 \\
\hline 0.3 & 0.3 & 0.3 & 0.3 & 0.3 & 0.3 & 0.3 & 0.3 & 0.3 \\
\hline 0.5 & 0.5 & 0.6 & 0.6 & 0.6 & 0.6 & 0.5 & 0.5 & 0.4 \\
\hline 0.5 & 0.5 & 0.5 & 0.5 & 0.5 & 0.5 & 0.5 & 0.4 & 0.4 \\
\hline 0.2 & 0.2 & 0.2 & 0.2 & 0.2 & 0.3 & 0.2 & 0.2 & 0.2 \\
\hline 0.3 & 0.3 & 0.3 & 0.3 & 0.3 & 0.3 & 0.3 & 0.2 & 0.2 \\
\hline 0.1 & 0.1 & 0.1 & 0.1 & 0.1 & 0.2 & 0.1 & 0.2 & 0.1 \\
\hline 0.2 & 0.2 & 0.2 & 0.2 & 0.2 & 0.2 & 0.2 & 0.2 & 0.2 \\
\hline 0.2 & 0.3 & 0.3 & 0.3 & 0.3 & 0.3 & 0.3 & 0.2 & 0.2 \\
\hline 0.3 & 0.3 & 0.3 & 0.3 & 0.3 & 0.3 & 0.3 & 0.2 & 0.2 \\
\hline 0.2 & 0.2 & 0.2 & 0.3 & 0.3 & 0.2 & 0.2 & 0.2 & 0.2 \\
\hline 0.2 & 0.2 & 0.2 & 0.2 & 0.2 & 0.2 & 0.2 & 0.2 & 0.2 \\
\hline 0.2 & 0.1 & 0.2 & 0.2 & 0.1 & 0.2 & 0.2 & 0.2 & 0.2 \\
\hline 0.2 & 0.2 & 0.2 & 0.2 & 0.1 & 0.2 & 0.2 & 0.1 & 0.1 \\
\hline 0.2 & 0.2 & 0.3 & 0.2 & 0.2 & 0.2 & 0.2 & 0.2 & 0.2 \\
\hline 0.2 & 0.2 & 0.2 & 0.2 & 0.2 & 0.2 & 0.2 & 0.2 & 0.2 \\
\hline 0.1 & 0.1 & 0.1 & 0.1 & 0.1 & 0.1 & 0.1 & 0.1 & 0.1 \\
\hline
\end{tabular}


wbheat

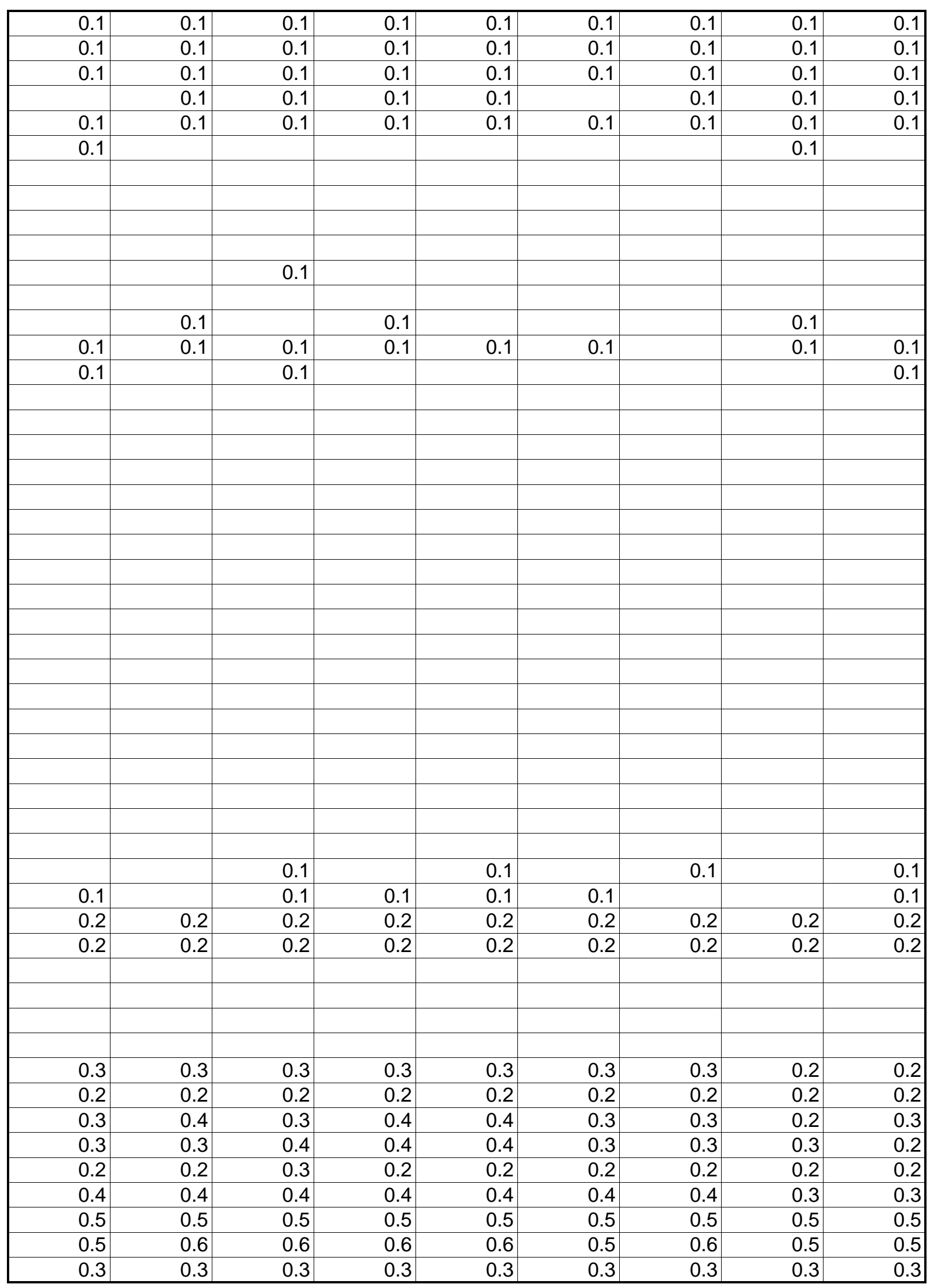


wbheat

\begin{tabular}{|c|c|c|c|c|c|c|c|c|}
\hline 0.2 & 0.2 & 0.2 & 0.2 & 0.3 & 0.3 & 0.2 & 0.2 & 0.2 \\
\hline 0.4 & 0.4 & 0.4 & 0.4 & 0.4 & 0.4 & 0.4 & 0.3 & 0.4 \\
\hline 0.7 & 0.7 & 0.7 & 0.7 & 0.7 & 0.7 & 0.7 & 0.7 & 0.7 \\
\hline 0.4 & 0.4 & 0.4 & 0.5 & 0.4 & 0.4 & 0.4 & 0.4 & 0.3 \\
\hline 0.5 & 0.5 & 0.5 & 0.5 & 0.5 & 0.5 & 0.5 & 0.4 & 0.5 \\
\hline 0.2 & 0.3 & 0.2 & 0.3 & 0.3 & 0.3 & 0.2 & 0.2 & 0.2 \\
\hline 0.5 & 0.5 & 0.5 & 0.5 & 0.5 & 0.5 & 0.5 & 0.5 & 0.4 \\
\hline 0.6 & 0.6 & 0.6 & 0.6 & 0.6 & 0.6 & 0.6 & 0.5 & 0.5 \\
\hline 0.6 & 0.6 & 0.6 & 0.6 & 0.6 & 0.5 & 0.4 & 0.4 & 0.4 \\
\hline 0.5 & 0.5 & 0.5 & 0.5 & 0.5 & 0.5 & 0.4 & 0.4 & 0.3 \\
\hline 0.5 & 0.5 & 0.6 & 0.5 & 0.5 & 0.5 & 0.4 & 0.4 & 0.3 \\
\hline 0.4 & 0.4 & 0.4 & 0.4 & 0.4 & 0.4 & 0.4 & 0.4 & 0.4 \\
\hline 0.4 & 0.3 & 0.4 & 0.4 & 0.4 & 0.4 & 0.4 & 0.4 & 0.4 \\
\hline 0.3 & 0.3 & 0.3 & 0.3 & 0.3 & 0.3 & 0.3 & 0.2 & 0.2 \\
\hline 0.2 & 0.2 & 0.2 & 0.2 & 0.2 & 0.2 & 0.2 & 0.2 & 0.2 \\
\hline 0.5 & 0.5 & 0.5 & 0.5 & 0.5 & 0.5 & 0.5 & 0.4 & 0.4 \\
\hline 0.4 & 0.3 & 0.4 & 0.4 & 0.4 & 0.4 & 0.4 & 0.4 & 0.4 \\
\hline 0.4 & 0.4 & 0.4 & 0.4 & 0.4 & 0.4 & 0.3 & 0.3 & 0.2 \\
\hline 0.3 & 0.4 & 0.3 & 0.4 & 0.4 & 0.3 & 0.3 & 0.3 & 0.2 \\
\hline 0.1 & 0.1 & 0.1 & 0.2 & 0.1 & 0.1 & 0.1 & 0.1 & 0.1 \\
\hline 0.1 & 0.1 & 0.1 & 0.1 & 0.1 & 0.1 & 0.1 & 0.1 & 0.1 \\
\hline 0.1 & 0.1 & 0.1 & 0.1 & 0.1 & 0.1 & 0.1 & 0.1 & 0.1 \\
\hline 0.1 & 0.1 & 0.2 & 0.1 & 0.2 & 0.2 & 0.2 & 0.2 & 0.2 \\
\hline \multirow[t]{3}{*}{0.1} & 0.1 & 0.1 & 0.1 & 0.1 & 0.1 & 0.1 & 0.1 & 0.1 \\
\hline & 0.1 & 0.1 & 0.1 & 0.1 & 0.1 & 0.1 & 0.1 & \\
\hline & 0.1 & 0.1 & 0.1 & 0.1 & 0.1 & 0.1 & 0.1 & \\
\hline 0.1 & 0.1 & 0.1 & 0.1 & 0.1 & 0.1 & 0.1 & 0.1 & 0.1 \\
\hline \multirow[t]{8}{*}{0.1} & 0.1 & & 0.1 & & & 0.1 & & \\
\hline & & & 0.1 & & & & 0.1 & \\
\hline & & & & & & & & \\
\hline & 0.1 & & & & & 0.1 & & 0.1 \\
\hline & & & & & & & & \\
\hline & & & & & & & & \\
\hline & 0.1 & & 0.1 & & & 0.1 & & \\
\hline & & & & & 0.1 & & & \\
\hline & & & & & & & & \\
\hline & & & & & & & & \\
\hline & & & & & & & & \\
\hline & & & & & & & & \\
\hline & & & & & & & & \\
\hline & & & & & & & & \\
\hline & & & & & & & & \\
\hline & & & & & & & & \\
\hline & & & & & & & & \\
\hline & & & & & & & & \\
\hline & & & & & & & & \\
\hline & & & & & & & & \\
\hline & & & & & & & & \\
\hline & & & & & & & & \\
\hline & & & & & & & & \\
\hline & & & & & & & & \\
\hline
\end{tabular}


wbheat

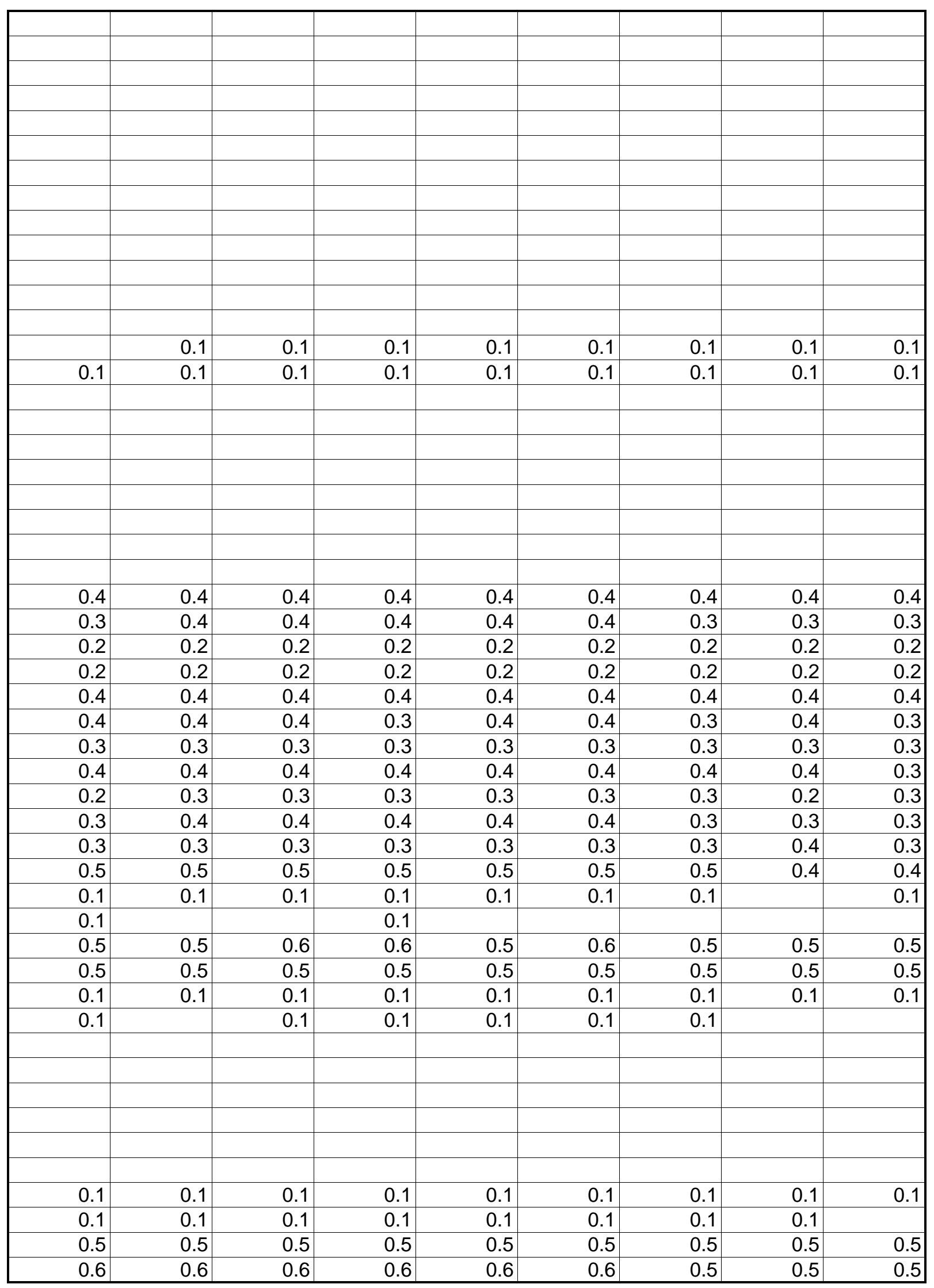


wbheat

\begin{tabular}{|c|c|c|c|c|c|c|c|c|}
\hline 0.7 & 0.7 & 0.7 & 0.7 & 0.7 & 0.7 & 0.6 & 0.6 & 0.5 \\
\hline 0.5 & 0.5 & 0.5 & 0.5 & 0.5 & 0.5 & 0.5 & 0.5 & 0.5 \\
\hline 0.5 & 0.5 & 0.5 & 0.6 & 0.5 & 0.5 & 0.5 & 0.5 & 0.5 \\
\hline 0.1 & 0.1 & 0.1 & 0.1 & 0.1 & 0.1 & 0.1 & 0.1 & 0.1 \\
\hline 0.1 & 0.1 & 0.1 & 0.1 & 0.1 & 0.1 & 0.1 & 0.1 & 0.1 \\
\hline 0.1 & 0.1 & 0.1 & 0.1 & 0.1 & 0.1 & 0.1 & 0.1 & 0.1 \\
\hline 0.1 & 0.1 & 0.1 & 0.1 & 0.1 & 0.1 & 0.1 & 0.1 & 0.1 \\
\hline 0.1 & 0.1 & 0.1 & 0.1 & 0.1 & 0.2 & 0.1 & 0.2 & 0.1 \\
\hline 0.7 & 0.7 & 0.8 & 0.7 & 0.7 & 0.7 & 0.6 & 0.6 & 0.5 \\
\hline 0.3 & 0.3 & 0.3 & 0.4 & 0.3 & 0.3 & 0.3 & 0.2 & 0.2 \\
\hline 0.2 & 0.2 & 0.2 & 0.2 & 0.2 & 0.2 & 0.2 & 0.2 & 0.1 \\
\hline 0.2 & 0.2 & 0.2 & 0.2 & 0.2 & 0.2 & 0.2 & 0.2 & 0.2 \\
\hline 0.2 & 0.2 & 0.2 & 0.2 & 0.2 & 0.2 & 0.2 & 0.2 & 0.1 \\
\hline 0.2 & 0.3 & 0.3 & 0.3 & 0.3 & 0.3 & 0.3 & 0.3 & 0.3 \\
\hline 0.4 & 0.4 & 0.4 & 0.4 & 0.4 & 0.3 & 0.3 & 0.3 & 0.3 \\
\hline & & & & & & & & \\
\hline & & & & & & & & \\
\hline & & & & & & & & \\
\hline & & & & & & & & \\
\hline & & & & & 0.1 & 0.1 & 0.1 & \\
\hline & & & & & & & & \\
\hline & & & & & & & & \\
\hline & & & & 0.1 & 0.1 & 0.1 & 0.1 & \\
\hline & 0.1 & 0.1 & & & & & & \\
\hline & & & & & 0.1 & & & \\
\hline & & & & & & & & \\
\hline & & & & & & & & \\
\hline & & & & & & & & \\
\hline & & & & & & & & \\
\hline & & & & & & & & \\
\hline & & & & & & & & \\
\hline & & & & & & & & \\
\hline & & & & & & & & \\
\hline & & & & & 0.1 & & & \\
\hline & & & & & & & & \\
\hline & & & & & & & & \\
\hline & & & & & & & & \\
\hline & & & & & & & & \\
\hline & & & & & & & & \\
\hline & & & & & & & 0.1 & \\
\hline & & & & & & & & \\
\hline & & & & & & & & \\
\hline & & & & & & & & \\
\hline & & & & & & & & \\
\hline & & & & 0.1 & & & & \\
\hline
\end{tabular}


wbheat

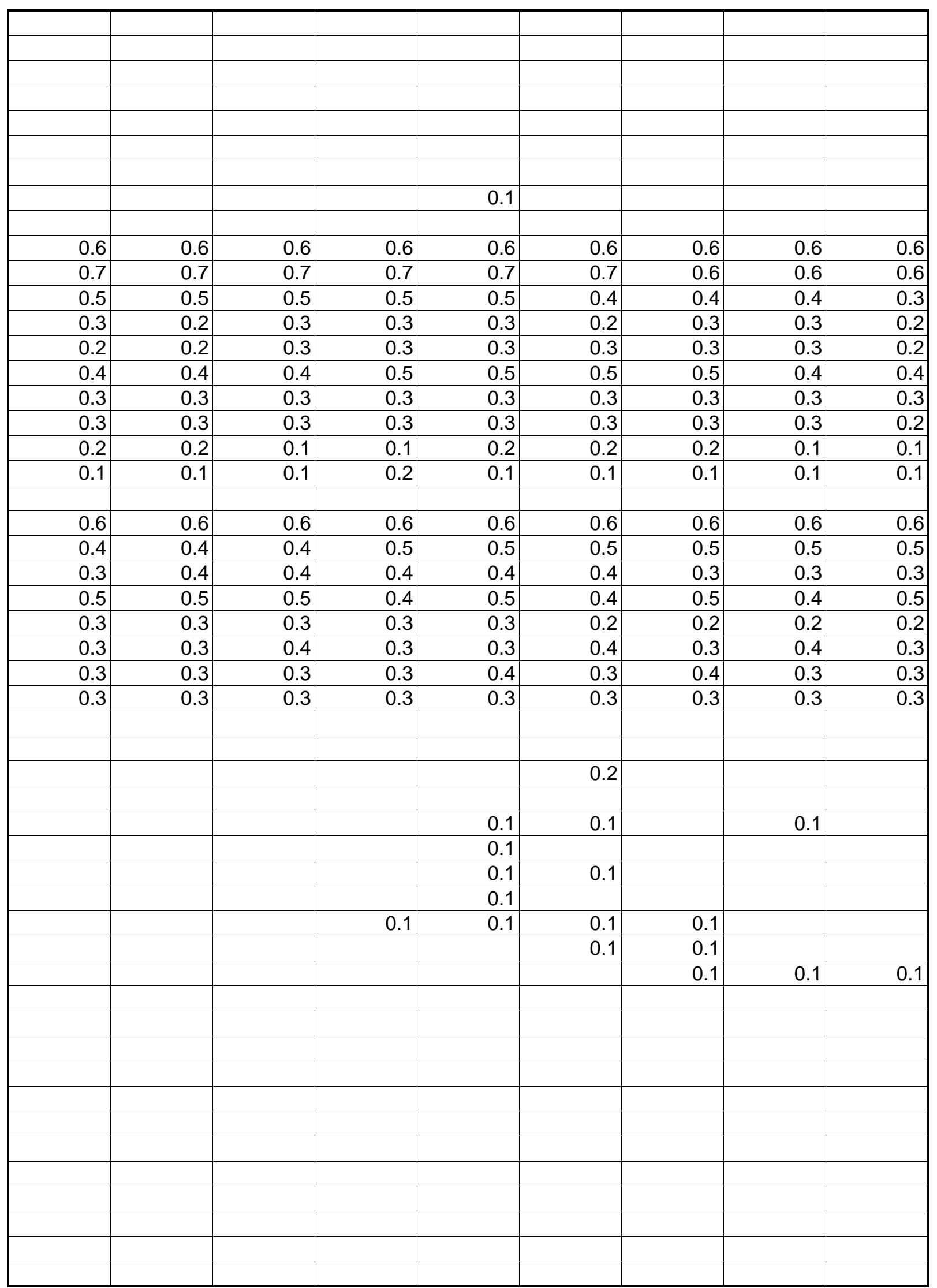


wbheat

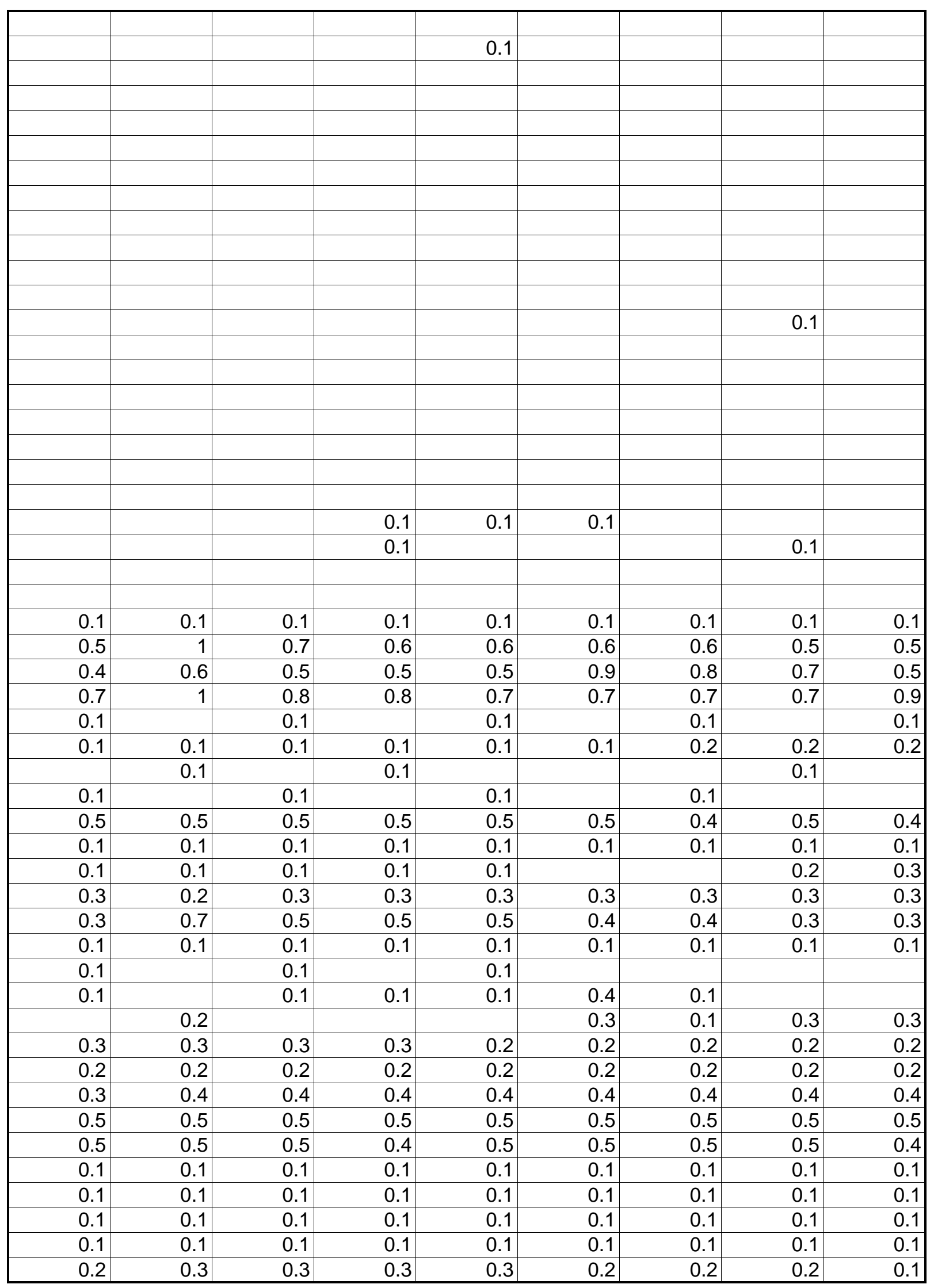


wbheat

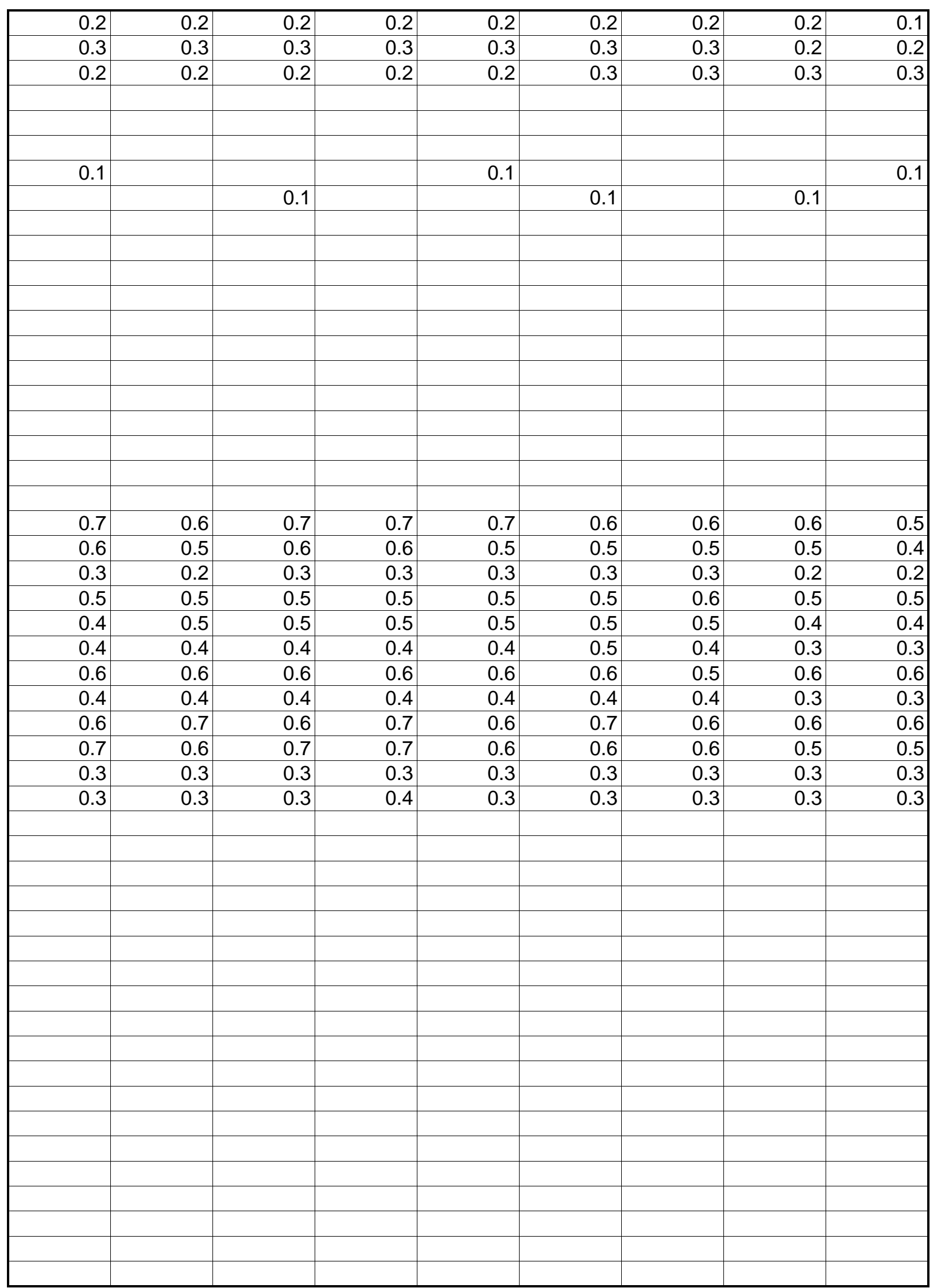


wbheat

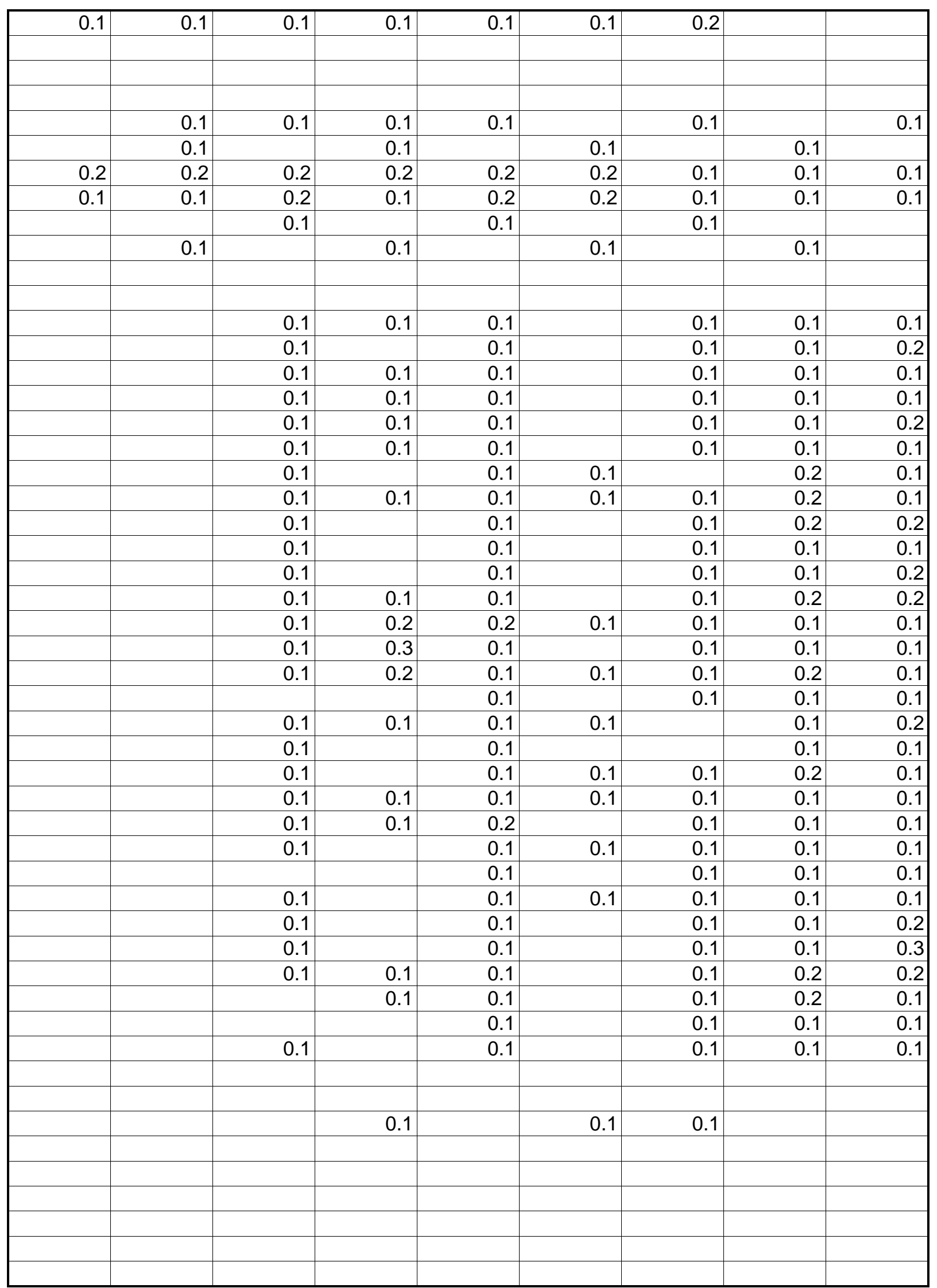


wbheat

\begin{tabular}{|c|c|c|c|c|c|c|c|c|}
\hline & & & & & & & & \\
\hline & & & & & & & & \\
\hline & & 0.1 & 0.1 & 0.1 & & & 0.1 & 0.1 \\
\hline & & 0.1 & & 0.1 & & 0.1 & 0.1 & 0.1 \\
\hline & & 0.1 & & 0.1 & & & 0.1 & 0.1 \\
\hline & & 0.1 & & 0.1 & & 0.1 & 0.1 & 0.2 \\
\hline & & 0.1 & & 0.1 & & 0.1 & 0.1 & 0.1 \\
\hline & & 0.1 & 0.1 & 0.1 & & 0.1 & & 0.1 \\
\hline & & & & 0.1 & & 0.1 & 0.1 & 0.1 \\
\hline & & 0.1 & & 0.1 & & 0.1 & 0.1 & 0.1 \\
\hline & & 0.1 & & 0.1 & & 0.1 & 0.1 & 0.1 \\
\hline & & 0.1 & & 0.1 & & 0.1 & & 0.1 \\
\hline & & & & 0.1 & & 0.1 & 0.1 & 0.1 \\
\hline & & 0.1 & & 0.1 & 0.1 & 0.1 & 0.1 & 0.1 \\
\hline & & 0.1 & 0.1 & 0.1 & & 0.1 & 0.1 & 0.1 \\
\hline & & & & 0.1 & 0.1 & 0.2 & 0.1 & 0.1 \\
\hline & & 0.1 & & 0.1 & & 0.1 & 0.1 & 0.1 \\
\hline & & 0.1 & & 0.1 & & & 0.1 & 0.1 \\
\hline & & 0.1 & & 0.1 & & & 0.1 & 0.1 \\
\hline & & 0.1 & & 0.1 & & 0.1 & 0.1 & 0.1 \\
\hline & & & & 0.1 & & 0.1 & 0.1 & 0.1 \\
\hline & & 0.1 & & 0.1 & & 0.1 & 0.1 & 0.1 \\
\hline & & 0.1 & & 0.1 & & 0.1 & 0.1 & 0.1 \\
\hline & & 0.1 & & 0.1 & & 0.1 & 0.1 & 0.1 \\
\hline & & 0.1 & & 0.2 & & 0.1 & 0.1 & 0.1 \\
\hline & & 0.1 & & 0.1 & & 0.1 & 0.2 & 0.1 \\
\hline & & & 0.1 & 0.1 & & & 0.1 & \\
\hline & & 0.1 & & 0.1 & & & 0.2 & \\
\hline & & 0.1 & & 0.2 & & 0.1 & 0.1 & 0.2 \\
\hline & & 0.1 & 0.1 & 0.1 & & 0.1 & 0.1 & 0.2 \\
\hline & & 0.1 & 0.1 & 0.1 & 0.1 & & 0.1 & 0.1 \\
\hline & & 0.1 & & 0.1 & & & 0.1 & 0.1 \\
\hline & & & & 0.2 & & 0.1 & 0.1 & 0.1 \\
\hline & & 0.1 & & 0.1 & 0.1 & 0.1 & 0.1 & 0.1 \\
\hline & & 0.093 & 0.041 & 0.196 & 0.031 & 0.072 & 0.093 & 0.093 \\
\hline & & 0.1 & & 0.1 & & 0.1 & 0.1 & 0.1 \\
\hline & & 0.1 & & 0.1 & & 0.1 & 0.1 & 0.1 \\
\hline & & 0.1 & & 0.1 & & & 0.1 & 0.1 \\
\hline & & 0.1 & & 0.2 & 0.1 & 0.1 & 0.1 & 0.1 \\
\hline & & 0.1 & & 0.1 & & & 0.1 & 0.1 \\
\hline & & & 0.2 & 0.1 & 0.1 & & & \\
\hline & & 0.1 & 0.2 & 0.2 & 0.1 & 0.1 & 0.1 & 0.1 \\
\hline & & 0.1 & 0.2 & 0.2 & & 0.1 & 0.1 & 0.1 \\
\hline & & 0.1 & 0.1 & 0.1 & & & 0.1 & \\
\hline & & 0.1 & & 0.2 & & 0.1 & 0.1 & 0.1 \\
\hline & & 0.1 & & 0.2 & & 0.1 & 0.1 & 0.1 \\
\hline & & 0.1 & 0.1 & 0.1 & & 0.1 & 0.1 & 0.2 \\
\hline & & 0.1 & & 0.1 & & 0.1 & 0.1 & 0.1 \\
\hline & & 0.1 & & 0.1 & 0.1 & 0.2 & 0.1 & 0.2 \\
\hline & & 0.1 & 0.1 & 0.2 & 0.1 & 0.1 & 0.2 & 0.2 \\
\hline
\end{tabular}


wbheat

\begin{tabular}{|c|c|c|c|c|c|c|c|c|}
\hline & & 0.1 & & 0.1 & & 0.3 & 0.1 & 0.1 \\
\hline & & 0.1 & & 0.2 & 0.1 & 0.1 & 0.1 & 0.1 \\
\hline & & 0.2 & 0.6 & 0.5 & 0.1 & 0.2 & 0.3 & 0.1 \\
\hline & & 0.2 & 0.5 & 0.4 & 0.2 & 0.2 & 0.1 & 0.1 \\
\hline & & 0.1 & 0.3 & 0.2 & 0.1 & 0.2 & 0.1 & 0.1 \\
\hline & & & 0.1 & 0.2 & & 0.1 & 0.1 & 0.2 \\
\hline & & 0.3 & 0.4 & 0.3 & 0.1 & 0.1 & 0.2 & 0.1 \\
\hline & & 0.1 & 0.2 & 0.2 & 0.1 & 0.1 & 0.1 & 0.2 \\
\hline & & 0.1 & & 0.2 & 0.1 & 0.1 & 0.1 & 0.2 \\
\hline & & 0.1 & & 0.1 & & 0.1 & 0.1 & 0.4 \\
\hline & & 0.2 & 0.5 & 0.4 & 0.1 & 0.1 & 0.1 & 0.1 \\
\hline & & 0.1 & 0.1 & 0.1 & & & 0.1 & 0.1 \\
\hline & & 0.1 & & 0.2 & 0.1 & 0.1 & 0.2 & 0.1 \\
\hline & & 0.3 & 0.4 & 0.2 & 0.1 & 0.1 & 0.2 & 0.2 \\
\hline & & 0.1 & 0.3 & 0.3 & 0.1 & 0.1 & 0.1 & 0.1 \\
\hline & & & & 0.1 & 0.1 & 0.1 & 0.1 & 0.1 \\
\hline & & 0.1 & 0.2 & 0.2 & 0.1 & 0.1 & 0.1 & 0.1 \\
\hline & & 0.3 & 0.6 & 0.5 & 0.3 & 0.2 & 0.2 & 0.1 \\
\hline & & 0.1 & 0.3 & 0.2 & 0.2 & 0.1 & 0.1 & 0.1 \\
\hline & & 0.2 & 0.6 & 0.4 & 0.3 & 0.2 & 0.2 & 0.1 \\
\hline & & 0.3 & 0.9 & 0.4 & 0.2 & 0.2 & 0.1 & 0.2 \\
\hline & & 0.2 & 0.6 & 0.4 & 0.1 & 0.2 & 0.1 & 0.2 \\
\hline & & & & & & & & \\
\hline & 0.1 & 0.9 & 1.3 & 0.8 & 0.4 & 0.4 & 0.3 & 0.3 \\
\hline & & 0.7 & 1.1 & 0.7 & 0.4 & 0.4 & 0.4 & 0.2 \\
\hline & 0.1 & 0.9 & 1.2 & 0.8 & 0.4 & 0.4 & 0.4 & 0.2 \\
\hline & & 0.6 & 1.1 & 0.8 & 0.3 & 0.3 & 0.2 & 0.2 \\
\hline & & 0.4 & 0.7 & 0.5 & 0.2 & 0.3 & 0.2 & 0.2 \\
\hline & & 0.2 & 0.5 & 0.3 & 0.2 & 0.2 & 0.2 & 0.2 \\
\hline & & 0.2 & 0.3 & 0.2 & 0.1 & 0.1 & 0.1 & 0.1 \\
\hline & & 0.1 & 0.3 & 0.3 & 0.2 & 0.2 & 0.2 & 0.2 \\
\hline & & 0.3 & 0.6 & 0.4 & 0.3 & 0.3 & 0.2 & 0.3 \\
\hline & & 0.7 & 1.1 & 0.8 & 0.6 & 0.6 & 0.7 & 0.6 \\
\hline \multirow[t]{5}{*}{0.2} & 1.1 & 2 & 1.9 & 1.4 & 1.1 & 0.9 & 0.6 & 0.6 \\
\hline & 0.1 & 1.1 & 1.3 & 0.9 & 0.7 & 0.5 & 0.5 & 0.4 \\
\hline & & 0.7 & 1 & 0.7 & 0.4 & 0.4 & 0.4 & 0.3 \\
\hline & & 0.5 & 0.9 & 0.6 & 0.3 & 0.4 & 0.4 & 0.3 \\
\hline & & 0.4 & 0.8 & 0.3 & 0.3 & 0.2 & 0.3 & 0.2 \\
\hline \multirow[t]{2}{*}{0.3} & 1.3 & 2 & 1.9 & 1.7 & 1.5 & 1.2 & 1 & 0.8 \\
\hline & 0.2 & 1.5 & 1.7 & 1.3 & 1 & 0.9 & 0.9 & 0.8 \\
\hline 0.1 & 0.7 & 1.7 & 1.7 & 1.4 & 0.6 & 1.1 & 1 & 0.9 \\
\hline \multirow[t]{10}{*}{0.1} & 0.8 & 1.8 & 1.9 & 1.6 & 1.3 & 1.2 & 1 & 0.9 \\
\hline & 0.3 & 1.6 & 1.7 & 1.2 & 0.8 & 0.7 & 0.7 & 0.5 \\
\hline & 0.6 & 2 & 2 & 1.5 & 0.9 & 0.9 & 0.7 & 0.5 \\
\hline & & 0.4 & 0.8 & 0.6 & 0.4 & 0.1 & & \\
\hline & & 0.2 & 0.8 & 0.5 & 0.1 & 0.1 & 0.1 & 0.1 \\
\hline & & 0.1 & & 0.1 & 0.1 & 0.1 & 0.1 & 0.2 \\
\hline & & 0.2 & 0.8 & 0.6 & 0.4 & 0.4 & 0.3 & 0.3 \\
\hline & & & & & & & & \\
\hline & & 1 & 1.1 & 0.8 & 0.6 & 0.6 & 0.5 & 0.5 \\
\hline & & 0.8 & 1.2 & 1 & 0.7 & 0.8 & 0.8 & 0.6 \\
\hline
\end{tabular}


wbheat

\begin{tabular}{|c|c|c|c|c|c|c|c|c|}
\hline & 0.2 & 1.4 & 1.7 & 1.4 & 0.9 & 0.8 & 0.6 & 0.5 \\
\hline & 0.1 & 1.3 & 1.6 & 1.2 & 0.9 & 0.8 & 0.5 & 0.3 \\
\hline \multirow[t]{4}{*}{0.2} & 1.3 & 2.2 & 2.1 & 1.8 & 1.5 & 1.2 & 1 & 0.8 \\
\hline & & 1 & 1.3 & 0.9 & 0.8 & 0.7 & 0.6 & 0.3 \\
\hline & & 0.5 & 1 & 0.7 & 0.5 & 0.3 & 0.2 & 0.3 \\
\hline & & 0.5 & 0.9 & 0.7 & 0.3 & 0.2 & 0.1 & 0.1 \\
\hline \multirow[t]{9}{*}{0.2} & 1 & 2.3 & 2.1 & 1.8 & 1.3 & 1.1 & 1 & 1 \\
\hline & 0.3 & 1.5 & 1.6 & 1.3 & 0.9 & 0.8 & 0.7 & 0.6 \\
\hline & 0.1 & 1 & 1.5 & 1.1 & 0.7 & 0.6 & 0.4 & 0.2 \\
\hline & & 0.7 & 1 & 0.7 & 0.3 & 0.1 & 0.1 & 0.1 \\
\hline & 0.6 & 2 & 1.8 & 1.4 & 1.1 & 0.9 & 0.8 & 0.6 \\
\hline & & 1.1 & 1.4 & 0.9 & 0.7 & 0.8 & 0.6 & 0.6 \\
\hline & 0.5 & 1.7 & 1.7 & 1.4 & 1.1 & 0.9 & 0.9 & 0.6 \\
\hline & & 1.1 & 1.2 & 0.9 & 0.7 & 0.6 & 0.5 & 0.4 \\
\hline & & 0.5 & 1.1 & 0.7 & 0.4 & 0.1 & 0.1 & 0.1 \\
\hline 0.2 & 1.4 & 2.2 & 2.1 & 1.9 & 1.4 & 1.3 & 1.2 & 1.1 \\
\hline \multirow[t]{12}{*}{0.1} & 0.6 & 1.9 & 1.8 & 1.4 & 1.1 & 1 & 1 & 0.9 \\
\hline & & 0.8 & 1 & 0.6 & 0.5 & 0.4 & 0.1 & 0.2 \\
\hline & & 1.1 & 1.3 & 1 & 1 & 0.9 & 0.8 & 0.8 \\
\hline & 0.2 & 1.4 & 1.6 & 1.2 & 0.8 & 0.8 & 0.6 & 0.5 \\
\hline & 0.4 & 1.8 & 2 & 1.4 & 1 & 0.2 & & \\
\hline & & 0.7 & 1.1 & 0.8 & 0.4 & 0.4 & 0.2 & 0.1 \\
\hline & & 0.4 & 0.9 & 0.5 & 0.4 & 0.2 & 0.1 & 0.2 \\
\hline & & 0.2 & 0.6 & 0.3 & 0.1 & 0.1 & 0.1 & 0.1 \\
\hline & & 0.1 & 0.6 & 0.3 & 0.2 & 0.2 & 0.1 & 0.1 \\
\hline & & 0.5 & 0.7 & 0.1 & 0.1 & 0.1 & 0.1 & 0.1 \\
\hline & & 0.3 & 0.9 & 0.7 & 0.5 & 0.6 & 0.6 & 0.6 \\
\hline & 0.2 & 1.4 & 1.7 & 1.3 & 0.9 & 0.9 & 0.9 & 0.9 \\
\hline 0.1 & 0.7 & 1.7 & 1.9 & 1.7 & 1.2 & 1.2 & 1.1 & 1.1 \\
\hline \multirow[t]{3}{*}{0.1} & 0.6 & 1.7 & 2 & 1.6 & 1.4 & 1.2 & 1.2 & 1 \\
\hline & 0.4 & 1.9 & 1.8 & 1.2 & 0.7 & 0.6 & 0.5 & 0.5 \\
\hline & 0.1 & 1.2 & 1.6 & 1.4 & 1 & 1 & 0.9 & 0.9 \\
\hline 0.3 & 0.8 & 1.8 & 1.9 & 1.7 & 1.4 & 1.2 & 1 & 0.9 \\
\hline \multirow[t]{18}{*}{0.1} & 0.5 & 1.6 & 1.8 & 1.5 & 1 & 0.7 & 0.7 & 0.7 \\
\hline & 0.2 & 1.3 & 1.5 & 0.9 & 0.7 & 0.6 & 0.5 & 0.4 \\
\hline & & & & 0.1 & 0.1 & & 0.1 & 0.1 \\
\hline & & 0.1 & 0.3 & 0.2 & & 0.1 & 0.2 & 0.1 \\
\hline & & 0.1 & 0.1 & 0.1 & & 0.1 & 0.1 & 0.1 \\
\hline & & & & 0.1 & & 0.1 & 0.1 & 0.1 \\
\hline & & & 0.1 & 0.1 & & 0.1 & 0.2 & 0.1 \\
\hline & & 0.5 & 0.9 & 0.6 & 0.4 & 0.3 & 0.2 & 0.3 \\
\hline & & 0.2 & 0.6 & 0.4 & 0.3 & 0.3 & 0.3 & 0.3 \\
\hline & & 0.6 & 0.9 & 0.5 & 0.4 & 0.4 & 0.4 & 0.3 \\
\hline & & 0.5 & 0.9 & 0.5 & 0.5 & 0.4 & 0.4 & 0.4 \\
\hline & & 0.4 & 0.7 & 0.5 & 0.3 & 0.3 & 0.2 & 0.2 \\
\hline & & & 0.1 & 0.2 & & 0.3 & 0.1 & 0.1 \\
\hline & & 0.1 & 0.4 & 0.3 & 0.2 & 0.1 & 0.2 & 0.1 \\
\hline & & 0.2 & 0.4 & 0.4 & & 0.2 & 0.1 & 0.2 \\
\hline & & 0.4 & 0.6 & 0.5 & 0.2 & 0.2 & 0.1 & 0.2 \\
\hline & & 0.2 & 0.4 & 0.3 & & 0.1 & 0.1 & 0.1 \\
\hline & & 0.1 & 0.1 & 0.1 & 0.1 & & 0.1 & 0.1 \\
\hline
\end{tabular}


wbheat

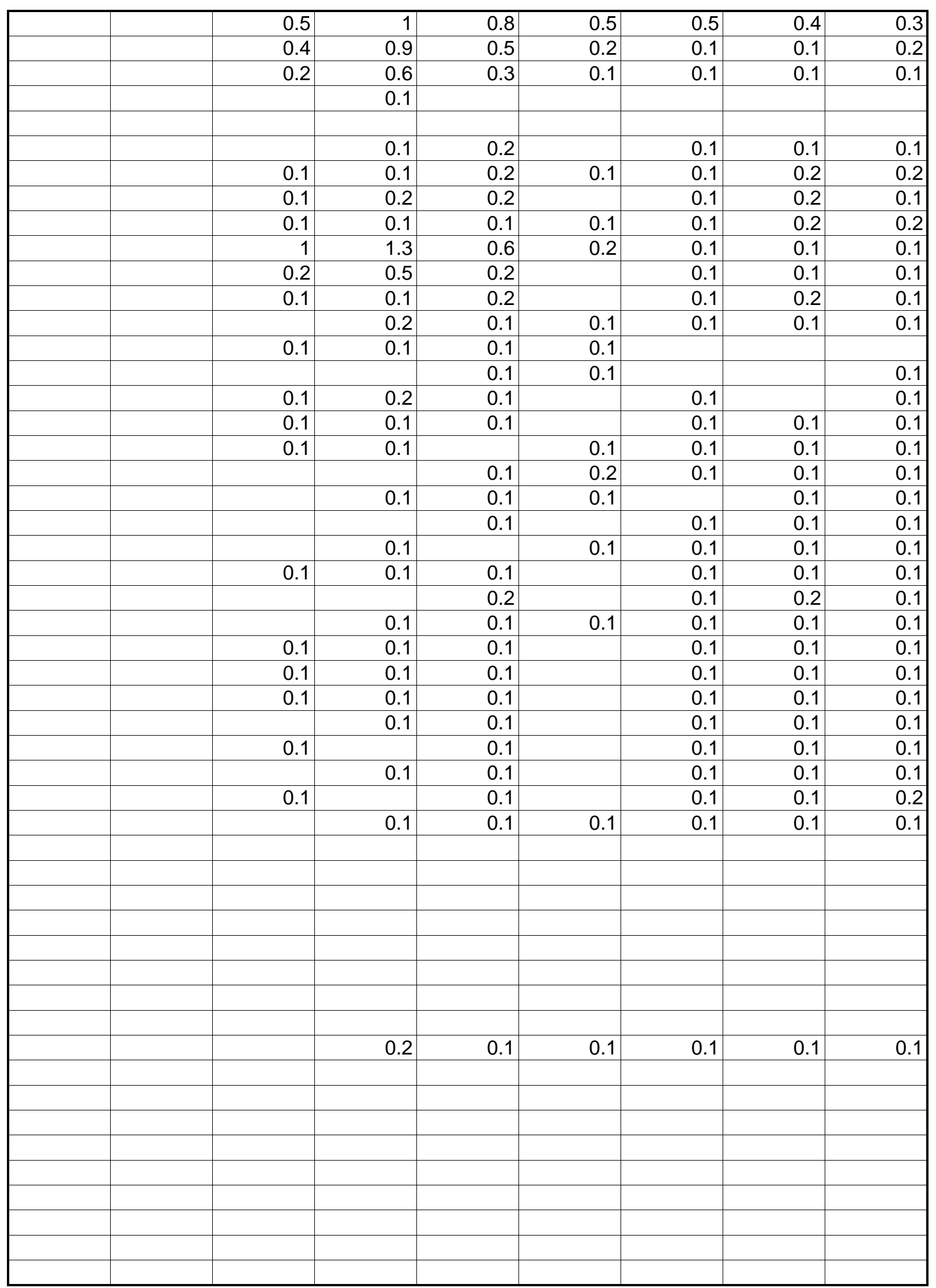




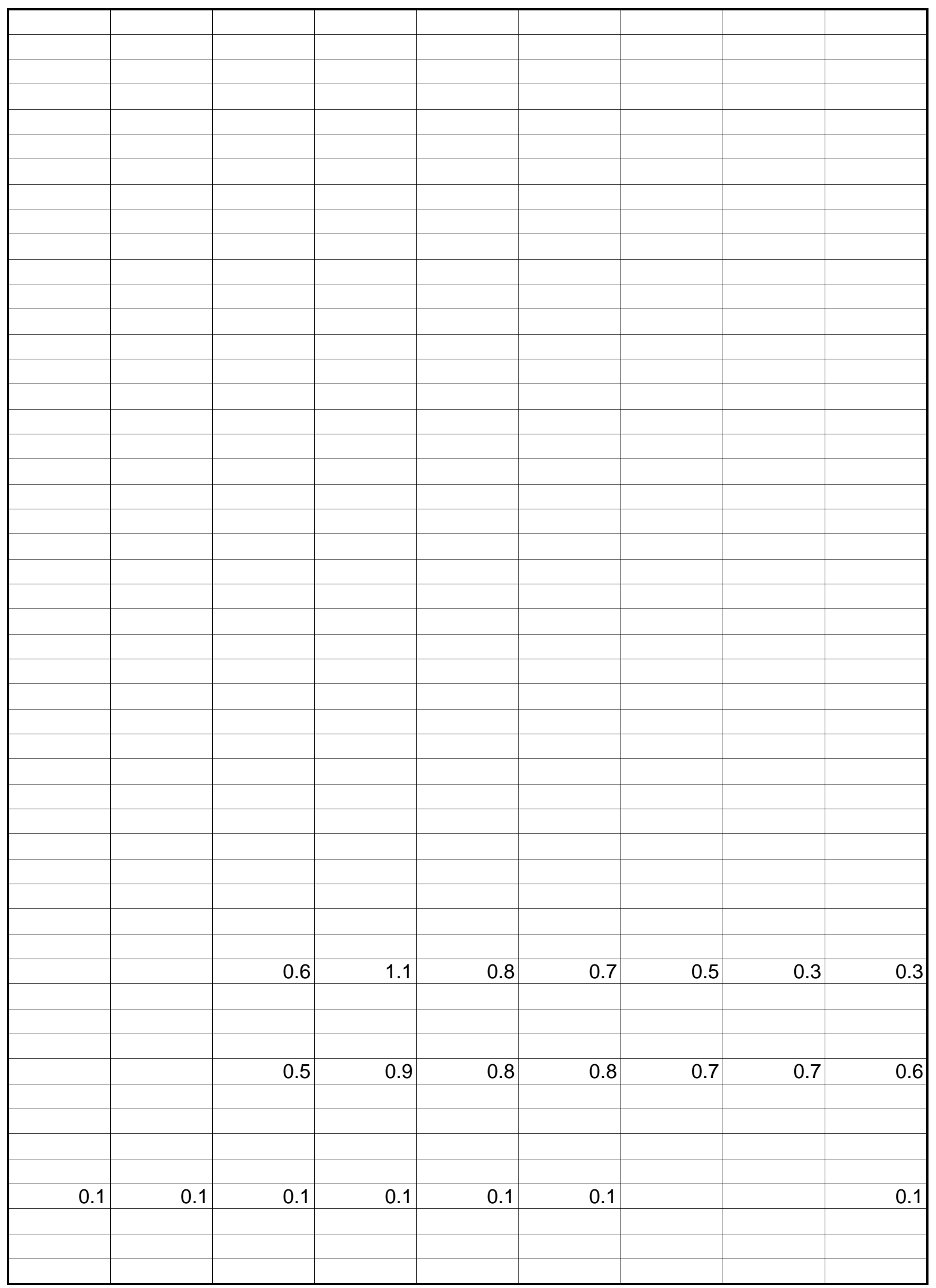


wbheat

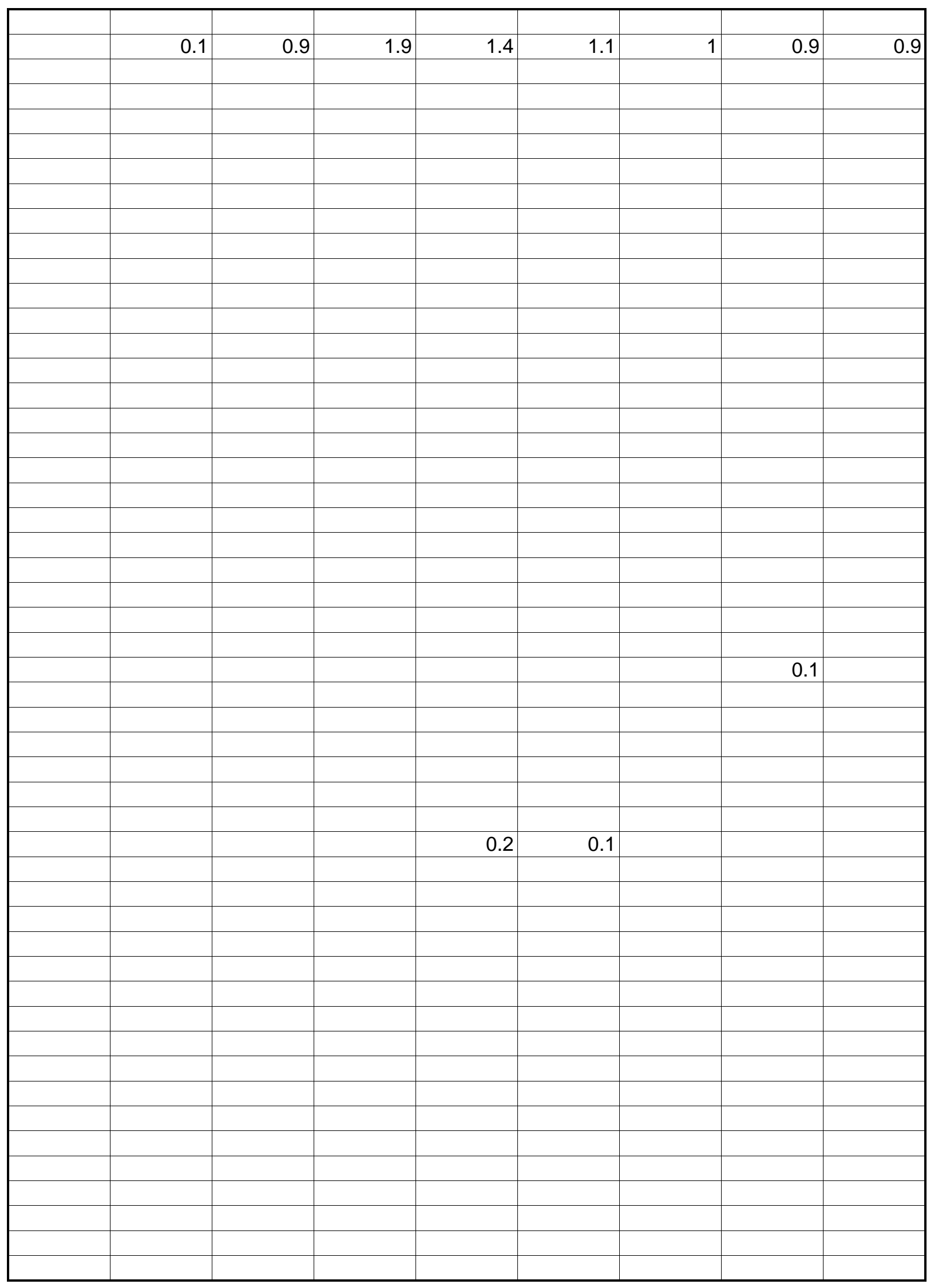

Page 53 
wbheat

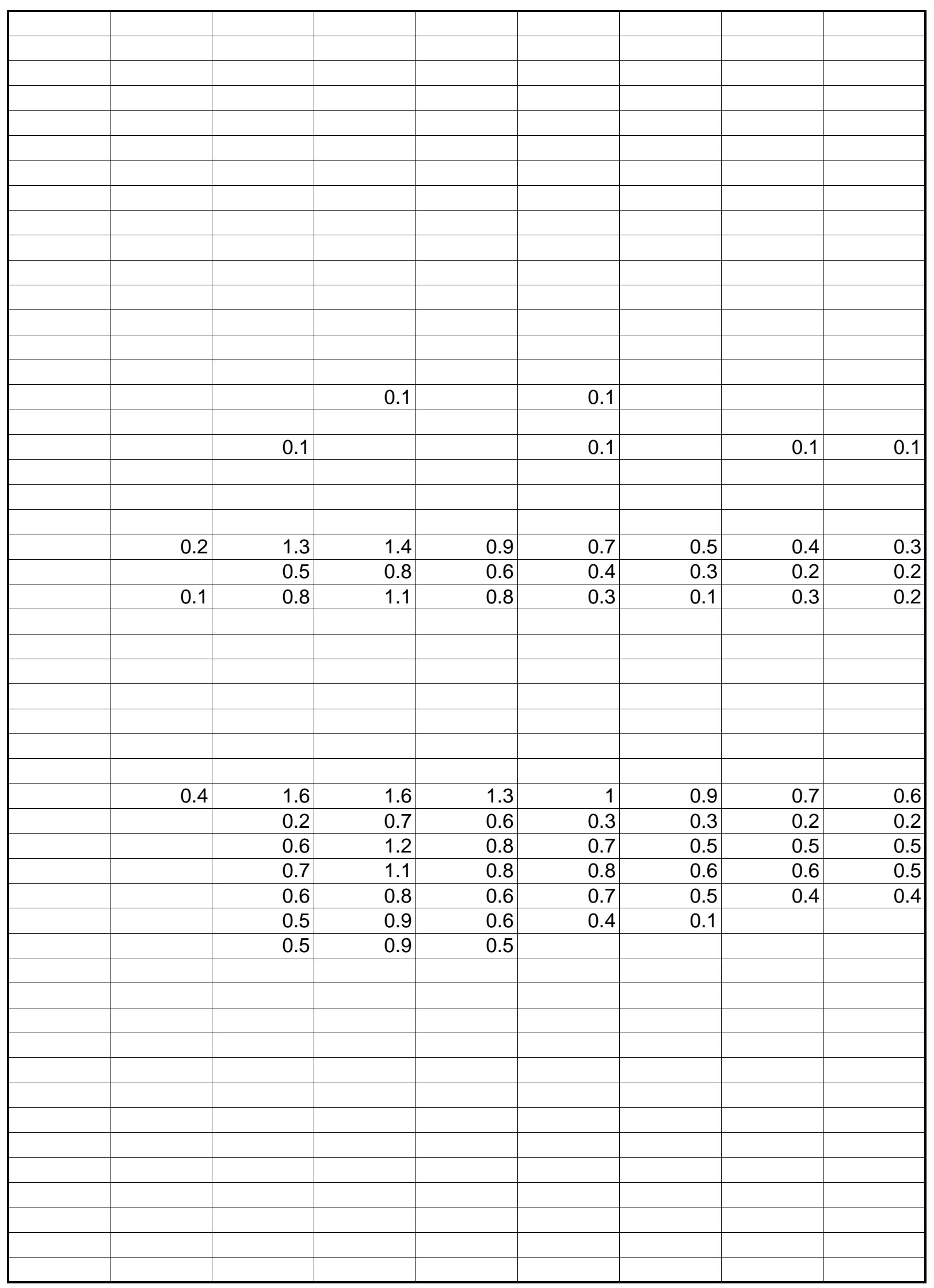


wbheat

\begin{tabular}{|c|c|c|c|c|c|c|c|c|}
\hline & & & & & & & & \\
\hline & & & & & & & & \\
\hline & & & & & & & & \\
\hline & & & & & & & & \\
\hline & & & & & & & & \\
\hline & & & & & & & & \\
\hline & & & & & & & & \\
\hline & & & & & & & & \\
\hline & & & & & & & & \\
\hline & & & & & & & & \\
\hline & & & & & & & & \\
\hline & & & & & & & & \\
\hline & & & & & & & & \\
\hline & & & & & & & & \\
\hline & & & & & & & & \\
\hline & & & & & & & & \\
\hline & & & & & & & & \\
\hline & & & & & & & & \\
\hline & & & & & & & & \\
\hline & & & & & & & & \\
\hline & & & & & & & & \\
\hline & & & & & & & & \\
\hline 0.35 & 0.39 & 0.35 & 0.37 & 0.34 & 0.28 & 0.26 & 0.28 & 0.28 \\
\hline 0.30 & 0.30 & 0.31 & 0.31 & 0.31 & 0.31 & 0.29 & 0.28 & 0.27 \\
\hline 0.32 & 0.34 & 0.30 & 0.31 & 0.28 & 0.31 & 0.29 & 0.28 & 0.28 \\
\hline \#DIV/0! & \#DIV/O! & 0.11 & 0.17 & 0.13 & 0.10 & 0.11 & 0.11 & 0.12 \\
\hline 0.17 & 0.52 & 0.70 & 0.87 & 0.60 & 0.49 & 0.38 & 0.34 & 0.30 \\
\hline 0.00 & 0.10 & 0.53 & 1.00 & 0.78 & 0.68 & 0.73 & 0.63 & 0.48 \\
\hline \#DIV/0! & 0.23 & 0.67 & 0.96 & 0.70 & 0.47 & 0.42 & 0.35 & 0.33 \\
\hline 0.00 & 0.00 & 0.00 & 0.00 & 0.00 & 0.00 & 0.00 & 0.00 & 0.00 \\
\hline & & & & & & & & \\
\hline & & & & & & & & \\
\hline & & & & & & & & \\
\hline & & & & & & & & \\
\hline 0.243 & 0.274 & 0.269 & 0.266 & 0.256 & 0.214 & 0.203 & 0.198 & 0.190 \\
\hline 0.174 & 0.180 & 0.185 & 0.184 & 0.180 & 0.175 & 0.168 & 0.155 & 0.153 \\
\hline 0.188 & 0.223 & 0.202 & 0.202 & 0.193 & 0.199 & 0.193 & 0.179 & 0.178 \\
\hline 0.000 & \#DIV/0! & 0.030 & 0.134 & 0.070 & 0.026 & 0.037 & 0.040 & 0.043 \\
\hline 0.078 & 0.394 & 0.660 & 0.642 & 0.515 & 0.405 & 0.355 & 0.314 & 0.272 \\
\hline 0.000 & 0.000 & 0.330 & 0.739 & 0.532 & 0.419 & 0.252 & 0.306 & 0.350 \\
\hline \#DIV/O! & 0.153 & 0.438 & 0.396 & 0.276 & 0.306 & 0.254 & 0.207 & 0.173 \\
\hline 0.000 & 0.000 & 0.000 & 0.000 & 0.000 & 0.000 & 0.000 & 0.000 & 0.000 \\
\hline & & & & & & & & \\
\hline & & & & & & & & \\
\hline & & & & & & & & \\
\hline & & & & & & & & \\
\hline 98.72 & 121.22 & 163.41 & 141.68 & 163.84 & 105.87 & 123.37 & 137.03 & 137.09 \\
\hline 35.30 & 36.70 & 37.70 & 38.10 & 37.20 & 37.00 & 35.40 & 33.10 & 31.40 \\
\hline 19.60 & 21.00 & 21.90 & 21.70 & 22.60 & 22.00 & 21.30 & 20.90 & 19.50 \\
\hline 0.00 & 0.00 & 6.79 & 4.84 & 10.00 & 2.33 & 6.97 & 8.39 & 8.59 \\
\hline 2.00 & 15.50 & 75.20 & 98.60 & 74.40 & 48.90 & 45.30 & 40.50 & 36.80 \\
\hline 0.10 & 0.20 & 2.10 & 4.00 & 3.10 & 2.70 & 2.20 & 1.90 & 1.90 \\
\hline
\end{tabular}


wbheat

\begin{tabular}{|r|r|r|r|r|r|r|r|r|}
\hline 0.00 & 0.70 & 7.40 & 10.60 & 7.70 & 5.60 & 3.80 & 3.50 & 3.00 \\
\hline 0.00 & 0.00 & 0.00 & 0.00 & 0.00 & 0.00 & 0.00 & 0.00 & 0.00 \\
\hline
\end{tabular}

Page 56 
wbheat

\begin{tabular}{|c|c|c|c|c|c|c|c|c|}
\hline 14 & 15 & 16 & 17 & 18 & 19 & 20 & 21 & 22 \\
\hline & & & & & & & & \\
\hline & & & & & & & & \\
\hline \multicolumn{9}{|l|}{0.1} \\
\hline \multicolumn{9}{|l|}{0.1} \\
\hline & & & & & & & & \\
\hline \multicolumn{9}{|l|}{0.1} \\
\hline & & & & & & & & \\
\hline \multicolumn{9}{|l|}{0.1} \\
\hline \multicolumn{9}{|l|}{0.1} \\
\hline \multicolumn{9}{|l|}{0.1} \\
\hline \multicolumn{9}{|l|}{0.1} \\
\hline & & & & & & & & \\
\hline & & & & & & & & \\
\hline \multicolumn{9}{|l|}{0.1} \\
\hline 0.2 & & & & 0.1 & 0.2 & 0.2 & 0.1 & 0.2 \\
\hline 0.1 & & & 0.4 & 0.4 & 0.4 & 0.4 & 0.4 & 0.4 \\
\hline 0.1 & & & & & & & & 0.1 \\
\hline & & & & & & & & \\
\hline \multicolumn{9}{|l|}{0.1} \\
\hline \multicolumn{9}{|l|}{0.2} \\
\hline & & & & & & & & \\
\hline 0.2 & 0.2 & 0.2 & 0.2 & 0.3 & 0.3 & 0.3 & 0.3 & 0.3 \\
\hline 0.6 & 0.3 & 0.2 & 0.2 & 0.2 & 0.3 & 0.3 & 0.3 & 0.3 \\
\hline 0.2 & 0.1 & 0.1 & 0.2 & 0.2 & 0.2 & 0.2 & 0.2 & 0.3 \\
\hline 0.3 & 0.4 & 0.4 & 0.4 & 0.4 & 0.4 & 0.4 & 0.4 & 0.4 \\
\hline 0.2 & 0.2 & 0.1 & 0.1 & 0.2 & 0.2 & 0.2 & 0.2 & 0.2 \\
\hline 0.2 & 0.1 & 0.1 & 0.1 & 0.1 & 0.1 & 0.1 & 0.1 & 0.1 \\
\hline 0.2 & 0.1 & 0.1 & 0.2 & 0.2 & 0.3 & 0.2 & 0.3 & 0.3 \\
\hline 0.5 & 0.2 & 0.2 & 0.2 & 0.2 & 0.3 & 0.3 & 0.3 & 0.4 \\
\hline 0.1 & 0.1 & 0.1 & 0.1 & 0.1 & 0.1 & 0.1 & 0.2 & 0.2 \\
\hline 0.1 & 0.1 & 0.1 & 0.1 & 0.1 & 0.1 & 0.1 & 0.1 & 0.1 \\
\hline 0.1 & & 0.1 & 0.1 & 0.1 & 0.1 & 0.1 & 0.1 & 0.1 \\
\hline 0.1 & & & & & 0.1 & & & \\
\hline \multicolumn{9}{|l|}{0.1} \\
\hline \multicolumn{9}{|l|}{0.4} \\
\hline 0.1 & & 0.1 & 0.1 & 0.1 & 0.1 & 0.1 & 0.1 & 0.1 \\
\hline 0.2 & 0.1 & 0.1 & 0.1 & 0.1 & 0.1 & 0.2 & 0.2 & 0.2 \\
\hline 0.2 & 0.1 & 0.1 & 0.1 & 0.1 & 0.1 & 0.1 & 0.1 & 0.2 \\
\hline 0.1 & & 0.1 & 0.1 & 0.1 & 0.1 & 0.1 & 0.1 & 0.2 \\
\hline 0.2 & 0.2 & 0.2 & 0.2 & 0.2 & 0.2 & 0.2 & 0.2 & 0.2 \\
\hline \multirow[t]{2}{*}{0.1} & 0.1 & 0.1 & 0.1 & 0.2 & 0.2 & 0.2 & 0.2 & 0.2 \\
\hline & & 0.1 & & 0.1 & & 0.1 & 0.1 & 0.1 \\
\hline 0.5 & 0.4 & 0.4 & 0.4 & 0.4 & 0.5 & 0.4 & 0.4 & 0.5 \\
\hline 0.3 & 0.3 & 0.2 & 0.3 & 0.3 & 0.4 & 0.3 & 0.4 & 0.4 \\
\hline 0.3 & 0.2 & 0.2 & 0.2 & 0.2 & 0.2 & 0.2 & 0.3 & 0.3 \\
\hline 0.2 & 0.1 & 0.2 & 0.2 & 0.2 & 0.2 & 0.2 & 0.2 & 0.2 \\
\hline 0.1 & 0.1 & 0.1 & 0.1 & 0.1 & 0.1 & 0.1 & 0.1 & 0.1 \\
\hline
\end{tabular}


wbheat

\begin{tabular}{|c|c|c|c|c|c|c|c|c|}
\hline 0.1 & 0.1 & & 0.1 & 0.1 & 0.1 & 0.1 & 0.1 & 0.1 \\
\hline 0.1 & 0.1 & 0.1 & 0.1 & 0.1 & 0.1 & 0.1 & 0.1 & 0.1 \\
\hline 0.1 & & 0.1 & 0.1 & 0.1 & 0.1 & 0.1 & 0.1 & 0.1 \\
\hline 0.1 & & & & & & 0.1 & 0.1 & 0.1 \\
\hline 0.2 & & & & 0.1 & & 0.1 & 0.1 & 0.1 \\
\hline 0.4 & 0.3 & 0.3 & 0.3 & 0.3 & 0.3 & 0.3 & 0.3 & 0.3 \\
\hline 0.2 & 0.1 & 0.1 & 0.2 & 0.1 & 0.2 & 0.2 & 0.2 & 0.2 \\
\hline 0.3 & 0.2 & 0.2 & 0.2 & 0.3 & 0.3 & 0.3 & 0.3 & 0.4 \\
\hline 0.5 & 0.3 & 0.4 & 0.4 & 0.4 & 0.4 & 0.4 & 0.4 & 0.4 \\
\hline 0.4 & 0.3 & 0.3 & 0.3 & 0.3 & 0.3 & 0.3 & 0.3 & 0.3 \\
\hline 0.5 & 0.4 & 0.4 & 0.4 & 0.4 & 0.4 & 0.4 & 0.4 & 0.5 \\
\hline 0.2 & 0.1 & 0.2 & 0.2 & 0.2 & 0.2 & 0.2 & 0.3 & 0.3 \\
\hline 0.3 & 0.2 & 0.2 & 0.2 & 0.2 & 0.3 & 0.3 & 0.3 & 0.4 \\
\hline 0.3 & 0.2 & 0.2 & 0.3 & 0.3 & 0.3 & 0.3 & 0.3 & 0.4 \\
\hline 0.3 & 0.2 & 0.2 & 0.2 & 0.2 & 0.3 & 0.3 & 0.3 & 0.3 \\
\hline 0.4 & 0.3 & 0.4 & 0.4 & 0.5 & 0.5 & 0.5 & 0.5 & 0.5 \\
\hline 0.4 & 0.3 & 0.3 & 0.3 & 0.3 & 0.4 & 0.4 & 0.4 & 0.4 \\
\hline 0.5 & 0.4 & 0.4 & 0.5 & 0.6 & 0.5 & 0.6 & 0.6 & 0.6 \\
\hline 0.6 & 0.4 & 0.5 & 0.5 & 0.5 & 0.5 & 0.5 & 0.5 & 0.5 \\
\hline 0.5 & 0.4 & 0.5 & 0.5 & 0.5 & 0.5 & 0.5 & 0.5 & 0.5 \\
\hline 0.3 & 0.2 & 0.3 & 0.3 & 0.4 & 0.4 & 0.4 & 0.4 & 0.4 \\
\hline 0.2 & 0.2 & 0.2 & 0.2 & 0.2 & 0.2 & 0.3 & 0.3 & 0.3 \\
\hline 0.2 & 0.2 & 0.2 & 0.2 & 0.2 & 0.3 & 0.3 & 0.3 & 0.3 \\
\hline 0.3 & 0.2 & 0.2 & 0.2 & 0.2 & 0.2 & 0.2 & 0.2 & 0.2 \\
\hline 0.3 & 0.2 & 0.2 & 0.3 & 0.3 & 0.3 & 0.3 & 0.4 & 0.4 \\
\hline 0.2 & 0.1 & 0.2 & 0.2 & 0.3 & 0.3 & 0.3 & 0.3 & 0.3 \\
\hline 0.2 & 0.1 & 0.1 & 0.2 & 0.3 & 0.3 & 0.2 & 0.3 & 0.3 \\
\hline 0.1 & 0.1 & 0.1 & 0.1 & 0.1 & 0.1 & 0.1 & 0.1 & 0.1 \\
\hline 0.2 & 0.2 & 0.2 & 0.2 & 0.2 & 0.2 & 0.2 & 0.3 & 0.3 \\
\hline 0.2 & 0.2 & 0.2 & 0.2 & 0.2 & 0.3 & 0.3 & 0.3 & 0.3 \\
\hline 0.4 & 0.3 & 0.3 & 0.3 & 0.3 & 0.4 & 0.4 & 0.4 & 0.4 \\
\hline 0.3 & 0.2 & 0.2 & 0.3 & 0.3 & 0.3 & 0.3 & 0.3 & 0.3 \\
\hline 0.4 & 0.2 & 0.2 & 0.2 & 0.2 & 0.2 & 0.3 & 0.3 & 0.3 \\
\hline 0.2 & 0.2 & 0.2 & 0.2 & 0.2 & 0.3 & 0.2 & 0.3 & 0.3 \\
\hline 0.2 & 0.1 & 0.1 & 0.1 & 0.1 & 0.1 & 0.1 & 0.2 & 0.2 \\
\hline 0.1 & & 0.1 & 0.1 & 0.1 & 0.1 & 0.1 & 0.1 & 0.2 \\
\hline 0.2 & 0.2 & 0.2 & 0.2 & 0.2 & 0.2 & 0.2 & 0.2 & 0.2 \\
\hline 0.2 & & & & & 0.1 & & 0.1 & 0.1 \\
\hline 0.1 & 0.1 & 0.1 & 0.1 & 0.1 & 0.1 & 0.2 & 0.1 & 0.1 \\
\hline 0.1 & 0.1 & 0.1 & 0.1 & 0.1 & 0.1 & 0.1 & 0.1 & 0.1 \\
\hline 0.2 & 0.1 & & 0.1 & 0.1 & 0.1 & 0.1 & 0.1 & 0.1 \\
\hline 0.1 & 0.1 & & 0.1 & 0.1 & 0.1 & 0.1 & 0.1 & 0.1 \\
\hline 0.1 & 0.1 & & 0.1 & 0.1 & & 0.1 & 0.1 & 0.1 \\
\hline 0.1 & 0.1 & 0.1 & 0.1 & 0.2 & 0.2 & 0.2 & 0.2 & 0.4 \\
\hline 0.4 & 0.4 & 0.4 & 0.4 & 0.4 & 0.5 & 0.4 & 0.5 & 0.5 \\
\hline 0.3 & 0.3 & 0.3 & 0.3 & 0.3 & 0.3 & 0.3 & 0.3 & 0.4 \\
\hline 0.2 & 0.2 & 0.1 & 0.2 & 0.2 & 0.2 & 0.2 & 0.2 & 0.2 \\
\hline 0.2 & 0.1 & 0.1 & 0.1 & 0.5 & 0.1 & 0.1 & 0.1 & 0.1 \\
\hline 0.1 & 0.1 & 0.1 & 0.1 & 0.1 & 0.1 & 0.1 & 0.1 & 0.1 \\
\hline 0.1 & 0.1 & 0.1 & 0.1 & 0.1 & 0.1 & 0.1 & 0.1 & 0.1 \\
\hline 0.2 & 0.1 & 0.1 & 0.1 & 0.1 & 0.2 & 0.2 & 0.2 & 0.2 \\
\hline
\end{tabular}


wbheat

\begin{tabular}{|c|c|c|c|c|c|c|c|c|}
\hline 0.2 & 0.1 & 0.1 & 0.1 & 0.1 & 0.1 & 0.1 & 0.1 & 0.1 \\
\hline 0.2 & 0.1 & 0.1 & 0.1 & 0.1 & 0.1 & 0.1 & 0.1 & 0.1 \\
\hline 0.2 & 0.1 & 0.1 & 0.1 & 0.1 & 0.1 & 0.1 & 0.2 & 0.2 \\
\hline 0.3 & 0.3 & 0.3 & 0.3 & 0.3 & 0.3 & 0.3 & 0.3 & 0.3 \\
\hline 0.4 & 0.2 & 0.2 & 0.2 & 0.2 & 0.2 & 0.2 & 0.3 & 0.3 \\
\hline 0.3 & 0.1 & 0.1 & 0.1 & 0.1 & 0.1 & 0.2 & 0.2 & 0.2 \\
\hline 0.2 & 0.1 & 0.1 & 0.1 & 0.1 & 0.1 & 0.1 & 0.2 & 0.1 \\
\hline 0.2 & 0.2 & 0.2 & 0.1 & 0.1 & 0.1 & 0.2 & 0.2 & 0.2 \\
\hline 0.2 & 0.1 & 0.1 & 0.2 & 0.1 & 0.1 & 0.1 & 0.2 & 0.1 \\
\hline 0.1 & 0.1 & 0.1 & 0.1 & 0.1 & 0.1 & 0.1 & 0.1 & 0.1 \\
\hline 0.1 & 0.1 & 0.1 & 0.1 & 0.1 & 0.1 & 0.1 & 0.1 & 0.1 \\
\hline 0.1 & 0.1 & 0.1 & 0.1 & 0.1 & 0.1 & 0.1 & 0.1 & 0.1 \\
\hline 0.2 & 0.1 & 0.1 & 0.1 & 0.1 & 0.1 & 0.2 & 0.2 & 0.2 \\
\hline 0.2 & 0.1 & 0.1 & 0.1 & 0.1 & 0.1 & 0.1 & 0.1 & 0.1 \\
\hline 0.2 & 0.2 & 0.2 & 0.2 & 0.2 & 0.3 & 0.3 & 0.2 & 0.3 \\
\hline 0.3 & 0.1 & 0.2 & 0.2 & 0.2 & 0.2 & 0.2 & 0.2 & 0.2 \\
\hline 0.2 & 0.1 & 0.1 & 0.1 & 0.1 & 0.1 & 0.1 & 0.2 & 0.2 \\
\hline 0.1 & 0.1 & 0.1 & 0.1 & 0.1 & 0.2 & 0.2 & 0.1 & 0.2 \\
\hline 0.1 & 0.1 & & & 0.1 & 0.1 & & 0.1 & 0.1 \\
\hline 0.1 & & & & 0.1 & 0.1 & & & \\
\hline \multicolumn{9}{|l|}{0.1} \\
\hline 0.1 & & & & & 0.1 & & 0.1 & 0.1 \\
\hline \multirow[t]{2}{*}{0.1} & & & & & 0.1 & 0.1 & & \\
\hline & & & & & & & & \\
\hline \multicolumn{8}{|l|}{0.1} & \\
\hline 0.1 & & & & & & 0.1 & & \\
\hline 0.1 & & 0.1 & & & & 0.1 & & 0.1 \\
\hline 0.1 & 0.1 & & 0.1 & 0.1 & & 0.1 & & 0.1 \\
\hline \multirow[t]{2}{*}{0.1} & 0.1 & 0.1 & 0.1 & 0.1 & & & & 0.1 \\
\hline & & & & & & & & \\
\hline & & & & & & & & \\
\hline \multirow[t]{2}{*}{0.1} & & & & 0.1 & & & & \\
\hline & & & & 0.1 & 0.1 & 0.1 & & \\
\hline \multicolumn{9}{|l|}{0.1} \\
\hline \multicolumn{9}{|l|}{0.1} \\
\hline \multicolumn{9}{|l|}{0.1} \\
\hline \multirow[t]{3}{*}{0.2} & & & & & & & 0.1 & \\
\hline & & & & & & & & \\
\hline & & & & & & & & \\
\hline & & & & & & & & \\
\hline \multicolumn{9}{|l|}{0.2} \\
\hline \multirow[b]{2}{*}{ 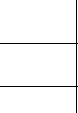 } & & & & & & & & \\
\hline & & & & & & & & \\
\hline & & & & & & & & \\
\hline \multirow{2}{*}{\multicolumn{9}{|c|}{0.1}} \\
\hline & & & & & & & & \\
\hline & & & & & & & & \\
\hline 0.1 & & & & & & & & \\
\hline & & & & & & & & \\
\hline
\end{tabular}


wbheat

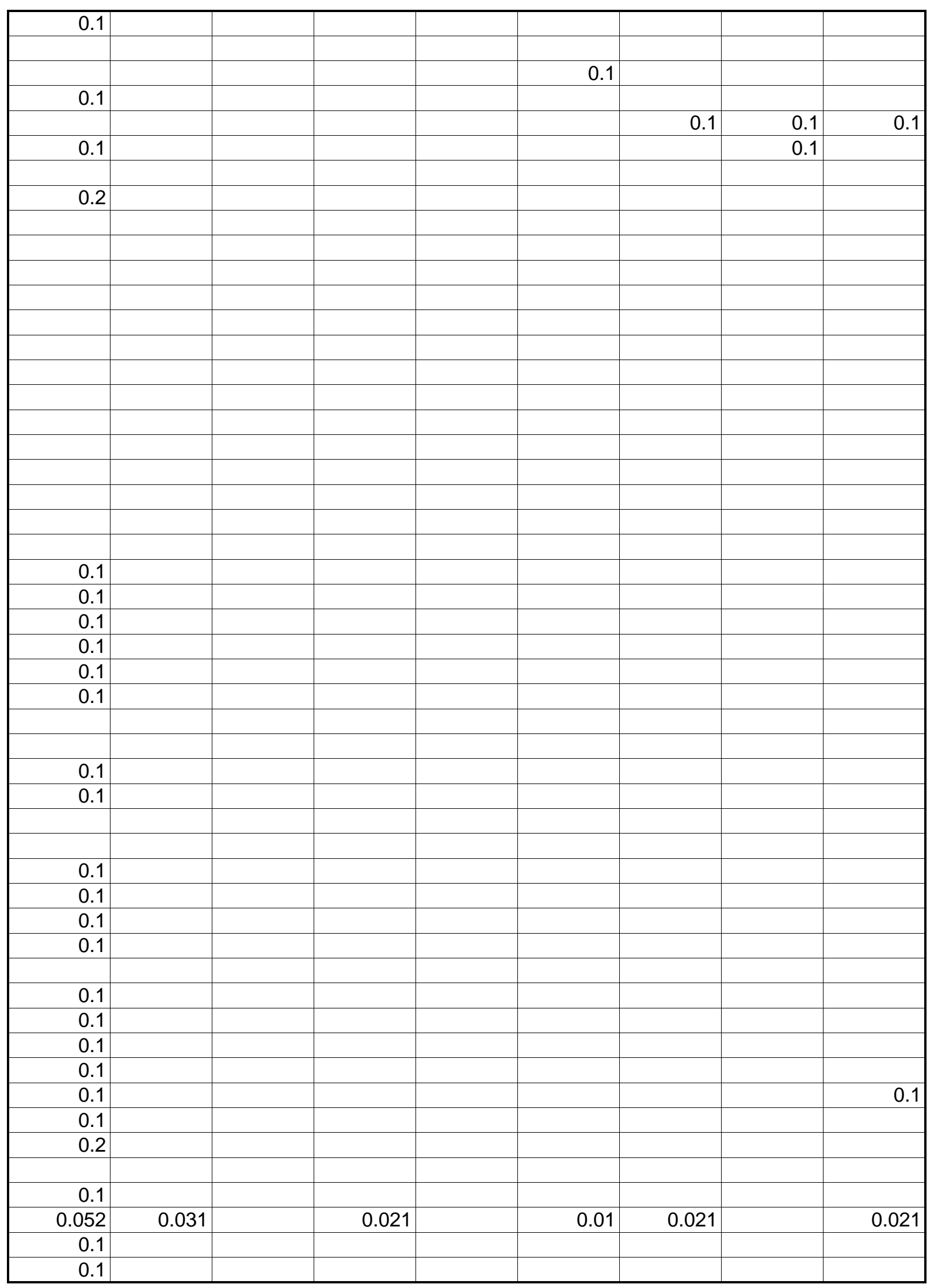


wbheat

\begin{tabular}{|c|c|c|c|c|c|c|c|c|}
\hline \multicolumn{9}{|l|}{0.1} \\
\hline 0.1 & & & & & & & & 0.1 \\
\hline \multicolumn{9}{|l|}{0.1} \\
\hline \multicolumn{9}{|l|}{0.1} \\
\hline \multirow[t]{2}{*}{0.1} & & & & & & & 0.1 & 0.1 \\
\hline & & & & 0.1 & & & & \\
\hline \multicolumn{9}{|l|}{0.1} \\
\hline \multicolumn{9}{|l|}{0.1} \\
\hline \multicolumn{9}{|l|}{0.1} \\
\hline 0.1 & & & & & & & 0.1 & 0.3 \\
\hline 0.2 & 0.1 & 0.1 & 0.1 & 0.1 & & & & \\
\hline \multirow[t]{2}{*}{0.1} & & & & 0.1 & & & & \\
\hline & & & & & & & & \\
\hline \multicolumn{9}{|l|}{0.1} \\
\hline \multicolumn{9}{|l|}{0.1} \\
\hline \multicolumn{9}{|l|}{0.1} \\
\hline \multicolumn{9}{|l|}{0.1} \\
\hline \multicolumn{9}{|l|}{0.1} \\
\hline \multicolumn{9}{|l|}{0.1} \\
\hline & & & & & & & & \\
\hline & & & & & & & & \\
\hline 0.1 & 0.1 & & 0.1 & 0.1 & 0.1 & & 0.1 & 0.1 \\
\hline 0.3 & 0.2 & 0.1 & 0.1 & 0.1 & 0.1 & 0.1 & 0.1 & 0.1 \\
\hline 0.4 & 0.2 & 0.1 & 0.2 & 0.2 & 0.2 & 0.2 & 0.3 & 0.2 \\
\hline 0.2 & 0.1 & 0.1 & 0.1 & 0.2 & 0.2 & 0.2 & 0.2 & 0.2 \\
\hline \multicolumn{9}{|l|}{0.1} \\
\hline 0.1 & & & & 0.1 & & & & \\
\hline 0.1 & & & & 0.1 & & & 0.1 & \\
\hline 0.2 & 0.1 & 0.1 & 0.1 & 0.1 & 0.1 & 0.1 & 0.1 & 0.1 \\
\hline 0.3 & 0.1 & 0.2 & 0.2 & 0.2 & 0.2 & 0.2 & 0.2 & 0.2 \\
\hline 0.1 & 0.1 & 0.1 & 0.1 & 0.1 & 0.1 & 0.2 & 0.3 & 0.2 \\
\hline 0.2 & & 0.1 & & & 0.1 & 0.1 & 0.1 & 0.1 \\
\hline 0.1 & & 0.1 & 0.1 & 0.1 & 0.1 & 0.1 & 0.1 & 0.1 \\
\hline 0.1 & 0.1 & 0.1 & 0.1 & 0.1 & 0.1 & 0.1 & 0.1 & 0.1 \\
\hline 0.2 & 0.1 & 0.1 & 0.1 & 0.1 & 0.1 & 0.1 & 0.2 & 0.2 \\
\hline 0.2 & 0.1 & 0.1 & 0.1 & 0.1 & 0.2 & 0.1 & 0.1 & 0.2 \\
\hline 0.2 & 0.1 & 0.1 & 0.1 & 0.2 & 0.2 & 0.2 & 0.3 & 0.2 \\
\hline 0.3 & 0.3 & 0.3 & 0.3 & 0.3 & 0.3 & 0.3 & 0.3 & 0.3 \\
\hline 0.4 & 0.3 & 0.4 & 0.4 & 0.3 & 0.3 & 0.3 & 0.3 & 0.3 \\
\hline 0.2 & 0.1 & 0.1 & 0.1 & 0.2 & 0.1 & 0.2 & 0.2 & 0.2 \\
\hline 0.3 & 0.1 & 0.2 & 0.2 & 0.2 & 0.2 & 0.2 & 0.2 & 0.3 \\
\hline 0.2 & 0.1 & 0.1 & 0.1 & 0.2 & 0.2 & 0.2 & 0.2 & 0.2 \\
\hline 0.3 & 0.1 & 0.2 & 0.2 & 0.2 & 0.3 & 0.3 & 0.3 & 0.3 \\
\hline 0.5 & 0.4 & 0.4 & 0.4 & 0.4 & 0.5 & 0.4 & 0.5 & 0.4 \\
\hline 0.4 & 0.3 & 0.3 & 0.3 & 0.4 & 0.4 & 0.4 & 0.4 & 0.4 \\
\hline 0.4 & 0.2 & 0.2 & 0.2 & 0.3 & 0.2 & 0.2 & 0.3 & 0.3 \\
\hline 0.2 & 0.2 & 0.2 & 0.2 & 0.3 & 0.2 & 0.3 & 0.3 & 0.3 \\
\hline 0.3 & 0.2 & 0.2 & 0.3 & 0.2 & 0.3 & 0.3 & 0.3 & 0.2 \\
\hline 0.3 & 0.2 & 0.3 & 0.3 & 0.5 & 0.5 & 0.5 & 0.5 & 0.5 \\
\hline 0.6 & 0.4 & 0.4 & 0.5 & 0.5 & 0.5 & 0.5 & 0.4 & 0.5 \\
\hline 0.4 & 0.3 & 0.3 & 0.3 & 0.3 & 0.4 & 0.4 & 0.4 & 0.4 \\
\hline
\end{tabular}


wbheat

\begin{tabular}{|c|c|c|c|c|c|c|c|c|}
\hline 0.5 & 0.3 & 0.3 & 0.3 & 0.4 & 0.4 & 0.4 & 0.4 & 0.4 \\
\hline 0.3 & 0.3 & 0.3 & 0.3 & 0.3 & 0.3 & 0.3 & 0.2 & 0.2 \\
\hline 0.3 & 0.2 & 0.2 & 0.2 & 0.3 & 0.3 & 0.3 & 0.4 & 0.4 \\
\hline 0.4 & 0.3 & 0.4 & 0.5 & 0.5 & 0.4 & 0.4 & 0.5 & 0.4 \\
\hline 0.3 & 0.2 & 0.3 & 0.3 & 0.4 & 0.4 & 0.4 & 0.4 & 0.4 \\
\hline 0.3 & 0.2 & 0.2 & 0.3 & 0.3 & 0.3 & 0.3 & 0.3 & 0.3 \\
\hline 0.3 & 0.2 & 0.2 & 0.2 & 0.3 & 0.3 & 0.3 & 0.3 & 0.3 \\
\hline 0.4 & 0.3 & 0.4 & 0.5 & 0.5 & 0.5 & 0.5 & 0.5 & 0.5 \\
\hline 0.2 & 0.2 & 0.1 & 0.2 & 0.2 & 0.3 & 0.3 & 0.3 & 0.3 \\
\hline 0.4 & 0.3 & 0.3 & 0.4 & 0.4 & 0.4 & 0.4 & 0.4 & 0.5 \\
\hline 0.4 & 0.3 & 0.3 & 0.3 & 0.4 & 0.4 & 0.4 & 0.4 & 0.3 \\
\hline 0.5 & 0.4 & 0.3 & 0.3 & 0.3 & 0.3 & 0.4 & 0.4 & 0.4 \\
\hline 0.4 & 0.4 & 0.4 & 0.5 & 0.5 & 0.5 & 0.5 & 0.5 & 0.6 \\
\hline 0.5 & 0.3 & 0.4 & 0.4 & 0.4 & 0.5 & 0.5 & 0.5 & 0.5 \\
\hline 0.3 & 0.1 & 0.1 & 0.2 & 0.1 & 0.2 & 0.2 & 0.2 & 0.2 \\
\hline 0.3 & 0.1 & 0.1 & 0.1 & 0.1 & 0.1 & 0.1 & 0.1 & 0.2 \\
\hline 0.3 & 0.2 & 0.2 & 0.3 & 0.3 & 0.3 & 0.3 & 0.3 & 0.4 \\
\hline 0.2 & 0.2 & 0.2 & 0.2 & 0.2 & 0.3 & 0.3 & 0.3 & 0.3 \\
\hline 0.2 & 0.2 & 0.1 & 0.2 & 0.2 & 0.2 & 0.2 & 0.2 & 0.3 \\
\hline 0.4 & 0.1 & 0.2 & 0.2 & 0.2 & 0.3 & 0.3 & 0.3 & 0.3 \\
\hline 0.4 & 0.3 & 0.3 & 0.4 & 0.4 & 0.5 & 0.5 & 0.5 & 0.5 \\
\hline 0.2 & 0.1 & 0.1 & 0.1 & 0.2 & 0.2 & 0.2 & 0.2 & 0.2 \\
\hline 0.2 & 0.1 & 0.2 & 0.1 & 0.2 & 0.2 & 0.1 & 0.2 & 0.2 \\
\hline 0.3 & 0.2 & 0.2 & 0.2 & 0.3 & 0.3 & 0.3 & 0.3 & 0.3 \\
\hline 0.3 & 0.2 & 0.2 & 0.2 & 0.2 & 0.2 & 0.2 & 0.3 & 0.2 \\
\hline 0.2 & 0.1 & 0.1 & 0.1 & 0.1 & 0.2 & 0.1 & 0.2 & 0.2 \\
\hline 0.2 & 0.1 & 0.1 & 0.1 & 0.2 & 0.1 & 0.1 & 0.2 & 0.1 \\
\hline 0.1 & 0.1 & 0.1 & 0.1 & 0.1 & 0.1 & 0.1 & 0.1 & 0.1 \\
\hline 0.3 & 0.2 & 0.1 & 0.2 & 0.2 & 0.2 & 0.2 & 0.3 & 0.3 \\
\hline 0.4 & 0.1 & 0.2 & 0.2 & 0.2 & 0.2 & 0.2 & 0.2 & 0.3 \\
\hline 0.3 & 0.3 & 0.2 & 0.2 & 0.3 & 0.2 & 0.3 & 0.3 & 0.3 \\
\hline 0.5 & 0.3 & 0.3 & 0.3 & 0.3 & 0.4 & 0.4 & 0.4 & 0.4 \\
\hline 0.4 & 0.3 & 0.4 & 0.4 & 0.5 & 0.5 & 0.5 & 0.5 & 0.6 \\
\hline 0.4 & 0.3 & 0.4 & 0.4 & 0.4 & 0.4 & 0.5 & 0.4 & 0.4 \\
\hline 0.3 & 0.1 & 0.1 & 0.1 & 0.1 & 0.2 & 0.2 & 0.2 & 0.2 \\
\hline 0.1 & 0.1 & 0.1 & 0.1 & 0.1 & 0.1 & 0.1 & 0.2 & 0.2 \\
\hline 0.2 & 0.1 & 0.1 & 0.1 & 0.1 & 0.1 & 0.1 & 0.2 & 0.1 \\
\hline 0.2 & 0.1 & 0.2 & 0.2 & 0.2 & 0.3 & 0.2 & 0.2 & 0.2 \\
\hline 0.2 & 0.1 & 0.1 & 0.1 & 0.1 & 0.2 & 0.2 & 0.2 & 0.2 \\
\hline 0.4 & 0.2 & 0.2 & 0.2 & 0.3 & 0.3 & 0.3 & 0.3 & 0.3 \\
\hline 0.3 & 0.2 & 0.2 & 0.2 & 0.2 & 0.2 & 0.3 & 0.3 & 0.3 \\
\hline 0.2 & 0.1 & 0.1 & 0.2 & 0.2 & 0.2 & 0.2 & 0.2 & 0.2 \\
\hline 0.2 & 0.2 & 0.2 & 0.1 & 0.2 & 0.2 & 0.2 & 0.2 & 0.2 \\
\hline 0.2 & 0.1 & 0.2 & 0.2 & 0.2 & 0.2 & 0.2 & 0.2 & 0.2 \\
\hline 0.3 & 0.1 & 0.2 & 0.1 & 0.2 & 0.2 & 0.2 & 0.2 & 0.2 \\
\hline 0.1 & 0.1 & 0.3 & & & 0.1 & 0.1 & 0.1 & 0.1 \\
\hline 0.1 & & & & 0.1 & & 0.1 & & \\
\hline 0.1 & & 0.1 & 0.1 & 0.1 & 0.1 & 0.1 & 0.1 & 0.1 \\
\hline 0.2 & 0.1 & 0.1 & 0.1 & 0.1 & 0.2 & 0.2 & 0.2 & 0.2 \\
\hline 0.4 & 0.3 & 0.3 & 0.3 & 0.3 & 0.4 & 0.4 & 0.4 & 0.4 \\
\hline 0.1 & 0.1 & & 0.1 & 0.1 & 0.1 & 0.1 & 0.1 & 0.1 \\
\hline
\end{tabular}


wbheat

\begin{tabular}{|c|c|c|c|c|c|c|c|c|}
\hline 0.2 & & 0.1 & 0.1 & & 0.1 & 0.1 & 0.1 & 0.1 \\
\hline 0.1 & & & & & & 0.1 & & \\
\hline 0.1 & & & & & & & 0.1 & \\
\hline 0.2 & & & & 0.1 & & 0.1 & & 0.1 \\
\hline 0.1 & & & & & & & 0.1 & 0.1 \\
\hline \multicolumn{9}{|l|}{0.1} \\
\hline 0.1 & & & & & & 0.1 & & \\
\hline 0.2 & 0.1 & 0.1 & & & & 0.1 & 0.1 & 0.1 \\
\hline 0.2 & & 0.1 & & & & 0.1 & & \\
\hline 0.2 & 0.1 & 0.1 & 0.1 & 0.1 & 0.1 & 0.1 & 0.1 & 0.1 \\
\hline 0.3 & 0.2 & 0.2 & 0.2 & 0.2 & 0.2 & 0.2 & 0.2 & 0.2 \\
\hline 0.1 & 0.1 & & & & & & & \\
\hline 0.2 & & 0.1 & 0.1 & & 0.1 & 0.1 & 0.1 & 0.1 \\
\hline \multirow[t]{2}{*}{0.1} & 0.1 & 0.1 & 0.1 & & 0.1 & & 0.1 & 0.1 \\
\hline & & & & & & & & 0.1 \\
\hline \multicolumn{9}{|l|}{0.1} \\
\hline 0.1 & & 0.1 & & 0.1 & 0.1 & 0.1 & 0.1 & 0.1 \\
\hline 0.2 & 0.1 & & 0.1 & 0.1 & & 0.1 & 0.1 & 0.1 \\
\hline \multirow[t]{2}{*}{0.1} & 0.1 & & & & & & & \\
\hline & & & & & & & & \\
\hline 0.1 & & & & & & & & 0.1 \\
\hline 0.1 & & & & & & & 0.1 & 0.1 \\
\hline \multirow[t]{2}{*}{0.2} & & & & & & & & 0.1 \\
\hline & & & & & & & & \\
\hline 0.1 & & & & & & & & 0.1 \\
\hline \multicolumn{9}{|l|}{0.1} \\
\hline \multicolumn{9}{|l|}{0.1} \\
\hline 0.1 & & & & & & & & 0.1 \\
\hline \multicolumn{9}{|l|}{0.1} \\
\hline \multicolumn{9}{|l|}{0.1} \\
\hline \multicolumn{9}{|l|}{0.1} \\
\hline 0.1 & & & & 0.1 & 0.2 & 0.1 & 0.2 & 0.2 \\
\hline \multicolumn{9}{|l|}{0.2} \\
\hline \multicolumn{9}{|l|}{0.1} \\
\hline \multicolumn{9}{|l|}{0.1} \\
\hline \multicolumn{9}{|l|}{0.1} \\
\hline \multicolumn{9}{|l|}{0.1} \\
\hline 0.1 & & & & & & & 0.1 & 0.1 \\
\hline \multicolumn{9}{|l|}{0.1} \\
\hline & & & & & & & & \\
\hline \multicolumn{9}{|l|}{0.1} \\
\hline \multicolumn{9}{|l|}{0.1} \\
\hline \multirow{2}{*}{\multicolumn{9}{|c|}{0.1}} \\
\hline & & & & & & & & \\
\hline & & & & & & & & \\
\hline 0.1 & & & & & & & & 0.1 \\
\hline 0.1 & & & & & & & & \\
\hline & & & & & & & & \\
\hline 0.1 & & & & & & & & \\
\hline 0.1 & & & & & & & & \\
\hline
\end{tabular}




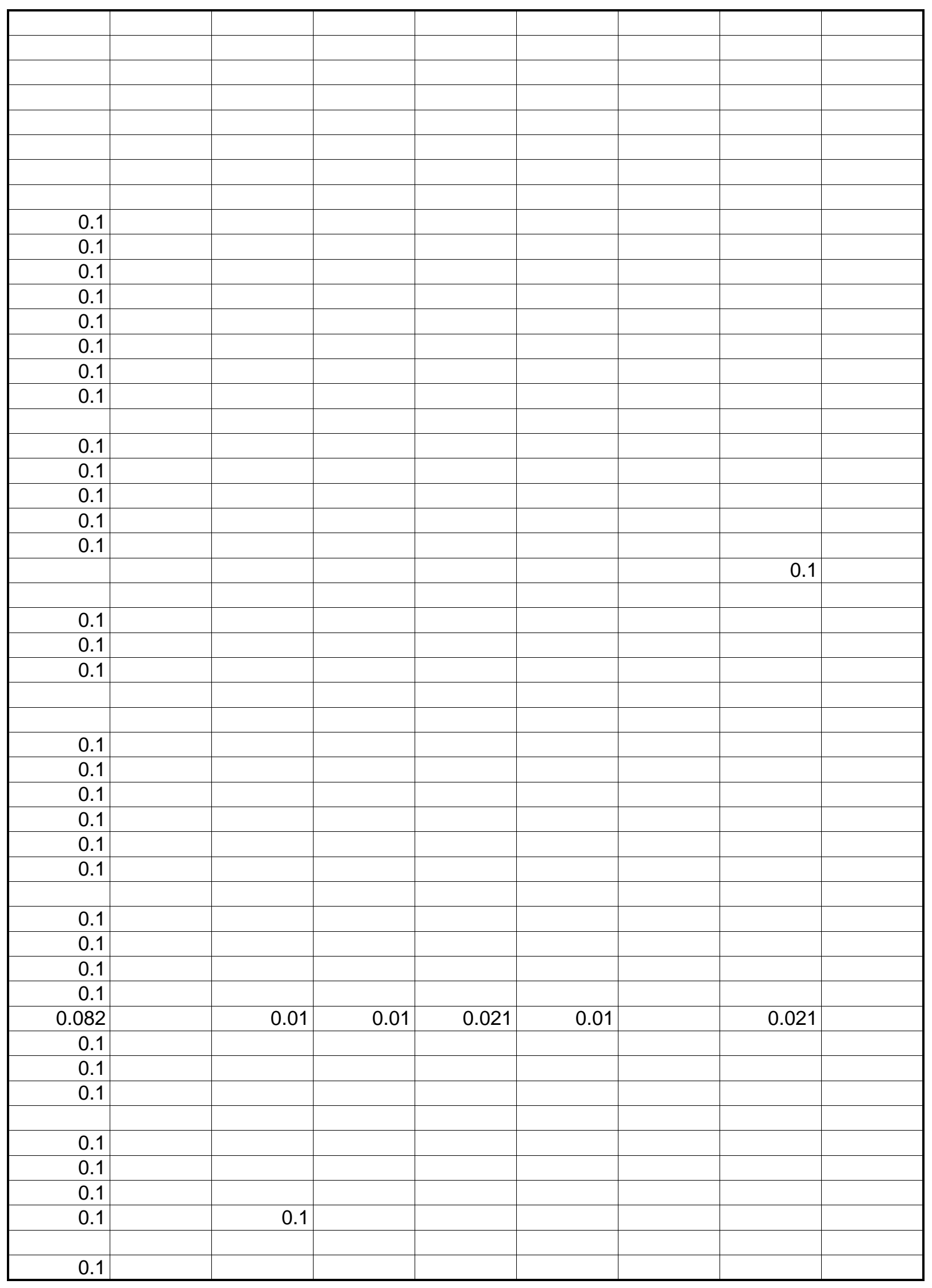


wbheat

\begin{tabular}{|c|c|c|c|c|c|c|c|c|}
\hline \multicolumn{9}{|l|}{0.1} \\
\hline \multicolumn{9}{|l|}{0.1} \\
\hline \multicolumn{9}{|l|}{0.1} \\
\hline \multicolumn{9}{|l|}{0.1} \\
\hline & & & & & & & & \\
\hline \multicolumn{9}{|l|}{0.1} \\
\hline \multicolumn{9}{|l|}{0.1} \\
\hline 0.1 & & 0.1 & & & & & & \\
\hline \multicolumn{9}{|l|}{0.1} \\
\hline & & 0.1 & & & & & & \\
\hline 0.1 & & 0.1 & & & & & & \\
\hline 0.3 & 0.2 & 0.3 & & & & & & \\
\hline 0.3 & 0.2 & 0.2 & & 0.1 & 0.1 & & 0.1 & \\
\hline \multicolumn{9}{|l|}{0.1} \\
\hline \multicolumn{9}{|l|}{0.1} \\
\hline 0.1 & 0.1 & 0.1 & & & & & & \\
\hline 0.4 & 0.2 & 0.3 & & & & & 0.1 & \\
\hline 0.3 & 0.2 & 0.1 & & & & & & 0.1 \\
\hline \multicolumn{9}{|l|}{0.1} \\
\hline 0.1 & 0.1 & & & & & & & \\
\hline \multicolumn{9}{|l|}{0.1} \\
\hline 0.2 & & 0.1 & & & & & & 0.1 \\
\hline 0.1 & 0.1 & 0.1 & & & & & & \\
\hline 0.3 & 0.1 & 0.1 & & & & & & \\
\hline \multirow[t]{2}{*}{0.1} & 0.1 & 0.1 & & 0.1 & & & & \\
\hline & 0.1 & & & & & & & \\
\hline 0.2 & 0.1 & 0.2 & & & & & & \\
\hline 0.1 & & 0.1 & & & & & & \\
\hline 0.2 & 0.2 & 0.2 & & & & & & \\
\hline 0.3 & 0.2 & 0.2 & & & & & & \\
\hline 0.2 & 0.1 & 0.2 & & & & & & \\
\hline 0.1 & 0.1 & 0.1 & & & & & & \\
\hline \multicolumn{9}{|l|}{0.1} \\
\hline 0.1 & & 0.1 & & & & & & \\
\hline 0.2 & 0.2 & 0.1 & & & & & & \\
\hline 0.2 & 0.1 & 0.1 & & & & & & \\
\hline 0.2 & 0.1 & 0.1 & & & & & & \\
\hline \multicolumn{9}{|l|}{0.1} \\
\hline 0.1 & & 0.1 & & & & & & \\
\hline 0.2 & 0.2 & 0.2 & & & & & & \\
\hline 0.2 & 0.2 & 0.2 & & & & & & \\
\hline 0.3 & 0.2 & 0.2 & & & & & & \\
\hline 0.4 & 0.2 & 0.2 & & & & & & \\
\hline 0.1 & 0.1 & 0.1 & & & & & & \\
\hline 0.4 & 0.3 & 0.3 & 0.2 & 0.1 & 0.2 & 0.2 & 0.2 & 0.2 \\
\hline 0.4 & 0.2 & 0.2 & 0.1 & 0.1 & 0.1 & 0.1 & 0.1 & 0.1 \\
\hline 0.6 & 0.3 & 0.3 & 0.2 & 0.2 & 0.2 & 0.2 & 0.2 & 0.2 \\
\hline 0.2 & 0.1 & 0.1 & 0.1 & & 0.1 & 0.1 & 0.1 & 0.1 \\
\hline 0.5 & 0.4 & 0.5 & 0.2 & 0.3 & 0.3 & 0.3 & 0.3 & 0.3 \\
\hline 0.3 & 0.2 & 0.2 & 0.2 & 0.1 & 0.1 & 0.1 & & 0.1 \\
\hline 0.2 & 0.1 & 0.1 & 0.1 & 0.1 & 0.1 & 0.1 & 0.1 & 0.1 \\
\hline
\end{tabular}


wbheat

\begin{tabular}{|c|c|c|c|c|c|c|c|c|}
\hline 0.3 & 0.2 & 0.2 & 0.1 & 0.1 & 0.1 & 0.1 & 0.1 & 0.1 \\
\hline 0.2 & 0.2 & 0.2 & 0.1 & 0.1 & 0.1 & 0.2 & 0.1 & 0.2 \\
\hline 0.3 & 0.1 & 0.1 & 0.1 & 0.1 & 0.1 & 0.1 & 0.1 & 0.1 \\
\hline 1.1 & 0.7 & 1 & 0.4 & 0.4 & 0.5 & 0.5 & 0.6 & 0.5 \\
\hline 0.7 & 0.5 & 0.6 & 0.3 & 0.4 & 0.2 & 0.2 & 0.2 & 0.2 \\
\hline 0.2 & 0.2 & 0.3 & 0.2 & 0.2 & 0.2 & 0.2 & 0.3 & 0.4 \\
\hline 0.7 & 0.5 & 0.5 & 0.3 & 0.4 & 0.4 & 0.4 & 0.4 & 0.4 \\
\hline 0.2 & 0.2 & 0.2 & 0.2 & 0.2 & 0.1 & 0.2 & 0.2 & 0.2 \\
\hline 0.1 & & & & & & & 0.1 & \\
\hline 0.1 & 0.1 & & 0.1 & & 0.1 & & 0.1 & 0.1 \\
\hline 0.3 & 0.2 & 0.2 & 0.1 & 0.1 & 0.2 & 0.2 & 0.2 & 0.2 \\
\hline 0.6 & 0.4 & 0.4 & 0.2 & 0.2 & 0.2 & 0.2 & 0.2 & 0.2 \\
\hline 0.9 & 0.7 & 0.7 & 0.5 & 0.5 & 0.5 & 0.5 & 0.5 & 0.5 \\
\hline 0.6 & 0.6 & 0.5 & 0.4 & 0.3 & 0.4 & 0.4 & 0.4 & 0.4 \\
\hline 0.5 & 0.3 & 0.3 & 0.3 & 0.3 & 0.3 & 0.3 & 0.3 & 0.3 \\
\hline 0.4 & 0.2 & 0.2 & 0.2 & 0.2 & 0.2 & 0.2 & 0.2 & 0.2 \\
\hline 0.1 & 0.1 & 0.1 & 0.1 & 0.1 & 0.1 & 0.1 & 0.1 & 0.1 \\
\hline \multicolumn{9}{|l|}{0.1} \\
\hline 0.9 & 0.7 & 1 & 0.6 & 0.6 & 0.6 & 0.6 & 0.6 & 0.6 \\
\hline 1.1 & 1.1 & 1.1 & 0.6 & 0.6 & 0.6 & 0.6 & 0.6 & 0.6 \\
\hline 0.7 & 0.5 & 0.4 & 0.4 & 0.4 & 0.4 & 0.4 & 0.4 & 0.4 \\
\hline 0.5 & 0.3 & 0.3 & 0.2 & 0.3 & 0.2 & 0.3 & 0.3 & 0.2 \\
\hline 0.2 & 0.2 & 0.2 & 0.2 & 0.2 & 0.2 & 0.2 & 0.2 & 0.2 \\
\hline 0.2 & 0.2 & 0.2 & 0.1 & 0.2 & 0.2 & 0.2 & 0.2 & 0.2 \\
\hline 0.1 & 0.1 & 0.1 & & 0.1 & & 0.1 & & 0.1 \\
\hline 0.2 & & & & 0.1 & 0.1 & & 0.1 & 0.1 \\
\hline 0.1 & 0.1 & 0.1 & 0.1 & 0.1 & 0.1 & 0.1 & 0.1 & 0.1 \\
\hline 0.1 & 0.1 & & 0.1 & & 0.1 & & 0.1 & 0.1 \\
\hline 0.5 & 0.4 & 0.4 & 0.3 & 0.3 & 0.3 & 0.4 & 0.5 & 0.5 \\
\hline 0.6 & 0.4 & 0.4 & 0.4 & 0.4 & 0.4 & 0.3 & 0.4 & 0.3 \\
\hline 0.5 & 0.3 & 0.4 & 0.4 & 0.4 & 0.4 & 0.4 & 0.4 & 0.4 \\
\hline 0.4 & 0.3 & 0.3 & 0.3 & 0.4 & 0.3 & 0.4 & 0.3 & 0.4 \\
\hline 0.5 & 0.4 & 0.4 & 0.3 & 0.4 & 0.4 & 0.4 & 0.4 & 0.4 \\
\hline 0.4 & 0.3 & 0.3 & 0.3 & 0.3 & 0.3 & 0.3 & 0.3 & 0.3 \\
\hline 0.3 & 0.2 & 0.2 & 0.1 & 0.1 & 0.1 & 0.1 & 0.1 & 0.1 \\
\hline 0.1 & & & & & 0.1 & & 0.1 & \\
\hline 0.1 & & & & & & & & \\
\hline \multicolumn{9}{|l|}{0.1} \\
\hline 0.5 & 0.3 & 0.4 & 0.2 & 0.2 & 0.2 & 0.2 & 0.4 & 0.4 \\
\hline 0.1 & 0.2 & 0.1 & 0.1 & 0.1 & & & 0.1 & \\
\hline 0.1 & 0.1 & 0.1 & & & & & & \\
\hline \multicolumn{9}{|l|}{0.1} \\
\hline \multicolumn{9}{|l|}{0.1} \\
\hline \multicolumn{9}{|l|}{0.1} \\
\hline \multicolumn{9}{|l|}{0.1} \\
\hline \multicolumn{9}{|l|}{0.2} \\
\hline 0.1 & 0.1 & 0.1 & & & & & & \\
\hline 0.1 & & & & & & & & \\
\hline & & & & & & & & \\
\hline & & & & & & & & \\
\hline
\end{tabular}




\begin{tabular}{|c|c|c|c|c|c|c|c|c|}
\hline & & & & & & & & \\
\hline & & & & & & & & \\
\hline 0.1 & 0.1 & 0.1 & & & & & & \\
\hline 0.1 & & & & & & & & \\
\hline 0.1 & & & & & & & & \\
\hline 0.1 & & & & & & & & \\
\hline 0.1 & & & & & & & & \\
\hline & & & & & & & & \\
\hline & & & & & & & & \\
\hline & & & & & & & & \\
\hline & & & & & & & & \\
\hline & & & & & & & & \\
\hline & & & & & & & & \\
\hline & & & & & & & & \\
\hline 0.4 & 0.3 & 0.3 & 0.3 & 0.3 & 0.4 & 0.4 & 0.4 & 0.4 \\
\hline 0.5 & 0.4 & 0.4 & 0.4 & 0.4 & 0.4 & 0.4 & 0.4 & 0.5 \\
\hline 0.4 & 0.3 & 0.3 & 0.3 & 0.3 & 0.3 & 0.4 & 0.4 & 0.4 \\
\hline 0.3 & 0.3 & 0.2 & 0.3 & 0.2 & 0.3 & 0.2 & 0.3 & 0.3 \\
\hline & & 0.1 & 0.1 & 0.1 & 0.1 & 0.1 & 0.1 & 0.1 \\
\hline 0.1 & 0.1 & 0.1 & 0.1 & 0.1 & 0.1 & 0.2 & 0.1 & 0.2 \\
\hline 0.4 & 0.4 & 0.3 & 0.3 & 0.4 & 0.4 & 0.4 & 0.4 & 0.4 \\
\hline 0.3 & 0.3 & 0.3 & 0.3 & 0.3 & 0.3 & 0.3 & 0.3 & 0.3 \\
\hline 0.1 & 0.1 & 0.1 & 0.1 & 0.1 & 0.1 & 0.1 & 0.1 & 0.1 \\
\hline 0.1 & 0.1 & & 0.1 & 0.1 & 0.1 & 0.1 & 0.1 & 0.1 \\
\hline 0.2 & 0.2 & 0.2 & 0.2 & 0.2 & 0.2 & 0.3 & 0.3 & 0.3 \\
\hline 0.4 & 0.3 & 0.3 & 0.3 & 0.3 & 0.3 & 0.3 & 0.3 & 0.3 \\
\hline 0.5 & 0.5 & 0.4 & 0.4 & 0.5 & 0.5 & 0.5 & 0.5 & 0.5 \\
\hline 0.4 & 0.4 & 0.4 & 0.3 & 0.4 & 0.4 & 0.4 & 0.4 & 0.4 \\
\hline 0.7 & 0.7 & 0.7 & 0.7 & 0.7 & 0.7 & 0.7 & 0.7 & 0.7 \\
\hline 0.6 & 0.6 & 0.5 & 0.5 & 0.5 & 0.6 & 0.5 & 0.6 & 0.6 \\
\hline 0.3 & 0.3 & 0.2 & 0.2 & 0.2 & 0.2 & 0.3 & 0.3 & 0.3 \\
\hline 0.4 & 0.4 & 0.4 & 0.4 & 0.4 & 0.4 & 0.4 & 0.4 & 0.5 \\
\hline 0.2 & 0.2 & 0.2 & 0.2 & 0.2 & 0.2 & 0.2 & 0.2 & 0.2 \\
\hline 0.3 & 0.3 & 0.3 & 0.2 & 0.3 & 0.3 & 0.3 & 0.3 & 0.3 \\
\hline 0.4 & 0.4 & 0.3 & 0.3 & 0.3 & 0.3 & 0.4 & 0.4 & 0.4 \\
\hline 0.5 & 0.4 & 0.4 & 0.4 & 0.4 & 0.4 & 0.4 & 0.4 & 0.4 \\
\hline 0.2 & 0.1 & 0.1 & 0.1 & 0.1 & 0.1 & 0.1 & 0.2 & 0.2 \\
\hline 0.2 & 0.2 & 0.2 & 0.2 & 0.2 & 0.2 & 0.2 & 0.2 & 0.2 \\
\hline 0.2 & 0.2 & 0.2 & 0.2 & 0.2 & 0.2 & 0.2 & 0.2 & 0.2 \\
\hline 0.2 & 0.2 & 0.2 & 0.1 & 0.2 & 0.2 & 0.2 & 0.2 & 0.2 \\
\hline 0.2 & 0.2 & 0.1 & 0.1 & 0.1 & 0.1 & 0.1 & 0.2 & 0.2 \\
\hline 0.2 & 0.1 & 0.1 & 0.1 & 0.1 & 0.1 & 0.1 & 0.1 & 0.2 \\
\hline 0.1 & 0.1 & 0.1 & 0.1 & 0.1 & 0.1 & 0.2 & 0.1 & 0.2 \\
\hline 0.2 & 0.2 & 0.2 & 0.2 & 0.2 & 0.2 & 0.2 & 0.2 & 0.2 \\
\hline 0.1 & 0.2 & 0.1 & 0.1 & 0.2 & 0.1 & 0.2 & 0.1 & 0.1 \\
\hline 0.1 & 0.1 & 0.1 & 0.1 & 0.1 & 0.1 & 0.1 & 0.1 & 0.1 \\
\hline 0.2 & 0.1 & 0.1 & 0.1 & 0.1 & 0.1 & 0.1 & 0.1 & 0.1 \\
\hline 0.2 & 0.2 & 0.2 & 0.2 & 0.2 & 0.2 & 0.2 & 0.2 & 0.2 \\
\hline 0.1 & & 0.1 & 0.1 & & & & 0.1 & \\
\hline
\end{tabular}


wbheat

\begin{tabular}{|c|c|c|c|c|c|c|c|c|}
\hline 0.1 & 0.1 & 0.1 & 0.1 & 0.1 & & 0.1 & 0.1 & 0.1 \\
\hline 0.1 & 0.1 & 0.1 & & & 0.1 & 0.1 & & 0.1 \\
\hline \multirow[t]{3}{*}{0.1} & 0.1 & 0.1 & 0.1 & 0.1 & 0.1 & 0.1 & 0.1 & 0.1 \\
\hline & & 0.1 & & & & & & \\
\hline & & 0.1 & & & & & & \\
\hline & & & & & & & & \\
\hline & & & & & & & & \\
\hline & & & & & & & & \\
\hline & & & & & & & & \\
\hline & & & & & & & & \\
\hline & & & & & & & & \\
\hline & & & & & & & & \\
\hline & & & & & & & 0.1 & \\
\hline & & & & & & & & \\
\hline & & 0.1 & & & & & & \\
\hline & & & & & & & & \\
\hline & & & & & & & & \\
\hline & & & & & & & & \\
\hline & & & & & 0.1 & 0.1 & & \\
\hline & & & & & & & & \\
\hline & & & & & & & & \\
\hline & & & & & & & & \\
\hline & & & & & & & & \\
\hline & & & & & & & & \\
\hline & & & & & & & & \\
\hline & & & & & & & & \\
\hline & & & & & & & & \\
\hline & & & & & & & & \\
\hline & & & & & & & & \\
\hline & & & & & & & & \\
\hline & & & & & & & & \\
\hline & & & & & & & & \\
\hline & & & & & 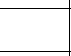 & & & \\
\hline & & & & & & & & \\
\hline & & & & 0.1 & & & & 0.1 \\
\hline & & & & & & 0.1 & & \\
\hline 0.2 & 0.2 & 0.2 & 0.2 & 0.1 & 0.2 & 0.1 & 0.2 & 0.2 \\
\hline 0.2 & 0.1 & 0.1 & 0.1 & 0.1 & 0.1 & 0.2 & 0.1 & 0.2 \\
\hline & & & & & & & & \\
\hline & & & & & & & & \\
\hline & & & & & & & & \\
\hline & & & & & & & & \\
\hline 0.2 & 0.2 & 0.2 & 0.1 & 0.1 & 0.2 & 0.2 & 0.2 & 0.2 \\
\hline 0.2 & 0.2 & 0.2 & 0.1 & 0.2 & 0.2 & 0.2 & 0.2 & 0.2 \\
\hline 0.2 & 0.2 & 0.2 & 0.2 & 0.2 & 0.2 & 0.2 & 0.2 & 0.2 \\
\hline 0.2 & 0.2 & 0.2 & 0.1 & 0.2 & 0.2 & 0.2 & 0.2 & 0.2 \\
\hline 0.2 & 0.2 & 0.2 & 0.2 & 0.2 & 0.2 & 0.2 & 0.2 & 0.3 \\
\hline 0.3 & 0.3 & 0.3 & 0.2 & 0.3 & 0.3 & 0.3 & 0.3 & 0.3 \\
\hline 0.5 & 0.5 & 0.5 & 0.5 & 0.5 & 0.6 & 0.5 & 0.5 & 0.6 \\
\hline 0.4 & 0.4 & 0.3 & 0.3 & 0.4 & 0.3 & 0.4 & 0.4 & 0.4 \\
\hline 0.3 & 0.3 & 0.2 & 0.3 & 0.2 & 0.2 & 0.2 & 0.2 & 0.3 \\
\hline
\end{tabular}


wbheat

\begin{tabular}{|c|c|c|c|c|c|c|c|c|}
\hline 0.2 & 0.2 & 0.2 & 0.2 & 0.2 & 0.3 & 0.3 & 0.3 & 0.3 \\
\hline 0.3 & 0.3 & 0.3 & 0.4 & 0.4 & 0.5 & 0.5 & 0.5 & 0.6 \\
\hline 0.7 & 0.7 & 0.7 & 0.7 & 0.7 & 0.7 & 0.7 & 0.7 & 0.7 \\
\hline 0.3 & 0.3 & 0.2 & 0.2 & 0.2 & 0.3 & 0.3 & 0.3 & 0.3 \\
\hline 0.4 & 0.4 & 0.4 & 0.4 & 0.4 & 0.4 & 0.4 & 0.4 & 0.4 \\
\hline 0.2 & 0.1 & 0.2 & 0.1 & 0.1 & 0.1 & 0.2 & 0.2 & 0.3 \\
\hline 0.4 & 0.4 & 0.3 & 0.3 & 0.3 & 0.4 & 0.4 & 0.4 & 0.4 \\
\hline 0.5 & 0.4 & 0.4 & 0.4 & 0.3 & 0.4 & 0.4 & 0.4 & 0.4 \\
\hline 0.3 & 0.3 & 0.3 & 0.3 & 0.3 & 0.3 & 0.3 & 0.3 & 0.3 \\
\hline 0.4 & 0.3 & 0.3 & 0.3 & 0.3 & 0.3 & 0.3 & 0.4 & 0.3 \\
\hline 0.3 & 0.3 & 0.2 & 0.2 & 0.2 & 0.3 & 0.3 & 0.3 & 0.3 \\
\hline 0.3 & 0.3 & 0.3 & 0.3 & 0.3 & 0.3 & 0.3 & 0.3 & 0.3 \\
\hline 0.4 & 0.4 & 0.4 & 0.4 & 0.4 & 0.4 & 0.4 & 0.4 & 0.4 \\
\hline 0.2 & 0.2 & 0.1 & 0.1 & 0.1 & 0.1 & 0.2 & 0.2 & 0.2 \\
\hline 0.1 & 0.1 & 0.1 & 0.1 & 0.1 & 0.1 & 0.2 & 0.2 & 0.2 \\
\hline 0.3 & 0.3 & 0.3 & 0.3 & 0.3 & 0.3 & 0.3 & 0.3 & 0.4 \\
\hline 0.4 & 0.4 & 0.4 & 0.4 & 0.4 & 0.4 & 0.4 & 0.4 & 0.4 \\
\hline 0.2 & 0.2 & 0.2 & 0.2 & 0.2 & 0.2 & 0.2 & 0.2 & 0.2 \\
\hline 0.2 & 0.2 & 0.2 & 0.1 & 0.1 & 0.1 & 0.1 & 0.1 & 0.2 \\
\hline 0.1 & 0.1 & 0.1 & 0.1 & & 0.1 & 0.1 & 0.1 & 0.1 \\
\hline 0.1 & 0.1 & 0.1 & 0.1 & 0.1 & 0.1 & 0.1 & 0.1 & 0.1 \\
\hline 0.1 & 0.1 & 0.1 & 0.1 & 0.1 & 0.1 & 0.1 & 0.1 & 0.1 \\
\hline 0.2 & 0.2 & 0.2 & 0.2 & 0.2 & 0.2 & 0.2 & 0.2 & 0.2 \\
\hline 0.1 & 0.1 & & & & & & & \\
\hline & & & & & 01 & & & 01 \\
\hline 0.1 & & 0.1 & 0.1 & & 0.1 & & & 0.1 \\
\hline & 0.1 & & & & & & & \\
\hline & & & & & & & & \\
\hline & & & & & & & & \\
\hline & & & & & & & & \\
\hline & & & 0.1 & & 0.1 & & 0.1 & 0.1 \\
\hline & & & & & & & & \\
\hline & & & & & & & & \\
\hline & & & & & & & & \\
\hline & & & & & & & & \\
\hline & & & & & & & & \\
\hline & & & & & & & & \\
\hline & & & & & & & & \\
\hline & & & & & & & & \\
\hline & & & & & & & & \\
\hline & & & & & & & & \\
\hline & & & & & & & & \\
\hline & & & & & & & & \\
\hline & & & & & & & & \\
\hline & & & & & & & & \\
\hline & & & & & & & & \\
\hline & & & & & & & & \\
\hline & & & & & & & & \\
\hline & & & & & & & & \\
\hline & & & & & & & & \\
\hline & & & & & & & & \\
\hline
\end{tabular}


wbheat

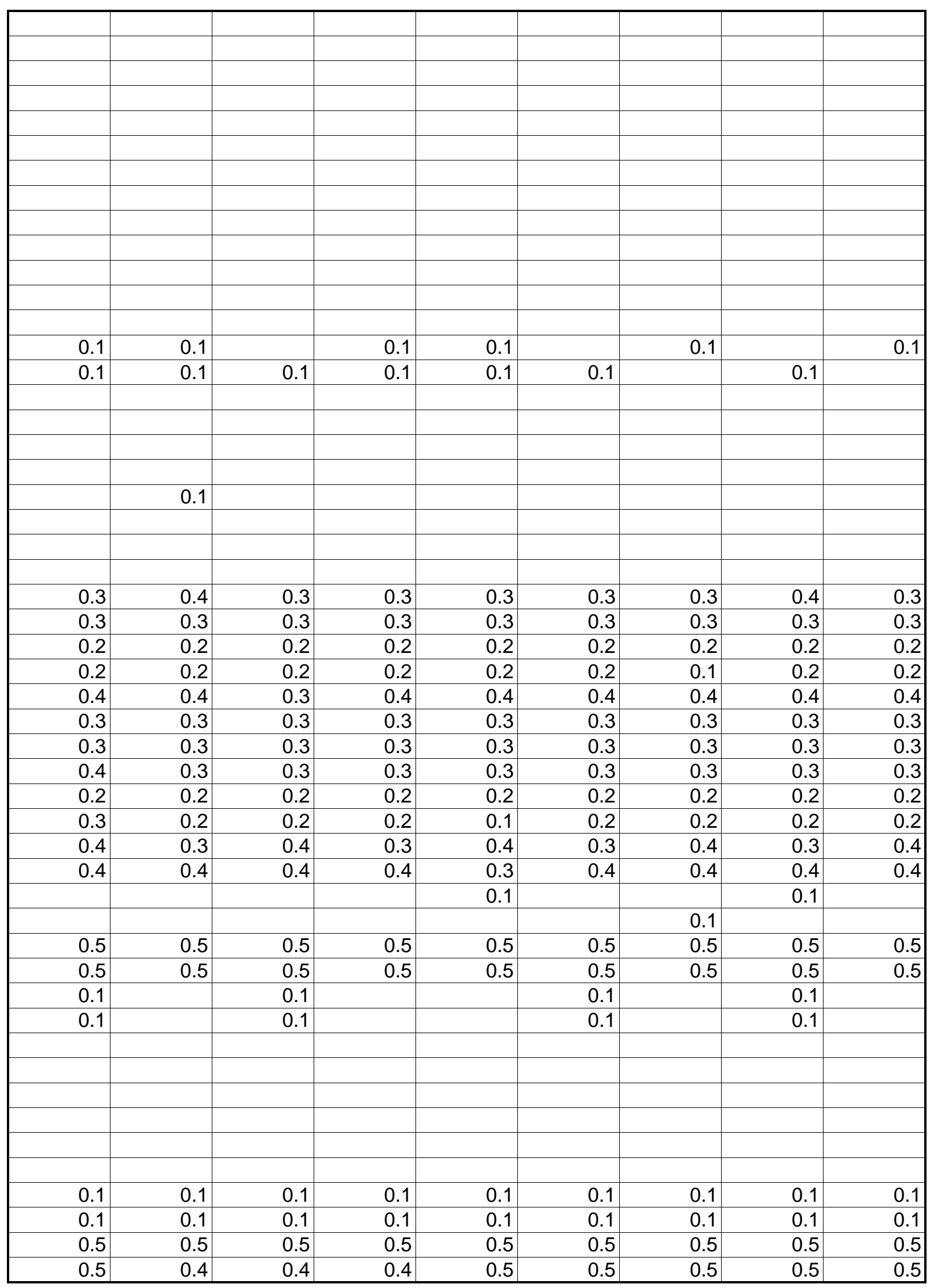


wbheat

\begin{tabular}{|c|c|c|c|c|c|c|c|c|}
\hline 0.5 & \begin{tabular}{l|l}
0.5 \\
\end{tabular} & \begin{tabular}{l|l}
0.5 \\
\end{tabular} & 0.5 & 0.5 & 0.5 & 0.5 & 0.5 & 0.5 \\
\hline 0.5 & 0.5 & 0.5 & 0.5 & 0.5 & 0.5 & 0.5 & 0.5 & 0.5 \\
\hline 0.2 & 0.1 & 0.1 & 0.1 & 0.1 & 0.1 & 0.1 & 0.1 & 0.1 \\
\hline 0.1 & 0.1 & 0.1 & 0.1 & 0.1 & 0.1 & 0.1 & 0.1 & 0.1 \\
\hline 0.1 & 0.1 & 0.1 & 0.1 & 0.1 & 0.1 & 0.1 & 0.1 & 0.1 \\
\hline 0.1 & 0.1 & 0.1 & 0.1 & 0.1 & 0.1 & 0.1 & 0.1 & 0.1 \\
\hline 0.1 & 0.1 & 0.1 & 0.1 & 0.1 & 0.1 & 0.1 & 0.1 & 0.1 \\
\hline 0.1 & 0.1 & 0.1 & 0.1 & 0.1 & 0.1 & 0.1 & 0.1 & 0.1 \\
\hline 0.5 & 0.4 & 0.4 & 0.4 & 0.4 & 0.4 & 0.4 & 0.4 & 0.5 \\
\hline 0.2 & 0.2 & 0.1 & 0.1 & 0.1 & 0.1 & 0.2 & 0.2 & 0.2 \\
\hline 0.1 & 0.1 & 0.1 & 0.1 & 0.1 & 0.1 & 0.1 & 0.1 & 0.1 \\
\hline 0.2 & 0.1 & 0.1 & 0.1 & 0.2 & 0.1 & 0.2 & 0.1 & 0.2 \\
\hline 0.1 & 0.1 & 0.1 & 0.1 & 0.1 & 0.1 & 0.1 & 0.1 & 0.1 \\
\hline 0.3 & 0.2 & 0.2 & 0.2 & 0.2 & 0.2 & 0.2 & 0.2 & 0.3 \\
\hline 0.2 & 0.2 & 0.2 & 0.2 & 0.2 & 0.2 & 0.2 & 0.2 & 0.2 \\
\hline & & & & & & & & \\
\hline & & & & & & & & \\
\hline & 0.1 & & & & & & & \\
\hline & & & & & & & & \\
\hline 0.1 & & & & & & & & \\
\hline & & & & & & & & \\
\hline & & & & & & & & \\
\hline & & & & & & & & \\
\hline & & 0.1 & & & & 0.1 & & \\
\hline & & & & & & & & \\
\hline & & & & & & & & \\
\hline & & & & & & & & \\
\hline & & & & & & & & \\
\hline & & & & & & & & \\
\hline & & & & & & & & \\
\hline & & & & & & & & \\
\hline & & & & & & & & \\
\hline & & & & & & & & \\
\hline & & & & & & & & \\
\hline & & & & & & & & \\
\hline & & & & & & & & \\
\hline & & & & & & & & \\
\hline & & & & & & & & \\
\hline & & & & & & & & \\
\hline & & & & & & & & \\
\hline & & & & & & & & \\
\hline & & & & & & & & \\
\hline & & & & & & & & \\
\hline & & & & & & & & \\
\hline & & & & & & & & \\
\hline
\end{tabular}


wbheat

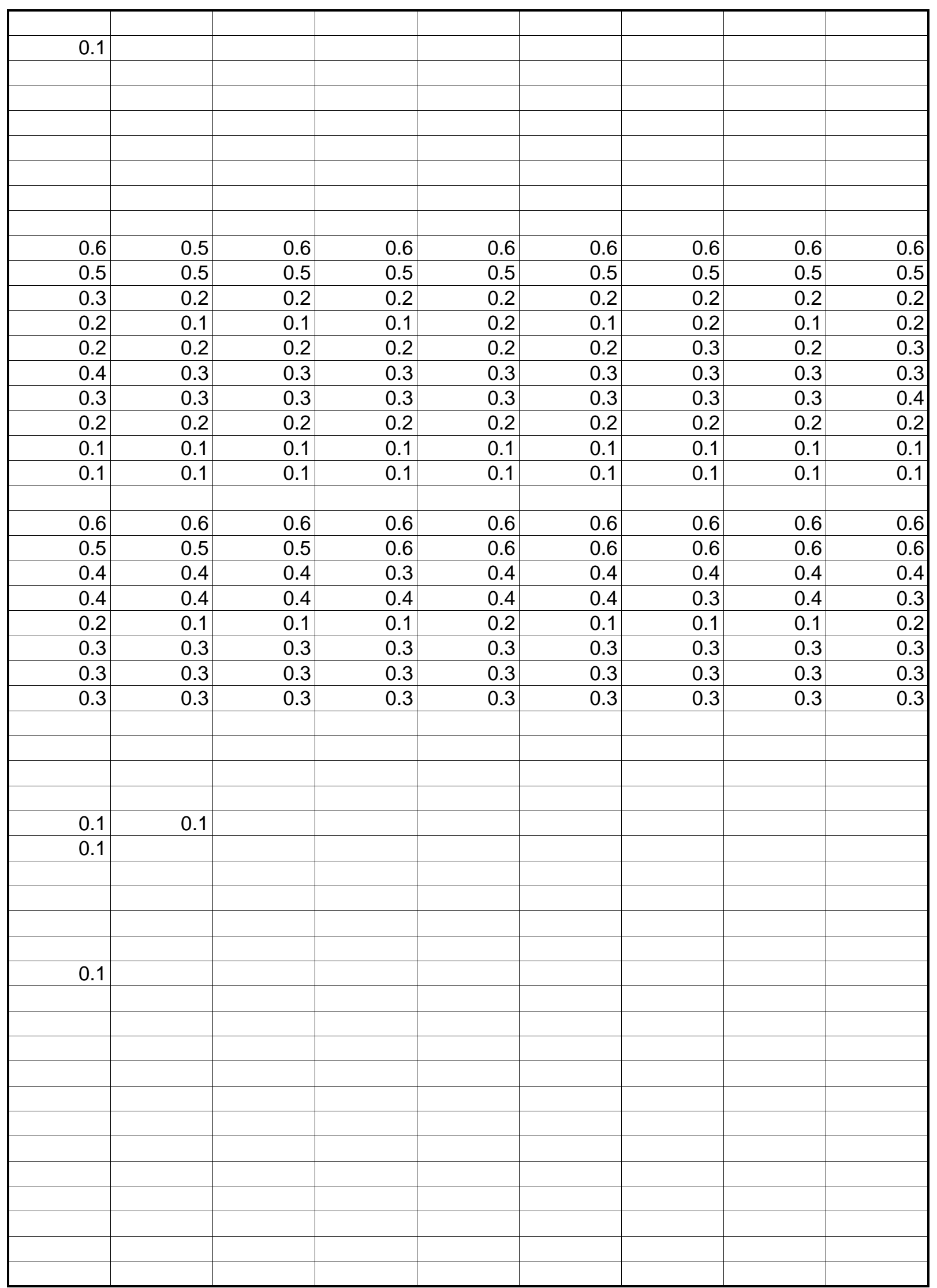


wbheat

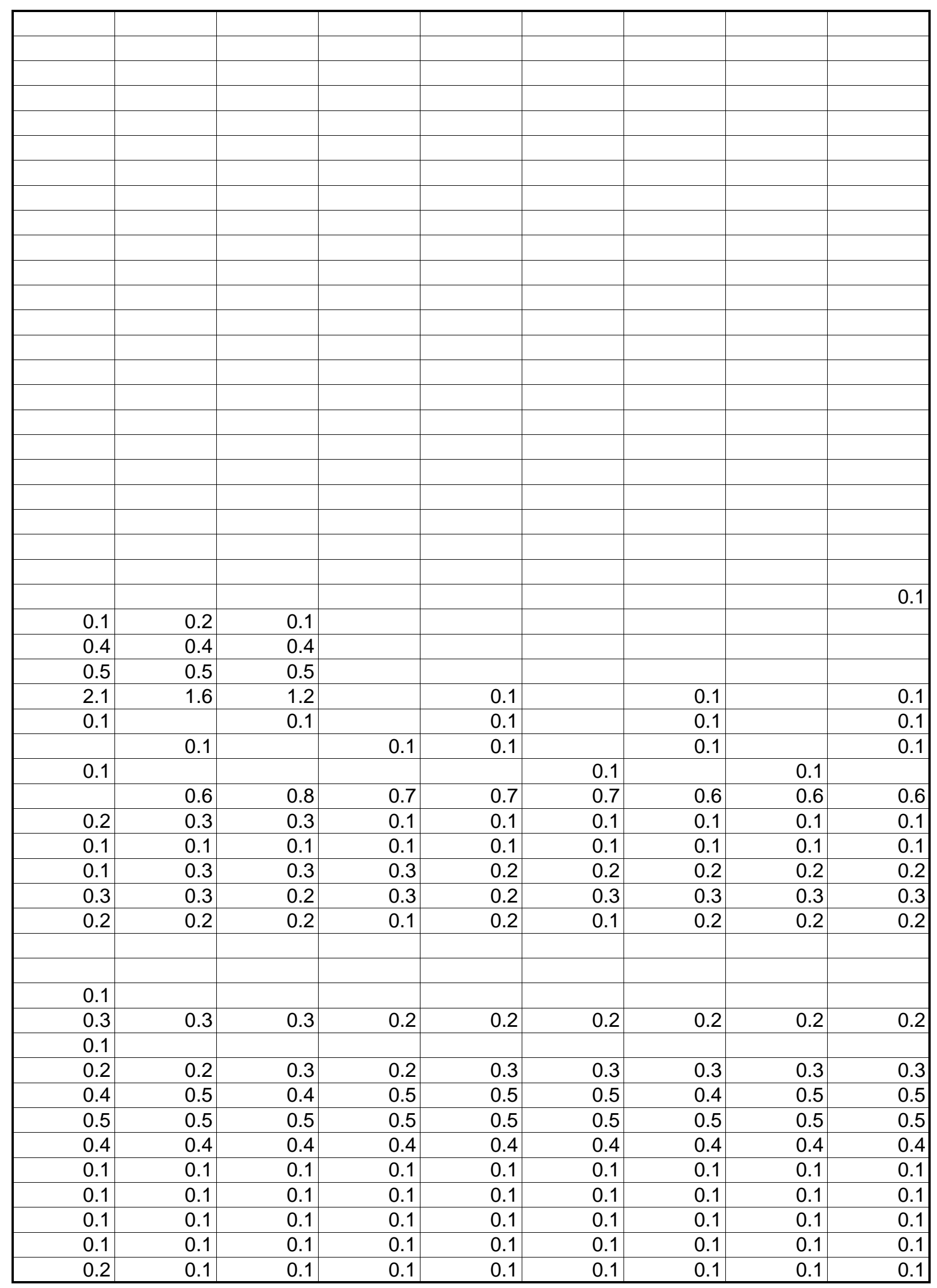


wbheat

\begin{tabular}{|c|c|c|c|c|c|c|c|c|}
\hline 0.1 & 0.1 & 0.1 & 0.1 & 0.1 & 0.1 & 0.1 & 0.1 & 0.1 \\
\hline 0.2 & 0.2 & 0.2 & 0.2 & 0.2 & 0.2 & 0.2 & 0.2 & 0.2 \\
\hline 0.3 & 0.3 & 0.3 & 0.3 & 0.3 & 0.3 & 0.3 & 0.3 & 0.3 \\
\hline & & & & & & & & \\
\hline & & & & & & & & \\
\hline & & & 0.1 & & & & & \\
\hline 0.1 & & 0.1 & & & & 0.1 & & \\
\hline & & & & & & & & \\
\hline & & & & & & & & \\
\hline & & & & & & & & \\
\hline & & & & & & & & \\
\hline & & & & & & & & \\
\hline & & & & & & & & \\
\hline & & & & & & & & \\
\hline & & & & & & & & \\
\hline & & & & & & & & \\
\hline & & & & & & & & \\
\hline 0.5 & 0.4 & 0.4 & 0.4 & 0.4 & 0.4 & 0.4 & 0.4 & 0.4 \\
\hline 0.4 & 0.4 & 0.3 & 0.3 & 0.3 & 0.3 & 0.3 & 0.3 & 0.4 \\
\hline 0.2 & 0.2 & 0.2 & 0.2 & 0.2 & 0.2 & 0.2 & 0.3 & 0.3 \\
\hline 0.5 & 0.5 & 0.5 & 0.5 & 0.5 & 0.5 & 0.5 & 0.5 & 0.5 \\
\hline 0.4 & 0.3 & 0.3 & 0.3 & 0.3 & 0.3 & 0.3 & 0.3 & 0.3 \\
\hline 0.3 & 0.3 & 0.2 & 0.3 & 0.3 & 0.2 & 0.3 & 0.3 & 0.3 \\
\hline 0.5 & 0.5 & 0.5 & 0.5 & 0.5 & 0.4 & 0.5 & 0.5 & 0.4 \\
\hline 0.3 & 0.3 & 0.3 & 0.3 & 0.3 & 0.2 & 0.3 & 0.2 & 0.2 \\
\hline 0.6 & 0.5 & 0.5 & 0.5 & 0.5 & 0.5 & 0.5 & 0.5 & 0.5 \\
\hline 0.4 & 0.4 & 0.4 & 0.3 & 0.4 & 0.4 & 0.4 & 0.4 & 0.4 \\
\hline 0.2 & 0.2 & 0.2 & 0.3 & 0.3 & 0.3 & 0.3 & 0.3 & 0.3 \\
\hline 0.2 & 0.2 & 0.1 & 0.1 & 0.1 & 0.1 & 0.2 & 0.2 & 0.1 \\
\hline & & & & & & & & \\
\hline & & & & & & & & \\
\hline & & & & & & & & \\
\hline & & & & & & & & \\
\hline & & & & & & & & \\
\hline & & & & & & & & \\
\hline & & & & & & & & \\
\hline & & & & & & & & \\
\hline & & & & & & & & \\
\hline & & & & & & & & \\
\hline & & & & & & & & \\
\hline & & & & & & & & \\
\hline & & & & & & & & \\
\hline & & & & & & & & \\
\hline & & & & & & & & \\
\hline & & & & & & & & \\
\hline
\end{tabular}




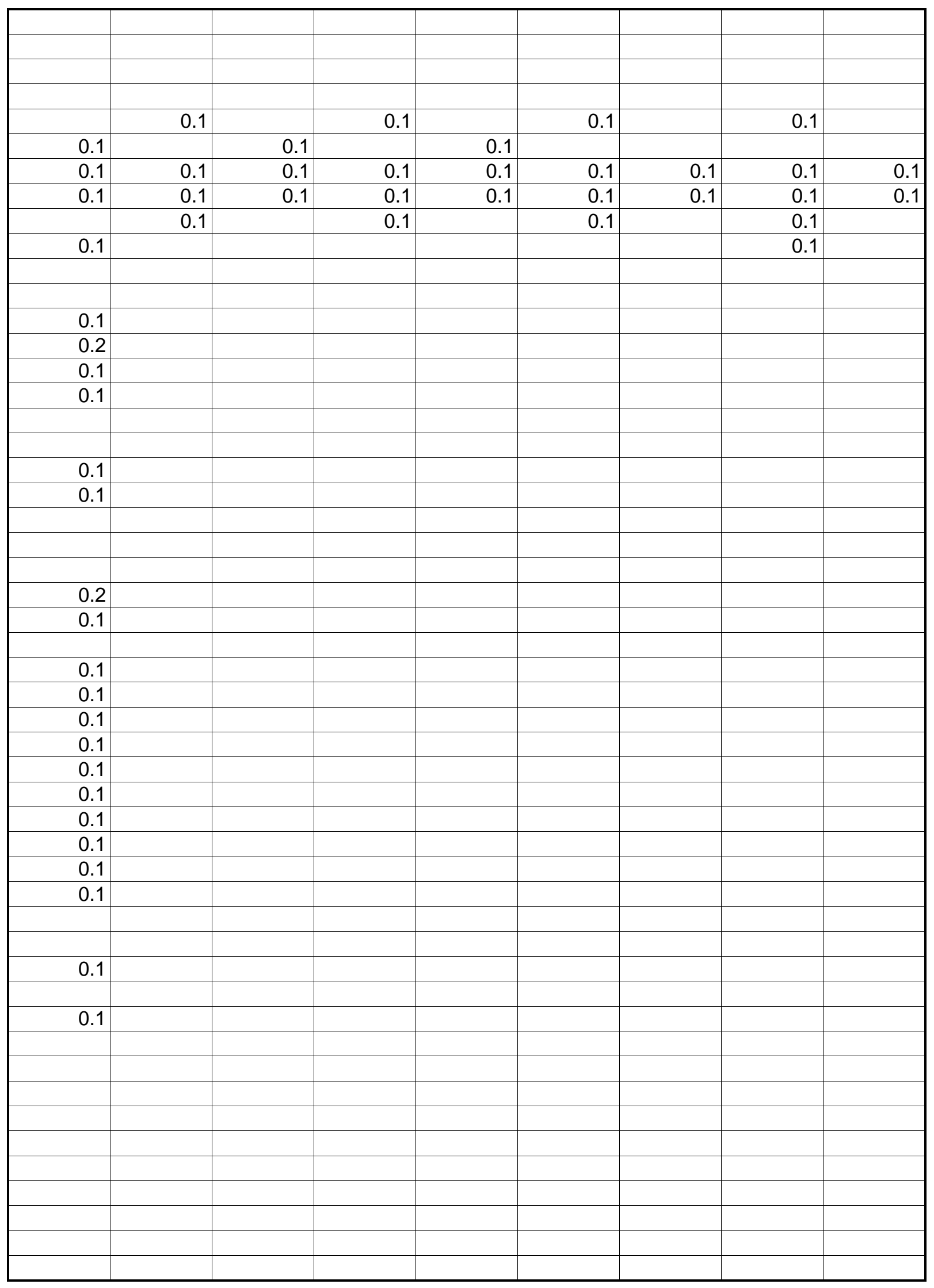




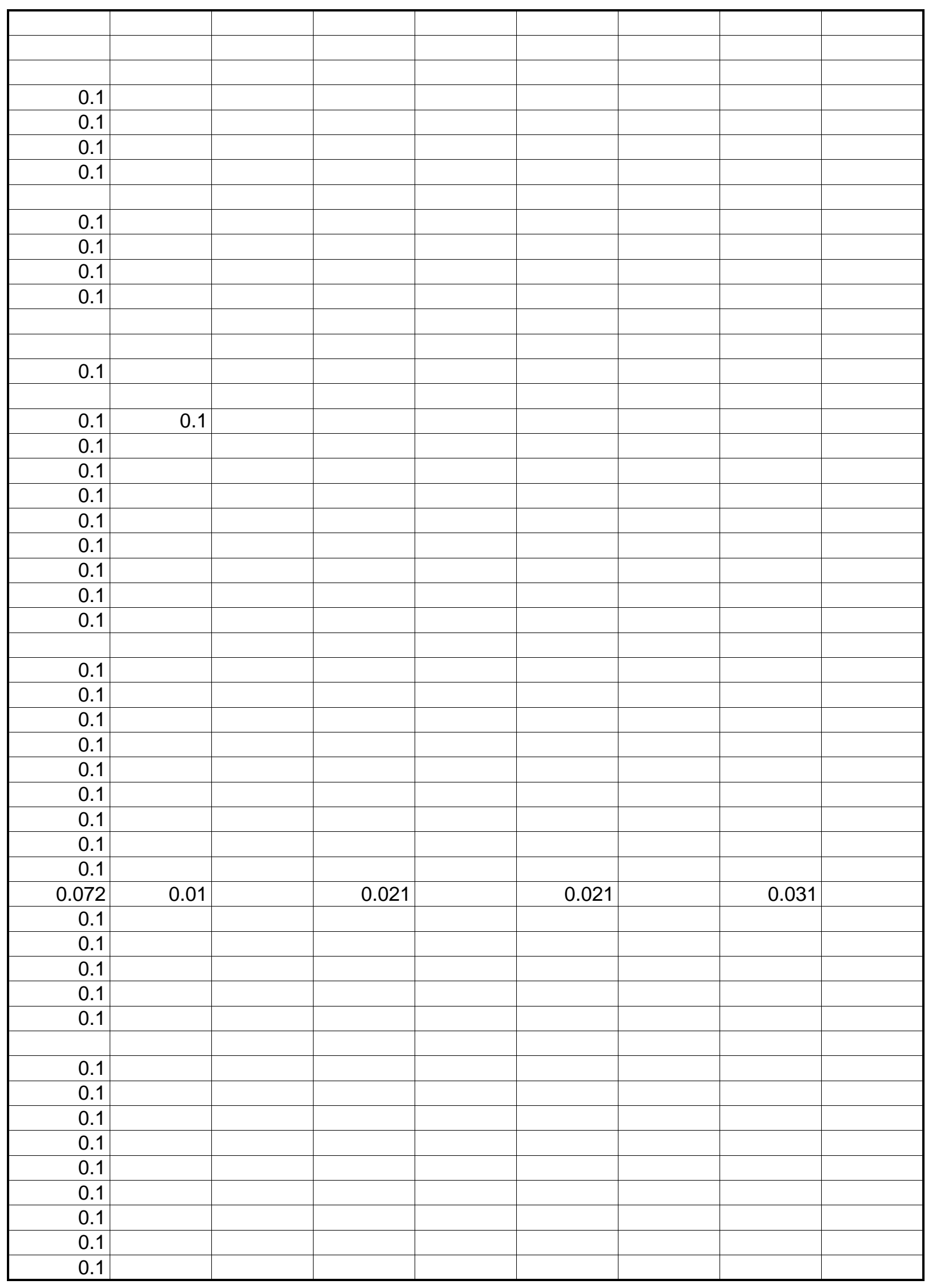


wbheat

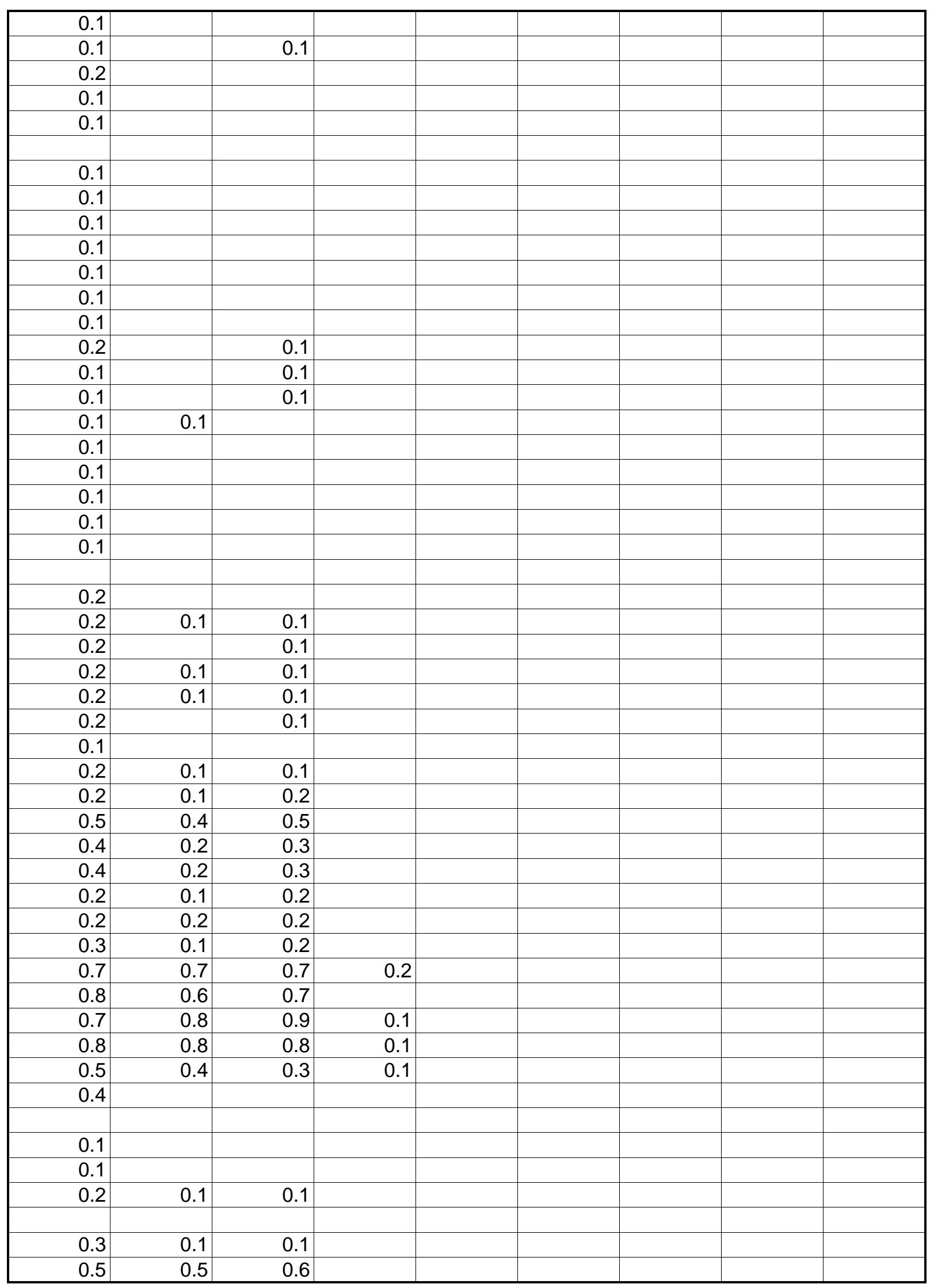


wbheat

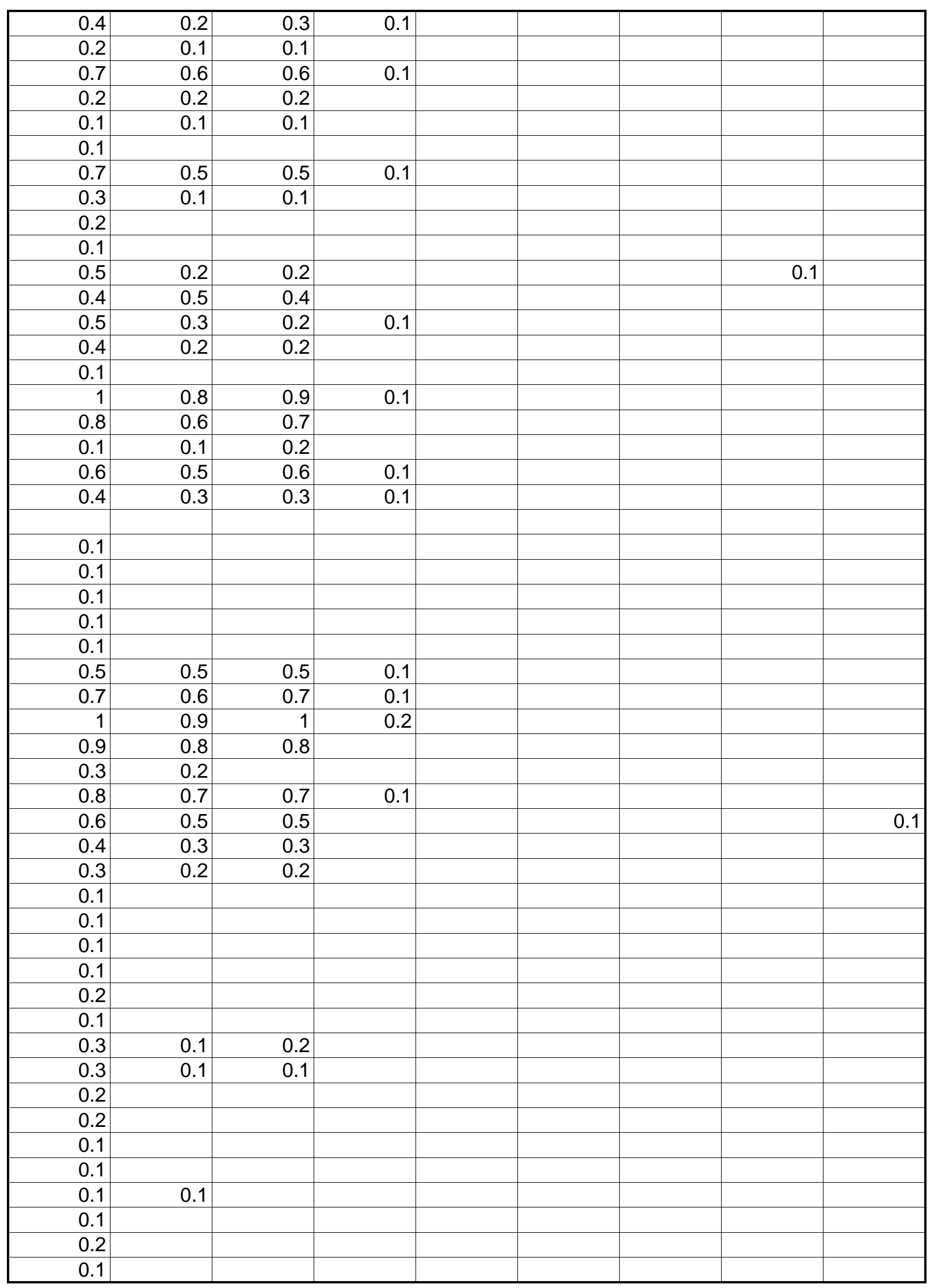


wbheat

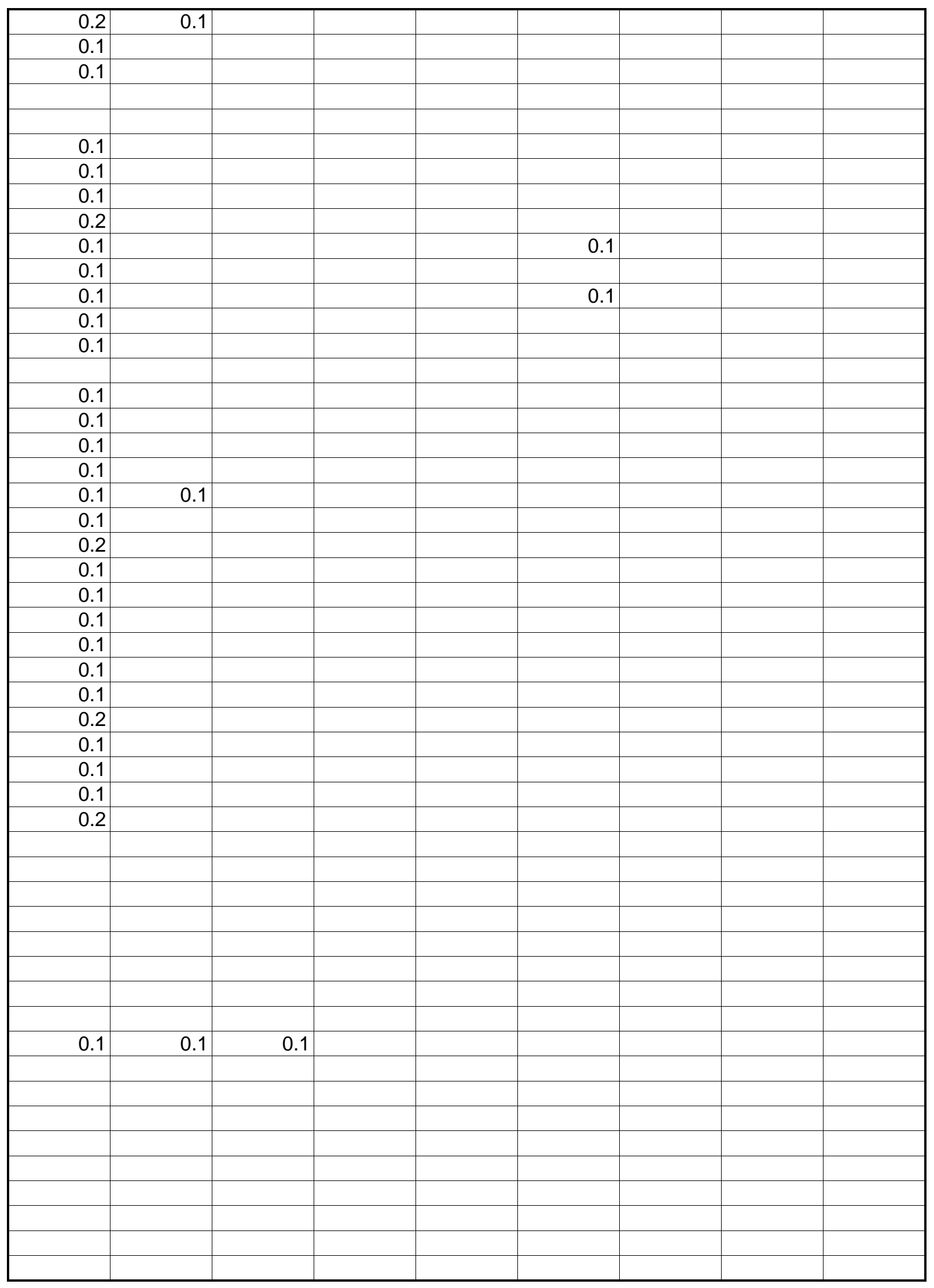




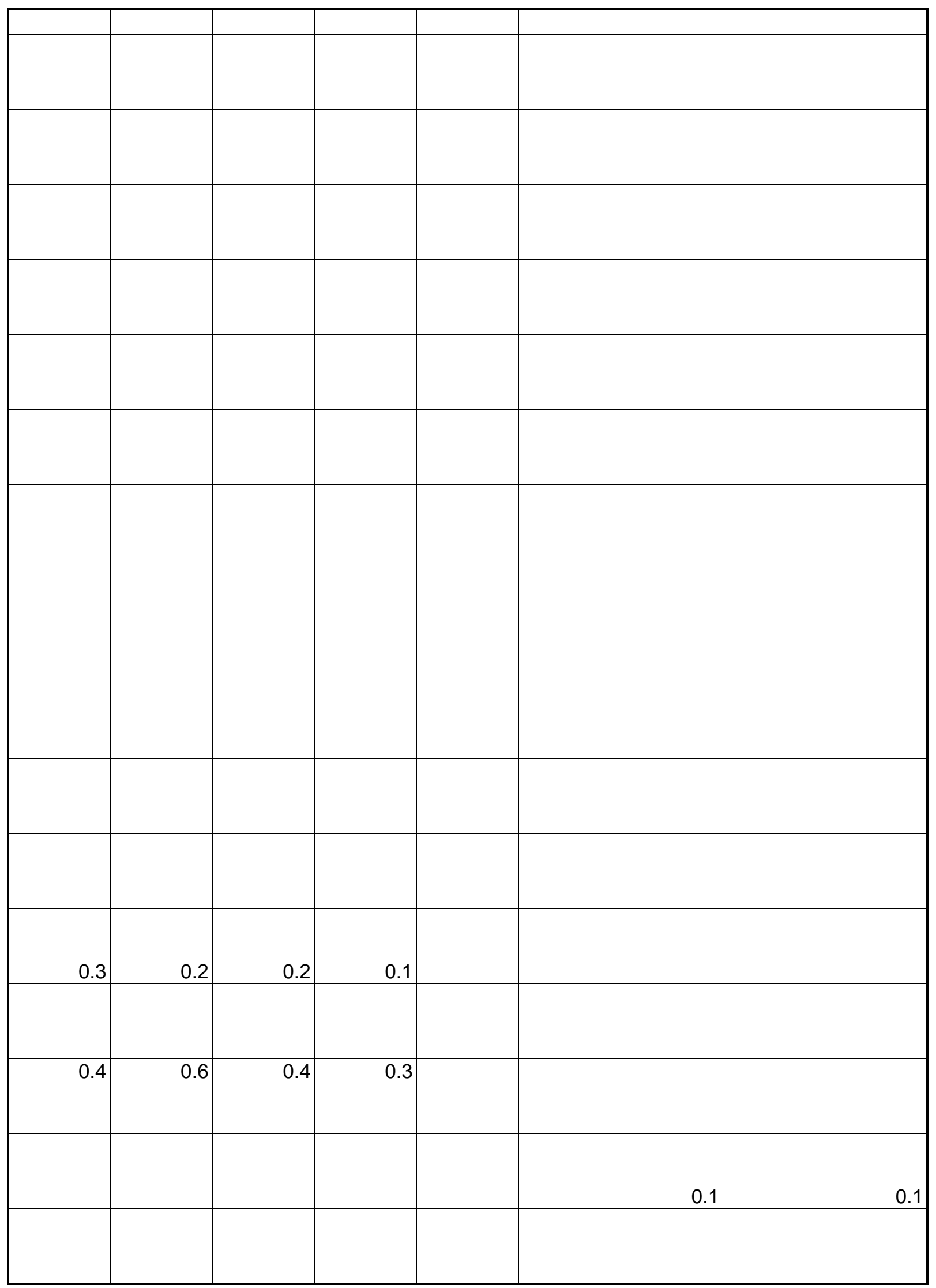


wbheat

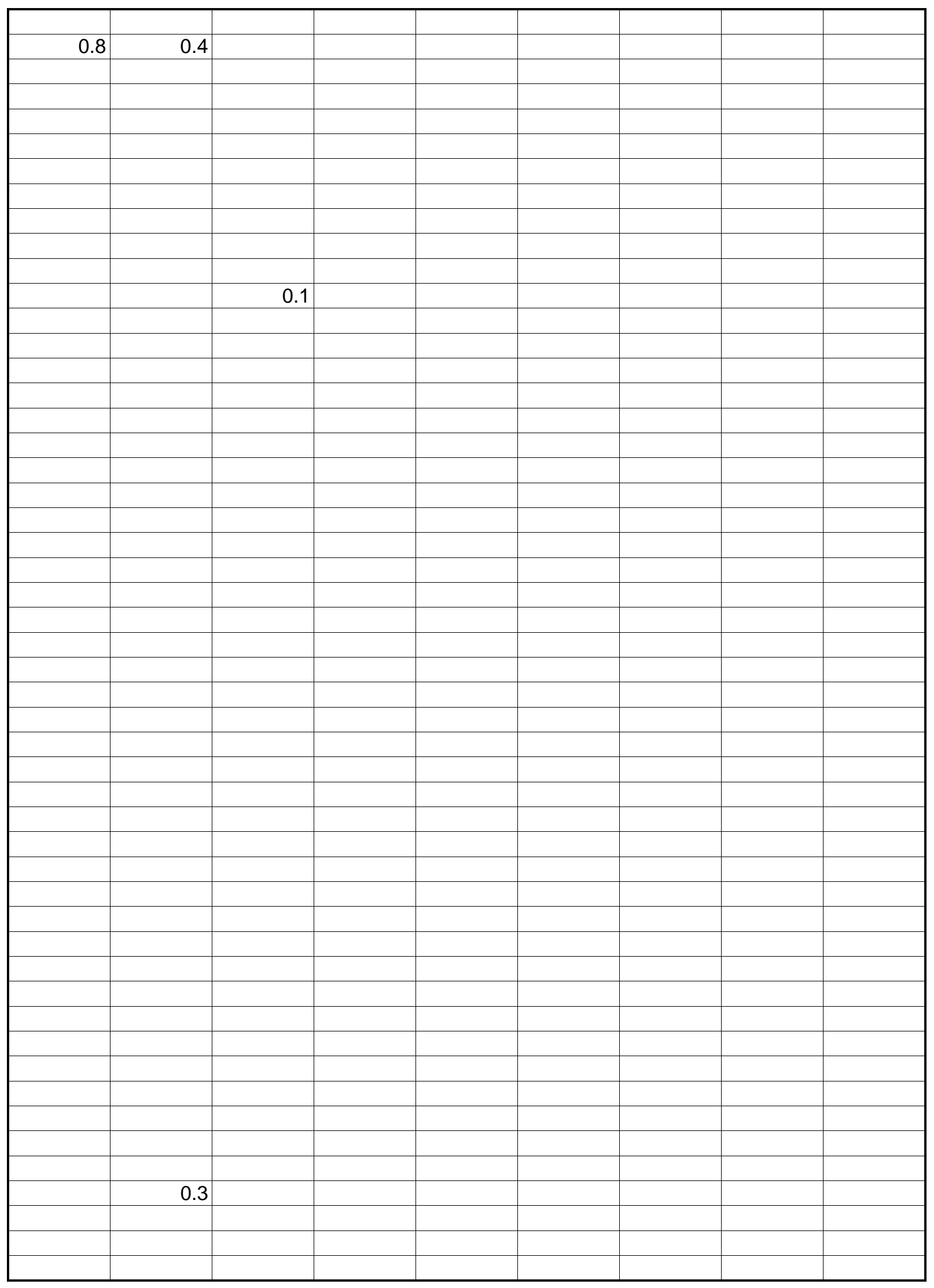

Page 81 
wbheat

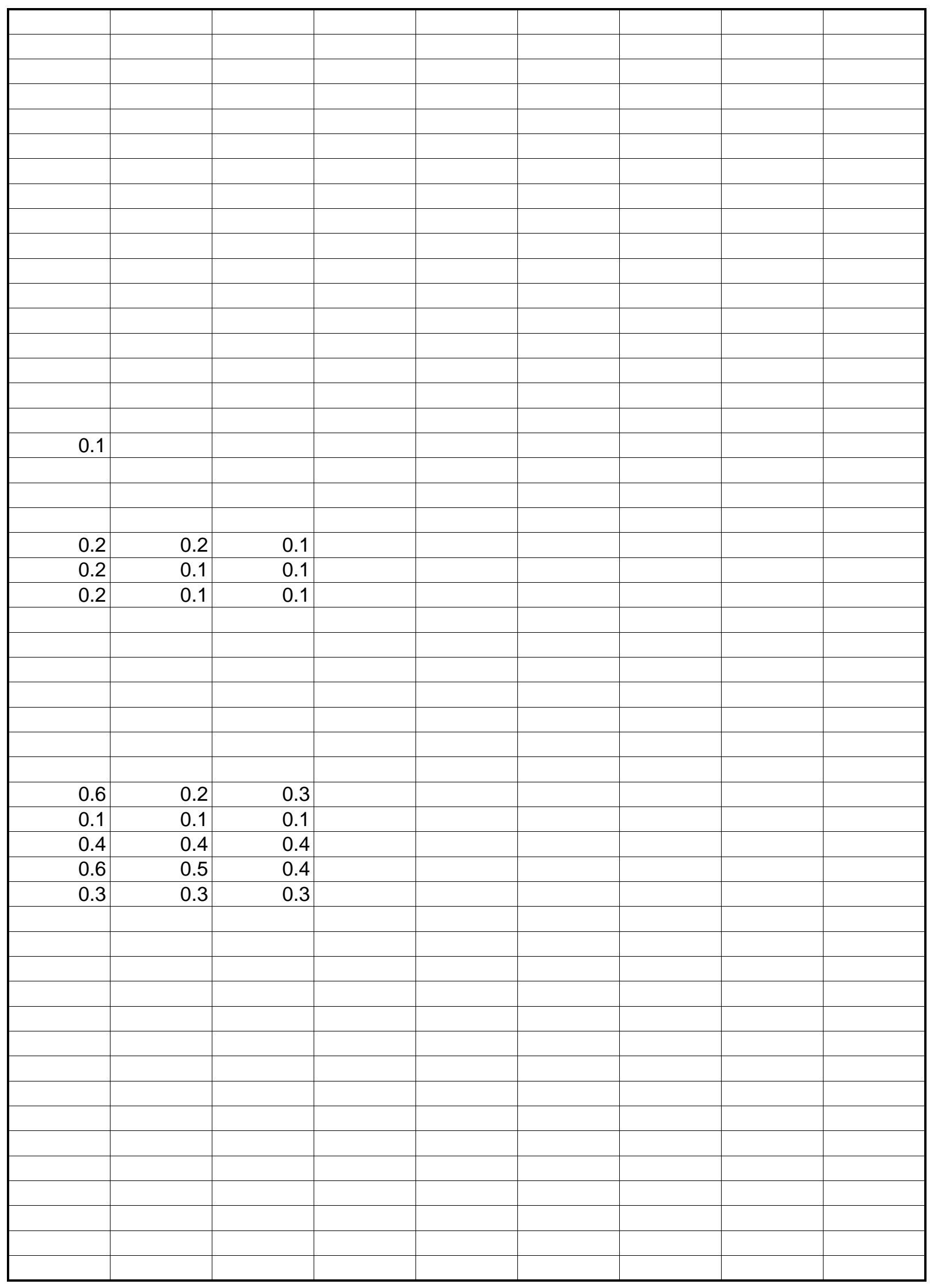




\begin{tabular}{|c|c|c|c|c|c|c|c|c|}
\hline & & & & & & & & \\
\hline & & & & & & & & \\
\hline & & & & & & & & \\
\hline & & & & & & & & \\
\hline & & & & & & & & \\
\hline & & & & & & & & \\
\hline & & & & & & & & \\
\hline & & & & & & & & \\
\hline & & & & & & & & \\
\hline & & & & & & & & \\
\hline & & & & & & & & \\
\hline & & & & & & & & \\
\hline & & & & & & & & \\
\hline & & & & & & & & \\
\hline & & & & & & & & \\
\hline & & & & & & & & \\
\hline & & & & & & & & \\
\hline & & & & & & & & \\
\hline & & & & & & & & \\
\hline & & & & & & & & \\
\hline & & & & & & & & \\
\hline & & & & & & & & \\
\hline 0.20 & 0.20 & 0.21 & 0.20 & 0.21 & 0.23 & 0.23 & 0.24 & 0.24 \\
\hline 0.26 & 0.25 & 0.23 & 0.23 & 0.00 & 0.25 & 0.00 & 0.26 & 0.27 \\
\hline 0.27 & 0.30 & 0.29 & 0.27 & 0.27 & 0.27 & 0.27 & 0.27 & 0.27 \\
\hline 0.10 & 0.06 & 0.10 & 0.02 & 0.00 & 0.00 & \#DIV/O! & 0.00 & \#DIV/0! \\
\hline 0.25 & 0.32 & 0.35 & 0.11 & \#DIV/O! & 0.10 & \#DIV/O! & 0.10 & 0.10 \\
\hline 0.50 & 0.40 & 0.23 & 0.00 & 0.00 & 0.00 & 0.00 & 0.00 & 0.00 \\
\hline 0.30 & 0.24 & 0.23 & \#DIV/0! & \#DIV/0! & \#DIV/O! & \#DIV/0! & \#DIV/0! & \#DIV/O! \\
\hline 0.00 & 0.00 & 0.00 & 0.00 & 0.00 & 0.00 & 0.00 & 0.00 & 0.00 \\
\hline & & & & & & & & \\
\hline & & & & & & & & \\
\hline & & & & & & & & \\
\hline & & & & & & & & \\
\hline 0.154 & 0.132 & 0.148 & 0.120 & 0.128 & 0.131 & 0.129 & 0.132 & 0.135 \\
\hline 0.149 & 0.144 & 0.139 & 0.142 & 0.000 & 0.151 & 0.000 & 0.148 & 0.000 \\
\hline 0.269 & 0.230 & 0.205 & 0.167 & 0.165 & 0.167 & 0.157 & 0.162 & 0.159 \\
\hline 0.018 & 0.064 & \#DIV/O! & \#DIV/0! & 0.000 & 0.000 & 0.000 & 0.000 & 0.000 \\
\hline 0.224 & 0.252 & 0.268 & 0.035 & \#DIV/O! & 0.000 & \#DIV/O! & \#DIV/O! & \#DIV/0! \\
\hline 0.265 & 0.200 & 0.153 & 0.000 & 0.000 & 0.000 & 0.000 & 0.000 & 0.000 \\
\hline 0.194 & 0.142 & 0.139 & \#DIV/O! & \#DIV/O! & \#DIV/O! & \#DIV/O! & \#DIV/O! & \#DIV/O! \\
\hline 0.000 & 0.000 & 0.000 & 0.000 & 0.000 & 0.000 & 0.000 & 0.000 & 0.000 \\
\hline & & & & & & & & \\
\hline & & & & & & & & \\
\hline & & & & & & & & \\
\hline & & & & & & & & \\
\hline 87.93 & 46.63 & 50.01 & 43.93 & 48.92 & 51.02 & 52.02 & 56.02 & 58.32 \\
\hline 29.40 & 27.40 & 26.20 & 25.20 & 25.70 & 27.00 & 28.00 & 28.30 & 29.60 \\
\hline 19.40 & 17.70 & 17.00 & 14.60 & 15.00 & 14.40 & 14.90 & 14.90 & 15.10 \\
\hline 6.37 & 0.11 & 0.10 & 0.02 & 0.00 & 0.02 & 0.00 & 0.03 & 0.00 \\
\hline 30.20 & 16.40 & 17.70 & 1.70 & 0.00 & 0.20 & 0.00 & 0.10 & 0.10 \\
\hline 1.50 & 1.20 & 0.70 & 0.40 & 0.00 & 0.00 & 0.10 & 0.00 & 0.10 \\
\hline
\end{tabular}


wbheat

\begin{tabular}{|l|l|l|l|l|l|l|l|l|}
\hline 2.70 & 2.20 & 1.80 & 0.00 & 0.00 & 0.00 & 0.00 & 0.00 & 0.00 \\
\hline 0.00 & 0.00 & 0.00 & 0.00 & 0.00 & 0.00 & 0.00 & 0.00 & 0.00 \\
\hline
\end{tabular}


wbheat

\begin{tabular}{|c|c|c|c|c|c|}
\hline 23 & $\begin{array}{l}\text { Daily Total } \\
\text { (Jday) }\end{array}$ & $\begin{array}{l}\text { Daily Total } \\
\text { (Post) }\end{array}$ & Monthly Total & $\begin{array}{l}\text { Monthly } \\
\text { Summary }\end{array}$ & \\
\hline & 0.5 & 0.5 & & Oct 91 & 94.1 \\
\hline & 0.6 & 0.6 & & Nov & 193.6 \\
\hline & 0.7 & 0.7 & & Dec & 254.4 \\
\hline & 0.5 & 0.5 & & Jan 92 & 158 \\
\hline & 0.5 & 0.5 & & Feb & 35.9 \\
\hline & 0.7 & 0.7 & & Mar & 13.7 \\
\hline & 0.6 & 0.6 & & Apr & 24.864 \\
\hline & 0.8 & 0.8 & & May & 126.2 \\
\hline & 0.8 & 0.8 & & Jun & 300.6 \\
\hline & 0.7 & 0.7 & & Jul & 202.3 \\
\hline & 0.6 & 0.6 & & Aug & 65.7 \\
\hline & 0.7 & 0.7 & & Sep & 19.2 \\
\hline & 0.7 & 0.7 & & Oct & 18.224 \\
\hline & 0.8 & 0.8 & & Nov & 61.6 \\
\hline 0.1 & 1.7 & 1.7 & & Dec & 156.3 \\
\hline 0.4 & 3.6 & 3.6 & & Jan 93 & 324.5 \\
\hline 0.3 & 1.2 & 1.2 & & Feb & 62.2 \\
\hline & 0.6 & 0.6 & & Mar & 207.6 \\
\hline & 0.7 & 0.7 & & Apr & 48 \\
\hline & 0.6 & 0.6 & & May & 81.7 \\
\hline & 0.5 & 0.5 & & Jun & 176.8 \\
\hline 0.3 & 5.4 & 5.4 & & Jul & 0.7 \\
\hline 0.4 & 8.9 & 8.9 & & Aug & 122.5 \\
\hline 0.3 & 8.1 & 8.1 & & Sep & 118.5 \\
\hline 0.5 & 11.5 & 11.5 & & Oct & 1.6 \\
\hline 0.3 & 9.5 & 9.5 & & Nov & 147.4 \\
\hline 0.2 & 6.4 & 6.4 & & Dec & 2.1 \\
\hline 0.3 & 5.6 & 5.6 & & Jan 94 & 77.4 \\
\hline 0.4 & 9.9 & 9.9 & & $\mathrm{Feb}$ & 67.9 \\
\hline 0.2 & 6.2 & 6.2 & & Mar & 118.3 \\
\hline 0.2 & 4.5 & 4.5 & 94.1 & Apr (pre) & 11.7 \\
\hline 0.1 & 3.8 & 3.8 & & Apr (post) & 12.7 \\
\hline & 1.9 & 1.9 & & May & 12.4 \\
\hline & 1.2 & 1.2 & & Jun & 17.946 \\
\hline & 5.7 & 5.7 & & Jul & 32.6 \\
\hline 0.1 & 2.4 & 2.4 & & Aug & 152 \\
\hline 0.2 & 4.4 & 4.4 & & Sep & 214.9 \\
\hline 0.1 & 5.7 & 5.7 & & Oct & 102.3 \\
\hline 0.2 & 4.6 & 4.6 & & Nov & 19.8 \\
\hline 0.2 & 8.5 & 8.5 & & Dec & 1 \\
\hline 0.2 & 6.5 & 6.5 & & Jan 95 & 12.9 \\
\hline 0.1 & 3.2 & 3.2 & & Feb & 9.6 \\
\hline 0.5 & 12 & 12 & & Mar & 0.6 \\
\hline 0.4 & 10.2 & 10.2 & & Apr & 46.4 \\
\hline 0.3 & 10.1 & 10.1 & & May & 1.9 \\
\hline 0.2 & 7.6 & 7.6 & & & \\
\hline 0.1 & 4.9 & 4.9 & & & 3930.634 \\
\hline
\end{tabular}


wbheat

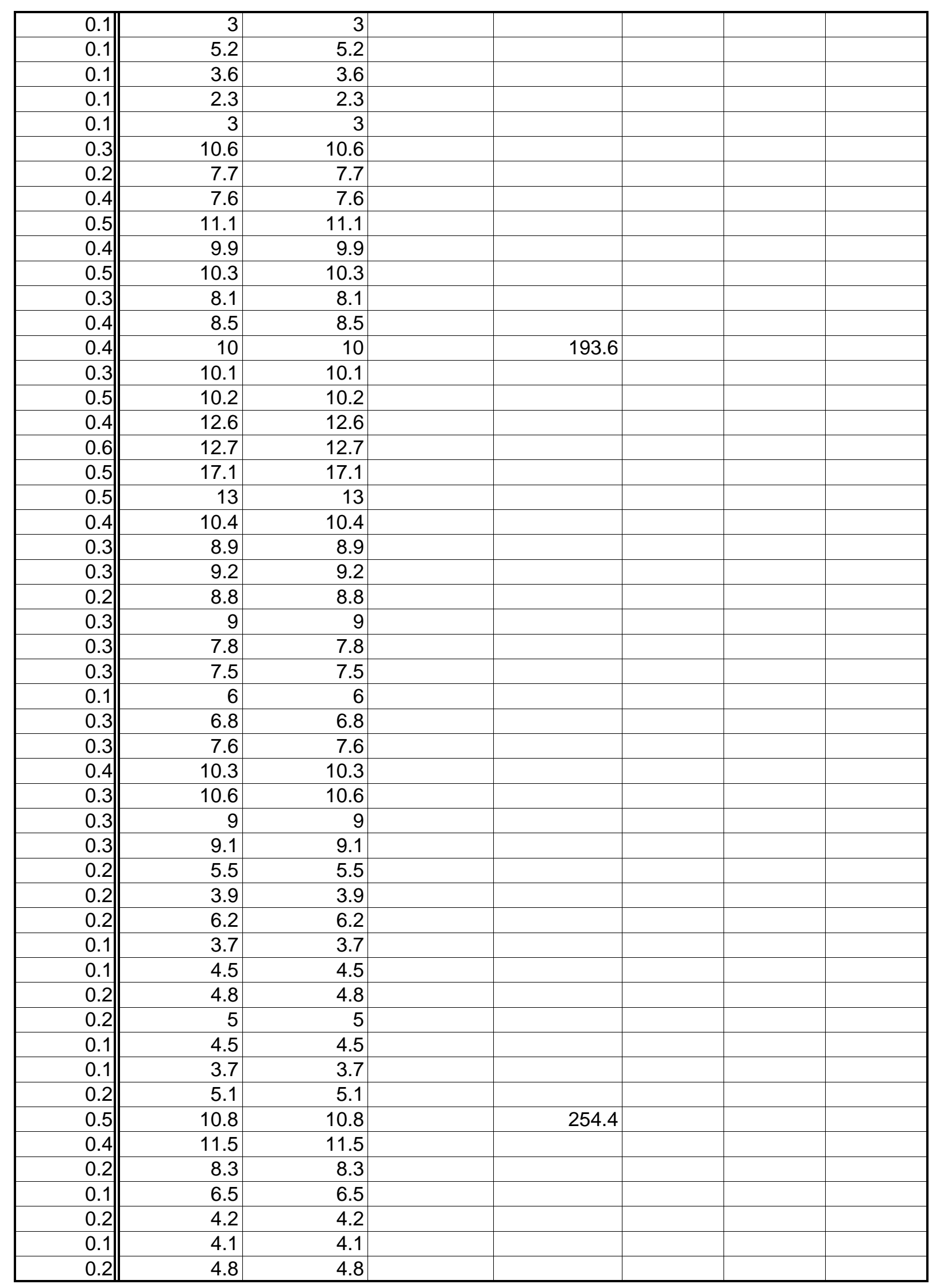


wbheat

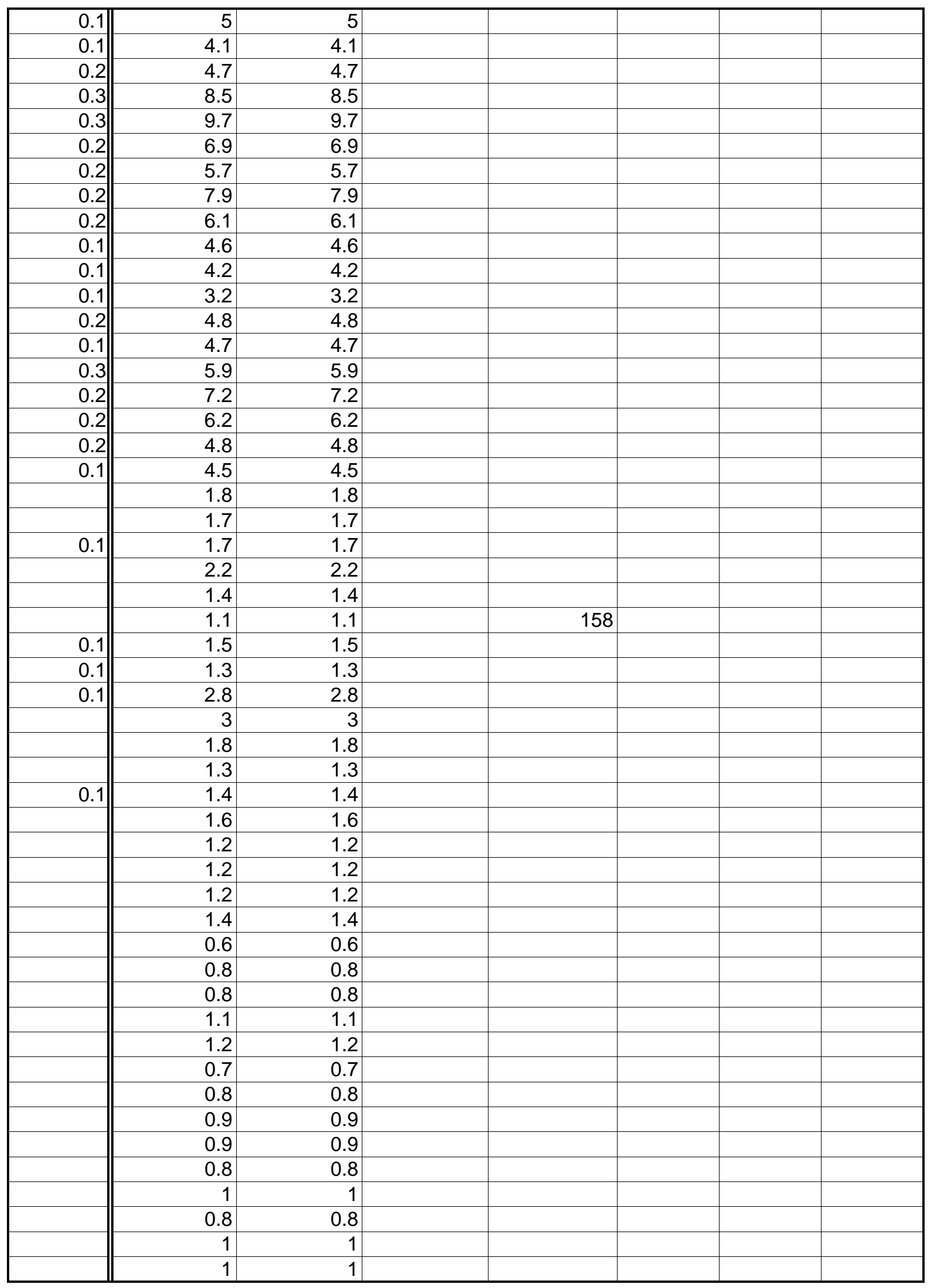


wbheat

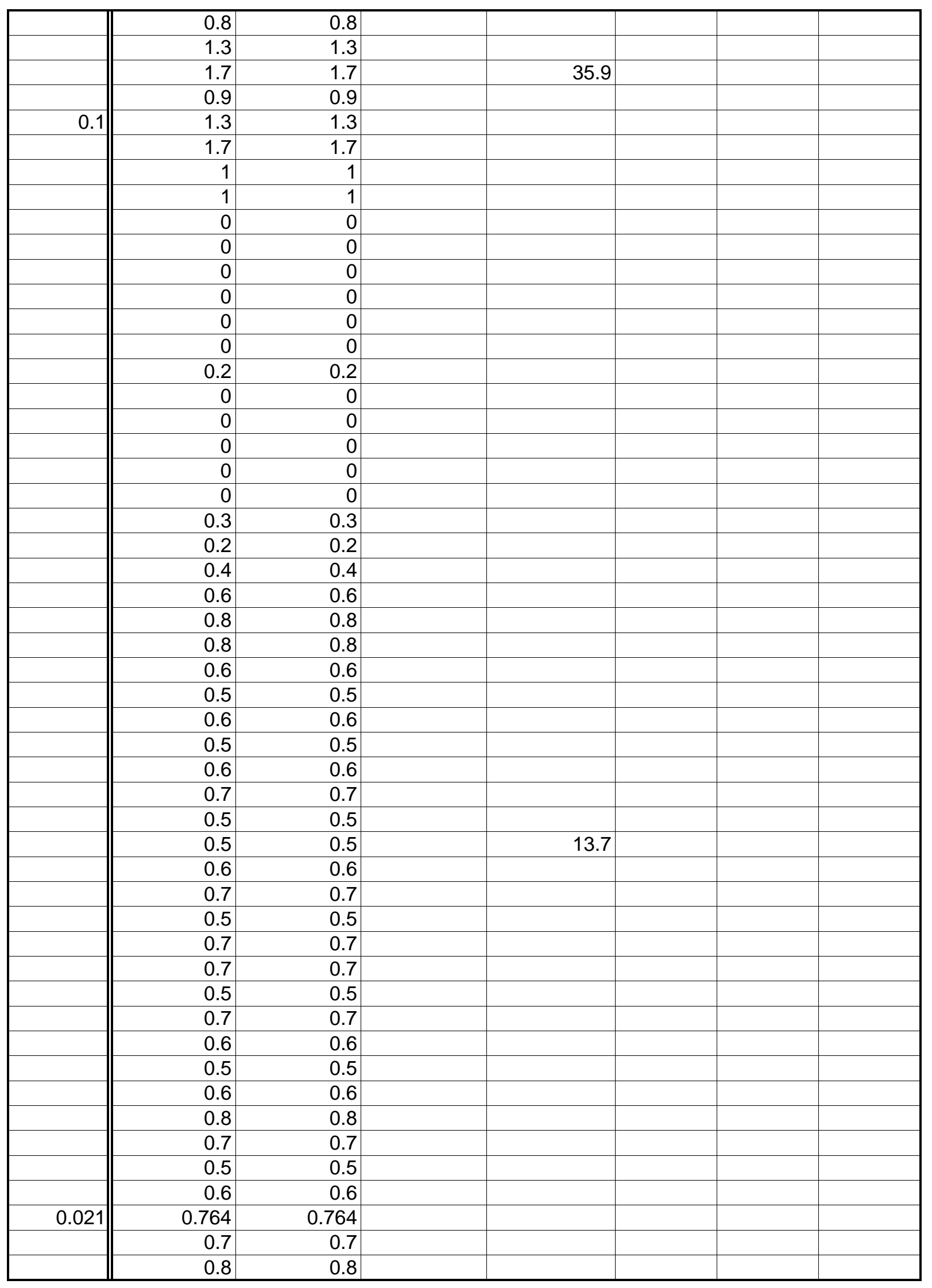


wbheat

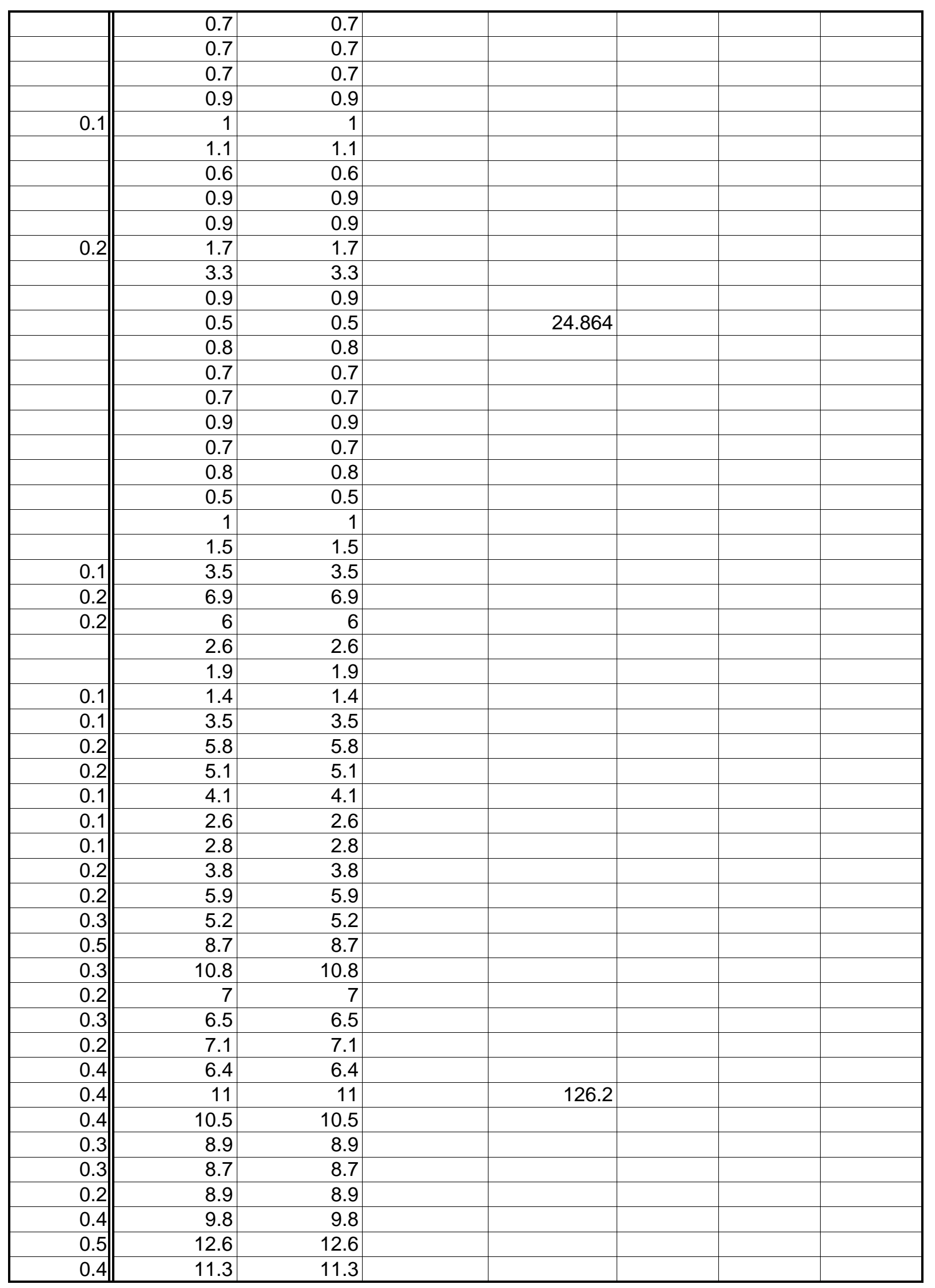


wbheat

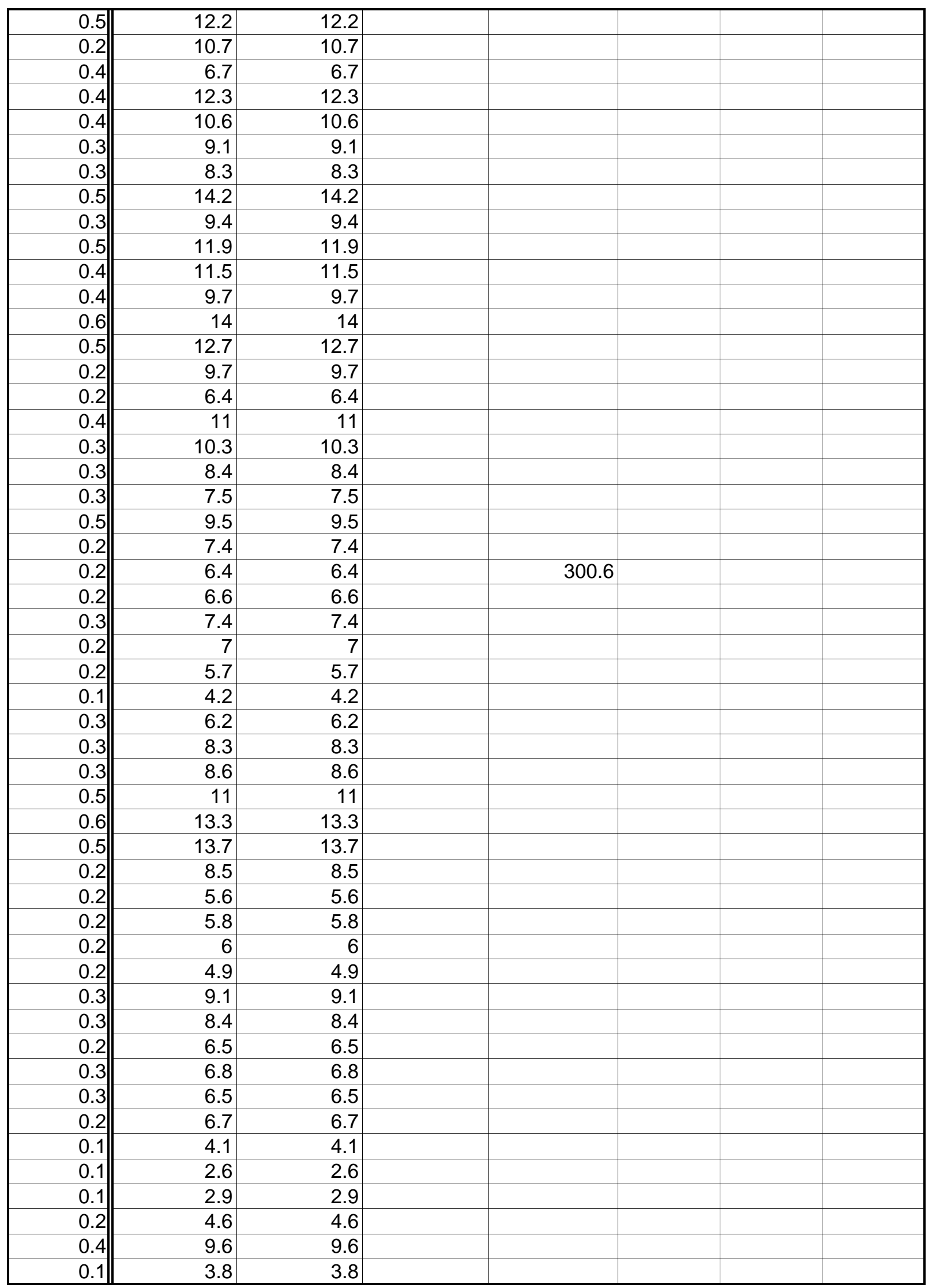


wbheat

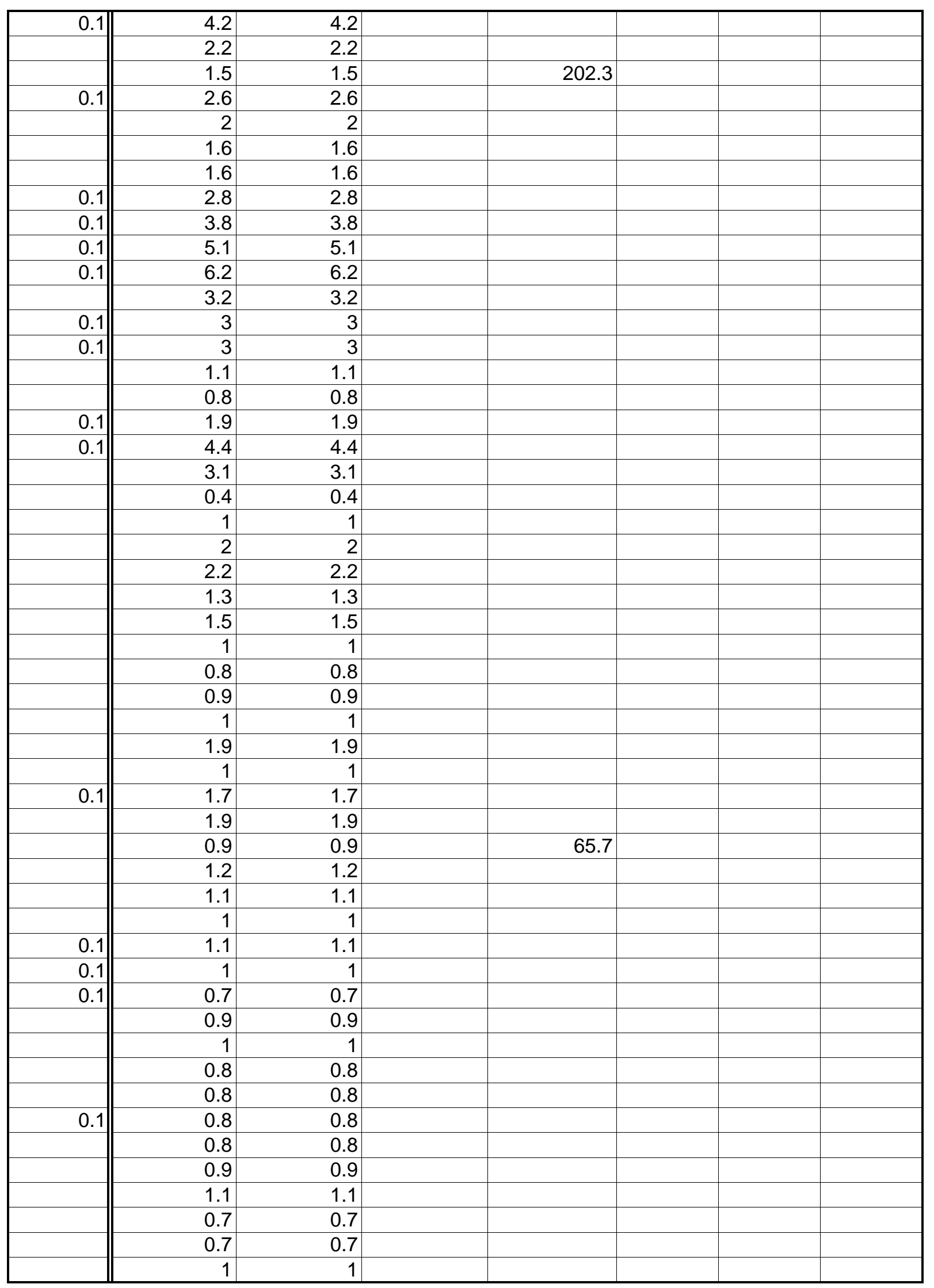


wbheat

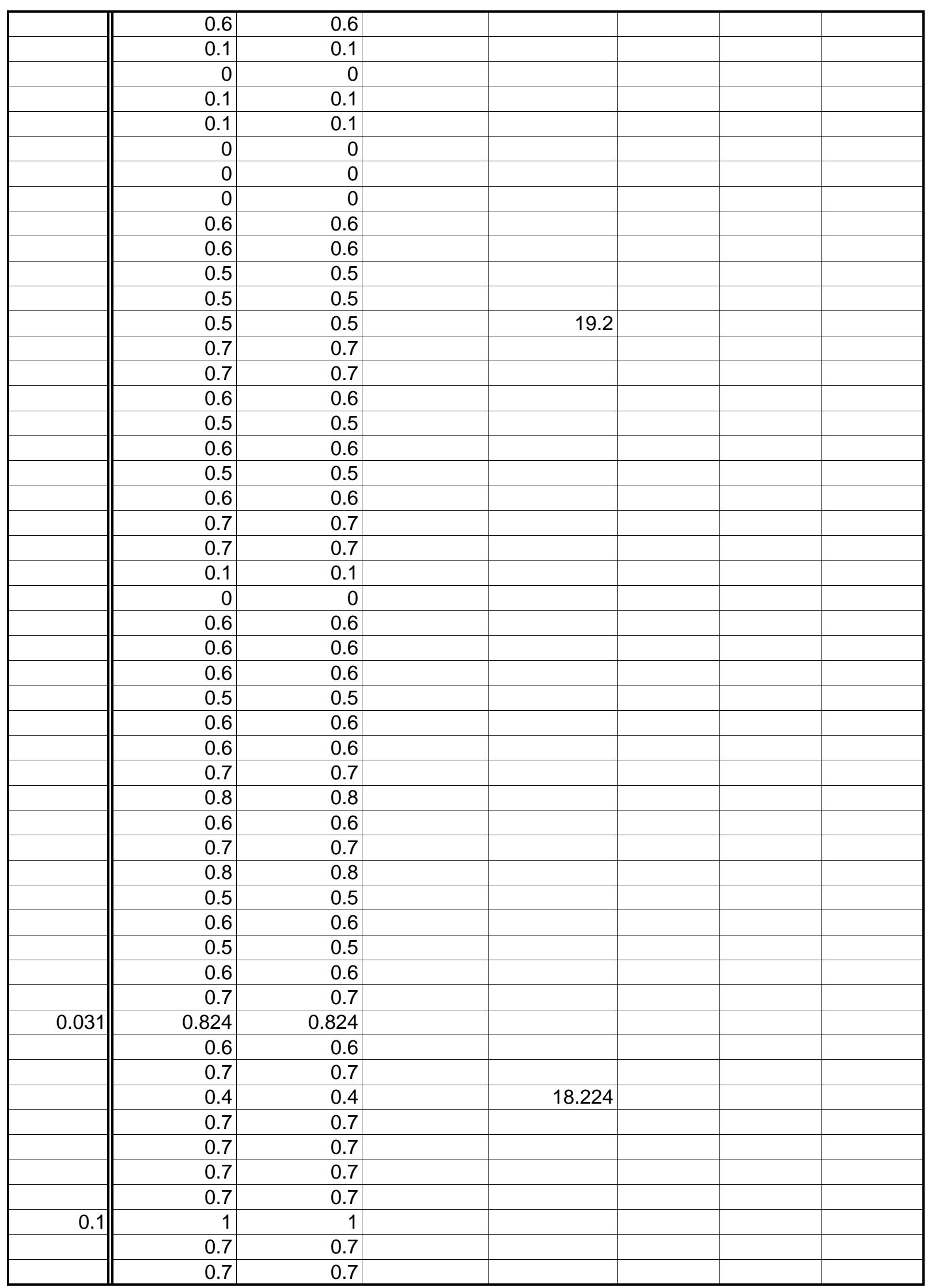


wbheat

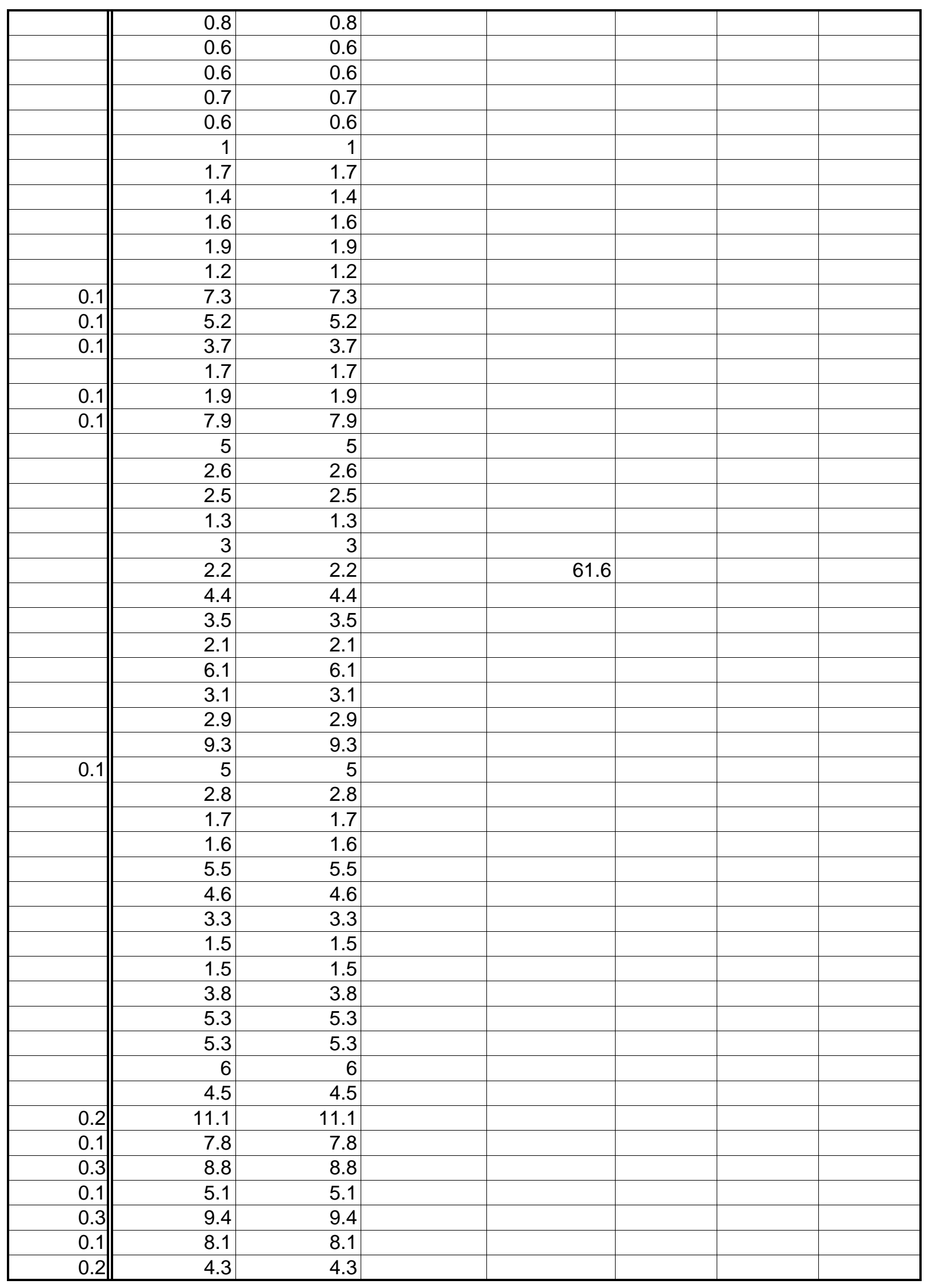


wbheat

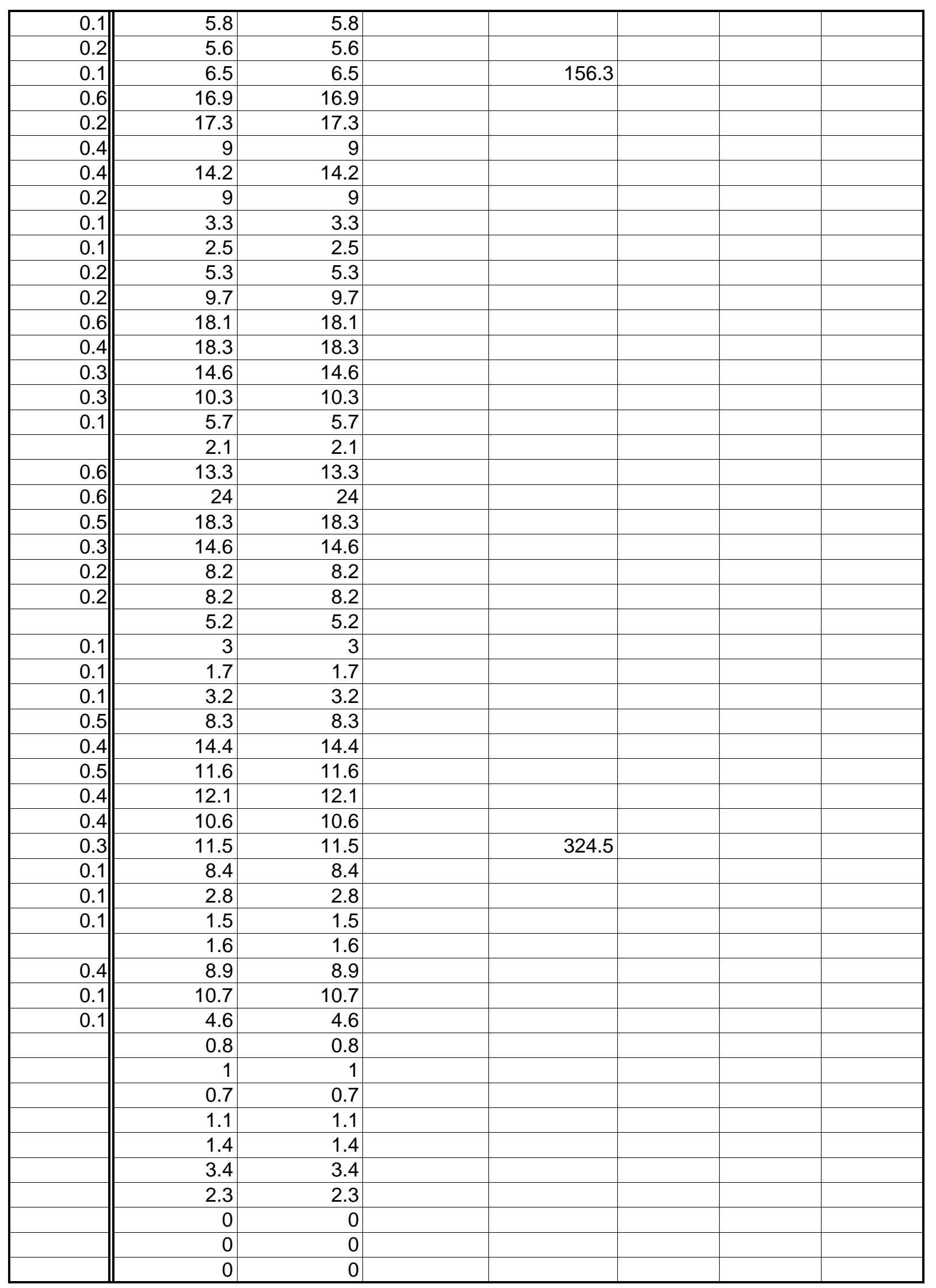


wbheat

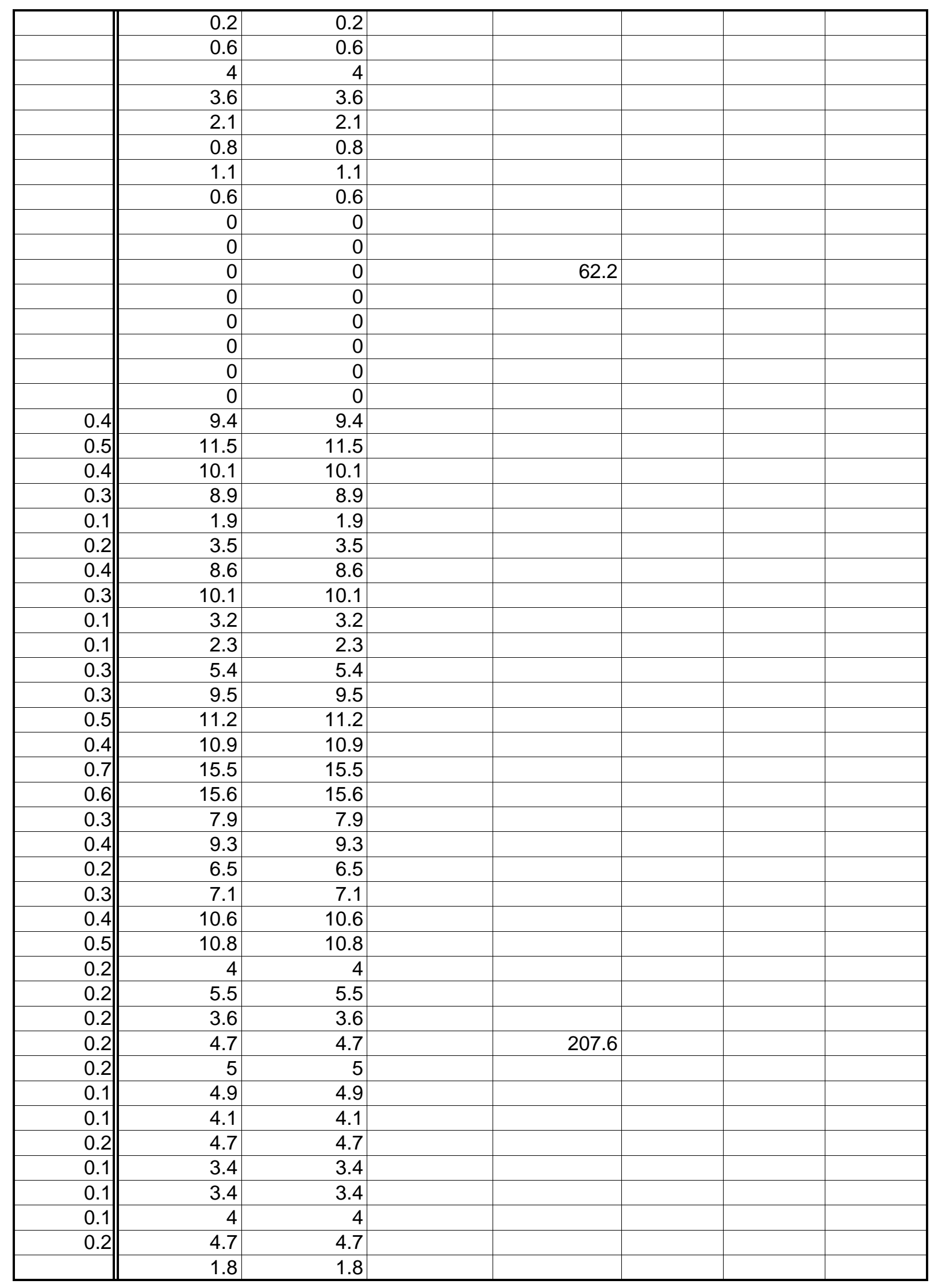


wbheat

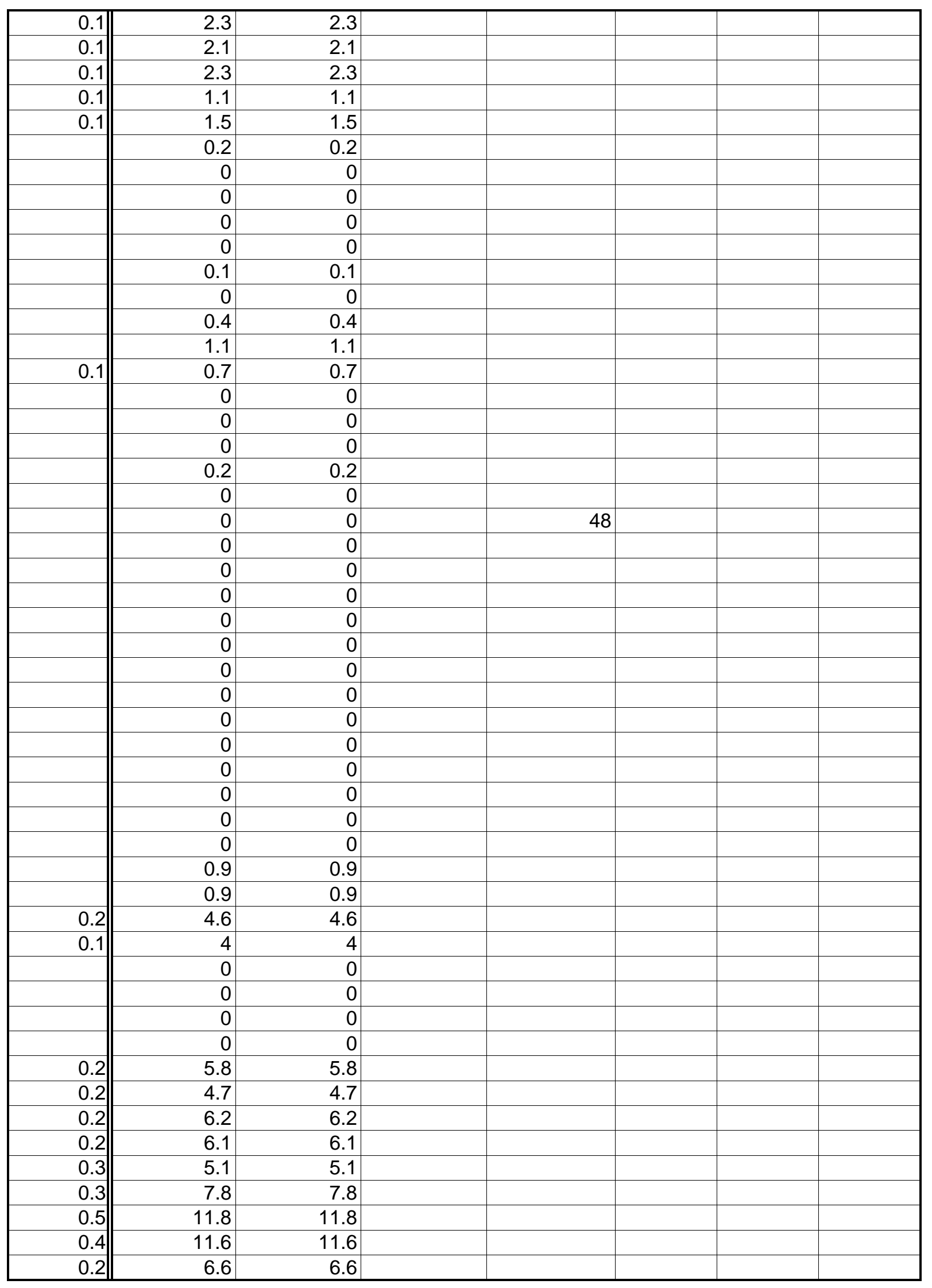


wbheat

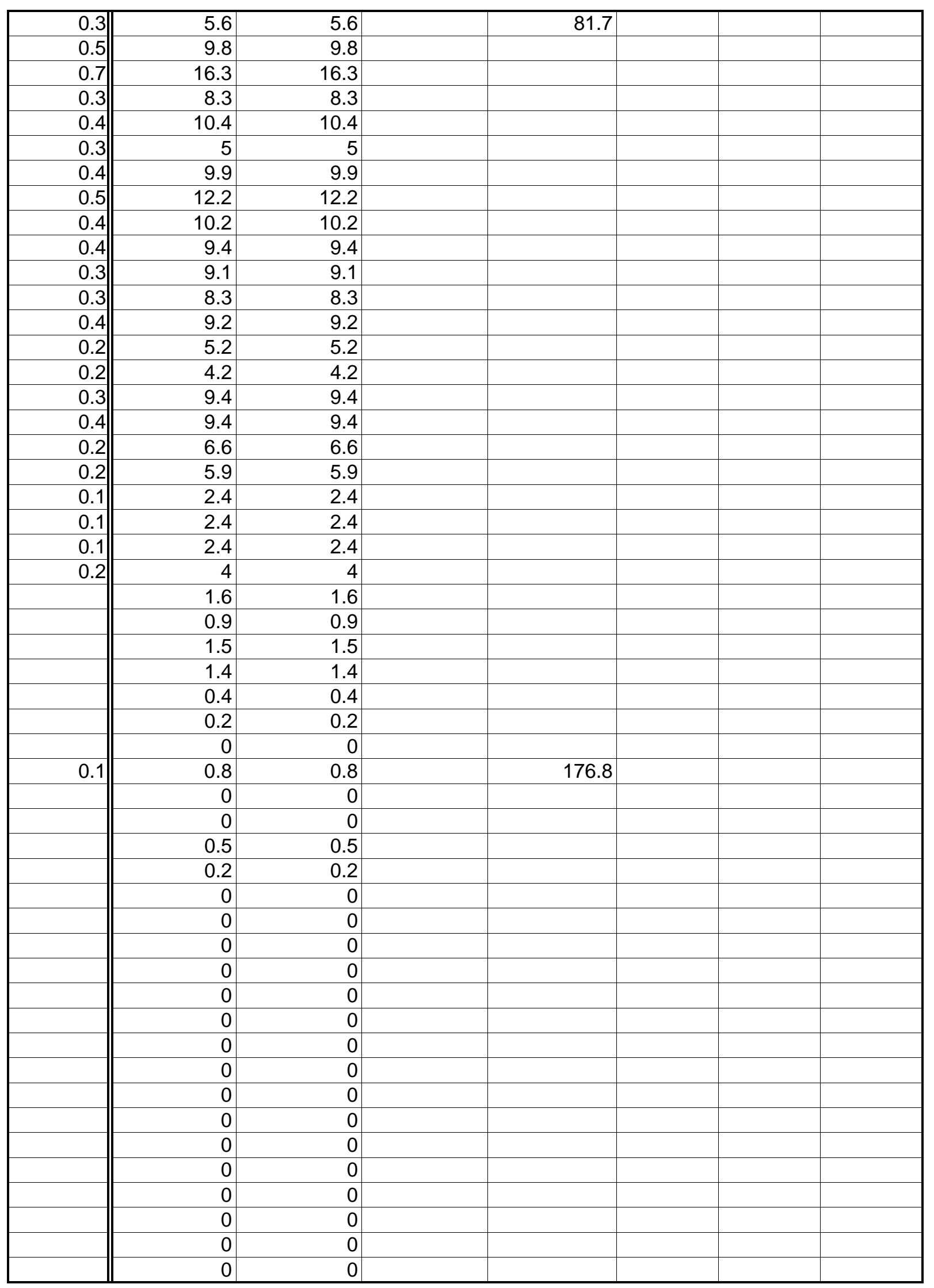




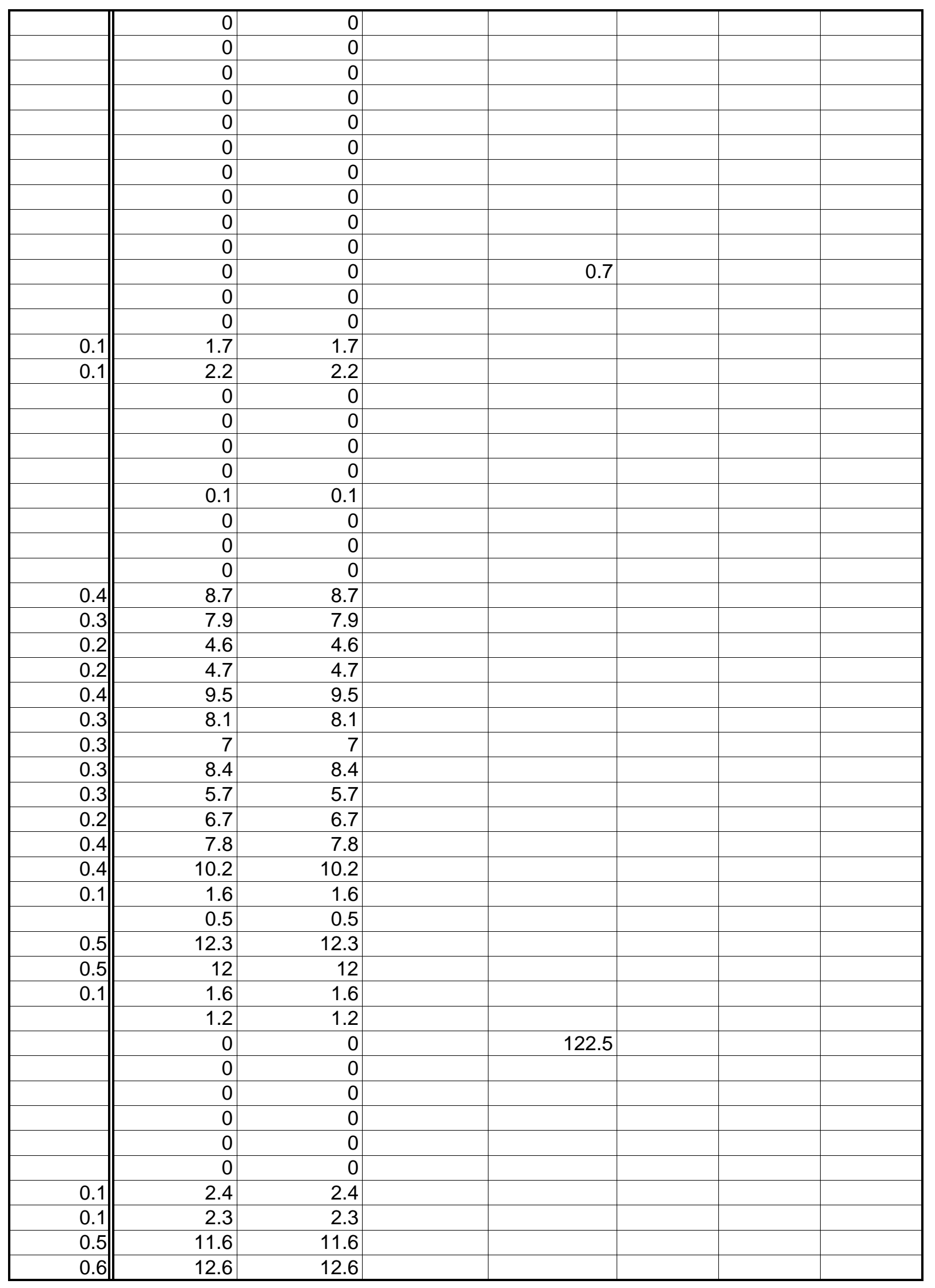


wbheat

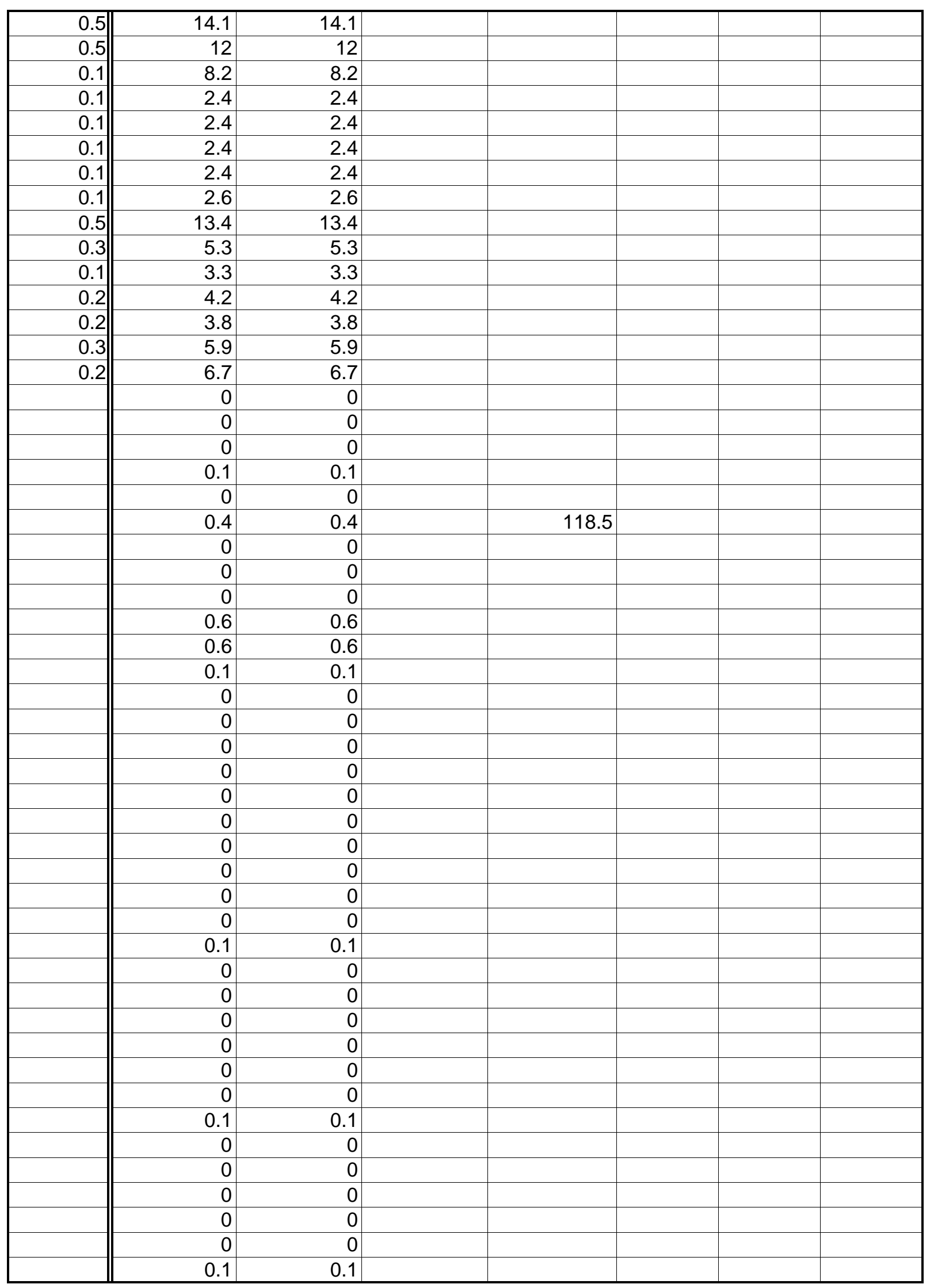


wbheat

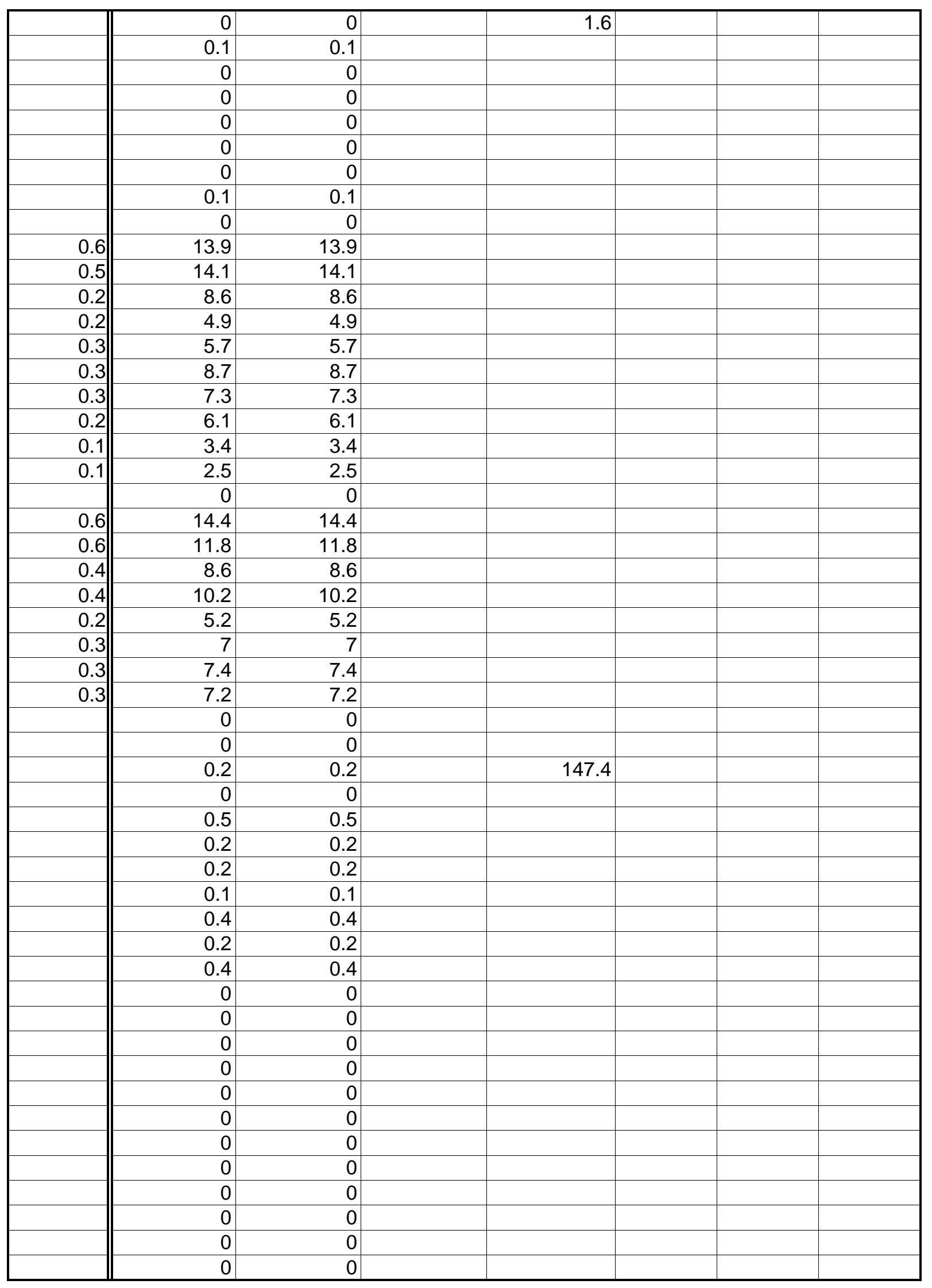




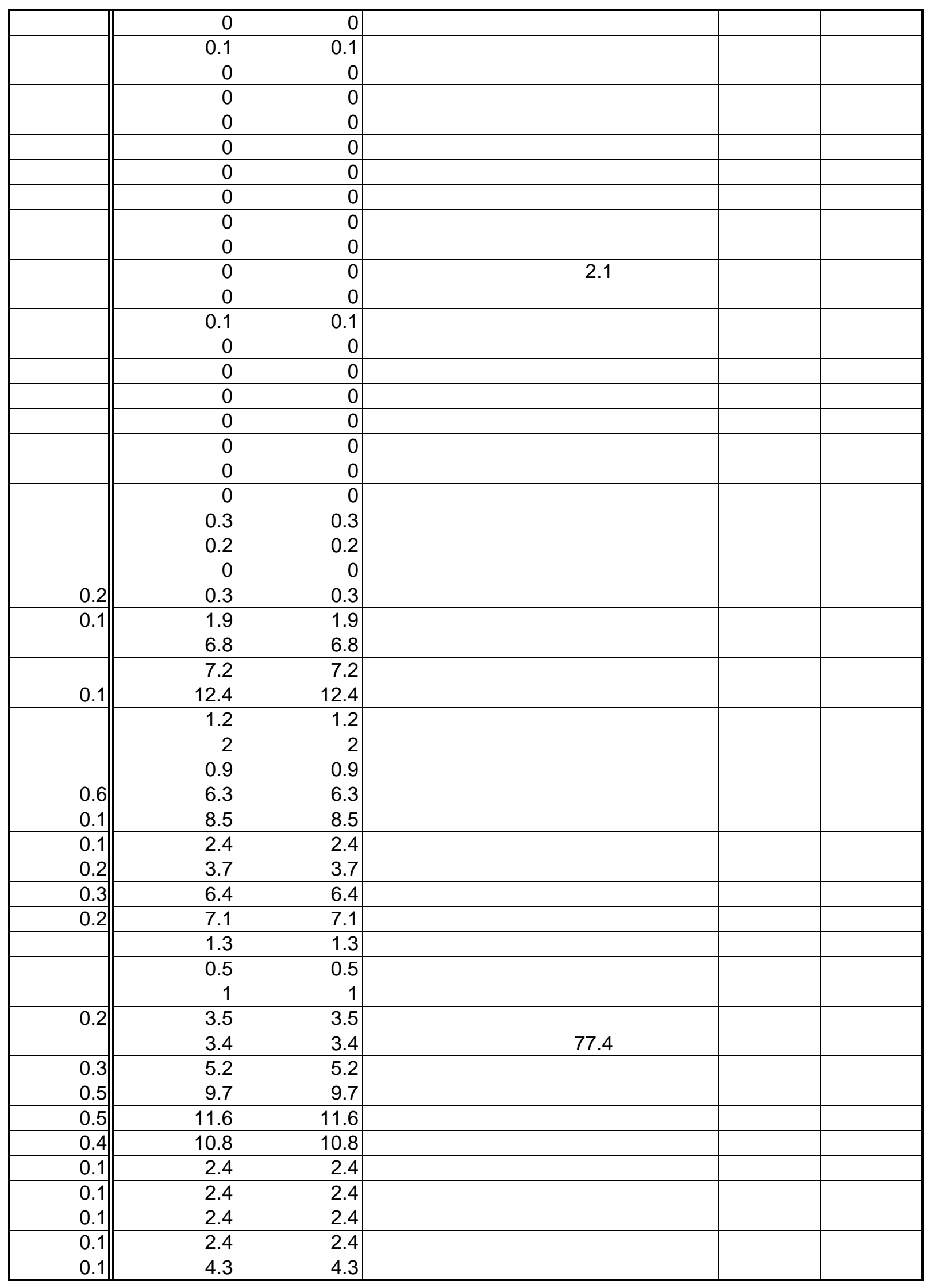


wbheat

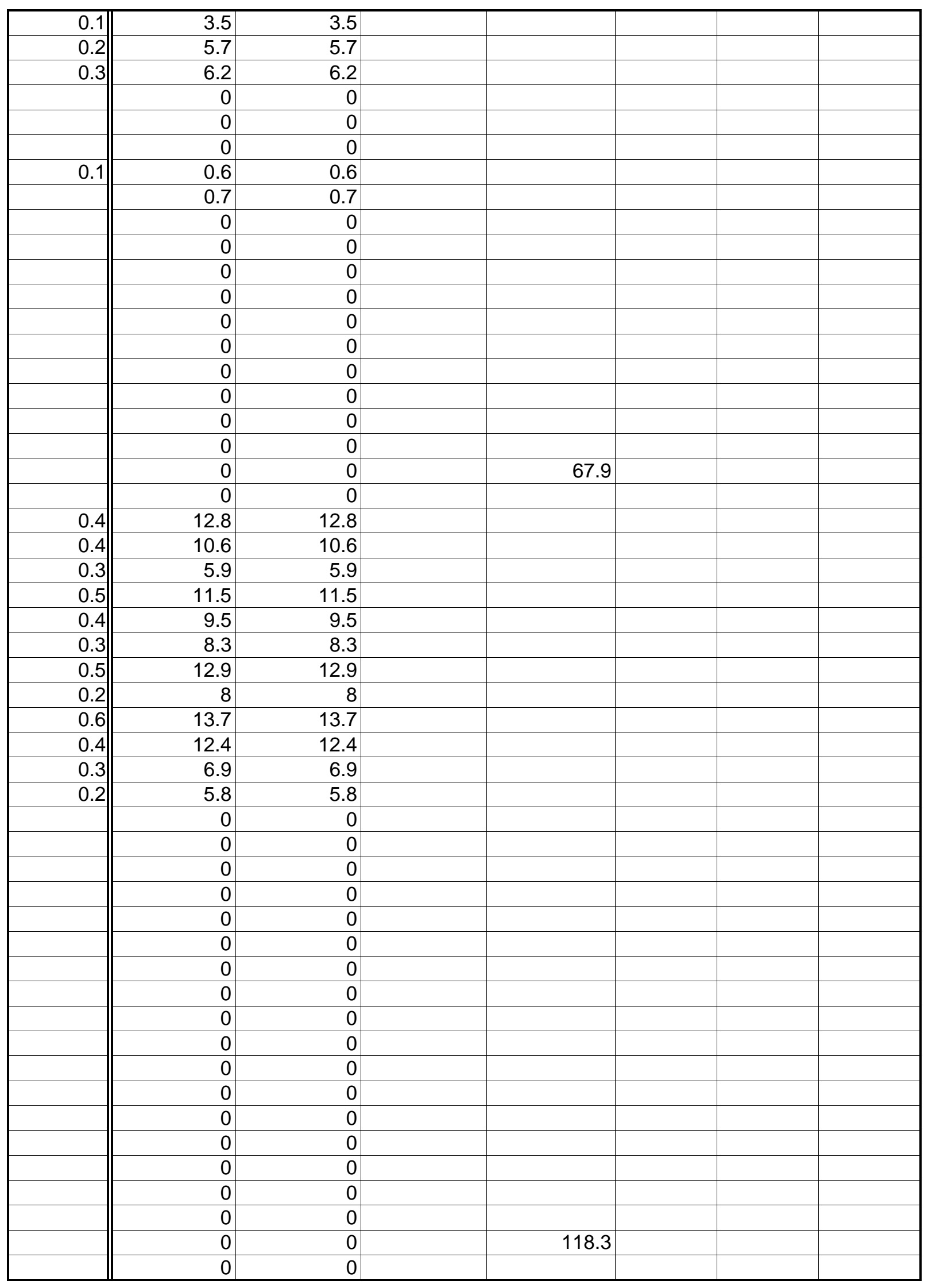


wbheat

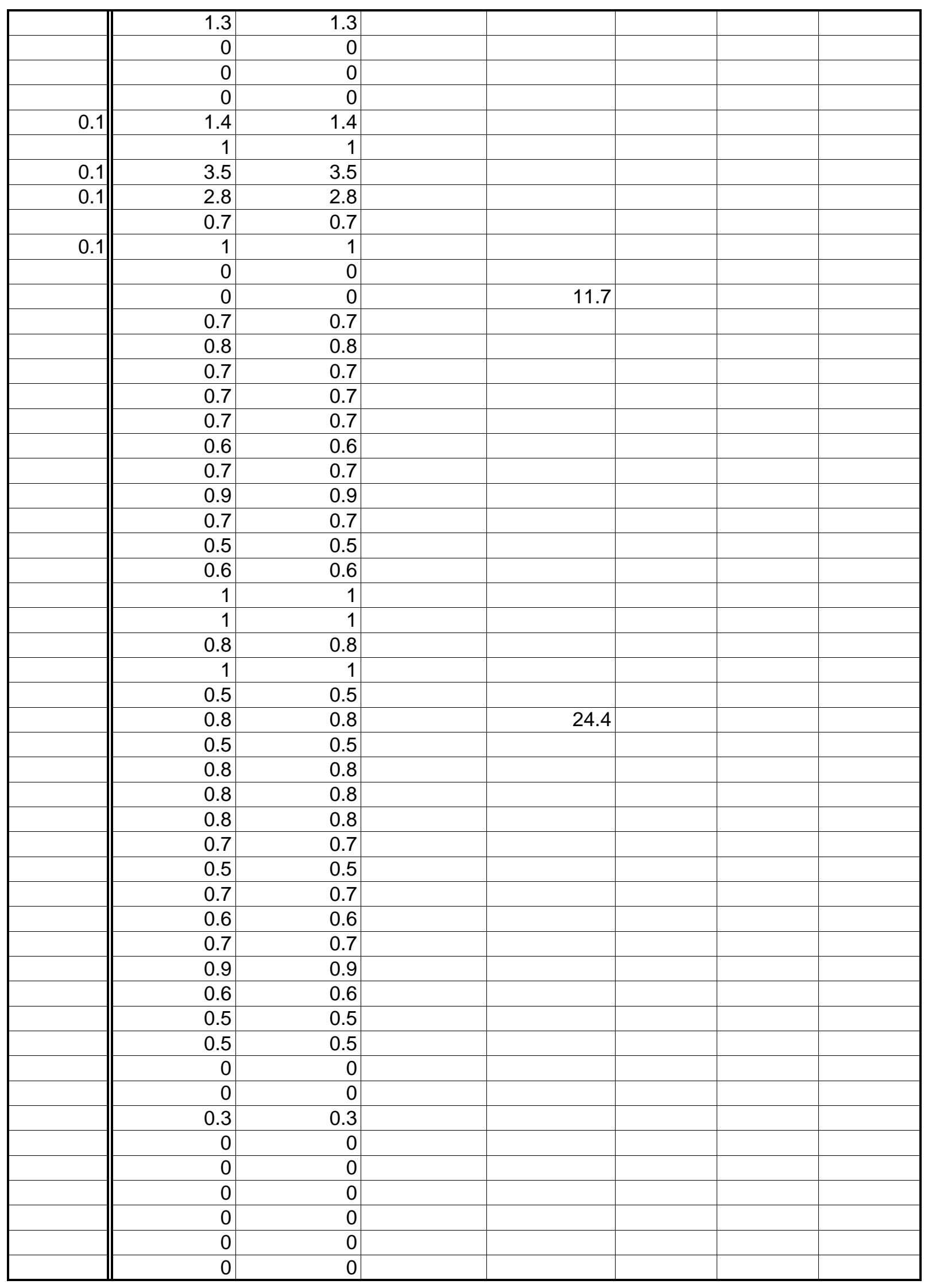




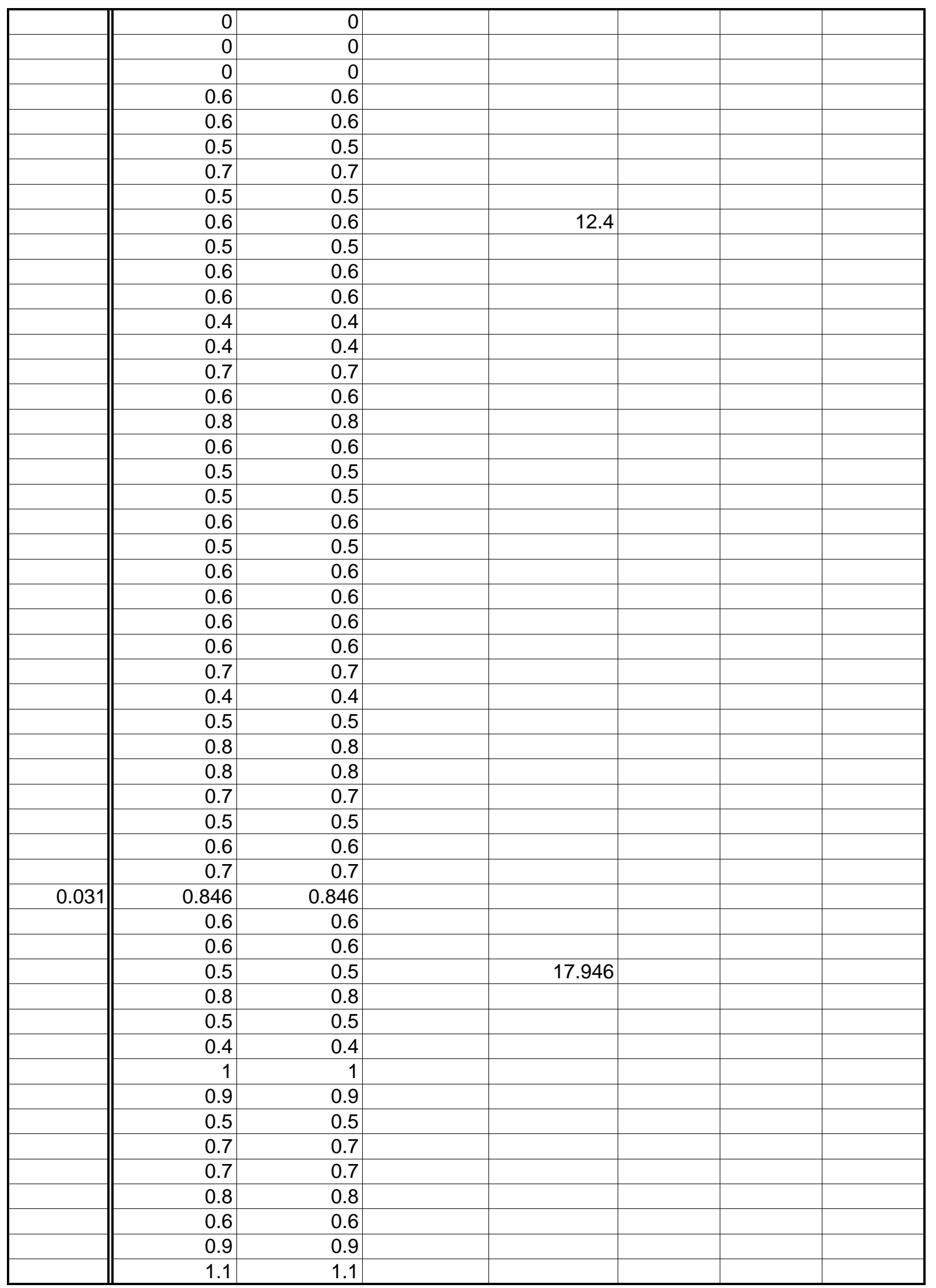


wbheat

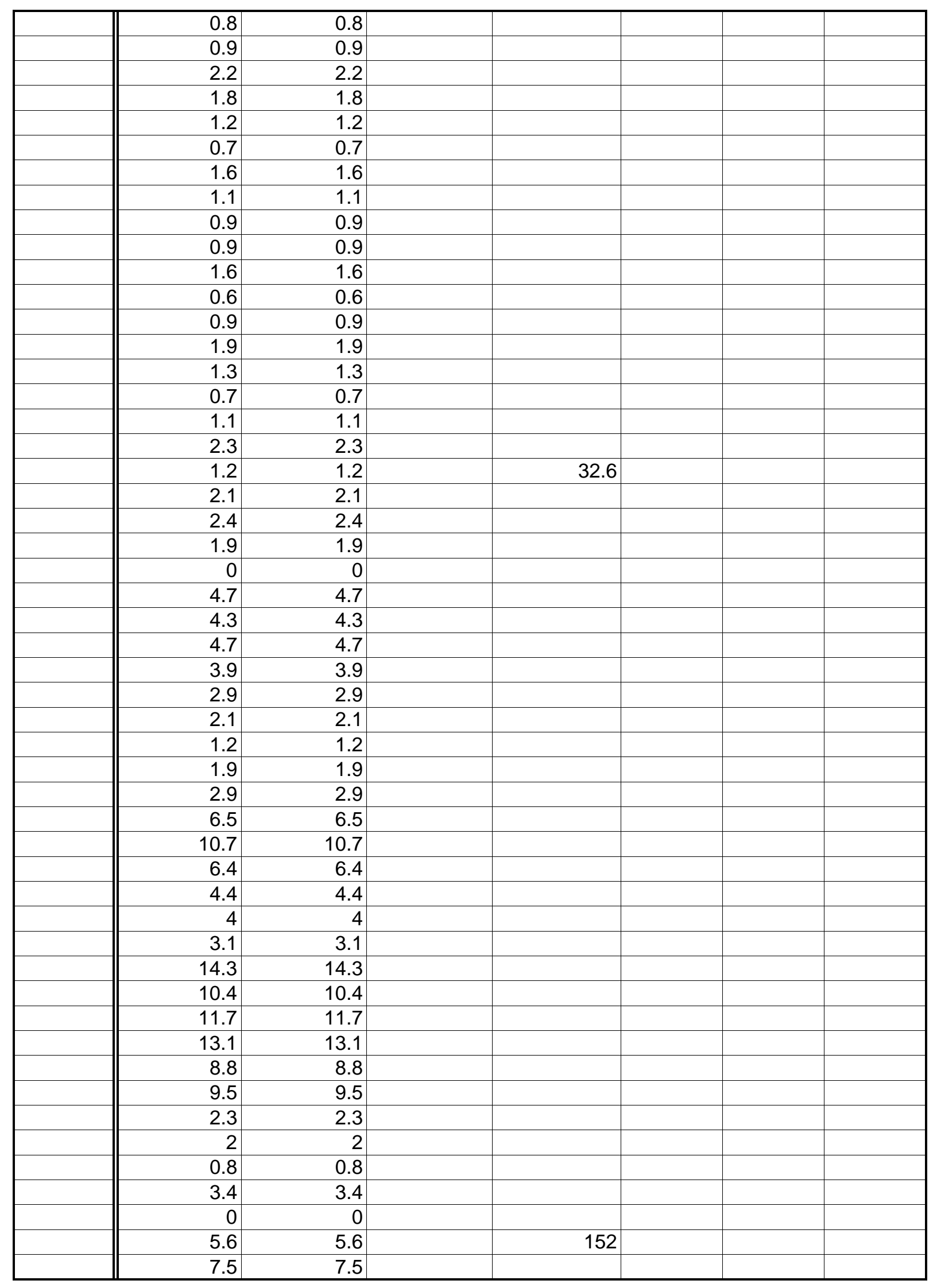


wbheat

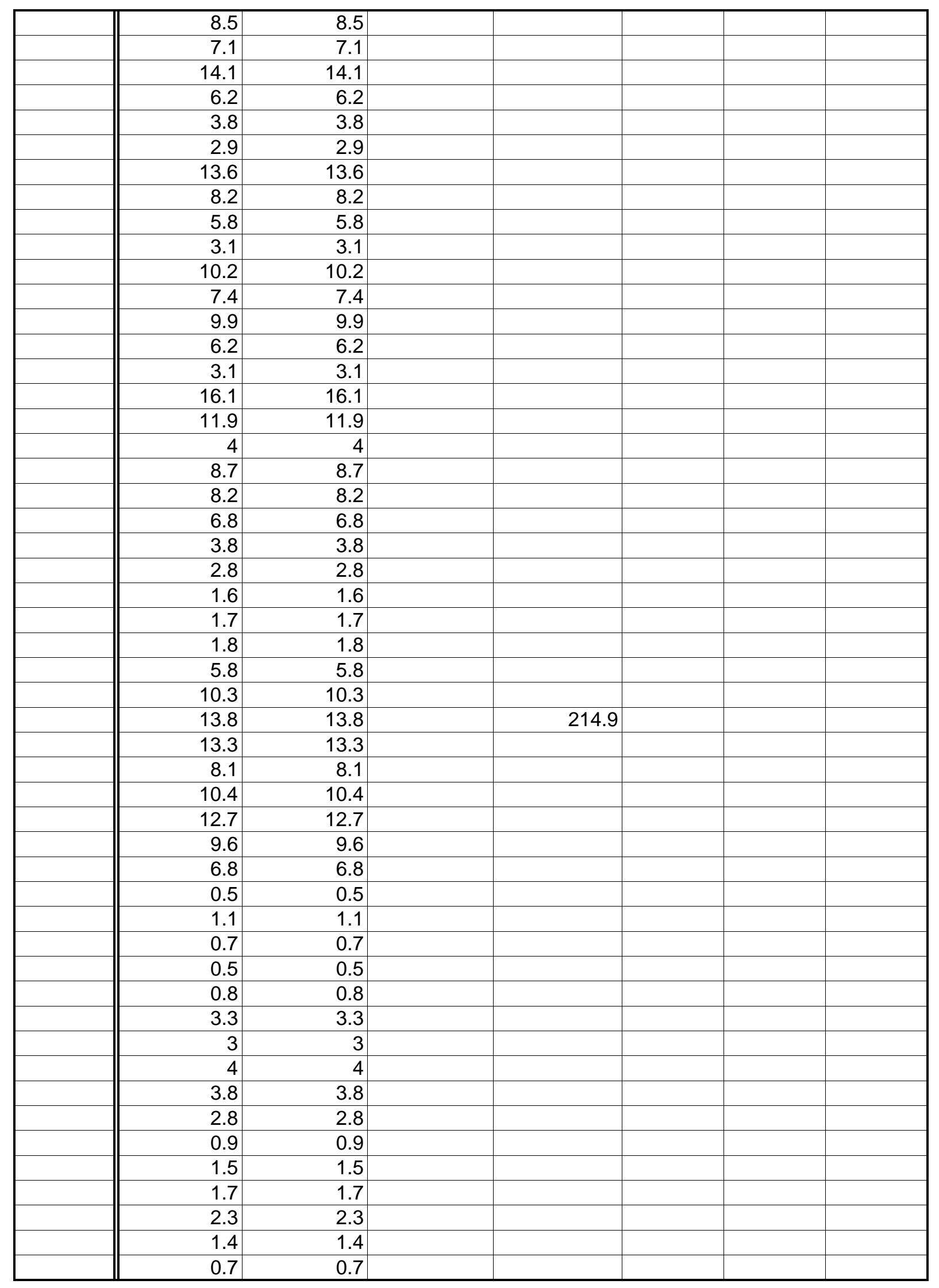


wbheat

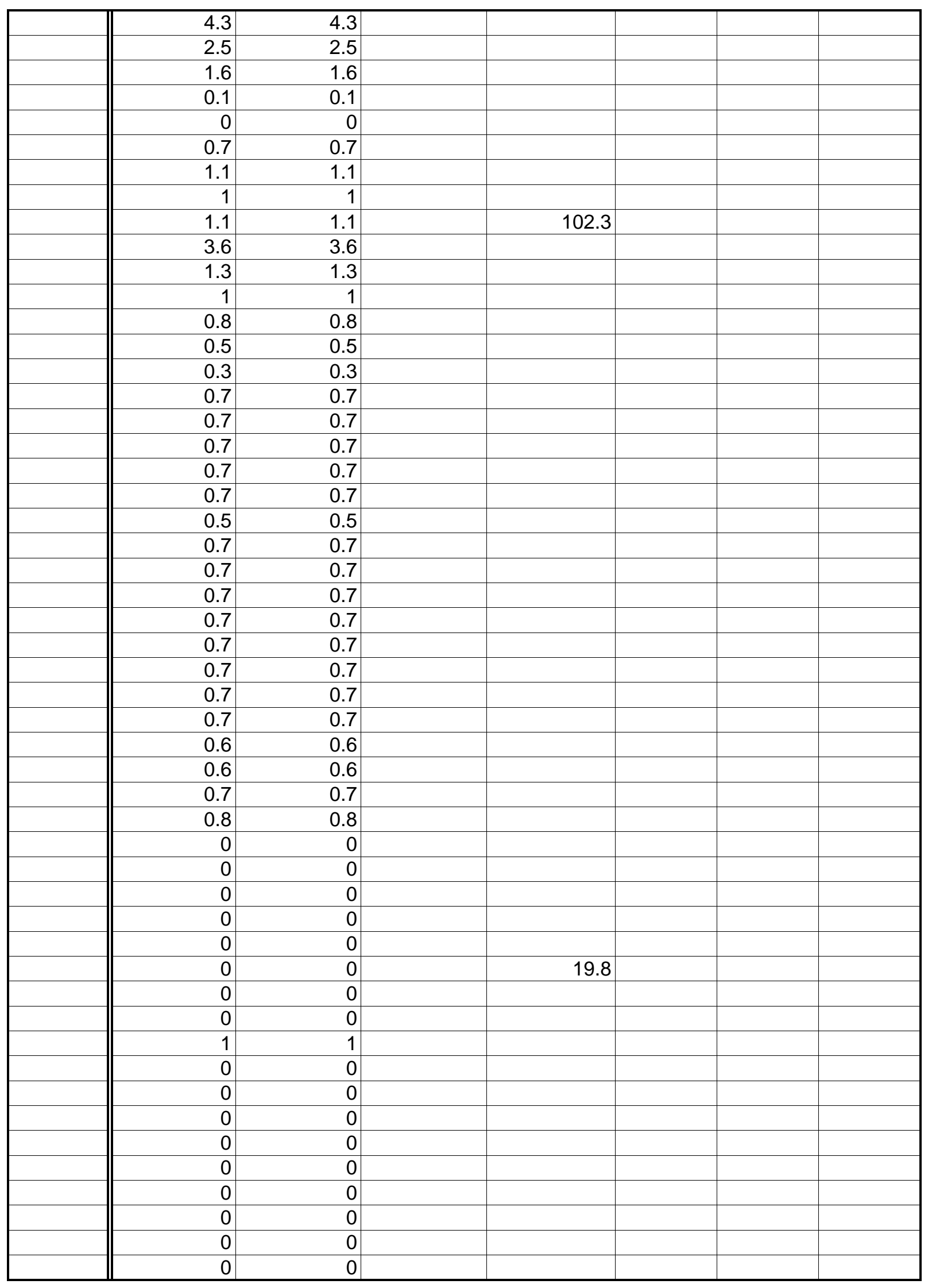




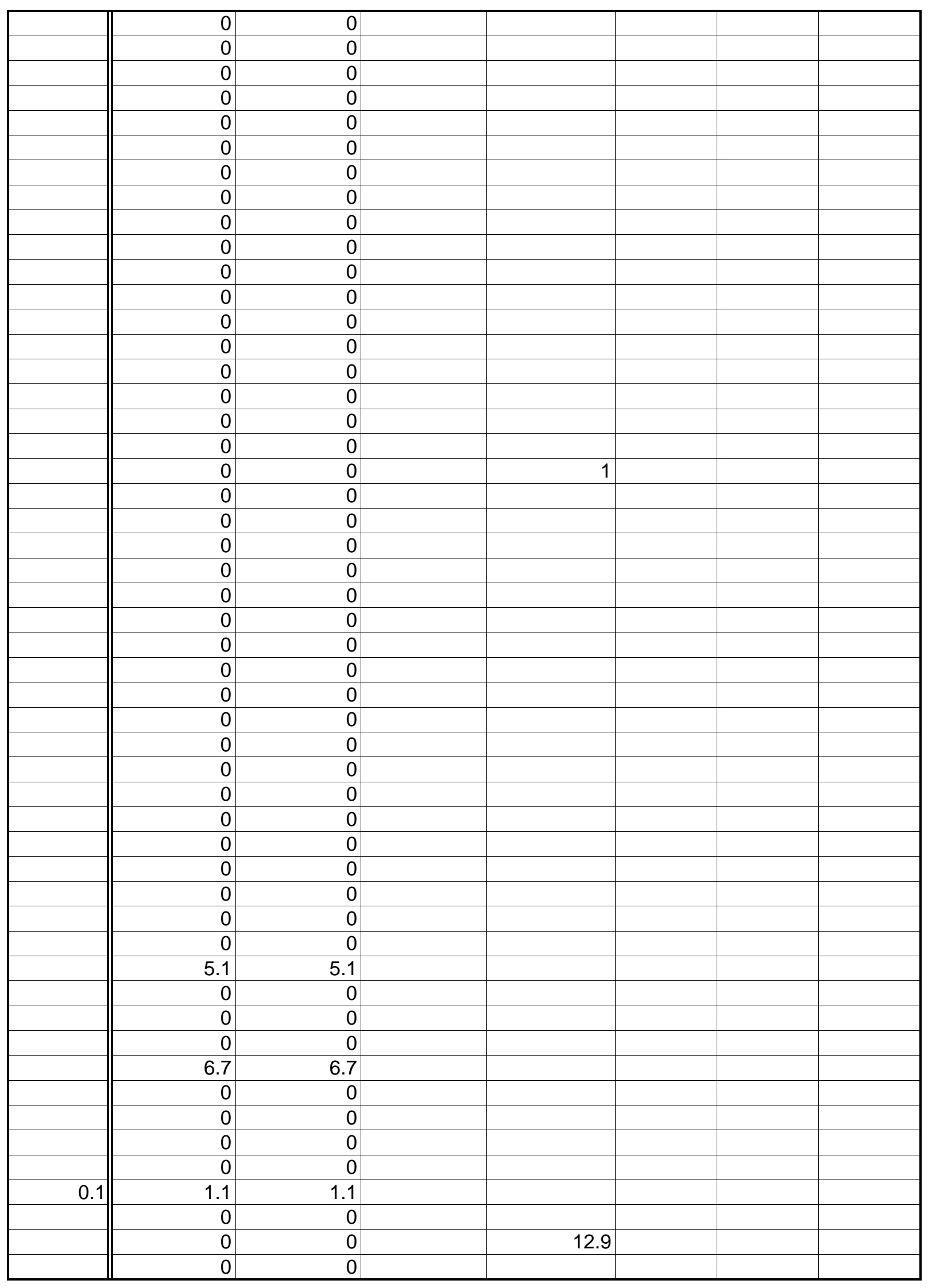




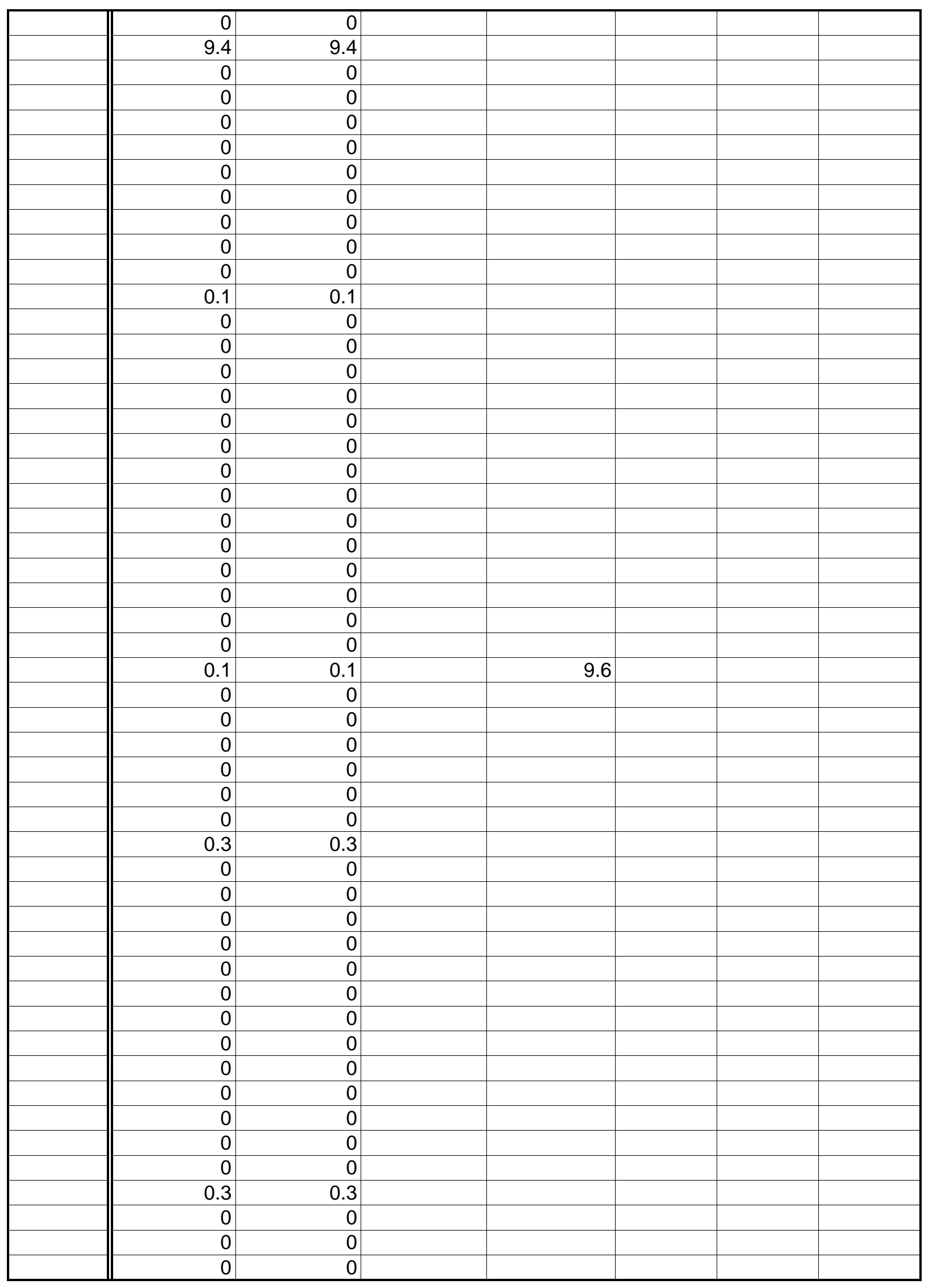




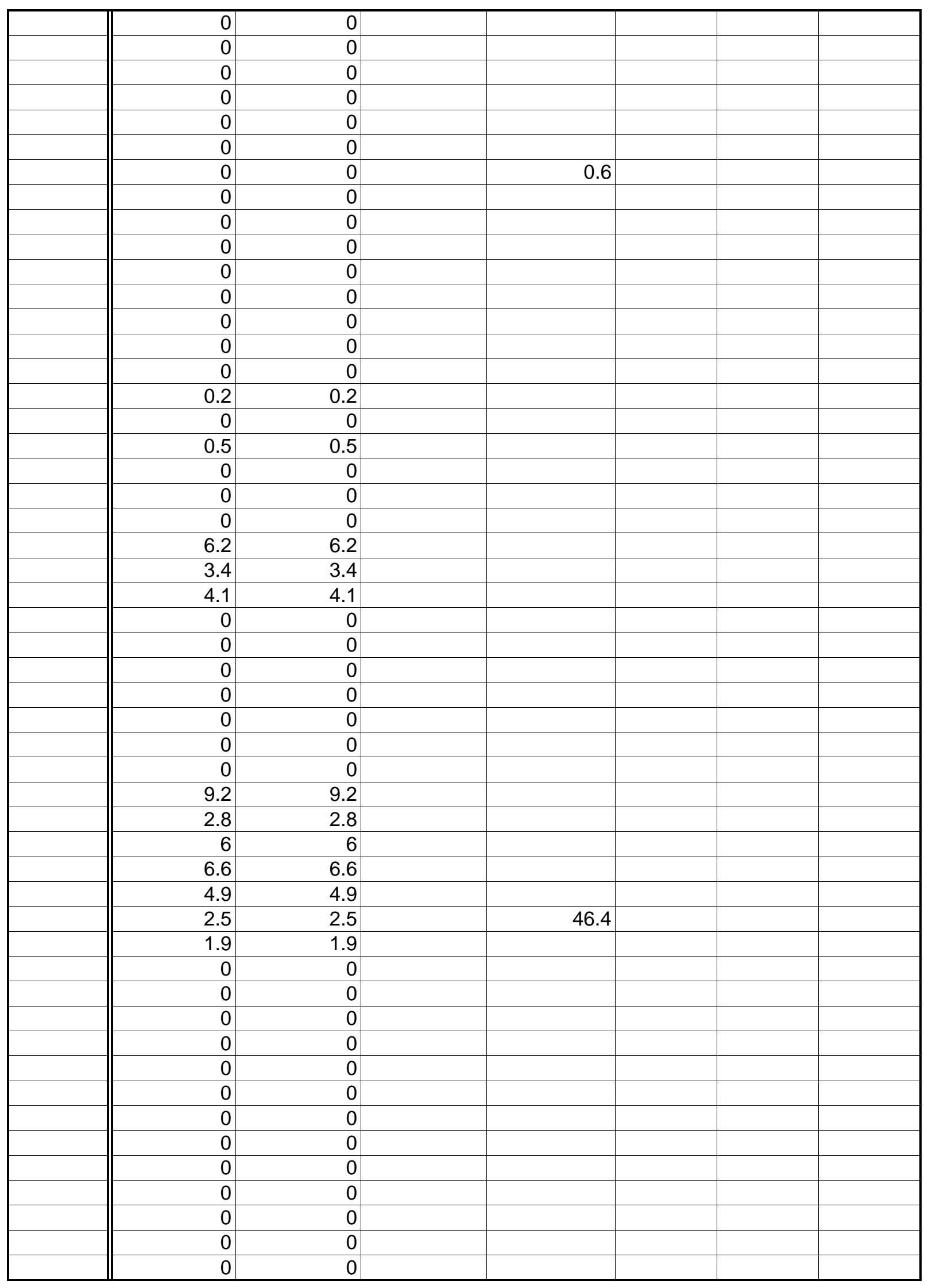


wbheat

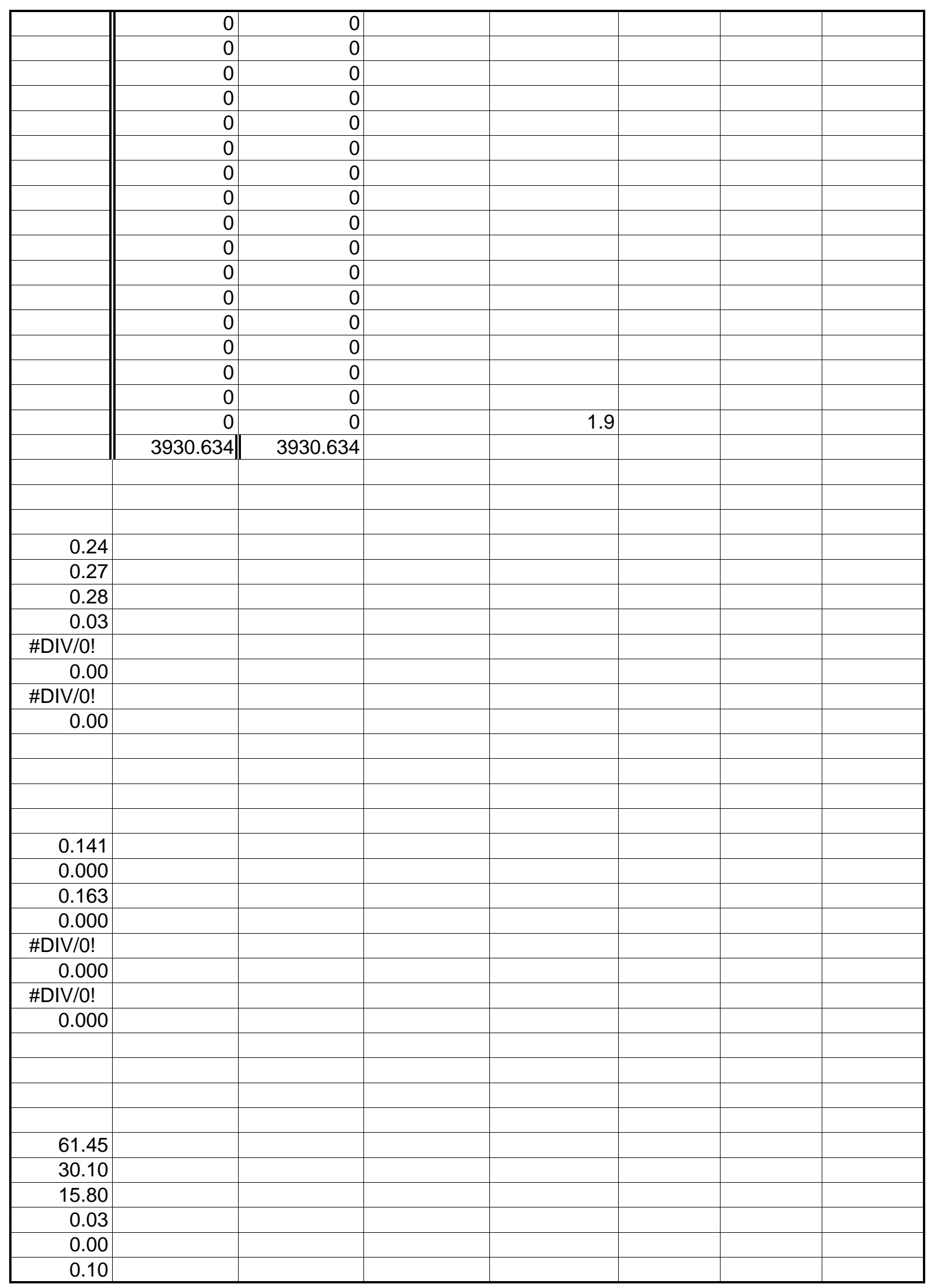


wbheat

\begin{tabular}{|l|l|l|l|l|l|l|l|}
\hline 0.00 & & & & & & & \\
\hline 0.00 & & & & & & & \\
\hline
\end{tabular}

Page 112 
wbheat

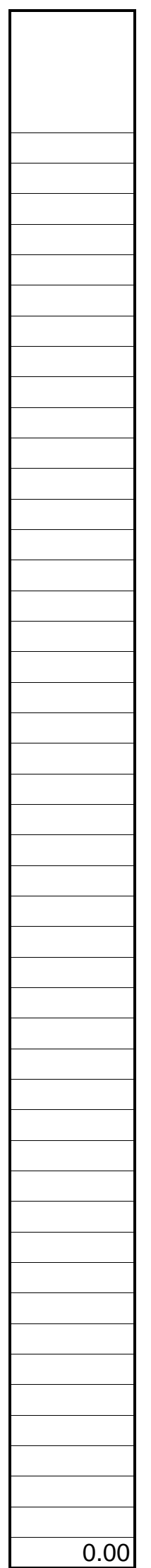

Page 113 


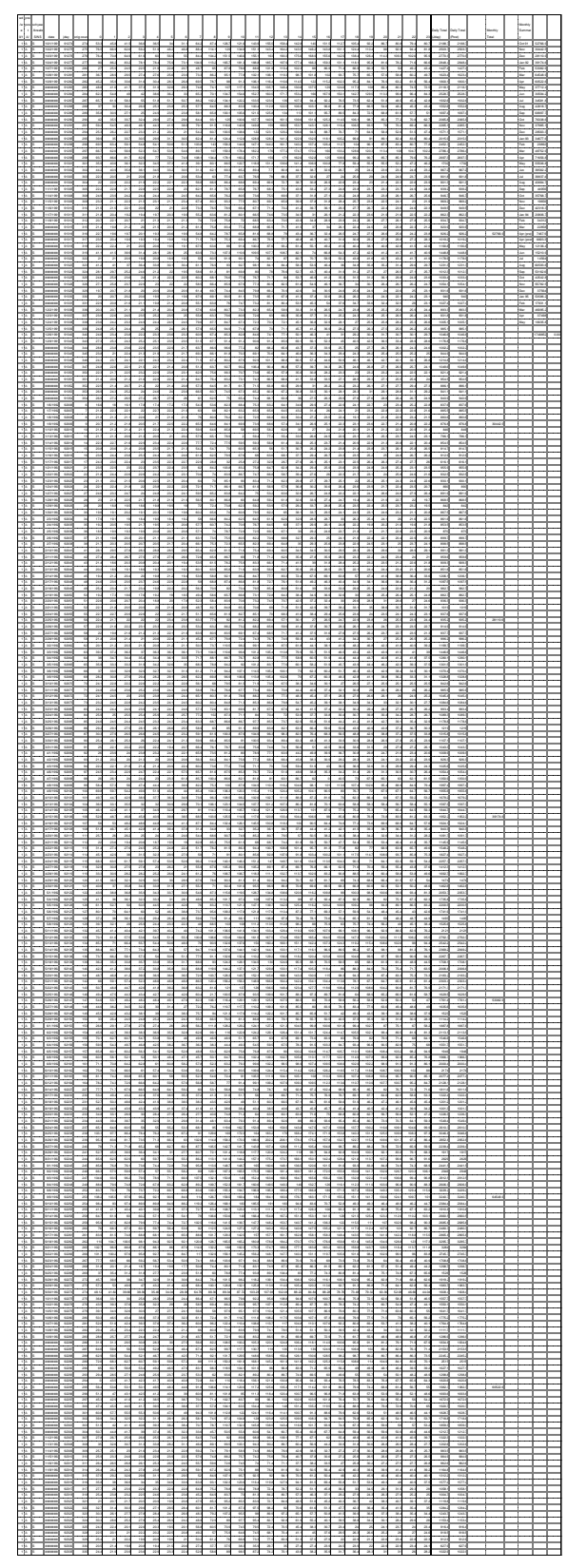




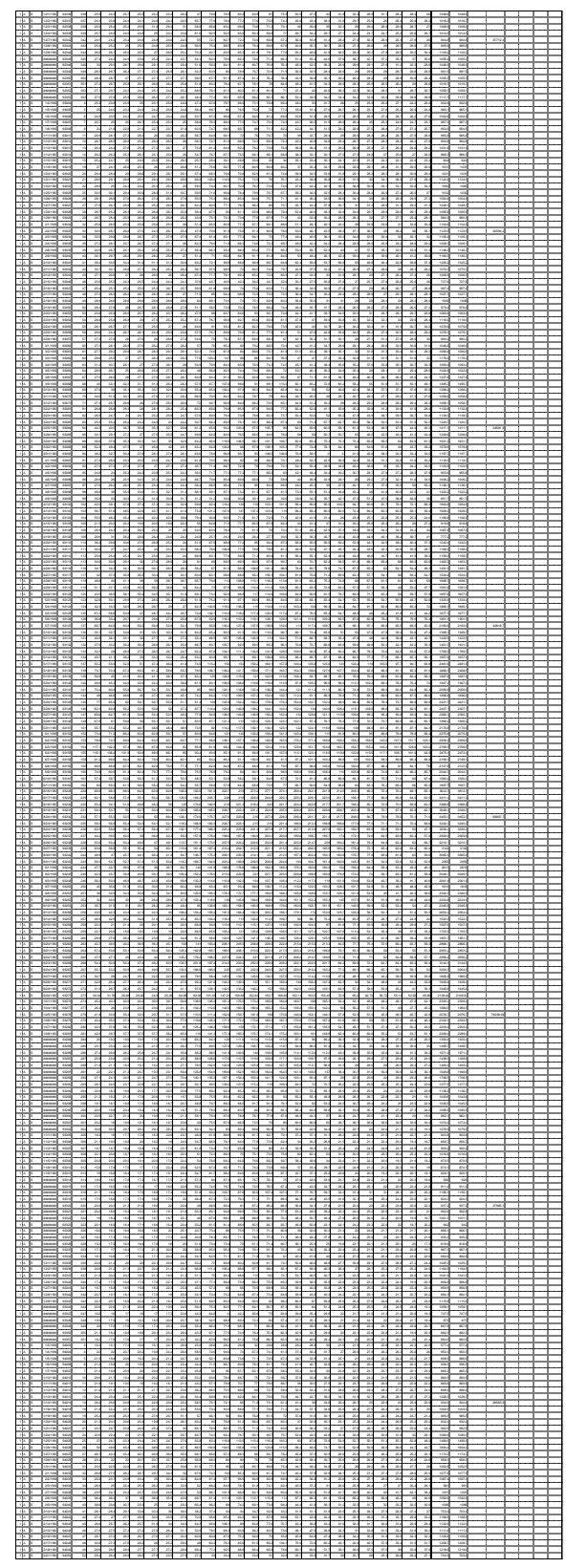




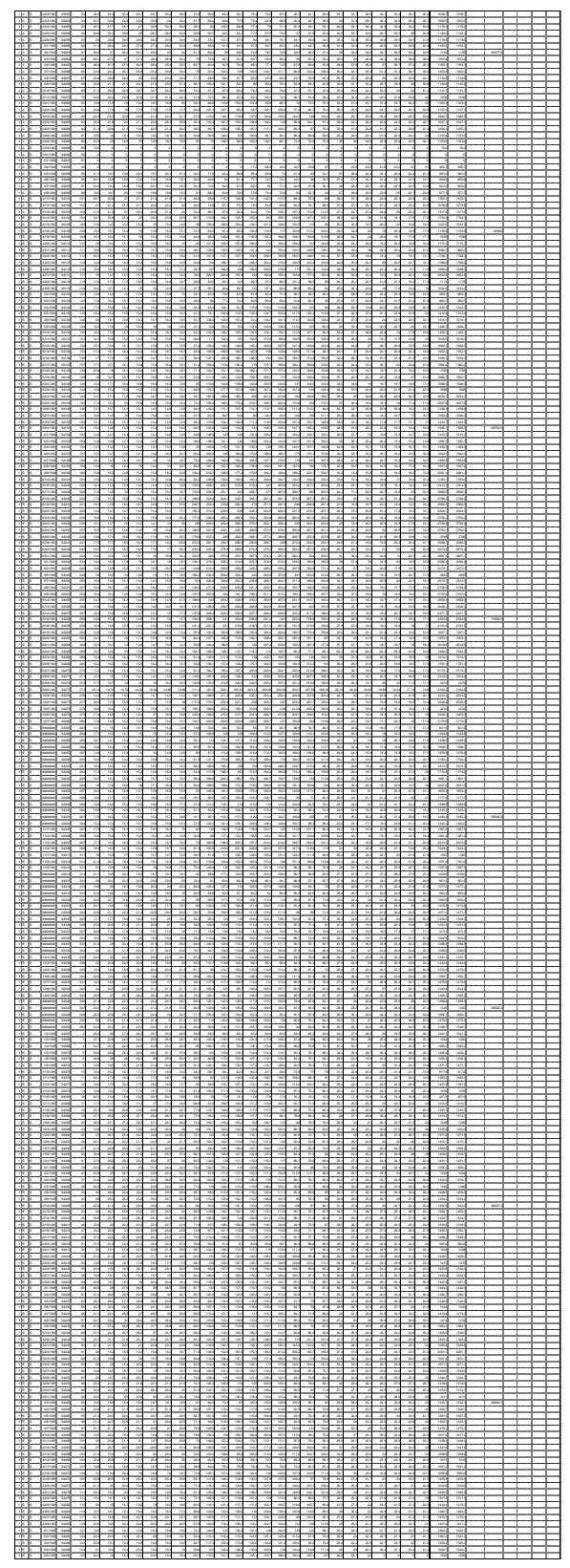




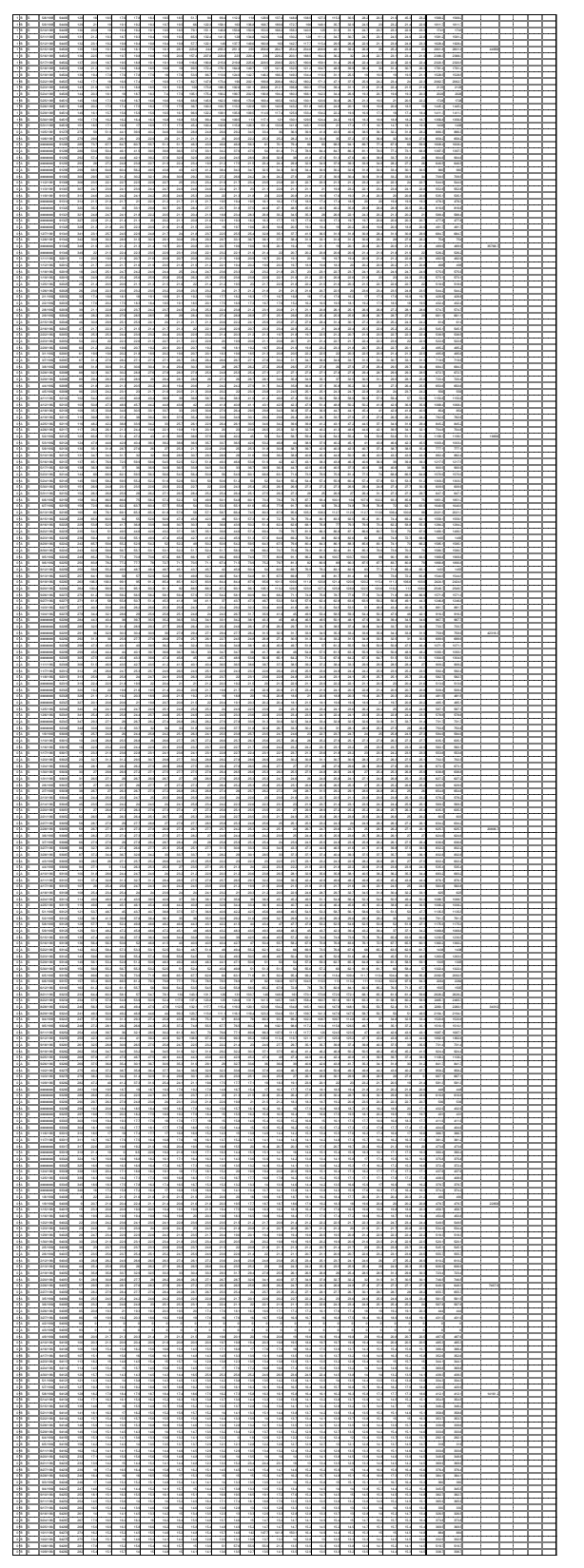




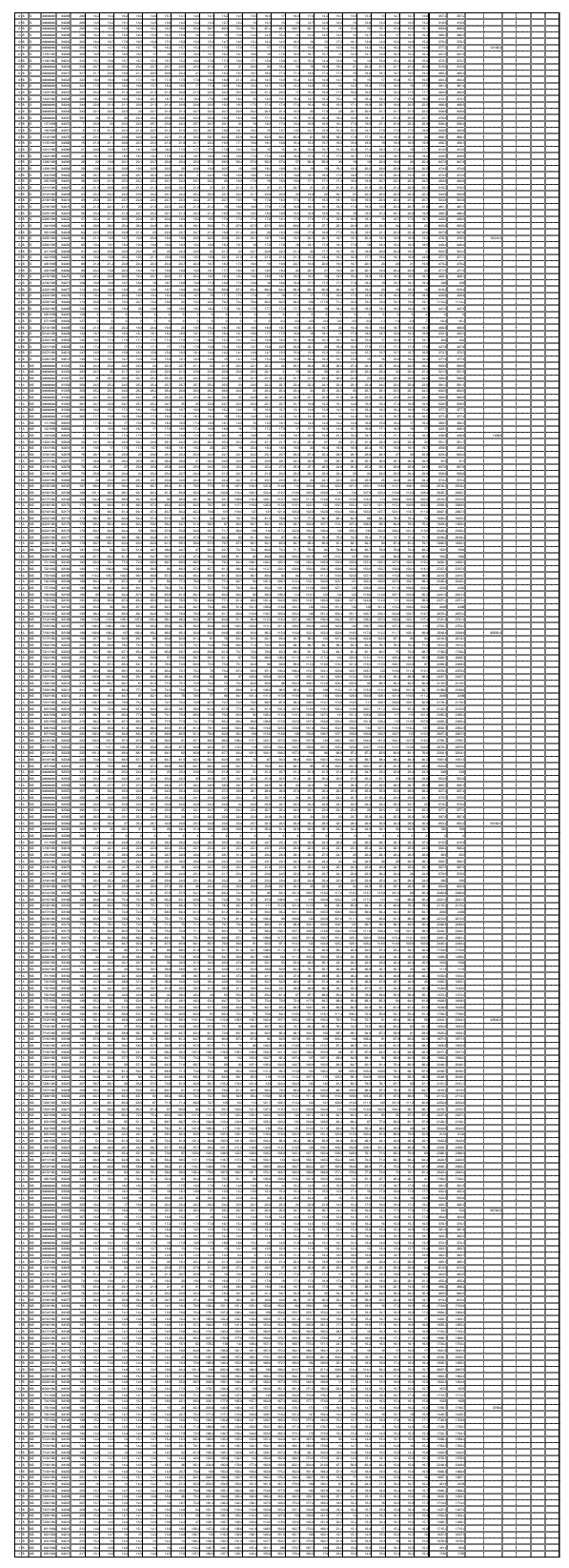




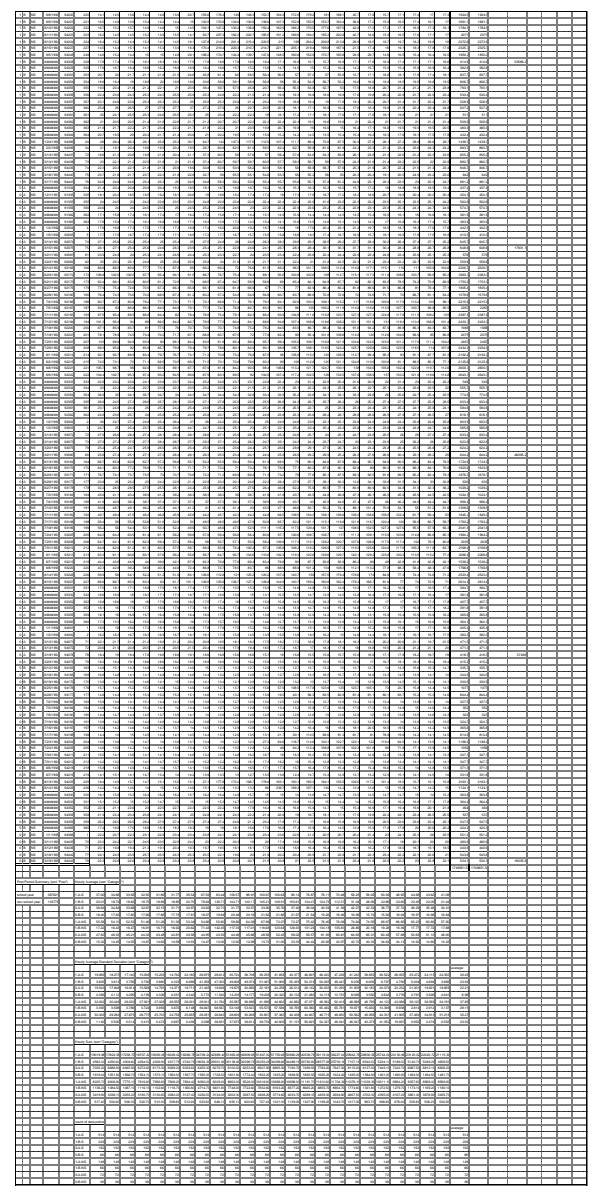


lights

\begin{tabular}{|c|c|c|c|c|c|c|c|c|c|c|}
\hline $\begin{array}{l}\text { wd/ } \\
\text { we } \\
0 / 1\end{array}$ & $\begin{array}{l}\text { pre/ } \\
\text { post } \\
\text { A/B }\end{array}$ & $\begin{array}{c}\text { schyear/ } \\
\text { breaks } \\
\text { S/NS }\end{array}$ & date & jday & $\begin{array}{c}\text { orig } \\
\text { count }\end{array}$ & & 1 & 2 & 3 & 4 \\
\hline 1 & A & $S$ & 10/1/1991 & 91274 & 274 & 17.7 & 12 & 12.7 & 12.6 & 12 \\
\hline 1 & $A$ & $S$ & 10/2/1991 & 91275 & 275 & 25 & 12.3 & 13 & 12.3 & 12.9 \\
\hline 1 & A & S & $10 / 3 / 1991$ & 91276 & 276 & 15.5 & 10.3 & 10.9 & 10.3 & 11 \\
\hline 1 & A & S & 10/4/1991 & 91277 & 277 & 20.9 & 9.3 & 9.9 & 10 & 9.9 \\
\hline 0 & $A$ & S & 10/5/1991 & 91278 & 278 & 17.1 & 12.4 & 11.8 & 12.4 & 11.9 \\
\hline 0 & A & S & 10/6/1991 & 91279 & 279 & 12.4 & 11 & 11.3 & 10.6 & 10.7 \\
\hline 1 & $A$ & $\mathrm{~S}$ & $10 / 7 / 1991$ & 91280 & 280 & 10.7 & 10.6 & 11.3 & 10.6 & 10.7 \\
\hline 1 & A & $S$ & 10/8/1991 & 91281 & 281 & 19.5 & 12.6 & 12.7 & 12 & 12.7 \\
\hline 1 & A & S & 10/9/1991 & 91282 & 282 & 18.9 & 10.2 & 10.3 & 10.2 & 10.2 \\
\hline 1 & A & S & \#\#\#\#\#\#\#\# & 91283 & 283 & 14 & 11.7 & 11.6 & 11.7 & 12.3 \\
\hline 1 & A & S & \#\#\#\#\#\#\#\# & 91284 & 284 & 20.5 & 12.6 & 13.2 & 12.7 & 13.2 \\
\hline 0 & A & $\mathrm{S}$ & \#\#\#\#\#\#\#\# & 91285 & 285 & 16.5 & 12 & 11.4 & 12 & 12 \\
\hline 0 & A & S & \#\#\#\#\#\#\#\# & 91286 & 286 & 11.9 & 12 & 12 & 12 & 11.4 \\
\hline 1 & A & S & \#\#\#\#\#\#\#\# & 91287 & 287 & 10.9 & 10.8 & 10.1 & 10.8 & 10.8 \\
\hline 1 & A & S & \#\#\#\#\#\#\#\# & 91288 & 288 & 14.6 & 10.8 & 10.2 & 10.8 & 10.2 \\
\hline 1 & A & S & \#\#\#\#\#\#\#\# & 91289 & 289 & 15.1 & 9.7 & 9.6 & 9.6 & 9.7 \\
\hline 1 & A & $S$ & \#\#\#\#\#\#\#\# & 91290 & 290 & 16.3 & 10.8 & 10.9 & 10.8 & 10.9 \\
\hline 1 & $A$ & S & \#\#\#\#\#\#\#\# & 91291 & 291 & 24.6 & 17.5 & 18.1 & 18 & 17.4 \\
\hline 0 & A & S & \#\#\#\#\#\#\#\# & 91292 & 292 & 13.4 & 11.4 & 11.4 & 11.4 & 11.4 \\
\hline 0 & A & S & \#\#\#\#\#\#\#\# & 91293 & 293 & 11.4 & 11.4 & 11.4 & 11.5 & 11.4 \\
\hline 1 & A & S & \#\#\#\#\#\#\#\# & 91294 & 294 & 11.4 & 11.5 & 11.4 & 11.5 & 11.4 \\
\hline 1 & A & S & \#\#\#\#\#\#\#\# & 91295 & 295 & 22.5 & 10.8 & 10.9 & 10.8 & 10.9 \\
\hline 1 & A & S & \#\#\#\#\#\#\#\# & 91296 & 296 & 16.7 & 14.4 & 13.9 & 14.3 & 13.8 \\
\hline 1 & A & S & \#\#\#\#\#\#\#\# & 91297 & 297 & 13.7 & 10.1 & 10.1 & 10.1 & 10.1 \\
\hline 1 & A & S & \#\#\#\#\#\#\#\# & 91298 & 298 & 21.3 & 13.2 & 13.1 & 12.6 & 13.2 \\
\hline 0 & A & $S$ & \#\#\#\#\#\#\#\# & 91299 & 299 & 17.5 & 10.5 & 10.4 & 10.5 & 10.5 \\
\hline 0 & $A$ & S & \#\#\#\#\#\#\#\# & 91300 & 300 & 11.1 & 10.5 & 10.6 & 11.1 & 10.6 \\
\hline 1 & A & S & \#\#\#\#\#\#\#\# & 91301 & 301 & 11.1 & 10.5 & 10.5 & 11.1 & 10.6 \\
\hline 1 & A & S & \#\#\#\#\#\#\#\# & 91302 & 302 & 33.1 & 21.7 & 12.9 & 12.9 & 13.5 \\
\hline 1 & A & S & \#\#\#\#\#\#\#\# & 91303 & 303 & 21.1 & 12.4 & 12.4 & 12.5 & 11.8 \\
\hline 1 & A & S & \#\#\#\#\#\#\#\# & 91304 & 304 & 20.7 & 11.5 & 10.9 & 11.5 & 11.4 \\
\hline 1 & A & S & 11/1/1991 & 91305 & 305 & 17.2 & 10.3 & 10.9 & 11 & 10.9 \\
\hline 0 & A & S & $11 / 2 / 1991$ & 91306 & 306 & 18.5 & 10.3 & 9.7 & 9.7 & 10.3 \\
\hline 0 & A & S & $11 / 3 / 1991$ & 91307 & 307 & 18 & 18.1 & 18.1 & 18.1 & 18.1 \\
\hline 1 & A & $S$ & $11 / 4 / 1991$ & 91308 & 308 & 18.1 & 18.1 & 18 & 18.1 & 18.1 \\
\hline 1 & A & S & $11 / 5 / 1991$ & 91309 & 309 & 27.8 & 10.8 & 10.3 & 10.9 & 10.3 \\
\hline 1 & A & S & $11 / 6 / 1991$ & 91310 & 310 & 20.7 & 10.9 & 10.3 & 10.8 & 10.9 \\
\hline 1 & A & S & $11 / 7 / 1991$ & 91311 & 311 & 22.4 & 11.5 & 11.5 & 12 & 11.5 \\
\hline 1 & A & S & $11 / 8 / 1991$ & 91312 & 312 & 18.9 & 8.6 & 9.3 & 9.2 & 8.7 \\
\hline 0 & A & S & 11/9/1991 & 91313 & 313 & 26.8 & 9.5 & 10.1 & 10 & 10.1 \\
\hline 0 & A & S & \#\#\#\#\#\#\#\# & 91314 & 314 & 8.3 & 8.2 & 8.3 & 8.3 & 7.7 \\
\hline 1 & A & S & \#\#\#\#\#\#\#\# & 91315 & 315 & 7.6 & 8.3 & 8.3 & 8.3 & 8.3 \\
\hline 1 & A & S & \#\#\#\#\#\#\#\# & 91316 & 316 & 25.3 & 12.1 & 12 & 12.1 & 12.1 \\
\hline 1 & A & $S$ & \#\#\#\#\#\#\#\# & 91317 & 317 & 25.9 & 10.8 & 10.8 & 10.9 & 10.8 \\
\hline 1 & A & S & \#\#\#\#\#\#\#\# & 91318 & 318 & 13.6 & 8.3 & 8.3 & 8.3 & 8.2 \\
\hline 1 & A & S & \#\#\#\#\#\#\#\# & 91319 & 319 & 67.6 & 65.8 & 51.5 & 12.5 & 11 \\
\hline 0 & A & S & \#\#\#\#\#\#\#\# & 91320 & 320 & 18.9 & 12.5 & 12.4 & 11.8 & 12.4 \\
\hline
\end{tabular}


lights

\begin{tabular}{|c|c|c|c|c|c|c|c|c|c|}
\hline \begin{tabular}{l|l}
0 & $A$ \\
\end{tabular} & $S$ & \#\#\#\#\#\#\#\# & 91321 & 321 & 8.8 & 9.4 & 8.8 & 8.9 & 8.8 \\
\hline $1 \mathrm{~A}$ & $\mathrm{~S}$ & \#\#\#\#\#\#\#\# & 91322 & 322 & 8 & 8 & 7.9 & 8 & 8 \\
\hline $1 \mathrm{~A}$ & S & \#\#\#\#\#\#\#\# & 91323 & 323 & 63.7 & 60 & 58.3 & 36.6 & 10.6 \\
\hline $1 \mathrm{~A}$ & $\mathrm{~S}$ & \#\#\#\#\#\#\#\# & 91324 & 324 & 55.9 & 48.1 & 46.3 & 21 & 8.3 \\
\hline $1 \mathrm{~A}$ & S & \#\#\#\#\#\#\#\# & 91325 & 325 & 39.7 & 30.1 & 18.4 & 9.8 & 10.5 \\
\hline $1 \mathrm{~A}$ & $\mathrm{~S}$ & \#\#\#\#\#\#\#\# & 91326 & 326 & 45.1 & 31.6 & 8.7 & 9.3 & 8.7 \\
\hline $0 \mathrm{~A}$ & $\mathrm{~S}$ & \#\#\#\#\#\#\#\# & 91327 & 327 & 12.5 & 8.1 & 8.1 & 7.5 & 8.1 \\
\hline $0 \mathrm{~A}$ & S & \#\#\#\#\#\#\#\# & 91328 & 328 & 7.5 & 8 & 8.1 & 7.5 & 8.1 \\
\hline $1 \mathrm{~A}$ & S & \#\#\#\#\#\#\#\# & 91329 & 329 & 8.1 & 8.1 & 7.5 & 8.1 & 8.1 \\
\hline $1 \mathrm{~A}$ & S & \#\#\#\#\#\#\#\# & 91330 & 330 & 17.4 & 6.4 & 6.4 & 7 & 6.3 \\
\hline $1 \mathrm{~A}$ & $\mathrm{~S}$ & \#\#\#\#\#\#\#\# & 91331 & 331 & 20.1 & 8 & 7.4 & 7.7 & 8 \\
\hline $1 \mathrm{~A}$ & NS & \#\#\#\#\#\#\#\# & 91332 & 332 & 7.3 & 6.7 & 6.7 & 7.3 & 6.7 \\
\hline $1 \mathrm{~A}$ & NS & \#\#\#\#\#\#\#\# & 91333 & 333 & 6.7 & 6.7 & 7.3 & 6.7 & 6.7 \\
\hline $0 \mathrm{~A}$ & NS & \#\#\#\#\#\#\#\# & 91334 & 334 & 6.7 & 6.7 & 7.3 & 6.7 & 6.7 \\
\hline $0 \mathrm{~A}$ & NS & $12 / 1 / 1991$ & 91335 & 335 & 7.3 & 6.7 & 6.7 & 7.4 & 6.7 \\
\hline $1 \mathrm{~A}$ & $\mathrm{~S}$ & $12 / 2 / 1991$ & 91336 & 336 & 7.3 & 6.7 & 6.7 & 7.4 & 6.7 \\
\hline $1 \mathrm{~A}$ & S & $12 / 3 / 1991$ & 91337 & 337 & 13.8 & 7.9 & 7.4 & 7.9 & 7.4 \\
\hline $1 \mathrm{~A}$ & S & $12 / 4 / 1991$ & 91338 & 338 & 19.8 & 6.8 & 7.4 & 6.8 & 6.8 \\
\hline $1 \mathrm{~A}$ & S & $12 / 5 / 1991$ & 91339 & 339 & 13.4 & 6.3 & 7 & 6.4 & 6.9 \\
\hline $1 \mathrm{~A}$ & S & $12 / 6 / 1991$ & 91340 & 340 & 9.2 & 7.1 & 7.2 & 7.1 & 7.7 \\
\hline $0 \mathrm{~A}$ & $S$ & 12/7/1991 & 91341 & 341 & 7.1 & 6.5 & 6.5 & 7.1 & 6.6 \\
\hline $0 \mathrm{~A}$ & S & 12/8/1991 & 91342 & 342 & 7.1 & 7.1 & 7.1 & 7.1 & 7.1 \\
\hline $1 \mathrm{~A}$ & S & $12 / 9 / 1991$ & 91343 & 343 & 6.1 & 6.6 & 6.7 & 6.7 & 6.1 \\
\hline $1 \mathrm{~A}$ & S & \#\#\#\#\#\#\#\# & 91344 & 344 & 16.8 & 7.2 & 7.1 & 7.1 & 7.2 \\
\hline $1 \mathrm{~A}$ & $\mathrm{~S}$ & \#\#\#\#\#\#\#\# & 91345 & 345 & 17 & 7.6 & 6.9 & 7 & 6.9 \\
\hline $1 \mathrm{~A}$ & S & \#\#\#\#\#\#\#\# & 91346 & 346 & 19.4 & 6.3 & 6.4 & 6.9 & 6.4 \\
\hline $1 \mathrm{~A}$ & S & \#\#\#\#\#\#\#\# & 91347 & 347 & 13.8 & 7.4 & 7.4 & 8 & 7.4 \\
\hline $0 \mathrm{~A}$ & S & \#\#\#\#\#\#\#\# & 91348 & 348 & 12.4 & 7.4 & 8.1 & 7.5 & 7.6 \\
\hline $0 \mathrm{~A}$ & $S$ & \#\#\#\#\#\#\#\# & 91349 & 349 & 7.5 & 7.5 & 8.1 & 7.5 & 7.5 \\
\hline $1 \mathrm{~A}$ & $S$ & \#\#\#\#\#\#\#\# & 91350 & 350 & 7.5 & 8.1 & 7.5 & 7.5 & 8.1 \\
\hline $1 \mathrm{~A}$ & S & \#\#\#\#\#\#\#\# & 91351 & 351 & 14.6 & 7.9 & 7.4 & 7.9 & 7.3 \\
\hline $1 \mathrm{~A}$ & S & \#\#\#\#\#\#\#\# & 91352 & 352 & 12.1 & 8.1 & 8.1 & 8.1 & 7.5 \\
\hline $1 \mathrm{~A}$ & S & \#\#\#\#\#\#\#\# & 91353 & 353 & 27.3 & 7.9 & 7.9 & 7.9 & 8 \\
\hline $1 \mathrm{~A}$ & S & \#\#\#\#\#\#\#\# & 91354 & 354 & 13.9 & 5.7 & 6.4 & 5.7 & 6.4 \\
\hline $0 \mathrm{~A}$ & NS & \#\#\#\#\#\#\#\# & 91355 & 355 & 12 & 8.4 & 8.4 & 8.4 & 8.4 \\
\hline $0 \mathrm{~A}$ & NS & \#\#\#\#\#\#\#\# & 91356 & 356 & 8.3 & 8.4 & 8.4 & 8.4 & 9.1 \\
\hline $1 \mathrm{~A}$ & NS & \#\#\#\#\#\#\#\# & 91357 & 357 & 8.4 & 8.4 & 8.4 & 8.4 & 8.4 \\
\hline $1 \mathrm{~A}$ & NS & \#\#\#\#\#\#\#\# & 91358 & 358 & 8.4 & 8.4 & 8.4 & 8.4 & 8.4 \\
\hline $1 \mathrm{~A}$ & NS & \#\#\#\#\#\#\#\# & 91359 & 359 & 8.4 & 8.4 & 9 & 8.4 & 8.4 \\
\hline $1 \mathrm{~A}$ & NS & \#\#\#\#\#\#\#\# & 91360 & 360 & 9 & 8.4 & 8.4 & 8.4 & 8.4 \\
\hline $1 \mathrm{~A}$ & NS & \#\#\#\#\#\#\#\# & 91361 & 361 & 8.4 & 8.4 & 8.4 & 8.4 & 8.4 \\
\hline $0 \mathrm{~A}$ & NS & \#\#\#\#\#\#\#\# & 91362 & 362 & 7.1 & 7.7 & 7.1 & 7.7 & 7.1 \\
\hline $0 \mathrm{~A}$ & NS & \#\#\#\#\#\#\#\# & 91363 & 363 & 7.1 & 7.7 & 7.1 & 7.7 & 7.7 \\
\hline $1 \mathrm{~A}$ & NS & \#\#\#\#\#\#\#\# & 91364 & 364 & 7.7 & 7.1 & 7.7 & 7.1 & 7.7 \\
\hline $1 \mathrm{~A}$ & NS & \#\#\#\#\#\#\#\# & 91365 & 365 & 7 & 7.7 & 7.7 & 7.2 & 7.7 \\
\hline $1 \mathrm{~A}$ & NS & $1 / 1 / 1992$ & 92001 & 1 & 7.6 & 7.7 & 7.7 & 7.7 & 7.7 \\
\hline $1 \mathrm{~A}$ & NS & $1 / 2 / 1992$ & 92002 & 2 & 7.7 & 7.7 & 7.7 & 7.7 & 7.7 \\
\hline $1 \mathrm{~A}$ & NS & $1 / 3 / 1992$ & 92003 & 3 & 7.6 & 8.2 & 7.6 & 8.2 & 8.2 \\
\hline $0 \mathrm{~A}$ & NS & 1/4/1992 & 92004 & 4 & 7.8 & 7.9 & 7.9 & 7.9 & 7.9 \\
\hline $0 \mathrm{~A}$ & NS & 1/5/1992 & 92005 & 5 & 7 & 7.7 & 7 & 7 & 7.6 \\
\hline $1 \mathrm{~A}$ & $S$ & 1/6/1992 & 92006 & 6 & 6 & 6 & 6.7 & 6 & 6.6 \\
\hline
\end{tabular}


lights

\begin{tabular}{|c|c|c|c|c|c|c|c|c|c|}
\hline $1 \mathrm{~A}$ & $\mathrm{~S}$ & $1 / 7 / 1992$ & 92007 & 7 & 16.4 & 7.9 & 7.9 & 8.5 & 8 \\
\hline $1 \mathrm{~A}$ & $\mathrm{~S}$ & $1 / 8 / 1992$ & 92008 & 8 & 10.4 & 6.8 & 6.7 & 6.2 & 6.8 \\
\hline $1 \mathrm{~A}$ & $S$ & 1/9/1992 & 92009 & 9 & 11.2 & 6.4 & 6.3 & 6.9 & 6.4 \\
\hline $1 \mathrm{~A}$ & $\mathrm{~S}$ & $1 / 10 / 1992$ & 92010 & 10 & 14.9 & 6.9 & 6.9 & 7 & 6.9 \\
\hline $0 \mathrm{~A}$ & $\mathrm{~S}$ & $1 / 11 / 1992$ & 92011 & 11 & 14.5 & 5.8 & 5.7 & 5.7 & 5.8 \\
\hline $0 \mathrm{~A}$ & $S$ & $1 / 12 / 1992$ & 92012 & 12 & 5.7 & 6.4 & 5.7 & 5.8 & 5.7 \\
\hline $1 \mathrm{~A}$ & $\mathrm{~S}$ & $1 / 13 / 1992$ & 92013 & 13 & 5.7 & 5.8 & 5.7 & 5.8 & 5.7 \\
\hline $1 \mathrm{~A}$ & $S$ & $1 / 14 / 1992$ & 92014 & 14 & 8.7 & 6.3 & 7 & 6.9 & 6.4 \\
\hline $1 \mathrm{~A}$ & $S$ & $1 / 15 / 1992$ & 92015 & 15 & 16.2 & 8.7 & 8.1 & 8.7 & 8.7 \\
\hline $1 \mathrm{~A}$ & $S$ & $1 / 16 / 1992$ & 92016 & 16 & 18.8 & 7.3 & 7.4 & 7.3 & 7.3 \\
\hline $1 \mathrm{~A}$ & $S$ & 1/17/1992 & 92017 & 17 & 31.4 & 8.9 & 7.1 & 6.5 & 7.2 \\
\hline $0 \mathrm{~A}$ & $\mathrm{~S}$ & $1 / 18 / 1992$ & 92018 & 18 & 17.3 & 7.3 & 7 & 7 & 7.5 \\
\hline $0 \mathrm{~A}$ & $\mathrm{~S}$ & $1 / 19 / 1992$ & 92019 & 19 & 6.3 & 6.4 & 6.3 & 7 & 6.3 \\
\hline $1 \mathrm{~A}$ & NS & $1 / 20 / 1992$ & 92020 & 20 & 6.4 & 6.3 & 6.4 & 6.9 & 6.4 \\
\hline $1 \mathrm{~A}$ & $S$ & $1 / 21 / 1992$ & 92021 & 21 & 6.3 & 6.4 & 6.3 & 6.4 & 6.3 \\
\hline $1 \mathrm{~A}$ & $S$ & $1 / 22 / 1992$ & 92022 & 22 & 17.1 & 8.2 & 8.1 & 8.1 & 7.5 \\
\hline $1 \mathrm{~A}$ & $S$ & $1 / 23 / 1992$ & 92023 & 23 & 11.5 & 7.9 & 7.3 & 8 & 7.3 \\
\hline $1 \mathrm{~A}$ & $S$ & $1 / 24 / 1992$ & 92024 & 24 & 18.7 & 13.4 & 12.8 & 12.8 & 12.7 \\
\hline $0 \mathrm{~A}$ & $S$ & $1 / 25 / 1992$ & 92025 & 25 & 19.2 & 6.6 & 6.7 & 6.7 & 7.3 \\
\hline $0 \mathrm{~A}$ & $S$ & $1 / 26 / 1992$ & 92026 & 26 & 6.6 & 6.7 & 6.7 & 6.6 & 6.7 \\
\hline $1 \mathrm{~A}$ & $S$ & $1 / 27 / 1992$ & 92027 & 27 & 6.6 & 6.7 & 6.7 & 6.7 & 6.6 \\
\hline $1 \mathrm{~A}$ & $\mathrm{~S}$ & $1 / 28 / 1992$ & 92028 & 28 & 19.2 & 7.6 & 7.7 & 7.6 & 7.7 \\
\hline $1 \mathrm{~A}$ & $S$ & 1/29/1992 & 92029 & 29 & 15.5 & 7.6 & 7.6 & 8.3 & 7.6 \\
\hline $1 \mathrm{~A}$ & $S$ & $1 / 30 / 1992$ & 92030 & 30 & 15.4 & 7.8 & 7.9 & 7.3 & 7.8 \\
\hline $1 \mathrm{~A}$ & NS & 1/31/1992 & 92031 & 31 & 27.8 & 8.4 & 9 & 8.4 & 9.1 \\
\hline $0 \mathrm{~A}$ & S & 2/1/1992 & 92032 & 32 & 13.1 & 7.3 & 7.3 & 7.2 & 7.3 \\
\hline $0 \mathrm{~A}$ & $S$ & $2 / 2 / 1992$ & 92033 & 33 & 6.6 & 6 & 6.7 & 6.7 & 6.6 \\
\hline $1 \mathrm{~A}$ & $S$ & $2 / 3 / 1992$ & 92034 & 34 & 6.6 & 6.1 & 6 & 6.1 & 6 \\
\hline $1 \mathrm{~A}$ & $S$ & 2/4/1992 & 92035 & 35 & 24.7 & 7.1 & 7.1 & 7.1 & 7.1 \\
\hline $1 \mathrm{~A}$ & $\mathrm{~S}$ & 2/5/1992 & 92036 & 36 & 14.5 & 9.1 & 9 & 9.1 & 9 \\
\hline $1 \mathrm{~A}$ & $S$ & 2/6/1992 & 92037 & 37 & 16.3 & 6.6 & 7.3 & 7.3 & 7.2 \\
\hline $1 \mathrm{~A}$ & $S$ & $2 / 7 / 1992$ & 92038 & 38 & 27.7 & 7.1 & 7.1 & 7.1 & 7.1 \\
\hline $0 \mathrm{~A}$ & S & 2/8/1992 & 92039 & 39 & 16.6 & 6.5 & 5.9 & 6.5 & 6.5 \\
\hline $0 \mathrm{~A}$ & $S$ & 2/9/1992 & 92040 & 40 & 5.9 & 6.5 & 6.5 & 6.5 & 6.5 \\
\hline $1 \mathrm{~A}$ & $S$ & $2 / 10 / 1992$ & 92041 & 41 & 6.5 & 6.5 & 6.5 & 6.5 & 6.5 \\
\hline $1 \mathrm{~A}$ & $S$ & 2/11/1992 & 92042 & 42 & 11 & 8.6 & 8.6 & 9.2 & 8.6 \\
\hline $1 \mathrm{~A}$ & $S$ & 2/12/1992 & 92043 & 43 & 14.6 & 8.8 & 8.3 & 8.2 & 8.2 \\
\hline $1 \mathrm{~A}$ & $S$ & 2/13/1992 & 92044 & 44 & 19.2 & 7 & 7.1 & 7.1 & 7.1 \\
\hline $1 \mathrm{~A}$ & $\mathrm{~S}$ & $2 / 14 / 1992$ & 92045 & 45 & 34.1 & 7.1 & 7 & 7.1 & 7.1 \\
\hline $0 \mathrm{~A}$ & $S$ & 2/15/1992 & 92046 & 46 & 18.9 & 7.3 & 7.3 & 7.2 & 7.3 \\
\hline $0 \mathrm{~A}$ & $S$ & $2 / 16 / 1992$ & 92047 & 47 & 7.3 & 7.2 & 7.3 & 7.3 & 7.2 \\
\hline $1 \mathrm{~A}$ & $S$ & $2 / 17 / 1992$ & 92048 & 48 & 7.3 & 7.3 & 7.2 & 7.3 & 7.3 \\
\hline $1 \mathrm{~A}$ & $S$ & 2/18/1992 & 92049 & 49 & 19.2 & 7.1 & 7.1 & 7.1 & 7.1 \\
\hline $1 \mathrm{~A}$ & $S$ & 2/19/1992 & 92050 & 50 & 27.4 & 7.4 & 7.3 & 7.4 & 7.4 \\
\hline $1 \mathrm{~A}$ & $S$ & 2/20/1992 & 92051 & 51 & 19.2 & 8.2 & 8.8 & 8.2 & 8.8 \\
\hline $1 \mathrm{~A}$ & $S$ & 2/21/1992 & 92052 & 52 & 11 & 7.2 & 7.3 & 7.2 & 7.8 \\
\hline $0 \mathrm{~A}$ & $S$ & $2 / 22 / 1992$ & 92053 & 53 & 17.4 & 7.8 & 7.3 & 7.8 & 7.8 \\
\hline $0 \mathrm{~A}$ & $\mathrm{~S}$ & 2/23/1992 & 92054 & 54 & 7.8 & 7.8 & 7.2 & 7.9 & 7.8 \\
\hline $1 \mathrm{~A}$ & $S$ & $2 / 24 / 1992$ & 92055 & 55 & 7.8 & 7.8 & 8.4 & 7.9 & 7.8 \\
\hline $1 \mathrm{~A}$ & $S$ & $2 / 25 / 1992$ & 92056 & 56 & 14.3 & 9 & 9 & 9 & 9 \\
\hline $1 \mathrm{~A}$ & $S$ & $2 / 26 / 1992$ & 92057 & 57 & 15 & 8.2 & 8 & 8.1 & 8.1 \\
\hline
\end{tabular}


lights

\begin{tabular}{|c|c|c|c|c|c|c|c|c|c|}
\hline $1 \mathrm{~A}$ & $S$ & $2 / 27 / 1992$ & 92058 & 58 & 27.4 & 8.8 & 8.2 & 8.1 & 8.2 \\
\hline $1 \mathrm{~A}$ & S & 2/28/1992 & 92059 & 59 & 23.2 & 13.2 & 14.1 & 13.6 & 13.5 \\
\hline $0 \mathrm{~A}$ & S & $2 / 29 / 1992$ & 92060 & 60 & 14.1 & 8.6 & 8 & 8.6 & 8.6 \\
\hline $0 \mathrm{~A}$ & $\mathrm{~S}$ & 3/1/1992 & 92061 & 61 & 8.7 & 8.7 & 8.1 & 8.7 & 8.7 \\
\hline $1 \mathrm{~A}$ & S & $3 / 2 / 1992$ & 92062 & 62 & 8.7 & 8.1 & 8.7 & 8.7 & 8.7 \\
\hline $1 \mathrm{~A}$ & $\mathrm{~S}$ & $3 / 3 / 1992$ & 92063 & 63 & 20.9 & 8.2 & 8.8 & 8.1 & 8.8 \\
\hline $1 \mathrm{~A}$ & $\mathrm{~S}$ & $3 / 4 / 1992$ & 92064 & 64 & 18.9 & 7.8 & 7.8 & 7.8 & 7.8 \\
\hline $1 \mathrm{~A}$ & S & $3 / 5 / 1992$ & 92065 & 65 & 22.7 & 8 & 8 & 7.9 & 8 \\
\hline $1 \mathrm{~A}$ & S & $3 / 6 / 1992$ & 92066 & 66 & 18.2 & 9 & 9.1 & 9.1 & 9.1 \\
\hline $0 \mathrm{~A}$ & S & $3 / 7 / 1992$ & 92067 & 67 & 10.1 & 8.9 & 8.8 & 8.9 & 8.9 \\
\hline $0 \mathrm{~A}$ & $\mathrm{~S}$ & $3 / 8 / 1992$ & 92068 & 68 & 8.3 & 8.2 & 8.2 & 8.3 & 8.2 \\
\hline $1 \mathrm{~A}$ & $S$ & 3/9/1992 & 92069 & 69 & 8.3 & 7.6 & 8.3 & 8.3 & 8.3 \\
\hline $1 \mathrm{~A}$ & S & 3/10/1992 & 92070 & 70 & 11.7 & 7.2 & 7.3 & 7.2 & 7.3 \\
\hline $1 \mathrm{~A}$ & S & $3 / 11 / 1992$ & 92071 & 71 & 17 & 7.4 & 7.4 & 7.4 & 7.4 \\
\hline $1 \mathrm{~A}$ & $\mathrm{~S}$ & $3 / 12 / 1992$ & 92072 & 72 & 20.1 & 7.2 & 7.2 & 7.2 & 7.2 \\
\hline $1 \mathrm{~A}$ & $\mathrm{~S}$ & $3 / 13 / 1992$ & 92073 & 73 & 15.9 & 8.1 & 8.2 & 8.2 & 8.2 \\
\hline $0 \mathrm{~A}$ & NS & 3/14/1992 & 92074 & 74 & 13.5 & 8 & 8.6 & 8 & 8.6 \\
\hline $0 \mathrm{~A}$ & NS & 3/15/1992 & 92075 & 75 & 8 & 7.9 & 8 & 8 & 8 \\
\hline $1 \mathrm{~A}$ & NS & 3/16/1992 & 92076 & 76 & 7.9 & 8 & 8 & 7.9 & 8 \\
\hline $1 \mathrm{~A}$ & NS & 3/17/1992 & 92077 & 77 & 8 & 7.4 & 7.9 & 7.5 & 8 \\
\hline $1 \mathrm{~A}$ & NS & 3/18/1992 & 92078 & 78 & 7.9 & 7.3 & 7.3 & 7.3 & 7.3 \\
\hline $1 \mathrm{~A}$ & NS & 3/19/1992 & 92079 & 79 & 7.3 & 7.3 & 7.3 & 7.9 & 7.4 \\
\hline $1 \mathrm{~A}$ & NS & $3 / 20 / 1992$ & 92080 & 80 & 7.2 & 7.3 & 7.3 & 7.3 & 7.3 \\
\hline $0 \mathrm{~A}$ & NS & 3/21/1992 & 92081 & 81 & 7.3 & 7.3 & 7.2 & 7.3 & 7.3 \\
\hline $0 \mathrm{~A}$ & NS & $3 / 22 / 1992$ & 92082 & 82 & 7.2 & 7.3 & 7.3 & 7.4 & 7.9 \\
\hline $1 \mathrm{~A}$ & $\mathrm{~S}$ & 3/23/1992 & 92083 & 83 & 7.2 & 7.3 & 7.3 & 7.3 & 7.3 \\
\hline $1 \mathrm{~A}$ & S & 3/24/1992 & 92084 & 84 & 21.6 & 7.9 & 7.9 & 8 & 7.9 \\
\hline $1 \mathrm{~A}$ & S & $3 / 25 / 1992$ & 92085 & 85 & 20.5 & 7.4 & 7.3 & 7.4 & 7.4 \\
\hline $1 \mathrm{~A}$ & $S$ & 3/26/1992 & 92086 & 86 & 18.8 & 9.1 & 9.2 & 9.1 & 9.2 \\
\hline $1 \mathrm{~A}$ & $S$ & 3/27/1992 & 92087 & 87 & 12.7 & 7.4 & 8.1 & 8 & 8.1 \\
\hline $0 \mathrm{~A}$ & S & 3/28/1992 & 92088 & 88 & 15.5 & 8.4 & 8.9 & 8.9 & 8.9 \\
\hline $0 \mathrm{~A}$ & S & 3/29/1992 & 92089 & 89 & 8.3 & 8.9 & 8.9 & 8.3 & 8.9 \\
\hline $1 \mathrm{~A}$ & S & 3/30/1992 & 92090 & 90 & 8.9 & 8.9 & 9 & 8.3 & 9 \\
\hline $1 \mathrm{~A}$ & S & $3 / 31 / 1992$ & 92091 & 91 & 17.7 & 9.6 & 9 & 9.7 & 9 \\
\hline $1 \mathrm{~A}$ & S & 4/1/1992 & 92092 & 92 & 13.1 & 8 & 8.6 & 7.9 & 8 \\
\hline $1 \mathrm{~A}$ & S & 4/2/1992 & 92093 & 93 & 19.6 & 9.8 & 9.8 & 9.2 & 9.8 \\
\hline $1 \mathrm{~A}$ & S & $4 / 3 / 1992$ & 92094 & 94 & 15 & 7.2 & 7.2 & 7.2 & 7.2 \\
\hline $0 \mathrm{~A}$ & $S$ & 4/4/1992 & 92095 & 95 & 12.3 & 8.2 & 8.9 & 8.3 & 8.8 \\
\hline $0 \mathrm{~A}$ & $S$ & 4/5/1992 & 92096 & 96 & 8.2 & 8.2 & 8.3 & 8.2 & 8.3 \\
\hline $1 \mathrm{~A}$ & S & 4/6/1992 & 92097 & 97 & 7.7 & 8.3 & 8.3 & 8.2 & 8.3 \\
\hline $1 \mathrm{~A}$ & S & 4/7/1992 & 92098 & 98 & 9.5 & 10.1 & 10.1 & 10.2 & 10.1 \\
\hline $1 \mathrm{~A}$ & S & 4/8/1992 & 92099 & 99 & 45.3 & 45.2 & 41.2 & 18.4 & 10.3 \\
\hline $1 \mathrm{~A}$ & $\mathrm{~S}$ & 4/9/1992 & 92100 & 100 & 23.3 & 7.3 & 8 & 8 & 7.9 \\
\hline $1 \mathrm{~A}$ & S & 4/10/1992 & 92101 & 101 & 16.5 & 7.4 & 7.3 & 7.4 & 7.4 \\
\hline $0 \mathrm{~A}$ & S & 4/11/1992 & 92102 & 102 & 19.2 & 10.2 & 9.5 & 9.6 & 9.6 \\
\hline $0 \mathrm{~A}$ & S & 4/12/1992 & 92103 & 103 & 9.7 & 9.6 & 9.7 & 9.7 & 9.8 \\
\hline $1 \mathrm{~A}$ & S & 4/13/1992 & 92104 & 104 & 10.5 & 10.6 & 11.1 & 10.5 & 10.5 \\
\hline $1 \mathrm{~A}$ & $S$ & 4/14/1992 & 92105 & 105 & 14.9 & 9.3 & 8.8 & 9.3 & 8.8 \\
\hline $1 \mathrm{~A}$ & S & 4/15/1992 & 92106 & 106 & 13.6 & 8.2 & 8.2 & 8.9 & 8.8 \\
\hline $1 \mathrm{~A}$ & S & 4/16/1992 & 92107 & 107 & 16.4 & 8.7 & 8.7 & 8.8 & 8.7 \\
\hline $1 \mathrm{~A}$ & $\mathrm{~S}$ & 4/17/1992 & 92108 & 108 & 11.1 & 7.1 & 7.2 & 7.1 & 6.6 \\
\hline
\end{tabular}


lights

\begin{tabular}{|c|c|c|c|c|c|c|c|c|c|}
\hline \begin{tabular}{l|l}
0 & $A$ \\
\end{tabular} & $S$ & 4/18/1992 & 92109 & 109 & 7.2 & 6.5 & 7.2 & 7.2 & 7.2 \\
\hline $\begin{array}{ll}\mathrm{A} \\
\end{array}$ & $\mathrm{S}$ & 4/19/1992 & 92110 & 110 & 6.5 & 7.2 & 7.2 & 7.1 & 6.6 \\
\hline $1 \mathrm{~A}$ & S & $4 / 20 / 1992$ & 92111 & 111 & 7.2 & 6.6 & 7.2 & 7.2 & 7.2 \\
\hline $1 \mathrm{~A}$ & $\mathrm{~S}$ & 4/21/1992 & 92112 & 112 & 14.5 & 8.1 & 8.2 & 8.2 & 8.2 \\
\hline $1 \mathrm{~A}$ & S & $4 / 22 / 1992$ & 92113 & 113 & 18.8 & 10 & 9.4 & 10.1 & 9.4 \\
\hline $1 \mathrm{~A}$ & S & 4/23/1992 & 92114 & 114 & 29.3 & 8.2 & 7.8 & 8.3 & 8.3 \\
\hline $1 \mathrm{~A}$ & $\mathrm{~S}$ & $4 / 24 / 1992$ & 92115 & 115 & 14.3 & 8 & 7.9 & 7.9 & 7.4 \\
\hline $0 \mathrm{~A}$ & S & 4/25/1992 & 92116 & 116 & 16.6 & 8.9 & 9 & 9.5 & 9 \\
\hline $0 \mathrm{~A}$ & S & 4/26/1992 & 92117 & 117 & 7.9 & 8 & 8 & 8 & 8 \\
\hline $1 \mathrm{~A}$ & S & 4/27/1992 & 92118 & 118 & 7.9 & 7.4 & 8 & 8 & 8 \\
\hline $1 \mathrm{~A}$ & S & $4 / 28 / 1992$ & 92119 & 119 & 16.6 & 8.9 & 9 & 9 & 8.9 \\
\hline $1 \mathrm{~A}$ & S & 4/29/1992 & 92120 & 120 & 7.9 & 8.1 & 8.7 & 8.1 & 8.1 \\
\hline $1 \mathrm{~A}$ & S & 4/30/1992 & 92121 & 121 & 20.1 & 15.8 & 15.8 & 15.9 & 15.6 \\
\hline $1 \mathrm{~A}$ & S & $5 / 1 / 1992$ & 92122 & 122 & 21.6 & 9.9 & 9.3 & 9.9 & 9.9 \\
\hline $0 \mathrm{~A}$ & $\mathrm{~S}$ & $5 / 2 / 1992$ & 92123 & 123 & 14.1 & 7.3 & 7.9 & 8 & 7.4 \\
\hline $0 \mathrm{~A}$ & S & $5 / 3 / 1992$ & 92124 & 124 & 8.1 & 7.5 & 8.1 & 8.1 & 7.5 \\
\hline $1 \mathrm{~A}$ & S & $5 / 4 / 1992$ & 92125 & 125 & 8 & 7.9 & 7.3 & 8 & 8 \\
\hline $1 \mathrm{~A}$ & S & $5 / 5 / 1992$ & 92126 & 126 & 17.6 & 9.5 & 9.5 & 9.5 & 9.5 \\
\hline $1 \mathrm{~A}$ & S & $5 / 6 / 1992$ & 92127 & 127 & 34.9 & 34.9 & 35.7 & 28.8 & 21.7 \\
\hline $1 \mathrm{~A}$ & S & $5 / 7 / 1992$ & 92128 & 128 & 30 & 29.7 & 29.7 & 29.6 & 15 \\
\hline $1 \mathrm{~A}$ & S & $5 / 8 / 1992$ & 92129 & 129 & 30.1 & 26.5 & 16.4 & 7.9 & 8.5 \\
\hline $0 \mathrm{~A}$ & S & $5 / 9 / 1992$ & 92130 & 130 & 6.4 & 7.1 & 7.1 & 6.5 & 7.1 \\
\hline $0 \mathrm{~A}$ & S & $5 / 10 / 1992$ & 92131 & 131 & 7.1 & 7 & 6.5 & 7.1 & 7.1 \\
\hline $1 \mathrm{~A}$ & $\mathrm{~S}$ & $5 / 11 / 1992$ & 92132 & 132 & 6.4 & 7.1 & 7.1 & 6.4 & 7.1 \\
\hline $1 \mathrm{~A}$ & S & $5 / 12 / 1992$ & 92133 & 133 & 12.2 & 7.8 & 7.8 & 7.8 & 7.2 \\
\hline $1 \mathrm{~A}$ & S & $5 / 13 / 1992$ & 92134 & 134 & 19.3 & 7 & 7 & 6.9 & 7 \\
\hline $1 \mathrm{~A}$ & S & $5 / 14 / 1992$ & 92135 & 135 & 11.4 & 7.9 & 8.6 & 8 & 7.9 \\
\hline $1 \mathrm{~A}$ & S & $5 / 15 / 1992$ & 92136 & 136 & 15.6 & 7 & 6.9 & 7 & 7 \\
\hline $0 \mathrm{~A}$ & S & $5 / 16 / 1992$ & 92137 & 137 & 18.1 & 7.3 & 7.2 & 7.3 & 7.2 \\
\hline $0 \mathrm{~A}$ & S & 5/17/1992 & 92138 & 138 & 7.1 & 6.5 & 6.4 & 6.5 & 6.5 \\
\hline $1 \mathrm{~A}$ & S & $5 / 18 / 1992$ & 92139 & 139 & 7.7 & 7.7 & 7.7 & 7.7 & 7.7 \\
\hline $1 \mathrm{~A}$ & S & $5 / 19 / 1992$ & 92140 & 140 & 32.8 & 12.6 & 7 & 7 & 7 \\
\hline $1 \mathrm{~A}$ & S & $5 / 20 / 1992$ & 92141 & 141 & 12 & 7.9 & 8 & 7.3 & 8 \\
\hline $1 \mathrm{~A}$ & S & $5 / 21 / 1992$ & 92142 & 142 & 19.4 & 15.8 & 16.3 & 15.9 & 16.2 \\
\hline $1 \mathrm{~A}$ & S & $5 / 22 / 1992$ & 92143 & 143 & 23 & 16 & 16 & 16 & 16 \\
\hline $0 \mathrm{~A}$ & S & $5 / 23 / 1992$ & 92144 & 144 & 14.8 & 8.9 & 8.9 & 8.9 & 9.5 \\
\hline $0 \mathrm{~A}$ & $\mathrm{~S}$ & $5 / 24 / 1992$ & 92145 & 145 & 8.9 & 9.5 & 8.9 & 8.9 & 8.8 \\
\hline $1 \mathrm{~A}$ & S & $5 / 25 / 1992$ & 92146 & 146 & 8.9 & 8.9 & 9.5 & 8.9 & 8.9 \\
\hline $1 \mathrm{~A}$ & $S$ & $5 / 26 / 1992$ & 92147 & 147 & 15.2 & 8.9 & 8.9 & 8.9 & 9 \\
\hline $1 \mathrm{~A}$ & S & $5 / 27 / 1992$ & 92148 & 148 & 19.5 & 9.1 & 9.2 & 9.2 & 9.1 \\
\hline $1 \mathrm{~A}$ & $\mathrm{~S}$ & $5 / 28 / 1992$ & 92149 & 149 & 10.2 & 9 & 8.4 & 8.9 & 9 \\
\hline $1 \mathrm{~A}$ & S & $5 / 29 / 1992$ & 92150 & 150 & 16.2 & 7.9 & 8.6 & 8.6 & 7.9 \\
\hline $0 \mathrm{~A}$ & S & $5 / 30 / 1992$ & 92151 & 151 & 12.7 & 8.8 & 8.1 & 8.7 & 8.8 \\
\hline $0 \mathrm{~A}$ & S & 5/31/1992 & 92152 & 152 & 8.7 & 8.7 & 8.2 & 8.7 & 8.1 \\
\hline $1 \mathrm{~A}$ & S & 6/1/1992 & 92153 & 153 & 8.7 & 8.1 & 8.7 & 8.8 & 8.1 \\
\hline $1 \mathrm{~A}$ & S & $6 / 2 / 1992$ & 92154 & 154 & 16 & 7.9 & 8 & 7.3 & 8 \\
\hline $1 \mathrm{~A}$ & S & 6/3/1992 & 92155 & 155 & 23.7 & 15.1 & 15.1 & 15.1 & 14.4 \\
\hline $1 \mathrm{~A}$ & $S$ & $6 / 4 / 1992$ & 92156 & 156 & 24.5 & 14.8 & 14.9 & 14.9 & 15.6 \\
\hline $1 \mathrm{~A}$ & S & $6 / 5 / 1992$ & 92157 & 157 & 16.8 & 17.4 & 16.8 & 17.4 & 16.9 \\
\hline $0 \mathrm{~A}$ & $\mathrm{~S}$ & 6/6/1992 & 92158 & 158 & 14.4 & 13.8 & 14.4 & 13.8 & 14.3 \\
\hline $0 \mathrm{~A}$ & S & 6/7/1992 & 92159 & 159 & 15.4 & 15.2 & 15.3 & 15.3 & 15.4 \\
\hline
\end{tabular}


lights

\begin{tabular}{|c|c|c|c|c|c|c|c|c|c|}
\hline $1 \mathrm{~A}$ & $S$ & $6 / 8 / 1992$ & 92160 & 160 & 15.3 & 15.3 & 15.3 & 15.4 & 15.4 \\
\hline $1 \mathrm{~A}$ & $\mathrm{~S}$ & 6/9/1992 & 92161 & 161 & 15.5 & 15.8 & 15.2 & 15.7 & 15.8 \\
\hline $1 \mathrm{~A}$ & S & 6/10/1992 & 92162 & 162 & 17.6 & 17.1 & 17.7 & 17.7 & 17.2 \\
\hline $1 \mathrm{~A}$ & S & 6/11/1992 & 92163 & 163 & 17.3 & 18 & 18 & 17 & 17.7 \\
\hline $1 \mathrm{~A}$ & S & $6 / 12 / 1992$ & 92164 & 164 & 16.1 & 16.4 & 16.6 & 16.9 & 16.7 \\
\hline $\begin{array}{ll}\mathrm{A} \\
\end{array}$ & $\mathrm{S}$ & $6 / 13 / 1992$ & 92165 & 165 & 17.9 & 17.5 & 17.8 & 17.5 & 17.3 \\
\hline $0 \mathrm{~A}$ & NS & 6/14/1992 & 92166 & 166 & 18 & 17.9 & 18 & 18.1 & 17.6 \\
\hline $1 \mathrm{~A}$ & NS & $6 / 15 / 1992$ & 92167 & 167 & 17.7 & 17.4 & 18.4 & 18 & 18 \\
\hline $1 \mathrm{~A}$ & NS & 6/16/1992 & 92168 & 168 & 14.8 & 15.5 & 15.5 & 15 & 15.1 \\
\hline $1 \mathrm{~A}$ & NS & 6/17/1992 & 92169 & 169 & 15.4 & 15.5 & 15.6 & 15 & 15.4 \\
\hline $1 \mathrm{~A}$ & NS & 6/18/1992 & 92170 & 170 & 16.2 & 16.2 & 16 & 15.8 & 16.1 \\
\hline $1 \mathrm{~A}$ & NS & 6/19/1992 & 92171 & 171 & 14.5 & 13.9 & 14.4 & 14.3 & 14.1 \\
\hline $0 \mathrm{~A}$ & NS & 6/20/1992 & 92172 & 172 & 14.4 & 14.5 & 14.2 & 14.1 & 15 \\
\hline $0 \mathrm{~A}$ & NS & 6/21/1992 & 92173 & 173 & 14.6 & 14.6 & 14.4 & 14.8 & 14.6 \\
\hline $1 \mathrm{~A}$ & NS & $6 / 22 / 1992$ & 92174 & 174 & 14.6 & 14.5 & 14.6 & 15.2 & 14.6 \\
\hline $1 \mathrm{~A}$ & NS & $6 / 23 / 1992$ & 92175 & 175 & 14.6 & 14.8 & 14.8 & 14.2 & 14.8 \\
\hline $1 \mathrm{~A}$ & NS & $6 / 24 / 1992$ & 92176 & 176 & 13.3 & 13.6 & 13.6 & 13.7 & 14.3 \\
\hline $1 \mathrm{~A}$ & NS & 6/25/1992 & 92177 & 177 & 13.7 & 13.1 & 13.7 & 13.6 & 13.7 \\
\hline $1 \mathrm{~A}$ & NS & $6 / 26 / 1992$ & 92178 & 178 & 13.1 & 14.1 & 14.1 & 14.1 & 14.1 \\
\hline $0 \mathrm{~A}$ & NS & 6/27/1992 & 92179 & 179 & 7.1 & 7.2 & 7.1 & 6.5 & 7.2 \\
\hline $0 \mathrm{~A}$ & NS & $6 / 28 / 1992$ & 92180 & 180 & 6.6 & 7.1 & 7.2 & 7.1 & 7.2 \\
\hline $1 \mathrm{~A}$ & NS & 6/29/1992 & 92181 & 181 & 7.1 & 7.2 & 6.5 & 7.2 & 7.2 \\
\hline $1 \mathrm{~A}$ & NS & 6/30/1992 & 92182 & 182 & 15.6 & 16.2 & 15.7 & 16.1 & 15.6 \\
\hline $1 \mathrm{~A}$ & NS & 7/1/1992 & 92183 & 183 & 17.9 & 17.9 & 17.5 & 17.9 & 18.7 \\
\hline $1 \mathrm{~A}$ & NS & $7 / 2 / 1992$ & 92184 & 184 & 14.3 & 14.4 & 13.8 & 14.4 & 14.5 \\
\hline $1 \mathrm{~A}$ & NS & $7 / 3 / 1992$ & 92185 & 185 & 14.2 & 13.3 & 14.4 & 13.9 & 14.6 \\
\hline $0 \mathrm{~A}$ & NS & $7 / 4 / 1992$ & 92186 & 186 & 14.4 & 14.5 & 14 & 14.6 & 14.6 \\
\hline $0 \mathrm{~A}$ & NS & $7 / 5 / 1992$ & 92187 & 187 & 13.9 & 14.5 & 13.9 & 14.5 & 14.6 \\
\hline $1 \mathrm{~A}$ & NS & $7 / 6 / 1992$ & 92188 & 188 & 13.9 & 14.5 & 14.6 & 13.6 & 14.2 \\
\hline $1 \mathrm{~A}$ & NS & 7/7/1992 & 92189 & 189 & 7.9 & 7.9 & 8.6 & 7.9 & 8 \\
\hline $1 \mathrm{~A}$ & NS & $7 / 8 / 1992$ & 92190 & 190 & 9.2 & 9.9 & 9.3 & 10 & 9.3 \\
\hline $1 \mathrm{~A}$ & NS & 7/9/1992 & 92191 & 191 & 8.9 & 8.9 & 8.8 & 9.6 & 8.9 \\
\hline $1 \mathrm{~A}$ & NS & 7/10/1992 & 92192 & 192 & 8.3 & 8.3 & 8.3 & 8.9 & 8.3 \\
\hline $0 \mathrm{~A}$ & NS & 7/11/1992 & 92193 & 193 & 9.1 & 9.2 & 9.1 & 9.2 & 9.2 \\
\hline $0 \mathrm{~A}$ & NS & $7 / 12 / 1992$ & 92194 & 194 & 9.1 & 9.2 & 8.5 & 9.2 & 9.7 \\
\hline $1 \mathrm{~A}$ & NS & 7/13/1992 & 92195 & 195 & 9.1 & 9.2 & 9.1 & 9.2 & 9.2 \\
\hline $1 \mathrm{~A}$ & NS & 7/14/1992 & 92196 & 196 & 9.3 & 9.3 & 9.9 & 9.3 & 9.9 \\
\hline $1 \mathrm{~A}$ & NS & 7/15/1992 & 92197 & 197 & 9.3 & 9.2 & 9.3 & 9.3 & 9.3 \\
\hline $1 \mathrm{~A}$ & NS & 7/16/1992 & 92198 & 198 & 8.8 & 8.9 & 8.2 & 8.9 & 9.5 \\
\hline $1 \mathrm{~A}$ & NS & $7 / 17 / 1992$ & 92199 & 199 & 8.1 & 8.7 & 8.1 & 8.7 & 8.2 \\
\hline $0 \mathrm{~A}$ & NS & 7/18/1992 & 92200 & 200 & 8.7 & 8.7 & 8.7 & 8.8 & 8.7 \\
\hline $0 \mathrm{~A}$ & NS & 7/19/1992 & 92201 & 201 & 8.8 & 8.7 & 9.3 & 8.7 & 8.8 \\
\hline $1 \mathrm{~A}$ & NS & 7/20/1992 & 92202 & 202 & 8.7 & 8.8 & 8.7 & 8.7 & 8.7 \\
\hline $1 \mathrm{~A}$ & NS & 7/21/1992 & 92203 & 203 & 9.3 & 9.9 & 9.9 & 10 & 9.9 \\
\hline $1 \mathrm{~A}$ & NS & 7/22/1992 & 92204 & 204 & 8.3 & 8.4 & 8.3 & 8.3 & 7.7 \\
\hline $1 \mathrm{~A}$ & NS & $7 / 23 / 1992$ & 92205 & 205 & 7.1 & 7.7 & 7.7 & 7.7 & 7.7 \\
\hline $1 \mathrm{~A}$ & NS & 7/24/1992 & 92206 & 206 & 10.4 & 10.5 & 11 & 10.5 & 10.4 \\
\hline $0 \mathrm{~A}$ & NS & 7/25/1992 & 92207 & 207 & 10 & 10.6 & 10.1 & 10 & 10.1 \\
\hline $0 \mathrm{~A}$ & NS & $7 / 26 / 1992$ & 92208 & 208 & 10.1 & 10 & 10 & 10 & 10.1 \\
\hline $1 \mathrm{~A}$ & NS & $7 / 27 / 1992$ & 92209 & 209 & 10 & 10 & 10 & 10.1 & 10 \\
\hline $1 \mathrm{~A}$ & NS & $7 / 28 / 1992$ & 92210 & 210 & 8.3 & 7.7 & 8.3 & 8.3 & 7.7 \\
\hline
\end{tabular}


lights

\begin{tabular}{|c|c|c|c|c|c|c|c|c|c|}
\hline $1 \mathrm{~A}$ & NS & $7 / 29 / 1992$ & 92211 & 211 & 9.1 & 9.2 & 9.2 & 9.2 & 9.2 \\
\hline $1 \mathrm{~A}$ & NS & $7 / 30 / 1992$ & 92212 & 212 & 10.7 & 11.2 & 11.3 & 10.7 & 11.3 \\
\hline $1 \mathrm{~A}$ & NS & $7 / 31 / 1992$ & 92213 & 213 & 8.3 & 8.3 & 8.9 & 8.3 & 8.4 \\
\hline $0 \mathrm{~A}$ & NS & 8/1/1992 & 92214 & 214 & 7.3 & 6.7 & 7.3 & 7.3 & 6.7 \\
\hline $0 \mathrm{~A}$ & NS & $8 / 2 / 1992$ & 92215 & 215 & 6.6 & 7.3 & 7.3 & 6.7 & 7.3 \\
\hline $1 \mathrm{~A}$ & NS & $8 / 3 / 1992$ & 92216 & 216 & 7.3 & 7.3 & 7.2 & 6.7 & 7.3 \\
\hline $1 \mathrm{~A}$ & NS & 8/4/1992 & 92217 & 217 & 7.1 & 7.1 & 6.5 & 7.1 & 7.1 \\
\hline $1 \mathrm{~A}$ & NS & $8 / 5 / 1992$ & 92218 & 218 & 7.7 & 7.2 & 7.7 & 7.1 & 7.8 \\
\hline $1 \mathrm{~A}$ & NS & 8/6/1992 & 92219 & 219 & 7.8 & 7.1 & 7.7 & 7.8 & 7.8 \\
\hline $1 \mathrm{~A}$ & NS & $8 / 7 / 1992$ & 92220 & 220 & 8.2 & 8.3 & 8.3 & 8.3 & 8.9 \\
\hline $0 \mathrm{~A}$ & NS & 8/8/1992 & 92221 & 221 & 8.1 & 8.1 & 8.1 & 8.1 & 8 \\
\hline $0 \mathrm{~A}$ & NS & 8/9/1992 & 92222 & 222 & 8.6 & 8.7 & 9.3 & 8.7 & 9.3 \\
\hline $1 \mathrm{~A}$ & NS & 8/10/1992 & 92223 & 223 & 8.1 & 8.7 & 8.1 & 8 & 8.7 \\
\hline $1 \mathrm{~A}$ & NS & $8 / 11 / 1992$ & 92224 & 224 & 14.6 & 14.7 & 14 & 14.7 & 14.6 \\
\hline $1 \mathrm{~A}$ & NS & 8/12/1992 & 92225 & 225 & 8.5 & 8.4 & 8.5 & 8.4 & 7.9 \\
\hline $1 \mathrm{~A}$ & NS & 8/13/1992 & 92226 & 226 & 8.7 & 8.7 & 8.6 & 8.7 & 9.3 \\
\hline $1 \mathrm{~A}$ & $\mathrm{~S}$ & 8/14/1992 & 92227 & 227 & 7.3 & 7.3 & 6.7 & 7.4 & 7.3 \\
\hline $0 \mathrm{~A}$ & S & 8/15/1992 & 92228 & 228 & 9.2 & 9.7 & 9.8 & 9.8 & 9.8 \\
\hline $0 \mathrm{~A}$ & S & 8/16/1992 & 92229 & 229 & 11.5 & 9.2 & 8.5 & 8.6 & 9.1 \\
\hline $1 \mathrm{~A}$ & $\mathrm{~S}$ & 8/17/1992 & 92230 & 230 & 8.5 & 9.2 & 8.6 & 8.5 & 9.2 \\
\hline $1 \mathrm{~A}$ & $S$ & 8/18/1992 & 92231 & 231 & 9.7 & 10.4 & 9.7 & 10.4 & 9.7 \\
\hline $1 \mathrm{~A}$ & S & 8/19/1992 & 92232 & 232 & 10.8 & 10.8 & 10.8 & 10.7 & 10.8 \\
\hline $1 \mathrm{~A}$ & $\mathrm{~S}$ & $8 / 20 / 1992$ & 92233 & 233 & 7 & 7.5 & 7.6 & 7 & 7.6 \\
\hline $1 \mathrm{~A}$ & S & 8/21/1992 & 92234 & 234 & 7.7 & 8.4 & 7.8 & 7.7 & 8.4 \\
\hline $0 \mathrm{~A}$ & $\mathrm{~S}$ & $8 / 22 / 1992$ & 92235 & 235 & 8.3 & 8.3 & 8.3 & 8.4 & 8.3 \\
\hline $0 \mathrm{~A}$ & S & 8/23/1992 & 92236 & 236 & 9.5 & 8.8 & 8.8 & 9.5 & 8.9 \\
\hline $1 \mathrm{~A}$ & S & 8/24/1992 & 92237 & 237 & 7.9 & 7.3 & 7.9 & 8 & 7.9 \\
\hline $1 \mathrm{~A}$ & S & 8/25/1992 & 92238 & 238 & 21.2 & 15.3 & 15.4 & 14.7 & 15.2 \\
\hline $1 \mathrm{~A}$ & $S$ & 8/26/1992 & 92239 & 239 & 16.3 & 7.4 & 6.9 & 7.4 & 7.4 \\
\hline $1 \mathrm{~A}$ & $S$ & 8/27/1992 & 92240 & 240 & 16.8 & 7.7 & 7.2 & 7.8 & 7.8 \\
\hline $1 \mathrm{~A}$ & S & 8/28/1992 & 92241 & 241 & 15.2 & 9.3 & 9.3 & 9.9 & 9.3 \\
\hline $0 \mathrm{~A}$ & S & 8/29/1992 & 92242 & 242 & 22.9 & 14.6 & 14.4 & 14.5 & 14.4 \\
\hline $0 \mathrm{~A}$ & S & 8/30/1992 & 92243 & 243 & 13.8 & 13.9 & 13.4 & 13.2 & 13.9 \\
\hline $1 \mathrm{~A}$ & S & 8/31/1992 & 92244 & 244 & 13.3 & 13.8 & 13.9 & 13.9 & 13.3 \\
\hline $1 \mathrm{~A}$ & S & 9/1/1992 & 92245 & 245 & 18.6 & 7.9 & 8.6 & 8.5 & 7.9 \\
\hline $1 \mathrm{~A}$ & S & 9/2/1992 & 92246 & 246 & 24.2 & 15.5 & 15.5 & 15.6 & 15.5 \\
\hline $1 \mathrm{~A}$ & S & 9/3/1992 & 92247 & 247 & 18.5 & 9.7 & 9.2 & 9.1 & 9.7 \\
\hline $1 \mathrm{~A}$ & S & 9/4/1992 & 92248 & 248 & 12.2 & 9.1 & 9.7 & 9.2 & 9.7 \\
\hline $0 \mathrm{~A}$ & $S$ & 9/5/1992 & 92249 & 249 & 18.7 & 8.2 & 7.6 & 8.2 & 7.6 \\
\hline $0 A$ & S & 9/6/1992 & 92250 & 250 & 7.6 & 7.7 & 8.2 & 8.2 & 7.7 \\
\hline $1 \mathrm{~A}$ & NS & 9/7/1992 & 92251 & 251 & 8.2 & 7.6 & 8.2 & 7.6 & 8.2 \\
\hline $1 A$ & S & 9/8/1992 & 92252 & 252 & 8.2 & 7.6 & 8.2 & 7.7 & 8.2 \\
\hline $1 \mathrm{~A}$ & S & 9/9/1992 & 92253 & 253 & 17.1 & 9 & 9.6 & 9.6 & 9.7 \\
\hline $1 A$ & S & 9/10/1992 & 92254 & 254 & 17 & 9.2 & 9.2 & 8.7 & 9.3 \\
\hline $1 \mathrm{~A}$ & S & 9/11/1992 & 92255 & 255 & 27.1 & 14.9 & 15.5 & 14.9 & 15.6 \\
\hline $0 A$ & S & 9/12/1992 & 92256 & 256 & 16.8 & 8.4 & 7.9 & 8.4 & 7.8 \\
\hline $0 A$ & S & 9/13/1992 & 92257 & 257 & 7.2 & 7.9 & 7.8 & 7.3 & 7.8 \\
\hline $1 A$ & $S$ & 9/14/1992 & 92258 & 258 & 7.8 & 7.2 & 7.9 & 7.2 & 7.9 \\
\hline $1 A$ & S & 9/15/1992 & 92259 & 259 & 17.6 & 7.8 & 7.2 & 7.9 & 6.6 \\
\hline $1 A$ & S & 9/16/1992 & 92260 & 260 & 18.6 & 9 & 8.5 & 9 & 8.5 \\
\hline $1 A$ & $S$ & 9/17/1992 & 92261 & 261 & 23.6 & 8 & 8.7 & 8 & 8 \\
\hline
\end{tabular}


lights

\begin{tabular}{|c|c|c|c|c|c|c|c|c|c|}
\hline $1 A$ & $S$ & 9/18/1992 & 92262 & 262 & 16.2 & 10 & 10.1 & 9.4 & 10.1 \\
\hline $0 A$ & $\mathrm{~S}$ & 9/19/1992 & 92263 & 263 & 16.1 & 10.1 & 10.1 & 10.1 & 10.1 \\
\hline $0 A$ & S & 9/20/1992 & 92264 & 264 & 10.7 & 10 & 10.6 & 10 & 10.6 \\
\hline $1 \mathrm{~A}$ & $\mathrm{~S}$ & 9/21/1992 & 92265 & 265 & 10.6 & 10.6 & 10.1 & 10.6 & 10.6 \\
\hline $1 A$ & S & 9/22/1992 & 92266 & 266 & 19.7 & 9.2 & 9.2 & 9.8 & 9.3 \\
\hline $1 A$ & S & 9/23/1992 & 92267 & 267 & 18.3 & 16.5 & 15.6 & 16.2 & 16 \\
\hline $1 A$ & $\mathrm{~S}$ & 9/24/1992 & 92268 & 268 & 18.8 & 9.9 & 9.3 & 9.9 & 9.9 \\
\hline $1 A$ & S & 9/25/1992 & 92269 & 269 & 18.4 & 9.5 & 9.6 & 10.2 & 9.5 \\
\hline $0 A$ & S & 9/26/1992 & 92270 & 270 & 16.6 & 10.5 & 10.4 & 10.5 & 10.4 \\
\hline $0 A$ & S & 9/27/1992 & 92271 & 271 & 10.4 & 10.4 & 10.4 & 10.5 & 10.5 \\
\hline $1 A$ & S & 9/28/1992 & 92272 & 272 & 10.5 & 10.4 & 10.5 & 11.1 & 10.5 \\
\hline $1 A$ & S & 9/29/1992 & 92273 & 273 & 14.3 & 10.1 & 9.6 & 10.1 & 10.1 \\
\hline $1 A$ & S & 9/30/1992 & 92274 & 274 & 21 & 11.7 & 11.7 & 11.8 & 12.3 \\
\hline $1 A$ & $S$ & 10/1/1992 & 92275 & 275 & 18.8 & 10.1 & 10.8 & 10.2 & 10.1 \\
\hline $1 \mathrm{~A}$ & $\mathrm{~S}$ & $10 / 2 / 1992$ & 92276 & 276 & 13.4 & 8.2 & 8.2 & 8.8 & 8.3 \\
\hline $0 A$ & S & $10 / 3 / 1992$ & 92277 & 277 & 21.4 & 8.8 & 8.8 & 8.8 & 8.9 \\
\hline $0 A$ & S & 10/4/1992 & 92278 & 278 & 8.8 & 8.2 & 8.8 & 8.2 & 8.2 \\
\hline $1 A$ & S & 10/5/1992 & 92279 & 279 & 8.2 & 8.8 & 8.2 & 8.2 & 8.8 \\
\hline $1 A$ & S & 10/6/1992 & 92280 & 280 & 21.7 & 8.7 & 9.2 & 8.6 & 9.3 \\
\hline $1 A$ & S & 10/7/1992 & 92281 & 281 & 18.2 & 9.4 & 9.4 & 9.4 & 9.4 \\
\hline $1 \mathrm{~A}$ & S & 10/8/1992 & 92282 & 282 & 16.4 & 8.2 & 8.8 & 8.2 & 8.8 \\
\hline $1 A$ & S & 10/9/1992 & 92283 & 283 & 16 & 8.1 & 7.6 & 8.2 & 7.5 \\
\hline $0 A$ & S & \#\#\#\#\#\#\#\# & 92284 & 284 & 22.7 & 9 & 8.3 & 9 & 8.4 \\
\hline $0 A$ & S & \#\#\#\#\#\#\#\# & 92285 & 285 & 9 & 8.4 & 9 & 9 & 8.4 \\
\hline $1 A$ & S & \#\#\#\#\#\#\#\# & 92286 & 286 & 8.4 & 9 & 8.4 & 9 & 9.1 \\
\hline $1 A$ & S & \#\#\#\#\#\#\#\# & 92287 & 287 & 24.7 & 16.3 & 16.5 & 16.5 & 16.8 \\
\hline $1 A$ & S & \#\#\#\#\#\#\#\# & 92288 & 288 & 18.2 & 8.9 & 9 & 9.6 & 9 \\
\hline $1 A$ & S & \#\#\#\#\#\#\#\# & 92289 & 289 & 18.8 & 9.5 & 9.5 & 9.4 & 9.5 \\
\hline $1 A$ & S & \#\#\#\#\#\#\#\# & 92290 & 290 & 18.3 & 10.8 & 10.9 & 10.9 & 10.3 \\
\hline $0 A$ & S & \#\#\#\#\#\#\#\# & 92291 & 291 & 13.9 & 10.1 & 9.5 & 10.2 & 10.1 \\
\hline $0 A$ & S & \#\#\#\#\#\#\#\# & 92292 & 292 & 9.5 & 10.1 & 10.1 & 10.2 & 10.1 \\
\hline $1 A$ & S & \#\#\#\#\#\#\#\# & 92293 & 293 & 9.5 & 10.1 & 10.1 & 9.5 & 10.1 \\
\hline $1 A$ & S & \#\#\#\#\#\#\#\# & 92294 & 294 & & 9.3 & 9.4 & 9.3 & 9.4 \\
\hline $1 A$ & S & \#\#\#\#\#\#\#\# & 92295 & 295 & 17.8 & 8.1 & 8.1 & 8.7 & 8.1 \\
\hline $1 A$ & S & \#\#\#\#\#\#\#\# & 92296 & 296 & 20.5 & 10.1 & 9.5 & 10.1 & 10.2 \\
\hline $1 A$ & S & \#\#\#\#\#\#\#\# & 92297 & 297 & 13.3 & 9.2 & 9.3 & 8.7 & 9.3 \\
\hline $0 A$ & $\mathrm{~S}$ & \#\#\#\#\#\#\#\# & 92298 & 298 & 28.3 & 16.3 & 16.9 & 16.4 & 16.9 \\
\hline $0 A$ & S & \#\#\#\#\#\#\#\# & 92299 & 299 & 16.1 & 15.8 & 16.2 & 16.3 & 15.7 \\
\hline $1 A$ & $S$ & \#\#\#\#\#\#\#\# & 92300 & 300 & 16 & 15.7 & 16.2 & 15.7 & 16.2 \\
\hline $1 A$ & S & \#\#\#\#\#\#\#\# & 92301 & 301 & 18.9 & 9.3 & 9.8 & 9.3 & 9.9 \\
\hline $1 \mathrm{~A}$ & $\mathrm{~S}$ & \#\#\#\#\#\#\#\# & 92302 & 302 & 17.9 & 8.3 & 8.3 & 8.4 & 8.3 \\
\hline $1 A$ & S & \#\#\#\#\#\#\#\# & 92303 & 303 & 21 & 9.4 & 9.3 & 8.8 & 9.4 \\
\hline $1 \mathrm{~A}$ & S & \#\#\#\#\#\#\#\# & 92304 & 304 & 17.7 & 9.2 & 9.2 & 9.2 & 8.7 \\
\hline $0 A$ & S & \#\#\#\#\#\#\#\# & 92305 & 305 & 21.9 & 15.9 & 15.5 & 15.7 & 16.6 \\
\hline $0 A$ & S & 11/1/1992 & 92306 & 306 & 15.7 & 15.3 & 15.8 & 14.9 & 15.8 \\
\hline $1 A$ & S & $11 / 2 / 1992$ & 92307 & 307 & 15.4 & 16 & 15.9 & 15.4 & 16 \\
\hline $1 \mathrm{~A}$ & S & $11 / 3 / 1992$ & 92308 & 308 & 14.4 & 10.1 & 10.8 & 10.7 & 10.1 \\
\hline $1 A$ & $S$ & 11/4/1992 & 92309 & 309 & 17.5 & 7.8 & 7.7 & 7.7 & 8.3 \\
\hline $1 A$ & S & $11 / 5 / 1992$ & 92310 & 310 & 12 & 9.1 & 9.2 & 9.2 & 9.1 \\
\hline $1 A$ & S & $11 / 6 / 1992$ & 92311 & 311 & 19.4 & 11.2 & 9.9 & 10.6 & 10.5 \\
\hline $0 A$ & $S$ & $11 / 7 / 1992$ & 92312 & 312 & 13.9 & 10.1 & 10.1 & 10.2 & 10.1 \\
\hline
\end{tabular}


lights

\begin{tabular}{|c|c|c|c|c|c|c|c|c|c|}
\hline \begin{tabular}{l|l}
0 & $A$ \\
\end{tabular} & $S$ & $11 / 8 / 1992$ & 92313 & 313 & 10.1 & 10.1 & 10.2 & 10.1 & 10.1 \\
\hline $1 \mathrm{~A}$ & $\mathrm{~S}$ & $11 / 9 / 1992$ & 92314 & 314 & 10.1 & 10.8 & 10.7 & 10.1 & 10.8 \\
\hline $1 \mathrm{~A}$ & S & \#\#\#\#\#\#\#\# & 92315 & 315 & 19.4 & 8.7 & 8.7 & 8.1 & 8.7 \\
\hline $1 \mathrm{~A}$ & S & \#\#\#\#\#\#\#\# & 92316 & 316 & 27.1 & 24.9 & 24.8 & 25.5 & 25 \\
\hline $1 \mathrm{~A}$ & S & \#\#\#\#\#\#\#\# & 92317 & 317 & 25.6 & 11.9 & 12.6 & 12 & 12 \\
\hline $1 \mathrm{~A}$ & $\mathrm{~S}$ & \#\#\#\#\#\#\#\# & 92318 & 318 & 23.8 & 17.5 & 18.1 & 17.6 & 17.4 \\
\hline $0 \mathrm{~A}$ & $\mathrm{~S}$ & \#\#\#\#\#\#\#\# & 92319 & 319 & 26.3 & 16.5 & 9.4 & 9.1 & 8.5 \\
\hline $0 \mathrm{~A}$ & S & \#\#\#\#\#\#\#\# & 92320 & 320 & 9.1 & 9 & 8.5 & 9.1 & 9.1 \\
\hline $1 \mathrm{~A}$ & S & \#\#\#\#\#\#\#\# & 92321 & 321 & 8.5 & 9.1 & 9 & 8.5 & 9.1 \\
\hline $1 \mathrm{~A}$ & $\mathrm{~S}$ & \#\#\#\#\#\#\#\# & 92322 & 322 & 18.9 & 9.4 & 9.3 & 9.3 & 9.4 \\
\hline $1 \mathrm{~A}$ & $\mathrm{~S}$ & \#\#\#\#\#\#\#\# & 92323 & 323 & 16.3 & 8.9 & 9.5 & 8.9 & 9 \\
\hline $1 \mathrm{~A}$ & $S$ & \#\#\#\#\#\#\#\# & 92324 & 324 & 14.8 & 9.3 & 9.3 & 9.4 & 9.9 \\
\hline $1 \mathrm{~A}$ & S & \#\#\#\#\#\#\#\# & 92325 & 325 & 14.1 & 9.5 & 10.1 & 9.6 & 9.5 \\
\hline $0 \mathrm{~A}$ & S & \#\#\#\#\#\#\#\# & 92326 & 326 & 18.3 & 9.2 & 9.1 & 9.2 & 9.2 \\
\hline $0 \mathrm{~A}$ & $\mathrm{~S}$ & \#\#\#\#\#\#\#\# & 92327 & 327 & 9.2 & 9.2 & 9.2 & 9.2 & 9.2 \\
\hline $1 \mathrm{~A}$ & $\mathrm{~S}$ & \#\#\#\#\#\#\#\# & 92328 & 328 & 9.2 & 9.2 & 9.2 & 9.2 & 9.1 \\
\hline $1 \mathrm{~A}$ & S & \#\#\#\#\#\#\#\# & 92329 & 329 & 18.8 & 9.7 & 9 & 9 & 9.6 \\
\hline $1 \mathrm{~A}$ & S & \#\#\#\#\#\#\#\# & 92330 & 330 & 22.2 & 9.8 & 10.4 & 10.4 & 10.4 \\
\hline $1 \mathrm{~A}$ & NS & \#\#\#\#\#\#\#\# & 92331 & 331 & 18.1 & 8.8 & 8.7 & 8.2 & 8.8 \\
\hline $1 \mathrm{~A}$ & NS & \#\#\#\#\#\#\#\# & 92332 & 332 & 8.8 & 8.7 & 8.8 & 8.1 & 8.8 \\
\hline $0 \mathrm{~A}$ & NS & \#\#\#\#\#\#\#\# & 92333 & 333 & 8.8 & 8.7 & 8.8 & 8.1 & 8.8 \\
\hline $0 \mathrm{~A}$ & NS & \#\#\#\#\#\#\#\# & 92334 & 334 & 8.7 & 8.8 & 8.1 & 8.7 & 8.8 \\
\hline $1 \mathrm{~A}$ & S & \#\#\#\#\#\#\#\# & 92335 & 335 & 8.2 & 8.8 & 8.7 & 8.8 & 8.8 \\
\hline $1 \mathrm{~A}$ & S & $12 / 1 / 1992$ & 92336 & 336 & 20.9 & 11.7 & 11 & 9.8 & 9.8 \\
\hline $1 \mathrm{~A}$ & $\mathrm{~S}$ & $12 / 2 / 1992$ & 92337 & 337 & 18.2 & 9.6 & 9 & 9.7 & 9 \\
\hline $1 \mathrm{~A}$ & S & $12 / 3 / 1992$ & 92338 & 338 & 19.9 & 9.9 & 9.8 & 9.9 & 10.4 \\
\hline $1 \mathrm{~A}$ & S & $12 / 4 / 1992$ & 92339 & 339 & 20.4 & 10.4 & 10.4 & 10.5 & 11 \\
\hline $0 \mathrm{~A}$ & S & $12 / 5 / 1992$ & 92340 & 340 & 14.4 & 9.7 & 9.7 & 9.6 & 9.7 \\
\hline $0 \mathrm{~A}$ & $S$ & $12 / 6 / 1992$ & 92341 & 341 & 9.6 & 9.7 & 9.7 & 9.7 & 9.6 \\
\hline $1 \mathrm{~A}$ & $S$ & 12/7/1992 & 92342 & 342 & 9.1 & 9.6 & 9.7 & 9.7 & 9 \\
\hline $1 \mathrm{~A}$ & S & 12/8/1992 & 92343 & 343 & 13 & 8.6 & 8.6 & 8.6 & 9.3 \\
\hline $1 \mathrm{~A}$ & S & $12 / 9 / 1992$ & 92344 & 344 & 21 & 8.5 & 8.5 & 9.1 & 8.5 \\
\hline $1 \mathrm{~A}$ & S & \#\#\#\#\#\#\#\# & 92345 & 345 & 18.4 & 11.3 & 11.8 & 11.9 & 11.3 \\
\hline $1 \mathrm{~A}$ & S & \#\#\#\#\#\#\#\# & 92346 & 346 & 16.4 & 10.6 & 10.7 & 10.6 & 10.7 \\
\hline $0 \mathrm{~A}$ & S & \#\#\#\#\#\#\#\# & 92347 & 347 & 25.7 & 17.5 & 17.4 & 17.5 & 16.4 \\
\hline $0 \mathrm{~A}$ & S & \#\#\#\#\#\#\#\# & 92348 & 348 & 17 & 17.2 & 17.4 & 17.4 & 16.7 \\
\hline $1 \mathrm{~A}$ & S & \#\#\#\#\#\#\#\# & 92349 & 349 & 18 & 17.5 & 17.6 & 17.1 & 17.5 \\
\hline $1 \mathrm{~A}$ & S & \#\#\#\#\#\#\#\# & 92350 & 350 & 19.1 & 10.2 & 10.2 & 10.8 & 10.3 \\
\hline $1 \mathrm{~A}$ & $S$ & \#\#\#\#\#\#\#\# & 92351 & 351 & 27.8 & 17.2 & 17.2 & 16.9 & 16.8 \\
\hline $1 A$ & S & \#\#\#\#\#\#\#\# & 92352 & 352 & 18.5 & 9.4 & 9.5 & 9.4 & 9.5 \\
\hline $1 \mathrm{~A}$ & $\mathrm{~S}$ & \#\#\#\#\#\#\#\# & 92353 & 353 & 21 & 14.6 & 15.1 & 15.2 & 15.2 \\
\hline $0 A$ & NS & \#\#\#\#\#\#\#\# & 92354 & 354 & 26.9 & 15.4 & 15.6 & 15.9 & 15.9 \\
\hline $0 A$ & NS & \#\#\#\#\#\#\#\# & 92355 & 355 & 16.1 & 16.3 & 15.8 & 16.8 & 16.1 \\
\hline $1 A$ & NS & \#\#\#\#\#\#\#\# & 92356 & 356 & 15.5 & 16.1 & 15.1 & 15.8 & 15.5 \\
\hline $1 \mathrm{~A}$ & NS & \#\#\#\#\#\#\#\# & 92357 & 357 & 15.6 & 16 & 16.1 & 15.5 & 16.2 \\
\hline $1 A$ & NS & \#\#\#\#\#\#\#\# & 92358 & 358 & 9 & 9 & 9.6 & 9 & 9.6 \\
\hline $1 \mathrm{~A}$ & NS & \#\#\#\#\#\#\#\# & 92359 & 359 & 9 & 9 & 9.6 & 8.9 & 9 \\
\hline $1 A$ & NS & \#\#\#\#\#\#\#\# & 92360 & 360 & 8.8 & 8.8 & 9.4 & 8.8 & 8.8 \\
\hline $0 A$ & NS & \#\#\#\#\#\#\#\# & 92361 & 361 & 9.4 & 8.8 & 9.4 & 8.8 & 8.8 \\
\hline $0 A$ & NS & \#\#\#\#\#\#\#\# & 92362 & 362 & 9.4 & 8.8 & 8.9 & 9.4 & 8.8 \\
\hline $1 A$ & NS & \#\#\#\#\#\#\#\# & 92363 & 363 & 8.8 & 9.4 & 8.8 & 9.5 & 8.8 \\
\hline
\end{tabular}


lights

\begin{tabular}{|c|c|c|c|c|c|c|c|c|c|}
\hline $1 \mathrm{~A}$ & NS & \#\#\#\#\#\#\#\# & 92364 & 364 & 9.4 & 8.8 & 8.8 & 9.4 & 8.8 \\
\hline $1 \mathrm{~A}$ & NS & \#\#\#\#\#\#\#\# & 92365 & 365 & 8.8 & 9.4 & 8.8 & 8.8 & 8.8 \\
\hline $1 \mathrm{~A}$ & NS & \#\#\#\#\#\#\#\# & 92366 & 366 & & & & & \\
\hline $1 \mathrm{~A}$ & NS & $1 / 1 / 1993$ & 93001 & 1 & 8.8 & 9.4 & 8.8 & 8.8 & 9.4 \\
\hline $0 \mathrm{~A}$ & NS & $1 / 2 / 1993$ & 93002 & 2 & 8.8 & 9.4 & 8.8 & 8.8 & 9.4 \\
\hline $\begin{array}{ll}\mathrm{A} \\
\end{array}$ & NS & $1 / 3 / 1993$ & 93003 & 3 & 9.4 & 8.8 & 8.9 & 9.4 & 8.8 \\
\hline $1 \mathrm{~A}$ & S & $1 / 4 / 1993$ & 93004 & 4 & 8.8 & 8.8 & 9.4 & 8.8 & 8.8 \\
\hline $1 \mathrm{~A}$ & S & $1 / 5 / 1993$ & 93005 & 5 & 19.4 & 9.1 & 9.8 & 9.2 & 9.2 \\
\hline $1 \mathrm{~A}$ & S & 1/6/1993 & 93006 & 6 & 24.3 & 9.9 & 10.5 & 10 & 9.9 \\
\hline $1 \mathrm{~A}$ & S & 1/7/1993 & 93007 & 7 & 27.3 & 18.2 & 17.7 & 17.7 & 17.6 \\
\hline $1 \mathrm{~A}$ & S & 1/8/1993 & 93008 & 8 & 17 & 8.7 & 8.6 & 9.3 & 8.7 \\
\hline $0 \mathrm{~A}$ & S & $1 / 9 / 1993$ & 93009 & 9 & 26.4 & 17 & 16.5 & 16.9 & 16 \\
\hline $0 \mathrm{~A}$ & S & 1/10/1993 & 93010 & 10 & 17.1 & 16.6 & 16.5 & 17.1 & 17.2 \\
\hline $1 \mathrm{~A}$ & S & $1 / 11 / 1993$ & 93011 & 11 & 17 & 16.5 & 17.2 & 17.1 & 16.4 \\
\hline $1 \mathrm{~A}$ & $\mathrm{~S}$ & $1 / 12 / 1993$ & 93012 & 12 & 15.8 & 10.2 & 10.2 & 10.8 & 10.1 \\
\hline $1 \mathrm{~A}$ & $\mathrm{~S}$ & $1 / 13 / 1993$ & 93013 & 13 & 22.6 & 16.4 & 15.6 & 15.4 & 15.8 \\
\hline $1 \mathrm{~A}$ & S & $1 / 14 / 1993$ & 93014 & 14 & 26.4 & 10.5 & 10.5 & 10.4 & 11.1 \\
\hline $1 \mathrm{~A}$ & S & $1 / 15 / 1993$ & 93015 & 15 & 35.8 & 17.5 & 17.2 & 17.6 & 17.7 \\
\hline $0 \mathrm{~A}$ & S & $1 / 16 / 1993$ & 93016 & 16 & 8.1 & 8.2 & 8.1 & 7.5 & 8.1 \\
\hline $0 \mathrm{~A}$ & S & 1/17/1993 & 93017 & 17 & 8.1 & 8.1 & 8.1 & 8.2 & 8.1 \\
\hline $1 \mathrm{~A}$ & NS & 1/18/1993 & 93018 & 18 & 8.1 & 8.1 & 7.6 & 8.1 & 8.1 \\
\hline $1 \mathrm{~A}$ & $S$ & 1/19/1993 & 93019 & 19 & 8.8 & 9.3 & 8.7 & 9.3 & 8.8 \\
\hline $1 \mathrm{~A}$ & S & $1 / 20 / 1993$ & 93020 & 20 & 17.9 & 12.2 & 11.6 & 12.2 & 11.7 \\
\hline $1 \mathrm{~A}$ & S & $1 / 21 / 1993$ & 93021 & 21 & 16.6 & 11.2 & 10.6 & 11.2 & 11.2 \\
\hline $1 \mathrm{~A}$ & $\mathrm{~S}$ & $1 / 22 / 1993$ & 93022 & 22 & 26.9 & 16 & 16.3 & 17.1 & 16.2 \\
\hline $0 \mathrm{~A}$ & S & $1 / 23 / 1993$ & 93023 & 23 & 22.3 & 15.3 & 15.1 & 16.3 & 15.4 \\
\hline $0 \mathrm{~A}$ & S & $1 / 24 / 1993$ & 93024 & 24 & 16.3 & 15.8 & 15.7 & 16 & 15.8 \\
\hline $1 \mathrm{~A}$ & S & $1 / 25 / 1993$ & 93025 & 25 & 15.8 & 16 & 15.7 & 15.9 & 16.5 \\
\hline $1 \mathrm{~A}$ & $S$ & $1 / 26 / 1993$ & 93026 & 26 & 28.8 & 10.8 & 9.6 & 10.3 & 9.6 \\
\hline $1 \mathrm{~A}$ & $S$ & 1/27/1993 & 93027 & 27 & 14.7 & 9.2 & 9.9 & 9.2 & 9.8 \\
\hline $1 \mathrm{~A}$ & S & $1 / 28 / 1993$ & 93028 & 28 & 16.7 & 7.9 & 8 & 8.6 & 8 \\
\hline $1 \mathrm{~A}$ & S & $1 / 29 / 1993$ & 93029 & 29 & 20.7 & 10 & 10.6 & 10 & 10.6 \\
\hline $0 \mathrm{~A}$ & S & $1 / 30 / 1993$ & 93030 & 30 & 17.6 & 7.8 & 7.9 & 7.8 & 7.9 \\
\hline $0 \mathrm{~A}$ & S & $1 / 31 / 1993$ & 93031 & 31 & 7.8 & 7.9 & 7.8 & 7.9 & 8.4 \\
\hline $1 \mathrm{~A}$ & S & 2/1/1993 & 93032 & 32 & 7.8 & 7.8 & 7.9 & 8.4 & 7.8 \\
\hline $1 \mathrm{~A}$ & S & $2 / 2 / 1993$ & 93033 & 33 & 19.9 & 9.8 & 9.2 & 9.2 & 9.8 \\
\hline $1 \mathrm{~A}$ & S & $2 / 3 / 1993$ & 93034 & 34 & 24.4 & 11 & 10.3 & 10.4 & 10.9 \\
\hline $1 \mathrm{~A}$ & $\mathrm{~S}$ & 2/4/1993 & 93035 & 35 & 23.9 & 17.7 & 18.2 & 17.9 & 18.2 \\
\hline $1 \mathrm{~A}$ & NS & 2/5/1993 & 93036 & 36 & 29.2 & 18.1 & 18.2 & 18 & 18.8 \\
\hline $0 \mathrm{~A}$ & $\mathrm{~S}$ & 2/6/1993 & 93037 & 37 & 7.6 & 8.3 & 7.6 & 8.3 & 7.6 \\
\hline $0 \mathrm{~A}$ & S & 2/7/1993 & 93038 & 38 & 7.6 & 7.6 & 8.3 & 7.6 & 8.2 \\
\hline $1 \mathrm{~A}$ & S & $2 / 8 / 1993$ & 93039 & 39 & 8.2 & 7.6 & 8.3 & 7.6 & 8.2 \\
\hline $1 \mathrm{~A}$ & S & 2/9/1993 & 93040 & 40 & 32.3 & 16.9 & 17.2 & 16.5 & 16.9 \\
\hline $1 \mathrm{~A}$ & S & 2/10/1993 & 93041 & 41 & 30 & 17.9 & 17.7 & 17.4 & 18.1 \\
\hline $1 \mathrm{~A}$ & S & 2/11/1993 & 93042 & 42 & 16.1 & 10.1 & 10.8 & 10.1 & 10.8 \\
\hline $1 \mathrm{~A}$ & S & $2 / 12 / 1993$ & 93043 & 43 & 24.7 & 18.3 & 18.4 & 18.6 & 18.5 \\
\hline $0 \mathrm{~A}$ & S & 2/13/1993 & 93044 & 44 & 23.4 & 16.1 & 16.3 & 17.1 & 16.3 \\
\hline $0 \mathrm{~A}$ & $S$ & 2/14/1993 & 93045 & 45 & 16.8 & 16 & 16.4 & 17 & 16.5 \\
\hline $1 \mathrm{~A}$ & S & 2/15/1993 & 93046 & 46 & 16.7 & 16.7 & 17 & 16.3 & 16.6 \\
\hline $1 \mathrm{~A}$ & $\mathrm{~S}$ & 2/16/1993 & 93047 & 47 & 17.8 & 8.5 & 8.6 & 7.9 & 8.5 \\
\hline $1 \mathrm{~A}$ & S & 2/17/1993 & 93048 & 48 & 23.2 & 16.2 & 16.3 & 15.8 & 15.9 \\
\hline
\end{tabular}


lights

\begin{tabular}{|c|c|c|c|c|c|c|c|c|c|}
\hline $1 \mathrm{~A}$ & S & 2/18/1993 & 93049 & 49 & 16 & 10 & 10 & 10 & 10 \\
\hline $1 \mathrm{~A}$ & S & 2/19/1993 & 93050 & 50 & 23 & 17.3 & 17.6 & 16.9 & 17.7 \\
\hline $0 A$ & $S$ & 2/20/1993 & 93051 & 51 & 28.7 & 17.6 & 17.2 & 17.1 & 17.5 \\
\hline $0 A$ & S & 2/21/1993 & 93052 & 52 & 15.3 & 15.5 & 15.7 & 15.8 & 15.9 \\
\hline $1 A$ & S & 2/22/1993 & 93053 & 53 & 15.8 & 16.1 & 15.3 & 15.8 & 15.8 \\
\hline $1 A$ & $\mathrm{~S}$ & $2 / 23 / 1993$ & 93054 & 54 & 23.8 & 9.2 & 9.9 & 9.8 & 9.2 \\
\hline $1 A$ & S & 2/24/1993 & 93055 & 55 & 22.5 & 17.9 & 18.5 & 17.7 & 17.9 \\
\hline $1 A$ & S & $2 / 25 / 1993$ & 93056 & 56 & 31.4 & 16.9 & 16.3 & 16.9 & 15.8 \\
\hline $1 A$ & S & 2/26/1993 & 93057 & 57 & 28.4 & 15.5 & 15.2 & 15.8 & 15.7 \\
\hline $0 A$ & $\mathrm{~S}$ & 2/27/1993 & 93058 & 58 & 23.4 & 16.4 & 16.4 & 16 & 16.1 \\
\hline $0 A$ & $\mathrm{~S}$ & 2/28/1993 & 93059 & 59 & 16.3 & 16.1 & 16.3 & 15.9 & 16.4 \\
\hline $1 A$ & $S$ & 3/1/1993 & 93060 & 60 & 14.5 & 15.1 & 14.1 & 14.6 & 15.1 \\
\hline $1 A$ & S & $3 / 2 / 1993$ & 93061 & 61 & 28.9 & 21.4 & 21 & 21.9 & 21.9 \\
\hline $1 A$ & S & $3 / 3 / 1993$ & 93062 & 62 & 45.1 & 18.4 & 17.6 & 17.4 & 17.6 \\
\hline $1 A$ & S & 3/4/1993 & 93063 & 63 & 28.3 & 19 & 17.3 & 16.9 & 16.8 \\
\hline $1 A$ & S & $3 / 5 / 1993$ & 93064 & 64 & 24.3 & 16 & 16.8 & 16.6 & 16.8 \\
\hline $0 A$ & S & $3 / 6 / 1993$ & 93065 & 65 & 28.7 & 16.2 & 16.3 & 16.1 & 16.2 \\
\hline $0 A$ & S & $3 / 7 / 1993$ & 93066 & 66 & 15.3 & 15.6 & 15.4 & 15.6 & 15.5 \\
\hline $1 A$ & $\mathrm{~S}$ & 3/8/1993 & 93067 & 67 & 15.5 & 15.5 & 15.2 & 15.4 & 15.4 \\
\hline $1 A$ & $\mathrm{~S}$ & $3 / 9 / 1993$ & 93068 & 68 & 18.8 & 11.3 & 11.4 & 11.3 & 11.4 \\
\hline $1 A$ & $S$ & 3/10/1993 & 93069 & 69 & 25.2 & 16.7 & 16.7 & 16.8 & 17.4 \\
\hline $1 A$ & S & 3/11/1993 & 93070 & 70 & 27.1 & 16.3 & 16.2 & 16.2 & 16.3 \\
\hline $1 A$ & S & $3 / 12 / 1993$ & 93071 & 71 & 15.7 & 7.7 & 7.7 & 7.6 & 7.7 \\
\hline $0 A$ & NS & 3/13/1993 & 93072 & 72 & 8.1 & 8.2 & 8.1 & 8.1 & 8.1 \\
\hline $0 A$ & NS & 3/14/1993 & 93073 & 73 & 8.1 & 8.1 & 8.7 & 8.1 & 8.1 \\
\hline $1 A$ & NS & $3 / 15 / 1993$ & 93074 & 74 & 8.1 & 8.2 & 8.1 & 8.7 & 8.1 \\
\hline $1 A$ & NS & 3/16/1993 & 93075 & 75 & 8.1 & 8.1 & 8.1 & 8.7 & 8.1 \\
\hline $1 A$ & NS & 3/17/1993 & 93076 & 76 & 8.7 & 8 & 8.7 & 8.7 & 7 \\
\hline $1 A$ & NS & 3/18/1993 & 93077 & 77 & 8.7 & 8.7 & 8.7 & 8.7 & 8.1 \\
\hline $1 A$ & NS & 3/19/1993 & 93078 & 78 & 8.1 & 8.7 & 8.8 & 8.7 & 8.7 \\
\hline $0 A$ & NS & 3/20/1993 & 93079 & 79 & 8.7 & 8.7 & 8.7 & 8.7 & 8.1 \\
\hline $0 A$ & NS & $3 / 21 / 1993$ & 93080 & 80 & 8 & 7.4 & 8 & 7.5 & 8 \\
\hline $1 A$ & S & $3 / 22 / 1993$ & 93081 & 81 & 7.4 & 8 & 7.4 & 8 & 7.4 \\
\hline $1 A$ & $\mathrm{~S}$ & $3 / 23 / 1993$ & 93082 & 82 & 17.4 & 9.4 & 9.4 & 9.4 & 9.4 \\
\hline $1 A$ & S & 3/24/1993 & 93083 & 83 & 20.7 & 14.9 & 14.8 & 15.4 & 14.7 \\
\hline $1 A$ & S & $3 / 25 / 1993$ & 93084 & 84 & 25.1 & 14.9 & 14.9 & 15.5 & 14.9 \\
\hline $1 A$ & S & 3/26/1993 & 93085 & 85 & 25 & 16 & 16.2 & 16.2 & 16.2 \\
\hline $0 A$ & $\mathrm{~S}$ & 3/27/1993 & 93086 & 86 & 27.1 & 16.1 & 16.3 & 16.8 & 16.2 \\
\hline $0 A$ & $S$ & 3/28/1993 & 93087 & 87 & 13.2 & 13.8 & 13 & 13.7 & 13.3 \\
\hline $1 A$ & S & 3/29/1993 & 93088 & 88 & 13.1 & 13.8 & 13.2 & 13.7 & 13.8 \\
\hline $1 A$ & S & 3/30/1993 & 93089 & 89 & 21.1 & 14.9 & 15.1 & 15.6 & 14.9 \\
\hline $1 A$ & S & 3/31/1993 & 93090 & 90 & 25.6 & 13.1 & 13 & 13.2 & 13.2 \\
\hline $1 A$ & S & 4/1/1993 & 93091 & 91 & 27.6 & 15.3 & 15.3 & 15.3 & 15.9 \\
\hline $1 A$ & S & 4/2/1993 & 93092 & 92 & 27.7 & 14.2 & 13.9 & 14.4 & 14.3 \\
\hline $0 A$ & S & 4/3/1993 & 93093 & 93 & 23.1 & 13.1 & 13.2 & 13.7 & 13 \\
\hline $0 A$ & S & 4/4/1993 & 93094 & 94 & 13 & & 6.8 & 13.1 & 13.6 \\
\hline $1 A$ & S & 4/5/1993 & 93095 & 95 & 13.4 & 13.4 & 13.4 & 14 & 13.4 \\
\hline $1 A$ & S & 4/6/1993 & 93096 & 96 & 22.6 & 16.2 & 15.8 & 16.3 & 15.7 \\
\hline $1 A$ & S & 4/7/1993 & 93097 & 97 & 29.5 & 14.6 & 14.7 & 14.7 & 15.2 \\
\hline $1 A$ & S & 4/8/1993 & 93098 & 98 & 20.8 & 14.3 & 14.2 & 14.2 & 14.8 \\
\hline $1 A$ & $S$ & 4/9/1993 & 93099 & 99 & 22.8 & 14.5 & 14 & 14.4 & 14 \\
\hline
\end{tabular}


lights

\begin{tabular}{|c|c|c|c|c|c|c|c|c|c|}
\hline \begin{tabular}{l|l}
0 & $A$ \\
\end{tabular} & $S$ & 4/10/1993 & 93100 & 100 & 22.2 & 13.6 & 13 & 13.7 & 13 \\
\hline $\begin{array}{ll}\mathrm{A} \\
\end{array}$ & $\mathrm{S}$ & $4 / 11 / 1993$ & 93101 & 101 & 13.5 & 13 & 12.9 & 13.6 & 13 \\
\hline $1 \mathrm{~A}$ & S & $4 / 12 / 1993$ & 93102 & 102 & 13.4 & 13 & 13.5 & 12.8 & 13 \\
\hline $1 \mathrm{~A}$ & $\mathrm{~S}$ & 4/13/1993 & 93103 & 103 & 20.3 & 11.8 & 12.5 & 12.6 & 11.9 \\
\hline $1 \mathrm{~A}$ & S & $4 / 14 / 1993$ & 93104 & 104 & 23 & 10.7 & 10.8 & 10.8 & 10.8 \\
\hline $1 \mathrm{~A}$ & S & $4 / 15 / 1993$ & 93105 & 105 & 21 & 11.2 & 11.1 & 11.1 & 11.2 \\
\hline $1 \mathrm{~A}$ & $\mathrm{~S}$ & 4/16/1993 & 93106 & 106 & 25.2 & 16.5 & 15.8 & 16.5 & 15.8 \\
\hline $0 \mathrm{~A}$ & S & 4/17/1993 & 93107 & 107 & 21 & 10.8 & 10.9 & 10.8 & 11.5 \\
\hline $0 \mathrm{~A}$ & S & 4/18/1993 & 93108 & 108 & 10.8 & 10.9 & 10.8 & 11.5 & 10.8 \\
\hline $1 \mathrm{~A}$ & S & 4/19/1993 & 93109 & 109 & 10.7 & 10.8 & 10.8 & 10.8 & 10.8 \\
\hline $1 \mathrm{~A}$ & $\mathrm{~S}$ & $4 / 20 / 1993$ & 93110 & 110 & 18.1 & 11.4 & 11.4 & 11.5 & 12.1 \\
\hline $1 \mathrm{~A}$ & $\mathrm{~S}$ & 4/21/1993 & 93111 & 111 & 23.4 & 10.2 & 10.3 & 10.2 & 10.3 \\
\hline $1 \mathrm{~A}$ & S & 4/22/1993 & 93112 & 112 & 23.1 & 12.3 & 13 & 12.3 & 12.9 \\
\hline $1 \mathrm{~A}$ & S & $4 / 23 / 1993$ & 93113 & 113 & 24.4 & 11.8 & 12.4 & 11.8 & 11.8 \\
\hline $0 \mathrm{~A}$ & $\mathrm{~S}$ & 4/24/1993 & 93114 & 114 & 22.6 & 10.9 & 10.8 & 11 & 10.9 \\
\hline $0 \mathrm{~A}$ & S & $4 / 25 / 1993$ & 93115 & 115 & 10.2 & 10.8 & 10.8 & 10.3 & 10.8 \\
\hline $1 \mathrm{~A}$ & S & 4/26/1993 & 93116 & 116 & 10.9 & 10.9 & 10.3 & 10.9 & 10.9 \\
\hline $1 \mathrm{~A}$ & S & 4/27/1993 & 93117 & 117 & 19.4 & 11.2 & 11.2 & 11.3 & 11.2 \\
\hline $1 \mathrm{~A}$ & S & 4/28/1993 & 93118 & 118 & 24.9 & 11.1 & 11.2 & 11.2 & 11.2 \\
\hline $1 \mathrm{~A}$ & S & 4/29/1993 & 93119 & 119 & 20.4 & 10.7 & 10.6 & 10.7 & 10 \\
\hline $1 \mathrm{~A}$ & S & 4/30/1993 & 93120 & 120 & 24.4 & 14.5 & 14 & 14.5 & 14.5 \\
\hline $0 \mathrm{~A}$ & S & $5 / 1 / 1993$ & 93121 & 121 & 27.7 & 13.5 & 12.8 & 12.9 & 13.5 \\
\hline $0 \mathrm{~A}$ & S & $5 / 2 / 1993$ & 93122 & 122 & 8.7 & 10.6 & 10.6 & 11.2 & 10.6 \\
\hline $1 \mathrm{~A}$ & $\mathrm{~S}$ & $5 / 3 / 1993$ & 93123 & 123 & 10.6 & 10.7 & 10.6 & 10.7 & 10.6 \\
\hline $1 \mathrm{~A}$ & S & $5 / 4 / 1993$ & 93124 & 124 & 23.2 & 10.1 & 10.7 & 10.6 & 10.7 \\
\hline $1 \mathrm{~A}$ & S & $5 / 5 / 1993$ & 93125 & 125 & 25 & 11 & 11.7 & 11.6 & 10.3 \\
\hline $1 \mathrm{~A}$ & S & $5 / 6 / 1993$ & 93126 & 126 & 25.4 & 14.1 & 14.7 & 14.6 & 14.6 \\
\hline $1 \mathrm{~A}$ & S & $5 / 7 / 1993$ & 93127 & 127 & 32.1 & 17 & 15 & 14.5 & 15 \\
\hline $0 \mathrm{~A}$ & S & 5/8/1993 & 93128 & 128 & 26.3 & 16.1 & 16.7 & 16.2 & 16.9 \\
\hline $0 \mathrm{~A}$ & S & 5/9/1993 & 93129 & 129 & 16.1 & 16.8 & 16.2 & 16.8 & 16.2 \\
\hline $1 \mathrm{~A}$ & S & $5 / 10 / 1993$ & 93130 & 130 & 17 & 16.3 & 17 & 17 & 16.3 \\
\hline $1 \mathrm{~A}$ & S & $5 / 11 / 1993$ & 93131 & 131 & 23.8 & 14.8 & 14.9 & 14.9 & 14.8 \\
\hline $1 \mathrm{~A}$ & S & $5 / 12 / 1993$ & 93132 & 132 & 23.7 & 13.6 & 14.4 & 14.2 & 13.7 \\
\hline $1 \mathrm{~A}$ & S & $5 / 13 / 1993$ & 93133 & 133 & 19.8 & 14.2 & 14.3 & 14.3 & 14.3 \\
\hline $1 \mathrm{~A}$ & S & $5 / 14 / 1993$ & 93134 & 134 & 22.5 & 14.6 & 14.6 & 14.7 & 14.7 \\
\hline $0 \mathrm{~A}$ & S & $5 / 15 / 1993$ & 93135 & 135 & 25.8 & 14.8 & 14.1 & 14.8 & 14.8 \\
\hline $0 \mathrm{~A}$ & $\mathrm{~S}$ & $5 / 16 / 1993$ & 93136 & 136 & 18.2 & 18.1 & 18.3 & 18.3 & 19 \\
\hline $1 \mathrm{~A}$ & S & $5 / 17 / 1993$ & 93137 & 137 & 19 & 18.6 & 19.3 & 18.6 & 19.3 \\
\hline $1 \mathrm{~A}$ & $S$ & $5 / 18 / 1993$ & 93138 & 138 & 22.3 & 15 & 14.6 & 15.1 & 14.6 \\
\hline $1 \mathrm{~A}$ & S & $5 / 19 / 1993$ & 93139 & 139 & 22.5 & 13.8 & 14.4 & 14.3 & 13.9 \\
\hline $1 \mathrm{~A}$ & $\mathrm{~S}$ & $5 / 20 / 1993$ & 93140 & 140 & 22.1 & 13.3 & 13.9 & 13.4 & 14 \\
\hline $1 \mathrm{~A}$ & S & $5 / 21 / 1993$ & 93141 & 141 & 31.8 & 17.3 & 16.3 & 16.3 & 16.3 \\
\hline $0 \mathrm{~A}$ & S & $5 / 22 / 1993$ & 93142 & 142 & 25.2 & 17 & 16.9 & 17.5 & 17 \\
\hline $0 \mathrm{~A}$ & S & $5 / 23 / 1993$ & 93143 & 143 & 15.3 & 15.2 & 15.2 & 15.2 & 15.1 \\
\hline $1 \mathrm{~A}$ & S & $5 / 24 / 1993$ & 93144 & 144 & 15.1 & 15.1 & 15.2 & 15.2 & 15.1 \\
\hline $1 \mathrm{~A}$ & S & $5 / 25 / 1993$ & 93145 & 145 & 21.6 & 14.3 & 13.6 & 14.2 & 14.3 \\
\hline $1 \mathrm{~A}$ & S & $5 / 26 / 1993$ & 93146 & 146 & 23.9 & 14.8 & 15.4 & 15.4 & 15.4 \\
\hline $1 \mathrm{~A}$ & $S$ & 5/27/1993 & 93147 & 147 & 19.9 & 13.9 & 13.9 & 14 & 13.3 \\
\hline $1 \mathrm{~A}$ & S & $5 / 28 / 1993$ & 93148 & 148 & 25.6 & 17.5 & 17 & 17 & 16.9 \\
\hline $0 \mathrm{~A}$ & $\mathrm{~S}$ & $5 / 29 / 1993$ & 93149 & 149 & 22.9 & 15.2 & 15.1 & 15.8 & 15.2 \\
\hline $0 \mathrm{~A}$ & $\mathrm{~S}$ & $5 / 30 / 1993$ & 93150 & 150 & 15.1 & 15.7 & 15.1 & 15.7 & 15.2 \\
\hline
\end{tabular}


lights

\begin{tabular}{|c|c|c|c|c|c|c|c|c|c|}
\hline $1 A$ & S & $5 / 31 / 1993$ & 93151 & 151 & 15.1 & 15.7 & 15.2 & 15.8 & 15.2 \\
\hline $1 A$ & S & 6/1/1993 & 93152 & 152 & 27.9 & 16.9 & 17.6 & 17 & 17.6 \\
\hline $1 A$ & $S$ & 6/2/1993 & 93153 & 153 & 29.2 & 18.4 & 19 & 18.9 & 19.1 \\
\hline $1 A$ & S & 6/3/1993 & 93154 & 154 & 31.9 & 14.7 & 14.7 & 14.7 & 14.6 \\
\hline $1 A$ & S & 6/4/1993 & 93155 & 155 & 24.5 & 12.2 & 12.9 & 12.8 & 12.8 \\
\hline $0 A$ & S & 6/5/1993 & 93156 & 156 & 22.7 & 13.2 & 13.7 & 13.9 & 13.3 \\
\hline $0 A$ & S & 6/6/1993 & 93157 & 157 & 13.9 & 13.2 & 14 & 13.8 & 13.1 \\
\hline $1 \mathrm{~A}$ & S & $6 / 7 / 1993$ & 93158 & 158 & 13.2 & 13.4 & 13.3 & 12.6 & 13.2 \\
\hline $1 A$ & S & 6/8/1993 & 93159 & 159 & 15.3 & 15.4 & 15.3 & 16.1 & 15.4 \\
\hline $1 A$ & S & 6/9/1993 & 93160 & 160 & 15.8 & 15.3 & 15.3 & 15.5 & 15.3 \\
\hline $1 \mathrm{~A}$ & $\mathrm{~S}$ & 6/10/1993 & 93161 & 161 & 14.8 & 14.3 & 14.8 & 14.3 & 14.9 \\
\hline $1 A$ & $S$ & 6/11/1993 & 93162 & 162 & 14 & 14 & 13.4 & 14.1 & 14.1 \\
\hline $0 A$ & S & 6/12/1993 & 93163 & 163 & 13.9 & 14 & 14 & 14.6 & 14 \\
\hline $0 A$ & NS & 6/13/1993 & 93164 & 164 & 13.9 & 13.9 & 14.7 & 13.9 & 14.1 \\
\hline $1 A$ & NS & $6 / 14 / 1993$ & 93165 & 165 & 13.9 & 13.9 & 14 & 14 & 14 \\
\hline $1 A$ & NS & $6 / 15 / 1993$ & 93166 & 166 & 15.8 & 15.2 & 15.1 & 15.7 & 15.1 \\
\hline $1 A$ & NS & 6/16/1993 & 93167 & 167 & 16.2 & 15.8 & 16.1 & 16.2 & 16.2 \\
\hline $1 A$ & NS & 6/17/1993 & 93168 & 168 & 16.4 & 16.4 & 16.4 & 16.5 & 16.4 \\
\hline $1 A$ & NS & 6/18/1993 & 93169 & 169 & 15.7 & 15.6 & 15.7 & 15.7 & 15.7 \\
\hline $0 A$ & NS & 6/19/1993 & 93170 & 170 & 14.5 & 14.5 & 14.5 & 14.4 & 14.5 \\
\hline $0 A$ & NS & 6/20/1993 & 93171 & 171 & 14.5 & 14.5 & 14.4 & 14.6 & 14.6 \\
\hline $1 A$ & NS & 6/21/1993 & 93172 & 172 & 14.4 & 14.5 & 14.6 & 14.4 & 14.5 \\
\hline $1 A$ & NS & $6 / 22 / 1993$ & 93173 & 173 & 18.5 & 18.9 & 18.9 & 18.4 & 19 \\
\hline $1 A$ & NS & 6/23/1993 & 93174 & 174 & 22.1 & 22.1 & 21.4 & 21.3 & 22 \\
\hline $1 A$ & NS & 6/24/1993 & 93175 & 175 & 18.7 & 19.2 & 18.6 & 19.1 & 18.6 \\
\hline $1 A$ & NS & $6 / 25 / 1993$ & 93176 & 176 & 18.3 & 18.9 & 19 & 18.7 & 18.3 \\
\hline $0 A$ & NS & 6/26/1993 & 93177 & 177 & 6.2 & 5.7 & 5.6 & 6.3 & 5.6 \\
\hline $0 A$ & NS & 6/27/1993 & 93178 & 178 & 5.7 & 5.6 & 5.6 & 5.6 & 5.7 \\
\hline $1 A$ & NS & $6 / 28 / 1993$ & 93179 & 179 & 5.7 & 5.6 & 5.6 & 5.6 & 5.7 \\
\hline $1 A$ & NS & 6/29/1993 & 93180 & 180 & 6.1 & 6.6 & 6.1 & 6.7 & 6.7 \\
\hline $1 A$ & NS & 6/30/1993 & 93181 & 181 & 7.5 & 7.4 & 6.2 & 6.3 & 5.6 \\
\hline $1 A$ & NS & 7/1/1993 & 93182 & 182 & 6.1 & 6.6 & 6.1 & 6.7 & 6.1 \\
\hline $1 A$ & NS & $7 / 2 / 1993$ & 93183 & 183 & 6.3 & 6.8 & 6.3 & 6.8 & 6.2 \\
\hline $0 A$ & NS & $7 / 3 / 1993$ & 93184 & 184 & 6.2 & 6.9 & 6.3 & 6.2 & 6.8 \\
\hline $0 A$ & NS & $7 / 4 / 1993$ & 93185 & 185 & 6.2 & 6.9 & 6.2 & 6.2 & 6.8 \\
\hline $1 A$ & NS & 7/5/1993 & 93186 & 186 & 6.9 & 6.3 & 6.8 & 6.3 & 6.9 \\
\hline $1 A$ & NS & $7 / 6 / 1993$ & 93187 & 187 & 6.8 & 6.3 & 6.8 & 6.3 & 6.9 \\
\hline $1 A$ & NS & 7/7/1993 & 93188 & 188 & 6.5 & 6.5 & 6.5 & 5.9 & 6.5 \\
\hline $1 A$ & NS & 7/8/1993 & 93189 & 189 & 11.6 & 12.3 & 12.3 & 11.6 & 12.1 \\
\hline $1 A$ & NS & $7 / 9 / 1993$ & 93190 & 190 & 13.4 & 13.4 & 12.9 & 13.3 & 12.7 \\
\hline $0 A$ & NS & 7/10/1993 & 93191 & 191 & 8 & 8.1 & 8 & 8 & 8 \\
\hline $0 A$ & NS & 7/11/1993 & 93192 & 192 & 6.9 & 6.8 & 7.5 & 6.8 & 7.4 \\
\hline $1 A$ & NS & 7/12/1993 & 93193 & 193 & 6.8 & 6.3 & 6.8 & 6.8 & 6.9 \\
\hline $1 A$ & NS & 7/13/1993 & 93194 & 194 & 6.4 & 7 & 6.4 & 7 & 7 \\
\hline $1 A$ & NS & 7/14/1993 & 93195 & 195 & 8.2 & 8.2 & 8.2 & 8.3 & 8.2 \\
\hline $1 A$ & NS & 7/15/1993 & 93196 & 196 & 10.7 & 10.1 & 10.1 & 10.7 & 10.1 \\
\hline $1 A$ & NS & $7 / 16 / 1993$ & 93197 & 197 & 7.3 & 7.2 & 7.3 & 7.2 & 7.3 \\
\hline $0 A$ & NS & 7/17/1993 & 93198 & 198 & 6.2 & 6.2 & 5.6 & 6.2 & 6.2 \\
\hline $0 A$ & NS & 7/18/1993 & 93199 & 199 & 6.2 & 6.2 & 6.2 & 6.2 & 6.2 \\
\hline $1 A$ & NS & 7/19/1993 & 93200 & 200 & 6.2 & 6.2 & 6.2 & 6.2 & 6.2 \\
\hline $1 A$ & NS & $7 / 20 / 1993$ & 93201 & 201 & 8.2 & 8.2 & 8.3 & 8.2 & 8.3 \\
\hline
\end{tabular}


lights

\begin{tabular}{|c|c|c|c|c|c|c|c|c|c|}
\hline $1 \mathrm{~A}$ & NS & $7 / 21 / 1993$ & 93202 & 202 & 11.3 & 11.4 & 11.4 & 11.5 & 11.5 \\
\hline $1 \mathrm{~A}$ & NS & $7 / 22 / 1993$ & 93203 & 203 & 7.2 & 6.6 & 7.2 & 6.6 & 7.3 \\
\hline $1 \mathrm{~A}$ & NS & $7 / 23 / 1993$ & 93204 & 204 & 7.4 & 7.4 & 6.7 & 7.4 & 7.4 \\
\hline $0 \mathrm{~A}$ & NS & $7 / 24 / 1993$ & 93205 & 205 & 6.8 & 6.2 & 6.8 & 6.8 & 6.8 \\
\hline $0 \mathrm{~A}$ & NS & $7 / 25 / 1993$ & 93206 & 206 & 6.8 & 6.8 & 6.8 & 6.1 & 6.8 \\
\hline $1 \mathrm{~A}$ & NS & $7 / 26 / 1993$ & 93207 & 207 & 6.8 & 6.7 & 6.8 & 6.8 & 6.2 \\
\hline $1 \mathrm{~A}$ & NS & $7 / 27 / 1993$ & 93208 & 208 & 7.2 & 7.2 & 7.3 & 7.8 & 7.2 \\
\hline $1 \mathrm{~A}$ & NS & $7 / 28 / 1993$ & 93209 & 209 & 6.7 & 6.7 & 6.8 & 6.9 & 6.8 \\
\hline $1 \mathrm{~A}$ & NS & 7/29/1993 & 93210 & 210 & 15.2 & 14.6 & 15.2 & 15.1 & 14.6 \\
\hline $1 \mathrm{~A}$ & NS & 7/30/1993 & 93211 & 211 & 6 & 6.6 & 6 & 6 & 6 \\
\hline $0 \mathrm{~A}$ & NS & $7 / 31 / 1993$ & 93212 & 212 & 6.5 & 6 & 7.2 & 6.6 & 6.7 \\
\hline $0 \mathrm{~A}$ & NS & 8/1/1993 & 93213 & 213 & 6.5 & 6.6 & 6.6 & 6.6 & 6.7 \\
\hline $1 \mathrm{~A}$ & NS & $8 / 2 / 1993$ & 93214 & 214 & 6.7 & 6.6 & 6.6 & 6.6 & 6.7 \\
\hline $1 \mathrm{~A}$ & NS & 8/3/1993 & 93215 & 215 & 9.3 & 10 & 9.3 & 9.3 & 9.9 \\
\hline $1 \mathrm{~A}$ & NS & $8 / 4 / 1993$ & 93216 & 216 & 9.3 & 9.9 & 10 & 9.3 & 10 \\
\hline $1 \mathrm{~A}$ & NS & 8/5/1993 & 93217 & 217 & 6.9 & 6.2 & 6.9 & 6.9 & 6.9 \\
\hline $1 \mathrm{~A}$ & NS & 8/6/1993 & 93218 & 218 & 7.1 & 6.6 & 6 & 6.6 & 6 \\
\hline $0 \mathrm{~A}$ & NS & 8/7/1993 & 93219 & 219 & 8.4 & 9 & 8.4 & 9 & 8.4 \\
\hline $0 \mathrm{~A}$ & NS & 8/8/1993 & 93220 & 220 & 8.3 & 7.6 & 8.3 & 8.2 & 8.2 \\
\hline $1 \mathrm{~A}$ & NS & 8/9/1993 & 93221 & 221 & 7.6 & 8.2 & 8.2 & 8.3 & 8.2 \\
\hline $1 \mathrm{~A}$ & NS & 8/10/1993 & 93222 & 222 & 7.9 & 8 & 8 & 8 & 8 \\
\hline $1 \mathrm{~A}$ & NS & 8/11/1993 & 93223 & 223 & 7.3 & 7.4 & 7.3 & 7.4 & 7.9 \\
\hline $1 \mathrm{~A}$ & NS & $8 / 12 / 1993$ & 93224 & 224 & 7.9 & 8.5 & 7.9 & 7.9 & 7.9 \\
\hline $1 \mathrm{~A}$ & NS & 8/13/1993 & 93225 & 225 & 8.4 & 8.9 & 9 & 8.3 & 9 \\
\hline $0 \mathrm{~A}$ & NS & 8/14/1993 & 93226 & 226 & 9.5 & 9.4 & 9.5 & 9.5 & 9.5 \\
\hline $0 \mathrm{~A}$ & NS & $8 / 15 / 1993$ & 93227 & 227 & 14.2 & 14.9 & 14.3 & 14.9 & 14.3 \\
\hline $1 \mathrm{~A}$ & S & 8/16/1993 & 93228 & 228 & 14.3 & 14.1 & 13.7 & 14.2 & 14.2 \\
\hline $1 \mathrm{~A}$ & S & 8/17/1993 & 93229 & 229 & 20.9 & 15.4 & 15.9 & 15.9 & 15.4 \\
\hline $1 \mathrm{~A}$ & S & 8/18/1993 & 93230 & 230 & 24.3 & 15 & 15.6 & 15 & 15.6 \\
\hline $1 \mathrm{~A}$ & $S$ & 8/19/1993 & 93231 & 231 & 18.2 & 13 & 13.6 & 13.1 & 12.9 \\
\hline $1 \mathrm{~A}$ & S & 8/20/1993 & 93232 & 232 & 18.5 & 12.4 & 13 & 13.1 & 13.1 \\
\hline $0 \mathrm{~A}$ & S & 8/21/1993 & 93233 & 233 & 17.9 & 12.9 & 12.9 & 12.8 & 13 \\
\hline $0 \mathrm{~A}$ & S & $8 / 22 / 1993$ & 93234 & 234 & 12.9 & 12.8 & 12.9 & 12.3 & 12.9 \\
\hline $1 \mathrm{~A}$ & S & 8/23/1993 & 93235 & 235 & 12.2 & 12.9 & 12.8 & 12.9 & 12.9 \\
\hline $1 \mathrm{~A}$ & S & $8 / 24 / 1993$ & 93236 & 236 & 21 & 13.6 & 13.6 & 13.6 & 13.7 \\
\hline $1 \mathrm{~A}$ & S & 8/25/1993 & 93237 & 237 & 20.8 & 13.3 & 14 & 13.3 & 13.3 \\
\hline $1 \mathrm{~A}$ & S & $8 / 26 / 1993$ & 93238 & 238 & 21.7 & 12.6 & 13.2 & 12.7 & 13.2 \\
\hline $1 \mathrm{~A}$ & S & 8/27/1993 & 93239 & 239 & 21.4 & 13.2 & 13.8 & 13.2 & 13.2 \\
\hline $0 \mathrm{~A}$ & $S$ & 8/28/1993 & 93240 & 240 & 23.9 & 13.2 & 13.2 & 13.7 & 13.3 \\
\hline $0 \mathrm{~A}$ & S & 8/29/1993 & 93241 & 241 & 13.6 & 12.9 & 13 & 13.7 & 13 \\
\hline $1 \mathrm{~A}$ & $\mathrm{~S}$ & 8/30/1993 & 93242 & 242 & 13.5 & 13 & 12.9 & 13.5 & 12.9 \\
\hline $1 \mathrm{~A}$ & S & 8/31/1993 & 93243 & 243 & 22.1 & 16.6 & 15.9 & 15.9 & 15.9 \\
\hline $1 \mathrm{~A}$ & S & 9/1/1993 & 93244 & 244 & 25.5 & 14.2 & 14.3 & 14.8 & 14.2 \\
\hline $1 \mathrm{~A}$ & S & 9/2/1993 & 93245 & 245 & 20 & 13.7 & 14.4 & 13.8 & 14.4 \\
\hline $1 \mathrm{~A}$ & S & 9/3/1993 & 93246 & 246 & 31.9 & 13.9 & 14.5 & 13.9 & 14.5 \\
\hline $0 \mathrm{~A}$ & S & 9/4/1993 & 93247 & 247 & 22.1 & 14.8 & 15.3 & 15.3 & 14.8 \\
\hline $0 \mathrm{~A}$ & $S$ & 9/5/1993 & 93248 & 248 & 15.3 & 15.3 & 14.8 & 15.4 & 15.5 \\
\hline $1 \mathrm{~A}$ & NS & 9/6/1993 & 93249 & 249 & 15.3 & 14.7 & 15.3 & 14.7 & 15.4 \\
\hline $1 \mathrm{~A}$ & S & 9/7/1993 & 93250 & 250 & 15.4 & 15.5 & 14.7 & 15.3 & 15.4 \\
\hline $1 \mathrm{~A}$ & S & 9/8/1993 & 93251 & 251 & 24.2 & 15.6 & 15.1 & 15.1 & 15.1 \\
\hline $1 \mathrm{~A}$ & S & 9/9/1993 & 93252 & 252 & 22.7 & 15.1 & 15.1 & 15.2 & 15.2 \\
\hline
\end{tabular}


lights

\begin{tabular}{|c|c|c|c|c|c|c|c|c|c|}
\hline $1 \mathrm{~A}$ & $S$ & 9/10/1993 & 93253 & 253 & 22.4 & 13.5 & 14.1 & 13.5 & 14.1 \\
\hline $\begin{array}{ll}\mathrm{A} \\
\end{array}$ & $\mathrm{S}$ & 9/11/1993 & 93254 & 254 & 22.2 & 13.6 & 14.2 & 14.2 & 14.2 \\
\hline $0 \mathrm{~A}$ & S & 9/12/1993 & 93255 & 255 & 12.9 & 13 & 12.9 & 13 & 13.6 \\
\hline $1 \mathrm{~A}$ & S & 9/13/1993 & 93256 & 256 & 12.9 & 12.9 & 13 & 12.9 & 13.6 \\
\hline $1 \mathrm{~A}$ & S & 9/14/1993 & 93257 & 257 & 24.6 & 13.5 & 14 & 13.4 & 13.5 \\
\hline $1 \mathrm{~A}$ & S & 9/15/1993 & 93258 & 258 & 22.9 & 14 & 13.4 & 13.4 & 14 \\
\hline $1 \mathrm{~A}$ & $\mathrm{~S}$ & 9/16/1993 & 93259 & 259 & 26.4 & 15.2 & 15.8 & 15.3 & 15.2 \\
\hline $1 \mathrm{~A}$ & S & 9/17/1993 & 93260 & 260 & 19.7 & 13.7 & 13.7 & 13.7 & 14.4 \\
\hline $0 \mathrm{~A}$ & S & 9/18/1993 & 93261 & 261 & 20.8 & 13.2 & 13.7 & 13.8 & 13.1 \\
\hline $0 \mathrm{~A}$ & S & 9/19/1993 & 93262 & 262 & 13.1 & 13.1 & 13.7 & 13.1 & 13 \\
\hline $1 \mathrm{~A}$ & $\mathrm{~S}$ & 9/20/1993 & 93263 & 263 & 13.7 & 14.3 & 13.8 & 14.4 & 13.6 \\
\hline $1 \mathrm{~A}$ & $\mathrm{~S}$ & 9/21/1993 & 93264 & 264 & 25.1 & 15.3 & 15.3 & 14.7 & 15.4 \\
\hline $1 \mathrm{~A}$ & S & 9/22/1993 & 93265 & 265 & 22.8 & 13.9 & 14 & 14.7 & 14.1 \\
\hline $1 \mathrm{~A}$ & S & 9/23/1993 & 93266 & 266 & 21.2 & 13.9 & 13.4 & 13.3 & 14 \\
\hline $1 \mathrm{~A}$ & $\mathrm{~S}$ & 9/24/1993 & 93267 & 267 & 23.6 & 13.3 & 13.2 & 13.2 & 13.2 \\
\hline $0 \mathrm{~A}$ & S & 9/25/1993 & 93268 & 268 & 20.5 & 14.3 & 14.4 & 14.3 & 14.4 \\
\hline $0 \mathrm{~A}$ & S & 9/26/1993 & 93269 & 269 & 14.3 & 14.3 & 15 & 14.3 & 14.4 \\
\hline $1 \mathrm{~A}$ & S & 9/27/1993 & 93270 & 270 & 14.4 & 14.5 & 14.4 & 15 & 14.5 \\
\hline $1 \mathrm{~A}$ & S & 9/28/1993 & 93271 & 271 & 20.4 & 13.2 & 13.8 & 13.8 & 13.9 \\
\hline $1 \mathrm{~A}$ & S & 9/29/1993 & 93272 & 272 & 19.9 & 13.9 & 14 & 13.4 & 14 \\
\hline $1 \mathrm{~A}$ & S & 9/30/1993 & 93273 & 273 & 21.5 & 13.6 & 14.2 & 13.7 & 13.6 \\
\hline $1 \mathrm{~A}$ & S & 10/1/1993 & 93274 & 274 & 22.5 & 14.2 & 14.3 & 14.3 & 14.3 \\
\hline $0 \mathrm{~A}$ & S & $10 / 2 / 1993$ & 93275 & 275 & 19.4 & 13.4 & 13.4 & 14.1 & 13.3 \\
\hline $0 \mathrm{~A}$ & $\mathrm{~S}$ & $10 / 3 / 1993$ & 93276 & 276 & 13.9 & 13.4 & 14 & 14 & 14.1 \\
\hline $1 \mathrm{~A}$ & S & $10 / 4 / 1993$ & 93277 & 277 & 12.7 & 13.6 & 14.1 & 13.7 & 14.1 \\
\hline $1 \mathrm{~A}$ & S & $10 / 5 / 1993$ & 93278 & 278 & 23.2 & 15.8 & 15.2 & 15.3 & 15.9 \\
\hline $1 \mathrm{~A}$ & S & $10 / 6 / 1993$ & 93279 & 279 & 21.3 & 13.5 & 13.5 & 13.6 & 14.2 \\
\hline $1 \mathrm{~A}$ & S & 10/7/1993 & 93280 & 280 & 28.5 & 14.3 & 15 & 14.4 & 14.4 \\
\hline $1 \mathrm{~A}$ & S & $10 / 8 / 1993$ & 93281 & 281 & 24.1 & 13.3 & 14.1 & 13.4 & 14.1 \\
\hline $0 \mathrm{~A}$ & S & 10/9/1993 & 93282 & 282 & 21.7 & 12.8 & 13.5 & 12.9 & 13.5 \\
\hline $0 \mathrm{~A}$ & S & \#\#\#\#\#\#\#\# & 93283 & 283 & 13 & 13.6 & 13.7 & 13.6 & 13 \\
\hline $1 \mathrm{~A}$ & S & \#\#\#\#\#\#\#\# & 93284 & 284 & 13.6 & 13.7 & 12.9 & 13.6 & 13.5 \\
\hline $1 \mathrm{~A}$ & S & \#\#\#\#\#\#\#\# & 93285 & 285 & 23.2 & 14 & 14.1 & 14.8 & 14.2 \\
\hline $1 \mathrm{~A}$ & S & \#\#\#\#\#\#\#\# & 93286 & 286 & 24.9 & 14.1 & 14.1 & 13.5 & 14.2 \\
\hline $1 \mathrm{~A}$ & S & \#\#\#\#\#\#\#\# & 93287 & 287 & 21.1 & 13.9 & 13.9 & 13.3 & 13.9 \\
\hline $1 \mathrm{~A}$ & S & \#\#\#\#\#\#\#\# & 93288 & 288 & 24 & 14.4 & 15 & 15 & 15.1 \\
\hline $0 \mathrm{~A}$ & S & \#\#\#\#\#\#\#\# & 93289 & 289 & 25.5 & 14.9 & 15 & 14.9 & 15.1 \\
\hline $0 \mathrm{~A}$ & S & \#\#\#\#\#\#\#\# & 93290 & 290 & 12.8 & 12.8 & 12.3 & 12.9 & 12.9 \\
\hline $1 \mathrm{~A}$ & $S$ & \#\#\#\#\#\#\#\# & 93291 & 291 & 11.8 & 12.5 & 12.5 & 13.1 & 12.4 \\
\hline $1 \mathrm{~A}$ & S & \#\#\#\#\#\#\#\# & 93292 & 292 & 22.3 & 13.8 & 13.2 & 13.9 & 13.2 \\
\hline $1 \mathrm{~A}$ & $\mathrm{~S}$ & \#\#\#\#\#\#\#\# & 93293 & 293 & 25.4 & 14.1 & 13.5 & 14.2 & 13.5 \\
\hline $1 \mathrm{~A}$ & S & \#\#\#\#\#\#\#\# & 93294 & 294 & 28 & 14.1 & 13.5 & 14.1 & 14.2 \\
\hline $1 \mathrm{~A}$ & S & \#\#\#\#\#\#\#\# & 93295 & 295 & 26.3 & 13.9 & 13.4 & 13.9 & 14 \\
\hline $0 \mathrm{~A}$ & S & \#\#\#\#\#\#\#\# & 93296 & 296 & 22.7 & 13.5 & 14.2 & 14.1 & 14.2 \\
\hline $0 \mathrm{~A}$ & S & \#\#\#\#\#\#\#\# & 93297 & 297 & 14 & 14.2 & 13.5 & 14.2 & 14.2 \\
\hline $1 \mathrm{~A}$ & S & \#\#\#\#\#\#\#\# & 93298 & 298 & 14.1 & 14.1 & 14.2 & 14.2 & 13.5 \\
\hline $1 \mathrm{~A}$ & S & \#\#\#\#\#\#\#\# & 93299 & 299 & 44.6 & 21.2 & 13 & 13.5 & 13.5 \\
\hline $1 \mathrm{~A}$ & $S$ & \#\#\#\#\#\#\#\# & 93300 & 300 & 26.7 & 13.5 & 14.1 & 14.1 & 14.2 \\
\hline $1 \mathrm{~A}$ & S & \#\#\#\#\#\#\#\# & 93301 & 301 & 26.4 & 15.1 & 13.6 & 14.3 & 14.3 \\
\hline $1 \mathrm{~A}$ & S & \#\#\#\#\#\#\#\# & 93302 & 302 & 27 & 18.2 & 18.3 & 18.3 & 18.2 \\
\hline $0 \mathrm{~A}$ & $\mathrm{~S}$ & \#\#\#\#\#\#\#\# & 93303 & 303 & 30.7 & 13.5 & 13.6 & 14.2 & 13.5 \\
\hline
\end{tabular}


lights

\begin{tabular}{|c|c|c|c|c|c|c|c|c|c|}
\hline $\begin{array}{ll}\mathrm{A} \\
\end{array}$ & $S$ & \#\#\#\#\#\#\#\# & 93304 & 304 & 14.1 & 14.1 & 14.2 & 14.2 & 14.1 \\
\hline $1 \mathrm{~A}$ & S & $11 / 1 / 1993$ & 93305 & 305 & 14.1 & 14.2 & 14.7 & 14.1 & 14.1 \\
\hline $1 \mathrm{~A}$ & $S$ & $11 / 2 / 1993$ & 93306 & 306 & 22.3 & 13.5 & 13.5 & 12.9 & 13.5 \\
\hline $1 \mathrm{~A}$ & $\mathrm{~S}$ & $11 / 3 / 1993$ & 93307 & 307 & 22.6 & 13.5 & 14.2 & 14.1 & 13.5 \\
\hline $1 \mathrm{~A}$ & S & $11 / 4 / 1993$ & 93308 & 308 & 24.5 & 15.4 & 15.6 & 15.5 & 15.5 \\
\hline \begin{tabular}{l|l}
$A$ \\
\end{tabular} & $\mathrm{~S}$ & $11 / 5 / 1993$ & 93309 & 309 & 19.8 & 14.1 & 14.1 & 13.5 & 14.1 \\
\hline $0 \mathrm{~A}$ & S & $11 / 6 / 1993$ & 93310 & 310 & 24 & 14 & 13.9 & 14 & 13.3 \\
\hline $0 \mathrm{~A}$ & S & $11 / 7 / 1993$ & 93311 & 311 & 13.1 & 13.1 & 12.6 & 13.2 & 13.1 \\
\hline $1 \mathrm{~A}$ & S & $11 / 8 / 1993$ & 93312 & 312 & 13.2 & 13.2 & 12.5 & 13.2 & 13.1 \\
\hline $1 \mathrm{~A}$ & $\mathrm{~S}$ & 11/9/1993 & 93313 & 313 & 24.7 & 15.1 & 14.5 & 15.1 & 14.5 \\
\hline $1 \mathrm{~A}$ & S & \#\#\#\#\#\#\#\# & 93314 & 314 & 23.5 & 15.7 & 15.5 & 15.1 & 15.5 \\
\hline $1 \mathrm{~A}$ & $S$ & \#\#\#\#\#\#\#\# & 93315 & 315 & 24.9 & 14.2 & 14.1 & 13.6 & 14.2 \\
\hline $1 \mathrm{~A}$ & S & \#\#\#\#\#\#\#\# & 93316 & 316 & 23.9 & 13.8 & 13.3 & 13.3 & 13.3 \\
\hline $0 \mathrm{~A}$ & S & \#\#\#\#\#\#\#\# & 93317 & 317 & 22.5 & 12.8 & 13.5 & 13.4 & 13.4 \\
\hline $\begin{array}{ll}\mathrm{A} \\
\end{array}$ & $\mathrm{S}$ & \#\#\#\#\#\#\#\# & 93318 & 318 & 13.4 & 13.4 & & 4.7 & 13.4 \\
\hline $1 \mathrm{~A}$ & S & \#\#\#\#\#\#\#\# & 93319 & 319 & 13.2 & 13.4 & 13.4 & 13.5 & 13.4 \\
\hline $1 \mathrm{~A}$ & S & \#\#\#\#\#\#\#\# & 93320 & 320 & 24.5 & 16.2 & 16.2 & 16.3 & 16.2 \\
\hline $1 \mathrm{~A}$ & S & \#\#\#\#\#\#\#\# & 93321 & 321 & 20.4 & 14.8 & 14.1 & 14.1 & 14.1 \\
\hline $1 \mathrm{~A}$ & S & \#\#\#\#\#\#\#\# & 93322 & 322 & 21.6 & 14.7 & 14.1 & 14.7 & 14.7 \\
\hline $1 \mathrm{~A}$ & S & \#\#\#\#\#\#\#\# & 93323 & 323 & 25.3 & 16 & 15.4 & 16 & 15.5 \\
\hline $0 \mathrm{~A}$ & S & \#\#\#\#\#\#\#\# & 93324 & 324 & 18.3 & 14.1 & 14.1 & 14.2 & 13.5 \\
\hline $0 \mathrm{~A}$ & S & \#\#\#\#\#\#\#\# & 93325 & 325 & 14.1 & 14.1 & 14.2 & 14.1 & 14.1 \\
\hline $1 \mathrm{~A}$ & $S$ & \#\#\#\#\#\#\#\# & 93326 & 326 & 14.1 & 14.2 & 14.1 & 13.6 & 14.1 \\
\hline $1 \mathrm{~A}$ & S & \#\#\#\#\#\#\#\# & 93327 & 327 & 18 & 10.5 & 9.9 & 10.6 & 10.6 \\
\hline $1 \mathrm{~A}$ & S & \#\#\#\#\#\#\#\# & 93328 & 328 & 19.4 & 10.7 & 10.7 & 10.1 & 10.8 \\
\hline $1 \mathrm{~A}$ & NS & \#\#\#\#\#\#\#\# & 93329 & 329 & 10 & 10.1 & 10.6 & 10.1 & 10 \\
\hline $1 \mathrm{~A}$ & NS & \#\#\#\#\#\#\#\# & 93330 & 330 & 10.1 & 10.6 & 10 & 10.1 & 10 \\
\hline $0 \mathrm{~A}$ & NS & \#\#\#\#\#\#\#\# & 93331 & 331 & 10.6 & 10 & 10.7 & 10 & 10.6 \\
\hline $0 \mathrm{~A}$ & NS & \#\#\#\#\#\#\#\# & 93332 & 332 & 9.8 & 10.5 & 10.4 & 9.8 & 10.5 \\
\hline $1 \mathrm{~A}$ & S & \#\#\#\#\#\#\#\# & 93333 & 333 & 9.8 & 10.4 & 10.5 & 9.8 & 10.5 \\
\hline $1 \mathrm{~A}$ & S & \#\#\#\#\#\#\#\# & 93334 & 334 & 18.4 & 10.5 & 9.9 & 10.4 & 10.5 \\
\hline $1 \mathrm{~A}$ & S & $12 / 1 / 1993$ & 93335 & 335 & 19 & 10.7 & 11.4 & 10.7 & 11.3 \\
\hline $1 \mathrm{~A}$ & S & $12 / 2 / 1993$ & 93336 & 336 & 19.6 & 10.7 & 10.1 & 10.7 & 10.7 \\
\hline $1 \mathrm{~A}$ & S & $12 / 3 / 1993$ & 93337 & 337 & 21.4 & 11.7 & 11.2 & 11.7 & 11.1 \\
\hline $0 \mathrm{~A}$ & S & $12 / 4 / 1993$ & 93338 & 338 & 22.3 & 10.6 & 10.7 & 10.7 & 10.6 \\
\hline $0 \mathrm{~A}$ & S & $12 / 5 / 1993$ & 93339 & 339 & 10.7 & 11.3 & 11.3 & 11.3 & 11.3 \\
\hline $1 \mathrm{~A}$ & S & $12 / 6 / 1993$ & 93340 & 340 & 9.2 & 9.9 & 9.8 & 9.9 & 9.8 \\
\hline $1 \mathrm{~A}$ & S & $12 / 7 / 1993$ & 93341 & 341 & 19.7 & 10.7 & 10.8 & 10.7 & 10.8 \\
\hline $1 \mathrm{~A}$ & $S$ & $12 / 8 / 1993$ & 93342 & 342 & 20.3 & 12.3 & 12.4 & 12.4 & 12.9 \\
\hline $1 \mathrm{~A}$ & S & $12 / 9 / 1993$ & 93343 & 343 & 20.2 & 13.4 & 14 & 13.4 & 13.5 \\
\hline $1 \mathrm{~A}$ & $\mathrm{~S}$ & \#\#\#\#\#\#\#\# & 93344 & 344 & 19.9 & 10.5 & 10.5 & 11.1 & 10.5 \\
\hline $0 \mathrm{~A}$ & S & \#\#\#\#\#\#\#\# & 93345 & 345 & 17.8 & 10 & 10.6 & 10 & 10.6 \\
\hline $0 \mathrm{~A}$ & S & \#\#\#\#\#\#\#\# & 93346 & 346 & 10.6 & 10.6 & 10.6 & 10.6 & 10.5 \\
\hline $1 \mathrm{~A}$ & S & \#\#\#\#\#\#\#\# & 93347 & 347 & 10.5 & 10.6 & 11.1 & 10.6 & 10.6 \\
\hline $1 \mathrm{~A}$ & S & \#\#\#\#\#\#\#\# & 93348 & 348 & 21.4 & 13.3 & 12.7 & 13.2 & 12.7 \\
\hline $1 \mathrm{~A}$ & $\mathrm{~S}$ & \#\#\#\#\#\#\#\# & 93349 & 349 & 21.3 & 13.8 & 13.8 & 13.9 & 13.8 \\
\hline $1 \mathrm{~A}$ & S & \#\#\#\#\#\#\#\# & 93350 & 350 & 17.8 & 14 & 13.5 & 13.5 & 13.5 \\
\hline $1 \mathrm{~A}$ & $S$ & \#\#\#\#\#\#\#\# & 93351 & 351 & 19.5 & 13.2 & 13.1 & 13.2 & 13.7 \\
\hline $0 \mathrm{~A}$ & NS & \#\#\#\#\#\#\#\# & 93352 & 352 & 25.6 & 14.5 & 14.4 & 14.4 & 14.5 \\
\hline $0 \mathrm{~A}$ & NS & \#\#\#\#\#\#\#\# & 93353 & 353 & 14.5 & 15 & 14.5 & 14.5 & 14.5 \\
\hline $1 \mathrm{~A}$ & NS & \#\#\#\#\#\#\#\# & 93354 & 354 & 14.4 & 15 & 14.4 & 14.4 & 14.4 \\
\hline
\end{tabular}


lights

\begin{tabular}{|c|c|c|c|c|c|c|c|c|c|}
\hline \begin{tabular}{l|l}
$1 A$ \\
\end{tabular} & NS & \#\#\#\#\#\#\#\# & 93355 & 355 & 27.4 & 14.6 & 14.7 & 14.6 & 14.6 \\
\hline $1 \mathrm{~A}$ & NS & \#\#\#\#\#\#\#\# & 93356 & 356 & 29.5 & 14 & 14.6 & 13.9 & 14.6 \\
\hline $1 \mathrm{~A}$ & NS & \#\#\#\#\#\#\#\# & 93357 & 357 & 24 & 7.7 & 7.8 & 7.2 & 7.8 \\
\hline $1 \mathrm{~A}$ & NS & \#\#\#\#\#\#\#\# & 93358 & 358 & 7.9 & 7.2 & 7.8 & 7.8 & 7.2 \\
\hline $0 \mathrm{~A}$ & NS & \#\#\#\#\#\#\#\# & 93359 & 359 & 7.2 & 7.8 & 7.2 & 7.8 & 7.8 \\
\hline $0 \mathrm{~A}$ & NS & \#\#\#\#\#\#\#\# & 93360 & 360 & 7.2 & 7.8 & 7.8 & 7.8 & 7.8 \\
\hline $1 \mathrm{~A}$ & NS & \#\#\#\#\#\#\#\# & 93361 & 361 & 7.8 & 7.8 & 7.8 & 7.8 & 7.8 \\
\hline $1 \mathrm{~A}$ & NS & \#\#\#\#\#\#\#\# & 93362 & 362 & 6.8 & 7.3 & 7.3 & 6.7 & 7.4 \\
\hline $1 \mathrm{~A}$ & NS & \#\#\#\#\#\#\#\# & 93363 & 363 & 7.9 & 7.3 & 7.9 & 7.2 & 7.9 \\
\hline $1 \mathrm{~A}$ & NS & \#\#\#\#\#\#\#\# & 93364 & 364 & 9.5 & 10.1 & 10.1 & 9.5 & 10.1 \\
\hline $1 \mathrm{~A}$ & NS & \#\#\#\#\#\#\#\# & 93365 & 365 & 16.7 & 16.1 & 16.2 & 16.1 & 16.7 \\
\hline $0 \mathrm{~A}$ & NS & $1 / 1 / 1994$ & 94001 & 1 & 9.1 & 9.1 & 8.5 & 9.1 & 9 \\
\hline $0 \mathrm{~A}$ & NS & $1 / 2 / 1994$ & 94002 & 2 & 9.1 & 9.1 & 8.5 & 9.1 & 9 \\
\hline $1 \mathrm{~A}$ & S & $1 / 3 / 1994$ & 94003 & 3 & 9.1 & 9.1 & 8.5 & 9.1 & 9.1 \\
\hline $1 \mathrm{~A}$ & S & $1 / 4 / 1994$ & 94004 & 4 & 33 & 17.4 & 12.7 & 13.2 & 13.3 \\
\hline $1 \mathrm{~A}$ & $\mathrm{~S}$ & $1 / 5 / 1994$ & 94005 & 5 & 17.9 & 13.6 & 13 & 13.6 & 13 \\
\hline $1 \mathrm{~A}$ & S & $1 / 6 / 1994$ & 94006 & 6 & 23.9 & 13.6 & 13.6 & 13.6 & 13.7 \\
\hline $1 \mathrm{~A}$ & S & $1 / 7 / 1994$ & 94007 & 7 & 24.4 & 21 & 22.8 & 22.7 & 23.4 \\
\hline $0 \mathrm{~A}$ & S & $1 / 8 / 1994$ & 94008 & 8 & 34.2 & 30.2 & 27.9 & 14.4 & 14.4 \\
\hline $0 \mathrm{~A}$ & $S$ & $1 / 9 / 1994$ & 94009 & 9 & 15.5 & 15.6 & 15.6 & 15 & 15.6 \\
\hline $1 \mathrm{~A}$ & $S$ & 1/10/1994 & 94010 & 10 & 15.6 & 15.7 & 15.6 & 15.6 & 15.1 \\
\hline $1 \mathrm{~A}$ & S & 1/11/1994 & 94011 & 11 & 23.5 & 13.8 & 14.3 & 13.6 & 13.9 \\
\hline $1 \mathrm{~A}$ & S & $1 / 12 / 1994$ & 94012 & 12 & 26.2 & 24.1 & 23.5 & 22.9 & 9.5 \\
\hline $1 \mathrm{~A}$ & S & $1 / 13 / 1994$ & 94013 & 13 & 17.1 & 12.4 & 12.4 & 11.9 & 12.4 \\
\hline $1 \mathrm{~A}$ & $\mathrm{~S}$ & $1 / 14 / 1994$ & 94014 & 14 & 21.6 & 13 & 12.9 & 13.7 & 13.2 \\
\hline $0 \mathrm{~A}$ & S & $1 / 15 / 1994$ & 94015 & 15 & 20.9 & 13.7 & 14.4 & 14.4 & 13.8 \\
\hline $0 \mathrm{~A}$ & S & $1 / 16 / 1994$ & 94016 & 16 & 14.4 & 14.4 & 13.9 & 14.4 & 13.8 \\
\hline $1 \mathrm{~A}$ & NS & $1 / 17 / 1994$ & 94017 & 17 & 13.9 & 14.4 & 13.8 & 14.4 & 14.4 \\
\hline $1 \mathrm{~A}$ & S & $1 / 18 / 1994$ & 94018 & 18 & 16.8 & 16.7 & 16.7 & 17.4 & 16.7 \\
\hline $1 \mathrm{~A}$ & $S$ & 1/19/1994 & 94019 & 19 & 18.7 & 13.6 & 13.1 & 13.7 & 13.2 \\
\hline $1 \mathrm{~A}$ & S & $1 / 20 / 1994$ & 94020 & 20 & 19.3 & 13.8 & 13.2 & 13.9 & 13.8 \\
\hline $1 \mathrm{~A}$ & S & $1 / 21 / 1994$ & 94021 & 21 & 26.6 & 15.9 & 16.5 & 15.9 & 15.9 \\
\hline $0 \mathrm{~A}$ & S & $1 / 22 / 1994$ & 94022 & 22 & 21.9 & 12 & 12.6 & 12.4 & 12 \\
\hline $0 \mathrm{~A}$ & S & $1 / 23 / 1994$ & 94023 & 23 & 13.6 & 13.7 & 14.4 & 13.8 & 13.7 \\
\hline $1 \mathrm{~A}$ & S & $1 / 24 / 1994$ & 94024 & 24 & 13.6 & 14.3 & 13.8 & 13.8 & 14.4 \\
\hline $1 \mathrm{~A}$ & S & $1 / 25 / 1994$ & 94025 & 25 & 19.3 & 13.7 & 13.9 & 13.8 & 13.9 \\
\hline $1 \mathrm{~A}$ & S & $1 / 26 / 1994$ & 94026 & 26 & 26.8 & 15.9 & 16 & 16.5 & 16 \\
\hline $1 \mathrm{~A}$ & S & $1 / 27 / 1994$ & 94027 & 27 & 23.7 & 14.1 & 13 & 12.9 & 13 \\
\hline $1 \mathrm{~A}$ & $S$ & $1 / 28 / 1994$ & 94028 & 28 & 22 & 12.3 & 12.8 & 12.9 & 12.9 \\
\hline $0 \mathrm{~A}$ & S & $1 / 29 / 1994$ & 94029 & 29 & 19.2 & 11.9 & 12.4 & 11.9 & 12.4 \\
\hline $0 \mathrm{~A}$ & S & $1 / 30 / 1994$ & 94030 & 30 & 11.9 & 12.4 & 11.9 & 11.9 & 12.5 \\
\hline $1 \mathrm{~A}$ & S & $1 / 31 / 1994$ & 94031 & 31 & 12.5 & 11.9 & 12.5 & 12.4 & 11.9 \\
\hline $1 \mathrm{~A}$ & S & 2/1/1994 & 94032 & 32 & 20.7 & 13.1 & 13.6 & 13.1 & 13 \\
\hline $1 \mathrm{~A}$ & S & 2/2/1994 & 94033 & 33 & 28.6 & 15.2 & 15.2 & 15.2 & 14.6 \\
\hline $1 \mathrm{~A}$ & S & $2 / 3 / 1994$ & 94034 & 34 & 22.3 & 12.9 & 13 & 12.9 & 12.9 \\
\hline $1 \mathrm{~A}$ & NS & 2/4/1994 & 94035 & 35 & 26.6 & 14.6 & 15.1 & 14.6 & 15.2 \\
\hline $0 \mathrm{~A}$ & S & 2/5/1994 & 94036 & 36 & 23.1 & 16.1 & 15.5 & 16.1 & 16.1 \\
\hline $0 \mathrm{~A}$ & $S$ & 2/6/1994 & 94037 & 37 & 16.1 & 15.4 & 16.1 & 16.1 & 15.5 \\
\hline $1 \mathrm{~A}$ & S & 2/7/1994 & 94038 & 38 & 16.1 & 16 & 15.5 & 16.1 & 16.1 \\
\hline $1 \mathrm{~A}$ & S & $2 / 8 / 1994$ & 94039 & 39 & 22.6 & 14 & 13.4 & 14 & 13.4 \\
\hline $1 \mathrm{~A}$ & $S$ & $2 / 9 / 1994$ & 94040 & 40 & 21.6 & 12.2 & 12.3 & 12.2 & 11.7 \\
\hline
\end{tabular}


lights

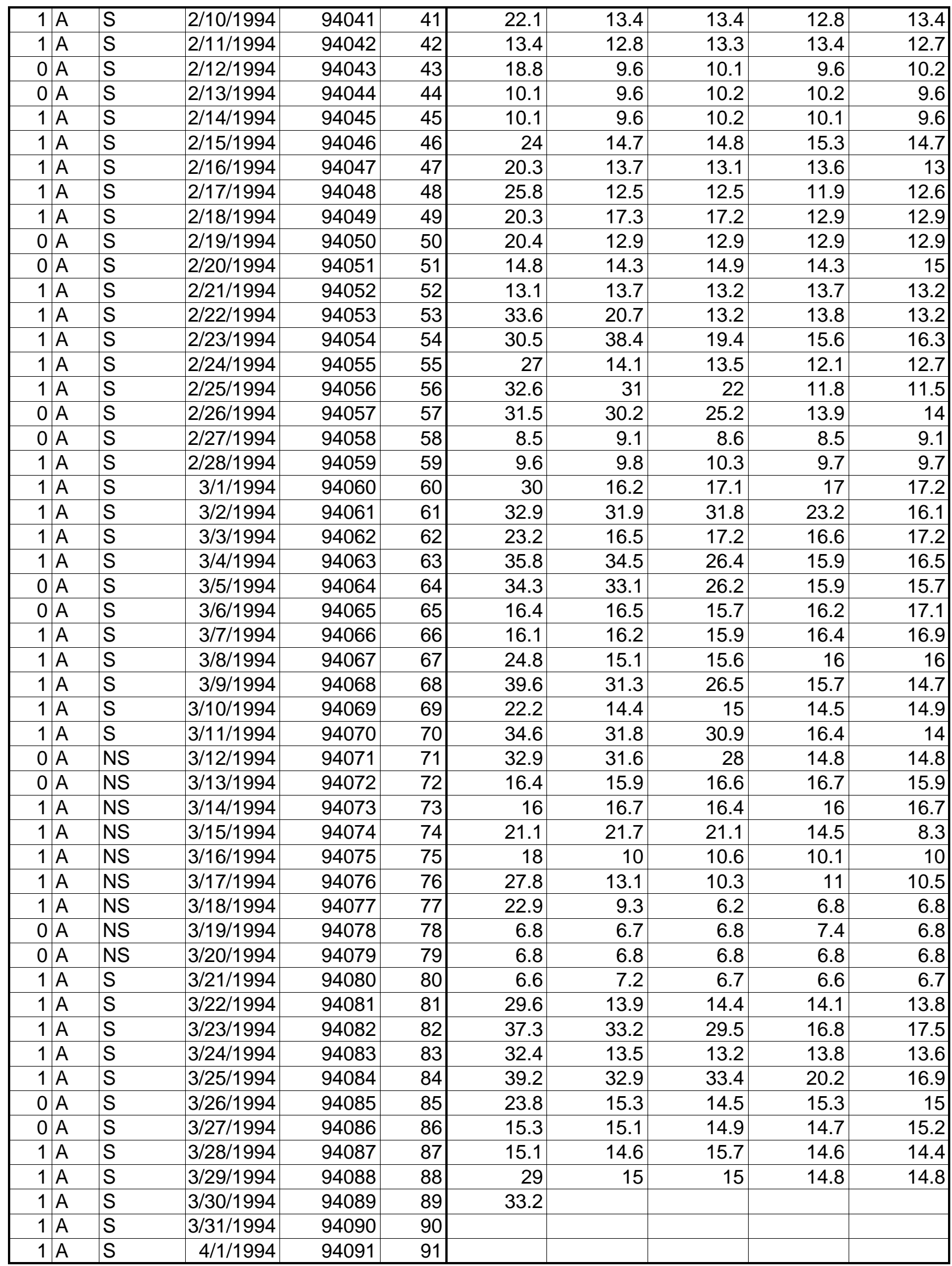


lights

\begin{tabular}{|c|c|c|c|c|c|c|c|c|c|}
\hline $0 \mathrm{~A}$ & $\mathrm{~S}$ & $4 / 2 / 1994$ & 94092 & 92 & & & & & \\
\hline $0 \mathrm{~A}$ & S & 4/3/1994 & 94093 & 93 & & & & & \\
\hline $1 \mathrm{~A}$ & S & 4/4/1994 & 94094 & 94 & & & & & \\
\hline $1 \mathrm{~A}$ & S & $4 / 5 / 1994$ & 94095 & 95 & 22.8 & 13.5 & 13 & 13.6 & 14.1 \\
\hline $1 \mathrm{~A}$ & S & 4/6/1994 & 94096 & 96 & 31.3 & 31.1 & 31.1 & 25.7 & 14.1 \\
\hline $1 \mathrm{~A}$ & $\mathrm{~S}$ & $4 / 7 / 1994$ & 94097 & 97 & 23.3 & 15.2 & 14.3 & 15.1 & 15.2 \\
\hline $1 \mathrm{~A}$ & S & 4/8/1994 & 94098 & 98 & 28 & 12.9 & 13.6 & 13.2 & 12.6 \\
\hline $0 \mathrm{~A}$ & S & $4 / 9 / 1994$ & 94099 & 99 & 23.3 & 15 & 15.4 & 15.9 & 15.8 \\
\hline $0 \mathrm{~A}$ & S & 4/10/1994 & 94100 & 100 & 15.5 & 15.5 & 15.3 & 15.8 & 15.3 \\
\hline $1 \mathrm{~A}$ & S & 4/11/1994 & 94101 & 101 & 15.5 & 15.2 & 15.5 & 14.9 & 16 \\
\hline $1 \mathrm{~A}$ & $\mathrm{~S}$ & 4/12/1994 & 94102 & 102 & 25.6 & 12.9 & 13.7 & 12.5 & 13.7 \\
\hline $1 \mathrm{~A}$ & $\mathrm{~S}$ & 4/13/1994 & 94103 & 103 & 32.8 & 29.7 & 29.4 & 20 & 13.2 \\
\hline $1 \mathrm{~B}$ & S & 4/14/1994 & 94104 & 104 & 33.6 & 15.8 & 15.7 & 15.9 & 15.4 \\
\hline $1 \mathrm{~B}$ & S & 4/15/1994 & 94105 & 105 & 14.4 & 2.3 & 2.2 & 2.3 & 2.3 \\
\hline $0 \mathrm{~B}$ & S & 4/16/1994 & 94106 & 106 & 16.7 & 2.4 & 2.4 & 1.8 & 2.4 \\
\hline $0 \mathrm{~B}$ & S & 4/17/1994 & 94107 & 107 & 2.4 & 1.8 & 2.4 & 1.8 & 2.4 \\
\hline $1 \mathrm{~B}$ & S & 4/18/1994 & 94108 & 108 & 1.8 & 2.4 & 2.4 & 1.8 & 2.4 \\
\hline $1 \mathrm{~B}$ & S & 4/19/1994 & 94109 & 109 & 12.9 & 1.8 & 2.4 & 1.8 & 2.4 \\
\hline $1 \mathrm{~B}$ & S & 4/20/1994 & 94110 & 110 & 21.4 & 1.8 & 2.4 & 1.8 & 2.4 \\
\hline $1 \mathrm{~B}$ & S & 4/21/1994 & 94111 & 111 & 18.2 & 2.4 & 1.8 & 2.4 & 1.8 \\
\hline $1 \mathrm{~B}$ & S & 4/22/1994 & 94112 & 112 & 15.9 & 1.8 & 2.4 & 2.2 & 1.7 \\
\hline $0 \mathrm{~B}$ & S & 4/23/1994 & 94113 & 113 & 16.4 & 3.5 & 3.4 & 3.5 & 3.6 \\
\hline $0 \mathrm{~B}$ & S & 4/24/1994 & 94114 & 114 & 2.3 & 1.6 & 2.5 & 1.8 & 2.3 \\
\hline $1 \mathrm{~B}$ & S & $4 / 25 / 1994$ & 94115 & 115 & 2.2 & 1.7 & 2.3 & 1.7 & 2.3 \\
\hline $1 \mathrm{~B}$ & S & 4/26/1994 & 94116 & 116 & 16.2 & 2.2 & 1.7 & 2.2 & 2.3 \\
\hline $1 \mathrm{~B}$ & S & 4/27/1994 & 94117 & 117 & 15 & 2.3 & 1.7 & 2.2 & 1.6 \\
\hline $1 \mathrm{~B}$ & S & 4/28/1994 & 94118 & 118 & 16.7 & 1.7 & 2.2 & 1.7 & 2.3 \\
\hline $1 \mathrm{~B}$ & S & 4/29/1994 & 94119 & 119 & 15.2 & 2.3 & 2.3 & 2.3 & 2.3 \\
\hline $0 \mathrm{~B}$ & S & 4/30/1994 & 94120 & 120 & 18.1 & 2.3 & 2.3 & 2.3 & 2.3 \\
\hline $0 \mathrm{~B}$ & S & 5/1/1994 & 94121 & 121 & 2.3 & 2.3 & 2.3 & 2.3 & 2.2 \\
\hline $1 \mathrm{~B}$ & S & $5 / 2 / 1994$ & 94122 & 122 & 2.2 & 2.3 & 2.3 & 2.9 & 2.2 \\
\hline $1 \mathrm{~B}$ & S & $5 / 3 / 1994$ & 94123 & 123 & 15.1 & 2.3 & 2.3 & 2.3 & 2.3 \\
\hline $1 \mathrm{~B}$ & S & $5 / 4 / 1994$ & 94124 & 124 & 18.3 & 3.4 & 3.5 & 3.4 & 3.5 \\
\hline $1 \mathrm{~B}$ & S & $5 / 5 / 1994$ & 94125 & 125 & 19 & 3.5 & 3.6 & 3.4 & 3.5 \\
\hline $1 \mathrm{~B}$ & S & $5 / 6 / 1994$ & 94126 & 126 & 17.2 & 3.5 & 3.5 & 3.5 & 3.5 \\
\hline $0 \mathrm{~B}$ & S & $5 / 7 / 1994$ & 94127 & 127 & 14.1 & 3.5 & 3.5 & 3.4 & 3.5 \\
\hline $0 \mathrm{~B}$ & S & $5 / 8 / 1994$ & 94128 & 128 & 3.6 & 3.6 & 3.4 & 3.5 & 3.6 \\
\hline $1 \mathrm{~B}$ & $\mathrm{~S}$ & 5/9/1994 & 94129 & 129 & 3.4 & 3.5 & 3.4 & 3.5 & 3.4 \\
\hline $1 \mathrm{~B}$ & $S$ & 5/10/1994 & 94130 & 130 & 13.5 & 3.5 & 3.5 & 3.4 & 3.5 \\
\hline $1 \mathrm{~B}$ & S & $5 / 11 / 1994$ & 94131 & 131 & 13.3 & 3.4 & 3.5 & 3.4 & 4.1 \\
\hline $1 \mathrm{~B}$ & S & $5 / 12 / 1994$ & 94132 & 132 & 14.7 & 3.6 & 3.6 & 3.5 & 3.5 \\
\hline $1 \mathrm{~B}$ & S & $5 / 13 / 1994$ & 94133 & 133 & 14 & 3.6 & 3.5 & 3.5 & 3.5 \\
\hline $0 \mathrm{~B}$ & S & $5 / 14 / 1994$ & 94134 & 134 & 16 & 3.6 & 3.6 & 3.5 & 3.5 \\
\hline $0 \mathrm{~B}$ & S & $5 / 15 / 1994$ & 94135 & 135 & 3.6 & 3 & 3.4 & 3.5 & 3.4 \\
\hline $1 \mathrm{~B}$ & S & $5 / 16 / 1994$ & 94136 & 136 & 14.2 & 15.8 & 15.8 & 4 & 3.5 \\
\hline $1 \mathrm{~B}$ & S & $5 / 17 / 1994$ & 94137 & 137 & 13.3 & 3.5 & 3.6 & 3.6 & 3.6 \\
\hline $1 \mathrm{~B}$ & S & $5 / 18 / 1994$ & 94138 & 138 & 17.1 & 3.4 & 3.5 & 3.4 & 3.5 \\
\hline $1 \mathrm{~B}$ & $S$ & 5/19/1994 & 94139 & 139 & 11.8 & 3.5 & 3.5 & 3.5 & 3.5 \\
\hline $1 \mathrm{~B}$ & S & $5 / 20 / 1994$ & 94140 & 140 & 15.6 & 3.5 & 3.5 & 3.6 & 3.5 \\
\hline $0 \mathrm{~B}$ & S & $5 / 21 / 1994$ & 94141 & 141 & 13.6 & 3.4 & 3.4 & 3.5 & 3.5 \\
\hline $0 \mathrm{~B}$ & $S$ & $5 / 22 / 1994$ & 94142 & 142 & 3.5 & 3.6 & 3.5 & 3.5 & 3.4 \\
\hline
\end{tabular}


lights

\begin{tabular}{|c|c|c|c|c|c|c|c|c|c|}
\hline $\begin{array}{ll}1 & B \\
\end{array}$ & $S$ & $5 / 23 / 1994$ & 94143 & 143 & 3.6 & 3.4 & 3.5 & 3.4 & 3.5 \\
\hline $1 \mathrm{~B}$ & $\mathrm{~S}$ & $5 / 24 / 1994$ & 94144 & 144 & 17.5 & 3.5 & 3.5 & 3.5 & 3.5 \\
\hline $1 \mathrm{~B}$ & S & $5 / 25 / 1994$ & 94145 & 145 & 15.5 & 3.4 & 3.5 & 3.4 & 3.5 \\
\hline $1 \mathrm{~B}$ & S & $5 / 26 / 1994$ & 94146 & 146 & 13.3 & 2.8 & 3.4 & 3.4 & 3.5 \\
\hline $1 \mathrm{~B}$ & S & $5 / 27 / 1994$ & 94147 & 147 & 17.4 & 3.4 & 3.5 & 3.4 & 3.4 \\
\hline $0 \mathrm{~B}$ & $\mathrm{~S}$ & $5 / 28 / 1994$ & 94148 & 148 & 12.9 & 3.4 & 3.5 & 3.4 & 16.6 \\
\hline $0 \mathrm{~B}$ & $\mathrm{~S}$ & $5 / 29 / 1994$ & 94149 & 149 & 3.4 & 3.4 & 3.5 & 3.5 & 3.4 \\
\hline $1 \mathrm{~B}$ & S & $5 / 30 / 1994$ & 94150 & 150 & 3.4 & 3.5 & 3.4 & 3.5 & 3.4 \\
\hline $1 \mathrm{~B}$ & S & 5/31/1994 & 94151 & 151 & 10.5 & 3.4 & 3.4 & 3.4 & 3.5 \\
\hline $1 \mathrm{~B}$ & S & 6/1/1994 & 94152 & 152 & 8.8 & 3.4 & 3.4 & 3.4 & 2.9 \\
\hline $1 \mathrm{~B}$ & $\mathrm{~S}$ & $6 / 2 / 1994$ & 94153 & 153 & 14.2 & 2.8 & 3.4 & 3.4 & 3.4 \\
\hline $1 \mathrm{~B}$ & $S$ & 6/3/1994 & 94154 & 154 & 8.7 & 3.4 & 3.4 & 2.8 & 3.5 \\
\hline $0 \mathrm{~B}$ & S & $6 / 4 / 1994$ & 94155 & 155 & 8.2 & 3.4 & 2.8 & 3.4 & 3.5 \\
\hline $0 \mathrm{~B}$ & S & $6 / 5 / 1994$ & 94156 & 156 & 3.3 & 3.3 & 2.7 & 3.3 & 3.3 \\
\hline \begin{tabular}{l|l}
1 & $B$ \\
\end{tabular} & $\mathrm{~S}$ & 6/6/1994 & 94157 & 157 & 3.3 & 3.3 & 3.3 & 3.2 & 2.7 \\
\hline $1 \mathrm{~B}$ & $\mathrm{~S}$ & $6 / 7 / 1994$ & 94158 & 158 & 9.3 & 3.5 & 3.4 & 2.9 & 3.5 \\
\hline $1 \mathrm{~B}$ & S & 6/8/1994 & 94159 & 159 & 8 & 3.5 & 3.4 & 3.5 & 3.4 \\
\hline $1 \mathrm{~B}$ & S & 6/9/1994 & 94160 & 160 & 8.4 & 3.5 & 3.4 & 3.4 & 3.5 \\
\hline $1 \mathrm{~B}$ & S & 6/10/1994 & 94161 & 161 & 8.2 & 3.4 & 3.5 & 3.5 & 3.4 \\
\hline $0 \mathrm{~B}$ & $S$ & 6/11/1994 & 94162 & 162 & 7.8 & 3.4 & 3.4 & 2.8 & 3.4 \\
\hline $0 \mathrm{~B}$ & NS & 6/12/1994 & 94163 & 163 & 3.4 & 3.4 & 3.5 & 3.4 & 3.4 \\
\hline $1 \mathrm{~B}$ & NS & 6/13/1994 & 94164 & 164 & 3.4 & 3.4 & 3.4 & 2.8 & 3.4 \\
\hline $1 \mathrm{~B}$ & NS & 6/14/1994 & 94165 & 165 & 7.6 & 3.4 & 3.4 & 3.4 & 3.5 \\
\hline \begin{tabular}{l|l}
1 & $B$ \\
\end{tabular} & NS & 6/15/1994 & 94166 & 166 & 7.9 & 2.8 & 3.5 & 3.4 & 4 \\
\hline $1 \mathrm{~B}$ & NS & 6/16/1994 & 94167 & 167 & 8.6 & 3.4 & 3.4 & 3.5 & 3.4 \\
\hline $1 \mathrm{~B}$ & NS & 6/17/1994 & 94168 & 168 & 8.7 & 2.8 & 3.4 & 3.4 & 3.4 \\
\hline $0 \mathrm{~B}$ & NS & 6/18/1994 & 94169 & 169 & 8.9 & 3.4 & 3.4 & 3.4 & 3.4 \\
\hline $0 \mathrm{~B}$ & NS & 6/19/1994 & 94170 & 170 & 3.4 & 3.4 & 3.3 & 3.4 & 3.4 \\
\hline $1 B$ & NS & $6 / 20 / 1994$ & 94171 & 171 & 3.4 & 3.4 & 2.8 & 3.4 & 3.4 \\
\hline $1 \mathrm{~B}$ & NS & 6/21/1994 & 94172 & 172 & 10.5 & 3.4 & 3.4 & 2.8 & 3.4 \\
\hline $1 \mathrm{~B}$ & NS & $6 / 22 / 1994$ & 94173 & 173 & 9.9 & 3.4 & 3.4 & 3.4 & 3.3 \\
\hline $1 \mathrm{~B}$ & NS & $6 / 23 / 1994$ & 94174 & 174 & 8.5 & 3.4 & 3.4 & 3.4 & 3.4 \\
\hline $1 \mathrm{~B}$ & NS & $6 / 24 / 1994$ & 94175 & 175 & 8.1 & 3.4 & 3.3 & 3.4 & 3.3 \\
\hline $0 \mathrm{~B}$ & NS & $6 / 25 / 1994$ & 94176 & 176 & 8.3 & 3.4 & 2.8 & 3.4 & 3.4 \\
\hline $0 \mathrm{~B}$ & NS & 6/26/1994 & 94177 & 177 & 3.4 & 3.4 & 3.4 & 3.4 & 2.8 \\
\hline $1 \mathrm{~B}$ & NS & 6/27/1994 & 94178 & 178 & 3.4 & 3.4 & 3.4 & 3.4 & 3.4 \\
\hline $1 \mathrm{~B}$ & NS & 6/28/1994 & 94179 & 179 & 8.5 & 3.4 & 3.4 & 3.4 & 3.4 \\
\hline $1 \mathrm{~B}$ & NS & 6/29/1994 & 94180 & 180 & 7.6 & 3.4 & 3.4 & 3.4 & 3.4 \\
\hline $1 \mathrm{~B}$ & NS & 6/30/1994 & 94181 & 181 & 7.5 & 3.4 & 3.4 & 3.4 & 3.4 \\
\hline $1 \mathrm{~B}$ & NS & $7 / 1 / 1994$ & 94182 & 182 & 7.5 & 3.4 & 3.4 & 3.5 & 3.4 \\
\hline $0 \mathrm{~B}$ & NS & $7 / 2 / 1994$ & 94183 & 183 & 7.9 & 3.4 & 3.4 & 3.4 & 3.4 \\
\hline $0 \mathrm{~B}$ & NS & $7 / 3 / 1994$ & 94184 & 184 & 3.4 & 3.4 & 3.4 & 2.8 & 3.4 \\
\hline $1 \mathrm{~B}$ & NS & $7 / 4 / 1994$ & 94185 & 185 & 3.4 & 3.5 & 3.4 & 3.4 & 3.4 \\
\hline $1 \mathrm{~B}$ & NS & $7 / 5 / 1994$ & 94186 & 186 & 7.5 & 3.4 & 3.3 & 3.4 & 3.4 \\
\hline $1 \mathrm{~B}$ & NS & $7 / 6 / 1994$ & 94187 & 187 & 7.2 & 3.4 & 3.4 & 3.4 & 3.4 \\
\hline $1 \mathrm{~B}$ & NS & 7/7/1994 & 94188 & 188 & 7.4 & 3.4 & 3.3 & 3.4 & 3.3 \\
\hline $1 \mathrm{~B}$ & NS & $7 / 8 / 1994$ & 94189 & 189 & 7.1 & 3.4 & 3.3 & 3.3 & 3.3 \\
\hline $0 \mathrm{~B}$ & NS & 7/9/1994 & 94190 & 190 & 7.9 & 2.8 & 3.4 & 3.3 & 3.3 \\
\hline $\begin{array}{ll}0 & B \\
\end{array}$ & NS & $7 / 10 / 1994$ & 94191 & 191 & 3.4 & 3.3 & 3.4 & 3.4 & 3.3 \\
\hline $1 \mathrm{~B}$ & NS & 7/11/1994 & 94192 & 192 & 3.4 & 3.3 & 3.4 & 3.3 & 3.4 \\
\hline $1 \mathrm{~B}$ & NS & $7 / 12 / 1994$ & 94193 & 193 & 10.3 & 3.4 & 3.3 & 3.4 & 3.3 \\
\hline
\end{tabular}


lights

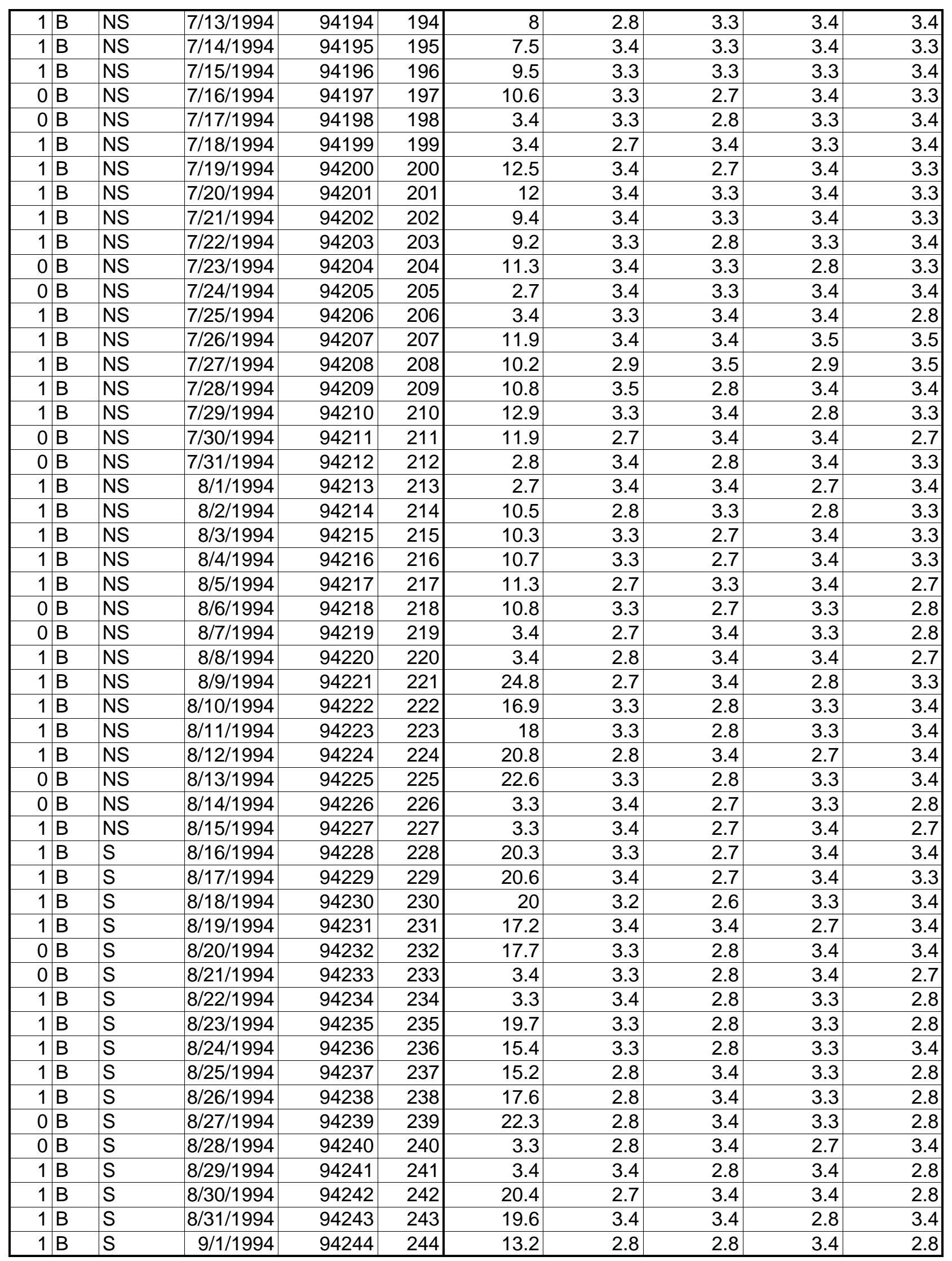


lights

\begin{tabular}{|c|c|c|c|c|c|c|c|c|c|}
\hline $\begin{array}{ll}1 & B \\
\end{array}$ & $S$ & 9/2/1994 & 94245 & 245 & 23.2 & 3.4 & 2.8 & 3.4 & 2.8 \\
\hline $\begin{array}{ll}0 & B \\
\end{array}$ & $\mathrm{~S}$ & $9 / 3 / 1994$ & 94246 & 246 & 18.3 & 2.9 & 3.4 & 2.8 & 3.4 \\
\hline $0 \mathrm{~B}$ & S & 9/4/1994 & 94247 & 247 & 3.4 & 2.8 & 2.9 & 3.4 & 2.8 \\
\hline $1 \mathrm{~B}$ & NS & 9/5/1994 & 94248 & 248 & 3.4 & 2.8 & 3.4 & 2.8 & 2.8 \\
\hline $1 \mathrm{~B}$ & $\mathrm{~S}$ & 9/6/1994 & 94249 & 249 & 11.6 & 2.9 & 3.5 & 2.9 & 3.5 \\
\hline $1 \mathrm{~B}$ & $S$ & 9/7/1994 & 94250 & 250 & 18.4 & 3.4 & 2.9 & 2.9 & 3.5 \\
\hline $1 \mathrm{~B}$ & $\mathrm{~S}$ & 9/8/1994 & 94251 & 251 & 18.1 & 3.5 & 2.9 & 3.5 & 2.9 \\
\hline $1 \mathrm{~B}$ & S & 9/9/1994 & 94252 & 252 & 17.2 & 2.9 & 2.9 & 3.6 & 2.9 \\
\hline $0 \mathrm{~B}$ & S & 9/10/1994 & 94253 & 253 & 20.5 & 3.6 & 2.9 & 3.5 & 2.9 \\
\hline $\begin{array}{ll}0 & B \\
\end{array}$ & $\mathrm{~S}$ & 9/11/1994 & 94254 & 254 & 3.5 & 2.9 & 2.9 & 3.5 & 2.9 \\
\hline $1 \mathrm{~B}$ & $\mathrm{~S}$ & 9/12/1994 & 94255 & 255 & 3.5 & 2.9 & 2.9 & 3.6 & 2.9 \\
\hline $1 \mathrm{~B}$ & S & 9/13/1994 & 94256 & 256 & 15.6 & 2.9 & 3.5 & 2.9 & 2.9 \\
\hline $1 \mathrm{~B}$ & S & 9/14/1994 & 94257 & 257 & 18.4 & 2.9 & 2.9 & 3.5 & 2.9 \\
\hline $1 \mathrm{~B}$ & $S$ & 9/15/1994 & 94258 & 258 & 17.5 & 3.5 & 2.9 & 3.5 & 2.9 \\
\hline \begin{tabular}{l|l}
1 & $B$ \\
\end{tabular} & $\mathrm{~S}$ & 9/16/1994 & 94259 & 259 & 18.4 & 2.9 & 3.5 & 2.9 & 3.5 \\
\hline $0 \mathrm{~B}$ & $\mathrm{~S}$ & 9/17/1994 & 94260 & 260 & 20.7 & 2.9 & 2.9 & 3.6 & 2.9 \\
\hline $0 \mathrm{~B}$ & S & 9/18/1994 & 94261 & 261 & 3 & 3.5 & 2.9 & 2.8 & 3.5 \\
\hline $1 \mathrm{~B}$ & S & 9/19/1994 & 94262 & 262 & 2.8 & 3.4 & 2.8 & 2.9 & 3.4 \\
\hline $1 \mathrm{~B}$ & S & 9/20/1994 & 94263 & 263 & 20.7 & 2.8 & 3.4 & 2.8 & 3.5 \\
\hline $1 \mathrm{~B}$ & S & 9/21/1994 & 94264 & 264 & 16.8 & 2.8 & 2.8 & 3.4 & 2.8 \\
\hline $1 \mathrm{~B}$ & $S$ & 9/22/1994 & 94265 & 265 & 20.7 & 3.4 & 2.8 & 2.8 & 3.4 \\
\hline $1 \mathrm{~B}$ & S & 9/23/1994 & 94266 & 266 & 22.2 & 2.8 & 2.8 & 3.5 & 2.8 \\
\hline $\begin{array}{ll}0 & B \\
\end{array}$ & $\mathrm{~S}$ & 9/24/1994 & 94267 & 267 & 16.2 & 3.4 & 2.8 & 2.9 & 3.4 \\
\hline \begin{tabular}{l|l}
0 & $B$ \\
\end{tabular} & $S$ & 9/25/1994 & 94268 & 268 & 3.4 & 2.8 & 2.9 & 3.4 & 2.8 \\
\hline $1 \mathrm{~B}$ & $\mathrm{~S}$ & 9/26/1994 & 94269 & 269 & 2.8 & 3.4 & 2.8 & 3.5 & 2.8 \\
\hline $1 \mathrm{~B}$ & S & 9/27/1994 & 94270 & 270 & 20 & 3.3 & 2.8 & 2.8 & 3.4 \\
\hline $1 \mathrm{~B}$ & S & 9/28/1994 & 94271 & 271 & 18.8 & 2.8 & 2.7 & 3.4 & 2.8 \\
\hline $1 \mathrm{~B}$ & S & 9/29/1994 & 94272 & 272 & 18.5 & 2.7 & 3.4 & 2.7 & 3.4 \\
\hline $1 B$ & $S$ & 9/30/1994 & 94273 & 273 & 20.1 & 3.3 & 2.8 & 3.3 & 2.8 \\
\hline $0 \mathrm{~B}$ & $S$ & 10/1/1994 & 94274 & 274 & 15.5 & 2.8 & 3.3 & 2.8 & 2.7 \\
\hline $0 \mathrm{~B}$ & S & 10/2/1994 & 94275 & 275 & 3.4 & 2.7 & 2.8 & 3.4 & 2.8 \\
\hline $1 \mathrm{~B}$ & S & $10 / 3 / 1994$ & 94276 & 276 & 2.7 & 2.8 & 3.3 & 2.8 & 3.3 \\
\hline $1 \mathrm{~B}$ & S & $10 / 4 / 1994$ & 94277 & 277 & 20.7 & 3.4 & 2.8 & 3.4 & 2.7 \\
\hline $1 \mathrm{~B}$ & S & $10 / 5 / 1994$ & 94278 & 278 & 21.1 & 2.8 & 2.8 & 3.4 & 2.7 \\
\hline $1 \mathrm{~B}$ & S & $10 / 6 / 1994$ & 94279 & 279 & 20.3 & 2.8 & 3.3 & 2.8 & 2.7 \\
\hline $1 \mathrm{~B}$ & S & $10 / 7 / 1994$ & 94280 & 280 & 15.1 & 3.3 & 2.8 & 3.4 & 2.7 \\
\hline $\begin{array}{ll}0 & B \\
\end{array}$ & S & $10 / 8 / 1994$ & 94281 & 281 & 23.2 & 2.8 & 8.8 & 9.4 & 10 \\
\hline $0 \mathrm{~B}$ & S & $10 / 9 / 1994$ & 94282 & 282 & 2.8 & 3.4 & 2.8 & 3.5 & 2.8 \\
\hline $1 \mathrm{~B}$ & $S$ & \#\#\#\#\#\#\#\# & 94283 & 283 & 3.4 & 2.8 & 3.4 & 2.9 & 3.4 \\
\hline $\begin{array}{ll}1 \mathrm{~B} \\
\end{array}$ & S & \#\#\#\#\#\#\#\# & 94284 & 284 & 22 & 2.8 & 3.4 & 2.8 & 3.5 \\
\hline $1 \mathrm{~B}$ & S & \#\#\#\#\#\#\#\# & 94285 & 285 & 19.8 & 3.5 & 2.8 & 3.4 & 2.9 \\
\hline $1 \mathrm{~B}$ & S & \#\#\#\#\#\#\#\# & 94286 & 286 & 22.1 & 3.4 & 2.8 & 3.5 & 2.8 \\
\hline $1 \mathrm{~B}$ & S & \#\#\#\#\#\#\#\# & 94287 & 287 & 21.2 & 2.8 & 3.4 & 2.8 & 2.8 \\
\hline $0 \mathrm{~B}$ & S & \#\#\#\#\#\#\#\# & 94288 & 288 & 20.3 & 3.4 & 2.8 & 3.4 & 2.9 \\
\hline $0 \mathrm{~B}$ & S & \#\#\#\#\#\#\#\# & 94289 & 289 & 2.8 & 3.4 & 2.9 & 3.4 & 2.8 \\
\hline \begin{tabular}{l|l}
$1 \mathrm{~B}$ \\
\end{tabular} & S & \#\#\#\#\#\#\#\# & 94290 & 290 & 2.8 & 2.7 & 3.4 & 2.8 & 2.8 \\
\hline $1 \mathrm{~B}$ & S & \#\#\#\#\#\#\#\# & 94291 & 291 & 21.9 & 3.4 & 2.8 & 2.7 & 3.4 \\
\hline $1 \mathrm{~B}$ & $S$ & \#\#\#\#\#\#\#\# & 94292 & 292 & 15.5 & 3.4 & 2.8 & 2.8 & 3.4 \\
\hline $\begin{array}{ll}1 \mathrm{~B} \\
\end{array}$ & S & \#\#\#\#\#\#\#\# & 94293 & 293 & 18.7 & 3.4 & 2.8 & 2.8 & 3.3 \\
\hline $1 \mathrm{~B}$ & S & \#\#\#\#\#\#\#\# & 94294 & 294 & 17.3 & 2.8 & 2.8 & 3.4 & 2.8 \\
\hline $0 \mathrm{~B}$ & $S$ & \#\#\#\#\#\#\#\# & 94295 & 295 & 17.5 & 2.8 & 3.4 & 2.8 & 2.8 \\
\hline
\end{tabular}


lights

\begin{tabular}{|c|c|c|c|c|c|c|c|c|c|}
\hline \begin{tabular}{l|l}
0 & $B$ \\
\end{tabular} & $S$ & \#\#\#\#\#\#\#\# & 94296 & 296 & 2.8 & 2.8 & 3.4 & 2.8 & 2.8 \\
\hline $1 \mathrm{~B}$ & $\mathrm{~S}$ & \#\#\#\#\#\#\#\# & 94297 & 297 & 2.8 & 3.3 & 2.8 & 2.8 & 3.4 \\
\hline $1 \mathrm{~B}$ & S & \#\#\#\#\#\#\#\# & 94298 & 298 & 21.3 & 2.8 & 3.5 & 2.8 & 3.4 \\
\hline \begin{tabular}{l|l}
1 & $B$ \\
\end{tabular} & $\mathrm{~S}$ & \#\#\#\#\#\#\#\# & 94299 & 299 & 20.7 & 2.8 & 2.9 & 3.5 & 2.8 \\
\hline $1 \mathrm{~B}$ & $S$ & \#\#\#\#\#\#\#\# & 94300 & 300 & 22 & 2.8 & 2.8 & 3.5 & 2.8 \\
\hline $1 \mathrm{~B}$ & $S$ & \#\#\#\#\#\#\#\# & 94301 & 301 & 22.4 & 2.8 & 3.5 & 2.8 & 2.8 \\
\hline $0 \mathrm{~B}$ & $\mathrm{~S}$ & \#\#\#\#\#\#\#\# & 94302 & 302 & 21.4 & 3.5 & 2.8 & 3.4 & 2.9 \\
\hline $0 \mathrm{~B}$ & S & \#\#\#\#\#\#\#\# & 94303 & 303 & 2.8 & 3.5 & 2.8 & 2.8 & 3.5 \\
\hline $1 \mathrm{~B}$ & S & \#\#\#\#\#\#\#\# & 94304 & 304 & 2.8 & 3.5 & 2.8 & 2.8 & 3.4 \\
\hline $1 \mathrm{~B}$ & S & 11/1/1994 & 94305 & 305 & 19.1 & 3.4 & 2.9 & 3.5 & 2.9 \\
\hline $1 \mathrm{~B}$ & $\mathrm{~S}$ & $11 / 2 / 1994$ & 94306 & 306 & 19.8 & 3.4 & 2.8 & 3.4 & 2.8 \\
\hline $1 \mathrm{~B}$ & S & $11 / 3 / 1994$ & 94307 & 307 & 19 & 3.4 & 2.8 & 2.8 & 3.4 \\
\hline $1 \mathrm{~B}$ & S & 11/4/1994 & 94308 & 308 & 20.8 & 3.4 & 2.7 & 2.8 & 3.4 \\
\hline $0 \mathrm{~B}$ & S & $11 / 5 / 1994$ & 94309 & 309 & 18.8 & 2.8 & 2.8 & 3.4 & 2.9 \\
\hline $\begin{array}{ll}0 & B \\
\end{array}$ & $\mathrm{~S}$ & $11 / 6 / 1994$ & 94310 & 310 & 3 & 3.6 & 3 & 3 & 3.6 \\
\hline $1 \mathrm{~B}$ & $\mathrm{~S}$ & $11 / 7 / 1994$ & 94311 & 311 & 3.4 & 2.8 & 2.9 & 3.4 & 2.8 \\
\hline $1 \mathrm{~B}$ & S & $11 / 8 / 1994$ & 94312 & 312 & 19.4 & 2.8 & 3.4 & 2.8 & 3.5 \\
\hline $1 \mathrm{~B}$ & S & $11 / 9 / 1994$ & 94313 & 313 & 27.2 & 2.8 & 2.9 & 3.4 & 2.8 \\
\hline $1 \mathrm{~B}$ & S & \#\#\#\#\#\#\#\# & 94314 & 314 & 19.7 & 3.6 & 3 & 3 & 3.6 \\
\hline $1 \mathrm{~B}$ & S & \#\#\#\#\#\#\#\# & 94315 & 315 & 26.8 & 3 & 3.5 & 2.8 & 2.8 \\
\hline $0 \mathrm{~B}$ & $S$ & \#\#\#\#\#\#\#\# & 94316 & 316 & 22.3 & 2.8 & 2.8 & 3.4 & 2.9 \\
\hline $0 \mathrm{~B}$ & S & \#\#\#\#\#\#\#\# & 94317 & 317 & 2.8 & 2.9 & 3.4 & 2.8 & 2.8 \\
\hline $1 \mathrm{~B}$ & S & \#\#\#\#\#\#\#\# & 94318 & 318 & 2.7 & 2.8 & 3.3 & 2.7 & 2.8 \\
\hline \begin{tabular}{l|l}
1 & $B$ \\
\end{tabular} & $S$ & \#\#\#\#\#\#\# & 94319 & 319 & 22.4 & 2.7 & 2.8 & 3.3 & 2.8 \\
\hline $1 \mathrm{~B}$ & $\mathrm{~S}$ & \#\#\#\#\#\#\#\# & 94320 & 320 & 23.3 & 2.7 & 3.4 & 2.8 & 3.3 \\
\hline $1 \mathrm{~B}$ & S & \#\#\#\#\#\#\#\# & 94321 & 321 & 18.7 & 2.8 & 3.3 & 2.8 & 2.7 \\
\hline $1 \mathrm{~B}$ & S & \#\#\#\#\#\#\#\# & 94322 & 322 & 22.8 & 2.7 & 2.8 & 3.3 & 2.8 \\
\hline $0 \mathrm{~B}$ & S & \#\#\#\#\#\#\#\# & 94323 & 323 & 21.3 & 2.7 & 2.8 & 3.3 & 2.8 \\
\hline $0 \mathrm{~B}$ & $S$ & \#\#\#\#\#\#\#\# & 94324 & 324 & 2.7 & 3.4 & 2.7 & 2.8 & 3.3 \\
\hline $1 \mathrm{~B}$ & $S$ & \#\#\#\#\#\#\#\# & 94325 & 325 & 3.4 & 2.7 & 2.7 & 3.4 & 2.7 \\
\hline $1 \mathrm{~B}$ & S & \#\#\#\#\#\#\#\# & 94326 & 326 & 18.5 & 3.4 & 2.8 & 2.7 & 3.4 \\
\hline $1 \mathrm{~B}$ & S & \#\#\#\#\#\#\#\# & 94327 & 327 & 9.2 & 1.1 & 0.4 & 0.4 & 0.4 \\
\hline $1 \mathrm{~B}$ & NS & \#\#\#\#\#\#\#\# & 94328 & 328 & 0.4 & 0.4 & 0.4 & 0.4 & 0.4 \\
\hline $1 \mathrm{~B}$ & NS & \#\#\#\#\#\#\#\# & 94329 & 329 & 0.4 & 1.1 & 0.4 & 0.4 & 0.4 \\
\hline $0 \mathrm{~B}$ & NS & \#\#\#\#\#\#\#\# & 94330 & 330 & 0.5 & 0.4 & 1 & 0.4 & 0.4 \\
\hline $0 \mathrm{~B}$ & NS & \#\#\#\#\#\#\#\# & 94331 & 331 & 0.5 & 0.4 & 0.4 & 0.4 & 0.4 \\
\hline $1 \mathrm{~B}$ & S & \#\#\#\#\#\#\#\# & 94332 & 332 & 0.4 & 0.4 & 0.5 & 0.4 & 0.4 \\
\hline $1 \mathrm{~B}$ & S & \#\#\#\#\#\#\#\# & 94333 & 333 & 21.8 & 2.8 & 2.7 & 3.4 & 2.8 \\
\hline $1 \mathrm{~B}$ & $S$ & \#\#\#\#\#\#\#\# & 94334 & 334 & 23.5 & 3.4 & 2.7 & 2.8 & 3.4 \\
\hline $\begin{array}{ll}1 \mathrm{~B} \\
\end{array}$ & S & 12/1/1994 & 94335 & 335 & 20.6 & 2.7 & 2.8 & 3.4 & 2.7 \\
\hline $1 \mathrm{~B}$ & S & $12 / 2 / 1994$ & 94336 & 336 & 19.8 & 2.7 & 2.8 & 3.4 & 2.7 \\
\hline $0 \mathrm{~B}$ & S & $12 / 3 / 1994$ & 94337 & 337 & 16.2 & 2.8 & 2.8 & 3.3 & 2.8 \\
\hline $0 \mathrm{~B}$ & $\mathrm{~S}$ & $12 / 4 / 1994$ & 94338 & 338 & 2.7 & 2.8 & 3.3 & 2.8 & 2.7 \\
\hline $1 \mathrm{~B}$ & S & $12 / 5 / 1994$ & 94339 & 339 & 2.7 & 3.4 & 2.8 & 2.7 & 3.4 \\
\hline $1 \mathrm{~B}$ & S & $12 / 6 / 1994$ & 94340 & 340 & 20.1 & 2.8 & 2.7 & 3.4 & 2.8 \\
\hline $1 \mathrm{~B}$ & S & $12 / 7 / 1994$ & 94341 & 341 & 21.8 & 2.8 & 2.8 & 3.3 & 2.8 \\
\hline $1 \mathrm{~B}$ & S & $12 / 8 / 1994$ & 94342 & 342 & 20.9 & 3.4 & 2.7 & 2.8 & 3.4 \\
\hline $1 \mathrm{~B}$ & $S$ & 12/9/1994 & 94343 & 343 & 17.4 & 2.8 & 3.3 & 2.8 & 3.4 \\
\hline $0 \mathrm{~B}$ & S & \#\#\#\#\#\#\#\# & 94344 & 344 & 21.8 & 2.8 & 2.7 & 3.4 & 2.7 \\
\hline $0 \mathrm{~B}$ & S & \#\#\#\#\#\#\#\# & 94345 & 345 & 3.3 & 2.8 & 2.7 & 3.4 & 2.7 \\
\hline $1 \mathrm{~B}$ & $S$ & \#\#\#\#\#\#\#\# & 94346 & 346 & 3.3 & 2.7 & 2.8 & 3.3 & 2.8 \\
\hline
\end{tabular}


lights

\begin{tabular}{|c|c|c|c|c|c|c|c|c|c|}
\hline 1 B & $S$ & \#\#\#\#\#\#\#\# & 94347 & 347 & 22.1 & 2.7 & 2.8 & 3.3 & 2.8 \\
\hline $1 \mathrm{~B}$ & $\mathrm{~S}$ & \#\#\#\#\#\#\#\# & 94348 & 348 & 20.3 & 2.8 & 3.3 & 2.8 & 2.7 \\
\hline $1 \mathrm{~B}$ & S & \#\#\#\#\#\#\#\# & 94349 & 349 & 24.5 & 2.8 & 3.3 & 2.8 & 2.8 \\
\hline $1 \mathrm{~B}$ & $\mathrm{~S}$ & \#\#\#\#\#\#\#\# & 94350 & 350 & 22 & 2.8 & 3.3 & 2.8 & 3.4 \\
\hline \begin{tabular}{l|l}
0 & $B$ \\
\end{tabular} & S & \#\#\#\#\#\#\#\# & 94351 & 351 & 19.9 & 3.4 & 2.7 & 2.8 & 3.4 \\
\hline \begin{tabular}{l|l}
0 & $B$
\end{tabular} & NS & \#\#\#\#\#\#\#\# & 94352 & 352 & 2.7 & 3.4 & 2.8 & 2.7 & 3.4 \\
\hline $1 \mathrm{~B}$ & NS & \#\#\#\#\#\#\#\# & 94353 & 353 & 2.8 & 3.3 & 2.8 & 2.7 & 3.4 \\
\hline $1 \mathrm{~B}$ & NS & \#\#\#\#\#\#\#\# & 94354 & 354 & 0.4 & 3.4 & 2.8 & 3.3 & 2.8 \\
\hline $1 \mathrm{~B}$ & NS & \#\#\#\#\#\#\#\# & 94355 & 355 & 0.4 & 3.4 & 2.7 & 2.8 & 3.3 \\
\hline $1 \mathrm{~B}$ & NS & \#\#\#\#\#\#\#\# & 94356 & 356 & 0.4 & 0.4 & 0.4 & 0.4 & 0.4 \\
\hline $1 \mathrm{~B}$ & NS & \#\#\#\#\#\#\#\# & 94357 & 357 & 0.4 & 0.4 & 0.4 & 0.4 & 0.4 \\
\hline 0 B & NS & \#\#\#\#\#\#\#\# & 94358 & 358 & 0.4 & 0.4 & 0.4 & 0.4 & 0.4 \\
\hline $\begin{array}{ll}0 & B \\
\end{array}$ & NS & \#\#\#\#\#\#\#\# & 94359 & 359 & 0.4 & 0.4 & 0.4 & 0.4 & 0.4 \\
\hline $1 \mathrm{~B}$ & NS & \#\#\#\#\#\#\#\# & 94360 & 360 & 0.4 & 0.4 & 0.4 & 0.4 & 0.4 \\
\hline \begin{tabular}{l|l}
1 & $B$ \\
\end{tabular} & NS & \#\#\#\#\#\#\#\# & 94361 & 361 & 0.4 & 0.4 & 0.4 & 0.4 & 0.4 \\
\hline $1 \mathrm{~B}$ & NS & \#\#\#\#\#\#\#\# & 94362 & 362 & 0.4 & 0.4 & 0.4 & 0.4 & 0.3 \\
\hline $1 \mathrm{~B}$ & NS & \#\#\#\#\#\#\#\# & 94363 & 363 & 0.4 & 0.4 & 0.4 & 0.4 & 0.4 \\
\hline $1 \mathrm{~B}$ & NS & \#\#\#\#\#\#\#\# & 94364 & 364 & 0.4 & 0.4 & 0.4 & 0.4 & 0.4 \\
\hline $0 \mathrm{~B}$ & NS & \#\#\#\#\#\#\#\# & 94365 & 365 & 0.4 & 0.4 & 0.4 & 0.4 & 0.4 \\
\hline $0 B$ & NS & $1 / 1 / 1995$ & 94366 & 1 & 0.4 & 0.4 & 0.4 & 0.4 & 0.4 \\
\hline $1 \mathrm{~B}$ & $S$ & 1/2/1995 & 94367 & 2 & 0.4 & 0.4 & 0.4 & 0.4 & 0.4 \\
\hline $1 \mathrm{~B}$ & S & $1 / 3 / 1995$ & 94368 & 3 & 24.3 & 3.6 & 2.9 & 3.6 & 3.5 \\
\hline $1 \mathrm{~B}$ & S & 1/4/1995 & 94369 & 4 & 20.4 & 3.6 & 2.9 & 3.6 & 2.9 \\
\hline \begin{tabular}{l|l}
1 & $B$ \\
\end{tabular} & $S$ & 1/5/1995 & 94370 & 5 & 21 & 3.5 & 3 & 3.5 & 3.5 \\
\hline $1 \mathrm{~B}$ & $\mathrm{~S}$ & 1/6/1995 & 94371 & 6 & 21.1 & 3.5 & 3.5 & 3 & 3.5 \\
\hline $0 \mathrm{~B}$ & S & 1/7/1995 & 94372 & 7 & 23.4 & 2.9 & 3.6 & 3.5 & 2.9 \\
\hline $0 \mathrm{~B}$ & S & 1/8/1995 & 94373 & 8 & 3.6 & 3.5 & 2.9 & 3.6 & 2.9 \\
\hline $1 \mathrm{~B}$ & S & 1/9/1995 & 94374 & 9 & 3.5 & 2.9 & 3.6 & 2.9 & 3.5 \\
\hline $1 \mathrm{~B}$ & S & $1 / 10 / 1995$ & 94375 & 10 & 20.9 & 3.5 & 3.5 & 3 & 3.5 \\
\hline $1 \mathrm{~B}$ & $S$ & 1/11/1995 & 94376 & 11 & 25 & 3.5 & 3.5 & 2.9 & 3.6 \\
\hline $1 \mathrm{~B}$ & S & $1 / 12 / 1995$ & 94377 & 12 & 23 & 2.9 & 3.6 & 2.9 & 2.9 \\
\hline $1 \mathrm{~B}$ & S & $1 / 13 / 1995$ & 94378 & 13 & 23 & 2.9 & 3.5 & 3 & 2.9 \\
\hline $0 \mathrm{~B}$ & S & 1/14/1995 & 94379 & 14 & 24.7 & 3.5 & 2.9 & 3.6 & 2.9 \\
\hline $0 \mathrm{~B}$ & S & $1 / 15 / 1995$ & 94380 & 15 & 2.9 & 2.9 & 3.6 & 2.9 & 2.9 \\
\hline $1 \mathrm{~B}$ & S & 1/16/1995 & 94381 & 16 & 2.9 & 3.6 & 2.9 & 3.5 & 2.9 \\
\hline $1 \mathrm{~B}$ & S & $1 / 17 / 1995$ & 94382 & 17 & 0.4 & 0.4 & 0.4 & 0.4 & 0.5 \\
\hline $1 \mathrm{~B}$ & S & $1 / 18 / 1995$ & 94383 & 18 & 22.9 & 3.5 & 2.9 & 3.6 & 2.9 \\
\hline $1 \mathrm{~B}$ & S & $1 / 19 / 1995$ & 94384 & 19 & 24.7 & 2.9 & 11.3 & 19.7 & 11.8 \\
\hline $1 \mathrm{~B}$ & $S$ & 1/20/1995 & 94385 & 20 & 27.4 & 2.9 & 2.9 & 3.5 & 2.9 \\
\hline $0 \mathrm{~B}$ & S & $1 / 21 / 1995$ & 94386 & 21 & 27.9 & 3.5 & 2.9 & 3 & 3.5 \\
\hline $\begin{array}{ll}0 & B \\
\end{array}$ & $\mathrm{~S}$ & $1 / 22 / 1995$ & 94387 & 22 & 3.5 & 3 & 2.9 & 3.5 & 2.9 \\
\hline $1 \mathrm{~B}$ & S & $1 / 23 / 1995$ & 94388 & 23 & 3.5 & 2.9 & 2.9 & 3.5 & 2.9 \\
\hline $1 \mathrm{~B}$ & NS & $1 / 24 / 1995$ & 94389 & 24 & 26.7 & 2.9 & 3.5 & 2.9 & 3.5 \\
\hline $1 \mathrm{~B}$ & S & $1 / 25 / 1995$ & 94390 & 25 & 22 & 3.5 & 2.9 & 2.9 & 3.5 \\
\hline $1 \mathrm{~B}$ & S & $1 / 26 / 1995$ & 94391 & 26 & 19.8 & 3.5 & 3 & 3.5 & 2.9 \\
\hline $1 \mathrm{~B}$ & S & $1 / 27 / 1995$ & 94392 & 27 & 26.8 & 2.9 & 2.9 & 3.6 & 2.9 \\
\hline $0 \mathrm{~B}$ & S & $1 / 28 / 1995$ & 94393 & 28 & 24.8 & 3.5 & 2.9 & 2.9 & 3.5 \\
\hline $0 \mathrm{~B}$ & $S$ & 1/29/1995 & 94394 & 29 & 3 & 3.5 & 2.9 & 3.5 & 2.9 \\
\hline $1 \mathrm{~B}$ & S & $1 / 30 / 1995$ & 94395 & 30 & 2.9 & 3.5 & 2.9 & 2.9 & 3.6 \\
\hline $1 \mathrm{~B}$ & S & $1 / 31 / 1995$ & 94396 & 31 & 25.1 & 2.9 & 3.5 & 2.9 & 2.9 \\
\hline $1 \mathrm{~B}$ & S & 2/1/1995 & 94397 & 32 & 22.9 & 2.9 & 3.5 & 2.9 & 3.5 \\
\hline
\end{tabular}


lights

\begin{tabular}{|c|c|c|c|c|c|c|c|c|c|}
\hline $1 B$ & S & $2 / 2 / 1995$ & 94398 & 33 & 15.3 & 2.9 & 3.5 & 3 & 2.9 \\
\hline $1 \mathrm{~B}$ & NS & 2/3/1995 & 94399 & 34 & 21.7 & 3.5 & 2.9 & 3 & 3.5 \\
\hline $\begin{array}{ll}0 & B \\
\end{array}$ & S & 2/4/1995 & 94400 & 35 & 22.3 & 2.9 & 3.5 & 2.9 & 3.5 \\
\hline $0 \mathrm{~B}$ & S & 2/5/1995 & 94401 & 36 & 3.5 & 2.9 & 2.9 & 3.5 & 3 \\
\hline $1 \mathrm{~B}$ & S & 2/6/1995 & 94402 & 37 & 2.9 & 3.5 & 3 & 3.5 & 2.9 \\
\hline $1 \mathrm{~B}$ & $\mathrm{~S}$ & $2 / 7 / 1995$ & 94403 & 38 & 23 & 3.5 & 2.9 & 3.5 & 2.9 \\
\hline $1 \mathrm{~B}$ & S & 2/8/1995 & 94404 & 39 & 21.1 & 2.9 & 3.5 & 3 & 3.5 \\
\hline $1 \mathrm{~B}$ & S & 2/9/1995 & 94405 & 40 & 22.4 & 3.5 & 2.9 & 3.5 & 2.9 \\
\hline $1 \mathrm{~B}$ & S & 2/10/1995 & 94406 & 41 & 21.7 & 3.5 & 2.9 & 2.9 & 3.5 \\
\hline $0 \mathrm{~B}$ & S & 2/11/1995 & 94407 & 42 & 22.1 & 2.9 & 2.9 & 3.5 & 2.9 \\
\hline $0 \mathrm{~B}$ & $\mathrm{~S}$ & 2/12/1995 & 94408 & 43 & 3.5 & 2.9 & 2.9 & 3.5 & 2.9 \\
\hline $1 \mathrm{~B}$ & $S$ & 2/13/1995 & 94409 & 44 & 2.9 & 3.5 & 3 & 2.9 & 3.5 \\
\hline $1 \mathrm{~B}$ & S & 2/14/1995 & 94410 & 45 & 25 & 2.9 & 3.5 & 2.9 & 3 \\
\hline $1 \mathrm{~B}$ & S & 2/15/1995 & 94411 & 46 & 22.7 & 2.9 & 3.5 & 2.9 & 2.9 \\
\hline $1 \mathrm{~B}$ & S & 2/16/1995 & 94412 & 47 & 23.1 & 3.6 & 2.9 & 2.9 & 3.5 \\
\hline $1 \mathrm{~B}$ & S & 2/17/1995 & 94413 & 48 & 26.1 & 2.9 & 2.9 & 3.5 & 3 \\
\hline $0 \mathrm{~B}$ & S & 2/18/1995 & 94414 & 49 & 23.4 & 3.5 & 2.9 & 3.5 & 2.9 \\
\hline $0 \mathrm{~B}$ & S & 2/19/1995 & 94415 & 50 & 2.9 & 2.9 & 3.5 & 2.9 & 3.5 \\
\hline $1 \mathrm{~B}$ & S & 2/20/1995 & 94416 & 51 & 3.5 & 2.9 & 2.9 & 3.5 & 2.9 \\
\hline $1 \mathrm{~B}$ & $\mathrm{~S}$ & 2/21/1995 & 94417 & 52 & 23.6 & 3.5 & 2.9 & 3.5 & 2.9 \\
\hline $1 \mathrm{~B}$ & $S$ & 2/22/1995 & 94418 & 53 & 22.8 & 2.9 & 3.5 & 2.9 & 3.5 \\
\hline $1 \mathrm{~B}$ & S & 2/23/1995 & 94419 & 54 & 22.8 & 3.5 & 2.9 & 3.5 & 2.9 \\
\hline $1 \mathrm{~B}$ & S & 2/24/1995 & 94420 & 55 & 22.1 & 2.9 & 3.5 & 2.9 & 2.9 \\
\hline $0 \mathrm{~B}$ & S & 2/25/1995 & 94421 & 56 & 22.4 & 2.9 & 3.5 & 2.9 & 3.5 \\
\hline $0 \mathrm{~B}$ & S & 2/26/1995 & 94422 & 57 & 2.9 & 2.9 & 3.5 & 2.9 & 3.5 \\
\hline $1 \mathrm{~B}$ & S & 2/27/1995 & 94423 & 58 & 2.9 & 3.5 & 2.9 & 2.9 & 3.5 \\
\hline $1 \mathrm{~B}$ & S & 2/28/1995 & 94424 & 59 & 26.7 & 2.9 & 2.9 & 3.6 & 2.9 \\
\hline $1 \mathrm{~B}$ & S & 3/1/1995 & 94425 & 60 & 17.6 & 3 & 2.9 & 3.5 & 2.9 \\
\hline $1 \mathrm{~B}$ & S & $3 / 2 / 1995$ & 94426 & 61 & 27.2 & 3.6 & 2.9 & 2.9 & 3.5 \\
\hline $1 \mathrm{~B}$ & $S$ & 3/3/1995 & 94427 & 62 & 21.9 & 2.9 & 3.5 & 2.9 & 2.9 \\
\hline $0 \mathrm{~B}$ & S & $3 / 4 / 1995$ & 94428 & 63 & 21.3 & 3.5 & 2.9 & 2.9 & 3.5 \\
\hline $0 \mathrm{~B}$ & S & $3 / 5 / 1995$ & 94429 & 64 & 3.6 & 2.9 & 2.9 & 3.5 & 2.9 \\
\hline $1 \mathrm{~B}$ & S & $3 / 6 / 1995$ & 94430 & 65 & 2.9 & 3.5 & 2.9 & 2.9 & 3.5 \\
\hline $1 \mathrm{~B}$ & S & $3 / 7 / 1995$ & 94431 & 66 & 25.2 & 2.9 & 3 & 3.5 & 2.9 \\
\hline $1 \mathrm{~B}$ & S & $3 / 8 / 1995$ & 94432 & 67 & 20.6 & 3.5 & 2.9 & 2.9 & 3.5 \\
\hline $1 \mathrm{~B}$ & S & 3/9/1995 & 94433 & 68 & 20.4 & 3.5 & 2.9 & 2.9 & 3.5 \\
\hline $1 \mathrm{~B}$ & S & 3/10/1995 & 94434 & 69 & 24 & 3.5 & 2.9 & 3.5 & 2.8 \\
\hline $\begin{array}{ll}0 & B \\
\end{array}$ & NS & 3/11/1995 & 94435 & 70 & 13.2 & 2.9 & 3.5 & 2.9 & 2.9 \\
\hline $0 \mathrm{~B}$ & NS & 3/12/1995 & 94436 & 71 & 2.9 & 3.5 & 2.9 & 2.9 & 3.5 \\
\hline $1 \mathrm{~B}$ & NS & 3/13/1995 & 94437 & 72 & 3.5 & 2.9 & 2.9 & 3.5 & 2.9 \\
\hline $1 \mathrm{~B}$ & NS & 3/14/1995 & 94438 & 73 & 14.7 & 2.9 & 3.5 & 2.9 & 2.9 \\
\hline $1 \mathrm{~B}$ & NS & 3/15/1995 & 94439 & 74 & 14.4 & 3.5 & 2.9 & 2.9 & 3.5 \\
\hline $1 \mathrm{~B}$ & NS & $3 / 16 / 1995$ & 94440 & 75 & 16.1 & 5.3 & 9.5 & 15.7 & 16.8 \\
\hline $1 \mathrm{~B}$ & NS & 3/17/1995 & 94441 & 76 & 21.8 & 21.8 & 22.2 & 21.9 & 22.2 \\
\hline $0 \mathrm{~B}$ & NS & 3/18/1995 & 94442 & 77 & 15.5 & 14.4 & 15 & 16.1 & 15.5 \\
\hline $0 \mathrm{~B}$ & NS & 3/19/1995 & 94443 & 78 & 14.4 & 13.6 & 14.4 & 14 & 14.1 \\
\hline $1 \mathrm{~B}$ & S & 3/20/1995 & 94444 & 79 & 13.7 & 14.1 & 15.1 & 14.8 & 15.1 \\
\hline $1 \mathrm{~B}$ & $S$ & 3/21/1995 & 94445 & 80 & 19.4 & 2.9 & 3.5 & 3 & 2.9 \\
\hline $1 \mathrm{~B}$ & S & 3/22/1995 & 94446 & 81 & 21.8 & 2.9 & 2.9 & 3.6 & 2.9 \\
\hline $1 \mathrm{~B}$ & S & 3/23/1995 & 94447 & 82 & 20.6 & 3 & 3.5 & 3 & 2.9 \\
\hline $1 \mathrm{~B}$ & $S$ & 3/24/1995 & 94448 & 83 & 22.9 & 3.5 & 3.6 & 3.5 & 3.6 \\
\hline
\end{tabular}


lights

\begin{tabular}{|c|c|c|c|c|c|c|c|c|c|}
\hline $0 \mathrm{~B}$ & $S$ & $3 / 25 / 1995$ & 94449 & 84 & 19.6 & 3.5 & 3.6 & 3.5 & 2.9 \\
\hline $\begin{array}{ll}0 \mathrm{~B} \\
\end{array}$ & $\mathrm{~S}$ & $3 / 26 / 1995$ & 94450 & 85 & 3.6 & 3.5 & 3.6 & 3.6 & 3.5 \\
\hline $1 \mathrm{~B}$ & $\mathrm{~S}$ & $3 / 27 / 1995$ & 94451 & 86 & 3.5 & 3.6 & 3.6 & 3.5 & 3.6 \\
\hline $1 \mathrm{~B}$ & $S$ & 3/28/1995 & 94452 & 87 & 22.6 & 3.6 & 3.6 & 3.5 & 3.6 \\
\hline $1 \mathrm{~B}$ & $S$ & 3/29/1995 & 94453 & 88 & 19.8 & 3.6 & 3.5 & 3.6 & 3.6 \\
\hline \begin{tabular}{l|l}
$1 B$ \\
\end{tabular} & $S$ & $3 / 30 / 1995$ & 94454 & 89 & 22.8 & 3.6 & 3.6 & 3.6 & 3.5 \\
\hline $1 \mathrm{~B}$ & $S$ & 3/31/1995 & 94455 & 90 & 15.6 & 3.6 & 3.6 & 3.5 & 3.6 \\
\hline $\begin{array}{ll}0 & B \\
\end{array}$ & $S$ & 4/1/1995 & 94456 & 91 & 20.8 & 3.5 & 3 & 3.5 & 3.6 \\
\hline $0 \mathrm{~B}$ & $S$ & $4 / 2 / 1995$ & 94457 & 92 & 3.5 & 3.5 & 3.6 & 3.5 & 3.6 \\
\hline $1 \mathrm{~B}$ & $S$ & 4/3/1995 & 94458 & 93 & 3.6 & 3.5 & 3.6 & 3.5 & 3.6 \\
\hline $1 \mathrm{~B}$ & $\mathrm{~S}$ & 4/4/1995 & 94459 & 94 & 20.8 & 3.5 & 3.6 & 3.6 & 3.6 \\
\hline $1 \mathrm{~B}$ & $\mathrm{~S}$ & 4/5/1995 & 94460 & 95 & 20 & 3.5 & 3.6 & 3.6 & 3.5 \\
\hline $1 \mathrm{~B}$ & $S$ & 4/6/1995 & 94461 & 96 & 20.8 & 3.5 & 3.6 & 3.6 & 3.5 \\
\hline $1 \mathrm{~B}$ & $S$ & $4 / 7 / 1995$ & 94462 & 97 & 19.6 & 3.6 & 3.5 & 3.6 & 3.6 \\
\hline $0 \mathrm{~B}$ & $S$ & 4/8/1995 & 94463 & 98 & 25.2 & 3.5 & 3 & 3.6 & 3.5 \\
\hline $0 \mathrm{~B}$ & $S$ & 4/9/1995 & 94464 & 99 & 3.6 & 3.5 & 3.5 & 3.6 & 3.5 \\
\hline $1 \mathrm{~B}$ & $S$ & 4/10/1995 & 94465 & 100 & 3.5 & 3.5 & 3.6 & 3.5 & 3.6 \\
\hline $1 \mathrm{~B}$ & $S$ & 4/11/1995 & 94466 & 101 & 21.9 & 3.6 & 3.6 & 3 & 3.6 \\
\hline $1 B$ & $S$ & 4/12/1995 & 94467 & 102 & 19.1 & 3.6 & 3.5 & 3.6 & 3.6 \\
\hline $1 \mathrm{~B}$ & $\mathrm{~S}$ & $4 / 13 / 1995$ & 94468 & 103 & 21.3 & 3.6 & 3.5 & 3.6 & 3.5 \\
\hline $1 \mathrm{~B}$ & $\mathrm{~S}$ & 4/14/1995 & 94469 & 104 & 24.1 & 3.6 & 3.5 & 3.6 & 3.5 \\
\hline $0 \mathrm{~B}$ & $S$ & 4/15/1995 & 94470 & 105 & 22 & 3.6 & 3.5 & 3.6 & 3.5 \\
\hline $0 \mathrm{~B}$ & $S$ & 4/16/1995 & 94471 & 106 & 3.5 & 3.5 & 2.9 & 3.5 & 3.6 \\
\hline $1 \mathrm{~B}$ & $S$ & 4/17/1995 & 94472 & 107 & 3.5 & 3.6 & 3.5 & 3.5 & 3.5 \\
\hline $1 \mathrm{~B}$ & $S$ & 4/18/1995 & 94473 & 108 & 12.4 & 3.6 & 3.5 & 3.6 & 3.5 \\
\hline $1 \mathrm{~B}$ & $S$ & $4 / 19 / 1995$ & 94474 & 109 & 20.6 & 3.5 & 3.6 & 3.6 & 3.5 \\
\hline $1 \mathrm{~B}$ & $S$ & 4/20/1995 & 94475 & 110 & 20.5 & 3.5 & 3.6 & 3.6 & 3.5 \\
\hline $1 \mathrm{~B}$ & $S$ & 4/21/1995 & 94476 & 111 & 21.2 & 3.6 & 3.5 & 3.6 & 3.5 \\
\hline $0 \mathrm{~B}$ & $\mathrm{~S}$ & $4 / 22 / 1995$ & 94477 & 112 & 21.6 & 3.5 & 3.6 & 3.5 & 3.6 \\
\hline $0 \mathrm{~B}$ & $\mathrm{~S}$ & 4/23/1995 & 94478 & 113 & 3.6 & 3.6 & 3.6 & 3.5 & 3.6 \\
\hline $1 \mathrm{~B}$ & $S$ & 4/24/1995 & 94479 & 114 & 3.6 & 3.5 & 3.6 & 3.6 & 2.9 \\
\hline $1 \mathrm{~B}$ & $S$ & $4 / 25 / 1995$ & 94480 & 115 & 21.5 & 3.6 & 3 & 3.5 & 3.6 \\
\hline $1 \mathrm{~B}$ & $S$ & $4 / 26 / 1995$ & 94481 & 116 & 19.7 & 3.6 & 3.5 & 3.6 & 3 \\
\hline $1 B$ & $S$ & 4/27/1995 & 94482 & 117 & 20.1 & 3.5 & 3.6 & 3.6 & 3.6 \\
\hline $1 \mathrm{~B}$ & $S$ & $4 / 28 / 1995$ & 94483 & 118 & 19.6 & 2.9 & 3.5 & 3.6 & 3.5 \\
\hline $0 \mathrm{~B}$ & $S$ & $4 / 29 / 1995$ & 94484 & 119 & 15.8 & 3.6 & 3.5 & 3.5 & 3.6 \\
\hline $0 \mathrm{~B}$ & $S$ & 4/30/1995 & 94485 & 120 & 3.5 & 3.5 & 3.6 & 3.5 & 3.5 \\
\hline $1 \mathrm{~B}$ & $\mathrm{~S}$ & 5/1/1995 & 94486 & 121 & 3.5 & 3.5 & 3.6 & 3.5 & 3.5 \\
\hline $1 B$ & $S$ & $5 / 2 / 1995$ & 94487 & 122 & 24.9 & 3.5 & 3.6 & 3.6 & 3.5 \\
\hline $1 \mathrm{~B}$ & $S$ & $5 / 3 / 1995$ & 94488 & 123 & 22.2 & 3.6 & 3.6 & 3.5 & 3.6 \\
\hline $1 \mathrm{~B}$ & $S$ & $5 / 4 / 1995$ & 94489 & 124 & 26.5 & 3.5 & 3.6 & 3.6 & 3.6 \\
\hline $1 \mathrm{~B}$ & $S$ & $5 / 5 / 1995$ & 94490 & 125 & 20.3 & 3.6 & 3.5 & 3.6 & 3 \\
\hline $\begin{array}{ll}0 B \\
\end{array}$ & $\mathrm{~S}$ & $5 / 6 / 1995$ & 94491 & 126 & & & & & \\
\hline $0 \mathrm{~B}$ & $S$ & $5 / 7 / 1995$ & 94492 & 127 & & & & & \\
\hline $1 \mathrm{~B}$ & $S$ & $5 / 8 / 1995$ & 94493 & 128 & 3.6 & 3.6 & 3.5 & 3.6 & 3.5 \\
\hline $1 \mathrm{~B}$ & $S$ & $5 / 9 / 1995$ & 94494 & 129 & 23.2 & 3.6 & 3.5 & 3.6 & 3.5 \\
\hline $1 \mathrm{~B}$ & $\mathrm{~S}$ & $5 / 10 / 1995$ & 94495 & 130 & 21.4 & 3.6 & 2.9 & 3.6 & 3.5 \\
\hline $1 \mathrm{~B}$ & $S$ & $5 / 11 / 1995$ & 94496 & 131 & 20.6 & 3.5 & 3.6 & 3.5 & 3.6 \\
\hline $1 \mathrm{~B}$ & $S$ & $5 / 12 / 1995$ & 94497 & 132 & 19.9 & 3.6 & 3.5 & 3.6 & 3.5 \\
\hline $0 \mathrm{~B}$ & $S$ & $5 / 13 / 1995$ & 94498 & 133 & 21 & 3.5 & 3.6 & 3.5 & 2.9 \\
\hline \begin{tabular}{l|l}
0 & $B$ \\
\end{tabular} & $S$ & $5 / 14 / 1995$ & 94499 & 134 & 2.9 & 3.5 & 3.5 & 3.5 & 3.5 \\
\hline
\end{tabular}


lights

\begin{tabular}{|c|c|c|c|c|c|c|c|c|c|}
\hline $1 \mathrm{E}$ & S & $5 / 15 / 1995$ & 94500 & 135 & 3.5 & 3 & 3.5 & 3.5 & 3.5 \\
\hline $1 \mathrm{E}$ & S & $5 / 16 / 1995$ & 94501 & 136 & 16.4 & 3.5 & 3.6 & 3.5 & 3.5 \\
\hline $1 \mathrm{E}$ & $\mathrm{S}$ & 5/17/1995 & 94502 & 137 & 17 & 3.6 & 3.5 & 3.5 & 3.5 \\
\hline $1 \mathrm{E}$ & S & 5/18/1995 & 94503 & 138 & 23.7 & 3.6 & 3.5 & 3.5 & 3.6 \\
\hline $1 \mathrm{E}$ & $\mathrm{S}$ & $5 / 19 / 1995$ & 94504 & 139 & 17.8 & 3.6 & 3.5 & 3.6 & 3.5 \\
\hline $0 \mathrm{E}$ & $\mathrm{S}$ & $5 / 20 / 1995$ & 94505 & 140 & 20.9 & 3.5 & 3 & 3.5 & 3.6 \\
\hline $0 \mathrm{E}$ & S & $5 / 21 / 1995$ & 94506 & 141 & 3.5 & 3.5 & 3.6 & 3.5 & 3.5 \\
\hline $1 \mathrm{E}$ & S & $5 / 22 / 1995$ & 94507 & 142 & 3.5 & 2.9 & 3.5 & 3.5 & 3.6 \\
\hline $1 \mathrm{E}$ & $S$ & $5 / 23 / 1995$ & 94508 & 143 & 19.4 & 3.6 & 3.5 & 3.5 & 3.6 \\
\hline $1 \mathrm{E}$ & $S$ & $5 / 24 / 1995$ & 94509 & 144 & 20.3 & 3.5 & 3.6 & 2.9 & 3.5 \\
\hline $1 \mathrm{E}$ & $\mathrm{S}$ & $5 / 25 / 1995$ & 94510 & 145 & 18.1 & 2.9 & 3.6 & 3.5 & 3.6 \\
\hline $1 \mathrm{E}$ & $\mathrm{S}$ & 5/26/1995 & 94511 & 146 & 16.3 & 3.6 & 3.5 & 3.6 & 2.9 \\
\hline $0 \mathrm{E}$ & S & $5 / 27 / 1995$ & 94512 & 147 & 14.9 & 3.5 & 3.6 & 2.9 & 3.6 \\
\hline $0 \mathrm{E}$ & S & $5 / 28 / 1995$ & 94513 & 148 & 3.6 & 3.5 & 3.5 & 3.6 & 3.6 \\
\hline $1 \mathrm{E}$ & $\mathrm{S}$ & $5 / 29 / 1995$ & 94514 & 149 & 3.6 & 3.5 & 3.6 & 3.5 & 3.5 \\
\hline $1 \mathrm{E}$ & S & $5 / 30 / 1995$ & 94515 & 150 & 11.4 & 3.5 & 3.5 & 3.5 & 3.6 \\
\hline $1 \mathrm{E}$ & $\mathrm{S}$ & $5 / 31 / 1995$ & 94516 & 151 & 11.7 & 3.5 & 3.5 & 3.6 & 3.5 \\
\hline & & & & & & & & & \\
\hline & & & & & & & & & \\
\hline \multicolumn{4}{|c|}{ Post Period Summary (sort "Post") } & \multicolumn{4}{|c|}{ Daily Average (sort "Category") } & & \\
\hline & & & & & & & & & \\
\hline \multicolumn{2}{|c|}{ school year } & 165493 & & $1-A-S$ & 15.13 & 10.60 & 10.43 & 10.18 & 10.06 \\
\hline \multicolumn{2}{|c|}{ non school year } & 58210 & & 1-B-S & 16.94 & 12.54 & 12.54 & 12.60 & 12.58 \\
\hline & & & & $0-A-S$ & 20.16 & 14.27 & 13.79 & 12.89 & 12.69 \\
\hline & & & & $0-B-S$ & 9.20 & 3.36 & 3.37 & 3.24 & 3.43 \\
\hline & & & & $1-A-N S$ & 13.70 & 2.98 & 2.95 & 3.08 & 2.99 \\
\hline & & & & 1-B-NS & 13.70 & 2.67 & 2.69 & 2.88 & 2.75 \\
\hline & & & & $0-A-N S$ & 15.61 & 4.10 & 4.17 & 4.28 & 4.29 \\
\hline & & & & $0-B-N S$ & 23.48 & 3.55 & 3.58 & 3.58 & 3.43 \\
\hline & & & & & & & & & \\
\hline & & & & & & & & & \\
\hline & & & & \multicolumn{5}{|c|}{ Daily Average Standard Deviation (sort "Category") } & \\
\hline & & & & & & & & & \\
\hline & & & & $1-A-S$ & 7.621 & 5.521 & 4.959 & 3.643 & 3.230 \\
\hline & & & & 1-B-S & 7.366 & 3.531 & 3.530 & 3.521 & 3.533 \\
\hline & & & & $0-A-S$ & 7.856 & 6.250 & 5.348 & 3.784 & 3.373 \\
\hline & & & & 0-B-S & 4.915 & 1.417 & 1.407 & 0.441 & 1.482 \\
\hline & & & & $1-\mathrm{A}-\mathrm{NS}$ & 8.000 & 0.573 & 0.748 & 0.810 & 0.838 \\
\hline & & & & 1-B-NS & 10.874 & 1.164 & 1.548 & 2.394 & 1.602 \\
\hline & & & & $0-A-N S$ & 8.160 & 3.031 & 3.243 & 3.454 & 3.520 \\
\hline & & & & 0-B-NS & 7.668 & 0.148 & 0.167 & 0.052 & 0.203 \\
\hline & & & & & & & & & \\
\hline & & & & & & & & & \\
\hline & & & & \multicolumn{3}{|c|}{ Daily Sum (sort "Category") } & & & \\
\hline & & & & & & & & & \\
\hline & & & & $1-A-S$ & 7744.70 & 5435.30 & 5351.30 & 5223.20 & 5160.50 \\
\hline & & & & 1-B-S & 3880.00 & 2859.90 & 2871.00 & 2886.50 & 2880.50 \\
\hline & & & & $0-A-S$ & 3769.10 & 2655.00 & 2551.70 & 2397.90 & 2360.50 \\
\hline & & & & $0-B-S$ & 809.50 & 295.70 & 296.70 & 285.30 & 301.80 \\
\hline & & & & 1-A-NS & 2028.30 & 440.60 & 436.20 & 456.40 & 442.90 \\
\hline & & & & 1-B-NS & 904.20 & 176.00 & 177.80 & 189.80 & 181.30 \\
\hline
\end{tabular}


lights

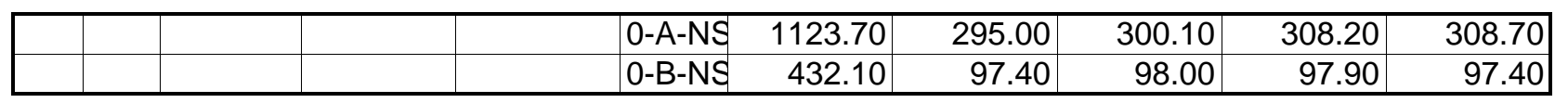




\begin{tabular}{|c|c|c|c|c|c|c|c|c|}
\hline 5 & 6 & 7 & 8 & 9 & 10 & 11 & 12 & 13 \\
\hline 12.6 & 12.6 & 17.4 & 55.9 & 100.6 & 102.6 & 89.4 & 78 & 88.1 \\
\hline 13 & 12.2 & 17.7 & 62.1 & 101.6 & 98.9 & 87.2 & 77.2 & 87.8 \\
\hline 10.3 & 10.3 & 15 & 58 & 101.5 & 98.9 & 84.7 & 79.2 & 88.1 \\
\hline 10 & 9.9 & 15.1 & 61.3 & 99 & 100.5 & 86.2 & 77.1 & 84.3 \\
\hline 12.4 & 12.5 & 11.8 & 12.4 & 10.8 & 13 & 14.2 & 13.6 & 13 \\
\hline 10.6 & 10.7 & 11.3 & 10.6 & 9 & 8.3 & 8.8 & 8.3 & 8.2 \\
\hline 10.6 & 10.7 & 17.3 & 62.9 & 102 & 99.2 & 82.5 & 72.8 & 89.9 \\
\hline 12.6 & 12.6 & 18.6 & 63.3 & 97.4 & 100.3 & 87.3 & 80.9 & 87.3 \\
\hline 10.3 & 10.2 & 15.7 & 59.3 & 97.4 & 93.7 & 83.5 & 73.2 & 86.7 \\
\hline 11.6 & 11.7 & 17.8 & 63.7 & 98.7 & 98.9 & 84.9 & 78.4 & 93.8 \\
\hline 13.2 & 12.6 & 18.9 & 61.4 & 99.8 & 98.4 & 86.1 & 75.9 & 88.2 \\
\hline 12.1 & 11.4 & 12 & 12 & 10.5 & 9.6 & 9 & 9.6 & 9.6 \\
\hline 12 & 12 & 12.1 & 12 & 10.4 & 9.6 & 9.6 & 9.6 & 9.6 \\
\hline 10.2 & 10.7 & 16.9 & 59.6 & 98.9 & 96.2 & 86.1 & 80.3 & 92.8 \\
\hline 10.8 & 10.2 & 16.2 & 61.5 & 100.3 & 102.8 & 87.5 & 81.4 & 93.3 \\
\hline 9.6 & 9.6 & 15.1 & 50.4 & 105.6 & 101.5 & 87.5 & 76.8 & 89.9 \\
\hline 10.8 & 10.2 & 18.4 & 58.6 & 101.8 & 85.9 & 86 & 81.1 & 89.5 \\
\hline 18 & 18 & 22.4 & 59.5 & 102.3 & 99.2 & 88 & 77.4 & 87.3 \\
\hline 11.4 & 11.5 & 10.8 & 11.4 & 9.9 & 9.6 & 9 & 9 & 9 \\
\hline 11.5 & 11.4 & 11.4 & 11.5 & 10 & 9.6 & 9 & 9 & 9 \\
\hline 11.5 & 11.4 & 17.1 & 62.4 & 102.3 & 99.5 & 89.2 & 80.7 & 91.1 \\
\hline 10.8 & 10.8 & 16.1 & 61.6 & 100.5 & 100.8 & 88.5 & 76.9 & 90.1 \\
\hline 14.4 & 13.8 & 21.2 & 66.3 & 102.3 & 101.9 & 89.1 & 78.5 & 86.7 \\
\hline 10.1 & 10 & 14.2 & 56.9 & 99.4 & 98.6 & 88.8 & 77.2 & 90.4 \\
\hline 13.2 & 12.5 & 18.6 & 58.6 & 101.7 & 99.7 & 88.9 & 78.6 & 85.3 \\
\hline 11.2 & 10.5 & 10.5 & 11.1 & 9.1 & 8.7 & 8.7 & 8.1 & 8.7 \\
\hline 11.2 & 10.6 & 10.6 & 11.1 & 9.8 & 8.2 & 8.7 & 8.8 & 8.7 \\
\hline 10.5 & 10.5 & 11.1 & 16.2 & 58.6 & 99.1 & 101.2 & 88.3 & 82.4 \\
\hline 12.9 & 12.9 & 12.9 & 18.1 & 62.7 & 101 & 100 & 11.7 & 102.6 \\
\hline 12.4 & 12.5 & 18.1 & 68.3 & 107.2 & 105.5 & 94 & 87.8 & 98.7 \\
\hline 11.5 & 11.5 & 16.8 & 65.8 & 105.4 & 100.6 & 89.7 & 82.3 & 96.3 \\
\hline 10.3 & 10.8 & 16.4 & 64.9 & 107.3 & 108.9 & 99.2 & 90.8 & 96.3 \\
\hline 9.7 & 9.6 & 9.7 & 9 & 18.7 & 19.8 & 19.3 & 19.2 & 19.9 \\
\hline 18 & 18.1 & 18.1 & 16.9 & 16.2 & 15.7 & 15.6 & 16.3 & 15.6 \\
\hline 18.1 & 18 & 30.8 & 65.2 & 107.6 & 105.7 & 95.1 & 88.9 & 95.5 \\
\hline 10.9 & 10.2 & 26 & 62.7 & 102.5 & 104.2 & 93.3 & 84.1 & 94 \\
\hline 10.9 & 10.8 & 22.5 & 64.1 & 108.3 & 101.6 & 83.9 & 79.3 & 92.8 \\
\hline 11.4 & 11.5 & 26.2 & 63.4 & 104.6 & 99.5 & 95.7 & 84.5 & 97.4 \\
\hline 9.3 & 9.2 & 22.2 & 62.8 & 103.5 & 104.9 & 94.2 & 82 & 92.5 \\
\hline 9.5 & 10.1 & 10 & 9 & 8.2 & 8.9 & 10.6 & 10.1 & 10.6 \\
\hline 8.3 & 8.3 & 8.3 & 7.8 & 5.9 & 5.8 & 5.9 & 5.9 & 6.5 \\
\hline 8.3 & 7.6 & 25.8 & 63.4 & 102.8 & 105.2 & 93 & 83.3 & 92.2 \\
\hline 12 & 11.5 & 31.8 & 65.9 & 102.7 & 99.3 & 87.4 & 82.4 & 86.4 \\
\hline 10.9 & 10.8 & 33.1 & 65.3 & 105.4 & 104.4 & 94 & 86.2 & 91.5 \\
\hline 8.3 & 8.3 & 27.5 & 64.6 & 104.7 & 103.8 & 91.5 & 84.5 & 92.1 \\
\hline 11 & 11 & 30.2 & 61.8 & 97.2 & 95.7 & 88 & 83.3 & 86.7 \\
\hline 12.5 & 11.8 & 12.4 & 11.4 & 15.3 & 38 & 40.5 & 46.1 & 49.9 \\
\hline
\end{tabular}


lights

\begin{tabular}{|c|c|c|c|c|c|c|c|c|}
\hline 8.8 & 8.9 & 9.4 & 8.5 & 10 & 40.2 & 47.3 & 59 & 60.2 \\
\hline 8 & 8.6 & 24.2 & 61.2 & 96.7 & 99.3 & 90.2 & 82.4 & 89.9 \\
\hline 11.2 & 10.6 & 16.9 & 53.7 & 83.2 & 81.8 & 76.4 & 72.4 & 76.4 \\
\hline 8.2 & 8.3 & 16.5 & 53.2 & 77.2 & 75.2 & 68.1 & 65.8 & 70.6 \\
\hline 10.4 & 10.4 & 21.4 & 50.7 & 71.2 & 73.1 & 68 & 61.7 & 64.6 \\
\hline 8.7 & 8.7 & 20.2 & 45 & 68.7 & 65.8 & 61.9 & 54 & 58.9 \\
\hline 8.1 & 7.5 & 8 & 7.9 & 5.6 & 5.7 & 5.6 & 5.7 & 5.6 \\
\hline 8.1 & 7.5 & 8 & 7.3 & 6.2 & 5.7 & 5.6 & 5.7 & 5.6 \\
\hline 7.5 & 12 & 32.9 & 45.3 & 67.7 & 67.4 & 61.3 & 55.4 & 62.1 \\
\hline 6.4 & 7 & 30 & 43.5 & 66.2 & 67.7 & 64.5 & 58.7 & 61.3 \\
\hline 7.4 & 7.8 & 34.7 & 44.9 & 68.2 & 68.3 & 63.8 & 58.3 & 60.2 \\
\hline 6.7 & 7.3 & 6.7 & 6.5 & 4.9 & 4.9 & 4.3 & 4.9 & 4.9 \\
\hline 7.3 & 6.7 & 6.6 & 7.2 & 4.9 & 4.2 & 4.9 & 4.3 & \\
\hline 7.3 & 6.7 & 7.3 & 6.7 & 5.2 & 4.9 & 4.9 & 4.3 & 4.9 \\
\hline 7.3 & 6.8 & 6.7 & 7.3 & 5.3 & 4.9 & 4.9 & 4.3 & 4.8 \\
\hline 6.7 & 6.6 & 10.8 & 44.6 & 69.1 & 67.6 & 60.7 & 58.4 & 62.5 \\
\hline 7.9 & 7.4 & 16.9 & 44.7 & 68.7 & 64.1 & 61.3 & 58.2 & 62.8 \\
\hline 6.7 & 7.9 & 32.2 & 44.1 & 69.1 & 69 & 62.8 & 56.9 & 61.6 \\
\hline 7 & 6.3 & 16 & 44.7 & 68.3 & 68 & 62.5 & 56.5 & 60.9 \\
\hline 7.1 & 7.2 & 20 & 44.2 & 68.1 & 67.4 & 61.3 & 58.6 & 59.5 \\
\hline 7.1 & 6.5 & 6.5 & 6.5 & 12.8 & 23.7 & 28.4 & 32.2 & 40.5 \\
\hline 7.1 & 7.1 & 7.1 & 7.7 & 10.9 & 24 & 26.4 & 26.9 & 26.3 \\
\hline 6.7 & 6.7 & 22.4 & 42.2 & 66.8 & 65 & 59.9 & 54.5 & 59.5 \\
\hline 7.1 & 7.1 & 21.7 & 43.2 & 67.3 & 65.8 & 61.3 & 55.4 & 59.9 \\
\hline 7 & 6.9 & 20.9 & 42.6 & 68.4 & 65.3 & 61.2 & 56.1 & 61.7 \\
\hline 6.3 & 6.9 & 17.3 & 43 & 66.4 & 65.9 & 60 & 52.6 & 57.6 \\
\hline 7.4 & 8 & 17.3 & 45.3 & 68.8 & 68 & 60.2 & 57.4 & 58.2 \\
\hline 8.1 & 7.5 & 7.5 & 8 & 6.9 & 5.1 & 5.6 & 5.7 & 5.1 \\
\hline 8.1 & 7.6 & 7.4 & 8.1 & 7 & 5 & 5.7 & 5.7 & 5.1 \\
\hline 7.6 & 7.4 & 19.7 & 44.4 & 68.2 & 67.5 & 60.3 & 53.2 & 58.8 \\
\hline 7.3 & 7.9 & 18.6 & 45.5 & 69.7 & 66.6 & 62.5 & 54.7 & 59.1 \\
\hline 8.1 & 8 & 18.8 & 44 & 69.7 & 66.5 & 61.9 & 59.1 & 61.3 \\
\hline 7.9 & 8 & 19.4 & 44.7 & 68.8 & 63 & 57.9 & 55.8 & 58.6 \\
\hline 5.7 & 6.4 & 9.1 & 33 & 66.1 & 67.6 & 63.8 & 62.8 & 63.9 \\
\hline 8.4 & 8.4 & 8.3 & 8.4 & 7.9 & 6.5 & 5.9 & 6.6 & 5.9 \\
\hline 8.4 & 8.4 & 8.4 & 8.4 & 7.9 & 6 & 6.5 & 6.6 & 5.9 \\
\hline 9 & 8.4 & 8.4 & 8.4 & 7.9 & 6 & 6.5 & 6.6 & 5.9 \\
\hline 8.4 & 8.4 & 8.4 & 8.4 & 7.9 & 6.5 & 6 & 6.6 & 6.6 \\
\hline 8.4 & 8.4 & 8.4 & 8.4 & 8 & 6.6 & 6 & 6.6 & 6.6 \\
\hline 8.4 & 8.4 & 8.4 & 8.3 & 8 & 6.5 & 6 & 6.5 & 6.6 \\
\hline 8.4 & 8.4 & 8.4 & 8.4 & 7.9 & 6.5 & 6 & 7.1 & 31.7 \\
\hline 7.7 & 7.8 & 7 & 7.7 & 6.7 & 5.3 & 5.8 & 5.3 & 5.2 \\
\hline 7.1 & 7.7 & 7.1 & 7.7 & 6.7 & 5.9 & 5.2 & 5.3 & 5.3 \\
\hline 7.7 & 7.1 & 7.6 & 7.1 & 7.3 & 5.3 & 5.2 & 5.3 & 5.4 \\
\hline 7.1 & 7.6 & 7.7 & 7.1 & 7.3 & 5.2 & 5.9 & 5.2 & 5.9 \\
\hline 7.7 & 7.7 & 7.1 & 7.7 & 7.4 & 5.9 & 5.2 & 5.9 & 5.3 \\
\hline 7.7 & 7.7 & 7.6 & 27.6 & 38.8 & 40.8 & 44.2 & 44.2 & 43.8 \\
\hline 7.7 & 8.2 & 7.5 & 24.4 & 28.9 & 30.2 & 35.6 & 35.7 & 35.6 \\
\hline 7.9 & 7.9 & 7.8 & 10.6 & 30.8 & 31.9 & 34.8 & 40.2 & 43.2 \\
\hline 7.1 & 7.6 & 7 & 7 & 7.3 & 5.2 & 5.1 & 8.7 & 8.8 \\
\hline 6.1 & 6 & 17.5 & 45 & 70.1 & 66.2 & 60.1 & 55.6 & 61.7 \\
\hline
\end{tabular}


lights

\begin{tabular}{|c|c|c|c|c|c|c|c|c|}
\hline 8.5 & 7.9 & 17.6 & 43.8 & 66.5 & 66.4 & 61.1 & 57.8 & 60.8 \\
\hline 6.7 & 6.2 & 15.4 & 44.1 & 68.8 & 65.3 & 60.5 & 54.2 & 61.4 \\
\hline 6.3 & 6.4 & 15.1 & 44.1 & 69.3 & 67.6 & 60.7 & 55 & 60 \\
\hline 7 & 6.9 & 13.5 & 45.1 & 67.8 & 67 & 61.5 & 55.3 & 56.9 \\
\hline 5.7 & 5.7 & 5.8 & 5.7 & 5.4 & 3.9 & 3.3 & 3.9 & 3.9 \\
\hline 5.8 & 5.8 & 5.7 & 5.7 & 5.5 & 3.9 & 3.3 & 3.9 & 3.9 \\
\hline 6.4 & 5.7 & 9.5 & 40.7 & 67.3 & 3.3 & 60.1 & 57.4 & 61.2 \\
\hline 6.9 & 6.9 & 11.3 & 44 & 66.5 & 65.3 & 60.5 & 57 & 58.4 \\
\hline 8.1 & 8.7 & 25.5 & 43.5 & 69.2 & 66.5 & 60.7 & 58 & 61.6 \\
\hline 7.4 & 7.3 & 19 & 42.9 & 66.3 & 68 & 63.5 & 56.4 & 60.3 \\
\hline 6.5 & 7.1 & 9.5 & 40.8 & 58.5 & 57.2 & 60.6 & 62 & 55.9 \\
\hline 7 & 6.9 & 7 & 7.5 & 6.6 & 4.5 & 5.1 & 5.1 & 5.1 \\
\hline 6.4 & 6.3 & 6.4 & 6.9 & 6 & 4.5 & 4 & 4.5 & 4.5 \\
\hline 6.3 & 6.3 & 6.4 & 6.9 & 6 & 4.7 & 3.9 & 5.1 & 5.7 \\
\hline 6.4 & 6.3 & 15 & 45.2 & 68.5 & 66.3 & 61.5 & 59.1 & 62.2 \\
\hline 8.2 & 7.4 & 15.6 & 45.3 & 68.7 & 64.5 & 57.3 & 55.8 & 59.6 \\
\hline 7.3 & 7.9 & 14.7 & 44.2 & 67.1 & 58.8 & 59.9 & 54.3 & 60.3 \\
\hline 12.8 & 13.3 & 20.8 & 45.4 & 67.7 & 63.4 & 60.1 & 58.3 & 61.1 \\
\hline 6.6 & 6.7 & 6.6 & 6.7 & 6.2 & 4.7 & 4.1 & 4.7 & 4.7 \\
\hline 6.6 & 6.7 & 6.7 & 6.6 & 6.2 & 4.7 & 4.7 & 4.1 & 4.7 \\
\hline 6.7 & 6.6 & 14.2 & 45.4 & 67.6 & 65.9 & 60.6 & 57.7 & 62.6 \\
\hline 7.6 & 7.6 & 16 & 45.2 & 68 & 65.5 & 60.7 & 57 & 62 \\
\hline 7.7 & 7.6 & 13.7 & 44 & 67.1 & 64.7 & 60.1 & 56.3 & 58.3 \\
\hline 7.9 & 7.8 & 14.2 & 44.8 & 66.9 & 64.6 & 60.4 & 56.6 & 57.4 \\
\hline 8.4 & 9 & 9.8 & 34 & 35.2 & 33.6 & 33.8 & 34.4 & 32.9 \\
\hline 7.3 & 7.2 & 6.7 & 7.2 & 6.8 & 4.1 & 4.6 & 4.7 & 4.7 \\
\hline 6.7 & 6.1 & 6 & 6.1 & 5.5 & 3.5 & 2.9 & 3.5 & 3.5 \\
\hline 6.1 & 6 & 13.5 & 45.5 & 67.2 & 67.6 & 63.4 & 61 & 62.7 \\
\hline 7.1 & 7 & 14.5 & 43.9 & 66.9 & 64 & 61.5 & 57.4 & 61.3 \\
\hline 9.1 & 9 & 17.8 & 46.2 & 68.4 & 65 & 61.7 & 58.4 & 60.8 \\
\hline 7.3 & 7.2 & 13.2 & 47.5 & 69.2 & 65.9 & 61.8 & 55.8 & 60.5 \\
\hline 7.1 & 7 & 14.5 & 45.4 & 67.9 & 61.5 & 56.4 & 52 & 55.4 \\
\hline 6.5 & 6.5 & 6.4 & 5.9 & 5.9 & 3.3 & 4 & 3.3 & 3.9 \\
\hline 6.5 & 6.5 & 6.4 & 5.9 & 6.2 & 3.4 & 3.9 & 3.9 & 3.9 \\
\hline 6.5 & 6 & 33.5 & 47.1 & 68 & 64.5 & 60.9 & 55.9 & 62.1 \\
\hline 8.6 & 8.6 & 18.6 & 45.5 & 67.6 & 66.1 & 62.4 & 56.5 & 59.3 \\
\hline 8.9 & 8.2 & 18.7 & 46 & 67.2 & 65 & 60.5 & 56.3 & 60.1 \\
\hline 7.1 & 7.1 & 16.2 & 45.7 & 63.7 & 62.8 & 56.1 & 52.2 & 59.2 \\
\hline 7.1 & 7 & 17.6 & 43.7 & 69.2 & 65.2 & 58.3 & 54.3 & 56.6 \\
\hline 7.2 & 7.3 & 6.6 & 7.3 & 6 & 4.7 & 4.7 & 4.1 & 4.6 \\
\hline 7.3 & 7.3 & 7.3 & 7.2 & 6.1 & 4.7 & 4.7 & 4.1 & 4.7 \\
\hline 7.3 & 7.2 & 20 & 43.3 & 66 & 64.4 & 59.1 & 55.9 & 60 \\
\hline 7.1 & 6.4 & 21 & 44.1 & 66.1 & 64 & 57.8 & 52.1 & 56.2 \\
\hline 7.4 & 7.4 & 21.6 & 44.4 & 67 & 66.4 & 60.3 & 56.1 & 59.8 \\
\hline 8.1 & 8.8 & 21.4 & 45 & 64.9 & 65.5 & 59.5 & 54.6 & 59.4 \\
\hline 7.2 & 7.2 & 16.8 & 48.1 & 64.2 & 64.5 & 58.8 & 54.8 & 56.6 \\
\hline 7.8 & 7.2 & 7.8 & 7.8 & 6 & 4.7 & 5.2 & 4.6 & 5.3 \\
\hline 7.8 & 7.8 & 7.2 & 7.9 & 5.9 & 5.3 & 4.6 & 5.3 & 4.6 \\
\hline 8.4 & 7.8 & 17.7 & 46 & 66.2 & 65.6 & 61.8 & 58.2 & 63 \\
\hline 9 & 8.4 & 20.7 & 44.7 & 66.6 & 67.4 & 63 & 59.5 & 61.5 \\
\hline 8.1 & 8.1 & 19 & 46.7 & 68.5 & 68.4 & 60.2 & 56.8 & 61.3 \\
\hline
\end{tabular}




\begin{tabular}{|c|c|c|c|c|c|c|c|c|}
\hline 8.1 & 8.8 & 16.7 & 45.8 & 67 & 55.7 & 59.6 & 55.3 & 59.8 \\
\hline 13.6 & 13.5 & 20.6 & 45.3 & 65.4 & 63.9 & 56.7 & 53.1 & 58.5 \\
\hline 8.7 & 8 & 8.6 & 8.6 & 6 & 6.1 & 6 & 5.5 & 6 \\
\hline 8.8 & 8.7 & 8.1 & 8.7 & 6.7 & 5.6 & 6.2 & 6.1 & 5.6 \\
\hline 8.1 & 8.7 & 19.5 & 46.1 & 65.9 & 66 & 58.6 & 52.8 & 58.4 \\
\hline 8.8 & 8.1 & 11.6 & 41.4 & 66 & 64.2 & 60.8 & 56.4 & 61 \\
\hline 7.8 & 7.8 & 20.5 & 45.3 & 67.5 & 66.6 & 61.4 & 58.9 & 61 \\
\hline 8 & 8 & 16.9 & 45.5 & 66.6 & 66.6 & 59.4 & 55 & 59.4 \\
\hline 9.1 & 8.4 & 18 & 45.6 & 69.2 & 66.9 & 61.2 & 56.3 & 60.2 \\
\hline 8.9 & 8.8 & 8.3 & 8.6 & 7 & 5.7 & 6.3 & 6.3 & 6.3 \\
\hline 7.7 & 8.3 & 8.3 & 8 & 6.3 & 5.2 & 5.7 & 5.7 & 5.1 \\
\hline 8.3 & 8.2 & 21.6 & 46.8 & 66.8 & 67.1 & 60.2 & 56.1 & 58.9 \\
\hline 7.2 & 7.2 & 18.1 & 46.7 & 68.3 & 67.1 & 59.9 & 55.6 & 61.9 \\
\hline 7.4 & 7.3 & 9.6 & 35.6 & 68.9 & 68.1 & 62.9 & 56.9 & 60 \\
\hline 7.2 & 6.6 & 16 & 43.3 & 66 & 67.7 & 60.8 & 55.4 & 59.3 \\
\hline 7.6 & 8.1 & 19.2 & 45.8 & 67.1 & 67.9 & 60.4 & 54.8 & 59.2 \\
\hline 8 & 8.6 & 8 & 8.1 & 5.4 & 5.4 & 6.1 & 6 & 6.6 \\
\hline 8 & 8 & 8 & 7.5 & 5.5 & 5.4 & 5.4 & 5.5 & 4.8 \\
\hline 8 & 8 & 7.9 & 7.5 & 5.4 & 5.4 & 6.3 & 7.8 & 9.6 \\
\hline 7.4 & 8 & 7.3 & 7.5 & 5.4 & 6 & 6.3 & 7.2 & 5.9 \\
\hline 7.3 & 7.3 & 7.9 & 6.7 & 4.8 & 4.7 & 4.7 & 4.7 & 4.7 \\
\hline 7.3 & 7.3 & 7.3 & 6.7 & 4.7 & 4.7 & 5 & 5.3 & 5.9 \\
\hline 7.2 & 7.3 & 7.3 & 6.7 & 4.7 & 4.7 & 4.8 & 5.3 & 5.3 \\
\hline 7.3 & 7.3 & 7.3 & 6.7 & 4.7 & 4.8 & 4.1 & 4.7 & 4.7 \\
\hline 7.3 & 7.3 & 7.3 & 6.7 & 4.8 & 4.1 & 4.7 & 4.8 & 4.7 \\
\hline 7.3 & 7.3 & 11.1 & 41.2 & 66.7 & 65.9 & 59.9 & 56.8 & 59.1 \\
\hline 7.3 & 7.9 & 20.7 & 44.2 & 66.1 & 66.4 & 61.9 & 56.3 & 61.4 \\
\hline 7.4 & 7.3 & 17.3 & 45.1 & 67.7 & 68.8 & 61.4 & 56.8 & 59 \\
\hline 9.2 & 9.1 & 20.8 & 45 & 66.4 & 65.9 & 59.4 & 58 & 59.7 \\
\hline 7.5 & 8 & 18.2 & 43.7 & 67.5 & 66.7 & 61.2 & 56.6 & 58.4 \\
\hline 8.4 & 8.9 & 8.9 & 7.5 & 6.4 & 5.7 & 6.3 & 5.8 & 6.3 \\
\hline 9 & 8.9 & 8.3 & 8.1 & 5.8 & 6.4 & 6.4 & 5.7 & 6.4 \\
\hline 9 & 8.9 & 19.1 & 44.7 & 66.9 & 67.2 & 61.3 & 56.6 & 60.1 \\
\hline 9.6 & 9.1 & 20.5 & 45.1 & 67.5 & 67.4 & 63.4 & 56.4 & 58.1 \\
\hline 8.6 & 7.9 & 28.7 & 44.1 & 64 & 62.6 & 59.8 & 57.2 & 60.9 \\
\hline 9.8 & 9.2 & 18.6 & 45 & 65.9 & 65.3 & 61 & 55.7 & 62 \\
\hline 7.2 & 7.1 & 19 & 44.2 & 65.8 & 63.4 & 59.9 & 55.3 & 57.3 \\
\hline 8.3 & 8.9 & 8.2 & 7.3 & 5.6 & 6.3 & 5.7 & 5.7 & 5.6 \\
\hline 8.2 & 8.3 & 8.2 & 6.6 & 5.7 & 5.1 & 5.7 & 5 & 5.7 \\
\hline 8.9 & 19.1 & 44.5 & 66.5 & 67.1 & 62.5 & 59.4 & 62.7 & 67.9 \\
\hline 10.1 & 19.6 & 45.3 & 68.3 & 68.4 & 61.4 & 54 & 56.8 & 67.2 \\
\hline 11 & 10.3 & 19.1 & 44 & 66.8 & 67.2 & 62.1 & 53.9 & 58 \\
\hline 8 & 7.4 & 16.8 & 43.5 & 65.5 & 66.9 & 60.5 & 53.8 & 56.3 \\
\hline 7.4 & 7.3 & 14.4 & 42.7 & 66.9 & 66.4 & 59.2 & 55.7 & 58.3 \\
\hline 9.6 & 9.5 & 9.5 & 11.2 & 8.9 & 9.2 & 9.2 & 9 & 7.2 \\
\hline 9.7 & 9.7 & 9.8 & 10.3 & 7.9 & 7.2 & 7.1 & 6.6 & 7.2 \\
\hline 11.1 & 10.5 & 17.7 & 46.8 & 66.7 & 67.8 & 61 & 56.7 & 60.5 \\
\hline 9.4 & 9.4 & 18.8 & 44.1 & 67.2 & 67.3 & 63.3 & 56.9 & 59.6 \\
\hline 8.9 & 8.8 & 16.5 & 43.2 & 66.5 & 67 & 61.7 & 57.8 & 55.2 \\
\hline 8.8 & 8.1 & 17.3 & 42.8 & 65.4 & 66.1 & 57.5 & 55.9 & 55 \\
\hline 7.2 & 7.1 & 7.2 & 6.5 & 5.2 & 4 & 4.6 & 4 & 4.6 \\
\hline
\end{tabular}


lights

\begin{tabular}{|c|c|c|c|c|c|c|c|c|}
\hline 7.1 & 6.6 & 7.2 & 7.1 & 5.2 & 4.1 & 4.6 & 4 & 4.6 \\
\hline 7.2 & 7.2 & 7.1 & 7.1 & 4.6 & 4.7 & 4 & 4.6 & 4.7 \\
\hline 7.2 & 7.1 & 18.8 & 44.4 & 65.8 & 66.9 & 60.7 & 55.4 & 58.3 \\
\hline 8.1 & 7.6 & 19.3 & 43.8 & 67.5 & 65.9 & 61.8 & 55.6 & 56.4 \\
\hline 9.5 & 10 & 21 & 44.2 & 67.3 & 65.9 & 60.9 & 54.1 & 56.3 \\
\hline 7.8 & 8.3 & 19 & 42.5 & 65.7 & 61 & 59.6 & 54.3 & 59.9 \\
\hline 7.9 & 7.9 & 20.4 & 41.4 & 66.1 & 65.9 & 58.6 & 52.9 & 54.3 \\
\hline 9 & 9.6 & 8.9 & 8.8 & 11.8 & 20.5 & 22.3 & 21.8 & 22.4 \\
\hline 7.4 & 8 & 8 & 7.7 & 18.6 & 19 & 19.8 & 18.4 & 19.1 \\
\hline 7.9 & 8 & 18.7 & 42.1 & 66.3 & 67 & 61.6 & 54.7 & 58.8 \\
\hline 8.4 & 9 & 11 & 36.8 & 67.1 & 67.1 & 60.7 & 55.2 & 58.1 \\
\hline 8.6 & 8.1 & 10.2 & 25.3 & 66.2 & 68 & 62.2 & 55.4 & 59.4 \\
\hline 15.9 & 15.9 & 26.8 & 43 & 65.5 & 67.6 & 60.3 & 55.5 & 60.4 \\
\hline 10 & 9.9 & 18.4 & 44.7 & 67.8 & 64.3 & 59.5 & 53.4 & 56 \\
\hline 7.9 & 8 & 8 & 6.9 & 8.8 & 10.4 & 10.7 & 11.6 & 10.9 \\
\hline 8.1 & 8.2 & 7.5 & 7.7 & 9.3 & 12.9 & 13.3 & 20.2 & 17.8 \\
\hline 7.9 & 7.4 & 18.1 & 44.3 & 67.5 & 65.5 & 60.7 & 53.4 & 60.3 \\
\hline 9.5 & 9.5 & 20.2 & 44.7 & 66.5 & 66.6 & 61.7 & 56.9 & 59.4 \\
\hline 10.6 & 9.9 & 19.4 & 43.8 & 67.1 & 65.8 & 61.3 & 53.5 & 59.8 \\
\hline 7.9 & 8 & 18.6 & 43 & 66.8 & 65.1 & 60.8 & 54.2 & 61.3 \\
\hline 7.9 & 8.4 & 20.9 & 42.9 & 66.9 & 63.4 & 58.5 & 52.3 & 53.8 \\
\hline 7.1 & 7.1 & 7.1 & 6.5 & 4 & 4.5 & 4.5 & 4 & 4.5 \\
\hline 7.1 & 7.1 & 6.5 & 6.5 & 4.5 & 4.6 & 3.9 & 4.6 & 3.9 \\
\hline 7.1 & 7 & 18.7 & 42.5 & 65.8 & 67.4 & 58.9 & 53.6 & 58 \\
\hline 7.7 & 7.8 & 19.2 & 42.4 & 64.8 & 63.3 & 59.5 & 53.3 & 57.8 \\
\hline 7 & 7 & 20.2 & 42.8 & 66.6 & 67.8 & 61 & 53.7 & 56 \\
\hline 8 & 8 & 20 & 44 & 66.4 & 67.6 & 61.4 & 58 & 60.6 \\
\hline 6.9 & 7 & 16.1 & 42.8 & 65.4 & 53.4 & 46.3 & 46.7 & 44.4 \\
\hline 7.2 & 7.3 & 7.2 & 6.5 & 4.7 & 12 & 34.5 & 37.1 & 37.7 \\
\hline 7.1 & 6.5 & 6.5 & 5.8 & 3.9 & 4 & 3.9 & 4 & 5.7 \\
\hline 7.6 & 7.7 & 22.8 & 41.6 & 64.1 & 66.5 & 58.7 & 54.8 & 58.7 \\
\hline 6.4 & 7 & 16.4 & 43.1 & 65.8 & 66.7 & 62.4 & 56.9 & 58.7 \\
\hline 8 & 7.9 & 16.1 & 44.1 & 66.8 & 66.5 & 57.7 & 55 & 60.4 \\
\hline 16 & 15.8 & 27.5 & 42.8 & 67.2 & 66.4 & 61.8 & 56.6 & 58.8 \\
\hline 16 & 16 & 24.9 & 41.5 & 65 & 56.7 & 56.6 & 56.2 & 59 \\
\hline 8.9 & 8.9 & 8.9 & 8 & 6.4 & 6.3 & 6.4 & 6.3 & 6.3 \\
\hline 8.9 & 8.8 & 9.5 & 8.1 & 6.3 & 6.4 & 6.4 & 6.3 & 6.4 \\
\hline 8.9 & 8.8 & 17.8 & 11.2 & 66.8 & 67.6 & 61.3 & 57.4 & 56.7 \\
\hline 8.3 & 8.8 & 19.7 & 43.2 & 67.9 & 67 & 59.8 & 53.8 & 60.7 \\
\hline 9.2 & 9.2 & 16.6 & 43.1 & 65.5 & 65.4 & 62.1 & 56.1 & 58.9 \\
\hline 8.4 & 9 & 18.9 & 42.6 & 60.9 & 61.6 & 54.5 & 54.4 & 59.1 \\
\hline 8.6 & 8.6 & 19.5 & 43.7 & 65.3 & 66.3 & 58.4 & 56.4 & 57.8 \\
\hline 8.1 & 8.8 & 8.7 & 7.3 & 5.5 & 6.2 & 5.6 & 6.2 & 5.6 \\
\hline 8.7 & 8.8 & 8.1 & 7.9 & 5.6 & 6.2 & 5.6 & 6.1 & 5.6 \\
\hline 8.7 & 8.1 & 19.4 & 42.8 & 66.7 & 66.2 & 60.5 & 54.5 & 57.3 \\
\hline 7.9 & 8 & 17.6 & 41.5 & 65.2 & 66.6 & 55.1 & 51.6 & 54.6 \\
\hline 15 & 15 & 15.4 & 28.5 & 52.9 & 58.7 & 59.3 & 55.3 & 53.6 \\
\hline 14.8 & 14.7 & 15.5 & 24.6 & 25.2 & 28.5 & 31.7 & 32.9 & 33.8 \\
\hline 17.5 & 16.8 & 17.4 & 26.6 & 36.5 & 42.5 & 43.7 & 44.9 & 43 \\
\hline 13.8 & 14.3 & 13.7 & 12.7 & 15.7 & 22.2 & 23.9 & 23.3 & 18.8 \\
\hline 15.3 & 15.4 & 15.4 & 13.6 & 12.9 & 12.8 & 12.1 & 12.8 & 12.7 \\
\hline
\end{tabular}


lights

\begin{tabular}{|c|c|c|c|c|c|c|c|c|}
\hline 15.4 & 15.3 & 15.3 & 28.4 & 42.1 & 42.8 & 43.3 & 41.7 & 35.1 \\
\hline 15.8 & 15.7 & 15.2 & 14.7 & 34.5 & 43.3 & 46.3 & 44.4 & 32.7 \\
\hline 17.8 & 17.7 & 17.1 & 17.4 & 39.6 & 43.8 & 47.1 & 47.4 & 36.8 \\
\hline 17.8 & 17.2 & 17.8 & 21.2 & 41.1 & 46.5 & 45.7 & 43.9 & 31 \\
\hline 16.7 & 16.6 & 16.6 & 19.8 & 41.6 & 43.5 & 42.5 & 40.3 & 30.3 \\
\hline 17.9 & 18 & 17.8 & 15.9 & 14.5 & 15.4 & 15 & 15.2 & 14.4 \\
\hline 18.5 & 17.9 & 17.9 & 16.5 & 15.1 & 16 & 15.5 & 15.4 & 15.1 \\
\hline 18.5 & 17.9 & 17.9 & 21 & 41.1 & 42.2 & 41.4 & 41.6 & 30.4 \\
\hline 15.3 & 15 & 15.5 & 18.3 & 40.6 & 42.3 & 40.7 & 39 & 29.8 \\
\hline 15.8 & 15.6 & 15.6 & 18.2 & 39.4 & 41.4 & 40.7 & 40.2 & 29.7 \\
\hline 16.2 & 16.2 & 16.1 & 17.5 & 37.3 & 40.2 & 39.3 & 38.1 & 25.8 \\
\hline 14.5 & 14.6 & 14.5 & 18.3 & 36.8 & 39.6 & 37.9 & 38.2 & 28 \\
\hline 14.6 & 14.6 & 14.6 & 13.3 & 11.7 & 12.1 & 12 & 12 & 12 \\
\hline 14.5 & 14.5 & 14.5 & 13.5 & 11.6 & 11.7 & 12 & 12 & 11.9 \\
\hline 14.5 & 14.5 & 15.7 & 25.3 & 39.7 & 37.1 & 36.7 & 36.7 & 31.6 \\
\hline 14.8 & 14.2 & 14.7 & 23.9 & 38.6 & 42.1 & 40.6 & 39.4 & 26 \\
\hline 13.7 & 13.6 & 13.7 & 22.1 & 42.1 & 48.4 & 47.8 & 46.6 & 35.4 \\
\hline 13.2 & 13.6 & 13.7 & 22 & 34.5 & 36.4 & 37.5 & 37.2 & 32 \\
\hline 14.1 & 14.1 & 14 & 22.2 & 36.7 & 40 & 40.1 & 37.3 & 25.9 \\
\hline 7.1 & 7.2 & 7.1 & 5.6 & 4.7 & 4.6 & 4 & 4.6 & 4.6 \\
\hline 7.1 & 7.2 & 6.5 & 6.2 & 4.6 & 4.1 & 4.6 & 4.6 & 4 \\
\hline 7.1 & 7.1 & 7.2 & 16.4 & 36.8 & 41.3 & 42.7 & 43.2 & 35.1 \\
\hline 16.1 & 15.5 & 15.5 & 24.9 & 38.1 & 43.7 & 42.7 & 43.4 & 41.1 \\
\hline 18.1 & 17.9 & 17.9 & 27.5 & 37.9 & 44.8 & 41 & 41.1 & 33.1 \\
\hline 13.9 & 14.4 & 14.4 & 24.1 & 27.3 & 27.6 & 27.9 & 28.6 & 28.5 \\
\hline 14.5 & 14 & 14.5 & 13.1 & 12.1 & 11.5 & 12.1 & 12.1 & 11.4 \\
\hline 13.5 & 14.2 & 14.6 & 13 & 11.9 & 11.5 & 12.1 & 11.4 & 12 \\
\hline 14 & 14.5 & 14.1 & 12.9 & 12 & 11.5 & 12 & 11.3 & 12 \\
\hline 14.6 & 13.9 & 14.5 & 18.9 & 17.4 & 17.9 & 17.9 & 18.6 & 18.5 \\
\hline 8 & 8.5 & 8 & 18.5 & 20.2 & 20.8 & 21.4 & 22 & 22.5 \\
\hline 9.9 & 9.3 & 9.9 & 18.1 & 20.4 & 21.6 & 23.6 & 22.9 & 22.9 \\
\hline 8.9 & 8.9 & 9.6 & 18.3 & 18.4 & 19.9 & 20.5 & 20 & 20.5 \\
\hline 8.4 & 8.3 & 8.9 & 17.1 & 20.8 & 21.7 & 20.9 & 21 & 21.6 \\
\hline 9.2 & 9.2 & 9.2 & 8.4 & 6.6 & 6.7 & 6.6 & 6.7 & 6.6 \\
\hline 9.2 & 9.2 & 9.2 & 8.4 & 6.7 & 6.6 & 6 & 6.7 & 6.6 \\
\hline 9.7 & 9.2 & 9.1 & 21.9 & 24.9 & 24.9 & 24.8 & 25.4 & 24.1 \\
\hline 9.4 & 9.3 & 9.9 & 14.7 & 23.6 & 25.2 & 25 & 25.5 & 24.8 \\
\hline 9.3 & 9.3 & 9.3 & 20 & 26.1 & 27.5 & 26 & 24.2 & 24.8 \\
\hline 8.9 & 8.8 & 8.9 & 21.7 & 24.7 & 25.1 & 25.1 & 24.4 & 24 \\
\hline 8.1 & 8.7 & 8.1 & 18.6 & 25.4 & 27.2 & 28.1 & 27.4 & 27.6 \\
\hline 8.7 & 8.7 & 8.8 & 8.5 & 5.6 & 6.2 & 6.2 & 6.2 & 6.3 \\
\hline 8.7 & 8.7 & 8.7 & 8 & 6.2 & 6.2 & 6.2 & 6.2 & 6.2 \\
\hline 8.8 & 8.7 & 8.7 & 13 & 12.9 & 13.9 & 15.6 & 21.5 & 23.3 \\
\hline 9.3 & 9.9 & 9.9 & 15 & 14.7 & 23.9 & 24.1 & 27.3 & 26.6 \\
\hline 8.3 & 8.3 & 8.3 & 16 & 27.5 & 27.6 & 27.7 & 27.8 & 26 \\
\hline 7.7 & 7.7 & 7.7 & 15.5 & 20 & 21.1 & 20.2 & 18.4 & 17.8 \\
\hline 10.5 & 11.1 & 10.4 & 17.2 & 25.5 & 25.7 & 25.6 & 27.7 & 26 \\
\hline 10.1 & 10.1 & 10.1 & 10.1 & 7.5 & 7.6 & 7.5 & 7.6 & 7.5 \\
\hline 10.7 & 10.1 & 10.1 & 9.5 & 8.2 & 7.5 & 7.6 & 7.5 & 6.9 \\
\hline 10.1 & 10 & 10 & 16.9 & 22.5 & 21.3 & 23 & 26.5 & 28 \\
\hline 8.3 & 7.7 & 8.3 & 12.7 & 22.5 & 25.3 & 23.9 & 32.6 & 31.1 \\
\hline
\end{tabular}


lights

\begin{tabular}{|c|c|c|c|c|c|c|c|c|}
\hline 9.2 & 9.2 & 9.2 & 17.3 & 25 & 24.5 & 27.9 & 27.9 & 23.9 \\
\hline 11.3 & 10.6 & 11.3 & 17.3 & 27 & 26.9 & 33 & 36.8 & 34.4 \\
\hline 8.9 & 8.3 & 8.4 & 15.4 & 28.7 & 31.7 & 32.9 & 31.9 & 29.5 \\
\hline 7.3 & 7.3 & 7.3 & 6.1 & 4.8 & 4.7 & 4.2 & 4.8 & 4.1 \\
\hline 7.3 & 7.3 & 6.7 & 6.7 & 4.8 & 4.2 & 4.7 & 4.2 & 4.8 \\
\hline 7.3 & 7.3 & 6.9 & 24.3 & 27.5 & 28.8 & 29.3 & 30.3 & 26 \\
\hline 7 & 7.1 & 7.1 & 15 & 19.4 & 18.6 & 23.2 & 25.5 & 25.6 \\
\hline 7.7 & 7.1 & 7.7 & 14.1 & 20.9 & 23.4 & 26.2 & 25.4 & 24.2 \\
\hline 7.2 & 7.7 & 7.9 & 14.5 & 23.9 & 27.5 & 25.8 & 27.2 & 26 \\
\hline 8.3 & 8.3 & 8.2 & 24.1 & 31.9 & 31.8 & 34.2 & 34.6 & 33.1 \\
\hline 8.1 & 8.1 & 8.1 & 7.7 & 5.6 & 6.2 & 6.7 & 7.4 & 6.8 \\
\hline 8.7 & 9.3 & 8.8 & 8.9 & 6.7 & 6.2 & 6.2 & 6.8 & 6.2 \\
\hline 8.1 & 8.1 & 8.7 & 18.5 & 31.8 & 33.3 & 33.1 & 32.5 & 31.6 \\
\hline 14.7 & 14.7 & 15.3 & 23.1 & 28.8 & 31.8 & 36.3 & 36.6 & 36.4 \\
\hline 8.5 & 8.5 & 9 & 27.1 & 35.9 & 37.6 & 42.3 & 42.1 & 41.3 \\
\hline 8.7 & 8.6 & 8.7 & 29.7 & 38.6 & 38.3 & 35 & 33.5 & 36.2 \\
\hline 6.7 & 7.3 & 6.7 & 21.5 & 26 & 28.1 & 29.6 & 29.5 & 30.6 \\
\hline 9.8 & 9.8 & 9.8 & 8.9 & 13.4 & 16 & 16.1 & 16.6 & 16.1 \\
\hline 8.6 & 9.1 & 8.6 & 8.3 & 6.6 & 6.7 & 6 & 6 & 6 \\
\hline 8.5 & 8.6 & 9.1 & 20.4 & 26.2 & 39.5 & 40.7 & 41.2 & 41.8 \\
\hline 10.4 & 9.7 & 10.9 & 25.6 & 28.9 & 32.6 & 35.5 & 37.9 & 40.5 \\
\hline 10.2 & 10.8 & 13.1 & 27.3 & 33.9 & 36.7 & 39.5 & 39.5 & 37.9 \\
\hline 7.5 & 7 & 7.6 & 25.6 & 44.2 & 57.4 & 63.2 & 62.3 & 55.1 \\
\hline 7.7 & 8.4 & 8 & 36.4 & 58 & 65.9 & 68.2 & 63 & 61.5 \\
\hline 8.4 & 8.3 & 8.3 & 8.1 & 9.9 & 15.2 & 29 & 30.9 & 26.6 \\
\hline 9.5 & 8.9 & 9.4 & 8.7 & 7.5 & 6.4 & 7.5 & 8.2 & 6.9 \\
\hline 7.9 & 7.9 & 25 & 48 & 66.2 & 65.8 & 64.5 & 60.4 & 59.9 \\
\hline 15.2 & 15.1 & 29.5 & 45.8 & 64 & 63.9 & 59 & 53 & 56.4 \\
\hline 7.4 & 6.8 & 25.1 & 44.8 & 63.9 & 66.1 & 59.1 & 55.6 & 58.9 \\
\hline 7.1 & 7.8 & 25.1 & 45.2 & 65.6 & 65.9 & 61.2 & 54.4 & 58.2 \\
\hline 9.3 & 9.3 & 23.1 & 45.3 & 64.2 & 66.2 & 59.2 & 52.5 & 54.6 \\
\hline 14.5 & 14.5 & 14.4 & 14.4 & 12.5 & 11.3 & 12 & 12 & 11.9 \\
\hline 13.9 & 13.8 & 13.8 & 13.8 & 10.9 & 11.1 & 10.8 & 11.3 & 11.4 \\
\hline 13.8 & 13.6 & 27.8 & 44.8 & 66.9 & 67 & 59.1 & 54.2 & 59.1 \\
\hline 8.6 & 7.9 & 27.8 & 45.9 & 64.2 & 65.6 & 59.4 & 55.4 & 61.7 \\
\hline 15.5 & 15.5 & 29.3 & 45.1 & 66.7 & 67.3 & 61.9 & 52.5 & 58.1 \\
\hline 9.2 & 9.7 & 13.5 & 46.2 & 64.5 & 66.5 & 58.8 & 51 & 58.5 \\
\hline 8.6 & 8.5 & 12.1 & 45.1 & 66.8 & 66.5 & 60.7 & 56.4 & 60.2 \\
\hline 8.2 & 7.6 & 8.1 & 7.6 & 6.3 & 5.1 & 5 & 5.1 & 5.7 \\
\hline 8.2 & 7.5 & 8.2 & 7.6 & 6.3 & 5.1 & 5.1 & 5 & 5.7 \\
\hline 7.6 & 8.2 & 7.6 & 8.2 & 6.3 & 5.1 & 5.1 & 5.7 & 5 \\
\hline 7.6 & 8.2 & 11.4 & 44.8 & 66.2 & 66.7 & 59.7 & 54.3 & 59.7 \\
\hline 9.6 & 9.7 & 15.8 & 43.9 & 66.1 & 67 & 59.4 & 54.7 & 60.4 \\
\hline 9.2 & 9.2 & 12.2 & 44.7 & 67.3 & 67.3 & 62.9 & 56.4 & 59.2 \\
\hline 14.9 & 15.5 & 19.2 & 45 & 65.3 & 66.1 & 61.1 & 54.1 & 56.5 \\
\hline 8.4 & 7.9 & 8.4 & 7.8 & 6.7 & 5.3 & 7.7 & 7.2 & 7.7 \\
\hline 7.2 & 7.8 & 7.3 & 7.8 & 6.1 & 4.7 & 4.7 & 5.3 & 4.7 \\
\hline 7.2 & 7.8 & $\begin{array}{l}3.7 \\
\end{array}$ & 43.6 & 66.2 & 66.1 & 58.7 & 54.2 & 58.9 \\
\hline 7.2 & 7.2 & 11.7 & 44.2 & 66.1 & 67.4 & 61.3 & 55.6 & 57.5 \\
\hline 8.4 & 9 & 13.6 & 43 & 64.4 & 64.6 & 59 & 54.4 & 56.9 \\
\hline 8.5 & 8 & 11.2 & 42.3 & 66.1 & 66.5 & 62.1 & 57.6 & 56.2 \\
\hline
\end{tabular}




\begin{tabular}{|c|c|c|c|c|c|c|c|c|}
\hline 10 & 9.4 & 14.8 & 44.1 & 67.1 & 67.1 & 60.9 & 55 & 55.1 \\
\hline 10.1 & 10.7 & 10.1 & 10.2 & 11.1 & 10.2 & 9.5 & 10.2 & 8.4 \\
\hline 10.7 & 10 & 10.6 & 10.6 & 9 & 7.5 & 8.1 & 7.5 & 7.5 \\
\hline 10 & 10.7 & 14.5 & 45.6 & 68.8 & 66.2 & 62.4 & 56.7 & 58.1 \\
\hline 9.2 & 9.9 & 18.8 & 40.1 & 65.7 & 66.5 & 60.5 & 54.3 & 57.5 \\
\hline 16 & 16.5 & 21.2 & 43.8 & 66.7 & 67.1 & 60.5 & 52.5 & 58.5 \\
\hline 9.4 & 9.9 & 12.3 & 43 & 66.5 & 67.4 & 61.6 & 56.6 & 56.2 \\
\hline 9.6 & 9.5 & 11.8 & 38.9 & 66.6 & 64.9 & 60.5 & 54.5 & 54 \\
\hline 10.5 & 10.4 & 9.8 & 10.5 & 9.4 & 7.9 & 7.3 & 7.9 & 7.9 \\
\hline 10.4 & 10.5 & 10.5 & 10.5 & 9.6 & 7.9 & 7.9 & 8 & 7.9 \\
\hline 10.6 & 10.4 & 16.9 & 46.5 & 69.1 & 68.6 & 63.2 & 55.1 & 57.8 \\
\hline 10.2 & 9.5 & 23.3 & 42.7 & 66.6 & 65.9 & 60.9 & 56.7 & 58.6 \\
\hline 11.8 & 11.7 & 23.8 & 44.6 & 68.1 & 67.8 & 62.8 & 58.1 & 60.4 \\
\hline 10.8 & 10.2 & 13 & 44.3 & 68.9 & 68.7 & 62.6 & 56.8 & 59.2 \\
\hline 8.2 & 8.8 & 12.5 & 42.7 & 68 & 67.2 & 61.5 & 55.1 & 56.6 \\
\hline 8.8 & 8.8 & 8.8 & 8.2 & 8 & 5.6 & 6.3 & 6.2 & 5.7 \\
\hline 8.8 & 8.3 & 8.8 & 8.2 & 7.4 & 6.2 & 5.7 & 5.6 & 5.7 \\
\hline 8.3 & 8.8 & 15.4 & 42.3 & 66.3 & 66.9 & 58.6 & 52.6 & 57.6 \\
\hline 8.6 & 9.2 & 15.2 & 44 & 67.2 & 66.7 & 61.4 & 59.1 & 59.6 \\
\hline 10.1 & 9.4 & 12.1 & 44.7 & 68.6 & 66 & 61.6 & 58.1 & 57.7 \\
\hline 8.2 & 8.8 & 12.5 & 44 & 67.3 & 64.2 & 56.9 & 54.1 & 55.9 \\
\hline 8.2 & 8.2 & 10.1 & 43.3 & 67.8 & 67.4 & 62.7 & 56.8 & 57.8 \\
\hline 9 & 8.4 & 9 & 8.4 & 8.2 & 5.9 & 5.8 & 6.4 & 5.9 \\
\hline 9 & 9.1 & 8.4 & 9 & 8.3 & 5.9 & 6.5 & 5.8 & 6.5 \\
\hline 8.4 & 9 & 14 & 46.2 & 67.9 & 67.1 & 61.5 & 56.8 & 58.1 \\
\hline 16.2 & 16.5 & 27.9 & 44.9 & 68.5 & 67.1 & 62 & 58.9 & 57.7 \\
\hline 9 & 9.6 & 24.1 & 43.7 & 68.9 & 67.3 & 60.4 & 56.8 & 55.5 \\
\hline 8.8 & 9.5 & 21.7 & 45.8 & 66.9 & 66.6 & 61.3 & 57.4 & 59.6 \\
\hline 10.9 & 13.3 & 26.6 & 43.8 & 69.2 & 67.5 & 61.7 & 58.3 & 55.8 \\
\hline 10.1 & 10.1 & 9.5 & 10.1 & 9.5 & 6.9 & 7.6 & 6.9 & 7.6 \\
\hline 9.6 & 10.1 & 10.1 & 10.1 & 9.5 & 6.9 & 7.6 & 6.9 & 7.6 \\
\hline 10.1 & 10.1 & 12 & 36.2 & 67.4 & 67.7 & 61.3 & 59.3 & 58.6 \\
\hline 9.3 & 9.4 & 21.5 & 44.5 & 67.8 & 67.8 & 60.7 & 57 & 57.8 \\
\hline 8.1 & 8.7 & 14.3 & 44.8 & 67.8 & 68.2 & 62.3 & 56.6 & 57.9 \\
\hline 10.1 & 9.5 & 13.5 & 46.8 & 69.8 & 68.9 & 62.5 & 57.8 & 58.2 \\
\hline 9.3 & 8.6 & 14.7 & 42.4 & 69.4 & 66.8 & 58.4 & 56.6 & 55 \\
\hline 15.9 & 16.9 & 16.3 & 16.8 & 15.8 & 14.2 & 13.3 & 13.7 & 14.4 \\
\hline 16.4 & 16.1 & 16 & 16.4 & 15.9 & 13.8 & 13.8 & 13.3 & 12.9 \\
\hline 15.4 & 15.9 & 16.3 & 23.1 & 47.3 & 33.9 & 61.9 & 58.3 & 58.9 \\
\hline 9.9 & 9.3 & 15.4 & 47.7 & 66.5 & 65.4 & 61.7 & 56.4 & 58.2 \\
\hline 8.3 & 8.3 & 14.4 & 46.9 & 67.2 & 66.3 & 58 & 56.7 & 58.3 \\
\hline 9.3 & 9.4 & 15 & 44.2 & 66.7 & 67.1 & 63.7 & 60.2 & 58 \\
\hline 9.2 & 9.2 & 18 & 44.5 & 45 & 43 & 43.6 & 43.5 & 44.4 \\
\hline 16 & 15.8 & 15.5 & 15.4 & 13.3 & 13.3 & 12.9 & 12.9 & 12.7 \\
\hline 15.4 & 15.9 & 15.3 & 15.5 & 13.4 & 12.2 & 12.5 & 12.7 & 13.3 \\
\hline 15.3 & 15.9 & 20.4 & 45.4 & 68.8 & 68.8 & 62 & 58.6 & 57.9 \\
\hline 10.7 & 10.2 & 14.4 & 44.6 & 68 & 66.3 & 62.2 & 57.2 & 58.4 \\
\hline 7.8 & 7.7 & 14 & 45.4 & 68.3 & 68.3 & 63.3 & 60 & 60.4 \\
\hline 9.2 & 9.1 & 14.2 & 46.2 & 69 & 68.5 & 62.1 & 59.8 & 59 \\
\hline 10 & 10.5 & 13.2 & 44.8 & 65.9 & 66.9 & 59.2 & 57 & 57.3 \\
\hline 10.1 & 10.2 & 10.1 & 9.7 & 8.2 & 7.6 & 7.7 & 8.6 & 7 \\
\hline
\end{tabular}


lights

\begin{tabular}{|c|c|c|c|c|c|c|c|c|}
\hline 10.2 & 10.1 & 10.2 & 9.8 & 8.2 & 7.5 & 7.6 & 7.6 & 8.1 \\
\hline 10.7 & 10.1 & 16 & 48.5 & 69.1 & 68.9 & 61.3 & 57.8 & 57.5 \\
\hline 8.7 & 8.7 & 13.3 & 46.1 & 66.4 & 62.2 & 57.7 & 59 & 59.5 \\
\hline 25.4 & 24.9 & 27.6 & 44.4 & 67.1 & 65.9 & 57.8 & 56.1 & 59.1 \\
\hline 11.9 & 12 & 17.3 & 47.9 & 70 & 69.3 & 63 & 58.6 & 59.5 \\
\hline 17.5 & 17.4 & 22.2 & 45.5 & 66.5 & 67.9 & 59.8 & 58.4 & 59.7 \\
\hline 9.1 & 9 & 8.5 & 8.8 & 6.6 & 6.5 & 5.9 & 5.9 & 6.6 \\
\hline 8.4 & 9.1 & 9.1 & 8.3 & 7.1 & 5.9 & 6.6 & 5.9 & 6.5 \\
\hline 9.1 & 8.5 & 17.8 & 44.8 & 67.3 & 67.4 & 60.1 & 61 & 60.1 \\
\hline 8.7 & 9.3 & 16.7 & 46.9 & 68 & 67.5 & 60.4 & 59.7 & 58.5 \\
\hline 9.5 & 8.9 & 16.3 & 45.8 & 66.8 & 68.2 & 62.6 & 60.3 & 60 \\
\hline 9.3 & 9.4 & 14.3 & 45.4 & 69.2 & 67.9 & 62.3 & 62 & 61.3 \\
\hline 9.5 & 10.1 & 18.6 & 45.8 & 68.5 & 67.3 & 57.5 & 58.2 & 60.5 \\
\hline 9.1 & 9.2 & 9.1 & 8.5 & 7.2 & 6.6 & 6.6 & 6 & 6.6 \\
\hline 9.1 & 9.2 & 9.2 & 9.2 & 7.2 & 6.6 & 6.6 & 6.7 & 6 \\
\hline 9.2 & 9.2 & 13.9 & 46.7 & 68.3 & 69.8 & 62.8 & 58.7 & 59 \\
\hline 9 & 9 & 15.8 & 45.1 & 69.7 & 66.4 & 62.3 & 62 & 59.9 \\
\hline 10.4 & 10.4 & 17 & 45.6 & 45.1 & 45.2 & 46.4 & 48.8 & 50.2 \\
\hline 8.7 & 8.8 & 8.1 & 8.8 & 6.8 & 6.2 & 5.6 & 6.2 & 5.6 \\
\hline 8.7 & 8.8 & 8.1 & 8.8 & 6.8 & 5.6 & 6.2 & 6.2 & 5.6 \\
\hline 8.7 & 8.7 & 8.2 & 8.7 & 6.8 & 5.6 & 6.2 & 6.2 & 5.6 \\
\hline 8.7 & 8.2 & 8.7 & 8.7 & 6.9 & 5.6 & 6.2 & 5.5 & 6.2 \\
\hline 8.1 & 8.8 & 11.7 & 47.5 & 67.7 & 67.1 & 58.2 & 59.7 & 58.8 \\
\hline 10.4 & 9.8 & 13 & 47.4 & 68.9 & 65.8 & 60.9 & 59.6 & 61.5 \\
\hline 9.1 & 9.6 & 14 & 45.2 & 69.2 & 67.3 & 61.6 & 57.6 & 58.7 \\
\hline 9.9 & 9.8 & 15.3 & 47.6 & 69.4 & 66.7 & 62.6 & 57.7 & 60.2 \\
\hline 10.4 & 10.5 & 15 & 45.1 & 69.7 & 63.9 & 56.7 & 55.2 & 57.4 \\
\hline 9.7 & 9.7 & 9.6 & 9.7 & 7.9 & 7 & 7.7 & 7.1 & 7.2 \\
\hline 9.7 & 9.7 & 9.7 & 9.6 & 7.9 & 6.5 & 7.1 & 7.1 & 7.2 \\
\hline 9.7 & 9.6 & 12.9 & 46.3 & 68.5 & 68.4 & 65.2 & 61.4 & 60.2 \\
\hline 8.6 & 8.6 & 11.7 & 41.5 & 66.8 & 69.1 & 62.9 & 60.4 & 61.3 \\
\hline 8.5 & 9.1 & 11.8 & 45.5 & 68.9 & 67.6 & 61.7 & 57.9 & 59.2 \\
\hline 11.8 & 11.3 & 15 & 46.2 & 68.4 & 66.6 & 60.4 & 59.2 & 59.1 \\
\hline 11.2 & 10.7 & 14.9 & 43.9 & 69.5 & 69.6 & 60.4 & 56.2 & 58 \\
\hline 17.2 & 17.4 & 17.4 & 17 & 15.5 & 14.9 & 14.8 & 14.8 & 14.9 \\
\hline 17.1 & 17.2 & 17.2 & 17.5 & 25.3 & 30.2 & 21.5 & 14.7 & 15.4 \\
\hline 18.1 & 17.3 & 22 & 44.8 & 69.6 & 66.8 & 61.8 & 61.6 & 60.8 \\
\hline 10.2 & 10.2 & 14.9 & 45.8 & 69.5 & 68.1 & 64.5 & 62.5 & 61.8 \\
\hline 17.2 & 17.1 & 20.2 & 45.3 & 68.7 & 67.7 & 62.5 & 61.2 & 62 \\
\hline 9.5 & 9.4 & 12 & 45.8 & 69.9 & 67.2 & 58.4 & 57.5 & 57.9 \\
\hline 15.1 & 14.5 & 28.2 & 44.5 & 66.4 & 68.3 & 62.2 & 56.1 & 65.3 \\
\hline 15.9 & 15.9 & 15.2 & 15.3 & 16.1 & 21.9 & 21.3 & 20.3 & 20.5 \\
\hline 16.1 & 16.2 & 15.9 & 16.1 & 15.1 & 14.1 & 13.4 & 13 & 14.1 \\
\hline 16.1 & 15.5 & 16.3 & 35.6 & 41.2 & 42.2 & 43.9 & 47.5 & 48.1 \\
\hline 15.6 & 15.9 & 16 & 33.7 & 38.6 & 37.7 & 42.1 & 42.4 & 43 \\
\hline 8.9 & 9 & 9 & 9.5 & 8.1 & 7.1 & 7 & 7 & 7.7 \\
\hline 9.6 & 9 & 9 & 8.9 & 8.8 & 7.4 & 6.8 & 6.9 & 6.8 \\
\hline 9.4 & 8.8 & 8.8 & 9.4 & 8 & 6.9 & 6.8 & 7.5 & 6.9 \\
\hline 9.4 & 8.9 & 8.8 & 9.4 & 7.9 & 6.9 & 6.8 & 7.5 & 6.9 \\
\hline 9.4 & 8.9 & 8.8 & 9.4 & 8.1 & 6.8 & 6.9 & 6.9 & 7.4 \\
\hline 8.9 & 9.4 & 8.8 & 8.8 & 8.6 & 6.8 & 6.9 & 6.9 & 6.8 \\
\hline
\end{tabular}




\begin{tabular}{|c|c|c|c|c|c|c|c|c|}
\hline 8.8 & 9.4 & 8.8 & 8.8 & 8 & 6.8 & 6.9 & 7.4 & 6.9 \\
\hline 9.4 & 8.8 & 8.7 & 8.8 & 8.6 & 6.9 & 7.4 & 6.9 & 6.8 \\
\hline & & & & & & & & \\
\hline 8.9 & 8.8 & 9.4 & 8.8 & 8.1 & 6.8 & 6.8 & 7.5 & 6.8 \\
\hline 8.9 & 8.8 & 8.8 & 9.4 & 8 & 6.8 & 6.9 & 6.8 & 7.5 \\
\hline 8.8 & 9.4 & 8.9 & 8.8 & 8 & 7.5 & 6.8 & 6.9 & 6.8 \\
\hline 9.4 & 8.8 & 13.3 & 46.2 & 68.4 & 67.4 & 61.3 & 59.3 & 61.9 \\
\hline 9.2 & 9.7 & 12.2 & 45.7 & 69.3 & 66.8 & 64.1 & 59.5 & 61.3 \\
\hline 9.9 & 10 & 12.8 & 45.5 & 67.5 & 68.4 & 63 & 60.2 & 61.2 \\
\hline 17.8 & 17.5 & 18.7 & 44.2 & 68.8 & 68.9 & 63.3 & 58.6 & 60.8 \\
\hline 8.7 & 9.2 & 11 & 43.4 & 69.7 & 68.8 & 62.5 & 59 & 60.9 \\
\hline 17.1 & 17.1 & 16.2 & 16.7 & 15.8 & 15.1 & 14.1 & 14.4 & 15.1 \\
\hline 16.1 & 16.9 & 16.5 & 16.7 & 15.6 & 15.1 & 14.3 & 14.7 & 14.6 \\
\hline 16.6 & 16.5 & 19.6 & 45.3 & 69.7 & 68.9 & 63.7 & 58.7 & 59.4 \\
\hline 10.2 & 10.2 & 12.8 & 42.3 & 68.4 & 66.2 & 60.8 & 57.6 & 58.7 \\
\hline 15.8 & 15.7 & 18.5 & 44.3 & 68.1 & 68.5 & 61.3 & 58.4 & 60.2 \\
\hline 10.4 & 10.5 & 13.2 & 45.7 & 69.3 & 68.5 & 62.5 & 59.7 & 60.6 \\
\hline 17.6 & 17.6 & 17.5 & 34.9 & 63.7 & 66.2 & 65.6 & 61.6 & 63.1 \\
\hline 8.1 & 8.2 & 8.1 & 7.5 & 7.3 & 5.6 & 6.1 & 6.2 & 5.6 \\
\hline 7.6 & 8.1 & 8.1 & 8.1 & 7.4 & 5.6 & 6.2 & 5.5 & 6.2 \\
\hline 8.1 & 8.2 & 7.5 & 8 & 7.4 & 5.5 & 6.2 & 7.3 & 7.3 \\
\hline 9.3 & 8.7 & 12.2 & 43.7 & 67.9 & 69.1 & 62.3 & 60.2 & 60.2 \\
\hline 11.6 & 12.2 & 14.6 & 47.4 & 69.4 & 67.4 & 60.9 & 56.7 & 57.9 \\
\hline 10.6 & 11.2 & 12.8 & 45 & 68.9 & 69 & 61.4 & 59.8 & 59.1 \\
\hline 15.9 & 16.3 & 19.2 & 43 & 66.8 & 67.4 & 61.9 & 57 & 57.6 \\
\hline 15.1 & 16.2 & 15.6 & 15.4 & 14.7 & 13.2 & 13.4 & 14.1 & 13.5 \\
\hline 15.9 & 15.8 & 16.4 & 17.1 & 21 & 19.5 & 17.2 & 14 & 13.9 \\
\hline 15.8 & 15.6 & 19 & 46.2 & 69.5 & 68.5 & 61.2 & 58.2 & 61.4 \\
\hline 9.6 & 9.6 & 12.5 & 46.4 & 69.8 & 69.4 & 62 & 57.7 & 58.9 \\
\hline 9.2 & 9.2 & 11.5 & 44.7 & 69.3 & 67.8 & 63.3 & 59.9 & 61.1 \\
\hline 8 & 8.5 & 10.2 & 43.1 & 67.3 & 60 & 62.9 & 59.6 & 61.7 \\
\hline 10.6 & 10 & 12.7 & 43 & 68.6 & 67.8 & 62.8 & 58.3 & 61.2 \\
\hline 8.5 & 7.8 & 7.8 & 7.9 & 7 & 5.9 & 5.8 & 5.9 & 5.9 \\
\hline 7.9 & 7.8 & 7.8 & 7.9 & 7 & 5.9 & 5.9 & 5.9 & 6.5 \\
\hline 7.9 & 7.8 & 12 & 44.7 & 68.6 & 69.3 & 61.1 & 59.6 & 60.4 \\
\hline 9.1 & 9.2 & 11.9 & 44.4 & 67.5 & 67.9 & 59.9 & 56.1 & 58.3 \\
\hline 10.3 & 10.3 & 13.2 & 45.3 & 67.6 & 68 & 62.9 & 60.7 & 60.6 \\
\hline 17.8 & 18.2 & 22.7 & 44.6 & 68.6 & 66.3 & 62.9 & 58.5 & 60.6 \\
\hline 17.7 & 17.7 & 18 & 31.9 & 35.6 & 36 & 36.9 & 34.7 & 34.8 \\
\hline 8.3 & 7.6 & 8.2 & 7.6 & 6.7 & 6.2 & 5.7 & 5.6 & 5.7 \\
\hline 7.6 & 8.2 & 7.6 & 8.2 & 6.7 & 5.7 & 6.2 & 5.7 & 5.7 \\
\hline 7.6 & 7.6 & 10.3 & 42.2 & 68.2 & 67.8 & 62.2 & 59.7 & 60.6 \\
\hline 17.4 & 16.7 & 19.6 & 46.8 & 68.8 & 67.7 & 60.3 & 58.6 & 60.2 \\
\hline 17.8 & 17.6 & 19 & 42.9 & 68.4 & 67.4 & 60.5 & 60.1 & 59.3 \\
\hline 10.1 & 10.8 & 12.4 & 45.1 & 69.3 & 68.6 & 60.9 & 57.3 & 59.7 \\
\hline 18.6 & 18.5 & 18.8 & 40.4 & 68.9 & 63.3 & 60.5 & 56 & 59.8 \\
\hline 16.5 & 16.4 & 16.3 & 16.8 & 14.9 & 14.4 & 14.4 & 14 & 14.3 \\
\hline 16.8 & 16 & 16.4 & 16.9 & 15.4 & 14.4 & 14.1 & 13.9 & 15.1 \\
\hline 16.9 & 17 & 17.6 & 31.9 & 49.1 & 51.7 & 52 & 52.7 & 49.2 \\
\hline 7.9 & 8.4 & 10.7 & 44.7 & 69.6 & 68.1 & 62.6 & 60.9 & 60.3 \\
\hline 15.6 & 16.2 & 18 & 44.4 & 67.8 & 67.3 & 62.9 & 60.2 & 60.7 \\
\hline
\end{tabular}




\begin{tabular}{|c|c|c|c|c|c|c|c|c|}
\hline 10 & 10 & 11.6 & 40.8 & 68.6 & 66.2 & 62.7 & 60.5 & 59.8 \\
\hline 17.5 & 17.6 & 19.2 & 42.5 & 66.6 & 67.3 & 61.7 & 59 & 56.4 \\
\hline 16.9 & 17.5 & 17.2 & 17.1 & 15.7 & 15.5 & 14.8 & 15.6 & 15.2 \\
\hline 15.3 & 15.8 & 15.7 & 15.3 & 14.3 & 13.8 & 13.7 & 14.1 & 13.3 \\
\hline 16.1 & 15.3 & 17.1 & 41.9 & 66.8 & 65.9 & 62 & 58 & 59.8 \\
\hline 9.8 & 9.8 & 11.6 & 47.6 & 69.2 & 69.5 & 63.7 & 59.6 & 58.2 \\
\hline 17.9 & 17.7 & 19.9 & 47.2 & 69.2 & 67.4 & 64.6 & 60.7 & 62.9 \\
\hline 16.6 & 16.3 & 17.6 & 44.3 & 68.6 & 67.8 & 59.9 & 55 & 59.7 \\
\hline 15.6 & 15.6 & 17.9 & 42.7 & 66.4 & 67.3 & 64 & 58.6 & 62.4 \\
\hline 16.4 & 17 & 16.2 & 15.9 & 13.8 & 13.8 & 13.8 & 13.8 & 13.9 \\
\hline 16.3 & 16.4 & 16.5 & 16.2 & 13.9 & 13.7 & 19.6 & 14 & 12.6 \\
\hline 14.6 & 15.1 & 15.7 & 42.8 & 66.7 & 65.5 & 63.8 & 58.4 & 60.2 \\
\hline 21.6 & 21.8 & 22.6 & 46.1 & 67.7 & 66.5 & 62 & 58.1 & 56.7 \\
\hline 17.4 & 17.5 & 19.6 & 45.4 & 68 & 65.2 & 63.5 & 60.4 & 61.6 \\
\hline 17.3 & 16.9 & 18.5 & 46.3 & 68.6 & 68.3 & 65.2 & 60.3 & 59.7 \\
\hline 16 & 16.8 & 18.2 & 41 & 67.3 & 68.4 & 62.4 & 56.6 & 57.6 \\
\hline 16.2 & 15.9 & 15.7 & 15.5 & 13.5 & 13.5 & 13.6 & 14.7 & 14.5 \\
\hline 15.5 & 15.5 & 15.6 & 15 & 12.8 & 12.3 & 13.1 & 13 & 13 \\
\hline 15.6 & 15.3 & 24.6 & 48.4 & 66.7 & 69.2 & 61.3 & 56.1 & 56.6 \\
\hline 11.4 & 11.3 & 13.5 & 45.5 & 67.8 & 66 & 59.7 & 57.9 & 56.6 \\
\hline 16.8 & 16.6 & 18.8 & 44 & 66.6 & 66.8 & 62.1 & 58.6 & 57.6 \\
\hline 16.1 & 16.2 & 23.3 & 42.1 & 67.4 & 70.4 & 60 & 56.6 & 60 \\
\hline 8.3 & 7.7 & 13.7 & 39.5 & 68.2 & 63.3 & 60.1 & 59.6 & 60.7 \\
\hline 8.1 & 8.1 & 8.1 & 7.2 & 5.5 & 5.6 & 5.5 & 6.1 & 5.6 \\
\hline 8.7 & 8.1 & 8.1 & 7.7 & 5.6 & 5.5 & 5.5 & 5.6 & 5.5 \\
\hline 8.1 & 8.1 & 8.7 & 7.1 & 5.7 & 5.5 & 6.2 & 5.5 & 5.5 \\
\hline 8 & 8.1 & 8.7 & 7 & 5.6 & 5.5 & 5.5 & 5.5 & 5.5 \\
\hline 9.3 & 8.7 & 8.7 & 8.3 & 6.1 & 6.1 & 6.7 & 7.4 & 5.5 \\
\hline 8.7 & 8.7 & 8.7 & 7.7 & 6.1 & 5.5 & 6.2 & 5.6 & 6.1 \\
\hline 8.7 & 8.7 & 8.1 & 8.2 & 5.6 & 6.1 & 5.5 & 6.2 & 6.1 \\
\hline 8.7 & 8.7 & 8.7 & 8.7 & 20.3 & 22.9 & 33.8 & 40.1 & 38.4 \\
\hline 7.4 & 8 & 7.5 & 6.9 & 8.3 & 10 & 9.4 & 15.9 & 19.2 \\
\hline 7.4 & 8 & 14.8 & 40.1 & 67.6 & 66.8 & 60.4 & 59 & 59.6 \\
\hline 9.4 & 9.4 & 15.4 & 40.5 & 68.4 & 67.8 & 58.4 & 58.1 & 58.8 \\
\hline 15.4 & 14.7 & 19.4 & 44.2 & 69 & 67.8 & 62.9 & 57.8 & 60 \\
\hline 14.8 & 14.8 & 19.9 & 43.6 & 67.6 & 66 & 61.1 & 57 & 55.9 \\
\hline 16.7 & 16.1 & 20.1 & 43.3 & 65.7 & 62.9 & 57.4 & 56 & 57.5 \\
\hline 16.2 & 16 & 16.2 & 21.5 & 21.3 & 21.2 & 21.4 & 21.3 & 21.2 \\
\hline 13.6 & 13.8 & 13.3 & 12.4 & 10.7 & 11.3 & 10.5 & 10.6 & 11.2 \\
\hline 13.2 & 13.7 & 18.6 & 45.3 & 66.3 & 67.5 & 61.8 & 58.1 & 57.7 \\
\hline 15.1 & 15 & 20.6 & 42.2 & 65.8 & 65.2 & 59.9 & 57.2 & 58.4 \\
\hline 13 & 13 & 16.6 & 40.7 & 65.6 & 65.9 & 58.9 & 54.8 & 55.9 \\
\hline 15.4 & 15.3 & 19.3 & 44.7 & 65.8 & 65.4 & 60.2 & 56.5 & 57.8 \\
\hline 14.4 & 14.4 & 17.7 & 41.4 & 64.7 & 65.6 & 60.4 & 55.6 & 58.9 \\
\hline 13.2 & 13.7 & 13.1 & 12.9 & 11.2 & 12.8 & 11.8 & 10.6 & 10.6 \\
\hline 13.1 & 13.1 & 13.7 & 13 & 13.1 & 10.6 & 10.6 & 12.4 & 15.1 \\
\hline 13.3 & 13.9 & 14.4 & 41.8 & 68.6 & 65.1 & 56.6 & 51.8 & 55.3 \\
\hline 15.7 & 16.4 & 18.7 & 43.8 & 69.6 & 62.2 & 57.4 & 53 & 62.6 \\
\hline 14.7 & 14.5 & 18.7 & 42.6 & 67.9 & 67.8 & 61.6 & 58.4 & 61.3 \\
\hline 14.3 & 14.1 & 16.9 & 45.5 & 68.5 & 65.3 & 59.6 & 56.2 & 59.2 \\
\hline 14.6 & 13.9 & 13.9 & 26.6 & 25.7 & 23.4 & 23.9 & 22.9 & 22.1 \\
\hline
\end{tabular}


lights

\begin{tabular}{|c|c|c|c|c|c|c|c|c|}
\hline 13.7 & 13 & 13.6 & 13.1 & 12.8 & 10.5 & 11.1 & 10.5 & 10.5 \\
\hline 13.6 & 13 & 13.5 & 13 & 12.7 & 10.5 & 10.5 & 11.2 & 10.3 \\
\hline 13.5 & 12.9 & 15.8 & 39.8 & 65.1 & 64 & 57.2 & 52.9 & 54.4 \\
\hline 12.5 & 12.6 & 16.4 & 43.7 & 69.3 & 68.8 & 61.5 & 55.4 & 58.6 \\
\hline 10.7 & 10.8 & 15.2 & 41 & 65.6 & 64.7 & 57.3 & 53.1 & 59.1 \\
\hline 11.1 & 11.1 & 12.9 & 40.8 & 65.1 & 64.9 & 59.5 & 55.2 & 57.5 \\
\hline 16.5 & 15.8 & 18.1 & 40.6 & 65.6 & 63.4 & 58.4 & 53.8 & 52.2 \\
\hline 10.9 & 10.8 & 10.8 & 10.8 & 9.7 & 8.3 & 8.9 & 8.3 & 8.4 \\
\hline 10.9 & 10.8 & 11.5 & 10.9 & 9.7 & 8.3 & 8.3 & 8.3 & 8.3 \\
\hline 10.7 & 10.7 & 10.7 & 18.5 & 19.9 & 18 & 18 & 18.6 & 18 \\
\hline 11.4 & 11.5 & 15.3 & 41.5 & 63.4 & 62 & 58.1 & 53 & 55.6 \\
\hline 10.2 & 10.3 & 11.9 & 37.9 & 65 & 63.9 & 58 & 54.9 & 55.3 \\
\hline 12.3 & 12.2 & 15.1 & 39.8 & 63 & 56.3 & 58.1 & 54.7 & 57.2 \\
\hline 11.7 & 11.8 & 15.8 & 40.1 & 64.8 & 50.7 & 48.2 & 43.9 & 41.5 \\
\hline 10.9 & 11.5 & 10.8 & 10.9 & 9.6 & 7.8 & 8.3 & 8.3 & 8.4 \\
\hline 10.9 & 10.8 & 10.3 & 10.9 & 9.6 & 8.3 & 8.4 & 8.3 & 7.8 \\
\hline 10.9 & 10.9 & 12.4 & 39.3 & 61.9 & 61.4 & 55.2 & 55.6 & 56.7 \\
\hline 10.7 & 11.2 & 12.9 & 40.3 & 63.2 & 62.1 & 54.7 & 52.1 & 54.5 \\
\hline 11.2 & 11.1 & 13 & 38.5 & 62 & 58.2 & 54.9 & 51.7 & 55.1 \\
\hline 11.4 & 10.6 & 11.7 & 38.8 & 62.9 & 61 & 55.7 & 54 & 55.2 \\
\hline 13.9 & 14.5 & 15.4 & 41 & 64.5 & 61.8 & 56.8 & 52.1 & 54.8 \\
\hline 12.8 & 13.4 & 12.8 & 13.7 & 22.2 & 23.1 & 22.3 & 23 & 22 \\
\hline 10.7 & 10.6 & 10.6 & 10.6 & 9.2 & 8.7 & 9.9 & 9.2 & 8.1 \\
\hline 11.3 & 10.6 & 12.4 & 39.8 & 61.6 & 61 & 56.8 & 53.3 & 55.4 \\
\hline 10.1 & 10.7 & 12.3 & 40.9 & 62 & 62.8 & 56.1 & 52.1 & 55.6 \\
\hline 10.8 & 10.9 & 13.3 & 38.5 & 61.3 & 63.7 & 58 & 55.1 & 57 \\
\hline 14.7 & 14.5 & 16.2 & 42.9 & 67.5 & 67.6 & 59.5 & 56.7 & 59.6 \\
\hline 14.9 & 14.5 & 17.3 & 44.1 & 68.5 & 67 & 59.5 & 54.7 & 55.8 \\
\hline 16.1 & 16.8 & 16.2 & 16.7 & 14 & 14.2 & 13.6 & 14.2 & 13.5 \\
\hline 16.2 & 16.8 & 16.3 & 16.9 & 14.6 & 13.6 & 14.3 & 13.6 & 14.1 \\
\hline 17 & 16.3 & 20.7 & 45.5 & 68.3 & 66.4 & 62 & 57.1 & 60.6 \\
\hline 14.8 & 14.1 & 17.1 & 43.3 & 68 & 66.4 & 59.7 & 55.9 & 58.7 \\
\hline 14.2 & 13.7 & 16.3 & 40.9 & 65.4 & 64.5 & 60.3 & 55.8 & 58.5 \\
\hline 14.3 & 14.2 & 18.5 & 43.7 & 65.8 & 65.5 & 60.2 & 53.6 & 53.4 \\
\hline 14.6 & 14.6 & 18.1 & 42.7 & 66.3 & 62.6 & 52.3 & 53 & 51.1 \\
\hline 14.8 & 14.8 & 14.2 & 16.5 & 17.5 & 17.4 & 18 & 17.9 & 18.1 \\
\hline 18 & 18.1 & 18.1 & 18.1 & 18.2 & 18 & 16.6 & 16.6 & 16.1 \\
\hline 18.7 & 19.2 & 20.9 & 42.3 & 64.1 & 65.2 & 60.2 & 55.2 & 58 \\
\hline 15.1 & 14.5 & 17.3 & 44 & 65.2 & 66.1 & 61.5 & 58.2 & 58.8 \\
\hline 14.3 & 13.8 & 17.6 & 43.5 & 65.9 & 63.7 & 56.6 & 53.3 & 54.1 \\
\hline 13.4 & 13.3 & 16 & 43.4 & 63.7 & 63.5 & 55.6 & 51.1 & 53.8 \\
\hline 16.9 & 16.2 & 18 & 41.4 & 64.9 & 59.7 & 58.3 & 56.7 & 58.2 \\
\hline 16.9 & 17.6 & 17.1 & 29.3 & 29.2 & 28.4 & 19.4 & 17.1 & 16.6 \\
\hline 15.1 & 14.5 & 15.1 & 15.2 & 12.6 & 12.6 & 12.6 & 12.6 & 12.5 \\
\hline 15.2 & 14.4 & 17.7 & 42.3 & 65.4 & 66.9 & 58.9 & 53.4 & 57.4 \\
\hline 14.3 & 13.6 & 18.7 & 42.2 & 66 & 60.8 & 57.6 & 54.2 & 56.4 \\
\hline 15.5 & 15.4 & 16.5 & 43 & 65.6 & 60.8 & 59.5 & 54.4 & 57.5 \\
\hline 14 & 13.9 & 16.4 & 43.3 & 64.9 & 60.6 & 55.6 & 52.8 & 54.7 \\
\hline 16.9 & 16.9 & 18.7 & 43.8 & 66 & 64.5 & 58.8 & 53.3 & 52.6 \\
\hline 15.8 & 15.1 & 15.8 & 15.1 & 13.3 & 12.7 & 13.2 & 12.6 & 13.1 \\
\hline 15.9 & 15.7 & 15.3 & 15.7 & 12.7 & 13.1 & 12.6 & 13.3 & 13.1 \\
\hline
\end{tabular}


lights

\begin{tabular}{|c|c|c|c|c|c|c|c|c|}
\hline 15.9 & 15.7 & 22.1 & 43.8 & 66.5 & 61.8 & 58.3 & 52.6 & 52.6 \\
\hline 17.6 & 16.9 & 21.2 & 45.1 & 64.3 & 62.9 & 60.4 & 54.8 & 55.5 \\
\hline 19.5 & 19 & 21.4 & 47 & 66 & 67.3 & 60.1 & 53.4 & 54.7 \\
\hline 14 & 14.5 & 14.8 & 39.5 & 60.2 & 64.1 & 62.9 & 56 & 49.5 \\
\hline 12.8 & 12.7 & 14.7 & 28.7 & 29.6 & 31.2 & 33.4 & 35.3 & 36.1 \\
\hline 13.8 & 13.9 & 13.2 & 13.7 & 11.4 & 11.3 & 10.7 & 11.2 & 11.3 \\
\hline 13.8 & 13.9 & 13.2 & 13.7 & 11.3 & 10.6 & 11.2 & 11.4 & 10.7 \\
\hline 13.2 & 12.5 & 13.9 & 24.1 & 28.2 & 27.7 & 27.7 & 28 & 28 \\
\hline 15.2 & 15.1 & 15.3 & 25.7 & 28.5 & 28 & 28.2 & 28.3 & 25.9 \\
\hline 16 & 15.3 & 15.5 & 25.6 & 29.5 & 31.9 & 29.2 & 30.5 & 30.2 \\
\hline 14.9 & 14.3 & 14.8 & 26.1 & 30.5 & 33.7 & 31.4 & 29.2 & 29.1 \\
\hline 14 & 14 & 14.6 & 25 & 27.6 & 27.5 & 27.2 & 25.1 & 24.8 \\
\hline 14 & 14 & 14.7 & 13.9 & 11.5 & 11.4 & 12.1 & 11.4 & 11.4 \\
\hline 14 & 14.6 & 14.1 & 13.9 & 11.5 & 12.1 & 11.3 & 11.4 & 11.4 \\
\hline 14.6 & 13.8 & 14.5 & 27.2 & 30.4 & 31.1 & 30.1 & 31.5 & 30.5 \\
\hline 15.7 & 15.1 & 16.8 & 25.7 & 27 & 27 & 25.8 & 27.2 & 25.1 \\
\hline 15.6 & 16.1 & 18.5 & 29.3 & 32.1 & 31.5 & 31.4 & 31.1 & 29.1 \\
\hline 16.5 & 16.4 & 17.8 & 28.6 & 31.7 & 32 & 31.2 & 30.8 & 30.5 \\
\hline 16.3 & 15.6 & 15.6 & 24.8 & 28.7 & 29.8 & 27.2 & 26.8 & 26 \\
\hline 14.6 & 14.5 & 14.5 & 13.7 & 12 & 12.5 & 12 & 12 & 11.9 \\
\hline 14.5 & 14.6 & 15.2 & 13.8 & 12.7 & 12 & 12 & 12 & 12 \\
\hline 14.5 & 14.5 & 14.4 & 23.7 & 31.9 & 32.2 & 30.7 & 30 & 28.8 \\
\hline 19 & 19 & 18.9 & 29.2 & 33.7 & 33.1 & 31.7 & 32.4 & 30.9 \\
\hline 21.3 & 21.9 & 21.5 & 26.9 & 29.4 & 27.9 & 27.5 & 27 & 27 \\
\hline 19.1 & 18.6 & 18.6 & 26 & 27.7 & 28.9 & 30.2 & 31.8 & 30.8 \\
\hline 18.8 & 18.8 & 18.1 & 23.1 & 27.3 & 29.5 & 28.3 & 27 & 25.4 \\
\hline 5.7 & 6.2 & 5.7 & 5.5 & 3.7 & 3.2 & 3.7 & 3.1 & 3.5 \\
\hline 5.6 & 5.6 & 6.3 & 5.6 & 3.1 & 3.1 & 3.1 & 3.1 & 3.1 \\
\hline 5.6 & 5.5 & 5 & 11.7 & 17.3 & 20.8 & 20.8 & 20.8 & 18.4 \\
\hline 6 & 6 & 6.7 & 17.3 & 23.7 & 23.7 & 22.7 & 24 & 22.6 \\
\hline 5.6 & 6.3 & 5.6 & 15.1 & 18.1 & 18.1 & 18.7 & 17.8 & 17.6 \\
\hline 6.6 & 6.1 & 6.2 & 17.8 & 21.9 & 24.6 & 24.8 & 23.5 & 23.1 \\
\hline 6.9 & 6.2 & 6.2 & 20 & 23.6 & 25.8 & 24.2 & 21.9 & 20.2 \\
\hline 6.2 & 6.8 & 6.3 & 6.8 & 3.7 & 4.3 & 3.8 & 4.3 & 3.8 \\
\hline 6.3 & 6.9 & 6.2 & 6.9 & 3.7 & 4.3 & 3.7 & 4.3 & 3.8 \\
\hline 6.2 & 6.8 & 6.3 & 6.8 & 3.8 & 4.3 & 3.7 & 4.3 & 3.8 \\
\hline 6.2 & 6.2 & 6.8 & 16.5 & 19.4 & 22.1 & 23.6 & 26 & 29.6 \\
\hline 6.5 & 5.9 & 6.4 & 20.5 & 37.8 & 35.8 & 35.4 & 34.8 & 30.1 \\
\hline 12.2 & 11.5 & 12.2 & 23.3 & 27.1 & 28.3 & 29.3 & 32.4 & 28.8 \\
\hline 13.3 & 12.7 & 13.5 & 25.4 & 31.3 & 32.3 & 32.8 & 32.6 & 29.5 \\
\hline 7.5 & 8 & 8 & 8 & 5.6 & 5.5 & 5.5 & 5.5 & 6.2 \\
\hline 6.9 & 6.8 & 7.4 & 6.9 & 5 & 4.3 & 4.9 & 4.3 & 4.3 \\
\hline 6.2 & 6.8 & 8 & 24 & 38.8 & 39.2 & 36.9 & 38.1 & 31.3 \\
\hline 6.4 & 7 & 9.1 & 27.3 & 38.1 & 39.6 & 35.6 & 37 & 32 \\
\hline 8.2 & 7.6 & 10.6 & 30.5 & 41.4 & 48 & 45.9 & 46 & 41.1 \\
\hline 10.1 & 10.6 & 12.1 & 29.3 & 38.7 & 40 & 37.4 & 39.4 & 34 \\
\hline 7.3 & 7.3 & 10.1 & 24.7 & 34.5 & 34.3 & 34.5 & 36.4 & 29.9 \\
\hline 6.2 & 6.2 & 6.2 & 6.2 & 3.8 & 4.3 & 3.7 & 3.7 & 3.7 \\
\hline 6.2 & 6.3 & 6.2 & 6.1 & 3.9 & 3.7 & 3.6 & 3.7 & 3.7 \\
\hline 6.2 & 5.6 & 8.4 & 26.2 & 33.3 & 33.9 & 32.3 & 34 & 28.1 \\
\hline 8.3 & 8.3 & 11.5 & 26.5 & 37.9 & 39.1 & 36.9 & 37.5 & 32.9 \\
\hline
\end{tabular}


lights

\begin{tabular}{|c|c|c|c|c|c|c|c|c|}
\hline 11.3 & 11.4 & 12.1 & 32.7 & 41.4 & 40.8 & 39.5 & 39.4 & 33.7 \\
\hline 6.6 & 7.2 & 8.5 & 29.8 & 37.3 & 40 & 38.3 & 36.7 & 30.1 \\
\hline 7.4 & 7.4 & 7.6 & 30.2 & 38.3 & 38.9 & 36 & 36.3 & 31.8 \\
\hline 6.8 & 6.2 & 6.8 & 6.8 & 4.6 & 4.3 & 4.2 & 4.3 & 4.3 \\
\hline 6.9 & 6.8 & 6.8 & 6.7 & 4.6 & 4.3 & 4.2 & 4.3 & 3.7 \\
\hline 6.8 & 6.7 & 7.4 & 27.3 & 37.2 & 38.2 & 36.8 & 36.3 & 31.6 \\
\hline 7.9 & 7.2 & 7.2 & 27.2 & 37.3 & 37.6 & 33.7 & 34.1 & 28 \\
\hline 6.7 & 6.8 & 7.3 & 23.7 & 30.1 & 37 & 36.2 & 40.5 & 35.1 \\
\hline 15.2 & 15.2 & 15.7 & 27.6 & 37.6 & 38.1 & 36.8 & 39.8 & 34.4 \\
\hline 6 & 6 & 6.6 & 26.4 & 37 & 38.7 & 36.7 & 36.9 & 31.9 \\
\hline 6.6 & 6.6 & 6.7 & 6.6 & 5.1 & 4.1 & 4.1 & 4.1 & 4.1 \\
\hline 7.2 & 6.6 & 6.7 & 6.6 & 5.1 & 4.1 & 4.1 & 4 & 4.1 \\
\hline 6.6 & 6.6 & 7.4 & 29.7 & 37.5 & 38.6 & 36.5 & 38.1 & 32.7 \\
\hline 9.3 & 9.3 & 10.3 & 32.5 & 40.9 & 40.8 & 40.4 & 41.7 & 33.8 \\
\hline 9.9 & 10 & 10.2 & 28.6 & 51.2 & 50.9 & 48.7 & 49.7 & 46.4 \\
\hline 6.2 & 6.9 & 6.9 & 30.9 & 38 & 38.4 & 36.2 & 38.8 & 35 \\
\hline 6 & 5.9 & 6.2 & 23.8 & 37.4 & 38.2 & 37.4 & 39 & 32.9 \\
\hline 9 & 8.4 & 8.4 & 9.8 & 17.6 & 21.3 & 24.6 & 23 & 20.3 \\
\hline 7.7 & 8.2 & 8.2 & 7.6 & 6.9 & 5.6 & 5.2 & 5.7 & 5.7 \\
\hline 8.3 & 7.6 & 8.3 & 31.4 & 39.2 & 41.9 & 41.2 & 43.2 & 40.3 \\
\hline 8 & 7.9 & 8 & 22 & 41.7 & 43.8 & 44.7 & 47.8 & 44.2 \\
\hline 7.3 & 7.3 & 7.2 & 22.4 & 31.8 & 36.7 & 37.7 & 43.4 & 45.6 \\
\hline 7.9 & 8.5 & 8 & 30.9 & 54.9 & 64.5 & 66 & 61.3 & 53.1 \\
\hline 8.9 & 8.3 & 11.8 & 35.2 & 60.9 & 47.4 & 46.6 & 58.1 & 56.4 \\
\hline 9.4 & 9.5 & 9.5 & 9.4 & 7.6 & 8.2 & 18 & 20.5 & 22.5 \\
\hline 14.4 & 14.8 & 14.3 & 14.3 & 13.5 & 11.7 & 12.4 & 11.6 & 11.7 \\
\hline 14.2 & 14.1 & 17.3 & 51 & 69.9 & 66.4 & 62.3 & 57.9 & 56.9 \\
\hline 15.8 & 15.2 & 18 & 45.8 & 67.5 & 65.4 & 58.5 & 55.2 & 57.6 \\
\hline 14.9 & 14.9 & 17.9 & 42.9 & 65.9 & 64.4 & 57.6 & 50.5 & 54.9 \\
\hline 13.6 & 12.8 & 15.4 & 43.1 & 68.3 & 66.3 & 60.1 & 52.9 & 57.1 \\
\hline 12.9 & 12.9 & 15.4 & 43.4 & 67.3 & 66.1 & 59 & 53.9 & 53.7 \\
\hline 12.9 & 12.8 & 12.2 & 12.8 & 11.5 & 10.4 & 10.3 & 10.2 & 9.7 \\
\hline 12.9 & 12.8 & 12.8 & 12.8 & 11.6 & 10.3 & 9.7 & 10.2 & 10.3 \\
\hline 12.8 & 12.8 & 14.8 & 44 & 67.3 & 66.1 & 59.6 & 56.3 & 57 \\
\hline 13.6 & 13.6 & 15.3 & 43.1 & 67.4 & 65 & 59.8 & 54 & 57.8 \\
\hline 13.2 & 13.3 & 16.1 & 41.7 & 67.8 & 66.5 & 58.5 & 53.7 & 57.1 \\
\hline 12.6 & 12.5 & 15 & 40.6 & 67.8 & 65.7 & 60 & 53.5 & 57.6 \\
\hline 13.3 & 13.1 & 15 & 41.2 & 67 & 67.2 & 59.5 & 54.4 & 56.9 \\
\hline 13.2 & 13.7 & 13.1 & 13.1 & 12 & 10.8 & 10.7 & 11.3 & 10.3 \\
\hline 13.6 & 13 & 12.9 & 12.9 & 11.8 & 11.1 & 10.4 & 10.4 & 10.3 \\
\hline 12.9 & 12.8 & 15.5 & 44.1 & 67.6 & 65.4 & 58.5 & 53.8 & 56.8 \\
\hline 15.9 & 16.4 & 17.3 & 42 & 65.6 & 64.9 & 59.8 & 55.5 & 59.2 \\
\hline 14.2 & 14 & 16 & 42.7 & 67.6 & 67.5 & 59.7 & 55.3 & 58.1 \\
\hline 13.8 & 13.6 & 15.6 & 42.6 & 66.4 & 67.3 & 57.9 & 54.3 & 55.3 \\
\hline 13.8 & 13.7 & 13.8 & 23.2 & 24.3 & 23 & 23.4 & 25.4 & 24.5 \\
\hline 15.5 & 15.4 & 14.7 & 15.4 & 14.4 & 12.8 & 12.2 & 12.9 & 12.3 \\
\hline 15.5 & 14.9 & 15.4 & 15.5 & 14.4 & 12.3 & 12.9 & 12.7 & 12.4 \\
\hline 15.4 & 14.8 & 15.3 & 14.8 & 14.5 & 12.9 & 12.8 & 12.3 & 13 \\
\hline 14.7 & 15.2 & 15.2 & 25.3 & 32.3 & 33.6 & 33.1 & 33.9 & 33.8 \\
\hline 15.7 & 14.9 & 17 & 44.6 & 67.7 & 67.8 & 60.1 & 57.1 & 57.8 \\
\hline 14.6 & 15.1 & 16.6 & 43.4 & 68.6 & 66.6 & 60.5 & 56.8 & 58.6 \\
\hline
\end{tabular}


lights

\begin{tabular}{|c|c|c|c|c|c|c|c|c|}
\hline 13.6 & 14 & 15.2 & 43 & 67.3 & 64.7 & 57.1 & 55.6 & 59.3 \\
\hline 14.2 & 14.1 & 14.1 & 13.5 & 13.9 & 12.2 & 12.2 & 12.9 & 12.3 \\
\hline 13 & 12.7 & 12.8 & 12.2 & 12.6 & 10.4 & 10.4 & 11 & 10.4 \\
\hline 12.8 & 13 & 15 & 42.5 & 67.5 & 66.6 & 61.6 & 58.8 & 60.9 \\
\hline 14.1 & 13.4 & 13.9 & 43.4 & 68.7 & 68.4 & 61.3 & 58.4 & 59.3 \\
\hline 13.4 & 13.3 & 18.6 & 39.8 & 67.8 & 67.7 & 61.8 & 57.7 & 58.4 \\
\hline 15.1 & 15.1 & 18 & 41.6 & 65.9 & 66 & 60.2 & 57.4 & 59.3 \\
\hline 13.7 & 13.7 & 16.6 & 40 & 66.2 & 66.9 & 61 & 59.9 & 59 \\
\hline 13.8 & 13.2 & 13.7 & 11.8 & 10.7 & 11.2 & 10.6 & 11.3 & 11.2 \\
\hline 13.7 & 13.2 & 13.7 & 11.6 & 10.6 & 11.2 & 10.7 & 10.7 & 11.2 \\
\hline 14.2 & 13.7 & 20.5 & 42.5 & 65.8 & 68.1 & 60.7 & 58.4 & 59 \\
\hline 15.4 & 15.2 & 18.2 & 43.7 & 65.4 & 66.3 & 58.5 & 56.8 & 56.6 \\
\hline 14.6 & 13.8 & 18 & 40.8 & 65.4 & 66.8 & 61.5 & 57.9 & 57.5 \\
\hline 13.3 & 13.2 & 16.8 & 40.1 & 63.9 & 65.6 & 58.3 & 59.2 & 57.2 \\
\hline 13.1 & 13.1 & 17.2 & 39.2 & 63 & 65.1 & 57.2 & 55.2 & 55.6 \\
\hline 14.4 & 14.4 & 14.3 & 12.3 & 11.9 & 12.5 & 11.9 & 11.9 & 12 \\
\hline 14.5 & 14.4 & 14.4 & 13.1 & 12 & 11.9 & 11.8 & 12.5 & 11.9 \\
\hline 14.5 & 14.3 & 17 & 43.2 & 67.9 & 68.1 & 60.2 & 56.3 & 58.8 \\
\hline 13.8 & 13.8 & 16 & 42.8 & 66.7 & 67 & 61 & 56.5 & 55.6 \\
\hline 14 & 13.9 & 16.6 & 44.7 & 65.6 & 66.7 & 61.2 & 56.8 & 57.1 \\
\hline 13.6 & 13.5 & 16.4 & 41.9 & 66.6 & 66.5 & 59.4 & 55.9 & 57 \\
\hline 14.3 & 14.1 & 17.3 & 41.7 & 65.1 & 67.1 & 60.3 & 58 & 58.3 \\
\hline 13.4 & 13.4 & 13.3 & 12 & 10.9 & 10.8 & 11.5 & 10.9 & 13.3 \\
\hline 14 & 14.1 & 14.1 & 12.8 & 11.6 & 11.6 & 11.6 & 11.5 & 11.5 \\
\hline 14.2 & 13.6 & 21.7 & 45.8 & 65.2 & 67.7 & 57.7 & 54.1 & 58.4 \\
\hline 15.3 & 15.7 & 18.4 & 43.9 & 65.8 & 66.3 & 57.6 & 55.8 & 56.4 \\
\hline 13.6 & 13.5 & 16.5 & 42.9 & 64.9 & 66.6 & 62.8 & 58.3 & 58.2 \\
\hline 15 & 14.3 & 17.9 & 43.5 & 66.2 & 65.9 & 62.1 & 59.4 & 59.2 \\
\hline 13.4 & 13.3 & 16.7 & 42.3 & 66.2 & 67.3 & 60.9 & 55.6 & 56.9 \\
\hline 13 & 13.6 & 13.5 & 11.8 & 11.1 & 10.4 & 11 & 10.5 & 11 \\
\hline 13.6 & 13.6 & 13 & 12.4 & 11.1 & 10.4 & 11 & 10.4 & 11.1 \\
\hline 13.1 & 13.5 & 14 & 39.8 & 68.5 & 68.2 & 63.1 & 56.2 & 59.1 \\
\hline 14.2 & 14.7 & 17.1 & 45.1 & 66.1 & 65.7 & 59.3 & 56.4 & 59.1 \\
\hline 14.1 & 14.1 & 16.3 & 44.1 & 66.2 & 66.2 & 60.6 & 58 & 60.3 \\
\hline 13.9 & 13.8 & 17.2 & 43.1 & 67.4 & 68.7 & 59.4 & 55.1 & 57.9 \\
\hline 14.5 & 14.9 & 17.3 & 42.5 & 67.8 & 67.4 & 63.6 & 60.7 & 61.2 \\
\hline 14.4 & 14.9 & 14.9 & 13.8 & 26.4 & 27.7 & 27.5 & 28.3 & 29.6 \\
\hline 13 & 12.9 & 12.9 & 12 & 22 & 25.6 & 26.8 & 31.8 & 35.2 \\
\hline 12.4 & 12.4 & 13.9 & 41.9 & 62.5 & 61.3 & 56 & 56.6 & 53.2 \\
\hline 13.8 & 13.1 & 15.4 & 40.4 & 65.9 & 65.7 & 58.9 & 58.9 & 60.3 \\
\hline 14.1 & 14.1 & 16.6 & 42.6 & 66 & 65.6 & 57.6 & 56.7 & 60.5 \\
\hline 13.6 & 14.2 & 24.7 & 44.2 & 67.2 & 66.7 & 60 & 57 & 56.4 \\
\hline 14 & 13.9 & 14.8 & 43.3 & 67.2 & 66.8 & 62.1 & 59.1 & 59.4 \\
\hline 14.1 & 13.6 & 14.1 & 13.2 & 11.5 & 10.9 & 11.5 & 11.6 & 10.9 \\
\hline 14.2 & 14.2 & 13.5 & 13.3 & 11.6 & 11.6 & 10.9 & 11.6 & 11.6 \\
\hline 14.2 & 14.1 & 15.8 & 43.4 & 68.5 & 68 & 63.8 & 57.6 & 58.9 \\
\hline 13 & 13.5 & 15 & 41.7 & 68.9 & 67.7 & 62.1 & 58.6 & 58.5 \\
\hline 13.6 & 14 & 15.6 & 40.8 & 67.4 & 66.6 & 60 & 56.1 & 60.1 \\
\hline 14.2 & 13.7 & 15.2 & 41.6 & 66.9 & 69.7 & 61.1 & 61.2 & 60.2 \\
\hline 18.8 & 18.2 & 19.2 & 44.4 & 67 & 67.6 & 61.8 & 59.4 & 58.7 \\
\hline 14.2 & 13.6 & 13.5 & 13.4 & 10.9 & 11.6 & 11 & 11.5 & 11.1 \\
\hline
\end{tabular}


lights

\begin{tabular}{|c|c|c|c|c|c|c|c|c|}
\hline 14.7 & 14.1 & 13.3 & 11.5 & 11.6 & 12.2 & 11.6 & 11.6 & 11.6 \\
\hline 14.1 & 14 & 16.2 & 47.1 & 66.7 & 68.5 & 62.1 & 58.4 & 60.5 \\
\hline 13.6 & 12.8 & 14.2 & 42.8 & 67.2 & 67 & 62.6 & 61.2 & 60.7 \\
\hline 14.1 & 13.4 & 16.4 & 44.7 & 68.3 & 67.6 & 63.7 & 59.4 & 60.1 \\
\hline 16.1 & 14.9 & 20 & 44.6 & 67.2 & 67.1 & 60.5 & 58.7 & 60.2 \\
\hline 14.1 & 14 & 17.3 & 45.8 & 64.2 & 50.9 & 61.8 & 58.5 & 60.2 \\
\hline 14 & 13.9 & 13.1 & 11.4 & 10.7 & 11.4 & 13.8 & 14.4 & 13 \\
\hline 13.2 & 12.6 & 13 & 10.1 & 10.5 & 10 & 10.5 & 9.9 & 10.6 \\
\hline 12.6 & 13 & 15.9 & 43 & 67.1 & 68.9 & 61.4 & 61 & 58.3 \\
\hline 14.5 & 15 & 18.5 & 43.1 & 66.1 & 66.8 & 60.7 & 58.9 & 57.6 \\
\hline 15.1 & 15.5 & 19.7 & 44.4 & 68.9 & 68.3 & 61.3 & 57.7 & 59.2 \\
\hline 14.1 & 14.2 & 18.5 & 43.3 & 66.6 & 68 & 61.9 & 56 & 57.6 \\
\hline 13.8 & 13.2 & 16.6 & 42.5 & 66 & 66.7 & 60.5 & 57.2 & 58.3 \\
\hline 13.5 & 13.4 & 13.5 & 10.2 & 11 & 10.9 & 10.2 & 11.4 & 12.7 \\
\hline 13.4 & 13.2 & 13.3 & 13.6 & 10.6 & 10.6 & 10.6 & 9.9 & 10.7 \\
\hline 13.4 & 13.3 & 17.2 & 46 & 68.1 & 67.9 & 61.8 & 59 & 61.3 \\
\hline 16.2 & 16.1 & 19 & 46.8 & 68.3 & 67.3 & 61.4 & 58.9 & 59.1 \\
\hline 14.7 & 14 & 16.9 & 45.7 & 66 & 65.5 & 61.2 & 58.4 & 59.9 \\
\hline 14.7 & 14 & 18.6 & 44.6 & 67.5 & 67.5 & 62.1 & 59.1 & 60.1 \\
\hline 16 & 15.4 & 19.6 & 45.5 & 68.4 & 65.4 & 57.3 & 54.7 & 57.3 \\
\hline 14.1 & 14.1 & 14.1 & 13.2 & 11.6 & 10.9 & 11.6 & 11.5 & 11.6 \\
\hline 14.2 & 13.5 & 14.1 & 13.3 & 11.5 & 11.7 & 11.5 & 11 & 11.6 \\
\hline 14.2 & 14 & 17.5 & 44.7 & 67.8 & 68.1 & 62.2 & 57.4 & 58.9 \\
\hline 10.5 & 10.5 & 14.4 & 41.9 & 64.3 & 63.4 & 57.5 & 56.3 & 56.9 \\
\hline 10.1 & 10.7 & 14.6 & 42 & 63.2 & 63 & 58.7 & 58.4 & 58.1 \\
\hline 10.6 & 10.1 & 10 & 9.8 & 7.5 & 8 & 7.5 & 7.4 & 8.1 \\
\hline 10.6 & 10 & 10 & 9.9 & 7.4 & 8.1 & 7.4 & 8.1 & 7.4 \\
\hline 10.7 & 10 & 10.6 & 9.8 & 7.5 & 7.5 & 7.9 & 7.3 & 8 \\
\hline 9.8 & 10.4 & 9.8 & 9.7 & 7.9 & 7.3 & 8 & 7.3 & 7.9 \\
\hline 9.8 & 10.4 & 13.5 & 43.1 & 66.2 & 66.4 & 58.7 & 53.9 & 57.6 \\
\hline 9.8 & 10.4 & 15.7 & 40.9 & 62.6 & 63.1 & 59.9 & 54.4 & 54 \\
\hline 10.8 & 11.3 & 13.8 & 43.9 & 66 & 66 & 60.2 & 57 & 57.1 \\
\hline 10 & 10.7 & 14.8 & 41.2 & 63.6 & 64.5 & 58.1 & 54.4 & 54.2 \\
\hline 11.1 & 11.1 & 16.1 & 39.5 & 60.3 & 63.9 & 58.3 & 58.3 & 57.8 \\
\hline 10.7 & 10.6 & 10.6 & 10.5 & 13.1 & 27 & 29.3 & 30 & 31.9 \\
\hline 11.3 & 11.3 & 10.7 & 11.2 & 9.4 & 14.3 & 17.6 & 20.2 & 19.6 \\
\hline 9.8 & 9.8 & 12.2 & 42.9 & 66.9 & 66.8 & 61.6 & 57.7 & 57 \\
\hline 10.8 & 10.8 & 14.3 & 41.9 & 65.6 & 65.6 & 57.4 & 56.4 & 56.6 \\
\hline 12.4 & 12.3 & 16.2 & 43.8 & 65.7 & 64.5 & 57.6 & 54.8 & 57.1 \\
\hline 14 & 13.3 & 17.7 & 42.8 & 65.7 & 62.2 & 57 & 54.4 & 55.2 \\
\hline 11.1 & 10.5 & 13.8 & 40.6 & 62.9 & 64.5 & 57.7 & 54.7 & 55.3 \\
\hline 10 & 10.6 & 9.9 & 10.6 & 7.5 & 7.4 & 8.1 & 7.4 & 8.7 \\
\hline 10.6 & 10.6 & 11.2 & 10.5 & 8.1 & 8 & 8 & 7.9 & 8 \\
\hline 10.5 & 10.5 & 13.3 & 40.1 & & 30.5 & 57.3 & 57.2 & 56.9 \\
\hline 13.3 & 13.2 & 15.4 & 44.3 & 68.9 & 66.9 & 58.7 & 57.7 & 58.3 \\
\hline 13.7 & 13.9 & 17.1 & 45.6 & 68.3 & 64.2 & 62 & 57.8 & 57.9 \\
\hline 14.1 & 13.4 & 16.7 & 44.5 & 69.9 & 65.3 & 58.2 & 56.8 & 60.3 \\
\hline 13.8 & 14.2 & 19.7 & 48.8 & 69.1 & 67.8 & 61.4 & 58.9 & 61.5 \\
\hline 14.3 & 14.4 & 14.4 & 15 & 14.4 & 14.8 & 13.6 & 13 & 12 \\
\hline 14.5 & 14.4 & 14.5 & 15 & 13.6 & 11.8 & 11.9 & 11.8 & 11.8 \\
\hline 14.4 & 14.3 & 14.3 & 36.4 & 40.2 & 42.5 & 42.6 & 41.5 & 43.2 \\
\hline
\end{tabular}


lights

\begin{tabular}{|c|c|c|c|c|c|c|c|c|}
\hline 15.2 & 14.5 & 14.5 & 26.4 & 33.9 & 31.9 & 33.3 & 32.6 & 31.9 \\
\hline 14 & 14.5 & 14 & 26.8 & 35.4 & 37.2 & 37.5 & 26.1 & 26.8 \\
\hline 7.2 & 7.8 & 7.2 & 7.8 & 7.1 & 5.2 & 4.6 & 5.2 & 5.2 \\
\hline 7.8 & 7.2 & 7.8 & 7.2 & 7.7 & 4.6 & 5.2 & 5.3 & 5.2 \\
\hline 7.2 & 7.8 & 7.8 & 7.2 & 7.7 & 5.3 & 5.2 & 4.7 & 5.2 \\
\hline 7.7 & 7.8 & 7.2 & 7.8 & 7.7 & 5.2 & 4.6 & 5.3 & 5.3 \\
\hline 7.2 & 7.8 & 7.7 & 7.8 & 7.1 & 5.3 & 6.4 & 6 & 7.4 \\
\hline 7.3 & 6.7 & 7.3 & 7.3 & 7 & 5.8 & 6.5 & 6.4 & 6.4 \\
\hline 7.2 & 7.9 & 7.2 & 7.9 & 8 & 12.2 & 14.2 & 14.8 & 14 \\
\hline 9.5 & 10 & 9.4 & 10 & 11.5 & 13.7 & 13.7 & 13.5 & 13.6 \\
\hline 16.1 & 16.1 & 16 & 16.7 & 15.7 & 12.6 & 12.6 & 12.6 & 13.1 \\
\hline 8.5 & 9.1 & 9.1 & 9.1 & 8.4 & 6.6 & 5.9 & 6.5 & 6 \\
\hline 8.5 & 9.1 & 9.1 & 8.4 & 9.1 & 5.9 & 6.5 & 5.9 & 6.6 \\
\hline 8.5 & 9.1 & 8.4 & 29.1 & 57.5 & 62.6 & 65.9 & 57.5 & 52.3 \\
\hline 12.6 & 13.2 & 16.1 & 44.3 & 69.3 & 66.9 & 60.6 & 56 & 60.5 \\
\hline 13.6 & 13 & 16.3 & 47.3 & 71.7 & 68 & 62.6 & 58 & 58.5 \\
\hline 13.6 & 13.6 & 16.6 & 42 & 69.8 & 66.5 & 58.5 & 57.6 & 59.8 \\
\hline 19.5 & 15 & 17.6 & 44.1 & 69.4 & 67.4 & 63.4 & 59.6 & 60.6 \\
\hline 14.3 & 15 & 14.3 & 14.3 & 14.9 & 12.4 & 13 & 13 & 12.5 \\
\hline 15.6 & 15.6 & 14.9 & 15.6 & 15.5 & 12.4 & 13 & 13 & 12.5 \\
\hline 15.5 & 15.6 & 18.1 & 45.6 & 70.6 & 67.6 & 62.3 & 59.9 & 59.8 \\
\hline 10.7 & 11.2 & 15.6 & 41.8 & 66.8 & 63 & 59.3 & 57.3 & 54 \\
\hline 7.8 & 7.8 & 11.2 & 37.9 & 69.5 & 66.6 & 61.8 & 58.1 & 60 \\
\hline 11.9 & 12.3 & 15.2 & 44 & 69.6 & 67.2 & 61.6 & 58.3 & 59.4 \\
\hline 13.1 & 13.2 & 16.6 & 42 & 69.7 & 64.8 & 59.2 & 56.3 & 59.3 \\
\hline 14.4 & 13.7 & 13.7 & 14.3 & 13.7 & 11.8 & 11.2 & 11.2 & 11.8 \\
\hline 14.4 & 14.5 & 13.8 & 14.3 & 13.8 & 11.2 & 11.8 & 11.2 & 11.9 \\
\hline 13.8 & 14.3 & 13.7 & 14.3 & 18.8 & 17 & 17.7 & 19.1 & 20.8 \\
\hline 16.7 & 16.8 & 20.1 & 45 & 71.2 & 67.2 & 62.8 & 61.1 & 59.4 \\
\hline 13.7 & 13.1 & 15.5 & 42.9 & 67.7 & 60.9 & 60 & 58.9 & 57.6 \\
\hline 13.2 & 13.7 & 16.5 & 42.8 & 68.6 & 65.7 & 61.2 & 60.1 & 61.1 \\
\hline 15.9 & 15.8 & 18.9 & 42.9 & 70.3 & 69 & 61.6 & 60.8 & 61.2 \\
\hline 12.6 & 12.5 & 11.9 & 12.5 & 11.8 & 9.9 & 9.3 & 10 & 9.3 \\
\hline 14.3 & 13.7 & 13.7 & 13.7 & 13.6 & 11.1 & 11.1 & 11.7 & 11.2 \\
\hline 13.7 & 13.7 & 16.8 & 42.1 & 69.1 & 65.4 & 61.9 & 60 & 59.9 \\
\hline 14.4 & 13.7 & 17.1 & 42.8 & 65.7 & 64.3 & 60.6 & 56.4 & 56.8 \\
\hline 16 & 15.8 & 18.9 & 44.6 & 68.8 & 63.8 & 61.3 & 58.6 & 60.2 \\
\hline 13.1 & 13.7 & 16.1 & 43.3 & 67.8 & 68.4 & 62.1 & 61.9 & 60 \\
\hline 12.8 & 12.8 & 15 & 42.1 & 67.1 & 67.6 & 62.5 & 61.9 & 60.7 \\
\hline 11.9 & 12.4 & 11.8 & 12.4 & 10.3 & 9.9 & 9.2 & 9.9 & 9.4 \\
\hline 11.9 & 12.5 & 11.8 & 11.9 & 10.9 & 9.3 & 10 & 9.3 & 10 \\
\hline 12.4 & 11.9 & 15.1 & 43.3 & 68.2 & 67.3 & 61.5 & 60.3 & 60.7 \\
\hline 13 & 13.6 & 22.6 & 44.7 & 69.6 & 67.3 & 61.9 & 60.3 & 55.1 \\
\hline 15.2 & 14.5 & 23.9 & 44.6 & 68.9 & 69.2 & 63.2 & 57 & 59.9 \\
\hline 13 & 12.2 & 22.9 & 45.1 & 68.6 & 65.1 & 57.5 & 54.5 & 55 \\
\hline 14.5 & 15.1 & 19 & 31.1 & 31.7 & 33.1 & 33.9 & 35 & 32.5 \\
\hline 15.5 & 16 & 15.4 & 16 & 13.8 & 13.5 & 13.5 & 14.2 & 13.7 \\
\hline 16.1 & 16.1 & 15.4 & 16.1 & 13.8 & 12.9 & 13.6 & 13.5 & 13 \\
\hline 15.4 & 16 & 19.7 & 44.8 & 66.6 & 68 & 60.2 & 57.8 & 59.2 \\
\hline 14 & 13.9 & 14.3 & 42.2 & 66.2 & 64.9 & 58.7 & 56 & 58.6 \\
\hline 12.2 & 12.2 & 21.7 & 43.7 & 69.6 & 69.3 & 62.4 & 61 & 60 \\
\hline
\end{tabular}


lights

\begin{tabular}{|c|c|c|c|c|c|c|c|c|}
\hline 13.4 & 12.8 & 13.4 & 12.8 & 11.6 & 10.2 & 10.9 & 10.8 & 10.2 \\
\hline 13.3 & 12.8 & 22.4 & 45.5 & 68.2 & 68.7 & 62.4 & 60 & 59.8 \\
\hline 10.2 & 9.6 & 10.1 & 9.5 & 7.8 & 7 & 7.6 & 7.1 & 7.6 \\
\hline 10.1 & 9.6 & 10.2 & 9.6 & 7.8 & 7.7 & 7 & 7.7 & 7.7 \\
\hline 10.2 & 9.5 & 21.4 & 42.9 & 63 & 65.6 & 58.1 & 54.2 & 56 \\
\hline 15.4 & 14.7 & 27.9 & 46.1 & 67.5 & 67.6 & 60.1 & 56.7 & 57.7 \\
\hline 13 & 13.6 & 26.2 & 44.7 & 67.4 & 64 & 58.1 & 55.3 & 57.2 \\
\hline 12.5 & 11.8 & 20.6 & 43.8 & 67.5 & 65 & 59.4 & 57.4 & 56.9 \\
\hline 12.9 & 12.9 & 26.4 & 46.6 & 67.6 & 67.7 & 59.5 & 58.9 & 59.5 \\
\hline 12.3 & 12.8 & 12.8 & 12.8 & 10.3 & 33.8 & 51.7 & 16.6 & 9.3 \\
\hline 14.3 & 14.9 & 14.3 & 23.1 & 38.8 & 46.4 & 49.5 & 48.5 & 45.7 \\
\hline 13.7 & 13.1 & 17.4 & 45.2 & 52 & 48.6 & 50.8 & 49.9 & 48.5 \\
\hline 13.2 & 13.7 & 25.9 & 44.3 & 68.5 & 68.9 & 64 & 61.3 & 61.1 \\
\hline 15.6 & 16.2 & 25.2 & 43.1 & 66.1 & 66.8 & 59.1 & 59.2 & 58.3 \\
\hline 12 & 12.1 & 23.3 & 44.3 & 68 & 67 & 60.5 & 58.8 & 59.9 \\
\hline 11.5 & 10.9 & 17.4 & 41.2 & 66.2 & 67.3 & 61.3 & 58.9 & 58.6 \\
\hline 13.9 & 13.9 & 13.8 & 14.3 & 11.9 & 12.4 & 12.1 & 13.3 & 10.3 \\
\hline 8.6 & 9.1 & 8.5 & 8.8 & 5.9 & 6 & 6.5 & 6 & 5.9 \\
\hline 9.7 & 9.6 & 19.9 & 43.3 & 69.4 & 68.9 & 63 & 60.9 & 62.3 \\
\hline 19.1 & 31.2 & 35.6 & 48.6 & 69.5 & 69.5 & 62 & 64.1 & 63 \\
\hline 15.5 & 16.2 & 18.6 & 47 & 69.2 & 69.4 & 63.6 & 62.9 & 62.2 \\
\hline 16.5 & 16.5 & 28.6 & 45.1 & 68.9 & 69.3 & 61.5 & 61.1 & 60.9 \\
\hline 16.7 & 16.1 & 20.6 & 45.4 & 69 & 69.6 & 60.4 & 59.7 & 58.5 \\
\hline 15.6 & 16.8 & 24.9 & 23.8 & 22.1 & 21.7 & 21.9 & 22.3 & 22.3 \\
\hline 16.4 & 16.2 & 16.3 & 16.1 & 13.6 & 13.9 & 13.5 & 13.9 & 13.6 \\
\hline 16.3 & 16.2 & 23 & 45.6 & 68.5 & 64.4 & 58 & 55.6 & 57.5 \\
\hline 16.2 & 15.7 & 24.1 & 46.7 & 67.9 & 67.1 & 62.2 & 59.6 & 60.2 \\
\hline 15.6 & 14.9 & 17.4 & 46.2 & 68.6 & 68.4 & 64 & 60.4 & 62.1 \\
\hline 14.2 & 14.3 & 20.1 & 45.3 & 69.2 & 66.4 & 61.5 & 56.6 & 58.7 \\
\hline 14.6 & 14.2 & 23 & 43.2 & 68.4 & 66.9 & 61.8 & 59.1 & 60.1 \\
\hline 13.8 & 14.6 & 13.7 & 12.9 & 12.9 & 14.1 & 14.7 & 14.4 & 15.4 \\
\hline 16.2 & 16.7 & 15.8 & 14.9 & 15.5 & 14.7 & 14.4 & 15.4 & 14.9 \\
\hline 16.1 & 15.8 & 16.6 & 14.6 & 13.7 & 13.9 & 15.8 & 9.1 & 8.6 \\
\hline 9 & 8.3 & 8.8 & 7 & 6 & 8.3 & 16.9 & 24.4 & 23.5 \\
\hline 10.7 & 10 & 10 & 8.6 & 8.3 & 12.7 & 17.4 & 21.2 & 21.2 \\
\hline 10.4 & 10.3 & 10.5 & 8.8 & 9 & 15.4 & 21.9 & 25.2 & 27 \\
\hline 6.8 & 6.8 & 6.8 & 4.7 & 9.2 & 14.2 & 19.5 & 20.6 & 21.7 \\
\hline 6.8 & 6.8 & 6.8 & 5.3 & 4.3 & 4.3 & 4.5 & 6.3 & 4.3 \\
\hline 6.7 & 7.4 & 6.8 & 5.4 & 4.3 & 4.2 & 4.3 & 4.2 & 4.3 \\
\hline 6.6 & 6.6 & 20.6 & 45.5 & 68.4 & 68.5 & 61.9 & 57.9 & 60.1 \\
\hline 14.2 & 14.7 & 24.7 & 44.8 & 69.2 & 68.5 & 25.4 & 52.9 & 53.6 \\
\hline 16.6 & 17.4 & 23.6 & 44.3 & 67.9 & 68 & 62.3 & 58.6 & 59.3 \\
\hline 13.1 & 13.9 & 24.7 & 42.8 & 65.8 & 64.9 & 57.8 & 57.2 & 54.6 \\
\hline 16.8 & 17.2 & 31.1 & 42.9 & 66.9 & 68 & 60.9 & 56.2 & 59.9 \\
\hline 14.5 & 15.3 & 15.5 & 24.4 & 23.4 & 24.4 & 23.8 & 24.6 & 24.2 \\
\hline 15.1 & 14.5 & 15.1 & 13.1 & 12.8 & 11.4 & 12.8 & 12.1 & 12.5 \\
\hline 15.4 & 14.5 & 27.6 & 44.3 & 68.8 & 69.7 & 61.9 & 60.5 & 58.5 \\
\hline 14.8 & 14.7 & 22.3 & 45.3 & 67.3 & 68.1 & 60.3 & 59.3 & 60.1 \\
\hline & & & & & & & & \\
\hline & & & & & & & & \\
\hline & & & & & & & & \\
\hline
\end{tabular}




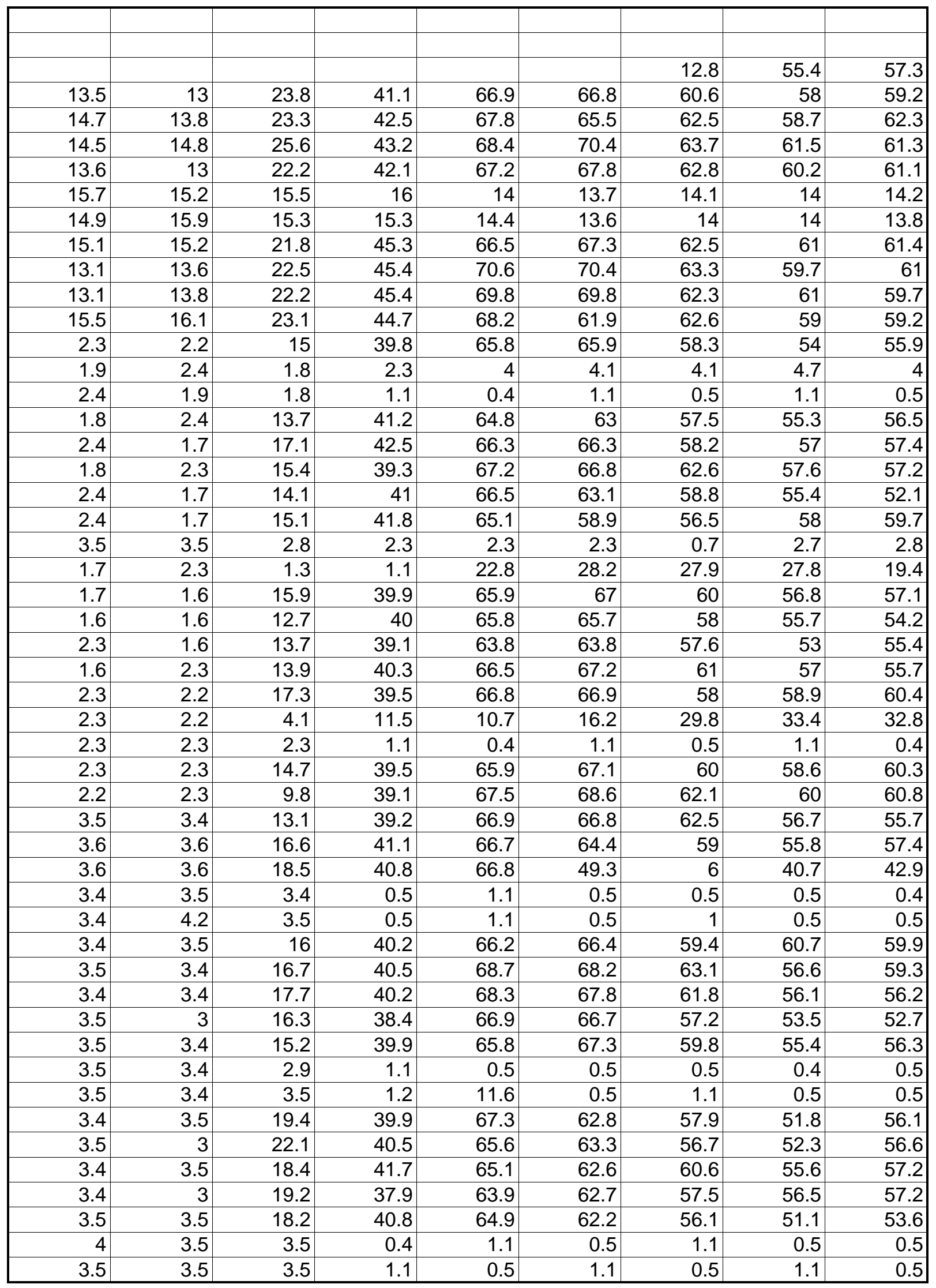


lights

\begin{tabular}{|c|c|c|c|c|c|c|c|c|}
\hline 3.4 & 3.5 & 18.3 & 42.3 & 63.5 & 54.4 & 57.1 & 55.7 & 55.6 \\
\hline 3.4 & 3.5 & 19.7 & 40.2 & 66.9 & 64.6 & 59.8 & 58.3 & 56.7 \\
\hline 2.8 & 3.5 & 20.5 & 39.4 & 67.2 & 66.8 & 59.7 & 54 & 58.1 \\
\hline 3.4 & 3.4 & 20.6 & 50.7 & 65.1 & 67.6 & 68.1 & 64.3 & 59.6 \\
\hline 3.5 & 3.4 & 18.1 & 23.5 & 27.5 & 28.6 & 33.8 & 33.7 & 32 \\
\hline 23.9 & 13.4 & 3.4 & 0.5 & 0.4 & 1.1 & 0.5 & 0.5 & 0.5 \\
\hline 3.5 & 3.4 & 3.5 & 0.4 & 0.5 & 0.5 & 1.1 & 0.5 & 0.4 \\
\hline 3.5 & 3.4 & 18.5 & 24.9 & 33.7 & 40.5 & 41.1 & 39.9 & 39.9 \\
\hline 3.4 & 3.4 & 15.2 & 24.2 & 41.9 & 43.2 & 44 & 43.6 & 40.5 \\
\hline 3.4 & 3.4 & 11.8 & 20.5 & 40.4 & 44 & 44.3 & 41.4 & 39.8 \\
\hline 3.4 & 3.5 & 18.3 & 30.1 & 43 & 45.3 & 45.9 & 43.7 & 37.2 \\
\hline 3.4 & 3.4 & 12.1 & 30.1 & 42.5 & 43.2 & 45.6 & 42.9 & 39.6 \\
\hline 3.4 & 3.4 & 3.4 & 0.5 & 0.5 & & 0.7 & 0.3 & 0.4 \\
\hline 3.3 & 3.3 & 3.3 & 0.3 & 0.4 & 0.9 & 0.4 & 0.3 & 0.4 \\
\hline 3.3 & 3.3 & 10 & 29.5 & 41.5 & 41.7 & 42 & 38.1 & 35.1 \\
\hline 3.5 & 3.4 & 11.6 & 26.4 & 38.3 & 39.4 & 38.4 & 35 & 31.2 \\
\hline 3.5 & 3.4 & 9.5 & 25.9 & 41.5 & 42.9 & 42.2 & 38.8 & 35 \\
\hline 3.4 & 3.4 & 12.2 & 35.7 & 40.7 & 41 & 40.9 & 38.8 & 34.7 \\
\hline 3.4 & 3.4 & 10.8 & 28 & 38.3 & 40.7 & 40.4 & 37.9 & 36.4 \\
\hline 3.4 & 3.4 & 3.3 & 0.5 & 0.5 & 0.5 & 0.4 & 0.5 & 0.5 \\
\hline 3.5 & 3.4 & 3.5 & 0.5 & 0.4 & 0.5 & 0.5 & 0.4 & 0.5 \\
\hline 3.4 & 3.4 & 9.9 & 25.3 & 39.5 & 41.8 & 41.3 & 36.1 & 32.8 \\
\hline 3.4 & 2.8 & 11 & 25.9 & 39 & 41.2 & 40.5 & 37.7 & 35 \\
\hline 3.4 & 2.8 & 13.1 & 27.1 & 40.6 & 41.2 & 41.2 & 38.3 & 32 \\
\hline 3.4 & 3.4 & 13.6 & 29.1 & 38.2 & 39.3 & 39 & 35.7 & 34.8 \\
\hline 3.5 & 3.4 & 13.8 & 30.1 & 39.1 & 41.3 & 40.2 & 37.7 & 35.4 \\
\hline 3.4 & 3.4 & 3.4 & 0.5 & 0.5 & 0.4 & 0.5 & 0.5 & 1.1 \\
\hline 2.8 & 3.4 & 3.4 & 0.5 & 1.1 & 0.4 & 0.5 & 0.5 & 0.5 \\
\hline 3.4 & 3.4 & 12.2 & 27.6 & 34.4 & 37.4 & 39.1 & 37.8 & 36.7 \\
\hline 3.4 & 3.4 & 17.9 & 35.7 & 39 & 40.4 & 40.2 & 37.5 & 38.7 \\
\hline 3.4 & 2.8 & 14.6 & 31.3 & 34.4 & 35.8 & 35.3 & 33.3 & 35.2 \\
\hline 3.5 & 2.7 & 12.4 & 27.7 & 31.9 & 29.3 & 30.3 & 29.6 & 33.8 \\
\hline 3.4 & 2.8 & 13.2 & 28.5 & 34.6 & 35.4 & 35.8 & 36.5 & 38.3 \\
\hline 3.3 & 3.4 & 3.4 & 0.5 & 0.5 & 0.5 & 0.5 & 0.4 & 0.5 \\
\hline 3.4 & 3.4 & 3.4 & 0.5 & 0.5 & 0.4 & 1.1 & 0.5 & 0.5 \\
\hline 3.4 & 3.4 & 11.7 & 29.8 & 34.5 & 35.7 & 36.8 & 35 & 35.2 \\
\hline 2.8 & 3.4 & 10.3 & 23 & 23.7 & 24.3 & 27.2 & 25.5 & 28.6 \\
\hline 3.4 & 3.4 & 10.1 & 19.9 & 21.1 & 21.2 & 21.2 & 23.1 & 23.3 \\
\hline 3.5 & 3.4 & 9.8 & 18.5 & 22.1 & 22.3 & 22.3 & 22.1 & 23.7 \\
\hline 3.4 & 3.4 & 11.6 & 18.4 & 19.1 & 18.8 & 21.3 & 24.1 & 25.4 \\
\hline 3.4 & 3.4 & 3.4 & 0.6 & 0.5 & 0.5 & 0.4 & 0.5 & 0.5 \\
\hline 3.5 & 3.4 & 3.4 & 1.2 & 0.5 & 0.4 & 0.5 & 0.5 & 0.5 \\
\hline 3.5 & 2.8 & 9 & 8.2 & 8.7 & 8 & 8.6 & 8 & 8.4 \\
\hline 2.8 & 3.3 & 9.9 & 18.2 & 19.5 & 20 & 19.6 & 21.3 & 24 \\
\hline 3.4 & 2.7 & 8.7 & 13.4 & 21.1 & 21 & 20.7 & 21.1 & 22.8 \\
\hline 3.4 & 3.3 & 10.1 & 19.8 & 21.4 & 21.6 & 22.2 & 21.7 & 23.9 \\
\hline 3.4 & 2.7 & 9.6 & 18.5 & 20.2 & 18.5 & 21.3 & 21.4 & 21 \\
\hline 3.4 & 3.4 & 3.3 & 0.4 & 0.4 & 0.4 & 0.5 & 0.5 & 0.4 \\
\hline 3.4 & 3.3 & 3.4 & 0.4 & 0.4 & 0.4 & 0.4 & 0.4 & 0.5 \\
\hline 3.4 & 3.3 & 10.1 & 19.1 & 21.3 & 27.6 & 28.9 & 29.4 & 30.5 \\
\hline 2.8 & 3.4 & 15.5 & 24.6 & 29.2 & 31.1 & 31.5 & 28 & 29.7 \\
\hline
\end{tabular}


lights

\begin{tabular}{|c|c|c|c|c|c|c|c|c|}
\hline 3.3 & 3.4 & 13.4 & 26.1 & 29.8 & 30.6 & 30.5 & 31.9 & 34.1 \\
\hline 2.8 & 3.4 & 9.6 & 10.4 & 18.3 & 18.4 & 20.6 & 20.4 & 3.2 \\
\hline 3.3 & 2.7 & 9.4 & 10.7 & 23.5 & 24.6 & 25.5 & 25.8 & 27.7 \\
\hline 3.3 & 3.3 & 3.3 & 0.4 & 0.5 & 0.4 & 0.4 & 0.4 & 0.4 \\
\hline 3.4 & 3.3 & 3.4 & 0.4 & 0.4 & 0.4 & 1.1 & 0.4 & 0.4 \\
\hline 3.3 & 3.4 & 10.3 & 29.7 & 31.8 & 32.2 & 32.4 & 32.9 & 33.9 \\
\hline 3.4 & 3.3 & 11.5 & 29.7 & 30.7 & 32.1 & 30.9 & 31 & 32.2 \\
\hline 3.4 & 3.3 & 10.7 & 22.3 & 26.1 & 26.1 & 26.7 & 28.9 & 29.8 \\
\hline 2.8 & 3.3 & 9.6 & 15.4 & 23.1 & 23 & 22.7 & 23.1 & 22.8 \\
\hline 3.4 & 3.3 & 10.9 & 25.6 & 29.9 & 29.2 & 28.9 & 30.4 & 32.3 \\
\hline 3.4 & 3.3 & 3.4 & 0.4 & 0.4 & 0.4 & 0.5 & 0.4 & 0.4 \\
\hline 3.4 & 3.4 & 3.3 & 0.6 & 0.4 & 0.4 & 1 & 0.4 & 0.4 \\
\hline 3.4 & 3.3 & 14.2 & 23.6 & 25.5 & 26 & 26.9 & 28.6 & 29.1 \\
\hline 3.5 & 2.9 & 10.9 & 22.1 & 24 & 25.2 & 23.9 & 24.1 & 26.4 \\
\hline 3.5 & 2.9 & 13.9 & 22.5 & 25.3 & 25.9 & 27.3 & 26.2 & 27.5 \\
\hline 2.8 & 3.3 & 12.6 & 25.6 & 26.6 & 27 & 26.7 & 25.2 & 25.8 \\
\hline 3.4 & 2.8 & 11.8 & 25 & 25.8 & 26.2 & 27.2 & 25.7 & 26 \\
\hline 3.4 & 2.7 & 3.4 & 0.5 & 14 & 21.2 & 23.9 & 26 & 28.1 \\
\hline 2.8 & 3.4 & 2.8 & 1 & 24.1 & 26.1 & 24.7 & 22.4 & 23.4 \\
\hline 3.4 & 2.8 & 10.7 & 21.5 & 26.7 & 27.8 & 26.7 & 25.5 & 25.7 \\
\hline 3.4 & 2.7 & 10 & 11.3 & 21.1 & 27.1 & 28.5 & 28.4 & 32.5 \\
\hline 2.8 & 3.3 & 10 & 20.4 & 30.1 & 33.1 & 33.2 & 38.5 & 44.5 \\
\hline 2.8 & 3.3 & 11.6 & 27.8 & 41.7 & 43.4 & 44 & 43.6 & 43.9 \\
\hline 3.3 & 2.8 & 10.1 & 27.3 & 32.5 & 34.9 & 37.3 & 34.2 & 35 \\
\hline 3.3 & 2.7 & 3.4 & 19.4 & 24 & 24.3 & 23.7 & 25.2 & 26.5 \\
\hline 3.3 & 2.8 & 3.4 & 0.4 & 0.4 & 0.5 & 0.4 & 0.4 & 1 \\
\hline 3.4 & 2.8 & 13.6 & 24.2 & 28.1 & 33.6 & 36.9 & 39.9 & 41.2 \\
\hline 3.4 & 2.8 & 17.3 & 27.2 & 37 & 43.6 & 44.1 & 43.7 & 40.2 \\
\hline 2.8 & 3.3 & 10.4 & 26.8 & 31.3 & 33.4 & 36.8 & 38.2 & 39.5 \\
\hline 2.7 & 3.4 & 11.5 & 36.1 & 47.9 & 49.1 & 50.9 & 58.9 & 58.3 \\
\hline 3.3 & 2.8 & 11.4 & 36.1 & 47.2 & 54.8 & 64.9 & 66.3 & 56.6 \\
\hline 2.7 & 3.4 & 12.3 & 30.3 & 35.1 & 39.1 & 42.4 & 43.9 & 46.5 \\
\hline 3.3 & 3.4 & 2.7 & 1.1 & 0.4 & 0.4 & 22.5 & 25.5 & 26.1 \\
\hline 3.4 & 3.3 & 21.6 & 48.3 & 66 & 65.1 & 57.9 & 53.1 & 55.1 \\
\hline 2.7 & 3.4 & 22.1 & 42.5 & 64.5 & 64.3 & 60.9 & 53.2 & 57.4 \\
\hline 2.7 & 3.4 & 14.9 & 34.9 & 64.5 & 64.9 & 59.3 & 53.5 & 56.4 \\
\hline 2.7 & 3.3 & 18.3 & 45.6 & 63.9 & 64.4 & 59.6 & 52.2 & 56.4 \\
\hline 3.4 & 2.7 & 14.5 & 41.6 & 63.5 & 63 & 57.3 & 52.5 & 54.8 \\
\hline 2.7 & 3.4 & 3.4 & 0.4 & 0.4 & 0.4 & 0.4 & 0.4 & 0.4 \\
\hline 3.4 & 3.4 & 2.7 & 1.1 & 0.4 & 0.4 & 1.6 & 2.2 & 2.8 \\
\hline 3.4 & 3.3 & 13.6 & 40.2 & 64.7 & 65.5 & 59.1 & 54.2 & 57.7 \\
\hline 3.3 & 3.4 & 12.5 & 38.6 & 64.6 & 64.1 & 56.9 & 55.1 & 53.6 \\
\hline 2.8 & 3.3 & 13.2 & 42.1 & 63.8 & 64.3 & 57.8 & 57.2 & 56.1 \\
\hline 3.4 & 2.7 & 16.1 & 41.8 & 64.7 & 65.3 & 58.4 & 56 & 55.9 \\
\hline 3.4 & 2.7 & 14.3 & 39.9 & 64.3 & 62 & 57.9 & 54 & 53.9 \\
\hline 3.4 & 3.3 & 2.8 & 0.5 & 0.4 & 0.4 & 0.4 & 0.4 & 0.4 \\
\hline 3.4 & 2.7 & 3.4 & 0.5 & 0.4 & 0.4 & 0.4 & 0.4 & 1.1 \\
\hline 3.4 & 2.7 & 14.6 & 41.4 & 64.7 & 64.9 & 58.2 & 56.6 & 54.8 \\
\hline 3.4 & 2.7 & 16.3 & 41.4 & 63.2 & 65.2 & 58.6 & 56.3 & 57.4 \\
\hline 2.9 & 3.5 & 20.1 & 39.9 & 64.7 & 64.9 & 58 & 55.9 & 58.2 \\
\hline 3.4 & 2.8 & 13.7 & 41.4 & 64.9 & 67.9 & 61.5 & 56.6 & 58.8 \\
\hline
\end{tabular}




\begin{tabular}{|c|c|c|c|c|c|c|c|c|}
\hline 2.7 & 3.4 & 20.4 & 41.3 & 64.7 & 62.5 & 57.4 & 55.6 & 60.4 \\
\hline 2.7 & 2.8 & 3.4 & 0.5 & 0.4 & 0.4 & 0.4 & 0.4 & 14.3 \\
\hline 3.4 & 2.8 & 2.8 & 1 & 0.5 & 0.4 & 0.4 & 0.4 & 0.4 \\
\hline 3.4 & 2.8 & 11 & 11.8 & 11.6 & 11.5 & 11.5 & 11.3 & 11.2 \\
\hline 2.9 & 2.9 & 17 & 40.9 & 65.5 & 66.2 & 58.5 & 57.8 & 59.6 \\
\hline 3 & 2.8 & 14.8 & 38.1 & 65.6 & 66.4 & 59.7 & 58.1 & 57.3 \\
\hline 2.9 & 3.5 & 15.5 & 40.3 & 64.8 & 65.2 & 60 & 56 & 58.4 \\
\hline 3.5 & 2.9 & 17.1 & 41.3 & 66.7 & 66.2 & 60.4 & 56.3 & 57.7 \\
\hline 2.9 & 3.6 & 2.9 & 0.4 & 13.6 & 26.8 & 30.7 & 29.6 & 29.8 \\
\hline 3.5 & 3 & 2.9 & 15.3 & 27.5 & 28.7 & 28 & 27 & 21.9 \\
\hline 3.5 & 2.9 & 15 & 40.6 & 64.4 & 67.1 & 60.7 & 55.9 & 56.9 \\
\hline 3.5 & 2.9 & 12.5 & 38 & 63.6 & 65.4 & 55.4 & 53.1 & 53.9 \\
\hline 3.5 & 2.9 & 16.5 & 40.9 & 63.8 & 65.4 & 58.4 & 54.7 & 54.9 \\
\hline 3 & 3.5 & 15.2 & 40.9 & 63.1 & 64.4 & 58.9 & 56.2 & 57.8 \\
\hline 2.9 & 2.9 & 14.6 & 40.6 & 62 & 62.8 & 58.4 & 57.3 & 58.2 \\
\hline 3.5 & 3 & 2.9 & 0.4 & 0.4 & 0.4 & 0.4 & 0.4 & 1.1 \\
\hline 2.8 & 2.8 & 3.4 & 0.4 & 1.1 & 0.4 & 0.4 & 0.5 & 0.4 \\
\hline 2.8 & 3.4 & 16.2 & 39 & 63.4 & 63.6 & 58 & 57.8 & 56.2 \\
\hline 2.8 & 3.4 & 15.4 & 40.9 & 66.2 & 66.3 & 59.3 & 57.9 & 57.3 \\
\hline 3.4 & 2.7 & 13.8 & 41.8 & 65.8 & 67.8 & 61.4 & 59.7 & 57.2 \\
\hline 2.7 & 3.4 & 20.5 & 41.3 & 65.7 & 67.6 & 61.7 & 60.2 & 59.9 \\
\hline 3.5 & 2.8 & 17.4 & 41.5 & 67 & 64.2 & 59.4 & 56 & 58.9 \\
\hline 2.8 & 3.5 & 2.8 & 1 & 0.4 & 0.5 & 0.4 & 0.4 & 0.4 \\
\hline 3.5 & 2.8 & 3.4 & 0.4 & 1 & 0.5 & 0.4 & 0.4 & 0.4 \\
\hline 2.8 & 3.4 & 22.4 & 43.3 & 67.2 & 67.3 & 62.6 & 58.7 & 57.7 \\
\hline 2.8 & 3.4 & 18 & 41.9 & 64.7 & 66.8 & 59.8 & 57.7 & 56.4 \\
\hline 3.4 & 2.7 & 13 & 41.4 & 66.6 & 65.6 & 57.6 & 58.7 & 55.6 \\
\hline 2.8 & 2.7 & 16.7 & 40.5 & 64.7 & 65.9 & 59.3 & 59.3 & 55.8 \\
\hline 2.8 & 3.3 & 17.1 & 41.7 & 65.1 & 66 & 56.5 & 53.8 & 56 \\
\hline 3.4 & 2.8 & 5.8 & 21.1 & 24.9 & 25.5 & 26 & 26.5 & 25.2 \\
\hline 3.4 & 2.8 & 2.7 & 1.1 & 0.4 & 0.4 & 0.4 & 0.5 & 0.4 \\
\hline 2.8 & 2.8 & 17.9 & 41.8 & 64.8 & 66.1 & 59.3 & 56.8 & 56.7 \\
\hline 2.8 & 3.4 & 16.1 & 40.6 & 65.9 & 64.9 & 60.8 & 55.2 & 57.6 \\
\hline 3.4 & 2.8 & 17.9 & 39.9 & 64.4 & 65.5 & 60.2 & 56.6 & 56.5 \\
\hline 3.4 & 2.8 & 16.3 & 38.7 & 65.4 & 65.7 & 57.6 & 56.8 & 56.8 \\
\hline 2.8 & 3.4 & 19.6 & 37.9 & 63.3 & 63.2 & 57.4 & 56 & 54.4 \\
\hline 9.4 & 10 & 10 & 8.9 & 16.9 & 18 & 18.5 & 16.6 & 4.1 \\
\hline 3.4 & 2.8 & 3.4 & 0.5 & 1 & 0.4 & 1 & 0.4 & 0.5 \\
\hline 2.8 & 3.4 & 9.7 & 22.7 & 25.7 & 30.4 & 34.8 & 38.9 & 37.9 \\
\hline 2.8 & 2.8 & 17.7 & 42.4 & 68.8 & 69.7 & 63 & 60.5 & 60 \\
\hline 3.4 & 2.9 & 18.6 & 39.8 & 66.5 & 67 & 62.4 & 60.1 & 59.4 \\
\hline 3.4 & 2.8 & 20.1 & 42.5 & 66.5 & 66.9 & 60.3 & 59.2 & 58.1 \\
\hline 3.4 & 2.8 & 17.3 & 43.3 & 65.9 & 64.6 & 59.8 & 56.6 & 57.5 \\
\hline 2.8 & 3.4 & 3.4 & 2.9 & 3.4 & 2.8 & 14.5 & 18.8 & 20 \\
\hline 3.4 & 2.8 & 3.4 & 0.4 & 0.5 & 1 & 0.4 & 0.4 & 0.4 \\
\hline 3.4 & 2.8 & 17.2 & 43.3 & 67.5 & 66.9 & 61.9 & 58.3 & 57.2 \\
\hline 2.8 & 2.8 & 18.5 & 44.4 & 65.6 & 58.1 & 62.1 & 59.8 & 57.1 \\
\hline 2.8 & 3.4 & 16.5 & 41.1 & 66.2 & 63.4 & 60 & 57.7 & 57 \\
\hline 2.8 & 3.4 & 24.6 & 40.4 & 65.9 & 61.5 & 62.3 & 61.1 & 59.3 \\
\hline 3.3 & 2.8 & 15.7 & 40.7 & 66.2 & 65.4 & 61 & 59 & 54.9 \\
\hline 3.4 & 2.8 & 8 & 23.9 & 24.4 & 25.3 & 24.6 & 24.7 & 24.7 \\
\hline
\end{tabular}


lights

\begin{tabular}{|c|c|c|c|c|c|c|c|c|}
\hline 3.4 & 2.8 & 3.4 & 0.5 & 0.4 & 1 & 0.5 & 0.4 & 0.4 \\
\hline 2.8 & 2.8 & 21.6 & 42.9 & 67.1 & 66.8 & 59.7 & 58.8 & 58 \\
\hline 2.8 & 2.9 & 19.5 & 39.5 & 66.6 & 67.2 & 62.4 & 59.3 & 61.6 \\
\hline 3.5 & 2.8 & 17.5 & 41.4 & 66.9 & 68.4 & 62.2 & 59.6 & 62.7 \\
\hline 3.5 & 2.8 & 16.9 & 42.1 & 67.1 & 68.2 & 61.1 & 58.7 & 58.4 \\
\hline 3.5 & 2.8 & 13.9 & 40 & 67.6 & 63.9 & 59 & 56.3 & 57.1 \\
\hline 2.9 & 3.4 & 2.9 & 0.5 & 0.4 & 0.4 & 0.4 & 0.4 & 9.7 \\
\hline 2.8 & 2.8 & 3.5 & 22.4 & 22.4 & 22.8 & 22.2 & 22.1 & 21.7 \\
\hline 2.9 & 2.9 & 3.5 & 41.4 & 67.8 & 65.7 & 61 & 56.8 & 56.8 \\
\hline 3 & 3.4 & 21.5 & 44.9 & 68.5 & 69.1 & 62.2 & 59.2 & 59.1 \\
\hline 2.9 & 3.4 & 16.6 & 41.4 & 66.7 & 68.2 & 60.2 & 57.6 & 56.7 \\
\hline 2.7 & 2.8 & 15.9 & 42.5 & 66.8 & 65.8 & 59 & 56.4 & 58.4 \\
\hline 2.8 & 2.8 & 26.3 & 43.1 & 68.3 & 67.7 & 61.9 & 58.1 & 62.7 \\
\hline 3.4 & 2.9 & 3 & 0.4 & 10.2 & 19.2 & 19.8 & 19 & 19.4 \\
\hline 3 & 3.5 & 3.3 & 13.6 & 17.7 & 18.1 & 17.5 & 11 & 0.4 \\
\hline 3.5 & 2.8 & 18.8 & 42.6 & 67.1 & 66 & 61.3 & 59.8 & 58.3 \\
\hline 2.8 & 2.8 & 12.4 & 39.9 & 66 & 66.9 & 59.1 & 58 & 56.6 \\
\hline 2.8 & 3.5 & 26 & 42 & 66.7 & 67.6 & 64.3 & 61.5 & 61.1 \\
\hline 3 & 3 & 19.4 & 41.5 & 66.6 & 64 & 60.9 & 57.5 & 59.2 \\
\hline 3.4 & 2.9 & 20 & 29 & 28.9 & 31.4 & 32.3 & 32.8 & 30.8 \\
\hline 3.4 & 2.9 & 2.9 & 0.4 & 0.4 & 0.4 & 0.4 & 0.4 & 0.5 \\
\hline 3.4 & 2.8 & 2.8 & 0.4 & 0.5 & 0.4 & 0.4 & 0.4 & 0.4 \\
\hline 3.3 & 2.7 & 15.6 & 41.7 & 67 & 67.6 & 64 & 61.1 & 59.9 \\
\hline 3.3 & 2.8 & 14.3 & 29.5 & 38.5 & 54.1 & 66.8 & 69 & 70 \\
\hline 2.8 & 2.7 & 21.8 & 41.9 & 69 & 68.7 & 61.5 & 58.9 & 58.7 \\
\hline 3.4 & 2.7 & 20.4 & 40.8 & 69.4 & 66.2 & 62 & 57.9 & 59.8 \\
\hline 2.7 & 3.4 & 18.8 & 41 & 68.6 & 66.8 & 60.5 & 56.9 & 57.1 \\
\hline 3.3 & 2.7 & 2.8 & 8.7 & 31.1 & 31.3 & 29 & 4.9 & 0.4 \\
\hline 2.8 & 2.7 & 3.4 & 0.4 & 0.5 & 0.4 & 0.4 & 0.4 & 0.4 \\
\hline 2.8 & 3.3 & 16.1 & 40.6 & 67.8 & 69.8 & 62.6 & 59.4 & 60.2 \\
\hline 2.8 & 2.7 & 15.8 & 39.7 & 65.7 & 67.3 & 58.6 & 56.9 & 58.4 \\
\hline 0.4 & 0.5 & 0.4 & 0.4 & 0.4 & 0.4 & 0.4 & 0.4 & 0.4 \\
\hline 0.4 & 0.5 & 0.4 & 0.4 & 0.4 & 0.4 & 0.4 & 0.4 & 0.4 \\
\hline 0.4 & 0.4 & 0.4 & 0.4 & 0.4 & 0.4 & 0.5 & 0.4 & 0.4 \\
\hline 0.4 & 0.4 & 0.4 & 0.4 & 0.5 & 0.4 & 0.4 & 0.4 & 0.4 \\
\hline 0.4 & 0.4 & 0.4 & 0.5 & 0.4 & 0.4 & 0.4 & 0.4 & 0.4 \\
\hline 0.4 & 1 & 15.2 & 40.4 & 66.7 & 68.9 & 61.1 & 61.2 & 57.8 \\
\hline 2.7 & 3.4 & 16.5 & 39.4 & 66.5 & 68.5 & 63.2 & 59 & 58.3 \\
\hline 2.7 & 2.8 & 21.5 & 41.2 & 68.1 & 68.9 & 63 & 56.4 & 59.1 \\
\hline 2.8 & 3.3 & 17.5 & 43.6 & 67.3 & 67.2 & 61 & 58.2 & 57.8 \\
\hline 3.4 & 2.8 & 19 & 40.7 & 67.9 & 68.7 & 62 & 61.8 & 62.5 \\
\hline 2.7 & 3.4 & 2.7 & 1 & 12.7 & 15.8 & 15 & 15.6 & 9 \\
\hline 3.4 & 2.7 & 3.4 & 0.4 & 0.4 & 0.4 & 0.4 & 0.4 & 0.5 \\
\hline 2.7 & 2.8 & 18.7 & 42.2 & 65.3 & 68.3 & 58.6 & 56.4 & 58.2 \\
\hline 3.4 & 2.7 & 15.8 & 41.2 & 66.7 & 68.9 & 62.1 & 55.6 & 58.6 \\
\hline 2.7 & 3.4 & 16.3 & 41.3 & 66.5 & 66.3 & 60.7 & 54.5 & 57.6 \\
\hline 2.7 & 3.4 & 21.5 & 37.5 & 67.5 & 68.8 & 63.8 & 60.6 & 60.3 \\
\hline 2.8 & 2.7 & 20.4 & 39.7 & 67 & 67.2 & 62.1 & 60.9 & 61.2 \\
\hline 2.8 & 3.3 & 2.7 & 0.4 & 0.4 & 0.4 & 0.5 & 0.4 & 0.4 \\
\hline 2.7 & 3.4 & 2.7 & 0.4 & 0.4 & 0.4 & 0.4 & 0.4 & 0.4 \\
\hline 2.7 & 3.3 & 13.3 & 36.7 & 68.2 & 66.4 & 61 & 60.2 & 59.3 \\
\hline
\end{tabular}


lights

\begin{tabular}{|c|c|c|c|c|c|c|c|c|}
\hline 2.7 & 3.4 & 17.7 & 40.5 & 67.9 & 66.3 & 63.1 & 59.7 & 58.9 \\
\hline 3.4 & 2.7 & 17.1 & 41 & 66 & 68.4 & 61.5 & 58.7 & 60.8 \\
\hline & 2.8 & 20.6 & 42.4 & 67.3 & 65.9 & 63.2 & 58.1 & 58.8 \\
\hline 2.7 & 2.8 & 21.1 & 42.5 & 66.7 & 67.1 & 63.9 & 58.6 & 61.3 \\
\hline 2.7 & 2.8 & 3.3 & 0.4 & 0.4 & 0.4 & 0.5 & 0.4 & 0.4 \\
\hline 2.7 & 3.4 & 2.7 & 0.4 & 0.4 & 0.4 & 0.4 & 0.5 & 0.4 \\
\hline 2.7 & 2.8 & 13.5 & 30.8 & 35.5 & 42.5 & 44.4 & 51.6 & 51.7 \\
\hline 2.7 & 3.4 & 17.5 & 35 & 37.6 & 36 & 39.7 & 37.1 & 34.4 \\
\hline 2.8 & 2.7 & 20.6 & 33.2 & 33.7 & 17.8 & 6.7 & 21.2 & 16.5 \\
\hline 0.4 & 0.5 & 0.4 & 0.4 & 0.4 & 0.3 & 0.4 & 0.5 & 0.4 \\
\hline 0.4 & 0.4 & 0.4 & 0.4 & 0.4 & 0.4 & 0.4 & 0.4 & 0.4 \\
\hline 0.4 & 0.4 & 0.4 & 0.4 & 0.4 & 0.4 & 0.4 & 0.4 & 0.4 \\
\hline 0.4 & 0.4 & 0.4 & 0.4 & 0.4 & 0.4 & 0.4 & 0.4 & 1 \\
\hline 0.4 & 0.4 & 0.4 & 0.4 & 0.4 & 0.4 & 0.4 & 0.4 & 0.4 \\
\hline 0.4 & 0.4 & 0.4 & 0.4 & 0.4 & 0.4 & 0.4 & 0.4 & 0.3 \\
\hline 0.4 & 0.4 & 0.4 & 0.4 & 0.4 & 0.4 & 0.4 & 0.4 & 0.4 \\
\hline 0.4 & 0.4 & 0.3 & 0.4 & 0.4 & 0.4 & 0.4 & 0.4 & 0.4 \\
\hline 0.4 & 0.3 & 0.4 & 0.4 & 0.4 & 0.4 & 0.4 & 0.4 & 0.4 \\
\hline 0.4 & 0.4 & 0.4 & 0.4 & 0.4 & 0.4 & 0.4 & 0.4 & 0.4 \\
\hline 0.4 & 0.4 & 0.4 & 0.4 & 0.4 & 0.4 & 0.4 & 0.4 & 0.4 \\
\hline 0.3 & 0.4 & 16.1 & 33.1 & 48 & 58.5 & 67 & 66.2 & 61.5 \\
\hline 2.9 & 3.5 & 20.1 & 42.3 & 67.9 & 68.7 & 63.5 & 61.2 & 60.4 \\
\hline 3.6 & 3.5 & 23.2 & 45.8 & 68.8 & 34.2 & 63.3 & 58.7 & 61.6 \\
\hline 3 & 3.5 & 20.1 & 43 & 67.2 & 66.3 & 61 & 60.7 & 59.7 \\
\hline 2.9 & 3.5 & 15 & 40.9 & 65.9 & 67.1 & 60.4 & 58.4 & 57.5 \\
\hline 3.6 & 3.5 & 2.9 & 0.5 & 0.4 & 0.4 & 0.4 & 0.4 & 0.4 \\
\hline 3.5 & 3.5 & 3 & 1.1 & 0.4 & 0.4 & 0.4 & 0.4 & 0.4 \\
\hline 3.5 & 2.9 & 20.9 & 42.6 & 65.5 & 64.2 & 63.1 & 59.9 & 58.2 \\
\hline 2.9 & 3.5 & 12.5 & 28.5 & 37.4 & 45.5 & 60.6 & 63.6 & 63.9 \\
\hline 3.5 & 2.9 & 18.2 & 40.8 & 66.9 & 68.2 & 61.3 & 55.5 & 58.6 \\
\hline 3.5 & 2.9 & 17 & 38.7 & 65.2 & 66.2 & 60.2 & 58.3 & 59.9 \\
\hline 3.5 & 3 & 17.4 & 41 & 66.4 & 57.8 & 56.6 & 57.6 & 59.3 \\
\hline 2.9 & 3.6 & 2.9 & 17.1 & 26.4 & 26.2 & 26.8 & 26.8 & 26.4 \\
\hline 3.6 & 2.9 & 3.5 & 0.4 & 0.4 & 0.4 & 0.4 & 0.5 & 0.4 \\
\hline 3 & 3.5 & 0.4 & 0.4 & 0.4 & 0.4 & 0.4 & 0.4 & 0.4 \\
\hline 0.4 & 0.4 & 24.2 & 41.6 & 66.4 & 66.5 & 63.3 & 59.2 & 60.5 \\
\hline 3 & 3.5 & 20.8 & 41.7 & 65.9 & 68.5 & 61.8 & 60 & 62.4 \\
\hline 2.9 & 3.5 & 20.7 & 43.1 & 66.3 & 68.1 & 62.3 & 58 & 59.1 \\
\hline 2.9 & 3.5 & 23.8 & 41.4 & 62 & 62.9 & 58.6 & 57.3 & 55.1 \\
\hline 2.9 & 3.5 & 2.9 & 0.5 & 0.4 & 0.4 & 0.4 & 0.4 & 0.4 \\
\hline 3.6 & 2.9 & 2.9 & 1.1 & 0.4 & 0.4 & 0.4 & 0.4 & 0.4 \\
\hline 2.9 & 3.5 & 18 & 38.5 & 64.5 & 63.8 & 60.2 & 56.9 & 58.4 \\
\hline 3 & 2.9 & 17.8 & 41.4 & 67.3 & 68.3 & 62 & 61.8 & 59.2 \\
\hline 2.9 & 2.9 & 20.1 & 42.2 & 66.8 & 61.2 & 58.1 & 58.9 & 57.4 \\
\hline 2.9 & 3.5 & 24.3 & 42.1 & 67.1 & 66.6 & 62.2 & 59.6 & 59.7 \\
\hline 3.5 & 2.9 & 25 & 43.2 & 67.2 & 65.3 & 57.3 & 53.3 & 54.7 \\
\hline 3 & 2.9 & 3.5 & 10.8 & 12.3 & 12.1 & 12.2 & 12.7 & 12 \\
\hline 3 & 3.5 & 2.9 & 0.4 & 0.4 & 0.4 & 0.4 & 0.4 & 0.4 \\
\hline 2.9 & 2.9 & 15.1 & 40.2 & 67.7 & 69.7 & 63.4 & 61.8 & 60.1 \\
\hline 3.5 & 2.9 & 19.5 & 40.6 & 66.4 & 62.7 & 61.2 & 56.3 & 55.6 \\
\hline 2.9 & 2.9 & 20.6 & 40.6 & 67.7 & 68.6 & 60.5 & 56.9 & 58.4 \\
\hline
\end{tabular}


lights

\begin{tabular}{|c|c|c|c|c|c|c|c|c|}
\hline 3.5 & 2.9 & 18.1 & 39.4 & 66 & 64.4 & 59.1 & 56 & 55.5 \\
\hline 2.9 & 3.5 & 11 & 28.5 & 30.4 & 30.3 & 32.4 & 35 & 35.9 \\
\hline 3 & 2.9 & & 0.4 & 0.4 & 0.4 & 0.4 & 0.4 & 0.4 \\
\hline 3.5 & 2.9 & 2.9 & 0.4 & 0.4 & 0.4 & 0.4 & 0.4 & 0.4 \\
\hline 2.9 & 3.5 & 17.2 & 39.3 & 66.5 & 67.3 & 62.6 & 61.2 & 58.6 \\
\hline 3 & 3.5 & 18.3 & 42.3 & 67.1 & 68 & 60.6 & 58.4 & 59.6 \\
\hline 2.9 & 2.9 & 17.3 & 40.5 & 67.2 & 66.8 & 60.9 & 59.6 & 58.6 \\
\hline 2.9 & 3.5 & 20.8 & 43.2 & 67 & 65.9 & 60.8 & 61 & 59 \\
\hline 2.9 & 2.8 & 15 & 40.6 & 66.7 & 65.7 & 59.6 & 57.4 & 58 \\
\hline 3.6 & 2.9 & 2.9 & 0.4 & 0.4 & 0.4 & 0.4 & 0.4 & 0.4 \\
\hline 3.5 & 3 & 2.9 & 0.4 & 0.4 & 0.4 & 0.4 & 0.3 & 0.4 \\
\hline 2.9 & 2.9 & 18.9 & 41.6 & 68.8 & 68.9 & 63.3 & 61.1 & 62.2 \\
\hline 3.5 & 2.9 & 17.8 & 44.5 & 64.5 & 66.5 & 62.1 & 61 & 60.7 \\
\hline 3.5 & 2.9 & 17.4 & 40.7 & 67.4 & 67.5 & 64 & 62 & 60.1 \\
\hline 3 & 3.5 & 15.3 & 34.1 & 64.8 & 68.4 & 62.1 & 60 & 60.8 \\
\hline 3.5 & 2.9 & 18.4 & 39.4 & 66.5 & 65 & 61.3 & 60 & 58.2 \\
\hline 2.9 & 3.6 & 2.9 & 1.1 & 0.4 & 0.4 & 0.4 & 0.4 & 0.4 \\
\hline 2.9 & 2.9 & 3.5 & 0.6 & 0.4 & 0.4 & 0.3 & 0.4 & 0.4 \\
\hline 3.5 & 2.9 & 15.4 & 29.9 & 29.9 & 31.2 & 32.6 & 33.7 & 40.6 \\
\hline 2.9 & 3.5 & 17.8 & 42 & 65.2 & 68.7 & 61.3 & 58.5 & 57.9 \\
\hline 2.9 & 2.9 & 21 & 42.2 & 68.6 & 67.8 & 60.6 & 58.6 & 57.2 \\
\hline 2.9 & 3.5 & 19.5 & 40.3 & 66.5 & 68 & 61.3 & 55.9 & 56.6 \\
\hline 3.5 & 2.9 & 18.4 & 40.2 & 66.3 & 53.9 & 57.4 & 55.9 & 55.4 \\
\hline 2.9 & 2.9 & 3.5 & 0.4 & 0.4 & 0.4 & 0.4 & 0.4 & 0.4 \\
\hline 2.9 & 2.9 & 3.5 & 0.5 & 0.3 & 0.4 & 0.4 & 0.4 & 0.4 \\
\hline 2.8 & 2.9 & 15.8 & 38.7 & 69.7 & 68.2 & 63.4 & 58.2 & 58.1 \\
\hline 2.9 & 3.5 & 17.6 & 38.5 & 65.5 & 67.4 & 60.8 & 60.5 & 58.6 \\
\hline 2.9 & 3.5 & 20.4 & 41.2 & 65.4 & 65 & 60.1 & 58.5 & 58.2 \\
\hline 2.9 & 2.9 & 16.3 & 34.9 & 59.9 & 65.7 & 63.4 & 62.2 & 60.2 \\
\hline 3.5 & 2.9 & 11.7 & 31.4 & 33.7 & 42.8 & 62 & 62.9 & 61.2 \\
\hline 2.9 & 2.9 & 3.4 & 6.3 & 24.6 & 27.4 & 27.3 & 27.3 & 27.5 \\
\hline 3.5 & 2.9 & 2.9 & 0.4 & 0.4 & 0.4 & 0.3 & 0.4 & 0.4 \\
\hline 2.9 & 2.9 & 21.6 & 39.9 & 66.4 & 64.1 & 62.5 & 58.7 & 58.7 \\
\hline 2.9 & 3.5 & 18.1 & 39.5 & 66 & 67.3 & 63.5 & 61.3 & 60.4 \\
\hline 2.9 & 2.9 & 19.3 & 39.7 & 67.7 & 67.5 & 62.2 & 59.7 & 59.2 \\
\hline 2.9 & 2.8 & 21.5 & 40.4 & 66.6 & 68.6 & 63.1 & 60.2 & 60 \\
\hline 2.9 & 3.5 & 18.7 & 40.2 & 65.6 & 65.4 & 58.3 & 56.7 & 57.5 \\
\hline 3.5 & 2.9 & 2.9 & 1.1 & 0.3 & 0.4 & 0.4 & 0.4 & 0.4 \\
\hline 2.9 & 3.5 & 2.9 & 0.4 & 0.4 & 0.4 & 0.4 & 0.4 & 0.4 \\
\hline 3.4 & 2.9 & 9.5 & 11 & 10.8 & 10.8 & 10.7 & 10.8 & 10.1 \\
\hline 3.5 & 2.9 & 13 & 15.6 & 15.4 & 15.4 & 14.6 & 15.3 & 16.1 \\
\hline 2.9 & 1.6 & 17.7 & 30.9 & 34.8 & 37.8 & 34.6 & 35 & 36.3 \\
\hline 16.8 & 15.8 & 13.1 & 14.2 & 14.1 & 14 & 14.8 & 15.8 & 16.2 \\
\hline 21.8 & 21.9 & 16.6 & 19.2 & 19.6 & 19.4 & 19.5 & 19.2 & 19.6 \\
\hline 16 & 14.5 & 15.1 & 14.9 & 15.2 & 14.6 & 14.7 & 14.8 & 14.9 \\
\hline 15.1 & 14.1 & 13.8 & 15.1 & 14.2 & 14.3 & 13.8 & 14.2 & 14.3 \\
\hline 14.9 & 14.7 & 20.9 & 40.5 & 59.4 & 61.9 & 57.8 & 53.2 & 54.3 \\
\hline 3.5 & 2.9 & 18.7 & 41.6 & 66.9 & 64.9 & 59.2 & 56.4 & 57.1 \\
\hline 2.9 & 3.4 & 22.5 & 40.9 & 65.7 & 66.2 & 58.9 & 56.8 & 59.9 \\
\hline 3.6 & 3 & 23.4 & 41 & 66.5 & 68.2 & 64 & 58.7 & 58.5 \\
\hline 3.5 & 3.5 & 27.2 & 41.2 & 66 & 66.4 & 61.1 & 58.6 & 57.9 \\
\hline
\end{tabular}


lights

\begin{tabular}{|c|c|c|c|c|c|c|c|c|}
\hline 3.6 & 3.5 & 9 & 19.2 & 20 & 20 & 19 & 18.7 & 18.4 \\
\hline 3.6 & 3.5 & 3.6 & 3.5 & 3.6 & 2.9 & 3.6 & 3.5 & 3.5 \\
\hline 3.6 & 3.5 & 21.3 & 44.6 & 70.5 & 71.6 & 63.1 & 60.4 & 59.5 \\
\hline 3.6 & 3 & 26.3 & 42.7 & 68.2 & 68.1 & 62.3 & 62.3 & 61.2 \\
\hline 3.6 & 3.6 & 23.7 & 41.9 & 69.3 & 68.1 & 63 & 60.5 & 58.3 \\
\hline 3.6 & 3.6 & 25.2 & 41.8 & 68.5 & 67.8 & 63.3 & 61 & 58.5 \\
\hline 3.6 & 3.5 & 25.6 & 41.4 & 67.4 & 67.5 & 64 & 60.7 & 60.2 \\
\hline 3.6 & 3.5 & 3.6 & 4.2 & 24.5 & 52.8 & 55.5 & 57.3 & 57.1 \\
\hline 3.6 & 3.5 & 0.4 & 19.3 & 40.3 & 45.2 & 47.3 & 49.1 & 50.9 \\
\hline 3.6 & 3.6 & 25.4 & 43.7 & 68.2 & 69.6 & 61 & 58.7 & 59.8 \\
\hline 3.5 & 3.7 & 25.2 & 41.2 & 67.3 & 69.2 & 61.5 & 59.2 & 58.4 \\
\hline 3.6 & 3.6 & 28.8 & 38.8 & 66 & 68.8 & 62.3 & 61.7 & 60.9 \\
\hline 3.6 & 3.7 & 26.3 & 41.1 & 66.9 & 67.3 & 57.5 & 56.7 & 56.1 \\
\hline 3.5 & 3.7 & 26.4 & 39.9 & 65.9 & 67.6 & 61.8 & 59.3 & 57 \\
\hline 3.6 & 3.5 & 3.5 & 0.5 & 0.4 & 0.5 & 0.5 & 0.4 & 0.5 \\
\hline 3.6 & 3.5 & 3.6 & 0.4 & 0.5 & 0.5 & 0.4 & 0.5 & 0.5 \\
\hline 3.5 & 3.7 & 25.1 & 41.9 & 67.3 & 68.8 & 63.6 & 58.9 & 57.3 \\
\hline 3.6 & 3.6 & 26.7 & 43.7 & 69.8 & 68.8 & 65.6 & 60.6 & 59.7 \\
\hline 3.5 & 3.6 & 25.1 & 43.6 & 70.4 & 69.1 & 62.8 & 60.6 & 60 \\
\hline 3.6 & 3.5 & 23.2 & 41.5 & 68.8 & 60.2 & 54.7 & 51.9 & 52.9 \\
\hline 3.6 & 3.5 & 18.3 & 28.2 & 33.2 & 33.6 & 32.8 & 33.6 & 34.8 \\
\hline 3.6 & 2.9 & 3.6 & 0.5 & 0.4 & 0.5 & 0.5 & 0.4 & 0.5 \\
\hline 3.6 & 3.5 & 3.5 & 0.5 & 0.5 & 0.4 & 0.5 & 0.5 & 0.4 \\
\hline 3.6 & 3.5 & 21.2 & 24.1 & 25.8 & 28.7 & 30.3 & 29.8 & 28.4 \\
\hline 3.6 & 3.6 & 21.7 & 31.7 & 46.9 & 64.3 & 61.9 & 58.4 & 55.9 \\
\hline 3.6 & 3.6 & 26.6 & 44 & 67.8 & 66.2 & 60.4 & 59 & 59.1 \\
\hline 3.6 & 3.6 & 26.5 & 42 & 67.4 & 66 & 62.7 & 58.7 & 56.6 \\
\hline 3 & 4.2 & 29.4 & 42.4 & 66.7 & 67.4 & 60.8 & 57.9 & 57 \\
\hline 3.6 & 3.5 & 3 & 20.9 & 34.6 & 36.4 & 37.3 & 38 & 37.6 \\
\hline 3.6 & 3.6 & 3.6 & 0.4 & 0.5 & 0.5 & 0.4 & 0.5 & 0.5 \\
\hline 3.6 & 4.4 & 33.2 & 43.1 & 70.1 & 69.7 & 63.6 & 58.5 & 58 \\
\hline 3.6 & 3.5 & 26.9 & 42.1 & 69.2 & 68.8 & 61.7 & 57.3 & 60 \\
\hline 3.5 & 3.6 & 27.4 & 41.5 & 68 & 69.1 & 61.5 & 59 & 57.1 \\
\hline 3.5 & 3.6 & 26.3 & 41.8 & 67.1 & 65.3 & 60.7 & 58 & 52.7 \\
\hline 3.6 & 3.5 & 21.5 & 41.6 & 66.2 & 46.1 & 38 & 40.6 & 41 \\
\hline 3.5 & 3 & 3.5 & 7.5 & 24.1 & 24.4 & 24.2 & 24.7 & 25.3 \\
\hline 3.6 & 3.5 & 3.6 & 0.5 & 0.5 & 0.4 & 0.5 & 0.5 & 0.4 \\
\hline 3 & 3.5 & 27.6 & 42.8 & 67.2 & 67.4 & 62 & 60 & 57.5 \\
\hline 3.6 & 3.6 & 26 & 42.8 & 67.8 & 67.2 & 61.4 & 59.1 & 58.4 \\
\hline 3.6 & 3.5 & 26.5 & 41.3 & 68.4 & 67.6 & 61.1 & 57.6 & 59.6 \\
\hline 3.5 & 3.6 & 23.9 & 40.1 & 67.9 & 68.9 & 64.8 & 61.2 & 58.6 \\
\hline \multirow[t]{3}{*}{3.6} & 3.6 & 23.2 & 42.4 & 68.2 & 65.5 & 61.1 & 55.8 & 56.3 \\
\hline & & & & & & & & \\
\hline & & & & & & & & 0.2 \\
\hline 3.6 & 3.6 & 9.7 & 24.1 & 42.9 & 58.1 & 61 & 57.5 & 52.7 \\
\hline 3.6 & 3.5 & 22.8 & 42.3 & 67.7 & 68.6 & 55 & 58.1 & 55.2 \\
\hline 3.5 & 3.6 & 23.6 & 43.1 & 65.1 & 69.4 & 62 & 58.2 & 57.9 \\
\hline 3.6 & 3.5 & 23.1 & 39.4 & 66.1 & 68.1 & 59.6 & 58.8 & 58.8 \\
\hline 3.6 & 3.5 & 21.7 & 39.9 & 62.2 & 54.5 & 58 & 59.3 & 57.1 \\
\hline 3.6 & 3.5 & 3.5 & 0.5 & 0.4 & 0.5 & 0.5 & 0.4 & 0.5 \\
\hline 2.9 & 3.6 & 3.5 & 0.5 & 0.4 & 0.5 & 0.4 & 0.5 & 0.5 \\
\hline
\end{tabular}


lights

\begin{tabular}{|c|c|c|c|c|c|c|c|c|}
\hline 3.5 & 2.9 & 22.2 & 39.2 & 64.4 & 64.3 & 60.1 & 57 & 53.1 \\
\hline 3.5 & 2.9 & 19 & 37.7 & 63.2 & 66.4 & 60 & 56.6 & 55.9 \\
\hline 3.5 & 3 & 20 & 38.6 & 65.6 & 68.2 & 61 & 58 & 56.7 \\
\hline 3.5 & 3.6 & 21.9 & 38.6 & 62.1 & 59.4 & 58.4 & 57.4 & 55.4 \\
\hline 3.6 & 3.5 & 20 & 37.9 & 67.5 & 60.6 & 60.6 & 56.5 & 57.9 \\
\hline 3.5 & 3.6 & 3.5 & 0.5 & 0.5 & 0.5 & 0.4 & 0.5 & 0.4 \\
\hline 3.6 & 3.5 & 3.6 & 0.5 & 0.4 & 0.5 & 0.4 & 0.5 & 0.5 \\
\hline 3.5 & 3.5 & 19.3 & 37.7 & 66.4 & 63 & 58.1 & 56 & 53.7 \\
\hline 3.5 & 3 & 18.7 & 38.4 & 65.1 & 62.8 & 58.4 & 56.7 & 56.7 \\
\hline 1.2 & 3.5 & 18.5 & 39.2 & 64.9 & 63.1 & 56.5 & 53.8 & 51.4 \\
\hline 3.6 & 3.5 & 19.8 & 38.4 & 64.3 & 65.1 & 62.2 & 59.1 & 56 \\
\hline 3.6 & 3.6 & 15.4 & 33.6 & 57.3 & 64.5 & 64.2 & 52.2 & 59.5 \\
\hline 3.6 & 3.5 & 3.6 & 0.5 & 0.4 & 0.5 & 0.5 & 0.4 & 0.5 \\
\hline 3.5 & 3.6 & 3.5 & 0.5 & 0.4 & 0.5 & 0.5 & 0.4 & 0.5 \\
\hline 2.9 & 3.6 & 14 & 18.7 & 25.8 & 30.9 & 35.9 & 35.8 & 34 \\
\hline 2.9 & 3.6 & 14.7 & 26.8 & 43.8 & 45.8 & 48.5 & 48 & 43.4 \\
\hline 3.5 & 2.9 & 15.1 & 24.9 & 43.8 & 46.9 & 47.7 & 48.1 & 41.6 \\
\hline & & & & & & & & \\
\hline & & & & & & & & \\
\hline & & & & & & & & \\
\hline & & & & & & & & \\
\hline 10.00 & 10.03 & 14.35 & 30.60 & 45.03 & 44.78 & 41.91 & 39.41 & 41.09 \\
\hline 12.58 & 12.51 & 13.99 & 28.75 & 40.71 & 40.39 & 37.69 & 36.23 & 36.21 \\
\hline 12.62 & 12.64 & 16.17 & 31.61 & 46.07 & 45.62 & 42.37 & 41.12 & 41.43 \\
\hline 3.46 & 3.30 & 10.79 & 21.88 & 32.32 & 32.77 & 30.85 & 29.86 & 29.58 \\
\hline 3.04 & 2.98 & 12.25 & 27.36 & 42.67 & 43.43 & 40.78 & 39.23 & 39.46 \\
\hline 2.57 & 2.62 & 11.94 & 24.11 & 37.38 & 36.83 & 35.21 & 34.38 & 34.24 \\
\hline 4.30 & 4.22 & 16.84 & 28.41 & 44.70 & 45.35 & 42.48 & 41.08 & 40.81 \\
\hline 3.58 & 3.58 & 24.90 & 41.65 & 68.08 & 67.30 & 62.10 & 58.43 & 46.62 \\
\hline & & & & & & & & \\
\hline & & & & & & & & \\
\hline & & & & & & & & \\
\hline & & & & & & & & \\
\hline 3.195 & 3.229 & 6.086 & 18.197 & 30.591 & 30.373 & 27.120 & 24.674 & 26.697 \\
\hline 3.547 & 3.511 & 4.253 & 13.893 & 24.833 & 24.513 & 21.845 & 20.174 & 20.889 \\
\hline 3.371 & 3.566 & 5.285 & 15.582 & 27.080 & 26.496 & 23.569 & 22.280 & 22.595 \\
\hline 2.255 & 1.189 & 5.769 & 15.751 & 25.056 & 24.650 & 22.803 & 21.330 & 21.557 \\
\hline 0.811 & 0.818 & 6.831 & 17.203 & 27.530 & 27.281 & 24.437 & 23.478 & 23.616 \\
\hline 1.132 & 1.142 & 8.737 & 19.375 & 31.186 & 31.054 & 29.376 & 28.216 & 28.217 \\
\hline 3.549 & 3.406 & 9.172 & 16.681 & 27.612 & 27.383 & 25.048 & 23.840 & 23.491 \\
\hline 0.172 & 0.255 & 7.264 & 13.889 & 22.629 & 22.388 & 20.545 & 19.677 & 22.197 \\
\hline & & & & & & & & \\
\hline & & & & & & & & \\
\hline & & & & & & & & \\
\hline 5131.70 & 5147.20 & 7359.20 & 15695.60 & 23098.90 & 22970.30 & 21497.30 & 20215.10 & 21038.40 \\
\hline 2880.50 & 2863.70 & 3204.70 & 6584.30 & 9322.30 & 9248.90 & 8630.20 & 8295.70 & 8292.80 \\
\hline 2347.00 & 2351.70 & 3007.80 & 5880.20 & 8523.80 & 8485.80 & 7923.50 & 7688.70 & 7747.50 \\
\hline 304.80 & 290.40 & 949.90 & 1925.10 & 2844.10 & 2850.90 & 2715.10 & 2627.40 & 2603.00 \\
\hline 449.40 & 441.50 & 1813.60 & 4048.70 & 6315.40 & 6427.40 & 6036.00 & 5806.60 & 5840.00 \\
\hline 167.00 & 172.80 & 776.00 & 1591.50 & 2466.90 & 2431.10 & 2323.70 & 2269.10 & 2260.00 \\
\hline
\end{tabular}


lights

\begin{tabular}{|r|r|r|r|r|r|r|r|r|}
\hline 309.50 & 304.10 & 1212.60 & 2045.30 & 3218.40 & 3264.90 & 3058.30 & 2957.40 & 2938.40 \\
\hline 95.20 & 96.40 & 460.30 & 808.10 & 1333.00 & 1351.90 & 1278.30 & 1223.50 & 1193.00 \\
\hline
\end{tabular}




\begin{tabular}{|c|c|c|c|c|c|c|c|c|}
\hline 14 & 15 & 16 & 17 & 18 & 19 & 20 & 21 & 22 \\
\hline 99.4 & 96.4 & 83.1 & 59.1 & 46.5 & 41.2 & 43.4 & 45.7 & 44.9 \\
\hline 98.7 & 100.8 & 86.8 & 64.7 & 61.6 & 49.4 & 49.4 & 53.7 & 54.4 \\
\hline 100.9 & 93.7 & 83.9 & 53.9 & 44.7 & 44.7 & 48.2 & 47.4 & 46.2 \\
\hline 96.9 & 95.3 & 83.3 & 60.2 & 48.7 & 39.3 & 39.1 & 38 & 38.8 \\
\hline 12.5 & 12.4 & 12.4 & 11.2 & 10 & 10 & 11.7 & 12.4 & 12.4 \\
\hline 8.8 & 8.3 & 8.2 & 8.2 & 8.2 & 10 & 11.2 & 10.7 & 10.6 \\
\hline 93.1 & 94.6 & 84 & 55.3 & 47.8 & 42.7 & 45.2 & 45.5 & 44.4 \\
\hline 97.4 & 95.2 & 79.4 & 62.1 & 49.1 & 44.5 & 45.8 & 44.9 & 45.4 \\
\hline 96.7 & 92.6 & 85.7 & 64.5 & 52.9 & 41.7 & 43.1 & 35.4 & 35.5 \\
\hline 100.8 & 95.5 & 81.5 & 61.4 & 55 & 49.7 & 48.8 & 49.8 & 51 \\
\hline 94 & 98.7 & 81.7 & 55.3 & 39.2 & 36.6 & 41.5 & 42.8 & 43.5 \\
\hline 9.6 & 11.4 & 11.4 & 11.4 & 10.3 & 9.7 & 12.1 & 12 & 11.5 \\
\hline 9 & 7.7 & 8.5 & 8.4 & 8.5 & 7.9 & 10.8 & 10.2 & 10.9 \\
\hline 102.7 & 88.7 & 77.7 & 52.6 & 42.2 & 34.3 & 36.5 & 40.8 & 44.4 \\
\hline 93.5 & 88.4 & 82.2 & 60.7 & 44.6 & 38.4 & 40 & 43 & 43.7 \\
\hline 98.9 & 95 & 85.7 & 75.1 & 68.1 & 63.8 & 61.5 & 66 & 63.7 \\
\hline 94.2 & 94.7 & 88.5 & 63.6 & 54.9 & 51.3 & 40.9 & 39.4 & 38.4 \\
\hline 97.6 & 95.9 & 74 & 56.2 & 41.8 & 36.1 & 36.2 & 36.1 & 36.9 \\
\hline 9 & 9.6 & 9 & 8.9 & 9 & 9.3 & 11.4 & 11.4 & 11.4 \\
\hline 9.6 & 9.1 & 9 & 9 & 8.9 & 10 & 10.8 & 11.4 & 11.4 \\
\hline 98.4 & 91.7 & 76.9 & 53.9 & 47.6 & 47.7 & 47 & 47.2 & 47.1 \\
\hline 103.9 & 97.5 & 80.9 & 54.9 & 59.3 & 59.7 & 61.4 & 63.3 & 56.1 \\
\hline 101.2 & 98.8 & 82.6 & 57 & 52.2 & 47.4 & 40.3 & 41.4 & 35.7 \\
\hline 103.9 & 100.5 & 84.6 & 63.8 & 49.7 & 42.4 & 41.6 & 44.9 & 46.3 \\
\hline 94.2 & 96.2 & 76.4 & 51.2 & 36.6 & 34.6 & 36.2 & 36.3 & 37 \\
\hline 8.7 & 8.1 & 8.7 & 8.7 & 8.2 & 9.2 & 11.1 & 10.5 & 10.5 \\
\hline 8.2 & 8.7 & 8.7 & 8.7 & 8.2 & 9.2 & 11.1 & 10.5 & 10.5 \\
\hline 91.9 & 104.1 & 99.1 & 76.3 & 66.4 & 50.9 & 49.6 & 51.2 & 43.3 \\
\hline 105.9 & 101 & 85 & 53.5 & 46.5 & 40.8 & 40.7 & 42.7 & 42.2 \\
\hline 102.2 & 104.4 & 85.8 & 49.2 & 45.4 & 43 & 44.7 & 44.7 & 43 \\
\hline 103.4 & 101.9 & 87.8 & 64 & 48.6 & 45.4 & 42 & 40.8 & 42.4 \\
\hline 98.7 & 99 & 86.9 & 59.9 & 46.3 & 46.6 & 47.5 & 43.7 & 38.9 \\
\hline 18.7 & 18.6 & 19.3 & 18.6 & 17 & 17.4 & 18.1 & 18 & 17.5 \\
\hline 16.3 & 15.6 & 15.7 & 16.2 & 16.4 & 18 & 18.1 & 17.4 & 18.1 \\
\hline 101.9 & 100.4 & 85.9 & 70.2 & 64.7 & 60.4 & 58 & 59.3 & 61.6 \\
\hline 101.5 & 99 & 82.9 & 62.8 & 53.7 & 43.6 & 42.8 & 44.1 & 44.7 \\
\hline 104.7 & 100.7 & 88.7 & 68.6 & 57.7 & 45.3 & 42.9 & 44.3 & 42.4 \\
\hline 96.5 & 101.2 & 81.9 & 61.1 & 50.7 & 42.4 & 38.3 & 40.1 & 37.7 \\
\hline 78.5 & 97.4 & 77.4 & 53.1 & 40.1 & 36.3 & 37.1 & 38.2 & 38.2 \\
\hline 9.9 & 8.9 & 8.2 & 5.9 & 7.3 & 7.6 & 8.3 & 8.3 & 8.2 \\
\hline 5.9 & 5.8 & 5.9 & 5.9 & 7.2 & 8.3 & 7.6 & 8.3 & 8.2 \\
\hline 104.9 & 95.7 & 72.3 & 55.5 & 44.6 & 42.2 & 42.3 & 42.8 & 43 \\
\hline 98.9 & 96.7 & 77.5 & 50.3 & 48.7 & 39.9 & 40.6 & 42.3 & 42.3 \\
\hline 104.8 & 101.6 & 79.5 & 57.2 & 48.8 & 46.9 & 47.1 & 47.7 & 44.1 \\
\hline 98.2 & 100.7 & 77.5 & 55.6 & 50.6 & 58.4 & 73.9 & 80.6 & 76.8 \\
\hline 92.6 & 89.4 & 74.8 & 61.4 & 43.3 & 38.1 & 40.2 & 41.3 & 42.1 \\
\hline 54.5 & 43.4 & 7 & 6.4 & 7.9 & 8.8 & 8.7 & 8.8 & 8.8 \\
\hline
\end{tabular}


lights

\begin{tabular}{|c|c|c|c|c|c|c|c|c|}
\hline 56.7 & 30.2 & 5.6 & 5.5 & 7.7 & 7.9 & 7.9 & 8 & 7.9 \\
\hline 92.4 & 91.8 & 74.2 & 51.8 & 44.7 & 63.3 & 69.6 & 66.5 & 64.1 \\
\hline 84.3 & 78.6 & 66.2 & 53 & 47 & 55.6 & 55.8 & 53.8 & 53.7 \\
\hline 76.4 & 75.1 & 60.4 & 46.1 & 46.8 & 49.8 & 46.9 & 45.7 & 40.3 \\
\hline 66.5 & 67.1 & 56 & 36.2 & 36.7 & 43.7 & 42.9 & 40.8 & 44.1 \\
\hline 62.5 & 62.5 & 53 & 47.5 & 41.6 & 38.9 & 39.8 & 39.8 & 39.8 \\
\hline 5.7 & 5.6 & 5.7 & 5.7 & 7.2 & 7.4 & 8 & 7.5 & 8 \\
\hline 5.7 & 5.7 & 5.6 & 5.7 & 7.2 & 7.5 & 8 & 7.4 & 8.1 \\
\hline 66.4 & 64.6 & 53 & 38 & 36.3 & 38.5 & 43.2 & 47 & 46.5 \\
\hline 67.9 & 65.1 & 51.2 & 34.2 & 31.9 & 31.5 & 35.7 & 38.1 & 33.9 \\
\hline 64.1 & 50.9 & 37.2 & 29.8 & 29.9 & 31 & 30.5 & 29.9 & 15 \\
\hline 4.3 & 4.8 & 4.9 & 4.3 & 6.6 & 6.6 & 6.7 & 7.2 & 6.7 \\
\hline 3.8 & 4.9 & 5 & 4.3 & 5.3 & 6.6 & 6.6 & 7.3 & 6.6 \\
\hline 4.8 & 4.3 & 4.9 & 4.9 & 5.4 & 6.7 & 6.6 & 7.3 & 6.7 \\
\hline 4.9 & 4.3 & 4.9 & 4.8 & 5.4 & 6.7 & 6.7 & 7.4 & 6.7 \\
\hline 67.5 & 63.1 & 55.8 & 44.4 & 36.7 & 34.5 & 33.8 & 33.7 & 33.1 \\
\hline 67.2 & 64.8 & 55.9 & 42.2 & 36.9 & 37.3 & 40.4 & 39.7 & 39.8 \\
\hline 66.7 & 63.5 & 53 & 34.8 & 32.3 & 31.9 & 38 & 33.2 & 31.6 \\
\hline 65.4 & 63.2 & 52.9 & 34.7 & 30.6 & 30.1 & 30.3 & 30.4 & 28 \\
\hline 66.5 & 64.3 & 54 & 40 & 31.5 & 32.7 & 32.9 & 34.8 & 40.9 \\
\hline 38.6 & 37.9 & 5.3 & 5.3 & 5.2 & 7.1 & 7.1 & 7 & 7.1 \\
\hline 18 & 6.7 & 4.3 & 4.2 & 4.8 & 6.6 & 6.7 & 6 & 6.7 \\
\hline 64.8 & 60.1 & 51.6 & 37.8 & 32 & 32.1 & 32.1 & 30.4 & 30.1 \\
\hline 62.6 & 61.3 & 52.5 & 37.4 & 35.2 & 38.8 & 40.5 & 39.9 & 37.6 \\
\hline 66 & 64 & 55.5 & 37.1 & 32.6 & 32.5 & 32.7 & 32.1 & 31.4 \\
\hline 62.6 & 62.8 & 50.9 & 40.1 & 35 & 33.6 & 33.6 & 31.2 & 32.4 \\
\hline 63.7 & 61.1 & 47.1 & 37.9 & 30.6 & 30.8 & 36.3 & 35.2 & 35 \\
\hline 5.6 & 5.7 & 5.1 & 5.7 & 6.1 & 7.5 & 8.1 & 7.4 & 7.5 \\
\hline 5.7 & 5.7 & 5.6 & 5.1 & 6.2 & 8.1 & 7.4 & 7.5 & 8.1 \\
\hline 60.2 & 54.4 & 47.6 & 41.3 & 30.8 & 31.5 & 32.5 & 31.6 & 30.1 \\
\hline 60.5 & 59.1 & 52.7 & 38.9 & 38 & 39.1 & 39.1 & 39.1 & 43 \\
\hline 62.3 & 64 & 50.1 & 40.1 & 39.9 & 37.3 & 37.5 & 38.1 & 37.6 \\
\hline 60.2 & 64.4 & 49.1 & 36.9 & 44.4 & 45.3 & 44.6 & 41.3 & 44 \\
\hline 67.2 & 53.1 & 39.2 & 49.8 & 51.6 & 50.2 & 47.8 & 48.5 & 36.9 \\
\hline 6.6 & 6.5 & 6 & 6.5 & 7 & 8.4 & 8.3 & 8.4 & 8.3 \\
\hline 6.6 & 6.6 & 6 & 6.5 & 7.1 & 8.3 & 8.3 & 8.3 & 8.4 \\
\hline 6.6 & 5.9 & 6.6 & 6.5 & 7 & 8.4 & 8.3 & 8.3 & 8.4 \\
\hline 5.9 & 6.6 & 6.6 & 6.6 & 6.4 & 8.3 & 8.4 & 8.4 & 8.3 \\
\hline 6.6 & 6 & 6.6 & 6.6 & 6.4 & 8.4 & 8.3 & 8.4 & 8.3 \\
\hline 5.9 & 6.6 & 5.9 & 6.7 & 6.9 & 8.4 & 8.3 & 8.3 & 8.3 \\
\hline 31.1 & 19 & 5.9 & 5.3 & 5.6 & 7.1 & 7.6 & 7.1 & 7.6 \\
\hline 5.3 & 5.3 & 5.9 & 5.2 & 5.7 & 7 & 7.7 & 7 & 7.7 \\
\hline 5.9 & 5.3 & 5.3 & 5.2 & 6.3 & 7 & 7.7 & 7 & 7.1 \\
\hline 5.9 & 5.3 & 5.3 & 5.2 & 5.6 & 7.6 & 7.1 & 7.6 & 7.1 \\
\hline 5.3 & 5.8 & 5.3 & 5.9 & 5.5 & 7.7 & 7.6 & 7.1 & 7.7 \\
\hline 5.2 & 5.9 & 5.3 & 5.8 & 5.6 & 7.6 & 7.7 & 7.6 & 7.7 \\
\hline 44.7 & 44.2 & 23.7 & 5.7 & 6.1 & 7.6 & 8.1 & 8.2 & 7.6 \\
\hline 35.7 & 35.8 & 34.7 & 34 & 35.1 & 17.4 & 7.8 & 7.9 & 7.8 \\
\hline 44.8 & 39.4 & 36.7 & 28.7 & 15.3 & 6.9 & 7 & 6.9 & 7.6 \\
\hline 10.1 & 7.1 & 3.6 & 4.2 & 4.5 & 6.6 & 5.9 & 6 & 6 \\
\hline 66 & 62.7 & 54.7 & 41.2 & 38.3 & 31.5 & 30.3 & 31.8 & 30 \\
\hline
\end{tabular}


lights

\begin{tabular}{|c|c|c|c|c|c|c|c|c|}
\hline 64.6 & 62.9 & 54.4 & 39.5 & 28.7 & 25.5 & 25.9 & 25.2 & 25.4 \\
\hline 63.7 & 63.8 & 52.3 & 35 & 27.2 & 25.5 & 26.2 & 26.1 & 25.8 \\
\hline 65.3 & 64.3 & 54.8 & 40.3 & 29.2 & 27.5 & 27.7 & 32.6 & 34.4 \\
\hline 62.9 & 63.2 & 51.8 & 37.2 & 26 & 25.9 & 29.9 & 30.5 & 30.5 \\
\hline 3.9 & 3.3 & 4 & 3.3 & 4.1 & 5.7 & 5.8 & 5.7 & 5.8 \\
\hline 3.3 & 3.9 & 3.9 & 4 & 3.5 & 5.7 & 5.7 & 5.7 & 5.7 \\
\hline 65.9 & 64.4 & 53.7 & 48.1 & 46.7 & 40.8 & 41.4 & 40.4 & 39.6 \\
\hline 66.9 & 62.8 & 56.2 & 48.2 & 42.2 & 42.4 & 42.5 & 42.6 & 38.9 \\
\hline 59.8 & 65 & 53.6 & 43.6 & 43.6 & 44.8 & 44.9 & 44.2 & 45.5 \\
\hline 64.5 & 62.8 & 54.7 & 47.3 & 48 & 49.4 & 48.4 & 46 & 46.7 \\
\hline 61.7 & 62.9 & 50 & 32.3 & 35.8 & 40.3 & 40.2 & 40.8 & 46.9 \\
\hline 5.1 & 5.1 & 5.2 & 5.1 & 5.8 & 6.9 & 6.4 & 6.3 & 7 \\
\hline 4.5 & 4.5 & 4.5 & 4.6 & 4 & 6.3 & 6.3 & 6.3 & 6.9 \\
\hline 5.7 & 5.7 & 5.2 & 4.5 & 3.9 & 6.4 & 6.3 & 5.7 & 6.4 \\
\hline 64.9 & 65.4 & 55.7 & 51.3 & 47.9 & 42.4 & 41.2 & 39.1 & 30.4 \\
\hline 64.8 & 62.2 & 55.4 & 47.3 & 40.2 & 40 & 38.2 & 37.1 & 38.3 \\
\hline 60.2 & 56.5 & 57.4 & 45.5 & 35.6 & 33.6 & 34.2 & 38.6 & 41.4 \\
\hline 53.9 & 55.5 & 52.9 & 47.3 & 46.2 & 44 & 42.4 & 43.1 & 42.7 \\
\hline 4.1 & 4.7 & 4.7 & 4.1 & 4.7 & 6.6 & 6.6 & 6 & 7.3 \\
\hline 4.1 & 4.6 & 4.7 & 4.1 & 4.7 & 6.6 & 6.6 & 6.7 & 6.6 \\
\hline 67 & 65.2 & 57.3 & 48.8 & 41.5 & 38.7 & 40.6 & 41.2 & 40.5 \\
\hline 64.8 & 63.8 & 58 & 46.6 & 36.8 & 36.1 & 39.5 & 39.7 & 40.4 \\
\hline 63 & 63.3 & 55.7 & 47.8 & 40.8 & 39.8 & 41.9 & 42.1 & 41.4 \\
\hline 59.8 & 59.7 & 55.5 & 51 & 49.4 & 49.1 & 51.5 & 52.2 & 52.1 \\
\hline 30.8 & 31.4 & 30.3 & 30.2 & 30.8 & 34.1 & 37.2 & 38 & 33.7 \\
\hline 4.1 & 4.9 & 5.9 & 5.9 & 4 & 5.8 & 6 & 6.6 & 6.6 \\
\hline 3.5 & 3.5 & 2.9 & 5.2 & 4.7 & 7 & 7.2 & 6 & 6 \\
\hline 63.6 & 64.9 & 54.6 & 50 & 40.4 & 38.4 & 40 & 39.5 & 38.9 \\
\hline 63.9 & 63 & 56 & 48.9 & 45 & 41.9 & 42.9 & 43 & 43.5 \\
\hline 67 & 65.1 & 57.2 & 51.5 & 41.3 & 37.8 & 38.1 & 39.6 & 38.9 \\
\hline 66.7 & 64.5 & 56.6 & 48.9 & 39.8 & 39.8 & 40.3 & 41.3 & 40.9 \\
\hline 63.9 & 63.1 & 54.8 & 49.4 & 45.7 & 42.7 & 41.5 & 39.8 & 39.7 \\
\hline 3.9 & 3.4 & 3.9 & 5.3 & 7.1 & 8.9 & 8.2 & 6.4 & 6.5 \\
\hline 3.4 & 3.9 & 3.9 & 3.9 & 3.4 & 5.4 & 6.4 & 5.9 & 6.5 \\
\hline 66.1 & 66.1 & 53.3 & 47.3 & 39.4 & 35 & 34.7 & 31.7 & 27.5 \\
\hline 64.5 & 65.3 & 51 & 41.6 & 36.7 & 36.8 & 38.1 & 39.3 & 38.2 \\
\hline 63.9 & 63.5 & 58.2 & 51 & 39.8 & 39.4 & 39.9 & 41.9 & 41 \\
\hline 62.7 & 63.7 & 58 & 50.7 & 43.2 & 40.2 & 41.6 & 42.4 & 42 \\
\hline 61.8 & 61.9 & 55.2 & 46.6 & 40.2 & 39 & 41.4 & 41.6 & 41.6 \\
\hline 4.7 & 4.1 & 5.4 & 5.8 & 5.9 & 7.4 & 7.2 & 7.2 & 6.7 \\
\hline 4.7 & 4.1 & 4.7 & 4.7 & 4.7 & 5.5 & 7.2 & 7.2 & 6.7 \\
\hline 65.3 & 61.4 & 53.8 & 47.4 & 36.6 & 37.2 & 40.9 & 44.6 & 45.2 \\
\hline 61.7 & 60.6 & 53.3 & 47.1 & 38.6 & 38.3 & 39 & 38.6 & 38.6 \\
\hline 63.6 & 60.1 & 55.3 & 47.3 & 42 & 41.8 & 44.2 & 43.7 & 44.3 \\
\hline 60.9 & 59 & 53.3 & 43.4 & 40.7 & 40.9 & 34.3 & 32.4 & 32.8 \\
\hline 64.2 & 61.7 & 52.9 & 40.3 & 41.8 & 41.3 & 43 & 42.1 & 42 \\
\hline 4.6 & 5.3 & 4.6 & 5.2 & 4.7 & 6.4 & 7.7 & 7.8 & 7.2 \\
\hline 5.2 & 4.7 & 5.2 & 4.7 & 5.2 & 7.6 & 7.7 & 7.8 & 7.8 \\
\hline 68.3 & 65.5 & 58.4 & 51.9 & 43.4 & 41 & 42 & 42.6 & 41.5 \\
\hline 67.6 & 67.3 & 54.9 & 52.1 & 44.1 & 40.5 & 38.8 & 38.8 & 39 \\
\hline 64.9 & 64.2 & 55.5 & 47.1 & 40.1 & 39.9 & 40.6 & 40.5 & 41.3 \\
\hline
\end{tabular}




\begin{tabular}{|c|c|c|c|c|c|c|c|c|}
\hline 63.7 & 66.2 & 56.7 & 49.7 & 46.7 & 44.8 & 45.2 & 45.2 & 40.6 \\
\hline 65.3 & 63.2 & 52.5 & 42.6 & 41.4 & 42.3 & 44 & 43.1 & 37.6 \\
\hline 5.5 & 6.1 & 6.8 & 6.9 & 6.8 & 7.6 & 9.3 & 11.6 & 8.7 \\
\hline 6.1 & 5.6 & 6.1 & 5.6 & 6.1 & 7 & 8.1 & 8.6 & 8 \\
\hline 63.7 & 61.7 & 51.4 & 39 & 31.7 & 31.2 & 32.6 & 32.8 & 30.4 \\
\hline 66.7 & 65 & 55.3 & 49.3 & 42.2 & 41 & 42.3 & 43.2 & 42.8 \\
\hline 63.3 & 62.9 & 57.1 & 43.9 & 36.5 & 39.1 & 38.1 & 39 & 38.4 \\
\hline 64.5 & 64 & 56.8 & 46.9 & 40.8 & 42 & 49.8 & 35.7 & 31.3 \\
\hline 63.8 & 60.1 & 53.1 & 37.8 & 32.7 & 31.6 & 37.3 & 38.2 & 31.3 \\
\hline 5.7 & 6.3 & 5 & 5.7 & 5.7 & 5.9 & 8.1 & 8.2 & 8.2 \\
\hline 5.7 & 5.6 & 5.1 & 5.7 & 5.7 & 6.5 & 7.6 & 8.2 & 8.2 \\
\hline 65.1 & 63.6 & 55.4 & 41.9 & 35.7 & 34.7 & 36.8 & 34.4 & 33.2 \\
\hline 66.1 & 64.8 & 55.8 & 50 & 49.3 & 48.3 & 52.6 & 52.4 & 51.1 \\
\hline 66.2 & 66.2 & 56 & 47.8 & 40.5 & 40.4 & 42.1 & 44.9 & 44.6 \\
\hline 63.5 & 62.8 & 55.3 & 40.1 & 35 & 33.9 & 35.6 & 35.7 & 36.3 \\
\hline 61 & 51 & 31.5 & 36.3 & 31.6 & 32.2 & 34.9 & 36.3 & 36.9 \\
\hline 6 & 4.9 & 5.4 & 5.4 & 5.4 & 5.6 & 7.9 & 7.9 & 7.9 \\
\hline 5.4 & 5.4 & 5.5 & 4.8 & 5.4 & 6.1 & 7.9 & 7.4 & 7.9 \\
\hline 9 & 7.2 & 4.8 & 5.4 & 4.8 & 5.5 & 8 & 7.3 & 7.3 \\
\hline 5.9 & 5.9 & 4.8 & 4.7 & 4.1 & 5.4 & 7.2 & 7.3 & 7.2 \\
\hline 4.8 & 4.1 & 4.7 & 4.7 & 4.8 & 5.3 & 7.3 & 7.2 & 7.3 \\
\hline 6 & 5.9 & 6.5 & 4.7 & 4.8 & 5.3 & 6.7 & 7.2 & 7.3 \\
\hline 4.1 & 4.8 & 4.7 & 4.7 & 4.7 & 4.8 & 7.2 & 7.3 & 7.2 \\
\hline 4.8 & 4.7 & 4.2 & 4.7 & 4.7 & 5.4 & 6.6 & 7.3 & 7.3 \\
\hline 4.7 & 4.8 & 4.1 & 4.8 & 4.7 & 5.3 & 7.3 & 7.3 & 7.3 \\
\hline 66.5 & 63.6 & 56.2 & 48.9 & 43.5 & 41.2 & 44.5 & 44.9 & 41.9 \\
\hline 66.8 & 64.3 & 55.7 & 49.8 & 44.7 & 43.2 & 46.4 & 46.4 & 38 \\
\hline 65.5 & 63 & 52.1 & 41.5 & 38 & 36 & 37.5 & 34.6 & 35.9 \\
\hline 67.5 & 62.7 & 53 & 38.7 & 31.7 & 31.6 & 32.5 & 31.9 & 30.7 \\
\hline 65.8 & 61.6 & 53.4 & 35.4 & 32.6 & 32.7 & 27.9 & 27.4 & 26.6 \\
\hline 5.8 & 6.3 & 5.8 & 6.3 & 5.8 & 6.8 & 8.3 & 8.3 & 8.9 \\
\hline 5.7 & 6.4 & 5.7 & 5.7 & 6.4 & 6.2 & 8.2 & 8.9 & 8.9 \\
\hline 65.3 & 61.4 & 53.5 & 38.6 & 31.7 & 29.3 & 31.4 & 33.2 & 34 \\
\hline 65.8 & 65.6 & 54 & 41.6 & 33.6 & 34 & 36 & 37.2 & 33.5 \\
\hline 65.2 & 66.1 & 55.9 & 43.7 & 35.4 & 34.3 & 36.5 & 37 & 37.8 \\
\hline 67.5 & 65.1 & 51.9 & 38.2 & 40.3 & 43.5 & 46.7 & 48 & 48.6 \\
\hline 61.6 & 58.3 & 53.7 & 40.8 & 34.6 & 35.6 & 38.5 & 40.3 & 40.9 \\
\hline 5.7 & 5.7 & 5.6 & 5.7 & 6.3 & 6.6 & 9.3 & 8.2 & 8.2 \\
\hline 5.7 & 5.1 & 5.6 & 5.7 & 5.1 & 6 & 7.6 & 8.2 & 8.2 \\
\hline 65.4 & 55 & 41.5 & 39.5 & 35.1 & 35.9 & 37.8 & 39.1 & 33.1 \\
\hline 61.2 & 53.9 & & 26.5 & 37.2 & 32.9 & 33.3 & 36.1 & 37.3 \\
\hline 65.3 & 61 & 52.1 & 40.4 & 36.6 & 33.5 & 34 & 37.3 & 39.2 \\
\hline 63 & 62.5 & 50.9 & 40.7 & 32.3 & 33.1 & 38.2 & 39.7 & 38 \\
\hline 61.9 & 59.2 & 50.6 & 40.9 & 39 & 35.8 & 34.9 & 36.1 & 33.7 \\
\hline 7.1 & 6.6 & 7.1 & 7.2 & 7.1 & 7.2 & 7.4 & 9 & 9.7 \\
\hline 7.1 & 7.1 & 7.2 & 7.1 & 7.6 & 9.6 & 9.8 & 10.4 & 11.1 \\
\hline 67.1 & 62.4 & 52.1 & 41.5 & 37 & 35.5 & 35.2 & 38.3 & 35.4 \\
\hline 65.6 & 63.2 & 51.1 & 41.1 & 34.5 & 31.7 & 31.8 & 35.6 & 36.8 \\
\hline 61.2 & 63.3 & 51 & 38 & 37.3 & 31.9 & 32.8 & 35.8 & 37.1 \\
\hline 60.3 & 59.5 & 48.3 & 32 & 38 & 35.8 & 33.1 & 35 & 34.4 \\
\hline 4 & 4.6 & 4.6 & 4 & 4.6 & 4.1 & 4.7 & 6 & 7.1 \\
\hline
\end{tabular}




\begin{tabular}{|c|c|c|c|c|c|c|c|c|}
\hline 4 & 4.6 & 4.6 & 4 & 4.6 & 4 & 4.8 & 5.9 & 7.2 \\
\hline 4 & 4.6 & 4.7 & 4 & 4.7 & 4.6 & 4.1 & 6.6 & 7.2 \\
\hline 67.3 & 62.8 & 55.4 & 45.5 & 44.9 & 44 & 46.6 & 50.8 & 47.5 \\
\hline 65.4 & 61 & 54.4 & 44.5 & 41.8 & 39.5 & 38.8 & 38.6 & 38.8 \\
\hline 61.1 & 62.2 & 54.5 & 46.8 & 45.2 & 41.8 & 40.5 & 41.5 & 38.5 \\
\hline 62.3 & 60.5 & 48.6 & 40.3 & 45.5 & 40.1 & 30.8 & 32.5 & 34 \\
\hline 60.5 & 60.4 & 51.9 & 42.1 & 36.7 & 34.8 & 35 & 37.7 & 38.6 \\
\hline 22.3 & 12.8 & 4.9 & 5.4 & 5.4 & 6.6 & 4.9 & 7.3 & 8 \\
\hline 13.7 & 11.5 & 7.3 & 4.8 & 5.4 & 4.8 & 5.4 & 6.8 & 7.9 \\
\hline 65.4 & 61.7 & 46.7 & 39.7 & 33.3 & 31.1 & 27.7 & 28.4 & 28.9 \\
\hline 65.6 & 61 & 52.6 & 46.5 & 40.6 & 39.6 & 39.9 & 40.6 & 38.1 \\
\hline 67.1 & 63.1 & 56.9 & 46.9 & 46.4 & 48.8 & 48.1 & 48.7 & 48.3 \\
\hline 66.7 & 65.5 & 52.7 & 43.3 & 45.5 & 41.9 & 41.1 & 38.3 & 37.2 \\
\hline 60.8 & 60.2 & 50 & 36.1 & 39.4 & 42.5 & 40 & 36.7 & 36.9 \\
\hline 12.8 & 12.1 & 8.4 & 5 & 5.6 & 5 & 5 & 7.4 & 7.5 \\
\hline 18.4 & 12.5 & 5.5 & 5.4 & 4.8 & 4.8 & 5.4 & 6.7 & 7.9 \\
\hline 62 & 61.5 & 48.5 & 38.5 & 34.5 & 33.3 & 32.7 & 33.9 & 33.3 \\
\hline 60.6 & 61.6 & 51.2 & 37.6 & 37.9 & 37.4 & 37.7 & 38.8 & 40.2 \\
\hline 62.5 & 62.1 & 51.4 & 34.8 & 29.6 & 27.8 & 27.2 & 29 & 30.4 \\
\hline 55 & 55.6 & 53.4 & 42.6 & 37.6 & 34.9 & 34.6 & 32.9 & 31.2 \\
\hline 57.1 & 60.8 & 45.8 & 36.8 & 29.9 & 27.6 & 24.7 & 26.8 & 26.4 \\
\hline 4 & 4.5 & 3.9 & 4.6 & 4.5 & 3.9 & 4.6 & 5.7 & 7.1 \\
\hline 4.6 & 4.5 & 3.9 & 4.6 & 3.9 & 4.5 & 4 & 5.7 & 7 \\
\hline 62.6 & 63.1 & 50.3 & 39.1 & 41.7 & 40.2 & 38.9 & 36.5 & 28.9 \\
\hline 59.9 & 58.3 & 52.7 & 38.1 & 35.3 & 34.5 & 36 & 39.2 & 40.7 \\
\hline 62.5 & 61.4 & 54.7 & 41.3 & 37.3 & 36 & 36.6 & 34.7 & 36.2 \\
\hline 64.3 & 62.9 & 52.4 & 38.4 & 38.4 & 38.2 & 36.1 & 37.7 & 37.3 \\
\hline 44.5 & 54.8 & 50.4 & 39.1 & 46.4 & 47.6 & 47.7 & 49.5 & 50.5 \\
\hline 38.4 & 20 & 3.9 & 4 & 3.9 & 4 & 3.9 & 5.6 & 6.5 \\
\hline 6.4 & 6.9 & 5.8 & 5.1 & 5.2 & 4.5 & 5.2 & 6.2 & 7.6 \\
\hline 64.1 & 63.6 & 52.6 & 45.4 & 43.5 & 42.3 & 45.9 & 47 & 44.9 \\
\hline 63.6 & 62 & 52.5 & 45 & 40.6 & 38.5 & 38.4 & 40 & 38.5 \\
\hline 63.8 & 63.5 & 57.8 & 50.3 & 44.4 & 40.5 & 40.8 & 41.5 & 42.3 \\
\hline 64.2 & 61.5 & 56.2 & 46.2 & 44.8 & 44.1 & 42.3 & 43.9 & 44.9 \\
\hline 53 & 53.5 & 50.4 & 43 & 38.3 & 34.9 & 35.2 & 35.6 & 29.9 \\
\hline 6.4 & 6.4 & 6.3 & 6.4 & 6.4 & 6.3 & 6.4 & 8 & 8.9 \\
\hline 6.3 & 6.3 & 6.4 & 6.3 & 6.3 & 6.4 & 6.3 & 7.9 & 8.9 \\
\hline 63.6 & 61.6 & 53.8 & 50.6 & 44.2 & 41.7 & 41.8 & 42 & 43 \\
\hline 63.4 & 63.5 & 54.2 & 41.5 & 40 & 36.9 & 35.7 & 37.1 & 38.2 \\
\hline 64.7 & 63.5 & 52.7 & 40.5 & 42.8 & 37.5 & 31 & 31 & 33.2 \\
\hline 65 & 63.6 & 52.5 & 35.3 & 34.5 & 34.7 & 32.9 & 33.5 & 34.1 \\
\hline 62.3 & 60 & 52.5 & 44.5 & 41.9 & 36.8 & 36.5 & 36.6 & 36.1 \\
\hline 6.2 & 5.6 & 6.2 & 5.6 & 6.2 & 5.6 & 6.2 & 6.4 & 8.8 \\
\hline 6.3 & 5.6 & 6.2 & 5.5 & 5.6 & 6.2 & 5.6 & 7 & 8.7 \\
\hline 61.6 & 60.5 & 53.7 & 42.7 & 41.5 & 36.5 & 34.9 & 36 & 39.3 \\
\hline 61.1 & 55.7 & 52 & 44.2 & 46.4 & 45.7 & 45.6 & 45.1 & 47.5 \\
\hline 55.5 & 55.5 & 43.5 & 34.8 & 35.6 & 35.6 & 35.1 & 35.2 & 36.9 \\
\hline 34 & 31.4 & 29.7 & 16.2 & 14.8 & 14.8 & 15.3 & 15 & 16.9 \\
\hline 43.3 & 38.9 & 28.8 & 11.9 & 11.3 & 11.8 & 11.4 & 11.5 & 14.1 \\
\hline 12.2 & 12.9 & 12.9 & 12.9 & 11.9 & 12.6 & 13 & 13 & 15.4 \\
\hline 12.9 & 12.2 & 12.9 & 12.8 & 12.8 & 12.2 & 12.7 & 13.4 & 14.6 \\
\hline
\end{tabular}


lights

\begin{tabular}{|c|c|c|c|c|c|c|c|c|}
\hline 30.2 & 27.9 & 23.9 & 18.5 & 13.3 & 12.9 & 13.4 & 13.4 & 15.9 \\
\hline 34.5 & 35.9 & 30.9 & 16.1 & 14.7 & 14.7 & 15.3 & 15.3 & 17.7 \\
\hline 38 & 39.6 & 31 & 16.6 & 14.8 & 14.8 & 14.4 & 15.9 & 17.3 \\
\hline 30.5 & 34.3 & 27.9 & 14.8 & 13.6 & 13.7 & 13.5 & 14.9 & 16.8 \\
\hline 29.9 & 33.8 & 27.4 & 14.3 & 15.3 & 14.9 & 14.9 & 15.9 & 16.9 \\
\hline 16 & 15.4 & 15.5 & 15 & 15 & 15.5 & 15.4 & 16 & 17.5 \\
\hline 14.9 & 15.4 & 15.3 & 15.3 & 14.9 & 15.2 & 15.3 & 16 & 17.9 \\
\hline 28.1 & 28 & 29.7 & 12.5 & 12.9 & 12.3 & 12.3 & 13.6 & 14.5 \\
\hline 30.4 & 30.8 & 21.8 & 15.9 & 13.1 & 12.3 & 12.9 & 13.5 & 14.3 \\
\hline 28.8 & 28 & 23.1 & 14.1 & 14.1 & 13.9 & 13.4 & 14.1 & 15.5 \\
\hline 25.4 & 23.3 & 20.8 & 12.3 & 11.4 & 12 & 11.3 & 12.1 & 13.5 \\
\hline 25.3 & 24.8 & 20.5 & 12.3 & 11.2 & 11.9 & 11.7 & 12 & 13.8 \\
\hline 11.6 & 11.5 & 12.1 & 11.4 & 12.1 & 12 & 11.9 & 12 & 14.3 \\
\hline 12 & 11.9 & 11.5 & 11.8 & 12.7 & 12 & 12.2 & 12.4 & 14 \\
\hline 28.5 & 30.3 & 22.9 & 12 & 12.4 & 11.7 & 12.4 & 12.3 & 14.8 \\
\hline 23.4 & 24.2 & 22.9 & 11.8 & 10.9 & 11.1 & 11.1 & 11.7 & 13.6 \\
\hline 33.4 & 39.9 & 23.3 & 11.2 & 10.6 & 11.2 & 10.5 & 11.3 & 13.1 \\
\hline 32.3 & 32.8 & 20.9 & 11.8 & 11.4 & 11.4 & 10.8 & 11.9 & 13.8 \\
\hline 25.9 & 27.4 & 12.5 & 4.6 & 4 & 4 & 4.6 & 4.5 & 7.1 \\
\hline 4 & 4.6 & 4 & 4.6 & 4 & 4 & 4.6 & 5.2 & 6.5 \\
\hline 4.6 & 4.6 & 4 & 4.6 & 4.1 & 4.6 & 4.6 & 4.6 & 7.1 \\
\hline 31.7 & 31.6 & 26.5 & 13.8 & 13 & 13 & 13.1 & 13.6 & 15.6 \\
\hline 38.4 & 37.5 & 36.3 & 23.3 & 15.4 & 14.9 & 14.4 & 15.9 & 17.9 \\
\hline 28 & 25.5 & 19.7 & 11.3 & 11.3 & 11.9 & 11.4 & 12.3 & 13.4 \\
\hline 26.9 & 27.4 & 23.1 & 12.8 & 11.3 & 11.3 & 12 & 11.7 & 13.8 \\
\hline 11.4 & 12 & 11.2 & 11.9 & 11.3 & 11.4 & 11.3 & 12.4 & 13.8 \\
\hline 11.4 & 11.2 & 12 & 11.2 & 11.3 & 11.3 & 11.9 & 11.7 & 13.4 \\
\hline 11.4 & 11.3 & 12 & 11.3 & 11.4 & 11.9 & 10.9 & 11.7 & 13.8 \\
\hline 18.4 & 16.1 & 7.7 & 5.4 & 5.5 & 5.4 & 4.8 & 5.9 & 8 \\
\hline 23.3 & 23.5 & 15.3 & 6.8 & 6.8 & 6.8 & 6.8 & 7.9 & 9.3 \\
\hline 23 & 23.5 & 12.9 & 6.4 & 6.4 & 5.7 & 6.4 & 6.9 & 8.8 \\
\hline 19.9 & 21.2 & 11.6 & 5.2 & 5.7 & 5.8 & 5.8 & 5.6 & 8.3 \\
\hline 22.2 & 22.8 & 15.6 & 6.6 & 6.1 & 6.6 & 6.6 & 6.5 & 9.1 \\
\hline 6 & 6.6 & 6.6 & 6.1 & 6.6 & 6.7 & 6 & 7.1 & 9.2 \\
\hline 6.6 & 6.7 & 6 & 6.7 & 6.6 & 6 & 6.6 & 7.2 & 8.5 \\
\hline 25.6 & 27.2 & 15.6 & 6.8 & 6.8 & 6.7 & 6.8 & 7.9 & 9.3 \\
\hline 23.6 & 22.5 & 14.2 & 6.8 & 6.7 & 6.2 & 6.8 & 7.4 & 8.7 \\
\hline 25.3 & 24.9 & 15 & 5.8 & 6.4 & 6.3 & 5.8 & 6.9 & 8.9 \\
\hline 24.6 & 23.8 & 13.5 & 5.6 & 5.6 & 5.5 & 5.6 & 6.1 & 8.2 \\
\hline 27.3 & 26.6 & 14.4 & 6.2 & 5.6 & 6.2 & 6.1 & 6.8 & 8 \\
\hline 6.2 & 6.2 & 6.2 & 5.6 & 6.1 & 6.2 & 6.2 & 6.8 & 8.1 \\
\hline 5.6 & 6.2 & 6.1 & 5.6 & 6.2 & 6.2 & 5.5 & 6.8 & 8.7 \\
\hline 24.5 & 24 & 15.3 & 6.8 & 6.8 & 7.3 & 6.8 & 7.9 & 9.3 \\
\hline 27.3 & 27.6 & 11.5 & 5.8 & 5.2 & 5.8 & 5.2 & 6.4 & 7.7 \\
\hline 25.1 & 25.2 & 11.5 & 5.2 & 4.5 & 5.2 & 4.6 & 5.7 & 7.7 \\
\hline 25.4 & 26.7 & 17.4 & 7.9 & 8 & 7.4 & 7.9 & 8.5 & 10.5 \\
\hline 26.1 & 24.7 & 14.4 & 7.6 & 6.9 & 7.5 & 7.6 & 8.1 & 10.1 \\
\hline 7.5 & 7 & 7.5 & 7.6 & 7.5 & 7.5 & 7 & 8.1 & 10 \\
\hline 7.6 & 7.5 & 7.6 & 7.5 & 6.9 & 7.6 & 7.5 & 8.2 & 10.1 \\
\hline 29.7 & 29.8 & 17.2 & 5.1 & 5.2 & 5.2 & 5.1 & 6.5 & 7.7 \\
\hline 30.1 & 22.5 & 13 & 6 & 6.7 & 6 & 6.7 & 6.7 & 9.2 \\
\hline
\end{tabular}


lights

\begin{tabular}{|c|c|c|c|c|c|c|c|c|}
\hline 21.5 & 20.9 & 13.5 & 8.1 & 8.1 & 8.1 & 8.8 & 8.8 & 10.7 \\
\hline 33.7 & 31.2 & 14.2 & 5.2 & 5.7 & 5.8 & 5.8 & 5.9 & 8.3 \\
\hline 28.8 & 25.9 & 14.1 & 4.2 & 4.2 & 4.7 & 4.2 & 5.5 & 6.7 \\
\hline 4.2 & 4.8 & 4.1 & 4.2 & 4.8 & 4.2 & 4.7 & 4.9 & 7.3 \\
\hline 4.1 & 4.8 & 4.2 & 4.1 & 4.8 & 4.2 & 4.7 & 4.9 & 7.3 \\
\hline 25.6 & 25 & 18.3 & 10.6 & 4 & 4.6 & 4 & 5.3 & 7.1 \\
\hline 24.9 & 23 & 14.5 & 5.8 & 4.6 & 4.6 & 4.6 & 5.9 & 7.1 \\
\hline 26.4 & 25.6 & 14.4 & 5.8 & 4.7 & 5.2 & 4.6 & 6 & 7.2 \\
\hline 25.7 & 24.2 & 12 & 7.5 & 7 & 5.7 & 5.2 & 6.5 & 8.3 \\
\hline 33.4 & 31.5 & 17 & 6.8 & 5 & 5.6 & 4.9 & 6.4 & 8.1 \\
\hline 6.2 & 6.1 & 6.2 & 6.2 & 6.1 & 6.2 & 6.8 & 7 & 8.7 \\
\hline 6.1 & 6.2 & 6.2 & 6.2 & 6.1 & 6.8 & 5 & 7 & 8.1 \\
\hline 30.6 & 31.3 & 20.9 & 12 & 12.1 & 11.6 & 11.6 & 13 & 14.7 \\
\hline 37.1 & 38.6 & 24.2 & 5.9 & 5.4 & 5.9 & 5.3 & 6.9 & 8.4 \\
\hline 40.3 & 40.3 & 28.6 & 10.5 & 6.2 & 6.2 & 6.2 & 7.7 & 8.7 \\
\hline 39.2 & 38.7 & 31.4 & 29.3 & 10.8 & 4.2 & 4.8 & 5.1 & 7.3 \\
\hline 29.4 & 30 & 23.4 & 10.3 & 6.7 & 7.2 & 7.3 & 8.3 & 9.8 \\
\hline 16.1 & 16.1 & 16.6 & 16.1 & 16.7 & 17.3 & 16.6 & 18.7 & 20.6 \\
\hline 6 & 6.1 & 6 & 6 & 6.1 & 6 & 6 & 7.6 & 8.6 \\
\hline 43.5 & 46.7 & 32.5 & 10.2 & 7.9 & 7.2 & 7.2 & 8.8 & 9.8 \\
\hline 42.1 & 44.7 & 35.8 & 21.3 & 8.3 & 8.3 & 8.2 & 9.3 & 10.8 \\
\hline 41.3 & 40.6 & 28.2 & 10.5 & 4.4 & 5.1 & 4.4 & 6.1 & 7.6 \\
\hline 59.5 & 60.9 & 58.9 & 30.6 & 5.9 & 5.2 & 5.2 & 7 & 8.4 \\
\hline 66.1 & 49.2 & 40.3 & 19.8 & 6.5 & 5.2 & 5.8 & 7.5 & 8.4 \\
\hline 26.6 & 24.9 & 12.3 & 10.4 & 9.9 & 9.4 & 6.4 & 8.1 & 9.4 \\
\hline 6.4 & 7 & 6.4 & 6.9 & 6.4 & 7 & 6.3 & 8.7 & 8.2 \\
\hline 66.2 & 61.3 & 56 & 41.6 & 37.7 & 32.3 & 31.7 & 33.5 & 34.4 \\
\hline 64.2 & 63.3 & 49.9 & 33.4 & 25.3 & 23.9 & 22.1 & 23.3 & 24.7 \\
\hline 64.1 & 61 & 50.3 & 37 & 30.7 & 28.2 & 26.2 & 26.9 & 28.3 \\
\hline 62.3 & 59.7 & 49 & 33.1 & 31.8 & 28.7 & 27.4 & 28.5 & 29.6 \\
\hline 59.4 & 58.9 & 53.3 & 46.2 & 40.1 & 37.5 & 37.7 & 38.8 & 39.5 \\
\hline 11.4 & 11.5 & 11.9 & 12.1 & 11.3 & 12.1 & 11 & 13 & 14 \\
\hline 10.8 & 11.2 & 10.4 & 11.4 & 10.8 & 11.4 & 10.9 & 13.3 & 13.7 \\
\hline 64.3 & 62.3 & 53.9 & 43.7 & 32.5 & 31 & 30.6 & 32.2 & 33.6 \\
\hline 65.1 & 65.2 & 53.3 & 48.8 & 39.3 & 34.5 & 34 & 35 & 35.6 \\
\hline 64.3 & 62.9 & 56.2 & 44 & 34.7 & 33.5 & 33.2 & 35.1 & 36.9 \\
\hline 63.5 & 61.4 & 50.7 & 40.6 & 38.3 & 33.8 & 32.6 & 33.1 & 32.6 \\
\hline 63 & 61 & 53.3 & 38.9 & 32.4 & 30.7 & 29.3 & 30.2 & 31.3 \\
\hline 5.1 & 5.1 & 5 & 5.1 & 5.7 & 5.1 & 5.2 & 7.6 & 7.6 \\
\hline 5.1 & 5.1 & 5.7 & 5.1 & 5 & 5.1 & 5.9 & 7 & 8.2 \\
\hline 5.2 & 5.1 & 5.6 & 5.1 & 5.1 & 5.1 & 5.9 & 7 & 8.2 \\
\hline 61.8 & 62.6 & 54.6 & 39.2 & 38.7 & 30.7 & 31.7 & 32.2 & 34 \\
\hline 66.2 & 63.9 & 55.5 & 37.9 & 31.3 & 29.9 & 30.2 & 32.9 & 32.9 \\
\hline 63.8 & 64.7 & 49.8 & 37.2 & 33 & 33.7 & 33.5 & 42.1 & 38 \\
\hline 62.4 & 60.9 & 50.7 & 39.4 & 36.1 & 32.7 & 33.2 & 30.5 & 29.9 \\
\hline 7.1 & 7.1 & 6.5 & 4.7 & 4.7 & 5.3 & 5.1 & 7.2 & 7.2 \\
\hline 4.8 & 5.3 & 4.7 & 4.7 & 4.8 & 5.3 & 5 & 7.3 & 7.2 \\
\hline 61.6 & 60.5 & 50 & 41 & 39.8 & 38.5 & 36.5 & 34.2 & 34.3 \\
\hline 61.6 & 61.8 & 52.8 & 42.2 & 40.5 & 36.8 & 36.8 & 38.9 & 38.4 \\
\hline 63.1 & 62.5 & 52.2 & 41.7 & 37.3 & 34 & 33.5 & 33.2 & 32.8 \\
\hline 62.3 & 60.8 & 50.7 & 37.1 & 33.1 & 37.9 & 50.1 & 38.8 & 28.3 \\
\hline
\end{tabular}


lights

\begin{tabular}{|c|c|c|c|c|c|c|c|c|}
\hline 60.2 & 57.5 & 49.4 & 37 & 28.6 & 33 & 35 & 36.1 & 36.6 \\
\hline 7.5 & 7.5 & 8.1 & 8 & 7.5 & 7.5 & 8 & 10.6 & 10 \\
\hline 8.1 & 7.4 & 7.5 & 8.1 & 7.5 & 8.1 & 8 & 10 & 10.6 \\
\hline 60.3 & 58.4 & 50.3 & 35.9 & 29.6 & 27.4 & 27.3 & 31.4 & 32.7 \\
\hline 61.8 & 62.6 & 56.2 & 46 & 46 & 49.4 & 39.7 & 42.1 & 39.4 \\
\hline 61.1 & 58.2 & 49.3 & 37.7 & 30.1 & 35.1 & 32.2 & 32.9 & 33 \\
\hline 61.5 & 62.7 & 51.2 & 36 & 30.2 & 32.1 & 36.4 & 36.9 & 37.7 \\
\hline 59.8 & 55.5 & 53.6 & 38 & 36.4 & 37.6 & 39.6 & 40.2 & 28.7 \\
\hline 7.9 & 7.3 & 7.9 & 7.9 & 7.4 & 7.9 & 8.6 & 10.5 & 10.5 \\
\hline 7.3 & 7.9 & 7.9 & 8 & 7.9 & 7.4 & 8.7 & 10.4 & 10.4 \\
\hline 62.6 & 60.8 & 49.5 & 39.1 & 41.2 & 37.8 & 37.2 & 39.8 & 39.6 \\
\hline 63.1 & 60.9 & 49.6 & 38.6 & 34.9 & 34.9 & 36.4 & 38.1 & 38.2 \\
\hline 62.5 & 61.8 & 51.4 & 37.4 & 31.4 & 36.6 & 34.8 & 28.8 & 29.9 \\
\hline 64 & 62 & 51.9 & 38.2 & 36.3 & 40.8 & 41.1 & 42.7 & 39.6 \\
\hline 60.6 & 57.5 & 49.5 & 40.5 & 37.2 & 40.7 & 46.3 & 48.6 & 48.6 \\
\hline 6.2 & 5.7 & 6.2 & 6.2 & 6.3 & 5.6 & 7.1 & 8.2 & 8.1 \\
\hline 5.6 & 5.7 & 5.6 & 5.6 & 5.7 & 5.6 & 6.6 & 8.7 & 8.2 \\
\hline 60.8 & 62.2 & 51.8 & 39.4 & 39.2 & 38.1 & 37.8 & 38.6 & 39.8 \\
\hline 64.2 & 61.4 & 47.2 & 39 & 35.5 & 33.1 & 35.3 & 35.1 & 34 \\
\hline 63.2 & 60.8 & 48.2 & 38.8 & 35.1 & 34.5 & 36.1 & 37.5 & 36.9 \\
\hline 56.4 & 59.1 & 49.1 & 36.6 & 41.6 & 36.7 & 35.6 & 34.7 & 34.1 \\
\hline 61.7 & 60.1 & 55.5 & 42.7 & 38.9 & 38.2 & 35.6 & 34.1 & 35.3 \\
\hline 5.8 & 5.8 & 5.9 & 5.8 & 6.4 & 5.9 & 7.5 & 8.4 & 8.9 \\
\hline 5.8 & 5.9 & 6.4 & 5.9 & 5.8 & 5.8 & 7.6 & 9 & 8.4 \\
\hline 62.8 & 60.9 & 53.6 & 46.3 & 43.1 & 40.1 & 39.8 & 37.3 & 34.7 \\
\hline 63.2 & 64 & 50.5 & 41.4 & 42.4 & 38.6 & 40.2 & 42.7 & 43.2 \\
\hline 58.2 & 59.5 & 53.4 & 37.4 & 31.9 & 32.2 & 39.5 & 40.3 & 33 \\
\hline 61.6 & 63.5 & 54.2 & 37.6 & 38.3 & 39.1 & 38.9 & 38.1 & 33.4 \\
\hline 60.8 & 59.1 & 49.3 & 40 & 35 & 39.1 & 43.8 & 38.6 & 39.2 \\
\hline 6.9 & 7.6 & 7 & 6.9 & 7.6 & 7 & 9.5 & 9.4 & 10.1 \\
\hline 6.9 & 7.6 & 6.9 & 7.6 & 6.9 & 7 & 9.5 & 9.5 & 10 \\
\hline 61.7 & 60.8 & 48.5 & 31.8 & 34 & 34.1 & 43.7 & 43.5 & 37 \\
\hline 63.8 & 62.5 & 49.6 & 34.6 & 34.8 & 36 & 43.2 & 44.4 & 45 \\
\hline 61.9 & 62 & 57 & 39 & 33 & 32.6 & 40.4 & 40.4 & 41 \\
\hline 64.2 & 60.7 & 51 & 39.1 & 35.6 & 31.5 & 35.9 & 40 & 36.5 \\
\hline 60.1 & 58.3 & 50.8 & 35.2 & 35.9 & 39.4 & 40.7 & 48.3 & 49.1 \\
\hline 14.4 & 13.5 & 12.6 & 13.9 & 13.1 & 13.7 & 15.2 & 16.2 & 15.6 \\
\hline 13.7 & 13.6 & 13.1 & 13.9 & 13 & 13.5 & 15.6 & 16.2 & 16.1 \\
\hline 60.8 & 61.5 & 45.1 & 33.1 & 33.7 & 43.9 & 44 & 44 & 44.4 \\
\hline 61.3 & 62.1 & 48.7 & 38.4 & 33.4 & 40.5 & 41.2 & 41.7 & 40.1 \\
\hline 63.3 & 61.9 & 49.5 & 35.6 & 47.9 & 48.4 & 48.3 & 48.9 & 48.2 \\
\hline 55.9 & 58.1 & 51.3 & 39.2 & 38.3 & 45.2 & 48.4 & 47.8 & 44 \\
\hline 39.9 & 40 & 42.9 & 37.7 & 37.8 & 39.2 & 38.6 & 37.7 & 35.4 \\
\hline 13.3 & 12.4 & 12.3 & 13.3 & 13.1 & 15.3 & 15.2 & 15.2 & 14.9 \\
\hline 12.6 & 12.2 & 13.4 & 12.7 & 13.3 & 15.7 & 15.2 & 15.9 & 15 \\
\hline 61.4 & 63.2 & 49.6 & 33.9 & 34 & 35.9 & 38.2 & 35.4 & 32.5 \\
\hline 63.2 & 63.6 & 53.2 & 39.3 & 38 & 35.9 & 36.1 & 29.7 & 29.4 \\
\hline 64.9 & 60.4 & 47.9 & 39.3 & 39.5 & 38.6 & 42.7 & 43.2 & 36.2 \\
\hline 59.6 & 62 & 47.4 & 35.4 & 28.6 & 31.6 & 33.4 & 36.5 & 36.1 \\
\hline 60.2 & 55.9 & 46.7 & 37.3 & 36.6 & 35.8 & 35.4 & 35.9 & 36.1 \\
\hline 7.6 & 7.6 & 7.6 & 7.6 & 8.2 & 10.1 & 10.1 & 10.2 & 10.1 \\
\hline
\end{tabular}


lights

\begin{tabular}{|c|c|c|c|c|c|c|c|c|}
\hline 7.6 & 8.2 & 7.6 & 8.1 & 8.3 & 10.7 & 10.1 & 10.7 & 10.1 \\
\hline 62.5 & 59.2 & 47.8 & 39.2 & 41.8 & 41.8 & 44.8 & 45.5 & 46.2 \\
\hline 64.3 & 63.8 & 52.3 & 35.8 & 35.1 & 39.8 & 39.8 & 40 & 38.8 \\
\hline 62.1 & 62.7 & 52.3 & 35.4 & 33 & 33 & 40.2 & 44.6 & 41.7 \\
\hline 64.2 & 63.9 & 56.9 & 47.2 & 53.6 & 53.3 & 50.6 & 49.9 & 46.8 \\
\hline 60.1 & 59.1 & 48.1 & 36.1 & 35.9 & 35.7 & 42.3 & 42.9 & 38.2 \\
\hline 5.9 & 6.5 & 5.9 & 6.6 & 6.7 & 8.4 & 9 & 8.4 & 9.1 \\
\hline 5.9 & 6.6 & 5.9 & 5.9 & 7.3 & 8.4 & 9.1 & 8.4 & 9.1 \\
\hline 56.1 & 57.8 & 52.2 & 40.8 & 45.1 & 43.9 & 44.3 & 43.1 & 41.5 \\
\hline 63.6 & 62.2 & 57.9 & 43 & 38.3 & 37 & 36.6 & 38.4 & 36.1 \\
\hline 65.9 & 60.9 & 48.6 & 35.5 & 32.3 & 40.7 & 40.2 & 39.6 & 34.2 \\
\hline 64.1 & 65.3 & 51.6 & 39 & 38.7 & 37.6 & 36.6 & 37.8 & 35.4 \\
\hline 62.1 & 56.7 & 50.7 & 40.9 & 40.6 & 42.2 & 42.4 & 43 & 43.7 \\
\hline 6.6 & 6.6 & 6 & 6.6 & 7.5 & 9.2 & 9.1 & 9.2 & 9.1 \\
\hline 6.6 & 6.6 & 6.6 & 6.6 & 7.5 & 9.2 & 8.5 & 9.1 & 9.2 \\
\hline 64 & 62.3 & 50.5 & 37 & 37.3 & 36.3 & 37.7 & 39.5 & 37.1 \\
\hline 66.5 & 63.3 & 52.3 & 38.7 & 35.7 & 41 & 43.2 & 42.8 & 43.4 \\
\hline 50.6 & 41.5 & 29.9 & 26.6 & 29.9 & 32.2 & 38.1 & 41.4 & 38.3 \\
\hline 6.2 & 6.2 & 5.6 & 6.2 & 7.1 & 8.2 & 8.7 & 8.8 & 8.8 \\
\hline 6.2 & 5.5 & 6.2 & 6.2 & 6.6 & 8.7 & 8.8 & 8.7 & 8.8 \\
\hline 6.2 & 5.6 & 6.2 & 5.5 & 7.2 & 8.7 & 8.8 & 8.7 & 8.1 \\
\hline 5.6 & 6.2 & 6.2 & 5.6 & 7.1 & 8.7 & 8.8 & 8.1 & 8.7 \\
\hline 64.2 & 62.8 & 49.5 & 34.3 & 33.4 & 37.7 & 44.6 & 44.6 & 44.6 \\
\hline 64 & 64.3 & 51.1 & 40.4 & 46.1 & 40.5 & 38.7 & 41.4 & 37.1 \\
\hline 65.8 & 65.1 & 53.4 & 38.7 & 36.3 & 35.3 & 42.4 & 42.5 & 42.6 \\
\hline 64.9 & 65 & 52.8 & 39.7 & 40.6 & 37.8 & 40.8 & 39.7 & 40.4 \\
\hline 62.6 & 65.2 & 51.9 & 39.6 & 39 & 42.8 & 39.9 & 40.3 & 40.2 \\
\hline 7.1 & 7.1 & 7.1 & 7.1 & 8.1 & 9.6 & 9.7 & 9.6 & 9.7 \\
\hline 7.1 & 6.5 & 7.1 & 7.1 & 8.1 & 9 & 9.5 & 9.6 & 9 \\
\hline 64.9 & 62.2 & 52.1 & 39.5 & 43.9 & 44.1 & 42 & 38.3 & 38.4 \\
\hline 64.3 & 60.7 & 46.3 & 37.6 & 45.4 & 44.7 & 44.5 & 45.7 & 45.8 \\
\hline 64.1 & 62 & 52.7 & 36 & 44.9 & 47 & 46.6 & 46.1 & 45.6 \\
\hline 60.2 & 59.3 & 52.4 & 46.9 & 46.3 & 43.3 & 40.1 & 42.2 & 40.8 \\
\hline 62 & 54.7 & 49 & 40.7 & 43.4 & 44.6 & 48.9 & 51.4 & 51.5 \\
\hline 13.8 & 14.6 & 14.8 & 14.7 & 15.7 & 16.8 & 17.2 & 17.3 & 16.7 \\
\hline 14.7 & 14.9 & 14.2 & 15.1 & 15.6 & 17.2 & 17.9 & 16.9 & 16.9 \\
\hline 65.8 & 66.4 & 48.6 & 35.2 & 50 & 51.5 & 50.4 & 50.5 & 50.6 \\
\hline 66.4 & 64.6 & 56.8 & 52.1 & 50.3 & 48.6 & 51.2 & 51.7 & 51 \\
\hline 66 & 65.8 & 52.1 & 34.7 & 35.5 & 37.5 & 42.1 & 42.7 & 42.8 \\
\hline 66.1 & 64.9 & 54.7 & 44.6 & 43.6 & 42.7 & 47.3 & 50.4 & 50.5 \\
\hline 66.8 & 53.2 & 42.4 & 41.9 & 44.3 & 47.1 & 49.9 & 52.1 & 52.6 \\
\hline 20.2 & 14.1 & 13.2 & 13.3 & 15 & 16 & 15.9 & 16.1 & 15.9 \\
\hline 13.6 & 14.1 & 13.4 & 13.1 & 14.9 & 16 & 15.9 & 16 & 16.6 \\
\hline 50.3 & 48.7 & 38.8 & 29.4 & 28.2 & 17 & 15.8 & 15.5 & 15.5 \\
\hline 39.8 & 20.5 & 7 & 7.1 & 7.9 & 9.6 & 8.9 & 9 & 8.9 \\
\hline 7 & 7 & 7.1 & 7 & 7.9 & 9.6 & 8.9 & 9 & 9 \\
\hline 7.5 & 6.8 & 6.9 & 6.9 & 7.7 & 9.4 & 8.8 & 8.8 & 9.4 \\
\hline 6.8 & 6.9 & 7.5 & 6.9 & 7.7 & 8.8 & 9.4 & 8.8 & 8.8 \\
\hline 6.8 & 6.9 & 6.9 & 7.4 & 7.8 & 8.8 & 9.4 & 8.8 & 8.8 \\
\hline 6.9 & 6.1 & 6.9 & 7.4 & 7.7 & 8.8 & 8.8 & 9.4 & 8.8 \\
\hline 6.9 & 7.4 & 6.9 & 6.8 & 7.7 & 8.8 & 9.4 & 8.8 & 8.8 \\
\hline
\end{tabular}


lights

\begin{tabular}{|c|c|c|c|c|c|c|c|c|}
\hline 6.8 & 6.9 & 6.8 & 6.9 & 7.6 & 8.7 & 8.8 & 9.4 & 8.8 \\
\hline 6.9 & 6.8 & 6.9 & 7.4 & 7.6 & 8.8 & 8.7 & 8.8 & 8.8 \\
\hline & & & & & & & & \\
\hline 6.9 & 6.8 & 6.9 & 6.8 & 8.2 & 8.8 & 8.8 & 8.9 & 9.4 \\
\hline 6.8 & 6.9 & 6.8 & 6.9 & 7.5 & 8.8 & 9.4 & 8.8 & 8.8 \\
\hline 6.9 & 6.8 & 6.9 & 7.4 & 7.6 & 8.8 & 8.7 & 8.8 & 9.4 \\
\hline 65 & 63 & 52.1 & 41.2 & 42.2 & 42.3 & 42.5 & 42.5 & 40.8 \\
\hline 64.7 & 63.6 & 53.7 & 42.6 & 40.4 & 39 & 38.6 & 40.3 & 42.7 \\
\hline 64.8 & 64.2 & 53.1 & 42.8 & 44.7 & 44.6 & 45.8 & 49.3 & 42.4 \\
\hline 63 & 63.1 & 51.1 & 36.4 & 36 & 38.8 & 46.2 & 45.4 & 46.1 \\
\hline 60.8 & 61.1 & 53.7 & 46 & 49.7 & 49.8 & 50.2 & 48.7 & 45.3 \\
\hline 14.5 & 14.9 & 14 & 15.2 & 15.1 & 16.3 & 16.8 & 16 & 17 \\
\hline 15.1 & 14.6 & 15.1 & 14.1 & 15.7 & 16.4 & 16.4 & 17.1 & 16.4 \\
\hline 63.8 & 65 & 51.5 & 35.1 & 37.3 & 42.3 & 42.2 & 43.1 & 41.5 \\
\hline 63.1 & 64.6 & 50.8 & 44 & 46.2 & 47.1 & 49 & 48.8 & 47.5 \\
\hline 63.7 & 64.4 & 52.8 & 34.2 & 35.4 & 35.8 & 35.8 & 37 & 39.6 \\
\hline 61.1 & 58.6 & 50.5 & 41.4 & 42.2 & 44.3 & 51.7 & 51 & 50.8 \\
\hline 66.9 & 66.4 & 38.1 & 8.3 & 6.1 & 8.1 & 7.4 & 8.1 & 8.1 \\
\hline 6.1 & 5.6 & 6.2 & 6.2 & 6.1 & 8.1 & 7.5 & 8 & 8.1 \\
\hline 5.6 & 6.1 & 6.2 & 5.6 & 6.6 & 7.5 & 8.1 & 8.2 & 7.5 \\
\hline 6.8 & 7.3 & 6.8 & 6.7 & 7.2 & 8.7 & 9.3 & 8.7 & 8.7 \\
\hline 64.5 & 66.4 & 55.3 & 39.3 & 38.6 & 38.8 & 35.1 & 35.9 & 30.5 \\
\hline 63.3 & 64.4 & 52.6 & 38 & 36.4 & 37.2 & 35.8 & 28.1 & 27.2 \\
\hline 62.9 & 63 & 52.7 & 45.6 & 44.4 & 42.7 & 41.3 & 39.9 & 44.6 \\
\hline 58.7 & 59.3 & 54.9 & 51.8 & 49.1 & 49.3 & 52.8 & 52.3 & 51 \\
\hline 13.7 & 15.3 & 19.8 & 16.8 & 14.2 & 15.6 & 15.7 & 15.2 & 15.5 \\
\hline 14 & 14.6 & 13.5 & 13.7 & 14.3 & 15.8 & 15.8 & 16.3 & 15.8 \\
\hline 63.4 & 62.1 & 51.5 & 37.9 & 38.7 & 42.9 & 43.4 & 42.2 & 38.1 \\
\hline 59.5 & 56.9 & 47.6 & 45.7 & 40.5 & 39.1 & 35.1 & 34.8 & 33 \\
\hline 64.2 & 62.4 & 48.6 & 35.6 & 32.4 & 37.8 & 37.3 & 37.3 & 37.9 \\
\hline 66 & 65 & 50 & 37.8 & 37.7 & 36.5 & 37.5 & 37.5 & 38.3 \\
\hline 62.4 & 63.6 & 55.5 & 43.2 & 40.3 & 38.1 & 36.1 & 35.9 & 31.8 \\
\hline 5.9 & 5.9 & 5.9 & 5.9 & 6.1 & 7.3 & 8.4 & 7.8 & 7.8 \\
\hline 5.9 & 5.9 & 5.9 & 5.9 & 6.1 & 7.2 & 7.8 & 7.8 & 7.8 \\
\hline 64.1 & 63.9 & 54.5 & 38.2 & 35.2 & 35.9 & 38.9 & 41.7 & 41 \\
\hline 64.7 & 61.7 & 53.7 & 44.9 & 43.3 & 46.3 & 52 & 52.5 & 46.1 \\
\hline 66.4 & 67.5 & 57 & 44.7 & 47.7 & 50 & 50.2 & 51.1 & 49.3 \\
\hline 62.3 & 62.7 & 56.8 & 50.3 & 51.8 & 49.6 & 54.4 & 55 & 56.2 \\
\hline 34.8 & 33 & 20.7 & 9.9 & 5.7 & 7.6 & 7.7 & 8.2 & 7.6 \\
\hline 6.3 & 5.6 & 6.3 & 5.7 & 5.7 & 7.6 & 7.6 & 8.2 & 7.5 \\
\hline 6.3 & 5.6 & 5.7 & 6.3 & 5.7 & 7.6 & 7.5 & 7.6 & 8.1 \\
\hline 63.5 & 62.8 & 52.1 & 44.8 & 41.3 & 46.3 & 49.9 & 47.9 & 45.5 \\
\hline 62.9 & 61.3 & 54.2 & 42.6 & 41.2 & 48.4 & 50.2 & 50.4 & 50 \\
\hline 61.2 & 61.3 & 54.3 & 48.3 & 47.7 & 46 & 46 & 45.3 & 38.9 \\
\hline 67 & 65.2 & 53.5 & 47.8 & 41.7 & 45.1 & 45.3 & 52.2 & 46.6 \\
\hline 64.5 & 66.1 & 59.4 & 50.3 & 50 & 49.5 & 52.1 & 52.8 & 48.9 \\
\hline 15.1 & 14.1 & 14.3 & 14.5 & 14.9 & 15.6 & 15.7 & 16.8 & 16.4 \\
\hline 15.6 & 13.9 & 14.9 & 15.1 & 14.3 & 15.7 & 16.7 & 16.9 & 16.3 \\
\hline 39.8 & 42.8 & 39 & 35.8 & 32.5 & 34.5 & 35.3 & 40.4 & 36.8 \\
\hline 67 & 62.7 & 52.9 & 45 & 49.3 & 47.3 & 45 & 44.6 & 42.4 \\
\hline 61.8 & 56.5 & 55 & 36.4 & 33.3 & 34.3 & 37.1 & 41.8 & 37.4 \\
\hline
\end{tabular}


lights

\begin{tabular}{|c|c|c|c|c|c|c|c|c|}
\hline 64.6 & 63.3 & 55.2 & 46.8 & 43 & 41.8 & 42.5 & 43.7 & 41.5 \\
\hline 61.6 & 62.7 & 53 & 44.7 & 46.2 & 45.7 & 44.2 & 43.2 & 38.4 \\
\hline 15.2 & 16.8 & 14.3 & 13.8 & 13.4 & 14.2 & 15.6 & 15.6 & 16.2 \\
\hline 13.7 & 13.7 & 13.8 & 13.3 & 13.4 & 14.7 & 15.5 & 15.2 & 15.3 \\
\hline 63.7 & 61.5 & 54.4 & 38.5 & 41.8 & 40.5 & 40.9 & 34.3 & 35.6 \\
\hline 64.5 & 57.6 & 53.5 & 48.2 & 52.9 & 51.8 & 52.9 & 53.5 & 46.2 \\
\hline 66.1 & 63.7 & 54.4 & 49.7 & 52.4 & 50.5 & 48.1 & 49.2 & 49.7 \\
\hline 66.1 & 61.6 & 52.7 & 49.2 & 50 & 50.5 & 51.1 & 50.1 & 52 \\
\hline 53.4 & 57.2 & 54 & 47.2 & 47.6 & 51.4 & 51.2 & 51.3 & 52.7 \\
\hline 13.8 & 13.6 & 13.5 & 13.9 & 13.7 & 15.2 & 15.4 & 16.2 & 15.8 \\
\hline 13.7 & 12.4 & 11.8 & 11.9 & 12.5 & 12.9 & 14.5 & 13.9 & 14.2 \\
\hline 65.5 & 64.6 & 53.9 & 44.1 & 46.3 & 47.4 & 48.8 & 49.3 & 46 \\
\hline 63.7 & 64.1 & 55.2 & 44.8 & 40.5 & 41.2 & 41.4 & 41.8 & 48.6 \\
\hline 66.8 & 64.8 & 58.8 & 55.3 & 53.5 & 50.3 & 49.9 & 47.3 & 47.6 \\
\hline 63.9 & 63.9 & 57.4 & 47.4 & 51.2 & 54.3 & 47 & 35.4 & 37 \\
\hline 63.2 & 62.6 & 55 & 49.4 & 43 & 42.8 & 42.9 & 47.2 & 49.6 \\
\hline 14.6 & 14.7 & 14.3 & 14.2 & 13.8 & 14.1 & 14.8 & 15.4 & 15.5 \\
\hline 13 & 12.5 & 12.9 & 13.1 & 12.8 & 14 & 14.6 & 15.4 & 15.5 \\
\hline 62.1 & 59.7 & 49.6 & 36 & 45.2 & 44.9 & 44.3 & 43 & 39.8 \\
\hline 60.2 & 62 & 51.2 & 41.3 & 47.8 & 51.1 & 49.9 & 51 & 45.4 \\
\hline 60.6 & 61.2 & 51.4 & 42 & 40.9 & 45.8 & 51.5 & 52.4 & 52.4 \\
\hline 66.2 & 63.5 & 50.3 & 36.8 & 43.7 & 46.4 & 45.7 & 45.4 & 43 \\
\hline 63.6 & 52.1 & 50.4 & 51.7 & 54.8 & 52.4 & 52.1 & 50.3 & 42.8 \\
\hline 5.5 & 5.5 & 5.6 & 5.5 & 5.5 & 6.9 & 8.1 & 8.6 & 8.1 \\
\hline 5.6 & 6.1 & 5.5 & 5.6 & 5.5 & 6.8 & 8.1 & 8.1 & 8.1 \\
\hline 5.5 & 5.5 & 5.6 & 5.5 & 5.5 & 6.9 & 8.1 & 8.1 & 8.1 \\
\hline 7.3 & 6.7 & 6.7 & 6.8 & 5.5 & 7.4 & 8 & 8.7 & 8 \\
\hline 6.1 & 6.1 & 5.6 & 6.1 & 6.2 & 6.7 & 8.1 & 8.7 & 8.1 \\
\hline 6.1 & 5.6 & 6.1 & 6.1 & 5.5 & 6.8 & 8.7 & 8.7 & 8.1 \\
\hline 5.5 & 6.2 & 6.1 & 5.5 & 6.2 & 6.7 & 8.7 & 8.7 & 8.1 \\
\hline 39.8 & 22.7 & 7.1 & 5.5 & 4.8 & 6.1 & 7.4 & 7.4 & 8 \\
\hline 22.4 & 16.9 & 8.9 & 5.4 & 4.8 & 6 & 7.3 & 7.4 & 7.3 \\
\hline 64.6 & 63.3 & 54.7 & 51 & 53.4 & 49.3 & 50.4 & 51.5 & 45.7 \\
\hline 63.6 & 60.2 & 57 & 50.4 & 48.4 & 48.3 & 48.6 & 47.9 & 44.2 \\
\hline 62.8 & 58.9 & 52.1 & 41.9 & 48.4 & 52.7 & 54.7 & 54.2 & 53.6 \\
\hline 61 & 59.7 & 55.5 & 48.9 & 49.3 & 47.6 & 48.2 & 48.5 & 47.8 \\
\hline 60.8 & 55.8 & 54.4 & 49.9 & 46 & 44 & 44.6 & 44.7 & 42.9 \\
\hline 22.2 & 18.2 & 12.5 & 11.8 & 10.5 & 11.6 & 13.1 & 13.7 & 13.1 \\
\hline 10.7 & 10.4 & 11.3 & 10.5 & 10.6 & 11.7 & 12.9 & 13.7 & 13.1 \\
\hline 60.9 & 59.6 & 53.8 & 43.3 & 45.4 & 42.4 & 43.2 & 42.3 & 42.5 \\
\hline 51 & 50.5 & 50.1 & 47.3 & 47.5 & 49.3 & 49 & 49.6 & 48.7 \\
\hline 60.4 & 59.6 & & & 54.6 & 61.9 & 59.1 & 55.8 & 54.7 \\
\hline 63.6 & 58.8 & 51.9 & 52.4 & 48.2 & 45.4 & 46 & 46.6 & 46.9 \\
\hline 57.3 & 56.6 & 52 & 49.2 & 48.6 & 46.2 & 47.6 & 49.8 & 50.5 \\
\hline 10.6 & 11.2 & 10.6 & 10.7 & 10.5 & 11.2 & 12 & 12.5 & 13.6 \\
\hline 12.1 & 11.4 & 10.9 & 10.8 & 10.7 & 11 & 10.8 & 12.2 & 13.3 \\
\hline 62.1 & 62.9 & 48.9 & 38.2 & 39.4 & 45.9 & 45.9 & 47.2 & 46.8 \\
\hline 65.8 & 62 & 54.5 & 47.5 & 51 & 57.4 & 58.1 & 58.1 & 57.7 \\
\hline 65.6 & 60.5 & 53.4 & 50.3 & 45.6 & 48.2 & 46.8 & 46.8 & 44.5 \\
\hline 64 & 59.9 & 54.9 & 48.9 & 43.8 & 46.6 & 45.8 & 47.4 & 49.2 \\
\hline 22.9 & 21.9 & 22.9 & 28.5 & 33.6 & 40.5 & 39.9 & 38.2 & 36.7 \\
\hline
\end{tabular}


lights

\begin{tabular}{|c|c|c|c|c|c|c|c|c|}
\hline 10.5 & 11 & 10.5 & 10.5 & 10.4 & 10.4 & 11 & 11.8 & 12.9 \\
\hline 10.6 & 10.4 & 11 & 10.4 & 10.4 & 10.4 & 10.4 & 11.7 & 12.8 \\
\hline 58 & 55.2 & 50.4 & 44.7 & 46.7 & 45.2 & 45.3 & 45.3 & 45.7 \\
\hline 58.8 & 59.2 & 48.7 & 45.5 & 45.4 & 46.2 & 45.7 & 45.8 & 47.3 \\
\hline 64.2 & 57 & 50.7 & 48.6 & 45.6 & 44.7 & 44.6 & 46.5 & 46.7 \\
\hline 62.2 & 58.9 & 50.8 & 45.6 & 44.4 & 43.5 & 42.8 & 43.4 & 44.5 \\
\hline 53.8 & 55.9 & 53.5 & 51.5 & 49.4 & 44.2 & 44.3 & 44.9 & 47.3 \\
\hline 8.3 & 8.3 & 8.3 & 8.3 & 8.4 & 8.3 & 8.3 & 9.6 & 10.8 \\
\hline 8.3 & 8.3 & 8.3 & 8.2 & 8.3 & 8.2 & 8.3 & 9.4 & 10.8 \\
\hline 18.1 & 20 & 19.3 & 18.8 & 18.2 & 19.4 & 19.2 & 22.6 & 33.7 \\
\hline 59.8 & 57.8 & 50.8 & 44.8 & 48.8 & 44.6 & 42.5 & 43.1 & 44.8 \\
\hline 60.5 & 57.8 & 51.3 & 47.3 & 50.1 & 50.5 & 49.4 & 48.7 & 50 \\
\hline 56.6 & 53.1 & 50.6 & 48.9 & 39 & 35.2 & 35.4 & 35.7 & 40.9 \\
\hline 42.1 & 52.2 & 48.4 & 43.7 & 42.3 & 43.5 & 48.2 & 43.7 & 45.2 \\
\hline 8.3 & 8.3 & 7.7 & 8.3 & 8.2 & 7.7 & 8.3 & 8.8 & 10.8 \\
\hline 8.3 & 8.3 & 8.4 & 8.3 & 7.7 & 8.3 & 8.4 & 9.4 & 10.2 \\
\hline 59.2 & 56.8 & 51.5 & 47.9 & 46.5 & 45.7 & 42.4 & 44.1 & 43.3 \\
\hline 59.3 & 55 & 49.5 & 46.6 & 42.3 & 38.3 & 39 & 39.3 & 40.2 \\
\hline 59.2 & 59.8 & 53.7 & 46.6 & 47.4 & 43.4 & 41.2 & 40.3 & 43.8 \\
\hline 58.7 & 56.4 & 48.4 & 41.7 & 42.4 & 43.5 & 43.1 & 40.1 & 40.8 \\
\hline 55.2 & 51.3 & 44 & 44.3 & 47.6 & 45.2 & 45.1 & 43.2 & 41.1 \\
\hline 9.3 & 8.6 & 9.1 & 9.2 & 9.2 & 7.9 & 8 & 9 & 10.5 \\
\hline 8.1 & 8.1 & 8.7 & 8.1 & 8.1 & 8.1 & 8 & 9.1 & 10.6 \\
\hline 59.5 & 58.6 & 48.9 & 44.3 & 47.3 & 48.3 & 48.3 & 49.8 & 51.9 \\
\hline 58.1 & 55.1 & 47 & 46.8 & 48.7 & 46.8 & 46.3 & 45.6 & 50.6 \\
\hline 59.4 & 60.1 & 54.4 & 52.1 & 47.8 & 46.2 & 45.8 & 46 & 44.7 \\
\hline 61 & 60.7 & 54 & 46.1 & 49.4 & 46.3 & 45.8 & 46.6 & 48.5 \\
\hline 56.7 & 51.8 & 51.6 & 43.6 & 38.5 & 41.1 & 45.2 & 46.8 & 43.3 \\
\hline 14.2 & 13.6 & 14.2 & 13.5 & 13.6 & 14.2 & 13.6 & 14.5 & 16 \\
\hline 13.6 & 14.3 & 13.7 & 14.3 & 14.4 & 13.7 & 14.4 & 14.6 & 16.2 \\
\hline 62.5 & 60.5 & 54.9 & 53.2 & 50.8 & 46.9 & 46.2 & 46.5 & 47.4 \\
\hline 64.3 & 63.1 & 53.4 & 49 & 46 & 47.8 & 47.3 & 47.6 & 49.3 \\
\hline 60.2 & 60.6 & 56.8 & 51.8 & 45.3 & 41.2 & 40.3 & 40.9 & 42 \\
\hline 58.1 & 59.7 & 51.8 & 52.4 & 48.4 & 44.3 & 42.4 & 43.8 & 46.3 \\
\hline 51.5 & 53.5 & 55.5 & 51.8 & 48.7 & 45.7 & 47 & 46.6 & 48.7 \\
\hline 18.6 & 17.9 & 18 & 18 & 16.8 & 15.7 & 15.7 & 15.8 & 18.2 \\
\hline 16.7 & 16.8 & 16.3 & 16.8 & 16 & 16.8 & 16.1 & 17 & 19 \\
\hline 64 & 59.8 & 55.8 & 53.4 & 45.7 & 41.6 & 40.3 & 40.5 & 41.1 \\
\hline 63.4 & 60.2 & 52.3 & 51.2 & 50.6 & 46.4 & 46.5 & 46.6 & 46.9 \\
\hline 56 & 56.3 & 52.2 & 49 & 48.1 & 48.1 & 46.6 & 44.7 & 45.4 \\
\hline 58.2 & 59.9 & 59 & 47.5 & 43 & 37.5 & 36.2 & 35.7 & 38.1 \\
\hline 51.8 & 52.5 & 52.9 & 43.1 & 37.5 & 33.1 & 31.7 & 31.5 & 33.1 \\
\hline 16.6 & 16.5 & 16.7 & 13.9 & 12.8 & 12.6 & 12.7 & 12.8 & 15.1 \\
\hline 12 & 12.6 & 12.5 & 12.7 & 12.5 & 12.6 & 12.6 & 12.8 & 14.3 \\
\hline 60 & 58.2 & 51.3 & 52.7 & 49.8 & 43.4 & 42.7 & 42.9 & 44.7 \\
\hline 58.9 & 60 & 51.4 & 45.1 & 41.4 & 38.5 & 37.3 & 37.4 & 39.9 \\
\hline 60.2 & 57.5 & 55.2 & 51.4 & 49.1 & 46.1 & 43 & 42.4 & 44.8 \\
\hline 59 & 56 & 51.5 & 47.4 & 43.4 & 43.1 & 41.8 & 41 & 44.1 \\
\hline 54.4 & 53.6 & 50.7 & 45.4 & 47.7 & 43.5 & 42.5 & 40.6 & 41.2 \\
\hline 13.2 & 12.5 & 12.5 & 13.1 & 12.5 & 13.2 & 12.5 & 12.6 & 15.7 \\
\hline 12.5 & 13.3 & 12.6 & 13 & 12.5 & 12.5 & 13.2 & 12.5 & 15 \\
\hline
\end{tabular}


lights

\begin{tabular}{|c|c|c|c|c|c|c|c|c|}
\hline 57.5 & 56.6 & 49.3 & 42.8 & 43.5 & 46.3 & 45.1 & 45.2 & 48.3 \\
\hline 54.1 & 53.9 & 51.8 & 49.6 & 49.6 & 48.5 & 47.3 & 47.2 & 49.1 \\
\hline 60 & 60.2 & 52.2 & 49.7 & 49.6 & 49.3 & 48.7 & 47.5 & 50 \\
\hline 60 & 59.5 & 50.4 & 42.1 & 39.8 & 38.2 & 38.7 & 38.7 & 41.2 \\
\hline 37.3 & 37.5 & 35.8 & 34.5 & 33.9 & 34.6 & 34.6 & 33.9 & 34.4 \\
\hline 11.4 & 10.7 & 11.3 & 10.7 & 11.3 & 11.4 & 10.7 & 11.3 & 13.8 \\
\hline 11.3 & 11.2 & 10.7 & 11.3 & 11.4 & 10.7 & 11.3 & 11.4 & 13.2 \\
\hline 25.7 & 26.8 & 22.2 & 12.8 & 12.8 & 12.9 & 12.8 & 12.7 & 15.2 \\
\hline 25.3 & 27.2 & 21.8 & 13 & 13 & 13.5 & 13 & 12.9 & 15.3 \\
\hline 29.7 & 29.6 & 18.8 & 11.7 & 12.3 & 11.8 & 11.1 & 12.2 & 14 \\
\hline 28.3 & 26.3 & 18.6 & 11.5 & 12.1 & 11.6 & 12.1 & 11.4 & 13.1 \\
\hline 24.9 & 24.6 & 17.4 & 11.4 & 11.4 & 11.6 & 12.1 & 11.4 & 13.7 \\
\hline 11.4 & 12.2 & 11.5 & 11.5 & 11.4 & 12.1 & 11.5 & 11.6 & 13.9 \\
\hline 12.1 & 11.6 & 11.5 & 11.4 & 12 & 11.5 & 11.5 & 11.5 & 13.8 \\
\hline 27.4 & 28.8 & 20.5 & 12.5 & 12.5 & 13.2 & 12.6 & 13.2 & 15 \\
\hline 26.2 & 28.8 & 21.3 & 14.2 & 13.6 & 13.6 & 13.7 & 13.1 & 15.4 \\
\hline 27.9 & 27.6 & 20.7 & 14.5 & 13.8 & 14.1 & 13.9 & 13.8 & 16.1 \\
\hline 29 & 29.7 & 19.5 & 14.4 & 13.8 & 13.2 & 13.3 & 13.9 & 15 \\
\hline 25.5 & 25.8 & 17.9 & 12.6 & 12 & 12 & 12 & 11.9 & 13.6 \\
\hline 12 & 12 & 12.1 & 12.1 & 12 & 12.7 & 12.1 & 12.1 & 13.8 \\
\hline 11.9 & 12.1 & 12.1 & 12.7 & 12 & 12.1 & 12 & 12 & 13.8 \\
\hline 30.4 & 28.9 & 24.1 & 16.5 & 15.9 & 16.5 & 16.6 & 16.5 & 18.1 \\
\hline 30.3 & 30.9 & 28.9 & 31.5 & 31.5 & 25.5 & 19.5 & 18.9 & 21.1 \\
\hline 27.2 & 27.4 & 23.6 & 30.5 & 34.7 & 21.5 & 16.7 & 16.1 & 18.5 \\
\hline 28.2 & 21.4 & 23.3 & 32 & 30.9 & 19.7 & 16.4 & 16.4 & 18 \\
\hline 19.5 & 18.9 & 15 & 17.6 & 17.3 & 10.3 & 3.6 & 3.1 & 5.4 \\
\hline 5.9 & 3.1 & 3.1 & 1.8 & 3.1 & 3.1 & 3.1 & 3.1 & 4.7 \\
\hline 3.1 & 3.1 & 3.1 & 3.1 & 3.1 & 3.1 & 3.1 & 3.1 & 4.7 \\
\hline 19.1 & 21.3 & 9.7 & 4.8 & 4.8 & 3.6 & 4.1 & 3.6 & 5.7 \\
\hline 22.5 & 17 & 6.9 & 3.7 & 4.3 & 4.9 & 4.9 & 4.9 & 6.5 \\
\hline 20.8 & 21.1 & 9.8 & 4.1 & 3.6 & 4.1 & 3.6 & 3.6 & 5.7 \\
\hline 22.2 & 20 & 9.2 & 4.3 & 3.7 & 4.4 & 3.7 & 4.3 & 5.4 \\
\hline 18.4 & 17.8 & 10 & 4.3 & 3.7 & 3.7 & 4.3 & 3.7 & 6 \\
\hline 4.3 & 3.7 & 4.3 & 3.8 & 4.3 & 3.7 & 4.3 & 3.8 & 5.9 \\
\hline 4.3 & 3.7 & 4.4 & 3.7 & 4.3 & 3.7 & 4.4 & 3.7 & 6 \\
\hline 4.3 & 3.7 & 3.7 & 4.3 & 3.8 & 4.3 & 3.7 & 4.3 & 5.9 \\
\hline 26.6 & 22.2 & 9.6 & 4 & 3.9 & 3.4 & 4 & 3.9 & 5.6 \\
\hline 29.3 & 28.7 & 18.1 & 9.7 & 9 & 9.7 & 9.6 & 9.2 & 11.3 \\
\hline 27.1 & 25.2 & 17.3 & 10.2 & 10.8 & 10.2 & 10.8 & 10.2 & 12.5 \\
\hline 30.1 & 26.6 & 15.4 & 5.5 & 5.5 & 5.6 & 5.4 & 5.5 & 7.1 \\
\hline 5.5 & 5.5 & 4.9 & 5.5 & 5.4 & 5.5 & 5.5 & 4.3 & 6.6 \\
\hline 4.9 & 4.3 & 4.3 & 4.3 & 4.3 & 5.5 & 5.5 & 5.5 & 7.1 \\
\hline 29.2 & 29.6 & 13.1 & 3.9 & 4.4 & 3.9 & 4.5 & 3.8 & 6.1 \\
\hline 29.1 & 29.8 & 20.6 & 5 & 5.7 & 5.7 & 5.6 & 5.7 & 7.3 \\
\hline 38.2 & 38.3 & 23 & 9.4 & 8.7 & 9.4 & 9.3 & 7.6 & 9.8 \\
\hline 30.6 & 24.6 & 9.2 & 4.7 & 4.7 & 4.7 & 4.8 & 4.7 & 7 \\
\hline 27.3 & 28 & 17 & 3.6 & 3.7 & 3.7 & 3.1 & 3.6 & 5.9 \\
\hline 3 & 3.7 & 3.6 & 3.7 & 3.7 & 3 & 3.7 & 4.3 & 5.8 \\
\hline 3.6 & 3.7 & 3.6 & 3.1 & 3.6 & 3.7 & 3.7 & 3.1 & 5.9 \\
\hline 26.7 & 26.5 & 15.5 & 5.8 & 5.8 & 5.8 & 5.7 & 5.8 & 7.4 \\
\hline 33.5 & 32.7 & 19.4 & 8.8 & 8.9 & 8.9 & 8.9 & 8.3 & 11.2 \\
\hline
\end{tabular}


lights

\begin{tabular}{|c|c|c|c|c|c|c|c|c|}
\hline 31.9 & 31.3 & 14.9 & 4.1 & 4.1 & 4.7 & 4.1 & 4.7 & 6.3 \\
\hline 29.6 & 27.1 & 15.7 & 4.9 & 4.3 & 4.8 & 4.9 & 4.9 & 6.5 \\
\hline 29.3 & 24.4 & 13.2 & 4.3 & 4.3 & 3.6 & 4.3 & 4.3 & 5.9 \\
\hline 3.6 & 4.3 & 4.2 & 3.7 & 4.2 & 4.3 & 3.7 & 4.2 & 6.6 \\
\hline 4.2 & 4.3 & 3.6 & 4.2 & 3.7 & 4.2 & 4.3 & 3.6 & 6.6 \\
\hline 29.8 & 30.6 & 18.6 & 4.7 & 4.7 & 4.7 & 4.7 & 5.3 & 7 \\
\hline 28.6 & 27 & 14.8 & 4.3 & 4.2 & 3.7 & 4.2 & 4.3 & 6.6 \\
\hline 36.2 & 36.7 & 23.9 & 12.7 & 12 & 12.7 & 12 & 12.7 & 14.4 \\
\hline 32 & 31.1 & 15.6 & 3.5 & 3.6 & 3.5 & 3.5 & 3.5 & 5.8 \\
\hline 28.8 & 29.3 & 14.8 & 4.1 & 4.1 & 4.1 & 4.1 & 4 & 6.5 \\
\hline 4 & 4.1 & 4.1 & 4.1 & 4 & 4.1 & 4.1 & 4.1 & 5.8 \\
\hline 4.1 & 4.1 & 4 & 4.1 & 3.5 & 4.1 & 4 & 4.1 & 6.5 \\
\hline 31.8 & 31.4 & 16 & 7.4 & 6.8 & 6.8 & 6.7 & 7.4 & 9.2 \\
\hline 31.7 & 34.3 & 18.9 & 8 & 6.8 & 7.4 & 7.4 & 6.8 & 9.8 \\
\hline 43.5 & 42.9 & 13.1 & 5.5 & 3.8 & 4.3 & 4.3 & 4.4 & 6 \\
\hline 35.6 & 33.9 & 20.5 & 5.2 & 5.3 & 4 & 4.1 & 4 & 7.2 \\
\hline 30.6 & 30.9 & 17.3 & 6.5 & 5.8 & 5.9 & 6.5 & 5.8 & 8.3 \\
\hline 21 & 20.4 & 21.5 & 22.2 & 23.3 & 23 & 20.6 & 12.4 & 13.4 \\
\hline 5.1 & 5.7 & 5 & 5.7 & 5.1 & 5.7 & 5.1 & 5.7 & 8.1 \\
\hline 39.4 & 39 & 27.1 & 16.8 & 16.4 & 17.2 & 13.9 & 5.4 & 8 \\
\hline 41.6 & 41.3 & 25.5 & 4.9 & 5.4 & 4.8 & 4.8 & 4.9 & 7.4 \\
\hline 44.4 & 45.1 & 26.8 & 6.1 & 5.4 & 5.4 & 6 & 5.5 & 7.9 \\
\hline 60.7 & 64.5 & 51 & 18.3 & 7.7 & 8.2 & 7.1 & 6.5 & 8.3 \\
\hline 64.8 & 66 & 48.6 & 7.2 & 6.4 & 7.6 & 7 & 7 & 8.9 \\
\hline 30.2 & 32.8 & 33 & 31.4 & 23.3 & 12.4 & 11.7 & 12.5 & 14.2 \\
\hline 11.7 & 11.7 & 11.6 & 12.3 & 11.6 & 11.8 & 11.8 & 11.7 & 14.2 \\
\hline 62.4 & 62.2 & 54.6 & 44.7 & 40.1 & 35.2 & 34.2 & 33.2 & 35.8 \\
\hline 59.9 & 62.4 & 54.2 & 43.8 & 38.9 & 38.7 & 36.2 & 36.9 & 35.4 \\
\hline 59.5 & 60.9 & 51.6 & 40.5 & 36.1 & 34.9 & 34.9 & 37.5 & 35.1 \\
\hline 59.8 & 61.7 & 53.2 & 46.7 & 42 & 38.8 & 36.6 & 37.2 & 38.1 \\
\hline 56 & 60.6 & 54.1 & 49.2 & 47.6 & 44.4 & 43.5 & 43.6 & 43.5 \\
\hline 10.4 & 10.4 & 9.7 & 10.2 & 10.3 & 10.4 & 10.5 & 10.6 & 13 \\
\hline 10.3 & 10.3 & 9.7 & 10.2 & 10.3 & 10.4 & 9.8 & 11.1 & 12.8 \\
\hline 59.3 & 59.9 & 52.7 & 50.9 & 41.3 & 37.7 & 36.4 & 36.1 & 37.8 \\
\hline 59.4 & 59.5 & 52.7 & 44.7 & 42.7 & 39 & 37.6 & 38.4 & 38.8 \\
\hline 58.4 & 58.6 & 52 & 43.2 & 43.4 & 41.8 & 41.2 & 40.8 & 39.1 \\
\hline 57.6 & 58.9 & 52.6 & 46.7 & 36.8 & 36.6 & 37.2 & 37.4 & 36.1 \\
\hline 56.1 & 56.7 & 53.6 & 45 & 42.2 & 39.7 & 37.1 & 36 & 37.6 \\
\hline 10.4 & 10.4 & 10.4 & 10.4 & 10.5 & 11.1 & 10.4 & 11.4 & 12.9 \\
\hline 10.4 & 10.3 & 10.4 & 10.3 & 10.4 & 10.4 & 11 & 11.4 & 12.8 \\
\hline 58.5 & 58.2 & 49.5 & 44.6 & 43.6 & 40.2 & 37.4 & 39.7 & 40 \\
\hline 60.9 & 60.4 & 53.9 & 46.7 & 43.2 & 43.7 & 42.3 & 43.6 & 44.6 \\
\hline 58.2 & 61.5 & 53.2 & 46.6 & 42.6 & 41.9 & 39.8 & 40.1 & 39.4 \\
\hline 58.5 & 58.7 & 53 & 48.6 & 48.9 & 49.1 & 49.4 & 49.8 & 51.1 \\
\hline 24.4 & 24.1 & 24.6 & 32 & 35.3 & 35.7 & 34.4 & 35.4 & 36.3 \\
\hline 13.1 & 12.4 & 13 & 12.9 & 12.4 & 13 & 12.4 & 14.1 & 15.5 \\
\hline 13 & 12.4 & 12.9 & 12.3 & 12.9 & 12.5 & 13.1 & 14.1 & 14.9 \\
\hline 12.3 & 13 & 12.3 & 12.8 & 12.3 & 13 & 12.3 & 14.1 & 15.4 \\
\hline 33.1 & 30.4 & 30.9 & 33.9 & 32.7 & 38.7 & 44 & 44.4 & 45.2 \\
\hline 59.4 & 59.2 & 54.6 & 50.7 & 45.7 & 46.3 & 44.3 & 44.7 & 41.8 \\
\hline 61.6 & 60.2 & 54.5 & 49.2 & 44.9 & 40.5 & 39.3 & 40.3 & 42.2 \\
\hline
\end{tabular}


lights

\begin{tabular}{|c|c|c|c|c|c|c|c|c|}
\hline 55.1 & 58 & 53.5 & 48.8 & 42.9 & 39.5 & 39 & 39.5 & 41.8 \\
\hline 12.6 & 13.1 & 13.2 & 13 & 11.4 & 11.8 & 11 & 12.4 & 13.5 \\
\hline 10.5 & 10.4 & 10.4 & 10.4 & 10.4 & 10.6 & 10.6 & 12.3 & 12.9 \\
\hline 63.3 & 63 & 57.5 & 50 & 44.8 & 42.1 & 40.9 & 40.2 & 40.5 \\
\hline 63.8 & 65.3 & 53 & 43.6 & 42.1 & 40.4 & 41.7 & 41.9 & 41 \\
\hline 61.8 & 61.5 & 55.6 & 49 & 46.8 & 51.1 & 50.3 & 49.3 & 48.4 \\
\hline 61.9 & 62.2 & 55.1 & 48.2 & 50.8 & 55.6 & 46 & 31.8 & 31.8 \\
\hline 60.8 & 61.7 & 55.2 & 43.1 & 36 & 36.8 & 34.8 & 34 & 34 \\
\hline 10.6 & 10.7 & 11.3 & 10.6 & 11.3 & 12 & 13.5 & 13.1 & 13.1 \\
\hline 10.7 & 11.2 & 10.7 & 11.3 & 11.2 & 13.1 & 14.4 & 14.4 & 13.8 \\
\hline 62.1 & 62.7 & 50.5 & 42.7 & 41.8 & 43.2 & 43.4 & 42.3 & 40.9 \\
\hline 59.4 & 63.5 & 54.2 & 42.5 & 46.6 & 44.8 & 41.4 & 40.2 & 39.6 \\
\hline 60.8 & 62.2 & 52.1 & 46.8 & 40 & 39.2 & 38.8 & 38.4 & 38.2 \\
\hline 59.9 & 59.8 & 54.1 & 49 & 45.9 & 43.2 & 43.5 & 43.4 & 42.9 \\
\hline 59.9 & 63.3 & 53.3 & 48.6 & 43.2 & 42.7 & 42.2 & 42.8 & 34.5 \\
\hline 11.9 & 12 & 13 & 13 & 12.5 & 13.4 & 14.3 & 14.3 & 14.4 \\
\hline 11.9 & 12 & 11.9 & 11.9 & 11.8 & 14.1 & 14.4 & 14.3 & 14.4 \\
\hline 53.7 & 60 & 53.6 & 44.5 & 39.5 & 39.6 & 39.3 & 39.2 & 38.8 \\
\hline 60 & 59.1 & 50.9 & 37.3 & 34.1 & 34.7 & 38.3 & 37.8 & 36.7 \\
\hline 61.7 & 61.2 & 54.3 & 54.9 & 46.4 & 42 & 41.6 & 41.4 & 39.9 \\
\hline 62.9 & 59.7 & 53.1 & 43.6 & 38.8 & 38.8 & 38.8 & 38.7 & 39.6 \\
\hline 60.7 & 60.3 & 53.1 & 40.6 & 42.4 & 44.6 & 43.9 & 43.1 & 42 \\
\hline 13.4 & 14 & 13.4 & 14 & 12.9 & 14.5 & 14 & 14 & 14 \\
\hline 11.6 & 11.6 & 11.5 & 12.1 & 12.8 & 13.7 & 13.3 & 14 & 13.5 \\
\hline 62.6 & 60.9 & 54.1 & 49.3 & 45.8 & 45.5 & 44.9 & 43.6 & 43 \\
\hline 60.3 & 59.5 & 55.3 & 50.7 & 42.1 & 41 & 39.8 & 40.4 & 41.7 \\
\hline 60.7 & 58.5 & 50 & 40.9 & 36.9 & 49 & 48.8 & 48.1 & 46.1 \\
\hline 62.8 & 60.3 & 48.6 & 44.4 & 40.9 & 42.4 & 43.1 & 42.7 & 44.6 \\
\hline 59.6 & 60 & 54.9 & 47 & 43.6 & 46 & 41.3 & 42 & 43.5 \\
\hline 11.1 & 10.4 & 11.1 & 10.4 & 11.1 & 12.8 & 13.6 & 12.9 & 13.6 \\
\hline 10.4 & 11 & 10.5 & 11 & 10.4 & 13.5 & 12.9 & 13.6 & 13.5 \\
\hline 62.8 & 63.8 & 56 & 45.1 & 42.2 & 42.9 & 42.5 & 41.9 & 41.9 \\
\hline 61.2 & 63.7 & 54 & 43.1 & 41.9 & 41.8 & 48.4 & 47.5 & 48.1 \\
\hline 61.1 & 60 & 56.5 & 48.4 & 44.3 & 46.7 & 43.5 & 42.4 & 44.1 \\
\hline 62.2 & 62.6 & 54.9 & 46.4 & 45.9 & 45.8 & 46.4 & 46.7 & 43.1 \\
\hline 63.9 & 63.9 & 53.4 & 51.7 & 47.1 & 45.7 & 44 & 46.3 & 46.5 \\
\hline 29.8 & 27.6 & 11.2 & 11.4 & 12.6 & 14.4 & 13.1 & 13.1 & 12.5 \\
\hline 21.3 & 12.9 & 12 & 9.8 & 10.6 & 12.3 & 12.3 & 12.3 & 12.3 \\
\hline 55.1 & 55.7 & 49 & 40.8 & 39.4 & 41.4 & 42.4 & 42.5 & 41.4 \\
\hline 62.5 & 60.8 & 53.9 & 48.3 & 44.9 & 47.4 & 46.6 & 48 & 46.2 \\
\hline 62.6 & 58.3 & 54.3 & 48 & 46.5 & 43.8 & 41.5 & 40.7 & 39.5 \\
\hline 59.4 & 60.3 & 51.8 & 47.9 & 50 & 50.7 & 46.8 & 43.8 & 42.8 \\
\hline 63.2 & 61.1 & 52.7 & 48.9 & 51.5 & 55.1 & 49.9 & 46.2 & 44.8 \\
\hline 11.6 & 11.5 & 11 & 11.5 & 12 & 13.5 & 14 & 14.2 & 13.4 \\
\hline 11 & 11.5 & 11 & 11.5 & 11.9 & 14.1 & 13.4 & 14.1 & 14.2 \\
\hline 62.6 & 62.6 & 57.7 & 51.1 & 50.7 & 47.6 & 39.2 & 36.4 & 35.5 \\
\hline 62.8 & 62.4 & 54 & 42.2 & 46.1 & 49.4 & 47.3 & 49.4 & 48.8 \\
\hline 64 & 63.7 & 57.1 & 49.9 & 48.5 & 53.9 & 47.8 & 43.8 & 44.3 \\
\hline 63.3 & 63.8 & 53.3 & 39.8 & 43.5 & 52 & 52 & 50.2 & 49.1 \\
\hline 62.6 & 62.8 & 57.8 & 51.2 & 49.3 & 50.4 & 50.3 & 46.1 & 43.9 \\
\hline 12.2 & 12.2 & 12.8 & 13.4 & 14.2 & 14.7 & 14 & 14.6 & 14.1 \\
\hline
\end{tabular}


lights

\begin{tabular}{|c|c|c|c|c|c|c|c|c|}
\hline 11.6 & 11.6 & 11.6 & 12.8 & 14.1 & 14.7 & 14.1 & 14 & 14 \\
\hline 63.4 & 61.3 & 54.3 & 52.2 & 49.4 & 47.4 & 46.4 & 47.6 & 44.7 \\
\hline 63.9 & 65 & 54 & 52.7 & 53.4 & 52.1 & 51 & 51 & 46.1 \\
\hline 63.1 & 63.1 & 54 & 52.4 & 49.9 & 44.9 & 44.5 & 48.7 & 48.3 \\
\hline 61.9 & 64.6 & 53.1 & 51.4 & 43.9 & 41.7 & 43.6 & 40.8 & 35.8 \\
\hline 62.6 & 64.1 & 55.8 & 52.7 & 49.5 & 45 & 40.3 & 43.3 & 39.8 \\
\hline 10.5 & 9.9 & 10.6 & 11.9 & 12.4 & 13.1 & 13.1 & 12.4 & 13 \\
\hline 9.9 & 10.6 & 10 & 11.8 & 13.2 & 13.1 & 12.5 & 13.1 & 13.2 \\
\hline 64 & 64.3 & 53.8 & 53.3 & 49.7 & 49.1 & 47.4 & 40.8 & 38.7 \\
\hline 64.4 & 62.3 & 53.6 & 51.8 & 48.2 & 45.3 & 44.9 & 44.2 & 42.8 \\
\hline 63.2 & 64.2 & 52.3 & 51.7 & 41.7 & 41.9 & 46 & 45.8 & 39.2 \\
\hline 63.8 & 62.1 & 55.3 & 49.1 & 45.2 & 42.8 & 43 & 43.7 & 41.6 \\
\hline 62.6 & 59.4 & 54.1 & 54.6 & 48.8 & 43.9 & 42.2 & 42.7 & 37.8 \\
\hline 12.6 & 11 & 10.8 & 12.2 & 13.4 & 12.8 & 13.4 & 13.3 & 13.5 \\
\hline 10.6 & 10.6 & 10.6 & 10 & 12.9 & 13.1 & 13.3 & 13.1 & 13.2 \\
\hline 61.3 & 64.5 & 52 & 46.9 & 43.8 & 43.8 & 44.2 & 44.2 & 43.5 \\
\hline 62.3 & 66 & 56.9 & 48.6 & 46.2 & 43.7 & 43.1 & 43.6 & 42.2 \\
\hline 63.6 & 66.2 & 56.8 & 52.6 & 50.7 & 44.5 & 43.4 & 46.3 & 44.3 \\
\hline 63.9 & 64.3 & 52.7 & 46.4 & 43.6 & 42.9 & 43.9 & 43.2 & 42.7 \\
\hline 60.5 & 60.1 & 53.6 & 48.1 & 48.3 & 47 & 45.3 & 42.3 & 35.8 \\
\hline 11 & 11.6 & 11.6 & 11.6 & 14 & 13.5 & 14 & 14 & 14 \\
\hline 11.6 & 11.6 & 11.6 & 11 & 14 & 14.1 & 14 & 13.5 & 14 \\
\hline 63.6 & 62.4 & 52.3 & 49.4 & 50.7 & 48.4 & 48.4 & 48.4 & 48.1 \\
\hline 60.8 & 62.2 & 50.8 & 42.3 & 40.1 & 38.9 & 39.6 & 39 & 40.3 \\
\hline 60.9 & 47.1 & 36.9 & 38 & 37.5 & 36.3 & 41.8 & 41.8 & 40.7 \\
\hline 7.4 & 8.1 & 7.5 & 7.5 & 10.5 & 10 & 10 & 10.7 & 10 \\
\hline 7.5 & 8.1 & 7.5 & 8 & 10 & 10.6 & 10 & 10.5 & 10 \\
\hline 7.3 & 7.9 & 7.4 & 7.9 & 9.8 & 10.4 & 9.7 & 10.4 & 9.7 \\
\hline 7.4 & 7.9 & 7.3 & 8 & 9.8 & 10.3 & 9.8 & 10.3 & 9.8 \\
\hline 62.3 & 59 & 52.9 & 47.4 & 50 & 46.9 & 47.5 & 43.3 & 38 \\
\hline 58.8 & 58.8 & 46.9 & 42.7 & 39 & 39 & 39.6 & 40.3 & 36.6 \\
\hline 61.7 & 63.6 & 55.4 & 49.1 & 44.3 & 40 & 37.7 & 39 & 36.9 \\
\hline 60.2 & 60.3 & 52.3 & 42.5 & 47.4 & 48.3 & 47.3 & 47.9 & 44.9 \\
\hline 63.4 & 61 & 59.4 & 45 & 50.2 & 50.5 & 49.9 & 51.1 & 52.3 \\
\hline 31.7 & 22.3 & 11.3 & 11.2 & 12.5 & 10.6 & 11.3 & 11.2 & 11.3 \\
\hline 19.8 & 15.7 & 7.4 & 7.3 & 9.8 & 9.8 & 9.8 & 9.8 & 9.7 \\
\hline 59 & 58.2 & 50.9 & 39.3 & 35.1 & 31.8 & 32.6 & 33.5 & 33.9 \\
\hline 60.5 & 57.3 & 50.2 & 47.9 & 49.9 & 47.5 & 45.4 & 45.9 & 47.2 \\
\hline 61.1 & 58.9 & 49.7 & 44 & 42.2 & 47.6 & 47.2 & 45.3 & 39.9 \\
\hline 58.9 & 58.5 & 49 & 39.7 & 47.5 & 48.7 & 50.3 & 49 & 49.4 \\
\hline 57.9 & 57 & 45.5 & 38.6 & 47.8 & 46 & 41.3 & 41.3 & 37.3 \\
\hline 8.7 & 8.6 & 8.1 & 8.1 & 10.5 & 10.5 & 10.6 & 10.5 & 10.6 \\
\hline 8 & 8 & 8 & 8 & 10.5 & 10.5 & 10.6 & 10.4 & 10.5 \\
\hline 66 & 63.7 & 54.4 & 41.6 & 37.3 & 43.9 & 43 & 43.6 & 42 \\
\hline 63.3 & 62.8 & 54.5 & 45 & 48.2 & 47.2 & 45 & 43.8 & 43.1 \\
\hline 57.8 & 62.3 & 52.5 & 40 & 43.8 & 45.5 & 44.9 & 43.3 & 40.5 \\
\hline 62.2 & 64.5 & 52.8 & 46.4 & 43.9 & 44.6 & 44.7 & 44.2 & 35.5 \\
\hline 66.9 & 57.3 & 48.4 & 44.7 & 46.2 & 43 & 41.8 & 41.9 & 46.2 \\
\hline 11.9 & 12 & 11.9 & 12 & 12 & 14.4 & 14.4 & 14.5 & 14.4 \\
\hline 11.9 & 11.8 & 11.8 & 11.8 & 12.6 & 14.4 & 14.4 & 14.3 & 14.3 \\
\hline 42.2 & 38.8 & 29.1 & 28.2 & 28.3 & 31.7 & 35.7 & 35.9 & 36 \\
\hline
\end{tabular}


lights

\begin{tabular}{|c|c|c|c|c|c|c|c|c|}
\hline 31.9 & 29.5 & 22.1 & 21.3 & 20.6 & 23.5 & 24 & 26.4 & 30.6 \\
\hline 24.8 & 22.3 & 22.7 & 22.8 & 22.3 & 25.6 & 25.3 & 24.3 & 24 \\
\hline 4.6 & 5.3 & 5.2 & 5.2 & 5.3 & 7.1 & 7.6 & 7.2 & 7.9 \\
\hline 5.2 & 4.7 & 5.2 & 5.2 & 5.4 & 7.6 & 7 & 7.6 & 7.1 \\
\hline 5.3 & 5.2 & 5.2 & 4.7 & 5.9 & 7.1 & 7.6 & 7.6 & 7.6 \\
\hline 5.2 & 5.3 & 4.6 & 5.3 & 5.3 & 7.6 & 7.7 & 7.6 & 7 \\
\hline 7.3 & 6.8 & 6.5 & 4.6 & 4.7 & 6.5 & 7 & 7 & 8.6 \\
\hline 5.8 & 7.9 & 7 & 4.7 & 5.4 & 7.7 & 7.8 & 7.8 & 7.8 \\
\hline 14.8 & 14.2 & 11.4 & 6.8 & 7.5 & 9.9 & 9.2 & 9.9 & 9.4 \\
\hline 13.6 & 13.6 & 13.5 & 13.6 & 13.5 & 16.5 & 15.9 & 15.9 & 16.1 \\
\hline 13.2 & 12.6 & 8.7 & 6.5 & 5.9 & 9 & 8.3 & 8.9 & 8.9 \\
\hline 6.5 & 6.5 & 5.9 & 6.6 & 5.9 & 8.9 & 8.9 & 8.4 & 9.1 \\
\hline 6.5 & 5.9 & 6.6 & 6 & 6.5 & 8.9 & 8.3 & 9.1 & 8.5 \\
\hline 64 & 70.8 & 60.7 & 45 & 39.5 & 39.3 & 39.4 & 40.5 & 38.9 \\
\hline 62.7 & 63.4 & 57.8 & 50.3 & 47.7 & 47.8 & 49.6 & 47.6 & 42.3 \\
\hline 63.7 & 61.3 & 55.2 & 43.3 & 36.8 & 38 & 42.2 & 42.8 & 44 \\
\hline 63 & 63 & 54 & 46.9 & 39.6 & 40 & 43 & 42.6 & 38 \\
\hline 63 & 64.2 & 57.8 & 48.2 & 38.5 & 37.3 & 37.7 & 39.2 & 39.1 \\
\hline 13 & 13.1 & 12.5 & 13 & 13 & 14.7 & 14.9 & 15.6 & 15.5 \\
\hline 13.1 & 13.1 & 12.5 & 13.1 & 13 & 15.2 & 14.9 & 15.5 & 15.6 \\
\hline 61.3 & 62.9 & 56.6 & 50.4 & 40.4 & 40.8 & 43.1 & 41.7 & 37.3 \\
\hline 58.1 & 59.6 & 51 & 49.6 & 46.9 & 43.3 & 41.5 & 40.4 & 35.5 \\
\hline 62.7 & 63.6 & 54.6 & 52.3 & 47.3 & 49.1 & 48.6 & 48.7 & 45.8 \\
\hline 62.8 & 62.7 & 53.8 & 50.3 & 44.2 & 44.1 & 43.9 & 40.3 & 36.3 \\
\hline 61.1 & 59.9 & 56.1 & 55.2 & 51.5 & 50.6 & 51 & 48.8 & 47.6 \\
\hline 11.2 & 11.9 & 11.2 & 11.2 & 11.8 & 13.3 & 13.6 & 13.6 & 14.3 \\
\hline 11.4 & 11.3 & 11.9 & 11.2 & 11.8 & 13.3 & 13.7 & 14.4 & 13.8 \\
\hline 18.3 & 16.6 & 14.9 & 14.2 & 14.1 & 16.2 & 16.6 & 16.6 & 17.4 \\
\hline 64.5 & 63.7 & 56.4 & 52.6 & 50.4 & 49.1 & 52.1 & 50.1 & 43.7 \\
\hline 64.6 & 63.7 & 55.1 & 45.3 & 39.4 & 38.5 & 37.9 & 39.9 & 38.7 \\
\hline 64.3 & 64.2 & 54.9 & 45.9 & 37.4 & 38 & 38.2 & 41 & 40.3 \\
\hline 65.5 & 66.3 & 60.6 & 54.6 & 50.4 & 47 & 41 & 41.6 & 42.3 \\
\hline 10 & 9.3 & 10 & 9.4 & 11.2 & 12.5 & 14.1 & 13.5 & 13.6 \\
\hline 11.1 & 11.2 & 11.2 & 11.2 & 11.7 & 12.4 & 13.5 & 14.1 & 13.5 \\
\hline 62.4 & 61.4 & 52.7 & 44.2 & 41.7 & 42.2 & 37.8 & 34.6 & 32.8 \\
\hline 60.8 & 61.2 & 56.3 & 50 & 43.5 & 44.9 & 45 & 44.8 & 44.7 \\
\hline 60 & 61.6 & 53.2 & 46.6 & 38.1 & 42.1 & 42.1 & 42 & 34.7 \\
\hline 61.7 & 63.7 & 58.9 & 52.1 & 49.9 & 51 & 52.5 & 50.8 & 43.7 \\
\hline 63.2 & 62.4 & 54.7 & 53.1 & 57.1 & 55 & 53.3 & 52.6 & 47.4 \\
\hline 9.9 & 9.4 & 10 & 9.3 & 10.2 & 12.4 & 11.9 & 11.8 & 12.4 \\
\hline 9.4 & 10 & 10 & 9.4 & 10.1 & 12.5 & 11.8 & 12.5 & 12.4 \\
\hline 65 & 63.6 & 54.2 & 43.4 & 43.8 & 51 & 40.5 & 36 & 35.2 \\
\hline 62.8 & 64.9 & 54.2 & 42.8 & 40.5 & 43.8 & 46.9 & 61.2 & 61.9 \\
\hline 63.6 & 65.9 & 53.8 & 41.9 & 40.8 & 42.7 & 41.3 & 41.9 & 41.2 \\
\hline 59.6 & 59.8 & 53.2 & 48.8 & 49.5 & 51.4 & 49.8 & 46.9 & 46.4 \\
\hline 33.9 & 32 & 31.5 & 32.8 & 31.7 & 33.5 & 32.8 & 32.9 & 32.9 \\
\hline 14.2 & 12.9 & 13.6 & 12.9 & 13.6 & 15.9 & 15.4 & 15.9 & 15.4 \\
\hline 13.5 & 12.9 & 13.5 & 12.9 & 13.6 & 15.3 & 15.9 & 15.3 & 16 \\
\hline 64.2 & 62.2 & 57.6 & 50 & 47.7 & 50.4 & 48.3 & 45.2 & 40.2 \\
\hline 61.3 & 61.5 & 57.6 & 51.8 & 47.3 & 43 & 42.1 & 42.1 & 41 \\
\hline 65.5 & 64.5 & 55.9 & 50.9 & 43.1 & 44.1 & 43.4 & 43.8 & 46.7 \\
\hline
\end{tabular}


lights

\begin{tabular}{|c|c|c|c|c|c|c|c|c|}
\hline 10.9 & 10.9 & 10.8 & 10.3 & 10.9 & 13.3 & 12.7 & 13.4 & 13.4 \\
\hline 62.2 & 64.2 & 56 & 50.2 & 47.1 & 47.3 & 47.3 & 45.5 & 37.2 \\
\hline 7.1 & 7.6 & 7.1 & 7.7 & 7.1 & 10 & 10.2 & 9.5 & 10.1 \\
\hline 7.1 & 7.7 & 7.1 & 7.7 & 7 & 10.1 & 9.5 & 10.1 & 10.2 \\
\hline 62 & 62.2 & 57.5 & 51.4 & 50.6 & 52.8 & 52.2 & 50.2 & 48.8 \\
\hline 61.1 & 60.6 & 54.3 & 50.3 & 46.5 & 46.6 & 46.3 & 43.4 & 43.6 \\
\hline 62.4 & 60.2 & 52.9 & 46.1 & 40.4 & 41.5 & 44 & 42.4 & 42.3 \\
\hline 58.3 & 61.3 & 54.8 & 49.5 & 47.2 & 46.8 & 42 & 42.1 & 46.5 \\
\hline 64.9 & 61.8 & 55.8 & 49.7 & 47.6 & 50.3 & 48.4 & 47 & 45.5 \\
\hline 9.9 & 9.3 & 9.9 & 9.3 & 9.9 & 11.6 & 13.5 & 14.1 & 14.8 \\
\hline 45.8 & 41.4 & 31.6 & 11.2 & 10.5 & 12.9 & 12.9 & 12.9 & 13.7 \\
\hline 51.4 & 50.8 & 48.9 & 40.6 & 41.3 & 42.1 & 42 & 42 & 43.9 \\
\hline 63.3 & 62.8 & 59.5 & 51.1 & 48.9 & 49.3 & 49.9 & 48.2 & 40.5 \\
\hline 63.3 & 63.9 & 56.2 & 49.4 & 47.8 & 47.3 & 47.2 & 48.5 & 46.7 \\
\hline 63.4 & 63.7 & 54.4 & 50.9 & 49 & 46.8 & 44.5 & 42.5 & 40.2 \\
\hline 63 & 61.6 & 56.4 & 49.6 & 47.8 & 47.5 & 42.9 & 37.6 & 35.5 \\
\hline 6 & 5.3 & 6 & 6.5 & 6.6 & 8.1 & 8.5 & 8.5 & 9.1 \\
\hline 6 & 8.9 & 8.4 & 9 & 8.3 & 9.5 & 9.7 & 9.7 & 9.7 \\
\hline 65.7 & 67.3 & 63.2 & 51.7 & 50.9 & 51 & 51.1 & 52.4 & 52.6 \\
\hline 66.5 & 64.3 & 60.1 & 46.9 & 43 & 42.3 & 42.9 & 38.5 & 36.3 \\
\hline 64.9 & 65.3 & 57.2 & 48.2 & 43.7 & 37.6 & 35.5 & 35.4 & 36.2 \\
\hline 63.8 & 64.1 & 55.9 & 48.7 & 44.4 & 44.4 & 42 & 39.5 & 41.2 \\
\hline 58.1 & 59.8 & 54.1 & 49.6 & 51.1 & 50.9 & 52 & 50.1 & 44.8 \\
\hline 20.3 & 19.6 & 13.5 & 13.7 & 13.6 & 15.2 & 16.3 & 16 & 15.7 \\
\hline 13.9 & 13.7 & 13.6 & 13.6 & 13.8 & 16 & 15.9 & 15.2 & 16.3 \\
\hline 60.6 & 60.9 & 53.4 & 46.6 & 42.2 & 42.1 & 43.4 & 43.5 & 41.6 \\
\hline 65.1 & 64.5 & 53.6 & 50.1 & 48.3 & 48.1 & 47.7 & 47.7 & 46.7 \\
\hline 66.6 & 65 & 59.3 & 56.2 & 58.2 & 58.7 & 56.8 & 56 & 51.8 \\
\hline 62.9 & 61.6 & 56.4 & 43.8 & 40.2 & 40.8 & 45.2 & 45.6 & 43.4 \\
\hline 62.6 & 52.8 & 47.5 & 53.8 & 54.2 & 49.9 & 49.7 & 49.2 & 49.3 \\
\hline 14.5 & 14.4 & 15.3 & 14.8 & 15.3 & 15.1 & 16.4 & 15.9 & 16.3 \\
\hline 15.2 & 14.8 & 15 & 14.6 & 15.5 & 15.3 & 16.2 & 15.8 & 15.9 \\
\hline 7.4 & 8.7 & 7.2 & 6.3 & 8.8 & 17 & 21.7 & 21 & 21 \\
\hline 23 & 22.1 & 17.9 & 18 & 12.6 & 11 & 11.5 & 12.1 & 20 \\
\hline 22.5 & 23.5 & 12.6 & 7.4 & 8.5 & 19.1 & 27.5 & 26.9 & 27.4 \\
\hline 26.6 & 25.9 & 12.8 & 11.5 & 9.8 & 7.3 & 6.2 & 17.1 & 22.2 \\
\hline 26 & 27.7 & 27.7 & 24.9 & 19.6 & 17.3 & 19.5 & 17 & 12.2 \\
\hline 4.2 & 4.3 & 4.2 & 4.3 & 4.2 & 5.5 & 6.6 & 6.8 & 6.7 \\
\hline 4.3 & 4.2 & 4.2 & 4.3 & 3.6 & 5.4 & 6.4 & 6.4 & 6.5 \\
\hline 60.5 & 62.6 & 56.7 & 48.5 & 47.5 & 44.8 & 45.3 & 42.1 & 41.5 \\
\hline 61.6 & 62.2 & 54.5 & 48.4 & 43.6 & 42.2 & 40.2 & 38.6 & 37.3 \\
\hline 60.9 & 63.6 & 53 & 53 & 48 & 47.9 & 49.5 & 46.3 & 40.3 \\
\hline 58.4 & 62 & 54 & 46 & 46.6 & 43.9 & 39.7 & 44 & 46.1 \\
\hline 59.9 & 57.5 & 53.5 & 45.1 & 41.9 & 41.5 & 43 & 43.8 & 42.1 \\
\hline 20.5 & 12.1 & 12.7 & 11.4 & 12.1 & 13.7 & 14.1 & 14.2 & 15 \\
\hline 11.9 & 11.9 & 12.6 & 12.2 & 15.1 & 17.4 & 14.6 & 14.5 & 14.5 \\
\hline 64.6 & 63 & 56.5 & 46.9 & 43 & 43.2 & 45.6 & 44.5 & 44.8 \\
\hline 61.5 & 64.3 & 56.6 & 51.4 & 51.8 & 50.7 & 47 & 42.1 & 41.2 \\
\hline & & & & & & & & \\
\hline & & & & & & & & \\
\hline & & & & & & & & \\
\hline
\end{tabular}


lights

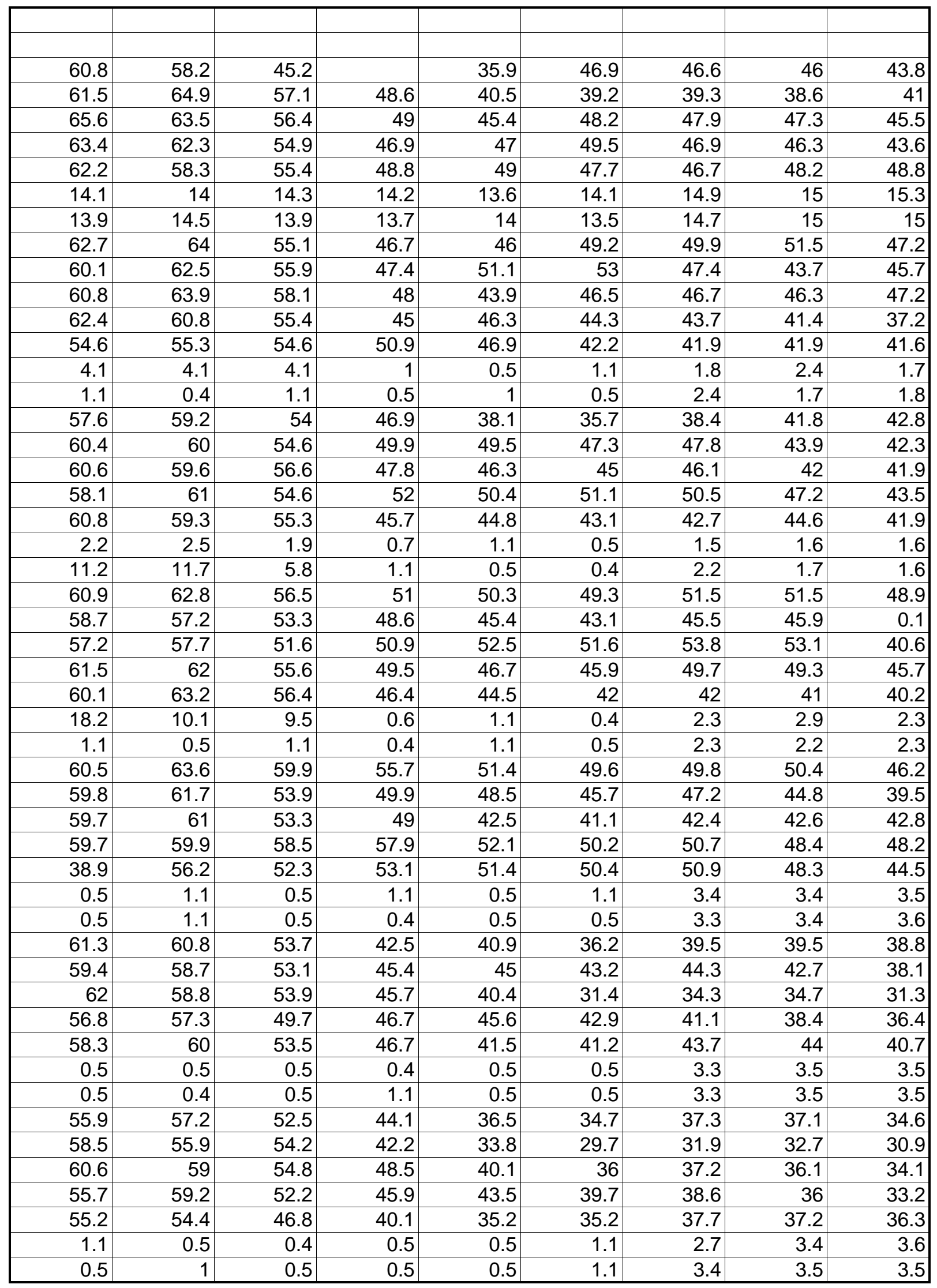


lights

\begin{tabular}{|c|c|c|c|c|c|c|c|c|}
\hline 52.8 & 52 & 55.5 & 52 & 49.1 & 45.4 & 44 & 43.5 & 39.6 \\
\hline 55.6 & 57.7 & 55.5 & 48.9 & 52.7 & 47.5 & 46.3 & 40.4 & 39 \\
\hline 63.5 & 61.3 & 58.5 & 48.7 & 41.8 & 38.8 & 38 & 35.5 & 33.5 \\
\hline 65.4 & 59.7 & 54.2 & 50.2 & 44.3 & 42.9 & 43.4 & 41.8 & 37.7 \\
\hline 29.2 & 32.1 & 28.6 & 27.7 & 25 & 21.3 & 21.9 & 22.4 & 22.8 \\
\hline 0.4 & 0.5 & 0.5 & 1.1 & 0.4 & 0.5 & 3.4 & 3.4 & 2.8 \\
\hline 0.5 & 0.5 & 0.5 & 0.5 & 0.4 & 1.1 & 3.4 & 2.8 & 3.4 \\
\hline 40.6 & 41.9 & 23.9 & 11.2 & 11 & 10.7 & 18.7 & 20 & 20.3 \\
\hline 38.2 & 35.5 & 31 & 14.5 & 11.2 & 12.4 & 14.2 & 14 & 13.8 \\
\hline 39.3 & 38.1 & 38.1 & 18.7 & 13.4 & 19.1 & 21.1 & 21.9 & 22 \\
\hline 33.5 & 32 & 28.5 & 14.9 & 10.9 & 11.3 & 14.2 & 13 & 13.5 \\
\hline 35.4 & 35.2 & 33.4 & 18.9 & 9.6 & 9.3 & 14.7 & 15.8 & 12 \\
\hline 2.9 & 0.3 & 1 & 0.3 & 1 & 0.3 & 3.3 & 3.3 & 3.2 \\
\hline 0.3 & 1 & 0.3 & 0.4 & 0.9 & 0.4 & 3.2 & 3.3 & 3.3 \\
\hline 34.5 & 33 & 30.1 & 18.1 & 11.3 & 12.4 & 14.1 & 13.8 & 14.3 \\
\hline 34.5 & 32 & 31.2 & 15.2 & 8.8 & 9.6 & 12.3 & 11.3 & 11.7 \\
\hline 35.4 & 25.7 & 21.2 & 15.8 & 10.2 & 10.7 & 13.3 & 13.1 & 12.9 \\
\hline 36.1 & 35.3 & 31.9 & 24.6 & 15.1 & 10.4 & 13 & 12.8 & 12.3 \\
\hline 33.6 & 34.2 & 28.3 & 19 & 11.8 & 10.6 & 12.7 & 11.8 & 12.1 \\
\hline 1 & 0.5 & 0.5 & 1.1 & 0.4 & 1.1 & 2.9 & 3.4 & 3.4 \\
\hline 0.5 & 0.5 & 0.4 & 0.5 & 0.5 & 0.4 & 3.5 & 3.4 & 3.4 \\
\hline 33.3 & 30.8 & 31.1 & 18.7 & 9.4 & 9.4 & 12.1 & 11.8 & 11.1 \\
\hline 34.7 & 33.5 & 28.5 & 13.9 & 9.3 & 9.9 & 12.1 & 11.8 & 11.5 \\
\hline 32.9 & 29.5 & 30.4 & 23.4 & 10.8 & 11.5 & 14 & 13.8 & 13.8 \\
\hline 32.8 & 34 & 33.2 & 16.5 & 10.5 & 11.3 & 12.9 & 12.7 & 13.2 \\
\hline 31.5 & 31.1 & 31.6 & 19.9 & 11.6 & 11.6 & 14.7 & 13.7 & 13.9 \\
\hline 0.4 & 0.5 & 1.1 & 0.5 & 0.4 & 1.1 & 2.8 & 3.4 & 3.4 \\
\hline 0.4 & 0.5 & 0.5 & 0.4 & 0.5 & 0.5 & 3.3 & 3.4 & 3.4 \\
\hline 40.1 & 38.8 & 34.2 & 19.8 & 15.2 & 14.8 & 17.9 & 17.5 & 16.6 \\
\hline 40.1 & 37.6 & 33.7 & 21.6 & 12.2 & 12.6 & 15.4 & 15.3 & 15.1 \\
\hline 37 & 35.1 & 31.9 & 20.9 & 10.6 & 10.4 & 13.5 & 13 & 12.4 \\
\hline 35.3 & 35 & 30.7 & 21.8 & 10.8 & 10.9 & 13.3 & 12.9 & 14 \\
\hline 39.1 & 36.6 & 34.7 & 24.3 & 9.6 & 9.9 & 12.2 & 11.4 & 11.9 \\
\hline 0.5 & 0.4 & 0.5 & 0.4 & 0.5 & 0.4 & 3.4 & 3.4 & 3.4 \\
\hline 0.4 & 0.5 & 0.5 & 0.4 & 0.5 & 0.5 & 3.3 & 2.8 & 3.4 \\
\hline 37.7 & 36.7 & 33.4 & 17.7 & 9.4 & 9.5 & 12.2 & 12.5 & 11.9 \\
\hline 30.5 & 28.6 & 23.7 & 17.3 & 9.4 & 9.1 & 12 & 11.7 & 11.9 \\
\hline 29.4 & 27.1 & 22.8 & 13.5 & 9.1 & 9.2 & 11.6 & 11.3 & 11.8 \\
\hline 25.1 & 22.7 & 22.1 & 14.4 & 7.7 & 8.8 & 10.6 & 11.4 & 10.7 \\
\hline 26.8 & 25.6 & 23.6 & 14.6 & 7.8 & 8.6 & 10.8 & 10.9 & 10 \\
\hline 1 & 0.5 & 0.5 & 0.5 & 0.4 & 0.5 & 3.3 & 3.4 & 3.4 \\
\hline 0.5 & 1 & 0.5 & 0.5 & 0.5 & 1.1 & 2.6 & 3.5 & 3.4 \\
\hline 8.6 & 8.1 & 8.5 & 8.4 & 8.8 & 8.4 & 10.9 & 11.5 & 10.3 \\
\hline 25.3 & 24.5 & 22.4 & 14.8 & 8.1 & 7.9 & 10.8 & 10.1 & 10.7 \\
\hline 24.2 & 25.6 & 23.4 & 13.9 & 9 & 8.8 & 11.8 & 12.1 & 10.9 \\
\hline 25.2 & 23.2 & 21.3 & 14.3 & 8.1 & 9 & 11 & 10.7 & 11.4 \\
\hline 25.1 & 24 & 22 & 14.5 & 9.4 & 9.5 & 11.6 & 11.9 & 11.7 \\
\hline 0.4 & 0.4 & 0.4 & 0.5 & 1 & 0.4 & 3.4 & 3.3 & 3.4 \\
\hline 0.4 & 0.4 & 0.4 & 0.4 & 0.4 & 1.1 & 3.3 & 3.4 & 2.8 \\
\hline 33.1 & 32.7 & 28.6 & 20.1 & 15.2 & 14.5 & 17.3 & 17.4 & 17.6 \\
\hline 30.5 & 32 & 27.9 & 17.4 & 9.2 & 9.9 & 12.2 & 11.7 & 11.9 \\
\hline
\end{tabular}


lights

\begin{tabular}{|c|c|c|c|c|c|c|c|c|}
\hline 34.6 & 32.8 & 30.9 & 18 & 9.1 & 8.8 & 11.5 & 11.8 & 11.1 \\
\hline 31.1 & 33 & 31.6 & 16.8 & 8.7 & 8.7 & 10.9 & 11 & 11.5 \\
\hline 27.6 & 26.3 & 26.4 & 16.8 & 10.1 & 9.9 & 12.2 & 12.6 & 12.7 \\
\hline 0.4 & 1 & 0.4 & 0.4 & 0.4 & 0.5 & 3.3 & 3.3 & 3.3 \\
\hline 0.4 & 0.4 & 0.4 & 0.4 & 0.4 & 0.4 & 3.4 & 3.3 & 3.4 \\
\hline 35.7 & 34.6 & 28.6 & 15.7 & 11.1 & 10.8 & 12.3 & 13.4 & 14.2 \\
\hline 33.9 & 30.8 & 26.5 & 18.7 & 10.1 & 10.4 & 13 & 13.1 & 13.4 \\
\hline 32 & 30.3 & 29.7 & 16.6 & 8.2 & 9 & 11.8 & 11.5 & 11.6 \\
\hline 25.1 & 26.6 & 25.9 & 15.2 & 8.5 & 8.3 & 11 & 11.6 & 11.1 \\
\hline 31 & 34.6 & 31.3 & 21.5 & 11.2 & 10.9 & 13.8 & 13.6 & 13.3 \\
\hline 0.4 & 0.4 & 0.4 & 0.3 & 0.4 & 0.4 & 3.2 & 3.4 & 3.3 \\
\hline 0.4 & 0.4 & 0.4 & 0.4 & 0.4 & 0.4 & 2.6 & 3.3 & 3.4 \\
\hline 28.3 & 28.5 & 27.7 & 17.3 & 10.4 & 9.4 & 12.3 & 13 & 12 \\
\hline 27.7 & 26.3 & 23.8 & 15.3 & 8.4 & 9.9 & 12.6 & 11.6 & 11.8 \\
\hline 26.3 & 26 & 25.5 & 17.6 & 10.2 & 11.4 & 13.6 & 13.5 & 12.4 \\
\hline 25.9 & 23.7 & 24.8 & 16.8 & 11 & 11.7 & 13.1 & 13.2 & 12.8 \\
\hline 27.1 & 28.4 & 28.2 & 26.8 & 19.1 & 12.5 & 12.9 & 12.7 & 12.7 \\
\hline 24.8 & 14.1 & 13.2 & 3 & 0.4 & 0.4 & 3.3 & 2.8 & 3.4 \\
\hline 16 & 0.4 & 0.4 & 0.4 & 0.4 & 0.4 & 3.4 & 3.4 & 2.7 \\
\hline 25.7 & 26.5 & 26.7 & 16.3 & 9.4 & 10.5 & 12.3 & 12.6 & 11.8 \\
\hline 34.4 & 37.2 & 33.7 & 15.9 & 9.4 & 10.5 & 12.7 & 12.3 & 12.8 \\
\hline 43.9 & 45.3 & 43.3 & 23.8 & 9.6 & 11.3 & 13.1 & 12.8 & 12.7 \\
\hline 44.2 & 47.7 & 48.9 & 25.8 & 10.6 & 10 & 13 & 12.7 & 13.1 \\
\hline 36.2 & 35.8 & 34.6 & 20.6 & 11.3 & 11.7 & 12.7 & 13.3 & 12.1 \\
\hline 26.6 & 26.4 & 22.5 & 25.5 & 25.2 & 19.9 & 10.1 & 3.3 & 2.7 \\
\hline 0.5 & 0.4 & 1 & 0.5 & 0.5 & 1 & 2.7 & 3.4 & 2.8 \\
\hline 41.1 & 42.4 & 39.4 & 32.1 & 24.9 & 23.9 & 26.6 & 26.5 & 26.2 \\
\hline 37.2 & 36.6 & 38.7 & 35.8 & 32.2 & 31.9 & 32.9 & 33 & 32.5 \\
\hline 40.2 & 38.9 & 38.6 & 36.8 & 32.6 & 31.8 & 31.1 & 31.3 & 22.8 \\
\hline 59.4 & 61.6 & 54.5 & 39.6 & 29 & 28.7 & 31 & 30.2 & 31.4 \\
\hline 60.3 & 62.1 & 62.9 & 52.4 & 45.6 & 40.3 & 42.2 & 39.9 & 35.9 \\
\hline 47.3 & 44.5 & 38 & 25.2 & 29.7 & 15.4 & 2.6 & 3.3 & 3.4 \\
\hline 25.9 & 11.6 & 0.4 & 0.4 & 0.4 & 0.4 & 3.2 & 2.8 & 3.3 \\
\hline 60.4 & 60.4 & 58.1 & 42.6 & 37.3 & 32.2 & 33.9 & 32.8 & 32.6 \\
\hline 62.1 & 60.2 & 52.9 & 42.2 & 39.4 & 36.9 & 37.6 & 36.6 & 35.8 \\
\hline 61.6 & 60.7 & 55.1 & 46.2 & 40.2 & 35 & 33.5 & 34.3 & 32.2 \\
\hline 61.7 & 58.8 & 54.9 & 46 & 34.2 & 30.2 & 32.1 & 30.6 & 31 \\
\hline 61 & 59.8 & 57.3 & 46.5 & 38.8 & 35.5 & 33.2 & 30.5 & 29 \\
\hline 0.4 & 0.4 & 0.5 & 0.4 & 0.4 & 0.4 & 3.4 & 2.7 & 3.4 \\
\hline 1 & 0.4 & 0.5 & 0.4 & 1 & 0.4 & 2.7 & 2.8 & 3.3 \\
\hline 59.6 & 59.6 & 54 & 46.6 & 36.5 & 32.2 & 32.1 & 32.1 & 31.3 \\
\hline 61 & 59.4 & 52.8 & 43.4 & 36.1 & 32.8 & 32.5 & 30.9 & 32 \\
\hline 62.1 & 61.3 & 54.8 & 42.7 & 37.5 & 36.6 & 38.6 & 36.4 & 34.3 \\
\hline 62.7 & 60.2 & 53.2 & 44 & 36.1 & 33.4 & 32.6 & 32 & 30.8 \\
\hline 58.5 & 56.6 & 51.8 & 43.3 & 39 & 39.5 & 37.7 & 37.8 & 34.2 \\
\hline 0.5 & 0.4 & 0.4 & 0.4 & 0.4 & 1 & 2.8 & 3.3 & 2.8 \\
\hline 0.4 & 0.4 & 0.4 & 1 & 0.5 & 0.4 & 2.8 & 3.3 & 3.4 \\
\hline 61.6 & 59.7 & 49 & 41.3 & 38.1 & 33.4 & 32.7 & 33.3 & 32.8 \\
\hline 61.8 & 59.3 & 52.8 & 44.4 & 36.7 & 33.6 & 32.8 & 32.3 & 29.2 \\
\hline 62.5 & 60.9 & 53.4 & 39.9 & 38.9 & 38.3 & 36.7 & & 37.9 \\
\hline 62.2 & 61.7 & 53.9 & 46.1 & 39.1 & 37.4 & 38.3 & 37.1 & 36.7 \\
\hline
\end{tabular}


lights

\begin{tabular}{|c|c|c|c|c|c|c|c|c|}
\hline 64.3 & 61.7 & 50.8 & 37.3 & 36.6 & 34.6 & 35.5 & 35.4 & 33.3 \\
\hline 24.4 & 28 & 27.9 & 27 & 27.1 & 27.4 & 30.5 & 30.1 & 17 \\
\hline 0.5 & 0.4 & 0.4 & 0.4 & 0.4 & 0.4 & 2.8 & 3.4 & 2.8 \\
\hline 11.5 & 11.5 & 11.3 & 12.1 & 11.3 & 11.3 & 14 & 14.4 & 13.6 \\
\hline 62.7 & 60.4 & 57.4 & 43.7 & 37.7 & 34.9 & 33.5 & 33.1 & 30.4 \\
\hline 61.2 & 61.3 & 54.3 & 45.3 & 43.1 & 37.5 & 34.5 & 33 & 33.2 \\
\hline 62.2 & 63.1 & 54.8 & 41.3 & 35.8 & 36 & 35.6 & 32 & 33.7 \\
\hline 60.9 & 59 & 54.8 & 46 & 41.4 & 39.4 & 36.4 & 34.6 & 33 \\
\hline 27.8 & 2.1 & 0.4 & 0.4 & 0.4 & 0.4 & 2.9 & 3.5 & 2.9 \\
\hline 2.1 & 0.4 & 0.4 & 0.4 & 0.5 & 0.4 & 2.9 & 3.5 & 2.9 \\
\hline 61.2 & 60.8 & 51.9 & 35.1 & 33.8 & 31.3 & 33.6 & 34.3 & 33.5 \\
\hline 59.8 & 58.8 & 54.4 & 41.9 & 35 & 30.7 & 32.3 & 32.6 & 30.3 \\
\hline 59.2 & 60.4 & 54.7 & 40.8 & 31.6 & 30.3 & 32.2 & 31.6 & 29.1 \\
\hline 61.4 & 57.3 & 51.8 & 40 & 36 & 35.2 & 35.8 & 35 & 33.3 \\
\hline 60 & 58.8 & 57.6 & 50 & 42.6 & 34.1 & 32.7 & 32.9 & 32.9 \\
\hline 0.4 & 0.4 & 0.4 & 0.4 & 0.4 & 0.4 & 2.8 & 2.8 & 2.9 \\
\hline 0.4 & 0.4 & 0.4 & 0.5 & 0.4 & 0.4 & 2.8 & 2.8 & 2.8 \\
\hline 60.1 & 59.8 & 51.4 & 42.3 & 39.1 & 34.3 & 33.4 & 33 & 32.2 \\
\hline 62.2 & 60.2 & 56.2 & 44.9 & 40.8 & 36.7 & 37 & 34.9 & 35 \\
\hline 62.9 & 62.4 & 56.4 & 47.1 & 44.7 & 36.9 & 37.2 & 35.1 & 34.1 \\
\hline 63.9 & 64.1 & 62.3 & 54.7 & 55.5 & 58 & 54 & 34.2 & 36.2 \\
\hline 60.6 & 58.2 & 53 & 42.3 & 38.1 & 34 & 36.2 & 35.5 & 29.4 \\
\hline 0.4 & 1 & 0.5 & 0.4 & 0.4 & 0.4 & 2.8 & 2.8 & 3.4 \\
\hline 0.4 & 0.4 & 0.4 & 0.4 & 0.4 & 0.4 & 3.4 & 2.8 & 2.8 \\
\hline 62.6 & 61.1 & 56 & 48.4 & 42.5 & 43 & 43.8 & 41.2 & 37.9 \\
\hline 59.1 & 61.4 & 57.5 & 49.1 & 47.4 & 42.6 & 39.2 & 36.7 & 31.8 \\
\hline 60.1 & 60.5 & 56.4 & 46.9 & 40.1 & 36.8 & 36.9 & 36 & 33.4 \\
\hline 61.4 & 58.9 & 54 & 45 & 41.1 & 39.7 & 40.7 & 39.3 & 34.2 \\
\hline 53.1 & 58.5 & 54.2 & 45.5 & 41.9 & 37.4 & 39.9 & 34.8 & 31.3 \\
\hline 20.8 & 19.8 & 19.9 & 21.1 & 21.5 & 17.1 & 16.9 & 10.2 & 2.8 \\
\hline 0.4 & 0.4 & 0.4 & 0.4 & 0.4 & 0.4 & 2.8 & 3.3 & 2.8 \\
\hline 59.2 & 58.4 & 50.3 & 40.7 & 37.8 & 38.1 & 41.5 & 39.4 & 36.7 \\
\hline 59.4 & 62.2 & 52.3 & 39.6 & 36.8 & 35 & 34.6 & 34.1 & 31.9 \\
\hline 61.2 & 62.1 & 54.7 & 47.7 & 42.6 & 40.4 & 41 & 40.3 & 37.4 \\
\hline 58.9 & 56.8 & 52.5 & 46.6 & 43.1 & 40.8 & 43.1 & 45.1 & 34.8 \\
\hline 59.1 & 59.9 & 54.5 & 45.2 & 42 & 43 & 43.7 & 42.3 & 42 \\
\hline 2.3 & 0.4 & 0.4 & 0.4 & 0.4 & 1.1 & 2.7 & 2.8 & 3.4 \\
\hline 0.4 & 1 & 0.4 & 0.4 & 0.5 & 0.4 & 2.8 & 3.4 & 2.7 \\
\hline 37.5 & 36 & 35.9 & 34.6 & 36.7 & 38.8 & 39.8 & 34.2 & 33.1 \\
\hline 62.2 & 63.4 & 55.8 & 44.4 & 39.5 & 36 & 34.8 & 33.4 & 31.8 \\
\hline 63.5 & 63.2 & 56.4 & 38.3 & 35.6 & 35.2 & 37.4 & 39.6 & 38.6 \\
\hline 61.4 & 61.8 & 55.5 & 42.9 & 39 & 36.3 & 36.8 & 36.5 & 33.7 \\
\hline 58.7 & 61.1 & 55.7 & 45.6 & 35.8 & 32.4 & 34.3 & 34 & 31 \\
\hline 21.1 & 20.1 & 1.4 & 1.1 & 0.4 & 0.4 & 3.3 & 2.8 & 2.8 \\
\hline 0.4 & 0.5 & 0.4 & 0.4 & 0.4 & 0.4 & 2.6 & 3.2 & 2.8 \\
\hline 61.2 & 60.2 & 53.7 & 48 & 44.4 & 38.2 & 37.7 & 35 & 33.1 \\
\hline 60.9 & 62.2 & 58.1 & 44.3 & 36.8 & 35.6 & 35.5 & 31.4 & 30.1 \\
\hline 60.1 & 60.9 & 54.2 & 42.9 & 37 & 32.9 & 34.6 & 33.9 & 33.9 \\
\hline 61.9 & 61.6 & 55.4 & 44.4 & 43.3 & 36.6 & 34.8 & 34 & 30.1 \\
\hline 60.2 & 61.8 & 52.9 & 37 & 34.4 & 30.8 & 31.9 & 31.7 & 30.3 \\
\hline 25.1 & 24 & 17.1 & 0.8 & 0.4 & 0.4 & 3.4 & 2.7 & 2.8 \\
\hline
\end{tabular}


lights

\begin{tabular}{|c|c|c|c|c|c|c|c|c|}
\hline 0.4 & 0.4 & 0.4 & 0.4 & 0.4 & 0.4 & 2.8 & 2.7 & 3.4 \\
\hline 63.8 & 64.9 & 52.2 & 44 & 35.5 & 37.1 & 42.4 & 39.9 & 36 \\
\hline 63.4 & 64.6 & 58.1 & 44.5 & 40.9 & 38.5 & 38.3 & 35.9 & 35.1 \\
\hline 64.1 & 63.3 & 50.7 & 43.5 & 39 & 32.8 & 34.2 & 34.6 & 35.9 \\
\hline 62.9 & 63.2 & 52.8 & 39.8 & 39.6 & 46.3 & 44.4 & 44.2 & 37.2 \\
\hline 59.8 & 63.4 & 56.4 & 48.1 & 45.4 & 46 & 44 & 39.3 & 38.5 \\
\hline 18.1 & 13.8 & 2.8 & 22.1 & 12.5 & 0.4 & 2.8 & 2.9 & 3.4 \\
\hline 21.9 & 0.8 & 0.4 & 0.4 & 0.4 & 0.4 & 3.4 & 2.8 & 2.8 \\
\hline 56.4 & 60.6 & 56.2 & 44.6 & 38.1 & 38.2 & 41.2 & 41.8 & 41.8 \\
\hline 63.3 & 61.6 & 58 & 43.1 & 40.3 & 42.2 & 40.6 & 38.2 & 36.7 \\
\hline 60.4 & 59.8 & 54.7 & 42.9 & 37.9 & 34.8 & 35.9 & 36.9 & 35.1 \\
\hline 61.3 & 63.7 & 56.7 & 48.6 & 43.6 & 45.5 & 46.2 & 42.7 & 36 \\
\hline 52.8 & 50.5 & 44.7 & 46.3 & 48 & 45.5 & 42.1 & 34.3 & 33 \\
\hline 13.6 & 0.8 & 0.4 & 0.4 & 0.5 & 0.4 & 3.4 & 3 & 3 \\
\hline 0.4 & 0.4 & 0.5 & 0.4 & 0.4 & 0.4 & 2.7 & 3.6 & 2.9 \\
\hline 64.3 & 64.1 & 53.2 & 41.5 & 38.3 & 33.1 & 31.8 & 31.8 & 29.1 \\
\hline 60.4 & 60.7 & 56.6 & 46.5 & 43.9 & 40.1 & 37.9 & 35.1 & 34.7 \\
\hline 64.6 & 65.1 & 57.8 & 42.4 & 39.3 & 34.7 & 34.2 & 37.9 & 37.8 \\
\hline 62.2 & 64.8 & 54.8 & 45.3 & 42.5 & 39.6 & 41.7 & 41.9 & 41.6 \\
\hline 30.5 & 29.4 & 32.2 & 33.4 & 31.3 & 31.2 & 35.3 & 34.2 & 33.6 \\
\hline 0.4 & 0.4 & 0.4 & 0.4 & 0.4 & 0.4 & 3.5 & 2.8 & 2.8 \\
\hline 0.4 & 0.4 & 0.4 & 0.4 & 0.4 & 0.4 & 3.5 & 2.7 & 2.8 \\
\hline 64.3 & 65.4 & 60.1 & 44.9 & 43.3 & 45.1 & 42.1 & 41 & 37 \\
\hline 69.6 & 63.3 & 66.9 & 67 & 60.3 & 40.9 & 36.7 & 35.7 & 34.7 \\
\hline 64.5 & 59.7 & 58.7 & 45 & 40.2 & 37.1 & 37 & 37.8 & 34.8 \\
\hline 61.5 & 64.1 & 56.4 & 42.1 & 41.9 & 40.2 & 41.7 & 37.7 & 35.3 \\
\hline 65.2 & 65.4 & 52.5 & 42.6 & 38.8 & 38.3 & 41.3 & 40.3 & 38.1 \\
\hline 0.4 & 0.4 & 0.5 & 0.4 & 0.4 & 0.4 & 2.7 & 2.7 & 3.4 \\
\hline 0.4 & 0.5 & 0.4 & 0.4 & 0.4 & 0.4 & 2.6 & 2.7 & 3.4 \\
\hline 62.7 & 63.8 & 48.9 & 37.7 & 45.2 & 42.2 & 48.9 & 47.8 & 46.8 \\
\hline 62.6 & 49.6 & 39.8 & 39.6 & 37.9 & 37.3 & 39.9 & 39.2 & 26.5 \\
\hline 0.5 & 0.4 & 0.4 & 0.4 & 0.4 & 0.4 & 0.4 & 0.4 & 0.5 \\
\hline 0.4 & 1.1 & 0.4 & 0.4 & 0.4 & 0.4 & 0.4 & 0.4 & 0.4 \\
\hline 0.4 & 0.4 & 0.4 & 0.4 & 0.4 & 0.4 & 0.4 & 0.4 & 0.4 \\
\hline 0.4 & 0.4 & 0.4 & 0.4 & 0.4 & 0.4 & 0.4 & 0.4 & 0.4 \\
\hline 0.4 & 0.4 & 0.5 & 0.4 & 0.4 & 0.4 & 0.4 & 0.4 & 0.4 \\
\hline 61.1 & 61.8 & 52 & 42 & 35.4 & 33.1 & 34.3 & 34.6 & 35.9 \\
\hline 63.4 & 64.5 & 55.6 & 41.3 & 38.5 & 34.5 & 37.9 & 37.5 & 36.6 \\
\hline 64.4 & 63.4 & 53 & 45.2 & 41.1 & 40.4 & 42.2 & 40.3 & 39.5 \\
\hline 63.9 & 63.2 & 55 & 44.9 & 39.6 & 37.2 & 38.3 & 36.6 & 34.7 \\
\hline 63.7 & 63.5 & 60.1 & 46 & 35.6 & 35.6 & 39.5 & 38.1 & 35.6 \\
\hline 0.5 & 0.4 & 0.4 & 0.4 & 0.4 & 0.4 & 3.4 & 2.7 & 2.7 \\
\hline 0.4 & 0.4 & 0.4 & 0.4 & 0.4 & 0.4 & 2.8 & 2.7 & 3.3 \\
\hline 63.9 & 62.6 & 56.4 & 48 & 38.9 & 32.1 & 34.1 & 35.3 & 34.5 \\
\hline 64.1 & 61.6 & 55.9 & 44.8 & 41.8 & 41 & 40 & 40 & 38.3 \\
\hline 60.6 & 63.4 & 56.3 & 44.4 & 37.5 & 36.4 & 39.3 & 38.8 & 34.3 \\
\hline 60.1 & 61.5 & 55.3 & 47 & 42.4 & 40 & 40.2 & 38.8 & 36 \\
\hline 63.4 & 62.3 & 55.9 & 43.1 & 39.5 & 41.4 & 45 & 44.7 & 41.1 \\
\hline 0.4 & 0.4 & 0.4 & 0.4 & 0.4 & 0.4 & 2.8 & 3.3 & 2.8 \\
\hline 0.4 & 0.4 & 0.4 & 0.4 & 0.4 & 0.4 & 2.8 & 3.3 & 2.7 \\
\hline 63.2 & 61.8 & 53.5 & 46.5 & 41 & 40.3 & 43 & 40.1 & 39.3 \\
\hline
\end{tabular}


lights

\begin{tabular}{|c|c|c|c|c|c|c|c|c|}
\hline 61.5 & 65.8 & 55.5 & 41.2 & 41.4 & 40.8 & 42.8 & 39.6 & 35.2 \\
\hline 61.3 & 60.7 & 52.9 & 42.9 & 39.2 & 37.8 & 40.4 & 38.6 & 36.4 \\
\hline 61 & 64.4 & 59.9 & 52.5 & 47.4 & 44.8 & 46.6 & 40.7 & 37 \\
\hline 65.1 & 58.2 & 46.9 & 42.6 & 39 & 40.8 & 44.5 & 43.8 & 39.8 \\
\hline 0.4 & 0.4 & 0.4 & 0.4 & 0.4 & 0.4 & 2.8 & 2.7 & 3.3 \\
\hline 0.4 & 0.4 & 0.4 & 0.4 & 0.4 & 0.4 & 2.7 & 2.7 & 3.4 \\
\hline 52.2 & 52.9 & 30.3 & 0.4 & 0.4 & 0.4 & 0.4 & 0.4 & 0.4 \\
\hline 35.3 & 35.7 & 25.3 & 0.4 & 0.4 & 0.4 & 0.5 & 0.4 & 0.4 \\
\hline 23.9 & 10.9 & 8.2 & 0.5 & 0.4 & 0.4 & 0.4 & 0.4 & 0.4 \\
\hline 0.4 & 0.4 & 0.4 & 0.4 & 0.4 & 0.4 & 0.4 & 0.4 & 0.4 \\
\hline 0.5 & 0.4 & 0.4 & 0.4 & 0.4 & 0.4 & 0.4 & 0.4 & 0.4 \\
\hline 1 & 0.4 & 0.4 & 1 & 0.4 & 0.4 & 0.4 & 0.4 & 0.4 \\
\hline 0.5 & 0.4 & 1 & 0.4 & 0.4 & 0.4 & 0.4 & 0.4 & 0.4 \\
\hline 0.4 & 0.4 & 0.4 & 0.4 & 0.4 & 0.4 & 0.4 & 0.4 & 0.4 \\
\hline 0.4 & 0.4 & 0.4 & 0.4 & 0.4 & 0.4 & 0.4 & 0.4 & 0.4 \\
\hline 0.4 & 0.3 & 0.4 & 0.4 & 0.4 & 0.4 & 0.4 & 0.4 & 0.4 \\
\hline 0.4 & 0.9 & 1.6 & 0.4 & 0.4 & 0.4 & 0.4 & 0.4 & 0.4 \\
\hline 0.4 & 0.4 & 0.4 & 0.4 & 0.4 & 0.4 & 0.4 & 0.4 & 0.3 \\
\hline 0.4 & 0.4 & 0.4 & 0.4 & 0.4 & 0.4 & 0.4 & 0.4 & 0.3 \\
\hline 0.4 & 0.4 & 0.4 & 0.4 & 0.4 & 0.3 & 0.4 & 0.4 & 0.4 \\
\hline 64.4 & 66 & 57.8 & 44.2 & 32.1 & 31.4 & 31.9 & 32 & 32.7 \\
\hline 63.4 & 65.2 & 55.2 & 39.7 & 36.3 & 33.3 & 42.5 & 44.1 & 43.2 \\
\hline 65.2 & 64.8 & 53.6 & 47.3 & 39.1 & 36.4 & 35.1 & 35.2 & 33.2 \\
\hline 63.5 & 64.5 & 54.8 & 43 & 40.7 & 39 & 34.9 & 32.6 & 32.5 \\
\hline 64.2 & 61.6 & 54 & 42.4 & 39.3 & 37.2 & 38.3 & 38.2 & 34.8 \\
\hline 0.4 & 0.4 & 0.4 & 0.4 & 0.4 & 0.4 & 3.4 & 2.9 & 3.5 \\
\hline 0.4 & 0.4 & 0.4 & 0.4 & 0.4 & 0.4 & 2.8 & 3.5 & 2.9 \\
\hline 63 & 64.7 & 57 & 48.1 & 43.8 & 40.2 & 41.3 & 38.8 & 35.6 \\
\hline 62.5 & 59.1 & 62.8 & 62 & 50.5 & 36 & 34.8 & 37.6 & 38.5 \\
\hline 62.8 & 61 & 57.6 & 49.4 & 42.8 & 41 & 44.4 & 43.1 & 41.6 \\
\hline 63.3 & 63.8 & 54 & 43 & 40.1 & 43.7 & 45.9 & 42.2 & 37.5 \\
\hline 61.1 & 62.6 & 54.9 & 48 & 43.9 & 41.6 & 43.2 & 41.9 & 40 \\
\hline 27 & 29.7 & 30 & 13.8 & 0.4 & 0.4 & 2.9 & 3.5 & 2.9 \\
\hline 0.4 & 0.4 & 0.4 & 0.4 & 0.5 & 0.4 & 2.8 & 2.9 & 3.6 \\
\hline 0.4 & 0.4 & 0.4 & 0.4 & 0.4 & 0.4 & 0.4 & 0.4 & 0.4 \\
\hline 62.6 & 63.5 & 55.8 & 46.2 & 41 & 36 & 38.9 & 35.7 & 31.9 \\
\hline 62.1 & 65.6 & 53.9 & 43.8 & 35.2 & 34.6 & 38.4 & 35.5 & 32.7 \\
\hline 60.6 & 61.4 & 57.2 & 44.5 & 47.3 & 44.5 & 41.9 & 41.6 & 41 \\
\hline 62.9 & 60.5 & 54.4 & 44.4 & 41 & 39 & 43.3 & 40.3 & 39.7 \\
\hline 0.5 & 0.4 & 0.4 & 0.4 & 0.4 & 0.4 & 2.8 & 3.5 & 2.9 \\
\hline 0.4 & 0.4 & 0.3 & 0.4 & 0.4 & 0.4 & 2.8 & 3 & 2.9 \\
\hline 62.5 & 55.2 & 54.1 & 40.1 & 42.3 & 42.9 & 45 & 44.5 & 41.6 \\
\hline 61.6 & 64.4 & 59 & 45.3 & 40.1 & 38.5 & 41.9 & 42.2 & 40.2 \\
\hline 62.8 & 65 & 59.6 & 47.3 & 38.9 & 35.8 & 39.7 & 41.2 & 37.1 \\
\hline 64 & 63.4 & 55.7 & 42.2 & 41.9 & 40.8 & 47 & 48.7 & 40.7 \\
\hline 57.6 & 57.5 & 55.1 & 43.9 & 40.8 & 41.6 & 44.2 & 45.2 & 39.9 \\
\hline 12.1 & 11.4 & 12 & 6.6 & 0.4 & 0.4 & 2.9 & 2.9 & 3.5 \\
\hline 0.4 & 0.4 & 0.4 & 0.4 & 0.4 & 0.4 & 3.4 & 3 & 2.9 \\
\hline 63.7 & 61.8 & 58.3 & 48.5 & 36.4 & 36.2 & 40.7 & 41.1 & 41.8 \\
\hline 58.9 & 59.9 & 56.6 & 44.7 & 39.5 & 35.6 & 36.6 & 35.7 & 33.3 \\
\hline 62.3 & 62.6 & 52.4 & 43.7 & 39 & 30.9 & 32.7 & 33.3 & 33.1 \\
\hline
\end{tabular}


lights

\begin{tabular}{|c|c|c|c|c|c|c|c|c|}
\hline 59.2 & 62.8 & 56.2 & 47 & 43 & 39.8 & 42.9 & 41.8 & 42.1 \\
\hline 34.8 & 33.9 & 34.1 & 34.4 & 34.3 & 34 & 37.3 & 38.8 & 39.3 \\
\hline 0.4 & 0.4 & 0.4 & 0.4 & 0.4 & 0.4 & 2.9 & 2.9 & 3.5 \\
\hline 0.4 & 0.4 & 0.4 & 0.4 & 0.4 & 0.4 & 3.5 & 2.9 & 3.4 \\
\hline 64.3 & 62 & 58.4 & 48.1 & 40.8 & 37.6 & 40.4 & 41.2 & 41.2 \\
\hline 63 & 62 & 55.7 & 44.9 & 43 & 46.5 & 47.9 & 47.4 & 45.1 \\
\hline 63.6 & 62.7 & 55 & 43 & 35.2 & 35.7 & 38.1 & 39.7 & 37.2 \\
\hline 61.6 & 63.4 & 56.3 & 46.1 & 39.3 & 36 & 39.2 & 38.5 & 36 \\
\hline 60.6 & 59.6 & 53.2 & 42.8 & 39.8 & 37.4 & 38.7 & 38.3 & 40.5 \\
\hline 0.4 & 0.4 & 0.4 & 0.4 & 0.4 & 0.4 & 2.8 & 3.5 & 3 \\
\hline 0.4 & 0.4 & 0.4 & 0.4 & 0.4 & 0.4 & 3.4 & 3 & 2.9 \\
\hline 66.1 & 64.5 & 59.7 & 49.1 & 46.5 & 45.5 & 46.9 & 49.4 & 48 \\
\hline 62.1 & 66.6 & 59.9 & 50.8 & 48.6 & 50 & 52.5 & 53.7 & 46.7 \\
\hline 61.3 & 63.5 & 58.3 & 52.9 & 50.7 & 51.3 & 51.2 & 43.4 & 38.6 \\
\hline 62.7 & 63.5 & 55.1 & 53.9 & 52.4 & 45.8 & 46.6 & 44.9 & 40.5 \\
\hline 59.4 & 58.3 & 58.3 & 48.3 & 49.2 & 48.1 & 51.4 & 49.4 & 47.8 \\
\hline 0.4 & 0.4 & 0.4 & 0.4 & 0.4 & 0.4 & 2.1 & 3.5 & 2.9 \\
\hline 0.4 & 0.4 & 0.4 & 0.4 & 0.4 & 0.4 & 2.7 & 2.9 & 3.5 \\
\hline 40.7 & 39.2 & 40.3 & 42.4 & 42.1 & 41.7 & 46.3 & 44.7 & 42 \\
\hline 62.1 & 64 & 59.1 & 47.9 & 42 & 41.7 & 41 & 38.5 & 36.2 \\
\hline 65.5 & 64 & 55 & 46.7 & 44.9 & 44.3 & 45.3 & 44.4 & 42.9 \\
\hline 60.8 & 63 & 55 & 49.6 & 44.8 & 43.7 & 46.5 & 45.3 & 45.2 \\
\hline 57.8 & 60.1 & 51.2 & 40.1 & 38.8 & 35.6 & 37.5 & 37.8 & 34.5 \\
\hline 0.4 & 0.4 & 0.4 & 0.4 & 0.4 & 0.4 & 2.8 & 2.9 & 2.9 \\
\hline 0.4 & 0.4 & 0.4 & 0.4 & 0.4 & 0.4 & 2.8 & 2.9 & 3.5 \\
\hline 62.3 & 60.5 & 56.1 & 42.4 & 37.2 & 34.3 & 37.6 & 39.9 & 36.6 \\
\hline 62.7 & 63 & 48.4 & 38 & 36 & 33.8 & 39.7 & 40.9 & 36.9 \\
\hline 63.1 & 63.4 & 51.6 & 42.3 & 36.7 & 38.2 & 42.4 & 45.3 & 44.4 \\
\hline 62.2 & 62.3 & 56.3 & 50.1 & 49.1 & 40.9 & 41.3 & 42 & 42.6 \\
\hline 63.8 & 66 & 56.1 & 47.1 & 47.5 & 50.5 & 51.8 & 48.9 & 45.6 \\
\hline 27.4 & 23.5 & 25.1 & 12.6 & 0.4 & 0.3 & 2.9 & 2.9 & 3.5 \\
\hline 0.4 & 0.4 & 0.4 & 0.3 & 0.4 & 0.4 & 3.4 & 2.9 & 3.5 \\
\hline 64.7 & 61.2 & 52.9 & 42.5 & 39.1 & 36.1 & 39.5 & 42.9 & 44.7 \\
\hline 60.1 & 64 & 54.8 & 43.1 & 38.8 & 35.4 & 36 & 36.9 & 37.4 \\
\hline 61.2 & 62.3 & 51 & 42.9 & 40.3 & 33.7 & 34 & 35.2 & 32.3 \\
\hline 64.9 & 61.5 & 57.2 & 47 & 45.6 & 46.1 & 46.7 & 44.4 & 42.1 \\
\hline 58.2 & 50.2 & 40.6 & 39.7 & 35.8 & 35.6 & 36.8 & 35.8 & 36.4 \\
\hline 0.4 & 0.4 & 0.4 & 0.4 & 0.3 & 0.4 & 2.9 & 2.9 & 2.8 \\
\hline 0.4 & 0.4 & 0.4 & 0.4 & 0.4 & 0.3 & 2.9 & 2.9 & 3.5 \\
\hline 10.7 & 10.7 & 11.3 & 14.9 & 15.8 & 15.2 & 16.8 & 17.9 & 17.8 \\
\hline 15.7 & 14.9 & 15.5 & 15.1 & 15.6 & 15.1 & 17.4 & 17.1 & 17.6 \\
\hline 40.3 & 39 & 24.5 & 12.6 & 13.1 & 12.2 & 14.3 & 14.7 & 15.9 \\
\hline 14.6 & 20.8 & 18.7 & 18.3 & 17.5 & 19.1 & 20.8 & 21.7 & 21.9 \\
\hline 21.1 & 21.6 & 18.7 & 12.2 & 12.9 & 14.5 & 14.4 & 15.4 & 15.4 \\
\hline 13.9 & 14.8 & 15.4 & 14.5 & 14.4 & 14.8 & 14 & 14.1 & 14.1 \\
\hline 13.7 & 14 & 14.1 & 14 & 14.9 & 13.8 & 13.8 & 14.2 & 13.9 \\
\hline 57.6 & 59.9 & 51.1 & 41.5 & 35.1 & 32 & 35.5 & 36.2 & 32.4 \\
\hline 62.7 & 61.3 & 56.1 & 41.2 & 41.1 & 39.7 & 40.5 & 40.8 & 37.5 \\
\hline 63.3 & 60.3 & 51.5 & 42.1 & 41.2 & 37.2 & 36.7 & 36.9 & 33.9 \\
\hline 61.7 & 62.6 & 54.2 & 49.4 & 46.4 & 44.7 & 41.2 & 42.4 & 40.5 \\
\hline 62.8 & 63.7 & 56.7 & 44.2 & 36.8 & 36.1 & 33.7 & 33.8 & 34 \\
\hline
\end{tabular}


lights

\begin{tabular}{|c|c|c|c|c|c|c|c|c|}
\hline 12.1 & 13.3 & 13.6 & 13.7 & 8.7 & 3.5 & 3.5 & 3.6 & 2.9 \\
\hline 3.6 & 3.5 & 3.6 & 3.5 & 3.5 & 3.6 & 3.5 & 2.9 & 3.5 \\
\hline 62.6 & 63.2 & 55.1 & 48.1 & 43.1 & 38.9 & 41 & 43.1 & 45.6 \\
\hline 65.1 & 64.8 & 56.5 & 50.1 & 46.9 & 42.9 & 47.7 & 48.6 & 42.6 \\
\hline 60.8 & 61.6 & 60.5 & 51.3 & 49.5 & 48.1 & 50.3 & 49.9 & 43.9 \\
\hline 63.3 & 63.2 & 57.3 & 51.5 & 45.1 & 41.6 & 42.3 & 40 & 35.7 \\
\hline 63.3 & 64.1 & 55.8 & 37.3 & 34.5 & 35.3 & 37.1 & 36.9 & 36.1 \\
\hline 44.8 & 40.4 & 1.5 & 0.5 & 0.4 & 3.5 & 2.9 & 3.6 & 3.5 \\
\hline 33.3 & 15.1 & 15.4 & 0.5 & 0.4 & 0.5 & 3.5 & 3.5 & 3 \\
\hline 61 & 63.1 & 56.9 & 46.5 & 45.3 & 45.5 & 47.9 & 46.9 & 42.2 \\
\hline 62.8 & 62 & 57.2 & 46.7 & 47.1 & 47.1 & 48.3 & 47.2 & 39.2 \\
\hline 63.8 & 65.5 & 58.9 & 44.2 & 41.4 & 37 & 38.8 & 37.9 & 38.5 \\
\hline 62.5 & 60.9 & 53.9 & 50.8 & 47.9 & 44.2 & 46.9 & 41.8 & 41 \\
\hline 62.2 & 59.9 & 46.4 & 37.9 & 42.1 & 42.7 & 46.5 & 46 & 42 \\
\hline 0.4 & 0.5 & 0.5 & 0.4 & 0.5 & 0.5 & 3.3 & 3.5 & 3.5 \\
\hline 0.4 & 0.5 & 0.4 & 0.5 & 0.5 & 0.5 & 3.4 & 2.9 & 3.6 \\
\hline 63.2 & 64.2 & 57.1 & 48 & 48.6 & 46.9 & 47.2 & 46.8 & 46.3 \\
\hline 65.3 & 64.4 & 59.1 & 50.9 & 44.1 & 43.8 & 42.7 & 42.5 & 40.3 \\
\hline 64.2 & 62.4 & 60.1 & 48 & 43.6 & 43.3 & 45 & 45.4 & 42 \\
\hline 58.1 & 62 & 52.7 & 44.2 & 40.2 & 37.1 & 40.3 & 39.4 & 39.5 \\
\hline 34 & 33.8 & 33.1 & 32.8 & 32.1 & 32.3 & 35.9 & 35 & 35.6 \\
\hline 0.4 & 0.5 & 0.4 & 0.5 & 0.5 & 0.4 & 3.5 & 3.5 & 3.5 \\
\hline 0.5 & 0.4 & 0.5 & 0.5 & 0.4 & 0.5 & 3.3 & 3.6 & 3.5 \\
\hline 24.4 & 22.6 & 24.2 & 28.8 & 28.5 & 28.7 & 27.4 & 31.5 & 28.8 \\
\hline 62 & 63.2 & 54.4 & 44.7 & 43.2 & 44.4 & 44.8 & 43.7 & 41.9 \\
\hline 60.5 & 62.8 & 54.8 & 44 & 41.6 & 40.7 & 42.1 & 40.6 & 36.5 \\
\hline 62.6 & 63.6 & 57.2 & 47.5 & 48.3 & 45.7 & 42.1 & 41.4 & 39.5 \\
\hline 60.8 & 60.4 & 56 & 46.6 & 41.6 & 39.4 & 39.7 & 40.7 & 39.2 \\
\hline 36.4 & 35.5 & 34.7 & 16 & 0.5 & 0.5 & 3.5 & 3.6 & 3.5 \\
\hline 0.4 & 0.5 & 0.5 & 0.4 & 0.5 & 0.5 & 3.5 & 3.5 & 3.6 \\
\hline 48.8 & 64.6 & 57.2 & 50.2 & 43.2 & 38.7 & 39.6 & 39.1 & 37.3 \\
\hline 62.7 & 64.1 & 57.5 & 42.7 & 38.1 & 38.4 & 41 & 41.6 & 38.1 \\
\hline 63.6 & 62.5 & 51.2 & 37.8 & 35.5 & 30.6 & 35.2 & 34.9 & 33.5 \\
\hline 61.4 & 60.5 & 54.1 & 45.6 & 36.4 & 34.7 & 36.7 & 34.3 & 32.6 \\
\hline 39.2 & 55.1 & 49.9 & 40.8 & 35.8 & 35.2 & 38 & 37 & 31.4 \\
\hline 25 & 24.6 & 24 & 11.6 & 0.5 & 0.4 & 3.5 & 3.5 & 2.9 \\
\hline 0.5 & 0.4 & 0.5 & 0.5 & 0.4 & 0.5 & 2.8 & 3.6 & 3.4 \\
\hline 62 & 62.6 & 53.3 & 40 & 38.6 & 35.5 & 39.7 & 38.5 & 38.2 \\
\hline 62 & 60.5 & 53.7 & 44 & 40.1 & 39.8 & 44.6 & 40.1 & 40.4 \\
\hline 62.5 & 63.4 & 54.6 & 43.8 & 44.5 & 45.1 & 48.2 & 47.8 & 45.2 \\
\hline 64.8 & 65.5 & 58.5 & 47.2 & 43.4 & 39 & 40.5 & 37.9 & 35.5 \\
\hline 60.8 & 60.9 & 54.9 & 47 & 41.3 & 40.2 & & & \\
\hline & & & & & & & & \\
\hline 0.4 & 0.5 & 0.4 & 0.5 & 0.5 & 0.4 & 1.2 & 1.9 & 3.4 \\
\hline 57.4 & 61.9 & 62.8 & 53.7 & 42.2 & 39.7 & 41.6 & 43.9 & 85 \\
\hline 60.8 & 59.8 & 55.9 & 44.7 & 41 & 39.6 & 42.5 & 42.3 & 40.3 \\
\hline 62.3 & 59.7 & 52.6 & 41.5 & 41.9 & 39.1 & 40.1 & 38.1 & 34.7 \\
\hline 63.7 & 62.3 & 56.5 & 45.7 & 39.4 & 36.5 & 37.9 & 37.7 & 36.3 \\
\hline 53.1 & 52 & 52.4 & 42.5 & 42.1 & 39.5 & 40.3 & 39.3 & 35.7 \\
\hline 0.4 & 0.5 & 0.5 & 0.4 & 0.5 & 0.4 & 3.4 & 3.6 & 3.5 \\
\hline 0.4 & 0.5 & 0.4 & 0.5 & 0.4 & 0.5 & 3.4 & 3.5 & 3.5 \\
\hline
\end{tabular}


lights

\begin{tabular}{|c|c|c|c|c|c|c|c|c|}
\hline 60.4 & 61.6 & 54.1 & 43.6 & 40 & 37 & 40 & 38.6 & 36.2 \\
\hline 59.7 & 59.7 & 53.1 & 42.8 & 37.8 & 36.4 & 37.8 & 37.6 & 35.6 \\
\hline 61.4 & 59.7 & 53.8 & 38.9 & 37.2 & 35.9 & 34.1 & 33.4 & 33 \\
\hline 55.9 & 63.1 & 52.5 & 42 & 38.1 & 36 & 34.6 & 34.1 & 32.2 \\
\hline 59.7 & 61.7 & 54.7 & 42.3 & 37.1 & 33.6 & 32.3 & 32.6 & 31.9 \\
\hline 0.5 & 0.4 & 0.5 & 0.5 & 0.4 & 0.5 & 3.4 & 3.5 & 3.5 \\
\hline 2.8 & 2.9 & 2.8 & 1.1 & 0.4 & 0.5 & 2.7 & 3.5 & 3.6 \\
\hline 59.7 & 58.1 & 55.7 & 47.7 & 39.8 & 34.6 & 34.6 & 35.9 & 35 \\
\hline 58.8 & 55.9 & 50.8 & 44.4 & 38.3 & 36.2 & 36.8 & 35 & 33.9 \\
\hline 56.6 & 58.1 & 55.5 & 43.8 & 39.1 & 37.3 & 37.6 & 36.8 & 28.7 \\
\hline 62.7 & 64.1 & 58.9 & 48.6 & 42.9 & 42.3 & 41.5 & 36.8 & 37.3 \\
\hline 64.2 & 62.5 & 57.5 & 44.3 & 37 & 35.6 & 36.6 & 32 & 31.2 \\
\hline 0.5 & 0.4 & 0.5 & 0.4 & 0.5 & 0.4 & 3.5 & 3.5 & 3.5 \\
\hline 0.5 & 0.4 & 0.5 & 0.4 & 0.5 & 0.4 & 3.4 & 2.8 & 3.5 \\
\hline 34.4 & 35.6 & 19.2 & 13.1 & 11.1 & 11.7 & 14.1 & 13.7 & 14.5 \\
\hline 42.9 & 41.1 & 37.6 & 35.1 & 22 & 12.2 & 14.8 & 14.6 & 14.4 \\
\hline 42.5 & 46.9 & 38.5 & 38.3 & 29.4 & 17.2 & 19 & 13.4 & 19.8 \\
\hline & & & & & & & & \\
\hline & & & & & & & & \\
\hline & & & & & & & & \\
\hline & & & & & & & & \\
\hline 43.34 & 42.25 & 35.33 & 27.40 & 25.38 & 25.14 & 25.67 & 26.12 & 25.87 \\
\hline 37.32 & 36.78 & 31.26 & 26.61 & 25.79 & 25.52 & 25.25 & 25.16 & 26.12 \\
\hline 43.40 & 43.02 & 37.86 & 33.63 & 32.52 & 33.00 & 32.72 & 32.41 & 31.40 \\
\hline 30.41 & 30.01 & 27.26 & 20.92 & 17.04 & 16.39 & 18.54 & 18.25 & 16.90 \\
\hline 41.26 & 40.37 & 36.19 & 29.02 & 25.59 & 23.93 & 24.95 & 23.98 & 22.69 \\
\hline 36.08 & 35.92 & 32.10 & 25.61 & 22.93 & 21.84 & 23.59 & 23.24 & 22.06 \\
\hline 42.44 & 42.59 & 37.32 & 30.59 & 28.13 & 26.98 & 28.70 & 28.62 & 27.30 \\
\hline 50.10 & 50.16 & 44.42 & 36.50 & 33.96 & 32.90 & 33.63 & 31.93 & 31.13 \\
\hline & & & & & & & & \\
\hline & & & & & & & & \\
\hline & & & & & & & & \\
\hline & & & & & & & & \\
\hline 29.132 & 28.586 & 24.408 & 18.834 & 17.115 & 16.354 & 16.689 & 16.676 & 15.881 \\
\hline 22.345 & 22.206 & 20.217 & 18.892 & 18.216 & 17.914 & 17.660 & 17.517 & 16.857 \\
\hline 24.357 & 24.470 & 21.448 & 18.738 & 17.496 & 16.770 & 16.283 & 15.894 & 15.055 \\
\hline 22.070 & 22.287 & 20.481 & 18.615 & 17.971 & 16.880 & 16.287 & 15.823 & 14.391 \\
\hline 25.493 & 26.152 & 23.705 & 19.441 & 18.019 & 17.001 & 16.349 & 15.695 & 14.793 \\
\hline 29.677 & 29.854 & 26.787 & 22.907 & 21.024 & 20.069 & 20.531 & 20.126 & 18.944 \\
\hline 25.323 & 25.678 & 22.767 & 19.214 & 18.529 & 17.772 & 17.535 & 17.285 & 16.218 \\
\hline 23.712 & 23.871 & 21.594 & 17.496 & 15.844 & 15.121 & 15.388 & 14.893 & 19.361 \\
\hline & & & & & & & & \\
\hline & & & & & & & & \\
\hline & & & & & & & & \\
\hline 22234.50 & 21675.40 & 18086.70 & 14055.00 & 13020.10 & 12895.30 & 13167.50 & 13397.40 & 13270.30 \\
\hline 8545.80 & 8422.70 & 7126.30 & 6067.60 & 5906.50 & 5844.10 & 5783.20 & 5762.60 & 5982.20 \\
\hline 8115.10 & 8045.40 & 7078.90 & 6254.40 & 6081.50 & 6171.40 & 6117.80 & 6060.60 & 5871.30 \\
\hline 2676.20 & 2640.90 & 2398.90 & 1841.00 & 1499.70 & 1442.50 & 1631.30 & 1605.70 & 1487.60 \\
\hline 6106.70 & 5975.30 & 5355.90 & 4295.30 & 3786.60 & 3540.90 & 3693.10 & 3525.50 & 3358.80 \\
\hline 2381.20 & 2370.50 & 2118.30 & 1690.40 & 1513.30 & 1441.40 & 1556.90 & 1534.10 & 1455.70 \\
\hline
\end{tabular}


lights

\begin{tabular}{|r|r|r|r|r|r|r|r|r|}
\hline 3056.00 & 3066.50 & 2686.90 & 2202.30 & 2025.30 & 1942.20 & 2066.30 & 2060.40 & 1965.80 \\
\hline 1271.80 & 1279.70 & 1149.40 & 938.80 & 828.90 & 767.60 & 770.50 & 743.90 & 761.30 \\
\hline
\end{tabular}


lights

\begin{tabular}{|c|c|c|c|c|c|}
\hline 23 & $\begin{array}{l}\text { Daily Total } \\
\text { (Jday) }\end{array}$ & $\begin{array}{l}\text { Daily Total } \\
\text { (Post) }\end{array}$ & Monthly Total & $\begin{array}{l}\text { Monthly } \\
\text { Summary }\end{array}$ & \\
\hline 41 & $\mid 1224.9$ & 1224.9 & & Oct 91 & 30111.1 \\
\hline 35.8 & 1288.5 & 1288.5 & & Nov & 25298.5 \\
\hline 39.2 & 1206.8 & 1206.8 & & Dec & 15933.8 \\
\hline 34.7 & 1177.7 & 1177.7 & & Jan 92 & 19072.4 \\
\hline 12.4 & 296.7 & 296.7 & & Feb & 19462.9 \\
\hline 10.6 & 236.6 & 236.6 & & Mar & 17249.6 \\
\hline 40.6 & 1195 & 1195 & & Apr & 20320.9 \\
\hline 35 & 1228.6 & 1228.6 & & May & 20348.3 \\
\hline 26.9 & 1164.8 & 1164.8 & & Jun & 14852.6 \\
\hline 49.2 & 1263.5 & 1263.5 & & Jul & 9536.2 \\
\hline 35 & 1195 & 1195 & & Aug & 13809.8 \\
\hline 12.1 & 271.2 & 271.2 & & Sep & 19931.7 \\
\hline 10.2 & 248.3 & 248.3 & & Oct & 21648.5 \\
\hline 41.4 & 1166.4 & 1166.4 & & Nov & 19361.8 \\
\hline 40.8 & 1195.9 & 1195.9 & & Dec & 17974.5 \\
\hline 49.8 & 1327.3 & 1327.3 & & Jan 93 & 20424 \\
\hline 31.6 & 1199.5 & 1199.5 & & Feb & 21439.9 \\
\hline 36.2 & 1214.7 & 1214.7 & & Mar & 20817.7 \\
\hline 10.8 & 250.4 & 250.4 & & Apr & 21789.6 \\
\hline 11.4 & 250.1 & 250.1 & & May & 23457.2 \\
\hline 44 & 1223.9 & 1223.9 & & Jun & 13639.6 \\
\hline 49.9 & 1308.9 & 1308.9 & & Jul & 9258.1 \\
\hline 35.2 & 1239.1 & 1239.1 & & Aug & 18188.4 \\
\hline 44 & 1221.4 & 1221.4 & & Sep & 21994.4 \\
\hline 33.2 & 1162.4 & 1162.4 & & Oct & 23621.5 \\
\hline 10.5 & 240.2 & 240.2 & & Nov & 21773.3 \\
\hline 10.6 & 236 & 236 & & Dec & 17399.4 \\
\hline 42.4 & 1206.9 & 1206.9 & & Jan 94 & 22462.4 \\
\hline 41.8 & 1129 & 1129 & & Feb & 21113.9 \\
\hline 39.5 & 1276.6 & 1276.6 & & Mar & 20615.2 \\
\hline 42.6 & 1264.8 & 1264.8 & 30111.1 & Apr (pre) & 8329.1 \\
\hline 39.5 & 1272.2 & 1272.2 & & Apr (post) & 11077.2 \\
\hline 18.1 & 374.7 & 374.7 & & May & 18114.8 \\
\hline 18 & 410.7 & 410.7 & & Jun & 10086.1 \\
\hline 57 & 1434.7 & 1434.7 & & Jul & 8129.9 \\
\hline 43.1 & 1276.2 & 1276.2 & & Aug & 17288.5 \\
\hline 38.2 & 1271.3 & 1271.3 & & Sep & 18626.2 \\
\hline 32 & 1245 & 1245 & & Oct & 18894.4 \\
\hline 37.6 & 1169.2 & 1169.2 & & Nov & 16681.7 \\
\hline 7.7 & 233.8 & 233.8 & & Dec & 11857.5 \\
\hline 8.3 & 174.9 & 174.9 & & Jan 95 & 18953.9 \\
\hline 45.3 & 1211 & 1211 & & Feb & 17559.8 \\
\hline 39.8 & 1230 & 1230 & & Mar & 19075.9 \\
\hline 37.9 & 1286.4 & 1286.4 & & Apr & 18637.5 \\
\hline 73.3 & 1377.6 & 1377.6 & & May & 18719.5 \\
\hline 37.6 & 1334.1 & 1334.1 & & & \\
\hline 8.8 & 469 & 469 & & & 814939.2 \\
\hline
\end{tabular}




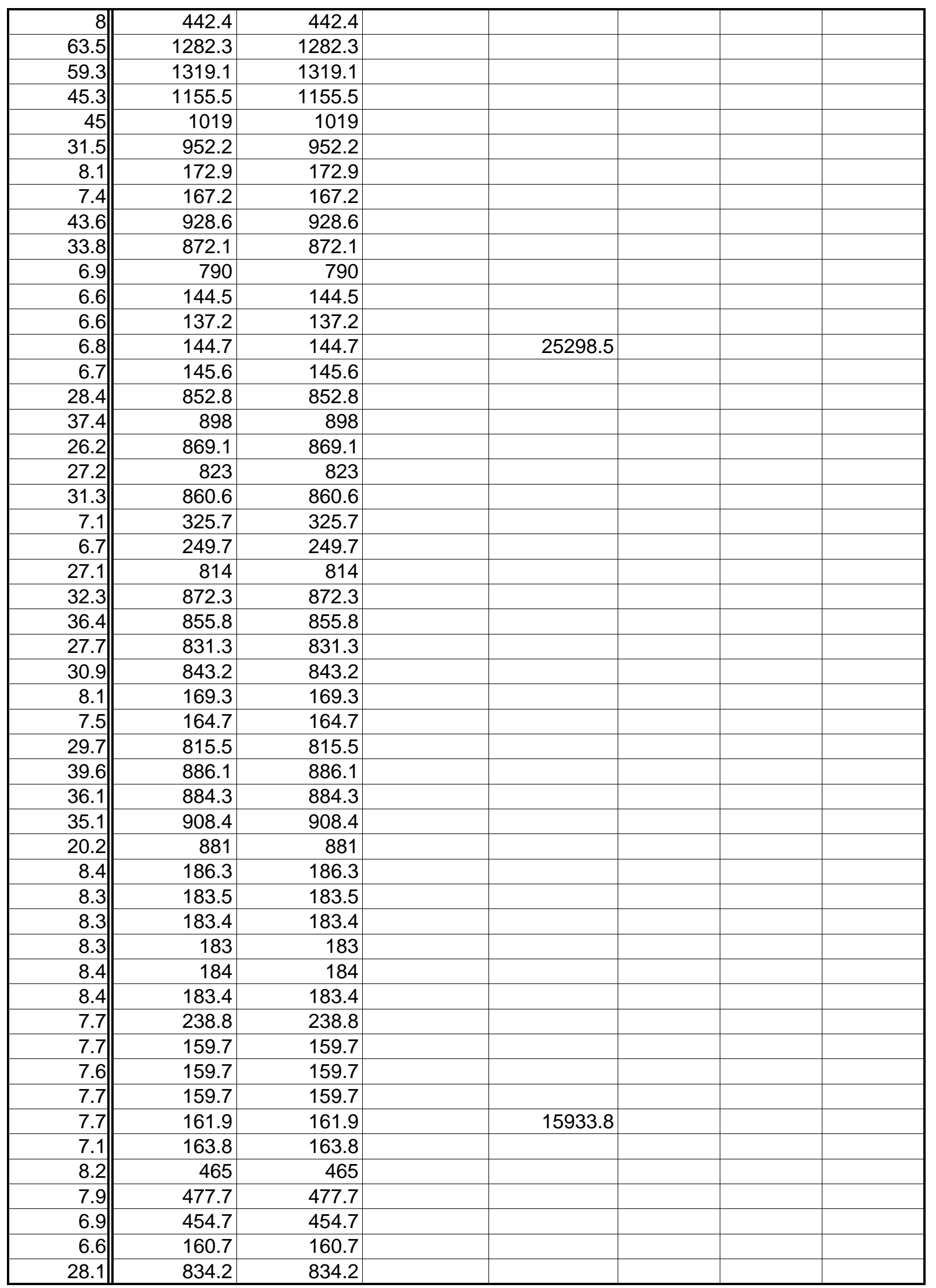




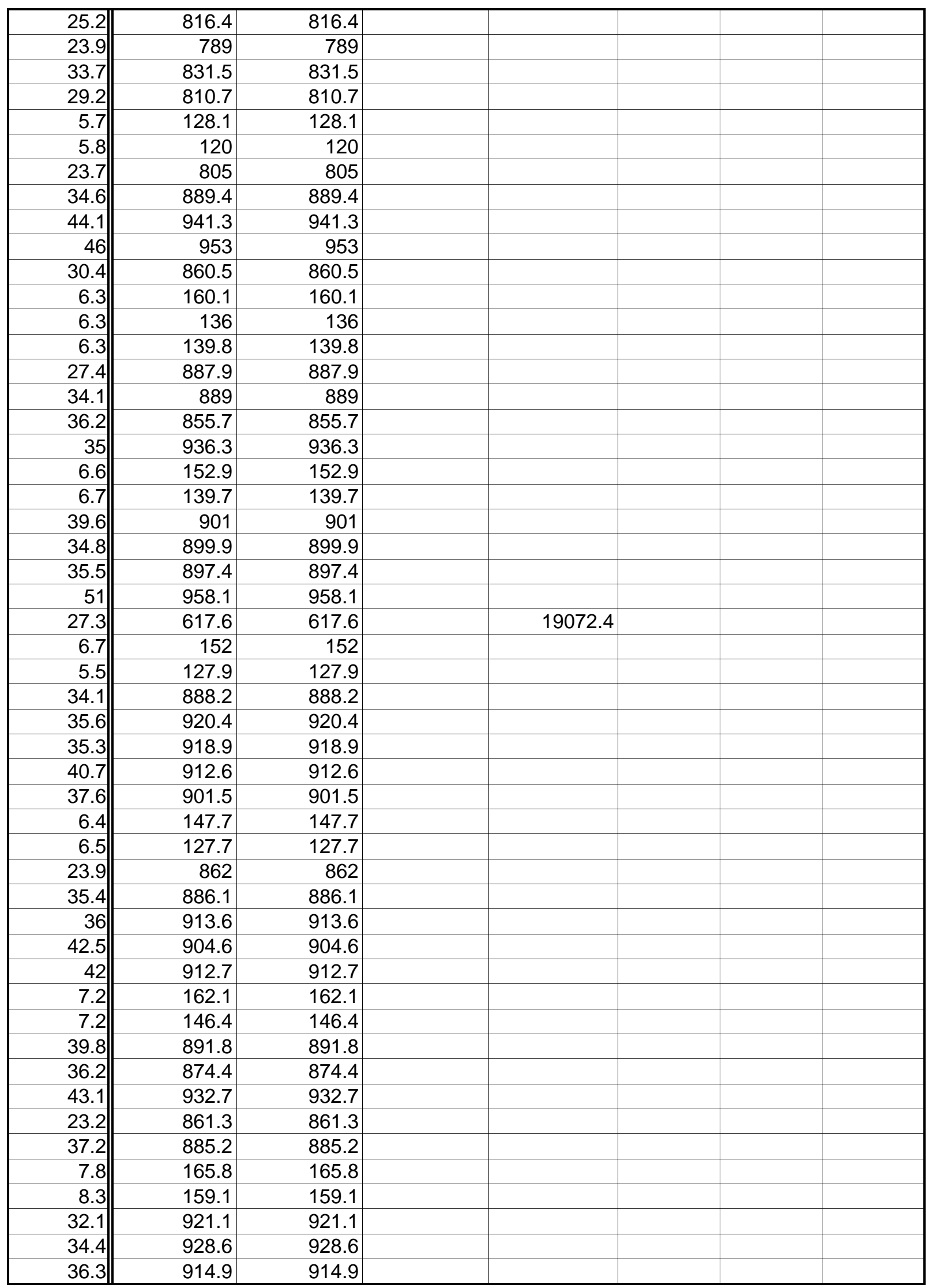


lights

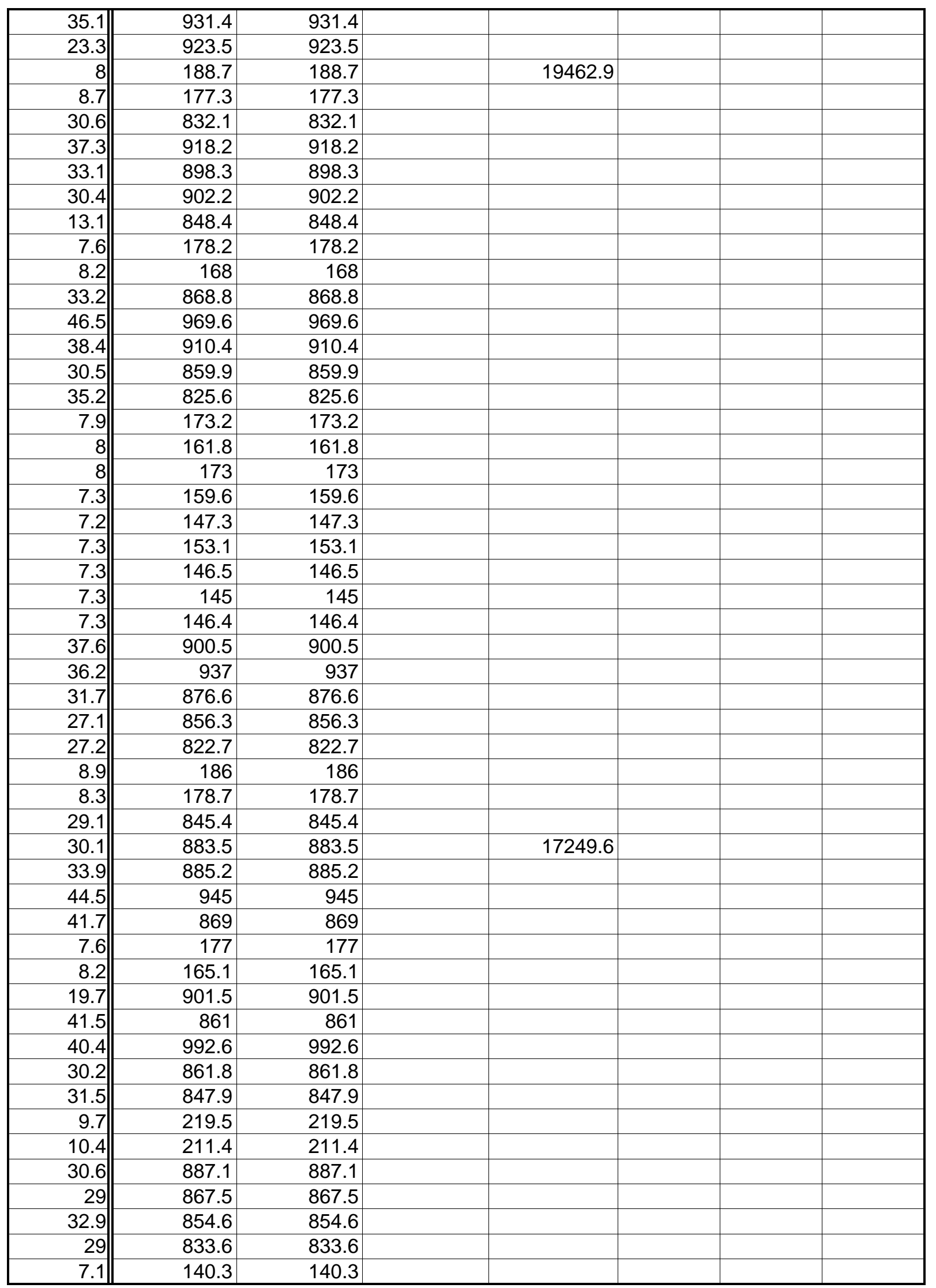




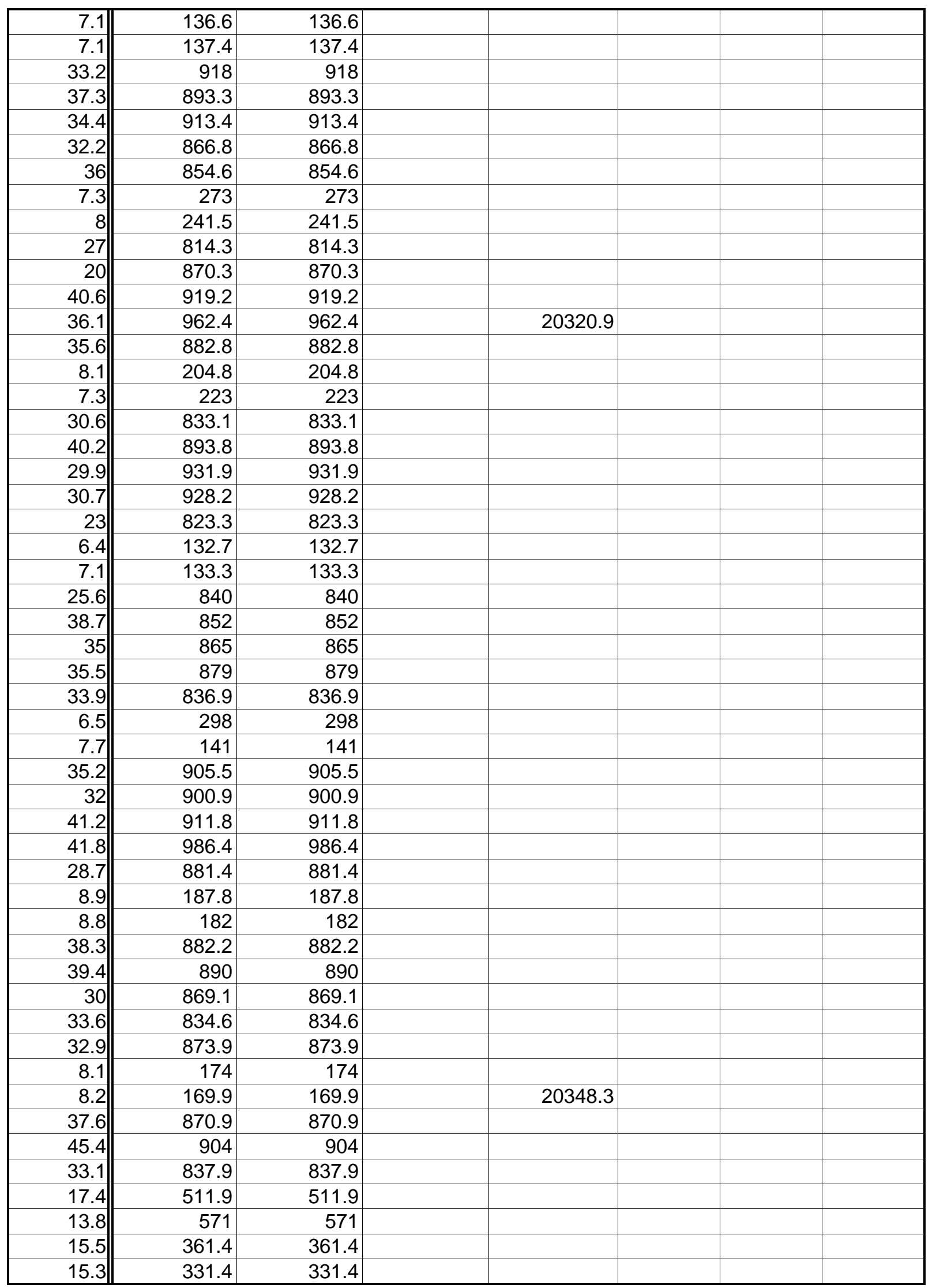




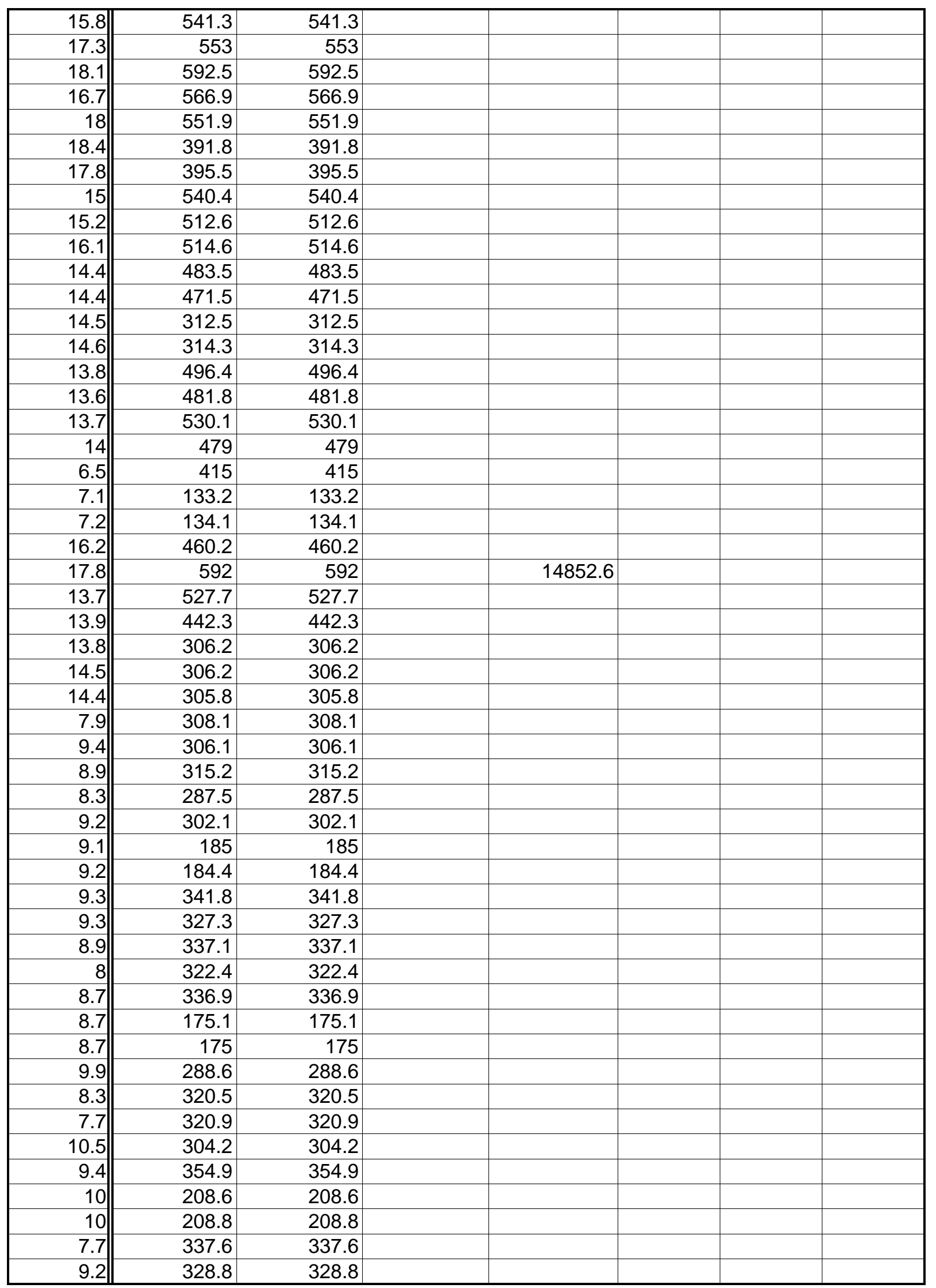




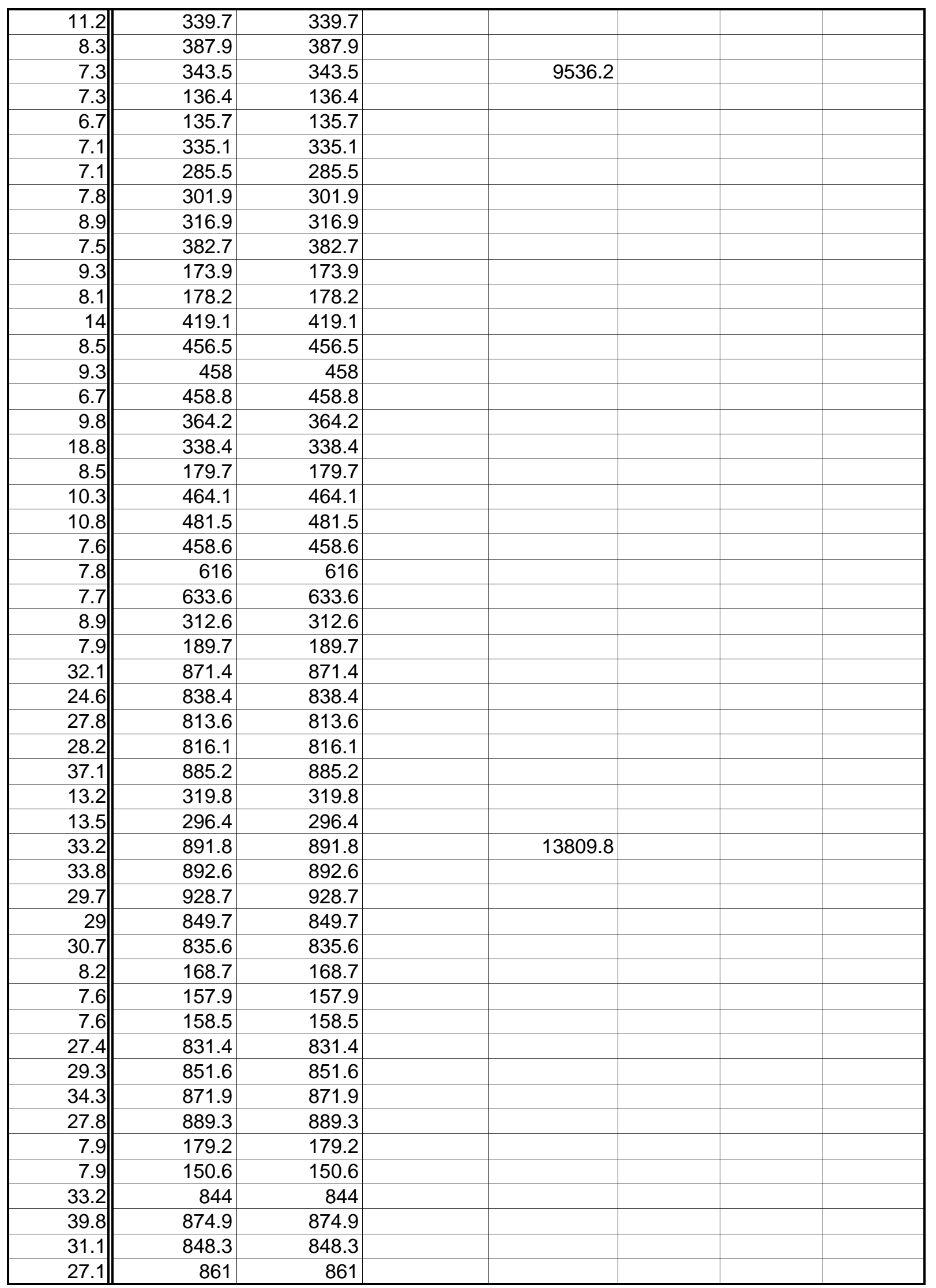




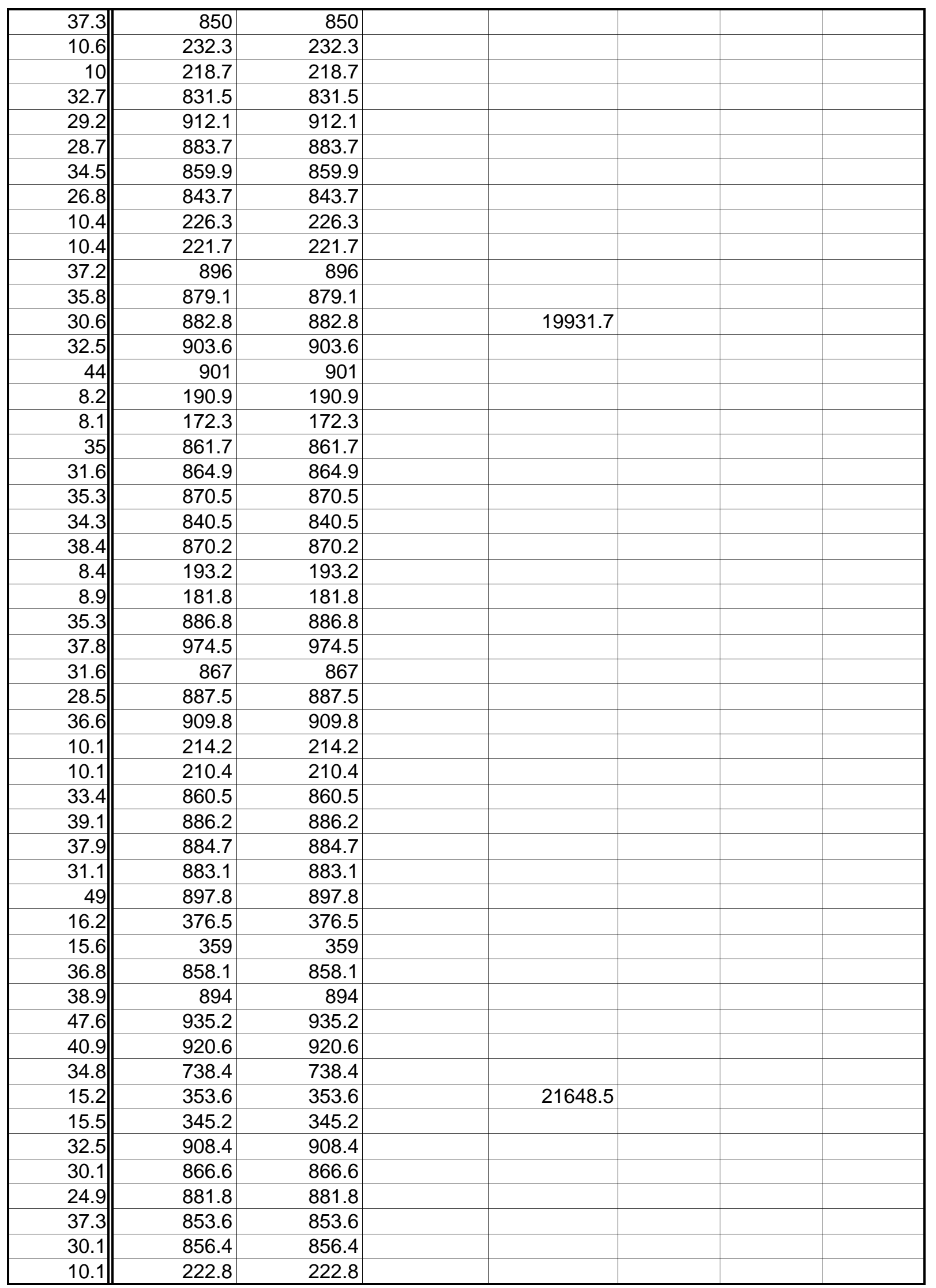




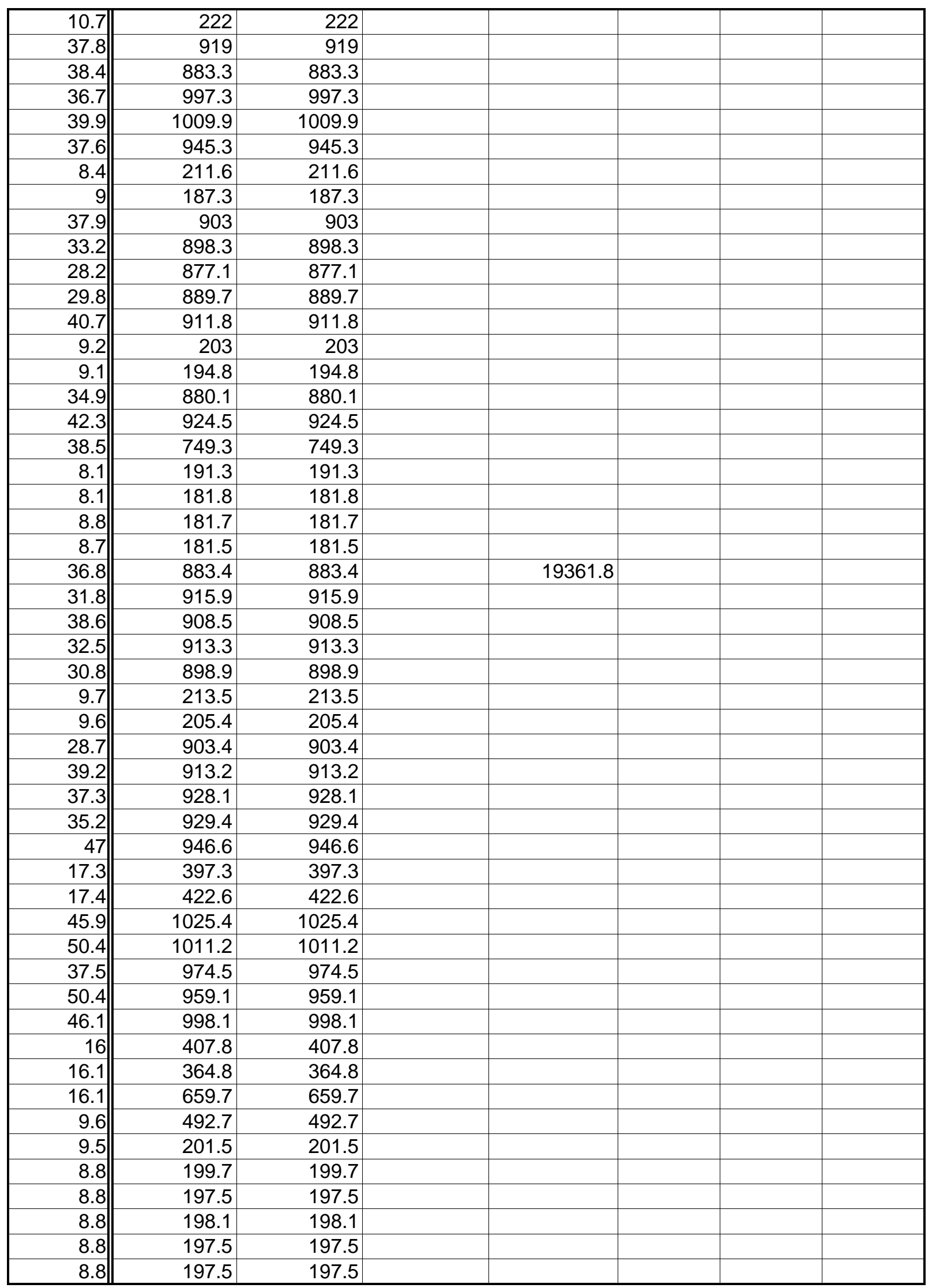


lights

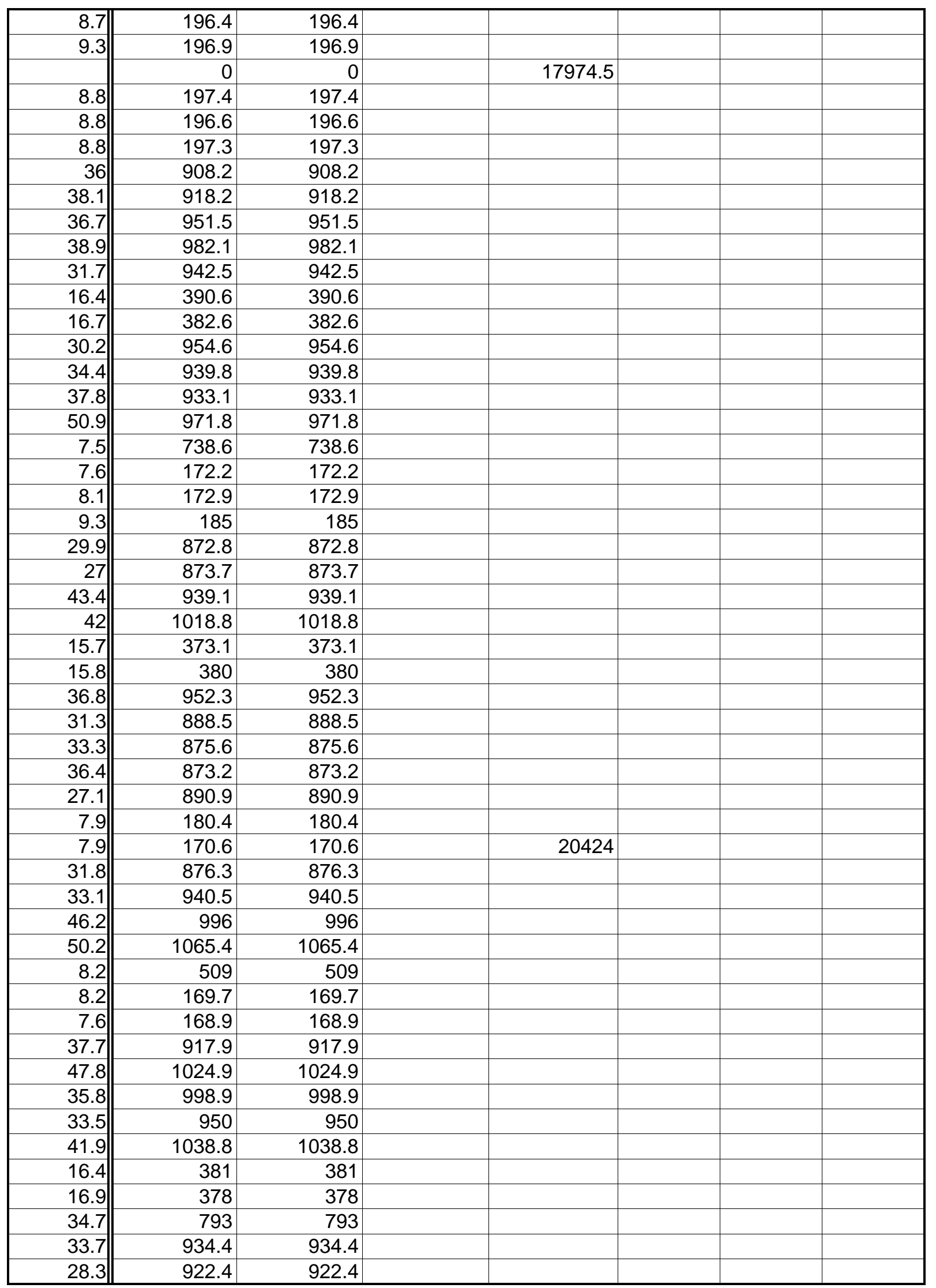




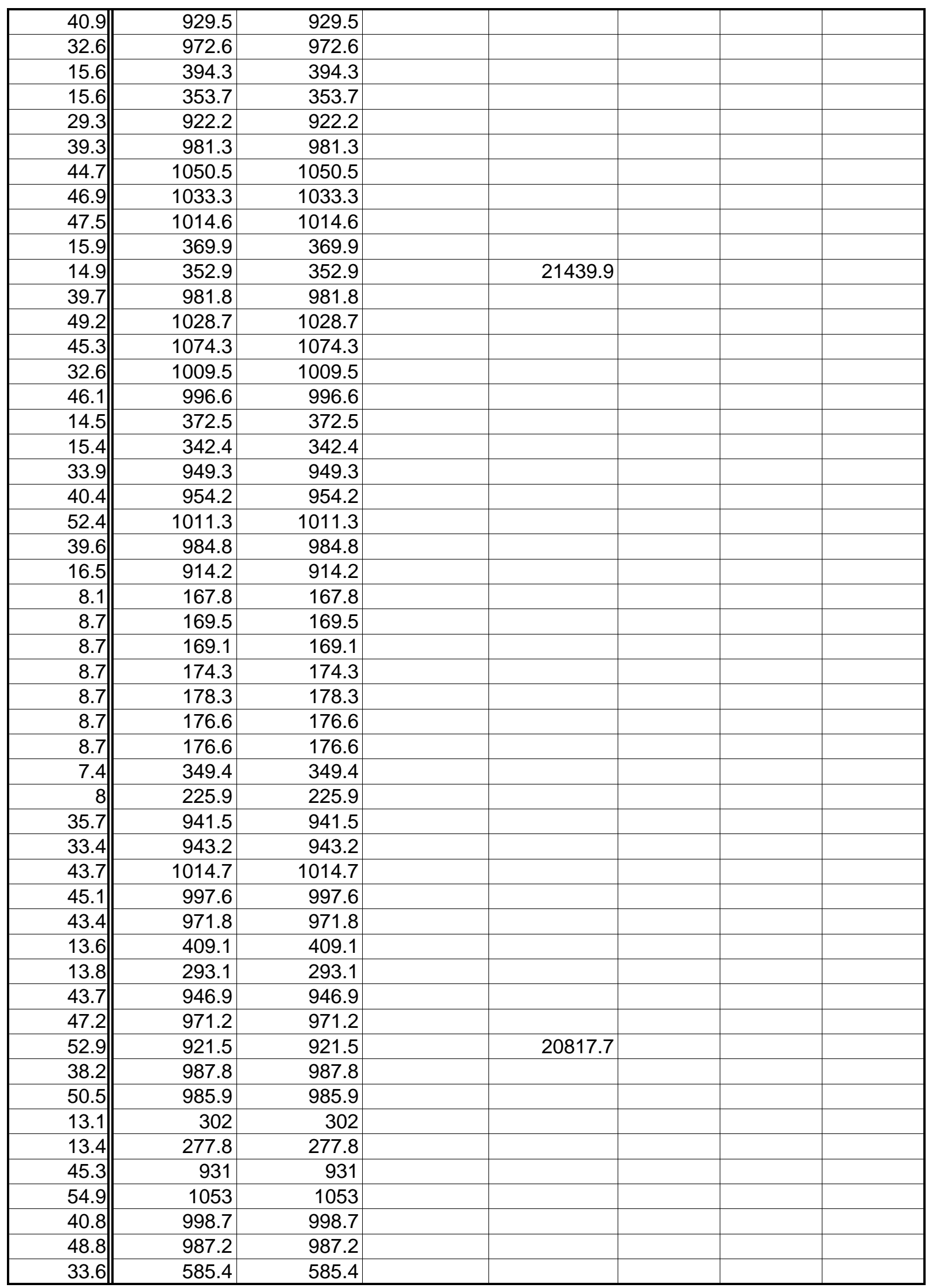




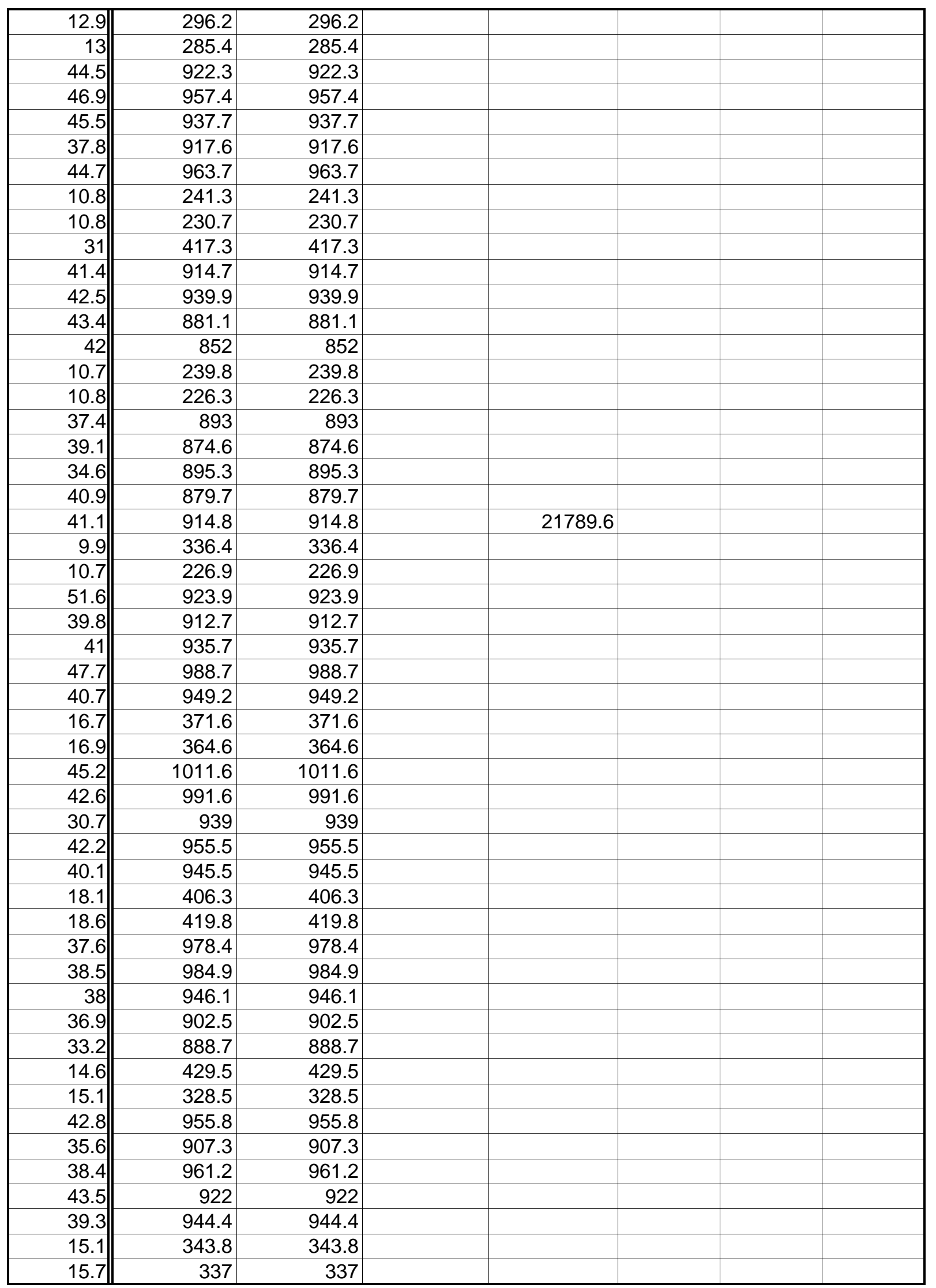


lights

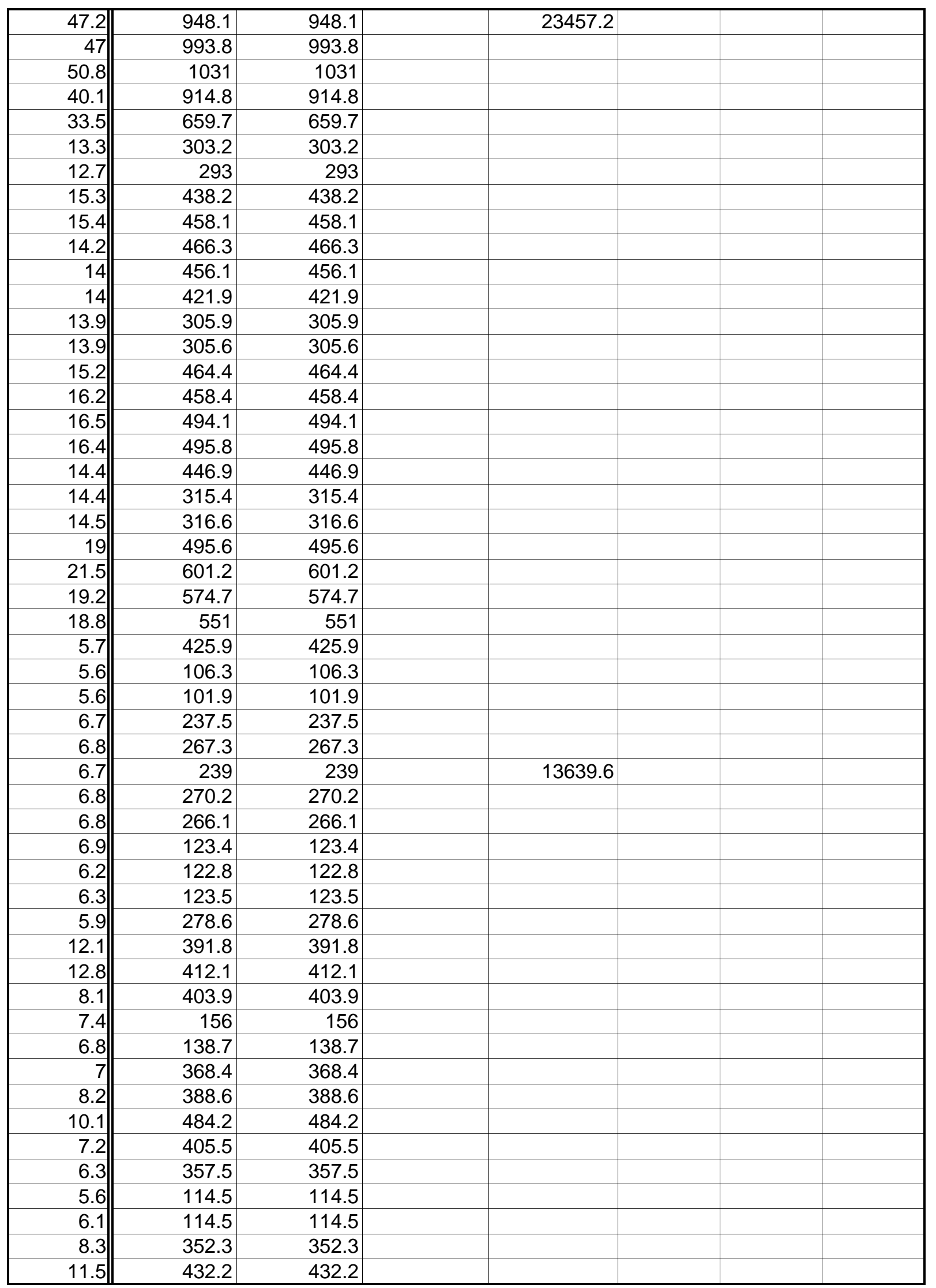




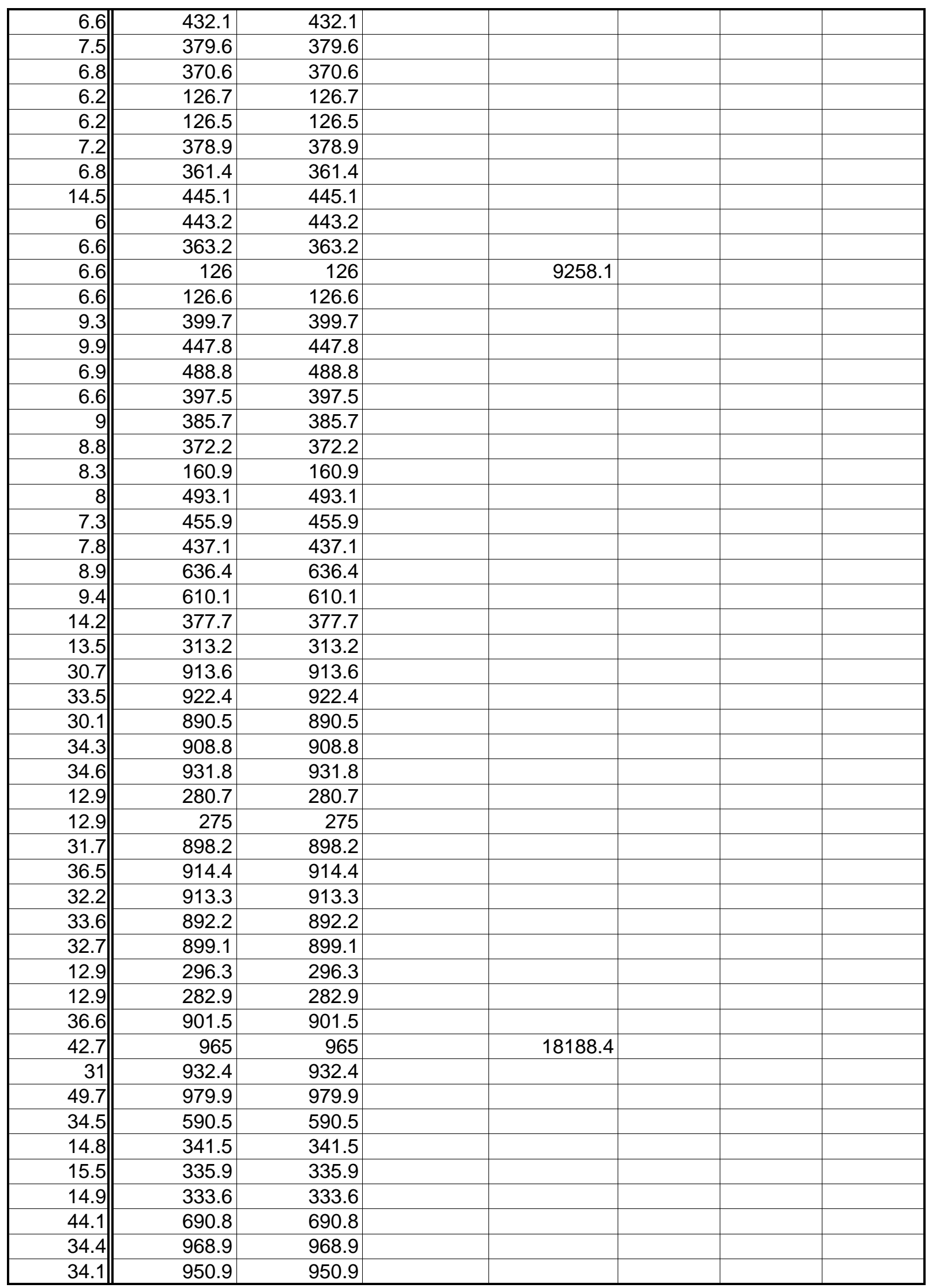




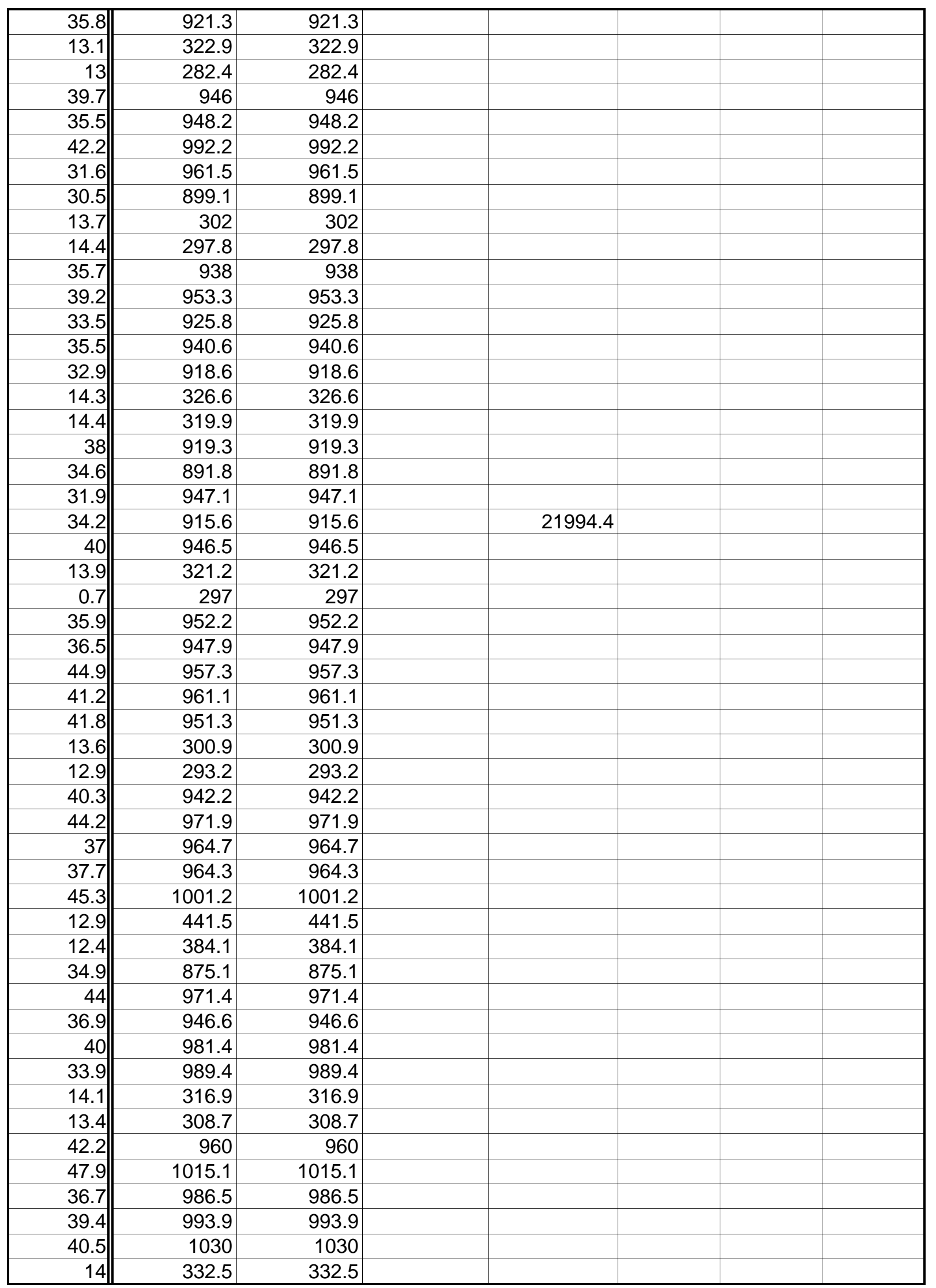


lights

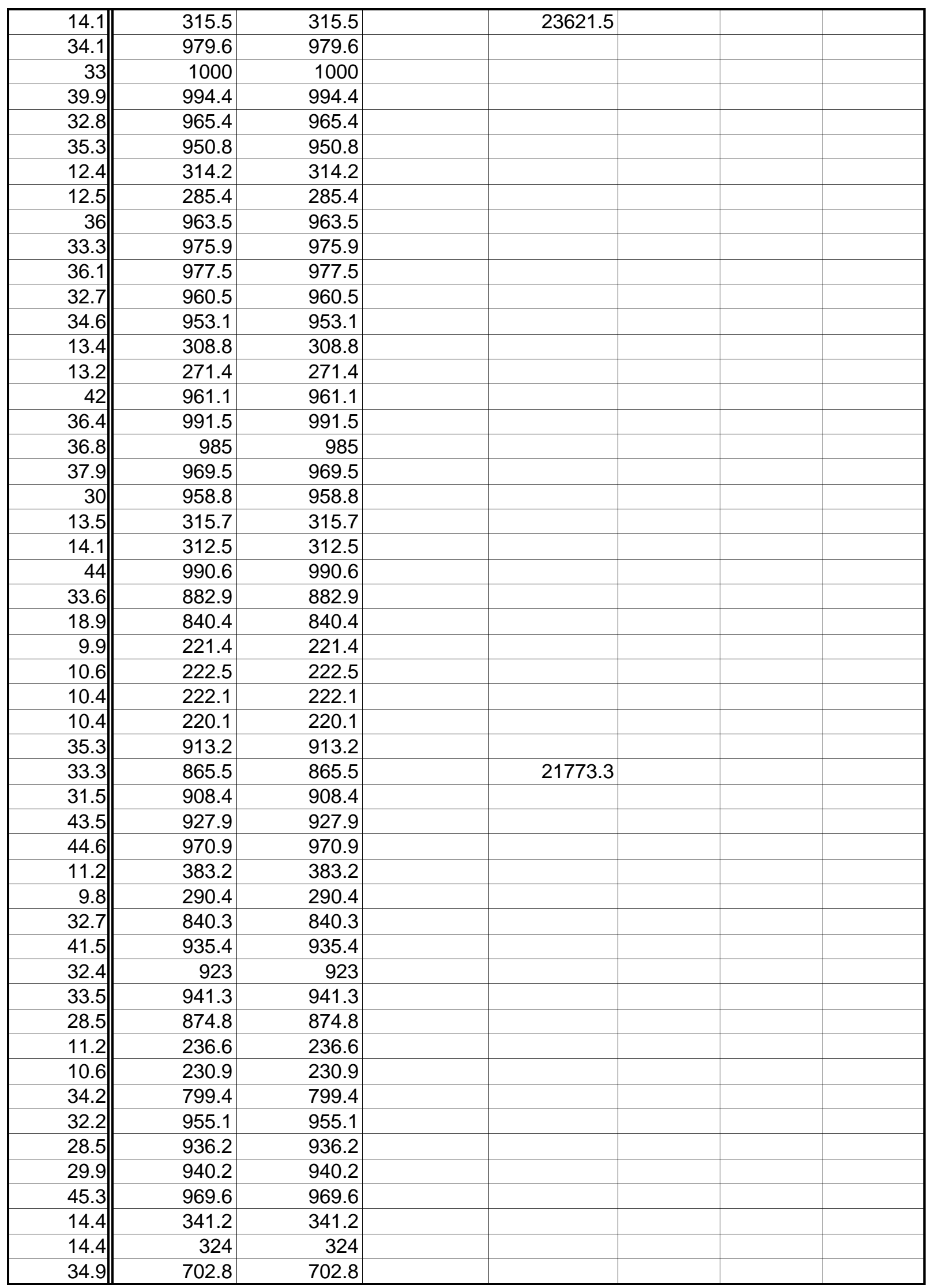




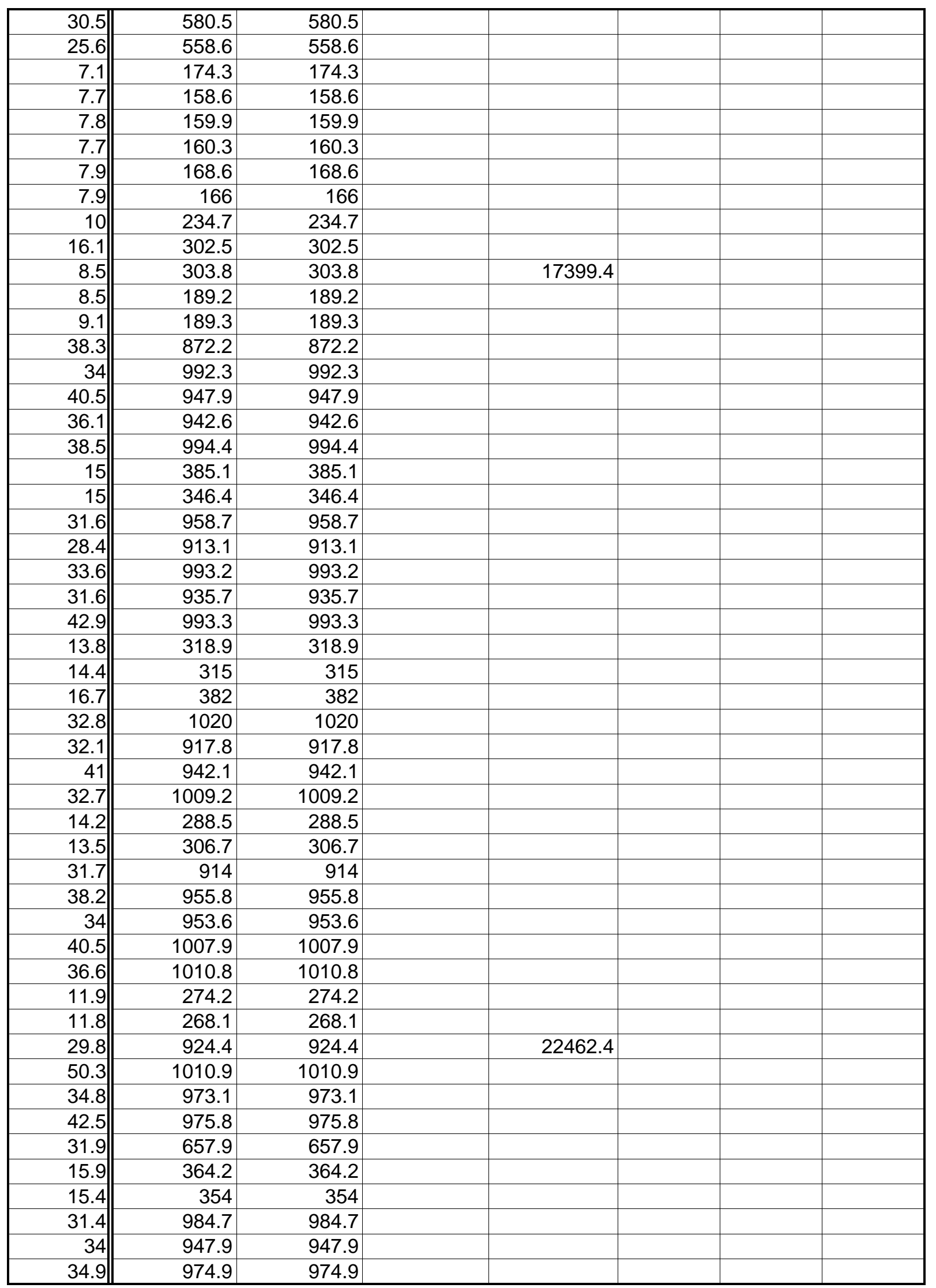




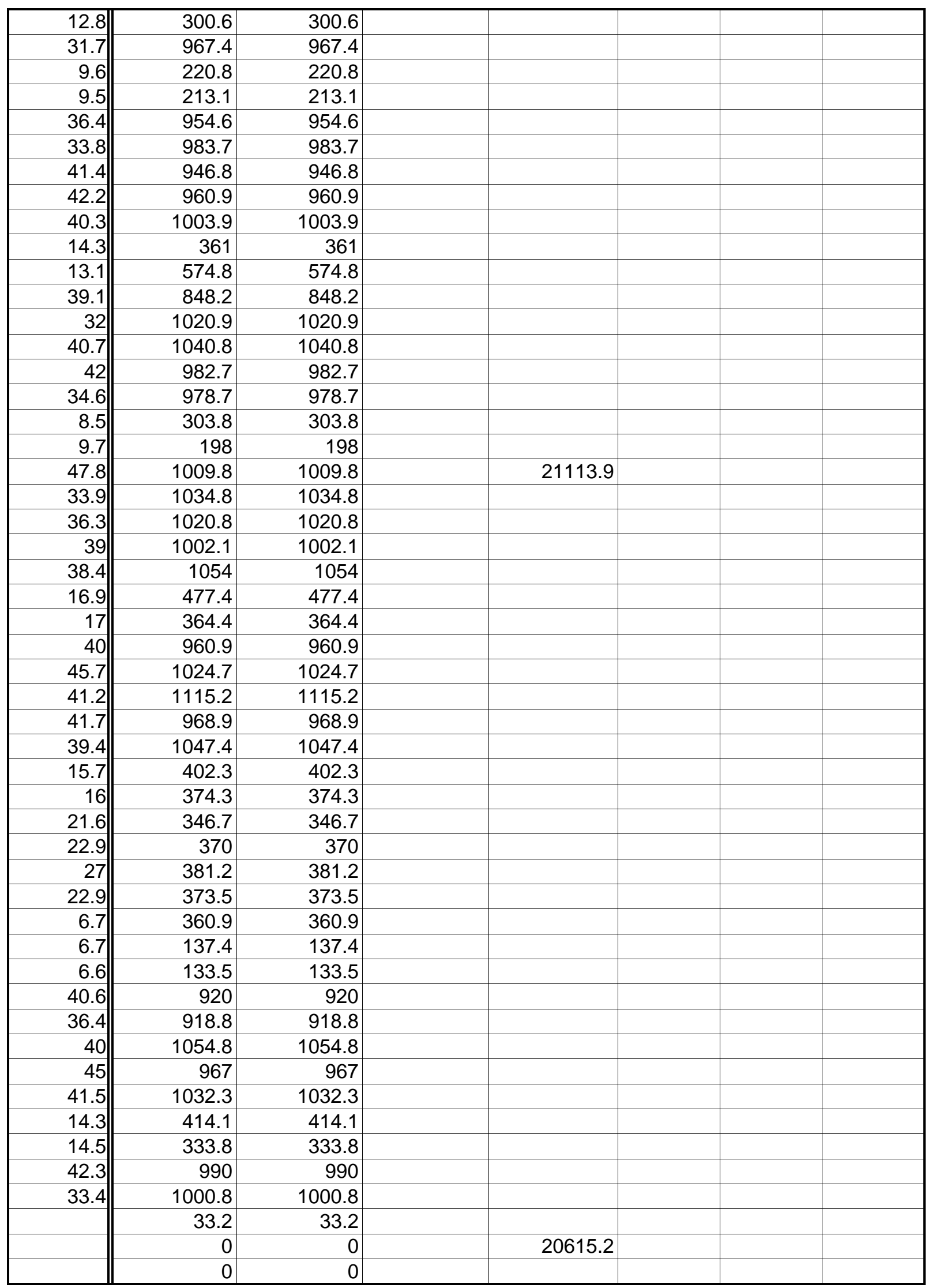




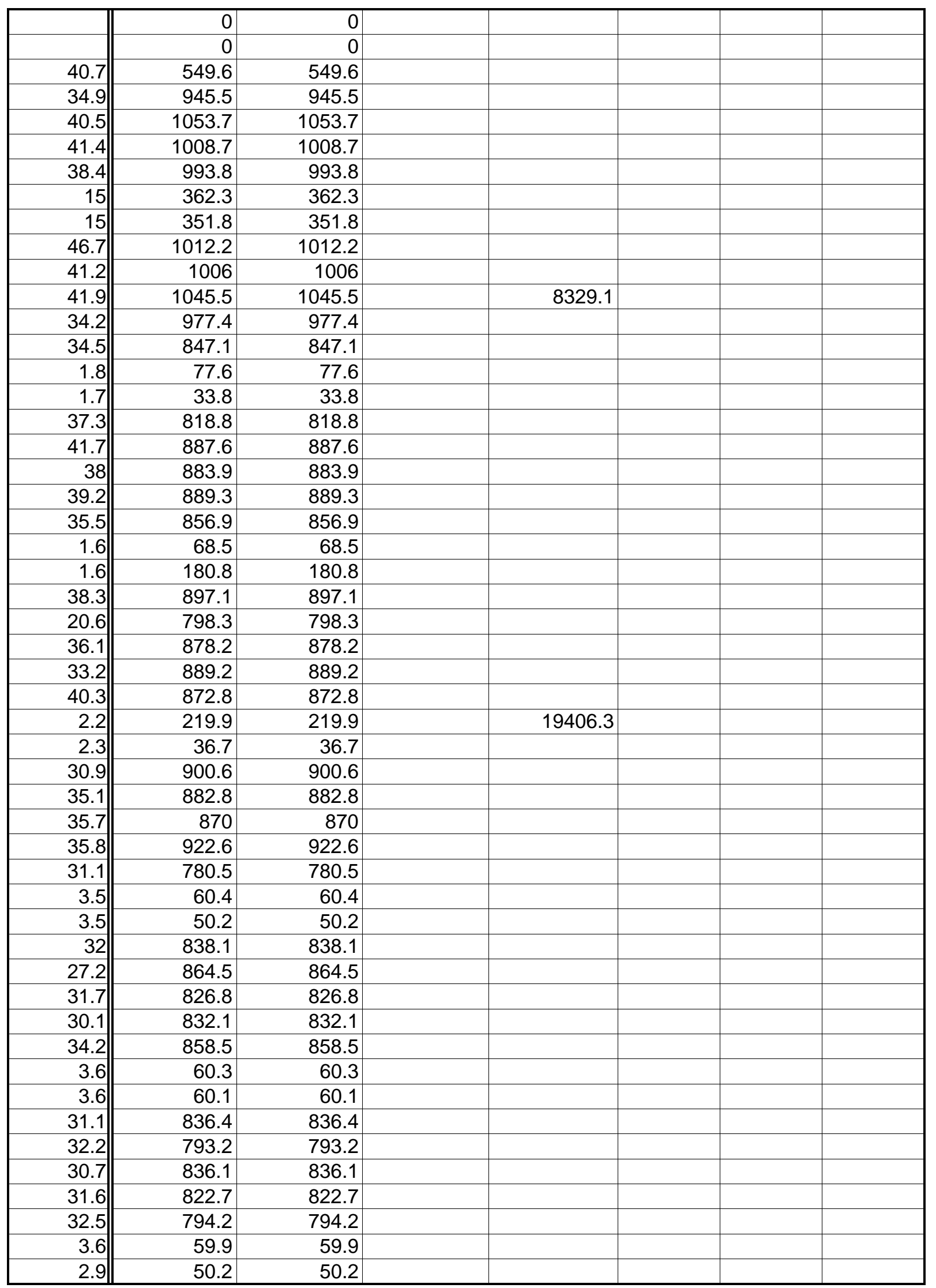




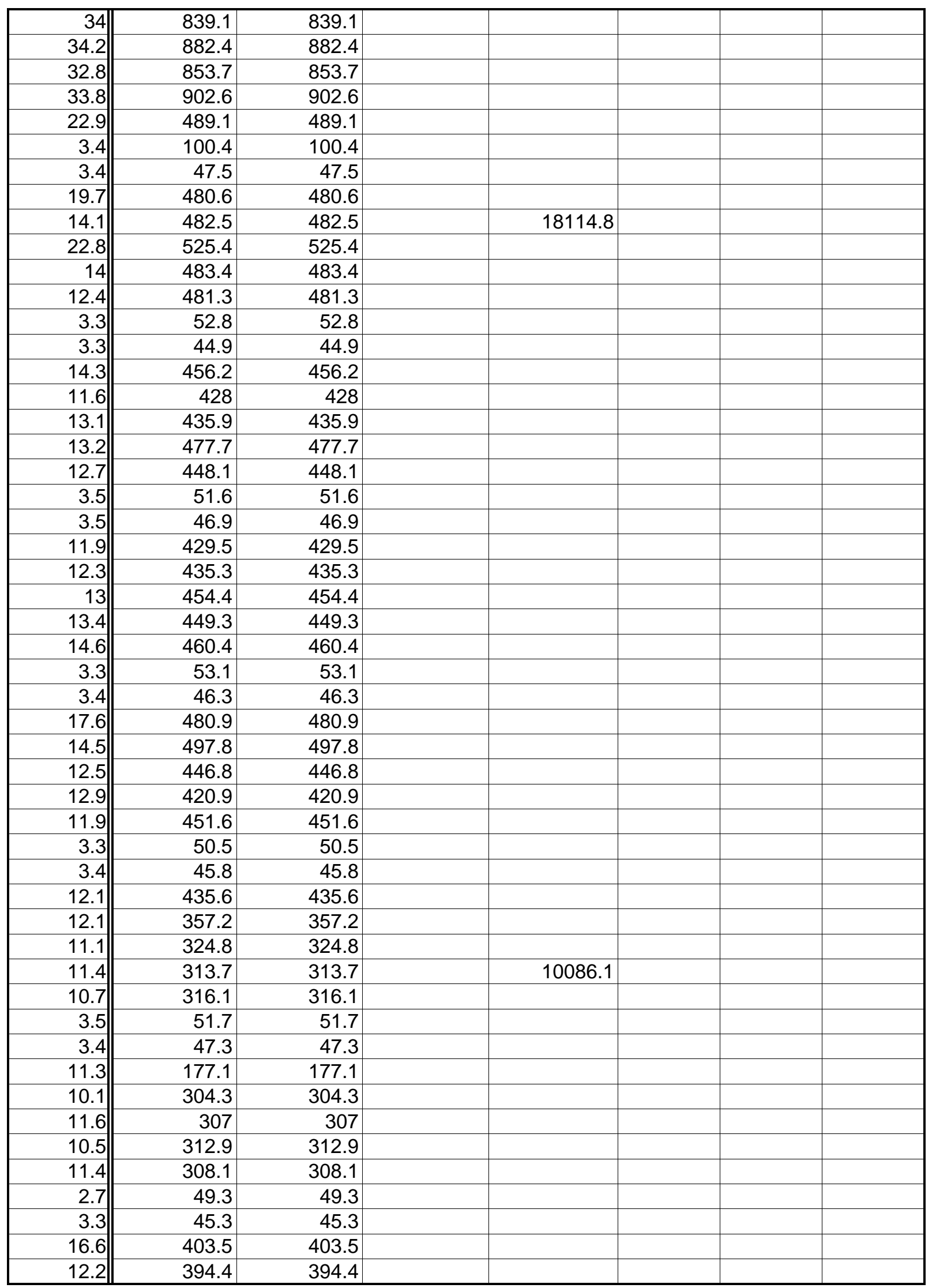




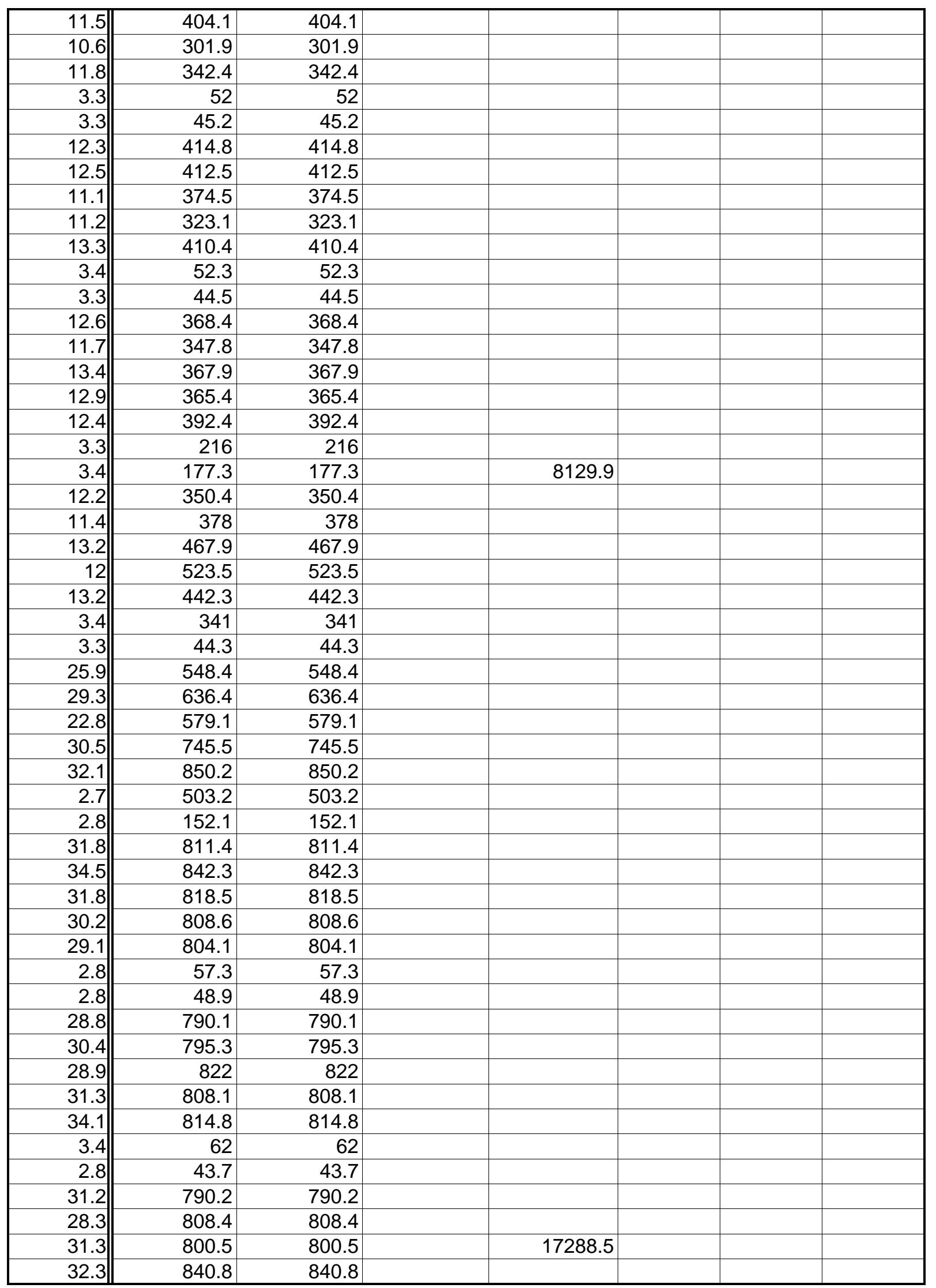




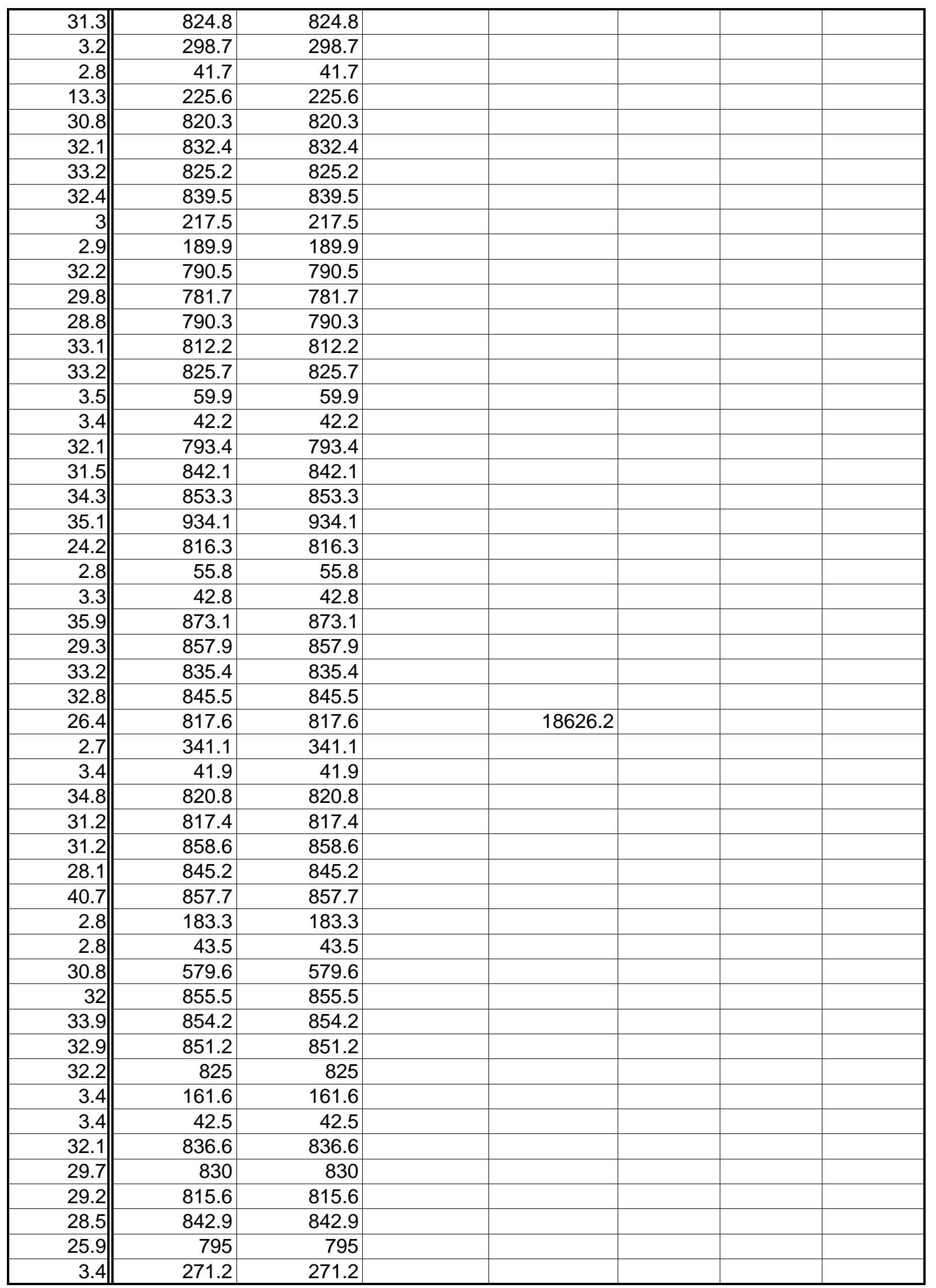




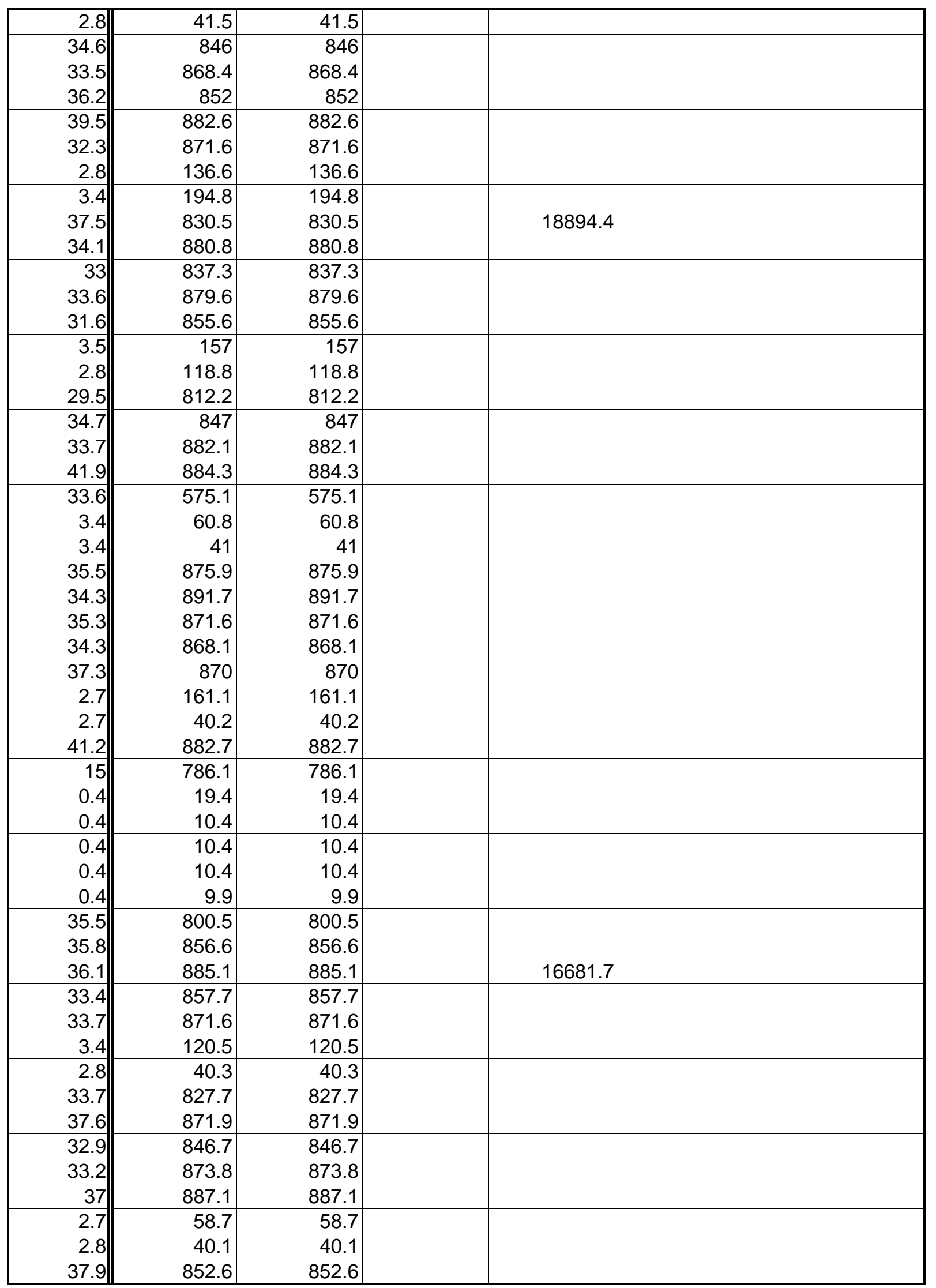




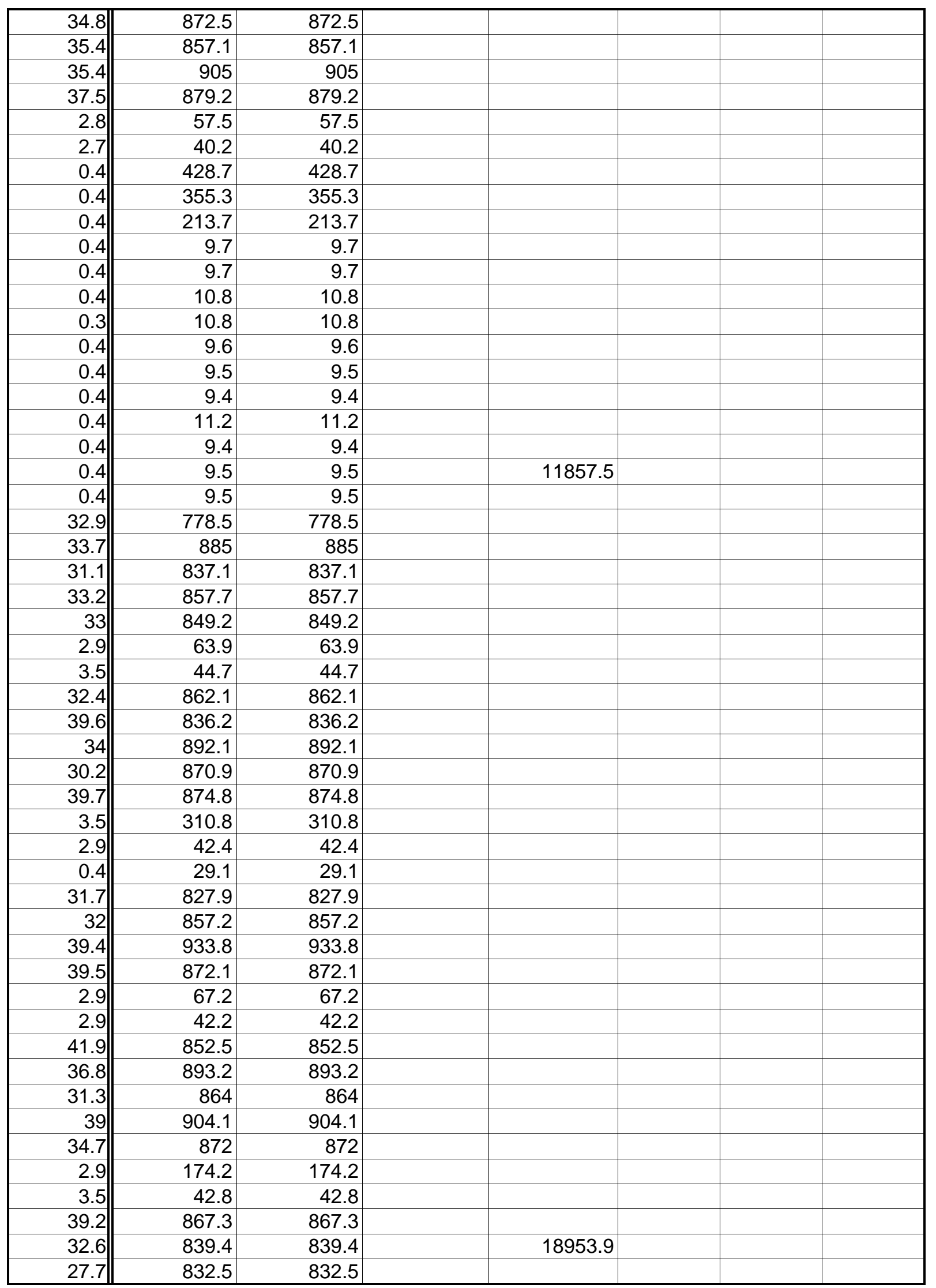




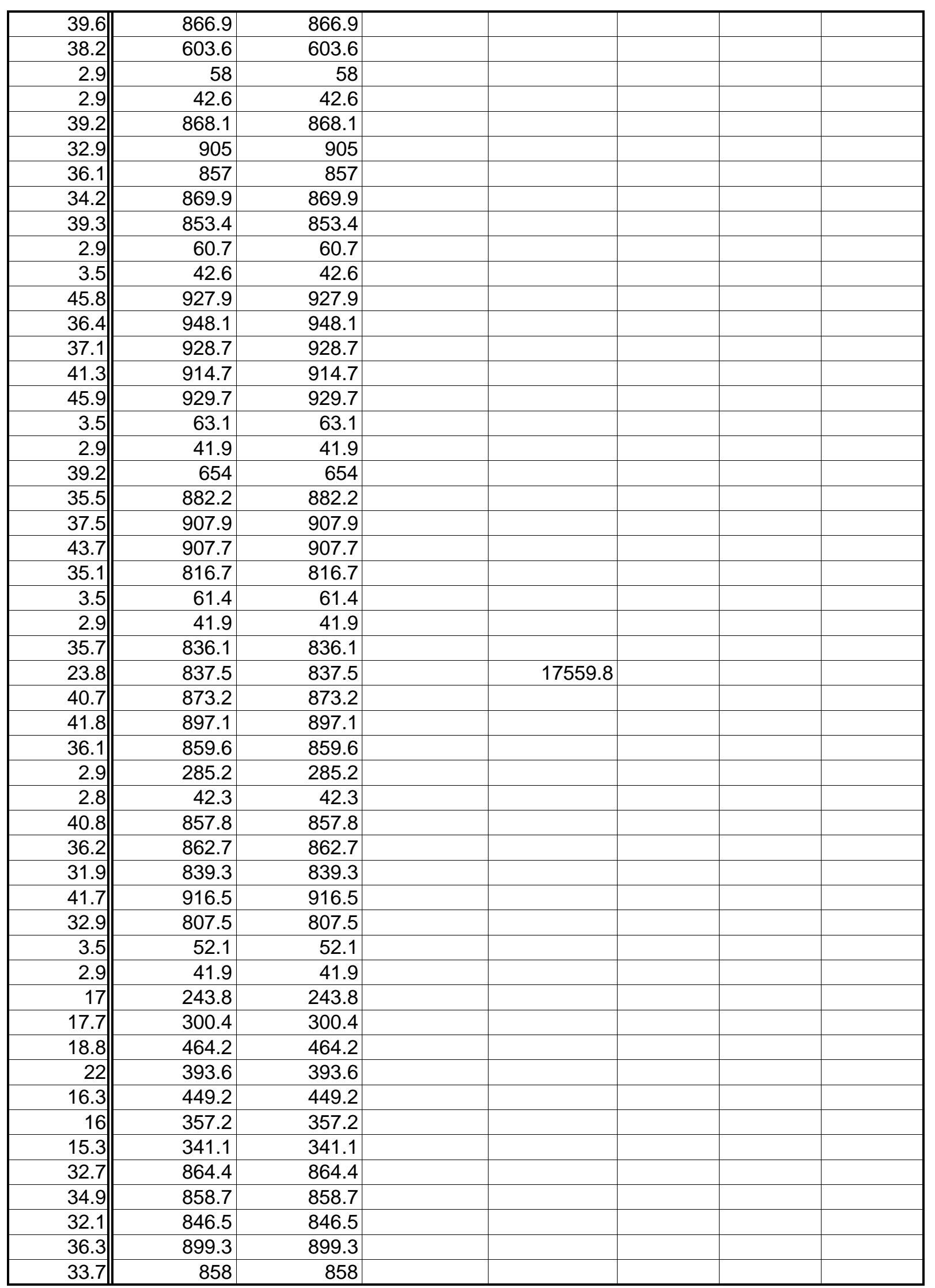




\begin{tabular}{|c|c|c|c|c|c|c|}
\hline 3.5 & 242.9 & 242.9 & & & & \\
\hline 3.6 & 83.9 & 83.9 & & & & \\
\hline 44.6 & 901.2 & 901.2 & & & & \\
\hline 37.2 & 937 & 937 & & & & \\
\hline 37.9 & 939.9 & 939.9 & & & & \\
\hline 30.5 & 900.9 & 900.9 & & & & \\
\hline 34.3 & 858.5 & 858.5 & 19075.9 & & & \\
\hline & 397.6 & 397.6 & & & & \\
\hline 3.5 & 356 & 356 & & & & \\
\hline 38 & 904.7 & 904.7 & & & & \\
\hline 34.6 & 916.5 & 916.5 & & & & \\
\hline 35.7 & 890.4 & 890.4 & & & & \\
\hline 38.6 & 902.7 & 902.7 & & & & \\
\hline 40.7 & 885.4 & 885.4 & & & & \\
\hline 2.9 & 68.2 & 68.2 & & & & \\
\hline 3.5 & 47.4 & 47.4 & & & & \\
\hline 43.8 & 919.9 & 919.9 & & & & \\
\hline 38 & 928.9 & 928.9 & & & & \\
\hline 37 & 923.1 & 923.1 & & & & \\
\hline 40.1 & 849.4 & 849.4 & & & & \\
\hline 35.3 & 599.8 & 599.8 & & & & \\
\hline 3.6 & 65.9 & 65.9 & & & & \\
\hline 2.9 & 46.5 & 46.5 & & & & \\
\hline 23.9 & 481.8 & 481.8 & & & & \\
\hline 40.4 & 857.3 & 857.3 & & & & \\
\hline 35.5 & 884.2 & 884.2 & & & & \\
\hline 36.6 & 906.3 & 906.3 & & & & \\
\hline 38.3 & 886.9 & 886.9 & & & & \\
\hline 3.6 & 388.5 & 388.5 & & & & \\
\hline 3.6 & 48.5 & 48.5 & & & & \\
\hline 31.8 & 871.9 & 871.9 & & & & \\
\hline 30.1 & 882.6 & 882.6 & & & & \\
\hline 34 & 842.9 & 842.9 & & & & \\
\hline 32.7 & 842.4 & 842.4 & & & & \\
\hline 27.2 & 724.8 & 724.8 & & & & \\
\hline 3.5 & 269.7 & 269.7 & & & & \\
\hline 3.6 & 47.3 & 47.3 & 18637.5 & & & \\
\hline 38.2 & 855.2 & 855.2 & & & & \\
\hline 39.9 & 894.1 & 894.1 & & & & \\
\hline 42 & 922.8 & 922.8 & & & & \\
\hline 34.2 & 899.8 & 899.8 & & & & \\
\hline & 718.8 & 718.8 & & & & \\
\hline & 0 & 0 & & & & \\
\hline 7 & 16.4 & 16.4 & & & & \\
\hline 39.2 & 858.4 & 858.4 & & & & \\
\hline 36.6 & 877.7 & 877.7 & & & & \\
\hline 32.1 & 863.5 & 863.5 & & & & \\
\hline 35.5 & 867.3 & 867.3 & & & & \\
\hline 33.6 & 824.4 & 824.4 & & & & \\
\hline 3.5 & 64.6 & 64.6 & & & & \\
\hline 3.5 & 46.3 & 46.3 & & & & \\
\hline
\end{tabular}


lights

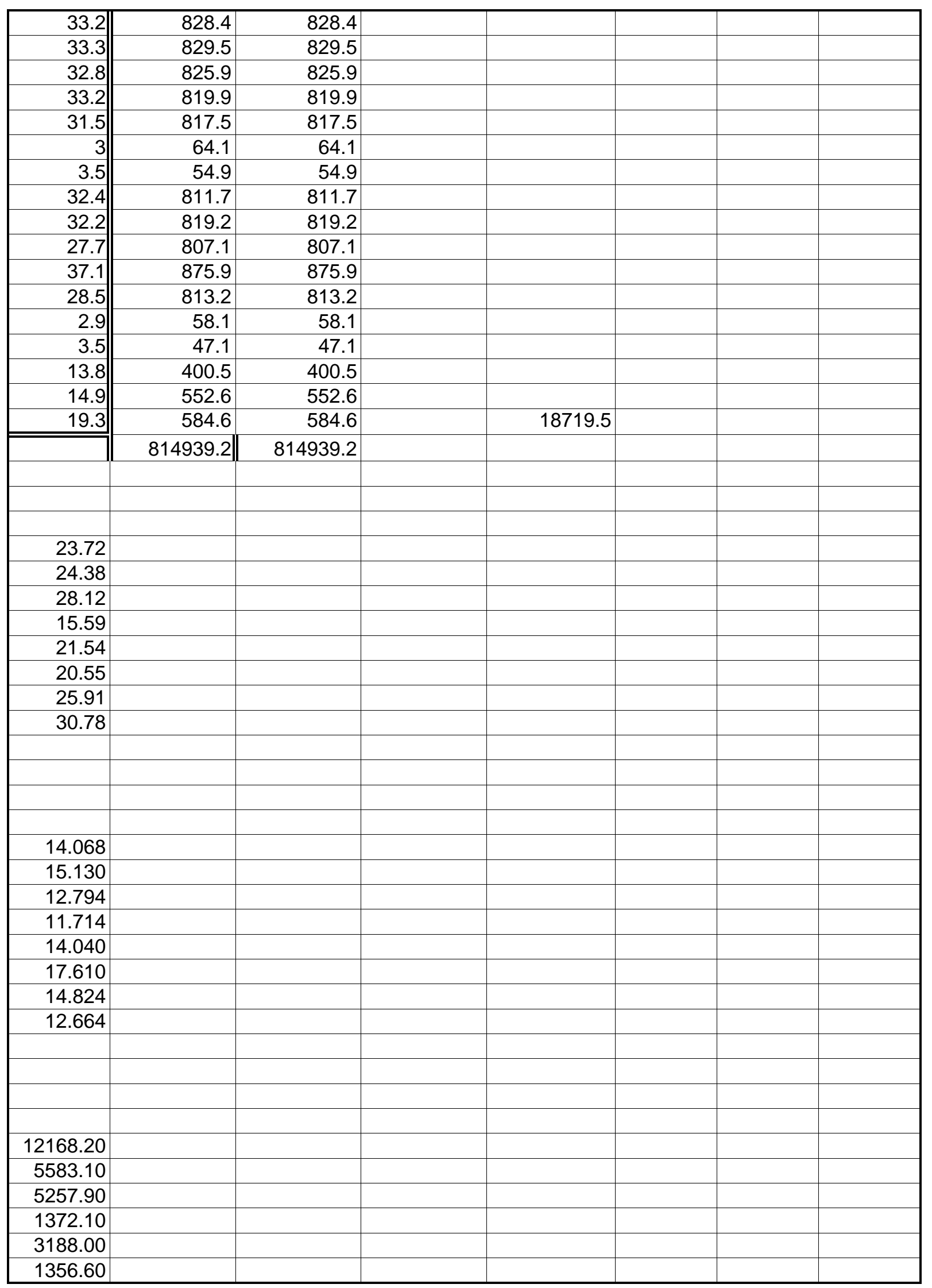


lights

\begin{tabular}{|r|l|l|l|l|l|l|l|}
\hline 1839.60 & & & & & & & \\
\hline 689.90 & & & & & & & \\
\hline
\end{tabular}

Page 112 
lights

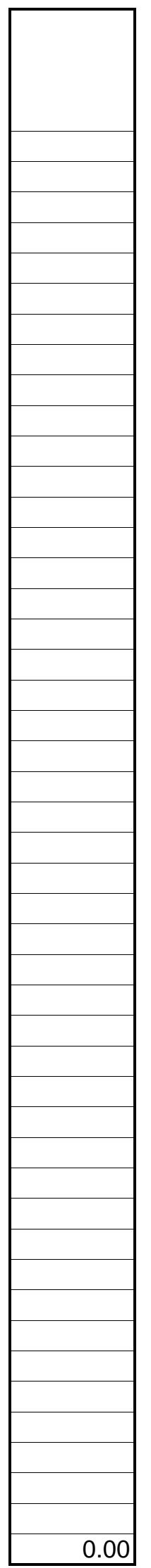

Page 113 


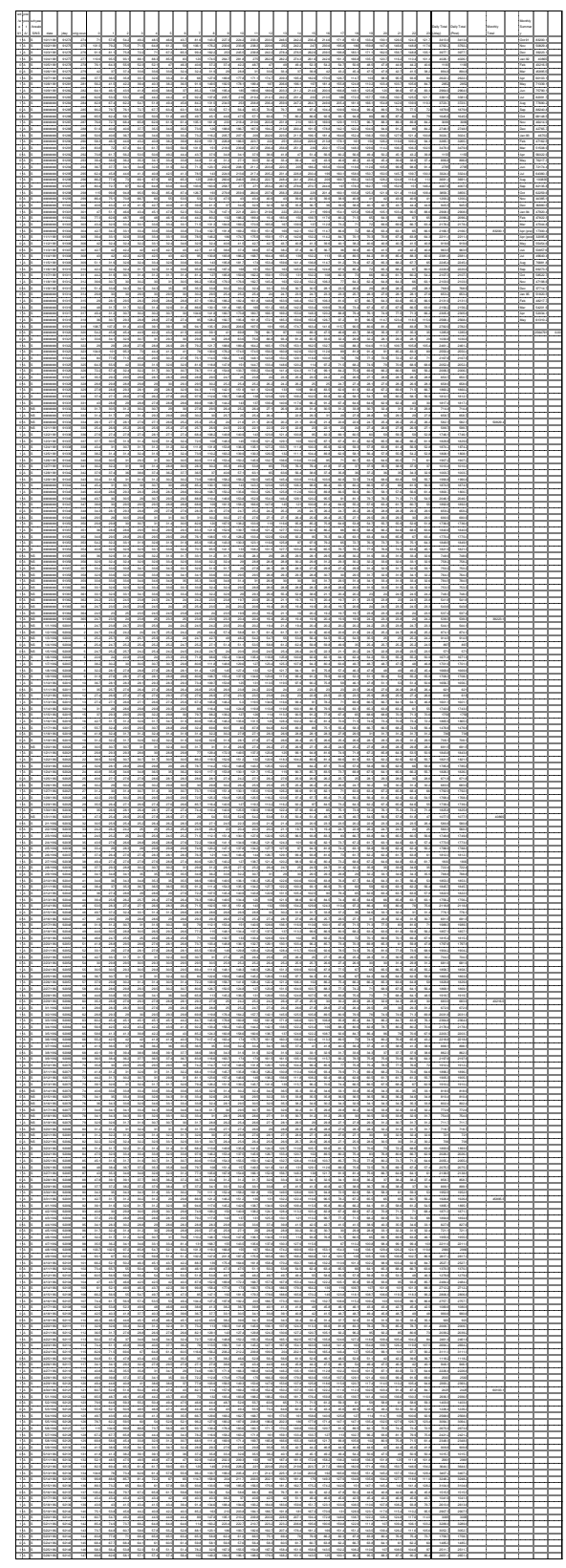




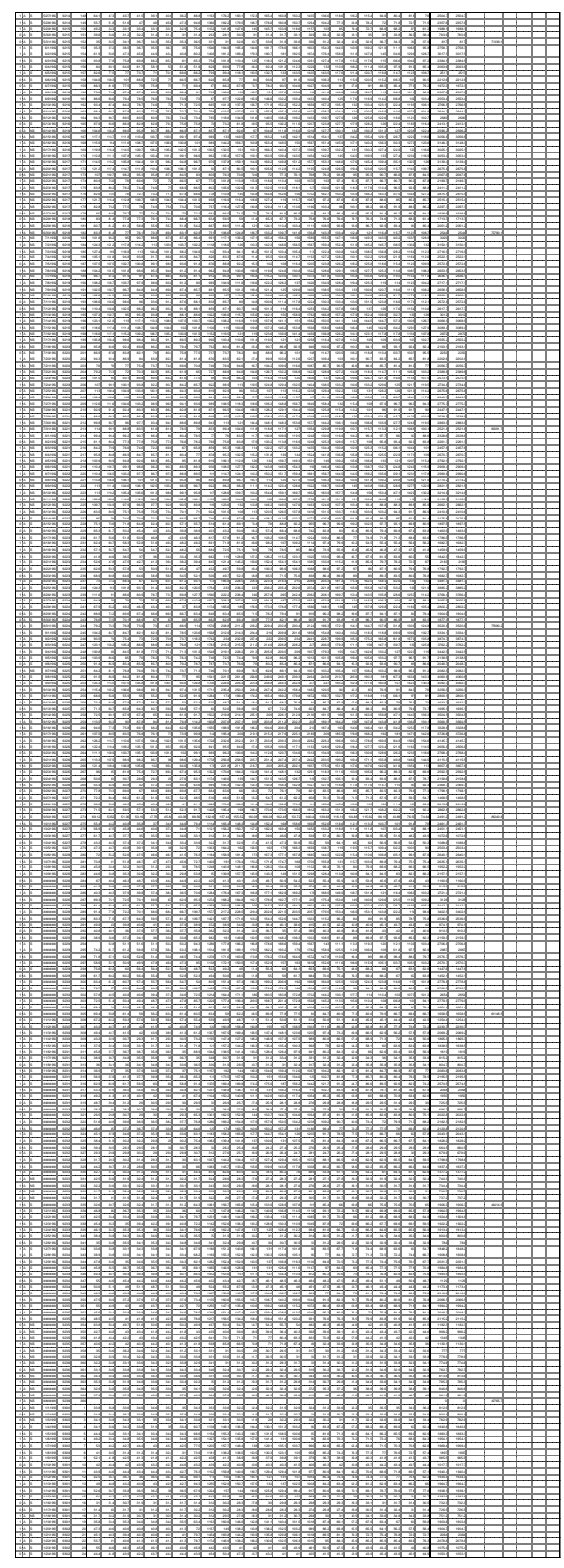




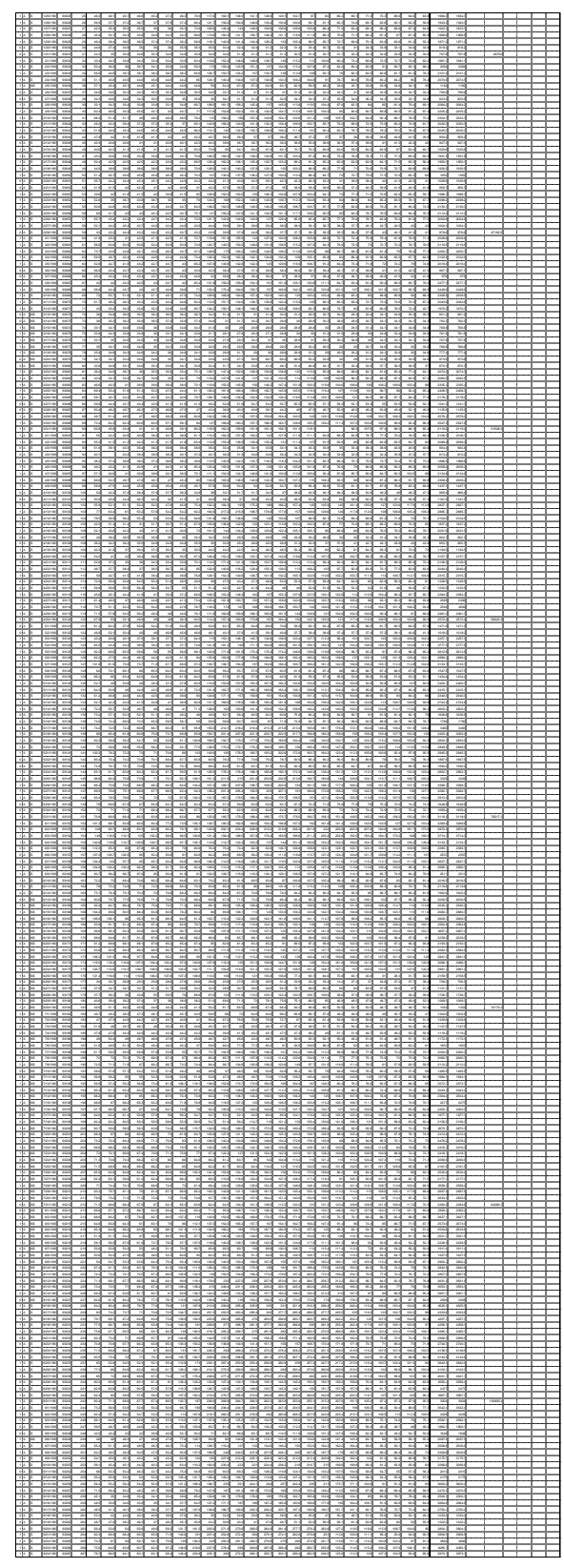




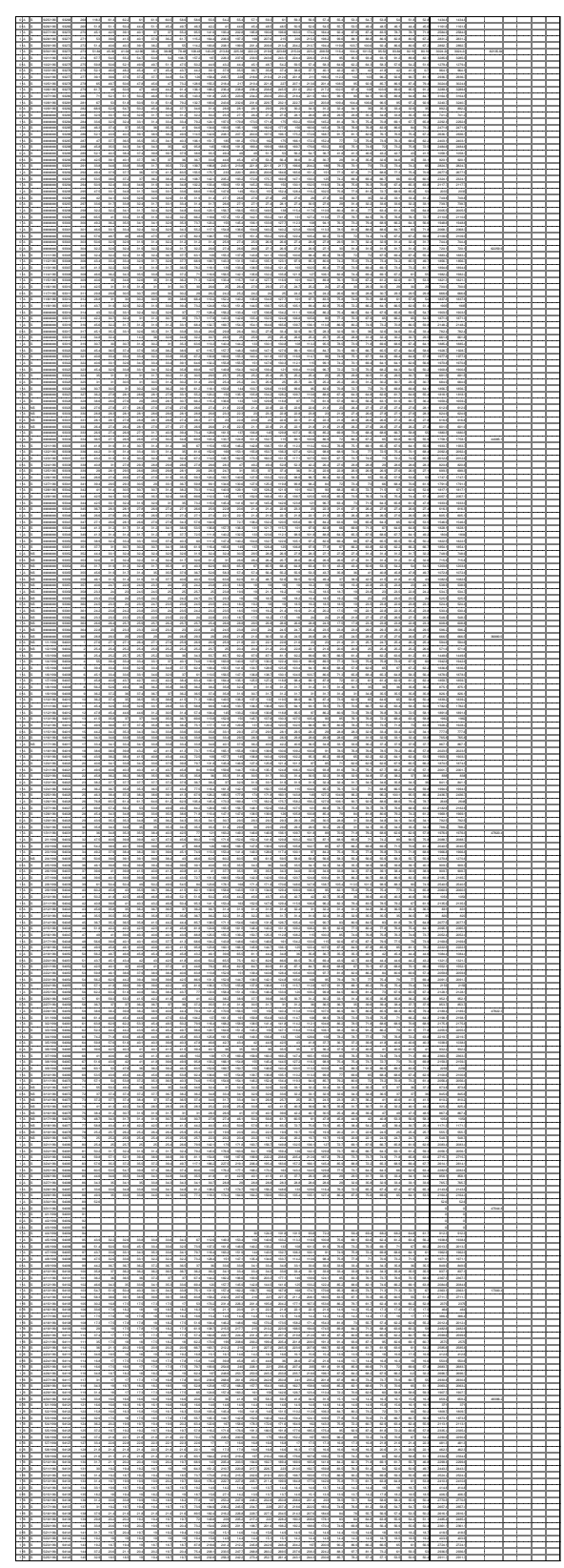




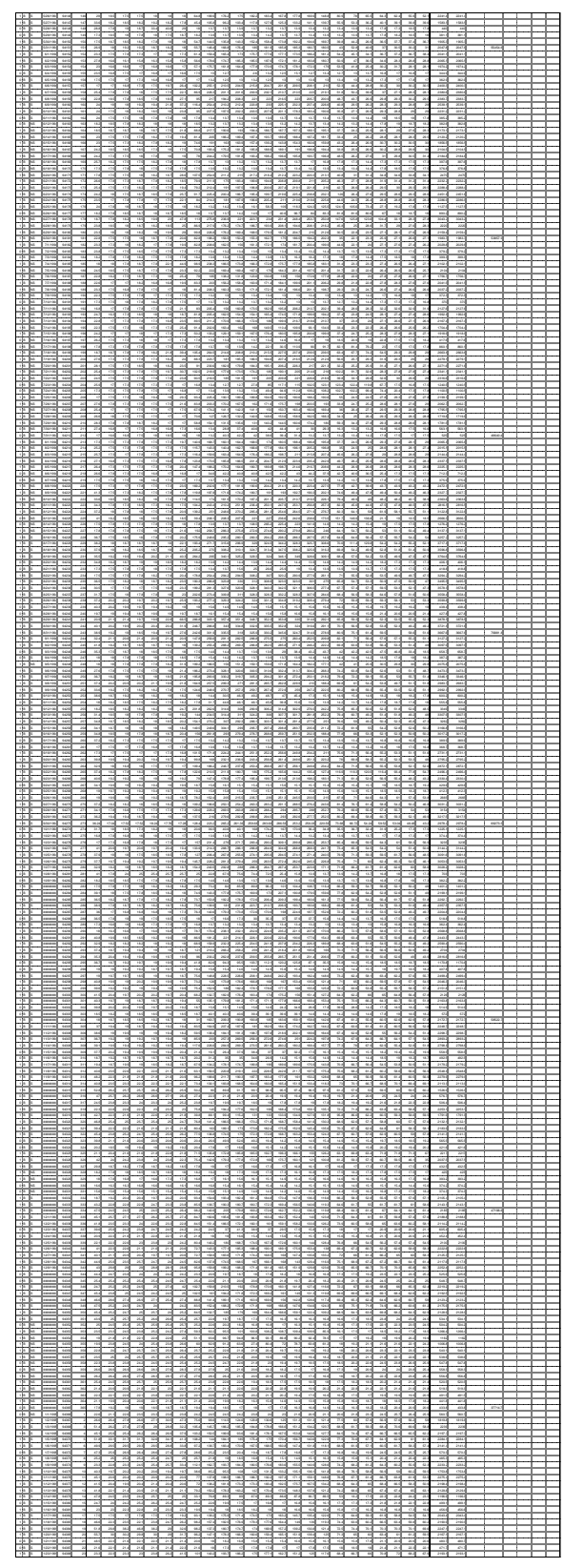




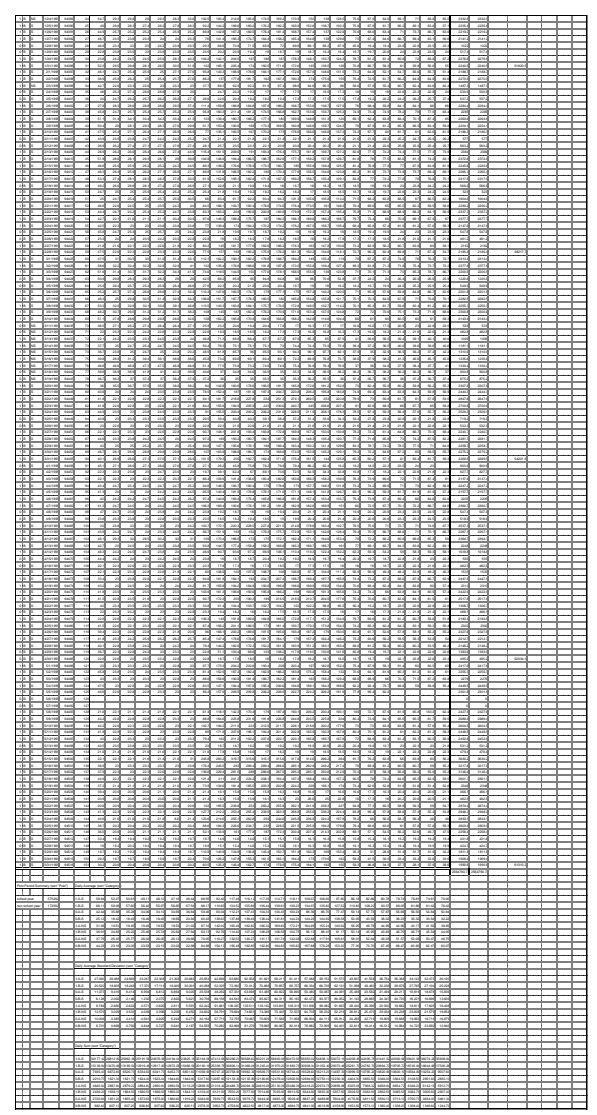




\begin{tabular}{|c|c|c|c|c|c|c|c|c|c|c|}
\hline $\begin{array}{l}\mathrm{wd} / \\
\text { we } \\
\text { o/1 }\end{array}$ & $\begin{array}{c}\text { pre } / p \\
\text { ost } \\
\text { A/B }\end{array}$ & $\begin{array}{l}\text { schyear/br } \\
\text { eaks S/NS }\end{array}$ & date & jday & $\begin{array}{l}\text { orig } \\
\text { count } \\
(156)\end{array}$ & 0 & 1 & 2 & 3 & 4 \\
\hline 1 & A & NS & 6/6/1991 & 91157 & 157 & & & & & \\
\hline 1 & A & NS & 6/7/1991 & 91158 & 158 & & & & & \\
\hline 0 & A & NS & 6/8/1991 & 91159 & 159 & & & & & \\
\hline 0 & A & NS & 6/9/1991 & 91160 & 160 & & & & & \\
\hline 1 & A & NS & 6/10/1991 & 91161 & 161 & & & & & \\
\hline 1 & A & NS & 6/11/1991 & 91162 & 162 & & & & & \\
\hline 1 & A & NS & $6 / 12 / 1991$ & 91163 & 163 & & & & & \\
\hline 1 & A & NS & 6/13/1991 & 91164 & 164 & & & & & \\
\hline 1 & A & NS & $6 / 14 / 1991$ & 91165 & 165 & & & & & \\
\hline 0 & A & NS & $6 / 15 / 1991$ & 91166 & 166 & & & & & \\
\hline 0 & A & NS & $6 / 16 / 1991$ & 91167 & 167 & & & & & \\
\hline 1 & A & NS & 6/17/1991 & 91168 & 168 & & & & & \\
\hline 1 & A & NS & 6/18/1991 & 91169 & 169 & & & & & \\
\hline 1 & A & NS & 6/19/1991 & 91170 & 170 & & & & & \\
\hline 1 & $\mathrm{~A}$ & NS & $6 / 20 / 1991$ & 91171 & 171 & & & & & \\
\hline 1 & A & NS & $6 / 21 / 1991$ & 91172 & 172 & & & & & \\
\hline 0 & A & NS & $6 / 22 / 1991$ & 91173 & 173 & & & & & \\
\hline 0 & A & NS & 6/23/1991 & 91174 & 174 & & & & & \\
\hline 1 & A & NS & $6 / 24 / 1991$ & 91175 & 175 & & & & & \\
\hline 1 & A & NS & $6 / 25 / 1991$ & 91176 & 176 & & & & & \\
\hline 1 & $\mathrm{~A}$ & NS & $6 / 26 / 1991$ & 91177 & 177 & & & & & \\
\hline 1 & A & NS & $6 / 27 / 1991$ & 91178 & 178 & & & & & \\
\hline 1 & A & NS & $6 / 28 / 1991$ & 91179 & 179 & & & & & \\
\hline 0 & A & NS & 6/29/1991 & 91180 & 180 & & & & & \\
\hline 0 & A & NS & $6 / 30 / 1991$ & 91181 & 181 & & & & & \\
\hline 1 & A & NS & $7 / 1 / 1991$ & 91182 & 182 & & & & & \\
\hline 1 & A & NS & 7/2/1991 & 91183 & 183 & & & & & \\
\hline 1 & A & NS & 7/3/1991 & 91184 & 184 & & & & & \\
\hline 1 & A & NS & 7/4/1991 & 91185 & 185 & & & & & \\
\hline 1. & A & NS & 7/5/1991 & 91186 & 186 & & & & & \\
\hline 0 & A & NS & 7/6/1991 & 91187 & 187 & & & & & \\
\hline 0 & A & NS & 7/7/1991 & 91188 & 188 & & & & & \\
\hline 1 & A & NS & 7/8/1991 & 91189 & 189 & & & & & \\
\hline 1 & A & NS & 7/9/1991 & 91190 & 190 & & & & & \\
\hline 1 & A & NS & $7 / 10 / 1991$ & 91191 & 191 & & & & & \\
\hline 1 & A & NS & $7 / 11 / 1991$ & 91192 & 192 & & & & & \\
\hline 1 & A & NS & 7/12/1991 & 91193 & 193 & & & & & \\
\hline 0 & A & NS & $7 / 13 / 1991$ & 91194 & 194 & & & & & \\
\hline 0 & A & NS & $7 / 14 / 1991$ & 91195 & 195 & & & & & \\
\hline 1 & A & NS & 7/15/1991 & 91196 & 196 & & & & & \\
\hline 1 & A & NS & $7 / 16 / 1991$ & 91197 & 197 & & & & & \\
\hline 1 & A & NS & 7/17/1991 & 91198 & 198 & & & & & \\
\hline 1 & A & NS & 7/18/1991 & 91199 & 199 & & & & & \\
\hline 1 & A & NS & $7 / 19 / 1991$ & 91200 & 200 & & & & & \\
\hline 0 & A & NS & $7 / 20 / 1991$ & 91201 & 201 & & & & & \\
\hline 0 & A & NS & 7/21/1991 & 91202 & 202 & & & & & \\
\hline 1 & A & NS & $7 / 22 / 1991$ & 91203 & 203 & & & & & \\
\hline
\end{tabular}




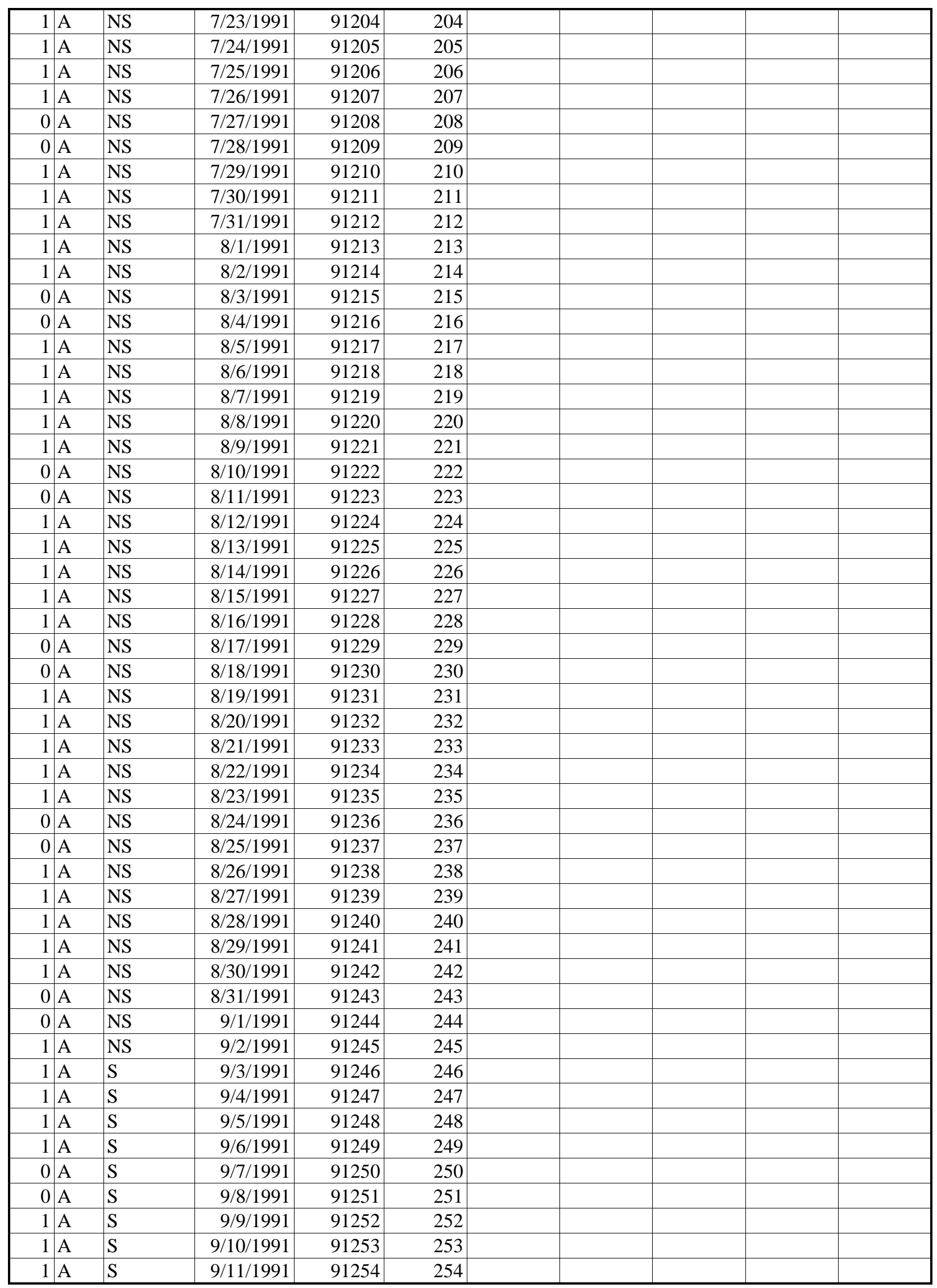




\begin{tabular}{|c|c|c|c|c|c|c|c|c|c|}
\hline $1 \mathrm{~A}$ & $\mathrm{~S}$ & 9/12/1991 & 91255 & 255 & & & & & \\
\hline $1 A$ & $S$ & 9/13/1991 & 91256 & 256 & & & & & \\
\hline $0 A$ & S & 9/14/1991 & 91257 & 257 & & & & & \\
\hline $0 A$ & $S$ & 9/15/1991 & 91258 & 258 & & & & & \\
\hline $1 A$ & S & 9/16/1991 & 91259 & 259 & & & & & \\
\hline $1 A$ & S & 9/17/1991 & 91260 & 260 & & & & & \\
\hline $1 \mid f$ & S & 9/18/1991 & 91261 & 261 & & & & & \\
\hline $1 A$ & S & 9/19/1991 & 91262 & 262 & & & & & \\
\hline $1 A$ & $S$ & 9/20/1991 & 91263 & 263 & & & & & \\
\hline $0 A$ & S & 9/21/1991 & 91264 & 264 & & & & & \\
\hline $0 A$ & S & $9 / 22 / 1991$ & 91265 & 265 & & & & & \\
\hline $1 A$ & S & 9/23/1991 & 91266 & 266 & & & & & \\
\hline $1 \mid f$ & S & 9/24/1991 & 91267 & 267 & & & & & \\
\hline $1 A$ & $S$ & 9/25/1991 & 91268 & 268 & & & & & \\
\hline $1 \mid f$ & $S$ & 9/26/1991 & 91269 & 269 & & & & & \\
\hline $1 \mathrm{f}$ & $S$ & 9/27/1991 & 91270 & 270 & & & & & \\
\hline $0 A$ & S & 9/28/1991 & 91271 & 271 & & & & & \\
\hline $0 A$ & $S$ & 9/29/1991 & 91272 & 272 & & & & & \\
\hline $1 A$ & S & 9/30/1991 & 91273 & 273 & & & & & \\
\hline $1 A$ & S & 10/1/1991 & 91274 & 274 & & & & & \\
\hline $1 \mid f$ & $S$ & $10 / 2 / 1991$ & 91275 & 275 & & & & & \\
\hline $1 A$ & S & 10/3/1991 & 91276 & 276 & 2 & 2 & 2 & 2 & 2 \\
\hline $1 A$ & S & 10/4/1991 & 91277 & 277 & 3 & 2 & 2 & 2 & 2 \\
\hline $0 A$ & $S$ & $10 / 5 / 1991$ & 91278 & 278 & 3 & 2 & 2 & 3 & 2 \\
\hline $0 A$ & S & $10 / 6 / 1991$ & 91279 & 279 & 2 & 2 & 2 & 2 & 2 \\
\hline $1 \mathrm{~A}$ & $S$ & 10/7/1991 & 91280 & 280 & 2 & 2 & 2 & 2 & 2 \\
\hline $1 A$ & $S$ & 10/8/1991 & 91281 & 281 & 0.8 & 0.8 & 0.8 & 0.4 & 0.8 \\
\hline $1 A$ & $S$ & 10/9/1991 & 91282 & 282 & 0.8 & 0.8 & 0.8 & 0.8 & 0.8 \\
\hline $1 A$ & S & 10/10/1991 & 91283 & 283 & 0.8 & 0.8 & 0.8 & 0.8 & 0.8 \\
\hline $1 A$ & $S$ & 10/11/1991 & 91284 & 284 & 0.8 & 0.4 & 1.2 & 0.8 & 0.8 \\
\hline $0 A$ & S & 10/12/1991 & 91285 & 285 & 0.8 & 0.8 & 0.8 & 0.8 & 0.8 \\
\hline $0 A$ & S & 10/13/1991 & 91286 & 286 & 0.8 & 0.8 & 0.8 & 0.8 & 0.8 \\
\hline $1 A$ & $S$ & 10/14/1991 & 91287 & 287 & 0.8 & 0.8 & 0.8 & 0.8 & 0.8 \\
\hline $1 f$ & $S$ & 10/15/1991 & 91288 & 288 & 0.8 & 0.8 & 0.8 & 0.8 & 0.8 \\
\hline $1 A$ & S & 10/16/1991 & 91289 & 289 & 0.8 & 0.8 & 0.8 & 0.8 & 0.8 \\
\hline $1 A$ & S & 10/17/1991 & 91290 & 290 & 0.8 & 0.8 & 0.8 & 0.8 & 0.8 \\
\hline $1 A$ & $S$ & 10/18/1991 & 91291 & 291 & 0.8 & 0.8 & 0.8 & 0.8 & 0.4 \\
\hline $0 \mathrm{~A}$ & S & 10/19/1991 & 91292 & 292 & 0.4 & 0.8 & 0.8 & 0.8 & 0.8 \\
\hline $0 \mathrm{~A}$ & S & 10/20/1991 & 91293 & 293 & 0.8 & 0.8 & 0.8 & 0.4 & 0.8 \\
\hline $1 f$ & $S$ & 10/21/1991 & 91294 & 294 & 0.4 & 0.8 & 0.8 & 0.8 & 0.4 \\
\hline $1 A$ & $S$ & 10/22/1991 & 91295 & 295 & 0.8 & 0.4 & 0.8 & 0.8 & 0.4 \\
\hline $1 A$ & NS & 10/23/1991 & 91296 & 296 & 0.8 & 0.8 & 0.4 & 0.8 & 0.8 \\
\hline $1 A$ & $S$ & 10/24/1991 & 91297 & 297 & 0.8 & 0.8 & 0.4 & 0.8 & 0.8 \\
\hline $1 A$ & S & 10/25/1991 & 91298 & 298 & 0.8 & 0.8 & 0.4 & 0.8 & 0.8 \\
\hline $0 A$ & S & 10/26/1991 & 91299 & 299 & 0.8 & 0.8 & 0.8 & 0.4 & 0.8 \\
\hline $0 A$ & $S$ & 10/27/1991 & 91300 & 300 & 0.8 & 0.8 & 0.8 & 0.4 & 0.8 \\
\hline $1 f$ & $S$ & 10/28/1991 & 91301 & 301 & 0.8 & 0.8 & 0.8 & 0.4 & 0.8 \\
\hline $1 A$ & S & 10/29/1991 & 91302 & 302 & 0.8 & 0.8 & 0.4 & 0.8 & 0.8 \\
\hline $1 A$ & S & 10/30/1991 & 91303 & 303 & 0.8 & 0.8 & 0.4 & 0.8 & 0.8 \\
\hline $1 A$ & S & 10/31/1991 & 91304 & 304 & 0.8 & 0.4 & 0.4 & 0.8 & 0.4 \\
\hline $1 A$ & $S$ & 11/1/1991 & 91305 & 305 & 0.4 & 0.4 & 0.8 & 0.4 & 0.4 \\
\hline
\end{tabular}




\begin{tabular}{|c|c|c|c|c|c|c|c|c|c|}
\hline $0 A$ & $S$ & $11 / 2 / 1991$ & 91306 & 306 & 0.8 & 0.4 & 0.4 & 0.8 & 0.4 \\
\hline $0 A$ & $S$ & $11 / 3 / 1991$ & 91307 & 307 & 0.4 & 0.4 & 0.8 & 0.4 & 0.4 \\
\hline $1 \mid A$ & $S$ & 11/4/1991 & 91308 & 308 & 0.4 & 0.8 & 0.4 & 0.4 & 0.8 \\
\hline $1 \mid A$ & $S$ & 11/5/1991 & 91309 & 309 & 0.8 & 0.4 & 0.8 & 0.4 & 0.4 \\
\hline $1 \mid A$ & $S$ & $11 / 6 / 1991$ & 91310 & 310 & 0.4 & 0.8 & 0.4 & 0.8 & 0.4 \\
\hline $1 A$ & $S$ & $11 / 7 / 1991$ & 91311 & 311 & 0.8 & 0.4 & 0.4 & 0.8 & 0.4 \\
\hline $1 A$ & $S$ & $11 / 8 / 1991$ & 91312 & 312 & 0.4 & 0.8 & 0.4 & 0.4 & 0.8 \\
\hline $0[A$ & $S$ & $11 / 9 / 1991$ & 91313 & 313 & 0.8 & 0.4 & 0.8 & 0.4 & 0.8 \\
\hline $0 \mathrm{~A}$ & $\mathrm{~S}$ & 11/10/1991 & 91314 & 314 & 0.4 & 0.8 & 0.4 & 0.8 & 0.4 \\
\hline $1 \mid A$ & $S$ & 11/11/1991 & 91315 & 315 & 0.8 & 0.8 & 0.8 & 0.4 & 0.8 \\
\hline $1 \mid A$ & $S$ & 11/12/1991 & 91316 & 316 & 0.4 & 0.4 & 0.8 & 0.4 & 0.4 \\
\hline $1 \mathrm{~A}$ & $S$ & 11/13/1991 & 91317 & 317 & 0.4 & 0.8 & 0.4 & 0.4 & 0.8 \\
\hline $1 A$ & $S$ & 11/14/1991 & 91318 & 318 & 0.4 & 0.4 & 0.8 & 0.4 & 0.4 \\
\hline $1 \mathrm{~A}$ & $S$ & 11/15/1991 & 91319 & 319 & 0.4 & 0.4 & 0.4 & 0.4 & 0.4 \\
\hline $0[A$ & $S$ & 11/16/1991 & 91320 & 320 & 0.4 & 0.4 & 0.4 & 0.4 & 0.4 \\
\hline $0 \mid A$ & $S$ & 11/17/1991 & 91321 & 321 & 0.4 & 0.4 & 0.4 & 0.8 & 0.4 \\
\hline $1 \mid A$ & $S$ & 11/18/1991 & 91322 & 322 & 0.4 & 0.8 & 0.4 & 0.4 & 0.4 \\
\hline $1 \mathrm{~A}$ & $S$ & 11/19/1991 & 91323 & 323 & 0.8 & 0.4 & 0.4 & 0.4 & 0.4 \\
\hline $1 \mathrm{~A}$ & S & 11/20/1991 & 91324 & 324 & 0.4 & 0.4 & 0.4 & 0.8 & 0.4 \\
\hline $1 \mathrm{~A}$ & $S$ & 11/21/1991 & 91325 & 325 & 0.4 & 0.8 & 0.4 & 0.4 & 0.4 \\
\hline $1 \mid A$ & $S$ & 11/22/1991 & 91326 & 326 & 0.4 & 0.4 & 0.4 & 0.4 & 0.4 \\
\hline $0 \mid A$ & $S$ & 11/23/1991 & 91327 & 327 & 0.4 & 0.4 & 0.8 & 0.4 & 0.4 \\
\hline $0 \mathrm{~A}$ & $S$ & 11/24/1991 & 91328 & 328 & 0.4 & 0.4 & 0.4 & 0.8 & 0.4 \\
\hline $1 \mid A$ & $S$ & 11/25/1991 & 91329 & 329 & 0.4 & 0.4 & 0.4 & 0.8 & 0.4 \\
\hline $1 \mathrm{~A}$ & S & 11/26/1991 & 91330 & 330 & 0.4 & 0.4 & 0.4 & 0.8 & 0.4 \\
\hline $1 A$ & NS & 11/27/1991 & 91331 & 331 & 0.4 & 0.8 & 0.4 & 0.4 & 0.4 \\
\hline $1 \mid A$ & NS & 11/28/1991 & 91332 & 332 & 0.4 & 0.4 & 0.4 & 0.4 & 0.8 \\
\hline $1 A$ & NS & 11/29/1991 & 91333 & 333 & 0.4 & 0.4 & 0.4 & 0.8 & \\
\hline $0 \mathrm{~A}$ & $\mathrm{~S}$ & 11/30/1991 & 91334 & 334 & 0.4 & 0.4 & 0.4 & 0.4 & 0.4 \\
\hline $0 A$ & $S$ & 12/1/1991 & 91335 & 335 & 0.8 & 0.4 & 0.4 & 0.4 & 0.8 \\
\hline $1 A$ & $S$ & 12/2/1991 & 91336 & 336 & 0.4 & 0.8 & 0.4 & 0.4 & 0.8 \\
\hline $1 A$ & $S$ & 12/3/1991 & 91337 & 337 & 0.8 & 0.4 & 0.4 & 0.4 & 0.4 \\
\hline $1 \mid A$ & $S$ & $12 / 4 / 1991$ & 91338 & 338 & 0.8 & 0.4 & 0.4 & 0.4 & 0.8 \\
\hline $1 A$ & $S$ & $12 / 5 / 1991$ & 91339 & 339 & 0.8 & 0.4 & 0.4 & 0.8 & 0.4 \\
\hline $1 A$ & $S$ & 12/6/1991 & 91340 & 340 & 0.8 & 0.4 & 0.4 & 0.4 & 0.4 \\
\hline $0 \mathrm{~A}$ & $S$ & 12/7/1991 & 91341 & 341 & 0.4 & 0.4 & 0.4 & 0.4 & 0.4 \\
\hline $0 \mid A$ & $S$ & $12 / 8 / 1991$ & 91342 & 342 & 0.4 & 0.4 & 0.4 & 0.4 & 0.4 \\
\hline $1 A$ & S & 12/9/1991 & 91343 & 343 & 0.4 & 0.4 & 0.4 & 0.4 & 0.4 \\
\hline $1 A$ & $S$ & 12/10/1991 & 91344 & 344 & 0.4 & 0.4 & 0.4 & 0.4 & 0.8 \\
\hline $1 \mathrm{~A}$ & $S$ & 12/11/1991 & 91345 & 345 & 0.4 & 0.4 & 0.4 & 0.4 & 0.4 \\
\hline $1 A$ & $S$ & 12/12/1991 & 91346 & 346 & 0.4 & 0.4 & 0.4 & 0.4 & 0.4 \\
\hline $1 A$ & $S$ & 12/13/1991 & 91347 & 347 & 0.4 & 0.4 & 0.4 & 0.4 & 0.4 \\
\hline $0 A$ & $S$ & 12/14/1991 & 91348 & 348 & 0.8 & 0.4 & 0.4 & 0.4 & 0.4 \\
\hline $0 \mathrm{~A}$ & S & 12/15/1991 & 91349 & 349 & 0.4 & 0.4 & 0.4 & 0.8 & 0.4 \\
\hline $1 A$ & $S$ & 12/16/1991 & 91350 & 350 & 0.4 & 0.4 & 0.4 & 0.8 & 0.4 \\
\hline $1 A$ & $S$ & 12/17/1991 & 91351 & 351 & 0.4 & 0.4 & 0.4 & 0.8 & 0.4 \\
\hline $1 A$ & $S$ & 12/18/1991 & 91352 & 352 & 0.4 & 0.4 & 0.8 & 0.4 & 0.4 \\
\hline $1 A$ & $S$ & 12/19/1991 & 91353 & 353 & 0.4 & 0.4 & 0.4 & 0.4 & 0.4 \\
\hline $1 A$ & NS & 12/20/1991 & 91354 & 354 & 0.4 & 0.4 & 0.4 & 0.4 & 0.4 \\
\hline $0 A$ & NS & 12/21/1991 & 91355 & 355 & 0.4 & 0.4 & 0.4 & 0.4 & 0.4 \\
\hline $0 A$ & NS & $12 / 22 / 1991$ & 91356 & 356 & 0.4 & 0.4 & 0.4 & 0.4 & 0.4 \\
\hline
\end{tabular}




\begin{tabular}{|c|c|c|c|c|c|c|c|c|c|}
\hline $1 \mathrm{~A}$ & NS & 12/23/1991 & 91357 & 357 & 0.4 & 0.4 & 0.4 & 0.4 & 0.8 \\
\hline $1 A$ & NS & 12/24/1991 & 91358 & 358 & 0.4 & 0.4 & 0.4 & 0.8 & 0.4 \\
\hline $1 \mathrm{~A}$ & NS & 12/25/1991 & 91359 & 359 & 0.4 & 0.8 & 0.4 & 0.4 & 0.4 \\
\hline $1 A$ & NS & 12/26/1991 & 91360 & 360 & 0.4 & 0.4 & 0.8 & 0.4 & 0.4 \\
\hline $1 \mid A$ & NS & $12 / 27 / 1991$ & 91361 & 361 & 0.4 & 0.4 & 0.4 & 0.8 & 0.4 \\
\hline $0 A$ & NS & 12/28/1991 & 91362 & 362 & 0.4 & 0.4 & 0.8 & 0.4 & 0.4 \\
\hline $0 \mathrm{~A}$ & NS & 12/29/1991 & 91363 & 363 & 0.4 & 0.8 & 0.4 & 0.4 & 0.4 \\
\hline $1 \mid A$ & NS & 12/30/1991 & 91364 & 364 & 0.4 & 0.4 & 0.8 & 0.4 & 0.4 \\
\hline $1 A$ & NS & 12/31/1991 & 91365 & 365 & 0.8 & 0.4 & 0.4 & 0.4 & 0.4 \\
\hline $1 \mid A$ & NS & 1/1/1992 & 92001 & 366 & & & & & \\
\hline $1 \mid A$ & NS & $1 / 2 / 1992$ & 92002 & 1 & 0.4 & 0.8 & 0.4 & 0.8 & 0.4 \\
\hline $1 \mathrm{~A}$ & NS & $1 / 3 / 1992$ & 92003 & 2 & 0.4 & 0.8 & 0.4 & 0.8 & 0.4 \\
\hline $0 \mathrm{~A}$ & NS & 1/4/1992 & 92004 & 3 & 0.4 & 0.4 & 0.8 & 0.4 & 0.4 \\
\hline $0 \mathrm{~A}$ & NS & 1/5/1992 & 92005 & 4 & 0.4 & 0.4 & 0.8 & 0.4 & 0.4 \\
\hline $1 \mathrm{~A}$ & NS & $1 / 6 / 1992$ & 92006 & 5 & 0.4 & 0.4 & 0.8 & 0.4 & 0.4 \\
\hline $1 A$ & S & 1/7/1992 & 92007 & 6 & 0.4 & 0.4 & 0.8 & 0.4 & 0.4 \\
\hline $1 A$ & $S$ & $1 / 8 / 1992$ & 92008 & 7 & 0.4 & 0.4 & 0.4 & 0.4 & 0.8 \\
\hline $1 \mathrm{~A}$ & $S$ & 1/9/1992 & 92009 & 8 & 0.4 & 0.4 & 0.4 & 0.4 & 0.4 \\
\hline $1 \mathrm{~A}$ & S & 1/10/1992 & 92010 & 9 & 0.8 & 0.4 & 0.4 & 0.4 & 0.4 \\
\hline $0 \mathrm{~A}$ & $S$ & 1/11/1992 & 92011 & 10 & 0.4 & 0.4 & 0.4 & 0.8 & 0.4 \\
\hline $0 \mid A$ & $S$ & $1 / 12 / 1992$ & 92012 & 11 & 0.4 & 0.4 & 0.4 & 0.8 & 0.4 \\
\hline $1 A$ & $S$ & $1 / 13 / 1992$ & 92013 & 12 & 0.4 & 0.4 & 0.4 & 0.8 & 0.4 \\
\hline $1 A$ & $S$ & $1 / 14 / 1992$ & 92014 & 13 & 0.4 & 0.4 & 0.4 & 0.8 & 0.4 \\
\hline $1 \mid A$ & $S$ & $1 / 15 / 1992$ & 92015 & 14 & 0.8 & 0.4 & 0.4 & 0.4 & 0.8 \\
\hline $1 \mathrm{~A}$ & S & 1/16/1992 & 92016 & 15 & 0.4 & 0.4 & 0.4 & 0.8 & 0.4 \\
\hline $1 A$ & NS & $1 / 17 / 1992$ & 92017 & 16 & 0.8 & 0.4 & 0.4 & 0.8 & 0.4 \\
\hline $0 \mid A$ & $\mathrm{~S}$ & $1 / 18 / 1992$ & 92018 & 17 & 0.4 & 0.4 & 0.4 & 0.4 & 0.8 \\
\hline $0 A$ & $S$ & 1/19/1992 & 92019 & 18 & 0.8 & 0.4 & 0.4 & 0.4 & 0.4 \\
\hline $1 f$ & $S$ & $1 / 20 / 1992$ & 92020 & 19 & 0.8 & 0.4 & 0.4 & 0.4 & 0.4 \\
\hline $1 \mid A$ & $S$ & $1 / 21 / 1992$ & 92021 & 20 & 0.4 & 0.4 & 0.4 & 0.4 & 0.8 \\
\hline $1 A$ & $S$ & $1 / 22 / 1992$ & 92022 & 21 & 0.8 & 0.4 & 0.4 & 0.4 & 0.4 \\
\hline $1 A$ & $S$ & $1 / 23 / 1992$ & 92023 & 22 & 0.4 & 0.4 & 0.4 & 0.4 & 0.4 \\
\hline $1 \mid A$ & $S$ & $1 / 24 / 1992$ & 92024 & 23 & 0.4 & 0.4 & 0.8 & 0.4 & 0.4 \\
\hline $0 \mathrm{~A}$ & $S$ & $1 / 25 / 1992$ & 92025 & 24 & 0.4 & 0.4 & 0.4 & 0.4 & 0.8 \\
\hline $0 \mid A$ & $S$ & 1/26/1992 & 92026 & 25 & 0.4 & 0.4 & 0.4 & 0.8 & 0.4 \\
\hline $1 \mid A$ & $S$ & $1 / 27 / 1992$ & 92027 & 26 & 0.4 & 0.4 & 0.4 & 0.4 & 0.4 \\
\hline $1 \mid A$ & $S$ & $1 / 28 / 1992$ & 92028 & 27 & 0.4 & 0.4 & 0.8 & 0.4 & 0.4 \\
\hline $1 A$ & S & 1/29/1992 & 92029 & 28 & 0.4 & 0.4 & 0.4 & 0.4 & 0.4 \\
\hline $1 A$ & $S$ & 1/30/1992 & 92030 & 29 & 0.4 & 0.4 & 0.4 & 0.4 & 0.4 \\
\hline $1 \mathrm{~A}$ & $S$ & 1/31/1992 & 92031 & 30 & 0.4 & 0.8 & 0.4 & 0.4 & 0.4 \\
\hline $0 \mathrm{E}$ & $S$ & 2/1/1992 & 92032 & 31 & 0.4 & 0.4 & 0.4 & 0.4 & 0.8 \\
\hline $0 \mathrm{E}$ & $S$ & $2 / 2 / 1992$ & 92033 & 32 & 0.4 & 0.8 & 0.4 & 0.4 & 0.4 \\
\hline $1 \mathrm{E}$ & $S$ & 2/3/1992 & 92034 & 33 & 0.8 & 0.4 & 0.4 & 0.4 & 0.4 \\
\hline $1 \mathrm{E}$ & S & 2/4/1992 & 92035 & 34 & 0.8 & 0.4 & 0.4 & 0.4 & 0.4 \\
\hline $1 \mathrm{E}$ & $S$ & 2/5/1992 & 92036 & 35 & 0.4 & 0.4 & 0.4 & 0.4 & 0.4 \\
\hline $1 \mathrm{E}$ & $S$ & 2/6/1992 & 92037 & 36 & 0.4 & 0.4 & 0.4 & 0.4 & 0.4 \\
\hline $1 \mathrm{E}$ & $S$ & 2/7/1992 & 92038 & 37 & 0.4 & 0.4 & 0.4 & 0.4 & 0.8 \\
\hline $0 \mathrm{E}$ & $S$ & 2/8/1992 & 92039 & 38 & 0.4 & 0.4 & 0.4 & 0.4 & 0.8 \\
\hline $0 \mathrm{E}$ & $S$ & 2/9/1992 & 92040 & 39 & 0.8 & 0.4 & 0.4 & 0.4 & 0.8 \\
\hline $1 \mathrm{E}$ & $S$ & 2/10/1992 & 92041 & 40 & 0.4 & 0.8 & 0.4 & 0.4 & 0.4 \\
\hline $1 \mathrm{~B}$ & S & $2 / 11 / 1992$ & 92042 & 41 & 0.8 & 0.4 & 0.4 & 0.4 & 0.4 \\
\hline
\end{tabular}




\begin{tabular}{|c|c|c|c|c|c|c|c|c|c|}
\hline $1 \mathrm{E}$ & $S$ & 2/12/1992 & 92043 & 42 & 0.4 & 0.4 & 0.4 & 0.8 & 0.4 \\
\hline $1 \mathrm{E}$ & $S$ & 2/13/1992 & 92044 & 43 & 0.4 & 0.8 & 0.4 & 0.4 & 0.4 \\
\hline $1 \mathrm{E}$ & $S$ & $2 / 14 / 1992$ & 92045 & 44 & 0.4 & & 0.8 & 0.4 & 0.4 \\
\hline $0 \mathrm{E}$ & $S$ & 2/15/1992 & 92046 & 45 & 0.4 & 0.4 & 0.4 & 0.8 & \\
\hline $0 \mathrm{E}$ & $S$ & 2/16/1992 & 92047 & 46 & 0.4 & 0.4 & 0.4 & 0.4 & 0.4 \\
\hline $1 \mathrm{E}$ & $S$ & 2/17/1992 & 92048 & 47 & 0.4 & 0.4 & 0.4 & 0.8 & 0.4 \\
\hline $1 \mathrm{E}$ & $S$ & 2/18/1992 & 92049 & 48 & 0.4 & 0.4 & 0.8 & 0.4 & 0.4 \\
\hline $1 \mathrm{E}$ & $S$ & 2/19/1992 & 92050 & 49 & 0.4 & 0.4 & 0.4 & 0.4 & 0.4 \\
\hline $1 \mathrm{~F}$ & $S$ & 2/20/1992 & 92051 & 50 & 0.4 & 0.4 & 0.4 & 0.4 & 0.4 \\
\hline $1 \mathrm{E}$ & $S$ & $2 / 21 / 1992$ & 92052 & 51 & 0.4 & 0.8 & 0.4 & 0.4 & 0.4 \\
\hline $0 \mathrm{E}$ & $S$ & 2/22/1992 & 92053 & 52 & 0.4 & 0.4 & 0.4 & 0.4 & 0.4 \\
\hline $0 \mathrm{E}$ & $S$ & 2/23/1992 & 92054 & 53 & 0.4 & 0.4 & 0.4 & 0.4 & 0.4 \\
\hline $1 \mathrm{E}$ & $S$ & $2 / 24 / 1992$ & 92055 & 54 & 0.4 & 0.4 & 0.4 & 0.4 & 0.4 \\
\hline $1 \mathrm{E}$ & $\mathrm{S}$ & 2/25/1992 & 92056 & 55 & 0.4 & 0.4 & 0.4 & 0.4 & 0.8 \\
\hline $1 \mathrm{E}$ & $S$ & 2/26/1992 & 92057 & 56 & 0.4 & 0.8 & 0.4 & 0.4 & 0.4 \\
\hline $1 \mathrm{E}$ & $S$ & $2 / 27 / 1992$ & 92058 & 57 & 0.4 & 0.4 & 0.4 & 0.4 & 0.8 \\
\hline $1 \mathrm{E}$ & $S$ & 2/28/1992 & 92059 & 58 & 0.4 & 0.4 & 0.4 & 0.4 & 0.8 \\
\hline $0 \mathrm{E}$ & $S$ & 2/29/1992 & 92060 & 59 & 0.4 & 0.4 & 0.4 & 0.4 & 0.8 \\
\hline $0 \mathrm{E}$ & $S$ & 3/1/1992 & 92061 & 60 & 0.4 & 0.4 & 0.4 & 0.8 & 0.4 \\
\hline $1 \mathrm{E}$ & $S$ & 3/2/1992 & 92062 & 61 & 0.4 & 0.4 & 0.4 & 0.8 & 0.4 \\
\hline $1 \mathrm{E}$ & $S$ & 3/3/1992 & 92063 & 62 & 0.4 & 0.4 & 0.4 & 0.4 & 0.4 \\
\hline $1 \mathrm{E}$ & $S$ & 3/4/1992 & 92064 & 63 & 0.4 & 0.4 & 0.4 & 0.4 & 0.4 \\
\hline $1 \mathrm{E}$ & $S$ & 3/5/1992 & 92065 & 64 & 0.4 & 0.4 & 0.4 & 0.4 & 0.4 \\
\hline $1 \mathrm{E}$ & $S$ & 3/6/1992 & 92066 & 65 & 0.4 & 0.8 & 0.4 & 0.4 & 0.4 \\
\hline $0 \mathrm{E}$ & $S$ & 3/7/1992 & 92067 & 66 & 0.4 & 0.4 & 0.4 & 0.4 & 0.4 \\
\hline $0 \mathrm{E}$ & $S$ & 3/8/1992 & 92068 & 67 & 0.4 & 0.4 & 0.4 & 0.4 & 0.4 \\
\hline $1 \mathrm{E}$ & $S$ & 3/9/1992 & 92069 & 68 & 0.4 & 0.4 & 0.4 & 0.8 & 0.4 \\
\hline $1 \mathrm{~F}$ & $S$ & 3/10/1992 & 92070 & 69 & 0.4 & 0.4 & 0.4 & 0.4 & 0.4 \\
\hline $1 \mathrm{E}$ & $S$ & 3/11/1992 & 92071 & 70 & 0.4 & 0.4 & 0.4 & 0.4 & 0.8 \\
\hline $1 \mathrm{E}$ & S & 3/12/1992 & 92072 & 71 & 0.4 & 0.4 & 0.4 & 0.4 & 0.4 \\
\hline $1 \mathrm{~F}$ & NS & 3/13/1992 & 92073 & 72 & 0.8 & 0.4 & 0.4 & 0.4 & 0.4 \\
\hline $0 \mathrm{E}$ & NS & 3/14/1992 & 92074 & 73 & 0.4 & 0.4 & 0.4 & 0.8 & 0.4 \\
\hline $0 \mathrm{E}$ & NS & 3/15/1992 & 92075 & 74 & 0.4 & 0.4 & 0.4 & 0.4 & 0.4 \\
\hline $1 \mathrm{~F}$ & NS & 3/16/1992 & 92076 & 75 & 0.4 & 0.4 & 0.8 & 0.4 & 0.4 \\
\hline $1 \mathrm{E}$ & NS & 3/17/1992 & 92077 & 76 & 0.8 & 0.4 & 0.4 & 0.4 & 0.4 \\
\hline $1 \mathrm{E}$ & NS & 3/18/1992 & 92078 & 77 & 0.4 & 0.4 & 0.4 & 0.4 & 0.4 \\
\hline $1 \mathrm{E}$ & NS & 3/19/1992 & 92079 & 78 & 0.4 & 0.4 & 0.4 & 0.8 & 0.4 \\
\hline $1 \mathrm{E}$ & NS & 3/20/1992 & 92080 & 79 & 0.4 & 0.4 & 0.4 & 0.4 & 0.8 \\
\hline $0 \mathrm{E}$ & NS & 3/21/1992 & 92081 & 80 & 0.4 & 0.8 & 0.4 & 0.4 & 0.4 \\
\hline $0 \mathrm{E}$ & NS & 3/22/1992 & 92082 & 81 & 0.4 & 0.4 & 0.4 & 0.4 & 0.4 \\
\hline $1 \mathrm{~F}$ & S & 3/23/1992 & 92083 & 82 & 0.4 & 0.4 & 0.8 & 0.4 & 0.4 \\
\hline $1 \mathrm{E}$ & $S$ & 3/24/1992 & 92084 & 83 & 0.4 & 0.4 & 0.4 & 0.8 & 0.4 \\
\hline $1 \mathrm{E}$ & $S$ & 3/25/1992 & 92085 & 84 & 0.4 & 0.4 & 0.4 & 0.4 & 0.4 \\
\hline $1 \mathrm{E}$ & $S$ & 3/26/1992 & 92086 & 85 & 0.4 & 0.4 & 0.4 & 0.4 & 0.4 \\
\hline $1 \mathrm{E}$ & $S$ & 3/27/1992 & 92087 & 86 & 0.4 & 0.4 & 0.4 & 0.4 & 0.4 \\
\hline $0 \mathrm{~B}$ & $S$ & 3/28/1992 & 92088 & 87 & 0.4 & 0.4 & 0.4 & 0.4 & 0.4 \\
\hline $0 \mathrm{~F}$ & $S$ & 3/29/1992 & 92089 & 88 & 0.4 & 0.4 & 0.4 & 0.4 & 0.4 \\
\hline $1 \mathrm{E}$ & $S$ & 3/30/1992 & 92090 & 89 & 0.4 & 0.4 & 0.4 & 0.8 & 0.4 \\
\hline $1 \mathrm{E}$ & $\mathrm{S}$ & 3/31/1992 & 92091 & 90 & 0.4 & 0.4 & 0.4 & 0.4 & 0.8 \\
\hline $1 \mathrm{E}$ & $S$ & 4/1/1992 & 92092 & 91 & 0.4 & 0.4 & 0.4 & 0.8 & 0.4 \\
\hline $1 \mathrm{E}$ & $\mathrm{S}$ & 4/2/1992 & 92093 & 92 & 0.4 & 0.4 & 0.4 & 0.4 & 0.4 \\
\hline
\end{tabular}




\begin{tabular}{|c|c|c|c|c|c|c|c|c|c|}
\hline $1 \mathrm{~B}$ & $S$ & 4/3/1992 & 92094 & 93 & 0.4 & 0.8 & 0.4 & 0.4 & 0.4 \\
\hline $0 \mathrm{~B}$ & $S$ & $4 / 4 / 1992$ & 92095 & 94 & 0.4 & 0.4 & 0.4 & 0.8 & 0.4 \\
\hline $0 \mathrm{~B}$ & $S$ & 4/5/1992 & 92096 & 95 & 0.8 & 0.4 & 0.4 & 0.4 & 0.4 \\
\hline $1 \mathrm{~B}$ & $S$ & 4/6/1992 & 92097 & 96 & 0.4 & 0.4 & 0.4 & 0.4 & 0.8 \\
\hline $1 \mathrm{~B}$ & $S$ & 4/7/1992 & 92098 & 97 & 0.8 & 0.4 & 0.4 & 0.4 & 0.4 \\
\hline 1 B & $S$ & 4/8/1992 & 92099 & 98 & 0.4 & 0.4 & 0.8 & 0.4 & 0.4 \\
\hline $1 \mathrm{~B}$ & $S$ & 4/9/1992 & 92100 & 99 & 0.4 & 0.4 & 0.4 & 0.4 & 0.4 \\
\hline $1 \mathrm{~B}$ & $S$ & 4/10/1992 & 92101 & 100 & 0.8 & 0.4 & 0.4 & 0.4 & 0.4 \\
\hline 0 B & $S$ & 4/11/1992 & 92102 & 101 & 0.4 & 0.4 & 0.8 & 0.4 & \\
\hline $0 \mathrm{~B}$ & $S$ & $4 / 12 / 1992$ & 92103 & 102 & 0.4 & 0.4 & 0.4 & 0.4 & 0.4 \\
\hline $1 \mathrm{~B}$ & $S$ & 4/13/1992 & 92104 & 103 & 0.4 & 0.8 & 0.4 & 0.4 & 0.4 \\
\hline 1 B & $S$ & 4/14/1992 & 92105 & 104 & 0.4 & 0.4 & 0.4 & 0.4 & 0.4 \\
\hline $1 \mathrm{~B}$ & $S$ & 4/15/1992 & 92106 & 105 & 0.4 & 0.4 & 0.8 & 0.4 & 0.4 \\
\hline $1 \mathrm{~B}$ & $\mathrm{~S}$ & 4/16/1992 & 92107 & 106 & 0.4 & 0.4 & 0.4 & 0.4 & 0.4 \\
\hline $1 \mathrm{~B}$ & NS & 4/17/1992 & 92108 & 107 & 0.4 & 0.4 & 0.4 & 0.4 & 0.4 \\
\hline $\begin{array}{lll}0 & B \\
\end{array}$ & NS & 4/18/1992 & 92109 & 108 & 0.4 & 0.4 & 0.4 & 0.4 & 0.4 \\
\hline $0 \mathrm{~B}$ & NS & 4/19/1992 & 92110 & 109 & 0.4 & 0.4 & 0.4 & 0.8 & 0.4 \\
\hline $1 \mathrm{~B}$ & NS & 4/20/1992 & 92111 & 110 & 0.4 & 0.4 & 0.4 & 0.4 & 0.8 \\
\hline $1 \mathrm{~B}$ & $\mathrm{~S}$ & $4 / 21 / 1992$ & 92112 & 111 & 0.4 & 0.4 & 0.8 & 0.4 & 0.4 \\
\hline $1 \mathrm{~B}$ & $\mathrm{~S}$ & 4/22/1992 & 92113 & 112 & 0.4 & 0.4 & 0.4 & 0.8 & 0.4 \\
\hline $1 \mathrm{~B}$ & $S$ & 4/23/1992 & 92114 & 113 & 0.4 & 0.4 & 0.4 & 0.4 & 0.8 \\
\hline $\begin{array}{ll}1 & B \\
\end{array}$ & $S$ & 4/24/1992 & 92115 & 114 & 0.8 & 0.4 & 0.4 & 0.4 & 0.4 \\
\hline $0 \mathrm{~B}$ & $S$ & 4/25/1992 & 92116 & 115 & 0.4 & 0.8 & 0.4 & 0.4 & 0.4 \\
\hline $0 \mathrm{~B}$ & $S$ & 4/26/1992 & 92117 & 116 & 0.4 & 0.4 & 0.4 & 0.4 & 0.4 \\
\hline $1 \mathrm{~B}$ & $S$ & 4/27/1992 & 92118 & 117 & 0.4 & 0.4 & 0.4 & 0.4 & 0.4 \\
\hline $1 \mathrm{~B}$ & NS & 4/28/1992 & 92119 & 118 & 0.8 & 0.4 & 0.4 & 0.4 & 0.4 \\
\hline $1 \mathrm{~B}$ & $S$ & 4/29/1992 & 92120 & 119 & & 0.8 & 0.4 & 0.4 & 0.4 \\
\hline $1 \mathrm{~B}$ & $S$ & 4/30/1992 & 92121 & 120 & 0.4 & 0.4 & 0.4 & 0.4 & 0.4 \\
\hline $1 \mathrm{~B}$ & $S$ & 5/1/1992 & 92122 & 121 & 0.4 & 0.4 & 0.4 & 0.4 & 0.4 \\
\hline $0 \mathrm{~B}$ & $S$ & 5/2/1992 & 92123 & 122 & 0.8 & 0.4 & 0.4 & 0.4 & 0.4 \\
\hline 0 B & $S$ & $5 / 3 / 1992$ & 92124 & 123 & 0.4 & 0.4 & 0.4 & 0.4 & 0.4 \\
\hline $1 \mathrm{~B}$ & $S$ & $5 / 4 / 1992$ & 92125 & 124 & 0.4 & 0.4 & 0.4 & 0.4 & 0.4 \\
\hline $1 \mathrm{~B}$ & $\mathrm{~S}$ & 5/5/1992 & 92126 & 125 & 0.4 & 0.4 & 0.8 & 0.4 & 0.4 \\
\hline $1 \mathrm{~B}$ & $S$ & 5/6/1992 & 92127 & 126 & 0.4 & 0.4 & 0.4 & 0.4 & 0.8 \\
\hline $1 \mathrm{~B}$ & $S$ & $5 / 7 / 1992$ & 92128 & 127 & 0.4 & 0.8 & 0.4 & 0.4 & 0.4 \\
\hline $1 \mathrm{~B}$ & $\mathrm{~S}$ & 5/8/1992 & 92129 & 128 & 0.8 & 0.4 & 0.4 & 0.4 & 0.4 \\
\hline $0 \mathrm{~B}$ & $S$ & 5/9/1992 & 92130 & 129 & 0.4 & 0.8 & 0.4 & 0.4 & 0.4 \\
\hline 0 B & $S$ & 5/10/1992 & 92131 & 130 & 0.4 & 0.4 & 0.4 & 0.8 & 0.4 \\
\hline $1 \mathrm{~B}$ & $\mathrm{~S}$ & 5/11/1992 & 92132 & 131 & 0.4 & 0.4 & 0.4 & 0.4 & 0.4 \\
\hline $1 \mathrm{~B}$ & $\mathrm{~S}$ & 5/12/1992 & 92133 & 132 & 0.8 & 0.4 & 0.4 & 0.4 & 0.4 \\
\hline $1 \mathrm{~B}$ & $S$ & 5/13/1992 & 92134 & 133 & 0.4 & 0.4 & 0.4 & 0.4 & 0.4 \\
\hline $1 \mathrm{~B}$ & $S$ & $5 / 14 / 1992$ & 92135 & 134 & 0.8 & 0.4 & 0.4 & 0.8 & 0.4 \\
\hline $1 B$ & $S$ & $5 / 15 / 1992$ & 92136 & 135 & 0.8 & 0.4 & 0.8 & 0.4 & 0.8 \\
\hline $0 \mathrm{~B}$ & $S$ & $5 / 16 / 1992$ & 92137 & 136 & 0.8 & 0.8 & 0.4 & 0.4 & 0.4 \\
\hline $0 \mathrm{~B}$ & $\mathrm{~S}$ & 5/17/1992 & 92138 & 137 & 0.4 & 0.4 & 0.4 & 0.4 & 0.4 \\
\hline $1 B$ & $S$ & $5 / 18 / 1992$ & 92139 & 138 & 0.4 & 0.4 & 0.4 & 0.4 & 0.4 \\
\hline $1 \mathrm{~B}$ & $S$ & 5/19/1992 & 92140 & 139 & 0.4 & 0.8 & & 0.8 & 0.4 \\
\hline $1 \mathrm{~B}$ & $S$ & 5/20/1992 & 92141 & 140 & 0.4 & 0.4 & 0.4 & 0.4 & 0.8 \\
\hline $1 \mathrm{~B}$ & $\mathrm{~S}$ & 5/21/1992 & 92142 & 141 & 0.4 & 0.4 & 0.4 & 0.4 & 0.4 \\
\hline $1 \mathrm{~B}$ & $S$ & $5 / 22 / 1992$ & 92143 & 142 & 0.4 & 0.4 & 0.4 & 0.4 & 0.4 \\
\hline $0 \mathrm{~B}$ & $\mathrm{~S}$ & $5 / 23 / 1992$ & 92144 & 143 & 0.4 & 0.4 & 0.4 & 0.4 & 0.4 \\
\hline
\end{tabular}




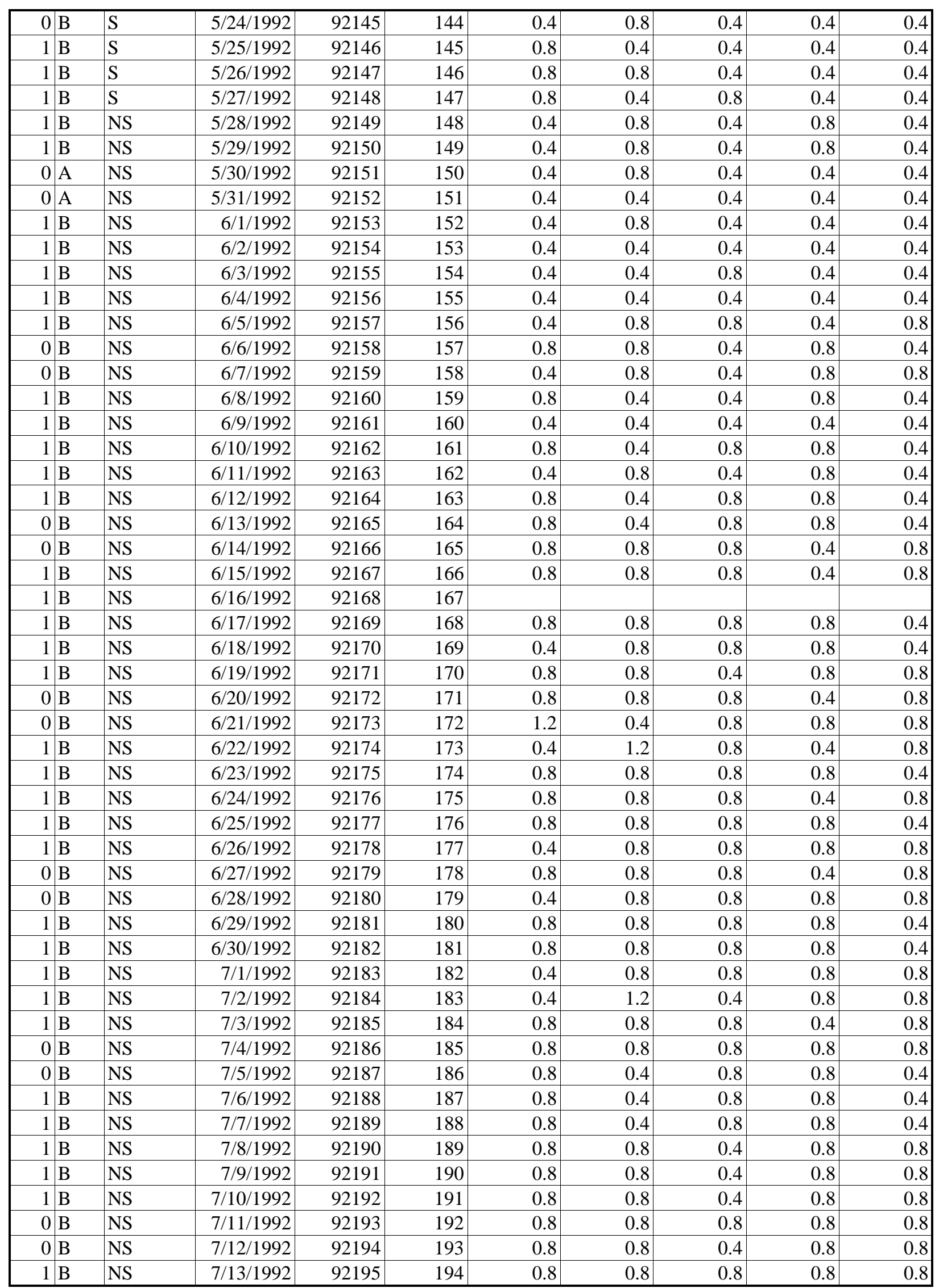




\begin{tabular}{|c|c|c|c|c|c|c|c|c|c|}
\hline $1 \mathrm{~B}$ & NS & 7/14/1992 & 92196 & 195 & 0.8 & 0.8 & 0.8 & 0.4 & 0.8 \\
\hline $1 \mathrm{~B}$ & NS & $7 / 15 / 1992$ & 92197 & 196 & 0.8 & 0.8 & 0.8 & 0.8 & 0.8 \\
\hline $1 \mathrm{~B}$ & NS & 7/16/1992 & 92198 & 197 & 0.4 & 0.8 & 0.8 & 0.8 & 0.8 \\
\hline $1 \mathrm{~B}$ & NS & 7/17/1992 & 92199 & 198 & 0.8 & 0.8 & 0.8 & 0.4 & 0.8 \\
\hline $\begin{array}{ll}0 & \mathrm{~B} \\
\end{array}$ & NS & $7 / 18 / 1992$ & 92200 & 199 & 0.8 & 0.8 & 0.8 & 0.4 & 0.8 \\
\hline $\begin{array}{ll}0 & B \\
\end{array}$ & NS & 7/19/1992 & 92201 & 200 & 0.8 & 0.8 & 0.4 & 0.8 & 0.8 \\
\hline $1 \mathrm{~B}$ & NS & 7/20/1992 & 92202 & 201 & 0.8 & 0.8 & 0.8 & 0.8 & 0.8 \\
\hline $1 \mathrm{~B}$ & NS & $7 / 21 / 1992$ & 92203 & 202 & 0.8 & 0.8 & 0.4 & 0.8 & 0.8 \\
\hline $1 \mathrm{~B}$ & NS & $7 / 22 / 1992$ & 92204 & 203 & 0.4 & 0.8 & 0.4 & 0.8 & 0.4 \\
\hline $1 \mathrm{~B}$ & NS & 7/23/1992 & 92205 & 204 & 0.8 & 0.8 & 0.4 & 0.8 & 0.4 \\
\hline $1 \mathrm{~B}$ & NS & $7 / 24 / 1992$ & 92206 & 205 & 0.4 & 0.8 & 0.8 & 0.4 & 0.8 \\
\hline $0 \mathrm{~B}$ & NS & $7 / 25 / 1992$ & 92207 & 206 & 0.8 & 0.8 & 0.4 & 0.8 & 0.8 \\
\hline $0 \mathrm{~B}$ & NS & 7/26/1992 & 92208 & 207 & 0.8 & 0.8 & 0.8 & 0.8 & 0.8 \\
\hline $1 \mathrm{~B}$ & NS & 7/27/1992 & 92209 & 208 & 0.8 & 0.8 & 0.4 & 0.8 & 0.8 \\
\hline $1 \mathrm{~B}$ & NS & $7 / 28 / 1992$ & 92210 & 209 & 0.8 & 1.2 & 0.4 & 0.8 & 0.8 \\
\hline $1 \mathrm{~B}$ & NS & 7/29/1992 & 92211 & 210 & 1.2 & 0.4 & 1.2 & 0.4 & 0.8 \\
\hline $1 \mathrm{~B}$ & NS & 7/30/1992 & 92212 & 211 & 0.8 & 0.4 & 0.8 & 0.8 & 0.8 \\
\hline $1 \mathrm{~B}$ & NS & $7 / 31 / 1992$ & 92213 & 212 & 0.8 & 0.8 & 0.8 & 0.8 & 0.4 \\
\hline $0 \mathrm{~B}$ & NS & 8/1/1992 & 92214 & 213 & 0.8 & 0.8 & 0.8 & 0.8 & 0.8 \\
\hline $0 \mathrm{~B}$ & NS & 8/2/1992 & 92215 & 214 & 0.8 & 0.8 & 0.4 & 0.8 & 0.8 \\
\hline $1 \mathrm{~B}$ & NS & $8 / 3 / 1992$ & 92216 & 215 & 0.8 & 0.8 & 0.4 & 0.8 & 0.8 \\
\hline $1 \mathrm{~B}$ & NS & 8/4/1992 & 92217 & 216 & 0.8 & 0.4 & 0.8 & 0.8 & 0.8 \\
\hline $1 \mathrm{~B}$ & NS & 8/5/1992 & 92218 & 217 & 0.8 & 0.4 & 0.8 & 0.8 & 0.4 \\
\hline $1 \mathrm{~B}$ & NS & $8 / 6 / 1992$ & 92219 & 218 & 0.8 & 0.8 & 0.8 & 0.8 & 0.4 \\
\hline $1 \mathrm{~B}$ & NS & 8/7/1992 & 92220 & 219 & 0.8 & 0.4 & 0.8 & 0.8 & 0.8 \\
\hline $0 \mathrm{~B}$ & NS & 8/8/1992 & 92221 & 220 & 0.8 & 0.8 & 0.8 & 0.8 & 0.8 \\
\hline $0 \mathrm{~B}$ & NS & 8/9/1992 & 92222 & 221 & 0.8 & 0.8 & 0.8 & 0.8 & 0.8 \\
\hline $1 \mathrm{~B}$ & NS & 8/10/1992 & 92223 & 222 & 0.8 & 0.8 & 0.4 & 0.8 & 0.8 \\
\hline $1 \mathrm{~B}$ & NS & $8 / 11 / 1992$ & 92224 & 223 & 0.8 & 0.8 & 0.8 & 0.8 & 0.8 \\
\hline $1 \mathrm{~B}$ & NS & $8 / 12 / 1992$ & 92225 & 224 & 0.8 & 0.8 & 0.8 & 0.8 & 0.8 \\
\hline $1 \mathrm{~B}$ & NS & 8/13/1992 & 92226 & 225 & 0.8 & 0.8 & 0.8 & 0.4 & 0.8 \\
\hline $1 \mathrm{~B}$ & NS & 8/14/1992 & 92227 & 226 & 0.8 & 0.8 & 0.4 & 0.8 & 0.8 \\
\hline $0 \mathrm{~B}$ & NS & $8 / 15 / 1992$ & 92228 & 227 & 0.8 & 0.4 & 0.8 & 0.8 & 0.4 \\
\hline $0 \mathrm{~B}$ & NS & 8/16/1992 & 92229 & 228 & 0.8 & 0.8 & 0.8 & 0.4 & 0.8 \\
\hline $1 \mathrm{~B}$ & NS & 8/17/1992 & 92230 & 229 & 0.4 & 0.8 & 0.8 & 0.4 & 0.8 \\
\hline $1 \mathrm{~B}$ & NS & 8/18/1992 & 92231 & 230 & 0.8 & 0.8 & 0.4 & 0.8 & 0.4 \\
\hline $1 \mathrm{~B}$ & NS & $8 / 19 / 1992$ & 92232 & 231 & 0.8 & 0.4 & 0.8 & 0.4 & 0.8 \\
\hline $1 \mathrm{~B}$ & NS & 8/20/1992 & 92233 & 232 & 0.4 & 0.8 & 0.8 & 0.8 & 0.4 \\
\hline $1 \mathrm{~B}$ & NS & 8/21/1992 & 92234 & 233 & 0.4 & 0.8 & 0.8 & 0.8 & 0.8 \\
\hline $\begin{array}{ll}0 & \mathrm{~B} \\
\end{array}$ & NS & $8 / 22 / 1992$ & 92235 & 234 & 0.8 & 0.4 & 0.8 & 0.4 & 0.8 \\
\hline $0 \mathrm{~B}$ & NS & 8/23/1992 & 92236 & 235 & 0.4 & 0.8 & 0.8 & 0.4 & 0.8 \\
\hline $1 \mathrm{~B}$ & NS & 8/24/1992 & 92237 & 236 & 0.8 & 0.8 & 0.4 & 0.8 & 0.8 \\
\hline $1 \mathrm{~B}$ & NS & $8 / 25 / 1992$ & 92238 & 237 & 0.8 & 0.8 & 0.4 & 0.8 & 0.4 \\
\hline $1 \mathrm{~B}$ & NS & 8/26/1992 & 92239 & 238 & 0.8 & 0.4 & 0.8 & 0.8 & 0.4 \\
\hline $1 \mathrm{~B}$ & NS & 8/27/1992 & 92240 & 239 & 0.8 & 0.8 & 0.8 & 0.8 & 0.4 \\
\hline $1 \mathrm{~B}$ & NS & $8 / 28 / 1992$ & 92241 & 240 & 0.8 & 0.8 & 0.8 & 0.4 & 0.8 \\
\hline $0 \mathrm{~B}$ & NS & 8/29/1992 & 92242 & 241 & 0.4 & 0.8 & 0.8 & 0.8 & 0.8 \\
\hline $0 \mathrm{~B}$ & NS & 8/30/1992 & 92243 & 242 & 0.8 & 0.8 & 0.8 & 0.4 & 0.8 \\
\hline $1 \mathrm{~B}$ & NS & $8 / 31 / 1992$ & 92244 & 243 & 0.8 & 0.8 & 0.4 & 0.8 & 0.8 \\
\hline $1 \mathrm{~B}$ & NS & 9/1/1992 & 92245 & 244 & 0.8 & 0.4 & 0.8 & 0.8 & 0.4 \\
\hline $1 \mathrm{~B}$ & NS & 9/2/1992 & 92246 & 245 & 0.8 & 0.8 & 0.4 & 0.8 & 0.8 \\
\hline
\end{tabular}




\begin{tabular}{|c|c|c|c|c|c|c|c|c|c|}
\hline $1 \mathrm{~B}$ & NS & 9/3/1992 & 92247 & 246 & 0.8 & 0.8 & 0.4 & 0.8 & 0.8 \\
\hline $1 \mathrm{~B}$ & NS & 9/4/1992 & 92248 & 247 & 0.4 & 0.8 & 0.8 & 0.8 & 0.8 \\
\hline \begin{tabular}{l|l}
0 & $B$ \\
\end{tabular} & NS & 9/5/1992 & 92249 & 248 & 0.8 & 0.8 & 0.8 & 0.8 & 0.4 \\
\hline $0 \mathrm{E}$ & NS & 9/6/1992 & 92250 & 249 & 0.8 & 0.8 & 0.8 & 0.8 & 0.4 \\
\hline $1 \mathrm{~B}$ & NS & 9/7/1992 & 92251 & 250 & 0.8 & 0.8 & 0.8 & 0.8 & 0.4 \\
\hline $1 \mathrm{~B}$ & $\mathrm{~S}$ & 9/8/1992 & 92252 & 251 & 0.8 & 0.8 & 0.4 & 0.8 & 0.8 \\
\hline $1 \mathrm{~B}$ & $S$ & 9/9/1992 & 92253 & 252 & 0.8 & 0.8 & 0.8 & 0.8 & 0.4 \\
\hline $1 \mathrm{~B}$ & $\mathrm{~S}$ & 9/10/1992 & 92254 & 253 & 0.8 & 0.4 & 0.8 & 0.8 & 0.4 \\
\hline $1 \mathrm{E}$ & $S$ & 9/11/1992 & 92255 & 254 & 0.8 & 0.8 & 0.8 & 0.4 & 0.8 \\
\hline $0 \mathrm{E}$ & $\mathrm{S}$ & 9/12/1992 & 92256 & 255 & 0.8 & 0.4 & 0.8 & 0.8 & 0.4 \\
\hline $0 \mathrm{E}$ & $\mathrm{S}$ & 9/13/1992 & 92257 & 256 & 0.8 & 0.8 & 0.4 & 0.8 & 0.8 \\
\hline $1 \mathrm{E}$ & $S$ & 9/14/1992 & 92258 & 257 & 0.4 & 0.8 & 0.8 & 0.4 & 0.8 \\
\hline $1 \mathrm{E}$ & NS & 9/15/1992 & 92259 & 258 & 0.8 & 0.4 & 0.8 & 0.4 & 0.8 \\
\hline $1 \mathrm{E}$ & S & 9/16/1992 & 92260 & 259 & 0.8 & 0.8 & 0.8 & 0.4 & 0.8 \\
\hline $1 \mathrm{E}$ & $S$ & 9/17/1992 & 92261 & 260 & 0.4 & 0.8 & 0.8 & 0.4 & 0.8 \\
\hline $1 \mathrm{E}$ & $S$ & 9/18/1992 & 92262 & 261 & 0.8 & 0.4 & 0.8 & 0.8 & 0.4 \\
\hline $0 \mathrm{E}$ & S & 9/19/1992 & 92263 & 262 & 0.8 & 0.4 & 0.8 & 0.8 & 0.4 \\
\hline $0 \mathrm{E}$ & $S$ & 9/20/1992 & 92264 & 263 & 0.8 & 0.8 & 0.8 & 0.8 & 0.4 \\
\hline $1 \mathrm{E}$ & S & 9/21/1992 & 92265 & 264 & 0.8 & 0.8 & 0.4 & 0.8 & 0.8 \\
\hline $1 \mathrm{E}$ & $S$ & 9/22/1992 & 92266 & 265 & 0.8 & 0.8 & 0.4 & 0.8 & 0.8 \\
\hline $1 \mathrm{E}$ & $S$ & 9/23/1992 & 92267 & 266 & 0.8 & 0.8 & 0.4 & 0.8 & 0.8 \\
\hline $1 \mathrm{E}$ & $S$ & 9/24/1992 & 92268 & 267 & 0.8 & 0.4 & 0.8 & 0.8 & 0.4 \\
\hline $1 \mathrm{E}$ & $S$ & 9/25/1992 & 92269 & 268 & 0.8 & 0.4 & 0.8 & 0.8 & 0.4 \\
\hline $0 \mathrm{E}$ & $S$ & 9/26/1992 & 92270 & 269 & 0.8 & 0.4 & 0.8 & 0.4 & 0.8 \\
\hline $0 \mathrm{E}$ & S & 9/27/1992 & 92271 & 270 & 0.4 & 0.8 & 0.4 & 0.8 & 0.8 \\
\hline $1 \mathrm{E}$ & $S$ & 9/28/1992 & 92272 & 271 & 0.8 & 0.8 & 0.8 & 0.4 & 0.8 \\
\hline $1 \mathrm{E}$ & $S$ & 9/29/1992 & 92273 & 272 & 0.8 & 0.8 & 0.8 & 0.8 & 0.4 \\
\hline $1 \mathrm{E}$ & $S$ & 9/30/1992 & 92274 & 273 & 0.8 & 0.4 & 0.8 & 0.4 & 0.8 \\
\hline $1 \mathrm{E}$ & $S$ & 10/1/1992 & 92275 & 274 & 0.8 & 0.4 & 0.8 & 0.4 & 0.8 \\
\hline $1 \mathrm{E}$ & $S$ & $10 / 2 / 1992$ & 92276 & 275 & 0.8 & 0.4 & 0.4 & 0.4 & 0.8 \\
\hline $0 \mathrm{E}$ & $S$ & 10/3/1992 & 92277 & 276 & 0.8 & 0.4 & 0.4 & 0.4 & 0.4 \\
\hline $0 \mathrm{E}$ & $S$ & 10/4/1992 & 92278 & 277 & 0.8 & 0.4 & 0.4 & 0.8 & 0.4 \\
\hline $1 \mathrm{E}$ & $S$ & $10 / 5 / 1992$ & 92279 & 278 & 0.4 & 0.8 & 0.4 & 0.4 & 0.4 \\
\hline $1 \mathrm{E}$ & $S$ & 10/6/1992 & 92280 & 279 & 0.4 & 0.8 & 0.8 & 0.4 & 0.4 \\
\hline $1 \mathrm{~F}$ & $S$ & 10/7/1992 & 92281 & 280 & 0.8 & 0.4 & 0.4 & 0.4 & 0.4 \\
\hline $1 \mathrm{~F}$ & $S$ & 10/8/1992 & 92282 & 281 & 0.4 & 0.4 & 0.4 & 0.8 & 0.4 \\
\hline $1 \mathrm{E}$ & $S$ & 10/9/1992 & 92283 & 282 & 0.8 & 0.8 & 0.4 & 0.4 & 0.4 \\
\hline $0 \mathrm{E}$ & S & 10/10/1992 & 92284 & 283 & 0.4 & 0.4 & 0.4 & 0.8 & 0.4 \\
\hline $0 \mathrm{E}$ & $S$ & 10/11/1992 & 92285 & 284 & 0.4 & 0.4 & 0.4 & 0.4 & 0.4 \\
\hline $1 \mathrm{E}$ & NS & 10/12/1992 & 92286 & 285 & 0.4 & 0.8 & 0.4 & 0.4 & 0.8 \\
\hline $1 \mathrm{E}$ & $\mathrm{S}$ & 10/13/1992 & 92287 & 286 & 0.8 & 0.4 & 0.8 & 0.4 & 0.4 \\
\hline $1 \mathrm{E}$ & $S$ & 10/14/1992 & 92288 & 287 & 0.4 & 0.8 & 0.4 & 0.4 & 0.4 \\
\hline $1 \mathrm{E}$ & $S$ & 10/15/1992 & 92289 & 288 & 0.8 & 0.4 & 0.8 & 0.4 & 0.8 \\
\hline $1 \mathrm{E}$ & S & 10/16/1992 & 92290 & 289 & 0.4 & 0.8 & 0.8 & 0.4 & 0.4 \\
\hline $0 \mathrm{E}$ & $S$ & 10/17/1992 & 92291 & 290 & 0.8 & 0.8 & 0.4 & 0.8 & 0.4 \\
\hline $0 \mathrm{E}$ & $S$ & 10/18/1992 & 92292 & 291 & 0.4 & 0.4 & 0.4 & 0.4 & 0.4 \\
\hline $1 \mathrm{E}$ & $S$ & 10/19/1992 & 92293 & 292 & 0.4 & 0.4 & 0.4 & 0.8 & 0.4 \\
\hline $1 \mathrm{E}$ & $S$ & 10/20/1992 & 92294 & 293 & 0.4 & 0.4 & 0.4 & 0.8 & 0.4 \\
\hline $1 \mathrm{E}$ & $S$ & 10/21/1992 & 92295 & 294 & & 0.4 & 0.4 & 0.4 & 0.4 \\
\hline $1 \mathrm{E}$ & $S$ & 10/22/1992 & 92296 & 295 & 0.4 & 0.4 & 0.4 & 0.4 & 0.4 \\
\hline $1 \mathrm{~B}$ & S & 10/23/1992 & 92297 & 296 & 0.8 & 0.4 & 0.4 & 0.4 & 0.4 \\
\hline
\end{tabular}




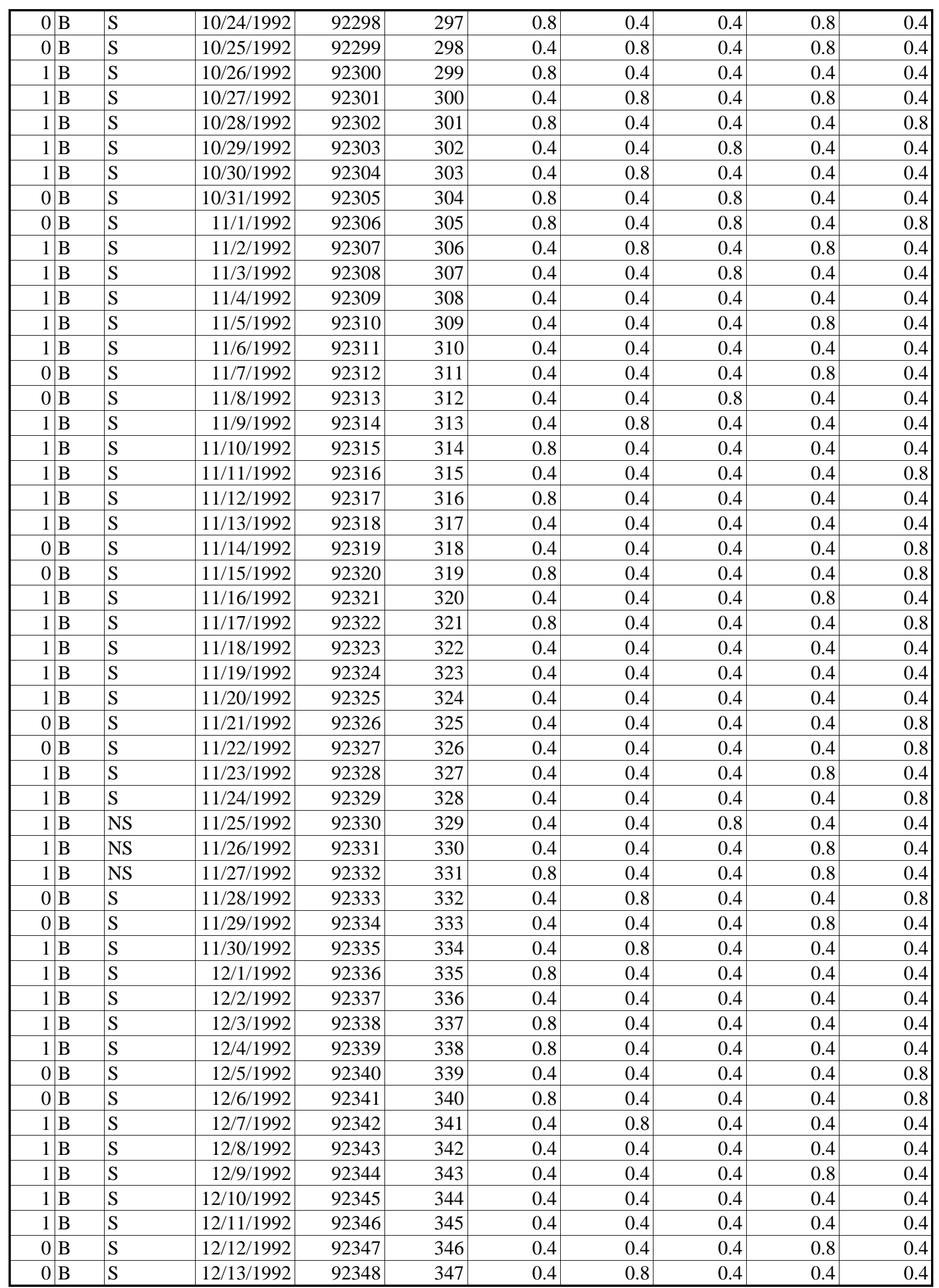




\begin{tabular}{|c|c|c|c|c|c|c|c|c|c|}
\hline $1 \mathrm{E}$ & $S$ & $12 / 14 / 1992$ & 92349 & 348 & 0.4 & 0.4 & 0.8 & 0.4 & 0.4 \\
\hline $1 \mathrm{E}$ & $S$ & 12/15/1992 & 92350 & 349 & 0.4 & 0.4 & 0.4 & 0.4 & 0.8 \\
\hline $1 \mathrm{E}$ & $S$ & 12/16/1992 & 92351 & 350 & 0.4 & 0.4 & 0.8 & 0.4 & 0.4 \\
\hline $1 \mathrm{E}$ & $S$ & 12/17/1992 & 92352 & 351 & 0.4 & 0.4 & 0.4 & 0.8 & 0.4 \\
\hline $1 \mathrm{E}$ & NS & 12/18/1992 & 92353 & 352 & 0.8 & 0.4 & 0.4 & 0.4 & 0.4 \\
\hline $0 \mathrm{E}$ & NS & 12/19/1992 & 92354 & 353 & 0.4 & 0.8 & 0.4 & 0.4 & 0.4 \\
\hline $0 \mathrm{E}$ & NS & 12/20/1992 & 92355 & 354 & 0.4 & 0.4 & 0.4 & 0.4 & 0.8 \\
\hline $1 \mathrm{E}$ & NS & 12/21/1992 & 92356 & 355 & 0.4 & 0.4 & 0.4 & 0.8 & 0.4 \\
\hline $1 \mathrm{~F}$ & NS & 12/22/1992 & 92357 & 356 & 0.4 & 0.4 & 0.8 & 0.4 & 0.4 \\
\hline $1 \mathrm{E}$ & NS & 12/23/1992 & 92358 & 357 & 0.4 & 0.4 & 0.4 & 0.8 & 0.4 \\
\hline $1 \mathrm{E}$ & NS & 12/24/1992 & 92359 & 358 & 0.4 & 0.8 & 0.4 & 0.4 & 0.4 \\
\hline $1 \mathrm{E}$ & NS & 12/25/1992 & 92360 & 359 & 0.4 & 0.4 & 0.4 & 0.8 & 0.4 \\
\hline $0 \mathrm{E}$ & NS & 12/26/1992 & 92361 & 360 & 0.4 & 0.4 & 0.8 & 0.4 & 0.4 \\
\hline $0 \mathrm{E}$ & NS & 12/27/1992 & 92362 & 361 & 0.8 & 0.4 & 0.4 & 0.8 & 0.4 \\
\hline $1 \mathrm{E}$ & NS & 12/28/1992 & 92363 & 362 & 0.4 & 0.4 & 0.8 & 0.4 & 0.4 \\
\hline $1 \mathrm{E}$ & NS & 12/29/1992 & 92364 & 363 & 0.8 & 0.4 & 0.4 & 0.4 & 0.4 \\
\hline $1 \mathrm{E}$ & NS & 12/30/1992 & 92365 & 364 & 0.8 & 0.4 & 0.4 & 0.4 & 0.4 \\
\hline $1 \mathrm{E}$ & NS & 12/31/1992 & 92366 & 365 & 0.8 & 0.4 & 0.4 & 0.4 & 0.4 \\
\hline $1 \mathrm{E}$ & NS & 1/1/1993 & 93001 & 1 & 0.4 & 0.8 & 0.4 & 0.4 & 0.8 \\
\hline $0 \mathrm{E}$ & NS & $1 / 2 / 1993$ & 93002 & 2 & 0.4 & 0.4 & 0.4 & 0.8 & 0.4 \\
\hline $0 \mathrm{E}$ & NS & $1 / 3 / 1993$ & 93003 & 3 & 0.4 & 0.8 & 0.4 & 0.4 & 0.4 \\
\hline $1 \mathrm{E}$ & NS & 1/4/1993 & 93004 & 4 & 0.4 & 0.4 & 0.4 & 0.4 & 0.4 \\
\hline $1 \mathrm{E}$ & S & $1 / 5 / 1993$ & 93005 & 5 & 0.8 & 0.4 & 0.4 & 0.4 & 0.4 \\
\hline $1 \mathrm{E}$ & $S$ & $1 / 6 / 1993$ & 93006 & 6 & 0.8 & 0.4 & 0.4 & 0.4 & 0.8 \\
\hline $1 \mathrm{H}$ & $S$ & 1/7/1993 & 93007 & 7 & 0.4 & 0.8 & 0.4 & 0.4 & 0.4 \\
\hline $1 \mathrm{E}$ & $S$ & $1 / 8 / 1993$ & 93008 & 8 & 0.4 & 0.4 & 0.8 & 0.4 & 0.4 \\
\hline $0 \mathrm{E}$ & $S$ & 1/9/1993 & 93009 & 9 & 0.8 & & 0.8 & 0.4 & 0.4 \\
\hline $0 \mathrm{~F}$ & $S$ & 1/10/1993 & 93010 & 10 & 0.4 & 0.8 & 0.4 & 0.4 & 0.8 \\
\hline $1 \mathrm{E}$ & $S$ & 1/11/1993 & 93011 & 11 & 0.8 & 0.4 & 0.4 & 0.4 & 0.8 \\
\hline $1 \mathrm{E}$ & $S$ & $1 / 12 / 1993$ & 93012 & 12 & 0.4 & 0.4 & 0.8 & 0.4 & 0.4 \\
\hline $1 \mathrm{~F}$ & $S$ & 1/13/1993 & 93013 & 13 & 0.4 & 0.4 & 0.4 & 0.4 & 0.4 \\
\hline $1 \mathrm{E}$ & $S$ & $1 / 14 / 1993$ & 93014 & 14 & 0.4 & 0.4 & 0.4 & 0.4 & 0.8 \\
\hline $1 \mathrm{E}$ & $\mathrm{S}$ & 1/15/1993 & 93015 & 15 & 0.4 & 0.4 & 0.4 & 0.4 & 0.8 \\
\hline $0 \mathrm{~F}$ & $S$ & 1/16/1993 & 93016 & 16 & 0.4 & 0.4 & 0.4 & 0.4 & 0.4 \\
\hline $0 \mathrm{E}$ & $S$ & $1 / 17 / 1993$ & 93017 & 17 & 0.8 & 0.4 & 0.4 & 0.4 & 0.4 \\
\hline $1 \mathrm{H}$ & $S$ & 1/18/1993 & 93018 & 18 & 0.4 & 0.4 & 0.4 & 0.4 & 0.4 \\
\hline $1 \mathrm{E}$ & $S$ & 1/19/1993 & 93019 & 19 & 0.4 & 0.4 & 0.4 & 0.4 & 0.8 \\
\hline $1 \mathrm{E}$ & $S$ & $1 / 20 / 1993$ & 93020 & 20 & 0.4 & 0.4 & 0.4 & 0.4 & 0.8 \\
\hline $1 \mathrm{E}$ & $\mathrm{S}$ & $1 / 21 / 1993$ & 93021 & 21 & 0.8 & 0.4 & 0.4 & 0.4 & 0.4 \\
\hline $1 \mathrm{E}$ & $S$ & 1/22/1993 & 93022 & 22 & 0.4 & 0.4 & 0.4 & 0.8 & 0.4 \\
\hline $0 \mathrm{E}$ & $S$ & 1/23/1993 & 93023 & 23 & 0.4 & 0.4 & 0.4 & 0.4 & 0.8 \\
\hline $0 \mathrm{E}$ & $S$ & 1/24/1993 & 93024 & 24 & 0.4 & 0.4 & 0.4 & 0.8 & 0.4 \\
\hline $1 \mathrm{E}$ & $S$ & $1 / 25 / 1993$ & 93025 & 25 & 0.4 & 0.4 & 0.8 & 0.4 & 0.8 \\
\hline $1 \mathrm{E}$ & $S$ & 1/26/1993 & 93026 & 26 & 0.4 & 0.8 & 0.4 & 0.4 & 0.4 \\
\hline $1 \mathrm{E}$ & $\mathrm{S}$ & $1 / 27 / 1993$ & 93027 & 27 & 0.4 & 0.8 & 0.4 & 0.4 & 0.4 \\
\hline $1 \mathrm{E}$ & $S$ & $1 / 28 / 1993$ & 93028 & 28 & 0.4 & 0.8 & 0.4 & 0.4 & 0.4 \\
\hline $1 \mathrm{E}$ & $S$ & 1/29/1993 & 93029 & 29 & 0.4 & 0.4 & 0.8 & 0.4 & 0.4 \\
\hline $0 \mathrm{E}$ & $S$ & 1/30/1993 & 93030 & 30 & 0.8 & 0.4 & 0.4 & 0.8 & 0.4 \\
\hline $0 \mathrm{E}$ & $S$ & $1 / 31 / 1993$ & 93031 & 31 & 0.4 & 0.8 & 0.4 & 0.4 & 0.8 \\
\hline $1 \mathrm{E}$ & $S$ & 2/1/1993 & 93032 & 32 & 0.4 & 0.4 & 0.8 & 0.4 & 0.4 \\
\hline $1 \mathrm{E}$ & $\mathrm{S}$ & 2/2/1993 & 93033 & 33 & 0.4 & 0.4 & 0.4 & 0.4 & 0.4 \\
\hline
\end{tabular}




\begin{tabular}{|c|c|c|c|c|c|c|c|c|c|}
\hline $1 \mathrm{~B}$ & $S$ & 2/3/1993 & 93034 & 34 & 0.4 & 0.4 & 0.4 & 0.4 & 0.8 \\
\hline $1 \mathrm{~B}$ & $S$ & 2/4/1993 & 93035 & 35 & 0.4 & 0.4 & 0.8 & 0.4 & 0.4 \\
\hline $1 \mathrm{~B}$ & S & 2/5/1993 & 93036 & 36 & 0.4 & 0.4 & 0.4 & 0.8 & 0.4 \\
\hline $0 \mathrm{E}$ & $S$ & 2/6/1993 & 93037 & 37 & 0.4 & 0.4 & 0.4 & 0.8 & 0.4 \\
\hline \begin{tabular}{l|l}
0 & $B$ \\
\end{tabular} & $S$ & 2/7/1993 & 93038 & 38 & 0.4 & 0.8 & 0.4 & 0.4 & 0.8 \\
\hline $1 \mathrm{~B}$ & $S$ & 2/8/1993 & 93039 & 39 & 0.4 & 0.8 & 0.4 & 0.4 & 0.4 \\
\hline $1 \mathrm{~B}$ & $S$ & 2/9/1993 & 93040 & 40 & 0.4 & 0.4 & 0.4 & 0.4 & 0.8 \\
\hline $1 \mathrm{~B}$ & $S$ & $2 / 10 / 1993$ & 93041 & 41 & 0.4 & 0.4 & 0.4 & 0.4 & 0.4 \\
\hline $1 \mathrm{E}$ & $\mathrm{S}$ & 2/11/1993 & 93042 & 42 & 0.4 & 0.4 & 0.4 & 0.4 & 0.8 \\
\hline $1 \mathrm{E}$ & $S$ & 2/12/1993 & 93043 & 43 & 0.8 & 0.4 & 0.4 & 0.4 & 0.4 \\
\hline $0 \mathrm{E}$ & $S$ & 2/13/1993 & 93044 & 44 & 0.8 & 0.4 & 0.4 & 0.4 & 0.4 \\
\hline \begin{tabular}{l|l}
0 & $B$ \\
\end{tabular} & $S$ & $2 / 14 / 1993$ & 93045 & 45 & 0.4 & 0.4 & 0.4 & 0.4 & 0.8 \\
\hline \begin{tabular}{l|l}
1 & $\mathrm{~B}$ \\
\end{tabular} & NS & 2/15/1993 & 93046 & 46 & 0.8 & 0.4 & 0.4 & 0.4 & 0.4 \\
\hline $1 \mathrm{E}$ & S & 2/16/1993 & 93047 & 47 & 0.8 & 0.4 & 0.4 & 0.4 & 0.4 \\
\hline $1 \mathrm{E}$ & $S$ & 2/17/1993 & 93048 & 48 & 0.4 & 0.4 & 0.4 & 0.8 & 0.4 \\
\hline $1 \mathrm{E}$ & $S$ & 2/18/1993 & 93049 & 49 & 0.4 & 0.4 & 0.8 & 0.4 & 0.4 \\
\hline $1 \mathrm{E}$ & S & 2/19/1993 & 93050 & 50 & 0.4 & 0.4 & 0.4 & 0.4 & 0.8 \\
\hline $0 \mathrm{E}$ & $S$ & $2 / 20 / 1993$ & 93051 & 51 & 0.4 & 0.4 & 0.4 & 0.4 & 0.4 \\
\hline $0 \mathrm{E}$ & S & 2/21/1993 & 93052 & 52 & 0.4 & 0.8 & 0.4 & 0.4 & 0.4 \\
\hline $1 \mathrm{E}$ & $S$ & 2/22/1993 & 93053 & 53 & 0.4 & 0.4 & 0.4 & 0.8 & 0.4 \\
\hline $1 \mathrm{E}$ & $S$ & $2 / 23 / 1993$ & 93054 & 54 & 0.4 & 0.4 & 0.8 & 0.4 & 0.4 \\
\hline $1 \mathrm{E}$ & $S$ & 2/24/1993 & 93055 & 55 & 0.8 & 0.4 & 0.4 & 0.4 & 0.4 \\
\hline $1 \mathrm{E}$ & $S$ & 2/25/1993 & 93056 & 56 & 0.4 & 0.4 & 0.4 & 0.4 & 0.4 \\
\hline $1 \mathrm{E}$ & $S$ & $2 / 26 / 1993$ & 93057 & 57 & 0.4 & 0.4 & 0.8 & 0.4 & 0.4 \\
\hline $0 \mathrm{E}$ & S & 2/27/1993 & 93058 & 58 & 0.4 & 0.8 & 0.4 & 0.4 & 0.4 \\
\hline $0 \mathrm{E}$ & $S$ & 2/28/1993 & 93059 & 59 & 0.4 & 0.4 & 0.4 & 0.4 & 0.8 \\
\hline $1 \mathrm{E}$ & $S$ & $3 / 1 / 1993$ & 93060 & 60 & 0.4 & 0.4 & 0.4 & 0.8 & 0.4 \\
\hline $1 \mathrm{E}$ & $S$ & $3 / 2 / 1993$ & 93061 & 61 & 0.4 & 0.4 & 0.4 & 0.4 & 0.4 \\
\hline $1 \mathrm{E}$ & $S$ & 3/3/1993 & 93062 & 62 & 0.4 & 0.4 & 0.4 & 0.4 & 0.4 \\
\hline $1 \mathrm{E}$ & $S$ & 3/4/1993 & 93063 & 63 & 0.4 & 0.4 & 0.8 & 0.4 & 0.4 \\
\hline $1 \mathrm{E}$ & $S$ & 3/5/1993 & 93064 & 64 & 0.8 & 0.4 & 0.4 & 0.4 & 0.8 \\
\hline $0 \mathrm{E}$ & $S$ & 3/6/1993 & 93065 & 65 & 0.8 & 0.4 & 0.4 & 0.4 & 0.4 \\
\hline $0 \mathrm{E}$ & $S$ & 3/7/1993 & 93066 & 66 & 0.4 & 0.4 & 0.8 & 0.4 & 0.4 \\
\hline $1 \mathrm{E}$ & $\mathrm{S}$ & 3/8/1993 & 93067 & 67 & 0.8 & 0.4 & 0.4 & 0.4 & 0.4 \\
\hline $1 \mathrm{~F}$ & $S$ & 3/9/1993 & 93068 & 68 & 0.4 & 0.8 & & 0.8 & 0.4 \\
\hline $1 \mathrm{E}$ & $S$ & 3/10/1993 & 93069 & 69 & 0.4 & 0.8 & 0.4 & 0.4 & 0.4 \\
\hline $1 \mathrm{E}$ & $S$ & $3 / 11 / 1993$ & 93070 & 70 & 0.8 & 0.4 & 0.4 & 0.4 & 0.4 \\
\hline $1 \mathrm{E}$ & S & 3/12/1993 & 93071 & 71 & 0.4 & 0.8 & 0.4 & 0.4 & 0.4 \\
\hline $0 \mathrm{E}$ & NS & 3/13/1993 & 93072 & 72 & 0.4 & 0.8 & 0.4 & 0.4 & 0.4 \\
\hline $0 \mathrm{E}$ & NS & $3 / 14 / 1993$ & 93073 & 73 & 0.8 & 0.4 & 0.4 & 0.4 & 0.8 \\
\hline $1 \mathrm{E}$ & NS & 3/15/1993 & 93074 & 74 & 0.4 & 0.4 & 0.4 & 0.4 & 0.8 \\
\hline $1 \mathrm{E}$ & NS & 3/16/1993 & 93075 & 75 & 0.4 & 0.8 & 0.4 & 0.4 & 0.4 \\
\hline $1 \mathrm{E}$ & NS & $3 / 17 / 1993$ & 93076 & 76 & 0.4 & 0.4 & 0.8 & 0.4 & 0.4 \\
\hline $1 \mathrm{E}$ & NS & 3/18/1993 & 93077 & 77 & 0.8 & 0.4 & 0.4 & 0.4 & 0.8 \\
\hline $1 \mathrm{E}$ & NS & 3/19/1993 & 93078 & 78 & 0.4 & 0.4 & 0.4 & 0.8 & 0.4 \\
\hline $0 \mathrm{E}$ & NS & $3 / 20 / 1993$ & 93079 & 79 & 0.8 & 0.4 & 0.4 & 0.4 & 0.4 \\
\hline $0 \mathrm{E}$ & NS & 3/21/1993 & 93080 & 80 & 0.4 & 0.4 & 0.4 & 0.4 & 0.8 \\
\hline $1 \mathrm{H}$ & $S$ & 3/22/1993 & 93081 & 81 & 0.4 & 0.4 & 0.4 & 0.4 & 0.8 \\
\hline $1 \mathrm{E}$ & $S$ & $3 / 23 / 1993$ & 93082 & 82 & 0.4 & 0.4 & 0.4 & 0.4 & 0.8 \\
\hline $1 \mathrm{E}$ & $S$ & 3/24/1993 & 93083 & 83 & 0.4 & 0.4 & 0.4 & 0.4 & 0.4 \\
\hline $1 \mathrm{~B}$ & S & 3/25/1993 & 93084 & 84 & 0.4 & 0.4 & 0.4 & 0.8 & 0.4 \\
\hline
\end{tabular}




\begin{tabular}{|c|c|c|c|c|c|c|c|c|c|}
\hline $1 \mathrm{~B}$ & $S$ & 3/26/1993 & 93085 & 85 & 0.4 & 0.8 & 0.4 & 0.4 & 0.4 \\
\hline \begin{tabular}{l|l}
$0 \mathrm{~B}$ \\
\end{tabular} & $S$ & $3 / 27 / 1993$ & 93086 & 86 & 0.4 & 0.4 & 0.8 & 0.4 & 0.4 \\
\hline $0 \mathrm{~B}$ & $S$ & 3/28/1993 & 93087 & 87 & 0.4 & 0.4 & 0.4 & 0.4 & 0.8 \\
\hline $1 \mathrm{E}$ & $S$ & 3/29/1993 & 93088 & 88 & 0.4 & 0.4 & 0.4 & 0.4 & 0.4 \\
\hline $1 \mathrm{~B}$ & $S$ & $3 / 30 / 1993$ & 93089 & 89 & 0.4 & 0.4 & 0.4 & 0.4 & 0.4 \\
\hline $1 \mathrm{~B}$ & $S$ & $3 / 31 / 1993$ & 93090 & 90 & 0.4 & 0.4 & 0.4 & 0.4 & 0.4 \\
\hline $1 \mathrm{~B}$ & $S$ & 4/1/1993 & 93091 & 91 & 0.8 & 0.4 & 0.4 & 0.4 & 0.4 \\
\hline $1 \mathrm{~B}$ & $S$ & $4 / 2 / 1993$ & 93092 & 92 & 0.4 & 0.8 & 0.4 & 0.4 & 0.4 \\
\hline $0 \mathrm{E}$ & $\mathrm{S}$ & 4/3/1993 & 93093 & 93 & 0.4 & 0.4 & 0.4 & 0.4 & 0.4 \\
\hline $0 \mathrm{E}$ & $S$ & 4/4/1993 & 93094 & 94 & 0.4 & & 0.4 & 0.4 & 0.4 \\
\hline $1 \mathrm{E}$ & $S$ & 4/5/1993 & 93095 & 95 & 0.4 & 0.4 & 0.4 & 0.8 & 0.4 \\
\hline $1 \mathrm{E}$ & $S$ & 4/6/1993 & 93096 & 96 & 0.4 & 0.4 & 0.8 & 0.4 & 0.4 \\
\hline $1 \mathrm{E}$ & $S$ & 4/7/1993 & 93097 & 97 & 0.4 & 0.4 & 0.4 & 0.4 & 0.4 \\
\hline $1 \mathrm{E}$ & $S$ & 4/8/1993 & 93098 & 98 & 0.4 & 0.4 & 0.4 & 0.4 & 0.4 \\
\hline $1 \mathrm{E}$ & NS & 4/9/1993 & 93099 & 99 & 0.4 & 0.4 & 0.4 & 0.8 & 0.4 \\
\hline $0 \mathrm{E}$ & NS & 4/10/1993 & 93100 & 100 & 0.4 & 0.4 & 0.4 & 0.4 & 0.4 \\
\hline $0 \mathrm{E}$ & NS & $4 / 11 / 1993$ & 93101 & 101 & 0.8 & 0.4 & 0.4 & 0.4 & 0.4 \\
\hline $1 \mathrm{E}$ & NS & 4/12/1993 & 93102 & 102 & 0.4 & 0.4 & 0.4 & 0.8 & 0.4 \\
\hline $1 \mathrm{E}$ & S & 4/13/1993 & 93103 & 103 & & 0.4 & 0.8 & 0.4 & 0.4 \\
\hline $1 \mathrm{E}$ & $S$ & 4/14/1993 & 93104 & 104 & 0.4 & 0.8 & & 0.4 & 0.8 \\
\hline $1 \mathrm{E}$ & $S$ & 4/15/1993 & 93105 & 105 & 0.8 & 0.4 & 0.4 & 0.4 & 0.8 \\
\hline $1 \mathrm{E}$ & $S$ & 4/16/1993 & 93106 & 106 & 0.4 & 0.4 & 0.4 & 0.4 & 0.8 \\
\hline $0 \mathrm{E}$ & $S$ & $4 / 17 / 1993$ & 93107 & 107 & 0.4 & 0.8 & & 0.8 & 0.4 \\
\hline $0 \mathrm{E}$ & $S$ & 4/18/1993 & 93108 & 108 & 0.4 & 0.4 & 0.8 & 0.4 & 0.4 \\
\hline $1 \mathrm{E}$ & S & 4/19/1993 & 93109 & 109 & 0.4 & 0.4 & 0.4 & 0.4 & 0.4 \\
\hline $1 \mathrm{E}$ & $S$ & 4/20/1993 & 93110 & 110 & 0.4 & 0.8 & 0.4 & 0.4 & 0.4 \\
\hline $1 \mathrm{E}$ & $S$ & $4 / 21 / 1993$ & 93111 & 111 & 0.4 & 0.8 & 0.4 & 0.4 & 0.4 \\
\hline $1 \mathrm{E}$ & $S$ & 4/22/1993 & 93112 & 112 & 0.4 & 0.4 & 0.4 & 0.4 & 0.8 \\
\hline $1 \mathrm{E}$ & $S$ & 4/23/1993 & 93113 & 113 & 0.4 & 0.8 & 0.4 & 0.4 & 0.4 \\
\hline $0 \mathrm{E}$ & $S$ & $4 / 24 / 1993$ & 93114 & 114 & 0.4 & 0.4 & 0.4 & 0.4 & 0.4 \\
\hline $0 \mathrm{E}$ & $S$ & 4/25/1993 & 93115 & 115 & 0.4 & 0.4 & 0.4 & 0.4 & 0.4 \\
\hline $1 \mathrm{~F}$ & $S$ & 4/26/1993 & 93116 & 116 & 0.4 & 0.4 & 0.4 & 0.4 & 0.4 \\
\hline $1 \mathrm{E}$ & $S$ & $4 / 27 / 1993$ & 93117 & 117 & 0.4 & 0.4 & 0.4 & 0.4 & 0.4 \\
\hline $1 \mathrm{E}$ & NS & 4/28/1993 & 93118 & 118 & 0.4 & 0.4 & 0.4 & 0.4 & 0.4 \\
\hline $1 \mathrm{~F}$ & S & 4/29/1993 & 93119 & 119 & 0.4 & 0.4 & 0.8 & & 0.8 \\
\hline $1 \mathrm{~F}$ & $S$ & 4/30/1993 & 93120 & 120 & 0.4 & 0.4 & 0.4 & 0.4 & 0.4 \\
\hline $0 \mathrm{E}$ & $S$ & $5 / 1 / 1993$ & 93121 & 121 & 0.4 & 0.4 & 0.4 & 0.8 & 0.4 \\
\hline $0 \mathrm{E}$ & S & $5 / 2 / 1993$ & 93122 & 122 & 0.4 & 0.4 & 0.4 & 0.4 & 0.4 \\
\hline $1 \mathrm{E}$ & $S$ & 5/3/1993 & 93123 & 123 & 0.4 & 0.8 & & 0.8 & 0.4 \\
\hline $1 \mathrm{E}$ & $\mathrm{S}$ & $5 / 4 / 1993$ & 93124 & 124 & 0.4 & 0.4 & 0.4 & 0.4 & 0.4 \\
\hline $1 \mathrm{E}$ & $S$ & 5/5/1993 & 93125 & 125 & 0.8 & & 0.8 & 0.4 & 0.4 \\
\hline $1 \mathrm{E}$ & $S$ & 5/6/1993 & 93126 & 126 & 0.4 & 0.4 & 0.4 & 0.4 & 0.4 \\
\hline $1 \mathrm{E}$ & $S$ & 5/7/1993 & 93127 & 127 & 0.4 & 0.4 & 0.4 & 0.4 & 0.4 \\
\hline $0 \mathrm{E}$ & S & 5/8/1993 & 93128 & 128 & 0.8 & & 0.4 & 0.8 & 0.4 \\
\hline $0 \mathrm{E}$ & $S$ & 5/9/1993 & 93129 & 129 & 0.4 & 0.4 & 0.4 & 0.4 & 0.4 \\
\hline $1 \mathrm{E}$ & $S$ & $5 / 10 / 1993$ & 93130 & 130 & 0.4 & 0.4 & 0.4 & 0.8 & 0.4 \\
\hline $1 \mathrm{E}$ & $S$ & $5 / 11 / 1993$ & 93131 & 131 & 0.4 & 0.4 & 0.4 & 0.4 & 0.8 \\
\hline $1 \mathrm{E}$ & $S$ & $5 / 12 / 1993$ & 93132 & 132 & 0.4 & 0.4 & 0.4 & 0.4 & 0.4 \\
\hline $1 \mathrm{E}$ & $S$ & $5 / 13 / 1993$ & 93133 & 133 & 0.4 & 0.4 & 0.4 & 0.4 & 0.4 \\
\hline $1 \mathrm{E}$ & $S$ & $5 / 14 / 1993$ & 93134 & 134 & 0.4 & 0.8 & 0.4 & 0.4 & 0.4 \\
\hline $0 \mathrm{~B}$ & S & 5/15/1993 & 93135 & 135 & 0.4 & 0.8 & 0.4 & 0.4 & 0.8 \\
\hline
\end{tabular}




\begin{tabular}{|c|c|c|c|c|c|c|c|c|c|}
\hline $0 \mathrm{~B}$ & $S$ & 5/16/1993 & 93136 & 136 & 0.4 & 0.8 & 0.4 & 0.8 & 0.4 \\
\hline $1 \mathrm{~B}$ & $S$ & $5 / 17 / 1993$ & 93137 & 137 & 0.8 & 0.4 & 0.8 & 0.8 & 0.4 \\
\hline $1 \mathrm{~B}$ & $S$ & $5 / 18 / 1993$ & 93138 & 138 & 0.4 & 0.4 & 0.8 & 0.4 & 0.8 \\
\hline $1 \mathrm{~B}$ & S & $5 / 19 / 1993$ & 93139 & 139 & 0.4 & 0.4 & 0.4 & 0.4 & 0.4 \\
\hline $1 \mathrm{~B}$ & $S$ & $5 / 20 / 1993$ & 93140 & 140 & 0.4 & 0.4 & 0.4 & 0.4 & 0.4 \\
\hline $1 \mathrm{~B}$ & $S$ & $5 / 21 / 1993$ & 93141 & 141 & 0.4 & 0.4 & 0.4 & 0.4 & 0.8 \\
\hline $0 \mathrm{~B}$ & $S$ & $5 / 22 / 1993$ & 93142 & 142 & 0.4 & 0.8 & 0.4 & 0.4 & 0.4 \\
\hline $\begin{array}{ll}0 & B \\
\end{array}$ & $S$ & $5 / 23 / 1993$ & 93143 & 143 & 0.8 & 0.4 & 0.4 & 0.4 & 0.4 \\
\hline $1 \mathrm{~B}$ & $S$ & $5 / 24 / 1993$ & 93144 & 144 & 0.4 & 0.4 & 0.4 & 0.4 & 0.4 \\
\hline $1 \mathrm{~B}$ & $S$ & 5/25/1993 & 93145 & 145 & 0.4 & 0.4 & 0.4 & 0.4 & 0.4 \\
\hline $1 \mathrm{~B}$ & $S$ & $5 / 26 / 1993$ & 93146 & 146 & 0.4 & 0.4 & 0.4 & 0.4 & 0.4 \\
\hline $1 \mathrm{~B}$ & NS & $5 / 27 / 1993$ & 93147 & 147 & 0.4 & 0.4 & 0.4 & 0.4 & 0.4 \\
\hline $1 \mathrm{~B}$ & NS & $5 / 28 / 1993$ & 93148 & 148 & 0.4 & 0.4 & 0.4 & 0.4 & 0.8 \\
\hline $0 \mathrm{~B}$ & NS & 5/29/1993 & 93149 & 149 & 0.4 & 0.4 & 0.4 & 0.4 & 0.8 \\
\hline $0 \mathrm{~B}$ & NS & $5 / 30 / 1993$ & 93150 & 150 & 0.4 & 0.4 & 0.4 & 0.4 & 0.8 \\
\hline $1 \mathrm{~B}$ & NS & 5/31/1993 & 93151 & 151 & 0.4 & 0.8 & 0.4 & 0.4 & 0.4 \\
\hline $1 \mathrm{~B}$ & NS & 6/1/1993 & 93152 & 152 & 0.4 & 0.8 & 0.4 & 0.8 & 0.4 \\
\hline $1 \mathrm{~B}$ & NS & $6 / 2 / 1993$ & 93153 & 153 & 0.4 & 0.8 & 0.4 & 0.8 & 0.4 \\
\hline $1 \mathrm{~B}$ & NS & 6/3/1993 & 93154 & 154 & 0.4 & 0.8 & 0.8 & 0.4 & 0.8 \\
\hline $1 \mathrm{~B}$ & NS & 6/4/1993 & 93155 & 155 & 0.4 & 0.8 & 0.8 & 0.4 & 0.8 \\
\hline $0 \mathrm{~B}$ & NS & $6 / 5 / 1993$ & 93156 & 156 & 0.4 & 0.8 & 0.4 & 0.8 & 0.8 \\
\hline $0 \mathrm{~B}$ & NS & 6/6/1993 & 93157 & 157 & 0.8 & 0.8 & 0.8 & 0.4 & 0.8 \\
\hline $1 \mathrm{~B}$ & NS & 6/7/1993 & 93158 & 158 & 0.8 & 0.8 & 0.8 & 0.4 & 0.8 \\
\hline $1 \mathrm{~B}$ & NS & 6/8/1993 & 93159 & 159 & 0.4 & 0.8 & 0.8 & 0.4 & 0.8 \\
\hline $1 \mathrm{~B}$ & NS & 6/9/1993 & 93160 & 160 & 0.8 & 0.4 & 0.8 & 0.8 & 0.4 \\
\hline $1 \mathrm{~B}$ & NS & $6 / 10 / 1993$ & 93161 & 161 & 0.8 & 0.8 & 0.4 & 0.8 & 0.8 \\
\hline $1 \mathrm{~B}$ & NS & $6 / 11 / 1993$ & 93162 & 162 & 0.4 & 0.4 & 0.4 & 0.4 & 0.4 \\
\hline $0 \mathrm{~B}$ & NS & $6 / 12 / 1993$ & 93163 & 163 & 0.4 & 0.4 & 0.4 & 0.4 & 0.4 \\
\hline $0 \mathrm{~B}$ & NS & $6 / 13 / 1993$ & 93164 & 164 & 0.8 & 0.4 & 0.4 & 0.4 & 0.4 \\
\hline $1 \mathrm{~B}$ & NS & 6/14/1993 & 93165 & 165 & 0.8 & 0.4 & 0.4 & 0.4 & 0.4 \\
\hline $1 \mathrm{~B}$ & NS & $6 / 15 / 1993$ & 93166 & 166 & 0.4 & 0.8 & 0.4 & 0.8 & 0.4 \\
\hline $1 \mathrm{~B}$ & NS & 6/16/1993 & 93167 & 167 & 0.8 & 0.4 & 0.8 & 0.4 & 0.8 \\
\hline $1 \mathrm{~B}$ & NS & 6/17/1993 & 93168 & 168 & 0.4 & 0.8 & 0.8 & 0.4 & 0.8 \\
\hline $1 \mathrm{~B}$ & NS & $6 / 18 / 1993$ & 93169 & 169 & 0.8 & 0.8 & 0.4 & 0.8 & 0.4 \\
\hline $\begin{array}{ll}0 & \mathrm{~B} \\
\end{array}$ & NS & 6/19/1993 & 93170 & 170 & 0.4 & 0.8 & 0.4 & 0.8 & 0.4 \\
\hline $0 \mathrm{~B}$ & NS & 6/20/1993 & 93171 & 171 & 0.4 & 0.4 & 0.4 & 0.4 & 0.4 \\
\hline $1 \mathrm{~B}$ & NS & $6 / 21 / 1993$ & 93172 & 172 & 0.4 & 0.4 & 0.4 & 0.4 & 0.4 \\
\hline $1 \mathrm{~B}$ & NS & 6/22/1993 & 93173 & 173 & 0.4 & 0.8 & 0.4 & 0.4 & 0.4 \\
\hline $1 \mathrm{~B}$ & NS & 6/23/1993 & 93174 & 174 & 0.8 & 0.4 & 0.8 & 0.4 & 0.4 \\
\hline $1 \mathrm{~B}$ & NS & $6 / 24 / 1993$ & 93175 & 175 & 0.4 & 0.8 & 0.4 & 0.8 & 0.8 \\
\hline $1 \mathrm{~B}$ & NS & 6/25/1993 & 93176 & 176 & 0.8 & 0.8 & 0.4 & 0.8 & 0.8 \\
\hline $0 \mathrm{~B}$ & NS & 6/26/1993 & 93177 & 177 & 0.8 & 0.4 & 0.8 & 0.8 & 0.4 \\
\hline $0 \mathrm{~B}$ & NS & $6 / 27 / 1993$ & 93178 & 178 & 0.8 & 0.4 & 0.8 & 0.4 & 0.4 \\
\hline $1 \mathrm{~B}$ & NS & 6/28/1993 & 93179 & 179 & 0.8 & 0.4 & 0.8 & 0.4 & 0.8 \\
\hline $1 \mathrm{~B}$ & NS & 6/29/1993 & 93180 & 180 & 0.8 & 0.8 & 0.8 & 0.4 & 0.8 \\
\hline $1 \mathrm{~B}$ & NS & $6 / 30 / 1993$ & 93181 & 181 & 0.8 & 0.8 & 0.4 & 0.8 & 0.4 \\
\hline $1 \mathrm{~B}$ & NS & 7/1/1993 & 93182 & 182 & 0.4 & 0.8 & 0.8 & 0.8 & 0.8 \\
\hline $1 \mathrm{~B}$ & NS & 7/2/1993 & 93183 & 183 & 0.4 & 0.8 & 0.8 & 0.4 & 0.8 \\
\hline $0 \mathrm{~B}$ & NS & 7/3/1993 & 93184 & 184 & 0.4 & 0.8 & 0.8 & 0.8 & 0.4 \\
\hline $0 \mathrm{~B}$ & NS & $7 / 4 / 1993$ & 93185 & 185 & 0.8 & 0.8 & 0.4 & 0.8 & 0.8 \\
\hline $1 \mathrm{~B}$ & NS & 7/5/1993 & 93186 & 186 & 0.8 & 0.4 & 0.8 & 0.8 & 0.8 \\
\hline
\end{tabular}




\begin{tabular}{|c|c|c|c|c|c|c|c|c|c|}
\hline \begin{tabular}{l|l}
1 & $B$ \\
\end{tabular} & NS & 7/6/1993 & \begin{tabular}{|l|}
93187 \\
\end{tabular} & 187 & & & & & \\
\hline $1 \mathrm{~B}$ & NS & 7/7/1993 & 93188 & 188 & & & & & \\
\hline $1 \mathrm{~B}$ & NS & 7/8/1993 & 93189 & 189 & & & & & \\
\hline $1 \mathrm{~B}$ & NS & 7/9/1993 & 93190 & 190 & & & & & \\
\hline $0 \mathrm{~B}$ & NS & 7/10/1993 & 93191 & 191 & & & & & \\
\hline $0 \mathrm{~B}$ & NS & 7/11/1993 & 93192 & 192 & & & & & \\
\hline $1 \mathrm{~B}$ & NS & 7/12/1993 & 93193 & 193 & & & & & \\
\hline $1 \mathrm{~B}$ & NS & 7/13/1993 & 93194 & 194 & & & & & \\
\hline $1 \mathrm{~B}$ & NS & 7/14/1993 & 93195 & 195 & & & & & \\
\hline $1 \mathrm{~B}$ & NS & 7/15/1993 & 93196 & 196 & & & & & \\
\hline $1 \mathrm{~B}$ & NS & $7 / 16 / 1993$ & 93197 & 197 & & & & & \\
\hline $0 \mathrm{~B}$ & NS & 7/17/1993 & 93198 & 198 & & & & & \\
\hline $0 \mathrm{~B}$ & NS & 7/18/1993 & 93199 & 199 & & & & & \\
\hline $1 \mathrm{~B}$ & NS & 7/19/1993 & 93200 & 200 & & & & & \\
\hline $1 \mathrm{~B}$ & NS & 7/20/1993 & 93201 & 201 & & & & & \\
\hline $1 \mathrm{~B}$ & NS & 7/21/1993 & 93202 & 202 & & & & & \\
\hline $1 \mathrm{~B}$ & NS & 7/22/1993 & 93203 & 203 & & & & & \\
\hline $1 \mathrm{~B}$ & NS & 7/23/1993 & 93204 & 204 & & & & & \\
\hline $0 \mathrm{~B}$ & NS & 7/24/1993 & 93205 & 205 & & & & & \\
\hline $0 \mathrm{~B}$ & NS & 7/25/1993 & 93206 & 206 & & & & & \\
\hline $1 \mathrm{~B}$ & NS & 7/26/1993 & 93207 & 207 & & & & & \\
\hline $1 \mathrm{~B}$ & NS & 7/27/1993 & 93208 & 208 & & & & & \\
\hline $1 \mathrm{~B}$ & NS & 7/28/1993 & 93209 & 209 & & & & & \\
\hline $1 \mathrm{~B}$ & NS & $7 / 29 / 1993$ & 93210 & 210 & & & & & \\
\hline $1 \mathrm{~B}$ & NS & 7/30/1993 & 93211 & 211 & & & & & \\
\hline $0 \mathrm{~B}$ & NS & 7/31/1993 & 93212 & 212 & & & & & \\
\hline $0 \mathrm{~B}$ & NS & 8/1/1993 & 93213 & 213 & & & & & \\
\hline $1 \mathrm{~B}$ & NS & $8 / 2 / 1993$ & 93214 & 214 & & & & & \\
\hline $1 \mathrm{~B}$ & NS & 8/3/1993 & 93215 & 215 & & & & & \\
\hline $1 \mathrm{~B}$ & NS & 8/4/1993 & 93216 & 216 & & & & & \\
\hline $1 \mathrm{~B}$ & NS & 8/5/1993 & 93217 & 217 & & & & & \\
\hline $1 \mathrm{~B}$ & NS & 8/6/1993 & 93218 & 218 & & & & & \\
\hline $0 \mathrm{~B}$ & NS & 8/7/1993 & 93219 & 219 & & & & & \\
\hline $0 \mathrm{~B}$ & NS & 8/8/1993 & 93220 & 220 & & & & & \\
\hline $1 \mathrm{~B}$ & NS & 8/9/1993 & 93221 & 221 & & & & & \\
\hline $1 \mathrm{~B}$ & NS & $8 / 10 / 1993$ & 93222 & 222 & & & & & \\
\hline $1 \mathrm{~B}$ & NS & $8 / 11 / 1993$ & 93223 & 223 & & & & & \\
\hline $1 \mathrm{~B}$ & NS & $8 / 12 / 1993$ & 93224 & 224 & & & & & \\
\hline $1 \mathrm{~B}$ & NS & $8 / 13 / 1993$ & 93225 & 225 & & & & & \\
\hline $0 \mathrm{~B}$ & NS & 8/14/1993 & 93226 & 226 & 0.8 & 0.8 & 0.8 & 0.8 & 0.4 \\
\hline \begin{tabular}{l|l}
0 & $B$ \\
\end{tabular} & NS & $8 / 15 / 1993$ & 93227 & 227 & 0.8 & 0.8 & 0.8 & 0.8 & 0.8 \\
\hline $1 \mathrm{~B}$ & NS & $8 / 16 / 1993$ & 93228 & 228 & 0.4 & 0.8 & 0.8 & 0.8 & 0.8 \\
\hline $1 \mathrm{~B}$ & NS & 8/17/1993 & 93229 & 229 & 0.8 & 0.8 & 0.8 & 0.8 & 0.8 \\
\hline $1 \mathrm{~B}$ & NS & 8/18/1993 & 93230 & 230 & 0.8 & 0.8 & 0.8 & 0.8 & 0.8 \\
\hline $1 \mathrm{~B}$ & NS & 8/19/1993 & 93231 & 231 & 0.8 & 0.8 & 0.4 & 0.8 & 0.8 \\
\hline $1 \mathrm{~B}$ & NS & 8/20/1993 & 93232 & 232 & 0.8 & 0.8 & 0.8 & 0.8 & 0.8 \\
\hline \begin{tabular}{l|l}
0 & $B$ \\
\end{tabular} & NS & $8 / 21 / 1993$ & 93233 & 233 & 0.8 & 0.8 & 0.8 & 0.4 & 0.8 \\
\hline $0 \mathrm{~B}$ & NS & 8/22/1993 & 93234 & 234 & 0.8 & 0.8 & 0.8 & 0.8 & 0.4 \\
\hline $1 \mathrm{~B}$ & NS & $8 / 23 / 1993$ & 93235 & 235 & 0.8 & 0.4 & 0.8 & 0.8 & 0.8 \\
\hline $1 \mathrm{~B}$ & NS & 8/24/1993 & 93236 & 236 & 0.8 & 0.8 & 0.8 & 0.4 & 0.8 \\
\hline $1 \mathrm{~B}$ & NS & 8/25/1993 & 93237 & 237 & 0.8 & 0.8 & 0.4 & 0.8 & 0.8 \\
\hline
\end{tabular}




\begin{tabular}{|c|c|c|c|c|c|c|c|c|c|}
\hline $1 \mathrm{E}$ & NS & 8/26/1993 & 93238 & 238 & 0.8 & 0.8 & 0.8 & 0.8 & 0.4 \\
\hline $1 \mathrm{E}$ & NS & $8 / 27 / 1993$ & 93239 & 239 & 0.8 & 0.8 & 0.4 & 0.8 & 0.8 \\
\hline $0 \mathrm{E}$ & NS & 8/28/1993 & 93240 & 240 & 0.8 & 0.8 & 0.4 & 0.8 & 0.4 \\
\hline $0 \mathrm{E}$ & NS & 8/29/1993 & 93241 & 241 & 0.4 & 0.8 & 0.8 & 0.4 & 0.8 \\
\hline $1 \mathrm{E}$ & NS & 8/30/1993 & 93242 & 242 & 0.8 & 0.8 & 0.4 & 0.8 & 0.8 \\
\hline $1 \mathrm{E}$ & NS & 8/31/1993 & 93243 & 243 & 0.8 & 0.8 & 0.4 & 0.8 & 0.8 \\
\hline $1 \mathrm{E}$ & NS & 9/1/1993 & 93244 & 244 & 0.8 & 0.8 & 0.4 & 0.8 & 0.8 \\
\hline $1 \mathrm{E}$ & NS & 9/2/1993 & 93245 & 245 & 0.8 & 0.8 & 0.8 & 0.4 & 0.8 \\
\hline $1 \mathrm{~F}$ & NS & 9/3/1993 & 93246 & 246 & 0.8 & 0.4 & 0.8 & 0.8 & 0.8 \\
\hline $0 \mathrm{E}$ & NS & 9/4/1993 & 93247 & 247 & 0.8 & 0.8 & 0.8 & 0.8 & 0.8 \\
\hline $0 \mathrm{E}$ & NS & 9/5/1993 & 93248 & 248 & 0.8 & 0.8 & 0.4 & 0.8 & 0.8 \\
\hline $1 \mathrm{E}$ & NS & 9/6/1993 & 93249 & 249 & 0.8 & 0.8 & 0.4 & 0.8 & 0.8 \\
\hline $1 \mathrm{E}$ & NS & 9/7/1993 & 93250 & 250 & 0.8 & 0.8 & 0.8 & 0.4 & 0.8 \\
\hline $1 \mathrm{E}$ & NS & 9/8/1993 & 93251 & 251 & 0.8 & 0.8 & 0.8 & 0.4 & 0.8 \\
\hline $1 \mathrm{E}$ & $S$ & 9/9/1993 & 93252 & 252 & 0.8 & 0.8 & 0.8 & 0.8 & 0.4 \\
\hline $1 \mathrm{~F}$ & $S$ & 9/10/1993 & 93253 & 253 & 0.8 & 0.8 & 0.8 & 0.8 & 0.4 \\
\hline $0 \mathrm{E}$ & $S$ & 9/11/1993 & 93254 & 254 & 0.8 & 0.8 & 0.8 & 0.4 & 0.8 \\
\hline $0 \mathrm{E}$ & $S$ & 9/12/1993 & 93255 & 255 & 0.8 & 0.8 & 0.4 & 0.8 & 0.4 \\
\hline $1 \mathrm{E}$ & $S$ & 9/13/1993 & 93256 & 256 & 0.4 & 0.8 & 0.8 & 0.8 & 0.4 \\
\hline $1 \mathrm{E}$ & $\mathrm{S}$ & 9/14/1993 & 93257 & 257 & 0.4 & 0.8 & 0.8 & 0.8 & 0.4 \\
\hline $1 \mathrm{E}$ & $S$ & 9/15/1993 & 93258 & 258 & 0.8 & 0.4 & 0.8 & 0.8 & 0.4 \\
\hline $1 \mathrm{E}$ & $S$ & 9/16/1993 & 93259 & 259 & 0.8 & 0.8 & 0.4 & 0.8 & 0.4 \\
\hline $1 \mathrm{E}$ & $S$ & 9/17/1993 & 93260 & 260 & 0.8 & 0.8 & 0.8 & 0.4 & 0.8 \\
\hline $0 \mathrm{E}$ & $S$ & 9/18/1993 & 93261 & 261 & 0.8 & 0.4 & 0.8 & 0.8 & 0.8 \\
\hline $0 \mathrm{E}$ & $S$ & 9/19/1993 & 93262 & 262 & 0.8 & 0.4 & 0.8 & 0.8 & 0.4 \\
\hline $1 \mathrm{E}$ & $\mathrm{S}$ & 9/20/1993 & 93263 & 263 & 0.4 & 0.8 & 0.8 & 0.4 & 0.8 \\
\hline $1 \mathrm{E}$ & $S$ & 9/21/1993 & 93264 & 264 & 0.8 & 0.4 & 0.8 & 0.4 & 0.8 \\
\hline $1 \mathrm{~F}$ & $S$ & 9/22/1993 & 93265 & 265 & 0.8 & 0.4 & 0.8 & 0.8 & 0.4 \\
\hline $1 \mathrm{E}$ & $S$ & 9/23/1993 & 93266 & 266 & 0.4 & 0.8 & 0.8 & 0.8 & 0.4 \\
\hline $1 \mathrm{E}$ & $S$ & 9/24/1993 & 93267 & 267 & 0.8 & 0.8 & 0.4 & 0.8 & 0.8 \\
\hline $0 \mathrm{E}$ & $S$ & 9/25/1993 & 93268 & 268 & 0.8 & 0.8 & 0.4 & 0.8 & 0.8 \\
\hline $0 \mathrm{E}$ & $S$ & 9/26/1993 & 93269 & 269 & 0.8 & 0.8 & 0.8 & 0.4 & 0.8 \\
\hline $1 \mathrm{E}$ & $\mathrm{S}$ & 9/27/1993 & 93270 & 270 & 0.8 & 0.8 & 0.4 & 0.8 & 0.8 \\
\hline $1 \mathrm{E}$ & $S$ & 9/28/1993 & 93271 & 271 & 0.8 & 0.4 & 0.8 & 0.8 & 0.4 \\
\hline $1 \mathrm{E}$ & $S$ & 9/29/1993 & 93272 & 272 & 0.8 & 0.8 & 0.4 & 0.8 & 0.8 \\
\hline $1 \mathrm{E}$ & $\mathrm{S}$ & 9/30/1993 & 93273 & 273 & 0.8 & 0.8 & 0.4 & 0.8 & 0.8 \\
\hline $1 \mathrm{E}$ & $S$ & 10/1/1993 & 93274 & 274 & 0.8 & 0.4 & 0.8 & 0.4 & 0.8 \\
\hline $0 \mathrm{E}$ & $S$ & 10/2/1993 & 93275 & 275 & 0.8 & 0.4 & 0.8 & 0.8 & 0.4 \\
\hline $0 \mathrm{E}$ & $\mathrm{S}$ & 10/3/1993 & 93276 & 276 & 0.4 & 0.8 & 0.8 & 0.8 & 0.4 \\
\hline $1 \mathrm{E}$ & $S$ & 10/4/1993 & 93277 & 277 & 0.8 & 0.8 & 0.4 & 0.8 & 0.8 \\
\hline $1 \mathrm{E}$ & $S$ & 10/5/1993 & 93278 & 278 & 0.8 & 0.8 & 0.4 & 0.8 & 0.8 \\
\hline $1 \mathrm{E}$ & $S$ & 10/6/1993 & 93279 & 279 & 0.8 & 0.8 & 0.4 & 0.8 & 0.4 \\
\hline $1 \mathrm{E}$ & $S$ & 10/7/1993 & 93280 & 280 & 0.8 & 0.8 & 0.4 & 0.8 & 0.4 \\
\hline $1 \mathrm{E}$ & $S$ & 10/8/1993 & 93281 & 281 & 0.8 & 0.4 & 0.8 & 0.4 & 0.8 \\
\hline $0 \mathrm{E}$ & $S$ & $10 / 9 / 1993$ & 93282 & 282 & 0.4 & 0.8 & 0.4 & 0.8 & 0.8 \\
\hline $0 \mathrm{~B}$ & $S$ & 10/10/1993 & 93283 & 283 & 0.8 & 0.8 & 0.8 & 0.4 & 0.8 \\
\hline $1 \mathrm{E}$ & $S$ & 10/11/1993 & 93284 & 284 & 0.4 & 0.8 & 0.4 & 0.8 & 0.4 \\
\hline $1 \mathrm{E}$ & $S$ & 10/12/1993 & 93285 & 285 & 0.8 & 0.4 & 0.4 & 0.8 & 0.4 \\
\hline $1 \mathrm{E}$ & $S$ & 10/13/1993 & 93286 & 286 & 0.8 & 0.4 & 0.8 & 0.4 & 0.4 \\
\hline $1 \mathrm{E}$ & $S$ & 10/14/1993 & 93287 & 287 & 0.4 & 0.4 & 0.4 & 0.8 & \\
\hline $1 \mathrm{E}$ & $S$ & 10/15/1993 & 93288 & 288 & 0.4 & 0.4 & 0.4 & 0.4 & 0.4 \\
\hline
\end{tabular}




\begin{tabular}{|c|c|c|c|c|c|c|c|c|c|}
\hline $0 \mathrm{~B}$ & $S$ & 10/16/1993 & 93289 & 289 & 0.4 & 0.8 & 0.4 & 0.8 & 0.4 \\
\hline $\begin{array}{ll}0 & \mathrm{~B} \\
\end{array}$ & $S$ & 10/17/1993 & 93290 & 290 & 0.4 & 0.4 & 0.8 & 0.4 & 0.8 \\
\hline $1 \mathrm{~B}$ & $S$ & 10/18/1993 & 93291 & 291 & 0.8 & 0.4 & 0.8 & 0.4 & 0.8 \\
\hline $1 \mathrm{~B}$ & S & 10/19/1993 & 93292 & 292 & 0.4 & 0.8 & 0.8 & 0.4 & 0.8 \\
\hline $1 \mathrm{~B}$ & $S$ & $10 / 20 / 1993$ & 93293 & 293 & 0.8 & 0.4 & 0.8 & 0.8 & 0.4 \\
\hline $1 \mathrm{~B}$ & $S$ & 10/21/1993 & 93294 & 294 & 0.8 & & 0.8 & 0.4 & 0.4 \\
\hline $1 \mathrm{~B}$ & $S$ & 10/22/1993 & 93295 & 295 & 0.8 & 0.4 & 0.4 & 0.4 & 0.4 \\
\hline $\begin{array}{ll}0 & B \\
\end{array}$ & $S$ & $10 / 23 / 1993$ & 93296 & 296 & 0.4 & 0.8 & 0.4 & 0.4 & 0.4 \\
\hline $0 \mathrm{~B}$ & $S$ & 10/24/1993 & 93297 & 297 & 0.4 & 0.8 & 0.4 & 0.4 & 0.4 \\
\hline $1 \mathrm{~B}$ & $S$ & 10/25/1993 & 93298 & 298 & 0.4 & 0.4 & 0.4 & 0.4 & 0.4 \\
\hline $1 \mathrm{~B}$ & $S$ & 10/26/1993 & 93299 & 299 & 0.4 & 0.4 & 0.4 & 0.4 & 0.4 \\
\hline $1 \mathrm{~B}$ & $S$ & 10/27/1993 & 93300 & 300 & 0.4 & 0.4 & 0.4 & 0.4 & 0.4 \\
\hline $1 \mathrm{~B}$ & $S$ & 10/28/1993 & 93301 & 301 & 0.8 & 0.8 & 1.2 & 0.8 & 1.2 \\
\hline $1 \mathrm{~B}$ & S & 10/29/1993 & 93302 & 302 & 0.8 & 1.2 & 1.2 & 0.8 & 1.2 \\
\hline $0 \mathrm{~B}$ & $S$ & $10 / 30 / 1993$ & 93303 & 303 & 1.2 & 1.6 & 1.2 & 1.2 & 1.2 \\
\hline 0 B & $S$ & 10/31/1993 & 93304 & 304 & 1.2 & 1.2 & 0.8 & 0.8 & 1.2 \\
\hline $1 \mathrm{~B}$ & $S$ & $11 / 1 / 1993$ & 93305 & 305 & 1.2 & 0.8 & 1.2 & 0.8 & 0.8 \\
\hline $1 \mathrm{~B}$ & $S$ & $11 / 2 / 1993$ & 93306 & 306 & 0.8 & 1.2 & 0.8 & 1.2 & 0.8 \\
\hline $1 \mathrm{~B}$ & S & 11/3/1993 & 93307 & 307 & 1.2 & 1.2 & 1.2 & 1.2 & 0.8 \\
\hline $1 \mathrm{~B}$ & $S$ & 11/4/1993 & 93308 & 308 & 1.2 & 1.2 & 0.8 & 1.2 & 0.8 \\
\hline $1 \mathrm{~B}$ & $S$ & $11 / 5 / 1993$ & 93309 & 309 & 1.2 & 1.2 & 0.8 & 1.2 & 0.8 \\
\hline $0 \mathrm{~B}$ & $S$ & 11/6/1993 & 93310 & 310 & 1.2 & 1.2 & 0.8 & 1.2 & 1.2 \\
\hline $0 \mathrm{~B}$ & S & $11 / 7 / 1993$ & 93311 & 311 & 0.8 & 1.2 & 0.8 & 1.2 & 0.8 \\
\hline $1 \mathrm{~B}$ & $S$ & 11/8/1993 & 93312 & 312 & 0.8 & 1.2 & 0.8 & 1.2 & 0.8 \\
\hline $1 \mathrm{~B}$ & S & 11/9/1993 & 93313 & 313 & 1.2 & 1.2 & 0.8 & 1.2 & 0.8 \\
\hline $1 \mathrm{~B}$ & $S$ & 11/10/1993 & 93314 & 314 & 1.2 & 0.8 & 1.2 & 1.2 & 0.8 \\
\hline $1 \mathrm{~B}$ & NS & $11 / 11 / 1993$ & 93315 & 315 & 1.2 & 0.8 & 1.2 & 0.8 & 1.2 \\
\hline $1 B$ & $S$ & 11/12/1993 & 93316 & 316 & 1.2 & 1.2 & 0.8 & 1.2 & 1.2 \\
\hline $0 \mathrm{~B}$ & S & 11/13/1993 & 93317 & 317 & 1.2 & 0.8 & 1.2 & 1.2 & 0.8 \\
\hline $0 \mathrm{~B}$ & $S$ & $11 / 14 / 1993$ & 93318 & 318 & 0.8 & 1.2 & 1.2 & 0.8 & 1.2 \\
\hline $1 \mathrm{~B}$ & $S$ & $11 / 15 / 1993$ & 93319 & 319 & 1.2 & 0.8 & 1.2 & 0.8 & 1.2 \\
\hline $1 \mathrm{~B}$ & $S$ & 11/16/1993 & 93320 & 320 & 0.8 & 1.2 & 1.2 & 0.8 & 1.2 \\
\hline $1 \mathrm{~B}$ & $S$ & 11/17/1993 & 93321 & 321 & 1.2 & 0.8 & 1.2 & 0.8 & 0.8 \\
\hline $1 \mathrm{~B}$ & $S$ & 11/18/1993 & 93322 & 322 & 0.8 & 1.2 & 0.8 & 1.2 & 0.8 \\
\hline $1 \mathrm{~B}$ & $S$ & 11/19/1993 & 93323 & 323 & 1.2 & 0.8 & 1.2 & 0.8 & 0.8 \\
\hline $0 \mathrm{~B}$ & S & 11/20/1993 & 93324 & 324 & 1.2 & 0.8 & 1.2 & 1.2 & 0.8 \\
\hline $\begin{array}{ll}0 & \mathrm{~B} \\
\end{array}$ & $S$ & $11 / 21 / 1993$ & 93325 & 325 & 1.2 & 0.8 & 1.2 & 0.8 & 0.8 \\
\hline $1 \mathrm{~B}$ & S & 11/22/1993 & 93326 & 326 & 0.8 & 1.2 & 0.8 & 0.8 & 1.2 \\
\hline $1 \mathrm{~B}$ & S & 11/23/1993 & 93327 & 327 & 0.8 & 1.2 & 0.8 & 0.8 & 1.2 \\
\hline $1 \mathrm{~B}$ & NS & $11 / 24 / 1993$ & 93328 & 328 & 0.8 & 1.2 & 0.8 & 1.2 & 0.8 \\
\hline $1 \mathrm{~B}$ & NS & $11 / 25 / 1993$ & 93329 & 329 & 0.8 & 1.2 & 1.2 & 0.8 & 1.2 \\
\hline $1 \mathrm{~B}$ & NS & 11/26/1993 & 93330 & 330 & 0.8 & 0.8 & 1.2 & 0.8 & 1.2 \\
\hline $0 \mathrm{~B}$ & NS & 11/27/1993 & 93331 & 331 & 0.8 & 0.8 & 0.8 & 0.8 & 0.8 \\
\hline $0 \mathrm{~B}$ & NS & 11/28/1993 & 93332 & 332 & 0.8 & 0.8 & 0.8 & 0.8 & 0.8 \\
\hline $1 \mathrm{~B}$ & $S$ & 11/29/1993 & 93333 & 333 & 0.8 & 1.2 & 0.8 & 0.8 & 0.8 \\
\hline $1 \mathrm{~B}$ & $S$ & $11 / 30 / 1993$ & 93334 & 334 & 0.8 & 1.2 & 0.8 & 0.8 & 0.8 \\
\hline $1 \mathrm{~B}$ & S & $12 / 1 / 1993$ & 93335 & 335 & 0.8 & 1.2 & 0.8 & 1.2 & 0.8 \\
\hline $1 \mathrm{~B}$ & S & $12 / 2 / 1993$ & 93336 & 336 & 1.2 & 0.8 & 0.8 & 1.2 & 0.8 \\
\hline $1 \mathrm{~B}$ & $S$ & $12 / 3 / 1993$ & 93337 & 337 & 0.8 & 1.2 & 0.8 & 0.8 & 1.2 \\
\hline $0 \mathrm{~B}$ & S & $12 / 4 / 1993$ & 93338 & 338 & 1.2 & 0.8 & 1.2 & 0.8 & 1.2 \\
\hline 0 B & $S$ & 12/5/1993 & 93339 & 339 & 1.2 & 0.8 & 0.8 & 0.8 & 0.8 \\
\hline
\end{tabular}




\begin{tabular}{|c|c|c|c|c|c|c|c|c|c|}
\hline $1 \mathrm{~B}$ & $S$ & $12 / 6 / 1993$ & 93340 & 340 & 0.8 & 1.2 & 0.8 & 0.8 & 0.8 \\
\hline $1 \mathrm{~B}$ & $S$ & $12 / 7 / 1993$ & 93341 & 341 & 0.8 & 0.8 & 0.8 & 1.2 & 0.8 \\
\hline $1 \mathrm{~B}$ & $S$ & 12/8/1993 & 93342 & 342 & 0.8 & 0.8 & 1.2 & 0.8 & 0.8 \\
\hline $1 \mathrm{~B}$ & S & 12/9/1993 & 93343 & 343 & 0.8 & 1.2 & 0.8 & 0.8 & 0.8 \\
\hline $1 \mathrm{~B}$ & $S$ & 12/10/1993 & 93344 & 344 & 1.2 & 0.8 & 1.2 & 0.8 & 0.8 \\
\hline $\begin{array}{ll}0 & B \\
\end{array}$ & $S$ & 12/11/1993 & 93345 & 345 & 0.8 & 1.2 & 0.8 & 1.2 & 0.8 \\
\hline $0 \mathrm{~B}$ & $S$ & 12/12/1993 & 93346 & 346 & 0.8 & 0.8 & 1.2 & 0.8 & 0.8 \\
\hline $1 \mathrm{~B}$ & $S$ & 12/13/1993 & 93347 & 347 & 0.8 & 1.2 & 0.8 & 1.2 & 0.8 \\
\hline $1 \mathrm{~B}$ & $S$ & 12/14/1993 & 93348 & 348 & 0.8 & 0.8 & 1.2 & 0.8 & 1.2 \\
\hline $1 \mathrm{~B}$ & $S$ & $12 / 15 / 1993$ & 93349 & 349 & 1.2 & 0.8 & 0.8 & 0.8 & 0.8 \\
\hline $1 \mathrm{~B}$ & $S$ & 12/16/1993 & 93350 & 350 & 1.2 & 0.8 & 0.8 & 1.2 & 0.8 \\
\hline $1 \mathrm{~B}$ & NS & 12/17/1993 & 93351 & 351 & 0.8 & 0.8 & 1.2 & 0.8 & 0.8 \\
\hline $0 \mathrm{~B}$ & NS & 12/18/1993 & 93352 & 352 & 1.2 & 0.8 & 1.2 & 0.8 & 1.2 \\
\hline $0 \mathrm{~B}$ & NS & 12/19/1993 & 93353 & 353 & 0.8 & 1.2 & 0.8 & 1.2 & 0.8 \\
\hline $1 \mathrm{~B}$ & NS & $12 / 20 / 1993$ & 93354 & 354 & 0.8 & 0.8 & 1.2 & 0.8 & 0.8 \\
\hline $1 \mathrm{~B}$ & NS & 12/21/1993 & 93355 & 355 & 0.8 & 1.2 & 0.8 & 1.2 & 0.8 \\
\hline $1 \mathrm{~B}$ & NS & 12/22/1993 & 93356 & 356 & 0.8 & 1.2 & 0.8 & 0.8 & 1.2 \\
\hline $1 \mathrm{~B}$ & NS & 12/23/1993 & 93357 & 357 & 1.2 & 0.8 & 1.2 & 0.8 & 0.8 \\
\hline $1 \mathrm{~B}$ & NS & 12/24/1993 & 93358 & 358 & 1.2 & 0.8 & 1.2 & 1.2 & 0.8 \\
\hline $0 \mathrm{~B}$ & NS & 12/25/1993 & 93359 & 359 & 0.8 & 0.8 & 0.8 & 0.8 & 0.8 \\
\hline $\begin{array}{ll}0 & \mathrm{~B} \\
\end{array}$ & NS & 12/26/1993 & 93360 & 360 & 0.8 & 1.2 & 0.8 & 0.8 & 0.8 \\
\hline $1 \mathrm{~B}$ & NS & 12/27/1993 & 93361 & 361 & 0.8 & 1.2 & 0.8 & 0.8 & 0.8 \\
\hline $1 \mathrm{~B}$ & NS & 12/28/1993 & 93362 & 362 & 0.8 & 0.8 & 1.2 & 0.8 & 0.8 \\
\hline $1 \mathrm{~B}$ & NS & 12/29/1993 & 93363 & 363 & 1.2 & 1.2 & 1.2 & 0.8 & 1.2 \\
\hline $1 \mathrm{~B}$ & NS & 12/30/1993 & 93364 & 364 & 0.8 & 1.2 & 0.8 & 1.2 & 0.8 \\
\hline $1 \mathrm{~B}$ & NS & 12/31/1993 & 93365 & 365 & 0.8 & 0.8 & 0.8 & 0.8 & 0.8 \\
\hline $0 \mathrm{~B}$ & NS & $1 / 1 / 1994$ & 94001 & 1 & 0.8 & 0.8 & 0.8 & 0.8 & 0.8 \\
\hline $0 \mathrm{~B}$ & NS & $1 / 2 / 1994$ & 94002 & 2 & 0.8 & 0.8 & 1.2 & 0.8 & 0.8 \\
\hline $1 \mathrm{~B}$ & NS & $1 / 3 / 1994$ & 94003 & 3 & 1.2 & 0.8 & 1.2 & 1.2 & 0.8 \\
\hline $1 \mathrm{~B}$ & NS & $1 / 4 / 1994$ & 94004 & 4 & 0.8 & 1.2 & 0.8 & 0.8 & 0.8 \\
\hline $1 \mathrm{~B}$ & NS & $1 / 5 / 1994$ & 94005 & 5 & 1.2 & 0.8 & 0.8 & 1.2 & 0.8 \\
\hline $1 \mathrm{~B}$ & $S$ & 1/6/1994 & 94006 & 6 & 0.8 & 1.2 & 1.2 & 0.8 & 1.2 \\
\hline $1 \mathrm{~B}$ & $S$ & 1/7/1994 & 94007 & 7 & 1.2 & 0.8 & 1.2 & 0.8 & 1.2 \\
\hline $0 \mathrm{~B}$ & $S$ & $1 / 8 / 1994$ & 94008 & 8 & 0.8 & 1.2 & 0.8 & 1.2 & 0.8 \\
\hline $\begin{array}{ll}0 & \mathrm{~B} \\
\end{array}$ & $S$ & 1/9/1994 & 94009 & 9 & 1.2 & 0.8 & 1.2 & 0.8 & 0.8 \\
\hline $1 \mathrm{~B}$ & S & $1 / 10 / 1994$ & 94010 & 10 & 1.2 & 0.8 & 0.8 & 1.2 & 0.8 \\
\hline $1 \mathrm{~B}$ & $S$ & $1 / 11 / 1994$ & 94011 & 11 & 1.2 & 0.8 & 1.2 & 0.8 & 0.8 \\
\hline $1 \mathrm{~B}$ & S & $1 / 12 / 1994$ & 94012 & 12 & 1.2 & 0.8 & 1.2 & 0.8 & 0.8 \\
\hline $1 \mathrm{~B}$ & S & $1 / 13 / 1994$ & 94013 & 13 & 1.2 & 1.2 & 0.8 & 1.2 & 1.2 \\
\hline $1 \mathrm{~B}$ & $S$ & $1 / 14 / 1994$ & 94014 & 14 & 0.8 & 1.2 & 0.8 & 0.8 & 0.8 \\
\hline $0 \mathrm{~B}$ & $S$ & 1/15/1994 & 94015 & 15 & 1.2 & 0.8 & 0.8 & 1.2 & 0.8 \\
\hline $0 \mathrm{~B}$ & S & $1 / 16 / 1994$ & 94016 & 16 & 0.8 & 1.2 & 0.8 & 1.2 & 0.8 \\
\hline $1 \mathrm{~B}$ & $S$ & $1 / 17 / 1994$ & 94017 & 17 & 0.8 & 0.8 & 1.2 & 0.8 & 0.8 \\
\hline $1 \mathrm{~B}$ & S & $1 / 18 / 1994$ & 94018 & 18 & 0.8 & 1.2 & 1.2 & 0.8 & 0.8 \\
\hline $1 \mathrm{~B}$ & $S$ & $1 / 19 / 1994$ & 94019 & 19 & 0.8 & 1.2 & 0.8 & 0.8 & 1.2 \\
\hline $1 \mathrm{~B}$ & $S$ & $1 / 20 / 1994$ & 94020 & 20 & 0.8 & 0.8 & 1.2 & 0.8 & 0.8 \\
\hline $1 \mathrm{~B}$ & S & $1 / 21 / 1994$ & 94021 & 21 & 1.2 & 0.8 & 1.2 & 1.2 & 0.8 \\
\hline $0 \mathrm{~B}$ & S & $1 / 22 / 1994$ & 94022 & 22 & 0.8 & 1.2 & 0.8 & 1.2 & 0.8 \\
\hline $0 \mathrm{~B}$ & $S$ & $1 / 23 / 1994$ & 94023 & 23 & 0.8 & 1.2 & 0.8 & 0.8 & 0.8 \\
\hline $1 \mathrm{~B}$ & S & $1 / 24 / 1994$ & 94024 & 24 & 0.8 & 0.8 & 1.2 & 0.8 & 0.8 \\
\hline $1 \mathrm{~B}$ & $S$ & $1 / 25 / 1994$ & 94025 & 25 & 1.2 & 0.8 & 0.8 & 0.8 & 1.2 \\
\hline
\end{tabular}




\begin{tabular}{|c|c|c|c|c|c|c|c|c|c|}
\hline $1 \mathrm{E}$ & $S$ & $1 / 26 / 1994$ & 94026 & 26 & 0.8 & 1.2 & 0.8 & 1.2 & 0.8 \\
\hline $1 \mathrm{E}$ & $S$ & $1 / 27 / 1994$ & 94027 & 27 & 0.8 & 1.2 & 0.8 & 1.2 & 1.2 \\
\hline $1 \mathrm{E}$ & $S$ & $1 / 28 / 1994$ & 94028 & 28 & 48.4 & 48.4 & 48 & 47.6 & 47.6 \\
\hline $0 \mathrm{E}$ & $S$ & $1 / 29 / 1994$ & 94029 & 29 & 0.8 & 0.8 & 1.2 & 0.8 & 0.8 \\
\hline $0 \mathrm{E}$ & $S$ & $1 / 30 / 1994$ & 94030 & 30 & 0.8 & 0.8 & 0.8 & 0.8 & 0.8 \\
\hline $1 \mathrm{E}$ & $S$ & $1 / 31 / 1994$ & 94031 & 31 & 0.8 & 0.8 & 0.8 & 0.8 & 1.2 \\
\hline $1 \mathrm{E}$ & $S$ & $2 / 1 / 1994$ & 94032 & 32 & 1.2 & 0.8 & 1.2 & 1.2 & 1.2 \\
\hline $1 \mathrm{E}$ & $S$ & $2 / 2 / 1994$ & 94033 & 33 & 0.8 & 0.8 & 1.2 & 0.8 & 0.8 \\
\hline $1 \mathrm{~F}$ & $S$ & 2/3/1994 & 94034 & 34 & 0.8 & 1.2 & 0.8 & 1.2 & 0.8 \\
\hline $1 \mathrm{E}$ & $S$ & 2/4/1994 & 94035 & 35 & 1.2 & 1.2 & 1.2 & 1.2 & 1.2 \\
\hline $0 \mathrm{E}$ & $S$ & 2/5/1994 & 94036 & 36 & 1.2 & 1.2 & 1.2 & 1.2 & 1.2 \\
\hline $0 \mathrm{E}$ & $S$ & 2/6/1994 & 94037 & 37 & 1.2 & 1.2 & 1.2 & 1.2 & 1.2 \\
\hline $1 \mathrm{E}$ & $S$ & 2/7/1994 & 94038 & 38 & 1.2 & 1.2 & 1.2 & 1.2 & 1.2 \\
\hline $1 \mathrm{E}$ & $\mathrm{S}$ & 2/8/1994 & 94039 & 39 & 1.2 & 1.2 & 1.2 & 1.2 & 1.2 \\
\hline $1 \mathrm{E}$ & $S$ & 2/9/1994 & 94040 & 40 & 1.2 & 1.6 & 1.2 & 1.2 & 1.2 \\
\hline $1 \mathrm{E}$ & $S$ & 2/10/1994 & 94041 & 41 & 1.2 & 1.6 & 1.2 & 1.2 & 1.2 \\
\hline $1 \mathrm{E}$ & $S$ & $2 / 11 / 1994$ & 94042 & 42 & 1.2 & 1.2 & 0.8 & 1.2 & 1.2 \\
\hline $0 \mathrm{E}$ & $S$ & $2 / 12 / 1994$ & 94043 & 43 & 1.2 & 0.8 & 1.2 & 1.2 & 0.8 \\
\hline $0 \mathrm{E}$ & $S$ & 2/13/1994 & 94044 & 44 & 1.2 & 1.2 & 1.2 & 1.2 & 1.6 \\
\hline $1 \mathrm{E}$ & $\mathrm{S}$ & 2/14/1994 & 94045 & 45 & 1.2 & 1.2 & 1.2 & 1.2 & 1.2 \\
\hline $1 \mathrm{E}$ & $S$ & $2 / 15 / 1994$ & 94046 & 46 & 1.2 & 1.2 & 1.2 & 1.2 & 1.2 \\
\hline $1 \mathrm{E}$ & $S$ & 2/16/1994 & 94047 & 47 & 1.2 & 1.2 & 1.2 & 1.2 & 1.2 \\
\hline $1 \mathrm{E}$ & $S$ & $2 / 17 / 1994$ & 94048 & 48 & 1.2 & 1.2 & 1.2 & 1.2 & 1.2 \\
\hline $1 \mathrm{E}$ & $S$ & 2/18/1994 & 94049 & 49 & 1.2 & 1.2 & 1.2 & 1.2 & 1.2 \\
\hline $0 \mathrm{E}$ & $S$ & 2/19/1994 & 94050 & 50 & 1.2 & 1.2 & 1.2 & 1.2 & 1.2 \\
\hline $0 \mathrm{E}$ & $\mathrm{S}$ & 2/20/1994 & 94051 & 51 & 1.2 & 1.2 & 1.2 & 1.2 & 1.2 \\
\hline $1 \mathrm{E}$ & NS & $2 / 21 / 1994$ & 94052 & 52 & 1.2 & 1.2 & 1.2 & 1.2 & 1.2 \\
\hline $1 \mathrm{~F}$ & S & 2/22/1994 & 94053 & 53 & 1.2 & 1.2 & 1.2 & 1.2 & 1.2 \\
\hline $1 \mathrm{E}$ & $S$ & 2/23/1994 & 94054 & 54 & 1.2 & 1.2 & 1.2 & 1.2 & 1.2 \\
\hline $1 \mathrm{E}$ & $S$ & $2 / 24 / 1994$ & 94055 & 55 & 1.2 & 1.2 & 1.2 & 1.2 & 1.2 \\
\hline $1 \mathrm{~F}$ & $S$ & 2/25/1994 & 94056 & 56 & 1.6 & 1.2 & 1.2 & 1.2 & 0.8 \\
\hline $0 \mathrm{E}$ & $S$ & 2/26/1994 & 94057 & 57 & 1.2 & 1.2 & 1.6 & 1.2 & 1.2 \\
\hline $0 \mathrm{E}$ & $\mathrm{S}$ & 2/27/1994 & 94058 & 58 & 1.2 & 1.2 & 1.2 & 1.2 & 1.2 \\
\hline $1 \mathrm{~F}$ & $S$ & 2/28/1994 & 94059 & 59 & 1.2 & 1.2 & 1.2 & 1.2 & 1.2 \\
\hline $1 \mathrm{E}$ & $S$ & $3 / 1 / 1994$ & 94060 & 60 & 0.8 & 1.2 & 1.2 & 1.2 & 1.2 \\
\hline $1 \mathrm{E}$ & $\mathrm{S}$ & 3/2/1994 & 94061 & 61 & 1.6 & 1.2 & 1.2 & 1.2 & 1.2 \\
\hline $1 \mathrm{E}$ & $S$ & $3 / 3 / 1994$ & 94062 & 62 & 1.2 & 0.8 & 1.2 & 1.2 & 1.2 \\
\hline $1 \mathrm{~F}$ & $S$ & $3 / 4 / 1994$ & 94063 & 63 & 1.2 & 1.2 & 1.2 & 1.2 & 1.2 \\
\hline $0 \mathrm{E}$ & $\mathrm{S}$ & 3/5/1994 & 94064 & 64 & 1.2 & 1.2 & 1.2 & 1.2 & 1.2 \\
\hline $0 \mathrm{E}$ & $\mathrm{S}$ & 3/6/1994 & 94065 & 65 & 0.8 & 1.2 & 1.2 & 1.2 & 1.2 \\
\hline $1 \mathrm{~F}$ & $S$ & $3 / 7 / 1994$ & 94066 & 66 & 1.2 & 1.2 & 1.2 & 1.2 & 1.2 \\
\hline $1 \mathrm{E}$ & $S$ & $3 / 8 / 1994$ & 94067 & 67 & 1.2 & 1.2 & 1.2 & 1.2 & 1.2 \\
\hline $1 \mathrm{E}$ & $S$ & $3 / 9 / 1994$ & 94068 & 68 & 1.2 & 1.2 & 1.2 & 1.6 & 1.6 \\
\hline $1 \mathrm{E}$ & $S$ & 3/10/1994 & 94069 & 69 & 1.6 & 1.2 & 1.2 & 1.2 & 1.6 \\
\hline $1 \mathrm{E}$ & NS & 3/11/1994 & 94070 & 70 & 1.6 & 1.2 & 1.2 & 1.6 & 1.2 \\
\hline $0 \mathrm{~B}$ & NS & $3 / 12 / 1994$ & 94071 & 71 & 1.2 & 1.2 & 1.6 & 1.2 & 1.2 \\
\hline $0 \mathrm{~F}$ & NS & 3/13/1994 & 94072 & 72 & 1.6 & 1.2 & 1.6 & 1.2 & 1.2 \\
\hline $1 \mathrm{E}$ & NS & $3 / 14 / 1994$ & 94073 & 73 & 1.6 & 1.6 & 1.6 & 1.2 & 1.6 \\
\hline $1 \mathrm{E}$ & NS & $3 / 15 / 1994$ & 94074 & 74 & 1.2 & 1.6 & 1.2 & 1.6 & 1.6 \\
\hline $1 \mathrm{E}$ & NS & 3/16/1994 & 94075 & 75 & 1.6 & 2 & 1.6 & 1.6 & 2 \\
\hline $1 \mathrm{E}$ & NS & 3/17/1994 & 94076 & 76 & 1.6 & 1.6 & 1.6 & 1.6 & 1.6 \\
\hline
\end{tabular}




\begin{tabular}{|c|c|c|c|c|c|c|c|c|c|}
\hline $1 \mathrm{~B}$ & NS & 3/18/1994 & 94077 & 77 & 2 & 1.6 & 2 & 2 & 1.6 \\
\hline $0 \mathrm{~B}$ & NS & 3/19/1994 & 94078 & 78 & 1.6 & 2 & 1.6 & 1.6 & 1.6 \\
\hline 0 B & NS & $3 / 20 / 1994$ & 94079 & 79 & 1.6 & 1.6 & 1.6 & 1.6 & 1.6 \\
\hline $1 \mathrm{~B}$ & $S$ & 3/21/1994 & 94080 & 80 & 1.6 & 1.6 & 1.6 & 1.6 & 1.6 \\
\hline $1 \mathrm{~B}$ & $S$ & $3 / 22 / 1994$ & 94081 & 81 & 1.6 & 1.6 & 1.2 & 1.6 & 1.6 \\
\hline $1 \mathrm{~B}$ & $S$ & 3/23/1994 & 94082 & 82 & 1.6 & 1.6 & 1.6 & 1.2 & 1.6 \\
\hline $1 \mathrm{~B}$ & $S$ & 3/24/1994 & 94083 & 83 & 1.6 & 1.6 & 1.6 & 1.6 & 1.6 \\
\hline $1 \mathrm{~B}$ & $S$ & $3 / 25 / 1994$ & 94084 & 84 & 1.6 & 1.6 & 1.6 & 1.6 & 1.6 \\
\hline $0 \mathrm{~B}$ & $S$ & 3/26/1994 & 94085 & 85 & 1.6 & 1.6 & 1.6 & 1.6 & 1.6 \\
\hline $0 \mathrm{~B}$ & $S$ & $3 / 27 / 1994$ & 94086 & 86 & 1.6 & 1.6 & 1.6 & 1.6 & 2 \\
\hline $1 \mathrm{~B}$ & $S$ & $3 / 28 / 1994$ & 94087 & 87 & 1.6 & 1.6 & 2 & 1.6 & 1.6 \\
\hline $1 \mathrm{~B}$ & $S$ & $3 / 29 / 1994$ & 94088 & 88 & 1.6 & 1.6 & 1.6 & 1.6 & 1.6 \\
\hline $1 \mathrm{~B}$ & $S$ & 3/30/1994 & 94089 & 89 & 2 & 1.6 & 1.6 & 1.6 & 1.6 \\
\hline $1 \mathrm{~B}$ & $\mathrm{~S}$ & 3/31/1994 & 94090 & 90 & 1.6 & 2 & 1.6 & 1.6 & 2 \\
\hline $1 \mathrm{~B}$ & NS & 4/1/1994 & 94091 & 91 & 2 & 1.6 & 1.6 & 1.6 & 1.6 \\
\hline $\begin{array}{lll}0 & B \\
\end{array}$ & NS & $4 / 2 / 1994$ & 94092 & 92 & 2 & 1.6 & 1.6 & 1.6 & 1.6 \\
\hline $0 \mathrm{~B}$ & NS & 4/3/1994 & 94093 & 93 & 2 & 1.6 & 1.6 & 1.6 & 1.6 \\
\hline $1 \mathrm{~B}$ & NS & $4 / 4 / 1994$ & 94094 & 94 & 1.6 & 2 & 1.6 & 1.6 & 1.6 \\
\hline $1 \mathrm{~B}$ & $\mathrm{~S}$ & $4 / 5 / 1994$ & 94095 & 95 & 2 & 1.6 & 2 & 1.6 & 2 \\
\hline $1 \mathrm{~B}$ & $S$ & 4/6/1994 & 94096 & 96 & 2 & 1.6 & 2 & 2 & 1.6 \\
\hline $1 \mathrm{~B}$ & $S$ & $4 / 7 / 1994$ & 94097 & 97 & 2 & 1.6 & 2 & 2 & 2 \\
\hline $1 \mathrm{~B}$ & $S$ & $4 / 8 / 1994$ & 94098 & 98 & & & & & \\
\hline $0 \mathrm{~B}$ & $S$ & 4/9/1994 & 94099 & 99 & & & & & \\
\hline $0 \mathrm{~B}$ & $S$ & 4/10/1994 & 94100 & 100 & & & & & \\
\hline $1 \mathrm{~B}$ & $S$ & $4 / 11 / 1994$ & 94101 & 101 & & & & & \\
\hline $1 \mathrm{~B}$ & $S$ & $4 / 12 / 1994$ & 94102 & 102 & 0.4 & 0.4 & 0.8 & & 0.8 \\
\hline $1 \mathrm{~B}$ & $S$ & 4/13/1994 & 94103 & 103 & & 0.8 & 0.4 & 0.4 & 0.4 \\
\hline $1 \mathrm{~B}$ & $S$ & $4 / 14 / 1994$ & 94104 & 104 & 0.4 & 0.4 & 0.4 & 0.4 & 0.4 \\
\hline $1 \mathrm{~B}$ & $S$ & $4 / 15 / 1994$ & 94105 & 105 & 0.4 & 0.4 & 0.4 & 0.4 & 0.4 \\
\hline $0 \mathrm{~B}$ & $S$ & $4 / 16 / 1994$ & 94106 & 106 & 0.4 & 0.4 & 0.8 & 0.4 & 0.4 \\
\hline $0 \mathrm{~B}$ & $S$ & $4 / 17 / 1994$ & 94107 & 107 & 0.4 & 0.4 & 0.4 & 0.8 & 0.4 \\
\hline $1 \mathrm{~B}$ & $S$ & 4/18/1994 & 94108 & 108 & 0.8 & 0.4 & 0.4 & 0.4 & 0.8 \\
\hline $1 \mathrm{~B}$ & $\mathrm{~S}$ & 4/19/1994 & 94109 & 109 & 0.8 & & 0.8 & 0.4 & 0.4 \\
\hline $1 \mathrm{~B}$ & $S$ & $4 / 20 / 1994$ & 94110 & 110 & 0.4 & 0.4 & 0.4 & 0.4 & 0.4 \\
\hline $1 \mathrm{~B}$ & $S$ & $4 / 21 / 1994$ & 94111 & 111 & 0.4 & 0.4 & 0.4 & 0.4 & 0.4 \\
\hline $1 \mathrm{~B}$ & $S$ & $4 / 22 / 1994$ & 94112 & 112 & 0.4 & 0.4 & 0.4 & 0.4 & 0.8 \\
\hline $0 \mathrm{~B}$ & $S$ & 4/23/1994 & 94113 & 113 & 0.4 & 0.4 & 0.4 & 0.8 & 0.4 \\
\hline $0 \mathrm{~B}$ & $S$ & $4 / 24 / 1994$ & 94114 & 114 & 0.4 & 0.4 & 0.4 & 0.4 & 0.8 \\
\hline $1 \mathrm{~B}$ & $S$ & $4 / 25 / 1994$ & 94115 & 115 & 0.8 & & 0.4 & 0.8 & 0.4 \\
\hline $1 \mathrm{~B}$ & $S$ & $4 / 26 / 1994$ & 94116 & 116 & 0.4 & 0.4 & 0.4 & 0.4 & 0.4 \\
\hline $1 \mathrm{~B}$ & $S$ & $4 / 27 / 1994$ & 94117 & 117 & 0.8 & 0.4 & 0.8 & 0.4 & 0.4 \\
\hline $1 \mathrm{~B}$ & $S$ & $4 / 28 / 1994$ & 94118 & 118 & 0.8 & 0.4 & 0.4 & 0.4 & 0.4 \\
\hline $1 \mathrm{~B}$ & $S$ & 4/29/1994 & 94119 & 119 & 0.4 & 0.4 & 0.4 & 0.4 & 0.4 \\
\hline $0 \mathrm{~B}$ & $S$ & 4/30/1994 & 94120 & 120 & 0.4 & 0.8 & 0.4 & 0.8 & 0.4 \\
\hline $0 \mathrm{~B}$ & $S$ & 5/1/1994 & 94121 & 121 & 0.4 & 0.4 & 0.4 & 0.4 & 0.8 \\
\hline $1 \mathrm{~B}$ & $S$ & $5 / 2 / 1994$ & 94122 & 122 & 0.4 & 0.4 & 0.4 & 0.4 & 0.8 \\
\hline $1 \mathrm{~B}$ & $S$ & 5/3/1994 & 94123 & 123 & 0.4 & 0.4 & 0.4 & 0.4 & 0.4 \\
\hline $1 \mathrm{~B}$ & $S$ & $5 / 4 / 1994$ & 94124 & 124 & 0.4 & 0.8 & 0.4 & 0.4 & 0.4 \\
\hline $1 \mathrm{~B}$ & $S$ & $5 / 5 / 1994$ & 94125 & 125 & 0.8 & 0.4 & 0.4 & 0.4 & 0.4 \\
\hline $1 \mathrm{~B}$ & $S$ & 5/6/1994 & 94126 & 126 & 0.4 & 0.4 & 0.4 & 0.4 & 0.4 \\
\hline $0 \mathrm{~B}$ & $S$ & 5/7/1994 & 94127 & 127 & 0.8 & 0.4 & 0.4 & 0.4 & 0.4 \\
\hline
\end{tabular}




\begin{tabular}{|c|c|c|c|c|c|c|c|c|c|c|}
\hline \begin{tabular}{l|l}
$0 \mathrm{E}$ \\
\end{tabular} & $B$ & $S$ & $5 / 8 / 1994$ & 94128 & 128 & 0.4 & 0.8 & 0.4 & 0.4 & 0.8 \\
\hline $1 \mathrm{E}$ & $\mathrm{B}$ & $S$ & $5 / 9 / 1994$ & 94129 & 129 & 0.4 & 0.8 & 0.8 & 0.4 & 0.8 \\
\hline $1 \mathrm{E}$ & $\mathrm{B}$ & $S$ & $5 / 10 / 1994$ & 94130 & 130 & 0.4 & 0.8 & 0.4 & 0.8 & 0.4 \\
\hline $1 \mathrm{E}$ & B & $S$ & $5 / 11 / 1994$ & 94131 & 131 & 0.8 & 0.4 & 0.8 & 0.4 & 0.8 \\
\hline $1 \mathrm{E}$ & $\mathrm{B}$ & S & $5 / 12 / 1994$ & 94132 & 132 & 0.8 & 0.4 & 0.8 & 0.4 & 0.4 \\
\hline $1 \mathrm{E}$ & $\mathrm{B}$ & $S$ & $5 / 13 / 1994$ & 94133 & 133 & 0.8 & 0.4 & 0.4 & 0.4 & 0.8 \\
\hline $0 \mathrm{E}$ & B & $\mathrm{S}$ & $5 / 14 / 1994$ & 94134 & 134 & 0.8 & 0.4 & 0.4 & 0.4 & 0.4 \\
\hline $0 \mathrm{E}$ & $\mathrm{B}$ & $S$ & $5 / 15 / 1994$ & 94135 & 135 & 0.4 & 0.4 & 0.4 & 0.4 & 0.4 \\
\hline $1 \mathrm{E}$ & B & $\mathrm{S}$ & $5 / 16 / 1994$ & 94136 & 136 & 0.8 & 0.4 & 0.4 & 0.4 & 0.4 \\
\hline $1 \mathrm{E}$ & B & $S$ & $5 / 17 / 1994$ & 94137 & 137 & 0.8 & 0.4 & 0.8 & 0.4 & 0.8 \\
\hline $1 \mathrm{~F}$ & B & $\mathrm{S}$ & $5 / 18 / 1994$ & 94138 & 138 & 0.8 & 0.4 & 0.8 & 0.8 & 0.4 \\
\hline $1 \mathrm{E}$ & B & $\mathrm{S}$ & 5/19/1994 & 94139 & 139 & 0.8 & 0.8 & 0.8 & 0.4 & 0.8 \\
\hline $1 \mathrm{~B}$ & $\mathrm{~B}$ & $\mathrm{~S}$ & $5 / 20 / 1994$ & 94140 & 140 & 0.4 & 0.8 & 0.8 & 0.4 & 0.8 \\
\hline \begin{tabular}{l|l}
0 & $B$ \\
\end{tabular} & B & $S$ & $5 / 21 / 1994$ & 94141 & 141 & 0.4 & 0.8 & 0.4 & 0.8 & 0.4 \\
\hline $0 \mathrm{~B}$ & B & $\mathrm{S}$ & $5 / 22 / 1994$ & 94142 & 142 & 0.8 & 0.4 & 0.4 & 0.8 & 0.8 \\
\hline $1 \mathrm{~B}$ & B & $\mathrm{S}$ & $5 / 23 / 1994$ & 94143 & 143 & 0.8 & 0.4 & 0.8 & 0.8 & 0.4 \\
\hline \begin{tabular}{l|l}
$1 \mathrm{~B}$ \\
\end{tabular} & B & $\mathrm{S}$ & $5 / 24 / 1994$ & 94144 & 144 & 0.4 & 0.8 & 0.4 & 0.8 & 0.8 \\
\hline $1 \mathrm{~B}$ & $\mathrm{~B}$ & $S$ & $5 / 25 / 1994$ & 94145 & 145 & 0.4 & 0.8 & 0.4 & 0.8 & 0.4 \\
\hline $1 \mathrm{~B}$ & $\mathrm{~B}$ & $S$ & $5 / 26 / 1994$ & 94146 & 146 & 0.8 & 0.8 & 0.4 & 0.8 & 0.4 \\
\hline \begin{tabular}{l|l}
1 & $B$ \\
\end{tabular} & B & $S$ & $5 / 27 / 1994$ & 94147 & 147 & 0.8 & 0.4 & 0.8 & 0.4 & 0.8 \\
\hline $\begin{array}{lll}0 & \mathrm{~B} \\
\end{array}$ & $\mathrm{~B}$ & NS & $5 / 28 / 1994$ & 94148 & 148 & 0.4 & 0.8 & 0.8 & 0.4 & 0.8 \\
\hline $0 \mathrm{~B}$ & $\mathrm{~B}$ & NS & $5 / 29 / 1994$ & 94149 & 149 & 0.8 & 0.4 & 0.8 & 0.8 & 0.4 \\
\hline \begin{tabular}{l|l}
$1 \mathrm{~B}$ \\
\end{tabular} & B & NS & $5 / 30 / 1994$ & 94150 & 150 & 0.4 & 0.8 & 0.8 & 0.8 & 0.4 \\
\hline \begin{tabular}{l|l}
1 & $B$ \\
\end{tabular} & B & NS & $5 / 31 / 1994$ & 94151 & 151 & 0.4 & 0.8 & 0.8 & 0.4 & 0.8 \\
\hline $1 \mathrm{~B}$ & B & NS & 6/1/1994 & 94152 & 152 & 0.4 & 0.8 & 0.4 & 0.8 & 0.8 \\
\hline $1 \mathrm{~B}$ & B & NS & 6/2/1994 & 94153 & 153 & 0.8 & 0.4 & 0.8 & 0.8 & 0.4 \\
\hline $1 \mathrm{~B}$ & $\mathrm{~B}$ & NS & 6/3/1994 & 94154 & 154 & 0.4 & 0.8 & 0.8 & 0.4 & 0.8 \\
\hline \begin{tabular}{l|l}
0 & $\mathrm{~B}$ \\
\end{tabular} & $\mathrm{~B}$ & NS & 6/4/1994 & 94155 & 155 & 0.4 & 0.8 & 0.4 & 0.8 & 0.4 \\
\hline & & & & & & & & & & \\
\hline & & & & & \multicolumn{2}{|c|}{ sort S/NS - 0/1 - A/B } & & & & \\
\hline & & & & & \multicolumn{2}{|c|}{ Daily Averages } & & & & \\
\hline & & & & & $1-A-S$ & 0 & 0 & 0 & 0 & 0 \\
\hline & & & & & $1-B-S$ & 0.604932 & 0.585205 & 0.568767 & 0.589617 & 0.567493 \\
\hline & & & & & $0-A-S$ & 0.525714 & 0.529577 & 0.519626 & 0.52028 & 0.505028 \\
\hline & & & & & $0-B-S$ & 0.49083 & 0.492035 & 0.467257 & 0.478603 & 0.506087 \\
\hline & & & & & 1-A-NS & 0.491139 & 0.538462 & 0.488312 & 0.501266 & 0.515 \\
\hline & & & & & 1-B-NS & 1.106818 & 1.124571 & 1.084091 & 1.081818 & 1.049143 \\
\hline & & & & & $0-\mathrm{A}-\mathrm{NS}$ & 1.152941 & 1.141176 & 1.164706 & 1.152941 & 1.141176 \\
\hline & & & & & 0-B-NS & 0.968182 & 0.937931 & 0.934831 & 0.922727 & 0.948315 \\
\hline \multicolumn{11}{|c|}{ sort JDAY } \\
\hline \multicolumn{4}{|c|}{ Table of Monthly Consumption } & & \multicolumn{2}{|c|}{ sort S/NS - 0/1 - A/B } & & & & \\
\hline & & & & & \multicolumn{4}{|c|}{ Standard Deviation of Daily Averages } & & \\
\hline & & Jun 91 & 0 & 0 & & & & & & \\
\hline & & Jul & 0 & 0 & 1-A-S & 0 & 0 & 0 & 0 & 0 \\
\hline & & Aug & 0 & 0 & 1-B-S & 0.29849 & 0.262491 & 0.259352 & 0.276973 & 0.256067 \\
\hline & & Sep & 0 & 0 & $0-\mathrm{A}-\mathrm{S}$ & 0.188403 & 0.188524 & 0.184001 & 0.18407 & 0.176506 \\
\hline & & Oct & 17072 & 17072.2 & $0-\mathrm{B}-\mathrm{S}$ & 0.167943 & 0.168729 & 0.149929 & 0.159291 & 0.176965 \\
\hline & & Nov & 5118 & 5117.6 & 1-A-NS & 0.16885 & 0.191529 & 0.166997 & 0.175041 & 0.182181 \\
\hline & & Dec & 4642 & 4642 & 1-B-NS & 3.592684 & 3.603163 & 3.566219 & 3.534856 & 3.546876 \\
\hline & & Jan 92 & 1225 & 1224.8 & $0-\mathrm{A}-\mathrm{NS}$ & 0.191066 & 0.200178 & 0.151522 & 0.130814 & 0.174282 \\
\hline & & Feb & 5518 & 5518 & 0-B-NS & 0.561514 & 0.534668 & 0.53917 & 0.529959 & 0.543999 \\
\hline
\end{tabular}




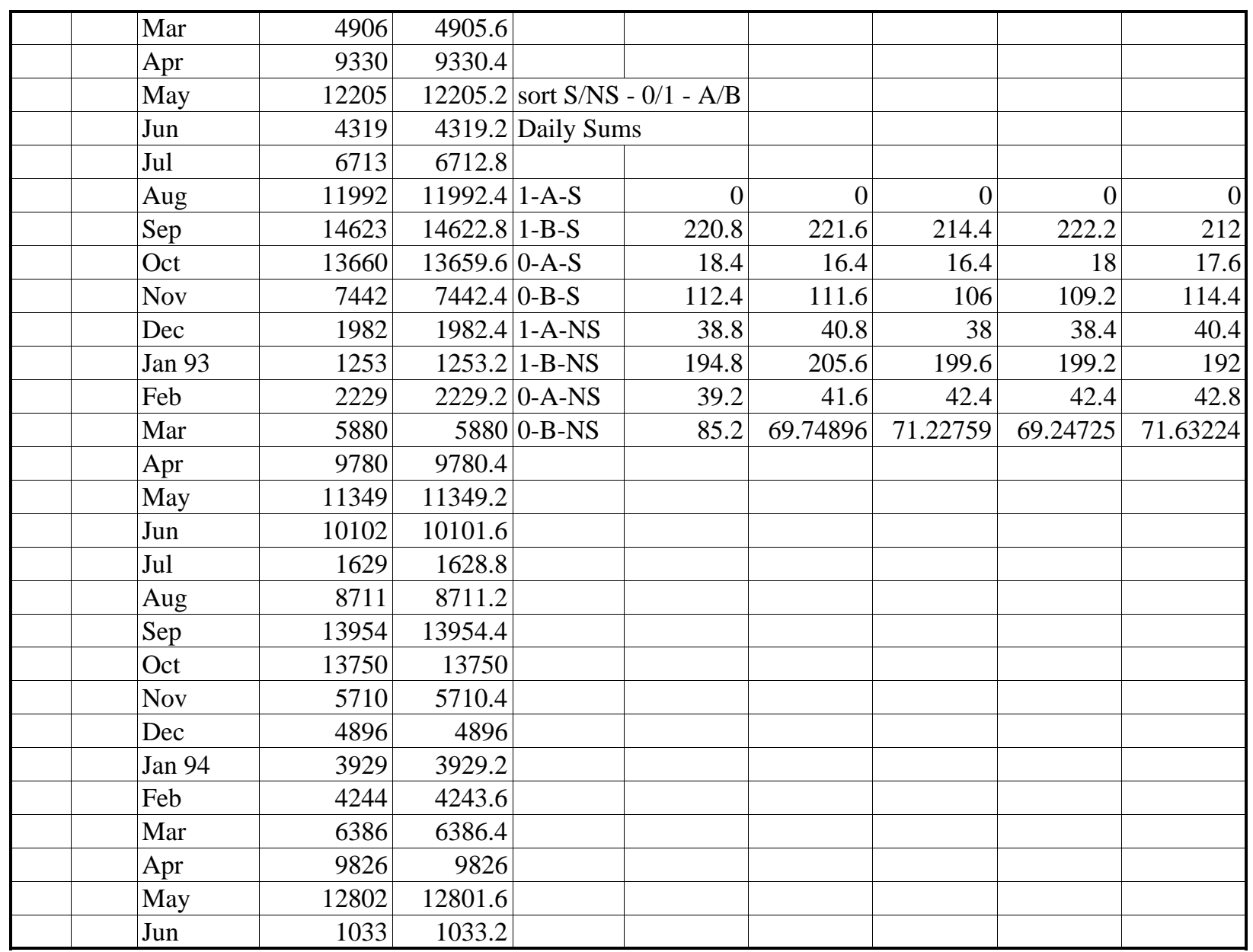


chlr

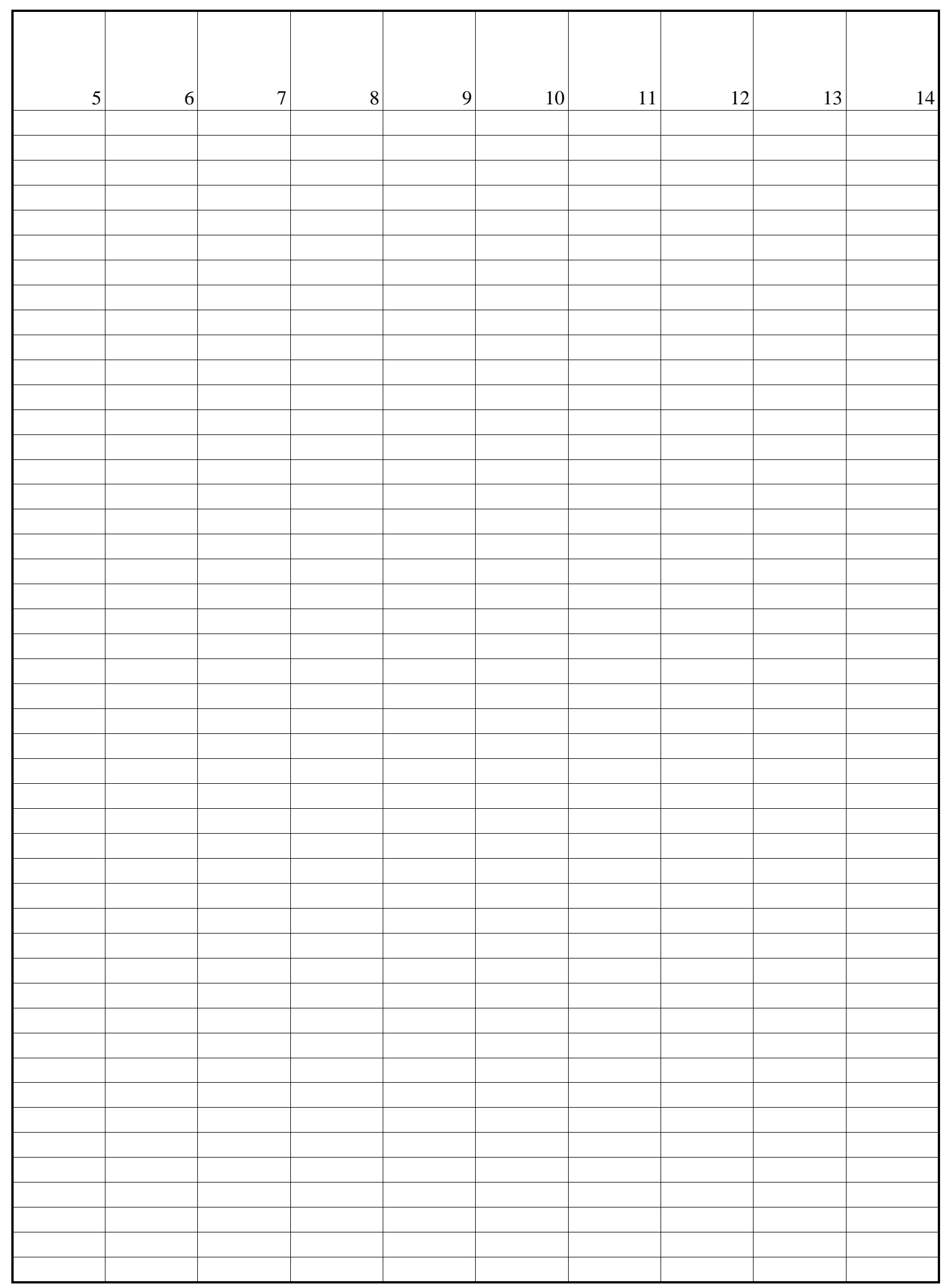

Page 24 


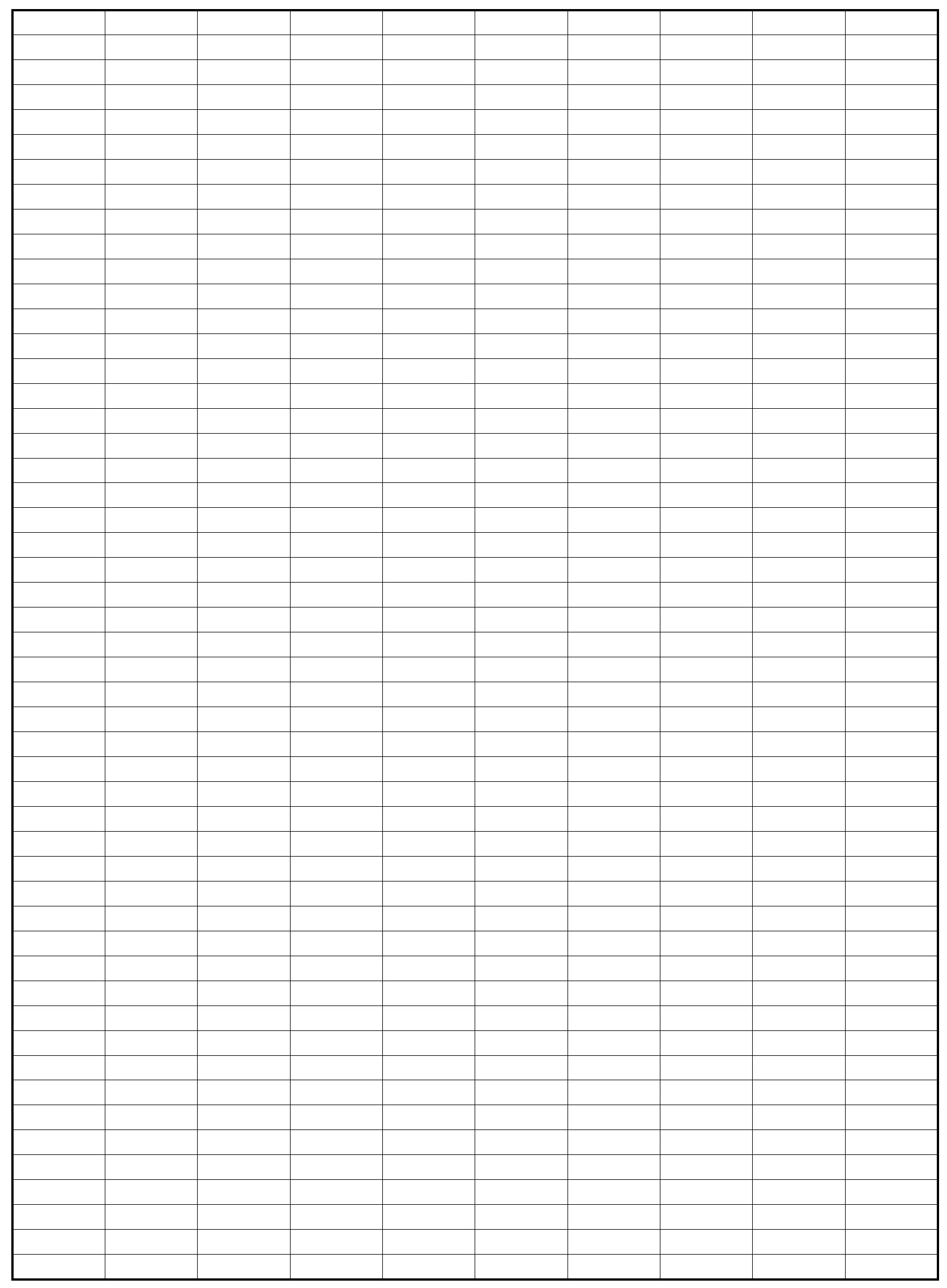

Page 25 


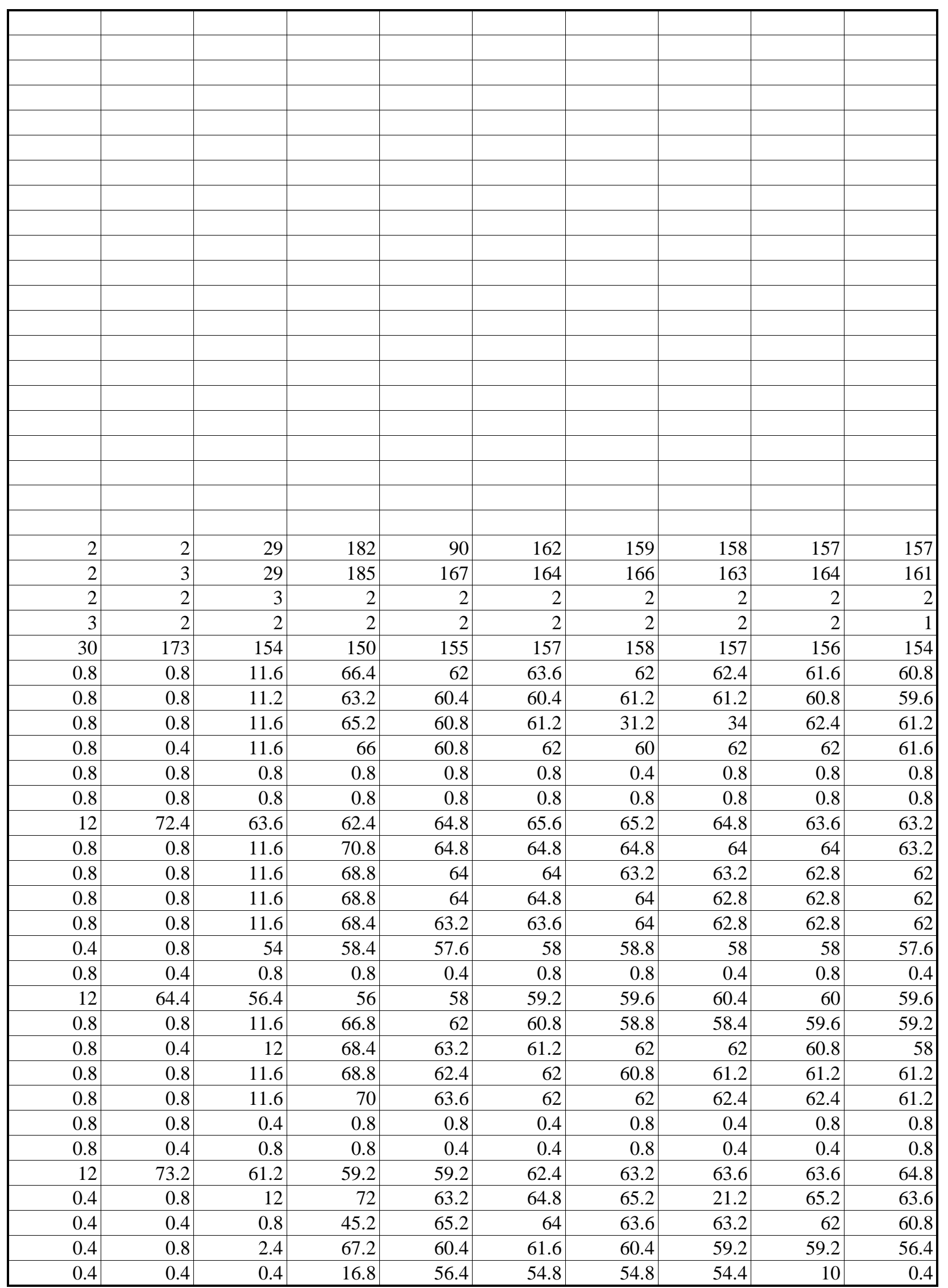




\begin{tabular}{|c|c|c|c|c|c|c|c|c|c|}
\hline 0.4 & 0.4 & 0.8 & 0.4 & 0.4 & 0.8 & 0.4 & 0.4 & 0.8 & 0.4 \\
\hline 0.8 & 0.4 & 0.8 & 0.4 & 0.4 & 0.8 & 0.4 & 0.4 & 0.8 & 0.4 \\
\hline 0.4 & 0.4 & 0.8 & 0.4 & 0.4 & 0.4 & 0.8 & 0.4 & 0.4 & 0.8 \\
\hline 0.8 & 0.4 & 0.8 & 0.4 & 0.4 & 0.8 & 0.4 & 0.8 & 0.4 & 0.8 \\
\hline 0.8 & 0.4 & 0.4 & 0.8 & 0.4 & 0.8 & 0.4 & 0.8 & 0.4 & 0.4 \\
\hline 0.4 & 0.4 & 0.4 & 0.8 & 51.6 & 61.6 & 60 & 59.6 & 59.2 & 58 \\
\hline 0.4 & 0.4 & 0.8 & 0.4 & 0.4 & 0.8 & 0.4 & 0.4 & 0.8 & 0.4 \\
\hline 0.4 & 0.4 & 0.8 & 0.4 & 0.8 & 0.4 & 0.4 & 0.8 & 0.4 & 0.4 \\
\hline 0.8 & 0.4 & 0.8 & 0.4 & 0.4 & 0.8 & 0.4 & 0.8 & 0.4 & 0.4 \\
\hline 0.8 & 0.4 & 0.8 & 0.4 & 0.8 & 0.4 & 2.8 & 4.4 & 6.4 & 5.2 \\
\hline 0.4 & 0.8 & 0.4 & 1.6 & 0.8 & 0.4 & 1.6 & 0.4 & 0.4 & 0.8 \\
\hline 0.4 & 0.4 & 0.4 & 0.8 & 0.4 & 0.4 & 0.4 & 1.6 & 1.2 & 4.4 \\
\hline 0.4 & 0.4 & 0.8 & 4.4 & 72.4 & 26.4 & 75.2 & 66.8 & 62.8 & 61.6 \\
\hline 0.4 & 0.4 & 0.4 & 61.6 & 62.8 & 62.8 & 61.2 & 60.8 & 61.2 & 59.6 \\
\hline 0.4 & 0.4 & 0.4 & 0.8 & 0.4 & 0.4 & 0.4 & 0.4 & 0.4 & 0.4 \\
\hline 0.4 & 0.4 & 0.4 & 0.4 & 0.4 & 0.8 & 0.4 & 0.4 & 0.4 & 0.4 \\
\hline 2.8 & 64.4 & 55.6 & 54 & 55.6 & 57.6 & 57.2 & 58.4 & 58.8 & 58 \\
\hline 0.4 & 0.4 & 2.8 & 64.8 & 58 & 58.4 & 58.4 & 59.2 & 59.6 & 59.2 \\
\hline 0.4 & 0.4 & 2.8 & 5.2 & 0.8 & 65.2 & 58.8 & 58 & 58 & 56 \\
\hline 0.4 & 0.8 & 2.4 & 58.4 & 54 & 54.4 & 55.6 & 54.8 & 54.4 & 54 \\
\hline 0.8 & 0.4 & 2.4 & 8.4 & 0.4 & 52.4 & 48 & 0.4 & 0.4 & 0.4 \\
\hline 0.4 & 0.8 & 0.4 & 0.4 & 0.4 & 0.8 & 0.4 & 0.4 & 0.4 & 0.4 \\
\hline 0.4 & 0.4 & 0.4 & 0.4 & 0.4 & 0.8 & 0.4 & 0.4 & 0.4 & 0.4 \\
\hline 0.4 & 0.4 & 0.4 & 0.4 & 0.8 & 0.4 & 0.4 & 0.4 & 0.4 & 0.4 \\
\hline 0.4 & 0.8 & 0.4 & 0.4 & 43.2 & 57.6 & 56.8 & 16.8 & 60.4 & 56 \\
\hline 0.4 & 0.4 & 0.4 & 50 & 56.4 & 58 & 58 & 58.4 & 56.8 & 21.6 \\
\hline 0.4 & 0.4 & 0.4 & 0.4 & 0.4 & 0.8 & 0.4 & 0.4 & 0.4 & 0.4 \\
\hline 0.8 & 0.4 & 0.4 & 0.4 & 0.4 & 0.8 & 0.4 & 0.4 & 0.4 & 0.4 \\
\hline 0.4 & 0.4 & 0.8 & 0.4 & 0.4 & 0.4 & 0.4 & 0.8 & 0.4 & 0.4 \\
\hline 0.4 & 0.4 & 0.8 & 0.4 & 0.4 & 0.8 & 0.4 & 0.4 & 0.4 & 0.8 \\
\hline 0.4 & 0.4 & 0.4 & 0.4 & 0.8 & 0.4 & 0.4 & 0.4 & 0.4 & 0.4 \\
\hline 0.4 & 0.4 & 0.8 & 0.4 & 0.4 & 0.4 & 0.4 & 0.8 & 0.4 & 0.4 \\
\hline 0.4 & 0.4 & 0.8 & 0.4 & 0.4 & 0.4 & 0.4 & 0.4 & 0.4 & 0.8 \\
\hline 0.4 & 0.4 & 0.8 & 0.4 & 0.4 & 0.4 & 0.8 & 0.4 & 0.4 & 0.4 \\
\hline 0.4 & 0.4 & 0.4 & 0.8 & 0.4 & 39.6 & 60.8 & 58.4 & 58 & 56.8 \\
\hline 0.4 & 0.4 & 0.8 & 0.4 & 0.4 & 0.4 & 0.4 & 0.4 & 0.4 & 0.4 \\
\hline 0.4 & 0.4 & 0.8 & 0.4 & 0.4 & 0.4 & 0.4 & 0.4 & 0.4 & 0.4 \\
\hline 0.4 & 0.4 & 0.8 & 29.2 & 64.4 & 62.8 & 61.2 & 61.2 & 60.8 & 59.6 \\
\hline 0.4 & 0.4 & 0.4 & 59.6 & 59.2 & 59.2 & 58.4 & 58.4 & 58 & 57.2 \\
\hline 0.4 & 0.4 & 0.4 & 58.8 & 59.6 & 60 & 59.2 & 59.2 & 59.2 & 58.8 \\
\hline 0.8 & 0.4 & 0.4 & 54.8 & 61.2 & 61.6 & 61.2 & 60 & 60 & 58.4 \\
\hline 0.4 & 0.4 & 2.8 & 68 & 60 & 60 & 59.6 & 58.8 & 58.8 & 57.2 \\
\hline 0.4 & 0.4 & 0.4 & 0.4 & 0.4 & 0.8 & 0.4 & 0.4 & 0.4 & 0.4 \\
\hline 0.4 & 0.4 & 0.4 & 0.4 & 0.8 & 0.4 & 0.4 & 0.4 & 0.4 & 0.4 \\
\hline 2.4 & 56 & 49.2 & 3.6 & 0.4 & 0.4 & 0.4 & 0.4 & 0.8 & 0.4 \\
\hline 0.4 & 0.4 & 0.4 & 0.8 & 0.4 & 0.4 & 0.8 & 0.4 & 0.4 & 0.4 \\
\hline 0.4 & 0.8 & 0.4 & 0.4 & 60.4 & 58.4 & 58.4 & 57.2 & 57.2 & 56 \\
\hline 0.8 & 0.4 & 2.4 & 54.8 & 58 & 57.6 & 57.2 & 56.8 & 56 & 55.2 \\
\hline 0.4 & 0.4 & 0.4 & 54.4 & 52.4 & 52.8 & 53.2 & 40.8 & 0.8 & 0.4 \\
\hline 0.8 & 0.4 & 0.4 & 0.4 & 0.4 & 0.4 & 0.4 & 0.4 & 0.4 & 0.4 \\
\hline 0.4 & 0.4 & 0.4 & 0.4 & 0.4 & 0.4 & 0.4 & 0.4 & 0.4 & 0.4 \\
\hline
\end{tabular}




\begin{tabular}{|c|c|c|c|c|c|c|c|c|c|}
\hline 0.4 & 0.4 & 0.4 & 0.4 & 0.4 & 0.4 & 0.8 & 0.4 & 0.4 & 0.4 \\
\hline 0.4 & 0.4 & 0.4 & 0.8 & 0.4 & 0.4 & 0.8 & 0.4 & 0.4 & 0.4 \\
\hline 0.4 & 0.4 & 0.4 & 0.8 & 0.4 & 0.4 & 0.4 & 0.4 & 0.8 & 0.4 \\
\hline 0.4 & 0.8 & 0.4 & 0.4 & 0.4 & 0.4 & 0.8 & 0.4 & 0.4 & 0.4 \\
\hline 0.4 & 0.4 & 0.4 & 0.8 & 0.4 & 0.4 & 0.4 & 0.8 & 0.4 & 0.4 \\
\hline 0.4 & 0.4 & 0.4 & 0.8 & 0.4 & 0.4 & 0.4 & 0.4 & 0.4 & 0.4 \\
\hline 0.4 & 0.4 & 0.8 & 0.4 & 0.4 & 0.4 & 0.4 & 0.4 & 0.8 & 0.4 \\
\hline 0.4 & 0.4 & 0.4 & 0.8 & 0.4 & 0.4 & 0.4 & 0.8 & 0.4 & 0.4 \\
\hline 0.4 & 0.8 & 0.4 & 0.4 & 0.4 & 0.8 & 0.4 & & 0.4 & 0.4 \\
\hline & & & & & & & & & \\
\hline 0.4 & 0.8 & 0.4 & 0.8 & 0.4 & 0.8 & 0.8 & 0.4 & 0.4 & 0.8 \\
\hline 0.8 & 0.8 & 0.4 & 0.4 & 0.8 & 0.4 & 0.4 & 0.4 & 0.4 & 0.8 \\
\hline 0.4 & 0.4 & 0.4 & 0.8 & 0.4 & 0.4 & 0.4 & 0.4 & 0.4 & 0.4 \\
\hline 0.4 & 0.4 & 0.8 & 0.4 & 0.4 & 0.4 & 0.4 & 0.4 & 0.4 & 0.8 \\
\hline 0.4 & 0.4 & 0.8 & 0.4 & 0.4 & 0.4 & 0.4 & 0.4 & 0.4 & 0.8 \\
\hline 0.4 & 0.4 & 0.4 & 0.8 & 0.4 & 0.4 & 0.4 & 0.4 & 0.4 & 0.8 \\
\hline 0.4 & 0.4 & 0.4 & 0.4 & 0.4 & 0.8 & 0.4 & 0.4 & 0.4 & 0.4 \\
\hline 0.4 & 0.4 & 0.4 & 0.8 & 0.4 & 12.8 & 4.4 & 3.2 & 0.4 & 30.4 \\
\hline 0.4 & 0.4 & 0.8 & 0.4 & 0.4 & 0.4 & 0.4 & 0.4 & 0.8 & 0.4 \\
\hline 0.4 & 0.4 & 0.8 & 0.4 & 0.4 & 0.4 & 24.4 & 58.4 & 55.6 & 54 \\
\hline 0.4 & 0.4 & 0.4 & 0.4 & 0.4 & 0.8 & 0.4 & 0.4 & 0.4 & 0.4 \\
\hline 0.4 & 0.4 & 0.4 & 0.4 & 0.4 & 0.8 & 0.4 & 0.4 & 0.4 & 0.4 \\
\hline 0.4 & 0.4 & 0.4 & 0.4 & 0.4 & 0.4 & 0.8 & 0.4 & 0.4 & 0.4 \\
\hline 0.4 & 0.4 & 0.8 & 0.4 & 0.4 & 0.4 & 0.4 & 0.8 & 0.4 & 0.4 \\
\hline 0.4 & 0.4 & 0.8 & 0.4 & 0.4 & 0.8 & 0.4 & 0.4 & 0.4 & 0.4 \\
\hline 0.4 & 0.8 & 0.4 & 0.4 & 0.4 & 0.8 & 0.4 & 0.4 & 0.4 & 0.4 \\
\hline 0.4 & 0.4 & 0.4 & 0.4 & 0.4 & 0.4 & 0.8 & 0.4 & 0.4 & 0.4 \\
\hline 0.4 & 0.4 & 0.8 & 0.4 & 0.4 & 0.4 & 0.4 & 0.4 & 0.4 & 0.8 \\
\hline 0.4 & 0.4 & 0.8 & 0.4 & 0.4 & 0.4 & 0.4 & 0.4 & 0.8 & 0.4 \\
\hline 0.4 & 0.4 & 0.4 & 0.4 & 0.4 & 0.4 & 0.4 & 0.4 & 0.4 & 0.4 \\
\hline 0.4 & 0.4 & 0.4 & 0.8 & 0.4 & 0.4 & 0.4 & 0.4 & 0.4 & 0.4 \\
\hline 0.8 & 0.4 & 0.4 & 0.4 & 0.4 & 0.4 & 0.4 & 0.4 & 0.4 & 0.4 \\
\hline 0.4 & 0.4 & 0.8 & 0.4 & 0.4 & 0.4 & 0.4 & 0.4 & 0.4 & 0.4 \\
\hline 0.4 & 0.4 & 0.4 & 0.4 & 0.4 & 0.8 & & 43.2 & 55.6 & 53.6 \\
\hline 0.4 & 0.4 & 0.4 & 0.4 & 0.4 & 0.4 & 0.4 & 0.8 & 0.4 & 0.4 \\
\hline 0.4 & 0.8 & 0.4 & 0.4 & 0.4 & 0.4 & 0.4 & 0.4 & 0.4 & 0.4 \\
\hline 0.4 & 0.4 & 0.4 & 0.4 & 0.8 & 0.4 & 0.4 & 0.4 & 0.4 & 0.4 \\
\hline 0.4 & 0.4 & 0.4 & 0.8 & 0.4 & 0.4 & 0.4 & 0.4 & 0.4 & 0.4 \\
\hline 0.4 & 0.4 & 0.4 & 0.8 & 0.4 & 0.4 & 0.4 & 0.4 & 0.4 & 0.4 \\
\hline 0.4 & 0.4 & 0.4 & 0.4 & 0.8 & 0.4 & 0.4 & 0.4 & 0.4 & 63.6 \\
\hline 0.4 & 0.4 & 0.4 & 0.4 & 0.4 & 0.4 & 0.4 & 36.8 & 59.2 & 56 \\
\hline 0.4 & 0.4 & 0.8 & 0.4 & 0.4 & 0.4 & 0.4 & 0.4 & 0.4 & 0.4 \\
\hline 0.4 & 0.8 & 0.4 & 0.4 & 0.4 & 0.4 & 0.4 & 0.4 & 0.4 & 0.4 \\
\hline 0.4 & 0.4 & 0.8 & 0.4 & 0.4 & 0.4 & 0.4 & 0.4 & 0.4 & 0.4 \\
\hline 0.4 & 0.8 & 0.4 & 0.4 & 0.4 & 42 & 59.2 & 56.8 & 55.6 & 54.4 \\
\hline 0.8 & 0.4 & 0.4 & 0.4 & 0.4 & 0.4 & 0.4 & 0.8 & 0.4 & 0.4 \\
\hline 0.4 & 0.4 & 0.4 & 0.4 & 0.8 & 0.4 & 0.4 & 0.4 & 0.4 & 0.4 \\
\hline 0.4 & 0.4 & 0.4 & 0.4 & 0.8 & 0.4 & 0.4 & 0.4 & 0.4 & 0.4 \\
\hline 0.4 & 0.4 & 0.4 & 0.4 & 0.8 & 0.4 & 0.4 & 0.4 & 0.4 & 0.4 \\
\hline 0.8 & 0.4 & 0.4 & 0.4 & 0.4 & 0.8 & 0.4 & 0.4 & 0.4 & 0.4 \\
\hline 0.4 & 0.8 & 0.4 & 0.4 & 0.4 & 0.4 & 0.4 & 0.4 & 0.4 & 0.8 \\
\hline
\end{tabular}




\begin{tabular}{|c|c|c|c|c|c|c|c|c|c|}
\hline 0.4 & 0.4 & 0.4 & 0.4 & 0.4 & 0.4 & 42.8 & 61.2 & 59.2 & 57.2 \\
\hline 0.4 & 0.4 & 0.4 & 64 & 58.4 & 58.4 & 58.8 & 58.8 & 58.4 & 57.2 \\
\hline 0.4 & 0.4 & 2.4 & 63.6 & 57.6 & 58 & 57.2 & 57.6 & 57.2 & 56 \\
\hline 0.8 & 0.4 & 2.4 & 64.8 & 57.2 & 57.6 & 57.6 & 57.6 & 57.2 & 56.8 \\
\hline 0.4 & 0.4 & 0.4 & 0.4 & 0.4 & 0.4 & 0.4 & 0.4 & 0.4 & 0.4 \\
\hline 0.4 & 0.4 & 0.4 & 0.4 & 0.4 & 0.4 & 0.4 & 0.4 & 0.8 & 0.4 \\
\hline 2.4 & 63.6 & 52.4 & 0.8 & 13.2 & 59.6 & 57.2 & 57.6 & 56 & 54 \\
\hline 0.4 & 0.8 & 2.4 & 60 & 53.6 & 54 & 53.6 & 53.6 & 52.8 & 51.6 \\
\hline 0.8 & 0.4 & 2.4 & 58 & 52.4 & 52.8 & 52.4 & 22.8 & 36 & 17.6 \\
\hline 0.4 & 0.4 & 0.4 & 0.4 & 0.8 & 0.4 & 0.4 & 40.4 & 57.2 & 54.4 \\
\hline 0.4 & 0.4 & 0.4 & 0.4 & 0.4 & 0.4 & 30.8 & 60 & 57.6 & 56 \\
\hline 0.4 & 0.8 & 0.4 & 0.4 & 0.4 & 0.4 & 0.4 & 0.4 & 0.4 & 0.4 \\
\hline 0.4 & 0.4 & 0.4 & 0.4 & 0.4 & 0.4 & 0.4 & 0.4 & 0.4 & 0.8 \\
\hline & 0.8 & 0.4 & 0.4 & 0.4 & 0.4 & 18.4 & 66 & 59.6 & 54.4 \\
\hline 0.4 & 0.4 & 0.4 & 0.4 & 0.8 & 0.4 & 0.4 & 0.4 & 0.4 & 0.4 \\
\hline 0.4 & 0.4 & 0.4 & 0.4 & 0.4 & 0.4 & 0.4 & 0.8 & 0.4 & 0.4 \\
\hline 0.4 & 0.4 & 0.4 & 0.4 & 0.4 & 0.4 & 39.6 & 54.8 & 53.6 & 21.2 \\
\hline 0.4 & 0.4 & 0.4 & 0.4 & 0.4 & 0.4 & 44.8 & 56.4 & 54 & 53.2 \\
\hline 0.4 & 0.4 & 0.4 & 0.4 & 0.4 & 3.2 & 56.8 & 50 & 50 & 49.2 \\
\hline 0.4 & 0.4 & 0.4 & 0.4 & 0.4 & 0.4 & 0.4 & 0.4 & 0.4 & 0.4 \\
\hline 0.4 & 0.4 & 0.4 & 0.8 & & 26 & 62.8 & 58.4 & 57.6 & 56.8 \\
\hline 0.4 & 0.4 & 0.4 & 0.4 & 0.4 & 0.4 & 37.2 & 36.8 & 64.8 & 44 \\
\hline 0.8 & 0.4 & 0.4 & 0.4 & 42 & 23.2 & 42.4 & 58.4 & 56.8 & 54.8 \\
\hline 0.4 & 0.4 & 0.4 & 0.4 & 52 & 58 & 56.8 & 55.6 & 54.8 & 54.4 \\
\hline 0.4 & 0.4 & 0.4 & 41.2 & 56.8 & 56.8 & 56.4 & 56.4 & 56 & 55.2 \\
\hline 0.4 & 0.4 & 0.8 & 0.4 & 53.2 & 56 & 53.2 & 53.2 & 32.4 & 7.6 \\
\hline 0.4 & 0.4 & 0.4 & 0.4 & 0.4 & 0.4 & 0.4 & 0.4 & 0.4 & 0.4 \\
\hline 0.4 & 0.4 & 0.4 & 47.6 & 58 & 57.6 & 57.2 & 57.2 & 56 & 55.2 \\
\hline 0.4 & 0.4 & 0.4 & 0.4 & 0.8 & 0.4 & 0.4 & 0.4 & 0.4 & 0.4 \\
\hline 0.8 & 0.4 & 0.4 & 0.4 & 0.4 & 0.4 & 0.4 & 0.8 & 0.4 & 0.4 \\
\hline 0.4 & 0.8 & 0.4 & 0.4 & 0.4 & 0.4 & 0.8 & 0.4 & 0.4 & 2.4 \\
\hline 0.4 & 0.4 & 0.4 & 0.4 & 0.8 & 0.4 & 0.4 & 0.4 & 0.4 & 0.4 \\
\hline 0.8 & 0.4 & 0.4 & 0.4 & 0.4 & 0.4 & 0.4 & 0.4 & 0.8 & 0.4 \\
\hline 0.4 & 0.4 & 0.4 & 0.8 & 0.4 & 0.4 & 0.4 & 0.4 & 0.4 & 0.4 \\
\hline 0.4 & 0.8 & 0.4 & 0.4 & 0.4 & 0.4 & 0.4 & 0.4 & 0.4 & 0.8 \\
\hline 0.4 & 0.8 & 0.4 & 0.4 & 0.4 & 0.4 & 0.4 & 0.4 & 0.4 & 0.4 \\
\hline 0.4 & 0.4 & 0.4 & 0.4 & 0.4 & 0.4 & 0.4 & 0.4 & 0.4 & 0.8 \\
\hline 0.4 & 0.4 & 0.4 & 0.4 & 0.4 & 0.4 & 0.4 & 0.8 & & 0.8 \\
\hline 0.8 & 0.4 & 0.4 & 0.4 & 0.4 & 0.8 & 0.4 & 0.4 & 0.4 & 0.4 \\
\hline 0.8 & 0.4 & 0.4 & 0.4 & 0.4 & 0.4 & 0.4 & 0.4 & 0.4 & 0.4 \\
\hline 0.4 & 0.4 & 0.4 & 0.4 & 0.4 & 0.4 & 0.4 & 0.4 & 0.4 & 0.8 \\
\hline 0.4 & 0.4 & 0.8 & 0.4 & 0.4 & 0.4 & 0.8 & 0.4 & 0.4 & 22 \\
\hline 0.4 & 0.8 & 0.4 & 0.4 & 0.4 & 0.4 & 0.4 & 13.2 & 62.8 & 56 \\
\hline 0.4 & 0.4 & 0.8 & 0.4 & 26.4 & 60 & 57.2 & 57.2 & 56.8 & 55.6 \\
\hline 0.8 & 0.4 & 0.4 & 0.4 & 22.8 & 61.2 & 57.6 & 57.2 & 56.4 & 55.6 \\
\hline 0.4 & 0.4 & 0.8 & 0.4 & 10 & 65.6 & 58 & 57.2 & 56 & 55.2 \\
\hline 0.4 & 0.4 & 0.4 & 0.4 & 0.4 & 0.4 & 0.4 & 0.4 & 0.4 & 0.8 \\
\hline 0.4 & 0.4 & 0.4 & 0.4 & 0.4 & 0.4 & 0.4 & 0.4 & 0.4 & 0.4 \\
\hline 0.4 & 0.4 & 0.4 & 0.4 & 1.2 & 2.8 & 2.4 & 46.8 & 0.8 & 60 \\
\hline 0.4 & 0.4 & 0.4 & 43.2 & 58 & 57.6 & 57.2 & 58 & 58 & 57.2 \\
\hline 0.4 & 0.8 & 0.4 & 10.8 & 63.6 & 58 & 58.4 & 58 & 57.6 & 56.4 \\
\hline
\end{tabular}




\begin{tabular}{|c|c|c|c|c|c|c|c|c|c|}
\hline 0.4 & 0.8 & 0.4 & 0.4 & 0.8 & 0.4 & 0.4 & 0.4 & 0.4 & 0.8 \\
\hline 0.4 & 0.4 & 0.4 & 0.8 & 0.4 & 0.4 & 0.4 & 0.4 & 0.8 & 0.4 \\
\hline 0.4 & 0.8 & 0.4 & 0.4 & 0.4 & 0.4 & 0.4 & 0.4 & 0.4 & 0.8 \\
\hline 0.4 & 0.4 & 0.4 & 0.4 & 0.4 & 0.8 & 0.4 & 0.4 & 0.4 & 0.4 \\
\hline 0.4 & 0.4 & 0.8 & 42.8 & 59.6 & 57.6 & 58 & 58 & 57.2 & 58.4 \\
\hline 0.4 & 0.4 & 0.8 & 63.6 & 59.6 & 59.2 & 59.2 & 59.2 & 58.8 & 59.6 \\
\hline 0.8 & 0.4 & 0.4 & 46 & 60.4 & 59.6 & 59.6 & 59.2 & 59.2 & 58.8 \\
\hline 0.4 & 0.4 & 0.4 & 0.8 & 16.8 & 68.4 & 62 & 56.4 & 56 & 54 \\
\hline 0.8 & 0.4 & 0.4 & 0.4 & 24.8 & 60.4 & 57.6 & 56.8 & 56 & 54.8 \\
\hline 0.4 & 0.4 & 0.4 & 0.4 & 0.4 & 0.4 & 0.4 & 0.4 & 0.4 & 0.4 \\
\hline 0.4 & 0.4 & 0.4 & 0.4 & 0.4 & 0.4 & 0.4 & 0.4 & 0.4 & 0.4 \\
\hline 0.4 & 0.4 & 0.8 & 0.4 & 53.6 & 60.8 & 59.2 & 58.8 & 58.4 & 56.4 \\
\hline 0.4 & 0.4 & 0.4 & 36.4 & 58.4 & 58.4 & 57.2 & 57.6 & 56.8 & 56.8 \\
\hline 0.4 & 0.4 & 0.8 & 37.2 & 58.4 & 58 & 58.4 & 57.6 & 57.2 & 56.4 \\
\hline 0.4 & 0.4 & 0.4 & 26.4 & 40.4 & 60 & 59.6 & 58.4 & 58 & 56.8 \\
\hline 0.4 & 0.8 & 0.4 & 0.4 & 0.4 & 0.4 & 0.4 & 0.4 & 0.4 & 0.4 \\
\hline 0.4 & 0.4 & 0.4 & 0.4 & 0.4 & 0.4 & 0.4 & 0.4 & 0.4 & 0.4 \\
\hline 0.4 & 0.4 & 0.4 & 0.4 & 0.4 & 0.4 & 0.4 & 0.4 & 0.4 & 0.4 \\
\hline 0.4 & 0.4 & 0.4 & 0.4 & 0.4 & 0.8 & 0.4 & 0.4 & 0.4 & 0.4 \\
\hline 0.4 & 0.4 & 0.4 & 0.4 & 68 & 59.6 & 58.8 & 58.4 & 58 & 56.8 \\
\hline 0.4 & 0.4 & 0.4 & 0.4 & 66 & 59.6 & 58.8 & 58.4 & 58 & 56 \\
\hline 0.4 & 0.4 & 0.4 & 31.6 & 60 & 58.4 & 57.2 & 59.2 & 60.4 & 60 \\
\hline 0.4 & 0.4 & 0.4 & 64.8 & 59.6 & 60 & 60 & 60 & 62 & 62 \\
\hline 0.4 & 0.4 & 0.4 & 12.4 & 65.6 & 58.4 & 56 & 55.2 & 54.4 & 35.2 \\
\hline 0.4 & 0.4 & 0.8 & 0.4 & 0.4 & 0.4 & 0.4 & 0.4 & 0.4 & 0.4 \\
\hline 0.4 & 0.4 & 12 & 60.4 & 55.6 & 56.4 & 55.6 & 56 & 55.6 & 54.4 \\
\hline 0.4 & 0.4 & 0.4 & 64.4 & 56.4 & 56.8 & 57.2 & 56.8 & 55.6 & 54 \\
\hline 0.4 & 0.4 & 0.4 & 66 & 57.6 & 58.4 & 57.6 & 57.2 & 56.8 & 55.2 \\
\hline 0.8 & 0.4 & 0.4 & 61.2 & 57.2 & 57.6 & 56.8 & 56.8 & 56.4 & 56 \\
\hline 0.4 & 0.4 & 0.4 & 63.2 & 56.8 & 57.2 & 56.8 & 57.6 & 56.8 & 56.4 \\
\hline 0.4 & 0.4 & 0.4 & 0.4 & 0.4 & 0.4 & 0.4 & 0.4 & 0.4 & 0.4 \\
\hline 0.4 & 0.4 & 0.4 & 0.4 & 0.8 & 0.4 & & 0.8 & 0.4 & 0.4 \\
\hline 0.4 & 0.4 & 0.4 & 35.2 & 62.8 & 60.8 & 60.8 & 60 & 59.2 & 58 \\
\hline 0.4 & 0.4 & 0.4 & 63.2 & 58.4 & 58.4 & 58.4 & 58.8 & 58 & 56.8 \\
\hline 0.4 & 0.4 & 0.8 & 62.4 & 58.8 & 58.4 & 58.4 & 58.4 & 58 & 56.8 \\
\hline 0.4 & 0.4 & 0.8 & 60.8 & 55.2 & 56 & 55.2 & 54.4 & 54.8 & 53.6 \\
\hline 0.4 & 0.4 & 0.4 & 60.8 & 55.2 & 56.4 & 56 & 55.6 & 54.4 & 54.4 \\
\hline 0.4 & 0.4 & 0.4 & 0.4 & 0.4 & 0.4 & 0.4 & 0.4 & 0.4 & 0.4 \\
\hline 0.4 & 0.4 & 0.4 & 0.8 & 0.4 & 0.4 & 0.4 & 0.4 & 0.4 & 0.4 \\
\hline 0.4 & 0.4 & 4.8 & 65.2 & 55.2 & 54.4 & 54.4 & 54.8 & 54.8 & 54 \\
\hline 0.4 & 0.4 & 0.4 & 62.8 & 55.2 & 56 & 28.8 & 60.4 & 58 & 58 \\
\hline 0.4 & 0.4 & 0.4 & 62.4 & 56.4 & 57.2 & 57.6 & 58.8 & 63.2 & 63.6 \\
\hline 0.4 & 0.4 & 0.4 & 66.8 & 61.6 & 62.8 & 62 & 61.6 & 62.4 & 62.4 \\
\hline 0.4 & 0.4 & 2 & 22.4 & 0.8 & 14.4 & 76 & 68.4 & 62 & 64 \\
\hline 0.4 & 0.4 & 0.4 & 0.4 & 0.4 & 0.4 & 0.4 & 0.4 & 0.4 & 0.4 \\
\hline 0.4 & 0.4 & 0.4 & 0.4 & 0.4 & 0.8 & 0.4 & 0.4 & 0.4 & 0.4 \\
\hline 0.4 & 0.4 & 0.4 & 72 & 64.4 & 61.2 & 59.2 & 60 & 60.4 & 60.4 \\
\hline 0.4 & 0.4 & 0.4 & 66 & 62.8 & 62 & 61.2 & 60.8 & 59.2 & 59.2 \\
\hline 0.4 & 0.4 & 0.4 & 66.8 & 60.4 & 60.8 & 61.2 & 60 & 59.2 & 60 \\
\hline 0.4 & 0.4 & 0.4 & 65.2 & 60 & 60.4 & 61.2 & 62 & 62 & 62 \\
\hline 0.4 & 0.4 & 0.8 & 66.4 & 60.4 & 60.8 & 60.4 & 60.4 & 59.6 & 59.6 \\
\hline
\end{tabular}




\begin{tabular}{|c|c|c|c|c|c|c|c|c|c|}
\hline 0.4 & 0.4 & 0.4 & 0.4 & 0.4 & 0.4 & 0.4 & 0.4 & 0.4 & 0.4 \\
\hline 0.4 & 0.4 & 0.4 & 0.4 & \begin{tabular}{l|l}
0.8 \\
\end{tabular} & 0.4 & 0.4 & 0.4 & 0.4 & 0.4 \\
\hline 0.8 & 0.4 & 5.2 & 72.4 & 65.2 & 62 & 62 & 60.8 & 61.2 & 60.4 \\
\hline 0.4 & 0.4 & 0.4 & 67.6 & 61.6 & 60.8 & 62.4 & 60.8 & 60.8 & 60.4 \\
\hline 0.4 & 0.4 & \begin{tabular}{l|l}
0.8 \\
\end{tabular} & 68.4 & 64.8 & 64.8 & 63.6 & 62.8 & 62 & 60 \\
\hline 0.4 & 0.4 & 0.4 & 68.8 & 61.6 & 61.6 & 62.4 & 61.6 & 62.8 & 63.2 \\
\hline 0.4 & 0.8 & 0.4 & 64.8 & 60.8 & 60.8 & 60.4 & 59.2 & 58.4 & 56.4 \\
\hline 0.4 & 0.8 & 0.4 & 10 & 64.4 & 58 & 56.8 & 56.4 & 28 & 0.4 \\
\hline 0.4 & 0.4 & 0.4 & 0.4 & 0.4 & 0.4 & 0.4 & 0.4 & 0.8 & 0.4 \\
\hline 0.4 & 0.4 & 0.4 & 12.4 & 66.8 & 56.8 & 56.8 & 56 & 57.6 & 57.6 \\
\hline 0.4 & 0.8 & 0.4 & 53.6 & 59.6 & 57.2 & 55.6 & 55.6 & 54 & 53.6 \\
\hline 0.4 & 0.4 & 0.4 & 51.2 & 54.4 & 52 & 50.8 & 51.2 & 47.6 & 48.4 \\
\hline 0.4 & 0.4 & 0.8 & 0.4 & 0.4 & 0.4 & 1.2 & 43.6 & 57.2 & 54.8 \\
\hline 0.8 & 0.8 & 0.4 & 0.4 & 0.8 & 0.4 & 0.8 & 0.4 & 0.8 & 0.8 \\
\hline 0.4 & 0.8 & 0.4 & 0.8 & 0.4 & 0.8 & 0.4 & 0.8 & 0.4 & 0.8 \\
\hline 0.4 & 0.8 & 0.4 & 0.4 & 0.4 & 0.4 & 0.4 & 0.4 & 0.4 & 0.4 \\
\hline 0.4 & 0.4 & 0.4 & 0.4 & 0.4 & 0.8 & 0.4 & & 0.8 & \\
\hline 0.8 & 0.4 & \begin{tabular}{l|l}
0.8 \\
\end{tabular} & 0.4 & 0.4 & 0.4 & 0.4 & 0.8 & 0.4 & 0.8 \\
\hline 0.8 & 0.4 & 0.8 & 0.4 & 0.4 & 0.4 & 0.4 & 0.4 & 0.8 & 0.8 \\
\hline 0.8 & 0.4 & 0.8 & 0.4 & 0.4 & 0.4 & 0.8 & 0.4 & 0.8 & 0.8 \\
\hline 0.8 & 0.8 & 0.4 & 0.8 & 0.4 & \begin{tabular}{|l|}
0.8 \\
\end{tabular} & 0.4 & 0.8 & 0.4 & 0.8 \\
\hline 0.8 & 0.4 & 0.8 & 0.4 & 0.8 & 0.4 & 0.8 & 0.8 & 0.4 & 0.8 \\
\hline 0.4 & 0.8 & 0.8 & 0.4 & 0.8 & 0.4 & 0.8 & 0.8 & 0.4 & 0.8 \\
\hline & & & & & & & & & \\
\hline 0.8 & 0.8 & 0.8 & 0.4 & 0.8 & 0.4 & 0.8 & 0.4 & 0.8 & 0.8 \\
\hline 0.8 & 0.8 & 0.4 & 0.8 & 0.8 & 0.4 & 0.8 & 0.4 & 0.8 & 0.8 \\
\hline 0.4 & 0.8 & \begin{tabular}{l|l}
0.8 \\
\end{tabular} & 0.8 & 0.4 & \begin{tabular}{|l|}
0.8 \\
\end{tabular} & 0.4 & 0.8 & 0.8 & 0.4 \\
\hline 0.8 & 0.4 & 0.8 & 0.8 & 0.8 & 0.4 & 0.8 & 0.4 & 0.8 & 0.8 \\
\hline 0.8 & 0.8 & 0.4 & 0.8 & 0.8 & 0.4 & 0.8 & 0.4 & 0.8 & 0.8 \\
\hline 0.8 & 0.4 & 0.8 & 0.8 & 0.8 & 0.4 & 0.8 & 0.8 & 0.8 & 0.4 \\
\hline 0.8 & 0.8 & 0.8 & 0.4 & 0.8 & 0.8 & 0.4 & 0.8 & 0.8 & 0.4 \\
\hline 0.8 & 0.8 & 0.8 & 10.4 & 79.6 & 66.4 & 64.4 & 62.8 & 61.6 & 60.8 \\
\hline 0.8 & 0.8 & 0.4 & 43.6 & 66.8 & 62.4 & 62.4 & 61.2 & 60.8 & 60.8 \\
\hline 0.8 & \begin{tabular}{|l|}
0.8 \\
\end{tabular} & 0.4 & 62 & 64.4 & 61.6 & 62 & 61.2 & 60.8 & 60.8 \\
\hline 0.8 & 0.8 & 0.4 & 0.8 & 0.8 & 0.8 & 0.4 & 0.8 & 0.8 & 0.8 \\
\hline 0.8 & 0.4 & 0.8 & 0.8 & 0.8 & 0.4 & 0.8 & 0.8 & 0.8 & 0.4 \\
\hline 0.8 & \begin{tabular}{l|l}
0.8 \\
\end{tabular} & 0.8 & 0.4 & 0.8 & \begin{tabular}{|l|}
0.8 \\
\end{tabular} & 0.8 & 0.4 & 0.8 & 0.8 \\
\hline 0.8 & 0.8 & 0.8 & 65.2 & 62 & 62.4 & 63.6 & 63.6 & 64.4 & 64 \\
\hline 0.8 & 0.4 & 0.8 & 60.8 & 52.4 & 24 & 10 & 76 & 66 & 64.4 \\
\hline 0.8 & 0.8 & 0.4 & 62 & 65.6 & 64 & 63.2 & 62.4 & 62.8 & 63.2 \\
\hline 0.8 & 0.8 & 0.8 & 0.4 & 8 & 76.8 & 66.4 & 64.8 & 63.2 & 62.4 \\
\hline 0.8 & 0.8 & 0.8 & 0.8 & 0.8 & 0.8 & 0.4 & 0.8 & 0.8 & 0.8 \\
\hline \begin{tabular}{|l|}
0.8 \\
\end{tabular} & 0.4 & 0.8 & 0.4 & 0.8 & 0.4 & 0.8 & 0.8 & 0.4 & 0.8 \\
\hline 0.8 & 0.8 & 0.8 & 0.4 & 0.8 & 0.4 & 0.8 & 0.8 & 0.8 & 0.4 \\
\hline 0.8 & 0.8 & 0.4 & 63.6 & 66.4 & 64.4 & 62.8 & 61.2 & 60.4 & 60 \\
\hline 0.8 & 0.4 & 0.8 & 59.6 & 69.6 & 65.6 & 64.8 & 64.4 & 64.4 & 64 \\
\hline 0.8 & 0.4 & 0.8 & 61.6 & 64.8 & 62.4 & 61.2 & 60.4 & 60.4 & 58.8 \\
\hline 0.8 & 0.8 & 0.8 & 26.4 & 71.6 & 63.6 & 61.6 & 61.2 & 60 & 60 \\
\hline 0.8 & 0.8 & 0.4 & 0.8 & 0.8 & 0.8 & 0.8 & 0.4 & 0.8 & 0.8 \\
\hline 0.8 & 0.4 & 0.8 & 0.8 & 0.4 & 0.8 & 0.8 & 0.4 & 0.8 & 0.8 \\
\hline 0.4 & 0.8 & 0.8 & 0.4 & 0.8 & 0.8 & 0.8 & 0.4 & 0.8 & 0.8 \\
\hline
\end{tabular}




\begin{tabular}{|c|c|c|c|c|c|c|c|c|c|}
\hline 0.8 & 0.8 & 0.4 & 38.4 & 75.6 & 66.4 & 65.2 & 65.2 & 65.2 & 65.6 \\
\hline 0.4 & 0.8 & 0.8 & 58 & 0.8 & 0.8 & 0.8 & 0.8 & 0.4 & 0.8 \\
\hline 0.8 & 0.8 & 0.8 & 0.4 & 0.8 & 0.8 & 0.8 & 0.4 & 0.8 & 20.8 \\
\hline 0.8 & 0.8 & 0.8 & 0.8 & 0.4 & 73.2 & 64.8 & 61.6 & 60 & 59.2 \\
\hline 0.8 & 0.8 & 0.4 & 0.8 & 0.8 & 0.8 & 0.4 & 0.8 & 0.8 & 0.8 \\
\hline 0.8 & 0.8 & 0.4 & 0.8 & 0.8 & 0.4 & 0.8 & 0.8 & 0.8 & 0.8 \\
\hline 0.4 & 0.8 & 0.8 & 0.8 & 0.4 & 0.8 & 0.8 & 0.4 & 0.8 & 0.8 \\
\hline 0.4 & 0.8 & 0.8 & 55.2 & 63.6 & 61.2 & 59.6 & 60 & 60 & 16 \\
\hline 0.8 & 0.8 & 0.4 & 0.4 & 0.8 & 0.4 & 57.6 & 69.6 & 63.2 & 64 \\
\hline 0.8 & 0.8 & 0.4 & 0.8 & 0.8 & 0.4 & 0.8 & 0.8 & 0.4 & 0.8 \\
\hline 0.8 & 0.8 & 0.4 & 28 & 75.2 & 65.6 & 63.2 & \begin{tabular}{ll|}
62.8 \\
\end{tabular} & 62.4 & 62 \\
\hline 0.8 & 0.8 & 0.8 & 0.8 & 0.8 & 0.4 & 0.8 & 0.8 & 0.8 & 0.8 \\
\hline 0.8 & 0.8 & 0.8 & 0.8 & 0.4 & 0.8 & 0.8 & 0.8 & 0.8 & 0.8 \\
\hline 0.8 & 0.8 & 0.8 & 0.8 & 0.4 & 0.8 & 0.8 & 0.8 & 0.8 & 0.8 \\
\hline 0.8 & 0.8 & 0.8 & 0.8 & 0.8 & 0.4 & 0.8 & 0.8 & 0.8 & 0.8 \\
\hline 0.8 & 0.8 & 0.4 & 0.8 & 0.8 & 0.4 & 0.8 & 0.8 & 0.8 & 0.4 \\
\hline 0.4 & 0.8 & 0.8 & 0.8 & 0.4 & 0.8 & 0.8 & 0.4 & 0.8 & 0.8 \\
\hline 0.8 & 0.8 & 0.8 & 0.4 & 0.8 & 0.4 & 0.8 & 0.8 & 0.4 & 0.8 \\
\hline 0.8 & 0.8 & 0.8 & 0.8 & 0.4 & 0.8 & 0.8 & 0.8 & 0.4 & 0.8 \\
\hline 0.4 & 0.8 & 0.8 & 0.4 & 0.8 & 0.8 & 0.4 & 0.8 & 0.8 & 0.4 \\
\hline 0.4 & 0.8 & 0.8 & 0.4 & 0.8 & 0.4 & 0.8 & 0.8 & 0.4 & 0.8 \\
\hline 0.4 & 0.8 & 0.8 & 0.4 & 0.4 & 70.8 & 64.4 & 62.8 & 62 & 61.2 \\
\hline 0.8 & 0.4 & 0.8 & 55.2 & 65.2 & 63.6 & 62 & 60.4 & 60 & 59.2 \\
\hline 0.8 & 0.8 & 0.4 & 54.4 & 63.2 & 61.6 & 61.2 & 61.2 & 60.4 & 60 \\
\hline 0.4 & 0.8 & 0.8 & 59.2 & 63.2 & 63.2 & 61.2 & 60.4 & 59.6 & 59.2 \\
\hline 0.8 & 0.4 & 0.8 & 31.6 & 74 & 68 & 66.8 & 65.6 & 65.6 & 64.4 \\
\hline 0.8 & 0.8 & 0.4 & 0.8 & 0.8 & 0.4 & 0.8 & 0.8 & 0.8 & 0.4 \\
\hline 0.8 & 0.4 & 0.8 & 0.8 & 0.8 & 0.4 & 0.8 & 0.8 & 0.8 & 0.4 \\
\hline 0.4 & 0.8 & 0.8 & 79.2 & 70.8 & 67.2 & 66.4 & 65.2 & 66 & 64.4 \\
\hline 0.8 & 0.4 & 0.8 & 62.4 & 67.2 & 65.6 & 63.6 & 63.6 & 63.6 & 62.8 \\
\hline 0.8 & 0.8 & 0.4 & 60.4 & 62.4 & 60.8 & 60 & 60 & 60.8 & 60.8 \\
\hline 0.4 & 0.8 & 0.4 & 54.4 & 62.4 & 61.2 & 61.2 & 61.2 & 62.8 & 61.2 \\
\hline 0.8 & 0.4 & 0.8 & 35.6 & 63.6 & 60.4 & 60.4 & 62 & 61.2 & 60.4 \\
\hline 0.8 & 0.8 & 0.4 & 0.8 & 0.8 & 0.4 & 0.8 & 0.8 & 0.4 & 0.8 \\
\hline 0.8 & 0.4 & 0.8 & 0.4 & 0.8 & 0.4 & 0.8 & 0.4 & 0.8 & 0.4 \\
\hline 0.8 & 0.8 & 0.4 & 25.2 & 71.2 & 63.6 & 62.8 & 61.2 & 60.8 & 60.4 \\
\hline 0.8 & 0.4 & 0.8 & 49.2 & 63.2 & 61.2 & 60 & 60.8 & 60.4 & 60.4 \\
\hline 0.8 & 0.4 & 0.8 & 58 & 65.6 & 64.8 & 62 & 62.4 & 62 & 61.6 \\
\hline 0.4 & 0.8 & 0.4 & 70 & 65.6 & 64.8 & 62.8 & 62 & 62.8 & 62 \\
\hline 0.8 & 0.4 & 0.4 & 69.2 & 63.6 & 65.2 & 65.2 & 64 & 63.6 & 62 \\
\hline 0.8 & 0.4 & 0.8 & 0.4 & 0.8 & 0.4 & 0.8 & 0.8 & 0.4 & 0.8 \\
\hline 0.4 & 0.8 & 0.4 & 0.8 & 0.8 & 0.4 & 0.8 & 0.4 & 0.8 & 0.8 \\
\hline 0.8 & 56.8 & 66 & 62.4 & 66 & 68 & 70 & 68 & 66.4 & 66 \\
\hline 0.8 & 0.4 & 55.6 & 66 & 67.2 & 69.6 & 70 & 68.4 & 67.6 & 67.2 \\
\hline 0.8 & 0.4 & 55.6 & 65.6 & 66.4 & 68 & 67.6 & 67.2 & 68.4 & 67.2 \\
\hline 0.8 & 0.8 & 55.2 & 64.8 & 64.8 & 67.2 & 68 & 67.2 & 67.2 & 66.8 \\
\hline 0.4 & 0.8 & 55.2 & 67.2 & 66.8 & 68 & 67.6 & 67.2 & 66.4 & 66 \\
\hline 0.8 & 0.4 & 0.8 & 0.8 & 0.4 & 0.8 & 0.4 & 0.8 & 0.8 & 0.4 \\
\hline 0.8 & 0.4 & 0.8 & 0.4 & 0.8 & 0.8 & 0.4 & 0.8 & 0.4 & 0.8 \\
\hline 0.8 & 57.6 & 68 & 62.4 & 64.4 & 67.6 & 69.6 & 69.6 & 68.4 & 67.2 \\
\hline 0.4 & 0.8 & 55.6 & 66 & 67.2 & 69.2 & 70.4 & 69.2 & 68.8 & 67.6 \\
\hline
\end{tabular}




\begin{tabular}{|c|c|c|c|c|c|c|c|c|c|}
\hline 0.4 & 0.8 & 56.4 & 68 & 68.4 & 70.8 & 70.8 & 69.6 & 69.6 & 67.6 \\
\hline 0.4 & \begin{tabular}{l|l}
0.8 \\
\end{tabular} & 56.8 & 69.6 & 70.8 & 72.4 & 72.8 & 71.6 & 72 & 70 \\
\hline 0.8 & 0.8 & 56.8 & 69.2 & 70.4 & 72.8 & 72 & 70.4 & 70 & 69.2 \\
\hline 0.8 & 0.8 & 0.4 & 0.8 & 0.8 & 0.4 & 0.8 & 0.8 & 0.4 & 0.8 \\
\hline 0.8 & \begin{tabular}{l|l}
0.8 \\
\end{tabular} & 0.4 & 0.8 & \begin{tabular}{ll|}
0.8 \\
\end{tabular} & 0.8 & 0.4 & 0.8 & 0.8 & 0.4 \\
\hline 0.4 & 1.2 & 0.4 & 0.8 & 0.4 & 0.8 & 0.4 & 0.8 & 0.8 & 0.4 \\
\hline 0.8 & 0.8 & 0.8 & 81.6 & 76.4 & 76.8 & 75.2 & 72 & 71.6 & 72 \\
\hline 0.8 & 0.8 & 57.6 & 70.4 & 72 & 73.6 & 73.6 & 72.8 & 72 & 70.4 \\
\hline 0.8 & 0.8 & 57.2 & 69.6 & 69.6 & 72 & 71.6 & 69.6 & 69.6 & 68 \\
\hline 0.8 & 0.8 & 55.2 & 66.4 & 64.4 & 66.4 & 68 & 68.4 & 67.6 & 66 \\
\hline 0.4 & 0.8 & 0.8 & 0.8 & 0.4 & 0.8 & 0.4 & 0.8 & 0.4 & 0.8 \\
\hline 0.8 & 0.4 & 0.8 & 0.8 & 0.4 & 0.8 & 0.4 & 0.8 & 0.4 & 0.8 \\
\hline 0.8 & 57.2 & 66 & 62 & 65.6 & 67.6 & 68.8 & 68 & 66 & 65.2 \\
\hline 0.8 & 0.4 & 54.4 & 63.2 & 64.4 & 68 & 68 & 67.2 & 65.6 & 60.4 \\
\hline 0.8 & 0.4 & 0.8 & 0.4 & 36.8 & 73.6 & 68.8 & 68.4 & 66.8 & 66 \\
\hline 0.8 & 0.4 & 54 & 65.6 & 63.6 & 65.2 & 66.4 & 64.8 & 64.8 & 64 \\
\hline 0.8 & 0.8 & 54.4 & 64 & 66 & 68 & 66 & 68 & 66.8 & 67.2 \\
\hline 0.8 & \begin{tabular}{l|l}
0.8 \\
\end{tabular} & 0.8 & 0.4 & 0.8 & 0.8 & 0.4 & 0.8 & 0.4 & 0.8 \\
\hline 0.8 & 0.4 & 0.8 & 0.8 & 0.4 & 0.8 & 0.8 & 0.4 & 0.8 & 0.8 \\
\hline 0.4 & 58 & 73.2 & 67.2 & 70 & 71.2 & 71.2 & 66.4 & 1.2 & 47.2 \\
\hline 0.8 & 0.8 & 56.8 & 69.2 & 66.4 & 67.6 & 69.2 & 70 & 70.4 & 68.8 \\
\hline 0.8 & 0.4 & 55.6 & 67.2 & 67.2 & 68 & 68 & 66.4 & 65.6 & 65.6 \\
\hline 0.8 & 0.4 & 52.4 & 63.2 & 62.8 & 64.4 & 64 & 63.2 & 63.6 & 54.8 \\
\hline 0.4 & 0.8 & 53.6 & 62.8 & 62.8 & 64 & 64.4 & 62.8 & 61.6 & 60.8 \\
\hline 0.4 & 0.8 & 0.8 & 0.4 & 0.4 & 0.8 & 0.4 & 0.8 & 0.4 & 0.4 \\
\hline 0.8 & 0.4 & 0.8 & 0.4 & 0.8 & 0.8 & 0.4 & 0.8 & 0.4 & 0.8 \\
\hline 0.8 & 57.6 & 69.2 & 65.2 & 66.8 & 68 & 67.6 & 66.8 & 67.6 & 65.6 \\
\hline 0.4 & 0.4 & 51.6 & 62.8 & 62.8 & 64 & 64.8 & 64.8 & 64.4 & 63.6 \\
\hline 0.4 & 0.4 & 0.8 & 67.6 & 60.8 & 61.2 & 60.8 & 58.4 & 57.6 & 56.4 \\
\hline 0.4 & 0.4 & 45.6 & 55.2 & 56.4 & 57.6 & 58 & 58 & 58 & 56.8 \\
\hline 0.8 & 0.4 & 46 & 55.6 & 56.4 & 58.4 & 58.4 & 58.8 & 57.6 & 56.8 \\
\hline 0.4 & 0.4 & 0.4 & 0.4 & 0.4 & 0.8 & 0.4 & 0.4 & 0.4 & 0.4 \\
\hline 0.8 & 0.4 & 0.4 & 0.4 & 0.4 & 0.4 & 0.8 & 0.4 & 0.4 & 0.4 \\
\hline 0.8 & 50 & 58.4 & 55.6 & 58.4 & 59.6 & 60.4 & 60 & 59.2 & 57.2 \\
\hline 0.4 & 0.4 & 48.8 & 56.8 & 58 & 60.4 & 60 & 60.8 & 59.6 & 58.4 \\
\hline 0.4 & 0.4 & 48 & 56.8 & 58.4 & 60 & 59.6 & 59.6 & 59.2 & 56.4 \\
\hline 0.4 & \begin{tabular}{l|l}
0.8 \\
\end{tabular} & 46.8 & 55.6 & 56 & 57.2 & 56.8 & 56.8 & 56.4 & 54.8 \\
\hline 0.4 & 0.4 & 45.2 & 53.6 & 54.8 & 56.8 & 56.8 & 57.6 & 58 & 57.2 \\
\hline 0.4 & 0.4 & 0.4 & 0.4 & 0.4 & 0.8 & 0.4 & 0.4 & 0.4 & 0.4 \\
\hline 0.4 & \begin{tabular}{l|l}
0.8 \\
\end{tabular} & 0.4 & 0.4 & 0.4 & 0.4 & 0.4 & 0.4 & 0.8 & 0.8 \\
\hline 0.4 & 50 & 57.2 & 55.2 & 57.6 & 59.2 & 59.2 & 59.2 & 58 & 55.6 \\
\hline 0.4 & 0.4 & 31.6 & 55.6 & 56 & 58.4 & 58 & 58.4 & 58.4 & 57.6 \\
\hline 0.4 & 0.4 & 33.6 & 57.6 & 58 & 59.2 & 58.8 & 59.2 & 58.8 & 55.6 \\
\hline 0.8 & 0.4 & 34.4 & 55.6 & 56 & 58 & 58.4 & 58.8 & 59.2 & 58.8 \\
\hline 0.8 & 0.4 & 50 & 57.2 & 57.6 & 60.4 & 60.4 & 60.4 & 60 & 59.2 \\
\hline 0.8 & 0.4 & 0.4 & 0.4 & 0.4 & 0.4 & 0.4 & 0.8 & 0.4 & 0.4 \\
\hline 0.4 & 0.4 & 0.4 & 0.4 & 0.4 & 0.8 & 0.4 & 0.4 & 0.4 & 0.4 \\
\hline 0.4 & 0.4 & 50.4 & 57.6 & 58.4 & 59.2 & 59.2 & 59.6 & 59.2 & 58 \\
\hline 0.8 & 0.4 & 48.4 & 57.2 & 58 & 59.6 & 59.6 & 58.8 & 58.4 & 57.2 \\
\hline 0.8 & 0.4 & 48 & 56.4 & 57.2 & 59.2 & 59.2 & 59.6 & 58.4 & 57.6 \\
\hline 0.4 & 0.8 & 48 & 58 & 58.4 & 59.6 & 58.4 & 56 & 58 & 58 \\
\hline
\end{tabular}




\begin{tabular}{|c|c|c|c|c|c|c|c|c|c|}
\hline 0.4 & 0.4 & 48 & 56.8 & 58.4 & 60 & 59.6 & 59.2 & 59.2 & 57.2 \\
\hline 0.4 & 0.4 & 0.4 & 0.4 & 0.4 & 0.8 & 0.4 & 0.4 & 0.4 & 0.4 \\
\hline 0.4 & 0.4 & 0.4 & 0.8 & 0.4 & 0.4 & 0.4 & 0.4 & 0.4 & 0.4 \\
\hline 0.4 & 0.4 & 50 & 57.6 & 55.6 & 30.8 & 60 & 59.6 & 59.6 & 58.8 \\
\hline 0.4 & 0.4 & 0.4 & 42.4 & 62.4 & 61.6 & 61.2 & 60 & 60.4 & 59.2 \\
\hline 0.4 & 0.4 & 48.8 & 58 & 59.6 & 60.4 & 58.8 & 59.6 & 58.8 & 58 \\
\hline 0.4 & 0.4 & 48.8 & 57.6 & 57.6 & 58 & 59.6 & 59.2 & 59.2 & 57.2 \\
\hline 0.8 & 0.4 & 48 & 56.4 & 58.8 & 60 & 60.8 & 60.4 & 59.6 & 59.2 \\
\hline 0.4 & 0.4 & 0.8 & 0.4 & 0.4 & 0.4 & 0.4 & 0.8 & 0.4 & 0.8 \\
\hline 0.4 & 0.8 & 0.4 & 0.4 & 0.4 & 0.8 & 0.4 & 0.4 & 0.4 & 0.4 \\
\hline 0.4 & 0.4 & 50 & 57.6 & 58 & 58.8 & 59.6 & 58.8 & 57.6 & 56.8 \\
\hline 0.4 & 0.8 & 46.8 & 56.8 & 58 & 57.2 & 56.4 & 57.2 & 56.8 & 56.8 \\
\hline 0.4 & 0.8 & 43.6 & 54 & 54.4 & 55.2 & 47.2 & 0.8 & 0.8 & 58 \\
\hline 0.8 & 0.4 & 40.8 & 50.4 & 51.6 & 53.2 & 53.2 & 51.6 & 52.8 & 8 \\
\hline 0.4 & 0.8 & 0.4 & 0.4 & 0.4 & 0.8 & 0.4 & 0.4 & 0.4 & 0.8 \\
\hline 0.4 & 0.8 & 0.4 & 0.4 & 0.4 & 0.8 & 0.4 & 0.4 & 0.4 & 0.4 \\
\hline 0.8 & 0.4 & 0.4 & 0.4 & 0.8 & 0.4 & 0.4 & 0.4 & 0.4 & 0.4 \\
\hline 0.4 & \begin{tabular}{l|l}
0.8 \\
\end{tabular} & 0.4 & 10 & 63.2 & 59.2 & 57.6 & 57.2 & 56.8 & 56 \\
\hline 0.4 & 0.4 & 45.6 & 55.2 & 57.2 & 58.4 & 58 & 56.8 & 56.8 & 55.6 \\
\hline 0.4 & 0.4 & 45.6 & 54.8 & 56.4 & 57.6 & 55.6 & 55.6 & 55.2 & 54.8 \\
\hline 0.8 & 0.4 & 45.6 & 54.4 & 54.4 & 55.2 & 56 & 55.2 & 54.4 & 53.6 \\
\hline 0.4 & 0.4 & 41.2 & 50.8 & 52.4 & 54 & 54 & 53.6 & 53.2 & 52 \\
\hline 0.4 & 0.4 & 0.4 & 0.4 & 0.4 & 0.4 & 0.8 & 0.4 & 0.4 & 0.4 \\
\hline 0.4 & 0.4 & 0.8 & 0.4 & 0.4 & 0.4 & 0.4 & 0.8 & 0.4 & 0.4 \\
\hline 0.4 & 0.4 & 43.2 & 50.8 & 52 & 53.2 & 27.2 & 0.4 & 0.8 & 0.4 \\
\hline 0.4 & 0.4 & 0.8 & 0.4 & 22.4 & 60.4 & 57.2 & 56.4 & 56.4 & 54.8 \\
\hline 0.8 & 0.4 & 43.6 & 52.8 & 54.4 & 56 & 57.2 & 56.4 & 55.6 & 55.6 \\
\hline 0.4 & 0.4 & 44.8 & 54 & 55.6 & 56.8 & 57.2 & 56.8 & 56 & 56 \\
\hline 0.4 & 0.4 & 43.6 & 52 & 53.6 & 53.6 & 54 & 53.2 & 53.2 & 52 \\
\hline 0.4 & 0.4 & 0.4 & 0.4 & 0.4 & 0.4 & 0.8 & 0.4 & 0.4 & 0.4 \\
\hline 0.4 & 0.4 & 0.4 & 0.8 & 0.4 & 0.4 & 0.4 & 0.4 & 0.4 & 0.4 \\
\hline 0.4 & 0.4 & 40.8 & 31.2 & 0.4 & 0.8 & 0.4 & 0.4 & 0.4 & 0.4 \\
\hline 0.4 & 0.4 & 0.4 & 0.4 & 0.8 & 0.4 & 0.4 & 40 & 56.8 & 54 \\
\hline 0.4 & 0.4 & 0.8 & 0.4 & 0.4 & 0.4 & 0.4 & 0.8 & 0.4 & 0.4 \\
\hline 0.4 & 0.8 & 0.4 & 0.4 & 0.8 & 0.4 & 0.4 & 0.4 & 0.8 & 0.4 \\
\hline 0.4 & 0.4 & 0.4 & 0.4 & 0.8 & 0.4 & 0.4 & 0.4 & 0.4 & 0.8 \\
\hline 0.4 & \begin{tabular}{l|l}
0.8 \\
\end{tabular} & 0.4 & 0.4 & 0.4 & 0.4 & 0.8 & 0.4 & 0.4 & 0.4 \\
\hline 0.8 & 0.4 & 0.4 & 0.4 & 0.4 & 0.8 & 0.4 & 0.4 & 0.4 & 0.4 \\
\hline 0.8 & 0.4 & 0.4 & 0.4 & 0.8 & 0.4 & 0.4 & 0.4 & 0.8 & 0.4 \\
\hline 0.4 & 0.4 & 0.4 & 0.8 & & 0.8 & 0.4 & 0.4 & 0.4 & 29.6 \\
\hline 0.8 & 0.4 & 31.6 & 0.8 & 0.4 & 0.8 & 0.4 & 15.6 & 56.4 & 53.2 \\
\hline 0.8 & 0.4 & 38.4 & 0.8 & 0.4 & 0.4 & 0.8 & 0.4 & 0.4 & 0.4 \\
\hline 0.4 & 0.4 & 46 & 54.4 & 53.6 & 55.6 & 55.6 & 55.6 & 54.8 & 54 \\
\hline 0.4 & 0.4 & 0.4 & 0.8 & 0.4 & 0.4 & 0.4 & 0.8 & 0.4 & 0.4 \\
\hline 0.8 & 0.4 & 0.4 & 0.4 & 0.4 & 0.4 & 0.8 & 0.4 & 0.4 & 0.4 \\
\hline 0.4 & 0.8 & 26.4 & 0.8 & 0.4 & 0.4 & 0.4 & 0.4 & 0.4 & 0.4 \\
\hline 0.4 & 0.4 & 0.8 & 0.4 & 0.4 & 0.4 & 0.4 & 0.4 & 0.4 & 0.8 \\
\hline 0.8 & 0.4 & 0.4 & 0.4 & 0.4 & 47.2 & 54 & 52.4 & 51.6 & 50.4 \\
\hline 0.4 & 0.4 & 0.4 & 0.4 & 0.8 & 0.4 & 0.4 & 0.4 & 0.4 & 9.2 \\
\hline 0.4 & 0.4 & 0.8 & 0.4 & 0.4 & 0.4 & 0.4 & 0.4 & 0.4 & 17.6 \\
\hline 0.4 & 0.4 & 0.4 & 0.8 & 0.4 & 0.4 & 0.4 & 0.4 & 0.4 & 0.4 \\
\hline
\end{tabular}




\begin{tabular}{|c|c|c|c|c|c|c|c|c|c|}
\hline 0.4 & 0.4 & 0.4 & 0.4 & 0.4 & 0.8 & 0.4 & 0.4 & 0.4 & 0.4 \\
\hline 0.4 & 0.4 & 0.4 & 0.4 & 0.4 & 0.4 & 0.4 & 0.8 & 0.4 & 0.4 \\
\hline 0.4 & 0.4 & 0.8 & 0.4 & 0.4 & 0.4 & 0.4 & 0.4 & 0.4 & 0.4 \\
\hline 0.4 & 0.4 & 0.4 & 0.4 & 0.4 & 0.4 & 0.8 & 0.4 & 0.4 & 0.4 \\
\hline 0.8 & 0.4 & 0.4 & 0.4 & 0.4 & 0.8 & 0.4 & 0.4 & 0.4 & 0.4 \\
\hline 0.8 & 0.4 & 0.4 & 0.4 & 0.4 & 0.8 & 0.4 & 0.4 & 0.4 & 0.4 \\
\hline 0.4 & 0.4 & 0.4 & 0.4 & 0.4 & 0.8 & 0.4 & 0.4 & 0.4 & 0.4 \\
\hline 0.4 & 0.4 & 0.4 & 0.8 & 0.4 & 0.4 & 0.8 & 0.4 & 0.4 & 0.4 \\
\hline 0.4 & 0.8 & 0.4 & 0.4 & 0.4 & 0.8 & 0.4 & 0.4 & 0.4 & 0.4 \\
\hline 0.4 & 0.4 & 0.4 & 0.8 & 0.4 & 0.4 & 0.4 & 0.4 & 0.4 & 0.8 \\
\hline 0.4 & 0.4 & 0.8 & 0.4 & 0.4 & 0.4 & 0.8 & 0.4 & 0.4 & 0.4 \\
\hline 0.4 & 0.4 & 0.8 & 0.4 & 0.4 & 0.8 & 0.4 & 0.4 & 0.4 & 0.8 \\
\hline 0.4 & 0.8 & 0.4 & 0.4 & 0.4 & 0.8 & 0.4 & 0.4 & 0.4 & 0.4 \\
\hline 0.4 & 0.4 & 0.4 & 0.8 & 0.4 & 0.4 & 0.8 & 0.4 & 0.4 & 0.4 \\
\hline 0.4 & 0.8 & 0.4 & 0.4 & 0.4 & 0.4 & 0.8 & 0.4 & 0.4 & 0.4 \\
\hline 0.8 & 0.4 & 0.4 & 0.4 & 0.8 & 0.4 & 0.4 & 0.4 & 0.4 & 0.8 \\
\hline 0.8 & & 0.8 & 0.4 & 0.4 & 0.4 & 0.8 & & 0.8 & 0.4 \\
\hline 0.4 & 0.8 & 0.4 & 0.4 & 0.4 & 0.4 & 0.4 & 0.4 & 0.8 & 0.4 \\
\hline 0.4 & 0.4 & 0.8 & 0.4 & 0.4 & 0.8 & 0.4 & 0.4 & 0.4 & 0.8 \\
\hline 0.4 & 0.4 & 0.8 & 0.4 & 0.4 & 0.4 & 0.4 & 0.8 & 0.4 & 0.4 \\
\hline 0.4 & 0.8 & 0.4 & 0.4 & 0.4 & 0.4 & 0.4 & 0.8 & 0.4 & 0.4 \\
\hline 0.8 & 0.4 & 0.4 & 0.4 & 0.4 & 0.4 & 0.8 & 0.4 & 0.4 & 0.4 \\
\hline 0.8 & 0.4 & 0.4 & 0.4 & 0.8 & 0.4 & 0.4 & 0.4 & 0.4 & 0.4 \\
\hline 0.4 & 0.4 & 0.4 & 0.4 & 0.4 & 0.8 & 0.4 & 0.4 & 0.4 & 0.4 \\
\hline 0.4 & 0.4 & 0.8 & 0.4 & 0.4 & 0.4 & 0.4 & 0.4 & 0.4 & 0.8 \\
\hline 0.4 & 0.4 & 0.4 & 0.4 & 0.4 & 0.8 & 0.4 & 0.4 & 0.4 & 0.4 \\
\hline 0.4 & 0.4 & 0.4 & 0.8 & & 0.8 & 0.4 & 0.4 & 0.4 & 0.4 \\
\hline 0.4 & 0.4 & 0.4 & 0.4 & 0.8 & 0.4 & 0.4 & 0.8 & 0.4 & 0.4 \\
\hline 0.4 & 0.4 & 0.8 & 0.4 & 0.4 & 0.4 & 0.4 & 0.4 & 0.4 & 0.4 \\
\hline 0.4 & 0.4 & 0.4 & 0.4 & 0.4 & 0.4 & 0.8 & 0.4 & 0.4 & \\
\hline 0.4 & 0.4 & 0.4 & 0.8 & 0.4 & 0.4 & 0.4 & 0.4 & 0.4 & 0.4 \\
\hline 0.4 & 0.4 & 0.4 & 0.4 & 0.4 & 0.8 & 0.4 & 0.4 & 0.4 & 0.4 \\
\hline 0.4 & 0.4 & 0.4 & 0.4 & 0.4 & 0.4 & 0.4 & 0.4 & 0.4 & 0.8 \\
\hline 0.8 & 0.4 & 0.4 & 0.4 & 0.4 & 0.4 & 0.4 & 0.4 & 0.4 & 0.4 \\
\hline 0.4 & 0.4 & 0.8 & 0.4 & 0.4 & 0.4 & 0.4 & 0.4 & 0.4 & 0.4 \\
\hline 0.4 & 0.4 & 0.4 & 0.4 & 0.8 & 0.4 & 0.4 & 53.6 & 56.4 & 54 \\
\hline 0.4 & 0.4 & 0.4 & 0.4 & 0.4 & 0.8 & 0.4 & 0.4 & 0.4 & 0.4 \\
\hline 0.4 & 0.4 & 0.4 & 0.4 & 0.4 & 0.4 & 0.8 & 0.4 & 0.4 & 0.4 \\
\hline 0.4 & 0.8 & 0.4 & 0.4 & 0.4 & 0.4 & 60.4 & 36.4 & 0.4 & 0.8 \\
\hline 0.4 & 0.4 & 0.4 & 0.8 & 0.4 & 16.8 & 55.6 & 53.2 & 53.2 & 51.6 \\
\hline 0.4 & 0.4 & 0.4 & 0.4 & 0.4 & 0.8 & 0.4 & 0.4 & 0.4 & 0.4 \\
\hline 0.4 & 0.4 & 0.8 & 0.4 & 0.4 & 0.4 & 0.8 & 0.4 & 0.4 & 0.8 \\
\hline 0.4 & 0.4 & 38.8 & 10.8 & 0.4 & 0.4 & 0.4 & 0.8 & 0.4 & 0.4 \\
\hline 0.8 & 0.4 & 0.4 & 0.4 & 0.8 & 0.4 & 0.4 & 0.4 & 0.8 & 0.4 \\
\hline 0.8 & 0.4 & 0.4 & 0.4 & 0.8 & 0.4 & 0.4 & 0.4 & 0.8 & 0.4 \\
\hline 0.8 & 0.4 & 0.4 & 0.8 & 0.4 & 0.4 & 0.4 & 0.8 & 0.4 & 0.4 \\
\hline 0.4 & 0.8 & 0.4 & 0.4 & 0.4 & 0.8 & 0.4 & 0.4 & 0.4 & 0.8 \\
\hline 0.4 & 0.8 & 0.4 & 0.4 & 0.4 & 0.8 & 0.4 & 0.4 & 0.4 & 0.4 \\
\hline 0.4 & 0.4 & 0.8 & 0.4 & 0.4 & 0.4 & 0.8 & 0.4 & 0.4 & 0.4 \\
\hline 0.8 & 0.4 & 0.4 & 0.4 & 0.8 & 0.4 & 0.4 & 0.4 & 0.4 & 0.4 \\
\hline 0.8 & 0.4 & 0.4 & 0.4 & 0.4 & 0.8 & 0.4 & 0.4 & 0.4 & 0.4 \\
\hline
\end{tabular}




\begin{tabular}{|c|c|c|c|c|c|c|c|c|c|}
\hline 0.4 & 0.4 & 0.4 & 0.4 & 0.4 & 0.4 & 0.4 & 0.8 & 0.4 & 0.4 \\
\hline 0.4 & 0.4 & 0.8 & 0.4 & 0.4 & 0.4 & 0.4 & 0.8 & 0.4 & 0.4 \\
\hline 0.4 & 0.4 & 0.8 & 0.4 & 0.4 & 0.8 & 0.4 & 0.4 & 0.4 & 0.4 \\
\hline 0.4 & 0.4 & 0.8 & 0.4 & 0.4 & 0.4 & 0.4 & 0.8 & 0.4 & 0.4 \\
\hline 0.4 & 0.4 & 0.4 & 0.8 & 0.4 & 0.4 & 0.4 & 0.4 & 0.4 & 0.8 \\
\hline 0.8 & 0.4 & 0.4 & 0.4 & 0.4 & 0.8 & 0.4 & 0.4 & 0.8 & 10.4 \\
\hline 0.4 & 0.4 & 0.4 & 0.4 & 0.4 & 23.2 & 0.8 & 0.8 & 0.4 & 27.6 \\
\hline 0.4 & 0.4 & 0.4 & 0.4 & 0.4 & 0.4 & 35.2 & 57.2 & 54.8 & 6.8 \\
\hline 0.4 & 0.4 & 0.4 & 0.4 & 0.4 & 53.6 & 54.4 & 48.4 & 0.8 & 32.8 \\
\hline 0.4 & 0.8 & 0.4 & 0.4 & 0.4 & 0.4 & 0.4 & 0.4 & 0.4 & 36.8 \\
\hline 0.8 & 0.4 & 0.4 & 0.4 & 0.8 & 0.4 & 0.4 & 0.4 & 0.4 & 0.4 \\
\hline 0.4 & 0.4 & 0.4 & 0.4 & 0.8 & 0.4 & 0.4 & 0.4 & 0.4 & 0.4 \\
\hline 0.4 & 0.8 & 0.4 & 0.4 & 0.4 & 0.4 & 0.4 & 0.4 & 0.4 & 0.4 \\
\hline 0.8 & 0.4 & 0.4 & 0.4 & 0.8 & 0.4 & 0.4 & 0.4 & 0.8 & 0.4 \\
\hline 0.4 & 0.8 & 0.4 & 0.4 & 0.8 & 0.4 & 0.4 & 0.8 & 0.4 & 0.4 \\
\hline 0.8 & 0.4 & 0.4 & 0.4 & 0.8 & 0.4 & 0.4 & 0.8 & 0.4 & 0.4 \\
\hline 0.4 & 0.4 & 0.4 & 0.8 & 0.4 & 0.4 & 0.4 & 0.4 & 0.4 & 0.4 \\
\hline 0.4 & 0.4 & 0.8 & 0.4 & 0.4 & 0.4 & 0.4 & 0.4 & 0.4 & 0.4 \\
\hline 0.4 & 0.4 & 0.4 & 0.4 & 0.4 & 0.8 & 0.4 & 0.4 & 0.4 & 0.4 \\
\hline 0.4 & 0.4 & 0.8 & 0.4 & 0.4 & 0.4 & 0.8 & 6.4 & 62 & 53.6 \\
\hline 0.4 & 0.8 & 0.4 & 0.4 & 0.4 & 0.4 & 21.2 & 56 & 53.6 & 52.4 \\
\hline 0.4 & 0.8 & 0.4 & 34.4 & 54 & 54.8 & 53.2 & 52.4 & 53.2 & 20.8 \\
\hline 0.4 & 0.4 & 0.8 & & 17.6 & 60.8 & 58 & 56.4 & 54.8 & 53.2 \\
\hline 0.4 & 0.4 & 0.8 & 0.4 & 0.4 & 0.4 & 0.4 & 0.8 & 0.4 & 0.4 \\
\hline 0.8 & 0.4 & 0.4 & 0.4 & 0.4 & 0.8 & 0.4 & 0.4 & 0.4 & 0.8 \\
\hline 0.4 & 0.4 & 0.4 & 0.8 & 0.4 & 0.4 & 0.4 & 0.4 & 0.4 & 0.4 \\
\hline 0.4 & 0.4 & 0.4 & 0.4 & 0.4 & 0.4 & 0.8 & 0.4 & 0.4 & 0.4 \\
\hline 0.4 & 0.4 & 0.4 & 0.8 & 0.4 & 0.4 & 0.4 & 0.4 & 0.4 & 0.4 \\
\hline 0.8 & 0.4 & 0.4 & 0.4 & 0.4 & 0.4 & 7.2 & 62.4 & 54 & 52.4 \\
\hline 0.4 & 0.4 & 0.4 & 0.8 & 0.4 & 32 & 51.6 & 52.4 & 51.6 & 50.8 \\
\hline 0.4 & 0.4 & 0.4 & 0.8 & 0.4 & 0.4 & 0.4 & 0.4 & 0.4 & 0.8 \\
\hline 0.4 & 0.8 & 0.4 & 0.4 & 0.4 & 0.4 & 0.4 & 0.4 & 0.4 & 0.4 \\
\hline 0.4 & 0.4 & 0.8 & 0.4 & 0.4 & 0.4 & 0.4 & 0.4 & 0.8 & 0.4 \\
\hline 0.4 & 0.4 & 0.8 & 0.4 & 0.4 & 18 & 56.4 & 53.6 & 52.8 & 52 \\
\hline 0.4 & 0.4 & 0.4 & 0.4 & 0.4 & 59.2 & 55.2 & 55.2 & 54 & 52.8 \\
\hline 0.4 & 0.4 & 0.4 & 0.4 & 55.2 & 56 & 54.8 & 54.8 & 55.2 & 53.6 \\
\hline 0.4 & 0.4 & 0.4 & 35.2 & 53.6 & 44 & 0.8 & 22.4 & 58 & 22.8 \\
\hline 0.8 & 0.4 & 0.4 & 0.8 & 0.4 & 0.4 & 0.4 & 0.8 & 0.4 & 0.4 \\
\hline 0.8 & 0.4 & 0.4 & 0.4 & 0.8 & 0.4 & 0.4 & 0.4 & 0.4 & 0.8 \\
\hline 0.4 & 0.4 & 0.4 & 0.8 & 0.4 & 0.4 & 0.4 & 0.4 & 0.4 & 0.8 \\
\hline 0.4 & 0.4 & 0.4 & 0.4 & 0.4 & 0.8 & 0.4 & 0.4 & 0.4 & 0.4 \\
\hline 0.4 & 0.4 & 0.8 & 0.4 & 0.4 & 0.4 & 0.4 & 0.8 & 0.4 & 0.4 \\
\hline 0.4 & 0.8 & 0.4 & 0.4 & 0.8 & 0.4 & 0.4 & 0.4 & 0.4 & 0.8 \\
\hline 0.4 & 0.4 & 0.4 & 0.4 & 0.4 & 0.8 & 0.4 & 0.4 & 0.4 & 0.8 \\
\hline 0.4 & 0.4 & 0.4 & 0.4 & 0.4 & 0.4 & 0.8 & 0.4 & 0.4 & 0.4 \\
\hline 0.4 & 0.8 & 0.4 & 0.4 & 0.4 & 0.4 & 0.8 & 0.4 & 0.4 & 0.4 \\
\hline 0.4 & 0.4 & 0.4 & 0.4 & 0.8 & 0.4 & 0.4 & 0.4 & 0.4 & 0.4 \\
\hline 0.4 & 0.4 & 0.4 & 0.4 & 32.8 & 56 & 54.8 & 54.8 & 55.6 & 28 \\
\hline 0.4 & 0.4 & 0.4 & 0.8 & 0.4 & 31.6 & 56 & 0.8 & 0.4 & 0.4 \\
\hline 0.8 & 0.4 & 0.4 & 15.2 & 57.6 & 54.8 & 54.8 & 54.8 & 54.8 & 53.6 \\
\hline 0.4 & 0.4 & 0.4 & 16.4 & 58.8 & 57.2 & 52.4 & 55.6 & 56 & 54.8 \\
\hline
\end{tabular}




\begin{tabular}{|c|c|c|c|c|c|c|c|c|c|}
\hline 0.4 & 0.4 & 0.4 & 48.8 & 56 & 55.2 & 54.8 & 54.8 & 53.6 & 53.2 \\
\hline 0.4 & 0.4 & 0.4 & 0.4 & 21.2 & 55.6 & 52.4 & 52.4 & 51.6 & 1.6 \\
\hline 0.4 & 0.4 & 0.4 & 0.4 & 0.4 & 0.4 & 0.4 & 0.8 & 0.4 & 0.4 \\
\hline 0.4 & 0.8 & 0.8 & 63.2 & 56 & 33.6 & 33.2 & 36.8 & 35.2 & 34.8 \\
\hline 0.8 & & \begin{tabular}{l|l}
0.8 \\
\end{tabular} & 49.6 & 56.8 & 56.8 & 57.2 & 56.8 & 55.6 & 55.2 \\
\hline \begin{tabular}{|l|}
0.8 \\
\end{tabular} & 0.4 & 0.4 & 4.8 & 61.2 & 56 & 54.8 & 54.4 & 54.4 & 53.6 \\
\hline 0.4 & 0.4 & 3.2 & 58.8 & 53.6 & 54 & 53.6 & 54 & 53.6 & 51.6 \\
\hline 0.4 & 0.4 & 33.2 & 49.6 & 52 & 52 & 52 & 51.6 & 52 & 50.8 \\
\hline 0.8 & 0.4 & 0.4 & 0.4 & 0.4 & 0.8 & 0.4 & 0.4 & 0.4 & 0.4 \\
\hline 0.4 & 0.4 & 0.8 & 0.4 & 0.4 & 0.4 & 0.4 & 0.4 & 0.4 & 0.8 \\
\hline 0.4 & 0.4 & 0.8 & 44.8 & 1.6 & 0.8 & 0.4 & 41.6 & 55.2 & 53.6 \\
\hline 0.4 & 0.4 & 0.8 & 14.8 & 56.4 & 54.8 & 55.2 & 55.6 & 54.8 & 54.4 \\
\hline 0.4 & 0.4 & 0.8 & 35.2 & 57.2 & 56 & 56.4 & 56 & 56 & 53.2 \\
\hline 0.8 & 0.4 & 0.4 & 33.6 & 54.4 & 54.8 & 54 & 48.8 & 52.8 & 11.2 \\
\hline 0.4 & 0.4 & 0.4 & 0.8 & 0.4 & 0.4 & 0.4 & 0.4 & 0.4 & 0.4 \\
\hline 0.8 & 0.4 & 0.4 & 0.4 & 0.4 & 0.4 & 0.4 & 0.8 & 0.4 & 0.4 \\
\hline 0.4 & 0.8 & 0.4 & 0.4 & 0.4 & 0.4 & 0.4 & 0.4 & 0.4 & 0.4 \\
\hline 0.4 & 0.4 & 0.4 & 0.4 & 0.4 & 8 & 0.8 & 0.4 & 0.4 & 0.4 \\
\hline 0.4 & 0.4 & 0.8 & 66.8 & 59.2 & 59.6 & 59.2 & 58.4 & 58 & 57.6 \\
\hline 0.4 & 0.4 & 0.4 & 52 & 59.6 & 59.2 & 58.8 & 57.6 & 57.2 & 18.4 \\
\hline 0.4 & 0.4 & 0.4 & 47.2 & 53.6 & 53.2 & 54 & 52 & 54 & 52 \\
\hline 0.4 & 0.4 & 0.4 & 0.4 & 0.4 & 53.2 & 54.4 & 53.6 & 53.6 & 52 \\
\hline 0.4 & 0.4 & 0.4 & 0.4 & 0.4 & 0.4 & 0.4 & 0.4 & 0.4 & 0.8 \\
\hline 0.4 & 0.4 & 0.4 & 0.4 & 0.4 & 0.8 & & 0.8 & 0.4 & 0.4 \\
\hline 0.4 & 0.4 & 0.4 & 58.8 & 57.6 & 56.8 & 57.2 & 56.4 & 56.4 & 54.8 \\
\hline 0.4 & 0.4 & 0.4 & 49.6 & 58.4 & 58.8 & 58.8 & 57.6 & 58 & 56.8 \\
\hline 0.4 & 0.4 & \begin{tabular}{l|l}
0.8 \\
\end{tabular} & 45.6 & 52 & 53.6 & 52.4 & 52.8 & 52.4 & 44.4 \\
\hline 0.4 & 0.4 & 0.4 & 43.6 & 50.8 & 51.2 & 51.2 & 52.4 & 51.2 & 51.2 \\
\hline 0.4 & 0.4 & 0.4 & 48 & 56 & 56 & 55.2 & 56 & 56.8 & 55.6 \\
\hline 0.4 & 0.4 & 0.4 & 0.4 & 0.8 & 0.4 & 0.4 & 0.4 & 0.4 & 0.4 \\
\hline 0.4 & 0.4 & 0.4 & 0.4 & \begin{tabular}{|l|}
0.8 \\
\end{tabular} & \begin{tabular}{|c|}
0.4 \\
\end{tabular} & 0.4 & 0.4 & 0.4 & 0.4 \\
\hline 0.8 & 0.4 & 0.4 & 54 & 59.2 & 57.2 & 57.6 & 58.8 & 59.2 & 61.6 \\
\hline 0.4 & 0.8 & 0.4 & 51.6 & 55.6 & 56 & 55.6 & 56 & 55.6 & 55.6 \\
\hline 0.8 & & 0.8 & 0.4 & 63.6 & 59.2 & 57.6 & 57.6 & 56.8 & 55.2 \\
\hline & 0.8 & 0.4 & 61.2 & 58.4 & 58.8 & 58.8 & 57.6 & 57.6 & 56.8 \\
\hline 0.4 & 0.4 & 0.4 & 58.4 & 57.2 & 56.4 & 66.4 & 81.2 & 80 & 76.8 \\
\hline 0.4 & 0.4 & 0.4 & 0.4 & 0.4 & 0.4 & 0.4 & 0.4 & 0.4 & 0.4 \\
\hline 0.4 & 0.8 & 0.4 & 0.4 & 0.4 & 0.4 & 0.4 & 0.8 & 0.4 & 0.4 \\
\hline 0.4 & 0.4 & 2.4 & 67.2 & 55.2 & 60 & 66 & 58 & 58.4 & 57.2 \\
\hline 0.4 & 0.4 & 0.4 & 61.6 & 59.2 & 58.4 & 58.8 & 59.6 & 57.2 & 54.4 \\
\hline 0.4 & 0.4 & 0.4 & 59.2 & 56 & 55.2 & 59.6 & 57.2 & 58.4 & 58 \\
\hline 0.4 & 0.4 & 0.4 & 58.4 & 55.2 & 55.6 & 55.6 & 53.2 & 44.8 & 40.4 \\
\hline 0.4 & 0.4 & 0.4 & 62 & 58 & 58.4 & 56 & 58 & 56.8 & 56.4 \\
\hline 0.4 & 0.4 & 0.4 & 0.4 & 0.4 & 0.4 & 0.4 & 0.4 & 0.4 & 0.4 \\
\hline 0.4 & 0.4 & 0.4 & 0.4 & 0.4 & 0.4 & 0.4 & 0.4 & 0.4 & 0.8 \\
\hline 0.4 & 0.4 & 21.6 & 62.4 & 58.4 & 62.4 & 16.8 & 59.6 & 59.6 & 56.8 \\
\hline 0.4 & 0.4 & 0.4 & 29.2 & 61.2 & 59.6 & 60 & 60.4 & 60.4 & 59.2 \\
\hline 0.4 & 0.8 & 3.6 & 61.2 & 55.6 & 56.4 & 57.2 & 59.2 & 59.2 & 60 \\
\hline 0.8 & 0.4 & 0.4 & 65.6 & 59.2 & 60 & 58.8 & 59.2 & 59.2 & 56.4 \\
\hline 0.4 & 0.4 & 10 & 60 & 57.6 & 58 & 58.4 & 58.4 & 58.8 & 56.8 \\
\hline 0.4 & 0.4 & 0.4 & 0.4 & 0.4 & 0.4 & 0.4 & 0.4 & 0.4 & 0.4 \\
\hline
\end{tabular}




\begin{tabular}{|c|c|c|c|c|c|c|c|c|c|}
\hline 0.8 & 0.4 & 0.4 & 0.4 & 0.4 & 0.4 & 0.4 & 0.4 & 0.8 & 0.4 \\
\hline 0.4 & 0.4 & 22 & 66.8 & 61.2 & 60 & 60 & 60 & 59.6 & 58 \\
\hline 0.4 & 0.4 & 0.8 & 22 & 68.4 & 60 & 60 & 64.8 & 66 & 64.4 \\
\hline 0.4 & 0.4 & 0.4 & 58.4 & 59.6 & 60.4 & 60.4 & 60 & 60 & 59.2 \\
\hline 0.8 & 0.4 & 0.4 & 56.4 & 58.8 & 60 & 60 & 59.2 & 59.6 & 58.4 \\
\hline 0.4 & 0.4 & 0.4 & 61.2 & 59.6 & 60 & 60.8 & 64.4 & 62.4 & 61.2 \\
\hline 0.8 & 0.4 & 0.4 & 0.4 & 0.4 & 0.4 & 0.4 & 0.4 & 0.4 & 0.4 \\
\hline 0.4 & 0.4 & 0.4 & 0.4 & 0.4 & 0.8 & 0.4 & 0.4 & 0.4 & 0.4 \\
\hline 0.4 & 0.4 & 21.6 & 68 & 62.8 & 63.2 & 64 & 62.4 & 16.8 & 63.6 \\
\hline 0.4 & 0.4 & 0.4 & 60 & 61.6 & 61.6 & 61.6 & 61.6 & 61.2 & 60.4 \\
\hline 0.4 & 0.4 & 0.8 & 60 & 59.6 & 60.8 & 60.8 & 60.8 & 59.6 & 60.4 \\
\hline 0.4 & 0.4 & 0.4 & 57.6 & 62.4 & 61.6 & 61.2 & 60.4 & 60 & 59.2 \\
\hline 0.4 & 0.4 & 0.4 & 56 & 57.2 & 56.4 & 54 & 54 & 52.4 & 52.8 \\
\hline 0.4 & 0.4 & 0.4 & 0.4 & 0.4 & 0.4 & 0.4 & 0.4 & 0.8 & \\
\hline 0.4 & 0.4 & 0.4 & 0.4 & 0.4 & 0.4 & 0.4 & 0.4 & 0.4 & 0.4 \\
\hline 0.4 & 0.8 & 18.8 & 35.6 & 64.4 & 61.2 & 56.4 & 56.8 & 56.4 & 52.8 \\
\hline 0.4 & 0.4 & 0.4 & 60.4 & 59.6 & 60 & 58.8 & 58.8 & 57.2 & 56.8 \\
\hline 0.8 & 0.4 & 0.4 & 58 & 59.2 & 54.4 & 56.4 & 55.6 & 56.8 & 57.6 \\
\hline 0.4 & 0.8 & 0.4 & 59.2 & 59.2 & 58 & 58 & 57.6 & 58.8 & 58.8 \\
\hline 0.4 & 0.4 & 0.8 & & 0.4 & 0.8 & 0.4 & 0.4 & 0.8 & 0.4 \\
\hline 0.4 & 0.8 & 0.4 & 0.8 & 0.4 & 0.8 & 0.4 & 0.4 & 0.8 & 0.8 \\
\hline 0.4 & 0.8 & 0.4 & 0.8 & 0.8 & 0.4 & 0.8 & 0.4 & 0.8 & 0.4 \\
\hline 0.8 & 0.4 & 0.8 & 72.4 & 69.2 & 66.4 & 65.6 & 64.8 & 64.4 & 64.4 \\
\hline 0.8 & 0.4 & 0.4 & 64 & 67.6 & 64.4 & 64.8 & 64 & 65.2 & 64.4 \\
\hline 0.8 & 0.4 & 0.4 & 64 & 64 & 64.8 & 64.4 & 64.8 & 65.6 & 64 \\
\hline 0.4 & 0.4 & 0.8 & 46.4 & 62 & 63.6 & 61.6 & 61.2 & 59.6 & 58 \\
\hline 0.8 & & \begin{tabular}{l|l}
0.8 \\
\end{tabular} & 0.4 & 0.4 & 0.4 & 0.4 & 0.4 & 0.4 & 0.8 \\
\hline 0.4 & 0.8 & & 0.8 & 0.4 & 0.4 & 0.4 & 0.4 & 0.4 & 0.4 \\
\hline 0.4 & 0.4 & 0.4 & 0.4 & 0.4 & 0.4 & 0.4 & 0.4 & 0.4 & 0.8 \\
\hline 0.4 & 0.4 & 0.4 & 0.4 & 70.8 & 64.4 & 62.8 & 62.4 & 62.4 & 61.6 \\
\hline 0.4 & 0.4 & 0.4 & 0.8 & 71.6 & 62 & 61.6 & 60.8 & 60 & 58.4 \\
\hline 0.4 & 0.8 & 3.2 & 72 & 62.4 & 63.6 & 63.2 & 62 & 62.8 & 60.8 \\
\hline 0.4 & 0.8 & 14.8 & 68.8 & 61.6 & 62.8 & 62.8 & 62.4 & 62 & 60.4 \\
\hline 0.8 & 0.4 & 15.2 & 69.6 & 61.2 & 60 & 60.4 & 58 & 57.6 & 58.8 \\
\hline 0.8 & 0.4 & 0.8 & 0.4 & 0.4 & 0.4 & 0.4 & 0.8 & 0.4 & 0.4 \\
\hline 0.4 & 0.4 & 0.4 & 0.4 & 0.4 & 0.4 & 0.4 & 0.8 & 0.4 & 0.4 \\
\hline 0.8 & 0.4 & 14.8 & 75.6 & 67.2 & 66 & 66.4 & 64.8 & 64.8 & 63.2 \\
\hline 0.4 & 0.8 & 14.4 & 70.8 & 64 & 64.4 & 63.6 & 62.8 & 62 & 61.2 \\
\hline 0.8 & 0.4 & 14.8 & 68.8 & 64 & 63.2 & 63.6 & 63.6 & 63.2 & 62.8 \\
\hline 0.4 & 0.4 & 15.2 & 70.8 & 64 & 63.2 & 63.2 & 64 & 63.6 & 64.8 \\
\hline 0.4 & 0.4 & 0.8 & 0.8 & 0.4 & 0.4 & 0.8 & 0.4 & 0.8 & 0.4 \\
\hline 0.8 & 0.4 & 0.8 & 0.4 & 0.8 & 0.4 & 0.8 & 0.4 & 0.4 & 0.8 \\
\hline \begin{tabular}{|l|}
0.8 \\
\end{tabular} & 0.4 & 0.8 & 0.4 & 0.4 & 0.4 & 0.4 & 0.4 & 0.8 & 0.4 \\
\hline 0.4 & 0.8 & 15.2 & 78.4 & 68.8 & 66.8 & 66.4 & 66.8 & 66 & 65.2 \\
\hline 0.8 & 0.4 & 15.2 & 74.8 & 68 & 66 & 65.6 & 66 & 67.2 & 66.4 \\
\hline 0.8 & 0.8 & 15.2 & 74 & 66 & 64.8 & 64 & 64.4 & 65.2 & 64.8 \\
\hline 0.4 & 0.8 & 15.2 & 71.6 & 64.8 & 63.2 & 62.8 & 62.8 & 62.4 & 62.8 \\
\hline 0.8 & 0.4 & 15.2 & 71.6 & 64 & 63.2 & 61.6 & 61.2 & 60.8 & 61.2 \\
\hline 0.8 & 0.8 & 0.4 & 0.8 & 0.4 & 0.8 & 0.8 & 0.4 & 0.8 & 0.8 \\
\hline 0.8 & 0.4 & 0.8 & 0.8 & 0.4 & 0.8 & 0.4 & 0.8 & 0.8 & 0.8 \\
\hline 0.4 & 0.8 & 15.2 & 78.4 & 67.6 & 65.6 & 64.4 & 65.2 & 64.8 & 64.8 \\
\hline
\end{tabular}




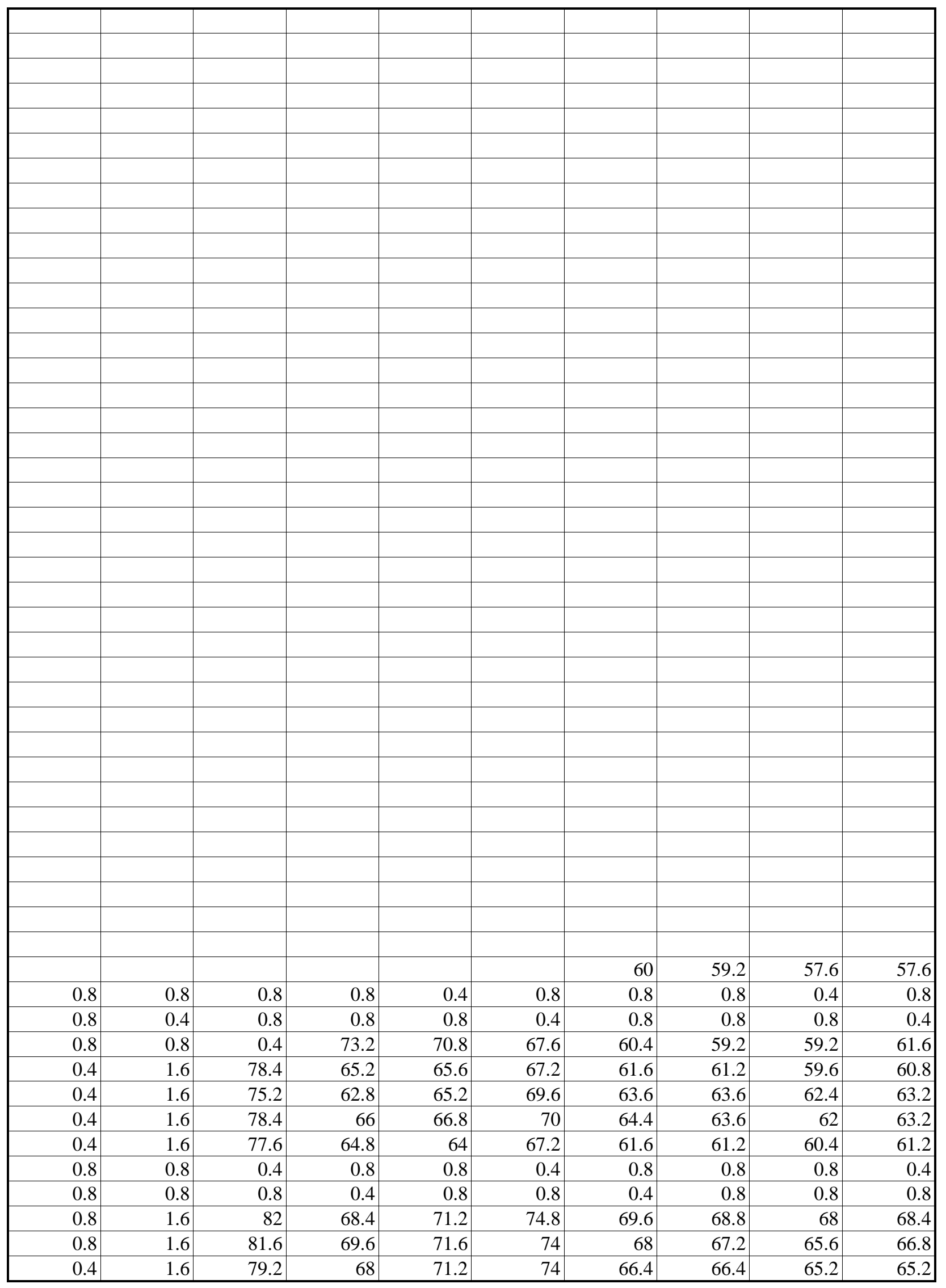




\begin{tabular}{|c|c|c|c|c|c|c|c|c|c|}
\hline 0.8 & 1.6 & 79.6 & 67.2 & 69.6 & 70.8 & 66 & 65.6 & 63.2 & 62.8 \\
\hline 0.8 & 1.2 & 79.6 & 67.2 & 68.4 & 70.8 & 65.6 & 66.4 & 64.8 & 65.2 \\
\hline 0.8 & 0.4 & 0.8 & 0.8 & 0.4 & 0.8 & 0.8 & 0.4 & 0.4 & 0.8 \\
\hline 0.8 & 0.4 & 0.8 & 0.8 & 0.4 & 0.8 & 0.4 & 0.8 & 0.4 & 0.8 \\
\hline 0.4 & 1.6 & 79.2 & 66 & 68.4 & 72 & 65.6 & 66 & 66 & 66 \\
\hline 0.8 & 1.2 & 78.4 & 65.6 & 70 & 72.4 & 64.8 & 65.2 & 63.6 & 64.8 \\
\hline 0.8 & 1.6 & 77.6 & 65.2 & 68 & 70.8 & 64.8 & 64.8 & 62.8 & 63.6 \\
\hline 0.8 & 1.6 & 78.4 & 66.8 & 69.2 & 72 & 65.2 & 65.6 & 63.6 & 62 \\
\hline 0.4 & 1.6 & 78.8 & 66.4 & 69.6 & 72.8 & 66 & 66.8 & 65.6 & 64.8 \\
\hline 0.4 & 0.8 & 0.8 & 0.8 & 0.4 & 0.8 & 0.8 & 0.4 & 0.8 & 0.8 \\
\hline 0.4 & 0.8 & 0.8 & 0.4 & 0.8 & 0.4 & 0.8 & 0.4 & 0.8 & 0.8 \\
\hline 0.8 & 0.4 & 0.8 & 0.8 & 0.4 & 0.8 & 0.8 & 0.8 & 0.4 & 0.8 \\
\hline 0.4 & 0.8 & 0.8 & 0.4 & 0.8 & 0.8 & 0.4 & 0.8 & 0.4 & 0.8 \\
\hline 0.8 & 0.4 & 10 & 78.8 & 66.4 & 64.8 & 64.4 & 61.2 & 60.8 & 60.4 \\
\hline 0.8 & 2 & 80 & 67.6 & 69.2 & 70.8 & 64 & 64.8 & 63.6 & 63.6 \\
\hline 0.8 & 1.6 & 80 & 68.4 & 70.4 & 74.4 & 67.6 & 66.8 & 65.2 & 64 \\
\hline 0.8 & 0.8 & 0.8 & 0.4 & 0.8 & 0.4 & 0.8 & 0.8 & 0.4 & 0.8 \\
\hline 0.8 & 0.4 & \begin{tabular}{l|l}
0.8 \\
\end{tabular} & 0.8 & 0.4 & 0.8 & 0.4 & 0.8 & 0.4 & 0.8 \\
\hline 0.8 & 1.6 & 84 & 77.2 & 77.6 & 80 & 72 & 71.2 & 71.2 & 71.6 \\
\hline 0.8 & 1.6 & 84.4 & 74.8 & 75.2 & 75.6 & 66.4 & 66 & 64.4 & 66 \\
\hline 0.8 & 1.6 & 74.8 & 65.2 & 66.4 & 68 & 63.6 & 64 & 62.8 & 63.2 \\
\hline 0.8 & 1.6 & 73.6 & 64 & 66 & 67.2 & 62.8 & 63.2 & 62.4 & 61.2 \\
\hline 0.8 & 1.6 & 73.6 & 64.4 & 66 & 66 & 61.6 & 63.2 & 59.6 & 59.2 \\
\hline 0.4 & 0.8 & 0.4 & 0.8 & 0.4 & 0.8 & 0.4 & 0.8 & 0.8 & 0.4 \\
\hline 0.8 & 0.8 & 0.4 & 0.8 & 0.4 & 0.8 & 0.4 & 0.8 & 0.4 & 0.8 \\
\hline 0.8 & 1.6 & 81.2 & 67.6 & 69.6 & 74.4 & 68.4 & 68.8 & 67.6 & 66 \\
\hline 0.8 & 1.6 & 80 & 67.2 & 71.2 & 74 & 69.6 & 68.4 & 68 & 67.6 \\
\hline 0.8 & 1.6 & 79.6 & 68 & 72.8 & 76 & 66.4 & 68.4 & 69.2 & 68.8 \\
\hline 0.8 & 1.6 & 80 & 68.4 & 70.4 & 74 & 68.8 & 68.8 & 67.2 & 66.4 \\
\hline 0.4 & 2 & 78.8 & 66.8 & 70 & 73.2 & 67.2 & 66.8 & 65.6 & 66 \\
\hline 0.4 & 0.8 & 0.8 & 0.4 & 0.8 & 0.8 & 0.4 & 0.8 & 0.4 & 0.8 \\
\hline 0.8 & 0.4 & 0.8 & 0.4 & 0.8 & 0.8 & 0.4 & 0.8 & 0.4 & 0.8 \\
\hline 0.8 & 1.6 & 80.8 & 68.4 & 68.4 & 70 & 65.6 & 65.6 & 64 & 63.6 \\
\hline 0.8 & 1.2 & 74 & 64.8 & 65.6 & 67.2 & 63.2 & 63.2 & 62 & 62 \\
\hline 0.4 & 1.6 & 75.2 & 65.2 & 66 & 67.2 & 63.6 & 63.6 & 62.4 & 62.4 \\
\hline 0.4 & 1.6 & 74.4 & 60.8 & 62 & 63.6 & 60.8 & 59.6 & 58.8 & 59.6 \\
\hline 0.8 & 1.6 & 71.2 & 60.8 & 60.8 & 60.8 & 61.6 & 61.2 & 60.4 & 60.8 \\
\hline 0.8 & 0.8 & 0.4 & 0.8 & 0.4 & 0.8 & 0.4 & 0.8 & 0.4 & 0.8 \\
\hline 0.8 & 0.4 & 0.8 & 0.8 & 0.4 & 0.8 & 0.4 & 0.8 & 0.4 & 0.8 \\
\hline 0.4 & 2 & 74.4 & 64 & 64 & 64.8 & 61.6 & 61.2 & 61.2 & 62.8 \\
\hline 0.4 & 2 & 72.8 & 63.2 & 63.2 & 63.6 & 61.2 & 61.6 & 60.4 & 59.6 \\
\hline 0.8 & 1.6 & 73.2 & 62.8 & 64 & 61.6 & 62.4 & 60.8 & 60.4 & 60.8 \\
\hline \begin{tabular}{|l|}
0.8 \\
\end{tabular} & 1.6 & 72.8 & 62.4 & 62.8 & 62 & 60.8 & 61.2 & 61.6 & 62.4 \\
\hline 0.4 & 2 & 72 & 61.6 & 60.4 & 62.4 & 63.2 & 62.4 & 61.2 & 61.2 \\
\hline 0.4 & 0.8 & 0.4 & 0.8 & 0.4 & 0.8 & 0.4 & 0.8 & 0.4 & 0.8 \\
\hline 0.4 & 0.8 & 0.4 & 0.4 & 0.4 & 0.8 & 0.4 & 0.4 & 0.4 & 0.4 \\
\hline 0.4 & 1.6 & 74 & 62 & 63.2 & 64.4 & 63.6 & 60.8 & 60 & 60 \\
\hline 0.4 & 1.6 & 70.4 & 58 & 60.4 & 62 & 62.4 & 63.2 & 62.4 & 63.6 \\
\hline 0.4 & 1.6 & 72 & 60.8 & 63.6 & 64 & 64.4 & 62.8 & 59.6 & 58.8 \\
\hline 0.8 & 1.6 & 71.2 & 60.8 & 62.4 & 63.6 & 63.2 & 63.2 & 61.6 & 61.2 \\
\hline 0.4 & 1.6 & 69.6 & 59.6 & 62 & 62.4 & 61.2 & 60.4 & 60.4 & 59.6 \\
\hline
\end{tabular}




\begin{tabular}{|c|c|c|c|c|c|c|c|c|c|}
\hline 0.4 & 0.4 & 0.4 & 0.4 & 0.8 & & 0.8 & 0.4 & 0.4 & 0.4 \\
\hline 0.4 & 0.8 & 0.4 & 0.4 & 0.4 & 0.4 & 0.4 & 0.8 & 0.4 & 0.8 \\
\hline 0.8 & 1.6 & 76 & 61.2 & 63.2 & 66.4 & 66 & 66 & 64 & 66 \\
\hline 0.4 & 2 & 74.4 & 61.6 & 64 & 66 & 66 & 66.4 & 65.2 & 66 \\
\hline 0.8 & 1.6 & 70.4 & 60 & 62.4 & 63.6 & 64.8 & 63.2 & 63.2 & 63.2 \\
\hline 0.4 & 1.6 & 70 & 59.6 & 60.8 & 62.4 & 62 & 61.6 & 60 & 60 \\
\hline 0.4 & 1.6 & 65.2 & 56.4 & 57.6 & 58.4 & 58 & 58 & 56.8 & 56.4 \\
\hline 0.8 & 0.4 & 50.8 & 53.2 & 52.4 & 52.8 & 52.8 & 52.4 & 52 & 52 \\
\hline 0.4 & 0.4 & 0.4 & 0.8 & 0.4 & 0.4 & 0.4 & 0.4 & 0.4 & 0.4 \\
\hline 0.8 & 0.4 & 0.4 & 0.4 & 0.8 & 57.2 & 57.6 & 57.6 & 55.2 & 54.8 \\
\hline 0.4 & 0.8 & 0.4 & 0.4 & 0.4 & 0.4 & 52.4 & 61.2 & 58.4 & 57.6 \\
\hline 0.8 & 0.4 & 0.4 & 0.4 & 0.4 & 66.8 & 62 & 57.2 & 56 & 54.8 \\
\hline 1.2 & 1.2 & 1.2 & 0.8 & 1.2 & 1.2 & 69.2 & 58.8 & 56 & 56 \\
\hline 1.2 & 1.2 & 0.8 & 1.2 & 39.2 & 61.2 & 59.2 & 58.8 & 57.2 & 57.2 \\
\hline 1.6 & 1.2 & 1.2 & 1.2 & 1.2 & 1.2 & 1.2 & 1.2 & 1.2 & 1.2 \\
\hline 0.8 & 1.2 & 0.8 & 0.8 & 1.2 & 0.8 & 0.8 & 0.8 & 1.2 & 0.8 \\
\hline 1.2 & 0.8 & 0.8 & 1.2 & 0.8 & 0.8 & 1.2 & 0.8 & 0.8 & 1.2 \\
\hline 1.2 & 0.8 & 1.2 & 0.8 & 1.2 & 0.8 & 1.2 & 0.8 & 1.2 & 62.4 \\
\hline 1.2 & 1.2 & 0.8 & 1.2 & 38 & 61.2 & 59.2 & 58.4 & 57.2 & 56 \\
\hline 1.2 & 0.8 & 1.2 & 0.8 & 38 & 61.2 & 59.2 & 58.4 & 57.6 & 56.8 \\
\hline 1.2 & 1.2 & 0.8 & 1.2 & 38.8 & 62.4 & 59.2 & 58 & 56.4 & 55.6 \\
\hline 1.2 & 1.2 & 1.2 & 1.2 & 1.2 & 0.8 & 1.2 & 1.2 & 1.2 & 0.8 \\
\hline 1.2 & 0.8 & 1.2 & 0.8 & 1.2 & 0.8 & 0.8 & 1.2 & 0.8 & 0.8 \\
\hline 0.8 & 1.2 & 0.8 & 0.8 & 1.2 & 0.8 & 0.8 & 1.2 & 0.8 & 1.2 \\
\hline 1.2 & 1.2 & 0.8 & 1.2 & 1.2 & 0.8 & 1.2 & 0.8 & 1.2 & 1.2 \\
\hline 1.2 & 1.2 & 0.8 & 1.2 & 36.4 & 50.4 & 1.2 & 1.2 & 1.2 & 39.6 \\
\hline 0.8 & 1.2 & 0.8 & 1.2 & 0.8 & 1.2 & 0.8 & 0.8 & 1.2 & 0.8 \\
\hline 0.8 & 1.2 & 1.2 & 0.8 & 39.2 & 62 & 59.6 & 59.2 & 59.2 & 59.2 \\
\hline 1.2 & 0.8 & 1.2 & 1.2 & 0.8 & 1.2 & 1.2 & 1.2 & 0.8 & 1.2 \\
\hline 1.2 & 0.8 & 1.2 & 0.8 & 1.2 & 1.2 & 1.2 & 1.2 & 0.8 & 1.2 \\
\hline 1.2 & 0.8 & 1.2 & 1.2 & 37.2 & 60 & 56.8 & 56.4 & 55.2 & 54 \\
\hline 0.8 & 1.2 & 0.8 & 1.2 & 36.4 & 58.4 & 56.8 & 56 & 54 & 53.2 \\
\hline 1.2 & 0.8 & 1.2 & 0.8 & 33.6 & 55.2 & 54 & 53.2 & 53.2 & 51.6 \\
\hline 1.2 & 0.8 & 1.2 & 0.8 & 32.4 & 54.4 & 53.2 & 53.2 & 51.6 & 50.8 \\
\hline 1.2 & 0.8 & 0.8 & 0.8 & 35.2 & 56.8 & 55.2 & 55.6 & 53.6 & 54 \\
\hline 1.2 & 0.8 & 1.2 & 0.8 & 1.2 & 0.8 & 1.2 & 0.8 & 0.8 & 1.2 \\
\hline 1.2 & 0.8 & 0.8 & 1.2 & 0.8 & 0.8 & 0.8 & 0.8 & 0.8 & 1.2 \\
\hline 0.8 & 1.2 & 0.8 & 0.8 & 1.2 & 0.8 & 0.8 & 39.6 & 51.2 & 49.6 \\
\hline 0.8 & 0.8 & 1.2 & 0.8 & 0.8 & 32.4 & 54.8 & 54 & 52.4 & 52.8 \\
\hline 0.8 & 1.2 & 0.8 & 0.8 & 1.2 & 36.4 & 56.4 & 55.2 & 53.2 & 1.2 \\
\hline 1.2 & 0.8 & 1.2 & 1.2 & 0.8 & 1.2 & 1.2 & 0.8 & 1.2 & 0.8 \\
\hline 0.8 & 0.8 & 1.2 & 0.8 & 0.8 & 0.8 & 1.2 & 0.8 & 0.8 & 0.8 \\
\hline 0.8 & 0.8 & 0.4 & 0.8 & 0.8 & 0.8 & 0.4 & 0.8 & 0.4 & 0.8 \\
\hline 0.8 & 0.8 & 0.8 & 0.4 & 0.8 & 0.4 & 0.8 & 0.8 & 0.4 & 0.8 \\
\hline 0.8 & 0.8 & 0.8 & 0.8 & 0.8 & 0.8 & 1.2 & 0.4 & 0.8 & 1.2 \\
\hline 1.2 & 0.8 & 1.2 & 0.8 & 0.8 & 0.8 & 0.8 & 0.8 & 1.2 & 0.8 \\
\hline 0.8 & 1.2 & 0.8 & 1.2 & 0.8 & 22 & 56.4 & 54.4 & 52.8 & 52.8 \\
\hline 1.2 & 0.8 & 0.8 & 1.2 & 37.2 & 55.2 & 54.4 & 53.6 & 53.2 & 52.4 \\
\hline 0.8 & 0.8 & 0.8 & 1.2 & 35.6 & 56.8 & 54.8 & 55.2 & 53.6 & 53.6 \\
\hline 0.8 & 0.8 & 1.2 & 0.8 & 0.8 & 1.2 & 0.8 & 1.2 & 0.8 & 0.8 \\
\hline 1.2 & 0.8 & 0.8 & 0.8 & 0.8 & 0.8 & 0.8 & 0.8 & 0.8 & 0.8 \\
\hline
\end{tabular}




\begin{tabular}{|c|c|c|c|c|c|c|c|c|c|}
\hline 0.8 & 1.2 & 0.8 & 0.8 & 1.2 & 0.8 & 0.8 & 20 & 54.8 & 6.4 \\
\hline 0.8 & 1.2 & 0.8 & 0.8 & \begin{tabular}{l|l}
0.8 \\
\end{tabular} & \begin{tabular}{|l|}
0.8 \\
\end{tabular} & 1.2 & 0.8 & 0.8 & 0.8 \\
\hline 0.8 & 1.2 & 0.8 & 0.8 & 28 & 58 & 56 & 54.8 & 53.6 & 53.2 \\
\hline 1.2 & 0.8 & 0.8 & 0.8 & 53.2 & 58.4 & 57.2 & 56.4 & 56 & 55.6 \\
\hline 1.2 & \begin{tabular}{l|l}
0.8 \\
\end{tabular} & \begin{tabular}{l|l}
0.8 \\
\end{tabular} & 0.8 & 37.2 & 59.2 & 57.2 & 57.6 & 55.6 & 54.8 \\
\hline 1.2 & 0.8 & 1.2 & 0.8 & $\begin{array}{l}0.8 \\
\end{array}$ & 1.2 & 0.8 & 0.8 & 1.2 & 0.8 \\
\hline 1.2 & 0.8 & 0.8 & 0.8 & 1.2 & 0.8 & 0.8 & 0.8 & 0.8 & 1.2 \\
\hline 0.8 & 0.8 & 1.2 & 0.8 & 45.6 & 56.4 & 54.8 & 52.8 & 52.8 & 51.6 \\
\hline 0.8 & 1.2 & 0.8 & 0.8 & 40.8 & 53.2 & 52.4 & 51.6 & 50.8 & 50.4 \\
\hline 1.2 & 0.8 & 0.8 & 0.8 & 27.2 & 1.2 & 0.8 & 0.8 & 29.6 & 51.2 \\
\hline 0.8 & 0.8 & 1.2 & 0.8 & 40 & 51.6 & 51.6 & 51.2 & 51.6 & 50.4 \\
\hline 0.8 & 1.2 & 0.8 & 0.8 & 42.4 & 53.2 & 53.6 & 52.4 & 52.4 & 42.4 \\
\hline 1.2 & 0.8 & 1.2 & 1.2 & 0.8 & 1.2 & 1.2 & 0.8 & 1.2 & 0.8 \\
\hline 1.2 & 0.8 & 1.2 & 0.8 & 0.8 & 1.2 & 0.8 & 1.2 & 0.8 & 0.8 \\
\hline 0.8 & 0.8 & 0.8 & 0.8 & 0.8 & 0.8 & 0.8 & 1.2 & 0.8 & 1.2 \\
\hline 0.8 & 1.2 & 0.8 & 0.8 & 0.8 & 0.8 & 1.2 & 0.8 & 0.8 & 0.8 \\
\hline 0.8 & 1.2 & 0.8 & 1.2 & 0.8 & 1.2 & 1.2 & 1.2 & 0.8 & 1.2 \\
\hline 0.8 & \begin{tabular}{l|l}
0.8 \\
\end{tabular} & \begin{tabular}{l|l}
0.8 \\
\end{tabular} & 0.8 & 0.8 & 1.2 & 0.4 & 1.2 & 0.8 & 0.8 \\
\hline 1.2 & 0.8 & 0.8 & 1.2 & 0.8 & 0.8 & 1.2 & 0.8 & 0.8 & 1.2 \\
\hline 0.8 & 0.8 & 0.8 & 0.8 & 0.8 & 0.8 & 0.8 & 0.8 & 0.8 & 0.8 \\
\hline 0.8 & 0.8 & 1.2 & 0.8 & \begin{tabular}{|l|}
0.8 \\
\end{tabular} & \begin{tabular}{|l|}
0.8 \\
\end{tabular} & 0.8 & 0.8 & 1.2 & 0.8 \\
\hline 1.2 & 0.8 & 0.8 & 0.8 & 0.8 & 0.8 & 0.8 & 0.8 & 1.2 & 0.8 \\
\hline 0.8 & 0.8 & 1.2 & 0.8 & 0.8 & 0.8 & 0.8 & 1.2 & 0.8 & 0.8 \\
\hline 1.6 & 1.2 & 1.2 & 0.8 & 1.2 & 1.2 & 0.8 & 1.2 & 1.2 & 0.8 \\
\hline 0.8 & 1.2 & 0.8 & 0.8 & 1.2 & 0.8 & 0.8 & 0.8 & 1.2 & 0.8 \\
\hline 0.8 & 0.8 & 0.8 & 0.8 & 0.8 & 0.8 & 0.8 & 1.2 & 0.8 & 0.4 \\
\hline 0.8 & 0.8 & \begin{tabular}{l|l}
0.8 \\
\end{tabular} & 0.8 & \begin{tabular}{|l|}
0.8 \\
\end{tabular} & \begin{tabular}{|l|}
0.8 \\
\end{tabular} & 0.8 & 0.8 & 0.8 & 0.8 \\
\hline 0.8 & 0.8 & 1.2 & 0.8 & 0.8 & 0.8 & 0.8 & 0.8 & 1.2 & 0.8 \\
\hline 1.2 & 0.8 & 0.8 & 1.2 & 0.8 & 0.8 & 0.8 & 1.2 & 0.8 & 0.8 \\
\hline 1.2 & 0.8 & 0.8 & 0.8 & 1.2 & 0.8 & 1.2 & 0.8 & 0.8 & 1.2 \\
\hline 1.2 & \begin{tabular}{|l|}
0.8 \\
\end{tabular} & \begin{tabular}{|l|}
0.8 \\
\end{tabular} & 1.2 & \begin{tabular}{|l|}
0.8 \\
\end{tabular} & \begin{tabular}{|l|}
0.8 \\
\end{tabular} & 0.8 & 1.2 & 0.8 & 0.8 \\
\hline 0.8 & 1.2 & 0.8 & 1.2 & 0.8 & 1.2 & 0.8 & 50.8 & 55.6 & 55.2 \\
\hline 1.2 & 1.2 & 1.2 & 1.2 & 1.2 & 1.2 & 1.2 & 0.8 & 1.2 & 1.2 \\
\hline 1.2 & 0.8 & 0.8 & 0.8 & 1.2 & 0.8 & 0.8 & 0.8 & 0.8 & 0.8 \\
\hline 1.2 & 0.8 & 1.2 & 0.8 & 0.8 & 0.8 & 0.8 & 0.8 & 1.2 & 1.2 \\
\hline 0.8 & 0.8 & 0.8 & 1.2 & 0.8 & 0.8 & 1.2 & 0.8 & 22 & 12.8 \\
\hline 1.2 & \begin{tabular}{l|l}
0.8 \\
\end{tabular} & 0.8 & 1.2 & 0.8 & 0.8 & 1.2 & 64.8 & 2 & 0.8 \\
\hline 1.2 & 0.8 & 1.2 & 0.8 & 1.2 & 1.2 & 1.2 & 56.8 & 56.4 & 1.2 \\
\hline 1.2 & 1.2 & 0.8 & 0.8 & 1.2 & 39.6 & 56.8 & 56 & 53.2 & 52.4 \\
\hline 1.2 & \begin{tabular}{l|l}
0.8 \\
\end{tabular} & 0.8 & 0.8 & \begin{tabular}{|l|}
0.8 \\
\end{tabular} & 34 & 54 & 52.4 & 51.6 & 50.8 \\
\hline 0.8 & 1.2 & 0.8 & 0.8 & 0.8 & 0.8 & 1.2 & 0.8 & 0.8 & 0.8 \\
\hline 0.8 & 1.2 & 0.8 & 0.8 & 0.8 & 0.8 & 1.2 & 0.8 & 1.2 & 0.8 \\
\hline 1.2 & 1.2 & 0.8 & 1.2 & 1.2 & 1.2 & 0.8 & 1.2 & 1.2 & 14.4 \\
\hline 1.2 & 1.2 & 0.8 & 0.8 & 1.2 & 0.8 & 1.2 & 0.8 & 0.8 & 0.8 \\
\hline 0.8 & 1.2 & 0.8 & 1.2 & 0.8 & 0.8 & 1.2 & 0.8 & 0.8 & 0.8 \\
\hline 0.8 & 0.8 & 0.8 & 1.2 & 0.8 & 0.8 & 0.8 & 0.8 & 0.8 & 1.2 \\
\hline 1.2 & 0.8 & 1.2 & 1.2 & 0.8 & 1.2 & 0.8 & 1.2 & 0.8 & 1.2 \\
\hline 1.2 & 0.8 & 0.8 & 1.2 & 0.8 & 1.2 & 0.8 & 0.8 & 1.2 & 0.8 \\
\hline 1.2 & 0.8 & 0.8 & 0.8 & 1.2 & 0.8 & 0.8 & 0.8 & 0.8 & 0.8 \\
\hline 0.8 & 1.2 & 0.8 & 0.8 & 0.8 & 0.8 & 0.8 & 1.2 & 0.8 & 1.2 \\
\hline 0.8 & 0.8 & 0.8 & 1.2 & 0.8 & 68.4 & 59.2 & 58 & 56.4 & 56.8 \\
\hline
\end{tabular}


chlr

\begin{tabular}{|c|c|c|c|c|c|c|c|c|c|}
\hline 1.2 & 0.8 & 1.2 & 0.8 & 47.6 & 60.4 & 58.4 & 58 & 56.4 & 56 \\
\hline 0.8 & 0.8 & 3.6 & 64.8 & 56.8 & 59.2 & 57.6 & 56.8 & 56.4 & 54.8 \\
\hline 48 & 47.2 & 47.2 & 38 & 1.2 & 1.2 & 0.8 & 1.2 & 0.8 & 1.2 \\
\hline 1.2 & 0.8 & 0.8 & 0.8 & 0.8 & 1.2 & 0.8 & 0.8 & 0.8 & 0.8 \\
\hline 0.8 & \begin{tabular}{l|l}
0.8 \\
\end{tabular} & \begin{tabular}{l|l}
0.8 \\
\end{tabular} & 0.8 & 0.8 & 0.8 & 0.8 & 0.8 & 0.8 & 0.4 \\
\hline 0.8 & 0.8 & 0.8 & 1.2 & 0.8 & 0.8 & 1.2 & 0.8 & 0.8 & 1.2 \\
\hline 1.2 & 0.8 & 1.2 & 1.2 & 1.2 & 0.8 & 1.2 & 0.8 & 1.2 & 1.2 \\
\hline 1.2 & 0.8 & 0.8 & 1.2 & 0.8 & 0.8 & 0.8 & 0.8 & 0.8 & 0.8 \\
\hline 1.2 & 0.8 & 0.8 & 1.2 & 1.2 & 0.8 & 1.2 & 0.8 & 1.2 & 1.2 \\
\hline 1.2 & 1.2 & 1.2 & 0.8 & 1.2 & 38.8 & 59.2 & 29.2 & 1.2 & 14.8 \\
\hline 1.2 & 1.2 & 0.8 & 1.2 & 1.2 & 1.2 & 1.2 & 1.2 & 1.2 & 0.8 \\
\hline 1.2 & 1.2 & 1.2 & 1.2 & 1.2 & 1.2 & 1.2 & 0.8 & 1.2 & 1.2 \\
\hline 0.8 & 1.2 & 1.2 & 20.8 & 68.8 & 62 & 61.2 & 60.4 & 60 & 33.2 \\
\hline 1.2 & 1.2 & 1.2 & 21.6 & 61.2 & 58.8 & 57.2 & 57.2 & 56.4 & 56.8 \\
\hline 1.2 & 1.2 & 1.2 & 21.2 & 60.8 & 58.4 & 57.6 & 57.2 & 54.4 & 6.8 \\
\hline 1.2 & 1.2 & 1.2 & 1.2 & 1.2 & 0.8 & 1.2 & 1.2 & 1.2 & 0.8 \\
\hline 0.8 & 1.2 & 0.8 & 1.2 & 1.2 & 0.8 & 1.2 & 0.8 & 1.2 & 0.8 \\
\hline 1.2 & 1.2 & \begin{tabular}{l|l}
0.8 \\
\end{tabular} & 1.2 & 0.8 & 1.2 & 0.8 & 1.2 & 1.2 & 0.8 \\
\hline 1.2 & 1.2 & 1.2 & 1.6 & 1.2 & 1.2 & 1.2 & 1.2 & 1.2 & 1.2 \\
\hline 1.2 & 1.2 & 1.2 & 1.2 & 1.2 & 0.8 & 1.2 & 1.2 & 0.8 & 1.2 \\
\hline 1.2 & 0.8 & 1.2 & 1.2 & 1.2 & 1.2 & 1.2 & 1.2 & 0.8 & 1.2 \\
\hline 1.2 & 1.2 & 1.2 & 1.2 & 1.2 & 1.2 & 0.8 & 22.8 & 49.6 & 46.4 \\
\hline 0.8 & 1.2 & 1.2 & 2.4 & 1.2 & 1.2 & 1.2 & 49.6 & 42 & 51.2 \\
\hline 1.2 & 1.2 & 0.8 & 1.2 & 1.2 & 26.4 & 58 & 46.4 & 42 & 42 \\
\hline 1.2 & 1.2 & 1.2 & 1.2 & 1.2 & 0.8 & 1.2 & 1.2 & 1.2 & 1.6 \\
\hline 1.2 & 1.2 & 1.2 & 1.2 & 0.8 & 1.6 & 1.2 & 1.2 & 0.8 & 1.6 \\
\hline 1.2 & 1.2 & 1.2 & 21.6 & 58 & 54 & 53.2 & 52.4 & 52.4 & 52.4 \\
\hline 1.2 & 1.2 & 1.2 & 20.8 & 58.8 & 57.6 & 56.8 & 57.2 & 55.2 & 51.6 \\
\hline 1.2 & 1.2 & 1.2 & 1.2 & 1.2 & 53.2 & 56.4 & 54.8 & 52.8 & 7.6 \\
\hline 1.2 & 1.2 & 1.2 & 1.2 & 1.2 & 1.2 & 0.8 & 35.2 & 52.8 & 30 \\
\hline 1.2 & 1.2 & 1.2 & 1.2 & 1.2 & 1.2 & 1.2 & 34.4 & 57.2 & 55.6 \\
\hline 1.2 & 1.2 & 1.6 & 1.2 & 1.2 & 1.2 & 1.2 & 1.2 & 1.2 & 1.2 \\
\hline 1.2 & 0.8 & 1.2 & 1.2 & 1.2 & 1.2 & 1.2 & 1.2 & 0.8 & 1.2 \\
\hline 1.2 & 0.8 & 1.2 & 1.2 & 1.2 & 1.2 & 1.2 & 1.2 & 0.8 & 1.2 \\
\hline 1.2 & 1.2 & 0.8 & 1.2 & 1.2 & 1.2 & 1.2 & 32 & 1.6 & 9.6 \\
\hline 0.8 & 1.2 & 1.2 & 1.2 & 1.2 & 0.8 & 1.2 & 1.2 & 1.2 & 46.4 \\
\hline 1.2 & 1.2 & 1.2 & 0.8 & 1.2 & 1.2 & 0.8 & 18 & 56.8 & 52.8 \\
\hline 0.8 & 1.2 & 1.2 & 1.2 & 1.2 & 1.2 & 49.6 & 39.6 & 30 & 30 \\
\hline 0.8 & 1.2 & 1.2 & 1.2 & 1.2 & 1.2 & 1.2 & 1.2 & 0.8 & 1.2 \\
\hline 1.2 & 1.2 & 1.2 & 1.2 & \begin{tabular}{|l|}
0.8 \\
\end{tabular} & 1.2 & 1.2 & 1.2 & 1.2 & 0.8 \\
\hline 1.2 & 1.2 & 1.2 & 1.2 & 49.2 & 57.2 & 54.8 & 55.2 & 54 & 53.6 \\
\hline 1.2 & 0.8 & 1.2 & 20.4 & 57.6 & 55.6 & 55.6 & 55.6 & 54.4 & 54.4 \\
\hline \begin{tabular}{|l|}
1.6 \\
\end{tabular} & 1.2 & 1.6 & 1.2 & 1.6 & 1.2 & 1.6 & 1.2 & 1.6 & 1.2 \\
\hline 1.2 & 1.2 & 1.2 & 1.2 & 1.2 & 1.2 & 1.2 & 1.2 & 1.2 & 1.6 \\
\hline 1.2 & 1.6 & 1.2 & 1.2 & 1.2 & 1.2 & 1.2 & 1.6 & 1.2 & 1.2 \\
\hline 1.6 & 1.2 & 1.2 & 1.6 & 1.2 & 1.2 & 1.2 & 1.6 & 1.2 & 1.2 \\
\hline 1.6 & 1.2 & 1.6 & 1.2 & 1.6 & 1.2 & 1.6 & 1.2 & 1.6 & 1.6 \\
\hline 1.6 & 1.6 & 1.2 & 1.6 & 1.6 & 1.2 & 1.6 & 1.6 & 1.2 & 1.6 \\
\hline 1.6 & 1.6 & 1.6 & 1.6 & 1.2 & 1.6 & 1.6 & 1.6 & 1.2 & 1.6 \\
\hline 1.6 & 1.6 & 1.6 & 1.6 & 2 & 1.6 & 1.6 & 1.6 & 1.6 & 1.6 \\
\hline 1.6 & 1.6 & 1.6 & 1.6 & 1.6 & 1.6 & 1.6 & 1.6 & 1.6 & 2 \\
\hline
\end{tabular}




\begin{tabular}{|c|c|c|c|c|c|c|c|c|c|}
\hline 1.6 & 2 & 1.6 & 1.6 & 1.6 & 1.6 & 1.6 & 1.6 & 2 & 1.6 \\
\hline 1.6 & 1.6 & \begin{tabular}{|l|}
1.6 \\
\end{tabular} & 1.6 & 1.6 & 1.6 & 1.6 & 1.6 & 1.6 & 1.6 \\
\hline 1.6 & 1.6 & 1.2 & 1.6 & 1.6 & 1.6 & 1.2 & 1.6 & 1.6 & 1.6 \\
\hline 1.2 & 1.6 & 1.6 & 34 & 57.2 & 56.8 & 56.8 & 55.6 & 54 & 52.8 \\
\hline 1.6 & 1.6 & 1.2 & 46 & 52 & 54.4 & 54.4 & 55.2 & 54.4 & 54 \\
\hline \begin{tabular}{|l|}
1.6 \\
\end{tabular} & 1.6 & 1.6 & 48.4 & 55.6 & 56.4 & 56 & 55.6 & 54.8 & 54 \\
\hline 1.2 & 1.6 & 1.6 & 49.2 & 55.6 & 56.4 & 56 & 55.6 & 54 & 54.8 \\
\hline 1.6 & 1.6 & 1.6 & 48 & 53.6 & 54 & 54 & 54.4 & 54 & 53.6 \\
\hline 1.6 & 1.6 & 2 & 1.2 & 1.6 & 1.6 & 2 & 1.6 & 1.6 & 1.6 \\
\hline 1.2 & 2 & 1.6 & 1.6 & 1.6 & 1.6 & 1.6 & 1.6 & 2 & 1.6 \\
\hline 2 & 1.6 & 1.6 & 2 & 31.2 & 53.2 & 52.8 & 52 & 50.8 & 50.4 \\
\hline 1.6 & 1.6 & 1.6 & 1.6 & 28.4 & 52.4 & 51.2 & 51.2 & 50 & 49.6 \\
\hline 1.6 & 1.6 & 1.6 & 1.6 & 28.8 & 52.8 & 51.2 & 51.6 & 50.4 & 50 \\
\hline 1.6 & 1.6 & 1.6 & 1.6 & 28 & 51.2 & 50.8 & 50.8 & 50.4 & 49.6 \\
\hline 1.6 & 1.6 & 1.6 & 1.6 & 21.2 & 2 & 1.6 & 1.6 & 1.6 & 1.6 \\
\hline 2 & 1.6 & 1.6 & 1.6 & 1.6 & 1.6 & 1.6 & 1.6 & 1.6 & 1.6 \\
\hline 2 & 1.6 & 1.6 & 1.6 & 2 & 1.6 & 1.6 & 1.6 & 2 & 1.6 \\
\hline 2 & 1.6 & \begin{tabular}{|l|}
1.6 \\
\end{tabular} & 1.6 & 40.8 & 53.6 & 52.4 & 53.2 & 51.6 & 51.6 \\
\hline 1.6 & 1.6 & 2 & 1.6 & 48.4 & 56.8 & 54.8 & 55.2 & 54.8 & 52.8 \\
\hline 2.4 & 1.6 & 2 & 2 & 43.6 & 52.4 & 50.8 & 50 & 49.2 & 48.4 \\
\hline \multirow[t]{5}{*}{2} & 2 & 1.6 & 2 & 40.4 & 48.8 & 48.8 & 48.8 & 48.4 & 47.6 \\
\hline & & & & 0.8 & 0.8 & 1.6 & & & \\
\hline & & & & & & & & & \\
\hline & & & & & & & & & \\
\hline & & & 28.4 & 59.2 & 57.6 & 56.8 & 56.4 & 53.6 & 50.4 \\
\hline 0.4 & 0.4 & 0.4 & 47.6 & 54.8 & 55.6 & 55.2 & 54.8 & 53.2 & 51.2 \\
\hline 0.4 & 0.4 & 0.4 & 42.8 & 49.6 & 52 & 52 & 52.4 & 50.8 & 51.2 \\
\hline 0.4 & 0.4 & 0.8 & 45.6 & 52.4 & 54.8 & 55.2 & 54.4 & 54 & 53.6 \\
\hline 0.4 & 0.4 & 33.2 & 50.4 & 48.8 & 52 & 51.6 & 52.4 & 50 & 50 \\
\hline 0.4 & 0.4 & 0.4 & 56 & 48 & 48.4 & 48 & 46.8 & 46.4 & 46 \\
\hline 0.4 & 0.4 & 0.4 & 0.8 & 0.4 & 0.4 & 0.4 & 0.4 & 0.4 & 0.4 \\
\hline 0.4 & 0.4 & 0.4 & 17.2 & 53.2 & 52 & 52.8 & 52.8 & 51.6 & 50.4 \\
\hline 0.4 & 0.4 & 32 & 48.4 & 50 & 53.6 & 53.6 & 53.2 & 52.8 & 52 \\
\hline 0.8 & & 32.4 & 49.6 & 51.6 & 54.8 & 54.8 & 54.8 & 53.2 & 52 \\
\hline \multirow[t]{2}{*}{0.4} & 0.4 & 30.8 & 47.6 & 49.2 & 52.4 & 52.4 & 52.4 & 51.2 & 50.4 \\
\hline & 0.8 & 31.2 & 48.4 & 49.2 & 52.4 & 52 & 52.4 & 52 & 50.8 \\
\hline 0.4 & 0.4 & 0.4 & 0.4 & $\begin{array}{l}0.8 \\
\end{array}$ & 0.4 & 0.4 & 0.4 & 0.4 & 0.8 \\
\hline 0.4 & 0.4 & 0.4 & 0.4 & 0.4 & 0.4 & 0.4 & 0.8 & 0.4 & 0.4 \\
\hline 0.4 & 0.4 & 0.4 & 0.4 & 44.8 & 57.6 & 55.6 & 54.4 & 54 & 52 \\
\hline 0.4 & 0.4 & 35.6 & 52 & 52.8 & 56.8 & 56.4 & 58.4 & 58 & 56.8 \\
\hline 0.4 & 0.4 & 36.8 & 54.8 & 59.2 & 63.2 & 61.6 & 62 & 61.6 & 60.8 \\
\hline 0.4 & 0.4 & 38.4 & 60.8 & 58.4 & 60.4 & 60 & 62 & 60 & 60.4 \\
\hline 0.4 & 0.8 & 38 & 60.4 & 59.2 & 59.6 & 58.8 & 59.2 & 59.2 & 56.8 \\
\hline 0.4 & 0.4 & 0.4 & 0.4 & 0.8 & 0.4 & 0.4 & 0.4 & 0.8 & 0.4 \\
\hline 0.4 & 0.4 & 0.4 & 0.4 & 0.8 & 0.4 & 0.4 & 0.4 & 0.8 & 0.4 \\
\hline 0.4 & 0.4 & 0.4 & 0.4 & 0.4 & 0.4 & 56 & 56.8 & 53.6 & 50.8 \\
\hline 0.8 & 0.4 & 34 & 52 & 53.2 & 56.8 & 53.2 & 54 & 52.4 & 52 \\
\hline 0.4 & 0.8 & 33.2 & 51.2 & 52.8 & 56.4 & 53.6 & 53.6 & 51.6 & 51.2 \\
\hline 0.4 & 0.4 & 34 & 51.6 & 54.8 & 58.4 & 54.4 & 55.6 & 53.6 & 53.2 \\
\hline 0.4 & 0.8 & 35.2 & 54.8 & 56 & 58 & 52.4 & 52.8 & 52 & 50 \\
\hline 0.4 & 0.4 & 0.4 & 0.8 & 0.4 & 0.4 & 0.4 & 0.4 & 0.4 & 0.4 \\
\hline
\end{tabular}




\begin{tabular}{|c|c|c|c|c|c|c|c|c|c|}
\hline 0.4 & 0.4 & 0.8 & 0.4 & 0.4 & 0.4 & 0.4 & 0.8 & 0.4 & 0.8 \\
\hline 0.4 & 0.8 & 40 & 65.6 & 62.8 & 64.8 & 60 & 60 & 58.8 & 57.2 \\
\hline 0.4 & 0.4 & 39.6 & 62.4 & 62.8 & 65.6 & 60.8 & 60.4 & 60 & 59.6 \\
\hline 0.4 & 0.4 & 38.8 & 60.8 & 60.8 & 65.2 & 61.6 & 60.4 & 59.2 & 58 \\
\hline 0.8 & 0.4 & 38.8 & 61.2 & 60.4 & 64 & 61.6 & 13.6 & 0.8 & 0.4 \\
\hline 0.4 & 0.4 & 39.6 & 68.4 & 68.8 & 73.6 & 73.6 & 72.4 & 71.2 & 67.2 \\
\hline 0.4 & 0.4 & 18.8 & 60 & 52 & 50 & 50 & 50 & 51.6 & 52 \\
\hline 0.4 & 0.8 & 0.4 & 0.4 & 0.4 & 0.4 & 0.4 & 0.4 & 0.4 & 0.4 \\
\hline 0.4 & 0.4 & 24.8 & 60 & 56.8 & 58.4 & 53.6 & 54.4 & 52.4 & 52 \\
\hline 0.4 & 0.4 & 38 & 55.2 & 56.8 & 59.2 & 56 & 56.8 & 55.2 & 54.4 \\
\hline 0.8 & 0.4 & 37.6 & 58.8 & 57.2 & 60.4 & 57.2 & 57.6 & 57.2 & 55.6 \\
\hline 0.8 & 0.4 & 38.4 & 60.4 & 60.4 & 63.6 & 60 & 59.2 & 58.8 & 57.6 \\
\hline 0.4 & 0.8 & 37.2 & 59.6 & 59.6 & 62.8 & 58.8 & 59.2 & 56.8 & 56.4 \\
\hline 0.8 & 0.8 & 0.4 & 0.4 & 0.4 & 0.4 & 0.4 & 0.4 & 0.4 & 0.8 \\
\hline 0.4 & 0.4 & 0.4 & 0.4 & 0.4 & 0.4 & 0.8 & 0.4 & 0.4 & 0.4 \\
\hline 0.4 & 0.8 & 39.2 & 63.6 & 62.8 & 63.6 & 60.4 & 60.8 & 58.8 & 57.6 \\
\hline 0.4 & 0.4 & 38 & 60.8 & 60.4 & 60.8 & 58 & 56.4 & 56 & 54.8 \\
\hline 0.8 & 0.4 & 38.4 & 60.8 & 56.4 & 59.6 & 56 & 56 & 54 & 54.4 \\
\hline 0.8 & 0.4 & 38.8 & 60 & 58.8 & 62.4 & 57.2 & 56.8 & 56.4 & 56.4 \\
\hline 0.4 & 0.8 & 38.4 & 59.6 & 58 & 61.6 & 57.2 & 55.2 & 57.2 & 56.8 \\
\hline 0.4 & 0.8 & 0.4 & 56.4 & 53.2 & 54.8 & 54 & 53.6 & 14.8 & 0.8 \\
\hline 0.8 & 0.4 & 0.8 & 0.8 & 0.4 & 0.8 & 0.4 & 0.8 & 0.4 & 0.8 \\
\hline 0.8 & 0.4 & 0.4 & 0.8 & 0.8 & 75.6 & 55.6 & 54.8 & 54.4 & 53.6 \\
\hline 0.8 & 0.4 & 0.8 & 68.8 & 62.8 & 62.4 & 55.6 & 54.4 & 52.4 & 52.4 \\
\hline 0.4 & 0.8 & 0.4 & 69.6 & 56 & 56 & 51.6 & 50.4 & 50 & 49.6 \\
\hline 0.8 & 0.4 & 0.8 & 67.6 & 54.8 & 56 & 51.2 & 49.6 & 49.2 & 48.8 \\
\hline 0.4 & 0.8 & 0.4 & 2.8 & 0.4 & 0.4 & 0.8 & 0.4 & 0.4 & 0.4 \\
\hline 0.8 & 0.8 & 0.4 & 0.4 & 0.4 & 0.4 & 0.8 & 0.4 & 0.8 & 0.8 \\
\hline & & & & & & & & & \\
\hline & & & & & & & & & \\
\hline & & & & & & & & & \\
\hline 0 & 0 & 0 & 0 & 0 & 0 & 0 & 0 & 0 & 0 \\
\hline 0.758904 & 2.872131 & 6.720765 & 22.87268 & 26.13041 & 28.00273 & 29.11484 & 29.73077 & 29.44493 & 29.03397 \\
\hline 0.506047 & 0.898805 & 4.546341 & 8.193189 & 11.22228 & 13.71646 & 15.83387 & 17.51863 & 18.9503 & 20.16475 \\
\hline 0.489083 & 0.460177 & 3.937118 & 16.91053 & 19.82632 & 21.44174 & 21.90218 & 22.79825 & 22.6713 & 22.12807 \\
\hline 0.491139 & 0.466667 & 2.83038 & 34.95696 & 37.92 & 38.255 & 38.14684 & 38.205 & 37.425 & 37.27089 \\
\hline 1.109091 & 1.320455 & 21.66818 & 21.83182 & 26.28636 & 30.98286 & 30.64633 & 31.72203 & 31.37175 & 31.10056 \\
\hline 1.129412 & 1.094118 & 1.094118 & 4.141176 & 10 & 12.84706 & 14.35294 & 18.45882 & 19.25882 & 16.98824 \\
\hline 0.913636 & 0.909091 & 12.29213 & 27.32889 & 32.6022 & 35.83297 & 35.63516 & 35.31111 & 34 & 33.33778 \\
\hline & & & & & & & & & \\
\hline & & & & & & & & & \\
\hline & & & & & & & & & \\
\hline & & & & & & & & & \\
\hline 0 & 0 & 0 & 0 & 0 & 0 & 0 & 0 & 0 & 0 \\
\hline 1.876798 & 13.81066 & 18.04875 & 31.8242 & 31.79656 & 32.58249 & 32.31284 & 32.07849 & 32.08587 & 31.40852 \\
\hline 0.17697 & 4.412831 & 13.24473 & 18.40619 & 21.44812 & 23.41391 & 24.79034 & 25.72417 & 26.39509 & 26.83378 \\
\hline 0.16679 & 0.143319 & 10.45231 & 25.69067 & 27.41789 & 27.44514 & 27.60753 & 27.73136 & 27.82719 & 26.68966 \\
\hline 0.16885 & 0.150036 & 5.784425 & 29.83582 & 29.41822 & 28.63513 & 28.66143 & 28.86989 & 28.68549 & 28.1225 \\
\hline 3.566051 & 3.501907 & 33.13185 & 30.61241 & 30.00745 & 31.85371 & 30.58535 & 30.1542 & 29.55404 & 29.45809 \\
\hline 0.154781 & 0.179124 & 0.204408 & 7.195879 & 21.76592 & 22.29103 & 24.28912 & 22.96646 & 25.23223 & 21.90453 \\
\hline 0.571986 & 0.541525 & 16.44514 & 27.47186 & 26.20089 & 28.00388 & 26.40092 & 26.17899 & 25.84086 & 25.56269 \\
\hline
\end{tabular}




\begin{tabular}{|c|c|c|c|c|c|c|c|c|c|}
\hline & & & & & & & & & \\
\hline & & & & & & & & & \\
\hline & & & & & & & & & \\
\hline & & & & & & & & & \\
\hline & & & & & & & & & \\
\hline 0 & 0 & 0 & 0 & 0 & 0 & 0 & 0 & 0 & 0 \\
\hline 283.8 & 1156.4 & 2915 & 8932.2 & 10109.6 & 10839.8 & 11187.8 & 11414 & 11335.8 & 11169.8 \\
\hline 17.6 & 17.2 & 980 & 1217.2 & 1333.2 & 1364 & 1352.4 & 1268 & 1262 & 1256.4 \\
\hline 111.2 & 103.6 & 638 & 3869.6 & 4397.6 & 4757.2 & 4865.6 & 5105.2 & 5102.8 & 4940 \\
\hline 38.4 & 36 & 310 & 2888.8 & 3090.4 & 3046.8 & 3002.4 & 3052.4 & 2990 & 2992.4 \\
\hline 204.4 & 240.4 & 3734.4 & 3466.4 & 4427.6 & 5257.2 & 5283.2 & 5439.2 & 5346 & 5238 \\
\hline 41.2 & 40.8 & 41.2 & 102.8 & 260.4 & 337.2 & 417.2 & 580 & 624.8 & 609.2 \\
\hline 68.59731 & 71.22144 & 1129.889 & 2559.435 & 3009.188 & 3315.079 & 3254.432 & 3207.345 & 3101.922 & 3038.824 \\
\hline & & & & & & & & & \\
\hline & & & & & & & & & \\
\hline & & & & & & & & & \\
\hline & & & & & & & & & \\
\hline & & & & & & & & & \\
\hline & & & & & & & & & \\
\hline & & & & & & & & & \\
\hline & & & & & & & & & \\
\hline & & & & & & & & & \\
\hline & & & & & & & & & \\
\hline & & & & & & & & & \\
\hline & & & & & & & & & \\
\hline & & & & & & & & & \\
\hline & & & & & & & & & \\
\hline & & & & & & & & & \\
\hline
\end{tabular}




\begin{tabular}{|c|c|c|c|c|c|c|c|c|c|}
\hline \multirow[t]{48}{*}{15} & 16 & 17 & 18 & 19 & 20 & 21 & 22 & 23 & \begin{tabular}{|l} 
DAILY \\
TOTAL
\end{tabular} \\
\hline & & & & & & & & & 0.00 \\
\hline & & & & & & & & & 0.00 \\
\hline & & & & & & & & & 0.00 \\
\hline & & & & & & & & & 0.00 \\
\hline & & & & & & & & & 0.00 \\
\hline & & & & & & & & & 0.00 \\
\hline & & & & & & & & & 0.00 \\
\hline & & & & & & . & & & 0.00 \\
\hline & & & & & & & & & 0.00 \\
\hline & & & & & & & & & 0.00 \\
\hline & & & & & & & & & 0.00 \\
\hline & & & & & & & & & 0.00 \\
\hline & & & & & & & & & 0.00 \\
\hline & & & & & & & & & 0.00 \\
\hline & & & & & & 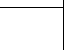 & & & 0.00 \\
\hline & & & & & & & & & 0.00 \\
\hline & & & & & & & & & 0.00 \\
\hline & & & & & & & & & 0.00 \\
\hline & & & & & & & & & 0.00 \\
\hline & & & & & & & & & 0.00 \\
\hline & & & & & & & & & 0.00 \\
\hline & & & & & & & & & 0.00 \\
\hline & & & & & & & & & 0.00 \\
\hline & & & & & & & & & 0.00 \\
\hline & & & & & & & & & 0.00 \\
\hline & & & & & & & & & 0.00 \\
\hline & & & & & & & & & 0.00 \\
\hline & & & & & & & & & 0.00 \\
\hline & & & & & & & & & 0.00 \\
\hline & & & & & & & & & 0.00 \\
\hline & & & & & & & & & 0.00 \\
\hline & & & & & & & & & 0.00 \\
\hline & & & & & & & & & 0.00 \\
\hline & & & & & & & & & 0.00 \\
\hline & & & & & & & & & 0.00 \\
\hline & & & & & & & & & 0.00 \\
\hline & & & & & & & & & 0.00 \\
\hline & & & & & & & & & 0.00 \\
\hline & & & & & & & & & 0.00 \\
\hline & & & & & & & & & 0.00 \\
\hline & & & & & & & & & 0.00 \\
\hline & & & & & & & & & 0.00 \\
\hline & & & & & & & & & 0.00 \\
\hline & & & & & & & & & 0.00 \\
\hline & & & & & & & & & 0.00 \\
\hline & & & & & & & & & 0.00 \\
\hline & & & & & & & & & 0.00 \\
\hline
\end{tabular}




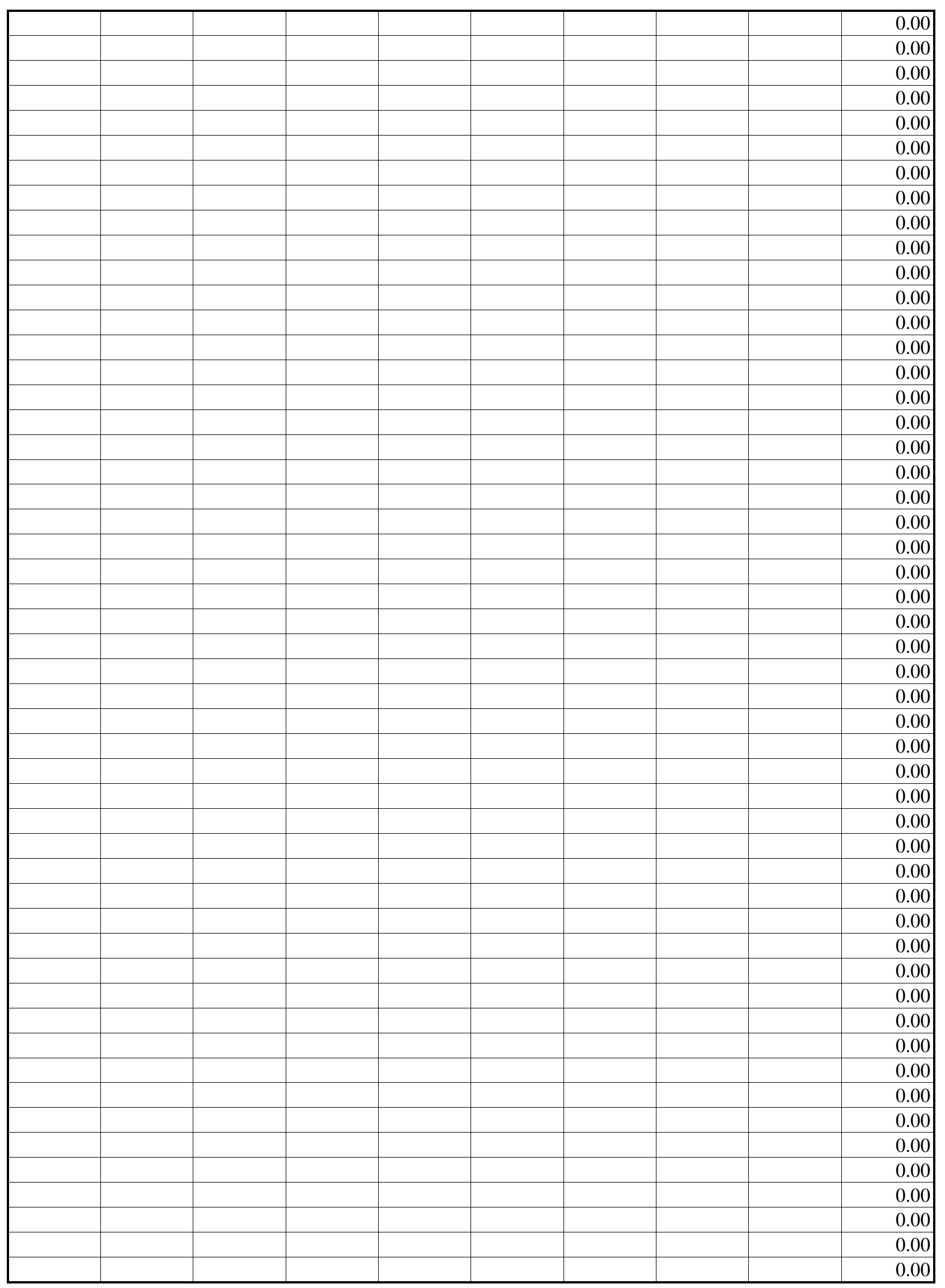




\begin{tabular}{|c|c|c|c|c|c|c|c|c|c|}
\hline & & & & & & & & & 0.00 \\
\hline & & & & & & & & & 0.00 \\
\hline & & & & & & & & & 0.00 \\
\hline & & & & & & & & & 0.00 \\
\hline & & & & & & & & & 0.00 \\
\hline & & & & & & & & & 0.00 \\
\hline & & & & & & & & & 0.00 \\
\hline & & & & & & & & & 0.00 \\
\hline & & & & & & & & & 0.00 \\
\hline & & & & & & & & & 0.00 \\
\hline & & & & & & & & & 0.00 \\
\hline & & & & & & & & & 0.00 \\
\hline & & & & & & & & & 0.00 \\
\hline & & & & & & & & & 0.00 \\
\hline & & & & & & & & & 0.00 \\
\hline & & & & & & & & & 0.00 \\
\hline & & & & & & & & & 0.00 \\
\hline & & & & & & & & & 0.00 \\
\hline & & & & & & & & & 0.00 \\
\hline & & & & & & & & & 0.00 \\
\hline & & & 1 & 2 & 2 & 2 & 2 & 2 & 11.00 \\
\hline 158 & 136 & 3 & 2 & 3 & 2 & 2 & 2 & 2 & 1418.00 \\
\hline 166 & 138 & 3 & 2 & 2 & 3 & 2 & 2 & 2 & 1535.00 \\
\hline 2 & 3 & 2 & 2 & 2 & 2 & 2 & 3 & 2 & 53.00 \\
\hline 2 & 2 & 2 & 2 & 2 & 2 & 2 & 2 & 2 & 48.00 \\
\hline 156 & 134 & 2 & 2 & 2 & 2 & 2 & 2 & 2 & 1758.00 \\
\hline 61.6 & 52.8 & 59.2 & 57.6 & 57.2 & 57.6 & 59.2 & 3.2 & 0.8 & 864.80 \\
\hline 61.2 & 52 & 1.2 & 0.8 & 0.8 & 0.8 & 0.8 & 0.8 & 0.8 & 562.80 \\
\hline 61.6 & 52.4 & 1.2 & 0.8 & 0.8 & 0.8 & 0.8 & 0.8 & 1.2 & 513.60 \\
\hline 62 & 52.8 & 1.2 & 0.8 & 0.8 & 1.2 & 0.8 & 0.8 & 0.8 & 572.40 \\
\hline 0.8 & 0.8 & 0.8 & 0.8 & 0.8 & 0.8 & 0.8 & 0.8 & 1.2 & 19.20 \\
\hline 0.8 & 0.8 & 0.8 & 0.8 & 0.8 & 0.8 & 1.2 & 0.8 & 0.8 & 19.60 \\
\hline 64.4 & 56 & 0.8 & 0.8 & 0.8 & 0.8 & 1.2 & 0.8 & 0.8 & 728.00 \\
\hline 64.4 & 55.2 & 1.2 & 0.8 & 0.8 & 0.8 & 0.8 & 0.8 & 0.8 & 599.20 \\
\hline 63.2 & 53.6 & 0.8 & 0.8 & 0.8 & 0.8 & 0.8 & 1.2 & 0.4 & 587.60 \\
\hline 63.2 & 53.6 & 1.2 & 0.8 & 0.8 & 0.8 & 0.8 & 0.8 & 0.8 & 589.20 \\
\hline 62.8 & 57.6 & 60.8 & 60.8 & 60 & 59.6 & 58.8 & 58.4 & 6.4 & 948.80 \\
\hline 58 & 57.6 & 58 & 57.6 & 58 & 57.6 & 56.8 & 57.2 & 12.4 & 938.40 \\
\hline 0.8 & 0.8 & 0.4 & 0.8 & 0.8 & 0.4 & 0.8 & 0.8 & 0.8 & 16.40 \\
\hline 60 & 51.6 & 0.8 & 0.8 & 0.8 & 0.8 & 0.8 & 0.4 & 0.8 & 665.60 \\
\hline 60.4 & 51.2 & 1.2 & 0.8 & 0.8 & 0.8 & 0.4 & 0.8 & 0.8 & 559.20 \\
\hline 57.6 & 48.8 & 0.8 & 1.2 & 0.4 & 0.8 & 0.8 & 0.8 & 0.8 & 564.40 \\
\hline 61.6 & 52.4 & 1.2 & 0.8 & 0.4 & 0.8 & 0.8 & 0.8 & 0.8 & 574.00 \\
\hline 62 & 51.6 & 0.8 & 0.8 & 0.8 & 0.8 & 0.8 & 0.8 & 0.4 & 579.20 \\
\hline 0.8 & 0.4 & 0.8 & 0.8 & 0.8 & 0.4 & 0.8 & 0.8 & 0.4 & 16.40 \\
\hline 0.8 & 0.8 & 0.4 & 0.8 & 0.8 & 0.8 & 0.8 & 0.4 & 0.8 & 16.00 \\
\hline 62.8 & 54 & 0.8 & 1.2 & 0.4 & 0.8 & 0.8 & 0.8 & 0.8 & 708.40 \\
\hline 61.6 & 56.4 & 1.2 & 0.8 & 0.4 & 1.2 & 0.8 & 0.8 & 0.4 & 555.60 \\
\hline 56.8 & 44.4 & 0.8 & 0.8 & 0.4 & 0.4 & 0.8 & 0.4 & 0.4 & 534.40 \\
\hline 57.2 & 24.4 & 0.4 & 0.4 & 0.8 & 0.4 & 0.4 & 0.8 & 0.4 & 516.00 \\
\hline 0.4 & 0.8 & 0.4 & 0.4 & 0.4 & 0.8 & 0.4 & 0.4 & 0.4 & 255.60 \\
\hline
\end{tabular}




\begin{tabular}{|c|c|c|c|c|c|c|c|c|c|}
\hline 0.4 & 0.8 & 0.4 & 0.4 & 0.4 & 0.8 & 0.4 & 0.8 & 0.4 & 12.80 \\
\hline 0.4 & 0.8 & 0.4 & 0.4 & 0.4 & 0.8 & 0.4 & 0.4 & 0.8 & 12.80 \\
\hline 0.4 & 0.4 & 0.8 & 0.4 & 0.8 & 0.4 & 0.4 & 0.8 & 0.4 & 12.80 \\
\hline 0.4 & 0.4 & 0.8 & 0.4 & 0.4 & 0.8 & 0.4 & 0.8 & 0.4 & 13.60 \\
\hline 0.4 & 0.8 & 0.4 & 0.4 & 0.4 & 0.8 & 0.4 & 0.4 & 0.4 & 12.80 \\
\hline 59.6 & 46.4 & 0.8 & 0.4 & 0.4 & 0.4 & 0.8 & 0.4 & 0.4 & 464.40 \\
\hline 0.4 & 0.8 & 0.4 & 0.4 & 0.8 & 0.4 & 0.4 & 0.8 & 0.4 & 12.80 \\
\hline 0.8 & 0.4 & 0.8 & 0.4 & 0.4 & 0.8 & 0.4 & 0.8 & 0.4 & 13.60 \\
\hline 0.8 & 0.4 & 0.4 & 0.8 & 0.8 & 0.4 & 0.8 & 0.8 & 0.4 & 14.00 \\
\hline 4.8 & 3.2 & 0.8 & 0.4 & 0.4 & 1.2 & 0.4 & 0.8 & 0.4 & 38.40 \\
\hline 0.4 & 0.4 & 0.4 & 0.8 & 0.4 & 0.4 & 0.8 & 0.4 & 0.4 & 14.40 \\
\hline 48.4 & 30.8 & 0.4 & 0.4 & 0.8 & 0.4 & 0.4 & 0.8 & 0.4 & 96.00 \\
\hline 62 & 58.8 & 0.8 & 0.4 & 0.4 & 0.8 & & 0.8 & 0.4 & 498.00 \\
\hline 61.2 & 52.4 & 0.8 & 0.4 & 0.4 & 0.4 & 0.4 & 0.4 & 0.4 & 550.00 \\
\hline 0.4 & 0.4 & 0.4 & 0.4 & 0.4 & 0.4 & 0.4 & 0.4 & 0.4 & 10.00 \\
\hline 0.4 & 0.4 & 0.8 & 0.4 & 0.4 & 0.4 & 0.4 & 0.4 & 0.4 & 10.80 \\
\hline 60 & 58 & 0.8 & 0.4 & 0.4 & 0.4 & 0.4 & 0.4 & 0.4 & 646.00 \\
\hline 60.4 & 58 & 0.8 & 0.4 & 0.4 & 0.8 & 0.4 & 0.4 & 0.4 & 545.60 \\
\hline 57.2 & 54.4 & 0.8 & 0.4 & 0.4 & 0.8 & 0.4 & 0.4 & 0.4 & 423.20 \\
\hline 55.6 & 52.4 & 0.8 & 0.8 & 0.4 & 0.4 & 0.4 & 0.4 & 0.8 & 503.60 \\
\hline 0.4 & 0.4 & 0.8 & 0.4 & 0.4 & 0.4 & 0.4 & 0.4 & 0.4 & 120.00 \\
\hline 0.8 & 0.4 & 0.4 & 0.4 & 0.8 & 0.4 & 0.4 & 0.4 & 0.8 & 12.00 \\
\hline 0.4 & 0.8 & 0.4 & 0.4 & 0.4 & 0.4 & 0.4 & 0.8 & 0.4 & 11.20 \\
\hline 0.4 & 0.8 & 0.4 & 0.4 & 0.4 & 0.8 & 0.4 & 0.4 & 0.8 & 11.60 \\
\hline 56.4 & 46 & 0.8 & 0.4 & 0.8 & 0.4 & 0.4 & 0.4 & 0.4 & 401.20 \\
\hline 0.8 & 0.4 & 0.4 & 0.4 & 0.4 & 0.4 & 0.8 & 0.4 & 0.4 & 367.20 \\
\hline 0.8 & & 0.8 & 0.4 & 0.4 & 0.4 & 0.4 & 0.8 & 0.4 & 11.20 \\
\hline 0.4 & 0.4 & 0.8 & 0.4 & 0.4 & 0.4 & 0.4 & 0.4 & 0.8 & 11.20 \\
\hline 0.4 & 0.4 & 0.4 & 0.4 & 0.4 & 0.8 & 0.4 & 0.4 & 0.4 & 10.80 \\
\hline 0.4 & 0.4 & 0.4 & 0.8 & 0.4 & 0.4 & 0.4 & 0.8 & 0.4 & 12.40 \\
\hline 0.4 & 0.4 & 0.4 & 0.4 & 0.8 & 0.4 & 0.4 & 0.4 & 0.4 & 11.20 \\
\hline 0.4 & 0.4 & 0.8 & 0.4 & 0.4 & 0.4 & 0.8 & 0.4 & 0.4 & 11.60 \\
\hline 0.4 & 0.4 & 0.4 & 0.4 & 0.8 & & 0.8 & 0.4 & 0.4 & 11.60 \\
\hline 28 & 38.4 & 0.8 & 0.4 & 0.4 & 0.4 & 0.4 & 0.4 & 0.4 & 77.20 \\
\hline 57.6 & 51.6 & 0.4 & 0.4 & 0.8 & 0.4 & 0.4 & 0.4 & 0.4 & 390.80 \\
\hline 0.4 & 0.4 & 0.4 & 0.8 & 0.4 & 0.4 & 0.4 & 0.4 & 0.4 & 10.40 \\
\hline 0.4 & 0.4 & 0.4 & 0.4 & 0.8 & 0.4 & 0.4 & 0.4 & 0.4 & 10.40 \\
\hline 60.8 & 47.6 & 0.4 & 0.4 & 0.4 & 0.4 & 0.8 & 0.4 & 0.4 & 514.40 \\
\hline 58.4 & 38.8 & 0.4 & 0.8 & 0.4 & 0.4 & 0.4 & 0.4 & 0.4 & 514.00 \\
\hline 59.2 & 38.8 & 0.8 & 0.4 & 0.4 & 0.4 & 0.4 & 0.4 & 0.4 & 519.20 \\
\hline 60 & 57.2 & 0.8 & 0.4 & 0.4 & 0.8 & & 0.8 & 0.4 & 541.60 \\
\hline 58 & 56 & 0.8 & 0.4 & 0.4 & 0.4 & 0.4 & 0.8 & & 545.20 \\
\hline 0.8 & 0.4 & 0.4 & 0.4 & 0.4 & 0.4 & 0.8 & 0.4 & 0.4 & 11.20 \\
\hline 0.8 & 0.4 & 0.4 & 0.4 & 0.4 & 0.4 & 0.4 & 0.8 & 0.4 & 11.20 \\
\hline 0.4 & 0.4 & 0.4 & 0.4 & 0.4 & 0.8 & 0.4 & 0.4 & 0.8 & 120.80 \\
\hline 0.8 & 0.4 & 0.4 & 0.4 & 0.8 & 0.4 & 0.4 & 0.4 & 0.8 & 12.00 \\
\hline 56.8 & 54.4 & 0.8 & 0.4 & 0.4 & 0.8 & & 0.8 & 0.4 & 466.80 \\
\hline 56 & 4 & 0.4 & 0.4 & 0.4 & 0.4 & 0.4 & 0.4 & 0.8 & 464.40 \\
\hline 0.4 & 0.4 & 0.4 & 0.4 & 0.4 & 0.4 & 0.4 & 0.4 & 0.4 & 261.60 \\
\hline 0.8 & 0.4 & 0.4 & 0.4 & 0.4 & 0.4 & 0.8 & & 0.8 & 10.80 \\
\hline 0.4 & 0.8 & 0.4 & 0.4 & 0.4 & 0.4 & 0.4 & 0.4 & 0.4 & 10.00 \\
\hline
\end{tabular}




\begin{tabular}{|c|c|c|c|c|c|c|c|c|c|}
\hline 0.4 & 0.4 & 0.4 & 0.8 & 0.4 & 0.4 & 0.4 & 0.4 & 0.8 & 11.20 \\
\hline 0.8 & 0.4 & 0.4 & 0.4 & 0.4 & 0.8 & 0.4 & 0.4 & 0.4 & 11.60 \\
\hline 0.4 & 0.4 & 0.4 & 0.4 & 0.8 & 0.4 & 0.4 & 0.4 & 0.4 & 11.20 \\
\hline 0.4 & 0.8 & 0.4 & 0.4 & 0.4 & 0.4 & 0.8 & 0.4 & 0.4 & 11.60 \\
\hline 0.4 & 0.8 & 0.4 & 0.4 & 0.4 & 0.4 & 0.8 & 0.4 & 0.4 & 11.60 \\
\hline 0.4 & 0.8 & 0.4 & 0.4 & 0.4 & 0.4 & 0.8 & 0.4 & 0.4 & 11.20 \\
\hline 0.4 & 0.4 & 0.4 & 0.4 & 0.4 & 0.8 & 0.4 & 0.4 & 0.4 & 11.20 \\
\hline 0.4 & 0.4 & 0.4 & 0.8 & 0.4 & 0.4 & 0.4 & 0.4 & 0.4 & 11.20 \\
\hline 0.8 & 0.4 & 0.8 & 0.4 & 0.8 & 0.4 & 0.8 & 0.4 & 0.8 & 12.40 \\
\hline & & & & & & & & & 0.00 \\
\hline 0.4 & 0.8 & 0.4 & 0.8 & 0.8 & 0.4 & 0.8 & 0.4 & 0.8 & 14.40 \\
\hline 0.4 & 0.4 & 0.4 & 0.4 & 0.4 & 0.8 & 0.4 & 0.4 & 0.4 & 12.40 \\
\hline 0.8 & 0.4 & 0.4 & 0.4 & 0.4 & 0.4 & 0.8 & 0.4 & 0.4 & 11.20 \\
\hline 0.4 & 0.4 & 0.4 & 0.4 & 0.4 & 0.4 & 0.8 & 0.4 & 0.4 & 11.20 \\
\hline 0.4 & 0.4 & 0.4 & 0.4 & 0.4 & 0.8 & 0.4 & 0.4 & 0.4 & 11.20 \\
\hline 0.4 & 0.4 & 0.4 & 0.4 & 0.4 & 0.4 & 0.8 & 0.4 & 0.4 & 11.20 \\
\hline 0.4 & 0.4 & 0.4 & 0.4 & 0.4 & 0.8 & & 0.8 & 0.4 & 10.80 \\
\hline 0.4 & 0.4 & 0.4 & 0.4 & 0.4 & 0.4 & 0.4 & 0.8 & & 59.20 \\
\hline 0.4 & 0.4 & 0.8 & 0.4 & 0.4 & 0.4 & 0.4 & 0.4 & 0.8 & 11.60 \\
\hline 54.8 & 41.2 & 0.4 & 0.4 & 0.4 & 0.8 & 0.4 & 0.4 & 0.4 & 296.80 \\
\hline 0.4 & 0.8 & 0.4 & 0.4 & 0.4 & 0.4 & 0.4 & 0.8 & 0.4 & 11.20 \\
\hline 0.4 & 0.4 & 0.4 & 0.4 & 0.8 & 0.4 & 0.4 & 0.4 & 0.4 & 10.80 \\
\hline 0.4 & 0.8 & 0.4 & 0.4 & 0.4 & 0.8 & 0.4 & 0.4 & 0.4 & 11.20 \\
\hline 0.4 & 0.4 & 0.8 & 0.4 & 0.4 & 0.4 & 0.4 & 0.8 & 0.4 & 12.00 \\
\hline 0.4 & 0.4 & 0.8 & 0.4 & 0.4 & 0.4 & 0.8 & 0.4 & 0.4 & 11.60 \\
\hline 0.4 & 0.4 & 0.4 & 0.8 & 0.4 & 0.4 & 0.4 & 0.4 & 0.4 & 11.60 \\
\hline 0.4 & 0.4 & 0.4 & 0.4 & 0.8 & 0.4 & 0.4 & 0.4 & 0.4 & 10.80 \\
\hline 0.4 & 0.4 & 0.4 & 0.4 & 0.8 & 0.4 & 0.4 & 0.4 & 0.4 & 11.20 \\
\hline 0.4 & 0.4 & 0.4 & 0.4 & 0.4 & 0.8 & 0.4 & 0.4 & 0.4 & 11.20 \\
\hline 0.4 & 0.4 & 0.4 & 0.4 & 0.4 & 0.4 & 0.4 & 0.4 & 0.4 & 10.00 \\
\hline 0.4 & 0.4 & 0.4 & 0.4 & 0.4 & 0.8 & 0.4 & 0.4 & 0.4 & 10.80 \\
\hline 56.8 & 42.4 & 0.4 & 0.4 & 0.4 & 0.4 & 0.4 & 0.4 & 0.4 & 108.40 \\
\hline 0.4 & 0.8 & 0.4 & 0.4 & 0.4 & 0.4 & 0.4 & 0.4 & 0.8 & 11.20 \\
\hline 54.4 & 40.4 & 0.4 & 0.8 & 0.4 & 0.4 & 0.4 & 0.4 & 0.4 & 255.60 \\
\hline 0.4 & 0.4 & 0.4 & 0.4 & 0.4 & 0.4 & 0.4 & 0.4 & 0.8 & 10.80 \\
\hline 0.8 & 0.4 & 0.4 & 0.4 & 0.4 & 0.4 & 0.4 & 0.4 & 0.4 & 10.40 \\
\hline 24 & 35.2 & 0.4 & 0.4 & 0.4 & 0.4 & 0.4 & 0.8 & 0.4 & 69.20 \\
\hline 0.4 & 0.8 & 0.4 & 0.4 & 0.4 & 0.4 & 0.4 & 0.4 & 0.8 & 10.80 \\
\hline 0.4 & 0.8 & 0.4 & 0.4 & 0.4 & 0.4 & 0.4 & 0.4 & 0.4 & 10.40 \\
\hline 57.6 & 34.8 & 0.4 & 0.4 & 0.4 & 0.4 & 0.8 & 0.4 & 0.4 & 165.60 \\
\hline 56.4 & 47.2 & 0.8 & 0.4 & 0.4 & 0.4 & 0.4 & 0.4 & 0.4 & 264.00 \\
\hline 0.4 & 0.8 & 0.4 & 0.4 & 0.4 & 0.4 & 0.4 & 0.4 & 0.4 & 10.80 \\
\hline 0.8 & 0.4 & 0.4 & 0.4 & 0.4 & 0.4 & 0.4 & 0.4 & 0.4 & 10.80 \\
\hline 0.8 & 0.4 & 0.4 & 0.4 & 0.4 & 0.4 & 0.4 & 0.4 & 0.8 & 11.20 \\
\hline 55.2 & 40.8 & 0.8 & 0.4 & 0.4 & 0.4 & 0.8 & 0.4 & 0.4 & 372.00 \\
\hline 0.4 & 0.4 & 0.4 & 0.4 & 0.8 & 0.4 & 0.4 & 0.4 & 0.8 & 11.20 \\
\hline 0.8 & 0.4 & 0.4 & 0.4 & 0.4 & 0.8 & 0.4 & 0.4 & 0.8 & 11.60 \\
\hline 0.8 & 0.4 & 0.4 & 0.4 & 0.4 & 0.8 & 0.4 & 0.4 & 0.4 & 11.20 \\
\hline 0.8 & 0.4 & 0.4 & 0.4 & 0.4 & 0.8 & 0.4 & 0.4 & 0.4 & 11.60 \\
\hline 0.4 & 0.4 & 0.8 & 0.4 & 0.4 & 0.4 & 0.4 & 0.4 & 0.4 & 11.20 \\
\hline 0.4 & 0.4 & 0.4 & 0.4 & 0.4 & 0.8 & 0.4 & 0.4 & 0.4 & 11.20 \\
\hline
\end{tabular}




\begin{tabular}{|c|c|c|c|c|c|c|c|c|c|}
\hline 58.8 & 37.6 & 0.4 & 0.4 & 0.4 & 0.4 & 0.4 & 0.4 & 0.4 & 324.40 \\
\hline 58.4 & 55.2 & 0.8 & & 0.4 & 0.4 & 0.4 & 0.4 & 0.8 & 534.40 \\
\hline 58 & 54.4 & 0.8 & 0.4 & 0.4 & 0.4 & 0.4 & 0.4 & 0.4 & 528.00 \\
\hline 57.6 & 55.2 & 0.8 & 0.4 & 0.4 & 0.4 & 0.4 & 0.4 & 0.4 & 530.40 \\
\hline 0.4 & 0.4 & 0.8 & 0.4 & 0.4 & 0.4 & 0.4 & 0.4 & 0.4 & 10.00 \\
\hline 0.4 & 0.4 & 0.4 & 0.4 & 0.4 & 0.4 & 0.4 & 0.4 & 0.4 & 10.40 \\
\hline 55.2 & 52.4 & 0.8 & 0.8 & 0.4 & 0.4 & 0.4 & 0.4 & 0.4 & 530.40 \\
\hline 53.2 & 51.2 & 0.8 & 0.4 & 0.4 & 0.8 & 0.4 & 0.4 & 0.4 & 492.80 \\
\hline 53.2 & 22.8 & 0.4 & 0.4 & 0.8 & 0.4 & 0.4 & 0.4 & 0.4 & 376.80 \\
\hline 55.6 & 30.8 & 0.4 & 0.4 & 0.4 & 0.4 & 0.4 & 0.4 & 0.4 & 246.80 \\
\hline 57.2 & 41.2 & 0.4 & 0.4 & 0.4 & 0.4 & 0.4 & 0.4 & 0.4 & 310.00 \\
\hline \multirow[t]{2}{*}{0.4} & 0.4 & 0.4 & 0.4 & 0.4 & 0.4 & 0.4 & 0.4 & 0.8 & 10.40 \\
\hline & 0.8 & 0.4 & 0.4 & 0.4 & 0.4 & 0.4 & 0.4 & 0.4 & 10.00 \\
\hline 57.2 & 29.6 & 0.8 & 0.4 & 0.4 & 0.4 & 0.4 & 0.4 & 0.4 & 293.20 \\
\hline 0.4 & 0.4 & 0.8 & 0.4 & 0.4 & 0.4 & 0.4 & 0.4 & 0.8 & 11.20 \\
\hline 0.4 & 0.4 & 0.8 & 0.4 & 0.4 & 0.4 & 0.4 & 0.8 & 0.4 & 11.20 \\
\hline 36.4 & 31.6 & 0.4 & 0.8 & 0.4 & 0.4 & 0.4 & 0.4 & 0.4 & 245.20 \\
\hline 54 & 35.2 & 0.4 & 0.4 & 0.4 & 0.4 & 0.8 & 0.4 & 0.4 & 305.60 \\
\hline 48.8 & 38.4 & 22.8 & 19.2 & 2.4 & 0.4 & 0.4 & 0.4 & 0.4 & 346.80 \\
\hline 0.4 & 0.4 & 0.4 & 0.4 & 0.8 & 0.4 & 0.4 & 0.4 & 0.4 & 10.40 \\
\hline 57.2 & 38 & 0.8 & 0.4 & 0.4 & 0.4 & 0.4 & 0.4 & 0.4 & 364.00 \\
\hline 0.8 & 0.4 & 0.4 & 0.4 & 0.4 & 0.4 & 0.4 & 0.4 & 0.4 & 191.20 \\
\hline 56 & 34.8 & 0.4 & 0.4 & 0.4 & 0.4 & 0.4 & 0.4 & 0.4 & 375.20 \\
\hline 56 & 26 & 0.8 & 0.4 & 0.4 & 0.4 & 0.4 & 0.4 & 0.4 & 420.80 \\
\hline 56.4 & 53.6 & 3.2 & 0.4 & 0.4 & 0.4 & 0.8 & 0.4 & 0.4 & 498.00 \\
\hline 0.8 & 0.4 & 0.4 & 0.4 & 0.4 & 0.4 & 0.4 & 0.4 & 0.4 & 263.60 \\
\hline 0.4 & 0.4 & 0.4 & 0.4 & 0.4 & 0.8 & 0.4 & & 0.8 & 10.40 \\
\hline 34.4 & & 1.2 & 0.4 & 0.4 & 0.4 & 0.4 & 0.4 & 0.4 & 430.00 \\
\hline 0.8 & 0.4 & 0.4 & 0.4 & 0.4 & 0.4 & 0.8 & 0.4 & 0.4 & 11.20 \\
\hline 0.4 & 0.4 & 0.4 & 0.4 & 0.4 & 0.8 & 0.4 & 0.4 & 0.4 & 10.80 \\
\hline 0.4 & 0.8 & 0.4 & 0.4 & 0.4 & 0.4 & 0.4 & 0.8 & 0.4 & 13.60 \\
\hline 0.4 & 0.4 & 0.8 & 0.4 & 0.4 & 0.4 & 0.4 & 0.8 & 0.4 & 11.20 \\
\hline 0.4 & 0.4 & 0.4 & 0.4 & 0.4 & 0.8 & 0.4 & 0.4 & 0.4 & 10.80 \\
\hline 0.4 & 0.8 & 0.4 & 0.4 & 0.4 & 0.4 & 0.4 & 0.4 & 0.4 & 10.80 \\
\hline 0.4 & 0.4 & 0.4 & 0.4 & 0.4 & 0.4 & 0.4 & 0.4 & 0.8 & 11.20 \\
\hline 0.4 & 0.4 & 0.8 & 0.4 & 0.4 & 0.4 & 0.4 & 0.4 & 0.4 & 10.40 \\
\hline 0.4 & 0.4 & 0.4 & 0.4 & 0.4 & 0.4 & 0.4 & 0.8 & 0.4 & 10.80 \\
\hline 0.4 & 0.4 & 0.4 & 0.4 & 0.4 & 0.8 & 0.4 & 0.4 & 0.4 & 10.80 \\
\hline 0.4 & 0.4 & 0.8 & 0.4 & 0.4 & 0.4 & 0.4 & 0.4 & 0.8 & 11.60 \\
\hline \multirow[t]{2}{*}{0.4} & 0.8 & 0.4 & 0.4 & 0.4 & 0.4 & 0.4 & 0.4 & 0.4 & 10.40 \\
\hline & 0.8 & 0.4 & 0.4 & 0.4 & 0.4 & 0.4 & 0.8 & 0.4 & 10.80 \\
\hline 58.4 & 40 & 0.8 & 0.4 & 0.4 & 0.4 & 0.4 & 0.4 & 0.8 & 130.80 \\
\hline 56.8 & 42.4 & 0.8 & 0.4 & 0.4 & 0.4 & 0.4 & 0.4 & 0.8 & 240.00 \\
\hline 56.8 & 43.6 & 0.4 & 0.4 & 0.8 & 0.4 & 0.4 & 0.4 & 0.4 & 420.80 \\
\hline 56.4 & 42 & 0.4 & 0.4 & 0.8 & & 0.8 & 0.4 & 0.4 & 416.40 \\
\hline 55.6 & 34.4 & 0.4 & 0.4 & 0.4 & 0.4 & 0.4 & 0.4 & 0.4 & 398.80 \\
\hline 0.4 & 0.4 & 0.4 & 0.4 & 0.4 & 0.4 & 0.4 & 0.4 & 0.4 & 10.00 \\
\hline 0.4 & 0.4 & 0.4 & 0.8 & 0.4 & 0.4 & 0.4 & 0.4 & 0.4 & 10.40 \\
\hline 59.2 & 42.8 & 0.8 & 0.4 & 0.4 & 0.4 & 0.8 & 0.4 & 0.4 & 223.60 \\
\hline 58 & 49.6 & 0.8 & 0.4 & 0.4 & 0.8 & 0.4 & 0.4 & 0.4 & 504.00 \\
\hline 57.6 & 48.4 & 0.8 & 0.4 & 0.4 & 0.4 & 0.4 & 0.4 & 0.4 & 475.60 \\
\hline
\end{tabular}




\begin{tabular}{|c|c|c|c|c|c|c|c|c|c|}
\hline 0.4 & 0.4 & 0.4 & 0.8 & 0.4 & 0.4 & 0.4 & 0.8 & 0.4 & 12.00 \\
\hline 0.4 & 0.4 & 0.4 & 0.4 & 0.8 & 0.4 & 0.4 & 0.4 & 0.4 & 11.20 \\
\hline 0.4 & 0.4 & 0.4 & 0.4 & 0.4 & 0.4 & 0.8 & 0.4 & 0.4 & 11.20 \\
\hline 0.4 & 0.4 & 0.8 & 0.4 & 0.4 & 0.4 & 0.4 & 0.4 & 0.4 & 10.80 \\
\hline 51.2 & 2.4 & 0.8 & 0.4 & 0.4 & 0.4 & 0.4 & 0.4 & 0.4 & 452.40 \\
\hline 45.2 & & 0.4 & 0.4 & 0.4 & 0.8 & 0.4 & 0.4 & 0.4 & 471.60 \\
\hline 60.4 & 55.6 & 0.8 & 0.4 & 0.4 & 0.4 & 0.4 & 0.4 & 0.4 & 525.60 \\
\hline 56 & 40 & 0.4 & 0.4 & 0.4 & 0.4 & 0.4 & 0.4 & 0.4 & 416.80 \\
\hline 56.4 & 55.2 & 5.6 & 0.4 & 0.4 & 0.4 & 0.4 & 0.4 & 0.4 & 434.00 \\
\hline 0.4 & 0.4 & 0.4 & 0.4 & 0.4 & 0.4 & 0.4 & 0.4 & 0.4 & 9.60 \\
\hline 0.4 & 0.4 & 0.4 & 0.4 & 0.4 & 0.8 & 0.4 & & 0.8 & 10.40 \\
\hline 58.4 & 58 & 5.6 & 0.8 & 0.4 & 0.8 & 0.4 & 0.4 & 0.4 & 476.40 \\
\hline 57.2 & 56.8 & 8.8 & 0.4 & 0.4 & 0.4 & 0.4 & 0.4 & 0.4 & 510.40 \\
\hline 57.6 & 56.4 & 8.4 & 0.8 & 0.4 & 0.4 & 0.4 & 0.4 & 0.4 & 512.00 \\
\hline 57.6 & 42.8 & 0.8 & 0.4 & 0.8 & 0.4 & 0.4 & 0.4 & 0.4 & 466.80 \\
\hline 0.4 & 0.4 & 0.4 & 0.8 & 0.4 & 0.4 & 0.4 & 0.4 & 0.4 & 10.40 \\
\hline 0.4 & 0.4 & 0.8 & 0.4 & 0.4 & 0.4 & 0.4 & 0.4 & 0.4 & 10.40 \\
\hline 0.4 & 0.4 & 0.4 & 0.8 & 0.4 & 0.4 & 0.4 & 0.4 & 0.4 & 10.40 \\
\hline 0.4 & 0.4 & 0.4 & 0.4 & 0.8 & & 0.8 & 0.4 & 0.4 & 10.80 \\
\hline 58.4 & 57.6 & 4 & 0.4 & 0.4 & 0.4 & 0.4 & 0.4 & 0.4 & 486.00 \\
\hline 56.8 & 55.6 & 3.2 & 0.4 & 0.8 & 0.4 & 0.4 & 0.4 & 0.4 & 479.20 \\
\hline 62.4 & 60.8 & 2.8 & 0.8 & 0.4 & 0.8 & 0.4 & 0.8 & 0.4 & 520.00 \\
\hline 63.6 & 62.8 & 3.2 & 0.8 & 0.4 & 0.8 & 0.4 & 0.8 & 0.4 & 565.20 \\
\hline 0.4 & 0.4 & 0.4 & 0.4 & 0.4 & 0.4 & 0.4 & 0.8 & 0.4 & 344.40 \\
\hline 0.8 & & 0.8 & 0.4 & 0.4 & 0.4 & 0.4 & 0.4 & 0.4 & 10.40 \\
\hline 55.2 & 55.2 & 2.8 & 0.4 & 0.4 & 0.8 & 0.4 & 0.4 & 0.4 & 525.20 \\
\hline 53.2 & 52.8 & 2.8 & 0.4 & 0.8 & 0.4 & 0.4 & 0.4 & 0.4 & 516.00 \\
\hline 57.2 & 56 & 2.8 & 0.8 & 0.4 & 0.4 & 0.8 & 0.4 & 0.4 & 531.20 \\
\hline 57.2 & 56.8 & 3.2 & 0.4 & 0.8 & 0.4 & 0.4 & 0.8 & & 525.60 \\
\hline 57.6 & 57.2 & 2.8 & 0.8 & 0.4 & 0.4 & 0.4 & 0.8 & 0.4 & 529.20 \\
\hline 0.4 & $\begin{array}{l}0.4 \\
\end{array}$ & 0.8 & & 0.8 & 0.4 & 0.4 & 0.4 & 0.4 & 10.00 \\
\hline 0.4 & 0.4 & 0.4 & 0.4 & 0.4 & 0.4 & 0.4 & 0.4 & 0.4 & 10.00 \\
\hline 58.4 & 58.4 & 3.2 & 0.4 & 0.4 & 0.4 & 0.4 & 0.4 & 0.4 & 522.80 \\
\hline 57.2 & 57.6 & 2.8 & 0.8 & 0.4 & 0.4 & 0.8 & 0.4 & 0.8 & 536.80 \\
\hline 57.6 & 57.6 & 3.2 & 0.4 & 0.8 & 0.4 & 0.8 & 0.4 & 0.4 & 536.80 \\
\hline 54.4 & 55.2 & 2.8 & 0.4 & 0.4 & 0.4 & 0.4 & 0.4 & 0.4 & 508.80 \\
\hline 55.6 & 56 & 2.8 & 0.4 & 0.4 & 0.4 & 0.4 & 0.4 & 0.4 & 513.20 \\
\hline 0.4 & 0.4 & 0.8 & 0.4 & 0.4 & 0.4 & 0.4 & 0.4 & 0.4 & 10.40 \\
\hline 0.4 & 0.4 & 0.4 & 0.4 & 0.4 & 0.4 & 0.4 & 0.4 & 0.4 & 10.00 \\
\hline 55.2 & 55.6 & 2.8 & 0.4 & 0.8 & 0.4 & 0.4 & 0.4 & 0.4 & 517.20 \\
\hline 59.2 & 59.6 & 2.8 & 0.8 & 0.4 & 0.8 & 0.4 & 0.8 & 0.4 & 507.60 \\
\hline 63.6 & 64 & 3.2 & 0.4 & 0.8 & 0.8 & 0.4 & 0.8 & 0.4 & 557.60 \\
\hline & 0.8 & 27.6 & 19.6 & 0.4 & 0.8 & 0.4 & 0.8 & 0.4 & 494.00 \\
\hline 64.4 & 64 & 2.8 & 0.8 & & 0.8 & & 0.8 & 0.4 & 446.80 \\
\hline 0.4 & 0.4 & 0.4 & 0.8 & 0.4 & 0.4 & 0.4 & 0.4 & 0.4 & 9.60 \\
\hline 0.4 & 0.4 & 0.4 & 0.4 & 0.4 & 0.4 & 0.4 & 0.4 & 0.4 & 9.60 \\
\hline 60 & 59.6 & 3.2 & 0.4 & 0.4 & 0.4 & 0.4 & 0.4 & 0.4 & 566.00 \\
\hline 59.6 & 58.8 & 2.8 & 0.8 & 0.4 & 0.8 & 0.4 & 0.4 & 0.4 & 558.80 \\
\hline 59.6 & 60.8 & 2.8 & 0.4 & 0.4 & 0.4 & 0.4 & 0.4 & 0.4 & 556.80 \\
\hline 61.2 & 60 & 2.8 & 0.8 & 0.4 & 0.8 & 0.4 & 0.4 & 0.4 & 562.80 \\
\hline 59.6 & 59.6 & 3.2 & 0.4 & 0.8 & 0.4 & 0.8 & 0.4 & 0.4 & 556.40 \\
\hline
\end{tabular}




\begin{tabular}{|c|c|c|c|c|c|c|c|c|c|}
\hline 0.4 & 0.8 & 0.4 & 0.8 & 0.4 & 0.8 & 0.4 & 0.4 & 0.4 & 10.80 \\
\hline 0.4 & 0.8 & 0.4 & 0.8 & 0.4 & 0.8 & 0.4 & 0.8 & 0.4 & 11.20 \\
\hline 61.2 & 60.8 & 3.2 & 0.4 & 0.8 & 0.8 & 0.4 & 0.8 & 0.4 & 581.20 \\
\hline 59.2 & 59.6 & 2.8 & 0.4 & 0.8 & 0.4 & 0.8 & 0.4 & 0.8 & 562.80 \\
\hline 58.8 & \begin{tabular}{|l|}
62.8 \\
\end{tabular} & 3.2 & 0.4 & 0.8 & 0.4 & 0.8 & 0.4 & 0.8 & 578.80 \\
\hline 64.8 & 64.4 & 3.2 & 0.8 & 0.4 & 0.8 & 0.8 & 0.4 & 0.8 & 582.00 \\
\hline 55.6 & 55.6 & 2.8 & & 0.8 & 0.4 & 0.4 & 0.4 & 0.4 & 540.80 \\
\hline \begin{tabular}{|l|}
0.4 \\
\end{tabular} & 0.4 & 0.4 & 0.4 & 0.4 & 0.4 & 0.4 & 0.4 & 0.4 & 280.80 \\
\hline 0.4 & 0.4 & 0.4 & 0.4 & 0.4 & 0.4 & 0.4 & 0.4 & 0.4 & 10.00 \\
\hline 57.2 & 54.8 & 16 & 0.4 & 0.8 & 0.4 & 0.8 & 0.4 & 0.8 & 498.40 \\
\hline 54.4 & 52 & 2.4 & 0.4 & 0.8 & 0.8 & 0.4 & 0.4 & 0.8 & 505.20 \\
\hline 47.6 & 47.6 & 2.8 & 0.4 & 0.8 & 0.8 & 0.8 & 0.4 & 0.8 & 460.40 \\
\hline 54.4 & 54 & 2.8 & 0.8 & 0.8 & 0.8 & 0.8 & 0.4 & 0.8 & 278.00 \\
\hline 0.4 & 0.8 & 0.4 & 0.8 & 0.8 & 0.4 & 0.8 & 0.8 & 0.8 & 14.80 \\
\hline 0.4 & 0.8 & 0.4 & 0.8 & 0.4 & 0.8 & 0.4 & 0.8 & 0.4 & 14.00 \\
\hline 0.4 & 0.4 & 0.4 & 0.8 & 0.4 & 0.4 & 0.8 & 0.4 & 0.4 & 10.80 \\
\hline 0.8 & 0.4 & 0.4 & 0.8 & 0.8 & 0.4 & 0.8 & 0.8 & 0.4 & 11.20 \\
\hline 0.4 & 0.8 & 0.4 & 0.8 & 0.4 & 0.8 & 0.8 & 0.4 & 0.8 & 13.60 \\
\hline 0.4 & 0.8 & 0.4 & 0.8 & 0.8 & 0.8 & 0.4 & 0.8 & 0.8 & 14.00 \\
\hline 0.4 & 0.8 & 0.4 & 0.8 & 0.8 & 0.8 & 0.4 & 0.8 & 0.8 & 14.40 \\
\hline 0.4 & 0.8 & 0.4 & 0.8 & 0.4 & 0.8 & 0.8 & 0.8 & 0.4 & 14.40 \\
\hline 0.4 & 0.8 & 0.8 & 0.4 & 0.8 & 0.8 & 0.8 & 0.8 & 0.4 & 15.20 \\
\hline 0.4 & 0.8 & 0.8 & 0.8 & 0.4 & 0.8 & 0.4 & & & 13.60 \\
\hline & & & 0.8 & 1.6 & 1.2 & 0.8 & 0.8 & 0.4 & 5.60 \\
\hline 0.4 & 0.8 & 0.8 & 0.8 & 0.8 & 0.4 & 0.8 & 0.8 & 0.8 & 16.00 \\
\hline 0.4 & 0.8 & 0.8 & 0.8 & 0.8 & 0.4 & 0.8 & 0.8 & 0.8 & 16.00 \\
\hline 0.8 & 0.8 & 0.4 & 0.8 & 0.8 & 0.8 & 0.8 & 0.8 & 0.8 & 16.00 \\
\hline 0.8 & 0.4 & 0.8 & 0.8 & 0.8 & 0.8 & 0.8 & 0.8 & 0.4 & 16.00 \\
\hline 0.8 & 0.4 & 0.8 & 0.8 & 0.8 & 0.8 & 0.8 & 0.8 & 0.8 & 16.40 \\
\hline 0.8 & 0.8 & 0.8 & 0.4 & 0.8 & 0.8 & 0.8 & 0.8 & 0.8 & 16.80 \\
\hline 0.8 & 0.8 & 2 & 0.8 & 0.4 & 0.8 & 0.8 & 0.8 & 0.8 & 17.60 \\
\hline \begin{tabular}{|l|}
61.2 \\
\end{tabular} & 60.8 & 5.2 & 0.4 & 0.8 & 0.8 & 0.8 & 0.8 & 0.8 & 542.80 \\
\hline 61.6 & 61.6 & 4.8 & 0.8 & 0.8 & 0.8 & 0.8 & 0.8 & 0.8 & 555.60 \\
\hline \begin{tabular}{|l|}
60.8 \\
\end{tabular} & 59.6 & 4.4 & 0.8 & 0.8 & 0.8 & 0.8 & 0.8 & 0.8 & 567.60 \\
\hline 0.4 & 0.8 & 0.8 & 0.8 & 0.8 & 0.8 & 0.8 & 0.8 & 0.8 & 16.80 \\
\hline 0.8 & 0.8 & 0.8 & 0.4 & 0.8 & 0.8 & 0.8 & 0.8 & 0.8 & 16.80 \\
\hline 0.8 & 0.4 & 0.8 & 0.8 & 0.8 & 0.8 & 0.4 & 0.8 & 0.8 & 16.40 \\
\hline 67.2 & 67.2 & 5.2 & 0.8 & 0.8 & 0.8 & 0.8 & 0.8 & 0.8 & 594.80 \\
\hline 64.4 & 64 & 4.8 & 0.8 & 0.8 & 0.8 & 0.8 & 0.8 & 0.8 & 496.80 \\
\hline 63.2 & \begin{tabular}{|l|}
62.8 \\
\end{tabular} & 4.8 & 0.8 & 0.8 & 0.8 & 0.8 & 0.8 & 0.8 & 584.00 \\
\hline 61.6 & 62 & 4.8 & 0.8 & 0.8 & 0.8 & 0.8 & 0.8 & 0.8 & 480.40 \\
\hline 0.8 & 0.8 & 0.8 & 0.4 & 0.8 & 0.8 & 0.8 & 0.4 & 0.8 & 17.20 \\
\hline 0.4 & 0.8 & 0.8 & 0.8 & 0.4 & 0.8 & 0.8 & 0.8 & 0.8 & 15.20 \\
\hline 0.8 & 0.8 & 0.4 & 0.8 & 0.8 & 0.8 & 0.8 & 0.8 & 0.8 & 16.00 \\
\hline 58 & 60.8 & 4.8 & 0.8 & 0.8 & 0.4 & 0.8 & 0.8 & 0.8 & 571.20 \\
\hline 64 & 63.2 & 5.2 & 0.4 & 0.8 & 0.8 & 0.8 & 0.8 & 0.8 & 594.00 \\
\hline 58.4 & 57.6 & 4.4 & 0.8 & 0.8 & 0.8 & 0.8 & 0.8 & 0.8 & 559.60 \\
\hline 61.2 & 60.4 & 4.8 & 0.8 & 0.8 & 0.8 & 0.8 & 0.8 & 0.4 & 540.40 \\
\hline \begin{tabular}{|l|}
0.4 \\
\end{tabular} & 0.8 & 0.8 & 0.8 & 0.8 & 0.8 & 0.4 & 0.8 & 0.8 & 16.80 \\
\hline 0.4 & 0.8 & 0.8 & 0.8 & 0.8 & 0.8 & 0.8 & 0.4 & 0.8 & 16.00 \\
\hline 0.8 & 0.4 & 0.8 & 0.8 & 0.8 & 0.8 & 0.8 & 0.8 & 0.4 & 16.40 \\
\hline
\end{tabular}




\begin{tabular}{|c|c|c|c|c|c|c|c|c|c|}
\hline 65.2 & 64 & 5.2 & 0.8 & 0.8 & 0.4 & 0.8 & 0.8 & 0.8 & 585.20 \\
\hline 0.8 & 0.8 & 0.8 & 0.8 & 0.8 & 0.8 & 0.8 & 0.8 & 0.8 & 74.80 \\
\hline 41.6 & 70 & 5.2 & 0.8 & 0.8 & 0.8 & 0.4 & 0.8 & 0.8 & 151.60 \\
\hline 59.2 & 59.2 & 4.8 & 0.8 & 0.4 & 0.8 & 0.8 & 0.8 & 0.8 & 452.80 \\
\hline 0.8 & 0.4 & 0.8 & 0.8 & 0.8 & 0.8 & 0.8 & 0.8 & 0.8 & 16.80 \\
\hline 0.4 & 0.8 & 0.8 & 0.8 & 0.8 & 0.8 & 0.4 & 0.8 & 0.8 & 16.40 \\
\hline 0.8 & 0.8 & 0.4 & 0.8 & 0.8 & 0.4 & 0.8 & 0.8 & 0.4 & 16.00 \\
\hline 0.8 & 0.4 & 0.8 & 0.4 & 0.8 & 0.8 & 0.8 & 0.4 & 0.8 & 386.40 \\
\hline 64.8 & 65.6 & 4.8 & 0.8 & 0.4 & 0.8 & 0.8 & 0.8 & 0.4 & 399.60 \\
\hline 0.4 & 0.8 & 0.8 & 0.8 & 0.4 & 0.8 & 0.8 & 0.8 & 0.8 & 15.60 \\
\hline 61.2 & 60.4 & 4.8 & 0.8 & 0.8 & 0.8 & 0.8 & 0.8 & 0.8 & 555.20 \\
\hline \begin{tabular}{|l|}
0.8 \\
\end{tabular} & \begin{tabular}{|l|}
0.4 \\
\end{tabular} & 0.8 & 0.8 & 0.8 & 0.8 & 0.8 & 0.8 & 0.8 & 17.20 \\
\hline 0.8 & 0.8 & 0.8 & 0.8 & 0.8 & 0.8 & 0.8 & 0.8 & 0.8 & 18.00 \\
\hline 0.4 & 0.8 & 0.8 & 0.8 & 0.8 & 0.8 & 0.8 & 0.8 & 0.8 & 17.20 \\
\hline 0.8 & 0.8 & 0.8 & 0.8 & 0.8 & 0.8 & 0.8 & 0.8 & 0.4 & 17.60 \\
\hline 0.8 & 0.8 & 0.8 & 0.8 & 0.4 & 0.8 & 0.8 & 0.8 & 0.8 & 16.40 \\
\hline 0.4 & 0.8 & 0.8 & 0.8 & 0.8 & 0.8 & 0.4 & 0.8 & 0.8 & 16.00 \\
\hline 0.8 & 0.4 & 0.8 & 0.8 & 0.8 & 0.8 & 0.8 & 0.8 & 0.4 & 16.00 \\
\hline 0.8 & 0.8 & 0.8 & 0.8 & 0.8 & 0.4 & 0.8 & 0.8 & 0.8 & 17.20 \\
\hline 0.8 & 0.8 & 0.8 & 0.8 & 0.8 & 0.4 & 0.8 & 0.8 & 0.8 & 16.00 \\
\hline 0.8 & 0.4 & 0.8 & 0.8 & 0.8 & 0.4 & 0.8 & 0.8 & 0.8 & 15.60 \\
\hline 59.2 & 32 & 0.8 & 0.4 & 0.8 & 0.8 & 0.4 & 0.8 & 0.8 & 422.80 \\
\hline 60 & 59.6 & 4.4 & 0.8 & 0.8 & 0.8 & 0.8 & 0.4 & 0.8 & 558.40 \\
\hline 60.4 & 52.8 & 1.2 & 0.4 & 0.8 & 0.8 & 0.8 & 0.8 & 0.8 & 545.60 \\
\hline 64.8 & 65.2 & 4.8 & 0.8 & 0.8 & 0.8 & 0.8 & 0.8 & 0.4 & 570.00 \\
\hline 64.4 & 65.2 & 4.8 & 0.8 & 0.8 & 0.8 & 0.4 & 0.8 & 0.8 & 580.00 \\
\hline 0.8 & 0.8 & 0.8 & 0.8 & 0.4 & 0.8 & 0.8 & 0.8 & 0.8 & 16.80 \\
\hline 0.8 & 0.8 & 0.8 & 0.4 & 0.8 & 0.8 & 0.8 & 0.8 & 0.4 & 16.00 \\
\hline 65.2 & 64.4 & 4.8 & 0.8 & 0.8 & 0.8 & 0.8 & 0.8 & 0.4 & 623.20 \\
\hline 61.6 & 62 & 4.4 & 0.8 & 0.8 & 0.8 & 0.8 & 0.4 & 0.8 & 586.40 \\
\hline \begin{tabular}{|l|}
60.8 \\
\end{tabular} & 60.4 & 4.8 & 0.8 & $\begin{array}{ll}0.8 \\
\end{array}$ & 0.4 & 0.8 & 0.8 & 0.4 & 560.00 \\
\hline 62 & 61.2 & 4.4 & 0.8 & 0.8 & 0.4 & 0.8 & 0.8 & 0.4 & 560.40 \\
\hline 60 & 59.6 & 4.4 & 0.8 & 0.8 & 0.4 & 0.8 & 0.8 & 0.8 & 536.40 \\
\hline 0.8 & \begin{tabular}{|l|}
0.4 \\
\end{tabular} & 0.8 & 0.8 & 0.4 & 0.8 & 0.8 & 0.4 & 0.8 & 15.60 \\
\hline 0.8 & 0.8 & 0.4 & 0.8 & 0.4 & 0.8 & 0.8 & 0.8 & 0.4 & 14.80 \\
\hline \begin{tabular}{|c|}
60.8 \\
\end{tabular} & \begin{tabular}{|c|}
60.8 \\
\end{tabular} & 4.4 & 0.4 & 0.8 & 0.8 & 0.8 & 0.4 & 0.8 & 539.60 \\
\hline 60.4 & 60 & 4.4 & 0.4 & 0.8 & 0.8 & 0.8 & 0.4 & 0.8 & 548.40 \\
\hline 61.6 & 61.2 & 4.4 & 0.8 & 0.8 & 0.4 & 0.8 & 0.8 & 0.8 & 572.80 \\
\hline 63.2 & 62.8 & 4.8 & 0.8 & 0.4 & 0.8 & 0.8 & 0.4 & 0.8 & 589.60 \\
\hline 63.6 & 63.6 & 4.8 & 0.8 & 0.8 & 0.4 & 0.8 & 0.8 & 0.8 & 593.20 \\
\hline 0.8 & 0.4 & 0.8 & 0.4 & 0.8 & 0.8 & 0.8 & 0.4 & 0.8 & 15.20 \\
\hline 0.4 & 0.8 & 0.8 & 0.8 & 0.4 & 0.8 & 0.8 & 0.8 & 0.4 & 15.20 \\
\hline 66.8 & 66.8 & 4.8 & 0.8 & 0.8 & 0.8 & 0.4 & 0.8 & 0.8 & 735.60 \\
\hline 67.6 & 67.6 & 5.2 & 0.4 & 0.8 & 0.8 & 0.8 & 0.8 & 0.4 & 679.60 \\
\hline 67.6 & 67.6 & 4.8 & 0.8 & 0.4 & 0.8 & 0.8 & 0.8 & 0.8 & 674.40 \\
\hline \begin{tabular}{ll|}
68 \\
\end{tabular} & 68.4 & 4.8 & 0.8 & 0.8 & 0.8 & 0.8 & 0.8 & 0.8 & 671.60 \\
\hline 66.4 & 66.4 & 4.8 & 0.8 & 0.8 & 0.8 & 0.8 & 0.8 & 0.4 & 670.80 \\
\hline 0.8 & 0.8 & 0.4 & 0.8 & 0.8 & 0.8 & 0.8 & 0.4 & 0.8 & 15.60 \\
\hline 0.4 & 0.8 & 0.8 & 0.8 & 0.8 & 0.4 & 0.8 & 0.8 & 0.8 & 15.60 \\
\hline 67.6 & 66.8 & 4.8 & 0.4 & 0.8 & 0.8 & 0.8 & 0.8 & 0.4 & 741.20 \\
\hline 68 & 68.4 & 4.8 & 0.4 & 0.8 & 0.8 & 0.8 & 0.8 & 0.8 & 683.60 \\
\hline
\end{tabular}




\begin{tabular}{|c|c|c|c|c|c|c|c|c|c|}
\hline 68 & 69.6 & 5.2 & 0.8 & 0.8 & 0.8 & 0.8 & 0.8 & 0.8 & 692.80 \\
\hline 70.8 & 72 & 5.2 & 0.8 & 0.8 & 0.8 & 0.8 & 0.8 & 0.4 & 712.80 \\
\hline 70.4 & 73.2 & 5.2 & 0.8 & 0.8 & 0.8 & 0.8 & 0.4 & 0.8 & 708.40 \\
\hline 0.8 & 0.4 & 0.8 & 0.8 & 0.8 & 0.8 & 0.8 & 0.8 & 0.4 & 16.00 \\
\hline 0.8 & 0.8 & 0.4 & 0.8 & 0.8 & 0.8 & 0.8 & 0.4 & 0.8 & 16.00 \\
\hline 0.8 & 0.4 & 0.8 & 0.8 & 0.8 & 0.8 & 0.8 & 0.4 & 0.8 & 15.60 \\
\hline 74.4 & 74.8 & 4.8 & 0.8 & 0.8 & 0.8 & 0.8 & 0.8 & 0.8 & 689.60 \\
\hline 73.2 & 73.2 & 5.2 & 0.8 & 0.8 & 0.4 & 0.8 & 0.8 & 0.8 & 722.40 \\
\hline 69.2 & 68.4 & 4.8 & 0.8 & 0.4 & 0.8 & 0.8 & 0.8 & 0.8 & 698.40 \\
\hline 66 & 65.2 & 4.8 & 0.8 & 0.4 & 0.8 & 0.8 & 0.8 & 0.4 & 666.40 \\
\hline 0.8 & 0.4 & 0.8 & 0.8 & 0.8 & 0.4 & 0.8 & 0.8 & 0.8 & 15.60 \\
\hline 0.4 & 0.8 & 0.8 & 0.4 & 0.8 & 0.4 & 0.8 & 0.8 & 0.4 & 14.80 \\
\hline 66.4 & 66.8 & 4.8 & 0.8 & 0.8 & 0.4 & 0.8 & 0.8 & 0.4 & 731.60 \\
\hline 60.4 & 59.6 & 4.4 & 0.8 & 0.8 & 0.4 & 0.8 & 0.8 & 0.8 & 644.00 \\
\hline 66.4 & 64 & 4.8 & 0.8 & 0.4 & 0.8 & 0.8 & 0.4 & 0.8 & 524.80 \\
\hline 64 & 66.8 & 4.8 & 0.8 & 0.8 & 0.4 & 0.8 & 0.8 & 0.8 & 652.00 \\
\hline 67.6 & 68.4 & 5.2 & 0.8 & 0.8 & 0.8 & 0.8 & 0.8 & 0.4 & 670.00 \\
\hline 0.8 & 0.8 & 0.8 & 0.8 & 0.8 & 0.4 & 0.8 & 0.8 & 0.8 & 16.40 \\
\hline 0.4 & 0.8 & 0.8 & 0.8 & 0.8 & 0.4 & 0.8 & 0.8 & 0.8 & 16.00 \\
\hline 79.6 & 74.8 & 5.2 & 0.8 & 0.8 & 0.4 & 0.8 & 0.8 & 0.8 & 692.80 \\
\hline 69.6 & 27.6 & 5.6 & 0.8 & 0.4 & 0.8 & 0.8 & 0.4 & 0.8 & 649.60 \\
\hline 65.6 & 64.8 & 4.8 & 0.4 & 0.8 & 0.8 & 0.8 & 0.4 & 0.8 & 666.40 \\
\hline 63.6 & 63.6 & 4.4 & 0.4 & 0.8 & 0.8 & 0.4 & 0.8 & 0.4 & 627.20 \\
\hline 62 & \begin{tabular}{|l|}
62.4 \\
\end{tabular} & 4.4 & 0.8 & 0.8 & 0.8 & 0.8 & 0.4 & 0.8 & 629.60 \\
\hline 0.8 & 0.4 & 0.8 & 0.8 & 0.8 & 0.4 & 0.8 & 0.8 & 0.4 & 14.40 \\
\hline 0.8 & 0.4 & 0.8 & 0.8 & 0.4 & 0.8 & 0.8 & 0.8 & 0.4 & 15.20 \\
\hline 67.2 & 66 & 4.4 & 0.8 & 0.4 & 0.8 & 0.8 & 0.4 & 0.8 & 739.60 \\
\hline 64.4 & 64.4 & 51.6 & 60.8 & 60 & 60.4 & 61.6 & 14 & 0.4 & 939.60 \\
\hline 57.6 & 57.2 & 4 & 0.4 & 0.8 & 0.8 & 0.4 & 0.8 & 0.4 & 549.20 \\
\hline 57.6 & 58 & 4 & 0.8 & 0.4 & 0.8 & 0.4 & 0.8 & & 571.20 \\
\hline 57.6 & 58 & 4 & 0.8 & 0.4 & 0.8 & 0.4 & 0.8 & 0.4 & 574.00 \\
\hline 0.4 & 0.4 & 0.8 & 0.4 & 0.8 & 0.4 & 0.8 & 0.4 & 0.8 & 11.60 \\
\hline 0.4 & 0.8 & 0.4 & 0.8 & 0.4 & 0.8 & 0.4 & 0.8 & 0.8 & 12.40 \\
\hline 58.8 & 58 & 4 & 0.8 & 0.8 & 0.4 & 0.8 & 0.4 & 0.8 & 646.80 \\
\hline 59.2 & 59.2 & 4 & 0.8 & 0.4 & 0.8 & 0.4 & 0.4 & 0.8 & 591.20 \\
\hline 56.4 & 58.4 & 4 & 0.8 & 0.4 & 0.8 & 0.4 & 0.8 & 0.4 & 583.20 \\
\hline 56 & 56 & 4 & 0.4 & 0.4 & 0.4 & 0.4 & 0.8 & 0.4 & 562.40 \\
\hline 58.4 & 58.4 & 3.6 & 0.8 & 0.4 & 0.4 & 0.4 & 0.4 & 0.8 & 566.40 \\
\hline 0.4 & 0.4 & 0.4 & 0.8 & 0.4 & 0.8 & 0.8 & 0.4 & 0.8 & 11.20 \\
\hline 0.4 & 0.4 & 0.8 & 0.4 & 0.8 & 0.8 & 0.4 & 0.8 & 0.4 & 12.80 \\
\hline 54.4 & 54 & 54.4 & 54.8 & 54 & 54 & 53.6 & 3.6 & 0.8 & 897.20 \\
\hline 58.8 & 58 & 4 & 0.8 & 0.4 & 0.8 & 0.4 & 0.8 & 0.4 & 561.20 \\
\hline 56 & 56.8 & 3.6 & 0.8 & 0.8 & 0.8 & 0.4 & 0.8 & 0.4 & 564.40 \\
\hline 60.4 & 60 & 4.4 & 0.8 & 0.4 & 0.8 & 0.8 & 0.8 & 0.4 & 571.60 \\
\hline 60 & 60 & 4 & 0.8 & 0.4 & 0.4 & 0.8 & 0.8 & 0.4 & 596.40 \\
\hline 0.4 & 0.4 & 0.4 & 0.4 & 0.8 & 0.4 & 0.4 & 0.4 & 0.4 & 10.40 \\
\hline 0.4 & 0.4 & 0.4 & 0.4 & 0.8 & 0.4 & 0.4 & 0.4 & 0.4 & 10.40 \\
\hline 58.4 & 58.8 & 4 & 0.8 & 0.4 & 0.4 & 0.4 & 0.8 & 0.4 & 588.80 \\
\hline 58.4 & 58.4 & 4 & 0.8 & 0.4 & 0.4 & 0.4 & 0.8 & 0.4 & 584.00 \\
\hline 58.4 & 58.8 & 4 & 0.4 & 0.8 & 0.8 & 0.4 & 0.8 & 0.4 & 583.20 \\
\hline 59.2 & 58.8 & 4.4 & 0.4 & 0.8 & 0.4 & 0.8 & 0.4 & 0.4 & 582.80 \\
\hline
\end{tabular}




\begin{tabular}{|c|c|c|c|c|c|c|c|c|c|}
\hline 58.4 & 58 & 4 & 0.8 & 0.4 & 0.8 & 0.8 & 0.4 & 0.8 & 585.60 \\
\hline 0.4 & 0.4 & 0.4 & 0.8 & 0.4 & 0.8 & 0.4 & 0.8 & 0.4 & 11.60 \\
\hline 0.4 & 0.8 & 0.4 & 0.8 & 0.8 & 0.4 & 0.8 & 0.4 & 0.8 & 11.60 \\
\hline 59.6 & 4 & 0.8 & 0.8 & 0.4 & 0.8 & 0.4 & 0.8 & 0.4 & 503.20 \\
\hline 60 & 60 & 4 & 0.4 & 0.8 & 0.8 & 0.4 & 0.4 & 0.8 & 538.00 \\
\hline 59.2 & 58.8 & 4.4 & 0.4 & 0.8 & 0.4 & 0.8 & 0.4 & 0.4 & 590.40 \\
\hline 57.6 & 56.8 & 4 & 0.8 & 0.4 & 0.8 & 0.4 & 0.8 & 0.4 & 582.00 \\
\hline 60.4 & 59.6 & 4 & 0.8 & 0.8 & 0.4 & 0.8 & 0.8 & 0.4 & 594.40 \\
\hline 0.4 & 0.8 & 0.4 & 0.8 & 0.8 & 0.4 & 0.8 & 0.8 & 0.4 & 13.20 \\
\hline 0.4 & 0.8 & 0.4 & 0.4 & 0.4 & 0.8 & 0.4 & 0.4 & 0.4 & 11.60 \\
\hline 57.2 & 57.6 & 4.4 & 0.4 & 0.4 & 0.4 & 0.4 & 0.8 & 0.4 & 582.00 \\
\hline 57.2 & 57.6 & 4 & 0.4 & 0.8 & 0.4 & 0.4 & 0.8 & 0.4 & 570.80 \\
\hline 54.8 & 54 & 3.6 & 0.4 & 0.4 & 0.4 & 0.4 & 0.8 & 0.4 & 432.40 \\
\hline 0.4 & 0.4 & 0.4 & 0.4 & 0.8 & 0.4 & 0.4 & 0.4 & 0.8 & 368.80 \\
\hline 0.4 & 0.4 & 0.8 & 0.4 & 0.4 & 0.4 & 0.8 & 0.4 & 0.4 & 11.60 \\
\hline 0.8 & 0.4 & 0.4 & 0.4 & 0.4 & 0.8 & 0.4 & 0.4 & 0.4 & 11.20 \\
\hline 0.4 & 0.8 & 0.4 & 0.4 & 0.4 & 0.4 & 0.4 & 0.8 & & 10.80 \\
\hline 57.6 & 57.6 & 4 & 0.4 & 0.4 & 0.8 & 0.4 & 0.4 & 0.4 & 485.20 \\
\hline 57.6 & 57.6 & 4 & 0.4 & 0.4 & 0.4 & 0.4 & 0.8 & & 568.00 \\
\hline 56 & 56.4 & 3.6 & 0.8 & 0.4 & 0.4 & 0.4 & 0.4 & 0.4 & 556.80 \\
\hline 54.8 & 54.8 & 3.6 & 0.4 & 0.4 & 0.4 & 0.4 & 0.4 & 0.8 & 547.60 \\
\hline 52.4 & 52 & 3.6 & 0.4 & 0.8 & 0.4 & 0.4 & 0.4 & 0.4 & 524.80 \\
\hline 0.4 & 0.4 & 0.8 & 0.4 & 0.4 & 0.4 & 0.4 & 0.4 & 0.8 & 10.80 \\
\hline 0.4 & 0.4 & 0.4 & 0.4 & 0.4 & 0.8 & 0.4 & 0.4 & 0.4 & 10.80 \\
\hline 0.4 & 0.4 & 0.4 & 0.4 & 0.4 & 0.4 & 0.4 & 0.8 & 0.4 & 234.80 \\
\hline 56 & 56 & 4 & 0.4 & 0.4 & 0.4 & 0.4 & 0.8 & 0.4 & 430.00 \\
\hline 56.4 & 56.8 & 4 & 0.4 & 0.4 & 0.4 & 0.4 & 0.4 & 0.8 & 554.40 \\
\hline 56 & 55.6 & 4.4 & 0.4 & 0.4 & 0.4 & 0.4 & 0.4 & 0.4 & 558.00 \\
\hline 52 & 51.6 & 4 & 0.4 & 0.4 & 0.4 & 0.8 & 0.4 & 0.4 & 528.40 \\
\hline 0.4 & 0.4 & 0.4 & 0.4 & 0.4 & 0.8 & 0.4 & 0.4 & 0.4 & 10.40 \\
\hline 0.4 & 0.4 & 0.8 & 0.4 & 0.4 & 0.4 & 0.4 & 0.8 & 0.4 & 10.80 \\
\hline 0.4 & 0.4 & 0.4 & 0.4 & 0.4 & 0.8 & 0.4 & 0.4 & 0.4 & 81.60 \\
\hline 54.8 & 46.4 & 0.8 & 0.4 & 0.4 & 0.4 & 0.4 & 0.4 & 0.8 & 260.80 \\
\hline 0.4 & 0.4 & 0.8 & 0.4 & 0.4 & 0.4 & 0.8 & 0.4 & 0.4 & 11.20 \\
\hline 0.4 & 0.8 & 0.4 & 0.4 & 0.4 & 0.8 & 0.4 & 0.4 & 0.4 & 11.60 \\
\hline 0.4 & 0.4 & 0.4 & 0.8 & 0.4 & 0.4 & 0.4 & 0.8 & 0.4 & 11.60 \\
\hline 0.4 & 0.4 & 0.8 & 0.4 & 0.4 & 0.4 & 0.4 & 0.8 & 0.4 & 11.20 \\
\hline 0.8 & 0.4 & 0.4 & 0.4 & 0.4 & 0.4 & 0.8 & 0.4 & 0.4 & 11.20 \\
\hline 0.4 & 0.4 & 0.4 & 0.4 & 0.4 & 0.4 & 0.4 & 0.4 & 0.4 & 10.40 \\
\hline 55.2 & 54 & 4 & 0.4 & 0.4 & 0.4 & 0.4 & 0.4 & 0.4 & 150.80 \\
\hline 53.6 & 54 & 3.6 & 0.4 & 0.4 & 0.8 & 0.4 & 0.4 & 0.4 & 276.00 \\
\hline 30 & 61.2 & 4 & 0.4 & 0.4 & 0.4 & 0.8 & 0.4 & 0.4 & 142.80 \\
\hline 55.2 & 53.2 & 4 & 0.4 & 0.4 & 0.8 & 0.4 & 0.4 & 0.4 & 547.60 \\
\hline 0.4 & 0.8 & 0.4 & 0.4 & 0.4 & 0.4 & 0.8 & 0.4 & 0.4 & 11.20 \\
\hline 0.4 & 0.4 & 0.8 & 0.4 & 0.4 & 0.4 & 0.4 & 0.4 & 0.8 & 11.20 \\
\hline 0.4 & 0.4 & 0.4 & 0.4 & 0.4 & 0.4 & 0.8 & 0.4 & 0.4 & 36.40 \\
\hline 0.4 & 0.4 & 0.4 & 0.4 & 0.4 & 0.4 & 0.8 & 0.4 & 0.4 & 10.80 \\
\hline 51.2 & 41.6 & 0.8 & 0.4 & 0.4 & 0.4 & 0.4 & 0.4 & 0.8 & 356.00 \\
\hline 58 & 35.6 & 0.8 & 0.4 & 0.4 & 0.4 & 0.4 & 0.8 & 0.4 & 112.00 \\
\hline 55.2 & 23.6 & 0.4 & 0.4 & 0.4 & 0.4 & 0.4 & 0.4 & 0.4 & 105.20 \\
\hline 0.4 & $\begin{array}{l}0.4 \\
\end{array}$ & 0.4 & 0.4 & 0.8 & 0.4 & 0.4 & 0.4 & 0.4 & 10.40 \\
\hline
\end{tabular}




\begin{tabular}{|c|c|c|c|c|c|c|c|c|c|}
\hline 0.4 & 0.4 & 0.4 & 0.8 & 0.4 & 0.4 & 0.4 & 0.4 & 0.4 & 10.40 \\
\hline 0.4 & 0.8 & 0.4 & 0.4 & 0.4 & 0.4 & 0.8 & 0.4 & 0.4 & 10.80 \\
\hline 0.4 & 0.4 & 0.4 & 0.4 & 0.8 & 0.4 & 0.4 & 0.4 & 0.4 & 10.40 \\
\hline 0.4 & 0.4 & 0.8 & 0.4 & 0.4 & 0.4 & 0.4 & 0.4 & 0.4 & 10.40 \\
\hline 0.4 & 0.8 & 0.4 & 0.4 & 0.4 & 0.8 & 0.4 & 0.4 & 0.4 & 10.80 \\
\hline 0.4 & 0.4 & 0.8 & 0.4 & 0.4 & 0.4 & 0.4 & 0.4 & 0.8 & 11.20 \\
\hline 0.4 & 0.8 & 0.4 & 0.4 & 0.4 & 0.4 & 0.8 & 0.4 & 0.4 & 10.80 \\
\hline 0.8 & 0.4 & 0.4 & 0.4 & 0.8 & 0.4 & 0.4 & 0.4 & 0.8 & 11.60 \\
\hline 0.8 & 0.4 & 0.4 & 0.4 & 0.4 & 0.4 & 0.8 & 0.4 & 0.4 & 11.20 \\
\hline 0.4 & 0.4 & 0.4 & 0.4 & 0.8 & 0.4 & 0.4 & 0.4 & 0.4 & 10.80 \\
\hline 0.4 & 0.4 & 0.4 & 0.8 & 0.4 & 0.4 & 0.4 & 0.4 & 0.8 & 11.60 \\
\hline 0.4 & 0.4 & 0.4 & 0.8 & 0.4 & 0.4 & 0.4 & 0.8 & 0.4 & 12.00 \\
\hline 0.8 & 0.4 & 0.4 & 0.4 & 0.4 & 0.8 & 0.4 & 0.4 & 0.4 & 11.60 \\
\hline 0.8 & 0.4 & 0.4 & 0.4 & 0.4 & 0.8 & 0.4 & 0.4 & 0.8 & 12.40 \\
\hline 0.8 & 0.4 & 0.4 & 0.4 & 0.8 & 0.4 & 0.4 & 0.4 & 0.4 & 11.60 \\
\hline 0.4 & 0.4 & 0.4 & 0.4 & 0.4 & 0.8 & 0.4 & 0.4 & 0.4 & 11.60 \\
\hline 0.4 & 0.4 & 0.4 & 0.4 & 0.8 & 0.4 & 0.4 & 0.4 & 0.4 & 11.20 \\
\hline 0.4 & 0.4 & 0.4 & 0.4 & 0.8 & 0.4 & 0.4 & 0.4 & 0.4 & 11.20 \\
\hline 0.4 & 0.4 & 0.4 & 0.8 & 0.4 & 0.4 & 0.4 & 0.8 & 0.4 & 12.40 \\
\hline 0.4 & 0.4 & 0.4 & 0.8 & 0.4 & 0.4 & 0.4 & 0.4 & 0.4 & 11.20 \\
\hline 0.4 & 0.4 & 0.4 & 0.8 & 0.4 & 0.4 & 0.4 & 0.4 & 0.8 & 11.60 \\
\hline 0.4 & 0.8 & 0.4 & 0.4 & 0.4 & 0.4 & 0.8 & 0.4 & 0.4 & 11.20 \\
\hline 0.4 & 0.4 & 0.8 & 0.4 & 0.4 & 0.4 & 0.4 & 0.4 & 0.4 & 11.20 \\
\hline 0.4 & 0.4 & 0.4 & 0.4 & 0.4 & 0.8 & 0.4 & 0.4 & 0.4 & 11.20 \\
\hline & 0.8 & 0.4 & 0.4 & 0.4 & 0.4 & 0.4 & 0.4 & 0.4 & 10.80 \\
\hline 0.4 & 0.4 & 0.8 & & 0.8 & 0.4 & 0.4 & 0.4 & 0.4 & 10.80 \\
\hline 0.4 & 0.4 & 0.4 & 0.8 & 0.4 & 0.4 & 0.4 & 0.4 & 0.4 & 10.80 \\
\hline 0.4 & 0.8 & 0.4 & 0.4 & 0.4 & 0.8 & 0.4 & 0.4 & 0.4 & 12.00 \\
\hline 0.8 & & 0.8 & 0.4 & 0.4 & 0.4 & 0.4 & 0.4 & 0.4 & 11.20 \\
\hline 57.6 & 49.2 & 0.8 & 0.4 & 0.4 & & 0.8 & 0.4 & 0.4 & 116.40 \\
\hline 0.8 & 0.4 & 0.4 & 0.4 & 0.4 & 0.4 & 0.4 & 0.8 & 0.4 & 10.80 \\
\hline 0.4 & 0.4 & 0.4 & 0.4 & 0.4 & 0.4 & 0.4 & 0.8 & 0.4 & 10.80 \\
\hline & 0.8 & 0.4 & 0.4 & 0.4 & 0.4 & 0.4 & 0.4 & 0.4 & 10.40 \\
\hline 0.4 & 0.4 & 0.4 & 0.4 & 0.4 & 0.4 & 0.4 & 0.8 & & 10.00 \\
\hline 0.4 & 0.4 & 0.4 & 0.4 & 0.4 & 0.4 & 0.8 & 0.4 & 0.4 & 10.80 \\
\hline 54.8 & 16.4 & 0.4 & 0.4 & 0.4 & 0.4 & 0.8 & 0.4 & 0.4 & 243.60 \\
\hline 0.4 & \begin{tabular}{|l|}
0.4 \\
\end{tabular} & 0.8 & 0.4 & 0.4 & 0.4 & 0.4 & 0.4 & 0.8 & 11.20 \\
\hline 0.4 & 0.4 & 0.8 & 0.4 & 0.4 & 0.4 & 0.4 & 0.4 & 0.4 & 10.80 \\
\hline 54.4 & 29.6 & 0.4 & 0.4 & 0.8 & 0.4 & 0.4 & 0.4 & 0.8 & 190.80 \\
\hline 52.8 & 52.4 & 3.6 & 0.4 & 0.4 & 0.4 & 0.8 & 0.4 & 0.4 & 346.80 \\
\hline 0.4 & 0.4 & 0.8 & 0.4 & 0.4 & 0.4 & 0.4 & 0.4 & 0.8 & 11.20 \\
\hline 0.4 & 0.4 & 0.4 & 0.4 & 0.8 & 0.4 & 0.4 & 0.8 & 0.4 & 12.00 \\
\hline 0.4 & 0.4 & 0.8 & 0.4 & 0.4 & 0.4 & 0.8 & 0.4 & 0.4 & 60.40 \\
\hline 0.4 & 0.4 & 0.8 & 0.4 & 0.4 & 0.4 & 0.8 & 0.4 & 0.4 & 12.00 \\
\hline 0.4 & 0.4 & 0.8 & 0.4 & 0.4 & 0.4 & 0.8 & 0.4 & 0.4 & 12.00 \\
\hline 0.4 & 0.4 & 0.4 & 0.8 & 0.4 & 0.4 & 0.4 & 0.8 & 0.4 & 12.00 \\
\hline 0.4 & 0.4 & 0.4 & 0.8 & 0.4 & 0.4 & 0.8 & 0.4 & 0.4 & 12.00 \\
\hline 0.8 & 0.4 & 0.4 & 0.8 & 0.4 & 0.4 & 0.8 & 0.4 & 0.4 & 12.40 \\
\hline 0.4 & 0.8 & 0.4 & 0.4 & 0.8 & 0.4 & 0.4 & 0.8 & 0.4 & 12.40 \\
\hline 0.8 & & 0.8 & 0.4 & 0.4 & 0.4 & 0.4 & 0.4 & 0.8 & 11.60 \\
\hline 0.4 & 0.4 & 0.4 & 0.8 & 0.4 & 0.4 & 0.4 & 0.4 & 0.4 & 10.80 \\
\hline
\end{tabular}




\begin{tabular}{|c|c|c|c|c|c|c|c|c|c|}
\hline 0.4 & 0.4 & 0.4 & 0.4 & 0.4 & 0.8 & 0.4 & 0.4 & 0.4 & 10.80 \\
\hline 0.4 & 0.4 & 0.4 & 0.8 & 0.4 & 0.4 & 0.4 & 0.8 & 0.4 & 11.60 \\
\hline 0.8 & 0.4 & 0.4 & 0.4 & 0.8 & 0.4 & 0.4 & 0.8 & 0.4 & 12.00 \\
\hline 0.4 & 0.8 & 0.4 & 0.4 & 0.4 & 0.8 & 0.4 & 0.4 & 0.4 & 11.60 \\
\hline 0.4 & 0.4 & 0.4 & 0.8 & 0.4 & 0.4 & 0.4 & 0.8 & 0.4 & 12.00 \\
\hline 0.4 & 0.4 & 0.4 & 0.4 & 0.4 & 0.4 & 0.8 & 0.4 & 0.4 & 21.60 \\
\hline 10.4 & 0.4 & 0.4 & 0.4 & 0.4 & 0.4 & 0.4 & 0.4 & 0.8 & 71.20 \\
\hline 0.4 & 0.4 & 0.4 & 0.4 & 0.4 & 0.4 & 0.4 & 0.4 & 0.4 & 162.00 \\
\hline 53.6 & 36.8 & 0.4 & 0.4 & 0.4 & 0.4 & 0.4 & 0.4 & 0.4 & 287.60 \\
\hline 53.2 & 42 & 0.8 & 0.4 & 0.4 & 0.8 & 0.4 & 0.4 & 0.4 & 142.00 \\
\hline \begin{tabular}{|l|}
0.4 \\
\end{tabular} & 0.4 & 0.8 & 0.4 & 0.4 & 0.4 & 0.4 & 0.8 & 0.4 & 11.60 \\
\hline 0.4 & 0.4 & 0.8 & 0.4 & 0.4 & 0.4 & 0.4 & 0.4 & 0.4 & 10.80 \\
\hline 0.8 & 0.4 & 0.4 & 0.4 & 0.4 & 0.4 & 0.4 & 0.4 & 0.4 & 10.80 \\
\hline 0.4 & 0.4 & 0.8 & 0.4 & 0.4 & 0.8 & 0.4 & 0.4 & 0.8 & 12.40 \\
\hline 0.4 & 0.8 & 0.4 & 0.4 & 0.4 & 0.8 & 0.4 & 0.4 & 0.8 & 12.40 \\
\hline 0.4 & 0.4 & 0.4 & 0.8 & 0.4 & 0.4 & 0.4 & 0.8 & 0.4 & 12.00 \\
\hline 0.8 & 0.4 & 0.4 & 0.4 & 0.4 & 0.4 & 0.4 & 0.4 & 0.8 & 11.20 \\
\hline 0.4 & 0.8 & 0.4 & 0.4 & 0.4 & 0.4 & 0.4 & 0.4 & 0.4 & 10.40 \\
\hline 0.4 & 0.4 & 0.4 & 0.8 & 0.4 & 0.4 & 0.4 & 0.4 & 0.8 & 11.20 \\
\hline 53.2 & 36.4 & 0.8 & 0.4 & 0.4 & 0.4 & 0.4 & 0.8 & 0.4 & 221.20 \\
\hline 52.4 & 53.6 & 4 & 0.4 & 0.4 & 0.4 & 0.4 & 0.4 & 0.4 & 300.80 \\
\hline 47.2 & 26.8 & 0.4 & 0.4 & 0.4 & 0.4 & 0.4 & 0.4 & 0.8 & 404.00 \\
\hline 53.6 & 39.6 & 0.8 & 0.4 & 0.4 & 0.4 & 0.4 & 0.4 & 0.4 & 400.80 \\
\hline \begin{tabular}{|l|}
0.4 \\
\end{tabular} & \begin{tabular}{|l|}
0.4 \\
\end{tabular} & 0.4 & 0.8 & 0.4 & 0.4 & 0.8 & 0.4 & 0.4 & 11.60 \\
\hline 0.4 & 0.4 & 0.4 & 0.8 & 0.4 & 0.4 & 0.4 & 0.4 & 0.8 & 12.00 \\
\hline 0.8 & 0.4 & 0.4 & 0.4 & 0.4 & 0.8 & 0.4 & 0.4 & 0.4 & 11.20 \\
\hline 49.6 & 42 & 0.8 & 0.4 & 0.4 & 0.4 & 0.4 & 0.4 & 0.8 & 102.00 \\
\hline 0.4 & 2 & 0.4 & 0.4 & 0.4 & 0.4 & 0.4 & 0.8 & 0.4 & 12.00 \\
\hline 54 & 51.2 & 0.8 & 0.4 & 0.4 & 0.4 & 0.8 & 0.4 & 0.4 & 289.60 \\
\hline 51.6 & 50.4 & 0.8 & 0.4 & 0.8 & 0.4 & 0.4 & 0.4 & 0.4 & 348.80 \\
\hline 0.4 & 0.4 & 0.4 & 0.4 & 0.8 & 0.4 & 0.4 & 0.4 & 0.4 & 11.60 \\
\hline 0.8 & 0.4 & 0.4 & 0.4 & 0.4 & 0.4 & 0.8 & 0.4 & 0.4 & 11.20 \\
\hline 0.4 & 0.4 & 0.4 & 0.4 & 0.4 & 0.8 & 0.4 & 0.4 & 0.4 & 11.20 \\
\hline 52.4 & 50.4 & 0.8 & 0.8 & 0.4 & 0.4 & 0.4 & 0.4 & 0.4 & 344.00 \\
\hline 53.6 & 52.8 & 0.8 & 0.4 & 0.4 & 0.4 & 0.4 & 0.4 & 0.4 & 390.40 \\
\hline 55.6 & 54 & 0.8 & 0.4 & 0.4 & 0.4 & 0.4 & 0.4 & 0.4 & 446.40 \\
\hline 0.4 & 0.4 & 0.4 & 0.8 & & 0.8 & 0.4 & 0.4 & 0.4 & 244.40 \\
\hline 0.8 & 0.4 & 0.4 & 0.8 & 0.4 & 0.4 & 0.8 & 0.4 & 0.4 & 12.40 \\
\hline 0.4 & 0.4 & 0.8 & 0.4 & 0.4 & 0.4 & 0.4 & 0.8 & 0.4 & 12.00 \\
\hline 0.4 & 0.4 & 0.4 & 0.4 & 0.8 & 0.4 & 0.4 & 0.4 & 0.8 & 12.00 \\
\hline 0.8 & 0.4 & 0.4 & 0.4 & 0.4 & 0.8 & 0.4 & 0.4 & 0.4 & 11.20 \\
\hline 0.4 & 0.4 & 0.8 & 0.4 & 0.4 & 0.4 & 0.8 & 0.4 & 0.4 & 11.60 \\
\hline 0.4 & 0.4 & 0.4 & 0.4 & 0.4 & 0.8 & 0.4 & 0.4 & 0.4 & 11.60 \\
\hline 0.4 & 0.4 & 0.4 & 0.4 & 0.4 & 0.4 & 0.8 & 0.4 & 0.4 & 11.60 \\
\hline 0.4 & 0.4 & 0.8 & & 0.8 & 0.4 & 0.4 & 0.4 & 0.4 & 10.80 \\
\hline 0.4 & 0.4 & 0.4 & 0.8 & 0.4 & 0.4 & 0.4 & 0.4 & 0.8 & 11.60 \\
\hline 0.4 & 0.8 & 0.4 & 0.4 & 0.4 & 0.4 & 0.4 & 0.8 & 0.4 & 11.20 \\
\hline 0.8 & & 0.8 & 0.4 & 0.4 & 0.4 & 0.4 & 0.8 & 0.4 & 290.40 \\
\hline 46 & 56.8 & 0.8 & 0.4 & 0.4 & 0.4 & 0.4 & 0.4 & 0.8 & 200.40 \\
\hline 56 & 54 & 0.8 & 0.8 & 0.4 & 0.4 & 0.4 & 0.4 & 0.4 & 462.80 \\
\hline 56.4 & 56.8 & 1.2 & 0.8 & 0.4 & 0.4 & 0.4 & 0.4 & 0.4 & 472.00 \\
\hline
\end{tabular}




\begin{tabular}{|c|c|c|c|c|c|c|c|c|c|}
\hline 54.4 & 54 & 1.2 & 0.8 & 0.4 & 0.4 & 0.4 & 0.4 & 0.4 & 492.40 \\
\hline 0.4 & 0.4 & 0.4 & 0.4 & 0.4 & 0.4 & 0.8 & 0.4 & 0.4 & 242.80 \\
\hline 0.4 & 0.4 & 0.4 & 0.4 & 0.4 & 0.4 & 0.8 & 0.4 & 0.4 & 10.80 \\
\hline 59.2 & 58.4 & 1.2 & 0.8 & 0.4 & 0.4 & 0.4 & 0.4 & 0.4 & 418.40 \\
\hline 56.8 & 55.6 & 1.2 & 0.4 & 0.8 & & 0.8 & 0.4 & 0.4 & 508.00 \\
\hline 53.6 & 54 & 1.2 & 0.8 & 0.4 & 0.4 & 0.4 & 0.4 & 0.4 & 454.40 \\
\hline 53.2 & 53.6 & 3.6 & 0.4 & 0.4 & 0.4 & 0.4 & 0.4 & 0.4 & 498.40 \\
\hline 51.6 & 51.6 & 4 & 0.4 & 0.4 & 0.4 & 0.4 & 0.8 & 0.4 & 506.40 \\
\hline 0.4 & 0.4 & 0.4 & 0.4 & 0.4 & 0.8 & 0.4 & 0.4 & 0.4 & 10.80 \\
\hline 0.4 & 0.4 & 0.4 & 0.8 & 0.4 & 0.4 & 0.4 & 0.4 & 0.8 & 10.80 \\
\hline 54 & 52.8 & 0.8 & 0.8 & 0.4 & 0.4 & 0.4 & 0.4 & 0.4 & 312.40 \\
\hline 54.8 & 53.2 & 1.2 & 0.4 & 0.4 & 0.4 & 0.4 & 0.4 & 0.4 & 461.60 \\
\hline 55.2 & 53.6 & 0.8 & 0.4 & 0.4 & 0.4 & 0.4 & 0.4 & 0.8 & 486.00 \\
\hline 44.8 & 52.8 & 0.8 & 0.4 & 0.4 & 0.8 & 0.4 & 0.4 & 0.4 & 414.40 \\
\hline 0.4 & 0.4 & 0.8 & 0.4 & 0.4 & 0.4 & 0.4 & 0.4 & 0.8 & 11.20 \\
\hline 0.4 & 0.4 & 0.4 & 0.4 & 0.8 & 0.4 & 0.4 & 0.4 & 0.4 & 10.80 \\
\hline 0.8 & 0.4 & 0.4 & 0.4 & 0.4 & 0.4 & 0.8 & 0.4 & 0.4 & 11.20 \\
\hline 0.4 & 0.4 & 0.4 & 0.4 & 0.4 & 0.4 & 0.4 & 0.4 & 0.8 & 18.40 \\
\hline 58.8 & 58.4 & 0.8 & 0.4 & 0.4 & 0.4 & 0.4 & 0.4 & 0.4 & 542.80 \\
\hline 61.2 & 57.2 & 1.2 & 0.4 & 0.4 & 0.4 & 0.4 & 0.4 & 0.4 & 488.40 \\
\hline 53.6 & 51.6 & 0.8 & 0.4 & 0.4 & 0.4 & 0.4 & 0.8 & 0.4 & 478.80 \\
\hline \multirow[t]{2}{*}{52.8} & 52.8 & 0.8 & 0.4 & 0.4 & 0.4 & 0.4 & 0.4 & 0.4 & 380.00 \\
\hline & 0.4 & 0.8 & 0.4 & 0.4 & 0.4 & 0.4 & 0.4 & 0.4 & 10.40 \\
\hline 0.4 & 0.4 & 0.4 & 0.4 & 0.4 & 0.4 & 0.4 & 0.8 & 0.4 & 10.80 \\
\hline 56 & 56.4 & 0.8 & 0.4 & 0.4 & 0.4 & 0.4 & 0.4 & 0.4 & 516.80 \\
\hline 57.2 & 56 & 0.8 & 0.4 & 0.4 & 0.4 & 0.4 & 0.4 & 0.4 & 518.00 \\
\hline 52.8 & 52.4 & 0.8 & 0.4 & 0.4 & 0.4 & 0.4 & 0.8 & 0.4 & 466.00 \\
\hline 52.4 & 52.4 & 0.8 & 0.8 & 0.4 & 0.4 & 0.4 & 0.4 & 0.4 & 463.60 \\
\hline 46.4 & 69.6 & 1.2 & 0.4 & 0.4 & 0.4 & 0.4 & 0.8 & 0.4 & 507.20 \\
\hline 0.4 & 0.4 & 0.4 & 0.4 & 0.4 & 0.4 & 0.8 & 0.4 & 0.4 & 10.40 \\
\hline 0.4 & 0.4 & 0.4 & 0.4 & 0.4 & 0.4 & 0.4 & 0.8 & 0.4 & 10.40 \\
\hline 64 & 75.2 & 1.2 & 0.4 & 0.8 & 0.4 & 0.4 & 0.4 & 0.8 & 554.80 \\
\hline 56.4 & 57.6 & 0.8 & 0.4 & 0.4 & 0.4 & 0.4 & 0.4 & 0.4 & 506.80 \\
\hline 54.4 & 54.8 & 0.8 & 0.4 & 0.4 & 0.4 & 0.4 & 0.4 & 0.4 & 466.40 \\
\hline 56.4 & 57.2 & 0.8 & 0.4 & 0.4 & 0.8 & & 0.8 & 0.4 & 530.00 \\
\hline 50.4 & 33.2 & 0.8 & 0.4 & 0.4 & 0.4 & 0.4 & 0.4 & 0.4 & 566.40 \\
\hline 0.4 & 0.8 & 0.4 & 0.4 & 0.4 & 0.4 & 0.4 & 0.4 & 0.8 & 10.80 \\
\hline 0.4 & 0.4 & 0.4 & 0.4 & 0.4 & 0.8 & 0.4 & 0.4 & 0.4 & 10.80 \\
\hline 58 & 57.2 & 0.8 & 0.8 & 0.4 & 0.4 & 0.4 & 0.4 & 0.8 & 546.80 \\
\hline 55.6 & 55.2 & 0.8 & 0.4 & 0.4 & 0.4 & 0.4 & 0.4 & 0.4 & 526.40 \\
\hline 58 & 56 & 0.4 & 0.4 & 0.4 & 0.4 & 0.4 & 0.4 & 0.4 & 524.00 \\
\hline 58.4 & 59.6 & 0.8 & 0.4 & 0.4 & 0.4 & 0.8 & 0.4 & 0.4 & 488.00 \\
\hline 57.6 & 56.8 & 0.8 & 0.4 & 0.4 & 0.4 & 0.4 & 0.4 & 0.4 & 526.40 \\
\hline \multirow[t]{2}{*}{0.4} & 0.4 & 0.4 & 0.4 & 0.8 & 0.4 & 0.4 & 0.4 & 0.4 & 10.40 \\
\hline & 0.8 & 0.4 & 0.4 & 0.4 & 0.8 & 0.4 & 0.4 & 0.4 & 10.40 \\
\hline 59.6 & 57.6 & 0.8 & 0.4 & 0.8 & 0.4 & 0.4 & 0.4 & 0.4 & 521.60 \\
\hline 56.8 & 56 & 0.8 & 0.8 & 0.4 & 0.4 & 0.8 & & 0.8 & 510.40 \\
\hline 59.6 & 56.4 & 0.8 & 0.8 & 0.4 & 0.8 & 0.4 & 0.4 & 0.8 & 536.00 \\
\hline 57.6 & 58.8 & 1.2 & 0.4 & 0.8 & 0.8 & 0.4 & 0.8 & 0.4 & 543.20 \\
\hline 56 & 57.6 & 0.8 & 0.8 & 0.4 & 0.8 & 0.4 & 0.8 & 0.8 & 539.60 \\
\hline 0.8 & 0.4 & 0.8 & 0.8 & 0.4 & 0.8 & 0.4 & 0.8 & 0.8 & 12.80 \\
\hline
\end{tabular}




\begin{tabular}{|c|c|c|c|c|c|c|c|c|c|}
\hline 0.8 & 0.4 & 0.8 & 0.4 & 0.8 & 0.4 & 0.8 & 0.8 & 0.4 & 13.20 \\
\hline 59.2 & \begin{tabular}{|l|}
58.8 \\
\end{tabular} & 1.2 & 0.4 & 0.8 & 0.8 & 0.4 & 0.8 & 0.8 & 574.80 \\
\hline 66.8 & 61.6 & 0.8 & 0.8 & 0.4 & 0.4 & 0.4 & 0.4 & 0.4 & 542.00 \\
\hline 59.2 & 58.8 & 1.2 & 0.8 & 0.4 & 0.8 & 0.4 & 0.4 & 0.8 & 544.00 \\
\hline 59.6 & 58.8 & 0.8 & 0.8 & 0.4 & 0.8 & 0.4 & 0.8 & 0.8 & 539.20 \\
\hline 62.4 & 62.8 & 0.8 & 0.8 & 0.4 & 0.8 & 0.4 & 0.8 & 0.4 & 562.80 \\
\hline 0.8 & 0.4 & 0.4 & 0.8 & 0.4 & 0.8 & 0.4 & 0.8 & 0.4 & 12.00 \\
\hline 0.4 & 0.4 & 0.8 & 0.4 & 0.4 & 0.4 & 0.4 & 0.4 & 0.8 & 11.20 \\
\hline 60.8 & 60.4 & 0.8 & 0.4 & 0.4 & & 0.8 & 0.4 & 0.4 & 549.60 \\
\hline 61.6 & 58 & 0.8 & 0.4 & 0.4 & 0.4 & 0.8 & 0.4 & 0.4 & 554.40 \\
\hline \begin{tabular}{|l|}
60.8 \\
\end{tabular} & 59.6 & 0.8 & 0.4 & 0.4 & 0.4 & 0.4 & 0.4 & 0.8 & 549.60 \\
\hline 58 & 54 & 1.2 & 0.4 & 0.4 & 0.4 & 0.4 & 0.4 & 0.4 & 541.20 \\
\hline 54 & 52 & 0.8 & 0.4 & 0.4 & 0.4 & 0.4 & 0.4 & 0.4 & 495.60 \\
\hline 0.4 & 0.8 & 0.4 & 0.4 & 0.4 & 0.8 & 0.4 & 0.4 & 0.4 & 10.80 \\
\hline 0.8 & 0.4 & 0.4 & 0.8 & 0.8 & 0.4 & 0.8 & 0.4 & 0.8 & 12.00 \\
\hline 54.4 & 54 & 1.2 & 0.4 & 0.8 & 0.4 & 0.8 & 0.8 & 0.4 & 519.20 \\
\hline 56 & 56 & 1.2 & 0.4 & 0.8 & 0.4 & 0.8 & 0.4 & 0.8 & 532.40 \\
\hline 58 & 56 & 1.2 & 0.4 & 0.8 & 0.8 & 0.8 & 0.4 & 0.8 & 521.60 \\
\hline 59.2 & 58.8 & 0.8 & 0.8 & 0.4 & 0.8 & 0.8 & 0.4 & 0.8 & 537.20 \\
\hline 0.8 & 0.4 & 0.8 & 0.4 & 0.8 & 0.8 & 0.4 & 0.8 & 0.8 & 14.00 \\
\hline 0.4 & 0.8 & 0.4 & 0.8 & 0.8 & 0.4 & 0.8 & 0.8 & 0.4 & 14.80 \\
\hline 0.8 & 0.4 & 0.8 & 0.8 & 0.8 & 0.4 & 0.8 & 0.8 & 0.4 & 15.60 \\
\hline 64.4 & 64.4 & 1.2 & 0.4 & 0.8 & 0.8 & 0.4 & 0.8 & 0.8 & 606.80 \\
\hline 64.4 & \begin{tabular}{|l|}
62.8 \\
\end{tabular} & 1.2 & 0.4 & 0.8 & 0.4 & 0.8 & 0.8 & 0.4 & 591.20 \\
\hline 58.8 & 3.6 & 0.4 & 0.8 & 0.8 & 0.4 & 0.8 & 0.8 & 0.4 & 523.20 \\
\hline 52 & 1.2 & 0.4 & 0.4 & 0.4 & 0.4 & 0.4 & 0.8 & 0.4 & 474.00 \\
\hline 0.4 & 0.8 & 0.4 & 0.4 & 0.8 & 0.4 & 0.4 & 0.8 & 0.4 & 11.60 \\
\hline 0.4 & 0.4 & 0.4 & 0.4 & 0.4 & 0.4 & 0.4 & 0.4 & 0.4 & 10.00 \\
\hline 0.4 & 0.4 & 0.4 & 0.4 & 0.4 & 0.4 & 0.4 & 0.4 & 0.4 & 10.40 \\
\hline 59.6 & 0.8 & 0.4 & 0.8 & 0.4 & 0.4 & 0.8 & 0.4 & 0.4 & 452.40 \\
\hline 49.2 & 0.8 & 0.8 & 0.4 & 0.8 & 0.4 & 0.8 & 0.4 & 0.8 & 433.60 \\
\hline 49.6 & 0.8 & 0.8 & 0.4 & 0.8 & 0.8 & 0.8 & 0.4 & 0.8 & 509.60 \\
\hline 50 & 0.8 & 0.8 & 0.4 & 0.8 & 0.8 & 0.4 & 0.8 & 0.4 & 515.20 \\
\hline 48 & 0.8 & 0.4 & 0.8 & 0.8 & 0.4 & 0.8 & 0.4 & 0.8 & 498.40 \\
\hline 0.4 & 0.4 & 0.4 & 0.4 & 0.8 & 0.4 & 0.4 & 0.4 & 0.4 & 12.00 \\
\hline 0.4 & 0.4 & 0.4 & 0.8 & 0.4 & 0.4 & 0.8 & 0.4 & 0.4 & 10.80 \\
\hline 50 & 0.8 & 0.8 & 0.4 & 0.8 & 0.4 & 0.8 & 0.4 & 0.8 & 541.20 \\
\hline 47.6 & 0.8 & 0.8 & 0.4 & 0.8 & 0.4 & 0.8 & 0.4 & 0.4 & 519.20 \\
\hline 50 & 0.8 & 0.8 & 0.4 & 0.8 & 0.8 & 0.8 & 0.4 & 0.8 & 523.60 \\
\hline 50 & 1.2 & 0.4 & 0.8 & 0.4 & 0.8 & 0.8 & 0.8 & 0.4 & 528.40 \\
\hline 0.8 & 0.8 & 0.4 & 0.8 & 0.8 & 0.8 & 0.4 & 0.8 & 0.8 & 15.60 \\
\hline 0.4 & 0.4 & 0.8 & 0.4 & 0.8 & 0.4 & 0.8 & 0.4 & 0.4 & 14.00 \\
\hline 0.8 & 0.4 & 0.8 & 0.4 & 0.8 & 0.4 & 0.8 & 0.8 & 0.4 & 13.60 \\
\hline 53.2 & 1.2 & 0.4 & 0.8 & 0.4 & 0.8 & 0.8 & 0.8 & 0.4 & 556.80 \\
\hline 52.8 & 1.2 & 0.4 & 0.8 & 0.8 & 0.8 & 0.4 & 0.8 & 0.8 & 552.80 \\
\hline 52.4 & 0.8 & 0.8 & 0.8 & 0.4 & 0.8 & 0.8 & 0.8 & 0.8 & 541.60 \\
\hline 50.4 & 1.2 & 0.8 & 0.4 & 0.8 & 0.8 & 0.8 & 0.8 & 0.8 & 527.20 \\
\hline 50 & 0.8 & 0.8 & 0.4 & 0.8 & 0.8 & 0.8 & 0.8 & 0.8 & 519.20 \\
\hline 0.8 & 0.4 & 0.8 & 0.8 & 0.8 & 0.8 & 0.8 & 0.8 & 0.4 & 16.40 \\
\hline 0.4 & 0.8 & 0.8 & 0.8 & 0.8 & 0.4 & 0.8 & 0.8 & 0.8 & 16.80 \\
\hline 52.4 & 1.2 & 0.8 & 0.4 & 0.8 & 0.8 & 0.8 & 0.4 & 0.8 & 549.20 \\
\hline
\end{tabular}




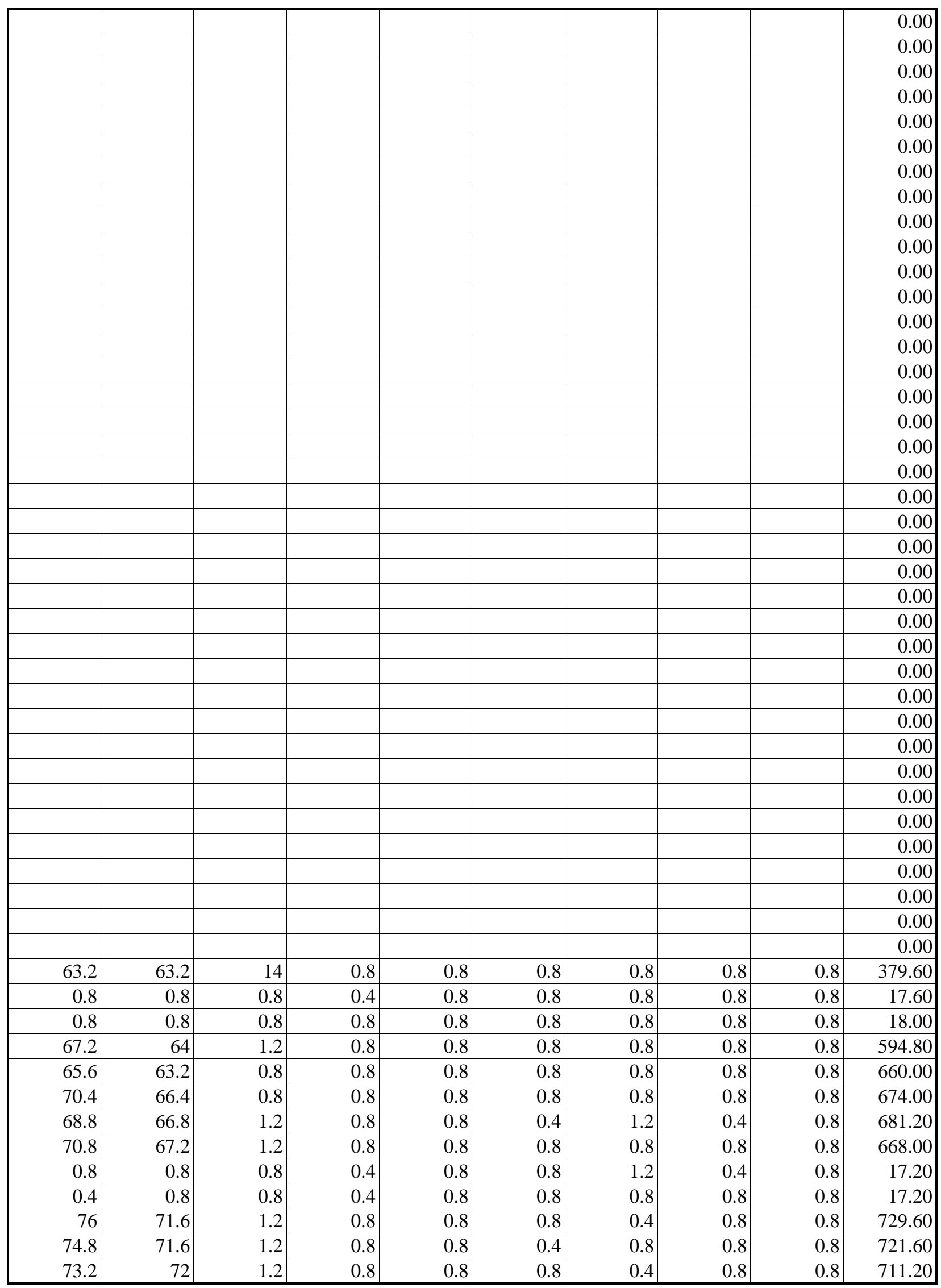




\begin{tabular}{|c|c|c|c|c|c|c|c|c|c|}
\hline 69.6 & 68 & 0.8 & 0.8 & 0.8 & 0.8 & 0.8 & 0.4 & 0.8 & 692.80 \\
\hline 72 & 69.2 & 1.2 & 0.8 & 0.4 & 0.8 & 0.8 & 0.4 & 0.8 & 699.20 \\
\hline 0.8 & 0.4 & 0.8 & 0.4 & 0.8 & 0.8 & 0.8 & 0.4 & 0.8 & 14.80 \\
\hline 0.8 & 0.4 & 0.8 & 0.8 & 0.4 & 0.8 & 0.8 & 0.8 & 0.4 & 15.20 \\
\hline 73.2 & 71.2 & 0.8 & 0.8 & 0.8 & 0.8 & 0.8 & 0.4 & 0.8 & 703.60 \\
\hline 72 & 68.4 & 0.8 & 0.8 & 0.8 & 0.8 & 0.8 & 0.8 & 0.8 & 695.60 \\
\hline 70 & 67.6 & 1.2 & 0.8 & 0.8 & 0.8 & 0.8 & 0.8 & 0.8 & 686.40 \\
\hline 69.2 & 65.2 & 1.2 & 0.8 & 0.8 & 0.8 & 0.8 & 0.8 & 0.8 & 688.40 \\
\hline 70.8 & 68.8 & 1.2 & 0.8 & 0.8 & 0.8 & 0.8 & 0.8 & 0.4 & 700.80 \\
\hline 0.4 & 0.8 & 0.8 & 0.4 & 0.8 & 0.8 & 0.8 & 0.4 & 0.8 & 16.00 \\
\hline 0.4 & 0.8 & 0.8 & 0.8 & 0.4 & 0.8 & 0.8 & 0.8 & 0.8 & 15.60 \\
\hline 0.4 & 0.8 & 0.8 & 0.8 & 0.4 & 0.8 & 0.8 & 0.8 & 0.4 & 15.60 \\
\hline 0.4 & 0.8 & 0.8 & 0.8 & 0.8 & 0.8 & 0.4 & 0.8 & 0.8 & 15.60 \\
\hline 60.8 & 59.2 & 1.2 & 0.8 & 0.8 & 0.4 & 0.8 & 0.8 & 0.8 & 596.40 \\
\hline 72.8 & 71.2 & 0.8 & 0.8 & 0.8 & 0.8 & 0.8 & 0.8 & 0.4 & 698.40 \\
\hline 72.4 & 69.6 & 1.2 & 0.8 & 0.8 & 0.8 & 0.8 & 0.4 & 0.8 & 709.60 \\
\hline 0.8 & 0.8 & 0.4 & 0.8 & 0.8 & 0.4 & 0.8 & 0.8 & 0.4 & 15.60 \\
\hline 0.8 & 0.4 & 0.8 & 0.8 & 0.8 & 0.4 & 0.8 & 0.8 & 0.8 & 15.20 \\
\hline 77.6 & 73.2 & 1.2 & 0.8 & 0.4 & 0.8 & 0.8 & 0.8 & 0.8 & 766.40 \\
\hline 72 & 68.4 & 1.2 & 0.4 & 0.8 & 0.8 & 0.4 & 0.8 & 0.8 & 723.60 \\
\hline 68.4 & 65.2 & 1.2 & 0.8 & 0.4 & 0.8 & 0.8 & 0.4 & 0.8 & 671.60 \\
\hline 66.8 & 63.6 & 1.2 & 0.8 & 0.4 & 0.8 & 0.8 & 0.8 & 0.4 & 660.80 \\
\hline 65.6 & 63.2 & 0.8 & 0.8 & 0.8 & 0.8 & 0.8 & 0.4 & 0.8 & 652.80 \\
\hline \begin{tabular}{|l|}
0.8 \\
\end{tabular} & \begin{tabular}{|l|}
0.4 \\
\end{tabular} & 0.8 & 0.8 & 0.8 & 0.4 & 0.8 & 0.8 & 0.8 & 15.20 \\
\hline 0.8 & 0.4 & 0.8 & 0.8 & 0.8 & 0.4 & 0.8 & 0.8 & 0.8 & 15.20 \\
\hline 74 & 71.2 & 0.8 & 0.8 & 0.8 & 0.8 & 0.4 & 0.8 & 0.8 & 719.20 \\
\hline 72.4 & 69.6 & 0.8 & 0.8 & 0.8 & 0.4 & 0.8 & 0.8 & 0.8 & 718.00 \\
\hline 76 & 74 & 1.2 & 0.8 & 0.8 & 0.4 & 0.8 & 0.8 & 0.8 & 729.60 \\
\hline 75.2 & 72 & 0.8 & 0.8 & 0.8 & 0.8 & 0.4 & 0.8 & 0.8 & 721.60 \\
\hline 72.8 & 70 & 1.2 & 0.8 & 0.8 & 0.8 & 0.4 & 0.8 & 0.8 & 708.00 \\
\hline \begin{tabular}{|l|}
0.8 \\
\end{tabular} & 0.8 & 0.4 & 0.8 & $\begin{array}{ll}0.8 \\
\end{array}$ & 0.8 & 0.8 & 0.8 & 0.4 & 15.60 \\
\hline 0.8 & 0.8 & 0.4 & 0.8 & 0.8 & 0.8 & 0.8 & 0.4 & 0.8 & 15.60 \\
\hline 68 & 66 & 1.2 & 0.4 & 0.8 & 0.8 & 0.8 & 0.4 & 0.8 & 690.80 \\
\hline 65.6 & \begin{tabular}{|l|}
64.8 \\
\end{tabular} & 0.8 & 0.8 & 0.8 & 0.4 & 0.8 & 0.8 & 0.4 & 661.60 \\
\hline 67.2 & 64.4 & 1.2 & 0.4 & 0.8 & 0.8 & 0.8 & 0.4 & 0.8 & 667.20 \\
\hline \begin{tabular}{|c|}
60.4 \\
\end{tabular} & 58 & 0.8 & 0.8 & 0.8 & 0.8 & 0.4 & 0.8 & 0.8 & 628.00 \\
\hline 62 & 60 & 0.8 & 0.8 & 0.8 & 0.8 & 0.4 & 0.8 & 0.8 & 629.60 \\
\hline 0.8 & 0.4 & 0.8 & 0.8 & 0.8 & 0.8 & 0.4 & 0.8 & 0.8 & 15.20 \\
\hline 0.4 & 0.8 & 0.8 & 0.4 & 0.8 & 0.8 & 0.8 & 0.4 & 0.8 & 15.20 \\
\hline 62 & \begin{tabular}{|l|}
60.8 \\
\end{tabular} & 1.2 & 0.4 & 0.8 & 0.8 & 0.8 & 0.4 & 0.8 & 647.20 \\
\hline 61.6 & 60.8 & 1.2 & 0.8 & 0.4 & 0.8 & 0.8 & 0.8 & 0.4 & 638.40 \\
\hline 63.6 & 62 & 0.8 & 0.8 & 0.8 & 0.4 & 0.8 & 0.8 & 0.4 & 641.20 \\
\hline \begin{tabular}{|l|}
62.8 \\
\end{tabular} & 58.8 & 0.8 & 0.8 & 0.4 & 0.8 & 0.8 & 0.4 & 0.8 & 637.20 \\
\hline 61.6 & 59.2 & 0.8 & 0.8 & 0.4 & 0.8 & 0.8 & 0.8 & 0.8 & 635.20 \\
\hline 0.4 & 0.8 & 0.4 & 0.8 & 0.8 & 0.8 & 0.4 & 0.8 & 0.4 & 14.40 \\
\hline 0.4 & 0.8 & 0.4 & 0.4 & 0.8 & 0.4 & 0.8 & 0.4 & 0.8 & 12.80 \\
\hline 62 & 60.4 & 0.8 & 0.8 & 0.8 & 0.4 & 0.8 & 0.4 & 0.4 & 639.20 \\
\hline 63.2 & 61.2 & 1.2 & 0.4 & 0.8 & 0.8 & 0.4 & 0.8 & 0.4 & 635.60 \\
\hline 61.6 & 59.6 & 0.8 & 0.8 & 0.4 & 0.4 & 0.8 & 0.4 & 0.8 & 635.60 \\
\hline 62.8 & 60.4 & 0.8 & 0.4 & 0.4 & 0.4 & 0.4 & 0.8 & 0.4 & 638.00 \\
\hline 61.6 & 58.8 & 1.2 & 0.4 & 0.8 & 0.4 & 0.8 & 0.4 & 0.8 & 624.00 \\
\hline
\end{tabular}




\begin{tabular}{|c|c|c|c|c|c|c|c|c|c|}
\hline 0.8 & 0.4 & 0.8 & 0.4 & 0.8 & 0.8 & 0.4 & 0.8 & 0.8 & 12.80 \\
\hline 0.4 & 0.8 & 0.8 & 0.4 & 0.8 & 0.8 & 0.8 & 0.4 & 0.8 & 13.60 \\
\hline 66.4 & 62.4 & 1.2 & 0.4 & 0.8 & 0.8 & 0.8 & 0.4 & 0.8 & 667.60 \\
\hline 67.2 & 65.6 & 63.6 & 61.6 & 60.4 & 60.4 & 60 & 48.4 & 0.8 & 1022.80 \\
\hline 64.4 & 62 & 0.8 & 0.8 & 0.4 & 0.4 & 0.4 & 0.4 & 0.4 & 645.60 \\
\hline 61.6 & 58.8 & 0.8 & 0.4 & 0.4 & 0.4 & 0.4 & 0.4 & 0.4 & 623.60 \\
\hline 57.6 & 56.8 & 54.4 & 53.6 & 53.6 & 53.6 & 52.8 & 43.2 & 0.4 & 896.40 \\
\hline 52 & 51.6 & 52.4 & 51.6 & 51.6 & 50.4 & 50 & 40.8 & 0.4 & 822.40 \\
\hline 0.4 & 0.4 & 0.8 & 0.4 & 0.4 & 0.4 & 0.4 & 0.4 & 0.8 & 10.80 \\
\hline 56 & 53.2 & 0.8 & 0.4 & 0.8 & 0.4 & 0.4 & 0.4 & 0.8 & 400.00 \\
\hline 58.8 & 56.4 & 0.8 & 0.8 & 0.4 & 0.8 & 0.4 & 0.8 & 0.4 & 353.60 \\
\hline 34.8 & 0.4 & 0.4 & 0.4 & 0.4 & 0.4 & 0.4 & 0.8 & 0.4 & 339.20 \\
\hline 56.4 & 54.8 & 1.2 & 1.2 & 1.2 & 1.2 & 0.8 & 1.2 & 1.2 & 370.00 \\
\hline 57.6 & 55.6 & 1.6 & 1.2 & 1.2 & 1.2 & 1.2 & 1.2 & 1.2 & 463.60 \\
\hline 1.2 & 1.2 & 0.8 & 1.2 & 1.2 & 1.2 & 0.8 & 1.2 & 0.8 & 27.20 \\
\hline 0.8 & 0.8 & 1.2 & 0.8 & 1.2 & 0.8 & 1.2 & 0.8 & 1.2 & 22.00 \\
\hline 0.8 & 1.2 & 0.8 & 1.2 & 0.8 & 1.2 & 1.2 & 0.8 & 1.2 & 22.40 \\
\hline 60 & 57.2 & 1.6 & 0.8 & 1.2 & 0.8 & 1.2 & 0.8 & 1.2 & 200.40 \\
\hline 57.2 & 55.2 & 1.6 & 1.2 & 0.8 & 1.2 & 0.8 & 1.2 & 0.8 & 458.80 \\
\hline 57.2 & 57.2 & 1.2 & 1.2 & 1.2 & 0.8 & 1.2 & 1.2 & 0.8 & 461.20 \\
\hline 56.8 & 55.6 & 1.2 & 1.2 & 1.2 & 1.2 & 1.2 & 1.2 & 1.2 & 459.60 \\
\hline 1.2 & 1.2 & 0.8 & 1.2 & 1.2 & 0.8 & 1.2 & 0.8 & 1.2 & 25.20 \\
\hline 0.8 & 1.2 & 0.8 & 1.2 & 0.8 & 1.2 & 0.8 & 1.2 & 0.8 & 22.40 \\
\hline 0.8 & 1.2 & 0.8 & 1.2 & 0.8 & 1.2 & 1.2 & 1.2 & 0.8 & 22.80 \\
\hline 62.4 & 58.4 & 1.6 & 0.8 & 1.2 & 1.2 & 1.2 & 0.8 & 1.2 & 143.60 \\
\hline 41.2 & 1.2 & 0.8 & 1.2 & 0.8 & 1.2 & 0.8 & 1.2 & 0.8 & 187.60 \\
\hline 1.2 & 1.2 & 1.2 & 0.8 & 1.2 & 0.8 & 1.2 & 1.2 & 0.8 & 23.20 \\
\hline 58 & 57.6 & 1.6 & 0.8 & 1.2 & 1.2 & 1.2 & 0.8 & 1.2 & 470.40 \\
\hline 1.2 & 1.2 & 0.8 & 1.2 & 1.2 & 0.8 & 1.2 & 1.2 & 1.2 & 24.80 \\
\hline 1.2 & 1.2 & 1.2 & 0.8 & 1.2 & 0.8 & 1.2 & 0.8 & 1.2 & 24.80 \\
\hline 54.8 & 54.4 & 1.6 & 1.2 & 0.8 & 1.2 & 0.8 & 1.2 & 1.2 & 445.20 \\
\hline 54.8 & 53.2 & 1.2 & 1.2 & 0.8 & 1.2 & 0.8 & 1.2 & 0.8 & 438.40 \\
\hline 52.8 & 52.8 & 1.2 & 0.8 & 1.2 & 0.8 & 1.2 & 0.8 & 1.2 & 421.20 \\
\hline 52.4 & 51.2 & 1.6 & 0.8 & 1.2 & 0.8 & 0.8 & 1.2 & 0.8 & 414.40 \\
\hline 54.8 & 52.8 & 1.2 & 1.2 & 1.2 & 0.8 & 1.2 & 0.8 & 1.2 & 432.80 \\
\hline 0.8 & 0.8 & 1.2 & 0.8 & 1.2 & 0.8 & 1.2 & 0.8 & 0.8 & 22.40 \\
\hline 0.8 & 0.8 & 0.8 & 0.8 & 1.2 & 0.8 & 0.8 & 1.2 & 0.8 & 20.80 \\
\hline 50.4 & 50 & 1.6 & 0.8 & 1.2 & 1.2 & 0.8 & 0.8 & 1.2 & 258.80 \\
\hline 53.2 & 52 & 1.2 & 1.2 & 0.8 & 1.2 & 0.8 & 1.2 & 0.8 & 367.20 \\
\hline 1.2 & 0.8 & 1.2 & 0.8 & 1.2 & 1.2 & 0.8 & 1.2 & 1.2 & 220.80 \\
\hline 1.2 & 0.8 & 1.2 & 0.8 & 1.2 & 0.8 & 1.2 & 0.8 & 1.2 & 24.00 \\
\hline 0.8 & 0.8 & 0.8 & 0.8 & 0.8 & 0.8 & 0.8 & 1.2 & 0.8 & 20.40 \\
\hline 0.8 & 0.8 & 0.8 & 0.8 & 0.8 & 0.8 & 0.8 & 0.8 & 0.8 & 17.20 \\
\hline 0.4 & 0.8 & 1.2 & 0.8 & 0.8 & 0.8 & 1.2 & 0.8 & 0.8 & 17.60 \\
\hline 0.8 & 0.8 & 0.8 & 1.2 & 0.8 & 1.2 & 0.8 & 0.8 & 1.2 & 20.40 \\
\hline 0.8 & 1.2 & 0.8 & 1.2 & 0.8 & 1.2 & 0.8 & 1.2 & 0.8 & 21.60 \\
\hline 53.2 & 53.2 & 1.2 & 0.8 & 1.2 & 0.8 & 1.2 & 0.8 & 0.8 & 360.40 \\
\hline 53.6 & 53.6 & 1.2 & 1.2 & 0.8 & 1.2 & 0.8 & 0.8 & 1.2 & 428.00 \\
\hline 54.8 & 53.2 & 1.6 & 0.8 & 0.8 & 1.2 & 0.8 & 0.8 & 1.2 & 432.40 \\
\hline 0.8 & 0.8 & 1.2 & 0.8 & 0.8 & 0.8 & 1.2 & 0.8 & 0.8 & 21.20 \\
\hline 0.8 & 1.2 & 0.8 & 0.8 & 0.8 & 0.8 & 1.2 & 0.8 & 0.8 & 19.60 \\
\hline
\end{tabular}




\begin{tabular}{|c|c|c|c|c|c|c|c|c|c|}
\hline 0.8 & 0.8 & 1.2 & 0.8 & 0.8 & 0.8 & 1.2 & 0.8 & 1.2 & 99.60 \\
\hline 29.2 & 57.2 & 1.2 & 0.8 & 0.8 & 0.8 & 0.8 & 1.2 & 0.8 & 105.20 \\
\hline 54.8 & 53.2 & 1.2 & 1.2 & 0.8 & 0.8 & 1.2 & 0.8 & 0.8 & 425.60 \\
\hline 56.4 & 56 & 1.6 & 0.8 & 0.8 & 1.2 & 0.8 & 1.2 & 0.8 & 463.60 \\
\hline 55.2 & 54.4 & 1.2 & 1.2 & 1.2 & 0.8 & 1.2 & 0.8 & 1.2 & 446.00 \\
\hline 0.8 & 0.8 & 1.2 & 0.8 & 0.8 & 1.2 & 0.8 & 0.8 & 1.2 & 22.00 \\
\hline 0.8 & 0.8 & 1.2 & 0.8 & 0.8 & 1.2 & 0.8 & 1.2 & 0.8 & 21.20 \\
\hline 52.4 & 52.4 & 1.2 & 1.2 & 0.8 & 1.2 & 1.2 & 0.8 & 1.2 & 434.00 \\
\hline 51.6 & 50.4 & 1.2 & 1.2 & 0.8 & 0.8 & 1.2 & 0.8 & 0.8 & 415.60 \\
\hline 50.8 & 1.2 & 0.8 & 0.8 & 0.8 & 0.8 & 1.2 & 0.8 & 0.8 & 175.60 \\
\hline 51.6 & 50.4 & 1.2 & 0.8 & 0.8 & 0.8 & 1.2 & 0.8 & 0.8 & 412.00 \\
\hline 1.6 & \begin{tabular}{|l|}
1.2 \\
\end{tabular} & 0.8 & 1.2 & 0.8 & 1.2 & 0.8 & 1.2 & 0.8 & 313.20 \\
\hline 1.2 & 1.2 & 1.2 & 0.8 & 1.2 & 1.2 & 0.8 & 1.2 & 1.2 & 24.40 \\
\hline 1.2 & 0.8 & 1.2 & 0.8 & 0.8 & 1.2 & 0.8 & 0.8 & 0.8 & 22.00 \\
\hline 0.8 & 1.2 & 0.8 & 1.2 & 1.2 & 0.8 & 1.2 & 0.8 & 1.2 & 21.60 \\
\hline 0.8 & 0.8 & 0.8 & 0.8 & 0.8 & 0.8 & 1.2 & 0.8 & 0.8 & 20.40 \\
\hline 0.8 & 1.2 & 0.8 & 1.2 & 1.2 & 0.8 & 1.2 & 1.2 & 0.8 & 23.60 \\
\hline 0.8 & 0.8 & 1.2 & 0.8 & 0.8 & 0.8 & 1.2 & 0.8 & 0.8 & 20.00 \\
\hline 0.8 & 0.8 & 1.2 & 0.8 & 0.8 & 0.8 & 0.8 & 1.2 & 0.8 & 21.60 \\
\hline 0.8 & 0.8 & 0.8 & 0.8 & 1.2 & 0.8 & 0.8 & 0.8 & 0.8 & 18.80 \\
\hline 0.8 & 1.2 & 0.8 & 1.2 & 0.8 & 1.2 & 0.8 & 0.8 & 1.2 & 21.20 \\
\hline 0.8 & 1.2 & 1.2 & 0.8 & 0.8 & 1.2 & 0.8 & 0.8 & 1.2 & 21.20 \\
\hline 0.8 & 0.8 & 1.2 & 0.8 & 1.2 & 1.2 & 1.2 & 1.2 & 0.8 & 21.60 \\
\hline 1.2 & 1.2 & 0.8 & 1.2 & 0.8 & 1.2 & 0.8 & 0.8 & 1.2 & 24.80 \\
\hline 0.8 & 0.8 & 0.8 & 0.8 & 0.8 & 0.8 & 0.8 & 0.8 & 0.8 & 20.40 \\
\hline 1.2 & 0.8 & 0.8 & 0.8 & 0.8 & 0.8 & 0.8 & 1.2 & 0.8 & 19.20 \\
\hline 0.8 & 1.2 & 0.8 & 0.8 & 0.8 & 1.2 & 0.8 & 0.8 & 1.2 & 19.60 \\
\hline 0.8 & 0.8 & 0.8 & 0.8 & 1.2 & 0.8 & 0.8 & 1.2 & 1.2 & 20.80 \\
\hline 1.2 & 0.8 & 0.8 & 0.8 & 1.2 & 0.8 & 0.8 & 1.2 & 0.8 & 21.60 \\
\hline 0.8 & 1.2 & 0.8 & 1.2 & 0.8 & 1.2 & 0.8 & 1.2 & 0.8 & 22.00 \\
\hline 1.2 & 0.8 & 1.2 & 1.2 & $\begin{array}{ll}0.8 \\
\end{array}$ & 1.2 & 0.8 & 1.2 & 1.2 & 22.40 \\
\hline 55.6 & 28.4 & 1.2 & 0.8 & 1.2 & 1.2 & 1.2 & 0.8 & 1.2 & 264.40 \\
\hline 1.2 & 1.2 & 1.2 & 1.2 & 0.8 & 1.2 & 1.2 & 0.8 & 1.2 & 25.60 \\
\hline 0.8 & 0.8 & 1.2 & 0.8 & 1.2 & 0.8 & 0.8 & 1.2 & 0.8 & 21.20 \\
\hline 0.8 & 0.8 & 1.2 & 1.2 & 0.8 & 0.8 & 1.2 & 0.8 & 0.8 & 21.60 \\
\hline 0.8 & 0.8 & 1.2 & 0.8 & 1.2 & 0.8 & 1.2 & 0.8 & 0.8 & 54.00 \\
\hline 36.8 & 16.4 & 1.2 & 0.8 & 1.2 & 0.8 & 0.8 & 1.2 & 0.8 & 139.20 \\
\hline 48.4 & 0.8 & 1.2 & 1.2 & 0.8 & 0.8 & 1.2 & 0.8 & 1.2 & 183.20 \\
\hline 54 & 1.2 & 1.2 & 0.8 & 1.2 & 0.8 & 0.8 & 1.2 & 0.8 & 330.80 \\
\hline 50.8 & 1.2 & 0.8 & 1.2 & 0.8 & 1.2 & 0.8 & 1.2 & 0.8 & 310.40 \\
\hline 1.2 & 0.8 & 0.8 & 0.8 & 1.2 & 0.8 & 1.2 & 0.8 & 1.2 & 22.40 \\
\hline 0.8 & 1.2 & 1.2 & 0.8 & 1.2 & 0.8 & 0.8 & 1.2 & 1.2 & 23.20 \\
\hline 48.4 & 1.2 & 0.8 & 1.2 & 1.2 & 0.8 & 1.2 & 0.8 & 1.2 & 85.60 \\
\hline 1.2 & 0.8 & 0.8 & 1.2 & 0.8 & 1.2 & 1.2 & 0.8 & 1.2 & 23.60 \\
\hline 0.8 & 0.8 & 1.2 & 0.8 & 0.8 & 0.8 & 1.2 & 0.8 & 0.8 & 22.00 \\
\hline 0.8 & 0.8 & 1.2 & 0.8 & 0.8 & 1.2 & 0.8 & 1.2 & 0.8 & 21.60 \\
\hline 0.8 & 0.8 & 1.2 & 0.8 & 0.8 & 1.2 & 0.8 & 1.2 & 0.8 & 24.00 \\
\hline 0.8 & 0.8 & 0.8 & 1.2 & 0.8 & 0.8 & 1.2 & 0.8 & 0.8 & 22.40 \\
\hline 1.2 & 0.8 & 0.8 & 1.2 & 0.8 & 0.8 & 0.8 & 1.2 & 0.8 & 21.60 \\
\hline 14 & 1.6 & 0.8 & 1.2 & 1.2 & 0.8 & 1.2 & 0.8 & 0.8 & 36.00 \\
\hline 57.2 & 1.2 & 1.2 & 0.8 & 1.2 & 0.8 & 1.2 & 0.8 & 1.2 & 373.60 \\
\hline
\end{tabular}




\begin{tabular}{|c|c|c|c|c|c|c|c|c|c|}
\hline 57.2 & 14.4 & 1.2 & 1.2 & 1.2 & 0.8 & 1.2 & 0.8 & 1.2 & 424.80 \\
\hline 55.2 & 53.2 & 51.2 & 50 & 49.6 & 50 & 49.6 & 48.8 & 49.2 & 873.60 \\
\hline 0.8 & 1.2 & 0.8 & 1.2 & 0.8 & 0.8 & 0.8 & 0.8 & 1.2 & 435.20 \\
\hline 1.2 & 0.8 & 0.8 & 0.8 & 0.8 & 0.8 & 0.8 & 0.8 & 0.8 & 20.80 \\
\hline 0.8 & 0.8 & 0.8 & 0.8 & 0.8 & 0.8 & 0.8 & 0.8 & 0.8 & 18.80 \\
\hline 0.8 & 0.8 & 1.2 & 1.2 & 1.2 & 0.8 & 1.2 & 1.2 & 1.2 & 23.20 \\
\hline 1.2 & 0.8 & 1.2 & 1.2 & 0.8 & 1.2 & 0.8 & 0.8 & 1.2 & 25.60 \\
\hline 0.8 & 1.2 & 0.8 & 1.2 & 0.8 & 1.2 & 0.8 & 1.2 & 1.2 & 22.40 \\
\hline 1.2 & 1.2 & 1.2 & 1.2 & 1.2 & 1.2 & 1.2 & 1.2 & 1.2 & 26.00 \\
\hline 65.2 & 1.6 & 1.2 & 1.2 & 1.2 & 0.8 & 1.6 & 0.8 & 1.2 & 229.60 \\
\hline 1.2 & 1.2 & 1.2 & 1.2 & 1.2 & 1.2 & 1.2 & 1.2 & 1.2 & 28.00 \\
\hline 1.2 & 0.8 & 1.2 & 1.2 & 1.2 & 1.2 & 1.2 & 1.2 & 0.8 & 27.60 \\
\hline 1.2 & 0.8 & 1.2 & 1.2 & 1.2 & 1.2 & 1.2 & 1.2 & 1.2 & 386.00 \\
\hline 57.2 & 15.6 & 1.2 & 1.6 & 1.2 & 1.2 & 1.2 & 1.2 & 1.2 & 460.40 \\
\hline 1.2 & 1.6 & 1.2 & 1.2 & 1.2 & 1.6 & 1.2 & 1.2 & 1.2 & 338.00 \\
\hline 1.2 & 1.2 & 0.8 & 1.2 & 1.2 & 1.2 & 1.2 & 0.8 & 1.2 & 27.60 \\
\hline 1.2 & 1.2 & 0.8 & 1.2 & 0.8 & 1.2 & 1.2 & 0.8 & 1.2 & 25.20 \\
\hline 1.2 & 1.2 & 1.2 & 0.8 & 1.6 & 0.8 & 1.2 & 1.2 & 1.6 & 26.40 \\
\hline 1.2 & 1.2 & 1.2 & 1.2 & 1.2 & 1.2 & 1.2 & 1.2 & 1.2 & 29.60 \\
\hline 1.2 & 1.2 & 1.2 & 1.2 & 1.2 & 0.8 & 1.2 & 1.2 & 1.2 & 27.60 \\
\hline 1.2 & 0.8 & 1.2 & 1.2 & 1.2 & 1.2 & 1.2 & 1.2 & 0.8 & 27.20 \\
\hline 47.6 & 12.8 & 1.2 & 1.2 & 1.2 & 1.2 & 1.2 & 1.2 & 1.2 & 201.60 \\
\hline 54.4 & 14.4 & 1.2 & 1.2 & 1.2 & 1.2 & 1.2 & 1.2 & 1.2 & 235.20 \\
\hline \begin{tabular}{|l|}
42.4 \\
\end{tabular} & 51.2 & 1.2 & 1.2 & 1.2 & 1.6 & 1.2 & 1.2 & 1.2 & 328.80 \\
\hline 0.8 & 1.6 & 1.2 & 1.2 & 1.2 & 1.2 & 1.2 & 1.2 & 1.2 & 28.80 \\
\hline 1.2 & 0.8 & 1.6 & 0.8 & 1.6 & 0.8 & 1.6 & 1.2 & 1.2 & 28.80 \\
\hline 52 & 47.2 & 1.6 & 1.2 & 1.2 & 1.2 & 1.2 & 1.6 & 1.2 & 462.00 \\
\hline 1.2 & 1.2 & 1.2 & 1.2 & 1.2 & 1.2 & 1.2 & 1.2 & 1.2 & 378.40 \\
\hline 29.6 & 49.6 & 1.6 & 1.2 & 1.2 & 1.2 & 1.2 & 1.2 & 1.2 & 324.80 \\
\hline 1.2 & 41.2 & 1.6 & 1.2 & 1.2 & 1.2 & 1.2 & 1.2 & 1.2 & 183.20 \\
\hline 56.8 & 52 & 1.6 & 1.2 & 1.2 & 1.2 & 1.2 & 1.6 & 1.2 & 279.60 \\
\hline 1.2 & 1.6 & 0.8 & 1.2 & 1.6 & 1.2 & 0.8 & 1.2 & 1.2 & 29.60 \\
\hline 1.2 & 1.2 & 1.2 & 1.2 & 1.2 & 1.2 & 1.2 & 1.2 & 0.8 & 27.60 \\
\hline 1.2 & 1.2 & 1.2 & 1.2 & 1.2 & 1.2 & 1.2 & 1.2 & 1.2 & 28.00 \\
\hline 62.8 & 47.6 & 1.2 & 1.2 & 1.2 & 1.2 & 1.6 & 1.2 & 0.8 & 175.60 \\
\hline 18.8 & 44.8 & 1.2 & 1.2 & 1.2 & 1.2 & 1.2 & 1.2 & 1.2 & 134.80 \\
\hline 53.6 & 44 & 1.6 & 1.2 & 1.2 & 1.2 & 1.2 & 1.2 & 1.2 & 247.20 \\
\hline 30.8 & 30 & 1.6 & 1.2 & 1.2 & 1.2 & 1.2 & 1.2 & 1.2 & 231.60 \\
\hline 1.2 & 1.2 & 1.2 & 1.2 & 1.2 & 1.2 & 1.2 & 0.8 & 1.6 & 28.00 \\
\hline 1.6 & 0.8 & 1.2 & 1.2 & 1.2 & 1.2 & 1.2 & 1.2 & 1.2 & 27.60 \\
\hline 54.8 & 45.2 & 1.2 & 1.2 & 1.2 & 1.2 & 1.2 & 1.2 & 1.2 & 443.20 \\
\hline 55.6 & 40.4 & 1.2 & 1.2 & 1.2 & 1.2 & 1.2 & 1.2 & 1.2 & 467.20 \\
\hline 1.2 & 1.6 & 1.2 & 1.6 & 1.2 & 1.2 & 1.6 & 1.2 & 1.2 & 32.80 \\
\hline 1.2 & 1.2 & 1.6 & 1.2 & 1.6 & 1.2 & 1.6 & 1.6 & 1.2 & 31.60 \\
\hline 1.2 & 1.2 & 1.6 & 1.2 & 1.2 & 1.6 & 1.2 & 1.2 & 1.6 & 31.60 \\
\hline 1.2 & 1.6 & 1.2 & 1.2 & 1.6 & 1.2 & 1.2 & 1.6 & 1.2 & 31.60 \\
\hline 1.2 & 1.6 & 1.2 & 1.6 & 1.2 & 2.4 & 1.6 & 1.6 & 1.6 & 35.20 \\
\hline 1.2 & 1.6 & 1.2 & 1.2 & 1.6 & 1.6 & 1.2 & 1.6 & 1.6 & 35.20 \\
\hline 1.6 & 1.6 & 2 & 1.6 & 1.6 & 1.6 & 2 & 2 & 2 & 38.40 \\
\hline 1.6 & 1.6 & 1.6 & 1.6 & 1.6 & 1.6 & 1.6 & 1.6 & 2 & 40.00 \\
\hline 2 & 2.4 & 2 & 2.4 & 2 & 2.4 & 2 & 2 & 1.6 & 43.20 \\
\hline
\end{tabular}




\begin{tabular}{|c|c|c|c|c|c|c|c|c|c|}
\hline 2 & 2 & 1.6 & 1.6 & 2 & 1.6 & 2 & 1.6 & 1.6 & 42.00 \\
\hline 1.6 & 1.6 & 1.6 & 1.6 & 1.6 & 1.6 & 1.6 & 1.6 & 1.6 & 38.80 \\
\hline 1.6 & 1.2 & 1.6 & 1.6 & 1.6 & 1.6 & 1.6 & 1.6 & 1.2 & 36.80 \\
\hline 55.2 & 44.4 & 2 & 1.6 & 1.6 & 1.2 & 1.6 & 1.6 & 1.6 & 490.40 \\
\hline 56 & 46 & 2 & 1.6 & 1.6 & 1.6 & 1.2 & 1.6 & 1.6 & 495.60 \\
\hline 55.6 & 46 & 1.6 & 1.6 & 1.6 & 1.6 & 1.6 & 1.2 & 1.6 & 505.60 \\
\hline 56.8 & 46.8 & 2 & 1.6 & 1.6 & 1.6 & 1.6 & 1.2 & 1.6 & 508.80 \\
\hline 54.4 & 44.8 & 2 & 1.6 & 1.6 & 1.6 & 1.6 & 1.6 & 1.6 & 495.20 \\
\hline 1.6 & 1.6 & 1.6 & 1.6 & 1.6 & 1.6 & 1.6 & 1.6 & 1.6 & 38.80 \\
\hline 1.6 & 1.6 & 1.6 & 1.6 & 2 & 1.6 & 1.6 & 1.6 & 2 & 40.00 \\
\hline 51.2 & 42.4 & 1.6 & 2 & 1.6 & 1.6 & 1.6 & 1.6 & 1.6 & 411.20 \\
\hline 50.8 & 42.4 & 2 & 1.6 & 2 & 1.6 & 1.6 & 1.6 & 1.6 & 402.40 \\
\hline 50.8 & 42.4 & 2 & 1.6 & 1.6 & 1.6 & 2 & 1.6 & 1.6 & 404.80 \\
\hline 50.8 & 42.4 & 2 & 1.6 & 2 & 1.6 & 1.6 & 1.6 & 1.6 & 401.20 \\
\hline 2 & 1.6 & 1.6 & 1.6 & 1.6 & 2 & 1.6 & 1.6 & 1.6 & 59.60 \\
\hline 2 & 1.6 & 1.6 & 1.6 & 2 & 1.6 & 1.6 & & 1.6 & 38.40 \\
\hline 1.6 & 2 & 1.6 & 1.6 & 2 & 1.6 & 1.6 & 2 & 1.6 & 41.20 \\
\hline 52.8 & 45.6 & 2 & 1.6 & 2 & 1.6 & 1.6 & 2 & 1.6 & 429.20 \\
\hline 55.2 & 47.2 & 2 & 1.6 & 2 & 1.6 & 2 & 1.6 & 2 & 454.00 \\
\hline 49.2 & 42.4 & 2 & 2 & 1.6 & 2 & 2 & 1.6 & 2 & 416.40 \\
\hline 49.2 & 42.4 & 2.4 & 2 & 2 & 1.2 & & & & 399.20 \\
\hline & & & & & & & & & 3.20 \\
\hline & & & & & & & & & 0.00 \\
\hline & & & & & & & & & 0.00 \\
\hline 53.2 & 45.2 & 0.4 & 0.4 & 0.4 & 0.4 & 0.4 & 0.4 & 0.4 & 463.60 \\
\hline 53.6 & 44.4 & 0.8 & 0.4 & 0.4 & 0.4 & 0.4 & 0.4 & 0.8 & 477.60 \\
\hline 53.2 & 45.6 & 0.8 & 0.4 & 0.4 & 0.4 & 0.4 & 0.8 & 0.4 & 456.40 \\
\hline 55.6 & 50 & 0.8 & 0.8 & 0.4 & 0.4 & 0.8 & & 0.8 & 483.20 \\
\hline 51.6 & 48.4 & 0.8 & 0.4 & 0.4 & 0.4 & 0.4 & 0.4 & 0.4 & 494.40 \\
\hline 10.4 & 0.4 & 0.4 & 0.4 & 0.4 & 0.4 & 0.8 & 0.4 & 0.4 & 357.20 \\
\hline 0.4 & 0.8 & 0.4 & 0.4 & 0.4 & 0.4 & 0.4 & 0.4 & 0.4 & 10.80 \\
\hline 53.6 & 49.6 & 0.8 & 0.4 & 0.4 & 0.4 & 0.4 & 0.4 & 0.4 & 440.40 \\
\hline 54.8 & 51.2 & 0.8 & 0.8 & & 0.4 & 0.8 & 0.4 & 0.4 & 508.40 \\
\hline 54 & 50 & 0.8 & 0.4 & 0.4 & 0.4 & 0.4 & 0.4 & 0.8 & 513.60 \\
\hline 53.2 & 50 & 0.8 & 0.4 & 0.4 & 0.8 & 0.4 & 0.4 & 0.4 & 496.00 \\
\hline 54 & 49.2 & 1.2 & 0.4 & 0.4 & 0.8 & 0.4 & 0.8 & 0.8 & 499.60 \\
\hline 0.4 & 0.8 & 0.4 & 0.8 & 0.4 & 0.8 & 0.8 & 0.4 & 0.8 & 12.80 \\
\hline 0.4 & 0.4 & 0.4 & 0.4 & 0.8 & 0.4 & 0.4 & 0.4 & 0.4 & 10.80 \\
\hline 56.4 & 51.6 & 0.8 & 0.4 & 0.8 & 0.4 & 0.4 & 0.4 & 0.4 & 434.00 \\
\hline 61.2 & 56 & 0.8 & 0.8 & 0.4 & 0.8 & 0.4 & 0.8 & 0.4 & 551.20 \\
\hline 62.8 & 59.2 & 0.8 & 0.8 & 0.4 & 0.8 & 0.4 & 0.8 & 0.4 & 590.00 \\
\hline 62 & 57.6 & 0.8 & 0.8 & 0.4 & 0.8 & 0.4 & 0.4 & 0.8 & 587.60 \\
\hline 59.6 & 55.2 & 0.8 & 0.4 & 0.8 & 0.4 & 0.8 & 0.8 & 0.4 & 573.60 \\
\hline 0.4 & 0.4 & 0.8 & 0.4 & 0.4 & 0.4 & 0.4 & 0.8 & 0.4 & 12.00 \\
\hline 0.4 & 0.4 & 0.4 & 0.4 & 0.8 & 0.4 & 0.4 & 0.4 & 0.8 & 11.60 \\
\hline 56.4 & 52.4 & 0.8 & 0.4 & 0.4 & 0.4 & 0.4 & 0.8 & 0.4 & 334.40 \\
\hline 58.4 & 53.6 & 0.8 & 0.4 & 0.8 & 0.4 & 0.4 & 0.4 & 0.4 & 526.40 \\
\hline 58 & 53.2 & 0.8 & 0.4 & 0.4 & 0.4 & 0.4 & 0.8 & & 521.60 \\
\hline 59.6 & 54.8 & 0.8 & 0.8 & 0.4 & 0.4 & 0.8 & 0.4 & 0.4 & 537.20 \\
\hline 59.2 & 55.2 & 0.8 & 0.8 & 0.4 & 0.4 & 0.8 & 0.4 & 0.4 & 532.80 \\
\hline 0.4 & 0.8 & 0.4 & 0.8 & 0.4 & 0.8 & 0.8 & 0.4 & 0.8 & 12.40 \\
\hline
\end{tabular}




\begin{tabular}{|c|c|c|c|c|c|c|c|c|c|}
\hline 0.4 & 0.8 & 0.4 & 0.8 & 0.4 & 0.8 & 0.8 & 0.4 & 0.8 & 13.60 \\
\hline 65.6 & 61.2 & 0.8 & 0.8 & 0.4 & 0.8 & 0.4 & 0.8 & 0.4 & 604.80 \\
\hline 65.2 & 60.4 & 1.2 & 0.4 & 0.8 & 0.8 & 0.4 & 0.8 & 0.4 & 605.20 \\
\hline 64.4 & 59.6 & 1.2 & 0.4 & 0.8 & 0.4 & 0.8 & 0.8 & 0.4 & 597.60 \\
\hline 6 & 77.6 & 0.8 & 0.8 & 0.4 & 0.8 & 0.4 & 0.8 & 0.4 & 392.80 \\
\hline 51.2 & 0.8 & 0.4 & 0.8 & 0.4 & 0.4 & 0.4 & 0.4 & 0.4 & 593.60 \\
\hline 50.8 & 49.2 & 18.4 & 0.4 & 0.4 & 0.4 & 0.8 & 0.4 & 0.4 & 508.80 \\
\hline 0.8 & 0.4 & 0.4 & 0.4 & 0.4 & 0.4 & 0.4 & 0.4 & 0.4 & 10.40 \\
\hline 12 & 63.6 & 0.8 & 0.8 & 0.4 & 0.8 & 0.4 & 0.8 & 0.4 & 495.60 \\
\hline 62.8 & 58.4 & 0.8 & 0.8 & 0.8 & 0.4 & 0.8 & 0.8 & 0.4 & 561.60 \\
\hline 64.4 & 60 & 0.8 & 0.8 & 0.8 & 0.4 & 0.8 & 0.8 & 0.4 & 575.20 \\
\hline 64 & 59.2 & 0.8 & 0.8 & 0.8 & 0.4 & 0.8 & 0.8 & 0.8 & 591.60 \\
\hline 63.2 & 58.4 & 1.2 & 0.4 & 0.8 & 0.8 & 0.4 & 0.8 & 0.8 & 581.60 \\
\hline 0.4 & 0.8 & 0.4 & 0.8 & 0.4 & 0.8 & 0.8 & 0.4 & 0.8 & 13.60 \\
\hline 0.8 & 0.4 & 0.8 & 0.4 & 0.8 & 0.8 & 0.4 & 0.8 & 0.4 & 13.20 \\
\hline 63.2 & 57.2 & 1.2 & 0.4 & 0.8 & 0.8 & 0.4 & 0.8 & 0.8 & 596.80 \\
\hline 62 & 59.2 & 0.8 & 0.8 & 0.8 & 0.8 & 0.4 & 0.8 & 0.8 & 575.60 \\
\hline 61.2 & 57.6 & 1.2 & 0.4 & 0.8 & 0.8 & 0.4 & 0.8 & 0.4 & 563.20 \\
\hline 63.6 & 58.8 & 0.8 & 0.8 & 0.8 & 0.4 & 0.8 & 0.8 & 0.4 & 578.40 \\
\hline 59.2 & 55.2 & 1.2 & 0.8 & 0.4 & 0.8 & 0.8 & 0.4 & 0.8 & 568.00 \\
\hline 0.8 & 0.8 & 0.4 & 0.8 & 0.8 & 0.8 & 0.4 & 0.8 & 0.8 & 298.80 \\
\hline 0.8 & 0.4 & 0.8 & 0.8 & 0.8 & 0.4 & 0.8 & 0.8 & 0.8 & 16.00 \\
\hline 54.4 & 54 & 20.4 & 0.8 & 0.8 & 0.8 & 0.4 & 0.8 & 0.8 & 433.60 \\
\hline 53.2 & 53.2 & 11.2 & 0.4 & 0.8 & 0.8 & 0.4 & 0.8 & 0.8 & 535.60 \\
\hline 49.2 & 49.2 & 10 & 0.8 & 0.8 & 0.4 & 0.8 & 0.8 & 0.4 & 500.40 \\
\hline 53.6 & 51.6 & 10.4 & 0.8 & 0.4 & 0.8 & 0.8 & 0.4 & 0.8 & 502.00 \\
\hline 0.4 & 0.8 & 0.4 & 0.8 & 0.4 & 0.8 & 0.4 & 0.8 & 0.8 & 16.00 \\
\hline 0.4 & 0.8 & 0.8 & 0.4 & 0.8 & 0.8 & 0.8 & 0.8 & 0.4 & 14.80 \\
\hline & & & & & & & & & \\
\hline & & & & & & & & & \\
\hline & & & & & & & & & \\
\hline 0 & 0 & 0 & 0 & 0 & 0 & 0 & 0 & 0 & \\
\hline 29.59669 & 26.67459 & 2.355191 & 1.340822 & 1.238692 & 1.250959 & 1.251791 & 0.958791 & 0.628099 & \\
\hline 21.23757 & 22.09296 & 21.08872 & 20.08902 & 19.18554 & 18.36927 & 17.62427 & 16.88803 & 16.2232 & \\
\hline 23.90973 & 20.37709 & 0.866087 & 0.489474 & 0.482096 & 0.482819 & 0.499563 & 0.492576 & 0.494737 & \\
\hline 37.02564 & 28.99 & 0.73 & 0.505 & 0.545 & 0.511392 & 0.536709 & 0.536709 & 0.53 & \\
\hline 32.87232 & 27.72655 & 2.298305 & 2.045198 & 2.047458 & 2.033898 & 2.042938 & 1.835028 & 1.118644 & \\
\hline 16.65882 & 13.12941 & 1.2 & 1.164706 & 1.176471 & 1.141176 & 1.176471 & 1.152941 & 1.141176 & \\
\hline 34.39556 & 31.84889 & 1.848889 & 0.968889 & 0.979775 & 0.96 & 0.939326 & 0.96092 & 0.945455 & \\
\hline & & & & & & & & & \\
\hline & & & & & & & & & \\
\hline & & & & & & & & & \\
\hline & & & & & & & & & \\
\hline 0 & 0 & 0 & 0 & 0 & 0 & 0 & 0 & 0 & \\
\hline 31.96366 & 29.63176 & 6.340681 & 6.262886 & 6.05966 & 6.074495 & 6.123261 & 4.300651 & 0.741794 & \\
\hline 27.21124 & 27.46748 & 27.08807 & 26.7807 & 26.46928 & 26.15678 & 25.84807 & 25.51353 & 25.19794 & \\
\hline 27.18148 & 26.10096 & 0.89158 & 0.167052 & 0.161905 & 0.162434 & 0.173331 & 0.169071 & 0.170432 & \\
\hline 27.00031 & 28.81743 & 0.268328 & 0.177108 & 0.193502 & 0.180446 & 0.190934 & 0.190934 & 0.188532 & \\
\hline 30.50842 & 30.10748 & 8.193543 & 7.984971 & 7.918707 & 7.891752 & 7.814727 & 6.652333 & 3.642262 & \\
\hline 24.06857 & 19.62101 & 0.241209 & 0.151522 & 0.195493 & 0.200178 & 0.195493 & 0.191066 & 0.174282 & \\
\hline 27.19316 & 25.35718 & 3.203132 & 0.522665 & 0.572113 & 0.53565 & 0.543059 & 0.501012 & 0.53967 & \\
\hline
\end{tabular}




\begin{tabular}{|c|c|c|c|c|c|c|c|c|c|}
\hline & & & & & & & & & \\
\hline & & & & & & & & & \\
\hline & & & & & & & & & \\
\hline & & & & & & & & & \\
\hline & & & & & & & & & \\
\hline 0 & 0 & 0 & 0 & 0 & 0 & 0 & 0 & 0 & 0 \\
\hline 11323.2 & 10236.6 & 953.6 & 553 & 514.6 & 518.6 & 514.4 & 360.6 & 235.6 & \\
\hline 1263.6 & 1206.8 & 91.2 & 18.4 & 19.2 & 18.8 & 17.6 & 20.4 & 16.4 & \\
\hline 5215.6 & 4133.2 & 167.6 & 111.2 & 110.4 & 109.2 & 114.8 & 112.4 & 112.4 & \\
\hline 2805.6 & 1939.6 & 54.8 & 40.4 & 43.6 & 41.2 & 42.8 & 42 & 42 & \\
\hline 5640.4 & 4928.4 & 415.2 & 372 & 370.8 & 368.4 & 370 & 332.4 & 206.8 & \\
\hline 586.4 & 547.6 & 44.8 & 41.6 & 43.6 & 42.8 & 43.6 & 43.6 & 43.2 & \\
\hline 3136.896 & 2907.639 & 178.7872 & 96.60311 & 95.25503 & 92.74952 & 89.67107 & 88.42499 & 85.48131 & \\
\hline & & & & & & & & & \\
\hline & & & & & & & & & \\
\hline & & & & & & & & & \\
\hline & & & & & & & & & \\
\hline & & & & & & & & & \\
\hline & & & & & & & & & \\
\hline & & & & & & & & & \\
\hline & & & & & & & & & \\
\hline & & & & & & & & & \\
\hline & & & & & & & & & \\
\hline & & & & & & & & & \\
\hline & & & & & & & & & \\
\hline & & & & & & & & & \\
\hline & & & & & & & & & \\
\hline & & & & & & & & & \\
\hline
\end{tabular}




\begin{tabular}{|c|c|c|c|c|c|}
\hline $\begin{array}{l}\text { DAILY } \\
\text { AVERAGE }\end{array}$ & $\begin{array}{l}\text { MONTHLY } \\
\text { TOTAL } \\
\text { formula }\end{array}$ & & & & \\
\hline \\
\hline & \multicolumn{5}{|c|}{ \#DIV/0! } \\
\hline \#DIV/0! & & & sort S/NS & sort JDAY & \\
\hline \#DIV/0! & & & 132810.60 & sum AE column & sum AG column \\
\hline \#DIV/0! & & & 115389.60 & & \\
\hline \#DIV/0! & & & 248200.20 & 248200.20 & 248211.8 \\
\hline \multicolumn{6}{|l|}{ \#DIV/0! } \\
\hline \#DIV/0! & & & sort $\mathrm{A} / \mathrm{B}, \mathrm{S} / \mathrm{NS}$ & & \\
\hline \#DIV/0! & & & B-S & 134638.40 & \\
\hline \#DIV/0! & & & B-NS & 85230.40 & \\
\hline \multicolumn{6}{|l|}{ \#DIV/0! } \\
\hline \multicolumn{6}{|l|}{ \#DIV/0! } \\
\hline \multicolumn{6}{|l|}{ \#DIV/0! } \\
\hline \multicolumn{6}{|l|}{ \#DIV/0! } \\
\hline \multicolumn{6}{|l|}{ \#DIV/0! } \\
\hline \multicolumn{6}{|l|}{ \#DIV/0! } \\
\hline \multicolumn{6}{|l|}{ \#DIV/0! } \\
\hline \multicolumn{6}{|l|}{ \#DIV/0! } \\
\hline \multicolumn{6}{|l|}{ \#DIV/0! } \\
\hline \multicolumn{6}{|l|}{ \#DIV/0! } \\
\hline \multicolumn{6}{|l|}{ \#DIV/0! } \\
\hline \multirow{2}{*}{\multicolumn{6}{|c|}{ \#DIV/0! }} \\
\hline \multicolumn{2}{|l|}{ \#DIV/0! } & & & & \\
\hline \multirow{2}{*}{\multicolumn{6}{|c|}{ \#DIV/0! }} \\
\hline \#DIV/0! & & & & & \\
\hline \multicolumn{6}{|l|}{ \#DIV/0! } \\
\hline \multicolumn{6}{|l|}{ \#DIV/0! } \\
\hline \multirow{2}{*}{\multicolumn{6}{|c|}{ \#DIV/0! }} \\
\hline \multirow{2}{*}{\multicolumn{6}{|c|}{$\begin{array}{l}\text { \#DIV/0! } \\
\text { \#DIV/0! }\end{array}$}} \\
\hline & & & & & \\
\hline \#DIV/0! & & & \multicolumn{2}{|c|}{ \#DIV/0! } & \\
\hline \#DIV/0! & & & & & \\
\hline \#DIV/0! & & & & & \\
\hline \#DIV/0! & & & & & \\
\hline \#DIV/0! & & & & & \\
\hline \#DIV/0! & & & & & \\
\hline \#DIV/0! & & & & & \\
\hline \#DIV/0! & & & & & \\
\hline \#DIV/0! & & & & & \\
\hline \#DIV/0! & & & & & \\
\hline \#DIV/0! & & & & & \\
\hline \#DIV/0! & & & & & \\
\hline \#DIV/0! & & & & & \\
\hline \#DIV/0! & & & & & \\
\hline \#DIV/0! & & & & & \\
\hline \#DIV/0! & & & & & \\
\hline \#DIV/0! & & & & & \\
\hline
\end{tabular}




\begin{tabular}{|l|l|l|l|l|l|}
\hline \#DIV/0! & & & & \\
\hline \#DIV/0! & & & & \\
\hline \#DIV/0! & & & & \\
\hline \#DIV/0! & & & & \\
\hline \#DIV/0! & & & & \\
\hline \#DIV/0! & & & & \\
\hline \#DIV/0! & & & & \\
\hline \#DIV/0! & & & & \\
\hline \#DIV/0! & & & & \\
\hline \#DIV/0! & & & & & \\
\hline \#DIV/0! & & & & & \\
\hline \#DIV/0! & & & & & \\
\hline \#DIV/0! & & & & & \\
\hline \#DIV/0! & & & & & \\
\hline \#DIV/0! & & & & & \\
\hline \#DIV/0! & & & & & \\
\hline \#DIV/0! & & & & & \\
\hline \#DIV/0! & & & & & \\
\hline \#DIV/0! & & & & & \\
\hline \#DIV/0! & & & & & \\
\hline \#DIV/0! & & & & & \\
\hline \#DIV/0! & & & & & \\
\hline \#DIV/0! & & & & & \\
\hline \#DIV/0! & & & & & \\
\hline \#DIV/0! & & & & & \\
\hline \#DIV/0! & & & & & \\
\hline \#DIV/0! & & & & & \\
\hline \#DIV/0! & & & & & \\
\hline \#DIV/0! & & & & & \\
\hline \#DIV/0! & & & & & \\
\hline \#DIV/0! & & & & & \\
\hline \#DIV/0! & & & & & \\
\hline \#DIV/0! & & & & & \\
\hline \#DIV/0! & & & & \\
\hline \#DIV/0! & & & & \\
\hline \#DIV/0! & & & & \\
\hline \#DIV/0! & & & & \\
\hline \#DIV/0! & & & & \\
\hline \#DIV/0! & & & & \\
\hline \#DIV/0! & & & & \\
\hline \#DIV/0! & & & & \\
\hline \#DIV/0! & & & & \\
\hline \#DIV/0! & & & & \\
\hline \#DIV/0! & & & & \\
\hline \#DIV/0! & & & & \\
\hline \#DIV/0! & & & & \\
\hline \#DIV/0! & \#DIV/0! & & & & \\
\hline \#DIV/0! & & & & \\
\hline \#DIV/0! & & & & \\
\hline
\end{tabular}




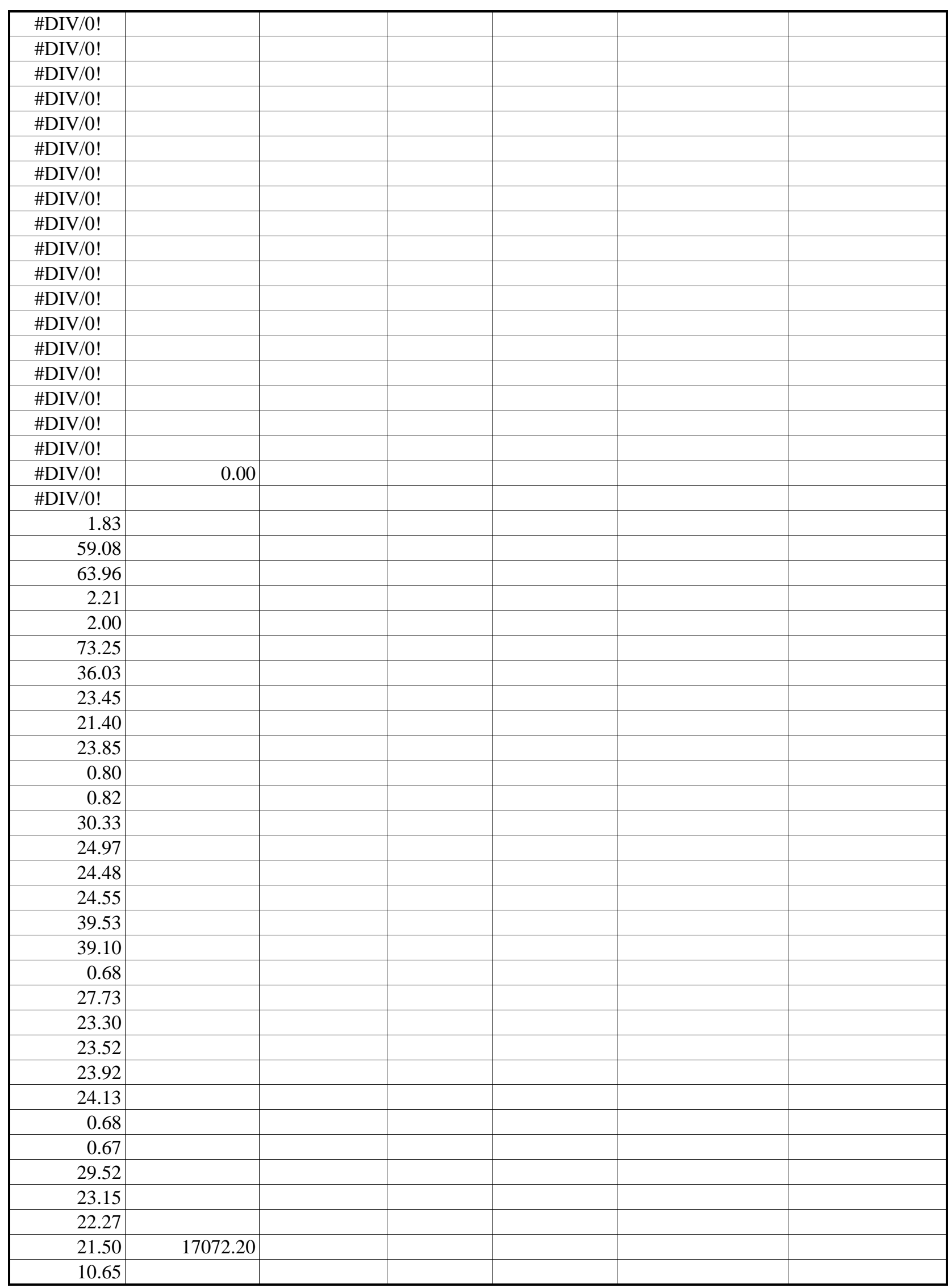




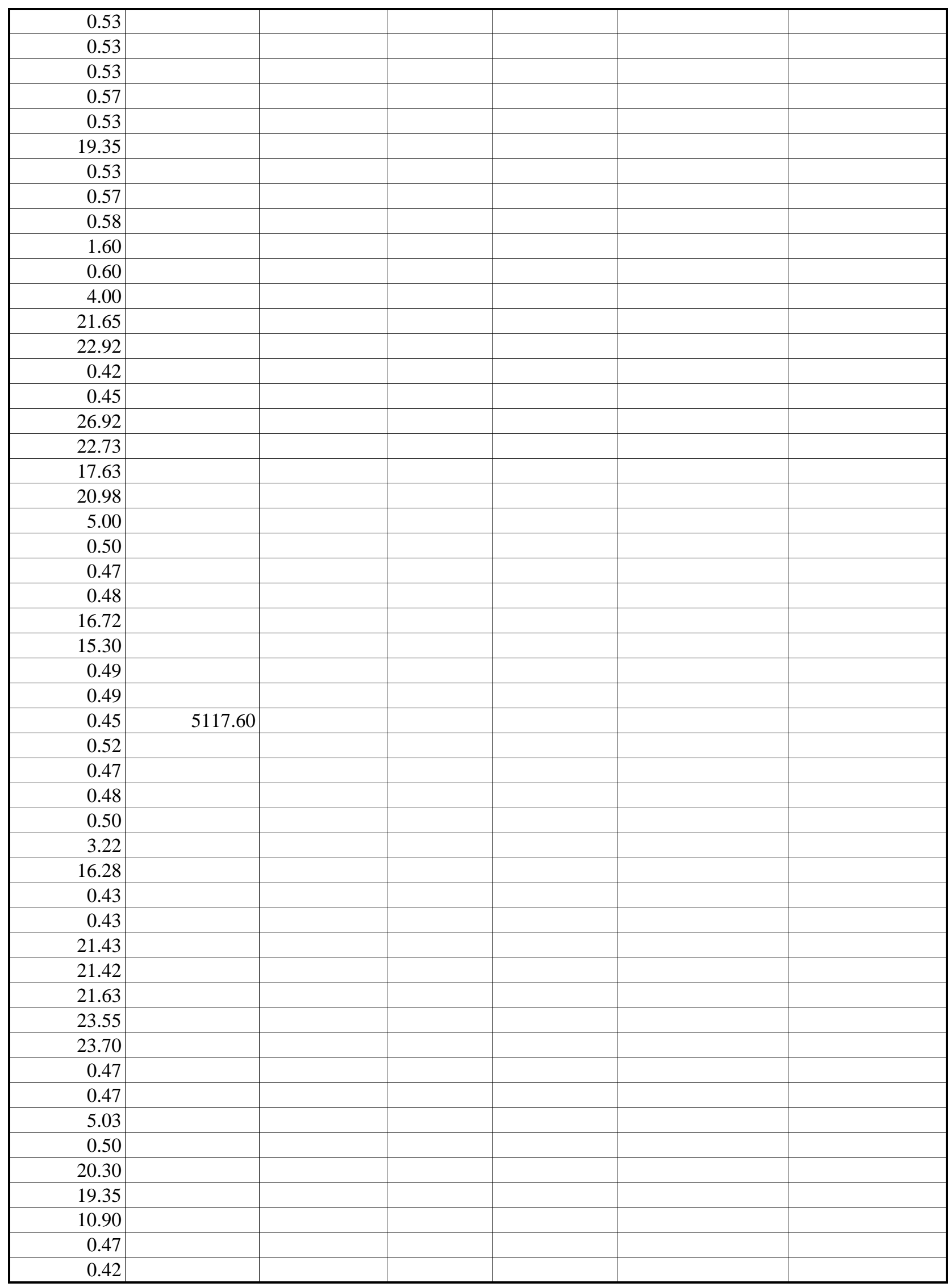




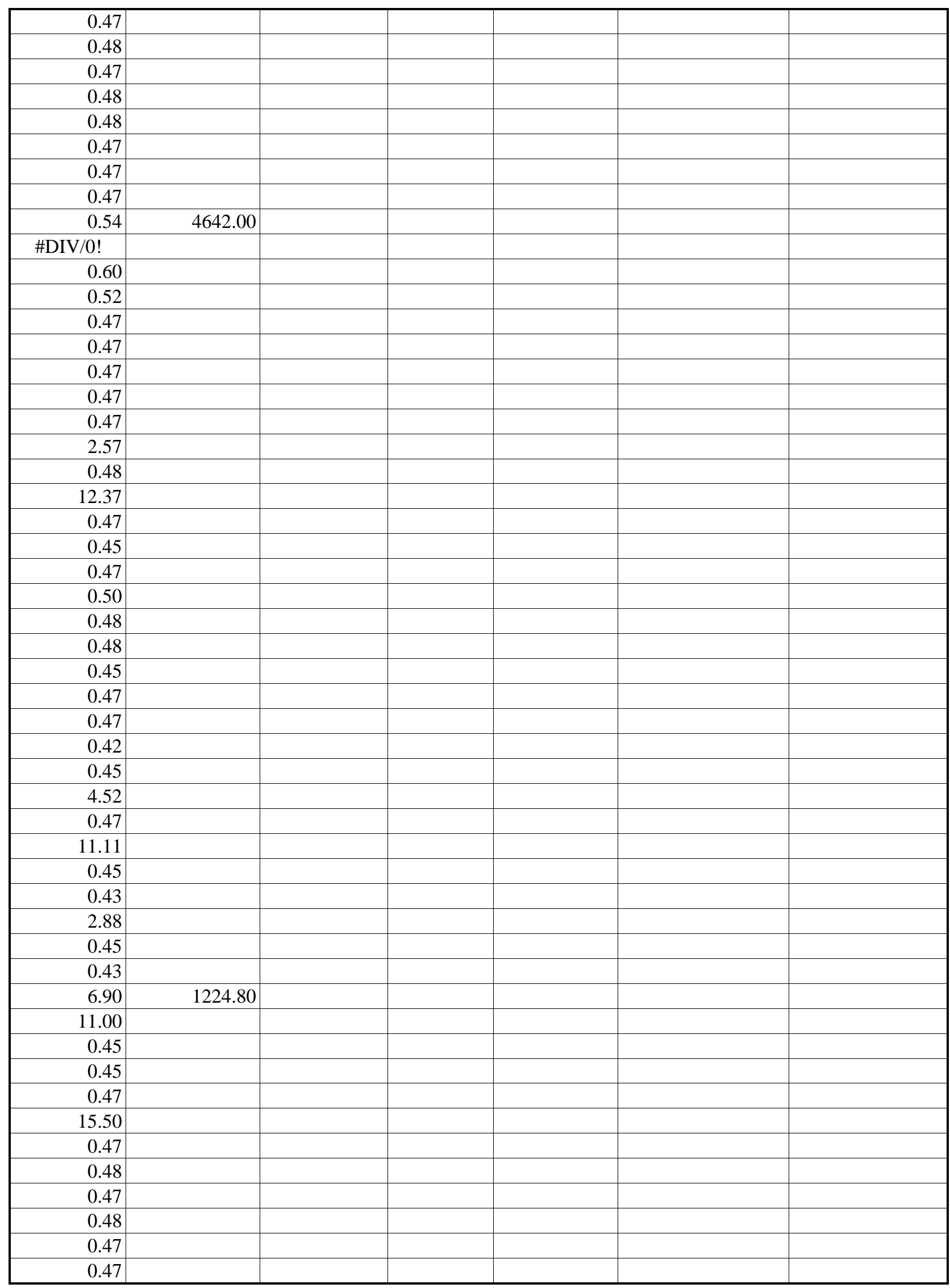




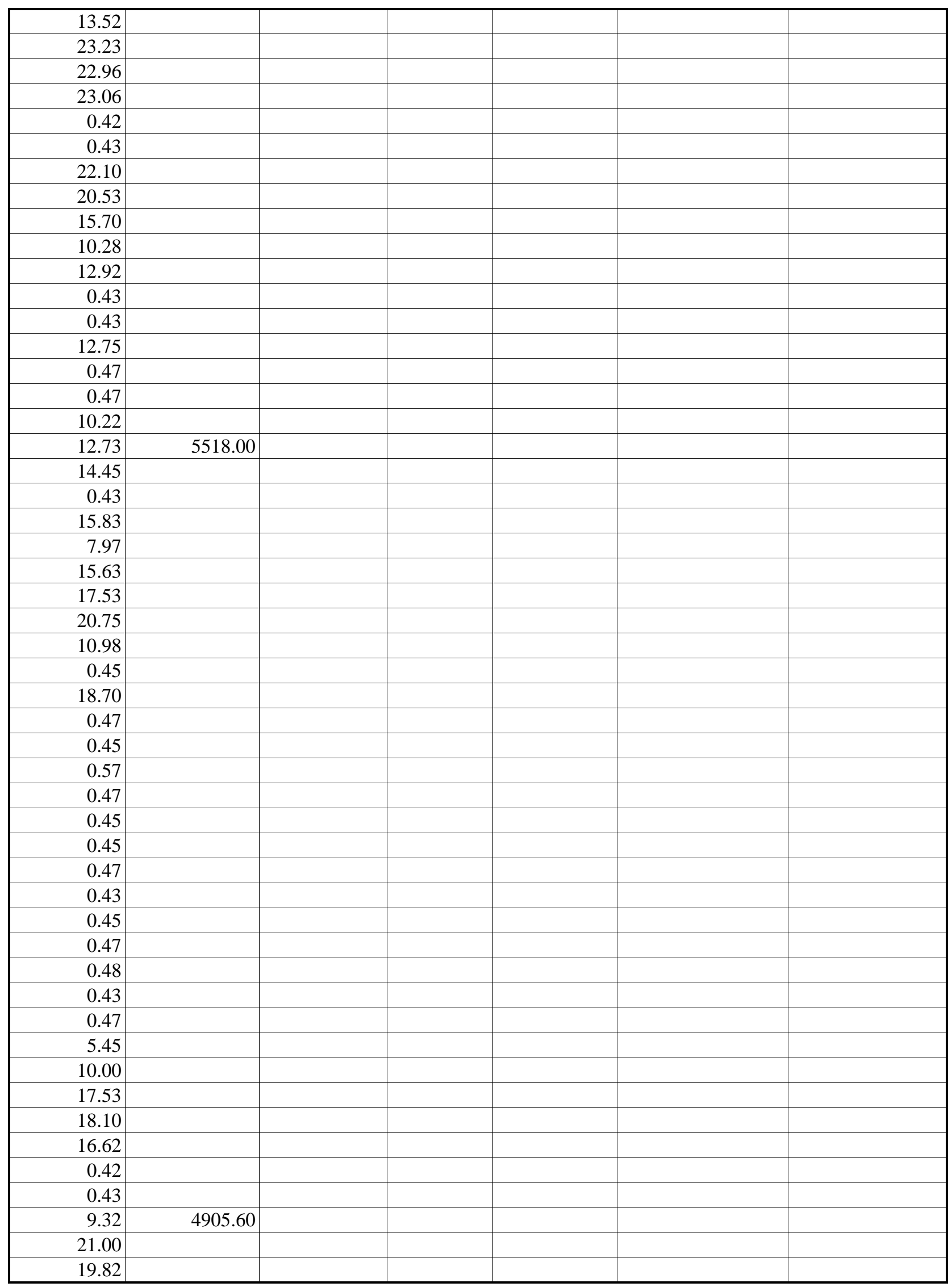




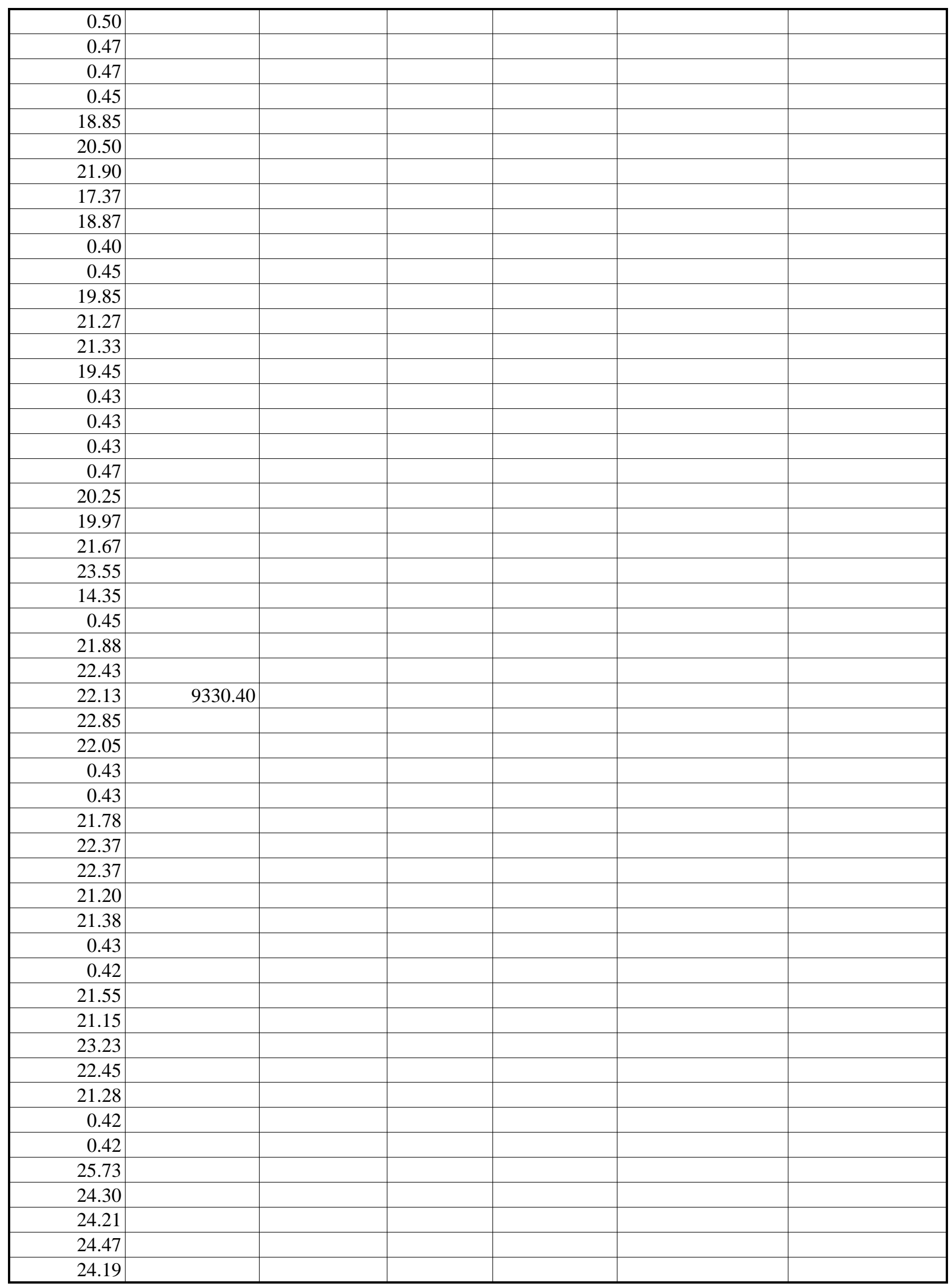




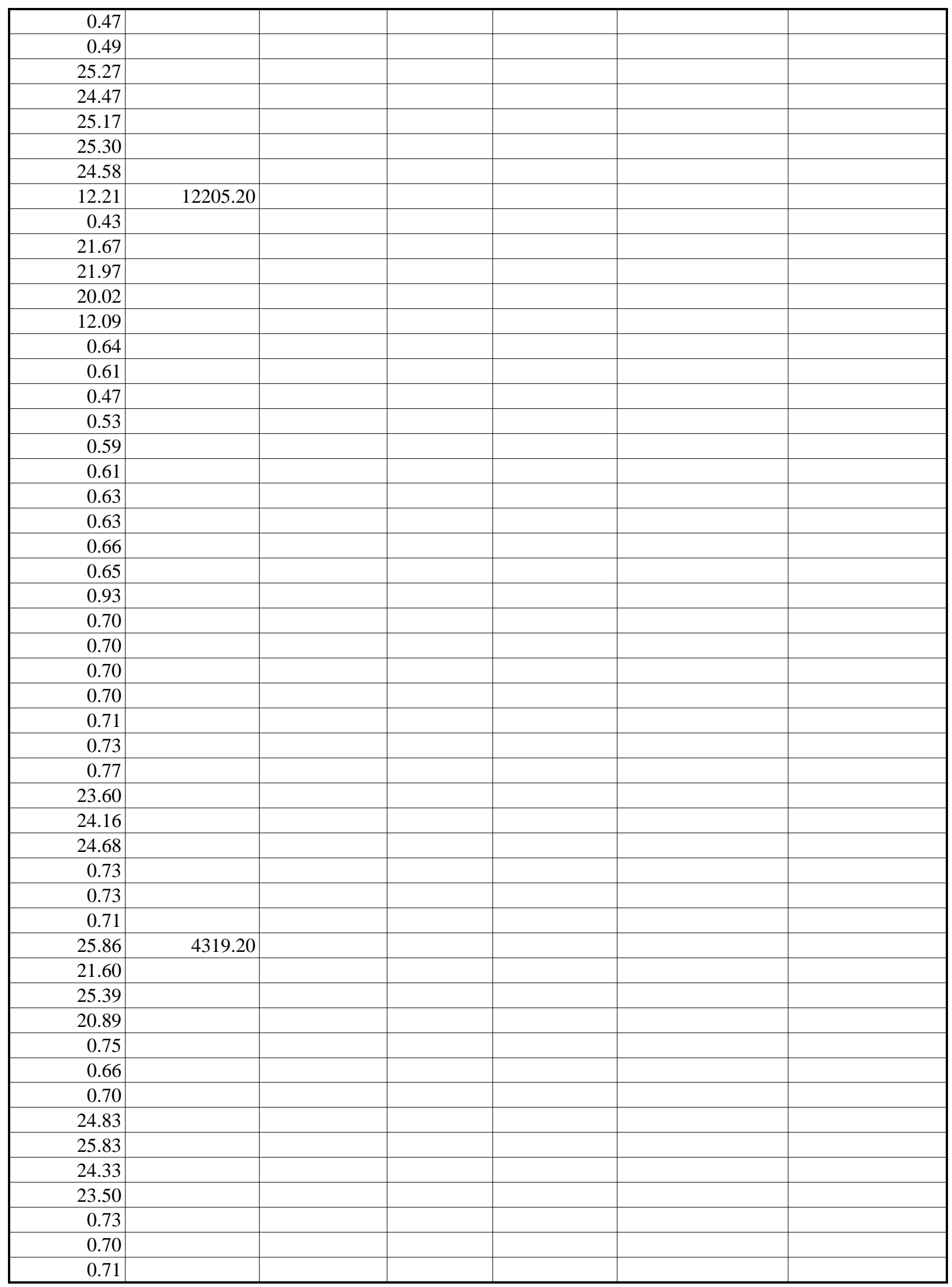




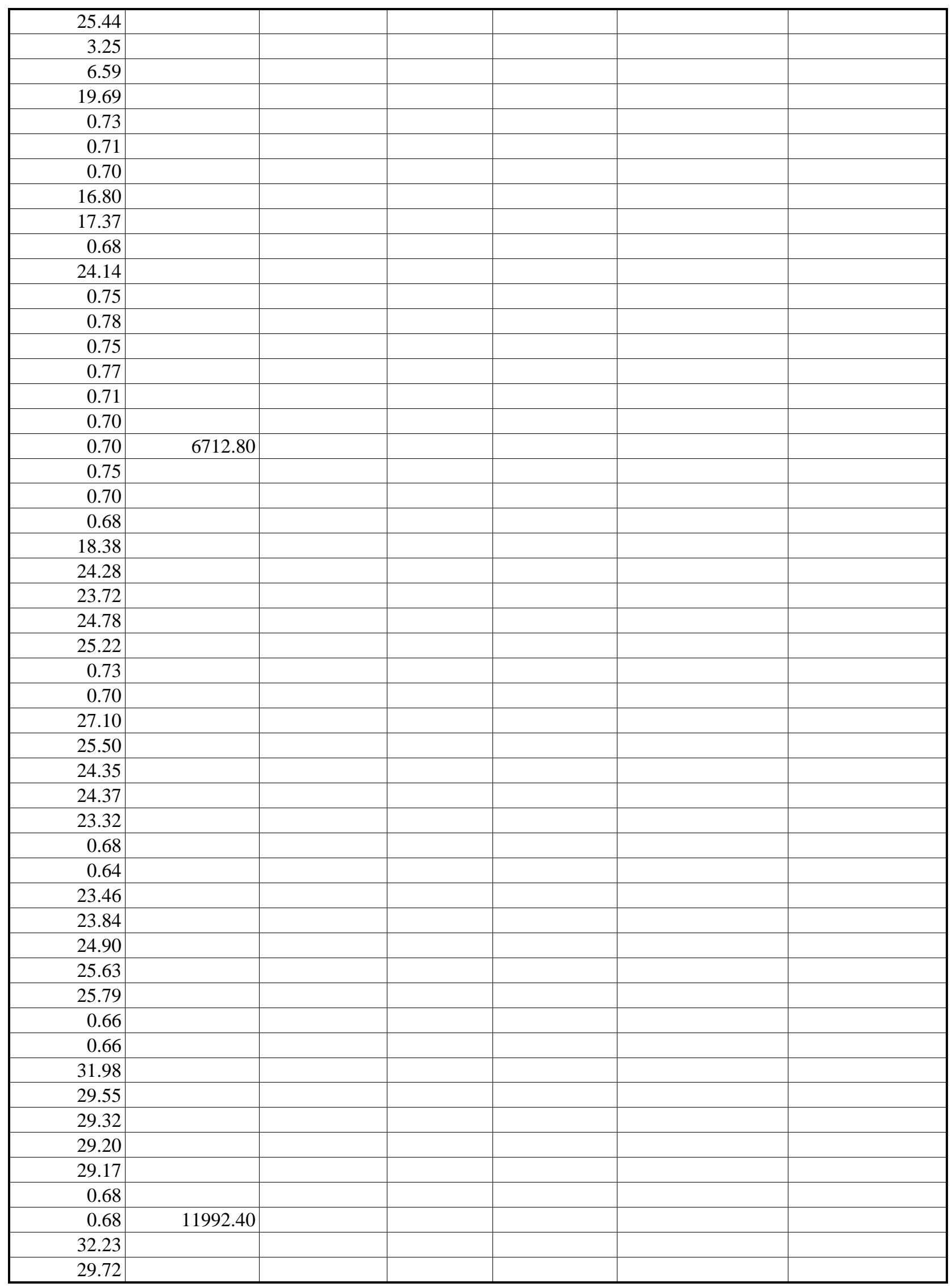




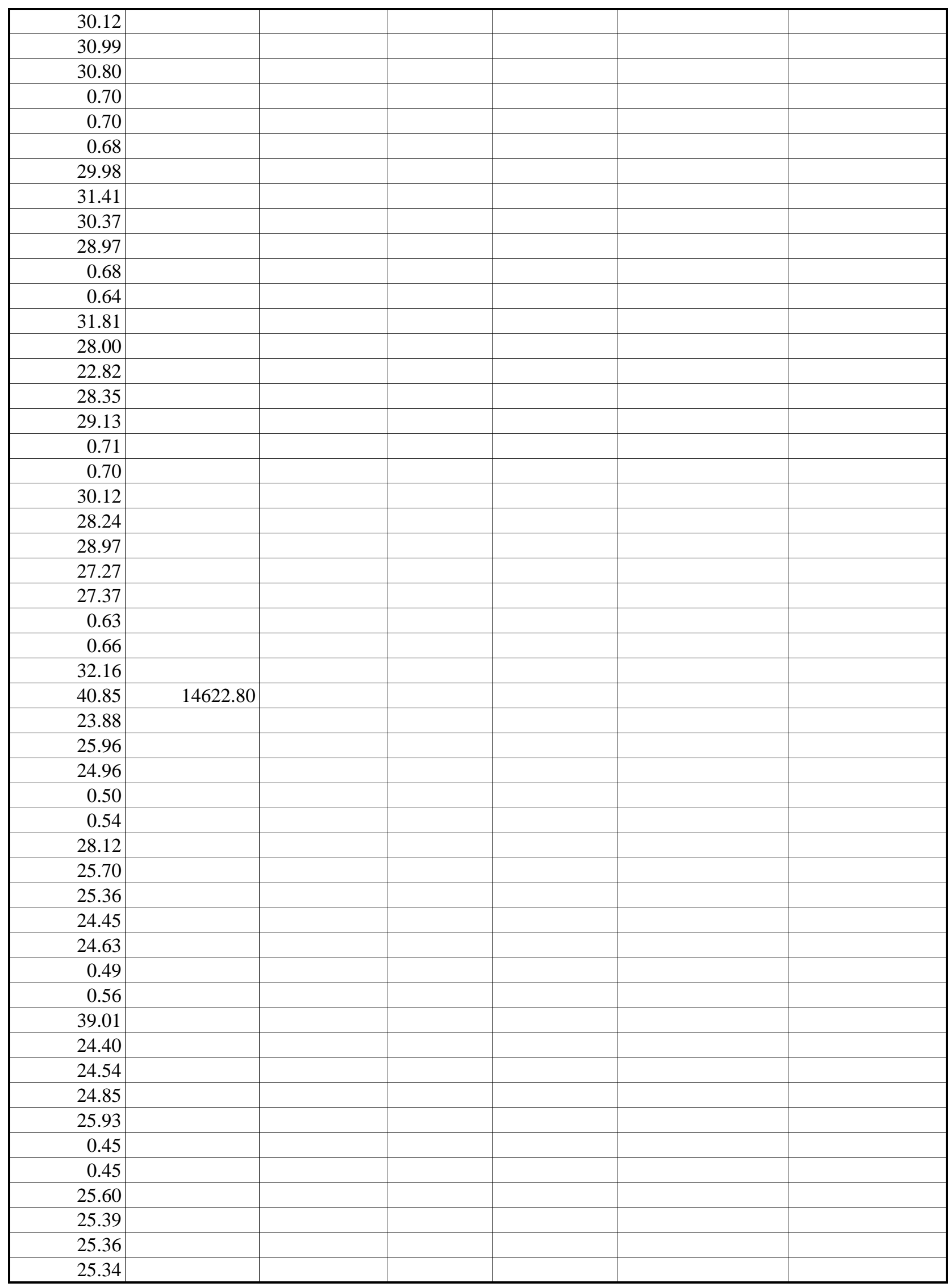




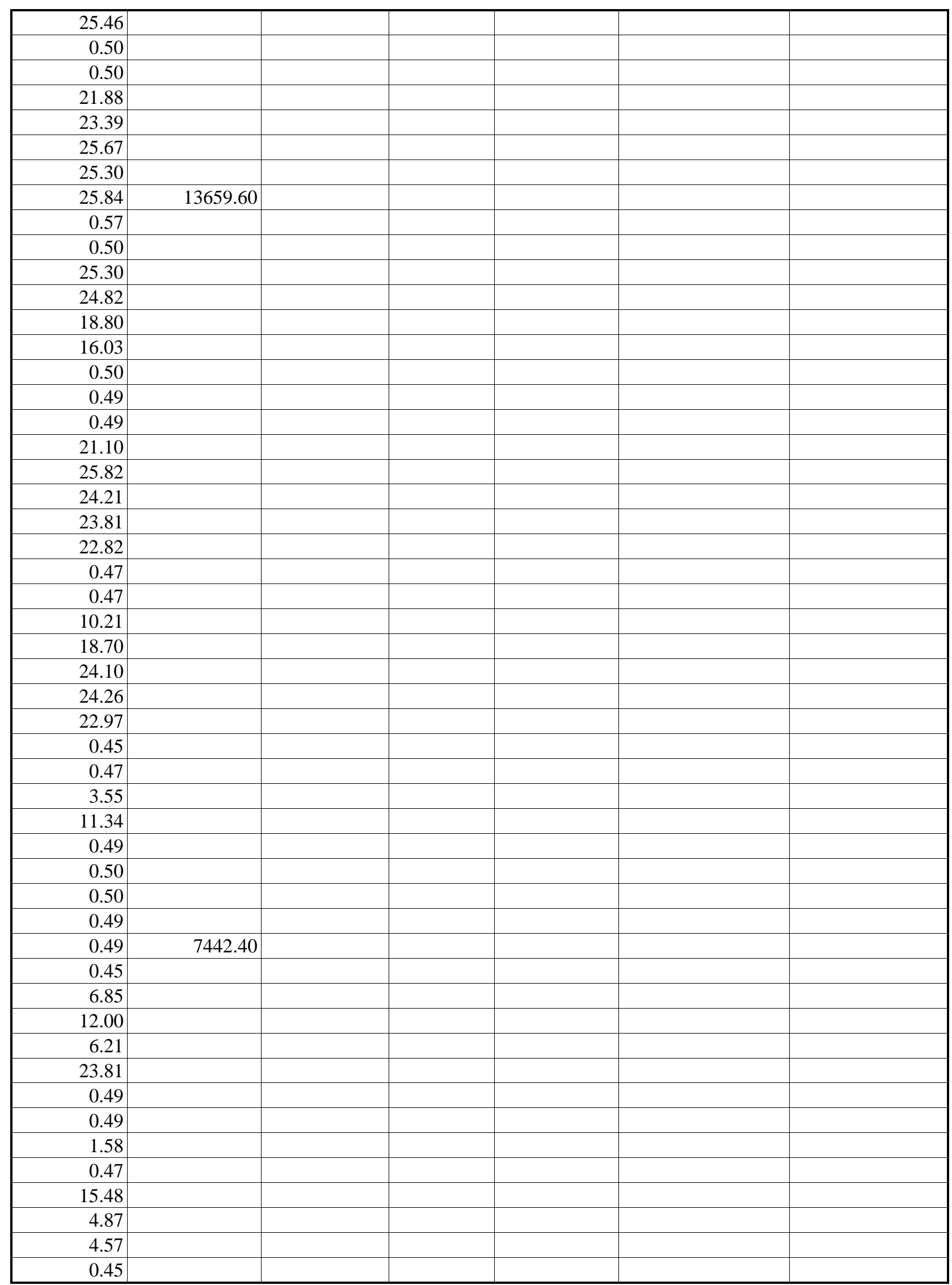




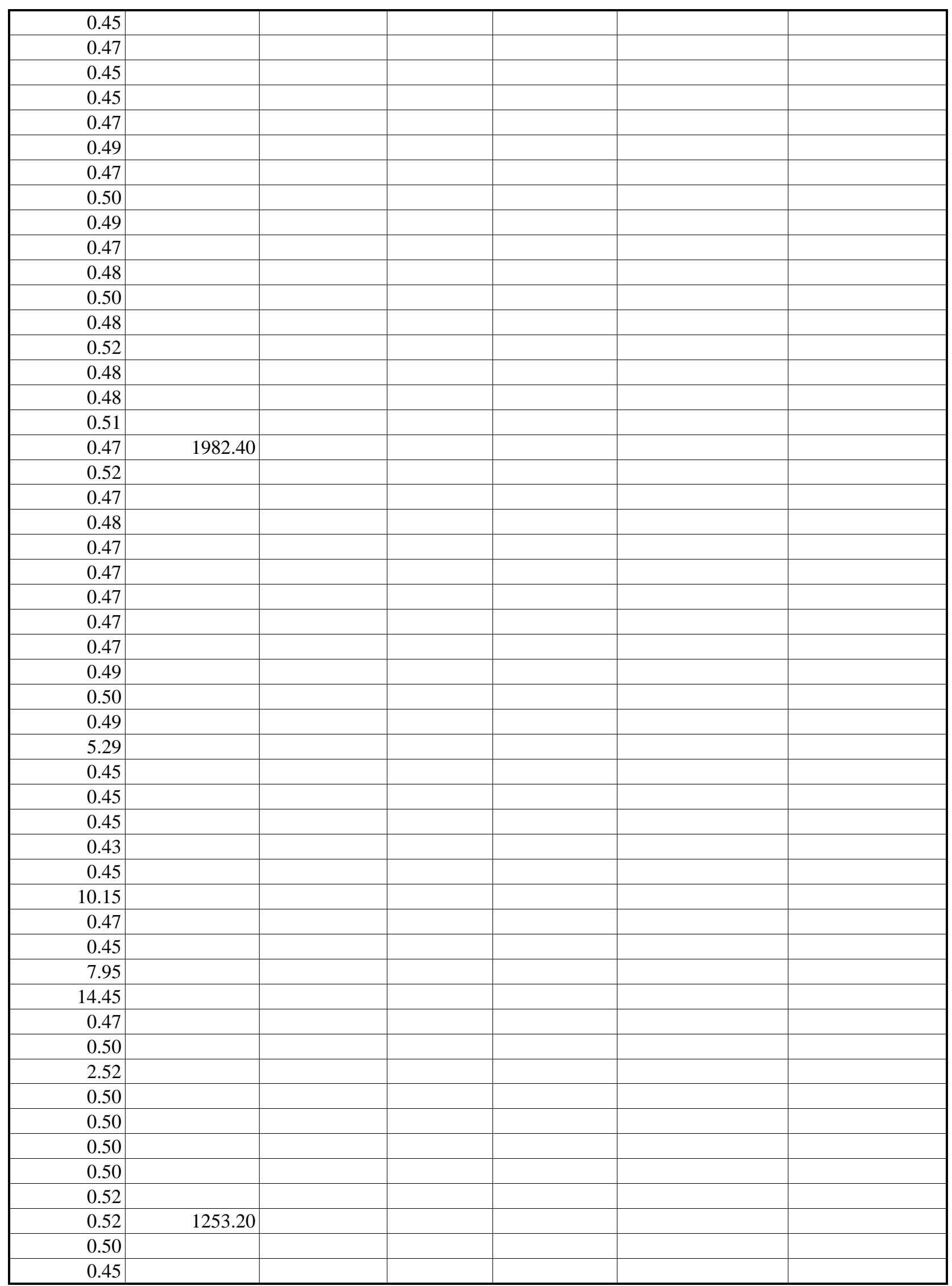




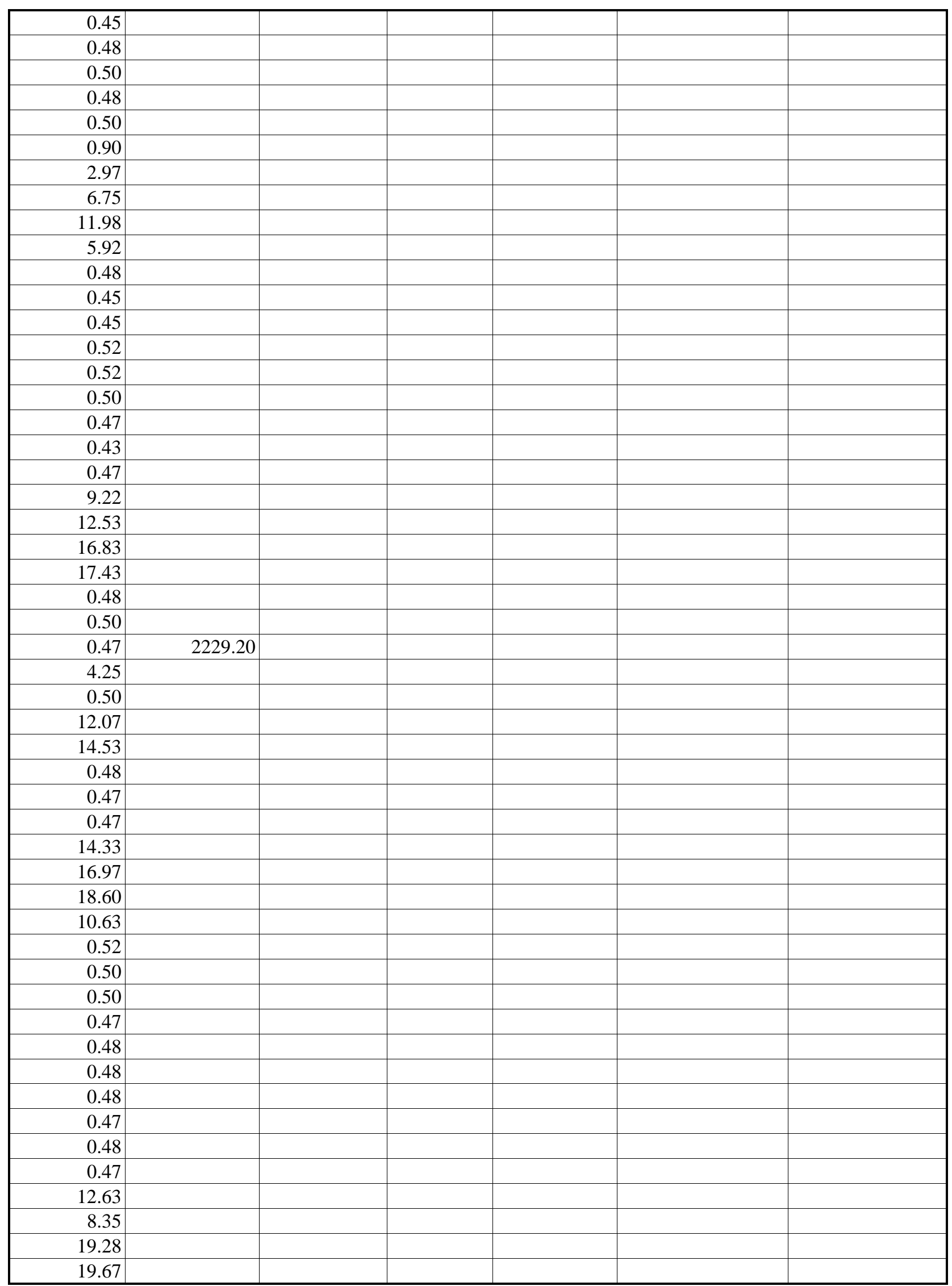




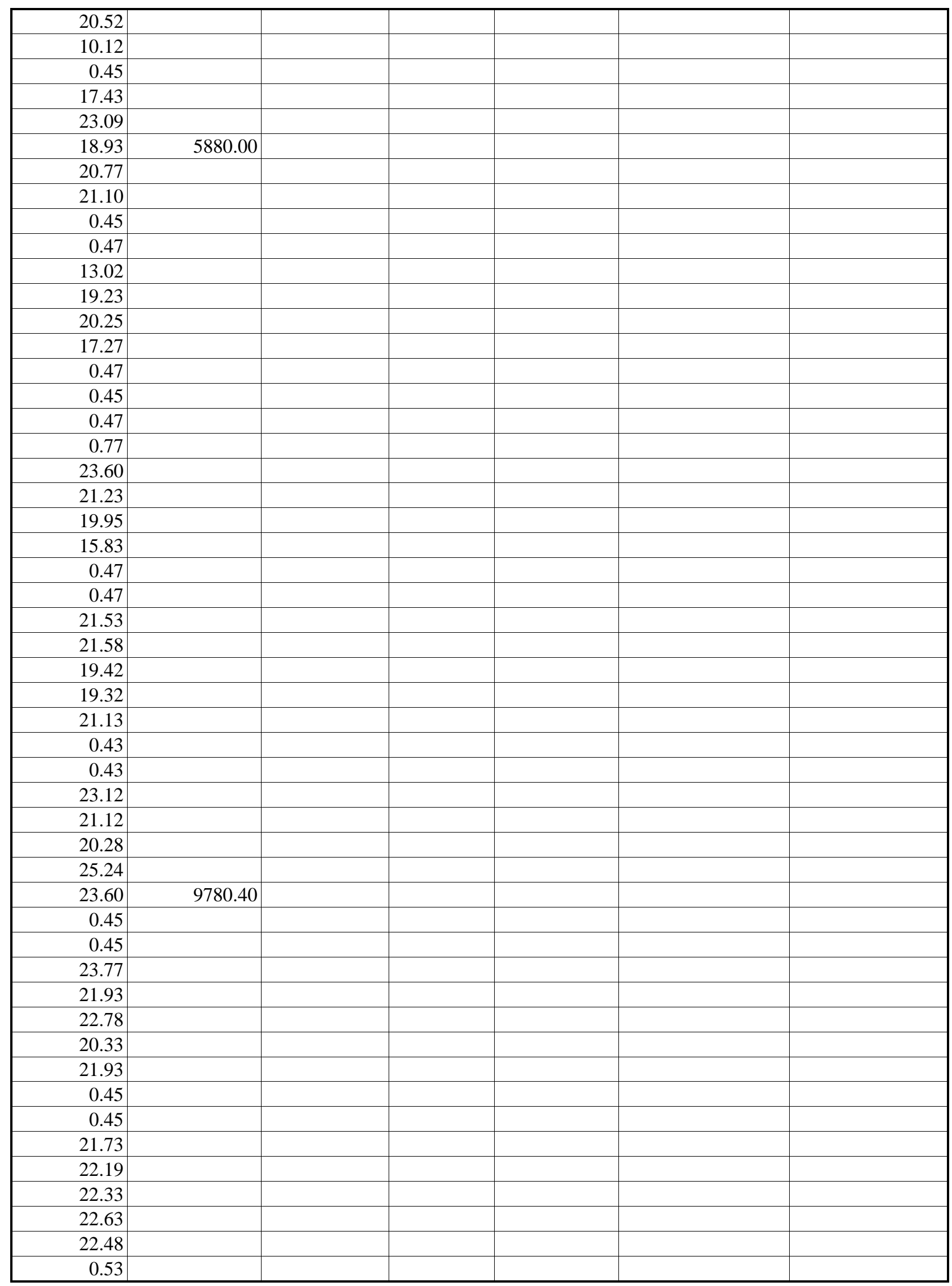




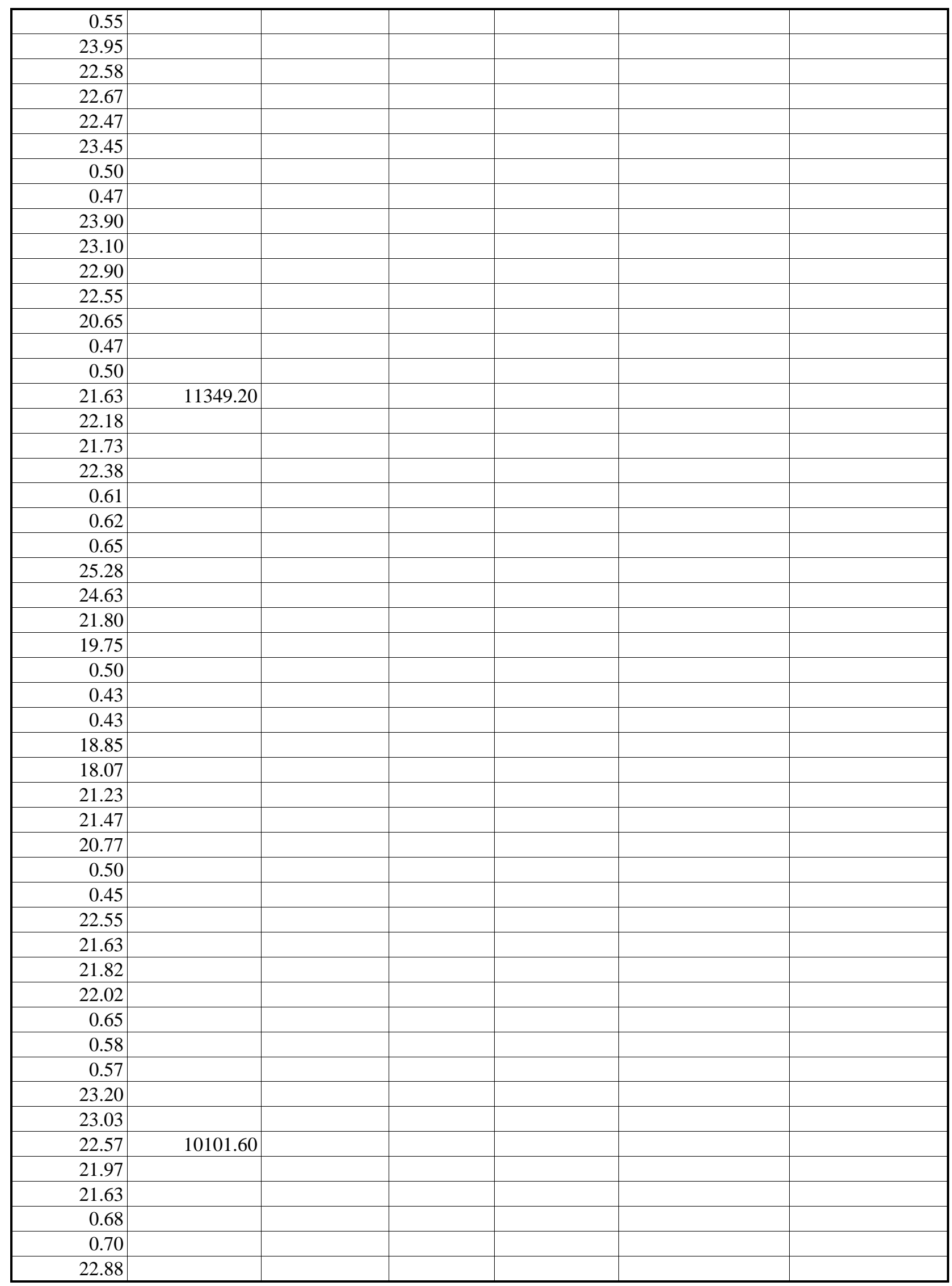




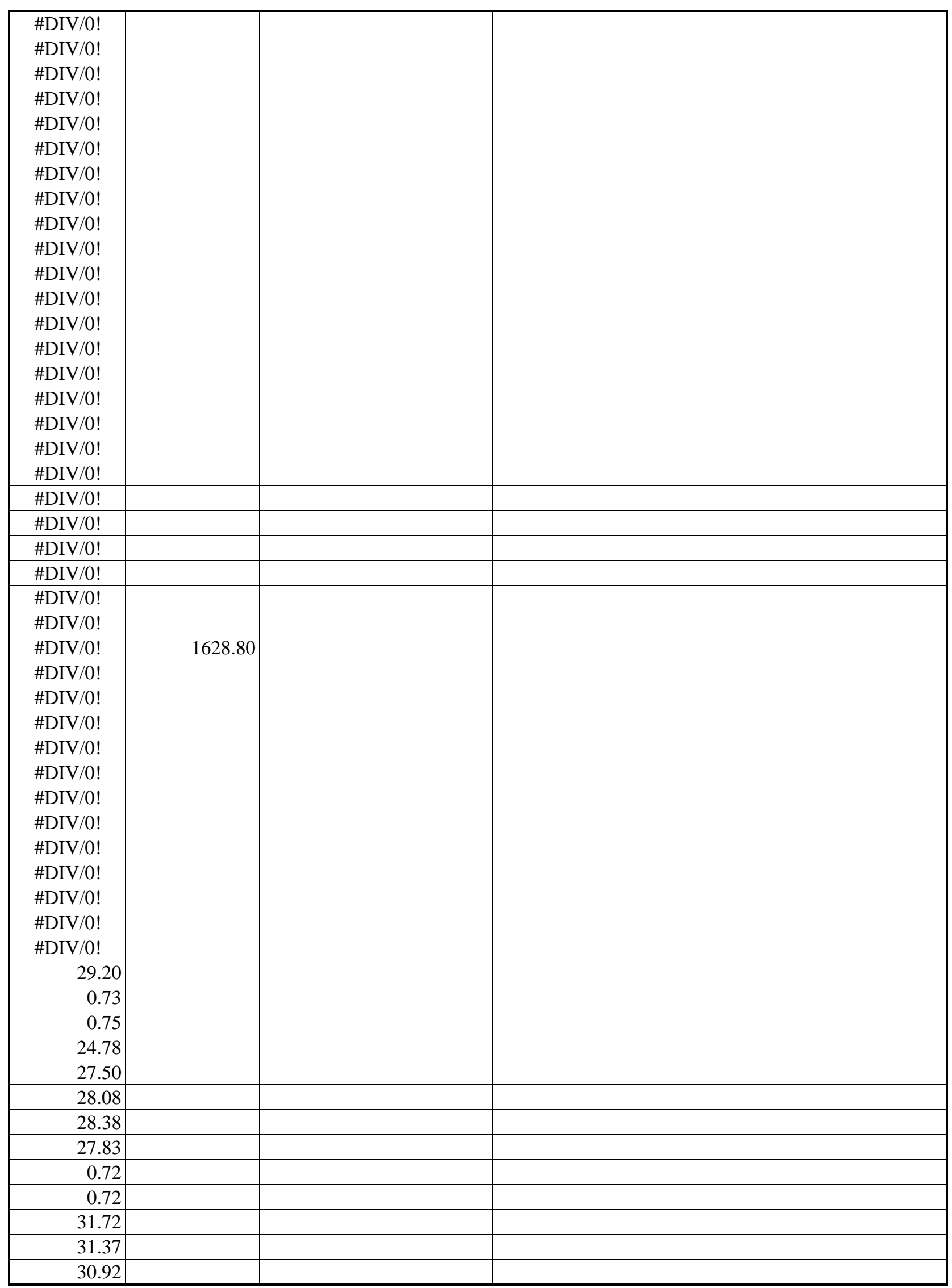




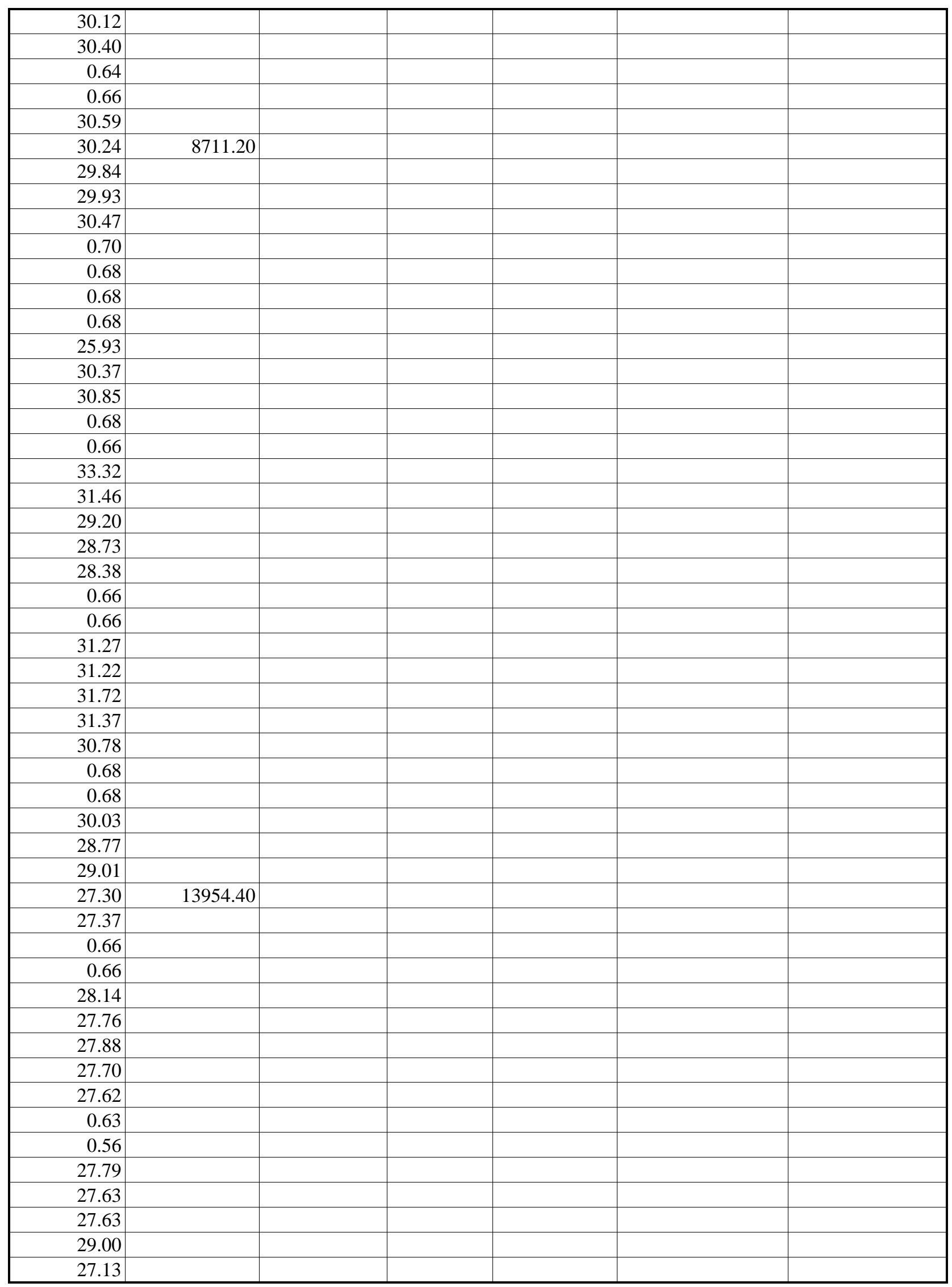




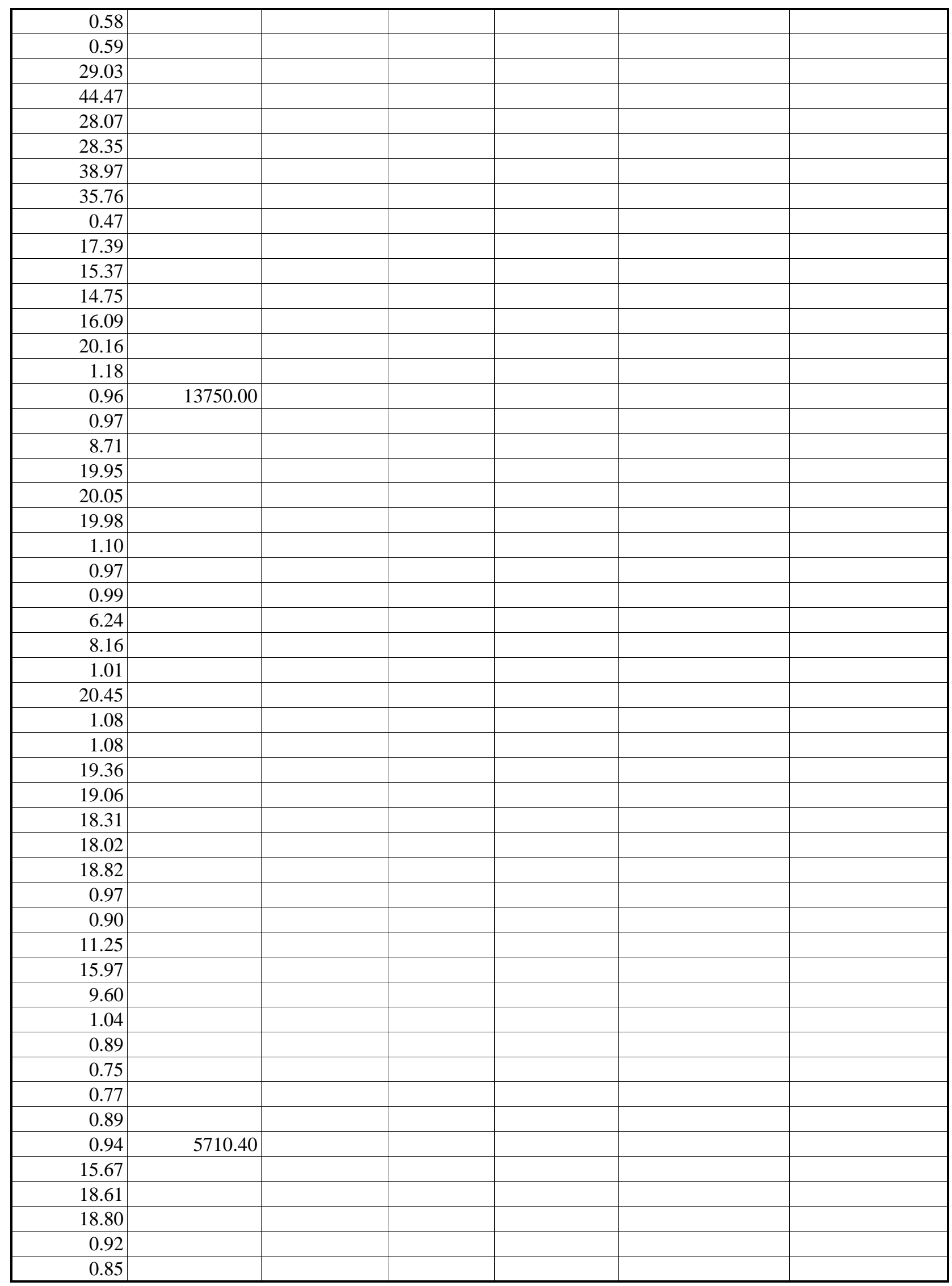




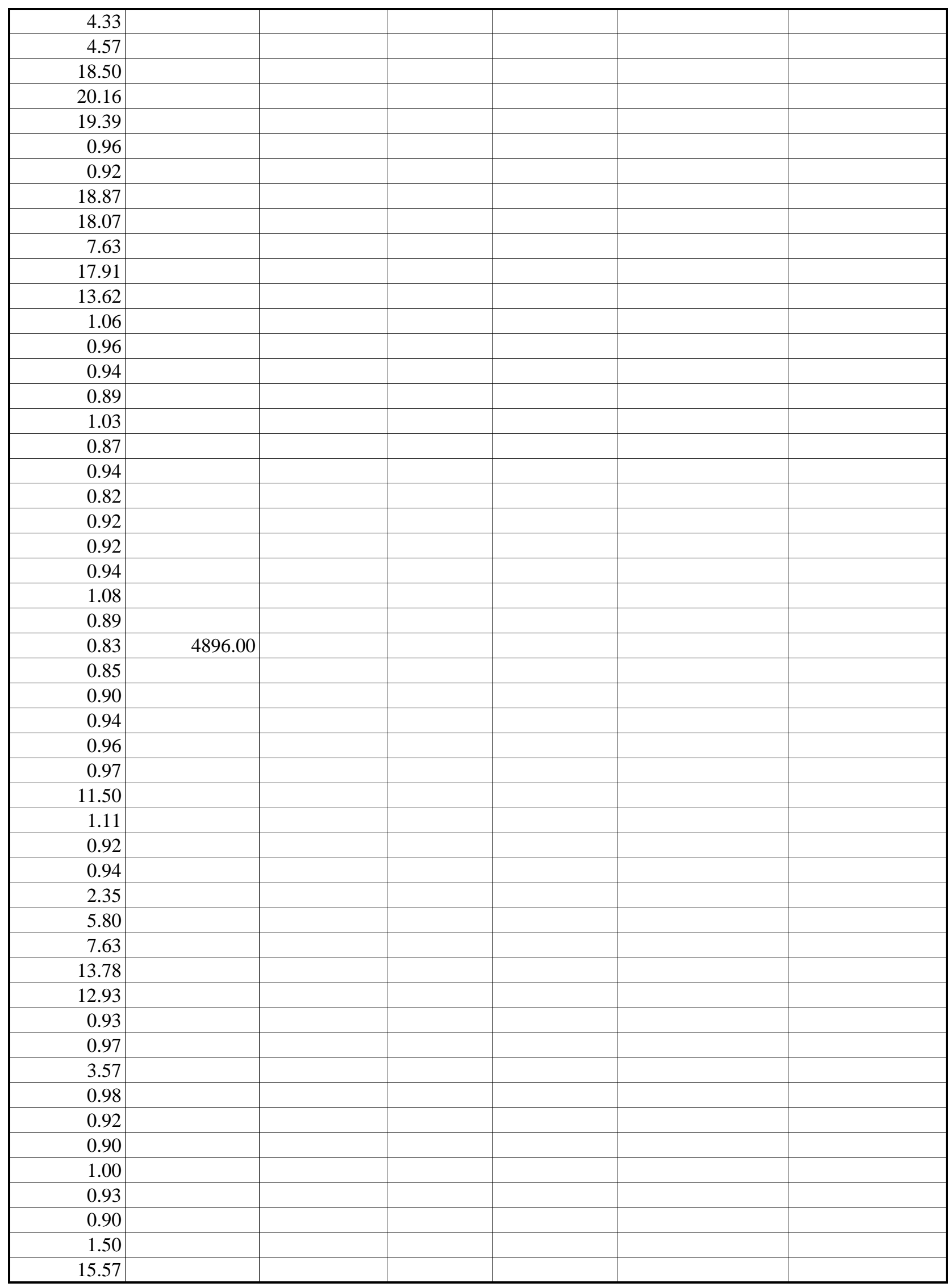




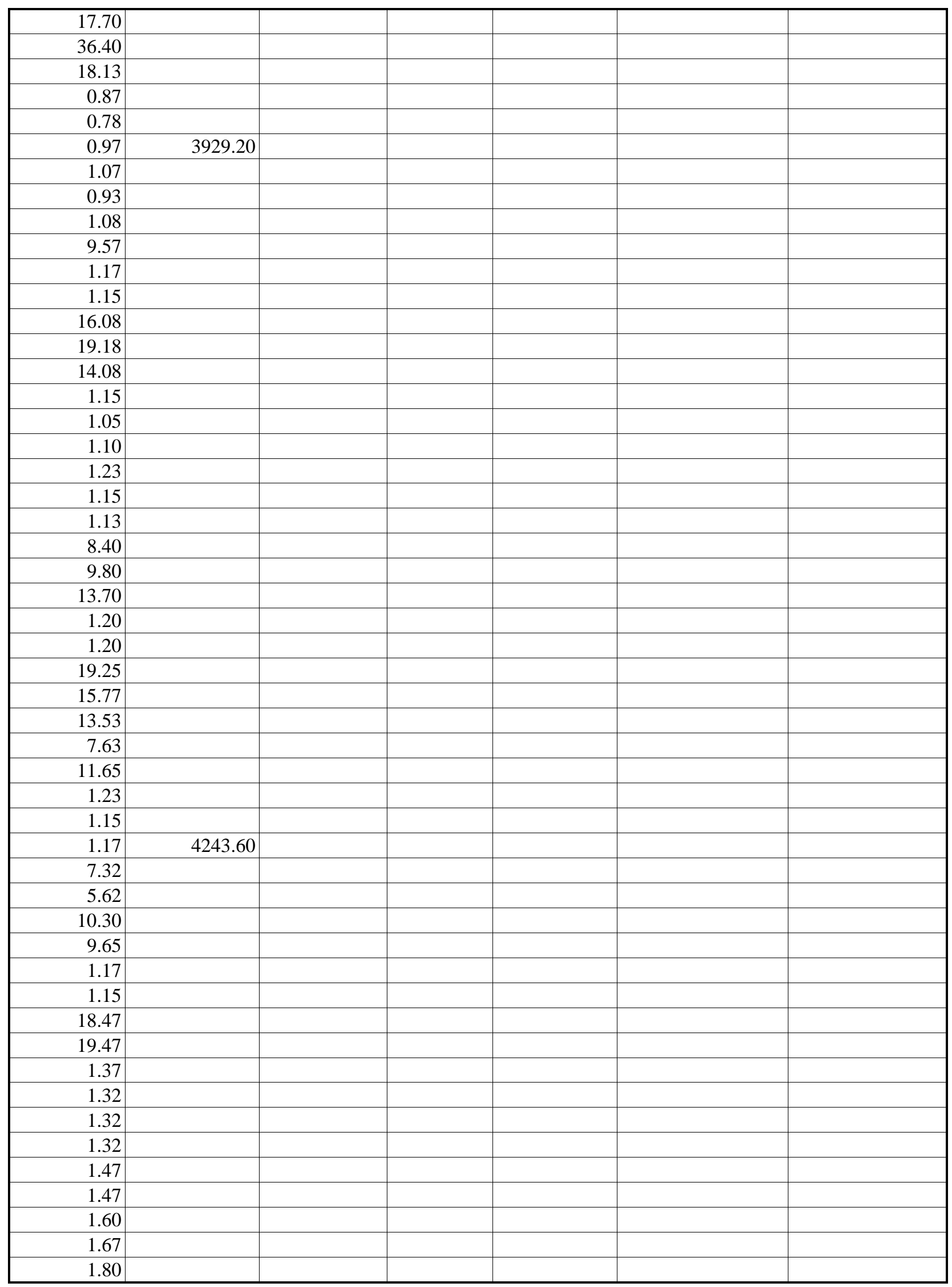




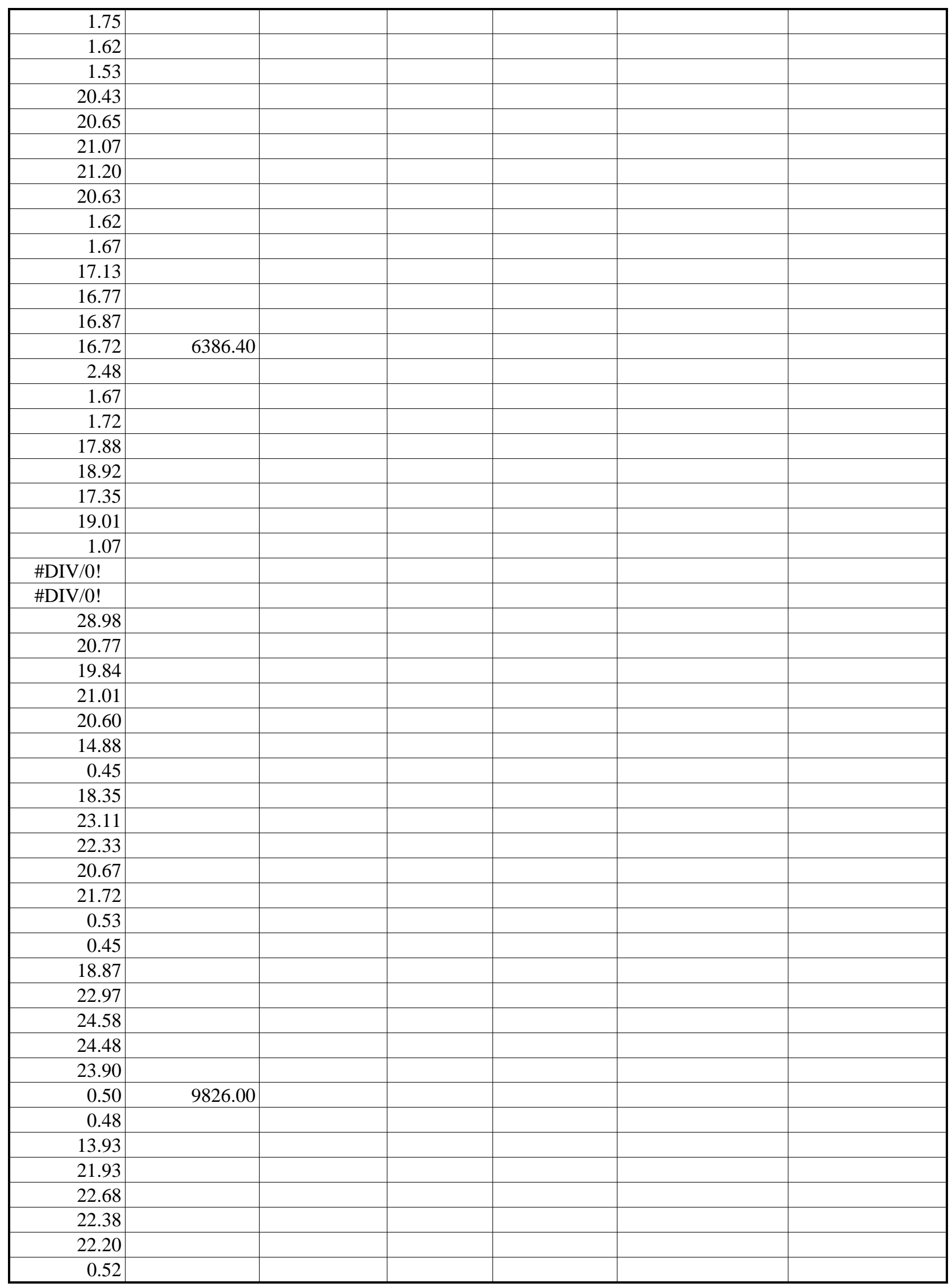




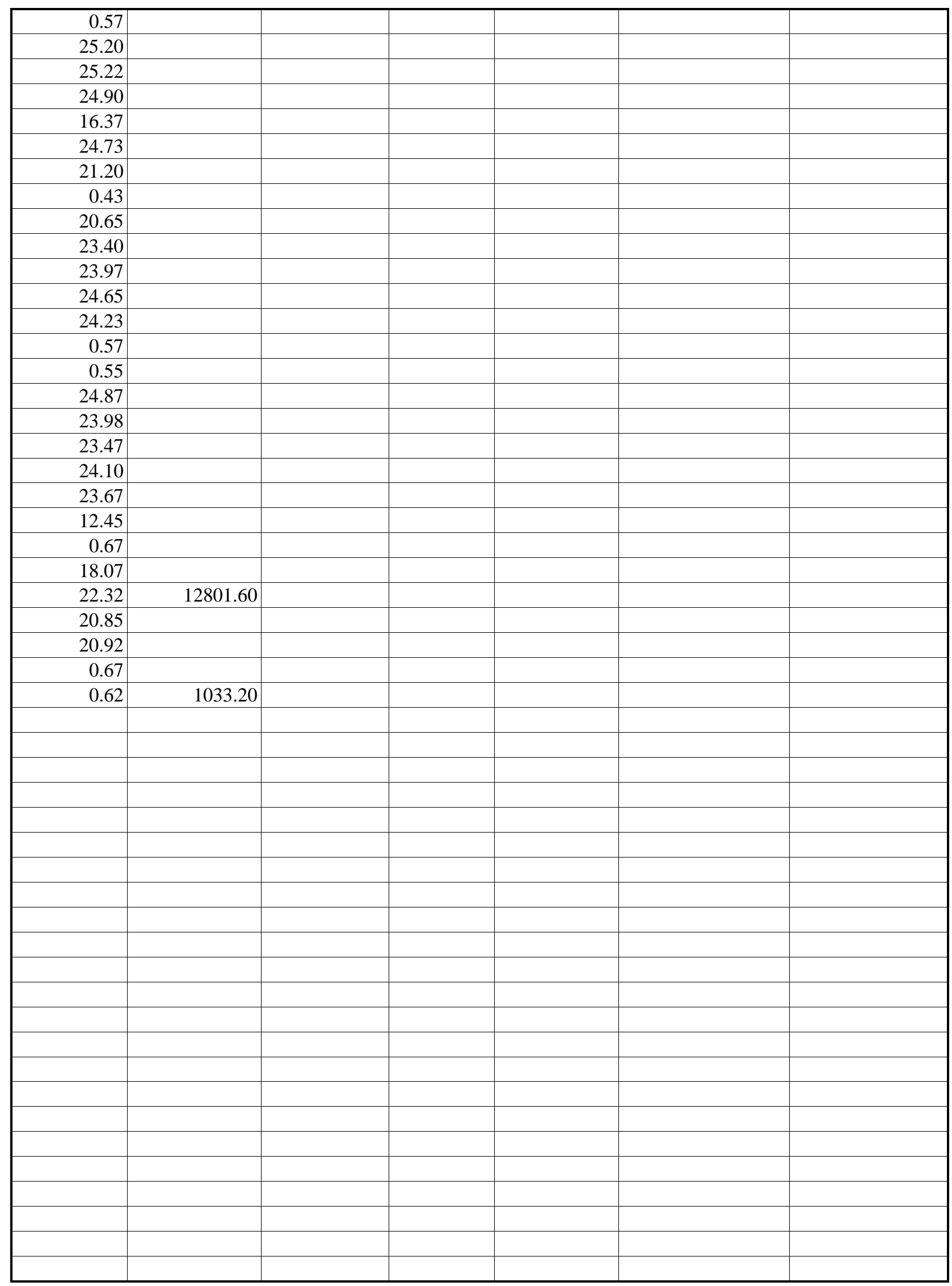


other

\begin{tabular}{|c|c|c|c|c|c|c|c|c|c|c|}
\hline $\begin{array}{c}\text { wd/ } \\
\text { we } \\
0 / 1\end{array}$ & $\begin{array}{c}\text { pre/p } \\
\text { ost } \\
\text { A/B }\end{array}$ & $\begin{array}{l}\text { schyear/br } \\
\text { eaks S/NS }\end{array}$ & date & jday & $\begin{array}{c}\text { orig } \\
\text { count } \\
\text { (156) }\end{array}$ & 0 & 1 & 2 & 3 & 4 \\
\hline 1 & A & S & 9/3/1991 & 91246 & 246 & 379.3 & 374.1 & 370.7 & 374.1 & 362 \\
\hline 1 & A & $S$ & 9/4/1991 & 91247 & 247 & 192.7 & 193.5 & 190.1 & 181.4 & 180.6 \\
\hline 1 & A & $S$ & 9/5/1991 & 91248 & 248 & 205.6 & 204.8 & 197.9 & 194.4 & 194.4 \\
\hline 1 & A & $S$ & 9/6/1991 & 91249 & 249 & 219.5 & 215.1 & 212.5 & 210 & 208.2 \\
\hline 1 . & A & $\mathrm{S}$ & 9/9/1991 & 91252 & 252 & 344.7 & 335.2 & 331.8 & 329.2 & 324.9 \\
\hline 1 & A & $S$ & 9/10/1991 & 91253 & 253 & 256.6 & 246.2 & 243.6 & 235 & 230.7 \\
\hline 1 . & A & $S$ & 9/11/1991 & 91254 & 254 & 248 & 238.5 & 230.7 & 227.2 & 223.8 \\
\hline 1 & A & $S$ & 9/12/1991 & 91255 & 255 & 227.2 & 222 & 216 & 218.6 & 213.4 \\
\hline 1 & A & $S$ & 9/13/1991 & 91256 & 256 & 238.5 & 230.7 & 232.4 & 227.2 & 221.2 \\
\hline 1 & A & $S$ & 9/16/1991 & 91259 & 259 & 360.3 & 354.2 & 341.3 & 339.6 & 331.8 \\
\hline 1 & A & $\mathrm{S}$ & 9/17/1991 & 91260 & 260 & 241.1 & 231.6 & 225.5 & 223.8 & 220.3 \\
\hline 1 & A & $S$ & 9/18/1991 & 91261 & 261 & 241.9 & 234.1 & 233.3 & 226.4 & 224.6 \\
\hline 1. & A & $S$ & 9/19/1991 & 91262 & 262 & 219.5 & 214.3 & 213.4 & 211.7 & 202.2 \\
\hline 1 & A & $S$ & 9/20/1991 & 91263 & 263 & 133.1 & 132.2 & 127.9 & 130.5 & 125.3 \\
\hline 1 & A & $S$ & 9/23/1991 & 91266 & 266 & 317.1 & 307.6 & 303.3 & 305 & 296.4 \\
\hline 1 & A & $S$ & 9/24/1991 & 91267 & 267 & 185.8 & 178.8 & 178.8 & 174.5 & 172.8 \\
\hline 1 & A & $S$ & 9/25/1991 & 91268 & 268 & 194.4 & 175.4 & 169.3 & 163.3 & 158.1 \\
\hline 1 & A & $S$ & 9/26/1991 & 91269 & 269 & 140 & 132.2 & 122.7 & 120.1 & 113.2 \\
\hline 1 & A & $S$ & 9/27/1991 & 91270 & 270 & 158.1 & 151.2 & 146 & 143.4 & 137.4 \\
\hline 1 & A & $S$ & 9/30/1991 & 91273 & 273 & 282.5 & 277.3 & 271.3 & 265.2 & 260.9 \\
\hline 1 . & A & $S$ & 10/1/1991 & 91274 & 274 & & & & & \\
\hline 1 & A & $S$ & 10/2/1991 & 91275 & 275 & & & & & \\
\hline 1 & A & $S$ & $10 / 3 / 1991$ & 91276 & 276 & 185.5 & 186.4 & 176 & 174.3 & 170.8 \\
\hline 1 & A & $S$ & 10/4/1991 & 91277 & 277 & 193.1 & 184.6 & 190.7 & 181.2 & 182 \\
\hline 1 & A & $S$ & 10/7/1991 & 91280 & 280 & 120.7 & 119 & 114.6 & 113.8 & 113.8 \\
\hline 1 & A & S & 10/8/1991 & 91281 & 281 & 124.5 & 119.3 & 116.7 & 113.6 & 110.7 \\
\hline 1 & A & $S$ & 10/9/1991 & 91282 & 282 & 124.5 & 120.2 & 119.3 & 113.2 & 113.2 \\
\hline 1 & A & S & 10/10/1991 & 91283 & 283 & 160.8 & 152.1 & 150.4 & 144.4 & 140.9 \\
\hline 1 & A & $\mathrm{S}$ & 10/11/1991 & 91284 & 284 & 153.9 & 148.2 & 147.4 & 140 & 139.2 \\
\hline 1 & A & $S$ & 10/14/1991 & 91287 & 287 & 173.7 & 173.7 & 169.4 & 167.7 & 167.7 \\
\hline 1 & A & $S$ & 10/15/1991 & 91288 & 288 & 192.7 & 186.7 & 175.5 & 162.5 & 160.8 \\
\hline 1 & A & $S$ & 10/16/1991 & 91289 & 289 & 176.3 & 163.4 & 159.9 & 154.7 & 150.4 \\
\hline 1 & A & $S$ & 10/17/1991 & 91290 & 290 & 159.9 & 153.9 & 152.1 & 150.4 & 145.2 \\
\hline 1 & A & $S$ & 10/18/1991 & 91291 & 291 & 161.6 & 150.4 & 144.4 & 142.6 & 139.6 \\
\hline 1 & A & $S$ & 10/21/1991 & 91294 & 294 & 135.2 & 127.9 & 128.8 & 125.3 & 123.2 \\
\hline 1 . & A & $S$ & 10/22/1991 & 91295 & 295 & 154.7 & 152.5 & 146.1 & 142.6 & 143 \\
\hline 1 & A & $S$ & 10/24/1991 & 91297 & 297 & 196.2 & 192.7 & 193.1 & 190.1 & 187.6 \\
\hline 1 & A & $S$ & 10/25/1991 & 91298 & 298 & 223 & 216.9 & 210.4 & 205.7 & 201.4 \\
\hline 1 & A & $\mathrm{S}$ & 10/28/1991 & 91301 & 301 & 350 & 348.3 & 337 & 333.1 & 329.2 \\
\hline 1 & A & $S$ & 10/29/1991 & 91302 & 302 & 258.4 & 253.2 & 245.8 & 246.3 & 238.5 \\
\hline 1 & A & S & 10/30/1991 & 91303 & 303 & 172 & 154.7 & 143 & 133.1 & 130.5 \\
\hline 1 & A & $S$ & 10/31/1991 & 91304 & 304 & 102 & 103.3 & 99.8 & 101.2 & 99.8 \\
\hline 1 & A & $\mathrm{S}$ & 11/1/1991 & 91305 & 305 & 93.8 & 91.2 & 90.8 & 90.3 & 88.6 \\
\hline 1 & A & $S$ & $11 / 4 / 1991$ & 91308 & 308 & 91.2 & 89.9 & 90.3 & 91.2 & 90.8 \\
\hline 1 & A & $\mathrm{S}$ & 11/5/1991 & 91309 & 309 & 178 & 179.3 & 178 & 176.7 & 177.6 \\
\hline
\end{tabular}


other

\begin{tabular}{|c|c|c|c|c|c|c|c|c|c|c|}
\hline 1 & A & $S$ & $11 / 6 / 1991$ & 91310 & 310 & 158.6 & 160.8 & 160.3 & 161.6 & 160.3 \\
\hline 1 & A & $S$ & 11/7/1991 & 91311 & 311 & 126.2 & 129.2 & 128.3 & 126.2 & 129.2 \\
\hline 1 & A & $S$ & 11/8/1991 & 91312 & 312 & 124.9 & 131.4 & 146.5 & 165.5 & 167.7 \\
\hline 1 & A & $S$ & 11/11/1991 & 91315 & 315 & 129.7 & 130.5 & 128.8 & 130.9 & 129.7 \\
\hline 1 & A & $S$ & 11/12/1991 & 91316 & 316 & 142.2 & 140.4 & 137.4 & 133.5 & 131.8 \\
\hline 1 & A & $S$ & 11/13/1991 & 91317 & 317 & 90.3 & 90.8 & 92 & 91.2 & 94.2 \\
\hline 1 & A & $S$ & 11/14/1991 & 91318 & 318 & 88.6 & 88.6 & 91.6 & 90.3 & 93.8 \\
\hline 1 & A & $S$ & 11/15/1991 & 91319 & 319 & 107.6 & 105 & 105.9 & 105.9 & 103.3 \\
\hline 1 & A & $S$ & 11/18/1991 & 91322 & 322 & 96.4 & 96.8 & 98.1 & 95.5 & 95.5 \\
\hline 1 & A & $S$ & 11/19/1991 & 91323 & 323 & 112.4 & 108.5 & 110.2 & 109.3 & 110.2 \\
\hline 1 & A & $S$ & 11/20/1991 & 91324 & 324 & 106.7 & 100.7 & 94.6 & 96 & 94.6 \\
\hline 1 & A & $S$ & 11/21/1991 & 91325 & 325 & 98.1 & 97.7 & 92 & 88.6 & 86 \\
\hline 1 & A & $S$ & 11/22/1991 & 91326 & 326 & 103.3 & 86.9 & 87.7 & 86 & 84.3 \\
\hline 1 & A & $S$ & 11/25/1991 & 91329 & 329 & 85.1 & 82.5 & 80.8 & 82.1 & 81.7 \\
\hline 1 & A & S & 11/26/1991 & 91330 & 330 & 132.7 & 115.4 & 114.5 & 112.4 & 114.5 \\
\hline 1 & A & $S$ & 12/2/1991 & 91336 & 336 & 93.8 & 96 & 95.5 & 95.5 & 95.1 \\
\hline 1 & A & $S$ & 12/3/1991 & 91337 & 337 & 172.9 & 164.6 & 148.2 & 138.7 & 141.3 \\
\hline 1 & A & S & 12/4/1991 & 91338 & 338 & 134.8 & 124.9 & 124.9 & 124.9 & 123.6 \\
\hline 1 & A & $S$ & $12 / 5 / 1991$ & 91339 & 339 & 122.8 & 125.7 & 123.2 & 124.5 & 126.6 \\
\hline 1 & A & $S$ & 12/6/1991 & 91340 & 340 & 130.5 & 124.9 & 101.6 & 105 & 102.4 \\
\hline 1 & A & $S$ & 12/9/1991 & 91343 & 343 & 130.1 & 125.7 & 125.7 & 126.6 & 127.5 \\
\hline 1 & A & S & 12/10/1991 & 91344 & 344 & 146.5 & 130.1 & 124.9 & 126.6 & 121.9 \\
\hline 1 & A & $S$ & 12/11/1991 & 91345 & 345 & 114.5 & 116.2 & 116.2 & 118 & 114.5 \\
\hline 1 & A & $S$ & 12/12/1991 & 91346 & 346 & 143 & 138.7 & 137 & 140.4 & 138.7 \\
\hline 1 & A & $S$ & 12/13/1991 & 91347 & 347 & 162 & 142.2 & 141.3 & 142.2 & 137 \\
\hline 1 & A & $S$ & 12/16/1991 & 91350 & 350 & 101.6 & 101.6 & 100.7 & 100.3 & 101.6 \\
\hline 1 & A & $S$ & 12/17/1991 & 91351 & 351 & 130.9 & 122.3 & 118 & 118.4 & 118.8 \\
\hline 1 & A & $S$ & 12/18/1991 & 91352 & 352 & 130.1 & 133.5 & 129.7 & 114.5 & 113.6 \\
\hline 1 & A & $S$ & 12/19/1991 & 91353 & 353 & 127.5 & 126.6 & 115.4 & 111.1 & 110.2 \\
\hline 1 & A & $S$ & 1/7/1992 & 92007 & 6 & 85.1 & 118.8 & 90.8 & 76.5 & 77.4 \\
\hline 1 & A & $S$ & 1/8/1992 & 92008 & 7 & 104.1 & 115.4 & 113.6 & 112.8 & 110.7 \\
\hline 1 & A & $\mathrm{S}$ & 1/9/1992 & 92009 & 8 & 135.2 & 118 & 120.6 & 118.8 & 127.5 \\
\hline 1 & A & $\mathrm{S}$ & 1/10/1992 & 92010 & 9 & 101.2 & 101.6 & 105.9 & 102.4 & 103.3 \\
\hline 1 & A & $S$ & 1/13/1992 & 92013 & 12 & 118.8 & 115.4 & 117.1 & 114.1 & 117.1 \\
\hline 1 & A & $\mathrm{S}$ & $1 / 14 / 1992$ & 92014 & 13 & 107.6 & 101.6 & 97.2 & 101.2 & 101.6 \\
\hline 1 & A & $S$ & 1/15/1992 & 92015 & 14 & 119.3 & 171.5 & 145.6 & 123.2 & 127.1 \\
\hline 1 & A & S & $1 / 16 / 1992$ & 92016 & 15 & 128.3 & 139.6 & 168.1 & 155.6 & 134.4 \\
\hline 1 & A & $S$ & 1/20/1992 & 92020 & 19 & 96.8 & 95.5 & 98.1 & 94.6 & 93.8 \\
\hline 1 & A & $S$ & 1/21/1992 & 92021 & 20 & 99 & 99 & 98.1 & 101.6 & 102 \\
\hline 1 & A & $S$ & 1/22/1992 & 92022 & 21 & 99.4 & 100.7 & 98.1 & 99.8 & 99 \\
\hline 1 & A & $S$ & $1 / 23 / 1992$ & 92023 & 22 & 96.4 & 98.1 & 96.4 & 97.2 & 94.6 \\
\hline 1 & A & $S$ & $1 / 24 / 1992$ & 92024 & 23 & 92 & 92.9 & 92.5 & 91.2 & 92 \\
\hline 1 & A & $S$ & $1 / 27 / 1992$ & 92027 & 26 & 70.4 & 68.7 & 70.4 & 69.6 & 70.4 \\
\hline 1 & A & $\mathrm{S}$ & $1 / 28 / 1992$ & 92028 & 27 & 71.3 & 71.3 & 71.8 & 71.3 & 71.3 \\
\hline 1 & A & $S$ & 1/29/1992 & 92029 & 28 & 91.2 & 91.2 & 92 & 90.3 & 91.2 \\
\hline 1 & A & $\mathrm{S}$ & $1 / 30 / 1992$ & 92030 & 29 & 103.3 & 99 & 99 & 98.1 & 97.2 \\
\hline 1 & A & $S$ & 1/31/1992 & 92031 & 30 & 95.5 & 93.4 & 95.5 & 94.6 & 94.6 \\
\hline 1 & B & $S$ & 2/3/1992 & 92034 & 33 & 83.9 & 81.7 & 82.5 & 82.5 & 81.7 \\
\hline 11 & B & $\mathrm{S}$ & 2/4/1992 & 92035 & 34 & 83 & 82.5 & 83.4 & 83.4 & 81.7 \\
\hline
\end{tabular}


other

\begin{tabular}{|c|c|c|c|c|c|c|c|c|c|c|}
\hline $1 \mathrm{~b}$ & B & $S$ & 2/5/1992 & 92036 & 35 & 93.8 & 92 & 92 & 92.9 & 91.2 \\
\hline $1 \mathrm{H}$ & $\mathrm{B}$ & $S$ & 2/6/1992 & 92037 & 36 & 93.8 & 92.9 & 91.2 & 92 & 90.3 \\
\hline $1 \mathrm{H}$ & B & $S$ & 2/7/1992 & 92038 & 37 & 105.9 & 105.9 & 106.7 & 106.7 & 106.3 \\
\hline $1 \mathrm{H}$ & B & $S$ & 2/10/1992 & 92041 & 40 & 86.9 & 88.2 & 82.5 & 85.1 & 86.9 \\
\hline $1 \mathrm{H}$ & B & $\mathrm{S}$ & $2 / 11 / 1992$ & 92042 & 41 & 92.5 & 89.5 & 88.6 & 89.5 & 85.1 \\
\hline $1 \mathrm{H}$ & B & $S$ & 2/12/1992 & 92043 & 42 & 96.4 & 93.8 & 92 & 92.5 & 92 \\
\hline $1 \mathrm{H}$ & B & $\mathrm{S}$ & $2 / 13 / 1992$ & 92044 & 43 & 100.7 & 92.5 & 92 & 92 & 92 \\
\hline $1 \mathrm{H}$ & B & $S$ & 2/14/1992 & 92045 & 44 & 108.5 & 106.3 & 102 & 96.4 & 92 \\
\hline $1 \mathrm{H}$ & B & $\mathrm{S}$ & $2 / 17 / 1992$ & 92048 & 47 & 106.7 & 90.3 & 90.3 & 90.8 & 91.2 \\
\hline $1 \mathrm{H}$ & $\mathrm{B}$ & $S$ & 2/18/1992 & 92049 & 48 & 83.4 & 84.3 & 83.9 & 84.3 & 83.4 \\
\hline $1 \mathrm{H}$ & $\mathrm{B}$ & $S$ & 2/19/1992 & 92050 & 49 & 93.8 & 93.8 & 97.2 & 94.6 & 93.8 \\
\hline $1 \mathrm{H}$ & $\mathrm{B}$ & $S$ & 2/20/1992 & 92051 & 50 & 87.7 & 80.8 & 80.8 & 80.8 & 80 \\
\hline $1 \mathrm{H}$ & $\mathrm{B}$ & $S$ & 2/21/1992 & 92052 & 51 & 90.3 & 86.5 & 86.9 & 80.8 & 80.8 \\
\hline $1 \mathrm{H}$ & $\mathrm{B}$ & $S$ & 2/24/1992 & 92055 & 54 & 95.5 & 90.3 & 81.7 & 81.7 & 81.7 \\
\hline $1 \mathrm{H}$ & $\mathrm{B}$ & $S$ & 2/25/1992 & 92056 & 55 & 83.4 & 85.1 & 83.4 & 83.4 & 83 \\
\hline $1 \mathrm{H}$ & $\mathrm{B}$ & $S$ & 2/26/1992 & 92057 & 56 & 78.2 & 77 & 77.4 & 76.5 & 76.5 \\
\hline $1 \mathrm{H}$ & $\mathrm{B}$ & $S$ & 2/27/1992 & 92058 & 57 & 85.1 & 85.1 & 86 & 85.1 & 85.6 \\
\hline $1 \mathrm{H}$ & $\mathrm{B}$ & $S$ & 2/28/1992 & 92059 & 58 & 84.3 & 84.3 & 84.3 & 84.3 & 83 \\
\hline $1 \mathrm{H}$ & B & $S$ & 3/2/1992 & 92062 & 61 & 73.9 & 78.2 & 75.6 & 77 & 76.5 \\
\hline $1 \mathrm{H}$ & B & $\mathrm{S}$ & $3 / 3 / 1992$ & 92063 & 62 & 86.9 & 85.1 & 84.3 & 85.1 & 85.1 \\
\hline $1 \mathrm{H}$ & B & $S$ & $3 / 4 / 1992$ & 92064 & 63 & 96.4 & 85.1 & 85.1 & 86.9 & 86.9 \\
\hline $1 \mathrm{H}$ & B & $\mathrm{S}$ & $3 / 5 / 1992$ & 92065 & 64 & 170.7 & 169.8 & 168.9 & 166.4 & 156 \\
\hline $1 \mathrm{H}$ & $\mathrm{B}$ & $S$ & 3/6/1992 & 92066 & 65 & 101.6 & 100.3 & 99 & 96.4 & 94.6 \\
\hline $1 \mathrm{H}$ & $\mathrm{B}$ & $S$ & 3/9/1992 & 92069 & 68 & 98.1 & 96.4 & 93.8 & 85.6 & 85.1 \\
\hline $1 \mathrm{H}$ & B & $S$ & 3/10/1992 & 92070 & 69 & 87.7 & 86.9 & 88.6 & 90.3 & 89.5 \\
\hline $1 \mathrm{H}$ & B & $\mathrm{S}$ & 3/11/1992 & 92071 & 70 & 98.1 & 92 & 92 & 93.8 & 89.1 \\
\hline $1 \mathrm{H}$ & B & $S$ & 3/12/1992 & 92072 & 71 & 83.4 & 81.7 & 81.7 & 82.5 & 82.5 \\
\hline $1 \mathrm{H}$ & B & $S$ & 3/23/1992 & 92083 & 82 & 88.6 & 87.7 & 88.2 & 89.5 & 87.7 \\
\hline $1 \mathrm{H}$ & B & $S$ & 3/24/1992 & 92084 & 83 & 87.7 & 87.7 & 88.6 & 87.3 & 87.7 \\
\hline $1 \mathrm{H}$ & B & $S$ & 3/25/1992 & 92085 & 84 & 93.8 & 90.3 & 91.2 & 90.3 & 90.3 \\
\hline $1 \mathrm{H}$ & B & $S$ & 3/26/1992 & 92086 & 85 & 76.5 & 74.8 & 75.6 & 75.6 & 76.5 \\
\hline $1 \mathrm{H}$ & B & $S$ & 3/27/1992 & 92087 & 86 & 81.7 & 80 & 80 & 79.1 & 80.8 \\
\hline $1 \mathrm{H}$ & B & $\mathrm{S}$ & $3 / 30 / 1992$ & 92090 & 89 & 88.6 & 89.5 & 88.6 & 89.9 & 88.6 \\
\hline $1 \mathrm{H}$ & B & $S$ & $3 / 31 / 1992$ & 92091 & 90 & 92.9 & 93.8 & 92.9 & 92 & 91.6 \\
\hline $1 \mathrm{H}$ & B & $S$ & 4/1/1992 & 92092 & 91 & 162.9 & 160.3 & 161.2 & 159 & 160.3 \\
\hline $1 \mathrm{H}$ & B & $S$ & 4/2/1992 & 92093 & 92 & 88.6 & 80.8 & 79.1 & 76.5 & 76.5 \\
\hline $1 \mathrm{H}$ & B & $S$ & 4/3/1992 & 92094 & 93 & 164.6 & 154.7 & 139.6 & 152.5 & 139.6 \\
\hline $1 \mathrm{H}$ & B & $S$ & $4 / 6 / 1992$ & 92097 & 96 & 80 & 79.1 & 80.8 & 79.1 & 77.8 \\
\hline $1 \mathrm{H}$ & B & $S$ & 4/7/1992 & 92098 & 97 & 79.6 & 78.2 & 80.8 & 80 & 80 \\
\hline $1 \mathrm{H}$ & B & $S$ & 4/8/1992 & 92099 & 98 & 91.2 & 88.6 & 86.5 & 86.9 & 86 \\
\hline $1 \mathrm{H}$ & B & $S$ & 4/9/1992 & 92100 & 99 & 106.7 & 105 & 102.4 & 102.4 & 103.3 \\
\hline $1 \mathrm{H}$ & B & $S$ & 4/10/1992 & 92101 & 100 & 102 & 101.6 & 89.5 & 86.9 & 86 \\
\hline $1 \mathrm{H}$ & B & $S$ & 4/13/1992 & 92104 & 103 & 105 & 108.1 & 105 & 107.6 & 104.1 \\
\hline $1 \mathrm{H}$ & B & $S$ & 4/14/1992 & 92105 & 104 & 102.4 & 109.3 & 124.9 & 121.4 & 117.1 \\
\hline $1 \mathrm{H}$ & B & $S$ & 4/15/1992 & 92106 & 105 & 120.6 & 117.1 & 104.6 & 101.6 & 105.9 \\
\hline $1 \mathrm{H}$ & B & $S$ & $4 / 16 / 1992$ & 92107 & 106 & 118.8 & 118 & 114.5 & 105.9 & 104.1 \\
\hline $1 \mathrm{H}$ & B & $\mathrm{S}$ & $4 / 21 / 1992$ & 92112 & 111 & 100.7 & 99 & 106.3 & 96.4 & 95.5 \\
\hline $1 \mathrm{H}$ & B & $S$ & 4/22/1992 & 92113 & 112 & 99.8 & 96.4 & 95.5 & 94.2 & 92.9 \\
\hline $1 \mathrm{H}$ & B & $S$ & 4/23/1992 & 92114 & 113 & 97.2 & 93.8 & 91.2 & 92.9 & 92.5 \\
\hline
\end{tabular}


other

\begin{tabular}{|c|c|c|c|c|c|c|c|c|c|c|}
\hline $1 \mathrm{l}$ & B & $S$ & 4/24/1992 & 92115 & 114 & 198.8 & 198.3 & 193.1 & 183.6 & 192.3 \\
\hline 11 & $\mathrm{~B}$ & $S$ & 4/27/1992 & 92118 & 117 & 97.2 & 96.4 & 96.4 & 95.5 & 95.5 \\
\hline $1 \mathrm{I}$ & $\mathrm{B}$ & $S$ & 4/29/1992 & 92120 & 119 & 112.3 & 110.7 & 110.2 & 108.5 & 108.5 \\
\hline $1 \mathrm{I}$ & $\mathrm{B}$ & $S$ & 4/30/1992 & 92121 & 120 & 120.6 & 114.5 & 116.2 & 116.2 & 112.8 \\
\hline $1 \mathrm{I}$ & $\mathrm{B}$ & $S$ & $5 / 1 / 1992$ & 92122 & 121 & 117.1 & 111.1 & 107.6 & 96.4 & 96.4 \\
\hline $1 \mathrm{I}$ & $\mathrm{B}$ & $S$ & $5 / 4 / 1992$ & 92125 & 124 & 105.9 & 105.9 & 106.7 & 105.9 & 105.9 \\
\hline 11 & $\mathrm{~B}$ & $S$ & $5 / 5 / 1992$ & 92126 & 125 & 105 & 103.3 & 104.6 & 103.3 & 101.6 \\
\hline $1 \mathrm{l}$ & B & $S$ & $5 / 6 / 1992$ & 92127 & 126 & 111.9 & 105.9 & 105 & 105 & 104.6 \\
\hline 11 & $\mathrm{~B}$ & $S$ & 5/7/1992 & 92128 & 127 & 192.3 & 196.2 & 193.1 & 185.4 & 173.3 \\
\hline $1 \mathrm{l}$ & B & $S$ & $5 / 8 / 1992$ & 92129 & 128 & 93.4 & 90.3 & 90.3 & 89.5 & 88.6 \\
\hline 11 & B & $S$ & $5 / 11 / 1992$ & 92132 & 131 & 88.6 & 88.6 & 87.7 & 87.7 & 86 \\
\hline $1 \mathrm{l}$ & B & $\mathrm{S}$ & $5 / 12 / 1992$ & 92133 & 132 & 96 & 93.8 & 98.1 & 98.1 & 99 \\
\hline $1 \mathrm{l}$ & B & $S$ & $5 / 13 / 1992$ & 92134 & 133 & 120.6 & 119.7 & 107.6 & 105 & 105.9 \\
\hline $1 \mathrm{l}$ & B & $\mathrm{S}$ & $5 / 14 / 1992$ & 92135 & 134 & 124.5 & 123.2 & 109.3 & 107.2 & 107.6 \\
\hline 11 & $\mathrm{~B}$ & $S$ & $5 / 15 / 1992$ & 92136 & 135 & 139.2 & 137 & 134.8 & 125.7 & 119.3 \\
\hline 11 & $\mathrm{~B}$ & $S$ & $5 / 18 / 1992$ & 92139 & 138 & 114.5 & 105 & 105 & 105.9 & 103.3 \\
\hline $1 \mathrm{I}$ & $\mathrm{B}$ & $S$ & $5 / 19 / 1992$ & 92140 & 139 & 95.5 & 94.2 & 96.8 & 94.2 & 95.5 \\
\hline $1 \mathrm{I}$ & $\mathrm{B}$ & $\mathrm{S}$ & $5 / 20 / 1992$ & 92141 & 140 & 105.9 & 99 & 92.9 & 98.1 & 92.5 \\
\hline $1 \mathrm{I}$ & $\mathrm{B}$ & $S$ & $5 / 21 / 1992$ & 92142 & 141 & 113.6 & 105.9 & 105.9 & 99 & 99 \\
\hline $1 \mathrm{I}$ & $\mathrm{B}$ & $S$ & $5 / 22 / 1992$ & 92143 & 142 & 99.8 & 99.8 & 103.3 & 103.3 & 97.2 \\
\hline 11 & $\mathrm{~B}$ & $S$ & $5 / 25 / 1992$ & 92146 & 145 & 120.2 & 118 & 118 & 113.6 & 114.5 \\
\hline 11 & $\mathrm{~B}$ & $S$ & $5 / 26 / 1992$ & 92147 & 146 & 125.3 & 122.8 & 120.6 & 118.8 & 119.7 \\
\hline 11 & $\mathrm{~B}$ & $S$ & $5 / 27 / 1992$ & 92148 & 147 & 128.8 & 126.6 & 125.3 & 116.2 & 112.8 \\
\hline 11 & $\mathrm{~B}$ & $S$ & 9/8/1992 & 92252 & 251 & 133.1 & 137.4 & 153.4 & 146.1 & 143.5 \\
\hline $1 \mathrm{H}$ & $\mathrm{B}$ & $S$ & 9/9/1992 & 92253 & 252 & 143.5 & 135.7 & 134 & 132.3 & 122.3 \\
\hline $1 \mathrm{H}$ & B & $S$ & 9/10/1992 & 92254 & 253 & 132.3 & 129.2 & 132.3 & 134.8 & 127.5 \\
\hline $1 \mathrm{H}$ & B & $S$ & $9 / 11 / 1992$ & 92255 & 254 & 140.9 & 134 & 138.3 & 134.4 & 128.8 \\
\hline $1 \mathrm{H}$ & $\mathrm{B}$ & $S$ & 9/14/1992 & 92258 & 257 & 107.6 & 102.9 & 102 & 102.4 & 99.4 \\
\hline $1 \mathrm{H}$ & B & $S$ & 9/16/1992 & 92260 & 259 & 96 & 92.5 & 89.9 & 90.3 & 85.6 \\
\hline $1 \mathrm{H}$ & B & $S$ & 9/17/1992 & 92261 & 260 & 101.6 & 98.6 & 101.2 & 101.6 & 98.6 \\
\hline $1 \mathrm{H}$ & B & $S$ & 9/18/1992 & 92262 & 261 & 87.3 & 87.7 & 88.2 & 89.1 & 86.9 \\
\hline $1 \mathrm{H}$ & $\mathrm{B}$ & $S$ & 9/21/1992 & 92265 & 264 & 127.9 & 115 & 121.4 & 114.1 & 118.4 \\
\hline $1 \mathrm{H}$ & $\mathrm{B}$ & $S$ & 9/22/1992 & 92266 & 265 & 127.1 & 167.7 & 162.9 & 154.7 & 154.7 \\
\hline $1 \mathrm{H}$ & $\mathrm{B}$ & $S$ & 9/23/1992 & 92267 & 266 & 159.9 & 134 & 133.5 & 142.6 & 128.8 \\
\hline $1 \mathrm{~b}$ & B & S & 9/24/1992 & 92268 & 267 & 96.8 & 98.1 & 100.3 & 98.6 & 99 \\
\hline $1 \mathrm{H}$ & $\mathrm{B}$ & $S$ & 9/25/1992 & 92269 & 268 & 92.5 & 90.3 & 91.6 & 89.9 & 89.5 \\
\hline $1 \mathrm{H}$ & $\mathrm{B}$ & $S$ & 9/28/1992 & 92272 & 271 & 115 & 103.7 & 102 & 101.6 & 101.2 \\
\hline $1 \mathrm{H}$ & $\mathrm{B}$ & $S$ & 9/29/1992 & 92273 & 272 & 96 & 101.2 & 108.1 & 107.2 & 109.3 \\
\hline $1 \mathrm{H}$ & $\mathrm{B}$ & $S$ & 9/30/1992 & 92274 & 273 & 96.8 & 94.6 & 97.7 & 94.6 & 95.1 \\
\hline $1 \mathrm{H}$ & $\mathrm{B}$ & $S$ & 10/1/1992 & 92275 & 274 & 74.4 & 71.3 & 79.6 & 78.2 & 70.9 \\
\hline $1 \mathrm{H}$ & $\mathrm{B}$ & $S$ & 10/2/1992 & 92276 & 275 & 73.5 & 73.9 & 75.6 & 75.6 & 73.5 \\
\hline $1 \mathrm{H}$ & B & $S$ & 10/5/1992 & 92279 & 278 & 82.5 & 80.4 & 80.8 & 80.8 & 80 \\
\hline $1 \mathrm{H}$ & $\mathrm{B}$ & $S$ & 10/6/1992 & 92280 & 279 & 86.9 & 83.9 & 102.9 & 98.1 & 91.2 \\
\hline $1 \mathrm{H}$ & $\mathrm{B}$ & $S$ & 10/7/1992 & 92281 & 280 & 80.4 & 214.7 & 184.5 & 168.9 & 168.9 \\
\hline $1 \mathrm{H}$ & $\mathrm{B}$ & $S$ & 10/8/1992 & 92282 & 281 & 160.3 & 94.6 & 83.4 & 83.9 & 83.4 \\
\hline $1 \mathrm{~F}$ & B & S & 10/9/1992 & 92283 & 282 & 85.6 & 85.6 & 90.3 & 88.6 & 83.4 \\
\hline $1 \mathrm{H}$ & $\mathrm{B}$ & $S$ & 10/13/1992 & 92287 & 286 & 81.3 & 91.2 & 115.8 & 112.8 & 110.2 \\
\hline $1 \mathrm{~b}$ & B & $S$ & 10/14/1992 & 92288 & 287 & 89.5 & 204.8 & 174.1 & 149.1 & 147.3 \\
\hline $1 \mathrm{~F}$ & B & $S$ & 10/15/1992 & 92289 & 288 & 161.6 & 104.1 & 102 & 95.5 & 91.6 \\
\hline
\end{tabular}


other

\begin{tabular}{|c|c|c|c|c|c|c|c|c|c|c|}
\hline $1 \mathrm{l}$ & B & $S$ & 10/16/1992 & 92290 & 289 & 103.3 & 99.4 & 116.7 & 112.8 & 107.6 \\
\hline 11 & $\mathrm{~B}$ & $S$ & 10/19/1992 & 92293 & 292 & 114.5 & 94.6 & 89.5 & 87.3 & 86.9 \\
\hline $1 \mathrm{I}$ & $\mathrm{B}$ & $S$ & 10/20/1992 & 92294 & 293 & 89.5 & 89.5 & 98.1 & 87.3 & 86.9 \\
\hline $1 \mathrm{I}$ & $\mathrm{B}$ & $S$ & 10/21/1992 & 92295 & 294 & & 156.8 & 139.6 & 131.8 & 133.5 \\
\hline $1 \mathrm{I}$ & $\mathrm{B}$ & $S$ & 10/22/1992 & 92296 & 295 & 151.7 & 99 & 96.4 & 89.5 & 86.9 \\
\hline 11 & B & $S$ & 10/23/1992 & 92297 & 296 & 93.4 & 86 & 92 & 91.2 & 88.6 \\
\hline 11 & $\mathrm{~B}$ & $S$ & 10/26/1992 & 92300 & 299 & 78.7 & 71.3 & 74.8 & 70.4 & 71.3 \\
\hline 11 & $\mathrm{~B}$ & $S$ & 10/27/1992 & 92301 & 300 & 81.7 & 79.6 & 86 & 107.2 & 106.7 \\
\hline 11 & $\mathrm{~B}$ & $S$ & 10/28/1992 & 92302 & 301 & 96.8 & 203.5 & 170.7 & 162 & 153 \\
\hline $1 \mathrm{l}$ & B & $S$ & 10/29/1992 & 92303 & 302 & 155.1 & 97.2 & 90.8 & 83.4 & 81.7 \\
\hline 11 & B & $S$ & 10/30/1992 & 92304 & 303 & 105 & 113.2 & 114.5 & 104.1 & 98.1 \\
\hline $1 \mathrm{l}$ & B & $\mathrm{S}$ & 11/2/1992 & 92307 & 306 & 99.8 & 94.2 & 93.8 & 89.9 & 90.3 \\
\hline 11 & B & $S$ & 11/3/1992 & 92308 & 307 & 75.6 & 76.5 & 81.3 & 74.8 & 74.8 \\
\hline $1 \mathrm{l}$ & B & $\mathrm{S}$ & 11/4/1992 & 92309 & 308 & 82.5 & 166.4 & 160.3 & 142.2 & 151.7 \\
\hline $1 \mathrm{l}$ & B & S & 11/5/1992 & 92310 & 309 & 128.3 & 93.8 & 80.8 & 80.4 & 79.1 \\
\hline $1 \mathrm{l}$ & B & $S$ & 11/6/1992 & 92311 & 310 & 80.8 & 80.8 & 80.8 & 80.8 & 83.4 \\
\hline $1 \mathrm{I}$ & $\mathrm{B}$ & $S$ & 11/9/1992 & 92314 & 313 & 97.2 & 96 & 99.8 & 96.4 & 97.2 \\
\hline $1 \mathrm{l}$ & B & S & 11/10/1992 & 92315 & 314 & 82.1 & 80.8 & 80 & 79.1 & 78.2 \\
\hline $1 \mathrm{I}$ & $\mathrm{B}$ & $S$ & 11/11/1992 & 92316 & 315 & 102.4 & 136.1 & 133.5 & 128.3 & 130.5 \\
\hline $1 \mathrm{I}$ & $\mathrm{B}$ & $S$ & 11/12/1992 & 92317 & 316 & 115.8 & 96.4 & 93.8 & 92 & 86 \\
\hline 11 & $\mathrm{~B}$ & $S$ & 11/13/1992 & 92318 & 317 & 100.7 & 103.3 & 107.6 & 102.4 & 99.8 \\
\hline 11 & $\mathrm{~B}$ & $S$ & 11/16/1992 & 92321 & 320 & 80 & 77.4 & 78.2 & 77 & 78.2 \\
\hline 11 & $\mathrm{~B}$ & $S$ & 11/17/1992 & 92322 & 321 & 71.8 & 80.8 & 85.1 & 84.3 & 83.9 \\
\hline 11 & $\mathrm{~B}$ & $S$ & 11/18/1992 & 92323 & 322 & 86.9 & 105 & 105 & 102.4 & 103.3 \\
\hline $1 \mathrm{H}$ & $\mathrm{B}$ & $S$ & 11/19/1992 & 92324 & 323 & 119.7 & 95.5 & 92.9 & 90.3 & 88.6 \\
\hline $1 \mathrm{H}$ & B & $S$ & 11/20/1992 & 92325 & 324 & 92.9 & 90.3 & 96.4 & 91.2 & 90.3 \\
\hline $1 \mathrm{H}$ & B & $S$ & 11/23/1992 & 92328 & 327 & 90.3 & 87.7 & 86.9 & 84.7 & 84.3 \\
\hline $1 \mathrm{H}$ & $\mathrm{B}$ & $S$ & 11/24/1992 & 92329 & 328 & 82.5 & 83.4 & 86 & 85.1 & 84.7 \\
\hline $1 \mathrm{H}$ & B & $S$ & 11/30/1992 & 92335 & 334 & 101.6 & 99.4 & 94.6 & 92 & 92 \\
\hline $1 \mathrm{H}$ & $\mathrm{B}$ & $S$ & $12 / 1 / 1992$ & 92336 & 335 & 95.1 & 93.8 & 104.1 & 105 & 104.1 \\
\hline $1 \mathrm{H}$ & B & $S$ & $12 / 2 / 1992$ & 92337 & 336 & 100.7 & 147.3 & 164.6 & 152.5 & 138.7 \\
\hline $1 \mathrm{H}$ & B & $\mathrm{S}$ & 12/3/1992 & 92338 & 337 & 123.6 & 97.2 & 90.3 & 88.6 & 87.7 \\
\hline $1 \mathrm{~b}$ & B & $\mathrm{S}$ & 12/4/1992 & 92339 & 338 & 90.8 & 83.4 & 87.7 & 86.9 & 86.9 \\
\hline $1 \mathrm{H}$ & $\mathrm{B}$ & $S$ & 12/7/1992 & 92342 & 341 & 98.1 & 92.5 & 95.5 & 76.5 & 74.8 \\
\hline $1 \mathrm{~b}$ & B & S & 12/8/1992 & 92343 & 342 & 74.8 & 73 & 77.4 & 76.5 & 77.4 \\
\hline $1 \mathrm{H}$ & B & $\mathrm{S}$ & $12 / 9 / 1992$ & 92344 & 343 & 103.3 & 132.7 & 145.6 & 115.8 & 118 \\
\hline $1 \mathrm{H}$ & $\mathrm{B}$ & $S$ & 12/10/1992 & 92345 & 344 & 116.2 & 94.6 & 88.6 & 87.7 & 86.9 \\
\hline $1 \mathrm{H}$ & B & $S$ & 12/11/1992 & 92346 & 345 & 73 & 70.4 & 72.2 & 72.2 & 71.3 \\
\hline $1 \mathrm{H}$ & B & S & 12/14/1992 & 92349 & 348 & 67.9 & 72.2 & 68.3 & 67 & 68.7 \\
\hline $1 \mathrm{H}$ & $\mathrm{B}$ & $S$ & 12/15/1992 & 92350 & 349 & 67 & 69.6 & 72.2 & 70.4 & 70.9 \\
\hline $1 \mathrm{H}$ & $\mathrm{B}$ & $S$ & 12/16/1992 & 92351 & 350 & 102.4 & 129.2 & 134.8 & 131.8 & 128.3 \\
\hline $1 \mathrm{H}$ & $\mathrm{B}$ & $S$ & 12/17/1992 & 92352 & 351 & 139.6 & 115.4 & 111.9 & 108.9 & 97.2 \\
\hline $1 \mathrm{H}$ & $\mathrm{B}$ & $S$ & $1 / 5 / 1993$ & 93005 & 5 & 73.5 & 105.9 & 114.5 & 111.9 & 97.2 \\
\hline $1 \mathrm{H}$ & $\mathrm{B}$ & $S$ & $1 / 6 / 1993$ & 93006 & 6 & 101.2 & 91.2 & 92 & 89.5 & 89.9 \\
\hline $1 \mathrm{H}$ & $\mathrm{B}$ & $S$ & $1 / 7 / 1993$ & 93007 & 7 & 97.2 & 92.5 & 91.2 & 90.3 & 89.5 \\
\hline $1 \mathrm{~F}$ & B & S & 1/8/1993 & 93008 & 8 & 87.7 & 88.6 & 86.5 & 85.1 & 85.1 \\
\hline $1 \mathrm{H}$ & $\mathrm{B}$ & $S$ & $1 / 11 / 1993$ & 93011 & 11 & 82.1 & 82.5 & 82.5 & 80 & 78.7 \\
\hline $1 \mathrm{~b}$ & B & $S$ & $1 / 12 / 1993$ & 93012 & 12 & 102.4 & 110.2 & 109.8 & 107.6 & 111.1 \\
\hline $1 \mathrm{~F}$ & B & $S$ & 1/13/1993 & 93013 & 13 & 111.9 & 94.6 & 86 & 85.1 & 83.4 \\
\hline
\end{tabular}


other

\begin{tabular}{|c|c|c|c|c|c|c|c|c|c|c|}
\hline $1 \mathrm{~F}$ & B & $\mathrm{S}$ & $1 / 14 / 1993$ & 93014 & 14 & 96.4 & 96.4 & 96.4 & 96.4 & 94.2 \\
\hline $1 \mathrm{H}$ & B & $S$ & 1/15/1993 & 93015 & 15 & 82.5 & 83.4 & 85.1 & 81.7 & 82.1 \\
\hline $1 \mathrm{H}$ & $\mathrm{B}$ & $S$ & 1/18/1993 & 93018 & 18 & 62.7 & 63.5 & 64.4 & 63.5 & 62.7 \\
\hline $1 \mathrm{H}$ & $\mathrm{B}$ & $\mathrm{S}$ & 1/19/1993 & 93019 & 19 & 60.9 & 64.4 & 67 & 65.3 & 64.9 \\
\hline $1 \mathrm{H}$ & B & $\mathrm{S}$ & $1 / 20 / 1993$ & 93020 & 20 & 119.7 & 97.2 & 96.4 & 94.6 & 92.5 \\
\hline $1 \mathrm{H}$ & $\mathrm{B}$ & $S$ & 1/21/1993 & 93021 & 21 & 99.4 & 99 & 100.7 & 100.7 & 99.8 \\
\hline $1 \mathrm{H}$ & B & $S$ & $1 / 22 / 1993$ & 93022 & 22 & 88.6 & 86.9 & 87.7 & 84.7 & 85.1 \\
\hline $1 \mathrm{H}$ & B & $S$ & $1 / 25 / 1993$ & 93025 & 25 & 86 & 86 & 86.5 & 85.1 & 84.7 \\
\hline $1 \mathrm{H}$ & B & $S$ & $1 / 26 / 1993$ & 93026 & 26 & 103.3 & 112.4 & 113.6 & 112.8 & 112.8 \\
\hline $1 \mathrm{~F}$ & $\mathrm{~B}$ & $S$ & $1 / 27 / 1993$ & 93027 & 27 & 105 & 102 & 105.9 & 102.4 & 102.4 \\
\hline $1 \mathrm{H}$ & B & $S$ & $1 / 28 / 1993$ & 93028 & 28 & 77.4 & 77.8 & 80 & 81.7 & 80.8 \\
\hline $1 \mathrm{H}$ & B & $S$ & $1 / 29 / 1993$ & 93029 & 29 & 79.1 & 80.8 & 80.4 & 78.2 & 78.2 \\
\hline $1 \mathrm{H}$ & $\mathrm{B}$ & $S$ & 2/1/1993 & 93032 & 32 & 85.1 & 88.6 & 86.5 & 85.1 & 85.1 \\
\hline $1 \mathrm{H}$ & B & $S$ & $2 / 2 / 1993$ & 93033 & 33 & 87.7 & 91.2 & 92.9 & 92.9 & 90.3 \\
\hline $1 \mathrm{~b}$ & B & S & 2/3/1993 & 93034 & 34 & 112.8 & 92 & 89.5 & 86 & 83.9 \\
\hline $1 \mathrm{H}$ & B & $S$ & 2/4/1993 & 93035 & 35 & 94.6 & 93.8 & 92.5 & 92 & 90.3 \\
\hline $1 \mathrm{H}$ & $\mathrm{B}$ & $\mathrm{S}$ & 2/5/1993 & 93036 & 36 & 86.9 & 87.7 & 89.5 & 84.7 & 86 \\
\hline $1 \mathrm{H}$ & B & $S$ & 2/8/1993 & 93039 & 39 & 84.3 & 85.6 & 85.1 & 85.1 & 83.4 \\
\hline $1 \mathrm{H}$ & $\mathrm{B}$ & $S$ & 2/9/1993 & 93040 & 40 & 137 & 92.9 & 93.8 & 92 & 89.9 \\
\hline $1 \mathrm{H}$ & B & $S$ & $2 / 10 / 1993$ & 93041 & 41 & 110.2 & 108.5 & 107.6 & 105.9 & 105.9 \\
\hline $1 \mathrm{H}$ & B & $S$ & 2/11/1993 & 93042 & 42 & 81.7 & 83.4 & 84.3 & 82.5 & 81.3 \\
\hline $1 \mathrm{H}$ & B & $S$ & $2 / 12 / 1993$ & 93043 & 43 & 79.6 & 81.7 & 81.7 & 80.8 & 80.8 \\
\hline $1 \mathrm{H}$ & $\mathrm{B}$ & $S$ & 2/16/1993 & 93047 & 47 & 61.4 & 69.6 & 71.3 & 68.7 & 66.1 \\
\hline $1 \mathrm{H}$ & $\mathrm{B}$ & $S$ & 2/17/1993 & 93048 & 48 & 111.9 & 93.8 & 89.5 & 89.1 & 86 \\
\hline $1 \mathrm{H}$ & B & $S$ & $2 / 18 / 1993$ & 93049 & 49 & 107.6 & 99 & 96.8 & 97.2 & 99.8 \\
\hline $1 \mathrm{H}$ & $\mathrm{B}$ & $\mathrm{S}$ & 2/19/1993 & 93050 & 50 & 139.6 & 146.5 & 123.2 & 95.5 & 93.4 \\
\hline $1 \mathrm{H}$ & B & $S$ & $2 / 22 / 1993$ & 93053 & 53 & 80.8 & 80.8 & 81.7 & 80.4 & 79.1 \\
\hline $1 \mathrm{~b}$ & B & S & 2/23/1993 & 93054 & 54 & 78.2 & 85.1 & 84.7 & 84.3 & 82.5 \\
\hline $1 \mathrm{H}$ & B & $S$ & $2 / 24 / 1993$ & 93055 & 55 & 66.6 & 63.5 & 62.7 & 61.8 & 60.9 \\
\hline $1 \mathrm{H}$ & B & $S$ & $2 / 25 / 1993$ & 93056 & 56 & 74.8 & 71.3 & 73 & 70.4 & 69.6 \\
\hline $1 \mathrm{H}$ & $\mathrm{B}$ & $\mathrm{S}$ & $2 / 26 / 1993$ & 93057 & 57 & 63.5 & 64.4 & 64 & 64.4 & 62.7 \\
\hline $1 \mathrm{H}$ & $\mathrm{B}$ & $\mathrm{S}$ & 3/1/1993 & 93060 & 60 & 64.4 & 66.1 & 68.7 & 66.6 & 65.3 \\
\hline $1 \mathrm{H}$ & $\mathrm{B}$ & $\mathrm{S}$ & $3 / 2 / 1993$ & 93061 & 61 & 86 & 79.1 & 80.8 & 77.4 & 77.4 \\
\hline $1 \mathrm{H}$ & $\mathrm{B}$ & $S$ & 3/3/1993 & 93062 & 62 & 70.4 & 69.6 & 74.8 & 69.6 & 69.6 \\
\hline $1 \mathrm{H}$ & B & $S$ & 3/4/1993 & 93063 & 63 & 85.1 & 76.5 & 73.5 & 63.5 & 64.4 \\
\hline $1 \mathrm{H}$ & B & $S$ & 3/5/1993 & 93064 & 64 & 72.6 & 71.3 & 70.4 & 68.7 & 67.5 \\
\hline $1 \mathrm{H}$ & $\mathrm{B}$ & $S$ & 3/8/1993 & 93067 & 67 & 71.8 & 74.8 & 75.6 & 74.8 & 73.9 \\
\hline $1 \mathrm{H}$ & B & $S$ & $3 / 9 / 1993$ & 93068 & 68 & 72.2 & 79.6 & 79.5 & 76.1 & 77.4 \\
\hline $1 \mathrm{H}$ & $\mathrm{B}$ & $\mathrm{S}$ & 3/10/1993 & 93069 & 69 & 73 & 68.3 & 67.9 & 66.1 & 67 \\
\hline $1 \mathrm{H}$ & $\mathrm{B}$ & $S$ & 3/11/1993 & 93070 & 70 & 82.1 & 80 & 80 & 79.1 & 78.2 \\
\hline $1 \mathrm{H}$ & B & $S$ & 3/12/1993 & 93071 & 71 & 91.2 & 85.6 & 85.1 & 83.4 & 80 \\
\hline $1 \mathrm{~F}$ & B & $S$ & $3 / 22 / 1993$ & 93081 & 81 & 69.6 & 69.6 & 70.4 & 68.7 & 68.3 \\
\hline $1 \mathrm{H}$ & B & $S$ & 3/23/1993 & 93082 & 82 & 69.6 & 76.5 & 75.6 & 73 & 73.5 \\
\hline $1 \mathrm{H}$ & B & $S$ & $3 / 24 / 1993$ & 93083 & 83 & 73 & 72.2 & 70.4 & 67.9 & 68.7 \\
\hline $1 \mathrm{H}$ & $\mathrm{B}$ & $\mathrm{S}$ & $3 / 25 / 1993$ & 93084 & 84 & 81.7 & 77.4 & 78.2 & 76.1 & 76.5 \\
\hline $1 \mathrm{H}$ & B & $S$ & $3 / 26 / 1993$ & 93085 & 85 & 86.9 & 87.3 & 87.7 & 86 & 86 \\
\hline $1 \mathrm{H}$ & $\mathrm{B}$ & $\mathrm{S}$ & 3/29/1993 & 93088 & 88 & 74.8 & 70.4 & 71.3 & 73 & 69.6 \\
\hline $1 \mathrm{H}$ & $\mathrm{B}$ & $S$ & 3/30/1993 & 93089 & 89 & 91.2 & 119.7 & 111.1 & 108.5 & 105.9 \\
\hline $1 \mathrm{~F}$ & B & $\mathrm{S}$ & $3 / 31 / 1993$ & 93090 & 90 & 108.5 & 96.4 & 92.9 & 90.3 & 91.2 \\
\hline
\end{tabular}


other

\begin{tabular}{|c|c|c|c|c|c|c|c|c|c|c|}
\hline $1 \mathrm{~F}$ & B & $S$ & 4/1/1993 & 93091 & 91 & 96 & 89.5 & 89.5 & 86.9 & 83.4 \\
\hline $1 \mathrm{~F}$ & B & S & 4/2/1993 & 93092 & 92 & 102.4 & 103.7 & 102.4 & 104.1 & 102.4 \\
\hline $1 \mathrm{H}$ & $\mathrm{B}$ & $S$ & $4 / 5 / 1993$ & 93095 & 95 & 74.8 & 73 & 75.6 & 73.5 & 73 \\
\hline $1 \mathrm{H}$ & $\mathrm{B}$ & S & $4 / 6 / 1993$ & 93096 & 96 & 68.7 & 75.6 & 75.2 & 73 & 72.2 \\
\hline $1 \mathrm{H}$ & $\mathrm{B}$ & S & 4/7/1993 & 93097 & 97 & 78.2 & 69.6 & 68.7 & 68.7 & 68.7 \\
\hline $1 \mathrm{H}$ & B & $S$ & 4/8/1993 & 93098 & 98 & 80 & 74.8 & 76.5 & 75.6 & 73.9 \\
\hline $1 \mathrm{H}$ & B & $S$ & 4/13/1993 & 93103 & 103 & 77.8 & 106.7 & 103.7 & 97.2 & 94.6 \\
\hline $1 \mathrm{H}$ & B & $S$ & 4/14/1993 & 93104 & 104 & 162.9 & 142.6 & 135.6 & 133.5 & 132.3 \\
\hline $1 \mathrm{H}$ & B & $S$ & $4 / 15 / 1993$ & 93105 & 105 & 86.5 & 80 & 79.1 & 77.4 & 72.6 \\
\hline $1 \mathrm{H}$ & B & S & $4 / 16 / 1993$ & 93106 & 106 & 80.8 & 80.8 & 80.8 & 76.5 & 73.5 \\
\hline $1 \mathrm{H}$ & B & $S$ & 4/19/1993 & 93109 & 109 & 72.2 & 69.6 & 72.2 & 71.3 & 70.4 \\
\hline $1 \mathrm{H}$ & B & S & $4 / 20 / 1993$ & 93110 & 110 & 99 & 129.7 & 122.3 & 117.1 & 107.6 \\
\hline $1 \mathrm{H}$ & B & $S$ & 4/21/1993 & 93111 & 111 & 93.8 & 85.6 & 85.1 & 85.1 & 83.4 \\
\hline $1 \mathrm{H}$ & B & $S$ & $4 / 22 / 1993$ & 93112 & 112 & 86 & 75.6 & 75.6 & 75.6 & 73.5 \\
\hline $1 \mathrm{~F}$ & B & S & 4/23/1993 & 93113 & 113 & 84.3 & 83 & 84.3 & 84.3 & 81.7 \\
\hline $1 \mathrm{~F}$ & B & S & $4 / 26 / 1993$ & 93116 & 116 & 74.8 & 75.6 & 75.6 & 75.6 & 75.6 \\
\hline $1 \mathrm{H}$ & $\mathrm{B}$ & S & $4 / 27 / 1993$ & 93117 & 117 & 81.7 & 114.5 & 106.7 & 99.8 & 97.2 \\
\hline $1 \mathrm{H}$ & B & S & 4/29/1993 & 93119 & 119 & 82.5 & 74.8 & 69.2 & 67.4 & 60.5 \\
\hline $1 \mathrm{H}$ & $\mathrm{B}$ & S & 4/30/1993 & 93120 & 120 & 75.6 & 73.9 & 76.5 & 76.5 & 74.8 \\
\hline $1 \mathrm{~F}$ & B & S & 5/3/1993 & 93123 & 123 & 74.8 & 74.4 & 76 & 76.1 & 73.9 \\
\hline $1 \mathrm{H}$ & B & $S$ & 5/4/1993 & 93124 & 124 & 73 & 86.9 & 84.3 & 83.4 & 79.1 \\
\hline $1 \mathrm{H}$ & B & $S$ & 5/5/1993 & 93125 & 125 & 89.1 & 80.4 & 79.6 & 81.7 & 79.1 \\
\hline $1 \mathrm{H}$ & B & $S$ & 5/6/1993 & 93126 & 126 & 78.2 & 81.7 & 78.2 & 76.5 & 80 \\
\hline $1 \mathrm{H}$ & B & $S$ & 5/7/1993 & 93127 & 127 & 81.7 & 81.7 & 81.7 & 80 & 80 \\
\hline $1 \mathrm{H}$ & B & $S$ & $5 / 10 / 1993$ & 93130 & 130 & 70.4 & 70.4 & 70.4 & 70.9 & 71.3 \\
\hline $1 \mathrm{H}$ & B & $S$ & $5 / 11 / 1993$ & 93131 & 131 & 86.9 & 98.1 & 91.2 & 90.3 & 91.6 \\
\hline $1 \mathrm{H}$ & B & $S$ & $5 / 12 / 1993$ & 93132 & 132 & 88.6 & 84.3 & 80.8 & 81.7 & 80.8 \\
\hline $1 \mathrm{H}$ & B & $S$ & $5 / 13 / 1993$ & 93133 & 133 & 99.8 & 93.8 & 88.6 & 86.9 & 87.7 \\
\hline $1 \mathrm{~F}$ & B & S & $5 / 14 / 1993$ & 93134 & 134 & 72.2 & 69.2 & 67.9 & 68.7 & 72.2 \\
\hline $1 \mathrm{H}$ & B & S & $5 / 17 / 1993$ & 93137 & 137 & 87.3 & 78.2 & 77.8 & 69.2 & 67.9 \\
\hline $1 \mathrm{H}$ & B & S & $5 / 18 / 1993$ & 93138 & 138 & 92 & 115.4 & 110.7 & 111.9 & 104.6 \\
\hline $1 \mathrm{H}$ & B & S & $5 / 19 / 1993$ & 93139 & 139 & 92 & 89.5 & 86.9 & 80 & 80 \\
\hline $1 \mathrm{H}$ & B & S & 5/20/1993 & 93140 & 140 & 100.7 & 82.5 & 82.5 & 80.8 & 78.2 \\
\hline $1 \mathrm{H}$ & B & $S$ & $5 / 21 / 1993$ & 93141 & 141 & 79.1 & 76.5 & 74.8 & 77.4 & 73.5 \\
\hline $1 \mathrm{H}$ & B & $S$ & 5/24/1993 & 93144 & 144 & 66.1 & 64.4 & 64.4 & 66.1 & 64.4 \\
\hline $1 \mathrm{~F}$ & B & S & 5/25/1993 & 93145 & 145 & 102.4 & 110.2 & 105 & 102.4 & 97.2 \\
\hline $1 \mathrm{H}$ & B & $S$ & $5 / 26 / 1993$ & 93146 & 146 & 92 & 82.5 & 80.8 & 84.3 & 80.8 \\
\hline $1 \mathrm{H}$ & B & $S$ & 9/9/1993 & 93252 & 252 & 84.7 & 78.7 & 76.1 & 77 & 73 \\
\hline $1 \mathrm{H}$ & B & $S$ & 9/10/1993 & 93253 & 253 & 82.1 & 82.1 & 79.6 & 78.7 & 78.2 \\
\hline $1 \mathrm{H}$ & B & $S$ & 9/13/1993 & 93256 & 256 & 86.9 & 86.5 & 86.5 & 85.6 & 86 \\
\hline $1 \mathrm{H}$ & B & $S$ & 9/14/1993 & 93257 & 257 & 83.4 & 82.1 & 81.3 & 80.4 & 81.7 \\
\hline $1 \mathrm{H}$ & B & S & 9/15/1993 & 93258 & 258 & 82.1 & 74.8 & 75.2 & 73.5 & 73 \\
\hline $1 \mathrm{H}$ & B & S & 9/16/1993 & 93259 & 259 & 71.8 & 67.5 & 67 & 65.7 & 66.1 \\
\hline $1 \mathrm{H}$ & B & $S$ & 9/17/1993 & 93260 & 260 & 76.1 & 74.4 & 73.5 & 73.9 & 72.6 \\
\hline $1 \mathrm{H}$ & B & $S$ & 9/20/1993 & 93263 & 263 & 90.3 & 85.6 & 85.6 & 84.3 & 83 \\
\hline $1 \mathrm{H}$ & B & S & 9/21/1993 & 93264 & 264 & 81.3 & 80.8 & 81.3 & 80 & 81.3 \\
\hline $1 \mathrm{H}$ & B & $S$ & 9/22/1993 & 93265 & 265 & 84.7 & 84.3 & 83.9 & 81.3 & 82.5 \\
\hline $1 \mathrm{H}$ & B & $S$ & 9/23/1993 & 93266 & 266 & 83.4 & 82.1 & 81.3 & 77.8 & 80 \\
\hline $1 \mathrm{H}$ & B & $S$ & 9/24/1993 & 93267 & 267 & 89.1 & 89.1 & 89.5 & 85.6 & 83.9 \\
\hline
\end{tabular}


other

\begin{tabular}{|c|c|c|c|c|c|c|c|c|c|c|}
\hline $1 \mid 1$ & B & $S$ & 9/27/1993 & 93270 & 270 & 78.7 & 86.5 & 85.1 & 84.7 & 83.9 \\
\hline $1 \mid 1$ & B & $S$ & 9/28/1993 & 93271 & 271 & 78.7 & 73.9 & 71.8 & 73.5 & 73 \\
\hline $1 \mathrm{l}$ & $\mathrm{B}$ & S & 9/29/1993 & 93272 & 272 & 83.9 & 83 & 80 & 81.3 & 77.8 \\
\hline 11 & $\mathrm{~B}$ & S & 9/30/1993 & 93273 & 273 & 76.1 & 75.2 & 76.5 & 74.4 & 73.5 \\
\hline $1 \mathrm{l}$ & $\mathrm{B}$ & S & 10/1/1993 & 93274 & 274 & 78.7 & 78.2 & 77 & 75.6 & 75.2 \\
\hline 11 & B & $S$ & 10/4/1993 & 93277 & 277 & 81.3 & 89.1 & 87.7 & 85.6 & 84.7 \\
\hline 11 & B & $S$ & 10/5/1993 & 93278 & 278 & 85.6 & 77.8 & 76.5 & 77 & 76.1 \\
\hline $1 \mathrm{l}$ & B & $S$ & 10/6/1993 & 93279 & 279 & 78.7 & 77.8 & 78.2 & 75.2 & 74.8 \\
\hline 11 & B & S & 10/7/1993 & 93280 & 280 & 77.8 & 77 & 75.6 & 76.1 & 74.8 \\
\hline 11 & B & S & 10/8/1993 & 93281 & 281 & 83.9 & 81.7 & 80.4 & 80.8 & 79.6 \\
\hline 11 & $\mathrm{~B}$ & S & 10/11/1993 & 93284 & 284 & 77.4 & 84.7 & 80.8 & 81.3 & 80 \\
\hline 11 & B & S & 10/12/1993 & 93285 & 285 & 81.3 & 75.6 & 74.8 & 72.6 & 73 \\
\hline 11 & B & S & 10/13/1993 & 93286 & 286 & 79.6 & 77.4 & 77 & 75.6 & 76.5 \\
\hline 11 & B & S & 10/14/1993 & 93287 & 287 & 90.3 & 79.1 & 74.8 & 70.9 & 71.7 \\
\hline 11 & B & S & 10/15/1993 & 93288 & 288 & 73 & 71.3 & 71.3 & 71.3 & 70.4 \\
\hline 11 & $\mathrm{~B}$ & S & 10/18/1993 & 93291 & 291 & 79.6 & 85.1 & 83 & 85.1 & 83 \\
\hline 11 & $\mathrm{~B}$ & S & 10/19/1993 & 93292 & 292 & 89.5 & 83 & 81.3 & 80 & 80.4 \\
\hline $1 \mathrm{l}$ & $\mathrm{B}$ & S & 10/20/1993 & 93293 & 293 & 96.8 & 94.6 & 96.8 & 95.1 & 92.9 \\
\hline $1 \mathrm{l}$ & $\mathrm{B}$ & S & 10/21/1993 & 93294 & 294 & 70.9 & 72.6 & 70 & 68.7 & 71.3 \\
\hline 11 & B & $S$ & 10/22/1993 & 93295 & 295 & 74.4 & 70.4 & 68.7 & 68.7 & 68.7 \\
\hline 11 & B & $S$ & 10/25/1993 & 93298 & 298 & 68.7 & 63.5 & 65.3 & 64.4 & 63.5 \\
\hline 11 & B & $S$ & 10/26/1993 & 93299 & 299 & 77.4 & 76.5 & 76.5 & 75.6 & 73.9 \\
\hline 11 & B & $S$ & 10/27/1993 & 93300 & 300 & 70.4 & 71.3 & 71.3 & 68.7 & 66.1 \\
\hline 11 & B & $S$ & 10/28/1993 & 93301 & 301 & 64 & 63.1 & 64.5 & 63.1 & 62.7 \\
\hline $1 \mathrm{H}$ & B & $S$ & 10/29/1993 & 93302 & 302 & 62.3 & 63.6 & 64.5 & 59.7 & 61 \\
\hline $1 \mathrm{H}$ & B & S & 11/1/1993 & 93305 & 305 & 64.5 & 64.9 & 62.7 & 64 & 63.1 \\
\hline $1 \mathrm{H}$ & B & $S$ & 11/2/1993 & 93306 & 306 & 82.1 & 80 & 80.4 & 79.2 & 80.4 \\
\hline $1 \mathrm{H}$ & B & $S$ & 11/3/1993 & 93307 & 307 & 69.6 & 67.9 & 66.2 & 65.3 & 67.5 \\
\hline $1 \mathrm{H}$ & B & S & 11/4/1993 & 93308 & 308 & 88.7 & 89.5 & 88.2 & 89.5 & 89.1 \\
\hline $1 \mathrm{~F}$ & B & S & $11 / 5 / 1993$ & 93309 & 309 & 71.4 & 72.2 & 71.8 & 71.4 & 69.2 \\
\hline $1 \mathrm{H}$ & B & S & 11/8/1993 & 93312 & 312 & 70.9 & 72.2 & 68.3 & 67.9 & 69.2 \\
\hline $1 \mathrm{~F}$ & B & S & 11/9/1993 & 93313 & 313 & 86.9 & 86.1 & 83.9 & 86.1 & 85.6 \\
\hline $1 \mathrm{H}$ & $\mathrm{B}$ & S & 11/10/1993 & 93314 & 314 & 85.2 & 81.3 & 79.2 & 81.7 & 81.3 \\
\hline $1 \mathrm{H}$ & B & $S$ & 11/12/1993 & 93316 & 316 & 61 & 61.9 & 60.5 & 59.3 & 59.3 \\
\hline $1 \mathrm{H}$ & B & $S$ & 11/15/1993 & 93319 & 319 & 61.9 & 58 & 60.1 & 59.7 & 58.4 \\
\hline $1 \mathrm{H}$ & B & $S$ & 11/16/1993 & 93320 & 320 & 60.5 & 60.1 & 60.1 & 62.3 & 61.9 \\
\hline $1 \mathrm{H}$ & B & $S$ & 11/17/1993 & 93321 & 321 & 65.3 & 65.7 & 67.9 & 64 & 64 \\
\hline $1 \mathrm{H}$ & B & $S$ & 11/18/1993 & 93322 & 322 & 64.9 & 64.5 & 64 & 63.6 & 63.1 \\
\hline $1 \mathrm{H}$ & B & $S$ & 11/19/1993 & 93323 & 323 & 78.3 & 68.3 & 68.8 & 67.5 & 66.6 \\
\hline $1 \mathrm{H}$ & B & $S$ & 11/22/1993 & 93326 & 326 & 77.8 & 76.6 & 78.7 & 76.1 & 75.7 \\
\hline $1 \mathrm{H}$ & B & $S$ & 11/23/1993 & 93327 & 327 & 68.3 & 67.1 & 66.6 & 64.9 & 66.2 \\
\hline $1 \mathrm{H}$ & B & S & 11/29/1993 & 93333 & 333 & 68.3 & 67.1 & 70 & 70 & 70.9 \\
\hline $1 \mathrm{~F}$ & B & S & 11/30/1993 & 93334 & 334 & 88.2 & 86.9 & 89.1 & 89.1 & 88.2 \\
\hline $1 \mathrm{H}$ & B & $S$ & $12 / 1 / 1993$ & 93335 & 335 & 79.6 & 79.2 & 80.4 & 78.3 & 79.6 \\
\hline $1 \mathrm{H}$ & B & S & $12 / 2 / 1993$ & 93336 & 336 & 65.3 & 63.1 & 63.1 & 61.9 & 61.4 \\
\hline $1 \mathrm{H}$ & B & $S$ & 12/3/1993 & 93337 & 337 & 92.5 & 85.2 & 70.9 & 66.6 & 64.5 \\
\hline $1 \mathrm{~F}$ & B & $S$ & $12 / 6 / 1993$ & 93340 & 340 & 62.3 & 60.1 & 60.5 & 61.4 & 62.3 \\
\hline $1 \mathrm{H}$ & B & $S$ & 12/7/1993 & 93341 & 341 & 78.7 & 77.8 & 79.6 & 80 & 78.7 \\
\hline $1 \mathrm{H}$ & B & $S$ & 12/8/1993 & 93342 & 342 & 95.1 & 85.6 & 83.5 & 84.7 & 83.9 \\
\hline
\end{tabular}


other

\begin{tabular}{|c|c|c|c|c|c|c|c|c|c|c|}
\hline $1 \mathrm{l}$ & B & $S$ & $12 / 9 / 1993$ & 93343 & 343 & 69.2 & 70.5 & 70 & 70 & 70 \\
\hline $1 \mathrm{l}$ & B & $S$ & 12/10/1993 & 93344 & 344 & 87.8 & 72.6 & 64.5 & 64 & 66.6 \\
\hline $1 \mathrm{I}$ & $\mathrm{B}$ & $S$ & 12/13/1993 & 93347 & 347 & 64 & 61.9 & 61.4 & 61.9 & 62.3 \\
\hline $1 \mathrm{I}$ & $\mathrm{B}$ & $S$ & 12/14/1993 & 93348 & 348 & 61.4 & 61.4 & 60.1 & 62.3 & 60.1 \\
\hline $1 \mathrm{I}$ & $\mathrm{B}$ & $S$ & 12/15/1993 & 93349 & 349 & 69.6 & 69.2 & 69.2 & 66.6 & 66.6 \\
\hline 11 & B & $S$ & 12/16/1993 & 93350 & 350 & 98.2 & 96 & 99.4 & 92.1 & 91.6 \\
\hline 11 & $\mathrm{~B}$ & $S$ & $1 / 6 / 1994$ & 94006 & 6 & 91.6 & 92.1 & 91.2 & 93.4 & 93 \\
\hline $1 \mathrm{l}$ & B & $S$ & $1 / 7 / 1994$ & 94007 & 7 & 98.2 & 96.8 & 95.6 & 94.2 & 92.1 \\
\hline 11 & $\mathrm{~B}$ & $S$ & $1 / 10 / 1994$ & 94010 & 10 & 99 & 97.7 & 99.4 & 99 & 98.6 \\
\hline $1 \mathrm{l}$ & B & $S$ & $1 / 11 / 1994$ & 94011 & 11 & 86.9 & 85.6 & 86.9 & 85.6 & 86.5 \\
\hline 11 & B & $S$ & $1 / 12 / 1994$ & 94012 & 12 & 74.8 & 63.1 & 61.9 & 62.3 & 61.4 \\
\hline $1 \mathrm{l}$ & B & $\mathrm{S}$ & $1 / 13 / 1994$ & 94013 & 13 & 71.4 & 71.4 & 70.9 & 71.4 & 72.2 \\
\hline 11 & B & $S$ & $1 / 14 / 1994$ & 94014 & 14 & 60.5 & 61.9 & 60.5 & 64 & 60.5 \\
\hline $1 \mathrm{l}$ & B & $\mathrm{S}$ & $1 / 17 / 1994$ & 94017 & 17 & 79.6 & 79.6 & 80.9 & 80.4 & 80.4 \\
\hline $1 \mathrm{l}$ & B & S & 1/18/1994 & 94018 & 18 & 110.7 & 111.1 & 110.3 & 110.7 & 109.8 \\
\hline $1 \mathrm{l}$ & B & $S$ & $1 / 19 / 1994$ & 94019 & 19 & 121 & 111.1 & 95.1 & 94.2 & 93.8 \\
\hline $1 \mathrm{I}$ & $\mathrm{B}$ & $S$ & $1 / 20 / 1994$ & 94020 & 20 & 87.3 & 88.2 & 86.9 & 87.3 & 88.2 \\
\hline $1 \mathrm{l}$ & B & S & $1 / 21 / 1994$ & 94021 & 21 & 84.3 & 83 & 84.3 & 85.2 & 83.9 \\
\hline $1 \mathrm{I}$ & $\mathrm{B}$ & $S$ & $1 / 24 / 1994$ & 94024 & 24 & 85.6 & 80.4 & 82.6 & 83.9 & 83 \\
\hline $1 \mathrm{I}$ & $\mathrm{B}$ & $S$ & $1 / 25 / 1994$ & 94025 & 25 & 77.4 & 77 & 77.8 & 74.4 & 76.6 \\
\hline 11 & $\mathrm{~B}$ & $S$ & $1 / 26 / 1994$ & 94026 & 26 & 102.9 & 83.5 & 81.3 & 76.6 & 77 \\
\hline 11 & $\mathrm{~B}$ & $S$ & $1 / 27 / 1994$ & 94027 & 27 & 70 & 69.6 & 69.2 & 68.8 & 70.5 \\
\hline 11 & $\mathrm{~B}$ & $S$ & $1 / 28 / 1994$ & 94028 & 28 & 112.3 & 113.2 & 111.8 & 107.9 & 104.5 \\
\hline 11 & $\mathrm{~B}$ & $S$ & $1 / 31 / 1994$ & 94031 & 31 & 106.3 & 105.5 & 107.2 & 107.2 & 108.5 \\
\hline $1 \mathrm{H}$ & $\mathrm{B}$ & $S$ & $2 / 1 / 1994$ & 94032 & 32 & 103.3 & 102 & 107.7 & 106.8 & 106.8 \\
\hline $1 \mathrm{H}$ & B & $S$ & $2 / 2 / 1994$ & 94033 & 33 & 136.6 & 119.3 & 121.5 & 121.9 & 121 \\
\hline $1 \mathrm{H}$ & B & $S$ & 2/3/1994 & 94034 & 34 & 91.6 & 93.8 & 96.8 & 96.4 & 96.8 \\
\hline $1 \mathrm{H}$ & $\mathrm{B}$ & $S$ & 2/4/1994 & 94035 & 35 & 90.4 & 88.7 & 93.8 & 92.1 & 90.4 \\
\hline $1 \mathrm{H}$ & B & $S$ & 2/7/1994 & 94038 & 38 & 62.7 & 62.7 & 64.5 & 63.6 & 61.9 \\
\hline $1 \mathrm{H}$ & $\mathrm{B}$ & $S$ & 2/8/1994 & 94039 & 39 & 68.8 & 69.6 & 69.6 & 69.6 & 68.8 \\
\hline $1 \mathrm{H}$ & B & $S$ & 2/9/1994 & 94040 & 40 & 96.4 & 78.8 & 79.2 & 70.5 & 70.5 \\
\hline $1 \mathrm{H}$ & B & $\mathrm{S}$ & 2/10/1994 & 94041 & 41 & 114.6 & 114.2 & 117.2 & 120.6 & 117.2 \\
\hline $1 \mathrm{~b}$ & B & $\mathrm{S}$ & 2/11/1994 & 94042 & 42 & 114.6 & 115.4 & 118.4 & 118 & 118.9 \\
\hline $1 \mathrm{H}$ & $\mathrm{B}$ & $S$ & $2 / 14 / 1994$ & 94045 & 45 & 100.8 & 102.5 & 103.3 & 102.5 & 100.8 \\
\hline $1 \mathrm{~b}$ & B & S & 2/15/1994 & 94046 & 46 & 96.4 & 95.6 & 94.7 & 94.7 & 94.7 \\
\hline $1 \mathrm{H}$ & B & $\mathrm{S}$ & 2/16/1994 & 94047 & 47 & 93 & 96.4 & 93.8 & 93.8 & 92.1 \\
\hline $1 \mathrm{H}$ & B & S & 2/17/1994 & 94048 & 48 & 68.8 & 70.5 & 68.8 & 67.9 & 68.8 \\
\hline $1 \mathrm{H}$ & B & $S$ & 2/18/1994 & 94049 & 49 & 67.1 & 65.3 & 63.6 & 64.5 & 63.6 \\
\hline $1 \mathrm{H}$ & B & S & 2/22/1994 & 94053 & 53 & 80 & 77.4 & 71.4 & 68.8 & 66.2 \\
\hline $1 \mathrm{H}$ & $\mathrm{B}$ & $S$ & 2/23/1994 & 94054 & 54 & 85.2 & 87.8 & 88.7 & 85.2 & 83.5 \\
\hline $1 \mathrm{H}$ & B & $\mathrm{S}$ & $2 / 24 / 1994$ & 94055 & 55 & 93 & 93.8 & 92.1 & 93 & 93 \\
\hline $1 \mathrm{H}$ & $\mathrm{B}$ & $S$ & 2/25/1994 & 94056 & 56 & 82.2 & 83.5 & 81.7 & 81.7 & 81.3 \\
\hline $1 \mathrm{H}$ & $\mathrm{B}$ & $S$ & $2 / 28 / 1994$ & 94059 & 59 & 71.4 & 73.1 & 74.8 & 74 & 71.4 \\
\hline $1 \mathrm{H}$ & $\mathrm{B}$ & $S$ & $3 / 1 / 1994$ & 94060 & 60 & 112.4 & 112 & 99.9 & 90.4 & 91.2 \\
\hline $1 \mathrm{H}$ & $\mathrm{B}$ & $\mathrm{S}$ & $3 / 2 / 1994$ & 94061 & 61 & 69.2 & 65.3 & 61 & 61 & 63.6 \\
\hline $1 \mathrm{~F}$ & B & S & 3/3/1994 & 94062 & 62 & 81.7 & 81.3 & 82.6 & 81.7 & 80.9 \\
\hline $1 \mathrm{H}$ & $\mathrm{B}$ & $S$ & 3/4/1994 & 94063 & 63 & 81.7 & 81.7 & 80.9 & 82.6 & 80.9 \\
\hline $1 \mathrm{~b}$ & B & $S$ & 3/7/1994 & 94066 & 66 & 63.6 & 64.5 & 63.6 & 66.2 & 65.3 \\
\hline $1 \mathrm{~F}$ & B & $S$ & 3/8/1994 & 94067 & 67 & 75.7 & 74 & 73.1 & 70.5 & 72.2 \\
\hline
\end{tabular}


other

\begin{tabular}{|c|c|c|c|c|c|c|c|c|c|c|}
\hline $1 \mathrm{l}$ & B & $S$ & $3 / 9 / 1994$ & 94068 & 68 & 75.7 & 75.7 & 74 & 72.7 & 71.8 \\
\hline $1 \mathrm{l}$ & B & S & 3/10/1994 & 94069 & 69 & 95.2 & 95.6 & 95.6 & 95.6 & 96 \\
\hline $1 \mathrm{I}$ & $\mathrm{B}$ & $S$ & 3/21/1994 & 94080 & 80 & 64.9 & 64.9 & 67.5 & 69.2 & 64.9 \\
\hline $1 \mathrm{I}$ & $\mathrm{B}$ & $S$ & $3 / 22 / 1994$ & 94081 & 81 & 66.7 & 67.5 & 68.8 & 67.5 & 61.5 \\
\hline $1 \mathrm{I}$ & $\mathrm{B}$ & $S$ & $3 / 23 / 1994$ & 94082 & 82 & 66.7 & 67.5 & 69.2 & 71.4 & 65.8 \\
\hline $1 \mathrm{I}$ & $\mathrm{B}$ & $S$ & $3 / 24 / 1994$ & 94083 & 83 & 71 & 74.4 & 73.6 & 74.4 & 70.1 \\
\hline 11 & $\mathrm{~B}$ & $S$ & $3 / 25 / 1994$ & 94084 & 84 & 68.4 & 68.4 & 71 & 68.4 & 65.8 \\
\hline $1 \mathrm{l}$ & B & $S$ & $3 / 28 / 1994$ & 94087 & 87 & 58 & 58.9 & 61.9 & 61.5 & 58.9 \\
\hline $1 \mathrm{I}$ & B & $S$ & 3/29/1994 & 94088 & 88 & 66.7 & 66.7 & 70.1 & 67.5 & 65.8 \\
\hline $1 \mathrm{l}$ & B & $S$ & $3 / 30 / 1994$ & 94089 & 89 & 66.3 & 65.8 & 68.4 & 66.7 & 64.1 \\
\hline 11 & B & $S$ & 3/31/1994 & 94090 & 90 & 67.5 & 69.7 & 69.2 & 69.2 & 64.5 \\
\hline $1 \mathrm{l}$ & B & $\mathrm{S}$ & $4 / 5 / 1994$ & 94095 & 95 & 69.7 & 70.1 & 70.6 & 71.8 & 71.4 \\
\hline 11 & B & $S$ & 4/6/1994 & 94096 & 96 & 76.6 & 76.2 & 74.9 & 76.6 & 77 \\
\hline $1 \mathrm{l}$ & B & $\mathrm{S}$ & 4/7/1994 & 94097 & 97 & 64.5 & 65.8 & 68 & 67.1 & 70.6 \\
\hline 11 & $\mathrm{~B}$ & $S$ & $4 / 8 / 1994$ & 94098 & 98 & 59.6 & 57.9 & 57.9 & 60.5 & 61.3 \\
\hline 11 & $\mathrm{~B}$ & $S$ & $4 / 11 / 1994$ & 94101 & 101 & 74.3 & 73.4 & 73.4 & 74.3 & 75.2 \\
\hline $1 \mathrm{I}$ & $\mathrm{B}$ & $S$ & $4 / 12 / 1994$ & 94102 & 102 & 72.2 & 70.4 & 67.5 & 71.7 & 70 \\
\hline $1 \mathrm{I}$ & $\mathrm{B}$ & $\mathrm{S}$ & $4 / 13 / 1994$ & 94103 & 103 & 70.8 & 68.3 & 64.4 & 65.3 & 65.3 \\
\hline 11 & B & $S$ & $4 / 14 / 1994$ & 94104 & 104 & 80.8 & 82.5 & 84.3 & 85.1 & 85.1 \\
\hline $1 \mathrm{I}$ & $\mathrm{B}$ & $S$ & 4/15/1994 & 94105 & 105 & 69.6 & 69.6 & 70.4 & 72.2 & 69.6 \\
\hline 11 & $\mathrm{~B}$ & $S$ & 4/18/1994 & 94108 & 108 & 65.7 & 67 & 67 & 69.6 & 69.2 \\
\hline 11 & $\mathrm{~B}$ & $S$ & 4/19/1994 & 94109 & 109 & 69.2 & 68.3 & 70 & 71.3 & 71.3 \\
\hline 11 & $\mathrm{~B}$ & $S$ & 4/20/1994 & 94110 & 110 & 63.5 & 63.5 & 64.4 & 65.3 & 65.3 \\
\hline 11 & $\mathrm{~B}$ & $S$ & 4/21/1994 & 94111 & 111 & 61.8 & 61.8 & 65.3 & 64.4 & 63.5 \\
\hline $1 \mathrm{H}$ & $\mathrm{B}$ & $S$ & 4/22/1994 & 94112 & 112 & 60.9 & 62.7 & 61.8 & 63.5 & 63.1 \\
\hline $1 \mathrm{H}$ & B & $S$ & $4 / 25 / 1994$ & 94115 & 115 & 64.9 & 70 & 67.9 & 68.3 & 68.7 \\
\hline $1 \mathrm{H}$ & B & $S$ & 4/26/1994 & 94116 & 116 & 64.4 & 63.5 & 63.5 & 67.9 & 64.4 \\
\hline $1 \mathrm{H}$ & $\mathrm{B}$ & $S$ & 4/27/1994 & 94117 & 117 & 72.6 & 73 & 72.6 & 74.8 & 73.9 \\
\hline $1 \mathrm{H}$ & B & $S$ & $4 / 28 / 1994$ & 94118 & 118 & 89.1 & 84.3 & 83.4 & 80.8 & 80.8 \\
\hline $1 \mathrm{H}$ & B & $S$ & $4 / 29 / 1994$ & 94119 & 119 & 70.4 & 74.8 & 73 & 70.4 & 71.3 \\
\hline $1 \mathrm{H}$ & B & $S$ & $5 / 2 / 1994$ & 94122 & 122 & 74.8 & 77.4 & 74.8 & 73 & 76.1 \\
\hline $1 \mathrm{H}$ & B & $\mathrm{S}$ & 5/3/1994 & 94123 & 123 & 82.5 & 79.1 & 81.7 & 80 & 76.5 \\
\hline $1 \mathrm{H}$ & $\mathrm{B}$ & $S$ & $5 / 4 / 1994$ & 94124 & 124 & 73.9 & 73.5 & 73 & 72.2 & 71.3 \\
\hline $1 \mathrm{H}$ & $\mathrm{B}$ & $S$ & $5 / 5 / 1994$ & 94125 & 125 & 70.9 & 73 & 73 & 71.3 & 72.2 \\
\hline $1 \mathrm{H}$ & $\mathrm{B}$ & $S$ & $5 / 6 / 1994$ & 94126 & 126 & 70.4 & 72.2 & 72.2 & 69.6 & 70.4 \\
\hline $1 \mathrm{H}$ & B & $\mathrm{S}$ & 5/9/1994 & 94129 & 129 & 67.9 & 68.3 & 69.2 & 67.9 & 67.5 \\
\hline $1 \mathrm{H}$ & $\mathrm{B}$ & $S$ & $5 / 10 / 1994$ & 94130 & 130 & 70.4 & 69.2 & 71.3 & 71.8 & 73 \\
\hline $1 \mathrm{H}$ & B & $S$ & $5 / 11 / 1994$ & 94131 & 131 & 94.2 & 92.9 & 92.5 & 92.9 & 92.5 \\
\hline $1 \mathrm{H}$ & B & $S$ & $5 / 12 / 1994$ & 94132 & 132 & 71.8 & 72.2 & 71.8 & 72.2 & 73 \\
\hline $1 \mathrm{H}$ & $\mathrm{B}$ & $S$ & $5 / 13 / 1994$ & 94133 & 133 & 75.2 & 74.8 & 75.6 & 73 & 71.8 \\
\hline $1 \mathrm{H}$ & $\mathrm{B}$ & $S$ & $5 / 16 / 1994$ & 94136 & 136 & 66.6 & 68.7 & 67.9 & 67.9 & 67.9 \\
\hline $1 \mathrm{H}$ & B & $S$ & $5 / 17 / 1994$ & 94137 & 137 & 69.2 & 70.4 & 70 & 69.6 & 68.3 \\
\hline $1 \mathrm{H}$ & $\mathrm{B}$ & $S$ & $5 / 18 / 1994$ & 94138 & 138 & 65.7 & 67 & 66.6 & 65.7 & 66.1 \\
\hline $1 \mathrm{H}$ & $\mathrm{B}$ & $S$ & $5 / 19 / 1994$ & 94139 & 139 & 72.6 & 73.5 & 71.8 & 71.3 & 70 \\
\hline $1 \mathrm{H}$ & $\mathrm{B}$ & $\mathrm{S}$ & $5 / 20 / 1994$ & 94140 & 140 & 79.1 & 77 & 75.2 & 75.6 & 75.2 \\
\hline $1 \mathrm{~F}$ & B & S & $5 / 23 / 1994$ & 94143 & 143 & 70 & 67 & 64.9 & 61.4 & 63.5 \\
\hline $1 \mathrm{~F}$ & B & $\mathrm{S}$ & $5 / 24 / 1994$ & 94144 & 144 & 80 & 81.3 & 80 & 80.4 & 78.7 \\
\hline $1 \mathrm{~b}$ & B & $S$ & $5 / 25 / 1994$ & 94145 & 145 & 76.5 & 77 & 76.5 & 74.4 & 74.8 \\
\hline $1 \mathrm{~F}$ & B & $S$ & $5 / 26 / 1994$ & 94146 & 146 & 74.4 & 72.6 & 73.9 & 70.9 & 72.2 \\
\hline
\end{tabular}


other

\begin{tabular}{|c|c|c|c|c|c|c|c|c|c|c|}
\hline 1 & B & $S$ & 5/27/1994 & 94147 & 147 & 81.3 & 80 & 82.1 & 82.5 & 78.7 \\
\hline 0 & A & $S$ & 9/7/1991 & 91250 & 250 & 214.3 & 210 & 212.5 & 203 & 197 \\
\hline 0 & A & $S$ & 9/8/1991 & 91251 & 251 & 196.1 & 192.7 & 194.4 & 194.4 & 197 \\
\hline 0 & A & $S$ & 9/14/1991 & 91257 & 257 & 240.2 & 237.6 & 239.3 & 222.9 & 215.1 \\
\hline 0 & A & $S$ & 9/15/1991 & 91258 & 258 & 221.2 & 219.5 & 214.3 & 214.3 & 214.3 \\
\hline 0 & A & $S$ & 9/21/1991 & 91264 & 264 & 140.8 & 135.6 & 130.5 & 121.8 & 116.6 \\
\hline 0 & A & $S$ & 9/22/1991 & 91265 & 265 & 163.3 & 156.4 & 155.5 & 154.7 & 150.3 \\
\hline 0 & A & $S$ & 9/28/1991 & 91271 & 271 & 146.9 & 139.1 & 141.7 & 130.5 & 129.6 \\
\hline 0 & A & S & 9/29/1991 & 91272 & 272 & 159 & 150.3 & 143.4 & 140.8 & 128.7 \\
\hline 0 & A & $S$ & 10/5/1991 & 91278 & 278 & 205.2 & 195.9 & 187.2 & 185.4 & 178.6 \\
\hline 0 & A & $S$ & 10/6/1991 & 91279 & 279 & 137.1 & 130.2 & 131.9 & 125.9 & 125.9 \\
\hline 0 & A & $S$ & 10/12/1991 & 91285 & 285 & 185 & 171.1 & 156.4 & 158.2 & 151.3 \\
\hline 0 & A & $S$ & 10/13/1991 & 91286 & 286 & 184.1 & 177.2 & 166 & 166.8 & 163.4 \\
\hline 0 & A & $S$ & 10/19/1991 & 91292 & 292 & 207 & 177.2 & 156.4 & 152.1 & 134.8 \\
\hline 0 & A & S & 10/20/1991 & 91293 & 293 & 149.5 & 139.2 & 132.3 & 122.3 & 122.8 \\
\hline 0 & A & $S$ & 10/26/1991 & 91299 & 299 & 216.1 & 203.1 & 189.3 & 189.7 & 183.2 \\
\hline 0 & A & $S$ & 10/27/1991 & 91300 & 300 & 231.6 & 229.9 & 215.2 & 207 & 197.9 \\
\hline 0 & A & S & 11/2/1991 & 91306 & 306 & 132.3 & 131.8 & 121.4 & 121.9 & 118.8 \\
\hline 0 & A & $S$ & 11/3/1991 & 91307 & 307 & 101.6 & 99.8 & 96.8 & 94.6 & 96.4 \\
\hline 0 & A & $S$ & 11/9/1991 & 91313 & 313 & 153 & 139.6 & 135.7 & 136.1 & 134 \\
\hline 0 & A & $S$ & 11/10/1991 & 91314 & 314 & 138.7 & 139.2 & 139.6 & 138.3 & 135.2 \\
\hline 0 & A & $S$ & 11/16/1991 & 91320 & 320 & 125.7 & 117.1 & 119.7 & 117.1 & 112.8 \\
\hline 0 & A & $S$ & 11/17/1991 & 91321 & 321 & 115.4 & 114.5 & 115.4 & 111.5 & 111.9 \\
\hline 0 & A & $S$ & 11/23/1991 & 91327 & 327 & 110.2 & 96.4 & 94.2 & 95.5 & 95.5 \\
\hline 0 & A & $S$ & 11/24/1991 & 91328 & 328 & 101.6 & 90.3 & 79.1 & 77.8 & 79.1 \\
\hline 0 & A & $S$ & 11/30/1991 & 91334 & 334 & 109.3 & 107.6 & 108.5 & 109.3 & 108.5 \\
\hline 0 & A & $S$ & $12 / 1 / 1991$ & 91335 & 335 & 99.4 & 99 & 98.1 & 98.1 & 97.7 \\
\hline 0 & A & $S$ & 12/7/1991 & 91341 & 341 & 153.4 & 132.7 & 128.3 & 122.3 & 124 \\
\hline 0 & A & $S$ & 12/8/1991 & 91342 & 342 & 144.8 & 131.8 & 131.8 & 130.9 & 131.8 \\
\hline 0 & A & $S$ & 12/14/1991 & 91348 & 348 & 154.7 & 129.2 & 126.6 & 116.2 & 117.1 \\
\hline 0 & A & $S$ & 12/15/1991 & 91349 & 349 & 105 & 100.7 & 92.9 & 90.8 & 91.2 \\
\hline 0 & A & $\mathrm{S}$ & 1/11/1992 & 92011 & 10 & 134.4 & 127.5 & 127.5 & 128.8 & 126.6 \\
\hline 0 & A & $\mathrm{S}$ & $1 / 12 / 1992$ & 92012 & 11 & 137.8 & 130.1 & 127.5 & 115 & 111.9 \\
\hline 0 & A & $S$ & 1/18/1992 & 92018 & 17 & 111.9 & 111.9 & 112.8 & 111.9 & 110.7 \\
\hline 0 & A & $\mathrm{S}$ & 1/19/1992 & 92019 & 18 & 108.1 & 97.2 & 99 & 99.8 & 98.1 \\
\hline 0 & A & $S$ & 1/25/1992 & 92025 & 24 & 96.4 & 96.4 & 96.4 & 95.5 & 96.8 \\
\hline 0 & A & $S$ & 1/26/1992 & 92026 & 25 & 77.4 & 71.3 & 73 & 70 & 69.6 \\
\hline 0 & $\mathrm{~B}$ & $S$ & 2/1/1992 & 92032 & 31 & 90.3 & 90.3 & 88.6 & 86.9 & 86.5 \\
\hline 0 & B & S & 2/2/1992 & 92033 & 32 & 88.6 & 77 & 77.4 & 77.4 & 78.2 \\
\hline 0 & $\mathrm{~B}$ & $S$ & 2/8/1992 & 92039 & 38 & 92.9 & 94.6 & 94.6 & 93.8 & 93.4 \\
\hline 0 & $\mathrm{~B}$ & $S$ & 2/9/1992 & 92040 & 39 & 99.4 & 82.5 & 81.7 & 81.7 & 80.4 \\
\hline 0 & $\mathrm{~B}$ & $S$ & 2/15/1992 & 92046 & 45 & 102.4 & 94.6 & 92.9 & 93.4 & 94.2 \\
\hline 0 & $\mathrm{~B}$ & $S$ & 2/16/1992 & 92047 & 46 & 102.4 & 93.8 & 92.9 & 92 & 92.9 \\
\hline 0 & $\mathrm{~B}$ & $S$ & 2/22/1992 & 92053 & 52 & 75.6 & 75.6 & 76.5 & 75.6 & 74.8 \\
\hline 0 & $\mathrm{~B}$ & $\mathrm{~S}$ & 2/23/1992 & 92054 & 53 & 97.2 & 93.8 & 95.5 & 85.1 & 84.3 \\
\hline 0 & B & S & 2/29/1992 & 92060 & 59 & 98.1 & 84.3 & 83.4 & 83.4 & 82.1 \\
\hline 0 & $\mathrm{~B}$ & $S$ & 3/1/1992 & 92061 & 60 & 76.5 & 76.5 & 75.6 & 77 & 76.5 \\
\hline 0 & B & $S$ & 3/7/1992 & 92067 & 66 & 108.5 & 92 & 91.2 & 90.3 & 90.3 \\
\hline $0 \mathrm{I}$ & B & $S$ & 3/8/1992 & 92068 & 67 & 124.9 & 102.4 & 99 & 100.7 & 99.8 \\
\hline
\end{tabular}


other

\begin{tabular}{|c|c|c|c|c|c|c|c|c|c|c|}
\hline $0 \mid \mathrm{l}$ & B & $S$ & $3 / 28 / 1992$ & 92088 & 87 & 80 & 78.2 & 79.1 & 79.1 & 78.2 \\
\hline 01 & B & S & 3/29/1992 & 92089 & 88 & 90.3 & 87.7 & 87.7 & 90.3 & 90.3 \\
\hline 01 & $\mathrm{~B}$ & $S$ & 4/4/1992 & 92095 & 94 & 88.6 & 86.9 & 88.6 & 86.5 & 86.9 \\
\hline 01 & $\mathrm{~B}$ & S & 4/5/1992 & 92096 & 95 & 81.3 & 77.4 & 78.2 & 77.4 & 77.4 \\
\hline 01 & $\mathrm{~B}$ & S & $4 / 11 / 1992$ & 92102 & 101 & 124 & 106.7 & 96.8 & 98.1 & 97.6 \\
\hline 01 & B & $S$ & 4/12/1992 & 92103 & 102 & 109.3 & 106.7 & 99.8 & 101.6 & 99 \\
\hline 01 & B & $S$ & $4 / 25 / 1992$ & 92116 & 115 & 122.3 & 115 & 111.1 & 109.3 & 111.9 \\
\hline 01 & B & $S$ & 4/26/1992 & 92117 & 116 & 124 & 129.2 & 123.2 & 124.9 & 122.3 \\
\hline 01 & B & $S$ & 5/2/1992 & 92123 & 122 & 117.6 & 116.2 & 112.8 & 113.6 & 109.3 \\
\hline 01 & B & S & $5 / 3 / 1992$ & 92124 & 123 & 119.7 & 125.7 & 129.2 & 124.9 & 120.6 \\
\hline 01 & B & $S$ & $5 / 9 / 1992$ & 92130 & 129 & 92 & 88.2 & 91.2 & 90.3 & 86 \\
\hline 01 & B & S & $5 / 10 / 1992$ & 92131 & 130 & 86.9 & 91.2 & 91.2 & 87.3 & 89.5 \\
\hline 01 & B & S & $5 / 16 / 1992$ & 92137 & 136 & 119.3 & 110.7 & 105.9 & 97.2 & 102.4 \\
\hline 01 & B & $S$ & $5 / 17 / 1992$ & 92138 & 137 & 115.4 & 116.2 & 101.6 & 99.8 & 104.1 \\
\hline 01 & B & S & $5 / 23 / 1992$ & 92144 & 143 & 104.1 & 99.8 & 92.9 & 92 & 92.9 \\
\hline 01 & $\mathrm{~B}$ & S & $5 / 24 / 1992$ & 92145 & 144 & 181 & 174.6 & 169.8 & 168.1 & 159.4 \\
\hline 01 & $\mathrm{~B}$ & S & 9/12/1992 & 92256 & 255 & 147.8 & 141.3 & 138.3 & 133.1 & 120.6 \\
\hline 01 & $\mathrm{~B}$ & S & 9/13/1992 & 92257 & 256 & 139.2 & 121 & 112.8 & 112.4 & 107.2 \\
\hline 01 & $\mathrm{~B}$ & S & 9/19/1992 & 92263 & 262 & 96.8 & 106.7 & 109.8 & 98.6 & 99 \\
\hline 01 & B & $S$ & 9/20/1992 & 92264 & 263 & 159.9 & 165.1 & 140 & 127.1 & 127.5 \\
\hline 01 & B & $S$ & 9/26/1992 & 92270 & 269 & 96.8 & 105 & 100.3 & 98.1 & 99.4 \\
\hline 01 & B & $S$ & 9/27/1992 & 92271 & 270 & 108.5 & 102.9 & 98.1 & 98.6 & 97.7 \\
\hline 01 & B & $S$ & 10/3/1992 & 92277 & 276 & 67.5 & 71.3 & 78.2 & 68.7 & 67 \\
\hline 01 & B & $S$ & 10/4/1992 & 92278 & 277 & 83.9 & 83.4 & 81.7 & 81.3 & 78.2 \\
\hline $0 \mathrm{H}$ & B & $S$ & 10/10/1992 & 92284 & 283 & 70.4 & 73 & 71.3 & 70.9 & 70.4 \\
\hline $0 \mathrm{H}$ & B & $S$ & 10/11/1992 & 92285 & 284 & 106.7 & 91.2 & 80 & 75.6 & 73.9 \\
\hline \begin{tabular}{l|l}
0 & $\mathrm{H}$ \\
\end{tabular} & B & $S$ & 10/17/1992 & 92291 & 290 & 88.2 & 84.7 & 103.3 & 93.4 & 92.9 \\
\hline $0 \mathrm{~F}$ & B & $S$ & 10/18/1992 & 92292 & 291 & 122.3 & 110.2 & 101.6 & 98.1 & 88.6 \\
\hline $\begin{array}{ll}\mathrm{P} \\
\mathrm{F}\end{array}$ & B & S & 10/24/1992 & 92298 & 297 & 111.5 & 106.7 & 110.2 & 102.9 & 100.7 \\
\hline $0 \mathrm{~F}$ & B & S & 10/25/1992 & 92299 & 298 & 77.4 & 80.4 & 76.5 & 71.8 & 73.9 \\
\hline $0 \mathrm{H}$ & B & S & 10/31/1992 & 92305 & 304 & 98.6 & 96.4 & 101.2 & 93.8 & 89.5 \\
\hline $0 \mathrm{~F}$ & B & S & 11/1/1992 & 92306 & 305 & 109.8 & 92 & 87.3 & 86 & 87.3 \\
\hline $0 \mathrm{H}$ & $\mathrm{B}$ & S & 11/7/1992 & 92312 & 311 & 96.4 & 99 & 100.7 & 100.3 & 99 \\
\hline $0 \mathrm{~F}$ & B & $S$ & $11 / 8 / 1992$ & 92313 & 312 & 116.2 & 110.2 & 109.8 & 109.3 & 105 \\
\hline $0 \mathrm{~F}$ & B & $S$ & 11/14/1992 & 92319 & 318 & 82.5 & 83.4 & 82.5 & 80.8 & 80.4 \\
\hline $0 \mathrm{~F}$ & B & $S$ & 11/15/1992 & 92320 & 319 & 83 & 83.4 & 83.4 & 85.1 & 79.6 \\
\hline $0 \mathrm{~F}$ & B & $S$ & 11/21/1992 & 92326 & 325 & 104.1 & 92.9 & 90.3 & 91.2 & 89.9 \\
\hline $0 \mathrm{~F}$ & B & $S$ & 11/22/1992 & 92327 & 326 & 79.1 & 71.3 & 66.1 & 66.1 & 70.9 \\
\hline $0 \mathrm{H}$ & B & $S$ & 11/28/1992 & 92333 & 332 & 92.9 & 93.4 & 95.5 & 95.5 & 94.2 \\
\hline \begin{tabular}{l|l}
0 & $\mathrm{~F}$ \\
\end{tabular} & B & $S$ & 11/29/1992 & 92334 & 333 & 103.3 & 97.2 & 97.2 & 97.7 & 97.2 \\
\hline \begin{tabular}{l|l}
0 & $\mathrm{H}$ \\
\end{tabular} & B & $S$ & $12 / 5 / 1992$ & 92340 & 339 & 118.8 & 111.1 & 113.6 & 93.8 & 92.5 \\
\hline $0 \mathrm{~F}$ & B & S & $12 / 6 / 1992$ & 92341 & 340 & 109.8 & 96.4 & 86 & 84.3 & 81.3 \\
\hline $0 \mathrm{~F}$ & B & S & $12 / 12 / 1992$ & 92347 & 346 & 111.1 & 87.7 & 89.5 & 86.5 & 86.9 \\
\hline $0 \mathrm{H}$ & B & $S$ & $12 / 13 / 1992$ & 92348 & 347 & 80 & 78.7 & 80.8 & 69.6 & 70.4 \\
\hline $0 \mathrm{H}$ & B & $S$ & 1/9/1993 & 93009 & 9 & 65.7 & 65.7 & 66.6 & 63.5 & 61.8 \\
\hline $0 \mathrm{~F}$ & B & S & 1/10/1993 & 93010 & 10 & 62.7 & 64.9 & 63.5 & 64.4 & 62.3 \\
\hline $0 \mathrm{~F}$ & B & $S$ & $1 / 16 / 1993$ & 93016 & 16 & 89.5 & 77.4 & 64.4 & 65.3 & 63.5 \\
\hline $0 \mathrm{H}$ & B & $S$ & $1 / 17 / 1993$ & 93017 & 17 & 59.7 & 63.5 & 61.8 & 60.9 & 60.1 \\
\hline $0 \mathrm{~F}$ & B & $S$ & 1/23/1993 & 93023 & 23 & 67.9 & 65.3 & 69.6 & 65.3 & 64 \\
\hline
\end{tabular}


other

\begin{tabular}{|c|c|c|c|c|c|c|c|c|c|c|}
\hline $0 \mathrm{I}$ & B & $S$ & $1 / 24 / 1993$ & 93024 & 24 & 66.1 & 67 & 67 & 66.6 & 64.4 \\
\hline $0 \mathrm{I}$ & $\mathrm{B}$ & $S$ & 1/30/1993 & 93030 & 30 & 111.5 & 110.2 & 91.2 & 89.1 & 86.9 \\
\hline $0 \mathrm{I}$ & $\mathrm{B}$ & $S$ & $1 / 31 / 1993$ & 93031 & 31 & 86.9 & 87.3 & 88.6 & 86.9 & 87.3 \\
\hline $0 \mathrm{I}$ & $\mathrm{B}$ & $S$ & $2 / 6 / 1993$ & 93037 & 37 & 86 & 86.9 & 88.6 & 85.6 & 88.6 \\
\hline $0 \mathrm{l}$ & B & S & 2/7/1993 & 93038 & 38 & 93.8 & 94.2 & 94.6 & 95.5 & 90.8 \\
\hline 01 & B & $S$ & 2/13/1993 & 93044 & 44 & 77 & 75.6 & 76.5 & 73.9 & 73 \\
\hline 01 & B & $S$ & 2/14/1993 & 93045 & 45 & 86.9 & 91.2 & 89.5 & 78.2 & 72.6 \\
\hline 01 & B & $S$ & $2 / 20 / 1993$ & 93051 & 51 & 185.4 & 185.4 & 112.8 & 86 & 86.9 \\
\hline 01 & $\mathrm{~B}$ & $S$ & 2/21/1993 & 93052 & 52 & 84.3 & 84.7 & 84.3 & 84.3 & 83.4 \\
\hline 01 & B & $S$ & $2 / 27 / 1993$ & 93058 & 58 & 70.4 & 70.9 & 66.1 & 67.9 & 66.1 \\
\hline 01 & $\mathrm{~B}$ & $S$ & $2 / 28 / 1993$ & 93059 & 59 & 67 & 68.7 & 67 & 66.1 & 66.6 \\
\hline 01 & B & $\mathrm{S}$ & $3 / 6 / 1993$ & 93065 & 65 & 69.2 & 73 & 76.5 & 72.2 & 72.2 \\
\hline 01 & B & $S$ & 3/7/1993 & 93066 & 66 & 71.3 & 73 & 73.5 & 72.2 & 72.2 \\
\hline 01 & B & $\mathrm{S}$ & $3 / 27 / 1993$ & 93086 & 86 & 68.7 & 65.3 & 70.9 & 68.7 & 64.4 \\
\hline 01 & B & S & 3/28/1993 & 93087 & 87 & 71.3 & 66.1 & 64.4 & 66.1 & 66.6 \\
\hline 01 & B & $S$ & $4 / 3 / 1993$ & 93093 & 93 & 134.4 & 133.5 & 132.7 & 130.1 & 130.1 \\
\hline 01 & B & $\mathrm{S}$ & 4/4/1993 & 93094 & 94 & 76.5 & & 39.3 & 76.5 & 75.6 \\
\hline $0 \mathrm{l}$ & B & S & 4/17/1993 & 93107 & 107 & 76.5 & 75.2 & 75.2 & 75.2 & 73.9 \\
\hline 01 & B & $S$ & $4 / 18 / 1993$ & 93108 & 108 & 71.3 & 70.4 & 70.9 & 70.4 & 68.7 \\
\hline 01 & $\mathrm{~B}$ & $S$ & 4/24/1993 & 93114 & 114 & 88.6 & 87.7 & 87.7 & 87.7 & 86.9 \\
\hline 01 & $\mathrm{~B}$ & $S$ & 4/25/1993 & 93115 & 115 & 88.6 & 85.1 & 86.9 & 86 & 86 \\
\hline 01 & $\mathrm{~B}$ & $S$ & $5 / 1 / 1993$ & 93121 & 121 & 86.9 & 86.9 & 87.7 & 86.5 & 86.9 \\
\hline 01 & $\mathrm{~B}$ & $S$ & $5 / 2 / 1993$ & 93122 & 122 & 89.5 & 84.3 & 78.2 & 75.6 & 73.9 \\
\hline 01 & $\mathrm{~B}$ & $S$ & 5/8/1993 & 93128 & 128 & 76.1 & 75.2 & 76.5 & 75.2 & 74.8 \\
\hline $0 \mathrm{H}$ & $\mathrm{B}$ & $S$ & 5/9/1993 & 93129 & 129 & 77.4 & 75.6 & 75.6 & 76.5 & 76.5 \\
\hline $0 \mathrm{H}$ & B & $S$ & $5 / 15 / 1993$ & 93135 & 135 & 97.2 & 89.9 & 82.5 & 80 & 70.9 \\
\hline $0 \mathrm{E}$ & B & $S$ & $5 / 16 / 1993$ & 93136 & 136 & 70.4 & 71.8 & 70.4 & 71.8 & 70.4 \\
\hline $0 \mathrm{~F}$ & $\mathrm{~B}$ & $S$ & $5 / 22 / 1993$ & 93142 & 142 & 112.8 & 98.6 & 90.3 & 91.2 & 86.9 \\
\hline \begin{tabular}{l|l}
0 & $\mathrm{~F}$ \\
\end{tabular} & B & $S$ & $5 / 23 / 1993$ & 93143 & 143 & 85.6 & 86.9 & 86.9 & 84.3 & 80 \\
\hline $0 \mathrm{H}$ & $\mathrm{B}$ & $S$ & 9/11/1993 & 93254 & 254 & 102 & 110.7 & 87.3 & 84.3 & 83.9 \\
\hline $0 \mathrm{~F}$ & B & $S$ & 9/12/1993 & 93255 & 255 & 70 & 70.9 & 69.6 & 72.6 & 70.4 \\
\hline $0 \mathrm{~F}$ & B & $\mathrm{S}$ & 9/18/1993 & 93261 & 261 & 130.5 & 105 & 94.2 & 89.1 & 91.6 \\
\hline $0 \mathrm{~F}$ & $\mathrm{~B}$ & $S$ & 9/19/1993 & 93262 & 262 & 93.4 & 89.5 & 93.4 & 86.5 & 88.6 \\
\hline $0 \mathrm{~F}$ & $\mathrm{~B}$ & $S$ & 9/25/1993 & 93268 & 268 & 123.6 & 121 & 98.1 & 97.7 & 96 \\
\hline $0 \mathrm{~F}$ & B & S & 9/26/1993 & 93269 & 269 & 90.8 & 89.1 & 86.5 & 86 & 84.7 \\
\hline $0 \mathrm{~F}$ & $\mathrm{~B}$ & $S$ & 10/2/1993 & 93275 & 275 & 88.2 & 92 & 89.9 & 80.4 & 86 \\
\hline $0 \mathrm{~F}$ & $\mathrm{~B}$ & $S$ & 10/3/1993 & 93276 & 276 & 130.1 & 105.5 & 87.3 & 83.9 & 80.8 \\
\hline $0 \mathrm{~F}$ & B & $S$ & 10/9/1993 & 93282 & 282 & 78.2 & 87.3 & 82.5 & 79.6 & 79.6 \\
\hline $0 \mathrm{~F}$ & B & $S$ & 10/10/1993 & 93283 & 283 & 80.4 & 80.4 & 81.3 & 76.5 & 74.4 \\
\hline $0 \mathrm{~F}$ & $\mathrm{~B}$ & $S$ & 10/16/1993 & 93289 & 289 & 73 & 77 & 73.9 & 73.5 & 73 \\
\hline $0 \mathrm{~F}$ & $\mathrm{~B}$ & $S$ & 10/17/1993 & 93290 & 290 & 77.4 & 77.4 & 77 & 75.6 & 76.1 \\
\hline $0 \mathrm{~F}$ & $\mathrm{~B}$ & $S$ & 10/23/1993 & 93296 & 296 & 69.6 & 68.3 & 67.9 & 69.6 & 67.9 \\
\hline $0 \mathrm{~F}$ & $\mathrm{~B}$ & $S$ & 10/24/1993 & 93297 & 297 & 73 & 71.8 & 70.4 & 70.4 & 70.4 \\
\hline $0 \mathrm{~F}$ & $\mathrm{~B}$ & $S$ & 10/30/1993 & 93303 & 303 & 64.5 & 60.6 & 58.4 & 60.1 & 58.4 \\
\hline $0 \mathrm{~F}$ & $\mathrm{~B}$ & $\mathrm{~S}$ & 10/31/1993 & 93304 & 304 & 69.6 & 66.2 & 64.9 & 64.9 & 62.7 \\
\hline $0 \mathrm{~F}$ & B & S & 11/6/1993 & 93310 & 310 & 71.4 & 67.1 & 68.3 & 67.1 & 65.3 \\
\hline $0 \mathrm{~F}$ & $\mathrm{~B}$ & $S$ & $11 / 7 / 1993$ & 93311 & 311 & 69.2 & 69.6 & 70.9 & 66.2 & 66.6 \\
\hline $0 \mathrm{E}$ & B & $S$ & 11/13/1993 & 93317 & 317 & 66.2 & 66.6 & 67.9 & 67.9 & 66.6 \\
\hline $0 \mathrm{~F}$ & B & $S$ & 11/14/1993 & 93318 & 318 & 83.9 & 79.2 & 80 & 79.6 & 79.2 \\
\hline
\end{tabular}


other

\begin{tabular}{|c|c|c|c|c|c|c|c|c|c|c|}
\hline $0 \mathrm{I}$ & B & $\mathrm{S}$ & 11/20/1993 & 93324 & 324 & 68.8 & 62.3 & 61 & 61 & 61.4 \\
\hline 01 & B & $S$ & 11/21/1993 & 93325 & 325 & 62.7 & 65.7 & 69.6 & 65.7 & 64.9 \\
\hline $0 \mathrm{I}$ & $\mathrm{B}$ & $S$ & 12/4/1993 & 93338 & 338 & 88.7 & 84.7 & 67.1 & 64.9 & 62.7 \\
\hline $0 \mathrm{I}$ & $\mathrm{B}$ & $\mathrm{S}$ & 12/5/1993 & 93339 & 339 & 63.6 & 62.3 & 61.4 & 63.1 & 65.7 \\
\hline 01 & B & $\mathrm{S}$ & 12/11/1993 & 93345 & 345 & 85.6 & 80.9 & 67.5 & $\begin{array}{l}65.3 \\
\end{array}$ & 66.6 \\
\hline 01 & $\mathrm{~B}$ & $S$ & 12/12/1993 & 93346 & 346 & 93.4 & 70.9 & 66.2 & 65.7 & 66.6 \\
\hline $0 \mathrm{I}$ & B & $S$ & $1 / 8 / 1994$ & 94008 & 8 & 116.7 & 108.5 & 108.9 & 108.5 & 108.1 \\
\hline 01 & B & $S$ & 1/9/1994 & 94009 & 9 & 105.1 & 103.7 & 102.5 & 103.7 & 104.6 \\
\hline $0 \mathrm{I}$ & B & $S$ & $1 / 15 / 1994$ & 94015 & 15 & 99.9 & 88.2 & 79.6 & 80.9 & 81.3 \\
\hline 01 & B & $S$ & $1 / 16 / 1994$ & 94016 & 16 & 78.7 & 79.2 & 80.4 & 80 & 79.6 \\
\hline 01 & $\mathrm{~B}$ & $S$ & $1 / 22 / 1994$ & 94022 & 22 & 90.8 & 80 & 71.8 & 69.6 & 72.6 \\
\hline 01 & B & $S$ & $1 / 23 / 1994$ & 94023 & 23 & 79.6 & 72.2 & 69.2 & 70 & 65.7 \\
\hline 01 & $\mathrm{~B}$ & $S$ & 1/29/1994 & 94029 & 29 & 135.7 & 147.8 & 147.4 & 143.5 & 127.1 \\
\hline 01 & B & $S$ & $1 / 30 / 1994$ & 94030 & 30 & 119.3 & 110.7 & 105.5 & 106.3 & 105.5 \\
\hline $0 \mathrm{l}$ & B & S & 2/5/1994 & 94036 & 36 & 81.7 & 69.6 & 69.6 & 67.9 & 67.1 \\
\hline 01 & B & $S$ & 2/6/1994 & 94037 & 37 & 64.5 & 63.6 & 64.5 & 62.7 & 61 \\
\hline 01 & $\mathrm{~B}$ & $\mathrm{~S}$ & $2 / 12 / 1994$ & 94043 & 43 & 102.5 & 109.8 & 109.4 & 108.5 & 104.6 \\
\hline 01 & B & $S$ & $2 / 13 / 1994$ & 94044 & 44 & 121.5 & 108.5 & 108.5 & 104.2 & 102.1 \\
\hline 01 & $\mathrm{~B}$ & $S$ & $2 / 19 / 1994$ & 94050 & 50 & 65.3 & 62.7 & 61.9 & 62.7 & 61.9 \\
\hline $0 \mathrm{I}$ & B & $S$ & $2 / 20 / 1994$ & 94051 & 51 & 63.6 & 64.5 & 63.6 & 61 & 63.6 \\
\hline $0 \mathrm{l}$ & B & $S$ & $2 / 26 / 1994$ & 94057 & 57 & 107.7 & 97.3 & 83.1 & 66.2 & 67.1 \\
\hline $0 \mathrm{l}$ & B & $S$ & $2 / 27 / 1994$ & 94058 & 58 & 74.8 & 74.8 & 74 & 74.8 & 72.2 \\
\hline $0 \mathrm{I}$ & $\mathrm{B}$ & $S$ & $3 / 5 / 1994$ & 94064 & 64 & 65.3 & 65.3 & 64.5 & 63.6 & 64.5 \\
\hline 01 & $\mathrm{~B}$ & $S$ & $3 / 6 / 1994$ & 94065 & 65 & 63.1 & 63.6 & 62.7 & 63.6 & 65.3 \\
\hline $0 \mathrm{~F}$ & B & $S$ & $3 / 26 / 1994$ & 94085 & 85 & 67.5 & 70.1 & 70.1 & 71.8 & 67.5 \\
\hline $0 \mathrm{~F}$ & $\mathrm{~B}$ & $\mathrm{~S}$ & $3 / 27 / 1994$ & 94086 & 86 & 64.9 & 64.9 & 67.5 & 67.5 & 62.8 \\
\hline $0 \mathrm{~F}$ & B & $S$ & $4 / 9 / 1994$ & 94099 & 99 & 65.7 & 66.5 & 67.4 & 70 & 68.3 \\
\hline $0 \mathrm{~F}$ & B & S & 4/10/1994 & 94100 & 100 & 70 & 68.3 & 70.8 & 73.4 & 72.6 \\
\hline $0 \mathrm{~F}$ & B & $S$ & $4 / 16 / 1994$ & 94106 & 106 & 66.1 & 67.9 & 66.6 & 69.6 & 67.9 \\
\hline $0 \mathrm{H}$ & B & $S$ & $4 / 17 / 1994$ & 94107 & 107 & 62.7 & 63.5 & 63.5 & 66.6 & 67 \\
\hline $0 \mathrm{H}$ & B & $S$ & $4 / 23 / 1994$ & 94113 & 113 & 75.6 & 65.3 & 66.1 & 68.3 & 68.7 \\
\hline $0 \mathrm{H}$ & $\mathrm{B}$ & $\mathrm{S}$ & $4 / 24 / 1994$ & 94114 & 114 & 61.8 & 66.1 & 64.4 & 67 & 65.7 \\
\hline $0 \mathrm{~F}$ & B & $S$ & 4/30/1994 & 94120 & 120 & 105 & 96.8 & 87.7 & 87.3 & 86.9 \\
\hline $0 \mathrm{H}$ & $\mathrm{B}$ & $S$ & $5 / 1 / 1994$ & 94121 & 121 & 74.8 & 74.8 & 75.6 & 74.8 & 74.4 \\
\hline $0 \mathrm{H}$ & $\mathrm{B}$ & $S$ & 5/7/1994 & 94127 & 127 & 74.4 & 73 & 73.9 & 72.2 & 72.2 \\
\hline $0 \mathrm{H}$ & $\mathrm{B}$ & $S$ & $5 / 8 / 1994$ & 94128 & 128 & 69.6 & 67.5 & 68.7 & 67.9 & 66.6 \\
\hline $\begin{array}{lll}0 & \mathrm{~F}\end{array}$ & $\mathrm{~B}$ & $S$ & $5 / 14 / 1994$ & 94134 & 134 & 76.1 & 77.4 & 79.1 & 77.4 & 78.2 \\
\hline $0 \mathrm{~F}$ & B & $S$ & $5 / 15 / 1994$ & 94135 & 135 & 76.5 & 75.6 & 76.5 & 75.6 & 74.8 \\
\hline $0 \mathrm{~F}$ & $\mathrm{~B}$ & $\mathrm{~S}$ & $5 / 21 / 1994$ & 94141 & 141 & 76.5 & 75.2 & 77.4 & 77 & 72.2 \\
\hline $0 \mathrm{~F}$ & B & $S$ & $5 / 22 / 1994$ & 94142 & 142 & 64 & 61.8 & 62.7 & 63.1 & 60.5 \\
\hline $1 A$ & A & NS & $6 / 6 / 1991$ & 91157 & 157 & 165.9 & 159 & 154.7 & 153.8 & 150.3 \\
\hline $1 A$ & A & NS & 6/7/1991 & 91158 & 158 & 176.3 & 177.1 & 171.1 & 171.1 & 170.2 \\
\hline $1 \mid f$ & A & NS & 6/10/1991 & 91161 & 161 & 139.1 & 139.1 & 136.5 & 139.1 & 138.2 \\
\hline $1 A$ & A & NS & $6 / 11 / 1991$ & 91162 & 162 & 133.9 & 133.1 & 133.1 & 131.3 & 129.6 \\
\hline $1 F$ & A & NS & $6 / 12 / 1991$ & 91163 & 163 & 142.6 & 143.4 & 140.8 & 144.3 & 139.1 \\
\hline 1$]$ & A & NS & $6 / 13 / 1991$ & 91164 & 164 & 164.2 & 154.7 & 158.1 & 157.2 & 152.9 \\
\hline $1 \mid f$ & A & NS & $6 / 14 / 1991$ & 91165 & 165 & 157.2 & 151.2 & 151.2 & 150.3 & 149.5 \\
\hline $1 \mid f$ & A & NS & $6 / 17 / 1991$ & 91168 & 168 & 152.1 & 151.2 & 149.5 & 148.6 & 146 \\
\hline $1 A$ & A & NS & $6 / 18 / 1991$ & 91169 & 169 & 161.6 & 158.1 & 153.8 & 153.8 & 146.9 \\
\hline
\end{tabular}


other

\begin{tabular}{|c|c|c|c|c|c|c|c|c|c|c|}
\hline 1 & A & NS & 6/19/1991 & \begin{tabular}{|l|l}
91170 \\
\end{tabular} & 170 & 212.5 & 206.5 & 197 & 191.8 & 186.6 \\
\hline 1 & A & NS & 6/20/1991 & 91171 & 171 & 146.9 & 142.6 & 143.4 & 140 & 142.6 \\
\hline 1 & A & NS & 6/21/1991 & 91172 & 172 & 158.1 & 152.9 & 145.2 & 145.2 & 141.7 \\
\hline 1 & A & NS & 6/24/1991 & 91175 & 175 & 139.1 & 139.1 & 136.5 & 135.6 & 135.6 \\
\hline 1 & A & NS & $6 / 25 / 1991$ & 91176 & 176 & 157.2 & 153.8 & 156.4 & 152.9 & 152.1 \\
\hline 1 & A & NS & 6/26/1991 & 91177 & 177 & 159 & 158.1 & 158.1 & 157.2 & 153.8 \\
\hline 1 & A & NS & 6/27/1991 & 91178 & 178 & 165.9 & 160.7 & 159 & 159.8 & 157.2 \\
\hline 1 & A & NS & 6/28/1991 & 91179 & 179 & 297.2 & 297.2 & 292 & 284.3 & 284.3 \\
\hline 1 & A & NS & 7/1/1991 & 91182 & 182 & 153.8 & 154.7 & 153.8 & 151.2 & 151.2 \\
\hline 1 & A & NS & 7/2/1991 & 91183 & 183 & 186.6 & 181.4 & 180.6 & 178 & 178 \\
\hline 1 & A & NS & 7/3/1991 & 91184 & 184 & 137.4 & 136.5 & 136.5 & 133.9 & 136.5 \\
\hline 1 & A & NS & 7/4/1991 & 91185 & 185 & 141.7 & 139.1 & 141.7 & 139.1 & 134.8 \\
\hline 1 & A & NS & 7/5/1991 & 91186 & 186 & 132.2 & 132.2 & 131.3 & 127.9 & 127 \\
\hline 1 & A & NS & 7/8/1991 & 91189 & 189 & 121 & 120.1 & 119.2 & 120.1 & 114.9 \\
\hline 1 & A & NS & 7/9/1991 & 91190 & 190 & 121.8 & 116.6 & 116.6 & 116.6 & 116.6 \\
\hline 1 & A & NS & $7 / 10 / 1991$ & 91191 & 191 & 124.4 & 122.7 & 123.6 & 122.7 & 120.1 \\
\hline 1 & A & NS & $7 / 11 / 1991$ & 91192 & 192 & 129.6 & 128.7 & 124.4 & 124.4 & 123.6 \\
\hline 1 & A & NS & $7 / 12 / 1991$ & 91193 & 193 & 121.8 & 122.7 & 121 & 120.1 & 119.2 \\
\hline 1 & A & NS & $7 / 15 / 1991$ & 91196 & 196 & 132.2 & 127 & 126.1 & 122.7 & 121 \\
\hline 1 & A & NS & 7/16/1991 & 91197 & 197 & 122.7 & 120.1 & 119.2 & 114 & 114.9 \\
\hline 1 & A & NS & 7/17/1991 & 91198 & 198 & 115.8 & 114.9 & 113.2 & 114 & 108.9 \\
\hline 1 & A & NS & 7/18/1991 & 91199 & 199 & 128.7 & 126.1 & 123.6 & 120.1 & 121 \\
\hline 1 & A & NS & $7 / 19 / 1991$ & 91200 & 200 & 114 & 110.6 & 108 & 106.3 & 107.1 \\
\hline 1 & A & NS & 7/22/1991 & 91203 & 203 & 119.2 & 116.6 & 114.9 & 114.9 & 114.9 \\
\hline 1 & A & NS & $7 / 23 / 1991$ & 91204 & 204 & 132.2 & 133.9 & 131.3 & 131.3 & 129.6 \\
\hline 1 & A & NS & 7/24/1991 & 91205 & 205 & 126.1 & 122.7 & 121.8 & 120.1 & 122.7 \\
\hline 1 & A & NS & $7 / 25 / 1991$ & 91206 & 206 & 148.6 & 145.2 & 143.4 & 141.7 & 139.1 \\
\hline 1 & A & NS & 7/26/1991 & 91207 & 207 & 153.8 & 154.7 & 148.6 & 149.5 & 148.6 \\
\hline 1 & A & NS & 7/29/1991 & 91210 & 210 & 151.2 & 147.7 & 146 & 144.3 & 144.3 \\
\hline 1 & A & NS & 7/30/1991 & 91211 & 211 & 147.7 & 147.7 & 146 & 146 & 143.4 \\
\hline 1 & A & NS & $7 / 31 / 1991$ & 91212 & 212 & 161.6 & 155.5 & 155.5 & 152.9 & 150.3 \\
\hline 1 & A & NS & 8/1/1991 & 91213 & 213 & 172.8 & 170.2 & 165.9 & 165 & 163.3 \\
\hline 1 & A & NS & 8/2/1991 & 91214 & 214 & 165.9 & 162.4 & 162.4 & 162.4 & 159.8 \\
\hline 1 & A & NS & 8/5/1991 & 91217 & 217 & 145.2 & 145.2 & 142.6 & 143.4 & 143.4 \\
\hline 1 & A & NS & 8/6/1991 & 91218 & 218 & 171.9 & 168.5 & 165 & 168.5 & 165.9 \\
\hline 1 & A & NS & 8/7/1991 & 91219 & 219 & 153.8 & 150.3 & 150.3 & 146.9 & 146 \\
\hline 1 & A & NS & 8/8/1991 & 91220 & 220 & 170.2 & 161.6 & 163.3 & 159.8 & 159.8 \\
\hline 1 & A & NS & 8/9/1991 & 91221 & 221 & 156.4 & 153.8 & 152.1 & 153.8 & 152.9 \\
\hline 1 & A & NS & 8/12/1991 & 91224 & 224 & 171.1 & 168.5 & 165 & 165.9 & 165.9 \\
\hline 1 & A & NS & $8 / 13 / 1991$ & 91225 & 225 & 198.7 & 194.4 & 195.3 & 191.8 & 187.5 \\
\hline 1 & A & NS & 8/14/1991 & 91226 & 226 & 248 & 244.5 & 246.2 & 240.2 & 241.1 \\
\hline 1 & A & NS & $8 / 15 / 1991$ & 91227 & 227 & 208.2 & 205.6 & 205.6 & 205.6 & 202.2 \\
\hline 1 & A & NS & 8/16/1991 & 91228 & 228 & 182.3 & 184 & 178.8 & 175.4 & 173.7 \\
\hline 1 & A & NS & $8 / 19 / 1991$ & 91231 & 231 & 219.5 & 213.4 & 214.3 & 204.8 & 203.9 \\
\hline 1 & A & NS & 8/20/1991 & 91232 & 232 & 284.3 & 278.2 & 269.6 & 264.4 & 257.5 \\
\hline 1 & A & NS & $8 / 21 / 1991$ & 91233 & 233 & 423.4 & 415.6 & 409.5 & 410.4 & 404.4 \\
\hline 1 & A & NS & $8 / 22 / 1991$ & 91234 & 234 & 420.8 & 413.9 & 412.1 & 404.4 & 398.3 \\
\hline 1 & A & NS & 8/23/1991 & 91235 & 235 & 400.9 & 398.3 & 385.3 & 384.5 & 372.4 \\
\hline 1 . & A & NS & $8 / 26 / 1991$ & 91238 & 238 & 229.8 & 226.4 & 218.6 & 214.3 & 212.5 \\
\hline
\end{tabular}


other

\begin{tabular}{|c|c|c|c|c|c|c|c|c|c|c|}
\hline $1 \mid$ & A & NS & 8/27/1991 & 91239 & 239 & 388.8 & 384.5 & 370.7 & 368.9 & 350.8 \\
\hline 11 & A & NS & 8/28/1991 & 91240 & 240 & 406.9 & 396.6 & 389.7 & 381 & 379.3 \\
\hline 11 & A & NS & 8/29/1991 & 91241 & 241 & 398.3 & 394.8 & 389.7 & 373.2 & 369.8 \\
\hline 11 & A & NS & 8/30/1991 & 91242 & 242 & 419 & 408.7 & 401.8 & 400 & 396.6 \\
\hline 11 & A & NS & 9/2/1991 & 91245 & 245 & 282.5 & 272.2 & 273 & 266.1 & 267.8 \\
\hline 11 & A & NS & 10/23/1991 & 91296 & 296 & 192.7 & 187.6 & 180.2 & 178 & 171.1 \\
\hline 11 & A & NS & 11/27/1991 & 91331 & 331 & 138.7 & 122.8 & 118 & 115.4 & 113.6 \\
\hline 11 & A & NS & 11/28/1991 & 91332 & 332 & 107.6 & 106.7 & 108.5 & 105.9 & 105.5 \\
\hline 11 & A & NS & 11/29/1991 & 91333 & 333 & 108.5 & 109.3 & 107.6 & 107.2 & 107.1 \\
\hline $1 / t$ & A & NS & 12/20/1991 & 91354 & 354 & 106.7 & 106.7 & 105.9 & 105.9 & 113.6 \\
\hline $1 t$ & A & NS & 12/23/1991 & 91357 & 357 & 92 & 91.2 & 91.2 & 92 & 92.5 \\
\hline 1 & A & NS & 12/24/1991 & 91358 & 358 & 88.6 & 93.8 & 100.7 & 85.6 & 86.9 \\
\hline 11 & A & NS & 12/25/1991 & 91359 & 359 & 87.7 & 89.1 & 89.5 & 88.6 & 89.5 \\
\hline $1 /$ & A & NS & 12/26/1991 & 91360 & 360 & 86 & 86.9 & 88.2 & 94.6 & 137 \\
\hline $1 \mid$ & A & NS & 12/27/1991 & 91361 & 361 & 84.3 & 97.2 & 118.8 & 81.3 & 84.3 \\
\hline 1 & A & NS & 12/30/1991 & 91364 & 364 & 82.5 & 111.9 & 125.3 & 83.4 & 79.1 \\
\hline 11 & A & NS & 12/31/1991 & 91365 & 365 & 87.3 & 120.6 & 92 & 85.1 & 89.5 \\
\hline $1 /$ & A & NS & 1/1/1992 & 92001 & 366 & & & & & \\
\hline 11 & A & NS & $1 / 2 / 1992$ & 92002 & 1 & 79.1 & 82.1 & 116.2 & 123.6 & 80.8 \\
\hline 11 & A & NS & $1 / 3 / 1992$ & 92003 & 2 & 118 & 98.6 & 78.2 & 78.7 & 80.8 \\
\hline 11 & A & NS & $1 / 6 / 1992$ & 92006 & 5 & 92 & 73 & 74.4 & 92.9 & 116.2 \\
\hline 11 & A & NS & 1/17/1992 & 92017 & 16 & 123.6 & 124 & 121.4 & 126.2 & 127.5 \\
\hline 11 & $\mathrm{~B}$ & NS & 3/13/1992 & 92073 & 72 & 97.7 & 97.2 & 97.2 & 97.2 & 97.2 \\
\hline 11 & $\mathrm{~B}$ & NS & 3/16/1992 & 92076 & 75 & 87.7 & 83.4 & 83 & 85.1 & 84.3 \\
\hline $1 \mathrm{H}$ & $\mathrm{B}$ & NS & 3/17/1992 & 92077 & 76 & 95.1 & 95.5 & 97.2 & 98.1 & 99 \\
\hline $1 \mathrm{H}$ & B & NS & 3/18/1992 & 92078 & 77 & 92 & 92 & 92 & 92 & 91.2 \\
\hline $1 \mathrm{H}$ & B & NS & 3/19/1992 & 92079 & 78 & 90.3 & 89.5 & 91.2 & 89.9 & 90.3 \\
\hline $1 \mathrm{H}$ & $\mathrm{B}$ & NS & 3/20/1992 & 92080 & 79 & 90.3 & 89.5 & 88.6 & 88.6 & 88.2 \\
\hline $1 \mathrm{H}$ & B & NS & $4 / 17 / 1992$ & 92108 & 107 & 111.1 & 111.9 & 108.5 & 108.5 & 108.5 \\
\hline $1 \mathrm{H}$ & $\mathrm{B}$ & NS & $4 / 20 / 1992$ & 92111 & 110 & 99.8 & 106.7 & 105 & 103.3 & 107.2 \\
\hline $1 \mathrm{H}$ & B & NS & $4 / 28 / 1992$ & 92119 & 118 & 96.8 & 97.2 & 97.2 & 95.5 & 94.6 \\
\hline $1 \mathrm{H}$ & B & NS & 5/28/1992 & 92149 & 148 & 130.9 & 127.1 & 129.2 & 126.2 & 121.4 \\
\hline $1 \mathrm{~b}$ & B & NS & $5 / 29 / 1992$ & 92150 & 149 & 127.5 & 125.3 & 123.2 & 110.7 & 105 \\
\hline $1 \mathrm{H}$ & $\mathrm{B}$ & NS & $6 / 1 / 1992$ & 92153 & 152 & 101.6 & 102.9 & 102.4 & 90.3 & 89.5 \\
\hline $1 \mathrm{~b}$ & B & NS & 6/2/1992 & 92154 & 153 & 93.8 & 92 & 89.5 & 88.6 & 88.6 \\
\hline $1 \mathrm{H}$ & B & NS & 6/3/1992 & 92155 & 154 & 113.6 & 105 & 102.9 & 108.5 & 108.5 \\
\hline $1 \mathrm{H}$ & $\mathrm{B}$ & NS & 6/4/1992 & 92156 & 155 & 103.3 & 107.6 & 105 & 113.6 & 110.2 \\
\hline $1 \mathrm{H}$ & $\mathrm{B}$ & NS & $6 / 5 / 1992$ & 92157 & 156 & 106.7 & 104.6 & 99.4 & 99 & 100.3 \\
\hline $1 \mathrm{H}$ & $\mathrm{B}$ & NS & 6/8/1992 & 92160 & 159 & 115 & 112.8 & 108.5 & 102.9 & 105.9 \\
\hline $1 \mathrm{H}$ & $\mathrm{B}$ & NS & 6/9/1992 & 92161 & 160 & 98.1 & 95.5 & 95.5 & 100.7 & 99.8 \\
\hline $1 \mathrm{H}$ & $\mathrm{B}$ & NS & 6/10/1992 & 92162 & 161 & 124.5 & 124 & 121 & 115.8 & 115.4 \\
\hline $1 \mathrm{H}$ & $\mathrm{B}$ & NS & $6 / 11 / 1992$ & 92163 & 162 & 107.6 & 111.5 & 106.7 & 102.9 & 100.7 \\
\hline $1 \mathrm{H}$ & $\mathrm{B}$ & NS & $6 / 12 / 1992$ & 92164 & 163 & 130.5 & 125.7 & 121.9 & 121 & 122.3 \\
\hline $1 \mathrm{H}$ & $\mathrm{B}$ & NS & 6/15/1992 & 92167 & 166 & 165.1 & 151.3 & 146.9 & 137.8 & 133.1 \\
\hline $1 \mathrm{H}$ & $\mathrm{B}$ & NS & 6/16/1992 & 92168 & 167 & 44.9 & 45.8 & 45.8 & 44.9 & 45.8 \\
\hline $1 \mathrm{~F}$ & B & NS & 6/17/1992 & 92169 & 168 & 143.5 & 140.9 & 134 & 131.4 & 136.1 \\
\hline $1 \mathrm{H}$ & $\mathrm{B}$ & NS & 6/18/1992 & 92170 & 169 & 146.5 & 150.4 & 141.8 & 134.8 & 130.9 \\
\hline $1 \mathrm{~b}$ & B & NS & $6 / 19 / 1992$ & 92171 & 170 & 166 & 141.8 & 130.1 & 128.8 & 126.2 \\
\hline $1 \mathrm{~F}$ & B & NS & $6 / 22 / 1992$ & 92174 & 173 & 143 & 133.6 & 133.1 & 123.2 & 120.2 \\
\hline
\end{tabular}


other

\begin{tabular}{|c|c|c|c|c|c|c|c|c|c|c|}
\hline $1 \mathrm{l}$ & B & NS & 6/23/1992 & 92175 & 174 & 135.7 & 134.8 & 130.5 & 126.2 & 119.7 \\
\hline $1 \mathrm{l}$ & B & NS & $6 / 24 / 1992$ & 92176 & 175 & 141.8 & 133.1 & 132.3 & 128.3 & 122.8 \\
\hline $1 \mathrm{I}$ & $\mathrm{B}$ & NS & $6 / 25 / 1992$ & 92177 & 176 & 122.8 & 114.1 & 108.9 & 108.1 & 103.3 \\
\hline $1 \mathrm{I}$ & $\mathrm{B}$ & NS & 6/26/1992 & 92178 & 177 & 124.9 & 109.8 & 102.9 & 103.7 & 96 \\
\hline $1 \mathrm{l}$ & B & NS & 6/29/1992 & 92181 & 180 & 115.8 & 112.4 & 105.5 & 97.7 & 94.6 \\
\hline $1 \mathrm{I}$ & $\mathrm{B}$ & NS & 6/30/1992 & 92182 & 181 & 106.3 & 110.7 & 91.6 & 84.7 & 81.7 \\
\hline 11 & $\mathrm{~B}$ & NS & 7/1/1992 & 92183 & 182 & 178.4 & 169.4 & 162.5 & 153.9 & 147.8 \\
\hline $1 \mathrm{l}$ & B & NS & $7 / 2 / 1992$ & 92184 & 183 & 139.6 & 132.7 & 134.4 & 128.8 & 126.2 \\
\hline $1 \mathrm{l}$ & B & NS & 7/3/1992 & 92185 & 184 & 150.4 & 146.1 & 137.4 & 126.6 & 127.9 \\
\hline $1 \mathrm{l}$ & B & NS & $7 / 6 / 1992$ & 92188 & 187 & 94.2 & 92 & 84.7 & 89.1 & 82.5 \\
\hline 11 & B & NS & 7/7/1992 & 92189 & 188 & 104.6 & 100.7 & 96.8 & 96 & 91.2 \\
\hline $1 \mathrm{l}$ & B & NS & 7/8/1992 & 92190 & 189 & 115 & 108.9 & 105.9 & 102 & 98.6 \\
\hline $1 \mathrm{l}$ & B & NS & 7/9/1992 & 92191 & 190 & 117.6 & 129.7 & 123.2 & 104.6 & 102.9 \\
\hline $1 \mathrm{l}$ & B & NS & $7 / 10 / 1992$ & 92192 & 191 & 112.4 & 110.7 & 106.7 & 102 & 100.3 \\
\hline $1 \mathrm{l}$ & B & NS & 7/13/1992 & 92195 & 194 & 107.2 & 105.5 & 102.9 & 93.4 & 96 \\
\hline $1 \mathrm{l}$ & B & NS & $7 / 14 / 1992$ & 92196 & 195 & 107.2 & 102.9 & 96.8 & 89.5 & 84.7 \\
\hline $1 \mathrm{I}$ & $\mathrm{B}$ & NS & $7 / 15 / 1992$ & 92197 & 196 & 113.2 & 112.4 & 104.6 & 106.3 & 94.2 \\
\hline $1 \mathrm{l}$ & B & NS & 7/16/1992 & 92198 & 197 & 105.9 & 115 & 105.5 & 109.8 & 104.6 \\
\hline $1 \mathrm{I}$ & $\mathrm{B}$ & NS & 7/17/1992 & 92199 & 198 & 127.9 & 115.8 & 108.1 & 112.8 & 104.6 \\
\hline $1 \mathrm{I}$ & $\mathrm{B}$ & NS & $7 / 20 / 1992$ & 92202 & 201 & 102.9 & 101.2 & 93.4 & 89.9 & 85.6 \\
\hline 11 & $\mathrm{~B}$ & NS & $7 / 21 / 1992$ & 92203 & 202 & 68.3 & 67.5 & 66.1 & 66.6 & 66.6 \\
\hline 11 & $\mathrm{~B}$ & NS & $7 / 22 / 1992$ & 92204 & 203 & 68.7 & 65.7 & 63.5 & 65.7 & 65.3 \\
\hline 11 & $\mathrm{~B}$ & NS & $7 / 23 / 1992$ & 92205 & 204 & 67.5 & 79.6 & 81.7 & 69.2 & 72.2 \\
\hline 11 & $\mathrm{~B}$ & NS & $7 / 24 / 1992$ & 92206 & 205 & 79.1 & 70.9 & 69.2 & 68.7 & 67.5 \\
\hline 11 & $\mathrm{~B}$ & NS & $7 / 27 / 1992$ & 92209 & 208 & 164.2 & 164.2 & 156.8 & 157.3 & 161.6 \\
\hline 11 & B & NS & $7 / 28 / 1992$ & 92210 & 209 & 173.7 & 175.1 & 174.1 & 172 & 172.9 \\
\hline 11 & B & NS & $7 / 29 / 1992$ & 92211 & 210 & 74 & 80 & 74 & 73 & 70 \\
\hline 11 & $\mathrm{~B}$ & NS & 7/30/1992 & 92212 & 211 & 90.8 & 95.5 & 92.5 & 81.3 & 70 \\
\hline $1 \mathrm{l}$ & B & NS & $7 / 31 / 1992$ & 92213 & 212 & 96 & 93.4 & 91.6 & 90.8 & 90.3 \\
\hline 11 & $\mathrm{~B}$ & NS & 8/3/1992 & 92216 & 215 & 89.1 & 86.5 & 82.5 & 83 & 81.3 \\
\hline $1 \mathrm{l}$ & B & NS & 8/4/1992 & 92217 & 216 & 103.7 & 97.2 & 92.5 & 89.1 & 87.3 \\
\hline $1 \mathrm{I}$ & $\mathrm{B}$ & NS & 8/5/1992 & 92218 & 217 & 88.2 & 80 & 82.1 & 78.7 & 77.4 \\
\hline $1 \mathrm{l}$ & B & NS & 8/6/1992 & 92219 & 218 & 98.6 & 98.6 & 89.1 & 91.6 & 86 \\
\hline $1 \mathrm{I}$ & $\mathrm{B}$ & NS & 8/7/1992 & 92220 & 219 & 101.2 & 101.6 & 91.6 & 90.8 & 85.6 \\
\hline $1 \mathrm{l}$ & B & NS & 8/10/1992 & 92223 & 222 & 120.2 & 121 & 118 & 113.2 & 113.2 \\
\hline 11 & $\mathrm{~B}$ & NS & 8/11/1992 & 92224 & 223 & 123.6 & 121.9 & 117.6 & 115 & 113.2 \\
\hline 11 & $\mathrm{~B}$ & NS & 8/12/1992 & 92225 & 224 & 134.8 & 127.1 & 125.3 & 123.6 & 122.8 \\
\hline 11 & B & NS & 8/13/1992 & 92226 & 225 & 125.3 & 122.8 & 125.3 & 121.4 & 120.2 \\
\hline 11 & B & NS & $8 / 14 / 1992$ & 92227 & 226 & 115.8 & 109.8 & 118.8 & 109.8 & 108.9 \\
\hline 11 & $\mathrm{~B}$ & NS & $8 / 17 / 1992$ & 92230 & 229 & 87.7 & 87.3 & 87.3 & 88.6 & 88.2 \\
\hline 11 & $\mathrm{~B}$ & NS & 8/18/1992 & 92231 & 230 & 91.6 & 86.5 & 86 & 87.3 & 84.3 \\
\hline 11 & B & NS & 8/19/1992 & 92232 & 231 & 111.5 & 101.6 & 102.9 & 99.8 & 95.1 \\
\hline 11 & $\mathrm{~B}$ & NS & $8 / 20 / 1992$ & 92233 & 232 & 114.5 & 113.2 & 108.9 & 100.3 & 104.1 \\
\hline 11 & $\mathrm{~B}$ & NS & $8 / 21 / 1992$ & 92234 & 233 & 95.5 & 95.1 & 84.7 & 83 & 90.8 \\
\hline 11 & $\mathrm{~B}$ & NS & $8 / 24 / 1992$ & 92237 & 236 & 105.5 & 97.7 & 97.2 & 97.7 & 98.6 \\
\hline $1 \mathrm{l}$ & B & NS & 8/25/1992 & 92238 & 237 & 100.3 & 110.7 & 111.1 & 110.7 & 110.2 \\
\hline $1 \mathrm{l}$ & B & NS & 8/26/1992 & 92239 & 238 & 122.8 & 111.1 & 104.6 & 108.9 & 101.6 \\
\hline $1 \mathrm{l}$ & B & NS & 8/27/1992 & 92240 & 239 & 127.9 & 120.2 & 114.1 & 103.7 & 111.9 \\
\hline $1 \mathrm{~F}$ & B & NS & 8/28/1992 & 92241 & 240 & 146.1 & 141.8 & 140 & 139.6 & 134 \\
\hline
\end{tabular}


other

\begin{tabular}{|c|c|c|c|c|c|c|c|c|c|c|}
\hline $1 \mathrm{l}$ & B & NS & 8/31/1992 & 92244 & 243 & 118.4 & 111.5 & 106.7 & 108.9 & 105.5 \\
\hline $1 \mathrm{l}$ & B & NS & 9/1/1992 & 92245 & 244 & 122.8 & 133.5 & 139.2 & 140 & 136.1 \\
\hline $1 \mathrm{I}$ & $\mathrm{B}$ & NS & 9/2/1992 & 92246 & 245 & 120.2 & 113.2 & 111.9 & 108.1 & 104.6 \\
\hline $1 \mathrm{I}$ & $\mathrm{B}$ & NS & 9/3/1992 & 92247 & 246 & 117.6 & 115 & 120.6 & 116.7 & 115.8 \\
\hline $1 \mathrm{l}$ & B & NS & 9/4/1992 & 92248 & 247 & 124.9 & 121 & 120.2 & 117.6 & 115 \\
\hline $1 \mathrm{I}$ & $\mathrm{B}$ & NS & 9/7/1992 & 92251 & 250 & 133.1 & 122.8 & 118.4 & 115 & 115.4 \\
\hline 11 & $\mathrm{~B}$ & NS & 9/15/1992 & 92259 & 258 & 101.2 & 113.6 & 115 & 113.6 & 102 \\
\hline $1 \mathrm{l}$ & B & NS & 10/12/1992 & 92286 & 285 & 87.7 & 83.9 & 83.4 & 85.1 & 83 \\
\hline $1 \mathrm{l}$ & B & NS & 11/25/1992 & 92330 & 329 & 93.8 & 117.1 & 121.9 & 118 & 119.7 \\
\hline $1 \mathrm{l}$ & B & NS & 11/26/1992 & 92331 & 330 & 141.3 & 105.9 & 99.8 & 99.4 & 99.8 \\
\hline 11 & B & NS & 11/27/1992 & 92332 & 331 & 104.6 & 101.6 & 107.6 & 107.2 & 106.7 \\
\hline $1 \mathrm{l}$ & B & NS & 12/18/1992 & 92353 & 352 & 91.6 & 90.3 & 92 & 92 & 90.3 \\
\hline 11 & B & NS & 12/21/1992 & 92356 & 355 & 97.2 & 80.8 & 81.7 & 79.6 & 80.8 \\
\hline $1 \mathrm{l}$ & B & NS & $12 / 22 / 1992$ & 92357 & 356 & 85.1 & 166.4 & 188.4 & 162.9 & 161.2 \\
\hline $1 \mathrm{l}$ & B & NS & 12/23/1992 & 92358 & 357 & 164.6 & 127.5 & 128.3 & 121 & 114.5 \\
\hline $1 \mathrm{l}$ & B & NS & 12/24/1992 & 92359 & 358 & 112.8 & 115 & 118.8 & 109.3 & 111.9 \\
\hline $1 \mathrm{I}$ & $\mathrm{B}$ & NS & 12/25/1992 & 92360 & 359 & 100.7 & 104.1 & 106.7 & 100.3 & 99 \\
\hline $1 \mathrm{l}$ & B & NS & 12/28/1992 & 92363 & 362 & 85.1 & 85.1 & 86.5 & 85.1 & 82.5 \\
\hline $1 \mathrm{I}$ & $\mathrm{B}$ & NS & 12/29/1992 & 92364 & 363 & 82.1 & 165.5 & 191.4 & 180.2 & 122.3 \\
\hline $1 \mathrm{I}$ & $\mathrm{B}$ & NS & 12/30/1992 & 92365 & 364 & 95.1 & 92.9 & 90.3 & 92.9 & 101.6 \\
\hline 11 & $\mathrm{~B}$ & NS & 12/31/1992 & 92366 & 365 & 85.6 & 86 & 86 & 85.1 & 84.3 \\
\hline 11 & $\mathrm{~B}$ & NS & 1/1/1993 & 93001 & 1 & 92 & 89.1 & 118.8 & 96.4 & 92.5 \\
\hline 11 & $\mathrm{~B}$ & NS & $1 / 4 / 1993$ & 93004 & 4 & 63.5 & 63.5 & 66.1 & 64.4 & 63.5 \\
\hline 11 & $\mathrm{~B}$ & NS & 2/15/1993 & 93046 & 46 & 79.6 & 77.4 & 75.6 & 74.8 & 76.5 \\
\hline $1 \mathrm{H}$ & $\mathrm{B}$ & NS & 3/15/1993 & 93074 & 74 & 97.2 & 92 & 91.2 & 89.5 & 90.8 \\
\hline $1 \mathrm{H}$ & B & NS & 3/16/1993 & 93075 & 75 & 92.9 & 99.4 & 98.1 & 96.4 & 96.4 \\
\hline $1 \mathrm{H}$ & B & NS & $3 / 17 / 1993$ & 93076 & 76 & 92 & 87.7 & 87.3 & 86.9 & 86.9 \\
\hline $1 \mathrm{H}$ & $\mathrm{B}$ & NS & $3 / 18 / 1993$ & 93077 & 77 & 74.4 & 75.6 & 77.4 & 73.9 & 74.4 \\
\hline $1 \mathrm{H}$ & B & NS & 3/19/1993 & 93078 & 78 & 68.7 & 67.9 & 67.9 & 66.6 & 67.9 \\
\hline $1 \mathrm{H}$ & $\mathrm{B}$ & NS & 4/9/1993 & 93099 & 99 & 92.9 & 110.2 & 103.3 & 101.2 & 100.7 \\
\hline $1 \mathrm{H}$ & B & NS & $4 / 12 / 1993$ & 93102 & 102 & 71.3 & 65.3 & 67 & 70.9 & 67 \\
\hline $1 \mathrm{H}$ & B & NS & 4/28/1993 & 93118 & 118 & 97.2 & 80.8 & 80.8 & 80.8 & 79.1 \\
\hline $1 \mathrm{~b}$ & B & NS & $5 / 27 / 1993$ & 93147 & 147 & 69.6 & 69.6 & 68.7 & 69.6 & 67.9 \\
\hline $1 \mathrm{H}$ & $\mathrm{B}$ & NS & $5 / 28 / 1993$ & 93148 & 148 & 71.3 & 69.6 & 67.9 & 64.4 & 63.1 \\
\hline $1 \mathrm{~b}$ & B & NS & 5/31/1993 & 93151 & 151 & 73.9 & 71.8 & 74.8 & 64.4 & 64.4 \\
\hline $1 \mathrm{H}$ & $\mathrm{B}$ & NS & $6 / 1 / 1993$ & 93152 & 152 & 78.2 & 79.6 & 75.6 & 76.1 & 75.6 \\
\hline $1 \mathrm{H}$ & B & NS & 6/2/1993 & 93153 & 153 & 92.9 & 76.1 & 72.2 & 70 & 67.9 \\
\hline $1 \mathrm{H}$ & B & NS & 6/3/1993 & 93154 & 154 & 93.8 & 77.8 & 73.5 & 72.2 & 70.9 \\
\hline $1 \mathrm{H}$ & B & NS & 6/4/1993 & 93155 & 155 & 98.1 & 78.7 & 77.8 & 77.4 & 68.3 \\
\hline $1 \mathrm{H}$ & $\mathrm{B}$ & NS & 6/7/1993 & 93158 & 158 & 102 & 91.6 & 93.4 & 92 & 86.5 \\
\hline $1 \mathrm{H}$ & $\mathrm{B}$ & NS & 6/8/1993 & 93159 & 159 & 111.9 & 98.6 & 102 & 93.8 & 93.4 \\
\hline $1 \mathrm{H}$ & B & NS & $6 / 9 / 1993$ & 93160 & 160 & 125.3 & 110.2 & 109.8 & 108.1 & 99 \\
\hline $1 \mathrm{H}$ & $\mathrm{B}$ & NS & 6/10/1993 & 93161 & 161 & 111.5 & 93.4 & 92 & 94.2 & 89.9 \\
\hline $1 \mathrm{H}$ & $\mathrm{B}$ & NS & $6 / 11 / 1993$ & 93162 & 162 & 90.3 & 71.3 & 70.4 & 70.4 & 70.4 \\
\hline $1 \mathrm{H}$ & $\mathrm{B}$ & NS & $6 / 14 / 1993$ & 93165 & 165 & 82.1 & 81.7 & 81.7 & 78.2 & 74.8 \\
\hline $1 \mathrm{H}$ & B & NS & 6/15/1993 & 93166 & 166 & 93.8 & 68.3 & 70.4 & 69.2 & 70.4 \\
\hline $1 \mathrm{H}$ & $\mathrm{B}$ & NS & $6 / 16 / 1993$ & 93167 & 167 & 87.3 & 71.3 & 71.8 & 72.2 & 70 \\
\hline $1 \mathrm{~b}$ & B & NS & $6 / 17 / 1993$ & 93168 & 168 & 93.8 & 74.4 & 72.6 & 73.9 & 73.5 \\
\hline $1 \mathrm{~F}$ & B & NS & 6/18/1993 & 93169 & 169 & 89.9 & 70 & 73 & 70.9 & 70.4 \\
\hline
\end{tabular}


other

\begin{tabular}{|c|c|c|c|c|c|c|c|c|c|c|}
\hline $1 \mathrm{l}$ & B & NS & 6/21/1993 & 93172 & 172 & 79.1 & 73 & 72.2 & 74.8 & 73 \\
\hline $1 \mathrm{H}$ & B & NS & $6 / 22 / 1993$ & 93173 & 173 & 77.4 & 63.1 & 62.7 & 64.4 & 63.5 \\
\hline $1 \mathrm{I}$ & $\mathrm{B}$ & NS & 6/23/1993 & 93174 & 174 & 90.8 & 70.4 & 66.6 & 66.1 & 68.7 \\
\hline $1 \mathrm{I}$ & $\mathrm{B}$ & NS & 6/24/1993 & 93175 & 175 & 86.9 & 64.9 & 65.3 & 64 & 64 \\
\hline $1 \mathrm{I}$ & $\mathrm{B}$ & NS & $6 / 25 / 1993$ & 93176 & 176 & 94.2 & 77 & 71.3 & 70.9 & 70.9 \\
\hline $1 \mathrm{I}$ & $\mathrm{B}$ & NS & 6/28/1993 & 93179 & 179 & 72.6 & 73.9 & 71.8 & 71.3 & 71.8 \\
\hline 11 & $\mathrm{~B}$ & NS & 6/29/1993 & 93180 & 180 & 93.4 & 76.1 & 70.9 & 69.6 & 67.5 \\
\hline 11 & $\mathrm{~B}$ & NS & 6/30/1993 & 93181 & 181 & 89.9 & 67.5 & 67 & 67.5 & 62.7 \\
\hline 11 & $\mathrm{~B}$ & NS & 7/1/1993 & 93182 & 182 & 91.2 & 67.5 & 64.9 & 62.3 & 59.7 \\
\hline $1 \mathrm{l}$ & B & NS & $7 / 2 / 1993$ & 93183 & 183 & 68.7 & 67.5 & 64 & 65.3 & 60.5 \\
\hline 11 & B & NS & 7/5/1993 & 93186 & 186 & 88.2 & 83.4 & 82.1 & 77.8 & 79.6 \\
\hline $1 \mathrm{l}$ & B & NS & 7/6/1993 & 93187 & 187 & & & & & \\
\hline 11 & B & NS & 7/7/1993 & 93188 & 188 & & & & & \\
\hline $1 \mathrm{l}$ & B & NS & 7/8/1993 & 93189 & 189 & & & & & \\
\hline 11 & B & NS & 7/9/1993 & 93190 & 190 & & & & & \\
\hline $1 \mathrm{l}$ & B & NS & $7 / 12 / 1993$ & 93193 & 193 & & & & & \\
\hline 11 & B & NS & 7/13/1993 & 93194 & 194 & & & & & \\
\hline $1 \mathrm{l}$ & B & NS & 7/14/1993 & 93195 & 195 & & & & & \\
\hline $1 \mathrm{l}$ & B & NS & 7/15/1993 & 93196 & 196 & & & & & \\
\hline $1 \mathrm{I}$ & $\mathrm{B}$ & NS & 7/16/1993 & 93197 & 197 & & & & & \\
\hline 11 & B & NS & $7 / 19 / 1993$ & 93200 & 200 & & & & & \\
\hline 11 & $\mathrm{~B}$ & NS & $7 / 20 / 1993$ & 93201 & 201 & & & & & \\
\hline 11 & $\mathrm{~B}$ & NS & $7 / 21 / 1993$ & 93202 & 202 & & & & & \\
\hline 11 & $\mathrm{~B}$ & NS & $7 / 22 / 1993$ & 93203 & 203 & & & & & \\
\hline 11 & $\mathrm{~B}$ & NS & 7/23/1993 & 93204 & 204 & & & & & \\
\hline 11 & B & NS & 7/26/1993 & 93207 & 207 & & & & & \\
\hline 11 & B & NS & 7/27/1993 & 93208 & 208 & & & & & \\
\hline 11 & $\mathrm{~B}$ & NS & $7 / 28 / 1993$ & 93209 & 209 & & & & & \\
\hline $1 \mathrm{l}$ & B & NS & $7 / 29 / 1993$ & 93210 & 210 & & & & & \\
\hline 11 & B & NS & 7/30/1993 & 93211 & 211 & & & & & \\
\hline 11 & $\mathrm{~B}$ & NS & 8/2/1993 & 93214 & 214 & & & & & \\
\hline 11 & $\mathrm{~B}$ & NS & 8/3/1993 & 93215 & 215 & & & & & \\
\hline $1 \mathrm{I}$ & $\mathrm{B}$ & NS & 8/4/1993 & 93216 & 216 & & & & & \\
\hline 11 & B & NS & $8 / 5 / 1993$ & 93217 & 217 & & & & & \\
\hline 11 & $\mathrm{~B}$ & NS & 8/6/1993 & 93218 & 218 & & & & & \\
\hline $1 \mathrm{l}$ & B & NS & 8/9/1993 & 93221 & 221 & & & & & \\
\hline 11 & $\mathrm{~B}$ & NS & $8 / 10 / 1993$ & 93222 & 222 & & & & & \\
\hline 11 & B & NS & $8 / 11 / 1993$ & 93223 & 223 & & & & & \\
\hline 11 & $\mathrm{~B}$ & NS & $8 / 12 / 1993$ & 93224 & 224 & & & & & \\
\hline 11 & $\mathrm{~B}$ & NS & $8 / 13 / 1993$ & 93225 & 225 & & & & & \\
\hline 11 & $\mathrm{~B}$ & NS & $8 / 16 / 1993$ & 93228 & 228 & 93.8 & 81.3 & 84.7 & 77.8 & 79.6 \\
\hline 11 & B & NS & $8 / 17 / 1993$ & 93229 & 229 & 89.9 & 89.1 & 88.2 & 87.3 & 87.3 \\
\hline 11 & B & NS & 8/18/1993 & 93230 & 230 & 85.6 & 89.9 & 88.2 & 86.5 & 86.5 \\
\hline 11 & $\mathrm{~B}$ & NS & $8 / 19 / 1993$ & 93231 & 231 & 79.6 & 82.1 & 80 & 77 & 76.1 \\
\hline 11 & $\mathrm{~B}$ & NS & $8 / 20 / 1993$ & 93232 & 232 & 77 & 77 & 75.2 & 75.2 & 72.6 \\
\hline $1 \mathrm{l}$ & B & NS & 8/23/1993 & 93235 & 235 & 96 & 97.2 & 91.6 & 89.9 & 88.2 \\
\hline 11 & B & NS & $8 / 24 / 1993$ & 93236 & 236 & 92.5 & 87.3 & 88.2 & 86.9 & 86.5 \\
\hline 11 & B & NS & $8 / 25 / 1993$ & 93237 & 237 & 85.6 & 83.9 & 83.4 & 81.3 & 80.4 \\
\hline $1 \mathrm{~F}$ & B & NS & 8/26/1993 & 93238 & 238 & 83 & 83 & 83.9 & 83 & 83.4 \\
\hline
\end{tabular}


other

\begin{tabular}{|c|c|c|c|c|c|c|c|c|c|c|}
\hline $1 \mathrm{l}$ & B & NS & \begin{tabular}{|l|}
$8 / 27 / 1993$ \\
\end{tabular} & 93239 & 239 & 94.2 & 80.4 & 88.6 & 83.9 & 80.4 \\
\hline 11 & B & NS & 8/30/1993 & 93242 & 242 & 76.1 & 78.7 & 80 & 77.8 & 74.4 \\
\hline 11 & $\mathrm{~B}$ & NS & 8/31/1993 & 93243 & 243 & 82.1 & 77.8 & 77.4 & 77.8 & 74.4 \\
\hline 11 & $\mathrm{~B}$ & NS & 9/1/1993 & 93244 & 244 & 87.3 & 84.7 & 86.9 & 83.9 & 84.7 \\
\hline 11 & B & NS & 9/2/1993 & 93245 & 245 & 79.6 & 79.6 & 78.7 & 78.2 & 75.2 \\
\hline 11 & B & NS & 9/3/1993 & 93246 & 246 & 78.7 & 79.1 & 77.8 & 76.1 & 74.4 \\
\hline 11 & B & NS & 9/6/1993 & 93249 & 249 & 81.3 & 72.6 & 73 & 71.8 & 72.6 \\
\hline 11 & B & NS & 9/7/1993 & 93250 & 250 & 74.4 & 72.6 & 72.6 & 72.2 & 73.5 \\
\hline 11 & B & NS & 9/8/1993 & 93251 & 251 & 77.8 & 74.4 & 73.5 & 73.9 & 74.4 \\
\hline 11 & $\mathrm{~B}$ & NS & 11/11/1993 & 93315 & 315 & 60.1 & 58 & 57.6 & 58.8 & 56.7 \\
\hline $1 \mathrm{I}$ & $\mathrm{B}$ & NS & 11/24/1993 & 93328 & 328 & 66.6 & 67.1 & 66.6 & 64.5 & 66.6 \\
\hline $1 \mathrm{l}$ & B & NS & 11/25/1993 & 93329 & 329 & 67.5 & 66.2 & 66.2 & 66.6 & 67.1 \\
\hline $1 \mathrm{I}$ & $\mathrm{B}$ & NS & 11/26/1993 & 93330 & 330 & 63.1 & 62.3 & 62.7 & 61.4 & 61 \\
\hline 11 & B & NS & 12/17/1993 & 93351 & 351 & 82.1 & 81.3 & 80.9 & 81.3 & 81.3 \\
\hline $1 \mathrm{l}$ & B & NS & 12/20/1993 & 93354 & 354 & 64 & 121 & 118 & 110.7 & 113.2 \\
\hline $1 \mathrm{l}$ & B & NS & 12/21/1993 & 93355 & 355 & 70.9 & 69.6 & 70 & 69.6 & 70.9 \\
\hline 11 & $\mathrm{~B}$ & NS & 12/22/1993 & 93356 & 356 & 69.2 & 70.5 & 70.9 & 68.3 & 69.6 \\
\hline 11 & $\mathrm{~B}$ & NS & 12/23/1993 & 93357 & 357 & 74 & 74.4 & 69.6 & 66.6 & 69.2 \\
\hline 11 & B & NS & 12/24/1993 & 93358 & 358 & 79.2 & 78.7 & 65.3 & 67.9 & 67.5 \\
\hline 11 & B & NS & 12/27/1993 & 93361 & 361 & 66.6 & 65.3 & 65.7 & 67.5 & 68.3 \\
\hline 11 & B & NS & 12/28/1993 & 93362 & 362 & 68.3 & 64.9 & 63.6 & 64.9 & 65.7 \\
\hline 11 & B & NS & 12/29/1993 & 93363 & 363 & 69.6 & 68.8 & 70.5 & 70 & 69.6 \\
\hline 11 & $\mathrm{~B}$ & NS & 12/30/1993 & 93364 & 364 & 85.6 & 87.8 & 82.1 & 81.7 & 83 \\
\hline 11 & $\mathrm{~B}$ & NS & 12/31/1993 & 93365 & 365 & 81.3 & 83 & 67.5 & 69.2 & 68.3 \\
\hline 11 & B & NS & $1 / 3 / 1994$ & 94003 & 3 & 64.5 & 62.3 & 59.3 & 61.9 & 58.8 \\
\hline $1 \mathrm{I}$ & $\mathrm{B}$ & NS & $1 / 4 / 1994$ & 94004 & 4 & 83 & 80.9 & 82.1 & 81.3 & 82.1 \\
\hline 11 & B & NS & $1 / 5 / 1994$ & 94005 & 5 & 93.8 & 84.7 & 83.9 & 84.3 & 85.6 \\
\hline $1 \mathrm{l}$ & B & NS & 2/21/1994 & 94052 & 52 & 61.9 & 62.7 & 61.9 & 61.9 & 63.6 \\
\hline $1 \mathrm{I}$ & B & NS & $3 / 11 / 1994$ & 94070 & 70 & 90.8 & 89.5 & 88.7 & 90 & 88.7 \\
\hline 11 & B & NS & $3 / 14 / 1994$ & 94073 & 73 & 87.4 & 140.1 & 129.7 & 124.1 & 121.1 \\
\hline $1 \mathrm{l}$ & B & NS & $3 / 15 / 1994$ & 94074 & 74 & 109.4 & 119.4 & 130.1 & 110.7 & 108.1 \\
\hline 11 & $\mathrm{~B}$ & NS & $3 / 16 / 1994$ & 94075 & 75 & 88.3 & 111.2 & 90.8 & 90 & 87.9 \\
\hline $1 \mathrm{I}$ & $\mathrm{B}$ & NS & $3 / 17 / 1994$ & 94076 & 76 & 90 & 93.4 & 116.8 & 92.6 & 105.5 \\
\hline 11 & $\mathrm{~B}$ & NS & $3 / 18 / 1994$ & 94077 & 77 & 87 & 88.3 & 88.7 & 88.7 & 86.5 \\
\hline 11 & B & NS & $4 / 1 / 1994$ & 94091 & 91 & 65.4 & 67.5 & 69.2 & 67.5 & 64.1 \\
\hline 11 & $\mathrm{~B}$ & NS & 4/4/1994 & 94094 & 94 & 64.1 & 62.8 & 62.3 & 65.8 & 64.9 \\
\hline $1 \mathrm{I}$ & $\mathrm{B}$ & NS & $5 / 30 / 1994$ & 94150 & 150 & 86 & 84.7 & 86.5 & 82.1 & 80.8 \\
\hline $1 \mathrm{I}$ & $\mathrm{B}$ & NS & $5 / 31 / 1994$ & 94151 & 151 & 82.5 & 79.6 & 78.7 & 80.8 & 79.6 \\
\hline $1 \mathrm{I}$ & $\mathrm{B}$ & NS & $6 / 1 / 1994$ & 94152 & 152 & 67.9 & 65.7 & 67 & 68.3 & 64 \\
\hline $1 \mathrm{I}$ & $\mathrm{B}$ & NS & $6 / 2 / 1994$ & 94153 & 153 & 92.5 & 93.8 & 91.6 & 85.6 & 85.1 \\
\hline $1 \mathrm{I}$ & $\mathrm{B}$ & NS & $6 / 3 / 1994$ & 94154 & 154 & 61.8 & 63.1 & 60.5 & 63.5 & 61.4 \\
\hline 0 & A & NS & 6/8/1991 & 91159 & 159 & 164.2 & 165.9 & 160.7 & 164.2 & 163.3 \\
\hline 0 & A & NS & 6/9/1991 & 91160 & 160 & 151.2 & 147.7 & 147.7 & 149.5 & 146 \\
\hline 0 & A & NS & $6 / 15 / 1991$ & 91166 & 166 & 151.2 & 151.2 & 152.9 & 152.1 & 152.9 \\
\hline 0 & A & NS & $6 / 16 / 1991$ & 91167 & 167 & 148.6 & 144.3 & 144.3 & 146.9 & 142.6 \\
\hline 0 & A & NS & $6 / 22 / 1991$ & 91173 & 173 & 152.9 & 152.9 & 152.9 & 147.7 & 149.5 \\
\hline 0 & A & NS & $6 / 23 / 1991$ & 91174 & 174 & 161.6 & 159.8 & 152.1 & 146.9 & 145.2 \\
\hline 0 & A & NS & 6/29/1991 & 91180 & 180 & 162.4 & 163.3 & 155.5 & 162.4 & 158.1 \\
\hline 0 & A & NS & 6/30/1991 & 91181 & 181 & 151.2 & 140.8 & 148.6 & 153.8 & 142.6 \\
\hline
\end{tabular}


other

\begin{tabular}{|c|c|c|c|c|c|c|c|c|c|c|}
\hline 0 & A & NS & 7/6/1991 & 91187 & 187 & 140.8 & 138.2 & 138.2 & 137.4 & 133.9 \\
\hline 0 & A & NS & 7/7/1991 & 91188 & 188 & 127 & 127.9 & 126.1 & 124.4 & 125.3 \\
\hline 0 & A & NS & $7 / 13 / 1991$ & 91194 & 194 & 127 & 121 & 115.8 & 117.5 & 116.6 \\
\hline 0 & A & NS & $7 / 14 / 1991$ & 91195 & 195 & 133.1 & 128.7 & 127 & 122.7 & 123.6 \\
\hline 0 & A & NS & $7 / 20 / 1991$ & 91201 & 201 & 132.2 & 127 & 126.1 & 123.6 & 125.3 \\
\hline 0 & A & NS & $7 / 21 / 1991$ & 91202 & 202 & 131.3 & 128.7 & 127 & 124.4 & 123.6 \\
\hline 0 & A & NS & $7 / 27 / 1991$ & 91208 & 208 & 157.2 & 152.1 & 151.2 & 149.5 & 150.3 \\
\hline 0 & A & NS & $7 / 28 / 1991$ & 91209 & 209 & 143.4 & 140.8 & 140 & 140 & 139.1 \\
\hline 0 & A & NS & $8 / 3 / 1991$ & 91215 & 215 & 166.8 & 163.3 & 160.7 & 161.6 & 159.8 \\
\hline 0 & A & NS & 8/4/1991 & 91216 & 216 & 160.7 & 159 & 159.8 & 157.2 & 157.2 \\
\hline 0 & A & NS & 8/10/1991 & 91222 & 222 & 189.2 & 181.4 & 185.8 & 184 & 169.3 \\
\hline 0 & A & NS & $8 / 11 / 1991$ & 91223 & 223 & 167.6 & 166.8 & 164.2 & 159 & 161.6 \\
\hline 0 & A & NS & $8 / 17 / 1991$ & 91229 & 229 & 221.2 & 215.1 & 210.8 & 210 & 205.6 \\
\hline 0 & A & NS & 8/18/1991 & 91230 & 230 & 220.3 & 214.3 & 216.9 & 207.4 & 206.5 \\
\hline 0 & A & NS & $8 / 24 / 1991$ & 91236 & 236 & 381.9 & 368.1 & 362 & 354.2 & 352.5 \\
\hline 0 & A & NS & $8 / 25 / 1991$ & 91237 & 237 & 389.7 & 385.3 & 368.1 & 362.9 & 353.4 \\
\hline 0 & A & NS & 8/31/1991 & 91243 & 243 & 425.1 & 419.9 & 414.7 & 395.7 & 385.3 \\
\hline 0 & A & NS & 9/1/1991 & 91244 & 244 & 387.1 & 378.4 & 368.1 & 368.9 & 363.7 \\
\hline 0 & A & NS & 12/21/1991 & 91355 & 355 & 117.1 & 121.4 & 112.8 & 107.6 & 108.5 \\
\hline 0 & A & NS & 12/22/1991 & 91356 & 356 & 115.4 & 108.5 & 105.9 & 106.7 & 104.1 \\
\hline 0 & A & NS & 12/28/1991 & 91362 & 362 & 143 & 134.4 & 131.4 & 143 & 108.5 \\
\hline 0 & A & NS & 12/29/1991 & 91363 & 363 & 122.3 & 83.9 & 99.8 & 137.8 & 105 \\
\hline 0 & A & NS & $1 / 4 / 1992$ & 92004 & 3 & 92.9 & 86 & 79.6 & 89.5 & 121.4 \\
\hline 0 & A & NS & $1 / 5 / 1992$ & 92005 & 4 & 86 & 115.4 & 101.2 & 83.4 & 84.3 \\
\hline 0 & A & NS & $5 / 30 / 1992$ & 92151 & 150 & 108.5 & 105.5 & 99 & 92.9 & 92.9 \\
\hline 0 & A & NS & $5 / 31 / 1992$ & 92152 & 151 & 95.5 & 95.5 & 95.5 & 91.2 & 90.3 \\
\hline 0 & B & NS & $3 / 14 / 1992$ & 92074 & 73 & 100.7 & 99.8 & 100.7 & 98.6 & 99.8 \\
\hline 0 & $\mathrm{~B}$ & NS & $3 / 15 / 1992$ & 92075 & 74 & 81.7 & 81.7 & 83.4 & 80.8 & 81.7 \\
\hline 0 & B & NS & $3 / 21 / 1992$ & 92081 & 80 & 74.8 & 76.1 & 77.4 & 77.4 & 78.2 \\
\hline 0 & B & NS & $3 / 22 / 1992$ & 92082 & 81 & 76.5 & 77.4 & 76.5 & 76.5 & 77.4 \\
\hline 0 & B & NS & $4 / 18 / 1992$ & 92109 & 108 & 111.1 & 105.9 & 106.7 & 103.3 & 100.7 \\
\hline 0 & B & NS & 4/19/1992 & 92110 & 109 & 92.9 & 93.8 & 93.8 & 92.5 & 92.9 \\
\hline 0 & $\mathrm{~B}$ & NS & $6 / 6 / 1992$ & 92158 & 157 & 121.9 & 118.4 & 119.7 & 113.2 & 117.1 \\
\hline 0 & $\mathrm{~B}$ & NS & 6/7/1992 & 92159 & 158 & 122.3 & 121.9 & 121.4 & 116.7 & 117.6 \\
\hline 0 & B & NS & 6/13/1992 & 92165 & 164 & 133.1 & 129.2 & 125.3 & 120.2 & 119.7 \\
\hline 0 & $\mathrm{~B}$ & NS & 6/14/1992 & 92166 & 165 & 146.9 & 138.3 & 135.7 & 129.2 & 130.5 \\
\hline 0 & $\mathrm{~B}$ & NS & 6/20/1992 & 92172 & 171 & 141.8 & 133.1 & 128.8 & 124.9 & 118.4 \\
\hline 0 & $\mathrm{~B}$ & NS & 6/21/1992 & 92173 & 172 & 144.8 & 133.5 & 123.6 & 120.2 & 118.4 \\
\hline 0 & $\mathrm{~B}$ & NS & 6/27/1992 & 92179 & 178 & 109.8 & 110.7 & 106.3 & 99.8 & 101.2 \\
\hline 0 & $\mathrm{~B}$ & NS & 6/28/1992 & 92180 & 179 & 125.7 & 125.3 & 114.1 & 113.2 & 110.7 \\
\hline 0 & $\mathrm{~B}$ & NS & 7/4/1992 & 92186 & 185 & 229.9 & 233.3 & 216.9 & 211.7 & 218.7 \\
\hline 0 & $\mathrm{~B}$ & NS & 7/5/1992 & 92187 & 186 & 83 & 80 & 78.7 & 77.8 & 75.6 \\
\hline 0 & $\mathrm{~B}$ & NS & 7/11/1992 & 92193 & 192 & 112.4 & 113.2 & 106.3 & 96.8 & 91.6 \\
\hline 0 & $\mathrm{~B}$ & NS & 7/12/1992 & 92194 & 193 & 101.2 & 92.5 & 82.5 & 83 & 78.7 \\
\hline 0 & $\mathrm{~B}$ & NS & 7/18/1992 & 92200 & 199 & 118.4 & 111.5 & 108.9 & 105 & 99.4 \\
\hline 0 & B & NS & 7/19/1992 & 92201 & 200 & 108.9 & 106.3 & 104.1 & 102 & 96 \\
\hline 0 & B & NS & 7/25/1992 & 92207 & 206 & 187.6 & 185 & 182.8 & 181.5 & 178 \\
\hline 0 & B & NS & 7/26/1992 & 92208 & 207 & 174.6 & 172.9 & 166.8 & 163.4 & 160.8 \\
\hline 0 & B & NS & 8/1/1992 & 92214 & 213 & 205.7 & 195.3 & 200.5 & 193.6 & 191 \\
\hline
\end{tabular}


other

\begin{tabular}{|c|c|c|c|c|c|c|c|c|c|c|}
\hline $0 \mathrm{I}$ & B & NS & 8/2/1992 & 92215 & 214 & 81.3 & 82.1 & 79.1 & 79.6 & 76.1 \\
\hline 01 & $\mathrm{~B}$ & NS & 8/8/1992 & 92221 & 220 & 117.6 & 113.2 & 107.2 & 108.1 & 104.6 \\
\hline 01 & $\mathrm{~B}$ & NS & 8/9/1992 & 92222 & 221 & 125.3 & 120.2 & 117.6 & 117.6 & 116.7 \\
\hline $0 \mathrm{I}$ & $\mathrm{B}$ & NS & 8/15/1992 & 92228 & 227 & 105.5 & 104.1 & 106.3 & 105.5 & 105.9 \\
\hline 01 & $\mathrm{~B}$ & NS & 8/16/1992 & 92229 & 228 & 133.1 & 129.7 & 122.8 & 120.6 & 121.9 \\
\hline 01 & $\mathrm{~B}$ & NS & 8/22/1992 & 92235 & 234 & 102 & 109.3 & 102.9 & 102.4 & 99.4 \\
\hline 01 & $\mathrm{~B}$ & NS & 8/23/1992 & 92236 & 235 & 91.2 & 77.8 & 82.1 & 76.5 & 82.1 \\
\hline 01 & $\mathrm{~B}$ & NS & 8/29/1992 & 92242 & 241 & 136.1 & 121.9 & 122.8 & 121 & 115 \\
\hline 01 & $\mathrm{~B}$ & NS & 8/30/1992 & 92243 & 242 & 115.8 & 111.5 & 104.6 & 105.9 & 96.8 \\
\hline 01 & B & NS & 9/5/1992 & 92249 & 248 & 152.1 & 152.1 & 152.1 & 146.1 & 142.2 \\
\hline 01 & B & NS & 9/6/1992 & 92250 & 249 & 175.5 & 144.4 & 130.5 & 127.9 & 119.7 \\
\hline 01 & B & NS & 12/19/1992 & 92354 & 353 & 85.1 & 84.7 & 86.9 & 86 & 84.3 \\
\hline 01 & B & NS & 12/20/1992 & 92355 & 354 & 103.3 & 91.2 & 84.3 & 80.8 & 78.7 \\
\hline 01 & B & NS & 12/26/1992 & 92361 & 360 & 113.6 & 117.1 & 115.8 & 118 & 116.2 \\
\hline 01 & B & NS & 12/27/1992 & 92362 & 361 & 113.2 & 92.9 & 86.9 & 83 & 82.5 \\
\hline 01 & B & NS & 1/2/1993 & 93002 & 2 & 140.4 & 100.7 & 79.1 & 76.1 & 76.5 \\
\hline 01 & $\mathrm{~B}$ & NS & $1 / 3 / 1993$ & 93003 & 3 & 65.3 & 65.7 & 63.5 & 63.5 & 64.4 \\
\hline $0 \mathrm{l}$ & B & NS & 3/13/1993 & 93072 & 72 & 111.1 & 109.8 & 111.9 & 110.2 & 110.2 \\
\hline 01 & B & NS & 3/14/1993 & 93073 & 73 & 94.2 & 96.4 & 96.4 & 96.4 & 96.8 \\
\hline $0 \mathrm{I}$ & B & NS & 3/20/1993 & 93079 & 79 & 71.8 & 73.9 & 72.2 & 73 & 72.2 \\
\hline 01 & $\mathrm{~B}$ & NS & 3/21/1993 & 93080 & 80 & 70.4 & 69.6 & 70.4 & 69.6 & 68.3 \\
\hline $0 \mathrm{I}$ & B & NS & 4/10/1993 & 93100 & 100 & 106.7 & 73.9 & 73.9 & 73 & 73.9 \\
\hline $0 \mathrm{l}$ & B & NS & 4/11/1993 & 93101 & 101 & 74.4 & 73.9 & 69.6 & 70.4 & 69.6 \\
\hline $0 \mathrm{l}$ & B & NS & 5/29/1993 & 93149 & 149 & 86.9 & 88.6 & 78.2 & 76.5 & 77.8 \\
\hline $0 \mathrm{H}$ & $\mathrm{B}$ & NS & 5/30/1993 & 93150 & 150 & 77.4 & 72.2 & 72.2 & 69.6 & 67.5 \\
\hline $0 \mathrm{H}$ & B & NS & 6/5/1993 & 93156 & 156 & 95.5 & 98.6 & 92.9 & 95.1 & 92.5 \\
\hline $0 \mathrm{H}$ & B & NS & 6/6/1993 & 93157 & 157 & 102 & 99.4 & 100.3 & 95.5 & 89.9 \\
\hline $0 \mathrm{H}$ & $\mathrm{B}$ & NS & $6 / 12 / 1993$ & 93163 & 163 & 93.8 & 81.7 & 81.7 & 80 & 80 \\
\hline $0 \mathrm{~F}$ & B & NS & 6/13/1993 & 93164 & 164 & 90.8 & 78.2 & 80 & 78.2 & 78.2 \\
\hline $0 \mathrm{H}$ & $\mathrm{B}$ & NS & 6/19/1993 & 93170 & 170 & 76.5 & 81.3 & 75.6 & 77 & 80.8 \\
\hline $0 \mathrm{~F}$ & B & NS & $6 / 20 / 1993$ & 93171 & 171 & 73.9 & 73 & 75.6 & 76.5 & 69.6 \\
\hline $0 \mathrm{H}$ & B & NS & 6/26/1993 & 93177 & 177 & 73.5 & 81.7 & 79.6 & 78.7 & 80.8 \\
\hline $0 \mathrm{~F}$ & B & NS & 6/27/1993 & 93178 & 178 & 69.2 & 70.4 & 73.5 & 76.5 & 70.4 \\
\hline $0 \mathrm{~F}$ & $\mathrm{~B}$ & NS & 7/3/1993 & 93184 & 184 & 71.3 & 81.3 & 73.5 & 75.2 & 74.8 \\
\hline $0 \mathrm{~F}$ & B & NS & 7/4/1993 & 93185 & 185 & 89.1 & 79.6 & 74.8 & 82.1 & 74.4 \\
\hline $0 \mathrm{~F}$ & B & NS & 7/10/1993 & 93191 & 191 & & & & & \\
\hline $0 \mathrm{H}$ & B & NS & 7/11/1993 & 93192 & 192 & & & & & \\
\hline $0 \mathrm{E}$ & B & NS & 7/17/1993 & 93198 & 198 & & & & & \\
\hline $0 \mathrm{~F}$ & B & NS & 7/18/1993 & 93199 & 199 & & & & & \\
\hline $0 \mathrm{~F}$ & $\mathrm{~B}$ & NS & $7 / 24 / 1993$ & 93205 & 205 & & & & & \\
\hline $0 \mathrm{~F}$ & $\mathrm{~B}$ & NS & $7 / 25 / 1993$ & 93206 & 206 & & & & & \\
\hline $0 \mathrm{~F}$ & $\mathrm{~B}$ & NS & 7/31/1993 & 93212 & 212 & & & & & \\
\hline $0 \mathrm{~F}$ & $\mathrm{~B}$ & NS & $8 / 1 / 1993$ & 93213 & 213 & & & & & \\
\hline $0 \mathrm{~F}$ & $\mathrm{~B}$ & NS & 8/7/1993 & 93219 & 219 & & & & & \\
\hline $0 \mathrm{~F}$ & B & NS & 8/8/1993 & 93220 & 220 & & & & & \\
\hline $0 \mathrm{~F}$ & B & NS & 8/14/1993 & 93226 & 226 & 73.5 & 78.7 & 78.7 & 78.7 & 71.3 \\
\hline $0 \mathrm{~F}$ & $\mathrm{~B}$ & NS & $8 / 15 / 1993$ & 93227 & 227 & 95.1 & 92.5 & 90.8 & 89.9 & 89.1 \\
\hline $0 \mathrm{E}$ & B & NS & $8 / 21 / 1993$ & 93233 & 233 & 80.4 & 86.5 & 85.6 & 83.4 & 78.7 \\
\hline $0 \mathrm{~F}$ & B & NS & 8/22/1993 & 93234 & 234 & 90.8 & 89.9 & 90.8 & 83.9 & 89.5 \\
\hline
\end{tabular}


other

\begin{tabular}{|c|c|c|c|c|c|c|c|c|c|c|}
\hline $0 \mathrm{~F}$ & B & NS & $8 / 28 / 1993$ & 93240 & 240 & 81.3 & 89.1 & 81.7 & 81.3 & 84.3 \\
\hline $0 \mathrm{~F}$ & B & NS & $8 / 29 / 1993$ & 93241 & 241 & 73.9 & 80.4 & 75.2 & 75.6 & 74.4 \\
\hline \begin{tabular}{l|l}
$0 \mathrm{~F}$ \\
\end{tabular} & B & NS & 9/4/1993 & 93247 & 247 & 74.4 & 83.9 & 84.7 & 80.4 & 77.8 \\
\hline $0 \mathrm{~F}$ & B & NS & 9/5/1993 & 93248 & 248 & 72.6 & 71.8 & 71.3 & 71.8 & 70 \\
\hline $0 \mathrm{~F}$ & B & NS & 11/27/1993 & 93331 & 331 & 71.8 & 71.8 & 73.5 & 72.6 & 71.8 \\
\hline \begin{tabular}{l|l}
$0 \mathrm{~F}$ \\
\end{tabular} & B & NS & $11 / 28 / 1993$ & 93332 & 332 & 71.8 & 71.8 & 71.8 & 70 & 74.4 \\
\hline $0 \mathrm{~F}$ & B & NS & 12/18/1993 & 93352 & 352 & 95.6 & 66.6 & 65.3 & 65.7 & 65.3 \\
\hline $0 \mathrm{~F}$ & B & NS & 12/19/1993 & 93353 & 353 & 68.3 & 66.2 & 70 & 66.2 & 67.5 \\
\hline \begin{tabular}{l|l}
0 & $\mathrm{H}$ \\
\end{tabular} & B & NS & $12 / 25 / 1993$ & 93359 & 359 & 78.7 & 72.6 & 68.3 & 67.5 & 66.6 \\
\hline \begin{tabular}{l|l}
$0 \mathrm{~F}$ \\
\end{tabular} & B & NS & $12 / 26 / 1993$ & 93360 & 360 & 65.7 & 67.1 & 67.5 & 67.5 & 67.5 \\
\hline \begin{tabular}{l|l}
0 & $\mathrm{H}$ \\
\end{tabular} & $\mathrm{B}$ & NS & $1 / 1 / 1994$ & 94001 & 1 & 77.8 & 78.7 & 63.1 & 61.4 & 66.6 \\
\hline \begin{tabular}{l|l}
0 & $\mathrm{H}$ \\
\end{tabular} & B & NS & $1 / 2 / 1994$ & 94002 & 2 & 64.9 & 64 & 63.6 & 62.3 & 62.3 \\
\hline \begin{tabular}{l|l}
0 & $\mathrm{H}$
\end{tabular} & B & NS & $3 / 12 / 1994$ & 94071 & 71 & 84.3 & 87.8 & 83.9 & 84.3 & 83.5 \\
\hline $\begin{array}{lll} & \mathrm{H}\end{array}$ & B & NS & 3/13/1994 & 94072 & 72 & 85.7 & 86.1 & 88.3 & 88.7 & 84.3 \\
\hline $\begin{array}{lll}0 & \mathrm{H}\end{array}$ & B & NS & 3/19/1994 & 94078 & 78 & 65.8 & 64.5 & 68.4 & 68.4 & 64.9 \\
\hline \begin{tabular}{l|l}
0 & $\mathrm{H}$
\end{tabular} & $\mathrm{B}$ & NS & $3 / 20 / 1994$ & 94079 & 79 & 66.7 & 65.8 & 70.1 & 70.1 & 65.8 \\
\hline $0 \mathrm{~F}$ & B & NS & $4 / 2 / 1994$ & 94092 & 92 & 62.8 & 63.2 & 65.8 & 65.8 & 64.9 \\
\hline \begin{tabular}{l|l}
0 & $\mathrm{~F}$ \\
\end{tabular} & $\mathrm{~B}$ & NS & $4 / 3 / 1994$ & 94093 & 93 & 68 & 67.5 & 66.7 & 64.9 & 66.7 \\
\hline $0 \mathrm{~F}$ & B & NS & $5 / 28 / 1994$ & 94148 & 148 & 100.7 & 102 & 99.4 & 97.2 & 94.2 \\
\hline $0 \mathrm{~F}$ & B & NS & 5/29/1994 & 94149 & 149 & 86.5 & 82.5 & 83.9 & 84.7 & 83.4 \\
\hline $0 \mathrm{~F}$ & B & NS & 6/4/1994 & 94155 & 155 & 76.5 & 75.2 & 73.9 & 77 & 73 \\
\hline & & & & & & & & & & \\
\hline & & & & & \multicolumn{2}{|c|}{ sort S/NS - 0/1 - A/B } & & & & \\
\hline & & & & & \multicolumn{2}{|c|}{ Hourly Averages } & & & & \\
\hline & & & & & 1-A-S & 155.8478 & 152.7411 & 149.7756 & 147.4356 & 145.5722 \\
\hline & & & & & 1-B-S & 89.31244 & 88.76608 & 88.22937 & 86.63089 & 85.54152 \\
\hline & & & & & $0-A-S$ & 150.2361 & 146.3556 & 143.7796 & 141.6625 & 139.8533 \\
\hline & & & & & 0-B-S & 118.9952 & 116.2991 & 113.6498 & 111.71 & 110.4886 \\
\hline & & & & & 1-A-NS & 172.7456 & 170.4848 & 168.6785 & 166.1165 & 164.7354 \\
\hline & & & & & 1-B-NS & 97.72634 & 95.59892 & 94.2586 & 91.89516 & 90.28548 \\
\hline & & & & & 0-A-NS & 174.2824 & 170.3676 & 167.7176 & 166.9412 & 163.7588 \\
\hline & & & & & 0-B-NS & 100.2916 & 97.1494 & 94.6988 & 93.16506 & 91.83855 \\
\hline \multicolumn{4}{|c|}{\begin{tabular}{l|l|l} 
sort JDAY & \\
\end{tabular}} & & & & & & & \\
\hline \multicolumn{4}{|c|}{ Table of Monthly Consumption } & & \multicolumn{2}{|c|}{ sort S/NS - 0/1 - A/B } & & & & \\
\hline & & & & & \multicolumn{4}{|c|}{ Standard Deviation ofHourly Averages } & & \\
\hline & & Jun 91 & 241636 & 241635.6 & & & & & & \\
\hline & & Jul & 220847 & 220846.6 & 1-A-S & 67.92734 & 65.84068 & 64.44604 & 64.03023 & 62.14249 \\
\hline & & Aug & 167456 & 167456 & 1-B-S & 21.68608 & 23.25114 & 21.88004 & 20.40185 & 19.73925 \\
\hline & & Sep & 167304 & 167304.1 & 0-A-S & 43.50569 & 43.00129 & 42.33532 & 41.75492 & 41.08912 \\
\hline & & Oct & 152268 & 152267.5 & 0-B-S & 66.47741 & 65.54043 & 64.2651 & 63.32119 & 62.24586 \\
\hline & & Nov & 136391 & 136391 & 1-A-NS & 86.02708 & 83.90454 & 81.77087 & 80.86727 & 78.90988 \\
\hline & & Dec & 219845 & 219844.5 & 1-B-NS & 24.34335 & 24.8851 & 25.18252 & 23.13703 & 22.02685 \\
\hline & & Jan 92 & 165440 & 165440.2 & 0-A-NS & 87.5901 & 86.1269 & 83.89355 & 81.095 & 79.38332 \\
\hline & & Feb & 143414 & 143413.9 & 0-B-NS & 33.3462 & 31.30629 & 29.98373 & 28.78991 & 28.68488 \\
\hline & & Mar & 161499 & 161499.4 & & & & & & \\
\hline & & Apr & 200369 & 200368.7 & & & & & & \\
\hline & & May & 244332 & 244331.7 & \multicolumn{2}{|c|}{ sort S/NS - 0/1 - A/B } & & & & \\
\hline & & Jun & 203001 & 203001.3 & \multicolumn{2}{|c|}{ Hourly Sums } & & & & \\
\hline & & Jul & 182240 & 182240 & & & & & & \\
\hline
\end{tabular}


other

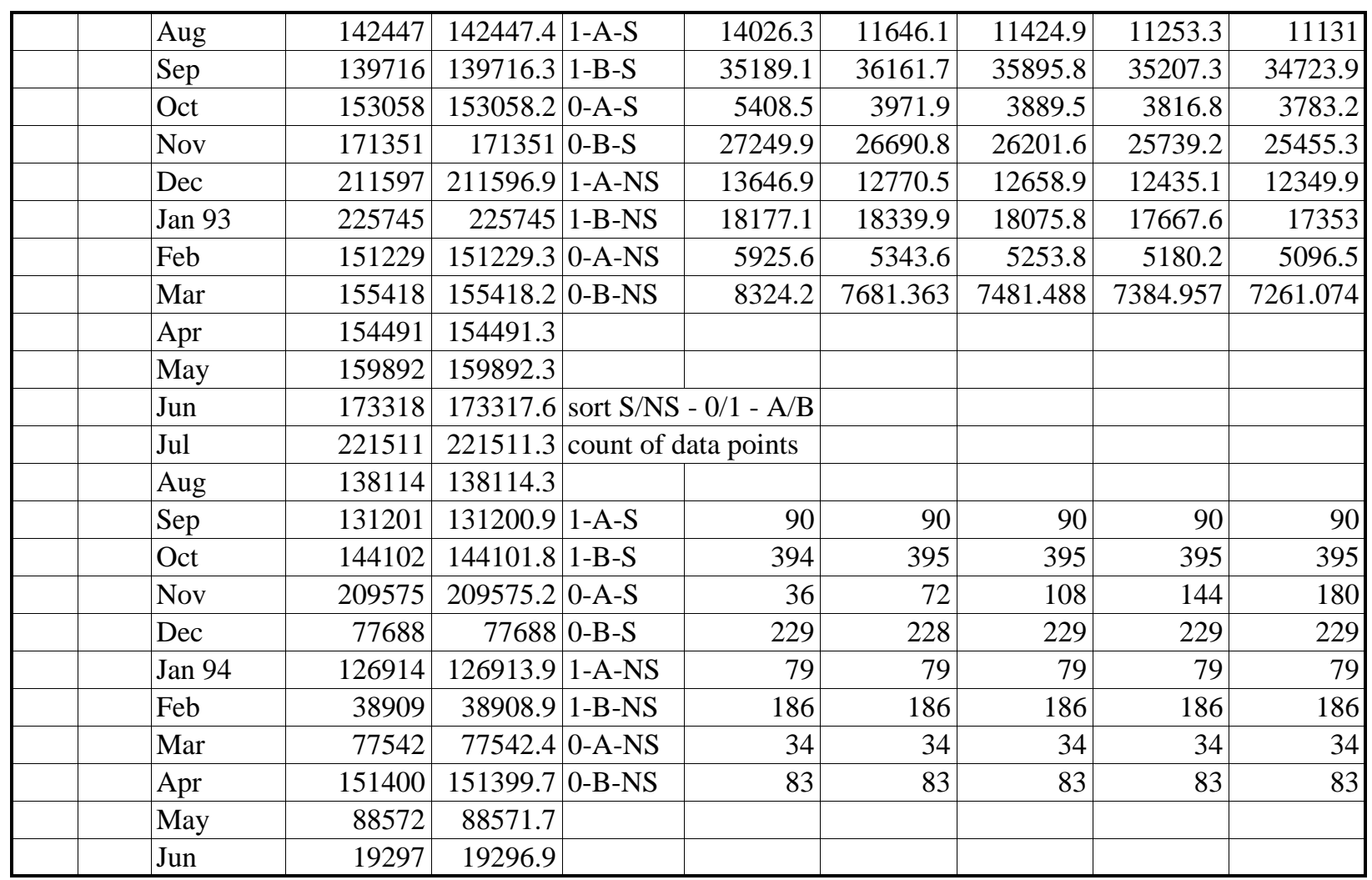




\begin{tabular}{|c|c|c|c|c|c|c|c|c|c|}
\hline 5 & 6 & 7 & 8 & 9 & 10 & 11 & 12 & 13 & 14 \\
\hline 363.7 & 361.2 & 393.1 & 613.4 & 758.6 & 785.4 & 803.5 & 851 & 846.7 & 821.7 \\
\hline 178 & 178 & 265.2 & 591 & 771.6 & 797.5 & 794.9 & 787.1 & 792.3 & 819.1 \\
\hline 191.8 & 184.9 & 266.1 & 605.7 & 768.1 & 776.7 & 788.8 & 851.9 & 841.5 & 845 \\
\hline 199.6 & 197 & 268.7 & 615.2 & 795.7 & 810.4 & 762.9 & 852.8 & 848.4 & 864 \\
\hline 341.3 & 436.3 & 462.2 & 631.6 & 797.5 & 842.4 & 858.8 & 887.3 & 868.3 & 884.7 \\
\hline 230.7 & 223.8 & 344.7 & 660.1 & 835.5 & 866.6 & 885.6 & 897.7 & 875.2 & 891.6 \\
\hline 217.7 & 216 & 315.4 & 649.7 & 784.5 & 828.6 & 861.4 & 882.1 & 866.6 & 872.6 \\
\hline 210 & 208.2 & 290.3 & 659.2 & 822.5 & 832 & 837.2 & 871.8 & 881.3 & 887.3 \\
\hline 217.7 & 218.6 & 295.5 & 671.3 & 820.8 & 863.1 & 878.7 & 886.5 & 873.5 & 892.5 \\
\hline 342.1 & 435.5 & 471.7 & 618.6 & 808.7 & 837.2 & 840.7 & 851 & 864.9 & 880.4 \\
\hline 215.1 & 210.8 & 306.7 & 656.6 & 810.4 & 849.3 & 860.5 & 869.2 & 865.7 & 883.9 \\
\hline 218.6 & 216 & 291.2 & 650.6 & 800.9 & 819.9 & 834.6 & 877.8 & 877 & 854.5 \\
\hline 191.8 & 184 & 250.6 & 586.7 & 740.4 & 730.9 & 738.7 & 729.2 & 724 & 743.9 \\
\hline 127 & 121.8 & 197.9 & 480.4 & 649.7 & 661 & 622.9 & 679.1 & 694.7 & 702.4 \\
\hline 305.9 & 397.4 & 404.4 & 557.3 & 782.8 & 804.4 & 837.2 & 833.8 & 836.4 & 847.6 \\
\hline 161.6 & 166.8 & 253.2 & 597.9 & 797.5 & 836.4 & 850.2 & 851.9 & 851 & 874.4 \\
\hline 147.7 & 140 & 174.5 & 540.9 & 692.1 & 703.3 & 714.5 & 727.5 & 743 & 736.1 \\
\hline 109.7 & 110.6 & 170.2 & 476.1 & 665.3 & 685.2 & 703.3 & 743 & 740.4 & 741.3 \\
\hline 132.2 & 128.7 & 159 & 512.4 & 678.2 & 716.3 & 731.8 & 750 & 753.4 & 749.1 \\
\hline 271.3 & 356.8 & 374.1 & 498.5 & 673.1 & 726.6 & 761.2 & 793.2 & 799.2 & 800.9 \\
\hline & & & & & & & & & \\
\hline 164.8 & 160.4 & 171.4 & 384.8 & 307.4 & 604.4 & 638.5 & 663.7 & 651.7 & 670.7 \\
\hline 179.4 & 169.8 & 175.8 & 402.5 & 588.1 & 634.3 & 650.5 & 637.1 & 656.8 & 659.8 \\
\hline 97.9 & 37.8 & 72.4 & 332.1 & 479.2 & 505.7 & 511.6 & 528.2 & 533.5 & 537.2 \\
\hline 106.3 & 106.3 & 132.7 & 406.2 & 540.2 & 583.5 & 597.2 & 604.6 & 617.5 & 627.8 \\
\hline 108.1 & 108.1 & 128.8 & 394.7 & 554.8 & 608.3 & 608.4 & 646.4 & 658 & 660.1 \\
\hline 137.4 & 134 & 156.9 & 425.6 & 568.2 & 583.3 & 577.9 & 627 & 644.4 & 657.6 \\
\hline 134 & 133.5 & 164.7 & 414.4 & 597.6 & 646.5 & 603.6 & 694 & 696.6 & 697.9 \\
\hline 164.3 & 192.8 & 210.3 & 527.7 & 721.4 & 725.8 & 726.2 & 742.2 & 765.8 & 765.4 \\
\hline 150.4 & 149.5 & 164.7 & 458 & 622.9 & 644.5 & 655.8 & 696.3 & 725.7 & 709.2 \\
\hline 144.4 & 142.6 & 168.1 & 450.5 & 598.7 & 625.5 & 647.9 & 663.4 & 682.8 & 697.5 \\
\hline 146.9 & 142.6 & 161.2 & 452.2 & 611.6 & 652.3 & 674.7 & 706.2 & 704.4 & 708.7 \\
\hline 138.3 & 130.5 & 155.2 & 444 & 608.1 & 672.5 & 678.2 & 688 & 717.4 & 714.7 \\
\hline 122.8 & 149 & 164.8 & 430.4 & 607.3 & 645 & 653.2 & 661 & 682.2 & 689.5 \\
\hline 139.2 & 139.2 & 164.7 & 453.3 & 634.4 & 703.8 & 713.6 & 707.1 & 736.1 & 746.9 \\
\hline 187.6 & 182.4 & 211.3 & 536.9 & 717.8 & 737.2 & 766.9 & 775.2 & 805.4 & 796.8 \\
\hline 194.5 & 185.8 & 215.6 & 527.9 & 703.6 & 751 & 752.8 & 779.1 & 795.6 & 791.6 \\
\hline 333.6 & 360.5 & 356.1 & 405.6 & 550.8 & 751.5 & 781.8 & 759.8 & 785.7 & 784.5 \\
\hline 234.6 & 229.9 & 232.5 & 310.8 & 631.5 & 800.9 & 843.7 & 273.4 & 829.9 & 842.7 \\
\hline 126.6 & 121.4 & 137.4 & 412.7 & 599.2 & 607.3 & 614.6 & 592.6 & 592 & 589.8 \\
\hline 99.8 & 98.6 & 132.4 & 390.7 & 562.5 & 585.5 & 591.9 & 557.7 & 535.2 & 500 \\
\hline 89.5 & 88.6 & 112.8 & 268.3 & 495.7 & 533.6 & 525.8 & 496.8 & 443.6 & 410.9 \\
\hline 91.2 & 92 & 137.4 & 310.6 & 523.2 & 550.8 & 561.7 & 550 & 536.1 & 494.3 \\
\hline 176.3 & 175 & 198.8 & 313.2 & 469.6 & 496 & 508.5 & 509 & 496.4 & 462.3 \\
\hline
\end{tabular}


other

\begin{tabular}{|c|c|c|c|c|c|c|c|c|c|}
\hline 161.6 & 160.3 & 188 & 285.2 & 436.8 & 456.3 & 475.7 & 462.3 & 444.6 & 430.7 \\
\hline 128.3 & 129.2 & 149.9 & 270.5 & 494.4 & 617.5 & 593.2 & 622.1 & 642.4 & 627.2 \\
\hline 164.6 & 161.2 & 159.9 & 262.3 & 403.1 & 468.4 & 486 & 509.4 & 488.2 & 461.8 \\
\hline 128.8 & 127.5 & 146.1 & 277.8 & 429.5 & 437.6 & 453.4 & 465.6 & 499 & 481.2 \\
\hline 133.5 & 134 & 159.4 & 263.6 & 426 & 468.8 & 474.5 & 441.1 & 442.8 & 451.9 \\
\hline 91.2 & 92 & 118.8 & 229 & 413.5 & 444.6 & 566.4 & 556.5 & 569 & 557.2 \\
\hline 92 & 90.3 & 115 & 340.3 & 538.4 & 550.8 & 579.7 & 592.4 & 592.1 & 587.3 \\
\hline 104.1 & 102.4 & 120.6 & 391.1 & 612 & 662.1 & 671.5 & 657.2 & 655.9 & 645.4 \\
\hline 98.3 & 120.5 & 144.8 & 329.6 & 526.7 & 558.4 & 542.4 & 529.1 & 519.2 & 556.3 \\
\hline 109.3 & 115.4 & 151 & 404.4 & 593.5 & 643.2 & 619.8 & 639.8 & 673.9 & 653.6 \\
\hline 94.6 & 99 & 121.6 & 235.9 & 396.6 & 481.7 & 489.8 & 578.8 & 578.8 & 553.1 \\
\hline 87.7 & 100.3 & 122 & 243.1 & 488.6 & 528.8 & 535.4 & 543.1 & 510.7 & 534.4 \\
\hline 83 & 81.7 & 97.8 & 230.9 & 386.7 & 463.4 & 487.7 & 454.9 & 448 & 437.6 \\
\hline 83.4 & 80 & 99.8 & 231.2 & 432.1 & 461.8 & 454.9 & 449.7 & 448.9 & 433.3 \\
\hline 116.2 & 112.4 & 126.6 & 239.8 & 445 & 479.8 & 484.9 & 468.8 & 474.4 & 465 \\
\hline 96.4 & 95.5 & 127.5 & 279.5 & 450.2 & 480 & 502.4 & 491.2 & 491.2 & 464.4 \\
\hline 142.2 & 139.6 & 165.1 & 284.7 & 449.7 & 466.2 & 464.4 & 451.9 & 437.6 & 417.8 \\
\hline 127.5 & 123.2 & 146.9 & 284.7 & 445.4 & 447.2 & 453.2 & 453.2 & 461 & 426.9 \\
\hline 127.5 & 127.5 & 140.9 & 267.4 & 431.6 & 445.4 & 436.4 & 430.7 & 429.9 & 405.7 \\
\hline 105 & 104.1 & 123.2 & 242 & 413.5 & 473.6 & 494.8 & 499.7 & 505.3 & 564.4 \\
\hline 127.5 & 125.7 & 154.7 & 427 & 641.5 & 685.4 & 697.4 & 699.1 & 718.5 & 708.5 \\
\hline 124 & 119.7 & 147.3 & 397.5 & 533.5 & 561.2 & 568.9 & 577.5 & 550.3 & 556.2 \\
\hline 113.6 & 111.1 & 134.4 & 377.5 & 574.6 & 612.2 & 624.2 & 625.1 & 608.7 & 635 \\
\hline 132.3 & 135.2 & 152.5 & 418.7 & 598.9 & 642.6 & 650.7 & 656.3 & 678.7 & 695.9 \\
\hline 137.8 & 135.2 & 145.8 & 412.4 & 591.5 & 609.6 & 608.3 & 627.2 & 603 & 596 \\
\hline 102.1 & 130.6 & 175.4 & 256.5 & 454.1 & 489.5 & 482.6 & 503.3 & 467.5 & 434.2 \\
\hline 122.3 & 117.1 & 137.8 & 264.4 & 416.9 & 442.8 & 450.2 & 463.6 & 462.7 & 433.3 \\
\hline 114.5 & 111.5 & 128.3 & 241.5 & 432.1 & 473.8 & 468.6 & 489.7 & 492.3 & 485.7 \\
\hline 110.7 & 108.5 & 137.6 & 319.3 & 534.7 & 573.1 & 563.2 & 575.6 & 555.7 & 555.6 \\
\hline 78.2 & 99 & 102.4 & 238.5 & 413.5 & 422.1 & 433.3 & 460.1 & 453.2 & 436.4 \\
\hline 114.5 & 113.6 & 130.1 & 245.8 & 419.5 & 453.7 & 456.7 & 452.3 & 454.9 & 447.2 \\
\hline 126.6 & 118.8 & 131.8 & 255.8 & 434.2 & 463.3 & 449.2 & 457.3 & 467.9 & 455.2 \\
\hline 106.7 & 111.9 & 140.9 & 279.5 & 431.6 & 454.9 & 458.4 & 461 & 451.1 & 433.3 \\
\hline 117.1 & 110.2 & 109.3 & 102.4 & 99 & 98.6 & 100.7 & 99.8 & 120.6 & 129.2 \\
\hline 101.6 & 96.4 & 112.8 & 244.1 & 428.1 & 429 & 445.9 & 445.4 & 442 & 414.3 \\
\hline 162.9 & 165.5 & 140 & 262.3 & 443.7 & 461.8 & 454.9 & 439.8 & 427.3 & 387.5 \\
\hline 131.8 & 154.3 & 178.9 & 257.9 & 426.4 & 448.5 & 442.8 & 442 & 431.6 & 403.1 \\
\hline 95.5 & 93.8 & 88.2 & 83.4 & 81.7 & 80 & 83.4 & 86 & 83.9 & 102.4 \\
\hline 104.1 & 102.4 & 119.7 & 356.4 & 543.9 & 537.9 & 542.2 & 521.5 & 487.8 & 450.6 \\
\hline 98.1 & 98.1 & 111.9 & 316.3 & 461 & 510.2 & 486.9 & 468.8 & 471.3 & 457.5 \\
\hline 96 & 94.6 & 105 & 307.2 & 453.2 & 456.7 & 464.4 & 454.1 & 470.5 & 457.5 \\
\hline 92.9 & 90.3 & 123.6 & 319.3 & 455.8 & 450.6 & 459.2 & 480 & 454.1 & 419.5 \\
\hline 70.4 & 69.2 & 65.3 & 61.8 & 58.4 & 62.7 & 60.1 & 59.2 & 63.5 & 73 \\
\hline 71.3 & 69.6 & 93.8 & 315 & 458.8 & 454.9 & 440.2 & 446.3 & 416 & 419.5 \\
\hline 89.5 & 87.7 & 110.2 & 341.3 & 464.4 & 485.2 & 487.8 & 484.3 & 470.5 & 439.4 \\
\hline 94.6 & 94.6 & 106.7 & 329.2 & 464.4 & 459.2 & 472.2 & 473.9 & 463.6 & 442.8 \\
\hline 93.8 & 93.8 & 122.3 & 363.3 & 457.1 & 450.6 & 479.1 & 499.9 & 446.3 & 453.9 \\
\hline 81.7 & 79.6 & 77.4 & 71.3 & 69.6 & 67.9 & 69.6 & 67 & 68.7 & 68.7 \\
\hline 81.7 & 81.7 & 90.8 & 274.4 & 452.3 & 471.3 & 455.8 & 461.8 & 457.5 & 435.9 \\
\hline
\end{tabular}




\begin{tabular}{|c|c|c|c|c|c|c|c|c|c|}
\hline 91.2 & 91.6 & 118 & 326.2 & 458.4 & 479 & 494.6 & 488.4 & 487.9 & 474.4 \\
\hline 89.9 & 90.3 & 116.2 & 338.3 & 443.7 & 467 & 463.6 & 448.5 & 446.3 & 416 \\
\hline 106.7 & 105.9 & 134.4 & 346.1 & 465.8 & 473.9 & 474.8 & 451.5 & 454.9 & 410 \\
\hline 83 & 85.1 & 80 & 77.4 & 74.8 & 80.4 & 96.4 & 100.7 & 92 & 87.7 \\
\hline 86 & 83.9 & 97.2 & 268.3 & 444.6 & 428.1 & 446.3 & 443.7 & 437.6 & 417.4 \\
\hline 92.9 & 92 & 99 & 296.8 & 430.7 & 448 & 480.8 & 501.3 & 476.5 & 468.1 \\
\hline 93.8 & 92 & 99 & 292.8 & 444.4 & 458.3 & 482.1 & 470.8 & 466.9 & 462.1 \\
\hline 92 & 92.9 & 117.7 & 306.2 & 448.7 & 476 & 486.3 & 481.5 & 485.4 & 486.6 \\
\hline 92 & 91.2 & 87.7 & 79.1 & 80.8 & 88.6 & 91.2 & 92 & 94.2 & 93.8 \\
\hline 85.7 & 113.5 & 124.7 & 245.4 & 417.9 & 571.1 & 556.2 & 588.7 & 586 & 582.8 \\
\hline 92.9 & 87.3 & 93.5 & 316.7 & 525.3 & 530.1 & 534.8 & 554.7 & 585.7 & 569.6 \\
\hline 81.3 & 80.8 & 97.8 & 247.9 & 436.6 & 533 & 525.6 & 539.7 & 548.1 & 509.4 \\
\hline 77.4 & 81.7 & 104.1 & 242.4 & 382.8 & 415.2 & 416 & 448.6 & 475 & 449.3 \\
\hline 80.8 & 80.8 & 78.2 & 73 & 94.6 & 94.6 & 73 & 77.4 & 88.6 & 85.6 \\
\hline 83.8 & 83 & 103.3 & 244.1 & 424.7 & 441.1 & 451.6 & 573.4 & 601.4 & 537.4 \\
\hline 78.2 & 76.5 & 89.5 & 224.2 & 405.3 & 439.4 & 454.1 & 451.5 & 452.3 & 433.3 \\
\hline 85.1 & 86 & 104.1 & 273.5 & 443.7 & 457.5 & 466.2 & 464.9 & 446.3 & 410.9 \\
\hline 86.9 & 84.3 & 100.7 & 261.4 & 431.6 & 446.3 & 460.7 & 479.2 & 470 & 415.1 \\
\hline 78.2 & 80 & 73 & 66.1 & 66.1 & 75.6 & 75.6 & 80 & 90.3 & 95.5 \\
\hline 85.1 & 83.4 & 99.8 & 247.2 & 412.1 & 447.5 & 470.3 & 469.5 & 469.4 & 496.2 \\
\hline 86.9 & 86 & 96.4 & 277.8 & 500.7 & 544.8 & 559 & 582.7 & 578.9 & 559.9 \\
\hline 158.2 & 158.6 & 174.1 & 315.8 & 510.1 & 547.9 & 555.5 & 591.3 & 605.9 & 590.6 \\
\hline 96.4 & 99 & 100.7 & 302 & 564.9 & 609 & 605.9 & 608.8 & 631.2 & 658.4 \\
\hline 86 & 86 & 85.1 & 73.9 & 75.6 & 76.5 & 79.1 & 80 & 77.4 & 88.6 \\
\hline 88.6 & 92 & 104.1 & 401.7 & 636.7 & 658.7 & 673.7 & 662.5 & 680.1 & 676.6 \\
\hline 88.6 & 86 & 100.7 & 228.6 & 375.9 & 425.6 & 428.1 & 435.1 & 419.5 & 401.4 \\
\hline 77 & 76.5 & 92 & 226 & 405.7 & 429.9 & 382.4 & 438.1 & 424.7 & 403.1 \\
\hline 88.6 & 90.3 & 85.1 & 79.1 & 80 & 80.8 & 80.8 & 79.1 & 86 & 91.6 \\
\hline 86.9 & 87.7 & 111.5 & 253.6 & 420.4 & 455.8 & 460.6 & 463.6 & 433.3 & 429 \\
\hline 90.3 & 92.5 & 104.1 & 238.1 & 406.5 & 444.6 & 447.2 & 443.9 & 474.6 & 481.4 \\
\hline 76.5 & 80.8 & 99.4 & 235.5 & 443.6 & 607 & 610.7 & 628 & 650 & 652 \\
\hline 77.8 & 84.3 & 92 & 257.9 & 500.8 & 599.8 & 638.8 & 663.4 & 675.4 & 684.8 \\
\hline 90.3 & 90.3 & 86.9 & 83.4 & 82.5 & 81.7 & 83.4 & 84.3 & 90.3 & 89.5 \\
\hline 89.5 & 92 & 111.9 & 230.3 & 487 & 497.5 & 445.2 & 527.8 & 543.5 & 559.5 \\
\hline 160.3 & 161.2 & 176.7 & 325.7 & 524.3 & 567.9 & 542.4 & 561.5 & 573.6 & 576.1 \\
\hline 75.6 & 79.6 & 89.5 & 295.9 & 498 & 548.5 & 547.3 & 599.5 & 590.4 & 588.1 \\
\hline 140.4 & 150.4 & 140.4 & 238.9 & 407.9 & 431.6 & 435.9 & 431.6 & 420.4 & 422.6 \\
\hline 78.2 & 77.4 & 73 & 68.7 & 68.7 & 69.2 & 68.7 & 70.4 & 73 & 95.5 \\
\hline 80.8 & 94.6 & 259.3 & 506.7 & 578.9 & 580.9 & 574.4 & 570.1 & 583.9 & 562 \\
\hline 87.7 & 100.7 & 291.2 & 517.9 & 507.2 & 550.8 & 576.7 & 600 & 616 & 609.1 \\
\hline 102 & 109.3 & 115.4 & 377.4 & 577.2 & 585.8 & 616 & 639.8 & 708.9 & 698.1 \\
\hline 86.9 & 94.6 & 98.1 & 358.6 & 582 & 628.8 & 662.9 & 661.6 & 681.9 & 692.5 \\
\hline 104.1 & 104.1 & 104.1 & 101.6 & 98.1 & 105 & 102.4 & 101.6 & 100.7 & 106.7 \\
\hline 113.6 & 119.7 & 122.8 & 451.5 & 683.4 & 664.1 & 746 & 759.4 & 798.7 & 807.6 \\
\hline 96.4 & 97.2 & 102.4 & 398.2 & 683.8 & 728.7 & 732.5 & 750.2 & 768.3 & 786.5 \\
\hline 104.1 & 115.4 & 120.2 & 451.8 & 688.1 & 726.5 & 750.3 & 745.1 & 777.4 & 765.3 \\
\hline 95.5 & 101.6 & 98.1 & 193.1 & 222.5 & 226.4 & 224.2 & 234.6 & 203.5 & 229.4 \\
\hline 93.8 & 93.8 & 106.7 & 361.6 & 586 & 627.3 & 651.4 & 657 & 675.5 & 694 \\
\hline 90.3 & 99 & 100.7 & 366.8 & 614.8 & 696.4 & 717.1 & 751.2 & 740.3 & 753.6 \\
\hline
\end{tabular}


other

\begin{tabular}{|c|c|c|c|c|c|c|c|c|c|}
\hline 188 & 199.2 & 217.3 & 457.4 & 670.9 & 717.5 & 736 & 766.8 & 726.7 & 797.1 \\
\hline 96.4 & 95.5 & 93.4 & 86.9 & 88.6 & 92.9 & 87.7 & 89.5 & 91.2 & 93.8 \\
\hline 105.9 & 118 & 124.9 & 376.2 & 576 & 612.8 & 622.8 & 656 & 584.6 & 482.5 \\
\hline 113.6 & 122.3 & 143 & 474.9 & 672.5 & 728.7 & 732.1 & 754.1 & 778.7 & 794.1 \\
\hline 95.1 & 105 & 114.5 & 441.6 & 657.3 & 669.9 & 699.2 & 717.3 & 735 & 755.3 \\
\hline 101.6 & 105 & 99.8 & 92.9 & 95.1 & 96.4 & 99.4 & 102.9 & 104.1 & 106.7 \\
\hline 101.6 & 108.5 & 129.2 & 497.9 & 702.7 & 738.4 & 728.9 & 708.1 & 723.6 & 742.9 \\
\hline 101.6 & 111.1 & 125.7 & 443.1 & 661.3 & 710.6 & 721.8 & 751.6 & 749.8 & 777 \\
\hline 168.1 & 165.5 & 181.5 & 458.6 & 642.8 & 690.7 & 693.3 & 722.7 & 722.2 & 745 \\
\hline 87.7 & 90.3 & 106.3 & 358.2 & 506.4 & 546.2 & 558.2 & 570.3 & 555.2 & 571.9 \\
\hline 79.1 & 82.5 & 76.5 & 81.3 & 81.7 & 86.9 & 88.6 & 91.2 & 92 & 93.8 \\
\hline 98.1 & 107.6 & 123.1 & 454.9 & 699.9 & 775 & 750 & 785 & 786.7 & 797.9 \\
\hline 109.3 & 116.2 & 132.7 & 564.5 & 770.8 & 820.1 & 726.3 & 842.5 & 851.8 & 868.2 \\
\hline 106.7 & 115.4 & 138.7 & 594.2 & 826.6 & 829.3 & 852.2 & 891.6 & 883.7 & 889.4 \\
\hline 113.6 & 130.9 & 147.3 & 588.1 & 795.5 & 860 & 862.5 & 897.4 & 894.9 & 930.3 \\
\hline 98.1 & 101.6 & 98.1 & 89.5 & 94.6 & 93.4 & 92 & 94.6 & 97.2 & 105 \\
\hline 94.6 & 104.1 & 111.1 & 348.8 & 601.7 & 702.6 & 758.1 & 762.5 & 774.2 & 784.6 \\
\hline 87.7 & 103.3 & 115.4 & 478.3 & 681.1 & 736.3 & 796.8 & 811.8 & 791 & 809.1 \\
\hline 96.4 & 106.7 & 121.4 & 512.1 & 733.6 & 792 & 709.5 & 775.5 & 789.2 & 795.4 \\
\hline 97.2 & 105.9 & 116.2 & 504.2 & 739.2 & 786.3 & 824.4 & 849.5 & 823.6 & 838.3 \\
\hline 113.6 & 106.7 & 100.7 & 98.1 & 101.2 & 110.2 & 117.1 & 121.4 & 123.2 & 130.9 \\
\hline 114.1 & 113.6 & 122.7 & 590.3 & 790.2 & 833.1 & 859 & 854.2 & 846.9 & 847.7 \\
\hline 112.8 & 119.7 & 134.4 & 613.2 & 801.5 & 810.1 & 833.6 & 831.7 & 830 & 833.8 \\
\hline 140.4 & 155.2 & 184.5 & 370.7 & 414.3 & 416.5 & 414.3 & 418.2 & 436.4 & 432.5 \\
\hline 127.9 & 132.3 & 297.3 & 654.5 & 820.4 & 923.7 & 928.8 & 957.9 & 946.2 & 946.7 \\
\hline 127.1 & 139.2 & 339 & 680.4 & 903.5 & 933.8 & 983.9 & 980.4 & 963.1 & 981.1 \\
\hline 127.1 & 126.2 & 329 & 657 & 879.1 & 916.4 & 935.8 & 960.3 & 959.4 & 955 \\
\hline 99.4 & 99.8 & 96.8 & 89.9 & 91.2 & 93.4 & 89.5 & 89.9 & 93.8 & 96.8 \\
\hline 89.9 & 96.4 & 298.1 & 605.5 & 793.6 & 836.6 & 868.6 & 876.3 & 769 & 729.3 \\
\hline 100.3 & 107.6 & 253.2 & 538.7 & 761.5 & 825.8 & 875.6 & 888 & 858.5 & 900 \\
\hline 88.2 & 93.8 & 288.1 & 591 & 793.5 & 829.9 & 889.2 & 895.1 & 894.2 & 905.4 \\
\hline 108.1 & 106.7 & 116.7 & 102.9 & 96.4 & 110.7 & 119.3 & 124.9 & 124.5 & 152.1 \\
\hline 149.9 & 291.9 & 387.3 & 685.3 & 907.2 & 920.7 & 940.5 & 945.3 & 413.5 & 984.4 \\
\hline 125.3 & 127.9 & 308.7 & 615.1 & 821.8 & 840.5 & 912.3 & 948.7 & 943.9 & 955.9 \\
\hline 96.8 & 105 & 226.9 & 541.1 & 783 & 788.2 & 796 & 842.5 & 827.8 & 829.5 \\
\hline 89.1 & 94.6 & 275.1 & 499.3 & 666.4 & 686.4 & 712.7 & 717.9 & 714 & 741.8 \\
\hline 98.6 & 98.1 & 85.6 & 83.4 & 82.1 & 81.3 & 82.5 & 87.3 & 95.5 & 109.8 \\
\hline 106.3 & 253.4 & 337.7 & 590.6 & 784.2 & 781.3 & 822.3 & 841.3 & 821.5 & 820 \\
\hline 92.9 & 100.7 & 256.8 & 453 & 635.3 & 684.2 & 724.9 & 742.2 & 727.9 & 746 \\
\hline 72.2 & 77.4 & 195.3 & 378.2 & 560.4 & 603.2 & 626.9 & 663 & 676.8 & 681.5 \\
\hline 72.2 & 78.2 & 211.9 & 360.4 & 537.2 & 586.9 & 609 & 677.3 & 695.4 & 705.2 \\
\hline 79.6 & 79.1 & 78.2 & 69.6 & 71.3 & 70.4 & 71.8 & 77.4 & 78.2 & 76.5 \\
\hline 89.1 & 216.1 & 284.6 & 454.2 & 627.6 & 677.4 & 742.3 & 785.9 & 768.5 & 792.1 \\
\hline 147.3 & 136.1 & 279.5 & 400.3 & 616.8 & 693 & 752.2 & 761.7 & 753.4 & 772.8 \\
\hline 82.5 & 88.6 & 250.1 & 427 & 658.7 & 712.4 & 773.3 & 775.9 & 760.7 & 807.6 \\
\hline 78.2 & 85.6 & 232.3 & 404.9 & 557.4 & 578.7 & 621.4 & 647.4 & 621.8 & 652 \\
\hline 105.9 & 230.8 & 295.3 & 468.4 & 631.9 & 683.8 & 711.5 & 714.1 & 642.7 & 598.4 \\
\hline 137.8 & 130.9 & 265.6 & 398.9 & 601.5 & 656.1 & 742.1 & 758.9 & 740.8 & 772.7 \\
\hline 91.2 & 97.2 & 257.6 & 456.5 & 659.1 & 775.4 & 824.2 & 816.9 & 811.2 & 843.8 \\
\hline
\end{tabular}




\begin{tabular}{|c|c|c|c|c|c|c|c|c|c|}
\hline 108.1 & 109.3 & 298.2 & 580.3 & 781.2 & 811.2 & 853.1 & 870 & 872.2 & 856.2 \\
\hline 87.7 & 87.7 & 84.3 & 78.2 & 83.4 & 86.5 & 90.3 & 86 & 80.8 & 78.2 \\
\hline 86.9 & 193.1 & 269.3 & 423.6 & 585.3 & 650.1 & 689 & 712 & 706.3 & 726.5 \\
\hline 127.9 & 124 & 269.6 & 397.3 & 594.3 & 661 & 729.2 & 749.9 & 777.1 & 776.6 \\
\hline 83.9 & 92.9 & 257.9 & 426.6 & 634.9 & 716.7 & 763.3 & 791.4 & 795.2 & 798.6 \\
\hline 81.7 & 87.3 & 255.3 & 482.9 & 679.5 & 718.9 & 771 & 823.6 & 770.6 & 793.9 \\
\hline 70.4 & 70.4 & 70.4 & 61.4 & 57.5 & 56.6 & 61.8 & 64.4 & 68.7 & 72.2 \\
\hline 96.4 & 92.9 & 229.1 & 313.9 & 565.6 & 404.7 & 822.1 & 855.4 & 837.2 & 876 \\
\hline 149.9 & 136.1 & 277.8 & 494.1 & 710 & 734.1 & 760.5 & 783.3 & 777.7 & 809.1 \\
\hline 80.8 & 87.7 & 259.6 & 510.5 & 688.6 & 762.1 & 757.7 & 806.1 & 814.7 & 824.1 \\
\hline 98.1 & 92.9 & 269.2 & 548.1 & 753.7 & 785.3 & 815.6 & 852.3 & 825.5 & 842.2 \\
\hline 86.9 & 87.3 & 81.7 & 69.6 & 66.1 & 64.9 & 66.1 & 66.1 & 71.3 & 75.6 \\
\hline 74.8 & 168.9 & 238.6 & 439.2 & 583.1 & 646.2 & 681.7 & 714.5 & 678.5 & 712.2 \\
\hline 140.4 & 137.4 & 289.3 & 442.6 & 724.8 & 756.7 & 793.8 & 830.1 & 826.2 & 841.8 \\
\hline 78.2 & 82.1 & 201.8 & 343.4 & 507.2 & 530.6 & 535.1 & 508.1 & 474.4 & 480.3 \\
\hline 79.6 & 80.8 & 145 & 306.4 & 489.3 & 477.3 & 491.1 & 507.4 & 478.6 & 437.8 \\
\hline 97.7 & 96.4 & 91.2 & 86.9 & 114.1 & 112.8 & 100.7 & 86.9 & 87.7 & 96.4 \\
\hline 75.6 & 90.8 & 135.2 & 335.6 & 550.2 & 573.2 & 547.2 & 547.6 & 572.2 & 662 \\
\hline 129.2 & 130.1 & 268.9 & 414.8 & 616.7 & 649.2 & 671.2 & 644.8 & 599.8 & 613.1 \\
\hline 80.8 & 89.5 & 209.3 & 346.1 & 567.4 & 630.1 & 709 & 737.6 & 718.9 & 736.6 \\
\hline 80.4 & 86 & 211 & 356 & 548.7 & 553.9 & 560 & 585 & 562.5 & 583.2 \\
\hline 78.2 & 76.5 & 72.6 & 67 & 60.9 & 57.5 & 58.4 & 62.3 & 58.4 & 59.2 \\
\hline 82.5 & 138.7 & 189.2 & 323.3 & 481.1 & 525.7 & 541.3 & 539.6 & 511.6 & 497.3 \\
\hline 102.4 & 98.1 & 176.3 & 308 & 471.8 & 536.6 & 569.2 & 608 & 614.9 & 592.3 \\
\hline 87.3 & 92.9 & 210.4 & 372.3 & 568.5 & 601.5 & 611.5 & 674.5 & 669.3 & 662.4 \\
\hline 92.9 & 95.5 & 242.9 & 434.2 & 620.9 & 658.6 & 680.7 & 671.6 & 657.7 & 643.8 \\
\hline 84.3 & 82.5 & 80 & 72.6 & 70.4 & 71.3 & 71.3 & 68.7 & 68.7 & 69.6 \\
\hline 82.5 & 130.9 & 210.6 & 344.6 & 451.5 & 497.7 & 473.1 & 483.4 & 460.1 & 461 \\
\hline 91.6 & 90.3 & 86.9 & 79.1 & 81.7 & 81.3 & 81.7 & 85.1 & 82.5 & 90.3 \\
\hline 101.2 & 102.4 & 168.1 & 357.3 & 496.9 & 492.1 & 509.4 & 494.7 & 467.5 & 473.9 \\
\hline 135.2 & 142.2 & 169.8 & 309.4 & 434.6 & 457.1 & 471.3 & 475.7 & 442.8 & 445.6 \\
\hline 85.6 & 85.1 & 138.6 & 300.7 & 430.7 & 451.1 & 465.3 & 480.3 & 491.4 & 477.3 \\
\hline 85.6 & 85.1 & 116.3 & 299.9 & 412.6 & 448.9 & 458 & 474.8 & 435.9 & 423 \\
\hline 74.4 & 74.8 & 71.3 & 64.4 & 63.5 & 60.9 & 61.4 & 71.3 & 61.8 & 65.3 \\
\hline 74.8 & 77 & 164.5 & 353.4 & 518.9 & 526.6 & 531.8 & 507.6 & 457.5 & 435.1 \\
\hline 118 & 126.6 & 150.4 & 296 & 455.8 & 473.9 & 461.8 & 454.9 & 426.4 & 429.5 \\
\hline 85.6 & 85.1 & 124 & 295.1 & 425.6 & 464.3 & 477.4 & 508.3 & 537.6 & 505.2 \\
\hline 69.6 & 69.6 & 86.9 & 284.7 & 451.1 & 431.6 & 459.2 & 461.8 & 441.1 & 428 \\
\hline 65.3 & 67 & 63.5 & 56.6 & 54 & 54.5 & 54 & 55.8 & 56.6 & 56.6 \\
\hline 67.9 & 74.8 & 85.1 & 227.7 & 384.9 & 427.3 & 448.9 & 464.9 & 437.6 & 433.3 \\
\hline 128.3 & 137 & 168.5 & 295.1 & 453.2 & 481.7 & 504.2 & 497.3 & 455.8 & 427.3 \\
\hline 94.6 & 132.7 & 164.6 & 253.6 & 432.5 & 461.8 & 475.3 & 486.9 & 478.3 & 426.4 \\
\hline 90.8 & 97.2 & 117.1 & 323.6 & 484.8 & 482.6 & 491.2 & 481.7 & 480 & 453.2 \\
\hline 90.3 & 90.3 & 110.2 & 310.6 & 464.4 & 449.3 & 448.9 & 452.3 & 442 & 415.2 \\
\hline 89.5 & 89.5 & 128.8 & 321.9 & 496.4 & 466.2 & 466.2 & 471.3 & 448 & 413.1 \\
\hline 84.3 & 83.4 & 93.8 & 275.2 & 466.2 & 457.1 & 460.1 & 461.8 & 431.6 & 412.6 \\
\hline 79.1 & 89.5 & 145.2 & 327.1 & 544.8 & 567.2 & 542.2 & 534.4 & 504.2 & 476.5 \\
\hline 109.3 & 111.1 & 130.9 & 312.4 & 500.7 & 523.2 & 507.2 & 492.9 & 423.8 & 410.4 \\
\hline 84.3 & 85.1 & 104.1 & 302.5 & 510.2 & 475.7 & 475.7 & 467.9 & 458.4 & 446.3 \\
\hline
\end{tabular}


other

\begin{tabular}{|c|c|c|c|c|c|c|c|c|c|}
\hline 93.8 & 96.4 & 151.7 & 352.1 & 519.7 & 480.4 & 499 & 480 & 464.4 & 436.8 \\
\hline 81.7 & 80.8 & 100.7 & 288.2 & 474.8 & 480.8 & 479.1 & 468.8 & 464.4 & 430.3 \\
\hline 61.8 & 80 & 112.8 & 306.3 & 447.6 & 414.3 & 429 & 442.3 & 422.3 & 460.9 \\
\hline 63.5 & 64.4 & 89.5 & 287.3 & 451.5 & 443.3 & 456.7 & 448.9 & 458.4 & 439.4 \\
\hline 92 & 92.9 & 106.7 & 320.1 & 486.9 & 494.7 & 496 & 480 & 480.8 & 426.4 \\
\hline 98.1 & 99.4 & 140.4 & 337.4 & 467 & 439.4 & 469.2 & 495 & 455.8 & 441.6 \\
\hline 82.5 & 83.4 & 97.2 & 259.3 & 444.6 & 425.6 & 487.9 & 479 & 457.4 & 444.3 \\
\hline 85.1 & 103.3 & 172 & 354.7 & 537 & 512.8 & 492.9 & 506.4 & 476.5 & 434.2 \\
\hline 111.5 & 162 & 195.7 & 289.9 & 446.8 & 448 & 474.8 & 471.3 & 448.5 & 417.8 \\
\hline 102 & 154.3 & 185.4 & 269.2 & 463.2 & 467 & 447.2 & 455.8 & 443.3 & 405.7 \\
\hline 81.3 & 149.9 & 207.8 & 341.3 & 455.8 & 465.3 & 480 & 454.5 & 456.7 & 405.7 \\
\hline 79.1 & 140.9 & 188 & 270.9 & 416.9 & 444.2 & 448 & 466.2 & 422.1 & 416.5 \\
\hline 85.6 & 137 & 176.7 & 329.6 & 503.8 & 509.4 & 518 & 507.6 & 458.4 & 422.1 \\
\hline 91.6 & 156.8 & 197.5 & 282.1 & 403.1 & 436.4 & 451.5 & 455.8 & 422.1 & 402.2 \\
\hline 85.1 & 130.1 & 164.6 & 297.7 & 447.2 & 447.2 & 469.6 & 458.8 & 453.2 & 416 \\
\hline 90.3 & 153.4 & 213.5 & 308 & 447.2 & 453.2 & 451.5 & 464.9 & 435.9 & 415.2 \\
\hline 85.1 & 149.1 & 175.5 & 272.6 & 435.1 & 445.9 & 466.2 & 464.4 & 444.6 & 417.8 \\
\hline 84.7 & 139.6 & 189.7 & 316.7 & 454.1 & 477.9 & 491.2 & 471.3 & 440.7 & 418.1 \\
\hline 90.3 & 126.6 & 162.9 & 305.5 & 435.9 & 449.4 & 480.4 & 486.5 & 463.6 & 447.6 \\
\hline 104.1 & 127.5 & 141.3 & 302 & 449.7 & 454.9 & 480.6 & 481.1 & 445.5 & 423.5 \\
\hline 80.8 & 116.2 & 137 & 277.8 & 399.6 & 437.2 & 445 & 451 & 421.7 & 424.3 \\
\hline 80 & 100.3 & 111.1 & 250.2 & 435.9 & 437.6 & 448 & 434.2 & 407.4 & 399.5 \\
\hline 66.6 & 82.5 & 118 & 274.4 & 443.3 & 451.5 & 447.2 & 435.9 & 430.3 & 396.2 \\
\hline 87.7 & 129.7 & 154.3 & 291.6 & 465.8 & 458.4 & 474.8 & 464.9 & 441.1 & 422.1 \\
\hline 100.3 & 144.8 & 167.2 & 309.8 & 454.5 & 485.2 & 512.8 & 500.3 & 485.2 & 448.9 \\
\hline 94.6 & 132.7 & 153.4 & 311.1 & 460.1 & 473.1 & 462.7 & 467.9 & 428.1 & 403.1 \\
\hline 79.1 & 121.4 & 140 & 253.6 & 410 & 424.7 & 432.9 & 444.6 & 451.2 & 456.2 \\
\hline 82.5 & 96.8 & 114.5 & 247.6 & 443.7 & 449.7 & 479.1 & 546.2 & 504.5 & 496.2 \\
\hline 60.9 & 141.8 & 134.4 & 288.7 & 480.8 & 480.9 & 505.8 & 513.5 & 504.1 & 486.4 \\
\hline 69.6 & 88.6 & 96.8 & 261.8 & 432.5 & 497.3 & 590 & 591.6 & 548.3 & 549.9 \\
\hline 63.5 & 80 & 77.8 & 245 & 426.4 & 443.7 & 448.9 & 440.7 & 433.3 & 396.2 \\
\hline 66.1 & 118.8 & 110.2 & 257.1 & 453.2 & 486.9 & 475.3 & 459.2 & 448.9 & 451.5 \\
\hline 77.4 & 92 & 101.6 & 284.3 & 416.9 & 446.3 & 451.5 & 466.2 & 447.2 & 452.3 \\
\hline 69.2 & 81.7 & 87.7 & 281.3 & 423 & 436.8 & 456.8 & 553.6 & 548.2 & 546.4 \\
\hline 63.5 & 83.4 & 180.2 & 260.1 & 417.8 & 440.6 & 419.3 & 533.4 & 540.2 & 500.4 \\
\hline 68.7 & 86.9 & 98.1 & 223.8 & 392.7 & 443.7 & 433.3 & 453.2 & 442 & 424.3 \\
\hline 75.6 & 128.3 & 138.3 & 278.7 & 428.1 & 467.6 & 515.6 & 578 & 552 & 548.5 \\
\hline 76.5 & 96.4 & 143 & 268.3 & 449.7 & 507.6 & 570.3 & 572.9 & 589.7 & 596.9 \\
\hline 67 & 99 & 107.6 & 241.5 & 497.8 & 576.4 & 566.4 & 652 & 672.3 & 683.4 \\
\hline 80 & 118.8 & 111.1 & 279.3 & 503.7 & 495.1 & 490 & 494.3 & 524.3 & 490.4 \\
\hline 77.8 & 92.9 & 99.8 & 255.8 & 455.8 & 464.4 & 461 & 504.6 & 467 & 439.4 \\
\hline 69.6 & 99 & 103.3 & 238.1 & 454.5 & 530.7 & 547.4 & 580.2 & 572.5 & 556.9 \\
\hline 73.9 & 98.1 & 101.6 & 263.6 & 425.6 & 459.2 & 465.9 & 458.8 & 433.3 & 416.9 \\
\hline 70 & 88.6 & 107.6 & 256.1 & 568.8 & 556 & 554.3 & 568.1 & 563 & 567.6 \\
\hline 76.5 & 84.3 & 173.3 & 330.1 & 523.5 & 545 & 559.3 & 646 & 665.4 & 681.3 \\
\hline 85.1 & 137.8 & 232.9 & 405.7 & 588.5 & 617.9 & 613.1 & 584.6 & 566.8 & 548.1 \\
\hline 67.9 & 155.6 & 167.7 & 416.3 & 606.7 & 630 & 638.1 & 694.1 & 716.5 & 684.9 \\
\hline 105.5 & 133.9 & 242 & 428.2 & 640.4 & 682.8 & 710 & 709.6 & 717.7 & 759.6 \\
\hline 89.9 & 118 & 224.2 & 327.8 & 541 & 555.7 & 572.5 & 578.9 & 569.4 & 614.3 \\
\hline
\end{tabular}


other

\begin{tabular}{|c|c|c|c|c|c|c|c|c|c|}
\hline 78.2 & 101.6 & 173.9 & 374.1 & 527.9 & 549.1 & 568.5 & 594.9 & 582.3 & 593.8 \\
\hline 101.6 & 119.7 & 198.4 & 296.9 & 426.7 & 533.8 & 570.1 & 582.6 & 555.4 & 554 \\
\hline 73.9 & 90.3 & 105.5 & 261.1 & 425.2 & 420 & 410.9 & 453.5 & 457.2 & 499.4 \\
\hline 70.4 & 77.4 & 172.9 & 305.7 & 463.7 & 537 & 540.1 & 562.2 & 524.9 & 539.2 \\
\hline 69.6 & 84.3 & 165.1 & 373.5 & 587.3 & 593.7 & 595.1 & 636.9 & 632.6 & 543.8 \\
\hline 74.4 & 85.1 & 159.4 & 330.1 & 515.8 & 513.7 & 565.5 & 582.8 & 545.1 & 511.5 \\
\hline 92.9 & 109.3 & 124.5 & 468.9 & 691.6 & 734.4 & 743.5 & 786.6 & 747.2 & 781.3 \\
\hline 132.7 & 260.5 & 254.5 & 530.3 & 706.8 & 728.8 & 692.9 & 694.1 & 697.1 & 674.5 \\
\hline 73 & 155.1 & 151.7 & 257.8 & 463.9 & 446.2 & 492.9 & 536.4 & 503.3 & 491.5 \\
\hline 72.2 & 122.3 & 134.4 & 242.4 & 428.1 & 490.3 & 505.5 & 559.8 & 576.3 & 580.4 \\
\hline 70.4 & 226.8 & 218.2 & 457.9 & 627.6 & 690.6 & 691 & 733.3 & 728.1 & 749.6 \\
\hline 109.3 & 226.8 & 236.3 & 479.2 & 664.8 & 696.3 & 716.2 & 745.1 & 660.8 & 640.4 \\
\hline 81.7 & 89.5 & 105.5 & 349.2 & 524.3 & 569.3 & 563.6 & 556.3 & 579.2 & 586.3 \\
\hline 73.9 & 85.1 & 92 & 327.1 & 503.9 & 523.4 & 529.4 & 579.2 & 588.2 & 586.4 \\
\hline 81.7 & 100.7 & 108.5 & 370.2 & 603.2 & 643.8 & 642 & 702.6 & 668.1 & 725.5 \\
\hline 74.4 & 164.6 & 156 & 486 & 676.1 & 717.8 & 742.5 & 760.3 & 740.9 & 749.7 \\
\hline 94.6 & 215.2 & 226 & 452.1 & 647.7 & 663.7 & 684 & 693.1 & 679.7 & 685.7 \\
\hline 63.9 & 216.9 & 216.5 & 469.3 & 655.3 & 680.8 & 616.8 & 642.2 & 626.7 & 592.9 \\
\hline 75.6 & 169.8 & 162 & 341.6 & 533.8 & 566.5 & 627.4 & 637.6 & 664.8 & 670.6 \\
\hline 73 & 121.4 & 124.6 & 409.7 & 564.3 & 626.9 & 660.6 & 688.5 & 682 & 701.4 \\
\hline 80.8 & 191.4 & 214.7 & 483.6 & 681.2 & 716.6 & 705 & 717.1 & 716.1 & 756 \\
\hline 79.1 & 194 & 217.3 & 525.7 & 724.2 & 734.5 & 711.1 & 738.5 & 695.9 & 732.6 \\
\hline 79.1 & 128.3 & 141.3 & 354.6 & 555.6 & 706.4 & 747.1 & 773.6 & 662.8 & 550.6 \\
\hline 77.4 & 194.9 & 217.3 & 521.2 & 728.2 & 765.9 & 765.7 & 780.9 & 753.6 & 741.9 \\
\hline 70.4 & 261.4 & 251.4 & 442.2 & 621.6 & 640 & 187.1 & 666.2 & 648 & 648.2 \\
\hline 88.6 & 197.5 & 213.9 & 401.9 & 618.8 & 656.7 & 690.8 & 693.9 & 687.8 & 697.7 \\
\hline 80.8 & 190.1 & 207.2 & 450.3 & 661.5 & 692.7 & 700.5 & 731.4 & 690.8 & 701.2 \\
\hline 86.5 & 196.6 & 215.6 & 449.3 & 642.4 & 657.1 & 661.8 & 721 & 712.4 & 726.4 \\
\hline 65.3 & 180.2 & 210.3 & 492.1 & 677.7 & 706.6 & 754.6 & 778 & 743.9 & 752.8 \\
\hline 67 & 291.6 & 308.9 & 533.7 & 766.5 & 803.1 & 814.4 & 840.3 & 830.3 & 846.6 \\
\hline 93.8 & 213 & 271.4 & 613.9 & 823.2 & 839.4 & 874 & 888.2 & 843.8 & 772.8 \\
\hline 77.4 & 192.3 & 210.4 & 454.8 & 654.1 & 692.1 & 704.2 & 709 & 703.8 & 728.8 \\
\hline 77.8 & 191.4 & 210.4 & 457.7 & 660 & 689.1 & 753 & 759 & 760.3 & 757.2 \\
\hline 71.3 & 189.7 & 226 & 517.7 & 694.7 & 746.1 & 770.4 & 791 & 768.8 & 771.7 \\
\hline 65.3 & 248.4 & 241.9 & 463.4 & 627.5 & 644.4 & 662.6 & 682.4 & 370.3 & 625 \\
\hline 86.9 & 195.7 & 206.1 & 442 & 652.1 & 684.9 & 665.9 & 698.7 & 662.8 & 674 \\
\hline 78.2 & 188.8 & 212.6 & 419.5 & 635.1 & 667.6 & 676.2 & 697.8 & 685.2 & 698.2 \\
\hline 73.5 & 93.9 & 374.5 & 770.5 & 937.4 & 934.9 & 945.2 & 957.3 & 940.4 & 961.1 \\
\hline 76.1 & 96.9 & 377.9 & 728.2 & 893 & 939.1 & 967.5 & 988.1 & 947.4 & 965 \\
\hline 83.9 & 249 & 627.1 & 754 & 943.6 & 966.3 & 989 & 983.7 & 957 & 995.4 \\
\hline 79.6 & 102.1 & 416.7 & 763.3 & 945.2 & 937 & 975.6 & 986.4 & 905.9 & 876.6 \\
\hline 69.2 & 88.3 & 296.7 & 496.4 & 658.5 & 691.5 & 695.9 & 729.2 & 725.2 & 740.3 \\
\hline 64.9 & 82.2 & 293.6 & 494.1 & 645.9 & 689.7 & 737.3 & 743.8 & 732.5 & 770.8 \\
\hline 72.6 & 92.6 & 326.4 & 524.8 & 716.8 & 683.1 & 741.1 & 779.2 & 742.2 & 788.4 \\
\hline 83.9 & 264.5 & 551.2 & 735.1 & 924.9 & 963.3 & 993.5 & 998.2 & 982.2 & 963.9 \\
\hline 79.6 & 101.2 & 397.8 & 723.4 & 897.3 & 898.9 & 946.5 & 971 & 955.8 & 969.2 \\
\hline 81.3 & 102.1 & 395.6 & 718.2 & 893.2 & 936.6 & 897.8 & 934.7 & 932.2 & 953.3 \\
\hline 78.7 & 100.4 & 406.4 & 723.9 & 861 & 921.3 & 966.3 & 971.5 & 947.1 & 953.1 \\
\hline 84.3 & 103.4 & 406.8 & 711.7 & 890.8 & 929 & 951.5 & 952.7 & 960.8 & 964.8 \\
\hline
\end{tabular}


other

\begin{tabular}{|c|c|c|c|c|c|c|c|c|c|}
\hline 83.9 & 242.9 & 527.5 & 599.5 & 768 & 781.9 & 798.4 & 804.4 & 791.4 & 800.4 \\
\hline 70.9 & 87.8 & 298.4 & 488.2 & 613.5 & 691.4 & 721.3 & 752.4 & 735.5 & 777.8 \\
\hline 80 & 96.9 & 298 & 480 & 627.8 & 675 & 732.1 & 747.7 & 761 & 789.5 \\
\hline 73.9 & 91.7 & 325.6 & 541.4 & 671.5 & 770.2 & 841.2 & 844.1 & 867.4 & 882.2 \\
\hline 76.1 & 95.2 & 339.2 & 576.8 & 728.9 & 798.9 & 844.7 & 856.4 & 842.5 & 903.4 \\
\hline 89.5 & 211.4 & 461.3 & 586.6 & 744.7 & 807 & 869.8 & 865 & 852.9 & 884.1 \\
\hline 75.6 & 94.8 & 358.3 & 609.9 & 744.6 & 818.5 & 883.2 & 863.7 & 850.3 & 886.5 \\
\hline 75.2 & 95.2 & 329.4 & 561 & 718.8 & 824 & 870.7 & 861.1 & 852 & 889.6 \\
\hline 74.4 & 91.7 & 350.6 & 601.2 & 747.6 & 814.1 & 868.9 & 850.3 & 851.6 & 866.4 \\
\hline 80 & 98.2 & 349.6 & 581.2 & 737.9 & 827.5 & 884.6 & 882.8 & 870.2 & 903.9 \\
\hline 84.3 & 193.7 & 414.2 & 497.9 & 702.3 & 761.6 & 816.8 & 817.9 & 806.6 & 837.7 \\
\hline 71.3 & 94.3 & 353.8 & 635.8 & 795 & 855.6 & 883.7 & 878.6 & 869 & 920.5 \\
\hline 73.9 & 96 & 332.4 & 558.7 & 705.4 & 749 & 738.3 & 752.8 & 759.5 & 817.3 \\
\hline 70 & 94.3 & 297.7 & 534.5 & 676.3 & 688.1 & 715.3 & 704 & \begin{tabular}{|c|}
672.8 \\
\end{tabular} & 693.1 \\
\hline 70.4 & 87.4 & 276.9 & 439.8 & 600.7 & 686.7 & 767.4 & 756.1 & 759.5 & 794 \\
\hline 88.2 & 242.9 & 534 & 670.6 & 851.8 & 909.1 & 920.7 & 950.9 & 913.2 & 922.4 \\
\hline 80 & 100.8 & 375.7 & 679.7 & 869.1 & 910.3 & 900.8 & 883.1 & 877.4 & 926.7 \\
\hline 90.8 & 111.6 & 385.8 & 696.9 & 858.6 & 887.7 & 898.6 & 804.3 & 717.9 & 739.5 \\
\hline 67.9 & 80.5 & 273.9 & 438.9 & 594.1 & 613.2 & 617.1 & 598.5 & 577.6 & 595.8 \\
\hline 67.9 & 80.5 & 204.4 & 365.2 & 496.2 & 542.1 & 547.7 & 524.3 & 533.3 & 532.8 \\
\hline 65.7 & 67.9 & 121.4 & 361.6 & 487.4 & 483.7 & 567.1 & 589.5 & 591.1 & 571.6 \\
\hline 75.6 & 75.2 & 112.8 & 303.7 & 454.1 & 548.2 & 631 & 632.6 & 619.8 & 662.1 \\
\hline 66.6 & 67.9 & 101.6 & 264 & 459.2 & 490.5 & 507.4 & 487.1 & 462.4 & 458.4 \\
\hline 63.6 & 73.1 & 107.7 & 267 & 415.2 & 411.8 & 480.3 & 534.8 & 503.9 & 525.5 \\
\hline 60.1 & 61 & 97.7 & 334 & 533.6 & 603.2 & 606.9 & 624.6 & 602 & 615.9 \\
\hline 65.3 & 85.6 & 170.3 & 304.7 & 463.2 & 489.1 & 493 & 471.8 & 439 & 441.2 \\
\hline 80.9 & 83.9 & 124.9 & 289.5 & 423.9 & 434.7 & 461 & 462.3 & 455.9 & 462 \\
\hline 67.9 & 80 & 113.2 & 265.8 & 421.6 & 539.3 & 572.4 & 561.1 & 540.7 & 539.3 \\
\hline 87.8 & 96.8 & 187.2 & 326.7 & 524.5 & 556.6 & 572.4 & 569.7 & 573.1 & 589.5 \\
\hline 71.4 & 82.6 & 125.3 & 302.9 & 596.2 & 626.2 & 590.5 & 603 & 583.8 & 557 \\
\hline 71.8 & 86.1 & 130.5 & 306.8 & 457.6 & 480.4 & 489.1 & 484.4 & 443.3 & 431.7 \\
\hline 86.9 & 86.9 & 115 & 297.7 & 435.1 & 465.8 & 473.1 & 465.8 & 432.5 & 439.4 \\
\hline 81.7 & 84.3 & 108.1 & 273.6 & 444.8 & 482.7 & 478.3 & 464.5 & 444.6 & 454.6 \\
\hline 60.5 & 70.5 & 117.2 & 323.2 & 515.5 & 572.2 & 607.4 & 627.7 & 633.7 & 657.1 \\
\hline 61 & 77 & 106.8 & 263.2 & 421.6 & 470.5 & 500.5 & 489.6 & 469.2 & 471.3 \\
\hline 61.4 & 67.1 & 104.6 & 246.8 & 437.1 & 581.8 & 548.9 & 528.1 & 504.1 & 516.2 \\
\hline 63.6 & 65.7 & 131 & 302.5 & 453.7 & 534.9 & 549.9 & 539.5 & 511.9 & 516 \\
\hline 62.7 & 66.6 & 157.8 & 315.4 & 486.9 & 464.9 & 470.4 & 452.2 & 482.4 & 524.6 \\
\hline 68.8 & 74.4 & 178 & 311.1 & 500.5 & 557.5 & 579.8 & 574.3 & 607.4 & 580.2 \\
\hline 78.7 & 99.9 & 144.4 & 325.8 & 456.7 & 479.6 & 493.4 & 525.5 & 532 & 514.6 \\
\hline 65.7 & 67.5 & 96.4 & 229 & 395.8 & 469.6 & 523.2 & 521.4 & 513.5 & 526.9 \\
\hline 73.5 & 127.1 & 229 & 342.2 & 489.1 & 485.6 & 531.9 & 499 & 468.4 & 452.4 \\
\hline 88.7 & 90.8 & 163 & 343.9 & 472.7 & 480.4 & 479.6 & 460.6 & 432.5 & 436.4 \\
\hline 77.8 & 80.9 & 121.9 & 279.6 & 432.1 & 453.2 & 487.1 & 496.8 & 481.2 & 485.5 \\
\hline 62.7 & 69.2 & 94.2 & 290.8 & 510.6 & 547 & 546.9 & 592.7 & 605.2 & 625.8 \\
\hline 64.9 & 72.6 & 167.7 & 334 & 518.2 & 571.3 & 618.3 & 617.9 & 635.9 & 612.5 \\
\hline 62.3 & 75.7 & 127.1 & 268.8 & 410.9 & 446.8 & 457.1 & 475.9 & 466.2 & 415.2 \\
\hline 78.7 & 80.9 & 106.3 & 270.5 & 450.2 & 470.9 & 465.4 & 458.8 & 434.7 & 428.6 \\
\hline 84.7 & 91.2 & 117.6 & 268.8 & 417 & 470.8 & 581.6 & 577.6 & 571.9 & 569.7 \\
\hline
\end{tabular}


other

\begin{tabular}{|c|c|c|c|c|c|c|c|c|c|}
\hline 68.8 & 78.7 & 183.2 & 335.3 & 533.5 & 586.1 & 606.4 & 583.8 & 603.2 & 601.9 \\
\hline 65.3 & 76.1 & 179.8 & 372.4 & 549.5 & 626 & 649.6 & 687.2 & 667.6 & 682.2 \\
\hline 62.3 & 82.1 & 217.4 & 351.7 & 510.8 & 521.6 & 534.4 & 503.6 & 553.7 & 549.7 \\
\hline 61.4 & 63.6 & 176.3 & 265.3 & 450.8 & 575.8 & 553.3 & 529.9 & 509.9 & 521.6 \\
\hline 67.9 & 68.3 & 86.5 & 261.9 & 467.9 & 495.6 & 480.4 & 462.3 & 441.3 & 449.9 \\
\hline 87.3 & 89.9 & 103.3 & 255.8 & 443 & 496.2 & 488.4 & 491.4 & 441.7 & 438.6 \\
\hline 92.5 & 112 & 150.4 & 327.1 & 458 & 470.5 & 478.7 & 490.9 & 474.9 & 453.7 \\
\hline 96.4 & 102.5 & 138.8 & 281.3 & 455.9 & 487 & 478.3 & 494.3 & 458.4 & 447.2 \\
\hline 99.4 & 186.7 & 212.6 & 345.3 & 505.5 & 513.3 & 506.8 & 502 & 468.8 & 462.4 \\
\hline 86.1 & 98.6 & 108.1 & 269.2 & 439.8 & 458 & 448.9 & 456.2 & 419.6 & 437.2 \\
\hline 65.3 & 68.3 & 86.9 & 238.5 & 410.1 & 463.6 & 476.6 & 552.3 & 508.7 & 473.1 \\
\hline 71.4 & 76.6 & 116.7 & 272.2 & 406.6 & 512.5 & 529.9 & 547.9 & 506.7 & 514.4 \\
\hline 61.9 & 70 & 105.5 & 271.4 & 442.4 & 484.4 & 504.1 & 489.3 & 444.3 & 440.8 \\
\hline 80.9 & 100.8 & 144.4 & 287.4 & 447.2 & 460.2 & 488.2 & 496.5 & 483.5 & 448.7 \\
\hline 110.3 & 117.2 & 149.5 & 333.6 & 492.1 & 509 & 530.2 & 515 & 463.2 & 467.5 \\
\hline 92.5 & 101.6 & 123.6 & 319.3 & 507.2 & 559.9 & 568.2 & 528 & 468.4 & 461.4 \\
\hline 86.5 & 94.2 & 149.5 & 351.3 & 477.9 & 496 & 476.1 & 490.8 & 448.5 & 428.2 \\
\hline 86.1 & 89.9 & 118.9 & 293.4 & 446.8 & 467.1 & 464.9 & 455 & 376.8 & 414.4 \\
\hline 85.6 & 131 & 154.7 & 296.4 & 477 & 528.8 & 534.9 & 531.9 & 490.8 & 492.1 \\
\hline 76.1 & 82.1 & 165.1 & 324.5 & 490 & 531.2 & 543 & 547.7 & 530.3 & 543.7 \\
\hline 80 & 81.3 & 107.7 & 304.2 & 504.5 & 534.9 & 593.1 & 600.4 & 570.9 & 582.5 \\
\hline 79.6 & 77 & 187.3 & 375.8 & 516.9 & 561.2 & 580.9 & 586.9 & 598.5 & 533.6 \\
\hline 103.2 & 122.1 & 197.3 & 307.6 & 473.1 & 506 & 515.9 & 512 & 469.2 & 460.2 \\
\hline 108.1 & 176.3 & 232.5 & 368.6 & 530.6 & 562.5 & 563.9 & 553.9 & 520.2 & 487 \\
\hline 105.9 & 127.9 & 172.5 & 376.4 & 587.2 & 601.4 & 588.9 & 593.6 & 520.7 & 524.1 \\
\hline 121.5 & 130.5 & 156.4 & 360.8 & 553.9 & 580.7 & 552.2 & 546.1 & 511.6 & 483 \\
\hline 96.4 & 109.8 & 159 & 360 & 555.2 & 582.4 & 577.7 & 564.3 & 503.4 & 492.1 \\
\hline 88.7 & 99.9 & 126.7 & 286.9 & 468.8 & 487.4 & 489.4 & 473.6 & 451.5 & 441.4 \\
\hline 70 & 85.2 & 149.1 & 279 & 480.7 & 573 & 552.2 & 576.4 & 553.4 & 569 \\
\hline 76.6 & 78.3 & 95.6 & 317.1 & 523.7 & 586.6 & 612.4 & 659.1 & 667.6 & 659.5 \\
\hline 84.3 & 98.2 & 118.9 & 393.5 & 593.2 & 630.2 & 619.8 & 536.4 & 529.7 & 464.9 \\
\hline 118 & 124.9 & 139.6 & 169.9 & 165.6 & 168.5 & 182.8 & 196.7 & 216.5 & 242 \\
\hline 120.2 & 126.7 & 158.2 & 340.9 & 543.1 & 542.7 & 561.3 & 563.4 & 511.2 & 454.5 \\
\hline 99 & 151.7 & 211.3 & 343.5 & 503.4 & 553.9 & 539.7 & 515.5 & 501.2 & 465.4 \\
\hline 95.6 & 100.3 & 129.3 & 325.4 & 509.4 & 514.6 & 485.2 & 487.8 & 459.7 & 438.6 \\
\hline 91.2 & 98.2 & 131.9 & 301.2 & 487.8 & 495.6 & 475.3 & 497.3 & 543.1 & 551.5 \\
\hline 70.9 & 75.7 & 183.7 & 287.9 & 455 & 452.4 & 442.9 & 506 & 549 & 527.7 \\
\hline 65.3 & 69.6 & 94.2 & 245.9 & 388.5 & 428.1 & 502.7 & 520.4 & 503.2 & 502.3 \\
\hline 71.4 & 77.4 & 169 & 327.4 & 542.5 & 576.6 & 593.8 & 612.4 & 584.2 & 574.8 \\
\hline 92.1 & 91.2 & 204.4 & 290.8 & 461 & 506.7 & 568.3 & 535.3 & 475.1 & 468.5 \\
\hline 93.8 & 99.9 & 129.3 & 283.1 & 455 & 501.6 & 502.9 & 532.4 & 540.8 & 518.6 \\
\hline 83.5 & 88.7 & 111.1 & 261.5 & 429.1 & 455 & 448.1 & 548.8 & 533.8 & 533.6 \\
\hline 73.1 & 127.1 & 225.2 & 288.2 & 443.8 & 477.5 & 475.7 & 484.4 & 458 & 440.3 \\
\hline 93 & 98.2 & 142.6 & 271 & 442 & 550.9 & 550 & 541.7 & 515.1 & 519.2 \\
\hline 67.5 & 81.7 & 139.6 & 296 & 462.8 & 474.4 & 468 & 472.3 & 441.2 & 478 \\
\hline 81.7 & 95.6 & 144 & 307.6 & 440.3 & 449.8 & 450.2 & 519.4 & 527.3 & 497.6 \\
\hline 83.9 & 86.9 & 110.3 & 252.8 & 423 & 457.6 & 493 & 551.4 & 524.7 & 548.9 \\
\hline 73.1 & 101.6 & 131.9 & 274.4 & 528 & 561.4 & 589.7 & 630 & 612.1 & 627.2 \\
\hline 81.7 & 102.9 & 142.2 & 368.4 & 587.8 & 612.3 & 641.6 & 641.6 & 655.8 & 627.3 \\
\hline
\end{tabular}


other

\begin{tabular}{|c|c|c|c|c|c|c|c|c|c|}
\hline 76.2 & 78.3 & 115.9 & 272.7 & 465 & 488.7 & 486.6 & 512.9 & 465 & 454.1 \\
\hline 97.3 & 105.9 & 131.9 & 302.1 & 473.1 & 506 & 485.2 & 494.7 & 455.9 & 426.1 \\
\hline 75.7 & 114.2 & 187.6 & 403.2 & 610.7 & 700.9 & 699.2 & 694.4 & 670.9 & 647.9 \\
\hline 71.8 & 76.2 & 174.2 & 341.9 & 508.7 & 602.2 & 608.3 & 676.6 & 684.3 & 686.4 \\
\hline 76.2 & 100.4 & 191.9 & 397.4 & 611.4 & 672 & 658.5 & 660.7 & 641.6 & 636.3 \\
\hline 80.9 & 117.6 & 217.9 & 412.2 & 636.5 & 691 & 711.2 & 738.4 & 744.3 & 753.9 \\
\hline 73.6 & 86.5 & 189.3 & 352 & 542.6 & 624.2 & 588.8 & 639.4 & 662.3 & 689.4 \\
\hline 64.5 & 74.4 & 185 & 222.6 & 418.9 & 479 & 480.3 & 523.4 & 515.1 & 509.5 \\
\hline 69.2 & 73.6 & 101.2 & 228.2 & 397.6 & 456.5 & 480.2 & 514.7 & 492.6 & 481.8 \\
\hline 68.4 & 72.7 & 172.9 & 231.7 & 401.5 & 548.5 & 559.6 & 564.4 & 574.3 & 549.6 \\
\hline 71 & 75.3 & 153.9 & 232.5 & 387.6 & 465.5 & 524.6 & 533.3 & 518.1 & 514.6 \\
\hline 77.9 & 99.5 & 113.8 & 373.4 & 573.7 & 633.5 & 670.1 & 687 & 691.7 & 680.7 \\
\hline 77.1 & 82.2 & 176 & 290.9 & 421.2 & 451.3 & 454.6 & 458.9 & 432 & 449.3 \\
\hline 67.1 & 74.9 & 150.5 & 233 & 412.3 & 463.6 & 448 & 500.7 & 506.3 & 495.9 \\
\hline 62.2 & 66.5 & 95.9 & 208.2 & 421.7 & 509.8 & 438.2 & 476.1 & 559.9 & 565.9 \\
\hline 78.6 & 184 & 306.7 & 458 & 703.7 & 733.8 & 751 & 756.6 & 724 & 730.7 \\
\hline 76.5 & 120.6 & 233.7 & 419 & 613.1 & 637.3 & 654.1 & 624.3 & 595.7 & 576.1 \\
\hline 71.3 & 80.8 & 165.5 & 343.4 & 516.3 & 603.8 & 630.6 & 670.8 & 658.5 & 669.4 \\
\hline 90.3 & 134.4 & 207.4 & 469.3 & 644.8 & 733.2 & 772.5 & 796.6 & 781.5 & 782.8 \\
\hline 76.5 & 130.1 & 292.5 & 530.2 & 683 & 733.4 & 746.7 & 736.4 & 725 & 733.6 \\
\hline 74.8 & 120.6 & 231.2 & 364.7 & 573.2 & 640.9 & 643.6 & 698.9 & 700.1 & 681.4 \\
\hline 76.5 & 118.8 & 241 & 432 & 625.6 & 682.5 & 754.2 & 765.9 & 730.8 & 773.1 \\
\hline 70 & 98.5 & 229.4 & 448.9 & 635.3 & 694.3 & 686.5 & 699.5 & 667.4 & 653 \\
\hline 66.1 & 70.4 & 193 & 371.4 & 561.6 & 623.2 & 663.9 & 668.2 & 652.1 & 661.5 \\
\hline 70.8 & 80.4 & 204.7 & 368 & 540.9 & 657.8 & 672 & 679.4 & 720.4 & 717.3 \\
\hline 73.9 & 174.1 & 349.5 & 498.1 & 710.3 & 796.9 & 787.7 & 798.4 & 786.7 & 784.4 \\
\hline 73.9 & 141.3 & 308.3 & 562.3 & 755.9 & 809.8 & 852.5 & 862.6 & 872.5 & 869.4 \\
\hline 84.3 & 157.7 & 346 & 605.3 & 787.5 & 869.1 & 866.3 & 886.7 & 875.8 & 890.5 \\
\hline 91.2 & 151.7 & 337.4 & 569.1 & 753.8 & 837.3 & 836 & 855.6 & 848.1 & 870.1 \\
\hline 80 & 140 & 319.7 & 528.8 & 718.4 & 810.4 & 817.3 & 849.7 & 846.3 & 865.1 \\
\hline 79.1 & 88.6 & 218.2 & 276.9 & 429.9 & 483.4 & 505.6 & 526.4 & 526.1 & 543.6 \\
\hline 74.4 & 86.9 & 168.2 & 384.3 & 553.3 & 669 & 677.7 & 673.5 & 690.6 & 711.8 \\
\hline 80.8 & 95.1 & 171.6 & 385.1 & 563.2 & 649.5 & 653.2 & 624.6 & 644.8 & 653 \\
\hline 78.2 & 114.5 & 193.2 & 459 & 658.9 & 701.1 & 745.7 & 770.4 & 784.5 & 780.6 \\
\hline 77.4 & 108.1 & 217.1 & 457.6 & 646.4 & 743.8 & 781.4 & 787 & 815.5 & 782 \\
\hline 77.4 & 241.1 & 413.6 & 651.5 & 846.1 & 898.6 & 876.6 & 895.6 & 884.7 & 871.6 \\
\hline 81.7 & 149.1 & 261.1 & 659.9 & 844.4 & 870.1 & 866.3 & 867.5 & 867.1 & 903.8 \\
\hline 100.7 & 163.8 & 259.3 & 601.9 & 830 & 874.8 & 875.8 & 883.1 & 880 & 892.4 \\
\hline 82.1 & 145.6 & 257.6 & 637.8 & 786.3 & 813 & 856.8 & 846.1 & 820 & 779.8 \\
\hline 81.7 & 134.4 & 235.2 & 584.8 & 796.1 & 827.6 & 850 & 870.2 & 871.4 & 891.8 \\
\hline 75.6 & 167.2 & 289.7 & 494.7 & 688 & 735.6 & 773.2 & 756.9 & 791.7 & 806 \\
\hline 78.2 & 136.1 & 215.2 & 598 & 783.9 & 817.8 & 833.9 & 872 & 826.9 & 855.4 \\
\hline 73.5 & 129.2 & 260.5 & 565 & 757.6 & 799.3 & 837 & 867.7 & 865.6 & 907.8 \\
\hline 77.8 & 123.2 & 217.3 & 572.9 & 706.8 & 763.2 & 789.3 & 810.8 & 822.5 & 841 \\
\hline 83.4 & 124.5 & 227.2 & 542.6 & 675.7 & 738.1 & 758.5 & 776.3 & 766.6 & 790.3 \\
\hline 70.4 & 191 & 283.1 & 530.8 & 737.3 & 808.2 & 818.3 & 855 & 836.3 & 855.6 \\
\hline 87.7 & 137.8 & 235 & 531.9 & 723.2 & 814.4 & 821.6 & 864.6 & 846 & 853.3 \\
\hline 82.1 & 141.3 & 284.7 & 569.9 & 781.7 & 819.1 & 822.7 & 837.4 & 809.1 & 821.7 \\
\hline 83 & 142.2 & 283.5 & 589.7 & 768 & 786 & 786.1 & 828.8 & 799 & 816.2 \\
\hline
\end{tabular}


other

\begin{tabular}{|c|c|c|c|c|c|c|c|c|c|}
\hline 80.8 & 140 & 260.5 & 558.2 & 749.8 & 823.1 & 824.1 & 793.2 & 805.1 & 806.3 \\
\hline 195.3 & 196.1 & 202.2 & 215.1 & 232.4 & 246.2 & 257.5 & 245.4 & 253.2 & 254 \\
\hline 192.7 & 189.2 & 188.4 & 188.4 & 195.3 & 206.5 & 221.2 & 224.6 & 230.7 & 299.8 \\
\hline 212.5 & 219.5 & 216.9 & 234.1 & 286 & 317.1 & 322.3 & 295.5 & 262.7 & 253.2 \\
\hline 207.4 & 204.8 & 204.8 & 198.7 & 207.4 & 220.3 & 222 & 225.5 & 230.7 & 241.1 \\
\hline 117.5 & 116.6 & 125.3 & 161.6 & 352.5 & 335.2 & 345.6 & 356.8 & 337 & 348.2 \\
\hline 150.3 & 147.7 & 146 & 147.7 & 140.8 & 150.3 & 163.3 & 165 & 165.9 & 182.3 \\
\hline 128.7 & 140.8 & 138.2 & 152.1 & 184 & 202.2 & 193.5 & 182.3 & 171.1 & 189.2 \\
\hline 124.4 & 121 & 118.4 & 110.6 & 111.5 & 117.5 & 134.8 & 144.3 & 146.9 & 151.2 \\
\hline 178.6 & 168.2 & 172.4 & 194.1 & 207.1 & 202.8 & 195 & 200.2 & 178.6 & 176 \\
\hline 120.6 & 121.6 & 118.1 & 112.9 & 111.2 & 112.9 & 119 & 117.2 & 125 & 133.8 \\
\hline 144.4 & 134 & 134 & 156.4 & 202.2 & 208.3 & 220.8 & 234.2 & 209.2 & 207.4 \\
\hline 159.9 & 158.2 & 154.7 & 134.8 & 152.1 & 153.9 & 163.4 & 170.3 & 172.9 & 185.8 \\
\hline 136.1 & 134 & 185.3 & 248.3 & 286.3 & 299.7 & 327.4 & 314.4 & 324.8 & 322.6 \\
\hline 117.6 & 120.6 & 112.4 & 108.9 & 120.6 & 123.6 & 142.6 & 138.7 & 141.8 & 142.2 \\
\hline 176.3 & 177.2 & 184.5 & 195.3 & 251.5 & 285.6 & 301.6 & 305.5 & 282.6 & 279.1 \\
\hline 182.4 & 184.5 & 182.4 & 169.4 & 176.7 & 51.4 & 229 & 213 & 218.2 & 219.5 \\
\hline 118.8 & 120.6 & 124.5 & 133.5 & 131.8 & 125.3 & 127.5 & 135.2 & 109.8 & 111.9 \\
\hline 91.6 & 92 & 89.9 & 81.7 & 86 & 85.6 & 87.7 & 80 & 80.4 & 82.5 \\
\hline 135.2 & 134.4 & 128.8 & 174.1 & 208.3 & 201.8 & 203.5 & 204 & 195.7 & 197.5 \\
\hline 134.8 & 135.2 & 130.5 & 124.9 & 130.1 & 133.1 & 134.4 & 133.1 & 135.2 & 132.7 \\
\hline 117.1 & 116.2 & 111.1 & 116.7 & 143.9 & 191.4 & 216.5 & 213.9 & 191.4 & 184.5 \\
\hline 115.4 & 107.6 & 99 & 87.7 & 88.6 & 86.5 & 89.5 & 86 & 85.1 & 90.3 \\
\hline 94.6 & 94.2 & 87.7 & 87.7 & 118.8 & 136.6 & 135.2 & 137 & 131.8 & 134.4 \\
\hline 80 & 80 & 73.9 & 72.2 & 74.8 & 73.5 & 81.7 & 83.4 & 81.7 & 80.8 \\
\hline 105.9 & 108.5 & 102 & 95.5 & 96.4 & 92.9 & 105.9 & 140.9 & 145.6 & 146.5 \\
\hline 97.2 & 97.2 & 92.5 & 86 & 81.7 & 83.9 & 83.4 & 84.3 & 82.5 & 82.1 \\
\hline 119.7 & 121.4 & 114.1 & 140.4 & 172.4 & 199.2 & 201.8 & 200.9 & 194 & 192.3 \\
\hline 129.2 & 130.9 & 124.5 & 117.1 & 119.7 & 118.8 & 115.4 & 112.8 & 111.1 & 120.6 \\
\hline 120.6 & 114.5 & 113.6 & 146.5 & 178.4 & 189.3 & 213.9 & 213 & 178.4 & 168.1 \\
\hline 92.9 & 92 & 87.7 & 78.2 & 83 & 82.5 & 88.6 & 91.2 & 89.5 & 109.3 \\
\hline 129.2 & 125.7 & 130.5 & 256.2 & 413.5 & 444.6 & 444.8 & 456.5 & 462.8 & 435 \\
\hline 109.3 & 111.1 & 107.6 & 113.6 & 127.5 & 128.8 & 131.8 & 140.4 & 119.7 & 130.1 \\
\hline 111.9 & 111.9 & 118.8 & 270 & 393.6 & 406.5 & 413.9 & 379.8 & 322.7 & 366.8 \\
\hline 99 & 97.2 & 132.3 & 135.2 & 116.2 & 128.3 & 145.6 & 155.1 & 139.6 & 120.2 \\
\hline 95.5 & 94.6 & 111.9 & 286.4 & 501.6 & 454.5 & 462.2 & 476.1 & 485.3 & 453.6 \\
\hline 70.4 & 68.7 & 66.1 & 66.1 & 112.8 & 113.6 & 114.5 & 110.7 & 69.6 & 97.2 \\
\hline 85.1 & 86.9 & 94.6 & 306.3 & 439.4 & 448 & 435.1 & 457.4 & 464.4 & 456.4 \\
\hline 74.8 & 77.4 & 70.9 & 72.2 & 136.1 & 149.1 & 162 & 137 & 111.9 & 120.6 \\
\hline 95.5 & 95.5 & 109.3 & 289 & 413.9 & 423 & 437.6 & 422.1 & 416.9 & 403.1 \\
\hline 83.4 & 82.5 & 80 & 79.1 & 101.2 & 104.1 & 111.1 & 105.9 & 97.2 & 110.2 \\
\hline 93.4 & 94.6 & 104.7 & 348.2 & 557.1 & 590.4 & 586.1 & 608.5 & 602.9 & 590.3 \\
\hline 92.9 & 92.9 & 89.5 & 210.4 & 235.5 & 277.8 & 285.6 & 281.3 & 263.1 & 264 \\
\hline 77.4 & 74.8 & 83.4 & 242.4 & 397.9 & 464.4 & 466.9 & 480 & 470.3 & 464.1 \\
\hline 82.5 & 83 & 80.8 & 73 & 124.9 & 148.2 & 178.4 & 180.2 & 157.7 & 147.3 \\
\hline 82.5 & 72.2 & 84.3 & 224.2 & 404 & 416 & 450.3 & 473.2 & 471.3 & 447.1 \\
\hline 75.6 & 77.4 & 76.5 & 104.1 & 152.5 & 173.9 & 198.1 & 178.1 & 164.3 & 171.1 \\
\hline 90.3 & 92.9 & 107.6 & 345.9 & 538.5 & 615.4 & 613.2 & 672 & 685.3 & 678.3 \\
\hline 99 & 100.7 & 97.7 & 149.9 & 294.1 & 299.1 & 295.9 & 285.5 & 270 & 185.1 \\
\hline
\end{tabular}


other

\begin{tabular}{|c|c|c|c|c|c|c|c|c|c|}
\hline 79.1 & 85.1 & 105.5 & 362.5 & 560.2 & 641.2 & 639.2 & 658.2 & 638.7 & 624.8 \\
\hline 92.9 & 92.9 & 88.6 & 89.5 & 115.4 & 118.8 & 124 & 116.2 & 90.3 & 92.5 \\
\hline 86.9 & 86.9 & 101.6 & 274 & 428.1 & 448.9 & 450.6 & 454.9 & 438.1 & 421.2 \\
\hline 77.4 & 79.6 & 75.6 & 70.4 & 99 & 110.2 & 111.9 & 106.7 & 87.7 & 100.3 \\
\hline 96 & 105 & 119.7 & 336.6 & 586.9 & 639.4 & 641.4 & 648.2 & 673.2 & 681.3 \\
\hline 100.7 & 103.3 & 99 & 93.8 & 118 & 123.2 & 130.1 & 134.4 & 105.9 & 106.7 \\
\hline 109.3 & 115.4 & 144.8 & 572 & 780.2 & 819.6 & 793.6 & 804.9 & 827.9 & 836.6 \\
\hline 114.5 & 118 & 117.1 & 201.9 & 251.5 & 247.5 & 258.5 & 245.5 & 202.2 & 178.2 \\
\hline 105.9 & 111.1 & 127.5 & 477.7 & 700.1 & 767.9 & 773.5 & 789.1 & 794.2 & 783.4 \\
\hline 119.7 & 118 & 111.9 & 103.3 & 122.3 & 132.7 & 137.8 & 135.2 & 112.8 & 119.7 \\
\hline 83.4 & 90.3 & 96.4 & 345.3 & 542.7 & 583 & 601.5 & 626.1 & 630.8 & 631.6 \\
\hline 90.3 & 92.9 & 109.3 & 148.2 & 181 & 160.3 & 162.9 & 140.4 & 99.8 & 94.6 \\
\hline 96.4 & 105 & 119.8 & 469.2 & 665.3 & 698.4 & 718.9 & 752.4 & 751.9 & 707.6 \\
\hline 108.5 & 99 & 103.3 & 92.9 & 115.4 & 122.3 & 131.8 & 135.2 & 131.8 & 135.2 \\
\hline 96.4 & 105 & 112.4 & 479.6 & 712.9 & 770.4 & 799.3 & 816.6 & 795.8 & 813.9 \\
\hline 143.9 & 143 & 137 & 121.4 & 117.1 & 139.6 & 128.3 & 116.2 & 116.2 & 115.4 \\
\hline 121.9 & 131.4 & 310.3 & 588.5 & 779.7 & 848.6 & 904 & 959.8 & 939.8 & 954.4 \\
\hline 96.4 & 93.4 & 119.3 & 123.6 & 181 & 195.3 & 214.7 & 216.9 & 190.5 & 193.6 \\
\hline 99.4 & 112.4 & 312.8 & 652.3 & 855 & 900.5 & 874 & 986.9 & 964.8 & 955.8 \\
\hline 119.3 & 119.3 & 141.8 & 194.9 & 301.6 & 312 & 454.9 & 465.8 & 456.7 & 450.2 \\
\hline 99 & 104.6 & 263.5 & 476.3 & 665.6 & 712.7 & 760.7 & 815 & 813.6 & 830 \\
\hline 94.6 & 98.6 & 131.4 & 143.9 & 202.6 & 181.5 & 192.3 & 207.4 & 188 & 177.6 \\
\hline 66.6 & 74.8 & 214.1 & 366 & 560.5 & 625 & 663.9 & 676.5 & 695.8 & 718.2 \\
\hline 77.4 & 80 & 88.6 & 86 & 122.3 & 115.8 & 120.6 & 127.5 & 124.9 & 131.8 \\
\hline 69.6 & 74.8 & 207.1 & 367.2 & 539.6 & 594.7 & 645.6 & 705.3 & 709.2 & 734.2 \\
\hline 73 & 80 & 97.2 & 97.2 & 97.2 & 104.6 & 112.8 & 111.1 & 112.8 & 122.3 \\
\hline 89.1 & 100.7 & 304.2 & 589.9 & 815 & 846.8 & 880.5 & 906.4 & 903.4 & 893.8 \\
\hline 88.2 & 87.7 & 92.9 & 89.5 & 128.3 & 141.3 & 146.5 & 152.1 & 144.8 & 126.6 \\
\hline 99 & 105.9 & 273.4 & 476.3 & 662.2 & 741.8 & 761.2 & 787.5 & 800.5 & 780.9 \\
\hline 72.2 & 71.3 & 78.2 & 93.8 & 114.5 & 124.5 & 130.1 & 131.8 & 129.2 & 142.2 \\
\hline 91.6 & 99.8 & 267.4 & 570.9 & 798.3 & 817 & 836 & 866.7 & 859.7 & 867 \\
\hline 83.4 & 81.7 & 120.2 & 177.6 & 198.3 & 230.3 & 238.1 & 238.5 & 219.1 & 210 \\
\hline 99 & 96 & 121.4 & 245.8 & 422.1 & 456.3 & 404 & 451.5 & 451.5 & 418.2 \\
\hline 100.7 & 103.7 & 122.3 & 187.1 & 205.2 & 203.1 & 203.5 & 194.9 & 174.1 & 175.9 \\
\hline 76.5 & 80.8 & 183.4 & 329.4 & 491.9 & 518 & 565.5 & 530.5 & 519.6 & 476.8 \\
\hline 80 & 78.2 & 75.6 & 76.5 & 92.9 & 120.6 & 131.4 & 124 & 116.2 & 114.5 \\
\hline 87.7 & 91.2 & 188 & 323 & 495.9 & 515.8 & 520.6 & 532.6 & 551.6 & 509.6 \\
\hline 68.7 & 67 & 67.9 & 63.5 & 111.1 & 131.8 & 140 & 146.5 & 136.1 & 130.1 \\
\hline 93.8 & 94.6 & 101.6 & 186.2 & 229.9 & 207 & 202.6 & 161.2 & 128.3 & 133.1 \\
\hline 94.6 & 95.1 & 93.8 & 86.9 & 89.5 & 87.7 & 102 & 114.5 & 113.6 & 111.9 \\
\hline 90.3 & 92 & 125.1 & 247.1 & 438.9 & 464.5 & 487 & 493.9 & 463.6 & 463.5 \\
\hline 73.9 & 73.9 & 71.3 & 68.3 & 73.9 & 108.5 & 111.9 & 111.5 & 107.6 & 104.1 \\
\hline 86 & 86.9 & 95.1 & 268.3 & 410 & 439.4 & 434.2 & 437.6 & 429 & 417.9 \\
\hline 68.7 & 67.9 & 66.1 & 86.5 & 124 & 137 & 134.4 & 140.4 & 115.4 & 102.4 \\
\hline 60.1 & 60.9 & 58.4 & 53.6 & 80.4 & 96.8 & 101.6 & 97.2 & 81.7 & 88.6 \\
\hline 63.5 & 61.8 & 60.9 & 54.9 & 50.2 & 50.6 & 54.9 & 67.5 & 68.7 & 69.6 \\
\hline 62.3 & 62.7 & 64.4 & 63.5 & 63.5 & 85.1 & 86.9 & 77.4 & 53.2 & 54.9 \\
\hline 59.2 & 61.8 & 58 & 51.4 & 48.8 & 48 & 50.6 & 47.1 & 47.1 & 54.9 \\
\hline 64.4 & 63.5 & 62.7 & 60.9 & 99 & 102 & 102.4 & 103.3 & 81.7 & 83.4 \\
\hline
\end{tabular}


other

\begin{tabular}{|c|c|c|c|c|c|c|c|c|c|}
\hline 65.3 & 65.3 & 63.1 & 54 & 52.3 & 52.3 & 52.8 & 52.3 & 53.2 & 55.4 \\
\hline 89.5 & 91.6 & 92 & 86.9 & 89.5 & 112.4 & 118 & 109.3 & 105 & 106.7 \\
\hline 86 & 86.9 & 84.7 & 75.6 & 75.6 & 73 & 74.4 & 71.3 & 80.8 & 92 \\
\hline 91.2 & 90.3 & 87.3 & 130.1 & 185.4 & 189.7 & 188.8 & 140.9 & 111.1 & 105.9 \\
\hline 92 & 92.9 & 90.3 & 83.9 & 80 & 79.1 & 76.5 & 77.4 & 73.9 & 83.9 \\
\hline 73.5 & 73 & 71.3 & 66.1 & 90.8 & 92 & 92.9 & 89.5 & 83.4 & 83.4 \\
\hline 73.9 & 74.8 & 72.2 & 81.7 & 77.8 & 63.5 & 64.4 & 63.5 & 66.1 & 78.2 \\
\hline 86 & 85.1 & 87.3 & 122.3 & 171.5 & 170.7 & 173.3 & 170.7 & 147.3 & 156.8 \\
\hline 82.5 & 83.4 & 81.7 & 73 & 73.9 & 72.6 & 73.9 & 72.2 & 84.3 & 92.9 \\
\hline 65.7 & 71.3 & 78.2 & 95.5 & 145.6 & 169.4 & 174.1 & 162.9 & 133.5 & 134 \\
\hline 66.1 & 65.3 & 63.5 & 56.2 & 56.6 & 69.6 & 71.3 & 71.3 & 74.8 & 75.6 \\
\hline 73 & 75.2 & 73 & 98.1 & 126.6 & 131.8 & 133.5 & 125.7 & 109.3 & 111.1 \\
\hline 72.2 & 73.9 & 69.2 & 63.5 & 65.3 & 62.7 & 65.3 & 64.4 & 63.1 & 65.3 \\
\hline 67 & 74.8 & 67 & 106.7 & 156.8 & 182 & 170.5 & 161.9 & 131.6 & 86.5 \\
\hline 65.3 & 65.3 & 63.5 & 54 & 54 & 64.4 & 59.2 & 56.2 & 59.2 & 66.1 \\
\hline 130.5 & 133.5 & 125.7 & 143.9 & 157.7 & 161.6 & 113.6 & 123.2 & 111.1 & 114.5 \\
\hline 74.8 & 74.8 & 75.2 & 73 & 70.4 & 72.2 & 70.4 & 71.3 & 71.3 & 89.1 \\
\hline 73.9 & 73.9 & 76.5 & 67 & 79.1 & 87.7 & 86.9 & 84.3 & 80 & 80.4 \\
\hline 67 & 66.1 & 65.3 & 58.4 & 59.2 & 57.1 & 61.3 & 61.4 & 62.7 & 65.3 \\
\hline 86.9 & 87.7 & 86.9 & 81.7 & 84.7 & 84.3 & 86.9 & 87.7 & 81.7 & 84.3 \\
\hline 86.9 & 86 & 85.1 & 73 & 78.7 & 79.1 & 68.7 & 65.3 & 66.1 & 66.1 \\
\hline 80.8 & 78.2 & 89.5 & 123.2 & 136.1 & 127.5 & 110.2 & 124 & 132.7 & 137.8 \\
\hline 77.4 & 73.5 & 71.3 & 73.9 & 70.4 & 69.6 & 71.3 & 71.8 & 66.1 & 68.7 \\
\hline 74.8 & 75.6 & 80 & 72.2 & 85.1 & 86 & 86 & 86 & 79.1 & 73 \\
\hline 73.9 & 69.6 & 66.1 & 60.9 & 62.7 & 60.9 & 60.1 & 62.7 & 67.9 & 66.6 \\
\hline 68.7 & 73 & 74.8 & 73 & 82.5 & 81.7 & 90.3 & 91.2 & 85.1 & 84.3 \\
\hline 69.2 & 69.6 & 78.2 & 75.6 & 73.9 & 75.6 & 75.6 & 78.2 & 81.3 & 85.1 \\
\hline 85.6 & 77.4 & 91.2 & 86.9 & 92 & 91.2 & 99 & 98.1 & 93.8 & 95.5 \\
\hline 81.7 & 84.3 & 84.3 & 77.4 & 73.9 & 75.2 & 73 & 70.4 & 69.6 & 74.8 \\
\hline 78.7 & 81.3 & 98.6 & 118 & 121 & 108.5 & 109.8 & 117.6 & 118.8 & 114.1 \\
\hline 68.3 & 70.4 & 102 & 114.1 & 113.6 & 102.9 & 108.5 & 114.1 & 110.2 & 113.2 \\
\hline 90.3 & 89.9 & 149.1 & 192.7 & 183.6 & 178.9 & 163.8 & 155.6 & 172.9 & 178.4 \\
\hline 86.5 & 90.8 & 96.4 & 107.2 & 114.5 & 101.2 & 97.2 & 99.4 & 105.9 & 110.7 \\
\hline 95.5 & 90.8 & 111.5 & 125.7 & 138.3 & 148.7 & 145.6 & 130.5 & 129.2 & 124.5 \\
\hline 86.5 & 86 & 96.8 & 105.9 & 108.1 & 95.1 & 102.4 & 103.7 & 107.6 & 108.9 \\
\hline 79.6 & 83 & 99.8 & 115.8 & 154.3 & 155.6 & 160.3 & 172 & 149.1 & 145.2 \\
\hline 78.7 & 80 & 89.9 & 98.6 & 98.1 & 91.6 & 103.3 & 96 & 92.9 & 99.4 \\
\hline 81.7 & 83 & 103.3 & 108.9 & 107.6 & 98.6 & 98.1 & 105.5 & 116.2 & 108.9 \\
\hline 75.6 & 74.4 & 80.8 & 83.4 & 74.8 & 72.6 & 70.4 & 73.9 & 80.8 & 78.2 \\
\hline 75.6 & 80 & 131.8 & 126.6 & 171.1 & 167.6 & 197.1 & 179.3 & 181.9 & 199.2 \\
\hline 75.6 & 74.4 & 84.3 & 91.2 & 88.6 & 81.7 & 86 & 85.6 & 87.7 & 89.9 \\
\hline 71.8 & 73.9 & 229.1 & 253.5 & 298.4 & 310.9 & 287.6 & 316.5 & 295.3 & 315.2 \\
\hline 69.6 & 70.4 & 70.4 & 61.4 & 80.8 & 73.9 & 67 & 67 & 66.1 & 58.4 \\
\hline 58 & 64.5 & 77.4 & 74 & 90.4 & 86.1 & 90.4 & 89.5 & 77.4 & 86.1 \\
\hline 64.9 & 64.5 & 59.7 & 56.2 & 57.6 & 57.1 & 59.7 & 56.2 & 56.7 & 58.8 \\
\hline 67.9 & 66.2 & 63.6 & 58.4 & 67.9 & 67.5 & 71.4 & 78.3 & 73.1 & 73.5 \\
\hline 67.1 & 67.5 & 61.9 & 60.5 & 59.3 & 60.5 & 61.4 & 65.3 & 66.6 & 70.9 \\
\hline 63.6 & 64.9 & 68.8 & 81.7 & 118.4 & 140.5 & 160.4 & 160.4 & 156.4 & 155.2 \\
\hline 79.2 & 78.7 & 78.3 & 83.9 & 80 & 62.7 & 63.6 & 62.7 & 65.7 & 62.7 \\
\hline
\end{tabular}


other

\begin{tabular}{|c|c|c|c|c|c|c|c|c|c|}
\hline 61 & 61.4 & 61 & 80.4 & 89.5 & 92.5 & 95.6 & 100.3 & 102 & 99 \\
\hline 64.5 & 62.3 & 58.8 & 53.2 & 53.6 & 48.4 & 50.2 & 66.6 & 67.5 & 49.8 \\
\hline 63.1 & 64 & 63.6 & 76.1 & 102.9 & 113.7 & 124.5 & 130.1 & 117.6 & 107.2 \\
\hline 62.7 & 59.7 & 58.8 & 52.8 & 52.8 & 51 & 55.4 & 62.3 & 65.7 & 70.9 \\
\hline 66.2 & 68.3 & 74.8 & 101.2 & 108.9 & 105.9 & 104.6 & 105.5 & 105.1 & 109.8 \\
\hline 66.2 & 65.7 & 64.9 & 58 & 57.6 & 56.2 & 58.8 & 57.1 & 55.4 & 58.4 \\
\hline 107.7 & 112.4 & 109.8 & 107.2 & 123.2 & 122.8 & 127.1 & 123.6 & 115 & 110.7 \\
\hline 102.5 & 103.7 & 99.9 & 94.2 & 91.6 & 90.8 & 89.9 & 89.1 & 92.1 & 96.4 \\
\hline 80.4 & 83.5 & 83 & 80.4 & 81.3 & 85.6 & 84.3 & 88.2 & 109.8 & 105.5 \\
\hline 80.4 & 76.6 & 77.8 & 68.3 & 72.6 & 72.6 & 72.2 & 74.4 & 71.4 & 72.6 \\
\hline 70.5 & 74.4 & 71.8 & 79.2 & 103.7 & 101.6 & 96 & 91.6 & 82.6 & 85.6 \\
\hline 70.5 & 69.2 & 65.7 & 70.9 & 60.1 & 60.5 & 58.8 & 62.3 & 58.8 & 82.1 \\
\hline 123.2 & 127.1 & 127.9 & 121 & 119.3 & 145.7 & 162.5 & 159.9 & 150.4 & 146.9 \\
\hline 107.2 & 109.8 & 106.3 & 96.8 & 96.8 & 95.1 & 97.7 & 93.4 & 93.4 & 99 \\
\hline 65.3 & 69.6 & 70 & 99 & 112.8 & 118.9 & 122.4 & 121.5 & 134.4 & 139.2 \\
\hline 62.7 & 61 & 61 & 53.2 & 52.4 & 52.4 & 56.7 & 56.2 & 57.6 & 79.2 \\
\hline 101.6 & 105.1 & 104.6 & 106.8 & 133.1 & 130.1 & 121 & 122.4 & 118 & 96.8 \\
\hline 100.8 & 101.6 & 101.6 & 100.4 & 102.5 & 101.6 & 97.3 & 104.2 & 108.5 & 120.6 \\
\hline 63.6 & 62.7 & 73.1 & 98.2 & 131.9 & 134.8 & 141.4 & 146.5 & 152.6 & 166 \\
\hline 64.5 & 63.6 & 63.6 & 60.1 & 55.4 & 52.8 & 51.5 & 53.2 & 53.6 & 75.3 \\
\hline 70.5 & 73.1 & 83.1 & 87.8 & 137.9 & 130.1 & 139.6 & 139.6 & 118 & 134.4 \\
\hline 74.8 & 75.2 & 73.1 & 67.9 & 74 & 75.7 & 72.2 & 71.4 & 70 & 91.2 \\
\hline 64 & 67.9 & 65.3 & 56.7 & 69.6 & 71.4 & 73.1 & 71.4 & 58 & 58.4 \\
\hline 61.9 & 63.6 & 62.7 & 54.1 & 54.5 & 55.8 & 64.5 & 66.2 & 68.8 & 66.6 \\
\hline 70.1 & 67.5 & 64.5 & 57.6 & 59.7 & 66.7 & 70.6 & 69.2 & 70.1 & 72.7 \\
\hline 67.1 & 63.7 & 61.5 & 54.6 & 52.8 & 53.7 & 54.6 & 53.7 & 54.2 & 56.3 \\
\hline 65.7 & 66.5 & 65.7 & 59.6 & 62.2 & 63.9 & 68.3 & 82.1 & 77.8 & 78.6 \\
\hline 69.1 & 70.8 & 70 & 60.5 & 60.5 & 61.3 & 64.8 & 63.9 & 65.7 & 65.7 \\
\hline 65.3 & 63.5 & 73.9 & 114.2 & 147.3 & 164.1 & 153.3 & 145.9 & 135 & 121.6 \\
\hline 62.7 & 62.7 & 62.7 & 53.6 & 53.2 & 54.9 & 54 & 54 & 57.5 & 65.3 \\
\hline 65.3 & 64.4 & 70.4 & 181 & 216.9 & 240.7 & 240.7 & 243.2 & 245 & 230.8 \\
\hline 63.5 & 62.7 & 60.9 & 53.2 & 55.8 & 54 & 57.5 & 58 & 58.4 & 60.9 \\
\hline 84.3 & 85.1 & 81.7 & 74.8 & 84.7 & 90.3 & 90.3 & 91.2 & 87.3 & 67.9 \\
\hline 73.9 & 73.9 & 70.4 & 62.7 & 60.5 & 61.8 & 62.7 & 65.3 & 64.9 & 68.7 \\
\hline 71.3 & 69.6 & 95.5 & 124.5 & 135.2 & 134.4 & 151.7 & 164.6 & 141.3 & 122.3 \\
\hline 67.9 & 68.7 & 67.5 & 60.9 & 59.2 & 59.2 & 64.4 & 66.6 & 69.6 & 70 \\
\hline 74.8 & 76.5 & 94.4 & 190.6 & 207.2 & 202.3 & 211.8 & 240.3 & 250.8 & 253 \\
\hline 71.3 & 70.9 & 70.4 & 62.7 & 60.9 & 61.8 & 60.9 & 64.4 & 65.3 & 70.4 \\
\hline 65.7 & 66.6 & 80 & 100.7 & 105 & 99 & 114.5 & 138.7 & 126.6 & 125.3 \\
\hline 62.7 & 61.8 & 58.4 & 53.2 & 52.3 & 57.5 & 57.1 & 59.2 & 64.4 & 72.2 \\
\hline 150.3 & 146 & 150.3 & 175.4 & 215.1 & 230.7 & 227.2 & 229 & 207.4 & 227.2 \\
\hline 168.5 & 166.8 & 164.2 & 168.5 & 183.2 & 193.5 & 200.4 & 201.3 & 194.4 & 187.5 \\
\hline 134.8 & 135.6 & 131.3 & 174.5 & 211.7 & 225.5 & 227.2 & 228.1 & 183.2 & 184.9 \\
\hline 125.3 & 126.1 & 125.3 & 165 & 205.6 & 220.3 & 222.9 & 221.2 & 187.5 & 198.7 \\
\hline 138.2 & 138.2 & 140 & 176.3 & 224.6 & 231.6 & 233.3 & 246.2 & 215.1 & 229.8 \\
\hline 149.5 & 146.9 & 156.4 & 192.7 & 235 & 253.2 & 250.6 & 253.2 & 229 & 300.7 \\
\hline 148.6 & 146.9 & 149.5 & 164.2 & 207.4 & 210 & 216 & 213.4 & 188.4 & 181.4 \\
\hline 146.9 & 140.8 & 139.1 & 292.9 & 323.1 & 342.1 & 350.8 & 343.9 & 309.3 & 316.2 \\
\hline 149.5 & 144.3 & 140.8 & 249.7 & 365.5 & 394.8 & 435.5 & 460.5 & 439.8 & 441.5 \\
\hline
\end{tabular}


other

\begin{tabular}{|c|c|c|c|c|c|c|c|c|c|}
\hline 184 & 182.3 & 186.6 & 348.2 & 400 & 407.8 & 400.9 & 402.6 & 287.7 & 370.7 \\
\hline 138.2 & 137.4 & 150.3 & 300.7 & 379.3 & 387.9 & 392.3 & 452.7 & 415.6 & 426 \\
\hline 143.4 & 140.8 & 144.3 & 162.4 & 205.6 & 221.2 & 235.9 & 241.1 & 208.2 & 204.8 \\
\hline 134.8 & 133.1 & 156.4 & 271.3 & 401.8 & 425.1 & 434.6 & 381.9 & 375 & 434.6 \\
\hline 150.3 & 150.3 & 153.8 & 328.3 & 355.1 & 356.8 & 365.5 & 361.2 & 345.6 & 350.8 \\
\hline 152.9 & 153.8 & 154.7 & 263.5 & 356.8 & 366.3 & 375.8 & 376.7 & 372.4 & 373.2 \\
\hline 156.4 & 153.8 & 154.7 & 313.6 & 360.3 & 381 & 380.2 & 395.7 & 391.4 & 391.4 \\
\hline 278.2 & 215.1 & 154.7 & 159 & 186.6 & 190.9 & 191.8 & 185.8 & 203.9 & 204.8 \\
\hline 148.6 & 148.6 & 159 & 314.5 & 337.8 & 368.1 & 353.4 & 356 & 348.2 & 350.8 \\
\hline 176.3 & 171.9 & 190.9 & 330.9 & 347.3 & 344.7 & 343 & 345.6 & 341.3 & 353.4 \\
\hline 133.9 & 132.2 & 138.2 & 297.2 & 310.2 & 305.9 & 309.3 & 309.3 & 305.9 & 324.9 \\
\hline 133.9 & 131.3 & 129.6 & 124.4 & 127.9 & 126.1 & 124.4 & 124.4 & 128.7 & 130.5 \\
\hline 123.6 & 126.1 & 124.4 & 126.1 & 123.6 & 128.7 & 127 & 133.9 & 140.8 & 145.2 \\
\hline 118.4 & 119.2 & 127 & 318.8 & 360.3 & 30.4 & 369.8 & 370.7 & 349.9 & 341.3 \\
\hline 114.9 & 112.3 & 117.5 & 284.3 & 356.8 & 367.2 & 375.8 & 367.2 & 332.6 & 343 \\
\hline 121.8 & 120.1 & 123.6 & 300.7 & 340.4 & 345.6 & 348.2 & 351.6 & 322.3 & 338.7 \\
\hline 123.6 & 119.2 & 123.6 & 309.3 & 331.8 & 341.3 & 343.9 & 352.5 & 318.8 & 342.1 \\
\hline 117.5 & 116.6 & 121 & 136.5 & 160.7 & 167.6 & 161.6 & 161.6 & 154.7 & 153.8 \\
\hline 120.1 & 122.7 & 134.8 & 308.4 & 317.1 & 316.2 & 319.7 & 324 & 323.1 & 328.3 \\
\hline 111.5 & 109.7 & 124.4 & 286 & 301.5 & 297.2 & 305 & 306.7 & 299.8 & 310.2 \\
\hline 110.6 & 108.9 & 116.6 & 285.1 & 295.5 & 300.7 & 303.3 & 309.3 & 312.8 & 309.3 \\
\hline 118.4 & 117.5 & 122.7 & 297.2 & 303.3 & 221.2 & 166.8 & 171.1 & 177.1 & 172.8 \\
\hline 103.7 & 104.5 & 126.1 & 132.2 & 133.9 & 140 & 146 & 149.5 & 147.7 & 149.5 \\
\hline 113.2 & 113.2 & 115.8 & 152.9 & 271.3 & 343 & 355.1 & 350.8 & 360.3 & 364.6 \\
\hline 129.6 & 125.3 & 132.2 & 299.8 & 287.7 & 292 & 288.6 & 298.1 & 253.2 & 223.8 \\
\hline 121.8 & 120.1 & 124.4 & 288.6 & 290.3 & 312.8 & 323.1 & 320.5 & 312.8 & 330.9 \\
\hline 139.1 & 136.5 & 140.8 & 289.4 & 299.8 & 310.2 & 315.4 & 324.9 & 330 & 342.1 \\
\hline 146 & 145.2 & 147.7 & 149.5 & 154.7 & 166.8 & 173.7 & 175.4 & 174.5 & 177.1 \\
\hline 142.6 & 142.6 & 145.2 & 323.1 & 318 & 328.3 & 324 & 324.9 & 327.5 & 342.1 \\
\hline 140.8 & 140 & 140.8 & 298.1 & 301.5 & & & 49.2 & 330.9 & 366.3 \\
\hline 151.2 & 145.2 & 160.7 & 315.4 & 335.2 & 361.2 & 373.2 & 367.2 & 351.6 & 368.1 \\
\hline 159.8 & 159.8 & 165 & 340.4 & 381 & 376.7 & 375 & 379.3 & 352.5 & 378.4 \\
\hline 159.8 & 159 & 162.4 & 171.1 & 187.5 & 209.1 & 232.4 & 232.4 & 220.3 & 223.8 \\
\hline 142.6 & 140 & 152.9 & 327.5 & 377.6 & 417.3 & 433.7 & 424.2 & 387.9 & 417.3 \\
\hline 160.7 & 165.9 & 169.3 & 343.9 & 393.1 & 408.7 & 432.9 & 432 & 368.9 & 370.7 \\
\hline 145.2 & 146 & 139.1 & 307.6 & 362 & 380.2 & 392.3 & 426.8 & 364.6 & 367.2 \\
\hline 156.4 & 165 & 175.4 & 396.6 & 407.8 & 420.8 & 432.9 & 432 & 381 & 410.4 \\
\hline 148.6 & 148.6 & 263.5 & 453.6 & 451.9 & 515.8 & 517.5 & 514.9 & 476.1 & 513.2 \\
\hline 159.8 & 156.4 & 305.9 & 464.8 & 504.6 & 539.1 & 567.6 & 574.6 & 530.5 & 557.3 \\
\hline 190.1 & 184 & 315.4 & 504.6 & 571.1 & 590.1 & 606.5 & 604.8 & 542.6 & 590.1 \\
\hline 233.3 & 234.1 & 380.2 & 545.2 & 580.6 & 624.7 & 639.4 & 642 & 563.3 & 599.6 \\
\hline 197.9 & 198.7 & 365.5 & 530.5 & 553 & 558.1 & 559 & 546.9 & 483 & 544.3 \\
\hline 176.3 & 181.4 & 381 & 484.7 & 445 & 471.7 & 489 & 480.4 & 450.1 & 464 \\
\hline 222 & 318 & 321.4 & 382.8 & 483 & 626.4 & 679.1 & 683.4 & 650.6 & 684.3 \\
\hline 253.2 & 245.4 & 297.2 & 539.1 & 566.8 & 675.6 & 655.8 & 658.4 & 617.8 & 721.4 \\
\hline 406.1 & 402.6 & 410.4 & 576.3 & 624.7 & 643.7 & 670.5 & 699.8 & 660.1 & 781.1 \\
\hline 388.8 & 388.8 & 400 & 648 & 757.7 & 853.6 & 830.3 & 844.1 & 851 & 836.4 \\
\hline 366.3 & 366.3 & 385.3 & 621.2 & 819.1 & 852.8 & 855.4 & 865.7 & 872.6 & 857.1 \\
\hline 225.5 & 318 & 359.4 & 622.9 & 807.8 & 821.7 & 838.1 & 871.8 & 865.7 & 861.4 \\
\hline
\end{tabular}


other

\begin{tabular}{|c|c|c|c|c|c|c|c|c|c|}
\hline 349.1 & 340.4 & 379.3 & 620.4 & 775.9 & 797.5 & 827.7 & 877 & 875.2 & 869.2 \\
\hline 375 & 366.3 & 390.5 & 585.8 & 797.5 & 818.2 & 839.8 & 869.2 & 871.8 & 855.4 \\
\hline 363.7 & 358.6 & 382.8 & 624.7 & 799.2 & 822.5 & 851 & 872.6 & 878.7 & 868.3 \\
\hline 393.1 & 389.7 & 420.8 & 629 & 824.3 & 835.5 & 835.5 & 873.5 & 924.5 & 909.8 \\
\hline 284.3 & 380.2 & 364.6 & 366.3 & 378.4 & 398.3 & 399.2 & 413.9 & 413.9 & 414.7 \\
\hline 168.5 & 169.8 & 201.4 & 525.2 & 667.7 & 683.6 & 713 & 733.7 & 652 & 583.1 \\
\hline 116.2 & 114.5 & 119.7 & 326.7 & 516.4 & 523.5 & 528.7 & 517 & 455.6 & 351.6 \\
\hline 108.5 & 105.9 & 101.6 & 94.6 & 97.2 & 102.9 & 130.9 & 120.6 & 99 & 97.2 \\
\hline 108.9 & 105.9 & 103.3 & 101.6 & 103.3 & 100.3 & 100.7 & 98.1 & 100.7 & 102.4 \\
\hline 114.5 & 105.9 & 116.2 & 355.1 & 511.8 & 533 & 538.6 & 508.7 & 323.2 & 227.7 \\
\hline 99.8 & 100.7 & 91.2 & 99 & 131.8 & 140.4 & 127.9 & 124 & 106.7 & 113.6 \\
\hline 89.5 & 99 & 88.6 & 83 & 97.2 & 106.7 & 96.8 & $\begin{array}{l}99.8 \\
\end{array}$ & 105.9 & 98.1 \\
\hline 88.6 & 86 & 86 & 78.7 & 77.4 & 75.6 & 77.4 & 74.8 & 74.4 & 77.4 \\
\hline 91.2 & 89.9 & 116.2 & 103.3 & 154.3 & 144.8 & 134 & 130.1 & 143 & 131.8 \\
\hline 131.8 & 88.6 & 81.7 & 138.3 & 169.8 & 157.7 & 167.2 & 140.9 & 112.8 & 115.4 \\
\hline 87.7 & 113.6 & 118 & 89.9 & 118.8 & 124.9 & 117.1 & 165.1 & 135.2 & 159.4 \\
\hline 80.8 & 103.7 & 107.6 & 125.7 & 159.4 & 125.3 & 156 & 9.5 & 113.6 & 141.3 \\
\hline 81.7 & 80.4 & 115.4 & 117.6 & 68.7 & 65.7 & 71.8 & 79.1 & 78.2 & 79.6 \\
\hline 94.2 & 117.6 & 96.4 & 105 & 137.4 & 156.8 & 166.4 & 154.3 & 131.8 & 128.8 \\
\hline 77.4 & 74.8 & 70 & 99.8 & 109.3 & 70.4 & 71.3 & 71.3 & 79.1 & 134 \\
\hline 126.6 & 126.2 & 133.5 & 330.5 & 496.4 & 466.6 & 479.1 & 474.8 & 472.2 & 425.6 \\
\hline 97.2 & 98.6 & 107.6 & 284.7 & 443.7 & 445.4 & 443.3 & 462.7 & 438.5 & 429.6 \\
\hline 84.3 & 86 & 80 & 73.5 & 83.4 & 88.6 & 87.7 & 87.7 & 87.7 & 86 \\
\hline 98.1 & 97.7 & 92 & 142.2 & 161.2 & 166.4 & 170.7 & 164.6 & 120.6 & 154.7 \\
\hline 92 & 91.6 & 89.5 & 147.3 & 174.1 & 174.1 & 174.1 & 177.6 & 145.6 & 156 \\
\hline 89.5 & 91.2 & 85.1 & 132.7 & 143 & 161.2 & 165.5 & 182.8 & 154.3 & 175.5 \\
\hline 89.5 & 96.4 & 87.7 & 143.9 & 155.1 & 162 & 165.5 & 173.7 & 150.3 & 178 \\
\hline 98.1 & 118.8 & 126.6 & 460 & 670.7 & 729.7 & 752.6 & 776.2 & 781.8 & 777.8 \\
\hline 102.4 & 104.1 & 104.1 & 95.5 & 95.5 & 96.4 & 97.2 & 98.1 & 115.4 & 119.7 \\
\hline 96.4 & 99 & 112.4 & 386.3 & 557.8 & 600.2 & 618.3 & 647.3 & 652.9 & 661.9 \\
\hline 119.7 & 124.9 & 127.1 & 555.4 & 768.1 & 787.1 & 763.2 & 793.4 & 827.9 & 836 \\
\hline 104.1 & 111.9 & 124.9 & 582.7 & 778.2 & 814.5 & 816.3 & 866.3 & 872.9 & 863.9 \\
\hline 90.3 & 88.6 & 84.3 & 81.7 & 80 & 80 & 77.4 & 78.2 & 92.5 & 90.3 \\
\hline 90.3 & 99 & 103.3 & 380.7 & 494.8 & 491.8 & 522.1 & 547.9 & 513.5 & 556.7 \\
\hline 105 & 107.2 & 113.6 & 453.6 & 476.9 & 457.7 & 459.3 & 493 & 463.5 & 488.1 \\
\hline 106.7 & 108.5 & 108.5 & 414.5 & 434.6 & 417.2 & 427 & 457.7 & 424.1 & 447.5 \\
\hline 100.7 & 102.4 & 94.2 & 375.4 & 424.7 & 451.5 & 463.6 & 502.4 & 490.6 & 495.6 \\
\hline 103.3 & 101.2 & 97.2 & 98.1 & 94.6 & 87.7 & 88.6 & 87.7 & 88.6 & 91.2 \\
\hline 93.8 & 94.6 & 104.1 & 300.3 & 306.3 & 320.6 & 340.9 & 329.2 & 278.3 & 306.7 \\
\hline 115 & 111.1 & 122.8 & 334.8 & 343.5 & 355.6 & 359.9 & 361.2 & 304.6 & 254.9 \\
\hline 99.4 & 104.1 & 105.5 & 287.3 & 335.7 & 423.8 & 440.2 & 445.4 & 413.1 & 426 \\
\hline 117.6 & 119.7 & 120.2 & 367.7 & 445.4 & 452.3 & 394 & 402.2 & 342.2 & 270.5 \\
\hline 133.5 & 129.7 & 117.6 & 121.4 & 134 & 149.9 & 153.9 & 167.7 & 189.7 & 203.1 \\
\hline 46.7 & 45.8 & 46.7 & 55.3 & 70.8 & 222.9 & 221.2 & 222.9 & 205.6 & 197.9 \\
\hline 127.9 & 121.9 & 128.8 & 245.8 & 280.9 & 296 & 293 & 299.4 & 259.3 & 270.5 \\
\hline 127.9 & 128.8 & 130.9 & 238.5 & 264.4 & 263.1 & 271.4 & 281.3 & 251.5 & 246.3 \\
\hline 124 & 117.6 & 127.9 & 223.8 & 251 & 261 & 262.3 & 267.9 & 241.1 & 245.8 \\
\hline 115.8 & 114.5 & 105.5 & 106.3 & 117.6 & 126.6 & 139.2 & 147.8 & 159 & 166.4 \\
\hline
\end{tabular}


other

\begin{tabular}{|c|c|c|c|c|c|c|c|c|c|}
\hline 113.2 & 110.7 & 121 & 214.7 & 246.3 & 274 & 269.2 & 269.6 & 250.6 & 270 \\
\hline 116.7 & 111.5 & 121 & 223.7 & 261.7 & 265.4 & 283.8 & 303.5 & 286.6 & 297.8 \\
\hline 103.7 & 97.7 & 114.5 & 188.8 & 206.2 & 220.1 & 252.1 & 280.9 & 257.2 & 277.9 \\
\hline 92.5 & 90.8 & 98.1 & 173.9 & 187.9 & 198.5 & 236.1 & 251.6 & 255.4 & 279.6 \\
\hline 91.6 & 89.1 & 84.7 & 80.8 & 95.1 & 115 & 122.8 & 129.2 & 127.1 & 140 \\
\hline 77.8 & 76.1 & 93.4 & 163.8 & 195.5 & 226.2 & 279.4 & 297.6 & 294.2 & 341.2 \\
\hline 146.1 & 140.4 & 153 & 214.8 & 242.2 & 269.8 & 305.4 & 337 & 324.5 & 337.4 \\
\hline 122.8 & 117.6 & 131.8 & 223.1 & 270.5 & 282.5 & 319.6 & 341.1 & 355.4 & 489.8 \\
\hline 127.9 & 121.9 & 130.5 & 186.2 & 292.7 & 331.9 & 376 & 404.4 & 418.9 & 438.7 \\
\hline 81.3 & 80.4 & 77 & 77.4 & 83 & 96.4 & 102 & 113.2 & 122.8 & 126.6 \\
\hline 87.3 & 88.2 & 102.4 & 266.4 & 318.1 & 326.1 & 357.1 & 377.7 & 344 & 359 \\
\hline 90.8 & 94.6 & 102 & 274.8 & 394.4 & 472.7 & 474.3 & 456.6 & 425.5 & 438.8 \\
\hline 102.9 & 99.8 & 108.9 & 411.9 & 472.6 & 469.8 & 486.6 & 479.6 & 437.3 & 459.6 \\
\hline 95.1 & 91.6 & 105.5 & 387.5 & 464.1 & 466 & 457.7 & 484 & 445.4 & 442.8 \\
\hline 87.7 & 84.7 & 83 & 78.2 & 84.7 & 89.1 & 101.2 & 123.2 & 125.3 & 129.7 \\
\hline 80.4 & 83 & 99 & 245.9 & 351.2 & 372.5 & 372 & 393.6 & 365.9 & 362.9 \\
\hline 99.8 & 96 & 110.7 & 223.7 & 309.4 & 330.1 & 368.1 & 373.3 & 355.6 & 374.2 \\
\hline 102 & 104.6 & 115 & 260.5 & 354.3 & 369 & 386.3 & 406.5 & 385.4 & 412.9 \\
\hline 96.8 & 101.2 & 114.1 & 159 & 211.3 & 330.3 & 365.5 & 354.8 & 341.8 & 359.8 \\
\hline 86 & 83.9 & 79.6 & 75.2 & 79.1 & 89.9 & 100.3 & 115.4 & 105.5 & 83.9 \\
\hline 66.1 & 67.5 & 89.9 & 265.3 & 333.8 & 337.1 & 340.4 & 342.6 & 313.2 & 285.5 \\
\hline 62.3 & 62.3 & 85.1 & 208.7 & 235.1 & 235.5 & 263.8 & 276 & 253.9 & 266 \\
\hline 74.4 & 70 & 89.5 & 121.9 & 165.1 & 171.5 & 166 & 162.5 & 156 & 174.6 \\
\hline 67.5 & 66.6 & 87.7 & 184.5 & 305 & 329.2 & 326.5 & 332.9 & 317.8 & 340.6 \\
\hline 157.3 & 154.7 & 153 & 153.9 & 158.6 & 160.8 & 174.6 & 182.4 & 193.6 & 193.6 \\
\hline 171.1 & 163.4 & 176.3 & 206.6 & 249.8 & 251 & 268.8 & 296.4 & 294.7 & 310.2 \\
\hline 70 & 66.6 & 71.3 & 134 & 258.4 & 253.6 & 261.9 & 267.9 & 280 & 293.4 \\
\hline 72.2 & 70.9 & 80.4 & 166 & 253.6 & 258.4 & 260.1 & 269.2 & 280 & 301.6 \\
\hline 90.8 & 86.5 & 101.2 & 159.4 & 273.1 & 283.9 & 291.2 & 303.3 & 309.8 & 318.9 \\
\hline 80 & 74.4 & 74.4 & 67.9 & 73.5 & 95.5 & 99.4 & 108.1 & 119.7 & 123.6 \\
\hline 77.4 & 78.7 & 90.8 & 141.3 & 315.8 & 383.7 & 378.8 & 396.8 & 362.2 & 395.9 \\
\hline 70.9 & 73 & 88.2 & 266.2 & 332.2 & 377 & 390.7 & 394.9 & 344.4 & 374.5 \\
\hline 87.3 & 83.9 & 98.1 & 264.4 & 353.2 & 375.6 & 401 & 409.7 & 390.6 & 390.1 \\
\hline 86.9 & 80.4 & 95.1 & 293.3 & 365.3 & 428.4 & 454.6 & 458.9 & 443.2 & 455.7 \\
\hline 107.2 & 105.9 & 96.8 & 90.8 & 98.6 & 112.8 & 116.7 & 118.4 & 117.6 & 129.2 \\
\hline 110.2 & 131.4 & 145.2 & 347.6 & 454.5 & 487.5 & 501.2 & 509.4 & 480.9 & 499.8 \\
\hline 118.4 & 124.9 & 134 & 355.8 & 477.1 & 497.7 & 531.7 & 531.7 & 476.4 & 505.7 \\
\hline 116.7 & 116.7 & 130.9 & 455.4 & 551 & 579.4 & 595.8 & 604.4 & 534.5 & 584.6 \\
\hline 109.3 & 116.7 & 137 & 464.9 & 576.1 & 579 & 596.3 & 583.3 & 529.9 & 529.8 \\
\hline 88.2 & 86.9 & 85.6 & 80 & 83 & 83.4 & 89.1 & 101.6 & 116.7 & 118 \\
\hline 83.9 & 115 & 135.2 & 437.9 & 501.6 & 513.6 & 531.6 & 530.6 & 481.8 & 579 \\
\hline 94.2 & 103.3 & 131.4 & 445 & 511.4 & 510.8 & 544.8 & 542.3 & 545.3 & 643.8 \\
\hline 103.7 & 102.4 & 130.5 & 535.6 & 759.5 & 762.9 & 813.2 & 848.3 & 842.6 & 855.1 \\
\hline 79.1 & 88.2 & 214.7 & 586.6 & 771.6 & 793.2 & 815 & 831.4 & 833.2 & 829.6 \\
\hline 98.1 & 96 & 90.3 & 83.9 & 92.5 & 103.3 & 106.3 & 113.6 & 106.3 & 123.6 \\
\hline 109.8 & 253.4 & 308.1 & 605.5 & 822.2 & 850.4 & 875.2 & 904.9 & 891.8 & 889.6 \\
\hline 101.2 & 101.6 & 265.8 & 614.8 & 846.9 & 867 & 895.1 & 917.4 & 901.8 & 912.6 \\
\hline 103.7 & 111.1 & 271.9 & 624.7 & 844.3 & 885.9 & 926.9 & 937.6 & 919.2 & 933.3 \\
\hline 127.1 & 131.4 & 293.9 & 642.8 & 864.9 & 906.5 & 942 & 948 & 942.8 & 935.4 \\
\hline
\end{tabular}


other

\begin{tabular}{|c|c|c|c|c|c|c|c|c|c|}
\hline 103.7 & 101.6 & 94.2 & 90.3 & 95.1 & 96.8 & 108.5 & 118.4 & 121.4 & 140.9 \\
\hline 133.1 & 185.2 & 364 & 657.3 & 872.2 & 885.4 & 926.6 & 944.7 & 887.2 & 903.9 \\
\hline 105 & 113.2 & 281.4 & 614 & 846 & 887.2 & 928.4 & 937.4 & 936.9 & 927.7 \\
\hline 113.6 & 120.2 & 306.5 & 653.4 & 883.7 & 925.4 & 962.5 & 967.2 & 949.1 & 954.5 \\
\hline 112.8 & 124.5 & 287.9 & 640.6 & 896.9 & 945.4 & 982.1 & 1007.5 & 988.1 & 984.1 \\
\hline 111.5 & 108.9 & 112.8 & 101.2 & 110.7 & 117.6 & 126.6 & 127.9 & 134.8 & 143 \\
\hline 101.2 & 257.3 & 348.7 & 605 & 799.3 & 831.8 & 855.7 & 873.8 & 846.4 & 877.4 \\
\hline 81.7 & 81.3 & 79.1 & 71.3 & 68.7 & 68.7 & 71.3 & 75.6 & 76.1 & 77 \\
\hline 120.6 & 114.5 & 140.4 & 243.2 & 420 & 452.3 & 460.1 & 466.3 & 478.9 & 470.4 \\
\hline 97.2 & 97.2 & 167.7 & 319.3 & 410 & 417.8 & 409.1 & 397.5 & 314.1 & 259.7 \\
\hline 104.1 & 99.4 & 99.8 & 171.5 & 206.6 & 224.2 & 200.9 & 143.9 & 155.6 & 177.6 \\
\hline 92.5 & 130.9 & 157.7 & 293.4 & 431.6 & 463.2 & 454.9 & 453.2 & 438.5 & 397 \\
\hline 80 & 79.1 & 78.2 & 70 & 68.7 & 67.9 & 71.8 & 73.9 & 73 & 73 \\
\hline 136.1 & 130.5 & 132.7 & 188.8 & 221.6 & 230.8 & 229.4 & 225.1 & 172.4 & 172.4 \\
\hline 115.4 & 117.1 & 123.2 & 166.8 & 168.9 & 170.7 & 188 & 178.4 & 132.7 & 149.5 \\
\hline 108.5 & 108.5 & 102 & 138.7 & 162.9 & 146.5 & 134 & 113.6 & 110.2 & 105 \\
\hline 99 & 99.8 & 98.6 & 104.1 & 124 & 100.3 & 101.6 & 94.6 & 99 & 103.7 \\
\hline 81.7 & 83.9 & 81.7 & 76.5 & 71.3 & 72.2 & 70.9 & 70.4 & 71.3 & 70.4 \\
\hline 118.4 & 130.9 & 160.3 & 156 & 208.3 & 190.5 & 183.6 & 180.2 & 137.8 & 174.6 \\
\hline 98.6 & 91.6 & 88.2 & 111.9 & 120.6 & 128.3 & 124.5 & 120.1 & 113.2 & 131.8 \\
\hline 84.3 & 83 & 84.3 & 120.6 & 149.9 & 146.5 & 149.1 & 167.2 & 134.8 & 162.9 \\
\hline 112.8 & 121.4 & 99.4 & 130.1 & 120.6 & 92.5 & 119.7 & 124.9 & 100.7 & 92.5 \\
\hline 62.3 & 70.4 & 80.8 & 221.6 & 343.5 & 347.8 & 342.2 & 308.9 & 238.9 & 302 \\
\hline 75.6 & 115 & 151.7 & 219.9 & 276.1 & 252.8 & 252.8 & 249.3 & 201.8 & 258.8 \\
\hline 88.6 & 154.3 & 149.9 & 181.9 & 213 & 208.3 & 191.4 & 182.8 & 153.4 & 180.2 \\
\hline 91.2 & 135.2 & 122.8 & 175.9 & 190.5 & 189.7 & 182.8 & 173.7 & 131.8 & 183.6 \\
\hline 87.7 & 105.5 & 101.6 & 169.8 & 172.9 & 163.8 & 165.5 & 153.4 & 105 & 143.5 \\
\hline 76.5 & 89.5 & 87.7 & 130.1 & 146.5 & 140 & 143.9 & 132.7 & 115.4 & 118.4 \\
\hline 66.1 & 80.8 & 78.2 & 115.4 & 140.4 & 142.2 & 121.9 & 124 & 96.4 & 102.4 \\
\hline 101.6 & 102.4 & 151.7 & 138.3 & 150.8 & 162 & 188.8 & 169.8 & 169.8 & 197.5 \\
\hline 65.3 & 141.3 & 105.9 & 162 & 185.4 & 198.5 & 211.7 & 224.2 & 213.9 & 236.3 \\
\hline 79.6 & 215.1 & 217.8 & 402.2 & 623.3 & 663.1 & 690.6 & 668.2 & 583.4 & 516.8 \\
\hline 68.7 & 180.2 & 222.5 & 461.7 & 648.7 & 683.2 & 685.3 & 712.9 & 630.3 & 549.1 \\
\hline 66.1 & 175.9 & 203.5 & 425.2 & 548.5 & 576.9 & 605.2 & 587.1 & 487.6 & 455.2 \\
\hline 64.4 & 286 & 294.8 & 310 & 413.4 & 408 & 432.6 & 427 & 369.6 & 428.4 \\
\hline 73.9 & 79.1 & 93.8 & 211.8 & 257.5 & 270.9 & 297.2 & 301.5 & 254.7 & 290.5 \\
\hline 67.5 & 75.6 & 126.6 & 242.7 & 263.9 & 292.1 & 297 & 296 & 290.5 & 404.6 \\
\hline 70.4 & 78.7 & 102.4 & 244.1 & 290.7 & 315.2 & 310.9 & 308.7 & 269.5 & 317 \\
\hline 68.7 & 76.5 & 102 & 162.4 & 184.5 & 185 & 196.6 & 193.1 & 178 & 187.1 \\
\hline 83.9 & 137 & 179.8 & 297.4 & 336.9 & 344 & 365.5 & 376.7 & 336.5 & 332.2 \\
\hline 89.1 & 100.7 & 132.7 & 313.6 & 373 & 384 & 422.5 & 458.7 & 370.3 & 352 \\
\hline 100.3 & 111.1 & 157.7 & 374 & 450.1 & 469.2 & 471.3 & 483 & 388.9 & 385.3 \\
\hline 85.1 & 93.8 & 134.8 & 341.5 & 427 & 439.2 & 391.1 & 378.6 & 309.3 & 304.9 \\
\hline 68.3 & 70.8 & 79.6 & 127.5 & 229.4 & 215.6 & 223.4 & 219.9 & 195.7 & 168.5 \\
\hline 76.5 & 78.2 & 126.6 & 207 & 351.7 & 345.1 & 354.5 & 348.9 & 310 & 296.1 \\
\hline 68.7 & 68.7 & 84.3 & 190.1 & 339.7 & 340.6 & 341 & 360 & 284.7 & 278.6 \\
\hline 71.3 & 83 & 135 & 338.4 & 382.6 & 386.5 & 419.8 & 414.1 & 325.1 & 306.4 \\
\hline 74.8 & 83.9 & 139.9 & 365.8 & 403.2 & 402.9 & 415 & 401.6 & 320.8 & 291.2 \\
\hline 71.8 & 82.5 & 136.9 & 277.7 & 265.4 & 267.5 & 255.8 & 235.8 & 180 & 198.7 \\
\hline
\end{tabular}


other

\begin{tabular}{|c|c|c|c|c|c|c|c|c|c|}
\hline 72.6 & 139.6 & 183.9 & 336.5 & 398.5 & 387.6 & 376 & 378.4 & 318.8 & 314.4 \\
\hline 63.5 & 74.4 & 130.8 & 323.2 & 362 & 365.9 & 382.2 & 377 & 287.1 & 293 \\
\hline 69.2 & 82.5 & 145.9 & 338.1 & 394.8 & 399 & 382.2 & 365.8 & 307.5 & 298.4 \\
\hline 66.1 & 79.1 & 128.2 & 310.2 & 376.6 & 359.3 & 366.2 & 373.2 & 306.2 & 295.5 \\
\hline 70.4 & 85.1 & 127.9 & 234.2 & 251.9 & 246.7 & 251.5 & 253.6 & 212.6 & 213 \\
\hline 72.2 & 116.7 & 178.3 & 345.8 & 423.7 & 423.1 & 423.5 & 419.6 & 334 & 324.5 \\
\hline 68.3 & 82.5 & 132.5 & 338.2 & 440 & 444.6 & 438.1 & 433.4 & 345.8 & 335.4 \\
\hline 66.6 & 74.4 & 129.1 & 333.8 & 433.4 & 451 & 452.7 & 445.4 & 358.2 & 352.5 \\
\hline 62.7 & 76.1 & 121.3 & 379.4 & 445.8 & 448.3 & 455.6 & 448.7 & 350.6 & 344.1 \\
\hline 64.9 & 74.8 & 126.5 & 297.3 & 351.6 & 388.7 & 372.1 & 349.2 & 262.3 & 267.1 \\
\hline 71.3 & 118.4 & 171.4 & 341.5 & 377.4 & 394.9 & 377.1 & 383.2 & 304.1 & 303.3 \\
\hline & & & & & & & & & \\
\hline & & & & & & & & & \\
\hline & & & & & & & & & \\
\hline & & & & & & & & & \\
\hline & & & & & & & & & \\
\hline & & & & & & & & & \\
\hline & & & & & & & & & \\
\hline & & & & & & & & & \\
\hline & & & & & & & & & \\
\hline & & & & & & & & & \\
\hline & & & & & & & & & \\
\hline & & & & & & & & & \\
\hline & & & & & & & & & \\
\hline & & & & & & & & & \\
\hline & & & & & & & & & \\
\hline & & & & & & & & & \\
\hline & & & & & & & & & \\
\hline & & & & & & & & & \\
\hline & & & & & & & & & \\
\hline & & & & & & & & & \\
\hline & & & & & & & & & \\
\hline & & & & & & & & & \\
\hline & & & & & & & & & \\
\hline & & & & & & & & & \\
\hline & & & & & & & & & \\
\hline & & & & & & & & & \\
\hline & & & & & & & & & \\
\hline & & & & & & & & & \\
\hline & & & & & & 652.8 & 657.9 & 591.3 & 635.3 \\
\hline 76.1 & 291.2 & 488.6 & 508.3 & 659.3 & 732.5 & 777.7 & 751.2 & 745.2 & 877.6 \\
\hline 86 & 107.3 & 325.1 & 683.9 & 821.7 & 850.4 & 868.9 & 789.8 & 732.7 & 832.6 \\
\hline 86.9 & 107.3 & 362 & 769.2 & 947.4 & 949.9 & 959.4 & 951.6 & 957.1 & 984.8 \\
\hline 77.4 & 97.8 & 357.9 & 753.1 & 942.4 & 972.8 & 991.4 & 956.8 & 951.5 & 972.7 \\
\hline 73 & 93.4 & 355.3 & 741.3 & 919.2 & 954.9 & 953.6 & 938.4 & 944.4 & 972.1 \\
\hline 86.5 & 331.9 & 604.9 & 760.2 & 953.5 & 1012.1 & 1031.1 & 1055.3 & 1020.6 & 1032.3 \\
\hline 86.5 & 108.1 & 369.4 & 776.3 & 977.3 & 981.8 & 1011.1 & 1005.9 & 976.4 & 1008 \\
\hline 80.8 & 102.9 & 372.7 & 726 & 960.4 & 987 & 1016.2 & 1007.6 & 975.9 & 1012.2 \\
\hline 79.6 & 100.4 & 374.9 & 763.1 & 926.6 & 944.4 & 982.9 & 987.6 & 929.5 & 936 \\
\hline
\end{tabular}


other

\begin{tabular}{|c|c|c|c|c|c|c|c|c|c|}
\hline 79.6 & 99.9 & 375.7 & 732 & 810.3 & 938.4 & 932.3 & 946.2 & 903.7 & 893 \\
\hline 85.1 & 326.7 & 524.7 & 668.4 & 896.7 & 912.1 & 958.2 & 953.5 & 939.7 & 957 \\
\hline 75.2 & 95.6 & 366.6 & 722.4 & 912.4 & 933.3 & 952.1 & 959.5 & 940.4 & 966 \\
\hline 84.7 & 104.7 & 371.7 & 725.4 & 904 & 935.8 & 965.1 & 940.9 & 944.6 & 960.2 \\
\hline 76.1 & 97.8 & 369.2 & 754 & 908.8 & 941.5 & 978.5 & 956.5 & 940.4 & 971.3 \\
\hline 73 & 95.2 & 333.3 & 740.6 & 945.6 & 965.7 & 1002.8 & 974.3 & 976.4 & 985.8 \\
\hline 77.8 & 157.7 & 180.6 & 180.6 & 193.1 & 207.4 & 210 & 211.7 & 219.9 & 204 \\
\hline 73 & 90.8 & 151.3 & 282.1 & 317.2 & 309.4 & 334.8 & 315.4 & 285.6 & 318 \\
\hline 73.5 & 94.6 & 155 & 316.9 & 352.6 & 357.7 & 359 & 353.5 & 325.4 & 375.1 \\
\hline 59.7 & 61 & 78.7 & 83.5 & 92.5 & 115.4 & 126.2 & 128.8 & 126.7 & 127.1 \\
\hline 66.6 & 68.8 & 159 & 287.8 & 428.2 & 467.3 & 483.6 & 485.7 & 418.5 & 264.9 \\
\hline 66.2 & 64.9 & 73.1 & 88.7 & 90.8 & 93 & 94.7 & 89.9 & 77.4 & 77.8 \\
\hline 63.1 & 62.3 & 80 & 130.5 & 135.7 & 159.9 & 115.4 & 125.3 & 121 & 112.4 \\
\hline 82.1 & 84.3 & 112.4 & 220.4 & 383.6 & 432.4 & 417.3 & 402.9 & 352.8 & 222.8 \\
\hline 111.5 & 125.3 & 127.1 & 170.3 & 202.2 & 215.2 & 229.9 & 207 & 134 & 163.8 \\
\hline 70 & 70.5 & 120.2 & 111.5 & 137.4 & 140.9 & 165.6 & 153.9 & 91.6 & 154.7 \\
\hline 71.8 & 68.8 & 138.3 & 100.8 & 131.4 & 131.9 & 131.9 & 127.5 & 75.2 & 119.8 \\
\hline 70 & 68.3 & 139.2 & 77.8 & 98.6 & 105.1 & 104.1 & 67.1 & 58 & 70.9 \\
\hline 67.1 & 66.6 & 136.6 & 67.1 & 59.7 & 59.7 & 61 & 58.8 & 58 & 67.1 \\
\hline 68.8 & 68.3 & 135.7 & 64 & 58.8 & 70.9 & 99.4 & 102.9 & 95.6 & 96 \\
\hline 65.7 & 63.1 & 126.7 & 115 & 140.9 & 139.2 & 150.4 & 133.6 & 99.4 & 147.8 \\
\hline 69.2 & 65.3 & 135.3 & 86.5 & 94.7 & 114.6 & 120.2 & 116.3 & 99.9 & 113.2 \\
\hline 83 & 80.9 & 149.5 & 126.2 & 141.4 & 142.6 & 134 & 115.8 & 96.4 & 137.4 \\
\hline 69.2 & 69.2 & 133.1 & 70.9 & 63.1 & 63.1 & 69.2 & 79.2 & 91.6 & 107.6 \\
\hline 63.6 & 115 & 135.7 & 183.7 & 342.2 & 398.4 & 403.6 & 364.3 & 270.5 & 337.9 \\
\hline 81.7 & 94.2 & 115 & 254.9 & 254.5 & 251.5 & 236.4 & 221.2 & 149.5 & 200.1 \\
\hline 85.2 & 93.4 & 118.4 & 264.9 & 282.6 & 268.8 & 254.9 & 237.3 & 191 & 248.9 \\
\hline 69.6 & 80.9 & 173.3 & 256.6 & 338.6 & 346 & 362.4 & 371.8 & 334.7 & 393.4 \\
\hline 87.8 & 95.2 & 122.4 & 220 & 288.2 & 296.9 & 243.3 & 238.6 & 186.3 & 203.6 \\
\hline 118.5 & 118.5 & 123.2 & 127.1 & 178.1 & 207 & 225.6 & 221.3 & 169.9 & 198 \\
\hline 124.5 & 121.1 & 118.5 & 152.2 & 185.4 & 196.3 & 174.7 & 161.7 & 144.8 & 173.8 \\
\hline 111.6 & 88.3 & 95.2 & 144.4 & 201.9 & 185.9 & 204.9 & 207.5 & 160.8 & 196.3 \\
\hline 100.4 & 90.8 & 120.2 & 128.9 & 151.3 & 168.6 & 182.4 & 160.8 & 118.5 & 134.5 \\
\hline 89.1 & 88.7 & 117.6 & 128 & 153.1 & 160 & 178.1 & 169.5 & 131.1 & 165.2 \\
\hline 68.4 & 73.6 & 82.2 & 89.1 & 97.2 & 68.8 & 69.2 & 71 & 70.1 & 71 \\
\hline 68.8 & 84.8 & 108.1 & 270.6 & 502.7 & 561.6 & 569.7 & 596.5 & 602.4 & 580.8 \\
\hline 83 & 204.4 & 300.3 & 318 & 355.2 & 412.6 & 427.4 & 444.6 & 390.6 & 384.4 \\
\hline 79.6 & 140.4 & 191.9 & 422 & 486.7 & 498.3 & 506 & 496 & 437.5 & 468.6 \\
\hline 65.3 & 103.7 & 157.7 & 367.6 & 419.2 & 455.5 & 478.9 & 453.3 & 407.9 & 418.7 \\
\hline 83.9 & 151.7 & 213.5 & 412.8 & 452.4 & 489.2 & 501.8 & 485.2 & 433.8 & 461 \\
\hline 71.3 & 136.6 & 200.9 & 331.6 & 376.3 & 359 & 350 & 366.8 & 345.2 & 331.4 \\
\hline 159 & 163.3 & 155.5 & 152.1 & 154.7 & 155.5 & 153.8 & 159 & 158.1 & 175.4 \\
\hline 147.7 & 146.9 & 145.2 & 139.1 & 135.6 & 139.1 & 139.1 & 140.8 & 139.1 & 146.9 \\
\hline 151.2 & 149.5 & 143.4 & 136.5 & 132.2 & 133.1 & 134.8 & 134.8 & 140.8 & 142.6 \\
\hline 142.6 & 143.4 & 136.5 & 134.8 & 143.4 & 140.8 & 144.3 & 146 & 150.3 & 151.2 \\
\hline 145.2 & 143.4 & 140 & 142.6 & 160.7 & 165 & 170.2 & 172.8 & 161.6 & 164.2 \\
\hline 146.9 & 142.6 & 139.1 & 135.6 & 138.2 & 144.3 & 148.6 & 150.3 & 156.4 & 157.2 \\
\hline 157.2 & 155.5 & 153.8 & 151.2 & 156.4 & 152.9 & 147.7 & 146.9 & 145.2 & 149.5 \\
\hline 150.3 & 152.1 & 142.6 & 146 & 146 & 150.3 & 158.1 & 155.5 & 159 & 154.7 \\
\hline
\end{tabular}


other

\begin{tabular}{|c|c|c|c|c|c|c|c|c|c|}
\hline 129.6 & 127.9 & 124.4 & 121.8 & 127.9 & 128.7 & 132.2 & 131.3 & 135.6 & 133.1 \\
\hline 121.8 & 121.8 & 118.4 & 116.6 & 118.4 & 122.7 & 124.4 & 118.4 & 117.5 & 120.1 \\
\hline 112.3 & 112.3 & 108 & 109.7 & 113.2 & 115.8 & 125.3 & 132.2 & 132.2 & 135.6 \\
\hline 120.1 & 119.2 & 115.8 & 113.2 & 116.6 & 122.7 & 123.6 & 126.1 & 130.5 & 133.9 \\
\hline 122.7 & 121 & 114.9 & 114.9 & 115.8 & 118.4 & 123.6 & 126.1 & 129.6 & 131.3 \\
\hline 120.1 & 120.1 & 113.2 & 113.2 & 118.4 & 121.8 & 128.7 & 122.7 & 123.6 & 127 \\
\hline 147.7 & 146.9 & 145.2 & 137.4 & 141.7 & 143.4 & 145.2 & 152.1 & 152.9 & 158.1 \\
\hline 137.4 & 136.5 & 133.1 & 130.5 & 133.1 & 136.5 & 141.7 & 147.7 & 150.3 & 152.1 \\
\hline 159 & 159 & 156.4 & 163.3 & 172.8 & 180.6 & 197 & 189.2 & 181.4 & 183.2 \\
\hline 152.9 & 149.5 & 149.5 & 149.5 & 159 & 162.4 & 168.5 & 172.8 & 178 & 168.5 \\
\hline 168.5 & 162.4 & 177.1 & 178 & 186.6 & 192.7 & 196.1 & 197 & 196.1 & 205.6 \\
\hline 157.2 & 154.7 & 149.5 & 148.6 & 149.5 & 156.4 & 154.7 & 165 & 170.2 & 171.1 \\
\hline 199.6 & 201.3 & 209.1 & 215.1 & 227.2 & 245.4 & 244.5 & 245.4 & 244.5 & 253.2 \\
\hline 202.2 & 203.9 & 193.5 & 194.4 & 201.3 & 206.5 & 215.1 & 217.7 & 224.6 & 231.6 \\
\hline 349.1 & 343.9 & 335.2 & 335.2 & 342.1 & 357.7 & 371.5 & 384.5 & 412.1 & 423.4 \\
\hline 347.3 & 343 & 335.2 & 330 & 334.4 & 349.1 & 367.2 & 374.1 & 384.5 & 390.5 \\
\hline 386.2 & 376.7 & 374.1 & 368.9 & 371.5 & 389.7 & 394.8 & 391.4 & 400 & 399.2 \\
\hline 362 & 356.8 & 350.8 & 282.5 & 256.6 & 264.4 & 274.8 & 279.1 & 278.2 & 286.8 \\
\hline 106.3 & 105.9 & 104.1 & 107.6 & 101.6 & 125.7 & 130.9 & 123.2 & 100.7 & 101.6 \\
\hline 110.2 & 112.8 & 103.3 & 98.1 & 102.4 & 104.1 & 106.7 & 111.1 & 109.3 & 109.3 \\
\hline 133.5 & 125.7 & 118.8 & 121.9 & 98.1 & 86.9 & 119.7 & 100.7 & 110.2 & 119.7 \\
\hline 86.9 & 124 & 124.5 & 89.5 & 81.7 & 101.6 & 111.9 & 73.9 & 66.6 & 70.4 \\
\hline 103.3 & 82.5 & 82.5 & 127.1 & 122.3 & 111.1 & 120.6 & 116.2 & 115.4 & 137.8 \\
\hline 94.6 & 124 & 97.7 & 72.2 & 85.1 & 102.4 & 105 & 109.3 & 67.9 & 75.2 \\
\hline 90.3 & 98.6 & 101.6 & 408.7 & 566.5 & 586.3 & 585.9 & 598.3 & 531.7 & 505.2 \\
\hline 84.3 & 85.6 & 85.1 & 168.8 & 431.5 & 460.4 & 441.7 & 416.2 & 271.8 & 130.9 \\
\hline 99.8 & 100.7 & 94.6 & 188.8 & 183.2 & 180.2 & 178.4 & 171.5 & 123.2 & 145.6 \\
\hline 82.1 & 86 & 77.4 & 73 & 81.7 & 85.1 & 86 & 89.5 & 87.3 & 89.5 \\
\hline 76.1 & 78.2 & 73 & 105.9 & 106.7 & 110.7 & 121.4 & 126.6 & 112.8 & 121.4 \\
\hline 77 & 77.4 & 74.8 & 72.2 & 68.7 & 73 & 79.1 & 80.8 & 80.8 & 80.8 \\
\hline 99.8 & 116.7 & 117.1 & 250.2 & 232 & 229.4 & 256.2 & 199.2 & 174.1 & 167.2 \\
\hline 91.2 & 86.9 & 79.1 & 73.9 & 89.5 & 90.3 & 104.1 & 114.5 & 116.2 & 115.4 \\
\hline 110.7 & 112.4 & 120.6 & 299.4 & 206.6 & 200 & 213.5 & 211.3 & 209.2 & 178.9 \\
\hline 114.5 & 117.6 & 123.2 & 127.9 & 131.8 & 139.2 & 144.8 & 150.4 & 130.9 & 127.9 \\
\hline 117.6 & 113.2 & 124 & 236.8 & 221.6 & 211.7 & 237.2 & 254.9 & 231.2 & 231.6 \\
\hline 119.3 & 123.2 & 124.5 & 130.1 & 159.9 & 168.1 & 165.1 & 172.9 & 187.1 & 193.6 \\
\hline 118.4 & 115.4 & 126.2 & 212.6 & 242 & 249.3 & 260.1 & 268.3 & 234.2 & 241.1 \\
\hline 116.7 & 108.9 & 120.6 & 124.5 & 127.9 & 140.4 & 153 & 161.2 & 162.5 & 177.2 \\
\hline 100.3 & 91.6 & 109.3 & 122.8 & 123.6 & 135.7 & 164.6 & 195.3 & 187.6 & 209.2 \\
\hline 104.6 & 101.6 & 108.1 & 108.9 & 108.9 & 109.3 & 127.9 & 135.7 & 159.9 & 163.8 \\
\hline 198.8 & 204.8 & 211.7 & 225.6 & 223.8 & 239.4 & 269.2 & 224.7 & 205.7 & 229 \\
\hline 73.5 & 74.8 & 80.4 & 85.1 & 87.3 & 100.7 & 97.7 & 109.8 & 125.7 & 121 \\
\hline 94.2 & 88.2 & 111.1 & 161.6 & 178.9 & 185 & 188.4 & 202.6 & 196.2 & 197.1 \\
\hline 76.1 & 100.7 & 104.6 & 127.9 & 149.1 & 161.6 & 181.5 & 196.6 & 191 & 196.2 \\
\hline 99.4 & 98.6 & 119.7 & 130.5 & 159.9 & 166 & 207.8 & 223 & 214.3 & 223.8 \\
\hline 91.6 & 90.8 & 118.8 & 136.6 & 161.6 & 161.2 & 182.4 & 176.3 & 191 & 204 \\
\hline 172.9 & 168.5 & 185 & 186.7 & 213.5 & 228.6 & 267 & 261.9 & 278.3 & 267 \\
\hline 160.8 & 157.3 & 185.8 & 191.9 & 214.7 & 214.3 & 235.9 & 235.9 & 245.4 & 267 \\
\hline 188.4 & 183.2 & 198.8 & 222.1 & 157.7 & 184.1 & 221.2 & 217.8 & 200 & 196.2 \\
\hline
\end{tabular}


other

\begin{tabular}{|c|c|c|c|c|c|c|c|c|c|}
\hline 74.8 & 75.2 & 95.1 & 108.5 & 121 & 135.7 & 155.1 & 154.7 & 153 & 163.8 \\
\hline 96 & 100.7 & 117.6 & 409.9 & 539.4 & 551.5 & 560.5 & 566 & 526.2 & 557.7 \\
\hline 108.1 & 102 & 108.5 & 116.7 & 139.2 & 134.4 & 153 & 154.7 & 163.4 & 174.1 \\
\hline 103.7 & 111.1 & 124.5 & 364.4 & 479 & 517.6 & 551.3 & 553.2 & 498.7 & 544.4 \\
\hline 116.7 & 117.6 & 113.6 & 113.2 & 108.9 & 113.6 & 121 & 133.1 & 134.4 & 133.1 \\
\hline 102.9 & 108.5 & 235.5 & 588.3 & 770.2 & 787.6 & 821.3 & 838.9 & 830.6 & 842.6 \\
\hline 75.2 & 78.2 & 85.6 & 102.4 & 125.3 & 127.5 & 146.1 & 146.9 & 143.9 & 146.1 \\
\hline 113.6 & 117.6 & 286.9 & 591.2 & 767.8 & 808.1 & 858.6 & 878.9 & 871 & 843.8 \\
\hline 96 & 97.2 & 117.6 & 105.5 & 120.6 & 135.7 & 146.5 & 149.5 & 147.8 & 154.3 \\
\hline 140 & 137.4 & 327.7 & 643.6 & 883.5 & 923.4 & 938 & 954.3 & 948.7 & 956.4 \\
\hline 112.4 & 110.7 & 149.1 & 158.2 & 164.2 & 168.9 & 175.5 & 185.8 & 196.6 & 192.7 \\
\hline 83 & 123.2 & 149.9 & 275.2 & 385.8 & 410.5 & 380.6 & 373.7 & 324.5 & 228.6 \\
\hline 80 & 80.8 & 101.6 & 96.4 & 106.7 & 107.2 & 105 & 81.7 & 94.6 & 101.6 \\
\hline 113.6 & 127.1 & 137.8 & 105.9 & 100.7 & 108.9 & 102.4 & 99 & 97.2 & 102.4 \\
\hline 81.7 & 82.5 & 82.5 & 76.1 & 73 & 72.2 & 72.6 & 71.3 & 71.3 & 73 \\
\hline 76.5 & 75.6 & 72.6 & 67 & 80 & 88.6 & 89.5 & 92.5 & 99 & 99.8 \\
\hline 61.8 & 63.1 & 60.1 & 54 & 51.4 & 50.6 & 49.7 & 53.6 & 54.9 & 54.9 \\
\hline 108.1 & 109.3 & 105 & 99.8 & 121 & 112.8 & 97.2 & 92 & 85.1 & 85.6 \\
\hline 96.4 & 97.2 & 93.8 & 85.6 & 83.4 & 85.1 & 80 & 78.2 & 78.2 & 78.7 \\
\hline 71.3 & 69.2 & 67.9 & 60.9 & 60.1 & 60.9 & 61.4 & 69.6 & 63.5 & 60.9 \\
\hline 68.7 & 69.6 & 64.4 & 60.9 & 60.5 & 61.8 & 59.2 & 59.2 & 60.1 & 64.4 \\
\hline 71.8 & 73 & 70.4 & 64.4 & 65.3 & 65.3 & 71.3 & 75.2 & 69.6 & 68.7 \\
\hline 69.6 & 69.2 & 68.7 & 61.8 & 62.7 & 57.5 & 56.6 & 60.1 & 64.4 & 60.1 \\
\hline 76.5 & 73.9 & 84.3 & 73 & 74.8 & 80 & 83.4 & 78.2 & 73.5 & 77.8 \\
\hline 71.3 & 69.6 & 84.3 & 75.6 & 71.3 & 76.5 & 76.5 & 80.8 & 85.1 & 98.1 \\
\hline 91.2 & 103.7 & 116.2 & 112.4 & 113.6 & 131.4 & 130.1 & 146.5 & 146.9 & 153 \\
\hline 92.9 & 89.1 & 105 & 104.6 & 110.7 & 116.2 & 128.8 & 133.5 & 132.3 & 133.5 \\
\hline 79.1 & 77 & 78.6 & 85.6 & 90.3 & 83.4 & 104.1 & 121.4 & 108.5 & 98.1 \\
\hline 79.1 & 78.2 & 77.4 & 88.6 & 93.8 & 91.2 & 92.9 & 92 & 78.2 & 76.1 \\
\hline 75.2 & 75.6 & 98.6 & 90.3 & 92.9 & 86.9 & 92 & 85.6 & 84.3 & 86.9 \\
\hline 79.1 & 72.2 & 92.9 & 85.1 & 83.4 & 80.8 & 74.8 & 76.1 & 80.8 & 92.9 \\
\hline 73.5 & 73 & 93.4 & 111.1 & 106.3 & 105 & 110.7 & 132.7 & 135.2 & 120.2 \\
\hline 69.2 & 71.3 & 89.9 & 90.3 & 82.5 & 76.5 & 90.3 & 92 & 94.2 & 95.5 \\
\hline 70 & 64 & 82.5 & 82.1 & 92.9 & 96.8 & 104.6 & 113.6 & 115 & 117.6 \\
\hline 70 & 68.7 & 93.4 & 89.1 & 94.6 & 105.5 & 113.6 & 119.3 & 115.8 & 128.8 \\
\hline & & & & & & & & & \\
\hline & & & & & & & & & \\
\hline & & & & & & & & & \\
\hline & & & & & & & & & \\
\hline & & & & & & & & & \\
\hline & & & & & & & & & \\
\hline & & & & & & & & & \\
\hline & & & & & & & & & \\
\hline & & & & & & & & & \\
\hline & & & & & & & & & \\
\hline 68.3 & 75.2 & 103.7 & 102 & 114.5 & 124.5 & 130.5 & 132.3 & 136.1 & 140 \\
\hline 85.6 & 92.9 & 108.1 & 104.6 & 106.3 & 111.9 & 115 & 121.9 & 125.3 & 144.8 \\
\hline 77 & 85.6 & 107.6 & 108.1 & 113.2 & 113.6 & 127.1 & 131.4 & 130.5 & 130.1 \\
\hline 83.9 & 91.6 & 103.7 & 105.9 & 96.8 & 103.7 & 108.5 & 108.9 & 120.2 & 127.1 \\
\hline
\end{tabular}


other

\begin{tabular}{|c|c|c|c|c|c|c|c|c|c|}
\hline 81.3 & 81.7 & 94.2 & 115 & 111.1 & 99.4 & 94.2 & 102.4 & 115.4 & 112.4 \\
\hline 75.2 & 76.5 & 88.2 & 108.1 & 108.5 & 92.5 & 95.5 & 88.2 & 98.1 & 97.7 \\
\hline 72.2 & 74.4 & 96 & 118.4 & 120.6 & 109.8 & 115.8 & 112.8 & 108.1 & 102.9 \\
\hline 70.4 & 72.6 & 85.6 & 101.6 & 101.2 & 88.6 & 96.8 & 96.4 & 98.6 & 99.4 \\
\hline 72.6 & 71.8 & 69.6 & 65.7 & 64.9 & 75.2 & 92 & 86.5 & 82.5 & 78.7 \\
\hline 70 & 68.3 & 66.6 & 61.8 & 61.4 & 54.9 & 55.4 & 54.5 & 54.9 & 58 \\
\hline 66.2 & 65.7 & 65.3 & 69.6 & 81.3 & 86.1 & 101.6 & 98.6 & 80 & 79.6 \\
\hline 67.9 & 64.9 & 63.6 & 60.5 & 56.2 & 53.2 & 55.4 & 53.2 & 55.4 & 58.8 \\
\hline 67.5 & 67.5 & 64 & 58 & 56.2 & 54.5 & 51 & 52.8 & 50.2 & 49.3 \\
\hline 68.3 & 68.3 & 67.1 & 54.5 & 51 & 55.4 & 51.9 & 51 & 49.8 & 50.2 \\
\hline 65.7 & 64 & 63.1 & 52.8 & 51 & 51 & 53.6 & 53.6 & 53.6 & 54.5 \\
\hline 63.1 & 63.1 & 61.9 & 51 & 51.9 & 51.9 & 53.6 & 51 & 52.4 & 54.5 \\
\hline 83.9 & 83.5 & 88.7 & 82.2 & 80 & 85.2 & 85.2 & 81.3 & 82.6 & 80.9 \\
\hline 85.7 & 84.3 & 84.8 & 76.6 & 77.9 & 79.2 & 77.9 & 79.2 & 78.8 & 76.2 \\
\hline 66.7 & 65.8 & 63.2 & 55.4 & 59.7 & 56.3 & 58 & 60.6 & 62.3 & 61.5 \\
\hline 66.7 & 66.7 & 62.7 & 55.4 & 56.3 & 56.3 & 59.3 & 60.6 & 60.6 & 62.3 \\
\hline 66.3 & 65.8 & 59.7 & 53.7 & 70.1 & 58 & 57.2 & 58 & 56.3 & 56.3 \\
\hline 61.9 & 63.2 & 63.2 & 55.4 & 50.7 & 52.8 & 54.6 & 52.8 & 53.3 & 52.8 \\
\hline 94.6 & 94.2 & 92 & 461.1 & 640.6 & 692.6 & 696.8 & 666.1 & 193.4 & 115.8 \\
\hline 82.1 & 80.8 & 76.1 & 71.8 & 87.7 & 94.2 & 96.4 & 99.4 & 103.3 & 89.9 \\
\hline 71.8 & 59.7 & 56.6 & 293.4 & 309.8 & 286.4 & 306.8 & 324.5 & 98.6 & 67.5 \\
\hline & & & & & & & & & \\
\hline & & & & & & & & & \\
\hline 144.9667 & 148.6356 & 177.9144 & 385.7067 & 550.3722 & 585.8678 & 593.8933 & 597.5478 & 602.9189 & 598.7422 \\
\hline 85.89013 & 112.8985 & 181.1843 & 365.0458 & 531.4911 & 566.4124 & 580.8182 & 594.3167 & 580.8433 & 583.0823 \\
\hline 138.3639 & 137.2004 & 136.4774 & 137.9741 & 142.5008 & 146.4912 & 150.8502 & 154.5609 & 157.1383 & 159.6657 \\
\hline 109.5664 & 111.0122 & 127.6799 & 199.8214 & 247.9293 & 259.5539 & 265.186 & 267.7734 & 258.7105 & 261.2576 \\
\hline 163.7266 & 165.6051 & 183.2089 & 300.4506 & 350.5038 & 365.7974 & 372.1628 & 370.3848 & 354.2342 & 363.2329 \\
\hline 89.50161 & 106.9328 & 153.1672 & 289.3108 & 363.5968 & 379.4683 & 390.7246 & 392.4877 & 364.0722 & 376.6465 \\
\hline 161.9176 & 162.1382 & 158.15 & 166.3118 & 180.6618 & 187.4824 & 192.5853 & 191.9941 & 186.0559 & 185.1794 \\
\hline 89.97229 & 91.0494 & 104.5373 & 140.1843 & 156.4614 & 161.0373 & 169.7205 & 171.7169 & 159.8964 & 160.3843 \\
\hline & & & & & & & & & \\
\hline & & & & & & & & & \\
\hline & & & & & & & & & \\
\hline 63.02108 & 76.62429 & 84.86851 & 143.0978 & 160.0605 & 164.0983 & 167.028 & 176.4669 & 178.2542 & 185.3368 \\
\hline 18.254 & 42.1464 & 89.97228 & 146.982 & 182.8566 & 194.2151 & 202.4603 & 205.4641 & 204.4085 & 212.6136 \\
\hline 40.36296 & 39.83378 & 39.62453 & 42.30218 & 52.91676 & 60.52508 & 66.51323 & 70.80132 & 73.74116 & 76.00214 \\
\hline 61.8243 & 63.872 & 80.70315 & 159.2085 & 210.6429 & 225.3422 & 230.1577 & 239.1506 & 235.443 & 236.0238 \\
\hline 78.39114 & 82.24223 & 98.17465 & 159.6581 & 197.3159 & 210.9832 & 215.4262 & 225.4947 & 217.6331 & 219.4239 \\
\hline 20.59879 & 43.2421 & 92.30675 & 192.8601 & 252.2756 & 261.3779 & 268.571 & 270.0534 & 268.785 & 270.7709 \\
\hline 79.11965 & 76.34657 & 76.04806 & 82.00638 & 108.2841 & 113.6487 & 112.1341 & 113.8832 & 104.7284 & 101.612 \\
\hline 26.93776 & 27.43542 & 47.32769 & 121.31 & 163.0854 & 171.2064 & 176.9169 & 178.1092 & 163.9776 & 165.1637 \\
\hline & & & & & & & & & \\
\hline & & & & & & & & & \\
\hline & & & & & & & & & \\
\hline & & & & & & & & & \\
\hline & & & & & & & & & \\
\hline
\end{tabular}


other

\begin{tabular}{|r|r|r|r|r|r|r|r|r|r|r|}
\hline 11098.4 & 11293.9 & 13126.5 & 29742.9 & 43975.3 & 47127.8 & 47836.6 & 47834.5 & 48376.9 & 47718.7 \\
\hline 34825.5 & 45459.6 & 72336.9 & 143208.9 & 207850.8 & 221466.1 & 227176.5 & 232480.2 & 227102.2 & 228289 \\
\hline 3756.1 & 3752.1 & 3811.1 & 5950.5 & 8283.2 & 8687.9 & 9130.5 & 9230.9 & 8868.5 & 8739.6 \\
\hline 25231.1 & 25613.7 & 29378.5 & 46418.5 & 57026.1 & 59225.1 & 60456.8 & 61437.2 & 59384.6 & 60251.7 \\
\hline 12274.3 & 12505.4 & 13863.6 & 24360.4 & 28737.5 & 29585.9 & 30055 & 30441.8 & 29497.8 & 30077.5 \\
\hline 17202 & 20380.6 & 28902.1 & 52059.3 & 64782.5 & 67573 & 70037.3 & 70178.9 & 64983.5 & 67242.5 \\
\hline 5022.1 & 5042 & 5003.9 & 5958.6 & 6358.6 & 6598.2 & 6885.7 & 6884 & 6507.1 & 6479.8 \\
\hline 7128.105 & 7264.372 & 8534.52 & 11602.01 & 13534.62 & 14005.11 & 14639.24 & 14796.88 & 13898.17 & 13920.69 \\
\hline & & & & & & & & & \\
\hline & & & & & & & & & \\
\hline & & & & & & & & & \\
\hline & & & & & & & & & \\
\hline & & & & & & & & & \\
\hline 90 & 90 & 90 & 90 & 90 & 90 & 90 & 90 & 90 & 90 \\
\hline 395 & 395 & 395 & 395 & 395 & 395 & 395 & 395 & 395 & 395 \\
\hline 216 & 252 & 288 & 324 & 360 & 396 & 432 & 468 & 504 & 540 \\
\hline 229 & 229 & 229 & 229 & 229 & 228 & 228 & 229 & 229 & 229 \\
\hline 79 & 79 & 79 & 79 & 79 & 78 & 78 & 79 & 79 & 79 \\
\hline 186 & 186 & 186 & 186 & 186 & 186 & 187 & 187 & 187 & 187 \\
\hline 34 & 34 & 34 & 34 & 34 & 34 & 34 & 34 & 34 & 34 \\
\hline 83 & 83 & 83 & 83 & 83 & 83 & 83 & 83 & 83 & 83 \\
\hline & & & & & & & & & & \\
\hline & & & & & & & & & \\
\hline
\end{tabular}




\begin{tabular}{|c|c|c|c|c|c|c|c|c|c|}
\hline 15 & 16 & 17 & 18 & 19 & 20 & 21 & 22 & 23 & $\begin{array}{l}\text { DAILY } \\
\text { TOTAL }\end{array}$ \\
\hline 769 & 730.1 & 549.5 & 470.9 & 333.5 & 287.7 & 244.5 & 223.8 & 201.3 & 12268.80 \\
\hline 807 & 756 & & & 32 & 319.7 & 252.3 & 221.2 & 215.1 & 9516.30 \\
\hline 827.7 & 717.1 & 431.1 & 499.4 & 371.5 & 294.6 & 265.2 & 264.4 & 232.4 & 11021.00 \\
\hline 839.8 & 767.2 & 388.8 & 325.7 & 291.2 & 273 & 241.9 & 229 & 216 & 10852.60 \\
\hline 867.5 & 811.3 & 460.5 & 387.9 & 355.1 & 346.5 & 311 & 286 & 262.7 & 5333.40 \\
\hline 892.5 & 821.7 & 457.9 & 385.3 & 357.7 & 339.6 & 292 & 269.6 & 245.4 & 6423.10 \\
\hline 851 & 772.4 & 459.6 & 370.7 & 333.5 & 287.7 & 257.5 & 253.2 & 235 & 12764.70 \\
\hline 861.4 & 778.5 & 458.8 & 382.8 & 353.4 & 311.9 & 273 & 260.1 & 245.4 & 11985.30 \\
\hline 858 & 812.2 & 589.2 & 510.6 & 479.5 & 448.4 & 415.6 & 265.2 & 241.9 & 11483.40 \\
\hline 861.4 & 790.6 & 450.1 & 365.5 & 339.6 & 326.6 & 274.8 & 266.1 & 237.6 & 11522.30 \\
\hline 860.5 & 820.8 & 451.9 & 380.2 & 334.4 & 306.7 & 273.9 & 260.9 & 247.1 & 12388.80 \\
\hline 838.1 & 807 & 460.5 & 373.2 & 330 & 285.1 & 267 & 258.3 & 235 & 6062.00 \\
\hline 710.2 & 651.5 & 325.7 & 254.9 & 224.6 & 190.1 & 162.4 & 146 & 133.9 & 6401.70 \\
\hline 651.5 & 589.2 & 311 & 247.1 & 210.8 & 187.5 & 162.4 & 158.1 & 154.7 & 12590.30 \\
\hline 819.1 & 735.3 & 400.9 & 324 & 291.2 & 261.8 & 220.3 & 210.8 & 191.8 & 11606.90 \\
\hline 854.5 & 829.4 & 591.8 & 502.8 & 346.5 & 271.3 & 251.4 & 236.7 & 213.4 & 11455.60 \\
\hline 724.9 & 679.1 & 356.8 & 277.3 & 245.4 & 197 & 164.2 & 162.4 & 145.2 & 9480.60 \\
\hline 711.1 & 675.6 & 368.9 & 291.2 & 271.3 & 279.1 & 226.4 & 198.7 & 168.5 & 8258.20 \\
\hline 729.2 & 678.2 & 351.6 & 265.2 & 263.5 & 235 & 193.5 & 182.3 & 154.7 & 5708.20 \\
\hline 773.3 & 727.5 & 406.9 & 328.3 & 264.4 & 223.8 & 181.4 & 160.7 & 153.8 & 4494.30 \\
\hline & & & & & & & & & 11591.80 \\
\hline & & & 100.1 & 288.3 & 254.6 & 220 & 204.5 & 191.5 & 11229.40 \\
\hline 643.8 & 636.4 & 429.9 & 369.5 & 324.5 & 279.7 & 242.5 & 213.1 & 200.2 & 9132.40 \\
\hline 651.3 & 623.2 & 426.4 & 360 & 329.8 & 296.8 & 267.6 & 249.4 & 227 & 8964.10 \\
\hline 496.3 & 488.9 & 322.9 & 256.3 & 245.1 & 198.4 & 173.4 & 149.2 & 128.5 & 9100.40 \\
\hline 624.4 & 590 & 506.7 & 420.2 & 437.9 & 566.2 & 551.6 & 185.2 & 132.3 & 3973.40 \\
\hline 633.5 & 601.2 & 375.5 & 305.1 & 262.7 & 238.5 & 208.3 & 185 & 166.8 & 3951.20 \\
\hline 635.6 & 601.6 & 370.3 & 335.3 & 300.7 & 263.6 & 224.7 & 194.5 & 167.3 & 10632.20 \\
\hline 680.2 & 669.5 & 380.7 & 331 & 307.6 & 246.8 & 218.7 & 212.6 & 214.3 & 0.00 \\
\hline 736.5 & 663.7 & 402.7 & 338.8 & 293 & 269.6 & 228.6 & 207.4 & 199.6 & 1259.00 \\
\hline 695.1 & 651.6 & 387.6 & 318.9 & 288.6 & 257.5 & 222.1 & 194.5 & 185 & 8650.40 \\
\hline 685 & 635 & 395.8 & 314.6 & 254.1 & 220.4 & 192.7 & 182.8 & 168.9 & 9117.20 \\
\hline 703.2 & 645.4 & 380.7 & 286.9 & 243.7 & 265.3 & 197.1 & 190.1 & 176.3 & 4314.60 \\
\hline 710.5 & 669 & 414.4 & 391.1 & 380.6 & 373.3 & 358.5 & 344.2 & 274.4 & 3051.40 \\
\hline 678.7 & 631 & 366.4 & 303.3 & 250.6 & 235.9 & 200.5 & 175.9 & 161.6 & 6676.50 \\
\hline 719.8 & 695.3 & 396.2 & 308.5 & 283.5 & 268.8 & 223.4 & 211.7 & 196.2 & 8921.60 \\
\hline 790.3 & 730.4 & 430.8 & 339.6 & 308.9 & 290.4 & 255.8 & 243.7 & 228.2 & 8042.70 \\
\hline 776.9 & 732 & 420 & 334.4 & 305.9 & 283.5 & 246.3 & 248.9 & 248.4 & 8354.50 \\
\hline 783.1 & 764.2 & 685.2 & 442 & 378 & 344.8 & 327.5 & 316.3 & 272.2 & 8772.90 \\
\hline 814.5 & 752.3 & 440.3 & 364.7 & 281.3 & 246.8 & 215.2 & 191.9 & 175.9 & 4556.80 \\
\hline 561.8 & 522.4 & 305.1 & 225.6 & 208.7 & 156.8 & 126.2 & 118 & 107.6 & 4197.60 \\
\hline 475.9 & 427.5 & 275.2 & 211.3 & 178.9 & 137.8 & 105 & 106.3 & 98.1 & 9734.00 \\
\hline 384.9 & 373.3 & 275.2 & 222.5 & 213.9 & 194.5 & 156.8 & 142.2 & 137 & 9056.10 \\
\hline 478.3 & 449.7 & 354.3 & 315 & 261 & 229.4 & 205.2 & 198.8 & 188.8 & 8675.40 \\
\hline 444.6 & 410.9 & 318.9 & 268.3 & 250.2 & 224.7 & 186.2 & 163.4 & 161.2 & 8811.00 \\
\hline
\end{tabular}


other

\begin{tabular}{|c|c|c|c|c|c|c|c|c|c|}
\hline 421.2 & 388.9 & 312.4 & 243.2 & 220.8 & 179.8 & 132.7 & 128.3 & 127.5 & 9601.50 \\
\hline 594.4 & 529.9 & 308.5 & 259.7 & 216.5 & 181.9 & 140.9 & 137 & 126.6 & 5737.70 \\
\hline 455.8 & 409.6 & 321.9 & 276.9 & 237.7 & 213 & 180.2 & 166 & 166.4 & 3256.70 \\
\hline 454.8 & 427.9 & 313.7 & 265.7 & 244.1 & 217.4 & 182.8 & 164.2 & 146.5 & 8449.50 \\
\hline 543.9 & 492.9 & 290.8 & 199.6 & 177.6 & 160.3 & 130.5 & 103.3 & 106.7 & 9180.60 \\
\hline 551.2 & 516.1 & 296.8 & 209.6 & 188.4 & 152.5 & 111.1 & 96 & 89.5 & 10295.30 \\
\hline 550.6 & 530.4 & 303.3 & 218.2 & 203.5 & 194.5 & 157.2 & 125.3 & 105 & 10351.20 \\
\hline 619.6 & 566.2 & 288.6 & 235.5 & 217.3 & 176.7 & 151.7 & 168.1 & 157.7 & 5769.90 \\
\hline 556.9 & 527.8 & 299 & 225.1 & 199.2 & 158.6 & 114.5 & 110.2 & 110.2 & 5619.90 \\
\hline 658.4 & 617.6 & 362.1 & 249.3 & 216.5 & 168.5 & 126.6 & 110.2 & 105.9 & 11880.80 \\
\hline 527.7 & 488.2 & 297.3 & 215.6 & 179.3 & 133.1 & 105 & 100.7 & 100.7 & 9955.00 \\
\hline 502.5 & 467.7 & 286.9 & 213.5 & 183.6 & 167.2 & 135.2 & 137 & 115.8 & 7459.10 \\
\hline 419.5 & 392.7 & 294.7 & 227.7 & 213 & 188 & 162.9 & 156.8 & 143 & 6576.40 \\
\hline 397.9 & 376.8 & 292.5 & 245.8 & 208.7 & 166.8 & 140.4 & 138.7 & 132.3 & 6020.60 \\
\hline 437.8 & 404.1 & 290.4 & 233.7 & 221.2 & 175 & 144.8 & 143.9 & 144.8 & 2817.80 \\
\hline 452.3 & 413.5 & 318.4 & 273.5 & 244.6 & 212.1 & 176.7 & 176.7 & 178.4 & 2129.80 \\
\hline 404 & 378 & 299 & 226 & 200 & 176.7 & 145.2 & 141.3 & 139.6 & 6981.20 \\
\hline 400.5 & 376.3 & 289 & 223.4 & 210 & 173.7 & 144.4 & 137.8 & 122.3 & 7123.10 \\
\hline 405.7 & 385.8 & 289.5 & 232 & 213.9 & 190.5 & 149.9 & 135.2 & 136.1 & 6457.90 \\
\hline 552.4 & 481.5 & 308 & 251.9 & 231.6 & 206.1 & 173.3 & 167.2 & 165.5 & 7409.20 \\
\hline 714.2 & 633.2 & 395.3 & 298.5 & 268.3 & 256.2 & 235.1 & 209.6 & 180.2 & 6728.40 \\
\hline 549.9 & 482.2 & 320.1 & 245.4 & 242.4 & 230.3 & 183.6 & 149.1 & 134.4 & 4154.40 \\
\hline 604.4 & 551.3 & 347.4 & 283.9 & 231.2 & 196.6 & 149.9 & 149.1 & 147.3 & 3282.10 \\
\hline 673.5 & 628.8 & 392.3 & 303.7 & 275.2 & 230.8 & 190.9 & 188.4 & 176.7 & 6513.20 \\
\hline 584 & 549.7 & 338.8 & 277.8 & 270.9 & 249.3 & 200.9 & 181.5 & 165 & 6286.50 \\
\hline 406.5 & 386.7 & 299.4 & 250.2 & 238.1 & 191 & 142.2 & 143 & 134.8 & 6307.90 \\
\hline 410.5 & 372.8 & 310.6 & 245.8 & 233.3 & 228.6 & 189.7 & 175 & 158.2 & 6919.20 \\
\hline 453.8 & 423.4 & 305.9 & 249.3 & 226.8 & 175.5 & 146 & 120.2 & 129.2 & 7731.40 \\
\hline 529.8 & 464.3 & 289.9 & 211.3 & 201.8 & 151.7 & 107.6 & 108.5 & 118.4 & 3313.80 \\
\hline 427.3 & 374.6 & 269.2 & 231.2 & 200.9 & 170.7 & 115 & 111.9 & 106.7 & 2421.40 \\
\hline 423 & 392.7 & 289.9 & 248.4 & 243.2 & 219.5 & 189.2 & 178 & 163.8 & 6709.10 \\
\hline 429 & 384.1 & 287.3 & 225.1 & 200.9 & 165.5 & 130.9 & 113.2 & 115.8 & 7769.60 \\
\hline 410.9 & 385.8 & 298.1 & 246.7 & 226.8 & 201.8 & 136.1 & 132.7 & 131.4 & 6270.10 \\
\hline 136.1 & 121.4 & 114.5 & 102.4 & 106.3 & 110.2 & 109.3 & 106.7 & 106.7 & 6365.90 \\
\hline 391 & 371.6 & 291.6 & 241.5 & 199.2 & 178 & 163.8 & 124 & 107.6 & 5818.20 \\
\hline 376.3 & 351.2 & 279.1 & 226.8 & 210.4 & 199.2 & 186.2 & 153 & 130.1 & 2774.30 \\
\hline 400.5 & 378.9 & 279.1 & 219.9 & 177.6 & 143 & 116.7 & 121.4 & 122.3 & 1962.40 \\
\hline 93.8 & 92 & 92 & 92.9 & 100.7 & 101.2 & 100.7 & 99 & 99.8 & 5687.20 \\
\hline 442 & 399.6 & 303.7 & 239.8 & 188.8 & 166.4 & 134.4 & 111.9 & 99.8 & 6198.10 \\
\hline 422.1 & 374.6 & 302.9 & 210.4 & 188 & 176.3 & 141.3 & 122.3 & 109.3 & 2690.40 \\
\hline 441.7 & 407.7 & 302 & 223.4 & 173.3 & 126.6 & 93.8 & 92.9 & 92.9 & 2176.90 \\
\hline 393.6 & 366.4 & 295.1 & 207 & 179.3 & 154.3 & 125.7 & 100.7 & 95.1 & 6400.40 \\
\hline 75.2 & 80 & 67 & 71.3 & 69.6 & 72.2 & 79.1 & 75.6 & 71.3 & 6294.70 \\
\hline 405.4 & 388.2 & 276.1 & 192.3 & 150.8 & 120.6 & 103.3 & 97.7 & 91.2 & 6079.70 \\
\hline 432.5 & 391.5 & 294.2 & 218.2 & 197.5 & 188 & 147.3 & 137.8 & 125.3 & 6004.40 \\
\hline 419.5 & 393.2 & 292.5 & 223.4 & 187.1 & 141.3 & 110.2 & 106.7 & 92.9 & 6627.50 \\
\hline 420.2 & 388.6 & 276.1 & 206.1 & 177.6 & 163.8 & 112.4 & 98.1 & 91.2 & 3948.60 \\
\hline 77.8 & 104.1 & 80 & 77.4 & 86 & 90.3 & 85.1 & 82.5 & 82.5 & 2979.90 \\
\hline 418.2 & 370.3 & 285.6 & 218.2 & 183.6 & 140.4 & 97.2 & 92 & 93.4 & 8811.50 \\
\hline
\end{tabular}


other

\begin{tabular}{|c|c|c|c|c|c|c|c|c|c|}
\hline 443.3 & 398.1 & 286 & 204.4 & 181 & 170.7 & 127.9 & 98.1 & 92 & 7323.50 \\
\hline 386.7 & 359.9 & 287.3 & 199.2 & 202.2 & 182.8 & 140.4 & 105.9 & 106.3 & 7656.90 \\
\hline 376.8 & 354.7 & 271.8 & 213.9 & 185.4 & 145.2 & 104.1 & 99.8 & 95.1 & 8519.90 \\
\hline 92 & 96.4 & 95.1 & 79.1 & 86 & 94.6 & 103.3 & 96.4 & 95.5 & 8009.40 \\
\hline 406.5 & 369.4 & 280.4 & 210.4 & 184.5 & 163.4 & 112.8 & 104.1 & 98.1 & 3529.40 \\
\hline 423.3 & 387.5 & 269.2 & 226 & 208.7 & 191.4 & 160.3 & 141.3 & 122.3 & 2349.00 \\
\hline 447 & 422.6 & 299.9 & 232.4 & 194.9 & 143 & 107.6 & 106.7 & 104.6 & 6193.50 \\
\hline 538.2 & 554.7 & 352.6 & 259.7 & 204.4 & 163.8 & 128.3 & 119.7 & 108.5 & 6244.00 \\
\hline 91.2 & 93.8 & 97.2 & 96.4 & 96.4 & 88.6 & 85.1 & 80.8 & 82.5 & 6289.50 \\
\hline 565.2 & 520.4 & 319.7 & 228.2 & 201.8 & 176.7 & 127.5 & 108.5 & 99.8 & 6808.10 \\
\hline 542.1 & 504.4 & 312 & 209.6 & 204.4 & 197.9 & 153.4 & 130.9 & 116.2 & 5592.80 \\
\hline 535.2 & 469.7 & 307.2 & 212.1 & 148.7 & 115.4 & 87.7 & 89.5 & 88.6 & 6192.60 \\
\hline 404 & 373.6 & 271.8 & 195.7 & 158.6 & 162 & 105 & 78.2 & 74.8 & 6032.00 \\
\hline 85.5 & 87.3 & 88.6 & 89.5 & 80.8 & 86 & 92.9 & 87.7 & 84.3 & 6014.00 \\
\hline 515.6 & 405.9 & 261 & 200 & 172.4 & 145.6 & 119.7 & 99 & 80 & 6112.30 \\
\hline 412.6 & 390.1 & 293.8 & 214.7 & 175.9 & 129.2 & 107.6 & 100.7 & 87.3 & 2842.50 \\
\hline 391 & 365.1 & 281.7 & 195.7 & 165.5 & 145.6 & 114.5 & 111.5 & 86 & 2683.00 \\
\hline 385.2 & 361.5 & 244.1 & 197.1 & 181 & 155.1 & 118 & 116.2 & 115.4 & 5737.10 \\
\hline 105.9 & 105.9 & 104.1 & 97.2 & 79.6 & 88.6 & 81.7 & 85.1 & 87.7 & 6144.70 \\
\hline 562.3 & 486.4 & 275.7 & 231.2 & 164.6 & 156 & 118.8 & 115.4 & 101.6 & 6002.70 \\
\hline 534.9 & 499.9 & 356.4 & 276.9 & 206.1 & 227.7 & 190.5 & 174.1 & 171.5 & 5167.80 \\
\hline 596.3 & 557.9 & 303.7 & 212.1 & 157.7 & 133.5 & 105 & 101.6 & 99.8 & 2883.90 \\
\hline 645.6 & 622.9 & 436.4 & 240.7 & 194 & 168.9 & 120.6 & 118 & 116.2 & 2229.20 \\
\hline 90.3 & 80.8 & 79.1 & 84.3 & 88.6 & 88.2 & 98.1 & 89 & 87.3 & 6352.60 \\
\hline 516.8 & & 147.4 & 257.1 & 184.5 & 158.6 & 128.3 & 117.1 & 99 & 6024.30 \\
\hline 388 & 359.9 & 257.1 & 197.5 & 156 & 143 & 104.6 & 85.1 & 81.7 & 5796.20 \\
\hline 385.8 & 363.3 & 264.8 & 191.4 & 156.8 & 127.9 & 101.6 & 97.2 & 97.2 & 5723.10 \\
\hline \begin{tabular}{|l|}
83.8 \\
\end{tabular} & 83 & 86 & 84.3 & 80.8 & 92 & 101.6 & 90.8 & 89.5 & 5710.40 \\
\hline 422 & 392 & 242 & 183.6 & 151.7 & 135.2 & 106.7 & 95.5 & 94.2 & 2048.60 \\
\hline 452.1 & 410.3 & 254.9 & 186.2 & 163.8 & 149.9 & 119.7 & 112.8 & 94.2 & 1654.40 \\
\hline 607.6 & 588 & 312.4 & 227.7 & 165.1 & 137 & 105 & 102.4 & 91.2 & 5368.00 \\
\hline 648.6 & 590.4 & 281.3 & 185.4 & 149.5 & 131.3 & 97.7 & 85.1 & 83.4 & 6048.50 \\
\hline 92 & 99 & 102.4 & 94.2 & 92.9 & 96.4 & 105.9 & 93.8 & 96.4 & 5864.60 \\
\hline 556.8 & 514.5 & 366.4 & 303.7 & 253.6 & 213 & 185.8 & 176.7 & 138.7 & 5867.80 \\
\hline 551.1 & 512 & 286.9 & 192.3 & 157.7 & 131.4 & 99.8 & 89.5 & 88.6 & 4720.00 \\
\hline 567.1 & 536.5 & 333.6 & 274.4 & 235.5 & 203.5 & 171.5 & 168.1 & 133.5 & 4274.30 \\
\hline 398.8 & 359.9 & 245.8 & 181.5 & 156 & 148.2 & 99 & 89.1 & 90.3 & 3794.90 \\
\hline 98.1 & 82.5 & 93.4 & 84.3 & 96.4 & 102.4 & 82.5 & 80.8 & 82.5 & 3556.20 \\
\hline 549.3 & 296.5 & 241.1 & 181.9 & 159.4 & 128.3 & 117.1 & 113.6 & 109.3 & 4172.10 \\
\hline 575.2 & & 194.9 & 219.9 & 173.3 & 156.4 & 129.2 & 116.2 & 114.5 & 4181.00 \\
\hline 661.9 & 640.8 & 343.9 & 213.9 & 180.2 & 156.8 & 105 & 105 & 102.4 & 4572.40 \\
\hline 665.4 & 614 & 329.6 & 220.8 & 181 & 166.4 & 137.8 & 128.3 & 124.9 & 5071.90 \\
\hline 111.9 & 112.8 & 113.6 & 107.6 & 106.7 & 106.3 & 113.6 & 114 & 102.9 & 4167.20 \\
\hline 790 & 735.2 & 398.8 & 263.6 & 198.3 & 174.6 & 145.6 & 131.8 & 121.4 & 3507.00 \\
\hline 779.2 & 727.7 & 393.8 & 262.3 & 214.7 & 189.7 & 151.7 & 137 & 119.7 & 3601.90 \\
\hline 739.9 & 747.1 & 408.9 & 245.4 & 183.6 & 157.7 & 144.8 & 132.7 & 116.2 & 5759.30 \\
\hline 238.1 & 233.7 & 138.7 & 112.8 & 111.5 & 115.8 & 121.9 & 118 & 100.7 & 6964.90 \\
\hline 682 & 661.2 & 366.7 & 228.6 & 191.4 & 168.9 & 130.1 & 130.9 & 101.6 & 6813.50 \\
\hline 698.3 & 668.4 & 452.1 & 317.6 & 274 & 246.7 & 213.9 & 203.5 & 200.9 & 6554.40 \\
\hline
\end{tabular}


other

\begin{tabular}{|c|c|c|c|c|c|c|c|c|c|}
\hline 807.6 & 754.8 & 397.2 & 248 & 204.4 & 182.4 & 143 & 125.3 & 123.2 & 4266.50 \\
\hline 97.7 & 115.8 & 115.8 & 107.6 & 98.1 & 96.4 & 99 & 102.4 & 97.2 & 3813.00 \\
\hline 527.4 & 510.5 & 284 & 180.2 & 125.3 & 131.8 & 128.3 & 122.3 & 117.1 & 3633.90 \\
\hline 766.2 & 738 & 393.8 & 255.8 & 188 & 175.9 & 133.1 & 130.9 & 121.4 & 6348.80 \\
\hline 740.3 & 707.8 & 370.9 & 229.4 & 169.4 & 145.6 & 118.8 & 120.2 & 117.5 & 6035.80 \\
\hline 105.9 & 107.6 & 107.6 & 101.6 & 99 & 103.3 & 112.8 & 113.6 & 105 & 6275.10 \\
\hline 769.3 & 706.2 & 371.8 & 240.7 & 194.9 & 176.7 & 142.2 & 136.1 & 120.6 & 7169.50 \\
\hline 770.5 & 736.4 & 468.9 & 313.7 & 270.9 & 256.2 & 216.9 & 200 & 207.4 & 5096.70 \\
\hline 727.8 & 683.7 & 356.2 & 217.3 & 170.3 & 146.5 & 105.5 & 105.9 & 90.3 & 3701.30 \\
\hline 644.6 & 585.9 & 313.4 & 184.5 & 151.7 & 134.4 & 109.3 & \begin{tabular}{|c|}
98.1 \\
\end{tabular} & 92.9 & 3691.70 \\
\hline 104.1 & 107.6 & 102.4 & 92.9 & 94.6 & 94.6 & 95.5 & 96.4 & 96.4 & 6183.80 \\
\hline 794.1 & 733.2 & 374.8 & 250.2 & 212.6 & 173.3 & 132.7 & 123.2 & 123.2 & 6004.00 \\
\hline 857.5 & 827.7 & 444.8 & 309.4 & 296 & 261.9 & 203.5 & 165.1 & 127.5 & 5319.60 \\
\hline \multirow{2}{*}{882.5} & 843.2 & \begin{tabular}{|l|}
422.8 \\
\end{tabular} & 294.2 & 235.1 & 218.7 & 161.2 & 149.5 & 144.8 & 3182.90 \\
\hline & 159.9 & 480.4 & 395.1 & 229.4 & 191.9 & 153.4 & 145.2 & 130.9 & 3260.70 \\
\hline 111.1 & 118 & 101.6 & 94.6 & 111.9 & 99 & 105 & 111.1 & 98.1 & 3198.20 \\
\hline 766 & 692.1 & 336.4 & 262.3 & 226 & 194 & 154.3 & 141.3 & 113.6 & 2971.20 \\
\hline 785.4 & 700.7 & 367 & 261.9 & 211.3 & 158.2 & 130.1 & 126.6 & 117.1 & 5629.70 \\
\hline 706.8 & 658.9 & 346.3 & 276.1 & 212.1 & 160.3 & 124.9 & 105.9 & 100.7 & 5542.20 \\
\hline 822.7 & 753 & 375.6 & 275.7 & 229.4 & 163.4 & 123.2 & 120.6 & 110.2 & 5541.00 \\
\hline 135.2 & 146.1 & 154.3 & 165.1 & 161.2 & 159 & 152.5 & 135.7 & 125.7 & 5364.60 \\
\hline 861.6 & 830.8 & 376.1 & 243.2 & 208.3 & 180.6 & 138.7 & 131.4 & 133.5 & 3317.10 \\
\hline 846.3 & 836.4 & 407.6 & 277.8 & 208.3 & 167.2 & 134 & 134.4 & 134 & 3047.30 \\
\hline 429.5 & 421.2 & 274.8 & 219.5 & 215.2 & 201.4 & 173.7 & 162.9 & 159 & 3096.60 \\
\hline 933 & 907.6 & 494.6 & 365.5 & 309.4 & 295.6 & 224.7 & 177.2 & 146.1 & 5188.20 \\
\hline 961 & 917.8 & 487.3 & 331 & 275.7 & 224.2 & 173.7 & 155.6 & 148.7 & 4870.20 \\
\hline 925.3 & 837.1 & 454.8 & 299.9 & 265.7 & 222.1 & 166 & 156.4 & 153 & 4825.50 \\
\hline 99.8 & 101.2 & 97.7 & 98.1 & 97.7 & 106.7 & 111.5 & 111.5 & 102.4 & 3894.20 \\
\hline 712.9 & 692.1 & 355 & 224.7 & 197.1 & 171.5 & 139.2 & 108.9 & 103.7 & 3227.90 \\
\hline 878 & 778.4 & 416 & 276.5 & 231.2 & 181.5 & 115.8 & 99 & 88.2 & 3087.10 \\
\hline 850.1 & 826.6 & 453.1 & 318 & 264.4 & 224.2 & 152.1 & 125.3 & 114.1 & 2928.10 \\
\hline 190.5 & 199.6 & 217.8 & 191.9 & 178.9 & 172.4 & 191 & 143.5 & 147.8 & 5375.00 \\
\hline 944.2 & 911.9 & 509.7 & 364.7 & 325.8 & 280.4 & 206.6 & 190.1 & 166.8 & 4759.70 \\
\hline 865.2 & 255.8 & 373.7 & 242 & 218.2 & 203.1 & 169.4 & 137.8 & 107.2 & 5229.80 \\
\hline 792.4 & 762 & 435 & 274.4 & 235.1 & 178 & 108.9 & 98.1 & 95.1 & 5412.10 \\
\hline 716.6 & 687.2 & 388.7 & 254.5 & 216.1 & 182.4 & 135.2 & 119.3 & 100.7 & 3919.30 \\
\hline 109.8 & 124 & 138.3 & 122.8 & 115.4 & 105.5 & 119.3 & 105.5 & 93.8 & 3686.60 \\
\hline 802 & 771.2 & 407.7 & 277.4 & 230.3 & 219.5 & 164.2 & 128.3 & 101.2 & 3549.30 \\
\hline 731.3 & 684.7 & 588.6 & 563.9 & 560.4 & 651.5 & 644.3 & 247.8 & 97.2 & 5344.00 \\
\hline 665.6 & 640.9 & 331.2 & 202.6 & 153 & 135.7 & 98.1 & 83.9 & 76.5 & 4671.40 \\
\hline 682 & 637.5 & 316.5 & 176.3 & 144.8 & 126.2 & 84.3 & 77 & 70 & 5975.40 \\
\hline 84.3 & 95.1 & \begin{tabular}{|c|}
99.8 \\
\end{tabular} & 94.2 & 93.8 & 99.4 & 105 & 97.7 & 84.7 & 6274.10 \\
\hline 750.8 & 725.6 & 390 & 238.5 & 198.8 & 194.9 & 137.4 & 99.8 & 85.6 & 4491.00 \\
\hline 752.1 & 726.2 & 466 & 361.2 & 314.1 & 278.3 & 217.3 & 197.5 & 161.6 & 4135.20 \\
\hline 786.9 & 749.4 & 390 & 226.4 & 188.8 & 169.4 & 129.2 & 104.6 & 94.6 & 3780.90 \\
\hline 656.8 & 630 & 331.2 & 209.6 & 186.2 & 158.6 & 123.2 & 125.3 & 88.6 & 6214.10 \\
\hline 585 & 557.7 & 321.4 & 212.2 & 192.2 & 213 & 216.8 & 133.8 & 98.6 & 6126.90 \\
\hline 751.6 & 730.8 & 484.2 & 342.2 & 294.2 & 298.1 & 235.5 & 189.3 & 167.2 & 6430.70 \\
\hline 818.4 & 788.2 & 419.8 & 250.6 & 201.4 & 196.2 & 144.8 & 123.6 & 109.3 & 6904.30 \\
\hline
\end{tabular}


other

\begin{tabular}{|c|c|c|c|c|c|c|c|c|c|}
\hline 801 & 827.3 & 411.2 & 254.1 & 214.7 & 191 & 134.8 & 120.2 & 115.4 & 7849.40 \\
\hline 84.3 & 89.5 & 92.9 & 84.3 & 82.1 & 100.7 & 105 & 96.4 & 88.6 & 4454.70 \\
\hline 685.5 & 656.6 & 346.8 & 221.2 & 193.1 & 200 & 154.3 & 126.2 & 94.6 & 3995.30 \\
\hline 750.3 & 746 & 497.1 & 338.8 & 291.6 & 278.7 & 225.1 & 192.7 & 162 & 7891.00 \\
\hline 746.8 & 724 & 402.1 & 247.6 & 227.3 & 186.7 & 145.6 & 125.3 & 101.6 & 8888.70 \\
\hline 767.6 & 724 & 391.3 & 257.1 & 209.2 & 207.8 & 154.7 & 143.9 & 125.7 & 9313.30 \\
\hline 70.4 & 74.4 & 80.8 & 78.7 & 77.8 & 104.1 & 89.1 & 86 & 79.6 & 8276.10 \\
\hline 816.5 & 730.4 & 430.3 & 279.1 & 255.3 & 227.3 & 188 & 143.5 & 112.8 & 7712.00 \\
\hline 784.1 & 757.3 & 516.1 & 375.4 & 326.7 & 276.5 & 222.5 & 181.9 & 166 & 5464.60 \\
\hline 784.1 & 722.3 & 418.1 & 276.9 & 243.7 & 175.9 & 134 & 123.2 & 112.8 & 5305.80 \\
\hline 822 & 771.8 & 411.6 & 284.3 & 235.5 & 202.2 & 133.5 & 117.6 & 106.7 & 9698.50 \\
\hline 89.5 & 80.4 & 76.5 & 72.2 & 77.4 & 81.3 & 92 & 89.5 & 77.4 & 11502.50 \\
\hline 684.1 & 659.5 & 359.3 & 225.1 & 194.9 & 182.8 & 115.4 & 91.6 & 84.3 & 13090.50 \\
\hline 819.8 & 763.2 & 509.2 & 359 & 284.3 & 256.2 & 186.2 & 153.9 & 133.5 & 13902.50 \\
\hline 465.3 & 426.4 & 272 & 217.3 & 178.4 & 124.9 & 99.8 & 89.1 & 84.3 & 13719.30 \\
\hline 412.6 & 390.1 & 289.9 & 206.1 & 183.2 & 156.8 & 119.7 & 105 & 98.6 & 9237.70 \\
\hline 79.1 & 86.5 & 86 & 78.2 & 83.4 & 83.4 & 84.3 & 82.1 & 81.2 & 8039.40 \\
\hline 644.8 & 618 & 352.8 & 243.2 & 218.2 & 168.5 & 137 & 118.8 & 105.9 & 12757.00 \\
\hline 586.9 & 571.4 & 342.5 & 277.8 & 235.5 & 226.8 & 173.3 & 130.5 & 121.8 & 13755.90 \\
\hline 727.6 & 684 & 388.7 & 259.3 & 227.7 & 169.8 & 124.9 & 108.5 & 102.4 & 13733.30 \\
\hline 574.2 & 520.6 & 323 & 188.8 & 142.2 & 130.1 & 105.9 & 93.8 & 86.5 & 13887.10 \\
\hline 81.7 & 80.8 & 70.4 & 69.6 & 78.2 & 85.6 & 98.1 & 92.9 & 75.6 & 14345.30 \\
\hline 490.4 & 457.5 & 291.6 & 202.6 & 190.5 & 162 & 126.6 & 118.4 & 92.9 & 9564.50 \\
\hline 577.3 & 539.3 & 333 & 244.1 & 227.7 & 218.2 & 191.4 & 157.3 & 140.4 & 7496.90 \\
\hline 640 & 605.9 & 358 & 239.8 & 193.1 & 147.3 & 105 & 99 & 96 & 8885.40 \\
\hline 646.4 & 575.1 & 320.5 & 237.2 & 204.4 & 181 & 143.9 & 118.8 & 105 & 8765.10 \\
\hline 69.6 & 69.6 & 70 & 70.4 & 80 & 85.1 & 81.7 & 82.1 & 80.8 & 5372.50 \\
\hline 448.9 & 441.1 & 304.6 & 232.9 & 208.7 & 174.6 & 124 & 106.7 & 99 & 2500.50 \\
\hline 100.3 & 99 & 100.7 & 96.4 & 92.9 & 105.9 & 112.4 & 108.5 & 99 & 2490.90 \\
\hline 445.4 & 402.2 & 290.8 & 226 & 203.5 & 181 & 150.8 & 130.1 & 110.2 & 5341.20 \\
\hline 445.1 & 412.6 & 325.2 & 251 & 209.6 & 175.9 & 130.1 & 123.2 & 124.9 & 2628.70 \\
\hline 457.9 & 422.9 & 279.8 & 207.8 & 166.4 & 122.8 & 92.9 & 92 & 92 & 2472.00 \\
\hline 434.8 & 429.6 & 290.6 & 211.3 & 185.4 & 188.8 & 128.8 & 128.3 & 127.5 & 2605.60 \\
\hline 67.9 & 73.9 & 83 & 68.7 & 74.8 & 83.4 & 92 & 75.6 & 73.5 & 2181.50 \\
\hline 414.3 & 388.4 & 276.9 & 210.4 & 196.6 & 176.7 & 140 & 108.5 & 107.6 & 2062.70 \\
\hline 406.5 & 392.7 & 256.2 & 199.2 & 191.4 & 203.5 & 161.6 & 122.3 & 118 & 2755.20 \\
\hline 500.9 & 453.5 & 254.1 & 200 & 175 & 131.8 & 88.6 & 84.3 & 75.2 & 3034.00 \\
\hline 428.4 & 391.2 & 248 & 190.5 & 168.9 & 150.8 & 120.6 & 128.8 & 125.7 & 2837.40 \\
\hline 57.5 & 61.8 & 60.1 & 61.4 & 68.7 & 72.2 & 70.4 & 70.4 & 66.1 & 2374.40 \\
\hline 417.8 & 404.4 & 285.6 & 213.9 & 189.7 & 171.5 & 127.1 & 111.9 & 107.6 & 2680.90 \\
\hline 404.8 & 375.4 & 284.7 & 226.8 & 193.6 & 160.3 & 137.8 & 149.1 & 143.9 & 2533.60 \\
\hline 411.7 & 391 & 286 & 200 & 149.1 & 136.1 & 114.5 & 99.8 & 96.4 & 0.00 \\
\hline 423.8 & 401.4 & 273.1 & 181 & 162 & 168.9 & 125.7 & 99 & 99 & 2107.50 \\
\hline 399.6 & 376.3 & 259.7 & 187.1 & 164.6 & 121 & 101.6 & 98.1 & 98.1 & 2907.20 \\
\hline 406.1 & 381.1 & 267.4 & 200 & 170.7 & 128.3 & 99 & 88.6 & 86.9 & 2560.10 \\
\hline 386.7 & 371.1 & 237.7 & 170.2 & 137.4 & 108.5 & 73.9 & 70.4 & 68.7 & 2441.70 \\
\hline 459.7 & 439.8 & 291.2 & 216.5 & 194 & 164.6 & 111.1 & 104.1 & 102.4 & 2116.70 \\
\hline 404.6 & 372.4 & 252.4 & 208.7 & 181 & 179.7 & 144.4 & 133.5 & 118.8 & 6245.40 \\
\hline 426 & 393.6 & 259.7 & 197.5 & 166.4 & 143 & 109.3 & 95.1 & 95.5 & 6479.80 \\
\hline
\end{tabular}


other

\begin{tabular}{|c|c|c|c|c|c|c|c|c|c|}
\hline 418.6 & 355.6 & 252.8 & 191.4 & 158.6 & 162 & 125.7 & 83.9 & 83.4 & 3979.90 \\
\hline 383.6 & 374.2 & 237.2 & 175.9 & 157.7 & 137.8 & 107.6 & 105.9 & 99.8 & 5688.10 \\
\hline 477.4 & 414.7 & 239.8 & 171.5 & 154.3 & 151.7 & 84.7 & 60.9 & 61.8 & 2331.00 \\
\hline 414.3 & 372 & 268.8 & 220.8 & 193.1 & 181.9 & 142.2 & 134.4 & 126.2 & 1899.50 \\
\hline 397 & 385.8 & 286 & 222.5 & 175.9 & 144.8 & 101.6 & 100.7 & 99.8 & 5576.10 \\
\hline 432.9 & 396.4 & 264 & 198.3 & 174.6 & 141.3 & 106.7 & 100.7 & 87.3 & 5973.10 \\
\hline 416.4 & 368.4 & 210.7 & 165.5 & 153.4 & 128.3 & 93.4 & 92.9 & 84.3 & 5750.70 \\
\hline 410 & 390.1 & 274.8 & 204.4 & 187.1 & 167.2 & 127.9 & 103.3 & 103.3 & 5802.30 \\
\hline 389.3 & 377.2 & 263.6 & 196.6 & 156 & 152.5 & 122.8 & 105 & 103.3 & 5399.80 \\
\hline 402.2 & 359.9 & 255.8 & 188 & 158.6 & 136.1 & 101.2 & 92.9 & 78.2 & 2194.20 \\
\hline 387.5 & 347.8 & 244.1 & 165.1 & 153.4 & 129.2 & 82.5 & 80.4 & 78.2 & 2125.50 \\
\hline 394.4 & 364.2 & 251.9 & 199.6 & 178.4 & 162 & 134 & 130.1 & 121.4 & 5527.90 \\
\hline 407.9 & 387.1 & 274 & 212.1 & 181.9 & 161.2 & 122.3 & 111.1 & 94.2 & 5982.80 \\
\hline 400.5 & 382.4 & 264.8 & 225.6 & 201.8 & 196.6 & 156.8 & 128.3 & 118 & 5890.10 \\
\hline 411.7 & 391.9 & 283.9 & 240.7 & 211.3 & 167.7 & 117.1 & 96.4 & 93.8 & 6408.40 \\
\hline 413.5 & 376.3 & 264 & 214.3 & 186.2 & 162 & 115.4 & 107.2 & 85.1 & 7597.90 \\
\hline 398.4 & 370.3 & 264 & 199.2 & 173.7 & 143.9 & 105.9 & 85.6 & 85.1 & 4628.10 \\
\hline 397 & 397 & 276.1 & 242.4 & 206.1 & 191.4 & 156.4 & 150.8 & 152.5 & 2171.90 \\
\hline 429.4 & 394.4 & 281.3 & 249.3 & 216.5 & 214.7 & 171.5 & 142.2 & 112.4 & 6639.10 \\
\hline 429 & 380.6 & 262.3 & 212.1 & 177.6 & 140.4 & 98.1 & 105 & 87.7 & 6734.70 \\
\hline 418.1 & 386.6 & 266.6 & 213.9 & 160.3 & 126.6 & 94.6 & 93.8 & 81.7 & 6064.40 \\
\hline 402.1 & 377.9 & 242.8 & 200 & 151.7 & 111.5 & 78.2 & 78.2 & 78.2 & 5341.50 \\
\hline 377.2 & 362.5 & 265.3 & 212.1 & 183.6 & 156.4 & 129.2 & 124 & 119.3 & 5592.70 \\
\hline 403.1 & 369 & 263.1 & 200.9 & 190.5 & 158.2 & 133.5 & 150.8 & 141.8 & 2709.50 \\
\hline 402.2 & 376.3 & 278.7 & 229 & 206.1 & 181 & 156.8 & 153 & 132.7 & 2040.10 \\
\hline 389.7 & 372.8 & 269.2 & 233.7 & 217.3 & 189.7 & 171.5 & 169.8 & 177.2 & 5961.30 \\
\hline 433.2 & 409.4 & 260.1 & 191.4 & 151.7 & 140.4 & 102.4 & 81.3 & 89.5 & 5401.80 \\
\hline 481.6 & 444.1 & 263.8 & 199.2 & 162.9 & 146.5 & 108.5 & 101.6 & 83.4 & 5521.70 \\
\hline 516.1 & 442.4 & 270 & 216.5 & 181 & 162.9 & 107.6 & 105 & 109.8 & 5530.00 \\
\hline 559 & 505.6 & 248 & 198.3 & 163.8 & 154.3 & 106.7 & 95.5 & 68.7 & 5363.10 \\
\hline 388.4 & 368.5 & 234.6 & 178 & 153.4 & 130.1 & 91.6 & 94.6 & 78.2 & 2741.90 \\
\hline 469.7 & 431.5 & 274.8 & 227.7 & 177.6 & 149.1 & 112.8 & 105 & 95.1 & 1997.40 \\
\hline 534.4 & 436 & 244.1 & 176.7 & 140.4 & 111.1 & 69.6 & 67.5 & 70.4 & 5919.00 \\
\hline 516.2 & 518.2 & 254.9 & 182.8 & 156 & 118.8 & 80.4 & 87.7 & 96.4 & 6951.50 \\
\hline 450.4 & 436.9 & 236.8 & 189.7 & 159 & 167.2 & 94.6 & 76.5 & 73.9 & 7307.40 \\
\hline 394.4 & 353.8 & 201.8 & 144.8 & 138.3 & 121.4 & 73 & 73.9 & 71.3 & 7431.50 \\
\hline 544.6 & 518.1 & 235.9 & 171.1 & 135.2 & 128.3 & 95.5 & 77.4 & 71.3 & 7902.60 \\
\hline 561.6 & 530.4 & 268.8 & 196.6 & 137 & 136.1 & 87.7 & 76.5 & 73.9 & 3638.40 \\
\hline 646.8 & 623.4 & 265.3 & 190.5 & 138.7 & 111.9 & 85.1 & 94.6 & 95.5 & 2052.90 \\
\hline 416 & 382.4 & 242.4 & 185.8 & 156.4 & 137.4 & 99.8 & 92.9 & 94.6 & 6726.50 \\
\hline 418.2 & 376.3 & 219.1 & 152.1 & 108.5 & 118 & 119.3 & 113.6 & 110.2 & 5227.40 \\
\hline 521.9 & 474.3 & 228.2 & 180.2 & 134.4 & 112.8 & 72.2 & 69.2 & 69.6 & 5153.20 \\
\hline 411.9 & 389.9 & 233.3 & 198.3 & 177.6 & 146.5 & 111.1 & 88.6 & 75.2 & 2074.90 \\
\hline 541 & 531.8 & 261.9 & 217.8 & 168.1 & 124.9 & 86.9 & 96.4 & 98.1 & 5464.30 \\
\hline 682.3 & 663.8 & 271.8 & 204.8 & 157.7 & 136.1 & 90.3 & 87.7 & 88.6 & 5623.00 \\
\hline 570.3 & 538.7 & 239 & 176.3 & 151.7 & 124 & 80 & 71.3 & 70.4 & 6798.90 \\
\hline 656.2 & 644 & 288.2 & 219.5 & 174.1 & 141.3 & 100.7 & 92.9 & 90.3 & 6929.30 \\
\hline 745 & 700.4 & 340.9 & 270.9 & 216.9 & 183.2 & 123.6 & 114.5 & 111.1 & 6864.90 \\
\hline 624.6 & 601.8 & 274.4 & 206.6 & 159.4 & 136.1 & 95.5 & 104.1 & 104.1 & 2504.20 \\
\hline
\end{tabular}


other

\begin{tabular}{|c|c|c|c|c|c|c|c|c|c|}
\hline 604.3 & 579.7 & 304 & 241.5 & 198.3 & 178.4 & 130.1 & 110.2 & 105.9 & 2180.80 \\
\hline 549.7 & 515.2 & 294.9 & 237.2 & 216.5 & 181 & 137 & 134.8 & 135.2 & 6756.60 \\
\hline 512.8 & 444 & 235.1 & 174.6 & 145.6 & 118 & 77.4 & 72.2 & 69.6 & 7082.70 \\
\hline 533.6 & 551.6 & 272.7 & 204.4 & 175.9 & 171.5 & 124 & 84.3 & 79.1 & 6937.60 \\
\hline 550.5 & 521.8 & 271.4 & 213 & 166.4 & 126.6 & 80 & 99 & 93.4 & 5739.70 \\
\hline 520.3 & 499.3 & 228.2 & 168.1 & 142.2 & 108.9 & 111.1 & 107.6 & 106.7 & 5274.10 \\
\hline 752.5 & 702.8 & 344.8 & 249.3 & 231.2 & 219.9 & 194.9 & 190.5 & 165.5 & 2120.30 \\
\hline 738 & 698.8 & 275.3 & 203.5 & 168.1 & 154.3 & 116.2 & 106.7 & 99 & 1942.50 \\
\hline 497.6 & 472.8 & 231.6 & 179.3 & 150.8 & 113.6 & 86 & 97.7 & 98.1 & 6686.70 \\
\hline 561.5 & 520.9 & 219.5 & 160.3 & 149.1 & 109.3 & 97.2 & 101.6 & 100.7 & 6576.10 \\
\hline 735.4 & 717.7 & 280.9 & 194.9 & 168.9 & 148.2 & 116.2 & 106.7 & 102.4 & 7559.60 \\
\hline 569.2 & 530.7 & 313.7 & 228.6 & 176.7 & 148.2 & 117.1 & 102.4 & 94.6 & 7582.10 \\
\hline 573.6 & 533.4 & 250.6 & 177.6 & 138.7 & 88.6 & 78.2 & 90.8 & 92.9 & 7451.60 \\
\hline 605.1 & 537.7 & 251.5 & 191 & 170.7 & 141.3 & 103.3 & 88.6 & 85.1 & 2580.80 \\
\hline 720.8 & 634.6 & 276.1 & 195.7 & 148.2 & 127.5 & 94.6 & 89.1 & 88.6 & 2547.60 \\
\hline 737.8 & 677.3 & 271.8 & 193.1 & 172 & 156.8 & 122.3 & 103.3 & 93.4 & 8801.20 \\
\hline 649.5 & 629.3 & 305.1 & 198.3 & 157.7 & 130.1 & 106.7 & 106.7 & 100.7 & 8669.80 \\
\hline 600.2 & 553.6 & 254.1 & 158.6 & 118.8 & 123.6 & 99.4 & 91.6 & 74.8 & 8681.80 \\
\hline 614 & 539.6 & 251.5 & 168.1 & 132.7 & 113.6 & 86 & 87.7 & 87.7 & 3618.00 \\
\hline 671.2 & 636.6 & 256.7 & 166.8 & 137.8 & 135.2 & 86.9 & 82.5 & 74.4 & 7687.30 \\
\hline 735.8 & 715.5 & 294.7 & 186.2 & 152.5 & 166.4 & 112.8 & 104.1 & 92.9 & 8673.20 \\
\hline 692 & 662.8 & 99 & 197.5 & 171.5 & 151.7 & 92 & 92 & 87.7 & 9428.90 \\
\hline 758.1 & 704.2 & 278.3 & 188.8 & 152.5 & 125.7 & 100.3 & 86.9 & 82.5 & 9644.00 \\
\hline 728.6 & 679.3 & 256.7 & 170.7 & 147.3 & 118.8 & 85.1 & 77.4 & 78.2 & 3611.30 \\
\hline 636.8 & 618 & 280 & 183.6 & 162.5 & 136.1 & 90.3 & 89.5 & 88.6 & 2326.90 \\
\hline 689.7 & 664.6 & 306.8 & 218.7 & 189.7 & 159.4 & 108.1 & 106.3 & 95.1 & 6936.80 \\
\hline 684.3 & 633.1 & 312 & 217.8 & 189.7 & 161.6 & 117.1 & 117.1 & 111.5 & 8797.40 \\
\hline 694.9 & 646.2 & 271 & 178.4 & 152.1 & 134 & 79.1 & 70 & 68.7 & 8238.70 \\
\hline 763.9 & 711.4 & 291.2 & 200.5 & 182.8 & 153.9 & 92.9 & 91.6 & 97.7 & 8845.10 \\
\hline 827.3 & 795.7 & 310.7 & 225.1 & 201.4 & 168.5 & 105 & 98.6 & 95.1 & 2846.40 \\
\hline 701.3 & 651.2 & 313.7 & 245.4 & 208.7 & 158.6 & 120.6 & 120.6 & 105.9 & 2490.60 \\
\hline 744.3 & 678.2 & 286.5 & 190.1 & 184.5 & 166.8 & 119.7 & 121.4 & \begin{tabular}{|l|}
109.8 \\
\end{tabular} & 8558.10 \\
\hline 724.9 & 703.2 & 282.6 & 193.6 & 162.9 & 137.4 & 80.8 & 77 & 77 & 9126.90 \\
\hline 745.4 & 720.8 & 293 & 235.1 & 211.3 & 162.5 & 125.7 & 134.8 & 127.5 & 8734.20 \\
\hline 661.5 & 646.4 & 301.6 & 197.5 & 159.4 & 128.7 & 104.6 & 103.3 & 99.8 & 6717.60 \\
\hline 656.4 & 632.3 & 279.1 & 194.9 & 149.9 & 131.8 & 98.6 & 97.2 & 98.1 & 6881.70 \\
\hline 684 & 654.1 & 274 & 174.1 & 135.2 & 104.1 & 78.2 & 92.9 & 90.8 & 2519.30 \\
\hline 919.1 & 862.8 & 399.2 & 281.7 & 229.9 & 205.7 & 117.6 & 85.6 & 87.7 & 2176.70 \\
\hline 901.3 & 861.8 & 343.5 & 224.7 & 196.2 & 166.8 & 114.1 & 116.2 & 117.6 & 8780.50 \\
\hline 933.3 & 868.6 & 398 & 257.5 & 227.7 & 219.5 & 149.5 & 102.9 & 82.1 & 9854.60 \\
\hline 810.1 & 732.5 & 361.7 & 280.4 & 336.2 & 357.8 & 307.2 & 110.7 & 98.6 & 10051.60 \\
\hline 732.5 & 694.3 & 342.7 & 209.2 & 159.4 & 135.7 & 81.3 & 77.4 & 71.8 & 8762.70 \\
\hline 754 & 687.2 & 327.1 & 245.4 & 199.2 & 191 & 98.6 & 83.9 & 76.5 & 8157.10 \\
\hline 841.6 & 771.4 & 335.3 & 253.2 & 223.8 & 171.1 & 164.2 & 159.4 & 148.7 & 2749.60 \\
\hline 885 & 855.9 & 390.6 & 255.8 & 221.2 & 199.6 & 115.4 & 89.1 & 83.9 & 2448.20 \\
\hline 921.2 & 855.7 & 408.7 & 316.3 & 365.5 & 373.7 & 312 & 96.8 & 89.1 & 8404.50 \\
\hline 890.8 & 853.9 & 379 & 229 & 182.4 & 162.9 & 96 & 89.9 & 84.7 & 8757.50 \\
\hline 883.8 & 855.1 & 382.8 & 243.7 & 240.3 & 228.2 & 154.3 & 97.7 & 92.5 & 8647.20 \\
\hline 925.1 & 834.6 & 362.5 & 257.5 & 209.2 & 168.5 & 151.7 & 142.6 & 152.1 & 9162.00 \\
\hline
\end{tabular}


other

\begin{tabular}{|c|c|c|c|c|c|c|c|c|c|}
\hline 748.5 & 709.9 & 307.2 & 217.3 & 180.6 & 172 & 101.2 & 96.4 & 81.3 & 9147.80 \\
\hline 738.8 & 717.1 & 351.7 & 284.3 & 593.6 & 657.1 & 658.4 & 462.3 & 94.6 & 3241.00 \\
\hline 738 & 727 & 341.8 & 234.6 & 191 & 197.9 & 142.6 & 88.6 & 78.7 & 3042.20 \\
\hline 836.4 & 788.7 & 342.2 & 205.7 & 231.6 & 195.3 & 142.2 & 89.9 & 80.4 & 9683.20 \\
\hline 846.9 & 783.3 & 346.5 & 224.7 & 210.9 & 187.6 & 131.8 & 121 & 117.6 & 9676.50 \\
\hline 834.8 & 765.2 & 372.9 & 237.2 & 202.2 & 183.2 & 107.2 & 88.6 & 85.6 & 6220.60 \\
\hline 843 & 747.9 & 337.5 & 288.6 & 378.9 & 388 & 324.9 & 195.3 & 80.8 & 11113.70 \\
\hline 816 & 733.7 & 347.4 & 197.9 & 166.8 & 158.6 & 89.1 & 78.7 & 76.5 & 11230.30 \\
\hline 785.6 & 705.8 & 332.7 & 231.6 & 201.8 & 172.9 & 120.2 & 86 & 83 & 10861.10 \\
\hline 843.9 & 788.4 & 317.2 & 205.7 & 165.5 & 163.4 & 126.2 & 116.7 & 104.6 & 10442.30 \\
\hline 796 & 718.1 & 350 & 253.2 & 239.4 & 222.5 & 160.8 & 86.9 & 80.8 & 3252.70 \\
\hline 871.6 & 797.6 & 379 & 257.9 & 318.9 & 302.5 & 253.6 & 196.2 & 81.7 & 2273.80 \\
\hline 752.3 & 712 & 338.8 & 202.2 & 173.3 & 143 & 96.8 & 93.8 & 91.6 & 9026.70 \\
\hline 649.1 & 625.6 & 296.4 & 177.6 & 155.1 & 150.8 & 90.3 & 78.7 & 77.4 & 9573.80 \\
\hline 748 & 691.2 & 265.8 & 165.5 & 141.8 & 131.8 & 97.7 & 87.7 & 83 & 9948.20 \\
\hline 876.2 & 768.8 & 353 & 232 & 230.8 & 213.5 & 131.4 & 99.8 & 93.4 & 10957.60 \\
\hline 862.5 & 818.3 & 398.6 & 278 & 299 & 280.9 & 238.9 & 154.6 & 99.4 & 5786.50 \\
\hline 692.5 & 632.7 & 287.8 & 183.2 & 152.5 & 131.8 & 84.3 & 77.4 & 74.8 & 3264.60 \\
\hline 560.5 & 518.4 & 235.1 & 173.3 & 157.7 & 122.3 & 84.3 & 80.8 & 82.5 & 11166.20 \\
\hline 529.1 & 494.4 & 248 & 196.1 & 206.5 & 197 & 162.3 & 146 & 89.5 & 9711.40 \\
\hline 605.8 & 554.2 & 421.7 & 241.5 & 192.7 & 145.6 & 106.7 & 85.1 & 76.1 & 9211.80 \\
\hline 623.8 & 605.4 & 375.9 & 248 & 194 & 151.3 & 111.1 & 86.5 & 69.6 & 8359.30 \\
\hline 423.1 & 377.2 & 276.1 & 169.8 & 122.3 & 99 & 76.5 & 65.7 & 62.7 & 8888.20 \\
\hline 516.4 & 467.1 & 327.1 & 182.8 & 127.5 & 104.2 & 70.9 & 61.9 & 61 & 3659.50 \\
\hline 564.5 & 552.7 & 357 & 182.8 & 141.4 & 125.8 & 103.3 & 73.1 & 68.8 & 2347.10 \\
\hline 413.1 & 379 & 254.1 & 194.9 & 179.8 & 143.1 & 92.1 & 82.1 & 80.9 & 9686.20 \\
\hline 429 & 389.5 & 221.3 & 191.9 & 175.1 & 148.7 & 96.4 & 77 & 68.8 & 10315.60 \\
\hline 520.8 & 503.8 & 280.1 & 218.3 & 193.6 & 157.8 & 103.7 & 91.2 & 90.8 & 7222.40 \\
\hline 587.3 & 541.6 & 265.8 & 217.4 & 172.5 & 143.5 & 90.4 & 84.3 & 73.5 & 7146.90 \\
\hline 529.9 & 389.4 & 235.5 & 194.1 & 191.5 & 155.2 & 108.5 & 94.7 & 76.6 & 7518.10 \\
\hline 412.2 & 390.2 & 248.9 & 194.1 & 175.5 & 159.5 & 134.4 & 98.2 & 88.2 & 2369.90 \\
\hline 439.6 & 391.7 & 249 & 215.2 & 219.1 & 190.6 & 142.2 & 114.1 & 86.1 & 1928.10 \\
\hline 409.8 & 353.9 & 224.7 & 181.1 & 160.8 & 120.6 & 67.5 & 64.5 & 61.4 & 8635.30 \\
\hline 633.2 & 523.9 & 261.1 & 193.6 & 172.5 & 138.8 & 80 & 74.4 & 74 & 9524.40 \\
\hline 517.2 & 433.8 & 224.8 & 193.2 & 186.7 & 143.1 & 85.6 & 74 & 63.6 & 8521.40 \\
\hline 497.3 & 443.6 & 246.8 & 195.8 & 191 & 170.7 & 147.8 & 116.3 & 81.3 & 7337.10 \\
\hline 498.4 & 479.4 & 245.9 & 204 & 176.8 & 147.8 & 80.9 & 67.5 & 65.3 & 7521.40 \\
\hline 517.8 & 467.2 & 258.5 & 191.9 & 194.1 & 176.3 & 119.3 & 109.4 & 90.8 & 2288.80 \\
\hline 542.2 & 479.4 & 235.5 & 196.7 & 173.3 & 149.5 & 105.9 & 102 & 86.1 & 8043.50 \\
\hline 496.5 & 430.4 & 249.8 & 185.8 & 181.1 & 132.7 & 86.5 & 74.4 & 67.1 & 9373.70 \\
\hline 485.9 & 457.8 & 225.2 & 194.9 & 181.5 & 146.5 & 89.1 & 66.2 & 64 & 9078.60 \\
\hline 420.8 & 378.5 & 248.9 & 193.2 & 171.1 & 149.1 & 96.8 & 91.6 & 89.5 & 9646.00 \\
\hline 405.3 & 350.4 & 234.2 & 193.2 & 188.4 & 152.6 & 96.8 & 86.1 & 78.7 & 9728.40 \\
\hline 447.9 & 385.7 & 234.7 & 209.2 & 179.4 & 143.5 & 82.6 & 77 & 65.7 & 2652.60 \\
\hline 591.8 & 527 & 289.1 & 214.8 & 199.6 & 162.1 & 108.1 & 103.7 & 98.2 & 2025.20 \\
\hline 598.4 & 507.5 & 252.4 & 215.2 & 206.6 & 163 & 124.5 & 109.8 & 99.9 & 8082.20 \\
\hline 392.3 & 347.4 & 245.9 & 207.4 & 195.3 & 148.7 & 93.8 & 83.9 & 80 & 9250.90 \\
\hline 416.6 & 392.1 & 247.6 & 225.6 & 223 & 198.8 & 151.3 & 118 & 104.6 & 8640.20 \\
\hline 548.3 & 511 & 263.2 & 192.3 & 188.4 & 153.9 & 92.1 & 77 & 73.5 & 8803.80 \\
\hline
\end{tabular}


other

\begin{tabular}{|c|c|c|c|c|c|c|c|c|c|}
\hline 589 & 554.8 & 280.9 & 225.6 & 220.4 & 188.9 & 133.1 & 125.8 & 99.4 & 8580.60 \\
\hline 630.8 & 579.8 & 270.1 & 197.5 & 171.6 & 149.5 & 123.2 & 118.4 & 98.2 & 2502.70 \\
\hline 520.4 & 492.8 & 247.6 & 198.4 & 180.6 & 150.9 & 128.4 & 96.8 & 64.5 & 1682.50 \\
\hline 498.8 & 452.4 & 239.9 & 199.2 & 193.6 & 159 & 117.2 & 81.3 & 68.3 & 8656.00 \\
\hline 418.4 & 356.5 & 235.9 & 205.7 & 189.3 & 148.7 & 99.9 & 100.3 & 101.2 & 9928.30 \\
\hline 425.3 & 381.6 & 245 & 196.2 & 169.4 & 131.4 & 81.7 & 82.1 & 83 & 8936.00 \\
\hline 436 & 398.4 & 269.2 & 216.9 & 206.2 & 175.1 & 144 & 94.2 & 94.7 & 9398.00 \\
\hline 428.2 & 372 & 255.4 & 231.2 & 214.3 & 194.9 & 154.3 & 144.4 & 126.7 & 9547.50 \\
\hline 446.8 & 362.1 & 275.3 & 191 & 158.6 & 121.9 & 112.8 & 93.4 & 86.5 & 3362.30 \\
\hline 497.2 & 381.9 & 239 & 204.8 & 200.1 & 153.9 & 132.3 & 102.5 & 84.7 & 1840.00 \\
\hline 487.3 & 360.4 & 239 & 200.1 & 148.7 & 111.5 & 74 & 73.5 & 70.5 & 7842.10 \\
\hline 497.2 & 395.4 & 220.8 & 191 & 170.7 & 135.7 & 83 & 81.7 & 60.5 & 9869.00 \\
\hline 486.6 & 360 & 229.9 & 182.8 & 180.6 & 167.3 & 124.5 & 105.9 & 101.2 & 6032.80 \\
\hline 430.3 & 369.5 & 261.9 & 232.1 & 210.5 & 193.6 & 159.5 & 125.3 & 111.1 & 5781.10 \\
\hline 431.7 & 377.6 & 275.7 & 222.6 & 218.7 & 174.2 & 130.1 & 127.1 & 123.2 & 5853.60 \\
\hline 439.8 & 401 & 282.2 & 191 & 172 & 140.9 & 94.7 & 91.6 & 88.2 & 3393.80 \\
\hline 396.6 & 362.1 & 261.5 & 185.8 & 158.2 & 122.4 & 87.3 & 86.9 & 85.6 & 2104.40 \\
\hline 379.4 & 322.3 & 226.9 & 210.9 & 183.2 & 157.8 & 103.7 & 101.6 & 94.2 & 7014.90 \\
\hline 472.4 & 421.8 & 240.3 & 218.3 & 202.7 & 174.6 & 120.6 & 103.7 & 75.2 & 7932.70 \\
\hline 532.9 & 401.4 & 243.3 & 238.5 & 201.8 & 191 & 159.5 & 127.1 & 100.8 & 7986.40 \\
\hline 555.4 & 474.6 & 287.4 & 226.9 & 203.6 & 163.4 & 106.8 & 92.5 & 76.6 & 6704.90 \\
\hline 508.1 & 462.6 & 297.9 & 254.1 & 232.9 & 198.8 & 131.8 & 113.6 & 115 & 5983.00 \\
\hline 432.9 & 397.1 & 297.3 & 250.2 & 236.8 & 212.6 & 147.8 & 147.8 & 143.1 & 2162.90 \\
\hline 478.7 & 419.1 & 304.7 & 269.2 & 232.9 & 193.6 & 130.1 & 109.4 & 104.2 & 1694.70 \\
\hline 512 & 458.8 & 317.6 & 277.9 & 262.7 & 232.1 & 173.7 & 148.7 & 137.9 & 6296.90 \\
\hline 441.6 & 388.5 & 291.2 & 248.5 & 216.9 & 179.4 & 104.6 & 97.3 & 92.1 & 7122.00 \\
\hline 466.2 & 408.3 & 304.7 & 240.7 & 226 & 203.6 & 138.8 & 100.8 & 89.5 & 7402.00 \\
\hline 429.9 & 356.1 & 245 & 208.8 & 203.6 & 166.8 & 104.7 & 96.8 & 84.3 & 7699.30 \\
\hline 553.5 & 451.1 & 302.9 & 234.7 & 203.6 & 159.5 & 100.8 & 74.8 & 70.5 & 6114.00 \\
\hline 636.6 & 509.7 & 318.5 & 233.4 & 213.9 & 186.3 & 131 & 112 & 99 & 2382.90 \\
\hline 455 & 389.8 & 287.4 & 237.3 & 217.4 & 171.2 & 118 & 118 & 115.4 & 1772.30 \\
\hline 225.2 & 256.3 & 218.7 & 186.3 & 168.1 & 158.6 & 144 & 124.5 & 114.6 & 6075.10 \\
\hline 424.8 & 386.7 & 280 & 232.1 & 203.1 & 174.2 & 132.7 & 114.1 & 99.9 & 3025.20 \\
\hline 421.3 & 391.1 & 282.2 & 239.9 & 220.8 & 193.6 & 116.3 & 99 & 97.3 & 2325.90 \\
\hline 417.8 & 380.2 & 270.1 & 228.6 & 220.8 & 177.6 & 123.2 & 98.2 & 92.5 & 2143.60 \\
\hline 515.7 & 459.8 & 334 & 213.9 & 188.9 & 140.5 & 74.8 & 70.5 & 69.6 & 6210.50 \\
\hline 496 & 391.7 & 259.7 & 216.5 & 194.9 & 173.3 & 142.2 & 111.1 & 90.4 & 6284.40 \\
\hline 501.1 & 462.9 & 360 & 319.3 & 299.5 & 266.2 & 212.2 & 188 & 79.2 & 5704.40 \\
\hline 565.6 & 544.8 & 419.6 & 235.5 & 201.8 & 147.4 & 97.3 & 92.1 & 89.5 & 5710.10 \\
\hline 440.4 & 397.1 & 282.7 & 222.6 & 186.3 & 158.6 & 116.3 & 116.3 & 96.4 & 5705.20 \\
\hline 479.2 & 411.5 & 248.1 & 201 & 175.9 & 148.3 & 105.9 & 98.2 & 96.4 & 2240.40 \\
\hline 534.2 & 478.5 & 339.7 & 228.6 & 203.6 & 175.1 & 125.8 & 126.3 & 110.3 & 1701.20 \\
\hline 417.8 & 371.2 & 237.3 & 208.8 & 191.5 & 176.8 & 131 & 123.2 & 111.1 & 5970.90 \\
\hline 513.5 & 444 & 264 & 194.1 & 154.3 & 127.5 & 83.1 & 71.4 & 70.9 & 5956.80 \\
\hline 491.8 & 404.5 & 252.8 & 208.8 & 180.2 & 137 & 84.3 & 83.5 & 84.3 & 5829.40 \\
\hline 445.8 & 405.3 & 261.9 & 184.6 & 173.3 & 137.9 & 81.7 & 82.6 & 83.5 & 5422.60 \\
\hline 507.5 & 437.4 & 233.4 & 159.5 & 132.7 & 108.5 & 65.3 & 67.1 & 69.6 & 5329.50 \\
\hline 615.7 & 527.6 & 304.7 & 211.3 & 174.2 & 160.4 & 101.6 & 84.3 & 75.7 & 2128.90 \\
\hline 592.4 & 540.2 & 291.7 & 207.9 & 180.2 & 163 & 99.9 & 85.2 & 79.2 & 1448.70 \\
\hline
\end{tabular}


other

\begin{tabular}{|c|c|c|c|c|c|c|c|c|c|}
\hline 440.3 & 395 & 293.4 & 230.8 & 178.5 & 153.5 & 107.3 & 105.1 & 105.1 & 5365.00 \\
\hline 390.2 & 356.5 & 249.8 & 194.1 & 174.7 & 148.3 & 94.3 & 90 & 92.1 & 6148.90 \\
\hline 609.2 & 539.7 & 354 & 202.3 & 160 & 137.9 & 90 & 72.7 & 67.5 & 5724.70 \\
\hline 651.6 & 593.4 & 372.1 & 191.9 & 168.6 & 139.2 & 87.8 & 71 & 66.7 & 5865.60 \\
\hline 592.4 & 544.1 & 375.1 & 200.6 & 164.3 & 122 & 72.7 & 72.2 & 71 & 5442.20 \\
\hline 696.6 & 641.8 & 410.1 & 220.4 & 189.3 & 129.7 & 73.6 & 70.5 & 68.4 & 5582.50 \\
\hline 681.7 & 611.8 & 373 & 186.8 & 150.5 & 113.3 & 70.1 & 69.2 & 67.5 & 5196.00 \\
\hline 500.9 & 447.5 & 236 & 169.1 & 153.9 & 137.5 & 83.1 & 64.9 & 64.9 & 1699.10 \\
\hline 468.5 & 465.6 & 315.1 & 172.9 & 154.4 & 132.3 & 79.6 & 66.7 & 65.8 & 1594.50 \\
\hline 548.8 & 483.8 & 337.6 & 194.5 & 149.6 & 116.8 & 70.6 & 68.4 & 67.5 & 6217.30 \\
\hline 511.7 & 431.9 & 227 & 165.2 & 146.6 & 115 & 72.7 & 69.2 & 71.8 & 5956.10 \\
\hline 609.2 & 590.4 & 424.8 & 255.9 & 231.3 & 189.3 & 106.9 & 90 & 80.1 & 5645.40 \\
\hline 486.5 & 437.1 & 310.8 & 188.1 & 153.1 & 121.6 & 68 & 67.5 & 65.4 & 5789.70 \\
\hline 490.8 & 458.7 & 255.9 & 158.7 & 160.4 & 125.8 & 63.9 & 59.6 & 58.8 & 5461.60 \\
\hline 536.5 & 497.7 & 349.9 & 186.6 & 155.5 & 120.1 & 69.1 & 66.5 & 67.4 & 1561.20 \\
\hline 708 & 640.8 & 404 & 245 & 194.9 & 162 & 135.2 & 99.8 & 73 & 1411.00 \\
\hline 560.7 & 496.5 & 333.6 & 194 & 160.3 & 146.5 & 101.6 & 77.4 & 71.8 & 5248.20 \\
\hline 621.6 & 540.2 & 346.5 & 226.8 & 200.9 & 152.5 & 85.1 & 83.9 & 80.8 & 5518.20 \\
\hline 727.2 & 608.4 & 395.8 & 209.2 & 168.9 & 136.1 & 81.3 & 71.7 & 69.2 & 5771.30 \\
\hline 705.3 & 610 & 382.8 & 190.5 & 156 & 115.4 & 70.4 & 71.3 & 70.4 & 5745.70 \\
\hline 691.2 & 683.1 & 395.8 & 216.5 & 193.1 & 155.1 & 108.5 & 86.9 & 69.6 & 5318.90 \\
\hline 741.8 & 690.1 & 439.8 & 235.1 & 193.5 & 156 & 98.6 & 80 & 63.5 & 1715.60 \\
\hline 633.7 & 574.7 & 373.3 & 209.6 & 166.4 & 124 & 65.3 & 62.7 & 61.4 & 1518.60 \\
\hline 633.7 & 625.6 & 408.7 & 194.9 & 160.3 & 111.5 & 62.7 & 62.7 & 63.5 & 5985.30 \\
\hline 711.5 & 678.3 & 452.4 & 339.2 & 299.4 & 247.2 & 190.5 & 186.7 & 86.5 & 5784.20 \\
\hline 794.6 & 689.7 & 464 & 239.8 & 223 & 207.8 & 149.9 & 86.9 & 66.1 & 5578.70 \\
\hline 847.7 & 763.9 & 476.1 & 211.7 & 165.5 & 142.6 & 86 & 77.8 & 73.9 & 5486.80 \\
\hline 828 & 783.2 & 488.2 & 235.9 & 184.5 & 152.1 & 101.6 & 91.6 & 91.2 & 5546.40 \\
\hline 821.9 & 765.8 & 491.7 & 213.5 & 168.1 & 123.6 & 80 & 80.8 & 72.6 & 2152.90 \\
\hline 820.8 & 745.7 & 458 & 226.8 & 187.6 & 156.8 & 121.9 & 123.6 & 122.3 & 1950.70 \\
\hline 675.4 & 589.6 & 360.4 & 226.8 & 195.7 & 136.1 & 87.7 & 82.1 & 82.5 & 5945.30 \\
\hline 704.5 & 637.6 & 340.5 & 232 & 200.5 & 149.9 & 90.3 & 84.3 & 80.8 & 5741.20 \\
\hline 609.9 & 565.4 & 336.2 & 208.7 & 173.3 & 140.4 & 96.4 & 91.6 & 76.9 & 5735.40 \\
\hline 738.7 & 719.3 & 412.2 & 269.6 & 238.9 & 182.8 & 120.2 & 96.4 & 72.2 & 5725.70 \\
\hline 772.8 & 656.7 & 377.6 & 246.3 & 222.5 & 173.3 & 103.7 & 80.8 & 73.9 & 5530.30 \\
\hline 836.4 & 799.3 & 458.8 & 317.2 & 289.9 & 253.2 & 176.7 & 93.4 & 71.3 & 2581.80 \\
\hline 831.6 & 760.4 & 452.4 & 282.1 & 229.9 & 192.7 & 132.7 & 99.4 & 94.6 & 1994.50 \\
\hline 815.2 & 739.6 & 427.3 & 264.8 & 207.4 & 160.3 & 99.4 & 76.1 & 73 & 5992.90 \\
\hline 688.7 & 686.2 & 356.9 & 246.3 & 209.6 & 153 & 98.1 & 79.6 & 75.6 & 6029.00 \\
\hline 841.3 & 727.6 & 384.9 & 225.6 & 169.8 & 127.5 & 89.5 & 80.8 & 77.4 & 5730.90 \\
\hline 743.1 & 708.8 & 401.8 & 254.9 & 233.7 & 178.9 & 140.4 & 84.7 & 71.3 & 5364.30 \\
\hline 805.5 & 755.5 & 426 & 255.8 & 200.5 & 161.2 & 97.7 & 73.5 & 68.7 & 5149.80 \\
\hline 844.5 & 747 & 474.4 & 300.7 & 242.8 & 178.4 & 134 & 102.9 & 79.1 & 1897.60 \\
\hline 772.4 & 743.5 & 445 & 286.9 & 229.9 & 168.9 & 115.8 & 90.8 & 81.3 & 1865.20 \\
\hline 772.3 & 707.1 & 436 & 219.1 & 182.4 & 148.7 & 109.3 & 90.8 & 77.8 & 5351.20 \\
\hline 807.7 & 730.8 & 479.2 & 267.4 & 207.4 & 166.8 & 109.3 & 86.5 & 81.3 & 5759.70 \\
\hline 808.9 & 714.9 & 431.2 & 231.6 & 196.2 & 161.6 & 112.8 & 89.9 & 83 & 6117.60 \\
\hline 799.3 & 762.3 & 405.7 & 247.6 & 194.5 & 154.7 & 118 & 92.5 & 75.6 & 6036.30 \\
\hline 794.4 & 698.9 & 380.2 & 222.1 & 176.3 & 149.9 & 113.2 & 92.5 & 83.4 & 2978.40 \\
\hline
\end{tabular}


other

\begin{tabular}{|c|c|c|c|c|c|c|c|c|c|}
\hline 749.5 & 704.3 & 449.8 & 200.5 & 132.7 & 125.3 & 131.4 & 105.9 & 102 & 1903.90 \\
\hline 243.6 & 250.6 & 235.9 & 232.4 & 218.6 & 215.1 & 203.9 & 200.4 & 198.7 & 5395.10 \\
\hline 378.4 & 377.6 & 373.2 & 379.3 & 366.3 & 360.3 & 369.8 & 361.2 & 345.6 & 5789.00 \\
\hline 269.6 & 273.9 & 264.4 & 265.2 & 259.2 & 249.7 & 241.1 & 237.6 & 226.4 & 5957.50 \\
\hline 262.7 & 266.1 & 389.7 & 391.4 & 382.8 & 371.5 & 366.3 & 368.1 & 356.8 & 6110.60 \\
\hline 337.8 & 343 & 340.4 & 323.1 & 305 & 298.9 & 188.4 & 165 & 165 & 5028.40 \\
\hline 194.4 & 210 & 203 & 198.7 & 190.9 & 182.3 & 340.4 & 322.3 & 312.8 & 2539.60 \\
\hline 190.9 & 193.5 & 178.8 & 183.2 & 184 & 177.1 & 168.5 & 168.5 & 159 & 1699.90 \\
\hline 171.1 & 170.2 & 189.2 & 180.6 & 182.3 & 168.5 & 291.2 & 300.7 & 294.6 & 5637.20 \\
\hline 169.9 & 179.3 & 173.4 & 166.5 & 176.8 & 179.4 & 156.1 & 150.8 & 137.1 & 5400.60 \\
\hline 139.7 & 144.9 & 138.8 & 139.7 & 132.8 & 132.8 & 131.9 & 125.9 & 121.6 & 5779.70 \\
\hline 224.7 & 225.6 & 223 & 215.2 & 216.1 & 206.6 & 198.8 & 189.3 & 184.6 & 5601.80 \\
\hline 193.6 & 195.3 & 205.7 & 205.7 & 202.2 & 194.5 & 178.5 & 180.6 & 178 & 4917.30 \\
\hline 304 & 282 & 274.6 & 268.1 & 270.3 & 263.8 & 256.8 & 240.9 & 170.8 & 2108.80 \\
\hline 143.5 & 153 & 152.5 & 152.1 & 153.9 & 147.3 & 148.7 & 136.6 & 134 & 1621.40 \\
\hline 282.6 & 275.2 & 275.7 & 270.5 & 273.1 & 250.2 & 245.4 & 239.4 & 237.2 & 5987.20 \\
\hline 227.3 & 229 & 230.3 & 240.3 & 303.3 & 379.4 & 368.1 & 373.7 & 360.4 & 6252.50 \\
\hline 118 & 110.7 & 101.6 & 99 & 106.7 & 110.7 & 102.4 & 101.2 & 102.4 & 6684.50 \\
\hline 84.3 & 84.7 & 84.3 & 82.5 & 86.9 & 89.1 & 90.3 & 90.3 & 90.8 & 5712.00 \\
\hline 211.7 & 231.2 & 224.7 & 217.3 & 217.3 & 152.1 & 139.6 & 139.2 & 139.6 & 5387.90 \\
\hline 152.1 & 154.3 & 149.1 & 134 & 140.9 & 141.3 & 131.4 & 130.5 & 133.5 & 5892.00 \\
\hline 153.4 & 143 & 124.9 & 117.1 & 115.4 & 117.1 & 116.2 & 116.2 & 115.4 & 5327.90 \\
\hline 96.4 & 108.5 & 115 & 104.1 & 98.1 & 99.8 & 99.8 & 97.2 & 98.1 & 6306.20 \\
\hline 131.4 & 125.7 & 126.6 & 130.1 & 130.5 & 132.7 & 128.3 & 118 & 101.2 & 6976.00 \\
\hline 80 & 78.7 & 73 & 75.6 & 87.7 & 88.6 & 95.5 & 89.1 & 84.3 & 6749.20 \\
\hline 132.7 & 132.7 & 115.4 & 99 & 105.9 & 108.1 & 108.5 & 104.1 & 100.7 & 2130.30 \\
\hline 86 & 91.2 & 86 & 86.5 & 92 & 92.9 & 92 & 93.4 & 93.8 & 1560.10 \\
\hline 188.8 & 185.4 & 180.2 & 191.9 & 200.9 & 189.7 & 168.1 & 168.1 & 158.6 & 7469.30 \\
\hline 125.7 & 130.1 & 122.3 & 111.9 & 116.7 & 122.3 & 128.3 & 125.7 & 125.7 & 8381.40 \\
\hline 162.5 & 147.3 & 149.1 & 148.2 & 151.7 & 155.1 & 122.8 & 110.2 & 102.4 & 6869.10 \\
\hline 113.2 & 111.9 & 110.2 & 111.9 & 116.2 & 111.9 & 99.8 & 99.4 & 99 & 6946.00 \\
\hline 416.1 & 385.6 & 265.7 & 217.3 & 217.3 & 196.2 & 172.4 & 152.5 & 145.6 & 6753.30 \\
\hline 110.2 & 106.3 & 104.1 & 106.7 & 114.5 & 115.4 & 115.4 & 111.5 & 116.2 & 2575.50 \\
\hline 375.4 & 319.3 & 238.1 & 130.9 & 130.5 & 140.4 & 128.3 & 127.5 & 122.3 & 1718.40 \\
\hline 113.6 & 96.4 & 115.4 & 147.3 & 145.2 & 135.2 & 126.6 & 118.8 & 114.5 & 5341.40 \\
\hline 412.2 & 370 & 239.8 & 173.7 & 156 & 134.4 & 112.8 & 109.3 & 99 & 6286.60 \\
\hline 104.1 & 99.8 & 105.9 & 99.8 & 92.9 & 82.5 & 73 & 70.4 & 69.2 & 6679.70 \\
\hline 436.1 & 396 & 284.3 & 202.6 & 177.6 & 160.3 & 112.8 & 107.6 & 94.6 & 6176.60 \\
\hline 119.7 & 119.3 & 100.7 & 80 & 74.8 & 80 & 81.7 & 82.5 & 81.7 & 8733.80 \\
\hline 382.8 & 349.5 & 263.1 & 176.7 & 159.4 & 147.8 & 123.2 & 118 & 104.1 & 8476.10 \\
\hline 111.5 & 104.1 & 74.8 & 74.8 & 82.5 & 90.8 & 90.3 & 95.5 & 89.5 & 5808.40 \\
\hline 582.6 & 548.7 & 428.6 & 333.1 & 267.4 & 272.6 & 239.8 & 153.4 & 118 & 6043.40 \\
\hline 264.8 & 262.3 & 280.9 & 270.9 & 249.3 & 235.5 & 226 & 139.6 & 131.8 & 1700.00 \\
\hline 443.9 & 380.4 & 275.2 & 210.4 & 183.6 & 164.6 & 100.7 & 118.8 & 115.4 & 1591.30 \\
\hline 123.2 & 109.3 & 111.1 & 111.1 & 118 & 118 & 111.1 & 100.7 & 95.1 & 8048.30 \\
\hline 425.5 & 399.4 & 249.3 & 161.2 & 149.9 & 143.9 & 96 & 92 & 89.5 & 7933.10 \\
\hline 178.4 & 174.1 & 125.8 & 98.3 & 82.3 & 84.3 & 85.1 & 85.1 & 74.8 & 6368.50 \\
\hline 691.8 & 622.9 & 332.9 & 255.3 & 227.7 & 240.7 & 227.3 & 214.7 & 177.6 & 6363.20 \\
\hline 170.3 & 149.1 & 117.1 & 104.1 & 96.4 & 99.8 & 103.3 & 98.1 & 96.4 & 7354.80 \\
\hline
\end{tabular}


other

\begin{tabular}{|c|c|c|c|c|c|c|c|c|c|}
\hline 563.9 & 500.4 & 266.6 & 179.3 & 150.8 & 124 & 98.1 & 98.1 & 94.6 & 2099.20 \\
\hline 121.4 & 152.5 & 165.5 & 118 & 108.5 & 100.7 & 91.2 & 89.5 & 89.5 & 1814.70 \\
\hline 370.3 & 338.3 & 232.9 & 188.8 & 149.5 & 124 & 80.8 & 80.8 & 80 & 8098.50 \\
\hline 104.1 & 102.4 & 94.6 & 86.9 & 86.9 & 85.1 & 83 & 82.5 & 84.3 & 7844.10 \\
\hline 665.9 & 597.1 & 330.5 & 196.6 & 149.9 & 132.7 & 107.6 & 108.5 & 111.9 & 7127.90 \\
\hline 105 & 106.7 & 105 & 103.3 & 106.7 & 104.1 & 105.9 & 107.6 & 105 & 6832.30 \\
\hline 819.4 & 791.7 & 431.4 & 292.1 & 226 & 207.4 & 171.5 & 172 & 158.6 & 2398.60 \\
\hline 139.6 & 144.8 & 123.2 & 111.1 & 109.3 & 109.3 & 111.9 & 105.5 & 98.1 & 1785.90 \\
\hline 751.1 & 742 & 392 & 229 & 191.4 & 175 & 124 & 121 & 119.7 & 7200.90 \\
\hline 111.9 & 111.1 & 110.7 & 114 & 115.8 & 111.1 & 113.6 & 112.8 & 111.9 & 8157.10 \\
\hline 608.8 & 594.6 & 272.8 & 175 & 147.3 & 125.7 & 99 & 92 & 87.7 & 7919.90 \\
\hline 96.4 & 96.4 & 84.7 & 80.8 & 82.5 & 85.1 & 88.6 & 89.5 & 89.5 & 7493.10 \\
\hline 658.8 & 636.7 & 328.1 & 223 & 190.1 & 156.4 & 118.4 & 115 & 110.2 & 8212.50 \\
\hline 135.2 & 129.2 & 111.1 & 116.7 & 110.2 & 107.6 & 106.7 & 107.6 & 112.8 & 1793.70 \\
\hline 797.5 & 762.1 & 375.2 & 264 & 235.1 & 197.5 & 164.2 & 182.8 & 185.4 & 1584.90 \\
\hline 111.1 & 117.6 & 124 & 126.2 & 132.7 & 132.3 & 123.2 & 123.2 & 119.7 & 7004.90 \\
\hline 908.6 & 804 & 413.4 & 283.5 & 252.8 & 217.8 & 172 & 162.5 & 156 & 7857.20 \\
\hline 172 & 156 & 141.8 & 137.4 & 113.2 & 111.9 & 115.8 & 115.8 & 110.7 & 7978.30 \\
\hline 934.6 & 918.3 & 494.2 & 365.5 & 329.2 & 253.2 & 187.6 & 166 & 181 & 7720.50 \\
\hline 449.3 & 448.5 & 283.5 & 247.2 & 191 & 163.8 & 150.4 & 133.1 & 143.5 & 8225.40 \\
\hline 815.8 & 776.5 & 378.4 & 240.3 & 207.4 & 172 & 128.8 & 114.5 & 110.7 & 1908.20 \\
\hline 188.4 & 210.4 & 219.5 & 216.1 & 208.3 & 179.3 & 160.8 & 143.5 & 118 & 2003.70 \\
\hline 717.4 & 673.8 & 349.4 & 204.8 & 178.4 & 154.7 & 107.6 & 95.1 & 90.3 & 9222.90 \\
\hline 133.5 & 130.1 & 127.9 & 127.5 & 101.2 & 93.8 & 86.5 & 86 & 83.9 & 9302.10 \\
\hline 715.7 & 671.7 & 324.7 & 185 & 190.5 & 155.1 & 112.8 & 115.4 & 117.6 & 8064.60 \\
\hline 121.4 & 130.1 & 129.2 & 112.4 & 94.6 & 95.1 & 95.1 & 92.9 & 89.1 & 8079.30 \\
\hline 867.1 & 768.6 & 372.7 & 216.9 & 185.4 & 181 & 155.6 & 146.1 & 130.9 & 8605.70 \\
\hline 122.3 & 124 & 126.6 & 118 & 115 & 111.1 & 106.7 & 115.4 & 117.1 & 2088.10 \\
\hline 768.4 & 696.3 & 354.6 & 208.3 & 167.2 & 159 & 118.4 & 99.8 & 99.4 & 1866.40 \\
\hline 156.8 & 163.8 & 175 & 161.6 & 128.3 & 82.1 & 84.3 & 80.4 & 80 & 7293.30 \\
\hline 825.2 & 776.8 & 421.1 & 267.9 & 226.4 & 180.2 & 134.8 & 122.8 & 137 & 7722.20 \\
\hline 203.5 & 195.3 & 145.6 & 126.2 & 121 & 115.4 & 103.7 & 103.7 & 98.1 & 7575.00 \\
\hline 404 & 377.2 & 310.2 & 268.3 & 226 & 194.9 & 184.1 & 182.8 & 141.3 & 10567.50 \\
\hline 173.7 & 155.1 & 144.8 & 149.1 & 156.8 & 155.6 & 149.1 & 106.7 & 98.1 & 10422.10 \\
\hline 498 & 448.3 & 243.5 & 162.9 & 132.3 & 119.7 & 98.1 & 93.8 & 86.9 & 2285.40 \\
\hline 111.9 & 124.9 & 94.2 & 80 & 85.1 & 85.1 & 82.5 & 79.1 & 78.7 & 2300.90 \\
\hline 496.6 & 472.8 & 263.8 & 175.9 & 137.8 & 123.2 & 94.2 & 86.9 & 82.5 & 11219.60 \\
\hline 142.2 & 140.4 & 132.7 & 121.4 & 120.6 & 107.2 & 104.1 & 89.5 & 87.7 & 10792.50 \\
\hline 162.9 & 160.3 & 101.6 & 94.2 & 98.1 & 111.9 & 137.8 & 134.8 & 106.7 & 8074.10 \\
\hline 110.2 & 108.5 & 93.4 & 101.6 & 108.5 & 106.7 & 104.1 & 105.5 & 108.5 & 8255.80 \\
\hline 458.9 & 390 & 241.4 & 173.3 & 166.4 & 136.6 & 120.6 & 122.3 & 118.8 & 8906.40 \\
\hline 105 & 109.8 & 106.7 & 108.5 & 112.8 & 115.4 & 113.2 & 111.1 & 105 & 3126.30 \\
\hline 420 & 359.2 & 238.1 & 187.1 & 181.9 & 159.4 & 121.4 & 106.7 & 100.7 & 2412.10 \\
\hline 93.8 & 90.3 & 90.3 & 96.4 & 99.4 & 84.3 & 81.7 & 80 & 70.4 & 10986.00 \\
\hline 86.9 & 88.6 & 90.3 & 77.8 & 62.7 & 64.4 & 65.3 & 62.7 & 63.5 & 11084.40 \\
\hline 86 & 90.8 & 75.6 & 76.5 & 81.7 & 80.4 & 82.5 & 80.8 & 82.5 & 10230.30 \\
\hline 79.1 & 68.7 & 71.3 & 63.5 & 72.2 & 69.6 & 68.7 & 61.4 & 62.2 & 10512.70 \\
\hline 68.7 & 60.9 & 60.9 & 63.5 & 62.7 & 85.1 & 90.8 & 80.8 & 64.4 & 10596.80 \\
\hline 89.5 & 86.9 & 64 & 58.4 & 65.3 & 66.1 & 66.1 & 66.1 & 65.7 & 2789.80 \\
\hline
\end{tabular}


other

\begin{tabular}{|c|c|c|c|c|c|c|c|c|c|}
\hline 72.2 & 75.6 & 60.9 & 60.9 & 69.2 & 81.7 & 95.5 & 91.6 & 80 & 2305.30 \\
\hline 105.5 & 83.4 & 75.6 & 77 & 85.1 & 87.7 & 86.5 & 86.9 & 86.9 & 9231.60 \\
\hline 78.2 & 80.4 & 79.1 & 92 & 100.3 & 104.1 & 92 & 87.3 & 86.9 & 10166.00 \\
\hline 105 & 113.2 & 124 & 100.7 & 98.1 & 90.8 & 92.9 & 93.8 & 92.9 & 8434.20 \\
\hline 92 & 80 & 75.6 & 75.2 & 79.1 & 92 & 106.7 & 102.9 & 86 & 9197.30 \\
\hline 82.5 & 82.5 & 80.4 & 86.9 & 95.5 & 96.4 & 86 & 86.5 & 86 & 9417.10 \\
\hline 90.3 & 90.3 & 84.7 & 71.3 & 76.5 & 88.6 & 101.6 & 110.2 & 104.1 & 3078.70 \\
\hline 156.8 & 157.3 & 153.4 & 134.4 & 118.8 & 116.2 & 102.4 & 111.9 & 85.1 & 2275.80 \\
\hline 89.5 & 86.9 & 91.2 & 87.3 & 80.8 & 91.2 & 90.3 & 80 & 79.6 & 9677.60 \\
\hline 124.9 & 130.9 & 145.6 & 147.8 & 135.2 & 120.6 & 97.2 & 68.7 & 67.5 & 10163.30 \\
\hline 83 & 92 & 86.9 & 80 & 72.2 & 83.9 & 99 & 92 & 72.2 & 9126.40 \\
\hline 109.8 & 107.6 & 93.8 & 78.2 & 73.9 & 76.5 & 72.6 & 73 & 72.2 & 9217.70 \\
\hline 65.3 & 67 & 64.4 & 64.4 & 68.7 & 81.3 & 95.5 & 87.7 & 71.3 & 9453.90 \\
\hline 67.9 & 67.9 & 67 & 68.7 & 71.3 & 77.4 & 78.7 & 78.2 & 79.1 & 2252.50 \\
\hline 66.1 & 61.8 & 66.1 & 73.9 & 67.9 & 82.5 & 102 & 97.2 & 72.2 & 1880.30 \\
\hline 106.7 & 92.9 & 75.6 & 73 & 73.9 & 77 & 79.1 & 78.2 & 77.4 & 9244.90 \\
\hline 87.7 & 88.6 & 88.6 & 71.8 & 76.5 & 91.2 & 102.4 & 100.7 & 77 & 10193.90 \\
\hline 77.8 & 62.7 & 61.4 & 63.5 & 65.3 & 65.3 & 69.6 & 71.3 & 73.9 & 8573.20 \\
\hline 71.3 & 70.4 & 69.6 & 72.2 & 73 & 75.6 & 89.5 & 89.9 & 75.6 & 7833.90 \\
\hline 81.7 & 76.5 & 93.8 & 92 & 98.1 & 111.1 & 124.5 & 119.7 & 99 & 8008.70 \\
\hline 67 & 67 & 67 & 68.7 & 76.5 & 83.4 & 106.7 & 99.4 & 80 & 3316.60 \\
\hline 140.4 & 118.4 & 111.1 & 103.3 & 93.8 & 85.1 & 85.1 & 86.9 & 86.5 & 2051.30 \\
\hline 77.4 & 88.6 & 84.3 & 72.2 & 73.9 & 85.6 & 96.4 & 100.7 & 80.8 & 10418.50 \\
\hline 85.1 & 81.7 & 80 & 72.2 & 73.5 & 72.2 & 77.4 & 76.5 & 75.6 & 10448.00 \\
\hline 66.5 & 64 & 67 & 68.7 & 68.7 & 71.8 & 77.4 & 72.2 & 73 & 8984.90 \\
\hline 83.9 & 87.7 & 91.6 & 106.3 & 99 & 83 & 77.4 & 77 & 74.4 & 6825.90 \\
\hline 91.6 & 99.8 & 110.7 & 133.5 & 122.8 & 111.1 & 102.9 & 98.6 & 86 & 6514.20 \\
\hline 93.4 & 95.5 & 99.8 & 94.2 & 84.3 & 80.4 & 85.1 & 89.1 & 88.6 & 5180.20 \\
\hline 72.2 & 86 & 85.6 & 83.4 & 91.2 & 89.5 & 92.9 & 92 & 70.9 & 1593.00 \\
\hline 102.9 & 94.2 & 93.8 & 90.8 & 77.8 & 71.3 & 76.1 & 72.6 & 71.3 & 6661.80 \\
\hline 118.4 & 118.8 & 117.6 & 115 & 95.1 & 91.2 & 94.2 & 89.9 & 89.9 & 6960.60 \\
\hline 167.7 & 137.8 & 132.3 & 117.6 & 98.6 & 104.1 & 104.6 & 99.4 & 98.6 & 5385.30 \\
\hline 104.6 & 114.5 & 121.9 & 118.4 & 108.1 & 95.5 & 105.5 & 94.2 & 88.2 & 5619.20 \\
\hline 124.5 & 122.8 & 127.5 & 131.4 & 106.3 & 102 & 111.5 & 94.2 & 92.9 & 6619.50 \\
\hline 110.7 & 113.2 & 111.9 & 105.5 & 86.5 & 83.9 & 89.9 & 86.9 & 78.7 & 1729.90 \\
\hline 156.4 & 156.8 & 157.3 & 154.7 & 146.1 & 137.4 & 143 & 136.6 & 139.2 & 1478.50 \\
\hline 94.6 & 99.4 & 104.6 & 107.6 & 93.4 & 90.8 & 97.7 & 90.3 & 81.3 & 5561.50 \\
\hline 108.5 & 110.7 & 99.8 & 100.3 & 79.6 & 87.3 & 86.9 & 81.3 & 79.1 & 5478.80 \\
\hline 86 & 83.9 & 84.3 & 78.2 & 69.2 & 76.5 & 86.5 & 79.1 & 78.7 & 6197.90 \\
\hline 204 & 219.9 & 229 & 208.7 & 186.7 & 125.3 & 103.3 & 82.1 & 77 & 6705.60 \\
\hline 96.4 & 99.4 & 101.2 & 101.6 & 83.9 & 89.1 & 89.1 & 82.5 & 79.6 & 6470.30 \\
\hline 304 & 322.5 & 299.2 & 307.8 & 292.3 & 300.4 & 272.3 & 210.6 & 75.6 & 1609.90 \\
\hline 60.9 & 60.1 & 58.8 & 60.9 & 55.8 & 59.2 & 65.3 & 66.1 & 64.9 & 1699.70 \\
\hline 89.5 & 79.2 & 64.9 & 61 & 61.9 & 65.3 & 70.9 & 71.4 & 70 & 5631.40 \\
\hline 59.7 & 61.4 & 59.3 & 61.4 & 62.7 & 61.4 & 63.6 & 64 & 65.3 & 5774.40 \\
\hline 60.1 & 62.7 & 60.5 & 60.1 & 63.6 & 65.7 & 70.5 & 69.2 & 70.5 & 5370.20 \\
\hline 79.6 & 86.1 & 87.3 & 82.6 & 79.6 & 76.6 & 78.7 & 74.8 & 70.9 & 6638.50 \\
\hline 147.4 & 134.4 & 104.6 & 96.4 & 98.2 & 89.1 & 85.2 & 83.5 & 84.3 & 2428.60 \\
\hline 67.1 & 76.6 & 64.5 & 58 & 63.6 & 60.5 & 60.1 & 61.4 & 61 & 1692.20 \\
\hline
\end{tabular}


other

\begin{tabular}{|c|c|c|c|c|c|c|c|c|c|}
\hline 87.3 & 90.8 & 86.1 & 86.5 & 88.7 & 85.6 & 83.5 & 82.1 & 77.8 & 5550.80 \\
\hline 51.9 & 51.9 & 48.4 & 53.6 & 61 & 61.4 & 81.3 & 80.9 & 80.4 & 5991.60 \\
\hline 91.6 & 92.5 & 84.3 & 98.6 & 96.8 & 86.5 & 80.9 & 71.8 & 64 & 5961.60 \\
\hline 72.6 & 60.1 & 55.4 & 59.7 & 64.9 & 64 & 61.9 & 60.5 & 62.3 & 5929.30 \\
\hline 97.7 & 99.4 & 106.8 & 108.1 & 111.5 & 107.7 & 115.8 & 101.2 & 92.1 & 6452.10 \\
\hline 59.7 & 59.7 & 59.3 & 62.3 & 69.2 & 67.1 & 68.3 & 64.5 & 64.9 & 1925.60 \\
\hline 115 & 118.4 & 123.2 & 96.8 & 102.5 & 102.9 & 104.6 & 102.5 & 102.9 & 1474.30 \\
\hline 98.6 & 104.6 & 100.8 & 94.7 & 98.6 & 97.7 & 99.9 & 98.6 & 100.3 & 5939.80 \\
\hline 78.3 & 81.3 & 77 & 75.2 & 80.9 & 79.6 & 78.3 & 80.4 & 80 & 5653.20 \\
\hline 71.8 & 74.8 & 78.3 & 77 & 80.9 & 78.7 & 82.1 & 80.9 & 82.6 & 5884.00 \\
\hline 84.7 & 91.6 & 81.3 & 68.8 & 69.2 & 71.8 & 76.6 & 76.1 & 79.6 & 5675.80 \\
\hline 87.8 & 85.6 & 86.5 & 83.5 & 84.7 & 83.9 & 81.3 & 82.6 & 83.9 & 5618.90 \\
\hline 142.2 & 130.5 & 134.8 & 131.4 & 115 & 118.4 & 118.4 & 115 & 119.3 & 6554.30 \\
\hline 98.6 & 95.1 & 97.7 & 99.4 & 102.9 & 105.5 & 106.3 & 107.2 & 107.2 & 6870.30 \\
\hline 135.3 & 126.7 & 124.9 & 94.7 & 66.2 & 65.3 & 64.5 & 63.6 & 64.5 & 2097.90 \\
\hline 84.3 & 78.7 & 61.9 & 65.3 & 67.9 & 67.1 & 63.6 & 62.7 & 64 & 1469.60 \\
\hline 93.8 & 95.6 & 106.8 & 115 & 122 & 125.3 & 122.4 & 130.1 & 131.5 & 5307.30 \\
\hline 121.5 & 123.2 & 113.7 & 109.4 & 106.8 & 104.2 & 102.5 & 105.1 & 103.3 & 5717.40 \\
\hline 171.1 & 166.9 & 154.3 & 125.8 & 84.3 & 79.2 & 74.8 & 62.7 & 65.3 & 6283.40 \\
\hline 76.6 & 73.5 & 77 & 86.5 & 87.4 & 69.2 & 70.1 & 67.1 & 63.6 & 6948.50 \\
\hline 131.9 & 137.5 & 152.1 & 151.7 & 143.6 & 124.9 & 115.8 & 107.7 & 93 & 7250.30 \\
\hline 99.9 & 94.7 & 95.6 & 76.6 & 71.4 & 77.4 & 82.6 & 78.3 & 74.4 & 2256.50 \\
\hline 61.9 & 60.1 & 61.9 & 59.3 & 65.3 & 65.3 & 63.6 & 65.7 & 65.8 & 1536.10 \\
\hline 71 & 72.6 & 66.2 & 66.2 & 68.8 & 66.2 & 65.3 & 64.5 & 65.3 & 6279.20 \\
\hline 72.7 & 61.5 & 60.6 & 59.7 & 61.5 & 65.8 & 67.5 & 66.7 & 66.7 & 6022.90 \\
\hline 60.6 & 59.7 & 60.6 & 59.7 & 59.3 & 67.5 & 59.7 & 60.6 & 60.2 & 5479.10 \\
\hline 74.3 & 76.9 & 63.9 & 63.1 & 64.8 & 66.5 & 70 & 69.1 & 68.3 & 5608.60 \\
\hline 70.8 & 70.8 & 74.3 & 70 & 71.7 & 70.8 & 76.9 & 76.9 & 76.9 & 6004.70 \\
\hline 68.2 & 62.7 & 63.5 & 63.5 & 65.3 & 61.8 & 66.6 & 67.9 & 67 & 6038.40 \\
\hline 65.3 & 64.9 & 67.9 & 70.4 & 66.1 & 64.4 & 66.1 & 66.1 & 67 & 2679.00 \\
\hline 213 & 203.1 & 200.9 & 194.5 & 183.6 & 74.4 & 64 & 67 & 63.1 & 2363.60 \\
\hline 60.9 & 61.8 & 67.9 & 67 & 62.3 & 60.9 & 64.4 & 64.4 & 65.3 & 6144.90 \\
\hline 66.1 & 63.5 & 64 & 66.1 & 65.3 & 67 & 74.8 & 75.2 & 75.6 & 5649.60 \\
\hline 67 & 64.4 & 67.9 & 65.3 & 66.6 & 69.6 & 77.4 & 77.4 & 74.4 & 5431.90 \\
\hline 109.3 & 93.4 & 95.5 & 97.7 & 97.2 & 91.6 & 89.9 & 85.1 & 76.1 & 5748.20 \\
\hline 73.9 & 76.1 & 73.9 & 75.2 & 76.5 & 73.5 & 73.5 & 73.9 & 67.5 & 5560.30 \\
\hline 238.6 & 247.2 & 132.8 & 76.5 & 73.9 & 80 & 81.3 & 80.8 & 78.2 & 2022.90 \\
\hline 63.1 & 67.9 & 62.7 & 63.5 & 69.6 & 61.8 & 65.3 & 69.6 & 66.1 & 1843.90 \\
\hline 125.7 & 112.4 & 104.1 & 71.8 & 69.6 & 73.5 & 73.5 & 73.9 & 63.1 & 5932.50 \\
\hline 75.2 & 73 & 75.2 & 75.6 & 73.5 & 70 & 72.2 & 70.9 & 71.3 & 6321.10 \\
\hline 243.6 & 247.1 & 244.5 & 242.8 & 217.7 & 216.9 & 203.9 & 180.6 & 180.6 & 6146.70 \\
\hline 190.1 & 189.2 & 180.6 & 171.9 & 172.8 & 175.4 & 169.3 & 165.9 & 165 & 5683.30 \\
\hline 203.9 & 199.6 & 198.7 & 212.5 & 190.9 & 183.2 & 172.8 & 148.6 & 133.1 & 5414.00 \\
\hline 199.6 & 210 & 210.8 & 230.7 & 197.9 & 195.3 & 180.6 & 149.5 & 147.7 & 1941.50 \\
\hline 229 & 233.3 & 235 & 242.8 & 213.4 & 214.3 & 190.1 & 166.8 & 164.2 & 1775.40 \\
\hline 332.6 & 295.5 & 305 & 265.2 & 201.3 & 197 & 188.4 & 174.5 & 158.1 & 6168.30 \\
\hline 202.2 & 205.6 & 199.6 & 181.4 & 164.2 & 165 & 155.5 & 153.8 & 154.7 & 6413.20 \\
\hline 329.2 & 348.2 & 341.3 & 326.6 & 208.2 & 210.8 & 196.1 & 181.4 & 165 & 6568.00 \\
\hline 438.9 & 449.3 & 449.3 & 423.4 & 268.7 & 259.2 & 248 & 218.6 & 213.4 & 6760.60 \\
\hline
\end{tabular}


other

\begin{tabular}{|c|c|c|c|c|c|c|c|c|c|}
\hline 434.6 & 451 & 454.5 & 404.4 & 197.9 & 193.5 & 184.9 & 171.1 & 156.4 & 6481.90 \\
\hline 434.6 & 443.2 & 444.1 & 389.7 & 203.9 & 203.9 & 197 & 179.7 & 162.4 & 3210.40 \\
\hline 197 & 196.1 & 194.4 & 208.2 & 171.1 & 171.1 & 161.6 & 159 & 157.2 & 2462.70 \\
\hline 423.4 & 401.8 & 396.6 & 330 & 208.2 & 224.6 & 210 & 163.3 & 156.4 & 6880.20 \\
\hline 352.5 & 337.8 & 357.7 & 335.2 & 208.2 & 218.6 & 206.5 & 165.9 & 163.3 & 7246.60 \\
\hline 387.1 & 391.4 & 396.6 & 371.5 & 227.2 & 231.6 & 201.3 & 169.3 & 166.8 & 6677.10 \\
\hline 391.4 & 389.7 & 392.3 & 400.9 & 367.2 & 368.9 & 357.7 & 320.5 & 299.8 & 6654.30 \\
\hline 201.3 & 208.2 & 193.5 & 190.9 & 189.2 & 172.8 & 177.1 & 171.1 & 166.8 & 5765.70 \\
\hline 350.8 & 346.5 & 366.3 & 353.4 & 227.2 & 248.8 & 235 & 216 & 190.1 & 2214.70 \\
\hline 337.8 & 325.7 & 338.7 & 317.1 & 180.6 & 188.4 & 183.2 & 142.6 & 140 & 1524.20 \\
\hline 322.3 & 321.4 & 324 & 305.9 & 159.8 & 179.7 & 160.7 & 150.3 & 147.7 & 6354.80 \\
\hline 130.5 & 133.1 & 135.6 & 133.9 & 133.1 & 134.8 & 135.6 & 135.6 & 133.1 & 7063.30 \\
\hline 140.8 & 139.1 & 144.3 & 149.5 & 156.4 & 148.6 & 147.7 & 144.3 & 140 & 6574.00 \\
\hline 352.5 & 344.7 & 342.1 & 328.3 & 193.5 & 193.5 & 162.4 & 148.6 & 121 & 3904.60 \\
\hline 351.6 & 349.1 & 350.8 & 342.1 & 174.5 & 169.3 & 164.2 & 152.9 & 127.9 & 6555.10 \\
\hline 354.2 & 358.6 & 356 & 322.3 & 172.8 & 180.6 & 173.7 & 159.8 & 136.5 & 2716.80 \\
\hline 345.6 & 321.4 & 325.7 & 307.6 & 163.3 & 157.2 & 149.5 & 130.5 & 127 & 2573.60 \\
\hline 146.9 & 146 & 146 & 145.2 & 145.2 & 142.6 & 132.2 & 129.6 & 127 & 6456.00 \\
\hline 328.3 & 322.3 & 321.4 & 298.1 & 135.6 & 141.7 & 136.5 & 139.1 & 121.8 & 6031.00 \\
\hline 317.1 & 315.4 & 314.5 & 267.8 & 126.1 & 126.1 & 121.8 & 120.1 & 118.4 & 6209.90 \\
\hline 186.6 & 322.3 & 328.3 & 264.4 & 150.3 & 155.5 & 137.4 & 133.9 & 127.9 & 5971.80 \\
\hline 187.5 & 191.8 & 184.9 & 176.3 & 151.2 & 143.4 & 129.6 & 125.3 & 116.6 & 6332.70 \\
\hline 150.3 & 158.1 & 158.1 & 159 & 159 & 152.1 & 142.6 & 137.4 & 132.2 & 2469.70 \\
\hline 375 & 368.1 & 372.4 & 348.2 & 151.2 & 176.3 & 182.3 & 146.9 & 133.9 & 1580.90 \\
\hline 300.7 & 313.6 & 290.3 & 170.2 & 144.3 & 152.9 & 143.4 & 129.6 & 126.1 & 6886.90 \\
\hline 352.5 & 345.6 & 339.6 & 198.7 & 190.1 & 185.8 & 159 & 151.2 & 148.6 & 6140.50 \\
\hline 343.9 & 340.4 & 319.7 & 202.2 & 179.7 & 191.8 & 168.5 & 161.6 & 158.1 & 6086.80 \\
\hline 188.4 & 191.8 & 177.1 & 172.8 & 171.9 & 170.2 & 164.2 & 160.7 & 156.4 & 6225.70 \\
\hline 346.5 & 341.3 & 330.9 & 191.8 & 19 & 167.6 & 179.7 & 165.9 & 149.5 & 2693.70 \\
\hline 368.1 & 363.7 & 359.4 & 210 & 178.8 & 175.4 & 165.9 & 178 & 173.7 & 1867.00 \\
\hline 386.2 & 378.4 & 352.5 & 211.7 & 203.9 & 200.4 & 185.8 & 175.4 & 176.3 & 5826.70 \\
\hline 395.7 & 400 & 398.3 & 244.5 & 196.1 & 202.2 & 189.2 & 174.5 & 168.5 & 6152.40 \\
\hline 227.2 & 222.9 & 209.1 & 186.6 & 183.2 & 180.6 & 178 & 166.8 & 165.9 & 5628.80 \\
\hline 431.1 & 422.5 & 233.3 & 223.8 & 214.3 & 210 & 189.2 & 176.3 & 172.8 & 5778.30 \\
\hline 392.3 & 358.6 & 197.9 & 204.8 & 207.4 & 199.6 & 162.4 & 161.6 & 156.4 & 5721.30 \\
\hline 405.2 & 400.9 & 417.3 & 421.6 & 268.7 & 203.9 & 194.4 & 172.8 & 167.6 & 1547.90 \\
\hline 504.6 & 504.6 & 343.9 & 325.7 & 331.8 & 203.9 & 170.2 & 166.8 & 159.8 & 1543.10 \\
\hline 544.3 & 517.5 & 382.8 & 373.2 & 358.6 & 299.8 & 203 & 199.6 & 197.9 & 6708.10 \\
\hline 573.7 & 528.8 & 260.1 & 244.5 & 229 & 223.8 & 216 & 215.1 & 203 & 7066.80 \\
\hline 626.4 & 602.2 & 308.4 & 292 & 294.6 & 292.9 & 285.1 & 265.2 & 254.9 & 5794.30 \\
\hline 593.6 & 543.5 & 385.3 & 349.9 & 267 & 245.4 & 238.5 & 213.4 & 214.3 & 5746.20 \\
\hline 582.3 & 542.6 & 265.2 & 236.7 & 231.6 & 234.1 & 226.4 & 203 & 190.1 & 7369.40 \\
\hline 475.2 & 456.2 & 321.4 & 281.7 & 275.6 & 279.1 & 247.1 & 234.1 & 223.8 & 7104.90 \\
\hline 688.6 & 642.8 & 358.6 & 338.7 & 326.6 & 333.5 & 320.5 & 295.5 & 286.8 & 7201.40 \\
\hline 776.7 & 740.4 & 598.8 & 497.7 & 489.9 & 470 & 462.2 & 450.1 & 432 & 7967.80 \\
\hline 829.4 & 814.8 & 642.8 & 547.8 & 505.4 & 472.6 & 451 & 450.1 & 438 & 7114.00 \\
\hline 829.4 & 788.8 & 592.7 & 568.5 & 514.9 & 483 & 436.3 & 427.7 & 413 & 1598.40 \\
\hline 851.9 & 807 & 614.3 & 526.2 & 465.7 & 440.6 & 416.4 & 405.2 & 388.8 & 1447.70 \\
\hline 843.3 & 809.6 & 609.1 & 532.2 & 502.8 & 480.4 & 454.5 & 432 & 399.2 & 5629.70 \\
\hline
\end{tabular}


other

\begin{tabular}{|c|c|c|c|c|c|c|c|c|c|}
\hline 851 & 810.4 & 636.8 & 547.8 & 520.1 & 494.2 & 461.4 & 442.4 & 416.4 & 5553.30 \\
\hline 871.8 & 811.3 & 591.8 & 515.8 & 487.3 & 465.7 & 444.1 & 416.4 & 406.1 & 6112.50 \\
\hline 845.9 & 816.5 & 616.9 & 540.9 & 500.3 & 480.4 & 461.4 & 445.8 & 431.1 & 5627.60 \\
\hline 889.9 & 831.2 & 632.4 & 565.1 & 520.1 & 497.7 & 469.2 & 447.6 & 430.3 & 7532.80 \\
\hline 413 & 396.6 & 407.8 & 427.7 & 438 & 432 & 417.3 & 394.8 & 382.8 & 5572.90 \\
\hline 545.5 & 556.9 & 305.1 & 252 & 251 & 224.7 & 214.3 & 209.2 & 198.8 & 5520.90 \\
\hline 212.6 & 161.2 & 141.3 & 116.2 & 115.4 & 115.4 & 115 & 108.5 & 108.5 & 5750.90 \\
\hline 97.7 & 102.8 & 94.2 & 92.9 & 102.4 & 102.4 & 105 & 104.6 & 105.9 & 1645.20 \\
\hline 99.8 & 101.6 & 99.4 & 99.8 & 102.4 & 105 & 105 & 106.7 & 106.3 & 1666.50 \\
\hline 225.1 & 226.8 & 205.2 & 164.6 & 137 & 141.3 & 130.9 & 120.6 & 116.2 & 8460.40 \\
\hline 125.7 & 148.2 & 141.3 & 123.6 & 108.5 & 99.8 & 88.6 & 87.7 & 88.2 & 7044.60 \\
\hline 89.1 & 76.5 & 80 & 80 & 84.3 & 88.2 & 88.6 & 87.7 & 86.9 & 7082.80 \\
\hline 77.4 & 78.2 & 77.4 & 76.5 & 87.3 & 92 & 104.1 & 132.7 & 96.4 & 8298.40 \\
\hline 140.4 & 151.3 & 86.9 & 90.3 & 111.1 & 120.6 & 102.9 & 121.4 & 99 & 8110.90 \\
\hline 118.8 & 139.2 & 128.3 & 142.2 & 147.3 & 162.9 & 146.1 & 141.3 & 137.8 & 2108.70 \\
\hline 163.8 & 154.3 & 93.8 & 79.6 & 118 & 106.7 & 81.7 & 92 & 79.1 & 1502.10 \\
\hline 157.3 & 153.4 & 93.4 & 69.6 & 73.5 & 76.5 & 120.2 & 111.9 & 80.4 & 7667.70 \\
\hline & & & & & & & & & 8248.90 \\
\hline 69.6 & 68.3 & 117.1 & 125.3 & 91.6 & 73.9 & 78.7 & 86 & 77 & 7475.90 \\
\hline 127.5 & 136.1 & 137.8 & 154.3 & 119.7 & 122.8 & 115.4 & 111.9 & 138.7 & 7171.80 \\
\hline 111.9 & 96.4 & 80.8 & 72.2 & 103.3 & 112.4 & 78.2 & 77.4 & 78.2 & 8215.40 \\
\hline 410 & 401.4 & 306.3 & 219.5 & 197.5 & 173.3 & 145.6 & 125.7 & 111.9 & 3406.00 \\
\hline 400.5 & 364.7 & 254.5 & 176.7 & 101.6 & 123.2 & 104.1 & 101.2 & 101.6 & 1484.80 \\
\hline 92.9 & 87.3 & 87.7 & 86 & 93.8 & 105 & 92.9 & 92 & 92.9 & 9021.60 \\
\hline 167.2 & 149.1 & 91.2 & 85.1 & 88.6 & 99.8 & 92.9 & 95.5 & 93.4 & 9277.40 \\
\hline 149.1 & 130.9 & 93.4 & 91.2 & 94.6 & 100.7 & 92.9 & 92.9 & 92 & 9692.40 \\
\hline 203.5 & 207 & 117.1 & 91.2 & 93.8 & 103.3 & 96.4 & 96.8 & 91.2 & 9386.70 \\
\hline 181 & 171.5 & 115.4 & 92.9 & 92.9 & 90.8 & 74.8 & 73 & 73.9 & 9299.10 \\
\hline 744.2 & 718.4 & 384.5 & 238.9 & 193.6 & 178.4 & 156 & 144.8 & 136.1 & 1918.90 \\
\hline 122.3 & 124.9 & 104.1 & 103.7 & 117.1 & 112.8 & 116.2 & 108.5 & 102.4 & 1669.20 \\
\hline 668 & 620.4 & 354 & 243.2 & 191.4 & 167.7 & 122.3 & 120.6 & 115.4 & 6490.20 \\
\hline 829.4 & 793.4 & 361.4 & 227.7 & 202.2 & 162.9 & 132.3 & 131.8 & 127.1 & 7609.90 \\
\hline 851 & 803.9 & 362.3 & 230.8 & 168.9 & 138.3 & 119.3 & 118.8 & \begin{tabular}{|l|}
109.8 \\
\end{tabular} & 7183.60 \\
\hline 83.4 & 83.4 & 82.5 & 81.7 & 86 & 94.6 & 94.6 & 105 & 86.9 & 8496.80 \\
\hline 573.5 & 556.9 & 403.9 & 331.4 & 204 & 178.4 & 156.4 & 152.5 & 126.2 & 8478.70 \\
\hline 466.6 & 467.3 & 341.5 & 264.8 & 178.9 & 159 & 143 & 118.8 & 105.5 & 2411.90 \\
\hline 457.8 & 490.7 & 364.4 & 303.7 & 196.2 & 168.5 & 156.4 & 154.3 & 115 & 1658.30 \\
\hline 519.3 & 517.1 & 368.7 & 323.2 & 203.1 & 178.9 & 159.9 & 153.4 & 127.9 & 10293.80 \\
\hline 102.4 & 113.6 & 111.1 & 117.6 & 113.6 & 111.1 & 107.2 & 111.9 & 95.5 & 9802.50 \\
\hline 334.4 & 328.8 & 239.8 & 229 & 204 & 177.6 & 160.8 & 153 & 126.6 & 9689.90 \\
\hline 280.4 & 306.8 & 219.1 & 208.3 & 172.4 & 157.3 & 139.2 & 133.5 & 109.8 & 8980.10 \\
\hline 438.5 & 432.1 & 354.7 & 245.4 & 194.5 & 183.2 & 172.4 & 162.5 & 134 & 9238.00 \\
\hline 285.6 & 306.8 & 279.5 & 247.2 & 203.1 & 188.4 & 183.6 & 166.8 & 141.8 & 3279.20 \\
\hline 223.4 & 233.3 & 233.3 & 214.3 & 212.1 & 201.4 & 107.6 & 44.9 & 45.8 & 1627.60 \\
\hline 179.7 & 190.1 & 165.9 & 172 & 287 & 251.1 & 215.2 & 200.5 & 153.4 & 8735.20 \\
\hline 276.9 & 281.7 & 250.6 & 239.4 & 231.6 & 226 & 199.6 & 183.2 & 152.1 & 9209.30 \\
\hline 261.4 & 267.9 & 254.9 & 267.9 & 253.2 & 232 & 205.7 & 202.2 & 165.1 & 9498.10 \\
\hline 254.9 & 256.7 & 232 & 244.6 & 230.8 & 215.2 & 179.8 & 160.8 & 137.4 & 9018.50 \\
\hline 181.5 & 185.8 & 198.8 & 212.1 & 181.5 & 172.9 & 159.9 & 153.9 & 149.5 & 8608.80 \\
\hline
\end{tabular}


other

\begin{tabular}{|c|c|c|c|c|c|c|c|c|c|}
\hline 275.7 & 280.9 & 295.2 & 288.6 & 251 & 238.5 & 213.5 & 185.8 & 151.3 & 2168.00 \\
\hline 328.5 & 330.6 & 283.4 & 249.3 & 229.9 & 197.1 & 181.5 & 165.1 & 130.5 & 1567.80 \\
\hline 291.8 & 307.3 & 293.3 & 292.1 & 241.1 & 211.7 & 191.9 & 163.4 & 132.3 & 9249.20 \\
\hline 296.9 & 297.2 & 274.7 & 267 & 223.8 & 206.6 & 172.9 & 144.4 & 120.2 & 9146.00 \\
\hline 159 & 172.4 & 196.2 & 192.7 & 177.2 & 155.6 & 135.2 & 122.8 & 120.2 & 9199.10 \\
\hline 354.4 & 379.5 & 379.3 & 360.4 & 299.9 & 271.4 & 236.8 & 230.8 & 181.5 & 8957.40 \\
\hline 371.1 & 349 & 311.4 & 283.5 & 221.2 & 216.9 & 189.3 & 175.5 & 143.5 & 8947.10 \\
\hline 472.5 & 467.7 & 429.8 & 336.2 & 257.5 & 223 & 200.5 & 197.1 & 155.6 & 5465.90 \\
\hline 443.8 & 446 & 410.8 & 388.9 & 350.8 & 312 & 289.5 & 264.4 & 248 & 2898.70 \\
\hline 140 & 141.8 & 160.3 & 154.7 & 158.2 & 141.8 & 121 & 118.4 & 106.3 & 2030.90 \\
\hline 366.2 & 372.1 & 350.3 & 258.4 & 185.8 & 182.8 & 159.9 & 130.5 & 121.9 & 2098.90 \\
\hline 452.7 & 449.2 & 406.1 & 271.8 & 156.4 & 158.2 & 134 & 118.4 & 114.1 & 2815.90 \\
\hline 432.4 & 453.9 & 419.8 & 297.3 & 154.7 & 154.7 & 147.8 & 125.3 & 110.7 & 2818.80 \\
\hline 447.7 & 459.7 & 426.3 & 304.2 & 148.7 & 140 & 116.7 & 107.2 & 111.9 & 2932.30 \\
\hline 146.1 & 155.1 & 168.5 & 164.2 & 145.2 & 146.1 & 128.8 & 120.2 & 112.8 & 2813.50 \\
\hline 365.9 & 373.2 & 352.5 & 247.2 & 178 & 153.4 & 135.7 & 126.2 & 115.8 & 2243.40 \\
\hline 392.3 & 407 & 375 & 210.9 & 163.4 & 154.7 & 127.9 & 110.7 & 115 & 1958.00 \\
\hline 436.2 & 441.5 & 304.1 & 248 & 211.7 & 185 & 143 & 136.6 & 132.3 & 8735.70 \\
\hline 406.5 & 475.6 & 447.9 & 343.9 & 225.1 & 210 & 171.1 & 148.7 & 124.5 & 3426.80 \\
\hline 81.3 & 79.6 & 78.2 & 76.1 & 69.2 & 69.6 & 68.3 & 70 & 69.6 & 2372.60 \\
\hline 272.2 & 266.6 & 254.1 & 164.6 & 106.3 & 102 & 75.2 & 75.6 & 70.9 & 2562.40 \\
\hline 286 & 280 & 233.7 & 166 & 101.6 & 90.8 & 68.3 & 68.3 & 67.9 & 7516.80 \\
\hline 185.4 & 187.6 & 180.6 & 142.6 & 137.8 & 114.1 & 88.2 & 84.7 & 77 & 9305.80 \\
\hline 348.3 & 359.5 & 324.4 & 316.3 & 299 & 292.1 & 228.2 & 221.2 & 196.2 & 9430.50 \\
\hline 200.9 & 203.1 & 210 & 223 & 231.6 & 206.6 & 189.3 & 178.9 & 179.8 & 2128.10 \\
\hline 328.4 & 322.3 & 305.1 & 172 & 161.6 & 157.3 & 129.7 & 96.8 & 77.4 & 6935.80 \\
\hline 311.1 & 295.6 & 194.5 & 178 & 154.3 & 144.4 & 121 & 114.1 & 91.6 & 6401.80 \\
\hline 310.6 & 308.5 & 299 & 279.1 & 184.1 & 163.4 & 124 & 108.1 & 109.8 & 6293.00 \\
\hline 331 & 351.2 & 330.1 & 320.6 & 344.8 & 312 & 267 & 254.9 & 215.6 & 6562.60 \\
\hline 143.5 & 159.4 & 174.6 & 170.3 & 157.3 & 139.6 & 121 & 115.8 & 113.2 & 3848.20 \\
\hline 416 & 363.7 & 183.2 & 158.6 & 166.8 & 152.1 & 101.6 & 95.1 & 89.1 & 2949.40 \\
\hline 394.5 & 381.9 & 223.7 & 202.2 & 197.1 & 172 & 127.9 & 117.1 & 104.6 & 2352.30 \\
\hline 395.8 & 395.6 & 240.7 & 220.8 & 180.6 & 164.2 & 139.2 & 119.3 & 108.9 & 4820.30 \\
\hline 480.4 & 570.7 & 265.6 & 228.2 & 192.7 & 196.2 & 146.9 & 123.6 & 122.3 & 4866.40 \\
\hline 144.4 & 152.1 & 165.1 & 187.1 & 185 & 157.3 & 139.2 & 132.3 & 130.1 & 5819.60 \\
\hline 525.8 & 517.9 & 276.9 & 233.3 & 238.5 & 216.1 & 168.5 & 148.7 & 141.3 & 5525.50 \\
\hline 629.6 & 568.7 & 267.8 & 233.3 & 226.4 & 229 & 181.5 & 153.4 & 137.4 & 4331.40 \\
\hline 585.5 & 573.8 & 245.8 & 189.3 & 186.7 & 171.5 & 146.1 & 122.8 & 119.7 & 3853.00 \\
\hline 567.9 & 534.1 & 223.7 & 169.4 & 155.6 & 168.9 & 131.4 & 115 & 109.3 & 3585.70 \\
\hline 123.6 & 114.1 & 114.5 & 115.8 & 111.1 & 115 & 104.6 & 105.5 & 92 & 3333.00 \\
\hline 634.7 & 608.8 & 325.6 & 219.1 & 199.6 & 206.6 & 173.7 & 134.4 & 115.8 & 4907.00 \\
\hline 655.9 & 654.5 & 384.4 & 252.8 & 214.3 & 192.7 & 146.9 & 129.2 & 120.2 & 4872.30 \\
\hline 818 & 774.3 & 425.9 & 254.9 & 195.3 & 176.7 & 124.5 & 105.5 & 102 & 4561.50 \\
\hline 816.4 & 791.7 & 425.5 & 264.4 & 219.1 & 182.4 & 130.5 & 115.4 & 115.8 & 4354.80 \\
\hline 132.7 & 124.5 & 107.2 & 112.4 & 112.8 & 107.2 & 103.7 & 102.9 & 98.1 & 3593.20 \\
\hline 870.6 & 841.3 & 462.6 & 318.9 & 292.1 & 285.2 & 248.4 & 165.1 & 132.3 & 3404.70 \\
\hline 885.4 & 843.9 & 464.8 & 306.3 & 266.2 & 225.6 & 166 & 144.4 & 135.2 & 4831.00 \\
\hline 939 & 908.7 & 479 & 331.8 & 283.9 & 233.3 & 172.9 & 152.1 & 146.9 & 4884.10 \\
\hline 927.3 & 888 & 471.3 & 315.4 & 280 & 225.6 & 179.8 & 162.5 & 163.4 & 4558.40 \\
\hline
\end{tabular}


other

\begin{tabular}{|c|c|c|c|c|c|c|c|c|c|}
\hline 155.1 & 161.6 & 154.7 & 147.8 & 151.3 & 149.9 & 144.4 & 136.6 & 127.1 & 4280.50 \\
\hline 888.8 & 837.8 & 489.4 & 311.5 & 264.4 & 250.6 & 190.1 & 159 & 131.8 & 3562.70 \\
\hline 921.3 & 882.9 & 515.3 & 313.2 & 271.4 & 265.3 & 188.4 & 139.2 & 127.9 & 3201.50 \\
\hline 915.2 & 913.6 & 546.9 & 333.6 & 272.2 & 216.9 & 158.2 & 145.2 & 141.8 & 2916.90 \\
\hline 976.4 & 921.6 & 533.9 & 324.1 & 308.5 & 259.3 & 185 & 169.4 & 157.7 & 5107.90 \\
\hline 136.6 & 159 & 177.6 & 188.4 & 179.8 & 160.8 & 177.2 & 162 & 140 & 5365.60 \\
\hline 839.1 & 808.4 & 412.5 & 286.9 & 235.1 & 250.2 & 167.7 & 138.3 & 105.9 & 5916.20 \\
\hline 87.7 & 88.6 & 89.9 & 80.8 & 87.3 & 84.7 & 84.3 & 81.3 & 82.5 & 6521.30 \\
\hline 453.2 & 415.8 & 303.3 & 246.7 & 219.1 & 202.6 & 166.4 & 158.6 & 146.9 & 4180.00 \\
\hline 228.6 & 201.8 & 154.7 & 137.8 & 121.4 & 118 & 108.1 & 108.5 & 108.5 & 2372.70 \\
\hline 168.9 & 149.5 & 111.9 & 99.8 & 105.9 & 104.6 & 103.3 & 93.8 & 93.8 & 2550.90 \\
\hline 362.5 & 352.6 & 258.8 & 186.2 & 165.5 & 131.4 & 91.2 & 86 & 85.1 & 5138.90 \\
\hline 70.9 & 72.2 & 78.2 & 80.8 & 84.7 & 84.3 & 85.1 & 85.1 & 83.9 & 5900.80 \\
\hline 150.4 & 124 & 157.7 & 173.3 & 161.2 & 149.1 & 152.1 & 156 & 140.4 & 6286.00 \\
\hline 163.8 & 134.4 & 104.1 & 99.8 & 111.5 & 110.2 & 111.9 & 111.1 & 111.9 & 6121.80 \\
\hline 91.2 & 88.6 & 88.6 & 91.6 & 99.8 & 99.8 & 101.6 & 102.4 & 101.2 & 3789.70 \\
\hline 100.7 & 103.3 & 99.8 & 108.1 & 110.2 & 122.3 & 122.3 & 134.8 & 137.8 & 3282.00 \\
\hline 68.3 & 76.5 & 73.9 & 73.9 & 81.3 & 81.7 & 82.5 & 82.5 & 83.4 & 2671.60 \\
\hline 142.2 & 113.6 & 84.3 & 91.2 & 93.8 & 94.2 & 113.6 & 111.9 & 104.1 & 5148.20 \\
\hline 131.8 & 112.8 & 105 & 90.3 & 84.7 & 86 & 86 & 84.3 & 86.9 & 5115.30 \\
\hline 152.5 & 108.5 & 96.4 & 98.1 & 97.7 & 90.3 & 84.3 & 90.3 & 83.4 & 5569.80 \\
\hline 105.9 & 141.3 & 113.6 & 110.7 & 129.2 & 147.3 & 133.5 & 114.1 & 107.6 & 5429.20 \\
\hline 333.1 & 296.4 & 137.8 & 114.5 & 118 & 111.9 & 91.6 & 76.5 & 73 & 3833.20 \\
\hline 262.7 & 254.5 & 124.9 & 91.2 & 88.6 & 83.4 & 70.4 & 67.9 & 63.5 & 3401.50 \\
\hline 193.6 & 176.7 & 100.7 & 98.1 & 92.9 & 95.1 & 99 & 92.9 & 95.5 & 1930.80 \\
\hline 192.3 & 169.8 & 86.5 & 88.6 & 86 & 90.3 & 97.7 & 89.5 & 90.3 & 4095.70 \\
\hline 149.1 & 148.2 & 63.5 & 66.1 & 67.9 & 77.8 & 84.3 & 92 & 90.3 & 3571.50 \\
\hline 107.6 & 99 & 60.9 & 61.8 & 62.7 & 67.9 & 67.5 & 68.7 & 67.9 & 2852.20 \\
\hline 98.1 & 101.6 & 67.5 & 65.7 & 70.9 & 74.8 & 72.2 & 73 & 73.9 & 5219.80 \\
\hline 174.1 & 184.5 & 125.3 & 129.2 & 134.4 & 137 & 137 & 141.3 & 136.6 & 5398.40 \\
\hline 249.3 & 219.1 & 99 & 92.9 & 95.5 & 92 & 88.6 & 81.7 & 78.7 & 4594.00 \\
\hline 469.2 & 475.7 & 187.6 & 127.5 & 120.6 & 101.6 & 75.6 & 92 & 90.3 & 4145.60 \\
\hline 607.3 & 604.4 & 226 & 123.2 & 82.5 & 92 & 94.6 & 94.6 & 76.5 & 4833.00 \\
\hline 416 & 402.5 & 117.6 & 89.5 & 78.2 & 79.1 & 73.9 & 76.5 & 75.6 & 3858.70 \\
\hline 362 & 293.3 & 199.2 & 189.7 & 128.8 & 124 & 95.1 & 95.1 & 96.4 & 4238.30 \\
\hline 300 & 273.2 & 215.7 & 209.6 & 157.3 & 150.8 & 122.8 & 131.8 & 121.9 & 5311.40 \\
\hline 394.7 & 383.8 & 350.4 & 357.3 & 188.4 & 172 & 121 & 136.1 & 125.3 & 4346.80 \\
\hline 309.7 & 291.1 & 240.3 & 225.6 & 187.1 & 178 & 127.1 & 136.1 & 125.3 & 2638.20 \\
\hline 188.4 & 188.8 & 172.9 & 168.1 & 154.7 & 150.4 & 106.7 & 115.8 & 115.8 & 2544.50 \\
\hline 332.2 & 330.4 & 268.4 & 281.3 & 226.4 & 217.8 & 166.4 & 169.4 & 146.9 & 4713.70 \\
\hline 340.8 & 332 & 259.7 & 257.9 & 223.8 & 196.6 & 148.7 & 163.4 & 160.3 & 4951.20 \\
\hline 341.2 & 285 & 255.3 & 257.5 & 198.8 & 187.1 & 144.4 & 151.3 & 143.9 & 5184.30 \\
\hline 281.5 & 215.7 & 204.4 & 209.6 & 127.5 & 115.4 & 104.1 & 119.3 & 115.4 & 5858.00 \\
\hline 145.6 & 126.2 & 109.3 & 123.2 & 115.8 & 92.9 & 96.4 & 79.6 & 97.2 & 6800.30 \\
\hline 275.6 & 215.2 & 145.6 & 164.2 & 153.4 & 128.3 & 119.3 & 103.3 & 99.8 & 3287.50 \\
\hline 263.6 & 210 & 179.8 & 171.5 & 134.8 & 131.8 & 108.9 & 111.9 & 100.3 & 2952.00 \\
\hline 278.7 & 190.1 & 199.6 & 209.6 & 172 & 161.6 & 115 & 118 & 103.7 & 6602.40 \\
\hline 237.7 & 209.2 & 189.3 & 207 & 159.9 & 148.7 & 105 & 111.5 & 101.6 & 6879.30 \\
\hline 185.3 & 149.5 & 130.1 & 134 & 121 & 110.2 & 72.6 & 80 & 81.3 & 7100.30 \\
\hline
\end{tabular}


other

\begin{tabular}{|c|c|c|c|c|c|c|c|c|c|}
\hline 277.5 & 206.6 & 201.4 & 187.1 & 141.8 & 134.4 & 109.8 & 108.5 & 89.1 & 6844.90 \\
\hline 245.3 & 185.8 & 156.4 & 163.8 & 135.7 & 143.9 & 107.2 & 107.6 & 99.8 & 6564.90 \\
\hline 248.9 & 200.5 & 192.7 & 190.5 & 151.3 & 151.3 & 102 & 106.7 & 94.2 & 2596.20 \\
\hline 255 & 200.1 & 190.5 & 214.3 & 168.1 & 151.3 & 107.2 & 112.4 & 103.3 & 2280.10 \\
\hline 173.7 & 193.6 & 172.4 & 166 & 137.4 & 138.3 & 83.4 & 81.3 & 80.4 & 6872.60 \\
\hline 284.6 & 228.6 & 245 & 240.3 & 173.3 & 170.3 & 114.1 & 115 & 105 & 7222.60 \\
\hline 307.5 & 246.8 & 242.4 & 244.6 & 178 & 173.7 & 118.8 & 115.8 & 102 & 9157.40 \\
\hline 342.4 & 262.7 & 238.5 & 240.3 & 168.9 & 153.9 & 106.3 & 121 & 111.5 & 9257.40 \\
\hline 321.1 & 244.2 & 222.1 & 208.7 & 134.8 & 128.8 & 74.4 & 90.8 & 87.3 & 9291.10 \\
\hline 251.5 & 207.4 & 207.4 & 228.6 & 143.5 & 140.9 & 89.1 & 100.3 & 98.6 & 2777.10 \\
\hline \multirow[t]{29}{*}{271.6} & 201.8 & 194.5 & 237.2 & 180.6 & 177.2 & 134 & 131.8 & 117.6 & 2406.60 \\
\hline & & & & & & & & & 10570.10 \\
\hline & & & & & & & & & 10288.20 \\
\hline & & & & & & & & & 10656.10 \\
\hline & & & & & & & & & 10903.50 \\
\hline & & & & & & & & & 9963.20 \\
\hline & & & & & & & & & 3114.90 \\
\hline & & & & & & & & & 2832.00 \\
\hline & & & & & & & & & 10831.80 \\
\hline & & & & & & & & & 10639.90 \\
\hline & & & & & & & & & 10947.80 \\
\hline & & & & & & & & & 11279.60 \\
\hline & & & & & & & & & 11061.90 \\
\hline & & & & & & & & & 3801.10 \\
\hline & & & & & & & & & 3148.00 \\
\hline & & & & & & & & & 10084.90 \\
\hline & & & & & & & & & 1853.30 \\
\hline & & & & & & & & & 6156.00 \\
\hline & & & & & & & & & 4581.90 \\
\hline & & & & & & & & & 3038.20 \\
\hline & & & & & & & & & 5396.80 \\
\hline & & & & & & & & & 4161.30 \\
\hline & & & & & & & & & 2355.10 \\
\hline & & & & & & & & & 1783.70 \\
\hline & & & & & & & & & 3882.90 \\
\hline & & & & & & & & & 3060.70 \\
\hline & & & & & & & & & 2549.70 \\
\hline & & & & & & & & & 2474.10 \\
\hline & & & & & & & & & 2575.50 \\
\hline 666 & 643.6 & 383.4 & 162.5 & 148.7 & 140 & 76.1 & 75.2 & 71.8 & 1818.90 \\
\hline 870.2 & 849.2 & 430.8 & 173.7 & 171.1 & 166.8 & 112.4 & 111.5 & 89.9 & 1793.50 \\
\hline 882.2 & 829.3 & 517.6 & 216.1 & 174.6 & 152.1 & 98.6 & 96.8 & 89.9 & 3248.90 \\
\hline 930.1 & 875.4 & 395.8 & 215.2 & 197.9 & 170.3 & 95.1 & 87.3 & 83 & 2374.30 \\
\hline 910.1 & 864.6 & 391.1 & 223 & 185.8 & 159.4 & 86.9 & 84.3 & 79.6 & 2525.50 \\
\hline 906.4 & 860.7 & 344.4 & 218.7 & 186.7 & 154.7 & 90.8 & 92.5 & 85.6 & 2614.60 \\
\hline 979.8 & 917.7 & 385.9 & 272.2 & 233.3 & 217.8 & 160.3 & 144.4 & 93.4 & 1881.50 \\
\hline 981.9 & 917.7 & 393.6 & 239.4 & 218.7 & 214.7 & 160.8 & 89.1 & 85.6 & 1410.70 \\
\hline 980 & 907.8 & 403.2 & 230.8 & 200.5 & 162.5 & 96.4 & 90.8 & 86.5 & 3928.70 \\
\hline 905 & 841.8 & 365.5 & 240.3 & 187.6 & 202.2 & 126.2 & 97.2 & 96.8 & 3465.20 \\
\hline
\end{tabular}


other

\begin{tabular}{|c|c|c|c|c|c|c|c|c|c|}
\hline 874.1 & 800.8 & 348.7 & 224.7 & 183.6 & 172 & 119.3 & 119.7 & 108.1 & 2260.50 \\
\hline 921.3 & 874 & 390.6 & 223 & 218.7 & 226.4 & 127.1 & 96.4 & 85.6 & 2078.90 \\
\hline 928.5 & 891.5 & 385.4 & 236.8 & 210 & 210.9 & 159 & 108.9 & 87.3 & 3111.80 \\
\hline 919.3 & 869 & 412.7 & 218.7 & 178 & 166 & 89.9 & 83.9 & 79.6 & 2958.50 \\
\hline 912.3 & 856.7 & 386.7 & 240.3 & 202.2 & 184.1 & 93.4 & 93.4 & 81.3 & 2556.70 \\
\hline 929.7 & 868.6 & 343.5 & 227.3 & 178.9 & 166 & 117.6 & 115 & 109.3 & 2146.00 \\
\hline 194.9 & 179.8 & 182.4 & 135.7 & 130.9 & 135.7 & 93.4 & 74.4 & 73 & 2035.80 \\
\hline 320.1 & 306.8 & 253.2 & 170.3 & 150.4 & 165.1 & 113.6 & 77.8 & 75.2 & 1520.90 \\
\hline 404.9 & 390.1 & 287.4 & 186.7 & 153.9 & 146.5 & 89.1 & 83 & 83.9 & 1563.40 \\
\hline 128.4 & 136.2 & 110.3 & 85.6 & 78.3 & 80.4 & 72.2 & 63.6 & 61.4 & 3247.50 \\
\hline 257.1 & 235.9 & 92.1 & 59.7 & 67.1 & 65.3 & 64 & 63.6 & 70.5 & 1648.50 \\
\hline 73.1 & 76.1 & 58.4 & 60.5 & 67.1 & 66.6 & 62.7 & 63.1 & 62.7 & 1543.20 \\
\hline 119.3 & 118.4 & 87.3 & 71.8 & 74.4 & 76.1 & 74.4 & 74 & 76.1 & 3111.50 \\
\hline 183.3 & 156 & 99.4 & 115.4 & 124.5 & 129.3 & 130.5 & 118.9 & 112.4 & 6721.70 \\
\hline 180.6 & 188.9 & 121 & 102.5 & 113.7 & 114.1 & 108.5 & 102 & 84.3 & 7119.50 \\
\hline 163.4 & 149.5 & 105.5 & 95.1 & 93.4 & 81.3 & 67.9 & 69.2 & 68.3 & 5805.10 \\
\hline 130.5 & 93.8 & 62.3 & 66.2 & 71.4 & 70.9 & 73.1 & 73.1 & 76.1 & 1874.00 \\
\hline 71.8 & 70.9 & 67.9 & 73.5 & 77 & 77 & 76.6 & 77.8 & 80.4 & 1867.70 \\
\hline 78.7 & 77.8 & 74.8 & 73.5 & 83.9 & 83.9 & 78.7 & 78.3 & 81.3 & 5293.20 \\
\hline 89.1 & 89.5 & 78.3 & 62.3 & 68.3 & 68.8 & 68.3 & 69.2 & 70.5 & 4120.90 \\
\hline 150.4 & 142.6 & 99 & 89.1 & 97.3 & 79.2 & 78.3 & 73.1 & 71.8 & 4871.70 \\
\hline 112.8 & 91.2 & 81.3 & 79.2 & 87.3 & 87.8 & 87.3 & 86.5 & 87.8 & 4422.30 \\
\hline 151.3 & 130.5 & 77 & 77.8 & 93.4 & 87.3 & 80.4 & 82.1 & 83 & 3197.70 \\
\hline 111.1 & 116.7 & 91.6 & 72.6 & 79.6 & 77.8 & 77.8 & 77.4 & 77.8 & 2895.10 \\
\hline 357.4 & 318.9 & 144.4 & 105.5 & 101.6 & 103.7 & 102.9 & 84.3 & 86.5 & 2860.50 \\
\hline 203.1 & 202.7 & 127.9 & 114.6 & 121 & 114.6 & 111.5 & 111.1 & 107.2 & 5394.60 \\
\hline 232.9 & 240.3 & 153.5 & 112.8 & 122.8 & 109.4 & 89.1 & 91.2 & 93.8 & 5579.50 \\
\hline 399.9 & 358.9 & 236 & 105.9 & 98.2 & 129.3 & 101.6 & 79.6 & 79.2 & 5907.80 \\
\hline 211.3 & 191.5 & 127.1 & 107.7 & 111.1 & 100.4 & 92.1 & 86.9 & 85.7 & 4879.20 \\
\hline 217.4 & 184.2 & 148.3 & 105.1 & 133.2 & 109.9 & 118 & 134.9 & 109 & 2957.70 \\
\hline 179.8 & 161.7 & 110.3 & 126.3 & 115 & 96.9 & 106 & 87.9 & 89.6 & 2107.80 \\
\hline 203.2 & 180.7 & 128 & 101.2 & 115 & 83.9 & 86.5 & 87.4 & 111.2 & 2020.90 \\
\hline 145.7 & 144.5 & 131.9 & 118.6 & 112.9 & 95.2 & 91.3 & 88.7 & 87.4 & 4297.80 \\
\hline 183.8 & 183.8 & 132.3 & 58.9 & 58.5 & 61.5 & 62.8 & 67.5 & 67.5 & 4141.10 \\
\hline 78.4 & 84.8 & 63.2 & 58 & 57.2 & 61.9 & 63.2 & 64.9 & 64.1 & 4783.10 \\
\hline 551.1 & 483.2 & 272.8 & 206.6 & 181.2 & 149.6 & 104.7 & 74.9 & 71 & 4757.20 \\
\hline 392.3 & 391.8 & 251.8 & 151.3 & 136.6 & 130.5 & 122.3 & 90.8 & 83 & 3410.30 \\
\hline 493.7 & 509.3 & 334.4 & 190.5 & 148.7 & 115.8 & 100.7 & 73.5 & 67.5 & 1959.70 \\
\hline 456.2 & 457.1 & 313.1 & 207.4 & 185 & 169.8 & 160.8 & 131.4 & 95.5 & 1947.80 \\
\hline 451.8 & 375.2 & 272.1 & 160.8 & 118 & 115.8 & 112.4 & 100.7 & 64 & 4734.60 \\
\hline 348.7 & 344.8 & 218.2 & 127.9 & 119.7 & 113.2 & 99.8 & 77.8 & 73.5 & 4335.70 \\
\hline 160.7 & 149.5 & 151.2 & 159 & 165.9 & 155.5 & 152.9 & 149.5 & 146 & 4584.10 \\
\hline 149.5 & 165.9 & 165 & 165.9 & 165 & 151.2 & 142.6 & 144.3 & 145.2 & 4507.90 \\
\hline 146.9 & 147.7 & 156.4 & 149.5 & 148.6 & 151.2 & 149.5 & 150.3 & 147.7 & 3557.70 \\
\hline 155.5 & 165 & 158.1 & 150.3 & 157.2 & 173.7 & 173.7 & 157.2 & 151.2 & 2293.00 \\
\hline 165 & 165.9 & 168.5 & 165.9 & 168.5 & 166.8 & 164.2 & 163.3 & 163.3 & 2017.30 \\
\hline 165 & 178 & 172.8 & 157.2 & 148.6 & 146 & 151.2 & 150.3 & 140 & 5099.00 \\
\hline 162.4 & 165 & 149.5 & 141.7 & 166.8 & 153.8 & 142.6 & 151.2 & 150.3 & 5165.90 \\
\hline 158.1 & 159.8 & 162.4 & 158.1 & 164.2 & 158.1 & 159.8 & 159.8 & 159.8 & 5197.20 \\
\hline
\end{tabular}


other

\begin{tabular}{|c|c|c|c|c|c|c|c|c|c|}
\hline 133.9 & 143.4 & 133.9 & 129.6 & 136.5 & 144.3 & 138.2 & 128.7 & 128.7 & 4990.40 \\
\hline 119.2 & 123.6 & 117.5 & 116.6 & 117.5 & 128.7 & 150.3 & 146 & 121 & 4347.80 \\
\hline 139.1 & 141.7 & 143.4 & 140.8 & 140.8 & 140 & 134.8 & 137.4 & 134.8 & 2393.10 \\
\hline 133.1 & 135.6 & 136.5 & 139.1 & 139.1 & 138.2 & 140.8 & 145.2 & 132.2 & 2505.20 \\
\hline 129.6 & 135.6 & 139.1 & 152.1 & 154.7 & 140.8 & 128.7 & 127 & 127 & 4900.00 \\
\hline 146 & 20.7 & 136.5 & 129.6 & 137.4 & 140.8 & 133.9 & 123.6 & 115.8 & 0.00 \\
\hline 152.9 & 169.3 & 178.8 & 165 & 165 & 173.7 & 156.4 & 148.6 & 146 & 0.00 \\
\hline 155.5 & 156.4 & 159.8 & 171.9 & 161.6 & 163.3 & 165 & 156.4 & 157.2 & 0.00 \\
\hline 190.1 & 190.9 & 181.4 & 177.1 & 175.4 & 170.2 & 166.8 & 165.9 & 163.3 & 0.00 \\
\hline 184.9 & 163.3 & 154.7 & 146.9 & 147.7 & 146 & 144.3 & 145.2 & 143.4 & 0.00 \\
\hline 203.9 & 197 & 197 & 197.9 & 197 & 183.2 & 168.5 & 171.9 & 168.5 & 0.00 \\
\hline 174.5 & 178 & 181.4 & 184 & 182.3 & 178 & 174.5 & 173.7 & 172.8 & 0.00 \\
\hline 244.5 & 243.6 & 236.7 & 241.1 & 236.7 & 234.1 & 227.2 & 229.8 & 222.9 & 0.00 \\
\hline 237.6 & 241.1 & 247.1 & 246.2 & 249.7 & 242.8 & 237.6 & 227.2 & 220.3 & 0.00 \\
\hline 431.1 & 432 & 428.5 & 429.4 & 424.2 & 411.3 & 405.2 & 402.6 & 400 & 0.00 \\
\hline 402.6 & 404.4 & 279.9 & 271.3 & 267.8 & 258.3 & 260.9 & 246.2 & 233.3 & 0.00 \\
\hline 413 & 420.8 & 414.7 & 415.6 & 412.1 & 401.8 & 404.4 & 399.2 & 389.7 & 0.00 \\
\hline 294.6 & 305 & 296.4 & 299.8 & 290.3 & 290.3 & 291.2 & 286.8 & 284.3 & 0.00 \\
\hline 105.5 & 102.4 & 104.1 & 110.2 & 114.5 & 104.1 & 102.9 & 106.3 & 103.7 & 0.00 \\
\hline 107.6 & 102 & 97.2 & 83.4 & 92.9 & 92 & 104.1 & 92.9 & 92 & 0.00 \\
\hline 150.8 & 115.8 & 99.8 & 114.5 & 148.2 & 122.3 & 81.3 & 80 & 129.2 & 0.00 \\
\hline 110.2 & 94.6 & 69.6 & 81.7 & 107.6 & 136.6 & 117.1 & 91.2 & 86 & 0.00 \\
\hline 135.7 & 138.7 & 103.3 & 102.4 & 124 & 130.9 & 78.7 & 81.7 & 76.5 & 0.00 \\
\hline 92.9 & 84.3 & 126.6 & 122.3 & 117.1 & 109.3 & 108.1 & 130.9 & 146.5 & 0.00 \\
\hline 540.6 & 491.3 & 251.2 & 125.3 & 111.5 & 96.4 & 94.6 & 99.8 & 97.2 & 0.00 \\
\hline 109.3 & 111.9 & 108.5 & 105.9 & 98.1 & 95.5 & 102.4 & 101.6 & 102.4 & 0.00 \\
\hline 168.1 & 154.3 & 92.5 & 86 & 82.5 & 89.5 & 91.2 & 87.3 & 81.7 & 0.00 \\
\hline 88.6 & 90.3 & 87.7 & 86.9 & 86.9 & 86.5 & 86 & 86 & 85.1 & 0.00 \\
\hline 136.1 & 124.9 & 94.2 & 88.6 & 72.2 & 78.2 & 78.2 & 78.2 & 76.1 & 0.00 \\
\hline 80 & 91.6 & 91.2 & 93.8 & 94.6 & 89.5 & 90.3 & 88.6 & 89.5 & 0.00 \\
\hline 175.9 & 165.5 & 127.5 & 105.5 & 101.6 & 90.3 & 99 & 96.4 & 95.5 & 0.00 \\
\hline 118 & 122.3 & 108.9 & 98.1 & 102.4 & 92.9 & 99 & 103.3 & 100.7 & 0.00 \\
\hline 194 & 185.8 & 200 & 194.5 & 203.1 & 153.4 & 131.4 & 127.1 & 127.9 & 0.00 \\
\hline 135.2 & 120.2 & 118.8 & 146.9 & 152.5 & 133.1 & 118.8 & 119.3 & 118.8 & 0.00 \\
\hline 244.1 & 267.9 & 245 & 230.8 & 224.2 & 178 & 162.5 & 155.6 & 149.1 & 0.00 \\
\hline 202.6 & 201.4 & 212.6 & 226.8 & 216.9 & 203.1 & 177.2 & 172 & 162.9 & 0.00 \\
\hline 246.3 & 248.4 & 211.7 & 217.8 & 210.9 & 189.3 & 153 & 156.4 & 148.2 & 0.00 \\
\hline 193.6 & 200 & 208.3 & 221.2 & 229 & 188.4 & 158.2 & 159 & 146.9 & 0.00 \\
\hline 217.3 & 229.9 & 235.1 & 229 & 207.4 & 177.2 & 137.4 & 146.1 & 125.3 & 0.00 \\
\hline 169.4 & 190.1 & 194.5 & 198.3 & 196.2 & 168.5 & 143.5 & 129.7 & 119.3 & 0.00 \\
\hline 208.3 & 149.5 & 134 & 120.6 & 103.7 & 98.6 & 83.9 & 85.1 & 83 & 0.00 \\
\hline 131.8 & 131.4 & 147.8 & 148.7 & 137.8 & 106.3 & 104.6 & 99.4 & 96.8 & 0.00 \\
\hline 207.8 & 231.6 & 221.2 & 254.1 & 214.3 & 200.5 & 174.1 & 146.1 & 128.8 & 0.00 \\
\hline 199.2 & 195.3 & 187.6 & 193.6 & 175.5 & 148.7 & 124.5 & 120.6 & 115 & 4904.60 \\
\hline 229 & 233.7 & 235.1 & 225.6 & 206.6 & 198.8 & 166 & 153.9 & 116.7 & 2705.60 \\
\hline 200 & 193.6 & 204.8 & 205.7 & 179.8 & 148.7 & 124 & 115 & 107.2 & 2809.90 \\
\hline 279.1 & 308 & 309.4 & 328.4 & 301.6 & 277.4 & 224.7 & 220.4 & 192.7 & 9300.50 \\
\hline 233.3 & 204 & 216.1 & 233.3 & 222.1 & 197.1 & 179.8 & 167.7 & 167.7 & 9597.40 \\
\hline 211.7 & 206.6 & 189.3 & 227.3 & 197.1 & 199.2 & 143.5 & 127.1 & 95.1 & 10562.40 \\
\hline
\end{tabular}


other

\begin{tabular}{|c|c|c|c|c|c|c|c|c|c|}
\hline 159.9 & 146.1 & 132.3 & 130.5 & 119.3 & 113.6 & 96.8 & 99.4 & 86.5 & 10453.40 \\
\hline 559.4 & 519.7 & 238 & 216.9 & 194.5 & 183.2 & 149.1 & 145.2 & 135.7 & 10263.10 \\
\hline 177.2 & 198.8 & 182.4 & 187.6 & 189.7 & 140.9 & 127.9 & 129.7 & 127.1 & 2582.60 \\
\hline 554.3 & 533.1 & 246.2 & 181.5 & 172 & 175 & 151.3 & 138.3 & 143.5 & 2606.80 \\
\hline 122.8 & 118 & 115.8 & 100.3 & 88.6 & 88.2 & 87.3 & 87.7 & 87.3 & 11756.10 \\
\hline 802.1 & 774.5 & 417.7 & 236.8 & 216.1 & 162.9 & 121.9 & 108.9 & 109.8 & 11043.70 \\
\hline 152.1 & 165.5 & 166.8 & 158.6 & 139.2 & 123.6 & 121.9 & 133.5 & 120.2 & 10814.80 \\
\hline 860.7 & 825.2 & 498 & 283.5 & 250.6 & 207.4 & 153 & 138.3 & 128.3 & 10503.90 \\
\hline 166 & 174.6 & 188.8 & 191.9 & 163.4 & 150.4 & 141.8 & 127.5 & 121 & 10089.60 \\
\hline 947.4 & 883.2 & 441.5 & 316.3 & 274.8 & 214.3 & 185 & 174.1 & 179.8 & 2314.80 \\
\hline 210 & 219.9 & 223.8 & 229 & 191.9 & 160.8 & 150.4 & 140.9 & 137.8 & 2089.90 \\
\hline 177.6 & 158.6 & 100.3 & 95.5 & 130.1 & 108.5 & 101.6 & 102.4 & 109.8 & 10772.20 \\
\hline 99 & 108.1 & 115.4 & 124.9 & 134.4 & 130.9 & 127.1 & 118.8 & 105.9 & 10531.30 \\
\hline 99.4 & 95.5 & 96.4 & 105 & 111.1 & 127.1 & 149.9 & 114.5 & 114.5 & 10381.70 \\
\hline 71.8 & 72.2 & 73 & 76.5 & 84.3 & 84.7 & 85.1 & 85.1 & 84.7 & 10435.80 \\
\hline 90.3 & 66.1 & 55.8 & 67.5 & 67.9 & 91.2 & 99 & 97.2 & 73 & 10534.70 \\
\hline 54 & 61.8 & 67.9 & 57.1 & 67 & 68.7 & 78.2 & 81.7 & 63.1 & 2311.00 \\
\hline 82.5 & 83.4 & 82.1 & 86.9 & 90.3 & 94.6 & 95.5 & 93.4 & 93.8 & 2110.00 \\
\hline 77.4 & 82.5 & 81.7 & 83.4 & 83.9 & 91.2 & 107.6 & 116.2 & 112.4 & 3414.30 \\
\hline 60.1 & 59.2 & 59.2 & 60.5 & 65.3 & 69.6 & 68.7 & 71.3 & 70 & 4475.40 \\
\hline 60.1 & 62.3 & 63.5 & 65.3 & 67 & 81.7 & 88.6 & 96 & 72.2 & 4962.80 \\
\hline 67.9 & 80 & 82.5 & 68.7 & 69.2 & 69.6 & 71.3 & 74.8 & 74.8 & 2107.20 \\
\hline 66.6 & 67 & 65.3 & 61.8 & 61.8 & 81.7 & 87.3 & 69.6 & 67.9 & 4437.10 \\
\hline 95.5 & 93.4 & 93.8 & 88.6 & 86.9 & 80.4 & 78.2 & 78.2 & 82.5 & 1740.40 \\
\hline 102 & 83.4 & 99 & 98.6 & 90.8 & 95.5 & 77.8 & 77.4 & 72.6 & 2187.90 \\
\hline 148.2 & 148.7 & 145.6 & 150.4 & 126.2 & 111.1 & 103.7 & 120.2 & 121.4 & 1744.50 \\
\hline 135.7 & 137 & 141.8 & 146.9 & 125.3 & 135.2 & 142.6 & 135.7 & 126.6 & 1554.00 \\
\hline 83.4 & 84.3 & 92.9 & 91.2 & 83.4 & 71.3 & 78.2 & 81.7 & 98.1 & 4287.60 \\
\hline 94.6 & 99 & \begin{tabular}{l|l}
99.8 \\
\end{tabular} & 83.4 & 78.2 & 81.7 & 74.8 & 79.1 & 77.4 & 1971.10 \\
\hline 83.4 & 83.4 & 72.2 & 86.9 & 79.6 & 67.9 & 70.4 & 75.6 & 80.8 & 1516.90 \\
\hline 85.1 & 86 & 76.5 & 81.3 & 70.4 & 81.7 & 102 & 99 & 79.1 & 3328.80 \\
\hline 116.2 & 116.2 & 102.9 & 91.2 & 81.3 & 75.6 & 79.6 & 94.6 & 80 & 2460.90 \\
\hline 106.3 & 112.8 & 104.6 & 94.6 & 77 & 70.4 & 74.4 & 87.3 & 78.2 & 2163.30 \\
\hline 127.9 & 130.1 & 140 & 138.3 & 130.5 & 121 & 92.5 & 98.6 & 99 & 1885.80 \\
\hline \multirow[t]{11}{*}{131.8} & 141.8 & 151.3 & 149.5 & 109.8 & 113.6 & 99.4 & 105.5 & 103.7 & 1771.20 \\
\hline & & & & & & & & & 1457.10 \\
\hline & & & & & & & & & 1430.40 \\
\hline & & & & & & & & & 1858.10 \\
\hline & & & & & & & & & 2390.00 \\
\hline & & & & & & & & & 2164.90 \\
\hline & & & & & & & & & 2490.20 \\
\hline & & & & & & & & & 1967.90 \\
\hline & & & & & & & & & 1455.10 \\
\hline & & & & & & & & & 1422.10 \\
\hline & & & & & & & & & 4326.90 \\
\hline 148.7 & 148.7 & 166 & 164.6 & 140 & 125.3 & 102 & 102.9 & 99.4 & 3482.10 \\
\hline 142.6 & 162.5 & 172 & 176.3 & 141.8 & 128.8 & 111.5 & 101.2 & 99.4 & 3723.50 \\
\hline 127.9 & 133.1 & 146.9 & 143.9 & 107.2 & 98.6 & 93 & 96.4 & 96.8 & 4627.90 \\
\hline 137 & 152.1 & 152.1 & 155.1 & 115.8 & 104.6 & 98.6 & 102.9 & 93.4 & 3543.80 \\
\hline
\end{tabular}


other

\begin{tabular}{|c|c|c|c|c|c|c|c|c|c|}
\hline 108.1 & 114.5 & 108.1 & 113.6 & 95.1 & 93.4 & 94.2 & 86.9 & 76.1 & 1990.70 \\
\hline 89.9 & 98.1 & 102 & 92.5 & 82.5 & 77 & 84.7 & 77.8 & 77.4 & 1972.20 \\
\hline 109.3 & 112.4 & 113.2 & 111.1 & 99.4 & 97.7 & 89.1 & 74.8 & 71.8 & 3549.60 \\
\hline 96.4 & 102 & 108.1 & 115.8 & 96.4 & 77 & 92.5 & 77 & 76.1 & 3204.20 \\
\hline 68.3 & 64.9 & 62.3 & 66.6 & 73.5 & 72.6 & 71.8 & 70.9 & 72.6 & 3162.10 \\
\hline 60.9 & 58.8 & 57.6 & 65.7 & 66.6 & 70 & 68.8 & 70 & 70 & 2870.90 \\
\hline 75.7 & 83.5 & 87.8 & 100.3 & 105.9 & 101.6 & 96 & 91.2 & 76.6 & 2696.20 \\
\hline 63.6 & 64.9 & 70.5 & 67.5 & 67.5 & 65.3 & 64 & 62.3 & 64 & 1516.50 \\
\hline 50.2 & 50.2 & 51 & 53.6 & 64.5 & 65.7 & 64 & 66.6 & 66.6 & 1504.70 \\
\hline 49.3 & 50.6 & 51 & 52.4 & 63.1 & 65.3 & 64 & 65.7 & 66.2 & 1690.00 \\
\hline 54.5 & 55 & 55.4 & 58.8 & 64 & 64.5 & 64 & 63.1 & 65.3 & 1411.40 \\
\hline 57.1 & 58 & 58.8 & 57.1 & 62.7 & 64.9 & 63.1 & 63.6 & 65.3 & 1423.20 \\
\hline 82.6 & 77 & 77.4 & 77.4 & 81.3 & 85.2 & 84.3 & 83.9 & 84.3 & 6361.00 \\
\hline 75.7 & 77 & 76.6 & 76.2 & 81.7 & 88.3 & 87.4 & 86.5 & 89.1 & 5109.50 \\
\hline 64.1 & 61.5 & 60.6 & 63.2 & 64.1 & 65.8 & 64.1 & 65.8 & 65.8 & 2116.20 \\
\hline 61.5 & 64.5 & 57.2 & 54.6 & 62.3 & 64.9 & 64.9 & 64.1 & 65.3 & 5491.00 \\
\hline 55.9 & 58 & 56.3 & 59.7 & 61.9 & 67.5 & 66.7 & & 61.5 & 6162.30 \\
\hline 53.7 & 55 & 54.6 & 56.3 & 59.3 & 60.6 & 63.2 & 63.7 & 62.3 & 5837.00 \\
\hline 126.2 & 116.7 & 99 & 91.6 & 89.1 & 86.5 & 85.1 & 87.3 & 87.3 & 5904.70 \\
\hline 87.3 & 88.6 & 88.2 & 94.2 & 97.7 & 91.2 & 89.1 & 89.9 & 87.3 & 4703.00 \\
\hline 71.3 & 73.5 & 66.6 & 64.4 & 64 & 64 & 64.9 & 67.5 & 65.3 & 2852.20 \\
\hline & & & & & & & & & \\
\hline & & & & & & & & & \\
\hline 5809527 & 54024 & 3483775 & 276 167 & 2472538 & 2332760 & 1893615 & 1713736 & 1588587 & \\
\hline \begin{tabular}{|l|}
500.9522 \\
563.0736
\end{tabular} & $\begin{array}{r}340.24 \\
518.8949\end{array}$ & 300.1294 & 213.9661 & 186.2623 & 162.1997 & 121.8235 & 106.1149 & 95.65595 & \\
\hline 162.0158 & 163.894 & 165.0974 & 165.7601 & 166.4178 & 166.8171 & 167.0696 & 167.0926 & 166.8375 & \\
\hline 260.1428 & 251.5694 & 198.8821 & 172.4642 & 149.8079 & 144.521 & 136.1297 & 129.0083 & 122.3419 & \\
\hline 367.9722 & 360.2241 & 304.8911 & 271.6025 & 222.2127 & 218.0772 & 204.757 & 193.1266 & 184.1709 & \\
\hline 374.0893 & 357.0952 & 235.5791 & 185.8909 & 160.1214 & 149.839 & 123.3888 & 114.9471 & 105.7818 & \\
\hline 191.2294 & 186.4765 & 176.6912 & 171.9794 & 174.5441 & 171.4471 & 166.3059 & 163.8735 & 161.7118 & \\
\hline 161.6373 & 160.2265 & 137.1976 & 131.0952 & 122.8687 & 114.0325 & 105.5627 & 103.6549 & 98.23735 & \\
\hline & & & & & & & & & \\
\hline & & & & & & & & & \\
\hline & & & & & & & & & \\
\hline 182.8285 & 171.084 & 93.84612 & 83.89115 & 73.04489 & 74.97594 & 74.21525 & 57.06808 & 49.98281 & \\
\hline 201.7983 & 192.0298 & 90.91028 & 54.49844 & 53.87327 & 55.53361 & 53.06113 & 33.81031 & 23.59944 & \\
\hline 77.8402 & 78.83675 & 79.09449 & 79.13571 & 79.09524 & 79.08732 & 79.29785 & 79.47921 & 79.49524 & \\
\hline 233.4913 & 219.4059 & 143.1721 & 118.8526 & 98.57096 & 91.73938 & 84.1196 & 78.50332 & 75.19148 & \\
\hline 221.8663 & 208.0452 & 152.2857 & 134.0336 & 121.7042 & 111.2659 & 105.3279 & 100.6155 & 97.33092 & \\
\hline 263.0763 & 252.2683 & 123.5556 & 80.50489 & 61.56968 & 53.87982 & 42.16425 & 37.10484 & 29.96835 & \\
\hline 105.0255 & 105.8376 & 80.81953 & 80.19874 & 76.65717 & 74.33304 & 76.4056 & 75.21209 & 73.05854 & \\
\hline 163.7218 & 155.3629 & 86.25115 & 68.1023 & 59.64136 & 47.67496 & 35.49156 & 32.10895 & 29.4605 & \\
\hline & & & & & & & & & \\
\hline & & & & & & & & & \\
\hline & & & & & & & & & \\
\hline & & & & & & & & & \\
\hline & & & & & & & & & \\
\hline
\end{tabular}


other

\begin{tabular}{|r|r|r|r|r|r|r|r|r|r|}
\hline 46249.9 & 43121.5 & 28758.8 & 22872.4 & 20583.4 & 18249.4 & 15233.8 & 13757.9 & 12804.1 & 629244.3 \\
\hline 220123.1 & 202501.4 & 118504.4 & 85210.8 & 74441.6 & 65120.6 & 49679 & 43560 & 39338.3 & 2715853 \\
\hline 8515.7 & 7985.5 & 6400.3 & 5505.5 & 5373.3 & 5254.7 & 4809.1 & 4526.4 & 4307 & 143757.8 \\
\hline 60144.2 & 58305.7 & 45445.6 & 39317 & 34014.8 & 32788.9 & 30929.4 & 29472.2 & 28002.1 & 974180 \\
\hline 30277.1 & 29399.6 & 23044 & 19907.5 & 16667.1 & 16273.8 & 15168.7 & 14477.6 & 13784.2 & 484260.1 \\
\hline 66806.7 & 63941.7 & 43195.6 & 34606.9 & 30187.6 & 28312.3 & 23551.9 & 21971.9 & 20279.5 & 945809.2 \\
\hline 6783.6 & 6566 & 6086.7 & 5947.4 & 5945.3 & 5630.3 & 5307.3 & 5236.8 & 5158.7 & 140201.8 \\
\hline 13877.21 & 13634.92 & 11220.75 & 10446.92 & 9643.389 & 9065.511 & 8393.899 & 8073.592 & 7739.595 & 249552.6 \\
\hline & & & & & & & & & \\
\hline & & & & & & & & & \\
\hline & & & & & & & & & \\
\hline & & & & & & & & & \\
\hline & & & & & & & & & \\
\hline 90 & 90 & 89 & 90 & 91 & 91 & 91 & 91 & 91 & 90 \\
\hline 394 & 393 & 395 & 395 & 395 & 395 & 395 & 395 & 395 & 395 \\
\hline 576 & 612 & 648 & 684 & 720 & 756 & 792 & 828 & 864 & 450 \\
\hline 229 & 229 & 229 & 229 & 229 & 229 & 229 & 229 & 229 & 229 \\
\hline 79 & 79 & 79 & 79 & 79 & 79 & 79 & 79 & 79 & 79 \\
\hline 187 & 187 & 187 & 187 & 187 & 187 & 187 & 187 & 187 & 187 \\
\hline 34 & 34 & 34 & 34 & 34 & 34 & 34 & 34 & 34 & 34 \\
\hline 83 & 83 & 83 & 83 & 83 & 83 & 83 & 82 & 83 & 83 \\
\hline & & & & & & & & & \\
\hline
\end{tabular}


other

\begin{tabular}{|c|c|c|c|c|c|}
\hline $\begin{array}{l}\text { DAILY } \\
\text { AVERAGE }\end{array}$ & $\begin{array}{l}\text { MONTHLY } \\
\text { TOTAL } \\
\text { formula }\end{array}$ & $\begin{array}{l}\text { MONTHLY } \\
\text { TOTAL value }\end{array}$ & & & \\
\hline \multirow{2}{*}{\multicolumn{6}{|c|}{$\begin{array}{l}\text { \#DIV/0! } \\
\text { \#DIV/0! }\end{array}$}} \\
\hline & \multicolumn{5}{|c|}{ \#DIV/0! } \\
\hline \#DIV/0! & & & sort S/NS & sort JDAY & \\
\hline \#DIV/0! & & & 3995113.20 & sum AE column & sum AG column \\
\hline \#DIV/0! & & & 1872558.70 & & \\
\hline \#DIV/0! & & & 5867671.90 & 5867671.90 & 5869129 \\
\hline \multicolumn{6}{|l|}{ \#DIV/0! } \\
\hline \#DIV/0! & & & sort $\mathrm{A} / \mathrm{B}, \mathrm{S} / \mathrm{NS}$ & & \\
\hline \#DIV/0! & & & B-S & 3184170.10 & \\
\hline \#DIV/0! & & & B-NS & 1201856.60 & \\
\hline \multicolumn{6}{|l|}{ \#DIV/0! } \\
\hline \multicolumn{6}{|l|}{ \#DIV/0! } \\
\hline \multicolumn{6}{|l|}{ \#DIV/0! } \\
\hline \multicolumn{6}{|l|}{ \#DIV/0! } \\
\hline \multicolumn{6}{|l|}{ \#DIV/0! } \\
\hline \multicolumn{6}{|l|}{ \#DIV/0! } \\
\hline \multicolumn{6}{|l|}{ \#DIV/0! } \\
\hline \multicolumn{6}{|l|}{ \#DIV/0! } \\
\hline \multicolumn{6}{|l|}{ \#DIV/0! } \\
\hline \multirow{2}{*}{\multicolumn{6}{|c|}{ \#DIV/0! }} \\
\hline \multirow{2}{*}{\multicolumn{2}{|c|}{$\begin{array}{l}\text { \#DIV/0! } \\
\text { \#DIV/0! }\end{array}$}} & & & & \\
\hline & & & & & \\
\hline \multicolumn{6}{|l|}{ \#DIV/0! } \\
\hline \multicolumn{6}{|l|}{ \#DIV/0! } \\
\hline \#DIV/0! & 241635.60 & 0 & & & \\
\hline \multicolumn{6}{|l|}{ \#DIV/0! } \\
\hline \multicolumn{6}{|l|}{ \#DIV/0! } \\
\hline \multirow{2}{*}{\multicolumn{6}{|c|}{ \#DIV/0! }} \\
\hline \multirow{2}{*}{\multicolumn{2}{|c|}{ \#DIV/0! }} & & & & \\
\hline \multirow{2}{*}{\multicolumn{6}{|c|}{\begin{tabular}{|l|}
209.83 \\
360.43
\end{tabular}}} \\
\hline \multirow{2}{*}{\multicolumn{6}{|c|}{\begin{tabular}{|l|l|}
360.43 \\
379.88
\end{tabular}}} \\
\hline & & & & & \\
\hline \multicolumn{6}{|l|}{179.78} \\
\hline 127.14 & & & & & \\
\hline 278.19 & & & & & \\
\hline 371.73 & & & & & \\
\hline 335.11 & & & & & \\
\hline 348.10 & & & & & \\
\hline 365.54 & & & & & \\
\hline 189.87 & & & & & \\
\hline 174.90 & & & & & \\
\hline 405.58 & & & & & \\
\hline 377.34 & & & & & \\
\hline 361.48 & & & & & \\
\hline 367.13 & & & & & \\
\hline
\end{tabular}


other

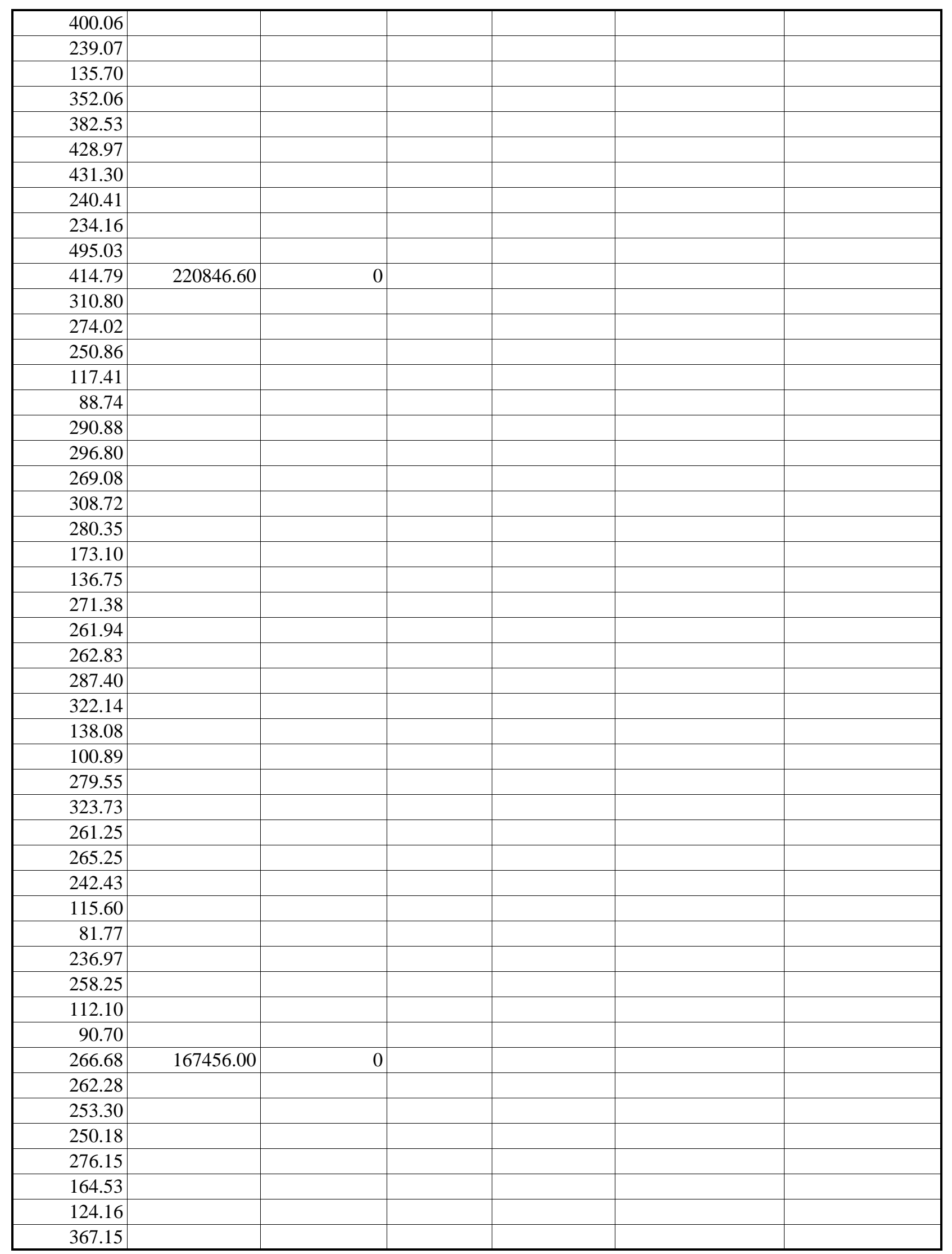


other

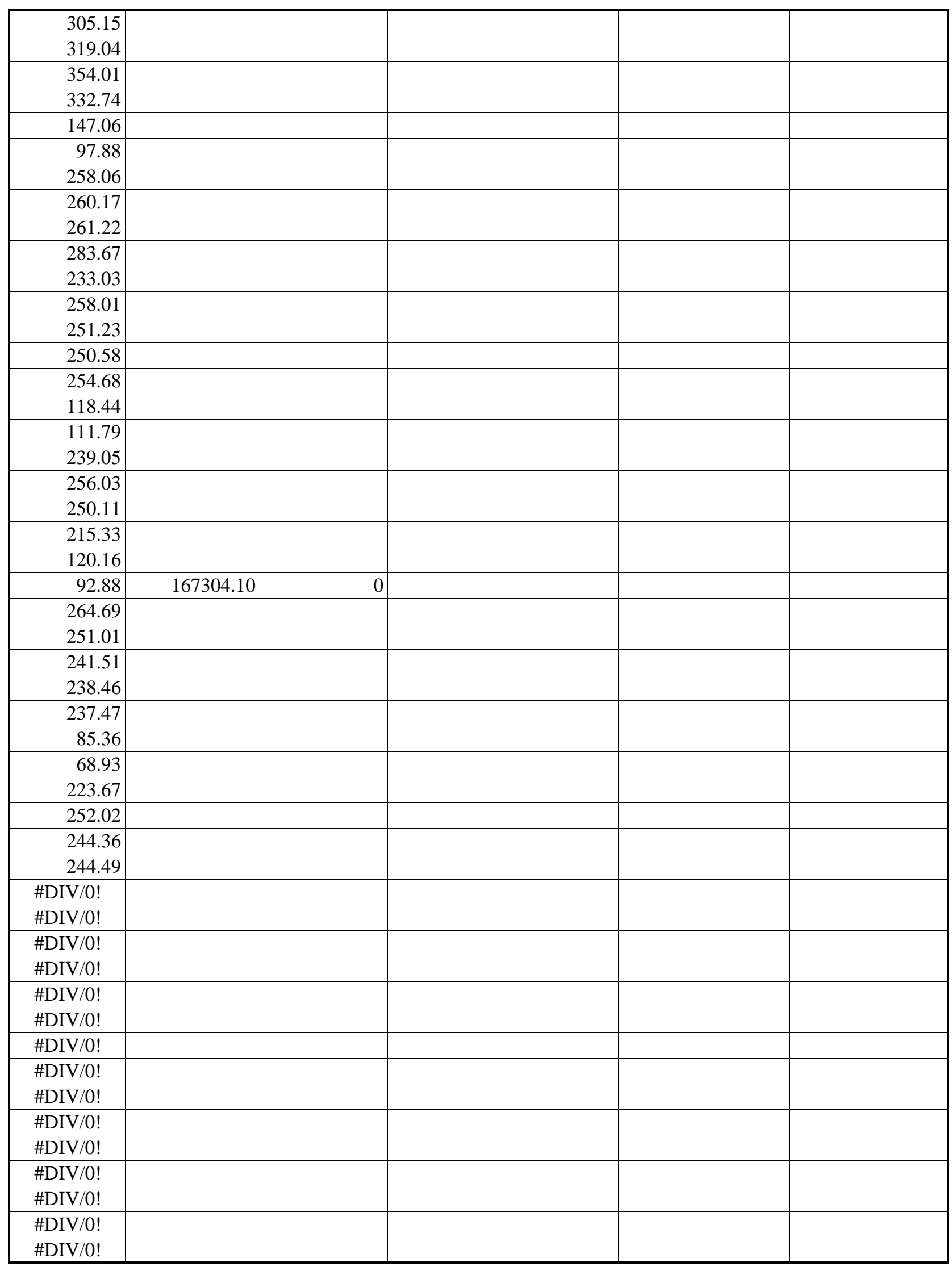


other

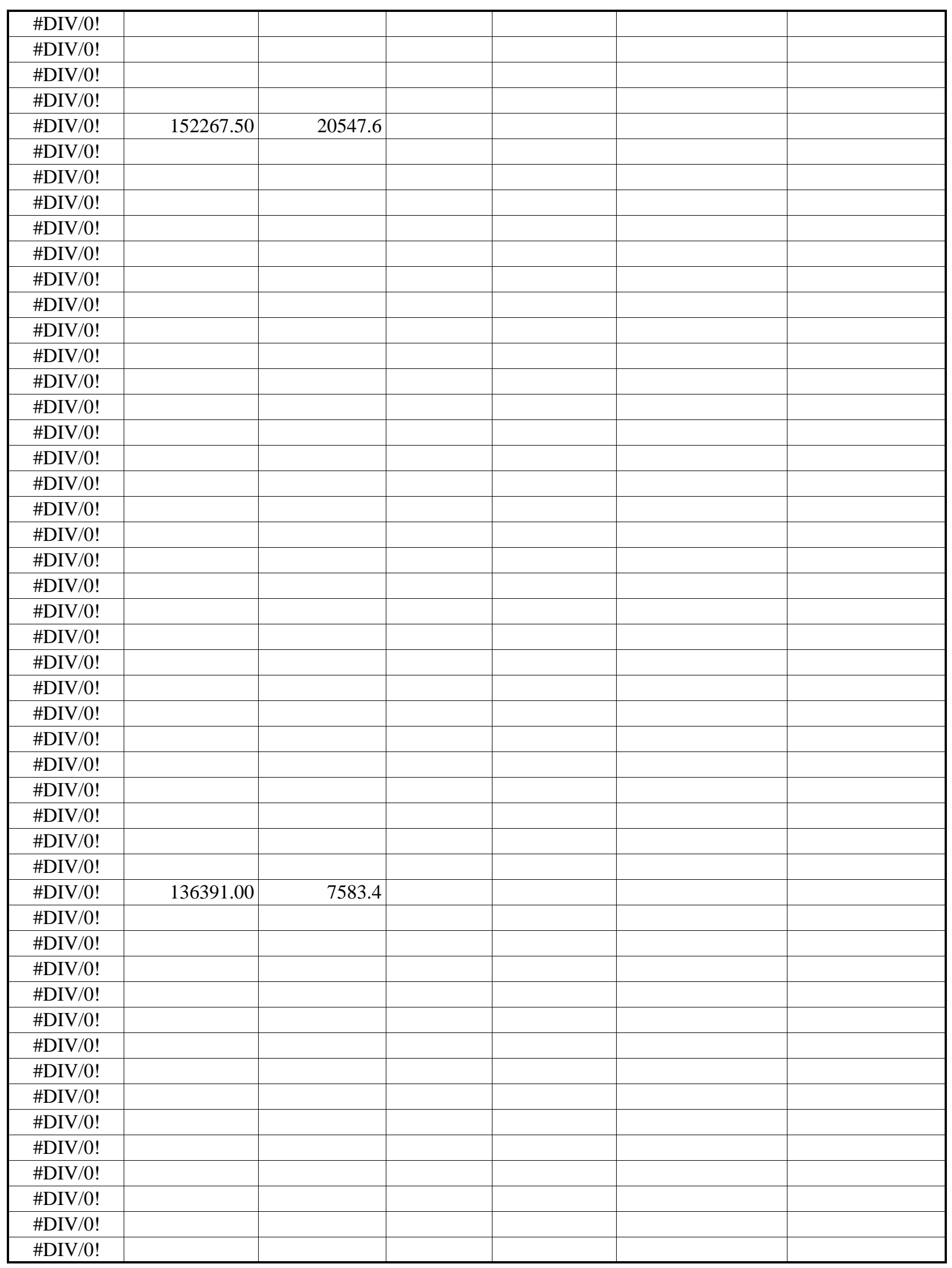


other

\begin{tabular}{|c|l|l|l|l|l|}
\hline \#DIV/0! & & & & \\
\hline \#DIV/0! & & & & \\
\hline \#DIV/0! & & & & \\
\hline \#DIV/0! & & & & \\
\hline \#DIV/0! & & & & \\
\hline \#DIV/0! & & & & \\
\hline \#DIV/0! & & & & \\
\hline \#DIV/0! & & & & \\
\hline \#DIV/0! & & & & \\
\hline \#DIV/0! & & & & \\
\hline \#DIV/0! & & & & \\
\hline \#DIV/0! & & & & \\
\hline \#DIV/0! & & & & \\
\hline \#DIV/0! & & & & \\
\hline \#DIV/0! & & & & \\
\hline \#DIV/0! & & & & \\
\hline \#DIV/0! & & & & \\
\hline \#DIV/0! & & & & \\
\hline \#DIV/0! & & & & \\
\hline \#DIV/0! & & & & \\
\hline \#DIV/0! & & & & \\
\hline \#DIV/0! & & & & & \\
\hline \#DIV/0! & & & & & \\
\hline \#DIV/0! & & & & & \\
\hline \#DIV/0! & & & & & \\
\hline 365.21 & & & & & \\
\hline 223.85 & & & & & \\
\hline 104.17 & & & & & \\
\hline 103.77 & & & & & \\
\hline 222.55 & & & & & \\
\hline 109.51 & & & & & \\
\hline 103.00 & & & & & \\
\hline 108.57 & & & & & \\
\hline 90.90 & & & & & \\
\hline 85.95 & & & & & \\
\hline 114.80 & & & & & \\
\hline 126.42 & & & & \\
\hline 118.23 & & & & \\
\hline 111.70 & & & & \\
\hline 105.54 & & & & \\
\hline \#DIV/0! & & & & \\
\hline 87.81 & & & & \\
\hline 121.13 & & & & \\
\hline 106.67 & & & & \\
\hline 101.74 & & & & & \\
\hline 260.20 & & & & & \\
\hline
\end{tabular}


other

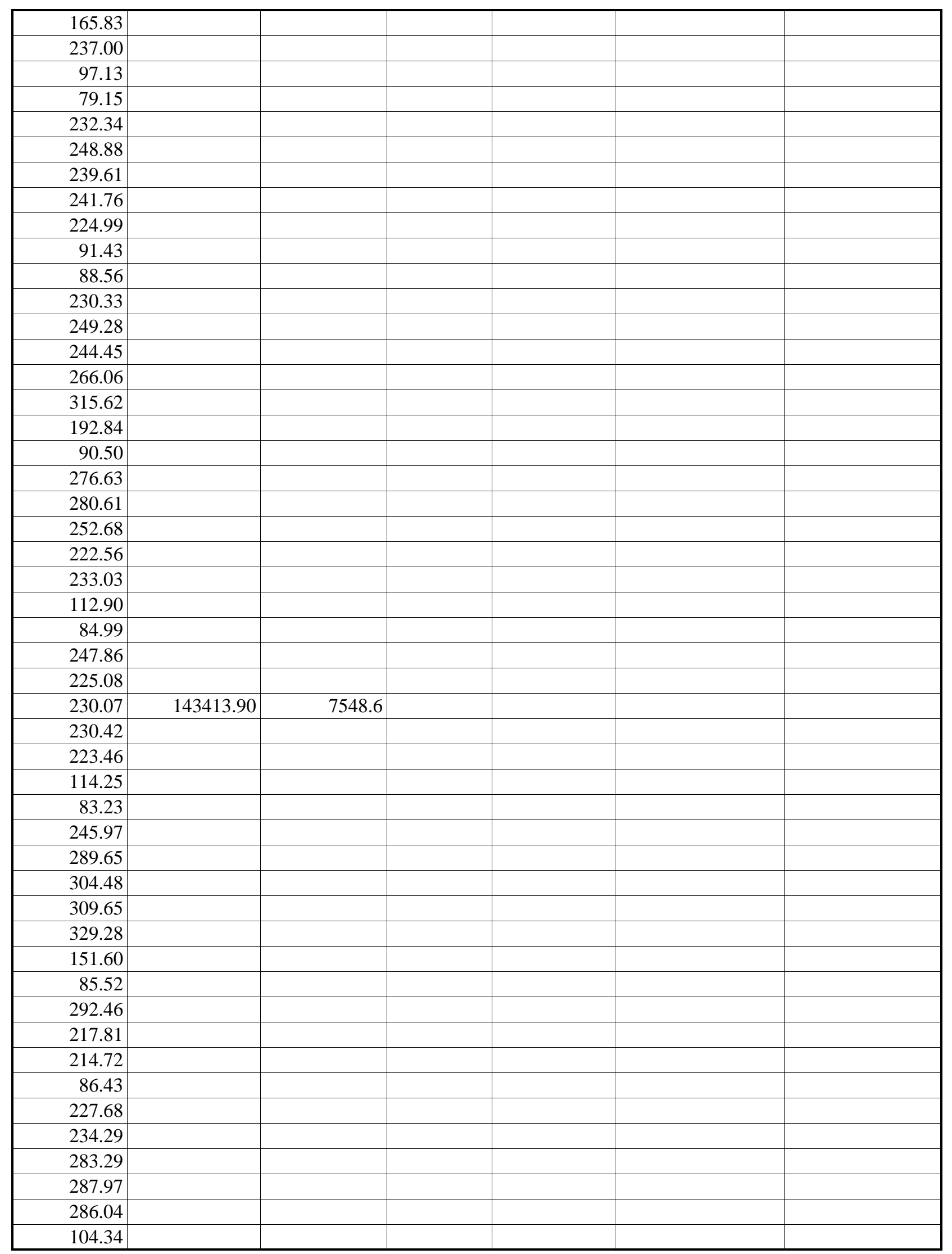


other

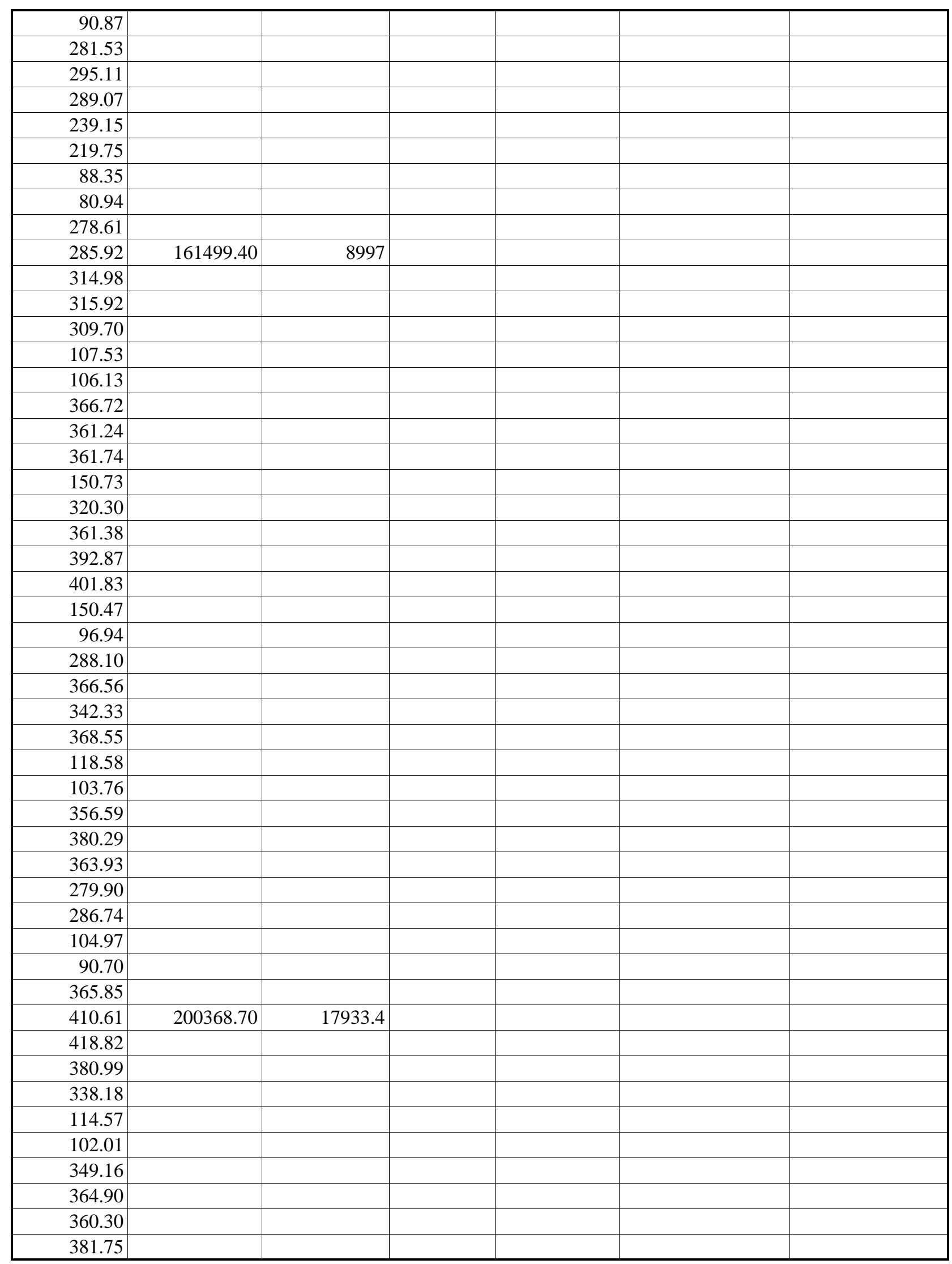


other

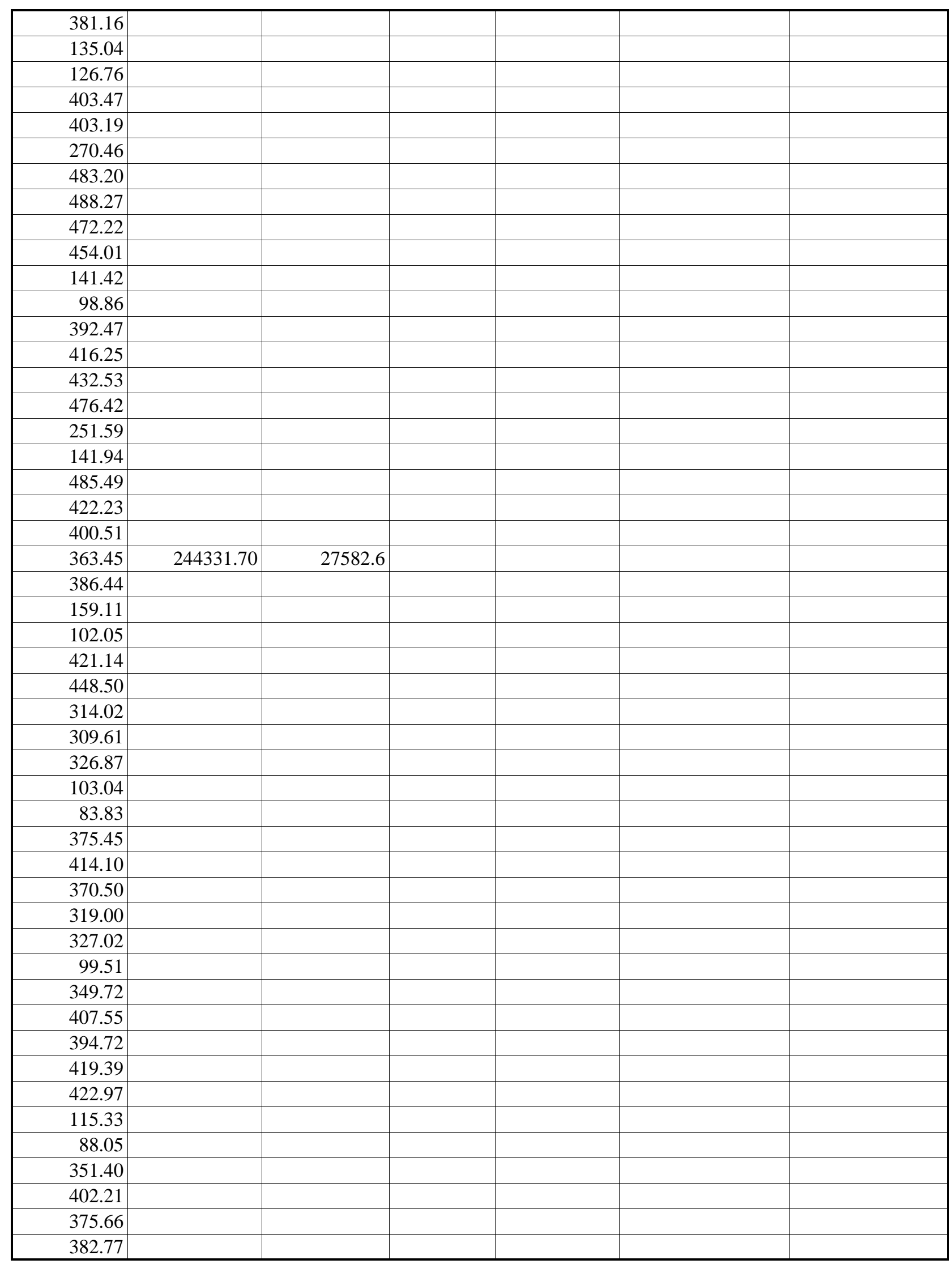


other

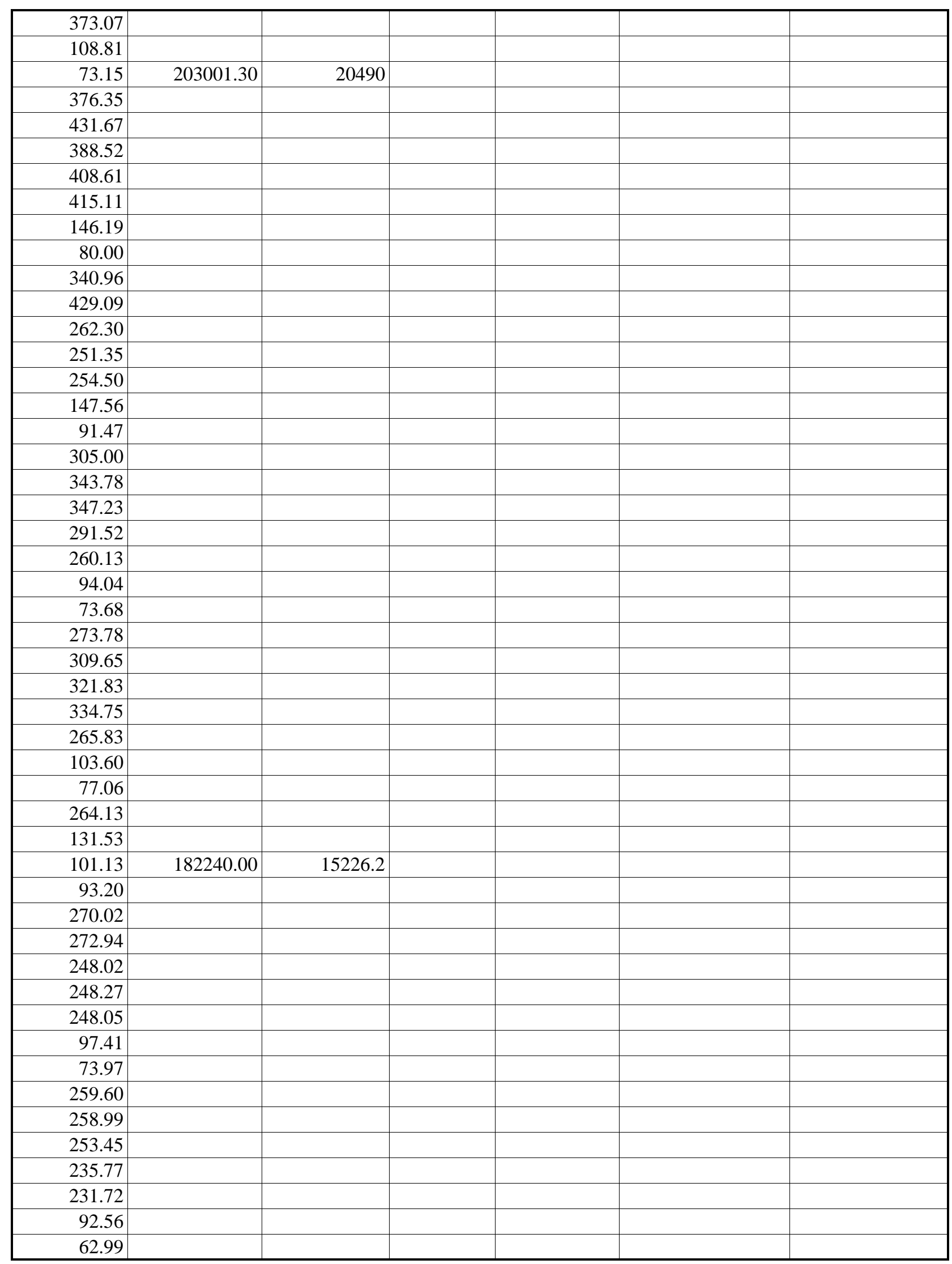


other

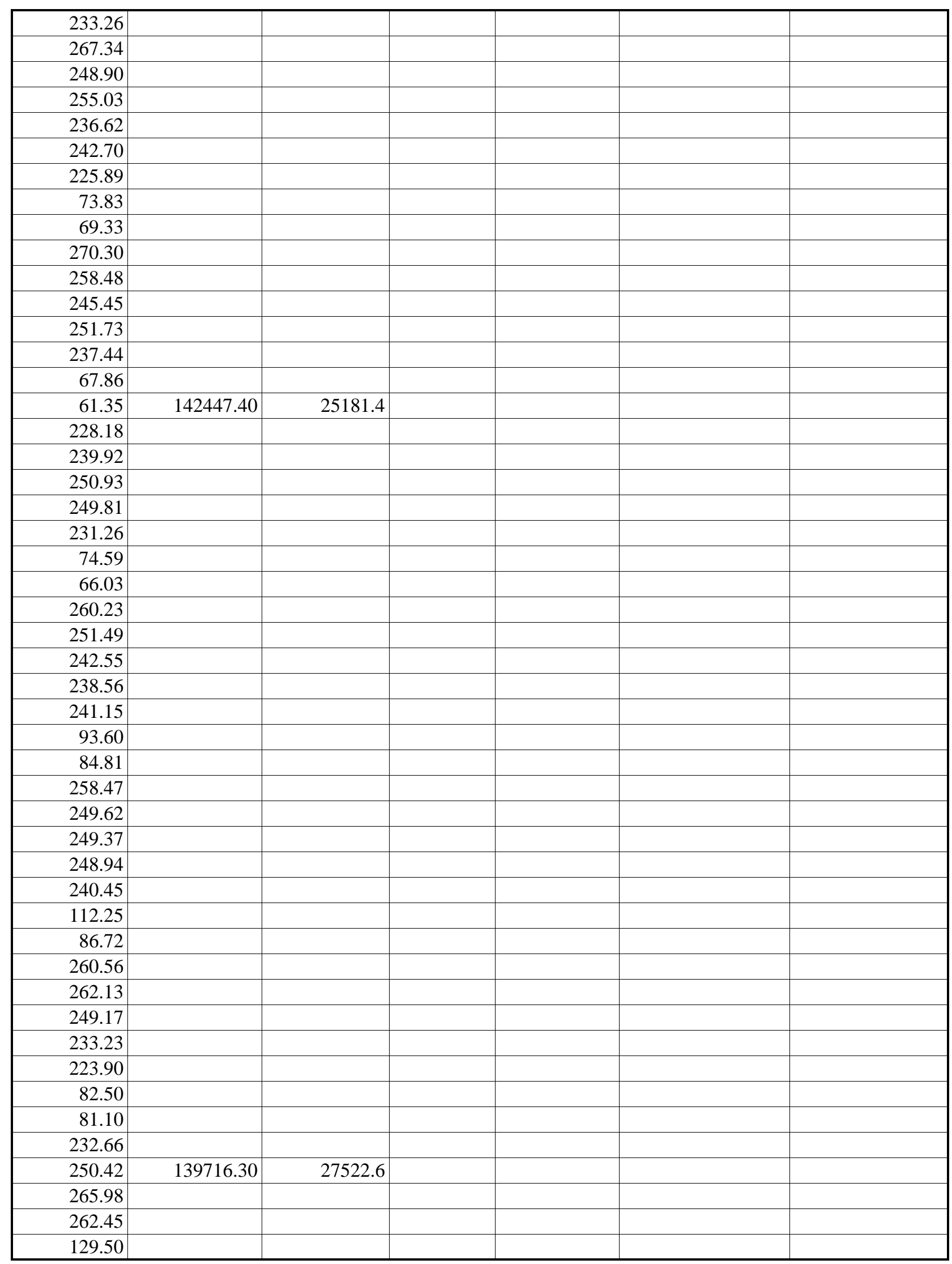


other

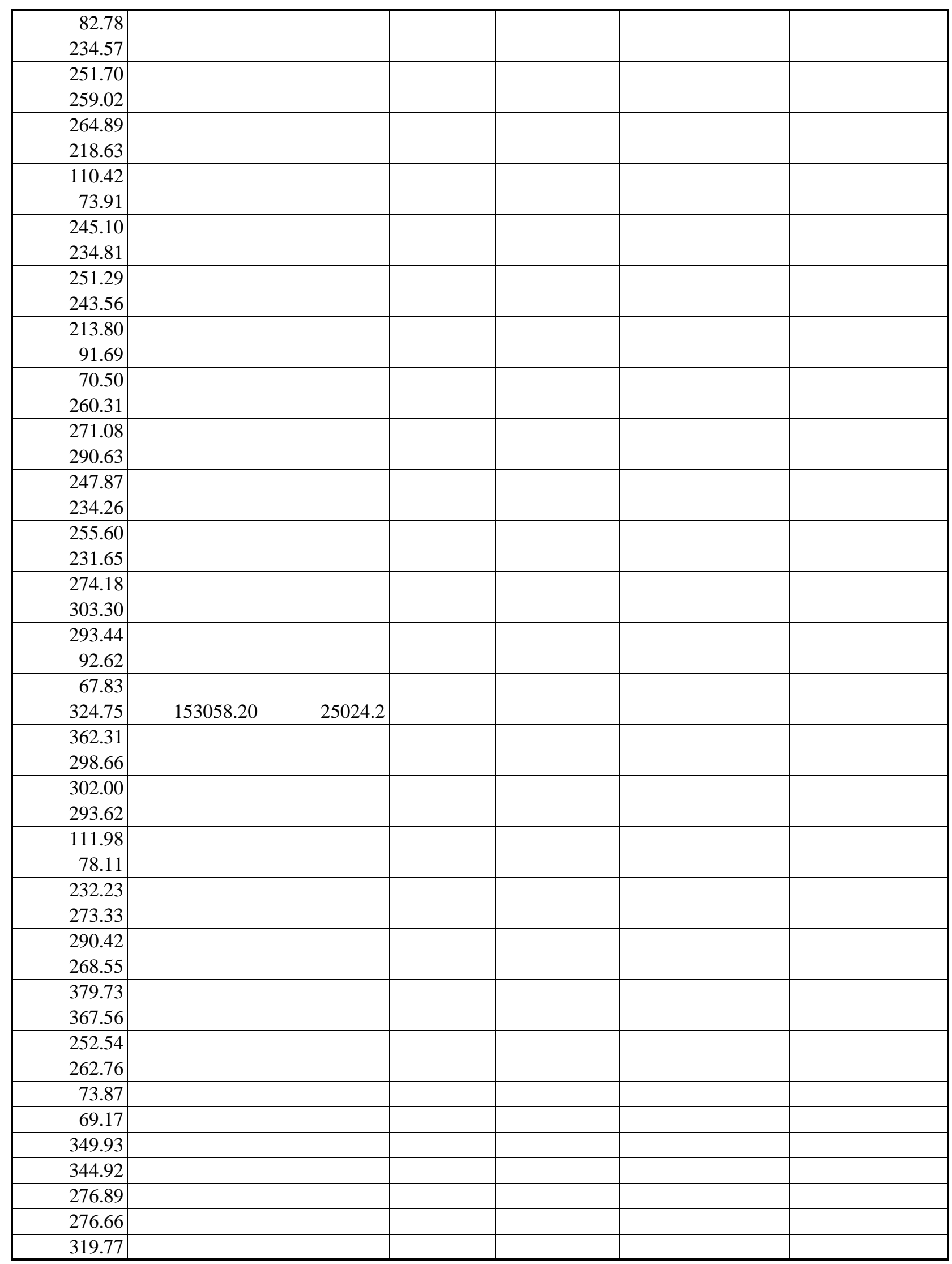


other

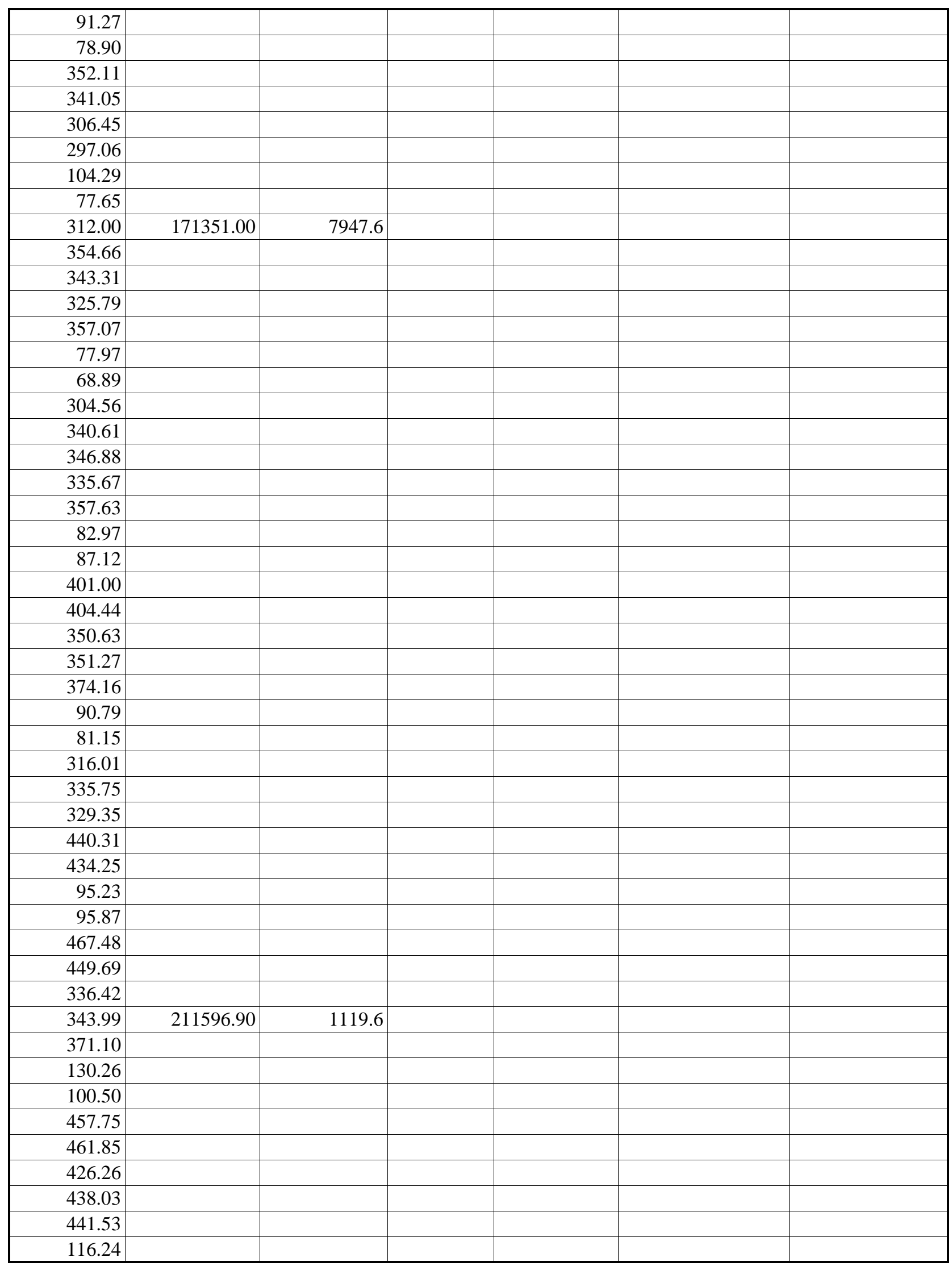


other

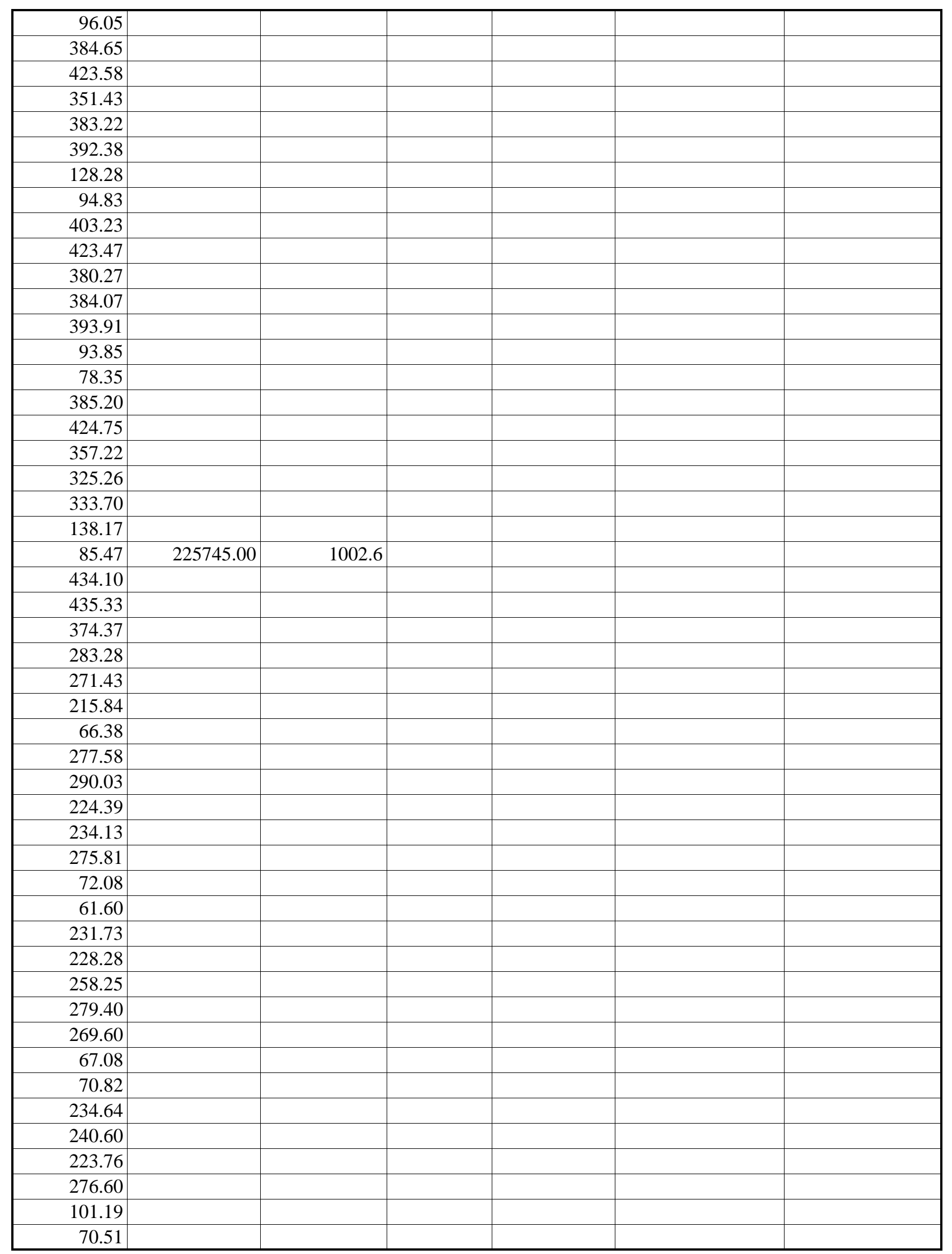


other

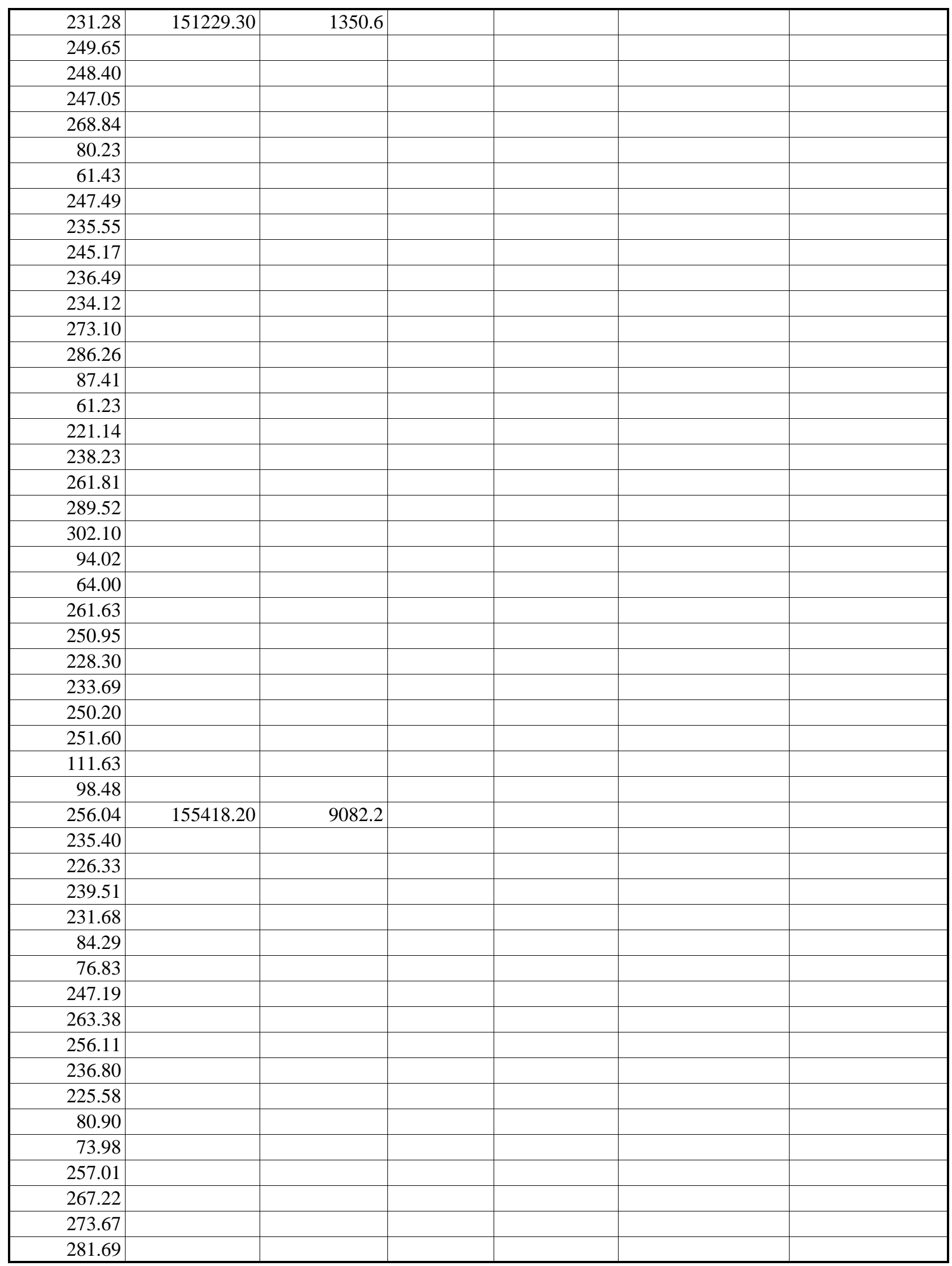


other

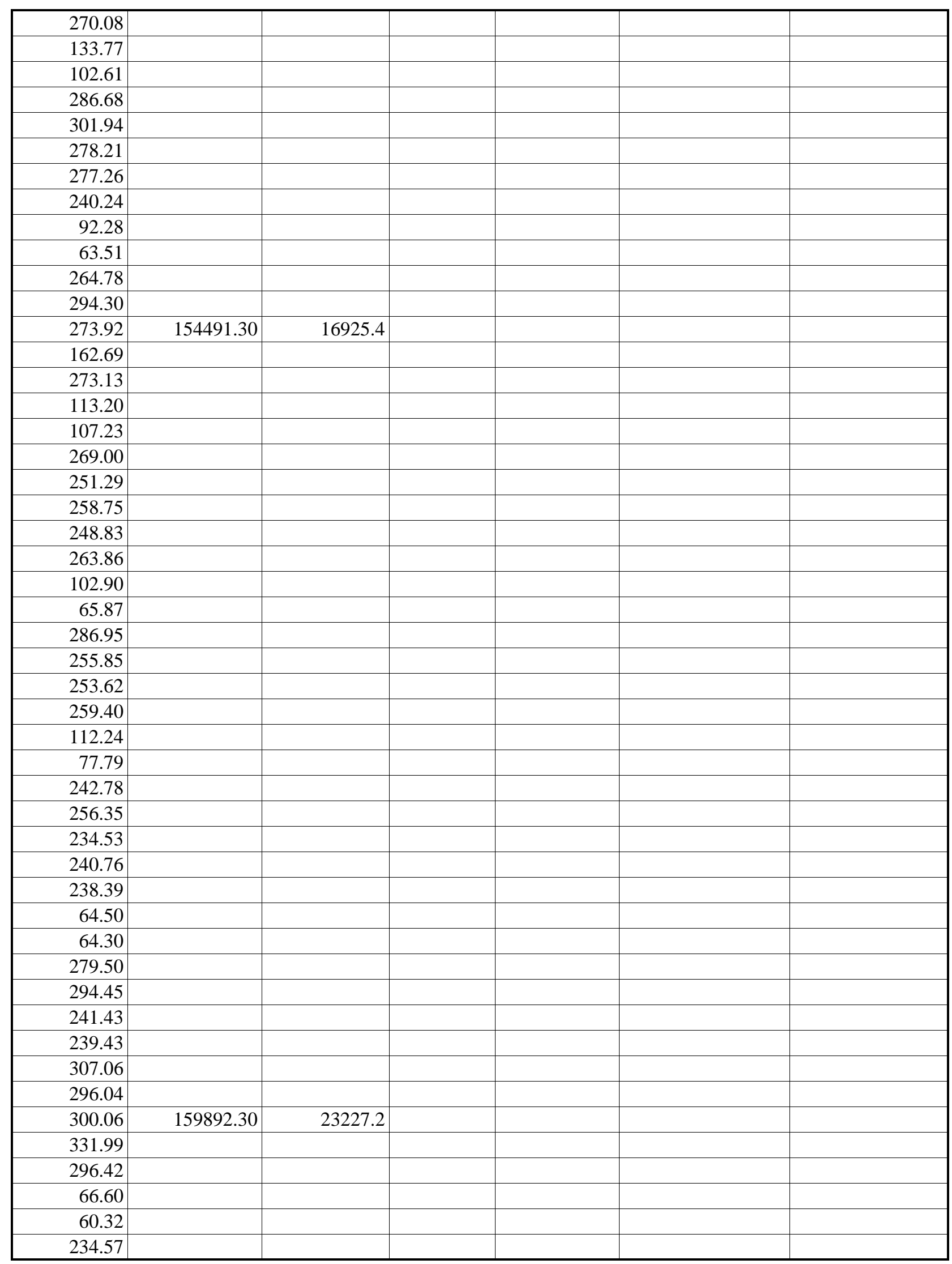


other

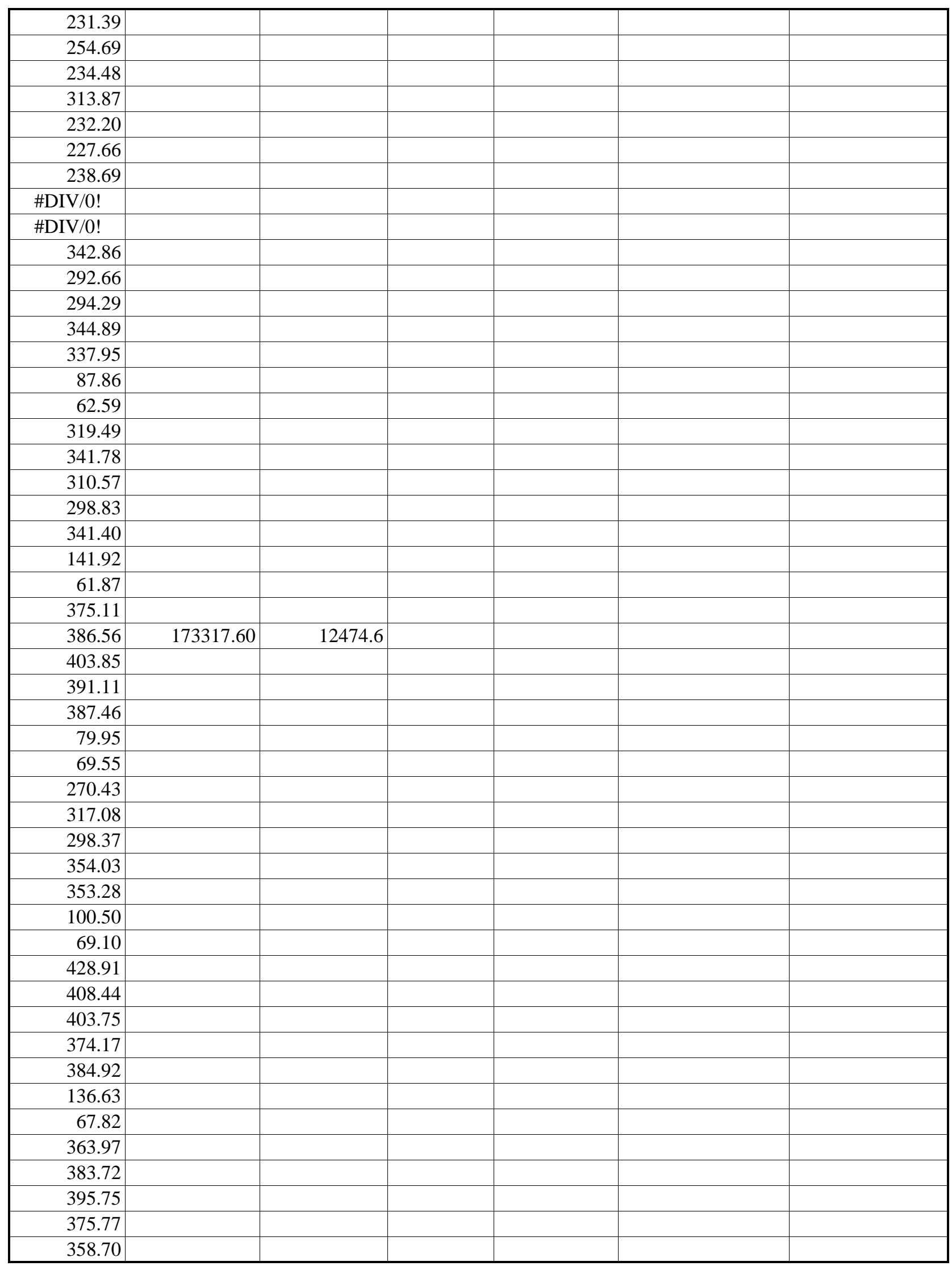


other

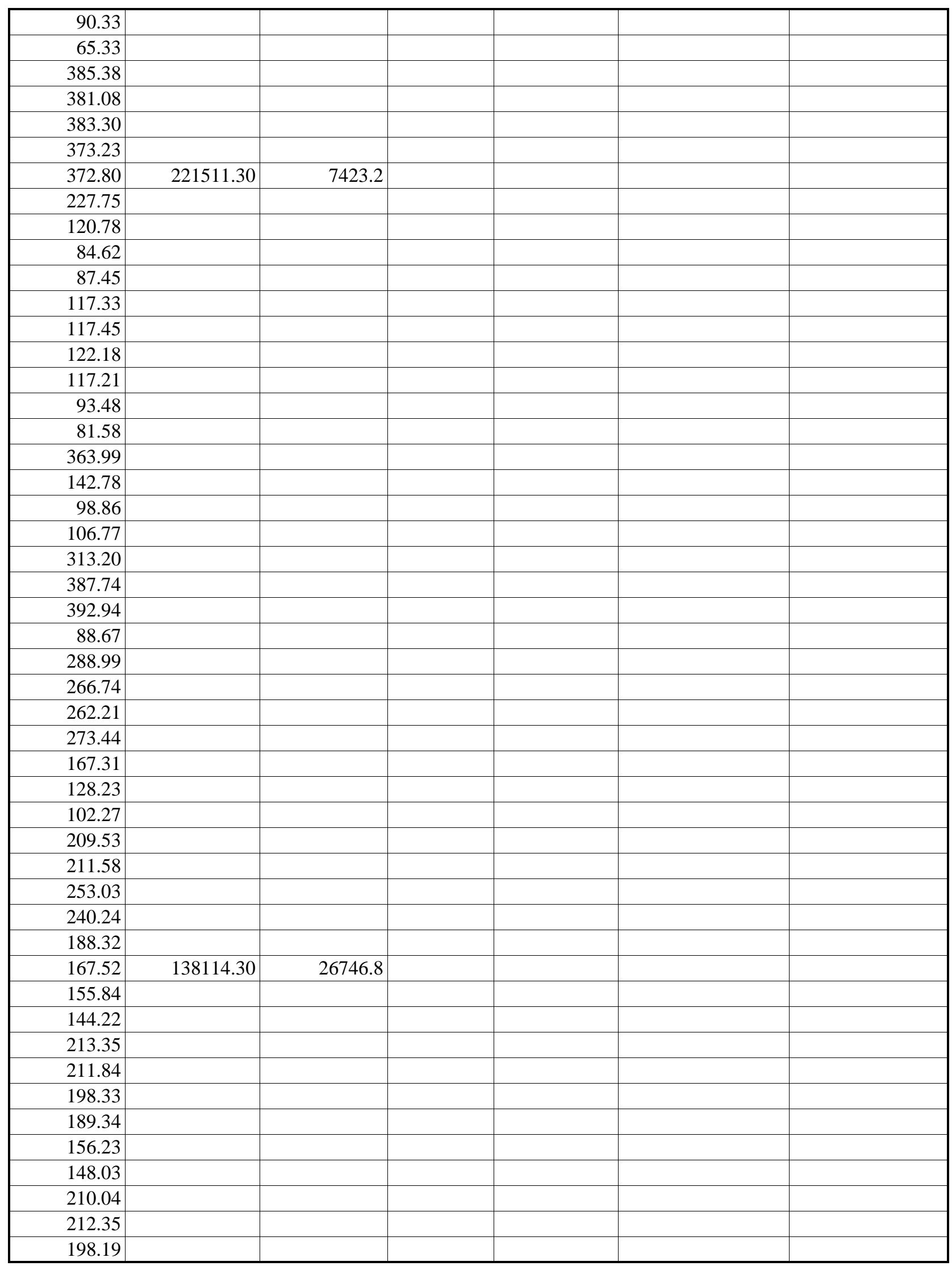


other

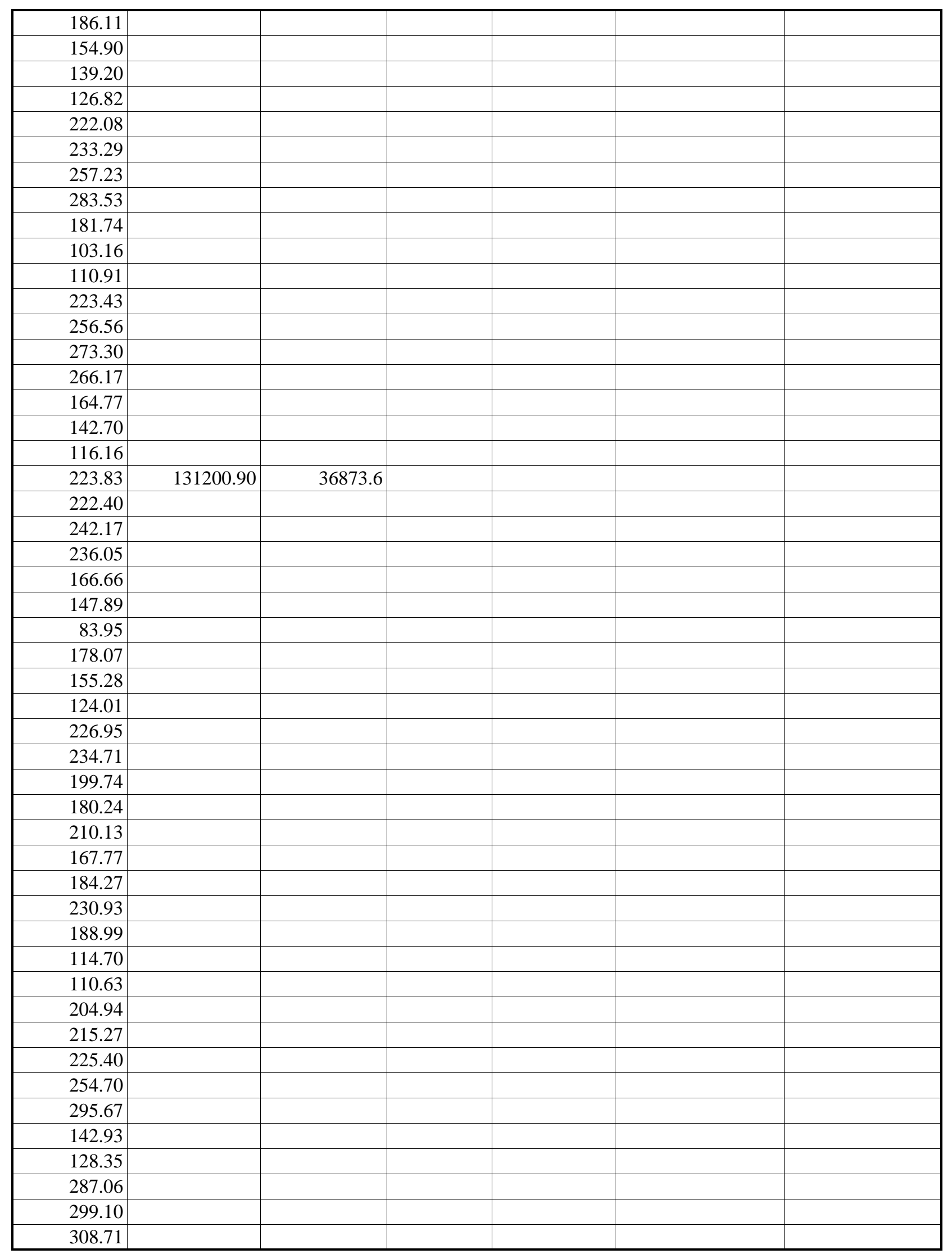


other

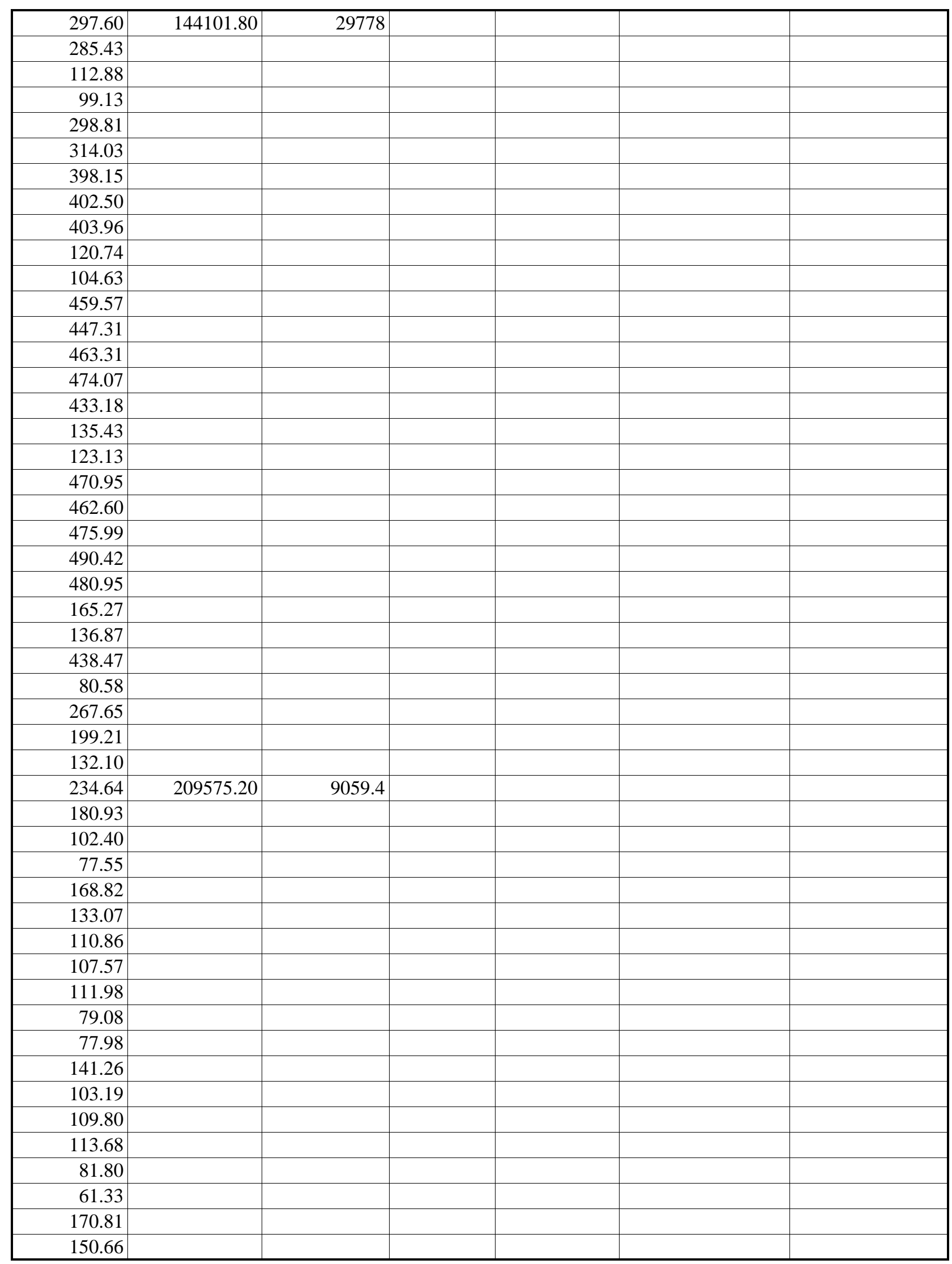


other

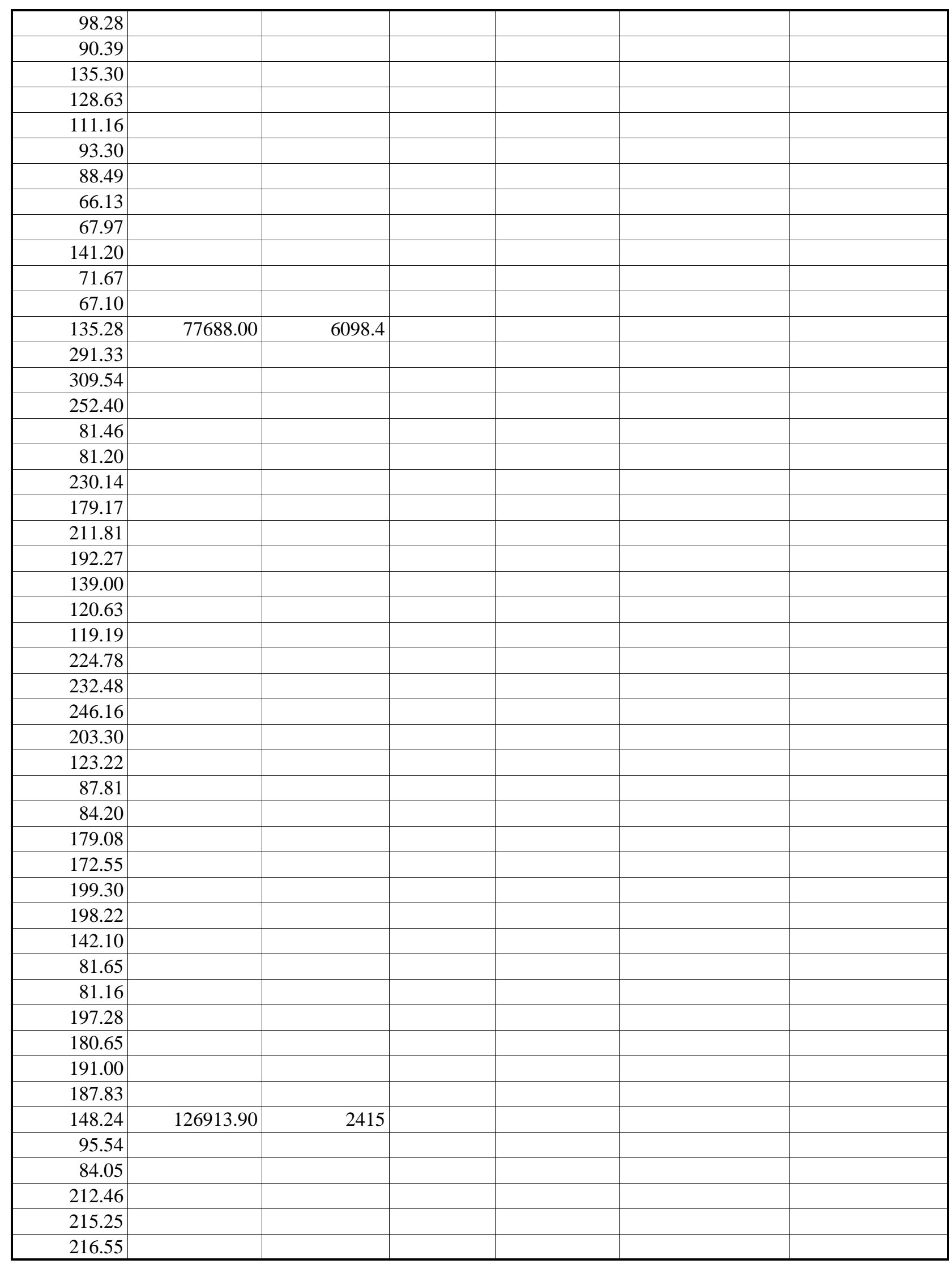


other

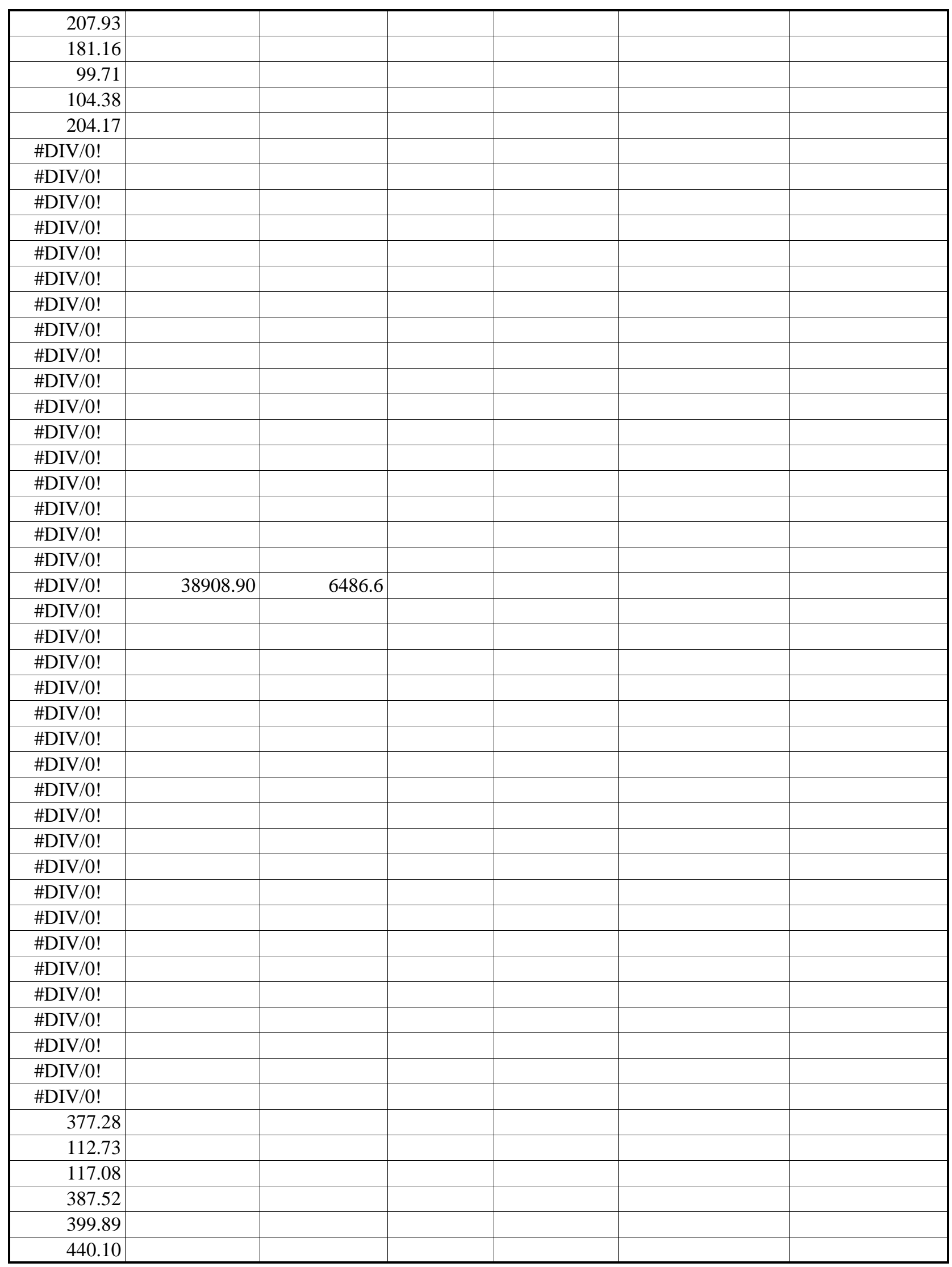


other

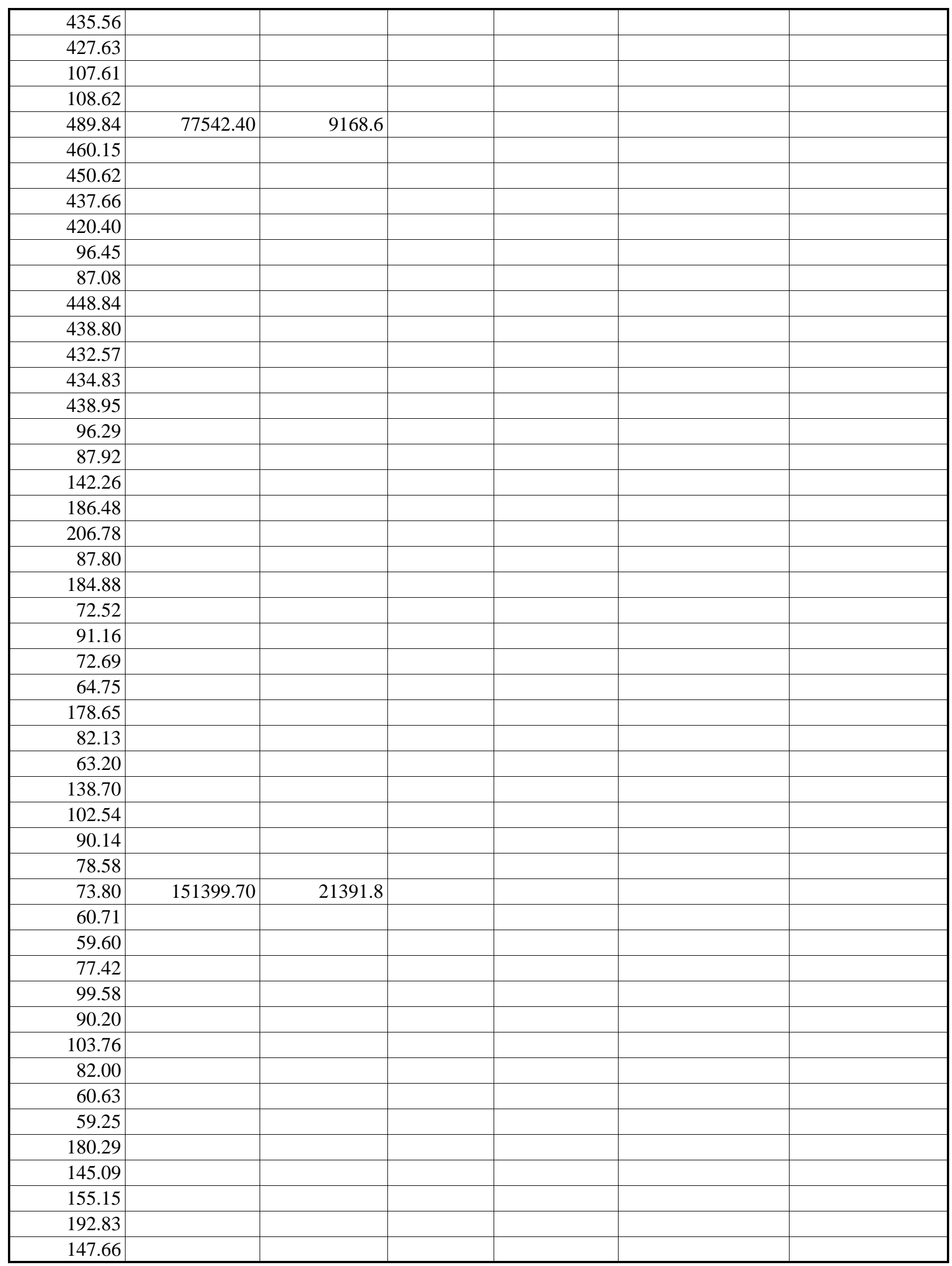


other

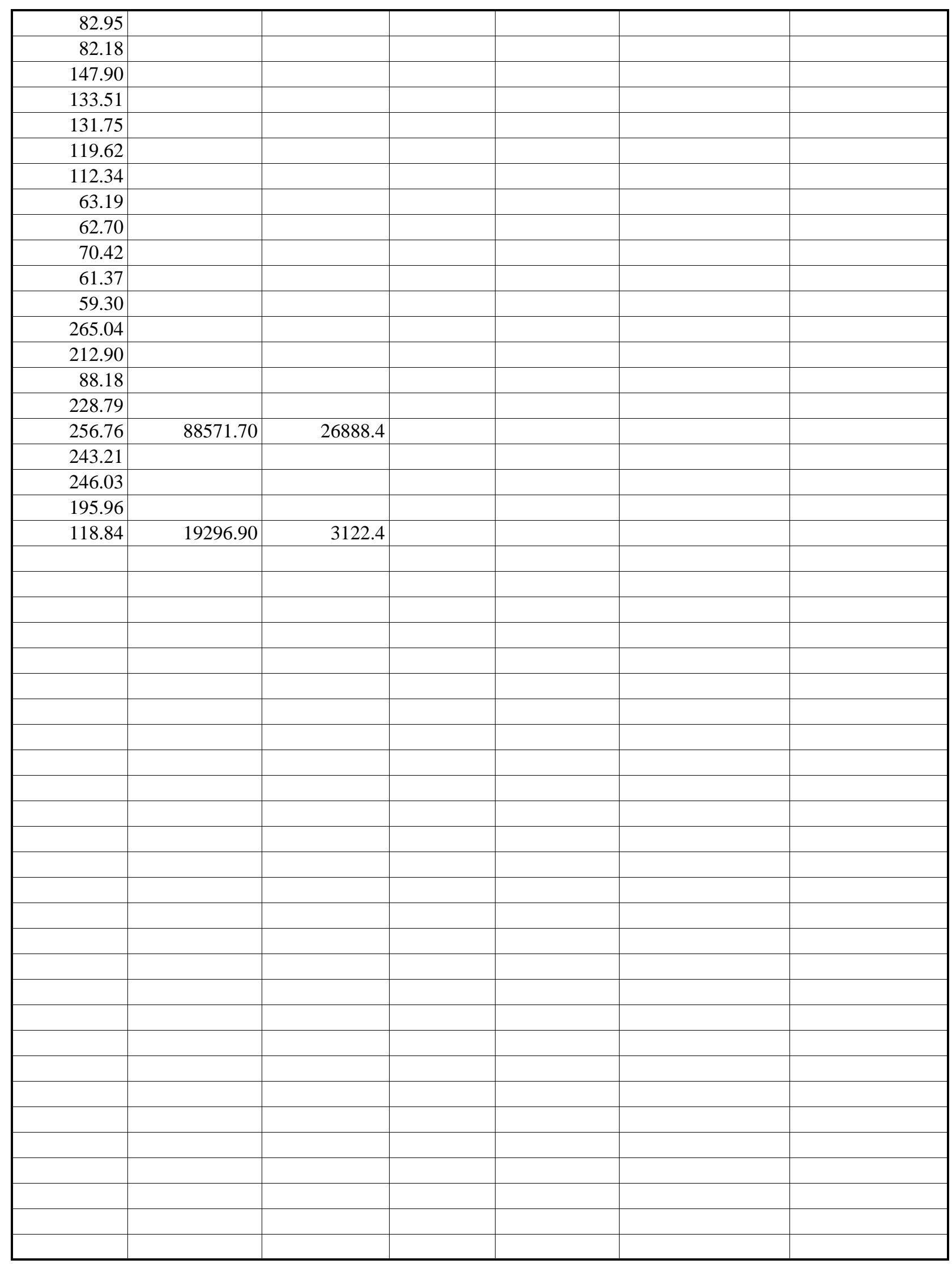




\begin{tabular}{|c|c|c|c|c|c|c|c|c|c|c|}
\hline $\begin{array}{l}\mathrm{wd} / \\
\text { we } \\
0 / 1\end{array}$ & $\begin{array}{c}\text { pre/p } \\
\text { ost } \\
\text { A/B }\end{array}$ & $\begin{array}{l}\text { schyear/br } \\
\text { eaks S/NS }\end{array}$ & date & jday & $\begin{array}{c}\text { orig count } \\
(156)\end{array}$ & 0 & 1 & 2 & 3 & 4 \\
\hline 1 & A & NS & 6/6/1991 & 91157 & 157 & & & & & \\
\hline 1 & A & NS & 6/7/1991 & 91158 & 158 & & & & & \\
\hline 0 & A & NS & 6/8/1991 & 91159 & 159 & & & & & \\
\hline 0 & A & NS & 6/9/1991 & 91160 & 160 & & & & & \\
\hline 1 & A & NS & 6/10/1991 & 91161 & 161 & & & & & \\
\hline 1 & A & NS & 6/11/1991 & 91162 & 162 & & & & & \\
\hline 1 & A & NS & $6 / 12 / 1991$ & 91163 & 163 & & & & & \\
\hline 1 & A & NS & 6/13/1991 & 91164 & 164 & & & & & \\
\hline 1 & A & NS & 6/14/1991 & 91165 & 165 & & & & & \\
\hline 0 & A & NS & 6/15/1991 & 91166 & 166 & & & & & \\
\hline 0 & A & NS & 6/16/1991 & 91167 & 167 & & & & & \\
\hline 1. & A & NS & 6/17/1991 & 91168 & 168 & & & & & \\
\hline 1 & A & NS & $6 / 18 / 1991$ & 91169 & 169 & & & & & \\
\hline 1. & A & NS & $6 / 19 / 1991$ & 91170 & 170 & & & & & \\
\hline 1 & A & NS & $6 / 20 / 1991$ & 91171 & 171 & & & & & \\
\hline 1 & A & NS & $6 / 21 / 1991$ & 91172 & 172 & & & & & \\
\hline 0 & A & NS & 6/22/1991 & 91173 & 173 & & & & & \\
\hline 0 & A & NS & $6 / 23 / 1991$ & 91174 & 174 & & & & & \\
\hline 1 & A & NS & $6 / 24 / 1991$ & 91175 & 175 & & & & & \\
\hline 1 & A & NS & $6 / 25 / 1991$ & 91176 & 176 & & & & & \\
\hline 1 & A & NS & $6 / 26 / 1991$ & 91177 & 177 & & & & & \\
\hline 1 & A & NS & $6 / 27 / 1991$ & 91178 & 178 & & & & & \\
\hline 1 & A & NS & $6 / 28 / 1991$ & 91179 & 179 & & & & & \\
\hline 0 & A & NS & 6/29/1991 & 91180 & 180 & & & & & \\
\hline 0 & A & NS & $6 / 30 / 1991$ & 91181 & 181 & & & & & \\
\hline 1 & A & NS & $7 / 1 / 1991$ & 91182 & 182 & & & & & \\
\hline 1 & A & NS & $7 / 2 / 1991$ & 91183 & 183 & & & & & \\
\hline 1 & A & NS & 7/3/1991 & 91184 & 184 & & & & & \\
\hline 1 & A & NS & 7/4/1991 & 91185 & 185 & & & & & \\
\hline 1 & A & NS & 7/5/1991 & 91186 & 186 & & & & & \\
\hline 0 & A & NS & $7 / 6 / 1991$ & 91187 & 187 & & & & & \\
\hline 0 & A & NS & 7/7/1991 & 91188 & 188 & & & & & \\
\hline 1 & A & NS & 7/8/1991 & 91189 & 189 & & & & & \\
\hline 1 & A & NS & 7/9/1991 & 91190 & 190 & & & & & \\
\hline 1 & A & NS & $7 / 10 / 1991$ & 91191 & 191 & & & & & \\
\hline 1 & A & NS & 7/11/1991 & 91192 & 192 & & & & & \\
\hline 1 & A & NS & 7/12/1991 & 91193 & 193 & & & & & \\
\hline 0 & A & NS & 7/13/1991 & 91194 & 194 & & & & & \\
\hline 0 & A & NS & 7/14/1991 & 91195 & 195 & & & & & \\
\hline 1 & A & NS & $7 / 15 / 1991$ & 91196 & 196 & & & & & \\
\hline 1 & A & NS & $7 / 16 / 1991$ & 91197 & 197 & & & & & \\
\hline 1 & A & NS & 7/17/1991 & 91198 & 198 & & & & & \\
\hline 1 & A & NS & 7/18/1991 & 91199 & 199 & & & & & \\
\hline 1 & A & NS & $7 / 19 / 1991$ & 91200 & 200 & & & & & \\
\hline 0 & A & NS & $7 / 20 / 1991$ & 91201 & 201 & & & & & \\
\hline 0 & A & NS & $7 / 21 / 1991$ & 91202 & 202 & & & & & \\
\hline 1 & A & NS & $7 / 22 / 1991$ & 91203 & 203 & & & & & \\
\hline
\end{tabular}




\begin{tabular}{|c|c|c|c|c|c|c|c|c|}
\hline \begin{tabular}{l|l}
$\mathrm{A}$ \\
\end{tabular} & NS & 7/23/1991 & 91204 & 204 & & & 0.5 & 0.5 \\
\hline $1 \mathrm{~A}$ & NS & 7/24/1991 & 91205 & 205 & & & & 0.5 \\
\hline $1 \mathrm{~A}$ & NS & $7 / 25 / 1991$ & 91206 & 206 & & & & \\
\hline $1 \mathrm{~A}$ & NS & 7/26/1991 & 91207 & 207 & & & & \\
\hline \begin{tabular}{l|l}
$0 \mathrm{~A}$ \\
\end{tabular} & NS & $7 / 27 / 1991$ & 91208 & 208 & & & & \\
\hline $0 \mathrm{~A}$ & NS & 7/28/1991 & 91209 & 209 & & & & \\
\hline $1 \mathrm{~A}$ & NS & 7/29/1991 & 91210 & 210 & & & & \\
\hline $1 \mathrm{~A}$ & NS & 7/30/1991 & 91211 & 211 & & & & \\
\hline $1 \mathrm{~A}$ & NS & 7/31/1991 & 91212 & 212 & & & & \\
\hline $1 \mathrm{~A}$ & NS & 8/1/1991 & 91213 & 213 & & & & \\
\hline $1 \mathrm{~A}$ & NS & 8/2/1991 & 91214 & 214 & & & & \\
\hline $0 \mathrm{~A}$ & NS & 8/3/1991 & 91215 & 215 & & & & \\
\hline $0 \mathrm{~A}$ & NS & 8/4/1991 & 91216 & 216 & & & & \\
\hline $1 \mathrm{~A}$ & NS & 8/5/1991 & 91217 & 217 & & & & \\
\hline $1 \mathrm{~A}$ & NS & 8/6/1991 & 91218 & 218 & & & & \\
\hline $1 \mathrm{~A}$ & NS & 8/7/1991 & 91219 & 219 & & & & \\
\hline $1 \mathrm{~A}$ & NS & 8/8/1991 & 91220 & 220 & & & & \\
\hline $1 \mathrm{~A}$ & NS & 8/9/1991 & 91221 & 221 & & & & \\
\hline $0 \mathrm{~A}$ & NS & $8 / 10 / 1991$ & 91222 & 222 & & & & \\
\hline $0 \mathrm{~A}$ & NS & 8/11/1991 & 91223 & 223 & & & & \\
\hline $1 \mathrm{~A}$ & NS & $8 / 12 / 1991$ & 91224 & 224 & & & & \\
\hline $1 \mathrm{~A}$ & NS & $8 / 13 / 1991$ & 91225 & 225 & & & & \\
\hline $1 \mathrm{~A}$ & NS & $8 / 14 / 1991$ & 91226 & 226 & & & & \\
\hline $1 \mathrm{~A}$ & NS & $8 / 15 / 1991$ & 91227 & 227 & & & & \\
\hline $1 \mathrm{~A}$ & NS & $8 / 16 / 1991$ & 91228 & 228 & & & & \\
\hline $0 \mathrm{~A}$ & NS & $8 / 17 / 1991$ & 91229 & 229 & & & & \\
\hline $0 \mathrm{~A}$ & NS & 8/18/1991 & 91230 & 230 & & & & \\
\hline $1 \mathrm{~A}$ & NS & $8 / 19 / 1991$ & 91231 & 231 & & & & \\
\hline $1 \mathrm{~A}$ & NS & $8 / 20 / 1991$ & 91232 & 232 & & & & \\
\hline $1 \mathrm{~A}$ & NS & $8 / 21 / 1991$ & 91233 & 233 & & & & \\
\hline $1 \mathrm{~A}$ & NS & $8 / 22 / 1991$ & 91234 & 234 & & & & \\
\hline $1 \mathrm{~A}$ & NS & 8/23/1991 & 91235 & 235 & & & & \\
\hline $0 \mathrm{~A}$ & NS & $8 / 24 / 1991$ & 91236 & 236 & & & & \\
\hline $0 \mathrm{~A}$ & NS & $8 / 25 / 1991$ & 91237 & 237 & & & & \\
\hline $1 \mathrm{~A}$ & NS & $8 / 26 / 1991$ & 91238 & 238 & & & & \\
\hline $1 \mathrm{~A}$ & NS & $8 / 27 / 1991$ & 91239 & 239 & & & & \\
\hline $1 \mathrm{~A}$ & NS & 8/28/1991 & 91240 & 240 & & & & \\
\hline $1 \mathrm{~A}$ & NS & $8 / 29 / 1991$ & 91241 & 241 & & & & \\
\hline $1 \mathrm{~A}$ & NS & 8/30/1991 & 91242 & 242 & & & & \\
\hline $0 \mathrm{~A}$ & NS & 8/31/1991 & 91243 & 243 & & & & \\
\hline $0 \mathrm{~A}$ & NS & 9/1/1991 & 91244 & 244 & & & & \\
\hline $1 \mathrm{~A}$ & NS & 9/2/1991 & 91245 & 245 & & & & \\
\hline $1 \mathrm{~A}$ & S & 9/3/1991 & 91246 & 246 & & & & \\
\hline $1 \mathrm{~A}$ & $S$ & 9/4/1991 & 91247 & 247 & & 0.5 & & \\
\hline $1 \mathrm{~A}$ & S & 9/5/1991 & 91248 & 248 & 0.5 & & & 0.5 \\
\hline $1 \mathrm{~A}$ & S & 9/6/1991 & 91249 & 249 & & & & \\
\hline $0 \mathrm{~A}$ & S & 9/7/1991 & 91250 & 250 & & 0.5 & & \\
\hline $0 \mathrm{~A}$ & S & 9/8/1991 & 91251 & 251 & & & & \\
\hline $1 \mathrm{~A}$ & S & 9/9/1991 & 91252 & 252 & & & & \\
\hline $1 \mathrm{~A}$ & $S$ & 9/10/1991 & 91253 & 253 & & & & \\
\hline $1 \mathrm{~A}$ & $S$ & 9/11/1991 & 91254 & 254 & & & & \\
\hline
\end{tabular}




\begin{tabular}{|c|c|c|c|c|c|c|c|c|c|c|}
\hline $1 A$ & A & $S$ & 9/12/1991 & 91255 & 255 & & & & & \\
\hline $1 A$ & A & S & 9/13/1991 & 91256 & 256 & & & & & \\
\hline $0 A$ & A & S & 9/14/1991 & 91257 & 257 & & & & & \\
\hline $0 A$ & A & S & 9/15/1991 & 91258 & 258 & & & & & \\
\hline $1 A$ & A & S & 9/16/1991 & 91259 & 259 & & & & & \\
\hline $1 A$ & A & S & 9/17/1991 & 91260 & 260 & & & & & \\
\hline $1 A$ & A & S & 9/18/1991 & 91261 & 261 & & & & & \\
\hline $1 A$ & A & S & 9/19/1991 & 91262 & 262 & & & & & \\
\hline $1 A$ & A & S & 9/20/1991 & 91263 & 263 & 0.5 & & & 0.5 & \\
\hline $0 A$ & A & S & 9/21/1991 & 91264 & 264 & & & 0.5 & & \\
\hline $0 A$ & A & S & 9/22/1991 & 91265 & 265 & 0.5 & & 0.5 & & \\
\hline $1 A$ & A & S & 9/23/1991 & 91266 & 266 & & & & & \\
\hline $1 A$ & A & S & 9/24/1991 & 91267 & 267 & & & & & \\
\hline $1 A$ & A & S & 9/25/1991 & 91268 & 268 & & & & & \\
\hline $1 A$ & A & S & 9/26/1991 & 91269 & 269 & & & & & \\
\hline $1 A$ & A & S & 9/27/1991 & 91270 & 270 & & 0.5 & & & \\
\hline $0 A$ & A & S & 9/28/1991 & 91271 & 271 & & & & & \\
\hline 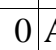 & A & S & 9/29/1991 & 91272 & 272 & & & & & \\
\hline $1 A$ & A & S & 9/30/1991 & 91273 & 273 & & & & & \\
\hline $1 A$ & A & S & 10/1/1991 & 91274 & 274 & & & & & \\
\hline $1 A$ & A & S & 10/2/1991 & 91275 & 275 & & & & & \\
\hline $1 A$ & A & S & 10/3/1991 & 91276 & 276 & & & & & \\
\hline $1 A$ & A & S & 10/4/1991 & 91277 & 277 & & & & & \\
\hline 0 : & A & S & 10/5/1991 & 91278 & 278 & & & & & \\
\hline $0 A$ & A & $S$ & $10 / 6 / 1991$ & 91279 & 279 & & & & & \\
\hline $1 \mid A$ & A & S & 10/7/1991 & 91280 & 280 & & & & & \\
\hline $1 A$ & A & S & 10/8/1991 & 91281 & 281 & & & & & \\
\hline $1 A$ & A & S & 10/9/1991 & 91282 & 282 & & & & & \\
\hline $1 A$ & A & S & 10/10/1991 & 91283 & 283 & & & & & \\
\hline $1 A$ & A & S & 10/11/1991 & 91284 & 284 & & & & & \\
\hline $0 A$ & $\mathrm{~A}$ & S & $10 / 12 / 1991$ & 91285 & 285 & & & & & \\
\hline $0 A$ & A & S & 10/13/1991 & 91286 & 286 & & & & & \\
\hline $1 A$ & A & S & 10/14/1991 & 91287 & 287 & & & & & \\
\hline $1 A$ & $\mathrm{~A}$ & S & $10 / 15 / 1991$ & 91288 & 288 & & & & & \\
\hline $1 A$ & A & S & 10/16/1991 & 91289 & 289 & & & & & \\
\hline $1 / A$ & A & S & 10/17/1991 & 91290 & 290 & & & & & \\
\hline $1 \mid A$ & A & S & 10/18/1991 & 91291 & 291 & & & & & \\
\hline $0 A$ & A & $S$ & $10 / 19 / 1991$ & 91292 & 292 & & & & & \\
\hline $0 A$ & A & S & 10/20/1991 & 91293 & 293 & & & & & \\
\hline $1 A$ & A & S & 10/21/1991 & 91294 & 294 & & & & & \\
\hline $1 A$ & A & S & 10/22/1991 & 91295 & 295 & & & & & \\
\hline $1 A$ & A & NS & 10/23/1991 & 91296 & 296 & & & & & \\
\hline $1 A$ & A & S & 10/24/1991 & 91297 & 297 & & & & & \\
\hline $1 A$ & A & $S$ & $10 / 25 / 1991$ & 91298 & 298 & & & & & \\
\hline 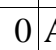 & $\mathrm{A}$ & S & 10/26/1991 & 91299 & 299 & & & & & \\
\hline $0 A$ & A & S & 10/27/1991 & 91300 & 300 & & & & & \\
\hline $1 A$ & $\mathrm{~A}$ & S & $10 / 28 / 1991$ & 91301 & 301 & & & & & \\
\hline $1 A$ & A & S & $10 / 29 / 1991$ & 91302 & 302 & & & & & \\
\hline $1 \mid A$ & A & S & 10/30/1991 & 91303 & 303 & & & & & \\
\hline $1 A$ & A & S & 10/31/1991 & 91304 & 304 & & & & & \\
\hline $1 / A$ & A & S & 11/1/1991 & 91305 & 305 & & & & & \\
\hline
\end{tabular}




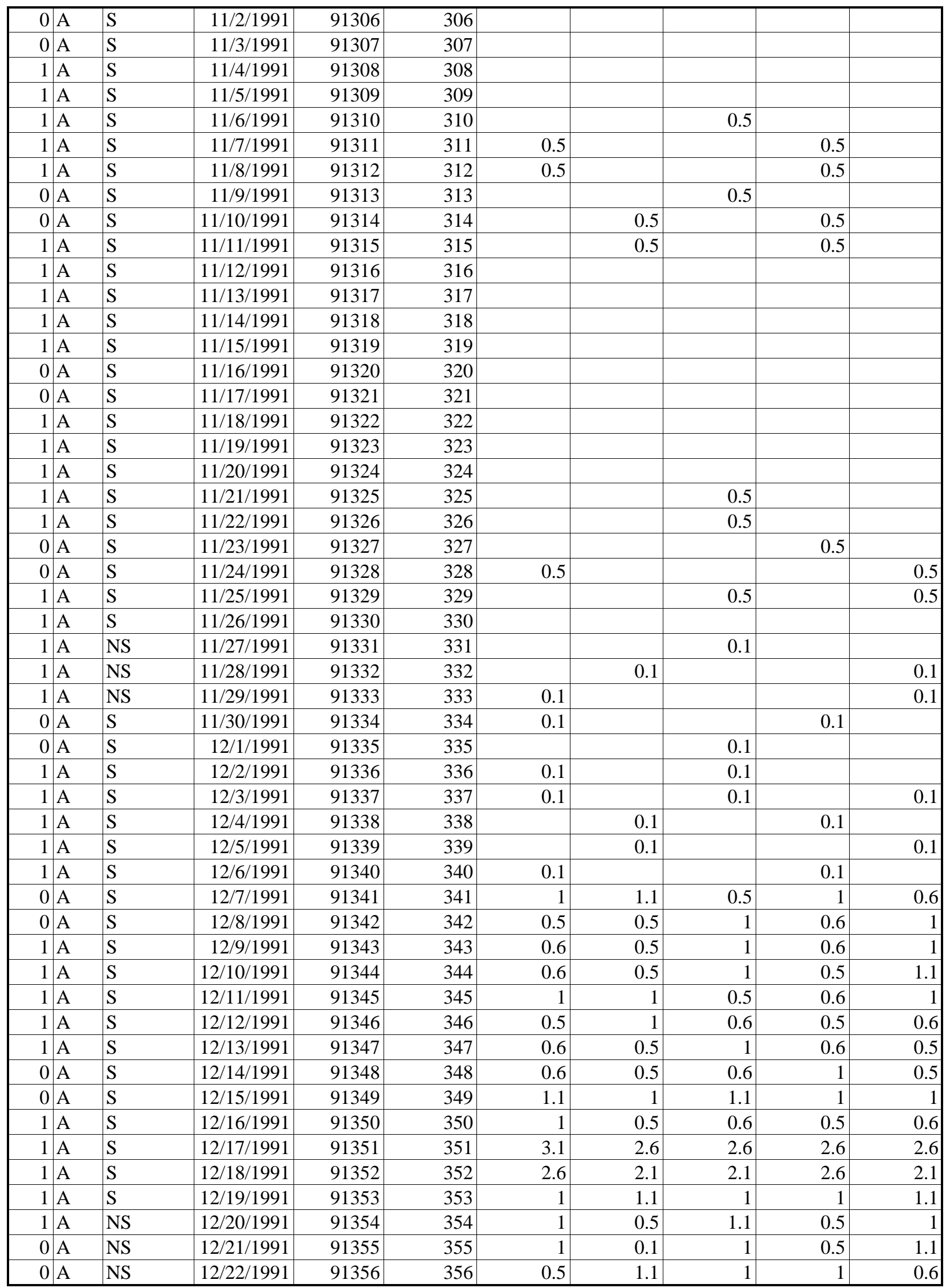




\begin{tabular}{|c|c|c|c|c|c|c|c|c|c|c|}
\hline $1 \mathrm{~A}$ & & NS & 12/23/1991 & 91357 & 357 & 0.5 & 1 & 0.5 & 0.5 & 1 \\
\hline $1 A$ & $\mathrm{~A}$ & NS & $12 / 24 / 1991$ & 91358 & 358 & & 0.5 & 0.5 & & 0.5 \\
\hline $1 A$ & A & NS & 12/25/1991 & 91359 & 359 & 0.1 & 0.5 & 1 & 0.5 & 0.5 \\
\hline $1 A$ & A & NS & 12/26/1991 & 91360 & 360 & 0.5 & 0.5 & 0.5 & 0.5 & 1.1 \\
\hline $1 A$ & A & NS & $12 / 27 / 1991$ & 91361 & 361 & 2.1 & 2.6 & 1.5 & 1.5 & 2.1 \\
\hline $0 A$ & $A$ & NS & 12/28/1991 & 91362 & 362 & 1 & 1.5 & 1.1 & 1.5 & 1 \\
\hline $0 A$ & A & NS & 12/29/1991 & 91363 & 363 & 1 & 1 & 1.5 & 1 & 1 \\
\hline $1 A$ & A & NS & 12/30/1991 & 91364 & 364 & 0.5 & 1 & 1 & 0.5 & 0.5 \\
\hline $1 A$ & $A$ & NS & $12 / 31 / 1991$ & 91365 & 365 & 0.5 & 0.5 & & 0.5 & 0.5 \\
\hline $1 A$ & A & NS & 1/1/1992 & 92001 & 366 & & & & & \\
\hline $1 \mathrm{~A}$ & A & NS & $1 / 2 / 1992$ & 92002 & 1 & 0.5 & 0.5 & 0.5 & 0.5 & 0.5 \\
\hline $1 A$ & A & NS & $1 / 3 / 1992$ & 92003 & 2 & & 0.5 & 0.1 & 0.5 & \\
\hline $0 A$ & A & NS & $1 / 4 / 1992$ & 92004 & 3 & 1 & 1 & 1 & 1 & 1 \\
\hline $0 A$ & A & NS & 1/5/1992 & 92005 & 4 & 1.5 & 1.5 & 1.5 & 1.6 & 1.5 \\
\hline $1 A$ & A & NS & $1 / 6 / 1992$ & 92006 & 5 & 0.5 & 1.5 & 1 & 1 & 1 \\
\hline $1 A$ & A & $S$ & 1/7/1992 & 92007 & 6 & 1.5 & 1 & 1.1 & 1 & 1 \\
\hline $1 A$ & A & $S$ & $1 / 8 / 1992$ & 92008 & 7 & 2.6 & 3.1 & 2.6 & 3.1 & 2.6 \\
\hline $1 A$ & A & S & $1 / 9 / 1992$ & 92009 & 8 & 2.6 & 2.6 & 2.6 & 2.1 & 2.6 \\
\hline $1 A$ & A & S & $1 / 10 / 1992$ & 92010 & 9 & 1.5 & 1.6 & 0.5 & 2.1 & 1.5 \\
\hline $0 A$ & A & S & $1 / 11 / 1992$ & 92011 & 10 & 3.1 & 3.1 & 3.1 & 3.1 & 3.1 \\
\hline $0 A$ & A & S & $1 / 12 / 1992$ & 92012 & 11 & 1.5 & 1 & 1.6 & 1 & 1.5 \\
\hline $1 A$ & A & S & $1 / 13 / 1992$ & 92013 & 12 & 1.5 & 1 & 1.6 & 1.5 & 1 \\
\hline $1 A$ & A & $S$ & $1 / 14 / 1992$ & 92014 & 13 & 1 & 1.1 & 1 & 1.5 & 1.6 \\
\hline $1 A$ & A & S & $1 / 15 / 1992$ & 92015 & 14 & 3.6 & 3.6 & 4.1 & 4.7 & 3.6 \\
\hline $1 A$ & A & S & 1/16/1992 & 92016 & 15 & 3.1 & 3.6 & 3.1 & 3.7 & 3.1 \\
\hline $1 A$ & A & NS & $1 / 17 / 1992$ & 92017 & 16 & 5.2 & 4.2 & 4.1 & 4.6 & 5.2 \\
\hline $0 A$ & A & $S$ & $1 / 18 / 1992$ & 92018 & 17 & 4.2 & 4.1 & 4.7 & 4.6 & 4.1 \\
\hline $0 A$ & A & S & $1 / 19 / 1992$ & 92019 & 18 & 2.6 & 2.1 & 2.6 & 2.1 & 2.1 \\
\hline $1 A$ & A & $S$ & 1/20/1992 & 92020 & 19 & 2.1 & 2.6 & 2.1 & 2.6 & 2.1 \\
\hline $1 A$ & A & $S$ & $1 / 21 / 1992$ & 92021 & 20 & 2.1 & 2.1 & 1.5 & 2.1 & 2.1 \\
\hline $1 A$ & A & S & $1 / 22 / 1992$ & 92022 & 21 & 3.6 & 3.1 & 3.6 & 3.6 & 3.6 \\
\hline $1 A$ & A & $S$ & 1/23/1992 & 92023 & 22 & 3.1 & 3.1 & 3.1 & 3.1 & 3.1 \\
\hline $1 A$ & A & $S$ & $1 / 24 / 1992$ & 92024 & 23 & 2.6 & 2.6 & 2.6 & 3.1 & 2.1 \\
\hline $0 A$ & A & S & $1 / 25 / 1992$ & 92025 & 24 & 3.1 & 3.1 & 3.1 & 2.6 & 3.1 \\
\hline $0 A$ & A & $S$ & 1/26/1992 & 92026 & 25 & 1 & 1 & 1 & 0.6 & 1 \\
\hline $1 A$ & A & $S$ & $1 / 27 / 1992$ & 92027 & 26 & 1 & 1 & 1 & 1 & 0.5 \\
\hline $1 A$ & A & S & $1 / 28 / 1992$ & 92028 & 27 & 1 & 0.5 & 1 & 0.5 & 1 \\
\hline $1 A$ & A & $\mathrm{S}$ & 1/29/1992 & 92029 & 28 & 1 & 1.1 & 1 & 1 & 1 \\
\hline $1 A$ & A & S & $1 / 30 / 1992$ & 92030 & 29 & 1.1 & 1.5 & 1.1 & 1.5 & 1.5 \\
\hline $1 A$ & A & S & $1 / 31 / 1992$ & 92031 & 30 & 1 & 1.6 & 1 & 1 & 1.6 \\
\hline $0 \mathrm{E}$ & $B$ & S & 2/1/1992 & 92032 & 31 & 0.1 & 0.5 & & 0.6 & 0.5 \\
\hline $0 \mathrm{E}$ & $B$ & $S$ & 2/2/1992 & 92033 & 32 & 1.5 & 0.6 & 0.5 & 1 & 1.1 \\
\hline $1 \mathrm{E}$ & B & S & 2/3/1992 & 92034 & 33 & 1.5 & 1 & 1.1 & 1 & 0.5 \\
\hline $1 \mathrm{E}$ & $\mathrm{B}$ & S & 2/4/1992 & 92035 & 34 & 1.1 & 1 & 1 & 0.5 & 1.1 \\
\hline $1 \mathrm{E}$ & $\mathrm{B}$ & S & 2/5/1992 & 92036 & 35 & 1 & 1 & 0.5 & 1.1 & 1 \\
\hline $1 \mathrm{E}$ & B & $S$ & 2/6/1992 & 92037 & 36 & 1 & 1.5 & 1.6 & 1 & 1.6 \\
\hline $1 \mathrm{E}$ & $B$ & S & 2/7/1992 & 92038 & 37 & 2.6 & 2.6 & 2.6 & 2.6 & 2.6 \\
\hline $0 \mathrm{E}$ & $B$ & $S$ & 2/8/1992 & 92039 & 38 & 1.5 & 1.1 & 1.5 & 1 & 1.6 \\
\hline $0 \mathrm{E}$ & $B$ & $S$ & 2/9/1992 & 92040 & 39 & 1 & 1.1 & 0.5 & 1 & 1.1 \\
\hline $1 \mathrm{E}$ & B & S & $2 / 10 / 1992$ & 92041 & 40 & 1.1 & 1 & 0.5 & 1.6 & 0.5 \\
\hline $1 \mathrm{~F}$ & B & $S$ & 2/11/1992 & 92042 & 41 & 1 & 1.6 & 1 & 1.5 & 1.1 \\
\hline
\end{tabular}




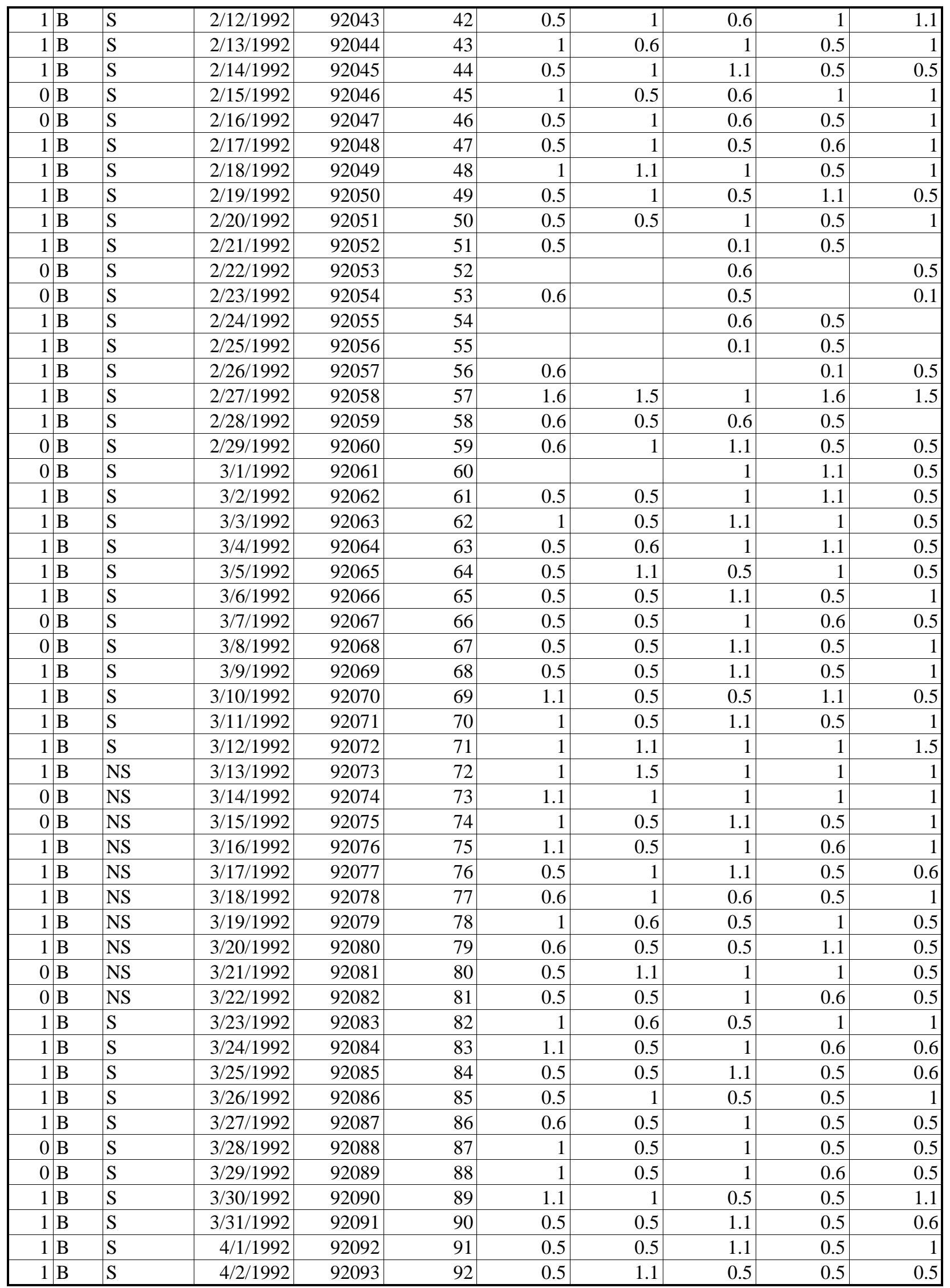




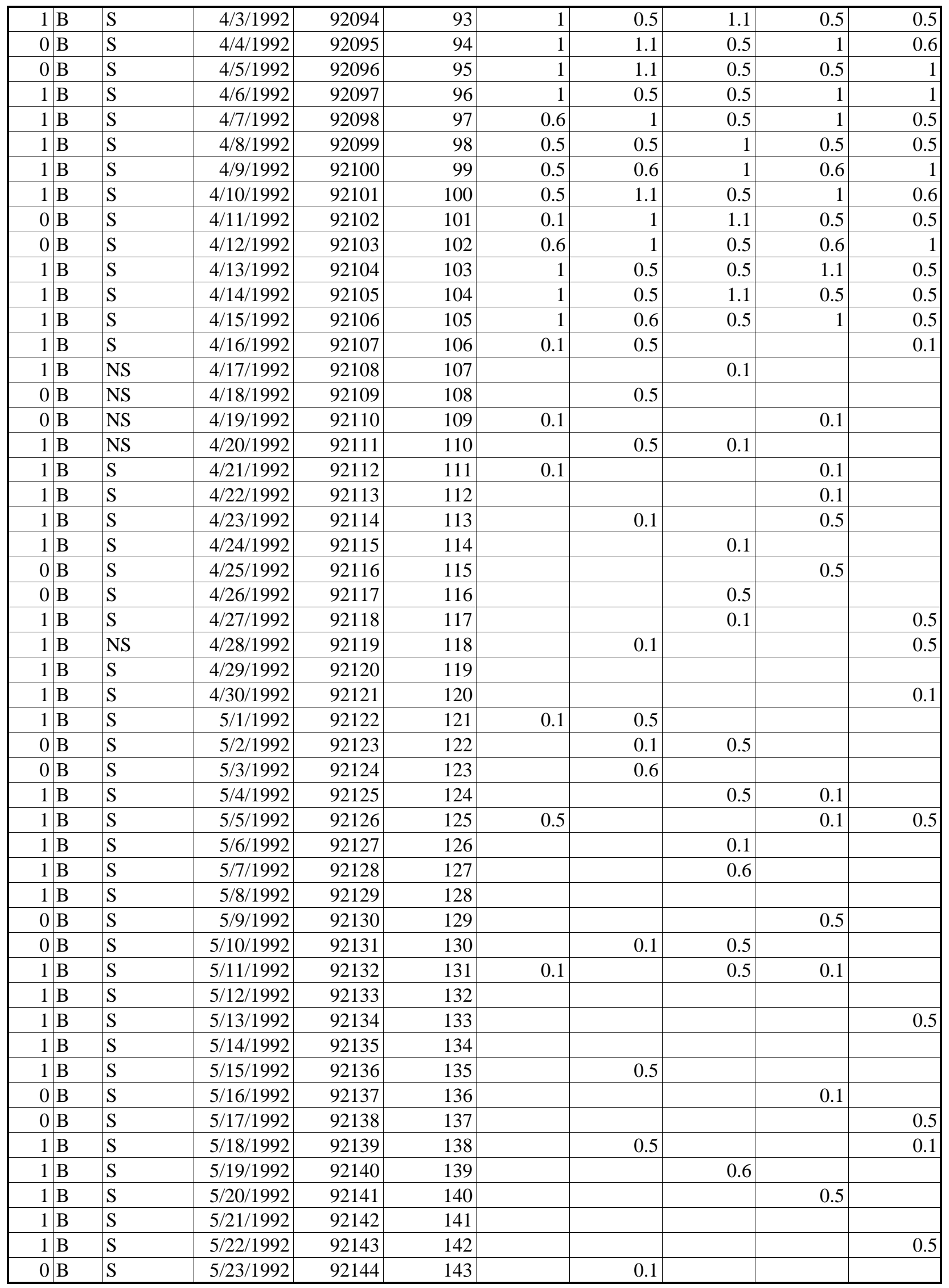




\begin{tabular}{|c|c|c|c|c|c|c|c|c|c|c|}
\hline \begin{tabular}{l|l}
$0 \mathrm{~F}$ \\
\end{tabular} & $\mathrm{~B}$ & $S$ & $5 / 24 / 1992$ & \begin{tabular}{|l|}
92145 \\
\end{tabular} & 144 & & & & 0.5 & \\
\hline $1 \mathrm{E}$ & B & S & 5/25/1992 & 92146 & 145 & 0.5 & & & 0.1 & \\
\hline $1 \mathrm{~F}$ & B & $S$ & $5 / 26 / 1992$ & 92147 & 146 & & 0.5 & & & \\
\hline $1 \mathrm{E}$ & B & S & $5 / 27 / 1992$ & 92148 & 147 & & & 0.5 & & \\
\hline $1 \mathrm{E}$ & B & NS & $5 / 28 / 1992$ & 92149 & 148 & & & & & 0.5 \\
\hline $1 \mathrm{~F}$ & B & NS & 5/29/1992 & 92150 & 149 & & & 0.5 & & \\
\hline $0 A$ & A & NS & $5 / 30 / 1992$ & 92151 & 150 & & & & 0.1 & 0.5 \\
\hline $0 A$ & A & NS & $5 / 31 / 1992$ & 92152 & 151 & 0.1 & & & & 0.1 \\
\hline $1 \mathrm{E}$ & B & NS & $6 / 1 / 1992$ & 92153 & 152 & & 0.5 & & 0.1 & \\
\hline $1 \mathrm{E}$ & B & NS & 6/2/1992 & 92154 & 153 & & 0.5 & & 0.1 & \\
\hline $1 \mathrm{~F}$ & B & NS & $6 / 3 / 1992$ & 92155 & 154 & & & & & \\
\hline $1 \mathrm{E}$ & B & NS & 6/4/1992 & 92156 & 155 & & 0.1 & & 0.5 & \\
\hline $1 \mathrm{E}$ & B & NS & 6/5/1992 & 92157 & 156 & & & & 0.5 & \\
\hline \begin{tabular}{l|l} 
& $\mathrm{E}$ \\
\end{tabular} & B & NS & 6/6/1992 & 92158 & 157 & & & & & \\
\hline $0 \mathrm{E}$ & B & NS & 6/7/1992 & 92159 & 158 & & & & 0.5 & \\
\hline $1 \mathrm{E}$ & B & NS & $6 / 8 / 1992$ & 92160 & 159 & & 0.5 & & 0.1 & \\
\hline $1 \mathrm{E}$ & B & NS & 6/9/1992 & 92161 & 160 & & & & & 0.1 \\
\hline \begin{tabular}{l|l}
$1 \mathrm{E}$ \\
\end{tabular} & B & NS & $6 / 10 / 1992$ & 92162 & 161 & & & & & \\
\hline $1 \mathrm{~F}$ & B & NS & 6/11/1992 & 92163 & 162 & & 0.5 & & & \\
\hline $1 \mathrm{E}$ & B & NS & 6/12/1992 & 92164 & 163 & & & & & \\
\hline $0 \mathrm{E}$ & B & NS & 6/13/1992 & 92165 & 164 & & & 0.5 & & \\
\hline \begin{tabular}{l|l}
0 & $\mathrm{E}$
\end{tabular} & B & NS & $6 / 14 / 1992$ & 92166 & 165 & & & & & \\
\hline $1 \mathrm{E}$ & B & NS & $6 / 15 / 1992$ & 92167 & 166 & & & & & \\
\hline \begin{tabular}{l|l}
1 & $\mathrm{E}$
\end{tabular} & B & NS & 6/16/1992 & 92168 & 167 & & & & 0.5 & \\
\hline $1 \mathrm{E}$ & B & NS & $6 / 17 / 1992$ & 92169 & 168 & & 0.5 & & & \\
\hline $1 \mathrm{E}$ & B & NS & 6/18/1992 & 92170 & 169 & & & & & \\
\hline $1 \mathrm{~F}$ & B & NS & $6 / 19 / 1992$ & 92171 & 170 & & & & & \\
\hline \begin{tabular}{l|l} 
& $\mathrm{E}$ \\
\end{tabular} & B & NS & $6 / 20 / 1992$ & 92172 & 171 & 0.5 & & & & \\
\hline \begin{tabular}{l|l}
$0 \mathrm{E}$ \\
\end{tabular} & B & NS & $6 / 21 / 1992$ & 92173 & 172 & & & & & 0.5 \\
\hline $1 \mathrm{E}$ & B & NS & $6 / 22 / 1992$ & 92174 & 173 & & & & & \\
\hline $1 \mathrm{E}$ & B & NS & $6 / 23 / 1992$ & 92175 & 174 & & & & & \\
\hline $1 \mathrm{~F}$ & B & NS & 6/24/1992 & 92176 & 175 & & & 0.5 & & \\
\hline $1 \mathrm{~F}$ & B & NS & $6 / 25 / 1992$ & 92177 & 176 & & & & & \\
\hline $1 \mathrm{E}$ & B & NS & 6/26/1992 & 92178 & 177 & 0.5 & & & & \\
\hline $0 \mathrm{E}$ & B & NS & 6/27/1992 & 92179 & 178 & & & & & 0.5 \\
\hline \begin{tabular}{l|l}
0 & $\mathrm{E}$ \\
\end{tabular} & B & NS & 6/28/1992 & 92180 & 179 & & & & 0.5 & \\
\hline \begin{tabular}{l|l}
1 & $\mathrm{E}$
\end{tabular} & B & NS & 6/29/1992 & 92181 & 180 & & & & 0.5 & \\
\hline $1 \mathrm{~F}$ & B & NS & $6 / 30 / 1992$ & 92182 & 181 & & & 0.5 & & \\
\hline $1 \mathrm{E}$ & B & NS & 7/1/1992 & 92183 & 182 & & & & & \\
\hline $1 \mathrm{E}$ & B & NS & $7 / 2 / 1992$ & 92184 & 183 & 0.5 & & & & \\
\hline $1 \mathrm{E}$ & B & NS & 7/3/1992 & 92185 & 184 & & & & & 0.5 \\
\hline \begin{tabular}{l|l}
0 & $\mathrm{E}$
\end{tabular} & B & NS & 7/4/1992 & 92186 & 185 & & & & & \\
\hline \begin{tabular}{l|l}
$\mathrm{E}$ \\
\end{tabular} & B & NS & 7/5/1992 & 92187 & 186 & & & & & \\
\hline $1 \mathrm{~F}$ & B & NS & 7/6/1992 & 92188 & 187 & & & 0.5 & & \\
\hline $1 \mathrm{E}$ & B & NS & 7/7/1992 & 92189 & 188 & & & & & \\
\hline $1 \mathrm{~F}$ & B & NS & 7/8/1992 & 92190 & 189 & & & & & \\
\hline $1 \mathrm{E}$ & B & NS & 7/9/1992 & 92191 & 190 & & & 0.5 & & \\
\hline $1 \mathrm{E}$ & B & NS & 7/10/1992 & 92192 & 191 & & & 0.5 & & \\
\hline \begin{tabular}{l|l}
0 & $\mathrm{E}$
\end{tabular} & B & NS & 7/11/1992 & 92193 & 192 & & & & & 0.5 \\
\hline \begin{tabular}{l|l}
0 & $\mathrm{E}$
\end{tabular} & B & NS & $7 / 12 / 1992$ & 92194 & 193 & & & & & \\
\hline $1 \mathrm{~F}$ & B & NS & 7/13/1992 & 92195 & 194 & & & 0.5 & & \\
\hline
\end{tabular}




\begin{tabular}{|c|c|c|c|c|c|c|c|c|c|c|}
\hline $1 \mathrm{~F}$ & B & NS & $7 / 14 / 1992$ & 92196 & 195 & & & & & \\
\hline $1 \mathrm{E}$ & B & NS & 7/15/1992 & 92197 & 196 & & & & 0.5 & \\
\hline $1 \mathrm{E}$ & B & NS & 7/16/1992 & 92198 & 197 & & & 0.5 & & \\
\hline $1 \mathrm{~F}$ & B & NS & 7/17/1992 & 92199 & 198 & & & & & \\
\hline $0 \mathrm{E}$ & B & NS & 7/18/1992 & 92200 & 199 & & & 0.5 & & \\
\hline $0 \mathrm{E}$ & B & NS & 7/19/1992 & 92201 & 200 & & & & & \\
\hline $1 \mathrm{E}$ & B & NS & $7 / 20 / 1992$ & 92202 & 201 & 0.5 & & & & \\
\hline $1 \mathrm{E}$ & B & NS & 7/21/1992 & 92203 & 202 & & & & & \\
\hline $1 \mathrm{E}$ & B & NS & 7/22/1992 & 92204 & 203 & & & & & \\
\hline $1 \mathrm{E}$ & B & NS & 7/23/1992 & 92205 & 204 & 0.5 & & & & \\
\hline $1 \mathrm{E}$ & B & NS & 7/24/1992 & 92206 & 205 & & & 0.5 & & \\
\hline $0 \mathrm{E}$ & B & NS & 7/25/1992 & 92207 & 206 & & 0.5 & & & \\
\hline $0 \mathrm{E}$ & $\mathrm{B}$ & NS & 7/26/1992 & 92208 & 207 & & & & & \\
\hline $1 \mathrm{E}$ & B & NS & 7/27/1992 & 92209 & 208 & & & & & 0.5 \\
\hline $1 \mathrm{E}$ & B & NS & 7/28/1992 & 92210 & 209 & & & & & \\
\hline $1 \mathrm{E}$ & B & NS & 7/29/1992 & 92211 & 210 & & & & & \\
\hline $1 \mathrm{E}$ & B & NS & 7/30/1992 & 92212 & 211 & & & & & 0.5 \\
\hline $1 \mathrm{E}$ & B & NS & 7/31/1992 & 92213 & 212 & & & & & \\
\hline $0 \mathrm{E}$ & $\mathrm{B}$ & NS & 8/1/1992 & 92214 & 213 & & & & & \\
\hline $0 \mathrm{E}$ & B & NS & $8 / 2 / 1992$ & 92215 & 214 & & & & 0.5 & \\
\hline $1 \mathrm{~F}$ & B & NS & 8/3/1992 & 92216 & 215 & & & 0.5 & & \\
\hline $1 \mathrm{E}$ & B & NS & 8/4/1992 & 92217 & 216 & & & & & 0.5 \\
\hline $1 \mathrm{E}$ & B & NS & 8/5/1992 & 92218 & 217 & & & & & \\
\hline $1 \mathrm{E}$ & B & NS & 8/6/1992 & 92219 & 218 & & 0.5 & & & \\
\hline $1 \mathrm{E}$ & B & NS & 8/7/1992 & 92220 & 219 & & & & & 0.5 \\
\hline \begin{tabular}{l|l}
$0 \mathrm{E}$ \\
\end{tabular} & B & NS & 8/8/1992 & 92221 & 220 & & & & & \\
\hline $0 \mathrm{E}$ & B & NS & 8/9/1992 & 92222 & 221 & & & & & \\
\hline $1 \mathrm{E}$ & B & NS & $8 / 10 / 1992$ & 92223 & 222 & & 0.5 & & & \\
\hline $1 \mathrm{E}$ & B & NS & 8/11/1992 & 92224 & 223 & & & & & \\
\hline $1 \mathrm{~F}$ & B & NS & $8 / 12 / 1992$ & 92225 & 224 & & 0.5 & & & 0.5 \\
\hline $1 \mathrm{E}$ & B & NS & $8 / 13 / 1992$ & 92226 & 225 & & & & & 0.5 \\
\hline $1 \mathrm{~F}$ & B & NS & 8/14/1992 & 92227 & 226 & & & & & \\
\hline $0 \mathrm{E}$ & B & NS & $8 / 15 / 1992$ & 92228 & 227 & & & & & \\
\hline $0 \mathrm{E}$ & B & NS & $8 / 16 / 1992$ & 92229 & 228 & & & & & \\
\hline $1 \mathrm{E}$ & B & NS & $8 / 17 / 1992$ & 92230 & 229 & & & & & \\
\hline $1 \mathrm{~F}$ & B & NS & $8 / 18 / 1992$ & 92231 & 230 & & & & & \\
\hline $1 \mathrm{E}$ & $\mathrm{B}$ & NS & $8 / 19 / 1992$ & 92232 & 231 & & & & & \\
\hline $1 \mathrm{~F}$ & B & NS & 8/20/1992 & 92233 & 232 & & & & & \\
\hline $1 \mathrm{E}$ & B & NS & 8/21/1992 & 92234 & 233 & & & & & 0.5 \\
\hline \begin{tabular}{l|l}
$0 \mathrm{~F}$ \\
\end{tabular} & B & NS & $8 / 22 / 1992$ & 92235 & 234 & & & & 0.5 & \\
\hline $0 \mathrm{E}$ & B & NS & $8 / 23 / 1992$ & 92236 & 235 & & & & & \\
\hline $1 \mathrm{E}$ & B & NS & 8/24/1992 & 92237 & 236 & & & & 0.5 & \\
\hline $1 \mathrm{E}$ & B & NS & $8 / 25 / 1992$ & 92238 & 237 & & & & 0.5 & \\
\hline $1 \mathrm{E}$ & B & NS & 8/26/1992 & 92239 & 238 & & & & & \\
\hline $1 \mathrm{~F}$ & B & NS & $8 / 27 / 1992$ & 92240 & 239 & & & & & \\
\hline $1 \mathrm{E}$ & B & NS & 8/28/1992 & 92241 & 240 & & & 0.5 & & \\
\hline $0 \mathrm{E}$ & $\mathrm{B}$ & NS & $8 / 29 / 1992$ & 92242 & 241 & & & & & \\
\hline \begin{tabular}{l|l}
0 & $\mathrm{E}$ \\
\end{tabular} & B & NS & 8/30/1992 & 92243 & 242 & 0.5 & & & 0.5 & \\
\hline $1 \mathrm{E}$ & $\mathrm{B}$ & NS & $8 / 31 / 1992$ & 92244 & 243 & 0.5 & 0.5 & & & 0.5 \\
\hline $1 \mathrm{E}$ & B & NS & 9/1/1992 & 92245 & 244 & 0.5 & & & 0.5 & 0.5 \\
\hline $1 \mathrm{E}$ & $\mathrm{B}$ & NS & 9/2/1992 & 92246 & 245 & & & & & 0.5 \\
\hline
\end{tabular}




\begin{tabular}{|c|c|c|c|c|c|c|c|c|c|c|}
\hline$\overline{1 \mathrm{~F}}$ & $\mathrm{~B}$ & NS & 9/3/1992 & 92247 & 246 & & & & & \\
\hline $1 \mathrm{E}$ & B & NS & 9/4/1992 & 92248 & 247 & & & & & \\
\hline $0 \mathrm{E}$ & B & NS & 9/5/1992 & 92249 & 248 & & & 0.5 & & \\
\hline \begin{tabular}{l|l}
$0 \mathrm{E}$ \\
\end{tabular} & B & NS & 9/6/1992 & 92250 & 249 & & & & & \\
\hline $1 \mathrm{E}$ & B & NS & 9/7/1992 & 92251 & 250 & & & & & \\
\hline $1 \mathrm{~F}$ & B & S & 9/8/1992 & 92252 & 251 & & 0.5 & & & \\
\hline $1 \mathrm{E}$ & B & S & 9/9/1992 & 92253 & 252 & & & & & \\
\hline $1 \mathrm{E}$ & B & S & 9/10/1992 & 92254 & 253 & & 0.5 & & & \\
\hline $1 \mathrm{E}$ & B & $S$ & 9/11/1992 & 92255 & 254 & & & & & \\
\hline $0 \mathrm{E}$ & B & S & 9/12/1992 & 92256 & 255 & & & 0.5 & & \\
\hline $0 \mathrm{~F}$ & B & S & 9/13/1992 & 92257 & 256 & 0.5 & & & & \\
\hline $1 \mathrm{E}$ & B & S & 9/14/1992 & 92258 & 257 & & & 0.5 & & \\
\hline $1 \mathrm{E}$ & B & NS & 9/15/1992 & 92259 & 258 & & & & & \\
\hline $1 \mathrm{E}$ & B & S & 9/16/1992 & 92260 & 259 & & & & & \\
\hline $1 \mathrm{~F}$ & B & S & 9/17/1992 & 92261 & 260 & & & & & \\
\hline $1 \mathrm{~F}$ & B & $S$ & 9/18/1992 & 92262 & 261 & 0.5 & & & & \\
\hline \begin{tabular}{l|l}
$0 \mathrm{E}$ \\
\end{tabular} & B & S & 9/19/1992 & 92263 & 262 & & & & 0.5 & \\
\hline \begin{tabular}{l|l}
0 & $\mathrm{E}$
\end{tabular} & B & S & 9/20/1992 & 92264 & 263 & & & 0.5 & & \\
\hline $1 \mathrm{~F}$ & B & S & 9/21/1992 & 92265 & 264 & 0.5 & & & & \\
\hline $1 \mathrm{E}$ & B & S & 9/22/1992 & 92266 & 265 & & & & & \\
\hline $1 \mathrm{~F}$ & B & S & 9/23/1992 & 92267 & 266 & & & 1 & & \\
\hline $1 \mathrm{~F}$ & B & S & 9/24/1992 & 92268 & 267 & & & & & \\
\hline $1 \mathrm{E}$ & B & S & 9/25/1992 & 92269 & 268 & & 0.5 & & & \\
\hline \begin{tabular}{l|l}
0 & $\mathrm{E}$
\end{tabular} & B & S & 9/26/1992 & 92270 & 269 & & 0.5 & & & \\
\hline \begin{tabular}{l|l}
$0 \mathrm{E}$ \\
\end{tabular} & B & S & 9/27/1992 & 92271 & 270 & & & & & \\
\hline $1 \mathrm{E}$ & B & S & 9/28/1992 & 92272 & 271 & & & 0.5 & & \\
\hline $1 \mathrm{~F}$ & B & S & 9/29/1992 & 92273 & 272 & & & & & \\
\hline $1 \mathrm{E}$ & B & S & 9/30/1992 & 92274 & 273 & & & 0.5 & & \\
\hline $1 \mathrm{E}$ & B & S & 10/1/1992 & 92275 & 274 & & & & & \\
\hline $1 \mathrm{E}$ & B & S & $10 / 2 / 1992$ & 92276 & 275 & & & & & \\
\hline $0 \mathrm{E}$ & B & S & $10 / 3 / 1992$ & 92277 & 276 & & 0.5 & & & \\
\hline $0 \mathrm{E}$ & B & S & 10/4/1992 & 92278 & 277 & & & & & \\
\hline $1 \mathrm{~F}$ & B & S & 10/5/1992 & 92279 & 278 & 0.5 & & & & \\
\hline $1 \mathrm{E}$ & B & S & 10/6/1992 & 92280 & 279 & & & 0.5 & & \\
\hline $1 \mathrm{E}$ & B & S & 10/7/1992 & 92281 & 280 & & & & & \\
\hline $1 \mathrm{~F}$ & B & S & 10/8/1992 & 92282 & 281 & & & & & \\
\hline $1 \mathrm{E}$ & B & S & $10 / 9 / 1992$ & 92283 & 282 & 0.5 & & & & \\
\hline \begin{tabular}{l|l}
0 & $\mathrm{E}$ \\
\end{tabular} & B & S & 10/10/1992 & 92284 & 283 & 1 & 0.5 & 1 & 0.5 & 0.5 \\
\hline \begin{tabular}{l|l}
$0 \mathrm{E}$ \\
\end{tabular} & B & S & 10/11/1992 & 92285 & 284 & 0.5 & 1 & 0.5 & 0.5 & 1 \\
\hline $1 \mathrm{E}$ & B & NS & $10 / 12 / 1992$ & 92286 & 285 & 0.5 & 0.5 & 1 & 0.5 & 0.5 \\
\hline $1 \mathrm{E}$ & B & S & 10/13/1992 & 92287 & 286 & 1 & 0.5 & 0.5 & 1 & 0.5 \\
\hline $1 \mathrm{E}$ & B & S & 10/14/1992 & 92288 & 287 & & & & & \\
\hline $1 \mathrm{~F}$ & B & S & 10/15/1992 & 92289 & 288 & & 0.5 & & & \\
\hline $1 \mathrm{~F}$ & B & S & 10/16/1992 & 92290 & 289 & & & & & 0.5 \\
\hline \begin{tabular}{l|l}
0 & $\mathrm{E}$ \\
\end{tabular} & B & S & 10/17/1992 & 92291 & 290 & & & & & \\
\hline \begin{tabular}{l|l}
$\mathrm{E}$ \\
\end{tabular} & B & S & 10/18/1992 & 92292 & 291 & & 0.5 & & & \\
\hline $1 \mathrm{E}$ & B & S & 10/19/1992 & 92293 & 292 & & & & & 1 \\
\hline $1 \mathrm{E}$ & B & $S$ & 10/20/1992 & 92294 & 293 & 0.5 & & & 0.5 & \\
\hline $1 \mathrm{~F}$ & B & S & 10/21/1992 & 92295 & 294 & & & 0.5 & & 0.5 \\
\hline \begin{tabular}{l|l}
$\mathrm{E}$ \\
\end{tabular} & B & S & 10/22/1992 & 92296 & 295 & & 0.5 & & 0.5 & \\
\hline $1 \mathrm{~F}$ & B & S & 10/23/1992 & 92297 & 296 & 0.5 & & & 1 & \\
\hline
\end{tabular}




\begin{tabular}{|c|c|c|c|c|c|c|c|c|c|c|}
\hline $0 \mathrm{E}$ & B & $S$ & $10 / 24 / 1992$ & 92298 & 297 & & 0.5 & & 0.5 & \\
\hline $0 \mathrm{E}$ & $\mathrm{B}$ & $S$ & $10 / 25 / 1992$ & 92299 & 298 & & 0.5 & & & 0.5 \\
\hline $1 \mathrm{~F}$ & B & $S$ & 10/26/1992 & 92300 & 299 & & 0.5 & & & 0.5 \\
\hline $1 \mathrm{E}$ & $\mathrm{B}$ & S & 10/27/1992 & 92301 & 300 & & & 0.5 & & 0.5 \\
\hline $1 \mathrm{E}$ & B & $S$ & 10/28/1992 & 92302 & 301 & & 0.5 & 0.5 & & \\
\hline $1 \mathrm{E}$ & $\mathrm{B}$ & S & 10/29/1992 & 92303 & 302 & & & 0.5 & & 0.5 \\
\hline $1 \mathrm{E}$ & $\mathrm{B}$ & S & 10/30/1992 & 92304 & 303 & & & & & \\
\hline $0 \mathrm{E}$ & $\mathrm{B}$ & $S$ & 10/31/1992 & 92305 & 304 & & & & 0.5 & \\
\hline $0 \mathrm{E}$ & B & S & 11/1/1992 & 92306 & 305 & & 0.5 & & & \\
\hline $1 \mathrm{E}$ & B & $S$ & $11 / 2 / 1992$ & 92307 & 306 & & & & 0.5 & \\
\hline $1 \mathrm{E}$ & $\mathrm{B}$ & S & $11 / 3 / 1992$ & 92308 & 307 & & & & & \\
\hline $1 \mathrm{E}$ & B & S & 11/4/1992 & 92309 & 308 & & 0.5 & & & \\
\hline $1 \mathrm{E}$ & $\mathrm{B}$ & S & 11/5/1992 & 92310 & 309 & 0.5 & 0.5 & 1 & 1 & 0.5 \\
\hline $1 \mathrm{E}$ & $\mathrm{B}$ & S & 11/6/1992 & 92311 & 310 & 1 & 1 & 1 & 1 & 1 \\
\hline $0 \mathrm{E}$ & B & $S$ & 11/7/1992 & 92312 & 311 & & 0.5 & & 0.5 & \\
\hline $0 \mathrm{E}$ & $\mathrm{B}$ & S & 11/8/1992 & 92313 & 312 & 1.5 & 0.5 & 1 & 1 & 1 \\
\hline $1 \mathrm{E}$ & $\mathrm{B}$ & $S$ & 11/9/1992 & 92314 & 313 & 1 & 1 & 0.5 & 0.5 & 1 \\
\hline $1 \mathrm{E}$ & B & S & 11/10/1992 & 92315 & 314 & 1 & 0.5 & 1 & 0.5 & 1 \\
\hline $1 \mathrm{E}$ & $\mathrm{B}$ & S & 11/11/1992 & 92316 & 315 & 1 & 0.5 & 0.5 & 1 & 0.5 \\
\hline $1 \mathrm{E}$ & $\mathrm{B}$ & $S$ & 11/12/1992 & 92317 & 316 & 1 & 0.5 & 0.5 & 1 & 0.5 \\
\hline $1 \mathrm{E}$ & B & $S$ & 11/13/1992 & 92318 & 317 & 1 & 0.5 & 0.5 & 0.5 & 0.5 \\
\hline $0 \mathrm{E}$ & $\mathrm{B}$ & S & 11/14/1992 & 92319 & 318 & 1 & 1 & 0.5 & 1 & 0.5 \\
\hline \begin{tabular}{l|l}
$0 \mathrm{E}$ \\
\end{tabular} & B & S & 11/15/1992 & 92320 & 319 & 1 & 1 & 0.5 & 0.5 & 1 \\
\hline $1 \mathrm{E}$ & B & S & 11/16/1992 & 92321 & 320 & 1 & 1 & 0.5 & 1 & 1 \\
\hline $1 \mathrm{E}$ & $\mathrm{B}$ & S & 11/17/1992 & 92322 & 321 & 1 & 1 & 1 & 1 & 1.5 \\
\hline $1 \mathrm{E}$ & $\mathrm{B}$ & $S$ & 11/18/1992 & 92323 & 322 & 1 & 0.5 & 1 & 1 & 0.5 \\
\hline $1 \mathrm{E}$ & B & $S$ & 11/19/1992 & 92324 & 323 & 0.5 & 1 & 0.5 & 1 & 0.5 \\
\hline $1 \mathrm{E}$ & $\mathrm{B}$ & S & 11/20/1992 & 92325 & 324 & & 1 & 0.5 & 0.5 & 1 \\
\hline \begin{tabular}{l|l}
$0 \mathrm{E}$ \\
\end{tabular} & B & S & 11/21/1992 & 92326 & 325 & 0.5 & 1 & 0.5 & 1 & 0.5 \\
\hline $0 \mathrm{E}$ & B & S & $11 / 22 / 1992$ & 92327 & 326 & 1 & 0.5 & 1 & 0.5 & 0.5 \\
\hline $1 \mathrm{E}$ & B & $S$ & $11 / 23 / 1992$ & 92328 & 327 & 0.5 & 0.5 & 1 & 1 & 0.5 \\
\hline $1 \mathrm{~F}$ & $\mathrm{~B}$ & S & $11 / 24 / 1992$ & 92329 & 328 & 0.5 & 0.5 & 1 & 1 & 1 \\
\hline $1 \mathrm{E}$ & $\mathrm{B}$ & NS & $11 / 25 / 1992$ & 92330 & 329 & 0.5 & 1 & 1 & 0.5 & 1 \\
\hline $1 \mathrm{E}$ & $\mathrm{B}$ & NS & 11/26/1992 & 92331 & 330 & 1 & 0.5 & 1 & 1 & 0.5 \\
\hline $1 \mathrm{E}$ & B & NS & 11/27/1992 & 92332 & 331 & 1.5 & 2.1 & 2.1 & 2.6 & 2.1 \\
\hline $0 \mathrm{E}$ & B & S & 11/28/1992 & 92333 & 332 & 2.1 & 2.1 & 2.6 & 2.6 & 1.5 \\
\hline $0 \mathrm{E}$ & B & $S$ & 11/29/1992 & 92334 & 333 & 2.1 & 2.1 & 2.1 & 2.1 & 2.1 \\
\hline $1 \mathrm{~F}$ & B & S & 11/30/1992 & 92335 & 334 & 1.5 & 1.5 & 1 & 1.5 & 1.5 \\
\hline $1 \mathrm{E}$ & B & S & 12/1/1992 & 92336 & 335 & 1 & 1.5 & 1.5 & 1 & 2.1 \\
\hline $1 \mathrm{~F}$ & B & $S$ & $12 / 2 / 1992$ & 92337 & 336 & 2.2 & 2.7 & 2.2 & 2.2 & 2.7 \\
\hline $1 \mathrm{E}$ & B & S & $12 / 3 / 1992$ & 92338 & 337 & 1.5 & 1 & 2.1 & 1 & 1.5 \\
\hline $1 \mathrm{E}$ & B & S & 12/4/1992 & 92339 & 338 & 1.5 & 1.5 & 2.1 & 1.5 & 1.5 \\
\hline $0 \mathrm{E}$ & B & $S$ & $12 / 5 / 1992$ & 92340 & 339 & 1.5 & 1 & 1 & 1.5 & 1 \\
\hline $0 \mathrm{E}$ & B & S & 12/6/1992 & 92341 & 340 & 1 & 1 & 1 & 1 & 0.5 \\
\hline $1 \mathrm{E}$ & $\mathrm{B}$ & S & 12/7/1992 & 92342 & 341 & 1 & 1 & 1 & 1 & 1 \\
\hline $1 \mathrm{E}$ & $\mathrm{B}$ & S & 12/8/1992 & 92343 & 342 & 1 & 1 & 1 & 1 & 1 \\
\hline $1 \mathrm{E}$ & $\mathrm{B}$ & $S$ & $12 / 9 / 1992$ & 92344 & 343 & 2.1 & 2.1 & 2.1 & 2.6 & 2.1 \\
\hline $1 \mathrm{E}$ & $\mathrm{B}$ & S & 12/10/1992 & 92345 & 344 & 1 & 1.5 & 1.5 & 1 & 1.5 \\
\hline $1 \mathrm{E}$ & B & $S$ & 12/11/1992 & 92346 & 345 & 1 & 1 & 0.5 & 0.5 & 1 \\
\hline $0 \mathrm{E}$ & B & S & $12 / 12 / 1992$ & 92347 & 346 & 0.5 & 0.5 & 1 & 1 & 1 \\
\hline \begin{tabular}{l|l}
0 & $\mathrm{E}$ \\
\end{tabular} & $\mathrm{B}$ & $\mathrm{S}$ & 12/13/1992 & 92348 & 347 & 1 & 1 & 0.5 & 1 & 0.5 \\
\hline
\end{tabular}




\begin{tabular}{|c|c|c|c|c|c|c|c|c|c|}
\hline $1 \mathrm{~B}$ & $S$ & 12/14/1992 & 92349 & 348 & 1 & 0.5 & 0.5 & 1 & 0.5 \\
\hline $1 \mathrm{~B}$ & $S$ & 12/15/1992 & 92350 & 349 & 1 & 0.5 & 1 & 0.5 & 1 \\
\hline $1 \mathrm{~B}$ & $S$ & 12/16/1992 & 92351 & 350 & 1.5 & 1.6 & 1.6 & 1.5 & 1.6 \\
\hline $1 \mathrm{~B}$ & $S$ & 12/17/1992 & 92352 & 351 & 1.5 & 1.5 & 1 & 1 & 1 \\
\hline $1 \mathrm{~B}$ & NS & 12/18/1992 & 92353 & 352 & 1 & 0.5 & 1 & 1 & 0.5 \\
\hline $\begin{array}{ll}0 & B \\
\end{array}$ & NS & 12/19/1992 & 92354 & 353 & 1 & 1 & 1 & 1 & 1 \\
\hline $0 \mathrm{~B}$ & NS & 12/20/1992 & 92355 & 354 & 1 & 0.5 & 3.1 & 1.5 & 0.5 \\
\hline $1 \mathrm{~B}$ & NS & 12/21/1992 & 92356 & 355 & 1 & 0.5 & 3.1 & 1.5 & 0.5 \\
\hline $1 \mathrm{~B}$ & NS & 12/22/1992 & 92357 & 356 & 0.5 & 2.1 & 4.2 & 2.6 & 1.1 \\
\hline $1 \mathrm{~B}$ & NS & $12 / 23 / 1992$ & 92358 & 357 & 1 & 1 & 3.6 & 2.1 & 0.6 \\
\hline $1 \mathrm{~B}$ & NS & 12/24/1992 & 92359 & 358 & 0.5 & 0.5 & 3.6 & 1.5 & 1 \\
\hline $1 \mathrm{~B}$ & NS & $12 / 25 / 1992$ & 92360 & 359 & 0.5 & 0.5 & 3.6 & 1.5 & 1 \\
\hline $0 \mathrm{~B}$ & NS & 12/26/1992 & 92361 & 360 & 1.5 & 1 & 3.6 & 1.5 & 1.1 \\
\hline $0 \mathrm{~B}$ & NS & 12/27/1992 & 92362 & 361 & 1 & 1 & 3.1 & 1.5 & 1 \\
\hline $1 \mathrm{~B}$ & NS & 12/28/1992 & 92363 & 362 & 0.5 & 1 & 3.6 & 1 & 1 \\
\hline $1 \mathrm{~B}$ & NS & 12/29/1992 & 92364 & 363 & 0.5 & 1.5 & 4.2 & 2.1 & 1.1 \\
\hline $1 \mathrm{~B}$ & NS & 12/30/1992 & 92365 & 364 & 0.5 & 1 & 3.1 & 0.5 & 1 \\
\hline $1 \mathrm{~B}$ & NS & 12/31/1992 & 92366 & 365 & 0.5 & & 2.6 & & \\
\hline $1 \mathrm{~B}$ & NS & $1 / 1 / 1993$ & 93001 & 1 & 1 & 1 & 3.6 & 1 & 1.5 \\
\hline $0 \mathrm{~B}$ & NS & $1 / 2 / 1993$ & 93002 & 2 & 2.1 & 2.1 & 4.6 & 1.5 & 1.5 \\
\hline $\begin{array}{ll}0 & \mathrm{~B} \\
\end{array}$ & NS & $1 / 3 / 1993$ & 93003 & 3 & 1 & 0.5 & 3.1 & 1 & 0.5 \\
\hline $1 \mathrm{~B}$ & NS & 1/4/1993 & 93004 & 4 & 0.5 & 0.5 & 0.5 & 1 & 0.5 \\
\hline $1 \mathrm{~B}$ & S & $1 / 5 / 1993$ & 93005 & 5 & 1 & 1 & 1.1 & 1 & 1 \\
\hline $1 \mathrm{~B}$ & $S$ & $1 / 6 / 1993$ & 93006 & 6 & 1.1 & 1 & 1 & 0.5 & 0.5 \\
\hline $1 \mathrm{~B}$ & S & 1/7/1993 & 93007 & 7 & 1 & 0.5 & 1 & 1 & 1 \\
\hline $1 \mathrm{~B}$ & S & $1 / 8 / 1993$ & 93008 & 8 & 0.5 & 1 & 0.5 & 0.5 & 1.5 \\
\hline $0 \mathrm{~B}$ & $S$ & $1 / 9 / 1993$ & 93009 & 9 & 1 & 0.5 & 1 & 0.5 & 1 \\
\hline $0 \mathrm{~B}$ & $S$ & $1 / 10 / 1993$ & 93010 & 10 & 1 & 1 & 0.5 & 1 & 1 \\
\hline $1 \mathrm{~B}$ & S & $1 / 11 / 1993$ & 93011 & 11 & 1 & 1 & 1 & 0.5 & \\
\hline $1 \mathrm{~B}$ & $S$ & $1 / 12 / 1993$ & 93012 & 12 & 1.5 & 1.5 & 1.5 & 1.5 & 2.1 \\
\hline $1 \mathrm{~B}$ & $S$ & 1/13/1993 & 93013 & 13 & 1 & 0.5 & 1 & 0.5 & 1 \\
\hline $1 \mathrm{~B}$ & $S$ & $1 / 14 / 1993$ & 93014 & 14 & 1.5 & 1.5 & 1.5 & 1.5 & 1.5 \\
\hline $1 \mathrm{~B}$ & $S$ & $1 / 15 / 1993$ & 93015 & 15 & 1.5 & 1.5 & 2.1 & 1.5 & 1.5 \\
\hline $0 \mathrm{~B}$ & $S$ & $1 / 16 / 1993$ & 93016 & 16 & 1 & 1 & 1 & 1 & 1 \\
\hline $\begin{array}{ll}0 & \mathrm{~B} \\
\end{array}$ & $S$ & 1/17/1993 & 93017 & 17 & & & & 0.5 & \\
\hline $1 \mathrm{~B}$ & S & 1/18/1993 & 93018 & 18 & 1 & 0.5 & 0.5 & 1 & 0.5 \\
\hline $1 \mathrm{~B}$ & $S$ & $1 / 19 / 1993$ & 93019 & 19 & 1 & 0.5 & 1 & 1 & 0.5 \\
\hline $1 \mathrm{~B}$ & S & 1/20/1993 & 93020 & 20 & 1.5 & 1.5 & 1.5 & 1.5 & 1 \\
\hline $1 \mathrm{~B}$ & S & 1/21/1993 & 93021 & 21 & 1.1 & 1 & 1.1 & 1 & 1.6 \\
\hline $1 \mathrm{~B}$ & $S$ & $1 / 22 / 1993$ & 93022 & 22 & 1 & 0.5 & 0.5 & 1 & 0.5 \\
\hline $0 \mathrm{~B}$ & $S$ & $1 / 23 / 1993$ & 93023 & 23 & & & & & \\
\hline $0 \mathrm{~B}$ & S & $1 / 24 / 1993$ & 93024 & 24 & & & & & \\
\hline $1 \mathrm{~B}$ & $S$ & $1 / 25 / 1993$ & 93025 & 25 & & & & & 0.5 \\
\hline $1 \mathrm{~B}$ & S & 1/26/1993 & 93026 & 26 & 2.1 & 3.1 & 2.6 & 2.6 & 2.6 \\
\hline $1 \mathrm{~B}$ & $S$ & 1/27/1993 & 93027 & 27 & 2.1 & 2.1 & 1.5 & 2.1 & 2.1 \\
\hline $1 \mathrm{~B}$ & $S$ & $1 / 28 / 1993$ & 93028 & 28 & 1 & 1 & 1 & 1 & 1 \\
\hline $1 \mathrm{~B}$ & S & $1 / 29 / 1993$ & 93029 & 29 & 0.5 & 1.5 & 0.5 & 1 & 0.5 \\
\hline $0 \mathrm{~B}$ & $S$ & 1/30/1993 & 93030 & 30 & 1 & 1.5 & 1 & 1 & 1 \\
\hline $0 \mathrm{~B}$ & $S$ & $1 / 31 / 1993$ & 93031 & 31 & 0.5 & 1 & 1 & 0.5 & 1 \\
\hline $1 \mathrm{~B}$ & S & 2/1/1993 & 93032 & 32 & 1 & 1 & 0.5 & 0.5 & 1 \\
\hline $1 \mathrm{~B}$ & $S$ & 2/2/1993 & 93033 & 33 & 1 & 0.5 & 1 & 1 & 0.5 \\
\hline
\end{tabular}




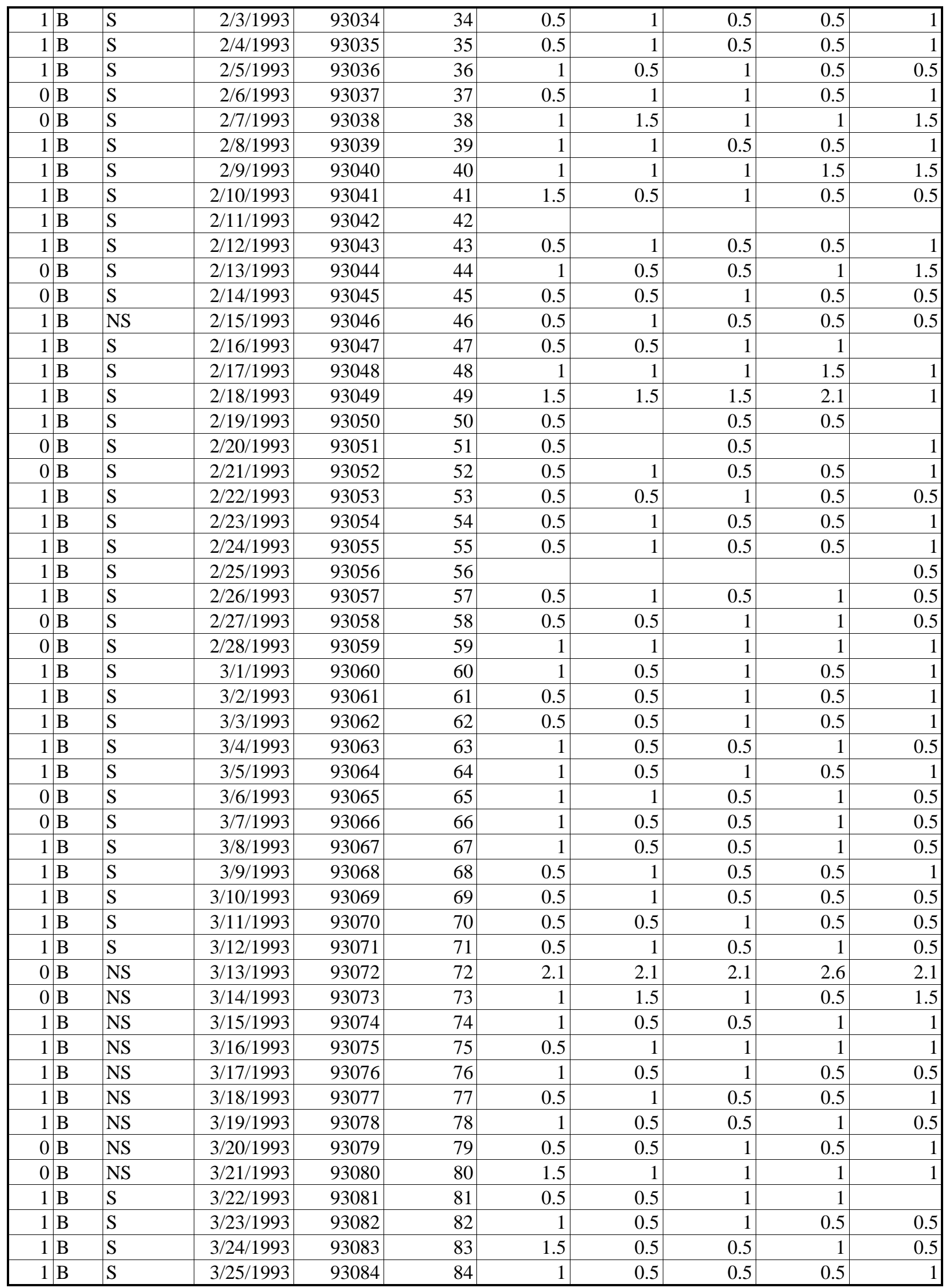




\begin{tabular}{|c|c|c|c|c|c|c|c|c|c|}
\hline \begin{tabular}{l|l}
1 & $B$ \\
\end{tabular} & $S$ & $3 / 26 / 1993$ & 93085 & 85 & 0.5 & 0.5 & 1 & 0.5 & 0.5 \\
\hline $0 \mathrm{~B}$ & S & 3/27/1993 & 93086 & 86 & 1 & 0.5 & 0.5 & 0.5 & 1 \\
\hline $0 \mathrm{~B}$ & $S$ & $3 / 28 / 1993$ & 93087 & 87 & 0.5 & 0.5 & 1 & 0.5 & 0.5 \\
\hline $1 \mathrm{~B}$ & S & $3 / 29 / 1993$ & 93088 & 88 & 0.5 & 0.5 & 1 & 0.5 & 0.5 \\
\hline $1 \mathrm{~B}$ & $S$ & $3 / 30 / 1993$ & 93089 & 89 & 0.5 & 1 & 0.5 & 0.5 & 0.5 \\
\hline $1 \mathrm{~B}$ & S & 3/31/1993 & 93090 & 90 & 0.5 & & & & \\
\hline $1 \mathrm{~B}$ & S & 4/1/1993 & 93091 & 91 & & & & 0.5 & \\
\hline $1 \mathrm{~B}$ & S & $4 / 2 / 1993$ & 93092 & 92 & 0.5 & & & & \\
\hline $0 \mathrm{~B}$ & $S$ & 4/3/1993 & 93093 & 93 & & & & & 0.5 \\
\hline $0 \mathrm{~B}$ & S & 4/4/1993 & 93094 & 94 & & & & & \\
\hline $1 \mathrm{~B}$ & $S$ & 4/5/1993 & 93095 & 95 & & & & & \\
\hline $1 \mathrm{~B}$ & S & 4/6/1993 & 93096 & 96 & & 0.5 & & & \\
\hline $1 \mathrm{~B}$ & S & 4/7/1993 & 93097 & 97 & & & 0.5 & & \\
\hline $1 \mathrm{~B}$ & S & 4/8/1993 & 93098 & 98 & & & & & 0.5 \\
\hline $1 \mathrm{~B}$ & NS & 4/9/1993 & 93099 & 99 & 0.5 & & & 0.5 & \\
\hline $0 \mathrm{~B}$ & NS & $4 / 10 / 1993$ & 93100 & 100 & & & 0.5 & & \\
\hline $0 \mathrm{~B}$ & NS & $4 / 11 / 1993$ & 93101 & 101 & & & & 0.5 & \\
\hline $1 \mathrm{~B}$ & NS & $4 / 12 / 1993$ & 93102 & 102 & 0.5 & & & & \\
\hline $1 \mathrm{~B}$ & $S$ & 4/13/1993 & 93103 & 103 & & 0.5 & & & \\
\hline $1 \mathrm{~B}$ & S & 4/14/1993 & 93104 & 104 & & & & & \\
\hline $1 \mathrm{~B}$ & $S$ & $4 / 15 / 1993$ & 93105 & 105 & 0.5 & & & & \\
\hline $1 \mathrm{~B}$ & $S$ & 4/16/1993 & 93106 & 106 & & & & & 0.5 \\
\hline $0 \mathrm{~B}$ & S & 4/17/1993 & 93107 & 107 & & & & & \\
\hline $0 \mathrm{~B}$ & $S$ & $4 / 18 / 1993$ & 93108 & 108 & & & 0.5 & & \\
\hline $1 \mathrm{~B}$ & S & $4 / 19 / 1993$ & 93109 & 109 & & & 0.5 & & \\
\hline $1 \mathrm{~B}$ & S & 4/20/1993 & 93110 & 110 & & & 0.5 & & \\
\hline $1 \mathrm{~B}$ & S & $4 / 21 / 1993$ & 93111 & 111 & & & & 0.5 & \\
\hline $1 \mathrm{~B}$ & S & $4 / 22 / 1993$ & 93112 & 112 & 0.5 & & & & \\
\hline $1 \mathrm{~B}$ & S & 4/23/1993 & 93113 & 113 & & & 0.5 & & \\
\hline $0 \mathrm{~B}$ & S & $4 / 24 / 1993$ & 93114 & 114 & 0.5 & & & & \\
\hline $0 \mathrm{~B}$ & S & $4 / 25 / 1993$ & 93115 & 115 & & & & 0.5 & \\
\hline $1 \mathrm{~B}$ & S & 4/26/1993 & 93116 & 116 & 0.5 & & & & 0.5 \\
\hline $1 \mathrm{~B}$ & S & 4/27/1993 & 93117 & 117 & 0.5 & & & & \\
\hline $1 \mathrm{~B}$ & NS & $4 / 28 / 1993$ & 93118 & 118 & & & & & 0.5 \\
\hline $1 \mathrm{~B}$ & $S$ & 4/29/1993 & 93119 & 119 & 0.5 & & & & \\
\hline $1 \mathrm{~B}$ & S & 4/30/1993 & 93120 & 120 & & 0.5 & & & \\
\hline $0 \mathrm{~B}$ & $S$ & 5/1/1993 & 93121 & 121 & & & & 0.5 & \\
\hline $0 \mathrm{~B}$ & S & $5 / 2 / 1993$ & 93122 & 122 & & & & 0.5 & \\
\hline $1 \mathrm{~B}$ & S & $5 / 3 / 1993$ & 93123 & 123 & & & 0.5 & & \\
\hline $1 \mathrm{~B}$ & S & $5 / 4 / 1993$ & 93124 & 124 & & & & 0.5 & \\
\hline $1 \mathrm{~B}$ & S & 5/5/1993 & 93125 & 125 & 0.5 & & & & \\
\hline $1 \mathrm{~B}$ & S & $5 / 6 / 1993$ & 93126 & 126 & 0.5 & & & 0.5 & \\
\hline $1 \mathrm{~B}$ & S & $5 / 7 / 1993$ & 93127 & 127 & 0.5 & & & & \\
\hline $0 \mathrm{~B}$ & S & $5 / 8 / 1993$ & 93128 & 128 & & 0.5 & & & \\
\hline $0 \mathrm{~B}$ & $S$ & 5/9/1993 & 93129 & 129 & & & & & \\
\hline $1 \mathrm{~B}$ & S & $5 / 10 / 1993$ & 93130 & 130 & & & & 0.5 & \\
\hline $1 \mathrm{~B}$ & S & $5 / 11 / 1993$ & 93131 & 131 & & & 0.5 & & \\
\hline $1 \mathrm{~B}$ & S & $5 / 12 / 1993$ & 93132 & 132 & 0.5 & & & 0.5 & \\
\hline $1 \mathrm{~B}$ & S & $5 / 13 / 1993$ & 93133 & 133 & & & & & 0.5 \\
\hline $1 \mathrm{~B}$ & $S$ & $5 / 14 / 1993$ & 93134 & 134 & & & & 0.5 & \\
\hline $0 \mathrm{~B}$ & $S$ & $5 / 15 / 1993$ & 93135 & 135 & 0.5 & & & & \\
\hline
\end{tabular}




\begin{tabular}{|c|c|c|c|c|c|c|c|c|c|c|}
\hline \begin{tabular}{l|l}
$0 \mathrm{~F}$ \\
\end{tabular} & $\mathrm{~B}$ & $S$ & 5/16/1993 & 93136 & 136 & & & & & \\
\hline $1 \mathrm{E}$ & B & S & $5 / 17 / 1993$ & 93137 & 137 & & & 0.5 & & \\
\hline $1 \mathrm{~F}$ & B & $S$ & $5 / 18 / 1993$ & 93138 & 138 & 0.5 & & & & \\
\hline $1 \mathrm{~F}$ & B & S & $5 / 19 / 1993$ & 93139 & 139 & & & & & 0.5 \\
\hline $1 \mathrm{E}$ & B & S & $5 / 20 / 1993$ & 93140 & 140 & & & & 0.5 & \\
\hline $1 \mathrm{~F}$ & B & S & $5 / 21 / 1993$ & 93141 & 141 & & & & & 0.5 \\
\hline $0 \mathrm{E}$ & B & S & $5 / 22 / 1993$ & 93142 & 142 & & & & 0.5 & \\
\hline \begin{tabular}{l|l}
0 & $\mathrm{E}$
\end{tabular} & B & S & $5 / 23 / 1993$ & 93143 & 143 & & 0.5 & & & \\
\hline $1 \mathrm{E}$ & B & $S$ & 5/24/1993 & 93144 & 144 & & 0.5 & & & \\
\hline $1 \mathrm{E}$ & B & S & $5 / 25 / 1993$ & 93145 & 145 & 0.5 & & & & \\
\hline $1 \mathrm{~F}$ & B & S & $5 / 26 / 1993$ & 93146 & 146 & & & & 0.5 & \\
\hline $1 \mathrm{E}$ & B & NS & 5/27/1993 & 93147 & 147 & & & & & 0.5 \\
\hline $1 \mathrm{~F}$ & B & NS & 5/28/1993 & 93148 & 148 & & & 0.5 & & \\
\hline \begin{tabular}{l|l} 
& $\mathrm{E}$ \\
\end{tabular} & B & NS & $5 / 29 / 1993$ & 93149 & 149 & & & 0.5 & & \\
\hline $0 \mathrm{E}$ & B & NS & $5 / 30 / 1993$ & 93150 & 150 & & & & & 0.5 \\
\hline $1 \mathrm{E}$ & B & NS & $5 / 31 / 1993$ & 93151 & 151 & 0.5 & & & & \\
\hline $1 \mathrm{E}$ & B & NS & 6/1/1993 & 93152 & 152 & & & & 0.5 & \\
\hline \begin{tabular}{l|l}
$1 \mathrm{E}$ \\
\end{tabular} & B & NS & 6/2/1993 & 93153 & 153 & & & & 0.5 & \\
\hline $1 \mathrm{E}$ & B & NS & 6/3/1993 & 93154 & 154 & & 0.5 & & & \\
\hline $1 \mathrm{E}$ & B & NS & 6/4/1993 & 93155 & 155 & & & & 0.5 & \\
\hline \begin{tabular}{l|l}
$0 \mathrm{~F}$ \\
\end{tabular} & B & NS & 6/5/1993 & 93156 & 156 & 0.5 & & & & \\
\hline \begin{tabular}{l|l}
0 & $\mathrm{E}$
\end{tabular} & B & NS & 6/6/1993 & 93157 & 157 & & & & 0.5 & \\
\hline $1 \mathrm{E}$ & B & NS & 6/7/1993 & 93158 & 158 & & & & 0.5 & \\
\hline \begin{tabular}{l|l}
1 & $\mathrm{E}$
\end{tabular} & B & NS & 6/8/1993 & 93159 & 159 & & & & & 0.5 \\
\hline $1 \mathrm{E}$ & B & NS & 6/9/1993 & 93160 & 160 & & & & 0.5 & \\
\hline $1 \mathrm{E}$ & B & NS & 6/10/1993 & 93161 & 161 & & & & & \\
\hline $1 \mathrm{~F}$ & B & NS & $6 / 11 / 1993$ & 93162 & 162 & & & 0.5 & & \\
\hline \begin{tabular}{l|l} 
& $\mathrm{E}$ \\
\end{tabular} & B & NS & $6 / 12 / 1993$ & 93163 & 163 & & & & 0.5 & \\
\hline \begin{tabular}{l|l}
$0 \mathrm{E}$ \\
\end{tabular} & B & NS & 6/13/1993 & 93164 & 164 & & & & 0.5 & \\
\hline $1 \mathrm{E}$ & B & NS & $6 / 14 / 1993$ & 93165 & 165 & & 0.5 & & & \\
\hline $1 \mathrm{E}$ & B & NS & $6 / 15 / 1993$ & 93166 & 166 & 0.5 & & & & \\
\hline $1 \mathrm{~F}$ & B & NS & 6/16/1993 & 93167 & 167 & & & & & 0.5 \\
\hline $1 \mathrm{E}$ & B & NS & 6/17/1993 & 93168 & 168 & 0.5 & & & & \\
\hline $1 \mathrm{E}$ & B & NS & 6/18/1993 & 93169 & 169 & & & 0.5 & & \\
\hline $0 \mathrm{E}$ & B & NS & $6 / 19 / 1993$ & 93170 & 170 & & & 0.5 & & \\
\hline \begin{tabular}{l|l}
0 & $\mathrm{E}$ \\
\end{tabular} & B & NS & 6/20/1993 & 93171 & 171 & & & & & 0.5 \\
\hline \begin{tabular}{l|l}
1 & $\mathrm{E}$
\end{tabular} & B & NS & 6/21/1993 & 93172 & 172 & & & & & \\
\hline $1 \mathrm{~F}$ & B & NS & $6 / 22 / 1993$ & 93173 & 173 & & & 0.5 & & \\
\hline $1 \mathrm{E}$ & B & NS & 6/23/1993 & 93174 & 174 & 0.5 & & & & 0.5 \\
\hline $1 \mathrm{E}$ & B & NS & 6/24/1993 & 93175 & 175 & 0.5 & & & & \\
\hline $1 \mathrm{E}$ & B & NS & $6 / 25 / 1993$ & 93176 & 176 & & 0.5 & & & \\
\hline \begin{tabular}{l|l}
0 & $\mathrm{E}$
\end{tabular} & B & NS & 6/26/1993 & 93177 & 177 & & & 0.5 & & \\
\hline \begin{tabular}{l|l}
$\mathrm{E}$ \\
\end{tabular} & B & NS & 6/27/1993 & 93178 & 178 & & & & & 0.5 \\
\hline $1 \mathrm{~F}$ & B & NS & 6/28/1993 & 93179 & 179 & & & 0.5 & & \\
\hline $1 \mathrm{E}$ & B & NS & 6/29/1993 & 93180 & 180 & & & 0.5 & & \\
\hline $1 \mathrm{~F}$ & B & NS & 6/30/1993 & 93181 & 181 & & & & & 0.5 \\
\hline $1 \mathrm{E}$ & B & NS & 7/1/1993 & 93182 & 182 & & & & & \\
\hline $1 \mathrm{E}$ & B & NS & 7/2/1993 & 93183 & 183 & & 0.5 & & & \\
\hline \begin{tabular}{l|l}
0 & $\mathrm{E}$
\end{tabular} & B & NS & 7/3/1993 & 93184 & 184 & 0.5 & & & & \\
\hline \begin{tabular}{l|l}
0 & $\mathrm{E}$
\end{tabular} & B & NS & 7/4/1993 & 93185 & 185 & & & & 0.5 & \\
\hline $1 \mathrm{~F}$ & B & NS & 7/5/1993 & 93186 & 186 & & & & & \\
\hline
\end{tabular}




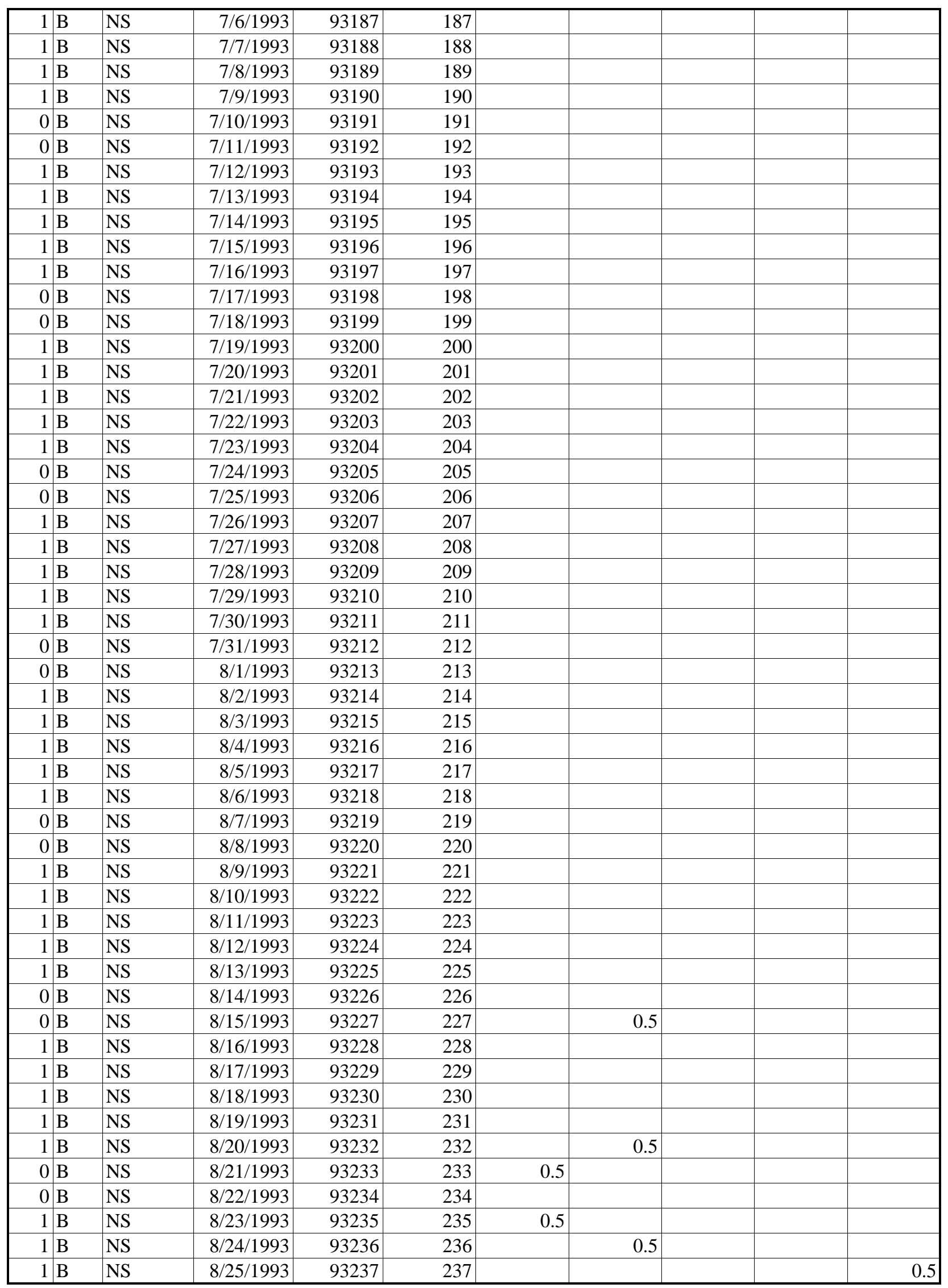




\begin{tabular}{|c|c|c|c|c|c|c|c|c|c|c|}
\hline$\overline{1 \mathrm{~F}}$ & $\mathrm{~B}$ & NS & 8/26/1993 & 93238 & 238 & & & & & \\
\hline $1 \mathrm{E}$ & B & NS & 8/27/1993 & 93239 & 239 & & 0.5 & & & \\
\hline $0 \mathrm{E}$ & B & NS & 8/28/1993 & 93240 & 240 & & 0.5 & & & \\
\hline \begin{tabular}{l|l}
$0 \mathrm{E}$ \\
\end{tabular} & B & NS & 8/29/1993 & 93241 & 241 & & & 0.5 & & \\
\hline $1 \mathrm{E}$ & B & NS & $8 / 30 / 1993$ & 93242 & 242 & & & & & \\
\hline $1 \mathrm{~F}$ & B & NS & 8/31/1993 & 93243 & 243 & & & & & 0.5 \\
\hline $1 \mathrm{E}$ & B & NS & 9/1/1993 & 93244 & 244 & & & & 0.5 & \\
\hline $1 \mathrm{E}$ & B & NS & 9/2/1993 & 93245 & 245 & 0.5 & & & & \\
\hline $1 \mathrm{E}$ & B & NS & 9/3/1993 & 93246 & 246 & & & & & \\
\hline $0 \mathrm{E}$ & B & NS & 9/4/1993 & 93247 & 247 & & & 0.5 & & \\
\hline $0 \mathrm{E}$ & B & NS & 9/5/1993 & 93248 & 248 & & & & & 0.5 \\
\hline $1 \mathrm{E}$ & B & NS & 9/6/1993 & 93249 & 249 & 0.5 & & & & \\
\hline $1 \mathrm{E}$ & B & NS & 9/7/1993 & 93250 & 250 & & 0.5 & & & \\
\hline $1 \mathrm{E}$ & B & NS & 9/8/1993 & 93251 & 251 & & & & & \\
\hline $1 \mathrm{E}$ & B & S & 9/9/1993 & 93252 & 252 & & 0.5 & & & \\
\hline $1 \mathrm{~F}$ & B & $S$ & 9/10/1993 & 93253 & 253 & & & & 0.5 & \\
\hline \begin{tabular}{l|l}
$0 \mathrm{E}$ \\
\end{tabular} & B & S & 9/11/1993 & 93254 & 254 & & & & & 0.5 \\
\hline \begin{tabular}{l|l}
0 & $\mathrm{E}$
\end{tabular} & B & S & 9/12/1993 & 93255 & 255 & & & & & \\
\hline $1 \mathrm{~F}$ & B & S & 9/13/1993 & 93256 & 256 & & & & & \\
\hline $1 \mathrm{E}$ & B & S & 9/14/1993 & 93257 & 257 & & & 0.5 & & \\
\hline $1 \mathrm{~F}$ & B & S & 9/15/1993 & 93258 & 258 & & & & & \\
\hline $1 \mathrm{~F}$ & B & S & 9/16/1993 & 93259 & 259 & 0.5 & & & & \\
\hline $1 \mathrm{E}$ & B & S & 9/17/1993 & 93260 & 260 & & & & & 0.5 \\
\hline \begin{tabular}{l|l}
0 & $\mathrm{E}$
\end{tabular} & B & S & 9/18/1993 & 93261 & 261 & & 0.5 & & & \\
\hline \begin{tabular}{l|l}
$0 \mathrm{E}$ \\
\end{tabular} & B & S & 9/19/1993 & 93262 & 262 & & & & & 0.5 \\
\hline $1 \mathrm{E}$ & B & S & 9/20/1993 & 93263 & 263 & & & 0.5 & & \\
\hline $1 \mathrm{~F}$ & B & S & 9/21/1993 & 93264 & 264 & & & & & 0.5 \\
\hline $1 \mathrm{E}$ & B & S & 9/22/1993 & 93265 & 265 & & & & & \\
\hline $1 \mathrm{E}$ & B & S & 9/23/1993 & 93266 & 266 & & 0.5 & & & \\
\hline $1 \mathrm{E}$ & B & S & 9/24/1993 & 93267 & 267 & & & & 0.5 & \\
\hline $0 \mathrm{E}$ & B & S & 9/25/1993 & 93268 & 268 & 0.5 & & & & \\
\hline $0 \mathrm{E}$ & B & S & 9/26/1993 & 93269 & 269 & & & & & 0.5 \\
\hline $1 \mathrm{~F}$ & B & S & 9/27/1993 & 93270 & 270 & & & 0.5 & & \\
\hline $1 \mathrm{E}$ & B & S & 9/28/1993 & 93271 & 271 & 0.5 & & & & \\
\hline $1 \mathrm{E}$ & B & $S$ & 9/29/1993 & 93272 & 272 & & & & 0.5 & \\
\hline $1 \mathrm{~F}$ & B & S & 9/30/1993 & 93273 & 273 & & & & & 0.5 \\
\hline \begin{tabular}{l|l}
1 & $\mathrm{E}$
\end{tabular} & B & S & 10/1/1993 & 93274 & 274 & & & & & \\
\hline \begin{tabular}{l|l}
0 & $\mathrm{E}$ \\
\end{tabular} & B & S & $10 / 2 / 1993$ & 93275 & 275 & 0.5 & & & & \\
\hline \begin{tabular}{l|l}
$0 \mathrm{E}$ \\
\end{tabular} & B & S & 10/3/1993 & 93276 & 276 & & & & & \\
\hline $1 \mathrm{~F}$ & B & S & 10/4/1993 & 93277 & 277 & & & & 0.5 & \\
\hline $1 \mathrm{E}$ & B & S & $10 / 5 / 1993$ & 93278 & 278 & & & & & 0.5 \\
\hline $1 \mathrm{E}$ & B & S & 10/6/1993 & 93279 & 279 & & 0.5 & & & \\
\hline $1 \mathrm{~F}$ & B & S & 10/7/1993 & 93280 & 280 & 0.5 & & & & \\
\hline $1 \mathrm{~F}$ & B & S & 10/8/1993 & 93281 & 281 & & 0.5 & & & \\
\hline \begin{tabular}{l|l}
0 & $\mathrm{E}$ \\
\end{tabular} & B & S & 10/9/1993 & 93282 & 282 & & & & & \\
\hline \begin{tabular}{l|l}
$\mathrm{E}$ \\
\end{tabular} & B & S & 10/10/1993 & 93283 & 283 & & & & & 0.5 \\
\hline $1 \mathrm{E}$ & B & S & 10/11/1993 & 93284 & 284 & & & 0.5 & & \\
\hline $1 \mathrm{E}$ & B & $S$ & 10/12/1993 & 93285 & 285 & & & & & \\
\hline $1 \mathrm{~F}$ & B & S & 10/13/1993 & 93286 & 286 & & & 0.5 & & \\
\hline \begin{tabular}{l|l}
$\mathrm{E}$ \\
\end{tabular} & B & S & 10/14/1993 & 93287 & 287 & & 0.5 & & & \\
\hline $1 \mathrm{~F}$ & B & S & 10/15/1993 & 93288 & 288 & 0.5 & & 0.5 & & \\
\hline
\end{tabular}




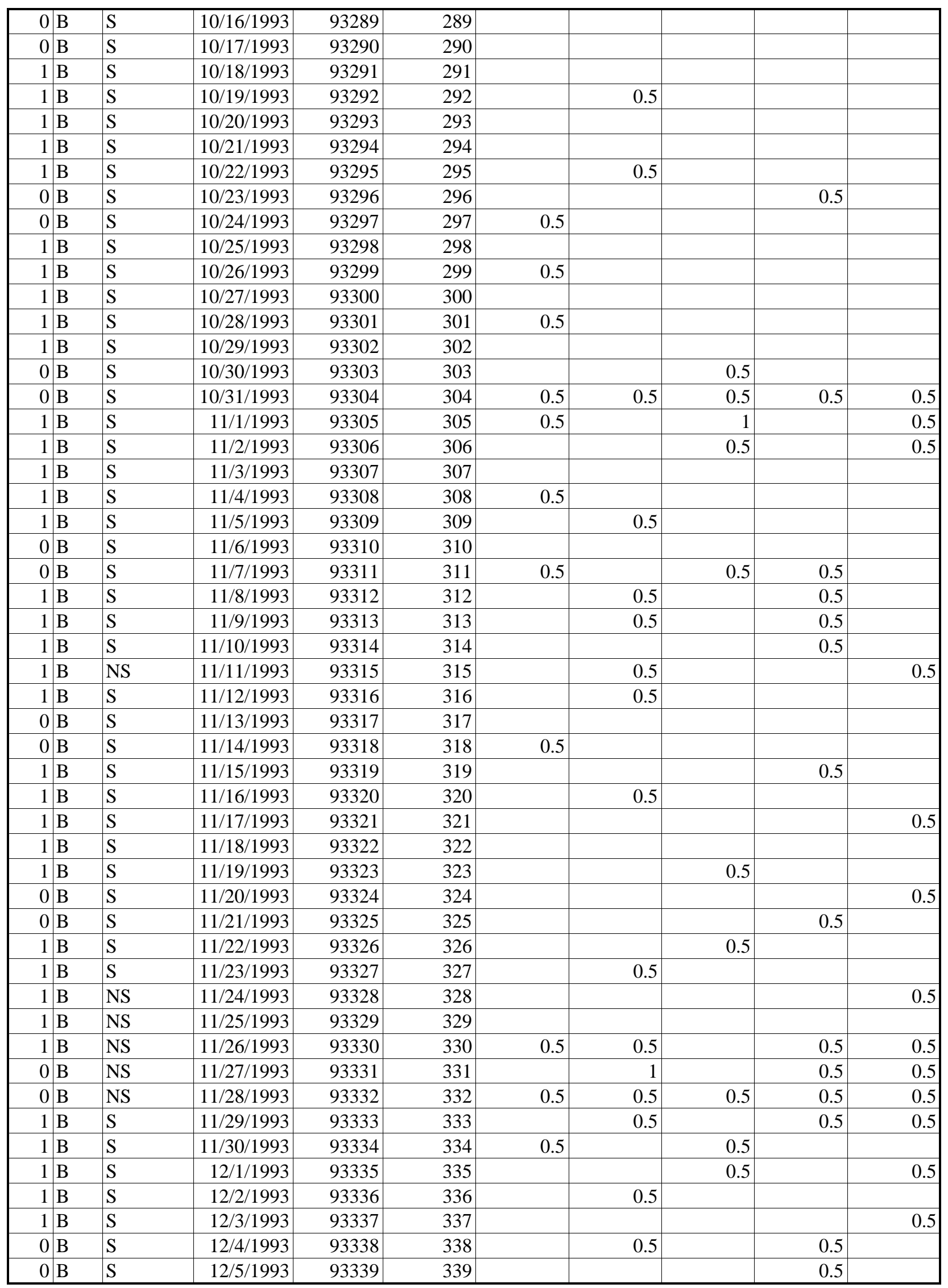




\begin{tabular}{|c|c|c|c|c|c|c|c|c|c|}
\hline $1 \mathrm{~B}$ & $S$ & 12/6/1993 & 93340 & 340 & & & & 0.5 & \\
\hline $1 \mathrm{~B}$ & S & 12/7/1993 & 93341 & 341 & & & 0.5 & & \\
\hline $1 \mathrm{~B}$ & S & 12/8/1993 & 93342 & 342 & & & & & \\
\hline $1 \mathrm{~B}$ & S & 12/9/1993 & 93343 & 343 & & & & 0.5 & \\
\hline \begin{tabular}{l|l}
$11 \mathrm{~B}$ \\
\end{tabular} & S & 12/10/1993 & 93344 & 344 & & & & & \\
\hline $0 \mathrm{~B}$ & S & 12/11/1993 & 93345 & 345 & & & 0.5 & & \\
\hline $0 \mathrm{~B}$ & S & $12 / 12 / 1993$ & 93346 & 346 & & & & & \\
\hline $1 \mathrm{~B}$ & S & $12 / 13 / 1993$ & 93347 & 347 & & & & & \\
\hline $1 \mathrm{~B}$ & $S$ & 12/14/1993 & 93348 & 348 & & & & 0.5 & \\
\hline $1 \mathrm{~B}$ & S & $12 / 15 / 1993$ & 93349 & 349 & & & 0.5 & & \\
\hline $1 \mathrm{~B}$ & S & 12/16/1993 & 93350 & 350 & 0.5 & & 0.5 & & 1 \\
\hline $1 \mathrm{~B}$ & NS & 12/17/1993 & 93351 & 351 & 0.5 & & & & 0.5 \\
\hline $0 \mathrm{~B}$ & NS & $12 / 18 / 1993$ & 93352 & 352 & 0.5 & & & & 0.5 \\
\hline $0 \mathrm{~B}$ & NS & 12/19/1993 & 93353 & 353 & & & & & 0.5 \\
\hline $1 \mathrm{~B}$ & NS & $12 / 20 / 1993$ & 93354 & 354 & 0.5 & 1.5 & 1 & 0.5 & 0.5 \\
\hline $1 \mathrm{~B}$ & NS & $12 / 21 / 1993$ & 93355 & 355 & & 0.1 & & 0.5 & 0.1 \\
\hline $1 \mathrm{~B}$ & NS & 12/22/1993 & 93356 & 356 & 0.1 & & & 0.6 & \\
\hline $1 \mathrm{~B}$ & NS & $12 / 23 / 1993$ & 93357 & 357 & 0.1 & 0.6 & 0.6 & & 0.1 \\
\hline $1 \mathrm{~B}$ & NS & $12 / 24 / 1993$ & 93358 & 358 & 0.6 & 0.1 & & 0.5 & 0.1 \\
\hline $0 \mathrm{~B}$ & NS & $12 / 25 / 1993$ & 93359 & 359 & 0.1 & 0.5 & 0.6 & & \\
\hline $0 \mathrm{~B}$ & NS & $12 / 26 / 1993$ & 93360 & 360 & & & 0.6 & 0.5 & \\
\hline $1 \mathrm{~B}$ & NS & $12 / 27 / 1993$ & 93361 & 361 & & & & & \\
\hline $1 \mathrm{~B}$ & NS & $12 / 28 / 1993$ & 93362 & 362 & & 0.5 & & & \\
\hline $1 \mathrm{~B}$ & NS & $12 / 29 / 1993$ & 93363 & 363 & & 0.5 & & & 0.1 \\
\hline $1 \mathrm{~B}$ & NS & 12/30/1993 & 93364 & 364 & 0.6 & 1 & 0.1 & 1 & 0.6 \\
\hline $1 \mathrm{~B}$ & NS & 12/31/1993 & 93365 & 365 & 0.1 & 0.1 & 0.5 & & \\
\hline \begin{tabular}{l|l}
0 & $\mathrm{~B}$ \\
\end{tabular} & NS & $1 / 1 / 1994$ & 94001 & 1 & 0.1 & 0.6 & & & 0.5 \\
\hline \begin{tabular}{l|l}
$0 \mathrm{~B}$ \\
\end{tabular} & NS & $1 / 2 / 1994$ & 94002 & 2 & & 0.1 & & & 0.5 \\
\hline $1 \mathrm{~B}$ & NS & $1 / 3 / 1994$ & 94003 & 3 & & 0.5 & & 0.1 & \\
\hline $1 \mathrm{~B}$ & NS & $1 / 4 / 1994$ & 94004 & 4 & & & & 0.5 & \\
\hline \begin{tabular}{l|l}
1 & $\mathrm{~B}$ \\
\end{tabular} & NS & $1 / 5 / 1994$ & 94005 & 5 & & 0.1 & & 0.5 & \\
\hline $1 \mathrm{~B}$ & S & $1 / 6 / 1994$ & 94006 & 6 & 1 & 0.5 & 1 & 1 & 0.5 \\
\hline $1 \mathrm{~B}$ & S & $1 / 7 / 1994$ & 94007 & 7 & 0.5 & 1 & & 1 & 0.5 \\
\hline \begin{tabular}{l|l}
0 & $\mathrm{~B}$ \\
\end{tabular} & S & $1 / 8 / 1994$ & 94008 & 8 & 0.5 & 0.5 & 0.5 & 1 & 0.5 \\
\hline $0 \mathrm{~B}$ & $S$ & $1 / 9 / 1994$ & 94009 & 9 & 0.5 & 0.5 & 0.5 & 0.5 & 0.5 \\
\hline $1 \mathrm{~B}$ & S & $1 / 10 / 1994$ & 94010 & 10 & 0.5 & 0.5 & 0.5 & 0.5 & 0.5 \\
\hline \begin{tabular}{l|l}
1 & $B$ \\
\end{tabular} & S & 1/11/1994 & 94011 & 11 & & & 0.5 & & \\
\hline $1 \mathrm{~B}$ & S & $1 / 12 / 1994$ & 94012 & 12 & 0.5 & & & 0.5 & \\
\hline \begin{tabular}{l|l}
$1 \mathrm{~B}$ \\
\end{tabular} & S & $1 / 13 / 1994$ & 94013 & 13 & & & & 0.5 & \\
\hline $1 \mathrm{~B}$ & S & $1 / 14 / 1994$ & 94014 & 14 & 0.5 & & & 0.5 & \\
\hline \begin{tabular}{l|l}
0 & $\mathrm{~B}$ \\
\end{tabular} & S & $1 / 15 / 1994$ & 94015 & 15 & & 0.5 & & & \\
\hline $0 \mathrm{~B}$ & S & $1 / 16 / 1994$ & 94016 & 16 & & & 0.5 & & \\
\hline \begin{tabular}{l|l}
1 & $B$ \\
\end{tabular} & S & 1/17/1994 & 94017 & 17 & & & & & \\
\hline \begin{tabular}{|l|l}
1 & $B$ \\
\end{tabular} & $S$ & $1 / 18 / 1994$ & 94018 & 18 & 0.5 & 1 & 1 & 0.5 & 1 \\
\hline $1 \mathrm{~B}$ & S & 1/19/1994 & 94019 & 19 & 0.5 & 0.5 & 1.1 & 1 & 0.5 \\
\hline $1 \mathrm{~B}$ & S & $1 / 20 / 1994$ & 94020 & 20 & 0.6 & 0.5 & 0.6 & 0.5 & 0.6 \\
\hline $1 \mathrm{~B}$ & S & $1 / 21 / 1994$ & 94021 & 21 & & 0.5 & & 0.1 & 0.5 \\
\hline $0 \mathrm{~B}$ & S & $1 / 22 / 1994$ & 94022 & 22 & 0.5 & 0.5 & 0.5 & 0.5 & 0.1 \\
\hline \begin{tabular}{l|l}
0 & $\mathrm{~B}$
\end{tabular} & S & $1 / 23 / 1994$ & 94023 & 23 & 0.5 & 0.1 & 0.5 & 0.6 & \\
\hline $1 \mathrm{~B}$ & S & $1 / 24 / 1994$ & 94024 & 24 & 0.6 & 0.6 & 0.1 & 0.6 & \\
\hline $1 \mathrm{~B}$ & S & $1 / 25 / 1994$ & 94025 & 25 & & & & 0.5 & \\
\hline
\end{tabular}




\begin{tabular}{|c|c|c|c|c|c|c|c|c|c|}
\hline $1 \mathrm{~B}$ & $S$ & 1/26/1994 & 94026 & 26 & 0.5 & & & 0.5 & \\
\hline $1 \mathrm{~B}$ & $S$ & 1/27/1994 & 94027 & 27 & & & 0.5 & & \\
\hline $1 \mathrm{~B}$ & $S$ & $1 / 28 / 1994$ & 94028 & 28 & 0.6 & 0.5 & 0.6 & 0.5 & 0.1 \\
\hline $0 \mathrm{~B}$ & $S$ & $1 / 29 / 1994$ & 94029 & 29 & 1.4 & 1.3 & 1.4 & 0.8 & 1.4 \\
\hline $0 \mathrm{~B}$ & S & $1 / 30 / 1994$ & 94030 & 30 & 0.8 & 1.4 & 0.8 & 0.8 & 0.9 \\
\hline $1 \mathrm{~B}$ & $S$ & $1 / 31 / 1994$ & 94031 & 31 & 0.9 & 0.8 & 0.9 & 0.9 & 0.9 \\
\hline $1 \mathrm{~B}$ & $S$ & 2/1/1994 & 94032 & 32 & 1.1 & 0.6 & 3.6 & 2.7 & 2.6 \\
\hline $1 B$ & $S$ & $2 / 2 / 1994$ & 94033 & 33 & 1.1 & 0.6 & 3.8 & 3.2 & 2.7 \\
\hline $1 \mathrm{~B}$ & $S$ & 2/3/1994 & 94034 & 34 & 0.6 & 0.6 & 4.2 & 2.6 & 2.7 \\
\hline $1 \mathrm{~B}$ & $S$ & 2/4/1994 & 94035 & 35 & 0.5 & 0.6 & 3.6 & 2.1 & 1.5 \\
\hline $0 \mathrm{~B}$ & S & 2/5/1994 & 94036 & 36 & 0.5 & & 0.5 & & 0.5 \\
\hline $0 \mathrm{~B}$ & $S$ & 2/6/1994 & 94037 & 37 & 0.5 & & & & \\
\hline $1 \mathrm{~B}$ & S & 2/7/1994 & 94038 & 38 & & & 3.6 & & 1 \\
\hline $1 \mathrm{~B}$ & $S$ & 2/8/1994 & 94039 & 39 & & & & 0.5 & \\
\hline $1 \mathrm{~B}$ & $S$ & $2 / 9 / 1994$ & 94040 & 40 & & 0.5 & & & \\
\hline $1 \mathrm{~B}$ & $S$ & 2/10/1994 & 94041 & 41 & 1.1 & 1.1 & 1.6 & 2.2 & 2.1 \\
\hline $1 \mathrm{~B}$ & $S$ & $2 / 11 / 1994$ & 94042 & 42 & 1.6 & 1.1 & 2.2 & 2.1 & 2.2 \\
\hline $0 \mathrm{~B}$ & $S$ & $2 / 12 / 1994$ & 94043 & 43 & 0.6 & 1.1 & 1.1 & 0.6 & 0.6 \\
\hline $0 \mathrm{~B}$ & S & 2/13/1994 & 94044 & 44 & 0.6 & 0.6 & 0.1 & 1.1 & 0.1 \\
\hline $1 \mathrm{~B}$ & $S$ & 2/14/1994 & 94045 & 45 & 0.1 & 1.1 & 0.6 & 0.6 & 1.1 \\
\hline $1 \mathrm{~B}$ & $S$ & 2/15/1994 & 94046 & 46 & 0.5 & & & 0.5 & \\
\hline $1 \mathrm{~B}$ & S & $2 / 16 / 1994$ & 94047 & 47 & 0.5 & & & & 0.5 \\
\hline $1 \mathrm{~B}$ & S & 2/17/1994 & 94048 & 48 & & & 0.5 & & 0.5 \\
\hline $1 B$ & $S$ & $2 / 18 / 1994$ & 94049 & 49 & 0.5 & & 0.5 & & \\
\hline $0 \mathrm{~B}$ & S & 2/19/1994 & 94050 & 50 & & & & & 0.5 \\
\hline $0 \mathrm{~B}$ & S & 2/20/1994 & 94051 & 51 & & 0.5 & & & \\
\hline $1 \mathrm{~B}$ & NS & $2 / 21 / 1994$ & 94052 & 52 & & 0.5 & & & \\
\hline $1 \mathrm{~B}$ & $S$ & 2/22/1994 & 94053 & 53 & & & & 0.5 & \\
\hline $1 \mathrm{~B}$ & S & 2/23/1994 & 94054 & 54 & & & 0.5 & & \\
\hline $1 \mathrm{~B}$ & $S$ & 2/24/1994 & 94055 & 55 & & 1 & & 0.5 & \\
\hline $1 \mathrm{~B}$ & S & $2 / 25 / 1994$ & 94056 & 56 & & & 0.5 & 0.5 & \\
\hline $0 \mathrm{~B}$ & $S$ & $2 / 26 / 1994$ & 94057 & 57 & & & & 0.5 & \\
\hline $0 \mathrm{~B}$ & $S$ & 2/27/1994 & 94058 & 58 & 0.5 & 0.5 & 0.5 & 0.5 & 0.5 \\
\hline $1 \mathrm{~B}$ & $S$ & $2 / 28 / 1994$ & 94059 & 59 & & 0.5 & 0.5 & & 0.5 \\
\hline $1 \mathrm{~B}$ & S & 3/1/1994 & 94060 & 60 & 0.5 & & & & \\
\hline $1 \mathrm{~B}$ & $S$ & 3/2/1994 & 94061 & 61 & & & & 0.5 & \\
\hline $1 \mathrm{~B}$ & $S$ & $3 / 3 / 1994$ & 94062 & 62 & & 0.5 & & 0.5 & \\
\hline $1 \mathrm{~B}$ & S & $3 / 4 / 1994$ & 94063 & 63 & & 0.5 & & & \\
\hline $0 \mathrm{~B}$ & S & $3 / 5 / 1994$ & 94064 & 64 & & & 0.5 & & \\
\hline $0 \mathrm{~B}$ & $S$ & $3 / 6 / 1994$ & 94065 & 65 & & 0.5 & & & \\
\hline $1 \mathrm{~B}$ & $S$ & $3 / 7 / 1994$ & 94066 & 66 & & & 0.5 & & \\
\hline $1 \mathrm{~B}$ & S & $3 / 8 / 1994$ & 94067 & 67 & & & & 0.5 & \\
\hline $1 \mathrm{~B}$ & S & 3/9/1994 & 94068 & 68 & & 0.5 & & & \\
\hline $1 \mathrm{~B}$ & $S$ & 3/10/1994 & 94069 & 69 & 0.5 & 0.5 & 0.5 & & 1 \\
\hline $1 \mathrm{~B}$ & NS & 3/11/1994 & 94070 & 70 & 0.5 & & 0.5 & 0.5 & 0.5 \\
\hline $0 \mathrm{~B}$ & NS & 3/12/1994 & 94071 & 71 & & 0.5 & & & \\
\hline $0 \mathrm{~B}$ & NS & 3/13/1994 & 94072 & 72 & 0.5 & & & 0.5 & \\
\hline $1 \mathrm{~B}$ & NS & $3 / 14 / 1994$ & 94073 & 73 & 0.5 & 1.5 & 1.5 & 0.5 & 1 \\
\hline $1 \mathrm{~B}$ & NS & $3 / 15 / 1994$ & 94074 & 74 & 0.5 & 0.5 & 0.5 & & 0.5 \\
\hline $1 \mathrm{~B}$ & NS & $3 / 16 / 1994$ & 94075 & 75 & & 0.5 & & 0.5 & \\
\hline $1 \mathrm{~B}$ & NS & 3/17/1994 & 94076 & 76 & & 0.5 & 0.5 & & 0.5 \\
\hline
\end{tabular}




\begin{tabular}{|c|c|c|c|c|c|c|c|c|c|c|}
\hline$\overline{1 \mathrm{~F}}$ & $\mathrm{~B}$ & NS & 3/18/1994 & \begin{tabular}{|c|}
94077 \\
\end{tabular} & 77 & 0.5 & 0.5 & & & 0.5 \\
\hline \begin{tabular}{l|l}
0 & $\mathrm{E}$
\end{tabular} & B & NS & $3 / 19 / 1994$ & 94078 & 78 & & & & 0.5 & \\
\hline $0 \mathrm{E}$ & B & NS & $3 / 20 / 1994$ & 94079 & 79 & & & 0.5 & & \\
\hline $1 \mathrm{~F}$ & B & S & $3 / 21 / 1994$ & 94080 & 80 & & & 0.5 & & \\
\hline $1 \mathrm{E}$ & B & S & $3 / 22 / 1994$ & 94081 & 81 & 0.5 & & & 0.5 & \\
\hline $1 \mathrm{~F}$ & B & S & $3 / 23 / 1994$ & 94082 & 82 & 0.5 & & & & \\
\hline $1 \mathrm{E}$ & B & S & $3 / 24 / 1994$ & 94083 & 83 & & & 0.5 & & \\
\hline $1 \mathrm{E}$ & B & S & $3 / 25 / 1994$ & 94084 & 84 & & & & 0.5 & \\
\hline $0 \mathrm{E}$ & B & $S$ & 3/26/1994 & 94085 & 85 & 0.5 & & & 0.5 & \\
\hline $0 \mathrm{E}$ & B & S & $3 / 27 / 1994$ & 94086 & 86 & 0.5 & & & 0.5 & \\
\hline $1 \mathrm{~F}$ & B & S & $3 / 28 / 1994$ & 94087 & 87 & & & & 0.5 & \\
\hline $1 \mathrm{E}$ & B & $S$ & $3 / 29 / 1994$ & 94088 & 88 & & & & & 0.5 \\
\hline $1 \mathrm{E}$ & B & S & 3/30/1994 & 94089 & 89 & & & & & \\
\hline $1 \mathrm{E}$ & B & S & 3/31/1994 & 94090 & 90 & 0.5 & & & & \\
\hline $1 \mathrm{E}$ & B & NS & 4/1/1994 & 94091 & 91 & & 0.5 & & & 0.5 \\
\hline $0 \mathrm{E}$ & B & NS & $4 / 2 / 1994$ & 94092 & 92 & & & 0.5 & & \\
\hline \begin{tabular}{l|l}
$0 \mathrm{E}$ \\
\end{tabular} & B & NS & $4 / 3 / 1994$ & 94093 & 93 & & 0.5 & & & \\
\hline $1 \mathrm{E}$ & B & NS & $4 / 4 / 1994$ & 94094 & 94 & & 0.5 & & & \\
\hline $1 \mathrm{E}$ & B & S & $4 / 5 / 1994$ & 94095 & 95 & & & 0.5 & & \\
\hline $1 \mathrm{E}$ & B & S & $4 / 6 / 1994$ & 94096 & 96 & & & & & 0.5 \\
\hline $1 \mathrm{E}$ & B & S & 4/7/1994 & 94097 & 97 & & & 0.5 & 0.5 & 1 \\
\hline $1 \mathrm{E}$ & B & S & 4/8/1994 & 94098 & 98 & & & 0.5 & & \\
\hline $0 \mathrm{E}$ & B & S & 4/9/1994 & 94099 & 99 & & & 0.5 & & \\
\hline \begin{tabular}{l|l}
0 & $\mathrm{E}$
\end{tabular} & B & S & $4 / 10 / 1994$ & 94100 & 100 & & & 0.5 & & \\
\hline $1 \mathrm{E}$ & B & S & 4/11/1994 & 94101 & 101 & 0.5 & & & 0.5 & \\
\hline $1 \mathrm{E}$ & B & S & 4/12/1994 & 94102 & 102 & & & 0.5 & & \\
\hline \begin{tabular}{l|l}
$1 \mathrm{~F}$ \\
\end{tabular} & B & S & 4/13/1994 & 94103 & 103 & & & & 0.5 & \\
\hline $1 \mathrm{E}$ & B & S & $4 / 14 / 1994$ & 94104 & 104 & & & & 0.5 & \\
\hline $1 \mathrm{E}$ & B & S & $4 / 15 / 1994$ & 94105 & 105 & & & & 0.5 & \\
\hline $0 \mathrm{E}$ & B & S & $4 / 16 / 1994$ & 94106 & 106 & & 0.5 & & & 0.5 \\
\hline $0 \mathrm{E}$ & B & S & $4 / 17 / 1994$ & 94107 & 107 & & & & & \\
\hline $1 \mathrm{~F}$ & B & S & 4/18/1994 & 94108 & 108 & & & 0.5 & & \\
\hline $1 \mathrm{~F}$ & B & S & $4 / 19 / 1994$ & 94109 & 109 & 0.5 & & & & \\
\hline $1 \mathrm{E}$ & B & S & $4 / 20 / 1994$ & 94110 & 110 & & & & 0.5 & \\
\hline $1 \mathrm{E}$ & B & S & $4 / 21 / 1994$ & 94111 & 111 & & 0.5 & & & \\
\hline $1 \mathrm{~F}$ & B & S & $4 / 22 / 1994$ & 94112 & 112 & & & & & 0.5 \\
\hline \begin{tabular}{l|l}
0 & $\mathrm{E}$
\end{tabular} & B & S & $4 / 23 / 1994$ & 94113 & 113 & & 0.5 & & & \\
\hline $0 \mathrm{~F}$ & B & S & $4 / 24 / 1994$ & 94114 & 114 & & & & & 0.5 \\
\hline $1 \mathrm{E}$ & B & S & $4 / 25 / 1994$ & 94115 & 115 & & & & 0.5 & \\
\hline $1 \mathrm{E}$ & B & S & 4/26/1994 & 94116 & 116 & & 0.5 & & & \\
\hline $1 \mathrm{E}$ & B & S & $4 / 27 / 1994$ & 94117 & 117 & & & & 0.5 & \\
\hline $1 \mathrm{E}$ & B & S & $4 / 28 / 1994$ & 94118 & 118 & & 0.5 & & & \\
\hline $1 \mathrm{~F}$ & B & S & $4 / 29 / 1994$ & 94119 & 119 & & & 0.5 & & \\
\hline \begin{tabular}{l|l}
0 & $\mathrm{E}$ \\
\end{tabular} & B & S & $4 / 30 / 1994$ & 94120 & 120 & & & & & 0.5 \\
\hline \begin{tabular}{l|l}
0 & $\mathrm{E}$
\end{tabular} & B & S & 5/1/1994 & 94121 & 121 & & & 0.5 & & \\
\hline $1 \mathrm{~F}$ & B & S & $5 / 2 / 1994$ & 94122 & 122 & & 1 & & & 1 \\
\hline $1 \mathrm{E}$ & B & S & $5 / 3 / 1994$ & 94123 & 123 & & & & & 0.5 \\
\hline $1 \mathrm{E}$ & B & S & $5 / 4 / 1994$ & 94124 & 124 & & & & & 0.5 \\
\hline $1 \mathrm{~F}$ & B & S & 5/5/1994 & 94125 & 125 & & 0.5 & & & \\
\hline \begin{tabular}{l|l}
$\mathrm{E}$ \\
\end{tabular} & B & S & 5/6/1994 & 94126 & 126 & & & & 0.5 & \\
\hline \begin{tabular}{l|l}
0 & $\mathrm{E}$ \\
\end{tabular} & B & S & 5/7/1994 & 94127 & 127 & & 0.5 & & & \\
\hline
\end{tabular}




\begin{tabular}{|c|c|c|c|c|c|c|c|c|c|c|}
\hline $0 \mathrm{~B}$ & B & $S$ & $5 / 8 / 1994$ & 94128 & 128 & & & & & \\
\hline \begin{tabular}{l|l}
1 & $B$ \\
\end{tabular} & B & $S$ & $5 / 9 / 1994$ & 94129 & 129 & & & & & \\
\hline $\begin{array}{ll}1 \mathrm{~B} \\
\end{array}$ & B & $S$ & $5 / 10 / 1994$ & 94130 & 130 & & & & 0.5 & \\
\hline $1 \mathrm{~B}$ & B & $S$ & $5 / 11 / 1994$ & 94131 & 131 & & & & 0.5 & \\
\hline \begin{tabular}{l|l}
1 & $B$ \\
\end{tabular} & B & $S$ & $5 / 12 / 1994$ & 94132 & 132 & 0.5 & & & & \\
\hline $1 \mathrm{~B}$ & $\mathrm{~B}$ & S & $5 / 13 / 1994$ & 94133 & 133 & & 0.5 & & & \\
\hline $0 \mathrm{~B}$ & B & S & $5 / 14 / 1994$ & 94134 & 134 & & & & & 0.5 \\
\hline \begin{tabular}{l|l}
0 & $B$ \\
\end{tabular} & B & $S$ & $5 / 15 / 1994$ & 94135 & 135 & 0.5 & & & & \\
\hline $1 \mathrm{~B}$ & B & $S$ & $5 / 16 / 1994$ & 94136 & 136 & & 0.5 & & & \\
\hline $1 \mathrm{~B}$ & B & $S$ & 5/17/1994 & 94137 & 137 & & & & & 0.5 \\
\hline $\begin{array}{ll}1 \mathrm{~B} \\
\end{array}$ & B & $S$ & $5 / 18 / 1994$ & 94138 & 138 & & & 0.5 & & \\
\hline \begin{tabular}{l|l}
11 & $B$ \\
\end{tabular} & B & $S$ & $5 / 19 / 1994$ & 94139 & 139 & 0.5 & & & & \\
\hline $1 \mathrm{~B}$ & $\mathrm{~B}$ & S & $5 / 20 / 1994$ & 94140 & 140 & & & & 0.5 & \\
\hline $0 \mathrm{~B}$ & $\mathrm{~B}$ & S & $5 / 21 / 1994$ & 94141 & 141 & 0.5 & & & & 0.5 \\
\hline \begin{tabular}{l|l}
0 & $B$ \\
\end{tabular} & B & $S$ & $5 / 22 / 1994$ & 94142 & 142 & 0.5 & & & & \\
\hline $1 \mathrm{~B}$ & B & $S$ & 5/23/1994 & 94143 & 143 & & & 0.5 & & \\
\hline $1 \mathrm{~B}$ & B & $S$ & $5 / 24 / 1994$ & 94144 & 144 & 0.5 & & & & \\
\hline \begin{tabular}{l|l}
1 & $B$ \\
\end{tabular} & B & $S$ & $5 / 25 / 1994$ & 94145 & 145 & & 0.5 & & & 0.5 \\
\hline \begin{tabular}{l|l}
$11 \mathrm{~B}$ \\
\end{tabular} & B & $S$ & $5 / 26 / 1994$ & 94146 & 146 & & 0.5 & & & \\
\hline $1 \mathrm{~B}$ & $\mathrm{~B}$ & S & $5 / 27 / 1994$ & 94147 & 147 & & & & & 0.5 \\
\hline \begin{tabular}{l|l}
0 & $B$ \\
\end{tabular} & B & NS & $5 / 28 / 1994$ & 94148 & 148 & & 0.5 & & & \\
\hline $0 \mathrm{~B}$ & B & NS & 5/29/1994 & 94149 & 149 & 0.5 & & & & \\
\hline $1 \mathrm{~B}$ & $\mathrm{~B}$ & NS & $5 / 30 / 1994$ & 94150 & 150 & & & & & 0.5 \\
\hline \begin{tabular}{l|l}
1 & $B$ \\
\end{tabular} & B & NS & $5 / 31 / 1994$ & 94151 & 151 & & & & & 0.5 \\
\hline \begin{tabular}{l|l}
1 & $B$ \\
\end{tabular} & B & NS & $6 / 1 / 1994$ & 94152 & 152 & 0.5 & & & & \\
\hline $1 \mathrm{~B}$ & $\mathrm{~B}$ & NS & $6 / 2 / 1994$ & 94153 & 153 & & & & 0.5 & \\
\hline $1 \mathrm{~B}$ & B & NS & 6/3/1994 & 94154 & 154 & & & & 0.5 & \\
\hline $0 \mathrm{~B}$ & B & NS & 6/4/1994 & 94155 & 155 & & & & & \\
\hline & & & & & & & & & & \\
\hline & & & & & \multicolumn{2}{|c|}{ sort S/NS - 0/1 - A/B } & & & & \\
\hline & & & & & \multicolumn{2}{|c|}{ Daily Averages } & & & & \\
\hline & & & & & $1-A-S$ & 0.5 & 0 & 0.5 & 0.5 & 0.5 \\
\hline & & & & & 1-B-S & 0.984967 & 0.974684 & 0.953488 & 0.928743 & 0.976582 \\
\hline & & & & & 0-A-S & 0.75 & 0.65 & 0.662791 & 0.663793 & 0.664384 \\
\hline & & & & & $0-\mathrm{B}-\mathrm{S}$ & 0.889172 & 0.92695 & 1.156291 & 0.928662 & 0.919595 \\
\hline & & & & & 1-A-NS & 0.5 & 0.5 & 0.5 & 0.5 & 0.5 \\
\hline & & & & & 1-B-NS & 0.479167 & 0.516071 & 0.552273 & 0.54 & 0.475 \\
\hline & & & & & 0-A-NS & 0.731579 & 0.784211 & 1.55 & 1.152381 & 1.2 \\
\hline & & & & & $0-\mathrm{B}-\mathrm{NS}$ & 0.5 & 0.557692 & 0.545455 & 0.5 & 0.583333 \\
\hline \multicolumn{11}{|c|}{ sort JDAY } \\
\hline \multicolumn{4}{|c|}{ Table of Monthly Consumption } & & \multicolumn{2}{|c|}{ sort S/NS - 0/1 - A/B } & & & & \\
\hline & & & & & \multicolumn{4}{|c|}{ Standard Deviation of Daily Averages } & & \\
\hline & & Jun 91 & 16 & 16.4 & & & & & & \\
\hline & & Jul & 21 & 20.5 & $1-\mathrm{A}-\mathrm{S}$ & \#DIV/0! & \#DIV/0! & \#DIV/0! & \#DIV/0! & 0 \\
\hline & & Aug & 1 & 0.5 & 1-B-S & 0.855614 & 0.811614 & 0.782289 & 0.861499 & 0.81414 \\
\hline & & Sep & 18 & 17.5 & $0-A-S$ & 0.3371 & 0.267492 & 0.260972 & 0.254571 & 0.25076 \\
\hline & & Oct & 16 & 16.4 & 0-B-S & 0.433024 & 0.505947 & 0.921345 & 0.532899 & 0.476475 \\
\hline & & Nov & 45 & 45.1 & 1-A-NS & 0 & 0 & 0 & 0 & \\
\hline & & Dec & 597 & 597 & 1-B-NS & 0.151529 & 0.232595 & 0.193485 & 0.176126 & 0.175645 \\
\hline & & Jan 92 & 1640 & 1640.1 & $0-\mathrm{A}-\mathrm{NS}$ & 0.374244 & 0.311383 & 1.396424 & 0.90367 & 0.8544 \\
\hline & & Feb & 775 & 775.2 & 0-B-NS & 0 & 0.215728 & 0.213201 & 0 & 0.190347 \\
\hline
\end{tabular}




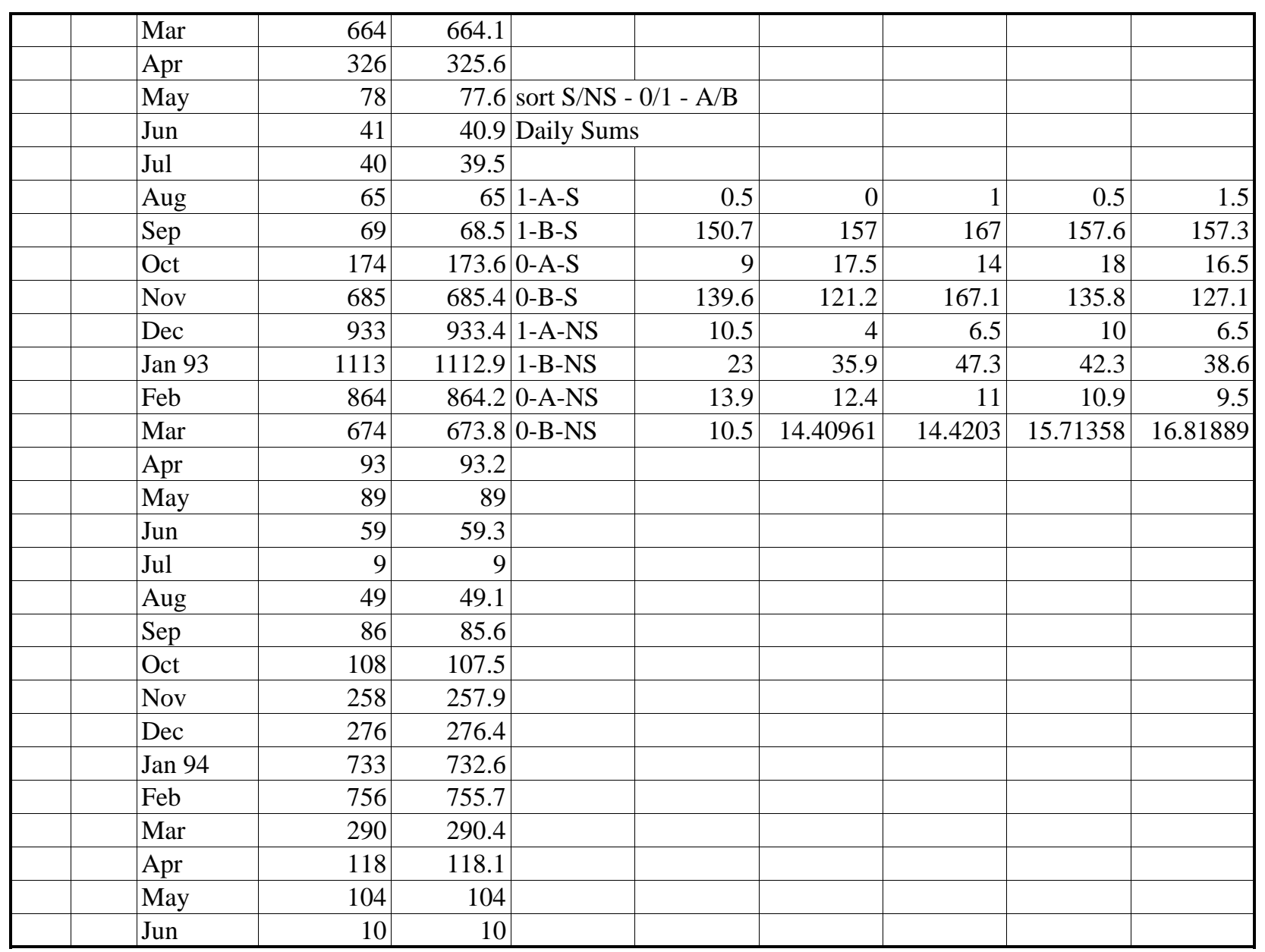




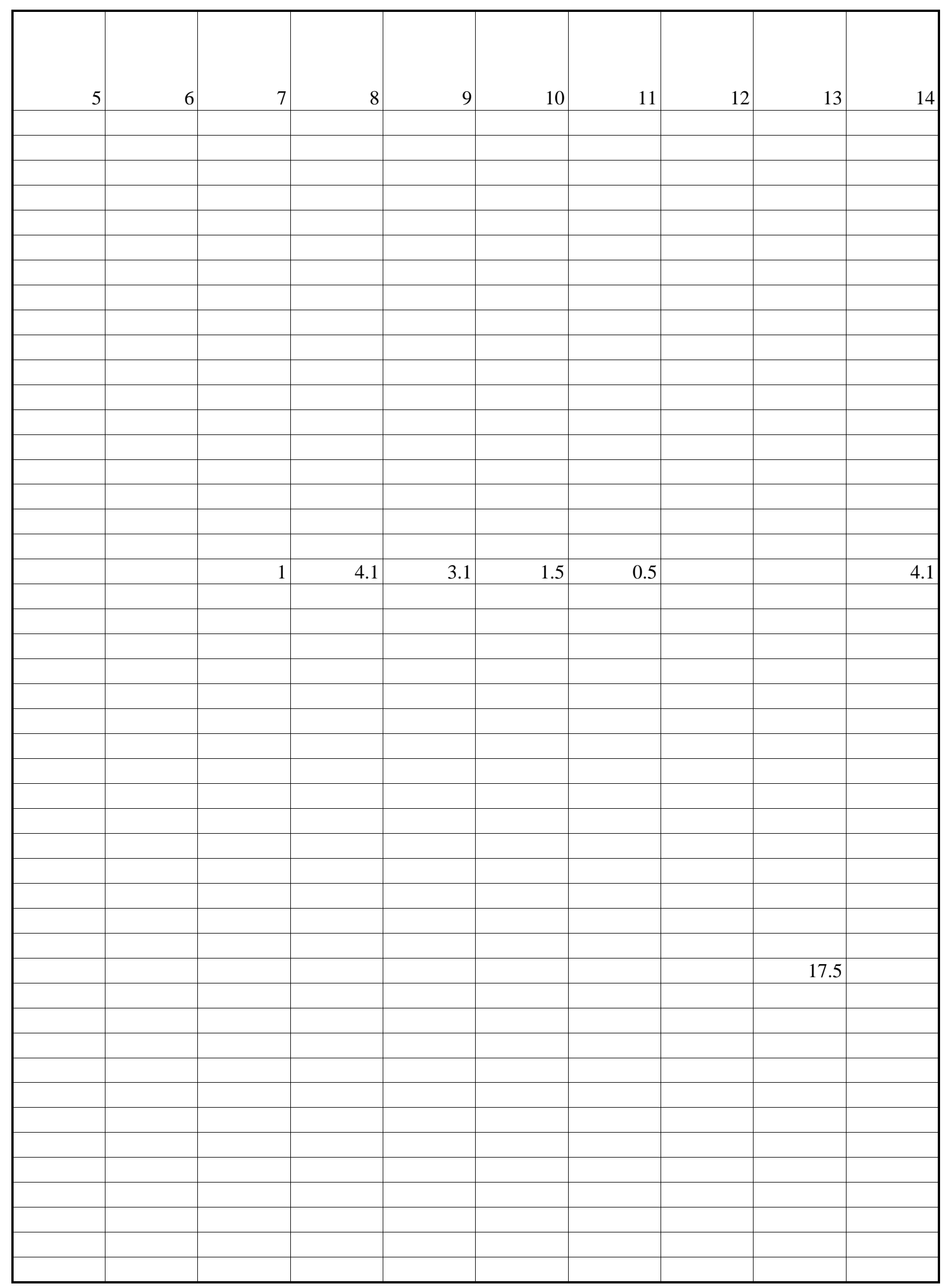

Page 24 


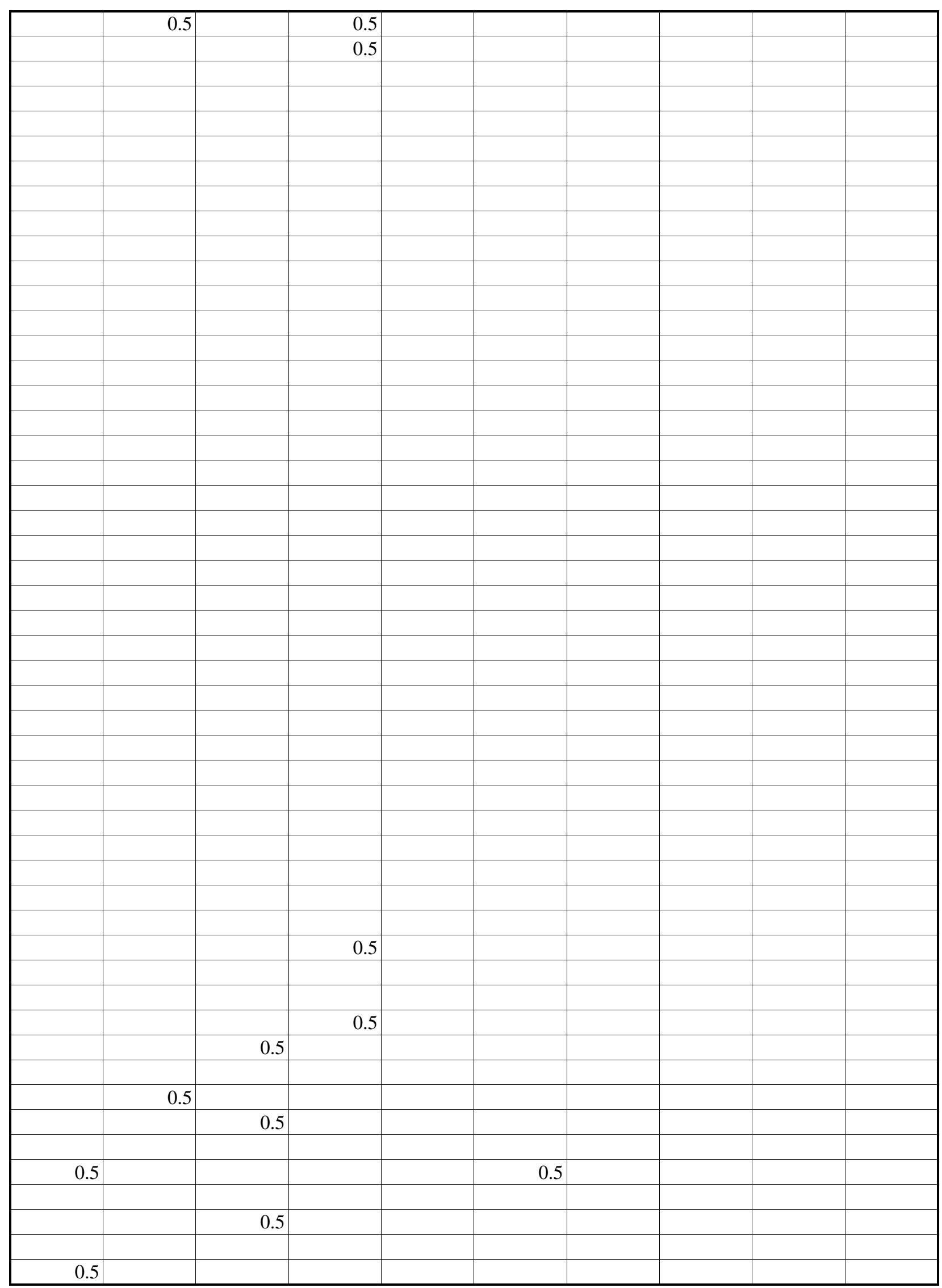

Page 25 
wbheat

\begin{tabular}{|c|c|c|c|c|c|c|c|c|c|}
\hline & & & & & & & & & \\
\hline & & & 0.5 & & & & & & \\
\hline & & & & & & & & & \\
\hline & & & 0.5 & & & & & & \\
\hline & & & & & & & & & \\
\hline 0.5 & & & 0.5 & & & & & & \\
\hline & 0.5 & & & & & & 0.5 & & \\
\hline & & & 0.5 & & & & & & \\
\hline & 0.5 & & & & 0.5 & & & & \\
\hline & 0.5 & & & 0.5 & & & & & \\
\hline & & & & & & & & & \\
\hline & & & & & & & & & \\
\hline & & & & & & & & & \\
\hline & & & & & & & & & \\
\hline & & & & & & & & & \\
\hline & & & & & & & & & \\
\hline & & & & & & & & & \\
\hline & & & & & & & & & \\
\hline & & & & & & & & - & \\
\hline & & & & & & & & & \\
\hline & & & & & & & & & \\
\hline & & & & & & & 13.9 & 0.5 & \\
\hline & & & & & & & & & \\
\hline & & & & & & & 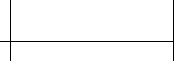 & & \\
\hline & & & & & & & 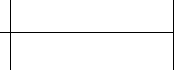 & 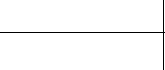 & \\
\hline & & & & & & & & & \\
\hline & & & & & & & & & \\
\hline & & & & & & & & & \\
\hline & & & & & & & & & 1.5 \\
\hline & & & & & & & & & \\
\hline & & & & & & & 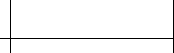 & & \\
\hline & & & & & & & 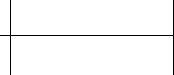 & & \\
\hline & & & & & & & & & \\
\hline & & & & & & & & & \\
\hline & & & & 南 & & & 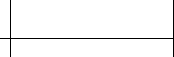 & & \\
\hline & & & & & & & & & \\
\hline & & & & & & & & & \\
\hline & & & & & & & 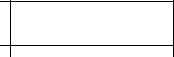 & & \\
\hline & & & & 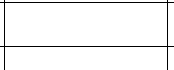 & & & 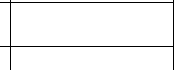 & & \\
\hline & & & & & & & 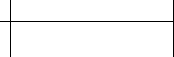 & & \\
\hline & & & & & & & & & \\
\hline & & & & & & & & & \\
\hline & & 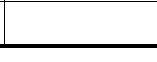 & & 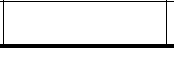 & 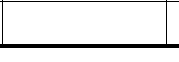 & & & 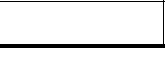 & 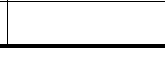 \\
\hline
\end{tabular}

Page 26 


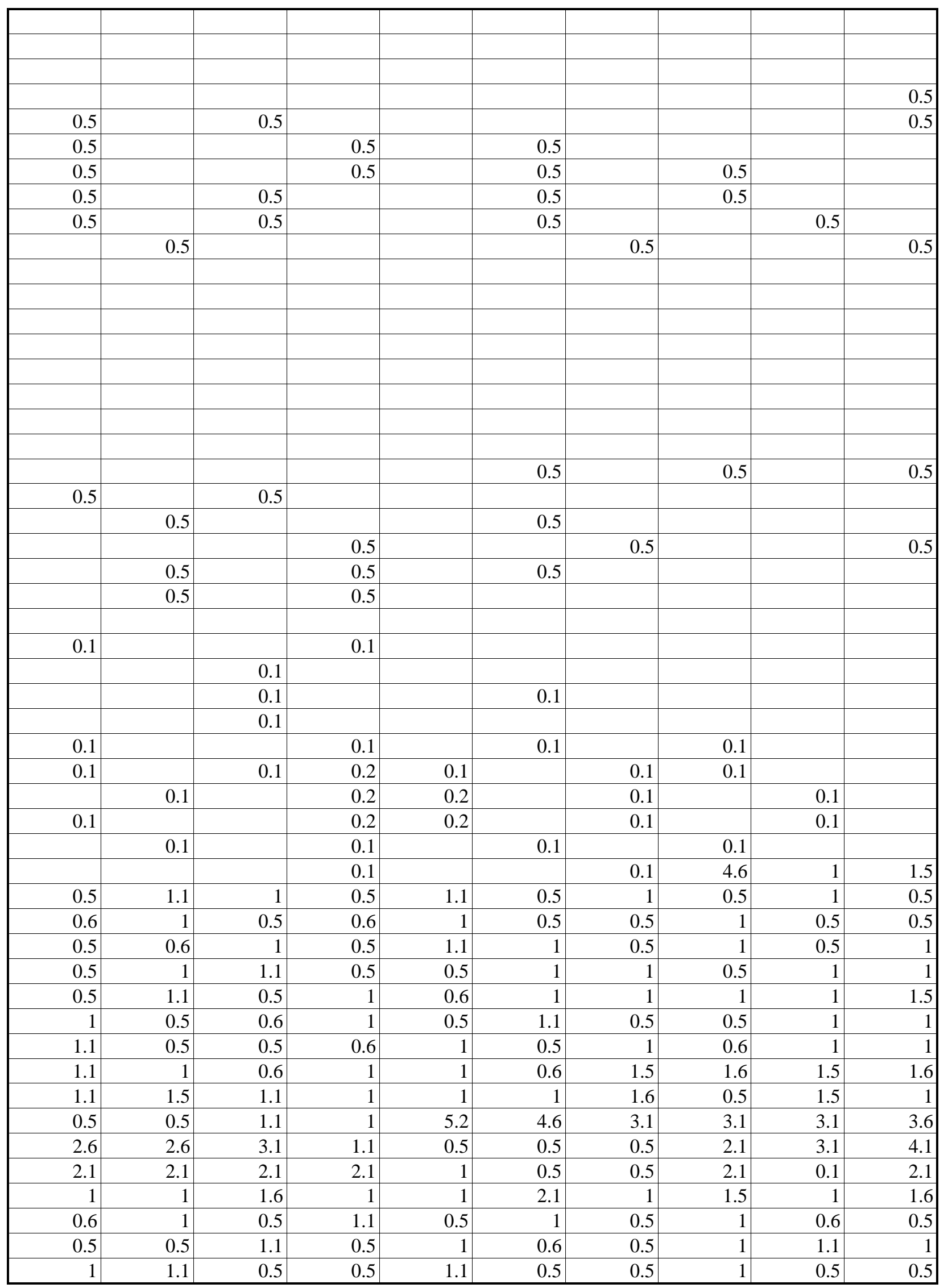




\begin{tabular}{|c|c|c|c|c|c|c|c|c|c|}
\hline 0.5 & 1 & 1 & 0.5 & 1.5 & 1 & 0.5 & 1 & 0.5 & 1.1 \\
\hline 0.5 & 0.6 & 0.5 & & 0.5 & 2.1 & 1 & 0.5 & & 0.5 \\
\hline 0.5 & 0.5 & 0.5 & 0.6 & 0.5 & 1 & 0.5 & & 1 & 0.6 \\
\hline 0.5 & 1 & 0.5 & 0.5 & 1 & 0.1 & 2.1 & 1.5 & 1 & 0.5 \\
\hline 2.1 & 2.6 & 1.5 & 1.5 & 0.6 & 1.5 & 1 & 0.5 & & 1 \\
\hline 1 & 1 & 1.6 & 1 & 1.5 & 1 & 0.6 & 1 & 0.5 & 0.5 \\
\hline 1 & 1.1 & 1.5 & 1 & 1.5 & 1.1 & 1 & 0.5 & 0.5 & 1 \\
\hline \multirow[t]{2}{*}{1} & 1 & 0.5 & 1.1 & 0.5 & 3.1 & 2.1 & 1 & 0.5 & 0.5 \\
\hline & 0.5 & 0.5 & 2.1 & 0.5 & 0.5 & 0.6 & & 0.5 & 0.5 \\
\hline & & & & & & & & & \\
\hline 0.5 & 0.5 & 0.5 & 0.1 & 0.5 & 0.5 & & 0.5 & 0.5 & 1 \\
\hline 0.5 & 0.5 & & 0.5 & 0.5 & 0.5 & 0.5 & 1 & 2.6 & \\
\hline 1.5 & 1.6 & 1 & 1 & 2.1 & 1 & 1 & 1.6 & 2.1 & 1.5 \\
\hline 1 & 2.1 & 1.5 & 1.5 & 1.6 & 2.1 & 2.1 & 1.5 & 1.5 & 1.5 \\
\hline 1 & 1 & 1 & 1 & 1.1 & 1.5 & 0.5 & 1 & 1 & 1 \\
\hline 1 & 1 & 1 & 4.6 & 3.6 & 1 & 4.7 & 3.7 & 3.6 & 3.6 \\
\hline 3.1 & 2.6 & 2.6 & 2.7 & 1.1 & 1 & 1 & 1 & 0.6 & 3.1 \\
\hline 2.6 & 2.1 & 2.6 & 0.6 & 1 & 1 & 1 & 0.5 & 1 & 1.5 \\
\hline 0.6 & 1.5 & 1 & 5.2 & 4.1 & 4.6 & 3.6 & 4.1 & 3.1 & 3.6 \\
\hline 3.1 & 3.6 & 3.1 & 3.1 & 3.1 & 3.6 & 2.6 & 2.1 & 1.5 & 1.5 \\
\hline 1 & 1.6 & 1 & 1.5 & 1.5 & 1 & 1.5 & 1 & 1.5 & 1.6 \\
\hline 1.5 & 1.1 & 1.5 & 1.5 & 1.1 & 1 & 1 & 1.5 & 1.6 & 1 \\
\hline 1 & 1 & 1.6 & 4.1 & 4.1 & 5.2 & 4.1 & 4.1 & 4.6 & 4.1 \\
\hline 4.1 & 4.1 & 5.2 & 4.2 & 4.2 & 3.1 & 3.6 & 3.1 & 3.1 & 2.6 \\
\hline 3.6 & 3.6 & 3.1 & 5.8 & 5.2 & 4.1 & 4.6 & 4.6 & 4.1 & 4.1 \\
\hline 4.7 & 4.1 & 4.6 & 6.3 & 5.8 & 6.3 & 5.2 & 6.3 & 5.7 & 5.7 \\
\hline 4.1 & 4.6 & 4.2 & 6.3 & 5.7 & 5.8 & 5.8 & 5.2 & 5.8 & 5.3 \\
\hline 2.6 & 2.1 & 2.6 & 2.1 & 2.6 & 2.1 & 2.1 & 2.6 & 2.6 & 2.6 \\
\hline 2.6 & 2.1 & 2.6 & 2.1 & 2.6 & 2.1 & 2.6 & 2.6 & 2.1 & 2.1 \\
\hline 1.5 & 2.1 & 2.6 & 7.4 & 7.3 & 7.3 & 6.2 & 6.8 & 5.3 & 5.7 \\
\hline 3.6 & 3.7 & 3.6 & 5.2 & 4.1 & 4.1 & 4.1 & 4.6 & 4.6 & 4.6 \\
\hline 3.1 & 3.1 & 3.1 & 4.1 & 3.7 & 3.7 & 3.7 & 4.1 & 4.6 & 2.1 \\
\hline 3.1 & 2.6 & 3.1 & 4.8 & 5.2 & 5.2 & 4.1 & 4.7 & 4.1 & 2.1 \\
\hline 3.1 & 3.1 & 3.1 & 2.7 & 2.7 & 2.6 & 1.5 & 2.6 & 1.5 & 1.5 \\
\hline 1 & 0.5 & 1 & 1 & 1 & 1.5 & 1 & 1 & 0.5 & 1 \\
\hline 1 & 0.6 & 1 & 1 & 1 & 1 & 1 & 0.5 & 1 & 1 \\
\hline 1 & 0.5 & 1 & 4.6 & 4.1 & 1.5 & 1 & 2.1 & 1.5 & 1.5 \\
\hline 0.6 & 1 & 1 & 5.7 & 3.1 & 2.6 & 4.1 & 4.7 & 3.1 & 2.1 \\
\hline 1.1 & 1 & 1.5 & 5.7 & 3.7 & 2.6 & 3.7 & 3.1 & 1.5 & 4.1 \\
\hline 1 & 1.1 & 2.6 & 6.7 & 3.7 & 2.1 & 1.5 & 1.6 & 1.5 & 1.5 \\
\hline 0.1 & & 0.6 & 3.1 & 4.6 & 1 & 1.6 & 2.1 & 1 & 1.5 \\
\hline 1 & 0.5 & 1.1 & 1 & 1.5 & 1 & 1 & 1 & 1 & 0.5 \\
\hline 1 & 1.1 & 0.5 & 1 & 1 & 0.6 & 1 & 1 & 1.1 & 1 \\
\hline 1.5 & 1 & 1 & 5.7 & 2.1 & 1 & 2.6 & 3.1 & 1 & 1.5 \\
\hline 1 & 1.6 & 1 & 4.6 & 3.6 & 4.7 & 1.5 & 2.1 & 1.1 & 4.1 \\
\hline 1.5 & 1.6 & 1.5 & 6.7 & 5.7 & 4.1 & 4.1 & 4.7 & 4.6 & 3.6 \\
\hline 2.6 & 3.1 & 3.1 & 3.1 & 3.1 & 5.2 & 5.2 & 4.2 & 1.5 & 2.1 \\
\hline 1 & 2.1 & 1.5 & 2.6 & 2.1 & 2.1 & 2.6 & 1.5 & 1.6 & 2.1 \\
\hline 1.5 & 1 & 1.5 & 0.6 & 1 & 1.5 & 1.1 & 0.5 & 1 & 1 \\
\hline 1 & 1 & 1.1 & 1.5 & 1 & 1.1 & 1.5 & 1.5 & 1.1 & 1 \\
\hline 1.5 & 1 & 1.6 & 3.1 & 2.1 & 2.1 & 2.1 & 2.1 & 1 & 1.5 \\
\hline
\end{tabular}




\begin{tabular}{|c|c|c|c|c|c|c|c|c|c|}
\hline 1.5 & 1.5 & 1 & 4.6 & 3.7 & 1 & 1 & 1.6 & 1 & 1 \\
\hline 1.1 & 1 & 1 & 1.6 & 1 & 0.5 & 1.1 & 0.5 & 1 & 1.5 \\
\hline 1 & 1.1 & 1 & 1 & 1.1 & 1 & 1.5 & 1.1 & 0.5 & 1.5 \\
\hline 0.6 & 1 & 0.5 & 0.6 & 1 & 0.5 & 1 & 1.1 & 0.5 & 1.5 \\
\hline 0.6 & 1 & 0.5 & 1.1 & 0.5 & 1 & 0.5 & 1 & 0.5 & 1 \\
\hline 0.5 & 1.1 & 1 & 0.5 & 1 & 0.6 & 1 & 0.5 & 1 & 0.6 \\
\hline 0.6 & 1 & 0.5 & 1 & 1 & 0.6 & 1 & 1 & 1 & 1 \\
\hline 1 & 0.6 & 1 & 1 & 1 & 0.5 & 0.5 & 1 & 1 & 1.5 \\
\hline 0.5 & 1 & 1 & 1 & 1 & 1 & 1 & 1 & 1 & 1 \\
\hline & 0.6 & & 0.5 & 0.1 & 0.5 & 0.5 & 0.5 & & 0.6 \\
\hline 0.1 & & 0.5 & 3.6 & 2.6 & & 0.5 & 0.5 & 0.5 & 1.1 \\
\hline & 0.5 & 0.1 & 0.5 & & 0.6 & & 0.5 & & 0.5 \\
\hline 0.1 & & & 0.6 & & 0.5 & 0.1 & & 0.5 & \\
\hline 0.6 & & & 0.6 & & 0.5 & & 0.6 & & 0.5 \\
\hline & 0.1 & 0.5 & 1.1 & & 3.1 & 5.2 & 5.7 & 2.1 & 3.1 \\
\hline 1.1 & 1.5 & 1.5 & 6.2 & 5.7 & 3.6 & 3.1 & 4.1 & 1.6 & 2.6 \\
\hline 0.6 & 0.5 & 0.6 & 6.2 & 3.6 & 2.1 & 1.5 & 1.5 & 1 & 1.5 \\
\hline 1 & 1.1 & 1 & 4.2 & 3.1 & 1.5 & 1 & 1 & 0.5 & 1.5 \\
\hline 1 & 1.1 & 0.5 & 1 & 0.5 & 1 & 0.6 & 1 & 1 & 1 \\
\hline 1 & 0.6 & 1 & 0.6 & 1 & 0.5 & 1 & 0.5 & 1 & 0.5 \\
\hline 1.1 & 0.5 & 1 & 0.5 & 1.1 & 0.5 & 1 & 1.1 & 1 & 1 \\
\hline 0.5 & 1 & 1 & 0.6 & 1 & 0.5 & 1.1 & 1 & 1 & 1 \\
\hline 1.1 & 0.5 & 1 & 0.6 & 1 & 1 & 0.5 & 1.1 & 0.5 & 1.5 \\
\hline 0.5 & 1.1 & 0.5 & 1 & 0.6 & 1 & 1 & 0.5 & 0.5 & 1.5 \\
\hline 1 & 1.1 & 0.5 & 0.6 & 1 & 1 & 0.5 & 1 & 0.5 & 1.5 \\
\hline 0.6 & 1 & 0.5 & 0.6 & 1 & 0.5 & 1 & 0.5 & 1 & 0.5 \\
\hline 0.5 & 1.1 & 0.5 & 1 & 0.6 & 1 & 0.5 & 0.5 & 1 & 1 \\
\hline 0.5 & 1 & 1.1 & 0.5 & 0.5 & 1.1 & 0.5 & 1 & 0.5 & 1.5 \\
\hline 1 & 0.6 & 1 & 0.5 & 1.6 & 1.5 & 2.1 & 2.1 & 2.6 & 2.6 \\
\hline 1 & 1.1 & 1.5 & 2.1 & 4.1 & 4.6 & 3.6 & 1.5 & 1 & 4.6 \\
\hline 1.1 & 1.5 & 1 & 6.2 & 3.6 & 2.6 & 3.1 & 4.1 & 2.1 & 1.5 \\
\hline 1.1 & 1 & 1 & 4.2 & 1.5 & 1 & 1 & 0.5 & 1 & 1 \\
\hline 0.5 & 1.1 & 0.5 & 0.5 & 1 & 1 & 0.6 & 1 & 0.5 & 1 \\
\hline 0.5 & 0.5 & 1 & 1 & 0.6 & 0.5 & 1 & 0.5 & 1 & 0.5 \\
\hline 1 & 0.5 & 1 & 0.6 & 1 & 0.6 & 0.5 & 1 & 0.5 & 1 \\
\hline 0.6 & 0.5 & 1.1 & 0.5 & 0.5 & 1 & 0.6 & 0.5 & 1.1 & 0.5 \\
\hline 0.6 & 1 & 0.6 & 0.5 & 1 & 1.5 & 0.5 & 1 & 1 & 0.5 \\
\hline 0.6 & 1 & 0.5 & 0.5 & 0.6 & 1 & 0.5 & 0.5 & 1 & 0.5 \\
\hline 0.6 & 0.5 & 1 & 0.5 & 1.5 & 0.5 & 0.6 & 1 & 0.6 & 0.5 \\
\hline 1 & 0.5 & 0.6 & 0.5 & 1 & 0.5 & 0.5 & 1.1 & 0.5 & 1.1 \\
\hline 0.6 & 0.5 & 1.1 & 0.5 & 0.5 & 0.6 & 1 & 0.5 & 0.5 & 0.5 \\
\hline 1 & 1 & 0.6 & 4.6 & 3.1 & 1.6 & 1.5 & 2.1 & 1.5 & 1.5 \\
\hline 1 & 0.5 & 0.6 & 1.1 & 1.5 & 2.6 & 0.5 & 1.6 & 1.5 & 1 \\
\hline 0.5 & 0.5 & 1.1 & 0.5 & 0.5 & 1 & 0.5 & 0.5 & 1 & 1 \\
\hline 1 & 0.5 & 0.5 & 1.1 & 0.5 & 1 & 0.5 & 0.5 & 0.5 & 1.5 \\
\hline 0.5 & 1 & 0.6 & 0.5 & 1 & 0.5 & 1 & 0.5 & 1 & 1.1 \\
\hline 1 & 0.6 & 1 & 0.6 & 0.5 & 1 & 1 & 0.6 & 0.5 & 0.6 \\
\hline 0.6 & 0.5 & 1 & 0.5 & 1.1 & 0.5 & 0.5 & 1 & 0.5 & 0.5 \\
\hline 1 & 1 & 0.6 & 0.5 & 1.1 & 1 & 1 & 0.5 & 1 & 1 \\
\hline 0.6 & 0.5 & 1 & 0.5 & 1.1 & 0.5 & 1 & 0.5 & 1 & 1 \\
\hline 1.1 & 0.5 & 0.5 & 1.1 & 1.5 & 1 & 1 & 1 & 1.5 & 1 \\
\hline
\end{tabular}




\begin{tabular}{|c|c|c|c|c|c|c|c|c|c|}
\hline 1.1 & 0.5 & 1.1 & 0.5 & 1 & 1.1 & 1 & 1 & 1.1 & 4.6 \\
\hline 1.1 & 0.5 & 1 & 5.2 & 2.1 & 1.5 & 1.6 & 1.5 & 1 & 2.1 \\
\hline 1 & 0.5 & 0.6 & 1 & 0.5 & 1 & 0.6 & 0.5 & 1 & 0.5 \\
\hline 0.6 & 1 & 0.5 & 1 & 0.5 & 1.1 & 1 & 0.5 & 1 & 0.5 \\
\hline 0.5 & 1.1 & 0.5 & 1 & 1 & 0.6 & 1 & 0.5 & 1 & 1 \\
\hline 1 & 0.5 & 1.1 & 0.5 & 0.5 & 1 & 0.5 & 0.5 & 1.5 & 1 \\
\hline 0.5 & 0.6 & 1 & 0.5 & 1.1 & 0.5 & 0.5 & 1 & 0.5 & 1 \\
\hline 1 & 0.5 & 0.5 & 1 & 0.5 & 1 & 0.5 & 1 & 0.5 & 1 \\
\hline 1.1 & 0.5 & 1 & 0.6 & 1 & 0.5 & 0.5 & 1 & 0.5 & 1 \\
\hline 0.5 & 0.6 & 1 & 0.6 & 0.5 & 1 & 0.5 & 0.5 & 1 & 0.5 \\
\hline 1.1 & & 1 & 1 & 0.6 & 0.5 & 0.5 & 1 & 0.5 & 1 \\
\hline 1.1 & 0.5 & 0.5 & 1.1 & 0.5 & 0.5 & 1 & 0.5 & 1 & 0.5 \\
\hline \multirow[t]{2}{*}{0.6} & 0.5 & 1 & 0.5 & & 0.6 & & & 0.5 & \\
\hline & & 0.1 & 0.5 & & & & & & 1 \\
\hline \multirow[t]{2}{*}{0.1} & 0.5 & & 0.1 & 0.5 & & & & & 0.5 \\
\hline & & 0.1 & & 0.5 & 0.1 & & & 0.1 & \\
\hline 0.5 & & 0.1 & & & 0.1 & 0.5 & & & \\
\hline 0.6 & & & 0.1 & & & & & & \\
\hline 0.5 & & 0.1 & & 0.5 & 0.1 & 0.5 & & & 0.1 \\
\hline 0.5 & 0.1 & & & 0.1 & & & & 0.5 & 0.5 \\
\hline 0.1 & & 0.5 & 0.1 & & & 0.5 & & & 0.5 \\
\hline \multirow[t]{4}{*}{0.1} & & 0.5 & & & 0.1 & 0.5 & & & 0.5 \\
\hline & & & & & 0.5 & & & & 0.5 \\
\hline & & & 0.5 & & 0.1 & & & 0.1 & 0.5 \\
\hline & 0.1 & 0.5 & & 0.1 & & & & & \\
\hline \multirow[t]{4}{*}{0.1} & & & 0.1 & & 0.5 & & & & \\
\hline & & 0.5 & & & & & & & \\
\hline & & 0.6 & & & 0.5 & & & & 0.5 \\
\hline & & & 0.5 & & & 0.5 & & & 0.5 \\
\hline 0.1 & & 0.5 & 0.1 & & & & & 0.5 & 0.5 \\
\hline \multirow[t]{5}{*}{0.1} & & 0.5 & 0.1 & & & & & & 0.5 \\
\hline & & 0.1 & & 0.5 & & & & & \\
\hline & & & & & 0.1 & 0.5 & & & 0.5 \\
\hline & 0.6 & & & & & 0.5 & & & 0.5 \\
\hline & & & 0.5 & & & & & 0.5 & 0.5 \\
\hline \multirow[t]{2}{*}{0.5} & & & & 0.5 & & & & & 0.5 \\
\hline & & & & & 0.5 & & & & 0.5 \\
\hline \multirow[t]{2}{*}{0.1} & 0.5 & & 0.1 & & & 0.5 & & & 0.5 \\
\hline & 0.1 & & & 0.6 & & & & & \\
\hline \multirow[t]{3}{*}{0.5} & & & & & 0.5 & & & & 0.5 \\
\hline & & & & & 0.5 & & 0.5 & & 0.5 \\
\hline & & & & 0.5 & & & 0.5 & & 0.5 \\
\hline 0.5 & & & & & 0.5 & & & 0.5 & 0.5 \\
\hline 0.5 & & 0.1 & & & & 0.5 & & & \\
\hline \multirow[t]{5}{*}{0.1} & & & & & & 0.5 & & & \\
\hline & & & 0.1 & 0.5 & & & & 0.1 & \\
\hline & 0.5 & 0.1 & & & & & & 0.5 & 0.5 \\
\hline & & & & 0.5 & & & & & 1 \\
\hline & 0.5 & & & & & & 0.5 & & 0.5 \\
\hline \multirow{2}{*}{0.1} & & & & & 0.5 & & & & 0.5 \\
\hline & 0.1 & & 0.5 & & & & & 0.5 & 0.5 \\
\hline
\end{tabular}




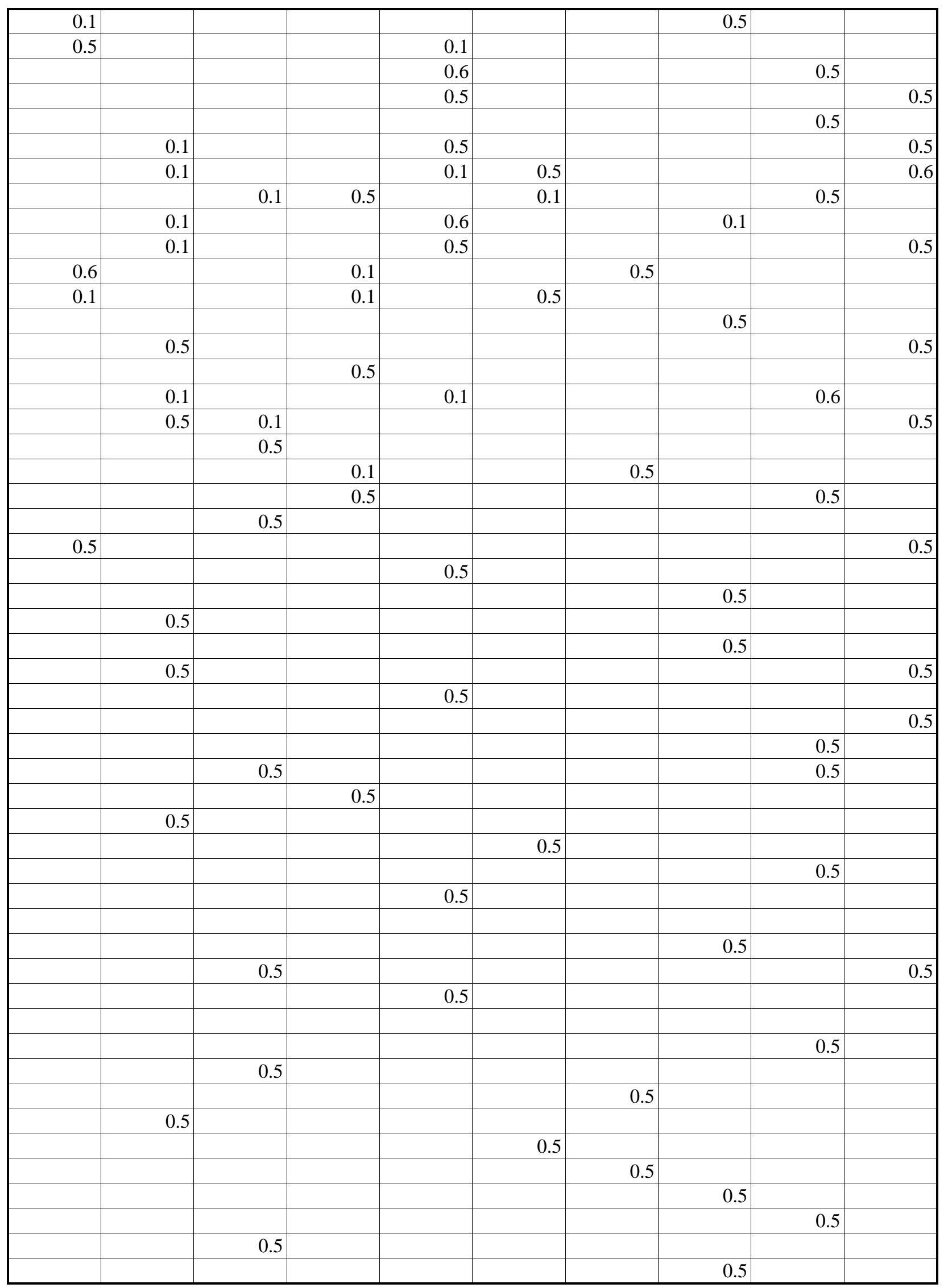




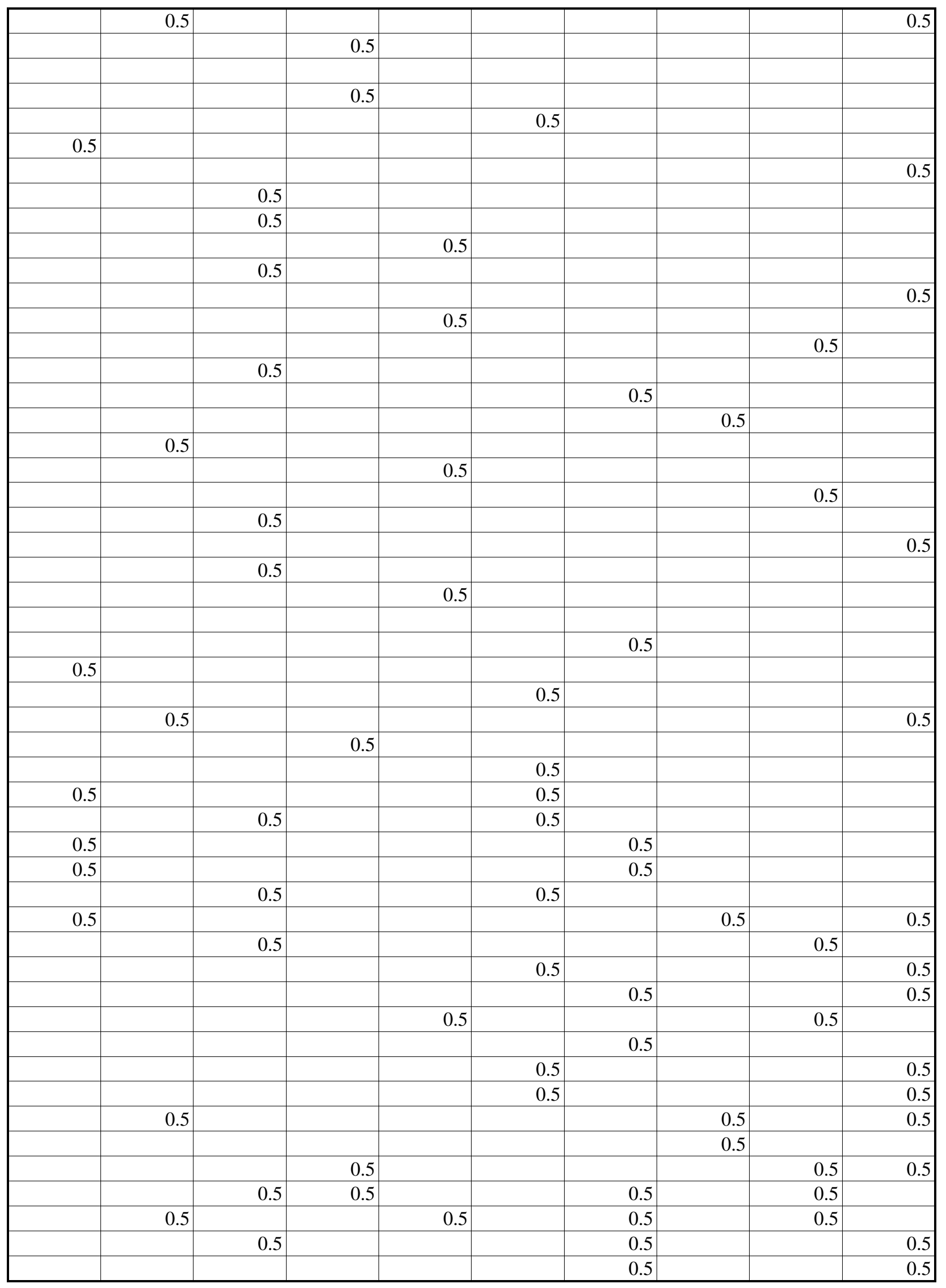

Page 32 


\begin{tabular}{|c|c|c|c|c|c|c|c|c|c|}
\hline & & & 0.5 & & & & & 0.5 & 0.5 \\
\hline & & 0.5 & & & & & 0.5 & & \\
\hline & & 0.5 & & & & & & & 0.5 \\
\hline \multirow[t]{9}{*}{0.5} & & & & & & & & 0.5 & \\
\hline & 0.5 & & & & & & & & \\
\hline & & & 0.5 & & & & & 0.5 & 0.5 \\
\hline & 0.5 & & & & & & 0.5 & & 0.5 \\
\hline & & & 0.5 & & & & & & 0.5 \\
\hline & & & 0.5 & & & & & 0.5 & 0.5 \\
\hline & & & & & & & 0.5 & & 0.5 \\
\hline & & & & 0.5 & & & & & \\
\hline & & & & & & 0.5 & & & \\
\hline 0.5 & & & & & & & & 0.5 & 0.5 \\
\hline \multirow[t]{8}{*}{0.5} & & & & & 0.5 & & & & 0.5 \\
\hline & & & & 0.5 & & & 0.5 & & \\
\hline & & & 0.5 & & & & & 0.5 & \\
\hline & & & & & & & & 0.5 & 0.5 \\
\hline & & & & & 0.5 & & & & \\
\hline & & & & & 0.5 & & & & \\
\hline & & & 0.5 & & & 0.5 & & & 0.5 \\
\hline & & & 0.5 & & & & & & 1 \\
\hline \multirow[t]{16}{*}{0.5} & & & & & & 0.5 & & & 0.5 \\
\hline & & & 0.5 & & & & & & 1 \\
\hline & & & 0.5 & & & & & 0.5 & \\
\hline & & 0.5 & & & & & & & 0.5 \\
\hline & & & & & & & & & 0.5 \\
\hline & & 0.5 & & & & & 0.5 & & 0.5 \\
\hline & & & & 0.5 & & & & 0.5 & \\
\hline & 0.5 & & & & & 0.5 & & & 0.5 \\
\hline & 0.5 & & & 0.5 & & & & & 0.5 \\
\hline & & & 0.5 & & & & & 0.5 & 0.5 \\
\hline & & 0.5 & & 0.5 & & & & 0.5 & \\
\hline & & & & 0.5 & & & & & \\
\hline & & & & 0.5 & & & & & 0.5 \\
\hline & & & 0.5 & & & & 0.5 & & 0.5 \\
\hline & & 0.5 & & & & & 0.5 & & 0.5 \\
\hline & & & 0.5 & 1 & 2.6 & 1 & 1.5 & 1 & 1.5 \\
\hline 0.5 & 1 & 0.5 & 1 & 0.5 & 0.5 & 1 & 0.5 & 1 & 1 \\
\hline 0.5 & 0.5 & 0.5 & 1 & 0.5 & 0.5 & 1 & 0.5 & 0.5 & 0.5 \\
\hline 1 & 0.5 & 1 & & 1 & 0.5 & 1 & 0.5 & 1 & 0.5 \\
\hline \multirow[t]{7}{*}{0.5} & 1 & 0.5 & 0.5 & 1 & 0.5 & 0.5 & & & 0.5 \\
\hline & & 0.5 & & & & & 0.5 & & 0.5 \\
\hline & & & & 0.5 & & & & 0.5 & 0.5 \\
\hline & & & & & & & 0.5 & & 0.5 \\
\hline & & & & & 0.5 & & & & 0.5 \\
\hline & & & & 0.5 & & & & & \\
\hline & & & & & & & & 0.5 & \\
\hline \multirow[t]{2}{*}{0.5} & & 0.5 & & 0.5 & & 0.5 & & & 1 \\
\hline & & 0.5 & & 0.5 & & 0.5 & & 0.5 & 0.5 \\
\hline 0.5 & & 0.5 & & 0.5 & & 0.5 & & 0.5 & 0.5 \\
\hline 0.5 & & 0.5 & & 0.5 & & & 0.5 & 0.5 & 0.5 \\
\hline
\end{tabular}




\begin{tabular}{|c|c|c|c|c|c|c|c|c|c|}
\hline & 0.5 & 0.5 & & & 0.5 & & 0.5 & 0.5 & 0.5 \\
\hline & 0.5 & & 0.5 & & 0.5 & & 0.5 & & 0.5 \\
\hline & 0.5 & & 0.5 & & 0.5 & & 0.5 & & 0.5 \\
\hline & 0.5 & & 0.5 & & 0.5 & & & 1 & \\
\hline \multirow[t]{2}{*}{0.5} & 0.5 & & & 0.5 & & 0.5 & & 0.5 & 0.5 \\
\hline & 0.5 & & 0.5 & 0.5 & & & & 0.5 & 0.5 \\
\hline \multirow[t]{6}{*}{0.5} & & & & 0.5 & & & 0.5 & & 0.5 \\
\hline & & & & & 0.5 & & 0.5 & & 0.5 \\
\hline & & 0.5 & & & & & 0.5 & & \\
\hline & & 0.5 & & & & & & & \\
\hline & 0.5 & & 0.5 & & & 0.5 & & 0.5 & 0.5 \\
\hline & & 0.5 & & & 0.5 & & & & 1 \\
\hline 1 & 0.5 & 1 & 1.5 & 0.5 & 1 & 2.1 & 1.5 & 1.5 & 2.1 \\
\hline \multirow[t]{2}{*}{1} & 0.5 & 1.5 & 1.5 & 3.6 & 3.6 & 2.1 & 3.1 & 4.6 & 2.1 \\
\hline & & 0.5 & 1.5 & 3.1 & 3.6 & 3.1 & 2.6 & 3.1 & 3.6 \\
\hline 0.5 & 1 & 1 & 1 & 0.5 & 1 & 1 & 1 & 0.5 & 0.5 \\
\hline 1 & 0.5 & 0.5 & 1 & 1 & 0.5 & 0.5 & 1 & 1 & 0.5 \\
\hline 1 & 0.5 & 1 & 0.5 & 1 & 0.5 & 1 & 1 & 1 & 1 \\
\hline 1 & 0.5 & 0.5 & 0.5 & 1 & 0.5 & 1 & 1 & 0.5 & 1.5 \\
\hline 1 & 0.5 & 0.5 & 1 & 0.5 & 0.5 & 1 & 1 & 0.5 & 1 \\
\hline 1 & 0.5 & 1 & 0.5 & 1 & 1 & 0.5 & 1 & 1.5 & 1.5 \\
\hline 1 & 1 & 0.5 & 1 & 0.5 & 1 & 1 & 1 & 1 & 1 \\
\hline 1 & 0.5 & 0.5 & 1 & 0.5 & 1 & 1 & 0.5 & 1 & 0.5 \\
\hline 0.5 & 1 & 1 & 1 & 1 & 1 & 1 & 0.5 & 1 & 1 \\
\hline 0.5 & 1 & 1 & 1 & 1 & 0.5 & 1 & 1 & 1 & 1 \\
\hline 0.5 & 1 & 0.5 & 0.5 & 1 & 1 & 1 & 0.5 & 1 & 1.5 \\
\hline 0.5 & 1 & 0.5 & 0.5 & 1 & 1 & 0.5 & 1 & 0.5 & 1 \\
\hline 0.5 & 0.5 & 1 & 0.5 & 0.5 & 1 & 1 & 0.5 & 0.5 & 1 \\
\hline 0.5 & 1 & 0.5 & 1 & 0.5 & 1 & 0.5 & 1 & 1 & 1 \\
\hline 1 & 0.5 & 0.5 & 1 & 1 & 1 & 0.5 & 1 & 0.5 & 0.5 \\
\hline 0.5 & 1 & 0.5 & 0.5 & 1 & 1 & 0.5 & 0.5 & 1 & 0.5 \\
\hline 0.5 & 0.5 & 1 & 2.1 & 0.5 & 5.2 & 2.6 & 2.1 & 2.6 & 1.5 \\
\hline 1 & 0.5 & 3.6 & 2.1 & 1.5 & 1.5 & 2.1 & 1 & 1 & 1 \\
\hline 1 & 0.5 & 3.6 & 3.6 & 2.6 & 2.6 & 2.6 & 2.1 & 2.6 & 2.1 \\
\hline 2.6 & 2.1 & 2.6 & 3.1 & 3.1 & 3.1 & 3.1 & 3.1 & 2.6 & 3.1 \\
\hline 1.5 & 1.5 & 1.5 & 2.1 & 3.7 & 2.1 & 2.1 & 2.1 & 2.1 & 2.1 \\
\hline 2.1 & 2.1 & 2.1 & 2.1 & 2.1 & 2.1 & 2.1 & 1.5 & 2.1 & 1.5 \\
\hline 1.5 & 1.5 & 1.5 & 1 & 1.5 & 1.5 & 1.5 & 1 & 1.5 & 1 \\
\hline 1 & 1.5 & 1.6 & 4.2 & 5.2 & 3.1 & 3.1 & 3.6 & 4.2 & 3.3 \\
\hline 2.2 & 2.2 & 2.8 & 2.6 & 2.1 & 1.5 & 2.1 & 1.5 & 1.5 & 1.5 \\
\hline 1.5 & 1.5 & 1.5 & 3.1 & 2.1 & 3.1 & 2.6 & 2.6 & 2.1 & 2.1 \\
\hline 1.5 & 1.5 & 2.1 & 2.1 & 2.1 & 2.1 & 2.1 & 1.5 & 1.5 & 1.5 \\
\hline 1 & 1.5 & 1 & 1 & 1 & 0.5 & 0.5 & 1 & 0.5 & 1 \\
\hline 1 & 1 & 1 & 1 & 1 & 1 & 1.5 & 1.5 & 1 & 1.5 \\
\hline 1 & 1 & 1 & 1.5 & 1 & 1 & 1 & 1 & 1 & 1 \\
\hline 1 & 1 & 1.7 & 7 & 5.3 & 4.1 & 3.7 & 3.9 & 2.1 & 2.6 \\
\hline 2.1 & 2.1 & 3.2 & 2.9 & 3.3 & 2.6 & 2.6 & 2.1 & 2.1 & 2.6 \\
\hline 1 & 1 & 1 & 4.7 & 1.5 & 1.5 & 0.5 & 1 & 1 & 1 \\
\hline 0.5 & 0.5 & 1.5 & 2.2 & 1 & 1 & 4.1 & 1 & 1 & 1 \\
\hline 1 & 1 & 1 & 4.7 & 3.6 & 2.1 & 2.1 & 1.5 & 1 & 1.5 \\
\hline 0.5 & 1 & 0.5 & 1 & 1 & 1.5 & 1 & 1 & 1 & 0.5 \\
\hline
\end{tabular}




\begin{tabular}{|c|c|c|c|c|c|c|c|c|c|}
\hline 0.5 & 1 & 0.5 & 1 & 0.5 & 0.5 & 1 & 0.5 & 0.5 & 1 \\
\hline 0.5 & 1 & 0.5 & 0.5 & 1 & 1 & 3.6 & 3.6 & 2.6 & 2.6 \\
\hline 2.1 & 4.7 & 4.7 & 3.3 & 3.1 & 3.1 & 4.1 & 2.6 & 2.6 & 2.6 \\
\hline 1.5 & 4.2 & 3.6 & 3.1 & 2.6 & 2.6 & 2.6 & 2.6 & 3.1 & 2.1 \\
\hline 1 & 4.1 & 3.1 & 3.1 & 3.1 & 3.1 & 2.6 & 2.1 & 2.6 & 2.6 \\
\hline 1 & 4.1 & 3.1 & 3.7 & 2.6 & 2.1 & 2.1 & 2.1 & 1.6 & 1.5 \\
\hline 0.5 & 0.5 & 1 & 0.5 & 0.5 & 1 & 0.5 & 0.5 & 1 & 0.5 \\
\hline 0.5 & 1 & 0.5 & 0.5 & 1 & 1 & 0.5 & 0.5 & 1 & 0.5 \\
\hline 1.5 & 1.6 & 1.5 & 1 & 2.1 & 2.1 & 1.5 & 2.6 & 1.5 & 2.1 \\
\hline 1 & 1 & 1 & 1 & 1 & 1 & 0.5 & 1 & 0.5 & 1 \\
\hline 0.5 & 0.5 & 1 & 1 & 0.5 & 0.5 & 1 & 0.5 & 0.5 & 1 \\
\hline 0.5 & 1 & 1 & 0.5 & 1 & 1 & 1.6 & 0.5 & 1.5 & 0.5 \\
\hline 1 & 1 & 1 & 1 & 1 & 1 & 1.1 & 0.5 & 1 & 0.5 \\
\hline 0.5 & 1 & 0.5 & 0.5 & 1 & 1 & 0.5 & 1 & 0.5 & 1 \\
\hline 0.5 & 1 & 0.5 & 1 & 0.5 & 1 & 0.5 & 0.5 & 1 & 0.5 \\
\hline 1.5 & 1 & 1 & 1 & 1 & 1 & 1 & 1 & 1 & 0.5 \\
\hline \multirow[t]{2}{*}{0.5} & 1 & 0.5 & 1 & 1 & & & 0.5 & & \\
\hline & & 0.5 & & 0.5 & & & 0.5 & & \\
\hline 1 & 1.1 & 1.5 & 2.1 & 1.1 & 1.5 & 1.5 & 1.1 & 1.5 & 1.5 \\
\hline 1.5 & 2.1 & 1.5 & 1.5 & 1.5 & 2.1 & 1.5 & 1.5 & 2.1 & 1 \\
\hline 1 & 0.5 & 0.5 & 1 & 0.5 & 1 & 0.5 & 1 & 0.5 & 0.5 \\
\hline 1 & 0.5 & 0.5 & 1.6 & 0.5 & 1 & 1 & 1 & 1 & 0.5 \\
\hline 1 & 1 & 1.1 & 5.2 & 5.2 & 3.7 & 3.8 & 2.1 & 2.1 & 2.1 \\
\hline 1 & 3.6 & 3.1 & 4.2 & 3.2 & 2.6 & 3.1 & 2.2 & 2.1 & 3.1 \\
\hline 1 & 3.6 & 2.6 & 3.2 & 3.2 & 2.6 & 3.1 & 2.6 & 3.1 & 3.1 \\
\hline 0.5 & 3.6 & 2.6 & 2.6 & 2.1 & 2.1 & 2.1 & 1.5 & 1.5 & 3.1 \\
\hline 0.5 & 0.5 & 1 & 0.5 & 0.5 & 1 & 0.5 & 0.5 & 1 & 0.5 \\
\hline 1 & 0.5 & 1 & 1.5 & 0.5 & 1 & 1 & 1.5 & 0.5 & 1 \\
\hline 1 & 3.6 & 4.1 & 4.8 & 5.2 & 4.7 & 4.7 & 4.8 & 5.2 & 4.7 \\
\hline 1.5 & 4.1 & 4.1 & 4.3 & 4.2 & 3.6 & 3.7 & 3.2 & 3.1 & 3.1 \\
\hline 1 & 3.1 & 3.1 & 3.2 & 3.1 & 3.1 & 3.1 & 2.6 & 3.1 & 3.1 \\
\hline 1 & 4.1 & 4.1 & 5.4 & 4.6 & 3.7 & 4.2 & 4.2 & 4.2 & 3.7 \\
\hline 2.1 & 3.6 & 1.5 & 3.2 & 2.7 & 3.2 & 5.7 & 3.1 & 3.1 & 3.1 \\
\hline \multirow[t]{2}{*}{1} & 1 & 1 & 1.5 & 1 & 0.5 & 0.5 & 1.5 & 0.5 & 0.5 \\
\hline & 0.5 & & & & & & 0.5 & & \\
\hline 1 & 0.5 & 1 & 4.6 & 1 & 1 & 1.5 & 1.5 & 1 & 2.1 \\
\hline 1 & 1 & 0.5 & 1.6 & 3.1 & 5.2 & 2.6 & 3.6 & 3.6 & 3.7 \\
\hline 1.5 & 4.1 & 3.6 & 4.2 & 4.6 & 3.6 & 4.1 & 4.1 & 4.1 & 4.1 \\
\hline 1.1 & 3.6 & 4.7 & 4.1 & 3.7 & 2.6 & 1.6 & 1.5 & 1 & 1 \\
\hline \multirow[t]{2}{*}{0.5} & 4.1 & 2.1 & 3.1 & 2.6 & 2.6 & 1.5 & 2.1 & 0.5 & 0.5 \\
\hline & 0.5 & & & & 0.5 & & & & \\
\hline \multirow[t]{2}{*}{0.5} & & & 0.5 & & & & & 0.5 & \\
\hline & 3.1 & 3.6 & 4.1 & 5.7 & 5.7 & 5.2 & 4.1 & 4.1 & 3.6 \\
\hline 2.6 & 6.7 & 6.7 & 5.2 & 5.2 & 4.6 & 4.1 & 4.6 & 4.6 & 4.1 \\
\hline 2.1 & 5.7 & 5.2 & 4.6 & 4.2 & 4.1 & 4.1 & 3.1 & 3.6 & 3.6 \\
\hline 1 & 4.6 & 4.1 & 3.6 & 3.6 & 3.1 & 3.1 & 3.1 & 2.1 & 3.1 \\
\hline 0.5 & 4.1 & 3.1 & 2.6 & 2.1 & 2.6 & 2.6 & 3.1 & 2.6 & 3.1 \\
\hline 1.5 & 1.5 & 1 & 1 & 1 & 1.5 & 1.5 & 1.5 & 1.5 & 0.5 \\
\hline 0.5 & 1 & 0.5 & 1 & 0.5 & 1 & 1 & 0.5 & 0.5 & 1 \\
\hline 0.5 & 5.2 & 4.7 & 4.1 & 4.1 & 3.8 & 3.1 & 2.7 & 2.1 & 2.6 \\
\hline 0.5 & 4.1 & 3.1 & 2.6 & 2.1 & 2.6 & 2.6 & 2.7 & 2.6 & 3.1 \\
\hline
\end{tabular}




\begin{tabular}{|c|c|c|c|c|c|c|c|c|c|}
\hline 0.5 & 4.1 & 3.1 & 3.7 & 3.6 & 2.6 & 3.1 & 2.1 & 2.1 & 2.6 \\
\hline 0.5 & 4.1 & 3.1 & 3.1 & 2.6 & 2.6 & 2.1 & 2.6 & 3.1 & 2.6 \\
\hline 1 & 4.1 & 3.1 & 3.1 & 3.1 & 3.6 & 2.6 & 3.1 & 3.6 & 3.6 \\
\hline 1 & 0.5 & 1 & 1 & 1.5 & 1.5 & 2.1 & 1.5 & 1 & 1.5 \\
\hline 1 & 1 & 1 & 1.5 & 1 & 1 & 1 & 1 & 1 & 1 \\
\hline 1 & 4.6 & 5.7 & 4.3 & 4.1 & 4.1 & 1.5 & 1.5 & 0.5 & 1.5 \\
\hline 1.5 & 4.1 & 3.6 & 3.8 & 2.6 & 2.6 & 2.1 & 2.1 & 2.1 & 2.6 \\
\hline 1 & 3.6 & 2.6 & 3.2 & 2.1 & 3.1 & 2.1 & 1 & 1.5 & 1.5 \\
\hline 0.5 & 3.1 & 2.1 & 2.1 & 1.5 & 2.1 & 2.1 & 1.5 & 1 & 0.5 \\
\hline 0.5 & 4.1 & 2.6 & 2.6 & 2.6 & 3.1 & 2.6 & 2.6 & 2.6 & 2.6 \\
\hline 0.5 & 0.5 & 1 & 0.5 & 1 & 0.5 & 1 & 1.5 & 0.5 & 1 \\
\hline 1 & 0.5 & 0.5 & 1 & 0.5 & 0.5 & 1 & 0.5 & 0.5 & 1 \\
\hline 1 & 3.6 & 3.6 & 1.5 & 1 & 0.5 & 1 & 1 & 0.5 & 0.5 \\
\hline 1 & 1 & 1 & 5.2 & 3.6 & 2.6 & 3.6 & 3.6 & 3.1 & 3.6 \\
\hline 1.5 & 4.6 & 3.6 & 3.6 & 3.6 & 3.6 & 3.6 & 3.1 & 2.6 & 2.1 \\
\hline 2.1 & 5.2 & 4.6 & 4.1 & 5.2 & 4.1 & 4.6 & 4.3 & 4.2 & 4.1 \\
\hline 1 & 3.6 & 3.1 & 3.7 & 2.6 & 4.6 & 3.6 & 4.1 & 3.1 & 3.1 \\
\hline 1 & 1 & 1 & 0.5 & 1 & 0.5 & 1 & 0.5 & 1 & 0.5 \\
\hline 0.5 & 1 & 0.5 & 0.5 & 1 & 0.5 & 1 & 0.5 & 1 & 0.5 \\
\hline 1 & 4.1 & 2.6 & 1 & 1 & 1.5 & 1.5 & 1.5 & 1 & 1 \\
\hline 1 & 0.5 & 1 & 1.5 & 1 & 1 & 1 & 1 & 1 & 1 \\
\hline \multirow[t]{2}{*}{0.5} & 0.5 & 1 & 1 & 1 & 0.5 & 0.5 & 1.5 & 1 & 1.1 \\
\hline & & 0.5 & 0.1 & 1.5 & 1.6 & 0.5 & 0.5 & 1 & 1.1 \\
\hline 0.5 & 1 & 1 & 6.2 & 2.1 & 2.1 & 2.1 & 1.6 & 1.5 & 1.6 \\
\hline 0.5 & 1 & 1.5 & 1 & 1.5 & 1.5 & 1.5 & 1.5 & 1 & 1.5 \\
\hline 1 & 1 & 1 & 1 & 1.5 & 0.5 & 1 & 0.5 & 1 & 1 \\
\hline 0.5 & 1 & 1 & 1.1 & 1 & 4.1 & 1 & 1 & 1 & 1 \\
\hline 1 & 0.5 & 1 & 5.2 & 1 & 1 & 1 & 1.1 & 0.6 & 1.5 \\
\hline 0.5 & 0.5 & 1 & 4.1 & 1 & 1 & 0.5 & 1 & 1 & 1.5 \\
\hline 0.5 & 1 & 1 & 5.7 & 2.1 & 0.5 & 0.5 & 1.1 & 1 & 1.5 \\
\hline 0.5 & 1 & 1 & 3.6 & 2.6 & 1.5 & 1.5 & 3.1 & 3.7 & 3.6 \\
\hline 1 & 0.5 & 1 & 1 & 1 & 0.5 & 0.5 & 1 & 0.5 & 0.5 \\
\hline 1 & 0.5 & 1 & 0.5 & 1 & 0.5 & 1 & 0.5 & 0.5 & 1 \\
\hline \multirow[t]{2}{*}{1} & 1 & 1 & 5.2 & 1 & 1 & 1.5 & 0.5 & 1 & 1 \\
\hline & 1 & 1 & 1 & 0.5 & 0.5 & 1 & 1 & 0.5 & 1 \\
\hline 1 & 0.5 & 1 & 0.5 & 1 & 0.5 & 1 & 0.5 & 1 & 1 \\
\hline 1 & 0.5 & 1 & 0.5 & 1 & 0.5 & 1 & 1 & 1 & 1 \\
\hline 1 & 1.5 & 1 & 5.7 & 3.1 & 3.6 & 4.1 & 3.6 & 3.6 & 4.7 \\
\hline 2.1 & 2.1 & 2.6 & 2.1 & 2.1 & 2.1 & 2.6 & 2.1 & 1.5 & 1.5 \\
\hline 1 & 1 & 0.5 & 0.5 & 1 & 1 & 1 & 0.5 & 1 & 0.5 \\
\hline 1.5 & 8.2 & 5.2 & 3.1 & 3.1 & 5.2 & 4.1 & 3.6 & 2.6 & 3.6 \\
\hline 1 & 4.6 & 3.1 & 1.5 & 1 & 1 & 1 & 0.5 & 1 & 1 \\
\hline 1 & 1 & 1 & 1.1 & 1 & 1 & 1 & 1 & 1 & 0.5 \\
\hline 0.5 & 1 & 1 & 1 & 1 & 1 & 1 & 1 & 0.5 & 1 \\
\hline 0.5 & 1 & 1 & 1.5 & 0.5 & 1 & 1 & 0.5 & 1 & 1 \\
\hline 1 & 0.5 & 0.5 & 1 & 0.5 & 1 & 0.5 & 1 & 1 & 1 \\
\hline 0.5 & 1 & 1 & 1 & 1 & 1 & 1 & 0.5 & 1 & 1 \\
\hline 1 & 1 & 1 & 1 & 1 & 0.5 & 1 & 1 & 0.5 & 1 \\
\hline 1 & 0.5 & 1 & 4.1 & 0.5 & 1 & 1 & 1 & 1 & 3.1 \\
\hline 1 & 1 & 0.5 & 1 & 0.5 & 1 & 1 & 0.5 & 1 & 1 \\
\hline 0.5 & 1 & 0.5 & 1 & 0.5 & 1 & 0.5 & 0.5 & 1 & 1 \\
\hline
\end{tabular}




\begin{tabular}{|c|c|c|c|c|c|c|c|c|c|}
\hline 1 & 0.5 & 0.5 & 1 & 0.5 & 1 & 0.5 & 1 & 0.5 & 1 \\
\hline 0.5 & 0.5 & 1 & 0.5 & 0.5 & 1 & 0.5 & 0.5 & 1 & 0.5 \\
\hline 1 & 0.5 & 0.5 & 0.5 & 1 & 0.5 & 0.5 & 1 & 0.5 & 0.5 \\
\hline 0.5 & 1 & 0.5 & 0.5 & 1 & 0.5 & 0.5 & 1 & 0.5 & 1 \\
\hline 0.5 & 0.5 & 1 & 0.5 & 0.5 & & & & 0.5 & 0.5 \\
\hline \multirow[t]{5}{*}{0.5} & & & & & 0.5 & & & 0.5 & \\
\hline & & 0.5 & & & 0.5 & & & & 1 \\
\hline & & 0.5 & 0.5 & & & & 0.5 & & 0.5 \\
\hline & & & 0.5 & & & & & & 0.5 \\
\hline & & 0.5 & & & & 0.5 & & & \\
\hline \multirow[t]{9}{*}{0.5} & & 0.5 & 2.6 & 1 & & 0.5 & 0.5 & & 1 \\
\hline & 0.5 & 0.5 & 0.5 & & & 0.1 & 0.5 & & 1 \\
\hline & & & 0.6 & & & 0.6 & 0.1 & 0.1 & 0.6 \\
\hline & & & 0.6 & & 0.5 & 0.1 & & 0.5 & 0.6 \\
\hline & & 0.5 & 0.6 & & 0.1 & 0.1 & 0.6 & & 0.1 \\
\hline & & & & & & & 0.5 & & \\
\hline & & & & 0.5 & & & & & \\
\hline & 0.5 & & & & & 0.5 & & & \\
\hline & & 0.5 & & & & 0.5 & & & 0.5 \\
\hline \multirow[t]{3}{*}{0.5} & & & & & & & 0.5 & & 0.5 \\
\hline & & 0.5 & & 0.5 & & & 0.5 & & 0.5 \\
\hline & & 0.5 & & & & 0.5 & 0.1 & 0.5 & 0.5 \\
\hline \multirow[t]{12}{*}{0.5} & & & & & & 0.5 & & & \\
\hline & & & & & 0.5 & & & & 0.5 \\
\hline & & & & 0.6 & & 0.5 & & & 0.5 \\
\hline & & & & 0.5 & & & 0.5 & & 0.5 \\
\hline & & 0.5 & & 0.5 & 1 & 0.5 & & 0.5 & 0.5 \\
\hline & & 0.5 & & 0.5 & & 0.5 & 0.6 & 0.5 & 0.5 \\
\hline & & 0.5 & & 0.1 & & 0.5 & & 0.5 & 0.5 \\
\hline & 0.5 & & & 0.5 & & & & & \\
\hline & & 0.5 & & & & & & 0.5 & \\
\hline & & & 0.1 & 0.1 & 0.6 & & 0.5 & & 0.5 \\
\hline & & 0.5 & 0.1 & 0.1 & 0.6 & & & 0.5 & 1 \\
\hline & & & 0.5 & & & & 0.5 & & \\
\hline \multirow[t]{17}{*}{0.5} & & & & & 0.5 & & 0.5 & & 0.5 \\
\hline & & 0.5 & & 0.5 & & & 0.5 & & 0.5 \\
\hline & & 0.5 & & & & & & 0.5 & \\
\hline & & 0.5 & & & 0.5 & & & & \\
\hline & & & 0.5 & & & 0.5 & & & 1 \\
\hline & & 0.5 & & & & & 0.5 & & 0.5 \\
\hline & & 0.5 & & & 0.5 & & & 0.5 & 0.5 \\
\hline & & 0.5 & & & 0.5 & & & 0.5 & \\
\hline & & 0.5 & & & 0.5 & & & 0.5 & 0.5 \\
\hline & & & 0.5 & & & 0.5 & & & \\
\hline & & 0.5 & & & 0.5 & & & & \\
\hline & & & 0.5 & & & 0.5 & & & 0.5 \\
\hline & & 0.5 & & 0.5 & & & & 0.5 & 0.5 \\
\hline & & & & 0.5 & & 0.5 & & & 1 \\
\hline & & & 0.5 & & & 0.5 & & 0.5 & 0.5 \\
\hline & & & 0.5 & & & 0.5 & & & 0.5 \\
\hline & 0.5 & & & & & 0.5 & & & \\
\hline
\end{tabular}


wbheat

\begin{tabular}{|c|c|c|c|c|c|c|c|c|c|}
\hline 0.5 & & & & & 0.5 & & & & \\
\hline & & 0.5 & & & & & 0.5 & & 0.5 \\
\hline \multirow[t]{7}{*}{0.5} & & & & 0.5 & & & & 0.5 & 0.5 \\
\hline & & & & 0.5 & & & 0.5 & & 0.5 \\
\hline & 0.5 & & & & & 0.5 & & 0.5 & \\
\hline & & & 0.5 & & & & 0.5 & & 0.5 \\
\hline & & & 0.5 & & & & & & 1 \\
\hline & & & 0.5 & & & & & & \\
\hline & & 0.5 & & & & 0.5 & & & 0.5 \\
\hline \multirow[t]{16}{*}{0.5} & & & & 0.5 & & & & 0.5 & \\
\hline & & & & & 0.5 & & & 0.5 & \\
\hline & & & & 0.5 & & & & & 0.5 \\
\hline & & & 0.5 & & & 0.5 & & & \\
\hline & & & & & & 0.5 & & & \\
\hline & & & & & & & 0.5 & & \\
\hline & 0.5 & & & & 0.5 & & & & \\
\hline & & & & & & 0.5 & & & \\
\hline & & & & & & & 0.5 & & \\
\hline & & 0.5 & & & & & & 0.5 & \\
\hline & & & & 0.5 & & & & & \\
\hline & & 0.5 & & & & & & & 0.5 \\
\hline & & & & & & & & 0.5 & \\
\hline & & & & 0.5 & & & & & \\
\hline & & 0.5 & & & & & & & \\
\hline & & & & 0.5 & & & . & & \\
\hline \multirow[t]{5}{*}{0.5} & & & & & 0.5 & & 0.8 & & \\
\hline & & & 0.5 & & & & & & \\
\hline & & & 0.5 & & & & & 0.5 & \\
\hline & & 0.5 & & & & & & & 0.5 \\
\hline & 0.5 & & & & & & 0.5 & & \\
\hline \multirow[t]{6}{*}{0.5} & & & & & & 0.5 & & & \\
\hline & & & & & & & 0.5 & & \\
\hline & 0.5 & & & & & 0.5 & & & \\
\hline & 0.5 & & & & & & & & 0.5 \\
\hline & & & 0.5 & & & & & & \\
\hline & & & & & 0.5 & & & & 0.5 \\
\hline \multirow[t]{7}{*}{0.5} & & & 0.5 & & & & & & \\
\hline & & & 0.5 & & & & 0.5 & & \\
\hline & & & & & 0.5 & & & & \\
\hline & 0.5 & & & & & 0.5 & & & \\
\hline & & & 0.5 & & & & & & 0.5 \\
\hline & & & & & 0.5 & & & & \\
\hline & & & & & 0.5 & & & & \\
\hline \multirow{3}{*}{0.5} & & & & & & & & & 0.5 \\
\hline & & 0.5 & & & & & & 0.5 & \\
\hline & & & & 0.5 & & & & & \\
\hline \multirow[t]{2}{*}{0.5} & & & & & & & 0.5 & & \\
\hline & & 0.5 & & & 0.5 & & & & 0.5 \\
\hline \multirow[t]{3}{*}{0.5} & & & & & & & & 0.5 & \\
\hline & & & 0.5 & & & & & & \\
\hline & & 0.5 & & & & & & & \\
\hline
\end{tabular}




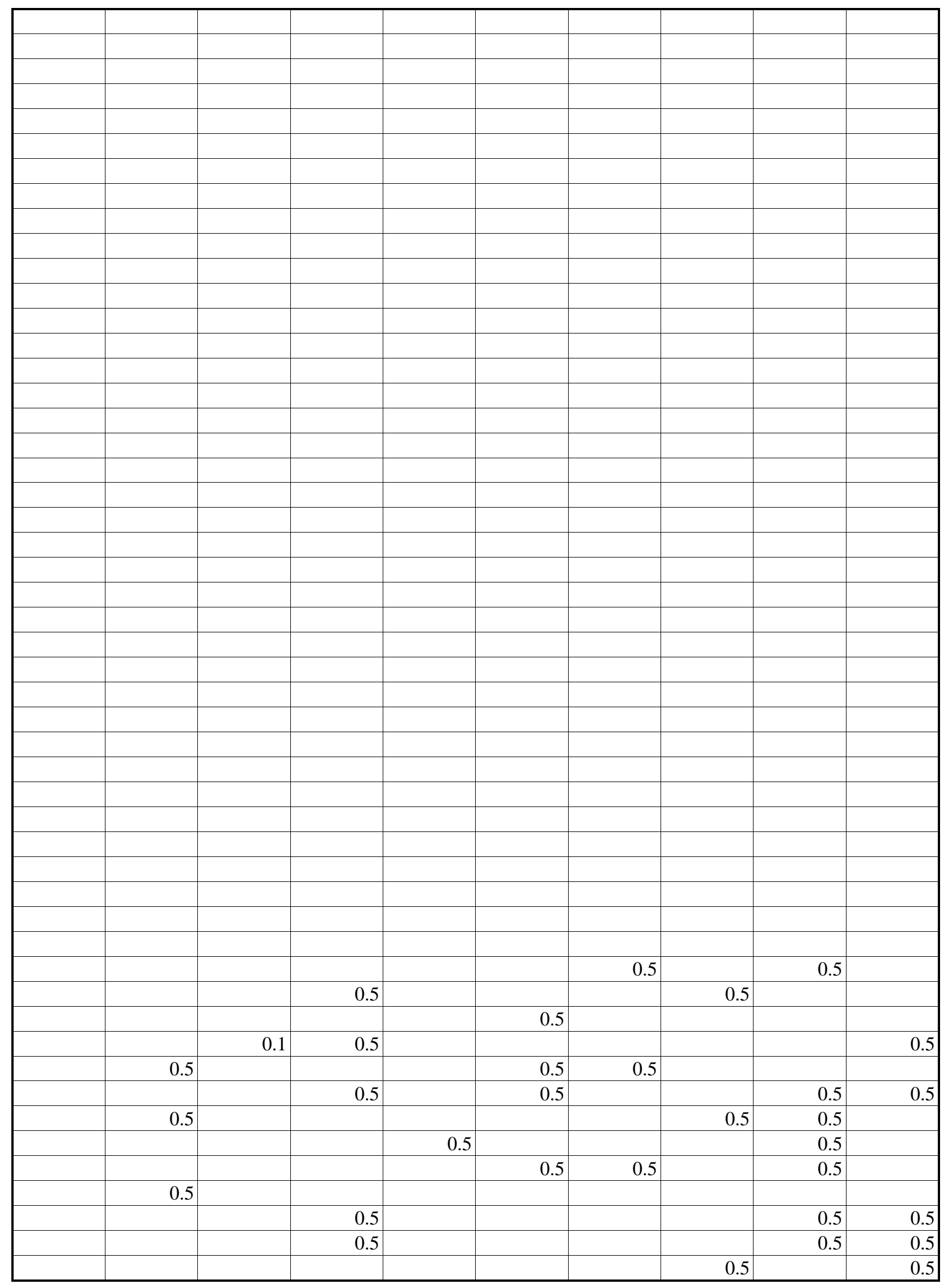

Page 39 
wbheat

\begin{tabular}{|c|c|c|c|c|c|c|c|c|c|}
\hline & & & 0.5 & & & & 0.5 & & \\
\hline & & & & & 0.5 & & & 0.5 & 0.5 \\
\hline & & & & 0.5 & & & & & \\
\hline & & & & & & & & & \\
\hline & & & 0.5 & & & & & 0.5 & 0.5 \\
\hline & & & & & 0.5 & & & 0.5 & \\
\hline & & & & 0.5 & & & & 0.5 & 0.5 \\
\hline & & & 0.5 & & & & 0.5 & & 0.5 \\
\hline & & & 0.5 & & & & 0.5 & 0.5 & 0.5 \\
\hline & & & 0.5 & & & & & 0.5 & \\
\hline & & & & & & 0.5 & & & \\
\hline & & & 0.5 & & & & & 0.5 & 0.5 \\
\hline & & & 0.5 & & & 0.5 & & & \\
\hline & 0.5 & & & & 0.5 & & & & \\
\hline & & & 0.5 & & & & & 0.5 & 0.5 \\
\hline & & & 0.5 & & & & & & 0.5 \\
\hline & & & & & 0.5 & & & & \\
\hline & & & & 0.5 & & & & & \\
\hline \multirow[t]{2}{*}{0.5} & & & & & & 0.5 & & 0.5 & \\
\hline & & & 0.5 & & & & & 0.5 & \\
\hline \multirow[t]{24}{*}{0.5} & & & 0.1 & 0.1 & 0.1 & 0.1 & & 0.5 & \\
\hline & & & 0.6 & 0.1 & 0.1 & & 0.5 & 0.5 & \\
\hline & & & 0.1 & 0.6 & 0.1 & & & 0.5 & \\
\hline & & & 0.5 & & & & & 0.5 & \\
\hline & & & & & 0.5 & & & 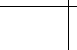 & \\
\hline & & & 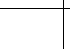 & 0.5 & & & & 0.5 & 0.5 \\
\hline & & & & 0.5 & & & & 0.5 & \\
\hline & 0.5 & & & 0.5 & & & & 0.5 & \\
\hline & & & & 0.5 & & & & 0.5 & \\
\hline & & & & & & 0.5 & & 0.5 & 0.5 \\
\hline & & & 0.5 & & & & 0.5 & & \\
\hline & & & & & 0.5 & & & & \\
\hline & 0.1 & & & & 0.5 & & & 0.5 & 0.5 \\
\hline & & & 0.5 & & & 0.5 & & & 1 \\
\hline & & & & & 0.5 & & & 0.5 & 0.5 \\
\hline & & & 0.5 & & & & 0.5 & & 0.5 \\
\hline & 0.5 & & & & 0.5 & & & 0.5 & \\
\hline & 0.5 & & & & & & 0.5 & & \\
\hline & 0.5 & & & & & & & 0.5 & \\
\hline & & & & 0.5 & & & 0.5 & & \\
\hline & & & 0.5 & & & & & 0.5 & 0.5 \\
\hline & & & 0.5 & & & 0.5 & & & 0.5 \\
\hline & & & & 0.5 & & & 0.5 & & 0.5 \\
\hline & & 0.5 & & & & & & 0.5 & 0.5 \\
\hline \multirow[t]{7}{*}{0.5} & & & & & & & & 0.5 & \\
\hline & & & & & 0.5 & & & & \\
\hline & 0.5 & & & & & & 0.5 & & 0.5 \\
\hline & & 0.5 & & & & 0.5 & & 0.5 & \\
\hline & & & 0.5 & & & & 0.5 & & 0.5 \\
\hline & & & 0.5 & & & & 0.5 & & 0.5 \\
\hline & & & & & 0.5 & & & 0.5 & \\
\hline
\end{tabular}




\begin{tabular}{|c|c|c|c|c|c|c|c|c|c|}
\hline & 0.5 & & & & & & 0.5 & & \\
\hline & & 0.5 & & & & & & & 0.5 \\
\hline \multirow[t]{3}{*}{0.5} & & & & 0.5 & & & & 0.5 & 0.5 \\
\hline & & & & 0.5 & & & 0.5 & & 0.5 \\
\hline & 0.5 & & & & 0.5 & & & 0.5 & \\
\hline 0.5 & & & & & 0.5 & & & 0.5 & 0.5 \\
\hline \multirow[t]{7}{*}{0.5} & & & & & & & 0.5 & 0.5 & 0.5 \\
\hline & & & & 0.5 & & & & 0.5 & \\
\hline & & & 0.5 & & & & & & \\
\hline & & & 0.5 & & & 0.5 & & & \\
\hline & & 0.5 & & & & 0.5 & & 0.5 & \\
\hline & 0.5 & & & 0.5 & & & & 0.5 & 0.5 \\
\hline & 0.5 & & & & 0.5 & & & 0.5 & 0.5 \\
\hline \multirow[t]{2}{*}{0.5} & & & & & 0.5 & & & 0.5 & 0.5 \\
\hline & 0.5 & & 0.5 & 0.5 & 0.5 & 0.5 & 1 & 1 & 1 \\
\hline 0.5 & 0.5 & 0.5 & 0.5 & 0.5 & 0.5 & 0.5 & 0.5 & & 0.5 \\
\hline \multirow[t]{5}{*}{0.5} & 0.5 & 1 & 1 & 1 & 1 & 0.6 & 2.1 & 3.1 & 2.1 \\
\hline & & 0.5 & 2.6 & 0.5 & & 0.5 & & 0.5 & 0.5 \\
\hline & 0.5 & & 0.5 & & 0.1 & 0.1 & 0.1 & 0.5 & 0.5 \\
\hline & 0.5 & & & & & 0.5 & & 0.5 & 0.5 \\
\hline & 0.5 & & & 0.5 & & 0.5 & & & 0.5 \\
\hline 0.5 & & & & 0.5 & & 0.5 & 0.5 & & 0.5 \\
\hline 0.5 & & 0.5 & 0.5 & 0.5 & 0.5 & & 0.5 & & 0.5 \\
\hline \multirow[t]{10}{*}{0.5} & & 0.5 & 4.6 & 2.6 & 1 & 1.5 & 1.5 & 1 & 1.5 \\
\hline & 3.6 & & 1.5 & 0.5 & 0.5 & & 0.5 & 0.5 & 2.6 \\
\hline & 3.1 & & 1.5 & & & 2.1 & 1 & 1.5 & 1 \\
\hline & & 0.5 & & & & & 0.5 & & \\
\hline & & & 0.5 & & 0.5 & & & 0.5 & 0.5 \\
\hline & 0.5 & & & & & 0.5 & & & \\
\hline & & & & 0.5 & & & & & 0.5 \\
\hline & & & & 0.5 & & & 0.5 & & 0.5 \\
\hline & & & 0.5 & & 0.5 & & 0.5 & & 0.5 \\
\hline & & & 0.5 & & & 0.5 & & 1 & 0.5 \\
\hline \multirow[t]{3}{*}{0.5} & & & 0.5 & 0.5 & 0.5 & 0.5 & 0.5 & & 0.5 \\
\hline & & 0.5 & & 0.5 & 0.5 & & & 0.5 & 0.5 \\
\hline & & & 0.5 & 1 & 0.5 & 1 & 1 & 1 & 0.5 \\
\hline \multirow[t]{2}{*}{0.5} & & & & & & & 0.5 & & \\
\hline & 3.6 & 2.6 & 1.5 & 1.5 & 1 & 1.5 & 1 & 1 & 0.5 \\
\hline \multirow[t]{2}{*}{0.5} & & & 1 & 0.5 & 0.5 & & 0.5 & 0.5 & \\
\hline & & & 0.5 & & & & 0.5 & 0.5 & \\
\hline 0.5 & & & 0.5 & 0.5 & 0.5 & 1 & 0.6 & 1 & 0.5 \\
\hline 0.5 & 0.5 & 1 & 1.6 & 2.2 & 1.1 & 1.1 & 1.1 & 1.1 & 1.1 \\
\hline 0.5 & 0.5 & 0.5 & 0.5 & 0.5 & 1 & 1 & 1 & 0.5 & 0.5 \\
\hline 0.5 & & 0.5 & 1 & 0.5 & 0.5 & 0.5 & & 0.5 & 0.5 \\
\hline 0.5 & 0.6 & 2.2 & 5.2 & 3.3 & 2.3 & 2.8 & 1.7 & 1 & 2.1 \\
\hline \multirow[t]{2}{*}{0.5} & 3.6 & 1.2 & 4.2 & 3.2 & 1.1 & 1 & & 1 & 0.5 \\
\hline & 3.1 & 1 & 1 & 2.6 & & & 0.5 & & 1 \\
\hline \multirow[t]{4}{*}{0.5} & & & & 0.5 & & & 0.5 & 0.5 & 0.5 \\
\hline & & & 0.5 & & & 0.5 & & 0.5 & 0.5 \\
\hline & & & 0.5 & & 0.5 & 1 & & & \\
\hline & & & & 0.5 & & & & & \\
\hline
\end{tabular}


wbheat

\begin{tabular}{|c|c|c|c|c|c|c|c|c|c|}
\hline & & 0.5 & & 0.5 & 3.6 & 1 & & 0.5 & 0.5 \\
\hline & & 1 & 2.6 & 1 & & & 0.5 & 0.5 & 1 \\
\hline & 0.5 & 0.5 & 0.5 & 0.5 & & 0.5 & & 0.5 & 0.5 \\
\hline & & 0.5 & 0.5 & & & & 0.5 & & 0.5 \\
\hline & 0.5 & & & & 0.5 & & & 0.5 & 0.5 \\
\hline \multirow[t]{9}{*}{0.5} & & & 0.5 & 0.5 & & & & & \\
\hline & 0.5 & & & & 0.5 & & & & \\
\hline & & & 0.5 & & 0.5 & & & 0.5 & \\
\hline & & 0.5 & 1 & 0.5 & 1 & 1 & & & 0.5 \\
\hline & 0.5 & 0.5 & 3.1 & 2.6 & 3.1 & 1 & 0.5 & 0.5 & 0.5 \\
\hline & 3.6 & 2.1 & 0.5 & 1.5 & 0.5 & 1 & 1.5 & & 0.5 \\
\hline & 3.1 & 2.1 & & 0.5 & & & 0.5 & & \\
\hline & & & & 0.5 & & & & 0.5 & \\
\hline & & & & 0.5 & & & & & 0.5 \\
\hline \multirow[t]{3}{*}{1} & 0.1 & 1.1 & 0.6 & 0.5 & & 0.5 & 1 & 0.1 & 0.6 \\
\hline & 0.1 & 1.5 & 0.6 & 0.5 & 0.5 & 1 & 2.1 & 1.5 & 1.5 \\
\hline & 0.1 & 1 & 0.6 & & 0.5 & 0.5 & 1 & 1.6 & 0.5 \\
\hline 1 & 0.6 & 1.6 & 0.6 & 1.5 & 1.6 & 1.5 & 1.1 & 1 & 1.1 \\
\hline 0.5 & 0.1 & 1.5 & 1.1 & 1 & 1.6 & 1 & 1.1 & 1 & 1.1 \\
\hline 0.6 & 0.6 & & 0.5 & 0.6 & 0.1 & 0.5 & & & 0.1 \\
\hline 0.6 & & 0.6 & & 0.6 & & 0.5 & & 0.1 & 0.5 \\
\hline 0.6 & 0.5 & 1 & 1.1 & 1 & 1 & 1.5 & 1.5 & 1 & \\
\hline 0.5 & & 0.5 & 1 & 1 & & 0.5 & & 0.5 & 0.5 \\
\hline 0.5 & & 1 & 1.6 & 1.5 & 2.1 & 1.5 & 2.1 & 1.1 & 1.6 \\
\hline 0.5 & 0.6 & 1.5 & 1.6 & 2.1 & 2.1 & 1.5 & 1.6 & 1.5 & 1.1 \\
\hline \multirow[t]{4}{*}{0.6} & & 0.5 & 1.5 & 1.1 & 1 & 1.1 & 1 & 1 & 0.6 \\
\hline & & 0.1 & & & 0.5 & & & & \\
\hline & 0.1 & & 0.5 & & 0.1 & & & 0.5 & \\
\hline & 3.1 & 1 & 2.1 & 3.1 & 4.1 & 2.8 & 2.3 & 1.8 & 1.8 \\
\hline 0.6 & 3.1 & 2.2 & 3 & 3.4 & 2.8 & 3.3 & 2.9 & 2.8 & 2.1 \\
\hline 0.1 & 3.6 & 2.6 & 4.1 & 4.4 & 3.9 & 3.2 & 2.6 & 2.1 & 1.5 \\
\hline 1 & 1 & 1 & 5.7 & 1.5 & 1 & 1 & 0.5 & 0.5 & \\
\hline 1 & 5.2 & 2.6 & 2.6 & 2.9 & 3.1 & 3.7 & 3.6 & 3.1 & 3.1 \\
\hline 0.5 & 1.5 & 1.5 & 2.1 & 2.1 & 3.1 & 2.1 & 1.5 & 1.5 & 1 \\
\hline 1 & 0.5 & 0.5 & 0.5 & 0.5 & 1 & 0.5 & 0.5 & 0.5 & 0.5 \\
\hline \multirow[t]{5}{*}{0.5} & 5 & 3.6 & 2.1 & 2.5 & 1.6 & 0.5 & 0.5 & & 0.5 \\
\hline & 4.1 & 1 & 1 & 0.5 & 0.5 & 0.5 & 0.5 & 0.5 & 0.5 \\
\hline & 2.6 & 0.5 & 2.1 & 1 & 1.5 & 0.5 & 0.5 & 0.5 & 1 \\
\hline & 2.6 & 1 & 2.5 & 1.5 & 1.5 & 1 & 1 & 1.5 & 1 \\
\hline & 2.6 & 1 & 2.1 & 2.6 & 1.5 & 4.1 & 1.5 & 0.5 & 0.5 \\
\hline \multirow[t]{2}{*}{0.5} & 0.5 & 1 & 1 & 1 & 0.5 & 0.5 & 0.5 & 0.5 & \\
\hline & 0.5 & & & & 0.5 & & & & 0.5 \\
\hline 0.5 & 3.1 & 2.1 & 3.1 & 2.1 & 1.5 & 1 & 1.5 & 2.6 & 2.1 \\
\hline 1 & 5.2 & 2.6 & 4.6 & 4.8 & 3.8 & 3.6 & 3.6 & 3.1 & 3.1 \\
\hline 0.5 & 5.2 & 3.1 & 4.9 & 4.8 & 4.1 & 3.1 & 2.6 & 2.6 & 3.1 \\
\hline \multirow[t]{2}{*}{1} & 4.4 & 2.1 & 4.4 & 2.6 & 2.6 & 2.2 & 1.5 & 2.1 & 2.1 \\
\hline & 3.6 & 1.3 & 3.4 & 3.1 & 3.1 & 2.1 & 1.5 & 1 & 1.5 \\
\hline 0.5 & 1.5 & 1 & 1.6 & 1 & 1.5 & 1.6 & 1.1 & & 0.6 \\
\hline 0.5 & 0.6 & & 1.1 & & 0.6 & 0.5 & 0.5 & 0.5 & 0.1 \\
\hline \multirow[t]{2}{*}{0.6} & 0.6 & 1.1 & 2.6 & 3.1 & 0.5 & 1 & 0.5 & 0.5 & 0.5 \\
\hline & & 0.5 & 1 & 0.5 & & & 0.5 & 0.5 & 0.5 \\
\hline
\end{tabular}




\begin{tabular}{|c|c|c|c|c|c|c|c|c|c|}
\hline & & & 1 & & & 0.5 & 0.5 & 0.5 & \\
\hline & & 0.5 & & & 0.5 & & 0.5 & 0.5 & 0.5 \\
\hline 0.5 & 1.1 & 0.6 & 6.2 & 3.8 & 3.6 & 3.9 & 3.9 & 4.4 & 3.8 \\
\hline 1.4 & 2.4 & 1.9 & 2.4 & 1.9 & 2.4 & 2.9 & 2.9 & 1.9 & 2.4 \\
\hline 1.4 & 0.9 & 0.9 & 1.4 & 0.8 & 0.8 & 0.8 & 0.8 & 0.9 & 0.8 \\
\hline 5 & 3 & 5 & 5.3 & 5.2 & 4.7 & 4.6 & 4.1 & 3.1 & 3.7 \\
\hline 2.7 & 3.7 & 3.7 & 5.7 & 4.7 & 5.7 & 4.6 & 4.7 & 4.7 & 4.6 \\
\hline 2.7 & 4.2 & 3.7 & 6.8 & 4.7 & 5.8 & 4.7 & 4.7 & 4.1 & 3.6 \\
\hline 2.6 & 3.1 & 4.2 & 5.2 & 5.2 & 4 & 4.4 & 3.7 & 2.6 & 3.1 \\
\hline \multirow{2}{*}{1.6} & 2.6 & 2.6 & 2.1 & 2.1 & 1.5 & 1 & 0.5 & 1.5 & 1 \\
\hline & 0.5 & & 0.5 & & 1 & 0.5 & 0.5 & & \\
\hline 0.5 & & 0.1 & & 0.5 & & & & 0.5 & \\
\hline \multirow[t]{3}{*}{0.5} & 1 & 0.5 & 0.5 & 0.5 & & & 0.5 & 0.5 & 0.5 \\
\hline & 0.5 & & 0.5 & & & 0.5 & & 0.5 & 1 \\
\hline & & & 0.5 & & 0.5 & & 1 & 1 & 4.6 \\
\hline 1.6 & 2.7 & 5.8 & 6.7 & 5.3 & 5.3 & 5.3 & 4.7 & 5.3 & 4.7 \\
\hline 2.2 & 3.2 & 6.8 & 8.1 & 6.1 & 5.4 & 4.2 & 5.3 & 5 & 3.9 \\
\hline 1.1 & 0.6 & 0.6 & 1.6 & 1.1 & 1.1 & 0.6 & 0.1 & 1 & 0.5 \\
\hline 1.1 & 0.1 & 1.1 & 0.1 & 1.1 & 0.1 & 1.1 & 0.1 & 0.6 & 0.6 \\
\hline \multirow[t]{6}{*}{0.1} & 1.1 & 5.8 & 6.3 & 4.5 & 3.2 & 4.1 & 3.1 & 3.1 & 2.3 \\
\hline & 0.5 & 3.6 & 4 & 3.3 & 2.8 & 2.6 & 2.1 & 3.1 & 2.1 \\
\hline & 0.5 & 3.6 & 4.4 & 2.7 & 2.6 & 2.1 & 2.1 & 1.5 & 1.5 \\
\hline & 0.5 & 2.6 & 3.6 & 2.6 & 2.6 & 2.1 & 1.5 & 1 & 1 \\
\hline & 0.5 & 2.1 & 2.6 & 1.5 & 1 & 0.5 & 1 & 0.5 & 0.5 \\
\hline & & & 1 & & & & 0.5 & & \\
\hline \multirow[t]{4}{*}{0.5} & & & & & 0.5 & & & & \\
\hline & 0.5 & & 0.5 & & & 0.5 & & & 0.5 \\
\hline & & & 0.5 & & 0.5 & & & 1 & \\
\hline & & 0.5 & 0.5 & 1 & 1 & 1 & 3.1 & 4.1 & 2.1 \\
\hline 0.5 & 0.5 & 3.6 & 5.2 & 3.6 & 2.1 & 2.6 & 1.5 & 1 & 1.5 \\
\hline 0.5 & 0.5 & 3.6 & 2.6 & 2.6 & 1 & 0.5 & 0.5 & 0.5 & 0.5 \\
\hline 0.5 & & 0.5 & 1 & 1.5 & 1 & 0.5 & 1.5 & 0.5 & 1 \\
\hline 0.5 & 1 & & 1.5 & 1 & 1.5 & 0.5 & 0.5 & 0.5 & 0.5 \\
\hline \multirow[t]{4}{*}{0.5} & 0.5 & 4.1 & 3.9 & 2.2 & 1 & 0.5 & 1 & 1 & 2.6 \\
\hline & 0.5 & 2.6 & 2.4 & 0.5 & 0.5 & & 0.5 & 0.5 & \\
\hline & 0.5 & 1 & 6.6 & 3.2 & 2.6 & 1.5 & 1 & 0.5 & 2.1 \\
\hline & 0.5 & 3.6 & 4.1 & 2.6 & 1.5 & 0.5 & 0.5 & 0.5 & 2.1 \\
\hline \multirow[t]{3}{*}{0.5} & & 3.1 & 2.5 & 2.1 & 0.5 & 1 & 1 & 1 & 1 \\
\hline & & 0.5 & & & & 0.5 & & & 0.5 \\
\hline & 0.5 & & & & 0.5 & & & & \\
\hline \multirow[t]{2}{*}{0.5} & 0.5 & & 0.5 & & & 0.6 & & 0.5 & 0.5 \\
\hline & & & 0.5 & & 0.5 & & & 0.5 & 0.5 \\
\hline 0.5 & & 0.5 & 4.6 & 2.6 & 2.1 & 1.5 & 3.1 & 2.6 & 2.6 \\
\hline 0.5 & 1 & 4.6 & 4.3 & 3.7 & 3.6 & 2.6 & 2.6 & 1.5 & 1.5 \\
\hline 0.5 & 0.5 & 4.1 & 3.2 & 3.2 & 1.5 & 1 & 1 & & 0.5 \\
\hline \multirow[t]{2}{*}{0.5} & & & 0.5 & & & 0.5 & & & 0.5 \\
\hline & & 0.5 & & & 0.5 & & & & 0.5 \\
\hline \multirow[t]{3}{*}{1.5} & 0.5 & 1 & 0.5 & 1.1 & 1.2 & 0.6 & 0.6 & 0.1 & 4.2 \\
\hline & 1 & 3.6 & 1.1 & 1.1 & 1 & 1 & 0.5 & 1 & 0.5 \\
\hline & 0.5 & 2.6 & 1 & 1 & 1 & 0.5 & 1 & 0.5 & 1 \\
\hline 0.5 & 0.5 & 2.6 & 1 & 1.5 & 0.5 & 1 & 0.5 & 0.5 & 1 \\
\hline
\end{tabular}




\begin{tabular}{|c|c|c|c|c|c|c|c|c|c|}
\hline & 0.5 & 2.1 & 1 & 1 & 0.5 & 0.5 & & & \\
\hline & & & 0.5 & & & & 0.5 & & \\
\hline & & 0.5 & & & & & & 0.5 & \\
\hline & & & 0.5 & & 0.5 & & & 1 & 0.5 \\
\hline \multirow[t]{8}{*}{0.5} & & & 0.5 & & & 0.5 & & 0.5 & 0.5 \\
\hline & 0.5 & & & & 0.5 & & 0.5 & & 1 \\
\hline & & & 0.5 & & & & 0.5 & 0.5 & 0.5 \\
\hline & & & 0.5 & & 0.5 & & & 0.5 & 0.5 \\
\hline & & & & 0.5 & & & & & 0.5 \\
\hline & . & & & 0.5 & & & & & 0.5 \\
\hline & . & 0.5 & & 0.5 & & 0.5 & & 1 & 0.5 \\
\hline & & 0.5 & 0.5 & 0.5 & & 0.5 & & 0.5 & 0.5 \\
\hline \multirow[t]{7}{*}{0.5} & & 0.5 & & 0.5 & & 0.5 & & 0.5 & 0.5 \\
\hline & 0.5 & 0.5 & & 0.5 & & 0.5 & & 0.5 & 0.5 \\
\hline & 0.5 & & 0.5 & & 0.5 & & & 0.5 & \\
\hline & & 0.5 & & & 0.5 & & & & \\
\hline & & 0.5 & & & & 0.5 & & & \\
\hline & 0.5 & & 0.5 & 0.5 & 0.5 & & & 0.5 & 0.5 \\
\hline & & & 0.5 & & 0.5 & . & & 0.5 & 0.5 \\
\hline \multirow[t]{23}{*}{0.5} & & 1 & 0.5 & 1 & 0.5 & 1 & 1 & 1 & 0.5 \\
\hline & 0.5 & 0.5 & 1 & 1 & 1 & & 1 & 0.5 & 0.5 \\
\hline & & 0.5 & & 0.5 & & 0.5 & & 0.5 & 0.5 \\
\hline & & 0.5 & & & & 0.5 & & & \\
\hline & & 0.5 & & & & & & & 0.5 \\
\hline & & & & 0.5 & & 0.5 & & 0.5 & 0.5 \\
\hline & & 0.5 & & & 0.5 & & & 0.5 & 0.5 \\
\hline & & & 0.5 & & & 0.5 & & 0.5 & 0.5 \\
\hline & & & 0.5 & & & 0.5 & & 0.5 & 0.5 \\
\hline & & & 0.5 & & & 0.5 & & 0.5 & \\
\hline & & & & & 0.5 & & & & \\
\hline & 0.5 & & 0.5 & & & & & & \\
\hline & 0.5 & & & & & 0.5 & & 0.5 & 0.5 \\
\hline & & 0.5 & & & 0.5 & & & 0.5 & 1 \\
\hline & & & 0.5 & & & 1.2 & 2.9 & 0.5 & 0.5 \\
\hline & 0.5 & & & & 0.5 & & & 0.5 & 0.5 \\
\hline & & & & 0.5 & & & 0.5 & 0.5 & 0.5 \\
\hline & 0.5 & & 0.5 & & & 0.5 & & & \\
\hline & & 0.5 & & & & & & & \\
\hline & & 0.5 & & & & & 0.5 & 0.5 & 0.5 \\
\hline & & & 0.5 & & & 0.5 & & 0.5 & 0.5 \\
\hline & 0.5 & & & & & & 0.5 & 0.5 & 0.5 \\
\hline & & & & 0.5 & & 0.5 & & & 1 \\
\hline \multirow[t]{9}{*}{0.5} & & & 0.5 & & & & 0.5 & & 0.5 \\
\hline & & 0.5 & & & & & & & 0.5 \\
\hline & & & 0.5 & & & & 0.5 & & \\
\hline & & & & 0.5 & 0.5 & & & 0.5 & 0.5 \\
\hline & 0.5 & & & & & 0.5 & & 0.5 & 0.5 \\
\hline & & & 0.5 & & & 0.5 & & 0.5 & 0.5 \\
\hline & & 0.5 & & & 0.5 & & & 0.5 & 0.5 \\
\hline & & & 0.5 & & & 0.5 & & 0.5 & 0.5 \\
\hline & & & & 0.5 & & & 0.5 & & \\
\hline
\end{tabular}




\begin{tabular}{|c|c|c|c|c|c|c|c|c|c|}
\hline & 0.5 & & & & 0.5 & & & & \\
\hline & 0.5 & & & 0.5 & & & 0.5 & 0.5 & \\
\hline & & & 0.5 & & & 0.5 & & 0.5 & 0.5 \\
\hline & & & 0.5 & & & 0.5 & & 0.5 & 0.5 \\
\hline & 0.5 & & & & 0.5 & & & 0.5 & 0.5 \\
\hline & & 0.5 & & & 0.5 & & 0.5 & & \\
\hline & & & & 0.5 & & & & & 0.5 \\
\hline \multirow[t]{9}{*}{0.5} & & & & & 0.5 & & & & 0.5 \\
\hline & 0.5 & & & 0.5 & & & & 1 & \\
\hline & & & & 0.5 & & & & 1 & \\
\hline & 0.5 & & & & 0.5 & & & 0.5 & 0.5 \\
\hline & 0.5 & & & 0.5 & & & 0.5 & 0.5 & \\
\hline & 0.5 & & & & & 0.5 & 0.5 & & 0.5 \\
\hline & & & & 0.5 & & & & & 0.5 \\
\hline & 0.5 & & & & & 0.5 & & & \\
\hline & & 0.5 & & & 0.5 & & & 0.5 & \\
\hline \multirow[t]{5}{*}{0.5} & & & & 0.5 & & & 0.5 & & \\
\hline & & & & & 0.5 & & 0.5 & & \\
\hline & 0.5 & & & & 0.5 & 0.5 & & & 0.5 \\
\hline & & & & 0.5 & 0.5 & & 0.5 & & \\
\hline & 0.5 & & & & & 0.5 & & & \\
\hline \multirow[t]{3}{*}{0.5} & & & & & 0.5 & & & & \\
\hline & & & 0.5 & & & & & & \\
\hline & 0.5 & & & & 0.5 & & & 0.5 & \\
\hline \multirow[t]{4}{*}{0.5} & & & & & 0.5 & & & & \\
\hline & 0.5 & & & & & & 0.5 & & \\
\hline & & & 0.5 & & & & 0.5 & & \\
\hline & 0.5 & & & 0.5 & & & & & \\
\hline & & & & & & & & & \\
\hline & & & & & & & & & \\
\hline & & & & & & & & & \\
\hline 0 & 0.5 & 0.666667 & 1.22 & 3.1 & 1.5 & 0.5 & 0 & 17.5 & 4.1 \\
\hline 0.943275 & 0.993865 & 0.992486 & 1.463889 & 1.449693 & 1.265698 & 1.2825 & 1.470968 & 1.153293 & 1.16878 \\
\hline 0.662791 & 0.653465 & 0.65 & 0.67037 & 0.696795 & 0.731214 & 0.756915 & 0.769231 & 0.79163 & 0.791829 \\
\hline 0.932877 & 1.731757 & 1.510778 & 1.919632 & 1.638365 & 1.644304 & 1.55061 & 1.440491 & 1.406289 & 1.386034 \\
\hline 0.5 & 0.5 & 0.5 & 0.463158 & 0.445 & 0.515789 & 0.483333 & 0.504762 & 0.5 & 0.544444 \\
\hline 0.571154 & 1.513433 & 1.086154 & 1.341414 & 1.244186 & 1.1 & 1.126316 & 0.984146 & 0.821622 & 0.807339 \\
\hline 1.375 & 1.337037 & 2.85 & 3.065625 & 2.666667 & 2.196552 & 2.025926 & 1.862069 & 1.75 & 1.975 \\
\hline 0.5625 & 0.530303 & 1.151613 & 0.9 & 0.918182 & 0.702564 & 0.666667 & 0.834483 & 0.619231 & 0.666102 \\
\hline & & & & & & & & & \\
\hline & & & & & & & & & \\
\hline & & & & & & & & & \\
\hline & & & & & & & & & \\
\hline \#DIV/0! & 0 & 0.288675 & 1.609969 & \#DIV/0! & \#DIV/0! & \#DIV/0! & \#DIV/0! & \#DIV/0! & \#DIV/0! \\
\hline 0.827824 & 0.840759 & 0.863 & 1.752501 & 1.447577 & 1.323224 & 1.266528 & 1.686076 & 1.094828 & 1.067616 \\
\hline 0.247836 & 0.242307 & 0.247678 & 0.27429 & 0.404239 & 0.518559 & 0.548313 & 0.569868 & 0.626883 & 0.633421 \\
\hline 0.472573 & 1.583161 & 1.322422 & 1.60727 & 1.340782 & 1.285415 & 1.228221 & 1.099811 & 1.077426 & 1.05781 \\
\hline 0 & 0 & 0 & 0.130002 & 0.15035 & 0.153707 & 0.08165 & 0.116087 & 0 & 0.176383 \\
\hline 0.182935 & 1.561652 & 0.776586 & 1.361947 & 1.140754 & 1.053286 & 0.963379 & 0.797909 & 0.709853 & 0.672021 \\
\hline 1.188486 & 1.230431 & 1.859086 & 2.370737 & 1.716884 & 1.749384 & 1.69787 & 1.627534 & 1.556909 & 1.402544 \\
\hline 0.25 & 0.121153 & 1.222803 & 1.01102 & 0.818709 & 0.580602 & 0.400219 & 0.730595 & 0.359214 & 0.571933 \\
\hline
\end{tabular}




\begin{tabular}{|r|r|r|r|r|r|r|r|r|r|}
\hline & & & & & & & & & \\
\hline & & & & & & & & & \\
\hline & & & & & & & & & \\
\hline & & & & & & & & & \\
\hline 1.5 & 1 & & & & & & & & \\
\hline 162.3 & 165 & 174.7 & 265.5 & 241.8 & 222.3 & 209.7 & 233 & 196.6 & 247.1 \\
\hline 16 & 15.5 & 17.5 & 19 & 23.2 & 23.7 & 22.3 & 23.7 & 26.7 & 29.8 \\
\hline 127.7 & 246.8 & 244.8 & 303.4 & 250.5 & 249.3 & 243.3 & 224.3 & 213.6 & 235.6 \\
\hline 5 & 4 & 11 & 8.7 & 6.8 & 8.7 & 8.5 & 8.4 & 9.5 & 16.5 \\
\hline 48.2 & 126 & 97.5 & 169.9 & 137.4 & 125.8 & 114.9 & 108.3 & 116.8 & 117 \\
\hline 12 & 16 & 66.2 & 79.2 & 55.9 & 44.4 & 35.2 & 35.7 & 33.6 & 39.1 \\
\hline 10.0476 & 20.75986 & 24.6077 & 26.54409 & 27.65889 & 24.65612 & 24.59227 & 22.26615 & 49.04207 & 36.93953 \\
\hline & & & & & & & & & \\
\hline & & & & & & & & & \\
\hline
\end{tabular}


wbheat

\begin{tabular}{|c|c|c|c|c|c|c|c|c|c|}
\hline \multirow[t]{19}{*}{15} & 16 & 17 & 18 & 19 & 20 & 21 & 22 & 23 & $\begin{array}{l}\text { DAILY } \\
\text { TOTAL }\end{array}$ \\
\hline & & & & & & & & & 0.00 \\
\hline & & & & & & & & & 0.00 \\
\hline & & & & & & & & & 0.00 \\
\hline & & & & & & & & & 0.00 \\
\hline & & & & & & & & & 0.00 \\
\hline & & & & & & & & & 0.00 \\
\hline & & & & & & & & & 0.00 \\
\hline & & & & & & & & & 0.00 \\
\hline & & & & & & & & & 0.00 \\
\hline & & & & & & & & & 0.00 \\
\hline & & & & & & & & & 0.00 \\
\hline & & & & & & & & & 0.00 \\
\hline & & & & & & & & & 0.00 \\
\hline & & & & & & & & & 0.00 \\
\hline & & & & & & & & & 0.00 \\
\hline & & & & & & & & & 0.00 \\
\hline & & & & & & & & & 0.00 \\
\hline & & & & & & & & & 0.00 \\
\hline \multirow[t]{29}{*}{2.1} & & & & & & & & & 16.40 \\
\hline & & & & & & & & & 0.00 \\
\hline & & & & & & & & & 0.00 \\
\hline & & & & & & & & & 0.00 \\
\hline & & & & & & & & & 0.00 \\
\hline & & & & & & & & & 0.00 \\
\hline & & & & & & & & & 0.00 \\
\hline & & & & & & & & & 0.00 \\
\hline & & & & & & & & & 0.00 \\
\hline & & & & & & & & & 0.00 \\
\hline & & & & & & & & & 0.00 \\
\hline & & & & & & & & & 0.00 \\
\hline & & & & & & & & & 0.00 \\
\hline & & & & & & & & & 0.00 \\
\hline & & & & & & & & & 0.00 \\
\hline & & & & & & & & & 0.00 \\
\hline & & & & & & & & & 17.50 \\
\hline & & & & & & & & & 0.00 \\
\hline & & & & & & & & & 0.00 \\
\hline & & & & & & & & & 0.00 \\
\hline & & & & & & & & & 0.00 \\
\hline & & & & & & & & & 0.00 \\
\hline & & & & & & & & & 0.00 \\
\hline & & & & & & & & & 0.00 \\
\hline & & & & & & & & & 0.00 \\
\hline & & & & & & & & & 0.00 \\
\hline & & & & & & & & & 0.00 \\
\hline & & & & & & & & & 0.00 \\
\hline & & & & & & & & & 0.00 \\
\hline
\end{tabular}




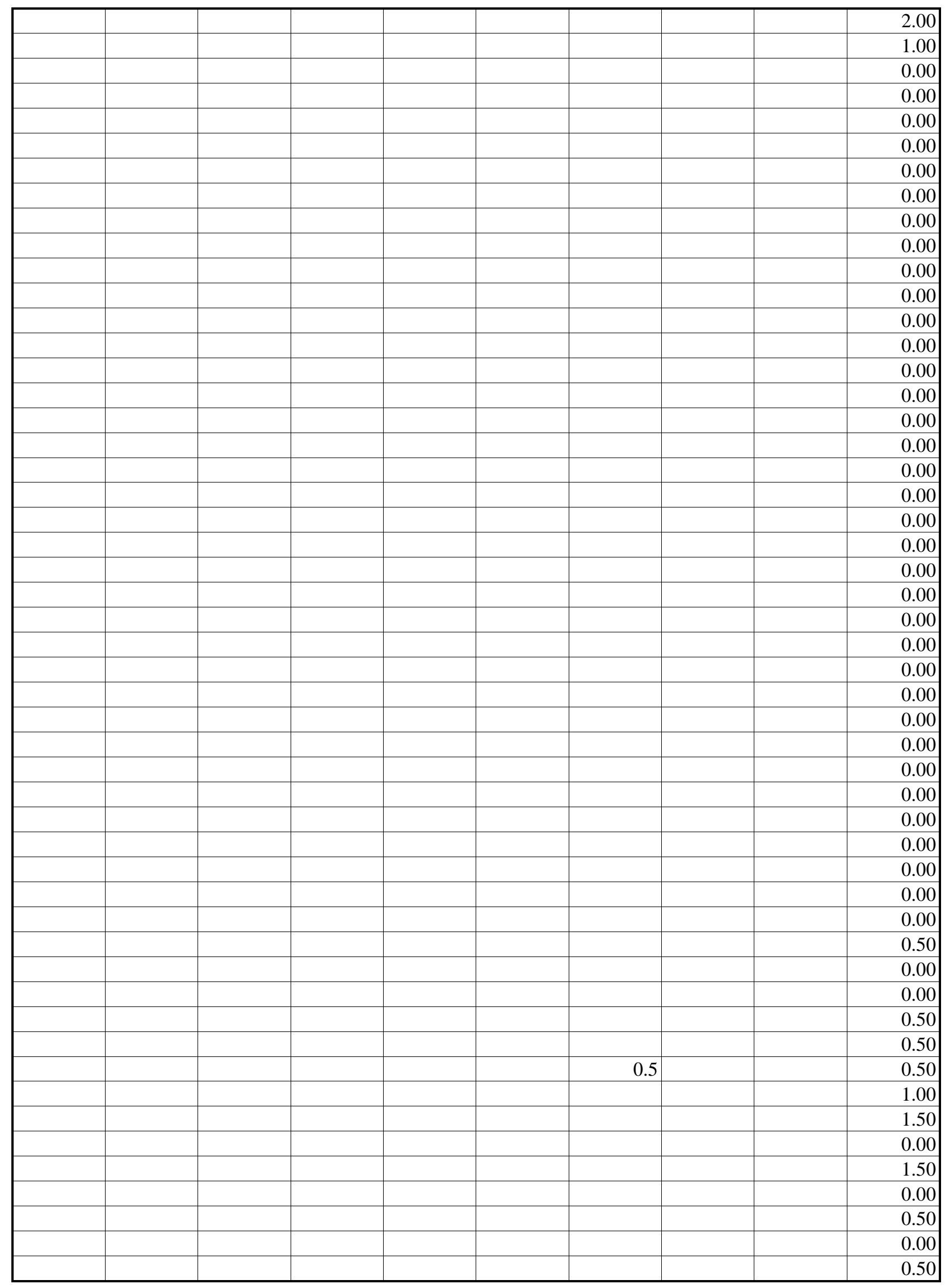




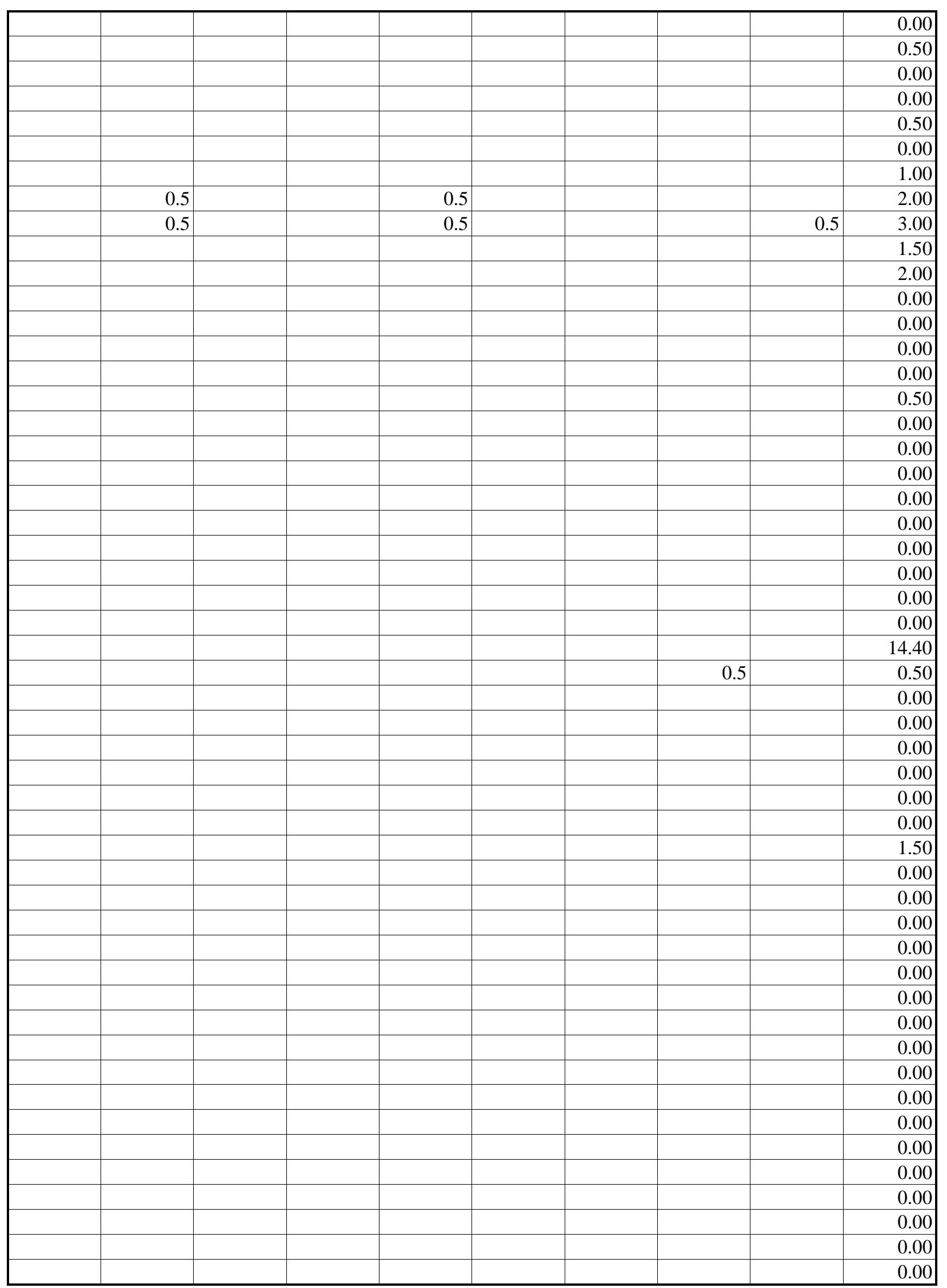




\begin{tabular}{|c|c|c|c|c|c|c|c|c|c|}
\hline & & & & & & & & & 0.00 \\
\hline & & & & & & & & & 0.00 \\
\hline & & & & & & & & & 0.00 \\
\hline & 0.5 & & 0.5 & & & 0.5 & & 0.5 & 2.50 \\
\hline & & & 0.5 & & & 0.5 & & & 3.00 \\
\hline & & 0.5 & & 0.5 & & 0.5 & & & 4.00 \\
\hline 0.5 & & & 0.5 & & & 0.5 & & 0.5 & 5.00 \\
\hline 0.5 & & 0.5 & & 0.5 & & & 0.5 & & 4.50 \\
\hline \multirow[t]{16}{*}{0.5} & & & 0.5 & & 0.5 & & 0.5 & & 5.00 \\
\hline & & & & & & & & & 2.50 \\
\hline & & & & & & & & & 0.00 \\
\hline & & & & & & & & & 0.00 \\
\hline & & & & & & & & & 0.00 \\
\hline & & & & & & & & & 0.00 \\
\hline & & & & & & & & & 0.00 \\
\hline & & & & & & & & & 0.00 \\
\hline & & & & & & & & & 0.00 \\
\hline & & & & & & & & & 0.00 \\
\hline & & 0.5 & & 0.5 & & & 0.5 & & 3.00 \\
\hline & & & 0.5 & & & & 0.5 & & 2.50 \\
\hline & & & & & 0.5 & & & 0.5 & 2.50 \\
\hline & & & 0.5 & & & 0.5 & & & 3.00 \\
\hline & & & 0.5 & & & 0.5 & & & 3.50 \\
\hline & & & & & & & & & 2.00 \\
\hline \multirow[t]{5}{*}{0.1} & & & & 0.1 & & & 0.1 & & 0.30 \\
\hline & & & & 0.1 & & & 0.1 & & 0.50 \\
\hline & & & 0.1 & & & 0.1 & & & 0.50 \\
\hline & & & & & & & & & 0.40 \\
\hline & & & & & & & & 0.1 & 0.40 \\
\hline 0.1 & & & 0.1 & & 0.1 & & 0.1 & & 0.90 \\
\hline \multirow[t]{3}{*}{0.1} & 0.1 & & & 0.1 & & 0.1 & & & 1.30 \\
\hline & & 0.1 & & 0.1 & & & & 0.1 & 1.30 \\
\hline & & 0.1 & & 0.1 & & 0.1 & & 0.1 & 1.30 \\
\hline 0.1 & & & 0.1 & & & 0.1 & & & 0.90 \\
\hline 1 & 0.5 & 1.1 & 0.5 & 1.1 & 0.5 & 0.5 & 1.1 & 0.5 & 14.30 \\
\hline 1 & 0.5 & 0.5 & 1.1 & 0.5 & 1 & 0.6 & 1 & 1.1 & 19.20 \\
\hline 1 & 0.5 & 1 & 0.5 & 0.5 & 0.5 & 1.1 & 0.5 & 1 & 16.90 \\
\hline 1 & 1 & 0.5 & 0.5 & 0.5 & 1 & 0.6 & 0.5 & 1 & 18.00 \\
\hline 1 & 1.1 & 0.5 & 1 & 0.5 & 0.6 & 1 & 0.5 & 0.6 & 18.60 \\
\hline 0.5 & 1 & 1 & 0.5 & 1.1 & 0.5 & 1.1 & & 1.1 & 20.10 \\
\hline 1 & 1 & 0.5 & 1 & 0.6 & 0.5 & 0.5 & 1.1 & 0.5 & 17.60 \\
\hline 1 & 0.6 & 1 & 0.5 & 0.6 & 1 & 0.6 & 0.5 & 1 & 17.80 \\
\hline 1 & 1.6 & 1 & 1.5 & 1.6 & 1 & 1 & 1.1 & 1 & 25.50 \\
\hline 0.6 & 1 & 1.1 & 1 & 1 & 0.6 & 0.5 & 0.5 & 0.6 & 23.40 \\
\hline 3.1 & 2.6 & 2.6 & 2.6 & 2.1 & 2.6 & 2.6 & 2.6 & 3.1 & 52.90 \\
\hline 2.6 & 2.1 & 2.6 & 2.6 & 2.1 & 2.1 & 2.1 & 2.1 & 2.1 & 54.10 \\
\hline 2.1 & 1.1 & 1.5 & 0.5 & 1.1 & 0.5 & 1 & 1.1 & 1 & 36.10 \\
\hline 1.5 & 1 & 1 & 0.5 & 1 & 1.1 & 0.5 & 1 & 0.6 & 26.20 \\
\hline 1 & 0.5 & 1 & 0.6 & 1 & 1 & 0.6 & 1 & 0.5 & 18.60 \\
\hline 0.5 & 1.1 & 0.5 & 1 & 1 & 0.6 & 1 & 0.6 & 1 & 18.80 \\
\hline 1 & 0.5 & 0.6 & 1 & 0.5 & 1 & 0.5 & 0.5 & 1 & 18.00 \\
\hline
\end{tabular}




\begin{tabular}{|c|c|c|c|c|c|c|c|c|c|}
\hline 0.5 & 1 & 0.5 & 1 & 0.5 & 0.5 & & 0.5 & & 16.60 \\
\hline 0.5 & 0.1 & 0.5 & 0.5 & 0.5 & 0.5 & 0.5 & 0.5 & 0.5 & 11.80 \\
\hline 0.5 & 1 & & 0.5 & 0.5 & 1 & 0.6 & 0.5 & 0.5 & 13.40 \\
\hline 3.6 & 2.6 & 2.1 & 2.1 & 1.5 & 2.1 & 2.1 & 2.1 & 1.5 & 31.50 \\
\hline 0.5 & 1 & 0.5 & 1 & 0.5 & 0.6 & 1 & 1 & 1 & 29.20 \\
\hline \multirow[t]{6}{*}{0.5} & 0.5 & 0.5 & 1 & 1.1 & 0.5 & 1 & 1 & 1 & 22.90 \\
\hline & 1 & 0.5 & 0.5 & 1 & 0.5 & 1.6 & 0.5 & 1 & 22.30 \\
\hline & 0.5 & 0.5 & & 0.5 & 0.5 & & 0.5 & 0.5 & 17.80 \\
\hline & & 0.5 & 0.5 & & 0.5 & & 0.5 & & 9.70 \\
\hline & & & & & & & & & 0.00 \\
\hline & & 0.5 & & 0.5 & & 0.5 & & 0.5 & 9.10 \\
\hline 1.5 & 1.5 & 1.5 & 1 & 1.5 & 1.5 & 1 & 2.1 & 2.1 & 21.40 \\
\hline 1.5 & 1 & 1 & 1.5 & 1.5 & 1.5 & 1 & 1.5 & 1 & 30.90 \\
\hline 1.5 & 1 & 1 & 2.1 & 1.5 & 1 & 1 & 1.1 & 1 & 35.20 \\
\hline 1 & 1 & 1 & 1 & 1 & 0.5 & 1 & 1.5 & 0.5 & 23.60 \\
\hline 3.1 & 3.1 & 3.1 & 3.6 & 3.1 & 3.1 & 3.6 & 2.6 & 3.1 & 61.80 \\
\hline 4.2 & 3.1 & 1.5 & 1 & 3.6 & 2.6 & 2.6 & 2.1 & 2.6 & 56.10 \\
\hline 1 & 1.5 & 1 & 1.5 & 1 & 1 & 1.1 & 1.5 & 0.5 & 36.50 \\
\hline 3.1 & 3.1 & 3.1 & 3.6 & 3.1 & 3.1 & 3.1 & 3.1 & 3.1 & 67.00 \\
\hline 1.5 & 1 & 1.5 & 1.1 & 1 & 1 & 1.5 & 1.1 & 1 & 53.50 \\
\hline 1 & 1 & 1.5 & 1.1 & 1.5 & 1 & 1.5 & 1.1 & 1.5 & 31.00 \\
\hline 1.5 & 1.1 & 1 & 1 & 1 & 1.1 & 1.5 & 1 & 1 & 29.60 \\
\hline 3.6 & 4.1 & 3.6 & 3.6 & 3.6 & 3.6 & 3.7 & 3.6 & 4.1 & 73.60 \\
\hline 3.1 & 2.6 & 3.1 & 3.1 & 3.6 & 3.1 & 3.1 & 3.1 & 3.1 & 84.80 \\
\hline 4.1 & 4.1 & 4.1 & 3.6 & 3.6 & 4.1 & 4.1 & 4.1 & 4.1 & 95.30 \\
\hline 5.8 & 5.2 & 4.6 & 4.1 & 4.2 & 4.1 & 4.1 & 3.6 & 4.6 & 118.30 \\
\hline 5.8 & 4.7 & 2.6 & 2.6 & 2.6 & 2.1 & 2.6 & 2.1 & 2.1 & 101.70 \\
\hline 2.6 & 3.1 & 2.1 & 2.6 & 2.1 & 3.1 & 2.1 & 2.1 & 2.1 & 57.40 \\
\hline 2.1 & 2.1 & 2.6 & 1.5 & 1.5 & 2.1 & 1.5 & 1.5 & 1.6 & 51.50 \\
\hline 4.7 & 4.6 & 4.6 & 4.1 & 4.1 & 4.1 & 3.1 & 3.6 & 3.6 & 98.60 \\
\hline 4.6 & 4.6 & 4.6 & 4.6 & 4.1 & 3.6 & 3.6 & 3.1 & 3.1 & 95.60 \\
\hline 2.1 & 2.6 & 4.1 & 2.6 & 3.1 & 2.6 & 2.6 & 3.1 & 2.6 & 76.20 \\
\hline 2.1 & 1.5 & 1.5 & 1.5 & 1 & 1.5 & 1 & 4.1 & 3.1 & 69.30 \\
\hline 1.6 & 2.1 & 1.5 & 1 & 0.5 & 1.5 & 1 & 1 & 0.5 & 50.10 \\
\hline 1 & 1.5 & 1.5 & 1 & 1 & 1 & 1 & 0.5 & 0.5 & 23.10 \\
\hline 1 & 1.1 & 0.5 & 1 & 1 & 1 & 1 & 0.5 & 1 & 21.70 \\
\hline 1 & 1 & 1 & 1 & 1 & 1.1 & 1 & 1.1 & 0.5 & 31.50 \\
\hline 2.1 & 1.6 & 2.1 & 2.1 & 1.1 & 1.5 & 1.5 & 1 & 1.5 & 47.60 \\
\hline 4.2 & 3.1 & 1.6 & 2.1 & 1 & 1.5 & 1.1 & 1 & 1.1 & 51.40 \\
\hline 1.5 & 1 & 1.1 & 0.5 & 1.5 & 0.5 & 0.6 & & 0.5 & 36.70 \\
\hline 1 & 1.5 & 0.5 & 1 & 1 & 1.1 & 1 & 1 & 0.5 & 25.90 \\
\hline 1 & 1.1 & 0.5 & 1.1 & 0.5 & 1 & 1 & 0.6 & 1 & 22.10 \\
\hline 1 & 1.1 & 1.5 & 0.5 & 1 & 1 & 1.1 & 1 & 0.5 & 23.10 \\
\hline 1 & 1.6 & 1 & 1 & 1.6 & 1.5 & 1 & 0.6 & 1 & 35.50 \\
\hline 2.1 & 1.6 & 1.5 & 1.5 & 1.5 & 1.6 & 1.5 & 1.1 & 1.5 & 43.80 \\
\hline 3.1 & 1.6 & 2.6 & 1.5 & 5.2 & 3.6 & 2.6 & 2.6 & 3.1 & 70.70 \\
\hline 1.6 & 1.5 & 1 & 4.2 & 3.1 & 2.1 & 1.5 & 1.6 & 1 & 63.80 \\
\hline 1.5 & 1.6 & 1.5 & 0.5 & 0.5 & 1 & 1 & 1.1 & 1 & 35.60 \\
\hline 0.5 & 1 & 1 & 1 & 1 & 1.5 & 1.1 & 0.5 & 1 & 24.00 \\
\hline 1.5 & 1 & 1.5 & 1.1 & 1.5 & 1 & 2.1 & 1.5 & 1.5 & 29.20 \\
\hline 1 & 1 & 1 & 1.1 & 0.5 & 1 & 0.6 & 1 & 1 & 32.50 \\
\hline
\end{tabular}




\begin{tabular}{|c|c|c|c|c|c|c|c|c|c|}
\hline 1.6 & 1 & 1 & 1 & 0.6 & 1 & 0.5 & 1.1 & 0.5 & 30.40 \\
\hline 1.1 & 1 & 1 & 1.1 & 1 & 0.5 & 1 & 1 & 0.6 & 22.70 \\
\hline 1 & 1 & 1 & 0.5 & 1.1 & 1 & 0.5 & 0.6 & 1 & 22.10 \\
\hline 1 & 1 & 1 & 0.5 & 1 & 0.5 & 1.1 & 0.5 & 1 & 20.00 \\
\hline 0.5 & 1 & 0.5 & 1 & 1 & 0.6 & 1 & 0.5 & 1.6 & 19.00 \\
\hline 0.5 & 1 & 0.5 & 0.6 & 1 & 1 & 0.6 & 1 & 0.5 & 18.10 \\
\hline 1 & 1 & 1 & 0.5 & 0.5 & 1.1 & 0.5 & 0.5 & 1.1 & 20.50 \\
\hline 1 & 0.5 & 1 & 1 & 0.5 & 1.1 & 0.5 & 1 & 1 & 20.30 \\
\hline 0.5 & 0.5 & & 0.5 & 0.1 & 0.5 & & 0.1 & & 15.20 \\
\hline 1 & & 0.6 & & & 0.5 & & 0.1 & 0.5 & 7.10 \\
\hline 0.5 & & 0.5 & 0.1 & & 0.5 & 0.1 & & & 12.20 \\
\hline & 0.5 & & & 0.1 & 0.5 & & 0.5 & 0.1 & 5.60 \\
\hline & 0.5 & & & 0.1 & 0.5 & & 0.1 & 0.5 & 4.60 \\
\hline 0.1 & 0.5 & 0.5 & & & 0.1 & 0.5 & & & 5.10 \\
\hline 2.6 & 2.6 & 2.1 & 2.1 & 2.1 & 1.5 & 1 & 1.6 & 1 & 38.70 \\
\hline 2.1 & 2.1 & 2.1 & 1.6 & 1.5 & 1.1 & 1.5 & 1 & & 51.20 \\
\hline 1 & 1 & 1 & 0.5 & 1.1 & 0.5 & 1 & 0.5 & 1 & 28.90 \\
\hline 1.5 & 0.5 & 1 & 0.5 & 1 & 1.1 & 0.5 & 0.5 & 1.1 & 27.30 \\
\hline 0.5 & 1 & 1 & 1 & 1 & 0.5 & 1 & 0.6 & 1 & 18.90 \\
\hline 1 & 0.5 & 1 & 0.5 & 1.1 & 0.5 & 1.5 & 0.6 & 1 & 19.00 \\
\hline 1 & 1 & 1.1 & 1 & 1 & 0.6 & 0.5 & 1 & 1 & 21.10 \\
\hline 1.1 & 1 & 1 & 0.6 & 1 & 0.5 & 1 & 0.6 & 1 & 20.20 \\
\hline 1 & 1 & 0.5 & 1 & 1.1 & 0.5 & 1 & 0.5 & 1.1 & 20.10 \\
\hline 1 & 1 & 1 & 1 & 0.5 & 0.5 & 0.5 & 1 & 1.1 & 19.40 \\
\hline 1 & 1 & 1 & 0.5 & 1 & 0.5 & 1 & 0.5 & 1.1 & 19.40 \\
\hline 1 & 0.5 & 1 & 1 & 1.1 & 0.5 & 1 & 0.5 & 1.1 & 18.50 \\
\hline 0.5 & 0.5 & 1 & 0.5 & 1 & 0.6 & 1.5 & 1 & 1 & 18.90 \\
\hline 0.5 & 0.5 & 1 & 1 & 0.5 & 1 & 0.5 & 1.1 & 0.5 & 18.50 \\
\hline 2.6 & 2.6 & 2.1 & 2.6 & 1.5 & 1.5 & 1.5 & 1 & 1 & 36.10 \\
\hline 3.1 & 1.5 & 2.1 & 1.5 & 2.1 & 0.6 & 1.5 & 1 & 1.1 & 45.20 \\
\hline 2.1 & 1.6 & 1.5 & 1 & 1 & 1.1 & 1 & 1 & 1 & 43.60 \\
\hline 0.5 & 0.5 & 1 & 1 & 0.6 & 1 & 0.6 & 1 & 0.5 & 25.10 \\
\hline 0.5 & 0.5 & 1 & 0.5 & 1.1 & 0.5 & 0.5 & 1 & 0.5 & 17.90 \\
\hline 1 & 0.5 & 0.5 & 1 & 0.5 & 1 & 0.6 & 1 & 0.6 & 18.00 \\
\hline 1 & 1 & 0.5 & 1.1 & 1 & 0.6 & 0.5 & 1 & 0.5 & 18.60 \\
\hline 0.5 & 1 & 0.6 & 0.5 & 1 & 1.1 & 1 & 0.5 & 0.6 & 17.40 \\
\hline 0.5 & 1 & 0.5 & 0.5 & 1 & 0.5 & 0.5 & 1.1 & 0.5 & 17.90 \\
\hline 1 & 1 & 0.5 & 0.5 & 1.1 & 0.5 & 0.6 & 1 & 0.5 & 16.60 \\
\hline 1 & 0.5 & 0.5 & 1.1 & 0.5 & 0.5 & 1.1 & 0.5 & 1 & 18.10 \\
\hline 1 & 0.5 & 1 & 0.6 & 0.5 & 0.6 & 1 & 0.5 & 0.6 & 16.70 \\
\hline 1 & 0.5 & 1 & 0.6 & 0.5 & 1 & 0.6 & 0.5 & 1 & 17.10 \\
\hline 1 & 1 & 0.5 & 1 & 0.5 & 1 & 0.6 & 1 & 0.6 & 29.50 \\
\hline 1.5 & 1 & 1 & 0.5 & 1 & 0.6 & 0.5 & 1 & 0.6 & 22.80 \\
\hline 1 & 1 & 1 & 0.5 & 1 & 0.6 & 1 & 0.5 & 1 & 18.20 \\
\hline 1 & 0.5 & 1 & 1 & 0.5 & 0.5 & 1 & 0.6 & 0.5 & 17.30 \\
\hline 1 & 1 & 1.1 & 1 & 0.5 & 1 & 0.6 & 1 & 0.6 & 19.00 \\
\hline 1 & 1 & 0.6 & 1 & 0.6 & 1 & 0.5 & 1.1 & 0.5 & 18.30 \\
\hline 0.5 & 1 & 0.5 & 0.5 & 0.5 & 1.1 & 0.5 & 0.6 & 1 & 17.10 \\
\hline 1.5 & 0.5 & 1 & 1 & 0.6 & 1 & 0.6 & 0.5 & 1 & 19.60 \\
\hline 1 & 1 & 1.5 & 0.6 & 1 & 0.5 & 1.1 & 1 & 0.5 & 19.50 \\
\hline 1.5 & 1.5 & 0.5 & 1 & 1 & 0.6 & 0.5 & 1 & 0.6 & 21.50 \\
\hline
\end{tabular}




\begin{tabular}{|c|c|c|c|c|c|c|c|c|c|}
\hline 5.2 & 2.6 & 1.5 & 1 & 1.1 & 1 & 1 & 0.6 & 0.5 & 31.10 \\
\hline 1 & 1 & 0.6 & 1 & 0.6 & 1 & 1 & 0.5 & 0.5 & 29.00 \\
\hline 1 & 0.5 & 0.6 & 0.5 & 1 & 0.6 & 0.5 & 1 & 0.6 & 17.60 \\
\hline 1.1 & 0.5 & 1 & 0.6 & 0.5 & 1.1 & 0.5 & 1 & 0.5 & 18.50 \\
\hline 1 & 1 & 0.5 & 1 & 0.5 & 1 & 0.5 & 0.5 & 1.1 & 18.90 \\
\hline 0.5 & & 0.5 & 0.5 & 1 & 1 & 0.5 & 0.6 & 1 & 16.70 \\
\hline 1 & 1 & 0.5 & 1 & 1 & 0.5 & 0.5 & 1.1 & 0.5 & 18.00 \\
\hline 1 & 1 & 0.5 & 1 & 0.5 & 1 & 1.1 & 0.5 & 1 & 18.80 \\
\hline 1 & 0.5 & 1 & 1 & 0.5 & 0.5 & 1 & 0.6 & 1 & 18.00 \\
\hline 1 & 0.5 & 0.5 & 1 & 0.5 & 0.5 & 1 & 0.5 & 0.5 & 16.40 \\
\hline 0.5 & 0.5 & 0.5 & 1 & 0.5 & 1 & 0.5 & 0.5 & 0.5 & 16.30 \\
\hline 1 & 1 & 0.5 & 0.5 & 0.5 & 0.5 & 1 & 0.5 & 0.5 & 16.80 \\
\hline \multirow[t]{2}{*}{0.5} & & & 0.5 & & & & & & 8.30 \\
\hline & & 0.5 & & 0.5 & & & & 0.1 & 3.40 \\
\hline \multirow[t]{2}{*}{0.5} & & & 0.5 & & & & & & 2.80 \\
\hline & 0.5 & 0.1 & & & 0.1 & & 0.5 & & 2.50 \\
\hline 0.5 & & & & & & & 0.1 & & 2.00 \\
\hline 0.5 & & & & & & 0.1 & 0.5 & & 2.40 \\
\hline \multirow[t]{2}{*}{0.5} & & & & & & & 0.5 & 0.1 & 3.10 \\
\hline & 0.5 & 0.5 & & & & & 0.1 & 0.5 & 3.40 \\
\hline 0.5 & & 0.5 & & & & & 0.1 & 0.5 & 3.90 \\
\hline 0.5 & & & & & 0.5 & & & & 2.80 \\
\hline \multirow[t]{3}{*}{0.5} & & & & & 0.5 & & & & 2.50 \\
\hline & 0.1 & & & & 0.6 & & & 0.1 & 2.50 \\
\hline & 0.5 & & & & & 0.1 & 0.5 & & 2.40 \\
\hline 0.5 & & & & & & & & 0.5 & 2.30 \\
\hline \multirow[t]{2}{*}{0.5} & 0.5 & & 0.5 & & & & & & 2.00 \\
\hline & & 0.5 & & & & & & & 2.20 \\
\hline \multirow[t]{3}{*}{0.5} & & 0.5 & & & & & 0.1 & & 3.20 \\
\hline & & & 0.5 & & & & & & 2.80 \\
\hline & & & & & 0.5 & & & & 2.30 \\
\hline \multirow[t]{4}{*}{0.5} & & & & & & 0.1 & & & 1.80 \\
\hline & & 0.5 & & & 0.5 & & & 0.5 & 3.70 \\
\hline & & 0.5 & & 0.5 & & & & & 2.70 \\
\hline & & & 0.5 & & 0.5 & & 0.1 & & 3.20 \\
\hline \multirow[t]{3}{*}{0.5} & & & & & & 0.5 & & & 2.50 \\
\hline & 0.5 & & & 0.5 & & & 0.1 & & 2.60 \\
\hline & & & & & 0.5 & 0.1 & & & 2.90 \\
\hline \multirow[t]{5}{*}{0.5} & & & & & & & 0.5 & & 2.40 \\
\hline & 0.5 & & & 0.5 & & & 0.5 & & 3.00 \\
\hline & & 0.5 & & 0.5 & & & 0.5 & & 3.50 \\
\hline & 0.5 & & & & & 0.5 & & & 2.50 \\
\hline & & & 0.5 & 0.5 & & & & & 3.50 \\
\hline \multirow[t]{8}{*}{0.5} & & & & & & 0.6 & & & 2.30 \\
\hline & & & 0.5 & & & & & & 1.60 \\
\hline & 0.5 & & & & 0.1 & & & & 1.90 \\
\hline & & & & 0.5 & & 0.5 & & & 3.20 \\
\hline & & & & 0.5 & 0.5 & & & & 3.00 \\
\hline & 0.5 & & & & 0.5 & & & & 2.50 \\
\hline & 0.5 & & & 0.5 & & & & 0.5 & 3.10 \\
\hline & & & & 0.5 & & & 0.5 & & 2.70 \\
\hline
\end{tabular}




\begin{tabular}{|c|c|c|c|c|c|c|c|c|c|}
\hline & & & & & & & & & 1.10 \\
\hline & & 0.5 & & & & & & & 1.70 \\
\hline & & & 0.5 & & & & & & 2.10 \\
\hline & & & & & & & & 0.5 & 2.00 \\
\hline \multirow[t]{4}{*}{0.5} & & & & & & & & & 1.50 \\
\hline & & & & 0.5 & 0.1 & & & 0.1 & 2.30 \\
\hline & & & & & & 0.5 & 0.1 & & 2.50 \\
\hline & & & & & & & & 0.1 & 1.50 \\
\hline \multirow[t]{2}{*}{0.1} & & 0.5 & & & & & & & 2.00 \\
\hline & & & & & & 0.5 & & & 2.20 \\
\hline \multirow[t]{34}{*}{0.5} & & & & & & & & & 1.70 \\
\hline & & & & 0.5 & & & & & 1.80 \\
\hline & & & & & 0.5 & & & & 1.50 \\
\hline & & & & & & & & & 1.00 \\
\hline & & & & & 0.5 & & & & 1.50 \\
\hline & & & & & & 0.5 & & & 1.90 \\
\hline & & & & & & & & 0.5 & 1.70 \\
\hline & & 0.5 & & & & & & & 1.00 \\
\hline & & & & 0.5 & & & & & 1.60 \\
\hline & & & & & & & & & 1.00 \\
\hline & & & & & 0.5 & & & & 1.50 \\
\hline & & & & & & & 0.5 & & 1.50 \\
\hline & & 0.5 & & & & & & & 1.00 \\
\hline & & & & & 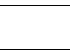 & . & & & 1.00 \\
\hline & & & 0.5 & & & & & 0.5 & 2.00 \\
\hline & & & & & & 0.5 & & & 1.00 \\
\hline & & & & & & & & & 1.00 \\
\hline & & & & 0.5 & & & & & 1.00 \\
\hline & & & & & & & & 0.5 & 1.50 \\
\hline & & & 0.5 & & & & & & 1.00 \\
\hline & & & & & & & & & 1.00 \\
\hline & & & & & & 0.5 & & & 1.50 \\
\hline & 0.5 & & & & & & & & 1.00 \\
\hline & & & 0.5 & & & & & & 1.00 \\
\hline & & & & & & & & & 1.00 \\
\hline & & & & & & & 0.5 & & 1.50 \\
\hline & & 0.5 & & & & & & & 1.00 \\
\hline & & & & & & 0.5 & & & 1.50 \\
\hline & & & & & & & & & 1.00 \\
\hline & & & 0.5 & & & & & & 1.00 \\
\hline & & 0.5 & & & & & & 0.5 & 1.50 \\
\hline & & 0.5 & & & & & & & 1.00 \\
\hline & 0.5 & & & & & & & & 1.00 \\
\hline & & & & & 0.5 & & & & 1.50 \\
\hline \multirow[t]{7}{*}{0.5} & & & & & & & & 0.5 & 1.50 \\
\hline & & 0.5 & & & & & & & 1.00 \\
\hline & & & & & 0.5 & & & & 1.50 \\
\hline & & & & 0.5 & & & & & 1.50 \\
\hline & & & & & & & 0.5 & & 1.50 \\
\hline & & 0.5 & & & & & & & 1.00 \\
\hline & & & & & 0.5 & & & & 1.50 \\
\hline
\end{tabular}




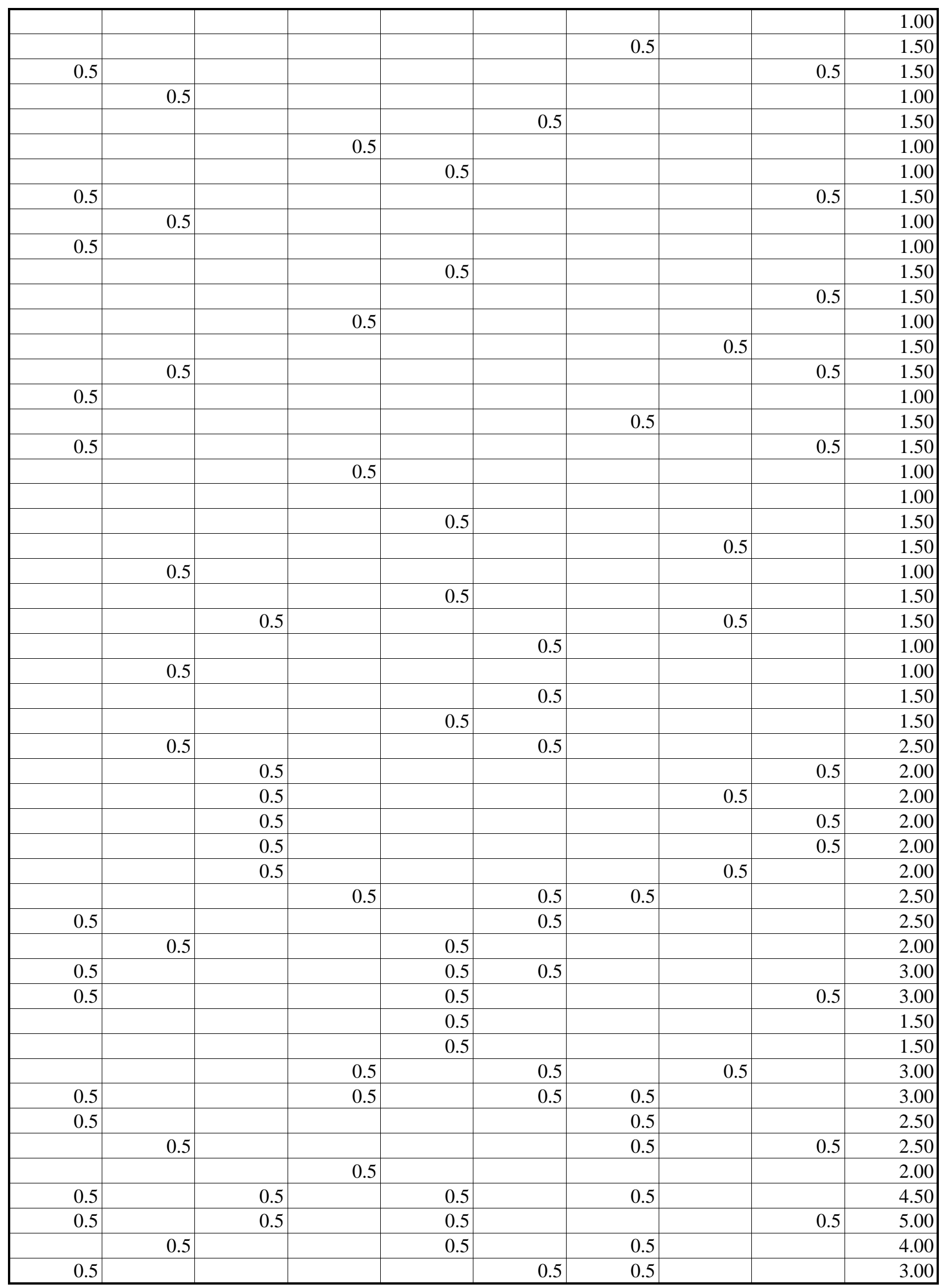




\begin{tabular}{|c|c|c|c|c|c|c|c|c|c|}
\hline & & & & 0.5 & & & 0.5 & & 2.50 \\
\hline \multirow[t]{3}{*}{0.5} & & & & & & & & & 1.50 \\
\hline & & & 0.5 & & & & & 0.5 & 2.50 \\
\hline & & & & & & 0.5 & & & 1.50 \\
\hline \multirow[t]{3}{*}{0.5} & & & & & & & & & 1.00 \\
\hline & & & & & & 0.5 & & & 2.50 \\
\hline & & & 0.5 & & 0.5 & & & & 2.50 \\
\hline \multirow[t]{7}{*}{0.5} & & & & & 0.5 & & & 0.5 & 3.00 \\
\hline & & & & 0.5 & 0.5 & & & & 2.50 \\
\hline & 0.5 & & & & & & & & 2.00 \\
\hline & & 0.5 & & & & & & & 1.00 \\
\hline & & & & & 0.5 & & & & 1.50 \\
\hline & & & & & 0.5 & 0.5 & & & 2.50 \\
\hline & 0.5 & & & 0.5 & & 0.5 & & & 3.00 \\
\hline 0.5 & & & & 0.5 & & & & & 2.00 \\
\hline \multirow[t]{6}{*}{0.5} & & & & & & & & 0.5 & 2.00 \\
\hline & 0.5 & & & & & & & 0.5 & 2.50 \\
\hline & 0.5 & & & & & & & & 1.50 \\
\hline & & & & 0.5 & & & & & 1.00 \\
\hline & & & 0.5 & & 0.5 & & 0.5 & & 3.00 \\
\hline & & & & 0.5 & 0.5 & & & & 3.50 \\
\hline \multirow[t]{2}{*}{0.5} & & & & 0.5 & 0.5 & & & & 3.00 \\
\hline & & & & & & 0.5 & & & 2.50 \\
\hline \multirow[t]{4}{*}{0.5} & & & & & & & & 0.5 & 2.50 \\
\hline & & & & 0.5 & & & & & 1.50 \\
\hline & & & & 0.5 & & & & & 1.50 \\
\hline & & 0.5 & & & 0.5 & & & & 2.50 \\
\hline 0.5 & & & & 0.5 & & & & 0.5 & 3.00 \\
\hline \multirow[t]{5}{*}{0.5} & & & & 0.5 & & & & & 2.50 \\
\hline & & 0.5 & & & & & & & 2.00 \\
\hline & & 0.5 & & & & & 0.5 & & 3.00 \\
\hline & & 0.5 & & & & & & & 2.00 \\
\hline & & & 0.5 & & & & & & 1.00 \\
\hline \multirow[t]{3}{*}{0.5} & & & & & 1 & & & & 3.00 \\
\hline & & & & 0.5 & & & & 0.5 & 2.50 \\
\hline & & 0.5 & & 0.5 & & & & & 2.50 \\
\hline 0.5 & 1 & 0.5 & 1 & 1 & 1 & 0.5 & 1.5 & 0.5 & 16.60 \\
\hline 0.5 & 1 & 0.5 & 1 & 0.5 & 1 & 0.5 & 0.5 & 1 & 16.50 \\
\hline 1 & 0.5 & 0.5 & 0.5 & 1 & 0.5 & 0.5 & 1 & 0.5 & 15.00 \\
\hline \multirow[t]{4}{*}{0.5} & 0.5 & 1 & 0.5 & 0.5 & 0.5 & 1 & 0.5 & 0.5 & 15.00 \\
\hline & & & & & & 0.5 & & & 8.00 \\
\hline & 0.5 & & & & 0.5 & & & & 2.50 \\
\hline & & 0.5 & & & 0.5 & & & & 3.00 \\
\hline 0.5 & & & 0.5 & & & & 0.5 & 0.5 & 3.50 \\
\hline \multirow[t]{2}{*}{0.5} & & & & & & & 0.5 & & 2.00 \\
\hline & & 0.5 & & 0.5 & & & & & 2.00 \\
\hline \multirow[t]{2}{*}{0.5} & & 0.5 & & & 0.5 & & 0.5 & & 3.50 \\
\hline & 0.5 & 0.5 & & 0.5 & 0.5 & & 0.5 & & 6.00 \\
\hline \multirow[t]{3}{*}{0.5} & & 0.5 & & 0.5 & 0.5 & 0.5 & & & 6.00 \\
\hline & 0.5 & 0.5 & & 0.5 & 0.5 & & 0.5 & & 6.50 \\
\hline & 0.5 & & 0.5 & & 0.5 & & 0.5 & 0.5 & 6.50 \\
\hline
\end{tabular}


wbheat

\begin{tabular}{|c|c|c|c|c|c|c|c|c|c|}
\hline & 0.5 & 0.5 & 0.5 & 0.5 & & & 0.5 & 0.5 & 7.00 \\
\hline & 0.5 & & & 0.5 & 0.5 & & & 0.5 & 5.50 \\
\hline & & 0.5 & 0.5 & & & 0.5 & & 0.5 & 5.50 \\
\hline 0.5 & & 0.5 & 0.5 & 0.5 & 0.5 & & 0.5 & & 6.50 \\
\hline \multirow[t]{6}{*}{0.5} & & & & 0.5 & 0.5 & 0.5 & & 0.5 & 6.50 \\
\hline & 0.5 & & & 0.5 & & & 0.5 & & 5.00 \\
\hline & 0.5 & & & & & & 0.5 & & 3.00 \\
\hline & 0.5 & & & & & 0.5 & & 0.5 & 3.50 \\
\hline & & 0.5 & & & & & & & 2.00 \\
\hline & 0.5 & & & & & & & 0.5 & 2.00 \\
\hline \multirow[t]{2}{*}{0.5} & & & & & 0.5 & & & & 3.50 \\
\hline & 2.1 & 1 & 1.5 & 1 & 0.5 & 1 & 1 & 1 & 11.60 \\
\hline 2.1 & 1.5 & 1 & 1.5 & 1.5 & 1 & 1 & 1 & 0.5 & 26.80 \\
\hline 2.1 & 1 & 1.5 & 1 & 1.5 & 0.5 & & 0.5 & & 35.70 \\
\hline 3.1 & 2.6 & 2.1 & 1.5 & 1.5 & 0.5 & 1 & 1 & 1.5 & 36.90 \\
\hline 1 & 1 & 0.5 & 1 & 0.5 & 1.5 & 0.5 & 1 & 0.5 & 19.00 \\
\hline 0.5 & 1 & 1 & 0.5 & 1 & 0.5 & 0.5 & 1 & 0.5 & 17.00 \\
\hline 1 & 0.5 & 1 & 1 & 1 & 1 & 0.5 & 1 & 0.5 & 19.00 \\
\hline 0.5 & 1 & 0.5 & 1 & 1 & 0.5 & 1 & 1 & 0.5 & 17.50 \\
\hline 1 & 0.5 & 1 & 0.5 & 1 & 0.5 & 1 & 0.5 & 0.5 & 16.50 \\
\hline 0.5 & 1 & 1 & 0.5 & 1 & 0.5 & 1 & 0.5 & 0.5 & 18.00 \\
\hline 1 & 0.5 & 1 & 0.5 & 1 & 1 & 0.5 & 1 & 0.5 & 19.00 \\
\hline 0.5 & 1 & 1 & 0.5 & 1 & 1 & 0.5 & 1 & 1 & 18.00 \\
\hline 1 & 1 & 0.5 & 1 & 1 & 1 & 0.5 & 1 & 1 & 20.50 \\
\hline 1 & 0.5 & 1 & 1 & 0.5 & 1 & 1 & 1 & 0.5 & 21.00 \\
\hline 0.5 & 1 & 0.5 & 1 & 1 & 1 & 0.5 & 0.5 & 1 & 18.50 \\
\hline 0.5 & 1 & 1 & 0.5 & 1 & 0.5 & 1 & 0.5 & 1 & 17.50 \\
\hline 1 & 1 & & 1 & 1 & 0.5 & 0.5 & 1 & 0.5 & 16.50 \\
\hline 0.5 & 1 & 0.5 & 0.5 & 1 & 1 & 0.5 & 1 & 0.5 & 17.50 \\
\hline 1 & 0.5 & 0.5 & 1 & 0.5 & 0.5 & 1 & 0.5 & 1 & 16.50 \\
\hline 0.5 & 1 & 0.5 & 0.5 & 1 & 0.5 & 0.5 & 1 & 1 & 16.50 \\
\hline 2.1 & 1.5 & 1 & 0.5 & 1.5 & 0.5 & 1 & 0.5 & 1 & 31.70 \\
\hline 1 & 2.1 & 1 & 1 & 1 & 1 & 1 & 1 & 0.5 & 28.40 \\
\hline 1.5 & 1.5 & 1.5 & 2.1 & 1.5 & 2.1 & 1.5 & 1.5 & 2.1 & 41.60 \\
\hline 2.6 & 3.1 & 3.1 & 2.1 & 2.1 & 2.6 & 2.1 & 2.1 & 2.6 & 59.80 \\
\hline 2.1 & 2.1 & 1 & 1.5 & 1.5 & 1.5 & 2.1 & 1.5 & 2.1 & 45.00 \\
\hline 2.1 & 1.5 & 1 & 1.5 & 1.5 & 1.5 & 1 & 1 & 2.1 & 41.40 \\
\hline 1.5 & 1 & 1.5 & 1.5 & 1 & 1 & 1.5 & 1.5 & 1 & 30.50 \\
\hline 3.5 & 2.9 & 3.5 & 2.9 & 3.5 & 2.3 & 2.8 & 2.2 & 2.2 & 62.70 \\
\hline 1.5 & 1 & 1.6 & 1.6 & 1 & 1.5 & 1 & 1.6 & 1.5 & 42.10 \\
\hline 2.1 & 2.1 & 2.1 & 1.5 & 2.1 & 1.5 & 1.5 & 1.5 & 1.5 & 43.70 \\
\hline 1.5 & 1.6 & 1.5 & 1 & 1 & 1.5 & 1 & 1.5 & 1 & 36.20 \\
\hline 1 & 1 & 0.5 & 1 & 0.5 & 1 & 1 & 1 & 0.5 & 21.00 \\
\hline 1.5 & 1.5 & 1 & 1.5 & 2.1 & 1.5 & 1.5 & 1.5 & 1 & 28.10 \\
\hline 1 & 0.5 & 1 & 1 & 1 & 1 & 1 & 1 & 1 & 23.00 \\
\hline 2.6 & 2.1 & 1.5 & 2.1 & 2.1 & 1.5 & 2.1 & 2.1 & 1.5 & 54.00 \\
\hline 2.6 & 2.1 & 1.5 & 1.5 & 1 & 2.1 & 1.5 & 1.5 & 2.1 & 50.40 \\
\hline 1 & 0.5 & 1 & 1 & 0.5 & 1 & 0.5 & 1 & 0.5 & 26.70 \\
\hline 0.5 & 1 & 1 & 0.5 & 0.5 & 1 & 0.5 & 0.5 & 1.5 & 23.80 \\
\hline 1 & 1 & 0.5 & 1 & 0.5 & 0.5 & 1 & 1 & 0.5 & 30.00 \\
\hline 1 & 0.5 & 0.5 & 1 & 0.5 & 0.5 & 1 & 0.5 & 0.5 & 18.00 \\
\hline
\end{tabular}




\begin{tabular}{|c|c|c|c|c|c|c|c|c|c|}
\hline 0.5 & 0.5 & 1 & 0.5 & 0.5 & 0.5 & 1 & 0.5 & 0.5 & 15.00 \\
\hline 2.6 & 2.6 & 2.1 & 1.5 & 1.5 & 1.5 & 2.1 & 1 & 1.5 & 36.30 \\
\hline 2.1 & 1.5 & 1.5 & 1.5 & 1 & 1.5 & 1.5 & 1.1 & 1 & 51.90 \\
\hline 2.1 & 2.1 & 1.5 & 1.5 & 0.5 & 1 & 0.5 & 1 & 0.5 & 43.20 \\
\hline 1.5 & 2.1 & 1.5 & 1 & 1.5 & 1 & 1 & 1 & 1 & 42.00 \\
\hline 1.5 & 2.1 & 1.1 & 0.5 & 1 & 1 & 1 & 0.5 & 1 & 37.60 \\
\hline 0.5 & 0.5 & 1 & 0.5 & 0.5 & 1 & 0.5 & 0.5 & 0.5 & 17.60 \\
\hline 1 & 0.5 & 1 & 1 & 1 & 0.5 & 1 & 0.5 & 1 & 20.10 \\
\hline 1 & 0.5 & 1 & 0.5 & 1 & 1.1 & 1 & 1 & 1 & 35.60 \\
\hline 1 & 0.5 & 0.5 & 1 & 0.5 & 1 & 0.5 & 1 & 1 & 23.30 \\
\hline 0.5 & 0.5 & 1 & 0.5 & 0.5 & 1 & 0.5 & 0.5 & 1 & 19.60 \\
\hline 1 & 0.5 & 1 & 1 & 0.5 & 0.6 & 1 & 1 & 1 & 23.30 \\
\hline 1 & 1 & 0.5 & 1 & 1 & 0.5 & 1 & 1.1 & 1 & 24.40 \\
\hline 0.5 & 0.5 & 1 & 1 & 0.5 & 0.5 & 1 & 0.5 & 1 & 20.60 \\
\hline 1 & 0.5 & 1 & 0.5 & 1 & 0.5 & 0.5 & 1 & 1 & 20.60 \\
\hline \multirow[t]{3}{*}{1} & 1 & 0.5 & 1 & 0.5 & 1 & 0.5 & 1 & 1 & 26.40 \\
\hline & 0.5 & & & & 0.5 & & & & 11.10 \\
\hline & 0.5 & & & & & & 0.5 & & 5.10 \\
\hline 1 & 1.6 & 1.5 & 1 & 2.6 & 2.1 & 2.1 & 2.6 & 2.1 & 37.60 \\
\hline 1 & 0.5 & 1 & 1 & 0.5 & 1 & 1 & 1 & 1 & 34.00 \\
\hline 0.5 & 1 & 0.5 & 1 & 0.5 & 0.5 & 1 & 0.5 & 1 & 18.60 \\
\hline 1.5 & 1 & 0.5 & 1 & 0.5 & 1 & 0.5 & 0.5 & 1 & 18.60 \\
\hline 1.5 & 2.1 & 1.5 & 1.5 & 1 & 1 & 1 & 1 & 0.5 & 42.50 \\
\hline 1.5 & 1 & 1 & 1 & 1 & 0.5 & 1 & 1 & 1 & 40.20 \\
\hline 2.1 & 2.1 & 1 & 1.5 & 1.5 & 1 & 0.5 & 0.5 & 1 & 42.80 \\
\hline 1 & 0.5 & 1.1 & 1 & 0.5 & 0.5 & 1 & 0.5 & 0.5 & 31.80 \\
\hline 0.5 & 1 & 0.5 & 0.5 & 1 & 0.5 & 1 & 0.5 & 1 & 16.00 \\
\hline 1 & 1 & 0.5 & 1 & 1 & 1 & 0.5 & 1 & 0.5 & 20.50 \\
\hline 3.7 & 2.7 & 2.1 & 2.6 & 2.1 & 1.5 & 1 & 1 & 1.5 & 64.50 \\
\hline 1.5 & 1 & 0.5 & 1 & 0.5 & 1 & 1 & 0.5 & 1 & 49.50 \\
\hline 2.6 & 2.1 & 1.5 & 1 & 1.5 & 1.5 & 1 & 1.5 & 1.5 & 45.70 \\
\hline 2.6 & 1.5 & 1 & 1.5 & 2.1 & 1.5 & 1.5 & 2.1 & 1.5 & 60.50 \\
\hline 2.6 & 2.1 & 1.5 & 1.5 & 1 & 1.5 & 1.5 & 1.5 & 1 & 52.10 \\
\hline \multirow[t]{2}{*}{1} & & & & 0.5 & & 0.5 & & & 15.00 \\
\hline & 2.1 & 1.5 & 0.5 & 0.5 & 1 & 0.5 & 1 & 0.5 & 9.10 \\
\hline 1 & 0.5 & 1 & 0.5 & 0.5 & 1 & 1 & 0.5 & 0.5 & 24.20 \\
\hline 2.6 & 1.6 & 1 & 2.1 & 1.5 & 2.1 & 1.5 & 1.5 & 1.5 & 44.30 \\
\hline 2.6 & 1.5 & 1.1 & 1.1 & 1.1 & 1.6 & 1 & 1.5 & 1.1 & 56.10 \\
\hline 1.5 & 0.5 & 1 & 0.5 & 0.5 & 1 & 0.5 & 0.5 & 1 & 36.60 \\
\hline \multirow[t]{3}{*}{0.5} & & & & 0.5 & & & & 0.5 & 23.60 \\
\hline & & & 0.5 & & & & & 0.5 & 2.00 \\
\hline & 0.5 & & & 0.5 & & & 0.5 & & 3.00 \\
\hline 2.6 & 2.1 & 1.5 & 1 & 2.1 & 2.6 & 2.1 & 2.1 & 2.1 & 57.90 \\
\hline 2.6 & 2.6 & 2.1 & 2.1 & 2.1 & 1.5 & 2.1 & 2.1 & 1.5 & 78.00 \\
\hline 2.6 & 1 & 2.1 & 1 & 1 & 1.5 & 1.5 & 1 & 1 & 60.80 \\
\hline 1.5 & 1 & 0.5 & 1 & 0.5 & 1 & 0.5 & 0.5 & 1 & 42.90 \\
\hline 2.1 & 1.5 & 1.5 & 1 & 1.5 & 1.5 & 1.5 & 1.5 & 1.5 & 43.50 \\
\hline 1 & 1 & 0.5 & 0.5 & 1 & 0.5 & 1 & 0.5 & 1 & 24.00 \\
\hline 0.5 & 0.5 & 1 & 1 & 0.5 & 0.5 & 1 & 0.5 & 0.5 & 17.00 \\
\hline 1.5 & 1 & 0.5 & 0.5 & 1 & 0.5 & 0.5 & 1 & 0.5 & 42.90 \\
\hline 2.1 & 1.5 & 1 & 1 & 1 & 1 & 1 & 1 & 1 & 39.60 \\
\hline
\end{tabular}




\begin{tabular}{|c|c|c|c|c|c|c|c|c|c|}
\hline 1.5 & 0.5 & 1 & 0.5 & 1 & 1.5 & 0.5 & 0.5 & 1 & 38.50 \\
\hline 1.6 & 1 & 1 & 1 & 1.5 & 1 & 0.5 & 1.5 & 0.5 & 39.00 \\
\hline 3.1 & 2.1 & 1.5 & 1.5 & 1.5 & 1.5 & 1 & 1 & 1 & 47.60 \\
\hline 1 & 1 & 1.5 & 1 & 1.5 & 1 & 1.5 & 1 & 1 & 26.60 \\
\hline 1 & 0.5 & 1 & 0.5 & 1 & 0.5 & 1 & 0.5 & 0.5 & 22.00 \\
\hline 1 & 1 & 0.5 & 1 & 1 & 1 & 1 & 1 & 1 & 40.30 \\
\hline 1 & 1 & 1 & 1 & 1 & 1 & 0.5 & 1 & 1 & 40.60 \\
\hline 0.5 & & & 0.5 & & & 0.5 & & & 25.70 \\
\hline 1 & 1 & 0.5 & 0.5 & 1 & 0.5 & 0.5 & 1 & 0.5 & 23.00 \\
\hline 1.5 & 1 & 1 & 0.5 & 1 & 0.5 & 0.5 & 1 & & 35.90 \\
\hline 0.5 & 0.5 & 1 & 0.5 & 0.5 & 1 & 0.5 & 1 & 0.5 & 17.50 \\
\hline & 1 & 0.5 & 1 & 0.5 & 0.5 & 0.5 & 1 & 0.5 & 15.00 \\
\hline 1 & 0.5 & 0.5 & 0.5 & 0.5 & 1 & 0.5 & 1 & 1 & 23.20 \\
\hline 2.1 & 2.1 & 1.5 & 2.1 & 1.5 & 1 & 1.5 & 1 & 1 & 44.60 \\
\hline 1.5 & 1 & 1 & 1 & 1 & 2.1 & 1.5 & 1.5 & 1.5 & 48.50 \\
\hline 2.6 & 2.6 & 1.5 & 2.1 & 1 & 1.5 & 1.5 & 1 & 0.5 & 62.90 \\
\hline 2.1 & 1 & 1 & 1 & 1 & 0.5 & 1 & 0.5 & 1 & 42.60 \\
\hline 1 & 0.5 & 0.5 & 1 & 1 & & 1 & 1 & 0.5 & 16.00 \\
\hline 0.5 & 1 & 0.5 & 0.5 & 0.5 & 1 & 0.5 & 0.5 & 1 & 16.00 \\
\hline 1 & 0.5 & 0.5 & 1 & 0.5 & 0.5 & 1 & 0.5 & 1 & 25.20 \\
\hline 1 & 0.5 & 0.5 & 1 & 0.5 & 1 & 0.5 & 1 & 0.5 & 19.50 \\
\hline 1 & 1 & 0.6 & 0.6 & 1.1 & 0.6 & 0.6 & 0.1 & 0.1 & 17.30 \\
\hline 1 & 1 & 1 & 0.5 & 1 & 0.5 & 0.5 & 0.5 & 1 & 14.30 \\
\hline 1.5 & 1 & 1 & 1 & 1 & 1 & 0.5 & 0.5 & 1 & 31.20 \\
\hline 1 & 1 & 1.5 & 1.5 & 2.1 & 2.6 & 1.5 & 1 & 1 & 28.70 \\
\hline 0.5 & 0.5 & 1.5 & 0.5 & 0.5 & 1 & 0.5 & 1 & 0.5 & 20.00 \\
\hline 1.1 & 1.6 & 2.1 & 0.5 & 1 & 0.5 & 0.5 & 1 & 1 & 25.00 \\
\hline 1 & 1 & 0.5 & 0.5 & 1 & 0.5 & 0.5 & 1 & 0.5 & 23.40 \\
\hline 1 & 0.5 & 0.5 & 1 & 0.5 & 0.5 & 1 & 0.5 & 0.5 & 21.10 \\
\hline 1 & 1 & 2.6 & 1 & 0.5 & 0.5 & 1 & 0.5 & 0.5 & 26.00 \\
\hline 2.6 & 1.5 & 1 & 0.5 & 1 & 0.5 & 1 & 0.5 & 0.5 & 34.20 \\
\hline 1 & 0.5 & 1 & 0.5 & 1 & 1 & 1 & 0.5 & 0.5 & 17.50 \\
\hline 0.5 & 1 & 0.5 & 1 & 0.5 & 0.5 & 1 & 0.5 & 0.5 & 16.00 \\
\hline 1 & 0.5 & 0.5 & 1 & 0.5 & 0.5 & 1 & 0.5 & 1 & 23.20 \\
\hline 1 & 0.5 & 1 & 0.5 & 0.5 & 1 & 0.5 & 0.5 & 1 & 17.00 \\
\hline 0.5 & 0.5 & 1 & 0.5 & 1 & 1 & 0.5 & 1 & 0.5 & 17.00 \\
\hline 1 & 0.5 & 0.5 & 1 & 0.5 & 1 & 0.5 & 1 & 0.5 & 17.50 \\
\hline 4.1 & 4.6 & 2.1 & 2.6 & 2.1 & 2.1 & 2.6 & 2.1 & 2.1 & 59.30 \\
\hline 1.5 & 0.5 & 1 & 0.5 & 1 & 0.5 & 0.5 & 1 & 0.5 & 36.70 \\
\hline 0.5 & 1 & 0.5 & 0.5 & 1 & 0.5 & 0.5 & 0.5 & 0.5 & 18.00 \\
\hline 3.1 & 2.6 & 1 & 1.5 & 0.5 & 1 & 1 & 0.5 & 1 & 55.40 \\
\hline 0.5 & 1 & 0.5 & 1 & 0.5 & 0.5 & 1 & 0.5 & 0.5 & 25.70 \\
\hline 1 & 0.5 & 1 & 1 & 0.5 & 1 & 0.5 & 0.5 & 1 & 19.10 \\
\hline 1 & 0.5 & 0.5 & 1 & 0.5 & 1 & 1 & 1 & 0.5 & 19.00 \\
\hline 0.5 & 1 & 0.5 & 1 & 0.5 & 0.5 & 1 & 0.5 & 1 & 18.00 \\
\hline 1 & 1 & 1 & 1 & 1 & 1 & 1 & 0.5 & 1 & 19.50 \\
\hline 0.5 & 1 & 0.5 & 0.5 & 1 & 0.5 & 1 & 0.5 & 1 & 19.50 \\
\hline 1 & 1 & 0.5 & 0.5 & 1 & 0.5 & 0.5 & 1 & 0.5 & 18.00 \\
\hline 1 & 0.5 & 1 & 0.5 & 1 & 0.5 & 0.5 & 1 & 0.5 & 23.20 \\
\hline 0.5 & 1 & 0.5 & 1 & 1 & 0.5 & 1 & 0.5 & 0.5 & 17.50 \\
\hline 0.5 & 1 & 0.5 & 0.5 & 0.5 & 1 & 0.5 & 0.5 & 1 & 16.00 \\
\hline
\end{tabular}




\begin{tabular}{|c|c|c|c|c|c|c|c|c|c|}
\hline 1 & 0.5 & 1 & 0.5 & 0.5 & 1 & 0.5 & 0.5 & 0.5 & 16.00 \\
\hline 0.5 & 1 & 0.5 & 0.5 & 1 & 0.5 & 0.5 & 1 & 0.5 & 15.00 \\
\hline 0.5 & 0.5 & 1 & 0.5 & 1 & 0.5 & 0.5 & 0.5 & 1 & 15.00 \\
\hline \multirow[t]{2}{*}{1} & 0.5 & 0.5 & 0.5 & 1 & 0.5 & 0.5 & 1 & 0.5 & 15.50 \\
\hline & & & & 0.5 & & & & & 7.00 \\
\hline \multirow[t]{7}{*}{0.5} & & & & 0.5 & & & & & 2.50 \\
\hline & & & & & 0.5 & & & & 3.00 \\
\hline & & 0.5 & & & 0.5 & & & & 3.00 \\
\hline & & & 0.5 & & & & & 0.5 & 2.50 \\
\hline & & & 0.5 & & & & & 0.5 & 2.00 \\
\hline & & & & & 0.5 & & & & 7.10 \\
\hline & 0.5 & & & & & 0.5 & & & 4.60 \\
\hline \multirow[t]{2}{*}{0.6} & 0.1 & 0.1 & 0.1 & & & & 0.5 & & 3.90 \\
\hline & 0.5 & & & & & & & & 3.30 \\
\hline \multirow[t]{6}{*}{0.5} & & & & & & & 0.6 & 0.1 & 3.70 \\
\hline & & & 0.5 & & & & & & 1.50 \\
\hline & & & 0.5 & & & & & & 1.50 \\
\hline & 0.5 & & & & & & & & 1.50 \\
\hline & 0.5 & & & & & & & 0.5 & 3.00 \\
\hline & & & & 0.5 & & & & & 2.00 \\
\hline 0.6 & & & & 0.5 & & & & & 3.10 \\
\hline \multirow[t]{3}{*}{0.5} & & 0.5 & & & 0.5 & & 0.5 & & 4.60 \\
\hline & & 0.5 & & & & 0.5 & & & 2.00 \\
\hline & & & & & & 0.5 & & & 2.00 \\
\hline \multirow[t]{2}{*}{0.5} & & & & & & & 0.5 & & 3.10 \\
\hline & 0.5 & & & & & & & 0.5 & 3.00 \\
\hline \multirow[t]{2}{*}{0.5} & & & & & 0.5 & & & & 5.00 \\
\hline & 0.5 & & & 0.5 & & & & & 4.10 \\
\hline \multirow[t]{3}{*}{0.5} & & & & 0.5 & & & & & 3.60 \\
\hline & 0.5 & & & & 0.5 & & & & 2.00 \\
\hline & & & 0.5 & & & & & & 2.00 \\
\hline \multirow[t]{6}{*}{0.5} & & & & & & 0.5 & & & 3.30 \\
\hline & & & 0.5 & & & 0.5 & & & 3.80 \\
\hline & & 0.5 & & 0.5 & & & & & 2.50 \\
\hline & 0.5 & & & & & 0.5 & & & 3.00 \\
\hline & 0.5 & & & 0.5 & & & & & 3.50 \\
\hline & & 0.5 & & 0.5 & & & & 0.5 & 3.00 \\
\hline \multirow[t]{2}{*}{0.5} & & & & & & & 0.5 & & 2.50 \\
\hline & 0.5 & & & 0.5 & 0.5 & & & & 4.00 \\
\hline \multirow[t]{2}{*}{0.5} & & & 0.5 & & 0.5 & & & & 3.50 \\
\hline & 0.5 & & & 0.5 & & & & & 3.00 \\
\hline \multirow[t]{4}{*}{0.5} & 0.5 & & & 0.5 & & 0.5 & & & 4.00 \\
\hline & 0.5 & & & 0.5 & 0.5 & & & & 3.50 \\
\hline & & & & 0.5 & & & & 0.5 & 2.50 \\
\hline & & & 0.5 & & & & 0.5 & & 2.00 \\
\hline \multirow[t]{4}{*}{1} & & & & 0.5 & & 0.5 & & & 4.00 \\
\hline & & & 0.5 & 0.5 & & & & & 3.50 \\
\hline & & & 0.5 & & & 0.5 & & & 3.50 \\
\hline & 0.5 & & & 0.5 & & & 0.5 & & 4.00 \\
\hline \multirow{2}{*}{0.5} & & & 0.5 & & 0.5 & & & & 3.50 \\
\hline & & & & 0.5 & & 0.5 & & & 2.00 \\
\hline
\end{tabular}


wbheat

\begin{tabular}{|c|c|c|c|c|c|c|c|c|c|}
\hline & 0.5 & & & & 0.5 & & & & 2.00 \\
\hline & 0.5 & & & 0.5 & & & & & 3.00 \\
\hline & 0.5 & & 0.5 & & & 0.5 & & & 3.50 \\
\hline & 0.5 & & & & 0.5 & & & & 3.00 \\
\hline \multirow[t]{3}{*}{0.5} & & 0.5 & & 0.5 & & & 0.5 & & 4.00 \\
\hline & & & & & 0.5 & 0.5 & & & 3.00 \\
\hline & & & & 0.5 & & & & & 2.50 \\
\hline \multirow[t]{5}{*}{0.5} & & & & & 0.5 & & & & 2.00 \\
\hline & & & & 0.5 & & & & & 2.50 \\
\hline & 0.5 & & & & & & & 0.5 & 2.50 \\
\hline & 0.5 & & & & & & 0.5 & & 2.50 \\
\hline & & & & 0.5 & & & & & 2.00 \\
\hline \multirow[t]{3}{*}{0.5} & & & & & & & & 0.5 & 2.50 \\
\hline & 0.5 & & & & & & 0.5 & & 2.00 \\
\hline & 0.5 & & & & & & & & 1.50 \\
\hline 0.5 & & & & & & & & 0.5 & 2.00 \\
\hline 0.5 & . & & & & & 0.5 & & & 2.00 \\
\hline \multirow[t]{5}{*}{0.5} & & & & & & & & & 1.50 \\
\hline & & & & 0.5 & & & & & 2.00 \\
\hline & & 0.5 & & & & & & & 1.50 \\
\hline & & & & & 0.5 & & & & 2.00 \\
\hline & & 0.5 & & & & & & & 1.50 \\
\hline 0.5 & & & & 0.5 & & & & & 2.00 \\
\hline 0.5 & & & & & 0.5 & & & & 2.00 \\
\hline 0.5 & & & & & 0.5 & & & & 2.00 \\
\hline 0.5 & & & & & & 0.5 & & & 2.80 \\
\hline \multirow[t]{4}{*}{0.5} & & & & & & 0.5 & & & 2.00 \\
\hline & & & & 0.5 & & & & & 2.00 \\
\hline & & & & & 0.5 & & & & 2.00 \\
\hline & & 0.5 & & & & & & & 2.00 \\
\hline \multirow[t]{6}{*}{0.5} & & & & & & 0.5 & & & 2.50 \\
\hline & & 0.5 & & & & & & & 1.50 \\
\hline & 0.5 & & & & & & & & 2.00 \\
\hline & & & & & & 0.5 & & & 2.00 \\
\hline & 0.5 & & & & 0.5 & & & & 2.00 \\
\hline & & & & & 0.5 & & & & 2.00 \\
\hline \multirow[t]{2}{*}{0.5} & & & & & 0.5 & & & & 2.00 \\
\hline & & 0.5 & & & & & & & 2.00 \\
\hline \multirow[t]{3}{*}{0.5} & & & & & 0.5 & & & & 2.50 \\
\hline & & & 0.5 & & & & & & 2.00 \\
\hline & & & & & 0.5 & & & & 2.00 \\
\hline \multirow{9}{*}{0.5} & & & & & & & 0.5 & & 2.00 \\
\hline & & 0.5 & & & & & & & 1.50 \\
\hline & & & 0.5 & & & & & & 2.00 \\
\hline & & & & 0.5 & & & & & 2.00 \\
\hline & & 0.5 & & & & & & 0.5 & 2.00 \\
\hline & & & 0.5 & & & & & & 1.50 \\
\hline & & & & & & & & & 2.00 \\
\hline & & & & 0.5 & & & & & 2.00 \\
\hline & & & 0.5 & & & & 0.5 & & 2.00 \\
\hline 0.5 & & & & & & & & 0.5 & 1.50 \\
\hline
\end{tabular}




\begin{tabular}{|c|c|c|c|c|c|c|c|c|c|}
\hline & & & & & & & & & 0.00 \\
\hline & & & & & & & & & 0.00 \\
\hline & & & & & & & & & 0.00 \\
\hline & & & & & & & & & 0.00 \\
\hline & & & & & & & & & 0.00 \\
\hline & & & & & & & & & 0.00 \\
\hline & & & & & & & & & 0.00 \\
\hline & & & & & & & & & 0.00 \\
\hline & & & & & & & & & 0.00 \\
\hline & & & & & & & & & 0.00 \\
\hline & & & & & & & & & 0.00 \\
\hline & & & & & & & & & 0.00 \\
\hline & & & & & & & & & 0.00 \\
\hline & & & & & & & & & 0.00 \\
\hline & & & & & & & & & 0.00 \\
\hline & & & & & & & & & 0.00 \\
\hline & & & & & & & & & 0.00 \\
\hline & & & & & & & & & 0.00 \\
\hline & & & & & & & & & 0.00 \\
\hline & & & & & & & & & 0.00 \\
\hline & & & & & & & & & 0.00 \\
\hline & & & & & & & & & 0.00 \\
\hline & & & & & & & & & 0.00 \\
\hline & & & & & & & & & 0.00 \\
\hline & & & & & & & & & 0.00 \\
\hline & & & & & & & & & 0.00 \\
\hline & & & & & & & & & 0.00 \\
\hline & & & & & & & & & 0.00 \\
\hline & & & & & & & & & 0.00 \\
\hline & & & & & & & & & 0.00 \\
\hline & & & & & & & & & 0.00 \\
\hline & & & & & & & & & 0.00 \\
\hline & & & & & & & & & 0.00 \\
\hline & & & & & & & & & 0.00 \\
\hline & & & & & & & & & 0.00 \\
\hline & & & & & & & & & 0.00 \\
\hline & & & & & & & & & 0.00 \\
\hline & & & & & & & & & 0.00 \\
\hline 0.5 & & & & & & & & 0.5 & 2.00 \\
\hline & 0.5 & & & & & & & & 1.50 \\
\hline & & & & 0.5 & & & & & 1.50 \\
\hline & & & & & 0.5 & & 0.5 & & 2.10 \\
\hline 0.5 & & & & & 0.5 & & & 0.5 & 3.00 \\
\hline & & & 0.5 & 0.5 & & & 0.5 & & 3.50 \\
\hline & 0.5 & & 0.5 & 0.5 & & & & & 3.00 \\
\hline 0.5 & & & 0.5 & 0.5 & & & & & 3.00 \\
\hline & 0.5 & & & & & & & & 2.50 \\
\hline 0.5 & & & & & & & & & 1.00 \\
\hline & & & 0.5 & 0.5 & & & & & 3.00 \\
\hline & & & 1 & & & 0.5 & & & 3.50 \\
\hline 0.5 & & 0.5 & & 0.5 & & & & 0.5 & 3.50 \\
\hline
\end{tabular}




\begin{tabular}{|c|c|c|c|c|c|c|c|c|c|}
\hline & & & 0.5 & & & & & & 1.50 \\
\hline & 0.5 & & & & & & & & 2.50 \\
\hline & & & 0.5 & & & & & & 1.50 \\
\hline 0.5 & & & & & & & & 0.5 & 1.50 \\
\hline 1 & 1.5 & & 0.5 & 0.5 & & 0.5 & & & 5.50 \\
\hline \multirow[t]{4}{*}{0.5} & & & 0.5 & 0.5 & & 0.5 & & & 3.50 \\
\hline & 0.5 & & 0.5 & & 0.5 & & & & 3.50 \\
\hline & & & 1 & & & & & 0.5 & 3.50 \\
\hline & & & & & 0.5 & & & & 2.50 \\
\hline \multirow[t]{8}{*}{0.5} & & & & 0.5 & & & & & 2.50 \\
\hline & & & 0.5 & & & & & & 1.50 \\
\hline & & & & & 0.5 & & & & 2.50 \\
\hline & & 0.5 & & & 0.5 & 0.5 & & & 3.00 \\
\hline & & & & 0.5 & 0.5 & & & & 2.00 \\
\hline & & & & 0.5 & & 0.5 & & & 3.00 \\
\hline & 0.5 & & & & & 0.5 & & 0.5 & 3.00 \\
\hline & 0.5 & & & & & & & 0.5 & 2.00 \\
\hline 0.5 & & & & & & & 0.5 & & 1.50 \\
\hline 0.5 & & & 0.5 & 0.5 & 0.5 & & & & 3.50 \\
\hline \multirow[t]{2}{*}{0.5} & & & & & & & 0.5 & & 2.50 \\
\hline & & 0.5 & & & & & & & 1.90 \\
\hline 0.5 & & & 0.5 & & 0.5 & & 0.5 & & 4.30 \\
\hline \multirow[t]{3}{*}{0.5} & & & & & & & 0.5 & & 2.80 \\
\hline & & 0.5 & & & & 0.5 & & & 2.50 \\
\hline & & & & & 0.5 & & & & 1.50 \\
\hline 0.5 & & & 0.5 & 0.5 & & & 0.5 & & 4.00 \\
\hline 0.5 & & & 0.5 & 0.5 & & & 0.5 & & 3.50 \\
\hline 0.5 & & & 0.5 & 0.5 & 0.5 & & & & 3.50 \\
\hline \multirow[t]{2}{*}{0.5} & & 0.5 & & & & 0.5 & & & 3.00 \\
\hline & 0.5 & & & & & & & 0.5 & 3.00 \\
\hline \multirow[t]{2}{*}{0.5} & & & & & 0.5 & & & & 2.50 \\
\hline & & & & 0.5 & & & & & 1.50 \\
\hline \multirow[t]{4}{*}{0.5} & & & 0.5 & 0.5 & 0.5 & & & & 4.10 \\
\hline & & & 0.5 & 0.5 & & & & 0.5 & 4.00 \\
\hline & & 0.5 & & 0.5 & 0.5 & & & & 3.50 \\
\hline & 0.5 & & & 0.5 & & & & 0.5 & 3.50 \\
\hline 0.5 & & 0.5 & & & & & & 0.5 & 3.00 \\
\hline \multirow[t]{4}{*}{0.5} & & & & & & & 0.5 & & 2.50 \\
\hline & & & & & & 0.5 & & & 1.50 \\
\hline & 0.5 & & & & & 0.5 & & & 2.50 \\
\hline & & & 0.5 & 0.5 & 0.5 & & & & 3.50 \\
\hline 0.5 & & 0.5 & & 0.5 & 0.5 & & & & 4.00 \\
\hline \multirow[t]{2}{*}{0.5} & & & 0.5 & 0.5 & & & & & 3.50 \\
\hline & 0.5 & & 0.5 & & & & & 0.5 & 3.50 \\
\hline \multirow[t]{2}{*}{0.5} & & & & & & & 0.5 & & 2.00 \\
\hline & & 0.5 & & & & & & & 1.50 \\
\hline 0.5 & & & 0.5 & & 0.5 & & 0.5 & & 4.00 \\
\hline \multirow[t]{4}{*}{0.5} & & & 0.5 & 0.5 & 0.5 & & & & 3.50 \\
\hline & & 0.5 & 0.5 & 0.5 & & & & & 3.50 \\
\hline & & & 0.5 & & 0.5 & & & & 3.00 \\
\hline & 0.5 & 0.5 & & & & & & 0.5 & 3.50 \\
\hline
\end{tabular}




\begin{tabular}{|c|c|c|c|c|c|c|c|c|c|}
\hline & & 0.5 & & & & & & 0.5 & 2.00 \\
\hline & & & & & & 0.5 & & & 1.50 \\
\hline & & 0.5 & & 0.5 & & 0.5 & & & 3.50 \\
\hline 0.5 & & & 0.5 & 0.5 & & & & 0.5 & 4.00 \\
\hline \multirow[t]{3}{*}{0.5} & & & 0.5 & & & & & 0.5 & 3.00 \\
\hline & 0.5 & & & & 0.5 & & 0.5 & & 3.50 \\
\hline & 0.5 & & 0.5 & & & & 0.5 & & 4.00 \\
\hline \multirow[t]{2}{*}{0.5} & & & & & & & & & 2.00 \\
\hline & & 0.5 & & & & 0.5 & & & 2.00 \\
\hline \multirow[t]{5}{*}{0.5} & 0.5 & & & 0.5 & 0.5 & & & & 3.00 \\
\hline & 0.5 & & 0.5 & 0.5 & & 0.5 & & & 4.00 \\
\hline & 0.5 & 0.5 & & 0.5 & & & & & 3.50 \\
\hline & 0.5 & & & & & & 0.5 & & 3.50 \\
\hline & 0.5 & & & & & 0.5 & & & 3.00 \\
\hline 0.5 & 0.5 & 0.5 & 0.5 & 0.5 & 0.5 & 0.5 & 0.5 & & 10.00 \\
\hline 0.5 & & 0.5 & & 0.5 & 0.5 & & 0.5 & 0.5 & 10.00 \\
\hline \multirow[t]{2}{*}{0.5} & 0.5 & 0.5 & 0.5 & & 0.5 & & 0.5 & & 17.90 \\
\hline & 0.5 & 0.5 & & 0.5 & & 0.5 & & & 8.10 \\
\hline \multirow[t]{3}{*}{0.5} & 0.5 & & & 1 & & & & & 4.30 \\
\hline & 0.5 & & 0.5 & & & 0.5 & & & 4.00 \\
\hline & 0.5 & & & 0.5 & & & & 0.5 & 4.00 \\
\hline \multirow[t]{2}{*}{0.5} & & 0.5 & 0.5 & & 0.5 & & 0.5 & 0.5 & 5.50 \\
\hline & 0.5 & & & 0.5 & 0.5 & & & 0.5 & 7.00 \\
\hline 1 & 0.5 & & 0.5 & 0.5 & 0.5 & & 0.5 & & 19.20 \\
\hline \multirow[t]{6}{*}{0.5} & & 0.5 & & 0.5 & 0.5 & & 0.5 & & 13.20 \\
\hline & 0.5 & & 0.5 & 0.5 & 0.5 & & & & 12.70 \\
\hline & 0.5 & & & & 0.5 & & & & 3.00 \\
\hline & & & & 0.5 & & & 0.5 & & 3.50 \\
\hline & & & & 0.5 & & & & & 1.50 \\
\hline & & & & & & & & 0.5 & 2.00 \\
\hline \multirow[t]{2}{*}{0.5} & & & & & 0.5 & & & & 3.00 \\
\hline & 0.5 & & 0.5 & 0.5 & & 0.5 & & & 4.50 \\
\hline \multirow[t]{4}{*}{0.5} & & & 0.5 & 0.5 & & 0.5 & & & 5.00 \\
\hline & 0.5 & & 0.5 & 0.5 & & & & & 5.00 \\
\hline & & 0.5 & & 0.5 & & & & 0.5 & 4.50 \\
\hline & & 0.5 & & & 0.5 & & & & 7.00 \\
\hline 0.5 & & & & & & & 0.5 & & 2.50 \\
\hline 0.5 & & & 0.5 & & & & & & 15.70 \\
\hline \multirow[t]{2}{*}{0.5} & & & 0.5 & 0.5 & 0.5 & & & & 6.00 \\
\hline & & & 0.5 & & & & & 0.5 & 3.00 \\
\hline 0.6 & 1 & 0.5 & 0.5 & & 0.5 & 0.5 & 0.5 & 0.5 & 9.70 \\
\hline 0.6 & 1.1 & 0.6 & 0.5 & 0.5 & 0.5 & & 0.5 & 1 & 18.60 \\
\hline \multirow[t]{2}{*}{0.5} & 0.5 & 0.5 & & 1 & & 0.5 & 0.5 & 0.5 & 12.50 \\
\hline & & 0.5 & & 0.5 & & 0.5 & & 0.5 & 9.00 \\
\hline 1.5 & 0.5 & & & 0.5 & & & 0.5 & & 26.20 \\
\hline \multirow[t]{2}{*}{0.5} & & & & 1 & & 0.5 & 0.5 & & 19.80 \\
\hline & & & 0.5 & 0.5 & & & 0.5 & & 11.70 \\
\hline \multirow[t]{3}{*}{0.5} & & & & & 0.5 & 0.5 & & 0.5 & 5.00 \\
\hline & & 0.5 & & & 0.5 & & & & 3.50 \\
\hline & & 0.5 & & & & 0.5 & & & 4.00 \\
\hline 0.5 & & & & & 0.5 & & & & 2.00 \\
\hline
\end{tabular}




\begin{tabular}{|c|c|c|c|c|c|c|c|c|c|}
\hline & 0.5 & & & & 0.5 & & & & 8.10 \\
\hline & 0.5 & 0.5 & 1 & 0.5 & & 0.5 & & & 10.10 \\
\hline 0.5 & & & 0.5 & 1 & & 0.5 & & & 6.00 \\
\hline 0.5 & & & & & 0.5 & 0.5 & & & 4.00 \\
\hline \multirow[t]{3}{*}{0.5} & & & 0.5 & & & & & & 3.00 \\
\hline & & & 0.5 & & & 0.5 & & & 3.00 \\
\hline & & & & 0.5 & & & 0.5 & & 2.00 \\
\hline \multirow[t]{4}{*}{0.5} & & & & & & 0.5 & & & 2.50 \\
\hline & 0.5 & & & & & & & 0.5 & 6.00 \\
\hline & & 0.5 & & & & 0.5 & & 0.5 & 14.30 \\
\hline & 0.5 & & 1 & & & & & & 14.70 \\
\hline \multirow[t]{3}{*}{0.5} & 0.5 & & 0.5 & & 0.5 & & 0.5 & & 9.70 \\
\hline & 0.5 & 1 & 1 & 1 & 0.5 & & & 0.5 & 6.50 \\
\hline & & 0.5 & & & & & & & 2.00 \\
\hline 0.6 & 1.1 & 0.6 & 0.5 & 0.5 & 0.5 & 0.5 & 0.1 & 0.5 & 14.40 \\
\hline 1.5 & 1 & 1 & 0.5 & 0.1 & & & 0.1 & 0.5 & 14.70 \\
\hline 1 & 1 & 0.1 & 0.5 & 0.1 & 0.1 & 0.6 & 0.1 & 0.1 & 10.10 \\
\hline 1.1 & 1.1 & 0.6 & 0.1 & 0.1 & 0.1 & 0.6 & 0.1 & 0.1 & 16.90 \\
\hline \multirow[t]{3}{*}{1.1} & 1 & 0.6 & 0.1 & 0.1 & 0.1 & 0.6 & 0.1 & 0.1 & 15.10 \\
\hline & 0.5 & & & 0.5 & & & 0.1 & 0.5 & 5.80 \\
\hline & & & & & 0.5 & & & 0.6 & 5.10 \\
\hline \multirow[t]{2}{*}{0.5} & & & & & & 0.5 & & & 10.20 \\
\hline & & & 0.5 & 1 & 0.5 & & 0.5 & & 7.50 \\
\hline 2.1 & 1.6 & 0.6 & 1.1 & 1 & 0.6 & 0.5 & 1.1 & 0.6 & 22.80 \\
\hline 1.1 & 1.1 & 0.1 & 0.6 & 0.6 & 0.6 & & 0.6 & 0.1 & 22.20 \\
\hline \multirow[t]{3}{*}{1.1} & 1.6 & 0.6 & 0.1 & 0.6 & 0.1 & 0.1 & 0.1 & 0.1 & 13.50 \\
\hline & & 0.5 & & & & & 0.5 & & 2.80 \\
\hline & & & & & & 0.5 & & & 2.30 \\
\hline 0.8 & 0.7 & 0.5 & 0.5 & & & 0.5 & & 0.5 & 26.20 \\
\hline 0.5 & 0.5 & 0.5 & & 0.5 & & & & 0.5 & 29.20 \\
\hline \multirow[t]{2}{*}{0.5} & 1 & 1 & 1 & 1 & 1 & 0.5 & 1 & 1 & 36.70 \\
\hline & 0.5 & & & 0.5 & 1 & & 1 & 0.5 & 20.70 \\
\hline 2.6 & 2.6 & 2.1 & 1.1 & 1.1 & 1.5 & 1.6 & 1 & 0.6 & 48.10 \\
\hline 0.5 & 1.5 & 0.5 & 0.5 & 0.5 & 0.5 & 0.5 & 0.5 & 1 & 25.90 \\
\hline \multirow[t]{3}{*}{0.5} & 0.5 & 0.5 & 0.5 & 0.5 & 0.5 & 0.5 & 0.5 & 0.5 & 13.00 \\
\hline & 0.5 & & & & & & 0.5 & & 20.30 \\
\hline & & 0.5 & & 0.5 & & & & & 10.60 \\
\hline 0.5 & 1 & 0.5 & 0.5 & 1 & & & 0.5 & & 15.20 \\
\hline 0.5 & 0.5 & & 0.5 & & & 0.5 & & & 16.10 \\
\hline 1 & & & & 0.5 & & & 0.5 & & 19.40 \\
\hline \multirow[t]{2}{*}{0.5} & 0.5 & & & 0.5 & & & 0.5 & & 8.50 \\
\hline & & & & 0.5 & & & 0.5 & & 3.00 \\
\hline 2.1 & 0.5 & 0.5 & 0.5 & 0.5 & 1.5 & 0.5 & 1 & 1 & 27.70 \\
\hline 3.1 & 2.6 & 1.5 & 1 & 1 & 2.1 & 1 & 0.5 & 1.1 & 53.30 \\
\hline 4.9 & 3.3 & 0.5 & 0.5 & 0.5 & 1.1 & 0.6 & 0.5 & 0.5 & 50.00 \\
\hline 1.5 & 1 & & & 0.5 & & & 0.5 & & 31.30 \\
\hline 1 & 1 & 0.5 & 0.5 & 0.5 & 0.5 & 0.5 & 0.5 & & 26.70 \\
\hline 1 & 0.5 & 0.5 & 0.1 & 0.5 & & 0.5 & 0.6 & 0.5 & 16.70 \\
\hline 0.5 & 0.6 & 0.6 & 0.1 & 0.5 & 0.6 & 0.1 & 0.6 & & 9.70 \\
\hline 0.5 & 0.5 & & & 0.5 & & & & 0.5 & 14.90 \\
\hline 0.5 & & 0.5 & & 0.5 & & & & & 5.50 \\
\hline
\end{tabular}




\begin{tabular}{|c|c|c|c|c|c|c|c|c|c|}
\hline 0.5 & & & 0.5 & & 0.5 & & & & 5.00 \\
\hline 1.5 & 1 & 0.5 & & 0.5 & 0.5 & 0.5 & 0.5 & 0.5 & 8.50 \\
\hline 3.7 & 3.8 & 1.3 & 1.9 & 2.3 & 1.8 & 0.9 & 1.4 & 0.8 & 52.00 \\
\hline 2.4 & 1.3 & 1.3 & 1.4 & 1.3 & 1.3 & 0.9 & 0.9 & 1.3 & 40.90 \\
\hline 0.9 & 0.8 & 0.8 & 0.8 & 0.3 & 1.4 & 0.8 & 0.9 & 0.9 & 21.80 \\
\hline 2.6 & 3.1 & 2.6 & 2.6 & 2.6 & 2.1 & 3.1 & 2.2 & 1.6 & 70.60 \\
\hline 5.3 & 3.7 & 4.6 & 4.2 & 4.2 & 3.1 & 2.6 & 2.6 & 2.2 & 87.90 \\
\hline 3.1 & 3.1 & 2.6 & 1 & 2.1 & 2.1 & 2.6 & 2.6 & 2.1 & 77.70 \\
\hline 3.6 & 2.6 & 2.1 & 2.1 & 2.1 & 1.5 & 1.5 & 2.6 & 1.6 & 68.50 \\
\hline 0.5 & 1 & 0.5 & 0.5 & 0.5 & 1 & 0.5 & 0.5 & 0.5 & 30.30 \\
\hline \multirow[t]{2}{*}{0.5} & & & & & & 0.5 & & & 5.50 \\
\hline & & 0.5 & & & & & 0.5 & & 3.10 \\
\hline \multirow[t]{2}{*}{0.5} & & & 0.5 & & & 0.5 & & & 10.60 \\
\hline & & 0.5 & & & & & 0.5 & & 4.50 \\
\hline 3.6 & 3.1 & 2.1 & 2.1 & 1.5 & 1.6 & 2.1 & 1.6 & 1.1 & 26.90 \\
\hline 4.2 & 4.2 & 2.1 & 1.6 & 2.1 & 1.1 & 2.6 & 2.1 & 1.1 & 76.60 \\
\hline \multirow[t]{2}{*}{3.5} & 3.4 & 1.6 & 1.1 & 0.6 & 1.1 & 1.1 & 0.6 & 0.6 & 73.00 \\
\hline & 0.5 & 0.1 & 0.6 & 0.1 & 0.6 & 0.1 & 0.6 & 0.6 & 15.50 \\
\hline 0.6 & 0.6 & 0.6 & 0.6 & 0.1 & 0.6 & 0.6 & 0.6 & 0.6 & 13.40 \\
\hline 1.8 & 1.3 & 0.5 & 1 & & 0.5 & 0.5 & 0.5 & & 43.20 \\
\hline 2.1 & 1 & & 1 & & 0.5 & 0.5 & & & 30.20 \\
\hline 1 & 1 & & & 0.5 & & 0.5 & & & 25.00 \\
\hline \multirow[t]{2}{*}{1} & 1 & & & & 0.5 & & 0.5 & & 21.50 \\
\hline & 0.5 & & 0.5 & & & & & 0.5 & 12.70 \\
\hline 0.5 & & & & & 0.5 & & & & 3.00 \\
\hline \multirow[t]{2}{*}{0.5} & & & & & 0.5 & & & & 2.50 \\
\hline & & & 0.5 & & & & & 0.5 & 3.50 \\
\hline 0.5 & & & 0.5 & & & & & 0.5 & 4.00 \\
\hline 2.1 & 1 & 0.5 & 1.5 & & 0.5 & 0.5 & 1 & & 20.90 \\
\hline 1 & 1.5 & 0.5 & 0.5 & & 0.5 & 0.5 & 0.5 & 0.5 & 29.10 \\
\hline 0.5 & & & & & 0.5 & & 0.5 & & 15.30 \\
\hline 1.5 & 1.5 & 0.5 & 1 & 1 & 1 & 1 & 0.5 & 0.5 & 17.00 \\
\hline 0.5 & & 0.5 & & & 0.5 & 0.5 & & & 12.00 \\
\hline 1.5 & 1 & & 0.5 & & & 0.5 & & & 22.30 \\
\hline 0.5 & 0.5 & & 0.5 & 0.5 & & 0.5 & & 0.5 & 11.00 \\
\hline 1 & 0.5 & & & 0.5 & & & 0.5 & & 22.00 \\
\hline \multirow[t]{4}{*}{1} & 0.5 & & 0.5 & & 0.5 & & 0.5 & & 19.90 \\
\hline & & & 0.5 & & & 0.5 & & & 14.20 \\
\hline & & & & & 0.5 & & & & 2.50 \\
\hline & 0.5 & & & 0.5 & & & & 0.5 & 3.00 \\
\hline 0.5 & & & 0.5 & & & & 0.5 & & 5.10 \\
\hline 0.5 & 0.5 & & & & 0.5 & & & & 4.00 \\
\hline 1 & 2.1 & 0.5 & 0.5 & 0.5 & 0.5 & 0.5 & & 0.5 & 26.70 \\
\hline 1.5 & 1.5 & 1 & & & 0.5 & 0.5 & & 0.5 & 33.90 \\
\hline \multirow[t]{3}{*}{0.5} & & 0.5 & 0.5 & & 0.5 & & & 0.5 & 20.00 \\
\hline & & & 0.5 & & 0.5 & & & & 3.50 \\
\hline & & & 0.5 & & & & 0.5 & & 3.50 \\
\hline 1.1 & 1.1 & & 1 & & 0.5 & & 0.5 & & 20.50 \\
\hline 1 & 0.5 & 0.5 & & 0.5 & & 0.5 & & 0.5 & 16.30 \\
\hline 0.5 & 1 & & & 0.5 & & 0.5 & & 0.5 & 13.10 \\
\hline 0.5 & 0.5 & 0.5 & & & 0.5 & & & & 13.10 \\
\hline
\end{tabular}




\begin{tabular}{|c|c|c|c|c|c|c|c|c|c|}
\hline \multirow[t]{10}{*}{0.5} & & & 0.5 & & & & & 0.5 & 8.60 \\
\hline & & 0.5 & & & & & 0.5 & & 2.50 \\
\hline & 0.5 & & & & & & & 0.5 & 2.50 \\
\hline & 0.5 & & 0.5 & & & & 0.5 & & 4.50 \\
\hline & & 0.5 & & 0.5 & & & & & 4.50 \\
\hline & & & 0.5 & & & & 0.5 & & 4.00 \\
\hline & 0.5 & & & & & 0.5 & & & 3.50 \\
\hline & & & & 0.5 & & & & & 3.00 \\
\hline & & & 0.5 & & & & & & 2.50 \\
\hline & & & & 0.5 & & & 0.5 & & 3.00 \\
\hline 0.5 & & & & 0.5 & & & & 0.5 & 5.00 \\
\hline 0.5 & & & & & 0.5 & & & 0.5 & 5.00 \\
\hline 0.5 & 0.5 & & & & & 0.5 & & & 4.50 \\
\hline \multirow[t]{2}{*}{0.5} & & & 0.5 & & & & 0.5 & & 5.00 \\
\hline & 0.5 & & & & & & 0.5 & & 4.00 \\
\hline 0.5 & & & & & 0.5 & & & & 2.50 \\
\hline 0.5 & & & & & 0.5 & & & & 2.50 \\
\hline \multirow[t]{2}{*}{0.5} & & & 0.5 & & & & & 0.5 & 5.00 \\
\hline & 0.5 & & & 0.5 & & & & 0.5 & 4.00 \\
\hline 0.5 & & & 0.5 & & & & 0.5 & 0.5 & 9.50 \\
\hline \multirow[t]{5}{*}{0.5} & & & 0.5 & & & & 0.5 & & 9.50 \\
\hline & 0.5 & & & & & 0.5 & & & 4.00 \\
\hline & 0.5 & & & & 0.5 & & & & 2.50 \\
\hline & 0.5 & & & & & & & & 2.00 \\
\hline & & 0.5 & & & & 0.5 & & & 4.00 \\
\hline \multirow[t]{3}{*}{0.5} & & & 0.5 & & & & 0.5 & & 4.00 \\
\hline & 0.5 & & & & & 0.5 & & & 3.50 \\
\hline & & & 0.5 & & & & 0.5 & & 3.50 \\
\hline 0.5 & & & 0.5 & & & & & & 3.00 \\
\hline \multirow[t]{2}{*}{0.5} & & & & & 0.5 & & 0.5 & & 3.00 \\
\hline & 0.5 & & & 0.5 & & & & & 2.00 \\
\hline \multirow[t]{3}{*}{0.5} & & & & & 0.5 & & & & 3.50 \\
\hline & & 0.5 & & & & & 0.5 & & 4.00 \\
\hline & 0.5 & & & 0.5 & & & & & 7.10 \\
\hline \multirow[t]{3}{*}{0.5} & & & 0.5 & & & & 0.5 & & 4.00 \\
\hline & & 0.5 & & & & & 0.5 & & 3.50 \\
\hline & & & 0.5 & & & 0.5 & & & 3.00 \\
\hline 0.5 & & & & 0.5 & & & & & 2.00 \\
\hline \multirow[t]{8}{*}{0.5} & & & & & & 0.5 & & & 3.50 \\
\hline & & 0.5 & & & & 0.5 & & & 3.50 \\
\hline & & 0.5 & & & & & 0.5 & & 3.50 \\
\hline & & & 0.5 & & & 0.5 & & & 3.50 \\
\hline & & & & 0.5 & & & 0.5 & & 3.50 \\
\hline & & 0.5 & & & & & & 0.5 & 2.50 \\
\hline & & 0.5 & & 0.5 & & & & & 2.50 \\
\hline & & 0.5 & & 0.5 & & & & & 5.00 \\
\hline \multirow[t]{2}{*}{0.5} & & & & 0.5 & 0.5 & & & 0.5 & 4.50 \\
\hline & 0.5 & & 0.5 & 0.5 & & 0.5 & & & 4.50 \\
\hline \multirow[t]{3}{*}{0.5} & & & 0.5 & 0.5 & & & & 0.5 & 4.50 \\
\hline & & 0.5 & & 0.5 & & & 0.5 & & 4.00 \\
\hline & & & & 0.5 & & & 0.5 & & 2.50 \\
\hline
\end{tabular}




\begin{tabular}{|c|c|c|c|c|c|c|c|c|c|}
\hline & & & 0.5 & & & & 0.5 & & 2.00 \\
\hline \multirow[t]{3}{*}{0.5} & 0.5 & & & 0.5 & 0.5 & & & & 4.00 \\
\hline & 0.5 & & 0.5 & 0.5 & & & 0.5 & & 4.50 \\
\hline & & 0.5 & 0.5 & & & & & & 3.50 \\
\hline 0.5 & & & 0.5 & & 0.5 & & & & 4.00 \\
\hline \multirow[t]{4}{*}{0.5} & & 0.5 & & & 0.5 & & 0.5 & & 4.00 \\
\hline & & & & 0.5 & & & & & 2.00 \\
\hline & & & & & 0.5 & & & & 2.50 \\
\hline & 0.5 & & 0.5 & 0.5 & & & & 0.5 & 4.50 \\
\hline 0.5 & 0.5 & & 0.5 & & 0.5 & & & & 4.00 \\
\hline 0.5 & & & & 0.5 & & & & & 3.50 \\
\hline \multirow[t]{4}{*}{0.5} & 0.5 & & 0.5 & & 0.5 & & & & 4.50 \\
\hline & & 0.5 & & & 0.5 & & 0.5 & & 4.00 \\
\hline & & & & & 0.5 & & & & 2.50 \\
\hline & 0.5 & & & & 0.5 & & & & 2.50 \\
\hline 0.5 & & & & 0.5 & & & & & 3.00 \\
\hline \multirow[t]{3}{*}{0.5} & & & 0.5 & & & & & & 3.00 \\
\hline & 0.5 & & & 0.5 & & & & & 3.00 \\
\hline & 0.5 & & & & & & 0.5 & & 3.50 \\
\hline \multirow[t]{12}{*}{0.5} & & & & & & 0.5 & & & 3.00 \\
\hline & & & 0.5 & & & & & & 2.00 \\
\hline & 0.5 & & & & & 0.5 & & & 2.50 \\
\hline & 0.5 & & & 0.5 & & & & & 2.00 \\
\hline & & & & 0.5 & & & & & 2.50 \\
\hline & 0.5 & & & & 0.5 & & & & 2.50 \\
\hline & & 0.5 & & & & & 1 & & 3.00 \\
\hline & & & & 0.5 & & & & 0.5 & 2.50 \\
\hline & 0.5 & & & & & 0.5 & & & 2.00 \\
\hline & & & & & & & & & \\
\hline & & & & & & & & & \\
\hline & & & & & & & & & \\
\hline 2.1 & 0 & 0 & 0 & 0 & 0 & 0.5 & 0 & 0 & \\
\hline 1.197093 & 1.156129 & 1.065432 & 1.055769 & 0.991429 & 0.973964 & 0.980745 & 0.95 & 0.957927 & \\
\hline 0.79854 & 0.802721 & 0.797143 & 0.797885 & 0.794318 & 0.785027 & 0.779897 & 0.77598 & 0.769014 & \\
\hline 1.174233 & 1.029814 & 0.975862 & 0.930872 & 0.909259 & 0.914103 & 0.892763 & 0.896575 & 0.890972 & \\
\hline 0.522222 & 0.5 & 0.5 & 0.5 & 0.5 & 0.5 & 0.5 & 0.51 & 0.45 & \\
\hline 0.793103 & 0.801389 & 0.58 & 0.553165 & 0.566667 & 0.58125 & 0.52963 & 0.516393 & 0.521053 & \\
\hline 1.665517 & 1.6125 & 1.289474 & 1.129167 & 1.25 & 1.021739 & 1.065217 & 1.059091 & 0.955556 & \\
\hline 0.57027 & 0.612121 & 0.525 & 0.515625 & 0.5 & 0.5 & 0.5 & 0.518519 & 0.5 & \\
\hline & & & & & & & & & \\
\hline & & & & & & & & & \\
\hline & & & & & & & & & \\
\hline & & & & & & & & & \\
\hline \#DIV/0! & \#DIV/0! & \#DIV/0! & \#DIV/0! & \#DIV/0! & \#DIV/0! & \#DIV/0! & \#DIV/0! & \#DIV/0! & \\
\hline 1.079605 & 0.950672 & 0.875712 & 0.869204 & 0.867938 & 0.801952 & 0.785216 & 0.798977 & 0.812176 & \\
\hline 0.64211 & 0.637984 & 0.625741 & 0.616413 & 0.604514 & 0.591214 & 0.582615 & 0.572044 & 0.563458 & \\
\hline 0.768242 & 0.687281 & 0.53984 & 0.500527 & 0.508292 & 0.499477 & 0.475785 & 0.455225 & 0.462875 & \\
\hline 0.097402 & 0 & 0 & 0 & 0 & 0 & 0 & 0.031623 & 0.141421 & \\
\hline 0.727179 & 0.64097 & 0.289828 & 0.253608 & 0.267321 & 0.341739 & 0.190956 & 0.234649 & 0.200657 & \\
\hline 1.343842 & 1.183331 & 1.144501 & 0.889787 & 1.126647 & 0.691478 & 0.885031 & 0.805138 & 0.578255 & \\
\hline 0.215851 & 0.342562 & 0.111803 & 0.088388 & 0 & 0 & 0 & 0.096225 & 0 & \\
\hline
\end{tabular}




\begin{tabular}{|c|c|c|c|c|c|c|c|c|c|}
\hline & & & & & & & & & \\
\hline & & & & & & & & & \\
\hline & & & & & & & & & \\
\hline & & & & & & & & & \\
\hline & & & & & & & & & \\
\hline 2.1 & 0 & 0 & 0 & 0 & 0 & 0.5 & 0 & 0 & ) \\
\hline 209.9 & 182.7 & 176.1 & 168.7 & 177.5 & 169.6 & 160.9 & 156.5 & 160.6 & \\
\hline 21.3 & 24.2 & 21.1 & 19 & 23.5 & 19 & 15.5 & 20 & 16.5 & \\
\hline 181.9 & 155.3 & 132.5 & 130.2 & 136.3 & 132.6 & 126.2 & 120.9 & 120.3 & \\
\hline 12 & 9.5 & 5 & 6 & 12 & 9 & 7 & 4 & 3.5 & \\
\hline 95.7 & 80.6 & 54 & 59 & 66.7 & 52.4 & 46.3 & 48 & 41.1 & \\
\hline 27.7 & 23 & 7.3 & 14.3 & 5.3 & 11.8 & 9.3 & 7.8 & 7.8 & \\
\hline 23.32098 & 19.51467 & 13.73291 & 17.98248 & 18.51167 & 14.27608 & 12.24825 & 17.72656 & 11.04452 & \\
\hline & & & & & & & & & \\
\hline & & & & & & & & & \\
\hline & & & & & & & & & \\
\hline & & & & & & & & & \\
\hline & & & & & & & & & \\
\hline & & & & & & & & & \\
\hline & & & & & & & & & \\
\hline & & & & & & & & & \\
\hline & & & & & & & & & \\
\hline & & & & & & & & & \\
\hline & & & & & & & & & \\
\hline & & & & & & & & & \\
\hline & & & & & & & & & \\
\hline & & & & & & & & & \\
\hline & & & & & & & & & \\
\hline
\end{tabular}




\begin{tabular}{|c|c|c|c|c|c|}
\hline $\begin{array}{l}\text { DAILY } \\
\text { AVERAGE }\end{array}$ & $\begin{array}{l}\text { MONTHLY } \\
\text { TOTAL } \\
\text { formula }\end{array}$ & $\begin{array}{l}\text { MONTHLY } \\
\text { TOTAL value }\end{array}$ & & & \\
\hline \multicolumn{6}{|l|}{$\begin{array}{l}\text { \#DIV/0! } \\
\text { \#DIV/0! }\end{array}$} \\
\hline \\
\hline \#DIV/0! & & & sort S/NS & sort JDAY & \\
\hline \#DIV/0! & & & 8882.60 & sum AE column & sum AG column \\
\hline \#DIV/0! & & & 3005.90 & & \\
\hline \#DIV/0! & & & 11888.50 & 11888.50 & 11891 \\
\hline \multicolumn{6}{|l|}{ \#DIV/0! } \\
\hline \#DIV/0! & & & sort $\mathrm{A} / \mathrm{B}, \mathrm{S} / \mathrm{NS}$ & & \\
\hline \#DIV/0! & & & B-S & 6702.20 & \\
\hline \#DIV/0! & & & B-NS & 2784.80 & \\
\hline \multicolumn{6}{|l|}{ \#DIV/0! } \\
\hline \multicolumn{6}{|l|}{ \#DIV/0! } \\
\hline \multicolumn{6}{|l|}{ \#DIV/0! } \\
\hline \multicolumn{6}{|l|}{ \#DIV/0! } \\
\hline \multicolumn{6}{|l|}{ \#DIV/0! } \\
\hline \multicolumn{6}{|l|}{ \#DIV/0! } \\
\hline \multicolumn{6}{|l|}{ \#DIV/0! } \\
\hline \multicolumn{6}{|l|}{ \#DIV/0! } \\
\hline \multicolumn{6}{|l|}{2.34} \\
\hline \multirow{2}{*}{\multicolumn{6}{|c|}{$\begin{array}{l}\text { \#DIV/0! } \\
\text { \#DIV/0! }\end{array}$}} \\
\hline & & & & & \\
\hline \multicolumn{6}{|l|}{ \#DIV/0! } \\
\hline \multicolumn{6}{|l|}{ \#DIV/0! } \\
\hline \multicolumn{6}{|l|}{ \#DIV/0! } \\
\hline \#DIV/0! & 16.40 & 0 & & & \\
\hline \multicolumn{6}{|l|}{ \#DIV/0! } \\
\hline \multicolumn{6}{|l|}{ \#DIV/0! } \\
\hline \multirow{2}{*}{\multicolumn{6}{|c|}{ \#DIV/0! }} \\
\hline \multirow{2}{*}{\multicolumn{6}{|c|}{$\begin{array}{l}\text { \#DIV/0! } \\
\text { \#DIV/0! }\end{array}$}} \\
\hline & & & & & \\
\hline \multirow{2}{*}{\multicolumn{6}{|c|}{ \#DIV/0! }} \\
\hline \#DIV/0! & & & & & \\
\hline \#DIV/0! & & & & & \\
\hline \#DIV/0! & & & & & \\
\hline 17.50 & & & & & \\
\hline \#DIV/0! & & & & & \\
\hline \#DIV/0! & & & & & \\
\hline \#DIV/0! & & & & & \\
\hline \#DIV/0! & & & & & \\
\hline \#DIV/0! & & & & & \\
\hline \#DIV/0! & & & & & \\
\hline \#DIV/0! & & & & & \\
\hline \#DIV/0! & & & & & \\
\hline \#DIV/0! & & & & & \\
\hline \#DIV/0! & & & & & \\
\hline \#DIV/0! & & & & & \\
\hline \#DIV/0! & & & & & \\
\hline
\end{tabular}




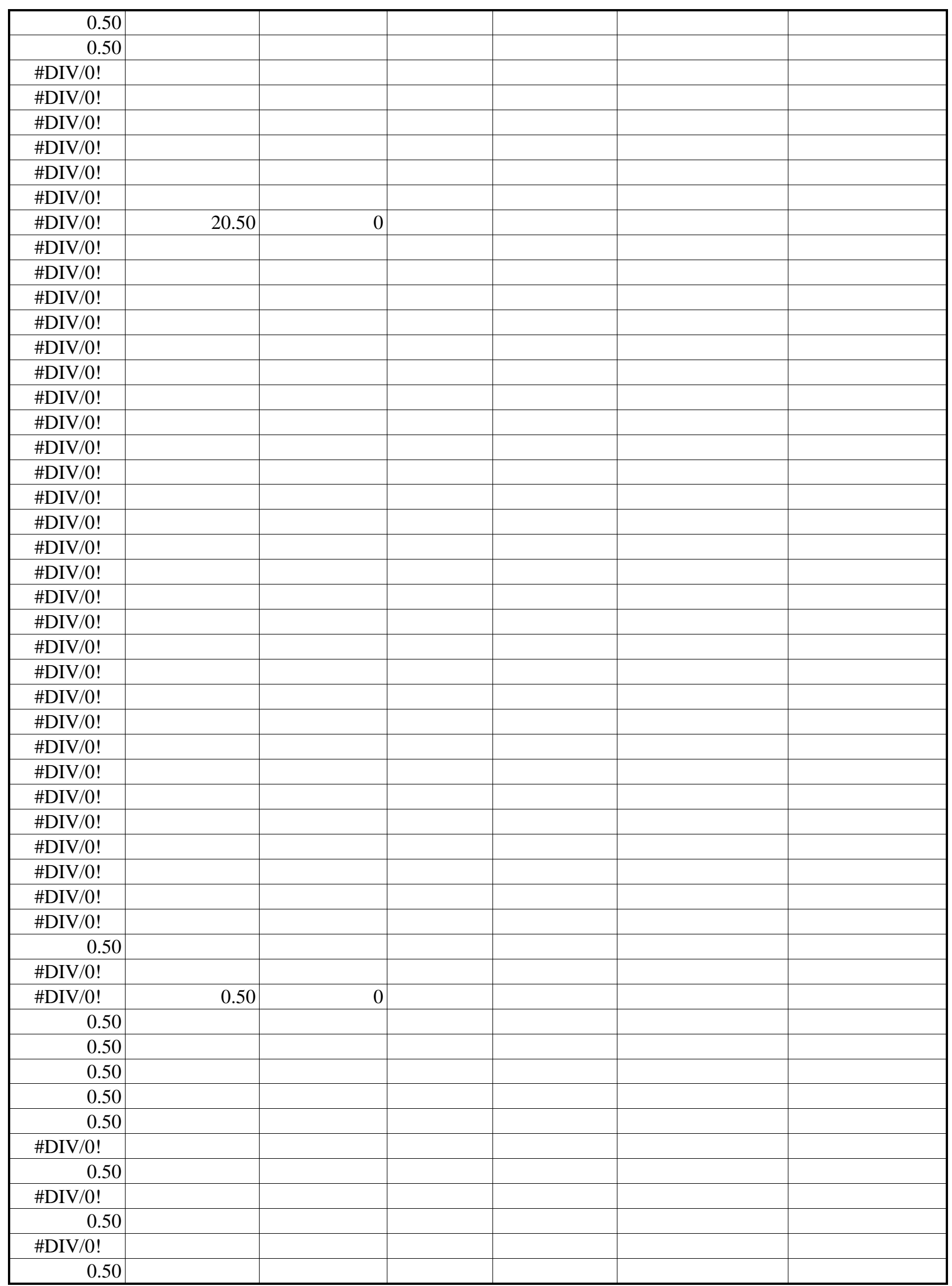




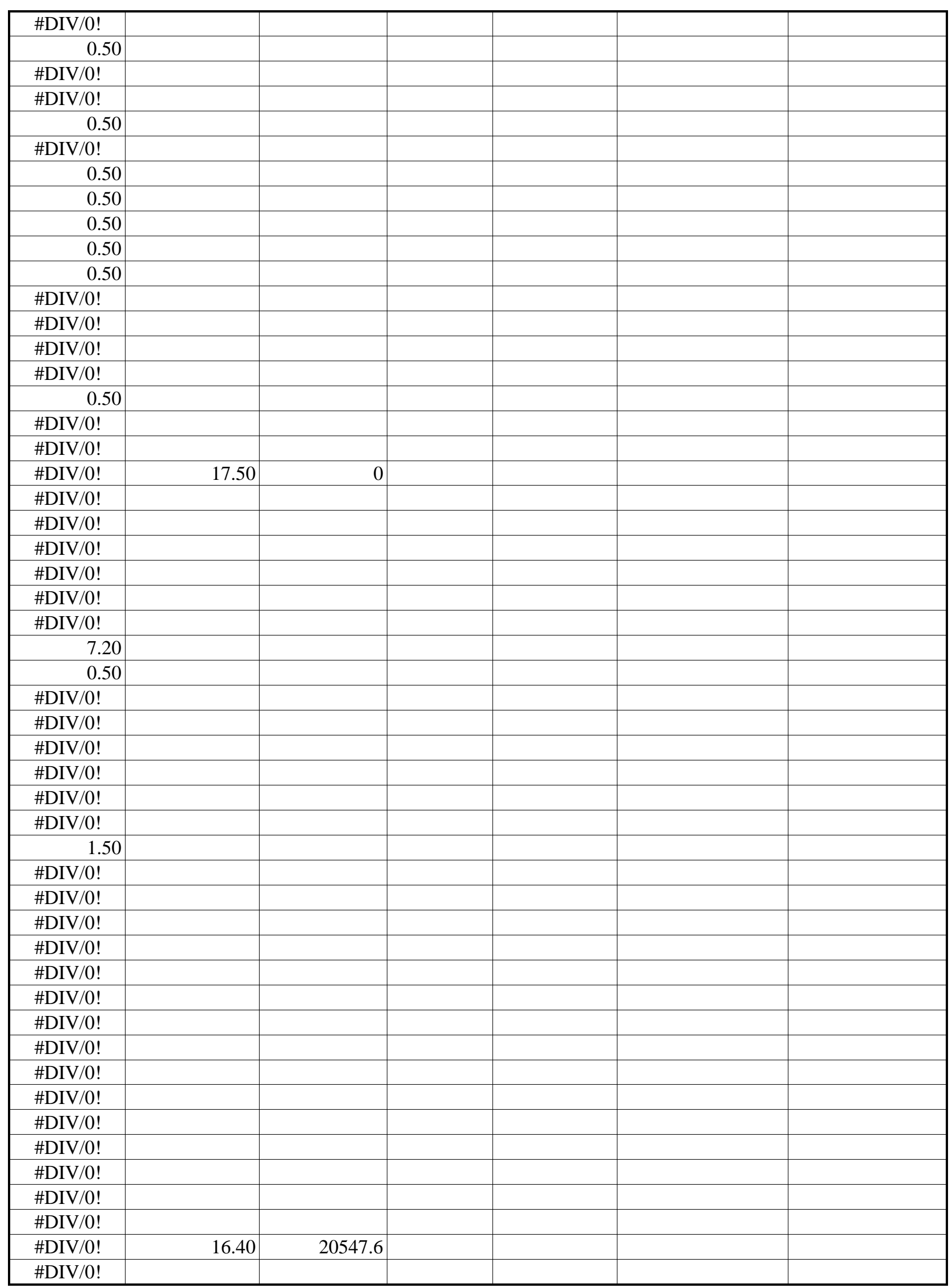




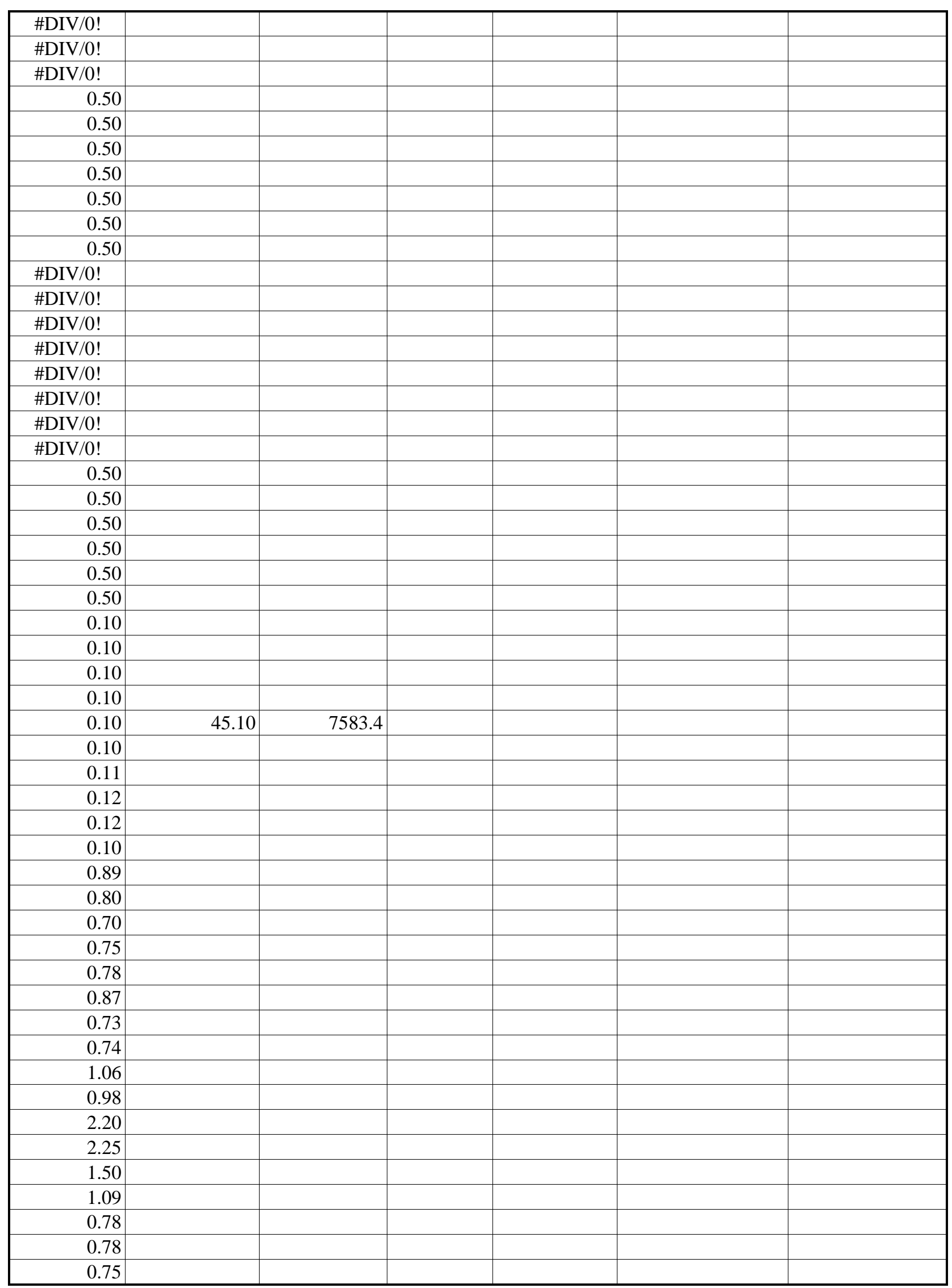


wbheat

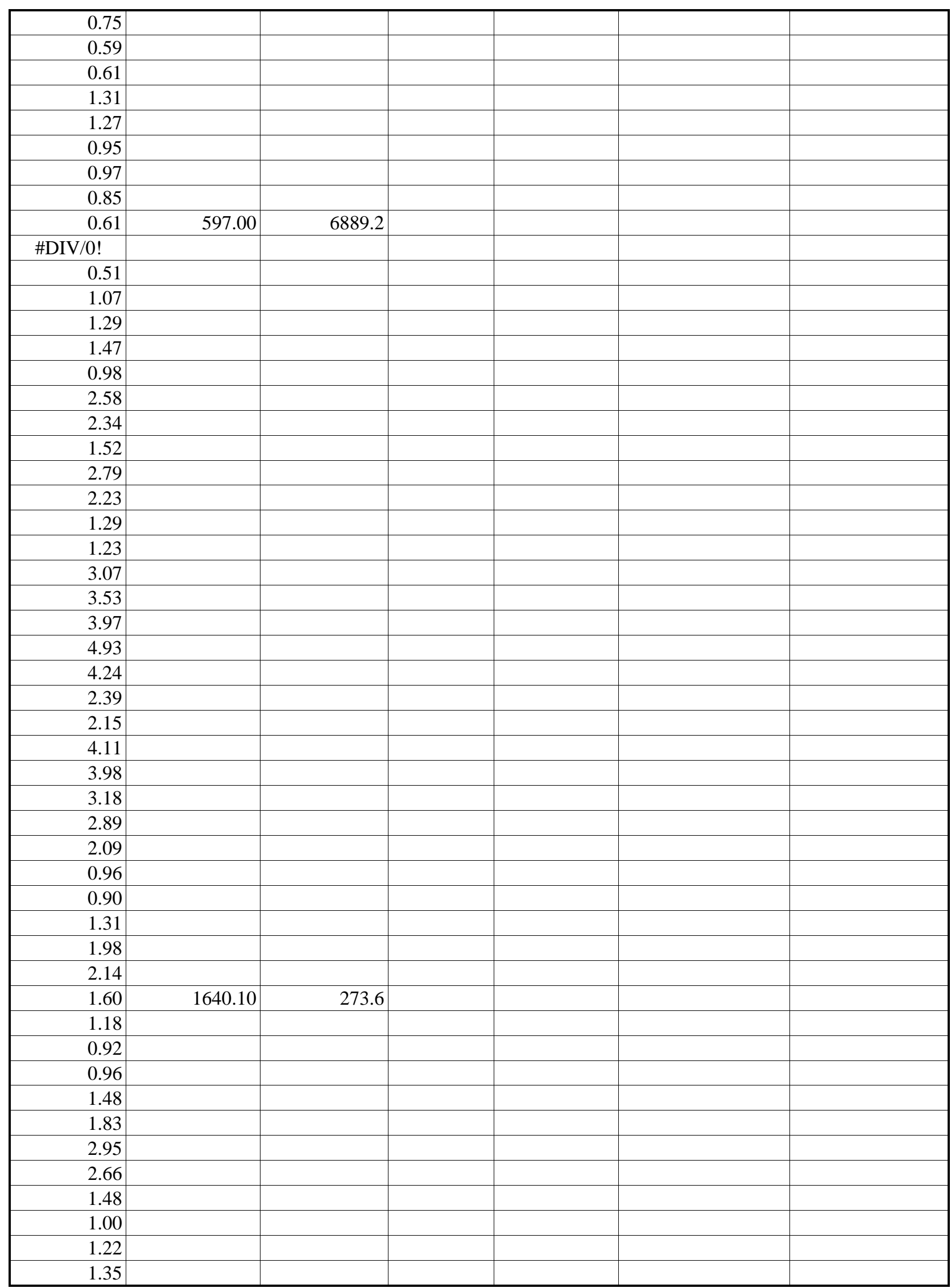


wbheat

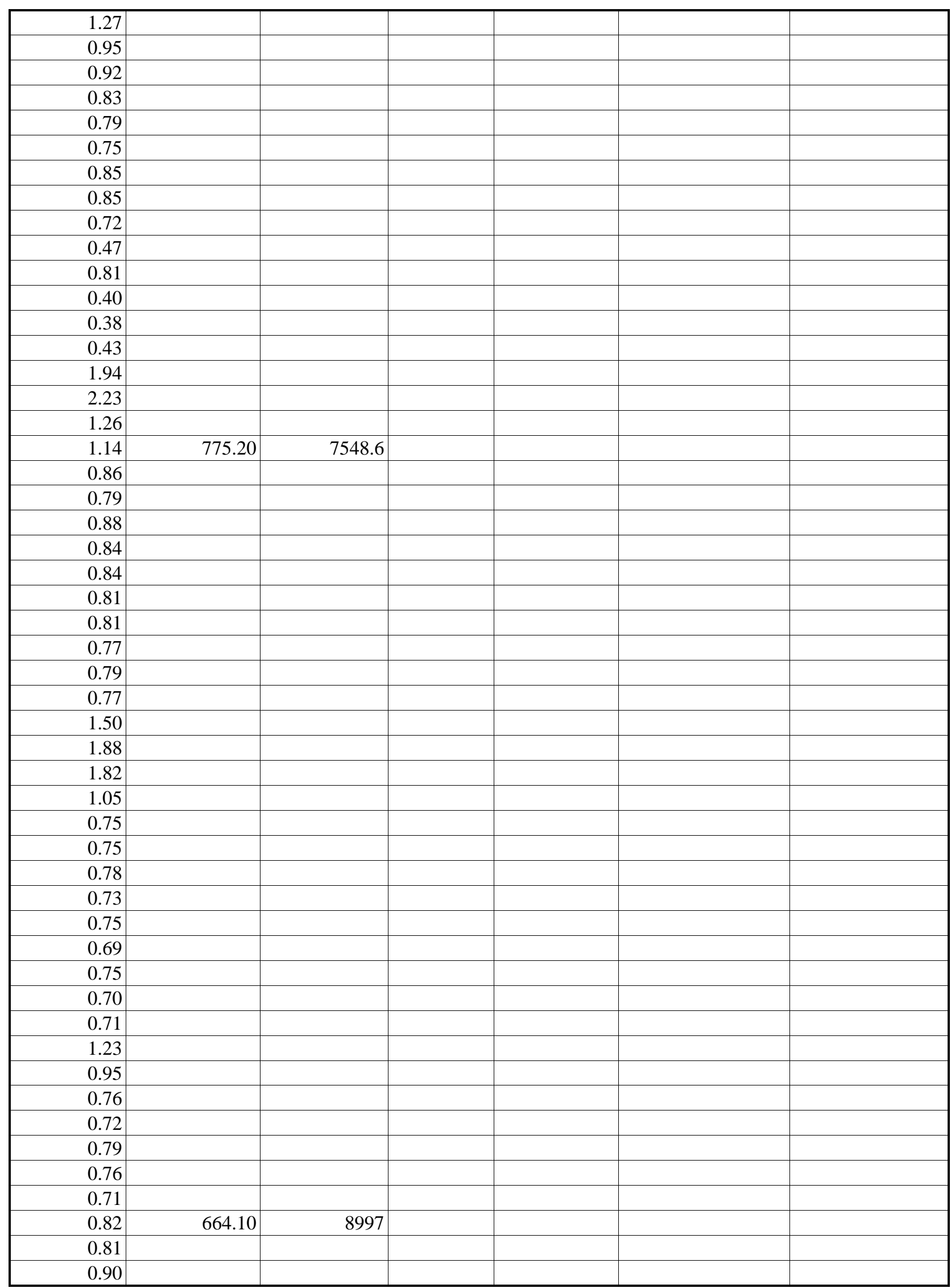


wbheat

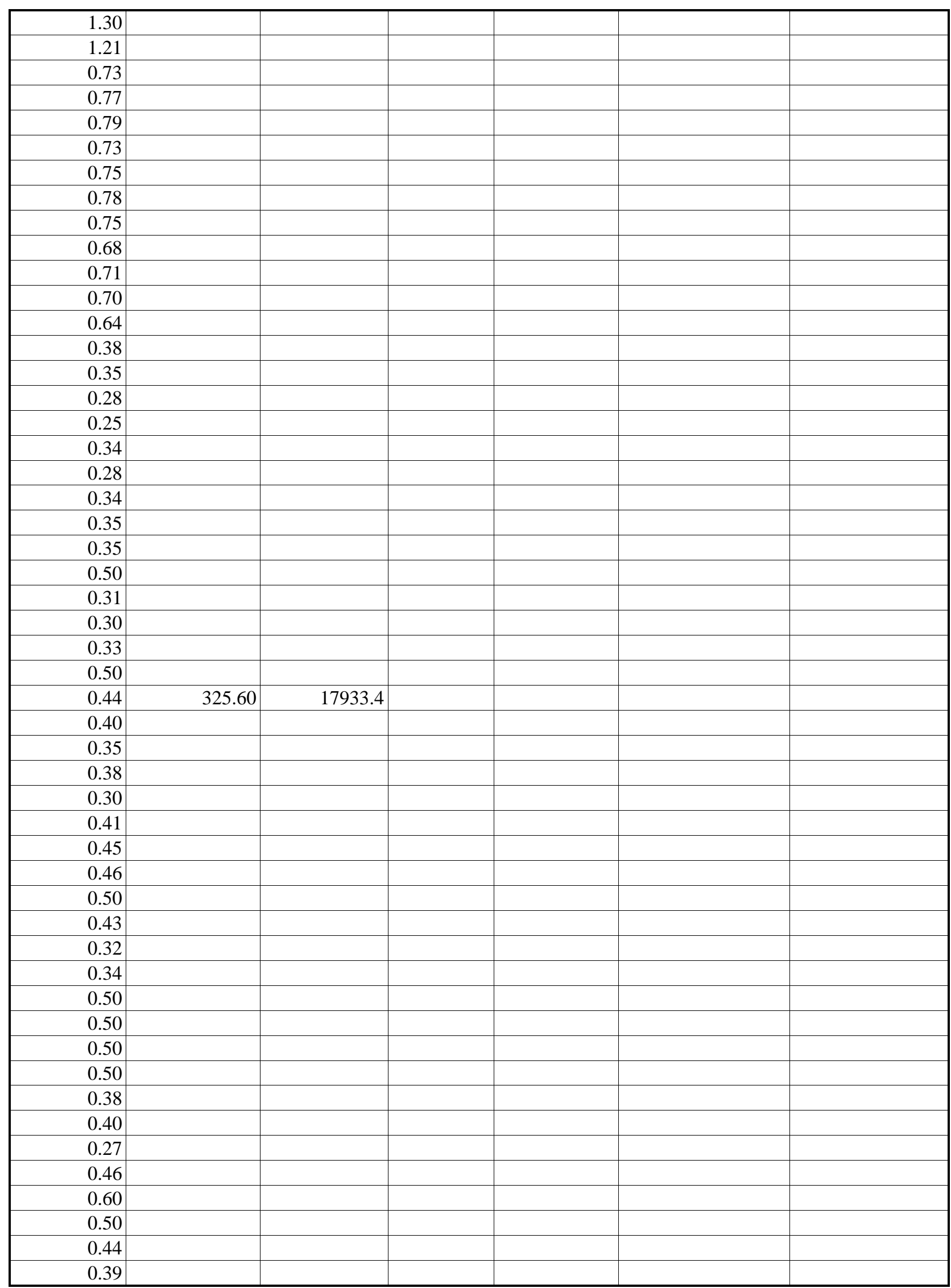


wbheat

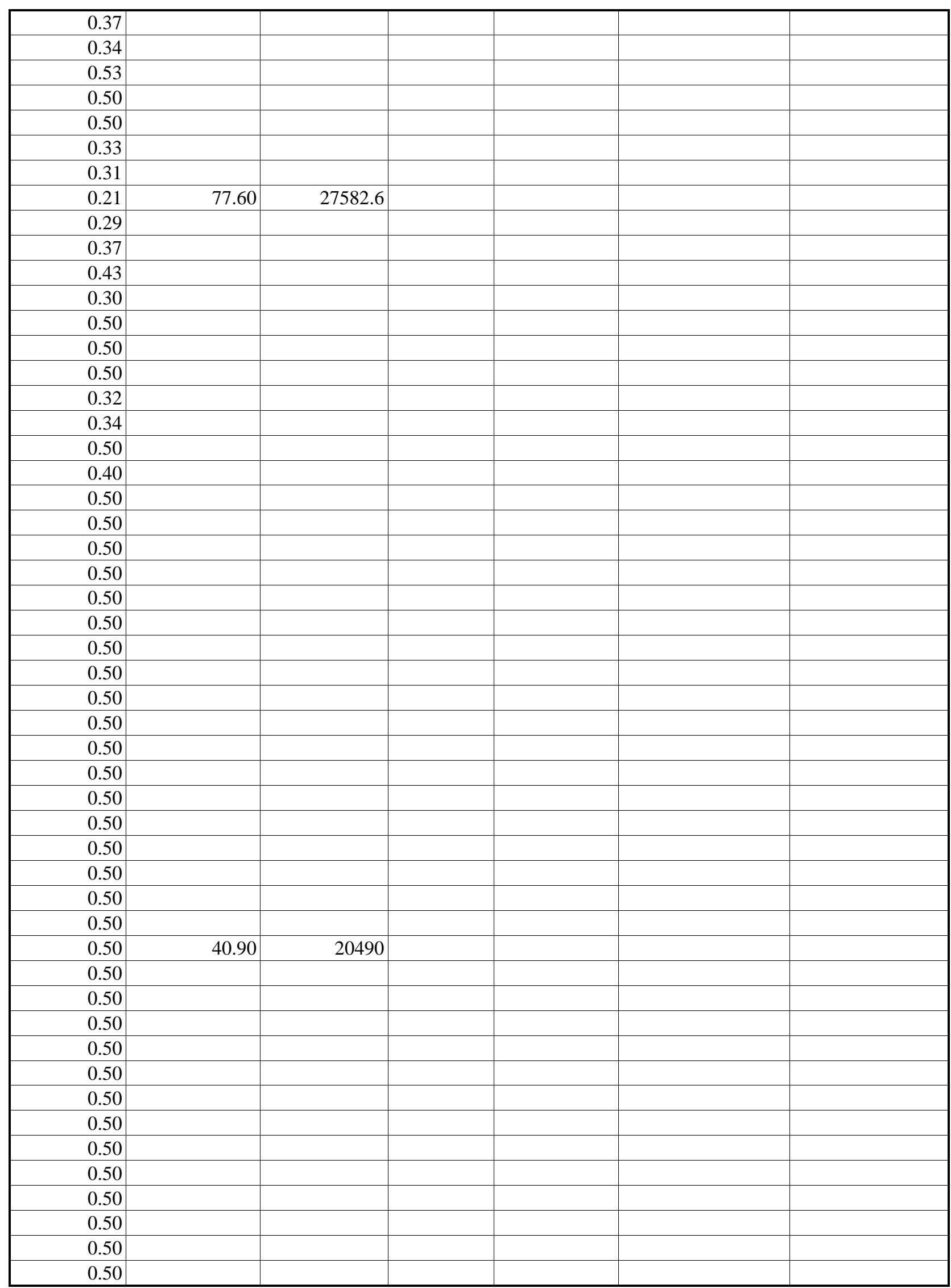

Page 77 
wbheat

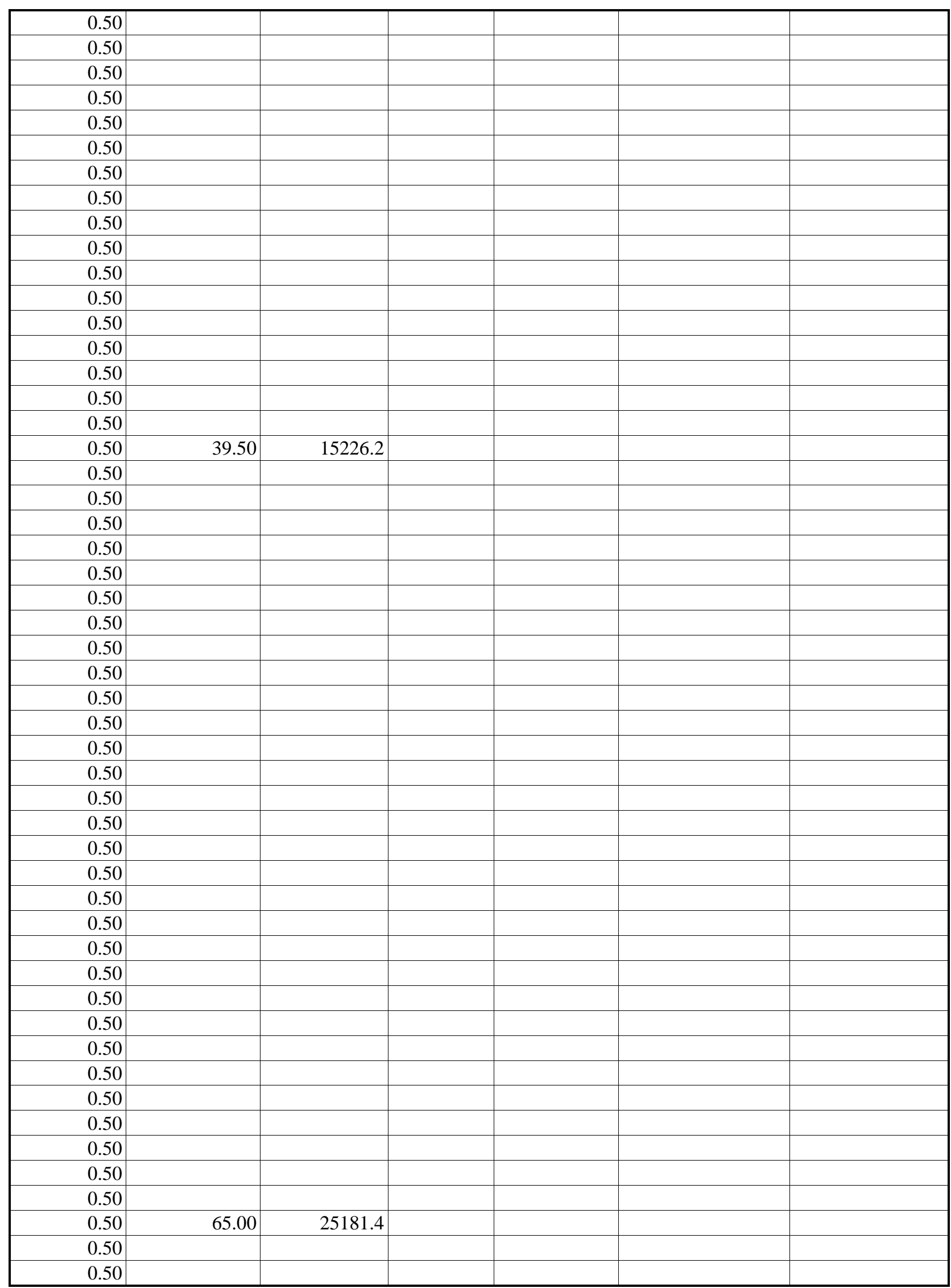


wbheat

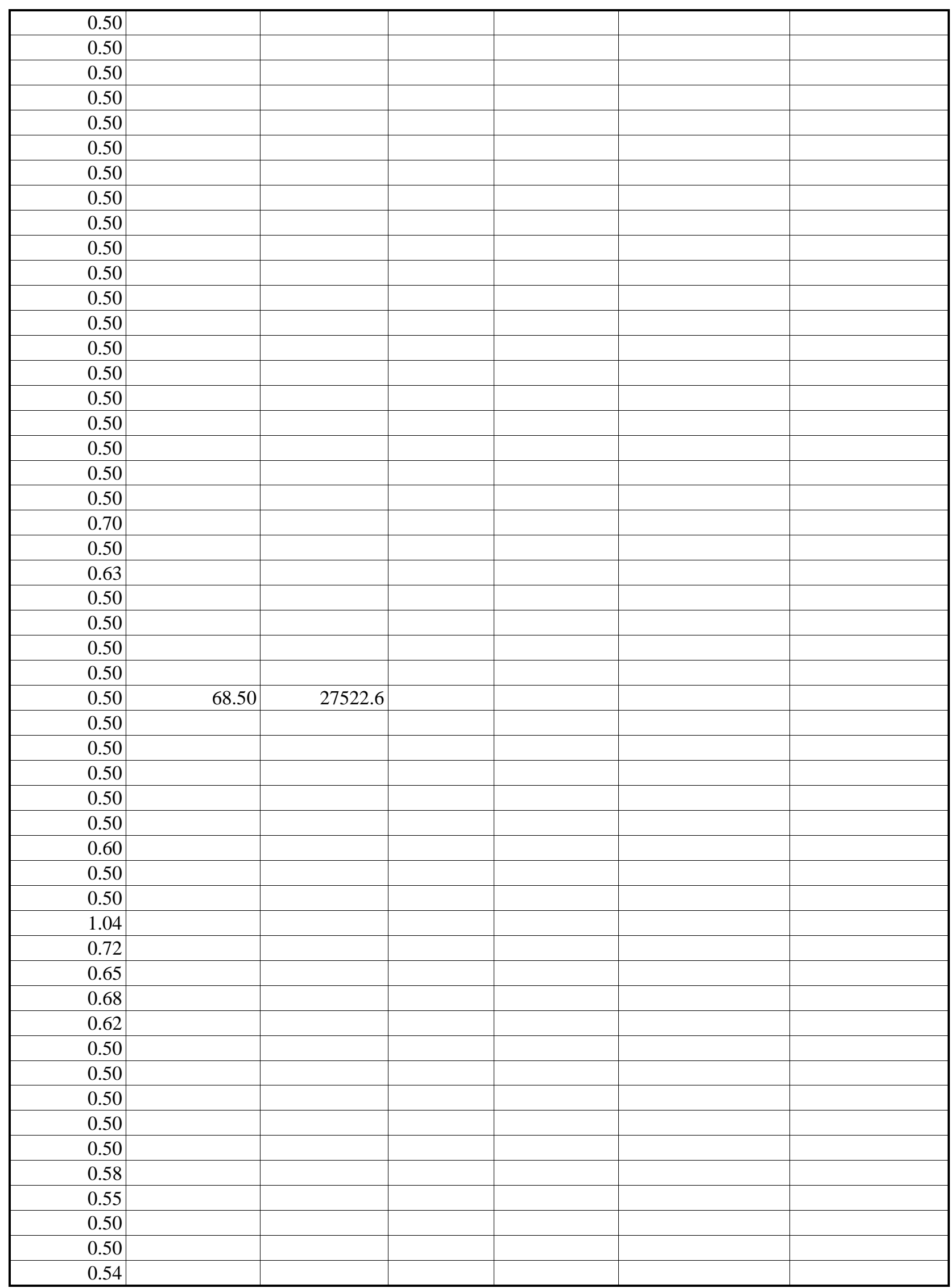


wbheat

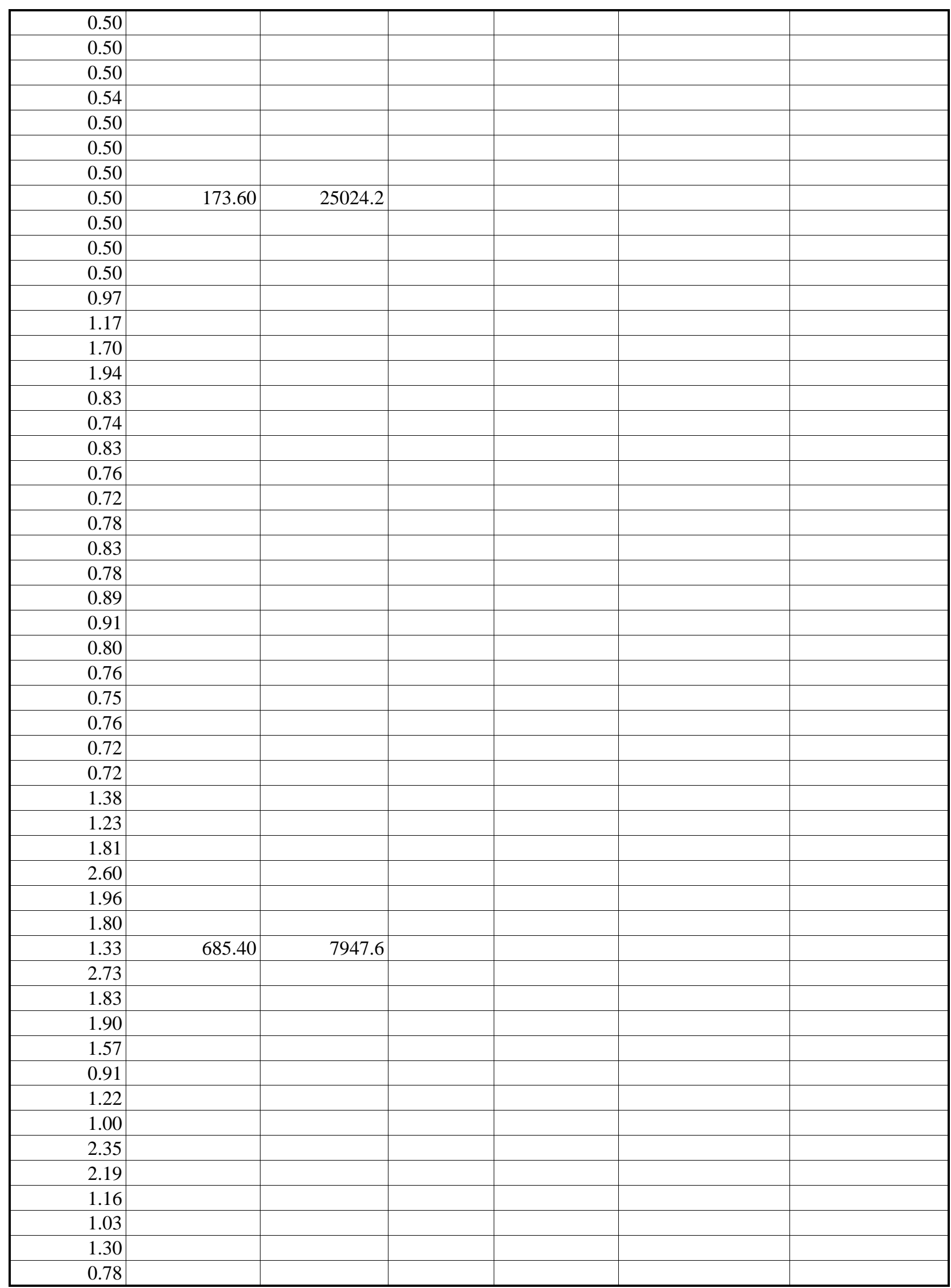


wbheat

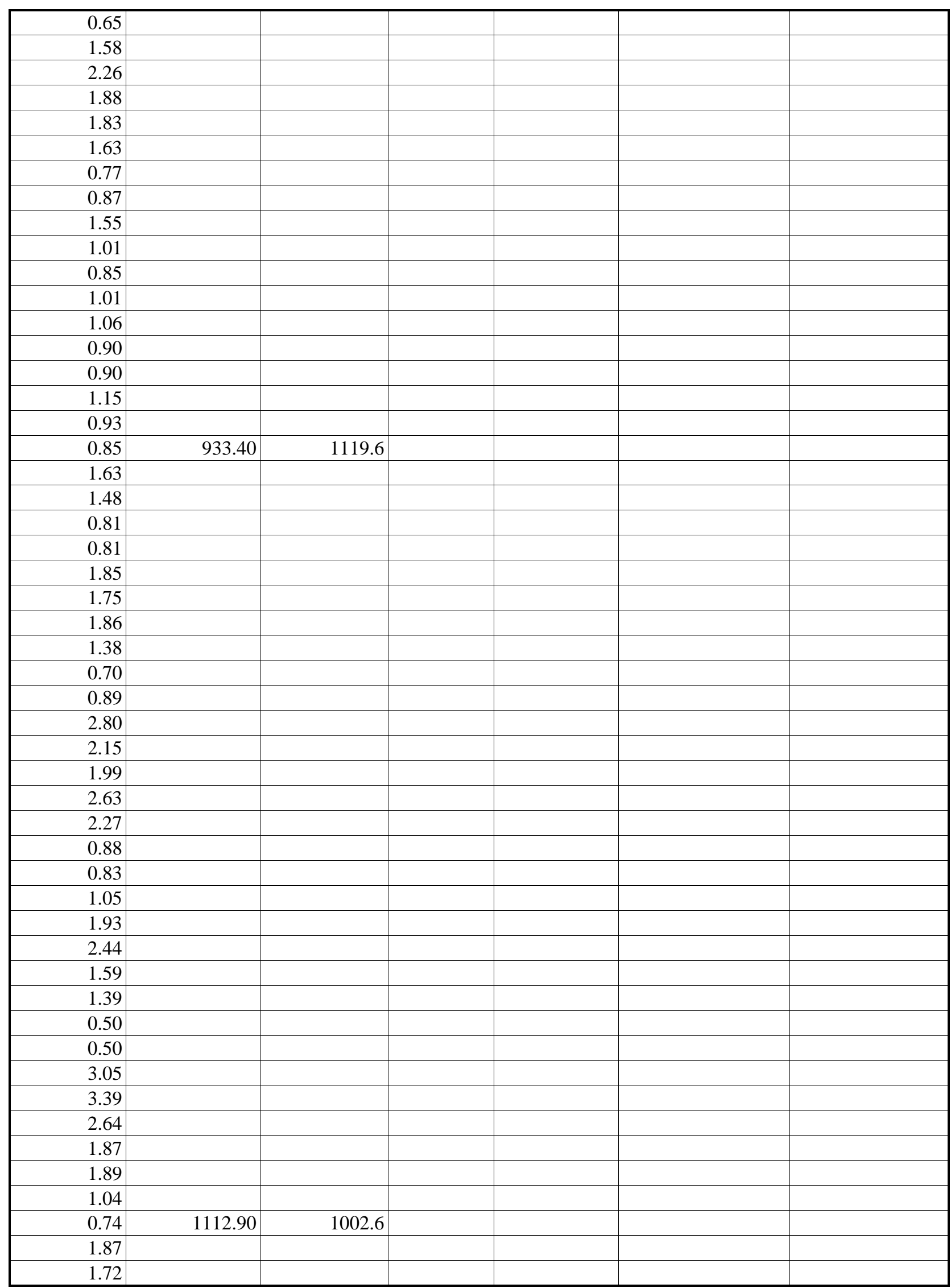


wbheat

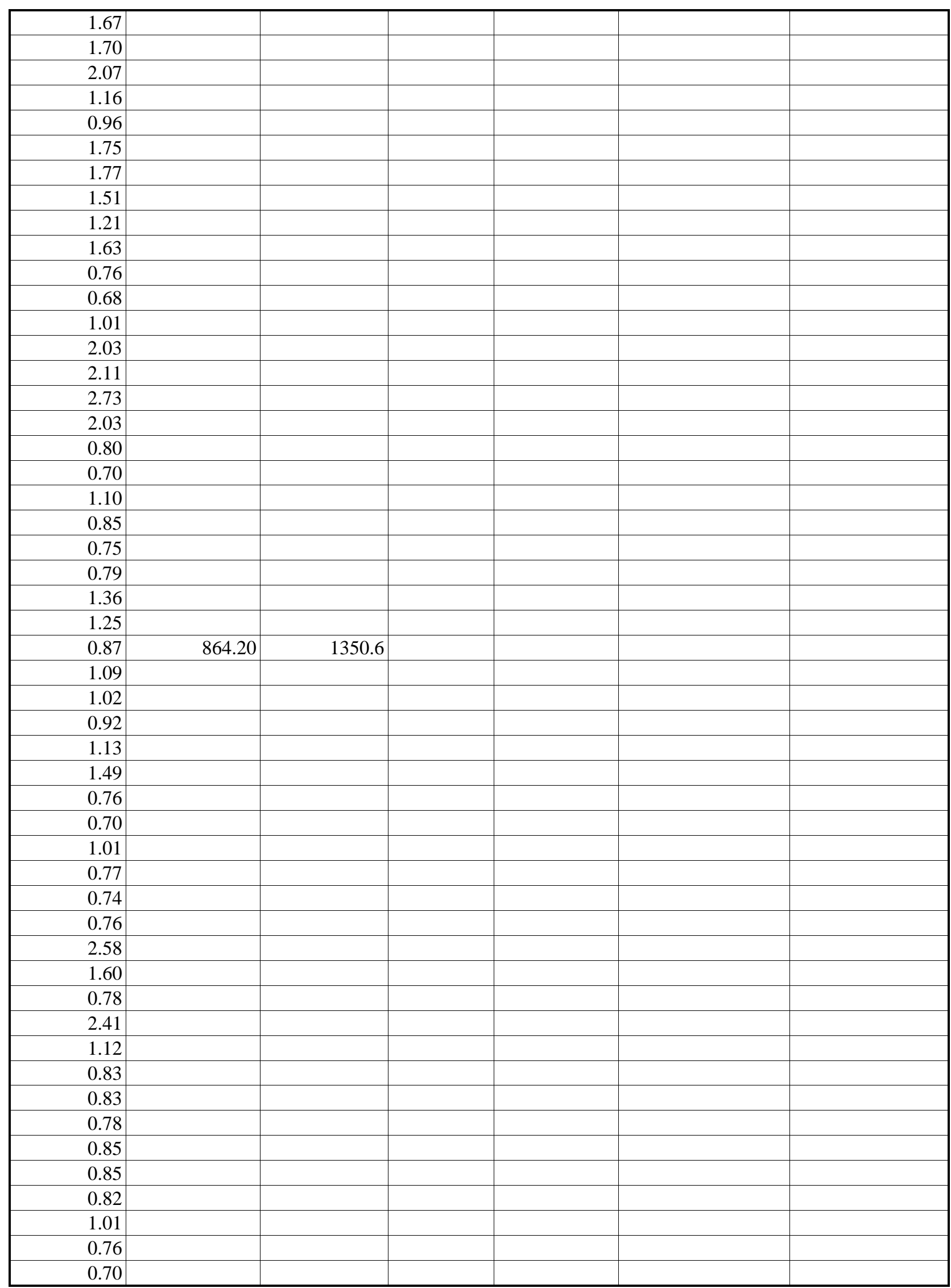

Page 82 
wbheat

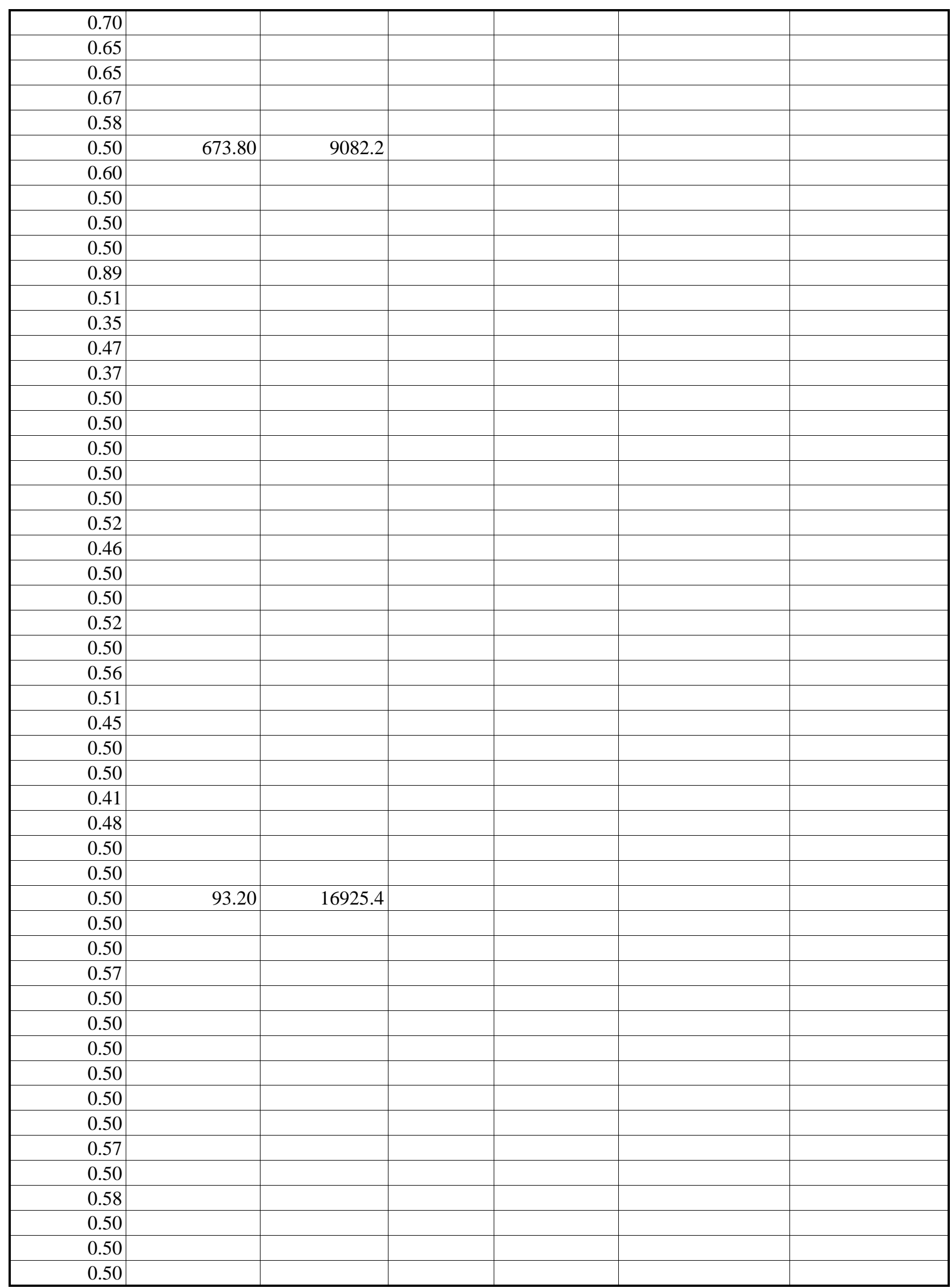


wbheat

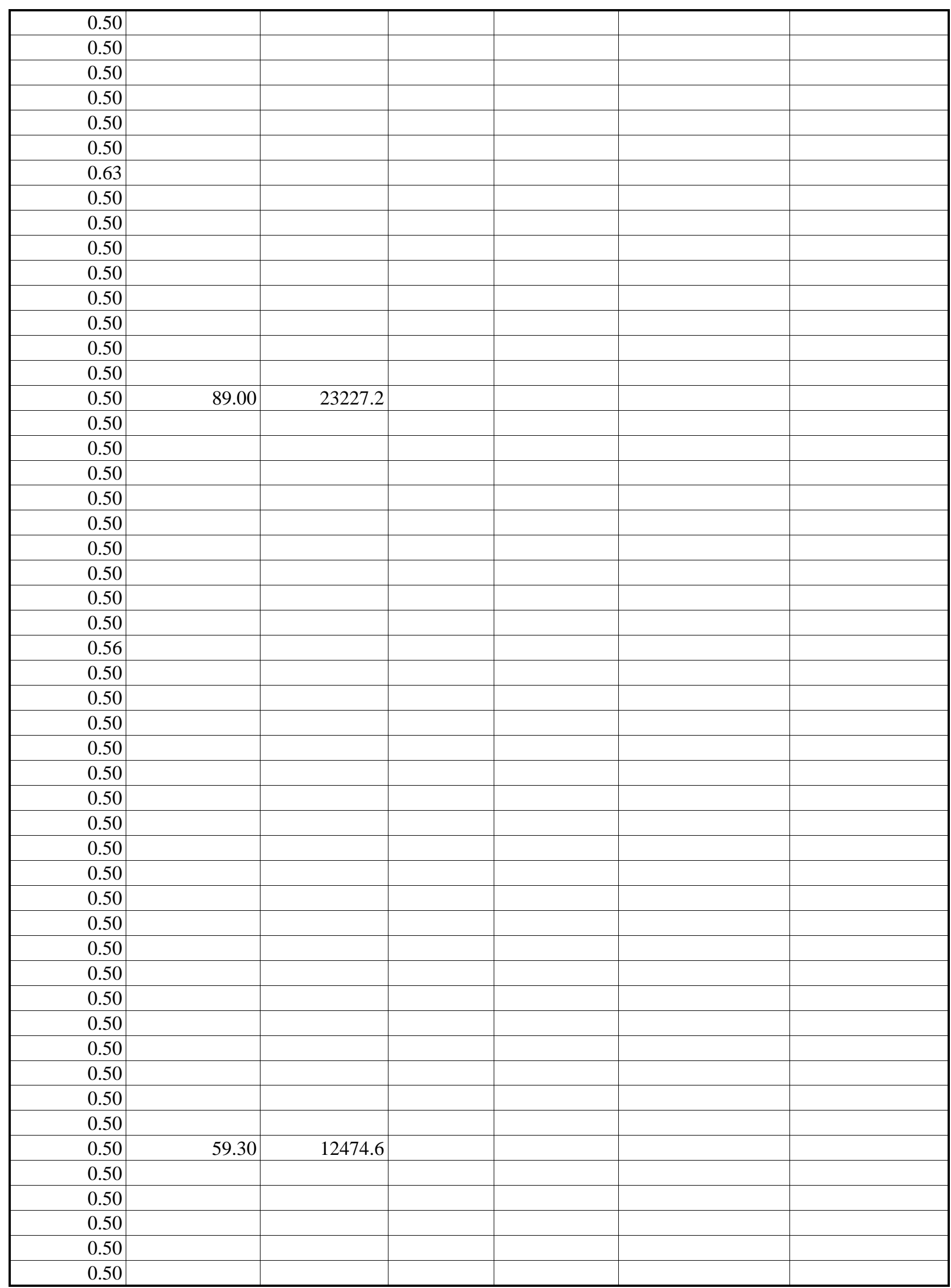




\begin{tabular}{|r|l|l|l|l|}
\hline \#DIV/0! & & & & \\
\hline \#DIV/0! & & & & \\
\hline \#DIV/0! & & & & \\
\hline \#DIV/0! & & & & \\
\hline \#DIV/0! & & & & \\
\hline \#DIV/0! & & & & \\
\hline \#DIV/0! & & & & \\
\hline \#DIV/0! & & & & \\
\hline \#DIV/0! & & & & \\
\hline \#DIV/0! & & & & \\
\hline \#DIV/0! & & & & \\
\hline \#DIV/0! & & & & \\
\hline \#DIV/0! & & & & \\
\hline \#DIV/0! & & & & \\
\hline \#DIV/0! & & & & \\
\hline \#DIV/0! & & & & \\
\hline \#DIV/0! & & & & \\
\hline \#DIV/0! & & & & \\
\hline \#DIV/0! & & & & \\
\hline \#DIV/0! & & & & \\
\hline \#DIV/0! & & & & \\
\hline \#DIV/0! & & & & \\
\hline \#DIV/0! & & & & \\
\hline \#DIV/0! & & & & \\
\hline \#DIV/0! & & & & \\
\hline \#DIV/0! & & & & \\
\hline \#DIV/0! & & & & \\
\hline \#DIV/0! & & & & \\
\hline \#DIV/0! & & & & \\
\hline \#DIV/0! & & & & \\
\hline \#DIV/0! & & & & \\
\hline \#DIV/0! & & & & \\
\hline \#DIV/0! & & & & \\
\hline \#DIV/0! & & & & \\
\hline \#DIV/0! & & & & \\
\hline \#DIV/0! & & & & \\
\hline \#DIV/0! & & & & \\
\hline 0.50 & & & & \\
\hline 0.50 & & & & \\
\hline 0.50 & & & & \\
\hline 0.42 & & & & \\
\hline 0.50 & & & & \\
\hline 0.50 & & & & \\
\hline 0.50 & & & & \\
\hline 0.50 & & & & \\
\hline 0.50 & & & & \\
\hline 0.50 & & & & \\
\hline
\end{tabular}


wbheat

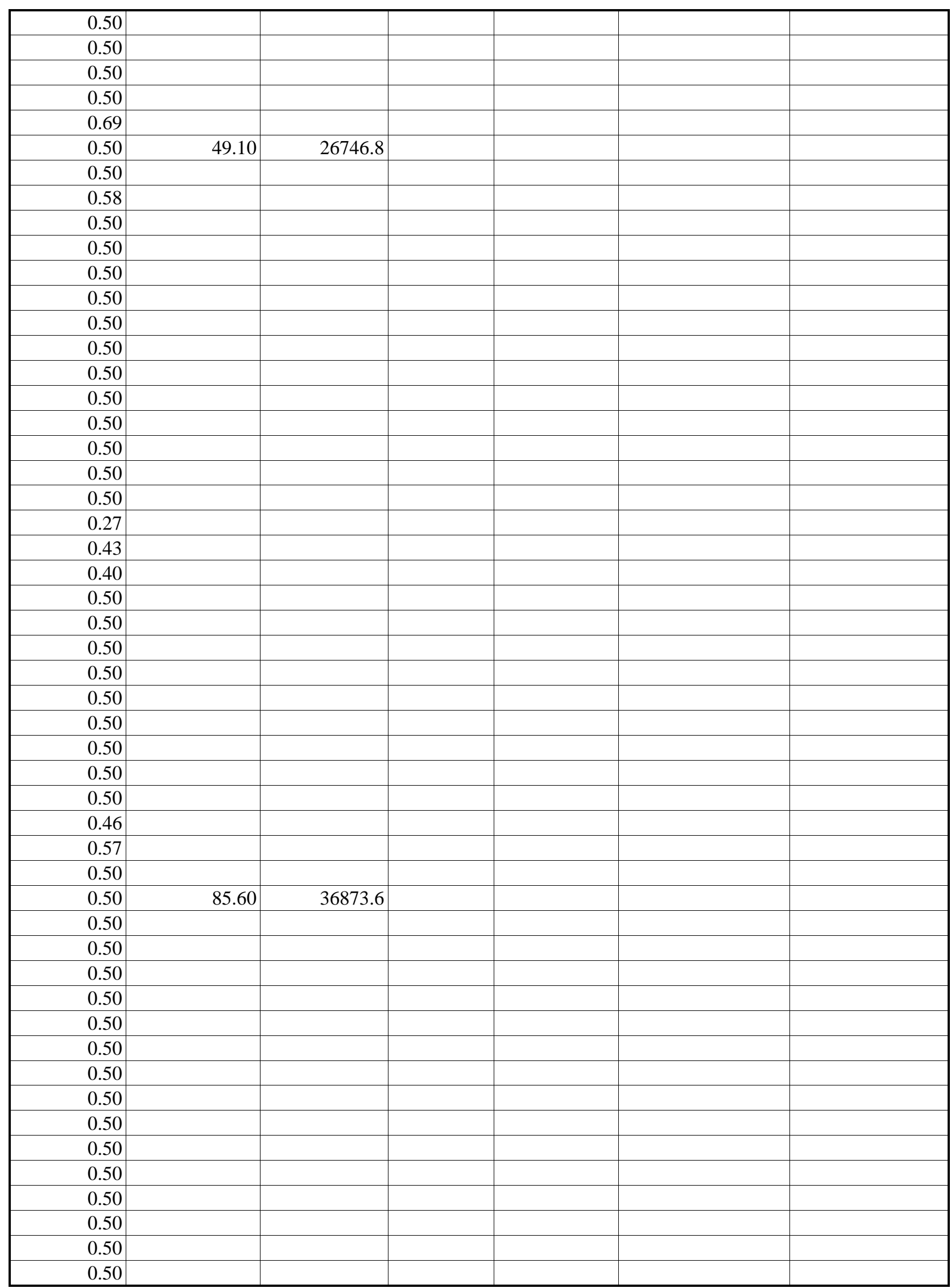


wbheat

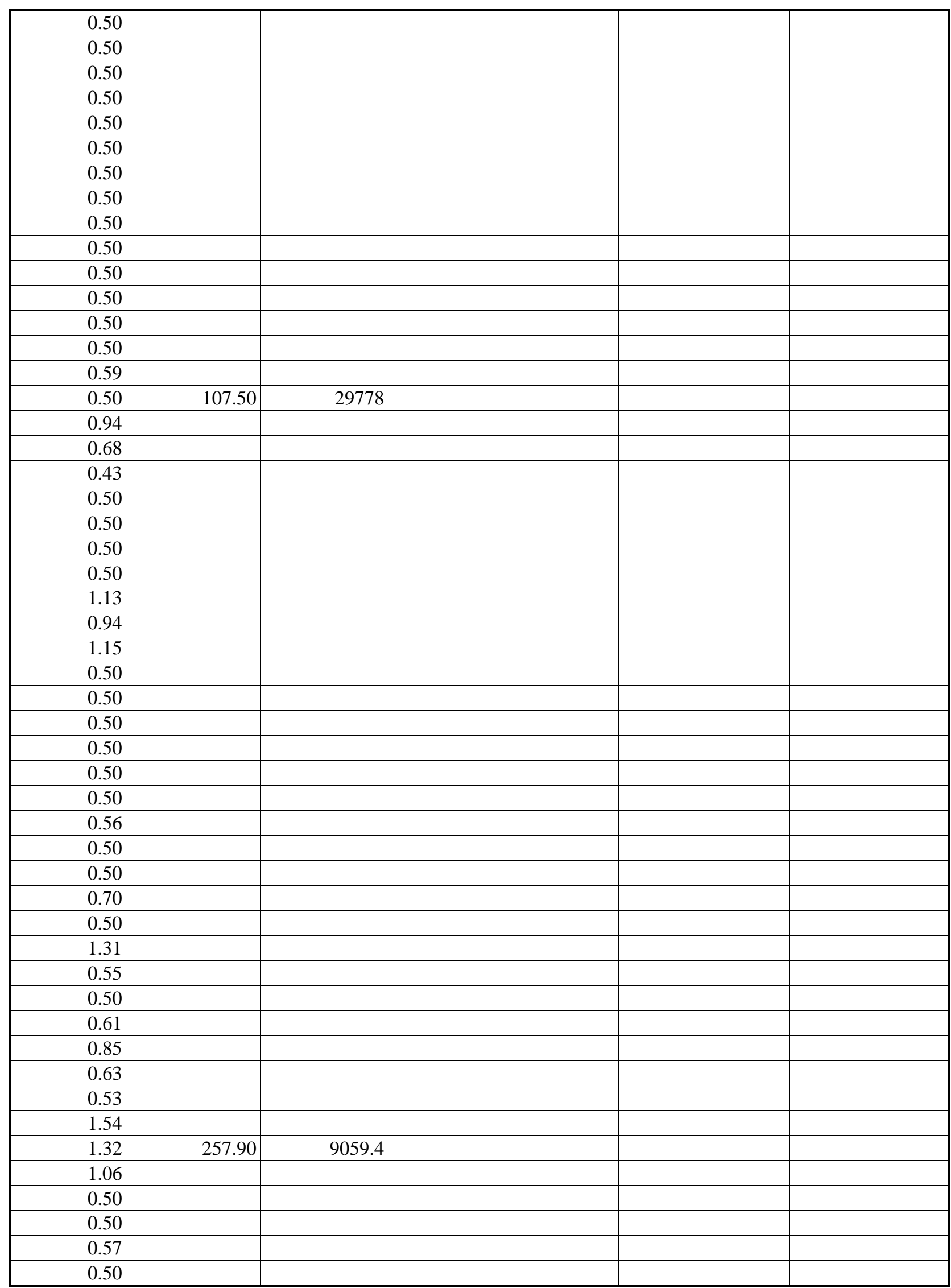


wbheat

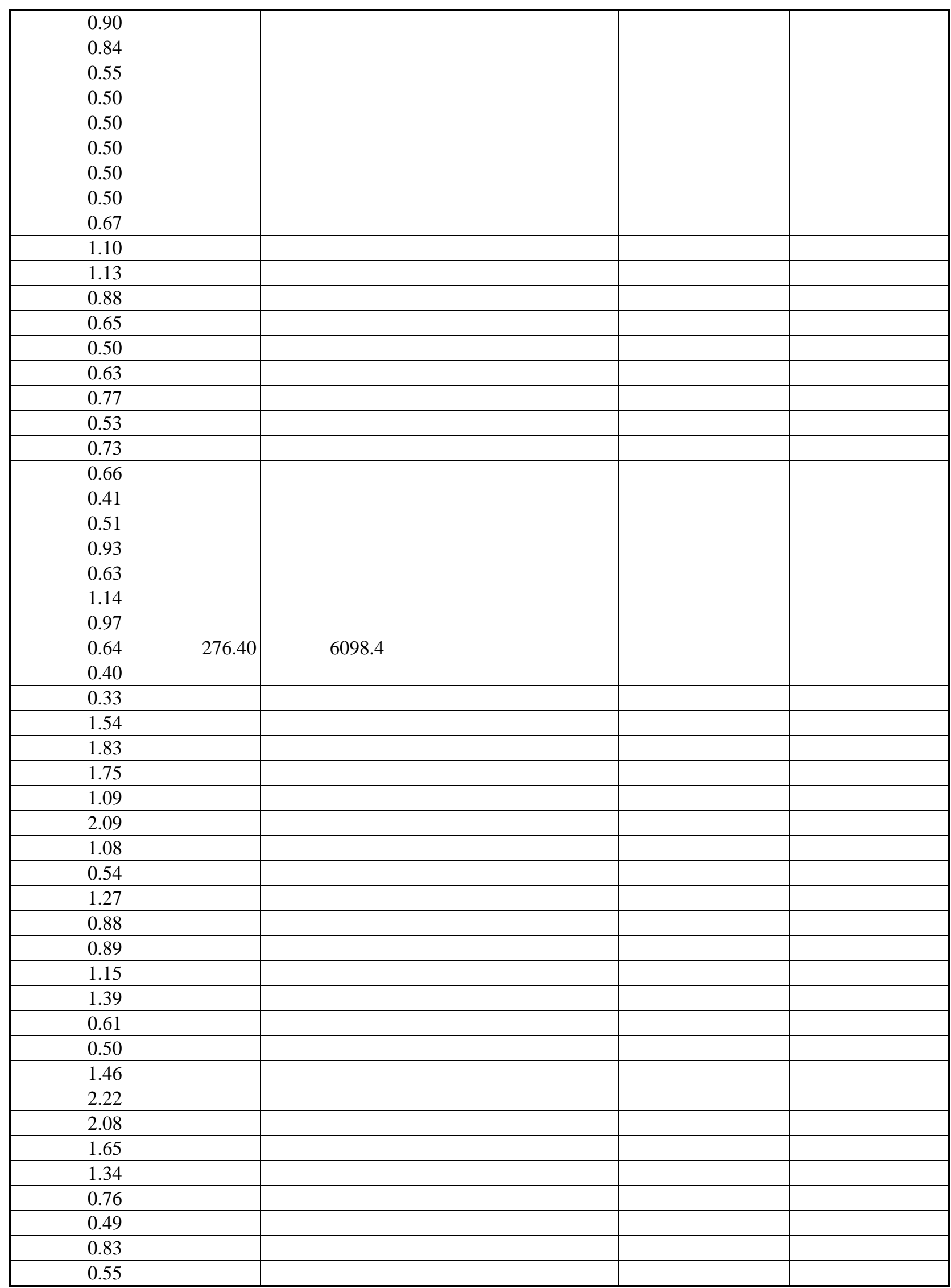


wbheat

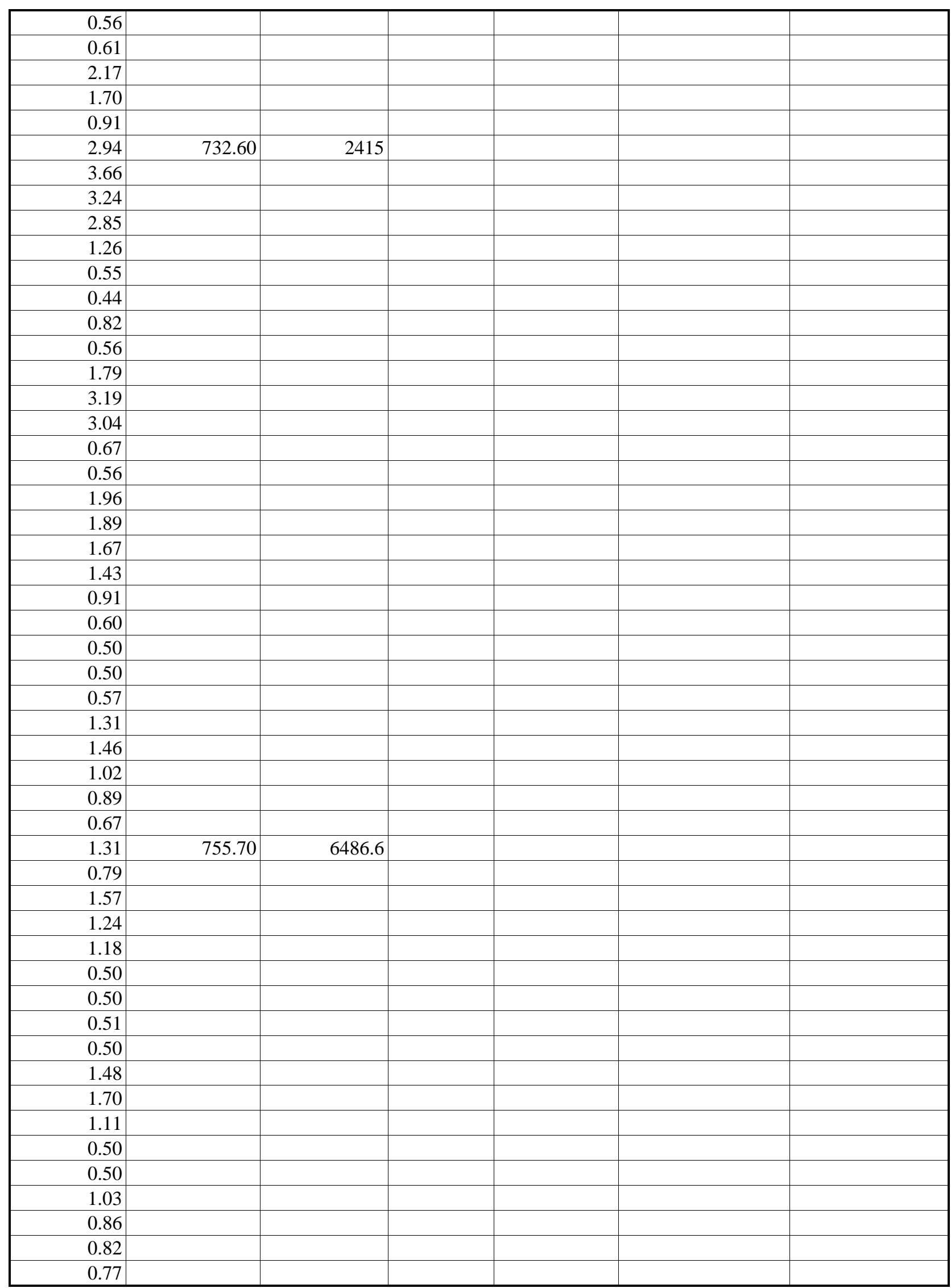




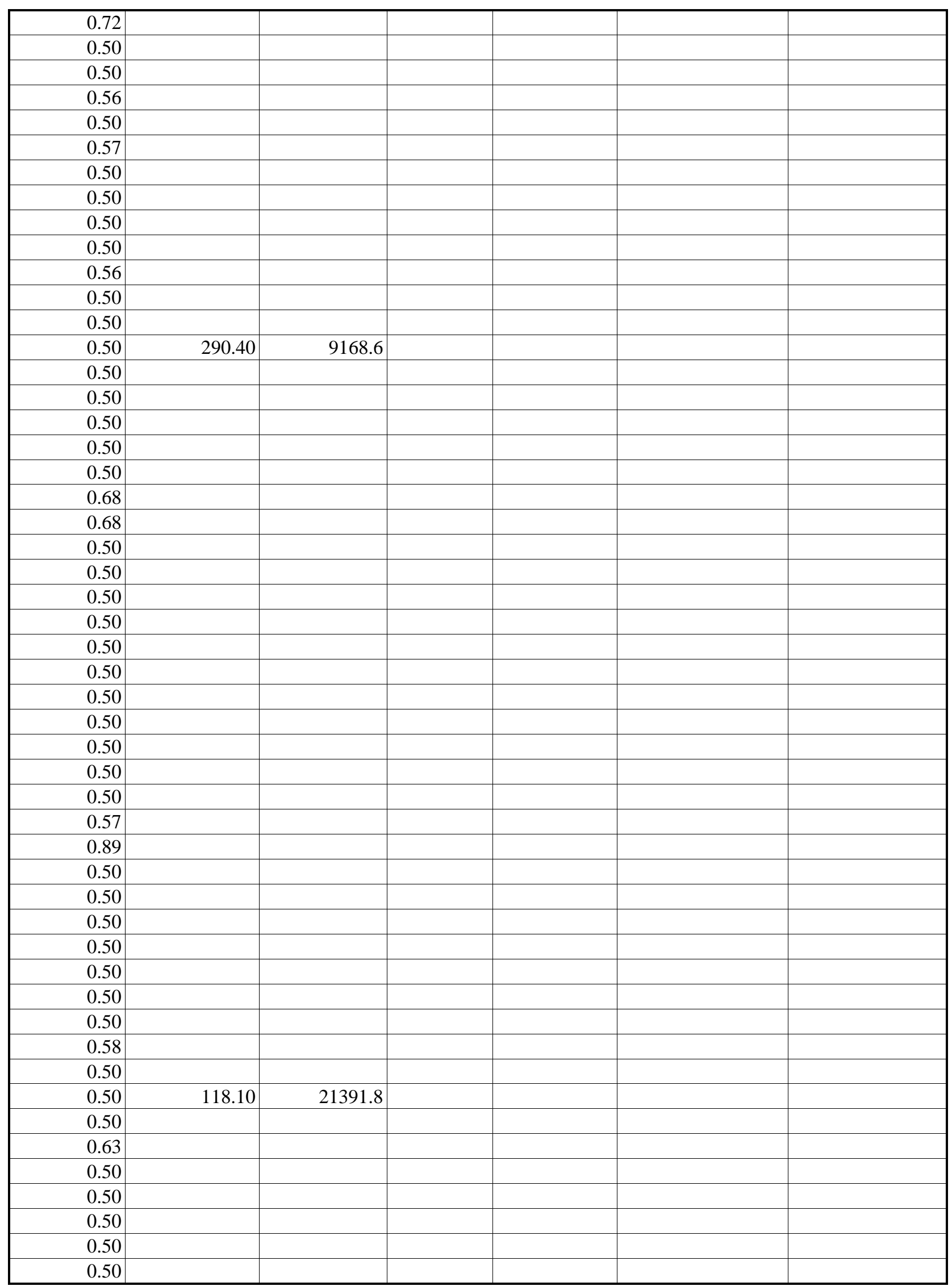


wbheat

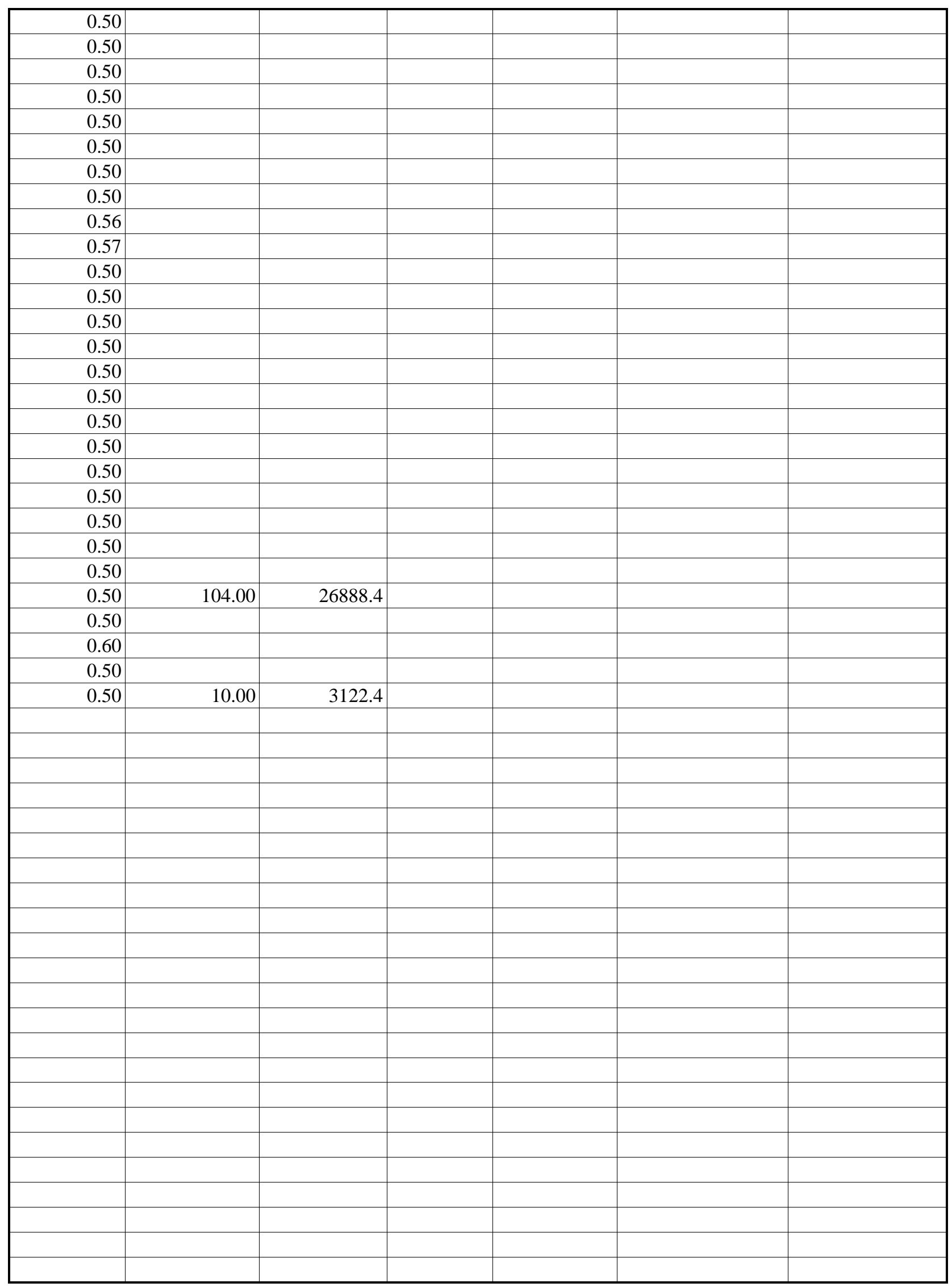

Page 91 